\title{
An atypical biotype I Actinobacillus pleuropneumoniae serotype 13 is present in North America
}

Gottschalk, Marcelo; Lacouture, Sonia; Angen, Øystein; Kokotovic, Branko

Published in:

Proceedings of the 21st IPVS Congress

Publication date:

2010

Document Version

Publisher's PDF, also known as Version of record

Link back to DTU Orbit

Citation (APA):

Gottschalk, M., Lacouture, S., Angen, Ø., \& Kokotovic, B. (2010). An atypical biotype I Actinobacillus pleuropneumoniae serotype 13 is present in North America. In Proceedings of the 21st IPVS Congress

\section{General rights}

Copyright and moral rights for the publications made accessible in the public portal are retained by the authors and/or other copyright owners and it is a condition of accessing publications that users recognise and abide by the legal requirements associated with these rights.

- Users may download and print one copy of any publication from the public portal for the purpose of private study or research.

- You may not further distribute the material or use it for any profit-making activity or commercial gain

- You may freely distribute the URL identifying the publication in the public portal 


\section{$21^{\text {ST }}$ INTERNATIONAL PIG VETERINARY SOCIETY (IPVS) CONGRESS}

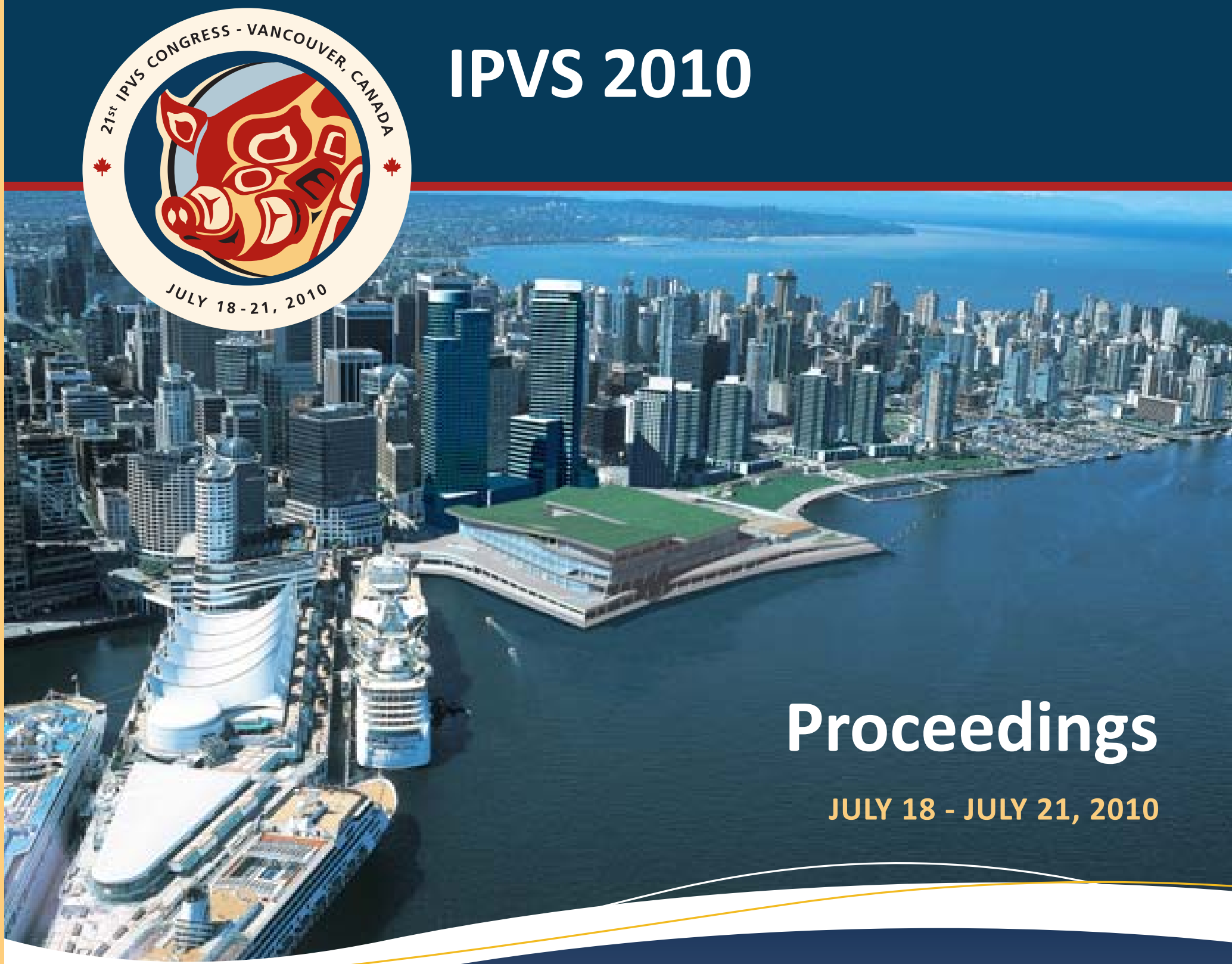

Vancouver Convention Centre, West Building Vancouver, British Columbia, Canada 


\section{Proceedings}

\section{of the \\ $21^{\text {st }}$ International Pig Veterinary \\ Society Congress}

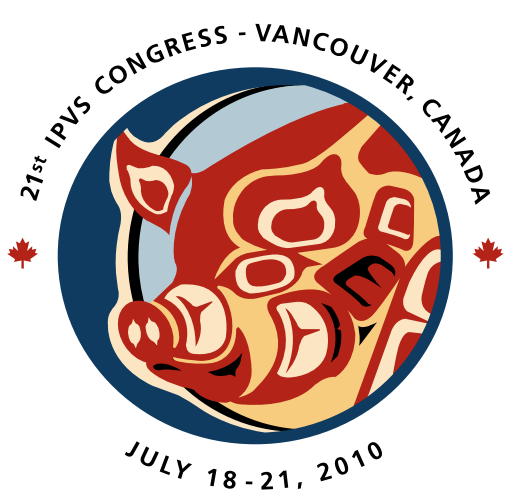

Vancouver, Canada

July $18-21,2010$

Edited by Sylvie D’Allaire and Robert Friendship 


\section{$21^{\text {st }}$ International Pig Veterinary Society Congress}

\section{Organizing Committee}

$\begin{array}{ll}\text { President: } & \text { Ernest Sanford } \\ \text { Vice-President/Secretary: } & \text { John Harding } \\ \text { Finance/Treasurer: } & \text { Al Theede } \\ \text { Scientific Committee Co-chairs: } & \text { Sylvie D'Allaire } \\ & \text { Robert Friendship } \\ \text { Sponsorship Committee Co-chairs: } & \text { Camille Moore } \\ & \text { Neil Shantz } \\ \text { Social Committee Co-chairs: } & \text { Chris Byra } \\ & \text { Gail Cunningham } \\ & \text { Julie Ménard } \\ \text { Technical Tours: } & \text { Chris Byra } \\ \text { Accompanying Persons Tour Program: } & \text { Rick Stanley } \\ & \text { Madonna Benjamin }\end{array}$

\section{Scientific Committee}

\section{Co-chairs:}

Sylvie D’Allaire, Faculté de médecine vétérinaire, Université de Montréal Robert Friendship, Ontario Veterinary College, University of Guelph

\section{Members:}

Ron Ball, Faculty of Agricultural, Life and Environmental Sciences, University of Alberta

Tim Blackwell, Ontario Ministry of Agriculture, Food and Rural Affairs

Cornelius F.M. de Lange, Ontario Agricultural College, University of Guelph

Jérôme del Castillo, Faculté de médecine vétérinaire, Université de Montréal

Cate Dewey, Ontario Veterinary College, University of Guelph

Richard Drolet, Faculté de médecine vétérinaire, Université de Montréal

George Foxcroft, Faculty of Agricultural, Life and Environmental Sciences, University of Alberta

Carl A. Gagnon, Faculté de médecine vétérinaire, Université de Montréal

Harold Gonyou, Prairie Swine Center, University of Saskatchewan

Marcelo Gottschalk, Faculté de médecine vétérinaire, Université de Montréal

Carlton Gyles, Ontario Veterinary College, University of Guelph

John Harding, Western College of Veterinary Medicine, University of Saskatchewan

Dan Hurnik, Atlantic Veterinary College, University of Prince Edward Island

Roy Kirkwood, College of Veterinary Medicine, Michigan State University

Ann Letellier, Faculté de médecine vétérinaire, Université de Montréal

Guy-Pierre Martineau, École Nationale Vétérinaire de Toulouse

Scott McEwen, Ontario Veterinary College, University of Guelph

Suzanne Millman, College of Veterinary Medicine, lowa State University

Zvonimir Poljak, Ontario Veterinary College, University of Guelph

Dongwan Yoo, College of Veterinary Medicine, University of Illinois 


\section{IPVS Board}
President 2010
Dr. Ernest Sanford - Canada
Vice President 2010
Dr. John Harding - Canada
President Elect 2012
Dr. Won Hyung Lee - Korea
President 2008
Dr. Peter Evans - South Africa
President 2006
Dr. Bent Nielsen - Denmark
President 2004
Dr. Henning Bossow - Germany
President 2002
Dr. Hank Harris - USA
President 2000
Dr. Ross Cutler - Australia
IPVS General Secretary
Dr. Francois van Niekerk - South Africa
Congress Secretary 2010
Dr. Al Theede - Canada 


\section{Preface}

The 21st International Pig Veterinary Society Congress is held in Vancouver, Canada, marking the first time Canadians have had the opportunity of hosting this meeting. As a tribute to one of the founders of the IPVS and one of its most influential members, the 21st Congress will begin with the Tom Alexander Memorial Lecture delivered by $\mathrm{Dr}$ Hank Harris. In a slight departure from the format of previous meetings there will be only two keynote speakers, both on the opening morning. To introduce major topics there will be eight lead speakers who will each present a talk at the beginning of the specific concurrent session matching their area of expertise. For the first time at an IPVS congress posters will be presented electronically. The number of abstracts for this conference exceeds that of any previous IPVS congresses and this provided the scientific chairs with a challenge in terms of organizing the submissions for oral and poster presentations.

We wish to thank the following reviewers for reading and scoring the abstracts and assisting with the organization of the program:

$\begin{array}{lllll}\text { Glen Almond } & \text { Janet Alsop } & \text { Oystein Angen } & \text { Marie Archambault } & \text { Poul Baekbo } \\ \text { David Benfield } & \text { Pat Blackall } & \text { Tim Blackwell } & \text { Thomas Blaha } & \text { Patrick Boerlin } \\ \text { Corrie Brown } & \text { Mary Buhr } & \text { William Caine } & \text { Susy Carman } & \text { Glen Cassar } \\ \text { Chan Hee Chae } & \text { Jan Dahl } & \text { Peter Davies } & \text { Martin de Jong } & \text { Kees deLange } \\ \text { Anne Deckert } & \text { Cate Dewey } & \text { Scott Dee } & \text { John Deen } & \text { Jérôme del Castillo } \\ \text { Dennis DiPietre } & \text { Mariano Domingo } & \text { Trish Dowling } & \text { Richard Drolet } & \text { Gerald Duhamel } \\ \text { Kay Faaberg } & \text { John Fairbrother } & \text { Ying Fang } & \text { George Foxcroft } & \text { Julie Funk } \\ \text { Carl Gagnon } & \text { Connie Gebhart } & \text { Harold Gonyou } & \text { Marcelo Gottschalk } & \text { Marie Gramer } \\ \text { Roberto Guedes } & \text { Carlton Gyles } & \text { Fred Haesebrouk } & \text { Pat Halbur } & \text { Derek Haley } \\ \text { David Hampson } & \text { Patricia Holyoake } & \text { Scott Hurd } & \text { Dan Hurnik } & \text { André Jestin } \\ \text { Han Soo Joo } & \text { Sven Jorsal } & \text { Roy Kirkwood } & \text { Robert Knox } & \text { Paul Langford } \\ \text { Lars Larsen } & \text { Martine Laitat } & \text { Arlette Laval } & \text { Ann Letellier } & \text { Paul Luimes } \\ \text { Joan Lunney } & \text { Janet Maclnnes } & \text { François Madec } & \text { Dominiek Maes } & \text { Guy-Pierre Martineau } \\ \text { Eric Mateu } & \text { Scott McEwen } & \text { Steven McOrist } & \text { B.M. Meehan } & \text { Suzanne Millman } \\ \text { Cora Miry } & \text { Soenke Moehn } & \text { Antonio Morilla } & \text { Bob Morrisson } & \text { Herve Morvan } \\ \text { Michael Murtaugh } & \text { Hans Nauwynck } & \text { Eric Nelson } & \text { Jens Peter Nielsen } & \text { John Patience } \\ \text { Cinta Prieto } & \text { Zvonimir Poljak } & \text { John Prescott } & \text { Sylvain Quessy } & \text { Simone Oliviera } \\ \text { Tanja Opriessnig } & \text { Andrijana Rajic } & \text { Jim Riviere } & \text { Nicolas Rose } & \text { J. M. Sanchez-Vizcaino } \\ \text { Joaquim Segales } & \text { Mariela Segura } & \text { Glenn Songer } & \text { Vibeke Sørensen } & \text { Jim Squires } \\ \text { Tomasz Stadejek } & \text { Katharina Stärk } & \text { Eileen Thacker } & \text { Flemming Thorup } & \text { Montserrat Torremorell } \\ \text { J.-P. Vaillancourt } & \text { Alain Villeneuve } & \text { Amy Vincent } & \text { Per Wallgren } & \text { Scott Weese } \\ \text { Michael Wendt } & \text { Kyoung-Jin Yoon } & \text { Ruurd Zijlstra } & \text { Jeff Zimmerman. } & \end{array}$

The final program consists of 266 oral presentations in addition to the 2 keynote and 8 lead presentations. There are roughly 890 posters. For the convenience of delegates the proceedings are divided into two parts representing oral presentations in part 1 arranged according to session and the posters in part 2 with papers grouped based on subject. For quick overview, a table of contents for both orals and posters is included at the beginning of the proceedings as well as an overall author index at the end

We sincerely hope all delegates will enjoy this time of coming together and sharing ideas and information and that the scientific and social programs will stimulate and encourage the advancement of knowledge and friendship.

Sylvie D’Allaire and Robert Friendship 


\section{Acknowledgements}

A congress the size and scope of IPVS 2010 would not have been possible without the financial, professional and moral support of our Congress Partners and Supporters.

\section{Partner Sponsors}

Bayer HealthCare AG

Boehringer Ingelheim Animal Health

Intervet Schering-Plough Animal Health

Merial

Novartis Animal Health

Pfizer Animal Health

\section{Supporter Sponsors}

Genetiporc

Harris Vaccines

Hypor

Janssen Animal Health

Newsham Choice Genetics

PIC

Uniferon 


\section{Locations of IPVS Congresses}

\begin{tabular}{ll}
1969 & Cambridge, UK \\
1972 & Hannover, Germany \\
1974 & Lyon, France \\
1976 & Ames, USA \\
1978 & Zagreb, Yugoslavia \\
1980 & Copenhagen, Denmark \\
1982 & Mexico City, Mexico \\
1984 & Ghent, Belgium \\
1986 & Barcelona, Spain \\
1988 & Rio de Janeiro, Brazil \\
\hline 1990 & Lausanne, Switzerland \\
\hline 1992 & The Hague, Netherlands \\
\hline 1994 & Bangkok, Thailand \\
1996 & Bologna, Italy \\
\hline 1998 & Birmingham, UK \\
2000 & Melbourne, Australia \\
2002 & Ames, USA \\
2004 & Hamburg, Germany \\
\hline 2006 & Copenhagen, Denmark \\
\hline 2008 & Durban, South Africa \\
\hline 10 & Vancouver, Canada \\
\hline 19
\end{tabular}




\section{Table of Contents}

\section{Invited Speakers}

\section{Tom Alexander Memorial Lecture}

Pig health assurance - underlying principles and future direction. $\ldots \ldots \ldots \ldots \ldots \ldots \ldots 1$ DL Hank Harris

\section{Keynote Address}

Animal welfare and the veterinary profession: 50 years of change $\ldots \ldots \ldots \ldots \ldots \ldots \ldots .7$ David Fraser

\section{Lead Speakers}

Role of nutrition and intestinal adaptation in weanling pig health

Douglas Burrin

Progress in Porcine Reproductive and Respiratory Syndrome

Virus (PRRSV): What we know about PRRSV, from basic to applied

science - A historical perspective...

Jane Christopher-Hennings

Pork safety: past achievements and future challenges........................ 15

Peter Davies

Porcine circovirus type 2: success and failure.

Caroline Fossum

Application of advanced Al technologies to improve the competitiveness of the pork industry.

George R. Foxcroft

Mycoplasma hyopneumoniae infections in pigs:

update on epidemiology and control.

Dominiek Maes

Social behavior in swine and its impact on welfare

Jeremy N. Marchant-Forde

Dysentery and ileitis - High time to tackle the difficult science behind these diseases

Steven McOrist

\section{Oral Presentations \\ Monday July 19}

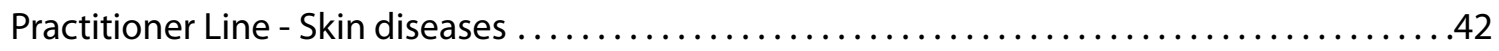

Practitioner Line - Diseases...................................... 47

Porcine Circovirus Associated Diseases - Molecular biology. ....................... 51

Porcine Circovirus Associated Diseases - Clinical aspects ........................54 
Nutrition/Growth ....................................................

Reproduction - Sow management. .....................................65

Reproduction - Semen and boar management. ...........................68

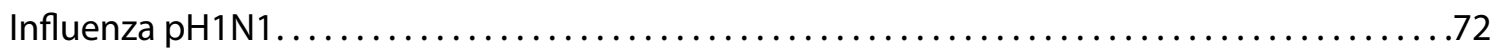

Swine Influenza. ..............................................77

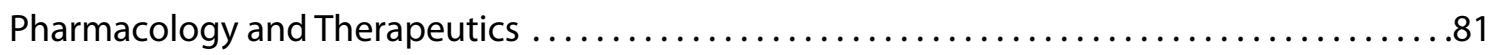

\section{Oral Presentations}

\section{Tuesday July 20}

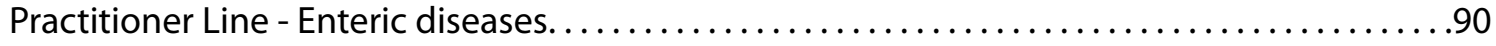

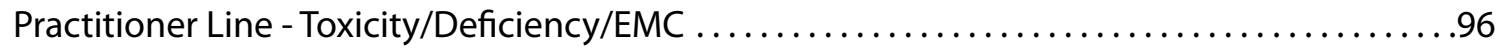

Porcine Circovirus Associated Diseases - Diagnostic aspects .................... 102

Porcine Circovirus Associated Diseases - Control ........................... 108

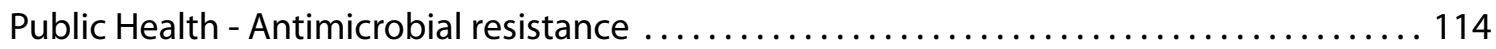

Public Health - MRSA ................................................. 118

Reproduction - Farrowing/neonatal ................................... 124

Reproduction - Sow management. .................................. 130

Respiratory Diseases - Mycoplasma hyopneumoniae........................ 136

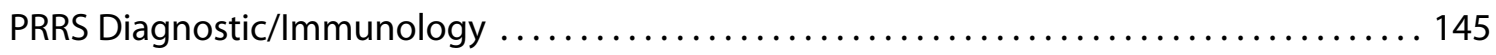

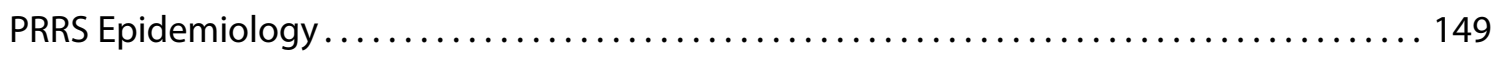

Practitioner Line - Sow management \& diseases .......................... 155

Practitioner Line - Piglet management. .............................. 160

Porcine Cirovirus Associated Diseases - Control. ............................ 164

Public Health - Salmonella ............................................ 173

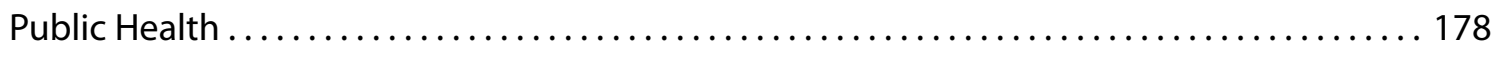

Production - Intact boars. ........................................ 182

Production - Sow management/disorders $\ldots \ldots \ldots \ldots \ldots \ldots \ldots \ldots \ldots \ldots \ldots \ldots \ldots \ldots \ldots \ldots \ldots \ldots \ldots \ldots \ldots \ldots$

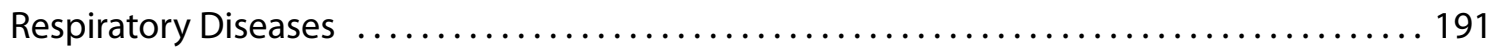

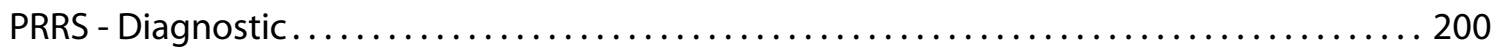

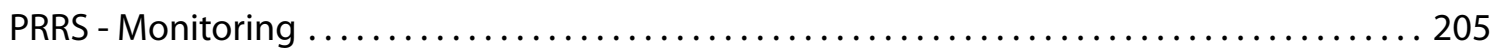




\section{Oral Presentations \\ Wednesday July 21}

Practitioner Line - Sow diseases ...................................... 209

Practitioner Line - Disease control . .................................... 215

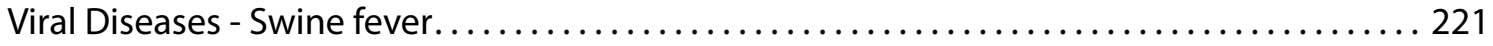

Viral Diseases - Miscellaneous ........................................ 227

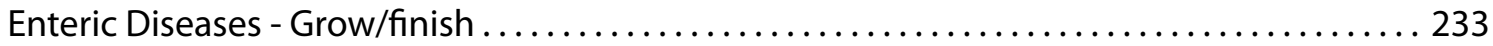

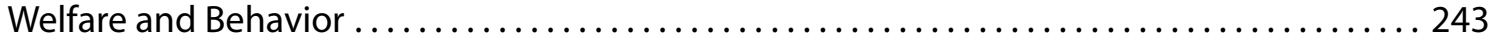

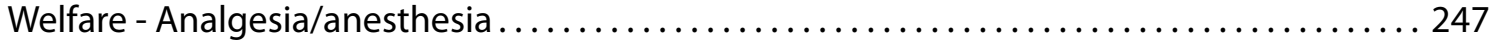

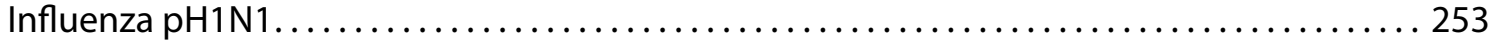

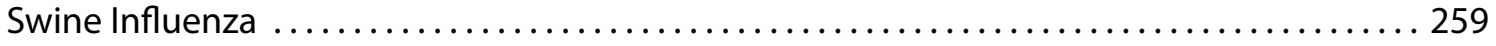

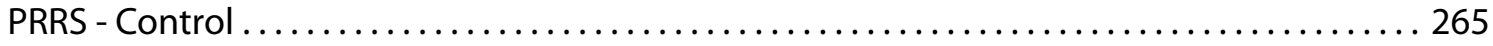

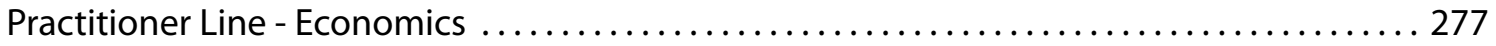

Porcine Circovirus Associated Diseases - General. .......................... 282

Enteric Diseases - Piglets and weanling pigs............................ 287

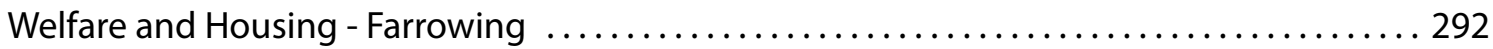

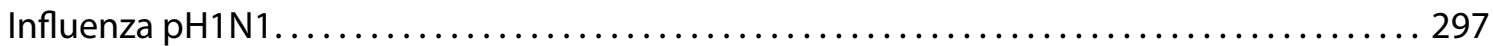

Disease Surveillance ................................................ 302

\section{Poster Presentations}

Porcine Circovirus Associated Diseases (PCVAD)

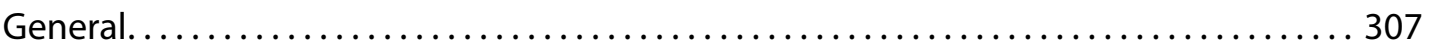

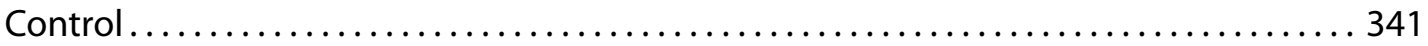

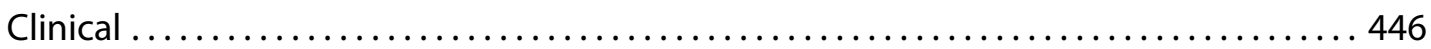

Porcine Reproductive and Respiratory Syndrome (PRRS)

Diagnostics................................................... 475

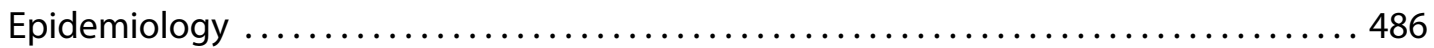

Genetics.................................................... 499

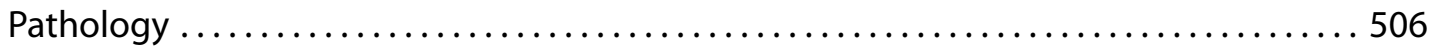

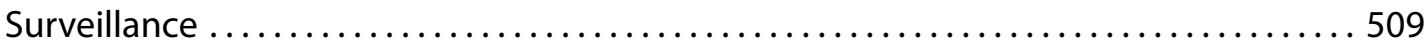

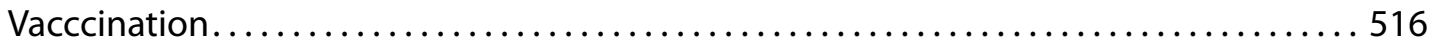

Control.................................................... 548

Respiratory diseases
$\quad$ Swine influenza. $\ldots \ldots \ldots \ldots \ldots \ldots \ldots \ldots \ldots \ldots \ldots \ldots \ldots \ldots \ldots \ldots \ldots \ldots \ldots \ldots \ldots, \ldots \ldots 2$

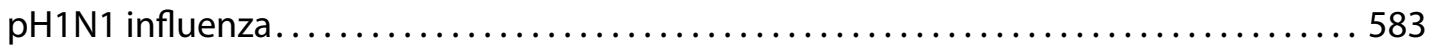

Actinobacillus pleuropneumoniae .................................... 593 
Mycoplasma hyopneumoniae ..................................... 620

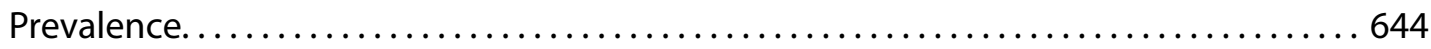

Immunology/Pathology............................................... 651

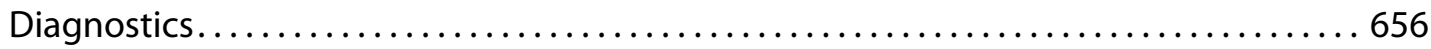

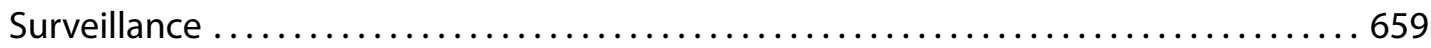

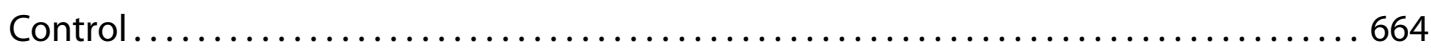

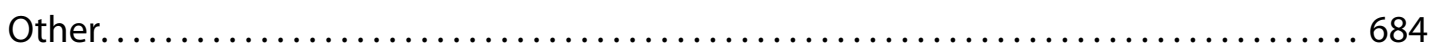

\section{Enteric diseases - Grower and finisher pigs}

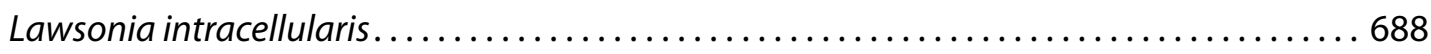

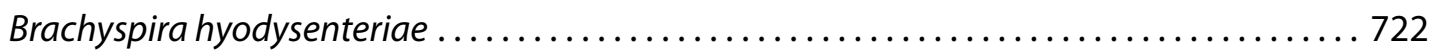

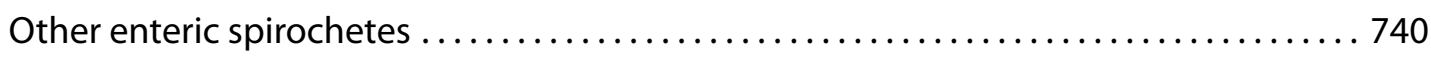

Hemorrhagic bowel syndrome.................................. 743

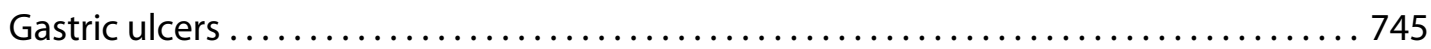

\section{Enteric diseases - Suckling and weanling pigs}

General................................................. 751

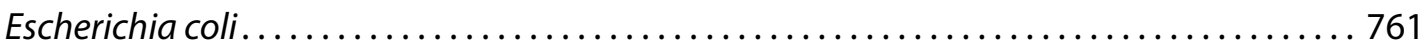

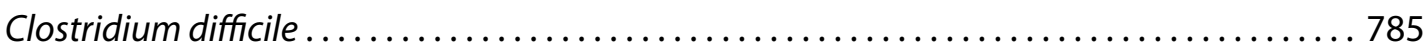

Clostridium perfringens ......................................... 787

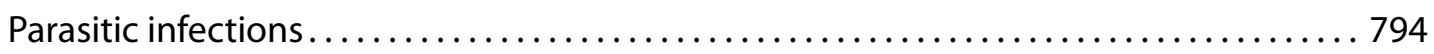

Viral enteric disease ............................................... 812

\section{Other bacterial diseases}

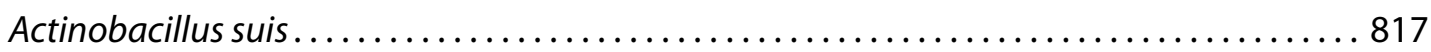

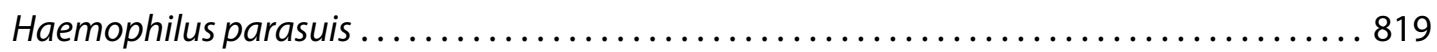

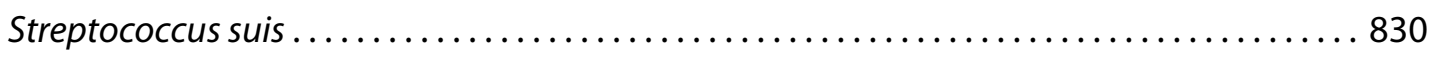

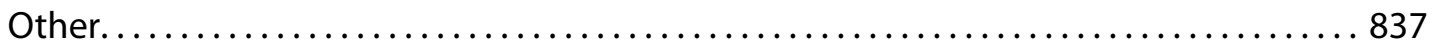

\section{Other viral diseases}

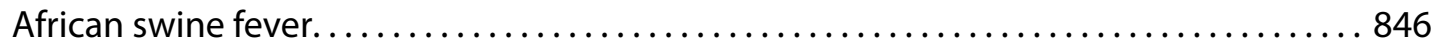

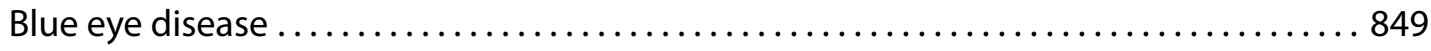

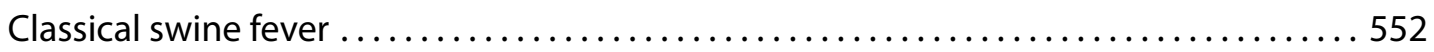

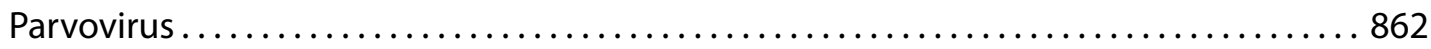

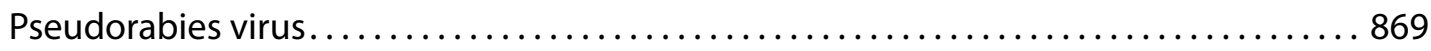

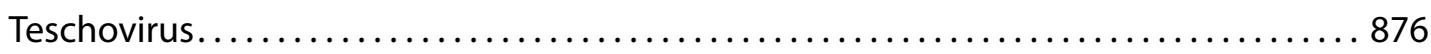

Torque Teno virus ...................................................... 879

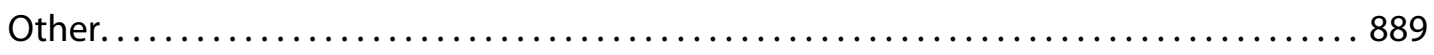




\section{Other diseases}

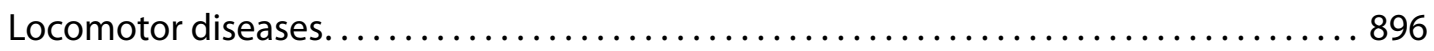

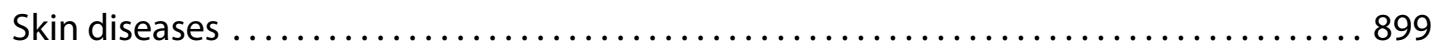

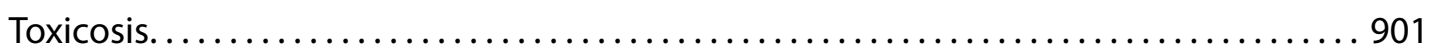

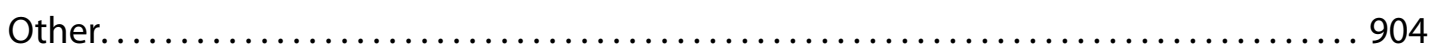

Genetics/Disease resistance/Immunology........................... 911

\section{Surveillance and Biosecurity}

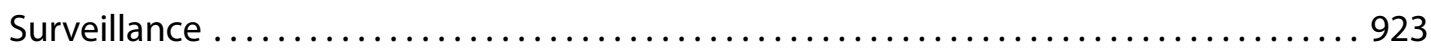

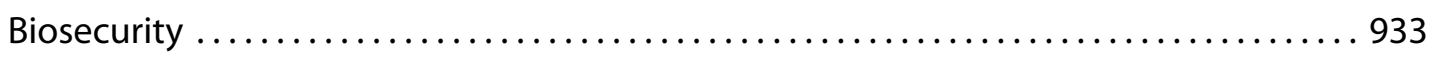

\section{Public health}

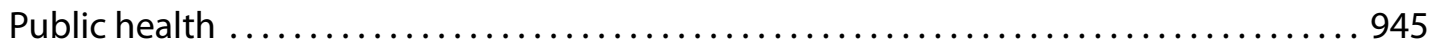

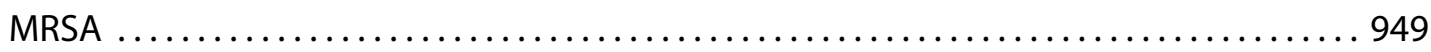

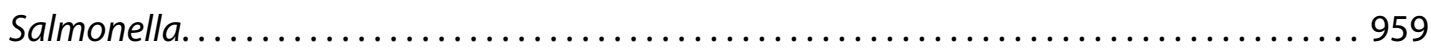

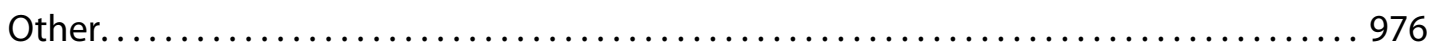

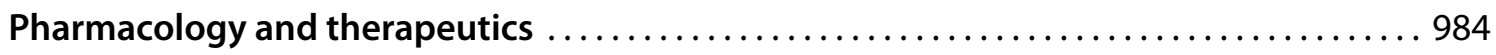

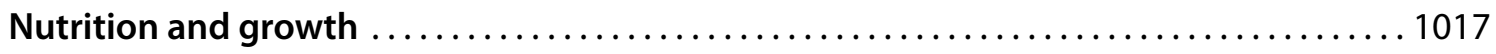

Reproduction

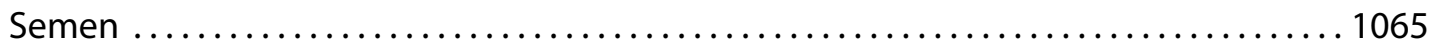

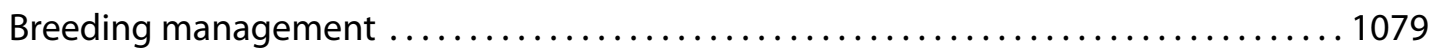

General..................................................... 1099

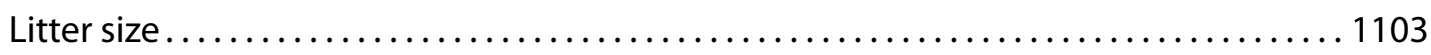

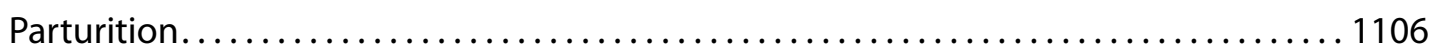

Disease...................................................... 1111

\section{Production}

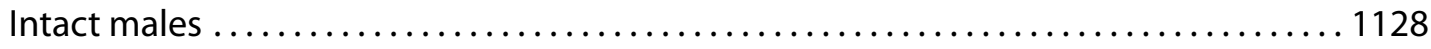

General and economics ........................................ 1141

\section{Welfare/Behaviour}

Analgesia/anesthesia ........................................... 1151

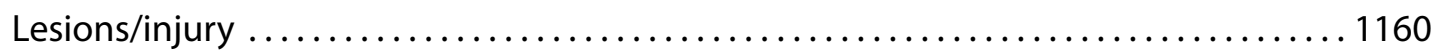

Lameness ................................................... 1164

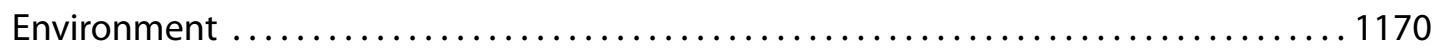

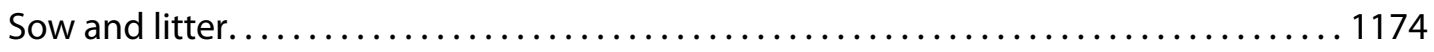

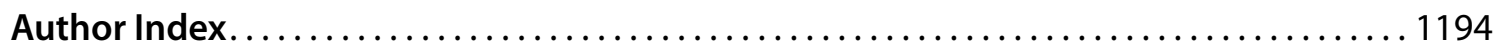




\title{
Pig health assurance - underlying principles and future direction
}

\author{
DL Hank Harris \\ Department of Animal Science, College of Life Sciences and Agriculture and Veterinary Diagnostic \\ and Production Animal Medicine, College of Veterinary Medicine, lowa State University, Ames, IA, USA
}

\begin{abstract}
The work of Tom Alexander centered on controlling swine diseases to benefit the breeding stock and commercial pig producer; and his discoveries and keen insight led to the principles of health assurance used world-wide in the industry today. An overview of his contributions to pig disease research and production, beginning with his involvement as an organizer of the first IPVS Congress in 1969, and exemplified by his paper delivered at that meeting concerning the development of diseasefree pigs, will be followed by a look to the future.
\end{abstract}

\section{Introduction}

"Important principles may, and must be inflexible" --Abraham Lincoln

"The principles of disease control must not be set in stone. They are really guidelines which should be under constant re-evaluation and modification. But modification of a principle or action contrary to a principle should not be taken by any department or country without good reason and only after consultation and agreement among the appropriate parties.

_- Tom Alexander, PIC Veterinary Liaison Meeting, Schlesvig, Germany $1991^{\prime \prime}$

The contradiction between these two quotations, one by a great politician and one by a great contributor to swine medicine exemplify Tom's career as both an academician and as an early proponent of what we now refer to as evidenced-based medicine $(1,6,52)$.

Let me explain by presenting an example - Tom was a student and early adopter of the Specific Pathogen-Free (SPF) program originated by George Young in the U.S. (61). In fact, Tom's paper presented at the first IPVS meeting in 1969 and co-authored by Ken Woolley (founder of Pig Improvement Company, now known as PIC) was entitled, SPF Swine Repopulation (15). By that time, Tom had designed and operated the first experimental primary SPF procurement unit in Ontario, Canada and his paper was the first introduction to the concept in Europe. It was Tom's association with Ken and PIC which caused him to realize that strict adherence to the SPF principles would not be practical for either a breeder or a commercial operation. And although SPF organizations are still in existence today in some countries such as Switzerland, Denmark and at a minor level in North America, they have had to adopt Tom's approach to flexibility in the principles of health assurance. One could further expand this example by stating that the SPF association is a political organization while practical health assurance programs are carried out under the context of a profitable business. To paraphrase Tom's philosophy, which has now been adopted by virtually all breeders and commercial operations of any size world-wide, health assurance principles must be adhered to as long a they don't bankrupt the business.
It seems only fitting that at the first IPVS meeting to be held in Canada that we honor Tom Alexander and give him credit for establishing the first SPF units in Canada and bringing these procedures to Europe via the first IPVS meeting held in Cambridge, England.

\section{Tom's Major Achievements and Contributions}

I'll return to more about Tom's approach to swine evidencedbased medicine but first let us look at his educational background and his many contributions to swine medicine as an academician.

After receiving his veterinary degree at London, Tom became house surgeon at the Large Animal Clinic in Ontario Veterinary College, finished a Master of Veterinary Science degree (Toronto) and served in various academic posts in Ontario through 1966. He completed his PhD degree at Cambridge during the years of 1962-65, returning for a short time to Ontario prior to accepting a lecturer position at Cambridge in 1966 where he remained until retirement in 2004. It was while in Canada that Tom met and married his wife Lenore.

Tom's work at Ontario regarding SPF and gnotobiotic pigs $(10,12,57)$ led to investigations into vomiting and wasting disease of pigs $(3,5,11,56)$. He was the first to describe its viral etiology, and picornaviruses became the subject of his PhD dissertation at Cambridge (8) under the mentorship of A.O. Betts.

David Taylor and Tom were the first to describe the etiologic agent of swine dysentery (SD) in 1971(58). Their work was simultaneously and independently confirmed by Bob Glock and my team at lowa State University (ISU) $(33,41)$. The first IPVS meeting I attended was in 1972 in Hanover, Germany where Tom's group and mine presented papers on the etiology and pathology of $\mathrm{SD}$. These discoveries by our two teams led to a lasting friendship, my consultative involvement with PIC, and scientific collaboration between Tom and me $(40,62)$. Tom subsequently made many other contributions to our knowledge of swine dysentery $(4,14,44)$.

Tom's pioneering work in primary SFP pigs, gnotobiotes and discovery of anaerobic spirochetes in the intestine of pigs led him to more sophisticated studies on the anaerobic microbiota of pigs, sheep and rabbits [9538;9556;9559]. Eventually, his group at Cambridge and mine at ISU combined efforts to discern the role of 'normal' flora such as Bacteroides and Fusobacteria in the pathogenesis of SD $(40,62)$. Tom and Dick Lysons were the first to develop gnotobiotic lambs with a defined functional rumen flora (46).

While Tom was completing his PhD work on picornaviruses, he conducted some collaborative investigations and experiments with S.D. Elliott and J.H. Thomas on streptococcal meningitis and arthritis (28). Eventually, when streptococcal meningitis reemerged in the British pig industry and became a threat to both pigs and humans around 1975, Tom led the effort to understand 
and control the disease. This work by Tom and his student and colleague, Feliciti Clifton-Hadley was his largest contribution to the scientific literature. These clinical investigations and experiments led to discoveries pertaining to pathogenesis, control measures including vaccine development, and epidemiology of the disease $(19,21-26,42,43,64)$.

The development of Medicated Early Weaning (MEW) was one of Tom's best known contributions to the pig industry (13). Until 1978, there were 3 methods of starting and maintaining high health status herds with new genetic material: 1) direct population from another high health status herd; 2) grafting desired genes by artificial insemination or embryo transfer or cross-fostering young piglets; and 3) population by primary SPF methods (1). In 1977, the main PIC nucleus herd (Fyfield Wicke) became infected with Mycoplasma hyopneumoniae. The herd had been established by surgical-derivation in 1962 and this was its first encounter with the organism. Ken Woolley asked Tom to try a different method other than surgical-derivation to set up a new nucleus herd free of $\mathrm{M}$. hyopneumoniae. Tom proposed the hypothesis that medication of both the sow and piglets combined with early weaning would result in pigs free of the infection. He enlisted Dick Lysons (then at Compton) and some PIC folks (mainly Graham Boon and Keith Thornton) to help with the design and implementation of his hypothesis. The MEW project was a huge success and resulted in not only the elimination of M. hyopneumoniae but also Bordetella bronchiseptica and some non-pathogenic colonic treponemes (now called Brachyspira). The MEW technique was soon adopted in PIC herds in France, Brazil and the U.S. with similar results. In an attempt to decrease the cost of repopulating herds even further, PIC modifed the procedures which became known by several synonyms; modified MEW (MMEW), Isowean, and Segregated Early Weaning (SEW) $(27,32,36-39,45,53)$. MEW or MMEW are now used to eliminate a variety of infectious agents including: pseudorabies virus, Porcine Reproductive Respiratory Syndrome Virus (PRRSV), Transmissible Gastroenteritis (TGE) Virus, Leptospira, Pasteurella multocida, Actinobacillus pleuropneumoniae, Streptococcus suis type II, and Haemophilus parasuis. The modifications applied to MEW were the basis for the I development of three site and multiple site production rearing systems $(37,39)$. Other breeding stock companies have used the techniques to eliminate various infectious agents.

In the late 90s, Tom became interested in zoonoses involved in pig to human xenotransplantation (54). One of his last presentations, at the 1998 IPVS, bore the title 'Zoonoses', in which he described all the pathogens of pigs of possible concern to human health. He concluded that the only pathogen of significance was Salmonella (2). His concern at the time was the possibility that transgenic pig hearts and kidneys would soon be transplanted into humans. This has not been forthcoming, unfortunately, for a variety of reasons unrelated to the risk of pig to human transmission of disease.

During the early days of his tenure as Lecturer at Cambridge University, Tom first met Ken Woolley, President of PIC. Becoming an advisor to PIC was crucial to Tom's development as both an academician, applied scientist and leader of evidenced-based swine medicine. The most important swine diseases at the time were atrophic rhinitis and enzootic pneumonia. Professor Betts in London was an early investigator in etiologic studies on enzootic pneumonia of pigs and pig producers in England were wanting pigs free of the disease. While Peter Whittlestone and Reg Goodwin at Cambridge and Bill Switzer and John Mare in
Ames were discovering the causative agent (called Mycoplasma hyopneumoniae $(48)$ in Ames and M. suipneumoniae $(34,35)$ in Cambridge), Tom was busy establishing enzootic pneumonia free herds for PIC. Goodwin was a proponent of the SPF approach which he later named 'Minimal Disease (MD) Program'. Through his experience in dealing with the practical aspects of advising a breeding stock company, Tom departed from the SPF and MD programs strict adherence to rigid rules concerning the presence/absence of specific pathogens. It was his departure from testing for the presence of specific pathogens to consideration of the practical difference between infection versus disease which set the stage for Tom's remarkable contributions to pig production world-wide (1).

Tom relied heavily on the discoveries being made by Betts, Goodwin, Whittlestone and Switzer in his advisory capacity to PIC, as it was during this period of time when respiratory diseases such as Mycoplasma pneumonia and atrophic rhinitis were key economic factors in commercial production Tom formulatedthe Veterinary Liaison Scheme for PIC and the implemented disease control strategies in that document have now become dogma in the swine industry world-wide.

\section{This was how they worked:}

Tom was one of the first veterinarians to recognize that pig density and farm location were important for disease control for both commercial operaters and breeding stock producers (reviewed by Tom in his Howard Dunne Memorial lecture at the American Association of Swine Practitioners in 1988 (16)and also published by the Pig Veterinary Society (7).

Tom found that the SPF and MD programs were too inflexible for the development of a breeding stock organization such as PIC. His insight led to concepts which are common terms/principles in the industry today: single sourcing of breeding stock and replacements, vet to vet interactions for matching the infectious disease status between source farm and the recipient farm, laboratory tests can be useful but never do a test if you aren't sure of how to interpret the results, a breeder cannot guarantee freedom from disease (the main downfall of the SPF/MD programs), when problems arise in a breeder herd, shipments must be put on 'hold' until an investigation can occur to protect downstream recipient herds, pig movement should only be one way, down a pyramid but never reversed or across a pyramid and the implementation of biosecurity measures including shower-in; shower-out. Without doubt, many breeders went out of business for failure to follow these rules. Today, most breeders follow these rules and many commercial operations who have become breeders in their own organizations should follow them more closely. There is no doubt that PIC would not enjoy its dominant position today in the breeding stock business if it had not been for Tom's insight and creativity in these early days of PIC (personal communication, Bill Christianson, CEO, Genus Americas).

In 1989, Tom organized a meeting of all the PIC associated veterinarians from around the world in Schlesvig, Germany. It was a 3 day meeting in which he led the 30 plus attendees in a discussion leading to the creation of a document entitled the Principles of Disease Control (later called The Principles which underlie the PIC Health Assurance Programme). The current version is reprinted here with the permission of PIC. One of the most entertaining aspects of the meeting was the 'role playing' performed by veterinarians from various countries which led to a better understanding of practical disease control approaches. 


\section{The Principles Which Underlie The PIC Health Assurance Programme}

1 Genetic improvement is a long term business and therefore the over-riding approach to disease control in PIC must be long term.

2 Every PIC herd should be set up with the healthiest pigs available that are appropriate for the purpose.

3 For insurance purposes there should be a contingency plan for the supply of suitable genetics to sustain the business.

4 It is both impossible and unnecessary to try to standardise the health status of all the herds throughout a breeding organisation.

5- PIC aims to protect customer health but cannot guarantee freedom from specific infectious agents.

6 PIC should adopt a continuous policy of maintaining high health status overall by such measures as closure of herds when they become unsatisfactory, the application of total or partial repopulation and the establishment of new high health status herds.

7 PIC must monitor for evidence of virulent pathogens over a wide spectrum of diseases and anticipate that new diseases will arise.

8 When a problem is suspected in a herd, sales must be put on hold while it is investigated.

9 Location is the most important factor in preventing contamination and herd breakdowns.

10 If a herd is situated in an excellent location the most important biosecurity factor is then the introduction and removal systems.

11 All pig transport and pig movement must meet PIC specifications

12 Pigs should move in one direction only, that is down the pyramids and not across it or up it.

13 Appropriate biosecurity measures must be taken on every farm to prevent contamination and should be proportional to the impact of a breakdown in that unit.

14The movement of people between herds or other sources of contamination must be controlled and appropriate downtime must be laid down for each herd.

15 To prevent dissemination of disease and because PIC cannot standardise health, PIC should aim at single sourcing and minimize source changes, keeping necessary changes within pyramids.

16 Laboratory tests can be very useful, but never do a test if you cannot be sure of interpreting the results.

17The implementation of the health assurance programme should be predominantly regional and local rather than central, and where appropriate PIC should use independent veterinarians.

18 Every new nucleus and multiplier herd should be totally populated with PIC stock and must be pre-approved by Health Assurance.

19 Because clinical signs of disease are a major component of monitoring and diagnosis, antibiotics or vaccines that mask clinical signs must not be used routinely in PIC herds.

20 Every herd that receives live animals should isolate and monitor them before introduction.

21 Boar studs should be considered equal to nucleus herds with respect to set-up, monitoring and biosecurity.
22 PIC should be open about the operation of the health assurance programme and should communicate with individual customers particularly when problems arise.

23 Welfare is a component of the health assurance programme and should be monitored regularly.

The principles must not be set in stone. They are really guidelines which should be under constant re-evaluation and modification. But modification of a principle or action contrary to a principle should not be taken by any department or country without good reason and after consultation and agreement with Health Assurance.

No matter where in the world a new infectious agent emerged or re-emerged, Tom would often play a role in discerning the economic importance of the agent in a particular region or country and then either direct or assist in developing disease control strategies. Many of these opportunities came about because of his senior advisory role to PIC but often veterinarians and geneticists from other breeding stock companies or SPF organizations would call on him for advice. There are numerous unpublished documents penned by Tom in the files of veterinarians world-wide. These deal with a variety of infectious agents from Pasteurella multocida to PRRSV and PCV-2. Many of these contributions were not necessarily credited to Tom. For example, he had a major role in the early development of procedures to control PRRSV in boar studs UK and US and in the elimination of Foot and Mouth Disease virus in a herds in China (55).

\section{Future Direction}

\section{Advancing Pig Health}

I commend the organizing and scientific committees of this meeting for choosing this theme. Since the first IPVS meeting in 1969 which Tom helped organize, this group has emphasized the importance of infectious and non-infectious diseases and their impact on pig health. As new agents emerge and old ones re-emerge, pig veterinarians and scientists must continually utilize field data, evidence-based analysis/conclusions and scientific discoveries to improve the health status of swine. Furthermore, economic constraints combined with management and structural changes such as vertical integration are continual threats to the health and well-being of swine. The scientific program committee has done an excellent job in fulfilling the theme of the congress.

Vaccines will become increasingly important for the control of infectious diseases especially when fewer and fewer pharmaceuticals are available for either growth promotion or prevention/therapy. Within the next 10 years, immunogenetics will become increasingly important not necessarily for the creation of disease resistant pigs but rather for the development of vaccine-ready pigs $(47,63)$. Evidence already exists in both swine and cattle that multi-disciplinary approaches combining immunobiology, quantitative and molecular genetics with reverse genetics related to infectious agents will lead to more powerful and cost effective prevention of infectious diseases. If regulatory agencies do not streamline approval processes and recognize molecular based technologic advances, the consolidation of the swine industry will result in integrators preparing their own sophisticated vaccines. Both virulent and avirulent (modified live) viruses have been used extensively in swine in an attempt to control PRRSV $(49-51,65)$. It has been clearly shown that the re-introduction of live PRRSV into swine induces new strains of the virus containing both virulent and vaccine genes 
$(29,66)$. These newly created viruses not easily contolled with currently available vaccines. Recently, it was reported that the use of attenuated Newcastle Vaccines has created and maintained a large population ofvaccine-related genotype viruses. The presence of these viruses has allowed for co-infection and recombination of vaccine and wild type strains (20).

I predict that by 2020, Porcine Reproductive Respiratory Syndrome (PRRS) virus will be eradicated from major swine rearing areas of the world. This will be due to industry recognition that live viruses (modified live or virulent) must not be used in control programs for the disease. Breeders have long since recognized the necessity of maintaining nucleus and multiplier herds free of PRRSV. In addition, it is well known and accepted that closed herds with high biosecurity measures often eliminate the virus if live viruses are not used in the herd (60) .

Swine influenza virus (SIV) will continue to infect and form reassortants in the adult breeding herds. The concern that these viruses pose a threat to human health will drive the industry to wean and rear pigs free of SIV. Isowean pigs can be procurred free of SIV(38) and a multi-site system has sucessfully elminated SIV without vaccination (59). Vaccines will be developed which override the interference of the SIV immune response due to maternal antibody.

Recombinant-based vaccines will become more commonly available for the prevention of swine diseases. At the present time, they are more costly to produce than traditional vaccines from live or inactivated viruses. It is important for the creation of stockpiles of vaccines for Foreign Animal Diseases (FAD) that recombinant based vaccines be created and approved for use in the U.S. For example, Foot and Mouth Disease vaccines are currently not produced in the U.S. due to difficulty in production of vaccines requiring growth of the virus. As the recombinant vaccines are improved and shown to provide a significant economic benefit, these vaccines will replace traditional vaccines. Some vaccines for Porcine Circovirus (PCV) are recombinant proteins (derived from baculovirus) and others are prepared from chimeric viruses $(30,31)$. There are no recombinant vector based vaccines for swine available in the U.S. Chimeric and vector based recombinant vaccines have a distinct advantage in that they induce both humoral and cell mediated responses $(17,18)$. Recombinant vaccines will be especially useful in the development of vaccines which differentiate infected from vaccinated animals (DIVA).

\section{Pig Health Assurance World-Wide}

Pigs world-wide will be reared in systems in which the newly weaned pig is placed in isolation away from the breeding and farrowing operations. This simple concept originated with Tom's concept of medicated early weaning (MEW) in 1978 (13). The modifications made to MEW eventually led to the development of three site and multi-site production systems which are utilized in every main pig rearing country in the world $(37,39)$. Even natural and organic pig rearing systems rely on this concept of isolated weaning for disease control.

The major disadvantage of multi-site rearing systems is the evolution of lowered herd immunity due to separation of the young growing and finisher pigs (37). This will be overcome by improved vaccines. However, as consolidation continues in the swine industry, a major restructuring of wean to finish buildings will occur. This will result in more strict adherence to all-in; all-out and single sourcing principles as advocated by Tom many years ago. Furthermore, commercial operations will follow the lead of breeders and pay closer attention to the value of well isolated sites in order to minimize disease during the growerfinisher stages.

Welfare and environmental issues will continually challenge the industry but the solutions to these problems will actual improve the health and productivity of pigs. Less crowding with better housing will lead to improved pig health andfinancial success. Furthermore, the environment will improve as the concentration of pigs per square mile decreases. The isolation/location of pig units will benefit from a biosecurity standpoint.

In the 1960/70s, the United Kingdom led the world in innovation and efficient swine production. This was soon followed by North American producers in the adoption of new technology, expansion, improvement in pigs per sow per year and feed efficiency and vertical integration especially in the 1980/90s. For 2010 and beyond, we'll witness huge technological advance and contribution to the pig industry in China. As Tom suggested, the Principles of Health Assurance will need to be flexible to meet these changing times and situations by country.

I would like to end on a personal note.

Tom was friend, mentor, and colleague to me but most of all I was the pupil. He came into my life at a most opportune time. I am forever grateful.

\section{When the pupil is ready to learn, a teacher will appear. -Zen Proverb}

\section{Acknowledgement}

Appreciation is expressed to Isabel Harris, Joel Harris, Dan Tucker, Joe Connor, Steve Henry, Bob Glock, and Bill Christianson for edits/comments.

\section{Conflict of interest disclosure}

DL Hank Harris has been either a consultant or fully employed by PIC since 1973. Dr. Harris is Founder and President of Harrisvaccines, Inc. d/b/a Sirrah Bios, a veterinary biologics company. 


\section{Reference List}

1. Alexander, T. J. L. 1995. The components of pig health, p. 1-25. In J. R. Hill and D. W. B. Sainsbury (ed.), The Heatlh of Pigs. Longman Scientific \& Technical, Essex.

2. Alexander, T. J. L. 1998. ZOONOSES. Proceedings of the 15th IPVS Congress, Birmingham, England, 5-9 July 1998 167-174.

3. Alexander, T. J. L. 1962. Viral Encephalomyelitis of Swine in Ontario Experimental and Natural Transmission. Am J Vet Res 23:756-762.

4. Alexander, T. J. L. 1969. The Clinical Signs, Diagnosis and Control of Swine Dysentery. The Veterinary Record 85:59-63.

5. Alexander, T. J. L. 1969. Vomiting and Wasting Disease (Clinical Aspects). The Veterinary Record 85:166-167.

6. Alexander, T. J. L. 1971. Changes in Pig Production in Britain and their Effects on the Veterinary Profession. The Veterinary Record 88:138-141.

7. Alexander, T. J. L. 1988. Disease Control - Present and Future. Proc Pig Vet Soc 21:155-165.

8. Alexander, T. J. L. and A. O. Betts. 1967. Further Studies of Porcine Enteroviruses Isolated at Cambridge - I Infections in SPF Pigs and Preparation of Monospecific Antisera. Res. in Vet. Sci. 8:321-329.

9. Alexander, T. J. L., G. Boon, and K. Thorton. 1981. The Establishment of a New Nucleus Herd by Medicated Early Weaning. Proc Pig Vet Soc 8:74-81.

10. Alexander, T. J. L., O. P. Miniats, D. G. Ingram, R. G. Thomson, and E. L. Thackeray. 1969. Gnotobiotic Pigs: Procurement, Microbial Flora, Serum Proteins and Lymphatic Tissues. Can. Vet. Jour. 10:98-105.

11. Alexander, T. J. L., W. P. C. Richards, and C. K. Roe. 1959. An Encephalomyelitis of Suckling Pigs in Ontario. Canadian Journal of Comparative Medicine 23:8-11.

12. Alexander, T. J. L. and C. K. Roe. 1962. Attempts at establishing swine herds from atrophic rhinitis and virus pnuemonia: 2. Repopulation with specific pathogen-free pigs. Can. Vet. Jour. 3:299-306.

13. Alexander, T. J. L., K. Thornton, G. Boon, R. J. Lysons, and A. F. Gush. 1980. Medicated Early Weaning to Obtain Pigs Free From Pathogens Endemic in the Herd of Origin. The Veterinary Record 106:114-119.

14. Alexander, T. J. L., P. D. Wellstead, and M. J. Hudson. 1976. Studies of Bacteria Other Than Treponema Hyodysenteriae Which May Contribute to the Lesion of Swine Dysentery. Proc Pig Vet Soc L.1.

15. Alexander, T. J. L. and K. W. Woolley. 1969. S.P.F. swine repopulation. Proceedings First International Pig Veterinary Society Congress 1:17.

16. Alexander, TJ. L. 1988. Disease Control - Present and Future. Proceedings American Association of Swine Practitioners 115-126.

17. Babiuk, L. A., S. L. Babiuk, B. I. Loehr, and S. van Drunnen Littel-van den Hurk. 2000. Nucleic acid vaccines: research tool or commercial reality. Veterinary Immunolgy and Immunopathology 76:1-23.

18. Bowersock, T. L. and S. Martin. 1999. Vaccine delivery to animals. Advanced Drug Delivery Reviews 38:167-194.

19. Chanter, N., P. W. Jones, and T. J. L. Alexander. 1993. Meningitis in pigs caused by Streptococcus suis - a speculative review. Vet Micro 36:39-55.

20. Chong, Y. L., A. Padhi, P. J. Hudson, and M. Poss. 2010. The Effect of Vaccination on the Evolution and Poplulation Dynamics of Avian Paramyxovirus-1. PLoS Pathogens 6:1.

21. Clifton-Hadley, F. and T. J. L. Alexander. 1991. Diagnosis of Streptococcus suis Infection in Pigs, p. 115-126. In Swine Practice.

22. Clifton-Hadley, F. A. and T. J. L. Alexander. 1980. The carrier site and carrier rate of Streptococcus suis type II in pigs. Vet Rec 107:40-41.

23. Clifton-Hadley, F. A., T. J. L. Alexander, and M. R. Enright. 2010. Survival of Streptococcus-Suis Type-2 in Pig Carcasses. Vet Rec 118:275.

24. Clifton-Hadley, F. A., T. J. L. Alexander, and M. R. Enright. 1986. Monitoring Herds for Streptococcus-Suis Type-2 - Chance Contamination of Slaughter Pigs. Vet Rec 118:274.
25. Clifton-Hadley, F. A., T. J. L. Alexander, M. R. Enright, and J. Guise. 1984. Monitoring Herds for Streptococcus-Suis Type-2 By Sampling Tonsils of Slaughter Pigs. Vet Rec 115:562-564.

26. Clifton-Hadley, F. A., T. J. L. Alexander, I. Upton, and W. P. H. Duffus. 1984. Further studies on the subclinical carrier state of streptococcus suit type 2 in pigs. Vet Rec 114:513-518.

27. Connor, J. 1990. Modified medicated early weaning. Proc Amer Assoc Swine Pract 261-265.

28. Elliott, S. D., T. J. L. Alexander, and J. H. Thomas. 1965. Streptococcal infection in young pigs !!. Epidemiology and experimental production of the disease. J. Hyg. , Camb. 64:213-220.

29. Faaberg, K., S. Yuan, C. Nelsen, B. Schmitt, and M. Murtaugh. 1998. Virual recombination between strains of porcine reproductive and respiratory syndrome virus. Proceedings Allen D. Feman Conference 55 .

30. Fachinger, V., R. Bischoff, S. B. Jedidia, A. Saalmuller, and K. Elbers. 2008. The effect of vaccination against porcine circovirus type 2 in pigs suffering from porcine respiratory disease complex. Vaccine 26:1488-1499.

31. Fenaux, M., T. Opriessnig, P. G. Halbur, F. Elvinger, and X. J. Meng. 2004. A Chimeric Porcine Circovirus (PCV) with the Immunogenic Capsid Gene of the Pathogenic PCV Type 2 (PCV2) Cloned into the Genomic Backbone of the Nonpathogenic PCV1 Induces Protective Immunity against PCV2 Infection in Pigs. Journal of Virology 78:6297-6303.

32. Geiger, J. O., D. L. Harris, S. L. Edgerton, W. Jackson, J. M. Kinyon, R. D. Glock, J. F. Connor, and D. E. Houx. 1992. Elimination of Toxic Pasteurella Utilizing Isowean Three-Site Production. Proc Amer Assoc Swine Pract 45-47.

33. Glock, R. D. and D. L. Harris. 1972. Swine dysentery - II. Veterinary Medicine/Small Animal Clinician 67:65-68.

34. Goodwin, R. F. W., A. P. Pomeroy, and P. Whittlestone. 1967. Characterization of Mycoplasma suipneumoniae: Mycoplasma causing enzootic pneumonia of pigs. J Hyg 65:85.

35. Goodwin, R. F. W., A. P. Pomeroy, and P. Whittlestone. 1965. Production of enzootic pneumonia in pigs with a Mycoplasma. Vet Rec 77:1247.

36. Harris, D. L. 1990. The Use of Isowean 3 Site Production to Upgrade Health Status. Proceedings - 11th Congress- IPVS 374.

37. Harris, D. L. 2000. Multi-Site Pig Production. lowa State University Press, Ames, IA 50010.

38. Harris, D. L. 1990. Isolated Weaning - Eliminating Endemic Disease and Improving Performance. Large Animal Veterinarian 10-12.

39. Harris, D. L. 1988. Alternative approaches to eliminating endemic diseases and improving performance of pigs. The Veterinary Record 123:422-423.

40. Harris, D. L., T. J. L. Alexander, S. C. Whipp, I. M. Robinson, R. D. Glock, and P. J. Matthews. 1978. Swine Dysentery: Studies of Gnotobiotic Pigs Inoculated with Treponema hyodysenteriae, Bacteriodes vulgatus, and Fusobacterium necrophorum. JAVMA 172:468-471.

41. Harris, D. L., R. D. Glock, C. R. Christensen, and J. M. Kinyon. 1972. Swine Dysentery - I. Veterinary Medicine/Small Animal Clinician 67:61-64

42. Holt, M. E., M. R. Enright, and T. J. L. Alexander. 1989. Studies of the Protective Effect of Different Fractions of Sera from Pigs Immune to Streptococcus suis Type 2 Infection. J. Comp. Path 100:435-442.

43. Holt, M. E., M. R. Enright, and T. J. L. Alexander. 1990. Protective Effect of Sera Raised against Different Fractions of Streptococcus suis Type 2. J. Comp. Path 103:85-94.

44. Hudson, M. J., T. J. L. Alexander, and J. F. Prescott. 1976. Swine-Dysentery - Protection of Pigs by Oral and Parental Immunization With Attenuated Treponema-Hyodysenteriae. Res. in Vet. Sci. 21:366-367.

45. Johnson, R. G. 1992. MMEW Post Weaning Performance: "Life in the Fast Lane". Proceedings of the American Association of Swine Practitioners 1992:463-467. 
46. Lysons, R. J. and T. J. L. Alexander. 1971. Establishment of a Limited Rumen Microflora in Gnotobiotic Lambs. Res. in Vet. Sci. 12:486-487.

47. Mallard, B. A., B. N. Wilkie, and B. W. Kennedy. 1989. The Influence of the Swine major Histocompatibility Genes (SLA) on Variation in Serum Immunoglobulin (Ig) Concentration. Veterinary Immunology and Immunopathology 21:139-151.

48. Mare, C. J. and W. P. Switzer. 1965. New species: Mycoplasma hyopneumoniae, a causative agent of virus pig pneumonia. Veterinary Medicine 60:841-846.

49. Mengeling, W. L. 2005. The porcine reproductive and repiratory syndrome quandary. Part II: Vaccines and vaccination strategy. Journal of Swine Health \& Production 13:152-156.

50. Mengeling, W. L. 2005. The porcine reproductive and respiratory syndrome quandary. Part 1: Fact versus speculation. Journal of Swine Health \& Production 13:91-95.

51. Mengeling, W. L., K. M. Lager, A. C. Vorwald, and D. F. Clouser. 2003. Comparative safety and efficacy of attenuated single-strain and multi-strain vaccines for porcine reproductive and respiratory syndrome. Vet Micro 93:25-38.

52. Muirhead, M. R. and T. J. L. Alexander. 1988. Educating the Future Pig Consultant and Specialist. Proc Pig Vet Soc 21:42-52.

53. Muirhead, S. 1994. MEW work at S. Dakota. Feedstuffs September 26:10-31.

54. Onions, D., D. K. C. Cooper, T. J. L. Alexander, C. Brown, E. Claassen, J. E. Foweraker, D. L. Harris, B. W. J. Mahy, P. D. Minor, A. D. M. E. Osterhaus, P.-P. Pastoret, and K. Yamanouchi. 2000. An approach to the control of disease transmission in pig-to-human xenotransplantaion. Xenotransplantation 7:143-155.

55. Poulin, M. C. and W. T. Christianson. 2006. On-Farm eradication of foot-and-mouth disease as an alternative to mass culling. The Veterinary Record 158:467-472.
56. Roe, C. K. and T. J. L. Alexander. 1958. A Disease of Nursing Pigs Previously Unreported in Ontario. Canadian Journal of Comparative Medicine 22:305-307.

57. Roe, C. K. and T. J. L. Alexander. 1961. Attempts at establishing swine herds free from atrophic rhinitis and virus pneumonia: 1. Review of intial work at the Ontario Veterinary College. Can Vet J 2:139-142.

58. Taylor, D. J. and T. J. L. Alexander. 1971. The Production of Dysentery in Swine by Feeding Cultures Containing a Spirochaete. Br. vet. J. 127:Iviii-Ixi.

59. Torremorell, M., A. Juarez, E. Chavez, J. Yescas, J. M. Doporto, and M. Gramer. 2009. Procedures to eliminate H3N2 swine influenza virus from a pig herd. Vet Rec 165:74-77.

60. Tubbs, R., B. Thompson, and D. Reetzke. 1999. PRRS elimination in 2-site farm, p. 81-82. In .

61. Underdahl, N. R. 1973. Specific Pathogen Free Swine. University of Nebraska Press, Lincoln.

62. Whipp, S. C., I. M. Robinson, D. L. Harris, R. D. Glock, P. J. Matthews, and T. J. L. Alexander. 1979. Pathogenic synergism between Treponema hyodysenteriae and other selected anaerobes in gnotobiotic pigs. Infection and Immunity 26:1042-1047.

63. Wilkie, B. and B. Mallard. 1999. Selection for high immune response: an alternative approach to animal health maintenance? Veterinary Immunology and Immunopathology 72:231-235.

64. Williams, A. E. B. W. F. and T. J. L. Alexander. 1988. A murine model of Streptococcus suis type 2 meningitis in the pig. Res. in Vet. Sci. 45:394-399.

65. Yeske, P. 2003. Analyzing the value of herd stabilization of disease elimination vs. depopulation and repopulation. American Association of Swine Veterinarians 445-449.

66. Yuan, S., NelsenC.J., M. P. Murtaugh, B. J. Schmitt, and K. S. Faaberg. 1999. Recombination between North American strains of porcine reproductive and respiratory syndrome virus. Virus Research 61:87-98. 


\title{
Animal welfare and the veterinary profession: $\mathbf{5 0}$ years of change
}

\author{
David Fraser \\ Animal Welfare Program, Faculty of Land and Food Systems, University of British Columbia, Vancouver, BC, Canada
}

\section{Introduction}

About 10 years ago I received an unexpected telephone call. It was from the Burger King Corporation to say that the company wanted to create a program to assure their customers about the welfare of the animals in their supply chain, and would I serve on the advisory committee. I admit to having been a trifle skeptical. Fast food restaurants as agents of social change? - it did not quite fit the popular stereotype. But I joined the committee and was pleasantly surprised to find myself in discussions about maintaining public trust and 'doing the right thing' for animals. To cut a long story short, Burger King's engagement in animal welfare led to some tangible changes. In fact Temple Grandin (2000), after years of inspecting slaughter plants with mediocre animal welfare standards, reported a dramatic improvement immediately after chain restaurants became involved.

This, of course, is just one example of the remarkable growth in attention being paid to animal welfare, in some cases by unexpected players:

- In 2005 the 170 member nations of the World Organization for Animal Health unanimously adopted 80 pages of animal welfare standards which now appear in the highly influential Terrestrial Animal Health Code.

- Around the same time the International Finance Corporation, the investment arm of the World Bank, called for animal welfare to be part of the business plan of the livestock companies in which they invest.

- In 2008 the FAO, the United Nations agency involved in agriculture and hunger reduction, held an international consultation on how to help countries, especially developing countries, to implement good animal welfare practices.

- And it is expected that a 'Universal Declaration on Animal Welfare' will soon be presented to the United Nations with the expressed support of many of the world's countries.

Nearly 40 years ago, when I began doing research on the welfare of pigs, animal welfare was on the very fringe of science. To most people the words 'research' and 'animal welfare' did not belong in the same sentence except perhaps to criticize animal welfare standards in laboratories. So how did it happen that animal welfare moved from being a fringe issue to something that attracts the attention of Burger King, the World Bank and the United Nations?

In this presentation I would like to trace this change by looking at four topics:

- some of the reasons behind the growing focus on animal welfare

- the debate about exactly what 'animal welfare' means

- some of the science that has been applied to animal welfare issues

- and the role of veterinarians in this rapidly changing world.

\section{The growing focus on animal welfare}

Every culture has an 'animal mythology' - a set of fundamental beliefs and values regarding animals - which we can often perceive through the art and stories of the culture (material in this section is from Fraser, 2008). In the creation story of the Ojibway culture of central Canada, people had fallen from the sky onto an earth that was covered in water, and they were able to survive only because the animals took pity on them, starting with the turtle who allowed its shell to form the base of the dry land. And throughout Ojibway legends and conduct, we see this fundamental assumption - perhaps not surprising for a hunting culture living in a harsh northern climate - that people are vulnerable beings whose survival is made possible by the cooperation of animals.

The first chapter of the Bible tells the very different creation story of a pastoralist culture where the ownership and care of animals was fundamental to the economy. In that story we read that life began when God created the natural world and filled it with plants and animals, and then, as a final act of creation, produced human beings and set them to rule over the natural world in god's place. And later in the story, when God flooded the world, it was the capable humans who saved the helpless animals from drowning, which is roughly the opposite of how the Ojibway saw the relationship.

In these two examples of animal mythology we see both empirical beliefs - about what animals are like and the history of our involvement with them - and evaluative or ethical beliefs about how important animals are and how they should be treated. The biblical creation story implied that people and animals are separated by a large gulf which included at least three components. One was appearance: people had been created in the 'image' of God, and therefore looked nothing like birds, fish or quadrupeds. Second, people had a different origin: they were created separately from the animals and for a different purpose. And third (although disagreement arose on this point) people were seen as having a unique inner, spiritual nature that animals did not share. Here, then, were three empirical beliefs - about different appearance, different origin, and different inner life that reinforced the idea of humans as fundamentally different from animals and helped to justify the use of animals for human purposes.

But in modern culture, empirical beliefs are not fixed by the constant retelling of traditional stories, but rather are subject to change in light of scientific discoveries and other developments. And indeed over the centuries we have seen these three empirical beliefs gradually chipped away, at least partly by science.

The first scientific development came from anatomy. Beginning in Italy during medieval times, the study of anatomy became one of the frontiers of scientific research for many centuries. Moreover, this research was communicated to the public in the many 'dissecting theatres' that sprouted up in the major centres of learning. These theatres allowed people to witness the dis- 
section of animals or even humans cut down from the gallows. And through this anatomical research and the remarkably direct form of public education, it came to be recognized that humans - organ for organ and bone for bone - are actually built on the same anatomical template as the other vertebrate animals.

This realization became common knowledge around the year 1700 , and it touched off two centuries when scientists and philosophers alike struggled to understand the implications of this new knowledge. The process culminated with the evolutionary biology of the 1800 s and the startling proposal that the reason why we and other species have the same anatomical structure is that we share a common evolutionary origin. Thus, by about a century ago people had lost both their unique appearance and their unique origin.

Late in the 1900s, I think the study of animal behaviour led to a further revision in our view of animals, this one centred on their mental and emotional lives. A classic example is the work of primatologist Jane Goodall who studied animals in such detail that she could describe their personalities and unique life histories. From Jane Goodall we learn about the young chimpanzee she called 'Flint' who, at the mature age of eight, remained so attached to his aging mother that when she died, he stayed near the place of her death until he himself died of starvation. Drawing together this and many other observations, primatologist Roger Fouts (1997) described the chimpanzee as a:

'highly intelligent, co-operative, and violent primate who nurtures family bonds, adopts orphans, mourns the death of mothers, practises self-medication, struggles for power, and wages war.'

Thus, with some species at least, even in our mental lives the gap between humans and animals has come to seem remarkably narrow.

To sum up, the large gap between people and animals that we saw in the Biblican creation story has narrowed through scientific research, so that many people today believe that humans and animals share a common anatomical form, a common evolutionary ancestry and, with some species, a complex mental and emotional life. This altered set of empirical beliefs, about what animals are like, has stimulated a massive rethinking of our ethical beliefs, about how animals should be treated. And because science has expanded from being an element of Western culture, and has become more an element of global culture, perhaps it is not surprising that animal welfare is rapidly becoming a global issue.

\section{What is animal welfare?}

As the current wave of concern about animal welfare began, roughly in the 1960s, a debate emerged over what animal welfare really involves.

The first major criticism of confinement production systems came in the book Animal Machines, by the English animal advocate Ruth Harrison (1964). She described cages for laying hens and crates for veal calves, and she claimed that these systems are so unnatural that they cause animals to lead miserable and unhealthy lives. She went on to ask:

How far have we the right to take our domination of the animal world? Have we the right to rob them of all pleasure in life simply to make more money more quickly out of their carcasses?
Later, in Animal Liberation, Australian philosopher Peter Singer (1990) adopted the principle that actions should be judged right or wrong on the basis of the pain or pleasure that they cause, and he claimed:

There can be no moral justification for regarding the pain (or pleasure) that animals feel as less important than the same amount of pain (or pleasure) felt by humans.

In these and many other quotations, a key concern centres on words like "pleasure","pain", "suffering", and "happiness". There is no simple English word to capture this class of concepts. They are sometimes called "feelings but that term seems too insubstantial for states like pain and suffering. They are sometimes called "emotions but emotions do not include states like hunger and thirst. Perhaps the most accurate, if rather technical, term is "affective states", a term that refers to emotions and other feelings that are experienced as either pleasant or unpleasant rather than hedonically neutral.

In discussing animal welfare, however, some people put the emphasis elsewhere. A British committee that was formed to evaluate the welfare of farm animals concluded:

In principle we disapprove of a degree of confinement of an animal which necessarily frustrates most of the major activities which make up its natural behaviour. (Brambell 1965)

Astrid Lindgren, the famous author of the Pippi Longstocking stories and a driving force behind animal welfare reform in Sweden, proposed:

Let [farm animals] see the sun just once, get away from the murderous roar of the fans. Let them get to breathe fresh air for once, instead of manure gas. (Anonymous 1989)

And American philosopher Bernard Rollin (1993) insisted that we need:

... a much increased concept of welfare. Not only will welfare mean control of pain and suffering, it will also entail nurturing and fulfilment of the animals' natures

In these quotations, although affective states were often involved implicitly or explicitly, the central concern was for a degree of "naturalness" in the lives of animals: that animals should be able to perform their natural behaviour, that there should be natural elements in their environment, and that we should respect the "nature" of the animals themselves.

All of the above quotations reflected the views of social critics and philosophers, but when farmers and veterinarians engaged in the debate, they brought a different focus. For example, one veterinarian defended confinement systems this way:

My experience has been that ... by-and-large the standard of welfare among animals kept in the so called "intensive" systems is higher. On balance I feel that the animal is better cared for; it is certainly much freer from disease and attack by its mates; it receives much better attention from the attendants, is sure of shelter and bedding and a reasonable amount of good food and water. (Taylor 1972)

Or as the veterinary educator David Sainsbury (1986) put it:

Good health is the birthright of every animal that we rear, whether intensively or otherwise. 
Here the primary emphasis is on the fairly traditional concerns of veterinarians and animal producers that animals should have freedom from disease and injury, plus food, water, shelter and other necessities of life - concerns that we might sum up as the basic health and functioning of the animals.

In these various quotations, then, we see a variety of concerns that can be grouped roughly under three broad headings: one centres on the affective states of animals, one on the ability of animals to lead reasonably natural lives, and one emphasizes basic health and functioning. These are not, of course, completely separate or mutually exclusive; in fact, they often go hand in hand. Letting a pig wallow in mud on a hot day is good for its welfare because the pig will presumably feel more comfortable (an affective state), because it can perform its natural cooling behaviour (natural living), and because it will have less disruption of its body processes caused by heat stress (basic health).

But the different criteria do not always go together. The gestation stall, where sows cannot walk or turn around for most of pregnancy, is a way of promoting healthy weight gain and avoiding injuries from aggression, but it is very unnatural and may create a life that is not very pleasurable. Feeding sows as much as they want may avoid unpleasant feelings of hunger, but can lead to unhealthy weight gain. Some outdoor systems look very natural, but involve parasitism and low piglet survival. Thus, genuine disagreements can arise when people try to create standards or practices to promote animal welfare based on any one of the three areas of concern.

\section{Animal welfare science}

As this debate unfolded in the 1960s and 1970s, people began doing scientific research on animal welfare. Partly the research was done for the practical goal of making production systems more efficient by making them better suited to the animals. And partly the research was done because people expected science to resolve the disagreements over the interpretation of animal welfare. Surely, if animal welfare standards were 'science-based', there could be no disagreements based on differing interpretations of animal welfare. The outcome, however, proved more interesting as the following simple examples show.

Some of the research used the basic health and functioning of animals as an indicator of animal welfare. In one elegantly simple example Ragnar Tauson and co-workers (1998) improved the design of cages for laying hens just by studying the health of birds in cages of different types. They found that the birds developed foot lesions if the slope of the floor was too steep, and neck lesions if the feed trough was too deep and installed too high for comfortable access. There was often feather damage that could be reduced by using solid side partitions, and overgrown claws that could be prevented by installing abrasive strips. By modifying cage designs accordingly, Tauson was able to make large improvements in animal welfare as well as production efficiency, and this research then formed the basis of animal welfare standards for cage design, first in Sweden and then in the European Union.

Other scientists tried to improve animal welfare by making living conditions more 'natural' for animals. On many commercial dairy farms, calves are separated from their mothers within the first day after birth, and are then fed milk from a bucket, usually in two fairly large meals per day. This, of course, is very unnatural. Under natural conditions, the calf would stay close to the cow for the first two weeks, and would feed many times per day in smaller meals. Leaving cows and calves together is not feasible on dairy farms, but calves can still be fed in a more natural way. First, if the calves suck from a teat rather than drink from a pail, the sucking action seems to stimulate certain digestive processes more effectively. Second, if the teat feeding system allows the calves to have many small meals per day (as they would if feeding from the cow), then they can consume a larger daily intake without developing digestive problems. As a result, such calves gain much more weight than calves fed twice a day by bucket (Appleby et al. 2001).

In other cases, research has tried to reduce unpleasant affective states in animals. Hot-iron disbudding of calves involves heating a ring-shaped iron to about 600 degrees Celcius and pressing it against the calf's head so that it burns through the nerves and blood vessels that would allow the horn to develop. Research in New Zealand by Stafford and Mellor (2005) used changes in plasma levels of the stress-related hormone cortisol as an indirect indicator of the resulting pain. Hot-iron disbudding was followed immediately by an increase in cortisol, but that reaction was blocked if lidocaine was used to freeze the area. Even with lidocaine, however, cortisol levels showed a clear increase several hours after the disbudding, probably because the area remained inflamed and painful after the freezing had worn off. However, if an analgesic such as ketoprofen is added to the calf's milk on the morning and evening of the dehorning, the second peak of cortisol is also eliminated. The results indicate that effective pain management requires both local freezing and a longer-acting analgesic.

In the early stages of the debate about animal welfare, some people expected that the science would eliminate any disagreement over the different elements of animal welfare by finding the one 'scientific' view. Instead, each of the different views of animal welfare led to research that provided different and complementary ways of improving animal welfare, and all three have led to practical improvements and science-based animal welfare standards. Thus, instead of the science resolving the different interpretations of animal welfare, the different interpretations actually came to underlie and enrich the science.

The take-home message for the animal-based industries is that all three views of animal welfare have a scientific basis, and that the single-minded pursuit of any one criterion of animal welfare may fail to promote animal welfare as judged by the other criteria. For standards and practices to be widely accepted as improving animal welfare, they need to make a reasonable accommodation to all three.

\section{The role of veterinarians}

What is the role of veterinarians in this world of changing values and emerging science?

First, we need to recognize that animal welfare research is not a mature field of science. Much of the research is still more conceptual than strategic. Much of it is done at a researchfarm scale, not a commercial scale. And much of it does not yet involve the multi-disciplinary teams that are needed to look at all the implications of new ideas. There is a great opportunity for veterinarians to work with animal welfare scientists to refine and broaden the science, and to apply it in practical ways. Here, then, is a scientific and technical role.

Second, the increased public concern about the welfare of animals has created an expectation that veterinarians will provide leadership in promoting animal welfare, not just as technical 
specialists working to prevent and treat disease (important as that is), but also as champions of animal welfare in a broader sense that includes the different areas of concern. Here is a social leadership role.

I believe there is some urgency about fulfilling these roles. Some highly intensive pig production emphasizes basic health but ignores other animal welfare concerns; these systems sometimes offend against the public's view of what constitutes decent treatment of animals. Unless scientists and veterinarians can deal with the problems, then legislators and referenda may do so instead, and the outcomes may be less than ideal for the animals and for producers. At the other extreme there is a growth in outdoor systems that tend to be seen by the public as high-welfare, more or less by definition, but may involve serious problems of basic health and functioning. There is a need for technical innovation and good standards to ensure that this well-intentioned development does lead to good welfare for the animals.

But why, in 2010, are such basic problems as these still waiting to be solved? A century ago, agricultural and veterinary students studied a subject called 'animal husbandry' which included the feeding, breeding, handling, management and housing of animals. Then certain branches of science became established - nutrition, physiology, genetics - and before long, animal husbandry was replaced by a handful of scientific specialties. But when that happened, some of the original components of animal husbandry - the handling and management and housing - seemed to be forgotten as areas for scientific research and training. I think animal welfare science is finally restoring these missing elements of animal husbandry, but with a scientific basis that was not available a century ago. Here we see an educational role for veterinarians: to use animal welfare science as a way of restoring animal husbandry in veterinary education.

\section{Conclusions}

What can we conclude from a presentation that has ranged from creation stories to studies of animal pain? Perhaps five things:

- First, the social concern about animal welfare that we see today has grown out of a long history of change in attitudes, driven to a large degree by scientific discoveries that narrowed the gap that we perceive between people and animals.

- Second, social concern about animal welfare includes three main elements: (1) the basic health and functioning of animals, (2) the affective states of animals, especially freedom from negative states such as pain and distress, and (3) the ability to live in a way that suits the animals' natural behaviour and other adaptations.

- Third, each of these elements of animal welfare has a scientific basis, and all three have given rise to practical improvements and science-based standards. The science did not arbitrate among the different views of animal welfare; rather the different views of animal welfare influenced the science and contributed to the richness of its ideas and approaches.

- Fourth, the single-minded pursuit of any one element of animal welfare does not guarantee a high level of animal welfare as judged by the others. For practices and standards to be widely accepted as improving animal welfare, they need to strike a balance among all three.
- Fifth, the increased focus on animal welfare, and the emerging science of animal welfare, have created expanded opportunities for veterinarians to take on new scientific and technical roles, new social leadership roles, and new educational roles in improving the lives of animals.

\section{Acknowledgements}

Much of the text is based on my book Understanding Animal Welfare: The Science in its Cultural Context, published by WileyBlackwell in 2008. I am grateful to the publisher for permission to rework that material here. I am also grateful for the support of the Natural Sciences and Engineering Research Council of Canada, and of the UBC Animal Welfare Program and its many donors.

\section{References}

Anonymous 1989 How Astrid Lindgren Achieved Enactment of the 1988 Law Protecting Farm Animals in Sweden. Animal Welfare Institute, Washington.

Appleby MC, Weary DM and Chua B 2001 Performance and feeding behaviour of calves on ad libitum milk from artificial teats. Applied Animal Behaviour Science 74: 191-201.

Brambell FWR (chairman) 1965 Report of the Technical Committee to Enquire into the Welfare of Animals kept under Intensive Livestock Husbandry Systems. Her Majesty's Stationery Office, London.

Fouts RS 1997 Next of Kin: What Chimpanzees have Taught Me about Who We Are. Morrow, New York.

Fraser D 2008 Understanding Animal Welfare: The Science in its Cultural Context. Wiley-Blackwell, Oxford.

Grandin T 2000 Effect of animal welfare audits of slaughter plants by a major fast food company on cattle handling and stunning practices. Journal of the American Veterinary Medical Association 216: 848-851

Harrison R 1964 Animal Machines. Vincent Stuart Ltd, London.

Singer P 1990 Animal Liberation, 2nd edition. Avon Books, New York.

Rollin BE 1993 Animal welfare, science, and value. Journal of Agricultural and Environmental Ethics 6 (Supplement 2): 44-50.

Sainsbury D 1986 Farm Animal Welfare. Cattle, Pigs and Poultry. Collins, London.

Stafford KJ and Mellor DJ 2005 Dehorning and disbudding distress and its alleviation in calves. The Veterinary Journal 169: 337-349.

Tauson R 1998 Health and production in improved cage designs. Poultry Science 77: 1820-1827.

Taylor GB 1972 One man's philosophy of welfare. Veterinary Record 91: 426-428. 


\title{
LS.1
}

\section{Role of nutrition and intestinal adaptation in weanling pig health}

\author{
Douglas Burrin \\ USDA-ARS, Children's Nutrition Research Center, Houston, TX, USA
}

\begin{abstract}
Summary
The growth rate of the pig is most rapid during the neonatal and weaning periods. Nutrition and gastrointestinal function play a critical role in the survival, health and growth of the young pig during this transition from suckling to weaning. The high metabolic rate and cellular turnover of gut tissues result in substantial first-pass utilization of dietary nutrients, especially amino acids, to maintain gut function. The changes in the diet composition and gut microbiota after weaning are associated with increased gut growth and metabolism, which may limit the systemic availability of dietary nutrients. Strategies aimed at optimizing gut metabolism and supplementing key gut-nutrients in support of gut function may improve growth.
\end{abstract}

\section{Early gut development and nutrition}

The neonatal and weaning phases occupy critical periods of postnatal growth and development in commercially reared pigs. The relative rates of growth are higher during the neonatal period than at any time during postnatal life. More importantly however, is that during the neonatal and weaning phases, the survival, health and subsequent growth rate of young pigs depends on their ability to adapt physiologically to significant changes in nutrition and the environment. Critically to this adaptation process is the development of the gastrointestinal tract, particularly digestive, absorptive and immune function. Nutrition is a key determinant in the functional development and growth of the gastrointestinal tract.

The gastrointestinal tract of the piglet increases nearly threefold between birth and two weeks after weaning, increasing from $2 \%$ to $6 \%$ body weight. A major stimulus for gut growth at birth is the initiation of suckling or enteral nutrition. Enterally nutrients have a major anabolic effect on the neonatal gut, but growth factors present in sow's colostrum and milk may also have trophic effects. Weaning is the second major milestone in gastrointestinal growth in the developing piglet. Nutrition plays a central role during weaning, first by limiting gut growth due to reduced feed intake and then providing a major stimulus due to increased dry matter intake and changes in diet composition. The weaning transition is marked by reduced villus height, increased crypt cell hyperplasia and expansion of the lamina propria cell population. The weight of the colon is increased approximately threefold within seven days after weaning, mainly due to increased fermentation of undigested dietary carbohydrates and fiber. The hyperplasia in the crypt epithelium and lamina propria is thought to be linked to gut hypersensitivity reactions associated with plant proteins in the weaning diet, such as soy glycinins and lectins.

The weanling pig is especially vulnerable to pathogenic infection because of the withdrawal of sow's milk and the increased colonization of commensal gut microorganisms. Pathogenic infection results in an activation of the mucosal immune system and release of proinflammatory cytokines (e.g. tumor necrosis factor" and interleukins) that have been shown to increase crypt cell proliferation, villus enterocyte apoptosis, and intestinal acute phase protein synthesis.

\section{The concept of gut nutrient requirements}

Most swine nutritionists have an extensive knowledge of the nutrition requirements for the pig growth. However, in recent years, the concept of nutrient requirements has evolved to include the needs specifically for the gut. Technical advances have enabled direct estimates of gut nutrient utilization and their impact on whole animal nutrient metabolism in vivo studies in pigs using isotopic tracers and measurements of trans-organ balance of substrates. These and other studies with cultured porcine intestinal epithelial cells have revealed important qualitative characteristics of the metabolic fate of key substrates and the underlying cellular basis for intestinal nutrient utilization.

A major concept that has emerged from studies with young pigs is that non-essential amino acids are major gut fuels, especially glutamate, glutamine and aspartate. In vivo studies in pigs show that roughly $70-80 \%$ of the dietary glutamate, glutamine and aspartate is taken up by the gut in first pass and metabolized to $\mathrm{CO}_{2}$. Glucose is also quantitatively an important oxidative fuel for the pig intestine, in absolute amounts; the intestinal utilization of glucose is similar to the combined total from glutamate, glutamine and aspartate. However, the proportional use is lower, such that only $\sim 20-30 \%$ of the dietary glucose is metabolized by the gut.

Studies with pigs have shown that the neonatal intestine plays a key role in the metabolism of amino acids involved in the urea cycle, particularly arginine, proline, and ornithine. There is extensive interconversion between these amino acids and the intestine represents an important site of net arginine synthesis in neonatal pigs. Studies in pigs have also demonstrated the extensive metabolism of other essential amino acids, including threonine, lysine, phenylalanine, branched-chain amino acids, and methionine. It is generally considered that the primary metabolic fate of essential amino acids taken up by the gut is incorporation into tissue proteins. However, studies show that extensive irreversible catabolism and oxidation of amino acids occurs in the gut. Moreover, many of these essential amino acids are metabolized to other intermediates involved in intestinal function. For example, threonine is believed to be channeled into mucin synthesis and secreted by goblet cells, because mucin peptides are rich in threonine. Methionine may be converted to cysteine or s-adenosyl-methionine used in polyamine synthesis. Cysteine is used as a precursor for glutathione synthesis and maintenance of mucosal anti-oxidant status. The oxidation of some essential amino acids, such as the branched-chains and lysine, in the gut maybe nutritionally significant. The high essential amino acid utilization rate by gut tissues can have a significant impact on the systemic availability for lean tissue growth. Historically, weanling pig diets have been formulated largely to overcome the limitations or immaturity in digestive function in 
order to maximize growth of the whole animal. However, with a new understanding of intestinal nutrient utilization, it is possible to now formulate weanling pig diets with the specific goal of optimizing the growth, function and health of the gut. From the foregoing discussion of intestinal nutrient utilization some of the most promising candidates are glutamine, glutamate and threonine.

\section{Nutrition and enteric health and function}

A critical factor influencing the growth of weanling pigs is the degree of colonization with commensal and pathogenic microbes. Exposure to these microbes and their toxins can adversely affect intestinal structure and function. The pro-inflammatory stimulus induced by bacteria, endotoxin and cytokines significantly increases the intestinal protein synthesis rate. Studies in sheep show that parasitic infection increases the rate of leucine utilization and oxidation by PDV tissues, thereby reducing the systemic availability of dietary amino acids by $20-30 \%$. Thus, it is likely that the degree of commensal and pathogenic microbial load directly affects the intestinal nutrient requirements, which in turn may consume dietary nutrients and limit growth of weanling pig.

Antimicrobial compounds are fed to weanling pigs in order to suppress the activity of the gut microflora and enhance growth. Antimicrobials are thought to act via four possible mechanisms, a) prevention of infection, b) reduced microbial nutrient use, c) enhanced nutrient absorption, and d) reduced growth-depressing microbial metabolite load. It is generally held that one or a combination of these mechanisms act to diminish the thickness and mass of the intestinal mucosa and associated lymphoid tissue. A critical mechanistic question regarding the site of dietary amino acid utilization in the gastrointestinal tract is whether this activity is associated with the luminal microbes or the cell populations of the mucosa.

Alternative nutritional strategies are being used to manipulate the gut microflora including probiotics, prebiotics, organic acids, spray-dried plasma, and high concentrations of copper and zinc. These approaches have become an increasingly important consideration in swine nutrition, given the evidence of their benefits in animals and humans coupled with the growing concern of antimicrobial resistance. Probiotics and prebiotics are used to induce the colonization of bacteria (e.g. lactobacillus and bifidobacteria) considered to be beneficial for the host. The consequences of these alternative nutritional strategies on gut metabolism and nutrient utilization are poorly understood and warrant further study.

\section{References}

Burrin, DG and B Stoll. Intestinal nutrient requirements in weanling pigs. In Weaning the Pig: Concepts and Consequences. Ed. JR Pluske, J. Le Dividich and MWA Verstegen. Wageningen Pers, The Netherlands. pp. 301-335, 2003.

Chen L, Li P, Wang J, Li X, Gao H, Yin Y, Hou Y, Wu G. (2009) Catabolism of nutritionally essential amino acids in developing porcine enterocytes. Amino Acids. 37:143-52.

Gaskins HR, Collier CT, Anderson DB (2002) Antibiotics as growth promotants: mode of action. Anim Biotechnol 13, 29-42.

Lay, DC, RL Matteri, JA Carroll, TJ Fangman, and TJ Safranski (2001) Preweaning survival in swine. J. Anim. Sci. 80(E. Suppl. 1),E94-E103.

Pluske, JR, DJ Hampson, and IH Williams (1997) Factors influencing the structure and function of the small intestine in the weaned pig: a review. Livest. Prod. Sci. 51, 215-236.

Riedijk, MA, B Stoll, S Chako, H Schierbeek, AL Sunehag, JB van Goudoever, DG Burrin. (2007) Methionine transmethylation and transsulfuration in the piglet gastrointestinal tract. Proc Natl Acad Sci. 104:3408-3413.

Stoll, B and DG Burrin (2006) Measuring splanchnic amino acid metabolism in vivo using stable isotope tracers. J. Anim. Sci. 84(E. Suppl.):E60-E72.

Stoll, B, J Henry, PJ Reeds, H Yu, F Jahoor and DG Burrin (1998) Catabolism dominates the first-pass intestinal metabolism of dietary essential amino acids in milk protein-fed piglets. J. Nutr. 128, 606-614.

Stoll, B, DG Burrin, J Henry, H Yu, F Jahoor and PJ Reeds (1999a) Substrate oxidation by the portal drained viscera of fed piglets. Am. J. Physiol. 277, E168-E175.

Van Goudoever, JB, Stoll, B, Burrin, DG, Henry, J, Reeds, PJ. (2000) Adaptive regulation of intestinal lysine metabolism. Proc. Natl. Acad. Sci. 97:11620-11625. 


\title{
LS.2
}

\section{Progress in Porcine Reproductive and Respiratory Syndrome Virus (PRRSV): What we know about PRRSV, from basic to applied science - A historical perspective}

\author{
Jane Christopher-Hennings \\ South Dakota State University, Veterinary and Biomedical Sciences, Brookings, SD, USA
}

It is an absolute honor to be asked to give the opening talk at the PRRSV session at the 21st International Pig Veterinary Society Congress in Vancouver. After looking through the list of subsequent speakers, I recognize the great expertise and innovative thinking that is present and want to recognize those that have contributed to our knowledge about PRRSV. Hopefully this will be an encouraging presentation by demonstrating how we have collaborated and innovated in the past and how we can continue the momentum in trying to prevent this disease.

This talk will show a historical and comparative viewpoint in the discovery of PRRSV and subsequent development of reagents and diagnostic applications. I think we should take some encouragement in knowing that PRRSV is still a relatively "young" pathogen in the swine population compared to others such as transmissible gastroenteritis virus (TGEV) which was described in the US in 1946; pseudorabies virus (PRV), first found to be filterable in 1813 in cattle, but recovered from swine in 1909; and foot and mouth disease virus (FMDV) which was identified as a filterable agent in 1897 [1]. In addition, PRRSV is not "latent" like PRV where there is a constant risk of previously infected animals transmitting the virus; PRRSV is very susceptible to disinfectants, heat and drying (compared to porcine circovirus 2 (PCV2) and some other viruses which are not as readily inactivated); it is not transmissible to or from people like swine influenza virus (SIV) and we also have new technologies to rapidly identify the virus which were not previously available for other disease eradication programs. In addition, we currently have a voluntary, coordinated, funded and focused effort in the United States http://www.prrs.org http://www.prrssymposium.org), Canada and other countries, to understand, control and eliminate PRRSV transmission.

PRRSV was first identified as the cause of "Mystery Swine Disease", "Blue-eared" Pig Disease or "Swine Infertility and Respiratory Syndrome" (SIRS), now known as "PRRSV" in 1991 in Europe and subsequently in the US. Even though PRRSV is not eradicated, it should be noted that the causative agent of PRRSV was first identified only about 20 years ago. By comparison, PRV was discovered in swine in 1909 with much of the eradication completed in 2005; classical swine fever virus (CSF/Hog cholera) was first reported in 1810 with much of the eradication completed in the US between 1962 and 1976 and Brucellosis was identified in 1887 and nearly eradicated at this time. In these cases, between 100-200 years passed before eradication or near eradication was completed after identification of these swine pathogens.

Persistence of PRRSV was initially described, but PRRSV is not "latent" whereby viral replication can be re-activated and shedding of the virus can recur. With PRRSV, transmission of virus from pigs infected in utero can be documented up to 112 days of age [2]. However, duration of shedding may be somewhat age dependent with 4 month old gilts not demonstrating viral shedding between 90-180 days post inoculation [3], whereas
90-100\% of PRRSV infected 3 week old pigs were still infected at 63-100 days [4]. Therefore, recommendations have been made to close herds for 200 days post clinical signs to prevent shedding to newly introduced pigs. These findings demonstrate that there is a finite time period of viral shedding, unless a new viral strain or PRRSV naïve pigs are introduced.

Since PRRSV is an enveloped virus (unlike PCV2), it is susceptible to heat, drying and disinfectants which allows for more efficient removal of the virus from the environment. In 1992, the characteristics of this virus were described in cell culture whereby virus infectivity was completely inactivated after 45 minutes at $56 \mathrm{C}$, but more stable after 1 month incubation at $4 \mathrm{C}$ or 4 months at -70 C [5]. This susceptibility to higher temperatures and stability at lower temperatures was further confirmed for a range of PRRSV strains [6]. This information is also useful for effective biosecurity protocols [7].

Transmission of PRRSV through other animal vectors or people has not been confirmed, although mechanical transmission could occur through insects and fomites $[8,9]$. This differs from SIV whereby pigs are susceptible to influenza that could be transmitted through people [10], making biosecurity more difficult.

Newer diagnostics such as rapid molecular tests (real-time, quantitative or isothermal PCR) were discovered and utilized after 1983 [11] for "same day" results. These tests were not previously available for other swine pathogen elimination programs, but should be an advantage for PRRSV elimination protocols.

More convenient surveillance methods are also available to obtain and test samples economically and with the welfare of the animal in mind. These include pooling of samples, obtaining oral fluids [12] or blood swabs [13] and using newer technologies to multiplex for identification of various swine disease antigens, antibodies or immune parameters simultaneously [14].

Coordinated efforts to control or eliminate PRRSV are becoming more prevalent worldwide. In the US, the PRRSV Coordinated Agriculture Program (CAP1 and CAP2) started by the USDA in 2004 are designed to enable "the collective talents of the stakeholder community of scientists, veterinarians, pork producers, and allied industry researchers to develop innovative strategies to lessen the impact of PRRS and lead to the eventual elimination of the virus." Research on vaccines that are effective against a wide-variety of PRRSV strains and that can be differentiated from field strains should continue. With these resources and findings, we should be further along on PRRSV elimination than historically has been the case with other swine pathogen elimination programs. 


\section{References.}

[1] Straw B, JJ Zimmerman, S D'Allaire, DJ Taylor. In: Diseases of Swine, 9th edition. 2006. pp 489, 581.

[2] Benfield DA, et al.1997. Proc AASP. Quebec City, Canada. 1997; 455-458.

[3] Batista L, et al. 2002. Can J Vet Res 66:196-200.

[4] Horter D, et al. 2002. Vet Microbiol 86:213-228

[5]. Benfield D, et al. 1992. J Vet Diagn Invest 4:127-133.

[6] Zimmerman J, et al. 2010. J Vet Diagn Invest 2:257-260.

[7] Dee S, et al. 2005. Can J Vet Res 69: 58-63.

[8]. Hooper CC, et al. 1994. J Vet Diagn Invest 6:13-15.

[9] Trincado C, et al. 2004. Vet Record 154:233-237.

[10] Lange E, et al., 2009. J Gen Virol 90 (2009), 2119-2123.

[11] Mullis, K. Process for Amplifying Nucleic Acids US 4,683,202 July 28, 1987.

[12] Prickett J, et al. 2008. J Vet Diagn Invest 20:156

[13] Reicks D, et al. 2006. J Swine Health Prod 14:258-264.

[14] Lawson S, et al. 2010. Vaccine. In press. 


\title{
LS.3
}

\section{Pork safety: past achievements and future challenges}

\author{
Peter Davies \\ University of Minnesota, St Paul, MN, USA
}

\section{Introduction}

Ever since the dawn of phagocytosis, eating has been a risky endeavor. Because internalizing portions of our external environment can never be entirely risk free, food safety is a relative concept. However, eating entails less risk than not eating, and the availability and cost of food influence the levels of protection or assurance that consumers demand for their food supply. Hunger and undernutrition are responsible for about 35\% of child deaths and $11 \%$ of the total global disease burden. ${ }^{1}$ At the other extreme, the World Health Organization projects that by 2015 more than 700 million adults will be obese, and 2.3 billion overweight. Overnutrition is imposing increasing burdens on health costs in many countries, with a recent estimate of $\$ 147$ billion annually for the USA. ${ }^{2}$ Paradoxically, obesity is also positively associated with poverty as energy dense foods of low nutritional value tend to be cheap. ${ }^{3}$ Like malnutrition and obesity, food safety presents another manifestation of global inequity linked to poverty and food security.

Pork has long been the most consumed meat globally, and projections of future global pork demand remain robust, largely driven by anticipated growth in developing economies. The fact that per capita meat consumption increases as wealth increases reflects that animal proteins are preferred protein sources across the majority of cultures. However, contemporary discussions of projected world population growth, its implications for land use, and the environmental impact of food production raise complex questions about the future of meat consumption. 4,5 McMichael et al (2007) calculated that global average meat consumption was 100 grams per person per day, but varied ten-fold between high-consuming and low-consuming populations.4 They proposed $90 \mathrm{~g}$ per day as a "working global target" but advocated more even distribution and that not more than $50 \mathrm{~g}$ per day should be from ruminants.5 Although some grass roots groups and celebrities have promoted campaigns to reduce meat consumption (e.g. meat-free Monday), it is unrealistic to expect material change will arise from 'enlightened' consumer behavior. Policy options that could influence meat consumption patterns are yet to be clearly articulated. Similar to the situation with greenhouse gas emissions, increasing tensions are likely as the force of growing demand for animal protein in the developing world strains against calls for restraint from the relatively well fed western world. Priority issues in food safety also vary geographically, and some foodborne hazards that remain major public health issues in developing countries have been reduced to insignificance in developed countries. However, in most countries biological hazards greatly outweigh physical and chemical hazards in terms of public health impact and consumer concern, and therefore will be the focus of this paper.

\section{How safe is pork today?}

It depends - whose pork and compared to what? Unlike crossing a road, where the hazards can be directly perceived, foodborne hazards are mostly invisible to consumers. Food safety is termed a 'credence attribute'. ${ }^{8}$ Opinions are forged from both personal experience and external information, and perceptions of safety are influenced by social processes, including the media and government agencies. Food is such an intimate element of the human experience that there are all manner of passionate opinions about what we should eat, and how it should be produced, procured and prepared. Engaging authors such as Michael Pollan and Eric Schlosser, and films like Food Inc., have inspired a mass of neoromantic 'locavores' who disparage almost every facet of the mainstream commercial food industry. Critics of 'industrialized production' and 'factory farming' habitually conflate diverse issues (social justice, environmentalism, animal welfare...), and food safety is usually prominent in the mix. ${ }^{5}$ Mostly, however, they tend to be vague about which hazards are of particular concern, and why those specific hazards present greater risk in modern systems of animal production. Ironically, the consumer pressures that propelled revision of Federal regulations of meat inspection in the USA in the $1990 s,{ }^{6}$ may have also contributed to integration in the pork industry. Market demands for assurance of safety and quality (including traceability) motivate stronger packer-producer alliances, vertical integration, and marketing of branded products. ${ }^{7}$

In the USA, the ritually cited estimate of the annual burden of foodborne illness is 76 million cases per year, 325,000 hospitalizations, and 5000 deaths (based on the 1997 population). 9 Certainly disturbing numbers, but denominators matter and those numbers translate to approximately one foodborne illness per 4,200 meals; one hospitalization for every 1 million meals; and one death for every 65 million meals. Food is consumed without any harm more than 3,999 times out of 4,000 meals, demonstrating that the US food supply is relatively safe. However, the considerable burden of foodborne disease reflects the huge impact of low risk events that are spread across entire populations, and guarantees that food safety will remain a pivotal issue across all sectors of the food industry and in international trade. Export markets will remain exquisitely sensitive to real or perceived concerns with pork safety.

\section{Pork safety - contemporary vs. historic risks}

Swine production in developed countries has evolved rapidly, and shifts towards larger farms and more 'industrial' pork production practices are also evident in some developing countries. I will avoid the perilous turf of contemporary international comparisons, but pork safety in the USA today is demonstrably safer that at any time in the past. The aspirations driving most innovation in pork production [e.g., all-in/all-out (AIAO) management; multiple site production] have been improved animal health, productivity and profitability. Factors likely to influence pathogen transmission include herd size, population structure and dynamics; sources and health status of incoming stock; area density of pigs and other species; biosecurity practices; group sizes and animal density; replacement practices in breeding herds; pig flow (e.g., AIAO vs. continuous flow); housing systems; 
ventilation systems and air quality; sources, quality and delivery systems of feed and water; hygiene and effluent management; nutritional programs; weaning age; and specific health interventions (e.g., vaccines). Although designed to control pathogens of swine, the collective modifications implemented in modern pig production logically will have had collateral impact on the risks of introduction and transmission foodborne pathogens. Available evidence indicates that foodborne risks to US pork consumers have declined markedly in recent decades.

\section{Parasitic hazards - achievements to celebrate}

A telling feature at last year's 8th International Symposium on Epidemiology and Control of Foodborne Pathogens in Pork (Safepork), in Quebec City, was the paucity of current research on parasitic hazards in pork. Primarily a gathering of scientists from developed countries, there was not one paper on Taenia solium, the most injurious porkborne pathogen in the world. Estimated to infect 50 million people worldwide and cause up to 50,000 deaths per year, 10,11 it is also largely responsible for the fourfold higher rates of epilepsy in developing versus developed countries. ${ }^{12}$ This parasite thrives where sanitation is poor and traditional, free-range/scavenging pig production is practiced, 13 and has a tragic impact on the poorer populations of many developing countries. It has been excluded from commercial pork production in developed countries. At Safepork, only two papers addressed pork's most infamous hazard, Trichinella spiralis, with one of these papers documenting the extremely low seroprevalence of T. spiralis in commercial pigs in the USA. ${ }^{14}$ The virtual elimination of $T$. spiralis from modern commercial swine production has relegated the once perennial 'poster child' of porkborne disease to little more than an historical footnote in the annals of US public health and the commercial swine industry. ${ }^{15}$

Toxoplasma gondii is one of three pathogens responsible for three-quarters of fatal foodborne infections in the United States. ${ }^{9}$ Congenital T. gondii infection occurs in 500 to 5000 of the 4.2 million US newborns annually and about 1.2 million Americans are affected by ocular toxoplasmosis. ${ }^{16}$ The societal cost of congenital toxoplasmosis alone was estimated in 1990 to be up to $\$ 8.8$ billion annually, ${ }^{17}$ and it has been inferred that about $50 \%$ of cases in the USA are foodborne. ${ }^{8}$ Less than 30 years ago T. gondii infection occurred in $40 \%$ of US sows and $20 \%$ of market hogs, ${ }^{18}$ and undercooked pork was considered an important risk for human infection. Subsequent studies of national scope indicate that $T$. gondii seroprevalence has been reduced by some $90 \%$ (Figure 1). ${ }^{18-22}$ Because Toxoplasma seroprevalence in the past was much higher in pigs than in other food animals in the USA, pork was conventionally viewed to be the highest risk meat. Whether true or not in the past, recent case-control studies in the USA and Serbia suggest that this is not the case. ${ }^{23,24}$

The parasitic triad of Taenia, Trichinella and Toxoplasma has arguably been responsible for the lion's share of the burden of porkborne illness throughout the history of mankind, and they remain obdurate problems in many developing countries. The reduction of these agents to a state of relative inconsequence in modern pork production in developed countries is a substantial, yet largely unheralded, public health accomplishment. Even for the more epidemiologically complex T. gondii, the virtual elimination of Toxoplasma risk from pork is a feasible goal in modern confinement systems. The inherent trade off between parasite risk and the desired attributes of non-confinement livestock systems is captured in the following quotation about the potential for producing Toxoplasma-free meat: "Modern production technologies have shown that this is feasible and have led to a marked decrease of T. gondii infections in meat producing animals such as pigs. Conversely, demand for animal friendly production systems may however lead to a re-emergence of T. gondii in pork and poultry". ${ }^{25}$ The first step towards effective management of T. gondii in less intensive pork production systems will be recognition of the inherently greater risk of exposure.

\section{Enteric foodborne pathogens - a challenge for the 21 st century?}

In contrast to the heady progress in preharvest control of parasitic agents, experiences with bacterial foodborne pathogens have been humbling. The bacterial pathogens of most concern to pork safety (Salmonella, Campylobacter, Listeria, and Yersinia enterocolitica) ${ }^{26}$ have their primary ecological niche in the intestinal tracts of healthy birds and mammals. Their presence on meat stems from contamination events that can occur anytime during harvest and processing until meat is served to the end consumer. Contamination risk during harvest and processing is clearly a function of both on-farm exposure and slaughter hygiene, therefore both preharvest and postharvest arenas are logical targets for interventions. ${ }^{27}$ Salmonella, remains the preeminent bacterial hazard in most pork industries and was the subject of some $40 \%$ of papers at Safepork in 2009. Apart from the northern Nordic countries (Sweden, Finland and Norway), where uncommonly low Salmonella prevalence supports the possibility of excluding Salmonella from pig populations, most countries identify reduction rather than elimination of Salmonella to be a more attainable goal in pork production. ${ }^{28-30}$

The body of epidemiologic knowledge and experience with preharvest control of Salmonella dwarfs those of other enteric bacterial pathogens. Even so, there remains a dearth of validated, evidence-based interventions for preharvest Salmonella control. Despite the appealing logic for preharvest control of foodborne pathogens, the task has proven daunting and opinions remain divided on the feasibility and cost-effectiveness of preharvest control programs for Salmonella in pigs. Investment in preharvest surveillance and control has been widespread in western Europe, but much less in North America where substantial reduction of Salmonella contamination of hog carcasses has been achieved by improvements in the post harvest sector (Figure 2). ${ }^{31}$ Over some 15 years, the wealth of data generated by the Danish National Salmonella control program has provided much insight into the challenge of preharvest control. ${ }^{32-34}$ The most recent analysis from this program indicated that benefits from preharvest control were most likely to accrue in low prevalence regions and small processing plants. It was concluded that in medium to high prevalence scenarios, even drastic reductions at herd level may yield only limited benefits in reducing the prevalence of positive carcasses. ${ }^{35}$ These findings reinforce the importance of 'downstream' exposure to Salmonella during transport and lairage which has been extensively documented but remains difficult to resolve. ${ }^{36}$ While advances in process control and new interventions during slaughter and processing have yielded measurable improvements in meat hygiene, considerable investments in preharvest control of Salmonella appear to have yielded only modest benefits. Epidemiologic knowledge about Campylobacter, Listeria, and Yersinia in swine production remains insufficient to adequately inform specific preharvest control measures for these pathogens. 
The most vocal proponents of preharvest control of foodborne enteric bacteria have combined a degree of epidemiological naïveté with a belief (regardless of the vacuum of supporting data) that 'the problem' was attributable to modern farming methods, and specifically confinement production. In my opinion, the least equivocal outcome from fifteen of so years of preharvest research is that elimination of organisms that are normal flora (Campylobacter coli), or common commensals (Salmonella, Listeria, Yersinia) of the swine intestinal tract will not be achieved by facile interventions in farm management. The complexity of the epidemiology of Salmonella and other enteric organisms is difficult to overstate, but dominated by the fact that these organisms are commonly carried by, and shed in the feces of, healthy animals. In a review of preharvest food safety, Oliver et al (2009) conveyed the likely magnitude of the challenge of control of foodborne bacteria by forecasting 'there is little doubt that solutions to these and many other complex issues will be delineated through science-based research that will be conducted during the next century. ${ }^{\prime 3}$ Development of valid and reliable preharvest interventions to control bacterial foodborne pathogens remains the preeminent challenge for researchers in pork safety, and one that may indeed endure for several decades even for the most researched pathogens. In contrast post harvest interventions, and most notably food irradiation, present proven, safe and relatively low cost options for risk reduction for multiple hazards which should be pursued for higher risk items such as ready-to-eat and comminuted meat products. 38,39 However, implementation of food irradiation in the meat industry remains enmeshed in ideological opposition. Those who might oppose irradiation of higher risk meat products of on the grounds that producers must take more responsibility for food safety must be more motivated to constrain industry than to protect consumers from foodborne illness.

\section{Into the future and the wild card of emerging pathogens}

Just a few decades back, several of today's headlining foodborne pathogens were either unknown (E. coli O157:H7) or not known to be transmitted in food (e.g. Listeria, Campylobacter). We can anticipate that both true emergence and apparent emergence (discovery of pre-existing agents through new diagnostic approaches) of pathogens will present new challenges to pork safety and risk communication for the swine industry. Currently topical examples are methicillin resistant Staphylococcus aureus and Clostridium difficile. ${ }^{40-42}$ These are major emerging pathogens in human medicine, and the presence of strains of these organisms in animal reservoirs, including pigs, is a valid cause for consternation. There is a need for much better understanding of the ecology and epidemiology of these organisms both in swine production and post harvest. Similarly ongoing discoveries of multihost viral agents in pigs (e.g., Hepatitis E and $B$ viruses, caliciviruses and noroviruses) prompt questions about their zoonotic and foodborne disease potential. For all multihost microbial species, both emerging and familiar, more profound understanding of host adaptation and host specificity is necessary and requires greater research investment. For newly recognized organisms, understanding the biological and epidemiological determinants of possible risk to human or animal health is not a trivial task. The likely complexity is exemplified by Hepatitis E viruses which are widespread in swine throughout the world, but vary in genotype geographically, by host species, and in zoonotic risk. ${ }^{43}$ In some countries swine reservoirs have been shown to make negligible contributions to the human disease, ${ }^{44,45}$ demonstrating the principle that prevalence of an agent alone is a highly inadequate measure of zoonotic or foodborne risk. Advances in sequencing, subtyping, and other tools for 'molecular epidemiology' are bringing new opportunities to explore host adaptation of potential porkborne pathogens, with the power to greatly enhance both our biological insight and the acuity of risk analysis and communication efforts. Unfortunately, the increasingly adversarial climate in the USA promises rapid (and therefore not necessarily well founded) accusations of industry culpability whenever any potential zoonotic or foodborne issue comes to light.

Looking forward, the international marketplace for pork will require incrementally more demanding standards for pork safety. In order to remain competitive in premium markets, exporters must achieve and assure very low risks for chemical, physical and microbial hazards, and any failures have the potential to incite crippling consequences for market access. Quality assurance programs implemented on farms should be adequate to manage the majority of physical and chemical hazards, as well as parasitic foodborne hazards. Unless major research breakthroughs occur in preharvest control of enteric bacteria, improved meat hygiene and slaughter processing will continue to be the mainstay for microbiological safety. As always, the emergence of new or previously unrecognized foodborne hazards (both real and perceived) remains the most unpredictable and potentially devastating food safety issue.

Figure 1: Toxoplasma gondii seroprevalence reported in large studies of market hogs in the USA. ${ }^{18-22}$

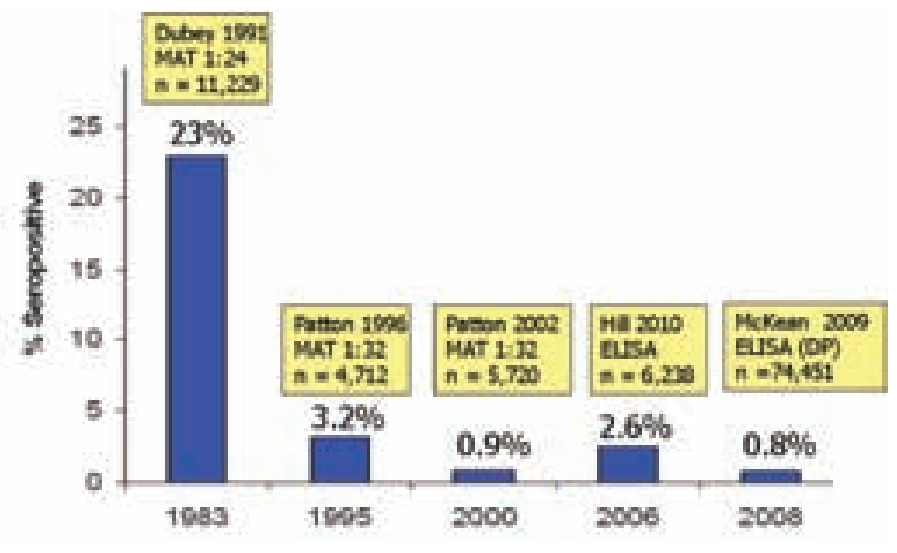

Figure 2: Salmonella prevalence on market hog carcasses in large, small and very small plants in the USA (1998-2009). ${ }^{30}$

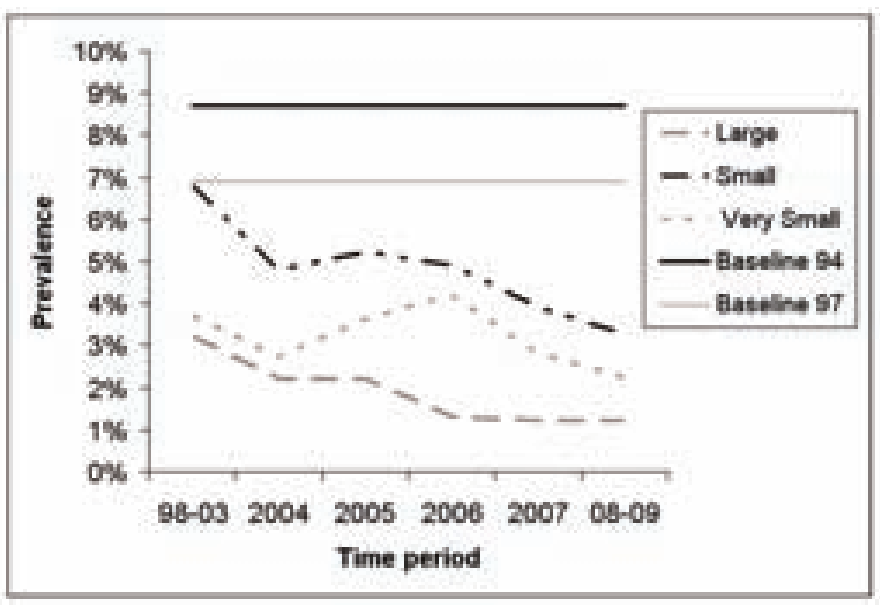




\section{References}

1. Black, R.E., Allen, L.H., Bhutta, Z.A., et al., (2008) Maternal and child undernutrition: global and regional exposures and health consequences. Lancet 371: 243-260.

2. Finkelstein, E.A., Trogdon, J.G., Cohen, J.W., Dietz, W. (2009). Annual medical spending attributable to obesity: payer-and service-specific estimates. Health Aff (Millwood). 28:w822-831.

3. Drewnowski, A. (2009) Obesity, diets, and social inequalities. Nutr Rev 67 Suppl 1:S36-9.

4. McMichael, A.J., Powles, J.W., Butler, C.D., Uauy, R. (2007) Food, livestock production, energy, climate change, and health. Lancet. 370:1253-1263.

5. Walker, P., Rhubart-Berg, P., McKenzie, S., Kelling, K., Lawrence, R.S. (2005) Public health implications of meat production and consumption. Public Health Nutr. 8:348-356.

6. Billy, T.J., Wachsmuth, I.K. (1997). Hazard analysis and critical control point systems in the United States Department of Agriculture regulatory policy. Rev Sci Tech. 16:342-348.

7. Davies, P.R. (1997) Food safety and access to domestic and export markets. Swine Health Prod 5:13-20.

8. de Jonge, J., Van Trijp, H. , Renes, R.J., Frewer, L.J . (2010) Consumer Confidence in the Safety of Food and Newspaper Coverage of Food Safety Issues: A Longitudinal Perspective. Risk Analysis 30: 125-142

9. Mead, P.S., Slutsker, L., Dietz, V., McCaig, L.F., Bresee, J.S., Shapiro, C., Griffin, P.M., Tauxe, R.V.1999. Food-related illness and death in the United States. Emerg Infect Dis 5:607-625

10. Eddi, C., Nari, A., Amanfu, W. (2003). Taenia solium cysticercosis/taeniosis: potential linkage with FAO activities; FAO support possibilities. Acta Trop. 87:145-148

11. Aubry, P., Bequet, D., Queguiner, P. (1995). Cysticercosis: a frequent and redoubtable parasitic disease. Med Trop (Mars) 55:79-87.

12. Quet, F., Guerchet, M., Pion, S.D. et al (2009). Meta-analysis of the association between cysticercosis and epilepsy in Africa. Epilepsia. (2009) (Epub ahead of print)

13. Phiri, I.K., Ngowi, H., Afonso, S., Ngoungou, E.B., Nicoletti, A., Preux, P.M. (2003). The emergence of Taenia solium cysticercosis in Eastern and Southern Africa as a serious agricultural problem and public health risk. Acta Trop. 87:13-23.

14. McKean, J., O'Connor, A.,Pyburn, D., Beary, J. (2009). Survey of market swine to determine prevalence of Trichinella antibodies in meat juice samples from selected abattoirs. Proceedings 8 th International Symposium: Epidemiology and Control of Foodborne Pathogens in Pork. Quebec City, Quebec City, pp.283-286.

15. Kennedy, E.D., Hall, R.L., Montgomery, S.P., Pyburn, D.G., Jones, .JL. 2009. Trichinellosis surveillance, United States, 2002-2007. MMWR Surveill Summ. 58(9):1-7

16. Jones, J.L., Dargelas, V., Roberts, J., Press, C., Remington, J.S., Montoya, J.G. (2009). Risk factors for Toxoplasma gondii infection in the United States. Clin Infect Dis. 49:878-884.

17. Roberts, T, Frenkel, J.K. (1990). Estimating income losses and other preventable costs caused by congenital toxoplasmosis in people in the United States. J. Am. Vet. Med. Assoc.196:249-256

18. Dubey, J.P. (1991). National seroprevalence of Toxoplasma gondii in pigs. J Parasitol 77:517-521.

19. Patton, S., Zimmerman, J., Roberts, T., Faulkner, C., Diderrich, V., Assadi-Rad, A., Davies, P. , Kliebenstein, J. (1996). Seroprevalence of Toxoplasma gondii in hogs in the National Animal Health Monitoring System (NAHMS). J Eukaryot Microbiol 43: S121.

20. Patton, S., Faulkner, C., Anderson A., Smedley K., Bush, E. (2002). Toxoplasma gondii infection in sows and market-weight pigs in the United States and its potential impact on consumer demand for pork. National Pork Board Report http://www.pork.org/PorkScience/ Documents/00-130\%20-Patton-UofTenn.pdf
21. Hill, D.E., Haley, C., Wagner, B., Gamble, H.R., Dubey, J.P. (2010). Seroprevalence of and risk factors for Toxoplasma gondii in the US swine herd using sera collected during the National Animal Health Monitoring Survey (Swine 2006). Zoonoses Public Health. 57:53-59

22. McKean, J., O'Connor, A., Pyburn, D., Beary, J. (2009). Survey of market swine to determine prevalence of Toxoplasma antibodies in meat juice samples from selected abattoirs. Proceedings 8th International Symposium: Epidemiology and Control of Foodborne Pathogens in Pork, Quebec City, pp.287-290.

23. Jones, J.L., Dargelas, V., Roberts, J., Press, C., Remington, J.S., Montoya, J.G. (2009). Risk factors for Toxoplasma gondii infection in the United States. Clin Infect Dis. 49:878-884.

24. Bobic', B., Nikolic', A., Klun, I., Vujanic', M., Djurkovic'-Djakovic', O. (2007). Undercooked meat consumption remains the major risk factor for Toxoplasma infection in Serbia. Parassitologia. 49:227-230.

25. Kijlstra, A., Jongert, E. (2009). Toxoplasma-safe meat: close to reality? Trends Parasitol. 25:18-22.

26. Fosse, J., Seegers, H., Magras, C. (2009). Prevalence and risk factors for bacterial food-borne zoonotic hazards in slaughter pigs: a review. Zoonoses Public Health. 2009 56:429-454.

27. Alban, L., Stärk, K.D. (2005). Where should the effort be put to reduce the Salmonella prevalence in the slaughtered swine carcass effectively? Prev Vet Med. 68:63-79.

28. Mousing, J., Jensen, P.T., Halgaard C, Bager F, Feld N, Nielsen B, Nielsen JP, Bech-Nielsen S. (1997). Nation-wide Salmonella enterica surveillance and control in Danish slaughter swine herds. Prev Vet Med. 29:247-261

29. Wegener, H.C., Hald, T., Lo Fo Wong, D., et al. (2003). Salmonella control programs in Denmark. Emerg. Infect. Dis. 9:774-780.

30. Davies, P.R., Hurd, H.S., Funk, J.A., Cray, P.J., Jones, F.T. (2004). The role of contaminated feed in the epidemiology and control of Salmonella enterica. Foodborne Pathog Dis. 1:202-215.

31. USDA Food Safety and Inspection Service. (2009). Salmonella Verification Testing Program for Raw Meat and Poultry. http://www.fsis. usda.gov/Science/Microbiology/index.asp\#Salmonella (accessed November 2, 2009).

32. Goldbach, S.G., Alban, L. (2006). A cost-benefit analysis of Salmonella-control strategies in Danish pork production. Prev Vet Med. 77:1-14.

33. Hurd, H.S., Enøe, C., Sørensen, L., Wachmann, H., Corns, S.M., Bryden, K.M., Greiner, M. (2008). Risk-based analysis of the Danish pork Salmonella program: past and future. Risk Anal. 28:341-351.

34. Baptista, F.M., Alban, L., Ersbøll, A.K., Nielsen, L.R. (2009). Factors affecting persistence of high Salmonella serology in Danish pig herds. Prev Vet Med. 92:301-308.

35. Dahl J. (2009). Risk factors for positive carcass swabs in Danish slaughterhouses. Proceedings 8th International Symposium: Epidemiology and Control of Foodborne Pathogens in Pork, Quebec City, pp.26-29.

36. Schmidt, P.L., O'Connor, A.M., McKean, J.D., Hurd, H.S. (2004). The association between cleaning and disinfection of lairage pens and the prevalence of Salmonella enterica in swine at harvest. J Food Prot. 67:1384-1388.

37. Oliver, S.P., Patel, D.A., Callaway, T.R., Torrence, M.E. (2009). Developments and future outlook for preharvest food safety J Anim Sci. 87:419-37.

38. Chun, H., Kim, J., Chung, K., Won, M., Song, K.B. (2009). Inactivation kinetics of Listeria monocytogenes, Salmonella enterica serovar Typhimurium, and Campylobacter jejuni in ready-to-eat sliced ham using UV-C irradiation. Meat Sci. 83:599-603.

39. Park JG, Yoon Y, Park JN, Han IJ, Song BS, Kim JH, Kim WG, Hwang HJ, Han SB, Lee JW. (2010). Effects of gamma irradiation and electron beam irradiation on quality, sensory, and bacterial populations in beef sausage patties. Meat Sci. 85:368-372. 
40. Wulf, M., Voss, A. (2008). MRSA in livestock animals-an epidemic waiting to happen? Clin Microbiol Infect. 14:519-521.

41. de Boer, E., Zwartkruis-Nahuis, J.T., Wit, B., Huijsdens, X.W., de Neeling, A.J., Bosch, T., van Oosterom, R.A., Vila, A., Heuvelink, A.E. (2009). Prevalence of methicillin-resistant Staphylococcus aureus in meat. Int J Food Microbiol 134:52-56.

42. Songer, J.G., Trinh, H.T., Killgore, G.E., Thompson, A.D., McDonald, L.C., Limbago, B.M. (2009). Clostridium difficile in retail meat products, USA, 2007. Emerg Infect Dis. 15:819-821

43. Aggarwal R, Naik S. (2009). Epidemiology of hepatitis E: current status. J Gastroenterol Hepatol. 24:1484-1493.

44. Zhang W, Yang S, Ren L, Shen Q, Cui L, Fan K, Huang F, Kang Y, Shan T, Wei J, Xiu H, Lou Y, Liu J, Yang Z, Zhu J, Hua X. (2009). Hepatitis E virus infection in central China reveals no evidence of cross-species transmission between human and swine in this area. PLoS One. 7;4(12):e8156.

45. Shukla P, Chauhan UK, Naik S, Anderson D, Aggarwal R. (2007). Hepatitis E virus infection among animals in northern India: an unlikely source of human disease. J Viral Hepat. 14:310-317 


\title{
Porcine circovirus type 2: success and failure
}

\author{
Caroline Fossum \\ Section of Immunology, Department of Biomedicine and Veterinary Public Health, SLU, Uppsala, Sweden
}

\begin{abstract}
Introduction
Vaccination against porcine circovirus type 2 (PCV2) reduces the incidence and severity of porcine circoviral disease (PCVD), in particular the postweaning multisystemic wasting syndrome (PMWS). This confirms that PCV2 is the essential infectious agent in this disease syndrome, but attempts to elucidate why PMWS became an emerging disease have failed. Much still remain to be clarified regarding the epidemiology of PCV2, its interaction with the host and the role of other infections in the development of PCVD. The current views on these issues are summarized below.
\end{abstract}

\section{Porcine circovirus}

Porcine circovirus (PCV) are small (approximately $1760 \mathrm{nt}$ ), single-stranded (ss) DNA virus that belong to the family Circoviridae. Two genotypes of PCV (PCV type 1 and PCV type 2) with similar genomic organisation and an approximate $80 \%$ homology at their nucleotide sequences are described (Meehan et al., 1998). PCV1 is regarded apathogenic whereas PCV2 has been associated with a number of disease syndromes of which PMWS is the best characterized. In 1999, an experimental model that evoked PMWS in colostrum-deprived piglets at co-inoculation of porcine parvovirus (PPV) and PCV2 was established (Allan et al., 1999).

The circular genome of PCV encodes a structural protein, the capsid (cap) protein, and a replicase protein with two splice variants (Rep and Rep'). Both Rep and Rep' are necessary for initiating the rolling-circle amplification used by PCV. In addition, a third open reading frame (ORF3) exists in PCV that is transcribed counter clockwise to ORF1 (rep, rep') and ORF2 (cap). The proposed apoptotic role of the gene product from ORF3 (Liu et al., 2005) or its importance for viral pathogenesis is not fully clarified but it is noteworthy that the greatest genomic differences between PCV1 and PCV2 are found within ORF3. Our current knowledge about the genomic organisation of PCV, their replication and interaction with host proteins are detailed in a recent review by Finsterbusch and Mankertz (2009).

\section{Porcine circovirus type 2 (PCV2) and diseases related to PCV2}

Despite many similarities between PCV1 and PCV2, only PCV2 has been associated with disease. Today, PCV2 is confirmed to be a necessary infectious agent for development of PMWS and demonstration of moderate to massive quantities of PCV2 within histopathological lesions in lymphoid tissues (lymphocyte depletion together with histiocytic infiltration and/or inclusion bodies and/or giant cells) is a major diagnostic criterium. Clinical signs of PMWS include progressive weight loss (wasting), enlarged inguinal lymph nodes, and in some cases jaundice, dyspnoea and/or diarrhoea. As reviewed (Ramamoorthy and Meng, 2009; Gillespie et al., 2009; Grau-Roma et al., 2010), PCV2 is also involved in a number of other syndromes including "res- piratory", "reproductive" and "enteric" disorders as well as porcine dermatitis and nephropathy syndrome (PDNS).

PCV2 isolated from pigs with various clinical conditions showed different replication kinetics when studied in vitro (Meerts et al., 2005), perhaps suggesting a variation in their virulence. Due to a high similarity between sequences from various PCV2 isolates (approximately $95 \%$ at the amino acid level) such a link is not very likely, but cannot be ruled out. Phylogenetic analysis performed in several countries however revealed a clustering of PCV2 into "genotypes / genogroups" with clade formations that could be related to "year of isolate" (Olvera et al., 2007). Analyses of these clades showes a relation between patterns in the PCV2 ORF2 sequence and cases of PMWS. Such a connection is further supported during an outbreak of PCVD in Canada in late 2004 - 2006 with more severe lesions and increased mortalities and the emergence of a new PCV2 variant, referred to as "RFLP type 321" (Gagnon et al., 2007; Carman et al., 2008). Indeed, reports from several countries describing a shift in genotype/genogroup of PCV2 accompanied by increased incidences of PMWS are accumulating as summarized in a recent review (Grau-Roma et al., 2010). To ease further comparative analyses a common nomenclature has been proposed with two main genotypes (PCV2a and $P(V 2 b)$ and a very rare genotype, recovered from pigs in Denmark, designated PCV2c (Segalés et al., 2008). In general, it appears from the epidemiological evidence that PCV $2 b$ is the genotype associated with outbreaks of severe PMWS post 2003.

The importance of PCV2 genotype for the pathogenicity of the virus is however still puzzling and results from experimental infections are not conclusive (Lager et al., 2007; Opriessnig et al., 2008; Saha et al., 2010). It appears that PCV2a and PCV2b on their own do not differ in virulence but a recent study highlights the importance of order of infection (Harding et al., 2010). In that experimental model, infection of gnotobiotic pigs with PCV2b seven days after a PCV2a infection, but not vice versa, caused PMWS. A similar approach but using eleven week old SPF-pigs that were repeatedly infected with infectious clones of heterologous PCV2a or with PCV2a followed by PCV2b did not induce any signs of clinical disease (Opriessnig et al., 2010a). Neither were any macroscopic lesions observed in the pigs although all seroconverted to PCV2 and PCV2 could be demonstrated at microscopic lesions in some samples. Both PCV2a and PCV2b DNA sequences were present in samples obtained from some of these dually infected pigs.

The simultaneous presence of PCV2a and PCV2b has been reported in field samples and also chimeric virus composed of an ORF1 region from PCV2a and an ORF2 region from $P C V 2 b$ is described (Hesse et al., 2008). Even more interesting is the demonstration of naturally occurring chimeric PCV2 genomes that are recombined within the ORF2 region (Cheung, 2009). Modifications within this region that encodes the cap gene can affect the structural capsid protein and thereby modify B-cell epitopes of the virus. Antigenic differences between PCV2 isolates have been demonstrated using monoclonal antibodies to the capsid 
protein (Lefebre et al., 2008) and to the N-terminal part of the Rep protein (Meng et al., 2010). Indeed, a new genomic variant of PCV, apparently composed of ORF 1 from PCV1 and ORF 2 from PCV2a, has been detected in Canada (Gagnon et al., 2010). The prevalence of this virus, designated PCV1/2a, is low and its origin not clear, although it is now recognised that this isolate may be linked to a partially inactivated chimeric PCV2 vaccine.

Taken together, some epidemiological evidence favours the hypotheses that various isolates of PCV2 differ in their pathogenicity as a shift in genotype of PCV2 has repeatedly been reported when PMWS occurs in a region. This appears also to be the case in Norway that has succeeded to stay free from PMWS until recently. Thereafter, sequence analyses of PCV2 in samples obtained from pigs on 27 healthy farms all grouped into PCV2a whereas PCV2b was detected in samples from 12 farms affected by PMWS (Sonja Ylving, National Veterinary Institute, Oslo, Norway, personal communication). However it should be noted that severe outbreaks of PMWS, associated with PCV2a, did occur from 1996 onwards in N America and Europe, with no recovery of PCV2b from these outbreaks.

\section{Porcine circovirus type 2 (PCV2) and other viral infections in PMWS}

It is generally accepted that herd management has a predominant impact on the incidence of PMWS. In particular animal hygiene and other general disease-preventing measures are important factors to reduce porcine circoviral disease (Madec et al., 2008). In a field study conducted in one Norwegian and two Swedish finishing farms the role of management became evident (Brunborg et al., 2010). One of the Swedish farms changed their recruitment routines and finishing pigs were during a period obtained from the open market and the time between batches was reduced to less than 24 hours. This shift coincided in time with an increased frequency of runts, wasting pigs and a subsequent diagnose with PMWS whereas pigs in the other Swedish farm and in the Norwegian farm performed normal.

Serological analyses revealed prominent differences in the levels of antibodies to PCV2 and number of PCV2 DNA copies in samples collected sequentially during the first five weeks of the finishing period in the three farms. The amount of PCV2 DNA copies was similar in samples obtained from pigs at arrival to the two Swedish finishing farms but was hardly detectable in samples collected in the Norwegian farm. The initial level of antibodies to PCV2 was comparatively low and increased with time in the PMWS affected farm but remained at a steady high level in samples collected from pigs in the healthy Swedish farm. The majority of the Norwegian pigs remained seronegative to PCV2 during the firs five weeks but an extended sampling revealed that these pigs had seroconverted after 9 weeks in the finishing herd and that the pigs then displayed a low number of PCV2 DNA copies in blood. This study was conducted when Norway was still free from PMWS and it is notable that the genotype of PCV2 determined for the Norwegian samples was PCV2a whereas pigs in the Swedish farms harboured PCV2b, irrespectively they originated from the healthy farm or from that affected by PMWS. Indeed, a high similarity within PCV2b genotype was determined in samples obtained form the two Swedish farms (full genome sequence $99.7 \%$ at the nucleotide level) regardless they were affected by PMWS or not.

Longitudinal case-control studies, analysing time for seroconversion and PCV2 DNA load, performed in Danish and Spanish herds showed that the PCV2 load increased with decreasing maternal immunity (Grau-Roma et al., 2009). PMWS developed when the PCV2 DNA load was at its highest which in Spain occurred at the beginning of the fattening period but in Denmark took place in the nursery phase. In another Danish study weaned pigs, aged 8-14 weeks, from PMWS affected herds were mingled with $4-6$ weeks old PCV2 positive pigs from PMWS unaffected herds (Kristensen et al., 2009). Three to four weeks later PMWS developed in pigs from the unaffected herds both in those that were mingled in the same pen and in those housed in pens adjacent to pigs from the PMWS-affected herds. Thus, PMWS can be induced in pigs from PMWS free herd following contact to pigs from farms with clinical PMWS.

In both studies referred to above other pathogens than PCV2 were present on the farms. In the Spanish/Danish study the presence of Mycoplasma hyopneumoniae, Actinobacillus pleuropneumoniae, Pasteurella multocida, Lawsonia intracellularis, Salmonella spp. PPV, porcine reproductive and respiratory virus (PRRSV), swine influenza virus and Aujeszky's disease virus (AD) was confirmed and in the latter Danish study the PMWS-affected herds were seropositive for M. hyopneumoniae and PRRSV. Therefore an attempt was made to isolate virus from organ material collected from the PMWS-affected pigs using a large panel of different cell-types (Lohse et al., 2008). However, no viral cofactor could be linked to the development of PMWS. Instead, a difference in nucleotide sequence was found for PCV2 obtained from PMWS-affected and from non-affected herds. It is thus demonstrated that PMWS can be transmitted from affected to non-affected animals but we have failed to identify the "agent" that spreads the disease and to understand the disease process.

An earlier field study in Spain has indicated that another circular single-stranded DNA virus, the Torque teno virus (TTV) belonging to the genus Anellovirus, family Circoviridae, and in particular genogroup 2 (g2) of porcine TTV is more frequent in PMWS-affected pigs (Kekarinen et al., 2006). An experimental co-infection with g1-TTV and PCV2 in gnotobiotic pigs supports the theory that TTV can act as a co-factor in the development of PMWS (Ellis et al., 2008). A metagenomic analyse was therefore performed on viral DNA (Blomström et al., 2009) recovered from lymph nodes collected from two of the pigs that succumbed from PMWS in the Norwegian/Swedish study referred to above. Random amplification and large-scale sequencing generated approximately 9000 unique sequences. The majority of these (99.6\%) were from PCV2 and only 12 sequences were of other viral origin; 7 representing TTV and 5 representing a parvo-like virus. Further analyses confirmed that both g1-TTV and g2-TTV were present and interestingly the parvovirus sequence showed a relationship to human bocavirus (HBoV) and phylogenetic studies approved that the sequence emanated from a porcine boca-like virus (pBo-likeV).

Human Boca-virus was discovered in 2005 and was given its name due to sequence homologies with bovine parvovirus and canine minute virus. This parvovirus is widespread in the human population and has been associated with respiratory tract diseases and gastrointestinal infection (Allander, 2008). Thus the finding of a porcine boca-like virus together with TTV and PCV2 in lymph nodes from clinical cases of PMWS was exiting. For a further study of the distribution of these viruses an archived material was used. The archived material consisted of lymph nodes collected during a phylogentic study of PCV2 carried out in Sweden before and after the enzootic outbreak of PMWS in 2004 (Timmusk et al., 2008) and was supplemented with more 
recently collected samples, including some from piglets with congenital tremor. In total, samples from 58 pigs, 34 with PMWS and 24 without PMWS, were analysed by PCR or QPCR for the presence of PCV2a, PCV2b, g1-TTV, g2-TTV and the porcine boca-like virus.

A discriminative $\mathrm{qPCR}$ confirmed earlier sequencing data (Timmusk et al., 2008) showing that PCV2b was present at high DNA copy numbers ( 2108 per $100 \mathrm{ng}$ DNA) in samples obtained from PMWS-affected pigs. In two of these pigs PCV2a was also detected but at a lower DNA copy number. PCV2b was also detected in samples from pigs not affected by PMWS but then always at DNA copy numbers below 106 per $100 \mathrm{ng}$ DNA. Regardless of health status TTV-1, TTV-2 and pBo-likeV were present in $70-90 \%$ of the samples, and PCV2 was demonstrated in the majority of samples although at higher levels in samples collected from pigs affected with PMWS than from the others. The most pronounced difference was that pBo-likeV was detected in more samples from PMWS affected pigs and the simultaneous detection of TTV-1, TTV-2 and pBo-likeV was more frequent in samples form PMWS affected pigs (Table 1).

Table 1. Percentage of samples positive for PCV2a, PCV2b, g1-TTV, g2$T T V-2$ or pBo-likeV as determined by qPCR or PCR among pigs affected by PMWS (PMWS+) or not (PMWS-). Also the percentage of samples simultaneously positive for g1-TTV, g2-TTV-2 and pBo-likeV is given

\begin{tabular}{|l|c|c|c|c|c|c|}
\hline & $\begin{array}{c}\text { PCV2a } \\
(\%)\end{array}$ & $\begin{array}{c}\text { PCV2b } \\
\text { (\%) }\end{array}$ & $\begin{array}{c}\text { g1-TTV } \\
\text { (\%) }\end{array}$ & $\begin{array}{c}\text { g2-TTV } \\
\text { (\%) }\end{array}$ & $\begin{array}{c}\text { pBo-likeV } \\
\text { (\%) }\end{array}$ & $\begin{array}{c}\text { g1-TTV/g1-TTV/ } \\
\text { pBo-likeV (\%) }\end{array}$ \\
\hline PMWS + & 6 & 100 & 77 & 94 & 88 & 71 \\
\hline PMWS - & 12 & 50 & 79 & 83 & 46 & 33 \\
\hline
\end{tabular}

A similar distribution of g1-TTV, g2-TTV-2 and pBo-likeV in pigs with and without PMWS has been described for samples collected in UK (Gordon Allan and Michael McMenamy, Dept of Veterinary Science, Queen's University Belfast, Belfast, Northern Ireland, personal communication). Thus, it is not possible from these studies to determine the role of TTV and the porcine Bocalike virus in the aetiology of PMWS. However, in a Chinese study PCV2, PRRS, CSFV and TTV2 were more frequent in samples collected from pigs that also were postive for pBo-likeV than among those negative for the boac-like virus (Zhai et al., 2010) suggesting that $\mathrm{pBo}-\mathrm{likeV}$ could act in synergy with other virus or predispose for other viral infections.

Interestingly, in vitro studies using DNA from human TTV genogroup 4 demonstrated that DNA of this TTV can induce murine immune cells to produce and secrete cytokines such as interferon- $\gamma$ (IFN- $\gamma$ ), interleuin-6 (IL-6), and IL-12 (Rocchi et al., 2009). Indeed, linearized DNA from porcine g1-TTV and g-2TTV could in vitro interfere with IFN- a production by porcine plasmacytoid dendritic cells (Martinez-Guinó et al., 2009).

\section{PCV2 and the immune response}

The possibility that small circular DNA virus could harbour immunomodulatory sequences, i.e., CpG-motifs, was first suggested for PCV2 (Hasslung et al., 2003). It was shown that the DNA genome of PCV2 contains sequences that in synthetic form (oligodeoxyribonucleotides; ODNs) could act immunomodulatory on porcine peripheral blood mononuclear cells (poPBMC) in vitro and e.g. inhibit the IFN- a response to other DNA- but not RNA-virus (Wikstöm et al., 2007). Further analyses of the PCV2 genome for presence of CpG motifs identified additional 18 motifs that could induce IFN- a production and three inhibitory motifs (Kekarainen et al., 2008a). Indeed, PCV2 propagated in PK15 cells, but not PCV1, could act immunomodulatory and reduce the IFN- $\gamma$ and IFN- $a$ responses to recall antigen stimulation (Kekarainen et al., 2008b). In addition, PCV2 and UV-inactivated PCV2 induce IL-10 and thereby down-regulates the production of IFN- $\gamma$ and IL-12. Thus, a reduced production of IFN- $\alpha$ in combination with IL-10 production is likely to suppress a Th-1 type of immune response and thereby promote virus persistence.

One of the most important factors in the defence against viral infections is IFN- $a$. This cytokine can be produced in low amounts by monocytes / macrophages and myeloid dendritic cells and in particular by plasmacytoid dendritic cells (PDC) that respond with high production of IFN- $a$ to certain stimuli. Inerferon- $\alpha$ is important during the early defence against viral infections because it can induce an antiviral state in neighbouring non-infected cells and it activates the killing activity by Natural killer (NK) cells. Furthermore, IFN- a together with IL-12 promotes the development of a Thelper cell (Th) type 1 immune response that is important in the defence against viral infections. The IFN- $a$ response is thus a strategic point for a virus to modify and demonstration of non-replicating PCV2 in cells of the myeloid lineage (monocytes, macrophages and dendritic cells) in infected pigs is therefore remarkable. Series of elegant studies performed at the Institute of Virology and Immunoprophylaxis, Mittelhäusern, Switzerland have outlined the interaction of PCV2 and /or the viral DNA with porcine dendritic cells and effects on their function that are summarized in a recent review (Kekarainen et al., 2010).

Against this background it is plausible that the replication of PCV2 in vitro is enhanced by addition of IFN- $a$ or IFN- $\gamma$ (Meerts et al., 2005). Indeed an interferon-stimulated response element (ISRE) is present in the PCV2 genome within the promoter region of the rep gene that seems to play a role in the response of PCV2 to IFNs (Ramamoothy et al, 2009) but the underlying mechanisms are far from outlined.

Using the PCV2/PPV co-infection experimental model a weak T lymphocyte response, measured as PCV2-specific IFN- $\gamma$ secreting cells at in vitro re-stimulation was evidenced (Steiner et al., 2009). This response coincided with viremia, approximately 8 days post infection whereas the specific antibody response was delayed until four weeks post infection. Indeed, a transient correlation exists between viremia and IL-10 expression in pigs subclinically infected with PCV2 (Darwich et al., 2008). Studies that further support these findings include the increased expression of IL-10 mRNA levels observed in PBMC obtained form pigs experimentally co-infected with PRRSV and PCV2 (Shi et al., 2010) and examination of IL-10 producing splenocytes obtained from naturally cases of PMWS (Crisci et al., 2010).

An important role of IL-10 is the regulation of B cell isotype switch. A number of field studies have documented the importance of neutralizing antibodies against PCV2 for protection against PMWS (for review see Kekarainen et al., 2010). As initially demonstrated by Meerts et al. (2006) protection is correlated to isotype switching and animals that remain IgM positive are those that are most likely to develop PMWS.

Taken together, the studies of PCV2 and its interaction with the porcine immune system has been a partial success and the knowledge that pigs can mount an immune response to the cap protein of PCV2 that protects against PCVD has been utilized in the vaccine development. 


\section{Vaccination against PCV2}

Vaccine development has so far focused on induction of immunity against the structural cap protein of PCV2. For instance, a chimeric viral particle composed of ORF1 from PCV1 and ORF2 from PCV2 is the source of antigen in the inactivated vaccine Suvaxyn ${ }^{\oplus}$ PCV2 from Fort Dodge. Also Ingelvac ${ }^{\circledR}$ CircoFLEX (Boehringer Ingelheim) and Porcilis ${ }^{\circledR}$ PCV (Intervet/ScheringPlough) contain at killed baculovirs vector carrying ORF2 whereas killed PCV2 is included in Circovac ${ }^{\circledR}$ (Merial). The latter vaccine is intended for vaccination of sows two week before farrowing. The immune sow will transfer a passive immunity to the piglet that is expected be protective during the subsequently development of an active immunity at exposure to PCV2 in the environment. The other three commercial vaccines are used for vaccination of pigs, approximately 3 weeks old. Despite differences in the source of antigen, adjuvant formulation and administration regime all these commercial vaccines have proven to protect efficiently against PMWS during field conditions. In addition, the sow vaccine improves farrowing rates and infertility problems (reviewed in Kekarainen et al., 2010).

A concern has been that PCV2a is the antigen source in the commercial vaccines listed above. Theoretically, amino acid changes in the cap protein of PCV2b could reduce the protective effect of an immune response elicited by PCV2a. Results obtained at experimental challenge of PCV2a-vaccinated pigs with PCV2b however imply a good "cross-protection" (Fort et al., 2009; Opriessnig et al., 2010b). Thus, development of vaccine against PCVD has been an outstanding success. Also immunologists are likely to benefit from these achievements. By scrutinizing the protective immune response elicited at vaccination against PCV2 insight will be gained about immune mechanisms necessary for induction of protection against porcine circovirus.

PCV as a contaminant in vaccines

Lately attention has been drawn to PCV as contaminants in vaccines for human use. Two licensed rotavirus vaccines, aimed to protect against enteric disease in infants, were found contaminated with PCV1 alone or in combination with PCV2, (http://www. fda.gov/BiologicsBloodVaccines/Vaccines/ApprovedProducts/ ucm211101.htm). In particular the PCV2 contamination urges for continual studies that increase our understanding of this virus, including its potential to replicate in human cells and the eventual immune modulating capacity of PCV2 DNA on human cells.

\section{References}

Allan GM, Kennedy S, McNeilly F, Foster JC, Ellis JA, Krakowka SJ, Meehan BM, Adair BM. 1999. Experimental reproduction of severe wasting disease by co-infection of pigs with porcine circovirus and porcine parvovirus. J Comp Pathol. 121: 1-11.

Allander T. 2008. Human bocavirus. J Clin Virol. 41: 29-33.

Blomström AL, Belák S, Fossum C, McKillen J, Allan G, Wallgren P, Berg M. 2009. Detection of a novel porcine boca-like virus in the background of porcine circovirus type 2 induced postweaning multisystemic wasting syndrome. Virus Res. 146: 125-129.

Blomström AL, Belák S, Fossum C, Fuxler L, Wallgren P, Berg M. 2010. Studies of porcine circovirus type 2, porcine boca-like virus and torque teno virus indicate the presence of multiple viral infections in postweaning multisystemic wasting syndrome pigs. Virus Res. In press.
Brunborg IM, Fossum C, Lium B, Blomqvist G, Merlot E, Jørgensen A, Eliasson-Selling L, Rimstad E, Jonassen CM, Wallgren P. 2010. Dynamics of serum antibodies to and load of porcine circovirus type 2 (PCV2) in pigs in three finishing herds, affected or not by postweaning multisystemic wasting syndrome. Acta Vet Scand. 19;52:22

Carman S, Cai HY, DeLay J, Youssef SA, McEwen BJ, Gagnon CA, Tremblay D, Hazlett M, Lusis P, Fairles J, Alexander HS, van Dreumel T. 2008. The emergence of a new strain of porcine circovirus-2 in Ontario and Quebec swine and its association with severe porcine circovirus associated disease-2004-2006. Can J Vet Res. 72: 259-268.

Cheung AK. 2009. Homologous recombination within the capsid gene of porcine circovirus type 2 subgroup viruses via natural co-infection. Arch Virol. 154: 531-534.

Crisci E, Ballester M, Domínguez J, Segalés J, Montoya M. 2010. Increased numbers of myeloid and lymphoid IL-10 producing cells in spleen of pigs with naturally occurring postweaning multisystemic wasting syndrome.Vet Immunol Immunopathol. In press.

Darwich L, Segalés J, Resendes A, Balasch M, Plana-Durán J, Mateu E. 2008. Transient correlation between viremia levels and IL-10 expression in pigs subclinically infected with porcine circovirus type 2 (PCV2). Res Vet Sci. 84: 194-198.

Ellis JA, Allan G, Krakowka S. 2008. Effect of coinfection with genogroup 1 porcine torque teno virus on porcine circovirus type 2-associated postweaning multisystemic wasting syndrome in gnotobiotic pigs. Am J Vet Res. 69: 1608-1614.

Finsterbusch T, Mankertz A. 2009. Porcine circoviruses - small but powerful. Virus Res. 143:177-183.

Fort M, Sibila M, Pérez-Martín E, Nofrarías M, Mateu E, Segalés J. 2009. One dose of a porcine circovirus 2 (PCV2) sub-unit vaccine administered to 3-week-old conventional piglets elicits cell-mediated immunity and significantly reduces PCV2 viremia in an experimental model. Vaccine. 27: 4031-4037.

Gagnon CA, Tremblay D, Tijssen P, Venne MH, Houde A, Elahi SM. 2007. The emergence of porcine circovirus $2 b$ genotype (PCV-2b) in swine in Canada. Can Vet J. 48: 811-819.

Gagnon CA, Music N, Fontaine G, Tremblay D, Harel J. 2010. Emergence of a new type of porcine circovirus in swine (PCV): A type 1 and type 2 PCV recombinant. Vet Microbiol. 144: 18-23.

Gillespie J, Opriessnig T, Meng XJ, Pelzer K, Buechner-Maxwell V. 2009. Porcine circovirus type 2 and porcine circovirus-associated disease. J Vet Intern Med. 23: 1151-1163.

Grau-Roma L, Hjulsager CK, Sibila M, Kristensen CS, López-Soria S, Enøe C, Casal J, Bøtner A, Nofrarías M, Bille-Hansen V, Fraile L, Baekbo P, Segalés J, Larsen LE. 2009.

Infection, excretion and seroconversion dynamics of porcine circovirus type 2 (PCV2) in pigs from post-weaning multisystemic wasting syndrome (PMWS) affected farms in Spain and Denmark. Vet Microbiol. 135: 272-82.

Grau-Roma L, Fraile L, Segalés J. 2010. Recent advances in the epidemiology, diagnosis and control of diseases caused by porcine circovirus type 2. Vet J. In Press.

Hasslung FC, Berg M, Allan GM, Meehan BM, McNeilly F, Fossum C. 2003. Identification of a sequence from the genome of porcine circovirus type 2 with an inhibitory effect on IFN-alpha production by porcine PBMCs. J Gen Virol. 84:2937-2945.

Harding JC, Ellis JA, McIntosh KA, Krakowka S. 2010. Dual heterologous porcine circovirus genogroup $2 \mathrm{a} / 2 \mathrm{~b}$ infection induces severe disease in germ-free pigs.

Vet Microbiol. In press.

Hesse R, Kerrigan M, Rowland RR. 2008. Evidence for recombination between PCV2a and PCV2b in the field. Virus Res.132: 201-207.

Kekarainen T, Sibila M, Segalés J. 2006. Prevalence of swine Torque teno virus in post-weaning multisystemic wasting syndrome (PMWS)affected and non-PMWS-affected pigs in Spain. J Gen Virol. 87: 833-837. 
Kekarainen T, Montoya M, Dominguez J, Mateu E, Segalés J. 2008a. Porcine circovirus type 2 (PCV2) viral components immunomodulate recall antigen responses. Vet Immunol Immunopathol. 124: 41-49.

Kekarainen T, Montoya M, Mateu E, Segalés J. 2008b. Porcine circovirus type 2-induced interleukin-10 modulates recall antigen responses. J Gen Virol. 89: 760-765.

Kekarainen T, McCullough K, Fort M, Fossum C, Segalés J, Allan GM. 2010. Immune responses and vaccine-induced immunity against Porcine circovirus type 2. Vet Immunol Immunopathol. In press.

Kristensen CS, Baekbo P, Bille-Hansen V, Bøtner A, Vigre H, Enøe C, Larsen LE. 2009. Induction of porcine post-weaning multisystemic wasting syndrome (PMWS) in pigs from PMWS unaffected herds following mingling with pigs from PMWS-affected herds.

Vet Microbiol. 138: 244-250.

Lager KM, Gauger PC, Vincent AL, Opriessnig T, Kehrli ME Jr, Cheung AK. 2007. Mortality in pigs given porcine circovirus type 2 subgroup 1 and 2 viruses derived from DNA clones. Vet Rec. 161: 428-429.

Lefebvre DJ, Costers S, Van Doorsselaere J, Misinzo G, Delputte PL, Nauwynck HJ. 2008. Antigenic differences among porcine circovirus type 2 strains, as demonstrated by the use of monoclonal antibodies. J Gen Virol. 89: 177-187.

Liu J, Chen I, Kwang J. 2005. Characterization of a previously unidentified viral protein in porcine circovirus type 2 -infected cells and its role in virus-induced apoptosis. J Virol. 79: 8262-8274.

Lohse L, Bøtner A, Hansen AS, Frederiksen T, Dupont K, Christensen CS, Baekbo P, Nielsen J. 2008. Examination for a viral co-factor in postweaning multisystemic wasting syndrome (PMWS). Vet Microbiol. 129: 97-107.

Madec F, Rose N, Grasland B, Cariolet R, Jestin A. 2008. Post-weaning multisystemic wasting syndrome and other PCV2-related problems in pigs: a 12-year experience.

Transbound Emerg Dis. 55: 273-283.

Martinez-Guinó, L., McCullough, K., Summerfield, A., Segalés, J., Kekarainen, T. Impact of Torque teno virus (TTV) on porcine dendritic cell function and activity. Abstract. 3rd European Veterinary Immunology Workshop, Berlin 10th-13th September 2009.

Meehan BM, McNeilly F, Todd D, Kennedy S, Jewhurst VA, Ellis JA, Hassard LE, Clark EG, Haines DM, Allan GM. 1998. Characterization of novel circovirus DNAs associated with wasting syndromes in pigs. J Gen Virol. 79: 2171-2179.

Meerts P, Misinzo G, McNeilly F, Nauwynck HJ. 2005. Replication kinetics of different porcine circovirus 2 strains in PK-15 cells, fetal cardiomyocytes and macrophages.

Arch Virol. 150: 427-441.

Meerts P, Misinzo G, Nauwynck HJ. 2005. Enhancement of porcine circovirus 2 replication in porcine cell lines by IFN-gamma before and after treatment and by IFN-alpha after treatment. J Interferon Cytokine Res. 25:684-693.

Meerts P, Misinzo G, Lefebvre D, Nielsen J, Bøtner A, Kristensen CS, Nauwynck HJ. 2006. Correlation between the presence of neutralizing antibodies against porcine circovirus 2 (PCV2) and protection against replication of the virus and development of PCV2-associated disease. BMC Vet Res. 2:6.

Meerts P, Van Gucht S, Cox E, Vandebosch A, Nauwynck HJ. 2005. Correlation between type of adaptive immune response against porcine circovirus type 2 and level of virus replication. Viral Immunol. 18: 333-341

Meng T, Jia Q, Liu S, Karuppannan AK, Chang CC, Kwang J. 2010. Characterization and epitope mapping of monoclonal antibodies recognizing N-terminus of Rep of porcine circovirus type 2. J Virol Methods. 165: 222-229.

Olvera A, Cortey M, Segalés J. 2007. Molecular evolution of porcine circovirus type 2 genomes: phylogeny and clonality. Virology. 357: 175-185.
Opriessnig T, Ramamoorthy S, Madson DM, Patterson AR, Pal N, Carman S, Meng XJ, Halbur PG. 2008. Differences in virulence among porcine circovirus type 2 isolates are unrelated to cluster type $2 \mathrm{a}$ or $2 \mathrm{~b}$ and prior infection provides heterologous protection.

J Gen Virol. 89: 2482-2491.

Opriessnig T, Prickett JR, Madson DM, Shen HG, Juhan NM, Pogranichniy RR, Meng XJ, Halbur PG. 2010a. Porcine circovirus type 2 (PCV2)infection and re-inoculation with homologous or heterologous strains: virological, serological, pathological and clinical effects in growing pigs. Vet Res. 41:31.

Opriessnig T, Patterson AR, Madson DM, Pal N, Ramamoorthy S, Meng XJ, Halbur PG. 2010b. Comparison of the effectiveness of passive (dam) versus active (piglet) immunization against porcine circovirus type 2 (PCV2) and impact of passively derived PCV2 vaccineinduced immunity on vaccination. Vet Microbiol. 14:177-83.

Ramamoorthy S, Meng XJ. 2009. Porcine circoviruses: a minuscule yet mammoth paradox. Anim Health Res Rev. 10:1-20.

Ramamoorthy S, Huang FF, Huang YW, Meng XJ. 2009. Interferon-mediated enhancement of in vitro replication of porcine circovirus type 2 is influenced by an interferon-stimulated response element in the PCV2 genome. Virus Res. 145: 236-243.

Rocchi J, Ricci V, Albani M, Lanini L, Andreoli E, Macera L, Pistello M, Ceccherini-Nelli L, Bendinelli M, Maggi F. 2009. Torquetenovirus DNA drives proinflammatory cytokines production and secretion by immune cells via toll-like receptor 9. Virology. 394: 235-242.

Saha D, Lefebvre DJ, Van Doorsselaere J, Atanasova K, Barbé F, Geldhof M, Karniychuk UU, Nauwynck HJ. 2010. Pathologic and virologic findings in mid-gestational porcine foetuses after experimental inoculation with PCV2a or PCV2b. Vet Microbiol. In press

Segalés J, Olvera A, Grau-Roma L, Charreyre C, Nauwynck H, Larsen L, Dupont K, McCullough K, Ellis J, Krakowka S, Mankertz A, Fredholm M, Fossum C, Timmusk S, Stockhofe-Zurwieden N, Beattie V, Armstrong D, Grassland B, Baekbo P, Allan G. 2008. PCV-2 genotype definition and nomenclature. Vet Rec. 162: 867-868.

Shi KC, Guo X, Ge XN, Liu Q, Yang HC. 2010. Cytokine mRNA expression profiles in peripheral blood mononuclear cells from piglets experimentally co-infected with porcine reproductive and respiratory syndrome virus and porcine circovirus type 2 .

Vet Microbiol. 140: 155-160.

Steiner E, Balmelli C, Gerber H, Summerfield A, McCullough K. 2009. Cellular adaptive immune response against porcine circovirus type 2 in subclinically infected pigs.

BMC Vet Res. 5:45.

Timmusk S, Wallgren P, Brunborg IM, Wikström FH, Allan G, Meehan B, McMenamy M, McNeilly F, Fuxler L, Belák K, Põdersoo D, Saar T, Berg M, Fossum C. 2008. Phylogenetic analysis of porcine circovirus type 2 (PCV2) pre- and post-epizootic postweaning multisystemic wasting syndrome (PMWS). Virus Genes. 36: 509-520.

Zhai S, Yue C, Wei Z, Long J, Ran D, Lin T, Deng Y, Huang L, Sun L, Zheng $\mathrm{H}$, Gao F, Zheng H, Chen S, Yuan S. 2010. High prevalence of a novel porcine bocavirus in weanling piglets with respiratory tract symptoms in China. Arch Virol. In press.

Wikström FH, Meehan BM, Berg M, Timmusk S, Elving J, Fuxler L, Magnusson M, Allan GM, McNeilly F, Fossum C. 2007. Structure-dependent modulation of alpha interferon production by porcine circovirus 2 oligodeoxyribonucleotide and CpG DNAs in porcine peripheral blood mononuclear cells. J Virol. 81: 4919-4927. 


\title{
LS.5
}

\section{Application of advanced Al technologies to improve the competitiveness of the pork industry}

\author{
George R. Foxcroft; Jenny Patterson; Audrey Cameron; Michael K. Dyck \\ Swine Reproduction-Development Program, Swine Research \& Technology Centre, University of Alberta, Edmonton, AB, Canada
}

\section{Introduction}

The use of artificial insemination (Al) in the pig has had a major impact on genetic improvement in the swine industry over the last 40 years. However, the overall production efficiency of the breeding herd is highly dependent on the reproductive capacity (fertility) of the boars used for breeding and the genetic merit of the boars for the performance of terminal line offspring. Given the polygamous structure of swine production, poor quality boars will affect the reproductive outcome of numerous females. In the case of Al this could be thousands of females. Although ejaculates collected for use in Al are subjected to standard semen analysis in commercial boar studs, the effectiveness of these evaluations is low compared to other food-animal species.

The following discussion of the need to use more advanced $\mathrm{Al}$ technologies to improve the impact of genetically elite boars is based on three assumptions:

1. Employing the use sub-fertile boars and low quality ejaculates reduces production efficiency.

2. The use of pooled semen from poorly defined males breaks the link between known genetic value of individual boars and the paternity of progeny produced.

3. The excessive number of sperm used per litter born (probably over 9 billion sperm using current practices), and hence the high numbers of boars needed for semen production, reduces the genetic impact of the best boars.

Collectively, these inefficiencies in Al use in the pork industry represent a major disadvantage to pork producers in a global food-animal marketplace. Given the recent advances in genomics technologies, our ability to identify boars with even greater potential EBVs will increase and the potential to optimize the use of these boars in efficient pork production systems will increase. Available estimates suggests that advanced Al technologies can increase the genetic value of every pig produced by as much as $\$ 1.00$.

\section{Strategic advantages resulting from improved evaluation of boar fertility.}

The ultimate measures of boar reproductive performance are pregnancy rate and litter size born. However, these are retrospective measures of boar fertility and can be highly influenced by breeding management and the quality of the gilts and sows bred (Colenbrander et al., 2003). A combination of a thorough physical examinations of the boar and conventional semen evaluation (concentration, morphology, motility) can provide an alternative to actual fertility data (Gibson, 1989). While these evaluations can establish that an animal is either sub-fertile or infertile, they fail to identify the relative fertility of boars that meet accepted industry standards for sperm and ejaculate quality (Ruiz-Sanchez, 2006). In general, the predictors of fertility currently applied in most commercial Al centers provide a very conservative estimate of the relative fertility of individual boars. Furthermore, the relatively high sperm numbers used in commercial Al practice (usually more than 3 billion total sperm per dose of extended semen), and the pooling of semen from multiple ejaculates, masks the limited fertility of some of these boars. Differences in fertility are more evident when lower numbers of sperm are used for Al and boars are evaluated on an individual basis.

As discussed by other reviewers, if the full economic impact of the highest genetically indexed boars is to be realized at commercial production level, the number of gilts and sows bred per boar must be maximized (Gerrits et al., 2005). A number of innovations in insemination technology, including post-cervical (Watson and Behan, 2002) and deep-uterine (Vazquez et al., 2005) insemination are conducive to the use of lower sperm numbers per insemination. The further possibility of using controlled ovulation techniques to achieve single fixed-time insemination protocols (Baer and Bilkei, 2004; Cassar et al., 2005) would also substantially increase the utilization of genetically superior boars. The combined application of post-cervical and fixed-time insemination (Pelland et al. 2008) could further promote dissemination of the most superior genetics.

Effective prediction of relative boar fertility is essential and will allow for the removal of less productive boars from commercial studs. This in turn will optimize the use of proven, high fertility, and genetically high indexed boars at lower sperm numbers per Al dose. At the nucleus level this will allow for increased selection pressure by increasing the number of offspring bred per collection from high ranking boars. At the level of terminal line production, this would allow for considerable improvements in production efficiency to be realized, by capitalizing on boars with a high index for traits such as growth rate, feed conversion efficiency and the carcass characteristics of their progeny. Even if the same costs were paid in genetic royalties, by purchasing fewer total doses of semen from genetically superior boars, the cost benefits realized by producers in grow-finish performance of the progeny and the value of the carcass sold appear to be substantial.

If these changes in production strategy are to be realized, it is critical to identify boars of relatively low fertility that will not perform well when used in the more challenging situations of reduced sperm numbers per Al dose or single fixed-time insemination. The very definition of "useable semen" changes in this more demanding context. Existing information, and recent research directed to achieving these more demanding criteria of useable semen, is presented below. This review will consider preliminary data that reinforces the need to identify substantial differences in boar fertility, and the "averaging effect" that results from the use of pooled semen doses from several boars. Finally, recent data supporting the case for a move towards single fixedtime $\mathrm{Al}$ and possibly post-cervical insemination as the critical "next steps" in applying our best pig genetics to more competitive pork production systems will be presented. 


\section{Approaches to boar semen evaluation}

There is a long history behind the search to find a single or combination of tests that can accurately predict male fertility from a semen sample (Amann, 1989). Unfortunately, there appears to be no simple answer to this very complex question (Rodriguez-Martinez, 2003). Laboratory assays often examine all of the sperm present in a sample for fertility, yet only one to 30 or so sperm are necessary to fertilize all available oocytes. Braundmeier and Miller (2001) suggested that the sperm that fertilize the oocytes in vivo may be a small (even a single), highly selected, sub-population that is not representative of the average sperm evaluated in the sample. They also suggest that, because sperm must meet many requirements for successful fertilization, testing a single attribute is unlikely to be a true measure of ultimate fertility. Using similar reasoning, RodriguezMartinez (2003) suggested that to accurately predict semen quality it is necessary to test all key sperm attributes within large and heterogeneous sperm populations that potentially affect fertilization and embryonic development. Nevertheless, the markers of relative fertility finally selected must ultimately predict the relative fertility of boars when using low sperm doses of extended semen for Al (Rodriguez-Martinez et al., 2009).

Braundmeier and Miller (2001) reviewed a number of functional and molecular tests used to assess male fertility. In this review they described two different sperm traits that affect fertility.

- Compensable traits are those that can be overcome by introducing large numbers of sperm during insemination. Problems with motility and morphology will reduce the number of sperm that are able to reach the oocyte, but by introducing large numbers of sperm the reduction in fertility can be minimized.

- Uncompensable traits are those that cannot be overcome by introducing larger numbers of sperm. These defects affect fertilization and embryo development and include nuclear vacuoles, sperm chromatin structure issues and morphological problems that do not inhibit fertilization.

To effectively predict fertility, it is essential to discriminate between compensable and uncompensable traits in an ejaculate. Evaluation of relative boar fertility in vivo using high sperm numbers per dose (e.g. 3 billion sperm) will mask differences in compensable traits and will not allow the industry to identify boars that will perform well in more demanding applications of Al.

Conventional semen evaluation generally includes a measure of seminal volume, sperm concentration, and the percentage progressively motile and morphologically normal sperm (Amann et al., 1995). Although some of these parameters are correlated with fertility in the boar (Flowers, 1997; Xu et al., 1998), several authors suggest that this information, while important, does not accurately predict whether a male is truly fertile (Brahmkshtri et al., 1999; Correa et al., 1997; Rawls et al., 1998). As shown in Table 1, existing analyses are also usually inadequate for predicting relative fertility in healthy boars with ejaculate quality that meets normal industry standards ( $>70 \%$ motility and $<30 \%$ abnormal sperm) (Flowers, 1997; Alm et al., 2006), even though the reproductive efficiency of these boars may still be substantially different (Flowers, 1997; Tardif et al., 1999; Popwell and Flowers, 2004; Ruiz-Sanchez, 2006). As illustrated in Table 2, differences in relative fertility become increasingly evident when low sperm doses ( $<2.0$ billion sperm) are used for Al (Tardif et al.,
1999; Watson and Behan, 2002; Ardon et al., 2003; Ruiz-Sanchez, 2006). This approach likely averts the compensatory effect of using excessive sperm numbers per Al dose (Saacke et al., 2000 ; Alm et al., 2006), thereby revealing important fertility differences among boars.

Table 1. Relationships between boar sperm motility, sperm penetration rates, farrowing rates and number of piglets born alive (from Flowers, 1997).

\begin{tabular}{|c|c|c|c|c|c|}
\hline $\begin{array}{c}\text { Motility } \\
(\%)^{\mathrm{a}}\end{array}$ & \multicolumn{2}{|c|}{$\begin{array}{l}\text { Sperm penetration } \\
{\text { rate }(\%)^{\mathrm{b}}}^{\mathrm{b}}\end{array}$} & \multicolumn{2}{|c|}{$\begin{array}{l}\text { Farrowing rate } \\
(\%)^{c}\end{array}$} & \multirow{2}{*}{$\begin{array}{c}\begin{array}{c}\text { Number born } \\
\text { alive }\end{array} \\
10.6^{\mathrm{v}} \\
\end{array}$} \\
\hline 94.7 & $89.5^{\mathrm{v}}$ & (58) & $86.9^{v}$ & $(460)$ & \\
\hline 82.3 & $81.7^{\mathrm{vw}}$ & (55) & $87.1^{\mathrm{v}}$ & (330) & $10.5^{\mathrm{v}}$ \\
\hline 76.1 & $84.3^{\mathrm{vw}}$ & (50) & $84.5^{\mathrm{v}}$ & $(300)$ & $10.5^{\mathrm{v}}$ \\
\hline 66.2 & $74.7^{\mathrm{w}}$ & (44) & $86.1_{v}$ & (264) & $10.1^{\mathrm{v}}$ \\
\hline 52.4 & $55.5^{x}$ & $(40)$ & $72.4^{w}$ & (201) & $9.2^{w}$ \\
\hline 44.2 & $34.7^{y}$ & $(28)$ & $72.3^{w}$ & (168) & $9.2^{\mathrm{w}}$ \\
\hline 32.6 & $21.3_{z}$ & (17) & $51.7^{x}$ & $(85)$ & $7.8^{x}$ \\
\hline SEM & & & & & 0.3 \\
\hline \multicolumn{6}{|c|}{$\begin{array}{l}\text { *Motility is expressed as the average number of motile spermatozoa within the following } \\
\text { classes: }>90 ; 80-89 ; 70-79 ; 60-69 ; 50-59 ; 40-49 ; \text { and } 30-39 \text {. }\end{array}$} \\
\hline \multicolumn{6}{|c|}{$\begin{array}{l}\text { b: Sperm penetration rate is defined as the percentage of eggs that were fertilized. The numbers } \\
\text { in parentheses represent the number of ejaculates within a motility category. }\end{array}$} \\
\hline \multicolumn{6}{|c|}{ : Number in parentheses represent the number of sows inseminated within a motility category. } \\
\hline \multicolumn{6}{|c|}{ SEM: Standard error of the mean. } \\
\hline \multicolumn{6}{|c|}{$\begin{array}{l}v, w, x, y, z: \text { Means within the same column with different superscripts are significantly different } \\
(P<0.05) \text {. }\end{array}$} \\
\hline
\end{tabular}

Table 2. In vivo fertility results from nine boars with acceptable semen characteristics (> 80\% sperm motility and $<15 \%$ abnormal sperm). Gilts inseminated with $1.5 \times 109$ morphologically normal, motile sperm per 50-mL dose. (from Ruiz-Sanchez, 2006).

\begin{tabular}{|c|c|c|c|c|c|c|c|}
\hline \multirow[b]{2}{*}{ Boar } & \multicolumn{3}{|c|}{ Number of Gilts } & \multicolumn{4}{|c|}{ Fertility in vivo } \\
\hline & Bred & $\begin{array}{l}\text { Preg- } \\
\text { nant }\end{array}$ & $\begin{array}{l}\text { Far- } \\
\text { rowed }\end{array}$ & $\begin{array}{c}\text { Preg- } \\
\text { nancy } \\
\text { rate }(\%)\end{array}$ & $\begin{array}{c}\text { Farrow- } \\
\text { ing rate } \\
(\%)\end{array}$ & $\begin{array}{c}\text { Total born } \\
\text { (mean } \pm \mathrm{SE} \text { ) }\end{array}$ & $\begin{array}{c}\text { Fertility } \\
\text { Index } \\
\text { (mean } \pm \text { SE) }\end{array}$ \\
\hline $\mathrm{R}-2$ & 51 & 50 & 50 & $98 x$ & $98^{x}$ & $11.7 \pm 0.4^{\mathrm{ab}}$ & $11.4 \pm 0.6^{\mathrm{a}}$ \\
\hline Y-2 & 53 & 48 & 47 & $91^{x y}$ & $89^{x y}$ & $12.0 \pm 0.5^{\mathrm{a}}$ & $10.9 \pm 0.6^{\mathrm{ab}}$ \\
\hline $\mathrm{Pu}-3$ & 57 & 54 & 52 & $95 x y$ & $91^{x y}$ & $11.2 \pm 0.5 \mathrm{abc}$ & $10.2 \pm 0.6^{\mathrm{ab}}$ \\
\hline B-1 & 55 & 54 & 52 & $98^{x}$ & $94^{x y}$ & $10.7 \pm 0.5$ abcd & $10.2 \pm 0.6^{\mathrm{ab}}$ \\
\hline $\mathrm{R}-3$ & 55 & 52 & 52 & $94^{x y}$ & $95^{x y}$ & $10.9 \pm 0.4$ abcd & $10.1 \pm 0.6^{\mathrm{ab}}$ \\
\hline$G-2$ & 45 & 42 & 41 & $93 x y$ & $91^{x y}$ & $10.1 \pm 0.5$ abcd & $9.5 \pm 0.6^{\mathrm{ab}}$ \\
\hline B-3 & 55 & 51 & 51 & $93^{x y}$ & $93^{x y}$ & $9.6 \pm 0.5^{\mathrm{cd}}$ & $8.8 \pm 0.6^{6 \mathrm{bc}}$ \\
\hline R-1 & 56 & 48 & 47 & $86^{y z}$ & $84^{y z}$ & $10.0 \pm 0.4^{\mathrm{bcd}}$ & $8.4 \pm 0.6^{\mathrm{bc}}$ \\
\hline $\mathrm{G}-1$ & 51 & 37 & 36 & $72^{2}$ & $71^{2}$ & $8.4 \pm 0.6^{\mathrm{d}}$ & $6.0 \pm 0.6^{c}$ \\
\hline & & & & $P=0.0003$ & $P=0.0003$ & $P<0.001$ & $P<0.0001$ \\
\hline
\end{tabular}

$x-z:$ Means with different superscripts within each column were different by $x 2$ analysis $(P<0.05)$.

a-b: LSM with different superscripts within each column were different $(P<0.05)$. Values in the table are least means $($ LSM \pm standard errors (SE) of LSM. P: probability of main effect of boar.

Although numerous other potential markers of semen quality and boar fertility have been investigated (see reviews of Foxcroft et al., 2008), and might eventually simplify the evaluation process, sufficient information already exists on which to base dramatic improvements in Al technology in the pork industry. 


\section{Evidence for differences in relative boar fertility in commer- cial studs}

The almost universal use of pooled semen doses in commercial boar studs severely limits the collection of data on relative boar fertility at production level. However, the limited data available continues to suggest a substantial range of fertility exists in contemporary populations of boars. Indeed, in the absence of routine procedures for identifying relative boar fertility, and hence an ability to effectively select stud boars for relative fertility at genetic nucleus level, a normal distribution of fertility traits should be expected. In recent discussions of overall breeding herd performance (Billy Flowers - personal communication) the point has also been made that limitations in Al technology may lead the industry to continually underestimate the existing productivity of contemporary commercial dam-lines. All these points are evident in recent data obtained from single-sire matings at the multiplication level (Figure 1).

Figure 1. Data on litter size born in sows bred to commercial Landrace boars using single-sire matings with 3 billion sperm per Al dose. (Tony Chandaruk - Personal communication)

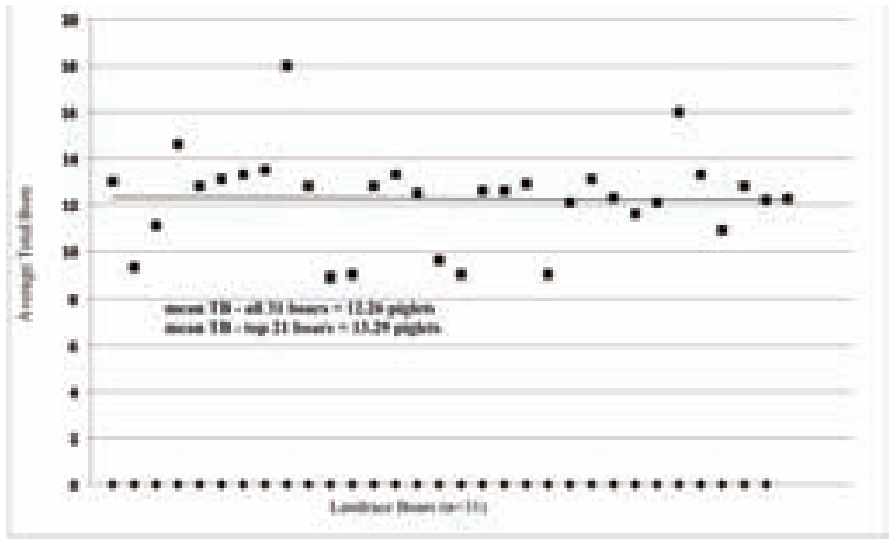

These results indicate that the productivity of the top two thirds of these boars is very high, and at an average of over 13 pigs total born, would allow ambitious targets for breeding herd performance to be achieved. However, when the productivity of the lower one third of these boars is included, overall productivity falls by over one pig born. This relatively inferior performance of 20 to $30 \%$ of boars evaluated is consistent with the more extensive data presented in Tables 1 and 2. With current Al practices, these substantial differences in boar productivity, and the link to known progeny produced by individual boars, are confounded by 1) the use of pooled semen and 2) high sperm numbers per Al dose.

\section{The problem of pooling semen when trying to optimize production efficiency.}

In one recent preliminary study, we evaluated the performance of two boars which routinely met normal criteria for acceptable semen quality (better than $80 \%$ motility and $<15 \%$ abnormal sperm) and had a history of good fertility when used in experiments requiring adequate numbers of pooled semen doses to normalize any confounding "boar effect" on the fertility of gilts and sows allocated to different experimental treatments. Before using these boars for homospermic (single sire) inseminations in gilts and sows in an experiment, we evaluated the performance of the two boars (Blue and Red) using both pooled (heterospermic) and single-sire (homospermic) Al protocols. In all cases, a total of 2 billion sperm per Al dose were used. As shown in Table 3, both boars were very productive with single-sire inseminations and still performed acceptably in Al with pooled semen. There was, nevertheless, a difference of 2.5 total embryos at day 30 of gestation between these boars, due to a $15 \%$ difference in either the fertilization rate and/or embryonic survival to this stage of pregnancy. Notably, the outstanding performance of the most fertile (Blue) boar was masked by using pooled semen and adoption of a single sire (homospermic) Al strategy would improve the total numbers of pigs born, by allowing the Blue boar to express his true reproductive potential. It seems reasonable to assume that a similar "averaging effect" results from the pooling of semen from the best boars shown in Figure 1 with less productive boars in this population.

Table 3 Results from two fertile boars when used in homospermic or heterospermic Al protocols with 2 billion sperm per Al dose. (SRTCunpublished data, 2009)

\begin{tabular}{|c|c|c|c|c|}
\hline Variable & Pooled & Al Doses & \begin{tabular}{|c|} 
Blue Boar \\
Single Sire AI
\end{tabular} & Red Boar \\
\hline \multicolumn{5}{|l|}{ Single Sire Al } \\
\hline \# sows bred & 32 & 11 & 14 & \\
\hline Ovulation Rate of sows bred ${ }^{\dagger}$ & 20.3 & 20.7 & 20.3 & \\
\hline Live Embryos at d30 $\dagger$ & 15.2 & 17.7 & 15.0 & \\
\hline Embryo Survival (\%)† & 75 & 85 & 75 & \\
\hline \multicolumn{5}{|l|}{$\mathrm{I}^{\dagger}$ mean values } \\
\hline
\end{tabular}

Data from another recent study, comparing seminal plasma protein profiles from two boars with dramatically different fertility (Novak et al., 2008), highlights the problem of linking the progeny born to boars used for Al when using pooled semen doses for commercial production. The fertilizing capacity the two boars studied (Boar A and B, Table 4), which had similar and traditionally acceptable semen parameters at collection, was initially tested with homospermic matings (i.e. single-sire Al). Based on approximately 30 mating records per boar, major differences between these boars in recorded farrowing rate and litter size born were evident. The semen from these boars was then pooled with 3 other boars with similar semen parameters and used for insemination (i.e. heterospermic semen from 5 boars in total, with equal numbers of sperm from each boar within the pooled Al dose). These pooled inseminations resulted in a $90 \%$ farrowing rate (9 pregnancies from 10 inseminations made) and 104 piglets were born these 9 litters. Paternity testing of the resulting piglets, using a panel of up to 84 single nucleotide polymorphism markers, identified Boar $B$ as the sire of $29.8 \%(31 / 104)$ of the piglets, while Boar A was identified as the sire for only a single piglet.

Table 4. Comparisons of homospermic vs. heterospermic inseminations with boars exhibiting different relative fertility (SRTC/Alberta Swine Genetics Corporation - unpublished data, Alberta, 2008)

\begin{tabular}{|c|c|c|}
\hline & \multicolumn{2}{|c|}{ Homospermic Al } \\
\hline Variable & Boar $A$ ( $n=31$ breedings) & Boar $B$ ( $n=27$ breedings) \\
\hline Farrowing Rate (\%) & 45 & 100 \\
\hline \multirow[t]{2}{*}{ Av. Total Born } & 9.22 & 12.04 \\
\hline & \multicolumn{2}{|c|}{$\begin{array}{l}\text { (Pool of } 5 \text { boars, including } A \text { and } B \text {, at equal sperm \#) } \\
\qquad(\mathbf{n = 1 0} \text { breedings) }\end{array}$} \\
\hline Farrowing Rate (\%) & \multicolumn{2}{|c|}{9} \\
\hline Av. Total Born ( $n=9$ ) & \multicolumn{2}{|c|}{11.56} \\
\hline Progeny sired (of 104 total) & 1 & 31 \\
\hline
\end{tabular}


(Simply from the perspective of optimizing breeding herd productivity, a move to single-sire Al programs seems to be justified. The very best boars will express their real potential, and overall herd productivity appears to increase. Furthermore, the small percentage of very inferior boars will quickly be identified and can be removed from commercial production. In time, it is realistic to suggest that both phenotypic and genomic markers will be developed that can be used prospectively to remove inferior boars before they are extensively used for commercial Al. However, the data presented above suggest that progress can be made by adopting single-sire mating strategies and evaluating boars on the basis of routine production criteria. Economically, there appears to be little down-side risk in adopting this strategy and considerable possibilities of positive benefits.

Even these preliminary data demonstrate the critical problems related to using heterospermic matings (pooled semen) in commercial practice:

1. As is evident from the results shown in Table 2 and Figure 1 above, even when semen appears to meet standard criteria for motility and morphology at collection, homospermic matings still identify some important differences among particular boars.

2. If inferior boars like Boar A in Table 4 are included in pooled semen doses, they produce very few of the terminal line progeny. Therefore, in practice, very high EBV boars may not pass on genetic potential in terms of pigs entering growfinish production if they are used in a "competitive" pooled semen Al protocol.

3. The disproportionate contribution of each boar to the litter progeny essentially means that the boars that do sire progeny are actually being required to do so at much lower than the total number of sperm originally included in the Al dose (say 1 to 2 billion, rather than 3 billion sperm).

\section{Application of advance Al technologies to optimize genetic transfer.}

Taking all of the above information into consideration, the logical conclusion regarding future developments in Al technology would be a move to single-sire inseminations with the lowest possible doses of semen using ejaculates from boars with high genetic value and proven fertility in a "low semen dose" environment. As in other domestic species, the logical way to achieve this outcome is with the introduction of single fixed-time Al programs and possibly linking this to post-cervical Al techniques. The substantial body of data describing the development of hormone treatment protocols for induced ovulation in the pig was extensively reviewed by Brüssow et al. (2009). Linked to this discussion is the interesting conclusion that contemporary commercial sows in well managed breeding herds show increasingly less variation in the weaning-to-estrus interval and may not even show a clear response to equine Chorionic Gonadotropin (eCG) treatment at weaning (Patterson et al., 2009). As a result, there are already reports of acceptable outcomes when exogenous hormones are used to induce ovulation in sows at a fixed time after weaning.

A recent study by Johnston et al. (2009), using a Gonadotropin Releasing Hormone $(\mathrm{GnRH})$ agonist which was applied to sows 4 days post-weaning and followed by a single insemination 24 hours later, resulted in farrowing rates and litter sizes comparable to sows receiving multiple inseminations during estrus (Table 5). It also resulted in a major improvement in the number of pigs born per insemination dose used and demonstrates the potential to maximize the use of a smaller number of high index boars.

Table 5. Fertility of sows bred by 10 days post-weaning (21-d lactation and average 10.7 pigs weaned) as Controls (no treatment) or after synchronization of ovulation with a GnRH agonist per vaginum in a gel-based vehicle (Ovugel). (From Johnson et al., 2009) $t=$ mean \pm S.E.

\begin{tabular}{|l|c|c|c|}
\hline & Control & OvuGel & Signif. \\
\hline Initial No. Sows & 150 & 150 & $*$ \\
\hline No. Sows Bred & 123 & 150 & $*$ \\
\hline In Estrus at Al (\%) & 100 & 83.3 & $<0.001$ \\
\hline No. of Services/Sow & 2.3 & 1.0 & $<0.001$ \\
\hline Wean to Estrus (days) $\dagger$ & $4.7 \pm 0.11$ & $4.4 \pm 0.11$ & 0.05 \\
\hline Sows Farrowed/Weaned (\%) & 72.7 & 76.7 & 0.43 \\
\hline Total Born Alive/Litter $\dagger$ & $10.9 \pm 0.29$ & $11.3 \pm 0.29$ & 0.37 \\
\hline Total Born/Semen Dose & 5.3 & 9.6 & $<0.001$ \\
\hline
\end{tabular}

Furthermore, Zak et al. (2009) have demonstrated that administration of porcine Luteinizing Hormone $(\mathrm{pLH})$ at the onset of behavioural estrus to control ovulation not only facilitated fixedtime insemination, but also resulted in reduced semen usage, less labour devoted to estrus detection, as well as improved sow productivity (see Table 6). These data demonstrate that administration of pLH at the time of estrus detection would allow for the application of a single fixed-timed administration 24-30 hours after pLH treatment.

Table 6. Fertility of sows bred as Controls (no treatment) or after synchronization of ovulation with a pLH at onset of estrus. (From Zak et al., 2009)

\begin{tabular}{|c|c|c|c|}
\hline C & Control & $\mathrm{pLH}$ & Signif. \\
\hline No. Sows & 156 & 163 & $*$ \\
\hline No. Inseminations & 2.2 & 2.0 & $\mathrm{P}<0.001$ \\
\hline Farrowing Rate (\%) & 82.3 & 87.4 & $\mathrm{P}=0.01$ \\
\hline Total Born/litter $\dagger$ & $11.7 \pm 0.3$ & $12.9 \pm 0.3$ & $\mathrm{P}<0.01$ \\
\hline Total Born Alive/Litter $\dagger$ & $10.8 \pm 0.3$ & $11.8 \pm 0.3$ & $\mathrm{P}<0.01$ \\
\hline$\dagger=$ mean \pm S.E.
\end{tabular}

In situations in which the synchrony of estrus after weaning may not allow the effective application of either pLH or GnRH at a fixed time after weaning, the alternative strategy of using ovulation-induction after an initial treatment at weaning with eCG continues to be explored with acceptable results (Cassar et al., 2005). The eCG/pLH protocol has been successfully applied in combination with post-cervical Al with reduced sperm numbers per Al dose, without adversely affecting sow fertility (Table 7).

Table 7. Reproductive performance of sows inseminated either cervically or post-cervically and with either 1 or 3 billion sperm per dose after synchronization of ovulation with eCG at weaning and pLH 80 hours later (from Pelland et al. 2008).

\begin{tabular}{|l|c|c|c|}
\hline & Cervical Al & \multicolumn{2}{|c|}{ Post-Cervical AI } \\
\hline No. Sperm/dose & 3 billion & 3 billion & 1 billion \\
\hline No. Sows bred & 104 & 102 & 101 \\
\hline Conception rate (\%) & 74.04 & 78.43 & 73.27 \\
\hline Farrowing rate (\%) & 67.31 & 68.63 & 68.32 \\
\hline Total litter size $\dagger$ & $10.93 \pm 3.08$ & $10.27 \pm 3.27$ & $10.79 \pm 2.83$ \\
\hline
\end{tabular}


The results of these studies has prompted work to evaluate the application of pLH at the onset of estrus followed by a single, low semen dose, 24-30 hours later using post-cervical Al (studies on-going). These and other results suggest that the implementation of single fixed-time Al programs in well managed sow herd can be a reality. Linked to the use of proven superior sires, postcervical insemination catheters and lower doses of semen, this fixed-time insemination will allow the pork production industry to apply the genetic value of elite boars to breeding programs that are competitive with other livestock species.

\section{Conclusions}

The evaluation of relative fertility amongst commercial Al boars and a move to single-boar Al programs holds the potential for significant economic benefit to the swine industry. Information provided by this approach would also be rapidly available so that elimination of less fertile boars could be achieved at an early stage. The characterization of Al boars that maintain high productivity at lower numbers of sperm per Al dose will also allow the industry to capitalize on established and emerging $\mathrm{Al}$ technologies like post-cervical insemination and single, fixedtime insemination. Moreover, all these changes can be made without any loss in productivity as measured in terms of pigs born per sow per year. The higher genetic merit of boars that could be used across an greater number of gilts and sows bred would provide substantial benefits to the producer in terms of the performance of terminal line progeny. These changes can be made without affecting, or perhaps even increasing, the total genetic royalties paid to breeding companies and commercial boar studs that are willing to facilitate these improvements in the efficiency and financial performance of the pork industry.

\section{References}

Alm, K., O. A. Peltoniemi, E. Koskinen, and M. Andersson. 2006. Porcine field fertility with two different insemination doses and the effect of sperm morphology. Reprod Domest Anim 41: 210-213.

Amann, R. P. 1989. Can the fertility potential of a seminal sample be predicted accurately? J Androl 10: 89-98.

Amann, R. P., D. F. Katz, and C. Wang. 1995. What is semen? How does semen analysis assist in understanding the reproductive status of the male? The handbook of andrology. American Society of Andrology, Schaumburg, IL.

Ardon, F., A. Dohring, X. Le Thi, K. F. Weitze, and D. Waberski. 2003. Assessing in vivo fertilizing capacity of liquid-preserved boar semen according to the 'hanover gilt model'. Reprod Domest Anim 38: 161-165.

Baer, C., and G. Bilkei. 2004. The effect of intravaginal applied gnrh-agonist on the time of ovulation and subsequent reproductive performance of weaned multiparous sows. Reprod Domest Anim 39: 293-297.

Brahmkshtri, B. P., M. J. Edwin, M. C. John, A. M. Nainar, and A. R. Krishnan. 1999. Relative efficacy of conventional sperm parameters and sperm penetration bioassay to assess bull fertility in vitro. Anim Reprod Sci 54: 159-168.

Braundmeier, A.G. and Miller DJ. 2001. The search is on: finding accurate molecular markers of male fertility. J. Dairy. Sci. 84:1915-1925.

Brüssow, K.-P., Schneider, F., Kanitz, W., Rátky, J., Kauffold, J. and Wähner, M. 2009. Studies on fixed-time ovulation induction in the pig. In: Control of Pig Reproduction VIII, Eds. H. Rodriguez-Martinez, J.L. Vallet and A.J. Ziecik, Nottingham Univ. Press, Nottingham, UK, Soc. Reprod. Fertil. Suppl. 66: 187-195.

Cassar, G., R. N. Kirkwood, K. Bennet-Steward, and R. M. Friendship. 2005. Towards single insemination in sows. In: Proceedings of the 36th Annual Meeting of teh American Association of Swine Veterinarians, Toronto, Ontario. p 353-356.

Colenbrander, B., B. M. Gadella, and T. A. Stout. 2003. The predictive value of semen analysis in the evaluation of stallion fertility. Reprod Domest Anim 38: 305-311.
Correa, J. R., M. M. Pace, and P. M. Zavos. 1997. Relationships among frozenthawed sperm characteristics assessed via the routine semen analysis, sperm functional tests and fertility of bulls in an artificial insemination program. Theriogenology 48: 721-731.

Flowers, W. L. 1997. Management of boars for efficient semen production. J Reprod Fertil Suppl 52: 67-78.

Foxcroft, G.R., Dyck, M.K., Ruiz-Sanchez, A., Novak, S. and Dixon, W.T. 2008. Identifying useable semen. Therio., 70 (8), 1324-1336.

Gerrits, R.J., Lunney, J.B., Johnson, L.A., Pursel. V.G., Kraeling, R.K., Rohrer, G.A. and Dobrinskya, J.R. 2005. Perspectives for artificial insemination and genomics to improve global swine populations. Theriogenology 63: 283-299.

Gibson, C. D. 1989. Examining for breeding soundness in boars. Veterinary medicine 84: 200.

Johnston, M.E., Gaines, A.M., Swanson M.E. and Webel, S.K. 2009. Timed insemination following $\mathrm{GnRH}$ agonist administration in weaned sows. Abstract, Conference Proceedings, VIIIth Int. Conf. Control of Pig Reproduction, Banff, Alberta. Canada.

Novak, S. et al. 2008. Fertility markers in boar semen. Advances in Pork Production 19: Abstract 28.

Patterson, J., Cameron, A., Smith, T., Kummer, R., Schott, R., Greiner, L., Connor, J. and Foxcroft, G. 2009. Responses to exogenous gonadotrophin treatment in contemporary weaned sows. In: Control of Pig Reproduction VIII, Eds. H. Rodriguez-Martinez, J.L. Vallet and A.J. Ziecik, Nottingham Univ. Press, Nottingham, UK, Soc. Reprod. Fertil. Suppl. 66: 303-304.

Pelland, C., Cassar, G., Kirkwood, R., Friendship, R. (2008) Fertility after intrauterine insemination with conventaional or low numbers of spermatozoa in sows with synchronized ovulation. J Swien Health Prod 16(4):188-192.

Popwell, J. M., and W. L. Flowers. 2004. Variability in relationships between semen quality and estimates of in vivo and in vitro fertility in boars. Anim Reprod Sci 81: 97-113.

Rawls, A. et al. 1998. Overlapping functions of the myogenic bhlh genes mrf4 and myod revealed in double mutant mice. Development 125: 2349-2358.

Rodriguez-Martinez, H. 2003. Laboratory semen assessment and prediction of fertility: Still utopia? Reprod Domest Anim 38: 312-318.

Rodríguez-Martínez, H., Kvist, U., Saravia, F., Wallgren, M., Johannisson, A., Sanz, L., Peña, F.J., Martínez, E.A., Roca, J., Vázquez, J.M. and Calvete, J.J. 2009. The physiological roles of the boar ejaculate. In: Control of Pig Reproduction VIII, Eds. H. Rodriguez-Martinez, J.L. Vallet and A.J. Ziecik, Nottingham Univ. Press, Nottingham, UK, Soc. Reprod. Fertil. Suppl. 66: $1-21$.

Ruiz-Sanchez, A. L. 2006. Semen assessment techniques for determining relative boar fertility, PhD Thesis, University of Alberta, Edmonton, Alberta.

Saacke, R. G., J. C. Dalton, S. Nadir, R. L. Nebel, and J. H. Bame. 2000. Relationship of seminal traits and insemination time to fertilization rate and embryo quality. Anim Reprod Sci 60-61: 663-677.

Tardif, S., J. P. Laforest, N. Cormier, and J. L. Bailey. 1999. The importance of porcine sperm parameters on fertility in vivo. Theriogenology 52: 447459.

Vazquez, J. M. et al. 2005. Improving the efficiency of sperm technologies in pigs: The value of deep intrauterine insemination. Theriogenology 63: 536-547.

Watson, P. F., and J. R. Behan. 2002. Intrauterine insemination of sow with reduced sperm numbers: Results of a commercially based field trial. Theriogenology 57: 1683-1693.

Xu, X. et al. 1998. In vitro maturation and fertilization techniques for assessment of semen quality and boar fertility. J Anim Sci 76: 3079-3089.

Zak, L.J., Patterson, J., Hancock, J., Hockley, D., Rogan, D. and Foxcroft, G.R. Benefits of synchronizing ovulation with porcine luteinizing hormone $(\mathrm{pLH})$ in a fixed time insemination protocol in weaned multiparous sows. In: Control of Pig Reproduction VIII, Eds. H. Rodriguez-Martinez, J.L. Vallet and A.J. Ziecik, Nottingham Univ. Press, Nottingham, UK, Soc. Reprod. Fertil. Suppl. 66: 305-306. 


\title{
LS.6
}

\section{Mycoplasma hyopneumoniae infections in pigs: update on epidemiology and control}

\author{
Dominiek Maes \\ Faculty of Veterinary Medicine, Ghent University, Belgium, Merelbeke, Belgium
}

\begin{abstract}
Introduction
Mycoplasma hyopneumoniae (M. hyopneumoniae) is the primary pathogen of enzootic pneumonia (EP), a chronic respiratory disease in pigs resulting from combined infections with $M$. hyopneumoniae and one or more secondary bacterial pathogens (Thacker, 2006). M. hyopneumoniae is also one of the primary agents involved in the porcine respiratory disease complex (PRDC). This respiratory complex includes both bacterial (Actinobacillus pleuropneumoniae, Pasteurella multocida, streptococci) and viral (porcine reproductive and respiratory syndrome virus (PRRSV), porcine circovirus type 2 (PCV2), Aujeszky's disease virus, swine influenza viruses (SIV) and porcine respiratory coronavirus) agents (Sibila et al., 2009). Both disease conditions cause major economic losses to the swine industry mainly due to reduced growth rate, poor feed conversion ratio, increased medication costs and increased mortality (Maes et al., 1996). Since elimination of $M$. hyopneumoniae from infected herds is difficult to achieve and also to maintain, most efforts are currently directed towards control of the disease. In the present paper, first some aspects of the epidemiology of M. hyopneumoniae infections will be reviewed, next interactions with other respiratory pathogens will be discussed, and finally an update will be given on control strategies.
\end{abstract}

\section{Transmission of M. hyopneumoniae}

Within a herd, $M$. hyopneumoniae is normally transmitted to susceptible pigs by direct contact with infected pigs or by sharing the same air-space with infected pigs. Piglets can become infected already in the farrowing unit by the sow either by direct nose-to-nose contact or by aerosols (vertical transmission). The chance of transmission from sow to offspring is higher in gilts and low parity sows (Fano et al., 2006), but also older sows up to the 7th parity may transmit the pathogen to the piglets (Calsamiglia and Pijoan, 2000). This is not surprising as infected animals are not able to efficiently and quickly clear the $M$. hyopneumoniae organisms from the respiratory tract. A recent study (Pieters et al., 2009) showed that pigs can remain infectious for at least 200 days post infection. The percentage of sows testing positive by $\mathrm{nPCR}$ varies depending on the study. Calsamiglia and Pijoan (2000) reported that between 24 and $56 \%$ of the sows were positive for $M$. hyopneumoniae in a multiple site system. Sibila et al. (2007) found lower prevalences namely between 0 and $11 \%$. The latter authors did not find any prevalence differences between sows from multiple site (MS) or farrow-to-finish (FTF) pig herds. Beilage et al. (2009) found that $65 \%$ of the sows were seropositive in 67 German pig herds, and that different management practices such as all-in all-out production in the farrowing units and acclimatization for replacement boars were associated with lower seroprevalences.

Also the percentage of infected pigs at weaning varies between studies. In most reports, approximately $5-20 \%$ of the piglets are positive at or shortly after weaning using nested PCR on nasal swabs (Sibila et al., 2008; Villarreal et al., 2010b), both in farrowto-finish and multi-site systems.

Dfferent infection and disease patterns have been reported in farrow-to-finish and multi-site systems (Sibila et al., 2004). In farrow-to-finish operations, infection of piglets at the nursery stage tends to be higher and the percentage of infected pigs increases progressively with advancing age. In multi-site systems, infections in nursery and growing pigs are less important than in single-site herds, and then may increase abruptly in the fattening unit (Sibila et al., 2004). In case of PRDC, clinical symptoms typically occur at 14 to 20 weeks of age (Dee, 1996).

Transmission does not only depend on the infectivity of infectious animals, but also on the susceptibility of not yet infected animals. In an experimental study aiming to quantify the spread of M. hyopneumoniae using reproduction ratios (Meyns et al., 2004), it was shown that during the nursery period, one infected pig will infect at least one penmate (Table 1). In the same study, it was also shown that the transmission rate tended to be higher with a highly virulent $M$. hyopneumoniae isolate than with a low virulent isolate (Vicca et al., 2003), but the difference was not statistically different. Villarreal et al. (2010a) found slightly lower transmission rates in pigs during the same period under field conditions. Morris et al. (1995) showed that pigs being in direct contact with other infected pigs are 7 times more likely to seroconvert than those having indirect contact.

Table 1. Results of transmission experiments of M. hyopneumoniae in nursery pigs (4-10 weeks old). Two out of 8 piglets were experimentally infected at the start of the experiment and the number of infected pigs was investigated 6 weeks later. Transmission rates are expressed using adjusted reproduction ratios $\left(R_{n}\right)$ values (Meyns et al., 2004)

\begin{tabular}{|c|c|c|c|c|c|}
\hline \multicolumn{2}{|c|}{$\begin{array}{c}\text { Highly virulent } \\
\text { M. hyopneumoniae isolate }\end{array}$} & \multicolumn{2}{c|}{$\begin{array}{c}\text { Low virulent M. hyopneu } \\
\text { M. hyopneumoniae moniae isolate }\end{array}$} \\
\hline \multicolumn{7}{|c|}{ \# of infected animals / \# of susceptible animals } \\
\hline Pen 1 & Pen 2 & Pen 3 & Pen 4 & Pen 5 & Pen 6 \\
\hline $2 / 6$ & $3 / 6$ & $5 / 6$ & $2 / 6$ & $2 / 6$ & $2 / 6$ \\
\hline \multicolumn{7}{|c|}{$R_{n}=1.47(0.68-5.38)$} & \multicolumn{4}{|c|}{$R_{n}=0.85(0.33-3.39)$} \\
\hline
\end{tabular}

The transmission of $M$. hyopneumoniae between herds can take place either by trade of (subclinically) infected pigs or by airborne transmission. The importance of airborne transmission has been illustrated in many field studies. Goodwin (1985) concluded that EP-free herds could be reinfected by airborne transmission of $M$. hyopneumoniae over a distance of $3.2 \mathrm{~km}$. Dee et al. (2009) have recently shown that airborne transport of $M$. hyopneumoniae organisms occurred out to a distance of $4.7 \mathrm{~km}$. Apart from direct contact and airborne transmission, indirect transmission of infection through fomites is not considered to be important.

\section{Interactions of $\boldsymbol{M}$. hyopneumoniae with other pathogens}

The interaction of $M$. hyopneumoniae with other pathogens has received much attention. In the past, studies mainly focussed on 
the interaction with parasitic and bacterial infections, whereas recently, the emphasis has been shifted towards the interactions with viral infections.

\section{Parasitic pathogens}

Lesions typical for EP are more severe in M. hyopneumoniae infected pigs that are concurrently infected with parasites such as Metastrongylus elongatus or Ascaris suum. Flesja and Ulvesaeter (1980) reported that the extent of pneumonia was associated with the presence of liver lesions due to migrating A. suum larvae. Other studies reported that the prevalence of pneumonia in a herd was positively associated with the prevalence of liver lesions in the herd. Steenhard et al. (2009) showed that experimental infections with $A$. suum may significantly compromise the immune response following $M$. hyopneumoniae vaccination.

\section{Bacterial pathogens}

M. hyopneumoniae predisposes pigs to infections with secondary bacteria. Different mechanisms may be involved in this phenomenon: damage of the epithelium, induction of cuffing lesions (massive infiltration of lymphohistiocytic cells), induction of thick, viscous mucus, and modulation of the immune system. Combined experimental infections with $M$. hyopneumoniae and either Pasteurella multocida (Sörensen et al., 1997) or Actinobacillus pleuropneumoniae (Marois et al., 2009) result in more severe lesions compared to the single infections. Co- or subsequent infections with P. multocida and A. pleuropneumoniae, and with other bacteria such as Bordetella bronchiseptica, Haemophilus parasuis, Arcanobacterium pyogenes, streptococci or staphylococci are commonly found in field outbreaks of EP. Different studies are inconsistent as to whether there is an association between turbinate atrophy and pneumonia.

\section{Viral pathogens}

Initial studies focussing on the interaction between M. hyopneumoniae and PRRSV could not demonstrate a potentiating effect of both pathogens (Van Alstine et al., 1996). Some years later however, it was shown under experimental conditions that M. hyopneumoniae significantly prolonged and increased the severity of PRRSV-induced pneumonia (Thacker et al., 1999). Dual infection studies with $M$. hyopneumoniae and SIV could not show the potentiating effects of both pathogens as observed with PRRSV. The effect was less pronounced and only transitory. Opriessnig et al. (2004) indicated using an experimental study that $M$. hyopneumoniae infection potentiates the severity of PCV2-associated lung and lymphoid lesions, increases the amount and prolongs the presence of PCV2-antigen, and increases the incidence of PMWS in pigs. On the contrary, the different experiments using dual infections with $M$. hyopneumoniae plus either PRRSV, SIV or PCV2 could not demonstrate a virus-dependent enhancement of mycoplasmal pneumonia. Combined infections under standardised experimental conditions may provide very useful information on the interactions of pathogens, but they only partially reflect the complexity of PRDC as occurring under field conditions. Many different pathogens may be involved under field circumstances, and environmental conditions such as management, breed and immunity of the animals, housing and air quality may largely influence the infection and disease course.

\section{Control of $M$. hyopneumoniae infections}

Optimization of management practices and housing conditions

Optimizing management and housing conditions is primordial in the control of M. hyopneumoniae infections and should be the first to be accomplished. Instituting management changes that reduce the possibilities of spreading $M$. hyopneumoniae or result in decreased lung damage by other pathogens may significantly improve the control of enzootic pneumonia. Additional factors different from housing and management conditions, such as strain differences, may determine the infection pattern and clinical course of the disease (Vicca et al., 2002). An overview of control measures for $M$. hyopneumoniae infections related to environmental and management factors has been published by Maes et al. (2008).

\section{Antimicrobial medication}

To control and treat respiratory disease including $M$. hyopneumoniae infections in pigs, tetracyclines and macrolides are most frequently used. Also, other potentially active antimicrobials against $M$. hyopneumoniae include lincosamides, pleuromutilins, fluoroquinolones, florfenicol, aminoglycosides and aminocyclitols. Fluoroquinolones and aminoglycosides have mycoplasmacidal effects. Since the organism lacks a cell wall, it is insensitive to $\beta$-lactamic antibiotics such as penicillins and cephalosporins. Although acquired antimicrobial resistance of M. hyopneumoniae has been reported to tetracyclines (Inamoto et al., 1994), and recently also to macrolides, lincosamides and fluoroquinoles (Vicca et al., 2004), it does not seem to constitute a major problem for treatment of $M$. hyopneumoniae infections to date.

An overview of peer reviewed studies assessing the efficacy of various antimicrobials used against $M$. hyopneumoniae infections under experimental as well as under field conditions is given by Vicca (2005). It can be concluded that for most antimicrobials tested, performance parameters were improved and lung lesions as well as clinical signs were decreased in treated animals. Treatment and control of enzootic pneumonia outbreaks may be disappointing because the symptoms may reappear after cessation of the therapy. Pulse medication in which medication is provided intermittently during critical production stages of the pigs, can also be used (Le Grand and Kobish, 1996). Pulse medication during extended periods of time as well as continuous medication during one or more production stages should be discouraged because of both the increased risk of spread of antimicrobial resistance and the possible risk for antimicrobial residues in the pig carcasses at slaughter.

In endemically infected farms, strategic medication of the reproductive herd is sometimes practiced as an attempt to decrease the bacterial shedding from sows to the newly introduced gilts. Antimicrobial medication of recently weaned pigs has been shown to reduce the number of $\mathrm{MM}$. hyopneumoniae organisms in the respiratory tract (Vicca et al., 2005; Thacker et al., 2006), but further research is necessary to quantify the shedding of $M$. hyopneumoniae organisms in sows receiving antimicrobial medication.

\section{Vaccination}

\section{Commercial vaccines}

Commercial vaccines, consisting of inactivated, adjuvanted whole-cell preparations, are widely applied worldwide. The major advantages of vaccination include improvement of daily 
weight gain (2-8\%), feed conversion ratio (2-5\%) and sometimes mortality rate. Additionally, shorter time to reach slaughter weight, reduced clinical signs, lung lesions and lower treatment costs are observed (Maes et al., 1998, 1999). Although protection against clinical pneumonia is often incomplete and vaccines do not prevent colonization, some studies indicate that the currently used vaccines may reduce the number of organisms in the respiratory tract (Meyns et al., 2006) and may decrease the infection level in a herd (Sibila et al., 2007). Transmission studies under experimental (Meyns et al., 2006) and field (Villarreal et al., 2010a) conditions showed that vaccination against $M$. hyopneumoniae with commercial vaccines induced only a limited and non-significant reduction in the spread of $M$. hyopneumoniae. Consequently, vaccination alone with the current vaccines will not be sufficient to eliminate $M$. hyopneumoniae from infected pig herds.

\section{Vaccination strategies}

Different vaccination strategies have been adopted, depending on the type of herd, the production system and management practices, the infection pattern and the preferences of the pig producer. Moreover, under field conditions, optimal vaccination strategies must balance the advantage of delayed vaccination with the need to induce immunity before exposure to pathogens. Since infections with $M$. hyopneumoniae may already occur during the first weeks of life, vaccination of piglets is most commonly used. Its efficacy has been demonstrated by means of numerous studies under experimental as well as field conditions (Jensen et al., 2002). Vaccination of suckling piglets (early vaccination; < 4 weeks of age) is more common in single-site herds, whereas vaccination of nursery/early fattening pigs (late vaccination; between 4 and 10 weeks) is more often practiced in three-site systems where late infections are more common.

Traditionally, double vaccination was the most frequent practice. During the last years, one-shot vaccines have been shown to confer similar benefits as two-shot vaccines and are more often used now (Baccaro et al., 2006). One-shot vaccination is especially popular because it requires less labor and it can be implemented more easily in routine management practices on the farm.

Vaccination of suckling piglets has the advantage that immunity can be induced before pigs become infected, and that less pathogens are present that can interfere with immune response. Possible disadvantages of vaccinating piglets before weaning include the presence of maternal antibodies and an increased risk for more severe PCV2 infections after weaning.

Vaccination of nursery pigs has no or less interference with possible maternally derived antibodies. However, nursery pigs may already be infected with $M$. hyopneumoniae. In addition, the age of infection or the age-window in which the piglets become infected may vary between successive groups within a herd (Sibila et al., 2004). Finally, many infections such as PRRSV or PCV2 mainly take place after weaning and may affect the general health status of the pigs, and consequently also interfere with proper immune responses after vaccination.

Only a few studies have assessed the effects of sow vaccination. Vaccination of sows at the end of gestation aims to both reduce the shedding of $M$. hyopneumoniae from the sow to the offspring and to protect the piglets against infection via maternally-derived immunity. It has been shown that vaccinating sows 5 and 3 weeks before farrowing was associated with a lower number of positive piglets at weaning using nested PCR on nasal swabs, both in farrow-to-finish operations and multisite production systems (Sibila et al., 2008). However, maternally derived antibodies only provide partial protection against lesion development and provide limited to no effect on colonization of M. hyopneumoniae (Thacker et al., 2000). The role of antigenspecific maternally derived immune cells in protection against M. hyopneumoniae is not known. Bandrick et al. (2006) showed in vivo response by delayed-type hypersensitivity and in vitro proliferation of maternally derived cells when newborn piglets were stimulated with $M$. hyopneumoniae antigen. Since piglets from vaccinated sows can still be infected, additional measures to control $M$. hyopneumoniae during the nursery and finishing phases may be warranted.

Vaccination of gilts is recommended in endemically infected herds to avoid destabilization of breeding stock immunity (Bargen et al., 2004). This is particularly the case when gilts are purchased from herds that are free from $M$. hyopneumoniae or from herds with a low infection level of $M$. hyopneumoniae.

Although vaccination confers beneficial effects in most infected herds, the effects are variable between herds. The variable results may be due to different factors such as improper vaccine storage conditions and injection technique, antigenic differences between field strains and vaccine strains, presence of disease at the time of vaccination, and interference of vaccine induced immune responses by maternally derived (colostral) antibodies.

\section{Experimental vaccines}

Investigation of new vaccines is actively occurring, including the use of aerosol and feed-based vaccines as well as subunit and DNA vaccines (Fagan et al., 2001; Lin et al., 2003; Murphy et al., 1993). Intradermal vaccination with a commercial bacterin has been shown to be efficacious (Jones et al., 2004). If M. hyopneumoniae vaccines could be delivered to the animals via aerosols or via the feed, this would provide an easy means for mass vaccination since it would substantially reduce labor costs and it would also be better for the welfare of the pigs as well as for stimulating a mucosal immune response at the respiratory tract. However, aerosol vaccination given 3 times with 2 weeks interval provided insufficient protection, in contrast with the intramuscular application of the same commercial vaccine which was efficacious (Murphy et al., 1993). On the other hand, Lin et al. (2003) showed that an oral micro-spheres experimental vaccine based on the PRIT-5 $M$. hyopneumoniae strain and prepared by a co-spray drying method significantly reduced pneumonia lesions following challenge infection with $M$. hyopneumoniae in pigs.

King et al. (1996) found only minimal and non-significant protection in a pig challenge infection model using a recombinant subunit vaccine based on the P97 adhesin of M. hyopneumoniae. Intranasal immunization of pigs with the attenuated Erysipelothrix rhusiopathiae YS-19 strain expressing a recombinant protein of M. hyopneumoniae $\mathrm{P} 97$ adhesin significantly reduced the severity of pneumonic lung lesions following challenge infection (Shimoji et al., 2003). However, apparently significant immune responses were not observed in the immunized pigs. Oral administration of the same recombinant protein in another live strain of E. rhusiopathiae (Ogawa et al., 2009) significantly reduced the severity of pneumonic lung lesions. Okamba et al. (2007) showed that a replication-defective adenovirus expressing the C-terminal portion of M. hyopneumoniae-P97 adhesin applied intranasally and intramuscularly in BALB/c mice, induced 
significant immune responses. Also several experimental DNA-vaccines have been developed and tested for immune responses in mice or pigs. Significant immune responses with DNA-vaccines were elicited in mice, based on the expression of a heat shock protein gene P42 (Chen et al., 2003), a ribonucleotide reductase $\mathrm{R} 2$ subunit gene fragment of $M$. hyopneumoniae (Chen et al., 2006), or the expression of different genes coding for several potential protective antigens (P36, P46, NrdF, P97, P97R1) (Chen et al., 2008). The studies suggest that these vaccines may represent new strategies for controlling $M$. hyopneumoniae infections in pigs, but they need to be validated in pigs under experimental and practical circumstances. Villarreal et al. (2009) showed that pigs inoculated with low virulent isolates of $M$. hyopneumoniae are not protected against a subsequent infection with a highly virulent $M$. hyopneumoniae isolate 4 weeks later and may even develop more severe disease signs. This may indicate that subsequent infections with different $M$. hyopneumoniae isolates may lead to more severe clinical disease in a pig herd.

Further studies are necessary for improving vaccines and vaccination strategies. From an immunological point of view, challenges include induction of immunity at the mucosal level. For rational design of vaccines, a comprehensive understanding of the pathobiology of $M$. hyopneumoniae infections and the molecular basis of pathogenicity of this micro-organism is required. Bacterial genes and antigens involved in survival of the bacterium in the host or that render the bacterium harmful to the host need to be identified. This may be facilitated by the fact that the genome of 3 different $M$. hyopneumoniae isolates has been sequenced (Minion et al., 2004; Vasconcelos et al., 2005).

\section{Preventive medication versus vaccination}

The use and efficacy of either vaccination or preventive (strategic) medication has been frequently discussed and the question arises whether medication and/or vaccination should be used. Advantages and disadvantages of both strategies are given in Table 2. Antimicrobials can be used in a flexible way, they are often effective against several (respiratory) pathogens and their administration is less labor-intensive since in-feed or in-water medication is mostly used. Vaccination, on the other hand, does not select for antimicrobial resistance in pathogenic bacteria and in bacteria belonging to the microbiota of the animal. It also avoids risks for antimicrobial residues in the pig carcasses at slaughter. While an immediate effect can be expected for antimicrobial treatment, the effect of vaccination of young piglets will only be evident at herd level if it is practiced for at least several months. Although vaccines are directed towards control of M. hyopneumoniae infections, also other secondary bacterial infections (Pasteurella multocida, Actinobacillus pleuropneumoniae) or lung lesions caused by these pathogens less frequently occur after vaccination (Maes et al., 1998; 1999; Meyns et al., 2006). In addition, it is very likely that combined vaccines will be used more frequently in the future. In this way, vaccination against different respiratory pathogens will be possible using one single application.

Neither vaccination nor preventive medication can prevent infection and adherence of $M$. hyopneumoniaeto the ciliated cells of the respiratory tract (Le Grand and Kobisch 1996). Finally, in case of high infection levels and/or in herds with poor management and housing conditions, the use of antimicrobials may remain necessary or may confer additional clinical and performance benefits in vaccinated herds (Mateusen et al., 2002).
Table 2. Comparison between vaccination and antimicrobial medication for the control of M. hyopneumoniae infections

\begin{tabular}{|l|c|c|}
\hline Parameter & Vaccination & Antimicrobial medication \\
\hline Strategy for use on farm & Long term & More flexible \\
\hline Labor & More laborious & Less laborious \\
\hline Efficacy & Against one pathogen & $\begin{array}{c}\text { Against different pathogens } \\
\text { (e.g. multiple disease chal- } \\
\text { lenges) }\end{array}$ \\
\hline Risk for residues & No & Yes (inappropriate use) \\
\hline Risk for antimicrobial resistance & No & Yes (inappropriate use) \\
\hline Prevention of colonization & No & No \\
\hline
\end{tabular}

\section{Conclusions}

M. hyopneumoniae infections occur worldwide and may affect pigs in various types of pig herds. The infection pattern, clinical course, the lung lesions and ultimately the financial losses vary largely depending on the herd, the management practices, the housing conditions, and also on the interactions between different respiratory pathogens. Control measures include optimizing management practices and housing conditions, the use of medication and vaccination. These measures can decrease the infection level in a herd and the number of organisms in the lungs, and improve health conditions of the animals but they do not guarantee the absence of $M$. hyopneumoniae. Further efforts are needed for development of more effective vaccines and vaccination strategies.

\section{References}

1. Baccaro M., Hirose F., Umehara O., Gonçalves L., Doto D., Paixão R., Shinya L., Moreno, A., 2006. Comparative efficacy of two single-dose bacterins in the control of Mycoplasma hyopneumoniae in swine raised under commercial conditions in Brazil. Vet J 172, 526-31.

2. Bandrick M., Pieters M., Pijoan C., Molitor T., 2006. Cellular immune response in piglets following sow vaccination wit Mycoplasma hyopneumoniae. In: Proc. Allen D. Leman Swine Conference, Vol. 33 Supplement. College of Veterinary Medicine, University of Minnesota, Minneapolis MN, USA, 11.

3. Bargen L., 2004. A system response to an outbreak of enzootic pneumonia in grow/finish pigs. Can Vet J 45, 856-9.

4. Beilage E., Rhode N., Krieter J., 2009. Seroprevalence and risk factors associated with seropositivity in sows from 67 herds in north-west Germany infected with Mycoplasma hyopneumoniae. Prev Vet Med 88, 255-63.

5. Calsamiglia M., Pijoan C., 2000. Colonisation state and colostral immunity to Mycoplasma hyopneumoniaeof different parity sows. Vet Rec 146, 530-2.

6. Chen A., Fry S., Daggard G., Mukkur T., 2008. Evaluation of immune response to recombinant potential protective antigens of $\mathrm{MycO}-$ plasma hyopneumoniae delivered as cocktail DNA and/or recombinant protein vaccines in mice. Vaccine $26,4372-8$.

7. Chen A., Fry S., Forbes-Faulkner J., Daggard G., Mukkur T., 2006. Comparative immunogenicity of Mycoplasma hyopneumoniae NrdF encoded in different expression systems delivered orally via attenuated S. Typhimurium aroA in mice. Vet Microbiol 114, 252-9.

8. Chen YL., Wang SN., Yang W., Chen YJ., Lin HH., Shiuan D., 2003. Expression and immunogenicity of Mycoplasma hyopneumoniae heat shock protein antigen P42 by DNA vaccination. Infect Immun 71, 1155-60.

9. Dee S., Otake S., Oliveira S., Deen J., 2009. Evidence of long distance airborne transport of porcine reproductive and respiratory syndrome virus and Mycoplasma hyopneumoniae. Vet Res 40, 39 (DOI: 10.1051/vetres/2009022). 
10. Dee S., 1996. The porcine respiraotry disease complex: are subpopulations important? Swine HIth Prod 4, 147-9.

11. Fagan P., Walker M., Chin J., Eamens G., Djordjevic S., 2001. Oral immunization of swine with attenuated Salmonella Typhimurium aroA SL3261 expressing a recombinant antigen of Mycoplasma hyopneumoniae (NrdF) primes the immune system for a NrDF specific secretory $\lg A$ response in the lungs. Microb Pathogenesis 30, 101-10.

12. Fano E., Pijoan C., Dee S., Torremorell M., 2006. Assessment of the effect of sow parity on the prevalence of Mycoplasma hyopneumoniae in piglets at weaning. In: 19th International Pig Veterinary Society, Copenhagen, Denmark, July 206, 96.

13. Flesja K., Ulvesaeter H., 1980. Pathological lesions in swine at slaughter. Acta Vet Scand [Suppl.] 74, 1-22.

14. Goodwin R., 1985. Apparent reinfection of enzootic-pneumonia-free pig herds: search for possible causes. Vet Rec 116, 690-4.

15. Inamoto T., Takahashi H., Yamamoto K., Nakai Y., Ogimoto K., 1994. Antibiotic susceptibility of Mycoplasma hyopneumoniae isolated from swine. J Vet Med Sci 56, 393-4.

16. Jensen D., Ersboll A., Nielsen J., 2002. A meta-analysis comparing the effect of vaccines against Mycoplasma hyopneumoniae on daily weight gain in pigs. Prev Vet Med 54, 265-78.

17. Jones G., Rapp-Gabrielson V., Wilke R., Thacker E., Thacker B., Gergen L., Sweeney D., Wasmoen T., 2004. Intradermal vaccination for Mycoplasma hyopneumoniae. J Swine HIth Prod 13, 19-27.

18. King K., Faulds D., Rosey E., Yancey R., 1996. Characterization of the gene encoding Mhp1 from Mycoplasma hyopneumoniae and examination of Mhp1's vaccine potential. Vaccine 15, 25-35.

19. Le Grand A, Kobisch M., 1996. Comparaison de l'utilisation d'un vaccin et d'un traitement antibiotique séquentiel dans un élevage infecté par Mycoplasma hyopneumoniae. Vet Med 27, 241-53.

20. Lin J., Weng C., Liao C., Yeh K., Pan M., 2003. Protective effects of oral microencapsulated Mycoplasma hyopneumoniae vaccine prepared by co-spray drying method. J Vet Med Sci 65, 69-74.

21. Maes D., Segales J., Meyns T., Sibila M., Pieters M., Haesebrouck F., 2008. Control of Mycoplasma hyopneumoniae infections in pigs. Vet Microbiol 126, 297-309.

22. Maes D., Verdonck M., Deluyker, de Kruif A., 1996. Enzootic pneumonia in pigs. Vet Q 18, 104-9.

23. Maes D., Deluyker H., Verdonck M., Castryck F., Miry C., Lein A., Vrijens B., de Kruif A., 1998. Effect of vaccination against Mycoplasma hyopneumoniae in pig herds with a continuous production system. J Vet Med B 45, 495-505.

24. Maes D., Deluyker H., Verdonck M., Castryck F., Miry C., Vrijens B., Verbeke W., Viaene J., de Kruif A., 1999. Effect of vaccination against Mycoplasma hyopneumoniae in pig herds with an all-in/all-out production system. Vaccine 17, 1024-34.

25. Marois C., Gottschalk M., Morvan H., Fablet C., Madec F., Kobisch M., 2009. Experimental infection of SPF pigs with Actinobacillus pleuropneumoniae serotype 9 alone or in association with Mycoplasma hyopneumoniae. Vet Microbiol 135, 283-91.

26. Mateusen B., Maes D., Van Goubergen M., Verdonck M., de Kruif A., 2002. Effectiveness of treatment with lincomycin hydrochloride and/or vaccination against Mycoplasma hyopneumoniae for controlling chronic respiratory disease in a herd of pigs. Vet Rec 151, 135-40.

27. Meyns T., Maes D., Dewulf J., Vicca J., Haesebrouck F., de Kruif A., 2004. Quantification of the spread of Mycoplasma hyopneumoniae in nursery pigs using transmission experiments. Prev Vet Med 66, 265-75.

28. Meyns T., Dewulf J., de Kruif A., Calus D., Haesebrouck F., Maes D., 2006. Comparison of transmission of Mycoplasma hyopneumoniae in vaccinated and non-vaccinated populations. Vaccine 24, 7081-6.
29. Minion F., Lefkowitz E., Madsen M., Cleary B., Swartzell S., Mahairas G., 2004. The genome sequence of Mycoplasma hyopneumoniae strain 232, the agent of swine mycoplasmosis. J Bacteriol 186, 712333.

30. Morris C., Gardner I., Hietala S., Carpenter T., Anderson R., Parker, K., 1995. Seroepidemiologic study of natural transmission of Mycoplasma hyopneumoniae in a swine herd. Prev Vet Med 21, 323-37.

31. Murphy D., Van Alstine W., Clark K., Albregts S., Knox K., 1993. Aerosol vaccination of pigs against Mycoplasma hyopneumoniae infection. Am. J. Vet. Res. 54, 1874-80.

32. Ogawa Y., Oishi E., Muneta Y., Sano A., Hikono H., Shibahara T., Yagi Y., Shimoij Y., 2009. Oral vaccination against Mycoplasmal pneumonia of swine using a live Eryselothrix rhusiopathiae vaccine strain as a vector. Vaccine 27, 4543-50.

33. Okamba F., Moreau E., Cheikh Saad Bouh K., Gagnon C., Massie B., Arella M., 2007. Immune responses induced by replication-defective adenovirus expressing the C-terminal portion of the Mycoplasma hyopneumoniae P97 adhesin. Clin Vaccine Immunol 14, 767-74.

34. Opriessnig T, Thacker E, Yu S, Fenaux M, Meng X, Halbur P., 2004. Experimental reproduction of postweaning multisystemic wasting syndrome in pigs by dual infection with Mycoplasma hyopneumoniae and porcine circovirus type 2. Vet Pathol 41, 624-40.

35. Pieters M., Pijoan C., Fano E., Dee S., 2009. An assessment of the duration of Mycoplasma hyopneumoniae infection in an experimentally infected population of pigs. Vet Microbiol 134, 261-6.

36. S?rensen V., Ahrens P., Barfod K., Feenstra A., Feld N., Friis N., BilleHansen V., Jensen N., Pedersen M., 1997. Mycoplasma hyopneumoniae infection in pigs: Duration of the disease and evaluation of four diagnostic assays. Vet Microbiol 54, 23-34.

37. Shimoji Y., Oishi E., Muneta Y., Nosaka H., Mori Y., 2003. Vaccine efficacy of the attenuated Erysipelothrix rhusiopathiae YS-19 expressing a recombinant protein of Mycoplasma hyopneumoniae P97 adhesin against mycoplasmal pneumonia of swine. Vaccine 21, 532-7.

38. Sibila M., Bernal R., Torrents D., Riera P., Llopart D., Calsamiglia M., Segalés J., 2008. Effect of sow vaccination against Mycoplasma hyopneumoniae on sow and piglet colonization and seroconversion, and pig lung lesions at slaughter. Vet Microbiol 127, 165-70.

39. Sibila M., Pieters M., Molitor T., Maes D., Haesebrouck F., Segales J., 2009. Current perspectives on the epidemiology of Mycoplasma hyopneumoniae infection.Vet J 181, 221-31.

40. Sibila M., Calsamiglia M., Nofrarías M., López-Soria S., Espinal A., Segalés J., Riera P., Llopart D., Artigas C., 2004. Longitudinal study of Mycoplasma hyopneumonicae infection in naturally infected pigs. In: Proc 18th IPVS congress, Hamburg Germany, 169.

41. Sibila M., Nofrarías M., López-Soria S., Segalés J., Valero O., Espinalm A., Calsamiglia M., 2007. Chronological study of Mycoplasma hyopneumoniae infection, seroconversion and associated lung lesions in vaccinated and non-vaccinated pigs. Vet Microbiol 122, 97-107.

42. Steenhard N., Jungersen G., Kokotovic B., Beshah E., Dawson H., Urban J., Roepstorff A., Thamsborg S., 2009. Ascaris suum infection negatively affects the response to a Mycoplasma hyopneumoniae vaccination and subsequent challenge infection in pigs. Vaccine 27 , 5161-9.

43. Thacker B., Thacker E., Halbur P., Minion C., Young T., Erickson B., Thanawonguwech T., 2000. The influence of maternally-derived antibodies on Mycoplasma hyopneumoniae infection. In: Proc 16th IPVS congress, Melbourne Australia, 454.

44. Thacker E., 2006. Mycoplasmal diseases. In: Diseases of Swine, 9th edition, B.E. Straw, J.J. Zimmerman, S. D'Allaire, D.J. Taylor (editors). Blacwell Publishing Ltd, Oxford, UK, pp. 701-17.

45. Thacker E., Halbur P., Ross R., Thanawongnuwech R., Thacker B., 1999. Mycoplasma hyopneumoniae potentiation of porcine reproductive and respiratory syndrome virus-induced pneumonia. J Clin Microbiol 37, 620-7. 
46. Thacker E., Nilubol D., Halbur P., 2006. Efficacy of Aureomycin ${ }^{\circledR}$ chlortetracycline (CTC) granulated premix in decreasing the potentiation of PRRSV pneumonia by Mycoplasma hyopneumoniae . In: Proc. 37th Annual Meeting Am. Assoc. Swine Vet., Kansas City, Missouri, 153-5.

47. Van Alstine W., Stevenson G., Kanitz C., 1996. Porcine reproductive and respiratory syndrome virus does not exacerbate Mycoplasma hyopneumoniae infection in young pigs. Vet Microbiol 49, 297-303.

48. Vasconcelos A., Ferreira H., Bizarro et al., 2005. Swine and poultry pathogens: the complete genome sequences of two strains of Mycoplasma hyopneumoniae and a strain of Mycoplasma synoviae. J Bacteriol 187, 5568-77.

49. Vicca J., Maes D., Jonker L., de Kruif A., Haesebrouck F., 2005. The efficacy of tylosin premix for the treatment and control of Mycoplasma hyopneumoniae infections. Vet Rec 156, 606-10.

50. Vicca J., Stakenborg T., Maes D., Butaye P., Peeters J., de Kruif A., Haesebrouck F., 2003. Evaluation of virulence of Mycoplasma hyopneumoniae field isolates. Vet Microbiol 97, 177-90.

51. Vicca J., Thermote L., Maes D., D'Hooghe I., Peeters J., Haesebrouck F., de Kruif A., 2002. Patterns of Mycoplasma hyopneumoniae infection in closed pig herds using serology and nested PCR on nasal samples. J Vet Med B 49, 349-353.
52. Vicca, J., 2005. Virulence and antimicrobial susceptibility of Mycoplasma hyopneumoniae isolates from pigs. $\mathrm{PhD}$ thesis, Faculty of Veterinary Medicine, Ghent University, ISBN 90-5864-086-8, pp. 219.

53. Vicca J., Stakenborg T., Maes D., Butaye P., Peeters J., de Kruif A., Haesebrouck F., 2004. In vitro susceptibilities of Mycoplasma hyopneumoniae field isolates. Antimicrob. Agents Chemother 48, 4470-2.

54. Villarreal I., Maes D., Meyns T., Gebruers F., Calus D., Pasmans F., Haesebrouck F., 2009. Infection with a low virulent Mycoplasma hyopneumoniae isolate does not protect piglets against subsequent infection with a highly virulent $M$. hyopneumoniae isolate. Vaccine $27,1875-9$.

55. Villarreal I., Meyns T., Dewulf J., Vranckx K., Calus D., Pasmans F. Haesebrouck F., Maes D., 2010a. Effect of vaccination against Mycoplasma hyopneumoniae on the transmission of $M$. hyopneumoniae under field conditions. Vet J, cond. Accepted.

56. Villarreal I., Vranckx K., Duchateau L., Pasmans F., Haesebrouck F., Jensen J., Maes D., 2010b. Prevalence and risk factors for early Mycoplasma hyopneumoniae infections in suckling piglets in different EU countries. In: Proc. 21st IPVS congress, Vancouver Canada. 


\title{
Social behavior in swine and its impact on welfare
}

\author{
Jeremy N. Marchant-Forde \\ USDA-ARS, Livestock Behavior Research Unit, Purdue University, West Lafayette, IN, USA
}

\begin{abstract}
Introduction
Pigs are social animals. From an evolutionary perspective, being social conveys a number of benefits, but potentially some disadvantages, especially for certain individuals within the group. Living in a social group can reduce predation, improve successful foraging, improve rearing of offspring, increase chances of mating and help thermoregulation. On the flip side, a group can be more conspicuous to a predator, competition within the group can reduce access to resources for some individuals, and may increase the risk of disease (1).

By definition, "social behavior is comprised of those patterns of behavior that involve two or more members of a species" (2).

Thus, social behavior includes sexual behavior and parental behavior. However, the emphasis of this manuscript will be on those behaviors that relate to formation and maintenance of social organization in swine, namely those centered on aggression and social dominance, as these are the aspects of social behavior in swine that have garnered most attention in relation to the animal's welfare. The animal's welfare can be defined as its state as regards its attempts to cope with its environment (3).
\end{abstract}

\section{Natural social organization}

In order to better understand the consequences of the systems in which we place pigs during commercial production, it is crucial to acknowledge the pig's origins and social behavior in a natural setting. The domestic pig is descended from the wild boar, but although they have changed greatly in terms of phenotype, their behavior, when given the opportunity, is extremely similar to their wild ancestors. The data from which we can conclude this comes from three main sources: 1) studies of wild boar in their natural habitat, 2) studies of feral populations of domestic pigs, and 3) studies of domestic pigs released into naturalistic enclosures.

The natural social organization of pigs centers on a core group or 'sounder' of 2-4 related sows plus their associated offspring of different sizes and ages $(4,5,6)$. Sows in the group are likely to be sisters or mother and daughters. Group size will be influenced by habitat and resource availability (especially food), as will the size of the home range, but can be as large as 6000 hectares (7). Home ranges may overlap with other sounders, but even when sharing home ranges, sounders will tend to actively avoid open confrontation with each other (6). As the offspring mature, the females split off to form their own sounders and the males split off to form adolescent bachelor groups, becoming solitary as mature boars. During the breeding season, mature boars may associate with sounders, becoming dominant to all sounder members. Within sounders, aggression is very rare. The group usually maintains a simple, linear social hierarchy, which is relatively stable over time. Position within the hierarchy is mostly determined by size and age, with large, mature, physically-strong sows being dominant over smaller sub-adults and juveniles (4). Aggression does occur during competition for resources, especially food, but most often, subordinate animals actively avoid conflict with dominant animals (8). Food will be scattered but available ad libitum in their complex environment, as long as the pigs forage. This social organization is such that pigs are not exposed to unrelated, unfamiliar pigs. New litters are integrated into the group early in life (7-14 days of age) when the sow returns to the group with her litter after isolation at farrowing, but no aggression has been observed during these interactions $(9,10)$.

In contrast, pigs housed in commercial systems may be housed individually (but in close proximity to others) or in groups ranging from small (4-5) to large (200+). Regardless of group size, there will be relatively limited space and a relatively simple environment and they may encounter frequent remixing. Access to food may be ad libitum or restricted. Unsurprisingly, aggression will be much more prevalent under commercial conditions than under natural conditions. How prevalent will be largely influenced by: 1) the degree of mixing/remixing, 2) the method of feeding, and 3) the amount and quality of space.

\section{Aggression and mixing}

When unacquainted pigs are mixed together, they often fight. The fight does not often break out immediately but can be a complex and gradual event as the pigs investigate each other using a series of specific and often reciprocal behaviors, characterized by nosing, sniffing and gentle nudging (11). This may then escalate into more vigorous pushing and pressing, bites, head-knocks and mounting, which continues until one pig withdraws, with or without being pursued. Most fighting takes place within $2 \mathrm{~h}$ of mixing and by 24-48 h post-mixing, the level of aggressive interactions should be basal, and a hierarchy established. The hierarchy is then maintained by threats, avoidance and withdrawal, or short-lived aggressive interactions.

A fight can result in injury to one or both parties and thus the potential cost of fighting can be high, particularly for the loser, both in terms of welfare of the pig but also economically for the producer. For the individual pig, the choice to engage in fight or not in the first place, or to know when to stop calls for the pig to be able to assess its fighting ability relative to the fighting ability of its opponent (12). If pigs were unable to carry out assessment of relative fighting ability, then we may expect that every pig would have to fight with every pig with which it is mixed in order for social order to be established. We know, however, that this is not the case in some instances. For example, a study examined mixing 4 pigs from one litter with 4 pigs from another litter, meaning that there were a total of 16 possible unacquainted pairs in each of 11 pens (12). The actual number of pairs which fought ranged from only 2 to 10 (median 6). The most supported explanation as to why this happens is that within each litter, the pigs had a clear idea of their own position within the hierarchy. Seeing the outcome of an interaction between a pen-mate and an unacquainted pig thus yields useful information about your own likelihood of success in a fight. In 
fact the study demonstrated a litter effect, with in each case, a 'dominant' litter moving freely around the pen, with a 'subordinate' litter huddling in one corner (12). In production, this is the most usual scenario - i.e. when pigs are mixed into a pen, there will already be existing pair-wise relationships between some members, which can help individual pigs gather information. We have examined close one-on-one encounters and looked at the sequences of behaviors that occur before a fight breaks out. We have found that escalation is more often preceded by the receiving pig ignoring the attention of the investigating pig. If the receiving pig turns to maintain nose-to-nose contact, then the chance of subsequent escalation is reduced (13).

Not surprisingly, there has been a great deal of research carried out into methods to reduce aggression at mixing (14). Many have little or no effect but there are some which clearly have an effect over the short or long term. Elements of pen design, such as a solid barrier in the center of the pen $(15,16)$ or the division of the lying area into distinct sub-areas can reduce aggression over the long term (17). Mixing at sunset $(18,19)$ and the use of drugs such as amperozide (19) and azaperone (20) can have short-term effects, but only so long as it remains dark or the drugs wear off. Mixing in the presence of a super-dominant animal - i.e. a boar - can reduce number of aggressive interactions, skin damage and flight distance (21). Other longer-term solutions include early social experience, repeated mixing, premixing and use of pre-exposure pens. Allowing piglets to mix before weaning can benefit their social development and enable them to form stable social hierarchies quicker $(22,23)$. Repeated mixing during growth may help replacement gilts acquire social skills that serve them better later in life (24). Pre-mixing of sows into groups at weaning can help them when they are subsequently mixed into a larger group post-service (25). Finally, placing pigs into adjacent pens or a small pen within a pen, so that they can have contact without the ability to fight, prior to mixing together can greatly reduce aggression (26).

What is clear is that any method that might facilitate communication, be it olfactory, vocal or, to a degree, physical will help the pig's ability to assess its chances better and thereby avoid fighting or at least avoid prolonged contests.

\section{Aggression and feeding}

Naturally, pigs tend to synchronize feeding and actively forage for relatively low quality food for many hours during the day, with peaks in activity around dawn and dusk. Again, this is potentially very different from the commercial situation. In production, pigs will have access to high quality feed, which can meet their nutritional requirements quickly and it may only be available for an extremely limited period of time each day. Whereas the grow/finish herd may have ad libitum access, but with restricted number of feeding spaces, the breeding herd usually has access to a single food drop a day, with food present for about 15-20 minutes every $24 \mathrm{~h}$. In many 'intensive' production systems, pigs do not have access to any alternative foraging substrate, such as straw, and thus, access to food becomes an important resource and one that may play a major role in determining the amount of aggression being displayed within a system. For sows, feeding systems that promote competition for access, such as floor feeding, can have relatively high levels of aggression. Feeding systems that reduce competition by enclosing sows in stalls or being available ad libitum, can have relatively low aggression.
Floor feeding may be cheap and 'low tech' in terms of equipment, but it is highly competitive (27) with dominant sows able to monopolize the feed if it is not widely distributed (28). The aggression elicited by floor feeding, and its production consequences, can be manipulated by ensuring that the feeding area is as widespread as possible and that group size is kept small and stable, with animals of similar body condition and nutritional needs (29). Trough feeding is another method of feeding a group simultaneously, but without any partitions, dominant animals can again monopolize large lengths of trough space, displacing subordinate sows, especially if food distribution along the trough is uneven. Aggression can be reduced by using wet feed (30), which flows better along the trough, and by using dividers to separate the trough into individual feeding spaces. Use of trickle feeders for delivery can also help to keep sows 'tied' to a single feeding space and reduce displacements (29).

Other feeding options for sows include individual feeding stalls into which the sows can be shut either manually or under their own control (free-access stalls) and thereby eat at their own rate without threat of displacement. Electronic Sow Feeder (ESF) systems have the big advantage of allowing each sow to eat an individual, stockperson-controlled allowance without fear of displacement, but sows have to feed sequentially. With a single feeder per 40-60 sows, the feeder station may be occupied for much of the day with sows queuing outside. Usually a fairly stable feeding order develops, with dominant sows accessing the feeder soon after the daily cycle begins and the more subordinate sows waiting to feeding towards the end of the cycle. However, the entrance to the feeder can become a focal point of activity for large parts of the day and hence, a focal point for aggression (31).

For the growing/finishing pig, feed is usually available ad libitum. Although feeding behavior and actual feed intake is stimulated by allowing pigs to feed simultaneously, there is still the need to have allocated individual feeding spaces incorporated into the feeder design to keep aggression as low as possible (32). There is also the question of how many feeding spaces are made available for the number of pigs in the pen, whether these should be in the form of one 'multi-space' feeder or several 'single-space' feeders and where the feeder or feeders should be placed in the pen $(33,34)$. The term 'social workload' has been used to describe the effort required and aggression encountered in negotiating a route through pen-mates to a feeder and displacing pigs which are either feeding or obstructing the feeder (35). Ad libitum feeding has also been investigated for sows using high fiber diets which have increased bulk and low energy. In general, increasing fiber doubles the amount of time that sows spend eating and reduces stereotypic behavior, restlessness and aggression (36).

\section{Aggression and space}

The amount of space that pigs have, and the quality of that space, can have a large impact on their behavioral repertoire, including agonistic social behavior. With sows, the minimum amount of space given to sows in current commercial systems is that encompassed by a gestation crate, which at about $1.25 \mathrm{~m} 2$ encloses the sow's static space requirement (37). The sow enclosed within this space has no pen-mates and thus, it is commonly assumed that she is free from the aggression attributed to group housing systems. In reality, this is not true. She may be free from the physical effects of aggression - i.e. the skin lesions and other injuries that group-housed sows may show - but 
several studies have shown that aggression between neighbors in crates can be high. Initial attack is more often followed by retaliation in crates, resulting in escalation in the intensity of aggression rather than the withdrawal and cessation of interaction most often seen in group housing systems $(38,39,40)$.

In pen systems, the amount of space given per animal will impact aggression. In general, as space allowance decreases, the total number of aggressive interactions increases (41). However, few studies have investigated the effects of space allowance as a single factor. In many comparative studies, when space allowance has varied, so have other aspects of the pen design or the group size, making drawing conclusion about space per se difficult. In some instances, space allowance may not show a linear relationship with aggression. For example, a study examining the effects of communal area space behind free-access sow stalls shows that decreasing communal space allowance may show in inverted U-shape relationship with aggression. At high space allowance, sows using the communal area can avoid each other and at low space allowance, they utilize the free-access stalls more, also reducing aggression. When the space allowance is intermediate, the sows are motivated to use the space, but are unable to avoid agonistic interactions so easily and thus, this treatment shows most aggression.

The other important aspect of space is its quality. Much of the current research in to group housing in North America involves the changing of stall systems to pen systems within similar types of building - i.e. into fully-slatted or part-slatted, non-bedded group housing. Usually, space is still fairly restricted and the environment offers no real 'enrichment' apart from pen-mates with which to interact. In these systems, there may be increases in skin lesions and aggression-associated lameness compared with sows in crates. However, if other enrichment is included, such as a foraging substrate and bedding, then aggression is often reduced compared with sows in non-enriched pens (42).

\section{Welfare impact of aggression}

The most obvious physical impact of aggression can be injury. This can take the form of lameness, skin lesions - which are often seen on the shoulders, flanks, hindquarters and ears - or vulva biting, seen in sows in particular. If occurring near slaughter, severe physical damage may lead to condemnation of parts of the carcass, thereby financially impacting the producer. Another economic impact can be decreased growth. If individuals are unable to access enough food to meet their requirements, then growth can be reduced. This can increase the weight variability within the pen and can become self-reinforcing, with the smaller pigs getting smaller and smaller relative to their pen-mates. Within the breeding herd, the timing of group formation and any post-service mixing can be critical. It is widely acknowledged that mixing around the time of implantation should be avoided and thus mixing at weaning and/or 5- 6 weeks postservice are recommended to maximize farrowing rate (43).

In terms of internal physiology, we also know that aggression activates both the sympathetic-adrenal-medullary axis and the hypothalamic-pituitary-adrenal axis, resulting in increased heart rate (31), increased plasma cortisol concentrations, increased plasma epinephrine and norepinephrine levels. For the breeding sow, this can explain the potential impact of stress due to aggression on fertility. For the slaughter pig, this can also help explain the effects of aggression on growth. With activation of the stress axes also comes a negative impact on immunity and thus disease incidence and rate of healing of any injury may be impacted. Finally, there is recent evidence that for the breeding sow, the stress due to aggression that she encounters during gestation can impact both the behavior and stress-reactivity of her offspring (44).

\section{References}

1. Mendl, M. T., Held, S. (2001) Living in groups: an evolutionary perspective. In. (eds. L. J. Keeling, H. W. Gonyou) Social Behaviour in Farm Animals, CABI Publishing, Wallingford, UK. pp7-36.

2. Banks, E. M., Heisey, J. A. (1977). Animal Behavior. Educational Methods, Chicago. 200pp

3. Broom, D. M. (1986) Indicators of poor welfare. Br Vet J 142: 524-526.

4. Mauget, R. (1981) Behavioural and reproductive strategies in wild forms of Sus scrofa (European wild boar and feral pigs) In. (ed. W. Sybesma). The Welfare of Pigs. The Hague, Martinus Nijhoff. 11, 3-13.

5. Graves, H.B. (1984) Behavior and ecology of wild and feral swine (Sus scrofa) J Anim Sci 58: 482-492.

6. Gabor, T. M., Hellgren, E. C., Van Den Bussche, R. A., Silvy, N. J. (1999) Demography, sociospatial behaviour and genetics of feral pigs (Sus scrofa) in a semi-arid environment. J Zool 247: 311-322.

7. Janeau, G., Spitz, F. (1984) L'espace chez le sanglier (Sus scrofa L.): Occupation et mode d'utilisation. Gibier Faune Sauvage 1: 76-89.

8. Jensen, P., Wood-Gush, D. G. M. (1984) Social interactions in a group of free-ranging sows. Appl Anim Behav Sci 12: 327-337.

9. Newberry, R. C., Wood-Gush, D. G. M. (1988) Development of some behaviour patterns in piglets under semi-natural conditions. Anim Prod 46: 103-109.

10. Petersen, H. V., Vestergaard, K., Jensen, P. (1989) Integration of piglets into social groups of free-ranging domestic pigs. Appl Anim Behav Sci 23: 223-236.

11. McGlone, J. J. (1985) A quantitative ethogram of aggressive and submissive behaviors in recently regrouped pigs. J Anim Sci 61: 559565.

12. Mendl, M., Erhard, H. W. (1997) Social choices in farm animals: to fight or not to fight? In. (eds. J. M. Forbes, T. L. J. Lawrence, R. G. Rodway, M. A. Varley) Animal Choices. BSAS, Penicuik, UK. pp45-53.

13. Marchant-Forde, J. N., Garner, J. P., Schenck, E. L., Johnson, A. K., Lay Jr., D. C. (2008) The social behavior carried out by unacquainted sows on mixing may predict the likelihood of escalation into aggression. J Anim Sci, 86 (E Suppl 2): 352

14. Marchant-Forde, J. N., Marchant-Forde, R. M. (2005) Methods to reduce aggression at mixing in swine. Pig News Info 26: 63N-73N.

15. Olesen, L. S., Nygaard, C. M., Friend, T. H., Bushong, D., Knabe, D. A., Vestergaard, K. S., Vaughan, R. K. (1996) Effect of partitioning pens on aggressive behavior of pigs regrouped at weaning. Appl Anim Behav Sci 46: 167-174.

16. Edwards, S. A., Mauchline, S., Stewart, A. H. (1993) Designing pens to minimize aggression when sows are mixed. Farm Build Prog 113: 20-23.

17. Bünger, B., Kallweit, E. (1994) Ethological studies on pregnant sows in a dynamic group housing system with rationed individual feeding by one electronic feeder station. Landbau Volk 44: 151-166.

18. Barnett, J. L., Cronin, G. M., McCallum, T. H., Newman, E. A. (1994) Effects of food and time of day on aggression when grouping unfamiliar adult pigs. Appl Animl Behav Sci 39: 339-347.

19. Barnett, J. L., Cronin, G. M., McCallum, T. H., Newman, E. A., Hennessy, D. P. (1996) Effects of grouping unfamiliar adult pigs after dark, after treatment with Amperozide and by using pens with stalls, on aggression, skin lesions and plasma cortisol concentrations. Appl Animl Behav Sci 50: 121-133. 
20. Luescher, U. A., Friendship, R. M., McKeown, D. B. (1990) Evaluation of methods to reduce fighting among regrouped gilts. Can J Anim Sci 70: 363-370.

21. Docking, C. M., Kay, R. M., Day, J. E. L., Chamberlain, H. L. (2001) The effect of stocking density, group size and boar presence on the behaviour, aggression and skin damage of sows in a specialized mixing pen at weaning. In: Proc BSAS p. 46.

22. D'Eath, R. B. (2004) Consistency of aggressive temperament in domestic pigs: The effects of social experience and social disruption. Agg Behav 30: 435-448.

23. D'Eath, R. B. (2005) Socialising piglets before weaning improves social hierarchy formation when pigs are mixed post-weaning. Appl Anim Behav Sci 93: 199-211.

24. van Putten, G., Bure, R. G. (1997) Preparing gilts for group housing by increasing their social skills. Appl Anim Behav Sci 54: 173-183.

25. Durrell, J. L., Beattie, V. E., Sneddon, I. A., Kilpatrick, D. (2003) Premixing as a technique for facilitating subgroup formation and reducing sow aggression in large dynamic groups. Appl Anim Behav Sci 84: 89-99.

26. Kennedy, M.J., Broom, D.M. (1996) Factors modulating aggression received by pigs mixed individually into groups. In: Proc 30th Int Cong ISAE. Centre for Study of Animal Welfare, Guelph. p 52 (Abstr.)

27. Csermely, D., Wood-Gush, D. G. M. (1986) Agonistic behaviour in grouped sows. I. The influence of feeding. Biol Behav 11: 244-252.

28. Csermely, D., Wood-Gush, D. G. M. (1990) Agonistic behaviour in grouped sows. Il. How social rank affects feeding and drinking behaviour. Bull di Zool 57: 55-58.

29. Gonyou, H. W. (2005) Experiences with alternative methods of sow housing. JAVMA 226:1336-1340.

30. Andersen, I. L., Boe, K. E., Kristiansen, A. L. (1999) The influence of different feeding arrangements and food type on competition at feeding in pregnant sows. Appl Anim Behav Sci 65: 91-104.

31. Marchant, J. N., Mendl, M. T., Rudd, A. R., Broom, D. M. (1995) The effect of agonistic interactions on the heart rate of group-housed sows. Appl Anim Behav Sci 46: 49-56.

32. O'Connell, N. E., Beattie, V. E.,Weatherup, R. N. (2002) Influence of feeder type on the performance and behaviour of weaned pigs. Livestock Prod Sci 74: 13-17.
33. Georgsson, L., Svendsen, J. (2002) Degree of competition at feeding differentially affects behaviour and performance of group-housed growing-finishing pigs of different relative weights. J Anim Sci 80: 376-383.

34. Walker, N., Donnelly, E., McCracken, K. J., Morrow, A. (1993) Improving Ad Lib Feeding for Pigs. ARINI, Occasional Publication No. 22.

35. Walker, N. (1995) A note on siting of single-space feeders for growing pigs. Irish J Agric Food Res 34: 57-61.

36. Meunier-Salaun, M. C., Edwards, S. A., Robert, S. (2001) Effect of dietary fibre on the behaviour and health of the restricted fed sow. Anim Feed Sci Tech 90: 53-69.

37. Baxter, M. R., Schwaller, C.E. (1983) Space requirements for sows in confinement. In: Farm Animal Housing and Welfare. Nijhoff, The Hague, The Netherlands, pp 181-195.

38. Barnett, J. L., Hemsworth, P. H., Winfield, C. G. (1987) The effects of design of individual stalls on the social behaviour and physiological responses related to the welfare of pregnant pigs. Appl Anim Behav Sci 18: 133-142.

39. Broom, D. M., Mendl, M. T., Zanella, A. J. (1995) A comparison of the welfare of sows in different housing systems. Anim Sci 61: 369-385.

40. Jensen, P. (1984) Effects of confinement on social-interaction patterns in dry sows. Appl Anim Behav Sci 12: 93-101.

41. Weng, R. C., Edwards, S. A., English, P. R. (1998) Behaviour, social interactions and lesion scores of group-housed sows in relation to floor space allowance. Appl Anim Behav Sci 59: 307-316.

42. Durrell, J. L., Sneddon, I. A., Beattie, V. E. (1997) Effects of enrichment and floor type on behaviour of cubicle housed sows. Anim Welfare 6: 297-308.

43. Kirkwood, R., Zanella, A. J. (2005) Influence of gestation housing on sow welfare and productivity. NPB Final Report.

44. Ison, S. H., D'Eath, R. B., Robson, S. K., Baxter, E. M., Ormandy, E., Douglas, A. J., Russell, J. A., Lawrence, A. B., Jarvis, S. (2010) 'Subordination style' in pigs? The response of pregnant sows to mixing stress affects their offspring's behaviour and stress reactivity. Appl Anim Behav Sci 124: 16-27. 


\title{
LS.8
}

\section{Dysentery and ileitis - High time to tackle the difficult science behind these diseases}

\author{
Steven McOrist \\ University of Nottingham, Sutton Bonington, UK
}

\section{Swine dysentery}

Clinical features of swine dysentery (SD) have been well described in the pig industry since the 1920's. There is a severe inflammation of the large intestine with bloody mucous diarrhoea. On affected farms, disease is usually common in fattening pigs from 12 to $75 \mathrm{~kg}$, but cases also occur regularly in gilts and sows. Spread of the disease through the herd is slow, building up in numbers as the dose rate of the agent builds up in the environment. The incubation period in field cases is normally 7 to 14 days. Pigs that recover only develop a mild immunity, but rarely suffer from the full disease again. The high economic cost of disease is associated with pig mortality, high morbidity, marked depression of growth and feed conversion efficiency, and the costs of continual in-feed medication. It is an oppressive disease for pig farmers, who rarely feel able to maintain a functional pig farm while a clinical presence continues.

Swine dysentery remains a major pig health issue in Europe and Asia-Pacific regions and is increasing in the USA, as carbadox usage declines. No useful vaccine or blood test is available and on-going medication is problematic for endemic SD - a major issue in central European pig farming is the high prevalence of tiamulin-resistant and pathogenic $B$ hyodysenteriae strains. The actions of regulatory authorities have removed many useful and effective anti-SD drugs (quinoxalines, metronidazoles, ionophores) across the pig raising world.

This major enteric disease is caused by the snake-like spirochaete bacterium, now called Brachyspira hyodysenteriae. This organism was first cultured and used to verify Koch's postulates by the groups of David J. Taylor in Cambridge and Hank Harris in lowa in 1970. They published a wide set of studies exploring the spirochaetal invasion of colonic epithelial cells and other key pathogenesis features of the early lesions (see Taylor and Blakemore 1971).

Microbiological diagnosis is critical because of the serious nature of SD, for example, a key issue surrounding the purchase of new breeding stock is whether any SD-free reassurance provided by a breeding company supplier is accurate. Diagnosis is therefore based on the history, the clinical picture, post-mortem examinations and laboratory tests involving both anaerobic isolation and PCR identification of haemolytic $B$ hyodysenteriae.

The key scientific questions regarding Brachyspira hyodysenteriae and SD are i/ those surrounding the pathogenesis of SD - how does this organism cause the lesions? and ii/ those of immunity - why is the immune and blood reaction so weak? The failure of the veterinary science community to answer these critical questions since 1970 has been regularly noted (see ter Huurne and Gaastra 1995) and I wish to note it again here. The genome has finally been elucidated (see Bellgard et al 2009) and it points to the existence of 6 likely haemolysins and 15 proteases. However, the few mechanistic studies on pathogenesis factors of motility, LPS outer coats and putative haemolysins do not amount to a real challenge-exposure based explanation about how this bac- terium produces such an impressive yet immunologically silent lesion and illness. The desired outcomes of vaccines and blood tests and new drugs for SD, which all became viable targets in 1971 , will presumably wait many further years until these basic microbiology challenge-exposure studies come about.

Part of this failure must lie with the lack of funding for basic studies of pig enteric diseases. Much of the previous funding in livestock microbiology research has ended up with the chicken and cattle sectors and focused largely on zoonotic/food industry issues such as Salmonella. Any left-over for pig studies have previously ended up in these food industry issues or studies in respiratory diseases such as PRRS.

\section{Ileitis - proliferative enteropathy}

Clinical features of proliferative enteropathy (PE, ileitis) have been well described in the pig industry since the 1930's. In growing pigs with uncomplicated proliferation of the mucosa, the condition is a chronic proliferative enteropathy, also known as porcine intestinal adenomatosis (PIA). Case lesions and clinical signs can vary from mild and sub-clinical, through to clinical diarrhoea and weight loss; or in more severe cases additional changes can be superimposed on this basic lesion, including necrotic enteritis, or an acute proliferative haemorrhagic enteropathy. All these forms of PE remain common and important enteric diseases. Estimates across the global swine industry show that around 96 percent of farm sites are infected, wherein around 30 percent of weaner-to-finisher pigs often have detectable lesions at some point, causing clear economic losses (see McOrist et al 2003).

The nature of PE was not clear until 1973, when lesions were examined ultrastructurally and small, curved intracellular vibrioid bacteria were consistently present only within the abnormal proliferating cells of the affected mucosa (see Rowland and Lawson 1974). The identity of these PE bacteria and their true aetiologic role were finally resolved in 1993 with successful co-culture of the intracellular organism and the reproduction of the disease in pigs using a pure culture of this agent (see Lawson et al. 1993 and McOrist et al. 1993). The name Lawsonia intracellularis was chosen to reflect the key role and persistence of the Scottish veterinarian, Gordon Lawson in its discovery. The unique feature of PE pathogenesis is therefore the clear causation of a monotypic epithelial cell proliferation by an infectious bacterium. To my knowledge, only two other bacteria (unrelated species of Bartonella and Citrobacter) have some parallels in this proliferative pathogenesis and the exact mechanism also remains unknown for them.

Lawsonia research has largely focused on issues important to the pig farming industry, such as the susceptibility of Lawsonia to various antibiotics, its epidemiology within and between pig farms and methods of diagnosis in live pigs and related topics (see Stege et al 2004). Lawsonia has a small, single circular genome and 3 mega-plasmids which are features commonly seen in 
other obligate and symbiont intracellular bacteria (see Gebhart and Kapur 2007 and Schmitz-Esser et al 2008). A vaccine for ileitis was first registered in 2001 (Enterisol lleitis, Boehringer Ingelheim) and is now widely registered and used around the world (see Guedes and Gebhart 2003 and Kroll et al 2004). Presumably, the choice of vaccine format (live attenuated oral delivery) is well-suited to this type of intracellular gut mucosal agent. This rapid ileitis vaccine development is in contrast to the slow pace of development for vaccines for other key enteric diseases of pigs, such as swine dysentery and post-weaning colibacillosis.

However, despite the interesting and unique nature of the proliferative nature of $\mathrm{PE}$, with perhaps insights to be gained into human colon cancer and the gut flora, no real progress has been made on the key scientific question regarding Lawsonia - how does this bacterial organism cause the monotypic proliferative lesions in the gut epithelium ? The intriguing mechanism whereby the bacteria cause infected epithelial cells to fail to mature, but continue to undergo mitosis, and form the hyperplastic to adenomatous crypts is not yet understood fully. This adenomatous effect probably reflects a Lawsonia-specific inhibition of the normal crypt cell differentiation process, as regulated locally at the crypt neck (see McOrist et al 2006 and Oh et al 2010). L intracellularis-infected intestinal crypts can become enormously elongated and often branched. Loss of body protein and amino acids into the intestinal lumen and the reduced nutrient absorption by the intestinal mucosa lacking mature enterocytes are the likely causes of the reduction in weight gain and feed conversion efficiency seen in pigs affected with chronic uncomplicated PE lesions (see Rowan and Lawrence 1982 and Gogolewski et al 1991). So far, further examination of the Lawsonia genome has proved of limited insight into exactly which genes cause this cell cycle/differentiation effect, with many unknown genes evident.

We therefore note again the failure of the veterinary science community to answer this critical question with basic studies in pig enteric disease. Presumably the reasons for this failure lie again in the lack of funding for basic studies of pig enteric diseases as part of the pots allocated for livestock microbiology research.

So what will all be saying at IPVS 2020 ...... So, Grandpa Ernie, what did all the old pig scientists find out about SD and PE ??? Lack of curiosity will kill the pig .....????

\section{Selected References:}

Bellgard, M.I., Wanchanthuek, P., La, T., Ryan, K., Moolhuijzen, P., et al. 2009. Genome sequence of the pathogenic intestinal spirochete Brachyspira hyodysenteriae reveals adaptations to its lifestyle in the porcine large intestine. PLoS ONE 4(3); e4641.

Gebhart, C.J. and Kapur, V. 2007. Lawsonia intracellularis genome -

http://www.ncbi.nlm.nih.gov/sites/entrez?Db=genomeprj\&cmd=Show DetailView\&TermToSearch $=35241$

Guedes, R. M.C.; and Gebhart, C.J. 2003. Onset and duration of fecal shedding, cell-mediated and humoral immune responses in pigs after challenge with a pathogenic isolate or attenuated vaccine strain of Lawsonia intracellularis. Vet Microbiol 91: 135-145.

Gogolewski, R.P., Cook, R.W., and Batterham, E.S. 1991. Suboptimal growth associated with porcine intestinal adenomatosis in pigs in nutritional studies. Aust Vet J 68:406-408.

Kroll, J.; Roof, M. B.; and McOrist, S. 2004. Evaluation of protective immunity in pigs following oral administration of an avirulent live vaccine of Lawsonia intracellularis. Am J Vet Res 65: 559-565.

Lawson, G. H. K.; McOrist, S.; Sabri, J.; and Mackie, R.A. 1993. Intracellular bacteria of porcine proliferative enteropathy: Cultivation and maintenance in vitro. J Clin Microbiol 31:1136-1142.

McOrist, S.; Jasni, S.; Mackie, R. A.; Maclntyre, N.; Neef, N.; and Lawson, G. H. K. 1993. Reproduction of porcine proliferative enteropathy with pure cultures of ileal symbiont intracellularis. Infect Immun 61:4286-4292.

McOrist S., Barcellos, D.E., and Wilson, R.J. 2003. Global patterns of porcine proliferative enteropathy. Pig J 51: 26-35

McOrist, S.; Gebhart, C.J.; and Bosworth, B.T. 2006. Evaluation of porcine ileum models of enterocyte infection by Lawsonia intracellularis. Can J Vet Res 70: 155-159.

Oh, Y-S.; Lee, J-B.; and McOrist, S. 2009. Microarray analysis of differential expression of cell cycle and cell differentiation genes in cells infected with Lawsonia intracellularis. Vet J 184: 340-345.

Rowan, T. G.; and Lawrence, T. L. J. 1982. Amino-acid digestibility in pigs with signs of porcine intestinal adenomatosis. Vet Rec 110: 306-307.

Rowland, A. C., and Lawson, G. H. K. 1974. Intestinal adenomatosis in the pig: Immunofluorescent and electron microscopic studies. Res Vet Sci 17:323-330.

Schmitz-Esser, S.; Haferkamp, I.; Knab, S.; Penz, T.; Ast, M.; Kohl, C.; Wagner, M.; and Horn, M. 2008. Lawsonia intracellularis contains a gene encoding a functional Rickettsia-like ATP/ADP translocase for host exploitation. J Bacteriol 190: 5746-5752.

Stege, H.; Jensen, T.K.; Moller, K.; Vestergaard, K.; Baekbo, P.; and Jorsal, S.E. 2004. Infection dynamics of Lawsonia intracellularis in pig herds. Vet Microbiol 104: 197-206.

Taylor, D.J., and Blakemore, W.F. 1971. Spirochaetal invasion of the colonic epithelium in swine dysentery. Res Vet Sci 12: 177-179.

Ter Huurne, A.A. and Gaastra, W. 1995. Swine dysentery: more unknown than known. Vet Microbiol 46: 347-360. 


\title{
0.001
}

\section{Exudative epidermitis is difficult to treat because of widespread antimicrobial resistant Staphylococcus hyicus}

\author{
Jeonghwa Park; Robert Friendship; Cate Dewey; Scott Weese; Zvonimir Poljak \\ University of Guelph, Guelph, ON, Canada
}

\section{Introduction}

Exudative epidermitis (EE) or greasy pig disease is caused by Staphylococcus hyicus. Certain strains of S. hyicus produce heatlabile exfoliative toxins that are important in the pathogenesis of the disease. Infection of skin wounds during the early suckling period is the typical presentation. Recently many herds have moved away from clipping needle teeth at birth. There may be a resultant, an increase in prevalence of EE. Traditionally, an injection of penicillin has been the treatment of choice but antimicrobial resistance to penicillin has been noted (1).

There are several studies determining the occurrence of different genes encoding antimicrobial resistance in S. hyicus (2) but there is limited information about the distribution of the mecA gene which encodes the low affinity penicillin-binding protein PBP 2A. The objectives of this study were to evaluate the extent of antimicrobial resistance of S.hyicus and to determine whether the mecA gene is present in S. hyicus in Ontario, Canada.

\section{Materials and Methods}

In a survey, farmers $(n=49)$ were asked how they treated EE and whether the treatment worked. In addition herds $(n=17)$ with EE problems were visited and skin swabs and scrapings were obtained from pigs with clinical signs of EE (generally 6 pigs per farm). Bacterial culture was performed on the samples with $S$. hyicus and S. aureus recovered from $77.3 \%(85 / 110)$ and $45.9 \%$ $(50 / 109)$ of the samples respectively. Susceptibility to penicillin (pen), ampicillin (amp), ceftiofur (cef), spectinomycin (spec), sulphonamides (sul), tetracycline (tet), tiamulin (tia) and trimethoprim/sulfa (tri/sul) was determined by the agar diffusion method defined by the Clinical and Laboratory Standards Institute (CLSI). In addition, molecular studies were conducted on isolates to determine the presence of the mecA gene using PCR and Pulsedfield gel electrophoresis (PFGE) analysis (3).

\section{Results}

Based on the survey, $69.4 \%$ (34/49) of farmers used topical treatments routinely to treat EE, including mixtures of topical antibiotics, antiseptics and mineral oil. In addition, 57\% of respondents said that they used injectable antibiotics and $82 \%$ indicated injectable penicillin as the treatment of choice. Many of the respondents expressed concern that treatment was of little value and that response was poor.

Antimicrobial resistance testing revealed that all isolates of $S$.hyicus and almost all isolates of $S$. aureus were resistant to penicillin and ampicillin and the majority were also resistant to ceftiofur (Figure 1).
Figure1. Percent of isolates resistant to antimicrobials

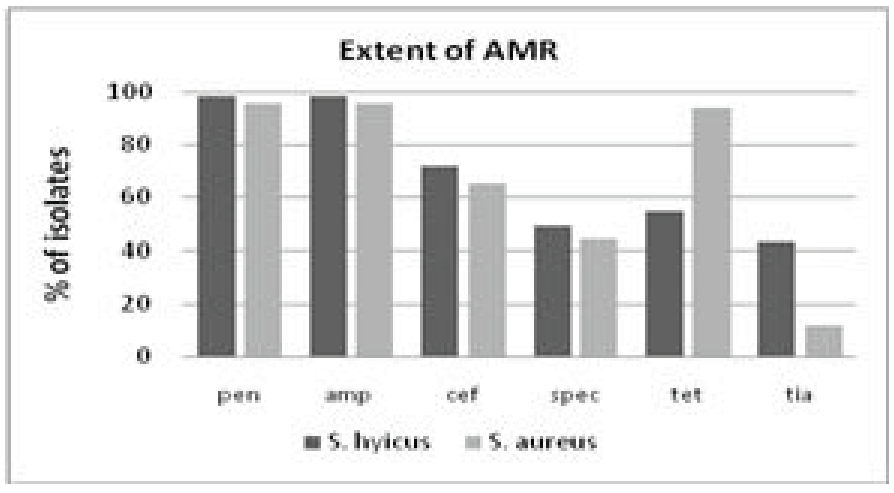

The percentage of isolates of S. hyicus and S. aureus from which the mecA gene was demonstrated was $2.73 \%$ (3/110), and $5.45 \%$ $(6 / 110)$, respectively. The mecA gene was present in $12.96 \%$ $(14 / 108)$ of the $S$. aureus isolated from nasal swabs from these same pigs.

\section{Discussion}

Penicillin was identified as the most common treatment choice by pig farmers and yet all isolates from cases of EE were shown to be resistant to not only penicillin but in most cases resistant to the entire family of beta-lactam antibiotics. These results explain why farmers reported a poor response to treatment of EE. Previous researchers have noted that penicillin-resistant isolates of $S$. hyicus containing the blaZ gene were relatively common in the pig population, affecting 50 to $75 \%$ of isolates (2). Methicillin-resistant $S$. aureus has been shown to be widespread in Ontario pig farms (4) and therefore it is not surprising that the mecA gene was isolated from $S$. aureus in these pigs. The relationship between S. aureus and S. hyicus and the possibility of gene transfer between these bacteria needs to be investigated further.

\section{References}

1. Wegener et al. (1993). Vet Microbiol, 34:363-72

2. Aarestrup et al (2002). Vet Microbiol, 89:83-94

3. Wielders et al (2002). J Clin Microb, 40: 3970-5

4. Khanna et al (2008). Vet Microbiol, 128:298-303 


\title{
0.002
}

\section{Etiology of the ear necrosis syndrome - investigation of infectious agents}

\author{
Christiane Lang ${ }^{1}$ Thomas Voglmayr $^{2}$ Franz Waxenecker $^{3}$ Ursula Hofstetter $^{3}$ \\ Herbert Weissenböck ${ }^{4}$ Mario Ogris ${ }^{5}$ Georg Bruns ${ }^{6}$ \\ Mathias Ritzmann ${ }^{1}$
}

1. Clinic for Swine, Vienna, Austria; 2. Traunkreis VetClinic, Ried im Traunkreis, Austria; 3. Biomin GmbH, Herzogenburg, Austria; 4. Institute of Pathology and Forensic Veterinary Medicine, University of Veterinary Medicine, Vienna, Austria; 5. Intervet GesmbH, Vienna, Austria; 6. Tieraerztliche Klinik Duemmerland, Steinfeld, Germany

\section{Introduction}

During the summer months lesions of the so-called ear necrosis syndrome can be observed frequently in weaners during the raising period. The stages range from mild epidermal changes to severe ulcers and necrosis (1). In the past, various pathogens have been discussed to be responsible for the development of ear necrosis, but the exact etiology of this disease could not be determined. Aim of this study was the evaluation of different infectious agents in pigs with ear necrosis syndrome.

\section{Materials and Methods}

A total of 96 piglets (age 6 to 10 weeks) were included in the study. Piglets in 9 of the 15 farms were sampled twice in the interval of a year; in the other 6 farms piglets were only sampled once. All piglets showed clinical signs of the ear necrosis syndrome in an initial stage. The total of piglets with ear necrosis on the respective farms ranged from 10 to $100 \%$. Collected samples and methods are summarized in table 1.

Table 1: Types of samples and methods

\begin{tabular}{|l|l|c|}
\hline \multicolumn{1}{|c|}{ Sample type } & n & Method \\
\hline Ear tissue & 96 & Pathohistology \\
\hline Ear tissue & 96 & In situ hybridization PCV2 \\
\hline Swab of lesion & 91 & Bacteriology \\
\hline Serum & 88 & PRRSV-PCR \\
\hline Serum & 96 & PCV2-PCR \\
\hline Serum & 96 & Mycoplasma suis-PCR \\
\hline Serum & 96 & Sarcoptes suis-Ab-ELISA \\
\hline Serum & 15 & Endotoxin \\
\hline Serum & 96 & Calcium, phosphorous \\
\hline
\end{tabular}

\section{Results}

The pathohistological findings included epidermal necrosis, epidermal hyperplasia, bacterial growth in superficial cell debris and intimal proliferation with consecutive vascular occlusion. In situ hybridization for PCV-2 was negative in all cases.

In 61 of the swabs streptococci could be found, in 48 of the swabs staphylococci were determined. 8 piglets were PRRSV positive, none of them was positive for PCV2. Only one piglet showed a positive result for Mycoplasma suis. 3 piglets showed antibodies against Sarcoptes suis. The measured endotoxin concentration ranged between 0.10 and $1.13 \mathrm{U} / \mathrm{ml}$. Calcium levels were increased in 20, decreased in 6 piglets, phosphorous concentrations were increased in 57 and decreased in 1 of the piglets.

\section{Discussion}

The pathohistological findings proved that ear necrosis did not only develop because of external inflicted injuries and ear biting. A multifactorial disease seemed more likely. The various infectious agents seemed to play a minor role, PCV2 could be completely excluded. Strepococci and staphylococci could be determined frequently in the swabs as well as pathohistologically, but it was unclear if they act in a sense of a primary or secondary infection. The endotoxin concentrations were in a noncritical range, but participation earlier in the course of infection can not be ruled out. Finally, the development of ear necrosis is caused by an interaction of several factors and infectious agents are definitely able to contribute to this disease pattern.

\section{Acknowledgment}

This study was supported by the Dres. Jutta und Georg-BrunsStiftung.

\section{References}

1 Mirt (1999): Vet. Rec. 144, 92-96. 


\title{
0.003
}

\section{The association between tail lesions and abscesses recorded at slaughter}

\author{
Marie Erika Busch; Niels Peter Baadsgaard \\ Pig Research Centre, Danish Agriculture \& Food Council, Copenhagen, Denmark
}

\section{Introduction}

The presence of tail lesions in slaughter pigs has been shown to be correlated to the risk of abscesses, including osteomyelitis (1, $2,3)$. However, the location of abscesses associated with tail lesions has been described in only one published paper (4), which describes significant correlations between tail lesions and abscesses in the spine, peritoneum, pelvis, legs, ribs and lungs. Abscesses are costly to the pig producer because of partial or total condemnations at slaughter. In addition, it is most likely that the disease process leading to abscesses causes reduced pig welfare and productivity, increased mortality and higher expenses for medical treatment $(5,6)$. The aim of the present study was to investigate the degree to which abscesses, including embolic pneumonia and osteomyelitis, could be attributed to tail lesions and to investigate the location of the abscesses associated with tail lesions. This would help the pig producer assess whether different kinds of abscesses recorded at slaughter are likely to be caused by tail lesions or whether other causes are more likely.

\section{Materials and Methods}

Data from the meat inspection was used for the analysis. The data set comprised records from 732,282 slaughter pigs (castrates and females) from 2,027 herds slaughtered at the largest Danish abattoir in Jan-Feb 2008. The meat inspection records the presence/absence of tail lesion for each individual pig as standard procedure. Similarly, the presence/absence of abscesses at different locations on the pig is recorded (see locations in Table 1). In the case of osteomyelitis, the location is not recorded. Each pig can have abscesses at several locations.

Statistical analysis: For each type of abscess, we calculated the relative risk (RR) of having an abscess when having a tail lesion compared to not having a tail lesion. The statistical significance of the interrelationships was tested by chi-square tests. In addition, the following were calculated:

- AF (attributable fraction): the proportion of abscesses in pigs with tail lesion attributable to tail lesions

- PAF (population attributable fraction): the proportion of abscesses in the population attributable to tail lesions

\section{Results}

The overall prevalence of tail lesions was $0.85 \%$. Table 1 shows an overview of the results.

Table 1. The association between tail lesions and abscesses recorded at slaughter

\begin{tabular}{|l|c|c|c|c|c|}
\hline & $\begin{array}{c}\text { Prevalence } \\
\text { of } \\
\text { +tail lesion }\end{array}$ & $\begin{array}{c}\text { lesions (\%) } \\
\text { - tail le- } \\
\text { sion }\end{array}$ & RR & AF & PAF \\
\hline Abscesses & & & & & \\
\hline Head & 0.96 & 0.99 & 0.97 & -3.0 & -0.03 \\
\hline Abdomen & 3.5 & 0.8 & $4.7^{*}$ & 78.5 & 3.0 \\
\hline Neck/breast & 4.9 & 1.3 & $3.9^{*}$ & 74.2 & 2.4 \\
\hline Limbs/toes & 4.2 & 1.0 & $4.1^{*}$ & 75.7 & 2.6 \\
\hline Hind part & 9.0 & 0.5 & $17.2^{*}$ & 94.2 & 12.1 \\
\hline Embolic pneum. & 1.2 & 0.05 & $25.9^{*}$ & 96.1 & 17.5 \\
\hline Osteomyelitis & 12.4 & 0.2 & $57.2^{*}$ & 98.3 & 32.3 \\
\hline
\end{tabular}

${ }^{*}=\mathrm{P}<<<0.05$

\section{Discussion}

The associations between tail lesion and all types of abscesses, except abscess in the head, were statistically significant. The highest RR, AF and PAF were found for osteomyelitis and embolic pneumonia. Osteomyelitis was found 57 times more often in pigs with tail lesion than in pigs without tail lesion, and 32 $\%$ of the osteomyelitis cases could be attributed to tail lesions. However, these numbers are probably underestimated, since many cases of healed tail lesions are probably not recorded by the meat inspection. The risks of abscesses in the abdomen, neck/breast and limbs/toes were also correlated with the presence of tail lesion (RR 3.9-4.7). However, as seen by the low PAFs (2.4-3.0), in most cases the causes of these abscesses should be found elsewhere. For example, they could be caused by unhygienic injection practice, hernias, foot injuries or other injuries serving as an entrance for bacteria. The potential associations among diseases recorded at slaughter may direct attention to preventive measures and hence help reduce costs of partial or total condemnation of the carcass for the producer.

\section{References}

1. Flesjå \& Ulvsæter, 1980. Acta vet scand, 21, Suppl. 74

2. Valros et al., 2004. Acta Agric Scand A, 54, 213-219

3. Kritas \& Morrison, 2007. Vet Rec, 160, 149-152

4. Huey, 1996. Vet Rec, 138, 511-514

5. Wallgren \& Lindahl, 1996. Acta vet scand, 37, 453-60

6. Flesjå et al., 1984. Acta vet scand, 25, 309-321 


\title{
0.004
}

\section{The development and consequences of ear necrosis in a weaner herd and two growing-finishing herds}

\author{
Marie Erika Busch ${ }^{1}$ Inger M. Jensen ${ }^{2}$ Jakob Korsgaard $^{3}$ \\ 1. Pig Research Centre, Danish Agriculture \& Food Council, Copenhagen, Denmark; \\ 2. Dyrlaegecentralen Sydvest A/S, Loegumkloster, Denmark; 3. Oe-Vet, Naestved, Denmark
}

\section{Introduction}

In Danish herds, ear necrosis (EN) in weaners and older pigs is mainly located along the ventral edge of the ear. Biting by other pigs and infections (possibly as a sequel to trauma) have been suggested as causes of EN $(1,2)$. The aim of this investigation was to explore 1) the development and healing of EN, 2) factors within the herd associated with EN and 3) the effect of EN on the health and growth of the pigs.

\section{Materials and Methods}

Three herds with high prevalences of EN were chosen for two studies. All study pigs were females or castrates. Study 1: Study 1 was carried out in a sow/weaner herd. A total of 136 randomly selected pigs from two batches were examined 2-3 times a week for 7 weeks beginning two days before weaning (day -2). EN was scored on a scale of 0-4 representing increasing severity (0: no EN; 4: loss of ear tissue and/or EN on $>20 \%$ of the ear). The average daily gain (ADG) of each pig during the observation period was measured. The study pigs were housed in 11 weaner pens with slatted floors and tube feeders (25 pigs/pen). The effects of weight at weaning, ear scratches on day 4, gender and sow parity on the binary 'EN score 2-4 on day 46' were analyzed in a logistic regression model. Study 2: Study 2 was carried out in two growing-finishing herds receiving pigs from the same sow herd (not the herd in study 1). The study comprised 218 pigs from one batch: 109 pigs without EN (controls) and 109 with EN (matched on pen, gender and weight). Pigs were examined once a week for 12 weeks from a weight of approx. $45 \mathrm{~kg}$. EN was scored on a scale of 0-4 (0: no EN; 4: bloody lesion with loss of ear tissue). ADG of each pig during the observation period was measured. Pens housed 11-22 pigs and had varying flooring and feeding systems. Meat inspection data for all pigs were collected. The effects of EN, herd, gender, weight at entrance and at slaughter on ADG were analyzed in a linear mixed model.

\section{Results}

Study 1: The prevalence of severe EN was highest at the end of the observation period (Figure 1). Weight at weaning, ear scratches on day 4, gender and sow parity were not found to have any effect on the risk of having EN on day 46. Pigs with and without EN had the same ADG ( $250 \mathrm{~g} /$ day). EN was not found to have any effect on signs of disease. Study 2: No effect of EN on $A D G$, lean mean percentage or mortality was found (overall mortality $2.8 \%$ ). Five pigs in the EN group and one control pig became unthrifty or lame, but the difference was not statistically significant $(P=0.1)$. At slaughter, abscesses were found in only two pigs, both from the EN group (corresponding to $1.8 \%$ of the EN pigs).
Figure 1. EN prevalence in the weaner herd (study 1) and in the EN group in the growing-finishing herds (study 2).

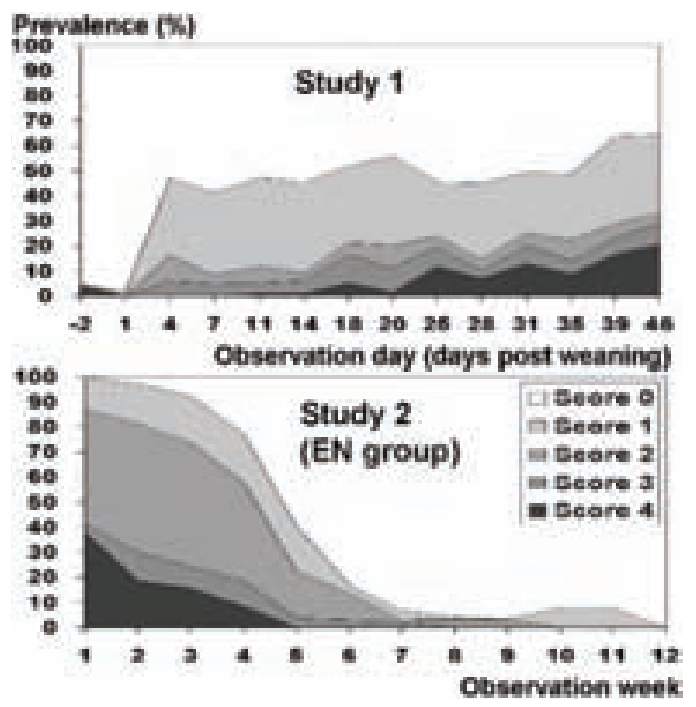

\section{Discussion}

There were no signs that small or weak pigs were predisposed to EN. EN was not found to have any effect on growth. Despite severe lesions, EN did not result in the amount of abscesses that are common in pigs with tail lesions (3). In growing-finishing pigs, more disease was found in pigs with EN, but a larger investigation would be necessary to determine whether the difference is statistically significant. The healing of EN seen in the growing-finishing pigs could be caused by immunological or behavioral changes in the pigs.

\section{References}

1. Blackshaw, JK (1981). Anim Prod 33:325-332

2. Richardson et al. (1984). Vet Pathol, 21, 152-157

3. Busch, ME, Baadsgaard, NP (2010). 21st IPVS Congr. 


\title{
0.005
}

\section{Idiopathic Vesicular Disease (IVD): a case report in the centre of Italy}

\author{
Marco Sensi ${ }^{1}$ Antonella Catalano ${ }^{2}$ Miriam Tinaro ${ }^{1}$ Caterina Mariotti ${ }^{1}$ Carmen Panzieri ${ }^{1}$ Silvia Marchi ${ }^{1}$ Silva Costarelli ${ }^{1}$ \\ 1. Istituto Zooprofilattico Sperimentale dell'Umbria e delle Marche, Perugia, Italy; 2. Azienda Sanitaria Locale 2, Perugia, Italy
}

\section{Introduction}

Several Swine Vesicular Disease (SVD) outbreacks were registered in central Italy (Umbria and Marche regions) during October 2008. In the same time vesicular lesions were observed in an high percentage of animals of a fattening unit (approx. 2000 fattening pigs), located the surveillance area.

The aim of this work is to describe the episode and explain the differential diagnostic pathway to rule out SVD and suspect Idiopathic Vesicular Disease (IVD)

\section{Materials and Methods}

A state veterinarian noted vesicular lesions on snout of several hogs during an inspective visit in a fattening farm, before their transport to slaughter house. The farm was immediately quarantined.

A trace back and trace forward investigation was performed by our Institute (Istituto Zooprofilattico Sperimentale dell'Umbria e delle Marche). Blood and fecal samples were collected from pigs with lesions and not and checked for exotic diseases (SVD and Foot and Mouth Disease - FMD) and for parvovirus.

Blood samples were investigated for blood count parameters.

Feed samples were collected from the hoppers and from the silo bean and tested for mycotoxines (Aflatoxines, Deoxynivalenol, Zearalenone, T2 toxin, Fumonisine) .

\section{Results and discussion}

Snout erosions were observed on $20 \%$ of the pig farm population. Few animals showed gingival and oral lesions, too. Coronary band was not interested.

Lesions were of 3-4 mm of diameter and circular or "V" shaped. (Fig. 1 and 2).

Rectal temperatures ranged from 38,5 to $39,3^{\circ} \mathrm{C}$ ruling out an infectious aetiology .

Feed was commercially purchased and the epidemiological trace back showed all the biosecurity measures were respected.

The last pig replacement happened six months before and no pigs were sold in the same time.

Lab test results were negative for SVD, FMD and parvovirosis. Blood parameters didn't reveal significative alterations and mycotoxins levels in the feed resulted within the normal ranges. Lesions resolved in nearly three weeks time.

In the last two years we observed, always in fattening farms and in the same season, two other episodes characterized by similar lesions.

The first case was referred to high levels of Deoxynilvalenol and the second one to a mistake in the organic acids inclusion in the feed.
In this case, looking the lab results and the symptomatology we reasonably suspect Idiopathic Vesicular Disease (IVD) even if a definitive diagnosis was impossible to achieve.

Idiopathic Vesicular Disease (IVD) is a sporadic condition affecting swine already reported in Australia, New Zealand, Canada and USA. It is characterized by a seasonal pattern (spring - autumn). Although, it isn't a severe disease for pigs and economically heavy for breeders, IVD represents an important differential diagnostic condition must be considered for SVD and FMD diagnosis.

We have to bear in mind predisposing factors can play an important role to exacerbate the clinical symptoms. In this case the particular shape of the nipple-drinkers could be contributed to complicate the symptoms.
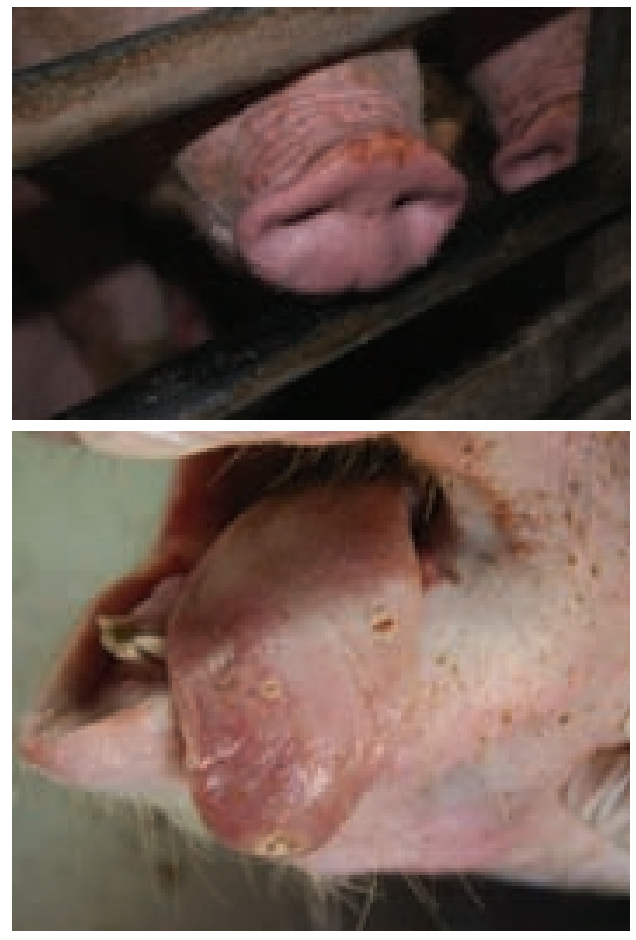

\section{References}

1. Montgomery JF, Oliver RE, Poole WSH. A vesiculo-bullous disease in pigs resembling foot and mouth disease. I. field cases. NZ Vet J 1987; 35.21-26.

2. Amass SF, Schneider JL, Miller CA, Shawky SA, Stevenson GW, Woodruff ME. Idiopathic vesicular disease in a swine herd in Indiana. J Swine Health prod 2004; 12(4):192-196.

3. Osweiler GD. Mycotoxins. In: Straw BE, Zimmerman JJ, D'Allaire SD, Taylor DJ, eds. Diseases of Swine. 9th ed. Ames: Blackwell publ, 2006:915-929.

4. Pasma T, Davidson S, Shaw SL. Idiopathic vesicular disease in swine in Manitoba. CVJ/Vol49/January 2008. 


\title{
0.006
}

\section{Prevalence and risk factors of lameness and claw lesions in two types of group housing for pregnant sows}

\author{
Liesbet M. Pluym ${ }^{1}$ Sebastiaan Van Hoorebeke ${ }^{1}$ Alfonso Lopez Rodriguez ${ }^{1}$ Jeroen Dewulf ${ }^{1}$ \\ Frédéric Vangroenweghe ${ }^{2}$ Frank Tuyttens $^{3}$ Dominiek Maes $^{1}$ \\ 1. Dept. of Reproduction, Obstetrics and Herd Health, Faculty of Veterinary Medicine, Ghent University, Merelbeke, Belgium; \\ 2. Dierengezondheidszorg Vlaanderen vzw, Torhout, Belgium; \\ 3. Animal Sciences Unit, Institute of Agricultural and Fisheries Research, Melle, Belgium
}

\section{Introduction}

Claw lesions and lameness in sows are an important welfare concern as well as a cause of considerable economic loss for pig producers. These problems are more common in group housing than in individual housing systems1. Given that group housing for pregnant sows will become mandatory in the EU from 2013 onwards, the aim of the present study was firstly to determine the prevalence of lameness and claw lesions, and secondly to analyze type of group housing and sow related risk factors associated with lameness and claw lesions.

\section{Materials and methods}

Eight Belgian pig herds with loose housing of pregnant sows were selected. In four of them, sows were housed in pens with electronic sow feeders, the other four herds used free access stalls (table 1). At the end of gestation, sows were investigated for lameness by visual judgement of their gait (lame or not lame). Within 3 days after farrowing, possible lesions of the claws of their hind limbs were scored using a standardized scoring system 2. Information about feed, housing conditions and culling (strategy) was collected as well as information about parity and breed of each sow. Logistic regression analysis was used to evaluate risk factors (type of group housing, breed, parity and lesion score) for lameness. The dataset of claw lesion scores was analyzed by using linear mixed model analyses. All analyses were performed using SPSS version 16.00. A P value $<0.05$ was considered significant.

Table 1: General characteristics of the 8 investigated herds (B1-B8)

\begin{tabular}{|c|c|c|c|c|c|c|c|c|}
\hline & B1 & B2 & B3 & B4 & B5 & B6 & B7 & B8 \\
\hline Herd size & 200 & 750 & 230 & 200 & 140 & 160 & 700 & 280 \\
\hline $\begin{array}{l}\text { No. of sows as- } \\
\text { sessed }\end{array}$ & 55 & 69 & 85 & 47 & 13 & 53 & 58 & 41 \\
\hline Breed & $\mathrm{T}$ & $T$ & $D$ & $\mathrm{H}$ & RS & $\mathrm{J}$ & $D$ & $\mathrm{~J}$ \\
\hline $\begin{array}{l}\text { Mean parity as- } \\
\text { sessed sows }\end{array}$ & 1.8 & 2.8 & 4.5 & 4.0 & 2.8 & 3.6 & 3.2 & 3.2 \\
\hline $\begin{array}{l}\text { \# week batch pro- } \\
\text { duction system }\end{array}$ & 3 & 2 & 4 & 3 & 1 & 5 & 4 & 3 \\
\hline $\begin{array}{l}\text { Type of group } \\
\text { housing }\end{array}$ & FA & FA & $F A$ & FA & ESF & ESF & ESF & ESF \\
\hline $\begin{array}{l}\text { Culling \% due to } \\
\text { locom. disorders }\end{array}$ & 6 & 0.6 & 3 & 4 & 9 & 15 & 12 & 7 \\
\hline Use of bedding & - & - & - & - & - & - & - & + \\
\hline
\end{tabular}

\section{Results}

Of the total of 421 sows that were investigated, $9.7 \%$ was lame (min. 2.4\% max. 23.1\%). No significant difference for lameness was found between the two types of group housing (mean prevalence: $7.4 \%$ in free access stalls, $13 \%$ in pens with electronic sow feeders). The prevalence of lameness significantly increased with increasing parity of sows. Claw lesions were seen most frequently on the wall and heel area of the claw - almost every sow had heel lesions - and there were significantly more lesions found on lateral claws than on medial claws. Only lesions on the heel area differed significantly between both types of group housing with higher scores in free access stalls (mean total score 8.3 versus 7.7). The prevalence of side wall lesions and overgrown (dew-)claws was increased with increasing parity. Breed was not significantly associated with lameness or claw lesions.

\section{Conclusions}

Lameness and claw lesions in sows housed in group during gestation are commonly found in Belgian pig herds, and their prevalence was higher in older sows. This indicates that a balanced parity distribution of sows is not only important for productivity reasons but also from a health and welfare point of view. Further research will focus on ways to control or prevent claw lesions in group housed sows.

\section{References}

1. Gjein H., Larssen R.B. (1995). Housing of pregnant sows in loose and confined systems- a field study. 2. Claw lesions: morphology, prevalence, location and relation to age. Acta Veterinaria Scandinavica. 36(4), 433-442.

2. http://www.verantwoordeveehouderij.nl/index.asp?netwerken/ enquete/zeugenklauwencheck/index.asp

\section{Acknowledgements}

We would like to thank "Veepeiler Varken" for sponsoring as well as the farmers for their co-operation in this study. 


\title{
0.007
}

\section{Kyphosis and lordosis: possible link to nutrition}

\author{
Jesus Borobia Belsué \\ MOSSVET, Portadown, UK
}

\section{Introduction}

In September 2008 two 600 sow birth to bacon units reported an increase of "humpy-backs" from 6 - 8 weeks of age and lameness from 6 weeks of age during the previous 5 months. There was no response to antibiotic treatment.

Farm B re-stocked from Farm A. Both units are closed to outside pig introductions other than semen.

Both units are negative to major pathogens other than Porcine Circovirosis and Mulberry Heart Disease.

\section{Materials and Methods}

Clinical investigation consisted of a farm visit in September 2008 and every 3 months thereafter. Three live 10 week old and one 16 week old pig were submitted to the laboratory (AFBI, Omagh, Northern Ireland). A blood sample was taken from the three 10 week old pigs for biochemistry (Ca, Mg, P, CPK, GPX, vitamin E). The vertebrae and ribs of all 4 pigs were analysed for $\mathrm{Ca}, \mathrm{Mg}, \mathrm{P}$ \& Ash.

Ca, P \& Mg were analysed in dry sow, lactating and grower diet in an independent laboratory (Anser Laboratories, Moy, Northern Ireland).

Vitamins A, E \& D3 were examined in all pig diets.

\section{Results}

Post-mortem examination of the pigs revealed lordosis from T9 to T10; T8 to T10; T5 to T12; T7 to T11 and kyphosis from T12 to $T 13 ; T 12$ to $L 1 ; T 13$ to $L 2 ; T 12$ to $L 2$. Midshaft bony exostoses were found half way between the costrochondral junctions and the vertebral bodies in 3 pigs and thickened costrochondral junctions in one animal.

No significant findings detected at histological examination. Immunofluorescence negative to PCV-2. No significant bacteria isolated.

Biochemistry of the blood was within normal range parameters apart of one pig with 3.72 mM P (upper limit: 3.4) and $7956 \mathrm{U} / \mathrm{I}$ CPK (upper limit: 5000).

Biochemistry of the bones detected values ranging from 18.6 to $27.0 \%$ Ash (Normal Values: $>50 \%$ ) and slightly high P values ranging from 19.6 to 20.6\% (Normal Values: $17-19 \%$ ).

Ca, P \& Mg in dry sow, lactating and grower diet were analysed (Ca Dry Sow: 1.35\%, Ca Lactating Sow: 1.12\%, Ca Grower: 1.01\%; P Dry Sow: 0.56\%, P Lactating Sow: 0.51\%, P Grower: 0.50\%; Mg Dry Sow: $0.21 \%$, Mg Lactating Sow: 0.14\%, Mg Grower: 0.14\%).

Vitamin A in Dry Sow diet was $15,000 \mathrm{mg} / \mathrm{kg}$.

Vitamin E in 1st \& 2nd stage diets were $124 \mathrm{mg} / \mathrm{kg} \mathrm{\&} 125 \mathrm{mg} / \mathrm{kg}$, respectively.

\section{Discussion}

Kyphosis in pigs is similar to Scheuermann's Disease in humans (Lowe, 1990). This lesion could be a factor in carcass downgrad- ing and limit the number of replacement gilts. "Humpy-back" is described as an idiopathic lumbar kyphosis with thoracic lordosis which has no hereditable basis and is associated with management and environmental factors (Penny et al., 1984). According to Lahrmann et al., 1993, it is secondary to a number of different primary lesions located in the vertebrae, e.g. osteomyelitis, fractures, neoplasm and metabolic diseases. Other possible causes are painful conditions of the legs and back (Lahrmann, 1990), musculo-mechanical stress on the lumbar spine (Corradi et al., 2004), early onset of puberty in male pigs (Done et al., 1988) and intrauterine viral infections (Done et al., 1999). Furthermore, there are suggestions of genetic involvement (Foster, 1986; Pearson et al., 2004; Laitat et al., 2006).

Infections, genetic, managerial and structural factors were removed as causes in this case. However, metabolic diseases could not be ruled out. This statement agrees with Lahrmann et al. (1993).

The thickening of costrochondral junctions is a failure of bone mineralisation and growth plate cartilage. Ca, P and Vit. D deficiencies are the common cause (Muirhead et al., 1997; Jackson et al., 2007).

$\mathrm{Ca}: \mathrm{P}$ ratio in bone ash analysed was normal. However, bone ash percentage was low. This decreased mineralisation could have been the cause of bony exostoses and Lordosis/Kyphosis. These diets had phytase incorporated in order to reduce addition of inorganic $P$ in the diet and reducing contamination. 500 UF (Phytase Units) $=0.8-1 \mathrm{~g} / \mathrm{kg}$ of $\mathrm{P}(0.08-0.1 \%)$. Heat treatments of the feed, such as pelleting, could destroy phytase in feed (Pérez, 2009). All these diets were pelleted. As a consequence of this, Ca \& P in the diets was re-formulated (Ca Dry Sow: 1.1\%, Ca Lactating Sow: 1.12\%, Ca Grower: 1.01\%; P Dry Sow: $0.6-0.8 \%$, P Lactating Sow: 0.7 -0.9\%, P Grower: 0.6 - 0.8\%).

High levels of vitamin A lead to lameness, decreased bone weight and narrow metaphyseal growth plates in the vertebrae (Taylor, 1995). Furthermore, it interferes with normal growth of epiphyseal plates leading to osteochondrosis (OCD) (Muirhead et al., 1997). OCD of vertebrae, with or without spondylosis, results in kyphosis (Hill, 1990).

European Food Safety Authority (EFSA) recommended the revision of maximum vitamin A level in feed for main food producing animals as a consequence of link to bone health problems (osteoporosis and bone fracture). The Panel proposed setting a level for fattening pigs at $1 / 2$ the current amount allowed by EU Regulation (EC) No 1831/2003.

Vitamin A in sow's diet was reduced to $8,500 \mathrm{mg} / \mathrm{kg}$.

Vitamin E in 1 st and 2nd stage was increased to $250 \& 200 \mathrm{mg} /$ $\mathrm{kg}$, respectively.

Genetics were not changed.

Control program was successful in this case. No further cases were seen since May 2009.. 


\title{
0.008
}

\section{Porcine Postweaning Wasting/Catabolic Syndrome (PWCS): pathological features}

\author{
John Harding ${ }^{1}$ Yanyun Huang ${ }^{1}$ Henry Gauvreau ${ }^{2}$ \\ 1. University of Saskatchewan, Saskatoon, SK, Canada; 2. Warman Veterinary Services, Warman, SK, Canada
}

\section{Introduction}

Porcine Postweaning Wasting/Catabolic Syndrome (PWCS) is an emerging condition causing elevated nursery mortality ${ }^{1}$. Affected pigs, weaned in robust body condition, develop anorexia and most become severely emaciated and die within 3 weeks of weaning. Mortality rates wax and wane and reach $10 \%$ in peak months. The cause of PWCS is unknown, but an infectious etiology suspected. Pathogens including PRRSV, porcine circovirus 2 , rotavirus, transmissible gastroenteritis virus, influenza virus, Lawsonia intracellularis, Mycoplasma hyopneumoniae, Helicobacter spp., Campylobacter spp., calicivirus and coccidia were not causally related to PWCS ${ }^{2}$ (and unpublished data). The objective of the current study was to characterize the pathologic changes in PWCS-affected pigs.

\section{Materials and Methods}

Five emaciated pigs (PWCS-E) and 3 age-matched healthy pigs (PWCS-H) from a PWCS-affected farm, and 4 healthy agematched pigs from each of two PWCS unaffected farms (CTR1, CTR2; 8 pigs in total) were selected 2 to 3 weeks postweaning. Necropsy examinations were performed immediately after humane euthanasia. Gross and histologic lesions were assessed and multiple fresh and fixed tissues processed routinely. Morphometrics of villi lengths of duodenum, jejunum and ileum (at least 10 villi from each section) were conducted on all PWCSE and PWCS-H pigs, 2 CTR1 and 4 CTR2 pigs. The average villi length was calculated for each tissue and pig. Potential group differences were examined using a Kruskal-Wallis $\mathrm{H}$ followed by a post-hoc Mann-Whitney $U$ test with $P<.05$ considered significant.

\section{Results}

No gross lesions were observed in any PWCS-H, CTR1 or CTR2 pig. Of PWCS-E pigs, 4/5 had severe thymic atrophy and $3 / 5$ had mild to moderate bronchopneumonia. Histologically, the thymic atrophy was mainly due to the reduced thickness of the cortex. Small intestinal villous atrophy was readily observed histologically in PWCS-E pigs. Mean villi lengths in duodenum, jejunum and ileum were significantly shorter in PWCS-E than CTR pigs (control considered normal) (Table 1). Mean villi lengths in jejunum, but not the duodenum or ileum ( $P=0.05$ for ileum), were significantly shorter in PWCS-E than PWCS-H. Colitis, characterized by multifocal to diffuse necrosis of the epithelium and/ or increase mononuclear infiltration in the lamina propria, was found in all PWCS-E and PWCS-H pigs, but not in any CTR pig. Subjective assessment concluded the colitis was more severe in PWCS-E than PWCS-H. No microscopic changes were found in any CTR pig.
Table 1. Mean intestinal villous lengths $(\mu m \pm S D)$ of emaciated (PWCS-E) and age-matched healthy (PWCS-H) pigs from a PWCS-affected farm, and from age-matched pigs (CTR) from 2 unaffected farms

\begin{tabular}{|l|c|c|c|c|}
\hline Groups & $\mathbf{n}$ & Duodenum & Jejunum & Ileum \\
\hline PWCS-E & 5 & $188.1(38.5)^{\mathrm{a}}$ & $166.4(31.3)^{\mathrm{a}}$ & $182.8(69.8)^{\mathrm{a}}$ \\
\hline PWCS-H & 3 & $251.1(81.1)^{\mathrm{a}}$ & $287.4(26.2)^{\mathrm{b}}$ & $299.6(40.3)^{\mathrm{a}}$ \\
\hline CTR (1 \& 2) & 6 & $516.0(64.6)^{\mathrm{b}}$ & $409.7(37.8)^{\mathrm{c}}$ & $401.2(50.8)^{\mathrm{b}}$ \\
\hline \multicolumn{5}{|l}{ Values within columns with different superscripted letters are statistically different at P $<0.05}$. \\
\hline
\end{tabular}

\section{Discussion}

The results of this study indicate the principle lesions associated with PWCS are thymic and intestinal villous atrophy, and colitis. Villous atrophy was highly prevalent but less severe in the PWCS-H compared to the PWCS-E pigs suggesting the former were either subclinically affected, in the early stages of the disease before notable loss of condition, or villous atrophy is a predisposing risk factor for PWCS. The presence of thymic atrophy in PWCS-E pigs may be the direct consequence of the condition, or an indirect result of prolonged anorexia. The latter is supported by the absence of thymic atrophy in PWCS-H pigs and in one PWCS-E pig that was only moderately affected. Colitis may be a direct result of the disease or indirectly associated with small intestinal pathology including villous atrophy. Bronchopneumonia was not observed previously in PWCS ${ }^{1}$. Its importance cannot be ruled out but it is less likely because clinical respiratory signs (coughing, dyspnea) were not observed. Further efforts to identify the cause of PWCS are underway.

\section{References}

1) Gauvreau H, Harding J. Proc Western Can Assoc Swine Vet Conf. Saskatoon, SK. October 2-3, 2008, p. 47-54.

2) Huang $Y$, Gauvreau H, Harding J. Proc 21st Int Pig Vet Soc Confr. Submitted. 


\title{
0.009
}

\section{Porcine Postweaning Wasting/Catabolic Syndrome (PWCS): the initial diagnostic investigation}

\author{
John Harding ${ }^{1}$ Yanyun Huang ${ }^{1}$ Henry Gauvreau² Brendan O'Connor ${ }^{3}$ \\ 1. University of Saskatchewan, Saskatoon, SK, Canada; 2. Warman Veterinary Services, Warman, SK, Canada; \\ 3. Prairie Diagnostic Services, Saskatoon, SK, Canada
}

\section{Introduction}

Since 2008, veterinarians have reported persistent and sometimes extensive nursery mortality associated with anorexia and weight loss in PCV2-vaccinated pigs ${ }^{1}$. We have performed a diagnostic investigation in an affected farm and have temporarily named the condition Postweaning Wasting/Catabolic Syndrome (PWCS). Evidence suggests PWCS is likely infectious. The etiology is unknown, but common porcine pathogens have been ruled out. The objective of this report is to summarize the diagnostic investigation in the PWCS-affected farm.

\section{Clinical Presentation and Interventions}

A well-managed, high health (PRRSV, Mycoplasma hyopneumoniae negative, PCV2 positive) 100-sow farrow-finish farm experienced elevated nursery mortality since 2007 (Table 1) almost exclusively due to PWCS. Affected pigs in good body condition at weaning develop anorexia, lethargy and emaciation shortly thereafter. Most PWCS-affected pigs die within 3 weeks. Survivors grow slower than their pen-mates. Management interventions targeting ventilation, piglet comfort, diet and water quality have not reduced PWCS-losses. PCV2 piglet vaccination and all antimicrobial strategies attempted have been unsuccessful. Losses have been partially mitigated following aggressive sanitation of farrowing and nursery rooms using either strong sodium hypochlorite solution or hydrated lime.

Table 1. Nursery mortality in the PWCS-affected farm.

\begin{tabular}{|c|c|c|c|}
\hline Year & Mortality (\%) & Year & Mortality (\%) \\
\hline 2005 & 1.7 & 2007 & 6.6 \\
\hline 2005 & 1.3 & 2008 & 6.3 \\
\hline 2006 & 2.6 & 2009 & 8.3 \\
\hline
\end{tabular}

\section{Diagnostic Investigation}

In response to the elevated mortality, live pigs or tissues from 4 pigs in poor body condition were submitted for routine diagnostics. Lesions were non-specific, the etiology was undetermined, and adjunct testing for PRRS (IHC), PCV2 (IHC), Leptospira spp. (silver staining) was negative. There was no evidence of coccidiosis. A specialized disease investigation team was recruited and more extensive diagnostics on 7 cases were undertaken. Histological examination revealed severe thymic atrophy (5/7 pigs), mild lymphocytic gastritis (7/7), severe small villous intestinal atrophy (7/7), moderate to severe colitis (6/7), mild interstitial thickening of lung (7/7), moderate to severe lymphoplasmacytic rhinitis (4/5) and mild lymphoid depletion (4/7). Bacteria culture of various organs did not yield significant findings in $7 / 7$ pigs. Complete blood counts in $3 / 3$ pigs were unremarkable. Serum chemistry in $3 / 3$ pigs was consistent with cholestasis, gastric stasis, anorexia and dehydration. Adjunct testing of appropriate tissues from selected pigs failed to conclusively identify common swine pathogens (Table 2 ). Feces subsequently submitted from 5 healthy suckling (1-18 day old), 5 thin and diarrheic nursery (25-35 day old), and 2 grow-finisher pigs was examined by electron microscopy (EM) to detect possible gastrointestinal pathogens. Six of 12 samples were negative. Calicivirus was observed in 4/12 (1 suckling, 3 nursery, 1 grower) samples. Rotavirus, coronavirus, and either parvovirus or enterovirus were observed in 1 nursery sample each.

Table 2. Microbiologic results of PWCS-affected pigs

\begin{tabular}{|l|c|c|c|}
\hline Pathogens & Assay & Tissue & \# pos /\# tested \\
\hline PRRSV & $P C R$ & lung & $0 / 7$ \\
\hline PCV2 & IHC & tonsil & $4 / 7$ \\
\hline Influenza & $P C R$ & lung & $0 / 7$ \\
\hline TGEV & IHC & intestine & $0 / 1$ \\
\hline Rotavirus & IHC & intestine & $0 / 3$ \\
\hline Helicobacter spp & $P C R$ & stomach & $0 / 3$ \\
\hline Campylobacter spp & $P C R$ & stomach & $0 / 3$ \\
\hline Enteric calicivirus & $P C R$ & intestine & $3 / 7$ \\
\hline
\end{tabular}

\section{Discussion and Conclusions}

Major porcine pathogens have been ruled out as the cause of PWCS. Scant PCV2-positive staining with/without mild lymphoid depletion is not uncommon in commercial pigs. Failure of PCV2 vaccination to control PWCS in this farm makes PCVD unlikely. Calicivirus is highly prevalent but not reported to cause disease in commercial pigs ${ }^{2}$. Moreover, porcine enteric Calicivirus DNA2 was demonstrated in $2 / 3$ healthy and $2 / 5$ sick pigs subsequently submitted from the farm. Further efforts to identify the cause of PWCS are underway.

\section{Reference}

1. Dufresne, L et al (2008) Proc AD Leman Swine Conf: 3579-85.

2. Wang, $\mathrm{QH}$ et al (2007) Vaccine. 25(30),5453-5466 


\title{
0.011
}

\section{Emergence of a new type of porcine circovirus (PCV1/2a) in swine in Canada}

\author{
Carl A. Gagnon; Nedzad Music; Guy Fontaine; Donald Tremblay; Josée Harel \\ Université de Montréal, Faculté de médecine vétérinaire, St-Hyacinthe, QC, Canada
}

\section{Introduction}

Between September 2008 and January 2009, three cases (FMV08-1114252, FMV08-1133505 and FMV09-1134568) were submitted to the Veterinary diagnostic service of the University of Montreal. None of the three submitted cases were related to "Porcine circovirus associated diseases" (PCVAD). Several diagnostic assays were performed including a multiplex real-time quantitative PCR assay (mrtqPCR) for the detection and differentiation of porcine circovirus (PCV) type $2 \mathrm{a}$ and $2 \mathrm{~b}$ genotypes in the lung and lymph nodes(1). Odds results were obtained with the mrtqPCR assay, suggesting genomic mutations in the viral genome.

\section{Materials and methods}

To confirm the genomic nature of the three PCV positive cases, specific PCR assays were performed to detect the ORF1 of PCV1 and the ORF2 of PCV2 genes. A third PCR assay was selected to amplify both ORF1 of PCV1 and ORF2 of PCV2 genes simultaneously. The entire PCV genome was amplified by PCR that produced 2 overlapping PCR products. Both strands of the purified DNA PCR products were sequenced(2). Virus isolation was conducted using the PK15A cell line as previously described(2) and an immunofluorescence assay (IFA) was performed to confirm the presence of PCV using an anti-PCV2 pig serum as described previously(3).

\section{Results}

All the obtained results converge to demonstrate the emergence of a new PCV. In addition, infectious viruses were isolated from 2 out of the 3 submitted cases and expression of the viral Cap protein encoded by the ORF2 gene was confirmed by IFA in PCV infected cells. The results indicate the presence of both PCV1 ORF1 and PCV2 ORF2 genes in the PCR products. To confirm the genomic nature of the new PCV, its viral genome was sequenced. The ORF1 was more genomically related to the ORF1 of PCV1 with a nucleotide (nt) identity of $99.7 \%$ compared to ORF1 of PCV2a and $2 \mathrm{~b}$ with nt identities of $82.5 \%$ and $82.8 \%$, respectively. On the other hand, the ORF2 was more genomically related to the ORF2 of PCV2a with a nt identity of $985 \%$ compared to ORF2 of PCV1 and PCV2b with nt identities of $66.7 \%$ and $92.5 \%$, respectively. The nt identity of the entire viral genome of the new PCV was $86.4 \%, 88.7 \%$ and $86.5 \%$ compared to PCV1, PCV2a and PCV2b, respectively.

\section{Discussion}

It is proposed to name this new PCV by taking into account previously described nomenclature(4) and by indicating the origin of the ORF1 at first and the origin of the ORF2 in second. Consequently, the name proposed for this new PCV is PCV1/2a. The prevalence of PCV1/2a seems to be very low in Quebec, Canada ( $2.5 \%$ of PCV positive cases), and its origin is now in debate. Two hypotheses could explain the emergence of the PCV1/2a in swine: (a) the natural genetic recombination between PCV1 and PCV2a in infected animals; or (b) a human made virus originating from a chimeric killed vaccine strain. Still, even if PCV1 and PCV2a are found in low amount in Quebec swine, the genetic recombination between PCV1 and PCV2a is a possible explanation. In early 2008 , a new vaccine was commercially available in Canada (Suvaxyn ${ }^{\circledR}$ PCV2). This vaccine uses a chimeric killed or inactivated PCV $(5,6)$. This chimeric PCV genome is composed of the ORF1 of PCV1 and the ORF2 of PCV2a. The nt sequences of the PCV1/2a strains were found to possess 5 to $6 \mathrm{nt}$ differences compared to the chimeric PCV vaccine strain with a nt identity of $99.6-99.7 \%$ and all nt differences were located in ORF1.

\section{References}

1. Gagnon et al., 2008 (J. Vet. Diagn. Invest. 20, 545-558). 2. Gagnon et al., 2007 (Can. Vet. J. 48, 811-819).

3. Racine et al., 2004 (Clin. Diagn. Lab. Immunol. 11, 736-741).

4. Segales et al., 2008 (Vet. Rec. 162, 867-868).

5. Gillespie et al., 2008 (Vaccine 26, 4231-4236).

6. Meng et al., 2007 (United States Patent number: 7,279,166).

\section{Acknowledgments}

This work was supported by NSERC, the Veterinary diagnostic service of the Faculté de médecine vétérinaire of the Université de Montréal, the FPPQ, the CIPQ, and the CDAQ. 


\title{
0.012
}

\section{Mutations of the PCV-2 capsid motif, specific of each PCV-2 genogroup, attenuate virulence in vivo}

\author{
Beatrice Grasland; Aude Allemandou; Anne-Cécile Nignol; Nicolas Rose;
} François Madec; André Kéranflec'h; Roland Cariolet; André Jestin

AFSSA, Ploufragan, France

\section{Introduction}

The porcine circovirus of type 2 (PCV-2) is a non-enveloped, small single-stranded DNA virus. It is the causal agent of postweaning multisystemic wasting syndrome (PMWS) and is involved in "porcine circovirus diseases". After the re-emergence of PMWS in North America in 2005, the presence of new circulating PCV-2 isolates belonging to PCV-2b genogroup raises the assumption that this genogroup is more virulent than the PCV-2a one. Viruses of each genogroup contain a distinct motif of amino acid in the capsid protein (amino acids 86 to 91), the unique component of the virus outer structure1. In this study, we investigate if PCV-2 genetic variation is responsible of virulence and check if the capsid motif contributes to this difference.

\section{Materials and Methods}

Two infectious clones representative of both PCV-2 genogroups were constructed (PCV-2a and PCV-2b clones). Two mutants were generated by directed mutagenesis. The PCV-2a (PCV-2b motif) mutant has the capsid motif of PCV2b genogroup into the backbone of PCV-2a and the PCV-2b (PCV-2a motif) mutant, the capsid motif of PCV-2a genogroup in the backbone of PCV$2 \mathrm{~b}$. The infectious clones and the mutants were characterized in vitro. Then their virulence was assessed by an experimental trial on PCV-2 free SPF (Specific pathogen free) piglets. Forty 6-weeks-old SPF piglets were divided into six groups as shown on Table 1. The experiment ended between 35 and 40 dpi.

Table1: Experimental protocol

\begin{tabular}{|l|c|c|c|c|c|c|}
\hline \multicolumn{1}{|c|}{$\begin{array}{c}\text { Group } \\
\text { number }\end{array}$} & $\mathbf{1}$ & $\mathbf{2}$ & $\mathbf{3}$ & $\mathbf{4}$ & $\mathbf{5}$ & $\mathbf{6}$ \\
\hline Inoculum & PBS & PBS & PCV-2a DNA & $\begin{array}{c}\text { PCV-2b } \\
\text { DNA }\end{array}$ & $\begin{array}{c}\text { PCV-2a (PCV- } \\
\text { 2b motif) } \\
\text { DNA }\end{array}$ & $\begin{array}{c}\text { PCV-2b (PCV- } \\
\text { 2a motif) } \\
\text { DNA }\end{array}$ \\
\hline Day 0: & & & $\begin{array}{c}400 \mu g \text { DNA/ } \\
\text { pig }\end{array}$ & $\begin{array}{c}400 \mu g \\
\text { DNA/pig }\end{array}$ & $\begin{array}{c}400 \mu g \text { DNA/ } \\
\text { pig }\end{array}$ & $\begin{array}{c}400 \mu g \text { DNA/ } \\
\text { pig }\end{array}$ \\
\hline Days 2, 7: & KLH/ICFA & $\begin{array}{c}\text { KLH/ICFA } \\
\text { by IM }\end{array}$ & $\begin{array}{c}\text { KLH/ICFA } \\
\text { by IM }\end{array}$ & $\begin{array}{c}\text { KLH/ICFA } \\
\text { by IM }\end{array}$ & $\begin{array}{c}\text { KLH/ICFA } \\
\text { by IM }\end{array}$ \\
\hline $\begin{array}{l}\text { Number of } \\
\text { pigs }\end{array}$ & 4 & 4 & 8 & 8 & 8 & 8 \\
\hline
\end{tabular}

Tissue samples were collected at necropsy. The PCV-2 seroconversion was monitored using ELISA test. Quantification of viral genomes and infectious particles were respectively assessed by quantitative PCR and by IPMA2.

\section{Results}

Both parental clones and both mutants were infectious in vitro. The results of the experimental trial are summarized in Table 2.

All the controls were negative for the presence of PCV-2.

Table 2: Results of the in vivo characterization of both parental strains and both capsid motif mutants.

\begin{tabular}{|l|c|c|c|c|}
\hline & $\begin{array}{c}\text { Parental } \\
\text { clone }\end{array}$ & $\begin{array}{c}\text { Parental } \\
\text { clone }\end{array}$ & $\begin{array}{c}\text { Mutant } \\
\text { clone }\end{array}$ & $\begin{array}{c}\text { Mutant } \\
\text { clone }\end{array}$ \\
\hline $\mathrm{PCV}-2 \mathrm{a}$ & $\mathrm{PCV}-2 \mathrm{~b}$ & $\begin{array}{c}\mathrm{PCV}-2 \mathrm{a}(\mathrm{PCV}- \\
\text { 2b motif) }\end{array}$ & $\begin{array}{c}\mathrm{PCV}-2 \mathrm{~b}(\mathrm{PCV}- \\
\text { 2a motif) }\end{array}$ \\
\hline $\begin{array}{l}\text { Score mean (hypertrophy of TB } \\
\text { lymph nodes) }\end{array}$ & $1.37 \mathrm{a}$ & $1.5 \mathrm{a}$ & $0.62 \mathrm{~b}$ & $0.87 \mathrm{ab}$ \\
\hline Seroconversion of all pigs & 28 to 35dpi & 21 to $28 \mathrm{dpi}$ & absence & 21 to 28dpi \\
\hline $\begin{array}{l}\text { Genomic load in serum (mean } \\
\text { in log10/ml) }\end{array}$ & $4.8 \mathrm{a}$ & $6.8 \mathrm{~b}$ & $0 \mathrm{c}$ & $4.1 \mathrm{a}$ \\
\hline
\end{tabular}

$a, b$, c indicates significant differences $(p<0.06)$

Moreover, infectious particles were detected in tissue of the four groups inoculated with the clones and mutants.

\section{Discussion and Conclusion}

In vitro, both PCV-2 parental clones and both capsid motif mutants were infectious. In vivo: (i) The PCV-2b strain induced an earlier seroconversion and a higher genomic load in serum than the PCV-2a strain. The PCV-2b strain was more virulent than the PCV-2a one. (ii) The PCV-2b (PCV-a motif) mutant showed similar features to the PCV-2a parental strain with a moderate virulence attenuation. (iii) The PCV-2a (PCV-2b motif) mutant was highly attenuated.

In conclusion, these results showed that the PCV-2 capsid motif specific of genogroup is a molecular marker of virulence of PCV2.

\section{References and acknowledgements \\ 1 Cheung et al, 2007, Arch Virol 152:1035-1044 \\ 2 Grasland et al, 2005, Vet Res $36: 685-697$}

This work was supported by the U.S. National Pork Board (project NPB 08-268). We thank Fort Dodge for the gift of the PCV-2a strain. 


\title{
0.013
}

\section{Cell-dependent susceptibility to Porcine circovirus ORF3-induced apoptosis}

\author{
Mark Chaiyakul ${ }^{1}$ Karolynn Hsu ${ }^{1}$ Avril Hatherell ${ }^{1}$ Rkia Dardari ${ }^{1}$ Frank Marshall ${ }^{1}{ }^{2}$ Markus Czub ${ }^{1}$ \\ 1. University of Calgary, Calgary, AB, Canada; 2. Swine Health Services, Calgary, AB, Canada
}

\section{Introduction}

Porcine circovirus associated disease (PCVAD) has caused considerable economic impact worldwide since its first description on a Canadian pig farm in the 1990s. PCVAD, a wasting and immunosuppressive disease in post-weaned pigs, is caused by Porcine circovirus type 2 (PCV2). Despite the availability of effective vaccines, little is known about the molecular pathogenesis of the disease. Belonging to the Circoviridae family, PCV2 and PCV1 are closely-related viruses with a $1.7 \mathrm{~kb}$, ambisensed and circular ssDNA genome ${ }^{1}$. While PCV2 is pathogenic, PCV1 is not. Investigating the differences in the genomic composition of these viruses will provide clues to the molecular pathogenesis of PCVAD. ORF3 is a good candidate as a determinant of PCV pathogenicity because PCV1 and PCV2 ORF3 share only $60.6 \%$ amino acid (aa) sequence identity in the translated region. In addition, a consistent single nucleotide (nt) substitution in the PCV2ORF3 coding region resulted in a stop codon, leading to PCV2ORF3 that is half the length of PCV1ORF3 (104aa versus 206aa). PCV2ORF3 was shown to induce apoptosis and a mutant virus devoid of ORF3 expression did not induce apoptosis in Cos7 and PK-15 cell lines ${ }^{2}$. Abrogation of ORF3 expression appeared to reduce PCV2 virulence in a specific-pathogen-free (SPF) piglet model $^{3}$. Due to the differences between PCV1 and PCV2 ORF3 and what is known about PCV2ORF3, it is hypothesized that ORF3 determines the pathogenicity of PCV. Thus, the objective of this study is to compare and contrast the pro-apoptotic capability of PCV1 and PCV2 ORF3.

\section{Materials and Methods}

PCV1 and PCV2 ORF3 genes were cloned into mammalian expression peGFP-C1 vector to create fusion proteins with eGFP at the N'terminus. Flow cytometry was used to assess cell viability and apoptosis. Immunoblot was used to detect protein expression and PARP cleavage associated with apoptosis. Z-Asp-CH2DCB was used as a caspase inhibitor. Three cell lines were used in this study: human epithelial 293T, porcine kidney epithelial PK-15 and porcine primary kidney (PPK) cells. Statistical analysis was performed, using ANOVA and Tukey's multiple comparison tests with $p<0.05$ considered as statistically significant.

\section{Results and Discussion}

In all three cell lines, eGFPPCV1ORF3 significantly induced higher cell death. Meanwhile, eGFPPCV2ORF3 significantly induced higher cell death only in PK-15 cells. It was also consistently observed that eGFPPCV1ORF3 was a more potent inducer of cell death than eGFPPCV2ORF3.

During apoptosis, a programmed cell death process, phosphatidylserine molecules become externalized to the outer surface of the cell membrane. This externalization is important for macrophage recognition, phagocytosis and clearance of apoptotic cells without causing an inflammatory response ${ }^{4}$. In addition, caspases become activated and cleave PARP molecules, DNA repair enzymes, leading to apoptosis ${ }^{5}$. In this study, significantly higher percentage of phosphatidylserine externalization as well as the presence of a cleaved PARP fragment was observed in cells transfected with eGFPPCV1ORF3. Moreover, addition of increasing concentration of a caspase inhibitor reduced the level of cell death induced by eGFPPCV1ORF3. Hence, cell death induced by PCV1ORF3 occurs via a caspasedependent apoptotic pathway, similar to what was reported for PCV2ORF3 2 .

While PCV1 does not cause disease, PCV2 causes PCVAD. Their distinctly different ORF3 protein was hypothesized to be the factor for their different pathogenicity. Although both PCV1 and PCV2 ORF3 can induce cell death via apoptosis, it is not completely surprising because they share $60.6 \%$ aa sequence identity. However, PCV1ORF3 appears more potent than PCV2ORF3 in inducing cell death despite the lack of cytopathic effects in cells infected with PCV1 ${ }^{6}$. It is speculated then that PCV1ORF3 expression is absent or is tightly regulated by other viral protein(s), incapacitating PCV1 from causing PCVAD in pigs. Different cell death profiles seen with different cell lines and even within the same cell population suggest a cellular-dependent response to ORF3-induced apoptosis.

\section{References}

(1) Opriessnig et al. 2007. J Vet Diagn Invest 19:591-615.

(2) Liu et al. 2005. J Virol 79: 8262-74.

(3) Karuppanan et al. 2009. Virol 383:338-47.

(4) Duvall et al. 1985. Immunol 56:351-8.

(5) Tewari et al. 1995. Cell 81:801-9. (6) Tischer et al. 1982. Nature 295:64-6.. 


\title{
0.014
}

\section{Clinical and virological outcome of experimental PCV2a and PCV2b infections in mid-gestational porcine foetuses}

\author{
Dipongkor Saha $^{1}$ D.J. Lefebvre', 2 J. Van Doorsselaere ${ }^{3}$ K. Atanasova ${ }^{1}$ F. Barbé ${ }^{1,4}$ M. Geldhof ${ }^{1}$ U.U. Karniychuk ${ }^{1}$ H.J. Nauwynck ${ }^{1}$ \\ 1. Laboratory of Virology, Faculty of Veterinary Medicine, Ghent University, Merelbeke, Belgium; 2. CODA-CERVA-VAR, Veterinary and Agrochemical \\ Research Centre, Virology Department, Groeselenberg, Brussels, Belgium; 3. Department of Health Care and Biotechnology, KATHO Catholic \\ University College of South-West Flanders, Roeselare, Belgium; 4. Animal Health Care Flanders, Torhout, Belgium
}

\section{Introduction}

PCV2 may cause reproductive failure in the field (1), and therefore the effects of experimental PCV2 infections, mainly PCV2a, on reproduction have been studied extensively (2). Recent field observations on the occurrence of PMWS suggest that PCV2b may be more pathogenic than PCV2a (3). The aims of the present study were to investigate if there are differences in viral replication and clinical outcome between PCV2a and PCV2b infections in porcine foetuses.

\section{Materials and Methods}

Four conventional sows were submitted to laparotomy at 55 days of gestation. In each of four sows, three foetuses were inoculated with PCV2 and one foetus was mock inoculated. Inoculations were performed by transuterine injection with $10^{4.3} \mathrm{TCID}_{50}$ of PCV2 into the peritoneal and amniotic cavities of the foetuses (4). In the first sow, two foetuses were inoculated with PCV2a strain Stoon-1010 and one with PCV2a strain 1121. In the second sow, two foetuses were inoculated with PCV2b strain 48285 and one with PCV2b strain 1147. In the third sow, one foetus was inoculated with PCV2a strain Stoon-1010, one with PCV2a strain 1121 and one with PCV2b strain 1147. In the fourth sow, one foetus was inoculated with PCV2a strain 1121, one with PCV2b strain 48285 and one with PCV2b strain 1147. Twenty-one days post inoculation (dpi), the sows were euthanized and all foetuses were collected. All foetuses were examined for gross lesions and tissue samples from various organs were taken for titration on PK-15 cells and for localization of viral antigens. In order to verify that the foetuses had been inoculated with the specific PCV2 strain, the capsid gene was amplified using heart and/or lung tissue from infected foetuses followed by sequencing. Abdominal fluids were tested for the presence of PCV2-specific antibodies by IPMA.

\section{Results}

All PCV2-inoculated foetuses, except one PCV2 (1147)-inoculated foetus, were oedematous and had distended abdomens (Fig.1.a). Haemorrhages and congestion in internal organs and liver enlargement were observed (Fig.1.b). One PCV2 (1147)-inoculated foetus had a normal external appearance, but liver enlargement and generalized lymph node enlargement were observed. Gross pathological lesions were not observed in mock-inoculated and non-inoculated foetuses. High PCV2 titres (> 10.3 $\mathrm{TCID}_{50} / \mathrm{g}$ tissue) were found in all PCV2-inoculated foetuses, especially in the heart, spleen and liver. High numbers of PCV2-infected cells (>1,000 infected cells / $10 \mathrm{~mm}^{2}$ tissue) were observed in the heart (Fig.1.C). PCR and DNA sequencing of the capsid gene recovered pure PCV2a and pure PCV2b sequences from PCV2a- and PCV2b-inoculated foetuses, respectively. No evidence for mixed samples (e.g. containing more than one PCV2 strain) was seen. All adjacent foetuses of PCV2-inoculated foetuses were negative in PCR assays with the exception of one adjacent foetus of Stoon-1010- inoculated foetus, which was positive in PCR assays and a pure Stoon-1010 sequence was obtained. All mock-inoculated foetuses and their adjacent foetuses were negative in PCR assays. Three PCV2-inoculated foetuses had a low anti-PCV2 Ab titre of 10 to 40.

\section{Conclusions}

From this model, it can be concluded that different PCV2 strains of both genotypes induced similar gross pathological lesions and replicated to similar high titres in different foetal organs.

\section{References}

1. West et al. (1999). J Vet Diagn Invest. 11, 530-532

2. Pensaert et al. (2004). Vet Microbiol. 98, 175-183

3. Grau-Roma et al. (2008). Vet. Microbiol. 128, 23-35

4. Sanchez et al. (2001). Vet. Microbiol. 83, 169-176

Fig. 1. Different aspects of PCV2 replication after inoculation of a 55day old foetus. a) Subcutaneous oedema and abdominal distension. b) Haemorrhages and congestieon in internal organs and liver enlargement. c) Immunofluorescent staining for the PCV2 capsid protein in the heart. Bar $=50 \mu \mathrm{m}$.

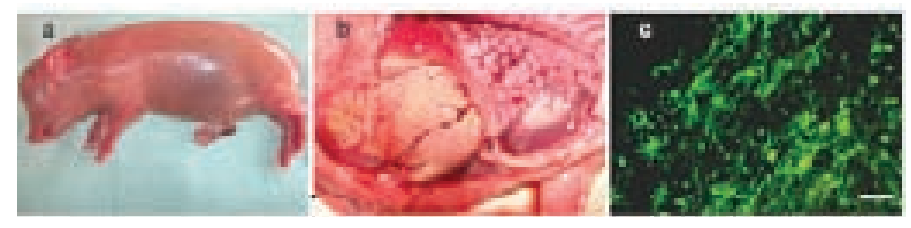




\title{
0.015
}

\section{Singular PCV2a or PCV2b infection results in apoptosis of hepatocytes in clinically affected gnotobiotic pigs}

\author{
Avanti Sinha ${ }^{1}$ Kelly Lager ${ }^{2}$ Chong Wang ${ }^{1}$ Tanja Opriessnig $^{1}$ \\ 1. lowa State University, Ames, IA, USA; 2. National Animal Disease Center, USDA-ARS, Ames, IA, USA
}

\section{Introduction}

Systemic infection with porcine circovirus type 2 (PCV2) is often clinically associated with respiratory signs, failure to thrive and diarrhea (1) Currently, PCV2 can be further subdivided into two main genotypes, PCV2a and PCV2b which under experimental conditions result in very similar macroscopic and microscopic lesions in conventional pigs (2). Viruses are often associated with apoptosis of host cells but there are conflicting results of the role of PCV2 in this pathway $(3,4)$. The objective of the present study was to assess the association of PCV2 antigen with apoptosis in liver tissues from gnotobiotic pigs experimentally inoculated with PCV2.

\section{Materials and Methods}

Forty-eight gnotobiotic pigs were separated into five groups based on inoculation status and development of clinical disease: (1) Non-inoculated, healthy $(n=4),(2)$ PCV2a, healthy $(n=10)$, (3) PCV2a, clinically affected $(n=6),(4)$ PCV2b, healthy, $(n=13)$ and (5) PCV2b, clinically affected $(n=15)$. Formalin-fixed and paraffin-embedded sections of liver from all pigs were subjected to a CCaspase-3 (CCasp-3) immunohistochemistry (IHC) assay, a TUNEL assay, and a PCV2 IHC (5). For each slide, 10 random 40x fields were scored for presence of characteristic staining. The scoring scheme used ranged from 0 (no signal) to 3 (high degree of staining in the majority of the cells and fields examined). All sections of liver tissue were also scored for the presence of the following microscopic lesions: Presence of neutrophils and presence and degree of necrosis, degeneration and inflammation. The sum scores were used for analysis. The following nine comparisons were made using the Fisher's Exact Test for dependency between the categorical variables in paired situations: (1) TUNEL score versus disease status, (2) CCaspase-3 score versus disease status, (3) PCV2 IHC score versus disease status, (4) PCV2 IHC versus TUNEL score, (5) PCV2 IHC versus CCaspase-3 score, (6) Lesion sum versus CCaspase- 3 score, (7) Lesion sum versus TUNEL score, (8) Lesion sum versus PCV IHC and (9) Lesion sum versus disease status. A statistically significant difference was defined as a P-value of less than 0.05 .

\section{Results}

Sixteen of 21 (76 2\%) clinically affected PCV2-inoculated pigs and $11 / 27$ (40.7\%) clinically unaffected PCV2 inoculated pigs had moderate-to-severe hepatic lesions characterized by severe diffuse lymphohistiocytic hepatitis associated with degeneration and loss of hepatocytes. Specific CCasp-3 staining and TUNEL labeling was detected in the nuclei of hepatocytes in PCV2a and PCV2b infected pigs with significantly $(P<0.05)$ higher levels of apoptotic cells in clinically affected pigs. Clinically affected pigs also had significantly $(P<0.05)$ higher levels of PCV2 IHC stain compared to non-affected pigs. The lesion sum score was also significant in the clinically affected pigs $(P<0.05)$.

\section{Discussion}

This experiment was conducted in order to delineate the role of PCV2a and PCV2b genotypes in apoptosis of hepatic cells in gnotobiotic pigs. According to the results obtained, the gnotobiotic pigs when experimentally inoculated with PCV2a or PCV2b developed clinical disease which was associated with severe hepatitis, abundant amount of PCV2 antigen in inflammatory cells and hepatocytes, and apoptosis occurred in hepatocyte-like cells detectable by CCasp3 stains and the TUNEL assay. The pig experiments were of limited duration and it is not known if the 11/27 infected, but healthy pigs that had hepatic lesions and minimal apoptosis, would manifest clinical disease with a significant increase in CCasp4 and TUNEL stained cells. In conclusion, PCV2 infection regardless of genotype resulted in apoptosis of hepatocytes in clinically affected gnotobiotic pigs and apoptosis appears to be one pathway by which PCV2 induces disease.

\section{References}

1. Opriessnig, T. et al. (2007) J. Vet. Diagn. Invest. 19, 591-615.

2. Opriessnig, T. et al. (2008) J. Gen. Virol. 89, 2482-2491.

3. Krakowka, S. et al. (2004) Vet. Pathol. 41, 471-481.

4. Mandrioli, L. et al. (2004) Vet. Immunol. Immuno. pathol. 97, 25-37.

5. Sorden, S. et al. (1999) J. Vet. Diagn. Invest. 11, 528-530. 


\title{
0.016
}

\section{High prevalence of porcine circovirus viremia in newborn piglets in five clinically normal swine breeding herds in North America}

\author{
Huigang Shen; Darin Madson; Tanja Opriessnig \\ lowa State University, Ames, IA, USA
}

\section{Introduction}

Porcine circovirus type 2 (PCV2) has been associated with a number of disease manifestations in pigs including reproductive failure (1). It has been demonstrated that PCV2 can be transmitted horizontally and vertically; however, it is unclear to what extent vertical PCV2 transmission occurs under field conditions especially in farms subclinically infected with PCV2. The objective of the current study was to evaluate the rate of intrauterine PCV2 infection in healthy piglets born on commercial farms in North America and to further characterize dam viremia, dam antibody levels, and piglet antibody levels.

\section{Materials and Methods}

Five commercial breeding herds were selected to participate in this study, with one farm located in Mexico (Farm A) and the remaining four farms located in the U.S. (Farms B-E). A total of 128 clinically healthy sows and 504 live-born normal appearing piglets born to the dams were randomly selected at a rate of three to four piglets per litter. Serum and colostrum samples of the dams were collected immediately after parturition and before suckling. Piglet serum samples were collected after birth and prior to colostrum uptake. All samples were tested for the presence of anti-PCV2 IgG antibodies using a PCV2 ORF2-based ELISA (2) and for the presence and amount of PCV2a and PCV2b DNA by a multiplex differential real-time PCR (3).

\section{Results}

All (128/128) dams and $21.2 \%(107 / 504)$ of the piglets were positive for anti-PCV2 IgG antibody. The overall PCV2 DNA prevalence was $66.4 \%(85 / 128)$ in dams and $43.1 \%(217 / 504)$ in piglets. In general, more dams and piglets were positive for PCV2b (71.8 and 75.1\%, respectively) compared to PCV2a (12.9 and $15.1 \%$, respectively). Concurrent PCV2a/b infection was detected in 13/128 dams and in 21/504 piglets on all five farms. Piglets born to dams with higher PCV2 DNA loads had increased frequency of PCV2 viremia and elevated PCV2 DNA copy numbers in serum. Higher amounts of PCV2 DNA were detected in PCV2 seronegative piglets compared to seropositive piglets indicating that some level of protective humoral immunity developed in utero.

\section{Discussion}

Vertical transmission is an important means of PCV2 transmission in clinically normal herds. In the selected farms, PCV2b was more prevalent than PCV2a in piglets, and virus transmission through breeding herds may result in continuous PCV2b exposure to grow-finish herds. Dam viremia was associated with the frequency and level of PCV2 infection in the piglets. The piglet anti-PCV2 antibody could alleviate PCV2 infection, indicating humoral immune responses occurring in the fetuses may play a protective role. Dam PCV2 antibodies did not necessarily alleviate PCV2 infection in fetuses.

\section{Acknowledgements}

We thank Boehringer Ingelheim Vetmedica Inc. for funding of this study.

\section{References}

1. Opriessnig, T. et al. (2007) J. Vet. Diagn. Invest. 19, 591-615.

2. Nawagitgul, P. et al. (2002) Clin. Diagn. Lab. Immun. 1, 33-40.

3. Opriessnig, T. et al. (2010) Vet. Res. 41:31. 


\title{
0.017
}

\section{Assessment and quantification of Post-weaning Multi-systemic Wasting Syndrome (PMWS) severity at farm level.}

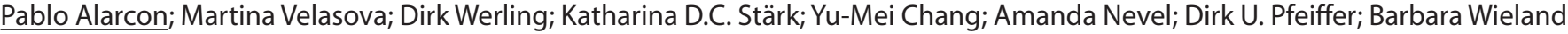

Royal Veterinary College, London, UK

\section{Introduction}

Post-weaning Multi-systemic Wasting Syndrome (PMWS) causes major economic losses in the English pig industry (1). Severity of clinical signs and economic impact vary considerably between affected farms. Appropriate diagnosis and determination of PMWS severity on the farm are crucial for informing decision about control measures. The present study aimed to define and quantify severity levels of PMWS observed on farms using a novel approach based on morbidity, mortality and presence of porcine circovirus type 2 (PCV2).

\section{Materials and Methods}

In 2008-2009, 147 pig farms across England and not vaccinating against PCV2 were enrolled in a cross-sectional study, and retrospective data on PMWS morbidity and production indicators were collected. Classical data collection methods were combined with tools used in participatory epidemiology. In addition, on each farm serum samples from 20 pigs of four different age groups were collected and tested for PCV2 antigen and antibodies against other important pathogens (2). After correcting for misclassification, factor analysis was used to generate a small number of factor variables representing biological meaningful aspects of variation among morbidity variables and these were then together with relevant risk factors variables subjected to principal component analysis to derive an algorithm for PMWS severity.

\section{Results}

Evidence of PCV2 presence was found on all farms enrolled in the study. Factor analysis performed on morbidity data resulted in two factors: Morbidity Factor 1 (MF1) representing mainly weaner and grower morbidity, and Morbidity Factor 2 (MF2) which mainly reflect variation in finisher morbidity. Principal component analysis was performed on the identified morbidity factors, all production variables and laboratory results. This resulted in the extraction of one component representing variation in MF1, post-weaning mortality and percentage of PCV2 PCR positive animals. The extracted component accounted for $60 \%$ of the total variance of these three variables. The distribution of the resulting values for each variable is shown in Figure 1.The scores from the resulting component were normalised within a value range from 0 to 10. Cut-offs values were identified and farms were classified into: non or slightly affected farms (24\%) with a score $<4$, highly affected farms $(23 \%)$ with a score $>6.5$ and, the majority of the farms (53\%), considered to be moderately affected farms with middle scores $(4-6.5)$.

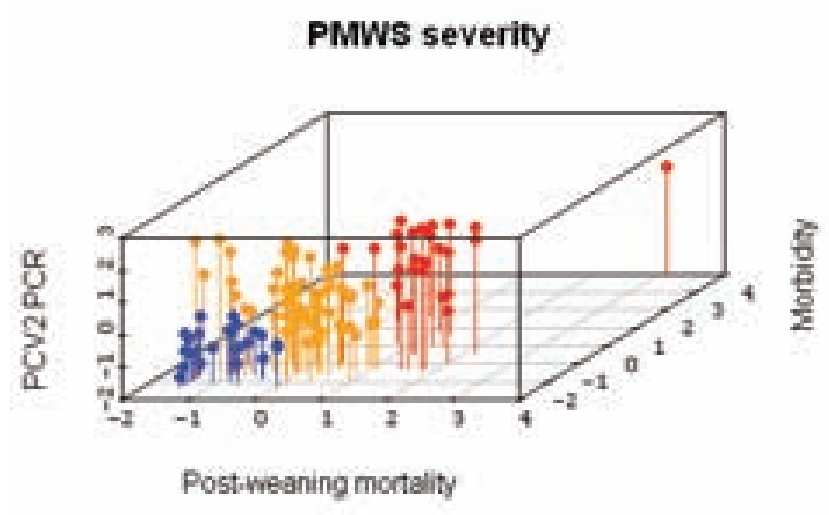

Figure 1: Contribution of morbidity, post-weaning mortality and PCV2 $P C R$ results to the PMWS severity score. In blue 'non or slightly affected' farms, orange 'moderately affected' and in red 'highly affected' farms.

\section{Discussion}

The factor analysis indicated a clustered pattern of PMWS morbidity, with farms either having high morbidity in weaners/ growers or mainly in finishers. The principal component measures PMWS severity on the farms and establishes weaners and growers as the main age groups affected by the disease. The distribution in the three severity categories is believed to reflect the situation of PMWS in the English pig population before the onset of PCV2 vaccination and will be used in further research to investigate risk factors associated with different PMWS severity levels and to measure PCV2 vaccination efficacy at each of these. The outcomes will then inform economic models for the development of cost-effective disease control measures for PMWS.

\section{References}

1. Armstrong et al. (2004) IPVS, Germany

2. Wieland et al. (2008), The Pig journal, in press for vol.23 


\title{
0.019
}

\section{Effects of supplementation of omega-3 fatty acids to gilts on subsequent reproductive performance and growth performance of their litters}

\author{
Miranda N. Smit ${ }^{1}$ Jennifer L. Patterson ${ }^{1}$ Steve K. Webel ${ }^{2}$ Rose A. O'Donoghue ${ }^{1}$ George R. Foxcroft ${ }^{1}$ \\ 1. University of Alberta, Faculty of Agricultural, Life and Environmental Sciences, Edmonton, AB, Canada; 2. JBS United Inc., Sheridan, IN, USA
}

\section{Introduction}

Dietary supplementation of omega-3 polyunsaturated fatty acids ( $\mathrm{n}-3$ PUFAs) during gestation and/or lactation is reported to increase litter size in pigs (Spencer et al., 2004; Webel et al., 2003) and is believed to be beneficial for reproductive performance in sows (Webel et al., 2004). However, results are inconsistent. This study re-assessed the effects of $n-3$ PUFA supplementation to gilts during late gestation and lactation on the subsequent reproductive performance and the growth performance of their litters.

\section{Material and Methods}

At day 60 (d60) of gestation 117 pregnant gilts were pairmatched by weight and litter of origin, and allocated to one of two treatments:

- Control gilts (CON, N=56) fed standard gestation and lactation diets, or

- Treated gilts (FP10, N=61) fed the standard diets supplemented with $84 \mathrm{~g} / \mathrm{d}$ of Sow Fat Pack 10 (JBS United, Sheridan, IN), a fish-oil based supplement rich in omega-3 fatty acids.

Gilts were fed CON or FP10 diets from d60 of first gestation, through a 21-d lactation, and until euthanasia at d30 of their second gestation. All sows were feed restricted during the last week of lactation (fed $60 \%$ of expected feed intake).

Sow body weight, and backfat and loin depth, were measured at $\mathrm{d} 60$ of gestation, $\mathrm{d} 1$ of lactation, 7 days before weaning, at weaning, after last breeding and at $\mathrm{d} 30$ of second gestation. Net energy balance was calculated as reported by Vinsky et al. (2006). Individual piglet weights were measured on d1 of lactation, 7 days before weaning and at weaning in all litters, and at the end of the nursery phase in a subset of litters.

After weaning, sows were heat checked twice daily and bred by Al using semen from the same boars for both treatments. Sows were euthanized on-site at $\mathrm{d} 30$ of gestation, reproductive tracts were recovered, and the number, crown-rump length and weight of embryos, placental fluid volume, ovulation rate $(\mathrm{CL}$ number) and CL weight were recorded, and embryonic survival was calculated.

\section{Results}

Net energy balance during lactation and sow body composition were not different between CON and FP10 sows. The total number of piglets born ( $13.0 \pm 0.4$ vs. $12.9 \pm 0.3)$ and average piglet birth weight per litter ( $1.32 \pm 0.03$ vs. $1.34 \pm 0.03 \mathrm{~kg})$ were also not different between treatments. Repeated measures analysis for average piglet weight per litter showed that litters from FP10 sows compared to litters from CON sows were heavier at weaning (5.57 \pm 0.10 vs. $5.88 \pm 0.10 \mathrm{~kg}$, respectively; $\mathrm{P}<0.05)$ and had improved overall growth performance to the end of the nursery stage $(\mathrm{P}<0.05)$. Weaning-to-estrus interval, ovulation rate, and embryo weight/crown-rump length and survival to d30, did not differ between treatments. However, average weight of the CL's at $\mathrm{d} 30$ was higher in FP10 compared to CON sows (378.1 \pm 6.9 vs. $341.0 \pm 7.1 \mathrm{mg}$, respectively; $\mathrm{P}<0.001$ ).

\section{Discussion}

Supplementing gilt diets with omega-3 fatty acids during late gestation and lactation positively impacted litter weight after birth, but did not affect the most common reproductive characteristics in sows after rebreeding.

\section{References}

Spencer J., Wilson L., Webel S.K., Moser R. and Webel D.M., 2004. Effect of feeding protected n-3 PUFAs (FertiliumTM) on litter size in gilts. J. Anim. Sc. 82 (Suppl. 2): 81

Vinsky M.D., Novak S., Dixon W.T., Dyck M.K. and Foxcroft G.R., 2006. Nutritional restriction in lactating primiparous sows selectively affects female embryo survival and overall litter development. Reprod. Fertil. Dev. 18, 347-355.

Webel S.K., Otto E.R., Webel D.M., Moser R.L., Spencer J.D. and Orr D.E., 2003. Effect of protected n-3 PUFAs (FertiliumTM) on litter size in sows. J. Anim. Sc. 81 (Suppl. 1): 18

Webel S.K., Otto-Tice E.R., Moser R.L. and Orr D.E., 2004. Effect of feeding duration of protected n-3 PUFAs (FertiliumTM) on litter size and embryo survival in sows. J. Anim. Sc. 82 (Suppl. 2): 81 


\title{
0.020
}

\section{Evaluation of an aluminosilicate to decrease the toxic effects of Aflatoxin B1 in swine}

\author{
Juan C. Medina ${ }^{1}$ Jose A. Fierro $^{1}$ Ruben Perez-Franco ${ }^{1}$ Elizabeth Rodriguez ${ }^{2}$ Leticia Duran ${ }^{1}$ Mariana Altamirano ${ }^{1}$ Veronica Moreno ${ }^{2}$ \\ 1. NUTEK S.A de C.V., Tehuacan, PUE, Mexico; 2. Investigación Aplicada S.A. de C.V., Tehuacan, PUE, Mexico
}

\section{Background}

Aflatoxin B1 (AFB1) is a mycotoxin with carcinogenic, teratogenic, mutagenic and immunosuppressant properties; it is produced by several species of the Aspergillus genus (flavus, parasiticus, nomiusy pseudotamarii). Its target organ is the liver (hepatotoxic). The effects of aflatoxins (AFs) in swine depend on the age, concentration in feed, and exposure periods. Problems related to contamination with these toxins have been reported in all stages of swine lifespan. Several aluminosilicates have been reported to reduce the effects of aflatoxins in swine diets (Ramos and Hernandez, 1997).

\section{Aim}

To evaluate the efficiency of a mycotoxin binder to decrease the toxic effects of a diet contaminated with $680 \mu \mathrm{g} / \mathrm{kg}(\mathrm{ppb})$ of AFB1 in swine balanced feeds.

\section{Materials and Methods}

18 swine were used, weaned at 21 days of age divided into 3 groups with 6 repetitions. They were housed in individual corrals with swine use floor. A week of contiditioning was considered. Commercial feeds were used, they were proven to be free of contamination with other mycotoxins (AFs, fumonisin B1, Ochratoxin A, DON and T.2). The treatments were: 1. Negative control diet, 2. Positive control diet with $680 \mathrm{ppb}$ of AFB1, 3. Challenge diet with adsorbent: $5 \mathrm{~kg} / \mathrm{t}+680 \mathrm{ppb}$ of AFB1. The experiment took 21 days. Swine were weighed at the beginning of the experiment and individual weights were recorded weekly. Feed conversion was calculated weekly. At the end of the experiment all swine were sacrificed; during the necropsy, samples of tonsils, lungs, livers, gallbladders, kidneys, spleens and mesenteric lymph nodes. All organs were previously weighed. Samples were submitted to histopathology studies. Fat, iron, vitamin A and AFM1 were quantified in livers. Biochemical parameters were analyzed in sera (aspartate aminotransferase, alanine aminotransferase, alkaline phosphatase, gamma-glutamyltransferase (GGT), glucose, local proteins, albumen, creatinine, triglycerides, cholesterol, urea, uric acid) and vitamin A. The information obtained was analyzed with the Tukey test using the SYSTAT statistical software; the significance value was based on a probability of 0.05 .

\section{Results and Discussion}

Ssignificative statistical differences were observed in animal weight gain, total weight, feed consumption, alkaline phosphatase, GGT and total proteins. In animals in which damages produced by aflatoxins were observed, a recovery was obtained from the challenge diet that included the adsorbent. The decrease in weight gain of the positive control group was of $31.2 \%$ compared to the negative control group. $52.3 \%$ of the weight gain difference was recovered in the challenge group. The rest of the evaluated parameters only showed numeric differences. The histopathological analysis showed the effects of consuming aflatoxins in livers and kidneys.

\section{Conclusion}

AFB1 at the concentrations used in the experiment affected animals. The increase of the concentration of alkaline phosphatase and GGT enzymes indicate the effects of AFB1 in swine. The decrease of these enzymes in the group given a diet with adsorbent is another proof of the protection afforded by the adsorbent Adding mycotoxin adsorbents in the diets of animals exposed to contamination with aflatoxins diminished economic losses attributable to these toxins, especially in large productive facilities. 


\subsection{1}

\section{Effect of energy intake during gestation on performance and blood parameters of primiparous sows}

Octávio H. Eckhardt ${ }^{1}$ Felipe C. Horta ${ }^{1}$ Larissa J. Parazzi ${ }^{1}$ Esther R. Afonso ${ }^{1}$ Simone M. Martins ${ }^{1}$ Carolina I. Silva ${ }^{1}$ José E. Junior ${ }^{2}$ Francisco P. Rennó ${ }^{2}$ Aníbal S. Moretti ${ }^{1}$

1. Laboratory of Swine Research, University of Sao Paulo, Depto. Nutrição e Produção Animal (VNP), Pirassununga, SP, Brazil; 2. Laboratory of Biochemistry and Animal Physiology, University of Sao Paulo, Depto. Nutrição e Produção Animal (VNP), Pirassununga, SP, Brazil

\section{Introduction}

The relationship between productivity and metabolic status, especially in gilts, is of great interest. Greater energy intakes during late gestation have been linked to lower lactational feed intake, resulting in catabolism and a poorer reproductive outcome in the next parity 1,2,3. The aim of the study was to investigate the effects of energy intake in primiparous sows' performance and metabolic state.

\section{Materials and Methods}

Twenty three hybrid gilts, with 75 days of gestation, were fed according to 2 treatments until farrowing: $(P, n=12) 2,9 \mathrm{~kg}$ of a pre-lactation diet (3203 kcalME/kg, 17,25\%CP), and (G) 2,5kg gestation diet (2930 kcalEM/kg, 16,43\%CP). Gilts were weighted at 75 days of gestation, 3 days before farrowing, at farrowing, and weekly during lactation until weaning. Animals were fed to appetite during lactation. Blood collections were made during gestation (days 82, 89, 96 and 103), at farrowing, lactation (days 7 and 14) and weaning, to evaluate HDL, LDL and VLDL cholesterol, triglyceride (TGL), and non-esterified fatty acids (NEFA) (Laborlab ${ }^{\circledast} /$ Randox $^{\circledast}$ kits, SBA200 Celm ${ }^{\circledast}$ analyzer/Asys Expert Plus microplate reader). In a random design the performance variables and blood parameters were analysed with the PROC GLM and MIXED of SAS4, respectively.

\section{Results}

Daily weight gain from d75 until farrowing was higher for treatment $P$ (Table 1). Energy intake during late gestation did not influence lactational feed intake or weights. Although there was not significant difference regarding the animal's weights during lactation, the $P$ treatment reflected in a weight loss while $\mathrm{G}$ treatment in gain. Blood parameters showed some significant differences among the treatments related to time variable, and some interactions effect as show table 2.

\section{Discussion}

Unlike other reports1, higher gestational energy intake did not influence fed intake and weight during lactation. HDL and LDL levels remained higher for $\mathrm{P}$ during gestation, as a result of a higher energy intake and thus a numerically higher TGL liver production, requiring transport through lipoproteins. Higher TGL concentrations for $G$ during lactation, as a result of high consumption, led to higher VLDL levels for transport from liver and HDL for reverse transport to that organ. Higher NEFA concentration for $\mathrm{P}$ is due to a peak during parturition, suggestion a higher response to lipolytic stimuli in heavier animals. In conclusion, blood parameters reflected the higher energy intake during gestation for treatment $\mathrm{P}$, and despite this treatment lost more weight during lactation, this fact did not affected the metabolic state of the females.
Table 1- Weight (Wt), Avarage Daily gain (ADG) and feed intake during late gestation and lactation (Lact)

\begin{tabular}{|l|c|c|c|}
\hline & G & P & P \\
\hline Wt d75 Gestation $(\mathrm{kg})$ & $186.4 \pm 9.6$ & $183,8 \pm 7.1$ & $\mathrm{~ns}$ \\
\hline Farrowing Wt $(\mathrm{kg})$ & $220.1 \pm 13.7$ & $229.1 \pm 10.2$ & ${ }^{* *}$ \\
\hline ADG 75-112d $(\mathrm{kg} / \mathrm{d})$ & $0.92 \pm 0.16$ & $1.23 \pm 0.16$ & ${ }^{*}$ \\
\hline Postpartum Wt $(\mathrm{kg})$ & $194.1 \pm 10.4$ & $199.7 \pm 8.8$ & ns \\
\hline Weaning Wt $(\mathrm{kg})$ & $197.0 \pm 15.2$ & $195.3 \pm 12.2$ & ns \\
\hline Feed intake Lact $(\mathrm{kg} / \mathrm{d})$ & $5.4 \pm 0.8$ & $5.2 \pm 0.9$ & ns \\
\hline${ }^{*} \mathrm{P}<0.05 ;{ }^{* *} \mathrm{P}<0.010$ & \multicolumn{4}{|l}{} \\
\hline
\end{tabular}

Table 2 - Blood parameters during gestation and farrowing (Gest) and lactation (Lact)

\begin{tabular}{|c|c|c|c|c|c|c|c|}
\hline & & G & $\mathbf{P}$ & VC & p Treat & p Time & p TXT \\
\hline \multirow[t]{2}{*}{ TGL 1} & Gest & 34.61 & 40.23 & $22.3 \%$ & 0.113 & * & 0.14 \\
\hline & Lact & 34.10 & 29.74 & $20.1 \%$ & 0.124 & * & 0.26 \\
\hline \multirow[t]{2}{*}{ HDL 1} & Gest & 23.26 & 26.83 & $10.4 \%$ & * & * & * \\
\hline & Lact & 29.14 & 26.76 & $12.1 \%$ & 0.143 & * & * \\
\hline \multirow[t]{2}{*}{ LDL 1} & Gest & 48.22 & 56.09 & $12.0 \%$ & * & * & 0.29 \\
\hline & Lact & 72.32 & 72.54 & $16.4 \%$ & 0.843 & * & 0.46 \\
\hline \multirow[t]{2}{*}{ VLDL 1} & Gest & 6.92 & 8.05 & $22.3 \%$ & 0.113 & * & 0.14 \\
\hline & Lact & 6.82 & 5.95 & $20.1 \%$ & 0.124 & * & 0.26 \\
\hline \multirow[t]{2}{*}{ NEFA 2} & Gest & 0.189 & 0.274 & $48.7 \%$ & * & * & * \\
\hline & Lact & 0.204 & 0.244 & $43.6 \%$ & 0.181 & * & 0.53 \\
\hline
\end{tabular}

\section{References}

1 Coffey et al., 1994. J Anim Sci, 72:4-9.

2 Weldon et al., 1994. J Anim Sci, 72:387-394.

3 Thaker and Bilkei, 2005. Anim Reprod Sci, 88:309-318.

4 SAS: software. Version 9.0. Cary: SAS Institute, 2002

\section{Acknowledgement}

FAPESP 


\title{
0.022
}

\section{Effects of feeding oregano essential oil to sows on performance of her offspring from weaning through market}

\author{
Claudia Ariza-Nieto ${ }^{2,3}$ Roger D. Walker ${ }^{2}$ Martha A. Mellencamp ${ }^{1}$ Samuel K. Baidoo ${ }^{2}$ \\ 1. Ralco Nutrition, Inc., Marshall, MN, USA; 2. Southern Research and Outreach Center, University of Minnesota, Waseca, MN, USA; \\ 3. CORPOICA, Bogata, Colombia
}

\section{Introduction}

It has long been acknowledged that some plant essential oils exhibit diverse functional activities. Oregano essential oil (OEO) has been shown to possess anti-bacterial, anti-fungal, and anti-oxidant activities (1). Analysis of the chemical components of OEO has shown that it contains high levels of carvacrol and thymol. The phenolic nature of these two compounds is responsible for the potent activities associated with OEO. Results from several studies have shown that OEO supplementation positively influences sow reproduction and litter health $(2,3)$. In this study, we investigated the effects of feeding OEO to sows on performance and carcass characteristics of her offspring.

\section{Materials and Methods}

This experiment was carried out at the University of Minnesota Southern Research and Outreach Center. A total of 384 early weaned piglets (d19) from sows fed control (corn-soy) or OEO (control+250 ppm OEO, Ralco Nutrition, Inc., Marshall, MN) diets during lactation and gestation were randomly assigned to 48 pens. Each pen was randomly assigned to one of four dietary treatments: control, antibiotic (Mecadox ${ }^{\circledR} 10 \mathrm{~kg} /$ ton phase 1 and 2), OEO (1000 ppm phase 1 and 2, 500 ppm phase 3, 250 ppm phase 4 and 5) and antibiotic+OEO. Pigs were weighed at weaning (19d), phase 1 (33d), phase $2(54 d)$, phase $3(89 d)$, phase 4 (131d) and phase 5 (152 d). Twenty-seven pigs per treatment were randomly selected for collection of carcass data. Pigs were shipped to a commercial abattoir for slaughter at the end of phase 5. Standard carcass measurements were collected, including carcass weight, carcass yield, fat depth, loin depth, lean percentage, carcass premium score and carcass value.

\section{Results}

Analysis of variance showed that sow treatment significantly affected ADG. At the end of phase 1, pigs from OEO-fed sows grew faster than controls (175 v. $153 \mathrm{~g} / \mathrm{d}$ ). When measured over the entire wean-to-finish period, pigs from OEO-sows grew faster than pigs from control sows $(817 \mathrm{v} .789 \mathrm{~g} / \mathrm{d})$ and ate more feed (1.92 v. $1.86 \mathrm{~kg} / \mathrm{d})$. Pig treatment effect was significant for OEO. Supplementation of OEO in pig diets showed an increase in ADG $(20 \mathrm{~g} / \mathrm{d})$ compared to pigs without supplementation.
Dietary supplementation with OEO to sows significantly increased body weight of offspring at slaughter (116 v. 112.6 $\mathrm{kg}$ ) (Table 1). Sow treatment effects were observed for several carcass traits. There was no pig treatment effect observed for body weight or carcass traits, except for a sow treatment by pig treatment interaction for carcass premium (grade index). Carcass value showed that offspring from OEO-sows earned an additional $\$ 4.90$ when compared with pigs from control sows.

\section{Discussion}

In this study, administration of OEO to sows significantly improved her offspring's post-weaning growth and overall carcass quality and value. The effect of OEO in sow diets was first apparent in the higher litter weaning weights compared with control offspring. OEO-sows had better quality colostrum (more IgG and lymphocytes) than control sows, and this may have contributed to improved weaning weights (2). Study of postweaning performance showed that the offspring of OEO-sows gained more weight during the first two weeks post-weaning than controls. Pig performance following weaning is a known predictor of subsequent performance. The underlying mechanism responsible for improved post-weaning performance seems likely related to gut structure. OEO supplementation was shown to reduce the negative morphological changes that occur in the small intestine during the post-weaning period (4). Feeding OEO in sow gestation and lactation diets supports nutritional programs designed to increase post-weaning pig performance and subsequent growth through slaughter. Pigs from OEO-fed sows showed clear benefits to the producer in higher carcass weight, greater loin depth, improved premium score and additional value.

\section{References}

1. Burt, S.A. 2005. Internat. J. Food Microbiol. 94:223-253.

2. Ariza-Nieto, C., et al. 2005. Proc. A. D. Leman Conf. 32:abstract 33.

3. Cabrera, R., et al. 2008. Proc. Amer. Assoc. Swine Veterinarians, 193196.

4. Ariza-Nieto, C., et al. 2006. 10th Sym. Dig. Dis. Physiol. Pigs, 2.62

Table 1. Effects of OEO in sow diet on offspring carcass characteristics and value.

\begin{tabular}{|l|c|c|c|c|c|c|c|c|c|}
\hline Sow diet & $\begin{array}{c}\text { Number of } \\
\text { pigs }\end{array}$ & \multicolumn{9}{|c|}{ Carcass characteristics } \\
\hline & & $\begin{array}{c}\text { Body weight } \\
(\mathrm{kg})\end{array}$ & $\begin{array}{c}\text { Carcass } \\
\text { weight } \\
(\mathrm{kg})\end{array}$ & Yield (\%) & $\begin{array}{c}\text { Fat depth } \\
(\mathrm{mm})\end{array}$ & $\begin{array}{c}\text { Loin depth } \\
(\mathrm{mm})\end{array}$ & $\begin{array}{c}\text { Lean } \\
(\%)\end{array}$ & $\begin{array}{c}\text { Grade } \\
\text { index }\end{array}$ & Carcass value (\$) \\
\hline OE0 & 108 & 116.0 & 88.2 & 76 & 0.77 & 2.85 & 55.5 & 6.6 & 136.80 \\
\hline Control & 108 & 112.6 & 85.6 & 76 & 0.77 & 2.75 & 55.1 & 6.1 & 131.70 \\
\hline P value & $\begin{array}{c}\text { not applic- } \\
\text { able }\end{array}$ & $\mathrm{P}<0.05$ & $\mathrm{P}<0.05$ & $\begin{array}{c}\text { not signifi- } \\
\text { cant }\end{array}$ & not significant & $\mathrm{P}<0.05$ & $\mathrm{P}<0.05$ & $\mathrm{P}<0.05$ & $\mathrm{P}<0.05$ \\
\hline
\end{tabular}




\title{
0.023
}

\section{Effects of multi-enzyme preparations in the growth performance and jejunum morphology in weaned pig diets}

\author{
Guillermo Cano ${ }^{2}$ Alain Kanora ${ }^{3}$ Daniel Villalba ${ }^{1}$ Alberto Morillo ${ }^{2}$ \\ 1. University of Lleida, Lleida, Spain; 2. Tests and Trials, Lleida, Spain; 3. Huvepharma N.V., Antwerp, Belgium
}

\section{Introduction and Objectives}

The exogenous enzyme use is an alternative to increase ingredients digestibility and interest is growing in the use of enzymes to improve animal performance. One of the responses to dietary changes at weaning is the modification of the epithelium structure ${ }^{1}$. Absorption in the small intestine depends on the villus-crypt relation. The objective of the present study was to investigate the effect of multi-enzyme preparations in the growth performance and jejunum morphology in weaned pigs fed a wheat-barley diet.

\section{Material and Methods}

269 pigs (Landrace $x$ Large White $x$ Pietrain) were assigned at weaning ( 28 days old) to one of three treatment diets (16 pen per treatment) during 14 days: Control without enzymes; 0.25 $\mathrm{g}$ per kg of Hostazym ${ }^{\circledR}$ suis 2000; and $0.50 \mathrm{~g}$ per kg of a commercial Multi-Enzyme Preparation (MEP). Individual Bodyweight (BW) was measured on days 0, 7 and 14 and Average Daily Gain (ADG), Average Daily Feed Intake (ADFI) and Gain to Feed (G:F) were calculated during first week and overall study. Two pigs per treatment were slaughtered on days 7 and 14 to collect intestinal jejunum samples, from the proximal (PJ) and distal portion (DJ), to measure Villus Height (VH) and Depth of Crypt (CD).

Data were analyzed with an ANOVA test. Pen was the experimental unit to productive variables and pig to morphological ones, the pig error term was used to perform statistical tests.

\section{Results and Discussion}

In pigs fed diets containing Hostazym ${ }^{\circledR}$, ADG was $45 \mathrm{~g} /$ day higher $(p<0.01)$ during first week and $49 \mathrm{~g} /$ day higher $(p<0.05)$ on overall compared with pigs fed the Control diet. These higher ADG changes conduces Hostazym ${ }^{\circledR}$ pigs to had significantly higher BW at days $7(p<0.01)$ and $14(p<0.05)$. There were no differences in productive performance between MEP and CONTROL diets. Compared with MEP treatment, diet containing Hostazym $^{\circledast}$ tended to had higher ADG during first week (+26 g/ day, $\mathrm{p}=0.09$ ) and BW on day 7 (184 g, $\mathrm{p}=0.09)$ but these statistical tendency disappeared in the overall. There were no differences ( $p>0.05$ ) in ADFI and G:F between groups.

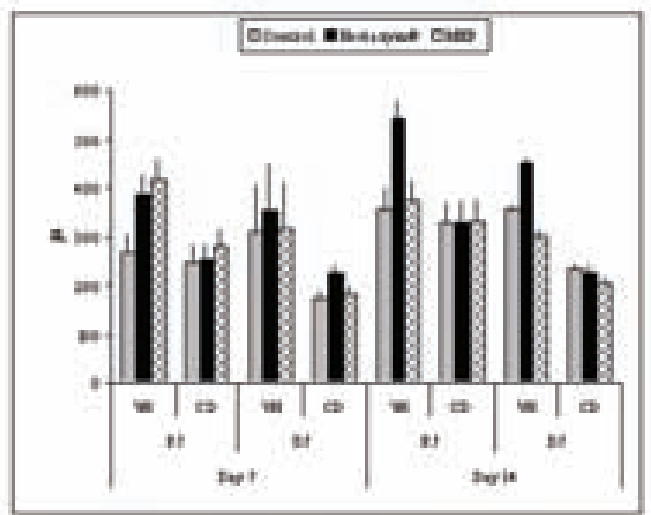

Figure L. Jejuntum morphology in mursery pigs.
Table 1. Growth performance in aursery ples.

\begin{tabular}{|c|c|c|c|c|}
\hline & Control & Hestaxyms & MEP & $\mathrm{SE}^{7}$ \\
\hline \multicolumn{5}{|l|}{ BW (ke) } \\
\hline Day 0 t & 8.3: & $8.1^{16}$ & $8.2^{\infty}$ & 0.1 \\
\hline Day 7 & $9.4^{\circ}$ & $9.8^{\circ}$ & $9.6^{t}$ & 0.1 \\
\hline Day 14 & $12.0^{n}$ & $12.7^{\circ}$ & $12.3^{n}$ & 0.2 \\
\hline \multicolumn{5}{|l|}{ ADG (g) } \\
\hline Days 0.7 & $181^{b}$ & $226^{\circ}$ & $200^{*}$ & 11 \\
\hline Days 0.14 & 295 & $343^{\circ}$ & $316^{k}$ & 13 \\
\hline \multicolumn{5}{|l|}{ ADFI (s) } \\
\hline Days 0-7 & $201^{\circ}$ & $223^{*}$ & $216^{*}$ & 10 \\
\hline Days 0-14 & $292^{\circ}$ & $313^{*}$ & $309:$ & 11 \\
\hline \multicolumn{5}{|l|}{ GaF (e/g) } \\
\hline Days 0.7 & $1.11^{4}$ & $1.00^{*}$ & $1.10^{\circ}$ & 0.04 \\
\hline Days 0-14 & 1.06 & $1.00^{\circ}$ & $1.00^{\circ}$ & 0.02 \\
\hline
\end{tabular}

Hostazym $^{\circledR}$ tended to have higher VH compared with Control on day 7 in proximal jejunum $(p=0.082)$. On day 14, Hostazym $^{\circledR}$ diet had longer villus than Control diet and MEP diet in proximal jejunum and in distal jejunum $(p<0.05)$. Depth of Crypt was not affected ( $p>0.05$ ). Villus Height and Depth of Crypt are important indicators of the digestive health and directly related to the absorption capacity of the mucus membrane ${ }^{2}$. Effects on $\mathrm{VH}$ may be due to enzyme supplementation reduces erosion of the villus ${ }^{3}$ due to improved digestibility of nutrients ${ }^{4}$, thus could improve ADG.

\section{References}

1. Pluske JR, Williams IH, Aherne FX. Villous height and cript depth in piglets in response to increases in the intake of cow's milk after weaning. Anim Sci. 1996;62:145-148.

2. Buddle JR, Bolton JR. The pathophysiology of diarrhoea in pigs. Pig News and information. 1992;13:41-45.

3. Nabuurs MJA. Microbilogical, structural and functional changes of the small intestine of pigs at weaning. Pig news and Information. 1995;16:93-97.

4. Omogbenigun FO, Nyachoti CM, Slominski BA. Dietary supplementation with multienzyme preparations improves nutrient utilization and growth performance in weaned pigs. J Anim Sci. 2004;82:10531061. 


\title{
0.024
}

\section{The use of bone metabolites excreted in urine as markers for evaluation of the phosphorus retention and status in grower-finisher pigs}

\author{
Kristina U. Sørensen; Hanne D. Poulsen \\ Dept. of Animal Health and Bioscience, Faculty of Agricultural Sciences, Aarhus University, Tjele, Denmark
}

\section{Introduction}

Phosphorus $(P)$ has several physiological functions and is vital for the maintenance of the skeletal tissue. Due to environmental concern, $\mathrm{P}$ supply in feed is lowered in order to reduce $\mathrm{P}$ excretion. However, pigs may develop hypophosphatemia if $P$ is not supplied in sufficient amounts resulting in reduced performance and bone strength. This project addresses the effect of lowering the dietary $\mathrm{P}$ content. The overall aim was to combine results on $P$ retention and excretion with physiological responses in urine and bones in order to evaluate if the urinary compounds are correlated to $P$ intake and thus may serve as markers for sufficient $P$ intake.

\section{Material and Methods}

Grower-finisher female pigs were fed LP (4.1), MP (6.2), or HP ( $8.8 \mathrm{~g} \mathrm{P} / \mathrm{kg}$ dry matter) from 40 to $105 \mathrm{~kg}$ body weight (BW). No microbial phytase was added. Determinations of $\mathrm{P}$, calcium (Ca) and nitrogen (N) balances were performed at 40, 70, and $105 \mathrm{~kg}$ BW. Furthermore, urine was analyzed for metabolites released during bone turnover reflecting the degradation of the bone collagen matrix. Bones were collected at slaughter (105 kg), defatted, dried, and analyzed for mineral content and bone mineral density.

\section{Results and Discussions}

The preliminary results show that $\mathrm{P}$ retention was lowered by $50 \%$ and up to $60 \%$ in pigs fed LP compared with MP and HP (Table 1).

Table 1. Phosphorus $(P)$, calcium $(C a)$, and nitrogen $(N)$ retention, $g$ day $^{-1}$. Mean values for three periods.

\begin{tabular}{|l|c|c|c|}
\hline & LP & MP & HP \\
\hline P retention, g day-1 & 2.87 & 5.96 & 7.12 \\
\hline Ca retention, g day-1 & 4.79 & 8.30 & 9.66 \\
\hline N retention, g day-1 & 23.0 & 27.7 & 27.2 \\
\hline
\end{tabular}

At $40 \mathrm{~kg} \mathrm{BW}$, P retention was identical for pigs fed MP and HP whereas $P$ retention was higher in pigs fed HP at 70 and 105 $\mathrm{kg} \mathrm{BW}$. These results indicate that the $P$ retention capacity was met at MP at $40 \mathrm{~kg}$ BW but not in the heavier pigs. Comparable results were obtained on $\mathrm{Ca}$ whereas the $\mathrm{N}$ retention was identical for pigs fed MP and HP but almost $20 \%$ lower in pigs fed LP. Seven different bone turnover metabolites were measured in urine; among these were pyridinoline, deoxypyridinoline, and hydroxylysine $(\mathrm{HYL})$. The results showed that some metabolites were affected by the dietary $\mathrm{P}$ content and the excretion was generally increased by $35-75 \%$ in pigs fed LP compared to HP. However, other traits were more affected by ageing showing an increase in urinary excretion by $15-75 \%$ from 40 to $105 \mathrm{~kg} \mathrm{BW}$.
Figure 1 summarizes results on dry weight of humerus, HYL, and daily $P$ retention as affected by $P$ intake.

Figure 1. Humerus dry weight, $g$, hydroxylysine (HYL), $\mu$ mol day ${ }^{-1}$, and Pretention, g day $^{-1}$.

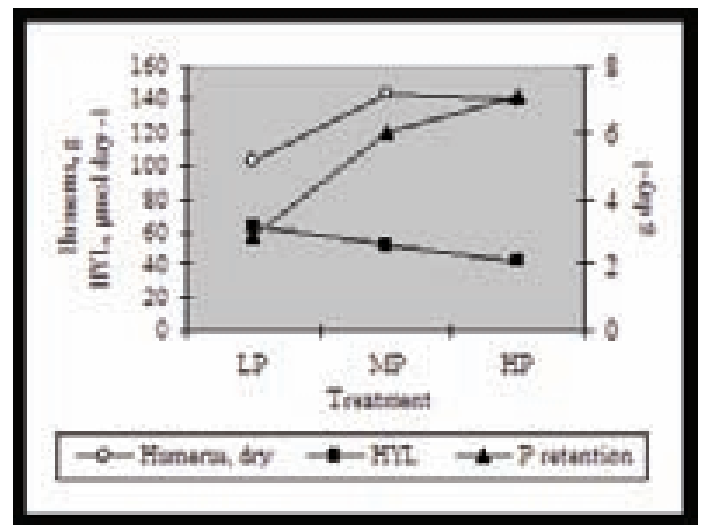

\section{Conclusions}

Lowering the dietary P supply greatly affect nutrient balances and bone turnover. There seems to be correlations between urinary metabolites and bone characteristics. Thus, these metabolites found in urine seem to be useful as non-invasive markers for the evaluation of sufficient $P$ supply to grower-finisher pigs.

\section{References}

Liesegang, A. 2003. Possibilities of monitoring bone metabolism in ruminants - an overview of the methods in use. Acta vet. Scand., suppl. 97, 23-28.

Nicodemo, M.L.F. et al., 1998. Effects of variations in dietary calcium and phosphorus supply on plasma and bone osteocalcin concentrations and bone mineralization in growing pigs. Exp. Physiol. 1998 83, 659-665. 


\title{
0.025
}

\section{Variation of nutrient content in a batch of growing pig meal due to segregation in the feeding silo and to transport with different feed conveyors}

\author{
An Cools; Dominiek Maes; Geert Janssens \\ Faculty of Veterinary Medicine, Ghent University, Merelbeke, Belgium
}

\section{Introduction}

Pig meal is a heterogeneous mixture of different particles. Each particle has a specific size but also a specific nutritional value. The particle size profile as well as average nutrient content of fattening meal is mostly quite similar between different batches. However, different manipulations of a batch such as emptying the feed silo or distribution of the meal throughout the stable by a feed conveyor can affect the particle profile of the feed. The particle size of pig feed is related to feed digestibility (Al-Rabadi et al, 2009) and voluntary feed intake (Anguita et al, 2007), but it also affects the incidence of gastric ulceration (Dirkzwager et al, 1998) and the occurrence of tail biting (Brumm et al, 2008). This study aimed to investigate possible variations in nutrient content of fattening pig meal between the point a new batch of meal is delivered in the silo and the point when the silo is almost empty. Secondly the effect of transport of meal by different types of conveyors on the nutrient content of feed was investigated.

\section{Materials and Methods}

The study was performed in four different farms. In each farm, feed samples were taken one day after the feed silo was filled with a new batch of growing pig meal (BEGIN) and again when the feed silo was almost empty (END). Every time, samples were taken in the feeding troughs in the pens on four different places along the feed conveyor: in the first pen of the first compartment (DISTANCE 1, 2, 3, 4). Two of the sampled farms used screw conveyors (SCREW) to transport the pig meal from the feed silo to the feed troughs in the pens, the two other farms used roundbottom drag conveyors (DRAG). Secondly, two of the farms offered their pigs standard meal (STANDARD), the other two farms added corn cob mix (CCM) on a daily basis to the standard meal before it was delivered to the different feeding troughs. The nutrient content of all feed samples was analysed for the following parameters by using near infrared spectroscopy: dry matter (DM), crude fibre (CF), crude fat (CFAT), crude protein (CP), crude ash (CA), sugar (SU) and starch (ST). All data were statistically analysed by using SPSS 17.0 statistical software.

\section{Results}

The amount of CP and ST was significantly higher when samples were taken shortly after filling the silo than samples taken when the silo was almost empty $(\mathrm{P}<0.001)$. For CFAT, $C F$ and $C A$ the opposite was true, these nutrients were lower in the beginning of the silo than at the end $(\mathrm{P}<0.001$ for CFAT and CF and $\mathrm{P}<$ 0.05 for $(A)$ ). The SU content of the feed samples was lower at the end of the silo than at the beginning, although this was not significantly $(0.1>P>0.05)$. The differences between BEGIN and END for CFAT, CF and CA were significantly larger for stables using DRAG than those using SCREW $(P<0.05)$. Also the difference is SU content was numerically larger for DRAG $(0.1>\mathrm{P}>$ 0.05). The differences in RE and ST were not affected by the conveyor type. Feed type did not significantly affect the differences in nutrient content between BEGIN and END but numerically, CCM had a more stable nutrient content than STANDARD. Distance, neither at BEGIN nor at END, significantly influenced the nutrient content of the feed. However, some numerical trends could be observed which are similar to those seen between BEGIN and END of the silo.

\section{Discussion}

The results indicate that manipulation of feed by emptying of the silo and distribution throughout the stable by automated feed conveyors can affect the nutrient content of the feed. Although differences are small from a first point of view, they can have an effect on the performance and health of the pigs in the different pens of a stable. There for the link of these differences in nutrient content will be further linked with differences in particle size and profile. In a following study the link between the variation in nutrient content throughout the stable will be studied in relation to feed intake and growth performance of pigs.

\section{References.}

Al-Rabadi, G.J.S. et al, 2009. Journal of Cereal Science 50: 198-204.

Anguita, M. et al, 2007. Livestock Science 107: 182-191.

Brumm, M.C. et al, 2008. Journal of Swine Health and Production 16: 72-80.

Dirkzwager, A. et al, 1998. Livestock Production Science 56: 53-60. 


\title{
0.027
}

\section{Effects of reduced stimulation of sows during insemination - a field trial}

\author{
Claus Hansen; Gunner Sørensen; Flemming Thorup; Anja K. Olesen; Niels Jorgen Kjeldsen \\ Pig Research Centre, Agriculture \& Food Council, Copenhagen, Denmark
}

\section{Introduction}

Efficient heat detection is crucial for correct timing of insemination. Before insemination, correct stimulation consists of the five point plan: 1. elevating the inguinal fold, 2. pushing the flanks, 3. applying pressure below the vulvae, 4 . applying pressure on the pelvis and 5. riding test, where the inseminator sits on the back of the sow. During this manual stimulation, a boar should be present in front of the sow. Stimulation increases contractions in the uterus [1], which is expected to support sperm transport. After inserting the catheter, the technician should sit on the back of the sow during the insemination. Some technicians exclude this during insemination. It has never been investigated if this reduced stimulation effects farrowing rate or litter size. The aim of this study was to investigate the effect of reduced stimulation during insemination of sows.

\section{Materials and Methods}

The experiment included 2912 multiparous Danbred L x Y sows in 4 commercial herds. The herds all displayed a farrowing rate above 86 per cent at start of the trial, to avoid management failures or infections to affect the study. Heat detection was performed once a day from day 4 after weaning. A boar was present in front of the sow during heat detection. Duration of heat detection using the five point plan was approximately 1 minute per sow If the sow was in standing heat, the five point plan was also the stimulation before introducing the insemination catheter.. Sows were assigned randomly into two groups.

Group 1:Traditional stimulation, where the technician sat on the back of the sow during insemination.

Group 2: Restricted stimulation. No stimulation of the sow during insemination. Semen entered the servix passively in both groups.

Group 2A: If semen did not enter within twenty minutes, sows in group 2 were stimulated by back pressure to achieve semen uptake. These sows were transferred to group $2 \mathrm{~A}$.

Date of farrowing and total born piglets were recorded for all sows in the experiment.

Significant difference between groups were analyzed using Mixed model for total number born and logistic regression was used to analyze for differences in farrowing rate.

\section{Results}

There was no statistically significant difference between groups when results from the four farms where pooled. Table 1 shows, that none of the farms experienced significant differences in farrowing rate nor total born piglets between the groups. There was a large difference between farms in percent sows transferred from group 2 to group $2 A$.
Table 1. Farrowing rate and total born for the three groups in the four farms.

\begin{tabular}{|l|c|c|c|}
\hline & Group 1 & Group 2 & Group 2a \\
\hline \multicolumn{4}{|c|}{ Farm 1 } \\
\hline No. of sows & 247 & 291 & 21 \\
\hline Farrowing rate & 87.6 & 89.5 & 95.5 \\
\hline Total born & 16.7 & 16.6 & 17.1 \\
\hline \multicolumn{5}{|c|}{ Farm 2 } \\
\hline No. of sows & 724 & 562 & 103 \\
\hline Farrowing rate & 88.4 & 90.9 & 92.0 \\
\hline Total born & 17.8 & 18.1 & 18.2 \\
\hline \multicolumn{5}{|l|}{ Farm 3 } \\
\hline No. of sows & 357 & 338 & 10 \\
\hline Farrowing rate & 93.9 & 94.2 & 100 \\
\hline Total born & 17.5 & 17.4 & 18 \\
\hline & 133 & 102 & 24 \\
\hline No. of sows & 90.5 & 82.3 & 88.9 \\
\hline Farrowing rate & 18.2 & 17.5 & 17.5 \\
\hline Total born &
\end{tabular}

\section{Discussion}

The results indicate that one minute of stimulation prior to insemination including use of the boar is enough stimulation to assure sperm transport. The number of sows in farm 4 was low, but a numerically lower farrowing percentage and number of total born in group 2 in this farm may indicate a problem in some farms, when sows are not stimulated during insemination. Farm 4 had diagnosed leptospirosis io the sow population during the experiment, which may have influenced the experiment, although this remains unknown.

\section{References}

Theriogenology, 2005; 63:2. 500-513. 


\title{
0.028
}

\section{Productivity in sows nursing large litters}

\author{
Flemming Thorup \\ Center for Pig Production, Agriculture and Food Council, Copenhagen, Denmark
}

\section{Introduction}

Litter size in Danish production herds increased from 11 to 14 live born piglets from 1996 to 2009. The average sow has 14.3 productive milk glands. Can they assert high piglet survival and growth, and still achieve high fertility in the following litter?

\section{Materials and Methods}

In two production herds three sows with at least 15 productive milk glands were selected at least 12 hours after farrowing. From these three sows 39 piglets were selected. The rest (the smallest piglets) were transferred to nurse sows (1). The 39 piglets were randomized back to the sows with 11,13 and 15 piglets per litter. This was repeated 44 and 43 times in the two herds, including 261 sows in the study. The next 22 days, the litters were managed following standard management in the two herds. In case a piglet fell behind, it was transferred to a runt nurse. No new piglets were added to the litters. Piglets were weighed at weaning, and the sows were followed through the next cycle.

\section{Results}

Data for piglet mortality and piglet removal is given in table one. Mortality includes mortality among piglets transferred to runt nurses. Herd 2 saw only minor differences in mortality between the three groups. The high piglet survival in this trial may be caused by the removal of the smallest piglets to nurse sows to achieve the low litter sizes. Also only healthy sows were included in the study. Figure 1 and 2 indicates how many piglets were still with the mother at 22 days, when the sow was given 11, 13 and 15 piglets. Table 2 gives the effect of litter size nursed on days to mating, farrowing rate and total born piglets in the next litter.

\section{Discussion}

Mortality and number of transferred piglets increased, when litter size was increased. The number of piglets weaned per sow and per pen did also increase. Most sows weaned 12 to 14 piglets. Only 10 per cent of the 87 sows nursing 15 piglets weaned all 15 piglets (Fig. 1 and 2). More knowledge to evaluate the capacity of the specific milk glands will give the manager a better chance to predict the optimal capacity of a specific sow. Glands not used in the previous lactation, were less productive in the succeeding lactation (2). Thus when increasing litter size at the sows, it is important first to challenge the youngest sows in the herd. Neither farrowing percentage nor litter size in the following litter was significantly affected by the number of piglets nursed.
Table 1. Piglet mortality and runt piglets in two herds

\begin{tabular}{|l|c|c|c|c|c|c|}
\hline & \multicolumn{3}{|c|}{ Herd 1 } & \multicolumn{3}{c|}{ Herd 2 } \\
\hline Piglets per litter & 11 & 13 & 15 & 11 & 13 & 15 \\
\hline Piglet mortality & 5 & 6 & 10 & 4 & 3 & 5 \\
\hline Runt Piglets & 4 & 5 & 8 & 4 & 9 & 12 \\
\hline
\end{tabular}

Table 2. Effect on fertility in the following cycle

\begin{tabular}{|l|c|c|c|c|c|c|}
\hline & \multicolumn{3}{|c|}{ Herd 1 } & \multicolumn{3}{c|}{ Herd 2 } \\
\hline Piglets/ litter & 11 & 13 & 15 & 11 & 13 & 125 \\
\hline Days to mating & 4 & 5 & 4 & 5 & 5 & 6 \\
\hline Farr. rate \% & 86 & 80 & 86 & 84 & 79 & 83 \\
\hline Tot. born pigs & 17.3 & 17.6 & 17.1 & 17.1 & 17.2 & 17.2 \\
\hline
\end{tabular}

Fig. 1. No. of litters with different no. of piglets weaned in herd 1
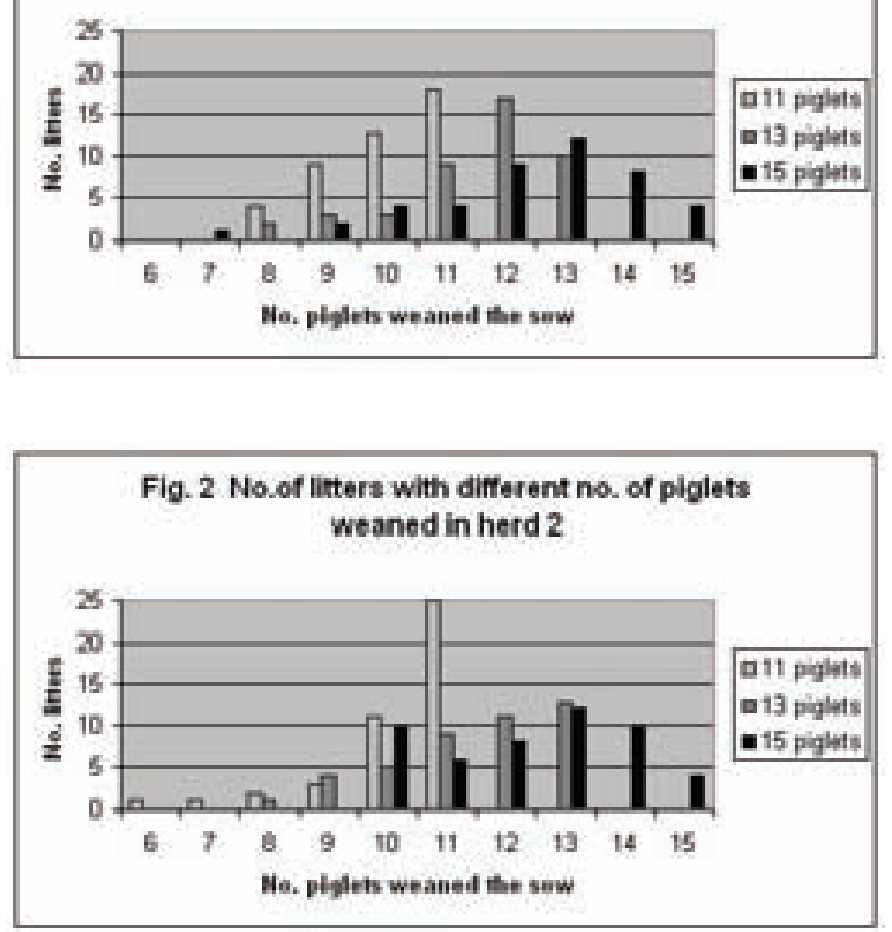


\title{
0.029
}

\section{Effect of early parturition on the reproductive performance in sows}

\author{
Caroline Vanderhaeghe ${ }^{1}$ Jeroen Dewulf ${ }^{1}$ Jan Jourquin ${ }^{2}$ Dominiek Maes ${ }^{1}$ \\ 1. Facutly of Veterinary Medicine, Department of Reproduction, Obstetrics and Herd Health, Merelbeke, Belgium; \\ 2. Janssen Animal Health, Beerse, Belgium
}

\section{Objectives}

The purpose of this study was to determine the incidence of early parturition ( $<114$ days of gestation) and to investigate the effect of early parturition on the reproductive performance in sows.

\section{Material and Methods}

This study was based on reproductive data records (Cercosoft NV, Oudenaarde, Belgium) of 37 pig herds that were willing to collaborate with the study. Two of these herds were excluded because the insemination date was not recorded for each individual sow and/or altrenogest was administered at the end of gestation. The database of the remaining 35 herds contained 87,978 farrowing records of which 26,988 records were excluded because induction of parturition was performed or due to missing information on farrowing data. Hence, 60,990 farrowing records of 17,401 sows were used for further analyses. The association of the outcome variables (total litter size, live born piglets, stillborn piglets) with the gestation length (GL) (categorized in 4 groups: 109-111d, 112-113d, 114-117d, 117-121d) was assessed using a multilevel linear mixed effect model, with herd included as random factor, and parity and breed as fixed factors. The influence of the duration of the previous gestation on the next was also determined. All statistical analyses were performed in MLwiN 2.0.

\section{Results}

The mean gestation length was $115.4 \pm 1.62$ days. Early parturition occurred in $10 \%$ of all farrowing events (Fig 1).

Table 1 shows that sows with $\mathrm{GL}<112$ d have more stillborn piglets (Odds ratio/OR: 5.8 ) and less piglets born alive (OR: 0.2) compared to those with GL 114-117d. A GL of 112-113d was associated with more total born piglets (OR: 1.7), more piglets born alive (OR: 1.1) and more stillborn piglets (OR: 1.5). Sows with $G L \geq 118 d$ were more likely to have less total born piglets (OR: 0.3 ) and less piglets born alive (OR: 0.3 ) compared to those with GL 114-117d. Sows with a gestation length of $<114 d$ were more likely to have a gestation length of $<114 \mathrm{~d}$ at the subsequent parity (OR: 1.2) as those with a gestation length of $\geq 114 \mathrm{~d}$ $(\mathrm{P}<0.05)$.

Tabel 1: Mean (s.d.) of total litter size, live born and stillborn piglets by gestation length (GL)

\begin{tabular}{|c|c|c|c|c|}
\hline GL (d) & $\mathbf{n}$ & Total litter size & Live born piglets & stillborn piglets \\
\hline $109-111$ & 576 & $13.1(3.7)$ & $10.5(4.4)^{*}$ & $2.6(3.4)^{*}$ \\
\hline $112-113$ & 5544 & $13.8(3.3)^{*}$ & $12.5(3.2)^{*}$ & $1.3(1.8)^{*}$ \\
\hline $\begin{array}{c}114-117=\text { refer- } \\
\text { ence }\end{array}$ & 48929 & $13.3(3.5)$ & $12.4(3.3)$ & $0.9(1.6)$ \\
\hline $118-121$ & 5941 & $11.9(3.9)^{*}$ & $10.9(3.8)^{*}$ & $1.0(1.7)$ \\
\hline
\end{tabular}

Fig 1: Frequency distribution (\%) of gestation length in 60,990 farrowing records.

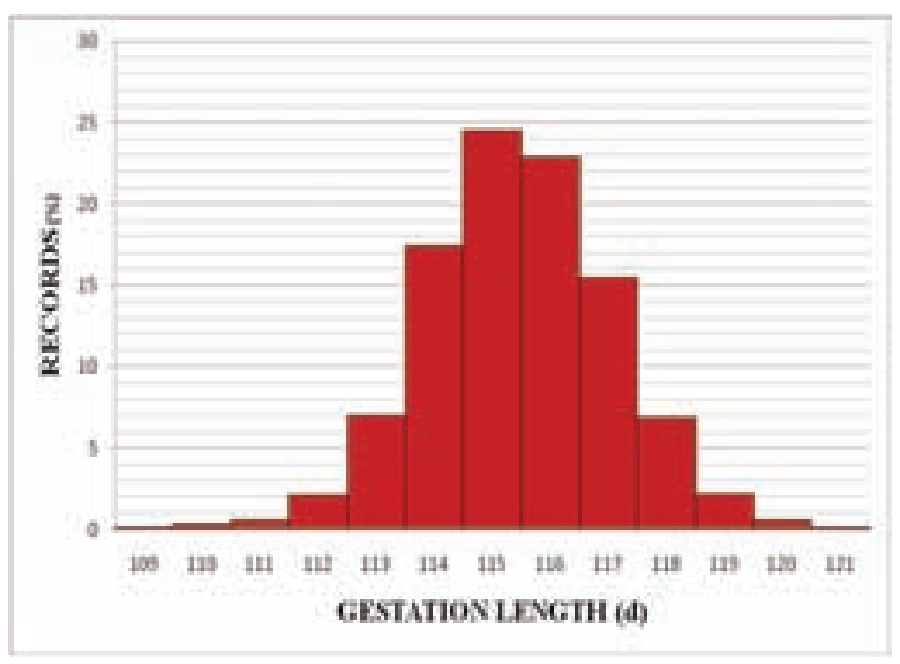

\section{Discussion and conclusion}

The present study showed that early parturition $(<114 d)$ occurred in $10 \%$ of the farrowing events. Early parturition significantly increased the risk for stillborn piglets, especially in sows with a $G L<112 d$. This is likely due to the relative immaturity of the piglets at parturition (1). Large litters and more piglets born alive were associated with a $G L<114 d$, while small litter size and low numbers of live born piglets were related to a $G L \geq 118 \mathrm{~d}$. This is in agreement with Sasaki and Koketsu (2007). Sows with a short gestation length were more likely to have an early parturition at the subsequent parity. This information may be important for producers to identify potential sows-at-risk. It may enable them to improve the reproductive performance by assisting farrowing or by preventing an early parturition.

\section{References}

1 Zaleski and Hacker (1993) Can. Vet. J. 34, 109-13.

2 Sasaki and Koketsu (2007) Theriogenology 68, 123-127. 


\title{
0.030
}

\section{Relationship between pre- and post freezing semen quality characteristics and field fertility}

\author{
Eveline Willems ${ }^{1}$ Hanneke Feitsma $^{2}$ Jascha Leenhouwers ${ }^{2}$ \\ 1. TOPIGS S\&D, Vught, Netherlands; 2. Institute for Pig Genetics, Beuningen, Netherlands
}

\section{Introduction}

In pig breeding, frozen semen is used for global linkage of nucleus herds. The objective of this study was to investigate the relationship between fresh and post thaw semen quality characteristics and fertility in sows.

\section{Materials and Methods}

A database (Cryobase) was set up with semen variables of ejaculates used for freezing. All ejaculates have been analyzed for several semen quality characteristics; Fresh ejaculates were evaluated for motility, progressive motility $(0,24 \& 72$ hours after collection) and morphology. Frozen-thawed ejaculates were evaluated for motility, progressive motility $(0,24 \& 48$ hours after thawing), morphology and for \% membrane intact cells ( 0 and 24 hours after thawing) using Nucleocounter SP100. Fertility results of TOPIGS nucleus herds are recorded and exchanged with a breeding database (PigBase) at the Institute for Pig Genetics. In order to analyze the fertility results of frozen semen PigBase and Cryobase were merged, corrected for outliers and standardized for farm, parity and boar line. This resulted in a dataset of 742 frozen ejaculates from 400 boars. The analysis was performed using SAS statistical package.

\section{Results}

Variables with a significant effect on farrowing rate (FR) and total number born (TNB) are found in this analysis. In table 1 the different $\mathrm{P}$-values for the variables tested are mentioned.

Table 1: $P$-values for the variables tested

\begin{tabular}{|l|c|c|}
\hline variable & FR & TNB \\
\hline fresh motility & 0,0001 & ns \\
\hline fresh progressive motility & 0,04 & ns \\
\hline fresh motility 24h & 0,001 & 0,004 \\
\hline fresh progressive motility 24h & 0,002 & 0,06 \\
\hline fresh motility 72h & 0,002 & 0,01 \\
\hline fresh progressive motility 72h & 0,03 & ns \\
\hline fresh morphology total & 0,001 & 0,01 \\
\hline post thaw motility & 0,009 & 0,01 \\
\hline post thaw progressive motility & ns & 0,07 \\
\hline post thaw motility $24 h$ & ns & 0,08 \\
\hline post thaw progressive motility $24 h$ & ns & ns \\
\hline post thaw motility 48h & ns & ns \\
\hline post thaw progressive motility 48h & ns & ns \\
\hline post thaw morphology total & ns & 0,01 \\
\hline post thaw \% intact cells & ns & 0,06 \\
\hline post thaw \% intact cells $24 h$ & ns & 0,04 \\
\hline
\end{tabular}

In general more fresh semen quality characteristics have a significant effect on fertility than post-thawing semen quality characteristics.
Correlations between certain semen quality characteristics and field fertility came forward during the analysis and some estimates of effect of variables are exposed in table 2.

Table 2: Regression coefficients of relationships between fertility and semen quality. Effects expressed as change in FR(\%) or TNB(\#) per percent increased morphologic abnormalities or percent membrane intact cells

\begin{tabular}{|l|c|c|}
\hline variable analyzed & FR & TNB \\
\hline fresh morphology total & $-2,41$ & $-0,26$ \\
\hline post thaw morphology total & ns & $-0,04$ \\
\hline post thaw membrane intact cells (0h) & ns & 0,06 \\
\hline post thaw membrane intact cells (24h) & ns & 0,04 \\
\hline
\end{tabular}

Some motility variables of the evaluated ejaculates shown to have a significant effect on field fertility. Mainly fresh motility variables have a significant effect on FR and TNB. For example the FR was $21,7 \%$ less when fresh semen had a progressive motility $24 \mathrm{~h}$ of $<70 \%$ compared to fresh semen progressive motility of $\geq 70 \%$.

\section{Discussion}

Significant relationships between fertility and fresh semen quality could lower the production costs of frozen semen, due to more adequate approval guidelines for evaluating suitability of ejaculates for freezing.

When the dataset increases, there may be more variables significantly important for field fertility.

UltiMateTM Computer Assisted Semen Analysis (CASA) systems are implemented since 2006 to check the motility and progressive motility of the ejaculates objectively, but not enough data were available for analysis yet.

\section{References}

Internal communications TOPIGS and IPG 


\title{
0.031
}

\section{Quality parameters assessment of boars seminal doses which were delivered to farms by artificial insemination centres in Spain during 2009}

\author{
Yahya Dahmani; René Mozo-Martín; Carmen Larraz; Juan Luís Úbeda \\ R\&D Department, Magapor SL, Ejea de los Caballeros. Zaragoza, Spain
}

\section{Introduction}

The methodology of analyses and process of swine ejaculated change among Artificial insemination centres in Spain. This change can affect the quality of semen doses delivered to farms for the insemination process. The analysis and control of the quality of seminal doses has a great importance as the productive improvement of the farms will depend on it. These parameters should comply minimum values required of semen quality standards for the swine artificial insemination (1). Therefore, the aim of this study is to assess quality parameters of boar seminal doses delivered to farms, by artificial insemination centres in Spain during 2009.

\section{Materials and methods}

For this study, we assessed 1678 boars seminal doses from 20 swine artificial insemination centres in Spain. The analyzed parameters of seminal doses were: concentration, $\mathrm{pH}$, osmolarity, agglutination, sperm motility, whole acrosomes, abnormal morphology and the hypo-osmotic (Host) test. All analyses were carried out by the same person and by microscopic standard examination. Data were processed by StatView 5.0 software(2). Normal distribution was checked by Kolmogorov Smirnov test. Variance of quality parameters of seminal doses between centres was analysed by ANOVA 2x2 way, and Fisher's PLSD test. Values are presented as means \pm standard deviation, and significant differences are considered when $\mathrm{p}<0.05$.

\section{Results}

ANOVA analysis of the different seminal parameters has showed a significant difference among Al centres $(P<0.0001)$. In the table 1 , we illustrated only the most important parameters: motility, concentration, abnormal morphology and Host test. Despite these differences among centres, almost all values of the parameters fulfil the recommended standards limits for the seminal doses analysis in swine, except for the motility parameter and abnormal morphology of sperm. Only in the centres C05 the seminal doses presented an average lower than the standard limit (64.6\%) for motility, meaning the possible rejection of $12.9 \%$ of all 1678 doses. However, differences in the parameter in an abnormal forms, have been very pronounced statistically. Values of abnormal forms sperm were higher than standard limit (>30\%) in the majority of centres. This result mean that $67.3 \%$ of total samples can be discarded. Concerning the concentration of doses $21.6 \%$ of samples showed values not conform with standard limit, however, the doses should not be rejected for this motif as we don't know if the samples were destined to post-cervical Al. The remaining parameters showed acceptable values and were within standard limits

\section{Discussion}

Values were within standard limits in most of the cases, but for the parameter of sperm abnormal forms. This result is coherent with the diversity in the methodology of work and process of swine semen doses. Besides, other factors interfere in this variability, such as the reception and transportation, temperature of doses, type of semen extender...etc. Nevertheless, it is surprising that the sperm abnormal forms parameter is out of the standard limits, since it depends on semen extender, temperature of transportation, boar and process of spermatogenesis. In addition to it , determination of this parameter was objective, contrary to others. Therefore, this anomaly would also be due to the high extraction rhythm of ejaculated, which can affect the sperm morphology.

Summing up, due to the fact that all seminal quality parameters should fulfil standard limits, without exception, it is necessary to maximize the precaution and to guarantee the quality of the seminal dose delivered to farms.

Table1. Means distribution of sperm motility, concentration, abnormal morphology and Host test, in 20 centres (CRI) from Spain. Values are presented as means \pm standard deviation. AF: Abnormal forms. Conc: concentration. LS: limits standard (1).

\begin{tabular}{|c|c|c|c|c|}
\hline CRI & Motility & Conc. x108/mI & AF & Host \\
\hline C01 & $81.2 \pm 18.5$ & $31.2 \pm 7.4$ & $27.7 \pm 12.6$ & $55.7 \pm 13.2$ \\
\hline C02 & $85.2 \pm 8.3$ & $36.7 \pm 10.3$ & $21.7 \pm 9.9$ & $55.6 \pm 11.1$ \\
\hline C03 & $83.9 \pm 12.4$ & $25.9 \pm 6.5$ & $26.7 \pm 15.5$ & $57.2 \pm 12.7$ \\
\hline C04 & $79.3 \pm 15.3$ & $52.3 \pm 27.6$ & $33.5 \pm 19.3$ & $53.2 \pm 13.5$ \\
\hline C05 & $64.4 \pm 26.8$ & $23.4 \pm 7.1$ & $23.8 \pm 13.1$ & $58.6 \pm 12.5$ \\
\hline C06 & $77.8 \pm 19.5$ & $39.03 \pm 11.2$ & $30.4 \pm 12.9$ & $56.8 \pm 14.4$ \\
\hline C07 & $74.5 \pm 21.8$ & $40.5 \pm 8.9$ & $39.9 \pm 15.2$ & $59.1 \pm 10.7$ \\
\hline C08 & $81.4 \pm 16.7$ & $27.6 \pm 10.5$ & $30.7 \pm 14.8$ & $54.8 \pm 12.2$ \\
\hline C09 & $76.4 \pm 17.8$ & $50.1 \pm 22.4$ & $31.8 \pm 19.2$ & $58.3 \pm 13.2$ \\
\hline C10 & $76.3 \pm 19.7$ & $45.6 \pm 18.3$ & $32.9 \pm 114.9$ & $51.9 \pm 13.8$ \\
\hline C11 & $81.8 \pm 12.9$ & $37.7 \pm 12.1$ & $37 \pm 18.2$ & $53.7 \pm 9.7$ \\
\hline C12 & $78 \pm 18.7$ & $34.14 \pm 7.1$ & $27 \pm 13.8$ & $65.1 \pm 10.3$ \\
\hline C13 & $85 \pm 15.5$ & $47.98 \pm 10.2$ & $26.2 \pm 11.8$ & $54.3 \pm 14.9$ \\
\hline C14 & $90 \pm 0.0$ & $38.5 \pm 12.6$ & $26.8 \pm 8.9$ & $61.5 \pm 2.4$ \\
\hline C15 & $82.1 \pm 11.9$ & $43 \pm 9.6$ & $37.5 \pm 16.6$ & $66.1 \pm 11.1$ \\
\hline C16 & $77.6 \pm 22.6$ & $43.5 \pm 20.6$ & $45.8 \pm 25.1$ & $58.7 \pm 12.3$ \\
\hline C17 & $75.5 \pm 22.8$ & $34.2 \pm 9.9$ & $37.8 \pm 15.1$ & $55.6 \pm 13.9$ \\
\hline C18 & $70.2 \pm 27.4$ & $42.6 \pm 13.1$ & $51.7 \pm 19.5$ & $54.1 \pm 12.8$ \\
\hline C19 & $80.9 \pm 15.1$ & $28.7 \pm 6.7$ & $22.8 \pm 9.4$ & $50.9 \pm 11.4$ \\
\hline C20 & $87.2 \pm 5.45$ & $31.8 \pm 8.8$ & $29.5 \pm 13.2$ & $58.9 \pm 14.6$ \\
\hline P & $<0.0001$ & $<0.0001$ & $<0.0001$ & $<0.0001$ \\
\hline LS & $\geq 65 \%$ & $3 \times 109$ & $\leq 25 \%$ & $\geq 50 \%$ \\
\hline
\end{tabular}

\section{References}

(1) Gonzalez-Urdiales R., et al. 2006. P. 22 In: Manual de técnicas de reproducción asistida en porcino. Ed. Universidad de Girona. Red temática nacional de reproducción porcina.

(2). StatView, SAS institute Inc. Version 5.0. 


\title{
0.032
}

\section{Influence of organic selenium supplementation on lipid peroxidation of boar sperm membranes}

\author{
Simone M. Martins ${ }^{2,1}$ André F. Andrade ${ }^{2}$ Larissa J. Parazzi ${ }^{1}$ Fabiane G. Zaffalon ${ }^{2}$ Carolina I. Silva ${ }^{1}$ \\ Andres M. Gallego ${ }^{2}$ Aníbal S. Moretti ${ }^{1}$ Rubens P. Arruda ${ }^{2}$ \\ 1. Laboratory of Swine Research, University of Sao Paulo, Depto. Nutrição e Produção Animal (VNP), Pirassununga, SP, Brazil; \\ 2. Laboratory of Semen Biotechnology and Andrology, University of Sao Paulo, Depto. Reprodução Animal (VRA), Pirassununga, SP, Brazil
}

\section{Introduction}

Boar spermatozoa are characterized by a high level of polyunsaturated fatty acids. Lipid peroxidation (LP) may be one of the mechanisms responsible for the negative biochemical and physiological changes during sperm storage. However, data on the changes in sperm lipid composition and their susceptibility to peroxidation during storage are limited1. Previous results demonstrated that boar spermatozoa contains glutathione peroxidase and a-tocopherol, suggesting that both selenium and vitamin $\mathrm{E}$ have a role in preventing peroxide damage to the sperm cell2. Therefore, the aim of this study was to evaluate the effect of dietary organic selenium on the membrane lipid peroxidation in the boar semen diluted with BTS and stored for 3 days.

\section{Materials and Methods}

Fifteen boars were kept on the same conditions and were fed the same standard diet during training. The animals were fed with three experimental diets: $0.3 p p m$ sodium selenite (Control); $0.5 \mathrm{ppm}$ sodium selenite (Inorganic) and $0.5 \mathrm{ppm}$ selenium yeast (Organic). Ejaculates were collected from each boar once per week using the gloved-hand technique. Semen was diluted with standard extender to $3 \times 10^{9}$ cells/dose and stored for 3 days at $18^{\circ} \mathrm{C}$. The peroxidation of the membranes was measured with the use of the fluorescent probe C11-BODIPY581/591. Semen aliquots were collected during the analysis steps to obtain samples. These were then stained with $0.5 \mu \mathrm{L}$ of probe $\mathrm{C} 11$ BODIPY581/591 (1 mg/mL, Molecular Probes) for 30 minutes at $37^{\circ} \mathrm{C}$. Then, $3 \mu \mathrm{L}$ of propidium iodide (PI) $(0.5 \mathrm{mg} / \mathrm{mL}$, Sigma) was added to the sample to mark the cells with damaged plasma membranes, when they were analysed by flow cytometry. The viable sperm cells (PI negative) were stained with probe C11BODIPY581/591 and analysed as the mean intensity of fluorescence emission (arbitrary units-a.u.) captured in the photomultiplier with a long pass of 502 and a band pass of $530 \pm 15 \mathrm{~nm} 3$. The experimental design was a randomised block. Data were analyzed using the PROC repeated from SAS4 and outcomes were compared among the three treatments by the orthogonal contrast method with $\mathrm{P}<.05$ level of significance.

\section{Results}

No interaction was found between the independent

variables time and treatment during the analysis of the dependent variable fluorescence intensity emitted by the sperm with plasma membrane that was intact and stained with the fluorescent probe C11-BODIPY581/591. Therefore, it was possible to carry out the analysis of the main effects separately. There were no changes in the fluorescence intensity emitted by viable cells independently of the treatment added. When analysing the time effect, it was verified that the fluorescence intensity emitted increased within 24 hours and decreased after that (Graphic 1).
Graphic 1 - Means \pm SEM of the fluorescence intensity of the sperm with intact plasma membrane and stained with a fluorescent probe C11-BODIPY581/591

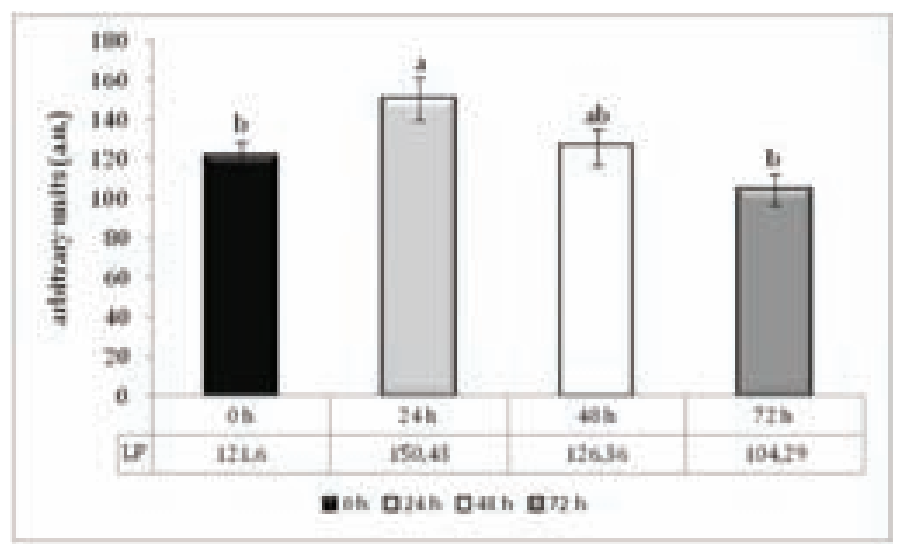

Values with a different letters differ $(\mathrm{P}<.05)$

\section{Discussion}

Higher lipid peroxidation was found within 24 hours, this was probably due to a high cell metabolism in the beginning of the cooling process 5 . The cooling led to a reduction in metabolism, which in turn, decreased lipid peroxidation after 48 hours, probably by the action of antioxidant systems of their own sperm. 5 In conclusion, the selenium yeast showed no better results in comparison to others treatments regarding lipid peroxidation.

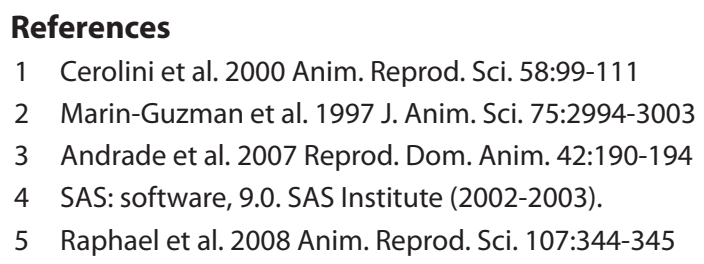

Acknowledgements: FAPESP Proc $n^{\circ} 07 / 55613-6$, Allech $^{\circledR}$ Inc., Agroceres ${ }^{\circledR}$ Nutrição Animal and Vet life ${ }^{\circledR}$ Veterinary Products. 


\title{
0.033
}

\section{Practical review on reproductive testis pathology in boar}

\author{
Juan Luís Úbeda ${ }^{1}$ María Victoria Falceto² Yahya Dahmani ${ }^{1}$ René Mozo-Martín ${ }^{1}$ José Antonio Bascuas ${ }^{2}$ \\ 1. R\&DDepartment, Magapor SL, Ejea de los Caballeros. Zaragoza, Spain; \\ 2. Departamento de Patologia Animal. University of Zaragoza, Zaragoza, Spain
}

\section{Introduction}

The boar low fertility depends on many factors like nutrition, stress, season, libido problems, anatomical problems of the reproductive organ, infections, as well as genetic transcendence causes.

Artificial insemination centres (AIC) discard boars when they start to present fertility problems or low seminal quality due to unknown causes, since animals maintenance supposes a considerable cost. In several cases, the reason for rejection is some reproductive pathology of testis misdiagnosed which is manifested as low seminal quality or bad fertility.

The aim of this study is to determine possible reproductive pathologies of testicle in culled boars from Al centres, which were sent to the slaughterhouse because of low fertility, low seminal quality or unknown causes. Seeking also a possible treatment, through morphological and histological studies of testicles.

\section{Material and methods}

More than 500 testis of slaughtered boars were collected. All animals were coming from AIC because of their low fertility or low semen quality or other unknown causes.

Biological material was conducted to the laboratory for processing and analyses. First morphologic exam of testis was performed. followed by processing of fixation and histological preparation (head, tail, epididymis body and the pampiniform plexus). Samples obtained were analyzed by microscopy illustrating every case by pictures.

\section{Results}

Several pathological reproductive incidences of the testicle were detected and registered (varicocele, fibrosis, inflammation, haemorrhages...etc). Figure 1 and 2 illustrate cases of varicocele and injuries with inflammation, respectively. The most frequent pathology was varicocele and lesions.

\section{Discussion}

Is very remarkable the frequency of the cases found of testicular pathology in the culled boars. This finding concord with some reported studies (1). In the majority of the cases in which boars were eliminated without specific cause, we found different degrees of fibrosis, as well as inflammatory focus.
Figure 1. Exam of testis with varicocele. From right to left, morphological, anatomical and histological assessment of the same testis respectively.

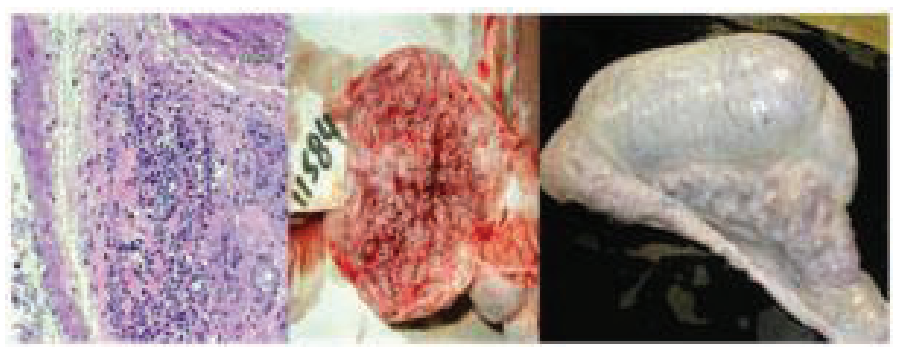

Figure 2. Exam of testis with lesion and inflammation. From right to left, anatomical and histological checking respectively.

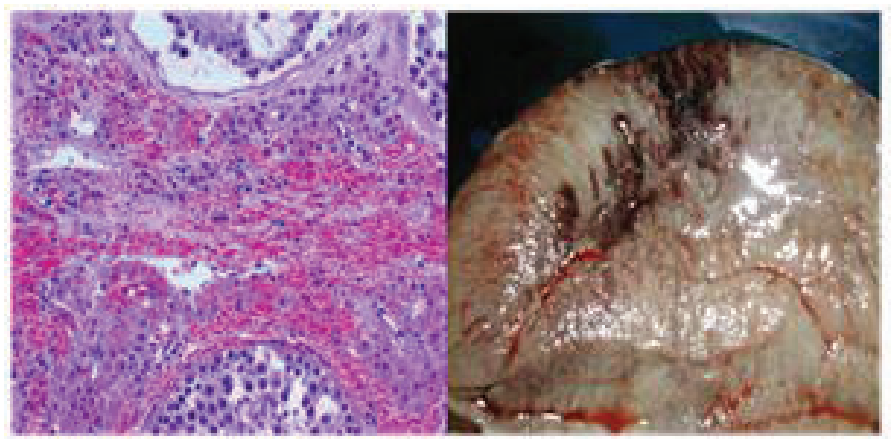

Theses symptoms are difficult to diagnose in farm without anatomy assessment and they are related to the low semen quality or low seminal production. This fact explains the lost of males productivity, the difficulty of covering seminal doses purchase orders from farms, as well as the dissatisfaction of the customers because of the seminal dose bad quality.

Similarly, testicular injuries take time to recover and many of them are chronic which involves a substantial increase in the final cost for the boar-stud. Finally, even though discarding animals seems to be the best solution in this case, we should increase inspection in slaughterhouse to examine the testicular pathologies in sacrificed boars, and try to give treatment to correct this fault and avoid animal sacrifice.

\section{References}

(1) Ubeda JL et al. 2006. Suis, n 26. 22-28. 


\title{
0.034
}

\section{Considerations in assessing the serological cross-reactivity of pig and novel H1N1 influenza viruses}

\author{
Jaime Maldonado ${ }^{1}$ Laura Valls $^{1}$ Eva Martínez $^{1}$ Montserrat Aguero² Dolores Buitrago² Enric Espunya ${ }^{1}$ \\ 1. Laboratorios Hipra, Amer, Spain; 2. Laboratorio Central de Veterinaria, Algete, Spain
}

\section{Introduction}

The novel human $\mathrm{H} 1 \mathrm{~N} 1$ ( $\mathrm{nH} 1 \mathrm{~N} 1$ ) virus can infect pigs and its occurrence in swine farms has been reported recently in several countries. Besides, it has been shown that $\mathrm{nH} 1 \mathrm{~N} 1$ cross-react with porcine viruses in the hemagglutination inhibition assay (HI)2. This work reports on the observed antigenic and genetic relatedness between $\mathrm{nH} 1 \mathrm{~N} 1$ and swine $\mathrm{H} 1 \mathrm{~N} 1$ viruses.

\section{Materials and Methods}

Two groups of $\mathrm{H} 1 \mathrm{~N} 1$ viruses isolated from swine (SIVs) in the 80s (early = 8) and 2000s (recent $=7$ ) in Spain, together with the Sw/ California/07/2009 virus of human origin were analyzed. Cross-HI was performed using antisera against each virus group. Partial nucleotide (nt) sequences (450nt) of the hemagglutinin segment $(\mathrm{HA})$ were obtained and analyzed by multiple alignments (MEGA 4, ClustalW and BLAST).

\section{Results and Discussion}

$\mathrm{HI}$ antibodies against early SIVs, recent SIVs and $\mathrm{nH} 1 \mathrm{~N} 1$ showed cross-reaction to viruses in each group to a variable extent (Table 1). Geometric Mean Titters (GMT) for early sera against early and recent SIVs were 269 and 98, respectively. GMTs for recent sera were 538 and 476 . HI titers for early and recent sera against $\mathrm{nH} 1 \mathrm{~N} 1$ were 160 and 80 respectively. $\mathrm{nH} 1 \mathrm{~N} 1$ antiserum also showed cross-reactivity against early and recent SIVs with GMTs of 59 and 21, respectively. These findings indicate that cross-reactivity among $\mathrm{nH} 1 \mathrm{~N} 1$ and SIVs occurs, regardless of the virus isolation period or the nature of antiserum tested in the $\mathrm{HI}$ assay (hyperimmune or convalescent in Table 1). Thus, the broad cross-reactivity of SwCA09H1N1 antiserum and SIVs isolated more than 20 years apart may suggest the existence of cross-reactive epitopes conserved between the hemagglutinins of influenza A H1N1 viruses. However these findings deserve further studies.

Nucleotide sequence analysis of the HA segment allowed clear differentiation among early, recent and the $\mathrm{nH} 1 \mathrm{~N} 1$ viruses. However, early SIVs showed slightly higher similarity to $\mathrm{nH} 1 \mathrm{~N} 1$ (73\%) than recent SIVs (range 69\%-71\%). Furthermore, BLAST search against all GenBank HA nucleotide entries returned significant (>90\% similaruty) matches to several avian species for early (1012 out of 100 hits) compared to recent (1 out of 100 hits) SIVs, suggesting adaptation to the swine host in recent SIVs1. To what extent the observed cross-reactivity is influenced by such differences is something that should be investigated further. Taken together, the additional data presented here support previous observations that demonstrate serologic cross-reactivity with $\mathrm{nH} 1 \mathrm{~N} 1$ virus in pigs in Europe. The repercussions that could have this in diagnosis and vaccination are yet to be assessed.
Table 1. Hemaglutination Inhibition titres of convalescent and hyperimmune antisera against swine and human influenza viruses

\begin{tabular}{|c|c|c|c|c|c|c|}
\hline \multirow{2}{*}{\multicolumn{2}{|c|}{ Swine and human viruses }} & \multirow{3}{*}{$\begin{array}{c}\text { Early } \\
\text { H1N1 } \\
\text { SwSp80a }\end{array}$} & \multicolumn{4}{|c|}{ Swine and human antisera } \\
\hline & & & \multirow{2}{*}{\begin{tabular}{|c|c|}
$\begin{array}{c}\text { Recent } \\
\text { H1N1 }\end{array}$ \\
SwSp04b
\end{tabular}} & \multirow{2}{*}{\begin{tabular}{|c} 
nH1N1 \\
CA09c,
\end{tabular}} & \multirow{2}{*}{$\begin{array}{c}\begin{array}{c}\text { H1N2 } \\
\text { SwSp04b }\end{array} \\
<20\end{array}$} & \multirow{2}{*}{\begin{tabular}{|c}
$\begin{array}{c}\text { H3N2 } \\
\text { SwSp04b }\end{array}$ \\
$<20$
\end{tabular}} \\
\hline $\begin{array}{l}\text { Early } \\
\text { H1N1 }\end{array}$ & A/sw/spain/1248/1988 & & & & & \\
\hline & A/sw/spain/1873-1/1987 & 160 & 320 & 80 & 20 & $<20$ \\
\hline & A/sw/spain/2688c/1989 & 320 & 640 & 80 & $<20$ & $<20$ \\
\hline & A/sw/spain/1877-4/1987 & 320 & 320 & 80 & $<20$ & $<20$ \\
\hline & A/sw/spain/Girona/1989 & 320 & 640 & NT & $<20$ & $<20$ \\
\hline & A/sw/spain/M87/1987 & 160 & 640 & 40 & $<20$ & $<20$ \\
\hline & $\begin{array}{c}\text { A/sw/ } \\
\text { spain/785Tarrag/1987 }\end{array}$ & 320 & 640 & 40 & $<20$ & $<20$ \\
\hline & $\begin{array}{l}\text { A/sw/spain/ } \\
\text { pp86BCN/1986 }\end{array}$ & 320 & 640 & 40 & $<20$ & $<20$ \\
\hline \multirow[t]{7}{*}{$\begin{array}{l}\text { Recent } \\
\text { H1N1 }\end{array}$} & A/sw/spain/45304/2003 & 80 & 320 & 20 & $<20$ & $<20$ \\
\hline & A/sw/spain/58788/2004 & 80 & 320 & 80 & $<20$ & $<20$ \\
\hline & A/sw/spain/59169/2004 & 160 & 640 & 40 & $<20$ & $<20$ \\
\hline & A/sw/spain/83649/2007 & 80 & 640 & 80 & $<20$ & $<20$ \\
\hline & A/sw/spain/50047/2003 & 160 & 640 & 20 & $<20$ & $<20$ \\
\hline & A/sw/spain/51915/2003 & 80 & 320 & $<20$ & $<20$ & $<20$ \\
\hline & A/sw/spain/53207/2004 & 80 & 640 & 20 & $<20$ & $<20$ \\
\hline nH1N1 & A/california/07/2009* & 160 & 80 & 320 & $<20$ & $<20$ \\
\hline H1N2 & A/sw/spain/40564/2004 & $<20$ & $<20$ & $<20$ & 1280 & $<20$ \\
\hline $\mathrm{H} 3 \mathrm{~N} 2$ & A/sw/spain/54008/2004 & $<20$ & $<20$ & $<20$ & $<20$ & 320 \\
\hline
\end{tabular}

\section{References}

1. Brown et al., J Gen Virol. 1997 78:553-562.

2. Kyriakis et al., Emerg Infect Dis. 2010 16:96-99. 


\title{
0.035
}

\section{Implications of clinical, virological and epidemiological findings in the first cases of pandemic (H1N1) 2009 virus infection in English pigs for the control of swine influenza}

\author{
Susanna Williamson ${ }^{1}$ A W. Tucker I S. McCrone ${ }^{2}$ J L. Wood ${ }^{2}$ H Habernoll ${ }^{3}$ K Yuill ${ }^{3}$ S C. Essen ${ }^{4}$ I H. Brown \\ 1. Veterinary Laboratories Agency, Bury St Edmunds, UK; 2. Dept. of Veterinary Medicine, Cambridge, UK; 3. BQP, Ltd, UK; \\ 4. Veterinary Laboratories Agency, New Haw, Surrey, UK
}

\section{Introduction:}

Since pandemic $\mathrm{H} 1 \mathrm{~N} 1$ infection in humans began its global spread, countries around the world have reported spread of infection to pigs. This paper describes the clinical, epidemiological and virological findings in the first three pig farms found to be infected with pandemic (H1N1) 2009 influenza virus in England. These showed interesting differences in disease presentation, spread and likely sources of infection and the findings have implications for the control of swine influenza infection in commercial breeding and growing pig units.

\section{Materials and Methods:}

Tonsil, trachea and lung samples were collected from pigs submitted for diagnostic postmortem examination with a history of respiratory disease or with respiratory pathology. Nasal swabs and sera were collected from pigs on farms where pandemic H1N1 was detected in submitted pigs. Nasal swabs and tissues were tested by influenza M gene PCR, positive samples were then tested by PCR for pandemic H1. Sera were tested for antibodies using the haemagglutination inhibition assay (HAIT).

\section{Results:}

The first three pandemic H1N1 infected units identified in England were an outdoor breeding unit (farm A) and two indoor nursery-finishers (farms $B$ and $C$ ). Morbidity ranged from 0 to $75 \%$ within groups and mortality was negligible or low. Clinical signs varied from inapparent infection to non-specific without respiratory signs in breeding pigs on farm $A$, to typical acute respiratory disease with rapidly spreading coughing highly suggestive of influenza infection in growing pigs (farms $B$ and C). The breeding unit from which growing pigs derived (which was not farm A) was suspected to be the source of infection for farms $B$ and $C$ which were weaned from the unit three weeks apart, however the origin of infection for this supply unit was not established and breeding sows on the unit showed no signs suspicious of influenza. Infection was detected on farm B for an 8-week period, as infection spread from one pig source to another; within any one shed of pigs the maximum duration of active infection detected was 4 weeks although pigs showed clinical recovery within 2 weeks. Circumstantial evidence suggests that a pig lorry driver was the possible origin of infection for the outdoor breeding unit (farm A). Active infection was present on this unit over at least a seven-week period at the end of which four week-old weaners moved to an indoor unit on which active infection was detectable three weeks later. On several occasions, PCR positive nasal swabs were obtained from pigs in which significant antibody titres could be detected. There was no or insignificant cross reactivity of HAIT antibody to pandemic virus with other UK endemic influenza strains (avian-like $\mathrm{H} 1 \mathrm{~N} 1, \mathrm{H} 1 \mathrm{~N} 2, \mathrm{H} 3 \mathrm{~N} 2$ ) in recovered pigs. The $\mathrm{H} 1 \mathrm{~N} 1$ virus isolated from all three farms was $99.7 \%$ identical to pandemic $\mathrm{H} 1 \mathrm{~N} 1$ (2009) circulating in the human population.

\section{Discussion:}

The main implications of these findings for the control of infection of pigs with pandemic (H1N1) 2009 are:

a) vigilance for clinical signs as a means of surveillance for suspect pandemic $\mathrm{H} 1 \mathrm{~N} 1$ infection in adult breeding pigs is unlikely to be effective as many undergo inapparent infection, or mild non-specific signs which could go unnoticed, particularly in an extensive outdoor setting.

b) in extensive outdoor situations where pigs are in the open air and stocking density is low, spread around the unit may occur more slowly and less predictably than on indoor units. Without the use of serology, it would be difficult to determine whether susceptible groups remain. In such situations, it may be appropriate to deliberately seed infection into potentially susceptible groups (for example unserved gilts) to avoid possible reproductive or respiratory problems during acute infection later.

c) influenza infection may persist within some types of unit for a more extended period than expected and movement of pigs on or off the unit should be undertaken in the knowledge that clinical recovery alone may not be sufficient evidence that the unit is no longer infected.

d) seropositive pigs can still shed virus during recovery and detection of seropositive groups does not necessarily mean that infection has fully resolved; infection can persist in batches of pigs weaned off site from sows following an outbreak. Further evaluation of these findings is in progress.

e) the serology results show that HAIT can be a useful means of surveillance for pandemic H1N1 influenza in pigs in some settings, but HAIT with endemic strains should be included to exclude cross reactions. 


\title{
0.036
}

\author{
Experimental challenge with two isolates of 2009 A/H1 N1 in weaned pigs \\ Amy L. Vincent ${ }^{1}$ Janice R. Ciacci-Zanella ${ }^{1}$ Alessio Lorusso ${ }^{1}$ Eraldo L. Zanella ${ }^{1}$ Kelly M. Lager ${ }^{1}$ \\ Philip C. Gauger ${ }^{2}$ Bruce H. Janke ${ }^{2}$ Marcus E. Kehrli ${ }^{1}$ \\ 1. USDA-ARS National Animal Disease Center, Ames, IA, USA; 2. lowa State University, Ames, IA, USA
}

\section{Introduction}

The gene constellation of the 2009 pandemic $\mathrm{H} 1 \mathrm{~N} 1$ virus is a unique combination from swine influenza A viruses (SIV) of North American and Eurasian lineages, but prior to April 2009 had never before been identified in swine or other species (1). Although the hemagglutinin gene is related to North American $\mathrm{H} 1 \mathrm{SIV}$, the infection potential in pigs was unknown immediately following the emergence of the virus in the human population. The objective of this study was to evaluate two isolates of 2009 pandemic $\mathrm{H} 1 \mathrm{~N} 1$ in a pathogenesis and transmission study in weaned pigs.

\section{Materials and methods}

Pathogenic and transmission characteristics between $\mathrm{A} /$ CA/04/2009 (CA/09) and A/Mexico/4108/2009 (MX/09) were compared in pigs. A total of 304 -week old pigs were inoculated with A/CA/04/2009 H1N1 ( $n=15$ pigs) or A/Mexico/4108/2009 H1N1 ( $n=15$ pigs) with approximately $2 \times 105 \mathrm{TCID} 50 / \mathrm{ml}$ of each respective virus. Pigs were sampled at $0,2,3,5,7$, and 9 days post infection (dpi) by nasal swab. Direct $(n=5)$ and indirect $(n=5)$ contact pigs were placed with each of the primary infected pig groups on 2 days post infection (dpi) and nasal swab samples were collected at $0,3,5,7$, and 9 days post contact $(\mathrm{dpc})$. Five primary challenged pigs were necropsied on days $3,5,7$, and 21 pi. Contact pigs were necropsied on day 20 pc. Hemagglutination inhibition ( $\mathrm{HI}$ ) assays were done with $21 \mathrm{dpi}$ and $20 \mathrm{dpc}$ to evaluate sero-conversion using challenge virus as well as a panel of North American H1 SIV isolates representing each phylogenetic cluster (2).

\section{Results}

Pigs experimentally infected with pandemic H1N1 2009 influenza A viruses developed similar respiratory disease and demonstrated equivalent macroscopic lung lesions on each given necropsy day. Virus titers in the lung were not different between CA/09 and MX/09 infected pigs, with mean titers of 104.8 and 104.5 respectively on $3 \mathrm{dpi}, 104.8$ and 104.8 respectively on $5 \mathrm{dpi}$, and undetectable on $7 \mathrm{dpi}$. However, differences in viral replication in nasal secretions and transmission to contact pigs were detected between CA/09 and MX/09 (Figure 1). $C A / 09$ inoculated pigs shed virus from the nose in a higher percentage of pigs as well as at higher viral titers. Additionally, direct and indirect contact pigs shed CA/09 virus at levels similar to primary inoculated pigs and for an extended time course. In contrast, MX/09 inoculated pigs shed virus in a lower percentage of pigs and at lower virus titers. A limited number of nasal swabs were positive from pigs in direct contact with $\mathrm{MX} / 09$ inoculated pigs and no samples from indirect contact pigs were found to be positive for MX/09 virus. Serologic cross-reactivity was demonstrated between the 2 pandemic isolates and with North American $\mathrm{H} 1$ isolates, although significant reductions in $\mathrm{HI}$ titers were detected.
Figure 1. Virus titers in nasal swab samples.

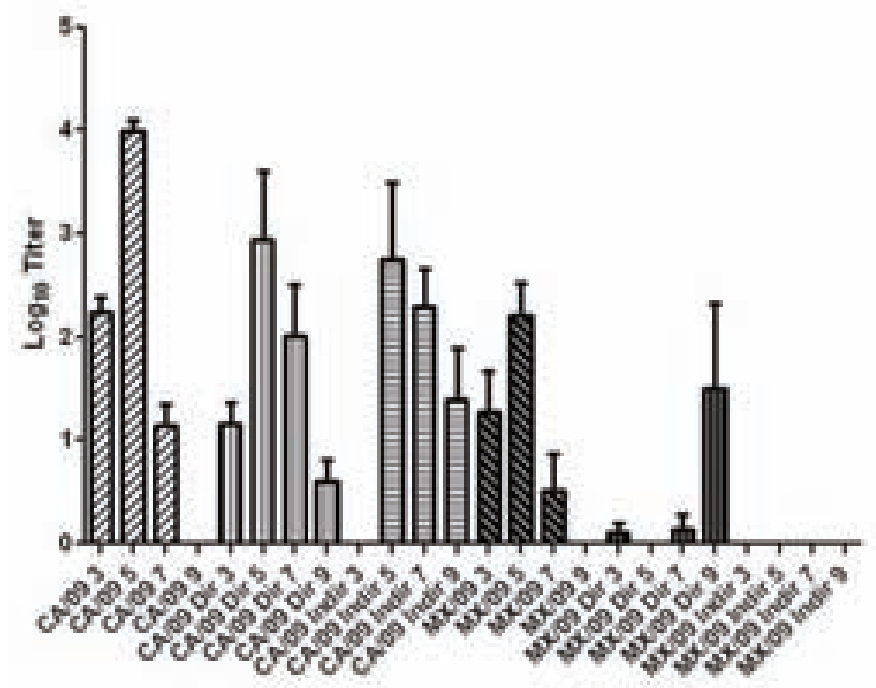

$C A / 09$ and $M X / 09=$ intratracheally inoculated pigs with each respective virus; Dir=sentinel pigs placed in direct contact with inoculated pigs; Indir=sentinel pigs placed in the same room, but without physical contact.

\section{Discussion}

Understanding the shedding and transmission patterns of different genotypes of H1N1 2009 in swine is important as several countries are now reporting outbreaks in commercial pig herds. The difference in the patterns of shedding and transmission may partially explain why some swine outbreaks have been relatively self-limiting. Virus variants that replicate to higher titers in the nose of pigs and easily transmit from pig to pig could present a greater threat to animal and human health. Based on sequence analysis, the HA genes from CA/09 and Mexico/09 differ by 4 or 5 amino acids depending on the reference sequences compared. Further work must be done to correlate the changes in the HA gene to the reduced shedding and transmission of the MX/09 virus.

\section{Acknowledgments}

We thank Michelle Harland, Brian Pottebaum and Jason Huegel for assistance with laboratory techniques and animal studies. Funding provided by USDA-ARS and DHHS-CDC.

\section{References}

1. R. J. Garten et al., Science 325, 197 (Jul 10, 2009).

2. A. L. Vincent et al., Virus Genes 39, 176 (2009). 


\subsection{7}

\section{Efficacy studies of a USDA conditionally-licensed vaccine for pandemic H1N1 swine influenza virus}

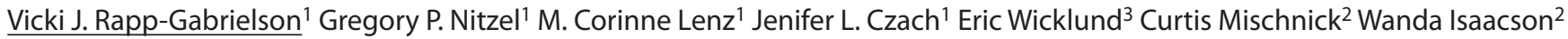
Michael A. Ellsworth ${ }^{3}$ Stephen K. Behan ${ }^{1}$ Therese K. Yonkers ${ }^{1}$ Lucas P. Taylor ${ }^{1}$ Shelly A. Zager Melissa Inman $^{4}$ Teresa Schieber $^{5}$

1. Veterinary Medicine Research and Development, Pfizer Animal Health, Kalamazoo, MI, USA; 2. Veterinary Medicine Research and Development, Pfizer Animal Health, Lincoln, NE, USA; 3. Pfizer Global Manufacturing, Pfizer Inc., Lincoln, NE, USA; 4. Benchmark Biolabs (BBL), Lincoln, NE, USA;

\section{Midwest Veterinary Services (MVS), Oakland, NE, USA}

\section{Introduction}

The Center for Veterinary Biologics (USDA/APHIS/ CVB) provided Master Seed Viruses (MSV) to veterinary biologics manufacturers for developing a conditionally-licensed pandemic H1N1 (pH1N1) swine influenza virus (SIV) vaccine for use in the US swine herd. ${ }^{1}$ The objective of these studies was to demonstrate a reasonable expectation of efficacy to support USDA requirements for conditional licensure and to evaluate the vaccine in a vaccinationchallenge model in pigs. ${ }^{2}$

\section{Materials and Methods}

Three studies were conducted in SIV negative pigs. ${ }^{3}$ In all studies, pigs were vaccinated on Days 0 and 14 and an adjuvanted placebo (T01) was included. In Study 1, pigs were vaccinated with adjuvanted, killed $\mathrm{pH} 1 \mathrm{~N} 1$ vaccines prepared from $\mathrm{A} /$ California/04/2009 (T02) or A/Mexico/ 4108/2009 (T03). In Study 2, pigs were vaccinated with low (T02), mid (T03) or high (T04) doses of $A / C A / 04 / 2009$ pH1N1 vaccine. Sera from both studies were tested for hemagglutination inhibition $(\mathrm{HI})$ antibodies to $\mathrm{pH} 1 \mathrm{~N} 1$. In Study 3, pigs were vaccinated with the A/CA/04/2009 pH1N1 vaccine formulated at a high (T02) or low (T03) dose. Lung lesions were scored at necropsy, 5 or 10 days after challenge with A/Mexico/ 4108/2009 pH1N1 virus. The studies were conducted in accordance with the Institutional Animal Care and Use Committees at the PAH, MVS or BBL sites.

\section{Results}

Cross-reactivity between the two $\mathrm{pH} 1 \mathrm{~N} 1$ viruses was apparent in Study 1 (Figure 1). In Study 2, the GMT, and individual HI titers of all vaccinated pigs, were $\geq 40$ in sera collected 7 or 14 days postrevaccination (data not shown). In Study 3, the mean percent lung lesion scores for both vaccine groups were significantly lower $(\mathrm{P} \leq 0.0001)$ than that of the placebo group (Figure 2$)$.

Figure 1: $\mathrm{HI}$ antibody titers of pigs to A/CAV04/2009 and AMX/4108/2009 pH1N1 (Study 1)

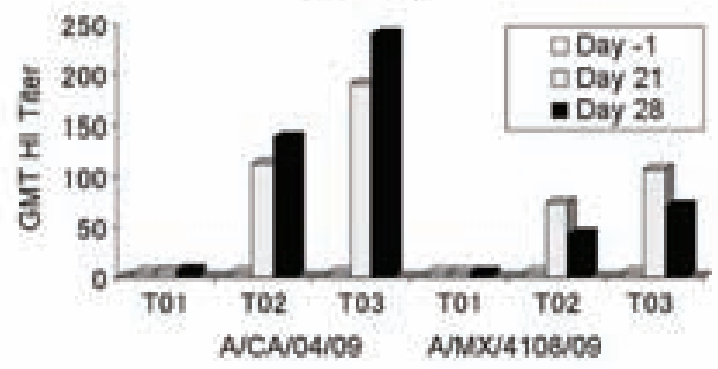

Figure 2: Mean lung lesions of pigs challenged with $\mathrm{A} M \mathrm{MX} / 4108 / 2009 \mathrm{pH} 1 \mathrm{~N} 1$ virus (Study 3)

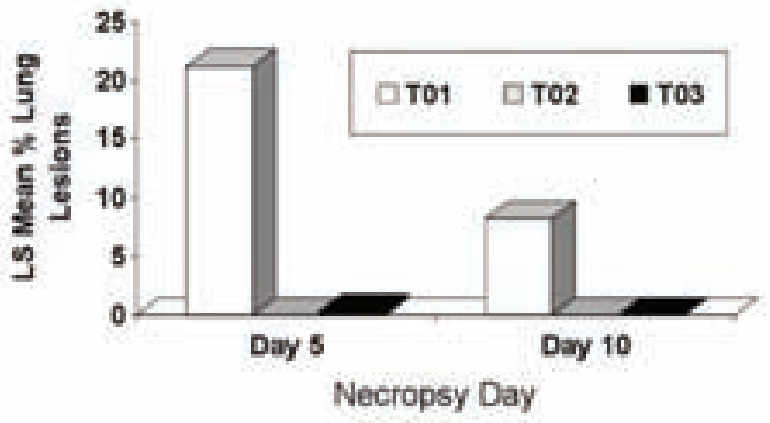

\section{Discussion}

Pigs vaccinated with the $A / C A / 04 / 2009 \mathrm{pH} 1 \mathrm{~N} 1$ vaccine developed $\mathrm{HI}$ titers of $\geq 40$, generally considered as a protective level. ${ }^{4,5}$ The vaccine also significantly reduced lung lesions in pigs challenged with a heterologous $\mathrm{pH} 1 \mathrm{~N} 1$ virus.

\section{References}

1. Center for Veterinary Biologics Notices No. 09-10, June 9, 2009 and No. 09-18, August 3, 2009.

2. Notice: This product license is conditional. Efficacy and/or potency of this product have not been fully demonstrated. Consultation with a veterinarian is recommended.

3. Study Report Nos. 3125R-60-09-764, 3125R-60-09-761, and 3121R60-09-792, Pfizer Inc.

4. Kuhn, M. and M.D. Ficken. 2003. Proceed. AASV. p. 231-233. Orlando, Florida.

5. Wasmoen, T. 2001. Pre-conference Seminar, Managing and Tracking SIV, p. 23-31. Allen D. Leman Swine Conference, St. Paul, Minnesota. 


\title{
0.038
}

\section{Experimental infection with H1N1 European Swine Influenza virus protects pigs from a subsequent infection with the $\mathbf{2 0 0 9}$ pandemic H1N1 human influenza virus}

\author{
Nuria Busquets ${ }^{1}$ Joaquim Segales ${ }^{1,2}$ Lorena Cordoba $^{1}$ Tufaria Mussa ${ }^{1}$ Elisa Crisci ${ }^{1}$ Gerard E. Martin-Valls ${ }^{1}$ \\ Meritxell Simon-Grifé ${ }^{1}$ Marta Pérez-Simó ${ }^{1}$ Monica Perez-Maillo ${ }^{1}$ Jose I. Nunez ${ }^{1}$ Francesc X. Abad ${ }^{1}$ Lorenzo Fraile $^{1}$ \\ Sonia Pina ${ }^{1}$ Natalia Majo ${ }^{1,2}$ Albert Bensaid $^{1}$ Mariano Domingo ${ }^{1,2}$ Maria Montoya ${ }^{1}$ \\ 1. Centre de Recerca en Sanitat Anima (CReSA), Bellaterra, Spain; \\ 2. Departament de Sanitat i Anatomia Animals, Facultat de Veterinaria, Universitat Autonoma de Barcelona, Bellaterra, Spain
}

\section{Introduction}

The recent pandemic human influenza virus $A(H 1 N 1) 2009$ contains gene segments from ancestors in North American and Eurasian swine lineages as well as from avian and human lineages. The emergence of this $\mathrm{A}(\mathrm{H} 1 \mathrm{~N} 1) 2009$ virus poses a potential global threat for human health. Importantly, the fact that it can infect other species, like pigs, favors a possible encounter with other influenza virus circulating in swine herds. In Europe, $\mathrm{H} 1 \mathrm{~N} 1, \mathrm{H} 1 \mathrm{~N} 2$ and $\mathrm{H} 3 \mathrm{~N} 2$ subtypes of swine influenza virus (SIV) currently have a high prevalence in commercial farms. To achieve a better assessment of the risk posed by the $\mathrm{A}(\mathrm{H} 1 \mathrm{~N} 1)$ 2009 virus in the actual situation of swine farms, it was analyzed whether a previous infection with a circulating European avianlike swine influenza virus generated cross-protective immunity against a subsequent infection with the new human influenza virus $A(\mathrm{H} 1 \mathrm{~N} 1) 2009$.

\section{Material and methods}

Two Influenza A virus isolates were used in this study: a circulating European avian-like swine influenza virus strain (A/ Swine/Spain/53207/2004 (H1N1)) (GenBank accession number CY010587 and hereafter referred to as V1) and the new human influenza virus A(H1N1) 2009 (A/Catalonia/63/2009 (H1N1)) (GenBank accession numbers GQ464405-GQ464411 and GQ168897, hereafter referred to as V2). Twenty-two snatchfarrowed, colostrum-deprived Large White $x$ Landrace piglets were obtained from a herd with a standard health status. At the age of 40 days, pigs were randomly distributed into four groups, namely C/C $(n=6)$, V1/C $(n=4), C / V 2(n=8)$ and V1/V2 $(n=4)$ balanced by sex and weight. Group V1/C animals were intranasally inoculated with $3.5 \mathrm{ml}$ of 107.04 tissue culture infectious doses $50 \%$ (TCID50) per $\mathrm{ml}$ of $\mathrm{V} 1$ on day 0 . Pigs from group C/V2 received $3.5 \mathrm{ml}$ of $106.15 \mathrm{TCID} 50$ per $\mathrm{ml}$ of $\mathrm{V} 2$ on day 21 . Finally, pigs in group V1/V2 were inoculated with both viruses, V1 on day 0 and $V 2$ on day 21 , whereas $C / C$ pigs served as control animals.

Pigs were clinically monitored daily until the necropsy day (28 days post-inoculation). Blood and nasal swabs were taken regularly during the experimental period. Anti-influenza A virus nucleoprotein (NP) antibodies (IgG in serum and IgA in nasal swabs) levels were also measured. Samples were analyzed by ID Screen ${ }^{\circledR}$ Influenza A Antibody Competition ELISA (ID-Vet, France). Primers and probes were modified to improve the European avian-like swine and new human influenza virus $A(\mathrm{H} 1 \mathrm{~N} 1)$ 2009 detection based on sequences database and previous reports (1). V1 and V2 viral loads in nasal swabs and lung tissue were assessed by a TaqMan quantitative RT-PCR. Inhibition of hemagglutination on serum was performed following standard procedures (2). The test was standardized at four hemagglutinin units and the starting dilution was 1:20.

\section{Results}

Pigs only infected with V2 had mild to moderate gross lesions (broncho-interstitial pneumonia). However, pigs inoculated with V1 virus and subsequently infected with V2 had very mild lung lesions, apparently attributed to the residual lesions caused by V1 infection. These later pigs also exhibited boosting levels of specific IgA antibodies in nasal swabs and IgG in sera. This boosting effect was also apparent for inhibition of V1 and V2 hemagglutination levels. Finally, in all animals from group V1/V2, neither virus shedding nor viral load in lungs or in nasal swabs were detected after challenge with V2, indicating a cross-protective effect in which specific anti-influenza antibodies seem to play a crucial role.

\section{Discussion}

A previous infection with one of the circulating European avianlike SIV H1N1 in the field was able to confer protective immunity to pigs against a challenge with the new variant influenza virus $\mathrm{A}(\mathrm{H} 1 \mathrm{~N} 1) 2009$ virus. These data pave the way for understanding the intimate immune responses generated between different influenza viral infections, within the same subtype. Further characterizations are required to understand the whole picture and all the mechanisms involved in protection.

\section{References}

1. Spackman, E. et al. J Clin Microbiol 40, 3256-60 (2002).

2. Webster, R. \& Krauss, S. (ed. WHO, W. H. O.) (Geneva, Switzerland, 2002). 


\title{
0.039
}

\section{Longitudinal study of swine influenza virus infection in a farrow to finish farm}

\author{
Meritxell Simon-Grifé ${ }^{1}$ Gerard E. Martín-Valls ${ }^{1}$ Maria J. Vilar ${ }^{1}$ Mercedes Mora ${ }^{1}$ \\ Margarita Martín ${ }^{2,1}$ Núria Busquets ${ }^{1}$ Enric Mateu²,1 Jordi Casal2, 1 \\ 1. Centre de Recerca en Sanitat Animal (CReSA), UAB-IRTA, Campus de la Universitat Autònoma de Barcelona, Bellaterra, Barcelona, Spain; \\ 2. Departament de Sanitat i d'Anatomia Animals, Universitat Autònoma de Barcelona, Bellaterra, Barcelona, Spain
}

\section{Introduction}

Pigs can be infected with avian, swine and human influenza $A$ viruses and, historically, swine was proposed to be the mixing vessel where reassortant influenza strains arise; however the H5N1 incidents showed that avian influenza viruses could be transmited directly to humans. The recent emergence of a human pandemic influenza A virus harbouring genes thought to be originally of swine influenza viruses (SIV) fuelled again the interest in the epidemiology of influenza in pigs. Swine influenza is a common respiratory pathogen of pigs causing a disease clinically similar to human influenza although, as suggested by others (1), subclinical infections might be common, particularly when considering the high seroprevalences (2) and the relatively low incidence of confirmed influenza outbreaks. It is worth to note, that the precise dynamic of SIV infection within a farm is scarcely known beyond the situation of an epidemic clinical outbreak. The present study was aimed to determine the infection dynamics of SIV in a farrow-to-finish farm.

\section{Material and methods}

A farrow-to-finish farm previously known to be positive for SIV antibodies was selected for the present study. The farm had 300 sows in stock and the annual replacement percentage in average was a 55\%. Farrowing batches were scheduled every 3 weeks. A whole weaning batch $(n=121)$ of 3 -week-old piglets was selected for the study. Nasal swabs and sera were taken weekly between the 3rd and the 13th week of age and afterwards, pigs were sampled again at 15, 17, 20 and 24 weeks of age. In each visit to the farm a clinical inspection of pigs was performed. Serum samples were tested for antibodies against swine influenza nucleoprotein using a commercial indirect ELISA (CIVTEST influenza, HIPRA, Spain). Samples were considered to be positive when the sample-to-positive control (S/P) ratio $\geq$ 0.2. SIV shedding was determined in nasal swabs by means of a TaqMan one-step qRT-PCR using a Fast7500 equipment (Applied Biosystems, Foster City, CA). Primers and probe were adapted from a previous report (3) and the design was based on sequences of European SIV strains retrieved from Genbank to improve the SIV matrix (M) gene detection.

\section{Results}

No clinical signs of influenza were observed for the duration of the study. However, seropositive animals were detected by ELISA at all sampling times (Figure 1). Initially, (3 weeks of age) seroprevalence was of $56.2 \%(68 / 121)$ declining until a low of $10.4 \%(12 / 115)$ at 6-weeks of age. Afterwards and until 13 weeks of age, seroprevalence showed ups and downs. From 15 weeks of age, all remaining seronegative animals seroconverted. Based on RT-PCR results, shedder pigs were observed at 3, 4, 7, 13, 15, 17,20 and 24 weeks of age with point prevalences ranging from 1 to $25 \%$. As shown in Figure 1, four waves of viral circulation were observed: weaning (3-4 weeks of age), 7 weeks of age, 13 weeks of age and 20 weeks of age. Interestingly the same animal could be shedding SIV in different infection waves.

Figure.1: Percentage of positive animals by ELISA and RT-PCR per week

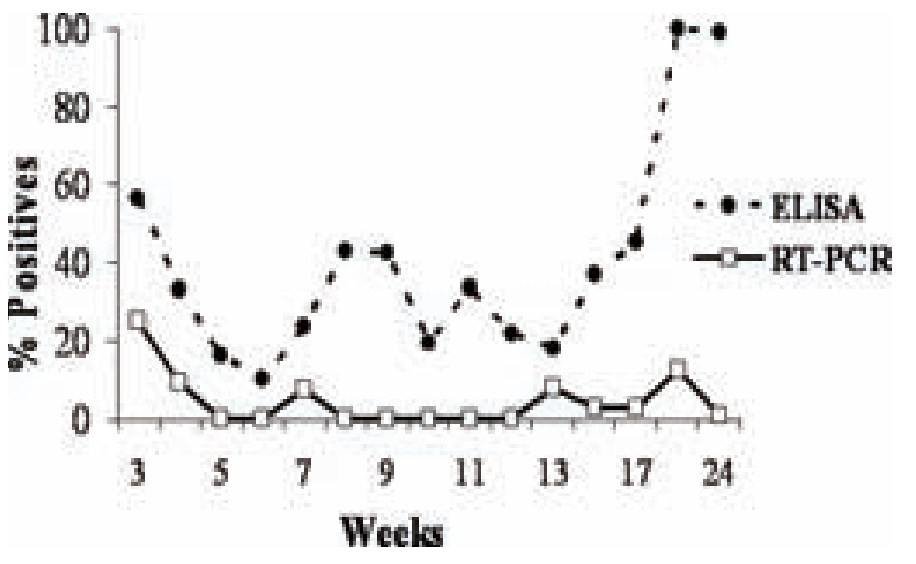

\section{Discussion}

Results of the present study showed that SIV may circulate subclinically in an endemic farm. Maternal-derived antibodies (MDA) probably persisted for at least 6 weeks and, most probably, very early infections became softened because of those MDA. The results also show different consecutive viral waves that were not translated to an explosive spread in the farm (general seroconversion) except for the last two waves, suggesting the introduction and circulation of a strain previously absent in the population in late stage fatteners and finishers. In summary, the present data suggests an endemic and subclinical circulation of SIV in consecutive viral waves.

\section{References}

1. Loeffen, WL. et al. Veterinary Microbiology. 91, 295-308 (2003).

2. Maldonado, J. et al. The Veterinary Journal. 172, 377-381(2006)

3. Spackman, E. et al. Journal of Clinical Microbiology 40, 3256-3260 (2002). 


\title{
0.040
}

\section{Development of an experimental aerosol infection model for swine influenza}

\author{
Ralf Dürrwald \\ IDT Biologika GmbH, Dessau-Roßlau, Germany
}

\section{Introduction}

The use of an appropriate challenge model is of considerable importance for any demonstration of the efficacy of a vaccine. Experimental induction of swine influenza is a difficult process. Pigs very often show no clinical signs of disease, or only a few such signs, upon experimental infection with influenza $A$ viruses. Intratracheal infection is the method recommended by the European Pharmacopoeia (1), but this method is not entirely satisfactory in terms of disease induction. Therefore a new aerosol challenge model for swine influenza was developed using a nebulizer.

\section{Materials and Methods}

The nebulizer (SAG-1) generates very fine droplets in a stable aerosol. Nebulization was performed in isolation rooms, with the generators suspended from the ceiling above the pigs, and all swine were in one room. The containers were filled with medium from tissue-cultivated virus (around 6 I of medium with 10 6.0-8.0 $\mathrm{TCID}_{50} / \mathrm{ml}$ on average), the doors to the isolation rooms were closed and an air pressure of 3-4 bar set on the generator. Principally the generator produces droplets in the range 0.5 to $20 \mathrm{mi}-$ crometers. Droplets in the range 5 to $20 \mu \mathrm{m}$ are deposited in the trachea, bronchi and alveoli. Smaller droplets of $2-5 \mu \mathrm{m}$ reach the alveoli and very small droplets of $0.2-0.5 \mu \mathrm{m}$ are exhaled again. Once nebulization was started the ventilation system was switched off so that the aerosol was constant for at least 1 hour.

Intratracheal challenge tests were also performed for comparison purposes by two different methods i) injection through a needle positioned close to the larynx in the cartilage without narcosis, ii) injection through a tube after intubation under narcosis.

In total, 60 infection trials with 2346 pigs using 46 strains of swine influenza $A$ viruses of subtypes $\mathrm{H} 1 \mathrm{~N} 1, \mathrm{H} 1 \mathrm{~N} 2$ and $\mathrm{H} 3 \mathrm{~N} 2$ including the new pandemic H1N1 2009 virus were carried out. Vaccinated and un vaccinated pigs were investigated in trials for proof of onset of immunity and duration of immunity as well as in dose titration trials comprising $>100$ pigs in one trial.

\section{Results}

Within 24 hours of aerosol nebulization the unvaccinated pigs developed elevated body temperatures of around $41^{\circ} \mathrm{C}$, tachypnea and dyspnea characterized by frequent labored abdominal breathing. The animals had a remarkable loss of appetite. Infected pigs generally recovered very soon. Body temperature and breathing normalized within 3-4 days of infection. Coughing was observed during that period and disappeared a few days later. Virus was shed over seven days. First antibodies occurred after 5-7 days. Vaccinated animals did not have elevated body temperature or only a low temperature rise. They were free of clinical signs of disease or had only very minor signs compared to unvaccinated pigs. Whereas unvaccinated pigs reached a plateau in body weight development, vaccinated pigs were not influenced by the infection. Strains of the subtypes differed in their virulence. Strains of subtype H3N2 were always virulent inducing severe respiratory disease and isolated cases of death. $\mathrm{H} 1 \mathrm{~N} 2$ strains were also virulent whereas $\mathrm{H} 1 \mathrm{~N} 1$ strains reflected a great variation in virulence but being in the majority of the experiments very mild (weak respiratory symptoms, no, or a very short period of fever induction). Aerosol infection did not harm the animals whereas after intratracheal infection single pigs suffered from the procedure due to either narcosis or injection which influenced the clinical outcome. Moreover, after intratracheal injection lungs had strong atypical lesions whereas lungs after aerosol injection reflected lesions in the apical parts of the lungs comparable to those after natural infection.

\section{Discussion}

The newly developed aerosol infection model is superior to intratracheal infection. It allows infecting a large number of pigs (up to 200) in one single trial within a very short period of time and without harm to the animals. Disease is induced within 24 hours and is either more severe after aerosol challenge in comparison to intratracheal challenge or comparable to it. In contrast to intratracheal infection there are no artificial lung lesions. The newly developed model is a valuable tool for vaccine development and research.

\section{References}

1 Porcine influenza vaccine (inactivated). In: European Pharmacopoeia. European Directorate for the Quality of Medicines 01/2005: 0963. 


\title{
0.041
}

\section{Susceptibility of swine to low pathogenic $\mathrm{H} 5$ and $\mathrm{H} 7$ avian influenza viruses}

\author{
Kelly M. Lager ${ }^{1}$ Amy L. Vincent ${ }^{1}$ Phillip C. Gauger ${ }^{2}{ }^{1}$ Dennis A. Senne ${ }^{3}$ David E. Swayne ${ }^{4}$ Laura C. Miller $^{1}$ Juergen A. Richt ${ }^{5,1}$ \\ Wenjun $\mathrm{Ma}^{5,1}$ David L. Suarez ${ }^{4}$ Marcus E. Kehrli ${ }^{1}$ \\ 1. USDA-ARS-NADC, Ames, IA, USA; 2. College of Veterinary Medicine, lowa State University, Ames, IA, USA; \\ 3. National Veterinary Services Laboratory, Ames, IA, USA; 4. Southeast Poultry Research Laboratory, Athens, GA, USA; \\ 5. College of Veterinary Medicine, Kansas State University, Manhattan, KS, USA
}

\section{Introduction}

The emergence of the 2009 pandemic H1N1 influenza virus from swine origin viruses 1 reinforced the concern about transmission of animal influenza viruses to man. This follows the transmission of highly pathogenic $\mathrm{H} 5 \mathrm{~N} 1$ viruses from birds to people identified in the late 1990s and continuing today.2 These two events demonstrate the transmission of novel influenza viruses to man. In addition, the discovery of a unique $\mathrm{H} 2 \mathrm{~N} 3$ reassortant virus in swine 3 provided insight into the introduction and adaptation of low pathogenic avian influenza virus (AIV) genes into a mammalian host. To explore the likelihood of swine serving as an indirect host for AIV, we tested the susceptibility of swine to low pathogenic $\mathrm{H} 5$ and $\mathrm{H} 7$ viruses isolated from North America.

\section{Materials and Methods}

Four-week-old influenza-free pigs were randomly allotted to one of 12 groups (Table 1). All pigs in each group $(n=12)$ were inoculated with a sham (Group 1) or virus inoculum (Groups 2-12). Virus challenge dose was about $1 \times 10^{6} \mathrm{EID}_{50}$ in a two ml volume that was dripped behind each lower eyelid and into each naris. Group 6 was given a swine virus to serve as a positive control; the remaining groups received either an $\mathrm{H} 5$ or $\mathrm{H} 7$ AIV.

At 3, 5, 7, and 28 days-post-inoculation (dpi), three pigs were euthanized in each group for virus detection and lesion evaluation. At 2 dpi, five contact pigs were comingled with each group and maintained as a group until $28 \mathrm{dpi}$ when all pigs were euthanized. Nasal swabs were collected on days 2, 4, and 6 pi from all live pigs in each group. Bronchioalveolar lavage fluid (BALF) was collected at necropsy. Nasal swabs and BALF were tested for viral nucleic acid with real-time RT-PCR.4 Day 28 pi sera was tested for influenza virus antibody with a commercially available ELISA.5

\section{Results}

No virus, antibody, or lesions were found in the negative control group, Group 1 pigs (Table 1). Challenge virus was only detected in NS from group 6 pigs challenged with the swine H1N1 at 2, 4, and 6 dpi (data not shown). Group 6 contact pigs were the only contact pigs that seroconverted by $28 \mathrm{dpi}$. AIV challenge virus was detected in the BALF from one or more pigs in each challenge group. Except for group 7, one or more inoculated pigs developed antibody by $28 \mathrm{dpi}$ : however no contact pigs in the AIV groups seroconverted. Mild lung lesions were observed in each group of pigs.
Table 1: Challenge group/subtype, virus detection in BALF, and seroconversion by ELISA.

\begin{tabular}{|c|c|c|c|c|c|c|}
\hline Group & & $3 \mathrm{dpi}$ & $5 \mathrm{dpi}$ & $7 \mathrm{dpi}$ & IN & DC \\
\hline 1 & Control & $0 / 0$ & $0 / 0$ & $0 / 0$ & 0 & 0 \\
\hline 2 & H7N9WF & $2 / 0.8$ & $0 / 2.9$ & $0 / 0.4$ & 2 & 0 \\
\hline 3 & H5N3 CK & $3 / 1.2$ & $1 / 0.7$ & $2 / 0.3$ & 1 & 0 \\
\hline 4 & H7N2 TY & $1 / 1.0$ & $0 / 0.3$ & $0 / 0.3$ & 1 & 0 \\
\hline 5 & H5N9TY & $2 / 3.7$ & $1 / 0.3$ & $0 / 0.0$ & 3 & 0 \\
\hline 6 & H1N1 SW & $3 / 11$ & $3 / 17$ & $3 / 13$ & 3 & 5 \\
\hline 7 & H5N9 EM & $2 / 3.7$ & $3 / 3.2$ & $2 / 4.3$ & 0 & 0 \\
\hline 8 & H5N3 MA & $1 / 3.9$ & $1 / 4.7$ & $0 / 2.1$ & 3 & 0 \\
\hline 9 & H7N8 MA & $3 / 2.4$ & 2/5.1 & $1 / 2.0$ & 3 & 0 \\
\hline 10 & H7N2 CK & $2 / 1.4$ & $2 / 2.2$ & $0 / 0.7$ & 3 & 0 \\
\hline 11 & $\mathrm{H} 5 \mathrm{~N} 2 \mathrm{PH}$ & 2/1.6 & $3 / 1.5$ & $1 / 3.0$ & 3 & 0 \\
\hline 12 & H7N2 QU & $2 / 0.8$ & $3 / 0.2$ & $0 / 0.4$ & 2 & 0 \\
\hline \multicolumn{7}{|c|}{$\begin{array}{l}\text { WF-waterfowl, CK-chicken, TY-turkey, SW-swine, EM-emu, MA-mallard, PH-pheasant, QU-quail. } \\
{ }^{*} \text { Number of PCR positive BALF samples out of } 3 \text { pigs/Mean lung lesion score for the } 3 \text { pigs. }{ }^{* *} I N= \\
\text { inoculated (positive out of } 3 \text { ) DC=direct contact pigs (positive out of 5) }\end{array}$} \\
\hline
\end{tabular}

\section{Discussion}

Challenge virus was detected in the BALF from one or more pigs in each of the AIV-challenged groups indicating that swine were susceptible to infection via a mucosal exposure. Although pigs were susceptible to infection, lung lesions were mild and variable. There was no virus detected from or serologic evidence of virus transmission from AIV-inoculated pigs to their respective contacts. In contrast, the contact pigs for group 6 were infected with the swine challenge virus and each pig seroconverted. Moreover, this was the only contact group from which viral nucleic acid was detected in nasal swabs. Some pigs were negative following mucosal challenge with AIV suggesting innate resistance or a challenge dose at the minimal threshold of infection.

\section{Acknowledgements}

Funding was provided by USDA ARS and a USDA APHIS AIV Supplemental Fund.

\section{References}

1. Garten, R. J. et al., Science 2009:325;197.

2. Duan, L. et al., Virology 2008:380;243.

3. Ma, W., et al., Proc Natl Acad Sci 2007:104;20949.

4. Spackman, E., et al., J Clin Microbiol 2002:40;3256.

5. Ciacci-Zanella, J., et al., J Vet Diagn Inv 2010:22;3. 


\title{
0.042
}

\section{Dynamics of swine influenza infections in the farrowing unit of a Danish sow herd}

\author{
Lars E. Larsen ${ }^{1}$ Nielsen K. Christian² Simon Aakerblom² Charlotte K. Hjulsager ${ }^{1}$ Jens P. Nielsen ${ }^{2}$ \\ Helle H. Stege ${ }^{2}$ Charlotte S. Kristensen ${ }^{3}$ Anders Elvstrom ${ }^{4}$ Leon Lau ${ }^{5}$ \\ 1. National Veterinary Institute, Copenhagen, Denmark; 2. University of Copenhagen, Faculty of Life Science, Copenhagen, Denmark; \\ 3. Pig Research Centre, Danish Agriculture and Food Council, Kjellerup, Denmark; 4. Odder Dyreklinik, Odder, Denmark;
} 5. Merial Norden A/S, Skovlunde, Denmark

\section{Introduction}

Swine influenza virus (SIV) is a common pathogen in pigs. In Denmark $90 \%$ of the sow herds are influenza A positive. The most common serotypes are $\mathrm{H} 1 \mathrm{~N} 1, \mathrm{H} 3 \mathrm{~N} 2$ and $\mathrm{H} 1 \mathrm{~N} 2$. Clinical signs are often manifested as acute respiratory disease with fever, anorexia, weight loss, lethargy, coughing, dyspnea, nasal, and ocular discharge. Reproductive failure and abortions can be seen in sows (1). Secondary infections and increased use of antibiotics are common sequelae (2). The economic importance, the within-herd infection dynamics and the clinical impact of the disease on piglets are, however, not fully elucidated. The aim of this study was to examine the clinical impact and the infection dynamics of SIV in a Danish sow herd. Furthermore, the effect of the age of the sows on SIV infection in the piglets was studied.

\section{Materials and Methods}

A total of 44 sows of Danish landrace and Yorkshire crossbreds and 199 piglets were included. From each sow, five randomly selected piglets were earmarked. Blood samples (serum) were collected from each sow prior to the transfer to the farrowing unit and 4 weeks after transfer. Nasal swabs were collected 5 days after transfer. Rectal temperature were taken at each sampling and the sows were monitored clinically daily during the first week after farrowing.

The piglets were observed clinically daily until weaning and a detailed clinical examination were performed 10-14 days after birth. The piglets were weighed 0-5 days after birth, 10-14 days after birth and at weaning (approx. day 28). From each piglet, nasal swabs were collected from both nostrils and pooled by litter (10 swabs per pool/litter). The nasal swabs were collected in 1 $\mathrm{ml}$ saline, send to the laboratory at the day of sampling and kept at -80 oC until test. Serum was separated from the blood samples by centrifugation and stored at $-20 \mathrm{oC}$. The nasal swabs were tested for SIV by real-time SYBR RT-PCR using primers previously described (3) and the serum samples were tested for antibodies by the standard haemagglutination inhibition test against the common SIV subtypes present in Denmark.

\section{Results}

SI virus was detected by RT-PCR in 12 out of the 43 tested litters (28.6\%). In contrast, SI virus was not detected in any of the 43 tested sows. Of the 43 sows, however, 12 (27.9\%) showed a significant (minimum 2-fold) increase in $\mathrm{HI}$ titer against at least one of the tested SIV subtypes. There was no significant relationship between SIV positive litter and increased antibody titer of the mother sow. The 1.st and 2.nd. parity sows had significantly more SIV positive litters compared to the older sows. Accordingly, the younger sows had a statistically significant higher risk of having a minimum 2-fold increase in antibodies against SIV in relation to farrowing. Apart from coughing, there were no significant differences in the incidences of individual clinical symptoms of SIV positive and negative litters, however, when the clinical signs were scored and summed up for each litter there was a significant higher score in litters with SIV positive piglets. The average daily weight gain was not different between SIV positive and negative litters.

\section{Discussion and conclusion}

The present study showed that a relatively high proportion of young piglets in a farrowing unit of a herd with circulating swine influenza virus were positive for SIV during the first weeks after birth. This was surprising since we expected that the piglets were protected by maternal antibodies. Interestingly, piglets from young sows had a higher risk of being infected by SIV compared to piglets from older sows. Since all the sows were negative for virus in nasal swabs and there was no correlation between seroconversion of a particular sow and the risk of her litter being positive, the piglets are probably not infected by their mother-sow. Thus, the increased risk of piglets from young sows being infected by SIV may be due to sub optimal quality of the passively acquired immunity. Thus, in relation to the handling of SIV in breeding units, the focus should be on the offspring of gilts and young sows.

\section{References}

1. F. Madec, C. Kaiser, J. M. Gourreau, F. Martinat-Botte, Comp Immunol. Microbiol. Infect. Dis. 12, 17 (1989).

2. W. L. Loeffen, W. A. Hunneman, J. Quak, J. H. Verheijden, J. A. Stegeman, Vet. Microbiol. 137, 45 (2009).

3. M. Munch, L. P. Nielsen, K. J. Handberg, P. H. Jorgensen, Arch. Virol. 146,87 (2001). 


\title{
0.043
}

\section{Tetracycline administered in water: pharmacokinetics and lessons for administration of other medications via water}

\author{
Sharon Mason ${ }^{1}$ Ronald Baynes ${ }^{1}$ Glen Almond ${ }^{1}$ Jim Riviere $^{1}$ Alan Scheidt ${ }^{2}$ \\ 1. North Carolina State University, Raleigh, NC, USA; 2. Pfizer Animal Health, Kalamazoo, MI, USA
}

\section{Introduction}

The use of medications dosed in water is a common occurrence in swine medicine. However, no published pharmacokinetic (PK) studies are available on tetracycline dosed in water in swine or the factors that determine the plasma concentration levels achieved in medicated swine.

\section{Materials and Methods}

Study. Twenty-four castrated male swine, 9 weeks of age (early grower), divided into 4 groups ( $n=6)$, were exposed to tetracycline powder administered exclusively in water at 0, 125, 250 and 500 ppm via a randomized block design (1). Blood samples were collected before and at 4, 8, 12, 24, 32, 48, 56, 72, 80, 96 and $104 \mathrm{~h}$ post-exposure. All plasma samples were assayed using HPLC-UV with a LOD of $0.05 \mu \mathrm{g} / \mathrm{mL}$ within 7 days of collection (1). Statistical analysis across groups and covariates using a one-way ANOVA (SAS 9.1, Cary NC) revealed no bias. All animals were healthy and the protocol met IACUC standards at NC State University College of Veterinary Medicine.

Pharmacokinetic analysis. Non-compartmental analysis was performed on plasma concentrations. Population PK analysis using a one-compartmental model was performed in Phoenix WinNonMix NLME 1.0 (Pharsight, Mountain View, CA). The developed model was improved and validated by two additional data sets from Dorr et al (2). Potential covariates considered were body weight, ambient temperature, water consumption, water concentration of tetracycline and health status of the animal.

\section{Results}

Non-compartmental PK analysis and statistical analysis showed that all plasma levels were above control (no exposure). Average plasma concentration levels for the 125- and 250-ppm groups were not significantly different from one another, but were significantly less than the 500-ppm group (Table 1). Population PK analysis showed that for tetracycline, health status did not improve the ability of the model to predict plasma concentrations. The population model included body weight, medication and water use. The population model was able to predict plasma concentrations of tetracycline and PK values $(\mathrm{Vd}, \mathrm{Cl})$ similar to previously published values. Bio-availability for the population model was similar to oral bioavailability (F) from the in vivo study.
Table 1: Pharmacokinetic parameters of tetracycline

\begin{tabular}{|c|c|c|c|}
\hline Parameter/Group ${ }^{\mathrm{a}}$ & 125 ppm & $250 \mathrm{ppm}$ & $500 \mathrm{ppm}$ \\
\hline Cmax, $\mu \mathrm{g} / \mathrm{ml}$ & 0.80 & 1.26 & 1.29 \\
\hline $\mathrm{Css}, \mu \mathrm{g} / \mathrm{ml}$ & 0.33 & 0.47 & 0.77 \\
\hline Half-life, h & 4.60 & 4.58 & 4.46 \\
\hline $\mathrm{Cl}, \mathrm{L} /\left(\mathrm{kg}{ }^{*} \mathrm{~h}\right)$ & 0.083 & 0.107 & 0.106 \\
\hline Vdss, L/kg & 0.554 & 0.646 & 0.769 \\
\hline F, apparent & 0.059 & 0.061 & 0.057 \\
\hline
\end{tabular}

\section{Discussion}

Tetracycline water medication PK parameters were consistent with values from the literature. Plasma levels were lower than those from observational studies in which animals were fasted. Low plasma concentrations may be due to low oral bioavailability and cation chelation of tetracycline by food.

Body weight, water consumption and water concentrations of the medication are the most important covariates for population PK analysis. Temperature was important in the initial study; however, ambient temperatures were not recorded for validation data. Temperatures appear important when large fluctuations are present, because this changes the baseline water consumption.

Population analysis on other medications is necessary for further validation of the above factors and to elucidate other potential covariates. In conclusion, tetracycline exemplifies how drugs with poor absorption have decreased ability to achieve adequate blood levels when dosed via water.

\section{References}

1. Mason et al. 2009, J. Anim. Sci., p 3179-3186.

2. Dorr et al. 2009, JAVMA, p 299-304. 


\title{
0.044
}

\section{Changes in antimicrobial sensitivity of fecal $E$. coli in pigs exposed to enrofloxacin by different dosing routes: evidence for the "Mutant Selection Window"}

\author{
Romain Béraud; Louis Huneault; Dave Bernier; Francis Beaudry; Ann Letellier; Jérôme R. del Castillo \\ Université de Montréal, St-Hyacinthe, QC, Canada
}

\begin{abstract}
Introduction
The fluoroquinolones (FQ) are synthetic, broad spectrum bactericidal antibiotics which use in Canada is restricted to companion animals and beef cattle. Since their introduction in veterinary medicine, FQ-resistant bacteria are emerging in domestic animals, but the relationship between drug use and drug resistance awaits elucidation. Recently, the "mutant selection window" (MSW) concept has been proposed to explain the enrichment of resistant bacteria during FQ exposure [1]. Because drug efflux transporters excrete FQ into the intestinal lumen, where drugresistant zoonotic pathogens may be selected, we hypothesized that the level of selection pressure will vary depending on the FQ dosing route. The study objective was to compare the changes in fecal E. coli population size and antimicrobial sensitivity of pigs treated with enrofloxacin (EFX) orally, intramuscularly, or by way of local delivery implants.
\end{abstract}

\section{Materials and Methods}

Parts of the study have been published elsewhere [2]. Briefly, 21 healthy pigs $(13.6 \pm 5.9 \mathrm{~kg})$ with no previous exposure to antibiotics, and instrumented with a jugular catheter were randomized across EFX-dosing groups: oral (O), intramuscular (I), or local (L: peri-femoral implants with $65 \mathrm{mg}$ EFX). Pigs in groups I and $\mathrm{O}$ were dosed daily for 15 days with ; $5.0 \mathrm{mg} \cdot \mathrm{kg}^{-1} \cdot \mathrm{d}^{-1} \mathrm{EFX}$. Feces were sampled rectally for 7 days prior to and during exposure to FQ. Jugular blood was sampled during treatment, and plasma EFX and ciprofloxacin (CFX) concentrations were measured with tandem mass spectrometry. Individual daily fecal $E$. coli counts, and disk diffusion testing of 12 antibiotics (3 colonies/pig) were performed daily. The effects of dosing route and time (as divided in first-exposure, bactericidal effect, and bacterial regrowth phases) on E. coli inhibition diameters were estimated with a repeated-measures linear mixed model. The effect of dosing route on the duration of FQ bactericidal phase was analysed with survival analysis. The a-level was set to 0.05 for all statistical tests.

\section{Results}

Average plasma EFX concentrations in pigs of group $L$ were $4.5 \%$ of those in the other two groups, and plasma CFX concentrations were $8.5 \%$ of those of EFX. Fecal E. coli were undetectable during the bactericidal phase in all 3 groups. The sensitivity of fecal E. coli to EFX (Fig. 1), CFX, nalidixic acid, ampicillin, and trimethoprim-sulfamethoxazole was significantly affected by dosing route $(P<0.05)$ and time $(P<0.05)$. In contrast, these factors had negligible effect on $E$. coli sensitivity to amoxicillin, ceftiofur, streptomycin, apramicin, gentamicin, florfenicol, and tetracycline.
Figure 1. Enrofloxacin inhibition diameters (least-square means \pm standard error) of Escherichia coli as a function of dosing route and exposure phase

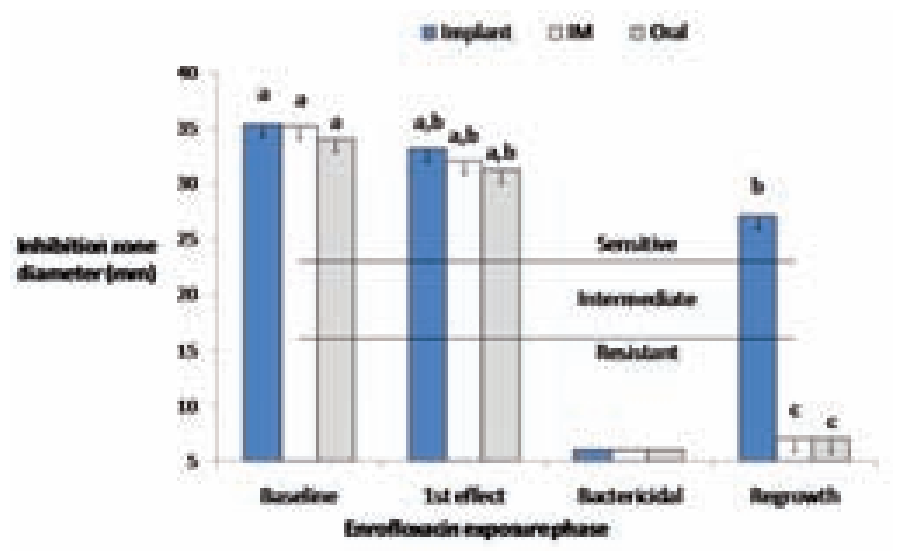

\section{Discussion}

Our hypothesis was verified: the local EFX delivery minimized both the intestinal efflux and selection of drug-resistant $E$. coli. Moreover, oral EFX maximized the bactericidal effect on E. coli, which retarded the resistance selection process. Exposure to EFX co-selected resistance to other antibiotic classes. The i.m. dosing of EFX causes significant resistance selection pressure on potentially zoonotic intestinal bacteria such as fecal E. coli. Because the intestinal FQ efflux exists in other mammals, these drugs should be used with caution.

\section{References}

1. Drlica K, \& Zhao X (2007). Clin. Infect. Dis., 44:681-8.

2. Béraud R, Huneault L, Bernier D, Beaudry F, \& del Castillo JRE (2008). Can. J. Vet. Res., 72:311-9. 


\title{
0.045
}

\section{Pleuromutilin susceptibility of Clostridium perfringens and Clostridium difficile isolates from pigs in Italy and Denmark}

\author{
Fabrizio Agnoletti ${ }^{1}$ Ilenia Drigo ${ }^{1}$ Luca Bano $^{1}$ Ulrich Klein ${ }^{2}$ Josef Szancer $^{3}$ \\ 1. Istituto Zooprofilattico Sperimentale delle Venezie, Treviso, Italy; 2. Novartis Animal Health Inc., Basel, Switzerland; \\ 3. Novartis Animal Health, Copenhagen, Denmark
}

\section{Introduction}

Clostridium perfringens type $A$ is a causative agent of diarrhoea in neonatal piglets with widespread occurrence in many pig producing areas; indeed the consensus $\beta 2$ toxin (cpb2 $2^{\text {con }}$ ) was recently considered the key virulence factor characterising the strains involved in neonatal enteritis of pigs $(1,2)$. The role of Clostridium difficile in neonatal diarrhea is increasing and becoming of greater clinical importance $(3,4)$ in many countries. Aim of the study was to assess the MIC values of toxigenic C. perfringens and $C$. difficile isolates from neonatal healthy piglets (less than 7 days old) or affected by enteritis, collected in Italian and Danish farms, against the pleuromutilin antibiotics, tiamulin and valnemulin (Denagard ${ }^{\circledR}$ \& Econor $^{\circledast}$ respectively - Novartis Animal Health Inc.)

\section{Materials and methods}

Strains: Sixty-eight C. perfringens isolates (30 strains from Italy, 38 strains from Denmark) were used for the study. The Italian strains were recovered from 30 different farms in 2007-2008 and the Danish strains from 20 different farms in 2006. Fifteen C. difficile strains (10 from Italy and 5 from Denmark) were recovered in 2007 -2008 and originated from different farms.

Genetic characterization of isolates: Clostridium perfringens strains were toxin-typed by a multiplex PCR for the detection of major toxin encoding genes (cpa, cpi, cpetx and $c p b)$ (5) and $\beta 2$ gene presence was evaluated according to Baums et al. (6). Cpb2 positive strains were further characterized according to Jost et al. (2) by PCR detection of consensus or atypical $c p b 2$ alleles. Clostridium difficile strains were tested for the presence of toxin $A$ and $B(t c d A$ e $t c d B)$ encoding gene and the presence of binary toxin genes ( $c d t A$ and $c d t B$ ) was also evaluated $(7,8)$. MIC testing: The MICs of tiamulin hydrogen fumarate and valnemulin hydrochloride were evaluated using the agar dilution method and concentrations ranged $0.016-256 \mu \mathrm{g} / \mathrm{ml}$. MIC testing was performed according to the CLSI guidelines (CLSI document M11-A7).

\section{Results}

The MIC values of the tested antimicrobial agents for C. perfringens and C. difficile isolates are shown in Tables $1 \& 2$.

Table1: Tiamulin MIC values.

\begin{tabular}{|l|c|c|c|}
\hline & $\begin{array}{c}\text { Italian C. perfrin- } \\
\text { gens type } \mathbf{A}(\mathbf{n}=30)\end{array}$ & $\begin{array}{c}\text { Danish C. perfrin- } \\
\text { gens type } \mathbf{A}(\mathbf{n}=38)\end{array}$ & C. difficile $(\mathbf{n}=\mathbf{1 5})$ \\
\hline$M_{50}$ & 4 & 2 & 8 \\
\hline$M_{90}$ & 64 & 4 & 16 \\
\hline Geometric mean & 4.6 & 2 & 8.4 \\
\hline MIC range & $0.125-128$ & $0.25-8$ & $0.125-16$ \\
\hline
\end{tabular}

Table2: Valnemulin MIC values.

\begin{tabular}{|l|c|c|c|}
\hline & $\begin{array}{c}\text { Italian C. perfrin- } \\
\text { gens type } \mathbf{A}(\mathbf{n}=\mathbf{3 0})\end{array}$ & $\begin{array}{c}\text { Danish C. perfrin- } \\
\text { gens type } \mathbf{A}(\mathbf{n}=\mathbf{3 8})\end{array}$ & C. difficile $(\mathbf{n}=\mathbf{1 5})$ \\
\hline $\mathrm{MIC}_{50}$ & 0.125 & 0.063 & 0.5 \\
\hline $\mathrm{MIC}_{90}$ & 8 & 0.125 & 1 \\
\hline Geometric mean & 0.3 & 0.06 & 0.5 \\
\hline MIC range & $0.063-32$ & $0.016-0.25$ & $0.063-1$ \\
\hline
\end{tabular}

\section{Discussion}

The Danish isolates of $C$. perfringens were more susceptible to pleuromutilins compared to the Italian isolates. Results for the tested C. perfringens and C. difficile strains show a higher susceptibility to valnemulin compared to tiamulin. Tiamulin pharmacokinetic data at doses of $15-20 \mathrm{mg} / \mathrm{kg}$ bodyweight/ day showed that after oral or parenteral application concentrations in colon contents between $13-18 \mu \mathrm{g} / \mathrm{ml}$ can be achieved $(9,10)$. Based on these data all Danish C. perfringens isolates could be considered susceptible to tiamulin. Twenty percent of the Italian C. perfringens isolates with MIC values between 32 \& $128 \mu \mathrm{g} / \mathrm{ml}$ were considered resistant to tiamulin. All Danish and $90 \%$ of the Italian strains of $C$. perfringens could be considered susceptible to valnemulin due to colon concentrations (1.7-5.2 $\mu \mathrm{g} / \mathrm{ml}$ ) achieved after oral application of valnemulin at doses of $3.8 \& 11.6 \mathrm{mg} / \mathrm{kg}$ bodyweight/day (11). All 15 C. difficile isolates showed susceptibility to valnemulin and tiamulin.

\section{References}

1.Bueschel D. M. et al. (2003).Vet Microbiol. 94,121-129.

2. Jost B.H. et al. (2005). Infect. Immunol 73, 652-656

3. Songer J.G., Anderson M.A. (2006). Anaerobe, 12,1-4

4. Songer J.G. (2004). Ani. H. Res.Rev. 5, 321-326

5. Han S.Y. et al. (1997). J. Clin Microbiol. 35, 228-232.

6. Baums C.G. et al. (2004). Vet. Microbiol. 100, 11-16.

7. Lemee L. et al.(2004). J. Clin Microb. 5710-5714.

8. Stubbs S. et al.(2000). FEMS Microb.Lett. 186: 307-312.

9. McKellar Q.A. et al. (2004). Proc. 18th IPVS, p. 622

10. Anderson M. et al (1994).Proc. AASV, 115-117

11. Burch D. \& Klein U. (2008). Proc. AAVM, 


\title{
0.046
}

\section{Evaluation of the effectiveness of carbonyl iron orally in the iron supplementation in piglets}

\author{
Fernando M. Toledano ${ }^{1}$ José Cristani ${ }^{2}$ Mere E. Saito ${ }^{2}$ Rogério F. Nunes ${ }^{1}$ Thiago K. Valerio $^{1}$ Priscyla C. Silva ${ }^{2}$ \\ 1. Ouro Fino Saúde Animal, Cravinhos, SP, Brazil; 2. Universidade do Estado de Santa Catarina, Lages, SC, Brazil
}

\begin{abstract}
Introduction
Iron is an essential mineral for composition of blood cells, muscle and various enzymes and it is directly related to the cellular respiration of the body, as well as enzymatic reactions and immune cells in the animal organism (1). The current system of intensive pig farming deprives the animal's access to soil, the main natural source of iron supplementation for this species. In order to prevent iron deficiency anemia cases, it is recommended the application of dextran iron injection at the 3rd day of life of piglets. However, this management practice can bring a lot of inconveniences to the development of these animals. In the practice of human medicine for over 3 decades is made of iron supplementation with oral presentations (capsules and tablets) in order to prevent various types of anemia. One of the molecules of iron commonly used for this purpose is the carbonyl iron, due to its high content if organic iron available and high intestinal absorption (2). The purpose of this study was to evaluate the effectiveness of iron carbonyl orally in the iron supplementation in piglets.
\end{abstract}

\section{Material and Methods}

The experiment was conducted in a farm producing piglets in the state of Santa Catarina - Brazil. A total of 60 piglets (initially $1.95 \pm 0.45 \mathrm{Kg}$ ) from 12 sows with same age and number of births and receiving the same management were used in this study. The experiment design was randomized blocks with 5 treatments and 12 repetitions, with each sow representing one experimental block. The treatments were: T1 - non iron supplementation, T2 - 2000mg dextran iron injectable the 3rd day, T3 - 150mg carbonyl iron orally the 3rd day, T4 - 200mg dextran iron injetable the 5 th day; T5 - 150mg carbonyl iron orally the 5 th day. No source of iron supplementation was used in addition to the treatments. The data were compared by Tukey test at a significance level of $5 \%$ for analysis of variance (ANOVA). The parameters evaluated were weight gain (at 21 and 57 days old) and blood parameters (hemoglobin, packed cell volume (at 21 days old).

\section{Results}

The evaluation of weight gain of piglets at 21 days of age showed a non-significant difference $(P>0.05)$ among animals not supplemented and supplemented (dextran iron and carbonyl iron). However, analysis of blood parameters in the same period showed that piglets which did not receive iron supplements had lower $(P<0.05)$ levels of hemoglobin (less 19.34\%) and packed cell volume (less 18.37\%) than those who received supplementation (dextran iron and carbonyl iron). Furthermore, piglets supplemented with dextran iron the 3rd day had higher $(\mathrm{P}<0.05)$ levels of hemoglobin (over $11.99 \%)$ and packed cell volume (over $9.25 \%$ ) than those who received carbonyl iron on the same day, but they were similar $(P>0.05)$ to piglets who received iron supplementation the 5 th day (dextran and carbonyl). Even at 57 days of age, data analysis showed that the piglets non iron supplemented had lower ( $P<0.05$ ) weight gain (less $10.31 \%$ ) than those who received dextran iron supplementation the $3 \mathrm{rd}$ day. There was not difference $(P>0.05)$ in weight gain of piglets according to the source of iron supplementation (dextran or carbonyl) and the timing of supplementation (the 3rd or 5th day).

\section{Discussion}

The data are consistent with (3), showing that piglets kept in intensive system of production and did not receive iron supplementation develop early iron deficiency anemia, evidenced by lower levels of hemoglobin and packed cell volume at 21 days old. However, the negative effect of livestock of iron deficiency in piglets may have a delayed response in relation to blood parameters, since the difference in weight gain among supplemented and non supplemented animals only became evident at 57 days old. The supplementation of carbonyl iron orally was shown to have a positive effect in preventing iron deficiency anemia in sucking piglets, and the administration the 5th day most appropriate. Even so, piglets supplemented with dextran iron the 3rd day had superior results for the blood parameters than piglets non supplemented and the supplemented with carbonyl iron.

\section{References}

1. LINDER, M.C. Nutritional Biochemistry and Metabolism with clinical applications. In: Nutrition and Metabolism of the trace elements. $2^{\mathrm{a}}$ ed. New York: Elsevier Science Publishing Company New York, p. 215-276. 1991.;

2. FRITZ, J. C.; PLA, G. W. Iron for Enrichment. Division of Nutrition, Univertity of Maryland, v. 49, n.2, p. 46-49, 1975.;

3. SVOBODA, M.; DRABEK, J.; KREJCI, J.; REJAKOVA, Z.; FALDYNA, M. Impairement of the peripheral lymphoid compartment in irondeficient piglets. Journal Veterinary Medicin, Berlim, v.51, p.231-237, 2004. 


\title{
0.047
}

\section{Effectiveness of tulathromycin injection for the control of porcine pleuropneumonia on a grower-finisher farm in an outbreak situation}

\author{
Kristen Reynolds; Zvonimir Poljak; Robert Friendship; Cate Dewey \\ Department of Population medicine, OVC, University of Guelph, Guelph, ON, Canada
}

\section{Introduction}

Outbreaks of diseases that result in acute death such as pleuropneumonia caused by Actinobacillus pleuropneumoniae are a great concern in some swine facilities. Porcine pleuopneumonia is partly controllable through good management practices $(1,2)$, though sudden outbreaks can periodically occur. Tulathromycin (DraxxinTM,Pfizer Animal Health) has been proven effective for the treatment of multifactorial swine respiratory disease $(3,4)$, as well as the treatment of porcine pleuropneumonia caused by A. pleuropneumoniae (5) if given up to 9 days prior to known exposure (6). The objective of this study was to evaluate the efficacy of tulathromycin in preventing losses in swine herds due to Actinobacillus pleuropneumoniae under field conditions.

\section{Materials and Methods}

Three field trials were carried out in a farrow-to-finish herd located in Ontario. The finisher barn had a continuous pig flow, and pigs of different ages were frequently housed in the neighbouring pens. The swine herd was endemically infected with a number of swine pathogens including Mycoplasma hyopneumoniae, Actinobacillus pleuropneumoniae, and PRRS virus. In late September 2007, the herd started to experience a sudden increase in mortality during the finisher phase, with both clinical signs and gross pathological lesions indicative of porcine pleropneumonia (PP). Presence of A. pleuropneumoniae (susceptible to penicillin and ceftiofur) was confirmed in typical lung lesions using bacteriological culturing.

Three trials performed in the finisher barn compared treatment with tulathromycin to treatment with long-acting penicillin, ceftiofur hydrochloride, or a combination of the two. Three different types of mortality (overall, reported and mortality due to A. pleuropneumoniae) were analyzed using logistic regression and Cox's proportional hazard models. Linear regression was used to analyze average daily gain (ADG).

\section{Results}

Serotypes 5A and 5B were detected in samples of pigs that died with typical PP lesions. The hazard of dying (Hazard Ratio $=$ HR) was higher in treatments other than tulathromycin in trials 1 $(\mathrm{HR}=2.36, \mathrm{P}=0.05)$ and $2(\mathrm{HR}=3.66 \mathrm{P}=0.02)$ for reported mortality. Similar results were attained for mortality due to A. pleuropneumoniae (Figure 1). Tulathromycin was found to increase average daily gain of pigs in trials 2 (ADG, Beta $=120 \mathrm{~g} /$ day, $\mathrm{P}<0.001)$ and 3 (ADG, Beta $=52.6 \mathrm{~g} /$ day, $\mathrm{P}<0.01)$.
Figure 1. Expected survival curves based on mortality due to $A$. pleuropneumoniae for pigs of average weight exposed to two interventions strategies in three consecutive field trials in a farm experiencing outbreak of porcine pleuropneumonia.

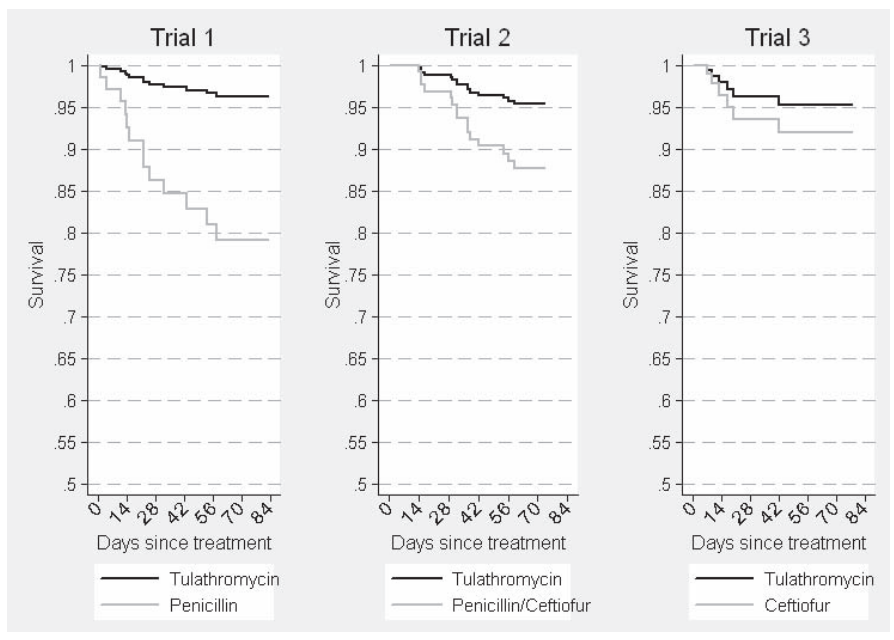

\section{Discussion}

It was concluded that under the conditions of this study and sufficient exposure to A. pleuropneumoniae, tulathromycin would significantly reduce pig mortality and improve growth performance relative to similar treatment with either penicillin or ceftiofur.

\section{References}

1. Veenhuizen MF. Compend Contin Educ Pract Vet 1998;20:S11.

2. Thacker, EL. Vet Clin North Am Food Anim Pract 2001;17:551.

3. Nanjiani IA et al. Vet Ther 2005;6:203-213.

4. Nutsch RG, et al. Vet Ther 2005;6:214-224.

5. Hart FJ, et al. Vet Rec 2006;158:433-436.

6. Waag TA, et al. J Swine Health Prod 2008;16:126-130. 


\title{
0.048
}

\section{Marbofloxacin achieves high concentration in pig tonsils according to a dose dependent fashion}

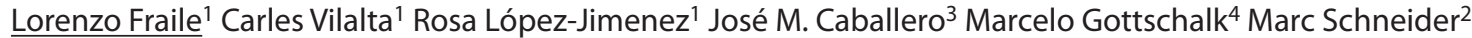 \\ 1. CReSA, Bellaterra, Spain; 2. Vetoquinol R\&D Department, Lure, France; 3. Vetoquinol E.V.S.A, San Fernando de Henares, Spain; \\ 4. Faculté de médicine vétérinarie, Québec, QC, Canada
}

\section{Introduction}

Actinobacillus pleuropneumonaie (APP) is the causative agent of porcine pleuropneumonia. Pigs can become asymptomatic carriers of the organism in their tonsils for long periods. Attempts to eradicate APP from pig herds have been made with antibiotics but there is a paucity of information about the penetration of antibiotics in tonsils. Marbofloxacin (MB) is a fluoroquinolone widely used in veterinary medicine to control APP under field conditions. The goal of this study was to quantify the MB penetration in tonsils after applying several MB regimen dosages.

\section{Material and methods}

Pigs were randomly divided in three groups (control, P1 and $\mathrm{P} 2$ ) of ten animals each one. The control group received $2 \mathrm{~mL}$ of serum saline. On the other hand, $\mathrm{P} 1$ group received a dose of 2 $\mathrm{mg}$ of $\mathrm{MB} / \mathrm{Kg}$ every day for three consecutive days and P2 group received a dose of $4 \mathrm{mg}$ of $\mathrm{MB} / \mathrm{Kg}$ every 48 hours two times. The animals were sacrificed 24 hours after finishing the treatment to obtain tonsils and serum samples. The concentration of marbofloxacin in sera and tonsils was quantified by an HPLC analytical method. Finally, It was also calculated the ratio MB tonsil concentration versus APP MIC90 value $(0.03 \mathrm{mg} / \mathrm{mL}$, ) determined following the CLSI recommendations $(4,7)$. This ratio is one of the PK/PD efficacy parameters described for fluoroquinolones (5).

\section{Results}

Average marbofloxacin serum concentration (AMSC) was 0.16 and $0.24 \mathrm{mg} / \mathrm{mL}$ for the P1 and P2 group, respectively. Average marbofloxacin tonsil concentration (AMTC) was 0.50 and $0.70 \mathrm{mg} / \mathrm{gr}$ for the P1 and P2 group, respectively (see figure). Thus, AMSC and AMTC increased in a dose-dependent fashion. However, the tonsil MB versus serum MB concentration ratio was close to 3 independently of the dose administered to animals.
Moreover, the MB tonsil concentration: APP MIC90 ratio was 16.6 and 23.3 for $\mathrm{P} 1$ and $\mathrm{P} 2$ groups, respectively.

\section{Discussion}

Marbofloxacin achieves a good penetration in tonsillar tissue, which compares favourably with

tonsil/plasma mean concentration ratios reported for other fluoroquinolones $(2,3)$. However, tonsil/plasma ratio observed for MB was very similar to the described by Esposito et al. (2006) for moxifloxacin in humans.

A great deal of information is now available on the PK/PD relationships for fluoroquinolones. Ratios of 125 for AUC0-24:MIC and 10 for Cmax:MIC have been recommended to achieve high clinical efficacy for concentration-dependent antimicrobial drugs like MB. The MB tonsil:MIC ratio described is above the threshold value (10) that is associated with clinical efficacy for all the doses studied (6). Thus, all the posology regimens tested might be efficacious to eliminate APP from tonsils. Obviously, the information provided here paves the way to carry out studies whose main goal could be to detect APP in tonsils of animals receiving several posology regimens of marbofloxacin.

\section{References}

1. Esposito, 2006. J Ant Chemoth. 57: 789-792.

2. Falser, 1998. Infection 16: 14-18.

3. Fish \& Chow,1997. Clin Pharmacokinet 32: 101-119.

4. NCCLS (2002). CLSI M31-A2, 2002. In http://www.clsi.org.

5. Sarasola, 2002 Ant Agent Chemoth.. 46(9):3013-3019.

6. Sidhu et al 2009. Res Vet Sci.. Ahead of print.

7. Valle, 2006. IPVS, Copenhagen, DK

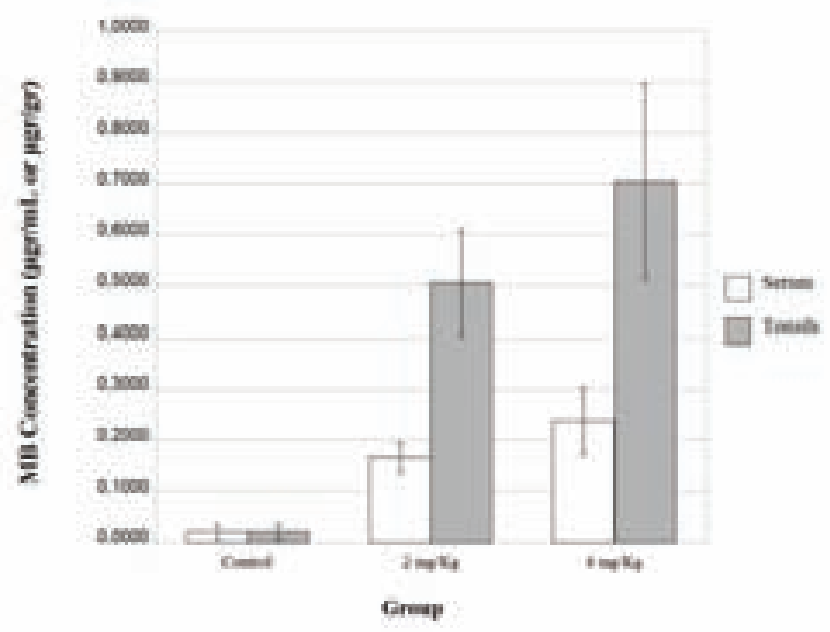




\title{
0.049
}

\section{Assessment of decisions pertaining to antimicrobials used in nursery pigs}

\author{
$\underline{\text { Rebecca Robbins }}^{1}$ Sam Copeland ${ }^{2}$ Glen Almond ${ }^{1}$ Maria Correa ${ }^{1}$ \\ 1. North Carolina State Univ. College of Veterinary Medicine, Raleigh, NC, USA; \\ 2. North Carolina State Univ. Department of Animal Science, Raleigh, NC, USA
}

\section{Introduction}

Judicious use of antimicrobials is a public health and civic responsibility and one of the most controversial of all therapeutics currently in use. Surveys of United States and Canadian pig producers indicate that the greatest proportions of antimicrobials are administered to nursery pigs 1,2. Guidance on antimicrobial use has been developed but no single treatment standard established. Thus, we conducted the first of a series of studies to determine patterns in antimicrobial treatment based on current practices in commercial nurseries.

\section{Materials and Methods}

The use of antimicrobials via injection or water was observed for 123 conventional health nursery barns. Barns housed pigs from weaning until 9-10 weeks of age and were isolated from farrowing or finishing barns. During the six month study period, 368 barns were observed. The unit of analysis was the barn (ecologic design). Antimicrobials delivered through the feed did not vary during the study and were not described. Reason for administration, clinical diagnosis, severity of disease, and antimicrobial regimen were recorded. Descriptive statistics were conducted in SAS (v. 9.1, Cary, NC, USA).

\section{Results}

There were 136 reports of barns that used an antimicrobial via water or injection representing $37 \%$ of nursery barns observed. Of the 136 medicated barns, 28 (20.6\%) were received antimicrobials more than once. Pigs $\leq 7$ weeks old received antimicrobials with the greatest frequency (63\%). Water was the favored route of administration at $61 \%$. We observed $65 \%$ of antimicrobials were for treating disease as opposed to metaphylaxis and $87 \%$ of disease was considered mild or moderate rather than severe. The majority (75\%) of clinical diagnoses had signs of respiratory disease; other diagnoses were systemic (18\%) or enteric (7\%) conditions. The antimicrobial regimens favored the use of chlortetracycline plus tiamulin (31\%) in water and enrofloxacin (29\%) by injection. The remaining $40 \%$ was spread over 8 other regimens. We also observed that the route of antimicrobial administration was influenced by disease severity $(p=0.002)$, purpose of administration $(p=0.019)$, and clinical diagnosis $(p<0.001)$. The relationships were further expressed in odds ratios (OR) with $95 \%$ confidence limits $(C L)$ and $p$-value reported in Table 1.

\begin{tabular}{|l|l|c|c|c|}
\hline \multicolumn{1}{|c|}{ Odds ratio if injection } & & $\mathbf{0 R}$ & $\mathbf{9 5 \%} \mathbf{C L}$ & $\mathbf{p}$-value \\
\hline \multirow{4}{*}{ Severity of disease } & Mild & - & - & - \\
\cline { 2 - 5 } & Moderate & 1.5 & $0.8,2.8$ & 0.26 \\
\cline { 2 - 5 } & Severe & 5.7 & $2.1,15.7$ & 0.00 \\
\hline \multirow{2}{*}{ Reason for administration } & Treatment & - & - & - \\
\cline { 2 - 5 } & Metaphylaxis & 2.1 & $1.1,3.8$ & 0.02 \\
\hline \multirow{4}{*}{ Clinical diagnosis } & Enteric & - & - & - \\
\cline { 2 - 5 } & Systemic & $*$ & $*$ & $*$ \\
\cline { 2 - 5 } & Respiratory & $*$ & $*$ & $*$ \\
\hline - reference category; ${ }^{*}$ non-rational outcome & $*$ & $*$ \\
\hline
\end{tabular}

\section{Discussion}

A previous report found that treatment frequency could approximate disease prevalence3. If this observation is valid, then we expect at least $1 / 3$ of nurseries to be diseased in a 6 month period. We would expect most disease to be respiratory in nature. The frequency of antimicrobial regimens applied also supports this expectation. Although we observed a preference for administration through water, the route was influenced by other factors. There is a strong preference for injectable antimicrobials when nurseries are severely diseased. Therefore, we believe that disease severity and route of administration are proxies of one another. Unfortunately, we could not obtain OR for clinical diagnosis due to sparse data. No enteric cases received antimicrobials by injection and this is likely due the exclusive use of single injection products having regulatory prohibitions in the U.S. as well as the pharmacodynamics of oral medications. In summary, decisions regarding antimicrobial use in pig populations are complex and are related to factors other than the antimicrobial or route of its administration.

\section{References}

1. National Animal Health Monitoring System. (2008).http://www. aphis.usda.gov/vs/ceah/ncahs/nahms/swine/swine2006/Swine2006 Partlll.pdf.

2. Dunlop RH, SA McEwan, AH Meek, RA Friendship, RC Clarke, WD Black. (1998). Can Vet J 39: 87-96.

3. Blaha T, P Dickhaus, D Meemken.(2006). Proceedings of the 19th IPVS Congresss: 189. 


\title{
0.050
}

\section{Compatibility of vaccines against atrophic rhinitis and neonatal E. coli diarrhea}

\author{
H.M.J.M. Swarts ${ }^{1}$ M. Murmans ${ }^{2}$ M. Witvliet $^{2}$ \\ 1. ISPAH, Global Marketing Swine, Boxmeer, Netherlands; 2. ISPAH, Microbiological R\&D, Boxmeer, Netherlands
}

\section{Introduction}

In modern swine production sows are vaccinated against several diseases, either to protect the sows or to protect the sows' progeny (or both). This leads to the fact that sow vaccination schemes can become very extended and very complex. To be able to work more practical and to reduce the number of injections for the sows, farmers are eager to look for options to combine vaccines without compromising on the efficacy.

Porcilis AR-T DF (1) and Porcilis Porcoli DF (2) are two vaccines that are given to pregnant sows to pass on passive immunity through MDA to their offspring to protect them against atrophic rhinitis and neonatal E. coli diarrhea, respectively. Porcilis AR-T DF contains a subunit of the P. multocida toxin (PMT) in combination with $\mathrm{B}$. bronchiseptica (Bb) cells and Porcilis Porcoli DF contains purified fimbrial adhesins (F4ab, F4ac, F5 and F6) and the E. coli heat labile toxin (LT). Since both vaccines require the same vaccination schedule (basic vaccination 8-6 weeks and booster / re-vaccination 4-2 weeks before farrowing) and both vaccine use Diluvac Forte (d,l- a-tocopheryl acetate) as the adjuvant, it was tested in the present study if injection of the vaccines after mixing would influence the antibody responses against the individual vaccine antigens.

\section{Materials and Methods}

Three groups of 10 pigs of 8 months of age with no or low antibody titers against the vaccine antigens were injected twice intramuscularly with a 4-week interval with Porcilis AR-T DF (2 $\mathrm{ml}$ ), Porcilis Porcoli DF (2 $\mathrm{ml}$ ) or both products after mixing (4 $\mathrm{ml}$ ). Two weeks after the second vaccination, serum samples were taken. Antibody titers were determined in a toxin-neutralization assay (PMT), a micro-agglutination test $(\mathrm{Bb})$ or by ELISA (E. coli antigens).

\section{Results}

The serological responses after vaccination with the two vaccines given individually or mixed are presented in Table 1.
Table 1. Antibody titers (log2) against both vaccine antigens at 2 weeks after the second vaccination.

\begin{tabular}{|l|c|c|c|}
\hline Vaccine antigen & \multicolumn{3}{|c|}{ Groups } \\
\hline & Porcilis AR-T DF & Porcilis Porcoli DF & Mixed \\
\hline PMT & $8.1^{\mathrm{a}} \pm 1.9$ & $<1^{\mathrm{b}}$ & $8.0^{\mathrm{a}} \pm 1.2^{\mathrm{a}}$ \\
\hline $\mathbf{B}^{\mathrm{b}}$ & $11.0^{\mathrm{a}} \pm 2.5^{\mathrm{a}}$ & $4.1^{\mathrm{b}} \pm 0.3$ & $11.5^{\mathrm{a}} \pm 0.8$ \\
\hline F4ab (K88 $\left.{ }^{\mathrm{ab}}\right)$ & $5.8^{\mathrm{a}} \pm 0.5$ & $12.1^{\mathrm{b}} \pm 1.2$ & $12.2^{\mathrm{b}} \pm 0.9$ \\
\hline F4ac (K88 $\left.{ }^{\mathrm{ac}}\right)$ & $6.2^{\mathrm{a}} \pm 0.9$ & $11.6^{\mathrm{b}} \pm 1.2$ & $11.0^{\mathrm{b}} \pm 0.9$ \\
\hline F5 (K99) & $7.5^{\mathrm{a}} \pm 1.0$ & $12.0^{\mathrm{b}} \pm 0.9$ & $11.0^{\mathrm{c}} \pm 0.9$ \\
\hline F6 (987P) & $8.1^{\mathrm{a}} \pm 0.8$ & $13.0^{\mathrm{b}} \pm 0.8$ & $12.6^{\mathrm{b}} \pm 0.6$ \\
\hline LT & $7.5^{\mathrm{a}} \pm 1.3$ & $11.9^{\mathrm{b}} \pm 0.9$ & $10.7^{\mathrm{c}} \pm 1.0$ \\
\hline For each antigen, groups with different superscripts are significantly different $(\mathrm{p}<0.05$, two- \\
sample t-test).
\end{tabular}

\section{Discussion and Conclusion}

For nearly all antigens, there was no significant difference between the antibody titer induced by the single vaccines or by the mixed products.

However, for the F5 and LT antigens, the antibody levels induced after mixing of the two vaccines were significantly lower than those induced by Porcilis Porcoli DF alone. In both the single and the combined group uniform titer values were reached after vaccination, which allowed for detecting significant difference between for the F5 and the LT titer values, however the serum antibody levels against these two antigens after mixed vaccination were still above the level that can be considered to provide protection via the colostrum (2).

\section{References}

1. Riising, H.-J, P. van Empel \& M. Witvliet (2002) Vet. Rec. 150, 569-571

2. Riising, H.-J, M. Murmans \& M. Witvliet (2005) J. Vet. Med. B 52, 296300 


\title{
0.051
}

\section{Possibilities of overcoming the inhibitory effect of colostrum-derived antibodies using intradermal immunization and immunization via mucosae}

\author{
Katerina Nechvatalova; Martin Faldyna; Eduard Gopfert; Pavlina Knotigova; Hana Kudlackova; Lenka Leva
}

Veterinary Research Institute Brno, Brno, Czech Republic

\begin{abstract}
Introduction and objectives
The colostrum-derived antibodies protect piglets in early life. Levels of these antibodies gradually decrease in sera of growing piglets and according to the prevailing opinion they decline so much at a certain time point that they cannot protect the organism against infection and even block the onset of active immunity. This period has been generally designated as the "immunity window" (1). On the other hand, our previous studies and studies of other authors (1-3) indicated that colostrum-derived antibodies do not completely prevent the immune response after immunization and that this period could be overcome or shortened by properly timed vaccination, local administration of antigens and/or by the use of proper type of adjuvant.
\end{abstract}

The aims of this study were to verify the possibility of overcoming the inhibitory effect of colostrum-derived antibodies in piglets using immunization via the skin or mucosae and to test the effect of higher proportions of adjuvant and/or higher concentrations of model antigen on quality and quantity of induced local and systemic immune responses.

\section{Materials and methods}

Piglets (5 animals in each group) with colostrum-derived antigen-specific antibodies were intranasally (i.n.), intratracheally (i.tr.) or intradermally (i.d.) immunized with increasing concentrations ( $3 \mathrm{mg}, 5 \mathrm{mg}$ or $10 \mathrm{mg} /$ piglet) of a model antigen - Keyhole Limpet Hemocyanin (KLH; Pierce, France) and increasing proportions of adjuvant Montanide IMS 1312 (Seppic, France) per immunization dose (50-75\%), i.e. at ratios of adjuvant to KLH from 1:1 to $3: 1$; the volume of each immunization dose was 1 $\mathrm{ml}$. Immunization of respective groups started at different age $(2,4$ or 6 weeks of life) i.e. at periods of high and subsequently decreasing levels of total and local antigen-specific antibodies. All piglets were reimmunized 3 weeks after the first administration of KLH.

Blood samples, nasal and bronchoalveolar lavage samples from all pigs were taken regularly for immunologic examination (isotype-specific indirect enzyme linked immunosorbent assays with purified KLH antigen and for the KLH-driven lymphocyte transformation test).

\section{Results}

The piglets were able to give a quantifiable (cell-mediated and antibody) primary immune response to i.n., i.tr., and i.d. immunization with KLH already at the age of 2 weeks of life in the presence of high levels of colostrum-derived antibodies, but only after use of higher concentrations of KLH and/or addition of higher amounts of adjuvant. Immunization led to production of local $\lg \mathrm{A}$ and $\operatorname{lgG}$ and systemic $\lg \mathrm{M}$ antibodies in these piglets. The levels of systemic lgG antibodies increased only in older piglets (aged 4 and 6 weeks) after i.d. and i.tr. immunization with $\mathrm{KLH}$, using the highest proportion of the adjuvant (75\%). Generally, the use of higher concentrations of KLH and higher proportions of adjuvant per immunization dose resulted in a faster and more intense immune response. On the other hand, the immunization dose containing $10 \mathrm{mg}$ of KLH elicited excessive but transient local inflammation of the respiratory tract. The immune response intensity after i.n. immunization was generally lower than after i.tr. immunization; moreover, the former provided weaker systemic immune response than i.d. immunization. After revaccination, no significant differences were observed in the immune responses of piglets with higher and lower levels of colostrum-derived antigen-specific antibodies at the time of primary vaccination.

\section{Discussion and conclusions}

The present study confirmed in accordance with other authors (4) that vaccination might be successful even in piglets with high levels of colostrum-derived antibodies at the time of immunization. Inhibitory effect of colostrum-derived antibodies was overcome in this study using local administration of antigen and proper balancing between the antigen concentration and adjuvant proportion in the immunization dose which were of crucial importance.

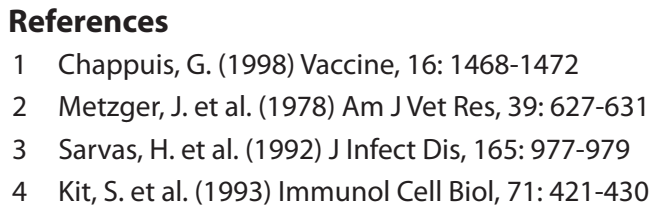

The study was supported by the Czech Science Foundation (524/06/P455), MZe CZ (MZe 0002716202) and MSMT CZ (CZ.1.05/2.1.00/01.0006 AdmireVet). 


\title{
0.052
}

\section{Time point for vaccination sows with $E$. coli vaccines may influence the protection of the offspring}

\author{
Thersa Salwén ${ }^{2}$ Per Wallgren ${ }^{1}$ \\ 1. Dept of Animal Health and Antimicrobial Strategies, Uppsala, Sweden; 2. Sanna Norrgården, Vintrosa, Sweden
}

\section{Introduction}

Neonatal diarrhoeas induced by E. coli are commonly prevented by vaccination of sows that are booster vaccinated 2-3 weeks before farrowing (1). However, the transfer of immunoglobulin from serum to colostrum is initiated at least four weeks prior to farrowing. (2). Therefore, vaccinating sows at an earlier time point appears tempting in herds that are affected by neonatal diarrhoeas caused by $E$. coli despite vaccinating sows.

\section{Materials and Methods}

The study was conducted in a multiplying herd with 160 landrace sows producing Landrace-Yorkshire hybrids. Forty sows farrowed every fourth week in an age segregated system, and the piglets were weaned at an age of 4 weeks. Despite vaccinating sows with E. coli vaccines (Porcovac, Plus vet/PorcilisPorcoli Diluvac Forte) during late pregnancy the herd had a high incidence of neonatal diarrhoeas. E. coli serotype $\mathrm{O} 149$ had repeatedly been diagnosed as the cause of these diarrhoeas by laboratory analyses.

The productivity of sows and piglets was documented in which the birth weight was standardised to $1.5 \mathrm{~kg}$. Piglets attended with diarrhoea were medically treated and recorded at an individual and litter level. 43 batches boostering sows with E coli vaccine two weeks before farrowing, were compared with 28 batches following altering the booster vaccination to six weeks before farrowing No other measures were carried out with the aim to control E. coli associated neonatal diarrhoeas. The statistical calculations were carried out using t-tests.

Figure 1. Productivity before and after switch of boostering sows with E. coli vaccine from two to six weeks ante partus

\begin{tabular}{lcccc}
\hline \multicolumn{2}{c}{ Vaccination } & $\begin{array}{c}2 \text { weeks } \\
43 \text { batches }\end{array}$ & $\begin{array}{c}6 \text { weeks } \\
28 \text { batches }\end{array}$ & Sign \\
\hline Sows, all & $\mathrm{n}$ & $39.0 \pm 2.9$ & $38.7 \pm 2.9$ & $\mathrm{~ns}$ \\
\% gilts & $\%$ & $28.9 \pm 9.0$ & $32.6 \pm 9.9$ & $\mathrm{~ns}$ \\
Live born & $\mathrm{n}$ & $11.6 \pm 1.0$ & $12.2 \pm 1.5$ & $\mathrm{~ns}$ \\
Weaned & $\mathrm{n}$ & $10.2 \pm 0.5$ & $10.4 \pm 0.8$ & $\mathrm{~ns}$ \\
dtweening & & & & \\
Age & days & $30.5 \pm 1.5$ & $29.1 \pm 1.3$ & $\ldots$ \\
Weight & $\mathrm{kg}$ & $9.0 \pm 0.6$ & $8.4 \pm 0.7$ & $\ldots$ \\
DWG & g/day & $248 \pm 21$ & $238 \pm 23$ & $\mathrm{~ns}$ \\
\hline
\end{tabular}

\section{Results}

Piglets were weaned at a somewhat higher $(p<0.001)$ weight before altering the time point of vaccinating sows. However, this was achieved by a higher $(p<0.001)$ weaning age as also deomnstrated by an equal daily weight gain of the two groups (Figure 1). Also the productivity of the sows was equal in the two goups.

The incidence of diarrhoeas during the sucklin period was decreased, both at individual $(p<0.001)$ and at litter $(p<0.05)$ level following the change of time for vaccinations (Figure 2 ).

Figure 2. The incidense of diarrhoea during the suckling period in the offspring to sows booster vaccinated with $E$ coli vaccine at two or six weeks prior to farrowing.

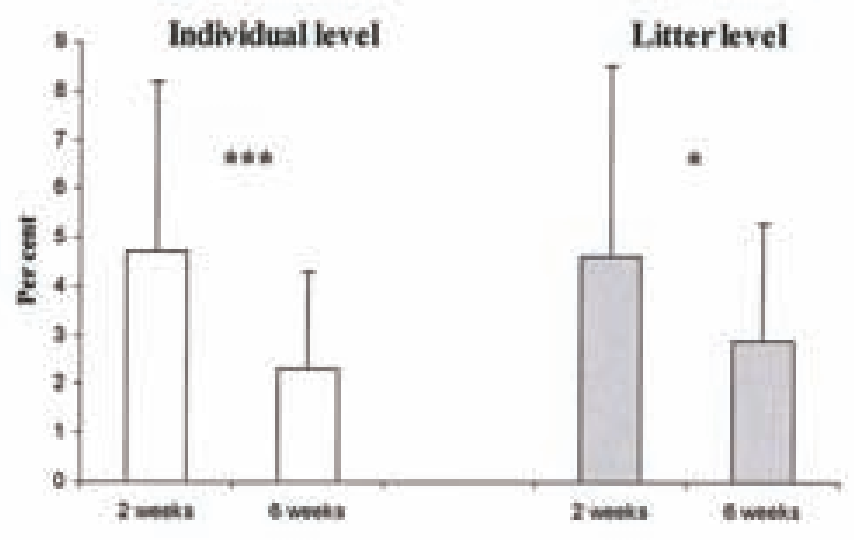

\section{Discussion and Conclusions}

The incidence of diarrhoea during the suckling period decreased following vaccinating sows at an earlier time point which was a positive welfare issue, both for the piglets and the staff. Thereby the use of antimicrobials also decreased, which ought to be favourable from an antimicrobial resistance point of view. Yet, the productivity in itself was not affected by the altered vaccination strategy, which probabaly could be explained by an alert staff treating piglets with neonatal diarrhoeas at an early stage - thereby preventing increased piglet mortality.

This work concludes that vaccinating sows at an earlier point of time than generally recomended could be worth testing in herds with $E$. coli associated neonatal diarrhioeas despite vaccinting sows.

\section{References}

1 Bertschinger \& Fairbrother. Dis. of swine, 8th edn.438-439.

2 Wallgren et al., 1998 Vet Microbiol 60:193-205. 


\title{
0.053
}

\section{Agreement between culture of intestinal and fecal samples for Escherichia coli and Brachyspira spp.}

\author{
Ken S. Pedersen ${ }^{1}$ Jens P. Nielsen ${ }^{1} \varnothing y s t e i n$ Angen² \\ 1. University of Copenhagen, Frederiksberg, Denmark; 2. National Veterinary Institute, Technical University of Denmark, Frederiksberg, Denmark
}

\section{Introduction}

Culture of fecal samples is often applied for examination of intestinal pathogens. Culture of feces may not reflect the intestinal microbiology and result in biased estimates of pathogen prevalence or incorrect diagnosis. The objective of the current study was to evaluate agreement between culture of intestinal and fecal samples for E. coli and Brachyspira spp.

\section{Materials and Methods}

Fecal samples and intestines were collected in a case control study conducted in 20 Danish herds. All herds were visited the day following notification from the farmer/veterinarian of an acute treatment requiring outbreak of diarrhea in pigs between 10 and 70 days post weaning. The farmer was not allowed to medicate before the pigs were examined. A sample of 80 pigs in each herd was selected by systematic random sampling among all pigs in the nursery room where the outbreak of acute diarrhea occurred. The pigs were subjected to a clinical examination and a fecal sample was collected. Among the examined pigs a simple random sample of 8 pigs with diarrhea and 8 pigs without diarrhea was selected and euthanized. Fecal samples from all euthanized pigs were examined for Escherichia coli and Brachyspira spp. by standard culturing methods. Small intestinal content was examined for E. coli and large intestinal content was examined for Brachyspira spp. Dry matter (DM\%) was determined for the fecal samples. DM\% $\leq 18.8$ was considered as diarrhea and was used (if necessary) to reclassify the pigs as diarrheic or non-diarrheic in the statistical analysis. For each Brachyspira spp., hemolytic E. coli, non-hemolytic E. coli (if found in pure culture or as the dominant bacterial flora) diagnostic sensitivity and specificity of the fecal culture were evaluated using the culture of the intestinal samples as reference standard. Further, differences between diagnostic sensitivity and specificity in pigs with or without diarrhea were evaluated using a Chi-sq test.

\section{Results}

Diagnostic sensitivity and specificity for fecal culture are displayed in table 1. In pigs with diarrhea and pigs without diarrhea the diagnostic sensitivity was significant different for hemolytic E. coli and borderline significant for non-hemolytic E. coli $(p=0.068)$ and B. innocens/B. murdochii $(p=0.066)$.

\section{Discussion}

A clear correlation between fecal and intestinal cultivation was only found for hemolytic E. coli. A difference in diagnostic sensitivity between pigs with and without diarrhea was also only observed for hemolytic E. coli. However, culture from intestinal content may not always be considered a true gold standard and the obtained estimates of sensitivity and specificity should therefore be interpreted with caution. The results imply that culture of fecal samples can either under or over estimate prevalence of these pathogens compared to culture of intestinal content. The study also demonstrates that diagnostic test have different performance in various subpopulations. The relatively low sensitivity for Brachyspira spp. may have implications for surveillance of swine dysentery.

Table 1. Diagnostic sensitivity and specificity of culture of fecal samples for E. coli and Brachyspira spp. using culture of intestinal content as reference test.

\begin{tabular}{|c|c|c|c|c|c|}
\hline & $\begin{array}{l}\text { Hemolytic } \\
\text { E. coli }\end{array}$ & $\begin{array}{c}\text { Non- } \\
\text { hemolytic } \\
\text { E. coli }\end{array}$ & $\begin{array}{l}\text { B. inter- } \\
\text { media }\end{array}$ & $\begin{array}{c}\text { B. inno- } \\
\text { cens or B. } \\
\text { murdochii }\end{array}$ & $\begin{array}{l}\text { B. pilosi- } \\
\text { coli }\end{array}$ \\
\hline $\begin{array}{l}\text { Pigs with diarrhea } \\
(\mathrm{DM} \% \leq 18.8)(\mathrm{n}=166)\end{array}$ & $\mathrm{n}=31$ & $\mathrm{n}=65$ & $n=13$ & $\mathrm{n}=48$ & $n=14$ \\
\hline Diagnostic sensitivity* & $\begin{array}{c}83.9 \#(66.3 \\
-94.5) \\
\end{array}$ & $\begin{array}{c}49.2(36.6- \\
61.9) \\
\end{array}$ & $\begin{array}{c}30.8(9.1- \\
61.4)\end{array}$ & \begin{tabular}{|c|}
$47.9(33.3-$ \\
$62.8)$ \\
\end{tabular} & $\begin{array}{c}28.6(8.4- \\
58.1)\end{array}$ \\
\hline Diagnostic specificity* & $\begin{array}{c}91.1(85.0- \\
95.3) \\
\end{array}$ & $\begin{array}{c}85.1(76.7- \\
91.4) \\
\end{array}$ & $\begin{array}{c}98.7(95.4- \\
99.8)\end{array}$ & \begin{tabular}{|c|}
$85.6(77.9-$ \\
$91.4)$
\end{tabular} & $\begin{array}{c}99.3(96.4- \\
100)\end{array}$ \\
\hline $\begin{array}{l}\text { Pigs without diarrhea } \\
\text { (DM\%>18.8) (n=146) }\end{array}$ & $n=15$ & $n=63$ & $n=9$ & $n=32$ & $n=6$ \\
\hline Diagnostic sensitivity* & $\begin{array}{c}40.0 \#(16.3 \\
-67.7) \\
\end{array}$ & $\begin{array}{c}33.3(22.0- \\
46.3) \\
\end{array}$ & $\begin{array}{c}33.3(7.5- \\
70.1) \\
\end{array}$ & \begin{tabular}{|c|}
$68.8(50.0-$ \\
$83.9)$ \\
\end{tabular} & $\begin{array}{c}16.7(0.4- \\
64.1) \\
\end{array}$ \\
\hline Diagnostic specificity* & $\begin{array}{c}96.2(91.4- \\
98.8)\end{array}$ & $\begin{array}{c}82.1(72.3- \\
89.6)\end{array}$ & $\begin{array}{c}97.8(93.7- \\
99.5)\end{array}$ & \begin{tabular}{|c|}
$89.5(82.3-$ \\
$94.4)$
\end{tabular} & $\begin{array}{c}100(97.4- \\
100)\end{array}$ \\
\hline \multicolumn{6}{|c|}{ All numbers in $\%, 95 \%$ confidence interval in brackets. } \\
\hline & & & & & \\
\hline
\end{tabular}




\title{
0.054
}

\section{Factors associated with the occurrence and the level of Isospora suis infection in Greek pig herds}

\author{
Vasilis Skampardonis ${ }^{1,2}$ Smaragda Sotiraki ${ }^{2}$ Polychronis Kostoulas ${ }^{1}$ Leonidas Leontides $^{1}$ \\ 1. University of Thessaly, Karditsa, Greece; 2. Veterinary Research Institute, National Agricultural Research Foundation, Thessaloniki, Greece
}

\section{Introduction}

Neonatal coccidiosis, which is due to Isospora suis parasitism, occurs in all types of farrowing facilities and under all types of management systems (Lindsay and Blagburn, 1994; Meyer et al., 1999; Mundt et al., 2005). In this study, we identified herd- and litter-level factors associated with the odds and the level of $I$. suis oocyst excretion in Greek pig herds.

\section{Materials and Methods}

Data were collected from 55 farrow-to-finish herds with continuous farrowing. From each herd, depending on the herd size, at least five and up to ten litters, in their second week of life, were coprologically sampled. Each litter-sample comprised a pool of individually collected faeces from half of the piglets. Oocyst excretion, expressed as oocysts per gram of faeces (OPG), was determined, by a modified McMaster technique, for all samples. A standarized risk-factor questionnaire was used to collect information on the use of toltrazuril (BAYCOX $5 \%$ ) and other herd- and litter-level factors that could be associated with I. suis infection. To simultaneously identify factors associated with the odds of and/or the amount of litter-level oocyst excretion we employed a two-part model (Liu et al., 2008). Presence or absence of oocyst excretion was the response variable in the logistic part of the model, while the natural logarithm of the nonzero OPG values was the response in the linear part (parts I and II, respectively). Initially, all factors were screened one by one in univariable models. Those with $P<0.25$ in any part of the model, were offered to the full model, which was further reduced by backwards elimination until only significant $(P<0.05)$ variables were left. In all models, the herd was incorporated in both parts as random-effects term and the likely cross-equation correlation ( $\rho_{\mathrm{CEC}}$ ) of the herd-level random-effects between parts I and II was accounted for. A weighting method, which adjusted for the unequal selection probabilities of litters in herds was applied in all models. Models were built in the aML software.

\section{Results and Discussion}

The odds of $I$. suis litter-level excretion were reduced when piglets were treated with toltrazuril, when the farrowing rooms were completely emptied and cleaned after weaning, when piglets were not cross-fostered between litters after the $1^{\text {st }}$ day of life and when the flooring of the pens was plastic. Further, larger farrowing rooms and more caretakers in the farrowing rooms reduced the odds of excretion. The level of OPG was decreased with toltrazuril-treatment, larger farrowing rooms and caretakers avoiding walking into farrowing pens. Thus, additionally to toltrazuril treatment, control of neonatal coccidiosis can be bettered by other factors.

\begin{tabular}{|c|c|c|c|}
\hline Logistic part (I) & & OR (95\%C.I.) & $p$ \\
\hline \multirow[t]{2}{*}{ Toltrazuril } & Yes & $0.2(0.1 ; 0.5)$ & 0.001 \\
\hline & No & 1 & \\
\hline Clean entire & Yes & $0.3(0.1 ; 0.9)$ & 0.039 \\
\hline farrowing room & No & 1 & \\
\hline Fostering after & Yes & $4(1.0 ; 16)$ & 0.045 \\
\hline 1 st day & No & 1 & \\
\hline \multirow[t]{2}{*}{ Metallic pen floor* } & Yes & $2.5(1.1 ; 5.7)$ & 0.034 \\
\hline & No & 1 & \\
\hline Pens per room ${ }^{*}$ & & $0.93(0.9 ; 0.97)$ & 0.001 \\
\hline Number of & & $0.6(0.5 ; 0.97)$ & 0.042 \\
\hline \multicolumn{4}{|l|}{ caretakers } \\
\hline Linear part (II) & & Coef. (95\% C.I.) & $P$ \\
\hline \multirow[t]{2}{*}{ Toltrazuril } & Yes & $-1.8(-0.6 ;-2.9)$ & 0.004 \\
\hline & No & ref. & \\
\hline Caretakers & Yes & $2.1(0.6 ; 3.6)$ & 0.009 \\
\hline entering in pens & No & ref. & \\
\hline Pens per room* & & $-0.04(-0.01 ;-0.08)$ & 0.023 \\
\hline$\rho$ CEC & & 0.99 & 0.005 \\
\hline \multicolumn{4}{|l|}{ * litter-level factors } \\
\hline
\end{tabular}

\section{References}

Lindsay, D.S., Blagburn, B.L., 1994. Parasitol. Today 10, 214-219.

Meyer et al., 1999. Vet. Parasitol. 82, 277-284.

Mundt et al., 2005. J. Vet. Med. B 52, 93-97.

Liu et al., 2008. Stat. Med. 27, 3528-3539.

aML Multilevel Multiprocess Statistical Software: Version 2.0 (www. applied-ml.com). 


\title{
0.055
}

\section{Likely carriage of Brachyspira hyodysenteriae in cockroaches and flies on pig farms}

\author{
Ruth Blunt; Laura Hancox; Ken Mellits; Steven McOrist \\ University of Nottingham, Sutton Bonington, UK
}

\section{Introduction}

Insects on pig farms can cause loss in various ways, including an ability to spread disease within and between farms. This may conceivably include re-infection of eradication project farms by insects travelling from adjacent farms when infected with the agent in question. Some 1980's research documented the occurrence of enteric pathogens, such as Brachyspira hyodysenteriae among possible vectors on pig farms. However, no information exists on a truly wide range of vectors or on the movement of exact strains or isolates around farms. Vector types may also have altered in pigs now facing PRRS, PMWS etc. Other studies have indicated that some floor-dwelling insects, such as cockroaches and beetles, may be capable of carrying other enteric agents such as Salmonella and E coli for significant periods on commercial pig and chicken farms. Increases in farm bedding usage will presumably also increase populations of potential infected vectors. This study aimed to investigate carriage of Brachyspira among farm insects.

\section{Materials and Methods}

We constructed a set of 15 case study farms in England and Ireland to include the following:- mixed-age farms, breeder only farms, recently depopulated farms, empty farms and various other scenarios. Most farms were Lawsonia and PCV-2 positive, as determined by clinical signs and faecal PCR results. Farms that are positive and negative for Brachyspira hyodysenteriae (swine dysentery $=$ SD) were targeted, as determined by clinical signs and faecal PCR and cultures.

At each farm, numerous longitudinal and age-group specific vector samples and population-stratified faecal samples were collected. Insects were collected via aerial sticky traps, motorised vacuum devices, floor and pitfall traps and sweep nets and identified by trained entomologists. DNA was extracted from the GI tract of larger insects and from whole body samples of smaller insects. PCR was performed on pig and insect origin DNA by published duplex species-specific methods for Brachyspira - both B hyodysenteriae and B pilosicoli. At present, strain or isolate typing methods are not readily available for $B$ hyodysenteriae.

We also conducted separate experimental challenge exposure studies of housed cockroaches and Musca domestica flies, dosed orally with 107 live $B$ hyodysenteriae derived from a confirmed SD case. We cultured and PCR tested both the intestinal tracts and faeces of dosed and control insects.

\section{Results}

A wide range of insects were found at varying levels in the 15 study farms. Prominent groups of flies were found - Diptera, including Musca domestica (house fly), Muscina stabulans (false stable fly), Ophyra spp (garbage fly), Stomoxys calcitrans (stable fly) and Syrphidae, including Eristalis sp (hover flies), as well as numerous floor-dwelling beetles associated with feed storage.

The oriental cockroach, Blatta orientalis was detected in considerable numbers on 2 farms. One of these farms was also positive for SD signs and B hyodysenteriae (by faecal PCR) in its pigs. $B$ hyodysenteriae $P C R$ products were also detected in the faeces of several pigs on 3 other swine dysentery-positive farms (with no cockroaches). No clear Brachyspira positive fly or other vectors were noted on these 3 farms. However, PCR products indicative of Brachyspira hyodysenteriae were also detected in 30 $\%$ of cockroaches, caught in association with the SD pig-positive farm. This agent was not detected in cockroaches on the other cockroach-positive but SD-negative farm.

Separate challenge exposure studies of Musca flies and cockroaches indicated that these potential vectors may hold B hyodysenteriae in their gastrointestinal tracts for periods of 1 to 3 days. We determined that these insects could not only retain these bacteria in their $\mathrm{Gl}$ tracts, but also excrete viable culturable $B$ hyodysenteriae for these periods, via their faeces.

Brachyspira pilosicoli was only noted in pig faeces collected on one farm, no clear positive B pilosicoli infection was noted in any potential vector.

\section{Discussion}

We found a wide range of flies, beetles, midges and cockroach activity on British and Irish pig farms - some of these appear to have the potential to carry and transmit pathogens, such as PCV2. In particular we found that cockroaches had the potential to carry Brachyspira hyodysenteriae for a potentially important period of 3 days. Cockroaches form large indestructible communities on pig farms when they are introduced - usually via food waste or other waste products. They may assist transmission of the SD agent within and between farms. This suggests that a program aimed at reducing infection levels or post-eradication re-infection with this agent should include not only appropriate biosecurity and pig medication, such as pleuromutilins, but also anti-insect population control measures, targeted at cockroaches and flies in association with pigs. 


\title{
0.056
}

\section{Sub-clinical ileitis: Technical and economic impact of nutrition and therapeutic measures implemented}

\author{
$\underline{\text { Annick Saives }}^{1}$ Jeanne Dupuis ${ }^{2}$ Alban Chauvin ${ }^{3}$ \\ 1. Elanco Europe, Brussels, Belgium; 2. Selas Veterinaire de la Hunaudaye, Lamballe, France; 3. Cooperl Arc'atlantique, Montfort Sur Meu, France
}

\section{Introduction}

Lawsonia intracellularis is responsible for an infection known as ileitis, of which the sub-clinical disease leads to decrease zootechnical performance (1). The symptoms are therefore nonspecific and differential diagnosis is essential for determining the prevention and treatment measures to be implemented. The purpose of this study is to describe the use of diagnostic tools in farms affected by sub-clinical ileitis, enabling therapeutic and zootechnical measures to be applied, and to evaluate their effect on zootechnical performances.

\section{Materials and methods}

A site 3 finishing barn with 320 pigs per batch, of a single animal origin and showing lack of uniformity at the entry of the animals (84th day of life) and also in the last part of the finishing stage, was selected (period 1, tab 1).

An anti-Lawsonia Ab serum profile was carried out in April 2008 using the IFAT (indirect fluorescence antibody technique (lleitest $^{\circledR}$ )) method, by collecting samples at 95, 125 and 167 days of age.

At 95 days of age, 10 out of 10 samples were negative; at 125 days of age, 10 out of 10 samples were positive; at 167 days of age, 5 samples out of 5 were positive.

Seroconversion occurs around 100 days of age, and according to experimental infection studies (2), spread probably primarily takes place at the entry.

An initial dose of Tylan was introduced into feed (100 ppm for 10 days) upon entry of the animals, followed by a second dose in the drinking water mid-finishing stage (10 days at $5 \mathrm{mg} / \mathrm{kg}$ ), due to the 2 nd period of heterogeneity.

After the treatment, an improvement in the ADG was seen (period 2, tab 1) but performances are still unsatisfactory at the end of the complete period. In October 2008, Lawsonia excretion FIRSTtest ${ }^{\oplus}$ was carried out to know if the problem persists. The FIRSTtest ${ }^{\circledR}$ is a single-use test for the colorimetric detection of Lawsonia using the ELISA technique, which identifies the bacterial surface antigen.10 samples were collected per age.

At 110 days of age, 9 out of 10 samples were negative and at 150 days, 10 out of 10 samples were negative.

As the herd was not excreting Lawsonia on this period, a change in feed rationing plan was decided in November 2008 (changes in formulation and schedule plan at the beginning of fattening period) and the 2 nd soluble Tylan treatment was reduced from 10 to 7 days ( period 3, tab 1).
78 animals per batch were weighed upon their arrival, 21 days later, and again, a further 21 days later, allowing to calculate 3 ADG periods. The slaughtering results per batch were also available and a comparison of results of the cold carcass weight per period was possible (period 1 before treatment, period 2 with the 2 Tylan treatments, period 3 with Tylan treatments changed and formulation adjustment).

\section{Results}

Tab 1: Progression of results per period

\begin{tabular}{|c|c|c|c|c|c|}
\hline & $\underset{1}{A D G}$ & $\underset{2}{A D G}$ & $\underset{3}{A D G}$ & $\underset{\text { total }}{\mathrm{ADG}}$ & $\begin{array}{l}\text { Average } \\
\text { cold } \\
\text { carcass } \\
\text { weight }\end{array}$ \\
\hline $\begin{array}{c}\text { Period } 1: 4 \text { batches } \\
\text { before treatment= } \\
1280 \text { pigs }\end{array}$ & $815 \mathrm{~g}$ & $880 \mathrm{~g}$ & $856 \mathrm{~g}$ & $832 \mathrm{~g}$ & $87 \mathrm{Kg}$ \\
\hline $\begin{array}{l}\text { Period 2:8 batches } \\
\text { with 2 Iylan } \\
\text { treatments }=2560 \\
\text { pigs }\end{array}$ & $923 \mathrm{~g}$ & $943 \mathrm{~g}$ & $833 \mathrm{~g}$ & $894 \mathrm{~g}$ & $88.6 \mathrm{Kg}$ \\
\hline $\begin{array}{l}\text { Period } 3: 8 \text { batches } \\
\text { writh Tylan } \\
\text { treatments reviewed } \\
\text { + fornulation } \\
\text { adjustment= } 2560 \\
\text { pigs }\end{array}$ & $913 \mathrm{~g}$ & $945 \mathrm{~g}$ & $835 \mathrm{~g}$ & $906 \mathrm{~g}$ & $89.7 \mathrm{~K}_{\mathrm{g}}$ \\
\hline
\end{tabular}

\section{Discussion and conclusion}

The 2 diagnostic tools described here are complementary. Serum testing made it possible to understand how Lawsonia circulates in the herd and to target the ideal treatment time. The FIRSTtest ${ }^{\circledast}$ made it possible to understand that the diarrhoea at the end of the finishing stage and heterogeneity were not related to Lawsonia and could therefore be improved by zootechnical measures (rationing and formulation).

Administration of Tylan treatments led to an improvement of 1.6 $\mathrm{kg}$ in average cold carcass weight and formulation adjustment led to a $1.1 \mathrm{~kg}$ gain.

\section{References}

1. Fourchon et al (2006) IPVS, $\mathrm{p} 62$

2. Guedes et al (2002) The Can J Vet Res, p258-263 


\title{
0.057
}

\section{Evaluation of efficacy of oral toltrazuril (Baycox $5 \%^{\circledR}$ ) for the improvement of post-weaning gut health in pigs in Spain}

\author{
Alberto M. Alujas ${ }^{2}$ Ruth Blunt $^{1}$ Steven McOrist ${ }^{1}$ \\ 1. University of Nottingham, Sutton Bonington, UK; 2. Tests and Trials Ltd, Lleida, Spain
}

\section{Introduction}

Isospora suis disease often occurs around the second week of life, through to the normal pig weaning age of 3-weeks-old. It is characterized by yellowish pasty diarrhoea for one to several days; with dehydration evident even though nursing from the mother often continues. Oocysts can survive outside the pig, in the farrowing area, for many months and are resistant to most disinfectants. The time for intervention in piglets with a control strategy is critical; use of a preventive drug usually take place at 3 to 4 days of age so that it can kill appropriate stages of the parasite within newly infected piglets. Various anti-coccidial drugs have been developed and tested against porcine neonatal isosporosis, such as sulphadimidine. However, in the European Union, the symmetrical triazintrione toltrazuril is the only coccidiocidal registered (since 2002) specifically for the control of porcine neonatal coccidiosis in a single oral dose format of $20 \mathrm{mg}$ per kg bodyweight. Clinical studies of toltrazuril after weaning have not been comprehensive and have not usually included information on the possible beneficial effects on gut health in the dosed pigs in the later post-weaner, grower or finisher stages. This is an important issue in pigs, which are considered to show limited compensatory growth in animals recovering from a previous illness or weight check around weaning age. The exact reasons for this are not clear, but could include better intestinal maturity and enzymatic activity and a lower susceptibility to further post-weaning infectious agents, in those heavier pigs at weaning. The aim of this on-farm study was to evaluate the efficacy of toltrazuril (Baycox ${ }^{\circledR} 5 \%$ oral suspension) for the improvement of gut health in a European grower-finisher pig herd.

\section{Methods}

Four-day-old matched piglets either received toltrazuril (Group $A, n=218$ ) or placebo (Group $B, n=216$ ) via blinded colour-coded medications. The subsequent diarrhoea scores, growth performance, gut health and entero-pathogen load in each group was monitored by blinded observations, feed and pig weighing, faecal PCR tests for Brachyspira and Lawsonia and PCV2, culture for coliforms and Clostridia, as well as Lawsonia immunofluorescence assay serology. Observations were made daily and measurements collected weekly from day 21 up to day 105 in the grower-finisher stage of farming.

\section{Results}

There was a markedly improved feed conversion in grower-finisher pigs in Group A, particularly in the finisher period, but similar weight gains throughout. Lawsonia serology results indicated an early (before day 56) and moderate on-farm exposure. Titres in Group B pigs were higher, with fewer negatives throughout $(p<0.01)$, suggesting that the intestines of these pigs were more receptive to pathogenic Lawsonia infections, just after weaning. Four Group B pigs, 77 to 105-days-old, but no Group A pigs, had putative positive faecal PCR results for Brachyspira hyodysenteriae (swine dysentery). Salmonella, PCV2, Brachyspira pilosicoli, E coli and Clostridia results were not noteworthy. There was more diarrhoea noted $(p<0.05)$ in the piglets up to weaning age in group $B$ (untreated), by blinded investigators.

\section{Discussion}

These results confirm the protective effect of toltrazuril $\left(\right.$ Baycox $^{\mathrm{TM}}$ ) for coccidiosis prior to weaning and demonstrate specific beneficial effects on gut health and feed conversion after weaning. We also found that post-weaning enteric diseases including both ileitis and swine dysentery due to Brachyspira hyodysenteriae were more severe in pigs untreated for coccidiosis.

We therefore suggest there are two main reasons why the lifetime effects of small weaning weight and peri-weaning infections of rotavirus and coccidia are so important and detrimental. First, there is an innate anatomical issue in that smaller, gut-damaged piglets being weaned with a limited supply of mature villi units, will grow more slowly and second, there is the likelihood that pre-weaning infections can carry forward into an increased number of post-weaning infections such as ETEC, Lawsonia and Brachyspira.

In the past 5 years, novel and effective preventive programs for Lawsonia and Isospora been introduced, related to an oral live attenuated vaccine (Enterisol ileitis ${ }^{\mathrm{TM}}$ ) and the oral anti-coccidial medication toltrazuril (Baycox ${ }^{\mathrm{TM}}$ ), respectively. Unlike coccidiosis in chickens, no major vaccines have been identified for coccidiosis in pigs. 


\title{
0.058
}

\section{Early farrowing, possibly induced by intoxication via feed, reduced piglet survival with $70 \%$}

\author{
Per Wallgren \\ Dept of Animal Health and Antimicrobial Strategies, Uppsala, Sweden
}

\section{Introduction}

Survival of piglets is depending of a full length gestation, and events leading to farrowing earlier than expected may seriously affect piglet survival. This report describes two groups of sows mated at the same time and standing in adjacent units, but with dissimilar results at farrowing.

\section{Materials and Methods}

Three weeks prior to farrowing, 69 sows belonging to the same group of sows at the central unit of a multisite production system (sow pool) were transported to a satellite herd and divided into two groups. Group A with 36 sows was placed in unit A, and Group B with 33 sows was placed in unit B. All sows were offered a wet feed from the same batch and prepared at the farm. Piglets born were registered as live born, and still alive at three, 35 (weaning) and 70 (allocation) days of life.

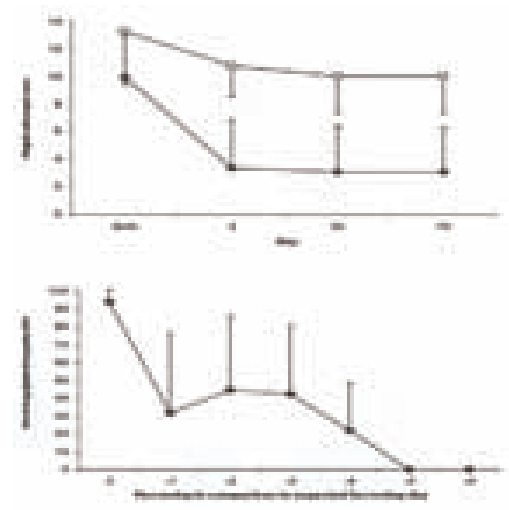

Figure 1. Number of live piglets per sow in group $A(O)$ and group $B$ $(\bullet)$, and the survival rate (\%) of piglets in group $B$ at three days of life in relation to day of farrowing in relation to expected day.

\section{Results}

Sows in group B started to farrow six days prior to expected farrowing with a mean of $-3.0 \pm 1.9$ days. Piglets born were premature and the mortality was significant. In mean these sows gave birth to $10.0 \pm 3.0$ live born piglets of which $3.4 \pm 3.5$ still were alive at three days of age. The mortality was $100 \%$ in litters born 5 or 6 days earlier than expected. The liquid feed was replaced with dry feed to the sows at the third day after the first farrowing, but only 4 out of 33 sows in group B farrowed at the expected day or later. The survival rate at 3 days of age in these 4 litters was $88 \%$ (Fig 1).
In contrast, sows in unit A farrowed at the expected time (at day $-0.1 \pm 1.1)$, with expected number of piglets born (13.3 \pm 3.8 ) and still alive at three days (10.8 \pm 2.2$)$. From the third day of life, mortality was near to zero in both groups (Fig 1).

Necropsy of 6 stillborn piglets including placenta in group B excluded infections as a cause of the incidence. Instead they supported, but did not prove, intoxication (of the sows).

\section{Discussion and Conclusions}

The incident in Group B clearly demonstrated the importance of the last days of the gestation for the survival rate of the piglets born.

It is also interesting that the sows in group A farrowed at the same time in the adjacent unit with perfectly normal results. Due to the necropsy results and the lack of clinical signs in any other unit, infections were concluded less likely to have caused the incident in group B. Instead the feed was focused.

Liquid feed systems hold $3 \mathrm{~kg}$ feed per meter and the systems are generally neither emptied nor cleaned. Instead, unwanted microbial growth is assumed to be controlled with a low $\mathrm{pH}$.

However, aggregations of food may arise, eg following curvatures, creating other conditions where growth of bacteria or fungi may take place. Considering the high water activity in the liquid feeding systems, bacterial growth probably appear first, with risk for bacterial toxins/endotoxins. With time, mould growth could also occur with subsequent mycotoxin formation.

If such an aggregate let go from the tube, pigs fed from the valves downstream will be exposed to its content, including endotoxins/mycotoxins which both have been proven able to induce abortion in sows $(1,2)$. However, as any toxin provocation will precede the abortions in time, a true relationship may be impossible to certify - even when strongly suspected as in this case. The feed loop to Unit B was opened by saw, cleaned and re-sealed. Subsequent farrowing batches have been normal.

\section{References}

1 Weaver et al., 1978. Mycotoxin-induced abortions in sows. Can Vet J. 19:72-74.

2. Wrathall et al., 1978. Experimentally induced bacterial endotoxaemia and abortion in pigs. Br. Vet. J. 134-225-230. 


\title{
0.059
}

\section{Acute selenium toxicity in finishing pigs due to a contaminated feed supplement}

\author{
Catriona M. Ritchie \\ SAC Veterinary Services, Aberdeen, UK
}

\section{Introduction}

Selenium toxicity in pigs is often due to accidental contamination of the diet (1-5). The acute form may present as sudden onset paralysis due to poliomyelomalacia of the spinal cord and it can be fatal. If finishing pigs ingest high levels of selenium the elevated tissue selenium levels present a potential risk to food safety. The mean blood selenium elimination half-life in pigs has been estimated to be 12 days (4). Selenium levels in liver and kidneys can still be elevated two months after a toxicity incident, with the kidney having the highest level (1). Livestock owners have a responsibility to produce food that is safe for human consumption. In the UK the Food Standards Agency (FSA) provides advice if there is a potential risk to the food chain, such as from a contamination incident. The financial losses due to selenium toxicity in finishing pigs are mainly due to culling of paralysed pigs, deaths and reduced marketability of exposed pigs. The consequences of the exposure are described and the loss due to acute selenium toxicosis in finishing pigs has been calculated.

\section{Materials and methods}

Fifty out of 316 twelve to fifteen-week-old pigs developed acute onset paralysis following the ingestion of a commercial, selenium contaminated, feed supplement. Two groups of pigs had clinical cases and a third group of 100 pigs, which had ingested the high selenium ration but for a shorter period, showed no clinical signs. Quadriplegia of alert pigs was the main finding. Affected pigs were usually willing to eat when presented with food. Alopecia developed in exposed pigs.

\section{Results}

Serum selenium levels in six pigs from the clinically affected groups ranged from 740 to $1880 \mathrm{ng} / \mathrm{ml}$, with a mean of $1310 \mathrm{ng} /$ $\mathrm{ml}$ (reference range 140-190ng/ml). Histopathological examination of the spinal cords from two pigs showed severe, bilaterally symmetrical lesions of poliomyelomalacia affecting the cervical and lumbar enlargements. The two, necropsied, quadriplegic pigs had pre-mortem serum selenium levels of 1370 and $1620 \mathrm{ng} / \mathrm{ml}$. Three other quadriplegic pigs had liver selenium levels of 9.49-13.2mg/kg DM (reference range $0.7-1.8 \mathrm{mg} . \mathrm{kg} \mathrm{DM}$ ).

Analysis of the commercial feed supplement detected a selenium content of $3090 \mathrm{mg} / \mathrm{kg}$, which was approximately 1000 fold higher than on the label. The complete diet was analysed and found to have a selenium content of $42.6 \mathrm{mg} / \mathrm{kg}$.

\section{Discussion}

The clinical signs, histopathological findings, blood and liver selenium levels were consistent with a diagnosis of selenium toxicity. The source was confirmed to be a feed supplement, which had been contaminated with a high level of selenium due to an error at the production plant.

As the pigs were destined for the food chain at 24 weeks of age the Food Standards Agency (Scotland) was informed of the incident and the potential risk to food safety. They advised a 114 day withdrawal period from the time the pigs last consumed the contaminated feed and if this was not possible the offal had to be discarded at slaughter. The long withdrawal period meant that the pigs would be overweight for their market specification and only suitable for the cull sow market but they would not have been suitable for the export market due to the selenium exposure. Removing the offal at the abattoir posed practical difficulties and the risk that misidentification could occur for which there could have been liability. There was also concern over potential damage to the reputation of the Scottish pig industry if it became known that the pigs had entered the food chain. All 416 exposed pigs were euthanased on farm and their carcases disposed of.

The cost incurred per pig was calculated as CAD\$39 (GBP23.32). The culled pigs were valued at CAD\$63500 (GBP38040). The compensated loss was CAD\$192 (GBP115) per exposed pig.

\section{References}

1. Penrith M.L. et al 1996. Ondersteport J Vet Res. 63(2):171-9

2. Hill J et al 1985. Aust Vet J. 62(6):207-9

3. Casteel et al 1985. J Am Vet Med Assoc. 186(10):1084-5

4. Davidson-York D et al 1999. J Vet Diag Invest.11(4):352-7

5. Casteignau A et al 2006. Proceedings of 19th IPVS Congress, Denmark.(2): 573 


\title{
0.060
}

\section{A case on chronic salt intoxication in growing pigs}

\author{
Carmen Alonso ${ }^{1}$ Nuria Llanes ${ }^{1}$ Joaquim Segalés ${ }^{2}$ \\ 1. Cooperativa d'Ivars, Ivars d'Urgell, Spain; 2. CRESA, Barcelona, Spain
}

\section{Introduction}

Sodium ( $\mathrm{Na}+)$ is necessary for the electrolytic balance in animals. Most ion sodium in the diet of different livestock species, including swine, is given by means of salt (sodium chloride). The standard recommendation of $0.2-0.25 \%$ meets the dietary $\mathrm{Na}+$ and $\mathrm{Cl}$ - requirements of growing and finishing pigs for most meal diets. Sodium ion toxicosis is also called water deprivation or salt poisoning, and it is rather well documented in swine (1). The occurrence of this toxicosis is inversely related to water intake, usually related to water deprivation due to inadequate water supply or changes in husbandry. Based on available data, salt intoxication results in an acute or sub-acute clinical problem. Chronic salt toxicity has not been reported so far in pigs. Therefore, the objective of the present study is to report the clinical, pathological and laboratorial findings of a chronic salt intoxication case in growing pigs.

\section{Materials and Methods}

Pigs from a single-origin fattening farm located in North Eastern Spain displayed increased mortality. Historical mortality records in the finishers ranged between 5-7\%, but in May 2008, mortality percentage increased dramatically to $49 \%$. Approximately, $30 \%$ of the pigs developed central nervous disorders and $70 \%$ of them respiratory problems. Affected pigs displayed hyperesthesia, paddling and, usually, lack of palpebral reflex, with no fever. Animals with respiratory clinical signs (dyspnea) adopted dogsitting position. Also, affected pigs showed paleness of the skin, and diarrhoea was identified occurring in all pens of diseased animals. A number of pigs were on-farm necropsied. Based on gross findings, oedema disease, postweaning multisystemic wasting syndrome (PMWS) and porcine reproductive and respiratory syndrome (PRRS) were suspected, besides the existence of concomitant bacterial-like pneumonia. Based on suspected etiologies, antibiotic was prescribed in water (neomycin) and several drugs were used parenterally (including enrofloxacyn, florfenicol and apramycin) in the different groups of affected animals. No significant response to antibiotic treatment was noted after 5 days of application. A number of laboratorial studies were implemented, including necropsy and histopathology of two pigs with central nervous clinical signs, antibody profile against PRRS virus, and urea and creatinine levels in serum. Further studies included determination in feed of heavy metals (cadmium and mercury), vitamin E, vitamin D, selenium, ochratoxine, oxalates and sodium chloride content were measured.

\section{Results}

The most significant pathological findings in both animals were a severe, bilateral, diffuse interstitial fibrosis in the middle and deep area of the renal cortex, with loss of glomeruli and glomerulosclerosis and a mild mononuclear multifocal meningoencephalitis with very occasional presence of eosinophils. Both pigs were also positive to PRRS virus detection in lungs. Both pigs had very high levels of both urea and creatinine. Obtained results so far, mainly the diffuse renal fibrosis, pointed out to a potential chronic toxicity scenario. Among the feed analyses performed, all determinations were within the normal ranges, but the sodium content of the ration, which was $221 \%$ on dry matter (those pigs ingested 30 times more sodium than that recommended for dry feeding with free access to drinking water (2). To determine the origin of these abnormally high salt levels, feed mix compounds were analysed for sodium chloride. Among 8 sources of whey, 2 of them had values of 17.2 and $21.3 \%$ of salt on dry matter; the remaining whey samples had values from 3.5 to $10.6 \%$ on dry matter.

\section{Discussion}

The present case report describes a complex situation in which clinical signs pointed towards an acute process, while kidney pathological features suggested a potential chronic toxicity process. Therefore, once the clinico-pathological findings were obtained, laboratorial investigations were directed towards a potential intoxication process. The confirmation of extremely high levels of salt in the diet, not previously reported in the literature as far as the authors' know, allowed confirming a diagnosis of salt toxicosis in the affected herd. Moreover, the presence of an apparent novel kidney lesion (characterized by diffuse cortical fibrosis and glomerulosclerosis) associated to this intoxication let us classify the clinical problem as chronic.

\section{References}

1. Carson TL. 2006. Diseases of Swine. pp,980.

2. Nutrient Requeriments of Swine, National Research Council, Tenth Revised Edition, 1998. 


\title{
0.061
}

\section{Carbon monoxide intoxication in weaned pigs}

\author{
Christina L. Planz ${ }^{1}$ Heiko Nathues ${ }^{1}$ Ulrich Brinkmann² Elisabeth Große Beilage ${ }^{1}$ \\ 1. University of Veterinary Medicine Hannover, Bakum, Germany; 2. Praxis an der Maiburg, Bippen, Germany
}

\section{Introduction}

Carbon monoxide intoxication in pigs, although not often observed, occurs in connection with dysfunction of heating devices and poorly ventilated compartments. Depending on the level of carbon monoxide in the air and the time of exposure it can take peracute to chronic courses. This case report describes the occurrence of acute carbon monoxide intoxication in weaned pigs. It points out the course of actions to approach the diagnosis.

\section{Materials and Methods}

In February 2009, on a piglet production farm almost all piglets of one batch, which was stocked in one compartment, died within few hours after weaning. The pig farmer had not noticed any symptoms of disease at the point of weaning. In addition to a clinical observation of the piglets in all compartments of the stable, six of the dead piglets were subjected to a pathological and histopathological examination. Furthermore, a thorough examination of the environment of the animals was conducted, comprising a simulation of the climatic conditions in the unit on the day of re-stocking. Therefore the gas-fired heater, which was used to heat up the compartment on that day and had been maintained recently, was put into operation. After some hours, the concentration of carbon monoxide in the air of the compartment was measured via gas detection pump under strict safety precautions, supervised by experts from the local fire department.

\section{Results}

The examination of the animals in the barn did not give any clinical evidence; the piglets did not show any specific signs of disease. Feed and water could be excluded as source of the disease for the piglets had still not received feed, and the water was the same as in other compartments. During pathological examination of six piglets there could not be detected any abnormalities that could have explained the cause of death. Especially the colour of blood and tissues could be ascertained as physiological. Also the findings in the histopathological examination were unspecific. The measurement of the carbon monoxide content in the air during simulation of the climatic conditions in the compartment revealed a concentration of $3000 \mathrm{ppm}$.

\section{Discussion}

Contrary to expectations, pathological as well as histopathological examination did not contribute to the diagnosis of carbon monoxide intoxication. None of the symptoms described in literature as typically being related to carbon monoxide poisoning, such as cherry-red blood, rose-coloured tissues or meningoencephalitis, could be detected in the examined animals. The diagnosis was only made after intensive examination of the environment, including running the gas-fired heater which was used in the relevant compartment. The measured concentration of 3000 ppm was clearly above the minimum lethal concentration of $1000 \mathrm{ppm}$. This demonstrates that in cases of suspected carbon monoxide intoxication it is indispensable to take even more elaborate diagnostic measures like the described reconstruction of the climatic conditions in the compartment: In the absence of clinical and (histo)pathological findings this might possibly be the only successful way to confirm and prove the diagnosis.

The excess in carbon monoxide in the unit was attributed to a malfunction of the gas-fired heater. Especially in combination with very low ventilation rates as common during winter time, this can easily lead to high carbon monoxide levels in the air of the compartment. Therefore veterinarians should urge pig farmers to exercise due care in the use of these devices, not only for reasons of animal welfare but also for their own safety.

\section{References}

Heinritzi K, Schweinekrankheiten. Ulmer UTB 2006 


\title{
0.062
}

\section{Assessment of vitamin E levels, selenium levels and presence of viral pathogens as the etiology of mulberry heart disease}

\author{
Paul Thomas ${ }^{1}$ Giacomo Bortoletto ${ }^{1}$ Peter Kirkland ${ }^{2}$ Wonil Kim¹ ${ }^{1}$ Alan Loynachan ${ }^{1}$ Steve Ensley ${ }^{1}$ Tanja Opriessnig $^{1}$ \\ 1. Iowa State University, Ames, IA, USA; 2. Elizabeth Macarthur Agricultural Institute, Camden, NSW, Australia
}

\section{Introduction}

Mulberry heart disease (MHD) in swine usually manifests as sudden death in young, fast-growing, apparently healthy pigs. The hallmark lesions are acute hemorrhagic myocarditis and myocardial necrosis resulting in heart failure. It has long been suspected that MHD is linked to deficient levels of vitamin E and/or selenium $(3,4,5,6)$. However, the exact relationship is still unclear and vitamin $\mathrm{E}$ and selenium deficiency are not observed in all cases of MHD (3). Furthermore, recent evidence suggests that infectious disease may be associated with similar heart lesions in pigs $(1,2)$. We investigated the relationship between MHD and vitamin $\mathrm{E}$, selenium, and 13 other mineral levels in heart and liver tissues. Additionally, several PCR assays were conducted on heart tissues to explore a possible relationship between viral pathogens and MHD.

\section{Materials and methods}

Tissues were collected at the lowa State University Veterinary Diagnostic Laboratory from 83 pigs representing 38 farms. On the basis of histological examination 42 of the pigs were classified as MHD-affected and 41 classified as MHD-unaffected. The farms ranged in size from 800 to 4,500 head and most had a history of increased incidence of MHD. Mineral panels were completed on all heart and liver tissues using a Varion 820 ICP-Mass Spectrometer. The panel included tissue levels for cadmium, calcium, chromium, cobalt, copper, iron, magnesium, manganese, molybdenum, phosphorus, potassium, selenium, sodium and zinc. In addition, vitamin E levels were also obtained from heart and liver tissues. Seventy-seven of the 83 heart tissues were tested using real-time and conventional PCR assays for pan-morbillivirus, pan-coronavirus, pan-pestivirus, porcine enterovirus (PEV), pan-herpesvirus, porcine circovirus type 1 (PCV1), PCV2, porcine parvovirus (PPV), European and North American porcine reproductive and respiratory syndrome virus (PRRSV), Bungowannah virus (BGWV) and West Nile virus (WNV). Pairwise t-test was used to analyze the data. A P value $<0.05$ was considered significant.

\section{Results}

Significant differences $(P<0.05)$ were observed in levels for sodium, copper and vitamin $\mathrm{E}$ in heart tissues and sodium, magnesium, phosphorus, selenium and vitamin $\mathrm{E}$ in liver tissues. Mean values were often higher in affected pigs compared to non-affected pigs; however, they were still within the normal parameters reported for tissue levels in healthy pigs (3). Vitamin E levels for both groups also resided within the normal range (3). Affected pigs had a trend for a higher incidence of PEV (P = $0.25)$, and pan-herpesvirus $(P=0.25)$

\section{Discussion}

The difference between affected and unaffected groups observed among heart vitamin $E$, liver vitamin $E$, and liver selenium is in agreement with the traditional etiology of $\operatorname{MHD}(3,4,5,6)$. While many of the pathogens were isolated from several pigs among the groups, they did not appear to be associated with MHD status in the pigs. Although selenium deficiency has not been shown to cause MHD, it has once again been illustrated that MHD is associated with decreased selenium levels. There does seem to be a relationship between MHD and PEV and herpesvirus. This needs to be further explored experimentally as it could provide researchers a better model to study MHD and eventually provide producers tools to prevent its occurrence.
References
1. Kirkland, P.D. et al. (2007) Virus Res 129:26-34.
2. Opriessnig, T. et al. (2006) J Comp Path 134:105-110.
3. Pallares, F.J. et al. (2002) J Vet Diagn Invest 14:412-414.
4. Sharp, B.A. et al. (1972) Can J Comp Med 36:371-376.
5. Sharp, B.A. et al. (1972) Can J Comp Med 36:393-397.
6. Van, F. et al. (1970) JAVMA 157:1208-1219. 


\title{
0.063
}

\section{First record of a clinical outbreak of EMCV in The Netherlands}

\author{
Manon Houben ${ }^{1}$ Leo van Leengoed ${ }^{2,1}$ Rudolf Raymakers ${ }^{3}$ Joep Gerards ${ }^{3}$ Norbert Stockhofe-Zurwierden ${ }^{4}$ Mark d. Groot $^{5}$ \\ 1. PorQ BV, Breugel, Netherlands; 2. Veterinary Faculty, Utrecht, Netherlands; 3. Veterinary Centre Someren, Someren, Netherlands; \\ 4. Central Veterinary Institute, Wageningen UR, Lelystad, Netherlands; 5. Intervet International, Boxmeer, Netherlands
}

\section{Introduction}

Encephalomyocarditis virus (EMCV) is endemic on many pig farms worldwide. EMCV is a Picornavirus and may infect a wide variety of animals like rodents, pigs and also man. Most important features in porcine EMCV infection are myocarditis and reproductive failure. Most clinical outbreaks in Europe were reported during the 1990's in Belgium, Italy, Greece and Cyprus (1). Many Dutch sow herds have antibodies against EMCV (2), but neither clinical signs nor pathological lesions were reported until now.

\section{Materials and methods}

The infected herd of this study has two separate sites located $80 \mathrm{~km}$ from each other in the southern Netherlands. The main location is a 1400 sow herd while the other location houses all breeding stock for replacement. Mated gilts are transported to the main farm and added to the herd after a six-week quarantine period. Signs of EMCV were noted from March 2008 on by farmer, veterinarian and veterinary consultant. Necropsies were performed at the Faculty of Veterinary Medicine in Utrecht and at the Central Veterinary Institute in Lelystad. EMCV RT-PCR was performed by CODA-CERVA (Veterinary And Agrochemical Research Centre, Belgium)

\section{Results}

In March 2008 a high mortality was observed in newborn piglets of multiparous sows. Apparently healthy well-grown piglets with good body condition died without previous clinical signs. Some litters stayed unaffected, while mortality of 2 to 4 days old piglets in other litters went up to $100 \%$. Sows showed no clinical signs. Cross-fostered piglets from unaffected litters stayed unaffected. Four piglets that died were necropsied. These piglets were pale, with severe hydrothorax, hydropericardium and pulmonary edema. Myocardia were severely pale with occasional petechia. Histological examination displayed a severe lymphohistiocytic myocarditis and a mild lymphocytic meningitis. EMCV was demonstrated in myocard by RT-PCR.

During March 16 abortions occurred, and involved 3 sows and $13(81 \%)$ gilts. Herd performance data were negatively influenced from March till May (figure 1). Surprisingly, only litters of multiparous sows were affected by sudden death of piglets, whereas none of the litters of gilts were affected.

Piglet mortality by sudden death persisted for 3 months. Thereafter, signs of EMCV-infection faded away and technical performance of the herd returned to pre-infection levels.
Figure 1: Technical performance data 2008

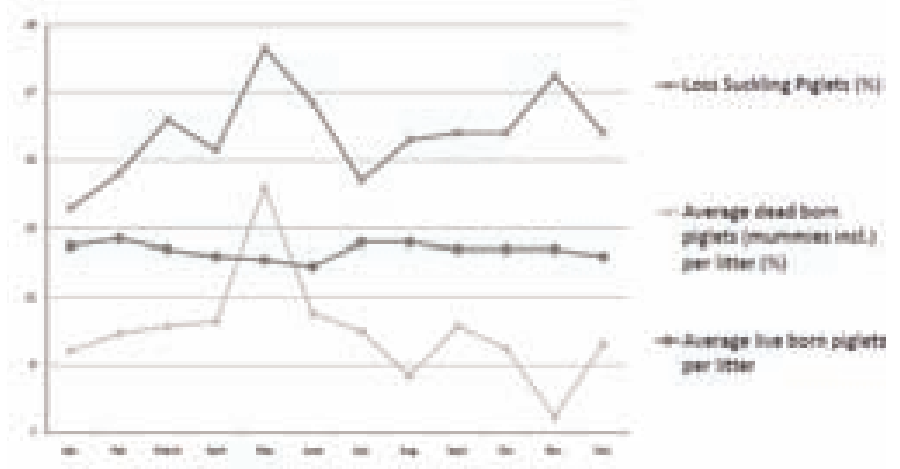

\section{Discussion}

Sudden death of suckling piglets from multiparous sows was the major clinical sign of this outbreak. Retrospectively, these signs appeared to be preceded by an increased abortion rate, mainly in gilts. No diagnosis was made upon the cause of abortions, and EMCV may be involved.

As litters of first parity sows stayed unaffected, we assume that these piglets were protected against EMCV by maternal derived antibodies.

In most cases rodents introduce and spread EMCV in swine herds (3). On this farm vermin is strictly controlled. Direct transmission of EMCV between pigs is in most cases limited, but may occasionally cause a large EMCV-outbreak (4). Although EMCV is endemic in many herds in The Netherlands, clinical signs of EMCV are rare. The outbreak of EMVC in this herd is most likely due to presence of high numbers of susceptible sows that were infected during late gestation. Theoretically, this can be an introduction of EMCV in a naïve herd, or an introduction of an EMCV strain that differs in pathogenicity and immunogenicity from endemic EMCV strains. Closer monitoring might elucidate whether a new more virulent strain of EMCV circulates in The Netherlands. Therefore, veterinarians and veterinary pathologists should consider EMCV as a differential diagnosis in case of mortality in suckling piglets and abortions.

\footnotetext{
References

1. Maurice H. et al., Epidemiology and Infection (133), 547-555

2. Augustijn M. et al., Tijdschrift v. Diergeneesk. (131) 40-44

3. Maurice $\mathrm{H}$ et al., Preventive Veterinary Medicine (78), 24-34

4. Maurice H. et al., Veterinary Microbiology (88), 301-314
} 


\title{
0.064
}

\section{Investigation of risk factors for porcine circo virus type $\mathbf{2}$ (PCV2) following an outbreak of Post- weaning Multi-systemic Wasting Syndrome (PMWS) on a large commercial pig farm}

\author{
Barbara Wieland; Dirk Werling; Dirk U. Pfeiffer; Amanda Nevel; Andrew Rycroft; Christopher M. Wathes
}

Royal Veterinary College, Hatfield, UK

\section{Introduction}

Porcine circo virus type 2 (PCV2) has been widely acknowledged as the necessary agent for post-weaning multi-systemic wasting syndrome (PWMS) in pigs. Various studies have identified potential risk factors, but it is still not fully understood what triggers the onset of this multi-factorial disease.

The present study aimed at evaluating the presence of porcine circovirus 2 (PCV2) and other pathogens before and during an outbreak of PMWS on a large commercial pig farm in the UK. Further we investigated the effect of environmental conditions and the parity and PCV2 immune and infection status of sows on presence of PCV2 in growing pigs.

\section{Material and Methods}

The study was based on data collected in two different research projects ongoing on a large commercial farm when a PWMS outbreak hit the farm. One project was a large scale experimental study on the impact of environmental conditions (dust and ammonia) on the onset of respiratory disease in 960 weaned pigs divided in 8 batches, batches were run one at a time over 2 years. At the end of the 42 day exposure experiment, serum samples were collected and post-mortem carried out on randomly selected pigs. The other project was a longitudinal study on the reproductive performance during the first three parities and involved 188 gilts originating from eight different breeders.

Serum samples of 371 growing pigs, aged 10-12 weeks, were tested for antibodies to PCV2 and other endemic pig pathogens. In addition, presence of PCV2 antigen was determined by semi-quantitative PCR. Fifty gilts and sows produced 135 of the 371 growing pigs of which serum samples had been collected. These 50 gilts and sows had been repeatedly sampled at different time points in the reproduction cycle resulting in a total of 296 samples which were tested for PCV2 antibodies and PCV2 antigen.

\section{Results}

Results show that PCV2 sero- and PCR-positive pigs were already present on the farm long before the actual PMWS outbreak. Prevalence of PCV2 antibodies and antigen in growing pigs was low in the first batches of the respiratory experiments, but reached up to $100 \%$ in the late batches. The weight gain in PCV2 PCR positive pigs was significantly lower than in negative pigs $(p<0.01)$ and PCV2 PCR positive pigs had a significantly higher overall pathological score in the post-mortem examination with a mean of 10.8 compared to 8.5 in PCR negative pigs $(p<0.01)$.

In sows, differences is sero-prevalence was observed for different stages in the reproduction cycle and sows in the second half of gestation and around farrowing seemed more likely to have measurable titres of PCV2 antibodies. Over a period of two years, most sows showed fluctuations in PCV2 antibody titres. When combining laboratory results of sows and growing pigs, growing pigs had higher odds to be PCV2 PCR positive if the sow tested positive for PCV2 antibodies in the time period around farrowing (OR 24.5, $p=0.01$ ), the sow was of parity 3 or higher (OR $40.9, p<0.01$.) and were less likely to test PCR positive if the sow originated from certain breeders (OR 0.04, $\mathrm{p}<0.01$ ).

\section{Discussion}

Through previous research it is well understood that maternal PCV2 antibodies prevent onset, or delay onset, of PMWS in the weaner. The results of this study further highlight the role of the sow for PCV2 in weaners (parity, PCV2 status around farrowing and breeding background). Our results indicate that in future studies on PMWS more sow data should be included. The trend of increased sero-prevalence in the second half of gestation and around farrowing may indicate latent PCV2 infection in sows and that increased stress levels result in reactivation of PCV2, followed by the observed PCV2 antibody response. 


\title{
0.065
}

\section{PCV2 in extended pooled semen from US boar studs}

\author{
Johannes Kauffold ${ }^{1}$ Friedrich Schmoll ${ }^{2}$ Gary C. Althouse ${ }^{1}$ \\ 1. Department of Clinical Studies - New Bolton Center, School of Veterinary Medicine, University of Pennsylvania, Kennett Square, PA, USA; \\ 2. Large Animal Clinic for Internal Medicine, University of Leipzig, Leipzig, Germany
}

\section{Introduction}

Prior studies have demonstrated PCV2 DNA and the virus itself in raw and extended semen from healthy naturally or experimentally infected boars [1-4]. To provide an epidemiological context to this finding, our group recently reported that of 472 German and Austrian raw semen samples screened for PCV2, 86 (18.2\%) were found to be positive [4]. In our continued work to provide a more global perspective of the epidemiology of PCV2 in boar semen, a cross-sectional study was performed to determine the occurrence of PCV2 in extended semen produced in US boar studs.

\section{Materials and Methods}

Randomly selected extended pooled semen samples $(n=198)$, 18-30 hours post-processing, originating from 17 studs located in different states of the US were tested for PCV2 using a nested PCR technique [4]. Extended-chilled samples submitted to the Andrology Laboratory at the University of Pennsylvania were centrifuged at 4,000 rpm for $10 \mathrm{~min}$ to produce sperm-rich and supernatant fractions. Fractions were cataloged and then stored at -20 degree $C$ until subsequent analysis. For all sample positives, all fragments obtained with the internal nested PCR were additionally sequenced to confirm PCV2 identity. A chi-square test was used to determine differences $(P<0.05)$.

\section{Results}

A total 49 (24.7\%) extended pooled semen samples were PCV2 positive (Table 1). Samples from 5 studs were negative; all other studs had at least one positive sample (range: $10-50 \%)$. Positive results were more often obtained with the sperm-rich pellet ( $n=34)$ than with the supernatant $(n=8)$. A small number of samples $(n=7)$ were positive for both the sperm-rich pellet and corresponding supernatant.

\section{Discussion}

This study confirms that PCV2 is shed in semen and can still be detected after semen is extended and stored for 18-30 hours. The percentage of samples found positive corresponds well with previous results from North America and Europe [1-4]. Of particular interest in the present data set was the differences observed between studs. Also, the fact that most samples were positive in sperm-rich pellets may indicate that the virus is sperm associated rather then a loose component in seminal plasma. All semen samples tested in this study were pooled semen originating from several boars. It is thus not known if one or more boars contributed to the PCV2 contamination. Also virus quantification was not performed which might have been necessary in order to better determine the risk of venereal transmission.
Table 1. Results of analysis of extended semen samples for PCV2 by nested PCR and sequencing $(n=198)$

\begin{tabular}{|l|c|c|c|c|}
\hline Stud ID & Pellet & S-tant* & Both\# & Total \\
\hline (n) & $\mathrm{n}$ & $\mathrm{n}$ & $\mathrm{n}$ & $\mathrm{n}$ \\
\hline $1(3)$ & 0 & 0 & 0 & 0 \\
\hline $2(17)$ & 3 & 0 & 0 & $3(17.6)$ \\
\hline $3(6)$ & 0 & 1 & 0 & $1(16.7)$ \\
\hline $4(21)$ & 3 & 1 & 0 & $4(19.0)$ \\
\hline $5(34)$ & 4 & 1 & 4 & $9(26.5)$ \\
\hline $6(28)$ & 5 & 4 & 0 & $9(32.1)$ \\
\hline $7(12)$ & 6 & 0 & 0 & $6(50.0)$ \\
\hline $8(12)$ & 5 & 0 & 1 & $6(50.0)$ \\
\hline $9(9)$ & 1 & 0 & 2 & $3(33.3)$ \\
\hline $10(6)$ & 0 & 0 & 0 & 0 \\
\hline $11(2)$ & 1 & 0 & 0 & $1(50.0)$ \\
\hline $12(4)$ & 1 & 0 & 0 & $1(25.0)$ \\
\hline $13(19)$ & 3 & 1 & 0 & $1(25.0)$ \\
\hline $14(5)$ & 0 & 0 & 0 & 0 \\
\hline $15(6)$ & 1 & 0 & 0 & $1(16.7)$ \\
\hline $16(4)$ & 0 & 0 & 0 & 0 \\
\hline $17(10)$ & 1 & 0 & 0 & $1(10.0)$ \\
\hline Total & $34^{\mathrm{a}}$ & $8^{\mathrm{b}}$ & $7^{\mathrm{b}}$ & $49(24.7)$ \\
\hline *Supernatant; \#Both supernatant and pellet were PCV2 positive; (a,b) P<0.05. \\
\hline
\end{tabular}

\section{References}

1. Kim et al. J Virol Meth 2001;98:25-31.

2. Larochelle et al. J Clin Microbiol 2000;38:4629-4632.

3. McIntosh et al. J Vet Diagn Invest 2006;18:380-384.

4. Schmoll et al. Theriogenology 2008;15:814-821. 


\title{
0.066
}

\section{Use of IgG and IgM differentiation of antibodies to detect primary and secondary immune response to Porcine circovirus type 2 (PCV2)}

\author{
Christoph Keller ${ }^{1}$ Takeshi Yamaguchi² Mali Miyashita $^{2}$ Ohlinger F. Volker ${ }^{1}$ \\ 1. bioScreen EVDMC GmbH, Münster, Germany; 2. Boehringer Ingelheim Vetmedica Japan Co.,LTD, Kawanishi, Japan
}

\section{Introduction}

PCV2 is the causative agent for porcine circovirus associated diseases (PCVAD), which clinically affect pig herds worldwide. 1 As antibody titres are usually high in affected and in not affected herds2 serological tests are not suitable for laboratory diagnostics for PCVAD. Recently commercial vaccines to protect pigs from PCVAD became available3. In this study lgG and IgM type antibodies to PCV2 in vaccinated and not vaccinated pigs were examined to demonstrate the effective priming of the immune system for contact to the pathogen.

\section{Materials and Methods}

In a GCP conform field trial on a Japanese pig farm a group of animals were vaccinated against PCV2 at 3 weeks of age (Ingelvac CircoFLEX ${ }^{\circledR}$, Boehringer Ingelheim). A not vaccinated control group was housed comingled. Blood samples from 20 randomly selected pigs each from both groups were taken at the time of vaccination ( 3 weeks of age) and 4, 6, 8, 10 and 12 weeks after vaccination. All samples were tested for antibodies specific to PCV2 ORF2 antigen using a commercially available ELISA kit (INGEZIM CIRCOVIRUS IgG/IgM ELISA, Ingenasa, Madrid, Spain). The ELISA was performed according to the manufacturers instructions. In addition PCV2 genome load was quantified in the samples using a quantitative real-time PCR according to the method published by Brunborg et al.4

Figure 1: Results of the ELISA ( $n$ positive results) and $q P C R(\% P C R$ positive pigs) tests for vaccinated and not vaccinated pigs ( $n=20 \mathrm{per}$ group).

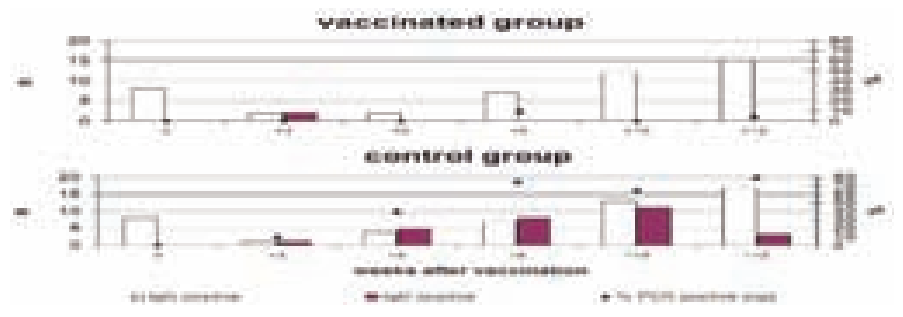

\section{Results}

Results of the tests are summarized in figure No. 1. Four weeks after the vaccination in both groups two animals were positive in the IgM specific ELISA. At this time the first samples from the control group were detected to be positive for PCV2 virus genome in the qPCR. In the following weeks the percentage of viremic animals in the control group increased. In parallel the number of IgM positive pigs rose in the control group. In the vaccinated group no more lgM positive samples were detected, but the number of IgG positive samples rises. Twelve weeks after vaccination still three samples from the control group were positive for IgM. The number of lgG positive samples was comparable in both groups at this time.

\section{Discussion}

The results presented in this study clearly demonstrate the effective priming of the porcine immune system after vaccination against PCV2. Not vaccinated pigs react against field virus exposure with the production of IgM (primary immune response), whereas vaccinated pigs react with a secondary immune response (predominantly lgG). The detection of many lgM positive animals in a group of pigs indicates a primary immune response. As the majority of vaccinated pigs does not react with $\lg M$, but with IgG (secondary immune response) to clinical exposure to PCV2, the differentiation of IgG and IgM against PCV2 could be used as a diagnostic tool to differentiate not vaccinated groups of pigs from vaccinated ones.

\section{References}

1. J. SEGALES et al. . Postweaning mulstisystemic wasting syndrome (PMWS) in pigs. A review. Veterinary quaterly. 2002: 24, 109-124.

2. R. Larochelle et al. Comparative serologic and virologic study of commercial swine herds with and without postweaning multisystemic wasting syndrome. Can J Vet Res. 2003: 67, 114-120.

3. M. Miyashita et al. Efficacy of a novel one-shot PCV2 vaccine under Japanese field conditions. Proc. 20th IPVS 2008, Durban, South Africa.

4. I. M. Brunborg et al. Quantitation of porcine circovirus type 2 isolated from serum/plasma and tissue samples of healthy pigs and pigs with postweaning multisystemic wasting syndrome using a TaqMan-based real-time PCR. J Virol Methods. 2004: 122, 171-178. 


\title{
0.067
}

\section{Diagnosis of Porcine Circovirus Diseases (PCVDs) by serology and qPCR}

\author{
Lars E. Larsen ${ }^{1}$ Peter Aastrup ${ }^{2}$ Charlotte K. Hjulsager ${ }^{1}$ Kirsten V. Larsen ${ }^{2}$ \\ 1. National Veterinary Institute, Copenhagen, Denmark; 2. Intervet/Schering-Plough Animal Health, Copenhagen, Denmark
}

\section{Introduction}

Porcine circovirus type 2 (PCV2) is considered the essential infectious agent of postweaning multisystemic wasting syndrome (PMWS). PCV2 is an ubiquitous virus and most if not all pigs become infected during their life, but only a proportion of them develop PMWS $(1,2)$. A threshold of 7 log10 PCV2 DNA copies/ $\mathrm{ml}$ serum has been suggested to establish a PMWS diagnosis (2-4), however limited information are available on the use of PCR in the diagnosis of other PCV2 related diseases (PCVDs). The present study describes the results of serological and virological profiling from 14 Danish herds with symptoms of PCVD.

\section{Material and Methods}

Sixteen Danish swine herds were included. Fourteen of herds had one or more of the following clinical symptoms in weaners, growers and/or finishers: Pneumonia, diarrhea or unspecific symptoms and/or wasting. None of the herds vaccinated the pigs against PCV2. The last two herds were control herds without any symptoms of PCV2 related diseases. In each herd, information on production type, health status, number of pig produced, mortality, weight gain, antibiotic usage and clinical symptoms were recorded. Blood samples were taken from 5 pigs from each of 6 age groups: 3-4 weeks after weaning, 2 weeks before transfer to the fattening unit, 1-2 weeks and 7-8 weeks after transfer and finally just before slaughter. Serum was separated and kept at $-80 \circ C$ until test for antibodies against PCV2 using an in house ELISA and for PCV2 virus load by qPCR (5)
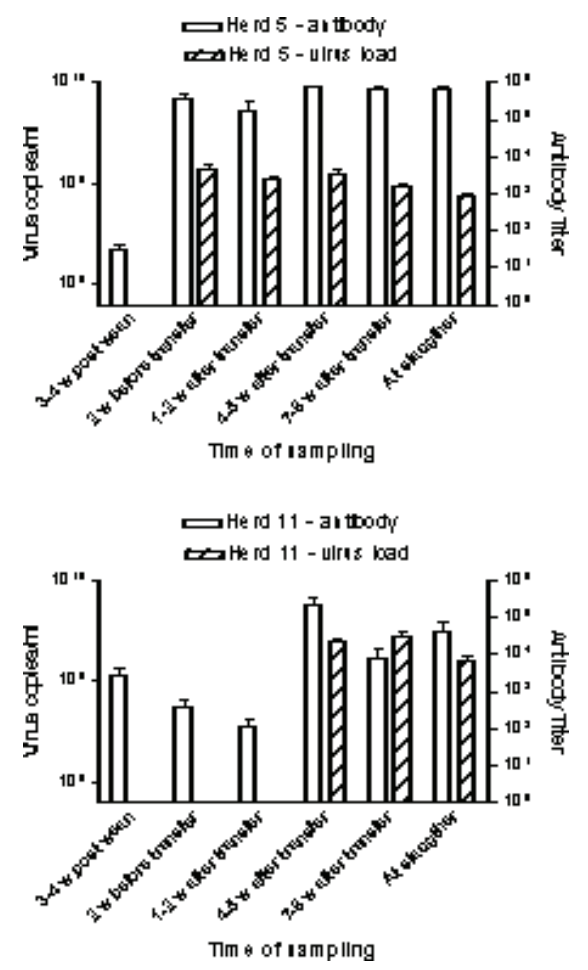

\section{Results}

Several different serological and virological profiles were found in the 16 herds, however basically the profiles could be grouped into two groups represented by herds 5 and 11 (figure). This grouping was independent of production type, size, health status etc. Apart from one herd, no virus was found in the samples taken 3-4 weeks after weaning. In the group of herds represented by herd 5, PCV2 viraemia and increased level of PCV2 antibodies were observed in weaners. In the other group of herds, represented by herd 11, viraemia and increased levels of antibodies were seen in older pigs - either in growers early after transfer or in finishers. In general, increased levels of antibodies were concurrent with increased PCV2 load. The level of antibody and virus in serum were very uniform within the sampling groups. The level of virus in serum were in most of the viraemic pigs between 4-6 log 10 DNA copies/ml, however, a few pigs in some groups had higher levels (7-9 log $10 / \mathrm{ml})$. In some herds increased viral load in serum were seen in the groups of pigs concurrent with appearance of clinical signs whereas no correlation was seen in other herds. Furthermore, comparable levels of viraemia were also seen in the two control herds.

\section{Discussion and conclusion}

The present study showed that PCV2 profiles on serum for the diagnosis of PCV2 related diseases in herds can be a valuable tool to confirm that PCV2 is circulating. However, the results of the profiling should be carefully accessed and related to herd management factors, the presence of other pathogens and the clinical symptoms. Increased serum PCV2 load in itself are not diagnostic for clinical PCVD.

\section{References}

1. G. G. Labarque, H. J. Nauwynck, A. P. Mesu, M. B. Pensaert, Vet. Q. 22, 234 (2000).

2. J. Segales et al., Vet. Microbiol. 111, 223 (2005).

3. I. M. Brunborg, T. Moldal, C. M. Jonassen, J. Virol. Methods 122, 171 (2004).

4. A. Olvera, M. Sibila, M. Calsamiglia, J. Segales, M. Domingo, J. Virol. Methods 117, 75 (2004).

5. C. K. Hjulsager et al., Vet. Microbiol. 133, 172 (2009). 


\title{
0.068
}

\section{Comparison of PCV2 viremia between heavy and light weight pigs at marketing age in farms with routine PCV2 vaccination}

\author{
Kwang Soo Lyoo ${ }^{1}$ Han Soo Joo ${ }^{1}$ Brian Caldwell ${ }^{2}$ Peter Davies ${ }^{1}$ \\ 1. University of Minnesota, St. Paul, MN, USA; 2. Choice Connection, Mapleton, MN, USA
}

\section{Introduction}

Because of the economic significance, PCV2 vaccine is now routinely used on most US swine farms. Although vaccinated pigs perform significantly better in terms of survivability and growth performance, subpopulations of light weight pigs in late finishing phase still are observed in farms with routine PCV2 vaccination. Swine producers and veterinarians have expressed concerns over this problem and questioned whether protection by the PCV2 vaccine may be inadequate to prevent poor growth in some cases. Therefore, it is hypothesized that a protection of light weight pigs could be attributed to PCV2 infection at late finishing age. This preliminary study was designed to compare PCV2 viremia and the antibody status of the lightest weight and the heaviest weight pigs at the marketing age in farms that routinely vaccinated against PCV2.

\section{Materials and Methods}

Seven different finishing farms that routinely used a commercial PCV2 vaccine and were experiencing no evident PCV2 associated clinical disease were selected. Within 2 weeks before the marketing on each farm, 30 lightest and 30 heaviest pigs in a group were identified visually by each farm manager, and blood samples from these 60 pigs in each farm were collected. PCV2 IFA titer was tested by a protocol used routinely in our laboratory. A differential nested PCR assay for PCV2a and $2 b$ was conducted as the method previously described ${ }^{1}$. For PCV2b specific real-time PCR, a PCV2b specific primer set was used, and the assay was performed using PerfeCTa SYBR Green SuperMix and Mx3005P. One-way repeated measure ANOVA and Student t-test was used to test for statistical significant differences.

\section{Results}

Overall, the IFA titers of the light pigs were higher than those of the heavy weight pigs (Fig. 1). Percentages of PCV2a and PCV2b viremic pigs in the 7 different finishing units tested by $\mathrm{nPCR}$ are shown in Table 1. There was no significant difference in PCV2a viremic pigs between the light and the heavy weight groups. However, percentages of PCV2b viremic pigs were higher in the light pigs than the heavy pigs in 6 of the 7 farms. The realtime PCR results for the pigs in each farm showed that average amount of PCV2b genomic DNA was higher in the light pigs than in the heavy pigs.
Fig. 1. Comparison of the mean PCV2 IFA titers between light and heavy weight pigs at marketing age.

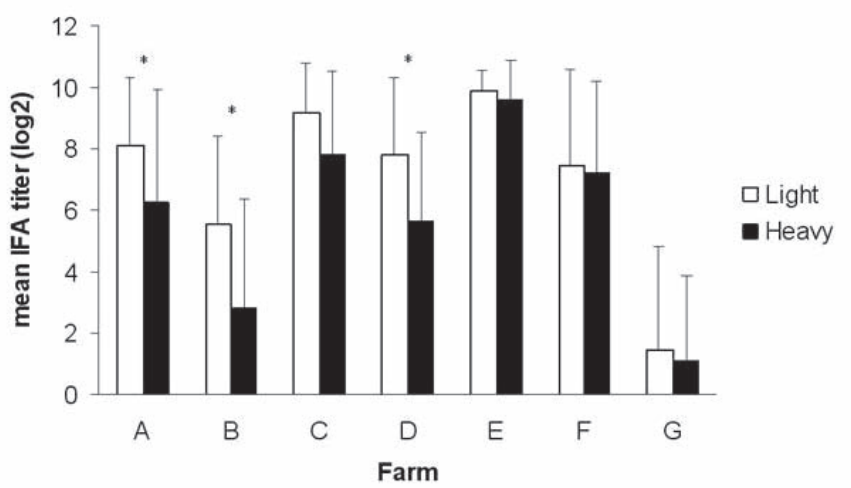

Table 1. Numbers and percentages of viremic pigs with PCV2a and $2 b$ in finishing pigs

\begin{tabular}{|c|c|c|c|c|c|c|}
\hline & \multicolumn{3}{|c|}{ PCY2testuserismit. } & \multicolumn{3}{|c|}{ PCVZ 1 mope sirmis } \\
\hline Exm & $\begin{array}{l}\text { Liglat } \\
\text { meinterion }\end{array}$ & $\begin{array}{l}\text { Mewy } \\
\text { mointerias }\end{array}$ & $\begin{array}{l}\text { Pr } \\
\text { ralles }\end{array}$ & $\begin{array}{l}\text { tidit } \\
\text { neitheris: }\end{array}$ & $\begin{array}{l}\text { Meviyy } \\
\text { mrichtopios. }\end{array}$ & notar \\
\hline$A$ & $3(10) 300^{*}$ & $5(17) 90$ & 0.157 & $5(27) 30$ & $2(7) 30$ & 0.014 \\
\hline B & $1(t) / 0$ & $1(3) 30$ & 10 & $1003) 30$ & $703) 30$ & 0.053 \\
\hline c & $3(10)$ o0 & $4(13) 90$ & 0337 & $25(93) 70$ & $25(9) 30$ & 10 \\
\hline D & $3(10)$ as & $0(0) / 23$ & 0.053 & $27(96) 25$ & $16(57) 28$ & 0.001 \\
\hline$E$ & 20129 & $0 \cos 29$ & 0.157 & $2597) 29$ & $3010) 29$ & 0.001 \\
\hline $\mathbf{F}$ & $2(7) .28$ & $2(0) 28$ & 10 & $16(57) 25$ & $22(45) 25$ & 0.046 \\
\hline$G$ & $001 / 30$ & $6(0) 30$ & 10 & 500930 & $4(13) 30$ & 0.157 \\
\hline Tetal & 1475208 & 126) 205 & 0.157 & $12300 y 265$ & 72059205 & 0.001 \\
\hline
\end{tabular}

\section{Discussion}

The present results indicate a potential association between PCV2 infection and the marketing weight in swine farms. Negative effects on production may still occur from PCV2 infection of vaccinated pigs when clinical diseases are not apparent. Although statistical significance was not always observed, higher PCV2 IFA titers and more viremic pigs were evident in the light weight pigs at marketing age. This finding also support a greater role of PCV2b compared with PCV2a suggesting that PCV2 vaccination should be focussed on the protection from PCV2b genotype infection. We could not determine when pigs may have been exposed to PCV2 on these farms but vaccination program may need to be adjusted to protect pigs from PCV2 infection until late in the finishing phase.

\section{References}

1. Lyoo KS et al 2008, J Vet Diagn Invest 20: 283-288. 


\title{
0.069
}

\section{Distribution of ORF2 and ORF3 genotypes of Porcine Circovirus Type 2 (PCV2) in wild boars and domestic pigs in Germany}

\author{
Gerald Reiner ${ }^{1}$ Bastian Bronnert ${ }^{1}$ Christina Fresen ${ }^{1}$ Corinna Hohloch ${ }^{2}$ Manfred Reinacher ${ }^{2}$ Stephan von Berg ${ }^{1}$ Hermann Willems ${ }^{1}$ \\ 1. Department of Veterinary Clinical Sciences, Justus-Liebig-University, Giessen, Germany; 2. Institute for Veterinary Pathology, Giessen, Germany
}

\section{Introduction}

Porcine circovirus 2 (PCV2), the essential infectious agent in PCVAD (porcine circovirus associated diseases) circulates at high rates among domestic pig and wild boar popula $\neg$ tions. Wild boars may be viremic and shed the virus with excretions and secretions, and thus serve as a reservoir for domestic pig PCV2 infection. We hypothesize that PCV2 strains circulating in wild boars and in domestic pigs are significantly different and thus, partially inde $\neg$ pendent. To prove this hypothesis, the distribution of ORF2 and ORF3 genotypes of PCV2 within wild boars and domestic pigs from overlapping greater areas of Germany was investigated.

\section{Materials and Methods}

Samples from 40 wild boars from 17 different hunting grounds and tissue samples of 60 apparent $\neg$ ly healthy domestic pigs from 18 provenances located in the same greater areas as the wild boars were analyzed. PCV2-specific DNA was ampli-fied by nested PCR ( $\mathrm{nPCR}$ ). PCV2 genotypes were classified based on ORF2 sequences as described by Olvera et al. (2007) and Segalés et al. (2008). ORF2 and ORF3 genotypes were detected by pyrosequencing on a PyroMark ID (Biotage, Sweden) system. Genotypes were compared with PCV2 sequences from the Genbank database (520 entries).

\section{Results}

Two ORF2 and nine ORF3 genotypes were detected in twelve combinations, with significant differences between wild boars and domestic pigs. Distribution among the federal states did not differ significantly within species. PCV2-2b was the most common genotype in both species. Almost $60 \%$ of the infected wild boars but only $4.8 \%$ of the infected domestic pigs carried the PCV2-2a subtype. ORF3 genotype frequencies differed also significantly between wild boars and domestic pigs. Some ORF3 genotypes were detected in domestic pigs only, others exclusively in wild boars. The genotypes of domestic pig PCV2 samples were dominated throughout the country by 2b/ORF3-1 and 2b/ORF3-3 types. PCV2 genotypes of the wild boars were, however, more heterogeneous than those of domestic pigs. Two different PCV2 genotypes in one animal have been isolated from $27 \%$ and $4 \%$ of wild boars and domestic pigs, respectively $(\mathrm{P}<0.001)$, and at least two different $\mathrm{PCV} 2$ genotypes have been isolated from $90 \%$ of hunting grounds and $50 \%$ of domestic pig provenances. The five Genbank database PCV2 sequences from German domestic pigs (from 1999/2000) differed significantly from the "domestic" sequences of the present study (2004/2007) and from 124 sequences obtained from different European countries. However, European and the "domestic" sequences of the present study were in good agreement. The six Genbank sequences of European wild boar isolates agree with the major genotypes of the wild boars of the present study. Some of the wild boar derived genotypes are not present or very rare among "domestic" and wild boar Genbank sequences worldwide.

\section{Discussion}

Differences in "domestic" PCV2 genotypes of the present study and Genbank entries from Germany may indicate the general switch from PCV2-2a to genotype PCV2-2b after the year 2003 that has been generally described by Dupont et al. (2008). Differences and conformity of PCV2 genotypes derived from wild boars and domestic pigs, either from this study or from European Genbank database entries, indicate that wild boar and domestic pig PCV2 do coexist with some exchange. More than $50 \%$ of the wild boar PCV2 genotypes are extremely rare in domestic pigs in Europe (Genbank) and Germany (this study), even worldwide, inferring a certain independence of wild boar and "domestic" PCV2 genotypes. The fact that genotype 2a is prevalent in wild boars (57.5\%), but rare in domestic pigs (4.8\%) and that genotype $2 \mathrm{~b}$ is the almost exclusive genotype in domestic pigs and also prevalent in wild boars, argues for the hypothesis that exchange of PCV2 between both species emerges primarily from domestic pigs to wild boars and less in the reverse direction. This hypothesis is supported by the finding that PCV2 loads of wild boars are significantly lower than those of domestic pigs (see abstract on the opposing page).

\section{References}

Olvera et al. 2007. Virology 357:175-185.

Segalés et al. 2008. Vet. Rec. 162: 867-868.

Dupont et al. 2008. Vet. Microbiol. 128: 56-64.. 


\title{
0.070
}

\section{Evaluation of alternative vaccination programs to control PCVAD}

\author{
Glen W. Almond ${ }^{1}$ Martin Liebstein ${ }^{1}$ Matthew Turner ${ }^{2}$ Patricia A. Routh ${ }^{1}$ \\ 1. College of Vet Med, North Carolina St Univ, Raleigh, NC, USA; 2. Prestage Farms, Clinton, NC, USA
}

\section{Introduction}

Porcine circovirus 2 (PCV2) is the primary pathogen associated with PMWS and porcine circovirus associated disease (PCVAD; 1 ). In North Carolina, the primary clinical manifestation of PCVAD was marked mortality in grower-finishing facilities. Producers dramatically reduced the mortality associated with PCV2 by vaccinating pigs; however, the costs of vaccinating nursery pigs can be overwhelming. Therefore, the primary goal of this study was to examine a less expensive approach by vaccinating sows with products intended for nursery pigs.

\section{Materials and Methods}

The study was conducted on commercial sow, nursery and finishing farms. Three commercial vaccines, approved for nursery pigs, were given to sows at 5 and 3 weeks prior to farrowing. For each vaccine, at least five gilts and five 4+ parity sows were vaccinated and farrowed. In addition, two control groups (6 sows/ parity) were injected with saline. At 7 days after farrowing, two lightweight piglets and two heavy piglets from each sow were weighed and ear tagged. Due to attrition in the sows from death or abortions prior to farrowing, only 45 sows farrowed and a total of 180 piglets were used in the study. Blood samples were collected from the sows prior to the first vaccination and before farrowing. Blood samples also were collected from piglets at approximately $7,14,52,70$ and 155 days of age. All serum samples were submitted to lowa State University for the determination of PCV-2 SP ratios.

\section{Results}

From birth until 155 days of age, mortalities for pigs from sows vaccinated with Vaccines 1, 2, 3 and 4 (controls) were 13.6\%, $10 \%, 18.2 \%$ and $19.2 \%$, respectively. The "heavy" and "light" pigs weighed $3.2+0.08 \mathrm{~kg}$ and $2.1+0.05 \mathrm{~kg}$ at 7-10 days of age, respectively. There were no differences in body weights among piglets from sows given the different vaccines. PCV2 titers did not differ between 4th parity sows and gilts prior to vaccination $(1.03+0.03)$ and sow titers increased slightly after vaccination $(1.10+0.04)$. Piglets from vaccinated sows had higher $(P<.05)$ titers than piglets from the control sows at 7-10 days after birth (Fig 1). With the notable exception of $155 \mathrm{~d}$, this difference was evident for most time points. At 14-16 days after birth, PCV2 titers were greater in piglets from sows vaccinated with Vaccine 2 than in piglets in the other three groups. This was also evident at $70 \mathrm{~d}$ ( $1 \mathrm{wk}$ after placement in the finishing sites). At 155 days of age, PCV2 titers were the lowest in pigs from sows vaccinated with the Vaccine 2, while pigs from control sows had the highest titers.
Fig 1. $P C V 2$ titers (SP ratios, mean+SEM) in pigs from sows receiving two vaccinations before farrowing. Means differed $(P<.05)$ between controls and vaccine groups at 7-10 days, and the four groups differed at $155 d$.

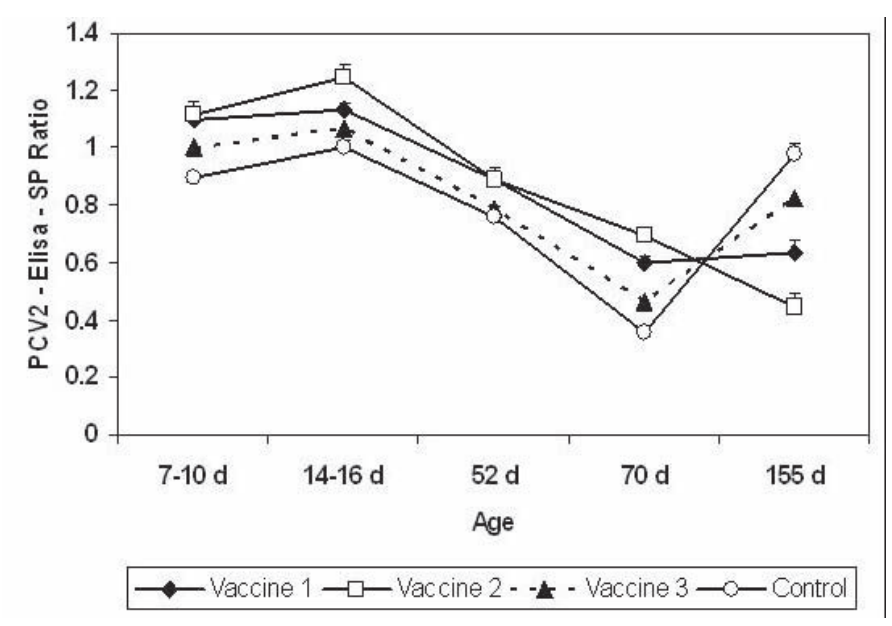

\section{Discussion}

The results indicate that gilts and older parity sows had prior exposure to PCV2. Based on a previous report (2), low sow antibody titers against PCV2 increased the risk of pigs subsequently dying from PCVAD. It was evident that the titers of young pigs (7-10 days) were greater when the sows were vaccinated for PCV2, particularly with Vaccine 2 . The decrease in titer with age represents declining maternal antibodies. Perhaps the most striking results were the PCV2 titers in pigs at 155 days of age. The PCV2 titers continued to decrease in pigs from sows vaccinated with Vaccine 2, thereby indicating that the pigs were not actively challenged with wild virus or that they were protected when exposed to wild virus. In contrast, the titers of control pigs and pigs from sows vaccinated with Vaccine 3, increased dramatically while in the finishing phase. Evidently, these pigs responded to exposure to wild virus during finishing. Overall, the results demonstrated that PCV2 vaccination of sows has potential to reduce losses associated with PCVAD.

\section{References}

1. Opriessnig, T et al. 2007. J Vet Diagn Invest 19:591-615.

2. Calsamiglia, M et al. 2007. Res Vet Sci 82: 299-304. 


\title{
0.071
}

\section{A comparison between sow and piglet PCV2 vaccines; effects on pig performance and viraemia in three crossbred types}

\author{
Helen M. Miller ${ }^{1}$ Stan Done ${ }^{2}$ \\ 1. University of Leeds, Leeds, UK; 2. Veterinary Laboratories Agency, Thirsk, UK
}

\section{Introduction}

The effects of PCV2 have devastated the pig industry in recent years. PCV2 infection increases mortality and feed costs and reduces growth rate and feed efficiency. Two different types of vaccine are now available to counteract this disease; one is given to the sow prior to farrowing, hence acting to provide passive immunity via colostrum, and the other is given directly to individual piglets. These vaccines were compared on a PCV2 affected farm where post weaning mortality had been steady at around $12 \%$ for the previous two years. Three crossbred genotypes, Large White, Hampshire and Pietrain, were used in the experiment to determine the impact of the vaccines on the health and performance of different genotypes. It has been suggested that PCV2 vaccination will be more beneficial to Large White type pigs as these are considered to be more susceptible to PCV2 associated diseases.

The objectives of the experiment were to compare the efficacy of the two vaccines when delivered either singly or in combination; to investigate the effect of vaccination on the virology of PCV2 and to compare the response of different genotype crossbreeds to vaccination.

\section{Materials and Methods}

The experimental design was a $2 \times 2$ factorial generating the following 4 treatments:

- Unvaccinated piglets from unvaccinated sows (unvaccinated control)

- Unvaccinated piglets from vaccinated sows

- Vaccinated piglets from unvaccinated sows

- Vaccinated piglets from vaccinated sows

Sows were batch farrowed in groups of approximately 24 every 3 weeks; sow vaccination status was applied across a whole batch generating 4 unvaccinated batches and 4 vaccinated batches. Within each litter in all batches every second piglet was vaccinated against PCV2 at 3 weeks of age. A total of 183 Large White $x$ Landrace sows were used in the experiment divided across the two vaccination treatments so that the parity profile was similar in both groups. Sows were mated to Large White, Pietrain or Hampshire type boars.

Cross-sectional blood sampling performed prior to the start of the experiment established that PCV2 viraemia occurred after 12 weeks of age in growing pigs. During the experiment six sows per batch were blood sampled on transfer to the farrowing house and 48 pigs per treatment were blood sampled at 3, 9, 12, 15 and 18 weeks of age.

\section{Results}

Both vaccines were effective in improving lifetime performance and shortening time to slaughter. Interestingly the effect of the sow vaccine was most pronounced during the early growth of the pig, whereas, piglet vaccination, but not sow vaccination, significantly improved growth rate between 7 weeks of age and slaughter. Maximum effect was gained when the two were used together which resulted in 6 days advantage in slaughter age over the unvaccinated animal. Using either vaccine singly halved this advantage.

Overall post weaning mortality declined from $12 \%$ to $6 \%$ during the experiment however there was no significant benefit associated with any particular vaccination strategy. PCR analysis indicated that peak viraemia was evident after 15 weeks of age (ie later than in the pre-experimental sampling) and that viral load was significantly reduced in animals which had received the piglet vaccine.

Both genotype and pig sex influenced the response to vaccination. In contrast to our initial hypothesis that Large White type pigs would benefit more from PCV2 vaccination in fact the opposite was observed: The Large White showed the poorest response to vaccination with a reduction in slaughter age of 5.7 days between unvaccinated female and double vaccinated male, a similar comparison in the Hampshire reduced time to slaughter by 12.4 days and in the Pietrain by 15 days.

\section{Discussion}

Vaccination resulted in improved performance throughout the growing period and not specifically during the period of maximum viral load in agreement with Cline et al. (2008). The best vaccination strategy for maximizing performance was to use the sow and piglet vaccines together, also reported by Pejsak et al. (2009). However the level of improvement that can be expected is further influenced by the pig genotype, therefore the cost effectiveness of a double versus a single vaccination strategy should be considered with this in mind.

\section{References}

Cline, G., et al. (2008) Veterinary Record, 163, 737-740.

Pejsak. Z, et al. (2009) Comparative Immunology, Microbiology and Infectious Disease. In Press 


\title{
0.072
}

\section{One-dose vaccination against Mycoplasma hyopneumoniae and Porcine Circovirus Type 2}

\author{
Alexander A. Eggen; Maarten Witvliet; Ulrike Schmidt; Maurice Raes \\ Intervet/Schering-Plough, Boxmeer, Netherlands
}

\begin{abstract}
Introduction
Mycoplasma hyopneumoniae (M.hyo) and Porcine Circovirus Type 2 (PCV2) are the two most prevalent pathogens that are encountered in the pig industry. Vaccination against M.hyo is already standard practice, and the same will be the case for PCV2. As vaccines against these two pathogens are preferably given at a young age, it would be advantageous if vaccinations against these two pathogens are combined. However, no interference between vaccinations is acceptable as that could lead to poor vaccine efficacy. In a previous study, it has been shown that the ISPAH vaccines Porcilis M Hyo and Porcilis PCV can be given concurrently without any negative consequences for the efficacy (1). Also, the efficacy and safety of simultaneous use (mixing) of both vaccines has been evaluated in the field without evidence of interference or apparent safety problems (2). Here, we report a series of studies on possible interference between vaccination with Porcilis PCV and M+PAC, and the efficacy of especially prepared ready-to-use M. hyo-PCV2 combination vaccines.
\end{abstract}

\section{Materials and Methods}

Groups of 8 conventional piglets, with maternally-derived antibodies against both M.hyo and PCV2, were vaccinated at weaning (3-4 weeks of age) with M+PAC (and Porcilis PCV), or with ready-to-use M.hyo-PCV2 combination vaccines. At 7 weeks of age, the pigs were infected with an M.hyo field isolate. Challenge infection was performed with a culture of strain 98 (obtained from Dr. N. Friis, Danish National Veterinary Laboratory) by intratracheal inoculation on two consecutive days, followed by necropsy to evaluate M.hyo-induced lung lesions (LLS) three weeks later.

Serum samples taken at the time of vaccination and at 10 weeks of age were analyzed for antibody titers against PCV2 in a specific ELISA.

The vaccines were based on M+PAC (Emunade adjuvant) and Porcilis PCV (X-solve adjuvant). Both adjuvants are emulsions of light mineral oil in water, but Emunade also contains alhydrogel, and d,l-a-tocopheryl acetate is part of the X-solve adjuvant. Combination vaccines were formulated with the same antigens that are used in the single vaccines. For M. hyo either the antigen that is used in M+PAC (Mh1) or in Porcilis M Hyo (Mh2) was used.

\section{Results and Discussion}

As shown in Table 1, all vaccines tested provided similar levels of protection against M.hyo (53\% - 61\% reduction in lung lesions) after challenge at 7 weeks of age. There were no significant differences between the vaccinated groups. Whereas the mean antibody titers against PCV2 had declined in the M+PAC only and control groups from $7.4 \log 2$ at the time of vaccination to 3.8 and 3.7 at 10 weeks of age, all animals that had been vaccinated against PCV2 showed a titer increase at 10 weeks. Piglets that have an antibody titer of $11.9 \pm 2.1$ at 10 weeks of age after vaccination with Porcilis PCV at 3 weeks are protected against PCV2 challenge at the end of the fattening period (3). In the present study, all groups were at or above that antibody level.

Table 1. M.hyo-induced lung lesions (LLS) and PCV2 antibody titers (log2) at 10 weeks of age. LLS according to Goodwin \& Whittlestone (1969).

\begin{tabular}{|l|c|c|c|}
\hline Vaccine(s) & M. hyo LLS & Reduction & PCV2 titer \\
\hline M+PAC & $7.0^{\mathrm{a}} \pm 5.4$ & $59 \%$ & $3.7^{\mathrm{a}} \pm 0.6$ \\
\hline M+PAC and Porcilis PCV separately & $8.0^{\mathrm{a}} \pm 4.4$ & $54 \%$ & $14.1^{\mathrm{b}} \pm 1.8$ \\
\hline Mh1+PCV/EMUNADE ready to use & $8.2^{\mathrm{a}} \pm 7.4$ & $53 \%$ & $12.5^{\mathrm{b}} \pm 1.7$ \\
\hline Mh1+PCV/XSolve ready to use & $7.1^{\mathrm{a}} \pm 4.3$ & $59 \%$ & $13.5^{\mathrm{b}} \pm 1.7$ \\
\hline Mh2+PCV/EMUNADE ready to use & $7.8^{\mathrm{a}} \pm 3.9$ & $55 \%$ & $11.8^{\mathrm{c}} \pm 1.7$ \\
\hline Mh2+PCV/XSolve ready to use & $6.8^{\mathrm{a}} \pm 5.6$ & $61 \%$ & $13.6^{\mathrm{b}} \pm 2.5$ \\
\hline Control & $17.3^{\mathrm{b}} \pm 6.4$ & - & $3.8^{\mathrm{a}} \pm 2.5$ \\
\hline $\begin{array}{l}\text { Groups with different superscripts are significantly different ( } \mathrm{p}<0.05 \text {, Mann Whitney U-test for } \\
\text { LLS and 2-sample t-test for PCV2 antibody titer). LLS were corrected for outliers. }\end{array}$ \\
\hline
\end{tabular}

It can therefore be concluded that there is no immunological interference between the M.hyo and PCV2 antigens of M+PAC, Porcilis M Hyo and Porcilis PCV, independent of whether they are given concurrently as separate single vaccines or combined in a ready-to-use vaccine with either Emunade or X-solve as the adjuvant.

Further proof for lack of interference comes from a recent field study in which M+PAC and Porcilis PCV were mixed just before administration (4). There was no significant difference between the anti-PCV2 antibody titers in groups of pigs that were vaccinated with the mixed products or that received the two vaccines by separate injections.

\author{
References \\ 1. Segales, Q. (2008) IPVS I/S-P satellite symposium \\ 2. Farreres, J. et al. (2010) IPVSa \\ 3. Eggen, A. et al. (2010) IPVS \\ 4. Farreres, J. et al. (2010) IPVSb
}




\title{
0.073
}

\section{Results of one-shot piglet vaccination at $0.5 \mathrm{ml}$ with Circovac ${ }^{\ominus}$ assessed by viremia and seroconversion in a farm affected by porcine circovirus type 2 diseases (PCVD)}

\author{
Bernhard Heissenberger; Christiane Lang; Andrea Ladinig; Christiane Püschel; Mathias Ritzmann
}

Clinic for Swine, Vienna, Austria

\section{Introduction}

Circovac $^{\circledR}$ (Merial, Lyon, France), an inactivated PCV2 vaccine, was previously evaluated under field conditions in piglets (1). In order to assess the effect of the vaccination on seroconversion and viremia in piglets, serum samples were analyzed serologically for the presence of PCV2 IgG and IgM antibodies and for the presence and the quantity of specific PCV2 DNA. Furthermore, the reduction of infection pressure after vaccination was investigated.

\section{Materials and Methods}

A controlled, blinded and randomized field study was performed on an Austrian 250-sow farrow-to-finish farm that experienced severe problems with PCV2 and 1105 piglets were included in this trial. Three consecutive batches were included at birth and piglets were randomly allocated to a Circovac ${ }^{\circ}$ or to a placebo treated control group. Vaccination was performed at three weeks of age. Control and vaccinated piglets stayed intermingled. Only batch 1 and batch 3 were chosen for collection of laboratory data in order to assess the impact of vaccination on viral pressure over time. Blood samples were collected regularly and serum samples of Circovac ${ }^{\circ}$ vaccinated ( $1^{\text {st }}$ batch: $n=20,3^{\text {rd }}$ batch: $n=18$ ) as well as of control animals ( $1^{\text {st }}$ batch: $n=20,3^{\text {rd }}$ batch: $n=15$ ) were analyzed via the Ingezim Circovirus lgG/lgM ELISA kit (Ingenasa, Madrid, Spain) and quantitative PCR (qPCR).

\section{Results}

The median logarithmic values of copies $/ \mathrm{ml}$ serum of the Circovac ${ }^{\circ}$ and the control group of both batches are presented in fig. 1. Between week 14 and 22, the pigs of the control group of batch 1 were viremic. The vaccinated pigs of batch 1 showed peaks only in week 16 and 20, which were lower than the one of the control group at the respective points of time. The control animals of batch 3 underwent a shorter and lower viremic phase than the ones of batch 1 . The median values of the vaccinated group of batch 3 remained completely at the baseline. According to the $\mathrm{qPCR}$ results the main viremia period occurred around the weeks 14 to 20. Fig. 2 a,b shows the percentage of pigs positive for specific PCV2 IgG and IgM antibodies of the vaccinated and the control group of batch 1, fig. 2 c,d for the animals of batch 3 . The vaccinated animals had a general lower incidence of IgM antibodies than the controls. Batch 3 showed a lower percentage of positive animals than batch 1 in both control and vaccinated pigs.
Figure 1: Median values of $q P C R$ of Circovac ${ }^{\circledR}$ and control animals of batch 1 (left) and batch 3 (right)

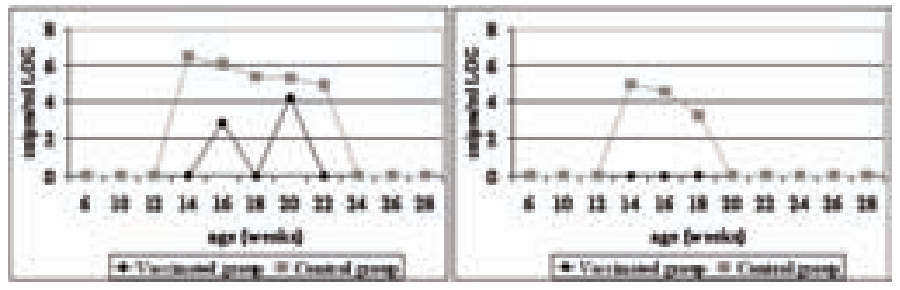

Figure 2: Percentage seropositive of $\operatorname{Circovac}^{\circledast}(a, c)$ and control $(b, d)$ animals of batch $1(a, b)$ and of batch $3(c, d)$

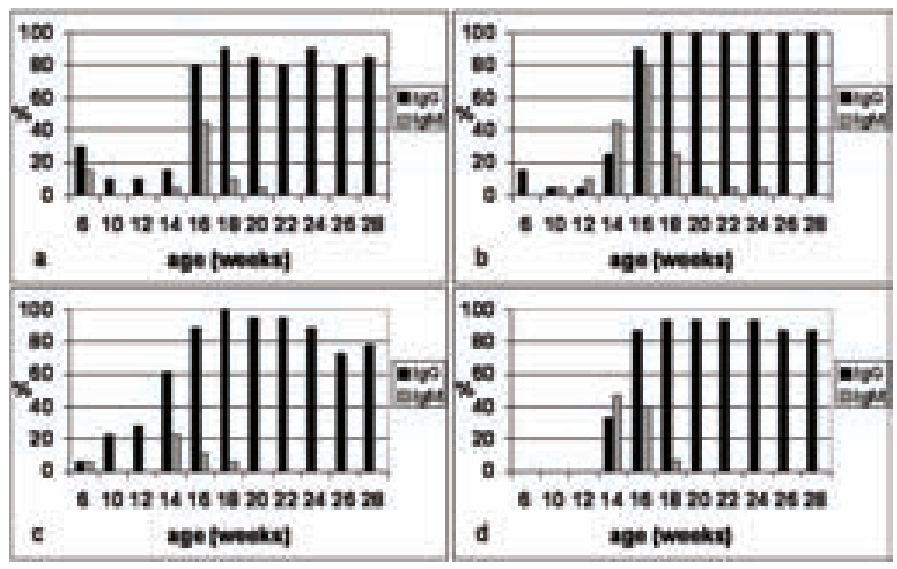

\section{Discussion}

According to viremia and seroconversion results, a positive effect of the vaccination of piglets with Circovac ${ }^{\circledR}$ has been observed. Vaccinated animals had a lower incidence for PCV2 specific IgM antibodies and the vaccination seemed to reduce the viral pressure in the facilities, as the occurrence of positive animals was in general lower in the $3^{\text {rd }}$ batch. This observation is supported by the qPCR data, which showed, that vaccinated animals had a lower serum viral load. Those facts suggest a reduction of virus shedding after vaccination in this trial.

\section{References}

1. Joisel F. et al. (2009): Proc. 4th Congr. Asian Pig Vet. Soc. p. 235. 


\title{
0.074
}

\section{Field Efficacy of Suvaxyn ${ }^{\circledast}$ PCV2 in a Large Commercial Piggery in South East Asia}

\author{
Ming Yong ${ }^{1}$ Fook K. $\mathrm{Ng}^{2}$ Philip Lehrbach ${ }^{3}$ \\ 1. Pfizer animal Health (Asia Pacific), Shanghai, China; 2. Swine Consultant, Singapore, Singapore; \\ 3. Pfizer Animal Health, Melbourne, VIC, Australia
}

\section{Introduction}

Porcine Respiratory Disease Complex (PRDC) is a continuing problem in the swine industry. PCV2-associated disease (PCVAD) such as post weaning multisystemic wasting syndrome (PMWS) has emerged as a serious complicating factor. Vaccination has been found to be effective in controlling PCVAD. The objective of this trial was to evaluate the field efficacy of Suvaxyn PCV2 based on reducing mortality and the cost of managing PRDC on a commercial farm in South East Asia.

\section{Material and Methods}

The trial involved a very large commercial piggery with a history of unacceptably high mortality in the grow-finish pigs (including respiratory distress and poor weight gain). Post mortem/laboratory testing indicated the presence of PRRS, MH and APP. In addition, histopathology and $P C R$ results indicated the presence of PCV2. Most of the pigs examined became viraemic with PCV-2 by 13 weeks of age.

There were 9,289 piglets enrolled in the study (see Table 1). All piglets in the vaccinated group were administered intramuscularly with a single $2 \mathrm{~mL}$ dose of Suvaxyn PCV2 at 5 weeks of age. Serum samples were collected just prior to vaccination and at 4 weeks post vaccination ( 9 weeks of age) for serological testing. PCV2 viraemia in sera was detected using the PCV2 specific PCR (serum from batch B pigs only). Average body weights were recorded at marketing age; pig mortality and rejects were also recorded.

Table 1. Suvaxyn PCV2 Field Efficacy Trial Design

\begin{tabular}{|l|c|c|c|c|c|}
\hline Herd & Barn & $\begin{array}{c}\text { Number of Pig } \\
\text { Enrolled }\end{array}$ & Treatment & Dose & Age \\
\hline Batch A & $1 \mathrm{a}$ & 979 & Suvaxyn PCV2 & $1 \times 2 \mathrm{~mL}$ & 5 weeks \\
\hline & $2 \mathrm{a}$ & 993 & Suvaxyn PCV2 & $1 \times 2 \mathrm{~mL}$ & 5 weeks \\
\hline Batch A & $1 \mathrm{~b}$ & 963 & Control & - & - \\
\hline & $2 \mathrm{~b}$ & 990 & Control & - & - \\
\hline Batch B & $3 \mathrm{a}$ & 2664 & Suvaxyn PCV2 & $1 \times 2 \mathrm{~mL}$ & 5 weeks \\
\hline & $3 \mathrm{~b}$ & 2700 & Control & - & - \\
\hline & & & & & \\
\hline
\end{tabular}

\section{Results and Discussion}

Herd performance

Vaccination improved the performance of the herds (see Table 2). All vaccinated groups demonstrated a remarkable decrease in mortality $(4.05 \%$ and $11.04 \%$ reduction for Batch $A$ and Batch $B$ respectively). The vaccinated group in Batch $B$ also showed better body weight gain (additional $4.11 \mathrm{~kg}$ per pig) and a lower number of rejected pigs. The calculated return on investment (ROI) was 2.7:1 and 18.2:1 for Batch $A$ and $B$ vaccinates respectively (based on Margin over feed costs $=50 \%$ ). There was a noticeable saving in antibiotic treatment, especially in Batch $A$ (US\$0.20 per pig).
Table 2. Average Body Weight, Percent Mortality and Number of Rejected Pigs at Marketing Age

\begin{tabular}{|l|c|c|c|c|c|}
\hline Herd & Group & $\begin{array}{c}\text { Number } \\
\text { of Pigs } \\
\text { Enrolled }\end{array}$ & $\begin{array}{c}\text { Number of } \\
\text { rejected } \\
\text { pigs }\end{array}$ & $\begin{array}{c}\text { Average } \\
\text { Harvest } \\
\text { Weight (Kg) }\end{array}$ & $\begin{array}{c}\text { Mortality (\%) Between } \\
\text { Vaccinates and } \\
\text { Controls }\end{array}$ \\
\hline Batch A & $\begin{array}{c}\text { Suvaxyn } \\
\text { PCV2 }\end{array}$ & 1972 & 0 & 80.87 & 4.05 \\
\hline & Control & 1953 & 0 & 80.92 & 11.04 \\
\hline Batch B & $\begin{array}{c}\text { Suavxyn } \\
\text { PCV2 }\end{array}$ & 2664 & 60 & 86.97 & \\
\hline \multicolumn{7}{|c|}{ Control } & 2700 & 140 & 82.86 & \\
\hline
\end{tabular}

The vaccinated groups showed stronger antibody production to PCV2 at 9 weeks of age (4 weeks post vaccination). The PCR results at 9 weeks of age demonstrated that the vaccinated groups had a much lower rate of PCV2 viraemia (17.2\%) as compared to the controls (77.8\%).

Figure 1. PCV2 Viraemia in Sera from Batch B Pigs at 9 weeks of age

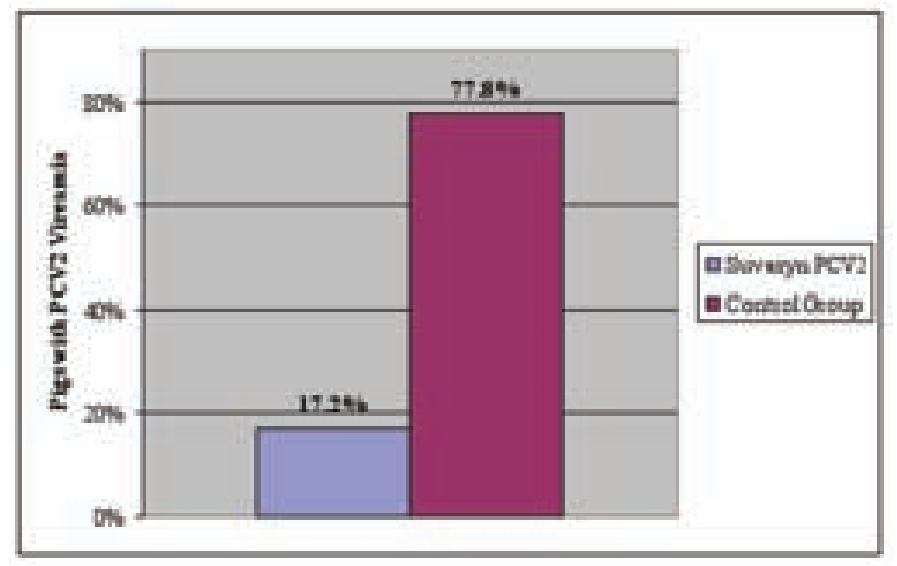

\section{Conclusion}

These results demonstrate that vaccination using Suvaxyn PCV2 in pigs, reduced PCV2 viraemia, total mortality and the cost of managing PRDC, as well as improving the growth performance on a commercial farm in South East Asia.

\section{References}

1. Kim J. et. al. 2003.Vet J. 166: 251-256

2. Opriessnig, T. et. al. 2008. Vet Microbiology, 13: 103-111 


\title{
0.075
}

\section{Long term evaluation of using PRRS MLV and a subunit PCV2 vaccine to reduce post-weaning loss in Thailand}

\author{
Jitjaroen Channarong; Sopon Kongtes; Nathaya Duangwhae; Lerdtida Meedacha
}

\author{
Boehringer Ingelheim (Thai), Bangkok, Thailand
}

\section{Introduction}

PRRSv and PCV2 are the two major pathogens causing swine production loss worldwide.

The objective of this study was to confirm the consistency and long-term efficacy of a PRRS MLV and a subunit PCV2 vaccine to reduce post-weaning losses (mortality and culls) on a commercial pig farm in Thailand.

\section{Materials and method}

The single site conventional commercial farm, 1,700 sows level was positive for PRRS. In November 2006 - March 2007 this farm experienced a PRRS outbreak in the breeding herd causing reproductive failure followed by high post weaning losses (mortality and culls) caused by PRDC in nursery pigs and grower-finishers from January to August 2007. Average nursery and grower-finishers loss in this period was $9.76 \%$ and $9.30 \%$ respectively. Clinical signs observed in nursery pigs started from 5-6 weeks of age with thumping, eyelid edema and conjunctivitis, in line with seroconversion in nursery pigs at 7-8 weeks as measured by PRRS ELISA (HerdChek PRRS 2XR, IDEXX laboratory). PRRS vaccination (Ingelvac ${ }^{\circledast}$ PRRS MLV) started in sows, gilts and piglets in September 2007. Piglets were vaccinated at an age of 10-14 days .

Breeding herd vaccination resolved the reproductive problems and reduced losses in nursery pigs. However the growerfinishers loss remained at a high level. Clinical signs, including wasting, paleness, PRDC, jaundice and diarrhea, started in 12-14 week old animals. Some pigs showed signs of PDNS skin lesion. Main lesions found in post mortems were spleen and kidneys enlargement, swelling of inguinal and enteric lymphnodes, indicating PCV2 infection.

As a consequence, PCV2 piglet vaccination (Ingelvac CircoFLEX ${ }^{\circledR}$ ) at 21 days old was introduced to control PCVAD problems in grower-finishers period.

Weekly post weaning losses of 124 batches (about 99,000 pigs) were evaluated between January 2007 to October 2009, divided into 3 phases: Jan 07-Aug 07 (before vaccination), Sep 07-Jul 08 (PRRS vaccination) and Aug 08 - Oct 09 (PRRS + PCV2 vaccination).

\section{Results}

Average nursery mortality decreased from $9.76 \%$ to $2.70 \%$ after introduction of PRRS MLV vaccination (Fig. 1), while average grow-finish mortality remained at $7.53 \%$ in PRRS vaccinated pigs (Fig. 2). In PRRS plus PCV2 vaccinated piglets, nursery mortality further decreased to an average of $1.83 \%$. A more pronounced effect was observed in grow-finish, with an average mortality of $2.84 \%$ in PRRS+PCV2 vaccinated piglets (Fig. 2).

Average post-weaning (wean-to-slaughter) losses were $19.2 \%$ in non-vaccinated batches, $10.2 \%$ in PRRS vaccinated and $4.6 \%$ in PRRS+PCV2 vaccinated batches.
Figure 1: Nursery mortality

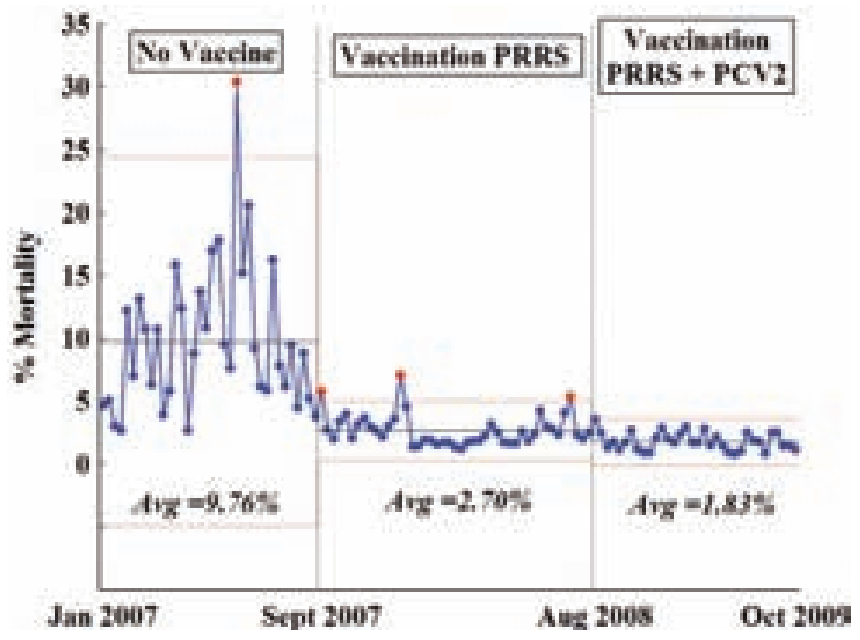

Figure 2: Grow-finisher mortality

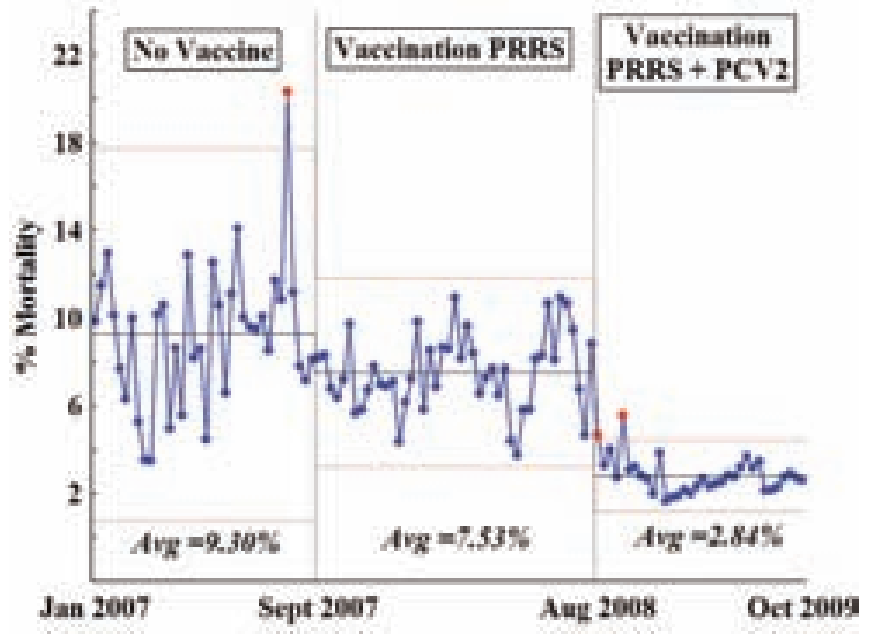

\section{Discussion and conclusion}

This farm was co-infected with PRRSv and PCV2. PRRS vaccination had a clear and consistent effect on nursery losses, but it could not control the losses in grow-finish due to PCV2 infection. Only when piglets were vaccinated against both, PCV2 and PRRSv, post-weaning mortality was reduced consistently. PRRS and PCV2 vaccination were shown to be very effective providing consistent protection in this long-term evaluation. 


\title{
0.077
}

\section{Antimicrobial resistance in E.coli and Salmonella from surveillance of Canadian swine at farm, abattoir and retail}

\author{
Anne E. Deckert $^{1,7}$ Sheryl Gow ${ }^{3,2}$ David Leger ${ }^{1}$ Brent Avery ${ }^{1}$ Lucie Dutil ${ }^{4,5}$ Danielle Daignault ${ }^{4}$ Daniel Leclair ${ }^{6}$ Richard Reid-Smith $^{1,7}$ \\ 1. Public Health Agency of Canada, Guelph, ON, Canada; 2. University of Saskatchewan, Saskatoon, SK, Canada; 3. Public Health Agency of \\ Canada, Saskatoon, SK, Canada; 4. Public Health Agency of Canada, Sainte-Hyacinthe, QC, Canada; 5. Universite de Montreal, Sainte-Hyacinthe, \\ QC, Canada; 6. Canadian Food Inspection Agency, Ottawa, ON, Canada; 7. University of Guelph, Guelph, ON, Canada
}

\section{Introduction}

Antimicrobial resistance (AMR) in the human and animal population is an issue of increasing public concern. The misuse of antimicrobials in human medicine has been associated with AMR but there is also concern with antimicrobial use in the food animal population. The Public Health Agency of Canada and its federal and provincial partners developed the Canadian Integrated Program for Antimicrobial Resistance Surveillance (ClPARS) to monitor AMR and antimicrobial use (AMU) in humans and the agri-food sector in Canada (1). The program includes active surveillance of cattle, swine and broiler chicken at abattoir and at retail. In addition, there is active surveillance of swine at the farm level. The primary organisms of interest are Salmonella, E.coli, Campylobacter, and Enterococcus. The focus of this work is antimicrobial resistance in E.coli and Salmonella from swine at the retail, abattoir and farm levels.

\section{Materials and Methods}

Samples and data for this work were collected in 2008 through CIPARS. CIPARS Retail conducts weekly/bi-weekly sampling of pork chops from retail food outlets in randomly selected census divisions, weighted by population, in the Atlantic Provinces, Quebec, Ontario, Saskatchewan and British Columbia. CIPARS Abattoir samples cecal contents from swine at 13 federally registered abattoirs in 8 provinces. The number of samples per abattoir is proportional to annual slaughter volume. CIPARS Farm is based on sentinel grower-finisher swine herds in the 5 major pork producing provinces. The number of herds per province is proportional to the number of grower-finisher units in each province. The program is administered through 29 herd veterinarians who have enrolled 108 herds. Pooled pen fecal samples are collected from pigs at $>175 \mathrm{lbs}$ once per year. All samples were cultured for generic E. coli and Salmonella using standard CIPARS methodology (1). Susceptibility testing was performed on 3 E. coli and 1 Salmonella isolate per sample. Minimum inhibitory concentrations (MIC) for 15 antimicrobials were established for all isolates using the Sensititre ${ }^{\circledR}$ Microbiology System (Trek Diagnostic Systems, Cleveland, OH, USA). CIPARS results are categorized according to the individual antimicrobial's importance to human medicine as determined by the Veterinary Drug Directorate (VDD) of Health Canada (2). The antimicrobials tested are categorized as Category I: Very High Importance (amoxicillinclavulanic acid, ceftiofur, ceftriaxone, ciprofloxacin), Category II: High Importance (amikacin, ampicillin, cefoxitin, gentamicin, kanamycin, nalidixic acid, streptomycin, trimethoprim-sulphamethoxazole), Category III: Medium Importance (chloramphenicol, sulfisoxazole, tetracycline), and Category IV: Low Importance (no antimicrobials tested).

\section{Results}

CIPARS 2008 surveillance of swine resulted in 318 E.coli isolates from Retail, 150 from Abattoir and 1425 from Farm. In addition there were 151 Salmonella isolates from Abattoir and 61 from Farm. The prevalence of Salmonella from retail pork in the CIPARS program was $2 \%$, which did not result in sufficient isolates for meaningful AMR evaluation. The top three Salmonella serovars from Abattoir were Derby (33 isolates, 21.9\%), Typhiumurium var 5. (31 isolates, 20.5\%), Typhimurium (17 isolates, $11.3 \%)$. The top three Salmonella serovars from Farm were Typhimurium var 5. (17 isolates, 27.9\%), Brandenburg (9 isolates, $14.8 \%$ ), and Derby (7 isolates, $11.5 \%$ ). Resistance to ceftriaxone and ciprofloxacin was only found in E.coli isolates from retail pork (prevalence $=0.3 \%$ ). There was no resistance to amikacin in any E.coli or Salmonella isolates. There was no resistance to nalidixic acid in Salmonella isolates from Farm or Abattoir and less than $2 \%$ resistance to this antimicrobial in E.coli isolates from Farm, Abattoir, and Retail. Less than $5 \%$ of E.coli and Salmonella isolates from all sectors were resistant to amoxicillin-clavulanic acid, ceftiofur, cefoxitin, and gentamicin. There was a substantial percentage of E.coli and Salmonella isolates that were resistant to kanamycin (range: 3.1-21.3\%), ampicillin (range: 17.3-34\%), and streptomycin (15.1-44.4\%). The most common resistance in E.coli and Salmonella from all sectors was to tetracycline (range: 37.4-84.7\%).

\section{Discussion}

The presence of resistance to all Category I antimicrobials in retail pork is of significance due to the proximity of these samples to the consumer and the importance of these drugs in human medicine. Current levels of resistance in these antimicrobials are very low, which is consistent with previous CIPARS results but continued monitoring is essential (1). The low prevalence of Salmonella in retail pork is important from a food safety perspective.

\section{References}

1. Government of Canada. Canadian Integrated Program for Antimicrobial Resistance Surveillance. Accessed February 11, 2010. http://www.phac-aspc.gc.ca/cipars-picra/index-eng.php

2. Government of Canada. Categorization of antimicrobial drugs based on importance in human medicine. Accessed February 11, 2010. http://www.hc-sc.gc.ca/dhp-mps/consultation/vet/consultations/ amr_ram_hum-med-rev-eng.php. 


\title{
0.078
}

\section{The association between antimicrobial resistance in fecal $E$. coli from swine at arrival in the finishing barn and close-to-market}

\author{
Anne E. Deckert ${ }^{1,2}$ Cara Sheppard ${ }^{2}$ Sheryl Gow ${ }^{3}$ David Leger ${ }^{1}$ Richard Reid-Smith ${ }^{1}$ Scott McEwen ${ }^{2}$ \\ 1. Public Health Agency of Canada, Guelph, ON, Canada; 2. University of Guelph, Guelph, ON, Canada; \\ 3. Public Health Agency of Canada, Saskatoon, SK, Canada
}

\section{Introduction}

Antimicrobial resistance (AMR) reduces our ability to effectively treat bacterial infections in animals and humans and is an issue of increasing public concern. AMR has been associated with the misuse of antibiotics in the human population but there is also concern with antimicrobial use (AMU) in agri-food production. The Public Health Agency of Canada and its federal and provincial partners developed the Canadian Integrated Program for Antimicrobial Resistance Surveillance (CIPARS) to monitor AMR and AMU in humans and the agri-food sector in Canada (1). The CIPARS Farm component was implemented in 2006 to provide information on AMR and AMU in swine at the farm level. It also has provided information on the role of AMR on arrival at the grower-finisher (GF) unit on AMR at Close-to-Market (CTM).

The objective of this study was to investigate the associations between AMR in fecal E. coli from groups of swine entering the GF unit and the same group of pigs at CTM.

\section{Materials and Methods}

Samples and data for this work were collected in 2007 through the CIPARS Farm Program. There were 108 herds enrolled in the 5 major pork producing provinces of Canada; $A B, S K, M B$, $\mathrm{ON}$ and QC. In each province, the number of CIPARS herds is proportional to the national total of GF units. To provide producer anonymity, herd veterinarians selected the producers and participated in the sample and data collection. Twenty-nine swine veterinarians from private and corporate practice have enrolled producers that are CQA ${ }^{\oplus}$ validated, produce more than 2000 market hogs/year, and are representative of herds in the veterinarian's swine practice. Herds that were regarded to be organic, feeding edible residual material or pasture-raised were excluded. These criteria helped ensure that the program was representative of the majority of hog production in Canada. In a subset of 66 herds, pooled fecal samples were collected from two pens of pigs at arrival in the finisher unit and from the same two pens at CTM (>175 lbs). In the remaining herds, samples were collected at CTM only and those results are not included in this analysis. All fecal samples were cultured for generic E. coli using standard CIPARS methodology (1). Susceptibility testing was performed on 3 E. coli per sample. Minimum inhibitory concentrations (MIC) for 15 antimicrobials were established for all isolates using the Sensititre ${ }^{\circledR}$ Microbiology System (Trek Diagnostic Systems, Cleveland, OH, USA). AMU data (feed, water and injectables), management and production information was collected using questionnaires. The relationship between AMR at Arrival and at CTM was investigated using multilevel logistic regression models with random intercepts for pen and herd. The outcome of interest was resistance to a specific antimicrobial in fecal $E$. coli from pens of CTM pigs, while the main potential risk factor of interest was resistance to the same antimicrobial among fecal E. coli from pens of pigs at arrival.Other potential risk factors that were investigated included season of sampling, barn capacity, number of pigs per pen, number of pigs at risk, biosecurity variables, barn flow (all-in-all-out or continuous) and the use of antimicrobials (feed, water, injection), the use of the same antimicrobial, the antimicrobial class and the number of antimicrobial classes used.

\section{Results}

There were 28 herds that provided matching Arrival and CTM samples in 2007, resulting in 164 Arrival and 168 CTM E.coli isolates. Twenty-one herds provided AMU data. No Arrival samples and only three CTM samples were susceptible to all antimicrobials tested. At the herd level, all herds were resistant to at least one antimicrobial at Arrival and at CTM. Resistance to ceftiofur(TIO), amikacin (AMK), cefoxitin (FOX), and gentamicin (GEN) was found in Arrival samples but not CTM samples. No resistance was found to ceftriaxone (CRO) and ciprofloxacin (CIP). Of 9 models that were examined only 3 models (kanamycin(KAN), trimethoprim-sulphamethoxazole(SXT), streptomycin(STR)) still included a variable representing AMR at Arrival either as a significant variable $(P<0.05)$ or a confounder ( $>20 \%$ change in the coefficient). The final model for KAN CTM isolate resistance included the number of antimicrobial classes used on farm and arrival pen resistance to KAN. Having two or more SXT resistant isolates in the arrival pen was the only significant variable for SXT resistance at CTM. Arrival pen resistance to STR was a confounder for the relationship between the use of antimicrobials in water and STR resistance at CTM.

\section{Discussion}

Based on these results, AMR data from pigs arriving in the GF unit provides limited information regarding AMR at CTM for specific antimicrobials. Resistance at Arrival is generally more prevalent than at CTM. The absence of resistance to CIP and CRO in CTM samples is of significance due to the proximity of these animals to the food chain and the importance of these drugs in human medicine.

\section{References}

1. Government of Canada. Canadian Integrated Program for Antimicrobial Resistance Surveillance. Accessed February 11, 2010. http://www.phac-aspc.gc.ca/cipars-picra/index-eng.php 


\title{
0.079
}

\section{Analysis of antimicrobial susceptibility profiles of Salmonella Serotypes using survival analysis}

\author{
Charles A. Haley ${ }^{1}$ Francisco Olea-Popelka² Bruce A. Wagner ${ }^{1}$ David Dargatz ${ }^{1}$ \\ 1. USDA:APHIS:VS:CEAH:NAHMS, Fort Collins, CO, USA; \\ 2. College of Veterinary Medicine and Biomedical Sciences, Colorado State University, Fort Collins, CO, USA
}

\begin{abstract}
Introduction.
Salmonella is an important cause of illness among swine and can cause foodborne illness in people ${ }^{1}$. There is growing global concern for the amount of resistance to antimicrobial drugs among Salmonella isolates. Many groups have called for more surveillance of antimicrobial resistance and the identification of factors that play a role in the emergence or amplification of resistance in populations including people, pets and animals used for food. Previous studies have been primarily confined to using established antimicrobial concentration cutoffs to declare isolates susceptible or resistant.
\end{abstract}

The objective of this study was to use survival analysis to identify factors associated with changes in concentrations of antimicrobials that inhibit Salmonella isolates with various MIC to better understand the ecology of antimicrobial drug resistance among salmonellae on swine farms.

\section{Materials and Methods}

Questionnaire data and fecal samples were collected as part of the National Animal Health Monitoring System's (NAHMS) Swine 2006 study. Fecal samples were collected from the pen floor for grower/finisher pigs. Up to 60 fecal samples per farm from a maximum of 10 pen floors containing late finishers (20 to 32 weeks of age) were collected and tested for Salmonella as previously described ${ }^{2}$.

Salmonella spp. antimicrobial susceptibility testing was conducted using a semi-automated system (SensititreTM, TREKTM Diagnostics, Inc., Cleveland, Ohio) as previously described ${ }^{2}$. S. Derby, the most common serotype isolated (29.6\% of isolates), was selected for this analysis. Two antimicrobials (Amoxicillin/ Clavulanic Acid and Ampicillin) were chosen from among a panel of 15 antimicrobials tested to demonstrate the technique. Survival analysis was done using Cox proportional hazards modeling using previously described methods ${ }^{3}$.

\section{Results}

Of the $173 \mathrm{~S}$. Derby isolates that were tested in increasing two fold concentrations (from 1 to $32 \mu \mathrm{g} / \mathrm{ml}$ ) most (134 for Amoxicillin/Clavulanic Acid and 129 for Ampicillin) were either inhibited at the first concentration or not at all even at the highest concentration $(32 \mu \mathrm{g} / \mathrm{ml})$. Because of this highly skewed distribution of the MIC values for these isolates, we dichotomized the MIC values. Concentration until event (inhibition) for each antibiotic was tested for change with farm level factors: herd size, total confinement of grower/ finisher pigs and usage of complimentary antimicrobials in the nursery or grow/finish phase in feed or water in the study. Farm of origin of the isolates were treated as random effects
For the S. Derby and Amoxicillin/Clavulanic Acid model, the hazard or rate of inhibition was reduced nearly $100 \%$ when grower/ finisher pigs were housed in total confinement or Amoxicillin was used during the nursery or grower/finisher phase. As herd size increased by 500 pigs the hazard of inhibiting S. Derby with Amoxicillin/Clavulanic Acid increased by 31\%. The proportion of variance at the farm level (intra-class correlation coefficient) or correlation of results within herds was 0.74 . The results with these variables were similar but less dramatic for the $\mathrm{S}$. Derby and Ampicillin model.

\section{Discussion}

Survival analysis methods show some promise for analysis of MIC data. Management confinement methods and the use of select antimicrobials in feed or water in the nursery or grow finisher phase increased survivability of S. Derby to these two antibiotics. Additional research is planned to continue analyzing antimicrobial use and inhibition data using these techniques.

\section{References}

1. Griffith R.W., Schwartz K.J., and Meyerholz D.K. "Salmonella" Diseases of Swine. Ed. Barbara E. Straw, Jeffery J. Zimmerman, Sylvie D'Allaire and David J. Taylor. Blackwell Publishing, 2006. 739-754.

2. Blau D.M., McCluskey B.J., Ladeley S.R., Dargatz D.A., Cray P.J., et al. Salmonella in Dairy Operations in the United States: Prevalence and Antimicrobial Drug Susceptibility. Journal of Food Protection 2005: Vol. 68, No. 4 pp. 696-702

3. Hosmer D.W., Lemeshow S. “Extensions of the Proportional Hazards Model-Truncated, Left Censored, and Interval Censored Data" Applied Survival Analysis: Regression Modeling of Time to Event Data. John Wiley and Sons, 1999. 253-269. 


\title{
0.080
}

\section{An experimental Helicobacter suis infection reduces daily weight gain in pigs}

\author{
Smitha Kumar ${ }^{1}$ Freddy Haesebrouck ${ }^{1}$ Frank Pasmans ${ }^{1}$ Bram Flahou ${ }^{1}$ Jeroen Dewulf ${ }^{2}$ Koen $_{\text {Chiers }}{ }^{1}$ Richard Ducatelle $^{1}$ \\ 1. UGent, Faculty of Veterinary Medicine, Department of Pathology, Bacteriology and Poultry Diseases, Merelbeke, Belgium; \\ 2. UGent, Faculty of Veterinary Medicine, Department of Reproduction, Obstetrics and Herd Health, Merelbeke, Belgium
}

\section{Introduction}

Helicobacter suis (H. suis) is a Gram negative, long, spiral-shaped bacterium colonizing the stomach of more than $60 \%$ of pigs at slaughter age3. The prevalence is very low in sucklings, increases from the time of weaning and is highest in adult animals. This bacterium is considered to be one of the risk factors associated with gastric ulcers in pigs2. Recently, $\mathrm{H}$. suis has been successfully cultured in vitro1. The purpose of this study was to evaluate the effect of an $\mathrm{H}$. suis infection on the daily weight gain in pigs.

\section{Materials and Methods}

In 5 different experimental set-ups, a total of 44 medicated early weaned piglets were intragastrically $(n=39)$ or orally $(n=5)$ inoculated once $(n=30)$ or thrice $(n=14)$ with a fresh $(n=24)$ or frozen and thawed $(n=20)$ culture of $H$. suis strain 5 at the age of $3(n=5), 6(n=14), 7(n=10), 8(n=5)$ and $9(n=10)$ weeks. All animals were euthanized $4(n=39)$ or $6(n=5)$ weeks postinoculation. All experimental set-ups included medicated early weaned animals as negative control groups $(\mathrm{N}=29)$ that were sham inoculated with sterile culture medium. The animals were fed a finely ground feed $(n=34)$, coarsely ground feed $(n=5)$ or milk replacement $(n=5)$. All animals were housed under identical environmental conditions. Each animal was weighed at the time of inoculation and at euthanasia. Daily weight gain was calculated over the period after inoculation. In the combined results of the 5 experiments, the effect of inoculation with $\mathrm{H}$. suis on the daily weight gain of inoculated animals was compared to the non-inoculated animals using a multivariable linear regression model including all variables that varied between the experiments as co-variables to correct for their effect (SPSS 17).

\section{Results}

After correcting for the effect of experimental set-up, type of feed, age at the time of inoculation, weight at the time of inoculation, number of inoculations, and the period after inoculation, there was a significant reduction of approximately $20 \mathrm{~g} /$ day (5\%) in the daily weight gain of experimentally infected animals compared to the non-infected control animals.

\section{Discussion}

Results of the present study indicate that an $\mathrm{H}$. suis infection reduces daily weight gain in pigs and thus may result in substantial economic losses. Clearance of infection with this bacterium may therefore have an economically beneficial effect.

\section{References}

1. Baele, M., Decostere, A., Vandamme, P., Ceelen, L., Hellemans, A., Mast, J., Chiers, K., Ducatelle, R., Haesebrouck, F., 2008. Isolation and characterization of Helicobacter suis sp. nov. from pig stomachs. Int. J. Syst. Evol. Microbiol. 58, 1350-1358.

2. Haesebrouck, F., Pasmans, F., Flahou, B., Chiers, K., Baele, M., Meyns, T., Decostere, A., Ducatelle, R., 2009. Gastric helicobacters in domestic animals and nonhuman primates and their significance for human health. Clin. Microbiol. Rev. 22, 202-223.

3. Hellemans, A., Chiers, K., Maes, D., De Bock, M., Decostere, A., Haesebrouck, F., Ducatelle, R., 2007. Prevalence of "Candidatus Helicobacter suis" in pigs of different ages. Vet. Rec. 161, 189-192. 


\subsection{1}

\section{Occurrence of MRSA in conventional and organic pig herds as well as in wild boars in Germany}

Thomas Blaha; Miriam Ostmeier; Birgit Brockers; Lars Meyer; Susanne Fischer; Ulrike Heine; Regina Tegeler; Diana Meemken Field Station for Epidemiology, University of Veterinary Medicine Hannover, Bakum, Germany

\section{Introduction}

The occurrence of MRSA (Methicillin-resistant S. aureus) as nasal colonizer of pigs in conventional herds has been reported in several countries with quite often a very high intra-herd prevalence $(1,2,3)$. Little is known about the frequency of MRSA in organic pig herds. Furthermore, there is only one report on a lower frequency of MRSA as nasal colonizer in "organic pig farmers" compared to "conventional pig farmers" (4). There are no publications on the occurrence of MRSA in wild boars. The objective of our study was to increase the knowledge on the occurrence of MRSA in different pig husbandry systems (organic and conventional) as well as in wild boars.

\section{Materials and Methods}

Our investigations on the occurrence of MRSA in different pig populations with different husbandry systems were carried out as follows:

- Occurrence of MRSA in collective dust samples (one pool of dust from different sample sites) from 85 conventional and 27 organic pig herds

- Occurrence of MRSA as nasal colonizer of pigs in 26 conventional and 20 organic pig herds by testing herd-size adjusted random samples to detect at least a $5 \%$ intra-herd prevalence of MRSA by testing 10 to 60 pigs per herd (in total 1760 nasal swabs), as well as of 120 hunted wild boars. All samples were collected, selectively enriched and cultured according to the EU- guidelines (SANCO/3100/2007 - Rev.2).

\section{Results}

The occurrence of MRSA in dust samples from conventional and organic pig herds are shown in Table 1.

Tab. 1: Occurrence of MRSA in collective dust samples

\begin{tabular}{|l|c|c|}
\hline Husbandry system & No. of tested herds & No. of MRSA-positive herds \\
\hline conventional & 85 & $67(79 \%)$ \\
\hline organic & 27 & $4(15 \%)$ \\
\hline
\end{tabular}

The frequency of MRSA-positive herds, identified by nasal swabs is demonstrated in Table 2. A herd was defined as MRSA-positive, if one nasal swab turned out to be MRSA-positive.
Tab. 2: Occurrence of MRSA in nasal swabs from conventional and organic pig herds, and from wild boars

\begin{tabular}{|l|c|c|}
\hline Pig population & No. & No. of MRSA-positive \\
\hline conventional & 26 herds & $24(92 \%)$ \\
\hline organic & 20 herds & $3(15 \%)$ \\
\hline wild boar & 120 animals & $0(0 \%)$ \\
\hline
\end{tabular}

Nine of the 120 tested wild boars (8\%) were carrier of Methicillinsensitive $\mathrm{S}$. aureus (MSSA) in their nasal cavity.

\section{Discussion}

In our still ongoing studies, the MRSA results (nasal swabs and dust samples) in conventional husbandry systems point to a measurably higher MRSA frequency than the MRSA results in organic husbandry systems. This preliminary assumption needs to be verified by further comparative studies. Possible reasons for the expected higher MRSA detection rate in conventional than in organic pig herds could be the circulation of MRSA between conventional herds (animal movements from conventional to organic herds are very seldom), the higher animal density in conventional herds (i.e. better transmission conditions), and the differences in the use of antibiotics in the two different husbandry systems. The fact that no MRSA, but MSSA were found in wild boars shows that there is no natural non-susceptibility of the wild boar for $S$. aureus. The differences in the MRSA frequency found in our studies do not jutify a direct link between the occurrence of MRSA and the actual use of antibiotics at a specific farm, since the spread of MRSA between conventional herds and the lack of this spread to organic pigs and to wild boar alone can sufficiently explain the differences as well. More detailed research into the risk factors of MRSA is presently carried out.

\section{References}

(1) Voss et al. (2005), Emerg. Infect. Dis 11:1965-1966

(2) Meemken et al. (2008), DTW 115: 132-139

(3) Khanna et al. (2008), Vet Microbial 128: 298-303

(4) Wulf et al. (2008), Proceedings 20th IPVS: 162

*This ongoing study is funded by the German Federal Ministry of Agriculture through the German Federal Office for Agriculture and Food (BLE). 


\title{
0.082
}

\section{MRSA in herds of fattening pigs in Germany: Typing results and factors associated with the detection in herds}

\author{
Bernd-Alois Tenhagen; Alexandra Fetsch; Katja Alt; Beatriz Guerra; Stefan Hertwig; \\ Jens A. Hammerl; Annemarie Kaesbohrer; Juliane Bräunig; Bernd Appel \\ Federal Institute for Risk Assessment (BfR), Berlin, Germany
}

\section{Introduction}

Methicillin-resistant Staphylococcus aureus (MRSA) has been demonstrated to be endemic in pig herds in Europe, Canada and the USA, posing a risk for colonization and infection to people in contact with these animals.

\section{Materials and Methods}

To assess the situation in herds of fattening pigs in Germany, dust samples $(n=5)$ of 290 farms from different regions in Germany were collected in 2008. A questionnaire provided information regarding herd characteristics and management practices.

Dust samples were pooled per farm and cultured using selective enrichment and chromogenic media. Presumptive MRSAisolates were confirmed by triplex-PCR for the detection of $16 \mathrm{~S}$ rRNA, nuc and mecA genes. Confirmed isolates ( $1 /$ herd) were tested for antimicrobial resistance, spa- and SCCmec typed. Selected isolates were also MLST-typed.

\section{Results}

MRSA were identified in 152 herds (52.4\%). Univariate analysis revealed an association of a number of factors with the detection of MRSA on a farm.

Larger herds were more likely to be positive for MRSA. Herds that had used antimicrobials on a group level, were also more likely to be positive. Tetracyclines ( $28.3 \%$ of herds), amoxicillin (19.7\%) and tylosin (17.6 \%) were most frequently used. The occurrence of diseases in the herd was not related to the detection of MRSA.

Buying pigs from several different farms or from weaner to grower operations was associated with MRSA in the herd. Type of pig farm was closely related to this factor. Farrow to finish units were less likely to buy pigs from several sources than weaner to finish or grower to finish operations.

Fully slatted floors and an all in / all out pig flow were associated with the isolation of MRSA from dust. Multivariate analysis revealed multicollinearity between variables. For example larger herds used antimicrobials more often and bought pigs from more sources. In the final model, only farm size and farm type remained as significant risk factors. However, it can be assumed, that other related factors contributed to the effect of these two factors.

Twelve different spa-types were identified in the tested isolates. All isolates were belonged to spa-types related to the multilocus sequence type ST398, with t011 (66.4\%) and t034 (23.0\%) as the predominant spa-types. There were substantial regional differences in the spa-type patterns. In one region, more than $90 \%$ of the isolates were spa-type t011 while in others, t011 and t034 were of similar frequency.
Most isolates (85\%) carried SCCmec-type V, $11 \%$ belonged to SCCmec-type III that has been shown to carry features of type $\mathrm{V}$ and type III.

\section{Discussion}

The results are in line with studies in slaughter pigs that were carried out in the North-West of Germany and with the results of the EU-baseline survey on MRSA in breeding herds (EFSA 2009, Tenhagen et al. 2009)

The latter has shown that MRSA is also prevalent in herds with breeding pigs in most European countries with an intensive pig production. From breeding herds, MRSA can easily be transmitted to fattening units via colonized weaners or growers. With a given proportion of positive herds in the population any further herd that pigs are bought from increases the risk, that one of the herds is positive for MRSA. Biosecurity protocols of pig farms so far did not include $S$. aureus as it was not regarded as a major pathogen for pigs. In fact, MRSA is only associated with clinical disease in pigs in exceptional cases and this study showed no relation between the disease status of herds and the prevalence of MRSA.

The risk factor having the strongest association with a positive test result was herd size. This might be due to buying more pigs in larger herds increases the risk of introduction of MRSA into the herd via colonized pigs.

Antimicrobial use was not a significant risk factor in the multivariate model. Apparently the introduction of the resistant clone is most likely to occur via colonized animals. Antimicrobials may add to the within herd spread, but this was not measured using the dust sample. In a Dutch study on veal calves that considered risk factors on animal level, antimicrobial use in the group proved to be a significant risk factor (Wagenaar and van de Giessen 2009).

\section{References}

EFSA 2009: The EFSA Journal 2009, 7(11), 82pp.

Wagenaar and van de Giessen2009: http://www.rivm.nl/cib/infectieziekten-A-Z/infectieziekten/MRSA/MRSA_publicaties.jsp

Tenhagen et al. 2009: Vet. Rec. 165:589-593 


\title{
0.083
}

\section{Prevalence study and risk factor analysis on MRSA in sow-herds}

\author{
Els M. Broens ${ }^{2}$ Elisabeth A. Graat ${ }^{2}$ Peter van der Wolf ${ }^{1}$ Arjen A. van de Giessen ${ }^{3}$ Mart C. de Jong ${ }^{2}$ \\ 1. Animal Health Service, Deventer, Netherlands; 2. Group of Quantitative Veterinary Epidemiology, \\ Wageningen University, Wageningen, Netherlands; 3. Centre for Infectious Disease Control Netherlands, \\ National Institute for Public Health and the Environment, Bilthoven, Netherlands
}

\section{Introduction}

In 2005 a distinct clone of methicillin resistant Staphylococcus aureus (MRSA ST398) was found in pigs and people in contact with pigs (1). Several studies report prevalences of MRSA-positive farms and identified risk factors for people working with pigs $(2,3)$. Little is known on risk factors associated with MRSA on pig farms. The objective of this study was to estimate a reliable prevalence for MRSA-positive farms and to identify and quantify risk factors.

\section{Materials and Methods}

During 2007 and 2008 a study on 202 pig farms, 171 farms with sows and 31 farms without sows, was performed. On these farms a nasal swab was taken from 60 , randomly selected, pigs. Additionally, 5 environmental samples were taken. On the first 50 farms, the people working and/or living on the farms and the sample takers were sampled (nasal swab) as well. These results were published by Van der Broek et al (2). Information was gathered on farm characteristics (e.g. management, hygiene and antimicrobial use). Microbiological analysis was done on individual environmental samples and pooled pig samples (6 swabs per pool; per pool one age-category). A farm was classified positive if at least one of the samples was MRSA-positive. Statistical analysis to identify risk factors was done on data from 171 sow-farms.

\section{Results}

The overall prevalence of MRSA-positive farms was $68.3 \%$; for farms without sows the prevalence was $71.0 \%$ and for sow-farms the prevalence was $67.8 \%$. On positive sow-farms, on average $63.3 \%$ of the pools and $33.8 \%$ of the environmental samples was MRSA-positive. In total, 18 different spa-types were identified, t011 and t108 being the most frequent ones, resp. 52.5\% and $34.1 \%$. Ninety-eight percent of the MRSA-isolates belonged to ST398. All ST398-isolates were sensitive to mupirocin and linezolid and resistant to tetracycline. Resistance to other antimicrobials varied between and within spa-types. In the final statistical model, 2 risk factors were associated with MRSA: herd size (number of sows) and time (months). Prevalence of MRSA- positive farms increased in time: from approximately $30 \%$ at the beginning of 2007 to approximately $75 \%$ at the end of 2008 . Prevalence of MRSA-positive farms increased when the number of sows on a farm increased: approximately $40 \%$ of small herds ( $<250$ sows) was MRSA-positive versus $>80 \%$ of large herds ( $>$ 500 sows). Other risk factors, e.g. presence of finishing pigs, purchase of gilts, rodent control and hygiene, dropped out of the statistical model but were associated with herd size. Antimicrobial use and MRSA on a farm were not significantly associated, though antimicrobial use was associated with herd size. Farms that apply antimicrobials as group medication have significantly more sows (486 sows) than farms that use antimicrobials just in case of disease ( 415 sows). Farms that use no antimicrobials at all were the smallest farms (355 sows).

\section{Discussion}

MRSA is present on a large number of Dutch sow-farms and the prevalence increases in time. Most likely MRSA was spreading among Dutch pig herds during the study period. A plausible transmission route seems to be the trade of animals, however farms without supply of pigs were found to be MRSA-positive as well $(4,5)$. Large herds have a higher risk, which can be explained by herd size itself and by the factors that are associated with herd size, e.g. antimicrobial use, the purchase of gilts and rodent control. The same positive association between herd size and disease has been reported for other swine diseases, e.g. influenza and pseudorabies (6-8). Herd size is therefore a compilation of several risk factors, which makes that larger farms are more often MRSA-positive.

\section{References}

1. Voss, A. et al (2005) Emerg Inf Dis 11, 1965-6.

2. Van der Broek, I.V.F. et al (2009) Epidemiol Infect 137, 700-8.

3. Smith T.C. et al (2009) PloS ONE 4, e4258.

4. Van Duijkeren et al (2008) Vet Mic 126, 383-9.

5. Broens et al (2008) Proc 20th IPVS OR.03.50, 165.

6. Poljak et al (2008) Vet Res 72, 7-17.

7. Morrison et al (1991) JAVMA 199, 580-3.

8. Gardner et al (2002) Anim Health Res Rev 3, 43-55. 


\title{
0.084
}

\section{Prevalence of MRSA among Danish participants at a conference for Danish pig producers}

\author{
Margit Andreasen ${ }^{1}$ Charlotte S. Kristensen ${ }^{1}$ Robert Skov² Elisabeth O. Nielsen ${ }^{1}$ \\ 1. Pig Research Centre, Copenhagen, Denmark; 2. Statens Serum Institut, Copenhagen, Denmark
}

\section{Introduction}

In 2008, the presence of MRSA CC398 was confirmed in several Danish pig herds. All participants at the yearly conference for Danish pig producers, were offered a test for MRSA, to gain an estimate of the nasal carrier status of MRSA among persons working in the Danish Pig Production. A total of 1945 persons working within various fields of pig production (pig producers, veterinarians, consultants etc.) participated in the conference.

\section{Materials and Methods}

Participation was on a strictly voluntary basis. Nasal sampling was performed by swabbing both nostrils. Prior to sampling a standardized questionnaire on profession (farmer, veterinarian, consultant or other), weekly hours of contact with pigs, working within sow units, finisher units or both, visits to pig units abroad and previous hospitalisation of the participant was completed. All swabs were screened for MRSA using incubation in enrichment broth followed by plating on chromogenic agar. MRSA was confirmed by PCR, typed by sequencing the staphylococcal protein A (spa) gene and susceptibility tested.

\section{Results}

Questionnaires and samples were obtained from 791 persons, of whom 22 were excluded ( 13 were foreign visitors and 9 did not complete the questionnaire). The remaining 769 persons comprised 487 farmers, 109 agricultural consultants, 25 veterinary practitioners and 150 persons working in other fields. The distribution of MRSA-positive persons on professions is shown in Table 1. Of the 19 CC398 isolates, 3 belonged to spatype t011, the remaining 16 to spatype t034. Two persons, both farmers, carried other MRSA-types (t032 and t044). All MRSA CC398 positive persons had weekly contact with pigs. However, the duration of contact with pigs was not statistically associated with the MRSA CC398 carrier status. Persons working only in finisher units had a significantly lower risk of carrying MRSA CC398 compared with persons working in sow units.
Table 1. Nasal carriers of MRSA among participants at the conference

\begin{tabular}{|l|c|c|c|}
\hline & n tested & MRSA CC398 & MRSA CC22 or CC80 \\
\hline Farmer & 487 & $15(3.1 \%)$ & $2(0.4 \%)$ \\
\hline Consultant & 109 & $2(1.8 \%)$ & $0(0 \%)$ \\
\hline Veterinarian & 25 & $0(0 \%)$ & $0(0 \%)$ \\
\hline Other fields & 150 & $2(1.4 \%)$ & $0(0 \%)$ \\
\hline Total & 769 & $19(2.5 \%)$ & $2(0.3 \%)$ \\
\hline
\end{tabular}

\section{Discussion and Conclusion}

The results implied that MRSA CC398 carriage is more frequent among persons working within the Danish pig industry than in the population in general. However, the CC398-prevalence of $3.1 \%$ among Danish pig farmers was much lower than those reported from e.g. Holland and Canada (app. 20\%) 1, 2. Also, a newly published report ${ }^{3}$ establishes that Danish herds have a low prevalence of MRSA compared to other comparable European countries. Working in sow units increased the risk of being an MRSA CC398 carrier. These findings support previous reports 4 , indicating that intensive and regular exposure to pigs increases the risk of humans getting colonized with CC398.

\section{References}

1. Khanna et al., 2008. Vet Microbiol., 128, 298-303

2. Voss et al., 2005. Emerging Inf Dis., 11, 1965-66

3. EFSA Journal 2009, 7 (11), 1376

4. Van den Brook et al., Epidemiol Infect. 2009, 137, 700-8 


\title{
0.085
}

\section{Prevalence of MRSA CC398 in pig holdings and human hospital patients in North Rhine-Westphalia, Germany}

\author{
Robin Köck ${ }^{2}$ ürgen Apel ${ }^{1}$ Christel Gerth ${ }^{1}$ Susanne Gundlach ${ }^{3}$ Claudia Lambrecht ${ }^{1}$ Theo Schulze-Horsel $^{1}$ Josef Schulte-Wuelver $^{4}$ Anita \\ Böcker $^{2}$ Alexander Friedrich ${ }^{2}$ Jürgen Harlizius $^{5}$ \\ 1. Animal Health Services, Landwirtschaftskammer NRW, Münster, Germany; 2. Universitätsklinikum, Münster, Germany; \\ 3. LUFA, Landwirtschaftskammer NRW, Münster, Germany; 4. Animal Health Services, Landwirtschaftskammer Niedersachsen, \\ Meppen, Germany; 5. Animal Health Services, Landwirtschaftskammer NRW, Bonn, Germany
}

\section{Introduction}

Recently, Methicillin-resistant Staphylococcus aureus (MRSA) have emerged in pigs in most European countries (1,3). Predominantly, porcine MRSA colonization and infection is caused by an MRSA clone characterized by clonal complex (CC) 398 as determined by multi-locus sequence typing (MLST) and Staphylococcus aureus protein A (spa) types t011, t034, t108 and relatives. This MRSA clone was hitherto rarely observed among patients in German hospitals (5). Since numerous studies indicated a frequent exchange of MRSA between animals and humans, we investigated the prevalence of MRSA CC398 in pig holdings in the area characterized by the highest pig density in North Rhine-Westphalia (NRW), Germany, and patients admitted to acute care hospitals in this region.

\section{Methods}

Within the framework of the Euregio project SafeGuard VetMednet, five dust samples were collected in a total of 86 pig holdings in the Euregio (NRW part) in 2009 using a sterile wipe and sampling a standardized area of $50 \mathrm{~cm} \times 50 \mathrm{~cm}$. MRSA diagnostic was performed using a double enrichment in selective broth (Phenol Red Mannitol Broth with $5 \mathrm{mg} / \mathrm{L}$ ceftizoxime, $75 \mathrm{mg} / \mathrm{L}$ aztreonam) and plating on a chromogenic medium (Brilliance MRSA, Oxoid, Wesel, Germany). MRSA was confirmed using coagulase tube test and mecA-PCR All MRSA isolates were typed using the Staphylococcus aureus protein A (spa) approach (4).

Human samples were derived from inpatients attending the acute care hospitals in the Euregio participating in a network for the prevention and control of MRSA (EUREGIO MRSA-net). In 2009, every first MRSA isolate per patient from nasopharyngeal and cutaneous screening specimens and from blood cultures was spa typed and entered into a common MRSA database (www.mrsa-net.eu). Clustering of spa types in spa clonal complexes (spa-CCs) was performed using the Based Upon Repeat Pattern (BURP) algorithm of the Ridom StaphType software.

\section{Results}

We identified MRSA in dust samples from 51 pig holdings (59\%). Among 103 MRSA isolates we found seven different spa types including t011 (69\%), t034 (20\%), t2510 (5\%), t1456 (3\%), and t108, t588, t1606 (each 1\%).

A total of 1,695 human isolates from screening specimens as well as $n=38$ blood culture isolates were obtained in the MRSAnet hospitals in 2009. Among the isolates from screenings 305 (18\%) were associated with spa-CC398 (spa types: t011, t034, $\mathrm{t} 108, \mathrm{t} 1197, \mathrm{t} 1451, \mathrm{t} 1580$, t1793, t2011, t2330, t2346, t2582,

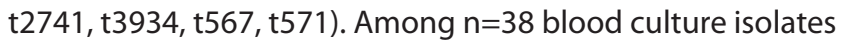
only one (2.6\%) was associated with spa-CC398 (t011).

\section{Discussion}

The prevalence of MRSA CC398 in environmental samples from pig holdings in the Euregio VetMed-net is higher than the overall prevalence for Germany observed in a recent report published by the European Food Safety Authority (1). As in other countries it was shown that the spa types t011 and t034 are predominant (3). This confirms the results of previous investigations (2).

MRSA CC398 are frequently introduced in to German hospitals located in an area with a high density of pig-production by inpatients colonized at admission. Despite the high burden of colonization, invasive human infections due to MRSA CC398 are rare until now.

\section{References}

1. The European Food Safety Authority, EFSA Journal 2009; 7(11):1376. [82 pp.]. doi:10.2903/j.efsa.2009.1376. Available online: www.efsa. europa.eu

2. Köck R, Harlizius J, Bressan N, Laerberg R, Wieler L H, Witte W, Deurenberg RH, Voss A, Becker K, Friedrich AW (2009) Eur J Clin Microbiol Infect Dis 28: 1375-1382.

3. Neeling de, A J, van den Broek M J, Spalburg E, C, van Santen-Verheuvel MG, Dam-Deisz W D, Boshuizen HC, van de Giessen AW, van Duijkeren E, Huijsdens XW. (2007) Vet Microbiol, 122:366-372.

4. Mellmann A, Friedrich AW, Rosenkötter N, Rothgänger J, Karch $\mathrm{H}$, Reintjes R, Harmsen D. (2006) PLoS Med, Ma;3(3):e.

5. Witte W, Strommenger B, Stanek C, Cuny C. (2007) Emerg Infect Dis, $13(2): 255-258$. 


\title{
0.086
}

\section{Efficiency of different sampling locations and methods to detect MRSA on pigs and in their housing environment}

\author{
Jochen Schulz ${ }^{2}$ Anika Friese ${ }^{3}$ Diana Meemken ${ }^{4}$ Uwe Rösler $^{5}$ Thomas Blaha $^{1}$ Jörg Hartung ${ }^{2}$ \\ 1. Station for Epidemiology, University of Veterinary Medicine Hannover, Foundation, Bakum, Germany; 2. Institute for Animal Hygiene, Animal \\ Welfare and Farm Animal Behaviour, University of Veterinary Medicine Hannover, Foundation, Hannover, Germany; 3. Institute of Animal Hygiene \\ and Environmental Health,Freie Universität Berlin, Berlin, Germany; 4. Field Station for Epidemiology, University of Veterinary Medicine Hannover, \\ Foundation, Bakum, Germany; 5. Institute of Animal Hygiene and Environmental Health,Freie Universität Berlin, Berlin, Germany
}

\section{Introduction}

Although Staphylococcus aureus belongs to the common skin flora of animals and humans this opportunistic pathogen can cause various skin and organ infections [1]. In particular methicillin resistant $S$. aureus strains (MRSA) gain more attention in recent years. In pig production units in Europe and Canada the special sequence type (ST) 398 was detected [2, 3] which can colonize animals and humans and is transmissible between them. To investigate the spread and transmission of MRSA fast and reliable methods are required by which these bacteria can be detected and monitored on pigs and in their housing environment. This paper reports about first results from investigations in piggeries comparing the efficiency of swab sampling at five locations on the pigs and of seven sampling methods to detect MRSA in the housing environment.

\section{Materials and Methods}

31 farms (15 fattening farms and 16 breeding farms) were presently sampled. Nasal swabs were taken from 60 randomly selected animals of all farms and examined as pooled samples (five swabs per pool). Skin swabs were taken from the neck region of 60 animals from 13 farms and were also analyzed as pool samples (five swabs per pool). Vaginal swabs were collected three to five times from four selected sows of 11 farms during the investigation period from September to December 2009. In three of these farms also the perianal region and the teats of the same sows were sampled with swabs.

From 26 piggeries pooled dust samples were taken from five different locations (sampling area approx. $500 \mathrm{~cm}^{2}$, at least $15 \mathrm{ml}$ dust per pool). Sedimentation dust was sampled in the center of four animal houses in two meters height on sterile surfaces with a defined area of $0.4 \mathrm{~m}^{2}$ (sampling time about 28 days). In 25 barns boot swabs were taken by walking with one pair of boots twice along the middle corridor. Pooled fecal samples and feed samples from feeders were taken from 13 farms and swabs from drinking nipples were sampled in 5 holdings. In 11 pig houses airborne bacteria were sampled by impingement (AGI-30) and a filtration method.

MRSA were grown after selective enrichment and direct plating on CHROMagar. Coagulase positive Staphylococci were identified with biochemical tests and the isolates were confirmed as MRSA by a triplex PCR method.

\section{Results}

MRSA was found in 27 (87\%) out of 31 investigated farms in at least one sample. The detection efficiencies of samplings from pigs and of samples from the housing environment in relation to positive tested farms were: Nasal swabs $=100 \%$; Skin swabs $=$ $100 \%$; Vaginal swabs $=73 \%$; Perianal swabs $=67 \%$; Teat swabs $=$ $67 \%$; Pooled dust $=78 \%$; Sedimentation dust $=33 \%$; Boot swabs $=91 \%$; Pooled fecal $=60 \%$; Drinking nipples $=0 \%$; Feed $=56 \%$; Air $=100 \%$.

\section{Discussion}

MRSA was found in $87 \%$ of the investigated piggeries. The efficiency of the sampling locations on the animals and the methods used for environmental sampling varied considerably. Nasal swabs and swabs of the neck seem to be more efficient to detect MRSA on a pig than samples from vagina and teats. $100 \%$ of the air samples were MRSA positive followed by boot sampling, pooled dust, feces and feed. Less successful was the detection in sedimentation dust which may has to do with the sampling period and the survival of Staphylococci in dust. No MRSA were found at drinking nipples.

The high air contamination and the contamination of surfaces in the direct environment of the pigs may contribute to the high positive results from nasal and skin swabs. Positive feed samples may also be a consequence of environmental contaminations. The results of this current study indicate that the most promising sampling locations to detect MRSA on pigs are the nose and the skin on the neck. For environmental sampling air, boot swabs and dust can be recommended.

The current investigations continuous and more detailed results will be presented on IPVS in 2010.

This study funded by the German Federal Ministry of Food, Agriculture and Consumer Protection.

\section{References}

1. Kloos et al. (1992), In: Balows et al., The Prokaryotes Vol. II: 13691420.

2. Wulf and Voss (2008), Clin. Microbiol. Infect. 14: 519-521

3. Khanna et al. (2008), Vet. Microbiol. 128: 298-303 


\title{
0.087
}

\section{The over-muscled sow syndrome: a new emerging syndrome in a hyperprolific sow herds: Preliminary observations on farrowing duration}

\author{
Thierry Solignac ${ }^{1}$ Alassane Keita ${ }^{2}$ Eric $_{\text {Pagot }}{ }^{2}$ G-P Martineau ${ }^{3}$ \\ 1. Coopagri Bretagne, Landerneau, France; 2. CTPA Zoopole, Ploufragan, France; 3. Ecole Vétérinaire, Toulouse, France
}

\section{Introduction}

Many factors can affect duration of farrowing such as breed, litter size, parity, body condition and housing (Farmer and Robert, 2002, Oliveiro et al., 2008). Fat sows are classically reported having long farrowing but the correlation was not very high (Oliveiro, 2009).

We reported the emergence of a new building syndrome that we call "Over-muscled sow syndrome" (Solignac and Martineau, 2010a). It emerges as a consequence of the selection for deposition of lean meat and hyperprolificay (Tribout et al., 2003, Bazin et al., 2003).

\section{Materials and Methods}

Duration of farrowing has been registered from 482 sows of 14 commercial herds in Brittany (France) with different hyperprolific genetic. For each sow, we measured body fat (BF) and body muscle (BL) (Noveko AC037L, 3.5 MHtz) on site P2 at farrowing. Each sow was then classified according to the BF and BL median values of data: $B L+$ when the value was above the median value and $\mathrm{BL}$ - when it was below. The same procedure was applied for BF (Table 1). Data were analysed using a 3 factorial (BF, BL and genetic line, coded A, B and C) ANOVA test with $p<0.05$ as level of significance.

Table 1: Morphotype characteristics of sows $(n=482)$ at farrowing

\begin{tabular}{|l|c|c|c|c|c|}
\hline Morphotype & Nb sows & \% Genetic(A/B/C) & $\begin{array}{c}\text { Mean } \\
\text { Parity }\end{array}$ & $\begin{array}{c}\text { Mean } \\
\text { BF(mm) })\end{array}$ & $\begin{array}{c}\text { Mean } \\
\text { BL(mm) }\end{array}$ \\
\hline $\mathrm{BF}+/ \mathrm{BL}+$ & 154 & $40 / 32 / 28$ & 3.2 & 24.5 & 57.7 \\
\hline $\mathrm{BF}+/ \mathrm{BL}-$ & 85 & $34 / 39 / 27$ & 2.9 & 23.5 & 49.1 \\
\hline $\mathrm{BF}-/ \mathrm{BF}+$ & 104 & $27 / 36 / 37$ & 2.9 & 17.1 & 57.1 \\
\hline $\mathrm{BF}-/ \mathrm{BL}-$ & 139 & $44 / 34 / 22$ & 2.5 & 15.9 & 47.6 \\
\hline
\end{tabular}

\section{Results}

Major results are reported in Figure 1. There is a statistically significant effect of $B L$ on duration of farrowing $(p=0.04)$. BL+ sows showed a farrowing duration of 3.9 versus 3.6 for $B L$ - ones. The effect of BF $(p=0.599)$ and that of genetic $(p=0.790)$ are not significant.

Figure 1: Morphotype and duration of farrowing

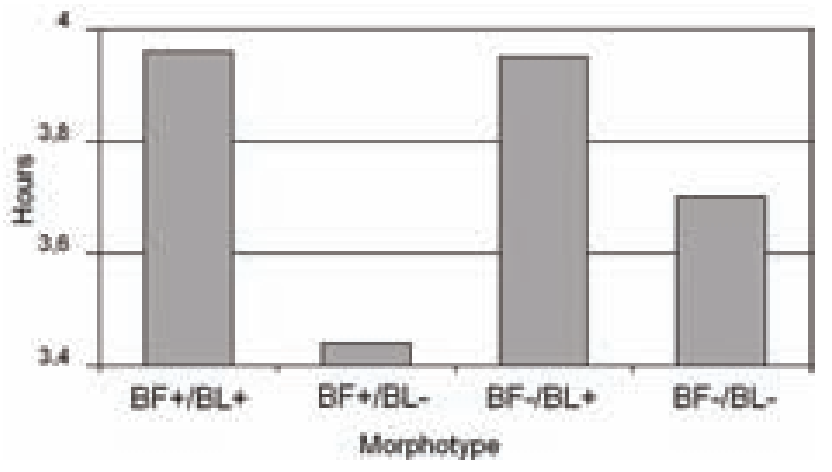

On the figure 2, is shown the distribution of Back lean (BL) according to Back fat (BF).

Figure 2: Correlation between BF $(x)$ and $B L(y)$

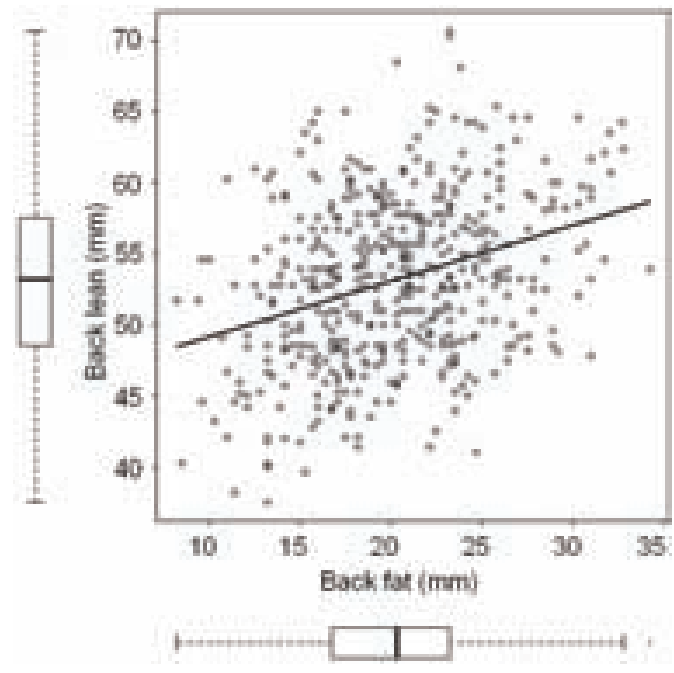

The correlation coefficient is 0.328 and the coefficient of determination is 0.108 , which means that only $10.8 \%$ of $\mathrm{BL}$ are explained by BF and vice versa.

\section{Discussion and conclusion}

In 2005, Foxcroft et al., wrote: "Accepting the risk of being considered some what heretical, most of our recent experiments with the lactating and weaned sow lead to the conclusion "that from a fertility and prolificacy perspective, fatness is simply not the key risk factor".

We would like to add that over-muscled sows are candidates to long farrowing. Management of lean deposition is of crucial importance. There is a need to modify our paradigmatic view of sow nutrition according only to their needs. Indeed, we have to take into account the fact that selection for high capacity of lean deposition has negative consequences on many parameters (Solignac and Martineau., 2010a,b).

\section{References}

Foxcroft G. et al., Proceedings AD Leman 2005; 130-138

Solignac T. and Martineau G-P. Proceedings IPVSa

Solignac T. and Martineau G-P. Proceedings IPVSb

Tribout T. et al., Journées Rech. Porcine en France, 2003 ; 35 : 285-292

Bazin C. et al., Journées Rech. Porcine en France, 2003 ; 35 : 277-284 


\title{
0.088
}

\section{Chronopart in catheterized commercial sows in conventional herds: Physiological, biochemical and hormonal follow-up in "easy" farrowing and "difficult" farrowing sows}

\author{
Jean-Noel Sialelli ${ }^{2}$ Fabien Vautrin² Pauline Bories ${ }^{1}$ Sylviane Boulot ${ }^{3}$ Marie-Christine Pere ${ }^{4}$ Guy-Pierre Martineau ${ }^{1}$ \\ 1. National Veterinary School, Toulouse, France; 2. Farm'apro, Lamballe, France; 3. IFIP, LeRheu, France; 4. INRA, St-Gilles, France
}

\section{Introduction}

Observational study on enzootic neonatal diarrhea (END) in Britanny lead to a link between END and the processus of parturition (Sialelli et al., 2009, Bories et al., 2010). Pre-partum investigations are mainly limited on experimental sows (Oliviero et al., 2007).

Intensive physiological investigation have been registered in 4 commercial hyperprolific herds (>14 TB) selected according to history of farrowing difficulties, high stillbirth (>1.2/litter), and good records.

\section{Material and methods}

Sows (19 P1 and 9 P2) were catheterized at day 109 (Figure 1) and intensively followed before and during farrowing. According to speed of farrowing, sows were classified in three groups: $<3 \mathrm{hrs}$ (SF for short farrowing), between 3 and 4 hrs (IF for intermediate farrowing) and $>4$ hrs (LF for Long farrowing). Stillborn and manual assistance were also taken into account.

Finally, sows classified with an "easy" farrowing are sows having a SF or IF without manual assistance and only stillbirth piglets at the end of farrowing. Sows with a "difficult" farrowing are LF and IF with manual intervention and stillbirth during farrowing (Table 1).

\begin{tabular}{|l|c|c|c|}
\hline Table I: data set & $\begin{array}{c}\text { Easy } \\
\text { farrowing }\end{array}$ & $\begin{array}{c}\text { Difficult } \\
\text { farrowing }\end{array}$ & P \\
\hline Number of sows (and PI) & $14(9)$ & $14(9)$ & NS \\
Sow's weight (kg) & 226.8 & 228.8 & NS \\
Back fat (mm) & 17.9 & 18.2 & NS \\
Gestation length (d) & 113.9 & 113.9 & NS \\
Total born & 14.1 & 15.3 & NS \\
\hline Live born & 13.8 & 13.9 & NS \\
Farrowing (min) & 173.2 & 297.0 & $<0.01$ \\
Total litter weight (kg) & 16.78 & 20.11 & $<0.05$ \\
\%piglets $>1.6 \mathrm{~kg}$ BW & 12 & 24.7 & NS \\
\%piglets < $1.25 \mathrm{~kg}$ BW & 57.4 & 46.7 & $<0.01$ \\
\hline
\end{tabular}

Sequence of sampling is reported on Figure 1A:Sampling procedure in catheterized sows; vertical arrow correspond to a blood sample.

Physiological, biochemical and hormonal investigations: Rectal temperature, hematocrit $(\mathrm{Ht})$ and Hemoglogin $(\mathrm{Hb}$, test Hémo$\mathrm{Cue}^{\circledR}$ ) were performed on farm. Plasma progesterone (P4), Estradiol (E2), Glucose (Glu), total magnesium (Mg), total calcium (Ca), Non Esterified Fatty Acid (NEFA), total protein (Prot), lactate (Lact), bicarbonate (HCO3) and creatine phospho-kinase (CK) have been determined using commercial kit.

\section{Results and discussion}

The progress of parturition (prolonged fast, uterine and muscular contractions, stress and hyperventilation) creates the conditions to a mobilization of the reserves and to a metabolic acidosis (fast increase of NEFA, lactate and bicarbonate in the beginning of birth, followed by a high level during all parturition). The significant correlation between the levels of lactate 3 hrs after the first piglet and the duration of parturition $(r=0,580$ $p<0,01$ and $r=0,326 p=0,09$ ) suggests a high risk of weakness in long farrowing.

Figure 1B: Follow-up of progesterone (left) and rectal temperature (right) by \% of sows with $t^{\circ}>39.5^{\circ} \mathrm{C}$ ) according to "easy" and "difficult" farrowing. Figure 1C: Calcium follow-up according to "easy" and "difficult" farrowing
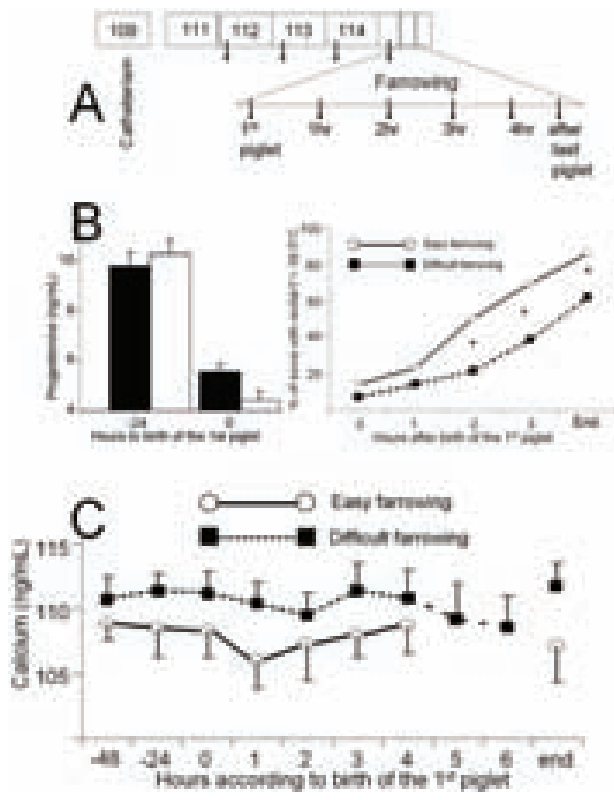

\section{Conclusion}

Pre-farrowing high blood progesterone may have consequences on uterus myometre and thereafter on farrowing. Other analysis are in progress (ocytocin, albumin, ...) and will be reported. Understanding of pathophysiology is probably the first step for the control.

\section{References}

Bories P. et al. Journ. Rech Porcine 2010; 42: in press

Oliviero et al., Anim. Reprod. Sci. $2008 ; 105: 365-377$

Sialelli J-N et al. Journ. Rech Porcine 2009; 41: 167-172 


\title{
0.089
}

\section{Birth weight implications for postnatal development of the reproductive tract in gilts}

\author{
Fernanda R. Almeida; Ana Luisa N. Alvarenga; Leticia P. Moreira; George R. Foxcroft; Helio C. Garcia \\ Universidade Federal De Minas Gerais, Belo Horizonte, MG, Brazil
}

\section{Introduction}

Selection for prolificacy appears to have created an imbalance between the number of conceptuses surviving to the postimplantation period and uterine capacity. These animals show characteristics of Intra-Uterine Growth Restriction (IUGR) due to a great competition among fetuses for nutrients and oxygen, resulting in lighter fetuses at term. The effects of IUGR on development of the reproductive tract in gilts have not been demonstrated so far. The present study evaluated those effects on the reproductive tract of light-weight offspring.

\section{Material and Methods}

New-born female pigs ( $n=28$; DanBred X PIC terminal line), born to 4th- 6th parity sows and in litters of 10 to 15 pigs, were identified as falling into two birth weight groups: high (HW: range 1.8 to $2.2 \mathrm{~kg}$ ) and low (LW: range 0.8 to $1.2 \mathrm{~kg}$ ). They were reared until the finishing period $(\sim 150 \mathrm{~d})$ and data on growth performance were collected. Gilts were slaughtered at 150 days of age and their reproductive tract was collected. The parameters measured were: length of the vaginal canal, uterine body and uterine horns. Additionally, the whole reproductive tract was weighed. Data were analyzed as a fully randomized design and the comparison between means was performed by t-test.

\section{Results}

HW gilts had a higher postnatal growth performance compared to their LW counterparts ( $\mathrm{P}<0.05$ - Table 1). Moreover, the biometric data of the reproductive tract showed that the vaginal canal length was lower in LW gilts $(P<0.05)$. However, the other parameters measured were similar between the experimental groups (Table 2).

\section{Discussion}

Evidence of a better growth performance in high birth weight animals has been previously demonstrated (1). Additionally, high growth rate gilts attain puberty earlier than low growth rate gilts (2). In fact, gilts that attain puberty earlier are the most fertile in the breeding herd. Moreover, these results suggest that light birth weight animals have a smaller vaginal canal. It has been shown a positive relationship between the vaginal canal length and the total number of pigs born (3). Therefore, light birth weight gilts may have poor reproductive performance.
Table 1 Mean body weight (wt) of HW and LW gilts from birth to 150 days

\begin{tabular}{|l|c|c|c|c|}
\hline \multicolumn{1}{|c|}{ Parameter } & HW (n=14) & LW (n=14) & SEM & P< \\
\hline Birth wt, $\mathrm{kg}$ & 2.0 & 1.0 & 0.03 & 0.01 \\
\hline Weaning wt, $\mathrm{kg}$ & 8.8 & 6.5 & 0.4 & 0.01 \\
\hline Wt at 63d, kg & 32.6 & 25.5 & 1.0 & 0.01 \\
\hline Wt at 110d, kg & 75.6 & 63.6 & 1.7 & 0.01 \\
\hline Wt at 150d, kg & 113.3 & 98.4 & 2.4 & 0.01 \\
\hline
\end{tabular}

Table 2 Biometric data of the reproductive tract (RT) in HW and LW gilts

\begin{tabular}{|l|c|c|c|c|}
\hline \multicolumn{1}{|c|}{ Parameter } & HW $\mathbf{n = 1 4}$ & LW $\mathbf{n}=\mathbf{1 4}$ & SEM & P \\
\hline RT wt, $\mathrm{kg}$ & 0.21 & 0.18 & 0.02 & 0.2 \\
\hline Mean ovarian wt, g & 4.3 & 3.7 & 0.2 & 0.06 \\
\hline Vaginal canal, cm & 32.9 & 29.5 & 0.7 & $<0.01$ \\
\hline Uterine body, cm & 32.6 & 25.5 & 1.0 & 0.2 \\
\hline Mean uterine horns, cm & 75.6 & 63.6 & 1.7 & 0.7 \\
\hline
\end{tabular}

\section{References:}

1) Gondret, F. et al. 2006 J. Anim. Sci. 84: 93-103.

2) Kummer, R. et al. 2009 Reprod. Domest. Anim. 44: 255-9.

3) Martin-Rillo, S. et al. 2001.Reprod.Domest. Anim. 36: 297-300. 


\title{
0.090
}

\section{Effects of birth weight and prenatal maternal dexamethasone treatment on leukocyte subsets in piglets}

\author{
John Harding; Crissie Auckland; Cheryl L. Waldner \\ University of Saskatchewan, Saskatoon, SK, Canada
}

\section{Introduction}

In humans, adverse events impacting fetal and infant growth are associated with the onset of metabolic ${ }^{1}$ and autoimmune ${ }^{2}$ diseases later in life. In developing nations, maternal malnutrition is associated with increased risk of infection-related death ${ }^{3}$, decreased response to $S$. typhi vaccination ${ }^{4}$, and decreased thymopoeitin production (a thymic growth marker) 5 in adolescence. In swine, prenatal maternal stress occurring in late gestation decreased the offsprings' lymphocyte proliferation response to $\mathrm{T}$ - and B-cell mitogens in early life ${ }^{6,7}$. The objective of this experiment was to assess the relationship between leukocyte subsets and birth weight, and to determine if prenatal dexamethasone treatment in early- and mid-gestation affects leukocyte numbers in 2 week old pigs.

\section{Materials and Methods}

Pregnant PIC sows were given dexamethasone at a high antiinflammatory dose ${ }^{8}(0.2 \mathrm{mg} / \mathrm{kg})$ IM daily for 7 days; on gestation days 30-36 (Dex30; $n=27$ ) or 60-66 (Dex60; $n=27$ ). Treated and non-treated control $(C T R ; n=25)$ sows were induced to farrow on day 115 . Judicious cross-fostering was encouraged to ensure the best perinatal conditions supporting piglet survival and growth. Blood was collected from 3 sibling piglets of low, average and high birth weights (BW) between 11 and 14 days of age. Complete blood counts and flow cytometry for $\mathrm{CD} 3^{+}, \mathrm{CD} 21^{+}, \mathrm{CD} 4^{+}$, $\mathrm{CD}^{+}$, monocyte, granulocyte and gamma-delta leukocytes were performed. The absolute number of leukocytes in each subset was calculated (subset proportion $x$ total WBC $\times 10^{9}$ ). Generalized linear mixed models were used to estimate the difference in cell numbers across treatment group. Estimates were adjusted for clustering within litter by including a random intercept for litter in the model (PROC MIXED). Potentially important co-factors including BW category and birth week were retained in the final model if they changed the regression coefficient by more than $10 \%$ or if statistically significant $(P<.05)$.

\section{Results}

CD8+ lymphocytes were significantly lower in the Dex60 $(0.50$ $\left.\mathrm{x} 10^{9} / \mathrm{L} ; P=0.01\right)$ and trended lower in the $\operatorname{Dex} 30\left(0.53 \times 10^{9} / \mathrm{L} ; P\right.$ $=0.07)$ compared to CRT $\left(0.63 \times 10^{9} / \mathrm{L}\right)$. No other dex-treatment group differences were found. Total white blood cell (WBC), $\mathrm{CD}^{+}, \mathrm{CD}_{21}{ }^{+}, \mathrm{CD}^{+}, \mathrm{CD}^{+}$and gamma-delta lymphocytes were significantly lower in low versus high BW piglets (Table 1). $\mathrm{CD}^{+}$, $\mathrm{CD}^{+}$and gamma-delta lymphocytes were significantly lower in average versus high BW piglets. Neither dex-treatment nor birth weight impacted monocyte or granulocyte number.
Table 1. Mean absolute leukocyte counts (x109/L) in low, average and heavy birth weight piglets

\begin{tabular}{|c|c|c|c|c|}
\hline & \multicolumn{3}{|c|}{ Birth Weight Category } & \multirow[b]{2}{*}{$P$ value } \\
\hline & Low & Avg & High & \\
\hline & $n=76$ & $n=76$ & $n=76$ & (model) \\
\hline Birth weight (kg) & 1.24 & 1.62 & 1.96 & \\
\hline Total WBC (no.) & $9.5 a$ & $10.1 \mathrm{ab}$ & $10.7 \mathrm{~b}$ & .046 \\
\hline CD3+ (T cells) & $1.77 a$ & $2.0 \mathrm{a}$ & $2.23 b$ & $<.001$ \\
\hline CD21+ (B cells $)$ & $0.37 a$ & $0.43 \mathrm{ab}$ & $0.47 \mathrm{~b}$ & .01 \\
\hline CD4+ (T-helper) & $0.80 \mathrm{a}$ & $0.86 a b$ & $0.93 b$ & .049 \\
\hline CD8+ (cytotoxic) & $0.49 \mathrm{a}$ & $0.51 \mathrm{a}$ & $0.62 \mathrm{~b}$ & .002 \\
\hline Gamma-delta & $0.44 a$ & $0.55 b$ & $0.64 c$ & $<.001$ \\
\hline
\end{tabular}

\section{Discussion}

While changes in absolute lymphocyte numbers may not reflect altered immune function, these results provide evidence that the immune response may be compromised in fetal growth retarded piglets. Moreover, dexamethasone treatment, a proxy for prenatal maternal stress, may adversely affect the development or maturation of CD8 ${ }^{+}$lymphocytes. More research is warranted to characterize functional immunologic changes associated with restricted fetal development. Immunologic programming has substantial implications for both the swine industry and public health in terms of disease control, food safety and zoonosis.

\section{References}

1. Hales,CN et al (2001) Br Med Bul. 60(1), 5-20.

2. Phillips, DIW (2006) Lupus. 15(11),794-800.

3. Moore, SE et al (1997) Nature. 388(6641),434.

4. McDade, TW et al (2001) Am J Clin Nutr. 74(4), 543-548.

5. McDade, TW et al (2001) J Nutr. 131(4),1225-1231.

6. Otten, $\mathrm{W}$ et al (2007) Livest Sci. 106(2-3), 261-270.

7. Tuchscherer, M et al (2002) Vet Immunol Immunopathol. 86(3/4),195-203.

8. Ferguson, DC et al. Glucorticoids. In: Adams H, R., ed. Vet Pharm. 8th ed. Ames: ISU Press, 2001. 


\title{
0.091
}

\section{Effects of short-term prenatal maternal dexamethasone treatment on piglet growth from birth to day 50}

\author{
John Harding; Crissie Auckland; Cheryl L. Waldner \\ University of Saskatchewan, Saskatoon, SK, Canada
}

\begin{abstract}
Introduction
The performance and health of livestock species is influenced by prenatal events, such as maternal malnutrition, stress and placental insufficiency that adversely affect embryonic and fetal development during sensitive periods. Humans at greatest risk of metabolic programming experience poor fetal and infant growth, rapid weight gain in childhood and obesity in later life ${ }^{1}$. These metabolic changes may be mediated directly or indirectly by excessive fetal glucocorticoid (GCC) exposure ${ }^{2}$. In swine, restricted fetal growth results in similar postnatal metabolic programming, affecting birth weight, survivability, growth rate, muscle development, glucose metabolism, blood pressure, and the stress response (as reviewed by Harding ${ }^{3}$ ). The objective of this experiment was to determine the effect of short-term prenatal dexamethasone treatment occurring in early- and midgestation on birth weight and early postnatal growth.
\end{abstract}

\section{Materials and Methods}

Pregnant PIC sows were given dexamethasone at a high antiinflammatory dose $4(0.2 \mathrm{mg} / \mathrm{kg})$ IM daily for 7 days; on gestation days 30-36 (Dex30; $n=27$ ) or 60-66 (Dex60; $n=27)$. Treated and non-treated control $(C T R ; n=25)$ sows were induced to farrow on day 115. On the day of birth (D0), and prior to any cross fostering, the litter size was recorded, and the body mass index (BMl; birth weight/crown rump length squared) determined. Judicious cross-fostering and split suckling was encouraged to ensure the best perinatal conditions supporting piglet survival and growth. Piglets were weaned at 19-22 days and piglets were sorted by size, treatment group and gender into nursery pens containing 10-13 pigs depending on the production week. Piglet weights were measured on day $0,20,34$, and 50 and the average daily gain (ADG) calculated. Generalized linear mixed models (SAS) were used to estimate the difference in birth weight and ADG across treatment groups. Estimates were adjusted for clustering within litter by including a random intercept for litter in the model (PROC MIXED). Biologically relevant co-factors were tested and retained in the final model if they changed the regression coefficient by more than $10 \%$ or if statistically significant $(P<.05)$.

\section{Results}

Birth weight, litter size and body mass index did not differ between groups. The ADG was higher in the Dex60 offspring than CTR between D20-50 and D0-50, and was higher in Dex60 than Dex30 between D34-50 and D0-50 (Table 1). The ADG of the Dex30 and CTR offspring did not differ statistically.
Table 1. Birth weight ( $\mathrm{kg}$ ) and average daily gain (ADG; grams/day) in offspring of dams treated with dexamethasone between gestation day 30-36 (Dex30) or 60-66 (Dex60)

\begin{tabular}{|l|c|c|c|c|}
\hline & Dex30 & Dex60 & CTR & P value \\
\hline & $\mathrm{n}=293$ & $\mathrm{n}=329$ & $\mathrm{n}=310$ & (model) \\
\hline Birth Weight & 1.64 & 1.48 & 1.53 & $\mathrm{~ns}$ \\
\hline ADG & & & & \\
\hline Day 0-20 & 285 & 269 & 261 & $\mathrm{~ns}$ \\
\hline Day 20-34 & 268 & 280 & 257 & 0.15 \\
\hline Day 34-50 & $544 \mathrm{a}$ & $575^{\mathrm{b}}$ & $546^{\mathrm{ab}}$ & 0.02 \\
\hline Day 20-50 & $415^{\mathrm{ab}}$ & $437^{\mathrm{b}}$ & $412^{\mathrm{a}}$ & 0.04 \\
\hline Day 0-50 & $365^{\mathrm{a}}$ & $372^{\mathrm{b}}$ & $353^{\mathrm{a}}$ & 0.02 \\
\hline Columns with different superscripts within rows differ statistically at $P<.05$ \\
\hline
\end{tabular}

\section{Discussion}

Excess fetal GCC exposure affects the development of all tissues and organ systems, and is the most likely hormone to metabolically program in-utero. Moreover, fetal GCC exposure permanently resets several endocrine axes, including those involved in fetal and postnatal growth (i.e. GH-IGF-1), potentially altering the postnatal growth trajectory in situations when caloric intake is restored ${ }^{5}$. This present research provides experimental evidence of "catch-up growth" during the first 30 days post-weaning in piglets exposed prenatally to a potent GCC for a short-term period. However, the effects of prenatal GCC exposure on post-natal growth appear to be gestation-stage dependent. Importantly, evidence of a prenatal mechanism causing an altered postnatal growth trajectory in pigs that is potentially independent of birth weight may further explain causes of biological variation in growth in commercial pork production.

\section{References}

1. Hales, CN et al (2001) Br Med Bull. 60(1),5-20.

2. Ozanne, SE (2001) Br Med Bull. 60(1),143-152.

3. Harding, JCS et al. (2009). Proc West Can Assoc Swine Vet.

4. Ferguson, DC et al. Glucorticoids. In: Adams H, R., ed. Vet Pharm. 8th ed. Ames: ISU Press, 2001.

5. Fowden, AL et al (2004) Reprod (Cambridge). 127(5),515-526. 


\title{
0.092
}

\section{Parity of the rearing dam can influence progeny innate immune responses}

\author{
$\underline{\text { Yvette J. Miller }}^{1}$ Alison M. Collins ${ }^{2}$ David Emery ${ }^{3}$ Douglas Begg ${ }^{3}$ Rob J. Smits ${ }^{4}$ Patricia K. Holyoake ${ }^{5}$ \\ 1. Portec Australia, Perth, WA, Australia; 2. Elizabeth Macarthur Agricultural Institute, Menangle, NSW, Australia; \\ 3. University of Sydney, Sydney, NSW, Australia; 4. Rivalea, Corowa, NSW, Australia; \\ 5. Wagga Wagga Agricultural Institute, Wagga Wagga, NSW, Australia
}

\section{Introduction}

Progeny from gilts have greater susceptibility to disease both pre- and post-weaning compared to sow progeny (Miller, 2006). This could result from differences in innate (non-specific) or adaptive immune responses in gilt compared to sow progeny. The aim of this experiment was to determine the innate immune responses of piglets born to or raised on gilts compared with sows. The null hypotheses were that innate immunity does not differ between progeny: (1) born to gilts and sows or (2) in the pre- and post-weaning period irrespective of the parity of the rearing dam.

\section{Materials and Methods}

This experiment was designed to differentiate between effects due to birth and rearing dam parity by cross-fostering progeny pre-suckling. Gilts (parity 0) and sows (parities 2-5) were induced to farrow on Day 114 of gestation. Piglets were collected presuckling and individually weighed and ear-tagged before being randomly transferred to a gilt or a sow. Sixteen gilt and 16 sow litters were assembled, each consisting of 5 gilt-born and 5 sowborn piglets. No piglets were returned to their birth dam. No deformed piglets or piglets $<900 \mathrm{~g}$ live weight were kept on trial. A blood sample was collected from 3 gilt-born and 3 sow-born piglets per litter at 2 and 7 weeks of age in lithium heparin blood tubes for the following tests:

Immune cell stimulation - Whole blood from each animal was stimulated with pokeweed mitogen (PWM; general immune cell stimulant) or phosphate buffered saline (PBS; negative control). The IFN $\gamma$ concentration was measured in the supernatant using a sandwich ELISA (Miller, 2008). A sample to positive (SP) ratio (\%) was calculated for each sample.

Phagocytic assay - Whole blood was mixed with a solution of killed yeast spores. A blood smear was stained with Diff Quik. The phagocytic index was determined, after counting 100 cells, from the proportion of cells containing yeast spores and the yeast spores/cell.

Differential cell count - Whole blood was mixed with Turks solution and the total number of white blood cells $/ \mathrm{mL}$ blood was counted. A blood smear was stained with Diff Quik and a differential cell count done on 200 white blood cells (morphologically identified).

All statistical analyses were done using REML linear regression in Genstat Release 10. For each analysis, birth dam parity, rearing dam parity, pre- or post-weaning, and birthweight were included as fixed effects. Random effects included litter and shed/ pen effects.

\section{Results and Discussion}

Birth dam parity had no significant effect $(P>0.05)$ on any piglet innate immune system measures indicating that adaptive im- munity or rearing dam parity was likely to be more important in disease susceptibility.

Rearing dam parity only influenced the phagocytic index; giltreared progeny had a significantly $(P<0.05)$ higher percentage of active phagocytes compared to sow-reared progeny both pre(39\% and 34\%) and post- (48\% and 43\%) weaning. Phagocyte activity can be stimulated by inflammation which also leads to the production of acute phase proteins (APP). These results may therefore support findings by Morales et al. (2006), where it was concluded that gilt-reared piglets, who had a higher concentration of APP compared to sow-reared progeny, had greater antigenic challenge to their immune system. While further research is required, this higher antigenic challenge could result from the comparatively reduced effectiveness of the maternal antibody and cell protection that progeny receive in colostrum and milk of gilts compared to older parity sows allowing disease to occur. Alternatively, there may be more pathogens being shed by gilts than sows but this is only likely to affect pre-weaning disease susceptibility.

Piglet age had a significant effect on all innate immunity measures with a greater number and activity of immune cells postweaning compared to pre-weaning (Table 1). This was expected as immune system maturity occurs over the first 7 weeks of life (Stokes et al, 2004) and confirmed that the tests were capable of detecting this change in innate immunity.

Table 1. Model-based means for piglet innate immune system parameters

\begin{tabular}{|c|c|c|}
\hline & 2 weeks old & 7 weeks old \\
\hline \multicolumn{3}{|l|}{ Number of cells (x106 cells/mL blood) } \\
\hline Lymphocytes & $5.2^{\mathrm{a}}$ & $6.2^{b}$ \\
\hline Phagocytes (monocytes + neutrophils) & $4.1^{\mathrm{a}}$ & $7.4^{\mathrm{b}}$ \\
\hline \multicolumn{3}{|l|}{$\begin{array}{l}\text { Immune cell responsiveness } \\
\text { (IFN ү; SP ratio \%) }\end{array}$} \\
\hline Pokeweed mitogen (PWM) & $34.7 \%$ a & $78.9 \%^{b}$ \\
\hline \multicolumn{3}{|l|}{ Phagocytic Index } \\
\hline \% Phagocytes with yeast spores & $36 \%$ a & $45 \%$ b \\
\hline Number of yeast spores/cell & $1.9^{\mathrm{a}}$ & $2.5^{b}$ \\
\hline
\end{tabular}

\section{Acknowledgements}

The authors acknowledge the support of the Pork CRC, Australian Pork Ltd, Rivalea and University of Sydney.

\section{References}

Miller, Y.J. 2008. PhD thesis. University of Sydney. Australia. Miller, Y.J et al. 2006. Proc. of the 19th IPVS Congress.

Morales, J. et al. 2006. J Anim Sci. 84: 292

Stokes et al. 2004. Anim Res. 53:325-334 


\title{
0.093
}

\section{Effect of housing system on reproductive performance of gestating sows}

\author{
Annop Suriyasomboon; Mongkol Techakumphu \\ Chulalongkorn University, Bangkok, Thailand
}

\section{Introduction}

There are several factors that influencing the pig reproduction such as breeds, parity number, nutrition, season, and temperature. The harsh climate condition was a major disadvantage for pig producing in tropical climate, such as Thailand, which resulted in lower reproductive performance of sows $(1,2,3)$. In recent years, a new closed housing system, evaporative system (EVAP), has been introduced to improve the microclimate for pig farms in Thailand. EVAP is aimed to reduce the temperature inside the building by a humidification process. Some pig farmers already applied this system with the boar (4). The gestating and lactating sows were also using this housing system. To my knowledge, no comprehensive study on the effect of temperature and humidity, based on daily recordings within the EVAP stable, on the reproductive performance of gestating sows has been performed. This study was to investigate the effect of housing systems on reproductive performance of gestating sows in Thailand.

\section{Materials and Methods}

Data were collected from 13 pig farms (range from 1,000-5,000 sows) in the intensive pig raising area in Thailand during the period September 2007 to August 2008. Seven of these farms had conventional (CON) housing system and six farms had EVAP housing system for the gestating sows. All herds had been operating for at least 5 years and the EVAP herds had used this system for at least 2 years before the start of this study. Temperature and humidity were recorded automatically inside the gestating housing by using $\mathrm{Hobo}^{\circledR}$ data logger. Reproductive data were obtained from the pig's herd management program i.e. PigLIVE (Version 2.0, Live Informatics Co., Ltd., Thailand). Data were statistically analyzed using the SAS program (Version 9, SAS Institute Inc., Cary, NC, USA). ANOVA was applied to the litter size, total number of piglets born, in relation to the climate at previous weaning, at mating (NTB-m), and at farrowing.

\section{Results}

There was a higher variation and range of the maximum temperature and the minimum humidity in the CON system than in the EVAP system in all three seasons. The average maximum temperature was lower, and the average minimum humidity was higher in the EVAP system than in the CON system. There was no significant effect of housing system on any of the reproductive variables analyzed. However, the interaction between housing system and 2-month periods was significant $(P<0.01)$. The litter size of sows kept in the EVAP system was higher than sows kept in the CON system over the year. Increasing temperature resulted in lower subsequent litter size and number of born alive piglets (NBA) $(\mathrm{P}<0.05)$. Increasing humidity in the housing resulted in decrease NBA and increase number of still born piglet $(P<0.05)$.
Figure 1. Combined effect of season and housing system on litter sizes of sows.

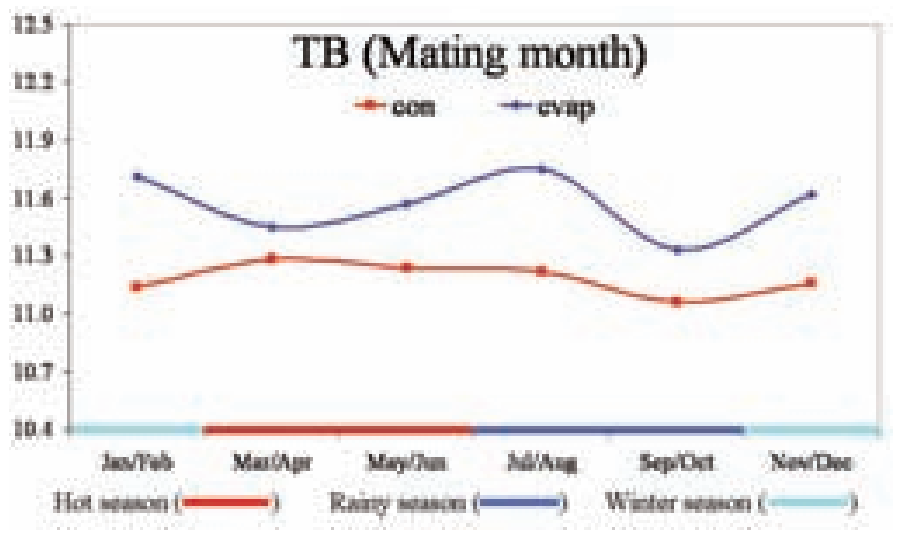

\section{Discussion}

The overall results reveal that not only elevated temperature but also elevated humidity has a negative impact on reproductive performance in sows. In this study, there was a significant interaction between housing system and 2-month periods on NTB-m, which indicates differences between seasonal variations in the ovarian function from the sows kept in the two housing systems. The smaller litter size in the CON system might be explained by a poor fertilization rate, high embryonic death, and more discomfort and/or heat stress of the sows. Thus, using the EVAP system might be an alternative choice for improve the reproductive performance of gestating sows.

\section{Acknowledgement}

This study was supported by The National Research Council of Thailand (2007).

\section{References}

1. Theriogenology 65: 606-628.

2. Theriogenology 54: 481-496.

3. Journal of Veterinary Medical Science 66, 477-482.

4. Livestock Production Science 89: 19-31. 


\title{
0.094
}

\section{A retrospective analysis of results from laboratory tests to assess the cause of reproductive failure in sows}

\author{
Heiko Nathues $^{1}$ Regina Tegeler $^{2}$ Elisabeth Grosse Beilage ${ }^{1}$ \\ 1. Field Station for Epidemiology, University of Veterinary Medicine Hannover, Bakum, Germany; \\ 2. Field Station for Epidemiology in Bakum, University of Veterinary Medicine Hannover, Bakum, Germany
}

\section{Introduction}

Diagnosis of the cause of reproductive failure in sows is seldom straightforward and is frequently unsuccessful (1). In order to confirm or to rule out infectious agents as the cause of a reproductive failure, practitioners often tend to submit material from abortions, cervical swabs, organs of the genitourinary system from slaughtered sows or serum samples for further examination to a laboratory. The aim of this study was to analyze data from a nationwide operating laboratory for routine diagnostics, where samples had been examined by standardized methods for various infectious agents that might be involved in reproductive diseases.

\section{Material and Methods}

A retrospective analysis was applied to data obtained by routine diagnostics over four consecutive years in Northwest Germany. All laboratory work was conducted and documented as specified in the ISO/IEC 17025:2005. Material from abortions (fetal lung, liver, kidney, spleen and heart muscle or placenta) was examined for the presence of PRRSV EU genotype and NA genotype (in Germany exclusively Ingelvac PRRS MLV), PCV2 and PPV, as for Leptospira and Chlamydia species by single PCR tests or by PCR screening (all five PCR tests from fetal liver). These PCR tests were performed by IVD-GmbH (Hannover, Germany). The cervical swabs and smears of organs from the genitourinary system were tested for presence of various bacteria by standard cultural methods. Serum samples were only submitted to PRRSV (EU and NA genotype) and PCV2 specific PCR tests. Data were stored in and later extracted from a SQL database.

\section{Results}

Material from abortions $(n=788)$ was examined as often as cervical swabs $(n=881)$. The detection rate for viral and bacterial pathogens by PCR was low in material from abortions (Tab. 1). On the opposite, $75.1 \%$ of all cervical swabs were tested positive for the presence of potentially pathogenic bacteria ( $E$. coli, Streptococci, Staphylococci, etc.). However, in $52.9 \%$ of all positive swabs 3 or more bacteria were identified (Fig. 1). The samples from the genitourinary organs were positive for one or two bacteria in $49.9 \%$ of all cases (Fig. 2). In serum samples, mainly originating from (weak born) suckling pigs, the average detection rate of PCV2, PRRSV EU genotype and PRRSV NA genotype was $41.6 \%, 23.1 \%$ and $12.9 \%$, respectively. During the same period, the detection rate of PCV2, PRRSV EU genotype and PRRSV NA genotype in material from abortions was $14.7 \%, 6.8 \%$ and $1.3 \%$, respectively.

\section{Discussion}

This study corroborates the difficulties in diagnosing the cause of reproductive failure by laboratory examination. When PRRSV is suspected causing reproductive failure, the impracticability of examining material from abortions compared to serum samples from (weak born) suckling pigs should be taken into account.
Considering the high number of cervical swabs contaminated with 3 or more bacterial agents, standard bacteriological examination is more feasible on genitourinary organs.

Tab. 1: Detection rate of different pathogens by PCR during 2006 and 2009

\begin{tabular}{|l|c|c|}
\hline Agent & $\begin{array}{c}\text { Average detection rate } \\
\text { (2006 to 2009) }\end{array}$ & $\begin{array}{c}\text { Range } \\
\text { (2006 to 2009) }\end{array}$ \\
\hline Chlamydia species & 1.4 & $0.0-7.7$ \\
\hline Leptospira species & 0.7 & $0.0-7.5$ \\
\hline PCV-2 & 16.3 & $3.8-37.5$ \\
\hline PPV & 2.7 & $0.0-2.9$ \\
\hline PRRSV (EU genotype) & 6.4 & $4.4-15.1$ \\
\hline PRRSV (NA genotype) & 1.3 & $0.0-4.8$ \\
\hline
\end{tabular}

Fig. 1: Results from bacteriological examination of cervical swabs

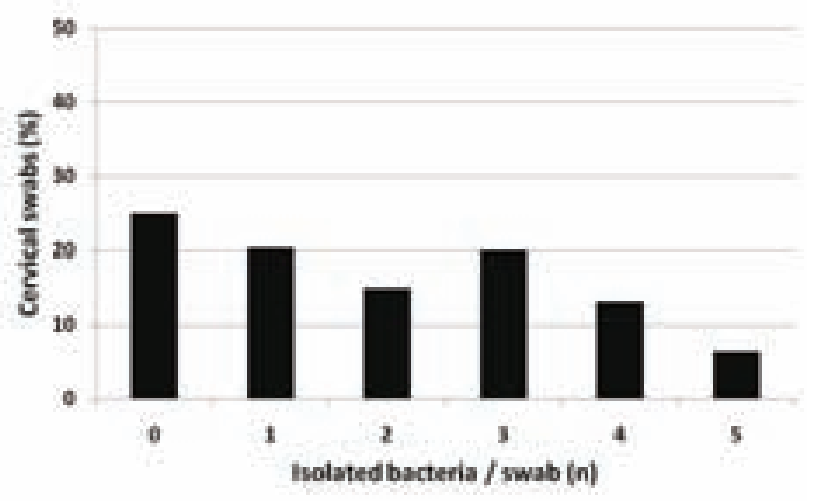

Fig. 2: Results from bacteriological examination of smears from genitourinary organs

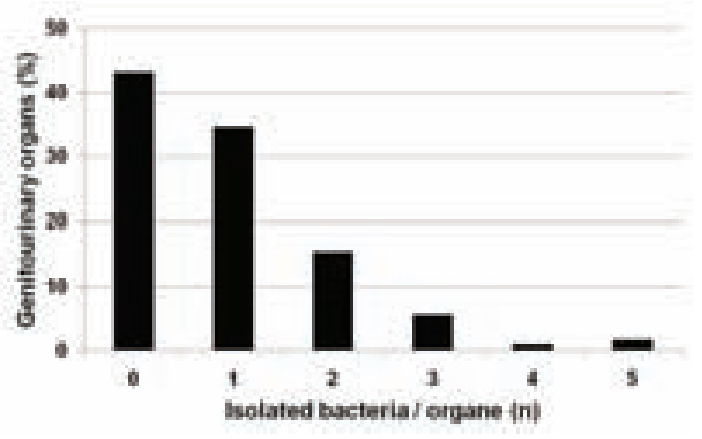

\section{References}

(1) Diseases of Swine (1999) p. 75 


\title{
0.095
}

\section{Synchronizing ovulation for fixed time artificial insemination in cyclic gilts with Porcine Luteinizing Hormone (pLH)}

\author{
$\underline{\text { Audrey C. Cameron }}{ }^{1}$ Jennifer Patterson ${ }^{1}$ Divakar J. Ambrose ${ }^{2}$ George R. Foxcroft ${ }^{1}$ Michael K. Dyck ${ }^{1}$ \\ 1. University of Alberta, Edmonton, AB, Canada; 2. Alberta Agriculture and Food, Edmonton, $A B$, Canada
}

\section{Introduction}

Optimal timing of artificial insemination (Al) in breeding programs is crucial to ensure successful fertilization and efficient production 1. Variation in the duration of standing estrus, which has direct implications for timing of ovulation, has been well established in swine2. Although a relationship between the time of ovulation after the onset of estrus, and estrus duration, has been reported in both sows 3 and gilts 4 , no other factors seem to relate to the timing of ovulation5. The recorded onset and duration of estrus also depends on the frequency of estrus detection and the observational skills of the stockperson 1. The use of exogenous hormones to control the timing of ovulation can eliminate the need for heat detection and facilitates the use of fixed-time Al procedures. Recent research has demonstrated that porcine luteinizing hormone ( $\mathrm{pLH}$ ) (Lutropin-V, Bioniche) can reliably synchronize ovulation in weaned sows 6 and gilts7 as a part of fixed time Al protocols. The primary objective of this study was to refine existing ovulation induction protocols to allow for implementation of a single fixed time artificial insemination (sFT-Al) program in cyclic gilts using porcine luteinizing hormones $(\mathrm{pLH})$.

\section{Materials and Methods}

A total of 100 known-cyclic gilts were synchronized using oral progestagens (altrenogest, $15 \mathrm{mg} / \mathrm{d}$ x 18/d; Regu-Mate, Intervet Canada) and were then randomly assigned to three different breeding protocols: 1) Control-1 (CON1) gilts received a single insemination at $158 \mathrm{~h}$ following the last altrenogest treatment (CON1, n=30); 2) Control-2 (CON2) gilts were bred using a standard 2 insemination breeding protocol. The first insemination occurred at the initial detection of standing estrus, and then again every $24 \mathrm{~h}$ later. $(\mathrm{n}=35) ; 3)$ Gilts in which ovulation was induced $(\mathrm{pLH})$ received $600 \mathrm{IU}$ equine chorionic gonadotropin (eCG; Folligon, Intervet Canada) 24 hrs after last altrenogest. They then received $5 \mathrm{mg}$ of pLH (Lutropin-V, Bioniche Animal Health Canada) 72 hours later and a sFT-Al performed 32 hours after the pLH injection $(n=35)$. Estrus detection and transcutaneous real-time ultrasonography were performed every $8 \mathrm{~h}$ $(8: 00,4: 00,12: 00)$ beginning $24 \mathrm{~h}$ after the last altrenogest treatment to determine time of onset of estrus and ovulation.

\section{Results}

LH gilts showed shorter intervals $(P<0.01)$ between the end of altrenogest treatment and the onset of standing estrus $(135.8 \pm 0.13 \mathrm{~h})$ compared to CON1 and CON2 gilts (155.0 \pm 0.13 and $153.7 \pm 0.12 \mathrm{~h}$, respectively) and between the end of altrenogest treatment and ovulation ( $154.8 \pm 0.12$ vs. $187.41 \pm 0.12$ and $187.1 \pm 0.17 \mathrm{~h}$, respectively). Furthermore, LH gilts show the least variation in the altrenogest to ovulation interval, with all gilts ovulating within a $31.9 \mathrm{~h}$ period, compared to $51.6 \mathrm{~h}$ and $72.2 \mathrm{~h}$ periods for the CON1 and CON2 animals. The Al to ovulation interval was also shorter $(\mathrm{P}<0.01)$ for the pLH animals when compared to the sFT-Al for CON1 and first Al for CON2 ( $23.4 \pm 2.75$ vs. $35.2 \pm 2.84$ and $31.4 \pm 2.74 \mathrm{~h}$, respectively). There was no significant difference in pregnancy rate between treatments with an overall average rate of $88 \%$. Similarly treatment did not affect litter size or the number of piglets born alive with overall averages of $12.5 \pm 0.7$ and $11.8 \pm 0.7$, respectively.

\section{Discussion}

These results indicate that pLH is appropriate for use in cyclic gilts, and can reliably synchronize ovulation, while shortening the period between Al and ovulation. Furthermore, the application of this ovulation induction protocol in combination with sFT-Al did not detrimentally influence pregnancy rate or litter size and therefore provided acceptable reproductive performance and efficiency. This suggests that pLH based sFT-AI protocols could be successfully applied in gilts, thereby decreasing the amount of semen needed to breed a single female and allow for the more efficient use of superior sires.
References
1. Soede et al. (1995) J. Reprod Fertil 1995;104:99.
2. Soede et al. (1997). J Reprod Fertil Suppl 52:91
3. Nissen et al. (1997) Theriogenology 47:1571.
4. Almeida et al. (2000). Theriogenology 53: 1389.
5. Langendijk et al. (2000). J Anim Sci 2000;78:3019
6. Cassar et al. (2005). J Swine Health Prod 2005;13:254
7. Degenstein et al. (2008) Theriogenology 70: 1075. 


\title{
0.096
}

\section{The effect of PG600 at weaning on primiparous sow performance}

\author{
Jennifer Patterson ${ }^{1}$ Audrey Cameron ${ }^{1}$ Taylor Smith ${ }^{1}$ Aline Kummer ${ }^{1}$ Rydell Schott ${ }^{2}$ Laura Greiner $^{3}$ Joseph Connor $^{3}$ George Foxcroft $^{1}$ \\ 1. University of Alberta, Edmonton, AB, Canada; 2. PIC North America, Hendersonville, TN, USA; 3. Innovative Swine Solutions, Carthage, IL, USA
}

Introduction. The principal goal of commercial breeding herds is to consistently meet weekly breeding targets. Weaned sows failing to return to estrus within $7 \mathrm{~d}$ after weaning contribute to missed breeding targets and increased non-productive sow days. However, in well managed contemporary sow farms, more than $90 \%$ of sows may return to estrus within 3 to 5 days after weaning (1). The primary objective of the present study was therefore to determine the response to gonadotrophin treatment (GT) at weaning in contemporary parity 1 commercial sows with lactation lengths typical of the North American swine industry.

\section{Materials and Methods}

Primiparous crossbred sows were blocked by genetic line (Pig Improvement Company (PIC) C22, $\mathrm{n}=261$; and PIC C29, $\mathrm{n}=114$ ), estimated post-farrowing weight and total born at parity 1 and then randomly allocated within block to either receive a combination dose of 400 IU eCG and 200 IU hCG (PG600, Intervet, USA, De Soto, KS) I.M. in the neck on the morning of weaning (PG group; $n=168$ ), or to be uninjected controls (CON group; $n$ $=207$ ). From the day after weaning (D1), all sows were provided twice daily fence-line contact with mature boars for stimulation and detection of estrus. Sows were bred by artificial insemination (AI) according to established herd protocols. Reproductive parameters analyzed were estrus synchronization rate (ESR) determined as the number of sows with first observed estrus within $7 \mathrm{~d}$ after weaning, weaning-to-estrus interval (WEI), proportion of sows bred that farrowed (FR), total litter size born (TB) and born alive piglets (BA) at farrowing. In addition, within each treatment, the synchrony of sows returning to estrus was determined as the proportion of sows in estrus within a 3-d period ( $d$ 4, 5 and 6 for CON vs $d$ 3, 4 and 5 for PG sows) (BRD3).

\section{Results}

Parity 1 total litter size ( $12.2 \pm 0.2$ vs $12.0 \pm 0.3)$ and estimated farrowing (194.0 \pm 1.7 vs $192.1 \pm 1.9 \mathrm{~kg})$ and weaning (189.6 \pm 1.4 vs $189.3 \pm 1.6 \mathrm{~kg}$ ) weights, were similar $(P>0.05)$ in $C O N$ and PG sows, respectively, indicating balanced allocation of sows across treatment. Lactation length ( $19.5 \pm 0.1$ vs $19.1 \pm 0.1 \mathrm{~d})$ and estimated weight loss in lactation ( $4.6 \pm 1.3$ vs $2.8 \pm 1 . \mathrm{kg})$ were also not different $(P>0.05)$ in CON and PG sows, respectively.
Considering data from all sows only results on the significant main effect of treatment are presented. Treatment did not affect ESR or BRD3 (Table 1). However, the timing of this 3-d breeding window ( $d 4,5$ and 6 for CON vs $d$ 3, 4 and 5 for PG sows) reflected a shorter $(\mathrm{P} \leq .001) \mathrm{WEI}$ in $\mathrm{PG}$ compared to CON sows. FR, TB and BA were not different. As an overall comparison of sow productivity between CON and PG sows, a combined Fertility Index (FI) was calculated and indicated no gain in productivity in response to GT treatment.

Table 1: Effects of treatment with exogenous gonadotropins (PG600) in primiparous sows at weaning or untreated controls (CON), on various parameters for those sows bred within 7 days after weaning.

\begin{tabular}{|l|c|c|c|}
\hline & CON & PG600 & P-Value \\
\hline ESR, \% & 87.4 & 92.3 & .26 \\
\hline WEI, d & $4.4 \pm 0.07$ & $4.0 \pm .08$ & $\leq .001$ \\
\hline BRD3, \% & 82.1 & 87.8 & .65 \\
\hline FR, \% & 88.8 & 87.7 & .85 \\
\hline TB & $12.8 \pm 0.3$ & $12.4 \pm 0.3$ & .36 \\
\hline BA & $12.1 \pm 0.3$ & $11.8 \pm 0.3$ & .47 \\
\hline SB & $0.5 \pm .06$ & $0.4 \pm .07$ & .30 \\
\hline FI & 993 & 1004 & - \\
\hline
\end{tabular}

\section{Discussion}

In the current study, excellent lactational and post-weaning management resulted in excellent fertility in untreated CON sows. Although GT treatment shortened the WEl, there was no effect on farrowing rates or subsequent litter sizes. GT treatment in more adverse times of the year may still be beneficial in terms of inducing more sows to show standing heat within the 7-day breeding week after weaning.

\section{Reference}

1. Poleze E. et al., Swine Health Prod. 2006;103:124-130. 


\title{
0.097
}

\section{Fertility and prolificacy following ovulation induction (by PG600 or hCG) and a single fixed time Al}

\author{
H.M J.M. Swarts ${ }^{1}$ M.A. Driancourt ${ }^{2}$ S. Ferchaud ${ }^{3}$ P. Guillouet ${ }^{3}$ K. Pere Per $^{3}$ \\ 1. ISPAH, Global Marketing Swine, Boxmeer, Netherlands; 2. ISPAH R\&D Pharmaceuticals, Beaucouze, France; 3. I.N.R.A., Rouillé, France
}

\section{Introduction}

The objective of the present study was to test the efficacy of two treatments for ovulation induction (PG600 ${ }^{\circledR}$ (400IU PMSG and 200IU HCG) and hCG (Chorulon $\left.{ }^{\circledR}\right)$ followed by a single artificial insemination (AI), on fertility and prolificacy of gilts previously synchronised with Matrix ${ }^{\circledast}$. Target is to obtain high reproductive performance while simplifying fertility-management (less time spent for oestrus detection, less Als and a more predictable timing of farrowing).

\section{Materials and methods}

The study was performed by INRA Rouillé (France). Thirty-six (36) Large White X Landrace pubertal gilts (129 kg average BW and 180 days old) were randomly assigned to three treatment groups, each including 12 animals. All gilts were synchronised by oral administration of Matrix ${ }^{\circledR}$ (15 mg altrenogest/animal) once daily for 14 consecutive days. Four days (96h) after the last administration of Matrix ${ }^{\circledR}$ (Day 0 ), animals were treated for ovulation induction as follows:

- Group 1: Non-treated animals (control)

- Group 2: I.m. administration of PG600

- Group 3: I.m. administration of hCG (500 IU)

At thirty-two hours after ovulation induction, gilts from groups 2 and 3 were inseminated once, while those of group 1 (control) were inseminated twice, 12 hours apart, at detected estrus. Ovaries were scanned (ultrasonography) starting immediately before treatment and then twice daily for the next 48 hours in order to check ovulations (based on the disappearance of the large preovulatory follicles present at earlier scans). Blood samples were collected before treatment, and 2, 4 and 7 days later, to assess changes in plasma progesterone concentrations and to document the quality of ovulation. Twenty-two days after $\mathrm{Al}(\mathrm{Day}+23)$, the pregnancy status of the gilts was checked by ultrasonography. At about thirty days after Al, all pregnant gilts were slaughtered, their ovaries collected and the numbers of total and normal foetuses were determined.

\section{Results}

A few gilts were not successfully synchronized by Matrix ${ }^{\circledR}$, reducing group size to $n=9$ in all three treatment groups.

At 48 hours after treatment, ultrasonographic scans demonstrated that ovulation was successfully induced over a $16 \mathrm{~h}$ time window in 100 and $78 \%$ of the animals treated with hCG and PG600 respectively. Only $22 \%$ of the control gilts ovulated during the same time window. Al was closely synchronized with ovulation in all hCG treated gilts and in 6/9 PG600 treated gilts. The remaining PG600 treated gilts ovulated respectively $12(\mathrm{~N}=2)$ and 24 hours $(n=1)$ later.
There was no treatment effect $(P=0.11)$ and no treatment by time interaction $(P=0.09)$ for changes in progesterone concentrations. Progesterone concentrations were low on day 0 and 2, and increased similarly in the three treatment groups to reach $22.8 \pm 6.6,18.8 \pm 4.2$ and $21.0 \pm 5.0 \mathrm{ng} / \mathrm{ml}$ in gilts treated with hCG, PG 600 and placebos respectively.

When the reproductive features of the gilts of the three groups were compared, no significant differences were demonstrated for fertility assessed by ultrasound 23 days after insemination or for the number of corpora lutea and live embryos observed 29 days after insemination.

A summary of the data describing ovulation rate, fertility, number of live embryos at slaughter and of embryonic survival (ratio between ovulation rate and total number of embryos at slaughter) is presented in the table below:

Table 1:

\begin{tabular}{|l|c|c|c|}
\hline PG600 & hCG & Control & \\
\hline $\begin{array}{l}\text { \% of ovulation 48hrs after } \\
\text { treatment }\end{array}$ & $78 \%$ & $100 \%$ & $22 \%$ \\
\hline $\begin{array}{l}\text { Fertility (ultrasound at } \\
\text { Day+23) }\end{array}$ & $8 / 9(89 \%)$ & $7 / 9(78 \%)$ & $9 / 9(100 \%)$ \\
\hline Nb of Corpora lutea & $19.0 \pm 2.2$ & $21.7 \pm 3.0$ & $21.7 \pm 3.1$ \\
\hline Nb of Live embryos & $15.3 \pm 5.1$ & $16.0 \pm 4.9$ & $17.0 \pm 5.3$ \\
\hline$\%$ embryonic survival & $80 \%$ & $74 \%$ & $78 \%$ \\
\hline
\end{tabular}

\section{Discussion and Conclusion:}

These results show the ability of an injection of PG600 or hCG to induce ovulation (when injected $96 \mathrm{~h}$ after the end of Matrix ${ }^{\circledR}$ ), hence allowing to obtain high fertility and prolificacy after a single fixed time Al (similar to that generated by multiple Als at detected estrus). Although these findings need to be confirmed on a larger sample, this study establishes that the LH activity intrinsic to the hCG and PMSG molecules is suitable for ovulation induction in swine. 


\title{
0.100
}

\section{Effect of vaccination against experimental infection with highly and low virulent Mycoplasma hyopneumoniae strains}

\author{
Iris Villarreal; Dominiek Maes; Katleen Vranckx; Dries Calus; Frank Pasmans; Freddy Haesebrouck
}

Faculty of Veterinary Medicine, Ghent University, Merelbeke, Belgium

\section{Introduction}

Mycoplasma hyopneumoniae (Mh) is the primary agent of enzootic pneumonia (EP), a contagious respiratory disease with major economical impact for the pig industry. Vaccination of piglets is widely practiced as a control strategy for this disease. However, the currently available vaccines do not induce a complete protection against colonization and transmission (2). Virulence differences ( 3 ) and protein variability (1) between Mh strains may indicate that vaccination efficacy could vary depending on the strain. The aim of this study was to assess the effect of vaccination with a commercial bacterin vaccine against low and highly virulent Mh strains at 4 and 8 weeks post infection (PI).

\section{Materials and Methods}

Ninety, 3-week-old, cross-bred (Rattlerow Seghers), Mh and PRRSV free, weaned piglets were moved to the experimental facilities of the Faculty of Veterinary Medicine, Ghent University, Belgium. After one week acclimatization, they were randomly allocated to 10 different groups housed in absolute filters (HEPA U15) to avoid spread of the pathogen between groups. Forty piglets were vaccinated $(v)$ with a one-shot vaccine (Stellamune One, Pfizer), 40 were left unvaccinated (nv) and 10 were assigned to the negative nv and non-challenged (NC) control group. The experimental design is summarized in Table 1. One low (LV) and one highly (HV) virulent Mh strain were used for experimental infection. After anesthesia, the pigs were inoculated intratracheally with $7 \times 107 \mathrm{CCU}$ of $\mathrm{Mh}$ in $7 \mathrm{ml}$ inoculum or with $7 \mathrm{ml}$ of sterile culture medium. Half of the piglets were euthanized at $4 \mathrm{w} \mathrm{PI}$ and the other half at $8 \mathrm{w}$ PI. The severity of coughing was assessed daily using a respiratory disease score (RDS) (values from 0-6). At necropsy, macroscopic lung lesions were quantified using the lung lesion score diagram of Hannan et al. (1982), ranging from 0-35. Additionally, lung tissue samples were taken from each pig to assess the severity of histopathological lesions (score from 0 to 5). Nasal swabs and bronchoalveolar lavage fluid (BALF) were collected after euthanasia and analyzed using $\mathrm{nPCR}$ to confirm presence of Mh.

\section{Results}

At $4 \mathrm{w} \mathrm{PI}$, the vHV group showed significantly milder respiratory symptoms and significantly lower macroscopic and microscopic lesion scores than the nvHV group $(P<0.05)$. The vLV group showed no significant differences for RDS, macroscopic and microscopic lesions scores, compared to the nvLV group. At $8 \mathrm{w} \mathrm{PI}$, both vHV and vLV groups had significantly lower RDS scores in comparison to the nvHV and nvLV groups, respectively $(\mathrm{P}<0.05)$. Only the vHV group showed significantly lower macroscopic lesions in relation to the nvHV group. Results are summarized in Table 2. As expected, all pigs, except for the nvNC group, were positive by $\mathrm{nPCR}$ on BALF and nasal swabs.

\section{Discussion and conclusions}

The results confirm the difference in virulence between the two strains (3), when measured during a period of either 4 or 8 weeks
PI. Vaccination significantly reduced the severity of disease in the vHV group at 4 or 8 weeks PI for most parameters. Vaccination was also beneficial in the LV groups, but the effects were less pronounced and depended on the measured parameter. Further research is warranted to assess the precise effect of vaccination on individual strains under field conditions, since combined infections might lead to more severe clinical disease in a pig herd (4).

Table1: Experimental design and number of pigs in each group.

\begin{tabular}{|l|c|c|c|c|c|}
\hline Group & Nb pigs & Vacc. & Challenge & Necropsy & \\
\hline & & D7 & D28 & 4wPI & 8wPI \\
\hline nvLV-4 & 10 & - & LV & + & NA \\
\hline nvLV-8 & 10 & - & LV & - & + \\
\hline nvLV-4 & 10 & - & HV & + & NA \\
\hline nvLV-8 & 10 & - & HV & - & + \\
\hline vLV-4 & 10 & + & LV & + & NA \\
\hline vLV-8 & 10 & + & LV & - & + \\
\hline vLV-4 & 10 & + & HV & + & NA \\
\hline vLV-8 & 10 & + & HV & - & + \\
\hline nvNC-4 & 5 & - & medium & + & NA \\
\hline nvNC-8 & 5 & - & medium & - & + \\
\hline $\begin{array}{l}\text { Pl: post infection; v vaccinated, nv non-vaccinated; LV low virulent strain, HV highly virulent } \\
\text { strain; NC non-challenged, NA not analyzed }\end{array}$ &
\end{tabular}

Table 2: Average respiratory disease score (RDS), macroscopic and microscopic lung lesions scores in the different groups

\begin{tabular}{|l|c|c|c|c|c|c|}
\hline Parameter & weeks PI & nvNC & nvLV & nvHV & vLV & vHV \\
\hline RDS & 4 & $0.00^{\mathrm{a}}$ & $0.07^{\mathrm{a}}$ & $0.45^{\mathrm{b}}$ & $0.07^{\mathrm{a}}$ & $0.12^{\mathrm{a}}$ \\
\hline & 8 & $0.00^{\mathrm{A}}$ & $0.24^{\mathrm{B}, \mathrm{D}}$ & $0.44^{\mathrm{B}}$ & $0.05^{\mathrm{A}}$ & $0.30^{\mathrm{C}, \mathrm{D}}$ \\
\hline $\begin{array}{l}\text { Macrosc. } \\
\text { Lesions }\end{array}$ & 4 & $0.00^{\mathrm{a}}$ & $2.38^{\mathrm{a}, \mathrm{b}}$ & $6.9^{\mathrm{b}}$ & $0.93^{\mathrm{a}}$ & $0.11^{\mathrm{a}}$ \\
\hline & 8 & $0.00^{\mathrm{A}}$ & $1.20^{\mathrm{A}}$ & $5.60^{\mathrm{B}}$ & $1.74^{\mathrm{A}}$ & $1.99^{\mathrm{A}}$ \\
\hline $\begin{array}{l}\text { Microsc. } \\
\text { Lesions }\end{array}$ & 4 & $1.17^{\mathrm{a}}$ & $2.90^{\mathrm{b}}$ & $4.04^{\mathrm{C}}$ & $3.10^{\mathrm{b}}$ & $3.02^{\mathrm{b}}$ \\
\hline & 8 & $1.17^{\mathrm{A}}$ & $2.92^{\mathrm{B}}$ & $3.62^{\mathrm{B}}$ & $3.41^{\mathrm{B}}$ & $3.35^{\mathrm{B}}$ \\
\hline $\begin{array}{l}\text { Different lowercase letters correspond to significantly different values between the groups at } \\
4 \mathrm{w} \text { PI; } \\
\text { different capital letters indicate significantly different values between the groups at } 8 \mathrm{~W} \mathrm{PI}\end{array}$ \\
\hline
\end{tabular}

\section{References}

1. Calus et al., 2007. Vet. Mic. 120: 284-291

2. Meyns et al., 2004. Prev Vet Med 66: 265-275

3. et al., 2003. Vet Mic. 97:177-190

4. Villarreal et al., 2009. Vaccine 27:1875-9

\section{Acknowledgements}

The study was financially supported by IWT project number 050642. Special thanks to Hanne Vereecke for all the help, effort and work dedicated to the project. 


\title{
0.101
}

\section{Prevalence and risk factors for early Mycoplasma hyopneumoniae infections in suckling piglets in different EU countries}

\author{
$\underline{\text { Iris Villarreal }}{ }^{1}$ Katleen Vranckx ${ }^{1}$ Luc Duchateau ${ }^{1}$ Frank Pasmans ${ }^{1}$ Freddy Haesebrouck ${ }^{1}$ Jens Jensen ${ }^{2}$ Ian Nanjiani $^{2}$ Dominiek Maes $^{1}$ \\ 1. Faculty of Veterinary Medicine, Ghent University, Merelbeke, Belgium; 2. Orion Pharma Animal Health, Nivå, Denmark
}

\section{Introduction}

Infections with Mycoplasma hyopneumoniae (Mh) occur worldwide and cause major economic losses to the pig industry. Knowledge about the infection pattern of this pathogen in young piglets is essential for the implementation of appropriate control measures and optimization of vaccination strategies. The aim of the present study was to investigate the prevalence of Mh infections in piglets at 3 weeks of age in different European countries by analyzing nasal swabs using nPCR. In addition, possible risk factors associated with $\mathrm{Mh}$ infection in young piglets were investigated.

\section{Materials and Methods}

The study was conducted between May 2008 and March 2009 in 9 European countries namely Belgium, Denmark, France, Germany, Hungary, Italy, Poland, Spain and The Netherlands. In each country, 6 single-site farrow-finish or sow herds were included (4 herds included in Germany). Selection criteria included herds of minimum 100 sows, presence of clinical respiratory problems related to $\mathrm{Mh}$ (coughing) in grow-finishing pigs, and no antimicrobials used against Mh in piglets less than 3 weeks of age. Thirty nasal swabs were collected per herd from piglets of $21 \pm 3$ days of age. The piglets were randomly selected from as many different sows as possible. A nested PCR ( $\mathrm{nPCR}$ ) based on a modified protocol (3) described by Stärk et al. (1998) was used for detection of Mh DNA. A questionnaire of 38 questions related to respiratory disease and herd management practices was completed during the herd visit. The questionnaire was subdivided in six categories including herd information, sow population, purchase policy, housing conditions, feeding management and health control strategies. The statistical analysis was based on a generalized mixed model using the PROC GLIMMIX procedure (SAS 9.2., 2008) with country and herd nested in country as random effects. Herd positivity was the dependent variable, whereas different potential risk factors served as independent predictor variables $(P \leq 0.10)$ in the univariate model, and were subsequently included in the multivariable analysis $(P \leq 0.05)$.

\section{Results}

In total, 52 herds and 1555 piglets were sampled for presence of Mh. The average percentage of positive pigs within the herds was $11 \%$ (95\% Cl 7.4-14.2). The min. and max. within-herd prevalence was 0 and $37 \%$. The percentage of herds with at least one positive tested pig was $68 \%$ (95\% Cl 51.7-83.4). In $32 \%$ of the herds, more than $10 \%$ of the piglets tested positive. France, The Netherlands, Italy and Germany had the highest percentage of Mh positive herds. Only in France, $100 \%$ of the herds were positive. The highest within-herd prevalences were found in Italy, France and Germany (Table 1). From the 38 variables analysed, only SIV sow vaccination was significant $(P \leq 0.05)$ and positively associated with a higher risk of a suckling piglet being $\mathrm{Mh}$ positive $(\mathrm{OR}=3.12 .95 \% \mathrm{Cl}$ 2.03-4.21).
Table 1: Prevalence of $M$. hyopneumoniae in nasal swabs of 3-week old pigs in different EU countries. Fifty-two herds were sampled, 30 piglets in each herd.

\begin{tabular}{|l|c|c|c|}
\hline Country & $\begin{array}{c}\text { Nb of positive herds/ } \\
\text { nb of herds sampled }\end{array}$ & $\begin{array}{c}\text { Nb (\%) of positive } \\
\text { piglets }\end{array}$ & $\begin{array}{c}\text { Min.-max. \% of posi- } \\
\text { tive pigs }\end{array}$ \\
\hline Belgium & $4 / 6$ & $6(3.3)$ & $0.0-10.0$ \\
\hline Denmark & $3 / 6$ & $3(1.7)$ & $0.0-03.3$ \\
\hline France & $6 / 6$ & $38(21.1)$ & $10.0-30.0$ \\
\hline Germany & $3 / 4$ & $23(18.7)$ & $0.0-26.7$ \\
\hline Hungary & $3 / 6$ & $18(10.0)$ & $0.0-30.0$ \\
\hline Italy & $5 / 6$ & $40(22.1)$ & $0.0-26.7$ \\
\hline Poland & $4 / 6$ & $17(9.7)$ & $0.0-20.0$ \\
\hline Spain & $2 / 6$ & $9(5.0)$ & $0.0-20.0$ \\
\hline The Netherlands & $5 / 6$ & $14(7.7)$ & $0.0-16.1$ \\
\hline Total & $\mathbf{3 5 / 5 2 ( 6 7 . 8 )}$ & $\mathbf{1 6 8 / 1 5 5 5 ( 1 0 . 7 )}$ & $\mathbf{0 . 0} \mathbf{0}-\mathbf{3 6 . 7}$ \\
\hline
\end{tabular}

\section{Discussion}

The results showed that in two thirds of the study herds, there was evidence of Mh infection in 3-week old piglets. In most of the positive herds, the prevalence was between 1 and 10\%. In some herds, more than $30 \%$ of the piglets was already positive. This suggests that in the selected herds, early infections with Mh are important $(1,2)$ and that infections commonly occur in European pig herds suffering from respiratory disease in the grow-finishing pigs. The higher risk of a piglet being Mh positive in case of SIV sow vaccination is difficult to explain, but it may be due to the fact that pig herds with respiratory symptoms may tend to vaccinate against SIV, overlooking the possible influence of other respiratory pathogens such as $\mathrm{Mh}$.

\section{References}

1. Fano et al., 2005. Swine Conf. Proceedings: 109-113.

2. Sibila et al., 2007.Vet Microbiol 121:352-6

3. Villarreal et al., 2009. Vaccine 27(12):1875-9

\section{Acknowledgements}

The study was financially supported by Pfizer.

Special thanks to Hanne Vereecke for all the help, effort and work dedicated to the project. 


\title{
0.102
}

\section{Safety of a novel temperature sensitive vaccine (Vaxsafe ${ }^{\circledR}$ MHP) as a tool for Mycoplasma hyopneumoniae control}

\author{
Horacio Lara-Puente $^{1}$ Francisco Quezada-Monroy ${ }^{1}$ Ruben Echeveste-Garcia de Alba ${ }^{1}$ Bernardo Lozano-Dubernard ${ }^{1}$ \\ Ernesto Soto-Priante ${ }^{1}$ David Sarfati-Mizrahi ${ }^{1}$ Abel Ciprian-Carrasco ${ }^{2}$ Joaquin Delgadillo-Alvarez $^{3}$ \\ Rima Youil ${ }^{4}$ David Tinworth ${ }^{4}$ Susana Mendoza-Elvira ${ }^{2}$ Yolanda Moreno-Morales ${ }^{3}$ Youssef Abs-EL Osta ${ }^{4}$ \\ 1. Laboratorio Avimex, Mexico City, DF, Mexico; 2. FES-C UNAM, Cuautitlan, MEX, Mexico; \\ 3. CENASA/SENASICA, Tecamac, MEX, Mexico; 4. Bioproperties Pty Ltd, Victoria, VIC, Australia
}

\section{Introduction}

Enzootic pneumonia (EP), also called mycoplasmal pneumonia, is recognized as one of the most economically significant swine respiratory diseases. The etiological agent of this disease ( $M$. hyopneumoniae, $\mathrm{Mh}$ ) attaches to the ciliated epithelial lining of the respiratory tract, resulting in impairment of the mucociliary mechanisms, predisposing to secondary bacterial infections. Vaxsafe ${ }^{\circledast} \mathrm{MHP}$ is a temperature sensitive Mh strain that is being developed by Bioproperties Pty Ltd (Australia) as the first live vaccine against EP.

\section{Materials and methods}

Forty (40) SPF pigs 3 weeks of age were used in this study. All pigs were ear tagged with an individual number, weighed and assigned to 4 study groups each containing 10 pigs. All groups were housed under the same conditions in negative pressure isolation rooms. Pigs were acclimatized for 3 days before treatment. Group 1 was the Negative control. Pigs within this group were administered vaccine diluent. Group 2 was vaccinated with Vaxsafe ${ }^{\circledR}$ MHP. Pigs within group 3 were vaccinated with a commercial inactivated vaccine. Group 4 was the positive control. Vaccination day was designated as "days post vaccination-zero" (DPV-0). Vaccination with Vaxsafe ${ }^{\circledR}$ MHP was performed by intranasal spray and the commercial vaccine by intramuscular injection. Vaccinated pigs were allowed to develop immunity over a period of 22 days and then challenged with live cultures of Mh IOWA strain 194 administered by aerosol at a dose of $\sim 10$ $6.0 \mathrm{ccu} / \mathrm{mL} / 45$ minutes in an aerosol chamber. On the first round pigs were challenged three times at 4 days apart DPV-22,-27 and -32 . All groups were then subjected to second round of challenge with a similar culture of Mh on 3 consecutive days (DPV$84,-85$ and -86). Post-mortems were performed 18 and 19 days after the second round of challenge. A number of parameters were measured including daily monitoring for clinical signs and temperature and periodic collection of nasal swabs (nPCR) and blood sampling (ELISA).

\section{Results}

Particular attention from a safety perspective was taken in the period between vaccination and immediately prior to the first challenge regime. During that period, no mortalities or clinical signs (coughing, sneezing, fever or loss of appetite) were observed in either the negative control or the vaccinated groups. Blood samples (2-5 ml) were collected from all pigs at the start of the study and at several intervals including post mortem. All samples were subjected to ELISA analysis to detect antibodies against $\mathrm{Mh}$.
Table 1. Clinical observations.

\begin{tabular}{|l|c|c|c|c|}
\hline Group & $\begin{array}{c}\text { Clinical signs at } \\
\text { various intervals }\end{array}$ & $\begin{array}{c}\text { Antibody } \\
\text { detection at } \\
\text { postmortem }\end{array}$ & $\begin{array}{c}\text { Detection in nasal } \\
\text { swabs (nPCR) } \\
8 \mathrm{dpv}\end{array}$ & $\begin{array}{c}\text { Fever (various } \\
\text { intervals) }\end{array}$ \\
\hline Negative Control & $0 \%$ & $0 \%$ & $0 \%$ & $0 \%$ \\
\hline Vaxsafe ${ }^{\oplus H P}$ & $0 \%$ & $12.5 \%$ & $55.5 \%$ & $0 \%$ \\
\hline $\begin{array}{l}\text { Commercial } \\
\text { vaccine }\end{array}$ & $0 \%$ & $77.8 \%$ & $0 \%$ & $0 \%$ \\
\hline Positive Control & $42 \%$ at 102 DPV & $42.9 \%$ & $0 \%$ & $0 \%$ \\
\hline
\end{tabular}

Nasal swabs were collected from all groups at the start of the study (DPV-0) and at DPV-8. Swabs were subjected to nPCR analysis. Mh was not detected at DPV-8 (before challenge) in pigs within the negative control group as well as groups 3 and 4. However, Mh was detected in $55.5 \%$ of pigs vaccinated with Vaxsafe ${ }^{\circledR}$ MHP (group 2) showing what appears to be a mild and transient colonization of the upper respiratory tract. (Table 1). None of the negative control pigs had detectable antibody. Pigs vaccinated with the inactivated commercial vaccine demonstrated serological response $(77.8 \%)$ while only $12.5 \%$ of the pigs vaccinated with Vaxsafe ${ }^{\circledR}$ MHP had demonstrable antibodies. In contrast $42.9 \%$ of the positive control group showed antibodies against Mh. at postmortem.

\section{Conclusions}

No adverse effects of any kind were observed during this study. Vaxsafe ${ }^{\circledR}$ MHP proved to be safe when applied in SPF pigs for the control of M. hyopneumoniae infection.

\section{Bibliography}

Lara, J.H. et al. Experimental infection with Mycoplasma hyopneumoniae in SPF pigs using an aerosol chamber. 20th International Pig Veterinary Society Congress. June 22-26, Durban, South Africa, (2008). 


\title{
0.103
}

\section{Diagnosis of Mycoplasma hyopneumoniae in growing pigs}

\author{
Erin Strait ${ }^{1}$ Cassandra Roe ${ }^{1}$ Nyssa Levy ${ }^{1}$ Carly Dorazio² Mike Kuhn $^{3}$ \\ 1. lowa State University, Ames, IA, USA; 2. TriOak Foods, West Burlington, IA, USA; 3. Pfizer Animal Health, New York, NY, USA
}

\section{Introduction}

Mycoplasma hyopneumoniae (M. hyo) is an important cause of pneumonia in swine around the world. M. hyo can be challenging to identify during the early stages of infection due to its often slow spread within a population. Currently, ante-mortem diagnosis relies primarily on detection of serum antibodies that may take weeks to months to become detectible by ELISA, or on nested PCR from nasal swabs which has lower sensitivity than samples collected from the lower respiratory tract ${ }^{1}$. Increasing the sensitivity of testing by nasal swabs would improve the utility of this sample. Recently, oral fluids have been described for the detection of PRRS in pig populations ${ }^{2}$. Oral fluids are easy to collect and capable of surveying a large number of pigs in a short period of time, but its usefulness for the detection of M.hyo has yet to be determined. This study sought to optimize testing for ante-mortem detection of M. hyo.

\section{Materials and Methods}

Samples were collected as part of a vaccine study in which 2 seeder pigs from each of 6 pens of 52 pigs were challenged with an intratracheal inoculum of strain 232 lung homogenate. Within each pen, serum and individual nasal swabs were collected from 10 pigs (excluding seeder pigs), and an oral fluid was collected from each pen at the following six time points: 4 weeks pre-challenge; at challenge; $2,4,8$, and 12 weeks post-challenge (oral fluids were not collected at 4 weeks pre-challenge). Oral fluids were collected in each pen by allowing pigs to chew on a cotton rope for 30 minutes. During the collection period the two challenged pigs were removed from the pen. Oral fluids were squeezed from the rope, transferred to a snap-cap tube and frozen until tested. Swabs and oral fluids were extracted by both a bead-based extraction method (Ambion Mag MAX-Viral Isolation Kit) and a spin-column extraction method (Qiagen QIAmp DNA mini kit). Extractions performed using the Ambion kit followed the oral fluid and total nucleic acid purification protocol. Extractions using the Qiagen kit followed the blood and body fluid protocol. Extractions were tested by a previously described primer and probe set targeting the mhp183 gene of M.hyo $^{3}$. PCR was performed using two different master mixes (Ambion VetMAX-Plus qPCR master mix or AB TaqMan Universal $P C R$ master mix) to further optimize the assay.

\section{Results}

The two extraction methods for oral fluids were compared and the spin-column kit resulted in lower $\mathrm{Ct}$ values (ie. greater sensitivity) as compared to the bead-based extraction method. The opposite was true when extraction methods for nasal swabs were compared. Based on these results, oral fluids were extracted using the Qiagen QIAmp DNA mini kit and nasal swabs were extracted using Ambion MagMAX kit. When the two different $P C R$ reagent mixes were compared the Ambion VetMAX-Plus qPCR master mix gave the most sensitive results regardless of sample type tested.

Significant numbers of PCR positives were not detected until 8 and 12 weeks post-challenge for both oral fluids and nasal swabs. Positives attributed to maternal antibody transfer were detected by ELISA at the earliest time points and the post vaccination/challenge antibodies appeared at 8 to 12 weeks, similar to the PCR results. Due to the limited number of collection time points in this study all 3 sample types detected positives post challenge at the same time point ( 8 weeks post challenge). Therefore, it was not possible to determine which of the sample types was able to detect positives at the earliest timepoint postinfection. However, differences in the sensitivities of these assays and sample types were observed. At eight weeks post challenge, $45 \%$ of serum, $72 \%$ of nasal swabs, and $100 \%$ of oral fluids were identified as positive. Stronger PCR positives were detected from oral fluid samples than from nasal swabs.

\section{Discussion}

In this study we optimized both the sample collection procedure and DNA extraction PCR protocols for nasal swabs and oral fluids and compared these results to serology. Future studies applying these techniques in the field will be needed to confirm these results, but demonstrate the potential for monitoring shedding of M.hyo through oral fluids or nasal swabs in the field.

\section{References}

1. Kurth KT, et al. 2002. J. Vet. Diag. Invest. 14, 463-469

2. Prickett J, et al. 2008. J. Vet. Diagn. Invest. 20, 156-163

3. Strait E, et al. 2008. J. Clin Microbiol, 46:8, 2491-2498 


\title{
0.104
}

\section{Increased performance after combined Mycoplasma and PCV2 vaccination}

\author{
$\underline{\text { Hanne Bak}}^{1}$ Erik Nörregård ${ }^{2}$ \\ 1. Boehringer Ingelheim AS, Copenhagen, Denmark; 2. Swedish Animal Health Service, Tommelilla, Sweden
}

\section{Introduction}

Vaccination against Mycoplasma hyopneumoniae ( $M$ hyo) is widely used in the pig industry to increase the performance of pigs. During the last years, vaccination against PCV2-virus has become more and more common, and in herds vaccinating against $P C V 2$, the usual $M$ hyo vaccination might seem unnecessary, because the pigs perform very well with PCV2 vaccination only. This side-by-side study evaluates the benefits of vaccinating against $M$ hyo in a Swedish herd already vaccinating against PCV2-virus.

\section{Material and Methods}

The study took place in a Swedish 2-site herd with groups of 45 sows farrowing every 5 th week. One batch of pigs was divided in 2 groups; 291 pigs were vaccinated against M Hyo and PCV2 (Ingelvac MycoFLEX ${ }^{\circledR}$ together with Ingelvac CircoFLEX ${ }^{\circledR}$ ) and 156 pigs were vaccinated against PCV2 only (Ingelvac Cir$\operatorname{CoFLEX}^{\circledR}$ ). Pigs were vaccinated at 3 weeks of age, weaned at 39 days of age and moved to the fattening site at 10.5 weeks of age. Each group of pigs was tattooed with different delivery numbers before slaughter. For each pig, slaughter date, carcass weight, meat $\%$ and carcass price was recorded at the slaughterhouse, and average daily weight gain (ADWG) (birth to slaughter) was calculated. Comparison of pigs vaccinated with 2 vaccines to pigs vaccinated with PCV2 only was done with Students t-test for ADWG, weight, meat $\%$, and price and with $\mathrm{W}-\mathrm{W}$ runs test for days (significance level $\mathrm{p}=0.05$ ).

Table 1: Performance from birth to slaughter.

\begin{tabular}{|l|c|c|c|}
\hline Vaccination & PCV2 + M hyo & PCV2 & Diff. \\
\hline ADWG (g/day) & 653.9 & 632.1 & $+21.8^{*}$ \\
\hline Time to slaught (days) & 172.3 & 175.6 & $-3.2^{*}$ \\
\hline Carcase weight $(\mathrm{kg})$ & 84.8 & 83.3 & $+1.5^{*}$ \\
\hline Price of carcase (€/pig) & 107.9 & 105.8 & $+2.1^{*}$ \\
\hline *=Statistically significant difference between means \\
\hline
\end{tabular}

\section{Results}

Pigs vaccinated with against both PCV2 and M Hyo showed a statistically significant improvement of performance measured as ADWG, time to slaughter, carcase weight and carcase price ( $p$ values $0.001,0.050,0.014$ and 0.011 ), compared to pigs vaccinated only against PCV2 (table 1). The meat percentage was not influenced by $M$ hyo vaccination $(p=0.558)$. In fig. 1 , the faster growth of pigs vaccinated with both vaccines is visualized. The increase in the price of the carcases was 3.6 times as high as the vaccine price.
Fig. 1: Number of days from birth to slaughter

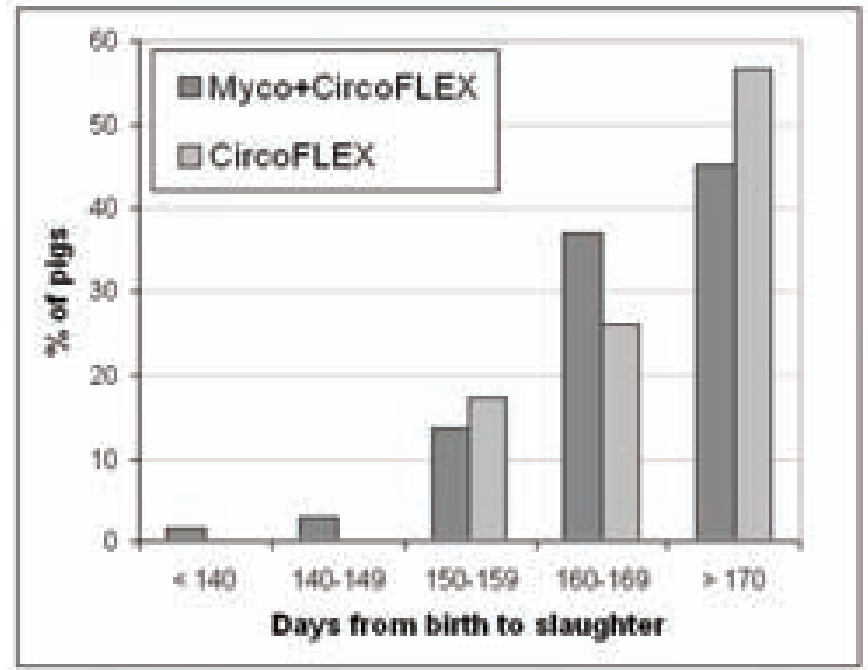

\section{Discussion and Conclusions}

The present herd had a good performance with PCV2 vaccination only, shown by an average daily weight gain of $632 \mathrm{~g} /$ day from birth to slaughter. There was no evidence of $M$ hyo problems, neither clinically or in the carcass remarks from the slaughterhouse.

Still, the performance was significantly improved by $M$ hyo vaccination, increasing the mean price of the carcass by $2.1 €$ per pig. Furthermore, the pigs grew faster, meaning that the farmer would also save food expenses and empty the barn sooner to put in new pigs.

Therefore, vaccination against $M$ hyo on top of the PCV2 vaccination gave a payback of more than 3.6 times the investment in the $M$ hyo vaccine. 


\title{
0.105
}

\section{Summary of Mycoplasma Hyopneumoniae Eradication Experiences Completed in Upper Midwest USA}

\author{
Paul E. Yeske
}

Swine Vet Center, St. Peter, MN, USA

\section{Introduction}

Mycoplasma hyopneumoniae continues to be an economically important respiratory infection in swine. There are a number of different ways to eradicate Mycoplasma from herds. Adaptations to the Swiss method have also been successful at eradication. These would include herd closures allowing the herds to continue to farrow during the process 1,2 . Vertical transmission studies demonstrate that transmission occurs up to 8 months post infection3. Herd closures have typically been 8-9 months duration and may or may not have off site breeding projects incorporated. These procedures have included a whole herd medication program at the end of the closure.

Another method is whole herd medication without herd closure using a long acting antibiotic such as Draxxin ${ }^{\circledR}(10 \%$ Tulathromycin, Pfizer), treating all pigs on site with an injection and repeating in 2 weeks4. The advantage of this approach is that the herd returns to negative status faster.

This study looked at results of herds that have been through various versions of Mycoplasma hyopneumoniae eradication procedures and the duration of time that the herds have stayed negative.

\section{Materials and Methods}

- Identified 23 herds that had been through a Mycoplasma hyopneumoniae eradication procedure.

o 16 herds used a herd closure and whole herd medication program.

o 7 herds did a whole herd injection of medication program and no closure.

- All farms had negative replacement breeding stock available.

- Herd's mycoplasma status where monitored using: pigs in the finishing flow and blood testing just prior to marketing.

o Clinical signs of Mycoplasma (coughing) in sow herds or finishing pigs.

o Serology of pigs at the end of the finishing phase.

o Using IDEXX test if vaccinated

\section{Results}

Herd closure and medication (C) and whole herd medication using Draxxin ${ }^{\oplus}(\mathrm{M})$ were $81 \%$ and $57 \%$ successful overall respectively, and $94 \%$ and $71 \%$ successful for a period greater than 1 year. The average "survivability" of herds in the study was 31 and 20 months respectively as shown in Table 1.

\section{Discussion}

Herd closure and medication (C) has been more successful, although both methods of Mycoplasma hyopneumoniae eradication were successful over time. However implementing herd closure takes longer to obtain Mycoplasma negative pigs, and depending on the economic advantage the whole herd medication may be more attractive. Each farm will have its own unique goals and limitations that must be considered but these 23 herds represent what can be done with the various programs being used in the field in pig dense areas of production.

Table 1. Mycoplasma hyopneumoniae eradication success in 23 farms by type: herd closure and medication (C); medication (M)

\begin{tabular}{|c|c|c|c|c|c|c|}
\hline Date & \# Sows & Type & Pos & Neg & $>1 \mathrm{Yr}$ & Months \\
\hline Dec 03 & 2500 & $C$ & & 1 & & 74 \\
\hline Apr 06 & 1200 & $C$ & & 1 & & 46 \\
\hline May 04 & 2500 & $C$ & & 0 & 1 & 49 \\
\hline Sep 06 & 2500 & $C$ & & 1 & & 41 \\
\hline Sep 06 & 600 & $C$ & & 1 & & 41 \\
\hline Mar 06 & 1350 & $C$ & & 0 & 1 & 18 \\
\hline Sep 07 & 5000 & $C$ & & 1 & & 29 \\
\hline Apr 06 & 2500 & $C$ & & 1 & & 46 \\
\hline Apr 06 & 900 & $C$ & 1 & 0 & & 3 \\
\hline Sep 06 & 2500 & $C$ & & 1 & & 41 \\
\hline Apr 07 & 900 & $C$ & & 1 & & 34 \\
\hline Sep 07 & 2500 & $C$ & & 1 & & 29 \\
\hline May 08 & 2500 & $C$ & & 1 & & 21 \\
\hline Sep 08 & 1700 & $C$ & & 1 & & 17 \\
\hline Sep 09 & 2500 & $C$ & & 1 & & 5 \\
\hline Sep 09 & 1350 & $C$ & & 1 & & 5 \\
\hline Total & 33000 & & 1 & 13 & 2 & 495 \\
\hline Success at herd level & & & & $81 \%$ & $94 \%$ & 31 \\
\hline Population & & & & 29150 & 32100 & \\
\hline $\begin{array}{l}\text { Success at population } \\
\text { level }\end{array}$ & & & & $88 \%$ & $97 \%$ & \\
\hline Aug 06 & 3500 & $M$ & & 1 & & 42 \\
\hline Sep 06 & 900 & M & & 0 & 1 & 13 \\
\hline Nov 06 & 3500 & $M$ & & 1 & & 39 \\
\hline Oct 07 & 1350 & $M$ & 1 & 0 & & 10 \\
\hline Nov 07 & 900 & $M$ & 1 & 0 & & 9 \\
\hline Sep 08 & 900 & $M$ & & 1 & & 17 \\
\hline Mar 09 & 900 & $M$ & & 1 & & 10 \\
\hline Total & 11950 & & & 4 & 1 & 139 \\
\hline Success at herd level & & & & $57 \%$ & $71 \%$ & 20 \\
\hline Population & & & & 8800 & 9700 & \\
\hline $\begin{array}{c}\text { Success at population } \\
\text { level }\end{array}$ & & & & $74 \%$ & $81 \%$ & \\
\hline
\end{tabular}

\section{References}

1. Schneider P. 2006. Proc. Allen D Leman Swine Conference, 82-86

2. Yeske P. 2007. Proc. American Association of Swine Veterinarians 367- 370

3. T orremorell M. et al. 2007 Proc. American Association of Swine Veterinarians 9-10

4. Yeske P 2008 Proc. IPVS Durban, OR.02.25 


\title{
0.106
}

\section{Effect of stopping Mycoplasma hyopneumoniae vaccination in pigs}

\author{
John Carr \\ Portec Australia, Belmont, WA, Australia
}

\section{Introduction}

The timing of the Mycoplasma hyopneumoniae vaccine was changed from weaning to $30 \mathrm{~kg}$ live weight and then the vaccine was stopped.

\section{Materials and methods}

A 140 sow weekly batch outdoor unit was selected for the trial. 1330 pigs a batch were weaned. The unit was PRRSv and SIV negative. No PMWS had been diagnosed on the farm. The farm was Mycoplasma hyopneumoniae and Actinobacillus pleuropneumoniae 1 and 15 positive. A few cases of Glässer's were seen. Post-weaning mortality was normally $3 \%$ primarily associated with enzootic pneumonia complicated with pasteurella and streptococci and hind limb weakness and lameness.

A change in vaccination timing from 4 weeks (farrowing) to 10 weeks of age (nursery) was made in response to stockpeople's request.

Following a 17 weeks trial the timing was moved back to 4 weeks of age. At this stage it was agreed to examine the impact of non vaccination - thus providing a 6 week non-vaccinated group. The pigs were monitored by clinical signs, post-weaning mortality and slaughterhouse examination.

\section{Results}

\section{Change of timing of vaccination}

The arrow indicates the start of the trial to administer vaccine at 10 weeks of age

There was no impact on postweaning mortality with vaccine timing changed from 4 to 10 weeks of age.

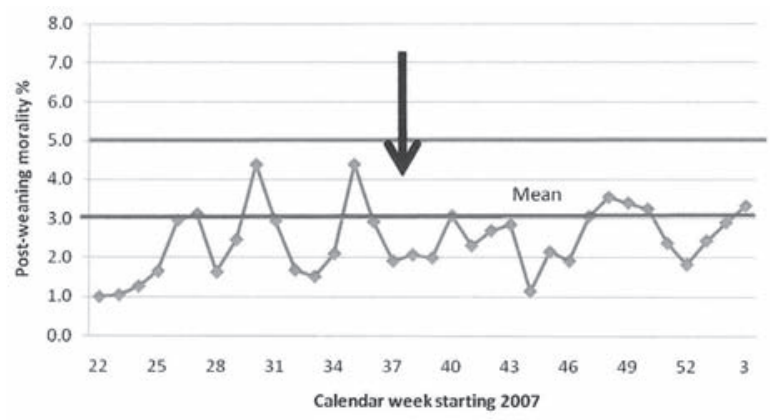

The lower line is the mean post-weaning mortality at 3\%, the upper line $2 \mathrm{sd}$ above the mean. At no point was the production system "out of control".

\section{Slaughterhouse examination}

There was no change in any slaughterhouse parameter associated with the change of timing from 4 to 10 weeks of age. However, the stockpeople complained of the increased effort in vaccination.

\section{Cessation of Mycoplasma vaccination}

To restore the vaccination at weaning a 6 week period of non vaccination was practiced.

The arrow indicates the 6 batches finishing without vaccine.

The lower line is the mean the upper line 2 sd above. The system was out of control 4 out of 6 batches (big dots). Over the 6 weeks a post-weaning mortality of $5.4 \%$ cf. normal $3 \%(p=0.007)$.

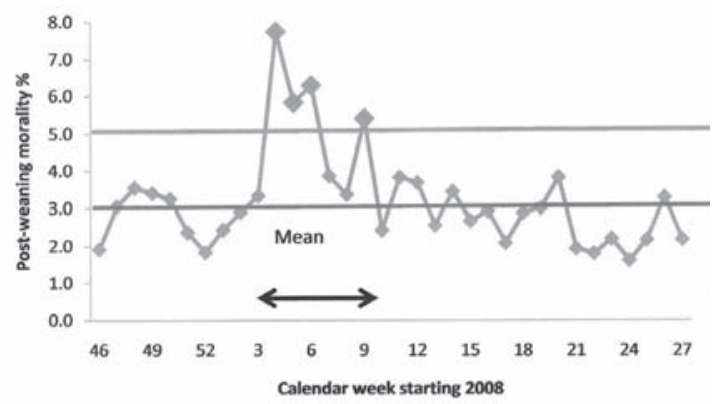

The mortality occurred in 85 to $95 \mathrm{~kg}$ pigs with clinical signs of severe complicated pneumonia.

\section{Slaughterhouse examination}

Enzootic pneumonia score: Q1 3 Q2 3 Q3 4 Q4 1.5 Q1 12 Q2 2.5 Q3 3.5 Q4 0.5

In addition to increase in enzootic/mycoplasma pneumonia scores, pleurisy increased to $35 \%$, normal less than $2 \%$. Pleuropneumonia like lesions increased to $10 \%$ normal less than $1 \%$. Pericarditis increased to $6 \%$ normally less than $1 \%$. There was no change in dermatitis, white spot, nephritis or ileitis scores.

Cost implication: $(\$ \mathrm{Au})$

Extra-mortality - loss of 2,605kg deadweight/ batches - @ $\$ 3.40$ a kg cost of production, removing the food not eaten - $\$ 7,781$ loss/batch

Loss of production - a 12 lung score equates to an increase in FCR of 0.15 . Thus an extra 21 tonnes per batch - @ $\$ 370$ a tonne $\$ 7,789$ extra per batch

Additional costs per batch - $\$ 15,869$ or $\$ 12.30$ a pig.

Cost of the Mycoplasma vaccine - $\$ 1.70$ (including labour and equipment) - with 1330 pigs a batch weaned - total costs $\$ 2261$ a batch.

Extra medication are not included in these costs.

\section{Discussion}

There was a 7:1 benefit of vaccination with the Mycoplasma hyopneumoniae vaccine. The benefit was shared between the cost of mortality of a nearly finished pig and the poorer food conversion of the remaining pigs. There was no impact, in a PRRSv negative herd, of vaccinating at weaning or 10 weeks of age. Please visit our website www.portec.com.au 


\title{
0.107
}

\section{Application of Mycoplasma hyopneumoniae P146 genotyping to an outbreak investigation}

\author{
Daniel Linhares ${ }^{1}$ Simone Oliveira ${ }^{2}$ Montserrat Torremorrell ${ }^{1}$ \\ 1. College of Veterinary Medicine, University of Minnesota, St Paul, MN, USA; \\ 2. Veterinary Diagnostic Laboratory, College of Veterinary Medicine. University of Minnesota, St Paul, MN, USA
}

\section{Introduction}

Enzootic pneumonia (EP) is an important respiratory disease of swine caused by Mycoplasma hyopneumoniae (Mh). EP impacts the performance of growing and finishing pigs by reducing growth rate, increasing mortality and worsening feed efficiency.

Mh negative pig farms have productivity advantages compared to positive systems. Therefore there is a premium in maintaining systems Mh negative. However, negative systems may become contaminated and in those cases it becomes critical to understand the source of Mh infection.

P146 is a lipoprotein that plays a role in Mh adhesion. It is located in the surface of the bacterial cell and contains a series of serine repeats. The gene that codes for these serine repeats was recently used for characterization and comparison of $\mathrm{Mh}$ isolates from different European herds and also from different continents (Mayor et al, 2007; 2008).

This study describes a case where Mh genotyping using sequencing of the P146 gene was used to investigate the source of contamination of a Mh negative system.

\section{Materials and Methods}

Herd $A$ is a 1,000 daughter nucleus sow herd that delivers gilts and boars to multiplier and commercial farms. Herd A was monitored on a regular basis by weekly veterinary visits and monthly serology screening including Mh. Additionally, slaughter checks were also performed on a monthly basis.

On August 2007, 2 out of 30 pigs tested positive by serology in the routine herd testing. No lung lesions or clinical signs were observed at that time. Discrete clinical signs were observed on nursery pigs in early September. Infection was confirmed when additional samples tested positive and clinical signs of Mh infection were evident by the end of September.

Herds B, C and D were 800,750 and 1,000 sow farms respectively that had Herd $A$ as the only source of replacement animals for at least 2 years. These herds were also Mh negative based on at least 4 years of regular serology and clinical monitoring. The last animal introduced in herd B was 5 boars (July 07), 63 gilts in herd C (June 07) and 30 gilts in herd D (May 07). Herds C and D did not have quarantine and received animals directly into their herds. Herd $B$ had a quarantine unit where animals were allowed to enter the herd after completion of Mh serology in $100 \%$ of the animals.
On September 07 herds C and D started developing clinical signs suggestive of Mh infection. Infection was confirmed by serology and positive PCRs of pneumonia lesions. In Herd B, however, no clinical signs were observed until December 07, when Mh infection was confirmed by serology and PCR of lungs of coughing pigs.

On December 07, lungs from two coughing pigs were collected in all aforementioned farms and also from eight $\mathrm{Mh}$ positive herds that were unrelated to those farms. Positive samples were sequenced by targeting the P146 gene segment and results were compared among all isolates. Diagnostic testing was conducted at the $U$ of Minnesota Veterinary Diagnostic Laboratory.

\section{Results and Discussion}

Considering that Herd $A$ was the only source of replacement animals for Herds B, C and D, Herd A was considered the most probable source of contamination for these herds. However, the timing of seroconversion and development of clinical signs did not match the current knowledge of Mh disease incubation, especially regarding Herd $B$ that received animals in July but clinical signs and evidence of infection was not detected until December. This delay may be explained by the low number of animals introduced, only 5 boars, and the slow spread of the infection in that population.

Considering this unusual situation, we needed a new approach to help the investigation and to answer whether the Herd A was indeed the source of infection of the other farms. The Mh isolate sequence of Herds B, C and D matched the sequence information of Herd A. These results suggested that the most likely source of infection in the studied herds was indeed the direct introduction of animals from Herd A. In addition, six out of the eight isolates recovered from the unrelated herds had a different sequence indicating that P146 gene sequencing had a good discriminatory ability to detect genetic differences among $\mathrm{Mh}$ isolates. In conclusion, P146 sequencing appears to be a useful tool in the aid of investigating $\mathrm{Mh}$ infections.

\section{References}

Mayor D et al. Vet Res. 2007;38(3):391-398.

Mayor D et al. Vet Microbiol. 2008;127(1-2):63-72. 


\title{
0.109
}

\section{Comparison of mycoplasmal lung lesion scoring methods}

\author{
Brad J. Thacker ${ }^{1}$ Erin L. Strait ${ }^{2}$ Lyle D. Kesl ${ }^{3}$ \\ 1. Intervet/Schering-Plough Animal Health, Desoto, KS, USA; 2. Veterinary Diagnostic Laboratory, \\ lowa State University, Ames, IA, USA; 3. Veterinary Resources, Ames, IA, USA
}

\section{Introduction}

There are several methods available for determining the level of pneumonia in swine lungs. These methods are often based on assessing the amount of visible lesions on the surface of the lung followed by calculating the percentage of the lung surface that exhibits the lesions to provide an overall lung score. The main methods evaluated in this paper included: 1) sketching the lesions onto a standard diagram followed by digital image analysis in order to determine the percentage of the lung surface that exhibits lesions ${ }^{1} ; 2$ ) the "PigMon" method where the extent of lesions in each of the seven lung lobes is estimated followed by placing these scores into a formula that represents the relative contribution of each lobe to the total lung $\mathrm{area}^{2}$; and 3) a method used by the first author where each lobe is scored separately followed by incorporation into a formula based on the relative weight of each of the lobes to the total lung weight. ${ }^{3}$ In addition, a different formula (BC) was used to calculate the total score from the individual lung lobe scores. ${ }^{4}$

\section{Materials and Methods}

The lungs ( $n=58$ ) evaluated in this study were part of a Mycoplasma hyopneumoniae vaccination/challenge study. At necropsy, lesion observations were done by: 1) Sketch- lesions were recorded onto a standard diagram; 2) VR (Veterinary Resources)- percent lesions in each lobe was recorded by the "PigMon" evaluator (third author); 3) BT- percent lesions in each lobe was recorded by the first author (BT) and 4) BTSK- sketches were scored using the BT observation method. This raw data was then incorporated into three formulas (PigMON, BT, BC) of which the contribution of each lobe to the total lung is presented in Table 1. An overall lung score was also assigned by the first author (BT/Total). The sketches were analyzed by digital imaging and the percent lesions generated by this method were considered the standard value for comparison with the other scoring methods. The scores generated using the various combinations of observation methods and overall score calculations were compared against the standard value on an individual pig basis. The percent differences between methods were calculated and correlations were performed.

\section{Results}

The overall lesion scores (\% pneumonia) by each method, differences between the methods and correlations to the standard values are presented in Table 2 . The overall percent pneumonia by the standard method (Sketch-Image) was $8.46 \%$. The average scores by the other methods varied from $5.99 \%(-2.47 \%)$ to $9.89 \%(+1.43)$. R-square values (correlation) varied from 0.844 to 0.986.

\section{Discussion}

The best combination of observation and calculation methods appeared to be the BT observation method from the sketch (BT-SK) along with the BC formula to generate the overall score. Accordingly, this method can be used in place of the digital image analysis which is fairly expense. In addition, the sketch provides a permanent record of the lung lesions which enables evaluation at a later time.

Table 1: Lung lobe contributions for calculations (\%)

\begin{tabular}{|l|c|c|c|}
\hline Lobe & PigMon & BT & BC \\
\hline Left Cranial & 10 & 4 & 5 \\
\hline Left Middle & 10 & 9 & 6 \\
\hline Left Caudal & 25 & 25 & 29 \\
\hline Accessory & 10 & 5 & 5 \\
\hline Right Cranial & 10 & 7 & 11 \\
\hline Right Middle & 10 & 15 & 10 \\
\hline Right Caudal & 25 & 35 & 34 \\
\hline
\end{tabular}

Table 2: Comparison of scoring methods

\begin{tabular}{|l|c|c|c|c|}
\hline Observation & Calculation & Pneum \% & Diff. vs. Image & $\begin{array}{c}\text { R-square vs. } \\
\text { Image }\end{array}$ \\
\hline Sketch & Image & 8.46 & NA & NA \\
\hline VR & PigMon & 7.44 & -1.02 & 0.922 \\
\hline BT & Total & 9.12 & +0.66 & 0.931 \\
\hline BT & BT & 9.48 & +1.02 & 0.948 \\
\hline BT-SK & BT & 9.89 & +1.43 & 0.986 \\
\hline VR & BT & 7.20 & -1.26 & 0.925 \\
\hline BT & BC & 8.37 & -0.09 & 0.950 \\
\hline BT-SK & BC & 8.76 & +0.30 & 0.986 \\
\hline VR & BC & 5.99 & -2.47 & 0.930 \\
\hline BT & PigMon & 9.71 & +1.25 & 0.844 \\
\hline BT-SK & PigMon & 9.73 & +1.27 & 0.978 \\
\hline
\end{tabular}

\section{References}

1. Thacker, EL, et al. JSHAP 6:107-112, 1998.

2. Davies, PR, et al. JAVMA 209:823-826, 1996.

3. Thacker BJ, et al. Proc IPVS, Rio de Janeiro, Brazil, p. 66, 1988.

4. Christensen, B. Diseases of Swine 8th ed.Ames, lowa: Blackwell Publishing; 1999:913-940. 


\title{
0.111
}

\section{Comparison of four ELISAs for detection of antibodies against different genetic subtypes of European genotype PRRSV}

\author{
Tomasz Stadejek; Ewelina Skrzypiec; Katarzyna Chabros; Zygmunt Pejsak; Katarzyna Podgorska
}

National Veterinary Research Institute, Pulawy, Poland

\section{Introduction}

Porcine reproductive and respiratory syndrome virus (PRRSV) strains belong to the two genotypes which are antigenically different. The genetic diversity in the European genotype (EUPRRSV) is much higher than in the North American genotype (NA-PRRSV) (3). The evidence of the restricted serological cross reactivity between the genetic subtypes exists $(1,3)$ but it is unknown whether it impacts the sensitivity or specificity of the ELISA diagnostic tests methods. To address this problem 175 sera from farms where different genotypes and genetic subtypes circulated were tested with three commercial and one in house ELISA tests.

\section{Materials and Methods}

Serum samples were obtained from 3 Polish, 4 Belarusian and 1 Ukrainian farms, where different genotypes and subtypes circulated as it was determined by PCR and DNA sequencing. In 2 of 3 Polish farms subtype 1 of EU-PRRSV circulated. In one farm the NA-PRRSV strain was present. In Belarusian and Ukrainian farms diverse East European subtypes circulated (3). Additionally, 31 samples from pigs from farms free from PRRSV were used. They included 17 sera from Sweden that earlier were found to react false positive in HerdChek PRRS 2XR (IDEXX) ELISA and negative in IPMA test. The samples were tested with HerdChek 2XR and $3 X$ (IDEXX), Ingezim PRRS DR (Ingenasa) and the in house test produced in our laboratory. The Ingezim test is specific for EUPRRSV while the remaining tests are specific for both genotypes. Results were compared for each pair of ELISA tests separately using McNemar's test.

\section{Results}

All ELISAs detected PRRSV specific antibodies in sera from all farms were EU-PRRSV infection was present. Both IDEXX tests and the in house ELISA detected antibodies to NA-PRRSV in all sera from the Polish farm were this genotype circulated while Ingenasa test detected seroconversion in only 5 of 12 of the samples. In case of samples from one Ukrainian farm infected by EU-PRRSV subtype 2 the Ingezim test appeared to be significantly more sensitive than PIWet and HerdChek X3 tests. The analysis of the specificity proved it to be highest in case of the HerdChek X3 test. The HerdChek 2XR test gave false positive results with most of the Swedish sera as it was found before.

\section{Discussion}

Very high genetic diversity of PRRSV poses a serious problem for PCR diagnosis, especially for the detection of the European genotype. However, our preliminary data show that it does not influence serological diagnosis by ELISA. All tests used in the study were sensitive in detection of antibodies induced by different, diverse genetic subtypes of EU-PRRSV. However, the $3 \mathrm{X}$ test seemed to be slightly less sensitive than the older $2 \mathrm{XR}$ test and the Ingezim DR. The $3 X$ test was the most specific test of all. Further studies are necessary to fully evaluate the impact of the antigenic diversity of the EU-PRRSV on sensitivity and the specificity of the serological methods of PRRS diagnosis.

\section{Acknowledgements}

The study was funded by a grant No N N308 265136 from the Polish Ministry of Science and Higher Education.

\section{References}

1. Karniychuk et al. Proc. 2009 International PRRS Symposium, Chicago, p. 74.

2. Stadejek et al. Medycyna Weterynaryjna 63, 1336-1341, 2007. [in Polish]

3. Stadejek et al. Arch Virol 153, 1479-1488, 2008.

\begin{tabular}{|l|c|c|c|c|c|}
\hline Farm and & Number of \\
genotype & samples & $\begin{array}{c}\text { \% of positive } \\
\text { samples }\end{array}$ & $\begin{array}{c}\text { HerdChek } \\
\text { 2XR }\end{array}$ & $\begin{array}{c}\text { HerdChek } \\
\text { X3 }\end{array}$ & Ingezim DR \\
\hline PL1 (EU sub 1) & 10 & 80.0 & 90.0 & 80.0 & 100.0 \\
\hline BY1 (EU) & 6 & 100.0 & 100.0 & 100.0 & 100.0 \\
\hline BY2 (EU sub 3) & 9 & 100.0 & 100.0 & 100.0 & 100.0 \\
\hline PL2 (EU sub 1) & 24 & 95.8 & 91.7 & 95.8 & 87.5 \\
\hline BY3 (EU) & 8 & 62.5 & 87.5 & 50.0 & 100.0 \\
\hline BY4 (EU sub 3) & 28 & 67.9 & 75.0 & 57.1 & 67.9 \\
\hline PL (NA) & 12 & 100.0 & 100.0 & 100.0 & 41.7 \\
\hline UA (EU sub 2) & 16 & 56.3 & 75.0 & 62.5 & 100.0 \\
\hline BY (EU) & 31 & 90.3 & 96.8 & 96.8 & 96.8 \\
\hline
\end{tabular}




\title{
0.112
}

\section{PRRSV-neutralization test - a tool for quality control?}

\author{
Jens Boettcher; Armin Gangl; Britta Janowetz; Michaela Alex; Hermann Niemeyer
}

Bavarian Animal Health Service, Poing, Germany

\begin{abstract}
Introduction
Vaccination against PRRSV is frequently practised in Germany to control PRRSV. Attenuated live vaccines of the North American (NA) and European (EU) genotype are used. Early after infection antibodies are detected in ELISA, however neutralizing antibodies $(\mathrm{nAb})$ are detected only at low titre and late $(>28$ days) after infection. Additionally, detection of nAb depends on the virus isolate. Assuming that induction of homologous $\mathrm{nAb}$ against vaccine virus after vaccination is a positive quality criterion, a PRRSV-neutralization test (NT) using vaccine virus was established. Data obtained by analysis of field cases will be presented.
\end{abstract}

\section{Material and Methods}

NT with 100 CID50 of NA- and EU-vaccine virus, respectively, was performed on Marc145 cells with $2 \%$ guinea pig complement (BÖTTCHER et al., 2006). Culture was checked for CPE until day 6. Results are given as titre: $<2,2,2.8,4,5.6,8, \geq 11.2$.

Sera were tested by PRRSV-ELISA (Idexx) according to manufacturer's instruction. A nested RT-PCR was performed according to PESCH et al. (2000). Blood was collected from gilts, sows at 1. and 5. parity and piglets after weaning 4, 8, 12 weeks of age. 10 animals per group were tested. For calculation of geometric mean of NT-titre a titre of $\geq 11.2$ was scored as 11.2.

\section{Results}

1. In herds with an acute outbreak of PRRSV and absence of vaccination with NA-PRRSV, sera with high EU-NT-titres $(\geq 11,2)$ were negative for $n A b s$ to NA-PRRSV. Sera with high NA-NT-titres $(\geq 11,2)$ from herds free from EU-PRRSV and vaccinated with NA-PRRSV were negative in EU-NT. Thus cross-neutralization between genotypes seems unlikely.

2. Comparison of ELISA and NT revealed that $30 \%$ of ELISAnegative sera were positive in at least one EU- or NA-NT; in contrast $67 \%$ of ELISA-positive sera were positive in NT, too. This difference between tests is explained by longer persistence and late induction of $n A b$, respectively.

3. As a consequence of an acute PRRSV-outbreak high NT-titres were observed in sows; maternally derived nAb (MDnAb) controlled infection in piglets until 12th weeks of age. PRRSV was detected in piglets with MDnAb by PCR when the geometric mean of the NT-titre dropped below 4.
4. Regarding vaccination $90 \%$ of piglets free of MDnAbs at vaccination (14-21 days p.n ) were positive in PCR until 8 weeks of age. A slight increase of NT-titre was observed until the 12 th week of age. In contrast, only $10 \%$ of piglets with a mean NT-titre of MDnAb of 7 at vaccination were positive in PCR two days p.vacc.

5. Low or no NT-titres were frequently detected month after vaccination.

6. Sows of 5th parity had generally lower titres than sows of 1 st parity, unless recent acute outbreaks in the herds were reported. Vaccination every 4 month was not effective in raising NT-titres in these animals. As a consequence offsprings of these sows are earlier susceptible to infection.

\section{Discussion and Conclusions}

PRRSV NT is a powerful tool in order to determine at least one side of PRRSV immunity; however, it is laborious and expensive. As a consequence NT should be substituted by an appropriate ELISA.

It is questionable if vaccination in the presence of homologous $\mathrm{nAb}$ is effective. This is especially important for piglets with MD$\mathrm{nAb}$. Thus prediction of an optimized time-point of vaccination is necessary. Additionally, gilts should be checked by NT before they are introduced into infected herds.

Extend of cross-neutralization within genotypes especially EU is still not sufficiently analysed. If antigenic heterogeneity of PRRSV isolates due to its high frequency of mutation is a real problem like in Influenzavirus A, Bluetonguevirus, Foot-and-Mouth-Disease Virus etc., serotypes have to be established. NT is the test of choice for establishment of serotypes.

\section{Acknowledgements}

This study was financially supported by the Free State of Bavaria and the Bavarian Joint Founding Scheme for the Control and Eradication of contagious Livestock Diseases.

\section{References}

Böttcher, J., M. Ritzmann, A. Gangl (2006): Felduntersuchungen mit einem "Porcine Reproductive and Respiratory Syndrome Virus" (PRRSV) Neutralisationstest. Tierärztl. Umschau, 61, 550 - 559

Pesch, S., C. Meyer, V.F. Ohlinger (2005): New insights into the genetic diversity of European porcine reproductive and respiratory syndrome virus (PRRSV). Vet. Microbiol. 107:31-48. 


\title{
0.113
}

\section{IL-10 and TGF $\beta$ expression in the lung and tonsil of pigs experimentally infected with porcine reproductive and respiratory syndrome virus}

\author{
Jaime Gómez-Laguna ${ }^{1}$ Irene M. Rodríguez-Gómez¹ Inmaculada Barrancoํㅜ Francisco J. Pallarés² \\ Francisco J. Salguero ${ }^{3}$ Antonio Bernabé2 Librado Carrasco ${ }^{1}$ \\ 1. Dept. of Anatomy and Comparative Pathology, Faculty of Veterinary Medicine, University of Córdoba, Córdoba, Spain; \\ 2. Dept. of Anatomy and Comparative Pathology, Faculty of Veterinary Medicine, University of Murcia, Murcia, Spain; \\ 3. Veterinary Laboratories Agency, New Haw, Addlestone, Surrey, UK
}

\section{Introduction}

Porcine Reproductive and Respiratory Syndrome (PRRS) is characterized by inducing an equivocal immune response (1). Immunomodulatory cytokines, namely IL-10 and TGF $\beta$, are able to inhibit the expression of several other cytokines and the onset of an efficient immune response (2), and they are considered as possible key mediators in the impairment of host immune response observed in PRRS (1). Therefore, the main goal of this study was to study the expression of IL-10 and TGF $\beta$ in the lung and tonsil of pigs experimentally with PRRS virus (PRRSV).

\section{Materials and Methods}

Thirty-two 5-weeks-old pigs from a high-healthy farm historically seronegative for PRRSV were used for the experimental infection. Twenty eight pigs were randomly distributed in batches of four, inoculated by the intramuscular route with 1 $\mathrm{ml}$ of 103.0 TCID50 PRRSV strain, and killed at 3, 7, 10, 14, 17, 21 and 24 days post-inoculation (dpi). The four remaining pigs were used as controls, inoculated with $1 \mathrm{ml}$ of sterile medium and killed at the end of the study ( $24 \mathrm{dpi}$ ). At the necropsy lung and tonsil samples were collected and fixed with $10 \%$ neutral buffered formalin and Bouin solution, for the histopathological and immunohistochemical studies, respectively. In the immunohistochemical study the following primary antibodies were used: anti-PRRSV (SDOW17/SR30; Rural Technologies Inc.); anti-porcine IL-10 (AF693; R\&D Systems Inc.); anti-TGF $\beta$ (AB101NA; R\&D Systems Inc.), as previously described (3). Immunolabelled cells were counted and morphologically identified as macrophages, lymphocytes or neutrophils.

\section{Results}

A higher expression of IL-10 was observed at $7 \mathrm{dpi}$ in the lung parenchyma of PRRSV-inoculated pigs, whereas TGF $\beta$ was enhanced at 3 and $10 \mathrm{dpi}$, and at the end of the study ( $24 \mathrm{dpi}$ ). In addition, the expression of PRRSV antigen peaked at 7dpi, showing a positive correlation with respect to the pulmonary expression of IL-10 and TGF $\beta$ ( $r=0.77$ and $r=0.76$, respectively).
On the other hand, both IL-10 and TGF $\beta$ showed an undulant expression in the tonsil of PRRSV-inoculated animals. Interestingly, PRRSV antigen displayed a similar kinetics than the one observed for IL-10 $(r=0.76)$, but no correlation was observed with respect to the expression of TGF $\beta$ in the tonsil.

IL-10 was mainly expressed in the cytoplasm of macrophages from the alveolar septa, however, TGF $\beta$ was chiefly expressed by pulmonary alveolar macrophages (PAMs). In the tonsil, the expression of immunomodulatory cytokines was observed in the cytoplasm of macrophages of the lymphoreticular areas, and secondly by lymphocytes. TGF $\beta$ was also expressed in the cytoplasm of few neutrophils.

\section{Discussion}

The correlation and similar kinetics observed between the expression of IL-10 and PRRSV antigen in lung and tonsil suggests that PRRSV replication may induce the expression of this cytokine. On the other hand, the expression of TGF $\beta$ and PRRSV antigens was correlated only in the lung but displayed a different kinetics. Thus, an indirect pathway to induce TGF $\beta$ may be activated by PRRSV. Nowadays, the regulatory T cells are considered as possible source of IL-10 and TGF $\beta$ and their role in the immune response is being studied in PRRS (4).

We have previously reported that septal macrophages were the main cells expressing IL-10 and proinflammatory cytokines (3). Interestingly, PRRSV and TGF $\beta$ antigens were chiefly expressed by pulmonary alveolar macrophages (PAMs). Thus, TGF $\beta$ may avoid the lung expression of proinflammatory cytokines and other cytokines by PAMs in PRRSV-infected pigs.
References
1. Mateu and Díaz, 2008. Vet J
2. Mitchell and Cotran, 2008. Basic Pathology. 8th Ed.
3. Gómez-Laguna et al., 2009. J Comp Pathol
4. Silva-Campa et al., 2009. Virology 


\title{
0.114
}

\section{Frequency of PRRS live vaccine virus (European and North American genotype) in vaccinated and non-vaccinated pigs submitted for respiratory tract diagnostics in North-Western Germany}

\author{
Elisabeth Grosse Beilage ${ }^{1} \underline{\text { Heiko Nathues }}^{1}$ Diana Meemken ${ }^{1}$ Timm C. Harder $^{2}$ Marcus G. Doherr ${ }^{3}$ Inga Grotha ${ }^{4}$ Irene Greiser-Wilke ${ }^{4}$ \\ 1. Field Station for Epidemiology, University of Veterinary Medicine Hannover, Bakum, Germany; \\ 2. Institute of Diagnostic Virology, Friedrich-Loeffler Institute, Greifswald - Insel Riems, Germany; \\ 3. Dept. Clinical Research \& Veterinary Public Health, Vetsuisse Faculty, University of Bern, Bern, Switzerland; \\ 4. Institute of Virology, Department of Infectious Diseases, University of Veterinary Medicine Hannover, Hannover, Germany
}

\section{Introduction}

A basic requirement of live vaccine virus strains is that natural transmission of vaccine virus is minimal or non-existent (3). The objectives of this study were (a) to determine the frequency of the EU and the NA genotype PRRS vaccine viruses in a large sample of pigs from North-Western Germany, (b) to assess the association between vaccine isolate detection and vaccination history and (c) to characterise the identified vaccine isolates considering their origin.

\section{Material and Methods}

During January and December 2007, 1,970 pigs were submitted to the Field Station for Epidemiology for post-mortem examination. Nine hundred and two of these pigs aged between 1 and 25 weeks of life had a history of respiratory disease and/or emaciation and were included in the study. The 902 pigs originated from 439 herds and were submitted by 141 different veterinary practices. Lung tissue from these pigs was tested for PRRSV by a nonaplex RT-PCR. Those samples positive for PRRSV were selected for further examination and genetic typing. The vaccination history was obtained from the veterinary surgeons who had submitted the pigs. Telephone interviews were conducted that followed a standardised questionnaire consisting of six closed (predefined range of answers or yes/no) questions on PRRS vaccination management/history of the herds of origin. It was hypothesised that these variables were related to the detection of the vaccine and/or wild-type viruses.

\section{Results}

Overall, $18.5 \%$ of the samples were positive for the EU wild-type virus. EU genotype vaccine virus was detected in $1.3 \%$ and the NA genotype vaccine virus in $8.9 \%$ of all samples. The production stage-specific detection rates for EU wild-type virus and NA genotype vaccine virus showed the highest values in weaning and growing pigs (Fig. 1).

Material from 168 of the 259 samples found positive in the diagnostic laboratory was available for genetic typing by amplifying and sequencing the ORF5. Of these, 104 were of the EU genotype, and 64 of the NA genotype. The ORF5 nucleotide sequence of 11 of the EU genotype isolates had $99.1-100 \%$, identity with the corresponding ORF of the Porcilis PRRS DV strain. Nucleotide identities of the remaining EU genotype isolates with the Porcilis PRRS-DV strain were between 85.3 and $91.7 \%$, allowing their classification as EU wild-type virus. Nucleotide identities of the ORF5 of the NA genotype isolates with the Ingelvac PRRS MLV vaccine strain were between 96 and $100 \%$. The detection of the EU vaccine was significantly higher in pigs vaccinated with the corresponding vaccine $(O R=9.4)$. Pigs vaccinated with NA genotype had significantly higher detection chances for the corresponding vaccine virus when compared to non-vaccinated animals $(\mathrm{OR}=3.34)$ animals, however, NA vaccine was also frequently detected in non-vaccinated pigs.

Fig. 1: Production stage-specific detection frequencies (nonaplex RT$P C R, D V-P C R$ ) for PRRS EU wild-type virus, PRRSV EU live vaccine virus and PRRSV NA live vaccine virus.

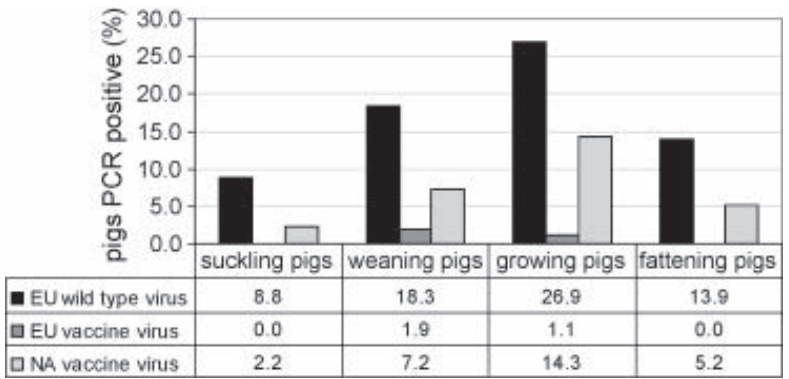

\section{Discussion}

The spread of vaccine virus within herds has been demonstrated in several studies $(1,2)$. However, data on age-dependent detection rates of live PRRS vaccine virus have not been published. The evident similarities in the dynamics of the NA genotype vaccine virus and the EU wild-type virus strongly support the contention that spontaneous transmission of the Ingelvac PRRS MLV is a common occurrence in those countries using this vaccine. Known that an eradication of PRRSV from infected herds would require a fundamental knowledge of the dynamics of PRRS virus infection and epidemiology, the results of this study show that this is not only needed for the PRRS wild-type virus but also for live vaccine viruses. Obvious differences in the potential of spontaneous spread of different PRRS vaccine viruses should be considered when choosing vaccines to be used in an eradication program.

\section{References}

(1) Botner A. et al. (1997) Vet Rec 141

(2) Botner A. et al. (1999) Vet Microbiol 68

(3) Mateu E. et al. (2008) Vet J 177 


\title{
0.115
}

\section{PRRS virus vertical transmission dynamics in a sow herd}

\author{
Jean P. Cano ${ }^{1}$ Scott Dee ${ }^{2}$ Albert Rovira² Claudia M. Zanzi ${ }^{2}$ Sukumarannair Anil ${ }^{2}$ Robert Morrison ${ }^{2}$ \\ 1. Boehringer Ingelheim Vetmedica, Inc., St Joseph, MO, USA; 2. College of Veterinary Medicine, University of Minnesota, St Paul, MN, USA
}

\section{Introduction}

After the introduction of porcine reproductive and respiratory virus (PRRSv) into a susceptible breeding herd, reproductive failure and an increase in pre-weaning mortality may be observed. Within a few months, the production parameters of the breeding herd may return to levels similar to those prior to infection. However, the presence of persistently infected individuals ${ }^{1}$ and the continuous introduction of susceptible pigs perpetuate viral transmission ${ }^{2}$. Questions regarding the prevalence of PRRSv shedding in endemically infected breeding herds and transmission of the virus in the farrowing room over time remain unanswered. Therefore, the objective of this study was to determine the prevalence of PRRSv infected piglets and litters at birth and at weaning in an endemically infected breeding herd.

\section{Materials and Methods}

A PRRSv-naïve 1500 sow farrow-to-wean farm became infected. The owners and the veterinarian decided to inject $1 \mathrm{~mL}$ of serum containing $10^{1.6} \mathrm{TCID}_{50}$ of the herd-specific PRRSv isolate to all sows in an attempt to hasten the spread of the virus ${ }^{3}$. During the 4th wk post-inoculation (PI), 59 sows farrowed. To estimate the prevalence of PRRSv infected litters, blood was collected from all pigs in a random sample of 38 litters at birth and again at weaning. The sample size was based on a desired confidence level of $95 \%$, an expected prevalence of $6.3 \%$, and $1.66 \%$ ( 1 positive / 60 litters) as the lowest possible prevalence (Epilnfo 6, Stone Mountain, GA). The expected prevalence of $6.3 \%$ infected litters per farrowing group was calculated based on detection of one positive pool by the "traditional sampling protocol", testing 4 pools comprised of 5 piglet samples by PCR (Pool Prevalence Calculator, AusVet, Animal Health Services, Australia). The sampling/testing process was repeated on litters from another group of farrowing sows 12 wks Pl. These two farrowing groups were selected as being representative of epidemic ( 4 wks PI) and endemic infection (12 wks PI). Serum samples were randomly pooled in groups of 5 and tested by RT-PCR. All samples in a positive pool were tested individually. Proportion of PCR-positive piglets and litters were compared using Fisher's Exact Test (Statistix $\left.{ }^{\circledR} 8\right)$.

\section{Results}

At 4 wks Pl, 26\% of the litters were PCR-positive at birth and $55 \%$ at weaning. At $12 \mathrm{wks} \mathrm{Pl}, 8 \%$ of the litters were positive at birth and $31 \%$ at weaning. A significant reduction in the prevalence of positive litters at birth $(P=0.03)$ and at weaning $(P=0.04)$ was observed from 4 to 12 wks PI. The prevalence of PCR-positive piglets at 12 wks PI was significantly lower at birth and weaning than at 4 wks $\mathrm{PI}(\mathrm{P}<0.01)$. Both at 4 and 12 wks $\mathrm{Pl}$, the prevalence of positive piglets $(P<0.05$, Figure 1$)$ and litters at weaning was significantly higher than at birth. Between $52 \%$ and $77 \%$ of the PCR-positive litters at birth or at weaning had only 1 or 2 positive piglets within the litter.
Figure 1. Prevalence of PRRSv PCR-positive piglets

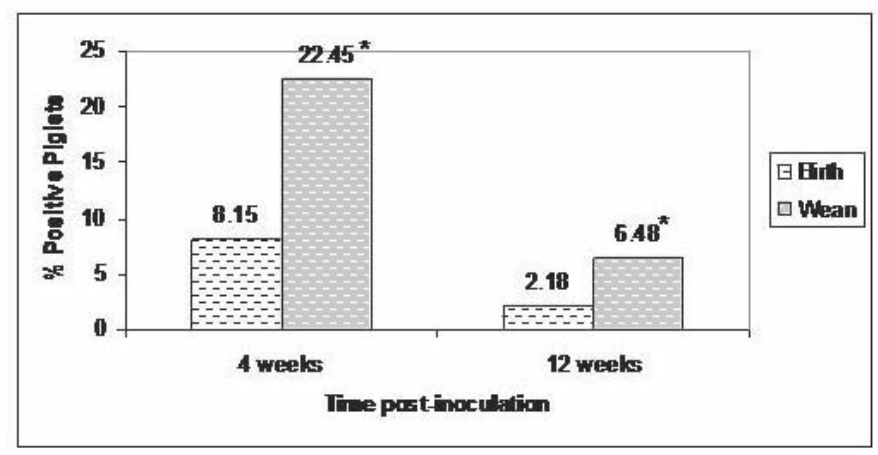

\section{Discussion}

The results demonstrate transmission among litters in the same farrowing room and support the sampling of piglets late during lactation as a tool to monitor PRRSv transmission. The fact that PRRSv vertical transmission may occur in as few as one newborn piglet of a litter, and that this very low within-litter prevalence of infection can be maintained until weaning, makes it necessary to reassess the sampling approach used today for breeding herds. The results of this study demonstrate that PRRSv infection prevalence in suckling piglets in an endemically infected breeding herd decrease to such levels that the "traditional monitoring protocols" will have insufficient sensitivity to determine the true infection status of the herd.

\section{References}

1. Wills, R. Vet Microbiol 1997 (55)231-40.

2. Dee, S. JAVMA 1994 (205-7)1017-18.

3. Batista L. IPVS 2004 (1)815. 


\title{
0.116
}

\section{Investigation of factors associated with porcine reproductive and respiratory syndrome (PRRS) herd status for production sites in a low density area of swine production in Quebec, Canada}

\author{
Marie-Ève Lambert ${ }^{1}$ Zvonimir Poljak $^{2}$ Sylvie D'Allaire ${ }^{1}$ \\ 1. University of Montreal, Faculty of Veterinary Medecine, St-Hyacinthe, QC, Canada; \\ 2. University of Guelph, Ontario Veterinary College, Guelph, ON, Canada
}

\section{Introduction}

Transmission of PRRS virus can occur by different ways and several factors may increase the risk of contamination of a herd. The objective of this exploratory study was to identify and quantify the effects of potential risk factors on PRRS status.

\section{Materials and Methods}

All premises containing sows in a low density area of swine production (Estrie) were included. A questionnaire was developed to assess the principal potential risks factors reported in literature for introduction of PRRS virus on a site or within a herd. The questionnaire was completed by the owner (independent farm) or by the employee (for farm under contract) and filled out by a single interviewer. Answers were obtained by phone and during a farm visit and included information about herd characteristics, geographical coordinates of the site and biosecurity requirements (layout of the site, entrance protocol, transportation of animals, dead pig disposal, pest management, feed and semen deliveries, manure management). Biosecurity was described as measures implemented by producer to limit introduction of diseases on the site and inside units. PRRS status was determined by ELISA (IDEXX HerdChek-PRRS 2XR) or qRT-PCR according to clinical signs observed. Associations with PRRS herd status were assessed by simple logistic regression for the following variables: herd size expressed in HPU as previously described (1), distance from the nearest site and from the public road, 22 biosecurity variables and a global biosecurity index (low or high: generated by two-step clustering procedure in SPSS with the 22 biosecurity variables). Two multivariable (backward selection) logistic models were built using SAS (SAS institute Inc., 1989) in order to compare the impact of a global biosecurity index vs. single biosecurity variables.

\section{Results}

On 54 production sites (participation rate of $79 \%), 15$ and 39 were negative and positive to PRRS virus, respectively. Significant variables associated with herd status in univariable analysis are shown in Table 1. Results of multiple logistic regression for both models are shown in Table 2.
Table 1: Factors associated with PRRS herd status using univariable logistic regression $(p<0.15)$

\begin{tabular}{|l|l|}
\hline \multicolumn{2}{|c|}{ Flori et al. 1995. Prev Vet Med 25: 51-62. } \\
\hline Variables & Description \\
\hline HPU $^{1,2}$ & $>300$ HPU ( 1000 sows) \\
\hline Neighbour 1,2 & $<1500$ m from nearest pig site \\
\hline Road $^{1,2}$ & Public road < 300 m from unit \\
\hline No Protocol & $\begin{array}{l}\text { Absence of shower or clear separation between contam- } \\
\text { inated and clean area }\end{array}$ \\
\hline Index $^{2}$ & Low biosecurity level \\
\hline $\begin{array}{l}1 \\
\text { Variable used in model 1. }\end{array}$ & \\
\hline
\end{tabular}

Table 2: Factors associated with PRRS herd status using multivariable logistic regression

\begin{tabular}{|c|c|c|c|c|}
\hline Model & Variables & Betas & OR & $95 \% \mathrm{Cl}(\mathrm{OR})$ \\
\hline \multirow[t]{2}{*}{1} & Neighbour & -1.83 & 6.2 & $1.2-33.3$ \\
\hline & No Protocol & -1.49 & 4.5 & $1.1-17.5$ \\
\hline \multirow[t]{2}{*}{2} & Neighbour & -2.02 & 7.5 & $1.4-40.0$ \\
\hline & HPU & -1.57 & 4.8 & $1.1-21.2$ \\
\hline
\end{tabular}

\section{Discussion}

For both models, having a site closer than 1500 m significantly increased the odds of being PRRS positive. From model 1, absence of an entrance protocol was also an important factor for positivity (OR=4.5). HPU was not kept in the final, having a $\mathrm{p}=0.0517$ in the last alternative model of the model building strategy ( $\mathrm{OR}=4.8, \mathrm{Cl}=1.0-23.8)$. In model 2, the association with the biosecurity index was not significant when adjusting for other predictors (HPU, Neighbour). The global index being a composite of 22 different variables of which several were not significantly associated in the univariable analysis might explain the lack of association with PRRS status in the final model.

\section{Conclusion}

In this low density area, sites without an entrance protocol or located within a short distance from neighbouring pig operations were more likely to be PRRS positive. Further analyses will be performed to investigate the effect of pig-site density in order to clarify associations found with neighbourhood.

\section{References}

1. Flori et al. 1995. Prev Vet Med 25: 51-62. 


\title{
0.117
}

\section{Dynamics of virus excretion and transmissibility of European-type PRRSV isolates of high and low virulence}

\author{
Francisco Javier Martínez-Lobo; Francisco Díez-Fuertes; Lidia Hornillos-Gumiel; \\ Patricia Aguilar-Calvo; Isabel Simarro; José María Castro; Cinta Prieto \\ Facultad de Veterinaria Universidad Complutense de Madrid, Madrid, Spain
}

\section{Introduction}

Porcine reproductive and respiratory syndrome (PRRS) is a disease causing high economic losses worldwide. The etiological agent, PRRS virus (PRRSV), is highly heterogenic. This variability of PRRSV has resulted in the emergence of strains differing in virulence. Previous studies carried out with American-type isolates have demonstrated that the degree of virus transmission and aerosol shedding are related to isolate pathogenicity (1). However, similar studies have never been done using European isolates. The objective of this study was to determine whether differences in virulence of European-type isolates significantly affect the frequency of shedding and transmission to sentinel pigs.

\section{Materials and Methods}

Four different isolates were used in this study: one highly virulent isolate, one isolate of low virulence, the vaccine strain included in PorcilisPRRS vaccine, and an attenuated version of the highly virulent isolate obtained after 20 passages on Marc145 cells.

Sixty 3-week old PRRSV seronegative piglets were randomly divided into 4 treatment groups. On day 0 of the experiment five pigs per group were isolated and inoculated intramuscularly with 105 TCID50 of one of the isolates used in this study. After two days of isolation, the remaining ten pigs of each group were housed with the inoculated pigs. Blood samples were collected from all pigs on days $0,3,6,10,14,19$ and 24 post-infection (p.i.). Nasal, oral and rectal swabs were obtained the days of blood collection from principal pigs. Virus isolation was carried out on porcine alveolar macrophages (PAM) or Marc-145 cultures depending on the challenge isolate. All swabs were tested by RT-PCR for the presence of PRRSV RNA. For antibody detection, serum samples collected on Day 24 were analyzed by a commercial ELISA.

\section{Results}

Viremia in principal pigs was a frequent finding in our study. Specifically, virulent isolate and its attenuated version were detected during the entire experimental period; virus of low virulence was mostly detected from 3 p.i. to day 14 p.i. and vaccine strain was detected only sporadically. In addition, PRRSV was shed by different routes by pigs of all experimental groups, although the frequency of shedding differed significantly between groups. In this sense the highly virulent isolate was detected in most of the swabs obtained between days 3 and 14 p.i. and less frequently thereafter. The highly virulent adapted to growth in Marc-145 cells was also frequently detected although the frequency of dtetion was lower than in its parental virus. On the contrary, low virulent isolate was only detected sporadically and mostly in days 6 and 10 p.i. Likewise, vaccine strain was detected sporadically being the less frequently isolated virus. Finally, transmission to in-contact pigs, measured either by detection of viremia or seroconversion, was detected in all experimental groups. However the frequency of transmission differed significantly between different experimental groups. Particularly, all and nine out of ten pigs were infected in groups exposed to highly virulent isolate and its attenuated version, respectively, while only two pigs exposed to low virulent isolate and vaccine strain became infected.

\section{Discussion}

The objective of this study was to evaluate the impact of PRRSV isolate virulence on shedding by different routes and transmission to in-contact pigs. Our initial hypothesis was that highly virulent isolates would replicate more efficiently in pigs and consequently would be shed in higher amounts and readily transmitted to susceptible animals. This theory was confirmed by the fact that all in-contact pigs exposed to pigs infected with highly virulent isolate became infected while only two were infected among those exposed to either pigs infected with an isolate of low virulence or vaccinated pigs. The highly virulent isolate was adapted to grow in Marc-145 cells with the purpose of limiting its ability to replicate in natural target cells, hampering in this way its transmission capability. However our results indicate that after 20 passages in Marc-145 cells the isolate retains its parental ability to replicate and be transmitted.

\section{Acknowledgements}

This study was supported by grant CSD-2006-00007 (Consolider Ingenio 2010).

\section{References}

1. Cho et al. 2006. Can J Vet Res, 70:297-301 


\title{
0.118
}

\section{The impact of respiratory disease on production in the pig industry in Great Britain}

\author{
Charlotte M. Evans $^{1}$ Graham F. Medley $^{2}$ Laura E. Green$^{2}$ \\ 1. BPEX, Kenilworth, Warwickshire, UK; 2. University of Warwick, Coventry, UK
}

\section{Introduction}

Respiratory disease is the most significant cause of morbidity and mortality in pigs post-weaning. This project used data gathered as part of a DEFRA/BPEX-funded project on post weaning multisystemic wasting syndrome (PMWS) to investigate respiratory diseases in 116 pig herds. The specific aims of the project were to: 1) Estimate the prevalence and incidence of common respiratory diseases in England and investigate their associations with one another and with post-weaning mortality; 2 ) Investigate antibody profiles for porcine reproductive and respiratory syndrome virus (PRRSV)-infected herds and to determine management factors associated with differences in profiles between herds; 3 ) Investigate how PRRSV could persist within infected herds using mathematical models and available data; 4) Determine the impact of PRRSV infection on disease and explore theoretically how this could be reduced by different control and elimination strategies

\section{Materials and Methods}

Questionnaires were completed by veterinarians that attended 116 pig herds in GB. Management factors were collected from on-farm interviews with unit managers. Management factors were investigated for their association with the within and between-herd variability in pig antibodies to PRRSV in 103 pig herds. To investigate the mechanisms that could lead to fade out of PRRSV, within-herd transmission dynamics were investigated using a mathematical model.

\section{Results}

From the results, it was shown that PRRS was frequently associated with raised mortality and morbidity when present with other disease causing agents in a herd. Antibody profiles for 103 herds were investigated (50 pigs were sampled in each herd). In 35 herds no pigs had PRRSV antibody. These were considered PRRSV negative. These herds were more likely to have less than 250 sows and be located more than two miles from another pig farm. Sixty eight herds had pigs with antibody to PRRSV (twenty seven of which were vaccination). Higher antibody levels were present in antibody positive herds that did not isolate gilts for $>6$ days. In $39 \%$ of antibody positive herds that were not using vaccination, only breeding sows were antibody positive, suggesting that there was antibody on the farm but no virus because the growing pigs were antibody negative. This is indicative of fade out of virus, i.e. its disappearance from a herd in which it has been present. In our mathematical models fade out was also more likely in small herds with little/no reintroduction of infectious stock.
To investigate the mechanisms that could lead to fade out of PRRSV, within-herd transmission dynamics were investigated using a mathematical model. When virus was introduced into a herd via gilts there was a high frequency of fade out in breeding pigs before the virus reached young stock. Once the virus had reached young stock there was an increased probability of virus persisting in the herd. This was also more likely in large herds, herds with increased contact between age groups and herds that had frequent re-introduction of virus (for example through purchasing infectious pigs, or wind borne spread). The results highlight the importance of determining whether a herd is truly infected or not and appropriate sourcing and isolation of new stock. The patterns observed suggest that the processes of re-introduction, persistence and fade-out of PRRSV play critical roles in the spread of PRRSV throughout the pig industry.

Mathematical models were also used to investigate the range of clinical disease observed as a result of infection with PRRSV and the result of strategies for control and elimination. PRRSV was difficult to eliminate without targeting both rearing pigs and sows. Rapid vaccination of sows once there was an increase in stillbirths reduced the risk of spread of virus to rearing pigs but not in all simulations.

\section{Discussion}

In areas where the density of herds is low it might be possible to control PRRSV by elimination in individual herds. In larger herds in pig dense regions, elimination might be difficult and control of disease might give more stability. The long-term benefits of elimination will depend on the risk of re-introduction of virus into the herd.

\section{References}

Evans, C.M. (2010) The impact of respiratory disease on production in the pig industry in Great Britain Ph.D. Thesis, University of Warwick

Evans, C.M., Medley, G.F., Green, L.E., (2008). Porcine reproductive and respiratory syndrome virus (PRRSV) in GB pig herds: farm characteristics associated with heterogeneity in seroprevalence. BMC Veterinary Research 4, 48

Evans, C.M., Medley, G.F., Creasey, S. J., Green., L. E. (2009). A stochastic mathematical model of the within-herd transmission dynamics of porcine reproductive and respiratory syndrome virus (PRRSV): fadeout and persistence. Preventive Veterinary Medicine 93, 248 - 257 


\title{
0.119
}

\section{Long distance airborne transport of viable PRRSV and Mycoplasma hyopneumoniae from a swine population infected with multiple viral variants}

\author{
Scott Dee; Satoshi Otake; Cesar Corzo; Simone Oliveira; John Deen \\ Swine Disease Eradication Center, University of Minnesota College of Veterinary Medicine,, St. Paul, MN, USA
}

\begin{abstract}
Introduction
Airborne transport of porcine reproductive and respiratory syndrome virus (PRRSV) and Mycoplasma hyopneumoniae (M hyo) has been reported out to $4.7 \mathrm{~km}$ (1). The objectives of this study were to simulate clinical conditions observed in the field through the creation of a mixed infection of heterologous PRRSV variants in conjunction with $M$ hyo in a population of growing pigs. We planned to use this unique model to evaluate the long distance airborne transport of these agents out to and beyond $4.7 \mathrm{~km}$ and assess their viability in long distance air samples. The study was based on the hypothesis that long distance airborne transport of PRRSV is not variant-specific.
\end{abstract}

\section{Materials and Methods}

To accomplish this goal, a mixed infection of 3 PRRSV variants (1-8-4, 1-18-2 and 1-26-2) and $M$ hyo 232 was established in a source population of growing pigs. Over 21-day period, air samples were collected from the source population and at designated distances from the herd, ranging from 1.4 to $10.2 \mathrm{~km}$ from the source facility. A cyclonic collector (Midwest Microtek, Brookings, SD, USA) capable of collecting $400 \mathrm{~L}$ of air per minute was used to collect air samples. The collector was placed downwind from the source facility in accordance with one of the 4 cardinal directions: north $(\mathrm{N})$, south $(\mathrm{S})$, east $(\mathrm{E})$ and west $(\mathrm{W})$ and the 4 intermediate directions: northwest (NW), northeast (NE), southwest (SW) and southeast (SE). Four long distance samples were collected each day, i.e. SW-4km, SW-3km, SW-2km and SW- $1 \mathrm{~km}$ from the source facility. Samples were tested for PRRSV RNA and $M$ hyo DNA by PCR and if positive, were sequenced (PRRSV: ORF 5, M hyo P146 gene) and quantified (PRRSV only). In addition, viability assessments were made of PCR-positive samples using modified swine bioassay $(2,3)$.

\section{Results}

On day $7 \mathrm{PI}$, all 3 PRRSV variants were detectedin the herd; however, by day 21 PI only PRRSV 1-8-4 was found in sera and tissues from source population animals. Cinical respiratory disease indicative of PRRS and enzootic pneumonia increased from 27 cases on day $0 \mathrm{PI}$ to 82 cases on day $10 \mathrm{PI}$. In exhaust air from the source population, PRRSV and $M$ hyo were detected in 21/21 and 8/21 air samples, respectively. Of the 21 samples, 18 contained PRRSV 1-8-4 and 3 contained PRRSV 1-18-2. The mean quantity of infectious virus across all 21 samples was $9.3 \times 10^{4}$
$\mathrm{TCID} 50 / \mathrm{mL}$ (range $=1.0 \times 10^{1} \mathrm{TCID} 50 / \mathrm{mL}$ to $6.3 \times 10^{5} \mathrm{TCID} 50 / \mathrm{mL}$ ). Five of 114 (4.4\%) long distance air samples were positive for PRRSV and 6 of 114 (5.2\%) were positive for M hyo. The 5 PRRSVpositive samples were collected at 2.3, 4.6, 6.6 and $9.1 \mathrm{~km}$ from the herd. All contained viable virus (range $=1.0 \times 10^{1} \mathrm{TCID} 50 / \mathrm{mL}$ to $2 \times 10^{2} \mathrm{TCID} 50 / \mathrm{mL}$ ) and were $>99.2 \%$ homologous to PRRSV 1-8-4. No evidence of PRRSV 1-18-2 or 1-26-2 was detected in long distance samples. All $6 \mathrm{M}$ hyo-positive samples were $99.9 \%$ homologous to M hyo 232 and 3 samples (collected at 3.5, 6.8 and $9.2 \mathrm{~km}$ from the herd) were infectious as determined by the presence of $\mathrm{M}$ hyo DNA in nasal swabs, tracheal and-bronchial swabs as well as evidence of gross and microscopic lesions suggestive of M. hyo infection in bioassay pigs

\section{Discussion}

These results are the first proof of airborne transport of these pathogens in a viable state over this distance. The origin of these pathogens was confirmed to be the source population, since the PRRSV 1-8-4 RNA and $M$ hyo 232 DNA present in long distance air samples was homologous to that found in experimentally inoculated animals and in exhausted air. In addition, the quantity of infectious PRRSV was determined by virus titration in all 5 samples and viability of both pathogens in long distance air samples was further confirmed by swine bioassay. Whether either agent could have been transported over greater distances than those reported here cannot be answered at this time; therefore, future studies are required. In conclusion, his new information will assist swine veterinarians in the development of sustainable area/regional programs for the control and eventual eradication of these economically significant diseases of pigs.
References
1. Dee et al., 2009. Vet Res, 40:39.
2. Swenson et al., 1994. JAVMA, 15:1943-1948.
3. Marois et al., 2007. Vet Microbiol,120:96-104. 


\title{
0.120
}

\section{The characteristic of High Pathogenic PRRS 2009 outbreak epidemiology and pathogenesis in middle Shandong area}

\author{
Sidang Liu; H Li; L Nie \\ College of Animal Science, Shandong Agricultural University, Shandong, China
}

Porcine reproductive and respiratory syndrome virus (PRRSV) is the causative agent of PRRS, a disease characterized by reproductive failure and respiratory distress in swine[1]. The RNA virus feature made PRRSV highly mutational and thus result various virulence differences among strains [2]. The so-called "High Fever Disease" emerged in southern China in June 2006 and rapidly spread to the whole country. This "High Fever Disease" was characterized by fast transmission, acute infection, high morbidity and mortality (20-30\% average and in some cases over $50 \%$ and even reach $100 \%$ ). Following research and diagnosis indicated the Disease is caused by "High Pathogenic Strain of PRRSV" with a 30 amino acids deletion in NSP2 [3].

After a two-year's stable phase, the High Pathogenic Strain of PRRS again busted out in the summer of 2009, including Jiangsu, Anhui, Henan, Shandong and Hebei provinces. In this report, we analyze the epidemiology of the disease in Shandong and lesions of infected pigs as to better understand the disease characters for future control.

Epidemiological investigation: Samples of 100 scaled pig farms and 200 backyards from 10 areas in Shandong were collected for serology analysis. Meanwhile, relevant information including vaccination procedure against PRRS, mortality and morbidity during the outbreak were also investigated by visit or by phone of the farms. The investigation clearly showed that the outbreak emerged in backyards below 50 sows first. These farms barely have any vaccination program against PRRS and the morbidity can reach up to $70 \%$. But, comparing with backyards, outbreaks in scaled farms (48/100) in general were postponed somehow. We listed the morbidity and mortality with the different types of farm with their PRRSV vaccine in use below (Table 1).

Clinical symptoms and lesions: Finishing and sow herd were infected first, infected pigs showed high body temperature, wasting and emaciation. Most infected pigs showed dark red skin (skins colors around belly and peripheral extremities even turned purple), ear cyanosis; thumping, coughing and irregular breathing; snivel and conjunctivitis; constipation, turbid and dark color urines. Pigs with long disease course would turn pale and show anemia, some could not stand till death. In some cases, sow abortions at 100-110 days post pregnancy, mummification and death fetus or weak piglets could also be observed.

\section{Conclusion and Discussions}

Comparing with the 2006 "High Pathogenic PRRS", the epidemic situation this time showed many differences: fewer farms were infected, low morbidity, salutatory spread, slow transmission, more sensitivity of pregnant sows and gilts, mortality varied much and had a longer disease course.. This can be explained by the acquired immunity of herds survived from last out break. However, earlier infections and more severe loss in household farm remain the same between two outbreaks, suggesting factors like poor feeding, management and bio-security measures, inappropriate medication program and confection with other diseases are also main cause of the diseases.

The macroscopical and microscopical lesions of the two breaks are almost the same, while herds vaccinated against PRRS or survived from last PRRS outbreak showing less severe and untypical lesions. Moreover, Mycoplasma hyopneumoniae, PCV2, CSFV coinfection lesions become quite common, while bacterial secondary infections, like Pericarditis serofibrinosa and pleuropneumonia, were rare. Rare bacterial secondary infections were due to the massive use of antibiotic in the epidemic.

Field experiences demonstrated that killed vaccines against PRRS are safe but the efficacy is limited. Despite the homogeneity difference between classical and high pathogenic strains, the field practice revealed the effective cross protection provided by classical strain vaccines. Especially, in our investigation, we found that farms vaccinated with Boehringer Ingelheim PRRS MLV have much easier days in the break. Whether the 2009 strain possesses new mutations regarding to virulence is still under study.

\section{Table 1.}

\begin{tabular}{|l|c|c|c|}
\hline & Type of farm (Number) & Morbidity \% & Mortality \% \\
\hline Scaled Farm & No vaccination (8) & 87.5 & 32.52 \\
\hline & Local mineral oil vaccine (10) & 70 & 27.10 \\
\hline & Local freeze dried vaccine (21) & 42.85 & 17.52 \\
\hline & BI PRRS MLV (12) & 16.67 & 9.32 \\
\hline & $\begin{array}{c}\text { BI PRRS MLV in sows; local freeze } \\
\text { dried vaccine in commercial herd } \\
\text { (49) }\end{array}$ & 26.53 & 13.21 \\
\hline Backyards & No vaccination (71) & 85.92 & 41.12 \\
\hline & local mineral oil vaccine (16) & 50 & 29.23 \\
\hline Total & local freeze dried vaccine (13) & 30.77 & 19.50 \\
\hline \multicolumn{2}{|c|}{ Scaled farms (100) } & 38 & $9.32-32.52$ \\
\hline BI PRRS MLV=Ingelvac PRRS MLV & 76 & $13.50-41.12$ \\
\hline
\end{tabular}

\section{References}

[1]. Done, SH, Paton D J Drew TW,et al,The pathology of experimental infection with UK isolates of porcine reproductive and respiratory syndrome virus in specific pathogen-free pigs[J].Pig J,1996,36:31-41

[2] Mateu E, Diaz I, Darwich L, Casal J, Martin M, et al.Evolution of ORF5 of Spanish porcine reproductive and respiratory syndrome virusstrains from 1991 to 2005[J]. Virus Res 115:198-206, 2006

[3]. Zhou, L., Zhang, J., Zeng, J., et al(2009). The 30-Amino-Acid Deletion in the nsp2 of Highly Pathogenic Porcine Reproductive and Respiratory Syndrome Virus Emerging in China is not related to its virulence. J. Virol. 83: 5156-5167 


\title{
0.121
}

\section{Prevalence of subclinical urinary tract infections in the Belgian sow population}

\author{
Frédéric A. Vangroenweghe ${ }^{1}$ Tamara Vandersmissen $^{2}$ Evelyne De Graef $^{1}$ Ellen Van Driessche ${ }^{1}$ Frans Castryck $^{1}$ \\ 1. DGZ-Vlaanderen, Torhout, Belgium; 2. DGZ-Vlaanderen, Lier, Belgium
}

\section{Introduction and Objective}

The prevalence of urinary tract infections (UTI) in sows in Belgium is not well known. In the neighbouring countries, the prevalence of (subclinical) UTI is estimated to be up to $20 \%$ (1). Urinary tract infections are responsible for sow mortality $(2,3)$ and also have an impact on the breeding performance of the sow $(1,4)$. Total born litter size, live born litter size and weaning litter size appeared to be lower in sows with UTI, while the weanto-oestrus interval and the wean-to-farrowing interval increases in these sows.

To investigate the prevalence of (subclinical) UTI in Belgium, a screening on Belgian sow herds was performed (project 'Veepeiler-varken' funded by the Sanitary Fund)

\section{Materials and Methods}

Twenty-five Belgian pig herds, with at least 100 sows and no clinical signs of UTI, were selected to participate in this screening. Drinking water supply was continuous in 11 farms, but it was restricted in 14 others. In each farm between 9 and 21 latepregnant sows were sampled. A total of 353 urine samples was collected. Urine samples were collected in the morning, the first jets of urine were avoided. Bacteriological study of the urine was performed by inoculating 2 different media: Columbia sheep blood agar (Oxoid) and McConkey agar (Oxoid). After overnight incubation at $37^{\circ} \mathrm{C}$ in an atmosphere with $5 \% \mathrm{CO} 2$, identification of grown colonies was performed. Quantitative examination was performed through dilution of the urine samples in maximum recovery diluent (Oxoid) and subsequent inoculation of plate count agar (Biorad). After overnight incubation at $37^{\circ} \mathrm{C}$ under aerobic conditions, colony counting was performed. Nitrite detection was performed using the strip-test (Merckoquant; Merck).

\section{Results}

The predominant germs in the urine were E. coli (27\%), Streptococcus sp. (15\%) and Staphylococcus sp. (14\%). In 38\% of the cases no growth could be observed. In total, $19 \%$ of the sows showed urine bacterial counts higher than $10 \mathrm{E} 5 \mathrm{CFU} / \mathrm{ml}$, which should be interpreted as indicative of UTI. In $16 \%$ of the cases, an UTI could be suspected since the urine bacterial counts varied between 10E4 to 10E5 CFU/ml (Figure 1). At herd level, 0 to $43 \%$ of the sampled sows could be demonstrated to suffer from a subclinical UTI. Comparing sows with continuous or restricted drinking water access, a slight but non-significant difference in the prevalences of urine bacterial counts of higher than $10 \mathrm{E} 5$ CFU/ml could be observed, with $17 \%$ and $20 \%$ of urine samples of sows with continuous and restricted access, respectively, showing counts indicative for UTI. The strip-test, which is indicative for an E. coli infection, demonstrated $10 \%$ nitrite positive urine samples.
Figure.1 Distribution of urine samples according to the urine bacterial count. Unsuspected (< 10E4 CFU/ml); suspected (10E4-10E5 CFU/ml); infected (> 10E5 CFU/ml)

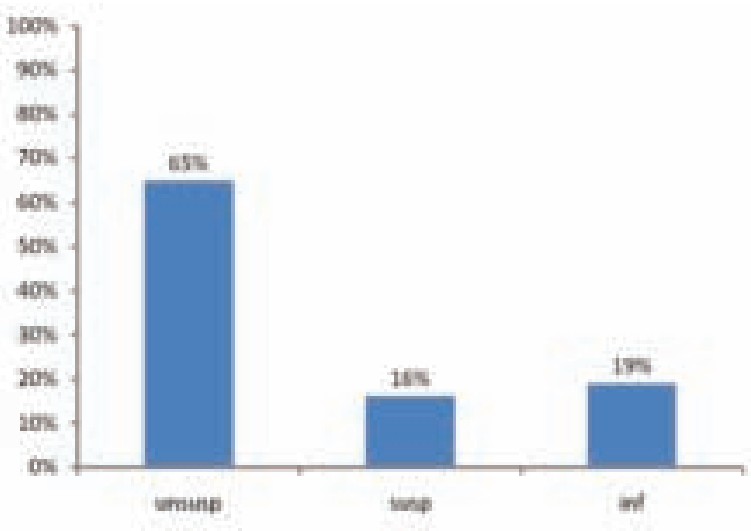

\section{Discussion and Conclusions}

The prevalence of subclinical UTI in the Belgian sow population, based on bacterial counts, is comparable to prevalence observed in neighbouring countries. Based on the strip-test, only half of the sows with a subclinical UTI can be detected. However, not all germs responsible for UTI have the capacity to produce nitrite. Essentially, E. coli can produce nitrite, whereas Streptococcus sp. and Staphylococcus sp., which can also provoke UTI, do not have a nitrite producing capacity.

\section{References}

1. Leneveu P.2007. Conventinal and emerging risk factors on farms with high health status. International Symposium Urinary tract Infections, Vétoquinol Academia, Lisbon, Portugal. P. 12-14

2. Abiven N., Seegers H., Beaudeau F., Laval A., Fourichon C. 1998. Risk factors for high sow mortality in French swine herds. Preventive Veterinary Medicine, 33: 109-19.

3. Vangroenweghe F., Miry C., Maes D., Castryck F. 2008. Diagnostic examination of increased sow mortality in Belgian pig farms. International Pig Veterinary Society, Durban, South-Africa. 22-25 juni 2008.

4. Mauch C., Bilkei G.. 2004. The influence of prepartal bacteriuria on the reproductive performance of the sow. Deutsche Tierarztliche Wochenschrifte, 111:166-9. 


\title{
0.122
}

\section{A new method for oestrus synchronizing of gilts in group housing systems}

\author{
Leo Suls; Jan Jourquin \\ Janssen Animal Heatlh, Beerse, Belgium
}

\section{Introduction}

Oestrus synchronization of breeding gilts with altrenogest is a common practice on many breeding farms, especially when a batch management system is used. The synchronization protocol implies a treatment of 18 consecutive days with an oral dose of $20 \mathrm{mg}$ oil-based altrenogest solution $(5 \mathrm{ml}$ product). It is imperative that a correct daily dose is administered. When the gilts are housed in individual stalls, the product is easily dosed on the feed. For welfare reasons, sow stalls are banned or limited to the first 28 days of gestation. This calls for alternative ways for successfully synchronization of the gilts.

A new application method has been developed for Regumate ${ }^{\circ}$ that allows individual treatment of gilts kept in groups.

\section{Materials and Methods}

For a farm expansion, 896 gilts were synchronized in a group housed situation administering altrenogest directly in the mouth.

The preconditions for a new method are demanding. Repetitive administration of the right dose to an individual animal, housed in group, without disturbing the group, but also convenient for the animal caretaker.

Janssen Animal Health developed a special dosing gun. This gun has a standard $5 \mathrm{ml}$ dose and can be easily connected to the 1 liter Regumate bottle. This gun allows a fast and accurate dosing with ample freedom of movement. Gilts have a very curious nature and they are very keen on soybean oil, the carrier of altrenogest. A 2-day training with a cheap vegetable oil prior to start of treatment is all that is needed. During the training period a person steps into the pen and waits until the gilts are calm and curious enough to approach. Gently one tries to squeeze a dose of oil into the mouth of all gilts. In the beginning this will take a bit of time, but after two days most animals will come spontaneously to ask for their dose of oil. After the short training, more than $99 \%$ of gilts will take the dose directly in the mouth.

\section{Results}

When the product is correctly applied, up to $95 \%$ of gilts come to oestrus within seven days after the last dose. 896 gilts were synchronized with the new method. Of these 844 gilts $(94.2 \%)$ came on heat within the seven day period. This is comparable to the results achieved in individual stalls (1).

Once the training period was over, there was no need to enter the pen as all gilts lined up, eager to get their dose, making this a convenient routine.
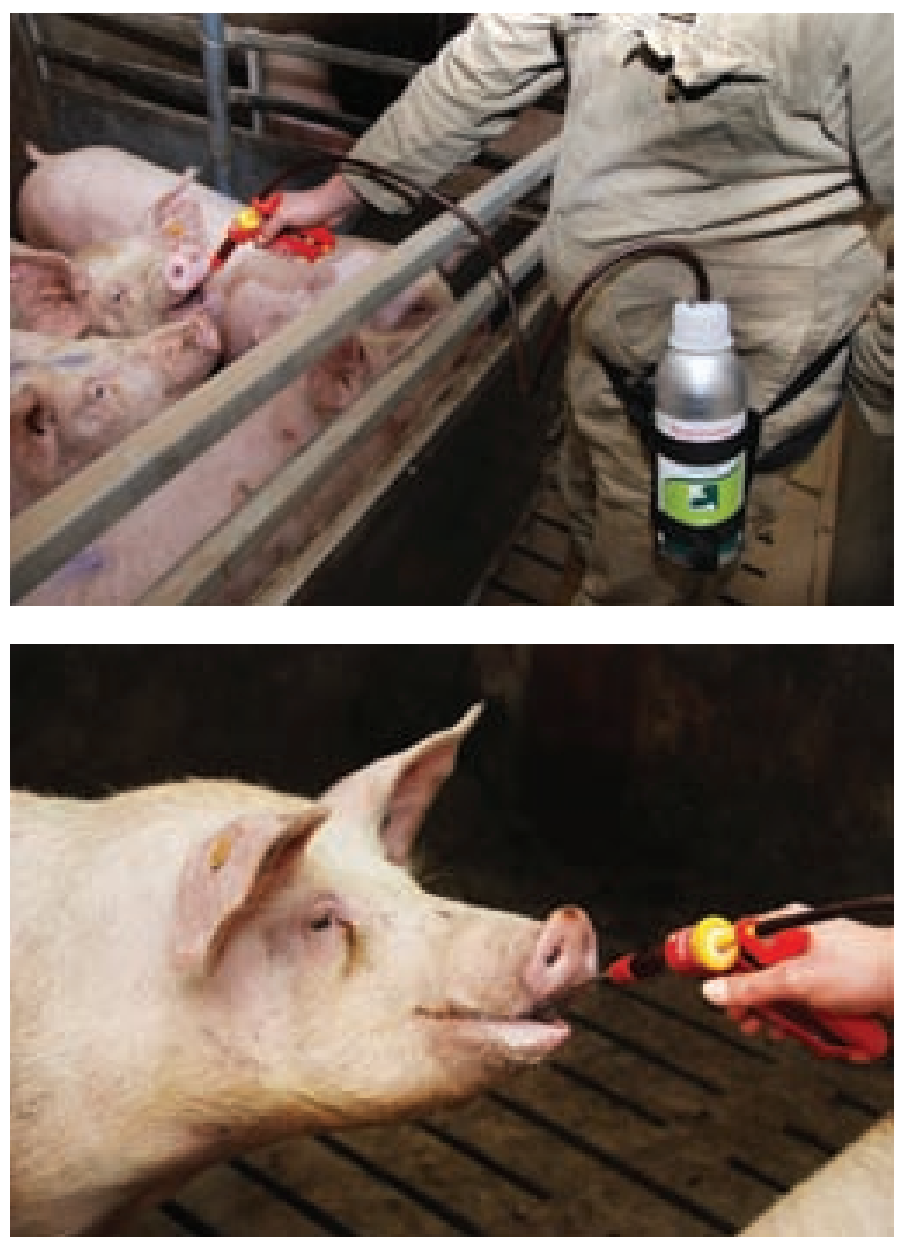

\section{Discussion}

This new method is a safe, accurate, effective and labor sparing alternative for gilt synchronization, and not only in group housed systems. It is also a convenient method where gilts are still kept in stalls.

Extensive experience in Germany, The Netherlands and England shows many advantages in the field. No product is spilled during application or leaks back from the mouth of the gilt. Administration is done very fast. Once the gilts are trained, 15 to 20 animals per minute can be dosed.

The oestrus synchronization results obtained with this new application method in practice appears to be at least as good as with the conventional on feed application.

An additional important benefit is the socializing aspect. During the short training and the 18 consecutive treatment days the gilts develop a very positive bond with the animal caretaker.

\section{References}

1. L. Suls et al., 20th IPVS proceedings, p. 431 


\title{
0.123
}

\section{Nitrite test for UTI diagnosis in sows: are we wrong?}

\author{
Guy-Pierre Martineau; Berengere Fabre; Herve Lefebvre
}

National Veterinary School, Toulouse, France

\section{Introduction}

In France, urinary tract infection (UTI) is routinely screened in sow herds using nitrite dipstick testing (Sialelli, 2004). Despite a low prevalence of clinical UTI, the rational for such screening remains controversial (Martineau et al., 2007). High water intake in pregnant sows, and consequently high urine dilution, may lead to false negative results. For unknown reasons, there is also an increase of positive results in late gestation (Sialelli, 2004).

The objectives of this study were i) to evaluate the day-to-day variability of the nitrite test and urine specific gravity (USG) in late gestating sows, ii) to compare nitritre test to semi-quantitative bacterial count, and iii) to assess the effect of urine dilution on the nitrite test results.

\section{Materials and Methods}

The herd-The study was performed in the same herd of 600 sows, free for clinical UTI, managed in a 7 batch farrowing system. Liquid feeding (dilution of $4 \mathrm{~L} / \mathrm{kg}$ of feed plus one extra "water meal" of $3 \mathrm{~L} /$ day for a given sow).

Selection of animals- For 3 consecutive weeks, urine was collected before feeding (before $6.00 \mathrm{am}$ ) in 130 late gestating sows. $15 / 130$ sows in which urine had a nitrite positive test and USG $\geq 1.015$ (refractometry) were included in the study.

Study design- For each included sow, urine was collected at least 3 times over the same week (same conditions as described above). USG and nitrite test was performed on each individual sample every day. The pink colour change of the reagent pad was assessed by the same investigator using a -/+/++/+++ scale. Moreover, a semi-quantitative microbiological culture (Count Exact $^{\oplus}$ ) was done on the first positive sample in each animal. Furthermore, a 2- and 4-fold dilution of each first sample with $\mathrm{NaCl} 0.9 \%$ was performed and nitrite test repeated again on the so diluted samples.

\section{Results and discussion}

There was no association between strong nitrite positive urine $(+++)$ and USG. Day-to-day variability of positive nitrite test results was high (Figure 1A) in $8 / 15$ sows, suggesting that diagnostic interpretation from a single sample may be misleading.

The relationship between nitrite test results and semi-quantitative bacteriological counts was poor (Table 1).

Some mildly nitrite positive urine has a relatively high bacteria counts and inversely.

Dilution induced also a decrease in positivity of the nitrite test (Figure 1B).
Table 1: Relationship between semi-quantitative bacteriological counts and nitrite test ( $n=15$ sows)

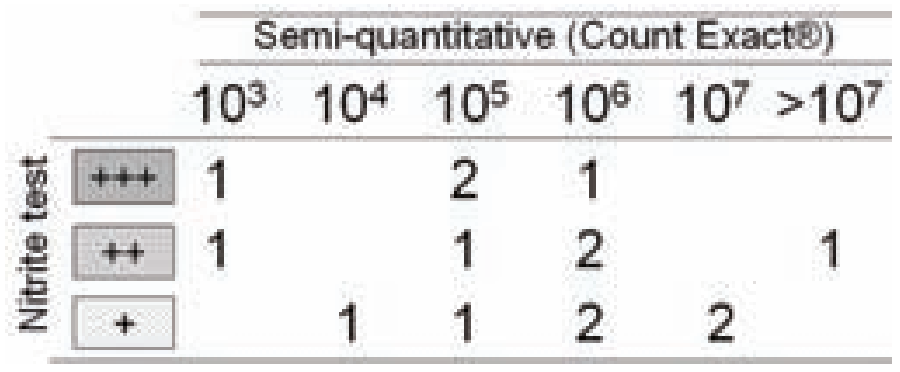

Figure 1A: Day-to-day variability of nitrite test ( $n=15$ sows) Figure 1B: Effect of dilution on nitrite test

\begin{tabular}{|c|c|c|c|c|}
\hline Mor & & vive & 11 & $F$ \\
\hline$0 1 \longdiv { 1 . 0 1 6 }$ & 1.011 & 1.010 & 1.014 & 1.0 \\
\hline 102 & & 1,017 & 012 & 1 \\
\hline \pm 03 & & 1.018 & 1.010 & 1 \\
\hline$\# 04$ & 1.021 & 1.014 & 1.014 & 1 \\
\hline 105 & 1.021 & 1.019 & 1.021 & 1.010 \\
\hline$\$ 0 6 \longdiv { 1 . 0 1 6 }$ & 1.011 & & 1.010 & 1 \\
\hline$0 7 \longdiv { 1 . 0 1 9 }$ & 1.020 & 1.013 & 1.021 & 1. \\
\hline 4081.018 & 1,017 & 1.016 & 1.019 & 1,0 \\
\hline 109 & 1.016 & 1.011 & 1011 & 1.0 \\
\hline$\# 10$ & 1.016 & 1.016 & 1.016 & 1.0 \\
\hline$\# 11$ 1 & 1.018 & 1.010 & 1.008 & \\
\hline$\$ 121.016$ & 1.018 & 1.020 & 1020 & \\
\hline$\$ 131.015$ & 1.009 & 1020 & 1.007 & L \\
\hline$\pm 1 4 \longdiv { 1 . 0 1 5 }$ & 1.016 & 1.011 & 1.011 & \\
\hline$\# 15$ & & 1.015 & 1.009 & 10.9 \\
\hline
\end{tabular}

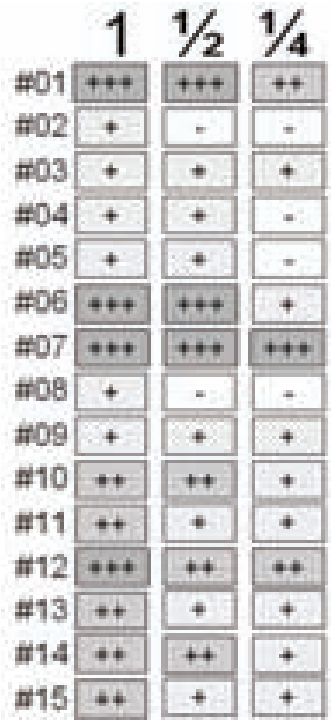

\section{Conclusion}

The poor relationship between positive nitrite test and semiquantitative as well as the large day-to-day intra-individual variability in nitrite positive test indicates that nitrite dipstick testing for screening UTI sows is questionable, especially when performed on a spot urine sample. Moreover, USG should be used to interpret adequately any positive results, as dilution of urine secondary to high water intake can lead to false negative results.

\section{References}

Almond G.A. et al., 2006. Proceed. IPVS, 125

Martineau G-P and G.A. Almond, 2008. CAB Reviews. 048

Martineau G-P et al.,2007. Journées Rech. Porcine en France. 39, 1-7

Sialelli J-N, 2004. Réussir porcs. 102, 40-43 


\title{
0.124
}

\section{Shoulder lesions in loose-housed lactating sows on partly slatted floors}

\author{
Nils Holmgren $^{2}$ Nils Lundeheim ${ }^{1}$ \\ 1. Swedish University of Agric. Science, Uppsala, Sweden; 2. Swedish Animal Health Service, Skara, Sweden
}

\section{Introduction}

Shoulder lesions (SL) were on average found in about $1 / 3$ of loose-housed lactating sows in 78 Swedish herds (3). Main risk factors are poor body condition, parity number, lameness and MMA $(1,2)$. In addition, environmental factors such as floor type may contribute to the development of SL during lactation $(1,2)$.

\section{Material and methods}

The trial was conducted as a prospective cohort study in a piglet producing herd. Consecutive batches of 40 loose-housed sows farrowed in pens with partly slatted floors. Half of the pens had $1.3 \mathrm{~m}^{2}$ concrete lying area for sows and $2.8 \mathrm{~m}^{2}$ plastic slatted floors (open area $34 \%$ ) with square formed $3 \mathrm{~mm}$ raised edges. Remaining pens in the same barn had $2.1 \mathrm{~m}^{2}$ concrete lying area and $2.8 \mathrm{~m}^{2}$ plane slatted floor (open area 34\%). Sows allocated into different pen types had no SL before farrowing, were matched for parity numbers and had all good body condition scores. Six times, once before farrowing and at weekly intervals until weaning at $5 \mathrm{w}$, body condition scores and SL were monitored. At this occasion, $1 \mathrm{~h}$ after morning feeding when all sows laid down, the lying position of the sows was recorded: heads and shoulders on concrete area or slatted floor located (SFL). Temperatures in barn were also measured. Differences in prevalences were studied by chi-square test.

\section{Results}

Prevalences of sows with SL increased linearly during lactation and reached $35-45 \%$ at weaning. Size of concrete lying area $\left(2.1 \mathrm{~m}^{2}\right.$ or $\left.1.3 \mathrm{~m}^{2}\right)$ and structure of slatted floor (edges or plane) did not significantly influence on the occurrence of SL.

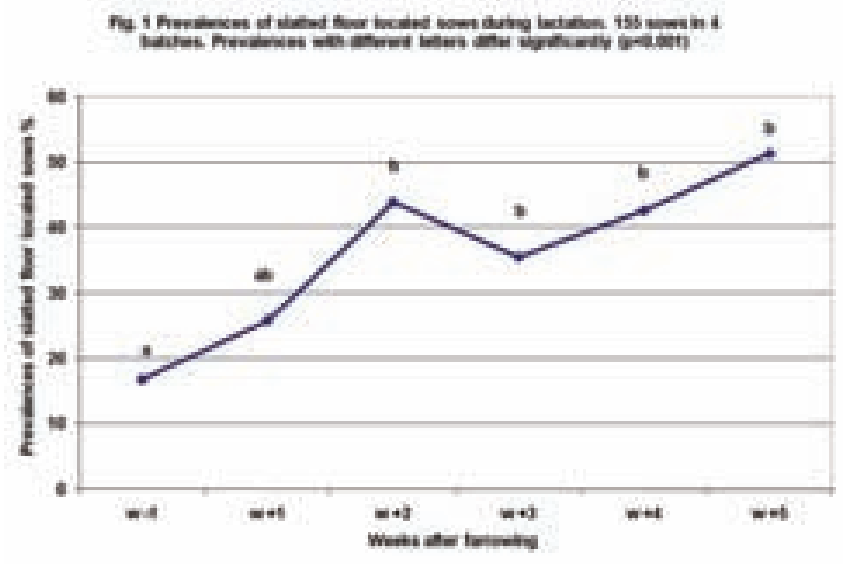

Before farrowing, $10-20 \%$ of the sows were SFL and from $2 \mathrm{w}$ after farrowing until weaning, $40-50 \%$ of the sows were SFL (Fig.1). When barn temperature was $18-21^{\circ} \mathrm{C}$ during lactation, significantly $(p<0.05)$ more SFL sows $(41 \%)$ were observed compared to when barn temperature was $17^{\circ} \mathrm{C},(31 \%)$.
For each sow, sum of SFL sows was calculated (0-6 for each sow). Among sows often in SFL-position, prevalence of SL at weaning was significantly higher compared to sows that were in SFLposition more seldom (Fig.2).

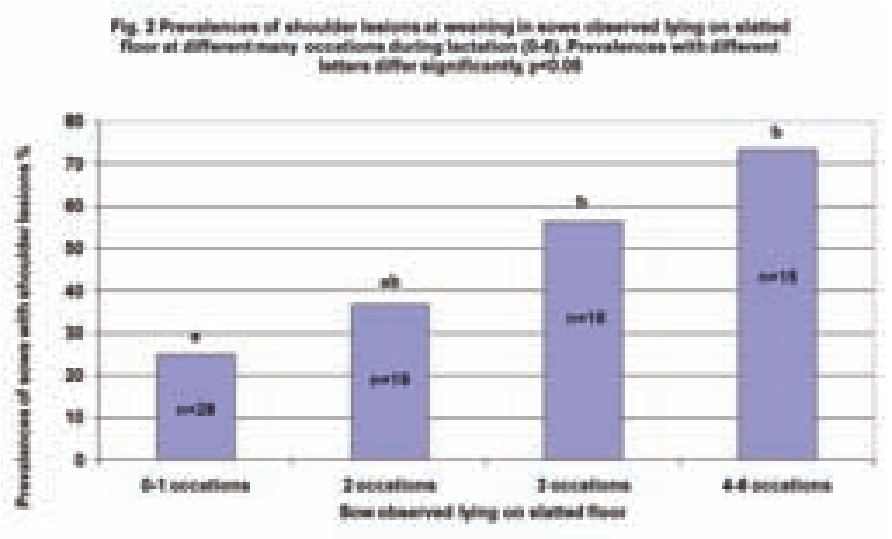

\section{Discussion}

At farrowing, sows prefer to lie on a warm area of the pen (5). Due to increased feed consumption and metabolic activity during lactation, the body temperature will increase (4). A high temperature in barn will contribute to this. To maintain thermal comfort during lactation the alternative for the sow is to lie on the slatted floor area. This is especially comfortable for loosehoused sows in modern farrowing pens as the slatted floor area is usually larger than the concrete lying area. However, increased pressure from slatted floors on shoulder tissues will result in SL (6).

In conclusion, to prevent SL in loose-housed lactating sows, slatted floor areas should be made uncomfortable to lie on and the concrete lying area should be made as large and comfortable as possible.
References
1. Davies PR et al. 1996. JAWMA 208;1058-62
2. Davies PR et al. 1997. JAWMA 210;1173-78
3. Ivarsson E et al. 2009. Pig Rapport $\mathrm{Nr} 42$
4. Littledike ET et al.1979. Lab Animal Science 29;621-4
5. Phillips PA et al.2000. Appl Anim Behav Sci 67; 59-65
6. Zurbrigg K. 2006. J Anim Sci 84; 2509-2514 


\title{
0.125
}

\section{Hemoglobin varies with parity and stage in cycle}

\author{
Inge Larsen ${ }^{1}$ Flemming Thorup ${ }^{2}$ \\ 1. LVK, Oestervraa, Denmark; 2. Pig Research Centre, Danish Agriculture and Food Council, Copenhagen, Denmark
}

\section{Introduction}

Various reference values are published for hemoglobin, however very few references distinguish between young and mature animals, nor to the different stages in a sows reproductive cycle.

Table 1. Reference values for hemoglobin from different sources.

\begin{tabular}{|c|c|}
\hline $\mathbf{g} / \mathbf{d L}$ & Reference \\
\hline $10.6-15.9$ & Central Laboratory, Life/ KU, 2009 (1) \\
\hline 12.6 & Schalm's Veterinary Haematology, 2008 (2) \\
\hline $9.0-13.0$ & The Merck Veterinary Manual, 2008 (3) \\
\hline $10.8-14.8$ & Idexx VetMedLab, 2007 (4) \\
\hline
\end{tabular}

The objective of this study was to determine the level of hemoglobin in Danish gilts and sows at different stages of the reproductive cycle.

\section{Material and Methods}

This study was performed in one Danish 360 sow herd participating in the Danish SPF programme. The farm was free from Mycoplasma hyopneumoniae, PRRS and Actinobacillus pleuroneumoniae (AP) type 2, but AP type 6 and 12 positive.

Blood was collected during pregnancy and lactation from 152 sows between April and December 2008. 900 bloodsamples were included in the calculations.

Bloodsamples were mailed by post, and analysed one or two days later at Idexx VetMedLab in Germany. Statistical analysis were carried out using Microsoft Office Excel 2007.

\section{Results}

The average level of hemoglobin of all bloodsamples in this study was $10.4 \mathrm{~g} / \mathrm{dL}$ (900 samples) with a standard deviation (st.d.) of 1.1.

The average level of hemoglobin in gilts was $11.0 \mathrm{~g} / \mathrm{dL}$ (st.d. = 1.0) (31 gilts, 193 samples). The average level of hemoglobin in pregnant 2nd to 9th litter sows was $10.2 \mathrm{~g} / \mathrm{dL}$ (st.d. = 1.0) (101 sows, 625 samples). The difference was statistical significant ( $p=$ $2 \times 10-21)$.
Figure 1. Hemoglobin $(\mathrm{g} / \mathrm{dL})$ in gilts and sows by day after first insemination. *

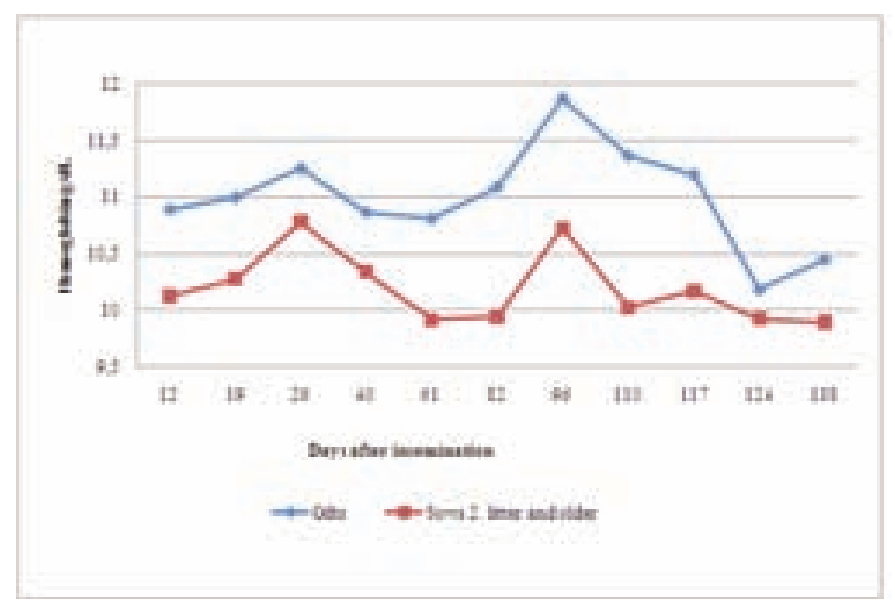

The level of hemoglobin was significantly higher in pregnant gilts than in sows at all points during gestation, while the level in gilts approaches the level of sows during lactation.

\section{Discussion}

When is a pig anemic?

To render a sow anemic or normal, should be based on an evaluation of what level of hemoglobin has an effect on sow welfare and productivity.

In this study no negative correlations were observed between level of hemoglobin end frequency MMA treatments, nor to the level of still born piglets.

The present experiment is extensive but performed in one herd only. A study in more herds, preferably covering more countries or continents are needed, to cover management, feeding and genetic differences as well.

\section{References}

1. Central Laboratory, Copenhagen University, Faculty of Life Sciences, Dyrlaegevej 48, 1870 Frederiksberg C, Denmark

2. Schalm's Veterinary Hematology, 5th edition 168,p. 1089-1095, Lea \& Feabiger

3. Merck \& Co Inc. 2008. http://www.merckvetmanual.com/mvm/htm/ present/mvm_mercklink.htm. Whitehouse Station, NJ USA.

4. Idexx VetMedLab. Idexx Laboratories 2007. Directory of Services 2007. Vet Med Labor GmbH. Mörikestr. 28/3, D-71636 Ludwigsburg, Germany. 


\title{
0.126
}

\section{Facilitation of nursing behavior in primiparous sows during colostrum phase improves piglet condition}

\author{
Jorge Miquet ${ }^{1}$ Gustavo Viana $^{2}$ \\ 1. I.P.B.-C.I.C.V.-INTA, Morón, Argentina; 2. Janssen Animal Health Argentina, Buenas Aires, Argentina
}

\section{Introduction}

The development and wellbeing of a piglet are determined by its ability to fulfill the need for homeostasis, growth and resistance to sanitary challenges. Early colostrum intake is the key for success. For the first two days of their lives, colostrum provides the required nutrients to the piglets and during their first weeks, the immunoglobulin (lg) fraction passively protects the piglets from disease (1). Critical factors for early life survival are the ability of the piglet to suckle, the available volume of colostrum, the udder quality and the behavior of the sow during the colostrum phase. For primiparous sows, farrowing is a stressful new experience which can result in savaging and crushing of neonatal piglets. The introduction of the group housing system potentially increases this problem, as primiparous sows get confined for the first time in their life at parturition. Azaperone is a sedative used to calm pigs at stressful moments, such as transport, regrouping and aggression.

In this study, the effect on piglet development after an injection of azaperone at the end of the partus of primiparous sows, was investigated in two farms with different housing systems. One farm had conventional farrowing crates (CF) the other outdoor huts $(\mathrm{OH})$.

\section{Materials and Methods}

50 primiparous sows per farm were included in the study. Both farms (BF) belonged to the same organization, with comparable genetics, nutrition and health care plan. Half of the sows were randomly allocated to either a azaperone treated group (AZA) or a non-treated reference group (NT). The AZA group was injected IM, $2 \mathrm{mg}$ azaperone per $\mathrm{kg}$ body weight (BW) (= $5 \mathrm{ml}$ Stresnil ${ }^{\circ}$ per $100 \mathrm{~kg} \mathrm{BW}$ ) at the moment of expulsion of the placenta. To assess the condition and health status of the piglets in field conditions, mortality rate and weight gain were used as parameters. The piglets were weighed and identified individually at birth and at weaning. Farrowing and weaning date, number of piglets born alive (BA), pre-weaning piglet mortality, mortality reason and number piglets weaned (W) were recorded.

A t-test was used for comparison of continuous variables and a Chi-square test for comparison of categorical variables (Statistix 1.0 for Windows).

\section{Results}

Lactation length and BA were comparable in both groups (Table 1). Mortality however, was higher in the NT group. Mortality reasons were similar for both groups, except for crushing (NT $n=35, A Z A n=16)$ and cannibalism (NT $n=7, A Z A n=0)$, which was significantly higher in the NT group $(p<0.01)$. There was an increase of $W$ and the average weaning weight of the piglets was higher in the AZA group.
Table 1: Piglet data comparison

\begin{tabular}{|c|c|c|c|c|c|c|}
\hline & & $\mathrm{BA}$ & w & $\begin{array}{l}\text { Lactation } \\
\text { length } \\
\text { (days) }\end{array}$ & $\begin{array}{c}\text { Weaning } \\
\text { weight }(\mathrm{kg})\end{array}$ & $\begin{array}{c}\text { Litter } \\
\text { weaning } \\
\text { weight }(\mathrm{kg})\end{array}$ \\
\hline \multirow[b]{2}{*}{$\mathrm{CF}$} & NT & 10.2 & 8.8 & 20.6 & 6.16 & 54.24 \\
\hline & AZA & 10.1 & 9.1 & 20.3 & 6.54 & 59.65 \\
\hline \multirow[b]{2}{*}{$\mathrm{OH}$} & NT & 9.8 & 7.8 & 20 & 5.88 & 45.91 \\
\hline & $A Z A$ & 10 & 8.4 & 20.7 & 6.16 & 51.83 \\
\hline \multirow[b]{2}{*}{$\mathrm{BF}$} & NT & 10 & 8.3 & 20.3 & 6.02 & 50.07 \\
\hline & AZA & 10 & 8.7 & 20.5 & 6.35 & 55.74 \\
\hline
\end{tabular}

Data presented in bold are statistically different $(p<0.05)$

This resulted in a significantly higher litter weight at weaning in the AZA group in both housing systems. The number of piglets weaned and the average individual weaning weight of the treated animals was significantly higher for AZA in BF.

\section{Discussion}

The present study showed the benefit of an azaperone injection of primiparous sows at the end of the partus. Piglet survival increased and they thrive better, resulting in a $11.3 \%$ litter weight increase at weaning.

In conventional farrowing crates, the difference was mainly due to an increased weight gain of the piglets, while in outdoor huts, a decrease of early mortality was the cause of the difference.

Calm sows in the first hours after farrowing are less likely to kill their piglets and they allow them access to the colostrum. Not only more piglets survive, but they develop better during lactation, a clear sign of their wellbeing.

\section{References}

1. J. Le Dividich et al., Journal of Agricultural Science 2005, 143, 469485 


\title{
0.127
}

\section{A study to evaluate the use of Elastrator ${ }^{\circledR}$ rings for treatment of umbilical hernia}

\author{
$\underline{\text { Kaj Vestergaard }}^{1}$ Kristian Viekilde² Poul Bækbo' \\ 1. Pig Research Centre, Danish Agriculture \& Food Council, Copenhagen, Denmark; 2. PORCUS Pig Practice, Ringe, Denmark
}

\section{Introduction}

Umbilical hernia is one of the most frequent developmental defects in pigs. Most investigators have hypothesized that hernia is inherited, although there is dispute about the mode of inheritance (1). Umbilical infections have also been claimed to cause umbilical hernias in pigs (2). In other species, breed and sex have been identified as risk factors, but no association between umbilical infection and umbilical hernias has been detected (3). The disease causes significant losses due to decreased productivity, including increased mortality, and, above all, umbilical hernia has important implications for pig welfare. For that reason, PORCUS Pig Practice and Pig Research Centre initiated a study to evaluate the efficacy of using Elastrator ${ }^{\circledR}$ rings to treat umbilical hernias in young pigs (4).

The purpose of the study was to determine whether surgical treatment of umbilical hernia using Elastrator ${ }^{\circledR}$ rings increased the pig's chance of reaching slaughter weight.

\section{Materials and Methods}

The study was conducted under the authorization of the Danish Animal Experiments Inspectorate. A total of 192 pigs with umbilical hernia were collected from herds throughout Denmark, independent of herd management and health status. On arrival at the research farm, the umbilical hernia of each pig was examined to determine whether the hernia was reducible through the abdominal wall. Pigs with irreducible hernias were excluded from the study. The pigs included in the study were randomly divided into two groups:

Group A: Treatment with Elastrator ${ }^{\circledR}$ rings

Group B: No treatment

An Elastrator ${ }^{\circledast}$ ring was fixed on the hernial sac close to the abdominal wall. All other medical treatments and causes of death among the pigs were recorded. The data analysis was conducted using $R$ version 2.9.2. A logistic regression model described the risk of death from umbilical hernia, with treatment, sex and weight as explanatory variables. The design of the study had a power of $80 \%$ to show a $50 \%$ reduction in death from complicated umbilical hernia.

\section{Results and Discussion}

The lower number of male pigs included in the study (Table 1) was mostly due to the risk of placing the Elastrator ${ }^{\circledR}$ ring close to the prepuce.

Table 1. No. of pigs with umbilical hernia

\begin{tabular}{|c|c|c|c|c|c|c|c|}
\hline & $\begin{array}{c}\text { Ex- } \\
\text { chnded }\end{array}$ & $\begin{array}{c}\text { AW } \\
\text { kz }\end{array}$ & $\begin{array}{c}\text { lis- } \\
\text { cluded }\end{array}$ & $\begin{array}{c}\text { Group } \\
\text { A }\end{array}$ & $\begin{array}{c}\text { AW } \\
\text { kz }\end{array}$ & $\begin{array}{c}\text { Grouip } \\
\text { B }\end{array}$ & $\begin{array}{c}\text { AW } \\
\text { kz }\end{array}$ \\
\hline Male & 57 & 16.8 & 16 & 7 & 17.0 & 9 & 16.0 \\
\hline Female & 70 & 17.3 & 47 & 23 & 17.2 & 24 & 17.3 \\
\hline Total & 127 & 17.2 & 63 & 30 & 17.1 & 33 & 16.8 \\
\hline
\end{tabular}

Table 2. No. of dead and surviving pigs in groups $A \& B$

\begin{tabular}{|c|c|c|c|}
\hline & Deaths & Survivals & Total \\
\hline Group A & 5 & 25 & 30 \\
\hline Group B & 15 & 18 & 33 \\
\hline & 20 & 43 & 63 \\
\hline
\end{tabular}

The results of the logistic regression showed a significant increase in mortality in the control group compared to the treatment group. The odds ratio was 4.75 (1.40, 18.72). Furthermore, the greater the start weight, the lower the risk of dying. The odds ratio was $0.87(0.78,0.95)$. Sex did not influence the risk of dying from umbilical hernia

Of the 20 pigs that died during the trial period, 9 were put down and 11 were found dead. The death of four pigs (two from each group) seemed to be unrelated to complications due to the umbilical hernia. The mortality among the excluded pigs (127; Table 1 ) was the same as for the pigs in group $B(45 \%)$. The 43 pigs that survived were all approved for slaughter, but they all received a remark by the meat inspectors at the slaughterhouse concerning internal signs of umbilical hernia.

Even though the study showed a significant result in favour of using Elastrator ${ }^{\circledR}$ rings, one has to be aware of the number of pigs that were excluded. According to this study, out of nearly 800,000 Danish pigs developing umbilical hernia every year, only one third ( 264,000 pigs) can be given the treatment. Nevertheless, the results from this study indicate an additional survival of 76,000 pigs per year, which can be estimated to provide the Danish pig farmers with a bonus of $€ 4,500,000$.

\section{References}

1. Angus K. Young GB. Vet Rec 1972; 90:245-247

2. Becker HN. Diseases of swine. 6th ed. Ames. lowa State University Press 1986; 861-862

3. Hayes HM Jr. Am J Vet Res 1974; 35:839-842

4. Pollicino P. et al. Use of Elastrator ${ }^{\circledR}$ rings to repair umbilical hernias in young swine. J. Swine Health Prod. 2007; 15(2). 92-95 


\title{
0.128
}

\section{Piglet serum IgG, a non disruptive method to measure colostrum distribution}

\author{
Jan Jourquin ${ }^{1}$ Marrit van Engen ${ }^{2}$ Lieve Goossens ${ }^{1}$ \\ 1. Janssen Animal Heatlh, Beerse, Belgium; 2. FarmulaOne, Best, Netherlands
}

\section{Introduction}

Colostrum intake is critical for the survival of the piglet. The first 2 days of their lives, colostrum provides the nutrients to fulfill the piglets needs for homeostasis and growth and during their first weeks, the immunoglobulin (lg) fraction passively protects the piglets from disease (1). It is estimated that a piglet needs 160 to $170 \mathrm{ml}$ of colostrum per kg bodyweight to survive (2). Immunoglobulin $\mathrm{G}(\mathrm{lgG})$ is absorbed by the gut wall during 24 to 48 hours, but the lgG content of the colostrum decreases quickly after birth (1). Next to total colostrum yield and IgG concentration, the distribution of the available quantity amongst the different littermates is becoming more important with increasing litter size. Several methods to estimate colostrum intake have been described in the past but they all share one disadvantage being the extensive manipulation of piglet and or sows during the colostrum intake phase. To eliminate the impact of manipulation on sow and piglet behavior, an alternative method without extra manipulations is required.

In this study, a method based on piglet serum IgG concentration was used to measure the impact of litter size on colostrum distribution.

\section{Materials and Methods}

In a commercial sows farm, 114 litters were included in the study. The farm data recording system was used to retrieve the relevant reproductive data. All normal farm procedures were continued. At tail docking (day 3 post partum) a blood drop of the remaining tail bud from six randomly chosen litter mates was sampled. These samples were analyzed with a DAS-ELISA test (3) (Colostrum Quality Counter ${ }^{\mathrm{TM}}$ ). There is a great variability in colostrum yield and colostrum IgG concentration between sows. Therefore the absolute IgG concentrations have poor predicting value on colostrum intake. Within the litter however, these variables are not relevant. In order to be able to compare the distribution for colostrum between sows, the coefficient of variation of the piglet serum $\lg G(C V \lg G)$ was calculated. This is the standard deviation divided by the average of that litter.

The statistical analysis was performed with the SAS program. A Poisson regression model on the number of died piglets per sow was used. The total number of piglets with the sow was included as covariate.

\section{Results}

It was demonstrated that the variation in lgG levels increased with litter size (Figure 1).

Figure 1: Impact of litter size on Ig G distribution and piglet mortality

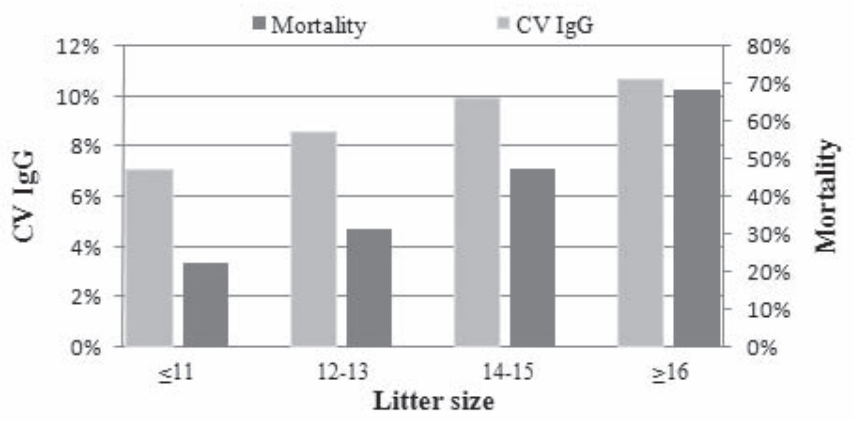

The mortality rate over all litters is strongly correlated with litter size $(p<0.0001)$. Lower birth weight and vitality compromise the colostrum intake. With an increasing number of piglets, total colostrum volume becomes a second critical factor. The distribution of that colostrum should therefore be optimal. However, the data show that the distribution gets worse with increasing litter size. All measures that increase the colostrum production and facilitate the distribution of the colostrum increases the chance of the piglets to survive.

\section{Discussion}

In this study, it is shown that by using serum IgG concentrations of three day old piglets, the distribution of the colostrum can be estimated. Both pre-weaning mortality and variation in colostrum uptake (represented as CV IgG) are positively correlated with litter size. This underlines the increasing importance of individual colostrum uptake with increasing productivity of our sow farms.

\section{References}

1. J. Le Dividich et al., Journal of Agricultural Science 2005, 143, 469485

2. N. Devillers et al., Animal 2007 1:7 p 1033-1041

3. A. Bianchi et al., Veterinary Immunology and Immunopathology 1995, 44, p 309-317 


\subsection{9}

\section{Comparison of body surface temperature and rectal temperature after administration of pyrogenic lipopolysaccharides}

Jan Bernardy

University of Veterinary and Pharmaceutical Sciences, Brno, Czech Republic

\section{Introduction}

Aim of the study was to contribute to the assess-ment of the feasibility of using infrared thermography as a method for routine diagnosing and treatment decision of diseased pigs and animal welfare monitoring. Infrared thermography is a non-invasive method, able to measure the surface temperature via emitted energy (infrared radiation) from the surface of the animal body(1).

However the method brings time-savings and facilitate handling with animals, it is still seldom used, partially due to conservative approach of veterinary surgeons, partially due to missing relevant data of veterinary infrared thermography. Lipopolysaccharid was used to induce pyrogenic reaction and compare core and surface temperature reactivity, and both values were compared.

\section{Material and Methods}

Twelve piglets 8 weeks old were used and randomly allocated into the two groups, a trial group and controls $(n=5)$. Temperature was measured at both groups either by infrared thermometer at thorax (measurement IR-LPS at trial group and IR-C at control group) or rectally (measurement TR-LPS at trial group and measurement TR-C at control group). Pyrogenic agent (lipopolysaccharide from Escherichia coli 0111:B4, lyophilized powder, sample No L2630, Sigma-Aldrich) was dissolved and administered intravenously to the trial group in amount of $0.1 \mathrm{ug}$ per kg of body weight. Temperature was measured every 30 minutes for 8 following hours. The data were evaluated by non-parametric Wilcoxon pair test, Spearman correlation and visualized by Statistica v.9 software.

\section{Results and Discussion}

Both values of temperatures measured rectally and superficially showed significant Spearman correlation rate $(0.703$, calculated at level of significance $p<.05)$, and difference between measurement at the hour 0 and 1 or between hour 1 and 8 showed significance higher than $\mathrm{p}<.05$ ). However some papers did not find correlation of skin and rectal temperature not satisfyinh enough, increased correlation in the postparturient period was noted (2) another papers assessing wound severity with colour and infrared imaging of reactive hyperemia (3), life-threatening complication (4) or significant linear relationship was observed between ambient tem-perature and mean body surface temperature at inoculated febrile pigs(5). The results obtained in the trial imply possibility to rely on correlation between rectal and superficial temperature. However there are some physical values to be considered, as airflow, humidity and some other values, refining and improvement the method of body surface temperature measurement in pigs would be helpful diagnostic tool.

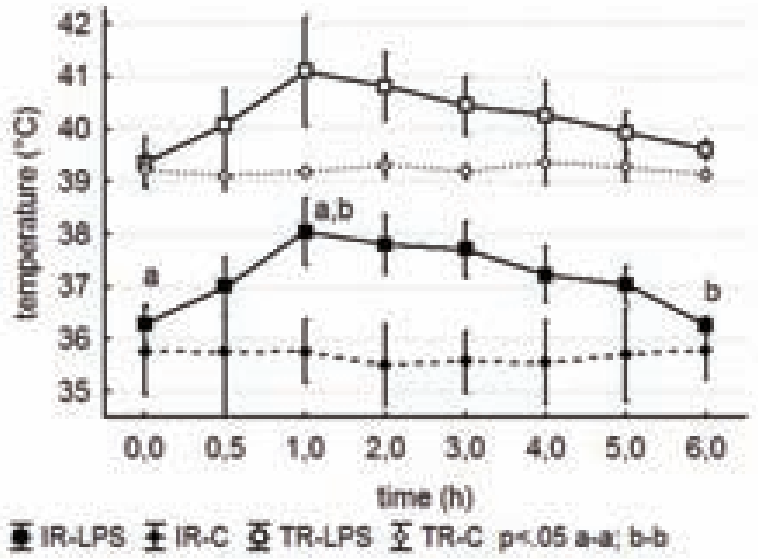

\section{Reference}

1 Speakman, J. R., and S. Ward, 1998, Zool Anal Commun Syst, 101:224

2 Zinn,K.R. et al., 1985, Am.J.Vet.Res.,46:1372

3 Hansen, G.L. et al., 1996, Wound.Repair Regen 4:386

4 Rich, P.B. et al., 2002, J.Surg.Res, 120:163

5 Loughmiller,J.A. et al.,2001, Am.J.Vet.Res., $62: 676$ 


\title{
0.130
}

\section{Evaluating PCV2 immune status of a herd and determining the value of vaccination}

\author{
Cristina Venegas-Vargas; Barbara Straw \\ Michigan State University, East Lansing, MI, USA
}

\section{Introduction}

Porcine Circovirus type 2 is an important viral pathogen worldwide. PCVAD was first described in Canada in the early 1990s as PMWS ${ }^{1,2}$. The Michigan State University swine herd experienced moderately severe PMWS accompanied by $5-10 \%$ nursery mortality. A vaccination program was initiated in 2007. Signs of clinical disease disappeared after implementing the program and remained inapparent for the next $2 \mathrm{yrs}$, and mortality of pigs in the nursery remained below $2 \%$. Potter et al. (2009) documented reduction in viral load following vaccination. There is the assumption that vaccination could minimize viral shedding and reduce environmental contamination to a no-effect level; allowing elimination of vaccination programs ${ }^{3,4}$. Two ways of determining if infectious levels of virus are present in a vaccinated herd were compared; monitoring disease in sentinels and recovery of virus through PCR.

\section{Materials and Methods}

Three replicates of 20 pigs each and one replicate of 22 pigs were weighed and ear tagged at weaning for use in the study. Pigs were matched in pairs by sex, weight and dam. Matched pairs of pigs were randomly allocated to sentinel (non-vaccinated) and control groups. Control pigs received a Killed Baculovirus vector PCV2 vaccine at weaning and 2 wks later per label directions. Blood samples were taken at weaning, 9 and 20 wks of age. ELISA, IFA-4 dilution and PCV2 PCR (Pools of 2 animals) were performed. Twelve ropes were placed in pens of common vaccinated pigs four times at one-month intervals. PCV2 PCR was performed. Pigs were weighed again at completion of the trial ( 20 wks of age) and ADG was calculated.

\section{Discussion}

Monitoring sentinels did not demonstrate presence of virus in the herd either through reduced pig performance, ELISA testing or PCR serum samples at normal cut offs (35 Ct). But it did through IFA-4 dilution, and PCR serum samples at a later $\mathrm{Ct}$ cut offs $(40 \mathrm{Ct}$ ). Sentinel monitoring required 4 months to complete while oral fluid PCR results were available immediately. Cut off value of PCR test on saliva and serum in a vaccinated herd may need to be changed.

\begin{tabular}{|l|c|c|c|c|c|c|}
\hline RESULTS & Week & Sentinel & & Control & & P-value \\
\hline ELISA (S/P) & 3 & 0.4499 & & 0.4602 & & 0.8591 \\
\hline & 9 & 0.1927 & & 0.7829 & & 0.0001 \\
\hline & 20 & 0.1067 & & 0.9630 & & 0.0001 \\
\hline IFA-4 dilution & 3 & & & & & 1.0 \\
\hline & 9 & & & & & 0.0001 \\
\hline & 20 & & & & & 0.0001 \\
\hline ADG (g) & & 713.5 & & 695.4 & & 0.4552 \\
\hline Mortality & & 7.32 & & 4.89 & & 1.0 \\
\hline PCR & & Sentinel & & Control & & \\
\hline & Serum Ct cut off & & 35 & 40 & 35 & 40 \\
\hline Positives & 3 & $0 / 15$ & $1 / 15$ & $0 / 14$ & $2 / 14$ & \\
\hline & 9 & $0 / 19$ & $1 / 19$ & $0 / 19$ & $2 / 19$ & \\
\hline & 20 & $0 / 18$ & $0 / 18$ & $0 / 19$ & $1 / 19$ & \\
\hline Oral Fluid & & $35 \mathrm{Ct}$ & & $40 \mathrm{Ct}$ & & \\
\hline Positives & $1^{\text {st }}$ & $1 / 12$ & & $9 / 12$ & & \\
\hline & $2^{\text {nd }}$ & $1 / 12$ & & $4 / 12$ & & \\
\hline & $3^{\text {rd }}$ & $3 / 11$ & & $6 / 11$ & & \\
\hline & $4^{\text {th }}$ & $0 / 11$ & & $0 / 11$ & & \\
\hline
\end{tabular}

\section{References}

${ }^{1}$ Rowland, R. et al., 2008. Proc. 39th AASV. ${ }^{2}$ Kixmoller M. et al., 2008. Vaccine, 26:3443-3451. ${ }^{3}$ Henry et al. 2008. Proc. $39^{\text {th }}$ AASV. ${ }^{4}$ Potter M. et al., 2009. Per comm. 


\title{
0.131
}

\section{Effect of $\mathrm{CIRCOVAC}^{\circledR}$ vaccination of sows on their reproductive performance}

\author{
John Hayden ${ }^{1}$ Ricardo Neto ${ }^{2}$ François Joisel ${ }^{3}$ \\ 1. Integra Veterinary Services, Mundford, Thetford, UK; 2. Merial Animal Health Ltd, Woking, UK; 3. Merial S.A.S., Lyon, France
}

\section{Introduction}

Recent studies have shown that PCV2 is involved not only in PMWS, but also in several other disease presentations, including reproductive disorders, deteriorating sow performance $(1,2)$. The objective of this study was to assess the impact of CIRCO$\mathrm{VAC}^{\circledR}$ on the reproductive performance of a sow breeding unit weaning pigs on-site and selling them at $35 \mathrm{~kg}$.

\section{Material and Methods}

A 1000-sow outdoor breeding unit started vaccinating the breeding animals in May 2007 with a PCV2 inactivated vaccine, CIRCOVAC. The first vaccinated sows farrowed in July 2007. PCVD has been clinically diagnosed on the nursery and finishing units. The number of pigs born alive per litter and weaned per litter was routinely recorded using herd management software.

Historical data, since 2005 were collected. The data were divided into blocks of 6 months from the start of vaccination for the periods before and after. The data were also compared for a period of 1 year before and after PCV2 vaccination was implemented. Statistical analyses were undertaken using the two sample t-test with unequal variances and a two-factor (month and year) analysis of variance. Tests were carried out using proprietary statistical software.

\section{Results}

Live born piglets per litter after CIRCOVAC vaccination were increased by 1.425 pigs, $\mathrm{p}<0.001$ (t-test) compared to the period before (2004 to July 2007).

Analyzing the data before and after July 2007 by a t-test shows that there is an improvement in the mean number of pigs of 1.066 pigs weaned per sow per litter after CIRCOVAC vaccination started.

Regarding pigs weaned per sow per litter, (Table 1) there was no significant difference between the first 6 months of 2005, 2006 and 2007 (before vaccinated sows started farrowing) but these were significantly different to the first 6 months of 2008 and 2009 (CIRCOVAC vaccinated sows started farrowing in July 2007), $p<0.001$. Similarly that there was no significant difference between the second 6 months of 2004, 2005 and 2006 (before vaccinated sows started farrowing) but these were significantly different to the second 6 months of 2007 and 2008.
Table 1: Six month periods with mean number pigs born alive per litter.

\begin{tabular}{|l|c|c|}
\hline & January - June & July - December \\
\hline 2004 & & $9.901^{\mathrm{a}}$ \\
\hline 2005 & $10.07^{\mathrm{a}}$ & $10.048^{\mathrm{a}}$ \\
\hline 2006 & $10.2^{\mathrm{a}}$ & $9.78^{\mathrm{a}}$ \\
\hline 2007 & $10.27^{\mathrm{a}}$ & $\mathbf{1 1 . 0 7}^{\mathrm{b}}$ \\
\hline 2008 & $\mathbf{1 1 . 6 9 ^ { \mathrm { b } }}$ & $\mathbf{1 1 . 2 4 ^ { \mathrm { b } }}$ \\
\hline 2009 & $\mathbf{1 1 . 8 2 ^ { \mathrm { b } }}$ & \\
\hline Different superscripts (a,b) indicate significant difference $(\mathrm{p}<0.001)$. \\
\hline
\end{tabular}

The use of CIRCOVAC resulted in an improvement of 0.8 pigs born alive between the 6 months before the use of vaccine and 6 months after, from 10.27 to 11.07 respectively.

The number of piglets weaned per sow per litter was significantly different between the first half of the years 2005, 2006, 2007 and the first half of 2008 and 2009, the mean number of pigs weaned per litter for the periods of July to December 2004 and 2006 was significantly different to the same periods in 2007 and 2008, Table 2.

Table 2: Six month periods with mean number pigs weaned per litter.

\begin{tabular}{|l|c|c|}
\hline & January - June & July - December \\
\hline 2004 & & $8.892^{\mathrm{a}}$ \\
\hline 2005 & $9.672^{\mathrm{a}}$ & 9.22 \\
\hline 2006 & $9.847^{\mathrm{a}}$ & $8.685^{\mathrm{a}}$ \\
\hline 2007 & $9.842^{\mathrm{a}}$ & $\mathbf{1 0 . 0 7}^{\mathrm{b}}$ \\
\hline 2008 & $\mathbf{1 0 . 7 6 7 ^ { \mathrm { b } }}$ & $\mathbf{1 0 . 1 4}^{\mathrm{b}}$ \\
\hline 2009 & $\mathbf{1 0 . 9 2 3 ^ { \mathrm { b } }}$ & \\
\hline \multicolumn{2}{|c|}{ Different superscripts $\left.{ }^{\mathrm{a}, \mathrm{b}}\right)$ indicate significant difference $(\mathrm{p}<0.001)}$. \\
\hline
\end{tabular}

\section{Discussion and conclusion}

This study shows the impact of using CIRCOVAC on the reproductive performance of vaccinated sows.

Improving the number of pigs weaned per sow per litter optimizes the sows' performance. This increase reduces the cost of production and considerably increases profitability.

The constant number of pigs born alive and weaned per litter during the post vaccination period indicates that the improvement achieved with CIRCOVAC vaccination resulted in a stable and sustained increase of the number of piglets born alive and weaned per litter.

\section{References}

1. O'Connor B. et al, (2001) Can Vet J; 42:551 - 553

2. West K.H. et al., (1999) J. Vet. D. Inv, 11, 6:530-532

${ }^{\circledR}$ CIRCOVAC is a registered trademark of Merial in Canada and elsewhere. 


\subsection{2}

\section{Efficacy against porcine circovirus type 2 of a single dose of Porcilis PCV given to MDA-positive piglets of either one or three weeks of age}

Andreas Palzer ${ }^{1}$ Beate Rist ${ }^{2}$ Mathias Ritzmann ${ }^{3,2}$ Christiane Lang $^{3}$ Stefan von Rueden ${ }^{4}$ Karl Heinritzi $^{2}$ Alex Eggen $^{5}$ Jos Smeets $^{5}$

1. Tierarztpraxis Scheidegg, Scheidegg, Germany; 2. Clinic for Swine of the LMU Munich, Oberschleissheim, Germany; 3. Clinic for Swine of the VUW, Vienna, Austria; 4. Intervet Deutschland GmbH, Unterschleissheim, Germany; 5. Intervet/Schering-Plough Animal Health, Boxmeer, Netherlands

\section{Introduction}

Infections with Porcine Circovirus type 2 (PCV2) are ubiquitous in the swine population and are associated with many disease complexes. The object of this study was to compare the efficacy of a single dose of Porcilis PCV (Intervet/Schering-Plough Animal Health), with a placebo, Diluvac Forte ${ }^{\circledR}$. The study was performed on a German 500-sow farm on which PMWS had been diagnosed clinically as well as by pathology, and the presence of PCV2 confirmed by serology. Vaccination of sows with Circovac $^{\circledR}$ had ceased 7 months before the trial began.

\section{Materials and Methods}

In this partly blinded study, a total of 416 piglets were selected to receive a $2 \mathrm{ml}$ dose i.m. of either Porcilis PCV or of placebo (Diluvac Forte ${ }^{\circledR}$ ) at 1 and 3 weeks of age (Table 1).

The piglets were weighed at 1, 3, 11 and 24 weeks of age. All the individual piglet treatments were recorded as a measure of morbidity. Post-mortem examination was performed on each piglet to determine the cause of death. Average daily weight gain (ADG), morbidity and mortality were compared between groups, analyzed using ANOVA and the Chi-square test.

Blood samples (serum) were taken from $20 \%$ of each group at intervals of 2 weeks until the start of the fattening period, and every 4 weeks thereafter. The sera were tested by ELISA for antibodies against PCV2 (log2) at the R\&D Service Laboratory (Boxmeer, The Netherlands) and by Ingezim Circovirus IgG/lgM (pos/ neg., Ingenasa, Madrid, Spain) at the Clinic for Swine, University of Veterinary Medicine, Vienna. Viremia was determined by qPCR (log10 copies $/ \mathrm{ml}$ ) at the Clinic for Swine in 17 samples from each group taken in weeks 3, 7, 11, 14, 18, 22 and 25.

\section{Results}

During the fattening period, pigs in group A (vaccine at 3 weeks old) performed significantly better than those in Group C (placebo) and group B (vaccinated at 1 week of age). The differences were $32.1 \mathrm{gm} /$ day $(p=0.0110)$ between groups $A$ and $C$, and 26.7 $\mathrm{gm} /$ day $(\mathrm{p}=0.0416)$ between Groups $A$ and $B$.

There were 9 deaths in each group. In the most cases the pigs were culled because of locomotion problems $(n=13)$. Group C (placebo) had more PCV2-related cases $(n=5)$ than the other two groups $(A: n=2 ; B: n=1)$.

The mean PCV2 antibody titer across all groups at 1 week of age was high at 12.4. It declined until week 18, but at that time was higher in Group A (6.3) than in Groups B (4.3) or C (2.7). Then, because of an active PCV2 infection the mean titer increased to 11.6 in week 25.
Only the piglets of Group A had a detectable lgM titer (Ingezim Circovirus IgG/IgM) between weeks 5 and 11 which was a reaction to vaccination. However, after the field infection, animals in Group A (vaccine at 3 weeks) had less IgM than those of Group C (placebo) or Group B (vaccine at 1 week).

Pigs of 22 weeks of age in the placebo group had the highest PCV2 viral load. In groups A and B, viremia was significantly reduced compared to the placebo group. These results demonstrate that there was a PCV2 infection towards the end of the fattening period, and that the vaccinated pigs were protected.

\begin{tabular}{|l|c|c|}
\hline Study Group & 1 week of age & 3 week of age \\
\hline A Porcilis PCV 3 weeks & Diluvac Forte & Porcilis PCV \\
\hline B Porcilis PCV 1 week & Porcilis PCV & Diluvac Forte \\
\hline C Placebo & Diluvac Forte & Diluvac Forte \\
\hline
\end{tabular}

\section{Discussion}

The efficacy of vaccination was clearly shown by the significantly improved weight gain during the growing period, as well as the low percentage of PCV2-PCR positive animals in the group vaccinated at 3 weeks.

The vaccination of piglets with Porcilis PCV at 3 weeks of age was more effective than that at the age of 1 week. The results of this study indicate that high levels of maternally derived antibody can interfere with early vaccination at 1 week of age. 


\title{
0.133
}

\section{Duration of Immunity induced by a single dose of $\mathbf{2} \mathbf{~ m l ~ P o r c i l i s ~}{ }^{\circledR}$ PCV when administered to piglets at 3 weeks of age}

\author{
Alexander A. Eggen ${ }^{1}$ Ulrike Schmidt ${ }^{1}$ Frank Roerink ${ }^{2}$ \\ 1. Intervet/Schering-Plough, Boxmeer, Netherlands; 2. Intervet/Schering-Plough, DeSoto, KS, USA
}

\section{Introduction}

Porcine Circovirus type 2 (PCV2) infection in pigs is regarded as the most important causative factor in the development of Porcine Circovirus Disease (PCVD). The first description of PCVD (initially called PMWS) involved young pigs (Harding 1998). More recent articles describe infection in older pigs and it is not uncommon to observe PCVD in late stage finishers (Palzer 2009). Under field conditions the timing of PCV2 virus infection is often unknown, therefore it is important that pigs are protected for the whole of the fattening period.

PCV2 virus is widespread, and infection in sows leads to varying antibody levels in blood and colostrum. Pigs can be vaccinated early in life against PCVD in the presence of MDA. The object of this study was to demonstrate the efficacy of Porcilis PCV in piglets with MDA against PCV2 virus, following a single dose of 2 $\mathrm{ml}$ at 3 weeks of age. At 25 weeks of age, vaccinated and control animals were challenged with wild type PCV2 virus to investigate the extent of protection.

\section{Materials and Methods}

Progeny of SPF sows with PCV2 antibodies (ranging from 7.1 to 11.4 ELISA log2 at farrowing) were studied. Piglets were assigned to different groups of 10 piglets, so that littermates were spread evenly over the groups, with equal numbers of males and females per group. At 3 weeks of age, Group 1 was vaccinated with $2 \mathrm{ml}$ of Porcilis PCV and Group 2 with placebo. At 25 weeks of age, the pigs were challenged intranasally with a heterologous wild type PCV2 challenge virus strain I-12/11.

All animals were observed daily for clinical signs and weighed several times during the study. Three weeks post challenge all animals were necropsied. At post mortem, mesenteric and inguinal lymph nodes, tonsil and spleen were sampled for the determination of PCV2 antigen and nucleic acid. Serum samples were taken at vaccination, at 10 weeks of age, at challenge and weekly thereafter until necropsy. Fecal swabs were taken at challenge and every week thereafter. Statistical analysis of the serology employed Tukey's multiple comparison. Q-PCR data was analyzed by ANOVA using a linear mixed model, assuming a constant correlation of the repeated measurements of a subject.

\section{Results}

No differences were found between the groups for clinical signs or bodyweight, either after vaccination or after challenge.

Serology; at the time of vaccination the average titer in the PCV vaccinated group was $7.2 \log 2$ and in the controls $73 \log 2$, both above the protective antibody level (Fort 2009). By the time of the challenge, the controls had no detectable antibodies, whereas the vaccinates had titers of at least $6.9 \log 2(P<0.0001)$. After challenge, a clear antibody response was seen in the controls but hardly any reaction in the vaccinated pigs (see table 1).
Table 1: Development of antibody titers

\begin{tabular}{|l|c|c|c|c|c|c|}
\hline Weeks of age & $\mathbf{3}$ & $\mathbf{1 0}$ & $\mathbf{2 5}$ & $\mathbf{2 6}$ & $\mathbf{2 7}$ & $\mathbf{2 8}$ \\
\hline Group/////wpc & & & $0 w p c^{*}$ & $1 w p c^{*}$ & $2 w p c^{*}$ & $3 w p c^{*}$ \\
\hline Porcilis PCV & $7.2 \pm 1.9$ & $11.9 \pm 2.1$ & $7.8 \pm 0.7$ & $8.1 \pm 0.9$ & $8.5 \pm 0.9$ & $9.0 \pm 1.1$ \\
\hline Control & $7.3 \pm 1.8$ & $3.0 \pm 1.9$ & $1.0 \pm 0$ & $1.0 \pm 0$ & $5.8 \pm 2.1$ & $10.2 \pm 3.4$ \\
\hline
\end{tabular}

Q-PCR on serum, fecal samples and lymphoid tissues. At the time of challenge, none of the samples of either group contained detectable amounts of PCV2 DNA. After challenge, a significant reduction in the PCV2 load was found in serum, inguinal lymph nodes, spleen and tonsils of the vaccinates $(P<0.05)$. No PCV2 DNA was detected in any of the feces samples from this group. However, PCV2 DNA was found in fecal swabs of the challenged control animals $(\mathrm{P}<0.0001)$. Similar findings in data generated under field conditions were reported by Toki (JSVSC 2008) and by Larsen (IPVS 2010).

\section{Discussion}

Porcilis PCV induces strong humoral and cellular immunity (Fort 20009, Martelli 2010 in press). Vaccination of MDA-positive piglets of 3 weeks of age with a single $2 \mathrm{ml}$ dose protects them from a heterologous challenge at 25 weeks of age. There was a significant reduction of virus shedding and PCV2 DNA load in lymphoid organs. The duration of immunity covering the whole fattening period, induced by a single dose, in the presence of MDA, is unique to Porcilis PCV. This feature has been confirmed by several field reports from different countries and forms part of the label claim for Porcilis PCV.

\section{References}

Harding; SHAP, 1998. Toki; 145th JSVSC 2008.

Palzer; Proc APVS 2009. Fort; Vaccine 2009.

Larsen; IPVS 2010. Martelli; in press 2010 


\title{
0.134
}

\section{Assessing the impact of maternally derived immunity on active immunization of pigs against PCV2}

\author{
Angela Baysinger $^{1}$ John Waddell ${ }^{2}$ Edgar Diaz $^{1}$ \\ 1. Boehringer Ingelheim Vetmedica, Inc., St Joseph, MO, USA; 2. Sutton Veterinary Clinic, Sutton, NE, USA
}

\section{Introduction}

Porcine circovirus associated disease (PCVAD) is caused by porcine circovirus type 2 (PCV2). Most sows and gilts develop antibodies after natural exposure to or immunization against PCV2 that become passively acquired by their nursing offspring. ${ }^{1}$ The objective of this study was to assess potential maternal interference with active immunization when vaccinating weaned piglets around 3 weeks of age against PCV2.

\section{Materials and Methods}

Piglets were weighed and ear tagged within 24 hours of birth and weaned at approximately 3 weeks of age. All pigs were individually blood sampled for PCV2 IFA antibody measurement and classification as low $(\leq 1: 320)$ or high $(\geq 1: 640)$ titer just prior to vaccination at approximately 3 weeks of age (Day 0 ) based upon previously reported experiments. ${ }^{2,3}$ Four treatment groups were created balancing for pig weight and gender: low pig titer/ non-vaccinated pigs (L-NV), high pig titer/non-vaccinated pigs (H-NV), low pig titer/vaccinated pigs (L-V), and high pig titer/ vaccinated pigs $(\mathrm{H}-\mathrm{V})$. Vaccinated groups were intramuscularly injected with $1 \mathrm{~mL}$ of Ingelvac CircoFLEX ${ }^{\circledR}$ (Boehringer Ingelheim Vetmedica, Inc., St Joseph, MO). Body weights were individually recorded at pre-determined intervals and a subset of pigs were serially blood sampled for diagnostic serology. Pigs from all groups were commingled within nursery and finishing pens. Individual pig was the experimental unit with Day 0-128 average daily gain as the primary outcome of interest. The main effect of Day 0 pig PCV2 IFA titer and pig treatment were assessed using ANCOVA with the model including dam parity, pig gender, and day 0 pig weights as co-variates. Pairwise comparisons utilized Tukey HSD (JMP v8.0).

\section{Results}

Mortality rate did not differ between treatment groups, consistent with the classification of this flow as subclinically affected by PCVAD. Vaccination of pigs significantly increased D0-128 weight gain parameters compared to non-vaccinates regardless of pig PCV2 IFA titer status at the time of vaccination at 3 weeks of age (L-V vs L-NV; H-V vs H-NV; Table 1). D0-128 weight gain parameters did not differ among vaccinated groups from low or high PCV2 IFA titer groups of pigs (L-V vs H-V; Table 1).

Table 1. Least square means for performance data by main effect of pig PCV2 IFA ( $L \leq 1: 320, H \geq 1: 640)$ titer at 3 weeks of age and pig treatment $(V=$ vaccinated; $N V=$ non-vaccinated $)$.

\begin{tabular}{|l|c|c|c|c|}
\hline Parameter & L-NV & H-NV & L-V & H-V \\
\hline Number of pigs & 239 & 222 & 174 & 286 \\
\hline Body weight, D128, Ibs & $218.86^{\mathrm{b}}$ & $219.25^{\mathrm{b}}$ & $226.57^{\mathrm{a}}$ & $225.61^{\mathrm{a}}$ \\
\hline ADG, D0-128, Ibs & $1.59^{\mathrm{b}}$ & $1.59^{\mathrm{b}}$ & $1.65^{\mathrm{a}}$ & $1.64^{\mathrm{a}}$ \\
\hline ab Means with different superscripts within a row differ significantly (Tukey HSD, P $\leq 0.05$ ). \\
\hline
\end{tabular}

\section{Discussion}

High levels of maternally derived PCV2 IFA antibodies ( $>1: 640$ or $>1: 320$ ) did not interfere with achieving significantly increased weight gain by active immunization of piglets with Ingelvac CircoFLEX at 3 weeks of age. Groups of pigs with subclinical PCVAD perform significantly better if vaccinated.

\section{References}

1. Fort, M. Vaccine 2009, (9203)1-7.

2. Hesse, R. Proc AASV 2009, 499-504.

3. Hesse, R. Proc Leman Swine Conf 2008, 68-71. 


\title{
0.135
}

\section{Impact of 1- OR 2-dose PCV2 vaccination regimens on wean-to-finish performance}

\author{
Keith Erlandson ${ }^{1}$ Jeff Okones $^{2}$ Roy Edler $^{2}$ Justin Rustvold ${ }^{2}$ Edgar Diaz $^{2}$ \\ 1. lowa Select Farms, lowa Falls, IA, USA; 2. Boehringer Ingelheim Vetmedica, Inc., St Joseph, MO, USA
}

\section{Introduction}

The objectives of this study were to compare wean-to-finish growth performance and efficiency in commercial pigs administered commercially available porcine circovirus type 2 (PCV2) vaccines used in either one or two dose vaccination regimens.

\section{Materials and Methods}

The study was conducted in a large production systems weanto-finish research site. A randomized block design was used to allocate pigs to pens by gender and weight. Approximately 2000 pigs were weaned at an average of 21 days of age from a single PRRS negative sow source and placed in 77 pens across two barns over a 4 day period. There were six full and one partial block in one barn and five full and two partial blocks in the other barn. Each pen contained approximately 26 pigs. Six treatment groups consisted of 12 pens of non-vaccinated controls and 13 pens of 5 different vaccinated groups with pen as the experimental unit (Table 1). Two pigs per pen were serially blood sampled every 4 weeks for serologic monitoring of PCV2 viremia by quantitative PCR.

Table 1. Study design

\begin{tabular}{|l|c|c|}
\hline Group & \# pens (n) & Treatment Description \\
\hline 1 (NVC) & 12 & Non-vaccinated Controls \\
\hline 2 (CF1) & 13 & Ingelvac CircoFLEX 1 full dose at 3 weeks of age \\
\hline 3 (CF2) & 13 & Ingelvac CircoFLEX 1/2 dose at 3 and 6 weeks of age \\
\hline 4 (CV2) & 13 & Circumvent 1 full dose at 3 and 6 weeks of age \\
\hline 5 (SV1) & 13 & Suvaxyn 1 full dose at 3 weeks of age \\
\hline 6 (SV2) & 13 & Suvaxyn $1 / 2$ dose at 3 and 6 weeks of age \\
\hline
\end{tabular}

Performance data was analyzed in a blinded manner using a randomized design. When the ANOVA P value was less than 0.05 for ADG and FCR, Tukey's HSD was used to discern differences between the vaccine treatments. The Chi square test was used to evaluate mortality rate.

\section{Results}

Natural exposure to PCV2 infection was confirmed by PCR. All vaccination regimens significantly reduced the incidence and magnitude of PCV2 viremia at 14, 18 and 22 weeks of age. All vaccination regimens except Suvaxyn 1 dose significantly improved the rate of day 0-178 average daily weight gain compared to non-vaccinated controls ( $\mathrm{P} \leq 0.05$, Table 2 ). Only Ingelvac CircoFLEX 1 dose significantly improved day 0-178 feed conversion efficiency compared to non-vaccinated controls ( $P \leq 0.05$, Table 2). Day 0-178 mortality rate was not affected by treatment.

Table 2. Performance results

\begin{tabular}{|l|c|c|c|}
\hline Group & Average Daily Gain, Ibs & Feed conversion ratio & Mortality rate, $\%$ \\
\hline 1 (NVC) & $1.26^{\mathrm{a}}$ & $2.85^{\mathrm{a}}$ & 7.69 \\
\hline 2 (CF1) & $1.36^{\mathrm{b}}$ & $2.73^{\mathrm{b}}$ & 4.14 \\
\hline 3 (CF2) & $1.33^{\mathrm{b}}$ & $2.78^{\mathrm{ab}}$ & 6.21 \\
\hline 4 (CV2) & $1.34^{\mathrm{b}}$ & $2.79^{\mathrm{ab}}$ & 4.71 \\
\hline 5 (SV1) & $1.32^{\mathrm{ab}}$ & $2.79^{\mathrm{ab}}$ & 7.03 \\
\hline 6 (SV2) & $1.33^{\mathrm{b}}$ & $2.76^{\mathrm{ab}}$ & 6.80 \\
\hline ab: means within a column differ significantly Tukey HSD P $\leq 0.05$. \\
\hline
\end{tabular}

\section{Discussion}

Ingelvac CircoFLEX administered as a single full dose to pigs at 3 weeks of age was the only vaccination regimen to significantly improve both rate of weight gain and feed conversion efficiency. Utilization of an effective single dose vaccine also maximizes time and labor utilization efficiency while imposing less stress on pigs. 


\title{
0.136
}

\section{Comparative efficacy of PCV2 and Mycoplasma vaccination regimens}

\author{
Joel Nerem \\ Pipestone Veterinary Clinic, Pipestone, MN, USA
}

\section{Introduction}

The objective of this study was to compare the efficacy of various vaccination programs against PCV2 and Mycoplasma hyopneumoniae. The study was supported by Boehringer Ingelheim Vetmedica, Inc.

\section{Materials and Methods}

A 3200 sow farrow to wean facility and a downstream nursery/ finishing site was used in the study. The system is PRRS virus positive, but stable (producing PRRS PCR negative weaned pigs) and is Mycoplasma hyopneumoniae ( $M$ hyo) positive. The off-site finishers had consistently suffered clinical porcine circovirus associated disease (PCVAD) losses with mortality rates of $10-15 \%$. The system historically used a combined half dose of RespiSure One (Pfizer 1-dose M hyo vaccine) with a full dose of Circumvent (Intervet 2-dose PCV2 vaccine) at processing (3-5 days of age) and weaning (17-22 days of age). To reduce labor and stress on the pigs, they wanted to evaluate using a 1-dose vaccination program with the Ingelvac ${ }^{\circ}$ CircoFLEX - MycoFLEX ${ }^{\mathrm{TM}}$ mixture , given either at weaning ( $\sim 21$ days of age) or processing ( $\sim 5$ days of age). They also wanted to evaluate the effectiveness of a split-dose program of $1 / 2$ dose of the Ingelvac ${ }^{\circ}$ CircoFLEX MycoFLEX ${ }^{\mathrm{TM}}$ mixture at processing and at weaning.

Vaccinations, tagging and the first two weights were taken at the sow farm. At weaning, pigs were moved to an off-site 3 barn nursery-finishing site. Pigs were first housed in a power ventilated nursery for 40 days. From day 40 to day 150, pigs were housed in two curtain-sided finishing barns.

Five treatment groups (Table 1) were balanced for sow-of-origin parity with approximately 400 pigs assigned to each treatment group. Individual pig was the experimental unit. Pigs were ear tagged at processing and randomized by pen placement in nurseries and finishers with all five treatment groups represented in each pen. Final weighing occurred just prior to first pigs being sold to market. Average daily gain was evaluated along with mortality rate, culling rate and \% prime marketed pigs.

Table 1: Study design

\begin{tabular}{|c|l|}
\hline Group & \multicolumn{1}{|c|}{ Treatment } \\
\hline 1 & Non-vaccinated Control \\
\hline 2 & Full dose Ingelvac CircoFLEX-MycoFLEX at weaning \\
\hline 3 & Full dose Ingelvac CircoFLEX-MycoFLEX at processing \\
\hline 4 & Half dose Ingelvac CircoFLEX-MycoFLEX at processing and weaning \\
\hline 5 & Full dose Circumvent - Half dose RespiSure One at processing and weaning \\
\hline
\end{tabular}

\section{Results}

No differences were observed between treatment groups during the nursery phase. Clinical PCVAD was observed in finishing and was confirmed diagnostically. Comparisons between the M. hyo vaccines used could not be made because clinical and serological results revealed no significant $M$. hyo exposure. The Ingelvac ${ }^{\circ}$ CircoFLEX - MycoFLEX ${ }^{\mathrm{TM}}$ mixture administered at 3-5 days of age was not statistically different than controls for any parameter (Table 2). All other treatment groups produced better rate of gain, cull rates and \% prime marketings than controls. Only Ingelvac ${ }^{\circ}$ CircoFLEX - MycoFLEX ${ }^{\mathrm{TM}}$ given at weaning reduced mortality rate.over controls.

Table 2: Finishing performance results

\begin{tabular}{|c|c|c|c|c|}
\hline Group & $\begin{array}{c}\text { Average Daily } \\
\text { Gain, lbs }\end{array}$ & Mortality, \% & Cull, \% & Prime Marketings, \% \\
\hline 1 & $1.45^{\mathrm{a}}$ & $3.7^{\mathrm{a}}$ & $15.3^{\mathrm{a}}$ & $79.40^{\mathrm{a}}$ \\
\hline 2 & $1.59^{\mathrm{b}}$ & $1.3^{\mathrm{b}}$ & $5.7^{\mathrm{b}}$ & $92.95^{\mathrm{b}}$ \\
\hline 3 & $1.50^{\mathrm{a}}$ & $3.8^{\mathrm{a}}$ & $12.2^{\mathrm{a}}$ & $84.07^{\mathrm{a}}$ \\
\hline 4 & $1.64^{\mathrm{b}}$ & $3.0^{\mathrm{ab}}$ & $3.7^{\mathrm{b}}$ & $93.37^{\mathrm{b}}$ \\
\hline 5 & $1.63^{\mathrm{b}}$ & $5.1^{\mathrm{a}}$ & $4.6^{\mathrm{b}}$ & $90.33^{\mathrm{b}}$ \\
\hline
\end{tabular}

\section{Discussion}

Vaccination at 5 days of age was not successful. The only group that performed significantly better than controls for all 4 performance parameters was a single dose of Ingelvac ${ }^{\circledR}$ CircoFLEX - MycoFLEX ${ }^{\mathrm{TM}}$ mixture at weaning. 


\title{
0.137
}

\section{The importance of on-farm monitoring after sow vaccination (CIRCOVAC $\left.{ }^{\circledR}\right)$ for successful control of porcine circovirus disease (PCVD)}

\author{
Keiri Tokuyama $^{1}$ M. Kagawa² Y. Saito ${ }^{3}$ S. Ogino ${ }^{3}$ \\ 1. Merial Japan Ltd, Tokyo, Japan; 2. Kagawa Livestock Clinic, Gunma, Japan; 3. Nippon Zenyaku Kogyo Co., Ltd., Fukushima, Japan
}

\section{Introduction}

When vaccination against PCV2 is implemented, failure of PCVD control is often conceived as lack of vaccine efficacy. Since it is known that multiple pathogens are usually involved in PCVD (1), it is important to continuously monitor post-vaccination status for successful control. The objective of this study is to show the benefit of sow vaccination and on-farm monitoring practices.

\section{Materials and methods}

A 120 sow farrow-to-finish farm located in central Japan, was selected for the study. Sows were mass-vaccinated twice 4-week apart for PCV2 with CIRCOVAC (Merial) thereafter 2-4 weeks before parturition. Blood samples from sows and pigs were tested for PCV2 antibodies (IFA), PPRSV (ELISA), M. hyo (ELISA), and $P C V 2$ viral load (RT-PCR). All clinical incidences, post-weaning mortality and days to market were recorded during the course of the study for approx. one year. Chi-test and Student-t test were used for statistical comparison of one year before and one year after vaccination.

\section{Results and Discussion}

Noticeable clinical signs were mainly due to diarrhea and/or digestive disorders during weaning, leading to increased mortality and runts. Although the mortality rate contained those losses, it stayed statistically lower than the previous year. Days to market were reduced by 10 days (Table 1 ).

Table 1: Post-weaning mortality and days to market

\begin{tabular}{|l|l|c|c|c|}
\hline & & $\mathbf{2 0 0 8}$ & $\mathbf{2 0 0 9}$ & Diff. \\
\hline Post-weaning mortality (\%) & Weaners & $13.6 \%$ & $4.1 \%^{*}$ & $-9.5 \%$ \\
\hline Post-weaning mortality (\%) & Finishers & $2.5 \%$ & $2.0 \%$ & $-0.5 \%$ \\
\hline Days to market & & $177 \pm 6.7$ & $167^{*} \pm 6.4$ & -10.0 \\
\hline${ }^{*}$ statistically significant $(\mathrm{P}<0.01)$ & \\
\hline
\end{tabular}

One year after vaccination sows had markedly lower PCV2 viral load and higher antibody titers (Fig. 1a). In pigs, PCV2 DNA was detected before vaccination at 8-13 wks, but one year after vaccination viremia was detected at the finishing stage (17-22 wks) (Fig.1b). Despite viremia at 21-22 wks, no particular clinical signs were observed, and days to market were reduced by 10 days. Mortality in the finishing period was reduced to $2.0 \%$.

As opposed to the pre-vaccination year, pigs seroconverted against PRRSV and $M$. hyo around mid-finishing in the year post vaccination. Both pathogens are known to negatively impact immune functions, and enhance PCV2 replication $(2,3)$. These co-factors may have played a role in the asymptomatic raise of PCV2 viremia in the late finishing stage. Mortality and growth improvements clearly indicate that PCVD was well controlled in this farm. The rise in M. hyo and PRRS ELISA titres indicate that continuous monitoring is necessary since farm epidemiology can change over time. Monitoring thus enables quick implementation of appropriate measures.

In terms of efficacy, cost and labor, sow vaccination is practically one of the most useful options when monitoring is carefully implemented as in the present study.

Figure 1: a) PCV2 IFA titer (left) and PCV2 viral load (right) in sows; b) PCV2 viral load (left: log 10) and PCV2 IFA titer (right) in fattening pigs

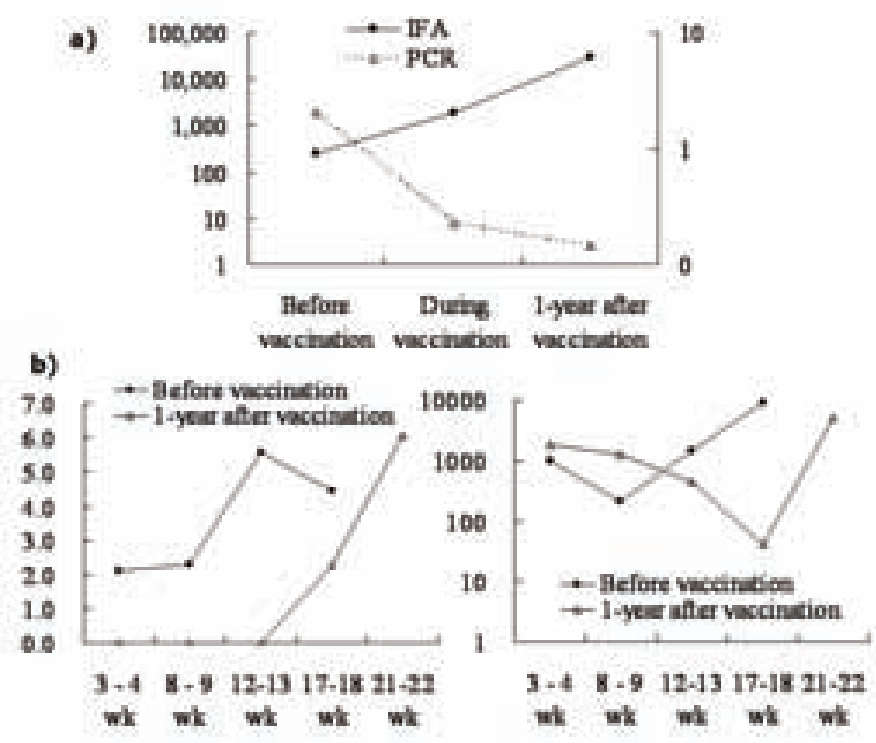

Figure 2: ELISA titers of PRRV and M. hyo.

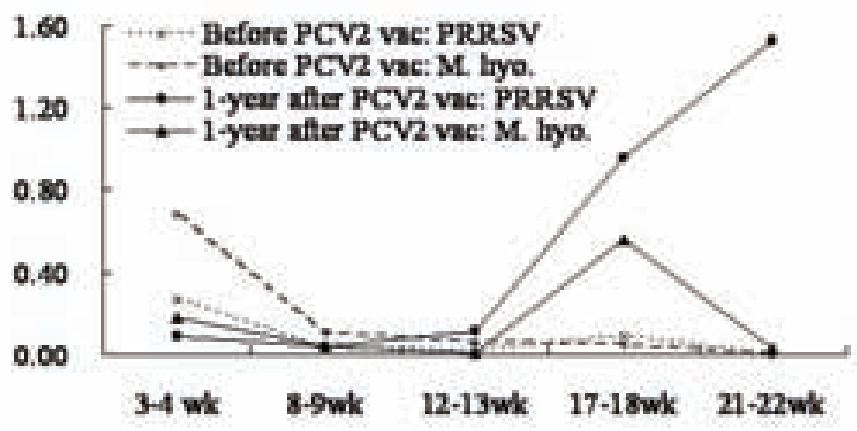

\section{References}

1. Charreyre et al. (2006) Proc.Jpn.Pig Vet.Soc., 49

2. Allan G. et al. (2000) Arch Virol, 145, 2421-2429

3. Oppriesnig T. et al. (2004) Vet Pathol, 41, 624-640

${ }^{\circledR} \mathrm{CIRCOVAC}$ is a registered trademark of Merial in the Canada and elsewhere. 


\title{
0.138
}

\section{Willingness to approach behaviour in weaned piglets following vaccination with two different PCV2 vaccines in a Japanese commercial farm}

\author{
Mali Miyashita ${ }^{1}$ Y Takahashi $^{2}$ Takeshi Yamaguchi $^{1}$ Yasuko Shimomura ${ }^{1}$ \\ 1. Boehringer Ingelheim Vetmedica Japan Co. Ltd., Tokyo, Japan; 2. Shokukanken Inc., Gunma, Japan
}

\section{Introduction}

Adapted from the animal observation section of the 2003 Swine Welfare Assurance Program (SWAP), willingness to approach observations have been used to evaluate pig behaviour after vaccination $(1,2)$. The objective of this study was to compare the willingness to approach behaviour in pigs following vaccination with two different PCV2 piglet vaccines that are commercially available in Japan.

\section{Materials and Methods}

A 450 sow PRRS positive farm with a past history of PMWS located in Ibaraki, Japan, was selected for the study. A total of 871 weaned piglets aged 3 to 4 weeks, were included for observation. At weaning pigs were allocated to pens of approximately 20 pigs each. After an average acclimation time of 1 week, half of the pens (22) received $1 \mathrm{ml}$ of Ingelvac CircoFLEX (group A), while the other half received $2 \mathrm{ml}$ of Porcilis PCV (group B). Vaccines were administered according to manufacturer instructions. There was no difference in average weight, age or sex between vaccine groups. An adaptation of the animal observation section of the 2003 SWAP was utilized to evaluate willingness to approach behaviour (WTA) of piglets pre- and post vaccination. After entering the pen, sitting down and waiting a 15 second period, the blinded observer counted all pigs that approached and/or were in eye contact to him. These counted pigs divided by the total number of pigs in the pen resulted in the \% willingness to approach (\% WTA). The initial observation (base line) was conducted at $4 \mathrm{pm}$ on the day prior to vaccination. On the following day, vaccination of pigs was completed by $10 \mathrm{am}$, and WTA observations were conducted at 6 hours $(4 \mathrm{pm})$ post vaccination. The change in post vaccination \% WTA was compared to the baseline for each vaccine group and between groups. The $\%$ WTA compared to baseline between groups was analyzed by the Pearson Chi-square test. Results were considered significant when $P$ values were equal to or less than 0.05 .

\section{Results}

Results are summarized in table 1 . Six hours post vaccination, there was a statistically significant difference $(p<0.0001)$ in the \% WTA compared to baseline between groups ( $83 \%$ and $27 \%$ for groups $A$ and $B$, respectively).

Table 1. Willingness to approach before and after PCV2 vaccination

\begin{tabular}{|c|c|c|}
\hline & Group A & Group B \\
\hline No. of pens observed & 22 & 22 \\
\hline No. of pigs observed & 423 & 448 \\
\hline Baseline pre-vaccination behaviour (\%WTA) & $69 \%(293 / 423)^{*}$ & $66 \%(297 / 448)^{*}$ \\
\hline Six hour post-vaccination behaviour (\%WTA) & $57 \%(242 / 423)^{*}$ & $18 \%(81 / 448)^{*}$ \\
\hline $\begin{array}{l}\text { Six hour post-vaccination behaviour compared } \\
\text { to baseline }\end{array}$ & $83 \%{ }^{A}(242 / 293)^{* *}$ & $27 \%{ }^{B}(81 / 297)^{* *}$ \\
\hline \multicolumn{3}{|c|}{$\begin{array}{l}A-B: p<0.0001 \\
{ }^{*} \text { Number of approaching pigs/Number of pigs observed }\end{array}$} \\
\hline
\end{tabular}

\section{Discussion and conclusion}

Using the willingness to approach model, it was previously shown that Ingelvac CircoFLEX was not different to saline (2), for which reason a control group was not included in the study protocol. In this study pigs from group A showed more willingness to approach 6 hours post vaccination, compared to those of group B, indicating that depending on the type of PCV2 piglet vaccine used, differences can occur in the behaviour of piglets at 6 hours after vaccination. Willingness to approach observations utilize the natural behaviour of pigs by assessing their level of interest to a person in the pen, thus providing a certain indication as to the well-being of those pigs. Based on the results of this study, willingness to approach observations may provide a useful tool to objectively assess the potential welfare impacts of different vaccines on pigs.

\section{References}

1. National Pork Board. Swine Welfare Assurance Program 2003. Animal Observation :12-17.

2. Bretey et al. (2009) An innovative method for quantifying animal behavior responses to various immunization protocols. A ASV Proceedings, USA, pp. 295-296. 


\title{
0.139
}

\section{Direct and indirect transmission of four Salmonella enterica serotypes in pigs}

\author{
Julia Österberg; Susanna S. Lewerin; Per Wallgren \\ National Veterinary Institute, Uppsala, Sweden
}

\begin{abstract}
Introduction
The Salmonella serotypes commonly detected in raw feed materials in Sweden often differ from the serotypes most often isolated from animals and humans. Why only few of the "feedassociated" serotypes seem to reach or spread within the animal population has not been sufficiently investigated. In the present study we have performed comparative examinations of two serotypes commonly isolated from pigs (S Typhimurium and S Derby) and two "feed associated" seotypes (S Yoruba and S Cubana) by studying their ability to infect pigs in a direct and indirect transmission situation aiming to investigate possible differences among serotypes.
\end{abstract}

\section{Materials and Methods}

In total, 48 ten-weeks-old pigs, deriving from a Swedish nucleus herd, were split into eight groups. Each group was housed in a separate room in a pen with solid concrete floor. Sawdust bedding covered half of the pen and the dung was cleared out twice daily.

Direct contact transmission (DT) was studied by mixing six pigs with two seeder pigs, aged 18 weeks, in each of four cleaned and disinfected pens. The two seeder pigs in each group had a well documented history of long-term faecal excretion of one of the four selected serotypes of Salmonella, as they had been inoculated with 109 colony forming units (cfu) of the serotype 8 weeks earlier $(1,2)$.

Indirect transmission (IT) was studied in six pigs in each of four pens were Salmonella infected pigs had previously been housed. The infected pigs had been inoculated with $109 \mathrm{cfu}$ of the serotype eight weeks earlier and their faecal excretion of Salmonella had been monitored for eight weeks before the naive pigs replaced them in the unwashed pens $(1,2)$.

All pigs in the eight rooms were monitored for two weeks with respect to their faecal excretion of Salmonella and the presence of serum antibodies. In total, eight faecal samples and three serum samples were collected from each pig. In addition, eight tissue samples per pig were collected at necropsy.

\section{Results}

The results from culturing the faecal samples and the samples collected post mortem are shown in Figure 1.

All pigs remained serologically negative throughout the study, except four pigs in the two Yoruba-groups that expressed antibody levels just above the cut-off in the in-house ELISA constructed to detect antibodies to $S$ Yoruba.
Figure 1. Number of Salmonella positive faecal samples per week and post mortem samples from eight tissues; tonsil (to), liver (li), spleen $(\mathrm{sp})$, colon wall (cw), three lymph nodes: mandibular ( $\mathrm{ml})$, ileocecal (il) and colonic $(\mathrm{cl})$, and cecum content (cc). DT=direct transmission trial, $I T=$ indirect transmission trial.

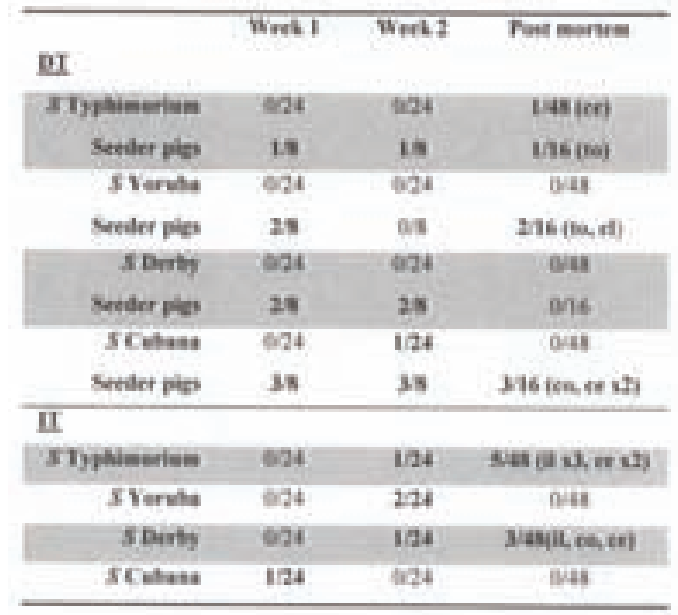

\section{Discussion and Conclusions}

The rate of transmission was apparently low in both the direct and the indirect transmission situation, and no differences between the four serotypes could be concluded with respect to the faecal shedding of Salmonella. In contrast, a difference between serotypes was indicated by the results from the IT post mortem samples, as S Typhimurium was re-isolated from four pigs housed in the contaminated environment.

In conclusion, the overall low level of transmission of all four serotypes in both trials most probably reflected a low dose exposure. Pigs shedding Salmonella at detectable but low levels may not necessarily transmit the pathogen to its pen mates, not even in a solid floor environment. This high-lights the importance of hygienic measures, in order to keep the shedding and re-circulation of Salmonella spp. low in infected herds. However, isolation of S Typhiurium from especially the ileocecal lymph nodes can be expected even in herds with a very low load of $S$ Typhimurium.

\section{References}

1. Österberg \& Wallgren (2008) Vet Rec 162: 580-586.

2. Österberg et al (2009) Vet Rec 165: 404-408. 


\title{
0.140
}

\section{A review on Salmonella control in fattening pigs through the use of potassium diformate (KDF) - European case studies}

\author{
Christian Lückstädt; Peter Theobald; Horst Auerbach
}

ADDCON, Bonn, Germany

Salmonella control has a high priority in European pork production. It is a significant cause of human Salmonellosis and causes major economic losses in the pork production chain, through reduced productivity, increased veterinary and hygiene control costs. Preventing the spread of salmonella to the consumer requires special control measures during slaughter and processing. The extra cost of these controls is increasingly being transferred back to the producer in the form of financial penalties or the loss of the market for contaminated pigs.

Gut health is increasingly being shown to be effective against intestinal pathogens, a strategy that has only really been made possible through the removal of antibiotic growth promoters in feed. Creating and maintaining a healthy intestinal environment has become essential to productivity and food safety programmes alike.

S. enteritica typhimurium is the predominant serotype found in pig carcasses in Europe, accounting for around $71 \%$ of cases. Several serotypes are resistant to antibiotics, putting increasing pressure on producers to prevent contamination. While salmonella cannot be eradicated in pig units, it can be controlled to minimise the risk to consumers. Biosecurity plays a significant role in salmonella control. In feed compounding, although heat treatment is effective in reducing contamination of feed leaving the feed mill, this effect does not persist during transport, storage and subsequent outfeeding. When conditions within the feed are less conducive to bacterial infection, salmonella contamination can be reduced. The next critical control point is within the pig's gut itself, where conditions for bacterial growth may once again be optimal. Salmonella growth requires warmth $\left(35-37^{\circ} \mathrm{C}\right.$ is optimal), a moisture content greater than $12 \%$ and a pH between $4.5-9.0$. It is no coincidence that the pig gut can provide salmonella everything it needs to thrive.

While biosecurity and hygiene in the feed mill and on farm are essential, the acidification of feed ingredients or finished feeds with organic acids also offers considerable benefits to salmonella control. Feed acidification is not only effective within the feed; possibly its biggest benefit occurs within the pig itself. Research trials in the UK, France and Ireland with $0.6 \%$ potassium diformate (KDF) feed additive, showed significantly reduced salmonella count in the feed as well as in the gut of pigs. This effect is particularly well illustrated by data collected on 12 farms in Ireland (Lynch et al., 2007). The main objective of this investigation was to evaluate the efficacy of salmonella control measures on highly infected farms. Salmonella control has been compulsory under Irish law since 2002 and farm status is categorised by the percentage of positive pigs in a herd according to the Danish mix-ELISA test. Category 3 (>50\% positive) farrow-to-finish farms and their associated fattening units were selected for the study. All the farms that were treated with KDF alone; or a combination of KDF with improved hygiene and biosecurity measures had notable improvements in both bacteriological and serological prevalence of Salmonella spp. All but one farm in which KDF was used ended the trial with a much improved salmonella status, with bacteriological prevalence also low on most farms. Using improved hygiene and biosecurity measures alone also improved salmonella status, but to a much lesser extent. The reduction in prevalence obtained by KDF alone, compared to the two farms which also implemented additional hygiene and biosecurity, demonstrates the additive's efficacy.

These findings are not unique, however. Studies by Dennis and Blanchard (2004) in the UK as well as most recently in France (Correge et al. 2010) concluded potassium diformate, to be an effective tool in a salmonella control strategy in commercial farms, reducing the percentage of salmonella positive pigs by $50 \%$ and in pork meat juice ELISA scores by $46 \%$, respectively in grower finisher pigs. The UK trial also showed an improvement in daily gain of $7.7 \%$, reduced mortality and a reduction in medicinal intervention compared to the rolling average for that unit. The economic benefit of implementing salmonella control was also evaluated.

Correge et al. (2010). Effect of acidifying fattening feed to reduce carriage of Salmonella in high prevalence pig herds. Journees Recherche Porcine, 217-218.

Dennis and Blanchard (2004). Effect of feeding potassium diformate on incidence of salmonella infection on a commercial unit. The Pig Journal 54, 157-160.

Lynch et al. (2007). Development of on-farm control measures for the reduction of Salmonellosis in slaughter pigs. Teagasc. 54 pp.. 


\title{
0.141
}

\section{Comparison of risk factors and bacteriological findings in the environment of pig herds with high or low Salmonella prevalence}

\author{
Verena Gotter ${ }^{1}$ Amely Ovelhey ${ }^{3}$ Sarah Kösters ${ }^{3}$ Thomas Blaha $^{1}$ Günter Klein ${ }^{2}$ \\ 1. University of Veterinary Medicine Hannover, Field Station for Epidemiology, Bakum, Germany; 2. University of Veterinary Medicine Hannover, \\ Institute for Food Quality and Food Safety, Hannover, Germany; 3. University of Veterinary Medicine Hannover, Department of Biometry, \\ Epidemiology and Information Processing, Hannover, Germany
}

\section{Introduction}

With the introduction of a national, serological Salmonella monitoring-program in Germany in 2007, the knowledge of risk factors for Salmonella infections in pigs in conventional husbandry systems reached a new level of meaning for the veterinary practitioner. In order to help those of his clients whose herds have a high Salmonella prevalence, he must be able to devise a strategy using the multitude of risk factors mentioned in literature as basis. However, the importance of individual risk factors is highly debated even among the scientific community (1), with few results from various studies being generally accepted, one being that since Salmonella ssp. does not require an animal host to survive, the indirect transmission plays a significant part $(2,3)$. The goal of this study was therefore to establish on a wide basis the validity and significance of known risk factors by comparing herds of high (category III) and low (category I) serological prevalence via questionnaire and to reinforce the findings with bacteriological samples from the environment of the animals. Following completion of the study the results are then to be published as guidelines for the veterinary practitioner.

\section{Material and Methods}

A 23page questionnaire with six parts and five attachments was validated and sample sites were chosen as accordingly. In category I 67 herds were examined; in category III 103 herds. All herds were situated in an area of high pig herd density in northwestern Germany. Only a fraction of the asked questions is presented in this abstract:

1. Are the driving boards cleaned and disinfected before each fattening batch?

2. Are the loadings ramps cleaned and disinfected before each fattening batch?

3. Is the central hallway of the barn cleaned and disinfected before each fattening batch?

Samples were taken by swabbing the area in question with a sterile, BPW (buffered peptone water)-enriched tissue. The samples were examined via RT-PCR (real time polymerase chain reaction), after an 18 hour enrichment phase in $B P W$ at $37^{\circ} \mathrm{C}$ at the Field Station for Epidemiology in Bakum.

\section{Results}

An excerpt of the results of the samples is presented in Table 1.

\begin{tabular}{|l|c|c|c|c|}
\hline Sample & N & $\begin{array}{c}\text { Total \% posi- } \\
\text { tive }\end{array}$ & $\begin{array}{c}\text { Cat. I \% posi- } \\
\text { tive }\end{array}$ & $\begin{array}{c}\text { Cat. III \% } \\
\text { positive }\end{array}$ \\
\hline driving boards & 239 & 20.5 & 10.2 & 26.5 \\
\hline loading ramps & 54 & 27.8 & 15.0 & 35.3 \\
\hline central hallway & 175 & 30.9 & 17.5 & 37.3 \\
\hline
\end{tabular}

\section{Discussion}

These samples show that in herds in category III have about double the amount of positive samples (in percent) than herds in category I. This supports the belief that herds in category III indeed do have more Salmonella in the environment surrounding the pigs. These three exemplary risk factors may belong to the crucial group of risk factors, which must be considered when implementing a strategy against the Salmonella problem of a herd, as further results may show when the detailed inspection of all answers of the survey, cross-referenced with the sample results become available. This may also answer the critical question whether this discrepancy between the herds is due to the individual execution of cleaning and disinfection or to the existence of such procedures per se.

\section{References}

(1) Stärk et al. (2002), Prev. Vet. Med. 53:7-20

(2) Funk et al. (2001), BMTW 114: 335-338

(3) Weigel et al. (2007), Prev. Vet. Med. 81: 274-289

*This ongoing study is funded by the FAEN (Forschungsverbund Agrarund Ernährungswissenschaften Niedersachsen). 


\title{
0.142
}

\section{Does pre-slaughter stress affect pork safety risk?}

\author{
Marcos H. Rostagno; Susan D. Eicher; Donald C. Lay, Jr. \\ USDA-ARS-LBRU, West Lafayette, IN, USA
}

\section{Introduction}

Food safety is a defining issue in the global pork market today, and Salmonella contamination is a major concern for the pork industry all over the world. Although much of the Salmonella contamination of pork occurs within abattoirs, infected pigs leaving the farm are considered as the original source of abattoir contaminations. According to Berends et al.(1996), live animals that carry Salmonella at the time of slaughter are 3-4 times more likely to produce positive carcasses. Furthermore, it is reasonable to assume that the extent of carcass contamination is also determined by the levels of Salmonella entering the abattoir in the intestinal tract of slaughtered pigs. Therefore, two experiments were designed to determine if common stressors occurring prior to slaughter affect the prevalence and levels of Salmonella in market weight pigs, and consequently, the associated pork safety risk.

\section{Materials and Methods}

Initially, a field study (i.e., under commercial conditions) was conducted to determine the effect of transportation from the production farm to the abattoir, and pre-slaughter lairage on the frequency of Salmonella shedding in market pigs. In this study, 5 Salmonella-positive finishing lots were used. From each lot, 30 pigs were randomly selected from Salmonella-positive pens, transported in a clean and disinfected livestock trailer for 1.5 hour, and subjected to 2 hours of pre-slaughter lairage. Individual fecal samples were collected immediately prior and after transportation, and after lairage. All samples were processed for the isolation and identification of Salmonella enterica. A follow up study was conducted under controlled conditions, with the objective of determining if feed withdrawal and transportation affect the levels of Salmonella in the intestinal tract of marketweight pigs. Three replicates with 20 market-weight pigs each (5 pigs/treatment) were conducted, including the following treatments; Control (no stress), feed withdrawal (12 h), transportation ( $1 \mathrm{~h}$ ), and feed withdrawal (12 h) followed by transportation (1 h). Pigs were individually challenged with a Salmonella Typhimurium Nalidixic acid-resistant strain 3 days prior to the experiment, and euthanized immediately after the treatments for necropsy. All samples were processed for the isolation and enumeration of the challenge strain.

\section{Results}

In the first study, significant increase $(\mathrm{P}<0.05)$ of Salmonella shedding prevalence was observed from pre-transport (11.3\%) to post-transport (20\%), and from post-transport to post-lairage $(42 \%)$. In the second study, feed withdrawal by itself or combined with transportation caused increased levels of Salmonella in the ileum $(\mathrm{P}<0.05)$, whereas only the combination of feed withdrawal and transportation caused increased levels of Salmonella in the cecum of market-weight pigs $(P<0.05)$.

\section{Discussion}

Studies of the effects of pre-slaughter stressors on food safety risk in market pigs are scarce. Taken together, our studies show that a significant increase of Salmonella shedding and its levels in the intestinal tract of market pigs occurs between the production farms and the abattoirs. We have already shown that an increase of Salmonella prevalence occurs due to rapid infections during pre-slaughter lairage (Hurd et al.,2001; Rostagno et al.,2003). Besides of confirming this occurrence, these studies show that the process of moving market pigs from the production farm to the abattoir also contributes to increase pork safety risk. Although many can argue that transporting pigs from the farms to the abattoir is a process that cannot be avoided, it is important to keep our minds open to potential changes or modifications. For instance, transportation conditions can be manipulated to minimize stress, and/or the gastrointestinal microbial ecosystem can be made more resilient to changes (or stabilized) through the use of probiotics, prebiotics or other alternatives.

\section{References}

Berends et al.(1996). Int J Food Microbiol, 30, 37-53.

Hurd et al.(2001). Am J Vet Res, 62, 1194-1197.

Rostagno et al.(2003). Appl Environ Microbiol, 69,4489-4494. 


\title{
0.143
}

\section{Relationship between serological status of sows and the assignment as Salmonella risk farm in the Belgian Salmonella control program}

\author{
Frédéric A. Vangroenweghe ${ }^{1}$ Marieke Strubbe ${ }^{1}$ Ellen Van Driessche ${ }^{1}$ Estelle Méroc ${ }^{2}$ Yves Van Der Stede $^{2}$ \\ 1. DGZ-Vlaanderen, Torhout, Belgium; 2. Veterinary and Agrochemical Research Center, Brussels, Belgium
}

\section{Introduction}

Salmonella is considered as one of the most important food borne pathogens that has potential implications for human health (1). To control Salmonella at the pre-harvest stage, the implementation of a surveillance and control program has been established in the different EU Member States. Since 2005, the Belgian Federal Agency for the Safety of the Food Chain (FASFC) implemented a National Salmonella surveillance and control program in pigs, the Salmonella Action Plan (SAP), which became compulsory by means of a Royal act in July 2007 (2). Since July 2007, Belgian pig farms can be assigned as Salmonella-risk farms, based on serological analysis of blood samples collected from the fattening pigs.

This study was conducted to evaluate the serological status of the sows on Salmonella risk farms compared to non-assigned farms.

\section{Materials and methods}

With a 4-month interval, every Belgian pig farm needs to collect blood samples from 12 fattening pigs for the National Aujeszkydisease monitoring program. All samples are analyzed using an indirect LPS-Salmonella ELISA (Idexx). Since July 2007, farms are identified as risk farms if the mean S/P-ratio, from 12 fattening pigs, is equal to or higher than 0.6 for 3 successive sampling events.

For this study blood samples $(n=1138)$ of sows were randomly collected on 100 different farrow-to-finish herds. To this end, 583 samples were obtained from 50 Salmonella risk farms, identified as farms with a mean S/P-ratio equal to or higher than 0.6 for 3 successive sampling events; and 555 samples were obtained from non-risk farms, identified as farms with a mean S/P-value lower than 0.2 for 3 successive sampling events.

A statistical analysis (Mann-Whitney-Wilcoxon test) was performed to compare the mean S/P ratio in both sow groups.

\section{Results}

The results show that the mean S/P ratio obtained from the sows of the Salmonella risk-farms (1.138 \pm 0.026 SEM) was significantly higher $(P<0.0001)$ compared with the non-risk farms $(0.702$ \pm 0.021 SEM). The mean S/P-ratio for the 1138 sows was $0.925 \pm$ 0.018 SEM. In this study there was a presence of Salmonella antibodies in $98.7 \%$ of the sows. At a cut-off of $S / P=0.6$ we found $63.6 \%$ of the sows to be positive.
Table 1. Number of samples sows and their mean S/P value \pm SEM for Salmonella in blood

\begin{tabular}{|l|c|c|}
\hline & Number of sows & Mean S/P-value \\
\hline Risks farms & 583 & $1.138 \pm 0.026$ \\
\hline Non-risk farms & 555 & $0.702 \pm 0.021$ \\
\hline Total & 1138 & $0.925 \pm 0.018$ \\
\hline
\end{tabular}

\section{Discussion and conclusions}

Sows play an important role in the maintenance of Salmonella infections in farrow-to-finish herds (3). The increasing number of sows on a farrow-to-finish farm was recently identified as a risk factor associated with higher average S/P-values on a farm (4). In this study we could clearly show that the infection status of the sows plays a significant role in the assignment as a Salmonella risk farm in the Belgian Salmonella control program, which is based on sampling of fattening pigs. Control of Salmonella on farrow-to-finish herds is now almost only done by implementing measures in the fattening unit. It is clear that further studies are needed to evaluate intervention measures in the sow unit. Vaccination could be one of these intervention options.

In conclusion, it is clear that the role of the sows and their serological Salmonella status is a potential influencing factor for the assignment of Salmonella risk farms, which is principally based on sampling of fattening pigs.

\section{References}

1. MAO et al. 2003. Foodborne enteric infections. Curr Opin Gastroenterol 19: 11-22

2. ANONYMOUS, Royal Act 27 april 2007

3. NOLLET et al. 2005. Vet.erinary Research 36, 645-656

4. HAUTEKIET et al. 2008. Preventive Veterinary Medecine, 86: 75-92 


\title{
0.144
}

\section{Use of workshops and face-to-face interviews to teach smallholder pig farmers about how to prevent disease due to Taenia solium}

\author{
Cate E. Dewey ${ }^{1}$ Jared Wohlgemut ${ }^{1}$ Florence Mutua ${ }^{2}$ Mike Levy ${ }^{1}$ \\ 1. University of Guelph, Guelph, ON, Canada; 2. International Livestock Research Institute, Nairobi, Kenya
}

\section{Introduction}

Neurocysticercosis is the major preventable cause of adult-onset epilepsy in developing countries. Epilepsy is caused by the neurocysticercosis form of Taenia solium. The life cycle includes the pig as an intermediate host having larval cysts (Cysticercus cellulosae) and the human as the definitive host, having adult tapeworms (taeniasis). Humans also act as intermediate hosts if they consume T. solium eggs.

The purpose was to describe changes in and retention of knowledge and changes in behaviours of pig farmers after providing workshops using the training of the trainers model followed by one-on-one farmer training.

\section{Materials and Methods}

A random sample of 282 pig farmers in the Busia and Kakamega Districts of Western Kenya were included in the study beginning in 2006. Each farm was visited 3 times at 4-7 month intervals. Data was collected from farmers in face-to-face interviews at each farm visit. The authors presented a one-day workshop about pig management, feeding, housing, and the lifecycle of Taenia solium and its association with epilepsy to government livestock, veterinary and public health officers (staff). Then, the staff taught this material during one-day farmer workshops. Three farms visits that were 5 months apart enabled researchers to conduct one-on-one training. Farmers were asked what management changes they made as a result of the research and workshops during 2008 \&2009 workshops.

Proportions of correct answers to questions regarding the lifecycle and prevention of disease due to T solium in people and pigs were compared amongst three groups of farmers based on training; those who attended workshops and those who were given one-on-one training. Acquisition of knowledge was compared within respondent from the 2 nd to the 3 rd farm visit using a McNemar's chi-square test.

\section{Results}

Many farmers (51\%) have seen tape worm segments in their own stool. Most farmers (89\%) knew that if they saw these segments, they should seek medical treatment. However, few farmers knew how people became infected with the tapeworm. Male farmers were 9 times more likely to have seen a pig with cysts than female farmers.

Farmers were 3.7 times more likely to know how people become infected by $\mathrm{T}$ solium after the one-on-one training (60\%) than before this training (50\%). Farmers with at least a grade 8 education were more likely to understand the life cycle than farmers with less education. Farmers were more likely to tether their pigs $100 \%$ of the time after the workshop (51\%) than before the workshop (34\%) and still more likely after the one-on-one training $(63 \%)(P<0.02)$. At the end of the study, $79 \%$ of farmers were keeping their pigs tethered $75 \%$ of the time. More than half ( $59 \%$ ) of the farmers knew how to make the pork safe to eat if it was infected with cysts. This proportion was higher (66\%) for those farmers who received the one-on-one training.

\section{Discussion}

Education is the primary method of reducing the prevalence of diseases due to Taenia solium in developing countries. Workshops and one-on-one training increased the level of knowledge and changed the behavior regarding confining pigs. The life cycle of T solium is difficult to understand. Farmers with a grade 8 education and those with individual training were most likely to understand the cycle and therefore know how to prevent the disease. Individual training is expensive but may be necessary to reduce the prevalence of epilepsy due to this disease. 


\title{
0.145
}

\section{Toxoplasma gondii Seroprevalence on U.S. swine operations in 2000 and 2006 and factors associated with changes in prevalence in 2006}

\author{
Charles A. Haley ${ }^{1}$ Eric Bush ${ }^{1}$ Delores Hill ${ }^{2}$ \\ 1. USDA:APHIS:VS:CEAH:NAHMS, Fort Collins, CO, USA; 2. USDA:ARS: ANRI: Animal Parasitic Diseases Laboratory, BARC-East, Beltsville, MD, USA
}

\section{Introduction.}

Toxoplasma gondii is a protozoan parasite capable of infecting most warm blooded animals, potentially resulting in the disease toxoplasmosis. It is estimated that 60 million people in the U.S. are infected, although few demonstrate symptoms1. Cats can serve as the host and shed oocysts in the feces for approximately 1-2 weeks. These oocysts then sporulate in 1-5 days following deposition ${ }^{1,2}$. Swine, can be infected by ingesting material contaminated with the oocysts. It has been estimated that up to $25 \%$ of apparently healthy pigs in the United States have positive antibody titers ${ }^{3}$. While few pigs display clinical signs, the parasite can persist in the edible tissues of pigs.

Our first objective was to compare sample and farm level prevalence of exposure to Toxoplasma (as measured by antibodies) in 2000 and 2006 from the National Animal Health Monitoring System's (NAHMS) swine studies and associated herd factors. The second objective was to present factors associated with the number of positive Toxoplasma test results within farms sampled in the 2006 study.

\section{Materials and Methods}

Questionnaire data and blood samples were collected as part of the NAHMS Swine 2000 and 2006 studies. Producers participating in the NAHMS Swine 2000 and 2006 studies were able to submit up to 15 and 35 blood samples, respectively from finisher pigs to be tested for antibodies to Toxoplasma. In 2000, 5,718 samples were collected from 394 swine sites and tested using a modified agglutination test (MAT) test. In 2006, 6,238 samples were collected from 185 sites and tested using an enzyme linked immunosorbant assay (ELISA).

\section{Results}

Overall, $0.9 \%$ of grower/finisher pigs sampled were positive for Toxoplasma antibodies in 2000 and $2.7 \%$ in 2006. Smaller herds ( $<2 \mathrm{~K}$ head) appeared to have more positive samples than medium (2-4.9 K head) and larger herds ( $5 \mathrm{~K}$ head plus) in both years. The number of finisher pigs housed in total confinement comprised a lower percentage of positives in 2000 compared with 2006.
The average within herd prevalence in positive (had at least one positive sample) and negative herds in 2000 was $1.8 \%$ of finishers overall and in positive herds was $23.5 \%$. In 2006 the corresponding average within herd prevalence was $4.6 \%$ and $17.4 \%$, respectively. Overall, $7.5 \%$ of sites were positive for Toxoplasma antibodies in 2000 and $26.8 \%$ of sites were positive in 2006. Similar to the sample level results, smaller herds appear to be affected more often in both years but the prevalence in confinement vs. not confined herds seemed to be different only in 2000.

For the 2006 samples we used a method of modeling called zero inflation to detect herd level practices associated with counts of positive samples 4 . For farms that buried dead weaned pigs off site, the count of Toxoplasma positives was 7.2 times higher than when they were not buried off site. When a farm composted dead preweaned pigs on site the incidence rate of Toxoplasma positives went up 83 percent, while the use of traps, bait, poison, exterminator or some other method besides cats or dogs for rodent control on the farm reduced the prevalence of Toxoplasma by 63 percent.

\section{Discussion}

It is likely that since there was less sampling of finisher pigs in 2000 , the incidence has not gone up so much as there wasn't sufficient sampling in 2000 to find a difference. However, for 2000 and 2006 it would be possible to get estimates of true herd prevalence based on published test accuracies for the MAT and ELISA tests. The results of modeling suggest there is an unmeasured effect since one would expect that burying pigs off-site would decrease positive counts.

\section{References}

1. Toxoplasmosis Fact Sheet. Centers for Disease Control and Prevention. [www.cdc.gov].

2. T oxoplasmosis. Centers for Disease Control and Prevention, Division of Parasitic Diseases. [www.dpd.cdc.gov].

3. Dubey, JP. Swine Toxoplasmosis. Veterinary Division -Animal Health Programs. North Carolina Division of Agriculture and Consumer Services. [www.ncagr.gov].

4. Dohoo, I., Martin, W., Stryhn, H. Veterinary Epidemiologic Research. Atlantic Veterinary College, Charlottetown, PE, Canada, 2003. 


\title{
0.146
}

\section{Prevalence, detection and isolation of Toxoplasma gondii in swine tissue samples from a slautgerhouse in Bogota-Colombia}

\author{
Johanna M. Campos; Myriam A. Hortua; Barbara H. Zimmermann \\ Laboratorio de Bioquímica y Biología Molecular de Parasitos BBMP, Departamento de Ciencias Biológicas, Facultad de Ciencias, \\ Universidad de los Andes, Bogota, Colombia
}

\section{Introduction}

Toxoplasmosis is a zoonotic disease caused by infection with the protozoan Toxoplasma gondii. Infections by this parasite are widely prevalent in humans and other warm-blooded animals in all continents. In humans, infection occurs either by consumption of uncooked meat containing cysts or ingesting or drinking contaminated food with sporulated oocysts. Studies have demostrated that toxoplasmosis in pregnant women is generally related with uncooked meat consumption( Kapperud et al., 1996; Baril et al., 1999; Cook et al., 2000). It is important to study the prevalence of this protozoon in pork as it is a major source of infection. The seroprevalence in pigs was reported to be $29 \%$ worldwide. (Kim \& Weiss . 2008)

To guarantee the destruction of the parasite, the meat must be properly cooked until it reaches a temperature of $67^{\circ} \mathrm{C}$ or must be frozen at freezing temperature lower than $-10^{\circ} \mathrm{C}$ for a period of 48 hours. (Kijlstra \& Jongert, 2008)

\section{Materials and Methods}

80 pig samples of tongue, heart and blood were collected in a slaughterhouse in Bogotá- Colombia.

DNA extraction of tissues samples of heart and tongue was performed by the method of salting out. The amplification of a 532 bp fragment of B1 gene was performed by nested PCR to evaluate the presence of the parasite (Boothroyd \& Grigg 2001). For the isolation of native strains, subcutaneous inoculation of the processed material in CFW and Swiss mice was performed. Infection was assessed by analyses of mice sera by Western-blot technique using an anti-mouse IgG secondary antibody labeled with peroxidase.

Seroprevalence in pigs was assessed. The presence of antibodies againts T. gondii and Trichinella in blood samples of pigs were analyzed using an ELISA kit (ID Screen toxoplasmosis indirect multi-species, and ID Screen Trichinella indirect), produced by ID VET innovative diagnosis (Montpellier- FRANCE).

\section{Results}

Analysis of the samples obtained by B1 gene amplification showed a $40 \%$ prevalence of $T$. gondii in pig tissue in 66 samples analyzed. Seroprevalence analysis showed $37 \%$ presence of antibodies againts T. gondii in 80 swine serum samples, where 22 samples were positive by ELISA and PCR. Serum analysis for Trichinella gave negative in all cases.

The sera of mice infected with PCR positive swine samples were tested using Western blot to evaluate the possibility of infection. After 6 months only one mouse was Western blot positive, showing the presence of a native avirulent strain. This mouse serum showed reactivity to tachyzoite proteins of $\mathrm{RH}$ strain. The presence of cysts in brain were also detected in this mouse using PCR.

\section{Conclusions}

Using amplification of gene B1 it was possible to detect the presence of the parasite in 27 of the 66 samples from a slaughterhouse of Bogota, Colombia. This represents a $40 \%$ prevelence of $T$. gondii in swine tissue.

In the 80 samples analysed by the ELISA technique, the seroprevalence was $37 \%$, coinciding with previous reported studies.

The tecnification of the production plants has allowed better control of some diseases transmitted by swine. Nevertheless the present Colombian legislation for the proper handling of swine is still too lax to control infection by certain mechanisms of control of T. gondii, for example the presence of cats in the production area.

According to the FAOSTAT Studies done in Colombia in 2005, the total consumption of pork and its derivatives corresponds to $10 \%$ of the total meat consumption. This, together with the high prevalence of T. gondii in the country, shows the urgent need for better methods of control.

\section{References}

Baril, L., Ancelle, T., Goulet, V., Thulliez, P., Tirard-Fleury, V., Carme, B., 1999. Risk factors for Toxoplasma infection in pregnancy: a casecontrol study in France. Scand. J. Infect. Dis. 31, 305-309.

Cook, A.J.C., Gilbert, R.E., Buffolano, W ., Zufferey, J., Petersen, E., Jenum, P.A., Foulon, W., Semprini, A.E., Dunn, D.T., 2000. Sources of Toxoplasma infection in pregnant women: european multicentre casecontrol study. Br. Med. J. 321, 142-147.

Dubey J.P, Jones J.L, Toxoplasma gondii infection in humans and animals in the United States,

International Journal for Parasitology 38 (2008) 1257-1278

Grigg ME, Boothroyd JC, Rapid identification of virulent type I strains of the protozoan pathogen Toxoplasma gondii by PCR-restriction fragment length polymorphism analysis at the B1 gene. Journal of Clinical Microbiology 2001 Jan;39(1):398-400

Kapperud, G., Jenum, P.A., StrayPedersen, B., Melby, K.K., Eskild, A., Eng, J., 1996. Risk factors for Toxoplasma gondii infection in pregnancy - results of a prospective case-control study in Norway. Am. J. Epidemiol. 144, 405-412.

Kijlstra A, Jongert E, Control of the risk of human toxoplasmosis transmitted by meat Int J Parasitol. 2008 Oct;38(12):1359-70

Weiss LM and Kim K; Toxoplasma gondii The Model Apicomplexan: Perspectives and Methods; 2007, Elsevier Ltda 


\title{
0.147
}

\section{Isolation of immunogenic glycopeptidolipids of Mycobacterium avium subsp. hominissuis}

\author{
Henk J. Wisselink ${ }^{1}$ Najlaa Abou ${ }^{1}$ Conny B. van Solt ${ }^{1}$ Lourens Heres $^{2}$ Peter Willemsen ${ }^{1}$ \\ 1. Central Veterinary Institute of Wageningen UR, Lelystad, Netherlands; 2. VION Fresh Meat West, Boxtel, Netherlands
}

\section{Introduction}

Mycobacterium avium (MA) is an important pathogen in both animals and humans which belongs to the M. avium complex (MAC). Diagnosis of MA-infections in pigs is traditionally based on the detection of granulomatous lesions in lymph nodes seen by eye at slaughter. Since sensitivity and specificity of this approach is questioned (Komijn et al., 2007) we developed as an alternative a serodiagnostic ELISA assay on the basis of a polar lipid fraction from MA (Wisselink et al., 2009). We isolated glycopeptidolipids (GPLs) from M. avium subsp. hominissuis (MAH) and analyzed them for their suitability for serodiagnosis of MA infections in pigs.

\section{Materials and Methods}

MAH serotype 4 strain 17404 was grown at $37^{\circ} \mathrm{C}$ in Dorset Henry medium. Glycolipid antigens were extracted, analyzed by one and two dimensional thin layer chromatography (TLC) and further purified (Nishiuchi et al., 2004; Papa et al., 1993; Kitada et al., 2002). As reference, GPLs from a MAH serotype 4 strain were used (Kindly supplied by Y. Nishiuchi, Osaka, Japan). The immunogenicity of the GPLs was evaluated in ELISA tests using reference serum samples obtained from pigs held under experimental and field conditions.

\section{Results}

The TLC-pattern of GPLs from MAH strain 17404 and the reference GPL (Nishiuchi et al., 2004) showed both a Retention Factor (Rf) value of 0.42 (Fig. 1)

Fig.1. One - dimensional $(A)$ and two-dimensional TLC analysis ( $B$ and C). Reference GPLs from MAH serotype 4 (lane 1) and MAH serotype 4 strain 17404 (lane 2) had both a Rf value of 0,42. The purity of GPLs was examined by two-dimensional TLC (B: MAH strain 17404; C: reference $G P L)$.

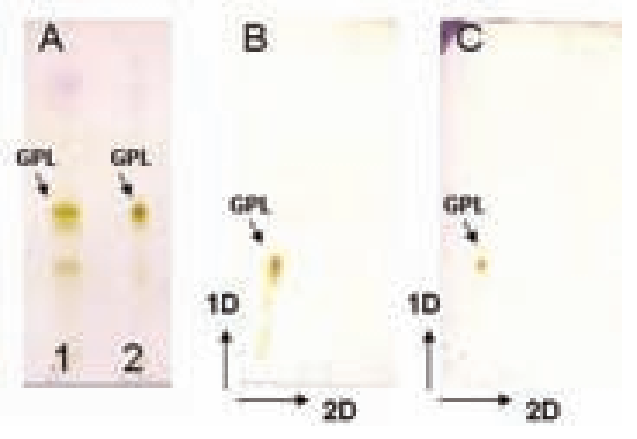

To evaluate the GPLs for their immunogenicity the cut-off value for negative and positive test results was calculated (Wisselink et al., 2009) which appeared to be 24.5 percentage positivity (PP). In all eight sera of pigs, experimentally infected with MA, high anti-GPL antibody titers were found. On the two infected farms $10.6 \%(11 / 104)$ of the pigs tested serologically positive and on the five farms, free for an MA infection 3.3\% (3/92) pigs (Fig. 2).

Fig. 2. Results of ELISA tests with 1 ug GPLs per well from MAH using serum samples from pigs experimentally infected with $M A(A ; n=8)$, from two farms with pigs known to be infected with $M A(B ; n=104))$ and pigs from five farms free for an MA infection ( $C ; n=92)$.

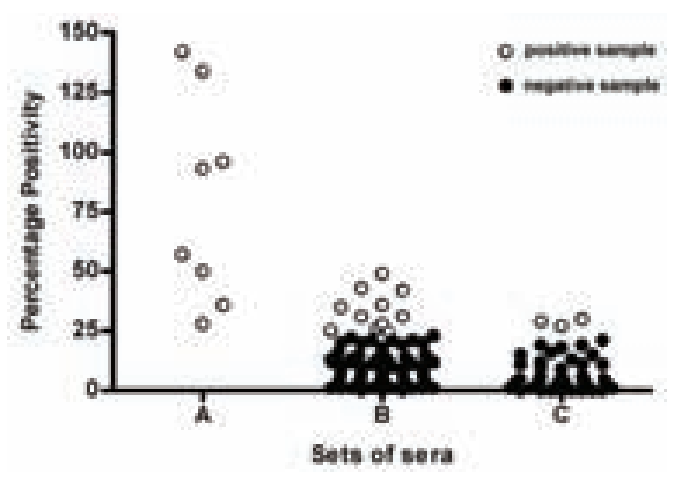

\section{Discussion}

Earlier we developed an ELISA test on the basis of polar lipids for serodiagnosis of MA infections in pigs. Here we show that purified GPLs can also be used as antigen in a serodiagnostic test because of its clear recognition by serum antibodies in experimentally infected pigs. For use under field conditions, the results of the GPL-ELISA indicate that MA-infected farms can be discriminated from MA-negative farms. Further work is needed to optimize and evaluate the GPL-ELISA for use in the field.

\footnotetext{
References

Kitada et al., 2002. Clin Infect Dis. 35: 1328-35

Komijn et al., 2007. Vet Microbiol. 120: 352-357

Nishiuchi et al., 2004. J Appl Microbiol. 97: 738-748

Papa et al., 1993. Res Microbiol 144: 91-99

Wisselink et al., 2009. Vet Microbiol. 2009.11.003
} 


\title{
0.148
}

\section{EU consumer reactions towards the vaccine method versus physical piglet castration with anesthesia: A quantitative cross-country study}

\author{
Wim Verbeke; Filiep Vanhonacker \\ Ghent University, Gent, Belgium
}

\section{Introduction}

In most countries, rearing male piglets are castrated to avoid boar taint, an unpleasant odor that can be released during the cooking of pork from entire boars. In the European Union (EU), physical castration is as yet the most common practice. This method is increasingly criticized for its violation of piglet welfare, integrity and health. Alternative methods are being researched and evaluated in terms of practical and economic feasibility, production efficiency and animal welfare.1 One promising alternative pertains to the vaccination with Improvac ( ${ }^{\circledR}$ registered trade mark of Pfizer). The vaccine stimulates the pig's immune system to produce specific antibodies against the Gonadotropin-Releasing Factor (GnRF), hence inhibiting the production and accumulation of androstenone and skatole that cause boar taint. This so-called 'vaccine method' has been shown to improve production efficiency, and to be beneficial from an animal welfare and environmental perspective. 2 The vaccine has received a marketing authorization of use in the EU market in March 2009. However, its successful introduction and adoption by stakeholders in pig supply chains is expected to depend among others on a favorable reception by consumers. Several qualitative3, sensory4 or quantitative single-country5,6 studies have already been reported. The present study adds to this body of evidence from the EU consumer point of view towards piglet castration, through providing a large-scale quantitative crosscountry approach, including the identification and profiling of cross-cultural pork market segments.

\section{Material and Methods}

A cross-sectional quantitative survey was conducted with representative consumer samples in Germany, France, the Netherlands and Belgium. In total 4,031 pork consumers responded on an online interview. The data is weighted per country to reflect the demographics of pork consumers in terms of age, gender and education. Key questions pertained to the awareness and acceptance of the method of castration (measured on a 7-point scale from $1=$ 'completely unacceptable' to $7=$ 'completely acceptable'), and the preference of the vaccine method relative to physical castration (measured on a 7-point scale from 1= 'strongly prefer physical castration'; 4='neutral'; 7='strongly prefer the vaccine method'). Participants were informed about boar taint, physical castration with anesthesia, and the vaccine method after some introductory questions.

\section{Results}

Significantly higher acceptance scores were reported for the vaccine method $(\mu=5.38, S D=1.61)$ versus physical castration with anesthesia ( $\mu=3.74, S D=1.74)$. Acceptance scores increased from Germany, over France and Belgium, to the Netherlands. About $70 \%$ preferred the vaccine method over physical castration with anesthesia; another $20 \%$ reported a neutral position. When informed about the two methods, $45 \%$ of the pork consumers intended to seek out pork produced using the vaccine method, while 33\% indicated to intend avoiding pork produced using physical castration. Three distinct market segments were identified based on importance attached to pork taste, price, health, animal welfare and environmental issues. The largest segment (59.2\%; relatively higher share of females and $>55$ years) reported a strong interest in ethical attributes and production method, along with interest in price, taste and health. Another segment (21.6\%; relatively higher shares of heavy pork consumers, males, $<35$ years, lower income, Dutch) focused mainly on price. The third segment (19.2\%; relatively higher shares of males, French and Germans) scored in between the previous segments on most attributes. The ethics oriented segment reported a lower confidence in the efficacy of physical castration, while the reported confidence levels that the vaccine method will avoid boar taint were similar across all segments. The two largest segments strongly rejected physical castration, while all three segments preferred the vaccine method to the same high degree.

\section{Discussion}

This cross-country study shows that uncertainties with respect to EU consumer acceptance of the vaccine method are void. A vast majority of pork consumers accept the vaccine method and strongly prefer this method over physical castration with anesthesia. The finding that confidence, acceptance and preference for the vaccine method do not differ between pork market segments indicates that the adoption of this method is unlikely to result in loss of any particular target market.

\footnotetext{
References

1 Prunier et al. (2006) Anim. Welf. 15:277-89.

2 Dunshea et al. (2001) Anim. Sci. 79:2524-35.

3 Hennessey \& Newbolt (2004) Proc. 18th IPVS.

4 Font i Furnols et al. (2008) Meat Sci. 80:1013-18.

5 Giffin et al. (2008) Proc. 20th IPVS.

6 Vanhonacker et al. (2009) Anim. Welf. 18:371-80.
} 


\title{
0.149
}

\section{Effects of GnRH down-regulation using deslorelin (Suprelorin ${ }^{\circledR}$ ) on sexual function in boars}

\author{
Johannes Kauffold ${ }^{1}$ Hartmut Rohrmann² Julia Boehm³ Axel Wehrend ${ }^{3}$ \\ 1. Department of Clinical Studies - New Bolton Center, School of Veterinary Medicine, University of Pennsylvania, Kennett Square, PA, USA; \\ 2. Rohrmann Affiliation, Rövershagen, Germany; 3. Clinic for Obstetrics, Gynecology and Andrology of Large and Small Animals, Faculty of \\ Veterinary Medicine, Justus-Liebig-University, Giessen, Germany
}

\section{Introduction}

Immunization against GnRH has been proven effective for boar taint removal [1], and long term treatment with GnRH analogues has been shown to down-regulate $\mathrm{GnRH}$ and to suppress $\mathrm{GnRH}$ dependent reproductive processes in several species [2]. This study was conducted to down-regulate $\mathrm{GnRH}$ in boars using Suprelorin ${ }^{\circledR}$ which is an implant that contains $4.7 \mathrm{mg}$ of the $\mathrm{GnRH}$ analogue deslorelin, and to test the effects on sexual function.

\section{Materials and Methods}

Five boars had the implant inserted intramuscularly at the age of 5 weeks and the effects studied until market age at 26-27 weeks. Surgically castrated $(n=4)$ and intact boars $(n=3)$ served as controls. Parameters assessed included health, testes growth and libido. Boars were trained to mount the dummy at the age of 180 days, and if mounted, were collected 3-6 times for semen analysis. Animals were bled twice at the age of 26 and 27 weeks, and serum analyzed for the concentration of testosterone, estrone, estrone sulphate and estradiol $17 \beta$, respectively. All intact and implanted animals were surgically castrated at the end of the experiment and the testes as well as the epidiymides subjected to histological evaluation.

\section{Results}

None of the boars that received the implant exhibited negative effects on general health or local reactions at the site of implant insertion. Four out of the 5 boars with the implant had only marginal testicular growth (Figure 1). These 4 boars did not show libido and did not mount, had low or undetectable concentrations of steroid hormones similar to what was observed in surgical castrates, and histological evidence of a ceased spermatogenesis. In the remaining fifth boar (\#9), the testes started to grow around week 17 (Figure 1). Steroid hormone concentrations were as high as in intact boars or higher. This boar also showed libido and could be collected; however, semen quality was bad for the first three ejaculates but then improved, reaching normality with the sixth ejaculate at the age of 34 weeks.
Figure 1. Mean $\left( \pm S D\right.$ ) testis length. Boars that received Suprelorin ${ }^{\circledast}$ had either marginal ( $n=4$; open squares) or distinct testis growth (boar \#9; triangles). Non-treated intact boars ( $n=3$; diamonds) tripled their testis size. Their testes were bigger then of boars with the implant (except boar \#9; $P<0.05-0.0001$ ).

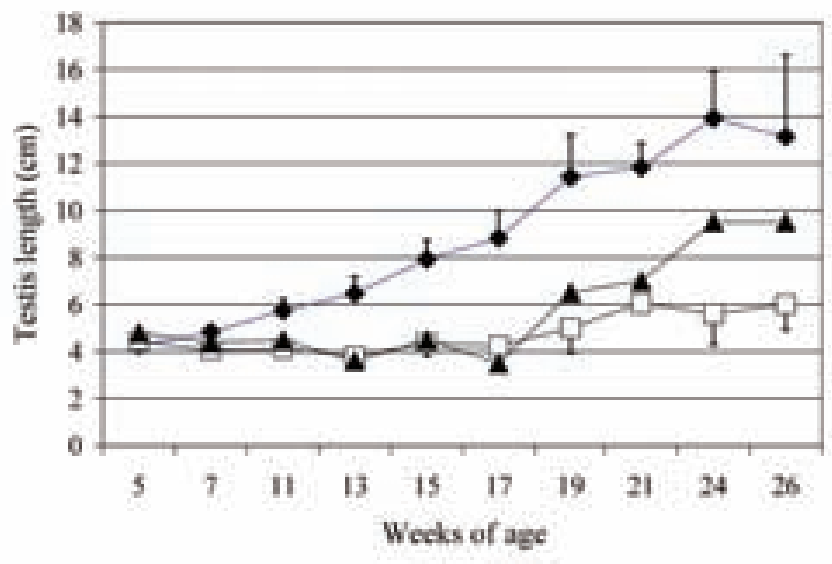

\section{Discussion}

This study has shown that, if five week old boars were treated with the implant Suprelorin ${ }^{\circledR}$ containing $4.7 \mathrm{mg}$ of the $\mathrm{GnRH}$ analogue deslorelin in order to down-regulate $\mathrm{GnRH}$, the testes only grow marginally and both the spermatogenesis as well as the steroidogenesis is inhibited. This is similar to what has been observed in male dogs [2]. Since these effects can last for an extended period up to the boars' market age, GnRH downregulation, as carried out in this study, may have potential for boar taint removal. However, since the effect does not seem to be sustained consistently in all boars, treatment may need to be refined.

\section{References}

1. Dunshea et al. J Anim Sci 2001;79:2524-2535.

2. Junaidi et al. Reprod Domest Anim 2009;44:725-734. 


\title{
0.150
}

\section{Effects of Ractopamine Hydrochloride on growth performance of Barrows, entire boars and boars vaccinated with Improvac ${ }^{\mathrm{Tm}}$}

\author{
Glauber S. Machado ${ }^{2}$ Luiz G. Peroni ${ }^{3}$ Sheerin Mathur ${ }^{1}$ Vickie L. King ${ }^{1}$ John P. Crane ${ }^{1}$ \\ 1. Pfizer Animal Health, Kalamazoo, MI, USA; 2. Integrall Soluções em Produção Animal Ltda., Patos de Minas, MG, Brazil; \\ 3. Perdigão Agroindustrial Ltda, Patos de Minas, MG, Brazil
}

\section{Introduction}

Ractopamine $(\mathrm{RH})$ is a $\beta$-adrenoceptor agonist known to enhance weight gain, leanness and feed efficiency in both barrows and gilts $(1,2)$. Barrows (physical castrates) are known to consume more feed, grow slower, and to have proportionally more fat compared to boars (3). An alternative to physical castration for the control of boar taint is vaccination against GnRF (also known as immunological castration) (4). Compared with barrows, boars that are raised with the use of vaccination are leaner and have better feed efficiency (5). The goal of the present study was to evaluate possible interactions between vaccination against GnRF and the use of ractopamine in finishing pigs.

\section{Materials and methods}

A total of 360 healthy crossbred Landrace $x$ Large-White pigs from DanBred genetics were randomly allocated to one of six treatments, soon after birth. The study had a randomized complete block design with sub-sampling, with body weight being the blocking factor. Treatments were arranged in a $3 \times 2$ factorial design: T1 (barrows + saline), T2 (barrows + $\mathrm{RH}+$ saline), T3 (vaccinated boars), T4 (vaccinated boars $+\mathrm{RH}$ ), T5 (boars $+\mathrm{RH}+$ saline) and T6 (boars + saline). Vaccination was performed using Improvac ${ }^{\mathrm{TM}}$ (Pfizer Animal Health) which was administered as two subcutaneous injections in the neck at 15 and 19 weeks of age. Day 0 was defined as the day when pigs were moved to the grower facility (approximately 9 weeks of age). Slaughter took place on Day 98 at approximately 23 weeks of age. The use of $\mathrm{RH}$ was in accordance with label recommendations, i.e., $5 \mathrm{ppm}$ in-feed during the last 28 days before slaughter. All animals were fed ad-libitum and weighed at 9, 15, 19, 21 and 23 weeks of age. Feed disappearance was measured daily. Diets fed during the last 28 days had 18\% Crude Protein (CP) and 1,0\% total Lysine for treatments T2 to T6, while the diet used for T1 had 15\% CP and $0,8 \%$ total Lysine.

\section{Results}

Following the second vaccination with Improvac, vaccinated animals showed higher feed intake $(p<0,05)$. In this same period, T4 (Improvac $+\mathrm{RH}$ ) had significantly higher daily gain than all other groups, being 18,6\% higher than T2 (barrows + $\mathrm{RH}+$ saline) and $8,5 \%$ higher than T3 (Improvac only). During this period, the addition of $\mathrm{RH}$ to the diet improved feed conversion of vaccinated boars by $12,2 \%$.
Analyzing the complete trial period (Day 0-98), entire boars were the most feed efficient (Table 1), but presented substantial incidence of boar taint at slaughter. Taking only vaccinated pigs and barrows (T1 to T4), the Improvac + RH group (T4) had significantly better feed efficiency than the others $(p<0,05)$. The administration of $\mathrm{RH}$ to Improvac vaccinated boars (T4) improved feed conversion by 4,3\% when compared to Improvac alone $(p=0,018)$. The use of Improvac and $\mathrm{RH}$ together improved feed efficiency by $6,9 \%(p=0,0007)$ when compared to barrows given $\mathrm{RH}$ (T2).

Table 1- Average daily gain (ADG), feed conversion and average daily feed intake among treatments for the whole trial period (Day 0 to Day 98)

\begin{tabular}{|c|c|c|c|c|}
\hline & $\begin{array}{c}\text { Weight day } 98 \\
(\mathbf{k g})\end{array}$ & $\begin{array}{c}\text { ADG } \\
\text { (kg/day) }\end{array}$ & Feed Conversion & $\begin{array}{c}\text { Feed Intake } \\
\text { (kg/day) }\end{array}$ \\
\hline T1 & $116,19^{a}$ & $0,980^{\mathrm{a}}$ & $2,62^{\mathrm{a}}$ & $2,55^{a, b}$ \\
\hline $\mathrm{T} 2$ & $119,44^{a, b, c}$ & $1,000^{a, b}$ & $2,60^{a, b}$ & $2,61^{\mathrm{a}}$ \\
\hline T3 & $119,95^{a, b, c}$ & $1,010^{a, b}$ & $2,53^{b}$ & $2,52^{b}$ \\
\hline $\mathrm{T} 4$ & $122,57^{c}$ & $1,040^{b}$ & $2,42^{c}$ & $2,49^{b}$ \\
\hline $\mathrm{T} 5$ & $121,17^{b, c}$ & $1,030^{b}$ & $2,30^{d}$ & $2,33^{c}$ \\
\hline T6 & $117,05^{a, b}$ & $0,980^{a}$ & $2,40^{c}$ & $2,34^{c}$ \\
\hline
\end{tabular}

\section{Discussion/Conclusions}

The addition of $\mathrm{RH}$ to the diet significantly improved feed efficiency of Improvac vaccinated pigs, when compared to pigs given Improvac alone. Besides that, the addition of $\mathrm{RH}$ during the last 28 days before slaughter produced similar qualitative effects in barrows, Improvac vaccinated pigs and entire boars, as described by a previous study (6). Comparing only the treatments which produced non-tainted meat, T4 (Improvac vaccinated boars $+\mathrm{RH}$ ) resulted in the best feed efficiency.

\section{References}

1 Xiao, R. J. et al. (1999) Anim. Feed Sci.Technol. 79:119-127.

2 Yen, J.T. et al. (1990) J. Anim. Sci. 68:3705-3712.

3 Campbell, R.G. et al. (1989) J. Anim. Sci. 67:177-186.

4 Oliver, W.T. et al. (2003) J. Anim. Sci. 81:1959-1966.

5 Dunshea, F.R. et al. (2001) J Anim. Sci. 79:2524-2535.

6 Rickard-Bell, C. et al. (2009) J. Anim. Sci. 87:3536-3543 


\title{
0.151
}

\section{Evaluation of the performance benefits from using Improvac ${ }^{\top \mathrm{TM}}$ in intact male pigs raised according to Canadian (Quebec) standards}

\author{
Christian Klopfenstein $^{2}$ Sheerin Mathur ${ }^{1}$ Vickie L. King ${ }^{1}$ Josee Daigneault ${ }^{1}$ \\ 1. Pfizer Animal Health, Kalamazoo, MI, USA; 2. Centre de développement du porc du Québec, Québec, QC, Canada
}

\section{Introduction}

Since the meat of boars is often tainted with an unpleasant odor, most countries of the world castrate pigs at a young age. However, as well as being a welfare concern, physical castration of young pigs impairs growth performance. Improvac is a novel immunological product which induces male pigs to develop antibodies to GnRF, consequently blocking testicular steroid synthesis and subsequent boar taint production prior to slaughter. ${ }^{1}$ Improvac is injected in the late stage of the grower-finisher period, and permits improved growth performance by retaining the advantages of intact males over most of the pig's life. ${ }^{1-2}$ The objective of this study was to evaluate the production benefits of using Improvac in male pigs raised under Canadian (Quebec) standards.

\section{Materials and Methods}

Animals and trial design: Male pigs from 4 high health commercial farrowing units from 4 different genetic lines (one per farm) were blocked by litter and randomly allocated to 2 treatment groups: 1.126 were physically castrated at 4 days of age; 2.126 dosed with Improvac (the second $2 \mathrm{~mL}$ dose given at least 4 weeks after the first dose and at least 4 weeks before slaughter). After weaning at 22 days of age, pigs were transferred to the research unit. Treatment groups were not commingled. During the trial period, pigs were tended according to the guidelines and principles outlined by the Canadian Council on animal care.

Growth performance: Pigs were individually weighed on Days 0 (23 days of age), 41 ( $1^{\text {st }}$ dose and transfer to grower-finisher), 71,99 ( 3 days after $2^{\text {nd }}$ dose in heavy pigs), 112 ( 2 days after $2^{\text {nd }}$ dose in light pigs), and Days $123,130,137$ and 144 (1 ${ }^{\text {st }}, 2^{\text {nd }}$, $3^{\text {rd }}$ and $4^{\text {th }}$ shipments to slaughter days). All pigs were fed ad libitum with feed rations meeting boar nutritional requirements. All the groups received the same rations during the same period. Feed consumption was recorded by pen.

Serology: Blood samples taken 1 day before second injection and 5 days before slaughter were assayed for anti-GnRF antibodies.

Behavior: Behavior assessment occurred 16-17 days post $2^{\text {nd }}$ dose (aggression, mounting, physical appearance).

Carcass assessment: Carcass quality was assessed by measurement of hot carcass weight, carcass yield, back fat thickness, lean yield, loin eye area, leg, loin, shoulder and belly yields, 24 hour loin and leg $\mathrm{pH}$, loin drip loss, ham yield and loin and leg Minolta color.

Statistical analysis: Data were analyzed using a general linear mixed model for repeated measures or for a single time point as appropriate.

\section{Results}

Growth performance: The mean live bodyweight at slaughter of Improvac pigs was significantly higher than for physically castrated pigs $(\mathrm{P}<0.05)$. Other growth data are given in Table 1.

Table 1. Growth performance - Least squares means

\begin{tabular}{|c|c|c|c|c|c|c|}
\hline & Group & $\begin{array}{c}\text { Days } \\
0-123\end{array}$ & $\begin{array}{l}\text { Days } \\
0-130\end{array}$ & $\begin{array}{l}\text { Days } \\
0-137\end{array}$ & $\begin{array}{l}\text { Days } \\
0-144\end{array}$ & $\begin{array}{l}\text { Days } 0 \text { - } \\
\text { slaughter }\end{array}$ \\
\hline \multirow[t]{2}{*}{$\begin{array}{l}\text { Daily gain } \\
\text { (kg/day) }\end{array}$} & 1 & $0,88^{a}$ & $0,88^{\mathrm{a}}$ & $0,89^{a}$ & $0,88^{a}$ & $\mathrm{~N} / \mathrm{D}$ \\
\hline & 2 & $0,90^{\mathrm{b}}$ & $0,92^{b}$ & $0,93^{b}$ & $0,94^{b}$ & $\mathrm{~N} / \mathrm{D}$ \\
\hline \multirow[t]{2}{*}{$\begin{array}{l}\text { Feed intake (kg/ } \\
\text { day) }\end{array}$} & 1 & $1,90^{\mathrm{a}}$ & $\mathrm{N} / \mathrm{D}$ & $\mathrm{N} / \mathrm{D}$ & $\mathrm{N} / \mathrm{D}$ & $1,98^{\mathrm{a}}$ \\
\hline & 2 & $1,77^{\mathrm{b}}$ & $\mathrm{N} / \mathrm{D}$ & $\mathrm{N} / \mathrm{D}$ & $\mathrm{N} / \mathrm{D}$ & $1,91^{b}$ \\
\hline \multirow[t]{2}{*}{ Feed conversion } & 1 & $2,18^{\mathrm{a}}$ & $\mathrm{N} / \mathrm{D}$ & $\mathrm{N} / \mathrm{D}$ & $\mathrm{N} / \mathrm{D}$ & $2,25^{\mathrm{a}}$ \\
\hline & 2 & $1,98^{b}$ & $\mathrm{~N} / \mathrm{D}$ & $\mathrm{N} / \mathrm{D}$ & $\mathrm{N} / \mathrm{D}$ & $2,09^{b}$ \\
\hline
\end{tabular}

Serology: The mean anti-GnRF antibody level at slaughter was significantly higher for the Improvac pigs than for the physically castrated pigs $(\mathrm{P}<0.05)$.

Behavior: None of the Improvac pigs showed any signs of boar behavior post $2^{\text {nd }}$ dose.

Carcass assessment: Improvac pigs had significantly lower carcass and belly yields than physically castrated pigs $(P<0.05)$. However, shoulder and ham yields were higher for the Improvac group $(P<0.05)$. No significant differences were observed for any other parameter.

\section{Discussion}

The results indicate that male pigs injected with Improvac developed a strong immune response to GnRF. The Improvac pigs had significantly better growth (live bodyweight, average daily gain, feed conversion) than physically castrated pigs without any effect on carcass quality.

\section{Conclusion}

The use of Improvac can allow productivity benefits in intact male pigs raised under Canadian standards without affecting carcass quality.

\section{References}

1. F. R. Dunshea et al., J. Anim. Sci. 79, 2524 (2001).

2. W. T. Oliver et al., J. Anim. Sci. 81, 1959 (2003). 


\title{
0.152
}

\section{Histomorphology of testes and epididymides of unvaccinated and Improvac ${ }^{\bullet}$ vaccinated slaughter boars}

\author{
Friedrich Schmoll ${ }^{1,3}$ Franz Schachinger ${ }^{2}$ Sabine Kölle ${ }^{2}$ \\ 1. Large Animal Clinic for Internal Medicine, Leipzig, Germany; 2. Institute of Anatomy, Histology and Embryology, VetmedUni, Vienna, Austria; \\ 3. Institute for Veterinary Public Health, VetmedUni, Vienna, Austria
}

\section{Introduction}

Active immunization against gonadotropin-releasing factor using a GnRF analogue-protein conjugate, (Improvac ${ }^{\circ}$ ) is an alternative to surgical castration. Usually, vaccination is recommended twice, at least 4 weeks apart, with the second injection given 4-6 weeks before slaughter (1). Studies suggest that the immune response is temporary. In pigs kept longer than 7-8 weeks after the second dose, anti-GnRF antibodies may decline below effective levels, allowing return of testicular function and the risk of boar taint accumulation. Therefore, the timing of vaccination in the fattening period is of high practical relevance.

The objective of this study was to evaluate testes and epididymides size and morphology as well as boar taint in intact and Improvac vaccinated boars receiving the $2^{\text {nd }}$ vaccination either 6 or 10 weeks prior to slaughter.

\section{Materials and Methods}

From a typical Austrian swine unit, 22 and 28 Improvac vaccinated males, receiving the $2^{\text {nd }}$ vaccination 6 and 10 weeks prior slaughter, respectively (2) were investigated. 17 intact boars were included as control animals. Testes and epididymides were collected at slaughter and measured (maximum length, width) using a calliper and all carcasses were organoleptically assessed (2). For histological evaluation, $1 * 1 \mathrm{~cm}$ pieces of testis and epididymidis were cut out, fixed, sectioned and stained with hematoxylin-eosin.

\section{Results}

Testes with and without adjacent epididymides were smallest in the group of the animals that were vaccinated 10 weeks before slaughter, followed by animals vaccinated 6 weeks before slaughter, and biggest in intact boars (table 1). Carcass weight and age of the animals at slaughter are shown in table 1.

Organoleptic abnormalites (i.e., pronounced sexual odour) were not detected in any of the carcasses from vaccinated animals. In contrast, carcasses of 4 of the 17 intact boars did have strong boar taint and were therefore condemned.

In the testes of all vaccinated boars, the number of spermatogonia and Sertoli cells in the Tubuli seminiferi was distinctly reduced. Leydig cells were degenerated and reduced in number. Spermatides and sperm were completely absent. Unlike boars vaccinated 6 weeks before slaughter, which had no spermatocytes, boars vaccinated 10 weeks prior to slaughter had single spermatocytes in the Tubuli seminiferi. In boars vaccinated 6 weeks before slaughter, the collagen fibers in the connective tissue between the Tubuli seminiferi showed a distinct reduction in number and size. In boars vaccinated 10 weeks before slaughter, beginning of the remodelling of the connective tissue was visible.
In the epididymides of all vaccinated boars no sperm was visible. In the major part of the Ductus the stereocilia were completely eliminated. Whereas secretion was completely absent in boars vaccinated 6 weeks prior to slaughter, it was present in boars vaccinated 10 weeks before slaughter.

Table 1: Carcass traits, testes size with and without epididymides in intact boars and boars that received the $2^{\text {nd }}$ Improvac dose 6 or 10 weeks prior to slaughter

\begin{tabular}{|l|c|c|c|}
\hline & $\begin{array}{c}\text { 6 weeks prior } \\
\text { slaughter }\end{array}$ & $\begin{array}{c}\text { 10 weeks prior } \\
\text { slaughter }\end{array}$ & intact boars \\
\hline days at fattening & 105 & 117 & 91 \\
\hline carcass weight $\mathrm{kg}$ & 104.0 & 106.0 & 91.9 \\
\hline \multicolumn{4}{|c|}{ testes with epididymides } \\
\hline length & 109.3 & 93.0 & 141.1 \\
\hline width & 56.0 & 52.2 & 75.5 \\
\hline & testes without epididymides \\
\hline length & 76.9 & 67.2 & 104.8 \\
\hline width & 48.2 & 41.1 & 66.5 \\
\hline
\end{tabular}

\section{Discussion}

Boar taint was completely eliminated in animals receiving the $2^{\text {nd }}$ Improvac dose 6 weeks or 10 weeks prior to slaughter. Vaccination induced a complete inhibition of testicular function. However, the effects of vacciantion seem to be reversible, since animals started to restore testicular and epidydimal function at week 10 after second vaccination.

\section{References}

1. Dunshea et al. J Anim Sci 2001;79:2514-2535.

2. Schmoll et al. J Swine Health Prod 2009;17:250-255. 


\title{
0.153
}

\section{Gestating sows' use of feeding stations and correlation between sows' deviant eating behaviour and heat/disease}

\author{
Lisbeth U. Hansen; Jens Vinter \\ Pig Research Centre, København, Denmark
}

\section{Introduction}

Electronic sow feeding (ESF) is a common feeding principle for group-housed gestating sows. With ESF it is possible to feed the sows individually and to check if the sows have been given their entire ration of feed. Studies have demonstrated that individual feeding of sows - particularly in the implantation period - is essential for satisfactory production results (1).

Pig houses with ESF often house large dynamic groups, which can make it difficult to supervise the individual animals. It is therefore necessary to have a tool that can provide the staff with information on sows with deviant behaviour, such as heat or disease.

\section{Materials and Methods}

In a one-year period in 5 production herds, data was compiled on the sows' use of the feeding stations, heat and health problems and production data.

The pens were equipped with large nesting boxes with bedding and slatted floor in the activity area. The sows were transferred to group-housing after service. There were $65-80$ sows per feeding station. Every 14 days, a technician from Pig Research Centre visited the herds.

\section{Results}

3.3 million observations/visits to the feeding stations were compiled corresponding to data from 18,300 cycles/ sows.

Visits to the feeding station

Regardless of the sow's age, the daily feed ration was eaten in one visit - typically the first. Gilts and young sows tended to have more visits to an empty feeder compared with older sows. This is most likely related to hierarchy.

\section{Time of visit to feeding station}

During the first 14 hours of the feed day, there was an even number of visits to the feeding stations. The sows had finished eating after approx. 19 hours. As the sows ate their feed ration, the frequency of unsuccessful visits to the feeding stations increased. Visits to the feeding station may have a facilitating effect on other sows in the group. Consequently, it must be expected that fewer unsuccessful visits reduce activity in the pen and thereby the risk of aggressive behaviour by the stations.

\section{Choice of feeding station}

Generally, the sows did not switch feeding stations from day to day in terms of feed visits. They stayed "loyal" to the feeding station they had chosen. In terms of unsuccessful visits, they were less "loyal" and used several feeding stations.

Number of sows per feeding station

More sows failed to eat their ration when there were more than 65 sows per feeding station.

Heat and health problems

A correlation was observed between changes in feeding time and heat. The sensitivity expressing the percentage of detected observations related to the "true" number of observations was 0.58-0.75. Unfortunately, the error rate (false alarms) was high, but can probably be reduced if surveillance was only made around expected heat.

The correlation between changes in feeding time and health problems only showed a low sensitivity, but can probably be improved by including information on sows' temperature and movement patterns (2)(3).

\section{Discussion}

More knowledge on sows' use of feeding stations coupled with more knowledge on sows' movement patterns and the use of GPS systems will give pig producers greater opportunities for detecting sows with deviant behaviour.

\section{References}

(1) Sørensen, G., (2003). Medd. 618. Den rullende Afprøvning. Danish Pig Production.

(2) Vinter, J., (2006). Detektion af brunst og helbredsproblemer ved dynamisk lineær modellering. Speciale ved KVL

(3) Cornou, C., Vinter, J., Kristensen, A.R., (2008). Automatic detection of oestrus and health disorders using data fra electronic sow feeders. Livestock Science 118 (2008), pp. 262-271 


\title{
0.154
}

\section{Effects of losing body condition scores during lactating period on blood concentrations of glucose, urea nitrogen and non-esterified fatty acid in sows}

\author{
Teera Rukkwamsuk; Supatee Kitkha; Pariwat Poolperm; Preeyaphan Udomprasert \\ Kasetsart University, Nakornpathom, Thailand
}

\section{Introduction}

The sows required higher energy during lactation for milk production. (1) At least $70 \%$ of energy requirement (2) and $90 \%$ of absorbed amino acids (3) needed for mammary growth and milk synthesis. Therefore, excessive mobilization of body reserved may occur if the sows inadequately intake nutrients, which resulting in poor lactation performance and infertility problems $(4,5)$

Body condition scoring, body weight loss during lactation can be practically evaluated. It is interesting to investigate if the loss of body condition score (BCS) observed on a farm can be monitored by using blood metabolites. The aim of this study wanted to compare the concentration of glucose, non-esterified fatty acid (NEFA) and blood urea nitrogen (BUN) between the sows losing $\geq 1$ point of condition score and the sows losing $<1$ point of condition score.

\section{Materials and Methods}

The study was conducted in a 1,000 sows farm with an opened system and traditional 2 site production during May - July 2009. Individual sow was given a BCS on farrowing day and weaning day and then categorized into two groups, based on the difference of BCS at weaning and farrowing date, for BCS loss $<1$ groups (group1) and BCS loss $\geq 1$ (group 2).

A total of 70 cross-bred sows with parity average of 2.8 were randomly selected. Blood samples were collected at 84 days of gestation, 2-3 days after farrowing and 1-2 days before weaning to evaluate the concentration of glucose, NEFA and BUN by using enzymatic spectrophotometry. Two-Sample t Test and correlation analysis were used for data analysis.

The gestation and lactation diet were formulated, based on broken rice, rice bran, soybean meal vitamin and mineral premixes, to contain $14.02 \%$ CP, 3,075 Kcal $/ \mathrm{kg} \mathrm{ME}$ and $0.85 \%$ Lys. and $18.1 \% \mathrm{CP}, 3,300 \mathrm{Kcal} / \mathrm{kg} \mathrm{ME}$ and $1.12 \%$ Lys, respectively.

\section{Results}

The concentration of glucose and NEFA between the sows in group 1 and the sows in group 2 were not different. However, the concentration of BUN in group 2 at 2-3 days after farrowing and at 1-2 days before weaning were lower than those of group $1(p=0.004$ and 0.030 respectively) (Table 1$)$.

Table 1 Blood glucose, BUN and NEFA concentrations during pregnancy, weaning and farrowing between 2 different BCS loss during lactating groups (Mean \pm SEM).
Table 1 Blood glucose, BUN and NEFA concentrations during pregnancy, weaning and farrowing between 2 different BCS loss during lactating groups (Mean \pm SEM).

\begin{tabular}{|l|c|c|c|}
\hline \multicolumn{1}{|c|}{ Blood metabolites } & BCS loss $<1$ & BCS loss $\geq 1$ & p - value \\
\hline & Glucose $(\mathrm{mg} / \mathrm{dl})$ & & \\
\hline 84 d of gestation & $89.40 \pm 2.25$ & $94.67 \pm 3.19$ & 0.194 \\
\hline 2-3 d after farrowing & $111.38 \pm 4.02$ & $104.27 \pm 3.97$ & 0.213 \\
\hline 1 - 2 d before weaning & $113.38 \pm 2.61$ & $110.35 \pm 3.86$ & 0.522 \\
\hline & NEFA (mmol/l) & & \\
\hline 84 d of gestation & $0.21 \pm 0.02$ & $0.21 \pm 0.03$ & 0.808 \\
\hline 2-3 d after farrowing & $0.24 \pm 0.02$ & $0.30 \pm 0.04$ & 0.212 \\
\hline 1 - 2 d before weaning & $0.38 \pm 0.04$ & $0.41 \pm 0.07$ & 0.719 \\
\hline & $B U N(\mathrm{mg} / \mathrm{dl})$ & & \\
\hline 84 d of gestation & $16.00 \pm 0.37$ & $16.84 \pm 0.64$ & 0.238 \\
\hline 2-3 d after farrowing & $20.05 \pm 0.69$ & $16.48 \pm 0.88$ & 0.004 \\
\hline 1 - 2 d before weaning & $20.34 \pm 0.44$ & $17.69 \pm 1.07$ & 0.030 \\
\hline
\end{tabular}

\section{Discussions}

BCS loss could reflect a negative energy balance condition of sows during lactation period. Sows in group 1 had less BCS loss than sows in group 2, and had higher serum BUN during lactation. The increased BUN might be caused by increased protein intake (6). In this study, sows having less BCS loss may have higher feed intake, consequently enhanced metabolism of protein which resulted in increasing of BUN.

\section{References}

1. Eissen,J. J., E. Kanis, and B. Kemp. 2000. Livest.Prod.Sci 64: 147-165.

2. Ahern,F. X. and I. H. Williams. 1992. Vet Clin North Am Food Anim Pract.8: 589.

3. Boyd,R. D., and K. J. Touchette. 1998. PIC USA Technical Memo: 171.

4. Whittemore,C. T., and C. A. Morgan. 1990. Livest.Prod.Sci: 1-37.

5. Clowes, E. J., F. X. Ahern, A. L. Schaefer, G. R. Foxcroft, and V. E. Baracos. 2003. J Anim Sci 81: 1517-1528.

6. http://en.wikipedia.org/wiki/Blood_urea_nitrogen access on $02 / 13 / 2010$ 


\title{
0.155
}

\section{Prevalence of lameness in farrowing and weaned gilts and sows in Ontario commercial hog operations}

\author{
Paisley Canning ${ }^{1,2}$ Tim Blackwell ${ }^{2}$ \\ 1. Animal Health Laboratory, University of Guelph, Guelph, ON, Canada; \\ 2. Veterinary Science and Policy, Ontario Ministry of Agriculture, Food and Rural Affairs, Elora, ON, Canada
}

\section{Introduction}

Lameness is a welfare and economic concern in hog production. Lame sows and gilts require treatment or removal from the herd. Early culling of sows is an economic cost for a hog producer. Lame sows are 1.7 times more likely than non-lame sows to be removed from a herd within 350 days of initial lameness assessment (Anil 2009). In 2007, American commercial herds participating in the PigCHAMP datashare program reported locomotor disorders including lameness and down sows as the reason for $14.5 \%$ of removals (Engblom 2009). Lame sows may also have poor reproductive performance including reduced number of pigs born alive compared to non-lame sows (Anil 2009). If lameness persists in a herd, it becomes a serious welfare issue and correcting it may require expensive modifications of housing areas. The prevalence and severity of lameness in gilts and sows has not been well documented. In the United Kingdom, the prevalence of abnormal gait in pregnant gilts and sows is $14.4 \%$ and $16.9 \%$ respectively but corresponding data for Ontario has not been collected (Kilbride 2009). To address this, the Ontario Ministry of Agriculture, Food and Rural Affairs has undertaken a surveillance project to determine the prevalence and severity of sow lameness on Ontario hog farms.

\section{Materials and Methods}

A scoring system was devised to score a sow or gilt's gait based on three categories: no lameness, mildly lame and clearly lame. To apply this scoring method, as sows and gilts enter and exit the farrowing room(s), they are scored a 0 (no lameness), 1 (mildly lame) or 2 (clearly lame). The scoring system focuses on the gait of the sow or gilt and does not ask the evaluator to identify the cause of lameness, if observed in the animal. A lameness scoring video was created that provides a tutorial on using this 0, 1, 2 lameness scoring system. The video is available from P. Canning (email address: canninp@gmail.com) and it helps standardize how producers score lameness on their farm. A survey form has been created for use with Episurveyor ${ }^{\circledR}$ on a Nokia 8086 and e71 cellular phones that asks producers to evaluate the soundness of sows and gilts as they move into and out of farrowing rooms each week, based on the $0,1,2$ system. If a sow or gilt is scored mildly lame (1) and clearly lame (2), her ID tag number is recorded to track changes in her lameness score over the course of the study. Producers complete the survey on the cell phone and then send in the data to an internet-based server for analysis each week. Participants may also email, fax or mail in a paper copy of the lameness survey. On each farm, the study will continue for at least six months.

\section{Results}

Currently we are receiving weekly data from six hog operations in southwestern Ontario. Participants indicated that they found the $0,1,2$ system and scoring video easy to understand and apply. Data has been collected on 1932 sows entering farrowing rooms and 1910 sows leaving the farrowing rooms. During the study period, 14 sows have died in the farrowing rooms for reasons unrelated to lameness. Below is a summary table of average prevalence data for enrolled herds.

\begin{tabular}{|l|c|c|}
\hline & $\begin{array}{c}\text { Average for all } \\
\text { herds }(\mathbf{n}=\mathbf{6})\end{array}$ & Range \\
\hline Females to farrow & & \\
\hline Score 0: no lameness & $97.67 \%$ & $94.64 \%-99.30 \%$ \\
\hline Score 1: mildly lame & $1.26 \%$ & $0.43 \%-4.17 \%$ \\
\hline Score 2: clearly lame & $0.51 \%$ & $0.00 \%-1.26 \%$ \\
\hline Females exiting farrowing room(s) & & \\
\hline Score 0: no lameness & $95.76 \%$ & $91.94 \%-98.53 \%$ \\
\hline Score 1: mildly lame & $2.36 \%$ & $0.61 \%-3.92 \%$ \\
\hline Score 2: clearly lame & $0.49 \%$ & $0.00 \%-1.61 \%$ \\
\hline
\end{tabular}

\section{Discussion}

The prevalence of lameness in gilts and sows in the six study farms is low. All herds experience some level of mild lameness, and a lower prevalence of clearly lame females. Comparatively, prevalence of abnormal gait in the U.K. for gilts and sows is $14.4 \%$ and $16.9 \%$ respectively (Engblom 2009). Although the scoring method used in this study differs from the $0,1,2$ system applied here, these results could indicate that in Ontario, lameness prevalence is comparatively low.

\section{References}

Anil, S.S., Anil, L., \& J. Deen. (2009). JAVMA Vol 235:6 734-738.

Engblom, L., Stalder, K \& J. Mabry. (2009). Benchmark Vol 4:1 24-25.

Kilbride, A. L., Gillman, C.E., \& L. E. Green. (2009). Animal Welfare Vol 18: $3215-224$. 


\title{
0.156
}

\section{Production performance among lactating loose housed Norwegian hybrid sows: Feed consumption, weight loss, litter gain and the effect of extra water access}

\author{
Signe Lovise Thingnes ${ }^{1,2}$ Ann Helen Gaustad ${ }^{1}$ Tore Framstad $^{2}$ \\ 1. Norsvin, Hamar, Norway; 2. Norwegian School of Veterinary Science, Oslo, Norway
}

\section{Introduction}

Farrowing crates for lactating sows have been prohibited in Norway since 2003. The Norwegian Landrace/Yorkshire (LY) sow is a highly productive sow that performs well under loose housed conditions, but factors such as parity number and water access can influence this performance. Leibbrandt (1989) found that limiting the water access to lactating sows led to reduced feed intake and the catabolism of body reserves to maintain milk production. The objective of this trial was to investigate the effect of parity number and extra water access on the sows' feed consumption, weight loss and production (in terms of litter gain) during lactation.

\section{Materials and Methods}

Data were obtained from 50 dry fed loose housed Norwegian $\mathrm{LY}$ sows from a commercial swine herd. Sows were fed using a pelleted lactation feed containing 9.86 MJ NE/kg feed and $8.26 \mathrm{~g}$ Lysin/kg feed. 25 sows were placed in pens with access to water only through drinking nipples in the rear part of the pen; and 25 sows had water access in the trough in addition to the water nipples. Sows were randomly distributed to the pens, but first and second parity sows were evenly distributed between the two treatments. Within 12-36 hours after parturition (day 1 ) number of liveborn piglets was recorded along with litter weight and weight of the sow. Sow and litter were weighed again on day 19, and the day before weaning and day of weaning, respectively. Daily feed consumption of the sows was recorded, and piglets were given access to creep feed from week two. The data was analysed using Proc GLM in SAS 9.1 2003 edition. One sow was emitted from the study due to abortion. Fixed effects included parity number and water strategy. Length of lactation was used as a covariate. Weight loss was corrected for weight at day 1.

\section{Results}

The average weight on day 1 for 1 st parity sows was $223.3 \pm 21.5$ $\mathrm{kg}$, for 2 nd parity sows $259.5 \pm 22.0 \mathrm{~kg}$ and older sows $279.8 \pm$ $24.4 \mathrm{~kg}$. Average number of weaned piglets was $11.2 \pm 1.0$ for 1st parity, $11.9 \pm 1.5$ for 2 nd parity and $11.7 \pm 1.7$ for older sows. Average length of lactation regardless of parity number and treatment was 31.7 days. Piglet mortality (not including stillbirths) was $8.2 \%$
Table 1: LS means for effect of parity number and water strategy on feed consumption, weight loss and litter gain.

\begin{tabular}{|c|c|c|c|c|c|c|}
\hline Parity: & $\mathbf{n}$ & \begin{tabular}{|c|} 
Average \\
feed cons. \\
(kg) during \\
lactation \\
\pm SE \\
\end{tabular} & 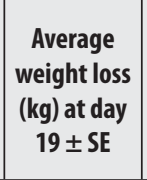 & \begin{tabular}{|c|} 
Average \\
weight \\
loss $(\mathrm{kg})$ at \\
weaning \\
$\pm \mathrm{SE}$ \\
\end{tabular} & $\begin{array}{l}\text { Average } \\
\text { litter gain } \\
\text { at day } 19 \\
(\mathbf{k g}) \pm \text { SE }\end{array}$ & $\begin{array}{c}\text { Average } \\
\text { litter gain } \\
\text { at weaning } \\
(\mathbf{k g}) \pm \mathrm{SE}\end{array}$ \\
\hline $1 s t$ & 15 & $214.3 \pm 6.9^{\mathrm{a}}$ & $21.0 \pm 2.9^{\mathrm{a}}$ & $26.5 \pm 4.0^{\mathrm{a}}$ & $52.9 \pm 2.3^{\mathrm{a}}$ & $91.0 \pm 2.9^{\mathrm{a}}$ \\
\hline 2nd & 14 & $\begin{array}{c}267.4 \pm 6.7 \\
b * * *\end{array}$ & $12.1 \pm 2.5^{b *}$ & $22.1 \pm 3.5^{\mathrm{a}}$ & $59.9 \pm 2.4 b *$ & $\begin{array}{c}113.2 \pm 2.9 \\
b * * *\end{array}$ \\
\hline 3rd-7th & 20 & $\begin{array}{c}276.2 \pm 5.7 \\
b * * *\end{array}$ & $4.9 \pm 2.3^{c * *}$ & $11.4 \pm 3.2^{b *}$ & $57.9 \pm 2.0^{\mathrm{ab}}$ & $\begin{array}{c}106.6 \pm 2.5 \\
b * * *\end{array}$ \\
\hline \multicolumn{7}{|c|}{ Treatment: } \\
\hline $\begin{array}{l}\text { Water in } \\
\text { trough }\end{array}$ & 25 & $255.6 \pm 5.0^{\mathrm{a}}$ & $12.5 \pm 2.0^{\mathrm{a}}$ & $20.2 \pm 2.7^{\mathrm{a}}$ & $57.8 \pm 1.8^{\mathrm{a}}$ & $106.1 \pm 2.2^{\mathrm{a}}$ \\
\hline $\begin{array}{l}\text { No extra } \\
\text { water }\end{array}$ & 24 & $249.6 \pm 5.4^{\mathrm{a}}$ & $12.8 \pm 2.0^{\mathrm{a}}$ & $19.8 \pm 2.7^{\mathrm{a}}$ & $56.0 \pm 1.9^{a}$ & $101.1 \pm 2.3^{a}$ \\
\hline
\end{tabular}

\section{Discussion}

1 st parity sows eat less $(p<0.0001)$, loose more weight $(p=0.02)$ and have a lower litter gain $(p<0.0001)$ during lactation, than 2nd parity and elder sows. This suggests a lower feed intake capacity in gilts than elder sows, and that one should be cautious not to overexert the sows during their first parity. Water access in the feed trough could not be shown to significantly affect the feed consumption and weight loss of the sows, nor did it affect the litter gain, although a numerical trend was present at weaning. The lack of a statistical difference in litter gain could be due to the limited number of sows in this trial.

\section{References}

Leibbrandt V. (1989). Swine report 1989, pp.14-17. University of Wisconsin, Madison. Seen in Fraser et.al., 1990. Water for piglets and lactating sows:quantity, quality and quandaries. Rec.Adv.Anim.Nutr. pp.137-160. 


\title{
0.157
}

\section{A tool to assess the economic impact of pleurisy in slaughter pigs}

\author{
Henrike J. Jager ${ }^{4}{ }^{1}$ TJ McKinley ${ }^{1}$ James L. Wood ${ }^{1}$ Gareth P. Pearce' ${ }^{1}$ Susanna M. Williamson² Nicholas Woodger ${ }^{2}$ \\ Benjamin Strugnell ${ }^{3}$ Stanley H. Done ${ }^{3}$ Henrike Habernoll ${ }^{5}$ Alexander W. Tucker $^{1}$ \\ 1. University of Cambridge, Cambridge, UK; 2. Veterinary Laboratories Agency, Bury St Edmunds, UK; \\ 3. Veterinary Laboratories Agency, Thirsk, UK; 4. Garth Partnership, Beeford, UK; 5. BQP Ltd, Stradbrooke, UK
}

Introduction: Pleurisy is an easily detectable indicator of poor respiratory health in pigs at slaughter and is a common reason for carcase trimming and rejection. Although prevalence in the UK was estimated, at individual pig level, to be around $12.5 \%$ for the period 2005-2009, surveillance data shows that for the same period around $87 \%$ of all assessed slaughter consignments $(18,648)$ were affected by pleurisy to some extent, with $38 \%$ of assessed batches recording pleurisy prevalence at $>10 \%$ (BPEX Pig Health Scheme). Recent work by Wellenberg et al (2010) proposed a 4 Euros cost per finisher in batches experiencing at $>10 \%$ prevalence, based on reduced daily live weight gain. Here we report the development of an economic tool for estimation of pleurisy associated costs based on average age at slaughter and post-trimming carcase weights. As part of the development we investigated the importance of recording pleurisy severity in addition to prevalence within the batch. Understanding the economic impact of pleurisy is essential to assist producers in justifying implementation of expensive disease control methods (e.g. all-in all-out production).

Materials and Methods: Correlation between pleurisy severity and individual carcase weight: 30 batches of slaughter pigs from grow to finish management systems were recruited over a 9 month period at a single abattoir and 40-50 pigs were assessed in each batch (a total of 1366 pigs). The presence and severity of pleurisy in each of the assessed pigs was scored as 0 (no pleurisy), 1 (visceral pleurisy), 2 (parietal pleurisy $<25 \%$ of dorsolateral (DL) surface area, 3 (parietal pleurisy $25-50 \%$ of DL surface area), 4 (parietal pleurisy $>50 \%$ of DL surface area). Mean posttrimming carcase weights were calculated for pigs affected by pleurisy of increasing severity using a multiple regression model (adjusted for the different mean weight in each batch using a zero-mean batch-level random effect).

Batch level correlation between pleurisy prevalence and on-farm performance: Eighty batches of slaughter pigs were recruited (a total of 96687 pigs in batches ranging from 198 - 4671 pigs). Three different integrators (each with different management systems) were represented including continuous flow (15 batches), wean to finish all-in all-out (35 batches) and grow to finish (30 batches). Performance data including average batch level post-trimming carcase weight $(\mathrm{kg})$ and average batch level age at slaughter (days) were recorded. One consignment of each batch was randomly selected for pathology assessment at slaughter with 50 pigs being assessed for the prevalence of pleurisy in the representative consignment of each batch. We calculated the means and 95\% confidence intervals for the effect of pleurisy prevalence on performance parameters using a multiple regression model including adjustments for an integratorlevel random effect and different variance components for each integrator.

\section{Results}

Analysis of the impact of increasing severity of pleurisy on posttrimming carcase weights revealed that the main effect on carcase weight was whether a pig had any type of pleurisy rather than the severity of that pleurisy. For this reason, in the batchlevel study, we focused only on pleurisy prevalence, and not severity. For the 80 batches studied, pleurisy prevalence ranged from $0 \%$ to $46 \%$, batch mean post-trim carcase weights ranged from $71.8-83.1 \mathrm{~kg}$, and batch mean slaughter age ranged from 139 - 183d. Our model indicated that for each $1 \%$ increase in pleurisy at batch level there was a $0.07 \mathrm{~kg}$ decrease in post-trim carcase weight ( $95 \% \mathrm{Cl}: 0.14-0.00 \mathrm{~kg} ; \mathrm{p}=0.04)$ and a $0.26 \mathrm{~d}(95 \%$ $\mathrm{Cl}: 0.09-0.44 \mathrm{~d} ; \mathrm{p}=0.00$ ) increase in mean slaughter age.

Discussion: We found that it was not necessary to record extensive information on the severity of pleurisy, beyond, simple prevalence, in order to detect a relationship between pleurisy and performance. This simple model of the impact of pleurisy on performance was based solely on UK data and different relationships might be found in other production contexts. However, based on prevailing price estimates in April 2009, it allowed us to estimate that for a pleurisy prevalence of $10 \%$ of a given batch the cost for every pig slaughtered in that batch was $£ 2.26$. At a $20 \%$ prevalence this cost increases to $£ 452$ per pig.

\section{Reference:}

Wellenberg GJ, Bouwkamp FT, PJvd Wolf et al. 2010. Vet. Microbiol. (in press). 


\title{
0.158
}

\section{Prior colonization with an avirulent Haemophilus parasuis isolate prevents disease from a subsequent virulent challenge with a heterologous isolate}

\author{
Susan L. Brockmeier; Crystal L. Loving; Michael A. Mullins; Karen B. Register; Tracy L. Nicholson \\ National Animal Disease Center, Ames, IA, USA
}

\section{Introduction}

Haemophilus parasuis causes Glasser's disease in swine characterized by systemic invasion and bacteremia resulting in a polyserositis syndrome. $H$. parasuis can also cause pneumonia in swine. Disease patterns suggest that maternal antibody may protect pigs early, allowing them to be colonized without resulting disease while developing an active immune response. This is supported by studies examining the practice of exposing neonatal piglets to the predominant virulent strain on the farm as a method of prophylaxis. ${ }^{1}$ Reports have been mixed as to the degree of heterologous protection among strains. ${ }^{2}$ This may be due to relatedness of the strains, exposure to live bacteria on a mucosal surface versus bacterins given parenterally, and colonization status of the animal.

\section{Materials and Methods}

Pigs ranging in age from 5 to 10 weeks of age were obtained from an experimental barrier herd that has tested free of known swine pathogens. The pigs were distributed into 5 groups with 5 pigs per group and challenged intranasally with $10^{6.5}$ CFU of 1 of 4 isolates of $H$. parasuis $(29755,12939, \mathrm{MN}-\mathrm{H}$, and SW114), all of differing serotypes, or sham inoculated with PBS. If pigs showed signs of Glasser's disease, they were euthanized and swabs of the respiratory tract and systemic sites were collected and cultured on Casman's plates with added NAD. One week after challenge, a control pig was placed in with a healthy appearing survivor in 2 of the groups. Three weeks after challenge all surviving pigs were rechallenged with isolate 12939.

\section{Results}

Pigs challenged with 3 of the $4 \mathrm{H}$. parasuis strains developed signs of Glasser's disease, including joint swelling and lameness, tremors, depression, and reluctance to move. All pigs challenged with isolate 12939 died peracutely or were euthanized due to signs of Glasser's disease and 4 of the 5 pigs inoculated with isolates 29755 or MN-H were euthanized due to signs of Glasser's disease between 2 and 5 days post infection. One pig each from the 29755 and $\mathrm{MN}-\mathrm{H}$ challenged groups and all pigs challenged with isolate SW114 remained healthy and showed no signs of disease throughout the experiment, but $H$. parasuis was isolated from nasal swabs of these pigs.

One week after challenge, one control pig was placed in with each of the survivors that had been challenged with isolate 29755 or $\mathrm{MN}-\mathrm{H}$ to determine if the infected but healthy pig would transmit $H$. parasuis to the naïve pig and whether disease would result. Five and 7 days after placing the control pigs in with the MN-H and 29755 survivors, respectively, the contact pigs showed signs of Glasser's disease and were euthanized and $H$. parasuis was isolated from systemic sites.
Three weeks after primary challenge, the 10 surviving pigs (3- control pigs, 5- SW114 pigs, 1- 29755 pig, and 1- MN-H pig) were all rechallenged intranasally with the 12939 isolate of $H$. parasuis. All control pigs were euthanized between 1 and 3 days post-challenge due to signs of Glasser's disease. Gross pathology findings and culture results were similar to those obtained from the pigs originally challenged with this isolate. The 2 pigs previously inoculated with isolates 29755 or $\mathrm{MN}-\mathrm{H}$ and the 5 pigs previously inoculated with SW114 remained healthy. These pigs were euthanized 10 days after secondary challenge, and no grossly visible lesions were noted. $H$. parasuis was isolated from the nasal cavity of 3 of these pigs and the lung of 1 of the pigs.

\section{Discussion}

Pigs from an experimental herd that is free from known swine pathogens were used in a study with several $H$. parasuis isolates in an effort to identify isolates of varying virulence. Three of the 4 isolates caused systemic disease, with pigs showing signs of disease from 1 to 5 days post infection. One isolate, SW114, caused no observable clinical signs; however, $H$. parasuis was isolated from nasal swabs from these pigs indicating they were colonized. The MN-H and 29755 survivors were able to directly transmit $H$. parasuis to naïve pigs which became ill with signs of Glasser's disease. The pigs colonized with SW114 were protected from challenge with a heterologous virulent strain. Thus, pigs within susceptible groups can remain healthy and serve as reservoirs for transmission, and heterologous protection using avirulent isolates is possible.

\section{References}

1. Oliveira, S., L. Batista, M. Torremorell, and C. Pijoan. 2001. Experimental colonization of piglets and gilts with systemic strains of Haemophilus parasuis and Streptococcus suis to prevent disease. Can J Vet Res 65:161-167.

2. Oliveira, S., and C. Pijoan. 2004. Haemophilus parasuis: new trends on diagnosis, epidemiology and control. Vet Microbiol 99:1-12. 


\title{
0.159
}

\section{Avirulent live Haemophilus parasuis vaccine induces cross-serotype immunity}

\author{
$\underline{\text { Russ Bey }}^{1,2}$ Jon Mahlberg ${ }^{1}$ Mark Titus ${ }^{1}$ Randy Simonson ${ }^{1}$ Doug Stine ${ }^{1}$ Simone Oliviera ${ }^{2}$ \\ 1. Newport Laboratories, Worthington, MN, USA; 2. University of Minnesota, St. Paul, MN, USA
}

\section{Introduction}

Haemophilus parasuis is the causative agent of Glässer's Disease, a primary problem for swine producers worldwide (1).

The National Animal Health Monitoring Service reports a sharp increased incidence of $H$. parasuis in U.S. swine herds from 2000-2006 (2). The incidence in nursery pigs has risen from $7.3 \%$ to $17.4 \%$, and it has risen in grower/finisher pigs from $5.4 \%$ to $18.7 \%$.

Fifteen serovars of $H$. parasuis have been defined. This diversity makes it difficult for the current commercial inactivated bacterins to provide cross-serotype protection. This lack of crossserotype protection led to the development of an avirulent live culture vaccine that is able to induce cross immunity for multiple serotypes.

\section{Materials and Methods}

An avirulent live culture $H$. parasuis vaccine was developed and evaluated for efficacy in susceptible pigs. Pigs 17-21 days of age were divided into two groups. One group was immunized once with the avirulent live culture vaccine; the control group received a placebo. All animals were challenged with virulent serotype 5 .

In a second study, three groups of susceptible pigs were vaccinated once with the live culture vaccine, while three other groups received a placebo. Animals were challenged with virulent $H$. parasuis serotype 4, 5, or 13.

In a third study, animals receiving oral antibiotic treatment (Carbadox or Denagard/chlortetracycline) were placed on the antibiotic seven days prior to vaccination. Two additional groups of pigs received either Draxxin or Excede one day prior to vaccination. Five weeks following vaccination, all animals were challenged with $H$. parasuis serotype 5 .

In these studies, pigs were observed daily for mortality and clinical signs of disease. Average daily gain was also determined.

\section{Results}

In these studies a typical, highly lethal Glässer's Disease was induced by experimental challenge of susceptible commercial pigs. A significant number $(p<0.05)$ of vaccinated animals were protected from death. Arthritis was significantly lower in vaccinated animals, as was the average total number of gross lesions. Seventy-five percent of animals receiving the placebo vaccine died.

The average daily gain in vaccinated animals ranged from 0.99 to $1.65 \mathrm{lbs} /$ day, compared to $-2.34 \mathrm{lbs} /$ day for surviving controls. No vaccinated animals had a fever following challenge.

Antibiotic treatment did not interfere with the efficacy of the vaccine, as $>90 \%$ of immunized animals were protected from death and lesions typical of $H$. parasuis infection. The mortality rate in those animals receiving the placebo was $80 \%$.

\section{Discussion}

A significant number of commercial pigs given ParaSail (the first avirulent live culture Haemophilus parasuis vaccine) were protected against experimental infection with $H$. parasuis serotypes 4,5 , and 13 . Immunized animals had reduced mortality and gross lesions as well as increased average daily gain compared to sham inoculated animals. These results suggest that the live vaccine expressed a wide variety of outer membrane proteins, which are thought to be required for the induction of crossprotective immunity $(3,4)$.

\section{References}

1. Olvera A., et al., 2006. Microbiology 152: 3683.

2. United States Department of Agriculture Animal and Plant Health Inspection Service. January, 2008.

3. Ruiz A., et al., 2001. J. Clin. Microbiol. 39: 1757.

4. Tadjine M., et al., 2004. Microbiol. 150: 3935. 


\title{
0.160
}

\section{Gene expression profiling in the lung of pigs with different susceptibilities to Glässer's disease}

\author{
Jamie M. Wilkinson ${ }^{1,4}$ Carole A. Sargent ${ }^{2}$ Lucina Galina-Pantoja ${ }^{3,5}$ Alexander W. Tucker $^{1}$ \\ 1. Department of Veterinary Medicine, University of Cambridge, Cambridge, UK; 2. Department of Pathology, University of Cambridge, \\ Cambridge, UK; 3. Pig Improvement Company, Hendersonville, TN, USA; 4. Department of Agricultural, Food, and Nutritional Science, \\ University of Alberta, Edmonton, AB, Canada; 5. Pfizer Animal Health, New York, NY, USA
}

\section{Introduction}

Glässer's disease is caused by the bacterium Haemophilus parasuis. Several commercial vaccines are available but none of these protects against all pathogenic strains. This is good justification to attempt the identification of genetic factors that affect susceptibility to this disease and utilize them in marker assisted selective breeding schemes. Previously, statistical differences in the severity of Glässer's disease in the offspring of different boars were observed, indicating a genetic component to susceptibility ${ }^{1}$. This project used microarrays to look for gene expression differences in the lung tissue of animals exhibiting different susceptibilities to Glässer's disease.

\section{Materials and Methods}

Offspring of 6 boars from a Landrace-Duroc breed cross were decontaminated, screened as free from $H$. parasuis, and reared without maternal colostrum in an isolation facility. Animals were inoculated at 21 days with a pathogenic strain of $H$. parasuis serovar 5 (strain 29755). Controls were mock-inoculated with saline. Pigs were scored for clinical signs of the disease by observation. Approximately equal numbers of pigs were euthanized at 24,48 and 72 hours post inoculation. At necropsy, animals were scored for the severity of lesions at affected sites and lung tissue samples stored in RNA Later (Ambion). In total, samples were obtained from 129 inoculated and 29 control animals ${ }^{1}$. The scores were used to classify animals into different susceptibility categories, and pigs from the two categories which exhibited the largest difference in susceptibility ('Fully Resistant' (FR) and 'Susceptible') were chosen for gene expression profiling by microarray.

RNA was isolated from lung samples of individual 'FR' and 'Susceptible' animals $(n=6)$ at two different time-points (24 and 72 hours), and from pools of 'Control' animals matched for sire and time-point. This was reverse transcribed into cDNA and amplified using the SMART-PCR system (BD Biosciences). The products were labelled with fluorescent $\mathrm{Cy}-3$ or $\mathrm{Cy}-5$ conjugated nucleotides and hybridized to pig oligonucleotide arrays (Operon). Labelled cDNA from individual 'FR' or 'Susceptible' pigs was hybridized to a reference sample from the pool of mockinoculated 'Control' animals. After hybridization, the arrays were washed to remove non-specific signal and scanned on an Axon A4100 scanner. Signal intensities were quantified using Bluefuse software and differentially expressed (DE) genes were identified using the programme 'limma'.

\section{Results}

Lists of DE genes produced by the analysis were used to identify the subsets of genes that were differentially expressed in ' $F R^{\prime}$ compared to both 'Control' and 'Susceptible' animals (the 'FR' gene expression profile) and in 'Susceptible' compared to both 'FR' and 'Control' animals (the 'Susceptible' expression profile) at both time-points. These genes were of most interest as they were potentially regulated differently in response to $H$. parasuis in 'FR' and 'Susceptible' animals. The expression profile of the 'FR' pigs contained 21 and 22 genes at 24 and 72 hours respectively. The expression profile of the 'Susceptible' animals contained 58 and 347 genes respectively.

\section{Discussion}

'FR' animals at 24 hours exhibited an increased expression of genes encoding extracellular matrix components, possibly indicative of tissue repair following the successful early resolution of infection. The gene expression profile of ' $F R^{\prime}$ animals at 72 hours supported the hypothesis that higher levels of antibacterial activity could have been responsible for the 'FR' phenotype, possibly due to an increase in the production of innate 'natural' antibodies and decreased signalling by the immunoregulatory transcription factor peroxisome proliferator-activated gamma (PPAR- $\gamma$ ). The expression profile of 'Susceptible' animals at both time-points was characterized by an imbalance in signalling between pro and anti-inflammatory cytokines, with the proinflammatory cytokine genes resistin (RETN) and interleukin 1-beta (IL1B) particularly highly expressed. At 72 hours, a reduction in the expression of genes involved in antigen presentation by both MHC class I and II molecules was observed, which could have contributed to the inability of 'Susceptible' animals to clear infection. In conclusion, this study identified several candidate genes and pathways associated with susceptibility to H.parasuis infection.

\section{References}

1. Blanco et al. (2008). Differences in susceptibility to H.parasuis infection in pigs. Can. J. Vet. Res. 72(3): 228-235. 


\title{
0.161
}

\section{The use of serum profiles for control of Haemophilus parasuis and Actinobacillus pleuropneumoniae}

\author{
Irena Golinar Oven; Zdravko Valenčak \\ Veterinary Faculty, Ljubljana, Slovenia
}

\section{Introduction}

Glässer's disease, caused by Haemophilus parasuis (HP), was once considered a sporadic disease of young pigs compromised by stress. Introduction of HP may result in systemic disease of high morbidity and mortality, affecting swine at any stage of production. HP is a contributor in the porcine respiratory disease complex (PRDC) as a predisposing agent, a secondary invader, or a primary pathogen involved in pneumonia (1).

Actinobacillus pleuropneumoniae (APP) is the etiological agent of pleuropneumonia in pigs and also the contributor in PRDC. It can cause pneumonia, clinical disease, or subclinical disease and causes losses from death, reduced production, and increased costs of medication or vaccination (2).

Treatment of PRDC varies with each individual production unit and system. Serology can gather information on which pathogens are circulating in a herd (3). Serum profile is testing groups of pigs at specified ages to detect when seroconversion to a pathogen is occurring in the unit. The most common method is to collect samples from several age groups on a single day. A less common but more accurate method is to follow individuals over time as they grow. The purpose of this study was to prepare serum profiles for HP and APP for one large farm.

\section{Materials and Methods}

39 serum samples from individual animals were tested on APP (ApxIV factor) and HP. Serum samples were taken at age of 2 , $4,6,8,11,14,17,22$ and 28 weeks. Pigs were raised in large Slovenian pig farm with 6000 breeding animals. For detection of specific antibodies against APP and HP commercial ELISA kits were used: The CHEKIT-APP-ApxIV ELISA test kit (IDEXX) and Haemophilus parasuis Antibody test kit (ELISA) Swinecheckâ HPS (Biovet).

\section{Results}

Colostral antibodies to HP were detected only in $5.1 \%$ at age 2 weeks. We detected antibodies in $25.6 \%$ at age 11 weeks, then prevalence increased to $94.7 \%$ at age 28 weeks (Figure 1). Antibodies to APP were detected in $100 \%$ till 4 weeks, then prevalence decreased to $53.8 \%$ at age 14 weeks, and then increased to $100 \%$ at age 22 and 28 weeks (Figure 2).
Figure 1. Serum profile of HP
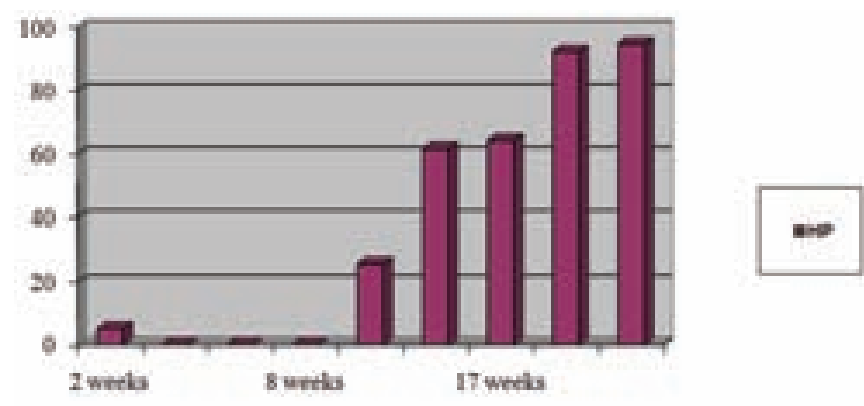

Figure 2. Serum profile of APP

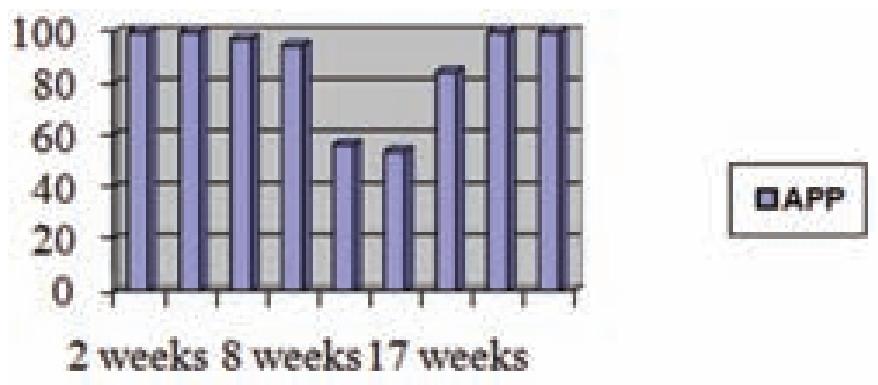

\section{Discussion}

Farm animals were not vaccinated against HP and APP. Colostral antibodies against HP were found only in $5.1 \%$ in 2 weeks old piglets. Circulating antibodies as consequence of natural infection were detected from 11 weeks (25,6\% prevalence) to 28 weeks old pigs ( $94,7 \%$ prevalence). Colostral antibodies against APP persisted for about almost 8 weeks (94,8\% prevalence). The lowest prevalence was detected in 14 weeks old pigs $(53,8 \%)$, and the highest in 22 and 28 weeks old pigs (100\%).

The results can enable the best time for vaccination which is 2-3 weeks before active antibodies appear. The best time for vaccination in this farm against HP is around 8-9 and against APP around 11-14 weeks of age.

Serum profile is a method which can help create an effective specific vaccination program on farm.

\section{References}

1. Rapp-Gabrielson et al (2006). In: Straw BE, eds. Diseases of swine. 9th ed. Ames: lowa State University Press, 681-90.

2. Gottschalk M, Taylor D (2006). In: Straw BE, eds. Diseases of swine. 9th ed. Ames: lowa State University Press, 563-75.

3. Brogden KA et al (2002). In: Polymicrobial diseases. ASM Press, 23152. 


\title{
0.162
}

\section{Porcine genetic markers for resistance to bacterial respiratory tract infections}

\author{
Doris Hoeltig $^{1}$ Gerald F. Gerlach $^{2}$ Karl-Heinz Waldmann ${ }^{1}$ Fugato Consortium IRAS 3 Fugato consortium RePoRI ${ }^{3}$ \\ 1. Clinic for Swine and Small Ruminants, Forensic Medicine and Ambulatory Service; University of Veterinary Medicine Hanover, Foundation, \\ Hannover, Germany; 2. IVD GmbH, Hannover, Germany; 3. University of Veterinary Medicine Hanover, Foundation, Hannover, Germany
}

\section{Introduction}

Respiratory tract infections are of major importance for the pig industry. One possibility of limiting the spread of respiratory tract disease may be the breeding of pigs with increased resistance to respiratory tract pathogens. Therefore the FUGATO-IRAS project and the follow-up project RePoRI work on the identification of genetic markers responsible for an increased resistance towards Actinobacillus (A.) pleuropneumoniae infection.

\section{Materials and Methods}

Within IRAS 106 pigs of 4 breeding lines (German Landrace, Piétrain, Hampshire, Large White) were infected. The aerosol infection was performed using A. pleuropneumoniae serotype 7 strain AP76. Sampling (BALF, blood) and phenotyping were carried out twice (day 4 and day 20 post infection [p.inf.]). Pigs were euthanized immediately after the sampling, lung lesions were scored during necropsy by the Lung Lesions Score according to Hannan et al. (1), and lung tissue was taken. For additional phenotyping a Respiratory Health Score (2) was used. Acute phase proteins were determined (blood, BALF), glycoproteins (BALF) were analyzed via differential two-dimensional gel electrophoresis (2D-DIGE) and RNA expression was investigated using the Affymetrix GeneChip ${ }^{\circledR}$ porcine Genome Array. In addition, a consumer study investigating the allele diversity within German pork was realised and SNPs for candidate genes, derived from known human genes, were detected and characterised.

The aim of the RePoRI-project (ongoing) is the validation of possible marker genes, the establishing of genetic markers and the modelling of the host reactions based on the IRAS results. To achieve these aims a QTL-analysis based on the F2 generation of a Hampshire (F0 male) -German Landrace (F0 female) cross, the development of a custom-buildt expression array (including candidate genes, selected ORFs of $A$. pleuropneumoniae and Mycoplasma hyopneumoniae) as well as pathway analysis using ConsensusPathDB and association studies by FAMHAP ${ }^{\circledR}$-software are performed.

\section{Results}

Within IRAS there were 7 main results: 1) Severity of disease is highly dependent upon the innate immune system. 2) The Hampshire-line came out to be the most resistant and the German-Landrace-line to be the most susceptible. 3) The level of haptoglobine in blood plasma correlates with the course of disease. 4) The level of hyaluronidase in BALF increases after pathogen contact and therefore might be a possible health marker. 5) The combination of a high level of Fetuin A and a low level of Surfactant-D-protein in BALF correlates with the absence of lung lesions and therefore might a marker for breeding selection. 6) There is no depletion of allele diversity within the German pig population. 7) 1597 potential genetic markers could be detected.

Within RePoRI so far, 188 microsatellites were established and mapped and an average distance of 14,5 cM could be realised. One Pathway (Toll like receptors) of importance was detected, and within the FAMHAP ${ }^{\circledR}$-Analysis of the genotypes there were significant differences $(p<0,05)$ found for 7 marker loci (mainly focussing on Transferrin- and Interferon receptors) between pigs with mild and severe pleuropneumonia.

\section{Discussion}

Although these studies for experimental reasons are limited to interactions between pigs and A. pleuropneumoniae they might be an essential first step in the development of genetic markers for DNA-based breeding selection aimed at the increased resistance towards lung infections. The first candidate markers might be available for testing under production condition within the next two years. Before applying these markers for selection in breeding programmes a thorough investigation is required to avoid unwanted adverse effects on wanted traits.

\section{References}

(1) Hannan, PC; Bhogal, BS and Fish, JP (1982): Tylosin tartrate and tiamutilin effects on experimental piglet pneumonia induced with pig lung homogenate containing mycoplasmas, bacteria and viruses. Res Vet Sci, 33: 76-88

(2) Hoeltig, D; Hennig-Pauka, I; Thies, K; Rehm, T; Beyerbach, M; Gerlach, GF and Waldmann KH (2009): A novel Respiratory Health Score supports a role of acute lunge damage and pig breed in the course of an Actinobacillus pleuropneumoniae infection. BMC Vet Res, 5: 14 


\title{
0.163
}

\section{Reduction of lung pathogens and clinical symptoms in nursery pigs suffering from PRDC by tiamulin (Denagard ${ }^{\oplus}$ ) treatment in a field trial}

\author{
Rebecca Langhoff ${ }^{1}$ Andrea Ladinig ${ }^{1}$ Andrea Barz ${ }^{1}$ Andreas Palzer ${ }^{2}$ Joachim Spergser ${ }^{3}$ Mathias Ritzmann ${ }^{1}$ \\ 1. Clinic for Swine, University of Veterinary Medicine Vienna, Vienna, Austria; 2. Clinic for Swine, Ludwig-Maximilians University, \\ Oberschleissheim, Germany; 3. Institute of Bacteriology, Mycology and Hygiene, University of Veterinary Medicine Vienna, Vienna, Austria
}

\section{Introduction}

Respiratory diseases in swine are often caused by several pathogens and triggered by a multitude of other environmental and management factors ${ }^{1}$. Different kinds of lesions in the lung and the surrounding tissue lead to a multi-factorial syndrome with respiratory symptoms and poor growth described as Porcine Respiratory Disease Complex (PRDC). The aim of this study was to investigate the effects on health and performance of a tiamulin (Denagard ${ }^{\circledR}$, Novartis Animal Health Inc.) treatment in nursery pigs suffering from PRDC.

\section{Materials and Methods}

The study was conducted at a farrow to finish farm. Piglets were vaccinated against PCV2 and Mycoplasma (M.) hyopneumoniae (two-shot). Respiratory symptoms occurred in the nursery between 8 and 12 weeks of age. 173 piglets at 9 weeks of age were randomly divided into two groups. Piglets of group TIA ( $\mathrm{n}$ $=88$ ) received $15 \mathrm{mg} / \mathrm{kg} /$ day tiamulin by water over 14 days. All animals of group CONT $(n=85)$ were left untreated. Both groups were housed in different pens within one room. All animals were weighed before treatment (day 0 ), at the last day of treatment (day 14) and on day 21. On day 0, 1, 2, 3, 5, 7, 9, 11, 13, 14, 15, 17,19 and 21 a cough index was calculated for both groups. 30 piglets from each group served as sampling animals (SAs). Bronchoalveolar lavage fluid (BALF) was collected from the SAs on day 0,14 and 21. BALF was used for bacteriological investigation (BI) of various lung pathogens and for PCR-testing to detect porcine mycoplasmas ${ }^{2}$.

\section{Results and Discussion}

In group TIA the amount of Haemophilus parasuis (HPS) isolated from BALF was significantly reduced on day 14 and 21 compared to day 0 . In addition (in group TIA) the amount of a-haemolytic Streptococci was significantly reduced on day 14 compared to day 0 and $M$. hyorhinis was detected in significantly higher amounts on day 14 than on day 21 . In group CONT only the amount of HPS was significantly higher on day 0 than on day 14 and day 21 . At day 0 the amounts of all pathogenes mentioned above were higher in group TIA compared to group CONT. Actinobacillus pleuropneumoniae, M. hyosynoviae and/or $\beta$-haemolytic Streptococci were only isolated in the BI of a few SAs on day 0 . All tested animals were free of those pathogens on day 14 and 21. The amount of other detected pathogens including Pasteurella multocida and Bordetella bronchiseptica in BALF did not differ significantly between the three sampling days. The cough index measured in both groups showed an estimated decrease of $50 \%$ after 6.1 days in group TIA and 25.9 days in group CONT, respectively. The decreasing rates differed significantly between the two groups.
Figure 1: Estimated cough index

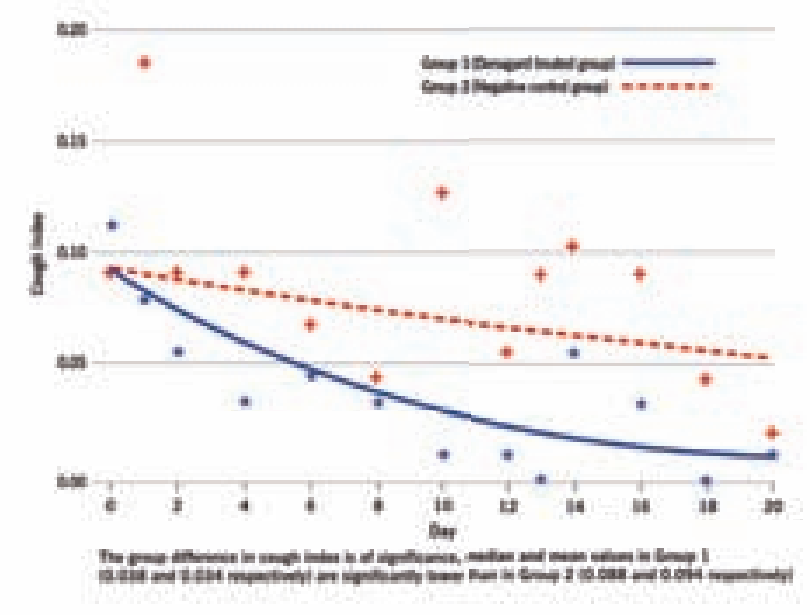

The mean body weight did not differ significantly between groups on day 0 (TIA: $20.10 \mathrm{~kg}$, CONT $19.22 \mathrm{~kg}$ ). During the trial period (day 0-14/0-21) the average daily weight gain was significantly higher $(\mathrm{p}<0.001)$ in group TIA $(731 / 722 \mathrm{~g} /$ day $)$ compared to group CONT (640/651 g/day).

The trial data verified the positive effect of tiamulin treatment on the health status and performance of nursery pigs suffering from PRDC. The lower pathogen burden after treatment correlated with a significantly faster reduction of the cough index and a significantly higher daily weight gain in group TIA. Treated and untreated animals were housed within one room. On one hand this might be an explanation why some positive effects could also be seen for the untreated animals (e.g. reduction of HPS). On the other hand this might have caused a constant reinfection of group TIA by the untreated animals (CONT) or other vectors, including the farmer.

\author{
References \\ 1. Sørensen, V., et al. (2006): Diseases of Swine, 9th \\ 2. Palzer, A., et al. (2008): Vet. Rec., 162, 267-271
}




\title{
0.164
}

\section{Novel immunization protocols to induce systemic and respiratory antibody responses in weaned pigs}

\author{
Marco A. Vega-López ${ }^{1}$ Gabriela Alvarado-Cesar² Carmen Ramírez-Estudillo Roberto Martínez ${ }^{3}$ Alejandro Vargas $^{3}$ \\ 1. CINVESTAV-IPN, Dept. Infectomics and Molecular Pathogenesis, México, DF, Mexico; \\ 2. FES-Cuautitlán-UNAM, Edo. de México, Mexico; 3. CEIEEP-UNAM, Jilotepec, MEX, Mexico
}

\section{Introduction}

Respiratory infections are an important problem in pig industry, especially at weaning, when passive maternal immunity decays and immune maturation is still incomplete (1-2). Vaccination is a successful strategy to prevent and control these maladies; however, intramuscular administration induces solid systemic immune response but poor mucosal protection. Mucosal vaccination has no practical use since it requires high doses of antigen, bacterial toxins as adjuvants or multiple doses (3). Thus, the design of effective mucosal immunization strategies is of paramount importance to control respiratory and mucosal diseases in the young pig.

\section{Aims}

To develop novel strategies of immunization to induce systemic and mucosal immune response in weaned pigs.

\section{Material and methods}

A total of 20 four weeks-old weaned, conventionally reared healthy pigs, from an experimental farm (CEIEPP-UNAM), free of zoonotic diseases and with no vaccination protocols running, compiling with academic and federal regulations of animal care, were divided in four groups of five animals each. Animals were immunized with ovoalbumin (OVA, Sigma, USA) three times, 10 days apart, by different routes as follows: subcutaneous (SC)/ transcutaneous (TC)/TC (8 mg/pig each time); SC/TC/intranasal (IN) and SC/SC/IN. A SC group was inoculated with PBS and adjuvant (GIBCO) as control. Samples of serum, nasal secretion (NS) and saliva were taken on days $0,10,20,27$ and 57 after the first inoculation and specific anti-OVA IgG and $\lg A$ were measured by quantitative ELISA.

\section{Results}

Anti-OVA lgG: All immunization protocols induced serum response after the second inoculation, peaking at 27d (SC/TC/ IN, $\mathrm{P}<0.05$ \& SC/TC/TC, $P<0.01$, Figure $1 \mathrm{~A}$ ). No IgG anti-OVA was detected in NS and saliva. Anti-OVA IgA: Again all protocols induced significant $\lg \mathrm{A}$ response in serum after the $2 \mathrm{nd}$ immunization, peaking at $27 \mathrm{~d}(\mathrm{P}<0.01)$. The $\mathrm{SC} / \mathrm{TC} / \mathrm{IN}$ group kept significance until day $57(\mathrm{P}<0.001$, Figure 1B). In NS the response was evident after the $3 \mathrm{rd}$ immunization in groups SC/TC/TC $(\mathrm{P}<0.023)$ and SC/SC/IN $(\mathrm{P}<0.037)$ and maintained differences to control until 57d (Figure 1C). All protocols induced immune response in saliva after the 2 nd inoculation, with peaks at $27 d$ in groups SC/SC/IN and SC/TC/IN $(P<0.001)$, which kept the response until $57 d(P<0.03)$ (Figure 1D).

\section{Discussion}

It has been reported that systemic and local immunization with live modified vaccines induces immune response in serum and mucosae (4). However, using inactivated vaccines the mucosal immune response is less successful (5). Our results show that using a combination of parenteral (SC/TC) and mucosal (IN) im- munization, systemic and local specific lgG and IgA are effectively induced in weaned pigs, even when a poorly immunogenic protein is used (OVA). IN and TC immunizations were effective only with previous parenteral sensibilization (SC), suggesting that circulating memory cells are able to migrate to mucosal sites after a local challenge. These immunization protocols may provide an effective way to induce integral (systemic/mucosal) immune response in young animals to prevent diseases.

Figure 1. Anti-OVA concentration of $\lg G(A), \lg A(B)$ in serum, $\lg A$ in nasal secretion $(C)$ and saliva $(D)$ from parenteral/mucosal immunized weaned pigs ( $n=5 /$ group). Each point is the mean plus/minus SEM. t-Student test. ${ }^{*} P<0.05,{ }^{* *} P<0.01,{ }^{* *} P<0.001$.

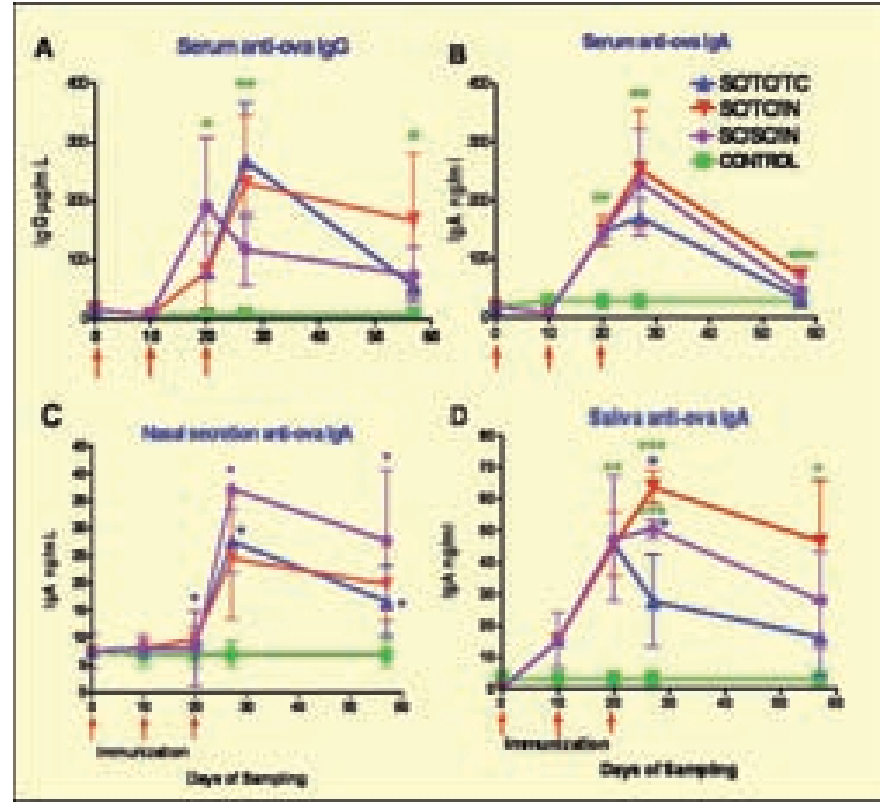

\section{Acknowledgments}

This project was partially funded by CONACYT-MÉXICO (60941) and the Instituto de Ciencia y Tecnología del Distrito Federal.

\section{References}

[1] Rothkötter HJ, Pabst R, 1989. Immunology 67:103-108.

[2] Stepanova $\mathrm{H}$, et al., 2007. Cellular Immunology 249:73-79

[3] Moyle PM, et al., 2004. Curr. Drug Deliv. 1:385-396.

[4] Ogra PL, et al., 2001. Clin. Microbiol. Rev. 14(2):430-445.

[5] Hur J, Hwa J, 2010.Veterinary Microbiology. In press. 


\subsection{5}

\section{M. hyorhinis vaccine prevents mycoplsmal lesion and disease}

Jung-Ah Lee ${ }^{1}$ Min-A Hwang ${ }^{1}$ Sang-Won Lee ${ }^{2}$ Jang-Hyuck Han ${ }^{3}$ Eun-Haeng Cho ${ }^{3}$

Sang-Shin Park ${ }^{4}$ Seung-Yong Park ${ }^{1}$ Chang-Seon Song ${ }^{1}$ In-Soo Choi ${ }^{1}$ Joong-Bok Lee $^{1}$

1. Konkuk University, Seoul, Korea; 2. Melborn University, Melborn, Australia; 3. KBNP, Inc., Seoul, Korea; 4. Texas A\&M, Texas, USA

\section{Introduction}

Porcine mycoplasmal pneumonia is one of well-known respiratory diseases. Mycoplasma hyorhinis is easily isolated in the upper respiratory tract and tonsil of swine unlike Mycoplasma hyopneumoniae. The ability of M. hyorhinis to cause pneumonia is unclear (1), but the pig with M. hyorhinis is simultaneously infected with porcine reproductive and respiratory syndrome virus (PRRSV). In previous study, we could find that $M$. hyorhinis may potentiate PRRSV-induced pneumonia in vivo. The aim of this study was to see if mycoplsmal lesion is prevented by vaccination to M. hyorhinis.

\section{Materials and Methods}

Sixteen weaned pigs free of M. hyorhinis, PRRSV and M. hyopneumoniae antibodies were randomly divided into 3 groups as shown in Table 1. 3 X 1010 CCU of M. hyorhinis was immersed in the mixtures of oil and water with a ratio of 50:50.

Table 1. Experimental design

\begin{tabular}{|c|c|c|c|}
\hline Group & No. of pigs & Vaccination & Challenge \\
\hline A & 6 & + & M. hyorhinis \\
\hline B & 6 & - & M. hyorhinis \\
\hline C & 4 & - & - \\
\hline
\end{tabular}

Pigs in group A were intramuscularly vaccinated with $M$. hyorhinis vaccine. Pigs in groups $B$ and $C$ were received with saline. After 4 weeks, $1010 \mathrm{CCU} / \mathrm{ml}$ of the $\mathrm{M}$. hyorhinis was administered through trans-trachea to pigs of groups $A$ and $B$. Clinical signs of the infected pigs were observed for 4 weeks after vaccination. Sera were collected weekly. Antibody response was measured by ELISA. Animals were sacrificed 4 weeks after challenge and the macroscopic lung lesions were evaluated as described in previous study (2). We performed Mann-Whitney $U$ test and Fisher's exact test to show the statistical differences of clinical signs and macroscopic lung lesions, and antibody responses between groups $A$ and $B$ by using SAS 9.2 software package (SAS Institute Inc, Cary, NC).

\section{Results and Discussion}

All pigs in group $A$ and $C$ showed neither any clinical signs nor mortality, but pigs in group B showed significant symptoms of respiratory disease including dyspnea, cough, fever and rough hair coat (Table 2).

Especially one of pigs in group B died of pneumonia showing typical mycoplasmal lesion on the lung, and we found more serious macroscopic lung lesions in group B than group $A$.
Table 2. Clinical signs and macroscopic score

\begin{tabular}{|l|c|c|c|}
\hline & A & B & C \\
\hline Clinical signs & 0 & $5.53^{* *}$ & 0 \\
\hline Macroscopic score & 0.22 & $1.0^{*}$ & 0 \\
\hline${ }^{*} \mathrm{p}<0.01,{ }^{* *} \mathrm{p}<0.001$ for differences between A and B by Mann-Whitney U test \\
\hline
\end{tabular}

No pigs in group A developed glanuroma tissue and fever on the site of vaccination for the entire experiment period. Five pigs in group B did not develop antibodies until the end of this study, whereas only one pig showed immune response at 2 weeks after challenge and then its antibody disappeared 1 week later. However, pigs in group A showed antibody response at 2 weeks after vaccination, Those antibody titers were increased until 6 weeks after vaccination and then reached flat till end of the experiment. We found statistically significant differences from 7 weeks between groups $A$ and $B$.

Figure 1. Immune responses after vaccination to $M$. hyorhinis

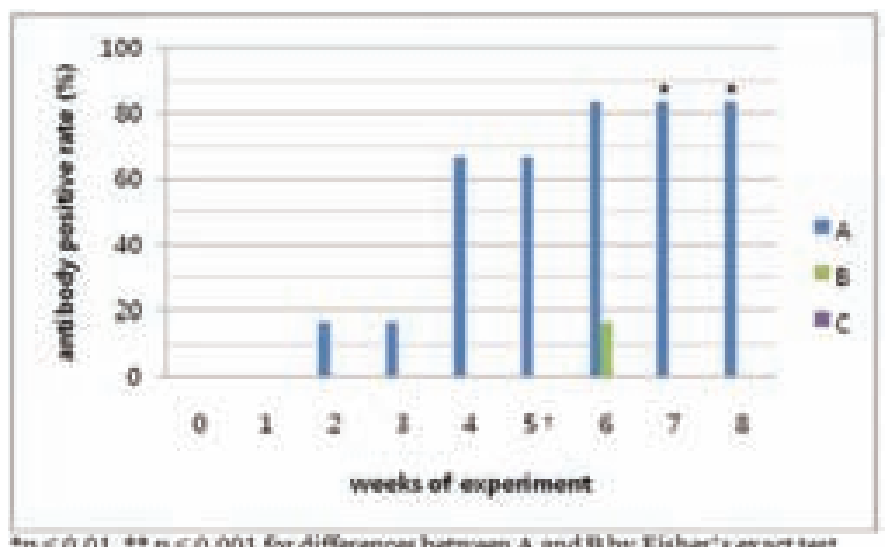

"p<0,01, ** $p<0,001$ for differences between $A$ and $B$ by Fisher's sexact teat t) hyorhinit inoctilation

The results indicated that mycoplasma vaccine may be used to prevent mycoplasmal lesion and disease.

\section{Reference}

1. Kawashima, K. et al. (1996). J. Comp. Pathol. 114, 315-323

2. Harbur, P.G., et al. (1995). Vet. Path. 32, 648-660 


\title{
0.166
}

\section{Regional evaluations for PRRS in market swine}

\author{
James D. McKean \\ lowa State University, Ames, IA, USA
}

\begin{abstract}
Introduction
PRRS eradication is a future USA pork industry goal. One consideration is the prevalence and distribution of infected animals in at-risk populations. This study from Midwestern (9) and nonMidwestern (3) market swine abattoirs assists understanding of area prevalence of PRRS generally and for defined sized producers within the population. This information refines the scope of epidemiological diverse populations for PRRS. An observed difference between regions or herd sizes relative to PRRS exposure potential offers the opportunity to consider appropriate control/ eradication strategies in larger groups than individual hers/sites. Two time periods were utilized to determine whether a seasonal effect on PRRS antibodies could be observed.
\end{abstract}

\section{Materials and Methods}

Each group of 10 or more swine (lot) at daily harvest on one shift in 12 cooperating abattoirs was sampled by USDA-APHISVS employees. As a convenience sample, 50-75 gm pieces of diaphragm (crus) of five carcasses per lot were obtained after evisceration, placed in whirl-pak bags and identified with lot number, date and plant number. In two shift plants, sampling rotated with each two week pay period to assure that over time most producers submitting to each plant were tested. Cooperating plants supplied lot identification, number per lot coupled to ownership information (name, mailing address and zip code) for lots daily. Such information enabled characterization of ownership and production size. A 12 week evaluation period was chosen for each sampling period to assure that low volume producers would submit multiple lots. All sampling was completed from November 2007 to October 2008. Meat juice samples were tested using the Herd Chek PRRS ELISA test, IDEXX Laboratories, Westbrook, Maine USA according to serum instructions. A positive lot contained one or more positive samples in the five tested for each lot.

\section{Results}

A total of 24,890 samples from 4978 lots were tested in the two time periods of fall-early winter and winter-spring timeframes. By aggregating number of infected lots and their source characteristics it was possible to develop information about distribution and prevalence of PRRS antibodies within the general population and for selected subpopulations. Estimated annual herd production, and geographic area differences were observed for PRRS antibody prevalence. As herd size increased, irrespective of geographic location, the percentage of positive lots increased (Table 1). Plants that obtained pigs from swine-dense production areas (Midwest $=9$ ) and North Carolina (1) had substantially higher prevalence than plants (2) drawing from less dense areas (Table 2).

\section{Discussion}

Antibodies to PRRS are common in market swine throughout the USA. There is general acceptance that higher swine density areas results in higher prevalence for PRRS. The results outlined in this large survey would indicate an inability to demonstrate seasonality in PRRS prevalence, but show differences in annual production/producer and in regional catchment. Rates of antibody prevalence increased with each production size category over the same period confirming the impression that increased system size maintains PRRS infections. Group categories above 2001-4000 are likely to be multi-site production systems. The plant data confirms the consideration that market swine from dense production areas are expected to be of higher prevalence. The plant in the North Eastern (NE) area had the lowest prevalence, followed by a catchment of primarily southeastern (SE) and north central USA areas. The Eastern la./III. plant is characterized by purchase of small lots vs. truckload lots at most of the other Midwest plants, and as such reflects the size but not location of production facilities serving the plant. The remainder of the Midwestern plants increased prevalence as catchment areas move into northern lowa and larger producers were suppliers. The North Carolina plant presents the highest prevalence, and was derived from larger production units. These observations may be useful in developing future PRRS surveillance and program development efforts.

Table 1 - \% positive lots by annual production classes

\begin{tabular}{|l|c|c|c|}
\hline \multicolumn{5}{|c|}{ \% positive lots } \\
\hline Ann. Prod. & PRRS 2-1 & PRRS 2-2 & Ave. \% pos. lots \\
\hline 1-1000 & 57.5 & 64.9 & 60.8 \\
\hline $1001-2000$ & 69.3 & 68.5 & 69.0 \\
\hline 2001-4000 & 71.1 & 71.7 & 71.4 \\
\hline $4001-9999$ & 75.6 & 75.8 & 75.7 \\
\hline $10000-1.5 \mathrm{M}$ & 70.7 & 75.3 & 72.6 \\
\hline Largest 40 & 85.0 & 78.0 & 83.3 \\
\hline
\end{tabular}

Table 2 - \% pos. lots by processing plant location

\begin{tabular}{|l|c|c|c|}
\hline Market area & PRRS 2-1 & PRRS 2-2 & Ave. \% plant \\
\hline NE & 40.1 & NA & 40.1 \\
\hline SE (not NC) & 61.2 & 53.8 & 57.6 \\
\hline Ea. la./III. & 69.3 & 69.2 & 69.3 \\
\hline Midwest & 76.4 & 71.4 & 74.3 \\
\hline Midwest & 78.0 & 80.0 & 78.7 \\
\hline Midwest & 80.6 & 81.0 & 80.7 \\
\hline Neb. area & 83.4 & 80.5 & 82.5 \\
\hline Midwest & 87.8 & 88.5 & 88.0 \\
\hline Midwest & 92.2 & 87.3 & 89.4 \\
\hline Midwest & 92.2 & 86.3 & 89.8 \\
\hline Midwest & 95.2 & 88.5 & 91.1 \\
\hline N. Car. & 97.4 & 93.9 & 95.9 \\
\hline
\end{tabular}

Acknowledgement: USDA-APHIS-VS for funding 


\title{
0.167
}

\section{Use of oral fluid samples to detect PRRSV infection in individual boars}

\author{
Apisit Kittawornrat ${ }^{1}$ Mark J. Engle ${ }^{2}$ Jeremy F. Johnson ${ }^{1}$ Wayne A. Chittick ${ }^{1}$ John R. Prickett ${ }^{1}$ Trevor J. Schwartz $^{1}$ Daniel J. Whitney ${ }^{1}$ \\ Chris W. Olsen ${ }^{1}$ Kent J. Schwartz ${ }^{1}$ Chong Wang ${ }^{4,3}$ Jeffrey J. Zimmerman ${ }^{1}$ \\ 1. lowa State University, Ames, IA, USA; 2. PIC North America, Endersonville, TN, USA; 3. Department of Statistics, lowa State University, \\ Ames, IA, USA; 4. Department of Veterinary Diagnostic and Production Animal Medicine, lowa State University, Ames, IA, USA
}

\section{Introduction}

Previously, we described the use of pen-based oral fluids to monitor PRRSV and/or PCV2 infections in wean-to-finish populations.1,2 The purpose of the present study was to evaluate the use of oral fluids for the detection of PRRSV infection in individually-housed boars.

\section{Materials and Methods}

The study was conducted using 72 boars ranging from 6 months to 3.6 years of age. Boar were under the ownership of PIC North America (Hendersonville, TN USA) and housing, study procedures, and protocols were approved and supervised by the PIC USA Health Assurance and Welfare department. Boars were assigned in equal numbers $(n=24)$ to three treatment trials $(I, I I, I I)$. Boars in Trial I were vaccinated intramuscularly (IM) once with $2 \mathrm{ml}$ of MLV (RespPRRS ${ }^{\circledR}$, Boehringer Ingelheim Vetmedica, Inc. St.Joseph, MO 64506). Boars in Trial II were inoculated IM with 2 $\mathrm{ml}$ of a Type 1 PRRSV. Boars in Trial III were inoculated IM with 2 $\mathrm{ml}$ of PRRSV strain MN-184.

Following exposure to PRRSV, boars were monitored for 21 days. Oral fluid samples were collected daily using 5/8", 3-strand, $100 \%$ cotton rope (Web Rigging Supply, Inc., Lake Barrington, IL 60010). Serum samples were collected from all boars on DPI -7, 0, 7, 14, 21. In addition, serum samples were collected from 4 randomly selected boars in each trial on DPIs 3, 5, 10, 17. Additional data collected on a daily basis included ambient temperature (maximum and minimum) in the facility and clinical observations. Serum and oral fluid were assayed for PRRSV RNA at lowa State University (Ames, IA USA) using nucleic acid extraction and PCR protocols specifically optimized for porcine oral fluids.

All statistical analyses were performed using SAS ${ }^{\circledR}$ Version 9.2 (SAS ${ }^{\circledR}$ Institute Inc., Cary, North Carolina, USA). Serum and oral fluids results for real-time qRT-PCR were analyzed using a repeated measures analysis of variance (ANOVA) model run with the GLIMMIX procedure in SAS.

\section{Results}

The analysis showed that PRRSV infection affected the volume of oral fluid samples collected from individual boars ( $p=0.0032$ ), but did not impact the success rate for oral fluid sample collection. That is, boars continued to provide oral fluid samples even during acute PRRSV infection, although less volume was collected.

Oral fluid samples were qRT-PCR-positive from DPI 1 to DPI 21. Positive serum samples were detected from DPI 3 (first collection) to DPI 21 . Overall, $92 \%$ of the results were in agreement, i.e., 218 serum samples and 238 oral fluid samples were PCR positive.

The concentration of virus in serum and oral fluid samples followed similar patterns over time, with oral fluid containing less virus than serum before $14 \mathrm{DPI}$ and equivalent levels of virus from 14 to $21 \mathrm{DPI}$. Boar age did not have a significant effect on the quantity of virus present in oral fluid samples, but the concentration of virus in both oral fluids and serum was affected both by the virus strain $(p=0.0001)$ and DPI $(p=0.0001)$.

\section{Discussion}

The data reported herein provide evidence that oral fluid samples are equivalent or superior to serum samples for the detection of acute PRRSV infection by qRT-PCR. The use of oral fluid samples offers a significant improvement in the ease, timeless, and cost of disease surveillance in commercial swine populations.

\section{References}

1. Prickett J, et al. 2008. Detection of PRRSV infection in porcine oral fluid samples: A longitudinal study under experimental conditions. J Vet Diagn Invest 20:156-163.

2. Prickett J, et al. 2008. Oral-fluid samples for surveillance of commercial growing pigs for PRRSV and PCV2 infections. J Swine Health Prod 16(2):86-91.

\section{Acknowledgements}

The study was supported in part by PIC North America and the PRRS CAP, USDA-NIFA Award 2008-55620-19132. 


\title{
0.168
}

\section{Histopathological findings in fetuses positive on porcine reproductive and respiratory syndrome by PCR or immunohistochemistry}

\author{
Dinko Novosel ${ }^{1}$ Tomasz Stadejek $^{2}$ Katarzyna Chrabos $^{2}$ Zoran Lipej$^{1}$ Besi Roic'1 Andreja Jungic'1 \\ 1. Croatian Veterinary Institute, Zagreb, Croatia; 2. National Veterinary Institute, Pulawy, Poland
}

\begin{abstract}
Introduction Porcine reproductive and respiratory syndrome virus (PRRSV) can cause reproductive disorder in naïve dams infected in the third trimester of pregnancy. Severity of gross and microscopic lesions in fetuses depends on the virus properties and the age of the fetus [1]. Segmental, a perivascular and transmural haemorrhages and multiple edemas with necrotic arteritis of umbilical cord together with lesions in myocardium, lungs, brain and liver usually can be seen $[2,3]$ but are infrequent. Detection of PRRSV in fetal tissue is usually done by RTPCR [1]. Immunohistochemistchemical (IHC) detection of PRRSV antigen in fetal thymus can be also used $[1,4,5]$.The aim of this study was to describe gross and microscopic lesion in fetuses from cases of abortion attributed to PRRSV and to evaluate the incidence of PRRSV detection by PCR and IHC
\end{abstract}

Material and methods For this study 83 fetuses from 8 large pig production units with significant reproductive losses, aborted in late gestation were used. Every fetus came from different sow.. In all of the farms presence of PRRSV was previously detected by serology, PCR and immunohistochemistry. During post-mortem examination, samples of brain, thymus, heart, lungs, liver, spleen, kidney, intestines, lymphnodes and umbilical cord (if was present) were collected and fixed in 10\% buffered formalin for histopathological examination and IHC. For PCR detection samples of lungs, spleen and liver were stored in $-70^{\circ} \mathrm{C}$. All tissue sections were stained with hematoxilin and eosin and were analysed by IHC with anti-PRRSV monoclonal antibodies (Ingenasa ${ }^{\circledR}$ ) according to previously described protocol [4]. For detection of PRRSV RT nested -PCR specific for ORF 7 was used on total RNA extracted from pooled homogenate of lung, liver and spleen as previously described [6].

Results PRRSV was detected by PCR in only 6 (7.23\%) fetuses. The IHC detected PRRSV only in thymus of 19 (23.89\%) fetuses. Three fetuses (3.61\%) were PRRSV positive in both methods of detection. Altogether, 22 (26.51\%) fetuses were positive in at least one of the methods while 61 (73.49\%) were negative. Viral antigen in thymus was detected in follicular area in triangular cells, monocytes/machrophages and in Hassel's bodies. A range of gross and microscopic lesions were observed in the fetuses (Table 1).

Discussion We were able to identify presence of PRRSV infection in only $26.51 \%$ of aborted fetuses from 5 Croatian pig production units where PRRSV circulation was previously shown. In three farms no evidence of PRRSV in aborted fetuses was found. Most frequent lesion found in PRRSV infected fetuses was edema and haemorrhages of umbilical cord, lymphocytic hepatitis and liver hyperaemia. All other lesions were equally frequent found in PRRSV positive and negative or even more in negative fetuses so it can't be considered as PRRSV related lesions. There is a clear discrepancy between the results obtained with the two PRRSV detection methods. This could be attributed to the fact that for PCR and IHC different tissues were used. Thymus that appeared to be the only organ where PRRSV antigen was found by IHC, was not used for PCR. This study indicates that the tissue selection is crucial for diagnosis of PRRSV in fetuses by PCR and $\mathrm{IHC}$.

\begin{tabular}{|c|c|c|c|c|c|}
\hline Findings & $\begin{array}{c}\% \text { of fetuses } \\
\text { with given } \\
\text { lesion }\end{array}$ & $\begin{array}{l}\% \text { of PRRSV } \\
\text { fetuses with } \\
\text { given lesion }\end{array}$ & $\begin{array}{c}\% \text { of } P C R \\
\text { postive } \\
\text { fetuses } \\
\text { with given } \\
\text { lesion }\end{array}$ & $\begin{array}{c}\% \text { of IHC pos- } \\
\text { tive fetuses } \\
\text { with given } \\
\text { lesion }\end{array}$ & $\begin{array}{c}\% \text { of } \\
\text { PRRSV } \\
\text { negative } \\
\text { fetuses } \\
\text { with } \\
\text { given } \\
\text { lesion }\end{array}$ \\
\hline $\begin{array}{l}\text { Edema and } \\
\text { haemorrhages of } \\
\text { umbilical cord of } \\
\text { umbilical cord }\end{array}$ & $51.51 \%$ & $100 \%$ & $100 \%$ & $100 \%$ & $18.18 \%$ \\
\hline $\begin{array}{l}\text { Myocardial } \\
\text { degeneration and } \\
\text { necrosis }\end{array}$ & $53.91 \%$ & $45.45 \%$ & $100 \%$ & $26.32 \%$ & $53.96 \%$ \\
\hline $\begin{array}{l}\text { Kidney cortex } \\
\text { haemorrhages }\end{array}$ & $72.29 \%$ & $59.09 \%$ & $100 \%$ & $42.85 \%$ & $74.60 \%$ \\
\hline Spleen hyperaemia & $31.33 \%$ & $27.27 \%$ & $100 \%$ & $0 \%$ & $31.74 \%$ \\
\hline $\begin{array}{l}\text { Microgliosis and } \\
\text { brain edema }\end{array}$ & $36.14 \%$ & $36.36 \%$ & $100 \%$ & $15.79 \%$ & $34.92 \%$ \\
\hline $\begin{array}{l}\text { Lymphocytic } \\
\text { hepatitis }\end{array}$ & $48.19 \%$ & $68.18 \%$ & $33 \%$ & $73.68 \%$ & $39.68 \%$ \\
\hline Lung edema & $61.46 \%$ & $54.54 \%$ & $0 \%$ & $73.68 \%$ & $61.90 \%$ \\
\hline Liver hyperaemia & $46.99 \%$ & $59.09 \%$ & $66 \%$ & $57.89 \%$ & $39.68 \%$ \\
\hline $\begin{array}{l}\text { Interstitial pneu- } \\
\text { monia }\end{array}$ & $46.99 \%$ & $36.36 \%$ & $0 \%$ & $42.85 \%$ & $49.29 \%$ \\
\hline $\begin{array}{l}\text { Lung edemaEpicar- } \\
\text { dial haemorrhages }\end{array}$ & $60.24 \%$ & $27.27 \%$ & $16.67 \%$ & $26.32 \%$ & $69.84 \%$ \\
\hline & & & & & \\
\hline
\end{tabular}

\section{References}

1. Benson, J.E., et al. (2002): J Vet Diagn Invest,. 14, p. 8-14.

2. Lager, K.M. and P.G. Halbur (1996): J Vet Diagn Invest. 8: p. 275-82.

3. Rossow, K.D., et al. (1996): Vet Pathol. 33: p. 95-9.

4. Halbur, P.G., et al. (1994) J Vet Diagn Invest. 6: p. 254-7.

5. Cheon, D.S. and C. Chae (2001) J Comp Pathol. 124: p. 231-7.

6. Stadejek, T., et al. (2006): J Gen Virol. 87: p. 1835-41. 


\title{
0.169
}

\section{Field trial of the specificity of an ELISA test for PRRS (IDEXX HERDCHEK ${ }^{\circledast}$ PRRS 2XR) high specificity confirmed, but varying behaviour according to the age of the animals}

\author{
Vincent Auvigne $^{1}$ Jean-Louis Pinsard ${ }^{2}$ Hervé Gourgues ${ }^{3}$ \\ 1. Ekipaj, Pozuelo de Alarcón, Spain; 2. Bio Chêne Vert, Chateaubourg, France; 3. IDEXX Laboratories, Cergy Pontoise, France
}

\section{Introduction}

The laboratory diagnosis of the Porcine Respiratory and Reproductive Syndrome (PRRS) is mainly based on the use of serological ELISA tests and PCR diagnostics. The aim of this study was to assess the specificity of a commercial ELISA test. In particular, the specificity of the test is compared in two different subpopulations: adult breeding sows and young pigs.

\section{Materials and methods}

The study takes into account a set of results carried out on French pig farms that are considered free of the PRRSV and with a low risk of contamination (areas with a low pig density or air filtration system). The farms are tested at least three times a year.

The IDEXX HerdChek ${ }^{\oplus}$ PRRS 2 XR is an indirect Elisa test for the detection of antibodies against the European and American strains of the PRRSV in the serum of pigs. Samples with an S/P ratio of 0.4 or more are positive. Those of less than 0.4 are negative. Only the first results were included in the study, before carrying out any confirmatory tests.

All farms with a positive result were checked to confirm that the farm was indeed considered as free from the disease, and to check if at least one subsequent negative result allowed excluding the hypothesis of a recent contamination.

\section{Results}

Test results carried out between December 2007 and March 2009 were taken into account. 641 adult animals (breeding sows) and 1017 young stock (between 80 and 150 days of age) were included in the study.

Specificity was significantly higher in young stock than in the adults (Chi2, $\mathrm{p}<0.02$, Table 1 ). Analysis of the $\mathrm{S} / \mathrm{P}$ ratio distributions of adult and young pigs showed a difference between the two sub-populations (Figure 1). They differed in the average (Mann \& Whitney test, $\mathrm{p}<0.001$ ) and in the standard deviation. The percentage of borderline-negative results (S/P between 0.2 and 0.4) was higher in adult pigs.

Table 1: Specificity of the test in both populations

\begin{tabular}{lllll} 
& Neg. & Pos. & Specificity & CI $-95 \%$ \\
\hline Mdult & 630 & II & $98.28 \%$ & {$[97.20-99.37]$} \\
\hline Young & 1012 & 5 & $99.51 \%$ & {$[99.03-99.99]$} \\
\hline
\end{tabular}

Figure 1: Distribution of S/P ratios according to the age

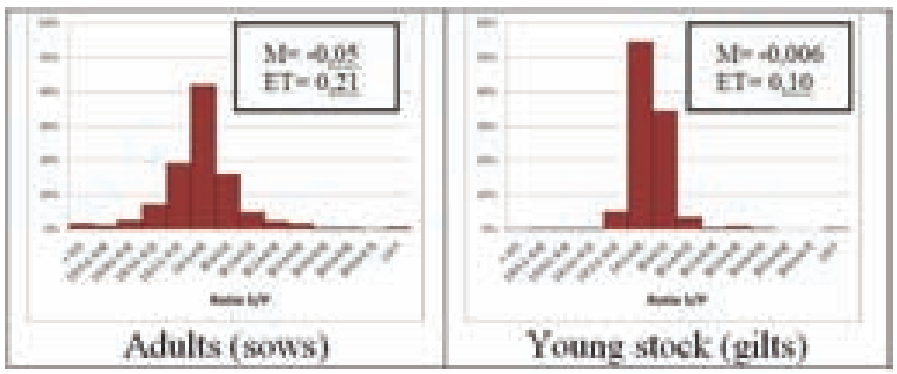

False positive results ranged from values very close to the limit (5 out of 16 with $S / P<0.45$ ) to clearly positive values (5 out of 16 false with $S / P>1$ ).

\section{Discussion}

The main difficulty of specificity studies in the absence of a reference tests is to deal with a disease-free study population. In this study, the combination of a history of controls and a low risk of contamination allowed affirming that the positive test results were false positives.

The observed values of specificity are high and confirm existing data obtained with a smaller sample (1). These specificity values are higher than those obtained with other tests in national control programmes in a disease-free environment (2). The specificity of a test should be put in balance with its sensitivity, as no test is $100 \%$ specific.

The difference in specificity between the two sub-populations (young stock and adult animals) studied is an original result. To our knowledge, this is the first time it has been described in the context of PRRS. One hypothesis to explain this difference between the two age groups is the existence of "immunological background noise", which increases with age due to the accumulation of non-specific antigenic stimulation. It is surprising to note that this lesser specificity of adult animals is not due to an increase of the S/P ratio but to their variability. It would be interesting to have equivalent data for other pathological agents of pigs in order to determine whether this is a systematic phenomenon or only linked to PRRS.

\section{References}

1. Idexx Laboratories (1999).

2. Corbellini L.G. et al. Vet Microbiol (2006).118, 267-73 


\title{
0.170
}

\section{Use of stillborn (pre-colostral) piglets as a sample source to assess viral circulation in PRRSv positive sow herds}

\author{
Dale Polson ${ }^{1}$ Brent Carmichael ${ }^{2}$ Montserrat Torremorell $^{3}$ Greg Hartsook ${ }^{1}$ \\ 1. Boehringer Ingelheim Vetmedica, Inc., St Joseph, MO, USA; 2. Iowa State University, Ames, IA, USA; 3. University of Minnesota, St Paul, MN, USA
}

\section{Introduction}

Production of PRRS virus (PRRSv) negative weaned pigs and elimination of virus from breeding herds has been successful using methods like herd closure, mass vaccination and rollover. ${ }^{1-5}$ When gestating breeding animals become infected, PRRSv can subsequently infect their fetuses. ${ }^{6}$ Weaning age piglet monitoring is a relatively common procedure used to detect virus circulation in the breeding herd, but this procedure alone is incapable of distinguishing farrowing area circulation of virus from gestational circulation where susceptible sub-populations of gestating animals may exist.

The objectives of this observational study were to develop and evaluate a procedure of monitoring stillborn piglets to detect gestational virus circulation. The combination of weaning age piglet monitoring and stillborn fetal piglet monitoring would support greater opportunities to detect and resolve sources of virus circulation within PRRSv-positive breeding herds.

\section{Materials and Methods}

PRRSv elimination projects utilizing herd closure and mass vaccination were conducted on three previously naïve sow farms that became infected with PRRS virus.

Ten to twenty stillborn fetuses and 60 weaning age piglets from each farm were sampled monthly, and all samples were individually tested by PRRS rtPCR. Selected stillborn fetuses were processed as follows: [1] swab both axillary spaces and place in a tube containing $1 \mathrm{~mL}$ saline, [2] clean axillary spaces with disposable disinfectant wipes and dry with paper towel, [3] using a new scalpel cut open both axillary spaces, [4] squeeze thorax hard so blood pools in axillary spaces, [5] use a disposable syringe to collect pooled blood, [6] transfer blood to serum separator tube, [7] open the thorax and check lungs for inflation, [8] incise stomach and check for milk. A fetus was considered acceptable for sampling if it had a wet umbilical cord, non-inflated lungs and no evidence of milk in the stomach.

\section{Results}

Serum and swab results from stillborn piglets from the three sow farms are shown in Table 1. Of the samples collected from 126 stillborn fetuses, $20.6 \%$ (26) of sera and $31.0 \%$ (39) of skin swab samples tested PCR positive. Interestingly, the percent of PCR positive stillborn serum samples declined over time at a similar rate as those of weaning age piglets (Table 2 ).
Table 1: Distribution of PRRSv PCR results for serum and skin swab samples collected from stillborn piglets.

\begin{tabular}{|l|c|c|c|}
\hline & Skin Swab - & Skin Swab + & Total \\
\hline Serum - & $83(65.9 \%)$ & $17(13.5 \%)$ & $100(79.4 \%)$ \\
\hline Serum + & $4(3.2 \%)$ & $22(17.5 \%)$ & $26(20.6 \%)$ \\
\hline Total & $87(69.0 \%)$ & $39(31.0 \%)$ & 126 \\
\hline
\end{tabular}

Table 2: Percent PRRSV PCR positive serum results by month for serum from stillborn and weaning age piglets.

\begin{tabular}{|l|c|c|c|}
\hline & Month 1 & Month 2 & Month 3 \\
\hline Stillbirths & $74.3 \%$ & $40.0 \%$ & $7.5 \%$ \\
\hline Weaners & $58.1 \%$ & $43.6 \%$ & $8.9 \%$ \\
\hline
\end{tabular}

\section{Discussion}

The axillary space was targeted for swabbing because of all the external surfaces on a stillborn piglet, the axillary space represents the cleanest skin surface to assess whether viral RNA acquired before or during parturition might be present on the exterior of a stillborn piglet.

As a herd's immunity develops following whole herd massvaccination, the level of in-utero infection would be expected to decline, fall to and remain at zero as long as new wild type virus introduction is prevented. If proper piglet management procedures are followed in the farrowing area7, the level and likelihood of new infection among and within farrowing rooms should also fall to and remain at zero.

The stillborn piglet sampling/testing procedure potentially complements PCR-testing of weaning age piglets to detect and pinpoint PRRSv circulation in the breeding herd.

\section{References}

1. Dee SA, et al.; Vet Rec; 1998;142:569-572.

2. Sanford SE; Proc 16th IPVS; 2000; 589.

3. Rajic A, et al.; J Swine Health Prod; 2001; 4:179-184.

4. Gillespie TG, Carroll AL; J Swine Health Prod; 2003; 6:291-295.

5. Polson D, Hartsook G; Proc 20th IPVS; 2008; 1:52.

6. Zimmerman J et al.; In Straw BA et al.; Diseases of Swine; pp 387417.

7. McCaw M; J Swine Health Prod; 2000; 8:15-21. 


\title{
0.171
}

\section{Evaluation of a methodology for collection and PRRSV detection at swine herd level}

\author{
Catherine Charreyre ${ }^{1}$ Andrea Delvecchio ${ }^{1}$ Valérie Cozette $^{1}$ Thaïs Vila $^{1}$ Mathieu Chevalier François Joisel $^{1}$ \\ 1. Merial S.A.S., Lyon, France; 2. Merial CRSV, Saint-Vulbas, France
}

\section{Introduction}

PRRS virus is usually detected in individual pig serum, but it is often difficult to do at the herd level because of cost and the labour. Researchers have recently developed a new methodology $(1,2,3)$ : the objective of this study was to compare this new method of detection of the virus in oral fluid (OF) with the classical detection of PRRSV in serum.

\section{Material and Methods}

Animals: 3 replicates of 10 commercial pigs, housed in 3 separate pens, in strict isolation.

Experimental design: at 12 weeks of age (Day 0) each pig received a 4-mL intranasal injection of the PRRSV challenge suspension.

Serum and OF samples collected at Day 0 were used to qualify the extraction method and the qRT-PCR test and to confirm the absence of PRRSV.

OF and blood were collected from each animal at D0 (PRRSV challenge) and at D3, 7, 10, 14, and 21. A cotton rope ("Sanicorde") tied up to each pen partition for 30 minutes was used to collect OF Pigs actively sought out and chewed the rope (2). The Sanicorde were wrung, OF were harvested and stored at $-80^{\circ} \mathrm{C}$ until use.

Lab investigations: Serum and OF samples collected at Day 0 were used to qualify the extraction method and the qRT-PCR test and to confirm the absence of PRRSV..extraction from OF and blood samples was performed with QIAamp ${ }^{\circledR}$ Viral RNA kit (Qiagen). OF samples were centrifuged to remove debris and PRRS virus load was evaluated by the qRT-PCR technique.

Statistical methods: McNemar's test for concordance between viremia and oral virus results; Binomial probability distribution to assess the minimum number of OF samples to detect at least 1 positive sample.

\section{Results}

All blood and OF samples collected prior to virus inoculation up to Day 0 and all case control tested negative.

Comparison showed that the levels of virus in serum and OF samples in different groups followed a similar pattern, with OF consistently containing a lower detectable concentration of virus (Figure 1).

Comparing the proportion of negative or positive results in OF and blood samples in the 3 groups, each as a whole, the correlation was significant $(p=0.025)$ with $72 \%$ of concordant responses between blood and OF.
Figure 1: Average detection by $q R T-P C R$ (maximum number of amplification cycles i.e. 40 - first detection amplification cycle $=40-C T$ ) of $P R R S V$ in serum and $O F$

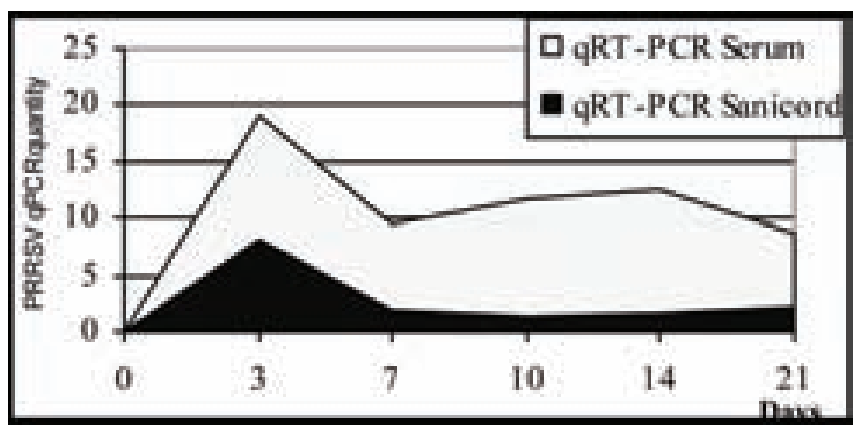

Ten of the 15 OF samples collected were found positive (67\%). Thus, we calculated the probability of having at least one positive sample (detection of PRRS virus in OF with Sanicorde) for a number of samples from 2 to 6 , and a 'true' percentage of positive pigs from $50 \%$ to $80 \%$.

With a 70\%-prevalence of positive pigs in a group, 3 sequential OF samples collected each 3 to 7 day, are necessary to obtain at least 1 positive result with $95 \%$ chance of success (Table 1).

Table 1: Probability to detect at least one positive sample

\begin{tabular}{|c|c|c|c|c|}
\hline & $\begin{array}{c}\text { True \% of posi- } \\
\text { tive samples in } \\
\text { the pig group }\end{array}$ & $\begin{array}{c}\text { True \% of posi- } \\
\text { tive samples in } \\
\text { the pig group }\end{array}$ & $\begin{array}{c}\text { True \% of posi- } \\
\text { tive samples in } \\
\text { the pig group }\end{array}$ & $\begin{array}{c}\text { True \% of posi- } \\
\text { tive samples in } \\
\text { the pig group }\end{array}$ \\
\hline No of samples & $50 \%$ & $60 \%$ & $70 \%$ & $80 \%$ \\
\hline 2 & 0.75 & 0.84 & 0.91 & 0.96 \\
\hline 3 & 0.88 & 0.94 & 0.97 & 0.99 \\
\hline 4 & 0.94 & 0.97 & 0.99 & 1 \\
\hline 5 & 0.97 & 0.99 & 1 & 1 \\
\hline 6 & 0.98 & 1 & 1 & 1 \\
\hline
\end{tabular}

\section{Discussion and Conclusion}

The qRT-PCR test we used was designed for blood tests; it had not been fully optimized for PRRSV detection in OF and requires improvements. However our results confirmed that the collection of OF samples could be a useful and cheaper methodology to detect and monitor PRRSV infection at herd level. The results of this method have to be confirmed in the field.

\section{References}

1. Hoffman P. et al., (2008) 38th AASV Proceedings, p. 301-302

2. Prickett J. et al., (2008a) J. Vet. Diagn. Invest. vol. 20, p. 156-163

3. Prickett J. et al., (2008b) J. Swine Health Prod., vol. 16, n. 2, p. 86-91 


\title{
0.172
}

\section{A comparison of individual versus pooled sample testing on pool-level and case-level PRRS virus PCR detection sensitivity and specificity}

\author{
Dale Polson $^{1}$ Greg Hartsook ${ }^{1}$ Brent Carmichael ${ }^{2}$ \\ 1. Boehringer Ingelheim Vetmedica, Inc., St Joseph, MO, USA; 2. lowa State University, Ames, IA, USA
}

\section{Introduction}

The time it takes to begin producing porcine reproductive and respiratory syndrome virus (PRRSv)-negative groups of weaned pigs following the new introduction of a non-resident PRRSv and related onset of a clinical reproductive PRRS outbreak in swine breeding herds has become an important means by which to assess the effectiveness of PRRS interventions applied to breeding herds. The measure "time-to-negative-pigs" (TTNP) is typically described as the number of weeks between the start of an intervention protocol (e.g., herd closure to new replacement introductions and/or mass vaccination) and the first "consistent" production of PCR-tested negative groups of weaned pigs. For estimating TTNP, the key measurement components are: sampling frame (e.g., piglets within 1 week or less of weaning), sample selection (e.g., smallest barrow from gilt litters, one piglet per litter) sampling method (jugular venipuncture, ear notch), sample type (e.g., blood-to-serum, swab-to-saline), sample size, sampling interval, sample pooling (e.g., pooling of $\mathrm{N}$ individual samples), shipping materials and method, PCR assay, laboratory procedures, and the number of consecutive testednegative samplings.

The objective of this study was to assess the effect of sample pooling of 5:1 and 10:1 on PCR detection sensitivity and specificity at both the pool and case levels when estimating TTNP.

\section{Materials and Methods}

Two 5,000 sow PRRS-naïve breeding herds became exposed and infected with a wild-type PRRSv. A sampling-pooling-PCR testing evaluation was conducted on both farms. Sixty (60) dueto-wean piglets were periodically sampled and tested by PRRSv PCR - initially per month, then, when the individual sample-level PCR prevalence was first noted to be approximately $30 \%$ and less, 60 piglets were sampled per week until zero individual PCR positives were detected for at least six consecutive weeks. Dueto-wean piglet selection targeted the smallest barrow suckling a gilt or youngest sow. ${ }^{1}$

PRRSv PCR testing was done on all individual samples collected as well as on pools composed of 5 and 10 individual samples. All pooling was done in the testing laboratory under a hood using special precautions to prevent cross-contamination. Where a pool contained one or more tested PCR-positive individual samples, the true state of the pools was defined as positive. Conversely, where a pool contained all tested PCR-negative individual samples, the true state of the pool was defined as negative. PCR detection sensitivity (Se) and specificity (Sp) were evaluated at both the pool-level and case-level. The Se and Sp of the tested pool sizes were compared to the individual sample PCR testing results of the samples making up the pools.

\section{Results}

The results show a comparable estimated pool-level sensitivity for pools of $5(84.5 \%, 95 \% \mathrm{Cl}=73.5 \%-91.6 \%, \mathrm{n}=274)$ and pools of $10(82.0 \%, 95 \% \mathrm{Cl}=68.1 \%-91.0 \%, \mathrm{n}=137)-$ a $15.5 \%$ and $18.0 \%$ relative reduction in sensitivity for samples pooled $5: 1$ and 10:1, respectively, as compared to the PCR testing results of the individual samples making up the pools. However, both the pools of 5 and 10 had $100 \%$ sensitivity and $100 \%$ specificity at the case-level.

At the pool level the specificity estimates were $99.0 \%$ for pools of 5 and $97.7 \%$ for pools of 10 . It is notable that all four "pool individuals-negative but pool-positive" (InPp) results occurred in the first two sets of cases pooled, and in every instance the InPp pool was preceded by a pool that contained one or more positive individual samples. After the pooling process was reviewed and adjustments made, no further occurrences of InPp were detected. This indicates the importance of the pooling process in obtaining accurate pool-level results.

\section{Discussion}

Based on these data, individual sample pooling up to 10:1 can offer comparable pool-level and case-level detection Se to that of pooling 5:1 and can further reduce testing costs when estimating TTNP.

\section{References}

1. Cano JP,et al.; Proc 39th AASV; pp 105-107; 2008. 


\title{
0.173
}

\section{Infection dynamics of Porcine Reproductive and Respiratory Syndrome Virus (PRRSV) in vaccinated and non-vaccinated pigs}

\author{
$\underline{\text { Helle H. Jessen }}{ }^{1}$ Charlotte K. Hjulsager ${ }^{2}$ Jens P. Nielsen ${ }^{3}$ Helle Stege ${ }^{3}$ Lars E. Larsen $^{2}$ Anders Elvstrøm 4 \\ 1. Danvet K/S, Hobro, Denmark; 2. National Veterinary Institute, Technical University of Denmark, Copenhagen, Denmark; \\ 3. University of Copenhagen, Faculty of Life Sciences, Copenhagen, Denmark; 4. Odder Dyreklinik, Odder, Denmark
}

\section{Introduction}

Piglets from a PRRSV (EU type) seropositive stabile sow herd were seronegative at weaning, but became infected with PRRSV EU after transfer to the finishing units located at the same site. To control PRRSV among the finishing pigs, vaccination with a modified live virus PRRSV EU vaccine (Intervac PRRS) all pigs prior to transfer to the finishing stables were initiated from October 2008. In July 2009 it was decided to terminate vaccination of pigs at transfer. The aim of this study was to investigate if nonvaccinated pigs could be inserted in an empty and clean stable without being infected despite the presence of vaccinated pigs in neighboring rooms.

\section{Materials and methods}

The study was performed as an intervention study with two groups of pigs including 58 vaccinated and 20 non-vaccinated Danish Landrace, Yorkshire and Duroc crossbreeds. All pigs were identified with ear tags. The vaccinated pigs were 8 weeks of age at the time of vaccination. Blood samples and nasal swabs were taken $0,1,2,3,8$ and 13 weeks after vaccination. The nonvaccinated pigs varied from 5 to 10 weeks of age at transfer to the finishing stable and were sampled 0, 5 and 10 weeks after transfer. Non-vaccinated pigs were transferred to a clean and disinfected stable three weeks after the vaccinated pigs were vaccinated. There was no physical contact between the two groups, that were separated by walls and a door that was kept closed during the study. All blood samples were examined for antibodies against the US and EU PRRSV types by a differentiating blocking ELISA (4) and for the EU PRRSV type by IPMA (1). A ratio (OD\%eu/OD\%us) was calculated if ELISA EU or ELISA US were positive (cut off: OD\% $\leq 44$ ). IPMA EU detected antibodies in four dilutions (1:50; 1:250; 1:1250; 1:6250). Serum samples and nasal swabs from vaccinated pigs were examined for PRRSV RNA by real time RT-PCR ( 3 ) and the ORF5 and ORF7 were sequenced from selected positive samples.

\section{Results}

None of the tested non-vaccinated pigs seroconverted to PRRSV after transfer to the fattening unit despite the simultaneous occurrence of viraemia in vaccinated pigs housed in a neighboring room. All vaccinated pigs were seronegative at the time of vaccination. After vaccination they developed an immune response, which resulted in $85.5 \%$ pigs with IPMA EU titers $\geq$ 1250 three weeks after vaccination. The mean ELISA OD\%eu in week three was 42.3. Eight weeks after vaccination, all pigs were seropositive (mean ELISA OD\%eu at 21.9) and $182 \%$ of the pigs had IPMA EU titers above 1250. PRRSV RNA was detected in serum samples 1 to 3 weeks after vaccination, but could not be detected in nasal swabs at any time. The level of PRRSV peaked 1 week after vaccination and then rapidly declined. None of the pigs had PRRSV viraemia at 8 and 13 weeks after vaccination. The virus was found to be identical to the vaccine virus (DV-MLV) by sequencing of ORF5 and ORF7. Thirteen weeks after vaccination, $94.5 \%$ of the pigs had IPMA EU titers above 1250, indicating an acute infection. No clinical symptoms were observed at this time. The mean ELISA ratio in week 13 was 0.6 indicating a previous PRRSV EU infection.

\section{Discussion}

The lack of transmission of PRRSV EU from vaccinated to noninfected pigs in neighboring rooms, is of importance for herd eradication programs in which PRRSV vaccination is carried out in order to reduce clinical effect. Until 8 weeks after vaccination, the vaccine induced an immune response comparable to a natural infection as previously described $(2,5)$. Virus was not detected in serum beyond week 3 after vaccination, and virus was not detected in nasal swabs at any time. Thus, it seems to be safe to mingle pigs 8 weeks after vaccination which is routinely used in Denmark. To our surprise, the IPMA EU titers markedly increased 13 weeks after vaccination indicating acute (re)infection. Although serum and nasal swabs were PCR-negative, this may be due to either an infection with a wild type virus or reactivation of the vaccine virus. Further studies are needed to elucidate if this is a general features in pigs vaccinated with live PRRSV vaccines.
References
1. Bøtner et al., Vet. Microbiol. 40 351-360 (1994)
2. Díaz et al., Virology $351249-259$ (2006)
3. Egli et al., J. Virol. Meth. 98 63-75 (2001)
4. Sørensen et al., Vet. Microbiol.60 169-177 (1998)
5. Zuckermann et al., Vet. Microbiol.123 69-85 (2007) 


\title{
0.174
}

\section{Serological, virological and productive monitoring to control PRRSV infection in an endemically infected breeding herd in North-Eastern Italy}

\author{
Giacomo Bortoletto $^{2}$ Marco Martini ${ }^{2}$ Stefano Nardelli ${ }^{1}$ Michele Drigo ${ }^{1}$ \\ 1. Istituto Zooprofilattico Sperimentale Venezie, Legnaro (PD), Italy; \\ 2. Dept. Public Health, Comparative Pathology and Veterinary Hygiene, Padua University, Legnaro, Italy
}

\section{Introduction}

In Italy PRRSV infection is widespread and circulation of several viral strains occurs. In breeding herds the key to break virus circulation is to expose replacement animals to the farm specific PRRSV strain to induce a good immune response prior to their introduction into the herd. Therefore, a rigorous bio-security policy is needed to avoid the incoming of new strains into an infected farm (Pesente et al. 2006).

The aim of this study was to monitor the serological, virological and productive trends of an endemically PRRSV infected breeding herd where the control of the infection was based on the implementation of an effective acclimatization program accompanied by strict bio-security measures.

\section{Materials and Methods}

The study was carried out in a 1500 sows farrow-to-wean farm, infected by PRRSV since 1995. As farmers decided not to vaccinate for PRRSV, an acclimatization program was improved over time. 52 3-week-old PRRSV negative gilts were introduced into the farm every month and at once exposed to PRRSV through the contact with weaned pigs, used as donor sources of virus. During the last 3 months of acclimatization viremic pigs were turned over every 2 weeks and taken away one month prior to the introduction of gilts into the stimulation unit. The aim of the program is to fertilize 8-month-old gilts after they become immune but no longer viremic.

For 24 consecutive months, from April 2007 to March 2009, sera samples were collected from 1185 gilts at the end of acclimatization. In the same period, 5 cross-sectional surveys were carried out almost seasonally, on a total of 607 pigs. Between October and November 2008 a PRRS outbreak occurred in the farm and thoracic fluid was collected from some aborted fetuses. PRRSV RNA was extracted from both sera and thoracic fluid samples, analyzed by RT-PCR, purified and sequenced in ORF7 region. Sera samples from gilts were also checked for the presence of a specific PRRSV immunity by an ELISA commercial kit. Performance Trend Analysis (PTA) was obtained using farm database, and analyzed statistically using Chi-square test for count data and one sample T-Test for continuous variables.

\section{Results}

After a first period of low efficacy of the acclimatization program, especially in July and November 2007, both prevalence of seropositive gilts and mean $\mathrm{s} / \mathrm{p}$ ratio increased in the subsequent months (Fig.1). This trend may be the consequence of seasonality and of a better management of the viremic pigs used as virus donors. The $100 \%$ virus positivity in pools from 3 groups of gilts during cold season together with the virological results of the 5 cross-sectional surveys (ranging from 2/16 positive pools in July 2008 to 23/23 in February 2009) confirms that season plays a key role in the viral circulation.
The outbreak consisted in a reproductive failure with 27 abortions and in a significant fall of the productive performance: number of farrowings, farrowing rate, piglets weaned, piglets weaned/sow/year, total born/litter, liveborn/litter, piglets weaned/litter. The comparison of these parameters before and after the outbreak didn't show significant differences, demonstrating that in a well acclimatization system the return to normality can be achieved rapidly. Phylogenetic analysis assessed the presence of a single homologous PRRSV variant on the farm and revealed the incoming of a new strain $(9,4-10,8 \%$ of nucleotide $\mathrm{p}$-distance) causing the outbreak and no longer detected in the following surveys.

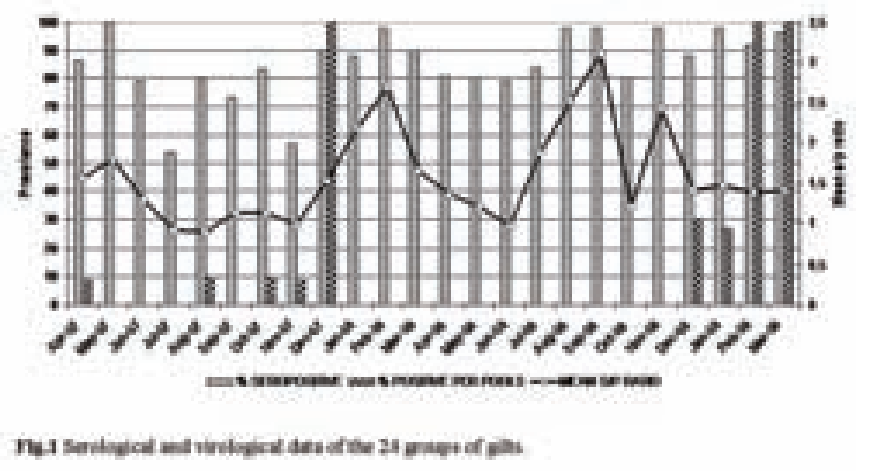

\section{Discussion}

This study confirms that an acclimatization program may be able to maintain the circulation in not reproductive units of a unique viral strain over time and that it may be able to restore promptly herd stability after an outbreak. Phylogenetic analysis is more and more often a useful tool to help farmers and veterinarians to understand the causes of the failures and to apply successful control measures.

\section{References}

Pesente P., Rebonato V., Sandri G., Giovanardi D., Sperati Ruffoni L., Torriani S. (2006). Phylogenetic analysis of ORF5 and ORF7 sequences of PRRSV from PRRS-positive Italian Farms: A show case for PRRSV epidemiology and its consequences on farm management. Vet. Microbiol. 114, 214-224. 


\title{
0.175
}

\section{IntraUterine Growth Retardation (IUGR): morphological and behavioral description}

\author{
Eric Chevaux ${ }^{1}$ Audrey Sacy ${ }^{1}$ Yannig Le Treut $^{1}$ Guy-Pierre Martineau ${ }^{2}$ \\ 1. LALLEMAND SAS, Blagnac, France; 2. Veterinary School of Toulouse, Toulouse, France
}

\section{Introduction}

Over the past 15 years, selection of hyperprolific sows has actually increased litter size but also increased heterogeneity of piglets' weight at birth. However, piglet's weight at birth by itself is not discriminating enough to predict the whole animal's productive life (Litten et al., 2003). Immaturity corresponds to piglets with intrauterine growth retardation (IUGR) after 114 gestational days, which present all the characteristics of runt piglets. The goal of this study was to determine a set of simple morphological criteria, backed-up by anatomical data, in order to allow the rapid identification of immature piglets in the farm.

\section{Materials and Methods}

924 piglets ( 837 born alive, 87 stillbirth and 29 mummified) from 67 Topigs sows (Great Yorkshire /Large White type) and N-line (Dutch Landrace), were monitored at birth in a French herd for birth and weaning weight, vitality score within the first 15 seconds of life (Baxter et al., 2008), and qualitative morphological signs of maturity including head morphology (on lateral and dorso-ventral observations, Figure 1), head/body, and behavior (after a pre-study performed by Sacy (2008)).

Figure 1: Dorso-ventral characteristics of normal (left), intermediate (middle) and IUGR (right) piglets

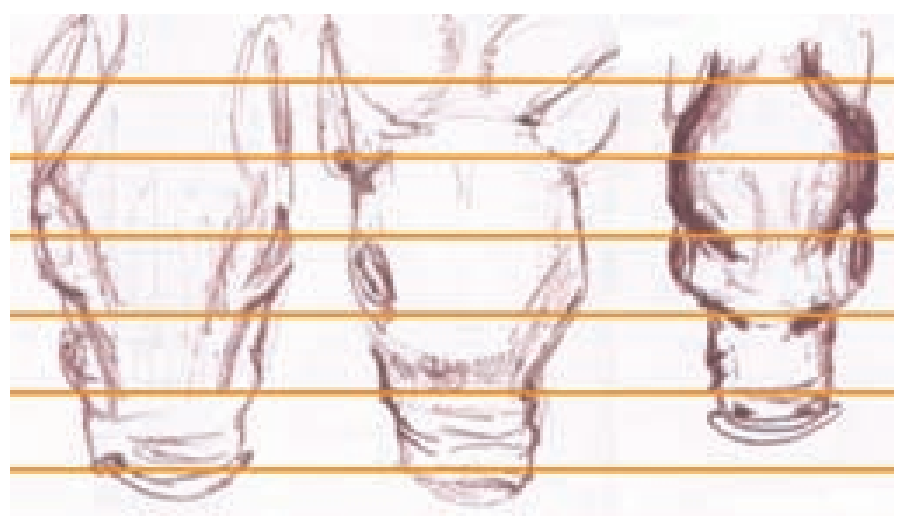

Piglets dead within 2 days post-farrowing (IUGR=147, control=282), including stillborn piglets, were necropsied and their vital organs weighed (brain, liver, heart and lungs). The brain is the first vital organ to attain maturity, while other organs such as liver mature later. Therefore, the brain/liver ratio is a good indicator of a piglet development stage. The higher the ratio, the more immature the piglet (Town et al., 2005):

\section{Results and discussion}

Morphology: IUGR piglets are generally lighter than the more mature ones ( $71 \%$ of IUGR piglets $<0.9 \mathrm{~kg}$ vs $2 \%$ of the mature ones $p<0.001$ ), but some light weight piglets exhibit the morphological traits of mature piglets.
Brain/Lung, Brain/Liver, and Brain/heart ratio: all ratios are highly significantly different between IUGR and control. Moreover, there is a correlation between the brain/lungs ratio criteria and morphological criteria of immaturity (Figure 2). Similar results were obtained with other vital organs such as heart: $5.21 \pm 1.95$ vs. $3.15 \pm 0.85(r=-0.677, p<0.001)$.

Behaviour: immature piglets exhibit typical behavioral criteria: they are usually found isolated from the other piglets, lying on their side, they have difficulties to suckle and are usually found where the farmer left them previously. They represented $11 \%$ of the live born piglets and it went up to $25 \%$ for older sows (parity $>5$ ).

Figure 2: Relationship between true stillborn piglets weight and their brain/lungs ratio $(r=-0.701, p<0.001)$

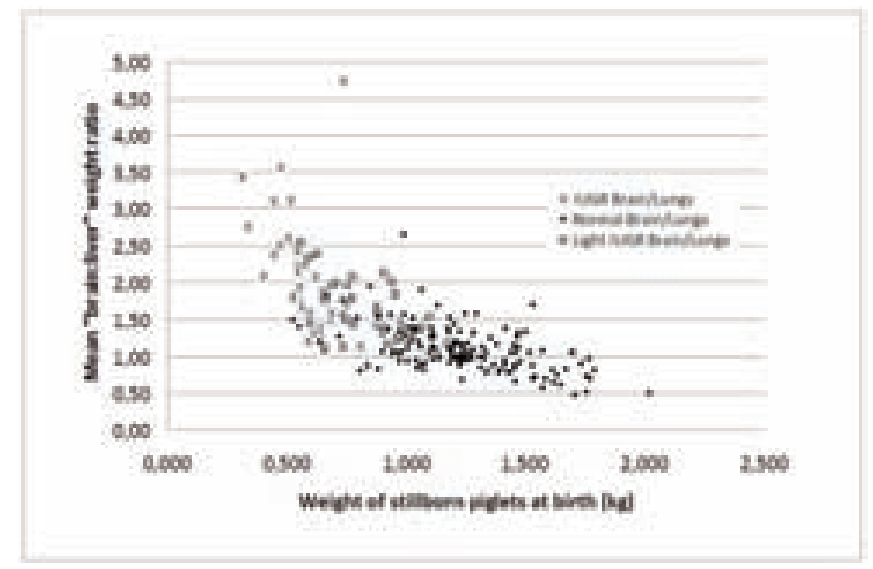

Growth and mortality: Survival rates of IUGR piglets in maternity was lower than for mature piglets (62\% vs. $89 \%$, p<0.01). At weaning ( 3 weeks), IUGR piglets $(n=54)$ have a lower live weight compared to control ( $n=642), 4.7 \pm 0.9 \mathrm{~kg}$ vs $5.7 \pm 1.2 \mathrm{~kg}, \mathrm{p}<0.01$.

\section{Conclusion}

Until now, IUGR assessment was based on anatomic criteria (Town et al., 2005). This study combines morphological and behavioral criteria that reflect piglets immaturity at birth, all criteria being validated by anatomical data.

From a producer point of view, quickly identification of immature piglets is essential.

However, it would be of interest to pursue this work with other genetic lines as the morphologic piglets criteria for immaturity might differ (according to the prolificacy).

\section{References}

Baxter et al. 2008, Theriogenology Apr 1;69(6):773-83.

Litten et al. 2003, Neonatology Vol. 84, No. 4.

Sacy 2008, MSc thesis, AgroParisTech, 103pp.

Town et al., 2005, Anim. Reprod. Sci. 85: 301-316. 


\title{
0.176
}

\section{Abortion in group housed sows with electronic feeders}

\author{
Bas Kolpa $^{2,1}$ Wikke Kuller ${ }^{1}$ Rick Janssen ${ }^{3}$ Theo Geudeke ${ }^{4}$ \\ 1. Department of Farm Animal Health, Faculty of Veterinary Medicine, Utrecht University, Utrecht, Netherlands; \\ 2. University Veterinary Practice for Farm Animals, Dorpstraat, Harmelen, Netherlands; \\ 3. Veterinary Centre Someren, Someren, Netherlands; 4. Animal Health Service, Deventer, Netherlands
}

\section{Introduction}

In the Netherlands, group housing of gestating sows will be obligatory from 2013 onwards. In 2008, a Dutch farm expanded from 280 to 450 sows and converted to group housing with electronic sow feeding. In the first 6 months of the new housing system, 59 animals aborted between 50 days until the end of gestation. The number of piglets weaned per sow per year dropped from 29 to 26.4 mainly caused by the abortions.

\section{Material and methods}

From 50 days of gestation onwards, 192 sows were housed in one group with four electronic sow feeders. Sows were customary vaccinated for PRRS, E.coli, Erisypelas, influenza and PPV. During the outbreak, different diagnostic examinations were performed (table) and a variety of experts visited the farm.

In the feeding stations, a feed data recorder was installed that made it possible to analyze feeding data.

\begin{tabular}{|c|c|c|}
\hline Material & Agent / substance & Method \\
\hline Blood & $\mathrm{H} 1 \mathrm{~N} 2, \mathrm{H} 1 \mathrm{~N} 2, \mathrm{H} 3 \mathrm{~N} 2$ & $\mathrm{HAR}^{1}$ \\
\hline Blood & PRRS, Leptospira, Aujeszky, BVD & ELISA $^{1}$ \\
\hline Blood & Hog Cholera & $P C R^{2}$ \\
\hline Blood & PCV2, PRRS PCR1 & $P C R^{1}$ \\
\hline Blood & EMCV & Virus isolation and $P C R^{3}$ \\
\hline Fetuses & PCV2, PRRS, PPV & $P C R^{1}$ \\
\hline Fetuses & & Pathology, general histology $\mathrm{HE}^{1}$ \\
\hline Fetuses & Chlamydophilia & Chlamydophilia histology, IHC' \\
\hline Placenta & & General histology, $\mathrm{HE}^{1}$ \\
\hline aborted sows & & General histology, $\mathrm{HE}^{1}$ \\
\hline Feed sample & $\begin{array}{l}\text { DON, NIV, T2, Ochratoxine A, } \\
\text { Fumonisin B1 and B2, Zeara- } \\
\text { lenone, and Aflatoxin B1 }\end{array}$ & LC-MS 1 \\
\hline Water & Ammonium, Nitrite & $\mathrm{kca}^{1}$ \\
\hline Water & Nitrate, Chloride, Sulfate & $\mathrm{IC}^{1}$ \\
\hline Water & Iron, $\mathrm{Mn}, \mathrm{Na}$ & $I C P 1$ \\
\hline Water & $\mathrm{pH}$ & \\
\hline Water & E. coli & Bacterial examination ${ }^{1}$ \\
\hline \multicolumn{3}{|c|}{ Animal Health Service, Deventer, The Netherlands } \\
\hline \multicolumn{3}{|c|}{${ }^{2}$ Central Veterinary Institute, Lelystad, The Netherlands } \\
\hline \multicolumn{3}{|c|}{${ }^{3}$ CODA-CERVA,Ukkel, Belgium } \\
\hline
\end{tabular}

\section{Results}

The animals that aborted were sometimes slightly inert but appeared clinically healthy. There were no signs of aggression. No influence of parity was found. The samples that were examined showed no (increase of) antibody concentration. Aborted fetuses within a sow were all in one stage of development. Litter size of the other sows was not affected. Feed data analysis showed that several animals frequently visited the feeder for a very short time, but probably were not provided with feed. Further investigation showed that sometimes the feeders had a random reset and had a mechanical malfunction. After adjustment of the feeders no more abortions were observed.

\section{Discussion}

Low feed intake is a risk factor during the first weeks of gestation (1). However, in this case abortion occurred later on. Since the sows were fed using electronic sow feeders, one would not expect a high risk of feed competition like in group housing systems with trough or floor feeding. (1). But due to the malfunctioning of the feeders, the feed intake of some sows could have been very low, which might affect pregnancy. Valuable information on real feed intake can be obtained by data recording systems that some manufacturers provide. Veterinarians should use this information.

Another aspect of this case is the effect of stress. The detrimental effect of stress on (re)productivity of sows is hard to establish in experimental settings (2). However, stress has an effect on the hypothalamic-pituitary-adrenocortical axis and therefore probably on the maintenance of pregnancy (2). The fact that the feeders quite often randomly malfunctioned probably resulted in a lot of agitation and thus in (long term) highly unpredictable stress. Examination of sow behaviour could have given a clue.

\section{References}

1 Spoolder et al. 2009. Livestock Science 125:1-14

2 Borell et al. 2007 Hormones and Behavior 52:130-138 


\title{
0.177
}

\section{Effects of zearalenone (ZEA) exposure of gilts during late gestation and lactation on metabolism and carry-over to piglets}

\author{
Johannes Kauffold ${ }^{1}$ Katharina Stephan ${ }^{2}$ Dina Knauf ${ }^{3}$ Frank F Bartol ${ }^{4}$ Martin Waehner ${ }^{2}$ \\ 1. Department of Clinical Studies - New Bolton Center, School of Veterinary Medicine, University of Pennsylvania, Kennett Square, PA, USA; \\ 2. Anhalt University of Applied Sciences, Bernburg, Germany; 3. Clinic for Obstetrics, Gynecology and Andrology of Large and Small Animals, \\ Faculty of Veterinary Medicine, Justus-Liebig-University, Giessen, Germany; 4. Departments of Anatomy, Physiology and Pharmacology, \\ College of Veterinary Medicine, Auburn University, Auburn, AL, USA
}

\section{Introduction}

ZEA is a mycotoxin that binds to the estrogen receptor and exerts estrogen-like effects. Pigs are highly sensitive to ZEA [1] and can exhibit reproductive disorders if consume it. There is indication that ZEA is carried over to piglets in utero and through colostrum/milk, and may cause hyperestrogenism [2]. However, neither of both routes of carry-over has been conclusively demonstrated. This study therefore focused on the metabolism and carry-over of ZEA to piglets when fed to late pregnant and lactating gilts.

\section{Materials and Methods}

Gilts ( $n=32$ ) were hormonally synchronized for estrus and ovulation, artificially inseminated and tested positive for pregnancy. They were fed crystalline ZEA (Sigma-Aldrich, Germany) from day 101 of gestation until days 20/21 of lactation in a concentration of either $1.500 \mu \mathrm{g} / \mathrm{kg}$ feed/day $(\mathrm{n}=7$; EXP-H) or $250 \mu \mathrm{g} /$ $\mathrm{kg}$ feed/day ( $n=9$; EXP-L), while eight gilts served as controls, respectively (CON-H, CON-L). For application, ZEA was dissolved in ethanol and injected into muffins that were individually fed to the gilts. CON animals received blank muffins. Prior to first colostrum uptake, female piglets were cross-fostered from the respective EXP to CON animals and vice versa, to obtain piglets that were differently exposed to ZEA: in utero only (IU), through colostrum/milk only (MI), in utero and through colostrum $/ \mathrm{milk}$ (IU \& MI), none (CON). Group size was 11-13 piglets, respectively. Colostrum/milk was collected four times in weekly intervals. First collection occurred after farrowing. Piglets were weaned on day 20/21 of lactation, then sacrificed and bile collected. Sows were sacrificed one day after weaning, and bile collected. All milk and bile samples were subjected to analysis for ZEA and its metabolites $\alpha$ - and $\beta$-zearalenol (ZOL) using HPLC and mass spectrometry.

\section{Results}

Median concentrations for both ZEA and a-ZOL in bile of CON-H and $L$ groups were around $5 \mathrm{ng} / \mathrm{ml}$ and $2 \mathrm{ng} / \mathrm{ml}$, respectively. Concentrations were $>250 x$ higher in in bile of EXP-H and 30-100 x higher in EXP-L gilts for both mycotoxins. ZEA, but not $a-$ and $\beta$-ZOL, was found in milk collected from EXP-H and $L$ but also from the respective $\mathrm{CON}$ animals. Mean concentrations of ZEA (average of the values obtained with all milk samples) were higher for the EXP-H and $\mathrm{L}$ than for the $\mathrm{CON}-\mathrm{H}$ and $\mathrm{L}$ group, respectively. In none of the piglets could $\beta$-ZOL be detected in bile. Almost all IU \& MI and MI piglets belonging to both the $\mathrm{H}$ and $\mathrm{L}$ experiment were tested positive for ZEA and $\mathrm{a}-\mathrm{ZOL}$ in bile. Only single piglets exposed to IU or belonging to CON were positive. Median concentrations of ZEA and a-ZOL were higher in IU \& MI and $\mathrm{MI}$ than in IU and CON piglets in both experiments, respectively $(P<0.0001)$.

\section{Discussion}

Results confirm that ZEA and its metabolites accumulate in bile of pigs and that the concentrations in bile depend on the amount of ZEA consumed [3]. The fact that non-exposed animals also had ZEA in bile albeit in very low concentrations indicates constant back-ground exposure. Data show clearly that ZEA can be encountered in colostrum/milk of lactating gilts. The fact that piglets nursed to ZEA-exposed gilts were preferentially ZEA positive in bile regardless of whether they were exposed also in utero or not, clearly demonstrates that carry-over of ZEA through colostrum/milk occurs in the pig. A recent study has shown altered expression of morphoregulatory important genes in those piglets [4].

\section{References}

1. Diekman \& Green. J Anim Sci;1992:1615-1627

2. Fink-Gremmels \& Malekinejad. Anim Feed Sci Technol 2009;137:326341

3. Dänicke et al. Mol Nutr Food Res 2005;49:932-943

4. Chen et al. Ann NY Acad Sci 2009;1160:188-199 


\title{
0.178
}

\section{Risk factors associated with frequency of abortions}

\author{
Nuno M. Rainho' Maria Aparicio ${ }^{1}$ Miguel A. de Andrés ${ }^{1}$ Joquin Morales ${ }^{1}$ Rafael Pallás$^{2}$ Vicente Rodríguez-Estévez ${ }^{3}$ Carlos Piñeiro $^{1}$ \\ 1. PigCHAMP Pro Europa, Segovia, Spain; 2. Kubus SA, Las Rozas, Madrid, Spain; \\ 3. Dpto. Producción Animal, Universidad de Córdoba, Córdoba, Spain
}

\section{Introduction}

Current swine production is everyday more linked to a proper analysis and monitoring to maintain competitiveness. Despite of the fact that every farm has to improve taking into account its own results, in many cases general standards can be useful to set a quick reference in order to help to define farm objectives and to compare performances through benchmarking processes. Literature has described references for most of the key indicators during the last decades, but some of the factors has not been recently updated or explained in depth including interactions between them. Those risk factors related to frequency of abortions through several years in a large herd population are presented.

\section{Materials and Methods}

The objectives of the present study were to investigate the relationship between the relative frequency of abortion (percentage, AP) and number of parity (NP), weaning to first mating interval (WSI), number of service (NS) and day of week for service (DW). The present study used more than 870,000 mating records corresponding to four years (from 2005 to 2008) of about 80,000 sows on 161 commercial farms from Spain, Portugal and Italy, monitored by PigCHAMP Pro Europa S.A. and registered with Pigchamp ${ }^{\circledR}$ software.

Each factor was categorized: four groups for NP: 1, 2, 3-6 and $\geq$ 7 parity; three groups for NS: first-serviced (FS), first-reserviced (FRS) and second-reserviced (SRS); four groups for WSI: $\leq 3,4-7$, 8-11 and 212 ; and two groups for DW: Weekday and Weekend. Data were analysed using the MIXED procedure of SAS ( $v 9.00$ ).

\section{Results}

Mean value ( \pm SEM) of AP was $0.78 \pm 0.03 \%$. The main effects studied resulted as follows: Regarding NP, mean AP in parities 1 and $\geq 6$ was higher than those in parities 2 and 3-6 ( $P=0.0001)$, showing a quadratic effect that explains the higher risks in both gilts and old sows (figure 1). A lineal effect was found for WSI, when WSI $\leq 7$ was lower than those of WSI $\geq 8(P=0.0001)$; furthermore, AP depended on DW and it was higher for matings during the weekend: 0.70 vs. $1.05 \%(P=0.0001)$. Finally the mean AP for NS increased with the number of oestrus repetition (figure 1), showing: $0.45,2.87,426 \%$ for FS, FRS, SRS respectively $(P=0.0001)$. Through the time, only year 2006 showed a lower AP (0.68) being the other years quite stable (around 0.80 all of them). Therefore factors associated with a higher AP were being gilts or old sows ( $\geq 7$ th parity), a reservice occurrence, prolonged WSI and service during the weekend.
Some interactions between the main factors studied were found. The most interesting one is probably the one between WSI and DW; sows mated $\leq 3 \mathrm{~d}$ after weaning showed no differences between weekday or weekend, while sows with normal WSI (4-7 d) showed a higher risk during weekends (0.63 vs 1.29, $\mathrm{p}<0.0001$ ), while later mated sows served 8-11 d post weaning performed better in matings during the weekend ( 1.24 vs 0.75 , $\mathrm{p}<0.0001)$.

Figure 1 - AP frequency (\%) depending on parity (left) and number of re-services (right)

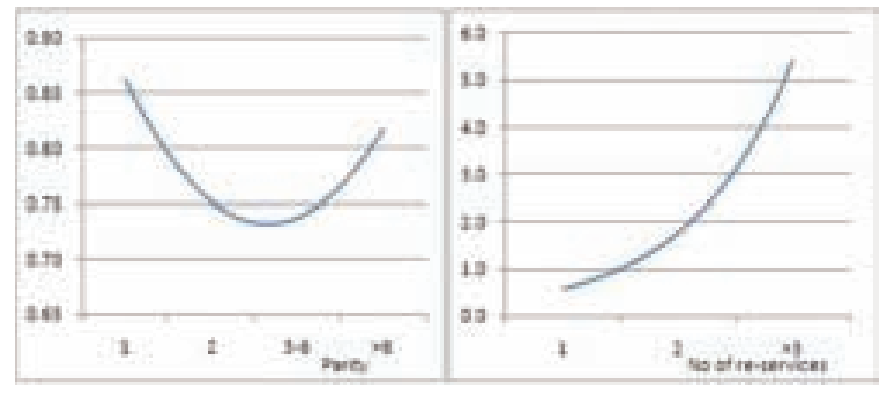

\section{Discussion}

Classical risk factors influencing.abortion and return to estrus are well known (e.g. Koketsu et al., 1997; Vargas et al., 2009). However these results add more information about the relative importance of some classical factors as NP, NS or WSI, and show a new factor as it is DW. Besides these results can be useful to define with more accuracy the objectives of every farm and to avoid those factors that are largely influencing poor performance in order to keep continuous improvement and competitiveness

\section{References}

Koketsu, Y, Dial, GD, King, VL. 1997. Theriogenology 47: 1347-63.

Vargas, AJ, Bernardi, ML, Bortolozzo, FP, Mellagi, AP, Wentz, I. 2009. Prev Vet Med. 89: 75-80. 


\title{
0.179
}

\section{Anaemia in the hyperprolific sow: Effect of injectable iron administration and relation with fattening score}

\author{
Vincent Auvigne $^{1}$ Hugues Perrin ${ }^{2}$ Arlette Laval ${ }^{2}$ Bénédicte Bertucat ${ }^{3}$ Valérie Normand ${ }^{4}$ \\ 1. Ekipaj, Pozuelo de Alarcón, Spain; 2. ENVN, Nantes, France; 3. Ceva Santé Animale, Libourne, France; 4. Porc.Spective, Pontivy, France
}

\section{Introduction}

Although anaemia in sows is less studied than in piglets, it can be supposed that it is a key factor of the sow's health status. The aim of this study is to describe levels of anaemia observed in hyperprolyfic farms in France and to study the effect of injectable iron (GLEPTOSIL ${ }^{\circledR}$ ) administration during gestation.

\section{Materials and Methods}

The study was carried out in 2009 in seven hyperprolyfic farms (13.8 to 15.6 born piglets/sow/year) with a high percentage of stillborn (> 8\%) and/or manual assistance at farrowing (> 15\%). Sows $(n=510)$ were individually randomised into two groups (Gleptoferron and Control), 7 weeks prior to farrowing after stratification according to parity and the initial haemoglobin level. Sows from the Gleptoferron group received two injections of GLEPTOSIL ${ }^{\circledR}$ at 7 and 4 weeks before farrowing $(10 \mathrm{ml}$ per injection, i.e. a total of $4 \mathrm{~g}$ of gleptoferron).

The level of anaemia was assessed by haemoglobinaemia, measured with the Hemocue ${ }^{\circledR} \mathrm{Hb} 201+$ device. (1). Kinetics was observed by measuring the levels at four stages of the reproductive cycle of each sow: seven and four weeks before farrowing, after farrowing (between 48h and 5 days) and on day 20 of lactation. Two measures of the backfat layer thickness (BFL) were carried out on each sow (four weeks before farrowing and at farrowing). Lme procedures ( $R$ 2.9.0) were used. Parity ranking and haemoglobin level at the start of the study were set as fixed effects, farm and batch as random effects.

\section{Results}

Haemoglobinaemia is linked to the parity rank and decreases all along the career of the sows. The average BFL decreases from the fourth litter onwards. Sows with high haemoglobinaemia levels are fatter (Figure 1). This relation is significant, even after adjustment for the parity $(p=0.02)$. A positive effect of the gleptoferron injection is observed 3 weeks after the first injection. However, there is an interaction between "Treatment" and "Parity" (Table 1).
Figure 1: Haemoglobin and BFL 4 weeks before farrowing

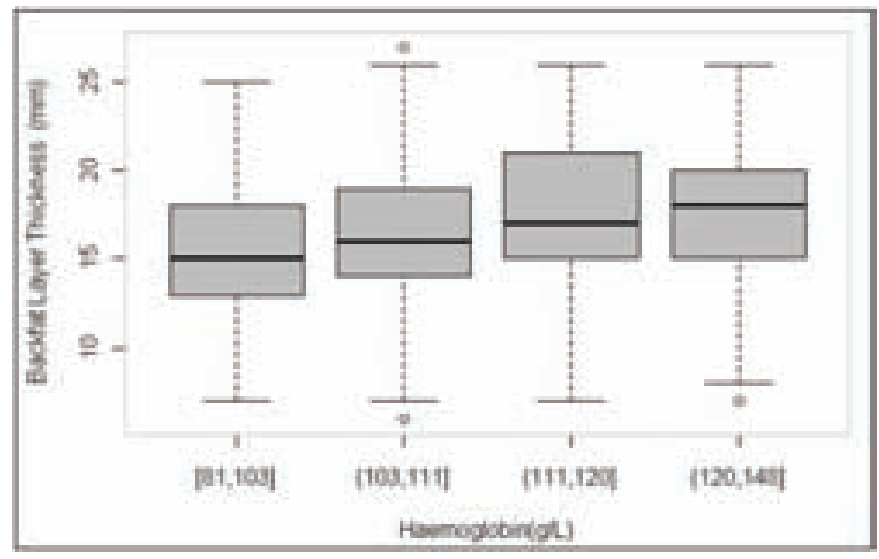

Table 1 - Treatment effect on haemoglobinaemia $(\mathrm{g} / \mathrm{L}) 4$ weeks before farrowing

\begin{tabular}{|c|c|c|c|c|c|c|}
\hline & \multicolumn{2}{|c|}{ Gleptofemon } & \multicolumn{2}{|c|}{ Control } & \multirow[b]{2}{*}{ diff. } & \multirow[b]{2}{*}{$\mathrm{p}$} \\
\hline Panity & $\mathrm{n}$ & average & $\mathrm{n}$ & average & & \\
\hline 1 & 36 & 117.3 & 43 & 116.8 & 0.5 & NS \\
\hline 2,3 & 85 & 114.4 & 83 & 110.9 & 3.5 & 0.05 \\
\hline 4,5 & 71 & 109.2 & 69 & 107.7 & 1.5 & NS \\
\hline$\geq 6$ & 63 & 107.7 & 60 & 107.6 & 0.1 & NS \\
\hline
\end{tabular}

\section{Discussion}

The drop in haemoglobinaemia with an increasing parity rank was not reported in a similar study carried out 30 years ago (2). This newly discovered relation might be due to the dramatic increase in prolificacy over the past decades. The relation between haemoglobinaemia and BFL is significant even after adjustment for the rank of parity, what strengthens its biological significance. Though, the study does not allow establishing a causal link we favour the hypothesis of an insufficient nutritional intake for both the build-up of body reserves and for restoring the haemoglobinaemia. The injection of gleptoferron allows partial compensation of the anaemia. This partial efficacy of gleptoferron could be explained by an insufficient quantity of iron injected.

\section{References}

1. von Schenck, H. et al. Clin Chem (1986).32, 526-529

2. Meissonier, E. et al. Journées Rech. Porcine (1980).31, 317-326 


\subsection{0}

\section{Sow Life ${ }^{\circledast}$ - demonstrating the quality of advisory teams and reducing sow mortality on Danish farms}

Kaj Vestergaard; Lisbeth U. Hansen; Elisabeth O. Nielsen; Hanne Nissen; Gunner Sørensen; Flemming Thorup; Heine Kristensen; Merete Studnitz; Lene K. Bruun; Verner Ruby; Michael G. Christiansen

Pig Research Centre, Danish Agriculture \& Food Council, Copenhagen, Denmark

\section{Introduction}

Sow mortality is generally neglected by pig advisers, despite the significant losses incurred. According to official reports submitted to the Danish Food Advisory Service by the Danish rendering plants, more than 156,000 sow carcases were processed during 2009 , indicating that Danish pig producers dispatched $14.4 \%$ of their sows to rendering plants. The economic output gained from reducing this number would be considerable for most herd managers in Denmark.

The reasons behind the high sow mortality have been investigated in Danish herds. The main purpose of Sow Life ${ }^{\circledR}$ was to gain an insight into the normal production strategy, to clarify the potential for improvements and then act upon this by establishing a one-year strategy for the herds.

\section{Materials and Methods}

A total of 17 farms with above average sow mortality were selected for the investigation. All farms recorded productivity levels electronically and had a herd manager who was willing to spend time together with an advisory team consisting of the herd veterinarian, a local pig production adviser and a specialist from the Pig Research Centre.

Over a 3-month period, data from each herd were analyzed, technical errors were corrected, and the advisory team together with the herd manager then decided on a strategy to reduce the sow mortality and thereby increase the economic output. The strategy included a fixed level for sow mortality. The production was closely monitored by the advisory team over the following 12 months. Minor changes to the strategy were made if necessary. If the goals of the strategy were achieved within the 12-month period, the herd manager and the advisory team would agree on new goals within the strategy.

\section{Results and Discussion}

As can be seen in Figure 1, it was possible to reduce sow mortality on all 17 farms. Figures on productivity are shown in Table 1. The three best farms increased their output by additional $€$ 59 - $€ 121$ per year sow. Three farms were unable to increase the output by reducing sow mortality, one suffered from problems in recruiting reliable personnel and the other two were put up for sale due to financial problems.
Figure 1. The black bars indicate the percentage of sows dying at the time of herd selection based on the results of 2007. The grey bars indicate the goals for sow mortality set by the herd manager together with the advisory team. The white bars show the results of 2009, after 12 months' work, of a strategy to reduce sow mortality.

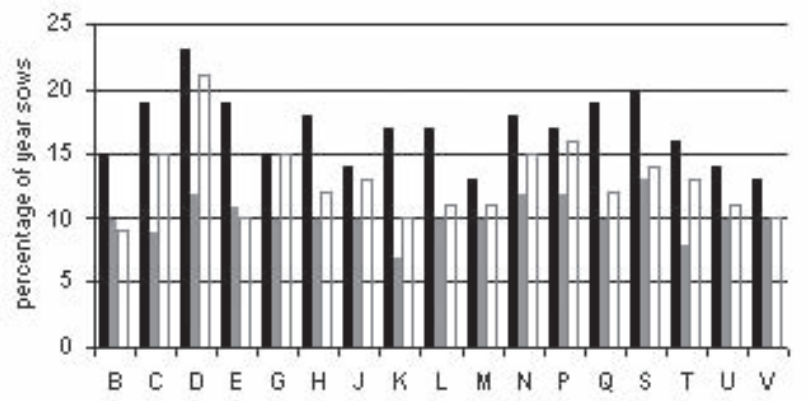

Table 1. Economic output on the three best farms before and after 12 months of Sow Life ${ }^{\circledast}$

\begin{tabular}{|c|c|c|c|c|c|c|}
\hline Farm & B & B & E & E & H & H \\
\hline Period & 2007 & 2009 & 2007 & 2009 & 2007 & 2009 \\
\hline Number of year sows & 686 & 690 & 2002 & 2015 & 669 & 644 \\
\hline $\begin{array}{c}\text { Number of live-bom } \\
\text { pigs per litter }\end{array}$ & 13.9 & 14.7 & 13.2 & 14.3 & 13.8 & 14.6 \\
\hline $\begin{array}{c}\text { Number of we aned } \\
\text { pigs per litter }\end{array}$ & 12.0 & 12.4 & 11.8 & 12.3 & 11.6 & 12.6 \\
\hline $\begin{array}{c}\text { Number of we aned } \\
\text { pigs per year sow }\end{array}$ & 25.7 & 27.2 & 25.6 & 28.5 & 26.3 & 28,9 \\
\hline \begin{tabular}{c} 
Farrowing percentage \\
\hline $\begin{array}{c}\text { Non-productive days } \\
\text { per litter }\end{array}$
\end{tabular} 193 & 88 & 86 & 92 & 90 & 91 \\
\hline $\begin{array}{c}\text { Sow mortality(\% of } \\
\text { year sows) }\end{array}$ & 15.4 & 9.6 & 18 & 11.2 & 17.9 & 11.5 \\
\hline $\begin{array}{c}\text { Additional } \\
\text { output per year sow }\end{array}$ & $\mathbf{€ 5 9}$ & $\mathbf{€ 1 2 1}$ & 10.7 & $\mathbf{€ 1 0 1}$ \\
\hline
\end{tabular}

When dealing with an issue such as sow mortality, it is important to agree on a specific goal and on how to achieve it. Despite different limits for sow welfare, 14 out of 17 farms achieved economic success through the planning process together with the advisory team.

The Sow Life ${ }^{\circledR}$ investigation has shown that by reducing sow mortality on Danish farms the herd managers would increase the output per year sow. 


\title{
0.181
}

\section{Reduction of total airborne bacteria and MRSA with an air cleaning device in a pig fattening unit - first results}

\author{
Jochen Schulz Richard Hölscher² Endong Bao ${ }^{3}$ Jörg Hartung ${ }^{1}$ \\ 1. Institute for Animal Hygiene, Animal Welfare and Farm Animal Behaviour, University of Veterinary Medicine Hannover, \\ Foundation, Hannover, Germany; 2. Hölscher + Leuschner Company, Emsbüren, Germany; \\ 3. College of Veterinary Medicine, Nanjing Agricultural University, Nanjing, China
}

\section{Introduction}

The air in modern pig fattening units contains large numbers of bacteria [1]. They give cause for concern threefold: firstly, high concentrations of airborne bacteria can impair the respiratory health of the animals. Secondly, the health of animal caretakers can be compromised by these air pollutants, and thirdly harmful bacteria can be transmitted via the exhaust air to neighboring animal houses and residential dwellings where they are supposed to cause infections and contaminations in animal and man [2]. Of particular interest are possible MRSA (methicillin resistant Staphylococcus aureus) emissions from piggeries. Therefore it seems useful to reduce the concentrations of airborne bacteria already in the animal house air.

This paper reports about experiments with a new air cleaning device in order to reduce the bacterial load incl. MRSA in the air of a commercial pig fattening unit.

\section{Materials and Methods}

The air cleaner consists of an air washer with an integrated UV (ultraviolet) irradiator (prototype developed by Hölscher \& Leuschner Comp., Emsbüren, Germany). The device was placed in the center of an animal house with 600 fattening pigs. For the investigations the air flow through the system was adjusted to approx. $7000 \mathrm{~m}^{3} \cdot \mathrm{h}^{-1}$. To warm up the UV lamps the system was started 10 min before each sampling. Two samplings (no. 1 and 2) were conducted in the 8th week, one in the 9th (no. 3) and one (no. 4) in the 10th week of the same fattening period. Airborne bacteria were sampled isokinatically and simultaneously using three impingers in the untreated air directly in front of the air cleaner and three impingers in the treated air behind the outlet of the system. Total airborne bacterial concentrations and airborne MRSA concentrations were determined as described by [3].

\section{Results}

In all samples a clear reduction of the total airborne bacteria concentrations were observed (about one log or average reduction rate and standard deviation of $93 \pm 2 \%$ (Table 1). Similar and even better results are achieved for MRSA which are reduced by nearly two log or $98 \pm 2 \%$ on average (Table 2 ).

Table 1: Total airborne bacterial concentrations (average and standard deviation) before and after treatment with an air cleaning device in a piggery

\begin{tabular}{|c|c|c|}
\hline sampling no. & untreated air [cfu/m $\left.{ }^{3}\right]$ & treated air [cfu/m $\left.{ }^{3}\right]$ \\
\hline 1 & $3.7 \times 10^{5} \pm 1.1 \times 10^{5}$ & $3.3 \times 10^{4} \pm 1.2 \times 10^{4}$ \\
\hline 2 & $2.8 \times 10^{5} \pm 1.5 \times 10^{5}$ & $1.9 \times 10^{4} \pm 1.1 \times 10^{4}$ \\
\hline 3 & $2.7 \times 10^{5} \pm 3.3 \times 10^{4}$ & $9.4 \times 10^{3} \pm 4.4 \times 10^{3}$ \\
\hline 4 & $3.1 \times 10^{5} \pm 6.6 \times 10^{4}$ & $1.2 \times 10^{4} \pm 2.0 \times 10^{3}$ \\
\hline
\end{tabular}

Table 2: Airborne MRSA concentrations (average and standard deviation) before and after treatment with an air cleaning device in a piggery

\begin{tabular}{|c|c|c|}
\hline sampling no. & untreated air [cfu/m $\left.{ }^{3}\right]$ & treated air $\left[\mathrm{cfu} / \mathrm{m}^{3}\right]$ \\
\hline 1 & $7.8 \times 10^{2} \pm 6.0 \times 10^{2}$ & $3.4 \times 10^{1} \pm 3.0 \times 10^{1}$ \\
\hline 2 & $9.1 \times 10^{2} \pm 1.2 \times 10^{3}$ & $1.8 \times 10^{1} \pm 2.1 \times 10^{1}$ \\
\hline 3 & $5.6 \times 10^{2} \pm 1.5 \times 10^{2}$ & 0 \\
\hline 4 & $7.4 \times 10^{2} \pm 4.5 \times 10^{2}$ & $4 \times 10^{0} \pm 6.0 \times 10^{0}$ \\
\hline
\end{tabular}

\section{Discussion}

The results demonstrate that the used air cleaning device is able to reduce the general bacterial load in the air of a commercial piggery significantly by $93 \%$ or about one log and MRSA by $98 \%$ or nearly two log. This air treatment technique offers the possibility to decrease bacterial loads in pig house air and bacterial emissions from pig farms. This would be beneficial for the health of the animals, the work force and the neighborhood.

\section{Conclusions}

The presented air cleaning device has obviously the potential to reduce the bacterial load in the air of piggeries significantly. Further investigations are necessary to confirm the results and to clarify the respective retention efficiency of the washer and the UV radiation unit.

\section{References}

1. Seedorf, J. et al. (1998), J. Agric. Eng. Res., 70: 97-109.

2. Hartung, J. and Schulz, J. (2008), Proc. Internat. Conf. Ragusa, Italy, 15.-17.09.08: 50 .

3. Schulz, J. and Hartung, J. (2009), Gefahrst. Reinhalt. L. 69: 348-352. 


\title{
0.182
}

\section{Livehaul accident response}

\author{
Jeremy S. Pittman ${ }^{1}$ Jennifer Woods ${ }^{2}$ \\ 1. Murphy-Brown, LLC, Waverly, VA, USA; 2. J. Woods Livestock Services, Blackie, AB, Canada
}

\section{Introduction}

A livehaul accident scene is never an easy sight, however a timely and organized response can improve a bad situation to better for the industry. Twenty-seven percent of all livehaul accidents involve swine, 80 percent of which involve market animals. ${ }^{1}$ The number of trailers carrying livestock in North America, makes a small percentage of accidents appear to occur frequently. For example, the 3 main pork processing plants in North Carolina and Virginia receive 72,000 market trucks per year. The yearly estimated number of livehaul accidents in NC and VA is 24, which is only $0.03 \%$. This paper outlines steps that should be followed when responding to a livestock accident. It is not a complete manual, but we have highlighted important areas based on principles of incident command, training documents and personal experiences.

Responsibilities of Responders - 1) Safety of the driver, all personnel and the general public; 2 ) the welfare and humane treatment of animals; 3 ) protection of property and 4) ensure a positive public perception of the industry.

Basics of Incident Command - 1) One person in command; 2) teamwork is necessary; 3 ) chain of command must be respected; 4) communication

Responders - Most emergency responders have little to no experience with handling livestock. Many transporters have little experience handling stressed livestock. Many livestock handlers have little to no experience in extraction. This requires teamwork, organization and a specially trained crew.

Local Responder Assistance - Law enforcement is responsible for public safety, traffic and crowd management. Fire departments are responsible for securing scene and vehicles, extrication if needed and water to cool animals. All others should be requested to leave the scene.

First on Scene - 1) Establish communication, exchange information with authorities, 2) establish lead person; 3) move loose animals off of the road; 4) provide assessment of scene to response team.
Rescue - It may take up to 2 hours before the actual extraction occurs, however response to the accident must occur prior to 2 hours. The average response time and clean up is 6 hours. Set up containment area and corral loose animals. Once containment area is set up, proper equipment and adequate responders are available, begin extraction of the animals inside the trailer, not before! Animals are loaded onto a transfer trailer in accordance with standard loading procedures and downer policy. All animals must be accounted for prior to leaving the accident scene. Sort boards and rattle paddles are used when loading animals. Electric prods are discouraged. Care should be taken when loading animals, take your time, show patience, composure, and use publicly accepted terminology.

Extrication - Rescue team should take ample time to assess accident scene and determine the best route/method of extrication. It must be determined if the trailer is stable to begin extrication, or a method of stabilization is determined. Never compromise the safety of the responders. Nearly $84 \%$ of trailers roll onto the right hand side ${ }^{1}$, making extraction more difficult. When trailers roll on their side, floor space is cut in half at best. Be sure to stagger work load to prevent exhaustion, accidents, injury.

Euthanasia - Safety comes first! One person, primarily a veterinarian or trained professional, is assigned all euthanasia duties for the incident. Injured animals outside the trailer are euthanized as soon as possible. Injured animals inside the trailer are euthanized as soon as they are safely accessible. Euthanize animals out of sight of the general public. Law enforcement should not be involved with euthanasia. Dead animals should be removed from the working area and covered.

Post Accident - Lead person should assess property damage, cleanup needs, removal of damaged vehicles and inspection of scene after completion. Response team completes accident reports and reviews incident and media response for recommendations on continued improvement. We learn something new every time and try to be prepared for any situation.

Summary - Ability to comply with the above processes leads to an improved accident scene, improved response time, minimal expense and a positive perception to an otherwise negative event. More information on training can be found at www. livestockhandling.net

\section{References}

1. Woods, J. 2007. Alberta Farm Animal Care. 


\title{
0.183
}

\section{Vaccination implementation and compliance in a large production system}

\author{
Jeremy S. Pittman ${ }^{1}$ Brad J. Thacker² John K. Johnson ${ }^{3}$ \\ 1. Murphy-Brown, LLC, Waverly, VA, USA; 2. Intervet/Schering-Plough Animal Health, Desoto, KS, USA; \\ 3. Iowa State University, Veterinary Diagnostic Laboratory, Ames, IA, USA
}

\begin{abstract}
Introduction- Porcine circovirus Type 2 (PCV2) in growing pigs is associated with severe systemic and respiratory disease leading to high mortality and decreased growth performance. Historically, vaccination for swine diseases has focused more on population immunity. However, the pathogenesis and epidemiology of PCV2 disease makes individual pig immunity important due in part to the ability of the virus to persist in the environment. Accordingly, any ability to measure and monitor compliance to PCV2 vaccination would be valuable for effectively controlling these diseases.
\end{abstract}

Compliance- Is defined as "the consistency and accuracy with which a patient follows the regimen prescribed by a physician or health professional." Compliance has been associated with four key factors: 1) patient's perceived susceptibility to the disease; 2) patient's perceived severity of the disease; 3 ) patient's belief that the benefits outweigh the costs; and 4) whether the patient receives "cues to action" or reinforcement of the regimen. ${ }^{1}$ The potential exists for non-compliance in any health or production protocol. ${ }^{2}$ The most detrimental type of non-compliance is complete neglect of the vaccination protocols where groups of pigs are left susceptible to disease. In most situations, compliance is based on employee adherence to the established protocols. Improving compliance is based on educating employees, monitoring the vaccination process and protocol reinforcement.

PCV2 vaccination protocol- Piglet vaccination protocols in this production system are based on vaccine efficacy, economic return and compliance. Circumvent ${ }^{\oplus} \mathrm{PCV}$, along with MycoSilencer ${ }^{\oplus} \mathrm{ONCE}$, is used on all farms, enabling sero-compliance monitoring. The first doses are given at weaning ( 21 days), when the entire farm staff is present. The second doses are given 21 days later, on the same day of the week, when the farm team is already in a "vaccination" mindset. Giving two vaccines requires multiple employees to be present, improving compliance by "witness motivation". The farm manager is mandated to be involved in the vaccination process, improving compliance by visual supervision and accountability.

Vaccine inventory management- A monthly report allows systematic monitoring of vaccine inventory, minimizes wastage, improves management of the product and reduces system vaccination cost.
Serological compliance- The PCV2 Differential ELISA test available at the lowa State University Veterinary Diagnostic Laboratory, Ames, IA, has been shown to be useful tool for compliance monitoring for the PCV2 vaccine used. ${ }^{3}$ Compliance is monitored monthly by testing 10, ten week-old nursery pigs; $<7$ positive samples would trigger the following actions: 1) detailed inventory accounting; 2 ) farm visit to review protocols; 3 ) additional serotesting to confirm previous results and to identify other non- or poorly vaccinated, "at risk" groups; and 4) personnel action such as a written warning.

Non-compliance examples- Monitoring of Farm A found $9 / 10$ and $10 / 10$ negative tests in subsequent months. Vaccine inventory review for the past 3 months discovered only $43 \%$ of required vaccine had been purchased. Farm visit revealed unacceptable practices by an employee. Manager was mandated to give all PCV2 vaccinations and subsequent testing returned to acceptable levels. Monitoring of Farm B found 10/10 pigs negative. Inventory report showed no issues. Farm investigation revealed the timing of second vaccination had drifted from 6 wks to 9 wks of age. Initial samples were collected only 2 days after the second dose and thus were negative.

Discussion- Overall compliance to health and production programs is a major concern in large integrated systems and the ability to measure, monitor and assign accountability to those programs, including vaccination protocols, is of considerable value. Our monitoring program has helped us identify several key areas of vaccine implementation that have improved compliance.

\section{References}

1. Health Belief Model. Encyclopedia of Public Health. Ed. Breslow, L., Cengage, G. 2002

2. Ohlinger, VF., Pesch, S., and Hense, I. Vet Res 31:147-148, 2000.

3. Pittman, JS., Thacker, B., Johnson, J. Proc AASV Ann. Mtg., Dallas, Texas, pp. 207-211, 2009. 


\title{
0.184
}

\section{Accuracy of dosing pumps: influence of type and maintenance}

\author{
Anne Hemonic; Isabelle Corrégé; Nicolas Berthelot
}

IFIP-Institut du porc, LeRheu, France

\section{Introduction}

Good practices when using medicines in farms are essential to get drug efficiency, to respect the maximum residue limits in meat and to prevent the development of antimicrobial resistance. In particular, water medication administered with a dosing pump requires a well-dissolved and homogeneous solution at drinkers (1), a good regulation of the flow at drinkers and a reliable equipment. The aim of this study was to evaluate the dosing accuracy at farm levels, according to type of pumps and maintenance.

\section{Materials and Methods}

Three equipments were compared:

- A hydraulic pump (HP1) used in a farm during 4 years without any maintenance.

- HP1 after maintenance procedure (HP2).

- An electric pump after maintenance procedure (EP).

Pumps were set up on an experimental pipe and tested with 9 combinations of 3 pressures (3, 1.5, 0.5 bars) and 3 water flows, matching farm values: $600 \mathrm{l} / \mathrm{h}$ simulated high fattening flows (10 drinkers set at $1 \mathrm{l} / \mathrm{min}$ and used simultaneously). 30 and $300 \mathrm{l} / \mathrm{h}$ simulated low and intermediate post-weaning flows ( 1 and 10 drinkers in use and set at $0.5 \mathrm{l} / \mathrm{min}$ ).

The dosing rate of the pumps was set at $5 \%$, as it is the most frequently used on French pig farms (2). EP was also set at $10 \%$ to check how it affected the accuracy.

During each test, after filling up the tank with water, the weights of the stock solution (W1) and of the water collected downstream (W2) were recorded every $10 \mathrm{~kg}$ (20 measures). For an expected dosing rate of $5 \%$, the observed one was calculated as: ((initial W1 - final W1) x 105)/W2.

We compared average observed dosing rates, coefficients of variation $(\mathrm{CV})$ and the percentage of the dosing rates, irrespective of the $5 \%$ margin error set by the manufacturer.

\section{Results and discussion}

At 300 and $600 \mathrm{l} / \mathrm{h}, \mathrm{HP} 1$ had average dosing rates between 4.96 and $5.10 \%$ with CV below $3 \%$ (Table 1), which is quite correct. On the opposite, at $30 \mathrm{l} / \mathrm{h}$, results lied between 1.52 and $3.75 \%$ and CV reached $69 \%$ at 3 bars. A hydraulic pump that has worked regularly without any maintenance will underdose at low flows, sometimes injecting less than one third of the expected volume.

HP2 was tested only at problem flow (30 l/h). Average dosing rates were between 5.17 and $527 \%$ with all CV below 3\%. So, maintenance clearly improved dosage accuracy.

$\mathrm{EP}$, set at $5 \%$, had correct results at 30 and $300 \mathrm{l} / \mathrm{h}(\mathrm{CV}<5 \%)$ with minor overdosing (between 2 and 5\%). But at $600 \mathrm{l} / \mathrm{h}$, EP underdosed and injected only $60 \%$ of expected volume. When the pump was set at $10 \%$, the trends were the same. So, with postweaning flows, electric pump was less accurate than hydraulic pump, though still acceptable. However, EP was not suitable with high fattening flows.

\section{Conclusion}

The regular maintenance of the pumps is crucial to get correct dosage accuracy. It consists in rinsing out the system after each use and performing yearly maintenance work on the pump.

Moreover, hydraulic pump is suitable with post- weaning and fattening flows. But, electric pump is accurate only with postweaning flows. It would be of interest to reset the rhythm of the electric pulse in order to be more compatible with higher fattening flows and to avoid underdosing.

\section{References}

1. Hémonic A., et al. (2010). J. Rech. Porcine, 42, 253.

2. Corrégé l., et al. (2008). Techniporc, 31, 17-21.

Table1: Accuracy of dosing pumps

\begin{tabular}{|c|c|c|c|c|c|c|c|c|c|c|}
\hline & flow $(I / h)$ & $30 \mathrm{I} / \mathrm{h}$ & $30 \mathrm{l} / \mathrm{h}$ & $30 \mathrm{I} / \mathrm{h}$ & $300 \mathrm{l} / \mathrm{h}$ & $300 \mathrm{l} / \mathrm{h}$ & $300 \mathrm{I} / \mathrm{h}$ & $600 \mathrm{I} / \mathrm{h}$ & $600 \mathrm{I} / \mathrm{h}$ & $600 \mathrm{l} / \mathrm{h}$ \\
\hline pump & pressure (bars) & 3 & 1.5 & 0.5 & 3 & 1.5 & 0.5 & 3 & 1.5 & 0.5 \\
\hline HP1 & Avg. dosing rate / CV (\%) & $1.52 / 69$ & $3.23 / 5$ & $3.75 / 14$ & $4.96 / 1$ & $5.02 / 2$ & $5.10 / 2$ & $5.0 / 1$ & $5.04 / 1$ & $5.09 / 2$ \\
\hline HP1 & $\%$ dos. rate $>5.25 \% /<4.75 \%$ & $0 / 100$ & $0 / 100$ & $5 / 95$ & $0 / 0$ & $0 / 0$ & $0 / 0$ & $0 / 0$ & $0 / 0$ & $10 / 0$ \\
\hline HP2 & Avg. dosing rate / CV (\%) & $5.17 / 2$ & $5.25 / 2$ & $5.27 / 1$ & not tested & not tested & not tested & not tested & not tested & not tested \\
\hline HP2 & $\%$ dos. rate $>5.25 \% /<4.75 \%$ & $5 / 0$ & $42 / 0$ & $55 / 0$ & not tested & not tested & not tested & not tested & not tested & not tested \\
\hline EP 5\% & Avg. dosing rate / CV (\%) & $5.10 / 2$ & $5.35 / 4$ & $5.39 / 3$ & $5.14 / 1$ & $5.37 / 1$ & $5.50 / 2$ & $2.87 / 23$ & $2.84 / 13$ & $2.86 / 4$ \\
\hline EP 5\% & $\%$ dos. rate $>5.25 \% /<4.75 \%$ & $20 / 0$ & $60 / 0$ & $55 / 0$ & $0 / 0$ & $85 / 0$ & $100 / 0$ & $0 / 95$ & $0 / 100$ & $0 / 100$ \\
\hline EP $10 \%$ & Avg. dosing rate / CV (\%) & not tested & not tested & not tested & $10.42 / 3$ & $10.8 / 1$ & $11.43 / 1$ & $7.52 / 25$ & $6.23 / 24$ & $6.14 / 2$ \\
\hline EP $10 \%$ & $\%$ dos. rate $>10.5 \% /<9.5 \%$ & not tested & not tested & not tested & $55 / 0$ & $100 / 0$ & $100 / 0$ & $10 / 80$ & $5 / 95$ & $0 / 100$ \\
\hline
\end{tabular}




\title{
0.185
}

\section{Vaccination against Erysipelothrix rhusiopathiae in outdoor swine production}

\author{
Fredrik Engström \\ Swedish Animal Health Service, Kristianstad, Sweden
}

\section{Introduction}

Joint disease is painful for the pig and causes $30 \%$ of all condemnations at slaughter in Sweden. Most common reasons for joint disease are infectious arthritis and osteochondrosis. Erysipelothrix rhusiophatiae is one of the most common bacterial agents involved in swine arthritis especially in outdoor pig production $(1,2)$. In comparison with conventional pig production, outdoor pigs suffer 3 times more observations for arthritis at slaughter (3). Despite this there are relatively few producers of outdoor pigs that vaccinate their slaughter pigs against erysipelas. The aim of this study was to investigate the effect of vaccinating outdoor slaughter pigs against erysipelas on the number of infectious arthritis seen at slaughter.

\section{Materials and Methods}

The trial was conducted as a prospective cohort study with vaccinated and control groups within 6 herds. Litters were split in half and groups were matched for gender and weight. In all, 903 pigs were given $2 \mathrm{ml}$ of Erysorb vet ${ }^{\circledR}$ s.C. at 8 and $11 \mathrm{w}$ of age and 905 unvaccinated pigs served as controls. Pigs were identified by ear tags and when slaughtered by tattooings. Those with acute arthritis were put to death and autopsied. Registrations for joint disease (infectious arthritis and osteochondrosis /arthrosis) were done at meat inspection. All data were collected from the abattoir and analysed by chi-square analysis.

\section{Results}

The pigs were slaughtered at two different abattoirs, 1200 and 598 pigs respectively. 10 pigs were put to death and autopsied due to acute arthritis.

Data from abattoirs and autopsy combined: Out of 903 vaccinated pigs, 33 (3.7 \%) were registered for arthritis, and 27 pigs (3.0 $\%)$ for osteochondrosis/arthrosis. 3 pigs were culled due to arthritis. Of the 905 non vaccinated pigs, 57 (6.3\%) were registered for arthritis, and 17 pigs (1.9\%) for osteochondrosis/arthrosis. 10 pigs were culled due to arthritis. Over all, the prevalence of arthritis was significantly lower among vaccinated pigs compared to among non-vaccinated pigs (Table 1).

In farms $A$ and $E$ the differences in number of observations for arthritis between groups were statistically significant. Concerning the other farms differences between groups were numerical or none.

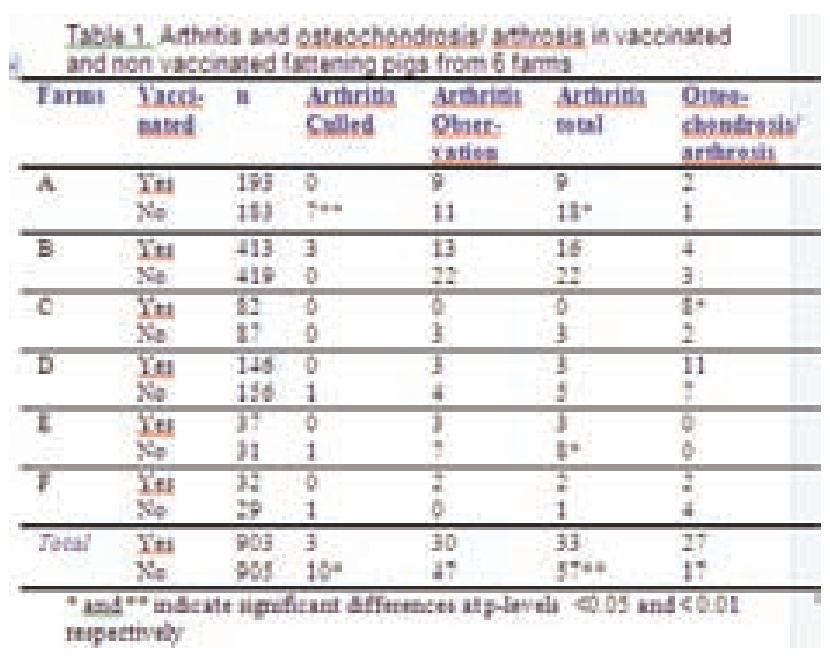

\section{Discussion}

There is doubt if vaccination has any major effect against chronic erysipelas (4). The relatively high number (33) of observations made for arthritis in the vaccinated group may be explained by arthritis due to other pathogens or chronic erysipelas in spite of vaccination. This study shows that vaccination against erysipelas in outdoor swine production significantly reduced the number of observations due to arthritis in pigs from some herds. Farmers that experience sudden onset of clinical joint disease and higher number of observations/culling due to arthritis at meat inspection are therefore advised to vaccinate against erysipelas.

\section{References}

1. Kugelberg C et al. 2001. Swed Vet J, 53, 4, 197-204

2. Friede I, Segall T. 1996.Swed Vet J, 48, 453-457

3. Heldmer E et al. 2006 Swed Vet J, 13, 13-19

4. Wood RL, Henderson LM. 2006. Erysipelas. In Leman AD et al, editors. Diseases of swine, 9th ed. Blackwell publishing; p. 629-638 


\title{
0.186
}

\section{Investigation of a case of PCV2 vaccine dilution and theft}

\author{
Gail L. Cunningham ${ }^{1}$ Mark Jacobson ${ }^{2} \mathrm{Ed} \mathrm{Krol}^{3}$ \\ 1. Boehringer Ingelheim Canada Ltd., Burlington, ON, Canada; 2. Warman Veterinary Services, Warman, SK, Canada; \\ 3. College of Pharmacy \& Nutrition University of Saskatchewan, Saskatoon, SK, Canada
}

\section{Introduction}

The use of pig vaccines to control porcine circovirus type 2 (PCV2) has proven to be both extremely effective in controlling disease and providing a return on investment to producers (1). This paper describes an unusual field case where clinical signs related to PCV2 infection were observed in a herd where pigs had seemingly been properly vaccinated with Ingelvac CircoFLEX ${ }^{\circledast}$. The investigation, triggered by the manager of the farm, revealed that the vaccine was not at fault.

Case History

Two farm sites in Western Canada were involved in this case investigation: Farm 1, a 5200-sow, farrow-to-feeder (22 kg) system and Farm 2, a 1300-sow, farrow-to-finish operation. These farms were exporting $22 \mathrm{~kg}$ feeder hogs to the United Stated for finishing. The following is an outline of the events of the case during 2009.

May 13-The manager of Farm 1 called the herd veterinarian and described irregularities in their Ingelvac CircoFLEX ${ }^{\oplus}$ vaccine on the farm. For several weeks, a number of vials out of an unused case of vaccine stored in the barn had the stoppers punctured as if they had been put on an automatic syringe. However, the punctured vials were just as full as those that appeared unopened. The vaccine in the punctured vials was also lighter in color than bottles that had an intact stopper but the change was subtle. Dilution of the vaccine was considered at the time but discounted as a probability.

May 22 - The herd veterinarian received information from the producer that the US feeding operations were seeing pigs with PCV2-associated disease in the feeders from the flow of pigs from Farm 1. Mortality was up to $7.5 \%$ in some groups. Boehringer Ingelheim, the vaccine manufacturer was contacted at that time to evaluate the integrity of the potentially altered vaccine from Barn 1.

June 1 - 8000 doses of Ingelvac CircoFLEX ${ }^{\circledR}$ are discovered missing from Barn 1. All vaccine is locked up in the main office and not put in the barn after this theft.

June 13-The office in Barn 1 has a break-in but no materials were missing. No Ingelvac CircoFLEX ${ }^{\circledR}$ was in the barn at the time. The veterinarian was then made aware that 2400 doses of Ingelvac CircoFLEX ${ }^{\circledR}$ and 1200 doses of Ingelvac MycoFLEX ${ }^{\circledR}$ had also gone missing from Farm2.

July 22- One vial of potentially altered vaccine from Barn 1 and an unopened vial of the same serial number of Ingelvac CircoFLEX $^{\circledast}$ were submitted by Boehringer Ingelheim to the College of Pharmacy and Nutrition at the University of Saskatchewan for evaluation.

\section{Materials and Methods}

The vaccine samples were analyzed using an ultraviolet-visible (UV-Vis) spectrophotometric measurement (2). The measurement technique is considered acceptable for determining the relative concentration of a protein. This technique allowed for the determination of relative protein concentrations of the original and the potentially altered sample but not for information on whether other contaminants had been introduced.

The assay utilizes absorbance readings at $260 \mathrm{~nm}$ and $280 \mathrm{~nm}$ in the following equation: [Protein $\mathrm{mg} / \mathrm{mL}]=(1.55 \times \mathrm{A} 280)-(0.76 \mathrm{x}$ A260), where " $A$ " is the absorbance reading.

The unopened sample of the same serial number as the potentially altered vaccine was used to prepare 10 to 200 fold dilutions using phosphate buffer. A calibration curve was prepared with the diluted samples of the original, $(y=1.5522 x+0.013$, $r 2=0.9655$ ), and the assay was determined to be linear in the indicated concentration range. The potentially altered sample was handled in an identical manner and analyzed by UV-Vis with the protein concentration determined using the above equation. The analysis was carried out in triplicate.

\section{Results and Discussion}

The potentially altered sample fell below the level of the calibration curve and it was concluded this sample was at least 5 times less concentrated than the unopened original sample.

It is hypothesized that the tampered bottles had $80 \%$ of the vaccine removed and replaced with liquid to make them appear full. It is estimated that this went on for a minimum of 8 weeks with 500 doses per week removed if the dilution was consistent. Between the diluted vaccine and that stolen from the barn, more than $\$ 22000$ CDN of product was stolen. The outcome of the dilution will never be fully known as the production system ceased operations shortly after this investigation.

Past investigations of cases of vaccine failure have been attributed to many causes including errors in shipping, storage, dosage and administration. Dilution may also now need to be considered when investigating perceived vaccine failure.

\section{References}

1. King, D. (2008) AASV Proc.; 159-162.

2. Bradley, JSC and J Markwell (2007) Curr. Protoc. Protein Sci.; 3.4.13.4.29. 


\title{
0.187
}

\section{Storage and detection of African Swine Fever Virus DNA in clinical material collected on FTA cards}

\author{
Jovita Fernández-Pinero; Carmina Gallardo; Ana Robles; Gómez Concepción; Alejandro Soler; Marisa Arias \\ Community Reference Laboratory for African Swine Fever, CISA-INIA, Madrid, Spain
}

\section{Introduction}

African Swine Fever (ASF) is a highly contagious disease that remains endemic in large parts of Sub-Saharan Africa and in Sardinia, producing great economic losses. In 2007, ASF emerged in the Caucasus for the first time, and has since spread to several countries in the region, being a major epidemic threat to neighbouring areas. As there is no vaccine available, rapid diagnosis of the disease is of outmost importance to contain its spread. Diagnosis is usually performed in equipped labs, although new tools for the on-site ASF virus (ASFV) detection are being developed. Key points of an accurate diagnosis include: appropriate collection and rapid convenient transport of suspicious samples to the lab, storage of samples in adequate conditions, and execution of suitable diagnostic techniques.

FTA cards (Whatman) are made of special filter paper that contains a chemically treated fiber matrix. It is described that once a biological sample is applied to the FTA card, chemicals rapidly lyse cells, inactivate proteins and stabilize the DNA. This kind of cards are described for the simple collection and storage of clinical samples at room temperature, allowing long-term stability and further detection of present DNA even for years (www. whatman.com).

\section{Materials and Methods}

Fresh EDTA-blood and serum samples collected at different days from experimentally ASFV infected pigs were applied to FTA cards. They were allowed to completely dry and subsequently stored at room temperature. Small disks were punched from sample area and put in a PCR plate well. Optimisation of the fast procedure for viral DNA purification on FTA cards was carried out to avoid cross contamination and to afterwards incorporate the PCR mix directly to the washed and dried disks. Conventional ${ }^{1}$ and real-time $P C R^{2,3}$ were run according to SOPs established in the lab (www.asf-referencelab.info). For a comparative study of the system convenience, aliquots of fresh collected blood and serum samples were also processed as usually to determine the presence of ASFV DNA following the reference PCR SOPs.

\section{Results}

Most of blood and serum samples collected on FTA cards, from different ASFV experimental inoculations, were properly identified by both conventional and real-time PCR. Some higher $\mathrm{Ct}$ values were observed when compared real-time PCR results from fresh and FTA-card samples analysis.

Analysing daily collected samples, positive results were obtained in fresh blood and serum samples from $1^{\text {st }}$ and $3^{\text {rd }}$ day postinoculation (dpi), respectively. Samples collected on FTA cards were ASFV positive from $2^{\text {nd }} \mathrm{dpi}$ in blood and $3^{\text {rd }} \mathrm{dpi}$ in serum after three years of room temperature storage.

Two ASFV positive EDTA-blood and serum samples were serially diluted in negative porcine blood and sera, respectively, and analysed for sensitivity studies, being detected as positive until $10^{-4}$ and $10^{-3}$, respectively. When FTA cards impregnated with these samples were analysed, $10^{-3}$ (real-time PCR) or $10^{-4}$ (conventional PCR) was the detection limit for blood sample, whereas $10^{-1}$ was the lowest positive dilution for sera.

\section{Discussion}

When clinical signs appear in ASFV infected animals and urgency for a rapid diagnosis is needed, the viraemia titre is sufficiently high to be detected by using FTA cards. The advantages of simple collection, transport and storage at room temperature, together with the non-infectious basis of samples collected on FTA cards, the easy treatment procedure and the potential combination with newly developed pen-side tests, show FTA cards as a possible convenient way to make real the ASFV DNA detection in basic/regional labs as a first line tool for the rapid on site diagnosis.

\section{References}

1. Agüero M., et al (2003) J Clin Microbiol, 41 (9): 4431-4434

2. Fernández J., et al (2007) Proceedings of the First Annual Meeting of Epizone, pp. 94

3. King D.P., et al (2003) J Virol Methods, 107: 53-61 


\title{
0.188
}

\author{
African swine fever: a high risk again \\ $\underline{\text { Lina Mur }}^{1}$ Beatriz Martinez-Lopez ${ }^{1}$ Marta Martinez-Aviles ${ }^{1,2}$ Jose Manuel Sanchez-Vizcaino $^{1}$ \\ 1. VISAVET, Animal Health Department, University Complutense, Madrid, Spain; \\ 2. Animal Health Research Centre. CISA-INIA, Valdeolmos, Madrid, Spain
}

\section{Introduction}

African swine fever (ASF) is one of the most important diseases of swine, due to the rapid and fatal spread causing great implications in international trade of swine products and animals (1). Since the eradication of the disease from the Iberian Peninsula in 1995, ASF has been restricted to Sub-Saharan countries and Sardinia (Italy). However, in the last decade, the epidemiology and distribution of the disease changed with the increased number of outbreaks in African countries, and the new affected areas in the European continent (Russian Federation and Caucasian countries).

The closeness of the European Union (EU) with these new affected territories and the high probability of Russian Federation to become endemic due to the presence of infected wild boars and the huge volume of unauthorized trade and transport in the area (2), convert ASF as a potential risk for EU.

In order to prevent the potential entrance and the negative impact of ASF within the EU borders, a risk assessment is necessary to detect the potential risk factors and to update the current surveillance and control programmes. This risk assessment will consider the following ways for the potential introduction of ASF in the EU: legal trade (live animals and products), illegal trade, wild animals, vectors and fomites. The study presented analyzes the risk of introduction of ASF in the EU by legal trade of live animals.

\section{Materials and Methods}

The presented model is a stochastic model to quantify the risk of introduction of ASF in EU by the legal trade of live pigs, adapted from the model used by Martinez et al. (3) to quantify the risk of introduction of Classical Swine Fever in Spain. The probability of having an outbreak of ASF in the EU by legal trade of an infected animal was estimated per month, country of origin of pigs, and destination country.

The input distributions were fitted using @Risk v5.5 (Paladise Corp.), and the model was run 10.000 times using a Monte Carlo approach with @Risk software on Microsoft Excel ${ }^{\circledR}$. The results obtained were mapped using the software ArcGIS 9.2 (ESRI $\left.{ }^{\circledR}\right)$.

\section{Results}

The results of the model indicate that the mean risk of introduction of ASFV in the EU is $5.07 \times 10^{-4}, 95 \%$ IP $\left(1.46 \times 10^{-5}, 1.85 \times 10^{-3}\right)$ which corresponds (if conditions remain constant), to approximately one outbreak in 1972 years. This risk is mainly concentrated in the countries of Slovenia, France and Belgium and in the month of May. The non-EU countries that mean a higher risk for the introduction of ASF in EU are Albania and Belarus.
Figure 1: Probability of introduction of ASFV in EU by legal trade of live animals.

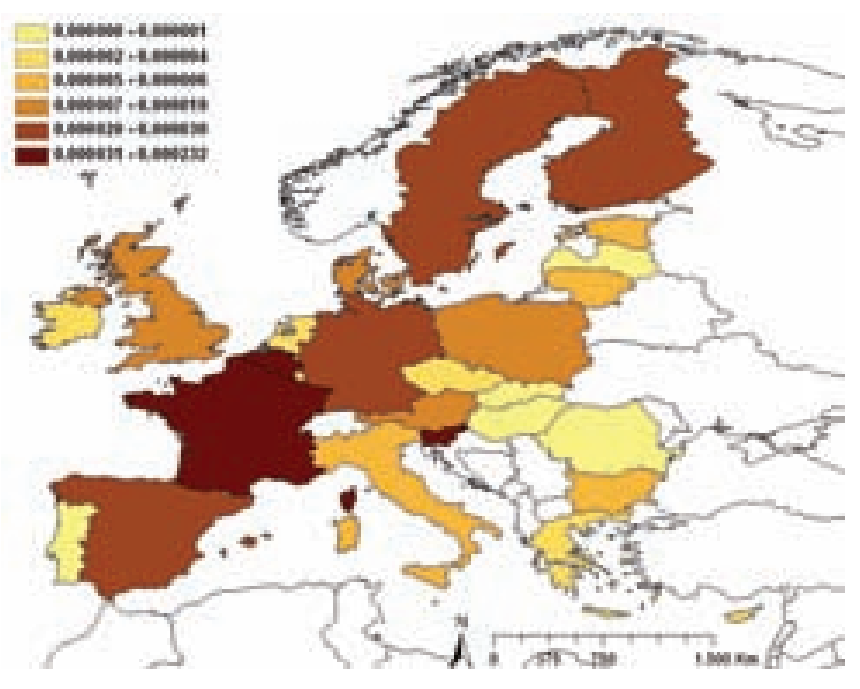

\section{Discussion}

Results from this study show that imports of live pigs do not represent a high risk for the introduction of ASFV in the EU. This result is consistent with previous epidemiological studies of the disease that identify contaminated garbage from airports and ports, as the main route for the introduction of ASFV in free areas (4). The other pathways of introduction such as illegal trade, wild boars, ticks and fomites have to be considered in further analyses in order to obtain a complete and useful risk assessment to prevent the introduction of ASF in the EU.

This work has been financed by the European Community's research project ASFRISK (FP7 KBBE-2007-1-3-05).

\section{References}

(1) Arias, M., Sanchez-Vizcaino, J.M. 2002. African Swine Fever. Trends in Emerging Viral Infections of Swine. Ed lowa State University Press: 119- 124.

(2) Beltran-Alcrudo, D. et al. 2009. Empres-Watch.FAO

(3) Martinez-Lopez, B. et al. 2009. Epid and Inf. 137, 1505-1515.

(4) Sanchez-Vizcaino, JM. 2006. African Swine Fever. Diseases of Swine, 9th Ed. Ames, lowa: 291-298. 


\title{
0.189
}

\section{Swine fever virus, a major health constraint to productivity of pig in Congo}

\author{
Tshibwabwa P. Nkambua
}

1. Laboratoire Veterinaire de Kinshasa, Kinshasa, Democratic Republic of the Congo; 2. Eau pour Tous, Kinshasa, Democratic Republic of the Congo

Porcine production represents an important component of the Congolese livestock production system, providing $12 \%$ of the value of livestock products consumed at the farm level and $48 \%$ of the cash income generated. Democratic Republic of Congo is third in Africa and seventh in the world in terms of pig population. The country has, however, benefited little from this enormous resource owing to a multitude of problems, disease being the most

important. Disease alone accounts for mortalities of 30\% in Congolese pig population. One of the diseases appears to be the African swine fever virus (ASFV). The ASFV is the main DNA arbovirus and the sole member of the family Asfarviridae. The pathogenesis of ASFV is characterized by a low infectious dose, lifelong infection, efficient transmission to pigs, and low mortality until after the first ovipositor. It causes a lethal, hemorrhagic disease in domestic pigs. Productivity losses attributable to the African swine fever virus are often substantial. A loss of US\$ 101.8 million is reported annually due to ASFV.
In a country confronted with challenges of an ever-rising human population and food shortage, such enormous losses caused by the swine fever virus are unacceptable. Therefore, swine fever virus control should receive special attention in poverty reduction strategies through improved productivity of livestock if the present and future challenges of food shortage are to be addressed.

This conference reviews extensive evidence demonstrating that swine fever virus is a major constraint to productivity of pig in Congo. It also discusses epidemiology and ecology of major swine fever virus in the country and suggests strategies for improved control in various agro-ecological zones and production system. 


\title{
0.190
}

\section{Analysis of antigenicity among various subgroups of classical swine fever virus}

\author{
Chia y. Chang ${ }^{1}$ Chin C. Huang ${ }^{1}$ Yu J. Lin ${ }^{1}$ Ming C. Deng ${ }^{1}$ Fun I. Wang ${ }^{2}$ \\ 1. Animal Health Research Institute, Taipei, Taiwan; 2. School of Veterinary Medicine, National Taiwan University, Taipei, Taiwan
}

\section{Introduction}

Classical swine fever virus (CSFV) is an enveloped positivestranded RNA virus in the genus Pestivirus of the Flaviviridae family. It is the causative agent of classical swine fever (CSF), a highly contagious disease in pigs. Major envelope glycoprotein E2 is exposed on the outer surface of the virion and induces neutralizing antibodies during infection. Four antigenic domains, $A$ to $D$, were identified at the $\mathrm{N}$-terminus of $E 2$ and formed two independent structural units; one composes of domain $B$ and $C$ and the other contains the domain A and D (1). Although CSFVs consist of a unique serotype, they can be differentiated by different patterns of reactivity with CSFV-specific monoclonal antibodies (mAbs) (2). The objective of this study was to further analyze the antigenic differences among various CSFV subgroups.

\section{Materials and Methods}

Three CSFV strains were used in this study, including a subgroup 2.1 virus (TD/96/TWN), a subgroup 3.4 virus (94.4/IL/94/TWN), and a subgroup 1.1 vaccine strain (LPC/AHRI). Several truncated and site-mutated E2 proteins of three CSFV strains were expressed using baculovirus system and tested for their reactions to a panel of mAbs against different epitopes and strains of CSFV. The reactivity was detected by indirect fluorescent assay (IFA).

\section{Results}

The antigenicities of E2 glycoproteins of different CSFV genotype subgroups can be distinguished by their variable reaction patterns between expressed $B / C$ domains of $E 2$ and a panel of $\mathrm{mAbs}$. The $\mathrm{mAbs}$ against $\mathrm{B} / \mathrm{C}$ domains were able to differentiate field viruses TD/96/TWN and 94.4/IL/94/TWN from the vaccine virus LPC/AHRI. The smallest integral antigenic unit of the $B / C$ domains was mapped to the $\mathrm{N}$-terminal 90 residues between amino acids 690 and 779 (Table 1). Site-directed mutagenesis further showed that the residues $693 \mathrm{C}, 737 \mathrm{C}, 771 \mathrm{~L}, 772 \mathrm{~L}, 773 \mathrm{~F}$ and D774 were critical for the reactivity of E2 protein with mAbs (Table 2). The D/A domains of various CSFVs were relatively conserved and recognized by all $m A$ bs against the A domain.

\section{Discussion}

Our study indicated that each of the four conserved residues, $771 \mathrm{~L}, 772 \mathrm{~L}, 773 \mathrm{~F}$ and $\mathrm{D} 774$ is important in maintaining the structural integrity of the $\mathrm{B} / \mathrm{C}$ domains. Mutation of either $693 \mathrm{C}$ or 737C abrogated the reaction with $\mathrm{mAb}$, indicating that the disulfide bond between $693 \mathrm{C}$ and $737 \mathrm{C}$ is essential for correct folding of E2 protein. Taken together, the reactivity of the epitope on $\mathrm{B} / \mathrm{C}$ domains with $\mathrm{mAbs}$ depends on both the disulfide bond and the motif 771 LLFD774 located at 34 amino acids downstream of the disulfide bond; the epitope is thus believed to be conformational and discontinuous.
Table1

\begin{tabular}{|c|c|c|c|c|}
\hline & & & $\begin{array}{c}\text { mAbs against } \\
\text { E2 }\end{array}$ & \\
\hline Domain & Strain & B/C domains & B/C domains & A domain \\
\hline & & Group 1 & Group 2 & \\
\hline & TD/96 & - & - & - \\
\hline B+C-80 & 94.4 & - & - & - \\
\hline & LPC & - & - & - \\
\hline & TD/96 & + & - & - \\
\hline B+C-90 & 94.4 & + & - & - \\
\hline & LPC & - & + & - \\
\hline & TD/96 & + & - & - \\
\hline B+C+D & 94.4 & + & - & - \\
\hline & LPC & - & + & - \\
\hline & TD/96 & - & - & + \\
\hline D+A & 94.4 & - & - & + \\
\hline & LPC & - & - & + \\
\hline
\end{tabular}

Table2

\begin{tabular}{|l|c|c|c|}
\hline \multicolumn{1}{|c|}{ Residue no. } & Mutation & mAbs against E2 & mAbs against E2 \\
\hline & & B/C domains & A domain \\
\hline None & None & + & + \\
\hline 693 & Cys to Gly & - & + \\
\hline 737 & Cys to Gly & - & + \\
\hline 770 & Glu to Gly & + & + \\
\hline 771 & Leu to Gly & - & + \\
\hline 772 & Leu to Gly & - & + \\
\hline 773 & Phe to Gly & \pm & + \\
\hline 774 & Asp to Gly & \pm & + \\
\hline & & & \\
\hline
\end{tabular}

\section{References}

1. van Rijn, P.A. et al. (1994). J. Virol. 68, 3934-3942.

2. Kosmidou, A. et al. (1995). Vet. Microbiol. 47, 111-118. 


\title{
0.191
}

\section{T cell and neutralizing antibody responses to synthetic dendrimeric peptides derived from E2 and NS2-3 proteins of classical swine fever virus}

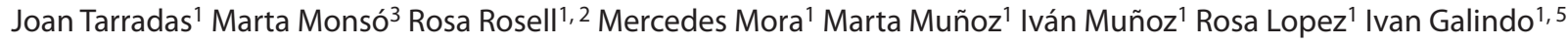 \\ Lorenzo Fraile $^{1,6}$ Mariano Domingo ${ }^{1,5}$ Beatriz G. De la Torre ${ }^{3}$ David Andreu³ Francisco Sobrino ${ }^{4}$ LLilianne Ganges $^{1}$ \\ 1. Centre de Recerca en Sanitat Animal (CReSA), Bellaterra, Barcelona, Spain; 2. Departament d'Agricultura, Alimentació i Acció Rural de la \\ Generalitat de Catalunya, Barcelona, Spain; 3. Departament de Ciències Experimentals i de la Salut, Universitat Pompeu Fabra, Barcelona, Spain; \\ 4. Centro de Biología Molecular Severo Ochoa, CSIC, Cantoblanco, Madrid, Spain; 5. Departament de Sanitat i d'Anatomia Animals, Universitat \\ Autònoma de Barcelona, Barcelona, Spain; 6. Institut de Recerca i Tecnologia Agroalimentàries (IRTA), Barcelona, Spain
}

\section{Introduction}

Classical swine fever virus (CSFV) is the causative agent of one of the most devastating porcine viral diseases; classical swine fever (CSF). There is a current need for efficient and safer marker vaccines to assist in the control of future CSF outbreaks (1).

Multimerization is a nature-mimicking strategy of antigen presentation. Indeed, pigs vaccinated with a dendrimeric peptide against foot-and-mouth disease virus, did not develop significant clinical signs upon challenge and inhibited replication and excretion of virus (2).

Here, we describe the design and construction of three dendrimeric peptide, that integrates B (three different epitopes from E2 protein) and T (from NS2-3 protein) cell epitopes, and their capability to induce $T$ cellular and neutralizing hummoral immune responses against CSFV challenge in domestic pigs.

\section{Material and methods}

The dendrimeric peptides were synthezised by Stepwise Solid Phase Peptide Synthesis on a Rink Amide ChemMatrix resin using standard Fmoc chemistry, P1: 4B (aa 694-712)_1T (14461460), P2: 4B (aa712-728)_1T and P3:4B (aa829-842)_1T. To evaluate the immune response induced by the dendrimeric peptides, four groups of six domestic pig were placed in four different section of the BSL3 animal house from CRESA (24 pigs in total). Three of these groups were inoculated twice, at day 0 and at day 21 , by intramuscular injection (i.m.) with $1.4 \mathrm{mg}$ of P1, P2 and $\mathrm{P3}$, respectively. Peptides were emulsified with Montanide V206 adjuvant (Seppic). The fourth group of animals was used as the control (non-vaccinated pigs).

Ten days after the last immunization, all pigs were challenged with 105 DICT50 of CSFV (strain Margarita) (3). The clinical signs of disease were scored daily and pigs were bled weekly to follow the specific neutralising antibody induction by neutralisation peroxidase-linked assay. Peptide specific antibodies were detected by indirect ELISA. E2 specific antibodies were detected using ELISA (IDEXX). Evaluation of the interferon gamma-producing cells was performed as described (4). The presence of CSFV RNA in serum was analyzed using the Q RT-PCR real time assay (5).

\section{Results}

Immunization of pigs with dendrimeric peptides induced dendrimer-specific antibodies in the three groups of immunized pigs, mainly in those that received dendrimer P1. These dendrimer-specific antibodies not recognize the E2 CSFV protein in ELISA, and were not neutralizing. On the other hand, the three dendrimeric constructions were able to induce IFN gamma-producing cells in immunized pigs after two doses, being the higher response also observed with dendrimer $\mathrm{P} 1$. After viral challenge, non vaccinated pigs developed fever and severe clinical signs of CSF from day 3 pi. Contrary, immunized groups showed lower score values, mainly in pigs immnunized with dendrimer P1 in two animals of this group. Induction of ELISA antibodies against E2 protein and neutralizing antibodies, as well as a decrease in viral charge at $13 \mathrm{dpi}$ were observed. On the other hand, the highest values of IFN gamma-producing cells in these animals was observed at $13 \mathrm{dpi}$.

\section{Discussion}

Multimerization of peptides, such as that provided by dendrimeric constructs, has been recognized as an effective tool for enhancing peptide immunogenicity (2). In this work, we report that inmunization of pigs with dendrimers P1, P2 and P3 induces non-neutralizing peptide-specific antibodies, as well as IFN gamma-producing cells, the latter probably due to the NS2-3 T cell epitope included in their formulation). With the three dendrimers, a significant lower score of CSF clinical signs after viral challenge was observed compared to that of naive animals. Interesetingly, two of the animals immunized with dendrimer P1 developed neutralizing antibodies and showed the highest values of IFN gamma- producing cells after CSFV challenge, suggesting that dendrimer P1 can induce efficient T cell and humoral responses that correlated with protection against CSFV.
References
1. Ganges et al., Vet J. 2008. 177:169-77
2. Cubillos et al., J Virol. 2008. 82:7223-30
3. Ganges et al.,Vaccine. 2005. 25:3741-52
4. Tarradas et al., Vet Microbiol. 2009. [Epub ahead of print]
5. Hofmann et al., J.Virol. Methods. 2005.130, 36-44 


\subsection{2}

\section{Classical Swine Fever vaccination: study of compatibility of mass vaccination in a sow herd and piglet vaccination}

Eric Brunier ${ }^{1}$ Rawat Chantong 2 Susichol Sittinuntn 2 T Sisom ${ }^{2}$ Achinee Runcharoen ${ }^{3}$

1. Ceva Animal Health Asia Pacific, Petaling Jaya, Malaysia; 2. Betagro LTC, Bangkok, Thailand; 3. Ceva Animal Health Thailand, Bangkok, Thailand

\section{Introduction:}

Classical Swine Fever (CSF) is a contagious infectious disease affecting swine classified in the A list of the OIE (International Office for Epizotics). Ceva Santé Animale has developed a live attenuated vaccine, COGLAPEST $^{\oplus}$, containing the Thiverval strain. This strain allows the vaccination of the sows during the gestation period 2 to 6 six weeks before farrow in order to enrich the colostrum with maternally derived antibodies (MDA). The timing for the piglet vaccination depends on the MDA level. The objective of this trial was to study whether, in mass vaccination conditions, a unique vaccination program can be defined for the piglets whenever the sows are vaccinated during the gestation.

\section{Material and method}

Iln a farrow to finish farm of 3000 sows in Thailand, three batches of 70 sows each have been constituted. The first batch has been vaccinated with COGLAPEST $^{\circledR}$ ( 2 ml intramuscularly) 100 days, the second batch 63 days, and the third batch 20 days before farrowing.

Different samples have been done: blood sample on the sows at farrowing and their five first born piglets at 14 days of age; colostrum after farrowing. The CSF antibody levels have been measured in all this samples by serumneutralization.

\section{Results:}

At farrowing, there was no significant difference among the three groups of sows concerning their serum VN titers (table 1). A significant difference existed between the groups vaccinated 63 or 100 days before gestation for the colostrum as well as for the piglets serum VN titers. The group vaccinated 63 days before gestation had the highest level of VN antibodies both in the colostrum and in the piglets serum.

Concerning the sows of first and second parity, there was no significant difference among the groups.

\section{Table 1}

\begin{tabular}{|l|c|c|c|c|c|}
\hline & & $\begin{array}{c}\text { Group 20 } \\
\text { days }\end{array}$ & $\begin{array}{c}\text { Group 63 } \\
\text { days }\end{array}$ & $\begin{array}{c}\text { Group 100 } \\
\text { days }\end{array}$ & $\mathbf{p}$ \\
\hline $\begin{array}{l}\text { Serology at } \\
\text { farrowing }\end{array}$ & Number animals & 70 & 70 & 70 & \\
\hline & $(\log 2)$ & $5.8 \mathrm{a}$ & $5.9 \mathrm{a}$ & $5.6 \mathrm{a}$ & $\mathrm{p}>0.16$ \\
\hline & St dev. & 1.46 & 1.59 & 1.41 & \\
\hline Colostrum & Number animals & 66 & 66 & 67 & \\
\hline & $(\log 2)$ & $9 \mathrm{ab}$ & $9.4 \mathrm{a}$ & $8.8 \mathrm{~b}$ & $\mathrm{p}<0.01$ \\
\hline & St dev. & 1.42 & 1.27 & 1.19 & \\
\hline Piglets & Number animals & 350 & 346 & 340 & \\
\hline & $(\log 2)$ & $5.8 \mathrm{ab}$ & $5.9 \mathrm{a}$ & $5.7 \mathrm{~b}$ & $\mathrm{p}=0.04$ \\
\hline & St dev. & 1.19 & 1.58 & 1.35 & \\
\hline
\end{tabular}

Correlation: (table 2). The three parameters studied - sow serology, colostrums, piglet serology - are not strongly correlated. The best correlation is between the antibody level of the sows at farrowing and of the piglets.

\section{Table 2}

\begin{tabular}{|l|c|c|c|}
\hline $\mathbf{r}=$ & serology sow & colostrum & serology piglet \\
\hline serology sow & 1 & 0.47 & 0.64 \\
\hline colostrum & 0.47 & 1 & 0.55 \\
\hline serology piglet & 0.64 & 0.55 & 1 \\
\hline
\end{tabular}

\section{Discussion and conclusion:}

The sow antibody level at farrowing is significantly higher when the vaccination is done at 63 days before farrowing compared with a vaccination done at 100 days as well as at 20 days before farrowing.

Studies done on piglets show the highest level of antibodies usually appears 50 days after vaccination (M. Launais et al.). It could be hence interesting to compare the vaccination of sows at 63 days and at 42 days before farrowing to define the best moment for the vaccination of piglets. The antibody level in piglets however is homogeneous enough in this trial to define a unique vaccination program for the piglets at 4 and 7 weeks of age (figure 1).

This trial shows that the mass vaccination against CSF with COGLAPEST $^{\circledR}$ is a possible practice that allows a common vaccination program for all the piglets.

\section{Figure 1}

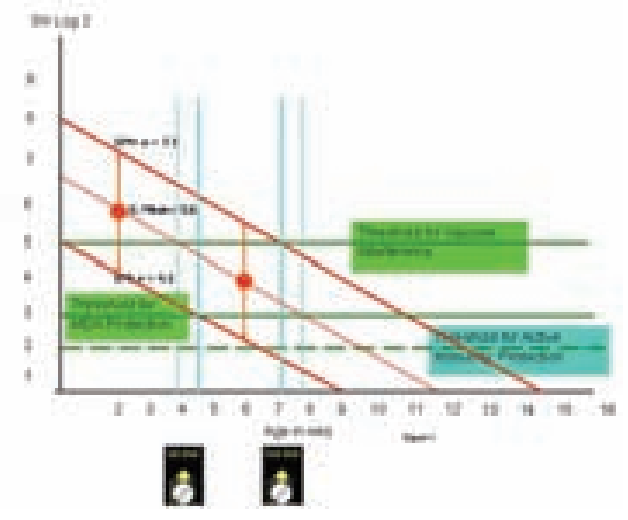

\section{References:}

1 Hog cholera virus: active immunization of piglets with the Thiverval strain in the presence and absence of colostral passive immunity - M. Launais, J.M. Aynaud, G. Corthier - Veterinary Microbiology, 3 (1978) 31-43 


\title{
0.193
}

\section{Isolation and initial characterisation of two unknown bocavirus-like viruses from swine in Northern Ireland}

\author{
John P. McKillen ${ }^{1}$ Francis McNeilly ${ }^{1}$ Catherine Duffy ${ }^{2}$ Michael McMenamy ${ }^{2}$ Irene McNair ${ }^{1}$ Bernt Hjertner ${ }^{2}$ \\ Andrena Millar2 Paula Lagan ${ }^{1}$ Karen McKay ${ }^{1}$ Brian Adair ${ }^{1}$ Gordon Allan ${ }^{1}$ \\ 1. Agri-Food \& Biosciences Institute, Belfast, UK; 2. Department of Veterinary Science, The Queen's University of Belfast, Belfast, UK
}

\section{Introduction}

The genus Bocavirus consists of three members; bovine parvovirus, canine minute virus and human bocavirus. Recently a novel porcine boca-like virus was identified in pigs in Sweden (PBo-likeV) ${ }^{1}$.

Recent research in our laboratories has isolated and identified 2 antigenically distinct novel bocavirus-like viruses in pigs ( $\mathrm{Sw}$ BoV1 and SwBoV2) from farms in Northern Ireland with clinical postweaning multisystemic wasting syndrome. Purification of the viruses followed by molecular cloning methods allowed partial sequencing of the two viruses.

\section{Materials and Methods}

Tissue and faeces isolates were grown up in primary pig kidney. Isolates that exhibited a cytopathic effect (CPE) were screened for a range of common viruses using PCR. Several PCR negative isolates were sucrose purified and put through a $1.16 \mathrm{~g} /$ $\mathrm{ml} \mathrm{CsCl}$ density gradient. An in-house antigen capture ELISA was developed and used to determine the virus-containing fractions. These were subject to electron microscopy (EM). DNA was isolated from the fractions using phenol/chloroform extraction and was electrophoresed on agarose. Bands were excised, purified, restricted with HAE III and cloned into TOPO TA vector. After transformation into E. coli and overnight growth on agar, colonies were grown in broth and plasmids were purified and sequenced with M13 primers. BLAST analysis was used to compare the sequences to GenBank. PCR primers were designed against the viral sequences and were used to produce amplicons spanning the clones. These were sequenced as before. Assembled sequences were analysed in NCBI ORF finder for presence of putative open reading frames. Nucleic acid and translated putative protein sequences were BLAST analysed and compared to known bocavirus sequences using Clustal W. Phylogenetic trees were produced from SWBoV sequences and those of known bocavirus sequences from GenBank using Mega 4. An antiswineFITC conjugate was used to screen 370 swine sera using virus infected coverslips. Monoclonal antibodies (MAbs) were produced for each of the SwBoVs.

\section{Results}

Two bocavirus-like viruses were isolated using virus culture. Small particles of about $20 \mathrm{~nm}$ could be seen by EM. After molecular characterisation they were found to be related to the genus bocavirus and were designated SwBoV1 and SwBoV2. Currently 5082 bps (SwBoV1) and 4215 bps (SwBoV2) have been sequenced. ORF analysis predicted 3 putative ORFs (1 partial
ORF for SwBoV2) with protein similarity corresponding to the NS1, NP1 and VP1/2 proteins of known bocaviruses. SwBoVs 1 and 2 share $84 \%$ homology to each other and 39-49\% homology with the other known bocaviruses, as determined by Clustal W analysis. Phylogeny of the partial DNA sequences grouped the SwBoVs with the other animal bocaviruses and that they were distinct from PBo-likeV (Fig. 1). Serological analysis showed that 32 and 33 out of 370 sera tested were +ve for SwBoV1 and 2 respectively. MAbs for the two SwBoVs do not cross-react.

Fig.1. Mega 4 bootstrapped neighbour joining phylogenetic tree of SWBoV partial sequences.

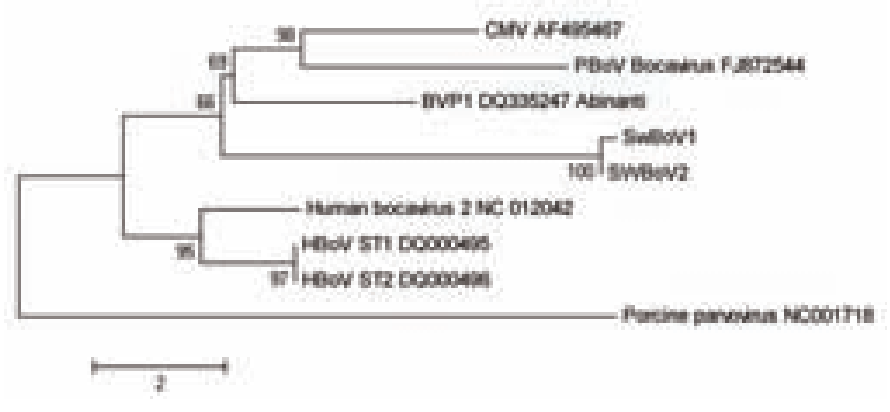

\section{Discussion}

Two-novel, antigenically distinct swine bocaviruses were isolated and partially characterised. These were related to the other known bocaviruses and distinct from another known boca-like virus, PBo-likeV from Sweden'. The prevalence, clinicopathological significance, epidemiology and genetic diversity of SwBoV within the swine population are currently being investigated.

\section{References}

1. Blomström AL., Belák S., Fossum C., McKillen J., Allan G., Wallgren P., Berg M., 2009. Virus Res. 146(1-2), 125-9 


\subsection{4}

\section{Genotype of Porcine Rubulavirus Isolates causing Blue Eye Disease}

Felipa Castro-Peralta; Francisco Quezada-Monroy; Ruben Echeveste-Garcia de Alba; Bernardo Lozano-Dubernard; David Sarfati-Mizrahi; Ernesto Soto-Priante; Refugio Cortes-Fernandez; Horacio Lara-Puente

Laboratorio Avi-Mex, S. A. de C. V., Mexico City, DF, Mexico

\section{Introduction}

Blue Eye Disease (BED) was reported at the beginning of the 80 's in farms near La Piedad Michoacan, Mexico, characterized for affecting pigs of different ages, generating nervous signs and high mortality in piglets, corneal opacity and reproductive alterations in adult animals (Stephanoet al1981). The first molecular characterization was done by Moreno et al,(1986) and named LPMV, this is considered as the prototype of the genus Rubulavirus (Santos et al, 2004). The objective of our study was to determine and compare the genetic sequence of 13 field isolates, but also to compare them with genetic information obtained from GenBank.

\section{Materials and Methods}

Viruses. 13 isolates obtained from 2000 to 2007 from 4 mexican states. The sequence for 7 viruses was gathered from GenBank (http://www.ncbi.nlm.nih.gov/) (see table 1).

Propagation. PK 15 cell monolayers were infected with each isolate and incubated at $37 \mathrm{C}$ for viral replication.

Identification by RT PCR. Amplification and transformation of genetic material (RNA) was done by RT PCR in order to obtain cDNA. From cDNA, the amplified fragment (HN) was visualized in agarose gel. Cortes et al(unpublished data).

Sequencing. The amplified fragment was purified and sequenced according to Cortes et al (unpublished data). The obtained results were compared utilizing the Vector NTI Advance program (Invitrogen Corp 2007) in order to align and generate a dendogram.

Results of viral presence were confirmed by Hemmaglutination Inhibition test (HI) and by RT PCR.

Table 1. Identification of virus samples

\begin{tabular}{|c|c|c|c|}
\hline Sample & Id GenBank & Sample & Id GenBank \\
\hline AVX1 & Lab Isolate & AVX11 & Lab Isolate \\
\hline AVX2 & Lab Isolate & AVX12 & Lab Isolate \\
\hline AVX3 & Lab Isolate & AVX13 & Lab Isolate \\
\hline AVX4 & Lab Isolate & LPHN1 & EF413172 \\
\hline AVX5 & Lab Isolate & LPHN2 & EF413173 \\
\hline AVX6 & Lab Isolate & LPHN3 & EF413174 \\
\hline AVX7 & Lab Isolate & LPHN4 & EF413175 \\
\hline AVX8 & Lab Isolate & LPHN5 & EF413176 \\
\hline AVX9 & Lab Isolate & LPHN6 & EF413177 \\
\hline AVX10 & Lab Isolate & LPHN7 & EF413178 \\
\hline
\end{tabular}

\section{Results and discussion}

Genetic homology is shown in table 2. The 13 field samples showed a high homology when compared against LPMV (ranging from $97.9 \%$ to $100 \%$ ) and from $98.0 \%$ to $100 \%$ for the 7 sequences obtained from GenBank. The consensus of the 20 sequenced data showed a high homology (98.94 to 100\%) when compared against the original LPMV sequence $\left(21^{\text {st }}\right.$ sequence shown in the dendogram).

Table 2. Homology percentage among isolates and LPVM (GenBank)

\begin{tabular}{|c|c|c|c|}
\hline Identification & Homology & Identification & Homology \\
\hline AVX1 & $99.7 \%$ & AVX11 & $99.0 \%$ \\
\hline AVX2 & $99.5 \%$ & AVX12 & $99.0 \%$ \\
\hline AVX3 & $99.4 \%$ & AVX13 & $98.0 \%$ \\
\hline AVX4 & $98.7 \%$ & LPHN1 & $99.0 \%$ \\
\hline AVX5 & $97.9 \%$ & LPHN2 & $98.0 \%$ \\
\hline AVX6 & $100 \%$ & LPHN3 & $100 \%$ \\
\hline AVX7 & $99.0 \%$ & LPHN4 & $99.0 \%$ \\
\hline AVX8 & $99.0 \%$ & LPHN5 & $99.0 \%$ \\
\hline AVX9 & $99.0 \%$ & LPHN6 & $99.0 \%$ \\
\hline AVX10 & $98.0 \%$ & LPHN7 & $99.0 \%$ \\
\hline
\end{tabular}

We could also detect the formation of 6 groups (clusters) among the isolates (figure 1). It is important to mention that individual genetic differences found in some of the 20 analized sequences are equal or higher than $2 \%$, when compared against the original sequence of LPMV, showing differing from previous report by Berg et al (1997), who reported differences of less than $2 \%$ comparing the same HN fragment.

Although we found differences up to $2.1 \%$, these differences are not expressed as in vivo antigenic changes (Echeveste et al (2009), similar to what is usually found when compared to other Paramixoviridae virus such as Newcastle disease virus (Anónimo, ANECA 2000).

Figure 1. Dendogram.

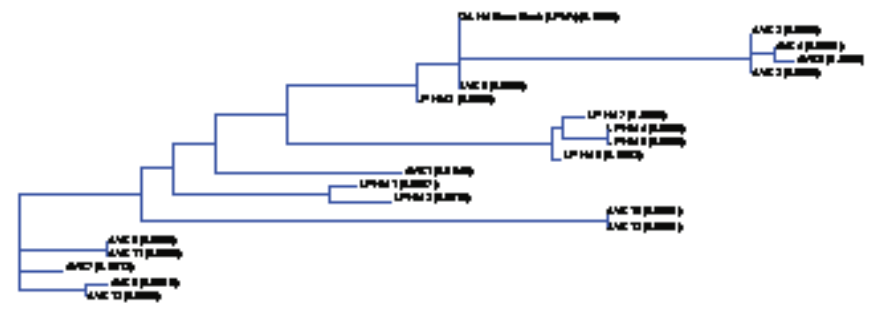

LPMV is considered the original sequence and thus used as comparative.

\section{Bibliography}

Anónimo. ANECA (2000) Enfermedades Emergentes: Enfermedad de Newcastle. Berg M. et al. Virus Genes 14:55-61 (1997). Stephano H. A. y cols. XVII AMVEC. pp46-47 (1981). Moreno J. L. et al. Arch. Virol; 91:221-231(1986).

Burleson G. F. Virology: A Laboratory Manual. Academic Press. New York. (2002) pp 86-92.

Santos-López G. et al. J. Arch. Med. Vet. v.36 n. 2 (2004).

Echeveste et al. AMVEC XLIV. p170 (2009). 


\title{
0.195
}

\section{Comparison between Virus Neutralization (VN) test, ELISA test and Hemmaglutination Inhibition (HI) test for Blue Eye Disease (BED) diagnosis}

\author{
Francisco Quezada-Monroy ${ }^{1}$ Ruben Echeveste-Garcia de Alba ${ }^{1}$ Efrain Verde-Rojo ${ }^{2}$ Bernardo Lozano-Dubernard ${ }^{1}$ \\ David Sarfati-Mizrahi ${ }^{1}$ Ernesto Soto-Priante ${ }^{1}$ Rosalba Carreon-Napoles ${ }^{3}$ Horacio Lara-Puente $^{1}$ \\ 1. Laboratorio Avi-Mex, S. A. de C. V., Mexico City, DF, Mexico; 2. Diagnosticos Clinicos Veterinarios, Mexico City, DF, Mexico; \\ 3. DPAC, FMVZ-UNAM, Mexico City, DF, Mexico
}

\section{Introduction}

Blue Eye Disease (BED) is caused by a Rubulavirus, characterized for producing nervous disorders in suckling pigs and reproductive problems in adult pigs. Serological diagnose is made through tests that quantify neutralizing or hemagglutinant antibodies (VN or $\mathrm{HI}$ tests), or based in a S/P relation (ELISA). The objective of this study was to compare the 3 diagnostic tests for BED.

\section{Materials and Methods}

Samples: Twenty three vaccinated animal sera, 17 negative sera from a BED free farm, along with 212 field sera.

Virus Neutralization (VN) Test: It was conducted according to Burlesson et al (2002), the samples were diluted, added to monolayer cell cultures and incubated at 37.0 C.

Hemmaglutination Inhibition Test (HI): Samples were inactivated and adsorbed in kaolin; bovine erythrocytes were applied according to the technique described by Ramirez et al (1996). Samples with a titer equal or higher than 1:16 were considered as positive.

Sera for ELISA were diluted 1:200 and tested according to the instruction of the kit developed by Aranda et al (unpublished data). Samples were considered positive when the $s / p$ was equal or higher than 0.4 .

All results were analyzed by descriptive statistics.

\section{Results}

Samples derived from vaccinated animals: On regards to sensitivity, VN test detected 22 samples as positive with a sensitivity of $95.65 \%$, while ELISA test detected less than $74 \%$. On regards to specificity, $\mathrm{VN}$ and $\mathrm{HI}$ resulted $100 \%$ negative with the negative samples while ELISA only 82.35\%, as shown in Table 1.

Field samples: VN test detected the highest percentage of positivity $(71.70 \%)$ compared to ELISA and $\mathrm{HI}$ tests, both with less than $47 \%$, as shown in Table 2.

Table 1. Results of sensitivity on positive samples obtained from vaccinated animals and specificity on negative samples obtained from a BED free farm.

\begin{tabular}{|c|c|c|c|c|}
\hline Test & $(+)$ & Sensitivity & $(-)$ & Specificity \\
\hline VN & $22 / 23$ & $95.65 \%$ & $17 / 17$ & $100 \%$ \\
\hline ELISA & $17 / 23$ & $73.91 \%$ & $14 / 17$ & $82.35 \%$ \\
\hline HI & $16 / 23$ & $70.83 \%$ & $17 / 17$ & $100 \%$ \\
\hline
\end{tabular}

Table 2. Results of tests on field sera samples.

\begin{tabular}{|c|c|c|}
\hline Test & Positive & Negative \\
\hline VN & $152(71.70 \%)$ & $60(28.30 \%)$ \\
\hline ELISA & $99(46.70 \%)$ & $113(53.30 \%)$ \\
\hline HI & $85(40.09 \%)$ & $127(59.91 \%)$ \\
\hline
\end{tabular}

\section{Discussion:}

In this study, VN showed the highest sensitivity in positive samples obtained from vaccinated animals, followed by ELISA and $\mathrm{HI}$ tests. This data agrees with previous reports. Hernandez et al (1992) obtained similar results with $\mathrm{VN}$ test from vaccinated animals in a disease free zone. Aranda et al (2006) found 77\% sensitivity in vaccinated animals at 30 days post vaccination using the ELISA test. Hernandez et al (1998) found that the $\mathrm{HI}$ test detects lower antibody levels compared to VN using sera derived from BED challenged pigs. Gonzalez et al (2002) using field sera samples were able to detect a higher number of positive samples using the ELISA test (58.18\%) compared to HI test (55.49\%).

\section{Conclusions.}

All serologic tests are important and have diagnostic applications. It's of capital importance to consider false positive or false negative results that every test can produce and this is why it's necessary to evaluate and compare results of samples using different tests. Not only sensitivity and specificity must rule our selection criteria, also other parameters such as Positive and Negative Predictive Values and Total Efficacy of the test in order to choose the one that best suits our requirements.

\section{Bibliography}

Burleson FG. et al Virology. A Laboratory Manual, Academic Press. p58 (2002).

Ramírez MH. et al Vet. Mex. 27(3):257-259 (1996).

Hernández JP. et al Vet. Mex. 23(3):217-222 (1992).

Aranda ME y cols. XLI AMVEC p196 (2006).

Hernández J. et al Veterinary Immunology and Immunopathology 64:367-381 (1998).

González VD. et al Trends in emerging viral infections of swine. lowa State Press. Pp55-57 (2002). 


\title{
0.196
}

\section{Efficacy of three different Modified Live Aujeszky's Disease vaccines under Asian field conditions}

\author{
Ming Yong ${ }^{1}$ Philip Lehrbach ${ }^{2}$ David Y. Ping- Cheng ${ }^{3}$ Wang Yao-Horng ${ }^{4}$ \\ 1. Pfizer Animal Health (Asia Pacific), Shanghai, China; 2. Pfizer Animal Health, Melbourne, VIC, Australia; \\ 3. Animal Technology Institute, Taipei, Taiwan; 4. College of Health Sciences, Yuanpei University, Hsinchu, Taiwan
}

\section{Introduction}

There is no official effort to eradicate Aujeszky's Disease (AD) in Asia but modified live vaccines are successfully used to control clinical disease. In recent years, due to the increased pressure of PRDC and the emergence of PCV2, there is renewed interest in looking at the efficacy of AD vaccines under field conditions in Asia. The objective of this trial was to evaluate the efficacy of three commercially available $A D$ vaccines.

\section{Material and Methods}

The trial was conducted in a commercial farm in Taiwan. 300 healthy piglets were randomly selected, ear tagged and assigned into 6 groups (see Table 1). Ten pigs were selected randomly from each group and transferred to the holding facility for laboratory challenge. Three commercial vaccines were used in the study: Suvaxyn Aujeszky IN/IM; Suvaxyn Aujeszky NIA 783 and Suvaxyn PRV gpl (3 groups, including with Suvaxyn O/W as diluent). The dose volume for all vaccines was $2 \mathrm{mLs}$. IN vaccination was done by the procedure described for administering the challenge virus.

All pigs were challenged with a local virulent strain (TNL) of AD at 28 days post-vaccination. Challenge dosage was 105 TCID50 in a $2 \mathrm{~mL}$ volume. Each animal was challenged with $1 \mathrm{~mL}$ of inoculum per nostril administered over a 30 second period. Mortality, clinical signs, serology and body weight were monitored.

Table 1. Aujeszky's disease Efficacy Trial Design

\begin{tabular}{|c|c|c|c|}
\hline Group & Vaccine & Doses/Route & Vacc.age \\
\hline 1 & Suvaxyn Aujeszky NIA3 783 & $1(\mathrm{IM})$ & 10 weeks \\
\hline 2 & Suvaxyn Aujeszky IN/IM & $1(\mathrm{IM})$ & 10 weeks \\
\hline 3 & Suvaxyn PRV gpl- & $1(\mathrm{IM})$ & 10 weeks \\
\hline 4 & Suvaxyn PRV gpl- & $1(\mathrm{IM})$ & 4 weeks \\
\hline 5 & Suvaxyn PRV gpl-(+0/W diluent) & $1(\mathrm{IM})$ & 10 weeks \\
\hline 6 & Normal Saline & $1(\mathrm{IM})$ & 10 weeks \\
\hline
\end{tabular}

\section{Results and Discussion}

Mortality and Clinical signs. Vaccinated groups survived the severe challenge ( $>80 \%$ ) while only one unvaccinated pig survived (Figure 1). Unvaccinated pigs developed high fever ( $>40 \mathrm{o}$ C) second day post challenge and showed respiratory (sneezing, dyspnoea, conjunctivitis) and nervous clinical signs (convulsion and paralysis). Post mortem findings were consistent with AD. Only mild fever was detected in a few of the vaccinated pigs and subsided quickly. The growth rate of the vaccinated and challenged pigs was not severely affected by the challenge and was comparable to vaccinated and unvaccinated unchallenged pigs on the farm, which remained seronegative ( $\mathrm{gE}$ antibody negative). In contrast, pigs challenged with virulent field strain of ADV seroconverted (gE antibody positive) 2 to 3 weeks postchallenge.
Figure 1. Survival curve of vaccinated and unvaccinated control pigs after virulent $A D$ challenge

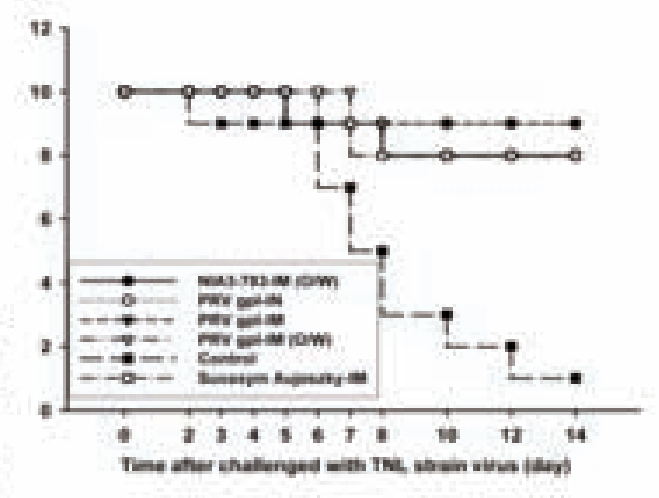

Serology All vaccinated groups showed a strong antibody response to AD 4 weeks post vaccination, with Suvaxyn Aujeszky NIA 783 stimulating the highest. Suvaxyn PRVgpl- in Suvaxyn $\mathrm{O} / \mathrm{W}$ diluent induced a higher response than the same vaccine in water.

Figure 2. SN titres following AD challenge

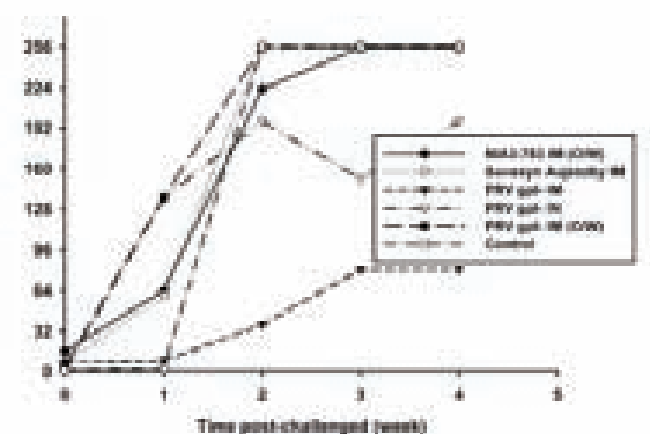

Despite the use of a very virulent challenge strain all 3 commercial vaccines were effective in reducing or preventing clinical signs, mortality and other production losses associated with AD.

\section{Acknowledgements}

Professor Yang PC and Prof Wang YH for laboratory support and compiling the results of the studies.

\section{References}

1. Vilnis, A. et al (1998) Vet Micro 62 81-96

2. Taft, A.C. (1999) AD Symp., Ploufragan, France

3. Pensaert, M.et al, (2000) Vet. Res. 31 141-145

4. Kimman, T.J. (1992) Vaccine, 10, 363-365. 


\title{
0.197
}

\section{Torque teno virus (TTV) detection in aborted and slaughterhouse collected foetuses}

\author{
Laura Martínez-Guinó ${ }^{1}$ Tuija Kekarainen ${ }^{1}$ Jaime Maldonado $^{2}$ Mario Aramouni ${ }^{1}$ Anna M. Llorens ${ }^{1}$ Joaquim Segales $^{1}$ \\ 1. Centre de Recerca en Sanitat Anima (CReSA), Bellaterra, Spain; 2. Laboratorios Hipra, S.A., 17170 Amer, Girona, Spain
}

\section{Introduction}

Torque teno virus (TTV) is a non-enveloped virus with circular single-stranded DNA genome belonging to the floating genus Anellovirus. TTV was discovered in humans and later identified in other species including pigs.

In pigs, two different genogroups have been described, genogroup 1 (TTV1) and genogroup 2 (TTV2). Swine TTV infection is ubiquitous and species-specific (1). However, its potential role on disease occurrence is yet to be defined. TTV infection has been associated with pigs affected by postweaning multisystemic wasting syndrome (PMWS) $(1,2)$ and linked with development of a porcine dermatitis and nephropathy syndrome (PDNS)-like condition in pigs co-infected with TTV1 and porcine reproductive and respiratory syndrome virus (PRRSV) (3). Faecaloral transmission route is considered the most common route of TTV dissemination but vertical transmission route may play an important role (4).

In the present work, a prospective study was designed to asses the prevalence of both swine TTVs in cases of abortions. Healthy foetuses collected from pregnant sows at slaughterhouse were used as age-matched controls.

\section{Material and Methods}

A total of 98 abortions cases from 22 different Spanish farms and 55 foetuses collected from pregnant sows (from 14 farms) at slaughterhouse were studied. Foetal age was estimated and foetuses collected were classified according first (A) or second half of gestation (B).

All foetuses were necropsied and relevant tissues were collected, pooled and submitted to DNA extraction. In order to evaluate the presence of TTV1 and TTV2, samples were analyzed by a specific polymerase chain reaction (PCR). Contingency tables were used to compare prevalence of TTV1 and TTV2 in different samples and association between gestation stage and TTV infection.

\section{Results}

Results on TTV prevalence are summarized in table 1. There were no statistically significant differences when comparing prevalence of swine TTVs by type of sample or by gestation stage.

Table 1. Prevalence of swine TTV1 and TTV2 in foetuses coming from abortions and from slaughtered sows with no reproductive problems. Foetuses were classified as from first ( $A$ ) and second half (B) of gestation.

\begin{tabular}{|l|c|c|c|c|}
\hline & Gestation & TTV1 & TTV2 & Co-infected \\
\hline Abortion cases $(n=98)$ & $A(n=10)$ & $2(20 \%)$ & $2(20 \%)$ & $0(0 \%)$ \\
\hline & $B(n=88)$ & $15(17.0 \%)$ & $27(30.7 \%)$ & $5(5.7 \%)$ \\
\hline Foetuses $(n=55)$ & $A(n=22)$ & $2(9.0 \%)$ & $8(36.4 \%)$ & $0(0 \%)$ \\
\hline & $B(n=33)$ & $4(12.0 \%)$ & $14(42.4 \%)$ & $3(9.0 \%)$ \\
\hline
\end{tabular}

\section{Discussion}

The present work represents the first description of swine TTV infection in pig foetuses at different stages of gestation. Results obtained confirm that vertical transmission is an important route of TTVs dissemination in swine. In addition, data obtained suggest that swine TTV infection is commonly present in aborted foetuses and foetuses collected from slaughtered sows. However, TTVs should not apparently be considered as infectious agents responsible for abortion occurrence.

\section{Acknowledgements}

This work was funded by grants AGL2006-02778 /GAN, TRT200600018 and CSD2006-00007 from Spanish government. T. Kekarainen was supported by Spanish Government, Ramón y Cajal program.

\section{References}

1. Kekarainen and Segales (2009) Vet. J. 180, 163-168

2. Ellis et al. (2008) Am. J. Vet. Res. 69, 1608-1614

3. Krakowka et al. (2008) Am. J. Vet. Res. 69, 1615-1622

4. Martínez-Guinó et al. (2009) Theriogenology 71, 1390-1395 


\title{
0.198
}

\section{Torque teno sus virus 1 and 2 viral loads in postweaning multisystemic wasting syndrome (PMWS) affected and non-affected pigs}

\author{
Mario Aramouni; Marina Sibila; Gerard E. Martin-Valls; David Nieto; Martí Cortey; Joaquim Segalés; Tuija Kekarainen
}

CReSA/UAB, Bellaterra (Barcelona), Spain

\section{Introduction}

Torque teno virus (TTV) is a small, non-enveloped virus with a circular single-stranded DNA genome, belonging to the family Anelloviridae, genus lotatorquevirus and widely distributed in different animal species and humans. In swine, two genetically distinct genogroups have been identified, Torque teno sus virus 1 (TTV1) and 2 (TTV2). No clear-cut pathogenic role of TTVs has been demonstrated so far. However, it has been shown that TTV2 prevalence is higher in postweaning multisystemic wasting syndrome (PMWS) affected pigs, and TTV1 has been linked to PMWS and a porcine dermatitis and nephropathy syndrome (PDNS)-like condition $(1,2,3)$. Nevertheless, the TTV viral load in diseased animals has not been investigated. Therefore, the aim of the present study was to quantify TTV1 and TTV2 viral load in serum of PMWS-affected and healthy animals using a newly developed quantitative PCR (qPCR) method.

\section{Materials and methods}

Serum samples of sixty animals ranging from 11 to 21 weeks of age included in a previous study on Porcine circovirus type 2 (PCV2) (5) were analyzed by TTV1 and TTV2 qPCRs. From them, 29 pigs were diagnosed as PMWS by histopathology and PCV2 in situ hybridization (ISH) (4), and 31 were healthy, age-matched animals.

A qPCR assay was developed using TTV1 and TTV2 D-Lux primers technology (Invitrogen). Standard curves were generated using ten-fold serial dilution (108 - 100 genomic equivalents) of purified TTV1 and TTV2 whole genome plasmids. Serum samples as well as standard dilutions were run in triplicates. The average log 10 copies of TTV1, TTV2 and PCV2 per $\mathrm{ml}$ of serum were compared globally, and between healthy and PMWS pigs, using Mann-Whitney's U and Kluskal-Wallis test. Significance was set at $\mathrm{P}<0.05$. $\mathrm{PCV} 2$ viral load was obtained from data generated in the abovementioned study (5).

\section{Results}

PCV2 log10 copies/ml from the previous published work (5) as well as TTV1 and TTV2 log10 DNA copies/ml of serum in PMWS and healthy pigs are represented in figure 1. TTV2 DNA was detected and quantified in all the PMWS affected animals but not in all healthy animals. TTV1 was detected in most but not all the animals of both groups. In general, TTV1 viral loads in serum were similar in healthy and PMWS animals, with values comparables to TTV2 viral loads in healthy animals. On the contrary, PMWS animals had significantly higher $(p<0.05)$ TTV2 viral load than healthy animals. PCV2 viral load in healthy animals was significantly lower than TTV2 loads. No significant differences were found between PCV2 and TTV2 loads in PMWS animals.

\section{Discussion}

Results from this study showed that TTV2 and TTV1 were highly prevalent among studied pigs. Moreover, the fact that TTV2 loads in serum were higher in PMWS affected than in healthy pigs would support the previously suggested association between TTV2 and PMWS (1). On the contrary, this relation was not observed for TTV1. However, results obtained in this study encourage further studies on the role of TTVs in pig diseases or disease triggering.

Figure 1: TTV1 and TTV2 log10 copies $/ \mathrm{ml}$ of serum in PMWS and healthy pigs. PCV2 viral loads of studied animals are from Grau-Roma et al.

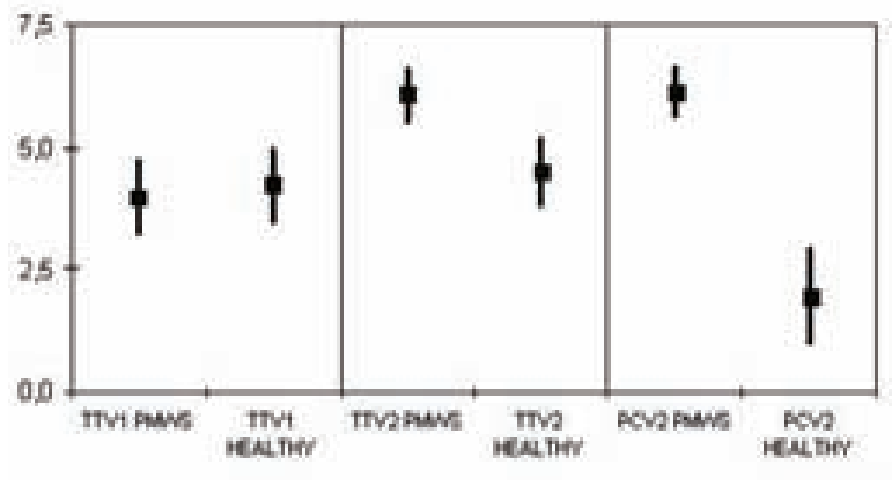

\section{Acknowledgements}

This work was funded by the grants AGL2006-02778/GAN, TRT2006-00018 and CSD2006-00007 from Spanish government. T. Kekarainen was supported by the Spanish Government, Ramón y Cajal programs.

\section{References}

1. Kekarainen et al (2006) J. Gen. Virol. 87:833-837.

2. Ellis et al (2008) Am. J. Vet. Res. 69:1608-1614.

3. Krakowka et al (2008) Am. J. Vet. Res. 69, 1615-1622.

4. Rosell et al (2000) Vet Rec. 146(2):40-43.

5. Grau-Roma et al (2009) Vet. Microbiol. 135, 272-282. 


\title{
0.200
}

\section{Simulation of the economic impact of Lawsonia intracellularis infection}

\author{
Patricia K. Holyoake ${ }^{1}$ Alison Collins ${ }^{2}$ Bruce Mullan $^{3}$ \\ 1. Wagga Wagga Agricultural Institute, Wagga Wagga, NSW, Australia; 2. Elizabeth Macarthur Agricultural Institute, Menangle, NSW, Australia; \\ 3. Department of Agriculture and Food, South Perth, WA, Australia
}

\section{Introduction}

Proliferative enteropathy (PE) is an enteric disease of pigs caused by the obligate intracellular bacterium Lawsonia intracellularis. ${ }^{1}$ Results of economic modelling conducted using the AUSPIG decision support system in 1996 estimated that PE cost pork producers up to $\$ 7.00$ per pig, with most of the cost attributed to using antibiotics in the pig diets to treat and control PE.. ${ }^{2,3}$ This model did not take into account the economic impact of subclinical PE in a herd, and the potential for reduced antibiotic costs through the use of vaccine (Enterisol ${ }^{\circledR}$ lleitis, Boehringer Ingelheim) to aid in the control of disease. This paper reports on recent simulations on the economic impact of $L$ intracellularis infection.

\section{Materials and Methods}

Growth and carcass data were from an experiment where 36 entire male pigs ( 18 challenged, 18 controls) were challenged at 63 days of age with $5.85 \times 10^{9} \mathrm{~L}$ intracellularis bacteria. Feed intake was measured on 2 consecutive days every week from 2 weeks before inoculation until 6 weeks after. Pigs were weighed each week from the day they arrived until slaughter at 106 days of age. Carcass composition data, including P2 backfat were sourced from CT scans conducted on pigs at 52, 85 and 106 days of age. Data on PE treatment and control costs were from $5 \mathrm{~L}$. intracellularis-infected farms of varying health status. The AUSPIG simulations were based on a 1000-sow farm with commercial Large White X Landrace genotype. Three infection categories (controls (non-challenged), sub-clinically and clinically-affected) were modelled. It was assumed that pigs were sold at a constant liveweight (approximately $104 \mathrm{~kg}$ ). The impact of infection at the herd level was modelled by altering the proportion of control, subclinical and clinical pigs in three simulations and including $50 \%$ females and $50 \%$ castrates. These proportions were based on results of a recent serological survey, where an average of $84.2 \%$ of pigs within herds in Australia had antibodies to $L$. intracellularis. ${ }^{4}$ The "subclinical" simulation included $20 \%$ control and $80 \%$ subclinical pigs. The "clinical" simulation included $10 \%$ control, $74 \%$ subclinical, $16 \%$ clinical and an increase in grower pig mortality of $0.5 \%$.

\section{Results}

The growth performance of pigs for the three infection herd categories is presented in Table 1. Profit (net revenue) in the control, non-infected herd was modelled at \$25.31AUD per pig. Subclinical $L$. intracellularis infection reduced net revenue by $\$ 8.33 A U D$, whilst clinical infection reduced net revenue by $\$ 13.00 A U D$, relative to the non-infected "herd". The cost of controlling PE was $\$ 2.70 A U D$ per pig.

Table 1: Results from the AUSPIG growth simulation for control (C), subclinical (SI) and clinical (CI) pigs infected with L. intracellularis.

\begin{tabular}{|l|c|c|c|c|c|c|}
\hline & Castrates & Castrates & Castrates & Females & Females & Females \\
\hline & $\mathrm{C}$ & $\mathrm{SI}$ & $\mathrm{Cl}$ & $\mathrm{C}$ & $\mathrm{SI}$ & $\mathrm{Cl}$ \\
\hline Slaughter age (d) & 147 & 147 & 192 & 147 & 147 & 189 \\
\hline Livewt. (kg) & 104.3 & 102.6 & 104.4 & 104.6 & 102.9 & 104 \\
\hline P2 (mm) & 12.1 & 12.2 & 14 & 12.6 & 12.8 & 14 \\
\hline FCR & 2.37 & 2.44 & 2.95 & 2.4 & 2.47 & 2.92 \\
\hline & & & & & & \\
\hline
\end{tabular}

\section{Discussion}

We used the AUSPIG decision support system to estimate the economic impact of $L$. intracellularis infection. This is similar to a previous estimate of subclinical PE costs. ${ }^{5}$ The impact of infection on growth and P2 backfat greatly out-weighed the PE control costs. Costs will vary among herds depending on the proportion of pigs infected, disease severity, treatment/control costs and effects on farm flow. Research is currently underway to refine the results of these simulations, including the growth response of pigs to vaccination/medication.

\author{
References \\ 1. McOrist S. et al.(1995) J Syst Bact 45:820-825 \\ 2. Black JL (1993) Aust J Agric Sc 44:541-555 \\ 3. Holyoake P. et al. (1996) Aust Vet J 73:89-92 \\ 4. Holyoake P. et al. (Accepted 2009). Aust. Vet J. \\ 5. Scholz AM. et al. (2008) IPVS P03.071
}

\section{Acknowledgements}

Financial and academic support was provided by the Australian Research Council, Boehringer Ingelheim, University of Sydney and Industry and Investment NSW. 


\title{
0.201
}

\section{Reduced muscle growth in pigs sub-clinically affected with proliferative enteropathy}

\author{
Alison M. Collins ${ }^{1}$ Shayne Fell ${ }^{1}$ Jolien van Straaten ${ }^{2}$ Nadine Bolsius ${ }^{2}$ \\ 1. Elizabeth Macarthur Agricultural Institute, Narellan, NSW, Australia; 2. University of Utrecht, Utrecht, Netherlands
}

\section{Introduction}

Pigs clinically affected with proliferative enteropathy (PE) suffer diarrhoea, reduced growth and poor feed efficiency, which can cost Australian producers as much as A\$7 per pig ${ }^{1}$. These costs do not include changes to body composition such as muscle growth. The impact of sub-clinical PE is difficult to estimate because many producers are unaware of the problem. In this study we examined the impact of sub-clinical PE in real time on body composition, muscle growth and P2 backfat depth.

\section{Materials and Methods}

Thirty six male hybrid weaner pigs (Large White $x$ Landrace) were randomly allocated at $13.8 \pm 1.0 \mathrm{~kg}$ into 2 treatments: pigs infected with L.intracellularis, and a cohort of uninfected pigs. Pigs were housed in individual pens, with strict quarantine between treatments (separate rooms). Serology and faecal PCR monitoring of pigs prior to challenge indicated that all pigs were naïve to L.intracellularis. At 9 weeks of age, one group of 18 pigs was orally challenged with $5.9 \times 10^{9}$ viable L.intracellularis extracted from a PE-affected mucosa, and the control group was inoculated with phosphate buffered saline. The consistency of pig's faeces was scored daily as either normal or diarrhoeic. Blood collected at 0, 28 and 38 days post inoculation (pi) was tested for L.intracellularis- specific IgG using an indirect fluorescent antibody test (IFAT). Faeces collected twice per week from 0 to 35 days pi was tested for L.intracellularis DNA by a conventional PCR. The body composition of pigs was determined at 14 days pre-inoculation and 21 and 42 days pi, using a Picker spiral CT scanner (Model PQ2000). Pigs were anaesthetized and cross-sectional images of the whole pig in $10 \mathrm{~mm}$ sections (reconstructed in 3 dimensions) were analysed to calculate tissue volumes of bone, muscle, fat, skin and water using described CT densities ${ }^{2}$. Muscle growth was defined as the difference in muscle volume divided by the number of days between CT scans. Tissue volumes were converted into a mass, using described tissue densities and calculated as a proportion of the total mass of the $\mathrm{pig}^{2}$. The P2 backfat was measured from the 42 days pi CT image 2 .

\section{Results}

L.intracellularis infection was demonstrated by faecal PCR and IFAT between 14 and 42 days pi in all pigs challenged with L.intracellularis. The majority of infected pigs ( $>80 \%)$ were subclinically affected, but pigs inoculated with L.intracellularis had a significantly higher probability of diarrhoea than control pigs between day 22 and 26 pi. L.intracellularis infection did not significantly alter the mean tissue weights or proportion of tissue types. However, L.intracellularis infection did significantly reduce the muscle growth in the late infection period.

The totals of CT tissue weights were accurate compared with the actual body weights of the pigs $( \pm 1 \%)$. Muscle growth was highly correlated with feed intake and weight gains of pigs $(r>0.80)$ and with the absence of diarrhoea $(r>0.72)$ throughout the trial. L.intracellularis infection did not significantly alter the P2 backfat depth.

\section{Discussion}

While sub-clinical PE did not affect body composition or P2 backfat depth, it did reduce muscle growth in experimentally challenged pigs. Animal genetics, gender, diet and pig weight all play a significant role in muscle growth. However, L.intracellularis-specific changes to the digestive and absorptive capacity of the intestine may also explain changes in muscle growth. Reduced amino acid absorption ${ }^{3}$ and reduced digestive enzyme activity ${ }^{4}$ have been described in pigs with PE.

\section{Acknowledgments}

Financial and academic support was provided by the Australian Research Council, Boehringer Ingelheim, the University of Sydney and NSW Department of Industry and Investment.

\section{References}

1. Holyoake, P. et al. (2000). Aust.Vet.J. 73, 89-92

2. Giles, L.R. et al.(2009). J.Anim.Sci. 87, 1648-58.

3. Rowan et al. (1982). Vet.Rec. 10, 306-307.

4. Collins, A.M. et al. (2009). In "Manipulating Pig Production XII", p.130, ed RJ Barneveld (Australasian Pig Sci. Association:Werribee). 


\title{
0.202
}

\section{Intestinal absorption and histomorphometry of Syrian hamsters (Mesocricetus auratus) experimentally infected with Lawsonia intracellularis}

\author{
Fabio A. Vannucci ${ }^{1}$ Elizabeth L. Borges ${ }^{2}$ Juliana S. Oliveira ${ }^{1}$ Roberto M. Guedes $^{1}$ \\ 1. Universidade Federal de Minas Gerais, Veterinary School, Belo Horizonte, MG, Brazil; \\ 2. Universidade Federal de Minas Gerais, Institute of Biological Science, Belo Horizonte, MG, Brazil
}

\section{Introduction}

L. intracellularis is an obligate intracellular bacterium and causative agent of porcine proliferative enteropathy (PPE) (1). PE has been experimentally reproduced in pigs and hamsters, and similar lesions have been observed in both species (2). So far, the physiopathological mechanisms responsible for diarrhea in PE are unclear (3). The aim of this study was to experimentally reproduce PE in hamsters ( $M$. auratus), evaluate the intestinal absorption and histomorphometric changes in infected animals.

\section{Materials and Methods}

Sixty Syrian hamsters were divided into two groups, inoculated $(n=30)$ and control $(n=30)$. The inoculum was prepared from segments of porcine intestines with PE lesions (1). Each hamster was intragastrically inoculated with $1.0 \mathrm{~mL}$ of inoculum using a gavage. On day 26 p.i., all animals were submitted to surgical procedures for evaluation of intestinal absorption. Two cannulae were introduced in the small intestine and Tyrode solution containing twice the concentration of glucose, $\mathrm{Na}+\mathrm{K}+$ and $\mathrm{Cl}$ was infused. Intestinal absorption was calculated by the difference among influx and efflux content for each 10-min interval. Gross and histological lesions were evaluated and one section was stained by IHC (1). The amount of positive antigen labeled in the sections was graded according to Table 1. Additionally, intact and well oriented intestinal villi were selected for morphometric evaluation.

\section{Results}

$\mathrm{IHC}$ results are summarized in Table 1. Five control and two inoculated hamsters died during the surgical procedure. Furthermore, three animals had no demonstrated positive antigen labeling and were excluded from the statistical analysis. The relevant results of intestinal absorption are demonstrated in Figure 1. There was no difference of villous height or crypt depth between groups. However, the infected group demonstrated positive correlation $(p<0.02)$ between the intensity of positive antigen label by IHC stain and crypt depth.

Table 1: Intensity of positive antigen label of L. intracellularis, in hamsters experimentally infected.

\begin{tabular}{|c|c|c|c|c|c|c|}
\hline Group & Grade $0 *$ & 1 & 2 & 3 & 4 & Total + \\
\hline Control & 30 & 0 & 0 & 0 & 0 & 0 \\
\hline Infected & 3 & 10 & 13 & 2 & 1 & 27 \\
\hline \multicolumn{7}{|c|}{$\begin{array}{l}\text { Grade } 0=\text { no positive antigen labeled; }(1)=\text { up to } 25 \% \text { of intestinal mucosa labeled; }(2)=25 \\
\text { to } 50 \% \text { of intestinal mucosa; }(3)=50 \text { to } 75 \% \text { of intestinal mucosa and }(4)=\text { more than } 75 \% \text { of } \\
\text { intestinal mucosa. }\end{array}$} \\
\hline
\end{tabular}

Fig. 1. Intestinal absorption. Comparison among: infected and control group $\left({ }^{*} p<0.05\right)$; control and IHC label grade $1+(a p<0.05)$; control and $\mathrm{IHC}$ label grade $2+(b p<0.05)$ and IHC label $1+$ and $2+(c p<0.05)$.

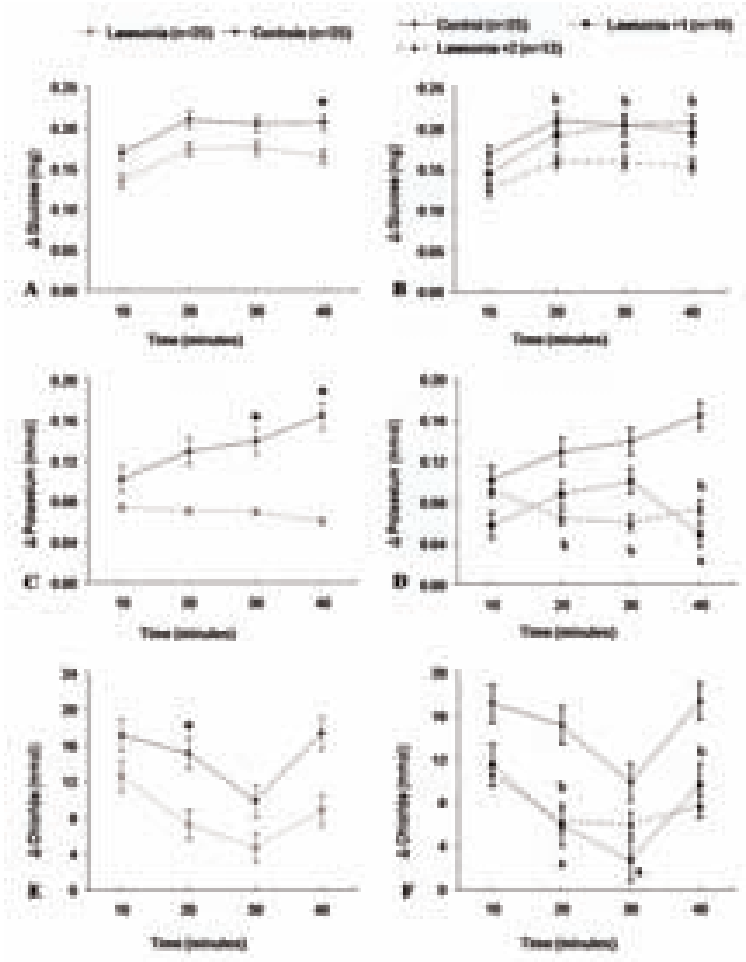

\section{Discussion}

This study described experimental reproduction of PE in hamsters which had significant reduction of intestinal absorption of glucose and electrolytes ( $\mathrm{K}+$ and $\mathrm{Cl}-$ ). We believe these findings are relevant for the understanding of $\mathrm{L}$. intracellularis pathogenesis. The poor intestinal absorption seems to be the main explanation for the lower performance of hamsters and pigs naturally affected with subclinical or clinical PE. Therefore, malabsorptive diarrhea appears to be the main process involved in physiopathology of PE diarrhea.

\section{References}

1. Guedes, R.M.C., Gebhart, C.J., Winkelman, N.A., et al., 2002. Comparison of different methods for diagnosis of Porcine Proliferative Enteropathy. Can. J. Vet. Res. 66, 99-107.

2. McOrist, S., Lawson, G.H.K., Rowland, A.C., et al. 1989. Early lesions of proliferative enteritis in pigs and hamsters. Vet. Pathol. 26, 260-264.

3. Moeser, A.J., Blikslager, A.T., 2007. Mechanisms of porcine diarrheal disease. J. Am. Vet. Med. Assoc. 231, 56-67.

\section{Acknowledgement}

CNPq - Conselho Nacional de Desenvolvimento Científico e Tecnológico 


\title{
0.203
}

\section{An alternative protocol for cultivation of Lawsonia intracellularis}

\author{
Fabio Vannucci ${ }^{1}$ Suphot Wattanaphansak ${ }^{2}$ Connie Gebhart ${ }^{1}$ \\ 1. University of Minnesota, Saint Paul, MN, USA; 2. Chulalongkorn University, Pathumwan, Thailand
}

\section{Introduction}

Lawsonia intracellularis is an obligate intracellular bacterium and causative agent of porcine proliferative enteropathy (PPE). The isolation and growth of $\mathrm{L}$. intracellularis in vitro require dividing eukaryotic cells in strict environmental conditions. The standard protocol for growth of this bacterium in monolayers has been described using a specific incubator (Tri-gas) with $83.2 \%$ nitrogen, $8.8 \%$ carbon dioxide and $8 \%$ oxygen and a temperature of $37 \mathrm{oC}(2)$. This requirement has limited the maintenance of this microorganism in vitro to only a few research institutes. The aim of this study was to describe an alternative protocol for cultivation of L. intracellularis in cell monolayers providing necessary growth conditions without Tri-gas incubators.

\section{Materials and Methods}

The L. intracellularis isolate PHE/MN1-00 previously isolated from a pig with the hemorrhagic form of PPE was used for evaluating growth under two different environmental conditions. The strain was grown in murine fibroblast-like McCoy cells, maintained in a cell culture system and stored at $-72^{\circ} \mathrm{C}$ until use, as described previously (1). The frozen bacteria were thawed and grown in cell culture for three continuous passages in specific conditions, which are described below, in order to allow the bacteria to recover from the frozen stage. The infection was monitored during every passage using immunoperoxidase staining with polyclonal antibody specific for L. intracellularis (1). After three passages, two 16-well tissue culture plates with one-dayold McCoy cells were infected with bacterial suspensions containing approximately $3.4 \times 107 \mathrm{~L}$. intracellularis organisms $/ \mathrm{ml}$. The method for quantification was performed by direct count as previously described (1). One plate was placed in an anaerobic jar and the air was evacuated by vacuum pump to $500 \mathrm{~mm} \mathrm{Hg}$ and replaced with pure hydrogen. The plate was then incubated at $37^{\circ} \mathrm{C}$ in a Tri-gas incubator for five days (2). The other plate was placed in a plastic bag (Original Space Bag ${ }^{\circledR}$ ) and hermetically closed. The air inside the bag was then removed by vacuum pump to $100 \mathrm{~mm} \mathrm{Hg}$. Afterward, the bag was inflated through a cuff connected to a gas cylinder containing 10\% hydrogen, $10 \%$ carbon dioxide, and $80 \%$ nitrogen. Finally, the bag was incubated in a conventional 37oC incubator for five days. Every 24 hours, the gas inside the bag was replaced. Carbon dioxide and oxygen percentages was monitored in both protocols using $\mathrm{CO} 2$ and $\mathrm{O} 2$ indicators (FYRITE ${ }^{\circledR}$ Gas Analyzer) at the initiation of incubation and every 24 hours during the five days of incubation.

\section{Results}

After five days, the McCoy cell monolayers in both plates were heavily infected by L. intracellularis demonstrating the success of the alternative incubation protocol. The infection rate was quantified by direct counting of the number of heavily infected cells after immunoperoxidase staining $(1,3)$. Four wells were counted for each incubation protocol. The average rate of infection of the monolayer was $78.9 \%(+1.3)$ in the Tri-gas incubator and $76.7 \%(+2.6 \%)$ in the bag incubation. The CO2 level measured between $11-12 \%$ in the Tri-gas incubator and $7.0-7.5 \%$ in the bag. The $\mathrm{O} 2$ level was $9.0 \%$ in the Tri-gas incubator in all measurements and ranged from 5.0 to $5.5 \%$ in the bag.

\section{Discussion}

Incubation in the bag provided environmental conditions for L. intracellularis to infect and multiply in the cells, similar to the published protocol (2). Based on these preliminary results, we believe this approach can be used for static cultivation of bacteria without requiring a Tri-gas incubator. This fact creates a option for in vitro studies and gives an opportunity to engage more research institutes in this area. Other isolates of L. intracellularis have been cultivated with this approach with the results being analyzed. In addition, studies using various conditions for growth and incubation in the bags are being conducted.

\section{References}

1. Guedes, RMC \& Gebhart, CJ. Vet. Microbiol. 91:135-145, 2003.

2. Lawson, GHK, et al. J. Clin Microbiol. 31 :1136-1142, 1993.

3. Wattanaphansak, S, et al. Can J Vet Res 69:265-271, 2005. 


\title{
0.204
}

\section{Effect of diarrhea on average daily weight gain in grower-finisher pigs}

\author{
Markku Johansen $^{3}$ Anders Stockmarr ${ }^{1}$ Charlotte S. Kristensen ${ }^{3}$ Poul Baekbo ${ }^{3}$ Jens Peter Nielsen ${ }^{2}$ Helle Stege $^{2}$ Ken S. Pedersen $^{2}$ \\ 1. Technical University of Denmark, Copenhagen, Denmark; 2. University of Copenhagen, Copenhagen, Denmark; \\ 3. Pig Research Centre, Danish Agriculture \& Food Council, Kjellerup, Denmark
}

\section{Introduction}

Diarrhea is an economically important disease, in growerfinishers. Pigs with diarrhea may have impaired growth and feed conversion. The objective of this study was to estimate the effect of diarrhea on the average daily weight gain in grower-finisher pigs in 5 herds with diarrhea.

\section{Materials and Methods}

A prospective cohort study was carried out in five growerfinisher herds with a history of diarrhea and seroconversion to Lawsonia intracellularis and PCV2 virus in the pigs. The herds were selected by convenience. In each herd 60 pigs from four pens were individually ear tagged. The pigs were weighed at the beginning of the study and at the end of the six-week observation period. A total of 298 pigs, all approximately 12 weeks old, were included in the study during the first week in the growerfinisher barn. No routine flock medication against diarrhea was used in the observation period. Faecal samples from each pig were collected in week 1, 3, 5 and 7 during the observation period and scored visually. A faecal score was assessed on 0-3 scale (normal=3, loose $=2$, fluid=1, fluid+discoloration=0). Diarrhea was defined as faecal score $\leq 1$.

The impact of diarrhea on average daily weight gain was estimated in a linear regression model with average daily weight gain (ADWG) as a dependent variable. Diarrhea at least once, diarrhea more than once, and start weight were included as systematic effects. The herd and pen were included as random effects. The analysis was carried out using the SASC-system, version 9.13, using Proc Mixed.

\section{Results}

The proportion of pigs with diarrhea in the observation period ranged from $3-28 \%$ in the five herds. A total of $9.7 \%$ of the pigs had diarrhea on one or more occasions during the six-week observation period. The proportion of pigs having diarrhea on one occasion was $7.4 \%$ while $23 \%$ had diarrhea on two or more occasions. The variation in prevalence during the observation period is shown in Figure 1. A total of 26 pigs had missing observations. The statistical analysis including 272 pigs showed a significant interaction between start weight and diarrhea on one or more occasions. $(\mathrm{P}<0.05)$. This means that the effect of diarrhea increases as the start weight increases. There was an additional effect of diarrhea more than once $(p<0,0001)$. The start weight $(P<0.0001)$, and squared start weight $(P<0.0001)$ had significant effects on the average daily gain. The results are shown in Figure 2.
The graph in Figure 2 indicates that the average effect of having diarrhea (fecal score $\leq 1$ ) on one or more occasions was a $118 \mathrm{~g} /$ day reduction of growth rate in the observation period for a pig with a start weight of $35 \mathrm{~kg}$. Pigs in the same weight category with a fecal score $\leq 1$ on two or more occasions had an average growth rate reduction of $477 \mathrm{~g} /$ day in the observation period.

Figure 1. Prevalence of diarrhea (faecal scores $\leq 1$.) in five growerfinisher herds

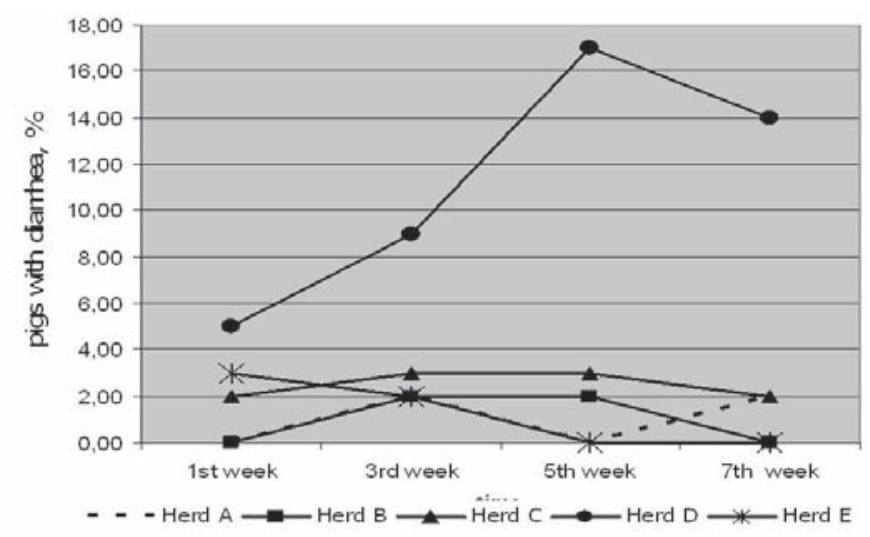

Figure 2. Effects of start weight and diarrhea on the estimated average daily weight gain

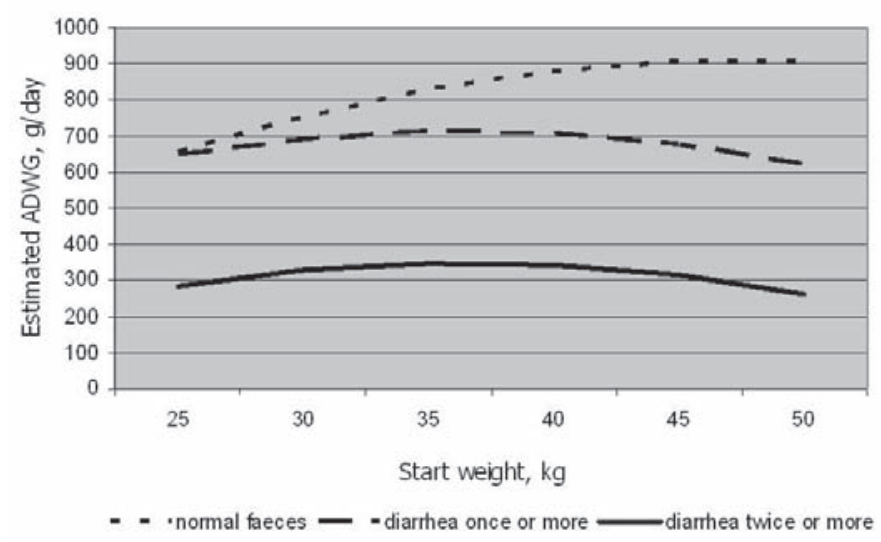

\section{Discussion}

It should be kept in mind that this study was based on a small number of herds and that most of the pigs with diarrhea originated from one herd only (fig 1). 


\title{
0.205
}

\section{Antimicrobial susceptibility of Belgian Brachyspira hyodysenteriae isolates}

\author{
Philip Vyt \\ Medic Lab, Diagnostic Laboratory, Aalst, Belgium
}

\section{Introduction}

Swine dysentery, a muco-hemorraghic colitis caused by Brachyspira hyodysenteriae, is responsible for substantial economic losses in affected herds. The prevalence of B. hyodysenteriae in Belgium has increased the last ten years1. Until 2004, isolates were characterized as susceptible to pleuromutilins. Since the appearance of pleuromutilin-resistant strains elimination attempts at farm level have become compromised. Susceptibility testing prior to the start of treatment protocols is now inevitable. The present study summarizes the antimicrobial susceptibility of B. hyodysenteriae isolates during 2008 and 2009.

\section{Materials and methods}

Sixty and 58 isolates of B. hyodysenteriae were collected for susceptibility testing in 2008 and 2009 respectively. Isolates were recovered from 49 and 52 different farms in 2008 and 2009. The strains were isolated on modified Trypticase Soy Agar (TSJ-BJ) with $5 \%$ sheep blood from feces or colon contents submitted for diagnostic purposes 1,2. Susceptibility was determined using the agar dilution technique against valnemulin, tiamulin, tylvalosin, lincomycin and doxycycline as described earlier 1,2. The tested concentrations $(\mu \mathrm{g} / \mathrm{ml})$ ranged in two-fold dilutions from 0.015 to 2 for valnemulin, 0.03 to 16 for tiamulin, 0.25 to 32 for tylvalosin, 2 to 128 for lincomycin and 0.062 to 16 for doxycycline.

\section{Results}

Table 1 outlines the results of 101 isolates from different farms. Similar results were obtained in 2008 and 2009. A high proportion of isolates with decreased susceptibility was observed for pleuromutilins: $40-45 \%$ had MIC for valnemulin >= $2 \mu \mathrm{g} / \mathrm{ml}$; $45-50 \%$ had MIC > = 1 for tiamulin. Results for both molecules were similar: a high MIC for tiamulin coincided with a high MIC for valnemulin. Forty isolates (40\%) had decreased susceptibility for both pleuromutilins. Only three isolates had a lower MIC for tiamulin compared to valnemulin, once with more than a twofold dilution.

For tylvalosin, MIC50 was $32 \mu \mathrm{g} / \mathrm{ml}$. Two-third of the isolates was categorized as susceptible for this molecule $(<=32 \mu \mathrm{g} / \mathrm{ml})$. In the case of lincomycin, 15 to $18 \%$ of the isolates were considered susceptible3. Twenty-eight isolates showed decreased susceptibility for both tylvalosin and lincomycin. Seventeen isolates, resistant to both pleuromutilins, had an MIC for tylvalosin of $<=32 \mu \mathrm{g} / \mathrm{ml}$. All four antimicrobials were considered ineffective in 21 isolates. Considering doxycyline, $61 \%$ had an $\mathrm{MIC}<=4 \mu \mathrm{g} /$ $\mathrm{ml}$ and were considered susceptible.

For the 17 farms from which two or more isolates were tested, susceptibility patterns of the isolates were within one dilution for all antimicrobials.
Table 1: MIC50, MIC90 $(\mu \mathrm{g} / \mathrm{ml})$ and susceptibility of B. hyodysenteriae isolates for valnemulin (V), tiamulin $(T)$, tylvalosin $(A)$, lincomycin $(L)$ and doxycycline (D)

\begin{tabular}{|l|c|c|c|c|c|c|}
\hline $\mathbf{2 0 0 8}$ & & V & T & A & L & \\
\hline $\mathrm{n}$ & & 49 & 49 & 49 & 49 & \\
\hline MIC50 & & 2 & 4 & 32 & 64 & \\
\hline MIC90 & & $>2$ & $>4$ & $>32$ & $>128$ & \\
\hline susceptible & $\mathrm{n}$ & 27 & 27 & 33 & 9 & \\
\hline & $\%$ & 55 & 55 & 67 & 18 & \\
\hline reduced susceptibility & $\mathrm{n}$ & 22 & 22 & 16 & 40 & \\
\hline & $\%$ & 45 & 45 & 33 & 82 & \\
\hline 2009 & & $\mathrm{~V}$ & $\mathrm{~T}$ & $\mathrm{~A}$ & $\mathrm{~L}$ & $\mathrm{D}$ \\
\hline $\mathrm{n}$ & & 52 & 52 & 52 & 52 & 33 \\
\hline MIC50 & & 0.25 & 0.5 & 32 & 64 & 4 \\
\hline MIC90 & & $>2$ & $>4$ & $>32$ & $>128$ & 8 \\
\hline susceptible & $\mathrm{n}$ & 31 & 26 & 36 & 8 & 20 \\
\hline & $\%$ & 60 & 50 & 69 & 15 & 61 \\
\hline reduced susceptibility & $\mathrm{n}$ & 21 & 26 & 16 & 44 & 13 \\
\hline & $\%$ & 40 & 50 & 31 & 85 & 39 \\
\hline
\end{tabular}

\section{Discussion}

The present data show the widespread distribution of decreased susceptibility to pleuromutilins in Belgian B. hyodysenteriae isolates. The decrease in susceptibility to both valnemulin and tiamulin reflects the problems on farm when treating animals infected with these strains (P. Vyt, personal observations). On those farms a complete susceptibility panel to all antibiotics may reveal other treatment options. Although limited sensitivity data was available for tylvalosin, recent $B$. hyodysenteriae isolates from Spain4 had lower MIC values than in Belgium. Doxycycline, based on a limited number of isolates, could be used in affected farms. These data demonstrate that in nearly half the cases with pleuromutilin resistance tylvalosin could be an alternative treatment.

\section{Literature}

1. Vyt, P. and Hommez, J. (2006), Flem. Vet. J.,75: 279.

2. Rasbäck et al (2005), Vet. Microb. 109: 229.

3. Rønne H.and Scanzer J. (1990), Proceedings IPVS Lausanne, Switzerland, 126.

4. Hidalgo et al, (2009). Proc 5th Int Conf on Spirochaetal Infect in Animals and Humans, Leon, Spain, abstract 20. 


\title{
0.206
}

\section{Comparison of three commercial vaccines for the control of Salmonella spp in pigs}

\author{
Juan M. Palacios ${ }^{1}$ Rosalba Carreon ${ }^{2}$ Celene Cruz ${ }^{2}$ \\ 1. Intervet Schering Plough AH, Huixquilucan, MEX, Mexico; 2. Veterinary Faculty UNAM, Mexico, DF, Mexico
}

\section{Introduction}

Salmonellosis is one of the most common infections in swine production, leading to important economic loss due to mortality, morbidity and the cost of non-specific treatment. Attenuated vaccines have been the main immunological basis for the control of these infectious diseases. In general, live attenuated vaccines induce a superior immunological response than the inactivated ones. Vaccination significantly reduces clinical signs, and the colonization and excretion of Salmonella sp. The available bacterin vaccines stimulate a strong humoral response providing protection against the septicemic phase 1,2. The aim of this study was to investigate the effect of three different products on the shedding of Salmonella spp in feces, and on standard production parameters.

\section{Materials and Methods}

The study was carried out on a farm in Mexico operating a continous production system. From weekly batches, pigs were randomly selected, individually identified and assigned to one of four treatment groups, each of 20 piglets: three vaccinated with different commercial vaccines, and an unvaccinated control group. Group 1: unvaccinated controls; Group 2: a modified vaccinea from $\mathrm{S}$. cholerae suis strain SC-54, for intranasal use in a single dose at one day old; Group 3: modified vaccineb against S. cholerae suis, for oral administration in a single dose at three weeks of age; Group 4: a bacterinc vaccine against S. cholerae suis and S.typhimurium, for parenteral administration in two doses at 3 and 5 weeks of age.

Rectal swabs were taken 5, 10 and 21 days post-vaccination for bacteriological analysis. Serum samples were collected at 70, 109 and 154 days of age for the detection of S. cholerae suis antibodies by ELISA (HerdCheck ${ }^{\mathrm{TM}}$, Idexx, EE.UU). Pigs were weighed at birth, 21, 70, and 105 days old and when sold. Average daily weight gain (ADG) and feed efficiency were calculated for a series of growing periods. Data were evaluated by variance analysis with a Tuckey test using SPSS 15.0 statistics software.

\section{Results}

Graph 1 shows S.cholerae suis fecal shedding after vaccination.

Chart 1 shows the production parameters (ADG by period, and feed efficiency), morbidity (signs of digestive disturbance) and mortality.

aBoheringer-IngelheimVetmedica,blntervet-Schering-Plough

c Novartis Animal Health Inc.

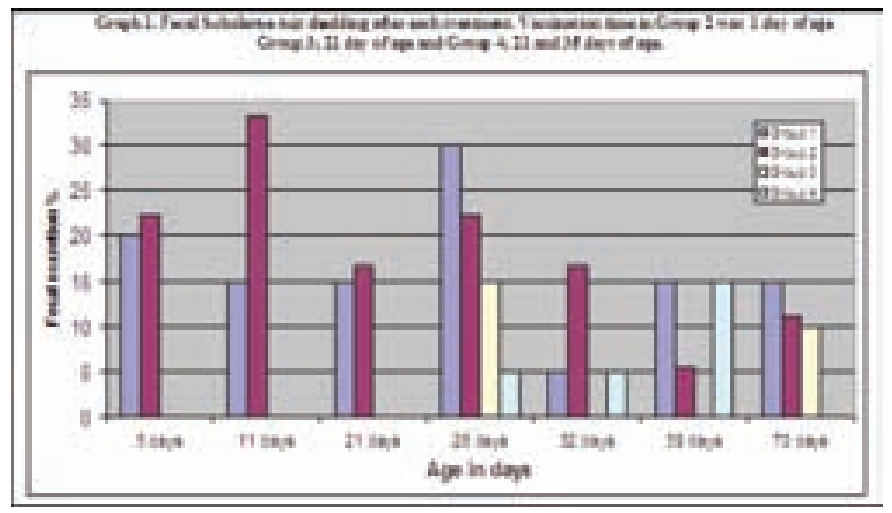

\begin{tabular}{|l|c|c|c|c|}
\hline Average daily gain (ADG) & Group 1 & Group 2 & Group 3 & Group 4 \\
\hline Lactation 1-21 days age $(\mathrm{kg})$. & 0.193 & 0.178 & 0.222 & 0.189 \\
\hline Weaning 21- 74 days age $(\mathrm{kg})$. & 0.705 & 0.649 & 0.634 & 0.622 \\
\hline Growing 74 - 145 days age $(\mathrm{kg})$. & 0.396 & 0.388 & 0.385 & 0.355 \\
\hline From 21 to 145 days & 0.48 & 0.453 & 0.452 & 0.429 \\
\hline Feed efficiency & & & & \\
\hline From 21 to 38 days age & 0.95 & 1 & 0.85 & 1.02 \\
\hline From 39 to 73 days age & 1.41 & 1.91 & 1.42 & 1.56 \\
\hline From 74 to 144 days age & 2 & 4.04 & 2.42 & 2.6 \\
\hline From 21 to 145 days & 1.71 & 2.92 & 1.82 & 1.99 \\
\hline Morbidity & & & & \\
\hline Digestive signs \% & 25 & 44.4 & 15 & 55 \\
\hline Mortality \% & 0 & 40 & 0 & 0 \\
\hline
\end{tabular}

\section{Discussion}

Shedding in Group 3 was less than in the control group thoughout the cycle. Although all products led to reduced shedding, the single dose vaccine on the first day of age (Group 2) applied to an immature intestine in the presence of high MDA seemed to compromise the health of the piglets, evident from low weight gains during the sucking and growing phases, with severe morbidity and mortality.

The vaccine given at weaning (Group 3) produced the best perfomance with resepct to ADG, morbidity, mortality and Salmonella sp shedding.

The use of a safe vaccine against Salmonella spp is a useful tool in the control of the disease. It helps reduce excretion and, together with biosecurity measures and good management, is the key to reducing damage and economic loss due to disease caused by Salmonella spp.

\section{References}

1. Kolb J. Procc.17th IPVS Ames lowa, USA, 2002:189

2. Erdman.MM.Procc.17thIPVS AmesUSA, 2002:574 


\title{
0.207
}

\section{Vaccination to control Salmonella and improve weight gain in pigs}

\author{
Vahab Farzan; Robert M. Friendship \\ University of Guelph, Guelph, ON, Canada
}

\section{Introduction}

Immunization appears to be one of the most promising approaches for control of Salmonella on swine farms (1). Salmonella Choleraesuis vaccines are commercially available (2-3), however S. Choleraesuis does not appear to be a common pathogen in Ontario swine herds any longer (4). On the other hand Salmonella Typhimurium var. Copenghagen has become the most frequent serovar recovered on Ontario swine farms (4). The objectives of the present study were i) to determine if an autogenous S. Typhimurium vaccine or a commercial live S. Choleraesuis vaccine can reduce the prevalence of Salmonella shedding in pigs, ii) to determine if Salmonella shedding is associated with weight gain in pigs.

\section{Materials and methods}

The trial was conducted on one farrow-to-finish pig operation with the history of salmonellosis. Nine cohorts of weaned pigs, with approximately 350 pigs in each group, were randomly assigned S. Typhimurium bacterin, S. Choleraesuis vaccine, or as the control group. A Salmonella Typhimurium var Copenhagen strain was used to prepare the autogenous vaccine. In each cohort, one pen was randomly selected in the nursery stage and 30 pigs were ear-tagged and weighed. The tagged pigs were weighed again when marketing. Pooled fecal samples were collected bi-weekly from manure found on the pen floor and cultured for Salmonella. A Generalized Linear Latent and Mixed Models was used to compare the presence of Salmonella in the pooled fecal samples collected from the pens in three groups. A mixed linear regression method was applied to compare the average daily gain in the vaccinated and the control pigs.

Figure 1: Proportion of Salmonella-positive samples in vaccinated and control pigs

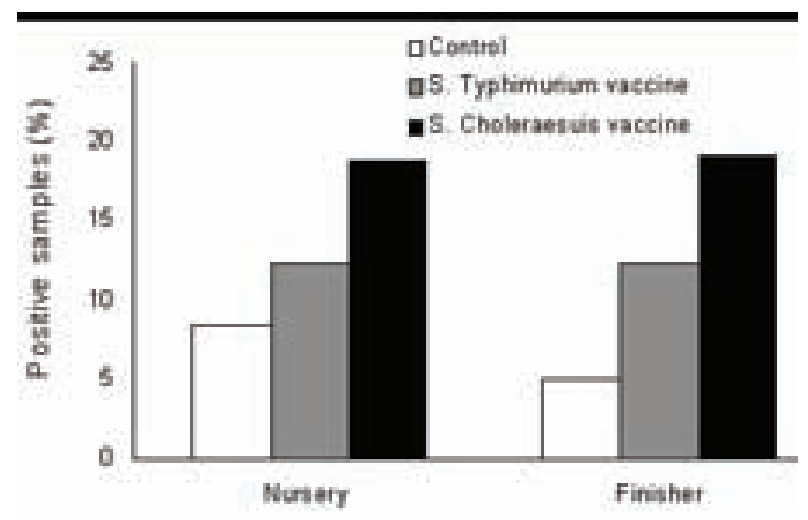

\section{Results}

The prevalence of Salmonella shedding in each group is shown in Figure 1. The odds of Salmonella shedding during finisher stage in S. Choleraesuis vaccinated pigs $[O R=5.2(1.8,15.5)]$ and S. Typhimurium vaccinated pigs $[\mathrm{OR}=3.3(1.1,9.9)]$ was higher than in the control group. The $\mathrm{S}$. Choleraesuis vaccinated pigs were more likely to shed Salmonella compared to pigs which were vaccinated with $\mathrm{S}$. Typhimurium bacterin. The control pigs which had the lowest average of Salmonella shedding showed the best growth performance compared to the vaccinated groups (Table 1). In addition, the pigs from pens with a higher Salmonella recovery rate were deemed to have a lower average daily gain.

Table 1: The impact of Salmonella vaccination on average daily gain in pigs

\begin{tabular}{|l|c|c|c|}
\hline \multicolumn{1}{|c|}{ Vaccine } & Coefficient & 95\% Cl & P value \\
\hline S. Choleraesuis & -26.6 & $-53.0,0.27$ & 0.048 \\
\hline S. Typhimurium & -90.8 & $-122.5,-59.0$ & $<0.001$ \\
\hline Control & Reference & & \\
\hline Weight at weaning $(\mathrm{Kg})$ & 4.7 & $0.7,8.7$ & 0.022 \\
\hline
\end{tabular}

\section{Discussion}

The prevalence of Salmonella shedding was initially lower in the control group. Younger pigs are more likely to shed Salmonella than finisher pigs (5) and therefore our estimate of vaccine effectiveness may be biased because the decrease in shedding might be due to age. It is possible that vaccination was performed after pigs had been exposed and that efficacy might have been improved had the pigs been vaccinated sooner. At least one study suggests that it might be prudent to establish a vaccination strategy for pregnant sows to control Salmonella in their piglets (6). Another significant finding of this study is that pigs that appeared clinically healthy but were found to be shedding Salmonella grew slower than pigs not shedding Salmonella. This suggests that there is an economic cost to subclinical Salmonella infection and provides an economic incentive for producers to implement Salmonella control measures.

\section{References}

1. Denagamage TN, et al. 2007. Foodborne Pathog Dis; 4: 539-49.

2. Maes D, et al. 2001. Berl Munch Tierarztl Wochenschr; 114: 339-41.

3. Roof MB, 1995. Am J Vet Res; 56: 39-44.

4. Farzan A, et al. 2008. Foodborne Pathog Dis; 5:579-88.

5. Rosendal T. 2007. MSc thesis University of Guelph.

6. Roesler U, et al. 2006. J Vet Med B Infect Dis Vet Public Health; 53: 224-8. 


\title{
0.208
}

\section{Clinical findings in outbreaks of acute diarrhea with indication of antibiotic treatment}

\author{
Ken S. Pedersen ${ }^{1}$ Sven E. Jorsal ${ }^{2}$ Helle Stege ${ }^{1}$ Poul Baekbo ${ }^{3}$ Jens P. Nielsen ${ }^{1}$ \\ 1. University of Copenhagen, Frederiksberg, Denmark; 2. National Veterinary Institute, Technical University of Denmark, Frederiksberg, Denmark; \\ 3. Pig Research Centre, Danish Agriculture and Food Council, Copenhagen, Denmark
}

\section{Introduction}

Gastrointestinal disease in weaners is the most common cause of antibiotic consumption in Danish pig production. Most antibiotics are used as recurring therapeutic batch medication of acute outbreaks of diarrhea. Clinical signs, pathology and microbiology of this type of outbreak have been poorly described. The objective of the current study was to estimate with-in batch prevalence of clinical signs in outbreaks of acute treatment requiring diarrhea in pigs between 10 and 70 days post weaning. Results of the pathological and microbiological investigations are reported elsewhere in these proceedings.

\section{Materials and Methods}

A cross sectional study was conducted. Herds were selected by multistage sampling. All herds serviced by six specialized swine veterinarians from the same vet practice at Zealand and fulfilling the inclusion criteria were selected. The criteria were recurring therapeutic use of in-feed or in-water medication for diarrhea at room level in pigs between 10 and 70 days post weaning. Only herds with modern intensive production systems were selected. One outbreak of acute diarrhea was investigated in each herd. Farmers and herd veterinarians notified the authors at initiation of an outbreak of acute diarrhea in a nursery room, where treatment by in-feed or in-water medication at the room level was necessary in their opinion. All herds were visited the day following notification and the farmer was not allowed to medicate any pigs before the pigs were examined. If the pigs had received antibiotic batch medication with-in the last 7 days of the examination day, the outbreak was excluded from the study. A sample of 80 pigs in each herd was selected for clinical examination by systematic random sampling among all pigs in the nursery room where the outbreak of acute diarrhea occurred. The selected pigs were subjected to a clinical examination by the same observer. The observer's diagnostic sensitivity and specificity for detection of diarrhea was estimated by fecal dry matter (DM\%) determination for a subset of the examined pigs ( $n=773)$. DM\% $\leq 18.8$ was considered as diarrhea (1).

\section{Results}

A total of 20 herds were examined during outbreaks of acute treatment requiring diarrhea in 2009. On average the pigs were 32 days post weaning (range: 12 to 63 days). Mean with-in batch prevalence of diarrhea (loose + watery) was 37\% (range: 25\% - 67\%), Table 1. For detection of diarrhea the observer had a diagnostic sensitivity $=0.88$ and specificity $=0.91$.

\section{Discussion}

The prevalence of diarrhea was higher than expected. The observer's diagnostic sensitivity and specificity gave an overestimated prevalence (true prevalence $=35 \%$, analysis not shown). The 24 hours between notification and examination may have contributed to the higher than expected prevalence. The with-in batch prevalence of other clinical signs than diarrhea was low. This may be due to the study of the early part of the outbreaks. The different with-in batch prevalence of clinical signs between herds may be due to different treatment thresholds for the individual farmers. It may also be the result of different etiology showing different clinical behavior. Further analyses are planed to explore these aspects. The small sample of herds investigated in the current study only represents modern intensive production systems. However, the results do imply that more pigs have diarrhea at initiation of antibiotic batch medication than is commonly believed.

\section{References}

1. Pedersen, K.S. et al., 2009. Proc 1st ESPHM: page 67.

Table 1. With-in batch prevalence of clinical signs in 20 herds with outbreaks of acute treatment requiring diarrhea 10 to 70 days post weaning ( $n=1585$ pigs)

\begin{tabular}{|c|c|c|c|c|}
\hline & mean & $\min$ & $\max$ & p-value* \\
\hline Diarrhea - Loose consistency & $24 \%$ & $16 \%$ & $34 \%$ & 1.0 \\
\hline Long hair coat & $14 \%$ & $5 \%$ & $39 \%$ & $<0.01$ \\
\hline Diarrhea - Watery consistency & $13 \%$ & $1 \%$ & $33 \%$ & $<0.01$ \\
\hline Mildly emaciated & $10 \%$ & $0 \%$ & $35 \%$ & $<0.01$ \\
\hline Perineal fecal stain & $9 \%$ & $0 \%$ & $29 \%$ & $<0.01$ \\
\hline Lumbar cavity & $8 \%$ & $1 \%$ & $31 \%$ & $<0.01$ \\
\hline Pot-bellied & $2 \%$ & $0 \%$ & $9 \%$ & 0.02 \\
\hline $\begin{array}{l}\text { Fecal blood, mucus or necrotic } \\
\text { material }\end{array}$ & $1.4 \%$ & $0 \%$ & $9 \%$ & $<0.01$ \\
\hline Pale & $0.3 \%$ & $0 \%$ & $3 \%$ & 1.0 \\
\hline \multicolumn{5}{|c|}{$\begin{array}{l}\text { Chi-sq or Fishers exact test for test of different prevalence between herds. P-value adjusted by } \\
\text { Bonferroni-correction for multiple comparisons. }\end{array}$} \\
\hline
\end{tabular}




\title{
0.209
}

\section{Intestinal pathology of acute diarrhea in weaners: A case control study in 20 Danish herds}

\author{
Ken S. Pedersen ${ }^{1}$ Roberto M. Guedes ${ }^{2}$ Sven E. Jorsal ${ }^{3}$ Helle Stege ${ }^{1}$ Jens P. Nielsen ${ }^{1}$ Tim K. Jensen ${ }^{3}$ \\ 1. University of Copenhagen, Frederiksberg, Denmark; 2. Veterinary School, Universidade Federal de Minas Gerais, Belo Horizonte, MG, Brazil; \\ 3. National Veterinary Institute, Technical University of Denmark, Frederiksberg, Denmark
}

\section{Introduction}

The most common cause of antibiotic consumption in Danish pig production is gastrointestinal disorders in weaners. The largest part of the consumption is used as recurring therapeutic batch medication of acute outbreaks of diarrhea. It is generally believed that proliferative enteropathy associated with Lawsonia intracellularis is the major cause of these outbreaks of diarrhea. The objective of the current study was to describe the gross pathology in outbreaks of acute treatment requiring diarrhea in pigs between 10 and 70 days post weaning. Results of clinical and microbiological investigations of these outbreaks are reported elsewhere in these proceedings.

\section{Materials and Methods}

A case control study was conducted. Herds were selected by multistage sampling. All herds serviced by six specialized swine veterinarians from the same vet practice at Zealand and fulfilling the inclusion criteria were selected. The criteria were recurring therapeutic use of in-feed or in-water medication for diarrhea at room level in pigs between 10 and 70 days post weaning. Only herds representing modern intensive production systems were selected. One outbreak of acute diarrhea was investigated in each herd. All herds were visited the day following notification from the farmer/veterinarian of an acute treatment requiring outbreak of diarrhea and the farmer was not allowed to medicate before the pigs were examined. If the pigs had received antibiotic batch medication with-in the last 7 days of the examination day, the outbreak was excluded from the study.

A sample of 80 pigs in each herd was selected by systematic random sampling among all pigs in the nursery room where the outbreak occurred. The selected pigs were subjected to a clinical examination and fecal samples were collected. Among the examined pigs a simple random sample of 8 pigs with diarrhea and 8 pigs without diarrhea was selected. The 16 pigs were killed and transported to DTU-VET for necropsy the following day. Fecal dry matter (DM\%) was determined. DM\% $\leq 18.8$ was considered as diarrhea (1) and was used (if necessary) to reclassify the pigs as diarrheic (cases) or non-diarrheic (controls) in the statistical analysis.

\section{Results}

Twenty-four non-diarrheic and 12 diarrheic pigs (determined at clinical examination) were reclassified according to the DM\% determination. Seven pigs were excluded from analysis. Results of the gross pathological examinations are displayed in Table 1.

\section{Discussion}

Small intestinal lymph node hyperplasia, large intestinal mucosa hyperemia and proliferative enteropathy-like changes were found at low but significantly higher levels in pigs with diarrhea than those without. The low prevalence of proliferative enteropathy-like changes implies that the importance of Lawsonia intracellularis in relation to diarrhea could be much lower than previously believed. Interestingly, obvious signs of infectious enteritis were rare and most of the other intestinal changes were equally prevalent in pigs with or without diarrhea.

Further conclusion awaits results of histological and microbiological examinations.

\section{References}

1. Pedersen, K.S. et al., 2009. Proc 1st ESPHM: page 67.

Table 1. Prevalence of gross intestinal changes* in 20 herds with outbreaks of acute treatment requiring diarrhea 10 to 70 days post weaning ( $n=313$ pigs)

\begin{tabular}{|c|c|c|}
\hline & $\begin{array}{l}\text { Pigs with diarrhea } \\
(\mathrm{DM} \% \leq 18.8)(\mathrm{n}=166)\end{array}$ & $\begin{array}{l}\text { Pigs without diarrhea } \\
(\mathrm{DM} \%>18.8)(\mathrm{n}=147)\end{array}$ \\
\hline \multicolumn{3}{|l|}{ Small intestine: } \\
\hline Hyperemia & $35 \%$ & $32 \%$ \\
\hline Flaccid & $23 \%$ & $24 \%$ \\
\hline Lymph node hyperplasia & $11 \% \#$ & 3\%\# \\
\hline Blood tingled contents & $6 \%$ & $5 \%$ \\
\hline Congestion of blood vessels & $5 \%$ & $4 \%$ \\
\hline Thickened intestinal wall & $5 \%$ & $0.7 \%$ \\
\hline \multicolumn{3}{|l|}{ Large intestine: } \\
\hline $\begin{array}{l}\text { Hyperplasia of colon associated } \\
\text { lymphatic tissue }\end{array}$ & $26 \%$ & $25 \%$ \\
\hline Mesenteric edema & $10 \%$ & $16 \%$ \\
\hline Lymph node hyperplasia & $5 \%$ & $3 \%$ \\
\hline Mucosa hyperemia & $5 \% \#$ & $0 \% \#$ \\
\hline $\begin{array}{l}\text { Mucosa erosions, ulcerations and/ } \\
\text { or necrosis }\end{array}$ & $5 \%$ & $3 \%$ \\
\hline Proliferative enteropathy-like: & $8 \% \#$ & $0.7 \% \#$ \\
\hline No intestinal changes: & $32 \%$ & $37 \%$ \\
\hline
\end{tabular}




\title{
0.211
}

\section{Validation of an animal-based welfare assessment tool for the New Zealand pork industry: a case-series of welfare conundrums}

\author{
Eric J. Neumann; Ian W. Barugh; William F. Hall; Daan Vink \\ Massey University, Palmerston North, New Zealand
}

\section{Introduction}

An animal-based welfare assessment tool was developed to quantify welfare outcomes for pigs in NZ. The current $2005 \mathrm{New}$ Zealand Code of Welfare (Pigs) ${ }^{1}$ was developed using traditional facility-based metrics that provides a means of collecting objective but indirect measures of the welfare of farmed pigs. As part of an ongoing effort to improve well-being of pigs, the pig industry in 2009 undertook a review of the current Code with the aim of developing an evaluation tool that would directly assess the well-being of the pigs. The tool combines both subjective and objective measures of welfare based on direct observations of the pigs. As the current Code is still in effect and represents the "minimum welfare standards", the proposed animal-based standards have been linked to applicable parts of the Code by which a farm would judged by a competent authority. As both the proposed animal-based criteria and the existing facilitybased criteria are visibly linked on the assessment tool, the assessor is able to conveniently judge the welfare of pigs using both systems concurrently. The animal-based welfare indicators include aspects of vocalisation, appearance, behaviour, mobility, and faecal consistency. Once the validation process has been completed by early 2010, a graduated approach to replacing the current Code with the new animal-based standard will be initiated.

\section{Materials and Methods}

Validation of the tool involved a one-day training session for the assessors. Following this, assessors visited farms having variable production practices, herd sizes, and housing systems in order to create a dataset suitable for an analysis of inter-rater reliability and repeatability. The tool allowed concurrent assessment of the farm based on the current welfare Code (PASS/FAIL grade on 20 facility-based standards - ie m2/metabolic bodyweight, number pigs /feeder space, water flow rate, gestation stall width, etc) and the proposed animal-based standards (use of a "traffic light" system of green, amber, and red).

\section{Results}

A total of 9 farms have been assessed and has included all stages production in both intensive and extensive production systems. The agreement between assessors was good but as all farms that have been assessed were in compliance with the current welfare regulations, statistical analysis of the data was uninformative.

\section{Discussion}

Analysis of the assessments demonstrated that, farms that appeared to have welfare issues were consistently identified by the assessors, and specific issues identified on farms by the assessors were similar.
Use of the animal-based standards (specifically, the ability to evaluate a situation with the traffic light scoring) was uniformly praised by assessors as they had the flexibility to interpret the welfare of a given situation in the context of the larger setting rather than having to singularly judge a pig or event as cause for PASS/FAIL of the entire farm. Examples of this included:

- Existence of a small proportion of gestation stalls $590 \mathrm{~mm}$ wide (currently less than the codified minimum width of $600 \mathrm{~mm}$ ) could still be given a "green light" as the farmer managed this by housing only gilts in the narrow stalls.

- Occurrence of dramatic vocalization by sows when an assessor entered a barn of limit-fed gestating sows could be given a "green light" when viewed in light of the sows' Pavlovian response to an anticipated feeding event.

However, use of the animal-based standards created several unanticipated conundrums for the assessors. Examples of this included:

- Reliance on the analogy that "good production levels must mean good welfare" had to be abandoned as its inverse ("poor production levels must mean poor welfare") was not necessarily valid. This was apparent in the case of a small and inefficient producer ( 30 sows producing only 7 pigs per sow per year) who's sows were fat, content, and in an enriched environment (sic broken down machinery, expansive wallows, and every-other-day waste-food feeding) would have failed the codified standards but received "green lights" across the board using the animal-based standards.

- In extensively housed sows, rock chewing was common on some farms. Assessors were unsure as to whether they should consider the behavior as stereotypic ("red light") or was simply the sow making use of environmental enrichment provided by the farmer ("green light").

\section{Conclusion}

It is unlikely that a purely animal-based or facility-based welfare assessment tool will accurately ascertain the true well-being of farmed pigs. A tool that uses a combination of these criteria is capable of identifying farms that compromise the welfare of pigs.

\section{References}

1. Anonymous. New Zealand Animal Welfare (Pigs) Code of Welfare 2005. In. Wellington, New Zealand: Ministry of Agriculture and Forestry - National Animal Welfare Advisory Committee; 2005:63. 


\title{
0.212
}

\section{Behavioural, clinical and clinical-chemical indices in pigs suffering from disease}

\author{
Gerald Reiner; Katrin Hübner; Hermann Willems \\ Department of Veterinary Clinical Sciences, Justus-Liebig-University, Giessen, Germany
}

\begin{abstract}
Introduction
In assessing the quality of life of farm animals, the absence of disease has been given highest priority (e.g. Dawkins 2006). However, a disease-free state cannot always be ensured. Thus, infectious diseases are major causes for pain and suffering (Hart 1988, Algers, 2004). During infection, sickness and suffering refer to a coordi $\neg$ nated set of behavioural and physiolo $\neg$ gical changes, leading to the concept of sickness behaviour. Behavioural indices are rarely assessed in conjunction with clinical diagnostics in more than a qualitative manner. Knowledge of the effects of model diseases on behaviour and on relationships among clinical/clinical-chemical parameters and behavioural indices would permit a better understanding and assessment of animal suffering due to infectious disease. Therefore, the aim of this study was to describe the physiological and behavioural patterns and relationships in pigs before, and during the acute, subclinical and chronic stages of a model disease.
\end{abstract}

\section{Materials and Methods}

Based on a well defined model disease (Sarcocystis miescheri$a n a$ ), we have studied behavioural patterns (lying inactive [LYWA], activity during lying [ADLY], feeding [FEED], drinking [DRNK], rooting [ROOT], walking [WALK], and social interactions [SOCB] of pigs during stages of health (day 0 , before infection), acute disease (day 14 post infection [p.i.]), subclinical disease (day 28 p.i.), and chronic disease (day 42 p.i.). Data were captured from video records of 139 F2 Meishan x Pietrain crossbred pigs between 09:30 a.m. to 11:30 a.m. The time interval for observation was chosen so as to overlap with the first activity peak in the morning and to avoid times of human intervention. Behavioural indices were correlated with a set of clinicalchemical parameters (red and white blood cells, serum enzymes, serum metabolites and electrolytes).

\section{Results}

Before infection, respective time fractions were $56 \%$ (LYWA), 21\% (ADLY), 10\% (FEED), 0.9\% (DRNK), 6.3\% (ROOT), 2\% (WALK), and $<1 \%$ (SOCB). This behavioural pattern changed distinctly during Sarcocys $\neg$ tosis. Overall activities were reduced from $44 \%$ (day 0 ) to $10 \%$ (day 14 p.i.), 34\% (day 28 p.i ), and 20\% (day 42 p.i.).

The development of different stages of disease was accompanied with distinct changes in clinical and clinical-chemical parameters. Deviation from the population's "standard" activity (before infection) could be predicted with clinical-chemical parameters (e.g. alkaline phosphatase [AP]).
Behavioural activities were generally reduced, whenever clinical/clinical-chemical traits changed to pathological values. A multivariate regression model revealed five predictors to explain e.g. total activity. The model was statistically significant with $\mathrm{P}<0.0001$ and explained $32 \%$ of the pigs' total variance for this indicator (correla $\neg$ tion coefficient 0564 ). AP was the most important predictor. A decrease of $100 \mathrm{lU} / \mathrm{I}$ was associated with a reduction in activity of $7.8 \%$ during the observation period. A $10 \%$ reduction in leukocytes or $10 \mathrm{mmol} / \mathrm{l}$ less serum sodium ions were each equivalent to a more than $5 \%$ increase in activity. Increases of $10 \mu \mathrm{mol} / \mathrm{l}$ in serum creatinine or $1000 \mathrm{IU} / \mathrm{l}$ in serum creatine kinase (CK) were associated with an increase in inactivity by another 1.4 and $5.1 \%$, respectively.

\section{Discussion}

To date, most welfare research has been concerned with identifying effects of housing conditions on welfare. The present study substantiates the role of infectious diseases in animal welfare. Taking into account the high prevalence of infectious diseases, it surprises that there is still very little research published on how different common diseases affect an animals' welfare (Algers, 2004). With more information about effects of defined model diseases on behaviour and on relationships among easily obtainable clinical/clinical-chemical parameters and behavioural indices, our understanding and assessment (eventually even under field conditions) of animal suffering due to infectious disease, even under subclinical conditions, might be decisively improved.

\section{References}

Algers, B., 2004. In: Proceedings of the OIE global conference on animal welfare, Paris, 23-25 February, 179-187.

Dawkins M.S., 2006. Trends Ecol. Evol. 21, 77-82.

Hart, B.L., 1988. Neurosci. Biobehav. Rev. 12, 123-137. 


\title{
0.213
}

\section{Impact of physical castration and anti-GnRH vaccination (Improvac ${ }^{\circledR}$ ) of boars on feeding behaviour and body lesion scores in single- and mixed-sex group housed fattening pigs}

\author{
Tatjana Schmidt ${ }^{1}$ Mario Grodzycki ${ }^{2}$ Marleen Paulick ${ }^{1}$ Franziska Rau Eberhard von Borell ${ }^{1}$ \\ 1. Institute of Agricultural and Nutritional Sciences, Halle (Saale), Germany; 2. Pfizer Animal Health, Berlin, Germany
}

\section{Introduction}

Physical castration of male piglets is common practice in many countries for the control of boar taint. A vaccine against $\mathrm{GnRH}$ (Improvac) is licensed in Europe as an alternative to this painful procedure. Vaccinated pigs behave like entire males up to the second vaccination and may potentially exhibit aggressive behaviour towards each other (von Borell et al, 2009). In this study we analysed feeding duration and indirectly measured agonistic interactions by shoulder lesion scores (Turner et al, 2006) of physically castrated pigs (PC) and pigs vaccinated with Improvac (IC). We also examined if penning with females would affect these parameters in males.

\section{Material and Methods}

PC and IC males (Pi x[German LRxLW]) were either penned in single-sex groups of 10 (treatment T1 and T2) or mixed with females (5:5, T3 and T4). A total of 160 animals started the experiment in four replicate groups. 11 pigs were excluded due to health problems. Pigs were mixed after weaning at $4 \mathrm{wks}$ of age and regrouped by weight at wk 11 for the start of the experiment (Vaccinations at 12 and 23 wks of age). An automatic single-space feeder (IVOG ${ }^{\circledR}$ ) per pen recorded the feeding duration (FD, minutes per day) of each pig. Shoulder lesions (LS) were scored weekly ranging from scratches (scores 1-2), abrasions (3-4) to severe wounds (5-6) and compared in 4 phases (P1-4). Mixed linear models with repeated measures design were used for statistical analysis with fixed effects of treatment, pen and replicate for feeding data and an additional fixed effect of observer for lesion score data as well as Tukey's HSD test (JMP® , SAS Institute).

\section{Results}

T1 showed a greater FD (Table 1) than T2 in wk 14 and than T2\&T4 in wk 20. There was no treatment effect in either FD in wk 25 or LS (Table 2) in P4. LS were high for all treatments in P1 but decreased in P2-4 (except for T2). T2 showed higher LS than T1 in $\mathrm{P} 2$ and $\mathrm{P} 3$.

Table 1: Feeding duration (FD, minutes)

\begin{tabular}{|c|c|c|c|}
\hline & wk 14 & wk 20 & wk 25 \\
\hline $\mathrm{T} 1$ & $65.9 \pm 3.54^{\mathrm{a}}$ & $54.8 \pm 3.45^{\mathrm{a}}$ & $37.7 \pm 3.58^{\mathrm{a}}$ \\
\hline $\mathrm{T} 2$ & $51.4 \pm 3.97^{\mathrm{b}}$ & $38.3 \pm 3.97^{\mathrm{b}}$ & $42.7 \pm 3.97^{\mathrm{a}}$ \\
\hline $\mathrm{T} 3$ & $55.1 \pm 5.66^{\mathrm{ab}}$ & $46.7 \pm 5.66^{\mathrm{ab}}$ & $33.5 \pm 5.66^{\mathrm{a}}$ \\
\hline $\mathrm{T} 4$ & $55.5 \pm 5.27^{\mathrm{ab}}$ & $40.4 \pm 5.27^{\mathrm{b}}$ & $38.0 \pm 5.51^{\mathrm{a}}$ \\
\hline $\begin{array}{l}\text { Least-Square means } \pm 95 \% \mathrm{Cl}^{\mathrm{ab}} \text { within wk, means lacking common superscript letter differ } \\
(\mathrm{p}<.05) .\end{array}$ \\
\hline
\end{tabular}

Table 2: Shoulder lesion scores (LS)

\begin{tabular}{|c|c|c|c|c|}
\hline wk & $11-13(P 1)$ & 14-18 (P2) & $19-23(\mathrm{P} 3)$ & 24-28 (P4) \\
\hline $\mathrm{T} 1$ & $1.75 \pm 0.16^{\mathrm{a}}$ & $1.60 \pm 0.15^{\mathrm{a}}$ & $1.63 \pm 0.15^{\mathrm{a}}$ & $1.62 \pm 0.15^{\mathrm{a}}$ \\
\hline $\mathrm{T} 2$ & $1.97 \pm 0.16^{\mathrm{a}}$ & $2.06 \pm 0.15^{b}$ & $2.02 \pm 0.15^{b}$ & $1.65 \pm 0.15^{\mathrm{a}}$ \\
\hline $\mathrm{T} 3$ & $2.01 \pm 0.18^{a}$ & $1.85 \pm 0.16^{a b}$ & $1.83 \pm 0.16^{a b}$ & $1.66 \pm 0.17^{a}$ \\
\hline T4 & $1.98 \pm 0.17^{\mathrm{a}}$ & $1.81 \pm 0.15^{a b}$ & $1.72 \pm 0.15^{a b}$ & $1.37 \pm 0.15^{\mathrm{a}}$ \\
\hline
\end{tabular}

\section{Discussion}

Georgsson \& Svendsen (2002) found that feeding time was lower in pig groups when high levels of competition occurred at the feeder. This could indicate that competition for feeder access reduced FD in IC groups in wk 14\&20. However, FD was only significantly higher in single-sex, not in mixed-sex PC males compared with IC groups.

In P1, shortly after regrouping of pigs, the LS were high in all treatments. Higher LS in single-sex IC males in P2 \& P3 indicate more agonistic interactions. In contrast, LS of mixed-sex IC groups were not greater than in the PC treatments suggesting less aggression in mixed-sex groups compared to IC single-sex groups. No differences occurred in FD and LS after effective castration of IC males. In all groups, mean LS were only marginally $>2$, indicating almost no severe injuries. Therefore, the results of this study suggest that the impact of increased aggression in the IC males does not seem to constitute a major welfare problem.

\section{References}

von Borell E, Baumgartner J, Giersing M, Jäggin N, Prunier A, Tuyttens FAM, Edwards SA (2009), Animal welfare implications of surgical castration and its alternatives in pigs, Animal 3: 1488 -1496

Georgsson L, Svendsen J (2002), Degree of competition at feeding differentially affects behavior and performance of group-housed growing-finishing pigs of different relative weights, Journal of Animal Science 80: $376-383$

Turner SP, Farnworth MJ, White IMS, Brotherstone S, Mendl M, Knap P, Penny P, Lawrence AB (2006), The accumulation of skin lesions and their use as a predictor of individual aggressiveness in pigs, Applied Animal Behaviour Science 96: 245 -259 


\title{
0.214
}

\section{Free space utilisation of group housed sows}

\author{
Fiona C. Rioja-Lang \\ Prairie Swine Centre, Saskatoon, SK, Canada
}

\section{Introduction}

With announcements by the largest producer/packers in both the USA and Canada that they will transition all of their production facilities to group housing for sows over the next ten years, all N. American producers are anticipating a change to group housing. This study investigates the implementation of walkin/lock-in stalls in group housed sows. There is some concern regarding the degree to which sows use the free space areas in this system, and how to avoid excessive competition and aggression.

The objectives of this study were to compare two different pen configurations by determining the proportion and type (size/ parity) of sows that are using the free space areas of the walk-in/ lock-in stalls, and also how sows utilize the free space areas.

\section{Methods}

Eight groups of 25 sows ( \pm 3 ; mean \pm SD) were used in the study, and were housed in walk-in/lock-in stall gestation pens at the Prairie Swine Centre, Saskatoon. Each of the groups were exposed to one of two configurations of free space areas. The first is referred to as the 'I-pen' as it consisted of an alley $(3.0 \mathrm{~m} x$ $10.7 \mathrm{~m}$ ) with slatted flooring running between two lanes of 16 stalls on each side. Any additional stalls, surplus to the group number, were locked off for the purpose of the trial. The second pen configuration is referred to as the 'T-pen' as it consisted of an identical alley with an additional solid floor loafing area at one end $(3.8 \mathrm{~m} \times 7.1 \mathrm{~m})$. Pigs were individually marked and photographs were taken from a mounted camera every 2 minutes over a $24 \mathrm{hr}$ period every week, for 11 weeks through gestation. Measurements recorded from the photographs include the percentage of time spent out of the stall over $24 \mathrm{hrs}$, and also the location and position of sows in the free space areas.

\section{Results}

The majority of sows did use the free space areas although not on a regular basis or for extended periods of time. Older, heavier sows appeared to use the free space area significantly more than younger, small sows $(\mathrm{P}<0.0001)$.

Table 1. Average percentage of time spent in free access area for sows housed in 'I-pen' configuration over $24 \mathrm{hr}$ period.

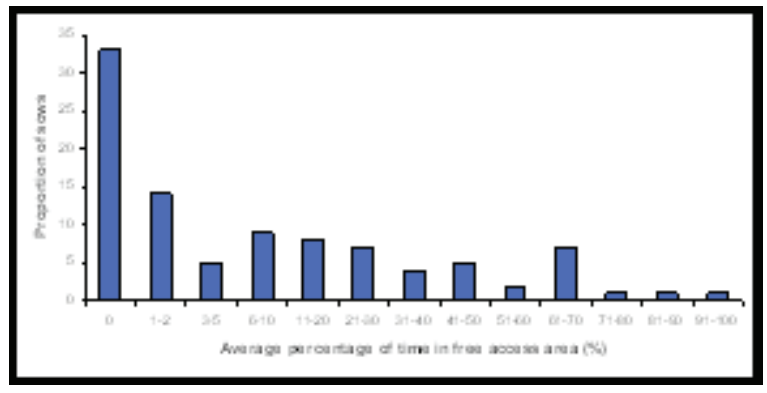

Table 2. Average percentage of time spent in free access area for sows housed in 'T-pen' configuration $24 \mathrm{hr}$ period.

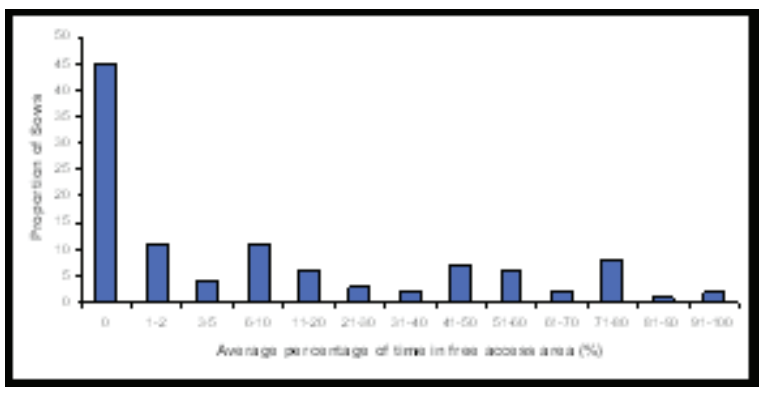

\section{Discussion}

Although many sows did use the free space, it was at a much lower level than expected. This could be due to several possibilities, such as lower ranking animals feeling threatened by higher ranking sows, or larger sows utilizing the free space due crowding in the stalls. With the impending ban of gestation stalls it is important that we conduct adequate research to design the optimum group housing system which will facilitate positive social interactions and minimize levels of aggression and competition.

Future research is likely to involve methods for enticing the sows into the free space areas, including making the free space more comfortable to lie on with rubber mats, providing environmental enrichment, or possibly allowing sows access to the free area in different social groups i.e. young and old.

\section{Acknowledgements}

Program funding to PSC from Sask Pork, Alberta Pork, Manitoba Pork council and Sask. Agriculture Development Fund is gratefully acknowledged. We also wish to thank technical staff from the PSC. 


\title{
0.215
}

\section{Examination of $\mathrm{CO}_{2}$ gas anesthesia during piglet castration}

\author{
Isabel Mühlbauer ${ }^{1}$ Susanne Zöls ${ }^{1}$ Winfried Otten ${ }^{2}$ Andreas Palzer ${ }^{1}$ Mathias Ritzmann ${ }^{3}$ Karl Heinritzi $^{1}$ \\ 1. Clinic for Swine, Oberschleißheim, Germany; 2. Leibniz Institute for Farm Animal Biology, Dummerstorf, Germany; \\ 3. Clinic for Swine Diseases, Vienna, Austria
}

\section{Introduction}

Whereas several countries are still seeking for an effective and practicable solution for piglet castration without any analgesic treatment , $\mathrm{CO}_{2} / \mathrm{O}_{2}$ gas anesthesia during castration is already performed in the Netherlands.

This study appraises stress and pain of castration without any analgesic treatment and a potentially positive effect of $\mathrm{CO}_{2}$ anesthesia by means of serum cortisol and plasma catecholamine measurements [1,3].

\section{Materials and Methods}

188 (n) three to five day old clinically healthy male piglets for cortisol (co) and 39 piglets for norepinephrine (ne) and epinephrine measurements were randomly assigned by weight to four treatment groups (tab. 1).

Table 1: Treatment groups

\begin{tabular}{|c|c|c|c|}
\hline group & treatment & co (n) & ne (n) \\
\hline I & Immobilisation & 47 & 10 \\
\hline II & Castration & 46 & 10 \\
\hline III & $\begin{array}{c}\text { Immobilisation under } \mathrm{CO}_{2} \text { gas } \\
\text { anesthesia }\end{array}$ & 48 & 9 \\
\hline IV & Castration under $\mathrm{CO}_{2}$ gas anesthesia & 47 & 10 \\
\hline
\end{tabular}

Inhalation anesthesia was introduced with a $70 \% \mathrm{CO}_{2}: 30 \% \mathrm{O}_{2}$ gas mixture using an anesthetic machine as in the Netherlands. After introduction time of $45 \mathrm{sec}$, piglets were anesthetized for a maximum of $30 \mathrm{sec}$ during castration or immobilisation.

To evaluate postoperative castration pain, blood samples for cortisol were taken from each piglet before as well as $30 \mathrm{~min}, 1$, 4 and $24 \mathrm{~h}$ after treatment. Due to the short half-value periods of epinephrine and norepinephrine, blood samples from those piglets were taken $15 \mathrm{~min}$ before, immediately and $2 \mathrm{~min}$ after treatment. Significant differences were tested with paired t-test.

\section{Results and Discussion}

Mean cortisol concentrations did not differ in basal values between the four treatment groups (Fig. 1). 30min after castration, mean cortisol concentrations of uncastrated groups I and III were significantly lower than mean concentrations of castrated groups II and IV. A significantly higher mean value was observed $1 \mathrm{~h}$ after castration in group II than in groups I, III and IV. $4 \mathrm{~h}$ after castration, cortisol levels of uncastrated groups I and III were still lower than of group II $(\mathrm{p} \leq 0.05)$.
Fig. 1: Mean serum cortisol concentrations (+SD) before as well as $30 \mathrm{~min}, 1,4$ and $24 \mathrm{~h}$ after treatment

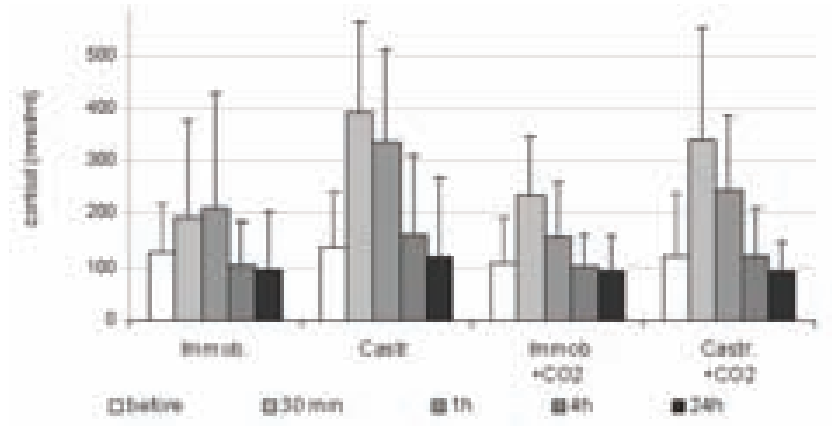

In accordance with results from Schulz et al. (2007), immobilisation (group I) and castration (group II) caused a twofold increase of plasma norepinephrine concentrations to $4471 \mathrm{pg} / \mathrm{ml}$ and respectively $4871 \mathrm{pg} / \mathrm{ml}$ immediately after treatment $(p \leq 0,001)$. In contrast, $\mathrm{CO} 2$ anesthesia induced a significant and nearly 50 fold increase of mean norepinephrine levels in groups III and IV immediately after treatment, and levels were still elevated $2 \mathrm{~min}$ after treatment. These results are confirmed by the results of epinephrine measurements.

Fig. 2: Mean plasma norepinephrine concentrations (+SD) before immediately after and 2 min after treatment

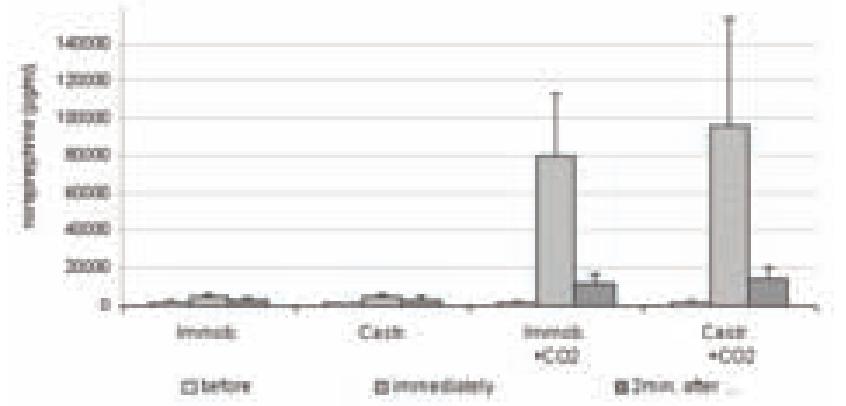

\section{Conclusion}

Serum cortisol concentrations $1 \mathrm{~h}$ after castration under $\mathrm{CO}_{2}$ anesthesia indicate a small but significant analgesic impact by the use of $\mathrm{CO}_{2}$. However, this effect was not detectable after $30 \mathrm{~min}$. In contrast, the analysis of plasma norepinephrine and epinephrine revealed a tremendous additional stress for piglets from both $\mathrm{CO}_{2}$ groups independent from castration. Considering that the aim of anesthesia is to reduce pain and stress, and that the consumers' demand for anesthesia during castration tends to increase animal welfare, $\mathrm{CO}_{2}$ anesthesia cannot fulfil the requirements to the full extent.

\section{References}

1) Heinritzi et al. (2006) Dtsch. Tierärztl. Wschr. 113 (3):94-7

2) Schulz et al. (2007) Dtsch. Tierärztl. Wschr. 114 (12):453-8

3) Vorwallner (2003) Vet med Diss 


\title{
0.216
}

\section{Pre-emptive meloxicam for postoperative analgesia in piglets undergoing surgical castration}

\author{
Alassane Keita; Eric Pagot \\ CTPA Zoopole développement, Ploufragan, France
}

\begin{abstract}
Introduction
The welfare issues associated with surgical castration procedures in piglets is a growing concern within the public and European lawmakers. Castration of male piglets is a routine practice in most European countries, carried out to avoid boar tainting of meat (an unpleasant smell that sometimes arises when the meat of boars is heated). Current European directives make anaesthesia and prolonged analgesia only mandatory for castration of pigs over 7 days of age, the procedure being carried out by a veterinary surgeon. Countries like Norway or Switzerland have, however, already banned surgical castration without anaesthesia and other countries may follow. It has been demonstrated that surgical castration was followed by an increase of plasma cortisol and plasma adrenaline via the activation of the adrenal and sympathetic axes [1,2]. This paper reports the outcome of a study having investigated the effect of a pre-operative intramuscular administration of meloxicam on postoperative stress and pain induced by surgical castration in piglets.
\end{abstract}

\section{Material and methods}

Overall 150 young (mean age of $5.1+1.3$ (SD) days and mean body weight of $2.4+0.6 \mathrm{~kg}$ ) healthy male piglets, coming from 3 different commercial farms, were included into the study. The piglets were ear-tagged and allocated at random within litters to groups to receive one of the following treatments between 10 and 30 minutes prior to surgical castration: a single intramuscular injection of $0.4 \mathrm{mg} / \mathrm{kg}$ meloxicam (i.e. $0.08 \mathrm{ml} / \mathrm{kg}$ of the product Metacam ${ }^{\circledast} 5 \mathrm{mg} / \mathrm{ml}$ solution for injection) or $0.08 \mathrm{ml} / \mathrm{kg}$ of a placebo (saline). Behavioural parameters (vocalisation, tremors, urine or faeces emission etc) were evaluated in blind conditions at time of castration up to $24 \mathrm{~h}$ post-castration. Blood samples were collected from 30 subjects selected at random from each group at 30 minutes and at 24 hours post castration. In addition blood samples were taken from 30 non-castrated contemporary piglets to provide baseline values for the concentrations of the evaluated hormones.

The piglets were individually weighed at the start of the study and again at weaning. The criteria were: blood cortisol and ACTH concentrations at 30 minutes post castration, blood Haptoglobin concentration $24 \mathrm{~h}$ post castration, behavioural alterations up to $24 \mathrm{~h}$ post castration, weight gain between castration and weaning. ANOVA, Kruskal-Wallis and Pearson Chi-square tests were used to analyse the data with $5 \%$ as level of significance.

\section{Results}

The mean age and body weight before castration were similar in the two groups.

The mean blood ACTH concentration at 30 minutes post castration (fig. 1) was significantly ( $p=0,007$ ) lower in meloxicam (17 pg/ml) and non-castrated $(18,1 \mathrm{pg} / \mathrm{ml})$ groups than in the placebo one $(35,8 \mathrm{pg} / \mathrm{ml})$.

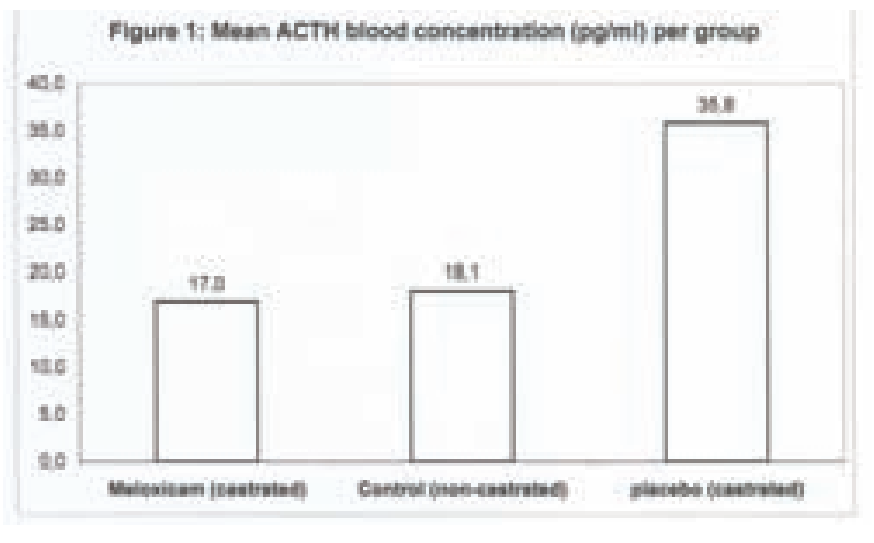

The mean blood cortisol concentration was significantly $(p<0.001)$ lower in the non-castrated group $(63 \mathrm{ng} / \mathrm{ml})$ than in the meloxicam (206 ng/ml) and placebo $(276 \mathrm{ng} / \mathrm{ml})$ groups. The difference between meloxicam and placebo was also significant.

At both 2 and 4 hours post castration, the proportion of piglets showing no behavioural alterations was significantly $(p<0.05)$ higher in the meloxicam group (83\%) than in placebo one (68\%).

No significant difference was observed between the mean blood Haptoglobin concentration and the weight gain of the two groups.

\section{Conclusion and discussion}

This study suggests that pre-emptive administration of meloxicam is able to produce an adequate postoperative analgesia after surgical castration of young piglet. Meloxicam can be proposed as a safe and effective solution to control stress and pain associated with routine castration in piglets.

\section{References}

1. Prunier et al., 2005, J. Anim. Sci., 83, 216-222

2. Keïta et al., 2010, Veterinary Anesthesia and Analgesia (accepted for publication)

\section{Aknowledgment}

The authors thank Boerhinger Ingelheim for its financial support 


\title{
0.217
}

\section{Comparison of anaesthetic effects during piglet castration by using commercial inhalation systems with carbon dioxide and isoflurane}

\author{
$\underline{\text { Karl-Heinz Waldmann }}{ }^{1}$ Maren Hoppe ${ }^{1}$ Maria Steigmann ${ }^{1}$ Arnim Andreae ${ }^{1}$ Sabine Kaestner ${ }^{2}$ \\ 1. Clinic for Swine and Small Ruminants, Hannover, Germany; 2. Small Animal Clinic, University of Veterinary Medicine, Hannover, Germany
}

\section{Introduction}

The common practice of castration of piglets without any anesthesia in the EU met with increasing criticism because of animal welfare reasons.

One of possible alternatives is the castration under general anesthesia using anesthetic gases [1, 2]. In the last years inhalation systems were established for automation the narcosis process. The present study should demonstrate the quality and depth of narcosis and the degree of reduction piglets' pain during castration under isoflurane- or carbon dioxide- anesthesia. Furthermore the influence of NSAIDs on the anesthesia and pain reactions was tested.

\section{Material and methods}

In the study exclusively healthy anatomically normal piglets at the age of one to seven days were used. All piglets had a bodyweight between 1.5 and $25 \mathrm{~kg}$.

Two kinds of commercial inhalation systems were tested; the first one used an isoflurane insufflation mixed with 30\% oxygen, while the second one worked with a gas mixture of $70 \%$ carbon dioxide and $30 \%$ oxygen.

Altogether 246 piglets in 18 groups were tested to compare the two different gas insufflations to traditionally not anesthetized piglets. Furthermore the influence of NSAIDs (Flunixine) in a common dosage was investigated. Each of the three anesthesia groups was split up in groups that were injected 60 minutes and 20 minutes before castration and a group without any NSAIDs. Additionally a sham group corresponding to every group existed, where the piglets were only handled in the same way without being castrated.

The most important method for recognizing the piglets' pain was the electroencephalogram (EEG). Due to frequency analysis an EEG score expressed the depth of anesthesia, whereat a score of 3 or more represent an adequate narcosis. Furthermore an electrocardiogram (ECG) was recorded continually. The pulse and oxygen saturation of the blood were recorded at the beginning and after the insufflation. We used a score to define the vocalization, the movements and the reactions to the manipulations. The wound healing was observed for three weeks.

\section{Results}

As measured by EEG score about $90 \%$ of the piglets of the carbon dioxide groups but only less than $40 \%$ of the piglets of the isoflurane groups did not show a sufficient narcosis. The treatment with NSAID had no influence on the depth of carbon dioxide narcosis. On the contrary NSAID influenced the depth of isoflurane narcosis significantly;. the group without any flunixine treatment showed a lower EEG score (score 2.7) than the treated group (score 3.0). In this context the moment of flunixine application, 20 or 60 minutes before castration, was indiscriminative.

Movements of the piglets, measured with EEG, which are longer than two seconds can not be pronounced as an unconscious reaction but point at a conscious defense reaction to the pain. Between both anesthetic groups a significant difference could be recognized. The reactions to the emasculation were much greater in the carbon dioxide group than in the isoflurane group. The ECG showed nearly no differences in pulse frequency and ECG waves between the status before and within the isoflurane anesthesia. In the contrary in many cases of carbon dioxide anesthesia a dramatic drop of pulse frequency from about 200$230 / \mathrm{min}$ before anesthesia to $20-40 / \mathrm{min} 30 \mathrm{sec}$ after carbon dioxide insufflation could be measured. Additionally characteristic ECG changes probably point at hyperkalemia as a consequence of acidosis.

\section{Conclusions}

The first results of our study showed that an isoflurane narcosis can reduce most of the pain originated in castration. It induces a mostly deep enough anesthesia, while the depth can be enhanced by the application of NSAIDs.

Carbon dioxide anesthesia - especially with this inhalation system - did not induce a sufficient narcosis in most cases. The consciousness is reduced but not enough to lock out the awareness of pain.

\section{References}

[1] Gerritzen M. A., M. Kluivers-Poodt 2008: Castration of piglets under CO2-gas anaesthesia. Animal (2008), 2:11, 1666-1673, The Animal Consortium.

[2] Walker, B., Jaegginn, N., Doherr, M., Schatzmann, U. (2004): Inhalation anaesthesia for castration of newborn piglets: experiences with isoflurane and isoflurane/N2O. J. Vet. Med. A 51, 


\title{
0.218
}

\section{Comparison of different analgesic treatments to reduce the pain associated with tail docking in pigs}

\author{
Tiffanie A. Brooks ${ }^{1}$ Mhairi A. Sutherland ${ }^{2}$ Brittany L. Davis ${ }^{1}$ John J. McGlone ${ }^{1}$ \\ 1. Texas Tech University, Lubbock, TX, USA; 2. Ruakura Research Centre, Hamilton, New Zealand
}

\section{Introduction}

Tail biting is a behavioural problem occurring in pigs that is a welfare problem and of economic concern for producers. Tail biting can not only affect the welfare of the pigs being bitten directly, but the procedure of tail docking as a means to control tail biting can cause acute pain which is also an animal welfare issue. Exactly what causes tail biting behaviour to break out among pigs remains elusive, but this behaviour has been attributed to an array of factors including the environment, nutrition, group size, genetics and lack of substrates. Tail docking is routinely used on farms in the US as a solution to this problem. The objective of this study was to evaluate the effectiveness of different analgesics to reduce the pain induced distress caused by tail docking in piglets.

\section{Materials and Methods}

At approximately 3 days of age, piglets were allocated to one of five treatment groups: 1) Sham tail docking (CON); 2) Tail docking using conventional cutting with side-cutting pliers (CUT); 3) Tail docking plus local anaesthetic administered immediately prior to tail docking (LA); 4) Tail docking plus topical anaesthetic applied to the wound after cutting (TOP); 5) Tail docking conducted while the piglets were anesthetized with carbon dioxide (100\% CO2 gas) using conventional cutting with side-cutting pliers (CO2).

Prior to (baseline), and 30,60, and 120 min after tail docking, piglets (10 pigs/treatment) were held in a supine position and blood was collected for a complete blood count and cortisol analysis. Piglet behaviour (10 pigs/treatment) was record using live observations for $60 \mathrm{~min}$ prior and $120 \mathrm{~min}$ after tail docking. Behaviours and postures measured included lying-alone, lyingtouching the sow or other piglets, nursing/massaging, standing, sitting, walking and pain-like behaviours and postures (e.g. hunching, scooting, and tail jamming).

Data were subjected to analysis of variance using the mixed model procedure of SAS version 9.1 (SAS Inst., Inc., Cary, NC).

\section{Results}

Leukocyte counts, differentials, and the neutrophil to lymphocyte ratio did not differ $(\mathrm{P}>0.05)$ among treatments.

Cortisol was greater $(P<0.05)$ in CUT, LA, TOP, and CO2 compared with CON piglets $30 \mathrm{~min}$ after tail docking. Cortisol did not differ $(P>0.05)$ among CUT, LA, and CO2 piglets 30 min after tail docking. At $30 \mathrm{~min}$, TOP piglets tended to have lower $(P=0.067)$ cortisol than CUT piglets. Cortisol did not differ $(P>0.05)$ among treatments at any other time point.

The percentage of time pigs spent performing nursing behaviour after tail docking tended to be greater $(P=0.062)$ in $\mathrm{CO} 2$ piglets compared with all other treatments. The percentage of time piglets spent lying alone was greater $(P<0.05)$ in CUT piglets compared with all other treatments 91-120 min after tail docking.

\section{Discussion}

Tail docking without analgesia caused increases in cortisol concentrations and performance of lying-alone behaviour, which may be indicative of pain. Cortisol concentrations tended to be reduced in piglets that had topical anaesthetic applied to the tail wound after tail docking, but not in piglets given local anaesthetic or carbon dioxide anaesthesia. Therefore, applying a topical anaesthetic to the tail wound may provide some relief from discomfort caused by tail docking in piglets.

Applying a topical anaesthetic to the tail wound immediately after tail docking may be an effective and easy way to reduce the pain caused by tail docking, but more research is needed to study the effectiveness of different analgesics that could be used to eliminate the pain caused by tail docking in piglets.

\section{Acknowledgements}

This research was funded by the National Pork Board, Des Moines, lowa, USA. 


\title{
0.219
}

\section{Periparturient analgesia: Does it improve neonatal survival and growth?}

\author{
Glen Cassar; Rocio Amezcua; Robert M. Friendship \\ Ontario Veterinary College, Univ of Guelph, Guelph, ON, Canada
}

\section{Introduction}

There has been an increase in the public concern over the welfare of farmed animals in recent times. Pain control is an important component of animal welfare and in swine production there are many areas where pain control could be addressed 1,2 The process of farrowing is associated with pain and could be a welfare issue for the sow as well as affecting her ability to adequately nurse piglets, thus affecting performance. Farrowing intervention could be painful and could result in reproductive tract infections. In cattle, it is now common for analgesics to be administered around the time of parturition, and this practice is considered to improve milk production. Research findings suggest that the peri-parturient sow experiences discomfort, which causes her to change positions frequently, increasing the chance that she will crush her piglets3. A major cause of pre-weaning mortality is due to maternal crushing of pigs. Pre-weaning mortality due to crushing has been estimated to be between 4.8 and 18 percent 4,5. Alleviating the sow's pain might lead to a decrease in body position changes and a consequent decrease in crushing, addressing both a welfare and economic issue. Reduced restlessness in the peri-parturient period could result in uninterrupted nursing by piglets and consequently improved weight gain. Ketoprofen, (Anafen, Merial Canada Inc) an injectable non-steroidal anti-inflammatory analgesic, is approved for use in swine in Canada.

The objective of the study was to evaluate the effect of analgesia to the sow at farrowing on piglet mortality and performance.

\section{Materials and Methods}

A total of 223 sows were randomly assigned to the following treatment groups.

a. Sows allowed to farrow with no analgesia $(n=105)$.

b. Sows treated with ketoprofen on the day before farrowing and on the day of farrowing $(n=118)$.

Anafen dosage was $1.5 \mathrm{ml}$ per $50 \mathrm{~kg}$ bodyweight. All piglets were ear notched for identification and weighed on the day of birth and again at 21 days of age to determine average daily weight gain (ADG). The number of stillborn piglets (SB) as well as preweaning mortality (PWM) were recorded.

\section{Results and Discussion:}

There was no difference in ADG, SB or PWM between the two groups. Table 1 shows the means for these parameters.

Table 1. Sow and piglet performance for sows given ketoprofen at farrowing and control sows.

\begin{tabular}{|l|c|c|}
\hline & Control & ketoprofen \\
\hline ADG $(\mathrm{kg})$ & $0.24 \pm 0.04$ & $0.25 \pm 0.05$ \\
\hline PWM $(\%)$ & 14 & 16 \\
\hline SB $(\%)$ & 12.7 & 13.3 \\
\hline$\%$ crushed & 3.3 & 2.7 \\
\hline
\end{tabular}

Based on the results in this trial there does not appear to be an economic incentive for producers to use analgesia routinely at farrowing. Possibly, if analgesia had been used only in the case of gilt farrowings or in the case of dystocia, a difference might have been observed.

\section{References}

1. Anil et al. J. of Applied Animal Welfare Science. 2005: 261-278.

2. Fisher AD. Proc NZ Soc. of An. Pdn. 2002:363-367.

3. Haussmann et al. J. An Sci.. 1999:2054-2059

4. Shaver et al. Proc AASV. 2008:281-283

5. Lay et al.. J Anim Sci.1999, Vol 77:2060-204 


\title{
0.220
}

\section{Behavioural and physiological differences in intensive stock-farming pigs}

\author{
Livia Moscati ${ }^{1}$ Marco Sensi ${ }^{1}$ Lorenzo Battistacci ${ }^{1}$ Augusto Vitale ${ }^{2}$ Arianna Manciocco ${ }^{2}$ \\ 1. Istituo Zooprofilattico Sperimentale Umbria e Marche, Perugia, Italy; 2. Istituo Superiore di Sanità, Roma, Italy
}

\section{Introduction}

The well-being of swine has become a relevant issue because the quality of life of pigs seems to be associated with the food production and meat quality (2). The heavy selection aimed to obtain individuals with elevated reproductive and productive performances has often conducted farm animals to a poor state of health and welfere(3). The aim of this work is to study the effects of the environmental enrichment on the normal and abnormal behaviors and hematological parameters in a commercial line of pigs.

\section{Materials and methods}

In the present study, rearing pigs housed in a commercial farm either in Standard or Enriched pens were followed up over a period of eleven weeks. 28 hybrid pigs with initial average weight of $32,5 \pm 2 \mathrm{~kg}$ were used in this study. The day of arrival at the farm, the animals were randomly assigned to two experimental groups. 14 individuals were housed in a physically enriched pen ('Enriched group') and 14 animals were housed in a conventional pen ('Standard group'). The pens contained a total of about 50 individuals. The enriched pen contained 6 chains, 5 tough, nontoxic plastic balls with stones inside, two hemp ropes hung from the ceiling and 6 rubber hoses placed on the floor. Behavioural data and blood samples were collected on day of arrival of the animals (T.0), 24 hours later (T.1), and two weeks (T.2), six weeks (T.3) and eleven weeks (T.4) from the arrival. These samples were used to determine a range of haematological parameters. All statistical analyses were conducted using Statview 5.0 (SAS Institute Inc.).

\section{Results}

Results are shown in Table 1. Pigs from Enriched housing spent significantly less time in abnormal behaviours than the animals raised in Standard condition. Blood samples from Enriched group showed lower levels of white blood cells (WBC) than the Standard counterpart (Tab.1).

\section{Discussion}

Swine exposed to physical enrichments presented important and consistent reduction of abnormal behaviors. Furthermore, pigs housed in the enriched pen had a six times lower probability than those from standard condition to present skin body lesions. This study gives a clear indication that the presence of manipulative objects provides welfare advantages to the rearing pigs. Pigs from Enriched housing showed a significant higher level of neutrophils than those from the Standard pen, whereas the percentages of circulating eosinophils in the blood samples of the Enriched individuals were significantly lower compared with swine housed in the Standard pen, this fact may be reasonably ascribed to a different parasitic status on individual basis (1). Both lymphocyte and monocyte levels were markedly lower in Enriched than in Standard group. Interestingly, the animals from Enriched housing showed a progressive reduction of these white blood cells from Time 2 to Time 4, whereas swine housed in the Standard pen showed an increment between Time 2 and 3. This profile can be a consequence of ongoing bacterial infections in progress, such as enzootic subclinical pulmonitis typical of intensive pig farming systems. With regard to haematological measures, all parameters considered showed significantly lower levels in the samples from Enriched pen than in those from the Standard group, except the concentration of Red Blood Cells (RBC) that was not affected at all. However, the levels recorded fall all within the range reported in the literature in intensive farmed pigs and may be interpreted as small perturbation induced by the sub-clinical ongoing inflammatory processes above reported. A monitoring of selected behavioral parameters and profile progression overtime and skin lesion checks may be a use-friendly strategy of management in farms with elevated animal density and limited numbers of workers.

\section{References}

1. Ellen, W. 2006. Evans Interpretation of Porcine Leukocyte Responses. Chapter 59. in Schalm's Veterinary Hematology. 5th ed Feldman, Zinkl, and Jain, Blackwell Publishing Oxford UK.

2. Mellor, D. J., and K. J. Stafford. 2001. Integrating practical, regulatory and ethical strategies for enhancing farm animal welfare. Australian Veterinary Journal 79: 762-768.

3. Stafford, K., and N. Gregory. 2008. Implications of intensification of pastoral animal production on animal welfare. N. Z. Vet. J. 56: 274280.

\section{Table 1}

\begin{tabular}{|l|c|c|}
\hline & Standard & Enriched \\
\hline WBC $(103 / \mu \mathrm{L})$ & $19.9 \pm 0.5$ & $17.8 \pm 0.6^{*}$ \\
\hline Neutrophils (\%) & $36.3 \pm 1.3$ & $42.4 \pm 1.5^{* *}$ \\
\hline Lymphocytes (\%) & $57.3 \pm 1.3$ & $52.8 \pm 1.4^{*}$ \\
\hline Eosinophils (\%) & $3.4 \pm 0.3$ & $2.4 \pm 0.2^{*}$ \\
\hline Monocytes (\%) & $2.5 \pm 0.2$ & $1.9 \pm 0.2^{*}$ \\
\hline Basophils (\%) & $0.3 \pm 0.1$ & $0.3 \pm 0.1$ \\
\hline HCT (\%) & $33.3 \pm 0.5$ & $31 \pm 0.7^{*}$ \\
\hline RBC (\%) & $6.9 \pm 0.1$ & $6.7 \pm 0.1$ \\
\hline MCV (fl) & $48.4 \pm 0.6$ & $45.5 \pm 1^{*}$ \\
\hline HGB (g/ld) & $10 \pm 0.1$ & $9.4 \pm 0.2^{*}$ \\
\hline Platelets (103/ $\mu \mathrm{L})$ & $470.2 \pm 10.8$ & $421.6 \pm 19.5$ \\
\hline $\begin{array}{l}\text { Means } \pm \text { SE of the haematological parameters } \\
\text { * Significant difference }(p<0.05) \\
\text { ** Highly significant difference }(\mathrm{p}<0.01)\end{array}$ \\
\hline
\end{tabular}




\title{
0.221
}

\section{Challenge of pigs with natural immunity to $\mathrm{H} 1$ and $\mathrm{H} 3$ swine influenza virus with pandemic 2009 H1N1 influenza virus}

\author{
Janice R. Ciacci-Zanella ${ }^{1}$ Kelly M. Lager ${ }^{1}$ Amy L. Vincent ${ }^{1}$ Eraldo L. Zanella ${ }^{1}$ Danielle Gava ${ }^{1,2}$ \\ 1. Virus and Prion Diseases Research Unit, NADC, ARS, USDA, Ames, IA, USA; \\ 2. College of Veterinary Medicine - Federal University of Rio Grande do Sul, Porto Alegre, RS, Brazil
}

\section{Introduction}

The emergence of the pandemic 2009 human H1N1 influenza A virus raised many questions about the implications for this virus in swine (1). One such question is, does prior exposure to influenza virus confer any protection against the new virus? This report describes a study to evaluate if natural exposure to swine influenza viruses confers protection against challenge with 2009 H1N1 virus.

\section{Materials and methods}

Conventionally raised pigs were purchased from a sow herd negative for swine influenza A virus. Due to purchasing and shipping logistics, the pigs had been weaned and transported to a nursery facility that was geographically separated from the sow farm. Within 7 days of arrival at NADC most of the pigs were seropositive by the Avian Influenza MultiS-Screen ELISA (2) and virus was detected in the nasal swabs from a few pigs. Multiplex RT-PCR assays using four specific primers for $\mathrm{H} 1$ and $\mathrm{H} 3$, and N1 and N2 were performed (3). Twenty group 1 (G1) pigs were housed for 5 additional weeks (until approximately 10 weeks of age) at which time they were challenged with $2 \times 10^{6}$ $\mathrm{TCID}_{50}$ ACalifornia042009 H1N1 (CA09) by the intranasal route. Naive-age-matched pigs ( $n=4$ pigs) or group 2 (G2) were also inoculated with CA09. Nasal swabs were taken on 0, 3 and 5 days post infection (dpi) to evaluate nasal shedding. Postmortem samples including serum, bronchoalveolar lavage fluid (BALF), lung, trachea and nasal swabs were collected at necropsy at day 5 pi. Serologic assays included hemagglutination inhibition $(\mathrm{HI})$ assay (4) and ELISA (2). HI assays were used to evaluate 0, 3 and 5 dpi serum samples against CA09 (H1N1), OH07 (H1N1), MN99 (H1N1), and TX98 (H3N2) viruses. Real-time PCR assays were performed for quantitation of nucleic acids in BALF of pandemic H1N1 influenza virus (5), and for PRRSV, PCV2 and Mycoplasma hyopneumoniae using in house assays (6).

\section{Results and discussion}

All pigs were negative for PRRSV, PCV2 and M. hyopneumoniae nucleic acids in BALF at necropsy. Genetic analysis of viruses isolated from nasal swabs and BALF samples from $\mathrm{G} 1$ pigs revealed the presence of simultaneous $\mathrm{H} 1$ and $\mathrm{H} 3$ virus infections prior to the start of the study at 5 weeks of age. After challenge with CA09, all naïve-challenged pigs (G2) had macroscopic lung lesions with an average lung involvement of $2.7 \%$. The multifocal lesions appeared typical with well demarcated margins, purple in color. Nine of the $20 \mathrm{G} 1$ pigs had detectable macroscopic lung lesions, but decreased in severity compared to the non-immune controls, with an average of $0.9 \%$. The character of the $\mathrm{G} 1$ lung lesions was different compared to $\mathrm{G} 2$. The macroscopic lesions were purple-grey in color; involving the tips of the apical and cardiac lobes with less distinct margins. G1 pigs had no detectable virus titers in the lung or nasal swabs ( 0,3 and 5 dpi). However, G2 pigs had CA09 viral titers in the lungs and nasal swabs with average titers of $10^{1.6} \mathrm{TCID}_{50} / \mathrm{ml}(3 \mathrm{dpi})$ and $10^{2.3} \mathrm{TCID}_{50} /$ $\mathrm{ml}(5 \mathrm{dpi})$ in nasal swabs samples, and of $10^{3.7} \mathrm{TCID}_{50} / \mathrm{ml} \mathrm{BALF}$ samples at 5 dpi. Real-time PCR for $\mathrm{H} 1 \mathrm{~N} 1$ pandemic virus in BALF (5 dpi) showed that $14 / 20$ naturally immune pigs (G1) and 4/4 naïve-challenged (G2) were positive for viral RNA. Quantitatively G2 samples presented an average of $9 \times 10^{6}$ copies of viral RNA / microliter of template. In contrast, G1 had an average of $1.4 \times 10^{3}$ copies, indicating a low viral replication in the lungs or residual RNA from challenge, confirming the negative results of virus isolation from nasal swabs and BALF. HI titers using the challenge virus (CA09) were not significant for either group at 0 and 5 dpi. However, significant $\mathrm{HI}$ titers against the $\mathrm{\gamma}$-cluster SIV $\mathrm{OH} 07$ were detected in $\mathrm{G} 1$ pigs at 0 dpi. H3N2 HI antibodies against TX98 were not detected in either group of pigs. These results indicate that pigs with natural immunity against $\gamma$-cluster SIV have significant protection against infection with 2009 pandemic H1N1.

\section{References}

1. Garten, R.J et al. 2009, Science, 325:197-201

2. Ciacci-Zanella, J. R., et al.2010 JVDI 22(1): 3-9

3. Choi, Y.K. 2002 Vir Research 87: 173-179.

4. Palmer D.F., et al.1975. Immun Series no 6, 51-52.

5. Lorusso, A., et al 2009 J. Virol. Methods (2009), doi:10.1016/j. jviromet.2009.12.002

6. Vincent et al. 2009. Influenza and Other Resp Vir DOI: 10.1111/j.1750-2659.2009.00121.x 


\title{
0.222
}

\section{Pandemic H1N1 influenza virus infection in a swine herd}

\author{
Julia M. Keenliside ${ }^{1}$ Craig Wilkinson $^{2}$ Sarah Forgie ${ }^{2}$ Patricia Lu ${ }^{1}$ Jennifer Patterson ${ }^{2}$ Eduardo Beltranena ${ }^{1}$ \\ Evelyn Stigger ${ }^{3}$ Richard Webby ${ }^{3}$ Lorne Babiuk $^{2}$ \\ 1. Alberta Agriculture and Rural Development, Edmonton, $A B$, Canada; 2. University of Alberta, Edmonton, AB, Canada; \\ 3. St Jude Children's Hospital, Memphis, TN, USA
}

\section{Introduction}

In April 2009, an outbreak of human influenza was reported due to a novel H1N1 virus. On June 11th , 2009, the World Health Organization declared a global human pandemic of H1N1 ( $\mathrm{pH} 1 \mathrm{~N} 1)$. On May 2nd, 2009, the first case of pH1N1 infection in swine was reported in small herd in Alberta, Canada. Symptoms developed in swine after a barn worker had returned from Mexico with influenza-like illness. Epidemiologic evidence supported human to swine transmission $(1,2)$. Little was known about the pathogenicity of this novel virus in swine. The objective of this study was to describe the clinical progression and production impacts of second $\mathrm{pH} 1 \mathrm{~N} 1$ infection in a naturally infected sow herd.

\section{Materials and Methods}

On June 16th, pH1N1 was confirmed in two swine barn workers at a second farm in Alberta. Symptoms in swine began on June 19th. The herd was a high health breeding herd of 300 sows, free of all major pathogens including PRRS. Sows were routinely vaccinated for classical swine influenza $\mathrm{H} 1 \mathrm{~N} 1$ and $\mathrm{H} 3 \mathrm{~N} 2$ viruses (Flusure, Pfizer Animal Health, Kirkland, Quebec). The herd was well managed with excellent biosecurity and above average air quality. No new animals had been introduced since September 2008.

Clinical signs in swine and humans were recorded daily during the outbreak. Nasal swabs were taken from systematically selected swine on days $-1,11,15,25,53$ after the onset of symptoms. All swine nasal swabs were screened by RT-PCR at the Food Safety and Animal Health Division laboratory in Edmonton. Initial samples were sent to National Centre for Foreign Animal Disease (NCFAD) in Winnipeg, Manitoba for confirmation and molecular characterization. Molecular characterization and comparison to human virus isolates was done at St. Jude Children's Research Hospital, Memphis, Tennessee. Reproductive performance records, feed intake and carcass quality data were obtained.

\section{Results}

The first clinical signs were observed in swine on June 19th, seven days after contact with the first ill worker and four days after contact with the second affected worker. A non-productive cough with a moderate abdominal effort, mild to moderate depression, pyrexia and anorexia of 24-48 hours duration was observed in gestating sows. Most affected animals displayed anorexia and depression only. Animals previously affected completely recovered within 24-48 hours. By the second day, two percent $(29 / 1450)$ of the herd was affected. Over the next seven days new cases were seen daily in gestating and lactating sows and gilts. No clinical signs were observed in piglets, weaned pigs or growing pigs. The proportion of the herd affected never exceeded two percent and declined to 0.5 percent by day eight. After day 36 no clinical signs were seen. The herd made a complete recovery with no associated mortality. Positive nasal swabs indicated asymptomatic shedding and infection was likely occurring in all classes of swine.

Some reproductive impact on individual sows and boars was recorded.

The only symptoms of respiratory disease prior to this outbreak were in three animals in December 2008. At that time nasal swabs and lung tissue were negative for influenza by PCR. No other symptoms of respiratory disease have been recorded in this herd since 2003.

\section{Discussion}

The mild clinical signs in this herd are in contrast to the more severe symptoms reported in the first case of pH1N1 in swine (1). The absence of other respiratory pathogens, air quality and vaccination likely contributed to the milder clinical picture. This infection could escape detection in a less rigorously observed herd, creating challenges for surveillance. Subsequent growth and reproductive performance was negatively impacted in spite of lack of clinical signs. PCR results indicate that be infection and viral shedding may occur in asymptomatic animals.

Molecular characterization of the virus indicates the same virus infected both the workers and swine. The chronology of symptoms in humans and swine supports human to swine transmission.

\section{References}

1. Howden et al. Can Vet J. 2009 November; 50 (11): 1153-1161 (2010)

2. Keenliside J. (2010) Advances in Pork Production 21, 129-135 


\title{
0.223
}

\section{Enhanced pneumonia with pandemic 2009 A/H1 N1 swine influenza virus in pigs}

\author{
Phillip C. Gauger ${ }^{1}$ Amy L. Vincent ${ }^{2}$ Crystal L. Loving ${ }^{2}$ Kelly M. Lager ${ }^{2}$ \\ 1. lowa State University, Ames, IA, USA; 2. USDA Agricultural Research Service National Animal Disease Center, Ames, IA, USA
}

\section{Introduction}

Swine influenza A viruses (SIV) in the major swine producing regions of North America consist of multiple subtypes of endemic H1N1, H1N2, and H3N2 derived from swine, avian and human influenza viruses with a triple reassortant internal gene (TRIG) constellation. ${ }^{1}$ Genetic drift and reassortment has resulted in four $\mathrm{H} 1$ phylogenetic clusters designated as $\alpha, \beta, \gamma$, and $\delta$ that co-circulate in U.S. swine. ${ }^{2}$ Control of SIV has relied on the use of commercially available or autogenous influenza vaccines in the U.S. swine industry. However, inactivated vaccines have demonstrated poor cross-protection against heterologous homosubtypic, or heterosubtypic SIV. The emergence of diverse genetic subtypes has increased the need for effective SIV vaccines with adequate cross-protection. In this report, we describe a vaccineheterologous virus challenge model demonstrating enhanced lung lesions in pigs given inactivated $\delta$-cluster $\mathrm{H} 1 \mathrm{~N} 2$ vaccine (human seasonal $\mathrm{H} 1$-lineage $\mathrm{HA}$ ) challenged with heterologous pandemic 2009 A/H1N1compared to non-vaccinated challenged pigs.

\section{Materials and Methods}

Twenty, three-week old conventional pigs free of SIV and PRRSV were randomly divided into two groups. The vaccinated and challenged (vax/challenge) group was vaccinated with $10^{6.5}$ $50 \%$ tissue culture infectious dose $\left(\mathrm{TCID}_{50}\right)$ units of $\mathrm{A} / \mathrm{SW} /$ MN/02011/08 ( $\delta$-cluster) inactivated by UV irradiation with the addition of a commercial adjuvant. Vaccine was administered intramuscularly at six and three weeks prior to challenge. Vax/ challenge and non-vaccinated/challenged (non-vax/challenge) pigs were inoculated intratracheally with $2 \mathrm{ml}$ of $1 \times 10^{5} \mathrm{TCID}_{50} /$ $\mathrm{ml} \mathrm{A/California/04/2009} \mathrm{H1N1} \mathrm{(CA/09).} \mathrm{Five} \mathrm{pigs} \mathrm{remained} \mathrm{non-}$ vaccinated, non-challenged controls. Pigs were observed daily for signs of clinical disease and fever. Serum samples were obtained at each vaccination, challenge and 5 days post-infection (dpi). Nasal swabs were taken on 0,3 , and 5 dpi to evaluate nasal virus shedding. All pigs were necropsied at $5 \mathrm{dpi}$. Lungs were removed and evaluated for the percentage of lesions typical of influenza virus infection in pigs. Each lung was lavaged with $50 \mathrm{ml}$ MEM to obtain bronchoalveolar lavage fluid (BALF).

\section{Results}

All pigs were free of influenza A virus and antibodies prior to vaccination. At 24 hours post-challenge, vax/challenge and nonvax/challenge pigs had significant $(P<0.05)$ febrile responses compared to controls. At 72 hours post challenge, vax/challenge pigs had a significantly elevated $(P<0.05)$ febrile response to infection that persisted to $5 \mathrm{dpi}$ compared to non-vax/challenge pigs. Febrile responses in non-vax/challenge pigs returned to normal at 72 hours. Vax/challenge pigs had significantly higher $(P<0.001)$ macroscopic pneumonia scores averaging 20.5 percent of the lung at $5 \mathrm{dpi}$ compared to an average of 8.9 percent of the lung in non-vax/challenge pigs. Macroscopic lesions were absent in negative controls. At $3 \mathrm{dpi}, 7 / 10$ and 5/10 pigs in the vax/challenge and non-vax/challenge groups, respectively, were positive for nasal shedding. By $5 \mathrm{dpi}$, both groups had 9/10 pigs positive for virus in the nasal cavity. Virus levels at $5 \mathrm{dpi}$ in nasal secretions were significantly less $(P<0.001)$ in the vax/challenge group compared to non-vax/challenge pigs. Virus levels in the lung were not significantly different. Isolate specific hemagglutination inhibition $(\mathrm{HI})$ titers developed only in pigs primed with the $\delta$-cluster killed vaccine and no cross-reactivity between sera from vaccinated pigs and CA/09 antigens were detected prior to challenge. Neutralizing antibodies (NA) against the $\delta$-cluster virus were detected prior to challenge in the sera from vaccinated pigs although NA responses were lacking against the $\mathrm{CA} / 09$ virus at the same time. Additional antibody assays are in progress.

\section{Discussion}

This study demonstrates an enhancement of pneumonia in vaccinated pigs challenged with a heterologous, homosubtypic virus, consistent with previous reports by our group. ${ }^{3}$ The enhanced pneumonia was significantly greater compared to pigs challenged with the same virus without previous vaccination or prior SIV specific antibodies. Therefore, it is speculated that the vaccine-induced immune response in the lung contributes to the enhanced pneumonia. In addition, virus replication in the nasal cavity and lung were lower in vax/challenge pigs in spite of the enhanced lung lesions. To date, this phenotype has been experimentally reproduced when pigs are administered an inactivated SIV H1 vaccine followed by heterologous homosubtypic challenge. This phenomenon has the potential to be realized in the U.S. swine population due to the exposure of genetically diverse $\mathrm{H} 1$ SIV currently circulating among swine vaccinated with inactivated heterologous virus vaccines.

\section{References}

1. A. L. Vincent et al., Adv Virus Res 72, 127 ( 2008).

2. A. L. Vincent et al., Virus Genes 39, 176 (2009).

3. A. L. Vincent et al., Vet Microbiol 126, 310 (2008). 


\title{
0.224
}

\section{Pandemic influenza A(H1N1) 2009 virus in swine herds: Outbreak in Norway 2009}

\author{
Chiek Er $^{1}$ Britt Gjerset ${ }^{1}$ Anna Germundsson ${ }^{1}$ Charlotte Hjulsager $^{2}$ Solfrid Åmdal ${ }^{3}$ Olav Hungnes ${ }^{4}$ \\ Merete Hofshagen ${ }^{1}$ Edgar Brun ${ }^{1}$ Bjørn Lium ${ }^{1}$ \\ 1. National Veterinary Institute, Oslo, Norway; 2. National Veterinary Institute, Technical University of Denmark, Copenhagen, Denmark; \\ 3. Norwegian Food Safety Authority, Oslo, Norway; 4. Norwegian Intsitute of Public Health, Oslo, Norway
}

\section{Introduction}

The first outbreak of infection with pandemic influenza $A(H 1 N 1)$ 2009 virus (H1N1/09 virus) in a Norwegian swine herd was recorded on 10th of October 2009. Until then the Norwegian pig population was considered free of swine influenza subtype $\mathrm{H} 1 \mathrm{~N} 1$ and H3N2, as documented by a serological surveillance program running since 1997 (1). This paper describes the results of a surveillance study in the Norwegian pig population from 10th of October to 31st of December 2009.

\section{Materials and methods}

Herds. To investigate the extent of the H1N1/09 virus outbreak in Norway in October 2009, the Norwegian Food Safety Authority initiated an increased surveillance targeted at the following categories:

- Herds where the pigs had been exposed to persons with verified $\mathrm{H} 1 \mathrm{~N} 1 / 09$ virus infection or to persons with influenza-like symptoms (ILI).

- Herds where pigs with ILI were observed.

- Herds with a history of contact with or close proximity to infected herds.

- Serological screening of nucleus and multiplier breeding herds.

Sampling and tests. Initially most herds were tested by nasal swabs from 20 pigs. Detection of H1N1/09 virus was done at the Norwegian National Veterinary Institute, by real-time RT-PCR to detect influenza $A$ virus (2). Positive samples were tested by H1N1/09 virus specific real-time RT-PCR (3). In addition, samples were tested at the National Veterinary Institute, Technical University of Denmark, by a H1N1/09 virus specific real-time RT-PCR (4). Later, many herds, including the nucleus and multiplier herds, were tested serologically by an enzyme-linked immunosorbent assay (ELISA, ID Screen ${ }^{\circledR}$ Influenza A Antibody Competition test, IDVET) and haemagglutination inhibition ( $\mathrm{HI}$ ) test to detect antibodies against influenza A virus on at least 20 pigs per herd. In addition, a few tracheal and bronchial swabs from pneumonic lungs, from the slaughter house were tested by RT-PCR.

Diagnostic criteria. One PCR-positive sample or five samples positive for antibodies against influenza A virus was defined as sufficient to confirm a farm as positive. If less than five samples were positive for antibodies with the ELISA, the herd was retested. The farm is declared negative when the re-tested second samples were all negative.

\section{Results}

Of the 114 herds tested by nasal swabs, 54 herds were positive for $\mathrm{H} 1 \mathrm{~N} 1 / 09$ virus, and 55 of the herds tested by serology were positive for antibodies against $\mathrm{H} 1 \mathrm{~N} 1 / 09$ virus as confirmed by $\mathrm{HI}$ test (Table 1).

Table1. Molecular and serology results for H1N1/09 virus surveillance in Norwegian swine herds from 10th of October to 31st of December 2009.

\begin{tabular}{|l|c|c|c|c|}
\hline Samples & Herds tested & $\begin{array}{c}\text { Herds positive } \\
\mathbf{n}(\%)\end{array}$ & Total samples & $\begin{array}{c}\text { Positive } \\
\text { samples }\end{array}$ \\
\hline Nasal swabs & 114 & 54 & 1628 & 440 \\
\hline Serology & 140 & 55 & 2659 & 659 \\
\hline *Total & 217 & 91 & 4287 & 1099 \\
\hline *Some herds were tested by both RT-PCR and serology. \\
\hline
\end{tabular}

Genome sequencing of the virus from one pig confirmed it as identical to the virus from a sick farm staff member at the same farm and that it is at least $99.8 \%$ similar to other pandemic viruses circulating in humans in Norway.

\section{Discussion}

The majority of the positive herds, especially among the herds tested first, had a history of contact between pigs and humans whom were diagnosed with pandemic influenza or people with ILI. This suggests infected humans to be the most likely source for transmission of H1N1/09 virus to positive pig herds. Ongoing studies will evaluate the risk factors for infection in pigs, and the hypothesis that humans transmitted $\mathrm{H} 1 \mathrm{~N} 1 / 09$ virus to pigs. The diversity of $\mathrm{H} 1 \mathrm{~N} 1 / 09$ virus in the Norwegian pig population will be studied by sequencing positive samples.

\section{References}

1. Lium B., et al. 2007. Surveillance and control programmes for terrestrial and aquatic animals in Norway. Annual report: NVI. p. 99-102.

2. World Health Organization.2009. http://www.who.int/csr/resources/

3. Robert Koch-Institut, Berlin. Germany. http://www.rki.de/cln_160/nn_200120/DE/Content/InfAZ/I/Influenza/IPV/Schweinegrippe_PCR.html.

4. Statens Serum Institut, Denmark, unpublished. 


\title{
0.225
}

\section{Towards risk-based surveillance for swine influenza virus}

\author{
Barbara Wieland ${ }^{1}$ Alexander Mastin ${ }^{1}$ Dirk U. Pfeiffer ${ }^{1}$ The COSI Consortium $^{2}$ \\ 1. Royal Veterinary College, Hatfield, UK; 2. Combating Swine Influenza Initiative consortium, Cambridge, UK
}

\section{Introduction}

Pigs are a significant reservoir of $\mathrm{H} 1 \mathrm{~N} 1, \mathrm{H} 1 \mathrm{~N} 2$ and $\mathrm{H} 3 \mathrm{~N} 2$ influenza viruses, strains of which are endemic in pigs worldwide. Swine influenza virus (SIV) can also contribute to substantial economic loss in pig production due to respiratory disease. In order to monitor the SIV strains present in the UK, a national swine influenza surveillance programme was implemented in 1991, since when avian-like swine H1N1 and H1N2 strains have been detected. The programme is based on passive surveillance and therefore the true proportion of farms in the UK exposed to the different SIV strains and the prevalence of subclinical infection is difficult to estimate. The emergence of the novel pandemic H1N1v strain in people in 2009 is also a substantial threat for the pig industry and infection studies have confirmed the susceptibility of pigs to the virus and the ability to transmit from pig to pig.

For the development of an effective active surveillance strategy to allow early detection of $\mathrm{H} 1 \mathrm{~N} 1 \mathrm{v}$ in pigs and to inform preventive and control measures, the epidemiology of SIV in England needs to be better understood. The study presented here aimed to provide further key knowledge by investigating farm level risk factors for SIV infection in pigs.

\section{Material and Methods}

In 2008/09, 146 farrow-to-finish farms were enrolled in a crosssectional study; the sample represented about $17 \%$ of the English pig herd. Of each farm serum samples of 20 animals of different age groups were tested for H1N1, H1N2 and H3N2 influenza viruses with haemagglutination inhibition $(\mathrm{HI})$ assays. At the time of the farm visit, extensive data on herd management, herd health, reproduction management, production parameters and the farm environment was collected. Factor analysis was used to reduce the number of variables and to account for co-linearity in the dataset. To identify herd level risk factors, the identified risk factors were tested for association with SIV infection using univariate and multivariate logistic regression models.

\section{Results}

Fifty-nine $\%$ of tested farms had at least one growing pig with a $\mathrm{HI}$ titre of $1 / 40$ or at least two animals with lower titres. Avianlike H1N1 was found to be the most common strain in England, followed by H1N2 and one farm tested positive for H3N2. Also $18 \%$ of farms tested positive for $\mathrm{H} 1 \mathrm{~N} 1$ and $\mathrm{H} 1 \mathrm{~N} 2$, which could either be cross-reaction or infection with both strains. H1N2 positive farms were found across the country. There was however, a significant cluster of $\mathrm{H} 1 \mathrm{~N} 1$ positive farms in the Northeast of the country with farms located within the cluster having a relative risk of $\mathrm{H} 1 \mathrm{~N} 1$ infection of 4.63. Risk factor analysis identified association of farm size, location, and biosecurity measures in place with increased risk of infection with SIV. Also SIV positive farms were more likely to be infected with other endemic pig pathogens and had poor respiratory scores in slaughterhouse monitoring programmes.

\section{Discussion}

In conclusion the findings of this study improve our understanding of the current swine influenza situation in England. The prevalence found is much higher than expected and highlights the importance of subclinical infection, respectively that the importance of SIV as a significant production disease has been underestimated in the past. The findings of the risk factor analysis provide insight in how spread of SIV in general, and spread of $\mathrm{H} 1 \mathrm{~N} 1 \mathrm{v}$ in particular, can be prevented and will inform future surveillance activities. 


\title{
0.226
}

\section{Antigenic and genetic diversity of delta cluster swine influenza virus H1N1 and H1N2 field isolates from the United States}

\author{
Vicki J. Rapp-Gabrielson ${ }^{1}$ Jonathan M. Evans ${ }^{1}$ Jenifer L. Czach ${ }^{1}$ Gregory P. Nitzel ${ }^{1}$ Therese K. Yonkers $^{1}$ \\ Eric Wicklund ${ }^{1}$ Stephen K. Behan ${ }^{1}$ Lucas P. Taylor ${ }^{1}$ Marie R. Gramer ${ }^{2}$ \\ 1. Veterinary Medicine Research and Development, Pfizer Animal Health, Kalamazoo, MI, USA; \\ 2. University of Minnesota Veterinary Diagnostic Laboratory, St. Paul, MN, USA
}

\section{Introduction}

Currently, four clusters of $\mathrm{H} 1$ swine influenza viruses (SIV) are circulating in the North American swine population. The most recent endemic $\mathrm{H} 1$ cluster to emerge is the human-like, or delta cluster, of $\mathrm{H} 1 \mathrm{~N} 1$ and $\mathrm{H} 1 \mathrm{~N} 2$ viruses that were first reported in the eastern and western USA in 2003 and 2005.,2 The delta cluster viruses, beta cluster viruses (reassortant-like $\mathrm{H} 1$ ) and gamma cluster viruses (H1N2-like) are currently co-circulating in the US swine population, while classic-like (alpha cluster) viruses are still occasionally isolated in the US and Canada., ${ }^{3,4}$

\section{Materials and Methods}

The hemagglutinin protein similarity of delta $\mathrm{H} 1$ viruses was determined by sequencing the HA1 portion of the gene then aligning with Clustal V. A neighbour-joining analysis was used to reconstruct phylogenetic trees with the DNASTAR computer program. The length of each pair of branches represents the distance between sequence pairs, while the units at the bottom of the tree indicate number of substitution events. To evaluate the association of genetic relatedness to antigenic crossreactivity, SIV-negative pigs were vaccinated with two doses of monovalent, adjuvanted and inactivated vaccines prepared from different delta cluster viruses. ${ }^{5}$ Sera were tested for hemagglutination inhibition ( $\mathrm{HI}$ ) antibody titers to homologous and heterologous delta $\mathrm{H} 1$ viruses. The animal phase of these studies was conducted according to the guidelines of Pfizer Animal Health's (PAH) Institutional Animal Care and Use Committee.

\section{Results}

There are genetic differences among contemporary delta $\mathrm{H} 1$ SIV (Figure 1). Viruses also differed in $\mathrm{HI}$ cross-reactivity to the homologous and heterologous viruses (Table 1).

Table 1: Hemagglutination inhibition cross-reactivity of delta $\mathrm{H} 1$ swine influenza viruses

\begin{tabular}{|l|c|c|c|c|c|}
\hline Virus & \multicolumn{5}{|c|}{ Antisera } \\
\hline Antigen & 031 & 718 & 082 & 708 & 726 \\
\hline 031 & $\mathbf{1 6 0}$ & $<10$ & 40 & 40 & 10 \\
\hline 718 & $<10$ & $\mathbf{6 4 0}$ & $<10$ & $<10$ & 640 \\
\hline 082 & $<10$ & $<10$ & $\mathbf{8 0}$ & 160 & $<10$ \\
\hline 708 & $<10$ & $<10$ & 160 & $\mathbf{4 0}$ & 10 \\
\hline 726 & 10 & 320 & 10 & 10 & $\mathbf{1 2 8 0}$ \\
\hline \multicolumn{7}{|l|}{ Values in bold are homologous titers. } \\
\hline
\end{tabular}

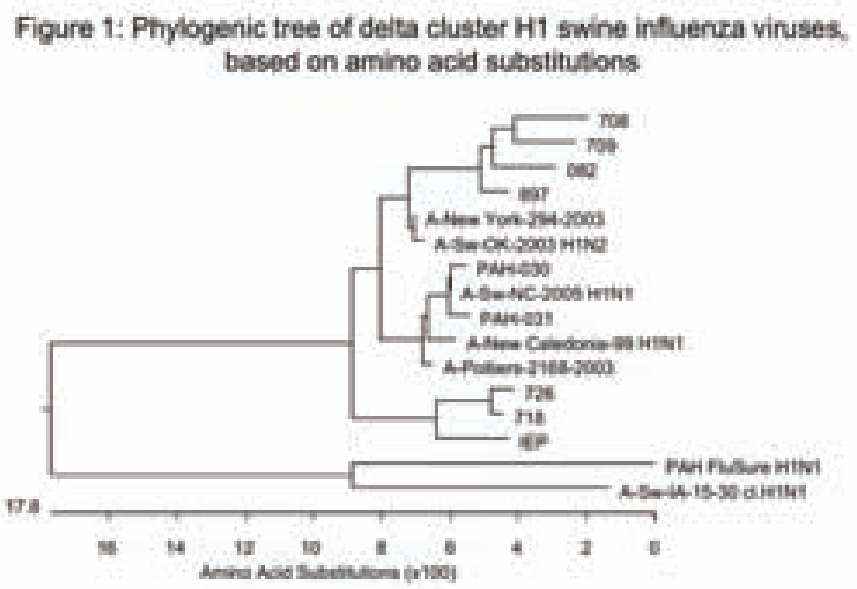

\section{Discussion}

We previously reported strong $\mathrm{HI}$ cross-reactivity between delta $\mathrm{H} 1$ viruses originating from US swine herds in 2005-2007.3,4 The current results, evaluating additional $\mathrm{H} 1$ field isolates from 2008-2009, indicate both genetic and antigenic diversity among viruses within this cluster. These data are consistent with a recent report that also demonstrated diversity among delta cluster viruses. ${ }^{6}$ Although $\mathrm{HI}$ cross-reactivity and HA similarity may be predictors of variability, vaccination and challenge studies are the best predictors of vaccine cross-protection.

\section{References}

1. Gramer, M. 2006. Proceed. 37th AASV, p. 463-464.

2. Karasin, A., S. Carman, C.W. Olsen. 2006. J Clin Microbiol. 44:1123-26.

3. Gramer, M., G. Nitzel, E. Wicklund et al. 2008. Proceed 39th AASV, p. 99-101.

4. Rapp-Gabrielson V., M. Gramer, G. Nitzel et al. 2008. Proceed. 20th IPVS, p. 24.

5. Study Reports No. 3121R-60-06-451, 3121W-60-08-682 and No. 3125W-60-09-795, Pfizer Inc.

6. Vincent, A., W. Ma, K. Lager et al. 2009. Virus Genes. 39 :176-185. 


\title{
0.227
}

\section{Genetic and antigenic characterization of $\mathbf{2 0 0 8}$ swine influenza viruses from the United States}

\author{
Amy L. Vincent ${ }^{1}$ Michelle L. Harland ${ }^{1}$ Alessio Lorusso ${ }^{1}$ David Alt ${ }^{1}$ Darrell O. Bayles ${ }^{1}$ Sabrina L. Swenson ${ }^{2}$ \\ Marie R. Gramer ${ }^{3}$ Kelly M. Lager ${ }^{1}$ Nicola S. Lewis ${ }^{4}$ \\ 1. USDA-ARS National Animal Disease Center, Ames, IA, USA; 2. USDA-APHIS National Veterinary Services Laboratories, Ames, IA, USA; \\ 3. University of Minnesota, St. Paul, MN, USA; 4. University of Cambridge, Cambridge, UK
}

\begin{abstract}
Introduction
Prior to the introduction of the 2009 pandemic H1N1 virus (1) from humans into pigs in the U.S., four phylogenetic clusters of the hemagglutinin $(\mathrm{HA})$ gene from $\mathrm{H} 1$ swine influenza viruses (SIV) co-circulated. Viruses from the classical H1N1 SIV lineage evolved over time to form $\alpha-, \beta-$, and $\gamma$-clusters. SIV with HA genes most similar to human seasonal $\mathrm{H} 1$ viruses emerged in 2003 to form the $\delta$-cluster (2). All four HA cluster gene types can be found with neuraminidase genes of either N1 or N2 subtype. Limited information was available regarding the 6 genes that make up the triple reassortant internal gene (TRIG) cassette in contemporary $\mathrm{H} 1 \mathrm{SIV}$. In addition, information regarding the antigenic relatedness of the $\mathrm{H} 1$ viruses and diagnostic reagent updates were in need due to the dynamic and variable nature of $\mathrm{H} 1 \mathrm{SIV}$. We characterized $12 \mathrm{H} 1$ isolates from 2008 by sequencing and phylogenetic analysis of all eight gene segments and by serologic cross-reactivity in the hemagglutination inhibition (HI) assay.
\end{abstract}

\section{Materials and methods}

Viruses. Swine influenza viruses isolated from outbreaks of respiratory disease in pigs were grown in Mardin-Darby Canine Kidney (MDCK) cells. Virus isolates were: A/SW/NC/02023/2008 H1N1, A/SW/OH/02026/2008 H1N1, A/SW/MO/02060/2008 H1N1, A/SW/IA/02096/2008 H1N1, A/SW/KY/02086/2008 H1N1, A/SW/MN/02011/2008 H1N2, A/SW/MN/02093/2008 H1N1, A/ SW/MN/02053/2008 H1N1, A/SW/NE/02013/2008 H1N1, A/SW/ NC/02084/2008 H1N1, A/SW/TX/01976/2008 H1N2, and A/SW/ IA/02039/2008 H1N2.

Antigenic characterization. Four-week-old cross-bred pigs were obtained from a herd free of influenza virus and antibodies. Two pigs per virus were immunized with inactivated virus combined with commercial adjuvant by the intramuscular route. Two or three vaccinations were given approximately 2-3 weeks apart until homologous $\mathrm{HI}$ titers reached $\geq 1: 80$.

$\mathrm{HI}$ assays were performed with turkey RBC's using panels of homologous and heterologous viruses and anti-sera with standard techniques. Quantitative analyses of the antigenic properties of swine influenza A H1 viruses were performed using antigenic cartography, previously used for human and swine influenza A (H3N2) viruses(3-4) and 2009 pandemic H1N1 (1).

Genetic characterization. Each virus isolate was concentrated and semi-purified on a sucrose cushion followed by library preparation for de novo pyrosequencing on a Genome Sequencer FLX system following the manufacturer's recommendations with modifications. Briefly, viral RNA was extracted, fragmented, reverse transcribed, and ligated to oligonucleotide adaptors containing multiplex identifier (MID) labels. Pools containing MID-labeled viral cDNA were used to prepare sequencing beads via Roche's GS-FLX standard chemistry emulsion-based PCR. Prepared beads were loaded into the small 16 regions on a GS-
FLX standard chemistry pico-titer plate and sequenced using the GS-FLX LR 70 standard chemistry. Sequencing reads were compared to an influenza database created from $>85,000$ influenza sequences extracted from GenBank in Dec. 2008. Using the BLAST results, sequencing reads were filtered, influenza specific reads extracted, and extracted reads were assembled with the Roche GS De Novo Assembler (Newbler) version 2.0 application. Gene segments with large gaps were closed by traditional primer based sequencing using an ABI 3100 genetic analyzer. The sequences were analyzed using SeqMan (DNASTAR). Phylogenetic analyses were conducted using MEGA version 4.

\section{Results}

Based on genetic analysis, each of the four previously described phylogenetic clusters of $\mathrm{H} 1$ SIV was represented in the 2008 panel. Additionally, it was demonstrated that the $\delta$-cluster HA diverged into sub-clusters $\delta-1$ and $\delta$-2. Serologic cross-reactivity paired with antigenic cartography demonstrated that the phylogenetic clusters are also divergent antigenically. The genes composing the TRIG were all North American in lineage, although genetic variation was apparent.

\section{Discussion}

In 2008, five genetic and antigenic clusters of H1 SIV co-circulated in the U.S. pig population with limited HI cross-reactivity between them. Genetic diversity was demonstrated in all gene segments, but most notably in the HA gene. Gene segments from the 2009 pandemic H1N1 formed clusters separate from North American swine lineage viruses, suggesting this virus was not present in U S. pigs prior to 2009. The HA variability has important implications for diagnostic testing as well as vaccine development.

\section{Acknowledgments}

We thank Brian Pottebaum and Jason Huegel for assistance with animal studies. Funding was provided by USDA/ARS and National Pork Checkoff.

\section{References}

1. R. J. Garten et al., Science 325, 197 (Jul 10, 2009).

2. A. L. Vincent et al., Virus Genes 39, 176 (2009).

3. D. J. Smith et al., Science 305, 371 (Jul 16, 2004).

4. J. C. de Jong et al., Journal of virology 81, 4315 (Apr, 2007). 


\title{
0.228
}

\section{Seroprevalence and risk factors of swine influenza in Spain}

\author{
Meritxell Simon-Grifé $^{3}$ Gerard E. Martín-Valls ${ }^{3}$ Maria J. Vilar ${ }^{3}$ Ignacio García-Bocanegra ${ }^{1}$ Mercedes Mora ${ }^{3}$ \\ Margarita Martín 2, 3 Enric Mateu², 3 Jordi Casal ${ }^{2,3}$ \\ 1. Departamento de Sanidad Animal, Universidad De Córdoba, UCO, Campus Universitarios de Rabanales, Córdoba, Spain; \\ 2. Departament de Sanitat i d'Anatomia Animals, Universitat Autònoma de Barcelona, Bellaterra, Barcelona, Spain; \\ 3. Centre de Recerca en Sanitat Animal (CReSA), UAB-IRTA, Campus de la Universitat Autònoma de Barcelona, Bellaterra, Barcelona, Spain
}

\section{Introduction}

Swine influenza is caused by type A influenza viruses. The three commonest swine influenza virus (SIV) subtypes are H1N1, H1N2 and $\mathrm{H} 3 \mathrm{~N} 2$ although other less frequent or sporadic subtypes can infect pig farms as well $\mathrm{H}_{4 \mathrm{~N} 6^{1}}$ In Spain, previous serological studies revealed high seroprevalences for $\mathrm{H} 1 \mathrm{~N} 1, \mathrm{H} 1 \mathrm{~N} 2$ and $\mathrm{H} 3 \mathrm{~N} 2$ viruses in sows ${ }^{2}$ and fattening pigs ${ }^{3}$. Nevertheless, available information indicating risk factors for the introduction and spread of the infection in farms is very much scarce. The main objectives of the present study were to estimate the seroprevalence of $\mathrm{H} 1 \mathrm{~N} 1, \mathrm{H} 1 \mathrm{~N} 2$ and $\mathrm{H} 3 \mathrm{~N} 2$ subtypes in sows and fattening pigs and to explore the potential risk factors associated with seropositivity to SIV.

\section{Material and methods}

Between 2007 and 2009 a cross-sectional survey was carried out in swine farms of pig-producing regions of Spain. Sampling was designed to guarantee a $95 \%$ probability of detecting at least one positive animal for an assumed within-herd seroprevalence of $50 \%$. As a result 100 swine farms located all over Spain were examined. Epidemiological data were gathered through on-farm interview of the farmer based on "closed-ended" questions. For each farm, blood samples were collected from 14 sows and 20 finishers, totaling 2,249 pigs for the whole survey.

Blood samples were analyzed by means of an indirect ELISA (CIVTEST Influenza, HIPRA) directed to detect antibodies against SIV nucleoprotein that was performed according to the manufacturer's instructions. A hemagglutination inhibition $(\mathrm{HI})$ test was standardized for H1N1 (A/swine/Neth/Best/96), H1N2 (strain A/swine/Gent/7625/99) and, H3N2 (strain A/swine/ Neth/St.Oedenrode/96) according to procedures previously described $^{4}$. IH titres $\geq 80$ were considered as positive. SPSS 14.0 statistical program was used to explore risk factors associated with swine influenza using the method of generalized estimating equations ${ }^{5}$ (GEE).

\section{Results}

By ELISA, all farms had at least one seropositive animal, and in total 1,435/2,249 animals yielded positive ELISA results (63.8\%; IC95\%: 61.8-65.8\%). Seroprevalence of sows was $77.8 \%\left(\mathrm{IC}_{95 \%}\right.$ : $75.5-79.9 \%)$ significantly higher $(p<0.05)$ than seroprevalence in fattening pigs $\left(40.9 \% ; \mathrm{IC}_{95 \%}: 37.6-44.2 \%\right)$. Average seroprevalence in positive farms was $68.3 \%$. A total of 1,231 animals (54.7\%; IC $95 \%$ : 52.7-56.8\%) were positive in the HI test. Again, sows had a significantly higher $(\mathrm{p}<0.05)$ seroprevalence compared to fattening pigs $\left(72.9 \% ; \mathrm{IC}_{95 \%}\right.$ : $70.6-76.3$ against $24.7 \%$; $\mathrm{IC}_{95 \%}$ : 21.8- 27.6). Table 1 summarizes the results obtained by individuals and age group. In the epidemiological analysis, four variables (open vs. solid partitions between pens, pig density, natural vs. forced ventilation and the percentage of replacement rate) were identified as risk factors.

\section{Table 1}

\begin{tabular}{|c|c|c|c|c|}
\hline \multirow{3}{*}{ Subtypes } & \multicolumn{4}{|c|}{$\begin{array}{l}\text { Number and percentage of } \\
\text { positive animals }\end{array}$} \\
\hline & \multicolumn{2}{|c|}{ Sows } & \multicolumn{2}{|c|}{ Fatteningpiss } \\
\hline & $\mathbf{N}^{\mathbf{P}}$ & $\%$ & $\mathbf{N}$ & $\%$ \\
\hline Hinlonly & 257 & 18.4 & 53 & 6.2 \\
\hline IIIEonly & 39 & 2.8 & 11 & 13 \\
\hline IBNQonly & 252 & 18 & 86 & 10.1 \\
\hline HINI+HINR & 40 & 2.8 & 20 & 2.4 \\
\hline $\mathrm{HIN1+HBNR}$ & 295 & 21.1 & 27 & 3.2 \\
\hline HINR+FBNR & 60 & 43 & 8 & 0.9 \\
\hline $\mathrm{H} I N 1+\mathrm{HINZ}+\mathrm{HBN2}$ & 78 & 5.6 & 5 & 0.6 \\
\hline Negative & 379 & 27 & 639 & 75.3 \\
\hline
\end{tabular}

\section{Discussion}

This results shows that SIV is widespread in Spain. The high seroprevalence observed against $\mathrm{H} 1 \mathrm{~N} 1$ and $\mathrm{H} 3 \mathrm{~N} 2$ compared to $\mathrm{H} 1 \mathrm{~N} 2$ could be explained by a reduced ability to spread the latter. The higher seroprevalence observed in sows could be explained by the fact that these animals have a long lifespan. As a consequence, sows would have more chances to be exposed to SIV. The epidemiological analysis also show results obtained in GEE have shown that major contact between animals favor SIV circulation. Low air renewal enhances a major concentration of SIV in environment, which explains higher seroprevalence in farms without forced ventilation. Finally, an increasing \% of replacement, could favor a higher number of susceptible animals to SIV infection.

\section{References}

1. Karasin et al. (2000) Can. Vet J. 41, 938-939.

2. Maldonado et al. (2006) Vet. J. 172, 377-381.

3. Fraile et al. (2009) Vet. J. doi:10.1016/j.tvjl.2009.03.029.

4. Webster and Krauss (2002) (ed. WHO, W. H. O.)(Geneva, Switzerland).

5. Liang and Zeger (1986). Biometrika 73, 13-22. 


\title{
0.229
}

\section{Searching for the universal H1N1 peptide-based vaccine}

\author{
Julia Vergara-Alert ${ }^{1}$ Jordi M. Argilaguet ${ }^{1}$ Núria Busquets ${ }^{1}$ Gerard E. Martín-Valls ${ }^{1}$ Raquel Rivas $^{1}$ Natàlia Majó ${ }^{1,2}$ \\ Sergio López-Soria ${ }^{1}$ Joaquim Segalés ${ }^{1}$ Fernando Rodríguez ${ }^{1}$ Veljko Veljkovic ${ }^{3}$ Ayub Darji ${ }^{1}$ \\ 1. Centre de Recerca en Sanitat Animal (CReSA), Bellaterra, Spain; 2. Departament de Sanitat i d'Anatomia Animals, Universitat Autònoma de \\ Barcelona (UAB), Bellaterra, Spain; 3. Center for Multidisciplinary Research, Institute of Nuclear Sciences VINCA, Belgrade, Serbia
}

\section{Introduction}

Pigs may act as "mixing vessels" and generate recombinant viruses of unknown pathogenicity for animals and humans, as reflected with the last human $\mathrm{H} 1 \mathrm{~N} 1$ influenza pandemia. One of the main problems to obtain efficient vaccines against flu is the high variability of the virus, limiting the protection to highly homologous viruses.

In an attempt to identify potential cross-reactive epitopes within the $\mathrm{H} 1$ haemagglutinin $(\mathrm{HA})$, a conformational spectrum analysis was done using sequences available in the GenBank database. One highly conserved peptide of 34 amino acids mapped in the flanking region of the $\mathrm{HA}$ binding site from human $\mathrm{A} / \mathrm{South}$ Carolina/1/18 (H1N1) (NF-34 peptide), was selected ${ }^{1}$. The objective of this study was to test the protective effect of the synthetic $\mathrm{HA}$-peptide based vaccine in vivo.

\section{Materials and Methods}

A total of eight 8 week-old conventional crossbreed pigs seronegative against swine influenza viruses (SIV) were randomly divided into two groups (four pigs each). Each group was intramuscularly injected three times either with the NF-34 peptide (10-15 $\mu \mathrm{g}$ per dose) (referred to as G1) or with phosphate buffer saline (referred to as G2) two weeks apart. One month after last dose, pigs were intranasally inoculated with $10^{6}$ tissue culture infectious doses $50 \%\left(\mathrm{TCID}_{50}\right)$ of the human $\mathrm{H} 1 \mathrm{~N} 1$ pandemic virus (A/Catalonia/63/2009 strain). Six days later, pigs were sacrificed. Serum and peripheral blood mononuclear cells (PBMCs) taken before each immunization, before challenge and at 6 days post-challenge (necropsy day) were used to detect specific humoral and cellular responses, respectively. Specific antibodies were detected using a peptide-based ELISA or by the haemagglutination inhibition assay, while cellular responses were determined by IFN $\gamma$-ELISPOT after overnight stimulation with either the NF-34 peptide or with inactivated virus. After challenge with pandemic $\mathrm{H} 1 \mathrm{~N} 1$ virus, pigs were sacrificed at day 6 post-challenge and viral loads checked by a real-time qRT-PCR in bronchoalveolar lavage (BAL).

\section{Results}

Pigs immunized with the NF-34 synthetic peptide developed peptide-specific antibodies. Also, G1 animals showed an antibody boost at day 6 post-challenge (lines in figure 1). Similarly, peptide-specific IFN $\gamma$-secreting cells were detected by ELISPOT before challenge, showing a clear boost by day 6 post-challenge (bars in figure 1). Specific T-cells did not only recognize the NF34 peptide but also the inactivated pandemic virus (data not shown).

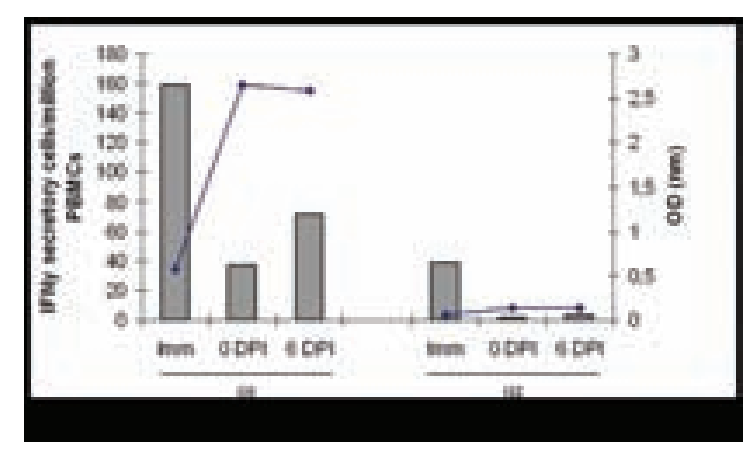

Despite the haemagglutination inhibitory antibodies induced after vaccination were relatively weak (data not shown), 50\% (2/4) of G1 pigs showed lower viral load in the BALs compared with $\mathrm{G} 2$ pigs (Figure 2).

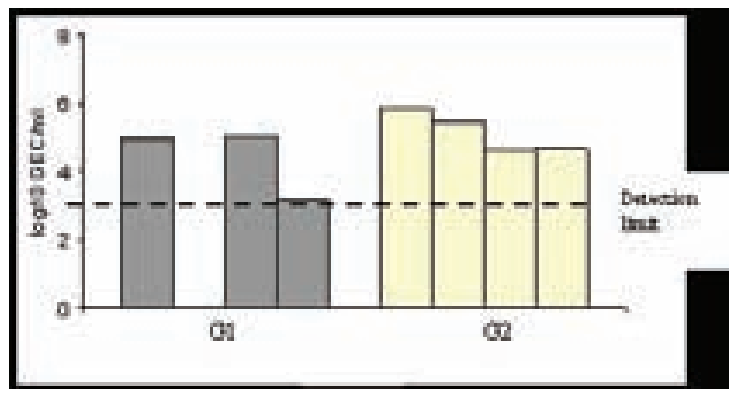

\section{Discussion}

Current vaccines against influenza (including those against seasonal influenza in humans) are restricted to the predicted circulating strain at a given time in a specific world location. It is important to notice that even though only partial protection was afforded in the present pig model, this was obtained against one of the most divergent $\mathrm{H} 1 \mathrm{~N} 1$ strains, with an HA differing in 3 aminoacids from the peptide used to vaccinate. We suggest that this strategy could be appropriated to immunize against divergent strains by incorporating to the vaccine epitopes able to induce neutralizing antibodies enabling to obtain safe and efficacious subunit flu vaccines.

\section{Acknowledgments}

Authors would like to thank Maria Ballester, Mercedes Mora, Joan Pujols, Virginia Aragón and Tuija Kekarainen from CReSA for their help and support and also for their invaluable discussions.

\section{References}

1. Veljkovic et al. BMC Struct Biol. 2009, 9:62. 


\title{
0.230
}

\section{Human-like H1 ( $\delta$-cluster) swine influenza virus (SIV) can efficiently replicate, transmit, cause lung pathology and induce humoral immune responses in the swine host}

\author{
Janice R. Ciacci-Zanella ${ }^{1,3}$ Amy L. Vincent ${ }^{1}$ Eraldo L. Zanella ${ }^{1,2}$ Susan Brockmeier ${ }^{1}$ \\ 1. Virus and Prion Diseases Research Unit, NADC, ARS, USDA, Ames, IA, USA; 2. College of Agronomy and Veterinary Medicine \\ - University of Passo Fundo, Passo Fundo, RS, Brazil; 3. Labex-USA, EMBRAPA, Brasilia, DF, Brazil
}

\section{Introduction}

Genomic characterization of recently identified $\mathrm{H} 1$ influenza $\mathrm{A}$ viruses demonstrated the viruses were triple reassortants with an internal gene constellation similar to contemporary U S. swine influenza virus (SIV) but hemagglutinin (HA) and neuraminidase (NA) most similar to human seasonal influenza virus lineages $(1,2)$. The HA gene of these viruses form the $\delta$-cluster in phylogenetic analyses of HA genes from North American SIV. $\mathrm{PB} 1-\mathrm{F} 2$ is an influenza $A$ virus protein encoded on gene segment 2 and described to be a virulence factor in other host species, but its role in swine is currently unknown (3). The $\delta$-cluster $\mathrm{H} 1$ SIV was demonstrated to contain segment 2 encoding PB1-F2 proteins with and without premature truncations (3). The objective of this work was to study the pathogenesis and transmission of $\delta$-cluster H1 SIV in swine.

\section{Materials and methods}

Three $\delta$-cluster $\mathrm{H} 1$ SIV grown in MDCK cells were used in this study. Two isolates, A/sw/MN/07002083/07 H1N1 (MN07) and A/sw/IL/00685/05 H1N1 (IL05), were chosen due the absence of the full-length coding sequence for PB1-F2, whereas A/sw/ TX/01976/08 H1N2 (TX08) contains the full-length PB1-F2 sequence. Forty-nine pigs were divided in seven groups composed of 3 primary inoculated pig groups with each $\delta$-cluster virus, three contact groups and one uninfected control group. Primary pigs $(n=10)$ were infected intratracheally with SIV and naïve contact pigs $(n=5)$ were co-mingled after 48 hours to be infected via natural transmission routes. Nasal swabs were taken on 0,3 , 5,7 and 9 days post infection (dpi) or days post contact (dpc) to evaluate nasal shedding. Oral fluids were also collected on days 3-10, 14, 18 and $20 \mathrm{dpi}$ as described previously (4). Postmortem samples including serum, bronchoalveolar lavage fluid (BALF), lung, trachea and nasal swabs were collected at necropsy at days 5 and 21 . Non-challenged age-matched negative control pigs were necropsied at $5 \mathrm{dpi}$ ( $\mathrm{n}=5$ pigs). Serologic assays included the hemagglutination inhibition (HI) (5) assay and the Avian Influenza MultiS-Screen ELISA (6). In addition to homologous $\mathrm{HI}$ assays, $21 \mathrm{dpi}$ and $19 \mathrm{dpc}$ serum were evaluated against the studied viruses (TX08, MN07 and IL05) as well as a previously generated $\mathrm{H} 1$ serum panel in heterologous $\mathrm{HI}$ assays. Real-time PCR assays were performed for detection of SIV (oral fluids) and of PRRSV, PCV2 and Mycoplasma hyopneumoniae nucleic acids in BALF using in house assays (3).

\section{Results and discussion}

All pigs were seronegative for specific antibodies to SIV by the HI assay and by ELISA prior to the start of the study. Pigs were also negative for PRRSV, PCV2 and M. hyopneumoniae nucleic acids in BALF at necropsy. All viruses induced SIV illness; however, clinical signs and macroscopic lesions induced by MN07 were reduced in severity as compared to TX08 group pigs in this study. MN07 infected pigs had lower virus titers in the lung and nasal swabs (day 5) than the TX08 and IL05 groups. However, on day $7 \mathrm{dpi}$ all of the nasal swab samples from MN07 and IL05 inoculated pigs were negative. In comparison, the TX08 inoculated group had $80 \%$ of the pigs shedding virus in nasal swab samples on $7 \mathrm{dpi}$, with average titers of $101.2 \mathrm{TCID} 50 / \mathrm{ml}$. In contrast to TX08, only contact pigs were still shedding on $7 \mathrm{dpc}$ in MN07 (10\%) and IL05 (20\%) groups. These data demonstrate that all viruses were able to replicate and be shed, however TX08 was more efficient in nasal shedding with higher titers and an extended duration of shedding. Oral fluids samples also showed lower however longer viral titers for MN07 (4-9 dpi) and IL05 (3-8 dpi) in comparison to TX08. Pigs in this study mounted a more robust humoral response to MN07 and IL-05 $\delta$-cluster H1N1 compared to the TX08. This was statistically significant for $\mathrm{HI}$ titers at days 14 and 21. Cross-HI using heterologous virus demonstrated little cross-reactivity between the TX08 anti-sera and the MN07 and IL05 $\delta$-cluster $\mathrm{H} 1$ viruses. However, there was cross-reactivity between the MN07 virus and IL05 anti-sera. This is consistent with phylogenetic evidence of 2 sub-clusters of $\delta$-H1 SIV. This study indicates that the $\delta$-cluster H1 SIV can efficiently replicate, transmit, cause lung pathology and induce humoral immune responses in the swine host. However, the kinetics of virus replication, pathogenesis, as well as host humoral immune response differed between the 3 viruses. Two sub-clusters of the $\delta$-cluster SIV appear to have emerged simultaneously with limited serologic cross-reactivity between them. What role (if any) the full-length PB1-F2 present in TX08 played in the higher nasal shedding of virus remains to be determined.
References
1. Olsen, C. W. 2002. Vir Research 85(2): 199-210.
2. Vincent, A.L., et al. 2008. Adv Vir Res 72: 127-54.
3. Vincent, A.L., et al. 2009. Vir Genes 39: 176-185.
4. Prickett, J. 2008. JVDI 20:156-163.
5. Palmer D.F., et al.1975. Immun Series no 6, 51-52.
6. Ciacci-Zanella, J. R., et al.2010 JVDI. 22(1): 3-9 


\title{
0.231
}

\section{Selection of influenza vaccine strains and rapid method for producing antigenically homologous vaccine}

\author{
$\underline{\text { DL Hank Harris }}{ }^{1}$ Marie Gramer ${ }^{2}$ Ryan L. Vander Veen ${ }^{1}$ Mark A. Mogler ${ }^{1}$ \\ 1. lowa State University, Ames, IA, USA; 2. University of Minnesota Veterinary Diagnostic Laboratory, St. Paul, MN, USA
}

\section{Introduction}

There are a wide variety of swine influenza viruses (SIV) occurring in the United States swine population. The subtypes and variants present include 4 clusters of $\mathrm{H} 1$ - alpha, beta, gamma (which includes the pandemic $\mathrm{H} 1 \mathrm{~N} 1$ virus) and $\mathrm{Hu} \mathrm{H} 1$ or delta, and one dominant H3N2 (cluster 4). 1,2 Traditionally, nucleotide sequences of the first 900 nucleotides ( 5 ' end) of the hemagglutinin (HA) gene are utilized to classify subtypes of viruses; however, genetic similarity does not necessarily correlate with antigenic relatedness. When selecting vaccine strains used to prevent seasonal influenza in humans, a vaccine strain is updated when there is an antigenic difference between the vaccine strain and a newly emerged strain of greater than 2 units (4-fold dilution) in the hemagglutination-inhibition ( $\mathrm{HI})$ assay. ${ }^{3}$ We report here the results of preparing antisera in pigs to recombinant protein subunit $\mathrm{HA}$ antigens and determining the antigenic relatedness of field strains of SIV by $\mathrm{HI}$ assay.

\section{Materials and Methods}

Swine influenza virus negative pigs were vaccinated twice with adjuvanted vaccine consisting of either recombinant $\mathrm{H} 1$ beta, delta, gamma, pandemic, or Cluster 4 H3 HA subunit proteins for the preparation of cluster-specific antiserum. Serum samples with reciprocal HI titers of 160-320 were used for further HI testing against field strains of SIV.

\section{Results}

Table 1 shows the $\mathrm{HI}$ results of $2 \mathrm{H} 1 \mathrm{~N} 1$ beta viruses, $\mathrm{A}$ and $\mathrm{B}$, from different farms. Hemagglutination inhibition titers are similar for Farm A and Harrisvaccines (HV) Beta viruses (within 2 antigenic units of the homologous reaction). Although all antigens were genetically similar, $\mathrm{HI}$ titers for Farm B are $>2$ antigenic units different from the homologous titers. Table 2 shows the $\mathrm{HI}$ results of cluster $4 \mathrm{H} 3 \mathrm{~N} 2$ antigens. Again, the HV, Farm $C$ and Farm $D$ antigens are genetically similar; however, in this case, there is little antigenic difference discernable by $\mathrm{HI}$.

Table 1. Reciprocal HI titers for antisera 1 and 2 against viruses Harrisvaccine (HV) Beta 2009, Farm A 2007, and Farm B 2009. Percent nucleotide similarities to HV Beta 2009 virus are also shown. Homologous reactions are in bold.

\begin{tabular}{|l|c|c|c|}
\hline & HV Beta Virus (2009) & Farm A Virus (2007) & Farm B Virus (2009) \\
\hline Antiserum 1 & $\mathbf{1 6 0}$ & 160 & 10 \\
\hline Antiserum 2 & $\mathbf{3 2 0}$ & 320 & 20 \\
\hline$\%$ Similarity & $100 \%$ & $97 \%$ & $96 \%$ \\
\hline
\end{tabular}

Table 2. Reciprocal HI titers for antisera 1 and 2 against viruses HV Cluster 4 H3N2 2007, Farm C 2007, and Farm D 2009. Percent nucleotide similarities to HV Cluster 4 H3N2 2007 virus are also shown. Homologous reactions are in bold.

\begin{tabular}{|l|c|c|c|}
\hline & $\begin{array}{c}\text { HV Cluster 4 H3N2 } \\
\text { Virus (2007) }\end{array}$ & Farm C Virus (2007) & Farm D Virus (2008) \\
\hline Antiserum 1 & $\mathbf{3 2 0}$ & 320 & 160 \\
\hline Antiserum 2 & 320 & 320 & 320 \\
\hline \% Similarity & $100 \%$ & $97 \%$ & $96 \%$ \\
\hline
\end{tabular}

\section{Discussion}

These antigenic results suggest that the HV beta vaccine would protect against a challenge with virus from Farm $A$ but not Farm B, despite the high genetic similarity of the three viruses. Antigenic drift was detectable in $\mathrm{H} 1$ strains, but was limited in the $\mathrm{H} 3$ viruses evaluated in this study. The $\mathrm{H} 3$ viruses were both antigenically and genetically similar, suggesting that vaccination with the HV Cluster $4 \mathrm{H} 3 \mathrm{~N} 2$ vaccine would provide crossprotection against isolates from Farm $C$ or Farm $D$. These results demonstrate that genetic similarity is not the sole indicator of possible vaccine protection, and should be combined with antigenic analysis to determine if a vaccine will be protective against field isolates. Because HA genes can be rapidly exchanged for constructs used to produce recombinant HA protein subunits, ${ }^{4}$ vaccines for prevention of influenza can be created quickly to match the strain occurring in the farm of origin.

\section{Acknowledgements}

The authors would like to thank Drs. Joe Connor, Steve Henry, James Lowe, and Tom Petznick for their input and assistance.

\section{References}

1. Vincent A,et al. Virus Genes 2009;39.

2. Gramer MR. American Association of Swine Veterinarians 2009: 471473.

3. Smith DJ,et al. Science 2004;305:371-6.

4. Vander Veen R,et al. PLoS Currents Influenza 2009 October 29 RRN1123. 


\title{
0.232
}

\section{Prior swine influenza virus infection enhances pulmonary responses to secondary Haemophilus parasuis infection}

\author{
Susan L. Brockmeier; Crystal L. Loving; Amy L. Vincent
}

National Animal Disease Center, Ames, IA, USA

\section{Introduction}

Swine Influenza virus (SIV) infection and associated complications are a leading cause of morbidity and mortality. It is appreciated that SIV is often complicated by secondary bacterial infection. Tracheal epithelial cells (TEC) and pulmonary cells respond to infection with proinflammatory cytokines for controlling pathogen spread; however, exacerbated responses can lead to immunopathology that may be detrimental to the host. The extent to which prior SIV infection alters host responses to secondary stimulation is poorly understood. Haemophilus parasuis is an important and common respiratory pathogen of pigs. The innate immune responses of the respiratory tract of pigs infected with SIV 5 days prior to secondary infection with $H$. parasuis were examined.

\section{Materials and Methods}

Groups of 4 pigs were infected with SIV and 5-days later infected with H. parasuis (SIV/Hps). Non-infected, SIV-only and $H$. parasuis-only groups were included as controls. Pigs were necropsied 1 day following $H$. parasuis inoculation. Pigs were inoculated intranasally with $2 \times 10^{6} \mathrm{CCID}_{50}$ of SIV (A/SW/ $\mathrm{OH} / 511445 / 2007 \mathrm{H} 1 \mathrm{~N} 1$ ) and $10^{6} \mathrm{CFU}$ of $H$. parasuis strain 12939. Nasal wash, tracheal wash and broncho-alveolar lavage (BAL) were performed and number of CFU of $H$. parasuis per $\mathrm{ml}$ of wash was determined. Levels of IL-8, IL- 6 , and IL-1 $\beta$ in BAL fluid and tracheal wash were determined by ELISA. mRNA expression of the cytokine genes from tracheal epithelium and BAL cells were determined using real-time RT-PCR.

\section{Results and Discussion}

H. parasuis colonization was higher in the nose and lungs of SIV/ Hps pigs compared to Hps-only pigs (Figure 1). These results indicate that influenza virus infection contributes enhanced bacterial colonization.
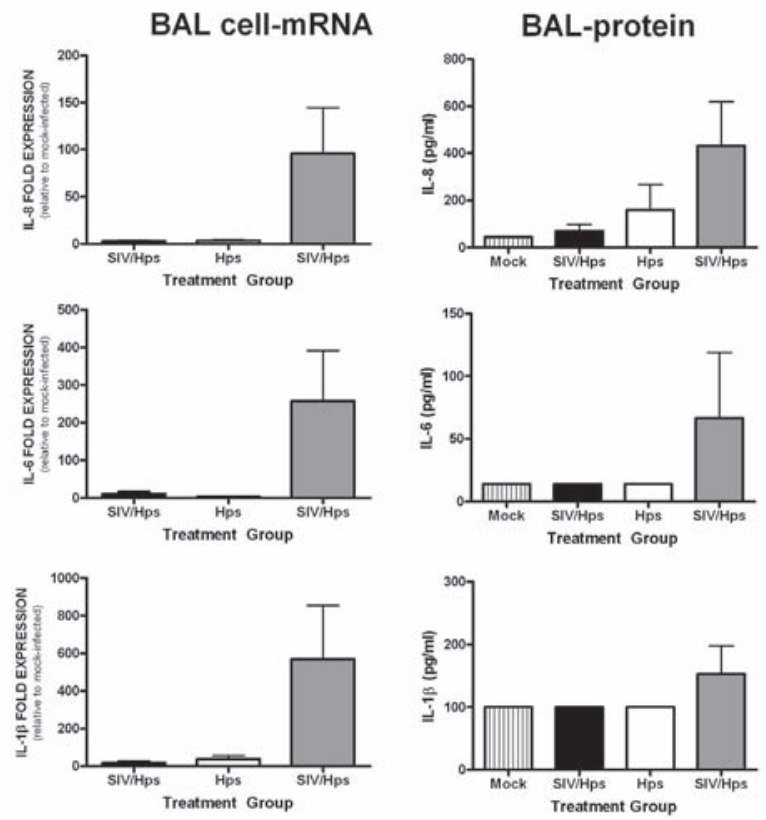

In SIV/Hps pigs, IL-8, IL- 6 and IL-1 $\beta$ protein levels were increased in the tracheal wash and BAL, and TEC and BAL cells IL-8, IL-6 and IL-1 $\beta$ mRNA expression levels were significantly increased over SIV-only and Hps-only pigs (Figure 2).

These results support the hypothesis that prior influenza infection alters subsequent immune responses. In addition, the data provide evidence of a synergistic response to secondary bacterial stimulation in animals previously infected with influenza virus.

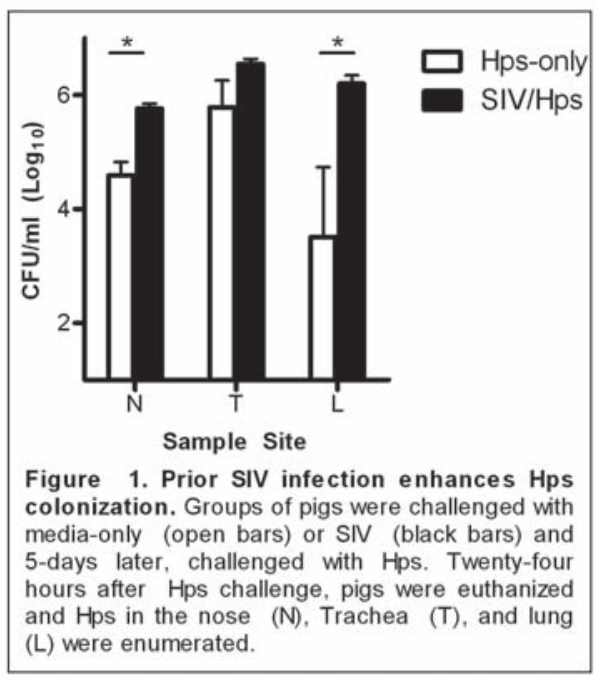




\title{
Standard herd classification and related terminology for PRRS virus
}

\author{
Derald J. Holtkamp ${ }^{1}$ Dale D. Polson ${ }^{2}$ \\ 1. lowa State University, Ames, IA, USA; 2. Boehringer Ingelheim Vetmedica, Inc., Ankeny, IA, USA
}

\begin{abstract}
Introduction
For over two decades producers, veterinarians and researchers have spent, and continue to spend, impressive amounts of time communicating about porcine reproductive and respiratory (PRRS) virus. And yet the lack of standard clearly defined terminology, related to the virus as well as the PRRS virus status of herds continues to create confusion and inefficiency. The purpose of this paper is to provide a standard herd classification scheme describing the PRRS virus status of herds, and a set of related definitions that is consistent with the biology and ecology of PRRS virus.
\end{abstract}

Development was jointly undertaken by the American Association of Swine Veterinarians (AASV) and the United States Department of Agriculture (USDA) PRRS-CAP. A definitions committee chaired by Dr. Bob Morrison was formed to complete the task. The committee was composed of researchers and veterinarians from private practice, universities and industry as well as representatives from AASV and the National Pork Board (NPB) in the U.S.

\section{Method for classification of herds}

For breeding herds, with or without growing pigs on the same premises there are four categories, I to IV. Category II is further divided into two sub-categories (II-A and II-B). For premises with only growing pigs present there are two categories.

The herd classification is based upon the shedding and antibody status of the herd. The shedding status is classified as negative, unknown or positive. An unknown shedding status means that diagnostic evidence is available to confirm the sustained absence of viremia and there have been no clinical signs over a period of time. However, the possibility that virus may be present in other tissue of and shed from the tested animals or other animals in the herd cannot be ruled out with certainty and therefore the shedding status is unknown. The antibody status is classified as negative or positive.

\section{Results - Classification of breeding herds}

Category I ("positive unstable") breeding herds have a positive shedding and antibody status. It is the default category when a status has not been confirmed.

Category II ("positive stable") breeding herds have an unknown shedding and positive antibody status. An absence of clinical signs of PRRS in the breeding herd and confirmation of a sustained lack of viremia in piglets, and if present growing pigs, for a minimum of 90 days is required. The antibody status of the herd remains positive. The possibility that animals in category II herds may still be infected and later shed the virus cannot be ruled out. A distinction is made between category II herds that are not undergoing PRRS virus elimination (II-A) and those that are (II-B). An elimination procedure begins when the herd is closed to introduction of breeding replacements that are not negative. Confidence that the piglets and growing pigs in category II-B breeding herds are not shedding virus is higher than for II-B since additional steps are being taken to eliminate shedding.

Category III ("provisional negative") breeding herds have a negative shedding status and positive antibody status. Sustained introduction of negative breeding replacements without seroconversion to the PRRS virus is required. Entering negative breeding replacements that have been in contact with previously positive animals and remain seronegative by ELISA for a minimum of 60 days is strong enough evidence to confirm that the virus is no longer being shed in the herd. Some adult breeding animals in the herd may still have antibodies to the PRRS virus due to prior infections. If growing pigs are present, confirmation of a negative antibody status in that subpopulation is also required. Category III herds include those intentionally pursuing elimination via herd rollover by entering negative breeding replacements.

Category IV ("negative") breeding herds have a negative shedding and antibody status. For herds established negative by herd rollover, all previously infected animals have been removed from the herd or alternatively all adult breeding animals in the herd are seronegative by ELISA. If growing pigs are present, confirmation of a negative antibody status in that subpopulation is also required. As with category III herds, sustained introduction of negative breeding replacements that remain seronegative is required. It also includes new premises startups that were populated with negative breeding replacements or premises that were completely depopulated and repopulated with negative breeding replacements that have remained seronegative by ELISA.

\section{Results - Classification of growing pig herds}

Herds with growing pigs only are classified as either positive or negative. Positive herds have a positive shedding and antibody status. It is the default category when a negative status cannot be confirmed. Negative herds have a negative shedding and antibody status.

\section{Discussion}

A standard, universally accepted herd classification scheme and definitions related to PRRS virus are required to facilitate regional and national elimination efforts. Equally important, they will improve communications between researchers, veterinarians, producers, genetic companies and other industry vendors by decreasing the amount of time required to communicate and reducing miscommunications. 


\title{
0.234
}

\section{Modelling PRRSv control within a pyramidal network of pig movements}

\author{
Catherine Belloc ${ }^{1}$ Amandine Lurette ${ }^{2}$ Matt Keeling ${ }^{3}$ \\ 1. INRA-ONIRIS Vet School, Nantes, France; 2. INRA, Montpellier, France; 3. University of Warwick, Coventry, UK
}

\section{Introduction}

Epidemiological models can help to improve pathogen control through the ex ante assessment of control measure efficacy at several scales. The pork primary production chain consists in pyramidal structures with herds having specific links between them, some being animal suppliers for others. Since animal movements are a key issue for pathogen spread, we elaborated a dynamic network model describing between-herd animal flows. We then tested by simulation the implementation of a control program combining both intra-herd control measures and restriction of animal movements depending on the sanitary status of herds for an important pathogen of pigs: the PRRSv.

\section{Materials and Methods}

A stochastic network model with a one-week discrete time step was elaborated based on data collected by a group of French producers over 12 months. 256 herds were included in the model (4 nucleus herds, 17 multiplier herds and 235 production herds). They were divided into four types: breeding $(n=7)$, farrow-to-finish ( $n=176)$, post-weaning-finishing $(n=5)$ and finishing ones $(n=68)$. Animals concerned by between-herd movements were $8 \mathrm{~kg}$-piglets ( $\mathrm{n}=346$ movements during the study period), $25 \mathrm{~kg}$-piglets ( $n=371)$, reproductive gilts $(n=2440)$. Herds were randomly categorized according to their PRRSv status (negative or infected). Nucleus herds were all negative and prevalence of herd infection was assumed to be 0.2 and 0.6 for multiplier and production herds respectively (Lebret, 2008). This status was evaluated every six months. To simulate the implementation of movement restrictions we generated two subnetworks based on most connected herds using Unicet software version 6: "restricted" ( $n=127$ herds) and "non-restricted" $(n=129)$ sub-networks. In the "restricted" sub-network, the infectious status of herds was checked before each movement and a PRRSv negative herd can be supplied by negative herds only. Moreover, herds of this sub-network may implement control measures aiming at reducing the probability of within-herd PRRSv persistence. We assumed that these measures were of variable efficacy depending on their real implementation under field conditions: probability of persistence of PRRSv between two tests (assumed to be equal to 1 without measure) could be reduced to $0.95,0.9$ or 0.8 .

\section{Results}

The numbers of infected herds observed after 520 weeks of control implementation for 100 simulations by scenario are presented in Figure 1. The restriction of movements taking into account the PRRSv status of herds leads to a slight decrease in the number of infected herds (mean values from 180.9 to 152.4). Additionally when movement restriction was combined with within-herd persistence reduction, the decrease was more important, leading to a negative status of all herds of the "restricted" network after 10 years if the persistence probability did not exceed 0.8 .
Figure 1 : Distribution of the total infected herds and infected herds in the 'non-restricted' sub-network after 520 weeks of simulation under four scenarii: no measure, restricted purchases between herds in the 'restricted sub-network', both restriction and a $0.95,0.9$ or 0.8 probability of the within-herd infection persistence in the 'restricted subnetwork'

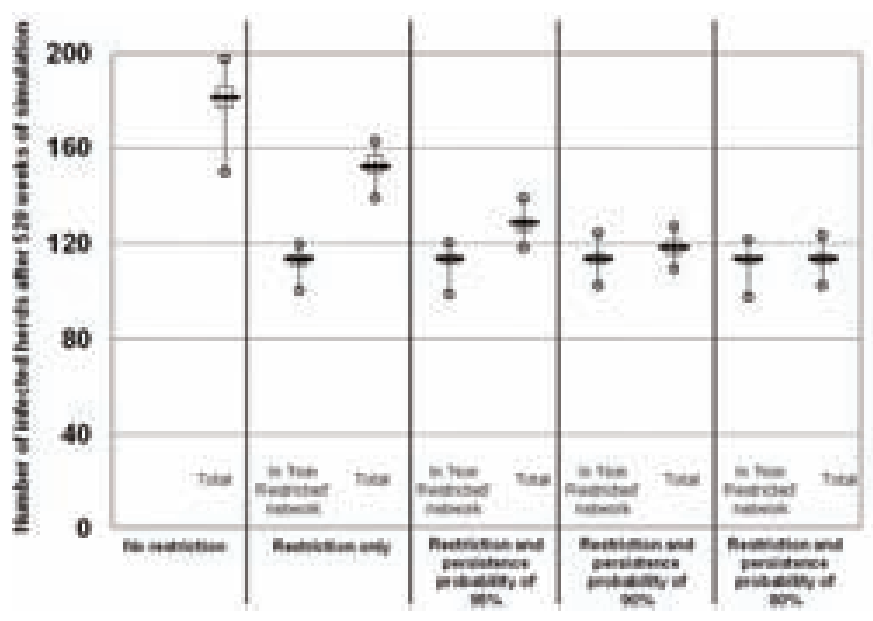

\section{Discussion}

Few data are available about the influence of control measures (biosecurity/vaccination) on the evolution of herd status over time. This model is a tool that can be improved if parameters of viral persistence can be more precisely determined. To reach this objective, data are currently collected. In the same way, although animal movements are crucial for herd contamination, other ways of viral spread will be considered. Another perspective is to combine this model with another one representing the within-herd viral dynamic. This new combined model will allow us to assess control measures implemented at both herd and production chain levels.

\section{References}

1: Lebret et al., 2008, Bulletin des GTV, 21, 28 


\title{
0.235
}

\section{MCREBEL as a key component for the successful elimination of PRRS virus from very large swine breeding herds}

\author{
Dale Polson $^{1}$ Greg Hartsook ${ }^{1}$ Kate Dion² \\ 1. Boehringer Ingelheim Vetmedica, Inc., St Joseph, MO, USA; 2. lowa State University, Ames, IA, USA
}

\section{Introduction}

Successful elimination of PRRS virus (PRRSv) from large swine breeding herds using herd closure, mass vaccination and rollover has been previously described. ${ }^{1}$ While use of these methods have been shown to result in successful elimination of PRRS virus from very large breeding herds, consistent elimination success in very large breeding herds may also require use of other management methods that minimize opportunities for persistent circulation of virus among subpopulations of susceptible animals. An area within breeding herds that may be prone to persistent virus circulation is the farrowing area. A set of concurrently applied farrowing area management practices (McREBEL) have been previously described for use in breeding herds to reduce suckling piglet losses during clinical outbreaks of PRRS. ${ }^{2}$

The objective of this observational study was to describe two cases where the discontinuation of McREBEL led to a recurrence of farrowing area PRRS virus circulation and the subsequent resumption of McREBEL resulted in the disappearance of virus circulation, and ultimately virus elimination in both farms.

\section{Materials and Methods}

In April 2008 two PRRSv naïve 5000 sow farrow to wean farms within the same production system and located $1.2 \mathrm{~km}$ apart identified PRRSv positive serum samples collected from gilts, sows and piglets by PCR and ELISA. Both farms instituted a herd closure, mass vaccination and rollover protocol to eliminate the virus. Also, strict McREBEL management practices were implemented in an effort to control PRRSv circulation in the farrowing area of both farms.

To assess progress with virus elimination, intensive sampling and PCR testing of breeding animal, stillborn fetus and dueto-wean piglet sera was initiated. Sixty individual due-to-wean piglet samples from each farm were tested by PRRS PCR and the prevalence of positive samples was followed over time utilizing the method described by Cano et al. ${ }^{3}$

Initial sampling and testing was conducted monthly, then as prevalence decreased to below $20 \%$ a weekly sampling and testing frequency was initiated.

\section{Results}

Following initial herd closure and mass vaccinations, the percent of PCR positive piglets declined on both farms to $13.6 \%$ at Farm A and 3.3\% at Farm B. During this time piglets throughout farrowing appeared healthy, exhibiting no detectable clinical signs of PRRS so farm personnel relaxed McREBEL practices. At the subsequent two monitoring periods, the percentage of PCR positive piglets on Farm B rose to $12.7 \%$ and $33.3 \%$. For the same two periods at Farm A the percentage of positives declined to $6.8 \%$ then rose to $30 \%$. Even though piglet health remained excellent, McREBEL practices were resumed on both farms due to the increase in PCR positives at Farm B. Following the resump- tion of McREBEL practices, the percentage of PCR positive piglets resumed a downward direction on both farms (Figure 1). MCREBEL practices were continued thereafter until there were no detected PCR positive samples found in the sampled dueto-wean piglets on each farm for at least six consecutive weekly samplings.

Figure 1: Combined percent of $P C R$-positive individual piglets in two previously naïve 5000 sow farms newly infected with wild-type PRRS virus.

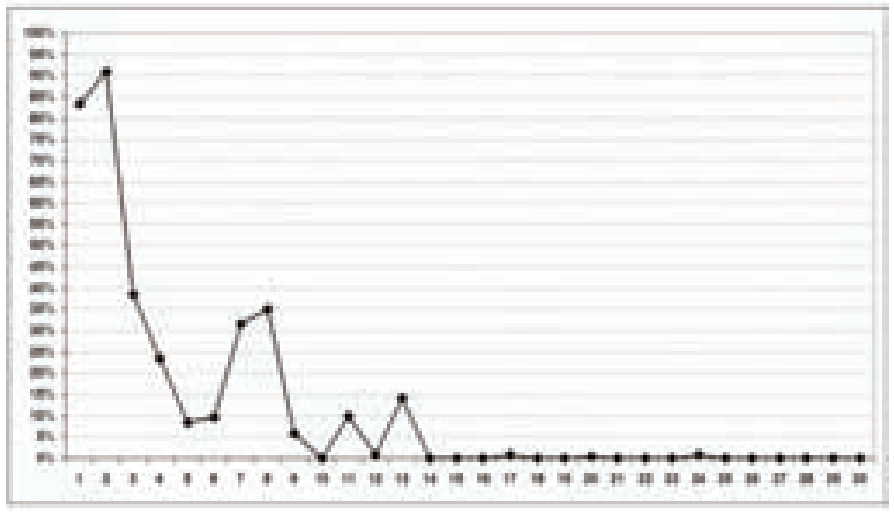

\section{Discussion}

Individual piglet monitoring provided evidence that prematurely relaxing McREBEL protocols during PRRSv elimination can enable resumed farrowing area virus circulation among otherwise healthy appearing piglets and lead to an increase in PCR positive weaned piglets, thus extending the time to negative weaned pig interval.

\section{References}

1. Polson D, Hartsook G; Proc 20th IPVS; 2008; v1p52.

2. McCaw M; J Swine Health Prod; 2000; 8:15-21.

3. Cano JP, et al.; Proc AASV; 2008; 105-107. 


\title{
0.236
}

\section{Combining sow herd stabilization and nursery PRRS virus control methods to achieve improved nursery performance}

\author{
Jose Angulo; John Kolb; Reid Philips \\ Boehringer Ingelheim Vetmedica, Inc., St Joseph, MO, USA
}

\section{Introduction}

Porcine Reproductive and Respiratory Syndrome Virus (PRRSv) is capable of causing severely negative biological and economic impact in nursery pigs. A recent analysis suggests a negative impact of US\$3.35-\$9.12/pig during the nursery phase. ${ }^{1}$ Nursery depopulation and PRRS modified live virus (MLV) vaccination of pigs are effective tools to minimize the clinical and economic impact of PRRSv infection. ${ }^{2,3}$ The objective of this study was to evaluate the impact of sow herd stabilization, nursery depopulation, and PRRS MLV pig vaccination on nursery performance.

\section{Materials and Methods}

The study was conducted in a 3,000 sow three-site production system. The sow herd stabilization program included loading the farm with its anticipated 170-day need for gilts, closing the sow herd for 170 days and mass vaccination of the sow herd with Ingelvac ${ }^{\circledR}$ PRRS MLV (BIVI) soon after herd closure. Pig interventions included PCV2 vaccination (Ingelvac CircoFLEX ${ }^{\circledR}$, BIVI), Mycoplasma hyopneumoniae (Ingelvac MycoFLEX ${ }^{\circledR}, \mathrm{BIVI}$ ) and PRRS MLV vaccinations in the nursery. Blood sampling was conducted monthly on 30 piglets from poor quality litters (1 piglet sampled per litter) at the sow site, and 20 pigs at 10 weeks of age from hospital pens at the nursery site. Along with the pig vaccination process, the nursery site was depopulated and remained empty for 4 weeks. PRRS RT-PCR testing was conducted on these sera including RFLP and ORF5 sequencing on PCR positive samples to differentiate wild type and MLV virus. The first blood sampling was conducted one month before starting the interventions. Mortality rates and average daily gain were assessed with statistical process behavior charting using MINITAB 15 software. A process change was inserted at the time of nursery depopulation and the onset of pig PRRS vaccination, with 93 groups closeout data prior to and 77 groups closeout data charted after the interventions.

\section{Results}

Wild type PRRSv was detected in 17\% (5/30) of pre-weaning pigs and $100 \%$ of nursery pigs $(n=20)$ the month pre-intervention but was not detected in any pigs during the 9 months post-intervention. MLV PRRSv was detected in nursery but not pre-weaning pigs post-intervention. The mean nursery mortality rate decreased from $2.90 \%$ pre- intervention to $2.15 \%$ post-intervention (Figure 1). Mean average daily gain (ADG) increased from 0.822 pre-intervention to $0.936 \mathrm{lb} /$ day post-intervention (Figure 2).
Figure 1. Percent nursery mortality pre-and post-intervention.

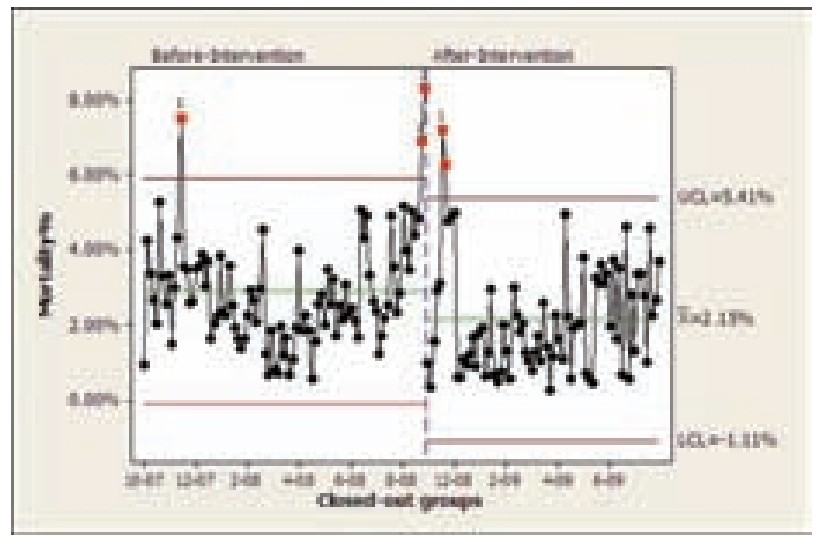

Figure 2. Average daily weight gain (Ibs) pre- and post-intervention.

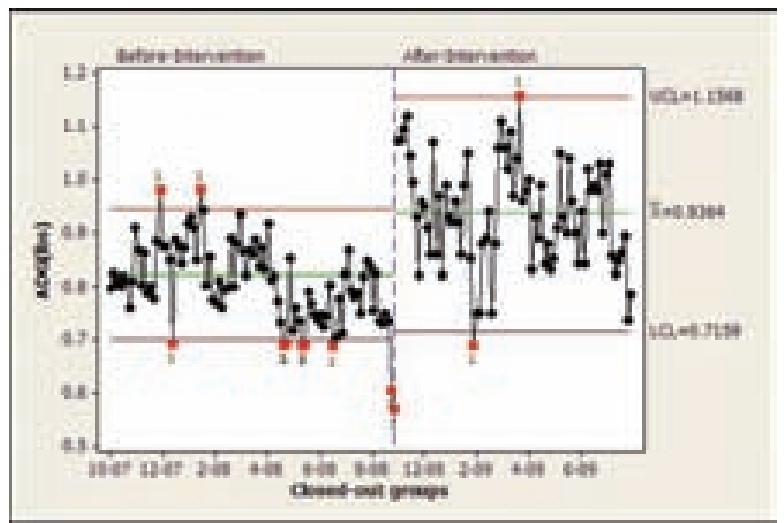

\section{Discussion}

Sow herd stabilization, nursery depopulation and on-going MLV PRRS vaccination of nursery pigs were successful at controlling wild type PRRSv.

\section{References}

1. Neumann E. et al 2005. JAVMA 227:385-392.

2. Gillespie et al. 2003. Proc AASV:549.

3. Spronk et al. 2009. Proc Leman Swine Conf Suppl:181. 


\title{
0.237
}

\section{Herd closure as a strategy to eradicate PRRSV not always a success in large sow herds}

\author{
Giampietro Sandri ${ }^{1}$ Patrizia Pesente ${ }^{2}$ Davide Giovanardi² \\ 1. Agricola Tre Valli, Quinto di Valpantena - Verona, Italy; 2. Laboratorio Tre Valli, S.Martino Buonalbergo, Italy
}

\section{Introduction}

Understanding the dynamics of PRRSv persistence is essential for the development of successful PRRS control and eradication programs1. Among these, "Herd Closure" - a months long closure of the sow herd - is considered to be one of the most successful. It relies on consistent natural exposure that should provide a "sterilizing immunity" to all breeding stock on the farm leading to the natural disappearance of the virus by replacement of positive animals over time2. We decided to use Herd Closure as initial strategy to eradicate PRRS from three large sow herds (site one) in northern Italy with some disappointing results.

\section{Materials, methods and case description}

Serum samples at different stages and places were tested for PRRSv antibodies by IDEXX PRRS Elisa. A conventional one step RT-PCR developed by Laboratorio Tre Valli was used for viral Rna detection. Sequencing in the ORF 7 region followed.

Farm M. (7.500 sows)

After experiencing a major PRRS outbreak during summer 2004 and instability throughout December of the same year, gilt replacement was finally discontinued in January 2005. The last group of gilts was introduced in mid January 2005 and the following were all diverted to outside projects. The first animals (pregnant gilts) were re-introduced on Dec.15 2005 leading to a total closure period of 11 months.

Farm SQ. (6.500 sows closed herd)

Major PRRS outbreak from October 2005 to January 2006. Internal gilt production was discontinued and off site nursery and rearing projects were started with purchased PRRS negative animals starting from March 15th 2006. A Wean \& Removal3 program was started in September 2006. PRRS negative animals/ gilts were again introduced on March 1st 2007 leading to a complete herd closure time of 12 months.

Farm A. (4.500 sow)

A severe PRRS outbreak occurred in this herd starting in AprilMay 2008. It was soon decided to discontinue gilts replacement in order to stabilize the herd and to prepare a possible future PRRS eradication project. The last 3 weeks old gilts were introduced on May 19th 2008 and soon were exposed (serum inoculated) - together with all the other gilts present in the nursery compartment - to the farm strain. After this point in time all replacement gilts were diverted to off site nursery, growing and breeding projects.The first group of PRRS negative pregnant gilts entered in this herd again on April 27th 2009 after a complete closure more than 11 months long.

\section{Results}

After closure Farm M. reached a reasonably good production level without significant clinical problem but was never able to produce again PRRS negative piglets. When PRRS neg. pregnant animals returned to farrow on farm a contingency plan allowed good segregation of that population but unfortunately PRRSv started to re-circulate causing clinical problems early in April '06. Farm S.Q. was more successful because stabilized reasonably soon and as early as July '06 was able to consistently produce groups of PRRS neg. piglets with the exception of a few batches in Dec.'06 when a little re-circulation occurred. Thank to the intensive Wean \& Removal program4 in place PRRS eradication was achieved anyhow. Also Farm A. reached a good stabilization after Herd Closure but was never able to produce again PRRS negative piglets. The PRRSv strains recovered from different nursery sites always showed an almost complete homology with the farm strain. Not long after the first pregnant gilts started to farrow on farm they showed some PRRS clinical signs. The PRRSv recovered showed over $99 \%$ homology with the previous one. In spite of Wean \& Removal PRRS eradication did not succeed in this farm.

\section{Conclusions}

Herd Closure after a major PRRS outbreak is definitely a good measure to speed up the return to clinical stability. Although in a number of different occasions Herd Closure proved to be a successful PRRS eradication tool it was not the case in our experiences. Under the conditions described before, even after very long closure periods, the specific PRRS viruses were still present. Though we have no evidence that any single animal can carry PRRSv for such a long time (11 mo.), we think that large herds with a lot of buildings and compartments certainly provide a "favourable environment" for the virus to "survive and move around" end eventually start new outbreaks when large naïve populations are introduced again.

\footnotetext{
References

1 Wills et al 2003 J. Clinical Microbiology; Jan. 58-62

2 Torremorel et al 2002 Advances in Pork Production Vol.

3 Sandri GP et al. Proc. of the 19th IPVS Congress (2006)
} 


\title{
0.238
}

\section{Clusters of biosecurity in Ontario sow herds and their geographical distribution}

\author{
Zvonimir Poljak ${ }^{1}$ Cate Dewey ${ }^{1}$ Robert Friendship ${ }^{1}$ Rob Deardon² Derald Holtkamp ${ }^{3}$ \\ 1. Department of Population Medicine, OVC, University of Guelph, Guelph, ON, Canada; 2. Department of Mathematics and Statistics, University of \\ Guelph, Guelph, ON, Canada; 3. Department of Veterinary Diagnostics and Production Animal Medicine, lowa State University, Ames, IA, USA
}

\begin{abstract}
Introduction
Implementing good biosecurity has direct benefits at the herd level since it is critical in reducing the likelihood of introduction and spread of emerging or endemic animal pathogens. In addition, biosecurity standards are increasingly having an impact at the industry level. Management practices related to biosecurity could be used to define a subpopulation and provide a basis for compartmentalization (1), to assist in the design of risk-based surveillance (2), or as an aid in regional disease control and eradication strategies. Studying biosecurity, however, is not without challenges. Several approaches and resulting assessments have been previously reported. The aim of this work was to assess biosecurity practices in Ontario sow herds including the determination of best number of biosecurity classes, characteristics of these classes, and their geographical distribution and association with herd density.
\end{abstract}

\section{Materials and Methods}

Questionnaire: The questionnaire used for this study was a part of the PRRS (Porcine reproductive and respiratory syndrome) Risk Assessment for the Breeding Herd (version 2) developed elsewhere, owned by the American Association of Swine Veterinarians and used extensively across North America (3). Three evaluators were trained and performed personal interviews with owners and herd veterinarians of 161 sow herds in Ontario during the summer of 2007. Herds were selected conveniently through herd veterinarians. The original questionnaire contained 155 questions divided into internal and external risk sections and 24 questions related to the demographics of the site.

Selection of variables for multivariate analysis: Out of all questions available, only 24 categorical variables were included in the statistical analysis. Variables were included if they were indicative of the introduction of pathogens from external sources including replacement animals and semen, management options related to acclimation and isolation units, site policies related to visitors, tools, sanitation, and protocols related to transportation of animals, feed, and people. Variables related to density of pig herds as well as variables related to herd type and demographics were excluded from this initial classification step. All variables were categorical with 2 to 4 discrete levels.

Statistical analysis: Two classification methods were used to classify the data: (i) two-step hierarchical clustering (SPSS 17) and (ii) latent class analysis (SAS 9.2, proc lca). Both of these methods have advantages over other classification methods in that they use statistical criteria to determine the best number of subclasses and because they can directly analyze nominal variables. Best number of classes was determined using both methods, examined descriptively and agreement was assessed using the kappa statistic. Generalized additive models using logistic regression ( $R$, package $m g c v$ ) were then used to map each of the biosecurity groups by displaying expected risk on a 10x10 km grid of Ontario. In addition, multinomial logistic regression (Stata
10) was used to investigate the association between biosecurity groupings of sow herds and variables indicative of herd density obtained through the questionnaire.

\section{Results}

Three classes of biosecurity practices exist in Ontario sow herds. This number, determined using the two different methods and classification of herds was in almost perfect agreement (kappa=0.83). Best description of these three is: (i) “High" biosecurity sow herds, (ii) "Low to moderate" biosecurity sow herds, and (iii) "Closed" sow herds. Proportion of sow herds that were classified in the high biosecurity group was higher in areas of high swine herd density. This result was confirmed independently by regressing the biosecurity group classification against the number of swine herds within a $8 \mathrm{~km}$ radius from each site (Overall $\mathrm{P}=0.03$ ). The probability of being a high biosecurity herd was lower than the probability of being a low biosecurity herd in areas of low pig-herd density. In contrast, the probability of being a high biosecurity herd was higher than the probability of being a low biosecurity herd if number of neighbouring herds was high.

\section{Discussion}

These results contribute to our understanding of the types of herds that currently exist in Ontario, and the identification of practices in each group that should be prioritized for further improvement. Another important finding was the association between high biosecurity and high density of pig herds. The establishment of a high biosecurity herd in a pig-dense area may be facilitated through two different processes: (i) necessity of protecting animal populations in a herd located in a pig dense area, (ii) available expertise typically present in such areas. However this finding could impact the design of regional disease control strategies and needs to be evaluated in other swine producing regions.
References:
1) Terrestrial Animal health Code. Accessed Jan 222010
2) Stark KD et al. BMC Health Serv Res. 2006; 6
3) Holtkamp, D et al. Proc of the 20th IPVS 


\title{
0.239
}

\section{Using air filtration to reduce the risk of PRRS introduction}

\author{
Darwin L. Reicks \\ Swine Vet Center, St. Peter, MN, USA
}

\section{Introduction}

There is a significant economic advantage to maintaining a PRRS negative herd, whether it is a boar stud, sow farm, nursery, or finish. As aerosol transmission of PRRS, Swine Influenza, and Mycoplasma diseases are being accepted as reality, any means to prevent aerosol transmission of these agents is of great interest. Our practice has taken an aggressive approach with our producers on air filtration systems. Six farms implemented filtration in 2005, five in 2006, eleven in 2007, and twelve in 2008, two in 2009, and two in 2010.

\section{Materials and Methods}

There are 21 boar studs, 12 sow farms, 3 research barns, and two finishing sites. Small and large farms have been filtered. Nine of the sow farms are over 2500 sows. The other three are 700 - 1200 sows. Six of the boar studs are air conditioned. Aerosol transmission of PRRS virus can be repeated experimentally and it is becoming widely accepted to be a common way that negative farms become re-infected.1,2 One challenge for implementation of air filtration is the number of filters needed to obtain adequate air flow through a barn during summer time ventilation. Fifteen of the filtered farms are partial filtered, where air is not filtered during the summer months when ventilation rates are high. Rather than using a true HEPA filter, we have utilized a filter with slightly lower efficiency, either the L9 or L6 filter from Camfil Farr. This is the same filter used successfully in experiments.1,2 The cost to filter a farm depends on the filter used (L9 is more restrictive so more filters are needed), and whether air enters the farm the same way year round or whether there is a different ventilation system for summer (i.e. tunnel ventilation) than for winter. In general, filters last about 3 years, and the cost per pig over a 10 year period ranges from $\$ 1.00$ to $\$ 1.60$ per pig in US\$.

\section{Results}

The first boar studs in our practice were filtered in August 2005. Since implementation of air filtration, we have had four PRRS breaks on farms using partial filtration. Three were infected when the air was not being filtered, and one was when the air was filtered, but a significant amount of air was back drafting through non-operational fans. We have had two PRRS breaks on farms with $100 \%$ filtration, although we believe that was the result of a transportation biosecurity breach or the result of having a number of heavily damaged filters in use. We have had two
Swine Influenza breaks on $100 \%$ filtered farms. We have not had any Mycoplasma hyopneumoniae breaks on any of the filtered farms, although most are vaccinated. An important consideration for us from the start has been that we shouldn't expect to never have a PRRS break just because we installed air filtration. However, if we can reduce the incidence of PRRS breaks significantly, it would be good return on the investment. This has proven to be the case, in our experience. Most of the farms that have filtration had a previous history of multiple PRRS breaks. I have been tracking the results of filter farms for the 5 years prior to, and after filtration and updating the results quarterly. The following table shows the results through 1st quarter 2010 for 38 Swine Vet Center client farms. The \# of Farm Years indicates the number of years the farm was in existence in the five years prior to implementing filtration, since some farms were less than five years old at the time of filtration.

\begin{tabular}{|l|c|}
\hline \# of Farms & $\mathbf{3 8}$ \\
\hline \# Farm years prior to filtration & 152 \\
\hline \# PRRS breaks in 5 years prior to filtration & 51 \\
\hline Break Rate per year & $34 \%$ \\
\hline \# Farm Years with 100\% Filtration & 50 \\
\hline \# PRRS breaks in 100\% filtered farms & 2 \\
\hline Break Rate per year 100\% filtered farms & $4 \%$ \\
\hline \# Farm Years with partial filtration & 51 \\
\hline \# PRRS breaks in partial filtered farms & 4 \\
\hline Break Rate per year partial filtration & $8 \%$ \\
\hline
\end{tabular}

\section{Discussion}

Air filtration systems have been in place in our practice for nearly 5 years, in a wide variety of farms. For boar studs, filtration has become the standard. To date, filtration has reduced the annual break rate on 38 farms from $34 \%$ prior to filtration to $8 \%$ after filtration for partial filtered farms and to $4 \%$ for $100 \%$ filtered farms.

\section{References}

1. Dee, S., Area spread of PRRSV: End of story? Proceedings from the 2007 Allen D. Leman Swine Conference p. 3-11.

2. Pitkin, A., Deen, J, Dee, S., Aerosol transmission of PRRSV: Application to the field. Proceedings from the 2007 Allen D. Leman Swine Conference p. 85-88. 


\title{
0.240
}

\section{A PRRS vaccination strategy to create PRRS-negative growers/gilts in SPF herds (re)infected with PRRS}

\author{
Geertjan van Groenland ${ }^{1}$ Toine Cruijsen ${ }^{2}$ Victor Geurts ${ }^{2}$ \\ 1. TOPIGS S\&D, Helvoirt, Netherlands; 2. Intervet-Shering Plough, Boxmeer, Netherlands
}

\section{Introduction:}

PRRS infections still lead to severe economic losses in the pig industry (1). It is possible to create PRRS-negative farms by depopulation and repopulation with negative piglets and gilts from PRRS-free farms. Despite stringent hygienic procedures on these farms, it is difficult to keep them PRRS-free in pig dense countries like the Netherlands. Between Febr-2006 and May-2008, 5 farms in the Netherlands became PRRS-free by depopulation and repopulation with SPF- pigs or by introducing SPF pigs in a new farm, and all of them became infected after 6 to 22 months. The PRRS-infection-route could not always be identified. Therefore, farmers choose to produce PRRS-negative growers and gilts via a system of vaccination together with testing and optimization of biosecurity. Vaccination of piglets was ceased as soon as there could not be detected PRRS-fields virus anymore in the outflow (by serological and virological tests). A conventional boar-producing farm followed the same strategy. The chosen PRRS vaccine (Porcilis ${ }^{\circledR}$ PRRS) reduces vertical transmission and reduces virus circulation in the population following vaccination $[$ Ro $<1](3,4)$. Furthermore, to reduce the risk of transmission between pigs by needle injection, a needleless vaccination system was used (IDAL ${ }^{\circledR}$, intra dermal applicator of liquids, Intervet/Schering-Plough animal health). The vaccine had already been effective in eradicating PRRS from farms(5).

\section{Materials and methods:}

Step 1. Once PRRS infection had been diagnosed on SPF farms, a cross-section of sows, piglets, fatteners and breeding gilts were sampled in combination with pooled virus isolation or PCR in order to investigate the spread. All biosecurity measures were checked and optimized and all pigs older than 2-weeks were vaccinated (herd vaccination, i.e. all sows, gilts, piglets, growers, fatteners). Sows were vaccinated 2-4 times with intervals of 2-4 weeks, thereafter all sows/gilts were vaccinated in mid-gestation (day 60) and during lactation (day 6). After the initial herd vaccination piglet vaccination was started at 14-21days of age. On farm 3, only (pregnant) gilts and boars were present that were vaccinated only once.

Step 2. After 3-6 months, again sows, breeding gilts, and pigs of $4,10,16$, and 22-26 weeks of age were sampled for serological, and virus isolation or PCR. When the tests for PRRS field virus were negative, piglet vaccination was ceased.

Step 3. Farms which had stopped piglet vaccination were monitored by the same sampling procedure as in Step 2, every 4 months.

\section{Results:}

Until now (February 2010), five out of six farms succeeded in producing PRRS negative gilts/growers/boars. Only one farm got re-infected during the trial, the isolated strain was an US-type PRRS strain. No vaccinations were added after this herd became re-infected (sow/gilts vaccination was continued) partly due to the fact that no clinical signs were seen after re-infection and the spread was limited (most cohorts were PRRS negative). From September on sera from 10-weeks-old pigs tested negative for PRRS.

\begin{tabular}{|l|c|c|c|c|c|c|l|}
\hline Farm & PRRS-free & Re-inf. & Type & Source & $\begin{array}{c}\text { Neg. } \\
\text { outflow } \\
\text { again }\end{array}$ & $\begin{array}{c}\text { Re- } \\
\text { infected }\end{array}$ & Type \\
\hline 1 New & Feb-06 & Mar-08 & EU & $?$ & Jan-10 & no & \\
\hline 2 Convent. & & Mar-06 & EU & $?$ & Dec-07 & no & \\
\hline 3 New & Nov-05 & Aug-06 & EU & $?$ & May-07 & no & \\
\hline $\begin{array}{l}\text { 4 De-pop } \\
\text { Re-pop }\end{array}$ & Aug-06 & Dec-07 & EU & $?$ & May-08 & Dec-08 & US \\
\hline $\begin{array}{l}\text { 5 De-pop } \\
\text { Re-pop }\end{array}$ & Feb-08 & Feb-08 & EU & Neighbour & Q04-2009 & no & \\
\hline $\begin{array}{l}\text { 6 De-pop } \\
\text { Re-pop }\end{array}$ & May-08 & Jan-09 & EU & Gilt Suppl. & 0ct-09 & no & \\
\hline
\end{tabular}

\section{Conclusion and discussion:}

Via testing, adjustments to biosecurity and targeted vaccination, it was possible to create PRRS-negative pigs from SPF farms that were infected with PRRS. In a pig dense country as the Netherlands where the estimated introduction risk of PRRS is high, this system proved to be effective in controlling PRRS.

On all farms, except for farm 3, sow vaccination (day 6- day 60) was continued to minimize the risk of vertical transmission because viremic born piglets will further spread the infection through the herds.

Sequence analyses on farms helps to identify the likely routes of re-infection. Two such routes were discovered during this trial/ survey: (i) an aerosol infection originating from a neighboring farm and (ii) via infected replacement gilts. Because almost all SPF-farms in the Netherlands became infected after depopulation/repopulation it seems that (i) either regional approach or (ii) air-filtration of incoming air will be necessary for farms in pig dense areas such as The Netherlands to remain PRRS-free(2). In the meantime vaccination of sows and gilts, together with well-defined internal biosecurity can be used to produce PRRSnegative offspring in infected breeding farms as well as in farms that are "at risk".

\section{References}

1. Neumann.et al,(2005) J..Am.Vet.Med.Ass. 227, no3,

2. Dee S.et al,(2008) Proc.20th IPVS congr., Vol.1, p57

3. Astrup P.et al, (2002) Proc.17th IPVS congr.,p380

4. Grosse Beilage E.et al, (2009) Prev.Vet.Med. 92:31-37

5. Voglmayr T.et al (2006)Tierärzt.Praxis 34,241-248 


\title{
0.241
}

\section{PRRS modified-live virus mass vaccination and its potential role in regional control programs}

\author{
Jean P. Cano; Laura Batista; Enrique Mondaca; Reid Philips; Dale Polson \\ Boehringer Ingelheim Vetmedica, Inc., St Joseph, MO, USA
}

\begin{abstract}
Introduction
Mass vaccination combined with unidirectional flow, herd closure and/or partial depopulation has been used successfully for porcine reproductive and respiratory syndrome (PRRS) control and eradication. ${ }^{1-3}$ However, the effects of therapeutic vaccination on the infection dynamics at a population level had not been studied. Two large scale experiments were conducted under commercial conditions in order to evaluate the effect of PRRS modified live virus (MLV) mass vaccination on wild-type virus transmission and pig response to a highly virulent heterologous challenge. ${ }^{4,5}$ In these studies, pigs were infected with a homologous 4 or heterologous5 PRRS virus (PRRSv) isolate prior to therapeutic MLV vaccination. The model also simulated the introduction of a new highly virulent strain to pig populations with diverse "exposure backgrounds".4,5
\end{abstract}

\section{Materials and Methods}

For the heterologous study, 80 PRRSv-naïve pigs per group (A-D) were inoculated intranasally (IN) with PRRSv MN 30-100. At 7 days post-inoculation (DPI), pigs were vaccinated IM with Ingelvac $^{\circledR}$ PRRS MLV vaccine. Group E was designated as the negative control. Pigs in group F received only vaccine. To evaluate viral transmission, sentinel pigs were introduced periodically to each group and sacrificed-sampled 30 days later. To evaluate persistence, 10 pigs per group were sacrificed-sampled at different $\mathrm{DPI}$. At $98 \mathrm{DPI}, 10$ pigs per group were transported to isolation facilities and inoculated IN with PRRSv MN 184. A naïve pig was introduced to each of the rooms with 5 inoculated pigs 3 days after the MN 184 challenge.

\section{Results}

Consistent with the homologous study, but in a more field relevant scenario, heterologous therapeutic vaccination:

- Avoided wild-type virus transmission to naïve-sentinel pigs after $97 \mathrm{DPI}$.

- Did not "displace" the wild-type virus from the population, or reduce the proportion of persistently infected pigs within 127 DPI.

- Did not reduce the viral load in lymphoid tissues of persistently infected pigs.

Previous exposure to PRRSv, including one dose of Ingelvac PRRS MLV (group F), significantly reduced clinical signs and increased post-challenge weight gain, but infection or shedding of a highly virulent heterologous isolate was not prevented. Pigs in group E remained ELISA and PCR negative through the duration of the study.

\section{Discussion}

These results represent a detailed and controlled evaluation of the benefits and limitations of the use of PRRS MLV mass vaccination under controlled field conditions involving a large population of pigs. Therapeutic vaccine intervention prevented viral shedding to naïve pigs and significantly improved clinical protection against a highly virulent heterologous challenge. Therefore, therapeutic vaccination may be used in combination with other strategies to reduce duration of PRRSv transmission and to increase the resistance of pig herds under certain scenarios in the field. This is especially relevant in a regional control context, where reducing shedding from infected populations and increasing resistance of susceptible populations is crucial for success. Potential applications of PRRS MLV mass vaccination intervention in regional control programs include:

- sow-herd mass exposure prior to a herd closure strategy,

- gilt acclimation programs,

- reduction of susceptibility of PRRS naïve-pigs placed into high prevalence areas, and

- reduction of infectiousness (shedding of wild-type virus) of PRRS infected-pigs placed into low prevalence areas.

Further epidemiological investigations need to be conducted to better understand the benefits and limitations of PRRS MLV mass vaccination intervention on a regional scale.

\section{References}

1. Dee, S. JSHAP 1998 (1)21-25.

2. Gillespie, T. JSHAP 2003 (11-6)291-5.

3. Philips, R. Proc Symp Emerg Pig Dis 2003: 129.

4. Cano, JP. Am J Vet Res 2007 (68-5)565-71.

5. Cano, JP. Am J Vet Res 2007 (25-22)4382-91. 


\title{
0.242
}

\section{Advancing PRRS area regional control in the state of Sonora, Mexico}

\author{
$\underline{\text { Laura Batista }}^{1}$ Marcela Fernandez ${ }^{2}$ Jesus Miramontes $^{2}$ Ramon Pliego $^{2}$ Enrique Mondaca $^{1}$ \\ 1. Boehringer Ingelheim Vetmedica, Inc., St Joseph, MO, USA; 2. Comite de Sanidad Porcina de Sonora, Sonora, SON, Mexico
}

\section{Introduction}

The global swine industry faces economically devastating diseases such as porcine reproductive and respiratory syndrome $(\mathrm{PRRS})^{1}$, porcine circovirus associated diseases (PCVAD), and new variants of influenza. Consequently, veterinarians have developed different means for individual or groups of farms to control and possibly eradicate diseases. However the risk of re-infection remains high even with the best current biosecurity practices. ${ }^{2}$ This has led to the consensus that more coordinated, or 'regional', approaches must be taken to combat PRRS and other emerging swine diseases, rather than isolated efforts on individual farms which are often frustrated by the reappearance of a disease. Therefore, the objective of this study was to establish a PRRS regional control program in the State of Sonora, Mexico to reduce the economic impact of this disease in the Sonoran swine industry.

\section{Materials and Methods}

In September 2008, producers from the state of Sonora in northwest Mexico decided to organize to implement a regional PRRS control program. The region of $182,554 \mathrm{sq} \mathrm{km}$, included 74 independently owned production systems with various production structures (i.e. farrow to finish, three site, and multisite production) with a total of 134,000 sows. The program was divided in three phases: phase 1 (September 2008-March 2009), data collection, understanding of the state's production industry organization, mapping, clusters and working groups establishment; phase 2 (April -September 2009) estimation of PRRS prevalence and virus sequencing analysis, individual farm risk assessment using the AASV PADRAP tool ${ }^{3}$, establishment of the program's website, and monthly continuous education and communication meetings with producers and veterinarians; phase 3 (October 2009-September 2011), pig and transportation route planning and implementation of farm and region control strategies.

\section{Results}

As of October of 2009, phases 1 and 2 have been successfully completed. The state was divided into three geographical regions with a $95 \%$ state PRRS prevalence. Twenty three working groups, including 91 clusters, were established and sequencing information allowed the identification of 5 major PRRS virus families. One hundred and twenty four PADRAP evaluations were completed and individual and regional analyses were performed.

\section{Discussion}

Information collection is a cumbersome and sometimes frustrating task, however, without the completion of phases 1 and 2 it would have been very difficult to establish solid bases to design and implement farm and regional PRRS control strategies. Disease control programs rely on accurate epidemiologic surveillance, access to appropriate tools for diagnosis and control, and adequate financial and human resources. To date, we have identified 3 major components leading to the successful advancement of this program. One, producer and veterinarian leadership and involvement; 2 , regular informational meetings with local producers and veterinarians addressing advances of the program, continuous education, issues related to confidentiality, access to data, etc.; and 3, the establishment of a producer-paid economic fund, in the case of Sonora a fee is paid per sacrificed animal, which allows the advancement of the program. If we are able to foster producers participation, set aside individual interests and use valid knowledge and understanding of pathogen transmission and how to prevent it, understand the impact of geographical location and implement effective rather than "psychological" biosecurity strategies, we can help our producers with the battle against PRRS virus and other economically important pathogens. Successful implementation of PRRS area regional control holds the potential to restore success and profitability to the swine industry of Sonora.

\section{References}

1. Neumann EJ et al. 2005. J Am Vet Med Assoc 227:385-392.

2. Davies P. 2007. MN Pork Congress

3. Polson D et al. 2005. George A Young Swine Conference Proc., 64-87. 


\title{
0.243
}

\section{Cost of PRRS outbreak in a sow herd and economic analysis of control strategies}

\author{
Zvonimir Poljak ${ }^{1}$ Isabelle Moreau² Martin Misener ${ }^{3}$ Doug MacDougald $^{3}$ Cate Dewey ${ }^{1}$ \\ 1. Department of Population medicine, OVC, University of Guelph, Guelph, ON, Canada; 2. Elanco Animal Health, Guelph, ON, Canada; \\ 3. OSHAB, Stratford, ON, Canada
}

\begin{abstract}
Introduction
Swine producers and veterinarians have several options to manage a herd when facing a PRRS outbreak. Some will allow the disease to follow its course while trying to address the clinical signs with individual or population treatments, others will use vaccination with commercial vaccines or expose all sows to PRRS virus recovered during the outbreak, while others will use tilmicosin in the immediate 4 weeks following the PRRS onset with or without virus exposure. The objective of this project was to evaluate production loses and economic efficiency of common intervention strategies.
\end{abstract}

\section{Materials and Methods}

Veterinarians in Ontario and Quebec (Canada) were asked to provide computer records from sow farms that experienced PRRS outbreaks since January 2004 and to fill a questionnaire with demographic, diagnostic and intervention data. Computer records were extracted and collapsed to weekly averages or counts, as appropriate. Temporal iterative multivariate scan statistics (SatScan 7.0) was used to determine onset and duration of outbreaks. This was confirmed with the questionnaire data. Weekly data were then summarized to three periods for each farm: (i) 6-months pre-outbreak, (ii) outbreak, (iii) 6 months post-outbreak. Proportional change in number of pigs weaned was calculated for each period, relative to pre-outbreak level. Cost of intervention was calculated from quantity of substances spend in the sow herd. Best fitting distributions were determined within each intervention category and stochastic partial budgeting model was constructed (@Risk 5.0). Proportion of sows that farrow per week and average number of pigs weaned per litter were estimated using random intercept linear model; intercept (mean) and residual standard deviation were used in the economic model to obtain a range of scenarios. Scenario evaluated was based on fixed herd size of 1000 sows, 36\$CND for a weaned pig, and 32 weeks after the start of outbreak. In economic model, "Late homologous exposure" and "Tilmicosin with or without homologous exposure" strategies were evaluated using "No intervention" strategy as a baseline. Although other interventions strategies were used, they were not included because of insufficient number of cases available to produce input distributions.

\section{Results}

Assuming each piglet would be sold at $\$ 36$, we estimated that if a farm chooses to do nothing, a PRRS outbreak will typically result in a loss of $\$ 80 /$ sow/outbreak in potential revenue from reduced weaned pig sales over a period of 6 months. At the extremes, in the best case a farm will lose as little as $\$ 29 /$ sow/ outbreak while the worst case, a farm will lose as much as $\$ 158$ / sow/outbreak when no treatment is selected. The farms under study that chose to do nothing still incurred additional expenses from increased number of individual treatment. These comfort treatments cost on average $\$ 2.60 /$ sow/outbreak. In farms that chose to try a PRRSV exposure control strategy, the PRRS outbreak either was shorter in duration or fewer piglets were lost. This resulted in higher revenue from wean piglets sale. The farms that exposed sows to PRRS virus, showed $\$ 20$ higher revenue/ sow/outbreak on average, than farms that chose to do nothing, while farms that included tilmicosin in the gestation diet after the outbreak, showed an average of $\$ 55$ higher revenue/sow/ outbreak than farms that chose to do nothing. Total intervention expenses related to individual animal treatment on farms that chose to expose sows with the PRRS virus averaged $\$ 2.90 /$ sow/ outbreak while farms that chose Pulmotil showed total average medication expenses of $\$ 12.80 /$ sow/outbreak. Herds applying late homologous exposure strategy were expected to have lower revenue than herds without any intervention in 35\% of cases. Herds applying tilmicosin strategy, with or without homologous exposure, were expected to have lower revenue than herds without any intervention in $13 \%$ of cases.

\section{Conclusions}

Under conditions evaluated in this study, no-intervention strategy seemed to be the most costly approach of managing PRRS outbreak in a sow herd. Management of PRRS under alternative, and more costly strategies, was expected to yield higher revenues on average; although none of the strategies is better under all scenarios. Under ideal conditions, collection of data that could be used for economic analysis would be a part of every routine outbreak investigation in swine herds. 


\title{
0.244
}

\section{Efficacy of AMERVAC - PRRS after heterologous challenge with a pathogenic Chinese-like PRRS virus}

\author{
Merce Roca ${ }^{1}$ Sergi Bruguera' ${ }^{1}$ Mariona Gimeno ${ }^{2,3}$ Ivan Diaz ${ }^{2}$ Ivan Galindo ${ }^{2,3}$ Joaquim Segales $^{2,3}$ Enric Mateu $^{2,3}$ \\ Natalia Bello ${ }^{1}$ David Llopart ${ }^{1}$ Jaime Maldonado ${ }^{1}$ Ricard March ${ }^{1}$ \\ 1. HIPRA, Amer (Girona), Spain; 2. Centre de Recerca en Sanitat Animal (CReSA), Bellaterra (Barcelona), Spain; \\ 3. Departament de Sanitat i Anatomia Animals UAB, Bellaterra (Barcelona), Spain
}

\section{Introduction}

Throughout the second half of 2006, large-scale, devastating outbreaks of porcine reproductive and respiratory syndrome (PRRS) affected several provinces of China ${ }^{1}$. That epidemic was linked to a hypervirulent genotype-II PRRS virus (PRRSV) harboring a distinctive deletion ${ }^{1}$. Economic losses from those outbreaks results from deaths but also from loss weight and impaired performance. Control of such outbreaks proved difficult and those hypervirulent strains spread in Asia. At present, genotype I strains are also present in different countries of Asia. AMERVAC -PRRS (a genotype-I vaccine) is being marketed in Asia for the prevention PRRS virus infections. Is feasible that some herds vaccinated with AMERVAC ${ }^{\oplus}$-PRRS could confront the infection with a hypervirulent Asian strain. The present study was intended to determine if vaccination with AMERVAC ${ }^{\circ}$-PRRS could produce some beneficial effect on performance of pigs infected with the hypervirulent strain.

\section{Material and Methods}

Twenty-one 4-week-old, PRRSV-naïve pigs were randomly divided into three groups ( $G 1 n=8, G 2 n=8$ and $G 3 n=5)$ and housed separately in isolation facilities (BSL3 at CReSA, Barcelona, Spain). Animals in G1 received intramuscularly (day 0) $2 \mathrm{ml}$ of a European-type modified live PRRS vaccine (AMERVAC ${ }^{\circ}$-PRRS containing $10^{4.6} \mathrm{TCID}_{50} / \mathrm{ml}$ ). $\mathrm{G} 2$ and $\mathrm{G} 3$ pigs remained unvaccinated. At day +32 , pigs in $\mathrm{G} 1$ and $\mathrm{G} 2$ were intranasally inoculated with $2 \mathrm{ml}$ of $1 \times 10^{5} \mathrm{TCID}_{50} / \mathrm{ml}$ of the strain PRRSV21 that was isolated from a highly virulent case of PRRS in Asia and share 99\% similarity in ORF5 with other highly virulent genotype-II Chinese PRRSV strains. Pigs in G3 remained uninfected along the trial. Weekly body weight (BW), average daily feed intake (ADFI) and clinical signs were assessed during the 21 days of the challenge phase of the study. Statistical analyses were done using a non-parametric Mann-Whitney test.

\section{Results}

After challenge, pigs in G2 suffered an evident respiratory distress starting the $5^{\text {th }}$ day post-inoculation (dpi) and lasting in most animals until the end of the trial. In G2, two animals died before the end of the study (25\%). In G1 pigs showed milder respiratory signs and suffered no losses. Regarding BW, groups were equal before the challenge but by $7 \mathrm{dpi}$ animals in $\mathrm{G} 2$ showed a lower BW compared to G1 and G3 $(p<0.05)$ and these differences remained statistically significant along the trial. Significant reduction in ADFI was recorded in $\mathrm{G} 2$ pigs from $7 \mathrm{dpi}$ to the end of the study. ADFI differences were remarkably high at the end of the trial $(\mathrm{G} 2=0.44 ; \mathrm{G} 1=1.57$ and $\mathrm{G} 3=1.97 \mathrm{~kg} / \mathrm{day} /$ animal). Thus, on average, comparing $\mathrm{G} 1$ pigs with unvaccinated-challenged pigs, vaccine prevented mortality (0/8 vs. 2/8) and allowed $8.9 \mathrm{~kg}$ more of weight gain for the 21 days period examined.
Figure 1: Body weight of pigs vaccinated once with AMERVAC ${ }^{\circledR}-P R R S$ and challenged with PRRS21 strain, compared with unvaccinated and PRRSV-naïve pigs.

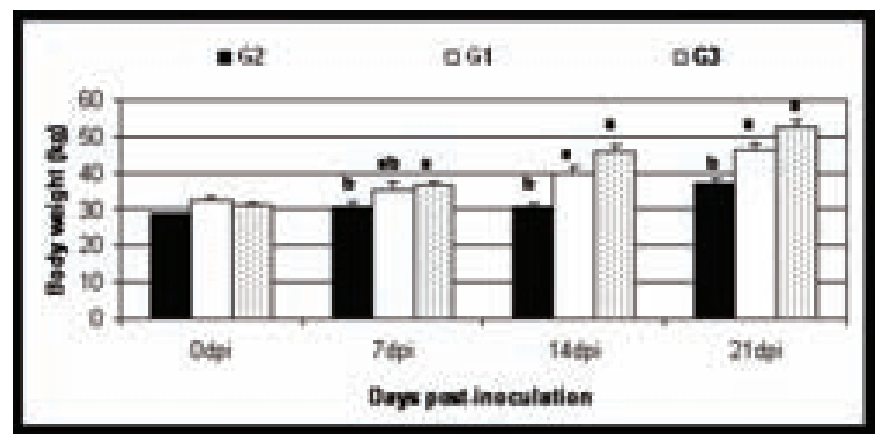

$\mathrm{a}, \mathrm{b}=\mathrm{p}<0.05 ; \mathrm{G} 1=$ vaccinated-challenged; $\mathrm{G} 2=$ Non-vaccinatedchallenged; $\mathrm{G} 3=$ Non-vaccinated-non-challenged.

\section{Discussion}

PRRS21 infection severely affected the growth performance of non-vaccinated pigs (G2), as expected from the virulent nature of that strain. In contrast, vaccinated pigs (G1) showed a better performance and lesser mortality indicating that vaccination with AMERVAC ${ }^{\natural}$-PRRS was able to keep productive performance in the challenge model. The present results suggest that under field conditions, this vaccination may provide beneficial effects in economic terms if a vaccinated herd should confront an infection with a hypervirulent genotype-II strain.

\section{References}

1. Tian K, et al. 2007. PLoS One. 2(6):e526. 


\title{
0.245
}

\section{Health status in organic pig herds in Europe}

\author{
Albert Sundrum ${ }^{1}$ Amke Goebel ${ }^{1}$ Davide Bochicchio ${ }^{2}$ Marianne Bonde ${ }^{3}$ Aude Bourgoin ${ }^{4}$ Gérald Cartaud $^{4}$ Klaas Dietze $^{1}$ \\ Sabine Dippel ${ }^{5}$ Stefan Gunnarsson ${ }^{6}$ Lene Hegelund ${ }^{3}$ Christine Leeb ${ }^{5}$ Kristina Lindgren ${ }^{6}$ Armelle Prunier $^{7}$ Sofia Wiberg ${ }^{8}$ \\ 1. University of Kassel, Witzenhausen, Germany; 2. C.R.A. SUI, Modena, Italy; 3. Aarhus University, Tjele, Denmark; \\ 4. Inter Bio Bretagne, Rennes, France; 5. University of Natural Resources and Applied Life Sciences, Vienna, Austria; \\ 6. Institute of Agricultural and Environmental Enginering, Uppsala, Sweden; \\ 7. INRA Institut National de Recherches Agronomiques, Rennes, France; 8. Swedish University of Agricultural Sciences, Skara, Sweden
}

\section{Introduction}

Organic pig production is still a small-scale produc-tion, achieving, however, increased interest by con-sumers who are concerned about the conventional production method. The EURegulation (EEC-No. 1804/1999) on organic livestock production, now replaced by EEC-No 834/2007, was introduced to provide a framework ensuring living conditions for organic livestock to be better than those in conven-tional systems and to harmonize the rules across member states. Scientifically based information on how and to what degree the EU-Regulation contrib-utes to the objective of a high status of pig health in organic farming is scarce. Further information is needed to assess whether consumer expectations in organic pig production in Europe are met.

\section{Material and Methods}

An epidemiological study was performed on 101 organic sow herds in 6 European countries (Denmark, Germany, Austria, Sweden, Italy and France). 13 to 20 farms per country were assessed. On-farm data included a comprehensive interview with the herd manager in relation to housing conditions, feeding regime, data acquisition, cleaning and disinfection measures, medicine usage, culling reasons, vaccina-tion protocols, etc.. Recorded livestock data included: liveborn and stillborn piglets/ farrowing, weaned pig-lets/sow/year, piglet losses/farrowing, losses of weaned piglets, litters /sow lifetime, and the prevalence of diseases according to the treatment records. Data from all countries were merged into one joint database and analysed statistically.

\section{Results}

Although dedicated to the same minimum standards, the farm structures were characterised by a huge heterogeneity. 67 farms were keeping their sows outdoors while 34 farms were indoors with outside runs. Group suckling was implemented on $52 \%$ of the farms. The degree of specialisation was compara-tively low. About $40 \%$ of the farms had another economically relevant livestock enterprise on the farm apart from keeping pigs. Farmers differed widely in their feeding regimes, in making use of phase feeding and diet calculations. Moreover, the farms varied in the cleaning management and in the implementation of disinfection measures. The study also revealed large differences in animal health management with respect to the use of quarantine, availability of hospital pens, presence of the farmer during farrowing, and regarding routine measures such as castration, teeth reduction, and iron supple-mentation of piglets. For 50 $\%$ of the farms the abattoirs provided feedback of pathological findings for slaughtered sows, and $80 \%$ for fattening pigs. Huge differences were detected in the perception of the farmers with regard to health problems on their farm and with respect to the availability of laboratory results.
Mean mortality rates of piglets until and after wean-ing averaged $19.7 \pm 9.7 \%$ and $4.9 \pm 5.4 \%$ resp., showing clear differences between countries and farms. Mean values for the annual replacement rates of sows and litters per sow lifetime were 32.4 $\pm 14.3 \%$ and $6.3 \pm 2.3$ resp. According to the treatment records, metritis and mastitis appeared as the predominant diseases of the sows, nearly all treated with antibiotics, followed by cases of lameness and abscesses. In the case of suckling piglets, diarrhoea, arthritis and respiratory diseases were predominant. Also the weaned piglets suffered primarily from diarrhoea. Farms showed an incidence of $27.6 \pm 34.3 \%$. of piglets treated with antibiotics against diar-rhoea.

\section{Conclusions}

The results indicated that in general the status of animal health in organic pig herds was comparable to data found on conventional farms. Differences between farms appear to be greater than those between production methods. Obviously, the production method defined by standards does not provide a homogenous outcome with respect to the animal health status. Differences in management practices and in feedback and control mechanisms within the farm system might be the main reasons for substantial variation in the prevalence rates of diseases. Any improvements in organic pig production require the use of feedback mechanisms and a clear definition of the expected results with respect to the outcome of the farm system. Conse-quently, there is a need for a change in the paradigm from standard and resource oriented to a result and outcome oriented approach. The implementation of a monitoring system including on farm clinical assessment, abattoir data and review of treatment data, concepts to deal with potential problems (e.g. health planning strategies, HACCP protocols, etc.) and a definition of the expected results with respect to the output of the farm system might be a way forward for organic farming ensuring a high status of animal health and welfare. 


\title{
0.246
}

\section{Pig management in rural villages of Busia and Kakamega District, Western Kenya}

\author{
Florence K. Mutua ${ }^{1}$ Cate Dewey ${ }^{2}$ Samuel Arimi ${ }^{3}$ Michael Levy ${ }^{2}$ William Ogara ${ }^{3}$ Esther Schelling ${ }^{4}$ \\ 1. International Livestock Research Institute, Nairobi, Kenya; 2. University of Guelph, Guelph, ON, Canada; \\ 3. University of Nairobi, Nairobi, Kenya; 4. Swiss Tropical Institute, Basel, Switzerland
}

\section{Introduction}

Intensive pig farming in many developing countries is thought to be stagnant (1). Most pigs in Kenya are exotic breeds raised on commercial piggeries $(2,3)$. Farmers in Western Kenya keep local pig breeds that are raised using traditional methods of tethering and free ranging (4). These breeds possess a number of advantages, among them good adaptation to prevailing conditions (5). Understanding how such pigs are managed aids in farm planning and decision making. Improved pig productivity will greatly enhance the income of resource-poor farmers. In this paper, we discuss pig management practices in two districts of Western Kenya. We also explore opportunities for improved local pig production while safeguarding human health.

\section{Materials and methods}

A total of 288 randomly selected pig farms were visited three times in the course of the study period (June 2006 to October 2008). We used questionnaires administered in face-to-face interviews to gather data on how farmers managed their pigs. Pigs were examined for Taenia solium cysts using the lingual palpation method. Data were analysed in Stata ${ }^{\circledR}$.

\section{Results}

Most farms had no pig barn (73\%; 209/288). These farmers said they lacked the skills to build the barn (11\%; 23/209) and had no money to purchase construction materials (45\%; 93/209). Kakamega farmers were more likely to construct pig barns than those in Busia ( $\mathrm{OR}=5.4 ; \mathrm{Cl} 3.1-9.7)$. Tethering of pigs was more frequent than free ranging or confinement to barns during the planting (91\%; 263 / 290), growing (90\%; 263 / 290) and crop harvesting seasons (78 \%; 227 / 290). Prevalence of cysticercosis in pigs was $4 \%(52 / 1290)$. Average land size per farm was $2.33(\mathrm{SD}=2.01)$ acres. Pork was the second most preferred meat (31\%; 91/288) after beef. Most families consumed pork (74 \%; 212 / 288), 40 $\%(n=212)$ included families that consumed pork once a week. The mean number of pigs owned per farm was $4.6(\mathrm{SD}=3.4)$, $1.8(\mathrm{SD}=1.2)$ and $1.5(\mathrm{SD}=0.9)$ for the pre-weaned, growing and adult pig categories. Farmers in Busia had been keeping pigs for fewer years (6.3 SD=5.6) than those in Kakamega (11.4 $\mathrm{SD}=8.7)(\mathrm{P}<0.05)$. Spraying $(29 \% ; 128 / 438)$, dipping $(24 \% ; 101$ / 438) and deworming (26\%; 116 / 438) were among the most frequent routine practices observed in Busia District.

\section{Discussion}

Improving pig management practices leads to improvements in farm performance and reduces costs in production. Failure to confine pigs is a risk factor for important zoonotic diseases such as T. solium (6). Small-holder farming is important in enhancing rural livelihoods; addressing issues related to pig feeding, tethering, marketing, disease and sow breeding will greatly promote pig farming in rural districts Western Kenya.

\author{
References \\ 1. Lekule and Kyvsgaard (2003). Acta Tropica, 87; 111-117 \\ 2 Kagira et al (2008). Livestock Res for Rural Dev, 20(10) \\ 3. Wabacha et al (2004). Pre Vet Med, 63; 197-210 \\ 4. Mutua et al (2007). J Swine HIth Prod, 15(4); 206-212 \\ 5. Rodriguez and Preston (1997). Livestock Res for Rural Dev, 9 (2) \\ 6. Phiri et al (2003). Acta Tropica, 87; 13-23
}




\title{
0.247
}

\section{Health bonus after transition to 4- or 5-week batch management systems}

\author{
Frédéric A. Vangroenweghe ${ }^{1}$ Leo Suls $^{2}$ Ellen Van Driessche ${ }^{1}$ Tamara Vandersmissen $^{3}$ Els Goyvaerts $^{3}$ Frans Castryck $^{1}$ \\ 1. DGZ-Vlaanderen, Torhout, Belgium; 2. Janssen Animal Health, Beerse, Belgium; 3. DGZ-Vlaanderen, Lier, Belgium
}

\section{Introduction}

Sow batch management systems (BMS) become more popular because of advantages in labour planning, batch size of piglets, strict all-in all-out practices and health management. The 4and 5-week BMS have advantages compared to a 3-week BMS, especially with regard to general health (1). Weaning piglets at 3 weeks of age in large batches creates possibilities for a significant improvement of the general farm health status. However, exact data on a health bonus with these management systems are not available. Therefore, a field study (project 'Veepeilervarken' funded by Sanitary Fund) was designed to measure the health bonus for pig farms when changing from a conventional 1-week management system to a 4- or 5-week group management.

\section{Materials and Methods}

Ten Flemish wean-to-finish pig farms, changing from a conventional 1-week management system to a 4- or 5-week BMS were included in the trial. The average farm size was 227 sows, with a minimum of 130 and a maximum of 500 . Samples were collected before and 2 years after finishing transition to the BMS. The type of samples and the different analysis are described in Table 1.

Table 1. Sampling schedule before and 2 years after transition

\begin{tabular}{|l|l|l|c|}
\hline \multicolumn{1}{|c|}{ Sample type } & Animal category & \multicolumn{1}{c|}{ Type of analysis** } & Number of samples \\
\hline blood/serum & gilts & PIA - PRRS - Mhyo & 10 \\
\hline & sows ( $>3$ litters) & Mhyo & 10 \\
\hline & piglets (10 wks) & PIA - PRRS - App - Mhyo & 10 \\
\hline & growers (14 wks) & PIA - PRSS - App - Mhyo & 10 \\
\hline & finishers ( $>80 \mathrm{~kg})$ & PIA - PRRS - App - Mhyo & 10 \\
\hline nasal swabs & piglets (6 wks) & PCR DNT* & 12 \\
\hline & piglets (10 wks) & PCR DNT* & 12 \\
\hline & growers (14 wks) & PCR DNT* & 12 \\
\hline $\begin{array}{l}\text { mixed fecal samples } \\
\text { (max. } 5 \text { animals) }\end{array}$ & growers (14 wks) & PCR Brachyspira sp. & 1 \\
\hline & finishers (> 80 kg) & PCR Brachyspira sp. & 1 \\
\hline
\end{tabular}

* DNT = Pasteurella multocida dermonecrotic toxine

\section{** ELISA test on serum samples}

The following ELISA-tests were used: PIA - Bioscreen Enterisol ${ }^{\circledR}$ Ileitis ELISA, PRRS - Idexx HerdChek PRRSv Ab test, M. hyopneumoniae - Idexx HerdChek M. hyopn. Ab testkit and A. pleuropneumoniae - Idexx Chekit - App - ApxIV ELISA.

\section{Results}

The results of the serological screening are presented in Table 2. The nasal swabs were all negative for Pasteurella multocida DNT on all herds screened. No clinical signs of atrophic rhinitis were present on these herds. All faecal samples were negative for Brachyspira hyodysenteriae. Nevertheless, minor pathogenic species of Brachyspira were detected, both before and after the transition.
Table 2. Results of serological analyses, expressed as percentage of positive animals before and after transition to a 4- or 5-week batch management systes and \% change

\begin{tabular}{|l|l|c|c|c|}
\hline Disease & Animal group & $\begin{array}{c}\% \text { postive } \\
\text { before } \\
\text { transition }\end{array}$ & $\begin{array}{c}\% \text { positive } \\
\text { after transi- } \\
\text { tion }\end{array}$ & $\begin{array}{c}\% \text { change } \\
\text { before-after }\end{array}$ \\
\hline PIA & gilts & 90 & 90 & $0 \%$ \\
\hline & piglets (10 wks) & 22 & 5 & $-77 \%$ \\
\hline & growers (14 wks) & 52 & 30 & $-38 \%$ \\
\hline PRRS & finishers (>80 kg) & 64 & 70 & $+9 \%$ \\
\hline & gilts & 90 & 83 & $-8 \%$ \\
\hline & piglets (10 wks) & 54 & 61 & $+13 \%$ \\
\hline & growers (14 wks) & 80 & 72 & $-10 \%$ \\
\hline M. hyopneumoniae & finishers (>80 kg) & 80 & 89 & $+11 \%$ \\
\hline & gilts & 51 & 49 & $-4 \%$ \\
\hline & sows (>3 litters) & 16 & 36 & $+125 \%$ \\
\hline & growers (14 wks) & 51 & 32 & $-37 \%$ \\
\hline & finishers (>80 kg) & 71 & 46 & $-40 \%$ \\
\hline A. pleuropneumoniae & piglets (10 wks) & 55 & 65 & $+18 \%$ \\
\hline & growers (14 wks) & 40 & 35 & $-13 \%$ \\
\hline & finishers (>80 kg) & 74 & 51 & $-31 \%$ \\
\hline
\end{tabular}

\section{Discussion and conclusions}

Transition to BMS seems to postpone and or slow down the seroconversion for PIA, M. hyo and App. This allows the farmer to vaccinate the pigs before they come in contact with the pathogen. It also allows more time for the pigs to build up immunity. Weaning age, maternal protection and transmission of pathogens from the sows to the piglets determine the speed and level of horizontal transmission within the batch. The degree of separation of the batches from each other then determines the vertical transmission between the batches.

In conclusion, the results show a clear health bonus for pig herds when changing from a 1-week BMS to a 4- or 5-week BMS.

\section{References}

1. Mekerke B., Leneveu P. 2006 AFMVP 7-8/12/2006, Toulouse, France. p. 49-64. 


\title{
0.248
}

\section{Economic impact of intensive consultancy on 35 Danish finisher farms}

\author{
Elisabeth O. Nielsen; Lisbeth Jørgensen; Henriette Steinmetz \\ Pig Research Centre, Danish Agricultural \& Food Council, Copenhagen, Denmark
}

\begin{abstract}
Introduction
Variation in productivity among Danish finisher farms implies that improvements can be made on many farms. Although Danish pig producers normally receive veterinary advice on a monthly basis, specific consultancy on feeding and housing is rarely provided. Cooperation between veterinarians and agricultural advisers is effective in some areas - such cooperation and thereby coordinated intensive consultancy formed the core of a project called "Plus $€ 3.3$ per produced pig". Our aim was to improve the productivity of finisher farms and thereby improve the economic output of the pig producer.
\end{abstract}

\section{Materials and Methods}

A total of 35 Danish finisher farms participated. Records of the productivity were required for participation. The intensive consultancy was carried out over a one-year period by a veterinarian and an agricultural adviser for each farmer. The project plan included three visits by both, and three single visits by the agricultural adviser and nine single visits by the veterinarian. The farmer paid for the intensive consultancy. Prior to the year of intensive consultancy, all farms were visited by a team from the Pig Research Centre (PRC) consisting of a specialist on feeding, a specialist on housing and ventilation and a veterinarian. This visit was part of the project, at no cost to the pig producer. The PRC team and the two local advisers drew up a detailed action plan for improved productivity for each farm. The purpose was to improve productivity (feed conversion, mortality, growth rate and slaughter weight). Only minor investments were suggested. The year of intensive consultancy was initiated when the pig producer had approved the plan of action. The consultancies were initiated during the year 2007 and ran to the end of 2008. The production results in the year of intensive consultancy were compared with those of the previous year.

\section{Results}

Twelve pig producers achieved an improvement in productivity of more than $€ 3.3$ per produced pig. The average economic improvement was $€ 2.5$ per produced pig. Increased consultancy and investments amounted to $€ 0.9$, resulting in a net improvement of $€ 1.6$ per produced pig. The farms produced between 2,000 pigs and 11,500 pigs per year. The action plan included between 10 and 31 separate actions: some involved changes of daily routine, while others needed to be addressed just once. On average, $71 \%$ of the separate actions in a plan were carried out. The plans were unique, though the following features were included in many of the plans: improving feed hygiene (especially in liquid feed systems), making the unit ready (clean, dry and warm) for new pigs, and putting more effort into the daily surveillance for diseased pigs or pigs in need of special attention. Eighteen out of 35 pig producers received four or more advisory visits from the agricultural adviser; the remaining seventeen pig producers received only two or three visits. All herds received a monthly visit from the veterinarian.

Table 1. Average productivity in 35 Danish finisher herds

\begin{tabular}{|l|c|c|c|}
\hline & One year before & Consultancy year & Change \\
\hline Start weight (kg) & 30.4 & 31.1 & +0.7 \\
\hline Slaughter weight (kg) & 80.9 & 82.1 & +1.0 \\
\hline Daily gain (gram) & 817 & 883 & +66 \\
\hline Feed conversion (kg feed/kg gain) & 2.65 & 2.57 & -0.08 \\
\hline Mortality (\%) & 4.9 & 4.0 & -0.9 \\
\hline $\begin{array}{l}\text { Economic output } \\
\text { (euro/prod. finisher*) }\end{array}$ & 14.7 & 16.3 & +1.6 \\
\hline *A five-year average of prices is used & & \\
\hline
\end{tabular}

\section{Discussion}

The whole action plan had to be cost-effective. However, it was, for example, easier to convince producers of better feeding regimes than to improve the climate by better ventilation. The individual actions had to be simple and practical, and the personnel carrying them out had to recognize their importance. We did not achieve this in all cases, since on average only $71 \%$ of the separate actions were carried out. Half of the farmers chose to have only two or three agricultural consultancies, which may have resulted in poorer productivity results. A certain degree of cooperation between more veterinary and agricultural advisers was achieved. This will improve the quality of the veterinary and agricultural consultancy. 


\title{
0.249
}

\section{Variation: an underestimated economic factor in pig production}

\author{
Dennis DiPietre ${ }^{2}$ Matthias J. Adam ${ }^{1}$ \\ 1. Boehringer Ingelheim Animal Health, Ingelheim am Rhein, Germany; 2. KnowledgeVentures, LLC, Columbia, MO, USA
}

\section{Introduction}

Modern swine production systems rarely undertake economic analysis using anything other than the arithmetic mean for production or economic performance metrics when needed in calculations. This is because modern systems are built and operated in such a way that collecting the data necessary to measure the variance of almost any metric is not practical in a commercial setting (e.g., measuring individual daily feed intake and weight gain for each animal in finishing) 1.

While farms measure and report their average feed efficiency, average daily gain, average market weight, cost of production etc, very few if any know the underlying variance of these distributions. For this reason, farms evaluate a host of economic decisions such as target market weight, the adoption of a new technology, vaccine and/or pharmaceutical, whether to replace aging equipment, etc. by comparing the average expected benefit per unit (head, kg, cwt) of production with the average additional cost per unit. A question arises as to how important the variance of the distribution generating the average is in making the correct decision.

\section{Materials and Methods}

To gain insight into this issue, a comprehensive simulation model was used to test the effect on net price received, total feed cost and return over feed cost associated with changes in the variance of pig weights at marketing. The weight and lean percent of ten thousand pigs randomly drawn from correlated distributions fit to annual marketing's of a large U.S. farm were used for the analysis. Average cost per ton (US) for each diet, wean-to-finish, was obtained from the producer and total feed cost per head was estimated from slaughter weight, a standard growth curve (ADG) and feed efficiency average curve (FCR) adjusted to match the historical closeout data of the farm (2.5 $\mathrm{kg} / \mathrm{kg}$ as FCR, $774 \mathrm{~g} /$ day as ADG - Average feed cost/US ton $W-F \$ 180 ;$ base carcass price: $\$ 1.43 / \mathrm{kg}$ ). The actual average live weight and standard deviation of live weights marketed by the farm over the test year were $122.4 \mathrm{~kg} / 12.28 \mathrm{~kg}$.

Different scenarios of reduction in variation of the weights at marketing from 5\%, 10\%, 20\% \& 30\% were simulated (table 1 \& 2 ). The change was carried out by reducing the distance to the mean by $\mathrm{x} \%$ for each individual animal weight in the dataset so the reduction in variation was symmetrical to the mean. Estimated individual animal lean and yield percents were each adjusted to the new weights by a separate constant estimated by regression.

\section{Results}

Table 1: Variance of the production metrics for each simulation (average remains identical)

\begin{tabular}{|c|c|c|c|}
\hline & Weights & FCR & ADG \\
\hline Actual & 12.28 & 0.18 & 36.64 \\
\hline StDev -5\% & 11.67 & 0.17 & 34.91 \\
\hline StDev $-10 \%$ & 11.04 & 0.16 & 33.17 \\
\hline StDev $-20 \%$ & 9.79 & 0.14 & 29.64 \\
\hline StDev -30\% & 8.50 & 0.13 & 26.02 \\
\hline
\end{tabular}

Table 2: Financial results of the different simulation

\begin{tabular}{|l|c|c|c|}
\hline & $\begin{array}{c}\text { Avg Net Carcass } \\
\text { Price Received/kg }\end{array}$ & Feed Cost /hd \$ & $\begin{array}{c}\text { Return over Feed } \\
\text { Cost /hd \$ }\end{array}$ \\
\hline Actual & $\$ 1.539$ & $\$ 58.59$ & $\$ 85.65$ \\
\hline StDev -5\% & $\$ 1.541$ & $\$ 58.54$ & $\$ 85.99$ \\
\hline StDev-10\% & $\$ 1.544$ & $\$ 58.41$ & $\$ 86.43$ \\
\hline StDev -20\% & $\$ 1.549$ & $\$ 58.15$ & $\$ 87.16$ \\
\hline StDev -30\% & $\$ 1.553$ & $\$ 57.82$ & $\$ 87.74$ \\
\hline
\end{tabular}

\section{Discussion}

The simulation model focuses on the slaughter weight as it is the most common source of individual data in pig production. Thereafter each individual pig can be assigned their individual metrics (FCR \& ADG) based on the adapted standard curves.

Simulation suggests that the standard deviations of the distribution of FCR \& ADG are important determinants of cost of production and therefore influence profitability. A reduction by $10 \%$ of the standard deviation of slaughter weights improves return over feed cost by $\$ 0.78 / \mathrm{hd}$. In addition, reducing variance results in a higher optimal weight which has not been accounted here.

\section{Conclusion}

By only accounting for the mean and changes in the mean in economic calculations, producers are missing important economic impacts associated with treatments or strategies which affect the variance of marketing weights.

\section{References}

1. Deen, J. (2005) Solutions To Control Variation, published by National Hog farmer, April 15, 2005 


\title{
0.250
}

\section{Why PCV2 viremia matters to swine practitioners}

\author{
Cate E. Dewey ${ }^{1}$ Kevin Vilacaㄹ Melissa Reidl ${ }^{1}$ Francisco de Grau³ Karen Richardson ${ }^{1}$ Zvonimir Poljak \\ 1. University of Guelph, Guelph, ON, Canada; \\ 2. Maitland Professional Veterinary Corp, Listowel, ON, Canada; 3. Intervet Canada Corp, Kirkland, QC, Canada
}

\section{Introduction}

Viremia results in immune system activation that leads to a redirection of nutrients that may result in a decrease in ADG and/ or an increase in feed-to-gain ratio. 1 Subclinical infections with PCV2 are common2,3 They are known to occur if vaccination does not prevent viral replication4. These PCV2 subclinical infections do not require co-infections.

The objective of this paper is to describe the prevalence and production impact of viremia in pigs while comparing two commercial circovirus vaccines or a placebo under field conditions.

\section{Materials and Methods}

A PRRS- and M. hyopneumoniae-free system was used for the study. At weaning, pigs were randomly assigned to be vaccinated with a one dose commercial PCV2 vaccine $(n=1026)$ (1D); a two dose commercial PCV2 vaccine $(n=1020)(2 D)$; or saline as a placebo $(n=100)$ (control). Both PCV2 vaccines were used according to manufacturer's label recommendations. Individual pig weights were collected when pigs were 3, 11 and 19 weeks of age and just prior to market at about $110 \mathrm{~kg}$. Individual ADG was determined between each pair of weights.To determine extent of PCV2 viremia, blood was collected from a random sample of 122 pigs at 3,9,15, 19, and 23 weeks of age and during the final week of shipping to market. Serum was tested with a qPCR. The proportion of viremic pigs at each sampling point by treatment group was determined. Difference in proportions was determined with a chi-square test. ADG was compared by group and by viremia status using a multiple linear regression after controlling for weaning cohort and starting weight.

\section{Results}

Starting weights did not differ by group. More pigs in the 1D $(47 \%)$ and control groups (85\%) were viremic than in the $2 \mathrm{D}$ group $(8 \%)(p<0.01)$. More control pigs $(73 \%)$ were viremic on at least 2 samples than 2D pigs $(0 \%)$ or $1 D$ pigs $(24 \%)(p<0.05)$. Pigs that were viremic more than once, tended to have a lower ADG (by $33.8 \mathrm{gm} / \mathrm{d}$ ) than pigs that were never viremic $(p=0.11)$. The proportion of viremic pigs remained at $3 \%$ to $4 \%$ for the entire grower-finisher phase for the 2D pigs. However, this proportion increased from $11 \%$ to $39 \%$ for the 1D pigs and from $53 \%$ to $63 \%$ for the control pigs from 15 to 19 weeks of age. The ADG in the $2 \mathrm{D}$ group was $42 \mathrm{~g}$ / day higher than in the $1 \mathrm{D}$ group from 19 weeks to market age $(p<0.01)$. The coefficient of variation of ADG in the 2D group (19\%) was lower than that of the 1D group $(28 \%)$ indicating that there was more variation in the growth of 1D group.

\section{Implications and Conclusions}

The proportion of viremic pigs was higher in the control group than in the vaccinated pigs. The pigs receiving the $2 \mathrm{D}$ vaccine maintained a low level of viremia throughout the finisher phase whereas the pigs in the 1D group exhibited a rising proportion of viremia toward the end of the finishing phase. This viremia was associated with a lower ADG and more variation in growth performance, particularly during the final phase of production. This is the least efficient time of growth, feed conversion and facility use. This variation extends the time for marketing. Hence, viremia contributes to the overall cost of production in the 1D group.

\section{References}

1. Colditz, I.G. 2002. Effects of immune system on metabolism: implications for production and disease resistance in livestock Livest Prod Sci. 75(3): 257-268.

2. Darwich, L., et al. 2008. Transient correlation between viremia levels and IL-10 expression in pigs subclinically infected with porcine circovirus type 2 (PCV2). Res Vet Sci. 84: 194-198.

3. Krakowka, S., et al., 2005. Features of porcine circovirus-2 disease: correlations between lesions, amount and distribution of virus, and clinical outcome. J Vet Diagn Invest. 17: 213-222.

4. Fort, M., et al., 2009. Development of cell-mediated immunity to porcine circovirus type 2 (PCV2) in caesarean-derived, colostrumdeprived piglets. Vet Immunol and Immunop. 129: 101-107. 


\title{
0.251
}

\section{Conventional sows inseminated with artificially PCV2-infected semen: II. Post mortem results}

\author{
Federico Morandi ${ }^{1}$ B. Bacci ${ }^{1}$ S. Panarese ${ }^{1}$ D. Ferrara ${ }^{1}$ Laura Fusaro $^{1}$ M.L. Bacci ${ }^{1}$ M. Dottori ${ }^{2}$ P. Bonilauri ${ }^{2}$ \\ Giorgio Leotti ${ }^{3}$ Thaïs Vila $^{4}$ François Joisel ${ }^{4}$ F. Ostanello ${ }^{1}$ Giuseppe Sarli ${ }^{1}$ \\ 1. School of Veterinary Medicine, University of Bologna, Bologna, Italy; 2. IZSLER, Reggio Emilia, Italy; \\ 3. Merial, Milano, Italy; 4. Merial S.A.S., Lyon, France
}

\section{Introduction}

Experimental infections, using several routes of virus inoculation in pregnant animals brought at parturition $(1,2,3,4)$, demonstrate the role of the PCV2 on reproductive pathology. It is known that in utero death of foetuses producing dead or mummified piglets at delivery infected with PCV2 can be assessed in tissues $(1,3,5)$. In PCV2 associated reproductive pathology the presence of uterine or placental lesions are scantly documented. We developed an original experimental model of PCV2 infection by artificial insemination of conventional sows with PCV2 infected semen and in this paper we present and discuss the PCV2 tissue distribution and the associated lesions of the sow and foetuses after post-mortem samplings.

\section{Material and Methods}

The experimental design and the results of the in vivo investigation are contextually presented (6). Necropsy was performed on all the not pregnant $(n=3)$ and pregnant $(n=6)$ animals. Several tissues of the sows and of each foetus (heart, lung, liver, placenta and amniotic fluid) were collected (Table 1) for real time-PCR, histology and immunohistochemistry (IHC). The corresponding uterine tract and the placenta were collected together with foetus sampling. Real time-PCR analyses were in accordance to the method by Olvera et al. (7). Samples for histology were stained with haematoxylin and eosin (H-E). IHC was performed using 1/100 PBS-diluted PCV2 antibody (Mab F217, by Dr. G. Allan (Belfast, UK)), as previously described (8) but using a streptavidin-biotin-peroxidase polymeric complex (SuperPicture kit peroxidise, Zymed $^{\circledR}$ Lab).

\section{Results}

In the sows, at necropsy, the only lesions recorded included mild to moderate chronic fibrous pleuritis and/or pericarditis. One animal showed enzootic pneumonia. One case of a mild sero-fibrinous peritonitis and two cases of white spotted liver were observed. Two out of the 3 non-pregnant sows showed congestion and oedema of uterine mucosa. Three out of 6 infected sows were pregnant and had respectively 16, 11 and 7 foetuses, whereas two out of the 3 controls 11 and 8 . One control sow aborted but any PCV2-DNA was evidenced in the 3 aborted foetuses. In only one placenta (positive for PCV2 DNA with moderately high viral load, $>108$ genome copies $/ \mathrm{ml}$ ) mild focal necrosis of the chorionic epithelium resulted positive by IHC for PCV2 antigen localized in the cytoplasm of the cells. The foetus showed also IHC positive reaction in the cytoplasm of hepatocytes.
Table 1: $R T-P C R$ results of tissue sampling

\begin{tabular}{|l|c|c|c|c|c|c|c|c|c|}
\hline & \multicolumn{5}{|c|}{ Infected } & \multicolumn{3}{c|}{ Control } \\
\hline & \multicolumn{3}{|c|}{ pregnant } & \multicolumn{2}{c|}{ not pregnant } & \multicolumn{3}{c|}{ pregnant } \\
\hline Sow ID & 25 & 26 & 29 & 27 & 32 & 33 & 28 & 30 & 31 \\
\hline Number of foetuses & 16 & 11 & 7 & 0 & 0 & 0 & 11 & $0 *$ & 8 \\
\hline Tonsil & - & - & - & + & - & - & - & - & - \\
\hline Uterine left lymph node & + & - & - & - & - & - & - & - & - \\
\hline Uterine right lymph node & + & - & - & + & - & - & - & - & - \\
\hline $\begin{array}{l}\text { Tracheo-bronchial lymph } \\
\text { node }\end{array}$ & + & - & - & $/$ & - & $/$ & - & - & - \\
\hline Cervix & - & - & - & - & + & - & - & - & - \\
\hline Foetuses (pos./total) & $10 / 16$ & - & $3 / 7$ & - & - & - & - & - & - \\
\hline Amniotic fluids (pos./total) & - & $1 / 11$ & - & - & - & - & - & - & - \\
\hline $\begin{array}{l}\text { Foetus and corresponding } \\
\text { uterine tract (left) }\end{array}$ & 7 & - & - & - & - & + & - & - & - \\
\hline $\begin{array}{l}\text { Foetus and corresponding } \\
\text { uterine tract (right) }\end{array}$ & 1 & - & - & - & - & - & - & - & - \\
\hline * Aborted 3 foetuses before necropsy & & & & & & & & \\
\hline
\end{tabular}

\section{Discussion}

The present investigation suggests the protective role of the anti-PCV2 antibodies in preventing foetal infection even in case of intrauterine route of infection. Sow n. 25, with the lowest anti-PCV2 titre (beginning of the experiment) (6) and viremia for two successive samplings, had also the highest number of positive foetuses and concordance with corresponding uterine tract positivity. Moreover, the results seem to demonstrate that PCV2 replicates also in chorionic epithelium where it was the possible cause of necrosis. As well as viral load in foetal tissues, also placenta lesions can account for foetus death. This study evidenced that not all foetuses are positive for PCV2, it should be recommended to send the whole litter to the lab for a correct diagnosis of PCV2-related reproductive failures.

\section{References}

1. Johnson C, et al., 1999. J Vet Diagn Invest 14 507-12

2. Cariolet R, et al., 2001. Proc. ssDNA viruses of plants, birds, pigs and primates, 128

3. Park JS, 2005, J Comp Pathol. 132:139-44

4. Rose N, et al., 2007. J Comp Pathol 136:133-44

5. West H, et al., 1999. J Vet Diagn Invest 11:530-32

6. Sarli G, et al., 2010. Proc 21st IPVS

7. Olvera A, et al., 2004. J Virol Methods 117:75-80

8. Sarli G, et al., 2009. Vet Rec 164:519-23 


\title{
0.252
}

\section{A preliminary study of the association between Porcine Circovirus Type 2, Lawsonia intracellularis and diarrhoea in growing pigs}

\author{
Patricia K. Holyoake ${ }^{1}$ Charlotte Hjulsager ${ }^{2}$ Lars E. Larsen $^{2}$ Ken S. Pedersen ${ }^{3}$ Markku Johansen ${ }^{4}$ \\ Helle Stege ${ }^{3}$ Beverley Orchard ${ }^{1}$ Marie Stahl' ${ }^{2}$ Oystein Angen ${ }^{2}$ Jens P. Nielsen ${ }^{3}$ \\ 1. Wagga Wagga Agricultural Institute, Wagga Wagga, NSW, Australia; 2. National Veterinary Institute, Technical University of Denmark, \\ Copenhagen, Denmark; 3. University of Copenhagen, Copenhagen, Denmark; 4. Danish Pig Research Centre, Copenhagen, Denmark
}

\section{Introduction}

Porcine Circovirus Type 2 (PCV2) infection is endemic in pig herds in most countries. Initially, PCV2 was linked to post-weaning multi-systemic wasting syndrome (PMWS). More recently, other disease syndromes have been associated with PCV2, and PCV2 has been reported to cause lesions similar to those of Lawsonia intracellularis (L. intracellularis). ${ }^{1}$ Our hypothesis was that there is an association between the quantities of PCV2 in serum and faeces, L. intracellularis in the faeces and diarrhoea in grower (>10 weeks) pigs.

\section{Materials and Methods}

Forty grower pigs were selected on six farms in Denmark for a case-control study. Each pig was ear-tagged and assessed for faecal consistency (normal, loose, fluid). Approximately half of the pigs had loose or fluid faeces and were classed as "diarrhoeic". Blood and faecal samples were collected from each pig and tested using real-time PCR specific for PCV2 (blood and faeces) and $L$. intracellularis (faeces only). 2,3 The associations between diarrhoea (+/-) and serum and faecal PCV2 quantity (negative/low versus moderate/massive), and between diarrhoea and $L$. intracellularis bacterial quantity (negative/low versus moderate/massive) were analysed. The association between the quantity of PCV2 in blood and PCV2 in faeces, and between the quantity of PCV2 in faeces and L. intracellularis in faeces were analysed. All analyses were conducted using generalized linear mixed models, with farm as a random effect (Genstat 11 th edition).

\section{Results}

Two farms had no pigs with moderate/massive PCV2 results in blood or faeces. One farm had no pigs with a negative/low faecal PCV2 result. Two farms had pigs with no moderate/massive $L$. intracellularis results. Pigs with diarrhoea were no more likely than pigs without diarrhoea to have moderate/massive quantities of PCV2 in blood or faeces ( $p>0.05)$. Pigs without diarrhoea were no more likely than pigs with diarrhoea to have negative/low quantities of PCV2 ( $p>0.05$ ). Pigs with moderate/ massive quantities of $L$. intracellularis had 1.91 times the odds $(95 \% \mathrm{Cl} 1.043 .49)$ of having diarrhoea than pigs without diarrhoea $(p<0.05)$. The farms where no pigs had moderate/massive quantities of PCV2 and/or L. intracellularis were excluded from analyses of associations between the two pathogens. Pigs with moderate/massive quantities of PCV2 in serum had 14.4 times the odds $(95 \% \mathrm{Cl} 1.08191 .6)$ of having moderate/massive quantities of PCV2 in faeces than pigs with low/negative PCV2 serum results $(p<0.05)$. There were no significant $(p>0.05)$ associations between PCV2 quantities in blood or faeces, and $L$. intracellularis quantities in faeces.

\section{Discussion}

These preliminary results suggest PCV2 was not associated with diarrhoea in pigs on the six farms studied Pigs with diarrhoea shed moderate/massive quantities of $L$. intracellularis in their faeces. There was no evidence of co-infection between PCV2 and $L$. intracellularis. PCV2 results for blood and faeces from the same pig suggested that either clinical sample may be tested with a similar result. As there was evidence of Brachyspira spp. infection in euthanized diarrhoeic pigs on all farms (data not shown $)^{4}$, the interaction between $L$. intracellularis and Brachyspira spp. warrants investigation.

\section{References}

1. Jensen TK. et al. (2006). J Comp. Path. 135:176-182

2. Hjulsager CK. et al. (2009). Vet Microbiol. 133:172-178

3. Ståhl M. et al. (2008). Abstract, Advances in qPCR

4. Holyoake PK. et al (2009). Proceedings of the Australasian Pig Science Association Conference:129

\section{Acknowledgements}

This project was supported by the University of Sydney, University of Copenhagen, Danish Pig Research Centre, Technical University of Denmark and Australian Pork Ltd. We thank the participating producers and veterinarians for their assistance. 


\title{
0.253
}

\section{Maternal immunity towards Porcine circovirus type $\mathbf{2}$ (PCV2)}

\author{
Per Wallgren ${ }^{1}$ Maria Persson ${ }^{1}$ Gunilla Blomqvist ${ }^{1}$ Caroline Fossum $^{2}$ \\ 1. Dept of Animal Health and Antimicrobial Strategies, Uppsala, Sweden; \\ 2. Swedish University of Agricultural Sciences, Uppsala, Sweden
}

\section{Introduction}

Postweaning Multisystemic Wasting Syndrome (PMWS) is associated to porcine circovirus type 2 (PCV2), and as neutralising antibodies prevent pigs from developing PMWS (1) transfer of maternal antibodies to PCV2 may protect the offspring from developing PMWS. We therefore scrutinised the maternal immunity to PCV2 in two herds diagnosed with PCV2, but not with PMWS.

\section{Materials and Methods}

The studies were carried out in a nucleus herd with 180 Yorkshire sows, and in an SPF herd established by caesareans in 1988 with 170 Landrace, Yorkshire and hybrid sows. (2). The SPF herd was infected by PCV2a in 1993, certainly through semen. The herd experienced a reproduction disorders for around six months following the introduction of PCV2 (3), but has stayed healthy since then. PCV2a was demonstrated in both herds prior to initiating the studies. Neither sows nor piglets were vaccinated against PCV2, and PMWS had not been diagnosed in any pig.

Six piglets from 4 litters in each herd were given an identity. Blood was collected from these piglets weekly from birth to 9 weeks of age. Presence of serum antibodies to PCV2 was analysed in two-fold dilutions with an IPMA-technique. The amounts of antibodies are presented as Log10-values.

\section{Results}

As seen in figure 1, piglets in both herds received maternal antibodies to PCV2 with a mean log10-titre of $3.8 \pm 0.6$. The titres decreased significantly in both herds, but the decrease was more pronounced in the SPF herd from 5 weeks and onwards. Four piglets from two litters in the conventional herd seroconverted at 6, 7, 7 and 9 weeks of age, whereas only one pig in the SPF herd seroconverted to PCV2 at the age of 9 weeks.

Figure 1. Mean antibody titres to PCV2 in 24 piglets from 4 litters in an SPF herd ( $O$ ) and in a conventional herd ( $\square$ ).

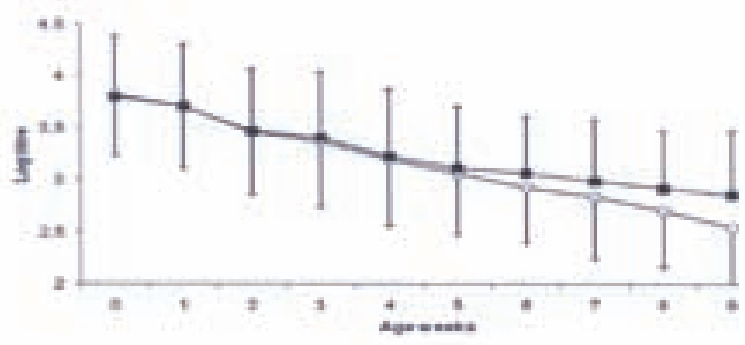

The mean antibody titres never differed between the herds, but when the offspring to different sows were compared significant differences between litters were at hand within both herds (Fig 2).
Figure 2. Mean antibody titres to PCV2 in piglets from different litters in an SPF herd (upper part) and in a conventional herd (lower part). Breed of the dams; $O=$ Landrace, $\nabla=$ Yorkshire, $\square=L Y$-hybrid. Open symbols = 1st parity, grey symbols = 3rd parity and black symbol = 7th parity. Dotted lines $=$ pure bred litter, Full lines $=$ crossbred litter.
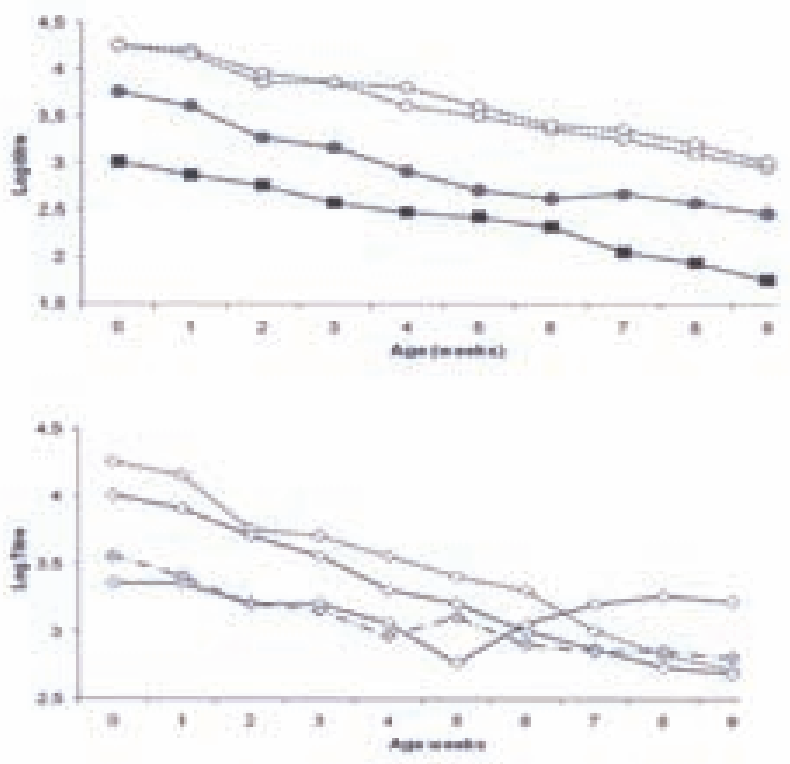

\section{Discussion and Conclusions}

The results showed that all piglets received a maternal transfer of antibodies to PCV2, and that the level of antibodies not was influenced by the rearing system. Nor was any influence of breed indicated since maximal levels of antibodies ( $\log 10=4.3$ ) was measured in offspring to both pure bred Landrace and Yorkshire sows.

In contrast, significant differences in antibody levels were recorded between litters belonging to the same farrowing batch within each herd, and the lowest levels of maternal antibodies was measured in the offspring to the 7th parity sow. In the conventional herd, piglets that received low amounts of antibodies commenced to seroconvert to PCV2 already at 6 weeks of age, indicating an active infection with PCV2. Such piglets may thus increase the PCV2 load concurrently to the decline of maternal immunity in other litters. Therefore sows that transfer a low maternal immunity may jeopardise the health status of a herd because piglets will meet the PCV2-infection at a younger age. The results obtained indicate that old sows may contribute to such risks, and vaccination of old sows may be of particular interest.
References
1 Meerts et al., 2005. Virol Immunol 18:333-341.
2. Wallgren \& Vallgårda. 1993. Sw Vet J. 45:733-735.
3. Wattrang et al., 2002. Vet Microbiol. 86:281-293. 


\title{
0.254
}

\section{Postweaning multisystemic wasting syndrome (PMWS) age-shift presentation is not supported by an 11-year retrospective study in Spain}

\author{
Joaquim Segales; Martí Cortey \\ Centre de Recerca en Sanitat Anima (CReSA), Bellaterra, Spain
}

\section{Introduction}

Postweaning multisystemic wasting syndrome (PMWS) was firstly diagnosed in Spain in 1997 (3). Since then, the number of disease diagnoses increased significantly, reaching a peak between years 2000-01 (2), and then decreased over time. After 2007, the number of PMWS diagnoses started increasing again, basically due to the advent of porcine circovirus type 2 (PCV2) vaccines in the market. Therefore, it seems that the number of PMWS diagnoses in a given period depends on several factors. Firstly, a diagnosis in front of a novel disease was needed (mainly between 1997 and 2001). Secondly, once the disease was known and no practical solutions did exist, the interest of laboratorial diagnosis decreased (2002-2006). This latter situation was coupled with the evolution of severe disease outbreaks to a more enzootic, chronic form of the disease. However, with the abovementioned introduction of PCV2 vaccines, the interest of a precise diagnosis of PMWS raised again among veterinarians (2007 to date). In such framework, a general perception was that the initial acute disease largely died down over time and since then veterinarians and producers face with the chronic effects of PMWS. Such situation leaded to the opinion that PMWS resulting mortality occurred at older ages in the chronic form of the disease (3). Therefore, the objective of the present study was to assess if such PMWS age-shift presentation took place over the years in Spain.

\section{Material and methods}

A total of 650 pigs diagnosed as PMWS at the Pathology Diagnostic Service at the Universitat Autònoma de Barcelona (Spain) with an unequivocally known age were included in this study. These animals were diagnosed as PMWS based on the three internationally accepted case definition criteria (4) between 1998 and 2008. The number of pigs available per year was variable, ranging from 9 in 2006 and 134 in 1999. The average number of pigs per year was around 60. Different ages were measured in months and for statistical purposes, a single number was given for all age groups included (i.e, age 1 corresponded to pigs between 1 and $<2$ months of age, age 2 corresponded to animals between 2 and $<3$ months of age, and so on).

Differences in the PMWS age presentation among years, and between the overall and yearly average age were tested by means of several chi-square and Kluskal-Wallis tests. Statistical significance level was set at $p<0.05$. Confidence intervals (95\%) for every year are presented in Fig. 1

\section{Results}

The youngest age recorded corresponded to 11 1-month-old piglets, while the oldest animals diagnosed as PMWS included 136 -month-old pigs. More than $80 \%$ of the pigs fall between 2 and $<4$ months of age (532 out of 650 animals). Fig. 1 shows the evolution of the average age of pigs diagnosed as PMWS between 1998 and 2008. Significant differences in age presentation were overall obtained, mainly due to the average age in two years: younger pigs in 2001 and older pigs in 2005. However, the average age evolution between 1998 and 2008 did not significantly increase.

Fig. 1. Average age evolution of pigs diagnosed as PMWS between 1998 and 2008.

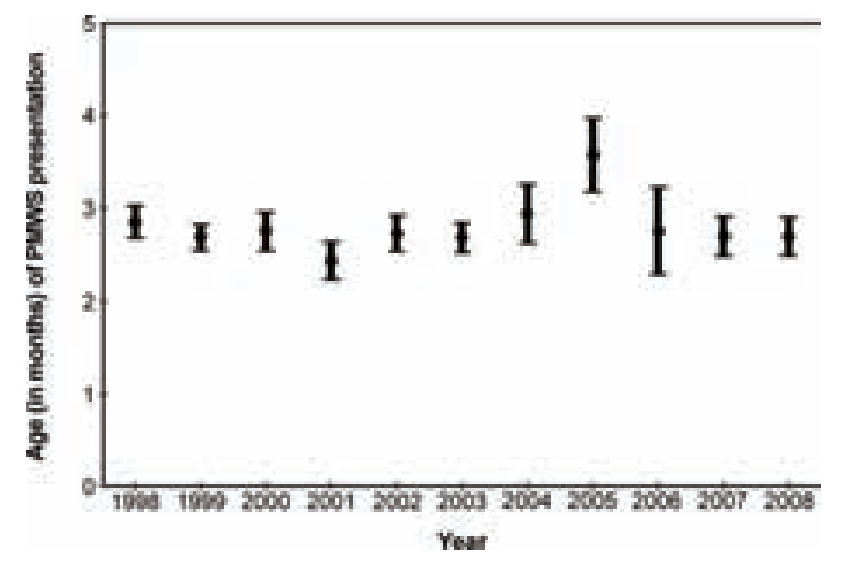

\section{Discussion}

With the emergence of new diseases, it is expected that initial severe outbreaks are followed by a more chronic, not as severe form of the clinical problem. Such situation has been described for PMWS. It has been claimed that the development of the so-called "herd immunity" has been the main reason for disease evolution as well as the assumed shift in age presentation (1). However, the present data do not fit with this general opinion, and points to a relatively constant average PMWS age presentation throughout time.

\section{References}

1. Burch D. 2008. Pig Progress, 24: 7-9.

2. Cortey et al. 2010. Vet J, in press.

3. Segalés et al. 1997. Vet Rec 141:600-601.

4. Segalés and Domingo 2002. Vet Q 24: 109-124. 


\title{
0.255
}

\section{Identification of the receptor for F18+ E. coli: new perspectives towards development of a prophylactic treatment against F18+ E. coli infections in pigs}

\author{
Annelies Coddens ${ }^{1}$ Susann Teneberg ${ }^{2}$ Eric Cox $^{1}$ \\ 1. University of Ghent, Faculty of Veterinary Medicine, Lab of Immunology, Merelbeke, Belgium; \\ 2. University of Gothenburg, Institute of Biomedicine, Department of Medical Biochemistry and Cell Biology, Gothenburg, Sweden
}

\section{Introduction}

F18-fimbriated Escherichia coli are associated with porcine postweaning diarrhoea (PWD) and oedema disease. Adhesion of F18fimbriated bacteria to the small intestine of susceptible pigs is a primary event in the infection process. However, the target cell receptor for $\mathrm{F} 18+\mathrm{E}$. coli (F18R) has remained unidentified. The objective of our study was to unravel the carbohydrate-binding specificity of F18-fimbriated E. coli and to examine whether these F18R molecules can inhibit the interaction between F18+ E. coli and the pig small intestine.

\section{Materials and methods}

To identify the F18R molecules, mixtures of glycosphingolipids (GSLs) isolated from pig intestines were separated on thin-layer plates, followed by a chromatogram binding assay with radiolabeled F18+ E. coli. In addition, the inhibitory capacity of the F18R molecules was assessed using an in vitro villous adhesion assay.

\section{Results}

We report that F18-fimbriated E. coli selectively interact with GSLs having blood group $\mathrm{ABH}$ determinants on type 1 core chains, and blood group A type 4 heptaglycosylceramide. F18binding GSLs were isolated from the small intestinal epithelium of blood group $\mathrm{O}$ and $\mathrm{A}$ pigs, and characterized by mass spectrometry and proton NMR. The only GSL with F18 binding activity of the blood group $\mathrm{O}$ pig was an $\mathrm{H}$ type 1 pentaglycosylceramide (Fuc a2Gal $\beta 3$ GlcNAc $\beta 3$ Gal $\beta 4$ Glc $\beta 1$ Cer). In contrast, the blood group A pig had a number of F18-binding GSLs, with the A type 1 hexaglycosylceramide (GalNAc a3(Fuc a2)Gal $\beta 3$ GlcNAc $\beta 3 \mathrm{Gal} \beta 4 \mathrm{Glc} \beta 1 \mathrm{Cer})$ being the predominant one.
The minimal binding epitope was identified as the blood group $\mathrm{H}$ type 1 determinant (Fuc a2Gal $\beta 3 \mathrm{GlcNAc}$ ), while an optimal binding epitope was created by addition of the terminal a3linked galactose or $\mathrm{N}$-acetylgalactosamine of the blood group $\mathrm{B}$ type 1 determinant (Gal a3(Fuc a2)Gal $\beta 3$ GlcNAc) and the blood group A type 1 determinant (GalNAc a3(Fuc a2)Gal $\beta 3$ GlcNAc), respectively.

Using the in vitro villous adhesion assay, the inhibitory capacity of several blood group sugars on F18+ E. coli adhesion was examined. Strong inhibition of F18+ E. coli adherence was observed after pre-incubation of F18+ E. coli with $1 \mathrm{mg}$ of the blood group H (52.4\%, S.D. = 19.9), blood group A (72.9\%, S.D. = $15.5)$ and blood group B sugar $(86.8 \%$, S.D. $=11.4)$, whereas the negative control sugar lacto-N-tetraose did not inhibit $(-2.8 \%$, S.D. $=12.4)$ the interaction of F18+ E. coli with the intestinal villi.

\section{Discussion}

A highly specific interaction of the F18-fimbriated bacteria with GSLs having blood group ABH determinants on type 1 core chains was demonstrated. In addition, we showed that F18R molecules could be used to inhibit the interaction of F18+ E. coli with the porcine intestinal epithelium using an in vitro assay. In our future work, in vivo experiments will be performed to investigate whether these F18R molecules, when supplemented in pig feed, could provide a safe and efficient anti-adhesive therapy against F18+ E. coli infections in pigs.

\section{References}

Coddens A, Diswall M, Angström J, Breimer ME, Goddeeris B, Cox E, Teneberg S, 2009. Recognition of blood group ABH type 1 determinants by the FedF adhesin of F18-fimbriated Escherichia coli. J Biol Chem. 284(15):9713-26. 


\title{
0.256
}

\section{Effect of toltrazuril in Isospora suis oocyst excretion from naturally infected nursing piglets}

\author{
Vasilis Skampardonis ${ }^{1,2}$ Smaragda Sotiraki² Polychronis Kostoulas ${ }^{1}$ Leonidas Leontides ${ }^{1}$ \\ 1. University of Thessaly, Karditsa, Greece; 2. Veterinary Research Institute, National Agricultural Research Foundation, Thessaloniki, Greece
}

\section{Introduction}

Currently, farm control of Isospora suis infection depends almost completely on the early routine treatment of piglets with toltrazuril. Its efficacy has been demonstrated mostly in experimentally infected piglets (Mundt et al., 2003). In this field trial we simultaneously evaluated toltrazuril efficacy in reducing both the odds and the level of oocyst excretion, from the 6th day post-farrowing (pf) until weaning at 29 days pf.

\section{Materials and Methods}

The study was conducted in a commercial farrow-to-finish herd with 300 sows, farrowing weekly all year round. The farm had a history of clinical diarrhoea in piglets, but no anti-coccidial compounds had been used. Prior to the initiation of the study, the presence of $I$. suis parasitism on the farm was parasitologically confirmed. Twenty-five litters with a total of 246 piglets were randomly allocated to either the treated (13 litters-128 piglets) or the untreated (12 litters-118 piglets) group. Piglets in the former, on the 3rd day pf, were orally treated with toltrazuril (BAYCOX ${ }^{\circ}$ \%) at the dose rate of $20 \mathrm{mg}$ per $\mathrm{kg}$ bw, whereas those in the latter litters received no treatment. At least half a gram of faeces was collected individually from the rectum of each piglet daily from 6- until 13-days-old and thereon, every second day until weaning (i.e., 15 samples/piglet). Oocyst excretion (expressed as oocysts per gram of faeces (OPG)) was recorded for all samples. Because OPG counts were semi-continuous data (Liu et al., 2008), to evaluate the effect of treatment on the longitudinal OPG counts we fitted a two-part model in the aML software. Presence or absence of oocyst excretion was the response variable in the first part of the model, while the natural logarithm of the non-zero OPG values was used in the second part. Treatment and time were included as covariates in the model. Random effects were incorporated in the model to account for the within litter and within piglet correlation of observations. We adjusted for the cross-equation correlation ( $\rho C E C)$ of the litter- and piglet-level random effects between the logistic and linear part of the model to capture the biologically plausible fact that higher rates of excretion may also have higher mean OPG counts. Subsequently, we quantified the litter- and piglet-level unexplained variation via estimation of variance partition coefficients (VPC)(Kostoulas et al., 2009).

\section{Results and Discussion}

Parameter estimates from the model are in the table. Treated piglets had 66.5 (95\% C.I.:10-500, $\mathrm{P}<0.001)$ times lower odds of excretion than the untreated ones. The mean difference in the amount of OPG in any day between treated and untreated piglets was 55.5 (95\% C.I.:6-518, P<0.001). Piglets with higher odds of excretion tended to excrete more oocysts ( $\rho C E C=0.99$, $\mathrm{P}<0.001)$. Litters with higher odds of excretion also tended to have more oocysts excreted ( $\rho C E C=0.55, P<0.001)$. VPCs were 40 and $23 \%$ and 26 and $17 \%$ for the litter- and piglet-level variance for the logistic and linear part of the model, respectively.

Toltrazuril treatment reduced both the odds of excretion and the mean amount of excreted oocysts. Estimated VPCs revealed that the greater portion of unexplained heterogeneity of oocyst excretion lies at the litter-level, highlighting the need of studies to identify litter-level risk factors for l.suis control.

The effect of toltrazuril treatment on the odds and level of oocyst excretion (logistic and linear part of the model, respectively) adjusting for piglet's age.

\begin{tabular}{|l|c|c|c|}
\hline & \multicolumn{3}{|c|}{ Logistic } \\
\hline & Coef. & $95 \%$ C.I. & $P$ \\
\hline Treatment & -4.2 & $-6.2 ;-2.3$ & $<0.001$ \\
\hline Piglet age & 0.10 & $0.08 ; 0.11$ & $<0.05$ \\
\hline & \multicolumn{4}{|c|}{ Linear* } \\
\hline Treatment & -4.0 & $-6.2 ;-1.9$ & $<0.001$ \\
\hline Piglet age & -0.01 & $-0.05 ; 0.04$ & $>0.05$ \\
\hline *Estimates of the linear part of the model were based on the analysis of the natural logarithm \\
of OPG.
\end{tabular}

\section{References}

Kostoulas et al. 2009. Prev Vet Med, 89: 155-162.

Liu et al. 2008. Stat Med, 27: 3528-3539.

Mundt et al. 2003. Parasitol Res, 90:160-162.

aML Multilevel Multiprocess Statistical Software: Version 2.0 (www. applied-ml.com). 


\title{
0.257
}

\section{Evaluation of the colostrum phase in 10 French pig herds dealing with enzootic neonatal diarrhea}

\author{
Thomas Gin $^{1}$ Eric Pagot ${ }^{2}$ Guy-Pierre Martineau ${ }^{1}$ \\ 1. National veterinary school, Toulouse, France; 2. CTPA, Ploufragan, France
}

\section{Introduction}

Enzootic neonatal diarrhea (END) is occurring in 15 to $20 \%$ of French farrow-to-finish pig farms1. Dealing with this issue, veterinarians have to work on the aggression side i.e. control of pathogens and on the defense side i.e. control of immunity. During the first weeks of life, piglet immunity is mainly providing by colostral antibodies and cells. In this paper, we focus on the colostrum phase in pig farms dealing with END.

\section{Materials and Methods}

The study was conducted in 10 French farrow-to-finish pig farms during 2006 and 2007. Selection of farms was performed according to their history of END for more than 6 months. For the purpose of this study, one of us (TG) spent 7 consecutive days on each farm, starting the first day of farrowing as all farms operate on batch farrowing system.

For sows farrowing from 7 am to $7 \mathrm{pm}$, colostrum were manually taken from cranial mammary glands before the 3rd piglet's birth and kept at $5^{\circ} \mathrm{C}$ until laboratory analysis. For each farrowing, data were recorded: length of gestation (calculated from the first day of $\mathrm{Al}$ ), parity and emergence of neonatal diarrhea (determined by a clinical examination 3 times a day and during 7 consecutive days). Clinical as well as laboratory investigations are also registered2. At the end of the investigation week, 4 whole litters were blood-sampled, 2 control litters and 2 litters with diarrhea. In each farm, we select those 4 litters on 3 criteria: litters for which the sow was sampled for colostrum, litters that were 4 to 6 days old and litters in which we had less than 4 crossfostering piglets.

IgG dosages were performed at LDA22 (Ploufragan, Côtes d'Armor, France) using a Pig IgG ELISA Quantification Kit ${ }^{\oplus}$ (BETHYL Laboratories, inc. Montgomery, Texas, USA).

\section{Results}

Piglet's sera from 16 "diarrhea litters" ( $n=178)$ and from 22 "control litters" ( $n=244)$ were collected for evaluation of lgG ([lgG] sera, $\mathrm{mg} / \mathrm{ml}$ ). The mean of [lgG] sera for "diarrhea litters" is 22.2 $\mathrm{mg} / \mathrm{ml}(\mathrm{SE}=0.84 ; \mathrm{CV}=0.50)$ while the one for "control litters" is $24.8 \mathrm{mg} / \mathrm{ml}(\mathrm{SE}=0.83 ; \mathrm{CV}=0.52)$. There is no significant difference (p>0.05).

Colostrum ( $n=135)$ from mixed-parities and mixed-genetics sows were collected for evaluation of lgG ([lgG]colostrum, mg/ $\mathrm{ml}$ ). When data from the 10 herds are pooled, we found that the mean of [lgG]colostrum is $81.3 \mathrm{mg} / \mathrm{ml}(\mathrm{SE}=2.5 ; \mathrm{CV}=0.32)$. There is a significant difference $(p=0.005)$ in the $[\mathrm{lgG}]$ colostrum between sows with "diarrhea litters" (41 sows, mean=70.8 mg/ml, SE=3.5) and sows with "control litters" (94 sows, mean $=85.8 \mathrm{mg} / \mathrm{ml}$, $\mathrm{SE}=3.1)$. There is also a significant difference $(\mathrm{p}=0.001)$ in the $[\mathrm{lgG}]$ colostrum between sows farrowing at 114 days (mean $=72.6$ $\mathrm{mg} / \mathrm{ml}, \mathrm{SE}=4.1$ ) and sows farrowing at 116 days (mean $=104.8$ $\mathrm{mg} / \mathrm{ml}, \mathrm{SE}=6.7)$. Finally, there is no parity effect $(\mathrm{p}>0.05)$ regarding $[\mathrm{lgG}]$ colostrum. As expected, there is a good positive correlation $(r 2=0.73)$ between $[\mathrm{lgG}]$ colostrum and average [lgG] sera of the litter.

\section{Discussion}

In our study, the colostrum phase was evaluated indirectly by the $[\mathrm{lgG}]$ sera of piglets. Within the same litter, [lgG]sera are in the $2 / 1$ to $3 / 1$ scale. This variation is not limited to affected litters or in some special litters. It is of concern in all litters. How can we explain this very important variation of colostrum intake between piglets? Two studies3,4 found that piglet's vitality, birth weight and birth order are factors explaining a part of colostrum intake variation between piglets.

In our study, the colostrum immune quality was evaluated by the $[\mathrm{lgG}]$ colostrum. First, we found a relationship between low $[\mathrm{lgG}]$ colostrum and occurrence of diarrhea in the litter. Then, neonatal diarrhea is generally more frequent in gilts like for neonatal E. coli diarrhea5. However, in our study, there is no parity effect $(p>0.05)$ regarding $[\mathrm{lgG}]$ colostrum. Another study 3 showed that parity rank explains only a small part of the colostrum yield variation. Finally, Devillers et al.3 show an impact of farrowing induction (probably by an indirect effect on gestation length) on colostrum yield variation. Our study tends to show the same impact on [lgG]colostrum but with the same confusion between gestation length and farrowing induction.

During the colostrum phase, a lot of changes occur for the sows and for the piglets. The regulation of these changes is called homeorhesis i.e. orchestrated changes for the priorities of a physiological state. A better understand of the colostrum phase is needed to improve the performance in farrowing units.

\section{References}

1. Sialelli et al. Proceed. Journées Recherche Porcine. 2009; 41 : 167172.

2. Gin et al. Proceed. IPVS 2010.

3. Devillers et al. Animal. 2007; 7:1033-1041.

4. Le Dividich et al. Proceed. Journées Recherche Porcine. 2004; 36 : 451-456.

5. Fairbrother J. In: Diseases of Swine. 9th edition. 2006: 641-648. 


\title{
0.258
}

\author{
Neonatal diarrhoea in piglets from E. coli vaccinated sows in Sweden \\ $\underline{\text { Lennart Melin }}^{1}$ Per Wallgren ${ }^{1}$ Sigbrit Mattsson ${ }^{1}$ Michael Stampe ${ }^{2}$ Monika Löfstedt ${ }^{2}$ \\ 1. National Veterinary Institute (SVA), Uppsala, Sweden; 2. Swedish Animal Health Service, Skara, Sweden
}

\section{Introduction}

The importance of virulent $E$. coli in the pathogenesis of neonatal diarrhoea in piglets has been well established since the 1960 's. The development of vaccines and proper vaccination routines of sows combined with good hygiene standards have reduced the problems with neonatal diarrhoeas to a minimum. However, despite proper vaccination routines the incidence of neonatal diarrhoeas has increased during the last years in several countries. This syndrome have been suggested the name New Neonatal Porcine Diarrhoea (NNPD). The aim of the present study was to search for possible causes for such diarrhoeas in Sweden.

\section{Materials and Methods}

Faecal samples, collected by The Swedish Animal Health Service, were taken from pens with clinically manifested diarrhoea in piglets less than 3 days of age despite $E$. coli vaccinations of their sows. In total 17 samples from 6 herds were investigated ( 3 samples from 5 herds and 2 samples from 1 herd).

The following analyses were performed;

- Defining the 4 most abundant aerobic bacterial species in each pen sample.

- Presence of 9 virulence factors (F4, F5, F6, F18, F41, LT, STa, $\mathrm{STb}, \mathrm{VT} 2)$ by PCR in the two dominating $E$. coli isolates/pen.

- Presence of 75 virulence factors in the two dominating E. coli isolates/pen using a microarray kit (Identibac EC Array Tube).

- The coliform floras were biochemically fingerprinted to define eventual common clones (Pheneplate ${ }^{\circ}$ ) (1)

- Determination of the resistance pattern of the two dominating $E$. coli isolates from each herd (Vetmic ${ }^{\circ}$ ).

- Presence of Clostridium perfringens type A (CpA) and Clostridium perfringens type $C(C P C)$ by anaerobic culturing and PCR.

- Presence of genes for enterotoxin (cpe) or beta2 toxin (cpb2) in isolated CPA by PCR (2).

\section{Results}

No unexpected bacterium was found in any sample. E. coli, Enteroccoccus spp. and $C p A$ were found in abundance in all samples.

As for $E$. coli no common virulence factor was found. In fact, only one of all the isolates was diagnosed with a virulence factor (STb) by the PCR technique. The microarray did not reveal any common pattern among the $E$. coli isolates. In total 18 virulence factors of the 75 possible were found in a random pattern among the isolates. Furthermore, no common phenotypic clone of $E$. coli could be demonstrated neither between nor within herds. The resistance pattern of all tested isolates of $E$. coli was normal for Swedish conditions (3).

All isolated $C p A$ had genes for $c p b 2$ but no one had genes for $c p e . C p C$ was not isolated from any sample.

\section{Discussion}

In an international perspective neonatal diarrhoea is associated to infection/overgrowth of E. coli, $C p C, C p A$, Salmonella or corona viruses such as TGEV and PED. Neither $C p C$ nor Salmonella was found in the herds included in this trial and TGEV and PED have never been isolated in Sweden.

Piglets are born with a sterile gastrointestinal tract (GIT) and the low production of gastric acid $(\mathrm{HCl})$ and $\mathrm{pH}$ dependent gastric proteases give almost free access for invading microbes to colonise the animals GIT. They are therefore dependent on the colostrum from the sow to be able to establish a normal gastro intestinal flora. To accomplish this sows are vaccinated against pathogenic E. coli 5-3 weeks prepartum.

Despite the well known importance of $E$. coli in neonatal diarrhoea, no common E. coli associated explanation to the diarrhoeas could be found in the study.

The role of $C p A$ on the other hand is not all clear. The bacterium is ubiquitous in gut content and soil and part of the normal flora. The genetic ability to produce $c p b 2$ may be a sign of pathogenicity (4), but the importance of $c p b 2$ is still under discussion.

So far, our results have excluded a number of microbes as likely causes of the neonatal diarrhoeas recently diagnosed in these herds with $E$. coli vaccinated sows.

Considering that this phenomenon has been observed in several countries, efforts to define microbes associated to such diarrhoeas ought to be intensified.

\section{References}

1) Kühn et al., 1995. Microecol \& Therapy. 23: 140-148

2) Engström et al., 2003, Vet microbial. 94: 225-235.

3) www.sva.se (Serach for SVARM REPORT)

4) Båverud et al., Proc IPVS 18. (1) 327. 


\title{
0.259
}

\section{The effect of Saccharomyces cerevisiae on the occurrence of enteropathogenic $E$. coli in weaned piglets}

\author{
Jan Bernardy ${ }^{1}$ Dana Kumprechtova ${ }^{1}$ Pavel Alexa ${ }^{2}$ Eric Auclair ${ }^{3}$ \\ 1. University of Veterinary and Pharmaceutical Sciences, Brno, Czech Republic; \\ 2. Veterinary Research Institute, Brno, Czech Republic; 3. Lesaffre Feed Additive, Marq en Baroeul, France
}

\section{Introduction}

Probiotics, including live yeast and lactic acid bacteria cultures, have been reported to improve performance of weaned piglets $(1,2)$. Piglets fed by milk replacer supplemented with Saccharomyces cerevisiae Sc 47 (SC) show an increased postweaning growth and reduced TNF- a expression in the intestinal and liver tissues, suggesting an important immunomodulatory role of the treatment (3).

E. van Heugen et al. evaluated the effects of SC supplementation on faecal microflora and assessed whether live yeast could replace antibiotics and growth-promoting effect of $\mathrm{Zn}$ and $\mathrm{Cu}$ in nursery pigs. They found variable responses, and determined the conditions under which a measurable response can be expected (4). The objective of the present study was to evaluate the effect of SC dietary supplementation on the presence of enterotoxigenic E. coli (ETEC) strains and Clostridium perfringens in faeces, general signs of diarrhea and performance of postweaning piglets.

\section{Material and methods}

The experiment was conducted under field conditions at the farrow to finish unit with 600 sows, with farrowing, post-weaning and finishing departments placed in separate buildings. The trial included two batches of piglets, from weaning at 25 days of age to the end of the post-weaning period at approximately 80 days of age. The piglets were housed in rooms with pens ( 25 piglets per pen) and slatted floor. The first batch (SC treatment) received mash liquid feed supplemented with SC culture at 1000 ppm (Actisaf Sc47, Lesaffre Feed Additives, France). The second batch (Control) was fed the same mash liquid feed without SC supplementation. Each treatment included 200 piglets. From each treatment, 10 piglets were randomly selected, ear-tagged and subjected to closer monitoring. They were weighed and taken rectal swabs on weekly basis, starting 11 days (Day 1) after the weaning. All the piglets in each treatment were subjected to daily diarrhea scoring (score 1 - no diarrhea signs; score 2 - pasty faeces, active piglets; score 3 - watery faeces, active piglets; score 4 - severe diarrhea, apathy piglets. Each entire batch was weighed at the beginning and end of the post-weaning period. The culling and mortality rates were summarized after the departure of the experimental piglets for the finishing department.

\section{Results}

The diarrhoea in weaned piglets is mainly caused by ETEC, how the results confirmed. The pathogenic strains $E$. coli were found in two piglets on Day 14 in the control group 0141:F18, VT2e+ and 0149:F4, STa+, LT+. In SC group no pathogenic strains were found.

Piglets were diagnosed in 98,12\% Clostridium perfringens positive in the first sampling and $100 \%$ positive in the later samples regardless of treatment.
Table 1

\begin{tabular}{|l|c|c|c|}
\hline Group & Days (d) & ADG (g) & No of piglets (n) \\
\hline SC & 55 & 413 & 280 \\
\hline Control & 56 & 405 & 206 \\
\hline
\end{tabular}

\section{Discussion}

In contrast to our findings (5), some researchers (6) re-ported that the yeast strain in their studies did not seem to affect any of the microbial groups tested incl. E. coli.

The mash liquid diet supplemented with SC showed a tendency $(p<0.05)$ to increase daily weight gain by $8 \mathrm{~g}$ as compared with the untreated Control group (Table 1). The SC dietary supplementation may have reduced pathogenic $E$. coli strains and diarrhea occurrence. The diarrhea scores of the SC group differed significantly from those of the Control on days of the trial 11 to 13 (Figure 1).

\section{Figure 1}

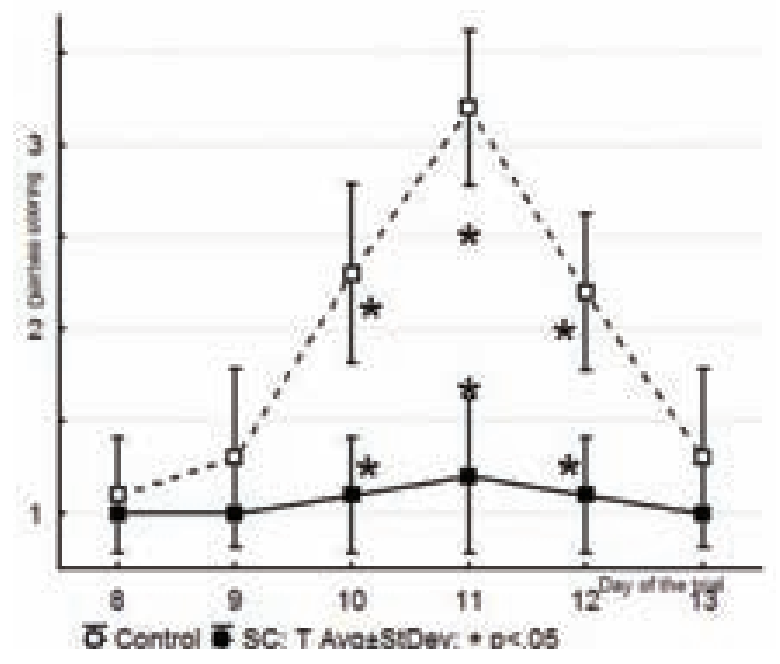

\section{References}

1 Lessard, M., and G. J. Brisson (1987) Can. J. Anim. Sci. 67:509-516.

2 Kornegay, E. T. et al. J. Anim. Sci. 73:1381-1389.

3 Eicher,S.D. et al. (2006) J Anim Sci 84(9): 2352

4 van Heugten,E. et al.(2003) J Anim Sci 1 (4): 104

5 Bernardy, J. et al. (2008) Proc 20th IPVS Congress:443

6 Mathew,A.G. et al. (1998) J Anim Sci 76 (8):2138 


\title{
0.260
}

\section{Factors associated with pre-weaning piglet mortality due to crushing}

\author{
Leena Anil ${ }^{1}$ Sukumarannair S. Anil ${ }^{2}$ Sam K. Baidoo ${ }^{1}$ John Deen² Mark E. Wilson ${ }^{3}$ Terry L. Ward ${ }^{3}$ \\ 1. University of Minnesota, Southern Research and Outreach Center, Waseca, MN, USA; \\ 2. University of Minnesota, Veterinary Population Medicine, St Paul, MN, USA; 3. Zinpro Corporation, Eden Prairie, MN, USA
}

\section{Introduction}

The average preaweaning piglet mortality in US herds in the first quarter of 2009 is reported to be $12.2 \% 1$. It has been reported 2 that one-fifth of all fetuses fully formed at the end of gestation die before weaning. Thus preweaning piglet mortality is an important cause for economic loss in swine production systems. Injuries and crushing due to the sow falling on the piglets while making postural changes constitute a major cause for preweaning piglet death. Claw lesions in pigs are putatively painful. Sows with severe claw lesions may be restricted in making the necessary postural adjustments while attempting to lie down within the limited space inside the farrowing crate. In pigs the support role is more important in the front limbs which are nearer to the center of gravity. Further, observation of a sow lying down also suggests more movements associated with front limbs than the hind limbs. It is important to understand the factors associated with piglet death due to crushing to minimize the loss of live born piglets. The objective of the present study was to analyze the association of preweaning piglet death due to crushing with factors such as claw lesions, parity of the sow and gestation housing system.

\section{Material and methods}

This study involving 611 parity records of sows housed in group pens with electronic sow feeders (ESF) and 399 parity records of sows housed in conventional gestation stalls was conducted at the University of Minnesota, Southern Research and Outreach Center at Waseca. The lesions in the claws of these sows were examined at mid-gestation using a mechanical restraint designed for the purpose in one, two or three consecutive parities. The total claw lesion scores for front and hind limbs were obtained 3 by adding the lesion scores in different claw areas (side wall, heel, sole, heel-sole junction, and white line) of lateral and medial claws. Information on reasons for preweaning piglet mortality, parity of the sow and housing system were collected from the PigCHAMP database of the unit. The association of number of preweaning piglet mortalities due to crushing with the parity of the sow, claw lesion scores in front and hind limbs, and gestation housing system was analyzed (Proc genmod, SAS v. 9.1) analysis with sow as a repeated measure. For analysis, parity of the sow was categorized into parities 1 and 2, 3 to 5 and $>5$.

\section{Results}

In this study $24 \%$ of preweaning piglet mortality was found to be due to crushing. Analysis indicated significant association between number of preweaning piglet deaths and parity of the sow, claw lesions and housing system. Sows of parities 1 and 2 and parities 3 to 5 had 61 and 36\% lower piglet death due to crushing respectively, compared to sows of parity $>5$ ( $P<0.05$ for both). For every unit increase in claw lesions in front limbs, the number of preweaning piglet death due to crushing increased by $8 \%(P<0.05)$. A higher hind limb claw lesion score was also associated with higher number of piglet death due to crushing, though this association was not significant $(P>0.05)$. Sows housed in group pens had 39\% less $(P<0.05)$ piglet death due to crushing compared to sows housed in stalls during gestation.

\section{Discussion}

The positive association observed between parity and piglet death due to crushing could be due to the smaller litter size in younger parity sows. An increase in number of piglet deaths due to crushing being associated with an increase in front limb claw lesion score is interesting. This may be suggestive of restriction due to pain in making the necessary postural movements to avoid uncontrollable falling within the farrowing crate, thus leading to a higher number of piglets being crushed. A higher number of crushed piglets in stall-housed sows could be related to a lack of exercise and consequent decrease in muscle weight and bone strength4 of these sows.

\section{Conclusion}

This study indicated a positive association between higher preweaning piglet death due to crushing and factors such as higher parity, stall gestation housing system and higher claw lesion scores in front limbs of sows.

\section{References}

1. http://www.pigchamp.com/summary_archives.html.

2. Van der Lende et al., (2001). Prenatal development as a predisposing factor for perinatal losses in pigs. Reproduction 58(Suppl.):247-261.

3. Anil, S.S. et al., (2007). Factors associated with claw lesions in gestating sows. J SHAP 15: 78-83.

4. Marchant, J.N. and Broom, D.M. (1996). Effects of dry sow housing conditions on muscle weight and bone strength. Animal Science 62:105-13. 


\title{
Claw lesion effects on piglets born alive of stall-housed sows in two sow herds
}

\author{
A. Da Silva; S.S. Anil; J. Deen; R.B. Morrison \\ University of Minnesota, Saint Paul, MN, USA
}

\section{Introduction}

The number of piglets born alive per litter directly influences the average number of piglets weaned per sow per year (1). The aim of the present study was to evaluate the association of the productivity of stall-housed sows in terms of low litter size ( $\leq 10$ piglets) with claw lesions scores, also considering stillborn, mummies, parity and sow herd.

\section{Materials and methods}

The lesions of these sows were collected from a convenience sample of 343 stall-housed sows (parities 1 to 13) belonging to two sow herds (Herd A, $n=145$, Herd $B, n=198$; of 1500 sows each herd). These sows were scored for lesions in different claw areas (side wall, heel, including overgrown heels, white line, heel-sole junction, and overgrown toes and dew claws). The claws were examined while the sows were in a recumbent position as there was no mechanical restraint to facilitate lesion scoring. The total score for each claw area was obtained by adding the scores for that area in the claws of front and hind limbs. For analysis, parity was categorized into parities 1 and 2, 3 to 5 and $>5$. A multivariate logistic regression analysis was performed to assess the association of lesion scores of different claw areas, stillborn, mummies, parity and sow herd with number of piglets born alive ( $\leq 10$ vs. $>10$ ) (Proc Logistic, SAS v 9.2).

\section{Results}

A total of 105 sows ( $30.6 \%$, mean $=6.9$, standard deviation $=2.8$ ) had $\leq 10$ piglets born alive. The number of sows that had $>10$ piglets born alive was 238 (69.4\%, mean $=13.2$, standard deviation=2.16). The total lesion score for all claw areas ranged from 0 to 27 and only two sows had no lesions. White line lesions (odds ratio 1.151, range of lesions scores $=0$ to 10 ) and overgrown toes (odds ratio 1.154 , range of lesions scores $=0$ to 10 ) were positively associated ( $P<0.05$ for both) with the likelihood of having $\leq 10$ piglets born alive. The likelihood of having $\leq 10$ piglets born alive increased with an increase in the severity of lesions in other claw areas as well (heel-sole crack and side wall), though the associations were not statistically significant. The numbers of stillborn piglets were positively associated with the likelihood of having $\leq 10$ piglets born alive (odds ratio 1.298, $\mathrm{P}<0.01$ ). Parity, number of mummies and sow herd were not found to be associated with piglets born alive in this study.

\section{Discussions}

The small number of sows with no lesions is in agreement with a previous study which indicated a high number of stalled housed sows that had at least one claw lesion (2). White line lesions and overgrown toes were positively associated with the likelihood of having $\leq 10$ piglets born alive; suggesting that productivity of sows can be negatively influenced by the development of severe claw lesions in sows. The results of this study are indicative of potential adverse effects of claw lesions on the productivity of sows. It is important to identify factors affecting sow productivity and to reduce early removal of sows due to low productivity.

\section{References}

1. Wilson, M.R. et al. 1986. A survey of productivity and its component interrelationships in Canadian swine herds. J. Anim. Sci. 62:576-582.

2. Anil, S.S. et al. 2007. Factors associated with claw lesions in gestating sows. J Swine Health Prod. 15(2):78-83.

\section{Acknowledgements}

The authors thank Barry Nienhaus for assistance in claw lesion scoring. 


\title{
0.262
}

\section{Better colostrum distribution increases piglet survival in high prolific sows}

\author{
$\underline{\text { Jan Jourquin }^{1} \text { Johan Biermann }}{ }^{2}$ Rainier van Gelderen ${ }^{1}$ Lieve Goossens ${ }^{1}$ \\ 1. Janssen Animal Heatlh, Beerse, Belgium; 2. DAP Helmond, Helmond, Netherlands
}

\section{Introduction}

Colostrum intake is critical for the survival of the piglet. During the first days, the colostrum provides the nutrients for homeostasis and growth. For a much longer period the colostrum immunoglobulin fraction passively protects the piglets from disease (1). Only during the first 24 to 48 hours of life, these colostrums IgG's can pass the piglets gut. The colostrum intake per $\mathrm{kg}$ body weight (BW) ranges between 210 to 370 gram, but the capacity of a piglet to ingest colostrum is exceeding $450 \mathrm{gram} /$ $\mathrm{kg} \mathrm{BW}$ (2). The total colostrum yield of sows varies between 1.91 and $5.31 \mathrm{~kg}$ (3). Because litter size does not affect colostrum yield (3), in hyper prolific sow breeds, a high variation in colostrums uptake is difficult to avoid.

The aim of this study was to investigate the impact of colostrum distribution on pre-weaning piglet survival in high prolific sows.

\section{Materials and Methods}

In a 1600 sow farm, the litters of 220 sows were included in the study. At 3 days of age, individual blood samples were retrieved from the docked tail of six randomly chosen litter mates. The IgG titre of the samples was measured with a DAS-ELISA test (Colostrum Quality Counter ${ }^{\mathrm{TM}}$ ). This IgG titre, determined after the phase of colostrums IgG passage through the gut, can be used as a measure of colostrum intake (4). Of each litter, the average IgG titre and the standard deviation was calculated. By dividing the standard deviation by the average, the coefficient of variance of piglet serum lgG (CV lgG) was calculated. This coefficient is a measure for variation in IgG intake within the litter, and therefore represents the variation of colostrum intake. During the lactation period piglet mortality in each litter was recorded. The statistical analysis was performed with the SAS ${ }^{\oplus}$ program. A Poisson regression model on the number of dead piglets per sow was used. The total number of piglets was included as covariate.

\section{Results}

The CV IgG amongst litter mates ranged from 18 to $208 \%$, with an average of $55 \%$. In Table 1, the impact of colostrum distribution on piglet mortality is shown. Sows with a CV IgG of less than $50 \%$ (average $36 \%$ ) were compared to those with a CV IgG of more than $50 \%$ (average $76 \%$ ). With the same average litter size, the mortality was found to be statistically significant lower in litters with a low CV IgG.
Table 1: Impact of colostrum distribution on pre-weaning piglet mortality

\begin{tabular}{|c|c|c|c|}
\hline CV IgG & Number of litters & Piglets with sow & Mortality (\%) \\
\hline$\leq 50 \%$ & 117 & 12.5 & $5.1^{\text {a }}$ \\
\hline$>50 \%$ & 103 & 12.6 & $7.7^{\mathrm{b}}$ \\
\hline $\mathrm{ab}$ : difference with p-value of 0.0259 & \\
\hline
\end{tabular}

The mortality rate over all litters was highly correlated with the number of piglets with the sow $(p<0.0001)$.

\section{Discussion}

These results clearly demonstrate that an even distribution of the available colostrum amongst littermates has a positive impact on the pre-weaning survival rate. The high number of piglets born alive, often in combination with low birth weight and low vitality as seen in highly prolific sows, reduces the chances of equal colostrums uptake amongst littermates. This leads to piglet loss mainly in the first days after birth. The ability of the sow to give all her piglets equal drinking opportunity is therefore a key factor. Even with a good colostrum distribution, mortality increases with the litter size, demonstrating that an increasing number of piglets calls for a higher colostrum yield, an increasing number of functional teats and sufficient drinking space. On the other hand, piglet suckling might also be promoted by reducing or eliminating all stress related behavior during the colostrum intake phase enabling the sow to optimally nurse her offspring.

All measures that improve the transfer and equal distribution of the available colostrum to each piglet should be taken into account to improve piglet survival.

\section{References}

1. J. Le Dividich et al., Journal of Agricultural Science 2005, 143, 469485

2. N. Devillers et al., Animal Science $2004,78,305-313$

3. N. Devillers et al., Animal 2007 1:7, 1033-1041

4. J.P. Nielson et al., Sammendrag 2008 


\title{
Effect of a softer surface in the farrowing crate on the behavior of lactating sows
}

\author{
A. Da Silva; S.S. Anil; J. Deen; S.K. Baidoo \\ University of Minnesota, Saint Paul, MN, USA
}

\begin{abstract}
Introduction
Lameness in swine herds is a common problem. Activity levels and frequency of standing may be higher during lactation (1). However, lameness, being putatively painful, may influence the frequency and/or duration of postural behaviors of the sow during lactation. Limited ability to make postural changes could in turn affect the quantity of feed and water consumed by the sow. Inadequate water intake has been suggested to cause low milk production (2) and reduced weight gain/death of piglets. Similarly, low lactation feed intake can affect subsequent reproductive performance of the sow. The quality and type of the floor are important factors influencing sows' standing and lying-down behavior (3). However, studies on the effect of floor type on lameness and its effects in behavior of sows are rare. The present study aims to explore the effect of providing rubber mats in farrowing crates as a measure to minimize the adverse effects of lameness on behavior.
\end{abstract}

\section{Materials and methods}

This study involved 70 lame and 70 non-lame gestating sows (gestation stall $n=63$ and group pens with ESF $n=77$ ) identified based on their ability to bear weight on all limbs without favoring any particular limb when they were moved from the gestation housing systems to farrowing rooms. Lameness in all the sows was assessed by the same person. Equal numbers of sows were randomly allocated to farrowing crates with cast iron floor or to crates with the cast iron floor covered with rubber mats ( $3 \mathrm{~cm}$ thickness) on the posterior half. The behaviors of the sows while in the farrowing crates on day 3,9 and 15 post-farrowing were recorded using cameras and a time-lapse VCR. The frequencies of behaviors (getting up, dog sitting, drinking water and eating) of lame and non-lame sows with and without rubber mats on these days were compared using separately models, controlling for the effect of housing system (Proc Genmod, SAS v 9.2).

\section{Results}

The frequencies of getting up, dog-sitting posture, drinking water and eating on day 3, 9 and 15 post-farrowing were (0-31, $2-48,2-41,0-29)$ respectively. The frequency of getting up was $3 \%$ lower and $15 \%$ higher ( $P<0.05$ for both) in stall-housed sows on d 3 and d 15 post-farrowing. Lame sows got up 12\% fewer times on d9 post-farrowing. These sows also tended to have
$(P=0.05) 16 \%$ lower frequency of getting up on $d 3$. On $d 9$ post farrowing, the frequency of dog-sitting posture was 13\% lower $(\mathrm{P}<0.05)$ in sows with rubber mat. Stall-housed sows adopted dog-sitting posture $16 \%$ and $44 \%$ more times ( $P<0.05$ for both) on $\mathrm{d} 9$ and d 15 post-farrowing. However, lame sows tended $(P=0.08)$ to show this postural behavior less frequently than nonlame-sows on $\mathrm{d} 9$. The frequency of drinking water tended to be lower for lame sows in comparison to non-lame sows $(P=0.09)$ and higher for those sows housed in gestation stalls $(P=0.08)$ on d3 post-farrowing. The frequency of drinking water was $14 \%$ lesser $(\mathrm{P}<0.05)$ among sows with rubber mats on $\mathrm{d} 9$ post-farrowing. Sows housed in stalls drank $39 \%$ more times $(P<0.05)$ than the group-housed sows on $\mathrm{d} 15$ post-farrowing. The frequency of drinking water tended to be higher in both lame sows $(P=0.07)$ and those housed in stalls $(P=0.07)$ on $d 9$ post-farrowing. The frequency of eating was $11 \%$ less $(P<0.05)$ among lame sows on d9 post-farrowing and the frequency tended to be less $(P=0.08)$ on $\mathrm{d} 3$ post farrowing.

\section{Discussions}

In general, the results are indicative of the adverse effect of lameness on postural behavior and feed and water consumption during lactation. However, post-partum behavior of the sow and familiarity of stall-housed sows to postural changes in restricted space could have influenced the results.

\section{References}

1. Valros, A. et al. 2003. Sow activity level, frequency of standing-tolying posture changes and anticrushing behaviour-within sowrepeatability and interactions with nursing behaviour and piglet performance. Applied Animal Behavior Science. 83:29-40.

2. Anonymous. 1987. Dead pigs by the bucketfuls. National Hog Farmer. 32 (11):32-39.

3. Leonard, F.C. et al. 1997. Behaviour, skin and foot lesions in sows and piglets on different farrowing house floors. In: Bottcher RW, Hoff SJ, (Eds.), Livestock Environment 5. American Society of Agricultural Engineers, St. Joseph, Michigan, US, pp.242- 249.

\section{Acnowledgements}

Funding was provided by the National Pork Board. The authors thank Alejandra Pinto, Aaron Nystrom, Mauricio Gonzalez, and Gustavo Lopez for technical help. 


\title{
0.264
}

\section{Use of paper strips as nesting material in sows: effects on abnormal behaviours, salivary cortisol and productive data}

\author{
Paolo Candotti; Daniele Zappulla; Ivonne Archetti; Sara Rota Nodari
}

Izsler, Brescia, Italy

\section{Introduction}

In both wild and domestic pigs nest building is a natural, inherited behaviour that begins $24 \mathrm{~h}$ before farrowing. Stalling in single barren farrowing crates inhibits this behaviour and represents a source of mental stress in sows (Lawrence A.B. et al., 1994). The aim of this study was to compare salivary cortisol, abnormal behaviours and productive data of the litters in sows kept in barren farrowing crates and in sows given paper strips.

\section{Material and Methods}

91 sows of a farrow to finish farm of Northern Italy were selected for the study. The sows of the treated group $(n=36)$ were given $2 \mathrm{~kg}$ of strips of paper at day 112 of gestation; the control group $(n=55)$ were left without. Both groups were kept in traditional farrowing crates. Salivary cortisol was evaluated at day 101, 107,112 and 113 of gestation and at day 1 and 28 of lactation. Abnormal behaviours were recorded at day 107, 112-115 of gestation and at the 1 st day of lactation. Productive data of the litters were also recorded.

\section{Results and Discussion}

Results reported in figure 1 show less abnormal behaviours in treated sows compared to control sows. Particularly, the treatment was able to reduce oral stereotypes.

Fig.1. Percentage of sows displaying abnormal behaviours in the observed period

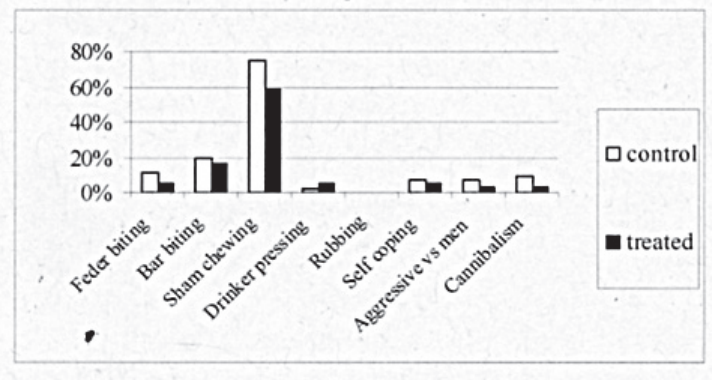

The salivary cortisol measured at day 101 of gestation was compared to the salivary cortisol measured at the other samplings time points. Treated sows showed a lower increase in cortisol compared to control sows. In figure 2 the average level at day 101 of gestation and the difference with this basal level at the other samplings is reported. These findings confirm previous reports (Jarvis et al., 2001) that showed a positive effect of environmental enrichment on animals' cortisol.
Fig 2. Average salivary cortisol in the two groups of treatments at $\mathrm{G} 101$ and average difference from that level at the other samplings $(\mathrm{G}=$ gestation, $\mathrm{L}=$ Lactation $)$

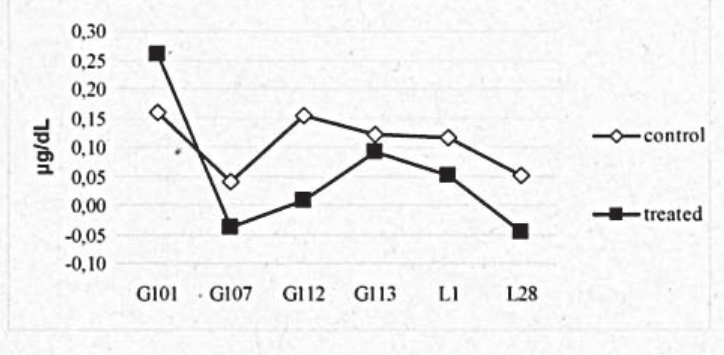

In table 1 the positive effect of treatment on the productive data of the litters is reported.

Table 1. Performance data of the litters

\begin{tabular}{|l|c|c|c|c|}
\hline \multicolumn{1}{|c|}{ Group } & BA Mean (SD) & \% CP/BA & \% SAV/BA & \% WP/BA \\
\hline control & $13.24(3.21)$ & 14 & 2.8 & 81 \\
\hline treated & $12.25(3.38)$ & 9.3 & 0.4 & 89 \\
\hline $\begin{array}{l}\text { Legend: } B A=\text { number of piglets born alive; } \mathrm{CP}=\text { crushed piglets; } S \mathrm{SV}=\text { piglets savaged by } \\
\text { their sow; WP = total weaned piglets; } \mathrm{SD}=\text { standard deviation }\end{array}$ \\
\hline
\end{tabular}

The overall effect of paper strips was positive on all the parameters recorded without adverse effects on the slurry system.

\section{References}

Jarvis S., Van Der Vegt B.J., Lawrence A.B., Mclean K.A., Deans L.A., Chirnside J. \& Calvert S.K. (2001) The effect of parity and environmental restriction on behavioural and physiological responses of pre-parturient pigs. Applied Animal Behaviour Science 71, 203-216.

Lawrence A., Petherick J., Mclean K., Deans L., Chirnside J., Gaughan A., Clutton E. \& Terlouw E. (1994) The effect of environment on behaviour, plasma cortisol and prolactin in parturient sows. Applied Animal Behaviour Science 39, 313-330. 


\title{
0.265
}

\section{Cross-reactivity and efficacy of a monovalent swine flu vaccine containing the pandemic H1N1 2009 virus}

\author{
$\underline{\text { Ralf Dürrwald }}{ }^{1}$ Michael Schlegel ${ }^{1}$ Hans-Joachim Selbitz ${ }^{1}$ Andi Krumbholz ${ }^{2}$ Roland Zell ${ }^{2}$ \\ 1. IDT Biologika GmbH, Dessau-Roßlau, Germany; 2. Universitätsklinikum Jena, Institut für Virologie und Antivirale Therapie, Jena, Germany
}

\section{Introduction}

Since its first emergence in the human population in spring 2009 (1) infections with the pandemic H1N1 virus (cH1N1v) have been reported in pigs and other animal species in several regions of the world $(2,3)$. The pandemic virus can be experimentally transmitted between pigs (4). Although vaccine strains of authorised swine flu vaccines are cross-reactive to $\mathrm{cH} 1 \mathrm{~N} 1 \mathrm{v}(5)$ a swine vaccine containing $\mathrm{CH} 1 \mathrm{~N} 1 \mathrm{v}$ may be superior to the available vaccines. In order to investigate this in more detail hyper immune sera, post infection sera and a monovalent vaccine containing $\mathrm{cH} 1 \mathrm{~N} 1 \mathrm{v}$ were produced and investigated for efficacy.

\section{Materials and Methods}

Hyper immune sera were established using a fourfold vaccination of pigs with antigens of $\mathrm{H} 1 \mathrm{~N} 1$ vaccine strains and a strain of the pandemic H1N1 virus using Freund's adjuvant. Blood samples were taken 14 days after last immunization. A vaccine containing the pandemic H1N1 virus was produced. Swine flu vaccines available in central Europe and the newly produced $\mathrm{cH} 1 \mathrm{~N} 1 \mathrm{v}$ vaccine were administered to pigs. Blood was withdrawn 7 days after second administration.

Furthermore an experimental aerosol infection was carried out using the parental strain of the most recent avian-like H1N1 strain contained in a European swine flu vaccine. Ten days after infection, blood samples were taken. Sera were investigated applying a neutralization test and hemagglutination inhibition $(\mathrm{HI})$.

\section{Results}

The investigation of hyper immune sera revealed that there is neutralizing activity between the pandemic $\mathrm{H} 1 \mathrm{~N} 1$ virus and avian-like H1N1 European vaccine strains (A/sw/Netherlands/25/80, A/sw/IDT/Re230/92, A/sw/Haselünne/IDT2617/07) as well as with the New Jersey/8/76 (Fort Dix reassortant) strain at low level. A hyper immune serum established against the pandemic H1N1 showed cross-reactivity with European avianlike $\mathrm{H} 1 \mathrm{~N} 1$ virus and very high titres against several pandemic H1N1 strains. The vaccines (Gripovac, Suvaxyn Flu, RESPIPORC FLU, RESPIPORC FLU3/Gripovac 3) also induced neutralizing antibodies against the pandemic $\mathrm{H} 1 \mathrm{~N} 1$ virus. However, the titres were lower in comparison to those obtained for avian-like H1N1 and not all pigs did respond. The highest degree of cross neutralization was achieved by experimental batches of a vaccine which contained the pandemic H1N1 virus strain (Tab. 1).
Tab.1. Antibodies induced by a swine flu vaccine containing $\mathrm{CH} 1 \mathrm{~N} 1 \mathrm{v}$ in comparison to an approved trivalent vaccine, mean $\mathrm{HI}$ titres reciprocal (pigs positive); avH1N1 avian-like H1N1, huH1N2 human-like H1N2, huH3N2, human-like H3N2

\begin{tabular}{|l|l|l|l|l|l|}
\hline Vaccine & No. of pigs & \multicolumn{1}{c}{ cH1N1v } & \multicolumn{1}{|c|}{ avH1N1 } & \multicolumn{1}{c|}{ huH1N2 } & huH3N2 \\
\hline cH1N1v & 6 & $1093(6)$ & $13(1)$ & $<20(0)$ & $<20(0)$ \\
\hline $\begin{array}{l}\text { avH1N1, } \\
\text { huH1N2+huH3N2 }\end{array}$ & 5 & $8(1)$ & $464(5)$ & $199(5)$ & $656(5)$ \\
\hline
\end{tabular}

\section{Discussion}

It is concluded that despite the cross-reactivity between avianlike and classical swine $\mathrm{H} 1 \mathrm{~N} 1$ strains with the pandemic $\mathrm{H} 1 \mathrm{~N} 1$ virus of 2009 a vaccine consisting of $\mathrm{CH} 1 \mathrm{~N} 1 \mathrm{v}$ is superior in terms of efficacy in comparison to vaccines already licensed.

\section{References}

1. Itoh $\mathrm{Y}$, et al. In vitro and in vivo characterization of new swine-origin H1N1 influenza viruses. Nature. 2009;460:1021-5.

2. Influenza pandemic (H1N1), animal. Available from http://www. promedmail.org. Latest information on Influenza A (H1N1).

3. Hofshagen $M$, et al. Pandemic influenza $A(H 1 N 1) v$ : Human to pig transmission in Norway? Euro Surveill. 2009; 14(45):pii=19406. Available online: http://www.eurosurveillance.org/ViewArticle. aspx? Articleld=19406

4. Brookes $S M$, et al. Influenza $A(H 1 N 1)$ infection in pigs. Vet Rec. 2009;164:760-1.

5. Kyriakis SK, et al. Serologic cross-reactivity with pandemic (H1N1) 2009 virus in pigs, Europe. Emerg Infect Dis 2009;16: 96-99. 


\title{
0.266
}

\section{Transmission dynamics of a $\mathrm{H} 1 \mathrm{~N} 1$ swine influenza virus in a population previously infected with the 2009 pandemic H1N1 strain}

\author{
Anna Romagosa; Monserrat Torremorell; Marie Gramer; John Deen; Han S. Joo
}

University of Minnesota, St Paul, MN, USA

\section{Introduction}

Little is known about the transmission and spread of swine influenza virus (SIV) in pig populations previously infected with the 2009 pandemic H1N1 influenza strain. A transmission experiment was performed to asses the transmission pattern of a triple reassortant $\mathrm{H} 1 \mathrm{~N} 1$ virus in pigs previously infected with the 2009pH1N1 SIV strain.

\section{Material and Methods}

Two groups of ten 3-week-old pigs were placed in separate isolation rooms. The groups were designated as control (C) or previously infected (I). Pigs in the control group were neither vaccinated nor infected with SIV. Pigs in the I group had been naturally infected with the 2009pH1N1 SIV strain at about 4 weeks prior to the beginning of the study. These pigs were virus free and $\mathrm{HI}$ test positive (titers $\geq 1: 40$ ) against the $2009 \mathrm{pH} 1 \mathrm{~N} 1$ at the time the study started. However, they were $\mathrm{HI}$ negative against the challenge strain (A/Sw/IA/0239/04).

In a separate room 2 naive pigs were challenged with the triple reassortant A/Sw/IA/0239/04 H1N11 which was genetically different from the $2009 \mathrm{pH} 1 \mathrm{~N} 1$. Twenty four hours post challenge the challenged pigs were confirmed positive by nasal swab PCR. At $48 \mathrm{~h}$ one challenged pig was moved into each of the treatment rooms and placed in direct contact with the pigs in group $C$ and I. Transmission was evaluated by collecting nasal swabs from all pigs on a daily basis. Nasal swabs were tested individually by PCR until all pigs in each group tested positive or until 14 days post contact (DPC) when the study was terminated. Blood samples were also collected at the beginning and the end of the study.

Pigs were monitored daily and clinical scores based on coughing, sneezing, lethargy, fever, nasal discharge and dyspnea were calculated. At necropsy the percentage of macroscopic lung lesions was recorded and tissues from the trachea, lungs, nasal turbinate, tonsils and lymph nodes were collected for PCR, virus isolation and histopathology.

\section{Results}

The transmission results are shown in Figure 1. Influenza virus was detected by PCR in all pigs in the control group by 5 DPC and pigs remained positive until necropsy (8 DPC). In contrast influenza virus was detected by PCR in only one pig in group I at 1 DPC. Differences in transmission patterns between the groups were statistically significant at $p<0.001$.
Figure 1. Cumulative number of infected pigs by group after mixing with the challenged pig.

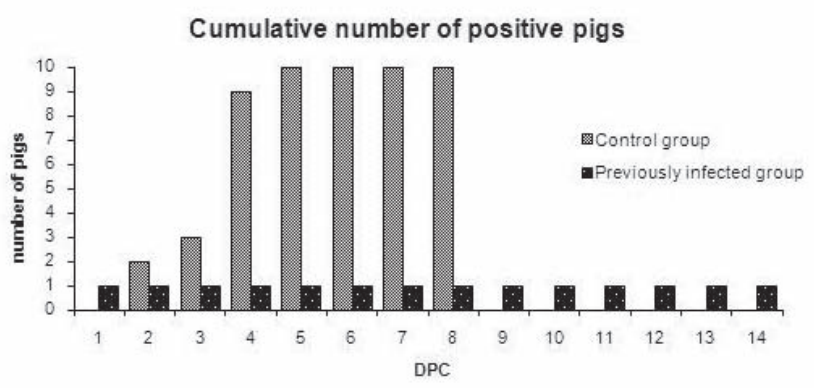

Figure 2. Average clinical scores for control and previously infected pigs.

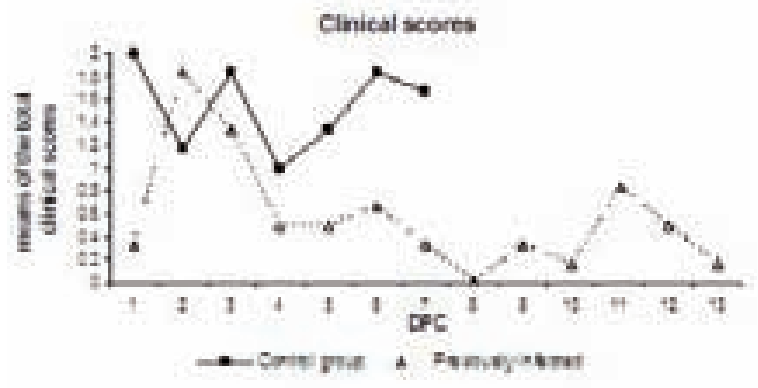

Differences in clinical scores (figure 2) and gross lung lesions were also observed. Pigs in group $C$ had an average clinical score of 1.54 compared to 0.78 in the I group $(p \leq 0.01)$. The percentage of macroscopic lung lesions at necropsy in the control group was $14 \% \pm 4.52$ and $2.38 \% \pm 1.38$ in the I group.

\section{Discussion}

Results indicate differences in transmission and spread of SIV between groups. Although SIV could spread rapidly in a naïve population (control group), transmission in the previously infected group with the 2009pH1N1 was limited and infection could not become established. This study represents preliminary work to understand the dynamics of SIV transmission in the field as represented by populations with different immune status.

\section{Acknowledgment}

Founding was provided by the NIH, NIAID, Centers of Excellence for Influenza Research and Suveillance and the SDEC at the UMN. Its contents are solely the responsibility of the authors and do not necessarily represent the official views of the $\mathrm{NIH}$.

\section{References}

1 A.L. Vincent et al. (2009) Virus genes 39:176-185. 


\title{
0.267
}

\section{Efficacy of inactivated swine influenza virus vaccines against 2009 H1N1 influenza virus in pigs}

\author{
Amy L. Vincent $^{1}{\text { Janice R. Ciacci-Zanella }{ }^{1} \text { Alessio Lorusso }{ }^{1} \text { Philip C. Gauger }{ }^{2} \text { Eraldo L. Zanella }}^{1}$ \\ Marcus E. Kehrli ${ }^{1}$ Bruce H. Janke ${ }^{2}$ Kelly M. Lager ${ }^{1}$ \\ 1. USDA-ARS National Animal Disease Center, Ames, IA, USA; 2. Iowa State University, Ames, IA, USA
}

\section{Introduction}

The gene constellation of the 2009 pandemic H1N1 virus is a unique combination from swine influenza A viruses (SIV) of North American and Eurasian lineages, but prior to April 2009 had never before been identified in swine or other species (1). Although its hemagglutinin gene is related to North American H1 SIV, it is unknown if vaccines currently used in U.S. swine would cross-protect against infection with pandemic H1N1. The objective of this study was to evaluate the efficacy of inactivated vaccines prepared with U.S. swine influenza viruses as well as an experimental homologous vaccine to prevent infection and disease from 2009 pandemic H1N1.

\section{Materials and methods}

Sixty three-week-old cross-bred pigs were obtained from a herd free of SIV and porcine reproductive and respiratory syndrome virus (PRRSV). A/California/04/2009 H1N1 (CA/09) was propagated in Madin-Darby Canine Kidney (MDCK) cells for use in the study described below. Three inactivated vaccines (Vaccines $A$, $B$, and D) were selected based on serologic evidence of crossreactivity in a previous study(2). Commercial vaccines were supplied by the manufacturers for use in the study and administered as per label. Vaccine A (FluSure ${ }^{\circledast} \mathrm{XP}$, Pfizer Animal Health, New York, NY) is a fully licensed trivalent commercial product containing cluster IV H3N2, $\gamma$-cluster H1N1, and $\delta$-cluster H1N1 SIV as vaccine seed viruses. Vaccine B (MaxiVac Excell ${ }^{\circledR}$ 5.0, Intervet/Schering-Plough, Boxmeer, The Netherlands) is a pentavalent product containing clusters I and IV H3N2 and $\beta_{-}^{-}, \gamma^{-}$, and $\delta$-cluster H1 SIV as vaccine seed viruses. Vaccine D (Newport Labs, Worthington, MN) is a bivalent autogenous vaccine containing $\beta$ - and $\gamma$-cluster $\mathrm{H} 1$ SIV as vaccine seed viruses. A monovalent experimental vaccine (Vaccine $\mathrm{E}$ ) was prepared from $\mathrm{CA} / 09$ at $8 \mathrm{HA}$ units per $50 \mu \mathrm{L}$ and $1 \mathrm{X} 106.550 \%$ tissue culture infectious dose (TCID50) per $\mathrm{mL}$ with inactivation by ultraviolet irradiation and addition of a commercial adjuvant. Pigs $(\mathrm{N}=10$ per group) were vaccinated with $2 \mathrm{~mL}$ of each vaccine by the intramuscular route at approximately 4 weeks of age, boosted at 7 weeks of age, and challenged at 10 weeks of age.

Nasal swabs were taken at 0,3 , and 5 days post infection (dpi). At necropsy, lungs were removed to estimate the percentage of macroscopic pneumonia. Each lung was lavaged to obtain bronchoalveolar fluid (BALF). Virus titers in BALF were determined by serial dilutions added to MDCK cells in 96-well plates, and virus from nasal swab samples were isolated on MDCK cells in 48-well plates and positive samples titrated in 96-well plates.

Hemagglutination inhibition $(\mathrm{HI})$ assays were done to evaluate sero-conversion using challenge virus as well as a panel of North American $\mathrm{H} 1 \mathrm{SIV}$ isolates representing each phylogenetic cluster (3).

\section{Results}

All vaccines tested provided partial protection ranging from reduction of pneumonia lesions to significant reduction in virus replication in the lung and nose. Although the commercial multivalent vaccines demonstrated partial protection, none were able to prevent all nasal shedding or clinical disease. An experimental homologous $2009 \mathrm{H} 1 \mathrm{~N} 1$ monovalent vaccine provided optimal protection with no virus detected from nose or lung at any time point in addition to amelioration of clinical disease. The $\mathrm{CA} / 09$ vaccine induced high $\mathrm{HI}$ titers against homologous $2009 \mathrm{H} 1 \mathrm{~N} 1$ as well as cross-reacting $\mathrm{HI}$ antibodies against $\alpha-, \beta-$, and $\gamma$-cluster H1N1 SIV. Commercial vaccines that induced $\gamma$-cluster $\mathrm{HI}$ antibodies demonstrated statistically significant reductions in virus titers.

\section{Discussion}

Based on cross-protection demonstrated with the vaccines evaluated in this study, the U.S. swine herd likely has significant immunity to $2009 \mathrm{H} 1 \mathrm{~N} 1$ from prior vaccination or natural exposure, especially those demonstrating immunity against $\gamma$-cluster SIV. However, consideration should be given for use of homologous vaccines to best protect naïve swine herds and limit the potential transmission of $2009 \mathrm{H} 1 \mathrm{~N} 1$ from herd to herd and from pigs to people.

\section{Acknowledgments}

We thank Michelle Harland, Brian Pottebaum and Jason Huegel for assistance with laboratory techniques and animal studies.

\section{References}

1. R. J. Garten et al., Science 325, 197 (Jul 10, 2009).

2. A. L. Vincent et al., Influenza and Other Respiratory Viruses in press, (2010).

3. A. L. Vincent et al., Virus Genes 39, 176 (2009). 


\title{
0.268
}

\section{Efficacy of swine influenza virus vaccines produced using the alphavirus replicon system}

\author{
Ryan L. Vander Veen ${ }^{1,2}$ Mark A. Mogler ${ }^{1,2}$ DL Hank Harris ${ }^{1,2}$ \\ 1. lowa State University, Ames, IA, USA; 2. Harrisvaccines, Inc., Ames, IA, USA
}

\section{Introduction}

Swine influenza virus (SIV) continues to circulate among most U.S. swine herds. The recent outbreak of pandemic H1N1 in the human population and its subsequent confirmation in pigs has again highlighted the zoonotic potential of these viruses. Using alphavirus replicon technology, a recombinant pandemic $\mathrm{H} 1 \mathrm{~N} 1$ hemagglutinin (HA) subunit vaccine and a cluster $4 \mathrm{H} 3 \mathrm{~N} 2$ replicon particle (RP) vaccine were developed and efficacy was determined in swine vaccination-challenge models.

\section{Materials and Methods}

Hemagglutinin genes derived from A/California/04/09 and a field strain of cluster $4 \mathrm{H} 3 \mathrm{~N} 2$ were commercially synthesized and inserted into the alphavirus replicon system as previously described. ${ }^{1,2}$ The replicon system was utilized to express HA subunit in vitro (pandemic $\mathrm{H} 1 \mathrm{~N} 1$ ) or to produce a replication-deficient particle that produces HA protein in vivo (cluster $4 \mathrm{H} 3 \mathrm{~N} 2$ ). Twenty pigs were randomized and divided equally among 4 groups. One group served as non-vaccinated controls, while the remaining 3 received varying doses (high, mid, low) of pandemic $\mathrm{H} 1$ subunit vaccine. Pigs were challenged intratracheally with $1 \times 10^{5} \mathrm{TCID}_{50}$ of A/California/04/2009 and necropsied 5 days post-challenge. The H3N2 study consisted of 50 animals, with 10 strict negative controls, 20 vaccinates and 20 non-vaccinates. The 20 RP vaccinated pigs received 2 doses of cluster 4 H3 RP vaccine. The 20 vaccinates and 20 non-vaccinates were challenged with $1 \times 10^{7} \mathrm{TCID}_{50}$ of homologous $\mathrm{H} 3 \mathrm{~N} 2$ virus. Necropsy was performed 4 days post-challenge. In both studies, vaccinated pigs received the first dose of vaccine at 3-4 weeks of age, with booster vaccination given 3 weeks later.

\section{Results}

\section{Pandemic H1N1 Study}

All of the vaccinated groups developed positive ( $>1: 40)$ mean homologous $\mathrm{HI}$ titers following two doses of HA subunit vaccine. At necropsy, lungs exhibited gross lesions typical of SIV. Lungs evaluated from the vaccinated groups had significantly lower gross lung lesions, as well as histopathological lesions. Live virus titration performed on post-challenge nasal swabs demonstrated that vaccinated pigs had reduced viral shedding when compared to non-vaccinated pigs. The group receiving the high dose HA subunit vaccine did not shed virus at a detectable level, while on days 3-5 post-challenge the low and mid dose vaccine groups demonstrated significantly reduced viral shedding when compared to the non-vaccinated controls. In addition to reduced lung lesions and viral shedding, all vaccinated groups demonstrated higher average daily gain than the non-vaccinated group following challenge.

\section{Cluster 4 H3N2 Study}

All vaccinated pigs developed robust homologous $\mathrm{HI}$ titers following two doses of SIV RP vaccine. At necropsy, lungs evaluated from vaccinated pigs exhibited significantly reduced gross lung lesions when compared to the non-vaccinated pigs. The non-vaccinated groups developed a febrile response 24 hours post-challenge, while the temperature of the vaccinated pigs was significantly lower at this time point. No live virus was detected in the broncho-alveolar lavage fluid (BALF) collected at necropsy in any of the vaccinated pigs, while all of the nonvaccinated pigs had detectable virus in the BALF samples. These results correlate with the reduced lung lesions observed in the vaccinated pigs. In addition, no live virus was detected from nasal swabs in any of the vaccinated pigs taken throughout the post-challenge period, while by days 3 and 4 post-challenge most of the non-vaccinated pigs had detectable live virus from nasal swab samples.

\section{Discussion}

These studies further demonstrate the applicability and efficacy of SIV vaccines produced utilizing the alphavirus replicon system. The pandemic H1N1 study demonstrated the rapidness with which this technology can be utilized to respond to emerging strains of influenza, as the pandemic vaccine was available in less than 2 months after the public announcement of infection in humans. This is much quicker than traditional methods of influenza vaccine preparation. The rapid exchange of HA influenza genes in this system enables us to produce different replicons which express the HA gene of all the different clusters currently circulating in the U.S. swine population, ${ }^{3}$ as well as respond quicker to observed antigenic drift among influenza isolates.

\section{Acknowledgements}

Thanks to AlphaVax Inc. (Research Triangle Park, NC) for constructing the replicon plasmids and the pandemic H1 RPs. Thanks to Dr. Alan Loynachan for serving as pathologist for these studies. Thanks also to Robyn Hoing (Harrisvaccines, Inc) for producing the H3 RPs. Grow lowa Values Fund provided funding for the pandemic H1N1 study.

\section{References}

1. Hooper JW, et al. Vaccine 2009 (28)2:494-511.

2. Kamrud Kl, et al. Virology 2007 360(2):376-87.

3. Harris DL, et al. AASV 41st Annual Meeting Proceedings 2010. 


\title{
0.269
}

\section{In vivo evaluation of vaccine efficacy against challenge with a contemporary field isolate from the alpha cluster of $\mathrm{H} 1 \mathrm{~N} 1$ swine influenza virus}

\author{
Susan E. Detmer ${ }^{1}$ Marie R. Gramer ${ }^{1}$ Sheerin Mathur ${ }^{2}$ Vicki J. Rapp-Gabrielson ${ }^{2}$ \\ 1. University of Minnesota, Department of Veterinary Population Medicine, St. Paul, MN, USA; 2. Pfizer Animal Health, VMRD, Kalamazoo, MI, USA
}

\section{Introduction}

Influenza A virus vaccines currently contain a mixture of field isolates that reflect the genetic and antigenic characteristics of the currently circulating strains. In order to continue to provide protection, these vaccines need to be regularly updated. For North American pigs, swine influenza is a year-round respiratory disease caused by $\mathrm{H} 1 \mathrm{~N} 1, \mathrm{H} 1 \mathrm{~N} 2$ and $\mathrm{H} 3 \mathrm{~N} 2$ subtypes and within the H1N1 subtype there are 4 clusters designated a (classical swine influenza), $\delta$ (human-like swine influenza), $\beta$ and $\gamma$ (reassortant swine influenza viruses). For Canadian pigs, most of the currently circulating influenza A viruses are in the $\mathrm{SwH} 1$ a cluster ${ }^{1}$. Therefore, this study was conducted to evaluate the efficacy of a vaccine containing a $\gamma \mathrm{H} 1 \mathrm{~N} 1$ virus (Flusure $\mathrm{XP}^{\mathrm{TM}}$ ) in pigs challenged with a contemporary a cluster field isolate of Canadian swine origin.

\section{Materials and Methods}

The animal phase of this study was conducted in accordance with the approval of the Institutional Care and Use Committee of the University of Minnesota.

Vaccines and vaccination: Flusure $\mathrm{XP}^{\mathrm{TM}}$ was administered as per manufacturer label instructions. Three-week-old swine from a high-health herd free of influenza, Mycoplasma hyopneumoniae and PRRS virus were vaccinated 14 days apart with a $2 \mathrm{ml}$ intramuscular dose and assigned to 2 treatment groups, and non-challenged pigs that were housed in 3 rooms in an isolation unit at the University of Minnesota as follows:

Table 1. Vaccination treatment groups.

\begin{tabular}{|c|c|c|c|}
\hline Room No. & Group & No. Swine & Challenge \\
\hline 1 & No treatment & 2 & No challenge \\
\hline 2 & No treatment & 1 & No challenge* \\
\hline & Vaccine & 5 & SwH1 a \\
\hline & Adjuvant alone & 5 & SwH1 a \\
\hline 3 & No treatment & 1 & No challenge* \\
\hline & Vaccine & 4 & SwH1 a \\
\hline & Adjuvant alone & 4 & SwH1 a \\
\hline
\end{tabular}

Challenge and post-challenge procedures: Immediately prior to challenge, the no treatment pigs $\left({ }^{*}\right)$ in rooms 2 and 3 were removed for euthanasia and necropsy. Two weeks following the second vaccination, swine were inoculated $2 \mathrm{ml}$ intratracheally and $2 \mathrm{ml}$ intranasally with $10^{5.5} \mathrm{TCID}_{50} / \mathrm{ml}$ of influenza A/Swine/ IL/02450/2008 (H1N1), an alpha-cluster H1 of Canadian swine origin. Swine were monitored daily for clinical signs and nasal swabs were taken for virus isolation on MDCK cells. On day 5 post-challenge, swine were euthanized for necropsy; bronchoalveolar lavage fluid (BALF) was collected, lungs were scored for gross lesions ${ }^{2}$ and lung samples were collected for histopathology $^{3}$ and virus isolation. All samples negative by virus isolation were retested by real time RT-PCR.
Hemagglutination Inhibition (HI) Antibodies: Blood was collected 12 and 2 days before the first vaccine, 1 day before the second vaccine, at challenge and 5 days after challenge. All sera were assayed for hemagglutination inhibition $(\mathrm{HI})$ titers against the homologous virus in each vaccine and against the challenge virus.

Statistical analysis: Gross lung scores, $\mathrm{HI}$ titers and virus titers were evaluated by a general linear repeated measures mixed model using SAS (SAS Institute, Cary, NC).

\section{Results and Discussion}

There were gross lesions present in the lungs of all pigs that were inoculated with the challenge virus. However, the challenge virus only produced mild clinical signs that included fever lasting 24-48 hours in a few pigs and rare coughing. The results of the nasal swab virus titers show that there was reduction in viral shedding for vaccinated swine compared to unvaccinated swine post-challenge. The virus titers for the BALF and lung samples were lower in the vaccinated swine than the unvaccinated swine. The vaccinated swine had $\mathrm{HI}$ titers $>40$ for all vaccine viruses on challenge day and 5 days after challenge. All swine had an $\mathrm{HI}$ titer $\leq 40$ for the challenge virus 5 days after challenge. These results indicate that vaccination with Flusure $X \mathrm{P}^{\mathrm{TM}}$ reduced virus isolation from nasal swabs, lungs and BALF in swine experimentally challenged with a $\mathrm{SwH} 1$ a cluster virus that is genetically similar to those currently circulating in Canadian swine.

\section{References}

1. A. L. Vincent et al., Virus Genes 39, 176 (2009).

2. P. G. Halbur et al., Vet. Pathol. 32, 648 (1995).

3. J. A. Richt et al., J. Clin. Microbiol. 41, 3198 (2003). 


\title{
0.270
}

\section{Nanoliter real-time PCR: A new molecular platform for simultaneous detection of multiple pathogens}

\author{
Wonil Kim² Leslie Bower ${ }^{1}$ Karen Harmon ${ }^{1}$ Kyoung-Jin Yoon ${ }^{1}$ \\ 1. lowa State University, Ames, IA, USA; 2. Chonbuk National University, Jeonju, Korea
}

\section{Introduction}

Many disease problems in swine production are often multifactorial, which can be a diagnostic challenge. A multiple pathogen detection system can be then of great help to accurate and rapid differential diagnosis, simultaneous surveillance for various infectious agents, and studying interactions among different pathogens involved in a disease complex (i.e., disease ecology). Although various multiplexing technologies have been developed and applied for these purposes, their applications have been somewhat limited due to the complexity of test development and maintenance: optimization of multiple assays in a multiplex system is very challenging; and addition of a new assay to the optimized system requires significant time and effort for additional validation. Therefore, a new detection system in which multiple individual assays can be optimized and run independently, yet simultaneously and economically, needs to be explored.

High-throughput nanoliter real-time PCR (OpenArrayTM, BioTrove) [1] uses conventional SYBR Green or TaqMan real-time PCR formats. For each sample the system can run real-time PCR assays for up to 64 different targets simultaneously as independent reactions in parallel. Each array slide can run 48 different samples at the same time. The system is also considered to be economical since the total reaction volume for each assay is only 33 nanoliters, which is nearly 1000 times less than that of conventional real-time PCR assay. The following study was conducted to evaluate its utility and feasibility for veterinary diagnostic use.

\section{Materials and Methods}

The nanoliter real-time PCR system was evaluated for multiple pathogen detection in comparison with individual TaqMan real-time PCR assays which had been routinely used by the lowa State University Veterinary Diagnostic Laboratory (ISUVDL) for various bacterial, viral and protozoan agents of various species.

Primers and probes labelled with FAM or VIC for 28 real-time assays were prepared in a plate (i.e., array) for the nanoliter PCR. The system was then validated and optimized using 86 clinical samples archived from previous submissions to ISUVDL and known positive control for each of the target agents. All procedures were performed as per manufacturer's recommended protocol.

\section{Results}

All of the 28 assays prepared in the nanoliter PCR simultaneously detected each target agent without generating false-positive results. Because preliminary evaluation revealed that the analytic sensitivity of the nanoliter PCR was 10-100 times lower than that of conventional TaqMan PCR assays, a universal extraction system was developed in this study to prepare concentrated RNA or DNA of viral, bacterial, and protozoan pathogens from various sample matrices such as serum, oral fluids, tissues, and feces. Combined with the universal extraction system, the nanoliter PCR showed sensitivity and specificity equivalent to the corresponding individual TaqMan PCR assays.

\section{Discussion}

The study demonstrated that a high-throughput nanoliter realtime PCR system can simultaneously detect multiple infectious agents in a variety of sample matrices. Since the system is based on PCR amplification, it can provide higher sensitivity as compared to a conventional microarray system which is based on hybridization, not to mention faster turnaround. For epidemiological or ecological research, substantial test cost saving can be expected with the nanoliter PCR system because of its capability for simultaneous detection of around 60 different pathogens in parallel, when compared to the cost incurred by all those individual real-time PCR assays. Overall the system appears to be a valuable tool for veterinary diagnostics and epidemiological or ecological research.

\section{References}

1. Stedtfeld R. D., S. W. Baushke, D. M. Tourlousse, et al. 2008. Development and experimental validation of a predictive threshold cycle equation for quantification of virulence and marker genes by highthroughput nanoliter-volume PCR on the OpenArray platform. Appl. Envir. Microbiol. 74:3831-3838. 


\title{
0.271
}

\author{
Herd health surveillance pilot project in Ontario commercial hog operations \\ Paisley Canning ${ }^{1,2}$ Tim Blackwell ${ }^{2}$ \\ 1. Animal Health Laboratory, University of Guelph, Guelph, ON, Canada; \\ 2. Veterinary Science and Policy, Ontario Ministry of Agriculture, Food and Rural Affairs, Elora, ON, Canada
}

\section{Introduction}

The American Veterinary Medical Association recently supported the establishment of a disease surveillance system for American swine operations (Snelson 2010). A comprehensive surveillance system of this type aims to detect emerging, zoonotic and food-borne diseases central to current public and animal health issues. Disease monitoring frequently only reflects reportable diseases and samples submitted to diagnostic labs. Certain subsets of swine producers in Ontario, however, do not typically utilize veterinary diagnostic services. Many of these producers also have limited access to modern means of communication such as a telephone or fax machine. Consequently, it is difficult to assess changes in mortality and to promptly identify disease outbreaks if they occur on these farms. This presents a challenge for potential national or provincial animal health surveillance systems. To address this, a pilot project was designed to assess the feasibility of a broad based surveillance system including conventional and low diagnostic service user farms.

\section{Materials and Methods}

Enrolled producers submit weekly mortality and morbidity data from all areas of their production system (including the breeding/gestation, farrowing, nursery, and grow/finish sections) using a mortality form. The recording form asks producers to record age of suckling pig deaths, number of piglets born alive, general comments, in addition to mortality rates in all applicable areas of production. Producers have the option to mail in hard copies of the form using provided postage paid envelopes or to use a Nokia 6086 or e71 cellular telephone to electronically submit the data. These phones operate an online surveillance software called Episurveyor ${ }^{\circledR}$ that allows users to create surveys accessible on the mobile phone. Producers record mortality data using this phone program and then send their completed weekly mortality forms to an internet database from the phone. Participants also fax their weekly submission to the OMAFRA office. Data received is compiled in a central database and analyzed weekly for increases in morbidity and mortality. If a change in herd health status is identified, the farm's veterinarian is informed. The veterinarian is compensated for professional services and laboratory diagnostics associated with the surveillance study (provided by Animal Health Laboratory (AHL), Guelph). Consequently, veterinary services are offered at a reduced cost to producers, thus significantly increasing contact between the veterinarians and producers. The study began in June 2009 and new producers from three different veterinary clinics are still enrolling.

\section{Results}

This pilot project focuses on evaluating if this submission design and set of incentives is sufficient to generate regular reporting of disease events each week. Secondly, from a surveillance standpoint, mortality data is analyzed for abnormal fluctuations as opposed to absolute numbers. Nine farms submit their data on time each week. For the remaining herds, receipt of data lags by one to three weeks between its completion on farm and arrival at the OMAFRA office. Currently, 14 of 24 producers utilized diagnostic services at least once through their veterinarian in response to mortality and morbidity changes in their herd. Submission to AHL through this program increased after a meeting was held for each clinic where producers received farm specific mortality data reports and spoke with study coordinators about the project. Direct interactions between veterinarians, producers and study coordinators appeared to help with compliance and highlighted the benefits of regular sample submission and veterinary contact.

\section{Discussion}

Currently, this pilot project is still active on participating farms and has generated useful data on herd health status. Due to the importance of catching emerging or zoonotic diseases early, timely data submission is crucial but has been problematic with some farms on this study. The Episurveyor ${ }^{\circledR}$ program has been functioning well on farm and data downloading has been successful. Compliance does remain a challenge and highlights the need for incentives and demonstrating the benefits of regular data submission to participating veterinarians and producers. Although more accessible to study participants, mailing-in data creates a larger lag between recording and collection. This can hinder effective disease surveillance but helps generate broad based surveillance data. The surveillance project continues to operate and is due to be completed in July 2010. A second producer meeting is planned in the spring to hold discussions on the surveillance methods in use and on mortality issues for each herd.

\section{References}

Snelson, H. (2010). News Story: AVMA Supports Comprehensive Surveillance System. Available online at http://swinevet.org/news/story. php?id=3987. 


\title{
0.272
}

\section{Testicles of castrated piglets an anachronism - but a powerful sample for disease surveilance in sow herds}

\author{
Jens Boettcher; Andreas Hensch; Armin Gangl; Britta Janowetz; Michaela Alex; Hermann Niemeyer; Gerd Wittkowski
} Bavarian Animal Health Service, Poing, Germany

\section{Introduction}

Collecting blood from adult sows is laborious; time consuming and sometimes hazardous to the veterinarian and/or the sow. Alternative samples would be of benefit. Some authors proposed colostrums as an alternative material, however antibody level in colostrums declines rapidly after parturition and due to high fat content samples have to be processed in the lab. Meat juice is a suitable sample for serodiagnosis in slaughtered animals (e.g. Salmonella enterica, PRRSV, Trichinella spp.). Therefore pieces of meat $(4 \mathrm{~cm} 3)$ are submitted to vials. After freezing and thawing meat juice is collected. As a general rule antibody concentration in meat juice is $1 / 20$ of that in blood. In the present study it was analysed if tissue fluid from testicles (TJ) of castrated piglets could serve as a sample to predict the dam's seroreactivity.

\section{Material \& Methods}

The following ELISAs (Dilution of TJ) were used: PRRSV (1/10), SIV H1N1 (1/10), Salmonella (1/2), Mycoplasma hyopneumoniae (Mhyo; 1/10) supplied by Idexx and PCV2 (1/20), PPV (1/40) supplied by Ingenasa. Except for the dilution of TJ tests were performed according to manufacturer's instructions. Results were expressed as titre (PCV2) or relative S/P-value after subtraction of the optical density of the negative control (other tests).

Blood and testicles were collected from piglets within the first week of life (not necessarily the same animal!) and blood was collected from the dam. Mean reactivity of OD or geometric mean of titre per litter were calculated and compared with the dam's value. Individual results were compared by kappa statistics. Finally, serological classification of herds based on testing TJ and dam's blood was compared.

\section{Results}

For each test a positive correlation between serum samples of dams and mean TJ reactivity of the litter was observed. Data for the comparison TJ versus dam's serum were summarized in the Table. Agreement of TJ with serum from piglets as determined by k-statistics was generally higher.

\begin{tabular}{|l|c|c|c|c|c|c|}
\hline & PRRSV & SIV & PPV & PCV2 & Mhyo & Salmonella \\
\hline Sow/litters & 246 & 180 & 221 & 193 & 181 & 266 \\
\hline herds & 31 & 23 & 25 & 28 & 21 & 32 \\
\hline TJ-Cut-off & $40 \%$ & $60 \%$ & $30 \%$ & 422 (titre) & $30 \%$ & $40 \%$ \\
\hline kappa & 0.786 & 0.784 & 0.491 & 0.59 & 0.694 & 0.587 \\
\hline
\end{tabular}

In the case of PRRSV, SIV and Mhyo $100 \%$ of the herds were correctly classified. Agreement between dam's serum and TJ was moderate for PPV, PCV2 and Salmonella. In the case of these infections pairs of samples with borderline reactivity tended to be misclassified.

\section{Discussions \& Conclusions}

$\mathrm{TJ}$ is an alternative sample for serology in sows. Although kvalues were sometimes below 0.8 one has to consider that mean reactivity per litter was compared. Agreement for PRRSV, SIV and Mhyo on herd level was excellent. Thus TJ-testing is a powerful tool especially for surveillance of PRRSV, SIV and Mhyo in sow herds.

In the case of Salmonella the ELISA-cut-off for meatjuice/serum was validated for slaughterhouse pigs and not for sows. The observation that ELISA-reactivity of piglets from negative sows (serum) was below $20 \%$ might indicate the need for an agerelated cut-off. Agreement was higher at a cut-off at $60 \%$ for sow serum and $40 \%$ for TJ. Comparison of sample materials in the case of PPV and PCV2 were complicated by high numbers of samples with borderline reactivity. This might be explained by vaccination (PPV) or frequent infection (PCV2). Application of ELISA tests for detection of protective antibodies (e.g. correlation with neutralizing antibodies) in the future might allow prediction of optimized time-points for vaccination of piglets.

\section{Acknowledgements}

This study was financially supported by the Free State of Bavaria and the Bavarian Joint Founding Scheme for the Control and Eradication of contagious Livestock Diseases.

\begin{tabular}{|l|c|c|c|c|c|c|}
\hline & PRRSV & SIV & PPV & PCV2 & Mhyo & Salmonella \\
\hline Sow/litters & 246 & 180 & 221 & 193 & 181 & 266 \\
\hline herds & 31 & 23 & 25 & 28 & 21 & 32 \\
\hline TJ-Cut-off & $40 \%$ & $60 \%$ & $30 \%$ & 422 (titre) & $30 \%$ & $40 \%$ \\
\hline kappa & 0.786 & 0.784 & 0.491 & 0.59 & 0.694 & 0.587 \\
\hline
\end{tabular}




\title{
0.273
}

\section{Pig health Surveillance in The Netherlands 2004-2009}

\author{
Hetty van Beers-Schreurs; Peter van der Wolf \\ GD-Animal Health Service, Deventer, Netherlands
}

\section{Introduction}

Surveillance of the health status of pigs is very important for Dutch Pig farmers and the Dutch Government. Five years ago the Dutch Ministry of Agriculture and the Dutch Livestock Board (PVV) built a surveillance system for pig health in The Netherlands. The AHS was asked to carry it out. The goals of this system are 1) watching trends and developments, 2) detecting outbreaks of notifiable diseases and 3 ) detecting unknown diseases/problems. This paper shows how it works.

\section{Materials and Methods}

The surveillance system consists of a helpdesk for veterinarians and farmers (telephone and website), pathology and laboratory service, and herd visits, combined with specific disease surveillance and data analysis (figure 1). Specific serological surveillance is done for Pseudo Rabies (PR), Classical Swine Fever (CSF), Swine Vesicular Disease (SVD), Salmonella, and Brucellosis in commercial pig farms and PR, CSF, SVD, CSF, Foot and Mouth Disease and Trichinella in wild boar.

Figure 1. Schematic representation of the Dutch system.

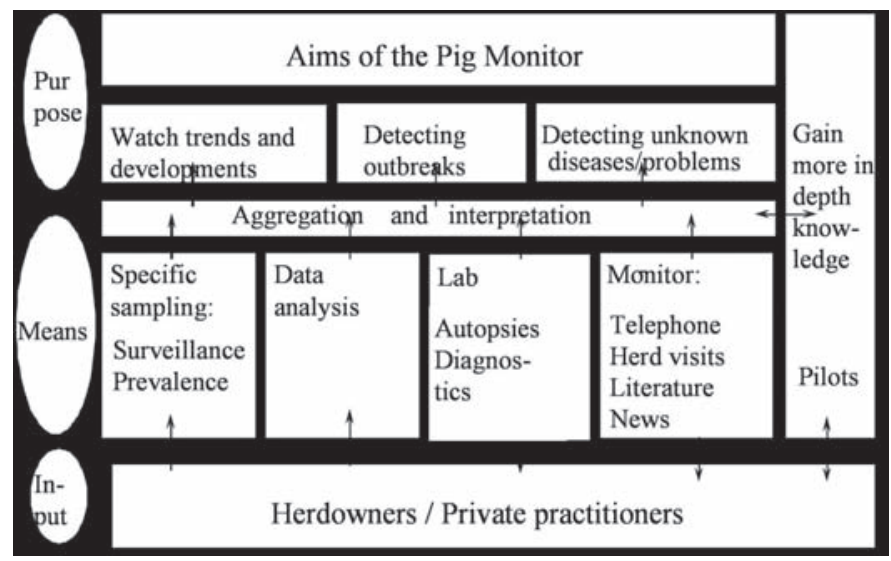

\section{Results}

In the years 2004 - 2009 there were 11824 questions (75\% by vets), 22706 autopsies and 696 herd visits.

The occurrence of the most common non-notifiable diseases has been determined, figure 2 e.g. shows the declining trend in the occurrence of weaning diarrhea (enterotoxicosis) in autopsies in weaned piglets. Many hundreds of thousands of samples have been tested for notifiable diseases and no outbreaks have been detected. Occasionally, serological positives have been found for $\mathrm{PR}(\mathrm{n}=2)$ in wild boar (close to the German and Belgium border), and for CSF from CSF vaccinated wild boar $(n=4)$. The PR and CSF virus have never been detected.

Special results were e.g. an outbreak of Talfan in weaned piglets, Porcine Ulcerative Dermatitis Syndrome in sows, progressive posterior paralysis in sows with unknown etiology, two (in- dependent) pigs with lymphosarcoma, broken legs as a result of low phytase in mixed feed and piglet mortality as a result of lack of vitamin $D$ in mixed feed.

Figure 2 Enterotoxicosis in weaned piglets (autopsies) in the period 2004 - 2009.

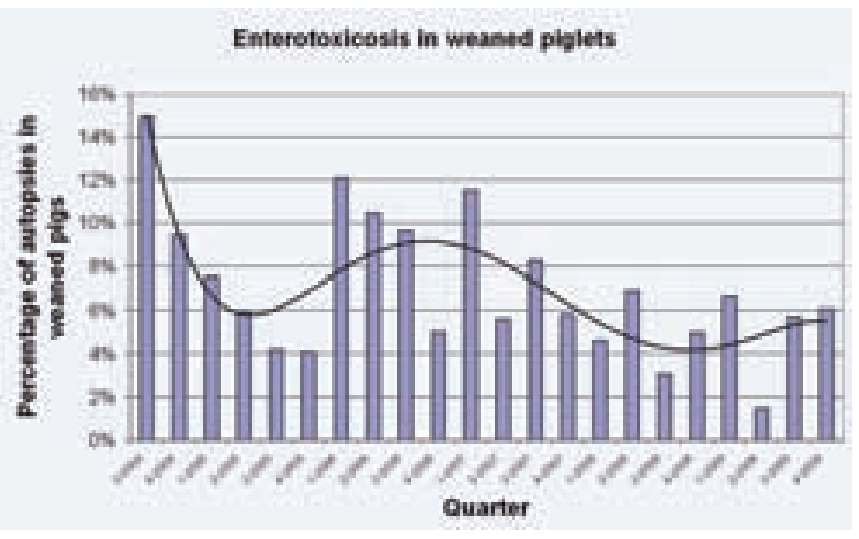

\section{Discussion}

In the past years the system has shown that it is capable of detecting isolated incidents as well as surveillance of pig health in general. Specific parameters show a declining trend resulting in an improvement of overall pig health.

Of course such a system has its strengths and weaknesses. Strengths are the aggregation of different kinds of data. One of the weaknesses of the system is the dependency on practitioners and farmers to send in animals for autopsy or blood/ faeces samples. In the next future attention will be paid to this "weakness". Autopsies and analysis of samples are not for free. The Government and the Dutch Livestock Board pay part of it. The results of diagnostics will not only provide the veterinarians and farmers more appropriate information about the cause of the problem, it will also give some information about the health status of Dutch pigs. In the case outbreaks of known diseases or occurrence of new syndromes, the Government, the Board, practitioners and farmers will be informed immediately, in order to make the right decisions. Reports will be handed over every 3 months 


\title{
0.274
}

\section{Evaluation of the Ontario Swine Veterinary-based Surveillance System (OSVS) - an update}

\author{
Maria R. Amezcua ${ }^{1}$ David L. Pearl ${ }^{1}$ Robert M. Friendship ${ }^{1}$ W. Bruce McNab² \\ 1. University of Guelph, Guelph, ON, Canada; 2. Ontario Ministry of Agriculture Food \& Rural Affairs, Guelph, ON, Canada
}

\section{Introduction}

There is an increasing interest in obtaining farm level data through veterinarians for real time surveillance, due to the growing concern of emerging and re-emerging diseases in swine populations. Practicing veterinarians are often the first to detect the initial cases of novel infectious diseases. Consequently, we undertook a twelve-month pilot study to determine the feasibility of a veterinary-based surveillance system for Ontario (1). This pilot program was evaluated based on the level of compliance among practitioners, and the timeliness and quality of data submitted to the program. Practitioners continued to participate in the program and the OSVS performance during the second year is presented in this study.

\section{Material and Methods}

Seven veterinarians from five clinics recorded and transmitted data from July 1, 2007 to June 31, 2008 using either paper forms or a personal digital assistant (PDA). In the second year of the study (July 2008 to June 2009), three additional practitioners joined the program. In addition, during the second year, OSVS recording forms were harmonized with clinic's recording forms and/or billing systems and technical personnel were involved in the transmission of the data.

The practitioners summarized the number of farm visits and calls according to the body systems affected and the manner in which production was affected. To estimate the level of compliance among practitioners, the OSVS calculated the weekly ratio of the OSVS submissions to submissions made by the same veterinarians to the AHL. Completeness of data was assessed by measuring the frequency of data entered for each of the form's fields/elements. The coverage of the program was assessed by determining the proportion of farms visited by practitioners compared to the number of farms registered in the Canadian Agricultural Census of 2006 in each agricultural region of Ontario. Timeliness was evaluated by calculating the difference between the date when the practitioners recorded the case and the date when the information was transmitted to the program coordinator.

\section{Results}

For the pilot study, the average time to availability of data (TAD) was 22.1 days and the quality of compliance was variable among veterinarians and months. Timeliness improved by 8 days in the second year of the study. However, timeliness and compliance continued to be highly variable among veterinarians particularly during the summer. Over the course of the pilot study, a total of 1,610 records were sent to the OSVS. The completeness of fields in the form ranged from $55-99 \%$. Actual estimates of morbidity and mortality for disease visits/calls were rarely recorded during the pilot study. A total of 2,226 records were sent to the program during the second year. The completeness of fields in the form in the second year ranged from $73 \%-100 \%$. Over the course of the pilot study, almost $24 \%$ of swine farms in Ontario were visited. After the pilot study, an increase in coverage of $13.6 \%$ was observed.

\section{Discussion}

Our results showed that it is feasible to administer a swine veterinary-based surveillance system in Ontario that is acceptable to practitioners, and can produce useful surveillance data. The use of friendly recording systems adapted to each clinic's management system and involving animal technicians in the regular transmission of clinicians' data helped to improve timeliness. However, financial incentives may be needed to improve compliance and achieve a "real-time" surveillance system using practitioner data.

Methods to assess different aspects of a veterinary-based surveillance program must be established to determine which aspects of the program's performance need improvement.

\section{References}

1. Amezcua R, Pearl, D.L., Friendship R.M., McNab W.B. Evaluation of a veterinary-based syndromic system implemented for swine. Can J Vet Res. In press 


\title{
P.001
}

\section{Profile and agreement of dam and piglet PCV2 IFA titers}

\author{
Angela Baysinger; Edgar Diaz; Roy Edler \\ Boehringer Ingelheim Vetmedica, Inc., St Joseph, MO, USA
}

\section{Introduction}

Porcine circovirus associated disease (PCVAD) is caused by porcine circovirus type 2 (PCV2). Following natural exposure to or immunization against PCV2, Most sows and gilts develop antibodies that become passively acquired by their nursing offspring. The objective of this evaluation was to illustrate the immunological status of a sow herd, and to assess the correlation of dam and offspring IFA antibody titers.

\section{Materials and Methods}

A 4,200 sow, PRRS-negative farm was selected for this evaluation. This herd had a young parity structure with no dams older than parity 3. Typical of commercial production, dams had been PCV2-vaccinated as weanling gilts but never booster vaccinated. Two hundred and five gestating dams (sows and gilts) were blood sampled 2 weeks prior to scheduled farrowing. Sera were tested by immunofluorescent antibody (IFA) assay at Kansas State University. Based upon these results, dams were assigned to either low titer $(\leq 160)$ or high titer $(\geq 1280)$ groups. Dams with intermediate titers (320-640), and their associated litters, were excluded from the evaluation. Piglets were ear tagged within 24 hours of birth and the existing cross-fostering practices of the farm were allowed with the following restrictions: pigs could only be cross-fostered after 24 hours of age, and only to a dam of like-titer status (i.e. high-titer dam to high-titer dam or low-titer dam to low-titer dam). All pigs were individually blood sampled for PCV2 IFA antibody measurement and classification as low $(\leq 1: 320)$ or high $(\geq 1: 640)$ titer at approximately 3 weeks of age based upon previously reported experiments. ${ }^{1,2} \mathrm{~A}$ contingency table was constructed and dam titer-pig titer percent agreement and Kappa statistic were calculated.

\section{Results}

The titer distributions of all dams are illustrated in Figure 1, while pig titers from only the designated low and high titer dam categories are represented.

Dam and pig titers by designated low/high categories appear in Table 1. Percent agreement of low dam-low pig and high damhigh pig titers [(305+349)/921] was $71 \%$ with a Kappa ${ }^{3}$ of 0.42 (SE 0.03).
Figure 1. Distribution of dam* and pig ${ }^{* *} P C V 2$ IFA titers.

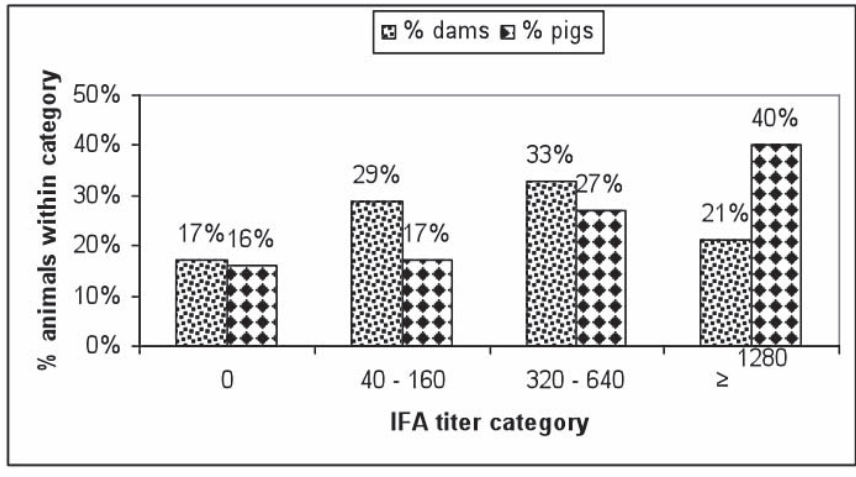

*All dams, $\mathrm{n}=205$. **Pigs from designated low and high dam litters only, $\mathrm{n}=921$.

Table 1. Contingency analysis between low and high dam and pig PCV2 IFA titers.

\begin{tabular}{|c|c|c|}
\cline { 2 - 3 } \multicolumn{1}{c|}{} & \multicolumn{2}{c|}{ Pig titer } \\
\hline Dam titer & Low $(\leq 1: 320)$ & High $(\geq 1: 640)$ \\
\hline $\begin{array}{c}\text { Low } \\
(\leq 160 ; n=94)\end{array}$ & $\begin{array}{c}66 \% \\
(n=305)\end{array}$ & $\begin{array}{c}34 \% \\
(n=159)\end{array}$ \\
\hline $\begin{array}{c}\text { High } \\
(\geq 1280 ; n=44)\end{array}$ & $\begin{array}{c}24 \% \\
(n=108)\end{array}$ & $\begin{array}{c}76 \% \\
(n=349)\end{array}$ \\
\hline
\end{tabular}

\section{Discussion}

There was moderate strength of agreement ${ }^{3}$ between low dam - low pig and high dam - high pig titers. Low titer pigs from high titer dams may be explained by insufficient passive transfer of maternal antibodies. High titer pigs from low titer dams are more difficult to explain. A likely contributing factor is the designated pig IFA titer limits being only a single dilution apart (320:640) and the inherent subjectivity of reading IFA results. It may also be possible that some of the piglet antibody measured was of active (pig) rather than passive (dam) origin. Another explanation could be a higher antibody concentration in colostrum than in sow serum and a high uptake of colostrum in the high titer pigs.

\section{References}

1. Fort, M. Vaccine 2009, (9203)1-7.

2. Hesse, R. Proc AASV 2009, 499-504.

3. Landis JR, Koch $\mathrm{G}$. The measurement of observer agreement for categorical data. Biometrics 1977, (33)159-174. 


\title{
P.002
}

\section{Qualitative and quantitative distribution of PCV2 in wild boars and domestic pigs in Germany}

\author{
Gerald Reiner $^{1}$ Bastian Bronnert ${ }^{1}$ Corinna Hohloch ${ }^{2}$ Christina Fresen ${ }^{1}$ Stephan von Berg ${ }^{1}$ Manfred Reinacher ${ }^{2}$ Hermann Willems $^{1}$ \\ 1. Department of Veterinary Clinical Sciences, Justus-Liebig-University, Giessen, Germany; 2. Institute for Veterinary Pathology, Giessen, Germany
}

\section{Introduction}

Porcine circovirus type 2 (PCV2) is considered the essential infectious agent of a series of diseases in swine that are now collectively termed porcine circovirus diseases (PCVD). Besides domestic pig production, PCV2 has also been detected in wild boars from several countries by serological studies, PCV2 antigen and DNA detection, indicating that PCV2 circulates at a high rate among the wild boar populations in Europe. PMWS reports in wild boars are scarce, however.

In domestic pigs, the high incidence of subclinical infection and the stability of the virus complicate diagnostics and appraisal of PCV2 infection and PCVD. Diagnostics and prognosis based just on serology or conventional PCR overestimate the risk of developing PCVD. However, several studies have provided evidence for the association between PCVD and the PCV2 load in serum or tissues of pigs (Brunborg et al., 2004; Olvera et al., 2004; Segales et al., 2005).

Neither the PMWS status nor the quantitative load of PCV2 has been documented comprehensively in wild boar populations. We hypothesize that the countrywide prevalence of PCV2infected wild boars is high with low incidence of PCVD, and that the discrepancy between both is associated with low loads of PCV2.

\section{Materials and Methods}

Samples from different tissues of 531 wild boars from 46 different hunting grounds and of 308 apparently healthy and 40 wasting (rejected at slaughter) domestic pigs from 48 provenances located in the same greater areas as the wild boars were analyzed. PCV2-specific DNA was detected independently by nested PCR (nPCR) and by quantitative realtime PCR (qPCR). $P C V 2-s p e c i f i c$ tissue alterations were examined by histological (HE) and immunohistochemical (IHC) standard procedures. Pigs were classified as PCVAD-affected (moderate to severe lymphocyte depletion and granulomatous inflammation of the lymphoid tissue, and moderate to high amounts of PCV2 genome equivalents [at least $5 \times 106$ copies of PCV2 DNA/ $\mu$ g extracted DNA in at least one of the tissues]), subclinically infected with PCV2 (no or only slight lymphoid lesions, and a lower amount of viral genome copies), and non-PCVD (no lymphoid lesions and negative in qPCR) (Segales et al., 2005). The threshold of 5x106 PCV2 DNA copies per $\mu \mathrm{g}$ of extracted DNA corresponds to 107 copies of other studies??? (e.g. Harding, 2008).

\section{Results}

The average prevalence for PCV2 infection, determined by nested PCR, was $63.1 \%$ in wild boars and $100 \%$ in domestic pigs ( $p<0.001$ ). Only $45.4 \%$ of the wild boars, but $98.8 \%$ of the domestic pigs were tested positive for PCV2 by qPCR. Wild boar prevalence in different areas of the country varied significantly between $36.2 \%$ and $87.5 \%$. PCV2 loads differed significantly $(p<0.001)$ between wild boars and domestic pigs. The average viral load was 102.8 in tissues of wild boars and 104.2 in those of domestic pigs. None of the 349 wild boars did definitely show clinical symptoms of PCVAD, but one of them (0.3\%) met the criteria for PCVD. Based on the same classification, $8.7 \%$ of the domestic pigs from 15 of the 48 provenances were found to be PCVD-affected. None of the wild boars and $7.1 \%$ of the domestic pigs had PCV2 loads above 5x106 DNA copies per $\mu \mathrm{g}$ of DNA. The number of PCVD-affected and non-affected pigs did not differ between pigs slaughtered and wasting pigs that were rejected at slaughter.

\section{Discussion}

In conclusion, this is the first study that describes the nationwide distribution of PCV2 in wild boars and domestic pigs qualitatively and quantitatively. As the level of PCV2 load appears to be the critical step in the development of severe PCVD, qPCR proved to be well suited for the evaluation of PCV2 infection and PCVD in wild boar and domestic pig populations.

\section{References}

Brunborg et al. 2004. J. Virol. Methods 122, 171-178.

Olvera et al. 2004. J. Virol. Methods 117, 75-80.

Segales et al.2005. Vet. Microbiol. 111, 223-229.

Harding 1996. In: Proceedings of the 27th Ann. Meet. West. Can. Assoc. Swine Pract., Saskatoon, p. 21. 


\title{
P.003
}

\section{Detection of Porcine Circovirus $\mathbf{2}$ in Mammary and Other Tissues from Experimentally Infected Sows}

\author{
Hwi Won Seo; Chan Hee Chae \\ Seoul National University, Seoul, Korea
}

\section{Introduction}

Porcine circovirus 2 (PCV2), a single stranded DNA virus within the family Circoviridae, is now recognized as the aetiological agent of post-weaning multisystemic wasting syndrome (PMWS) (Chae, 2004). The virus is shed in body fluids and has been isolated from oropharyngeal and nasal swabs, faeces, whole blood and serum taken from experimentally infected pigs. Recently, PCV shedding was detected by polymerase chain reaction (PCR) in colostrum and milk from experimentally infected sows ( $\mathrm{Ha}$ et al., 2008). The aim of the present study was to extend these observations by utilizing immunohistochemistry (IHC) and insitu hybridization (ISH) to determine the distribution of PCV2 in mammary tissue from experimentally infected sows.

\section{Materials and Methods}

Sows were randomly allocated to an infected $(n=6)$ or control group $(n=2)$. Each animal was housed separately within the facility. The viral inoculum used for infection contained the PCV2 strain SNUVR000470, at its third passage in PCV-free PK-15 cells. The six test animals were inoculated intra-nasally with $6 \mathrm{ml}$ of tissue culture fluid containing $1.2 \times 105$ tissue culture infective doses $50 \%$ (TCID50)/ml of virus. The inoculation was performed on day 93 of gestation. The two control sows were administered uninfected cell culture supernatant. Three days after parturition, the sows were sedated by intravenous injection of sodium pentobarbital and then euthanized by electrocution. The superficial inguinal lymph nodes, mammary gland, lung, liver, kidney, spleen, tonsil and ileum were also collected at necropsy and fixed in 10\% neutral-buffered formalin before processing. IHC was performed with a primary rabbit polyclonal anti-PCV2 antibody. ISH was performed with a PCR probe derived from the sequence of the open reading frame 2 (ORF2) of PCV2.

\section{Results}

PCV2 antigen and DNA were detected in the mammary tissue of all experimentally infected sows. Virus antigen was distributed throughout the mammary gland. Virus antigen and DNA was associated with cells with oval nuclei and abundant cytoplasm having morphological appearance consistent with macrophages. These cells were chiefly located within the lumina of the glandular tissue. The epithelial cells lining these glands did not contain DNA or antigen. Mammary tissue from control sows did not contain PCV2 DNA or antigen. Virus antigen and DNA were also detected in the lymph node, liver, spleen, tonsil and ileal Peyer's patches from all experimentally infected sows

\section{Discussion}

The results of this study confirm that the sows were successfully infected following intranasal inoculation with PCV2. The virus appeared to infect macrophage-like cells in the mammary gland of these sows. Although monocyte-macrophages may not be the primary target for PCV2 replication in vivo, the simultaneous detection of PCV2 protein and DNA reported here suggests that the virus infects and replicates within these cells. PCV2 has been shown previously to replicate within macrophages (Kim et al., 2003), and numerous macrophages may be found in the milk of sows.

PCV2 is known to replicate in the tonsil, entering the systemic circulation and the lymphoid organs such as lymph nodes and spleen (Kim et al., 2003). The virus is also present in circulating monocytes (Kim et al., 2003) suggesting that these cells act as vehicles for dissemination of the virus. We propose that these circulating monocytes may enter the mammary glandular tissue. Additional study is required to determine whether infectious PCV2 particles (as opposed to virus DNA) is present within the milk and whether any such virus may be shed directly within the milk whey or is contained within macrophages.

\section{References}

Chae, C. (2004). Postweaning multisystemic wasting syndrome; a review of aetiology, diagnosis and pathology. Veterinary Journal, 168, 4149.

Ha, Y., Ahn, K. K., Kim, B., Cho, K.-D., Lee, B. H., Oh, Y.-S., Kim, S.-H. and Chae, C. (2009). Evidence of shedding of porcine circovirus type 2 in milk from experimentally infected sows. Research in Veterinary Science, 86, 108-110.

Kim, J., Choi, C. and Chae, C. (2003). Pathogenesis of postweaning multisystemic wasting syndrome reproduced by co-infection with Korean isolates of porcine circovirus 2 and porcine parvovirus. Journal of Comparative Pathology, 128, 52-59. 


\title{
P.004
}

\section{Analysis of the genetic sequences of PCV2 virus in Mexico}

\author{
Francisco Robles $^{1}$ Ricardo Angulo² Jean Claude Chevez ${ }^{1}$ Edgar Diaz ${ }^{2}$ \\ 1. Boehringer Ingelheim Vetmedica S.A. de C.V., Guadalajara, JAL, Mexico; 2. Boehringer Ingelheim Inc., St. Joseph, MO, USA
}

\section{Introduction}

All pig-producing countries from around the world report the presence of Postweaning multisystemic wasting syndrome (PMWS.) This syndrome is regarded as a major problem in pig production. In America, PCV2 has been recognized in Canada, United States and Brazil. In Europe, France, Austria, Germany, United Kingdom and Sweden have detected the disease. In Asia, Japan, Taiwan and China have reported the disease's presence too $(1,2,3$.) Knowledge concerning the genetic sequences of PMWS has been very important in all these countries for establishing the homology level between the different isolations and for learning more about of the disease (4.) In Mexico, there is no report describing the genetic sequences and variations of the PCV2 virus present around the country. For this reason, the main objective was to describe the homology and genetic sequences of the PCV2 virus obtained from different regions of Mexico.

\section{Material and Methods}

10 samples from different regions of Mexico were analyzed. DNA was extracted and the PCR was performed to amplify a fragment of $506 \mathrm{pb}$ of the ORF2. The PCR products were cloned into a commercial plasmid vector and afterwards sequenced with $A B I$ automated DNA sequencer. The sequences were compiled and aligned with the published in the GENEBANK, and finally were analyzed with the Basic Genebee CLUSTAL W 1.83 program in order to obtain the phylogenetic tree.

Figure 1. Phylogenetic tree of Mexican PCV2 isolates and other PCV2 viruses from around the world. The red boxes indicate the sequences from Mexico pertaining to subgenotype $a$ and subgenotype $b$

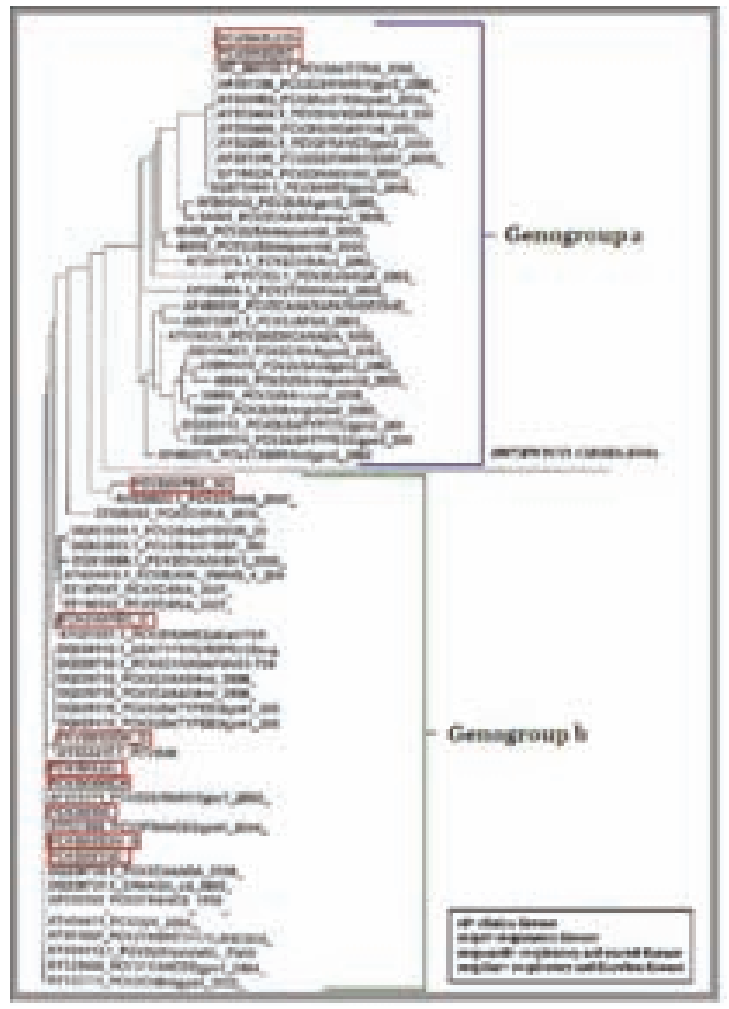

\section{Results}

10 sequences were identified, the homologies of the Mexican PCV2 virus range between $89 \%$ and $99 \%$. The majority of them are genotype "b" but the genotype "a" is also present. At the amino acids level, it ranges from $88 \%$ to $100 \%$.

The phylogenetic analysis (Figure 1) shows that the Mexican PCV2s are divided into 3 principal clusters. The majority of the sequences are closely related with European PCV2s in the PCV2 group a; and the virus inside of PCV2 group b have relation with European, Asian and Canadian virus.

The analysis of the main changes between amino acid sequences shows that there are 20 changes in the capsid protein; the majority of these changes are in the domain region situated in the position from aa59 to aa91. Table 1 shows these changes in detail.

Table 1. Most frequent changes of amino acids in PCV2 sequences from Mexico. One interesting finding is that the sequence PCV2MXP$B C \_S C$ is different in 8 amino acids when compared with the consensus sequences of the others samples that belong to genogroup $b$

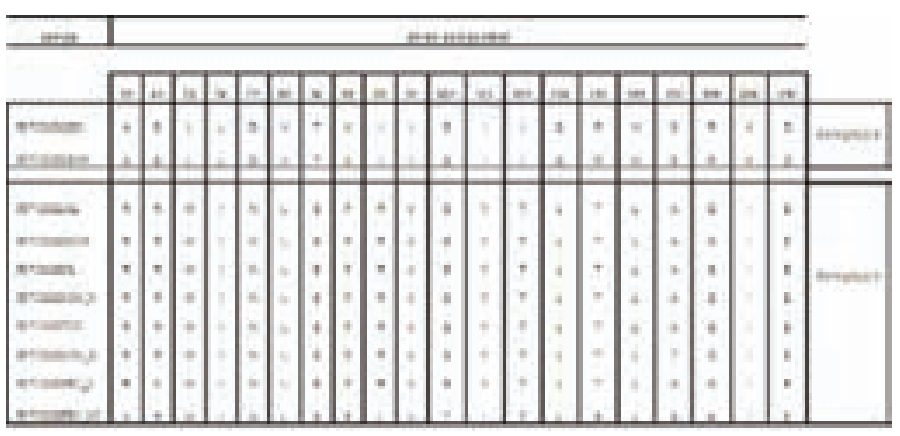

\section{Discussion}

Since PMWS was described in Canada (1997) genetic analysis of PCV2 has been done in different countries in order to understand the genetic variations and its implications (4.) According to our knowledge, there is not any report in Mexico about the genetic variations of Mexican PCV2. Our results show that the Mexican PCV2 has genetic variations; two strains show a high variation. The percentage of the heterology found in this investigation is higher than other reports described in America.

It is very important to establish whether the sequences of the Mexican PCV2 virus have relation with the pathogenicity of the strains.
References
1. Wallgren P. et al. (2004) Veterinary Quartely, 26:170-187.
2. Staebler S. et al. (2005) Veterinary Journal, 170:132-134.
3. Cao S. et al. (2005) Veterinary Research comm. 29:263-269.
4. Feneaux M. et al. (2000) Clinical Microbiology, 38:2494- 2503.
5. Opriessnig T. et al. (2008) J. Gen. Virol. 89: 2482-2491. 


\title{
P.005
}

\section{Heterologous expression of nuclear localization signal (NLS) truncated capsid protein of porcine circovirus type 2 (PCV2) in Escherichia coli and its potential of the specific antibodies induction}

\author{
Suphattra Jittimanee; Roongtham Kedkovid; Komkrich Teankum; Sanipa Suradhat; Roongroje Thanawongnuwech
}

Chulalongkorn University, Bangkok, Thailand

\section{Introduction}

PCV2 has been recognized as a causative pathogen of porcine circovirus associated disease (PCVAD), which is currently an important disease in the swine industry. The PCV2 capsid protein is the preferred antigen in a variety of serological tests and vaccines due to the strong immunogenicity (1). In this study, the recombinant NLS truncated capsid (NTC) protein was produced using E. coli expression system to prepare a subunit vaccine and examine the immunological response using an indirect in-house ELISA.

\section{Materials and Methods}

The NTC DNA fragment of PCV2 Thai isolate (07NP2) was amplified using PCR, cloned into the pGEX-5x-3 plasmid (GE Healthcare) and transformed into the E. coli BL21. For expression, 2 liters of the recombinant E. coli was induced with $0.1 \mathrm{mM}$ IPTG at $30{ }^{\circ} \mathrm{C}$ for $4 \mathrm{~h}$ (2). The bacteria was harvested and disrupted by sonication. The recombinant protein was purified using GST purification system (GE Healthcare). The expression of recombinant NTC protein was determined using SDS-PAGE and Western blot analysis. The concentration of the recombinant protein was measured using the Bradford assay.

To prepare a subunit vaccine, the purified recombinant protein was mixed with a commercial oil adjuvant. The vaccine efficacy was examined in the experimental pigs free from PCV2. Fourty-five, 4-week-old pigs were allocated into 5 groups: group $A(n=10)$ received a one-shot vaccination $(5 \mu \mathrm{g} /$ dose $)$; group $B(n=10)$ received a two-shot vaccination $(5 \mu \mathrm{g} /$ dose, 2 -wksinterval); group $C(n=10)$ received a one-shot vaccination $(50 \mu \mathrm{g} /$ dose); group $D(n=10)$ received a two-shot vaccination $(50 \mu \mathrm{g} /$ dose, 2-wks-interval). Group $\mathrm{E}(\mathrm{n}=5)$ was given only an adjuvant. The sera were collected before the vaccination ( $0 \mathrm{dpv}$ ) as the baseline and at 7, 14, 21, 28 and $42 \mathrm{dpv}$. Antibodies against recombinant NTC protein were detected using an in-house indirect ELISA (Chulalongkorn University-Veterinary Diagnostic Laboratory, CU-VDL).

\section{Results}

The expression of recombinant NTC protein from recombinant BL21 resulted in the production of the approximately $40 \mathrm{kDa}$ protein (Fig.1, lane 2 and 3). This protein also reacted with the rabbit anti-PCV2 antibody (Fig.1, lane 4). Results from the vaccine trial study revealed that the one-shot group received $50 \mu \mathrm{g}$ of the protein (group C) showed an increasing mean O.D values compared with the control group (Fig. 2). However, when
Figure 1: SDS-PAGE and Western blot analysis (arrow; 40 kDa marker)

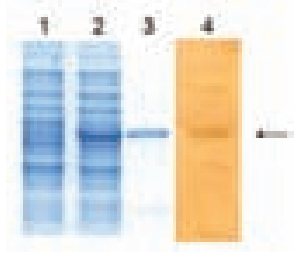

Figure 2: Mean O.D. ELISA of the vaccinated groups at 0, 7, 14, 21, 28 and 42 day post vaccination ( $d p v$.)

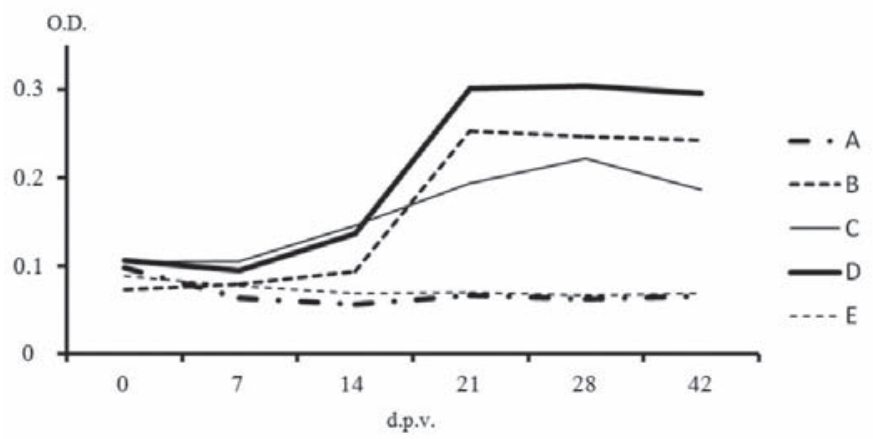

pigs were given a two-shot protocol, both the 5 - and $50-\mu \mathrm{g} /$ dose groups showed significantly increased mean O.D. (Fig. 2, B and D). After the second vaccination, the levels of antibodies in the two-shot vaccinated groups were significantly higher than those in the one-shot groups especially in the $50-\mu \mathrm{g} /$ dose group $(\mathrm{p}<$ 0.05).

\section{Discussion}

The results showed that the recombinant NTC protein expressed in the E. coli system could react with anti-PCV2 antibody and also induce the specific antibody responses in the experimental pigs especially in the two shot vaccinations of both concentrations. Interestingly, the immune response was also detected in the one-shot protocol when given the high concentration of the protein indicating of dose dependent. Our subunit vaccine could be used as a potential vaccine candidate. However, field trial of this subunit vaccine is needed for the future study.

\section{Acknowledgements}

This study was funded by National Research Council of Thailand 2009.

\section{References}

1. Marcekova, Z. et al. 2009. J Virol Methods; 162(1-2): 133-141.

2. Trundova, M. et al. 2007. Virus Genes 34(2): 199-204. 


\title{
P.006
}

\section{Comparison between an in house developed ELISA for the detecton of antibodies against PCV2 and the Synbiotics PCV2 ELISA}

\author{
Marc Martens; Alex Eggen; Hanny Swam \\ Intervet/Schering-Plough Animal Health, Boxmeer, Netherlands
}

\section{Introduction}

Porcine Circovirus type 2 (PCV2 infections )and its related Porcine Circovirus Disease (PCVD) is a major drawback in pig farms all over the world. Initially managerial measures were taken to reduce the impact. Last years the appearance of PCV- vaccines has been a milestone to really prevent the disease. Maternally derived antibodies (MDA) may interact with the take of the vaccine.(M Fort et all. Vaccine 2008, 2009) Also the moment of vaccination is critical: it should be weeks before the onset of infection. To be able to establish the optimum moment for vaccination it is of importance to have tools to measure antibodies against PCV2 Intervet Schering Plough Animal Health (ISPAH) developed an in house PCV2 antibody ELISA. Besides commercial ELISA manufacturers also produced ELISA kits for the determination of PCV2 antibodies. The use of all these different tests complicate the interpretation of articles and trials related to PCV2 infections and vaccination. ISPAH therefore compared its in house ELISA for the detection of antibodies against PCV2 with the commercial available ELISA test of Synbiotics which is widely used in the field.

\section{Materials and Methods}

77 serum samples of conventional sows that were known to have antibodies against PCV2 were tested in both tests in three independent runs.

Besides 20 sera of known negative PCV2 animals were tested in both ELISA's. The selected commercial test is from Synbiotics SERELISA ${ }^{\circledR}$ PCV2 Ab Mono Blocking ELISA. The ISPAH in house ELISA has been developed in R\&D of ISPAH and is not commercially available. Since the results of the inhouse ELISA are expressed in Log 2 titers also the Synbiotic results are expressed in Log2-titers for this comparison.

Figure 1: Mean titer ELISA PCV2 Synbiotics compared to mean titer ELISA PCV2 In House sorted ascending for ELISA PCV2 Synbiotics

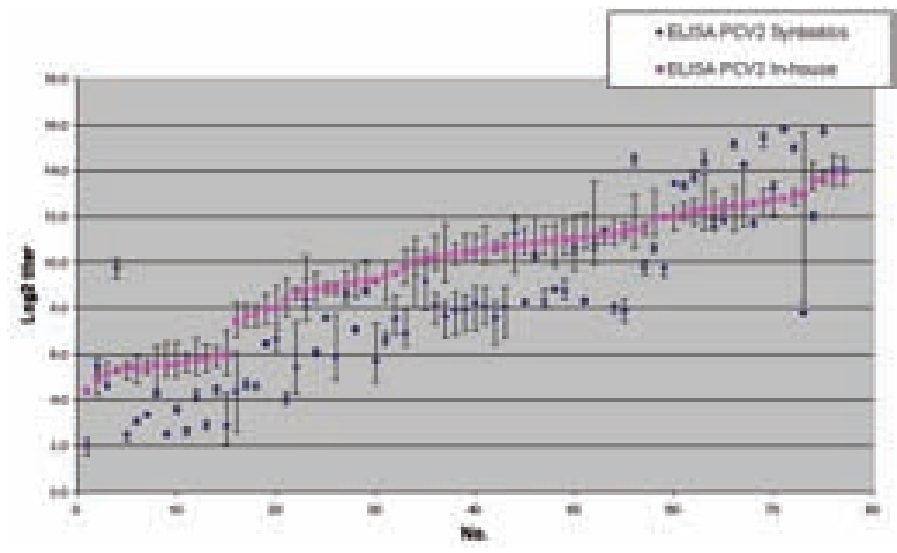

(Error bars show the titer range)

\section{Results}

In Figures 1 and 2 the mean results of the 77 sows in both ELISA's are presented. In both tests the sera show a regular increase of titer when sorted ascending, although the slope of the synbiotics test is more flat compared to the inhouse test. The in house ELISA shows a range of 2-16 log 2 The titer range in the synbiotics test was between 4 and $14 \log 2$.

In the in house ELISA all negative sera had a titer of $<2$ Log2. In the Synbiotics test this was $<4$ Log2. (individual data not shown)

\section{Discussion}

Both the ISAPH in house ELISA as well as the Synbiotics PCV2 ELISA show a regular increase of titer when sorting ascending. and both can be used to establish the antibody level in pigs. The slope of the two test differ which is important to realize when interpretating serological data; From in house data it is known that a 5Log2 titer in the ISPAH inhouse PCV2 antibody ELISA represents a protective level i.e. above 5 Log 2 pigs will be protected. Since the slope of the two tests differ probably higher titers in the Synbiotics test are needed for protection. From the ISPAH test it is known that high titers around 10-12 Log 2 at the moment of vaccination may lead to suboptimal vaccination results. It is to be expected that in the Synbiotics ELISA this can already be the case at a somewhat lower level.

\section{References}

Fort et al Vaccine 26 (2008) 1063-1071

Fort et al Vaccine 27 (2009) 4031-4037

Figure 2: Mean titer ELISA PCV2 Synbiotics compared too mean titer ELISA PCV2 In House sorted ascending for ELISA PCV2 In House

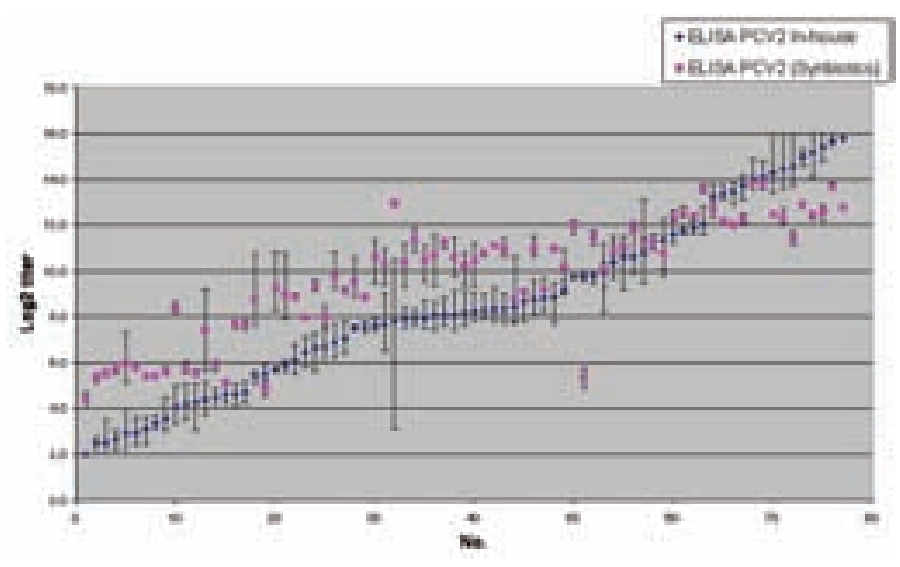

(Error bars show the titer range) 


\title{
P.007
}

\section{Detection and Genetic Characterization of Porcine Circovirus Type 2 (PCV2) in Pigs from Colombia}

\author{
María A. Rincón ${ }^{2}$ Jose D. Mogollon Víctor Vera ${ }^{1}$ Jairo Jaime Gloria C. Ramirez ${ }^{1}$ Michael Murtaugh ${ }^{3}$ Juan Abrahante $^{3}$ \\ 1. Universidad Nacional de Colombia, Bogota, Colombia; 2. Instituto Colombiano Agropecuario, \\ Bogota, Colombia; 3. University of Minnesota, St Paul, NM, USA
}

\section{Introduction}

Porcine circoviruses (PCV), members of the family Circoviridae, are composed of non-enveloped viruses with a single-stranded circular DNA. Two genetically and antigenically different viruses, PCV1 and PCV2, can be distinguished. PCV1 is nonpathogenic, and PCV2 is associated with postweaning multisystem wasting syndrome (PMWS) (1).

In Colombia, clinical PMWS associated with PCV2 infection was first reported in 2006.The PCV2 antigen was detected in the $91,2 \%$ of the 34 farms examined by IHC (2). This study reports the presence of PCV2 in Colombian swine herds prior and after PMWS outbreaks from 2006 - 2009.

\section{Materials and Methods}

Tissue samples (lymph nodes, lung, spleen, and kidney) were collected from nine farms with historical records of PMWS during 2006 - 2009. The individual diagnosis of PMWS was confirmed for each case (3). Twenty serum samples collected from March 2004 to November 2005 from four farms located in three different regions were also included. The samples were stored at $-80^{\circ} \mathrm{C}$ until use.

DNA extraction was carried out using the QIAamp ${ }^{\circledR}$ DNA Mini kit (Qiagen, USA) according to the manufacturer's instructions. The capsid gene was amplified and sequenced from nucleotides 998 to 1757 using specific primers capFw 5' CTT TTT TAT CAC TTC GTA ATG 3' and capRw 5' CGC ACT TCT TTC GTT TTC 3 (4). Amplicons were sequenced using an automatic sequencer (Applied Biosystems, Macrogen, Korea). Strain-specific sequence chromatograms generated using forward and reverse primers were assembled into individual contigs using SeqMan (DNASTAR). Resulting partial ORF2 sequences were aligned in ClustalX. The alignments was imported into CLC Sequence viewer for visual inspection, and the first $30 \mathrm{bp}$ and the last $20 \mathrm{bp}$ of the full length ORF2 region were excluded. Phylogenetic trees were generated in CLC Sequence

Viewer using the Unweighted Pair Group Method with Arithmetic mean (UPGMA) method.

\section{Results and Discussion}

Preliminary analysis of 14 tissue samples from the field cases and six serum samples from the retrospective survey were positive for PCV2 by PCR. The 20 sequences obtained could be divided into two main clusters supported by high confidence values that matched the two genotypes defined previously; PCV2b $(n=15)$ and PCV2a $(n=5)$. Interestingly, all of the PCV2a sequences but only one PCV2b sequence were found in serum collected during 2003-2005. All of the samples from 2006-2009 were PCV2b. The detection of PCV2b genotype since 2006 may explain the PMWS epizootic occurrence in farms of several states of Colombia. Additional sequencing is underway to expand these findings and provide a more complete picture of the genotype distribution of PCV2 and his association with PMWS in Colombian swine herds.

Figure 1. Phylogenetic tree of 20 Colombian samples based on ORF2 sequence.

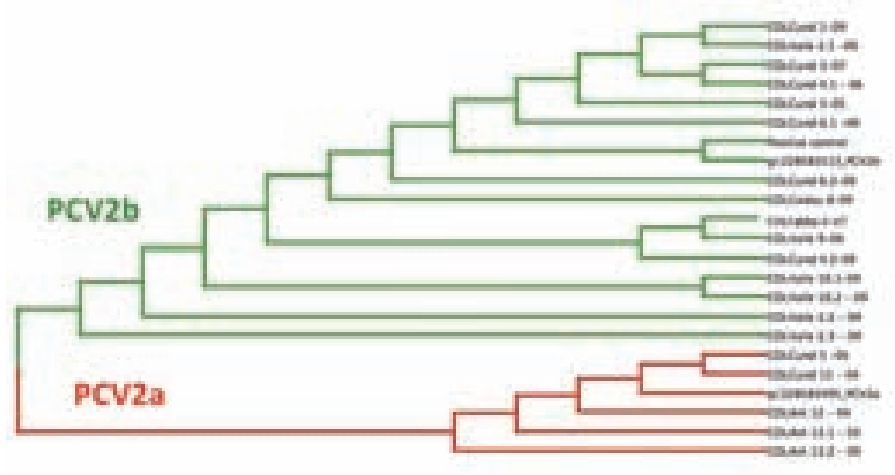

\section{References}

1. Todd, D. (2002) Avian Pathol 29, 373 - 394

2. Clavijo J et al (2008) Proceedings of the 20th IPVS Congress, Durban, South Africa, p 550 .

3. Segales J et al (2005)Anim Health Res Rev 6, 119-142)

4. Fort M et al (2007) Vet Microbiol 125, 244-255 


\title{
P.008
}

\section{Evidence of a genotypic shift in porcine circovirus type $\mathbf{2}$ (PCV2) from PCV2a to PCV2b after PMWS outbreaks in two Spanish farms}

\author{
Martí Cortey ${ }^{1}$ Emanuela Pileri ${ }^{1}$ Marina Sibila ${ }^{1} J_{\text {Joan Pujols }}{ }^{1}$ Mònica Balasch ${ }^{2}$ Joan Plana $^{2}$ Joaquim Segales $^{1}$ \\ 1. Centre de Recerca en Sanitat Anima (CReSA), Bellaterra, Spain; 2. Fort Dodge Veterinaria, S.A, Vall de Bianya, Spain
}

\section{Introduction}

PCV2 is considered to be the essential infectious agent of postweaning multisystemic wasting syndrome (PMWS). Many studies have not found any relationship between PCV2 strains and the occurrence of disease (1-3). Nonetheless, a growing number of publications (4-8) reported a shift from PCV-2a to PCV-2b that may be related to the occurrence of PMWS outbreaks.

Based on the existing evidence at different coutry levels, the main objective of this study was to follow up the evolution of PCV-2 genotypes in two farms in Spain that undergone a PMWS episode, to examine the dynamics of PCV2 infection and its relationship with the appearance of PMWS at herd level.

\section{Material and Methods}

A total of 51 samples (serum and PCV2 isolates from the PMWS outbreak) from Farm 1, collected from 1995 to 2009, and 87 samples from Farm 2 (serum samples), collected from 1996 to 2003, were analyzed. PMWS outbreak suspicion and diagnosis were confirmed on May 2007 and March-April 2001 in farm 1 and 2, respectively. Both were small experimental farrow-tofinish (40-60 sows) operations, free from most of common swine pathogens, including porcine reproductive and respiratory syndrome virus. A fragment of 652 located between the Rep and the Cap protein of the PCV-2 genome was sequenced on all available samples.

\section{Results}

A sudden genotype PCV2a to PCV2b overturn was observed on Farm 1: 34/37 sequences were PCV2a before the PMWS outbreak in 2007, whereas all sequences after the outbreak were PCV2b (Figure 1). On Farm 2, the frequency of PCV2a decreased steadily from 1996 to 2003 and the frequency of PCV2b increased (Figure 1). The chi-square analyses performed on both farm datasets yielded significant results, statistically supporting the change of genotype from PCV2a to PCV2b in both herds at the time of PMWS occurrence.

\section{Discussion}

The genetic shift linked to PMWS epizootics has been described in several countries from Europe $(5,7,8)$ and North-America $(4,6)$. However, this is the first report of a PCV2 genotype shift associated with PMWS appearance at individual farm level. Overall, the putative higher virulence of PCV2b strains seems to be supported by the present data. Both studies related the occurrence of PMWS epizootics to high frequencies of PCV2b and lower frequencies of PCV2a.
Figure 1. Historical histograms on PCV2 sequences detected in Farms 1 and 2.

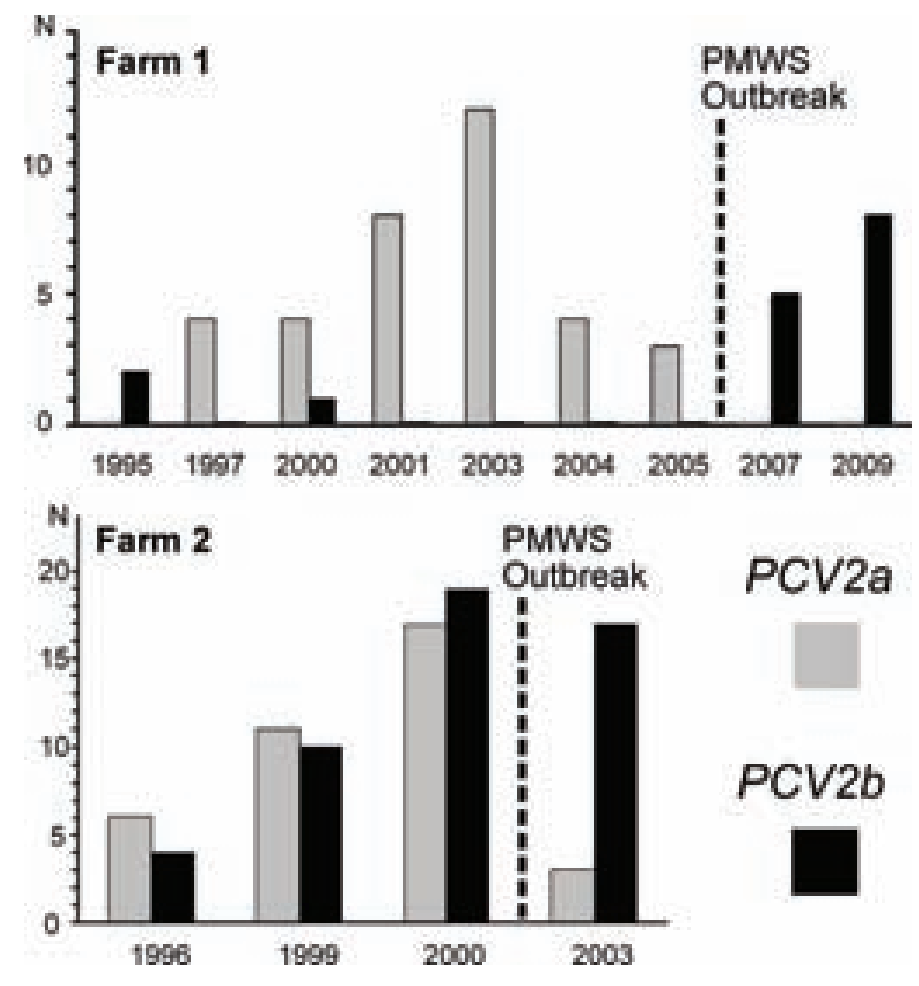

\section{Aknowledgements}

This work was funded by Projects No. 513928 from the Sixth Framework Programme of the EU Commission and Consolider Ingenio 2010-PORCIVIR (Spain). M. Cortey holds a Beatriu de Pinós grant from Catalan Government.

\section{References}

1. Allan et al. 2007. J Vet Diag Invest 336 19, 668-673.

2. de Boisseson et al. 2004. J Gen Virol 85, 293-304.

3. Wen et al. 2005. Vet Micro 110, 141-146.

4. Cheung et al. 2007. Arch Virol 152, 1035- 1044.

5. Dupont et al. 2008. Vet Micro 128, 56-64.

6. Gagnon et al. 2007. Vet J 48, 811-819.

7. Timmusk et al. 2008. Virus Genes 36, 509-520.

8. Wiederkehr et al. 2009. Vet Micro 136, 27-35. 


\section{P.009}

\section{Isolation and identification of Porcine Circovirus Type $\mathbf{2}$ (PCV2) from lymph node samples}

Francisco Quezada-Monroy; Bernardo Lozano-Dubernard; David Sarfati-Mizrahi; Ernesto SotoPriante; Refugio Cortes-Fernandez; Ruben Echeveste-Garcia de Alba; Horacio Lara-Puente

Laboratorio Avi-Mex, S. A. de C. V., Mexico City, DF, Mexico

\section{Introduction}

PCV2 is an icosahedrical $17 \mathrm{~nm}$ circular shaped, naked virus with single stranded ambivalent DNA (Fenaux 2004). Virus Isolation (VI) is done mainly in PK 15 cell line and it is particularly tedious due to the fact that the virus requires cells in active mitosis that provide it with DNA polymerase, needed for the viral replication process (Tischer et al 1987). Its presence is demonstrated by Immunoperoxidase (IP), Immunofluorescence or Molecular Biology techniques since it does not produce citopathic effect.

\section{Material and Method}

Cell line: PK 15 PCV2 free cells were employed, with 24 hrs growth and sensitized with D - Glucosamine.

Samples: Lymph nodes gathered from animals of 3 different farms, showing signs compatible with PCVAD. These samples were tested as 9 macerates for $\mathrm{VI}$ and 3 pools for PCRqrt.

Viral Isolation, all macerates were filtered at $0.22 \mu \mathrm{m}$ and used to infect PK 15 cell cultures with the after mentioned characteristics. Viral presence was confirmed by IP tests (NVRI, Pulaway: Poland 2007).

PCRqrt: genetic material extraction was performed from the pools; viral particles present were amplified and quantified using the protocol developed by Cortes et al (unpublished).

\section{Results}

VI: From the 9 macerates, 7 yielded positive and 2 negative by the IP test (Table 1).

PCRqrt: All 3 pools were positive with a viral load between $10^{2.0}$ and $10^{3.0}$ viral particles $/ \mathrm{mL}$ (Table 2 ).

\section{Discussion}

In this case PCV2 could be isolated and identified by IP and by PCRqrt showing an $80-100 \%$ positive respectively. These results confirm viral presence in Mexico and agree with seroprevalence results presented by Ramirez et al (2008) where $97 \%$ of the tested sera were positive, for PCRqrt Hernandez et al (2008) reported viral loads from $10^{3.0}$ to $10^{7.0} \mathrm{vp} / \mathrm{mL}$ from tissues. Other investigators results are also confirmed Trujano et al (2001) and Ramirez et al (2004), who reported viral detection by in situ hybridization and by viral isolation.It is noticeable the relation among the viral particles detected by PCRqrt with the positive results in $\mathrm{VI}$ and the stained nuclei detected by IP, where the higher the number of positive samples, higher the viral load and also the higher number of stained nuclei.
Table 1. Results by immunoperoxidase (IP).

\begin{tabular}{|c|c|c|}
\hline Sample & IP result & Stained nuclei \\
\hline 1 & Negative & - \\
\hline 2 & Positive & ++ \\
\hline 3 & Positive & ++ \\
\hline 4 & Positive & +++ \\
\hline 5 & Positive & + \\
\hline 6 & Negative & - \\
\hline 7 & Positive & ++ \\
\hline 8 & Positive & + \\
\hline 9 & Positive & +++ \\
\hline - Negative; + Slight; ++ Moderate; +++ Abundant \\
\hline
\end{tabular}

Table 2. PCRqt results.

\begin{tabular}{|c|c|c|}
\hline Sample & Farm pool & Quantification $(\mathbf{v p} / \mathbf{m l})$ \\
\hline 1 & Farm A & $1.7 \times 10^{2.0}$ \\
\hline 2 & Farm A & $1.7 \times 10^{2.0}$ \\
\hline 3 & Farm B & $4.1 \times 10^{3.0}$ \\
\hline 4 & Farm B & $4.1 \times 10^{3.0}$ \\
\hline 5 & Farm C & $2.1 \times 10^{3.0}$ \\
\hline 6 & Farm C & $2.1 \times 10^{3.0}$ \\
\hline 7 & Farm C & $2.1 \times 10^{3.0}$ \\
\hline 8 & Farm C & $2.1 \times 10^{3.0}$ \\
\hline 9 & Farm C & $2.1 \times 10^{3.0}$ \\
\hline
\end{tabular}

\section{Bibliography}

Fenaux M., PhD Thesis. Faculty of Virginia Polytechnic Institute, (2004). Tischer I. et al. Archives of Virology. 96:39-57 (1987).

National Veterinary Research Institute, Pulawy; Poland NMSACC - PCVD. 61-71. (2007).

Ramírez et al. VI Jornada Internacional de Producción Porcina (2008). Hernández et al VI Jornada Internacional de Producción Porcina (2008). Trujano et al. PCV-2 from emaciated pigs in Mexico Vet. Rec. 148:792. (2001).

Ramírez, MH et al. Memorias XXXIX congreso Nacional AMVEC (2004). 


\title{
P.010
}

\section{International immunohistochemistry ring trial for the detection of porcine circovirus type 2 (PCV2) antigen on formalin-fixed, paraffin-embedded tissues}

\author{
Joaquim Segales ${ }^{1}$ Katinka Belák ${ }^{2}$ Vivi Bille-Hansen ${ }^{3}$ Chan Hee Chae ${ }^{7}$ John Ellis ${ }^{8}$ Roberto M. Guedes ${ }^{9}$ Franits ${ }^{2}$ ek Kostka ${ }^{4}$ \\ Steven Krakowka ${ }^{10}$ Torfinn Moldal ${ }^{6}$ Hervé Morvan ${ }^{11}$ Tanja Opriessnig ${ }^{12}$ Manuel Quezada ${ }^{5}$ María Alejandra Quiroga ${ }^{13}$ \\ Jose Ramos-Vara ${ }^{14}$ Trpe Ristoski ${ }^{18}$ Norbert Stockhofe-Zurwieden ${ }^{15}$ Titus Sydler ${ }^{16}$ Tamas Tuboly ${ }^{17}$ Martí Cortey ${ }^{1}$ \\ 1. Centre de Recerca en Sanitat Anima (CReSA), Bellaterra, Spain; 2. Department of Pathology and Wildlife Diseases, Laboratory Veterinarian \\ National Veterinary Institute (SVA), Uppsala, Sweden; 3. National Veterinary Institute, Technical University of Denmark, Copenhagen, \\ Denmark; 4. State Veterinary Institute, Jihlava, Czech Republic; 5. Facultad de Ciencias Veterinarias, Universidad de Concepción, Chillán, Chile; \\ 6. Section for Pathology, National Veterinary Institute, Oslo, Norway; 7. Department of Veterinary Pathology, College of Veterinary Medicine, \\ Seoul National University, Seoul, Korea; 8. Department of Veterinary Microbiology, Western College of Veterinary Medicine, University of \\ Saskatchewan, Saskatoon, SK, Canada; 9. Department of Clinic and Surgery, Veterinary School, Universidade Federal de Minas Gerais, Belo \\ Horizonte, MG, Brazil; 10. Department of Veterinary Biosciences, College of Veterinary Medicine, The Ohio State University, Columbus, $\mathrm{OH}$, \\ USA; 11. ALDA22, Laboratoire de Développement et d'Analyses, Ploufragan, France; 12. Department of Veterinary Diagnostic and Production \\ Animal Medicine, College of Veterinary Medicine, lowa State University, Ames, IA, USA; 13. Laboratorio de Diagnóstico e Investigaciones \\ Bacteriológicas, Facultad de Ciencias Veterinarias, Universidad Nacional de La Plata, La Plata, Argentina; 14. School of Veterinary Medicine, \\ Purdue University, West Lafayette, IN, USA; 15. Central Veterinary Institute, Lelystad, Netherlands; 16. Institute of Veterinary Pathology, \\ University of Zurich, Vetsuisse Faculty Zurich, Zurich, Switzerland; 17. Department of Microbiology and Infectious Diseases, Faculty of Veterinary \\ Science, Szent István University, Budapest, Hungary; 18. Department of Pathology, Faculty of Veterinary Medicine, Skopje, Macedonia
}

\section{Introduction}

Porcine circovirus type 2 (PCV2) is the essential infectious agent of postweaning multisystemic wasting syndrome (PMWS). PMWS diagnosis is mainly established based on laboratorial criteria, including histopathology and detection of PCV2 in tissues (1). Therefore, the objective of the present abstract is to summarise the results of an international immunohistochemistry (IHC) ring trial performed in 18 different laboratories across the world.

\section{Materials and Methods}

The IHC ring trial was undertaken using 10, formalin-fixed, paraffin-embedded tissues, including lymph nodes $(n=5)$, lung $(n=3)$ and thymus $(n=2)$ from pigs with and without PMWS. The referral laboratory (laboratory 1 ) submitted the 10 tissue sections in duplicate to each laboratory (two laboratories contributed twice to the results). Each laboratory performed the IHC stain and evaluated the results blindly using a scale of 0 (negative), $1+$ (weak reactivity), $2+$ (moderate reactivity), and $3+$ (strong reactivity).

Secondly, a written survey detailing 28 technical aspects of the IHC assays were also sent to all participants. To analyse the results, two multiple correspondence analyses (MDS) were performed, one using the discrepancies of the ring trial a second one comparing differences among $\mathrm{IHC}$ technical details and results among laboratories.

\section{Results}

Sixteen out of 18 laboratories had a mean number of discrepant results between 0 and 1 among slides; such number was 2 or higher in the other two laboratories. Of all participant laboratories, only 14 sent the technique details survey completed. When the $\mathrm{IHC}$ result and $\mathrm{IHC}$ technique details were taken into account jointly, 12 out of the 14 submitting laboratories showed no significant differences among them.

\section{Discussion}

Overall, the present study indicates that most of the participant laboratories reported congruent results. Technical differences were not a major source of result disagreement.

\section{Acknowledgements}

This work was funded by project No. 513928 from the Sixth Framework Programme of the EU Commission

\section{References}

1. Segalés et al. 2005. Anim Health Res Rev 6: 119-142. 


\title{
P.011
}

\section{Pathological characterization of necrotizing lymphadenitis associated}

\author{
Ivan Galindo-Cardiel ${ }^{1,2}$ Llorenç Grau-Roma1, 2 Monica Perez-Maillo ${ }^{1}$ Joaquim Segales $^{1}$ \\ 1. Centre de Recerca en Sanitat Anima (CReSA), Bellaterra, Spain; 2. Departament de Sanitat i \\ Anatomia Animals, Universitat Autònoma de Barcelona, Bellaterra, Spain
}

\section{Introduction}

Necrotizing lymphadenitis is a pathological feature observed in about $2 \%$ of postweaning multisystemic wasting syndrome (PMWS)-affected pigs. The pathogenesis of the lesion has been linked to a Porcine circovirus type 2 (PCV2)-related apoptosis-induced mechanism (1), but no further studies have been focused on this topic. Therefore, the goal of this retrospective work was to gain further insights into PCV2-associated lymphoid necrosis pathogenesis in PMWS cases.

\section{Materials and Methods}

Three groups of animals were selected: PMWS-affected pigs with necrotizing lymphadenitis (group $1, n=5$ ), PMWS-affected pigs without necrotizing lymphadenitis (group 2, n=5) and healthy pigs with no PMWS-related lesions (group 3, n=5). Investigations performed included immunohistochemistry (IHC) of cleaved caspase-3 (to detect apoptosis) and von Willebrand factor (vWF, to detect this glycoprotein, which is involved in haemostasis), special stain for fibrin (Mallory stain) and in situ hybridization (ISH) to detect PCV2 genome.

\section{Results}

Table 1 includes individual results of histopathological findings. Fibrin deposition detected by Mallory stain was significantly higher in PMWS-affected lymph nodes with necrosis compared to those with PMWS or normal lymph nodes. Lymph nodes from PMWS-affected pigs had significantly higher PCV2 amount than those of healthy pigs. Otherwise, no significant differences were observed between the two PMWS-affected groups in regards intensity, location or amount of PCV2 genome detected by ISH. Staining pattern of VWF in the PMWS-affected pigs with follicular necrosis was endothelial (peri-membranous), subendothelial (collagen bound), cytoplasmic and intravascular (circulating). In regards apoptosis, although labelled cells from PMWS-affected pigs with follicular lymphoid necrosis had a strong intracytoplasm staining, CCasp-3 staining pattern did not show differences among the three groups of studied pigs.

\section{Discussion}

Necrotizing lesions in lymph nodes of severely PMWS-affected pigs usually show multifocal to coalescent areas of coagulative necrosis (in some cases, affecting the whole parenchyma of the lymph node) together with vascular thrombosis (3). This latter fact has been confirmed in the present study by the presence of fibrin thrombi detected by the Mallory stain. Taking into account that vascular injury seems to be the primary determinant of a thrombotic response (2), the existence of marked thrombosis in PMWS-associated necrotizing lymphadenitis suggests certain extension of vascular damage in these pigs. Such damage evidence was supported by the high endothelium venule hypertrophy and the over-expression of VWF in subendothelial (collagen bound), cytoplasmic and intravascular (circulating) locations. Such findings would account for the increase of intravascular fibrin deposition and, consequently, thrombosis. Therefore, obtained results would point towards a vascular accident with thrombi development and the subsequent follicular necrosis as the cause of necrotizing lymphadenitis in PMWS affected pigs.

Table 1. Results of histopathology, Mallory stain, PCV2 in situ hybridization, vWF and apoptosis scores in the three groups of studied pigs.

\begin{tabular}{|l|c|c|c|c|c|c|c|}
\hline Group & Case & PMWS & FN & ISH & CCasp3 & vWF & Mallory \\
\hline & 1 & +++ & +++ & +++ & +++ & +++ & ++ \\
\hline & 2 & +++ & ++ & +++ & + & ++ & ++ \\
\hline & 3 & +++ & +++ & +++ & ++ & +++ & ++ \\
\hline & 4 & +++ & + & ++ & + & + & + \\
\hline & 5 & +++ & + & +++ & + & + & + \\
\hline & 6 & ++ & - & +++ & ++ & - & - \\
\hline 2 & 7 & +++ & - & +++ & ++ & + & - \\
\hline & 9 & +++ & - & +++ & + & ++ & - \\
\hline & 10 & +++ & - & +++ & + & + & - \\
\hline & 11 & - & - & - & + & + & - \\
\hline & 12 & - & - & - & ++ & $+/-$ & $+/-$ \\
\hline 3 & 13 & - & - & - & $+/-$ & + & - \\
\hline & 14 & - & - & - & + & + & $+/-$ \\
\hline & 15 & - & - & - & + & + & $+/-$ \\
\hline
\end{tabular}

\section{References}

1. Kim and Chae (2005). Vet Rec 156: 177-178.

2. Nichols et al. (1991). Circulation 83: IV56-64.

3. Segalés et al. (2005). Anim Health Res Rev 6: 119-142. 


\title{
P.012
}

\section{Clinical incidence and frequency of lesions associated to porcine circovirus type 2 in pigs of a farm in the state of Yucatan, Mexico}

\author{
Jose L. Guillermo ${ }^{1}$ Jorge C. Rodriguez ${ }^{1}$ Marco A. Torres ${ }^{1}$ Monica Quintal2 Raquel Miranda ${ }^{1}$ Rafael Colin \\ 1. University of Yucatan, Merida, YUC, Mexico; 2. Patologia Animal, Merida, YUC, Mexico
}

\section{Introduction}

Serological studies have demonstrated that PCV2 is present in both affected and unaffected farms with postweaning multisystemic wasting syndrome (PMWS) with a seroprevalence nearly $100 \% 1$. However, the incidence of PMWS on a farm is typically low, suggesting that PCV2 is not the only agent involved and that other factors are required for the presence of the PMWS; animals infected with PCV2 and parvovirus or PRRSV, immunosuppressive or immunostimulating agents2, so that the incidence of PMWS is variable between regions and between farms3. The objectives were to estimate the cumulative and true incidence, the lethality rates and the frequency of macroscopic and microscopic lesions of PMWS and porcine dermatitis nephropathy syndrome (PDNS) in pigs of a farm in the state of Yucatan, Mexico.

\section{Materials and Methods}

The studied population was divided in 2 groups. Monitored group consisted of a production batch of 235 pigs, which were observed from week 4 to 22 of age to determine the number of new cases compatible with the PMWS and PDNS that occurred weekly and the number of pigs that died of these syndromes. Sacrificed group was formed gradually during each of the visits to the farm in this group the frequency of lesions compatible with the PMWS and PDNS was estimated, for which, post mortem studies were performed in all discarded pigs between 4-22 weeks of age and with signs compatible with the syndromes studied. Necropsy was performed in animals of both groups monitored and sacrificed. The organs inspected were: lymph nodes, spleen, liver, tonsils, ileum, lungs, kidneys and skin. Samples of all organs were obtained for histopathological studies

\section{Results}

14 pigs showed signs consistent with the syndromes mentioned, 7 with the PMWS and 7 with the PDNS. The cumulative incidence and incidence rate for both syndromes were 0.06 and 0.0034 respectively. 5 pigs died, lethality rates calculated for PMWS y PDNS were 0.4 and 0.3 respectively. Necropsy was performed at 80 pigs, 50 with signs compatibles with PMWS and 30 with PDNS. The most frequent macroscopic lesions in the PMWS were Lungs: lesions compatibles with interstitial pneumonia and supurative bronchopneumonia 94\%. Kidney: Nefromegaly with white spots $48 \%$. Lymph nodes: lymphadenomegaly and edema. Spleen decrease in size 68\%. PDNS: Lungs: compatible with interstitial pneumonia and supurative bronchopneumonia80\%. Skin: eritematous macules and plaques with crusted center $63 \%$ Kidney: nephromegaly with hemorrhage $87 \%$. Lymph nodes: lymphadenomegaly and congestion $63 \%$ and Spleen: Splenomegaly $60 \%$. There were no macroscopic lesions in liver, ileum and tonsils

Table: Frequency of microscopic lesions compatibles with PMWS and PDNS

\begin{tabular}{|c|c|c|}
\hline MICROSCOPIC LESIONS & PMWS $n=50$ & PDNS $n=30$ \\
\hline \multicolumn{3}{|l|}{ LUNGS } \\
\hline $\begin{array}{l}\text { Suppurative bronchopneumonia and histiocytic interstitial } \\
\text { pneumonia }\end{array}$ & $94 \%$ & $80 \%$ \\
\hline \multicolumn{3}{|l|}{ SKIN } \\
\hline Histiocytic dermatitis and neutrophilic vasculitis & -—- & $70 \%$ \\
\hline \multicolumn{3}{|l|}{ KIDNEYS } \\
\hline Histiocytic interstitial nephritis & $48 \%$ & -二 - \\
\hline Neutrophilic vasculitis & 一一一 & $37 \%$ \\
\hline \multicolumn{3}{|l|}{ LIVER } \\
\hline Histiocytic hepatitis with lipidosis & $78 \%$ & $57 \%$ \\
\hline \multicolumn{3}{|l|}{ LYMPH NODE } \\
\hline Histiocytic lymphadenitis and atrophy & $76 \%$ & $43 \%$ \\
\hline Histiocytic lymphadenitis and hyperplasia & $24 \%$ & $57 \%$ \\
\hline \multicolumn{3}{|l|}{ SPLEEN } \\
\hline Histiocytic splenitis and atrophy & $64 \%$ & $30 \%$ \\
\hline Histiocytic splenitis and hyperplasia & $18 \%$ & $63 \%$ \\
\hline \multicolumn{3}{|l|}{ TONSILS } \\
\hline Histiocytic tonsillitis and atrophy & $50 \%$ & $37 \%$ \\
\hline Histiocytic tonsillitis and hyperplasia & $30 \%$ & $47 \%$ \\
\hline \multicolumn{3}{|l|}{ ILEUM } \\
\hline Histiocytic ileitis and atrophy & $66 \%$ & $23 \%$ \\
\hline Histiocytic ileitis and hyperplasia & $20 \%$ & $67 \%$ \\
\hline
\end{tabular}

\section{Discussion}

The PMWS and PDNS affect the population studied; with a slow diffusion, high mortality and the organ most frequently affected by the both syndromes were the lymph nodes (100\%)

\author{
References \\ 1 Larochelle R, et al, (2003) Can. J. Vet. Res. 67: 114-120 \\ 2 Krakowka S, et al.,(2001). Vet Pathol. 38:31-42. \\ 3 Segalés, J., et al., (2003). Pigs New and Information.24:103-110
}




\title{
P.013
}

\section{Type of lesions and patterns associated on porcine circovirus type 2 in organs of positive swine for immunohistochemistry}

\author{
Monica Quintal ${ }^{2}$ Jose L. Guillermo ${ }^{1}$ Jorge C. Rodriguez ${ }^{1}$ Marco A. Torres ${ }^{1}$ Raquel Miranda ${ }^{1}$ Rafael Colin \\ 1. University of Yucatan, Merida, YUC, Mexico; 2. Patologia Animal, Merida, YUC, Mexico
}

\section{Introduction}

Porcine Circovirus type 2 (PCV2) is an emerging disease of swine that has been associated in the development of other diseases. Currently there are various diagnostic tools and techniques available for the detection of PCV2; however, the immunohistochemistry $(\mathrm{IHC})$ is considered the gold standard for the detection of this disease1.

The objectives of the present study were to demostrate the existence of the PCV2 antigen by $\mathrm{HHC}$, and to determine the distribution patterns of histopathological lesions suggestive to PCV2 in different organs and tissues.

\section{Materials and Methods}

An observational study was realized on a farm with semitecnificated intensive production system and full breed cycle. The type of sampling was not probabilistic for convenience. Sample sizes were 22 pigs. Whereas a population of 1400 pigs, an estimated prevalence of $30 \% 2,3$ and a confidence level of $99.5 \%$ using formula: $n=[1-(1-p) 1 / D][N-D / 2]+1] 64$.

Post mortem studies were performed in discarded pigs between 4-22 weeks of age. The method of euthanasia was electrostunning established by the NOM -033-ZOO 1997. Organs of 22 pigs with macroscopic lesions suggestive to PCV2 were sampled. The organs studied were lymph nodes, spleen and tonsils, lung, kidney, liver, skin and ileum. The tissue sections were fixed for 24 hours in $10 \%$ formalin $\mathrm{pH} 7.4$ processed by the standard method of embedding in paraffin, and stained with routine staining $\mathrm{H}$ $\& \mathrm{E}$, for subsequent microscopic observation. The PCV2 antigen was detected by IHC using the system avidin-biotin-peroxidase as described by Ramos-Vara5

\section{Results}

The microscopic lesions suggestive to PCV2 on lymphoid organs were: histiocytic infiltrate $68 \%(15 / 22)$ on spleen, $63 \%(14 / 22)$ lymph node and 54\% (12/22) tonsil, lymphoid depletion was observed $40 \%$ on lymph node, on $20 \%$ (4/22) spleen and tonsil. On epitelial organs the most common lesions were hepatitis lymphohistiocytic periportal, 77\% (17/22) interstitial lymphoplasmacytic nephritis with giant cells 36\% (8/22) membranoproliferative; glomerulonephritis 13\% (3/22), on ileum lymphoplasmacytic enteritis 59\% (13/22), depletion of mucosa associated lymphoid tissue (MALT) 22\% (5/22) and multinucleated giant cell
4.5\% (1/22); on skin lymphoplasmacytic histiocytic perivascular dermatitis 31\% (7/22); necrotizing neutrofilic leukocytoclastic vasculitis $22 \%$ (5/22); on lungs lymphoplasmacytic histiocytic bronquitis and bronchiolitis $72 \%(16 / 22)$ and interstitial pneumonia $31 \%(7 / 22)$

22 pigs evaluated by IHC technique, were positive, at least in one organ, where positive antigen-antibody reaction was detected on lymph node $98 \%(21 / 22)$, tonsils $98 \%(21 / 22)$, spleen $98 \%$ $(21 / 22)$, lung $68 \%$ (15/22), ileum 68\% (15/22), kidney 5\% (1/22), and liver $4 \%(1 / 22)$. All skin samples resulted negative.

The frequency of IHC positive cases by stage of production were: on weaning stage $8(36 \%)$ positive tissues of lymph nodes and spleen and 9 (41\%) tissues of tonsils. On feeding stage $11(50 \%)$ positive tissues of lymph nodes and spleen and 10 (45\%) tissues of tonsils

The grade of immunopositive observed in the lymphoid organs in weaning pigs were uniform for spleen, lymph nodes and tonsils, with ratings of mild, moderate and strong unlike feeding pigs where $27 \%(6 / 22)$ in the spleen, lymph nodes and tonsil, were strong grade.

The grade of immunopositive in epithelial organs were variable in ileums $27 \%(6 / 22)$ were moderate in the lungs 31\% (7/22) were weak in the kidneys there were not difference $9 \%(2 / 22)$ for three categories and the liver with $4 \%(1 / 22)$ that corresponded to grade of strong immunopositive, skin tissues without positive reaction

\section{Discussion}

The relevance of the present study has demonstrated that tissue samples from lymph node, spleen, tonsil, lung, liver, ileum, and kidney should be the collected for routine and specialized PCV2 analysis. This study is the first of its kind in Mexico

\section{References}

1 Segalés, J., et al. Diseases of swine. 2006. 299-307.

2 Torres, M.; Guillermo, L. et al. Memorias del XIV Congreso Nacional de Patología 2006, 265-278.

3 Segalés J. Memorias del XLI Congreso Nacional de AMVEC, 2006. 112-116.

4 Thrusfield, M. Veterinary Epidemiology 2005, 228-250.

5 Ramos-Vara, J.; Ackerman, J. Inmunohistochemical methods. 2007, 273-314 


\title{
P.014
}

\section{Correlation between qPCR and virus isolation for the quantification of porcine circovirus type $\mathbf{2}$ in tissue samples from naturally infected pigs}

\author{
Ann B. Cay ${ }^{1}$ DJ Lefebvre ${ }^{1,2}$ Y Van der Stede ${ }^{1} \mathrm{C}_{\text {Miry }}{ }^{3}$ HJ Nauwynck ${ }^{2}$ \\ 1. CODA-CERVA-VAR, Brussels, Belgium; 2. Laboratory of Virology, Faculty of Veterinary Medicine, Ghent \\ University, Merelbeke, Belgium; 3. DGZ, Animal Health Care Flanders, Torhout, Belgium
}

\section{Introduction}

Porcine circovirus type 2 (PCV2) is the causative agent of several diseases that are grouped as porcine circovirus(-associated) disease (PCV (A)D) (1). The diagnosis of PCVD is primarily based on the presence of high amounts of PCV2 antigen or nucleic acids associated with microscopic lesions in lymphoid tissues and/or the foetal/neonatal heart (1). For routine PCV2 quantification, immuno-histochemistry, immuno-fluorescence, antigen-capture ELISA, virus isolation (VI), in-situ hybridization (ISH) or quantitative PCR (qPCR) are used (1). The present study aims to define the correlation between $\mathrm{QPCR}$ and $\mathrm{VI}$ in tissues from naturally PCV2-infected pigs.

\section{Materials and Methods}

Thirty-one different tissue samples from weaners and growers that were suspected of PCVD based on clinical symptoms and historical findings, were collected and submitted to the regional laboratory for histopathological and virological analysis. Lymph nodes, spleen and/or lungs were homogenised and the tissue suspensions were titrated onto PK-15 cells. A titre of $>=104.5$ $\mathrm{TCID} 50 / \mathrm{g}$ tissue correlates well with histopathological lesions and was considered to be suggestive for PCVD (2). Simultaneously, tissue suspensions were analyzed by an ORF2-based real-time qPCR developed in the CODA-CERVA-VAR laboratory (home-made realtime-qPCR) and modified from previously described techniques $(3,4)$. A Pearson's correlation coefficient with a $95 \%$ confidence interval was calculated on the log 10 transformed values obtained in VI and RT-qPCR from all 32 samples.

\section{Results}

The Pearson correlation coefficient was between the $\log 10$ transformed values of VI and qRT-PCR was 0.72 [0.49-0.86] and can be considered as large (Fig1). The highest mean virus titre was found in the lymph nodes [ $4.5 \pm 1.9]$, followed by the lungs $[3.6 \pm 2.3]$ and the spleen [3.0 \pm 1.6$]$. The same pattern was observed for the qRT-PCR : highest mean viral load found in lymph nodes [8.5 \pm 3.5$]$, followed by the lungs [8.2 \pm 2.9 ] and the spleen [7.6 \pm 4.3$]$. However, the differences between the sample tissues were not statistically significant.

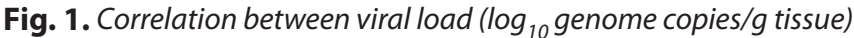
and virus titre $\left(\log _{10} T C I D_{50} / g\right.$ tissue)

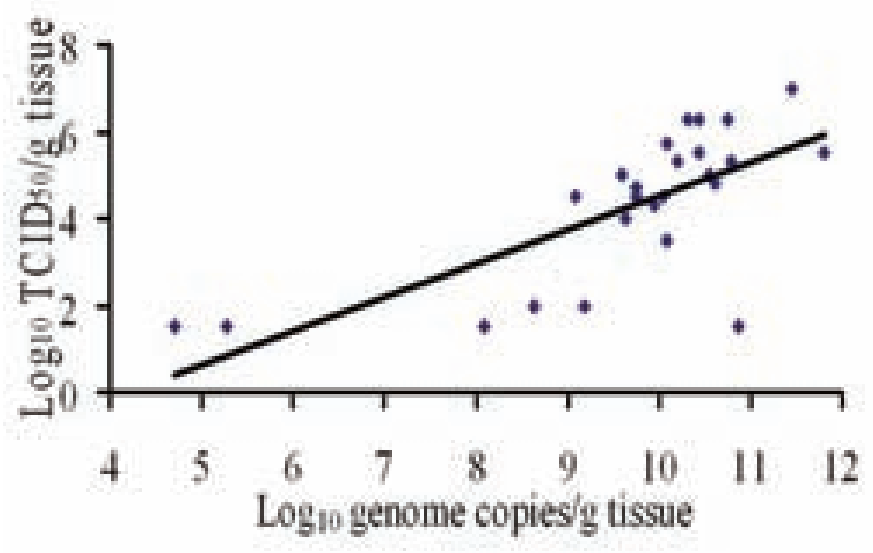

\section{Discussion}

Previously, it was shown that $>=10^{7}$ genome copies per $500 \mathrm{ng}$ DNA is suggestive for PCVD and correlates well with the results of ISH $(3,5)$. In this study, virus titres and the number of genome copies were both expressed per $g$ tissue. We could correlate a PCVD-suspected virus titre of $>=10^{4.5} \mathrm{TCID}_{50}$ with a viral load of $>=10^{10}$ genome copies, suggesting that our newly developed high throughput qPCR may be a valid alternative for time consuming VI. Although no significant differences could be found between the different tissue samples, the highest virus titre and viral load was found in the lymph nodes, confirming previous results (3).

\section{Conclusion}

Based on the correlation between $\mathrm{VI}$ and $\mathrm{qPCR}$ and given that $\mathrm{a}$ titre of $>=10^{4.5} \mathrm{TCID}_{50} / \mathrm{g}$ tissue is suggestive for PCVD (2), we can conclude from this study that a viral load of $>=10^{10}$ genomes $/ \mathrm{g}$ tissue can be suggestive for PCVD.

\section{References}

(1) Opriessnig et al, J Vet Diagn Invest, 2007, 19: 591-615.

(2) Sanchez et al, Vet Microbiol, 2003, 95 : 15-25.

(3) Brunborg et al, J Virol Methods, 2004, 122: 171-178.

(4) Olvera et al, J Virol Methods, 2004, 117: 75-80.

(5) Brunborg et al, J Vet Diagn Invest, 2007, 19: 368-375. 


\title{
P.015
}

\section{Clinical case: porcine circovirus type 2 (PCV2) and mycotoxine presence in an abortion case}

\author{
V. Muller ${ }^{1}$ Guillaume Perreul ${ }^{2}$ Jean-Bernard Herin ${ }^{3}$ Thaïs Vila ${ }^{4}$ François Joisel ${ }^{4}$ \\ 1. Réseau Cristal, SELARL Müller, Bazouges, France; 2. MERIAL, Lyon, France; 3. Merial S.A.S., Ancenis, France; 4. Merial S.A.S., Lyon, France
}

\section{Introduction}

Porcine circovirus type 2 (PCV2) associated to reproductive disorders must be included in the differential diagnosis of the reproduction infectious causes (1).

The objective of this clinical case description is to show the implication of PCV2 in a series of repeated abortions and the impact of CIRCOVAC ${ }^{\circledR}$ vaccination on this issue.

\section{Case description}

This PRRS-free 200-sow farrowing farm is well managed. Buildings are complying with the new welfare EU norms « 2012 » with boars and gestating sows loose on straw. Since 2007, around 20 late abortion cases (3-4 weeks to 3-4 days before expected farrowing date) per year, independently of the parity were recorded. The number of mummified foetuses was variable and could reach 0.6 per litter. Weaned piglets were well-formed but showed heterogeneity among litters. Post-weaning and fattening site are located offsite at a several-km distance from the maternity and did not experience any PMWS signs. Sow fertility was good (90\%). All laboratory investigation aiming at the detection of other infectious agents possibly involved in the reproductive disorders remained negative.

Early 2009, 2 sows aborted without fever. A PCV2 quantitative PCR was performed on sera from and on the hearts and livers of their aborted foetuses. Sera of the sows and thoracic liquid of their aborted foetuse were also submitted for PCV2 ELISA (SERELISA ${ }^{\oplus}$ PCV2 Ab Synbiotics, Lyon, France).

Mycotoxin i.e. zearalenone (ZEA) and deoxynivalenol (DON) quantification in straw was performed by a certified laboratory.

\section{Vaccination schedule}

Primovaccination against parvovirosis and erysipelas used to be performed systematically in quarantine followed by a booster during further lactations. After PCV2 diagnosis was confirmed, a vaccination with CIRCOVAC was carried out: 2 injections 3 weeks apart after pregnancy ultrasound check up due to practical sow containment aspects and a booster injection was administered 3 weeks before farrowing.

Abortions dramatically dropped down after primo-immunization from $3.6 \%$ to $1.3 \%$.

\section{Results}

A high level of mycotoxines was revealed in the straw (see table 1) in 2007 and 2008. After gestating sow feed was supplemented with appropriate concentration of anti-mycotoxines additives, mycotoxines level dropped down to acceptable levels.

A reduction in abortion rate was observed as soon as the first batch was vaccinated, and then no abortion cases were noticed anymore.
Table 1: Mycotoxine detection in straw

\begin{tabular}{|l|c|c|c|c|}
\hline Straw & $\mathbf{2 0 0 7}$ & $\mathbf{2 0 0 8}$ & $\mathbf{2 0 0 9}$ & Reference \\
\hline DON $(\mu \mathrm{g} / \mathrm{kg})$ & 5410 & 5330 & 1940 & 900 \\
\hline ZEA $(\mu \mathrm{g} / \mathrm{kg})$ & 1100 & 50 & 345 & 100 \\
\hline
\end{tabular}

Table 2: PCR and ELISA PCV2 detection in aborted foetuses and serum of aborted sows

\begin{tabular}{|l|l|l|c|}
\hline 70343 & Litter & Heart PCV2 PCR & + \\
\hline 70343 & Litter & mummified pool & - \\
\hline 70343 & Litter & 2 pools of normal aborted foetuses & + \\
\hline 70343 & Litter & Antibodies & - \\
\hline 70343 & Dam & Serum PCV2 PCR & - \\
\hline 70343 & Dam & ELISA (Log10) & 3.5 \\
\hline & & & \\
\hline 70344 & Litter & Heart and liver PCV2 & + \\
\hline 70344 & Litter & mummified pool & + \\
\hline 70344 & Litter & 2 pools of normal aborted foetuses & + \\
\hline 70344 & Litter & Antibodies & $1+/ 10$ \\
\hline 70344 & Dam & Serum PCV2 PCR & + \\
\hline 70344 & Dam & ELISA (Log10) & 3.2 \\
\hline
\end{tabular}

\section{Discussion}

Zearalenone is not known to be usually responsible for abortions even at as high doses as $50 \mathrm{mg} / \mathrm{kg}$ of feed which were far to be reached in this case (2). PCV2 is now well known to be a possible abortion agent ( 3 ) and diagnosis got on the farm showed its involvement in several aborted foetuses organ pools. Consequently, PCV2 can be considered as highly suspected to be responsible for the abortions.

\section{Conclusion}

Altogether abortions occurring late in gestation, a positive PCV2 diagnosis in sows and their aborted foetuses, poor antibody titres in sow sera along with no other pathogens are in favour of PCV2 implication in these abortions. The additional observation showing that abortions sharply stopped after CIRCOVAC vaccination contributed to incriminate PCV2 as the etiologic agent in this abortion episode.

\section{References}

1. Maes et al., (2008) Theriogenology, 20: 1337-1345

2. Rotter et al., (2006) J. Toxic. Envir. Health 48: 1-34

3. Cariolet et al., (2002) JRP 34: 317-323

\section{Acknowledgements}

The authors wish to thank Guillaume Vovard (ALIMAB society), Jérôme and Pascal, LDA22.

${ }^{\circledR}$ CIRCOVAC is a registered trademark of MERIAL in the United States of America and elsewhere. 


\title{
P.016
}

\section{Genotype analysis of Chinese porcine circovirus type 2 in 2008-2009}

\author{
Wenxiao Cui; Xin Guo; Xinna Ge; Hanchun Yang \\ China Agricultural University, Beijing, China
}

\section{Introduction}

Porcine circovirus type 2 (PCV2), a member of the Circoviridae family, is one of the most important pathogen affecting the swine industry worldwide.The genome of PCV2 is circular single stranded DNA with a size of 1767 or 1768 nucleotides $(1,2)$, which contains three major open reading frames (ORFs). The EU consortium proposes naming the three PCV2 genotypes as PCV-2a, PCV-2b and PCV-2c. Using this methodology, ORF2 sequences of PCV2 are assigned to different genotypes when the genetic distance between them is at least 0.035 (3). Our recent publication indicated that $P C V 2$ strains in China could be divided into 5 major groups PCV-2a, PCV-2b, PCV-2d and PCV-2e. In this study, we analyzed the genetic variation and genotype of 54 Chinese PCV2 sequences collected between 2008 and 2009.

\section{Materials and Methods}

Fifty-five sequences of PCV2 were collected in 2008-2009 from 11 pig-producing areas of China. Total DNA was extracted directly from each clinical sample. PCR was performed to amplify the complete genome of PCV2. The PCR product was purified with a QIA quick PCR purification kit (Qiagen, Germany) and cloned into the PGEM-T easy Vector System (Promega, USA) according to manufacturer's instructions. For each genomic DNA amplified, two clones from each of two independent amplifications were sequenced on both directions using the ABI Prism 3730 sequencer at Invitrogen (Beijing, China). The genomic homology analysis and comparison among the PCV2 sequences were performed using the Lasergene Package (DNASTAR Inc). The deduced amino acid sequences of the encoded capsid protein were aligned using the Clustal W method. Genotype determination was done by the criteria described previously (3).

\section{Results}

Sequence analysis. A total of 54 PCV2 sequences were obtained. Pairwise comparisons of PCV2 complete genomic sequence, ORF1 gene, ORF2 gene, ORF3 gene among 54 sequences exhibited that the nucleotide homology ranged from $94.7-100 \%$, 96.7-100\%, 90.0-100\%, 95.9-100\%, respectively and the ORF1, ORF2, ORF3 amino acid homology was $98.7-100 \%, 86.8-100 \%$, 87.6-100\% respectively.

Genotype analysis. Of the 54 Chinese PCV2 sequences in this study, 42 sequences belonged to genotype PCV-2d, 10 sequences were cluster in genotype PCV-2b, and 2 sequences were classified into genotype PCV-2e, indicating that genotype PCV-2d has become a dominating in China in 2008-2009.

\section{Discussion}

PCV-2d was firstly determined in China. This genotype of PCV2 has not been documented in other countries (4). ORF2 gene clustered in genotype PCV-2d encodes 234 amino acids, and the remaining genotypes encode 233 amino acids. Since the last codon of ORF2 gene (TGG) mutated to AAG, this resulted in the change of the reading frame and the addition of a lysine. In addition, the amino acid variations of ORF2 were found at three positions 89 (I/R to L), 90 (S to T) and 169 (S to R), which were unique for genotype PCV-2d. In our study, 42 sequences belonged to genotype PCV-2d, suggesting that genotype PCV$2 \mathrm{~d}$ became dominating in China in recent two years. Therefore, much attention should be paid to the pathogenicity of this genotype to pigs.

\section{References}

1. Chae, C.et al., (2004). Vet. J. 168, 41-49.

2. Mankertz, A., (2000). Virus Res. 66, 65-77.

3. Segales, J. et al ., (2008). Vet. Rec. 162, 867-868.

4. Wang, F. et al., (2009).Virus Res 145, 151-156. 


\title{
P.017
}

\section{Evaluation of a SYBR green real-time polymerase chain reaction assay for the quantification of porcine circovirus $\mathbf{2}$ genotype $b$ in serum}

\author{
Kwang Soo Lyoo; Seung W. Kang; Han Soo Joo \\ University of Minnesota, St. Paul, MN, USA
}

\section{Introduction}

Porcine circovirus 2 (PCV2) is identified as the major cause for post-weaning multi-systemic wasting syndrome (PMWS). The virus has now been classified into 2 genotypes of PCV2a and PCV2b. PCV2b genotype has been more frequently isolated from pigs with clinical PMWS1,2,3,4. Therefore, PCV2 $b$ has been suggested to be more pathogenic, although it is still debatable. The PCV2 genomic quantification test has become useful in the diagnosis of PMWS, because quantity of the viral genome in tissue samples from the affected pigs has been highly associated with the severity of PMWS. In this study, a SYBR green real-time PCR assay specific for PCV2b genotype was developed, and the results were compared to those by a nested PCR (nPCR). Although a PCV2 SYBR green real-time PCR assay was reported recently ${ }^{5}$, the assay was not aimed at differentiating between the two genotypes of PCV2.

\section{Materials and Methods}

Test sera were collected from 120 pigs in a Minnesota farm during severe clinical PMWS. A nPCR assay was conducted by the method as described previously ${ }^{4}$. For a PCV2b specific real-time PCR, the DNA fragment of 361 base-pair on ORF 2 was subcloned into PGEM-T Easy vector, and the plasmid was transformed in to JM109 cells. The copy number/ $\mu$ l was determined by the serially diluted plasmid. The real-time PCR assay was carried out using PerfeCTa SYBR Green SuperMix in a thermal cycler Mx3005P (Stratagene). All real-time PCR reactions were performed in triplicates in neighboring wells.

\section{Results}

The recombinant plasmid was constructed and the amount of input template copy numbers was calculated. The over range from $4.3 \times 10^{1}$ to $4.3 \times 10^{9}$ copies was obtained from the linear standard curve, which resulted in cycle threshold $(\mathrm{Ct})$ values from 38.5 to 10.8 cycles and on the slope value of $-3.49\left(R^{2}\right)$. The dilution factor from the original serum sample to real-time $P C R$ reaction was taken into consideration when PCV2b copy numbers were calculating. In a comparison between quantitative real-time PCR ( $q P C R$ ) and $n P C R$, the real-time PCR was found to be 1,000 times more sensitive than the nPCR assay. A com- parative result between the percentage of PCV2 viremic pigs by $\mathrm{nPCR}$ or $\mathrm{qPCR}$, and the mean genomic copy number of PCV2b by qPCR using the same samples is shown in Table 1. Of the 120 sera, 79 (65.8\%) and 13 (10.8\%) were positive by the nPCR for PCV2b and PCV2a genotype, respectively, whereas 98 (82\%) sera were detected as PCV2b positive by qPCR assay. The copy number of PCV2b DNA ranged $3.66-10.01 \log 10 \mathrm{in} 1 \mathrm{ml}$ of the serum samples.

Table 1. Detection of PCV2 genotypes by $n P C R$ or $q P C R$ from sera of farm during clinical outbreaks of PMWS

\begin{tabular}{|l|c|c|c|c|}
\hline & \multicolumn{2}{|c|}{ Age of pigs (wks) } & \multicolumn{2}{c|}{ Total } \\
\hline & $\mathbf{5 - 9}$ & $\mathbf{1 1 - 1 5}$ & Pos (\%) & Neg (\%) \\
\hline nPCR-PCV2a & 4 & 9 & $13(11)$ & $107(89)$ \\
\hline nPCR-PCV2b & 27 & 43 & $79(66)$ & $40(33)$ \\
\hline qPCR-PCV2b & 35 & 49 & $98(82)$ & $22(18)$ \\
\hline Mean DNA copy number $(\log 10)$ & 4.6 & 4.5 & \multicolumn{3}{|c}{ Total =120 } \\
\hline
\end{tabular}

\section{Discussion}

Higher prevalence of PCV2b genotype in association with clinical PMWS initially led us to develop a quantitative real-time PCR assay specific for PCV2b genotype. Most published realtime PCR assays for PCV2 infection were aimed at quantification of PCV2 without differentiation between the two genotypes. Development of a multiplex real-time PCR assay was reported to differentiate the two genotypes. However, the present SYBR green real-time PCR was found to be simpler to perform and less expensive when compared to the probe type in the multiplex PCR. In conclusion, the present assay could be highly valuable for PMWS diagnosis, especially if PCV2b genotype is more important factor for clinical PMWS.

\section{References}

1. Dupont K et al 2008. 128: 56-64

2. Gagnon CA et al 2007. Can Vet J $48: 811-819$

3. Grau-Roma L et al 2008. Vet Microbiol 128:23-35

4. Lyoo KS et al 2008. J Vet Diagn Invest $20: 283-288$

5. McIntosch KA et al 2009. Vet Microbiol 133:23-33 


\title{
P.018
}

\section{Epidemiologic observation of PCV2 genotype infection and seroprevalence during and after clinical outbreaks of PMWS in commercial farms}

\author{
Kwang Soo Lyoo; Nam H. So; Han Soo Joo; Peter Davies \\ University of Minnesota, St. Paul, MN, USA
}

\section{Introduction}

Post-weaning multi-systemic wasting syndrome (PMWS) has been an economically important disease in the U.S. since early 2006. Commercial PCV2 vaccines have been widely used on pig farms to prevent the development of PMWS and to reduce the economic losses. It has been suggested that PCV2 viremia and the antibody responses are integral to the development of PMWS in pigs. In order to better understand these relationships, we compared the prevalence of the viremic pigs with PCV2a and $2 \mathrm{~b}$ genotypes and PCV2 antibody titers during (2006) and after (2008) clinical PMWS outbreaks on commercial swine farms.

\section{Materials and Methods}

Five farms in Minnesota were selected based on the clinical histories of severe PMWS in 2006, and absence of clinical signs in 2008 following implementation of PCV2 vaccination. A total of 230 serum samples from pigs between 4 and 16 weeks age were collected from the 5 farms in 2006. In 2008, blood samples were collected from 322 pigs of the similar age groups at the same 5 farms. Differential nested PCR for PCV2 genotypes ${ }^{3}$ was performed to detect viremic pigs. PCV2 antibody titers were tested using both IFA and SN tests. The percentage of PCV2 viremic pigs, mean IFA and SN antibody levels were analyzed by parametric and non-parametric test, respectively, using SPSS software to test the differences during and after the clinical PMWS in the 5 farms.

\section{Results}

Across all 5 farms, the prevalence of PCV2a viremic pigs decreased from $11.1 \%$ in 2006 to $2.5 \%$ in 2008 . Prevalence of PCV2b viremic pigs decreased markedly from 66.6\% in 2006 to $11.0 \%$ in 2008 . Mean viremic pigs for both genotypes were significantly $(\mathrm{P}<0.001)$ lower in 2008 when compared to those in 2006. The mean IFA titer of the grow-finish pigs was significantly $(P<0.001)$ lower in $2008(7.6 \log 2)$ than that in $2006(10.7$ log2) (Fig. 1). The mean SN titers for the nursery and grow-finish phases in 2008 were significantly higher than those in 2006 $(\mathrm{P}<0.001$ and $\mathrm{P}<0.005$, respectively) (Fig. 2).
Fig. 1. Comparison of mean PCV2 IFA titers

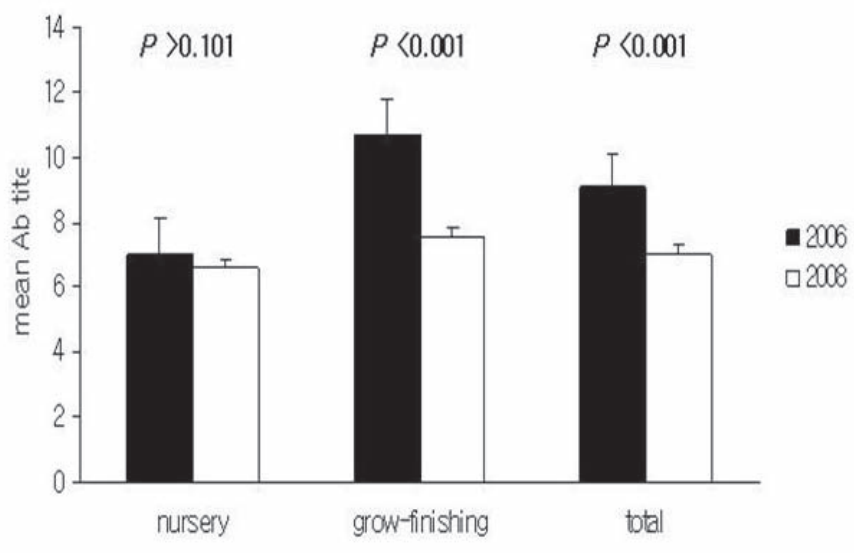

Fig. 2. Comparison of mean PCV2 SN antibody titers

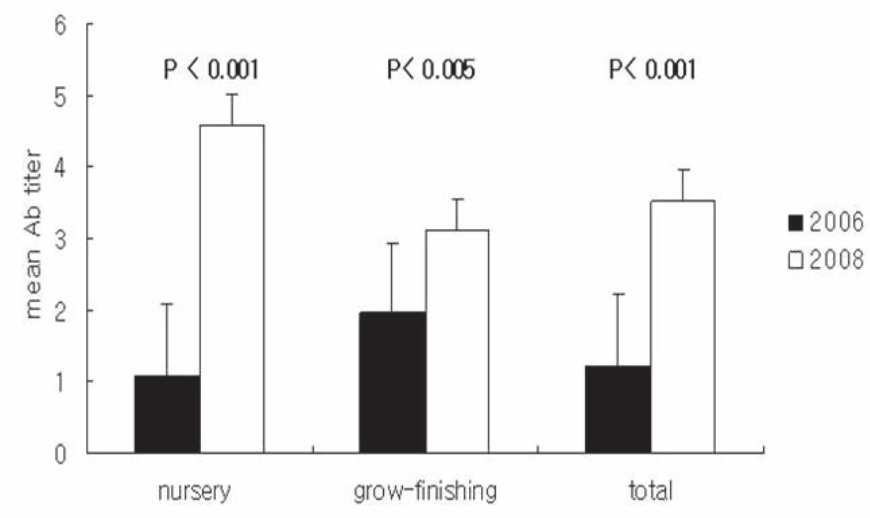

\section{Discussion}

Both the prevalence and level of viremia were significantly higher during clinical outbreaks in 2006 than following vaccination in 2008. The changes were more marked for PCV2b than PCV2a genotype supporting a greater role of PCV2b in the clinical outbreaks. This observation was similar to the previous findings by others ${ }^{1,2}$. The IFA antibody titers were higher during clinical PMWS in 2006 than in 2008 indicating evidence of PCV2 infection. In contrast, SN antibody titers were higher in 2008 in the absence of clinical disease suggesting a likely protection effect against clinical PMWS. The higher SN antibody levels in the nursery pigs could be due to high maternal antibody and/or recent $P C V 2$ vaccination.

\section{References}

1. Dupont K et al 2008, Vet Microb 128: 56-64

2. Gagnon CA et al 2007 , Can Vet J $48: 811-819$

3. Lyoo KS et al 2008, J Vet Diagn Invest 20: 283-288 


\title{
P.019
}

\section{Porcine Circovirus Type 2 (PCV2) In Utero Seroconversion, Early Viremia, And Serological Profile In Naturally Infected Piglets}

\author{
Priscilla Gerber; Flávia Garrôcho; Zélia Lobato \\ Universidade Federal de Minas Gerais, Belo Horizonte, MG, Brazil
}

\section{Introduction}

Reproductive disorders associated with PCV2 are characterized by abortion, stillbirth and mummified foetuses, with symptoms varying according to gestational age at the time of infection. Although PCV2 in utero infection was well characterized, little is known about its occurrence in naturally infected pigs and its consequence the onset of porcine circovirus diseases (PCVD) in the postnatal period.

The aims of this study were to describe PCV2 peripartum infection and PCV2 serological profile in naturally infected piglets.

\section{Materials and Methods}

Twenty PCV2 sows vaccinated with CIRCOVAC (Merial) were studied. One hundred piglets (5 pigs/sow) were ear-tagged at birth. Colostrum, serum and piglets' presuckle serum were collected at the day of parturition (DP). Milk samples were collected at day post-partum (DPP) 20. Serum samples were taken and piglets were weighed at DPP 1, 20, 42, 63, 84.

Colostrum and milk were evaluated for infectious PCV2[1], total (TA) and neutralising (NA) antibodies[2]. Serum samples were evaluated for PCV2 DNA[4], TA, NA, IgA and IgM anti-PCV2[3].

Non-normally distributed variables were analysed using the Mann-Whitney tests. Differences were considered significant when $\mathrm{P}<0.05$.

\section{Results}

Sows had high levels of TA and NA in serum (11.63 $\pm 1.32 \log 2$; $9.51 \pm 1.01 \log 2)$ and colostrum (12.32 $\pm 0.00 \log 2)$. However, 11 out of 20 and 5 out of 20 colostrum and milk samples had infectious PCV2 (1.96 $\pm 1.02 \log 10$ TCID50/50 $\mu$ land 00.30 $\pm 0.58 \log 10$

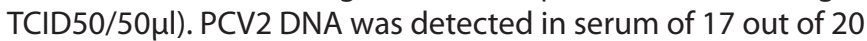
sows (2.02 \pm 0.89 PCV2 log10 copies/ml of serum) in DP.

Nine piglets were born with low levels of anti-PCV2 antibodies, ranging from 4.32 to 8.32 log2 TA titre. In DPP1, PCV2 DNA was detected in 29 piglets'serum samples, ranging from 2.00 to 3.97 PCV2 log 10 copies/ml of serum. There was no difference between the weight of viremic and non-viremic piglets (Table 1). Serological profile of TA, NA and IgM anti-PCV2 are shown in Fig. 1. IgM anti-PCV2 levels started to increase at DPP42, followed to seroconversion to TA and NA and decline of IgM levels at DPP84.

Table 1. Weight of piglets viremic and non-viremic at DPP1

\begin{tabular}{|c|c|c|c|c|}
\hline \multirow{2}{*}{ Group } & \multicolumn{4}{|c|}{ 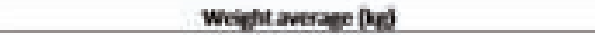 } \\
\hline & 21 & 42 & 63 & 84 \\
\hline Virentic (n 20$)$ & $584+1,54$ & 10,050201 & $2,31: 5,27$ & 372a inas \\
\hline Won viramk (n-71) & $691+1,10$ & $10,61+1,5$ & $23,46 \cdot 4,75$ & $77,69+791$ \\
\hline
\end{tabular}

Figure 1. Serological profile of TA, NA and IgM anti-PCV2 titres from DPP 0 to 84

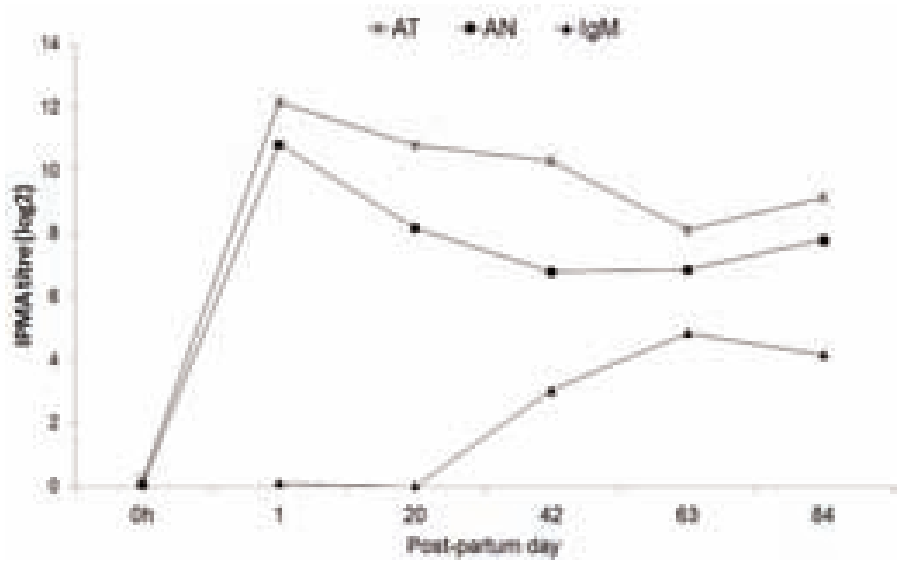

\section{Discussion and Conclusions}

Piglets were born with anti-PCV2 antibodies, indicating PCV2 in utero transmission after the period of immunocompetence. This result suggests occurrence of vertical transmission even in farms where sows have high titres of anti-PCV2 antibodies and in absence of reproductive failure associated to PCV2 (data not shown).

Vaccination of sows could not prevent PCV2 shedding into colostrum and sow viremia at DP even in the presence of high levels of PCV2 NA. PCV2 in utero transmission or early infection resulted in viremia in the first day of life of piglets. Even with the early PCV2 infection, piglets seropositive at birth and viremic at DPP1 had similar weight to non-viremic and seronegative piglets, maybe due to low PCV2 DNA levels found, and the absence of PCVD clinical signs.

Regardless of piglets' early viremia, PCV2 IgM raised only at DPP42, suggesting that PCV2 was able to stimulate the immune system only after the passive antibodies decay at subprotetor levels (Fig. 1). Seroconversion to PCV2 TA and NA at DPP84 coincides with the decline of IgM anti-PCV2 levels, apparently controlling PCV2 infection.

In conclusion, vertical transmission of PCV2 may occur generating viable piglets at birth without losses in growth rate. Sows vaccination may not prevent PCV2 viremia at parturition and virus shedding into colostrum.

\footnotetext{
References

1. Farnham, MW, et al. 2003. Can J Vet Res, 67(2):108-113.

2. Fort, $M$, et al. 2008. Vaccine 26, 1063-1071.

3. Meerts, P., et al. 2006. BMC Vet Res, v.2,6 p. 1-11.

4. Mclntosh, KA. et al. 2009. Vet Microbiol, v.133, p.23-33.
} 


\title{
Naturally porcine circovirus type 2 (PCV2) exposed pigs generate a specific antibody response able to neutralise different PCV2 isolates of different genotypes and geographic origins
}

\author{
Sherry Kurtz; Martí Cortey; Maria Fort; Marina Sibila; Joaquim Segales \\ Centre de Recerca en Sanitat Anima (CReSA), Bellaterra, Spain
}

\section{Introduction}

Porcine circovirus type 2 (PCV2) vaccines are able to induce a strong humoral immune response. Their neutralizing antibody response is apparently cross-protective against both predominant PCV2 genotypes (PCV2a and PCV2b) (2). Also, PCV2a infection protects against a subsequent PCV2b challenge, presumably through cross-neutralizing antibodies (NA) (5). It is also known that there are several epitopes (detected by monoclonal antibodies) in the PCV2 Cap protein that are shared between different genotypes, as well as PCV1 $(4,6)$, which represents a putative basis for cross-neutralization. However, there were several strains for which there was little or no neutralization using the same antibody panel. This suggests the existence of antigenic variability between PCV2 genotypes. However, these studies describing antigenic variation have been done using monoclonal antibodies, which are specific for unique epitopes. Therefore, the objective of the present study was to further insight on PCV2 antibody neutralization by using a pig sera obtained from field studies to assess the level of antigenic variation by cross-neutralization amongst different isolates of PCV2. Viral isolates used were from different geographical regions.

\section{Materials and Methods}

Serum samples. A total of 82 sera were used for the present study. A first group of animals corresponded to PMWS affected pigs $(n=20)$, which where found to be infected by PCV2b strains (3). A second group was composed of age-matched healthy pigs $(n=17)$ from the same farms, and a proportion of them also infected with PCV2b strains. Finally, in order to get sera samples from animals that would have been exposed to PCV2 in the past, randomly sampled slaughter age pigs $(n=45)$ were taken.

PCV2 neutralization test. Neutralization assays were carried out as described (1) using two PCV2a (Burgos and Stoon-1010) and two PCV2b (MO/S-06 and Sp-10-7-54-13) isolates. Burgos and Sp-10-7-54-13 strains were isolated from postweaning multisystemic wasting syndrome (PMWS) affected pigs in Spain, while Stoon-1010 and MO/S-06 were isolated from PMWS in Canada and United States, respectively.

Total PCV2 antibody test. An immunoperoxidase monolayer assay (IPMA) technique to detect antibodies to PCV2 (1) was also performed with all available sera and compared to the different neutralization values obtained.

\section{Results}

Most of the sera were able to neutralise the four PCV2 strains used in the test, even they differed in their antibody titre. Globally, PCV2a genotype strains used in the test originated significantly higher neutralising antibody titres when compared to those against the PCV2b genotype. No differences in NA titres were observed between PCV2 strains within the same genotype. In addition, NA titres detected in healthy and slaughterhouse animals were significantly higher compared to PMWS affected pigs. No statistical significant differences were detected in the neutralizing activity of sera obtained from either healthy, PMWS, or slaughterhouse pigs when tested to PCV2 strains of different geographical origin. Finally, there was no direct correlation between IPMA and neutralizing titres detected in any of the animal categories.

\section{Discussion}

The present study sought to examine the role of antigenic variability in the context of a natural infection. Pig sera of different animal categories were able to neutralize PCV2 strains from different genotypes and geographical regions. This point seems to be extremely important, since would explain the field evidence that PCV2 vaccines available in the market (all based on PCV2a strains) show efficacy in controlling PMWS independently of the causing genotype (2). However, the differences observed in the NA titres of the tested sera when either PCV2a or PCV2b strains were used as antigen in the test suggest the existence of antigenic variablility among those strains.

\section{Acknowledgements}

This work was funded by Projects No. 513928 from the Sixth Framework Programme (EU Commission), and Consolider Ingenio 2010 - PORCIVIR and TRT-2006/0035/C02/00 (Spain). Martí Cortey holds a Beatriu de Pinós Postdoctoral Grant (Government of Catalonia).
References
1. Fort et al. 2007. Vet Microbiol 125:244-55.
2. Fort et al. 2008. Vaccine 26:1063-71.
3. Grau-Roma et al. 2008. Vet Microbiol 128:23-35.
4. Lefebvre et al. 2008. J Gen Virol 89:177-87.
5. Opriessnig et al. 2008. J Gen Virol. 89:2482-91.
6. Shang et al. 2009. Mol Immunol. 2009 46:327-34. 
P.021

\section{Infectious risk factors for postweaning multisystemic wasting syndrome (PMWS) development}

Llorenç Grau-Roma ${ }^{1}$ Charlotte S. Kristensen ${ }^{2}$ Anders Stockmarr ${ }^{3}$ Claes Enoe $^{3}$ Sergio Lopez-Soria ${ }^{1}$ Miquel Nofrarias ${ }^{1}$ Vivi Bille-Hansen

${ }^{3}$ Charlotte K. Hjulsager ${ }^{3}$ Marina Sibila ${ }^{1}$ Sven E. Jorsal ${ }^{3}$ Lorenzo Fraile $^{1}$ Poul Baekbo ${ }^{2}$ Hakan Vigre ${ }^{3}$ Joaquim Segales $^{1}$ Lars E. Larsen $^{3}$

1. Centre de Recerca en Sanitat Anima (CReSA), Bellaterra, Spain; 2. Pig Research Centre, Danish Agriculture \& Food Council, Kjellerup, Denmark; 3. National Veterinary Institute, Technical University of Denmark, Copenhagen, Denmark

\section{Introduction}

Porcine circovirus type 2 (PCV2) is considered to be essential but not sufficient for postweaning multisystemic wasting syndrome (PMWS) development. Thus, several co-infections have been suggested as triggers for disease development in the field (1). The aim of the present study was to gain further insights on infectious agents as potential risk factors for PMWS development.

\section{Materials and Methods}

Two longitudinal case-control studies in 13 PMWS-affected farms from Spain and Denmark were performed as previously described (2). Blood samples from pigs (approximately 100 per batch coming from 10 sows) were longitudinally collected throughout their productive life. Pigs showing PMWS-compatible clinical signs were bled and euthanized together with healthy aged-matched pigs (ratio 2:1). PMWS was diagnosed and pigs were classified into three categories: i) PMWS cases; ii) wasted non-PMWS cases; iii) healthy pigs (2).

Serological analyses were performed in all collected serum samples to detect antibodies against Porcine reproductive and respiratory syndrome virus (PRRSV), Porcine parvovirus (PPV), Swine influenza virus (SIV) and Lawsonia intracellularis (Law) in both countries. Moreover, in Spain, antibodies against Mycoplasma hyopneumoniae, Aujeszky's disease virus and Salmonella spp. were also assessed.

A Cox proportional hazards model was used to investigate the simultaneous effects of seroconversion and maternal immunity against the studied pathogens in each country. Data were analysed within a survival analysis framework, where development of PMWS was considered as death/failure. All analyses were carried out using the Splus ${ }^{\circledR}$ software package, version 6.1.

\section{Results}

The number of studied pigs and their pathological classification is displayed in table 1.

Results from survival analysis showed that, for the Danish dataset, seroconversion against Law and PPV had a protecting effect. For animals that seroconverted against Law, the protective effect increased with the level of maternal immunity against this pathogen. Maternal immunity against PPV gave a protective effect in Spanish dataset. Moreover, high levels of maternal immunity against PCV2 had a protecting effect in both countries.
Table 1. Number of studied pigs within each pathological category.

\begin{tabular}{|l|c|c|c|c|}
\hline & PMWS & Healthy & Wasted non-PMWS & Total \\
\hline Spain & 35 & 29 & 43 & 107 \\
\hline Denmark & 55 & 31 & 47 & 133 \\
\hline
\end{tabular}

\section{Discussion}

Maternal PCV2 antibodies in both Danish and Spanish data showed a protecting effect against PMWS development, which is in agreement with previous studies (3). This situation would support the interest of PCV2 vaccination in sows in order to transfer a higher load of PCV2 antibodies to the offspring.

The protective effect of maternal immunity against PPV is in agreement with previous works suggesting PPV as a co-infectious trigger (4). Besides, the fact that seroconversion against PPV and Law resulted protective for PMWS development could seem contradictory. However, the present analysis was based on antibody levels, and not on direct evidences of pathogen infection. Since it is known that PMWS affected pigs are immunocompromised (5), seroconversion may indicate a well-developed immune system, which in turn will make the animal less likely to develop PMWS.

\section{Acknowledgements}

The authors acknowledge the financial support from the European Commission (project No. 513928).

\section{References}

1. Grau-Roma et al., 2010. Vet. J. in press.

2. Grau-Roma et al., 2009. Vet. Micorbiol. 135:272-82.

3. Calsamiglia et al., 2007. Res. Vet. Sci. 82, 299-304.

4. Allan et al., 1999. J. Comp. Pathol. 121, 1-11

5. Darwich et al., 2004. Arch Virol 149, 857-874. 


\title{
P.022
}

\section{Theoretical and experimental approaches to estimate the usefulness of pooled serum samples for the diagnosis of postweaning multisystemic wasting syndrome (PMWS)}

\author{
Martí Cortey; Sebastian Napp; Anna Alba; Emanuela Pileri; Llorenç Grau-Roma; Marina Sibila; Joaquim Segales \\ Centre de Recerca en Sanitat Anima (CReSA), Bellaterra, Spain
}

\section{Introduction}

Traditional postweaning multisystemic wasting syndrome (PMWS) diagnosis requires the necropsy of suspicious animals and the access to histopathological expertise (1). One of the major differences between PMWS-affected and non-affected pigs is porcine circovirus type 2 (PCV2) load in serum and tissues $(2,3,4)$. Consequently, real-time quantitative PCR (qPCR) has been proposed as a potential diagnostic alternative. However, sensitivity and/or specificity of qPCR on individual basis are relatively poor (5). The main objective of the present study was to analyze the applicability of qPCR to detect PCV2 loads in pooled serum samples, with the purpose of increasing the diagnostic capacity of the qPCR technique. A second objective was to develop an easy-to-use PMWS qPCR-based diagnostic recommendation at the herd level, without the need to perform post-mortem examinations.

\section{Material and Methods}

Firstly, a theoretical model based on two qPCR viral load thresholds $(3,4)$ to discriminate between PMWS affected and non-affected pigs was validated with an experimental pooling study considering pools of 3, 5, 10 and 20 randomly selected individuals. Secondly, pool sensitivities (PSe) and pool specificities (PSp) were estimated by means of Monte Carlo simulations. Such pooling included sera from diseased pigs with a PMWS diagnosis (PMWS), diseased pigs without fulfilling the diagnosis of PMWS (wasted-non-PMWS) and healthy (healthy) animals. Using only the PMWS diagnosed individuals and the wastednon-PMWS, a second pooling approach considering targeted sampling (animals showing clinical signs compatible with PMWS) was simulated as an option to increase sensitivity at herd level. If targeted sampling is used, the probability of detecting the disease would be theoretically increased. The PSe and PSp obtained previously were used to estimate the sensitivities and specificities at herd level (HSe and HSp) for both approaches. Finally, we evaluated the capability of the model to discriminate between PMWS-affected and non-affected farms using qPCR real and simulated pools.

\section{Results}

Several pools of 20 individuals did not report correct results in the experimental study, most probably caused by handling difficulties. Therefore, Monte Carlo simulations considered only pools of 3, 5 and 10 individuals. The best HSe and HSp estimations were obtained using a single pool of 10 individuals or two pools of 5 individuals under the random sampling scenario. Targeted sampling reported worst estimations than random sampling, mostly because wasted-non-PMWS individuals showed higher qPCR values than healthy ones, making in this case difficult the differentiation of truly affected and non-affected individuals. However, the model correctly discriminated and identified most of the PMWS-affected and PMWS-non-affected farms despite some false-positives and false-negatives were reported.

\section{Discussion}

Assuming that the qPCR data used $(3,4)$ are representative of the field situation, present results suggest that sample pooling may be a suitable strategy for PMWS diagnosis. Sera pooling offers several advantages compared with classical PMWS diagnostic methods. First, it represents a cost saving strategy, since more individuals can be analyzed using the same fixed analysis expenses. Second, the use of qPCR is performed in an easy-toget sample and, therefore, it does not require histopathological expertise, indispensable with a necropsy based method.

\section{Acknowledgements}

This work was funded by the Projects No. 513928 from the Sixth Framework Programme of the European Commission, GEN200320658-C05-02 (Spanish Government) and Consolider Ingenio 2010 - PORCIVIR (Spanish Government). Martí Cortey holds a Beatriu de Pinós Postdoctoral Research Grant funded by the Government of Catalonia.

\section{References}

1. Segalés et al. 2005. Anim Health Res Rev 6, 119-142.

2. Brunborg et al. 2004. J. Virol Methods 122, 171-178.

3. Grau-Roma et al. 2009. Vet Microbiol 135, 282-292.

4. Olvera et al. 2004. J Virol Methods 117, 75-80.

5. Bustin SA. 2002. J Mol Endocrinol 29, 23-39. 


\title{
P.023
}

\section{Results of one-shot Circovac ${ }^{\circ}$ vaccination at $0.5 \mathrm{ml}$ in piglets on weight and mortality parameters}

\author{
Bernhard Heissenberger; Andrea Ladinig; Christiane Lang; Christiane Püschel; Mathias Ritzmann \\ Clinic for Swine, Vienna, Austria
}

\section{Introduction}

Since the first report in 1996 (1) porcine circovirus type 2 (PCV2) diseases had a major impact on the swine industry worldwide. Since June 2007 Circovac $^{\oplus}$ (Merial, Lyon, France), an inactivated PCV2 vaccine for swine is centrally registered in Europe for protection of the offspring of vaccinated sows and gilts by passive immunization (2).

The objective of this field study was to evaluate the efficacy of Circovac ${ }^{\circ}$ administered once to three week old piglets at a dosage of $05 \mathrm{ml}$ intra-muscularly (i.m.).

\section{Materials and Methods}

The study was randomised, controlled, blinded and conducted on an Austrian 250-sow farrow-to-finish farm with severe problems especially during the fattening period caused by PCV2. In total 1105 piglets were included in this field trial. They were randomly allocated to the vaccination group, which received 0.5 $\mathrm{ml} \mathrm{Circovac}^{\oplus}$ i.m. at weaning, or to the placebo treated control group. Control and vaccinated piglets stayed intermingled. The body weight was determined at birth, weaning ( $3^{\text {rd }}$ week), the end of nursery (13 $3^{\text {th }}$ week), in the middle of the fattening period $\left(21^{\text {st }}\right.$ week) and at the end of the fattening period ( $28^{\text {th }}$ week). Wasting animals were defined as animals whose body weight was at least $25 \%$ lower than the average of the respective group. All animals were monitored for clinical symptoms and mortality was recorded during the whole study period.

\section{Results}

An important parameter of this study was the evaluation of average daily weight gain. A significant difference of the daily weight gain between both groups occurred during the entire fattening period in favor of the Circovac ${ }^{\circ}$ vaccinated group $(+41.43 \mathrm{~g} / \mathrm{d}$; $p<0.0001)$. The main viremia period on this farm occurred during the first half of fattening (from $13^{\text {th }}$ to $21^{\text {st }}$ week), when the difference in ADWG was most obvious (+64.83 g/day, $p<0.0001$ ), which is shown in figure 1. The number of wasting animals was reduced by $5.15 \%(p<0.044)$ in the Circovac vaccinated group during the first half of fattening $\left(13^{\text {th }}-21^{\text {st }}\right.$ week), the period of main viremia. The mortality rate during the fattening period was significantly reduced $(-7.4 \%)$ in the vaccinated group $(p<0.0001)$. As expected the difference in mortality was highest during the main viremia period. (Figure 2).
Figure 1: ADWG $13^{\text {th }}-21^{\text {st }}$ and $13^{\text {th }}-28^{\text {th }}$ week of life

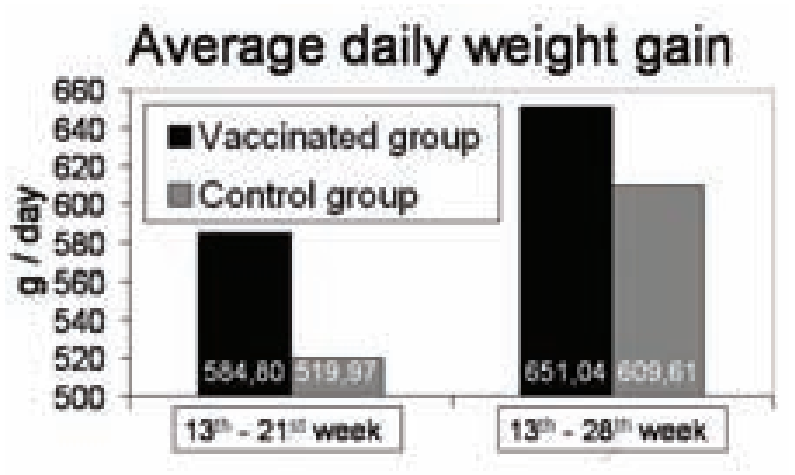

Figure 2: Mortality rate according to the main viremia period
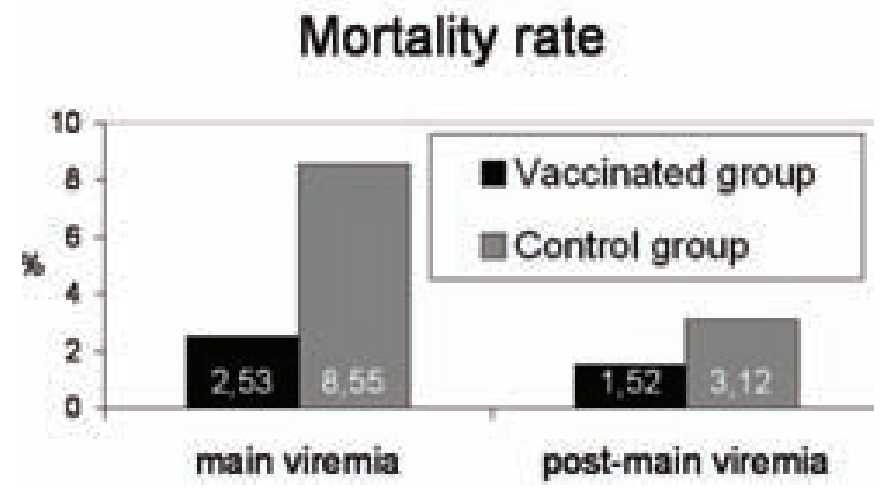

\section{Discussion}

Compared to a placebo treated control group, Circovac vaccinated animals showed significant improvements. Vaccination resulted in reduction of mortality rate, increase of daily weight gain and reduction of wasting animals during the course of the study and particularly during the main viremia period.

\section{References}

1. Harding, J. C. (1996): Proc. Western Canadian Assoc. Sw. Pract., 21.

2. Joisel, F. et al. (2007): Proc. $5^{\text {th }}$ Intern. Symp. Emerging and Re-emerging Pig Diseases, Krakow, Poland, 127. 


\title{
P.024
}

\section{Analysis of occurrence of porcine circovirus type 2 (PCV2) in PMWS-like pigs from farms in Slovak Republic}

\author{
Juraj Pistl; Tomas Csank; Michaela Vlasakova; Jana Pollakova; Anna Jackova; Stefan Vilček \\ University of Veterinary Medicine and Pharmacy, Košice, Slovak Republic
}

\section{Introduction}

Circoviruses are small DNA viruses, which infect number of animal species. The most important circovirus in swine is porcine circovirus type 2 (PCV2), whereas, the most frequent clinical form of circovirus infection is the post-weaning multisystemic wasting syndrome (PMWS). The disease has been reported in most of the European countries (Segalés, 2007). Hitherto, the epidemiological situation of PMWS in Slovakia was not well understood and the country held unknown PMWS status (Segalés, 2007). Any official data about PCV2 detection and surveillance were missing. On this background, the research was focused on the detection and isolation of PCV2 from pigs with PMWS-like symptoms based on generally accepted scientific criteria (biological and moleculo-genetic methods), and prevalence of specific antibodies.

\section{Materials and Methods}

86 post-weaning (42-50 days old, body weight 7.2-9.4 kg) Landrace and Slovak Large White pigs suffering from PMWS-like clinical symptoms were selected from twelve pig farms from different regions of Slovakia. More than $40 \%$ morbidity and up to $15 \%$ mortality in weanlings was observed in the farms with wasting and respiratory disorders. Immunohistochemistry allowed us to prove PCV2 in cryosections of lymph nodes. Immunoperoxidase test (IPMA) was used to confirm PCV2 in cell culture PK-15 (Sanchez et al., 2001). A 263bp fragment from the ORF2 genome region was amplified using primers CF8 and CR8 (LaRochelle et al., 1999). Nucleotide sequences of PCV2 amplified fragments were analyzed by BLASTn analysis to find out similar sequences in GenBank. IgM and IgG specific PCV2 antibodies were detected by ELISA (Ingezin circovirus lgM/lgG, Ingenasa, Spain).

\section{Results}

All investigated pigs were clinically characterized by severe growth retardation, emaciation and marked spine. 52 weanling pigs (60.5\%) were PCV2 positive. Massive occurrence of PCV2 infected cells was particularly seen in inguinal lymph nodes. PCV2 virus positive macrophages were located mainly in medullar cords along with paratrabecular sinuses of lymph nodes. The similarity search in BLASTn showed $100 \%$ sequence matches with Austrian AUT5 PCV2 strain (Accession number AY424405.1) and some Chinese strains as well. Based on the ratio of positivity/negativity, the coefficient of infection rate in individual farm was calculated. In four farms from twelve the coefficient of infection rate was 1.0, which indicates high prevalence of PCV2. In other farms coefficient was between $0.40-0.71$, which indices actual risk of PMWS. A zero coefficient was present in only one farm. These results show high prevalence of PCV2 with occurrence of PMWS in pig farms of Slovakia. Prevalence of specific PCV2 antibodies in animals tested was 50.5\%. In 51.5\% of samples both lgM+lgG antibodies were detected. $13.2 \%$ of samples showed presence of IgM, while $35.3 \%$ samples had IgG antibodies.

\section{Discussion}

In the Central Europe the diagnosis of PMWS and detection of PCV2 was reported first time in Hungary (Kiss et al., 2000). Later the disease was reported in Austria (Schmoll et al, 2002), Czech Republic (Celer jr and Carasová, 2002) and Poland (Stadejek et al., 2006). The first detection and isolation of PCV2 from PMWS animals in Slovakia based on the generally accepted diagnostic criteria (Sorden, 2000) was published in 2009 (Pistl et al., 2009).

Present study was focused to provide data on the evidence of PCV2 in PMWS-like pigs from other Central European country - Slovakia. Our work demonstrates the evidence of PCV2 in the PMWS-like affected pigs, determined on the basis of highly suggestive clinical and pathological symptoms, antigenic and genetic methods that clear the epidemiological situation of PCV2 in the pig farms of Slovakia.

\section{Acknowledgements}

This work was supported by the project INFEKTZOON - Center of excellence for infections of animals and zoonoses No. 26220120002, financed by European fund of regional development.

\section{References}

Celer V. jr., Carasová P.: J. Vet. Med. B 49, 2002.

LaRochelle R. et al.: J. Virol. Methods 80, 1999.

Kiss I. et al.: Acta Vet. Hung. 48, 2000.

Pistl J. et al: Dtsch. Tierärztl. Wochenschr., 116, 2009.

Sanchez R.E. jr. et al.: Vet. Microbiol. 83, 2001.

Schmoll F. et al: Wien. Tierärztl. Monatschr., 89, 2002.

Segalés J.: Advances in Pork Prod. 18, 2007.

Sorden S.D.: J. Swine Health Prod. 8, 2000.

Stadejek T. et al.: Med. Weterynaryjna 62, 2006. 


\title{
P.025
}

\section{Presence of viral pathogens in cases of porcine respiratory diseases complex (PRDC) in central Italy}

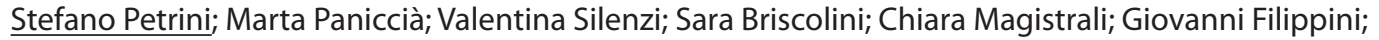 \\ Marco Sensi; Stefano Gavaudan; Francesca Ciuti; Manfredo Fortunati; Giovanni Pezzotti \\ Istituto Zooprofilattico Sperimentale dell'Umbria e delle Marche, Perugia, Italy
}

\section{Introduction}

Porcine respiratory disease complex (PRDC) in pigs is one of the most relevant health concern for swine production today. As with respiratory disease in humans and other species, this complex in swine is often the results of the combined action of primary and opportunistic infectious agents. Primary agents in pigs include viral agents (1-2-3), like European type of Porcine Reproductive and Respiratory Syndrome Virus (PRRSV-EU), Porcine Circovirus type 2 (PCV2), Swine Influenza Virus (SIV) and Suid herpesvirus type 1 (SuHV-1). The objective of the present work was investigate the presence of these major viral infection agents in cases of PRDC in central Italy (Umbria and Marche Regions).

\section{Materials and Methods}

A total of 527 pigs with PRDC were observed between years 2007 and 2009, originating from 58 herds were no vaccination against the above mentioned viral agents was adopted. There was no epidemiological relation between these herds; however, a common clinical history was reported, with animals showing severe symptoms of respiratory disorder, high mortality and increased number of culled pigs. Morbidity ranged from $30 \%$ to $70 \%$, with an average mortality of $4 \%$ to $6 \%$. Necropsy was carried out at the Istituto Zooprofilattico Sperimentale dell'Umbria e delle Marche where different organs (lung, kidney, liver, spleen, palatine tonsil, lymph node, small intestine, uterus, ovary, abortion) were collected for histology and virus isolation using NSK cell culture (4). In particular the presence of PRRSV-EU, PCV2, SIV, SuHV-1 was evaluated.

\section{Results}

Most of the animal examined appeared in poor body conditions. Macroscopic lesions were essentially observed in the apical lobes of the lungs, showing pneumonitis, bronchial and bronchiolar necrosis, purulent bronchopneumonia, pulmonary haemorrhages and fibrous/fibrinous pleuritis. In the palatine tonsils petechial haemorrhages were observed while the bronchial lymph nodes appeared enlarged and pale. Histology confirmed the presence of the lesions in the lungs, palatine tonsils and the lymphoid tissues were giant multinucleate cells, peribronchial mononuclear infiltrates, fibrous bronchiolitis, granolumatous inflammation, coagulative necrosis and haemorrhages were found. No significant lesions were observed in other organs. Viral pathogens detected from the samples are summarized in Table 1.
Table 1. Viral pathogen/s detection results in 527 pigs with PRDC lesions

\begin{tabular}{|l|c|c|}
\hline Pathogen/s & No.Positive & \% Positive \\
\hline PRRSV-EU only & 209 & 39,65 \\
\hline PCV2 only & 295 & 55,97 \\
\hline PCV2+PRRSV-EU & 13 & 2,46 \\
\hline SIV & N.I. & N.D. \\
\hline SuHV-1 & N.I. & N.D. \\
\hline N.I., Not isolated; N.D., Not determined \\
\hline
\end{tabular}

\section{Discussion}

The present study has contributed to clarify the role of the major viral agents in the occurrence of PRDC in pigs in the farms of central Italy. In particular, PCV2 was the most frequent agent, found in $55,97 \%$ of the cases followed by PRRSV in 39,65 \% of the animals. Only in few cases $(2,46 \%)$ an association between these two viruses was found. Significant differences have been therefore observed in respect to other geographical areas, such as Spain (5), where PCV2 has been found in lower percentages $(39,2 \%)$ or Korea (3) where, instead a frequent (55\%) co-infection of PCV2 and PRRSV has been described. No positive samples for SIV or SuHV-1 were recorded, demonstrating a minor epidemiological role of these agents in the epidemiology of respiratory disorders in this area. Also in respect to this, different situations have been described in Spain, where SIV occurred in 4,1\% of the cases and SuHV-1 in 1,4\% and in Korea, where 13,3\% of the samples from resulted positive to SIV.

\section{References}

1. Thacker E.L. et al., (2001). Pig J 48: 66-70.

2. Todd D. et al., (2001). Adv Virus Res 87:1-25-30.

3. Kim J. et al., (2003). Vet J 166:251-256.

4. Ferrari M. et al., (2003). J Virol Meth 107:205-212.

5. Grua-Roma L. et al., (2006). Proc IPVS Cong 1, 90. 


\title{
P.026
}

\section{Discovery of antinuclear antibodies in pigs infected with porcine circovirus type 2}

\author{
Marcus E. Kehrli, Jr ${ }^{1}$ Phillip C. Gauger ${ }^{1,2}$ Andrew K. Cheung ${ }^{1}$ Amy L. Vincent ${ }^{1}$ Kelly M. Lager ${ }^{1}$ \\ 1. National Animal Disease Center-USDA-ARS, Ames, IA, USA; 2. Department of Veterinary Diagnostic and \\ Production Animal Medicine, College of Veterinary Medicine, lowa State University, Ames, IA, USA
}

\section{Introduction}

Porcine circovirus type 2 (PCV2) causes post-weaning-multisystemic-wasting-syndrome (PMWS), a swine disease first observed in Canada in 1991. ${ }^{1}$ It is characterized by general wasting, respiratory disease, jaundice and pallor in young pigs resulting in production losses and variable mortality. Since the discovery of PCV2 in 1996, this virus has been linked to other disease syndromes (porcine dermatitis and nephropathy syndrome, reproductive failure, and enteritis) that are collectively known as porcine circovirus associated disease (PCVAD). PCV2 is ubiquitous in swine herds around the world and endemic in most herds. The majority of pigs remain clinically unaffected whereas a small proportion display severe clinical and sometimes fatal disease. We have experimentally reproduced PMWS using germfree piglets. ${ }^{2}$ An unusual feature of disease pathogenesis is a delayed onset of clinical disease following virus challenge and the subsequent rapid decline in the health of diseased piglets. Here we report evidence for development of antinuclear antibodies (ANA) in sera of germ-free pigs infected with PCV2.

\section{Materials and Methods}

A conserved amino acid sequence based on the published capsid protein sequence of PCV2a was used to make a synthetic peptide and to immunize rabbits for PCV2-specific antiserum production.

A series of animal studies were conducted with germ-free piglets derived aseptically from crossbred sows by cesarean section. ${ }^{2}$ Piglets were housed in sterile, stainless-steel pentub isolators (4 pigs/isolator) and fed a commercially-available pasteurized milk diet. Each pig within an isolator received the same treatment, either PCV2 or a sham inoculation at about 7-10 days of age. In comparative viral pathogenesis studies, different groups of pigs were similarly challenged with swine influenza virus.

ANA were measured by two independent methods: 1) a fluorescent microscope slide-based indirect immunofluorescence assay (IFA) (SCIMEDX HEp2 ANA IFA, Denville, NJ) and an ANA ELISA (QUANTA Lite ANA, INOVA Diagnostics, Inc., San Diego, CA) modified by using an anti-swine lgG secondary antibody (KPL, Inc., Gaithersburg, MD).

\section{Results}

To investigate PCV2 replication in tissue culture, a conserved peptide of the PCV2 nucleocapsid was produced to make polyclonal antisera in a rabbit. Initial testing with this antiserum revealed significant background staining of a porcine kidney cell line (PK-15) used to propagate PCV2 in our laboratory. The staining was most prominent in the nuclear region of PK-15 cells and was present regardless of the PCV2 infection status of the cells, suggesting the antisera was recognizing nuclear antigens unrelated to PCV2. This led us to investigate the presence of ANA in piglet sera from prior germ-free isolator studies with PCV2 and other viruses.

Thirty-eight pigs used in 3 separate studies were found to be free of ANA on the day of inoculation with virus. In these studies, 21 of 38 piglets infected with PCV2 isolates developed detectable ANA of the IgG isotype based on the Hep-2 IFA ANA test. Most piglets that developed ANA did so by 18-25 dpi with PCV2. In contrast, 7 piglets infected in 2 separate studies with a swine influenza virus remained free of detectable ANA. To confirm these semiquantitative findings, a subset of samples from one germ-free study were tested by an ELISA that collectively detects total ANA against chromatin (dsDNA and histones), Sm/RNP, SS-A, SS-B, Scl-70, centromere and PCNA, as well as antibodies against cytoplasmic antigens such as Jo-1, mitochondria (M-2) and ribosomal-P protein. ELISA results demonstrated development of increasing levels of ANA over the course of a study in 9 of 12 piglets infected with PCV2 but not in 4 of 4 control virusfree piglets.

\section{Discussion}

Antinuclear antibodies are directed against normal nuclear antigens and are detected in sera of patients with autoimmune disease. Development of ANA in piglets infected with PCV2 preceded onset of clinical disease but not all pigs with ANA died. Finding ANA and a PCV2 capsid peptide that induced antibodies cross-reactive with porcine nuclear antigens suggests an autoimmune component to the pathogenesis of PCV2-induced disease in the germ-free pig model.

\section{References}

1. Clark EG. Post-weaning multisystemic wasting syndrome. Proceedings of the American Association of Swine Practitioners 1997;499501.

2. Gauger P, Lager, KM, Vincent, AL, Opriessnig, T, Kehrli, ME, Cheung, AK: Reproduction of postweaning multisystemic wasting syndrome in germ-free swine with porcine circovirus type 2-group 1 and -group 2 virus. Vet Micro. (Submitted) 


\title{
P.027
}

\section{A study on the presence and DNA loads of porcine circovirus type 2 (PCV2) in feces samples}

\author{
Gerard J. Wellenberg; Frits Bouwkamp \\ Animal Health Service, Deventer, Netherlands
}

\section{Introduction}

Up till now, there is hardly any information on the role of porcine circovirus type 2 (PCV2) in the development of diarrhea (enteritis), or in differences in PCV2 DNA loads in feces collected from pigs with and without enteritis. This study was performed to examine the presence and PCV2 DNA loads in feces from pigs with and without diarrhea (enteritis).

\section{Materials and Methods}

Feces samples from 51 pigs ( $n=46$ farms) with diarrhea (enteritis) and from 11 pigs ( $\mathrm{n}=11$ farms) without diarrhea were examined for PCV2 DNA loads by quantitative PCR. Feces samples from pigs with diarrhea were also analyzed for the presence of Lawsonia intracellularis, Brachyspira hyodysenteriae and $B$. pilosicoli by PCR, E. coli, Salmonella spp by bacterial cultivation, coccidia and/or other parasites by microscopical examinations. The Student t-test and the one-sided Proportion test were used for statistical analyses.

\section{Results}

PCV2 DNA was detected in 23 (45\%) of the 51 feces samples from pigs with diarrhea and in 5 (45\%) of the 11 feces samples from pigs without diarrhea (Table 1).

Table 1: Results PCV2 DNA in feces from pigs with and without diarrhea (enteritis)

\begin{tabular}{|l|c|c|c|}
\hline & Diarrhea (enteritis) & Without diarrhea & P-value \\
\hline PCV2 DNA (n) & $23(45 \%)$ & $5(45 \%)$ & \\
\hline $\begin{array}{l}\text { Mean (log10 PCV2 DNA copies/ } \\
\text { mgr) }\end{array}$ & $4.43\left(\mathrm{se}^{*}: 0.24\right)$ & 3.03 (se: 0.28$)$ & 0.005 \\
\hline PCV2 DNA copies $>10^{4} / \mathrm{mgr}(\mathrm{n})$ & 12 & 0 & 0.037 \\
\hline *: se: standard error of the mean & \multicolumn{3}{|l}{} \\
\hline
\end{tabular}

Twelve feces samples from pigs with diarrhea (enteritis) and none of the feces samples from pigs without diarrhea had PCV2 DNA copy loads $>104 / \mathrm{mgr}(P=0.037)$. Co-infection of PCV2 with L. intracellularis, B hyodysenteriae, pathogenic E.coli, Salmonella spp. and coccidia were found in 13 (25.7\%) of the 51 feces samples from pigs with diarrhea.
Table 2: Results of the 51 feces samples collected from pigs with diarrhea (enteritis)

\begin{tabular}{|l|c|c|}
\hline Pathogens detected & Total & PCV2 DNA \\
\hline & (n) (\%) & copies/mgr $>10^{4}(\mathrm{n})$ \\
\hline No pathogens & $14(27.5 \%)$ & \\
\hline PCV2 & $10(19.6 \%)^{*}$ & 4 \\
\hline PCV2 + L. intracellularis & $5(9.8 \%)$ & 3 \\
\hline PCV2 + B. hyodysenteriae & $1(2.0 \%)$ & 1 \\
\hline PCV2 + L. intracellularis + B. hyodysenteriae & $1(2.0 \%)$ & 1 \\
\hline PCV2 + pathogenic E. coli & $1(2.0 \%)$ & 1 \\
\hline PCV2 + pathogenic E. coli + S. Typhimurium & $2(3.9 \%)$ & 1 \\
\hline PCV2 + pathogenic E. coli + B. hyodysenteriae & $1(2.0 \%)$ & \\
\hline PCV2 + Salmonella serovar B & $1(2.0 \%)$ & \\
\hline PCV2 + coccidia & $1(2.0 \%)$ & \\
\hline Pathogenic E. coli & $2(3.9 \%)$ & \\
\hline Pathogenic E. coli + B. hyodysenteriae & $1(2.0 \%)$ & \\
\hline E. coli + Salmonella serovar B & $1(2.0 \%)$ & \\
\hline L. intracellularis & $6(11.8 \%)$ & \\
\hline L. intracellularis + B. hyodysenteriae & $1(2.0 \%)$ & \\
\hline B. hyodysenteriae & $1(2.0 \%)$ & \\
\hline C. perfringens & $1(2.0 \%)$ & \\
\hline S. suis infection & $1(2.0 \%)$ & \\
\hline Totals & $51(100 \%)$ & \\
\hline *: 4 samples were examined for PCV2 only. & & \\
\hline
\end{tabular}

\section{Discussion}

PCV2 can be found in feces samples from pigs with and without diarrhea (enteritis), but;

- The mean PCV2 DNA copy load in PCV2 DNA positive feces was higher in pigs with diarrhea,

- and high PCV2 DNA copy loads (>104 copies/mgr) were found significantly more often in feces from pigs with diarrhea (enteritis)

\section{Acknowledgement}

This study was supported by the European Porcine Circovirus Research Award 2009 (Boehringer Ingelheim Animal Health). 


\title{
P.028
}

\section{Phylogenetic study of the ORF3 gene from Porcine Circovirus Type 2 in Brazil}

\author{
Juliana Amália F. Nascimento ${ }^{1}$ Leandro M. Freitas ${ }^{2}$ Wagner Carlos S. Magalhães ${ }^{2}$ Adrienny T. Costa $^{3}$ \\ Adriana Cortez ${ }^{4}$ Leonardo José Richtzenhain ${ }^{4}$ Marcos B. Heinemann ${ }^{1} \underline{\text { Zélia Inês P. Lobato }}^{1}$ \\ 1. Laboratório de Pesquisa em Virologia Animal, Escola de Veterinária, UFMG, Belo Horizonte, MG, Brazil; 2. \\ Departamento de Biologia Geral, Instituto de Ciências Biológicas, UFMG, Belo Horizonte, MG, Brazil; 3. Laboratório de \\ Pesquisas Veterinárias Especializadas Ltda (IPEVE), Belo Horizonte, MG, Brazil; 4. Laboratório de Biologia Molecular \\ Aplicada e Sorologia, Faculdade de Medicina Veterinária e Zootecnia, USP, São Paulo, SP, Brazil
}

\section{Introduction}

The correlation between the PCV-2 ORF3 gene and viral pathogenicity has been suggested previously. Three genogroups based on the ORF3 gene sequence (SG1, SG2 and SG3) were identified in Sweden and correlated to the PCVAD occurrence. The aims of this work were to determine the ORF3-based genotypes present in Brazilian herds, to establish their geographic distribution among main swine-producing states of Brazil and to correlate each genotype with lymphoid microscopic lesions.

\section{Materials and Methods}

Samples from 89 swines with clinical symptoms of PCVAD were collected in nine swine-producing Brazilian states. For histopathological evaluation, 44 samples were processed and the lesions were classified as discrete, moderate or severe. Thirtyone additional samples were collected from the same farms in a different time-point. DNA was extracted from the tissue suspension using guanidine thiocyanate and PCR product was purified and sequenced. The amino acid sequence alignment of the samples was made using Clustal W and phylogenetic tree was constructed based on the Neighbour Joining (NJ) method with 1000 bootstrap. Haplotype networks were constructed using the Network 4.2.0.1 software.

\section{Results}

The phylogenetic analysis of the ORF3 sequence allowed the identification of three genogroups (3.4\% SG1, 12.3\% SG2 and $84.3 \%$ SG3) and six subtypes (SG2A, SG2B, SG2C, SG2D, SG3A and SG3B). Furthermore, it was possible to observe that the majority of the samples were classified within subtypes SG3A and SG3B. The average distance of the amino acids was closer between the SG1 and SG2 genogroups. There was also a greater genetic proximity between the SG2A and SG2B subtypes and the SG3A and SG3B subtypes. Variations between SG1 and SG2 were detected at residues 41, 102, 103 and 104, and variations between SG1, SG2 and SG3 were detected at residues 41 and 103. Finally, the distance measured by the branch size was low, indicating a genetic proximity among the individuals. In samples from the same farm collected at a different time, $74.19 \%$ of the cases presented both samples were classified within the same subtypes (67.74\% in SG3B and 6.45\% in SG2D), whereas the remaining $25.81 \%$ of the samples were classified into different subtypes. When lesion severity was correlated with the ORF3 genogroup, subtypes SG1 and SG2 were associated with discrete lesions, SG3B was more frequently associated with discrete and moderate lesions, whereas SG3A was associated with moderate and severe lesions. Through the phylogeography analysis it was possible to group the sequences in 14 haplotypes. The $\mathrm{H} 11$ haplotype (from SG3B) grouped 55 (61.80\%) representative sequences from the nine states and was considered to be the ancestral haplotype for SG3 due its ample dispersion.

\section{Discussion}

Our findings consist in the first identification of subtypes in SG2 and SG3 based on amino acids 102 to 104 indicating the emergence of PCV-2 genotypes in Brazilian herds. The presence of different ORF3 genogroups and subtypes in the nine states studied indicated the wide circulation of the PCV-2 variants. The change of genogroup in $32.26 \%$ of the samples from the same farms collected at a different time confirms these results. The frequency of the histopathologic lesions corroborates literature results, which suggested that the genogroup classification can be based on the description of the PCVADs present on the farm. Moreover it was also possible to observe that SG3A variants were more pathogenic than those in subtype SG3B. The detection of the SG1 genogroup in only three samples, which were associated with discrete lesions, is likely indicative of the low prevalence of this genogroup among animals with clinical signs of PCVAD, further it supports the hypothesis that genogroup SG1 is less pathogenic. The major number of samples was classified in the SG3B subtype, and its wide distribution in the haplotypes suggests that this PCV-2 variant circulated widely with only minimal alterations in the ORF3 sequences.

\footnotetext{
References

Chianini et al., 2003. Vet. Immunol. Immunop. ,v.94, p.63-75;

Chomkzynski, P., 1993. Biotechniques, v.15, p.532-537;

Olvera et al., 2007. Virology, v.357, p.175-185;

Opriessnig et al., 2008. J. Gen. Virol., v.89, p.2482-2491;

Timmusk et al., 2008. Virus Genes, v.36, p.509-520.
} 


\title{
P.029
}

\section{Evidence of natural selection on Circovirus type 2 (PCV-2)}

\author{
Wagner Carlos S. Magalhães ${ }^{1}$ Juliana Amália F. Nascimento ${ }^{3}$ Leandro M. Freitas $^{1}$ Adrienny T. Costa ${ }^{2}$ \\ Adriana Cortez ${ }^{4}$ Leonardo José Richtzenhain ${ }^{4}$ Marcos B. Heinemann ${ }^{3}$ Zélia Inês P. Lobato $^{3}$ \\ 1. Departamento de Biologia Geral, Instituto de Ciências Biológicas, UFMG, Belo Horizonte, MG, Brazil; 2. Instituto \\ de Pesquisas Veterinárias Especializadas Ltda (IPEVE), Belo Horizonte, MG, Brazil; 3. Laboratório de Pesquisa em \\ Virologia Animal, Escola de Medicina Veterinária, UFMG, Belo Horizonte, MG, Brazil; 4. Laboratório de Biologia \\ Molecular Aplicada e Sorologia, Faculdade de Medicina Veterinária e Zootecnia, USP, São Paulo, SP, Brazil
}

\section{Introduction}

The evolution of the PCV-2 genetic diversity has been intensely studied for a better understanding of the pathogenesis of the associated syndromes and development of prophylactic measures. The nonsynonymous, amino acid-altering/synonymous, silent, substitution rate ratio $\left(\omega=d_{N} / d_{S}\right)$ provides a measure of natural selection at coding regions, where $\omega=1$ represents neutral evolution; $\omega>1$ states positive selection, caused by an excess of nonsynonymous polymorphism and consequently increasing the rate $(\omega)$, while $\omega<1$, indicating purifying selection, where the new mutations are eliminated, probably due their deleting nature.

\section{Materials and Methods}

Alignment of the 27 ORF2 PCV-2 sequences plus 7 Genbank sequences (AY484416; AY682995; AY556476; AF117753; AY146993; AY256458; AF408635) were used. The amino acid sequence alignment was made using the CLUSTAL W software. The phylogenetic tree was constructed based on the Neighbour Joining (NJ) using the MEGA 4.0 software. Different measures of polymorphism variability and several statistics employed in neutral tests using the program DNAsp v.4.50.3 were used. The program CODEML from the software package Phylogenetic Analysis by Maximum Likelihood v. 4.2b (PAML) was used to fit models of sequence evolution to our circovirus type 2 dataset.

\section{Results}

In ORF2 all values LRTs indicated significantly better fits for the selection models $M 2$ and M8 than the neutral models $M 1$ and $\mathrm{M} 7$ respectively (Table1). Also, at the model M3, different omega values along the sequence fits better than $\mathrm{MO}$ which consider the entire sequence, evolving under only one omega value. The codons that showed high values of omega are (69 R, $101 \mathrm{P}, 185 \mathrm{~S}$, with Probability of (omega $>1$ ) > 95\%). In addition, to the previous results provided for the likelihood analysis, tests of neutrality based on the sequence frequency spectrum (Tajima's D; Fu and Li's F) also provided evidences that corroborate these findings.

\section{Discussion}

Our work provided evidence that the ORF2 had undergone positive selection and identified 3 positively selected sites. Understanding the functional importance of these positively selected amino acid positions could help to predict possible changes in virulence, as well as responses to certain strains in future vaccination programs.

Table 1 Likelihood ratio test for the different models tested on the PCV2 Open reading frame $2\left({ }^{a} L R T=-2^{*}\left(H_{0}-H_{1}\right)\right.$, b degree freedom, c for 0.05)

\begin{tabular}{|l|c|c|c|c|}
\hline ORFs & Models compared & $-\mathbf{2} \mathbf{\Delta \mathbf { I } ^ { \mathbf { a } }}$ & d.f $^{\mathbf{b}}$ & p-value $^{\mathbf{c}}$ \\
\hline & M0 vs. M3 & $181.62^{* *}$ & 4 & $<0.00001$ \\
\hline ORF2 & M1 vs. M2 & $17.62^{*}$ & 2 & 0.000139 \\
\hline & M7 vs. M8 & $36.16^{* *}$ & 2 & $<0.00001$ \\
\hline
\end{tabular}

\section{References}

Fu and Li, 1993. Genetics, v.133, n.3, p.693-709;

Rozas et al., 2003. Bioinformatics, v.19, n.18, p.2496-2497;

Tajima, 1989. Genetics, v.123, n.3, p.585-95;

Yang, 2007. Mol. Biol. Evol. v.24, n. 8, p.1586-1591 (http://abacus.gene. ucl.ac.uk/software/paml.html). 


\title{
P.030
}

\section{Serological survey on porcine circovirus type 2 (PCV2) in commercial swine farms located in the Northeastern States of Brazil}

\author{
Clara N. Barbosa ${ }^{1}$ Janice R. Ciacci-Zanella ${ }^{2,5}$ Marisete F. Schiochet ${ }^{4}$ Giovani R. Bertani $^{3}$ \\ 1. Veterinary Clinic Department - Federal Rural University of Pernambuco - UFRPE, Recife, PE, Brazil; 2. Virus and Prion Disease \\ Research Unit. NADC. ARS. USDA, Ames, IA, USA; 3. Departament of Biochemistry - LIKA/ Federal University of Pernambuco - \\ UFPE, Recife, PE, Brazil; 4. Embrapa Swine and Poultry, Concórdia , SC, Brazil; 5. Labex-USA, EMBRAPA, Brasília, DF, Brazil
}

\section{Introduction}

Porcine circovirus type 2 (PCV2) is the cause of PCV2 associated diseases (PCVAD), a multifactorial disease that causes great economic impact on swine industry worldwide (2). Recently performed serological examinations indicated that this virus is widespread within the pig population (1). The aim of the present work was investigated the presence of antibodies against the PCV2 in pigs from commercial farms of the Northeastern States of Brazil detected by immunoperoxidase monolayer assay (IPMA).

\section{Materials and methods}

Herds and blood collection: 353 blood samples were collected from gilts with age between 20-28 weeks from 12 different farms of Northeastern States of Brazil. The major criteria used for the selection of the farms in the study were agreement permission the owners and no use of vaccines against PCV2. Second, the herd should be of at least 100 sows and monitored for the presence of clinical signs of the PCVAD.

Serologic analyses: Serum samples were evaluated for the presence of antibodies to PCV2 by IPMC (3). Serial dilutions of sera were performed from 1:20 to 1:5120 on 96 well plates containing fixed Swine Kidney (SK6) cells previously infected with PCV2. Titers equal or above 1:20 was considered positive and classified as low (1:20 to $1: 160)$, medium (1:320 to $1: 2560)$ and high (1:5120 or higher) titers.

Statistic analyses: The data were analyzed using the program $R$ (R Foundation for Statistical Computing, 2009).

\section{Results}

The results show that $306 / 353$ samples analyzed (86.7\%) reacted against PCV2 antigens (equal or above 1:20). The results are summarized in Table 1 and Figure 1.

\section{Discussion}

The present study represents the first antibody survey against PCV2 in pigs on farms of the Northeast of Brazil. This ubiquitous infectious agent circulates in all farms, regardless of syndrome being present (1). The antibody profile titer in non vaccinated farms can be used to guide vaccination programs. Lower titers against PCV2 can were observed between the ages of 23 and 25 weeks. This result suggests that this period can be identified as a window in the herd immunity and it is suitable to implement vaccination programs.
Table 1. Distribution of antibodies titers against $P C V 2^{a}$ per number of swine in farms located in Northeastern States of Brazil.

\begin{tabular}{|l|c|c|c|c|c|c|c|c|}
\hline & \multicolumn{7}{|c|}{ Classes of Antibody Titer } \\
\hline & NR $^{\mathbf{b}}$ & & Low & & Medium & & High & \\
\hline State & $\mathrm{n}$ & $\%$ & $\mathrm{n}$ & $\%$ & $\mathrm{n}$ & $\%$ & $\mathrm{n}$ & $\%$ \\
\hline BA & 11 & 23.4 & 47 & 38.5 & 45 & 27.6 & 6 & 30.0 \\
\hline CE & 7 & 14.9 & 14 & 11.5 & 22 & 13.5 & 5 & 25.0 \\
\hline PB & 7 & 14.9 & 8 & 6.5 & 16 & 9.2 & 2 & 10.0 \\
\hline PE & 11 & 23.4 & 25 & 20.5 & 29 & 17.8 & 2 & 10.0 \\
\hline RN & 11 & 23.4 & 28 & 23.0 & 52 & 31.9 & 5 & 25.0 \\
\hline Total & 47 & 100.0 & 122 & 100.0 & 164 & 100 & 20 & 100.0 \\
\hline
\end{tabular}

Figure 1. The relation between different ages in relation to antibody titer (log transformed multiplied by -1) against PCV2 in Northeastern Brazilian pig farms.

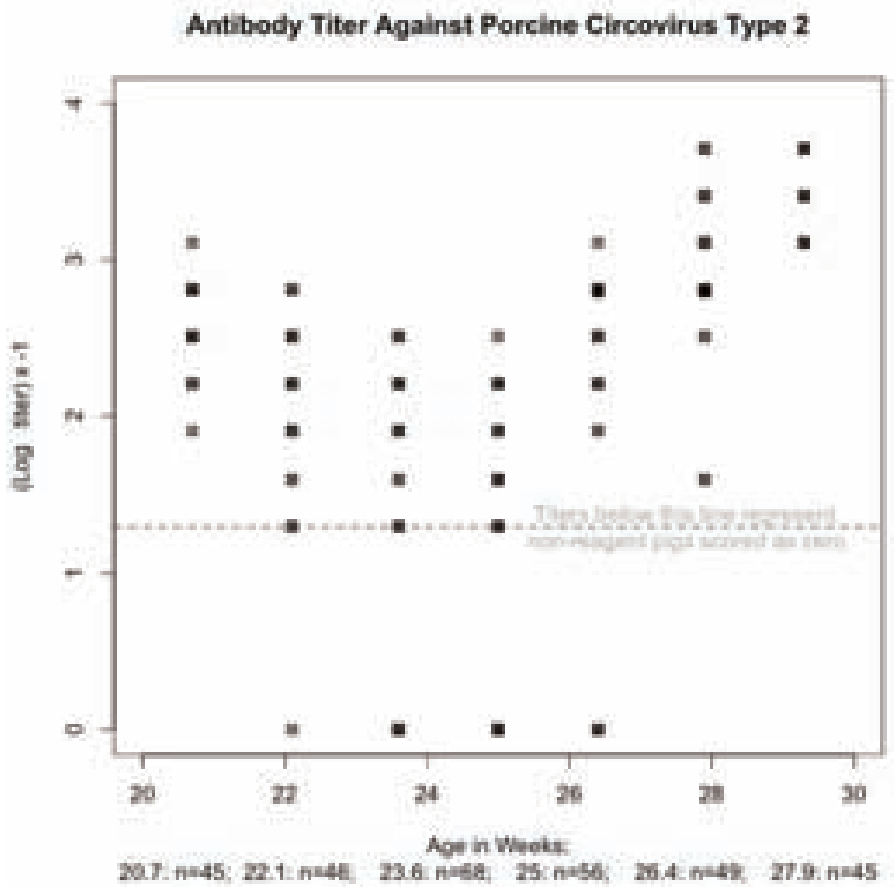

\section{References}

1. Fort, M., et al. 2007. Vet. Microbiol. 125,244- 255.

2. Segales, J., et al. 2005. Anim. Health Res. Rev.6, 119-142.

3. Rodriguez-Arrioja, G.M., et al. 2000. Vet. Rec. 146, 762-764. 


\title{
P.031
}

\section{Monitoring of PCV2 infections in Piedmont farms: from diagnosis to characterization of hystological phenotypes}

\author{
$\underline{\text { Katia Varello }}{ }^{1}$ Maria E. Careddu ${ }^{1}$ Giuliano Pisoni ${ }^{2}$ Marco Faccenda ${ }^{3}$ Franco Kobal ${ }^{3}$ Riccardo Madonna ${ }^{3}$ \\ Chiara Musella ${ }^{1}$ Pierluigi Acutis ${ }^{1}$ Fabio Zuccon ${ }^{1}$ Vittorio Sala² Elena Bozzetta ${ }^{1}$ \\ 1. Istituto Zooprofilattico Sperimentale di Torino, Turin, Italy; 2. Dipartimento di Patologia Animale, Igiene e Sanità \\ Pubblica Veterinaria - Università degli Studi di Milano, Milano, Italy; 3. Veterinary Practitioner, Cuneo, Italy
}

\section{Introduction}

Postweaning multisystemic wasting syndrome (PMWS) is a disease that affects late nursery and fattening pigs. Diagnosis of PMWS is based on the presence of compatible clinical signs (mainly wasting and respiratory distress), characteristic histopathological lesions, and detection of PCV2 within characteristic lesions. Microscopically, the most relevant features are lymphocyte depletion and histiocytic infiltrates in lymphoid tissues. Therefore, the sole detection of PCV2 antibodies in serum and/or viral DNA or antigen does not constitute PMWS diagnosis, since it only indicates evidence of PCV2 infection Histopathology and immunohistochemistry tests performed on a panel withdrawn from daily farm mortalities are fundamental tools for the diagnosis of PMWS. The objectives of this study were to evaluate the diffusion of PMWS in North-western Italy (Piedmont region) and the reliability of a histological grading in "farm diagnosis".

\section{Materials and Methods}

Fourteen farms with clinical signs related to PMWS were selected and a questionnaire to evaluate their clinical and management situation was drawn up.

Three to five animals (4-16 weeks old) were necropsied and samples from lung, tonsil, tracheobronchial and superficial inguinal lymph node, spleen and ileum were collected from all pigs for histopathological and PCR analysis for PCV2. Portions of tissues were fixed in 10\% neutral-buffered formalin and processed by standard paraffin wax techniques for histological analysis. Samples were cut in $4 \pm 2 \mu \mathrm{m}$ sections and stained with haematoxylin and eosin (HE) and immunohistochemically (IHC) using a monoclonal antibody against PCV2 (Ingenansa, Spain). Lung sections were scored for the presence and severity of interstitial pneumonia ranging from 0 (normal) to 6 (severe diffuse); lymphoid tissues sections were evaluated for the presence of lymphoid depletion ranging from 0 (normal) to 3 (severe) and for histiocytic inflammation and replacement of follicles ranging from 0 (normal) to 3 (severe).

In addition they were all tested by PCR for porcine reproductive and respiratory syndrome virus (PRRSV) and bacteriological and parasitological investigations were made on the basis of signs during necropsy.

\section{Results}

At post-mortem examinations some animals revealed mild to moderate hyperplasia in superficial lymph nodes and consolidation of lungs in association with broncopneumonia. Five of the 14 farms resulted positive for PMWS with at least one piglet showing typical lesions and immunohistochemical and molecular positivity. Lesion score for depletion target from 1 to 3 and for histiocytic inflammation from 1 to 2 . Further 9 farms resulted negative for PMWS and lesion score resulted 0 or 1 for both depletion and histiocytic inflammation. Lesion score for lung was quite variable in all farm analyzed whit values from 0 to 6 . PRRSV resulted also positive in all farms with PMWS and it was also present in eight of other farms. Only one showed no presence of viruses. Streptococcus suis, Pasteurella multocida, Haemophilus parasuis, Bordetella bronchiseptica, Salmonella tiphymurium were isolated form several collected organs.

\section{Discussion}

Histology and immunohistochemistry are considered essential for PMWS diagnosis. In this field the lesion score could be useful to understand the evolution of the pathology within the farms. It is noteworthy that lesions with score 2-3 of lymphoid depletion and 1-2 of histiocytic inflammation were more frequently associated with immunohistochemical positivity. In our cases histiocytic inflammation resulted from low to moderate but not severe and the depletion appeared to be the most frequent lesion. The lungs showed a variable score, in fact we observed severe interstitial pneumonia in subject with no also confirmed PMWS, probably related to the diffuse infection of PRRSV as described previously. The results obtained from the analysed farms suggest to emphasize the role of PRRSV and the importance to keep it under control.

\section{References}

1. Allan G.M., Ellis E.J.: 2000.J Vet Diagn Invest 12:3-14

2. Chae C. (2004). Vet J, 168.41-49.

3. Chae C. (2005). Vet J, 169. 326-336.

4. Opriessnig T., Thacker E.L., Yu S., Fenaux M., Meng X.-J., Halbur P.G. (2004). Vet Pathol 41:624-640.

5. G. Sarli, F. Ostanello, F. Morandi, L. Fusaro, M. Gnudi, B. Bacci, A. Nigrelli, L. Alborali, M. Dottori, F. Vezzoli, G. Barigazzi, L. Fiorentini, V. Sala, G. Leotti, F. Joisel (2009). Vet Rec. 164:519-23

This study was sponsored by Italian Ministry of Health within 2006 Research Program. 


\title{
P.032
}

\section{Commercial spray-dried porcine plasma contains high amounts of PCV2 DNA and high levels of anti-PCV2 antibodies but is not infective to naïve pigs}

\author{
$\underline{T a n j a ~ O p r i e s s n i g}^{1}$ Huigang Shen ${ }^{1}$ Shayleen Harrison ${ }^{1}$ Joy Campbell ${ }^{2}$ Terry Faidley $^{2}$ Louis Russell $^{2}$ Patrick Halbur $^{1}$ \\ 1. lowa State University, Ames, IA, USA; 2. APC, Inc., Ankeny, IA, USA
}

\begin{abstract}
Introduction
Porcine circovirus type 2 (PCV2) is a small DNA virus and is resistant to inactivation [1]. Spray-dried plasma (SDP) of porcine origin is widely used in modern swine production to improve growth and health. However, SDP has been proposed as a possible source of PCV2 transmission. Recently, PCV2 transmission was demonstrated using experimentally produced SDP from a clinically diseased pig and a laboratory spray-dryer [2]. Currently available results on infectivity of PCV2 present in commercial spray-dried products are conflicting and to date the importance and impact of this route of PCV2 transmission is not fully understood. The objectives of this study were (1) to determine the amount and variation of PCV2 DNA and antibodies in commercial spray-dried plasma products from different geographic regions in the U.S. and (2) to determine if naïve pigs fed a diet containing $4 \%$ inclusion rate of PCV2-PCR positive commercial SDP become infected with PCV2.
\end{abstract}

\section{Materials and Methods}

Samples from 2 spray-drying facilities located in the US were collected weekly for a 3 wk collection period. The samples included plasma from 14 different slaughterhouses (PSla) representing the major swine producing regions across the US. Samples were collected from liquid pooled plasma as it entered the spraydrying facility. In addition, concentrated plasma (Pliquid) and SDP (PDry) were collected at four different time points in the process. The samples represented approximately $60 \%$ of a single day's US swine slaughter. All samples were subjected to PCV2 ELISA and quantitative PCV2a/b real-time PCR [3]. A feeding trial was conducted in order to further evaluate the infectivity of the PCV2 DNA present in commercially produced spray-dried porcine plasma. Nine PCV2 naïve pigs ( $65 \mathrm{~kg} \mathrm{BW)} \mathrm{were} \mathrm{randomly}$ assigned to 1 of 3 groups of 3 pigs each including (1) negative controls, (2) positive controls, and (3) SDP pigs. The positive controls were inoculated with an infectious PCV2 stock inoculum. Negative and positive control pigs were fed a diet free of animal proteins. The SDP pigs received a diet with 4\% spray-dried porcine plasma for entire duration of the study. The SDP utilized in the feeding trial was selected based on presence of PCV2a and PCV2b DNA as determined by PCR. Blood was collected weekly from the pigs and the experiment was terminated 42 days post inoculation.

\section{Results}

All collected samples (PSla; PLiquid; PDry) were positive for antiPCV2 IgG when tested with the PCV2 ELISA. In general, there was no significant difference found between the $S / P$ values of the PSla, PLiquid and PDry samples. PCV2a DNA was found in 0/52, $0 / 32$ and $2 / 32$ of the PSla, PLiquid and PDry samples, respectively. In contrast, PCV2b DNA was found in 31/52, 22/32 and 16/32 of the PSla, PLiquid and PDry samples, respectively. Concurrent presence of PCV2a DNA and PCV2b DNA was found in 13/52, $1 / 32$, and $7 / 32$ of the PSla, PLiquid and PDry samples respectively. In the feeding trial, the positive control pigs became PCV2 DNA positive by 7 days post inoculation and remained positive. In contrast, negative control pigs and SDP pigs remained PCV2 PCR negative through 28 days of the trial. The trial is still ongoing at the time of abstract submission and final results will be presented.

\section{Discussion}

The high prevalence of PCV2 antibodies in pooled plasma collected in slaughter facilities from healthy animals across the U.S. is consistent with previous reports. Whereas PCV2a DNA was found only in a few of the SDP batches, most plasma samples tested were positive for PCV2b. This was similar across all stages in the SDP production process. This finding is especially interesting as most grow-finish pigs in the U.S. are vaccinated against PCV2 (using a PCV2a-based product) and potentially indicative of a lack of thorough cross-protection between genotypes. Preliminary results indicate that highly susceptible, PCV2 virus- and antibody-naive specific-pathogen-free pigs fed a high concentration of spray-dried PCV2 DNA positive plasma over at least 28 days remained PCV2-free. This evidence suggests that the commercial spray-drying process employed in the US is effective in eliminating the infectivity of PCV2, likely among the most difficult pathogens to inactivate.

\footnotetext{
References

1. Allan, G.M. et al. (2000) J. Vet Diagn Invest 12:21-27.

2. Patterson, A.R. et al. (2010). AASV proceedings.

3. Opriessnig, T. et al. (2010) Vet. Res. 41:31.
} 


\title{
P.033
}

\section{Retrospective investigation of porcine circovirus type 2 (PCV2) on archived porcine tissues from Goiás State, Brazil}

\author{
Patricia Soares ${ }^{2}$ Denise L. Barthasson² Tatyane P. Sales ${ }^{3}$ Duanne A. Silva ${ }^{3}$ Keili M. Souza 4 \\ Daniel Linhares $^{1}$ Eugenio G. Araujo ${ }^{3}$ Jurij Sobestiansky² Wilia M. Brito $^{4}$ \\ 1. College of Veterinary Medicine, University of Minnesota, Saint Paul, MN, USA; 2. Programa de Pós graduação em Ciência Animal, Escola de \\ Veterinária da Universidade Federal de Goiás/GO, Goiania, GO, Brazil; 3. Escola de Veterinaria - Universidade Federal de Goias, Goiania, GO, \\ Brazil; 4. Laboratório de Virologia Animal, Instituto de Medicina Tropical e Saúde Pública da Universidade Federal de Goiás, Goiania, GO, Brazil
}

\section{Introduction}

Porcine circovirus associated diseases (PCVAD), caused by porcine circovirus type 2 (PCV2), has been considered one of the most important disease in swine industry worldwide. The characteristic immunosuppressivity of the virus predisposes the host to secondary infections, increasing mortality in herds and the cost of production. Despite many efforts, there are many questions to answer about occurrence, pathogenesis and epidemiology of the infection yet, especially in Brazil. With the purpose to identify PCV2 DNA in the pigs from intensive production system in the State of Goiás, Brazil, a retrospective investigation on paraffin-embedded organs samples which had been submitted to histopathological diagnosis in Laboratory of Animal Pathology (LAP / UFG) of Veterinary School of Federal University of Goiás was performed.

\section{Materials and methods}

A total of 42 samples from 26 pigs necropsied during 2000 to 2002 , were chosen from the archival collection of the LAP / UFG based on typical histopathological lesions of PCVAD, such as lymphadenopathy, interstitial pneumonia, hepatitis, enteritis and/or interstitial nephritis. Blocks are cutted in sections of $10 \mu \mathrm{m}$ thin. First, sections were deparaffinized as described in Ciacci-Zanella et al (2006) and after, tissues were submitted to DNA extraction with standard phenol protocols. A polymerase chain reaction (PCR) using specific primers for the ORF2 of the PCV2 DNA according Kim et al. (2001) was used to amplify a PCV2 ORF2 fragment. A sample of PCV2 achieved from pig with clinical signs and lesions of porcine circovirosis (Barthasson, 2009) was used as positive control and ultrapure sterilized water was used as negative control.

\section{Results}

Specific PCV2 ORF2 DNA fragment were detected in 20 (47.61\%) out of 42 samples from $17(65,4 \%)$ animals. No samples of eight from 2000 analyzed showed positivity. In the samples obtained from 2001, 8 samples (66.7\%) from 15 blocks were positive.
These samples were picked up from 4 out of 5 pigs. Viral DNA was found in kidneys $(n=2)$, spleen $(n=2)$, liver $(n=2)$, lymph nodes ( $n=1)$, and lung $(n=1)$. In 2002, viral DNA has been found in $12(63.15 \%)$ out of 19 samples. Twelve out 13 pigs were positive. PCV2 DNA was found in lung $(n=3)$, lymph nodes $(n=3)$, liver $(n=2)$, spleen $(n=2)$, tonsils $(n=2)$ and intestine $(n=1)$ tissue fragments.

\section{Discussion}

The state of Goiás is the third largest swine's producer in Brazil. So far, porcine circovirosis was not confirmed by any molecular or virologic test in this region. The results found in our study, confirm the clinical and pathological evidence of PCV2 infection in Goiás. Although other Brazilians' studies showed that the PCV2 is present in the country since 1968, we did not analyze samples prior 2000, but we can point that PCV2 circulates in Goiás pig herds at least since 2001. Other study conducted in back yard pigs from Goiás had already identified PCV2 DNA in tissue samples, but to our knowledge, this is the first identification of PCV2 DNA in tissue from swine intensive production system in the state of Goiás with clinical and pathological evidence of PCVAD.

\section{References}

1- Barthasson, D. L. Identificação molecular do circovírus suíno tipo 2 e vírus da hepatite E em suínos de criações extensivas de Goiás. 2009. 90f. Tese (Doutorado em Ciência Animal) - Faculdade de Medicina Veterinária, Universidade Federal de Goiás, Goiânia.

2- Segalés, J.; Rosell, C.; Domingo, M. Pathological findings associated with naturally acquired porcine circovirus type 2 associated diseases. Veterinary Microbiology, v.98, p.137-149, 2004.

3- Silva Junior, A.; Braga, D. A. M.; Silva, F. M. F.; Myrra, L. V.; Fietto, J. L. R.; Vargas, M. I.; Nero, L. A.; Almeida, M. R. Detection on porcine circovirus 2 DNA by Real-Time polymerase chain reaction in 1978 Brazilians archived porcine tissues. Virus: Reviews and Research, v.14, supplement, p.108, 2009. 


\title{
P.034
}

\section{Phylogenetic characterization of porcine circovirus type 2 in Brazilians between 2002 and 2009}

\author{
Alessandra M. Castro ${ }^{1}$ Cíntia M. Baldin² Taís Nishikataํ Cíntia M. Faveroํ Sheila O. \\ Souza ${ }^{1}$ Karen L. Ferrari Paulo E. Brandão ${ }^{1}$ Leonardo J. Richtzenhain ${ }^{1}$ \\ 1. Faculdade de Medicina Veterinária e Zootecnia da Universidade de São Paulo, São \\ Paulo, Brazil; 2. Faculdade de Jaguariúna, Jaguariúna, SP, Brazil
}

\section{Introduction}

Porcine circovirus type 2 (PCV2) is a non-enveloped, circular, single-stranded DNA virus of approximately $1.76 \mathrm{~kb}$, belonging to the genus Circovirus in the family Circoviridae. The genome encodes three major open reading frames (ORFs) encoding the replicase proteins (ORF1), the viral capsid protein (ORF2), and a protein with suggested apoptotic activity (ORF3). The cap gene is considered a reliable phylogenetic marker for PCV2 strains since it was able to reconstruct the same tree as the whole viral genome (3). The aim of this study is to determine the clustering of PCV2 detected over this 7-year.

\section{Materials and Methods}

Twenty four ORF2 sequences were selected and multiplealigned with representative sequences of ORF-2 PCV2 available at the GenBank database using Clustal X v. 1.8. The analyses were with the following samples: 5 from 2002 (am2; am5; am13r; am22R; am25R); 6 from 2003 (am10; am3; am5; am9; am4; amC); 4 from 2004 (am13; am15; am16; am21); 5 from 2007 (am148k; am182k; am61k; am62k; am7k) and 3 from 2009 (CINamF3; CINamF4; CINamF7). The identity among sequences at nucleotide and aminoacid levels was determined using BioEdit v.7.0.5. The phylogenetic tree was performed using the distance based Neighbor-joining method and substitution Kimura 2-parameters model in the Mega v.2 package (Bootstrap 1000).

\section{Results}

Variations in the aminoacid composition among the 24 sequences were distributed across the whole capsid protein sequence, but more concentrated between the 57 and 91 position. In the sequences from this study the most substitutions was observed in $57,63,80,86,88,89,91,151,190$ and 191 position. Interestingly, these regions were almost located in putative epitope domains of PCV2 capsid protein (Fig 1). The 24 sequences are distributed within both subgroup (PCV2-1 and PCV2-2) since 2002 (e.g. am22r and am25R). Most of the sequences (14/24) are grouped in PCV2-1A/B group and one (am6) is in PCV2-2D group.

\section{Discussion}

This study confirms that both, PCV2-1 and PCV2-2 are circulating in Brazilians swine herds since 2002 (1). The sequences obtained from samples collected recently (2007 - 2009) are grouped in PCV2-1 group. However some sequences (old and new) are still without classification, creating a separated group within both PCV2 group (PCV2-1 and PCV2-2).
Figure 1: Variability of the amino acid sequence of ORF2 (Cap). The indicated sequence is a consensus from an alignment of the 24 Brazilians sequences of PCV2ORF-2. The grey block represents the immunogenic regions identified by PEPSCAN (2).

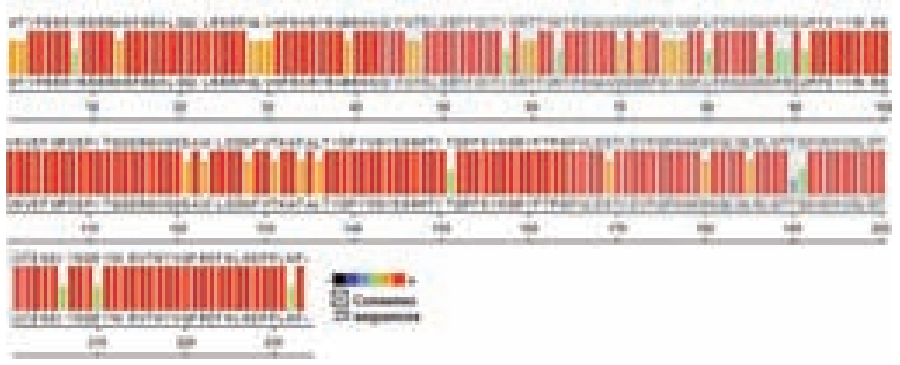

Figure 2: Neighbour-Joining tree of the PCV2 ORF-2 region. Numbers indicates values calculated on 1000 repeats of the alignment with the heuristic method. The following sequences were published (Am25R; Am13R;Am22R;AmC) (1)

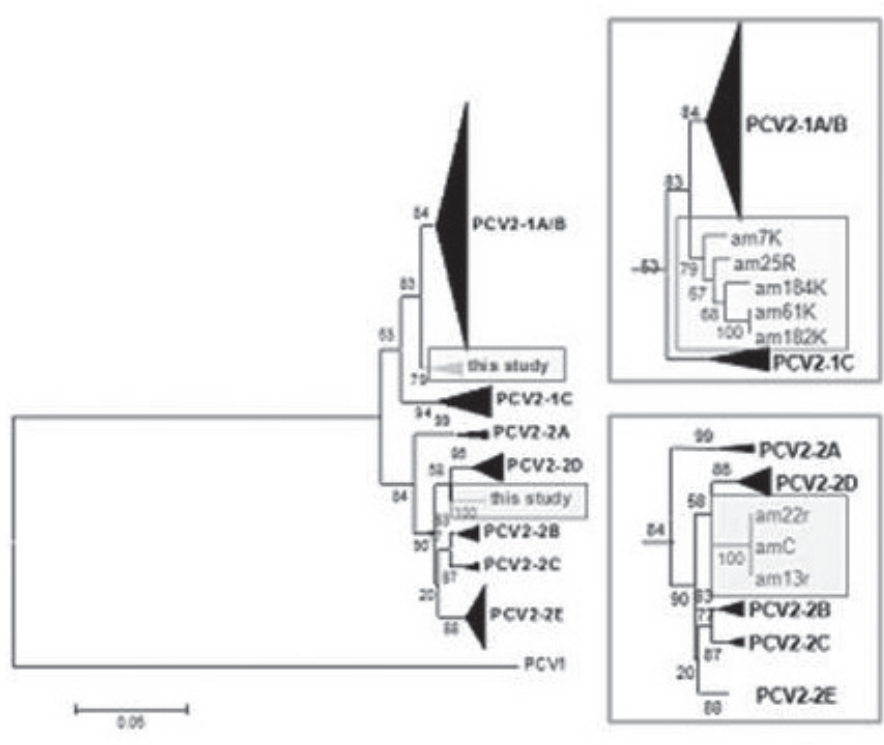

\section{References}

1. Castro, et al. (2007). Arch Viro 152 1435-1445.

2. Mahé, et al. (2009). J. Gen. Virol. 81, 1815-1824.

3. Olvera, et al. (2007). Virol. 357, 175-185. 


\title{
P.035
}

\section{Efficacy of Circumvent ${ }^{\circledR}$ PCV preventing PCVAD and maintain growth performance during an outbreak of PRRSv in a Canadian pig herd with a history PCVAD}

\author{
Charles Surprenant ${ }^{2}$ Francisco de Grau' Benoit Laplante ${ }^{2}$ Brad Thacker $^{3}$ Alex Eggen ${ }^{4}$ \\ 1. Intervet Canada Corp, Kirkland, QC, Canada; 2. Menard Inc, Ange-Gardien, QC, Canada; 3. Intervet, \\ Inc., Millsboro, DE, USA; 4. Intervet International BV, Boxmeer, Netherlands
}

\section{Introduction}

The efficacy of Circumvent ${ }^{\circledR}$ PCV (Intervet Schering Plough Animal Health) was demonstrated during the severe PCV2 disease outbreaks in Canada (de Grau et al., 2007). Many off these outbreaks involved co-infections with PRRSv, SIV, Mycoplasma hyopeneumoniae and other bacteria (Carman et al., 2006). PRRSv plays an important role in enhancing PCV2 replication and PCV2 viremia (Rovira et al., 2002). The objective of this field trial was to compare the efficacy and performance of Circumvent ${ }^{\circledR}$ PCV in comparison to unvaccinated control pigs. A severe PRRSv outbreak occurred during the vaccination trial.

\section{Materials and Methods}

The trial was carried out using a randomized, non-blinded and controlled design. In total, 350 pigs were selected and ear tagged at three days of age (D) and allocated to one of two treatment groups, vaccinated (VAC) or control (CON) $n=175$ pigs. Vaccinations were performed per label recommendations at weaning $(\sim 18 \mathrm{D})$ with a booster 3 weeks later. All pigs were weighed at 3 D, weaning, end of nursery (56 D), 106 D, 143 D and slaughter (165 D). Individual pig treatments were recorded for assessing morbidity. A post mortem was performed on every dead pig. Blood samples were collected from 12 pigs per group at $3 \mathrm{D}$, end of nursery, $120 \mathrm{D}$, and slaughter to evaluate antibody titers by ELISA and viremia by qPCR. Viral presence in the nose at $120 \mathrm{D}$ and slaughter and feces at slaughter was determined in the same pigs. All pigs were observed for local and systemic reactions during the first 72 hours after each vaccination. At slaughter, carcass quality information was retrieved.

\section{Results}

Due to the PRRSv outbreak in the nursery, birth to finish mortality was approximately $14 \%$ and did not differ between treatment groups. PRRSv was confirmed by laboratory analysis (PCR, genotyping, histopathology) and caused a mortality peak in the first third of the finisher stage. VAC pigs did not develop PCVAD and only CON pigs exhibited gross and microscopic lesions compatible with PCV2 infection. ADG, ELISA titers and viremia differed between the groups. In the finisher (20 to $120 \mathrm{Kg}$ ), ADG was $948 \mathrm{~g}$ for CON pigs vs. $973 \mathrm{~g}$ for VAC pigs ( $25 \mathrm{~g}$ difference). However, the difference in ADG between the groups during the highest PCV2 viremic period (106 D to slaughter) was $40 \mathrm{~g}$ $(1,053 \mathrm{~g}$ vs. $1,013 \mathrm{~g})$. Grower-finisher ADG was lower in viremic pigs in the sampled group $(925 \mathrm{~g})$ in comparison to non-viremic sampled pigs ( $994 \mathrm{~g}$ ). ELISA titer patterns differed between CON and VAC pigs as CON pigs were seronegative during the disease challenge. PCV2 viremia was first detected in CON pigs at 56 $D$ while no viremia was found in VAC pigs before the second dose or during the remaining of the grower-finisher period. At slaughter, $91 \%$ of CON pigs were viremic whereas low levels of PCV2 DNA were detected in 2 VAC pigs (17\%) (10E+04 genome copies) $(p<0.001)$. Nasal and fecal samples were qPCR positive for all pigs sampled. The CON pigs had much higher viral load in the nose.

\section{Discussion}

The animals used in this trial originated from a pig flow that had been vaccinated to prevent PCV2 infection for more than 18 months and PCV2 challenge appeared to remain high based on the reduced performance in CON pigs and the presence of PCV2 in feces and nose indicating a constant challenge due to shedding mainly by CON pigs. Vaccination appears to be an important tool to reduce shedding and prevent viremia even in presence of severe PRRS challenge. VAC pigs maintained an adequate ADG. Quantitative ELISA titers and qPCR levels appear to provide a good predictor and indicator, respectively of PCV2 viremia prevention in vaccinated pigs.

\section{References}

1. de Grau AF, et al., Proc 5th ISERPD. Krakow, Poland, pp.119-120, 2007.

2. Carman S, et al., Can Vet J. 47:761-2, 2006.

3. Rovira A, et al ., J Virol 76:3232-9, 2002 


\title{
P.036
}

\section{Comparative Efficacy of Ingelvac CircoFLEX ${ }^{\oplus}$-Ingelvac MycoFLEX ${ }^{\oplus}$ Combined vaccine versus Circumvent PCV2 $^{\mathrm{TM}}$ and Respisure $\mathrm{ONE}^{\circledR}$ in a Commercial Herd}

\author{
Martin Misener \\ Linwood Veterinary Services, Linwood, ON, Canada
}

\section{Introduction}

Mycoplasma hyopneumoniae (Mhyo) vaccines have been routinely used in the growing pig population in North America for nearly two decades. Porcine circovirus type 2 (PCV2) pig vaccines quickly became incorporated as standard vaccination for growing pigs once their availability was established. Since vaccination against Mhyo and PCV2 is widespread for the growing pig it was a logical extension that these two vaccines would be combined to be delivered in a single shot (1).

The objective of this study was to compare an off-label regimen which was being commonly used by veterinarians in Ontario at the time of the study to vaccinate against PCV2 and Mhyo with a combination recently licensed in Canada and the US, Ingelvac CircoFLEX-MycoFLEX. Efficacy and production parameters were evaluated in this study.

\section{Material and Methods}

This study was conducted in an 1500-sow, commercial production herd in Ontario, Canada, weaning about 600 pigs weekly at three weeks of age. A total of 1064, three-week-oil pigs were divided into two groups.

Group 1: On the day of weaning, 532 pigs were vaccinated with $1 \mathrm{~mL}$ of Circumvent PCV2 ${ }^{\mathrm{TM}}$ and $1 \mathrm{~mL}$ of Respisure ONE ${ }^{\circledR}$, both vaccines mixed in a single syringe and delivered as a single dose. Group 1 pigs were revaccinated

three weeks later with $1 \mathrm{~mL}$ of Circumvent PCV2 and $1 \mathrm{~mL}$ of Respisure ONE ${ }^{\circledR}$. Again, both vaccines were mixed in a single syringe and delivered as a single dose. Group 1 pigs received an off-label regimen which was being commonly used by veterinarians in Ontario at the time of the study.

Group 2: On the day of weaning 532 pigs were vaccinated with $1 \mathrm{~mL}$ of Ingelvac CircoFLEX ${ }^{\circledR}$ and $1 \mathrm{~mL}$ of Ingelvac MycoFLEX ${ }^{\circledR}$ as a single $2 \mathrm{~mL}$ combined dose. After vaccination pigs were moved, over a two-week period to fill a one-room finisher barn. Group 1 pigs were placed in pens on one side of the barn and Group 2 pigs placed in pens on the other side of the same room in the barn, all pigs sharing the same airspace, and feed delivery. Measurements were average daily gain total weight gain, (ADG), feed conversion (FC) mortality, and carcass grade.

\section{Results}

At marketing, the end of the study period, total weight, ADG, FC and mortality were $136.6 \mathrm{~kg}$ vs $133.2 \mathrm{~kg}, 880.5 \mathrm{~g} /$ day vs 822.8 g/day, 2.658 vs 2.742 and $2.63 \%$ vs $8.46 \%$, for Group 2 -vaccinated pigs vs Group 1 pigs, respectively (Table 1). Group 2 pigs attained a carcass grade of 103.0 vs 104.0 for Group 1 pigs. (Table 1). Group 2 pigs went to market 4.7 days faster than group 1 pigs. Cost/ kg of gain was calculated to be 92.4c for Group 2 pigs vs $95.3 \mathrm{c}$ for group 1 pigs (Table 1). The net advantage for vaccinating with the combined Ingelvac CircoFLEX-MycoFLEX ${ }^{\circledR}$ calculated to be $\$ 6.38$ per pig.

Table 1. Production parameters for Circumvent PCV2 ${ }^{\mathrm{TM}}$ and Respisure $O N E^{\oplus}$ vaccinated pigs (group 1) vs Ingelvac CircoFLEX-MycoFLEX ${ }^{\circledast}$ (group 2).

\begin{tabular}{|l|c|c|}
\hline Parameter & Group 1 & Group 2 \\
\hline \# of pigs & 532 & 532 \\
\hline Wt In (kg) & 26.7 & 26.7 \\
\hline Wt Out (kg) & 133.2 & 136.6 \\
\hline Wt Gain (kg) & 106.5 & 109.6 \\
\hline ADG (g) & 822.8 & 880.5 \\
\hline Feed conversion & 2.742 & 2.658 \\
\hline Days to market & 129.5 & 124.8 \\
\hline Grading & 104.0 & 103.0 \\
\hline Mortality (\%) & 8.46 & 2.63 \\
\hline Cost/kg gain (\$) & 0.953 & 0.924 \\
\hline Loss/pig (\$) & 28.60 & 21.45 \\
\hline Vaccine cost (\$) & 1.28 & 2.02 \\
\hline Extra labour (\$) & 0.10 & \\
\hline Net Advantage (\$) & - & 6.38 \\
\hline
\end{tabular}

\section{Discussion and Conclusions}

In this study, ADG was $57.7 \mathrm{~g}$ greater, total weight gain was 3.4 $\mathrm{kg}$ more and days to market were 4.7 days faster for the Ingelvac CircoFLEX-MycoFLEX ${ }^{\circledR}$-vaccinated pigs vs Circumvent PCV2 ${ }^{\mathrm{TM}}$ and Respisure $\mathrm{ONE}^{\circledR}$ vaccinated pigs, respectively resulting in an overall net advantage of $\$ 6.38$ for the Ingelvac CircoFLEXMycoFLEX ${ }^{\circledR}$-vaccinated pigs.

\section{References}

Nerem J. Comparative efficacy of various PCV2 and Mycoplasma hyopneumonniae vaccination protocols under conventional herd conditions. Leman Sw Conf. 36: 52-53, 2009. 


\title{
P.037
}

\section{Effect of Ingelvac CircoFLEX ${ }^{\circ}$ on the live weight and uniformity of pigs in a herd with mild PCVD}

\author{
Peggy De Backer; Guy Cluydts \\ SCS Boehringer Ingelheim Comm.V., Brussels, Belgium
}

\section{Introduction}

Infections with Porcine Circovirus Type 2 (PCV2) are widely distributed in the Belgian pig population, however in many cases without obvious clinical symptoms. The objective of this study was to determine the effect of PCV2 vaccination in a herd with mild PCVD.

\section{Materials and Methods}

The trial was performed in a Belgian gilt multiplying herd. Two years earlier, severe PCVD problems were observed in the herd. The problems concerned high mortality at the end of the nursery and early finishing, PMWS and diarrhea. Due to several changes in management such as batch farrowing and all-in allout management, the problem became milder and at the start of the trial there was an absence of clinical signs associated with PCV2.

Sows farrowed in batches, in a 3 week interval. In total 6 batches of piglets were involved in the trial. Of these batches, every other batch was vaccinated with Ingelvac CircoFLEX ${ }^{\circ}$ at an average age of $19.5+2.1$ days. The other batches were left as unvaccinated controls.

The female piglets (future gilts) were individually eartagged and followed during the growing period. Individual live weight registration of the gilts was performed at selection, at an average age of 176.7 days for the Ingelvac CircoFLEX ${ }^{\circ}$ and 176.3 days for the unvaccinated control group.

\section{Results}

At 25 weeks of age, the vaccinated pigs had a $7.5 \mathrm{~kg}$ higher live weight compared to the unvaccinated control animals $(P<0.001)$ and there was a trend towards a lower variance $(P=0.078)$ in the live weights of the vaccinated group (table 1). Both the higher live weight and better uniformity in the Ingelvac CircoFLEX ${ }^{\circ}$ group are reflected in figure 1. The distribution of live weights of the vaccinated animals is shifted to the right, indicating the $7.5 \mathrm{~kg}$ higher live weight and is narrower, indicating the better uniformity of the animals.
Table 1 Live weight of gilts at 25 weeks of age

\begin{tabular}{|l|c|c|c|}
\hline & Control & $\begin{array}{c}\text { Ingelvac } \\
\text { CircoFLEX }\end{array}$ & P-value \\
\hline Mean live weight $(\mathrm{kg})$ & 114.4 & 121.9 & $<0.001^{*}$ \\
\hline Variance & 185.9 & 157.2 & $0.078^{* *}$ \\
\hline Standard Deviation & 13.6 & 12.5 & - - \\
\hline No $^{\circ}$ of animals & 165 & 192 & - - \\
\hline${ }^{*}$ t-test ${ }^{* *}$ Levene test for homogeneity of variances \\
\hline
\end{tabular}

Figure 1 Live weight distribution of gilts at 25 weeks of age

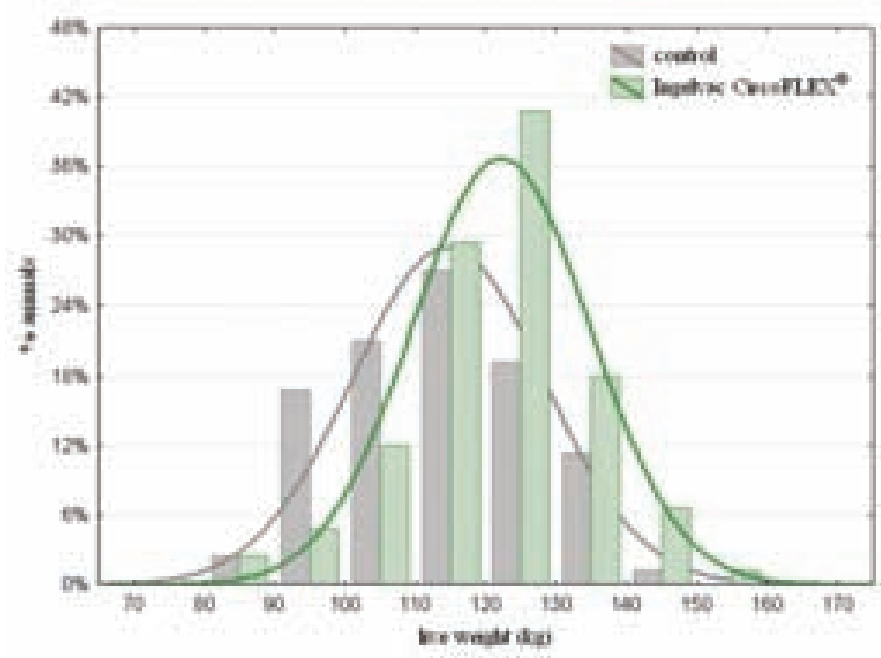

\section{Discussion}

The results of this field study show that even in mild cases of PCVD, with no apparent clinical signs, PCV2 vaccination can result in a significant improvement of production parameters such as daily gain. This better daily gain with tendency towards better uniformity may be a decisive argument for the use of the vaccine, in order to have an optimal expression of the genetic potential of gilts. 


\title{
P.038
}

\section{Safety of a single $2 \mathrm{ml}$ dose of Porcilis ${ }^{\circledR}$ PCV administered to pigs of 3 weeks of age under field conditions on a farm with confirmed PMWS}

\author{
Alexander A. Eggen ${ }^{1}$ Jos Smeets ${ }^{1}$ K. Kühnel ${ }^{2}$ J. Ellert ${ }^{2}$ \\ 1. Intervet/Schering-Plough, Boxmeer, Netherlands; 2. Tierärztliche Gemeinschaftpraxis Wilms-Schulze Kump, Visbek, Germany
}

\section{Introduction}

Porcine Circovirus type 2 (PCV2) infection is regarded as an important, but not the only, causative factor in the development of Porcine Circovirus Disease (PCVD) in piglets. Many other pathogens, management factors and as well as immunestimulating compounds (Krakowka, 2001; Grasland, 2005) have been mentioned as contributory to the clinical expression of the disease.

PCVD can occur in pigs of different ages and under field conditions, and the timing of the PCV2 virus infection is often unknown. Pigs are normally vaccinated early in life against PCVD all over the world.

The object of this study was to demonstrate the safety of a potent adjuvated vaccine (Porcilis PCV) in piglets of 3 weeks of age in the presence of PCV2 field virus.

\section{Materials and Methods}

The farm was selected because it had suffered an outbreak of PCVD, confirmed by clinical and post mortem investigations according to the guideline "PMWS case definition (herd level)". PCV2 DNA was detected in pigs by Q-PCR prior to and during the study.

In all, 2.5 production batches (517 piglets) were vaccinated and the parameters measured were compared with a reference group of 696 unvaccinated piglets from previous production batches.

Porcilis PCV is a sub-unit vaccine containing the capsular protein coded by ORF2 of PCV2 virus in an adjuvant consisting of a-tocopherol and light mineral oil (X-Solve ${ }^{\circledR}$ ). The product was allowed to reach room temperature (20-250C) and shaken before use. A $2 \mathrm{ml}$ dose was administered to piglets of 15-21 days of age in the farrowing unit, by intra-muscular injection with a needle $25 \mathrm{~mm}$ long.

Sows were routinely vaccinated against PRRS, Parvo, Erysipelas, Glässer, E.coli, Clostridium and Influenza. Piglets were treated with amoxicillin on day 1 and on days 3-5 and with iron. No other vaccines were given concurrently.

The piglets were scored for local and systemic (general health) reactions at the time of vaccination, 4 hours later, and on Day 7 and Day 14 following vaccination. Food intake was also scored during the same 14-day period.

The individual pig served as the statistical unit and descriptive statistics were used to present and summarize the data. Mortality data were recorded for the whole period up until slaughter, as a general indication of the effect of vaccination on performance
All management and other factors remained the same for the whole time the reference group and the trial group were on the farm.

\section{Results}

All piglets were healthy before the start of the study.

Local reactions: none of the 517 piglets vaccinated with Porcilis PCV showed any reaction during the 14-day observation period.

Systemic reactions and general health: none of the 517 piglets vaccinated with Porcilis PCV showed any systemic reaction or deviation from normal health during the 14-day observation period.

Feed intake was normal for the age of the pigs, which reinforced the assessment of their good general health.

Mortality: the mortality in the reference group $(n=696)$ was $5.19 \%, 6$ pigs died and 30 were culled. In the Porcilis PCV vaccinated group $(n=517), 11$ piglets died $(2.13 \%)$ and none were culled.

\section{Discussion}

Porcilis PCV is a highly efficacious vaccine based on the immunogenic capsular protein of PCV2 virus, with X-Solve as a potent adjuvant. This study shows that Porcilis PCV, although highly immunogenic, can be used safely on farms with confirmed PCVD (PMWS). No adverse effects of the immune-stimulation, as described by Krakowka, were noted, which accorded with Astrup's (IPVS 2010) report of a safety study executed in Denmark involving different breeds.

However, the package leaflet of Porcilis PCV (EMEA 2009) does contain a warning of possible local and systemic reactions following injection, and Astrup (IPVS 2010) found such reactions.

It appears that differences between farms, and the health of the piglets at the time of vaccination, account for the differences in the degree and extent of the side-effects of vaccination (data on file). Nevertheless, the incidence of these side-effects is low, and piglets recover completely when no other debilitating factors are present.

This study confirms the safety of Porcilis PCV. The importance of the handling and administration of the vaccine in strict accordance with the manufacturer's instructions cannot be over-estimated, and should always be brought to the attention of anyone involved in its administration.

\section{References}

Krakowka; Vet Path 2001.Grasland; Vet Res 2005.

Astrup, IPVS 2010. EMEA; 2009. 


\title{
P.039
}

\section{Immunogenicity of "DS Circo Pigvac" in the Various Levels of Maternal Antibodies against Porcine Circo Type 2 virus ( PCV-2 )}

\author{
Won Hur; Pill Soo Lee; Kang Soeng Joung; Yung Sik Mun; Sung D. Jung \\ Dae Sung Microbiologicals Co. Ltd., Eui Wang, Kyung Ki Do, Korea
}

\section{Introduction}

Currently available circo vaccines in the market are classified into two types.

One type is vaccine for piglet mostly at three weeks old; another type is claimed for sow.

The reasons for the claims can probably be attributed to the absence of the effective evaluating methods for the antibodies against circovirus after vaccination.

In order to evaluate the immunogenicity of "DS Circo Pigvac," we developed immunofluorescent assay (IFA) against circovirus and applied to the field test in post-weaning piglets with the various level of maternal antibodies after vaccination in the field.

\section{Materials and Methods}

1) Each group of piglets of 3 weeks old born from sero-positive sow against PCV-2 was selected and housed to estimate the level of the maternal antibodies in the isolator.

2) A total 60 piglets of 3 weeks old were tested for the initial level of the maternal antibody titers against PCV-2 and monitored for their antibody trends after vaccination.

\section{Results}

1) The maternal antibodies against PCV-2 were rapidly decreased in piglets. Initially log210 titer by IFA was decreased to $\log 27$ at 6 weeks old piglets. The piglets of negative titer showed the increase of antibody titer from 14 weeks due to the natural PCV-2 infection (Table 1).

Table 1: Comparison of PCV-2 Maternal Antibodies (MAb) between Acquired and Naturally Infected Titer in Piglets

\begin{tabular}{|l|c|c|c|c|c|c|c|}
\hline $\begin{array}{l}\text { Pig No./ } \\
\text { Weeks }\end{array}$ & & Maternal & $\begin{array}{c}\text { Anti- } \\
\text { bodies }\end{array}$ & Titer by & IFA(log2) & & \\
\hline & 3 & 6 & 8 & 10 & 12 & 14 & 16 \\
\hline 1 & 11 & 9 & 7 & 5 & $<3$ & $<3$ & $<3$ \\
\hline 2 & 10 & 7 & 6 & $<3$ & $<3$ & $<3$ & $<3$ \\
\hline 3 & 9 & 6 & $<3$ & $<3$ & $<3$ & 11 & 12 \\
\hline 4 & 8 & 5 & $<3$ & $<3$ & $<3$ & 12 & 12 \\
\hline 5 & 7 & $<3$ & $<3$ & $<3$ & $<3$ & 12 & $>12$ \\
\hline 6 & $<3$ & $<3$ & $<3$ & $<3$ & $<3$ & 11 & 12 \\
\hline
\end{tabular}

2) The immunogenicity of "DS Circo Pigvac" in 3 weeks old piglets revealed that while piglets with maternal antibodies titer lower than log 28 showed the increased antibody titers from 6 week, the group with higher than log29 from 10 week after vaccination ( Table 2 ).

Table 2. Immune Responses of "DS Circo Pigvac "Vaccination in 3 Week-Old Piglets Showing Different MAb Titer

\begin{tabular}{|l|c|c|c|c|}
\hline MAb / Weeks & Titer after & Vaccination by & IFA(log2) & \\
\hline & 3 & 6 & 10 & 16 \\
\hline $\log 2^{10}$ & 10.00 & 6.25 & 7.25 & 11.00 \\
\hline $\log 2^{9}$ & 9.00 & 4.75 & 1063 & 10.50 \\
\hline $\log 2^{8}$ & 8.00 & 8.43 & 10.71 & 10.86 \\
\hline $\log 2^{7}$ & 7.00 & 10.22 & 10.78 & 11.00 \\
\hline
\end{tabular}

\section{Discussions}

Since the maternal antibodies was rapidly disappeared and showed the variable antibody levels by the colostral intake, the preventive strategies through maternal antibodies is not proper.

Even in "DS Circo Pigvac" which has the enough antigen amount was also interfered with the immune responses of piglets about the maternal antibody titers more than log29.

However, it was found that the antibody titers were increased in piglets at 10 week mainly results from the oil adjuvant based formula. This result indicated the oil adjuvant efficacy in PCV-2 vaccination.

Moreover, the present data suggest the vaccination on pregnant sow is not proper strategy for the preventive measures against PCV-2.

For the effective PCV-2 protection in the sow claim, actually premating vaccination is recommended than pre-parturition from our side.

\section{References}

1. Kim, J. Choi, C. Chae, C. Pathogenesis of Post weaning Multisystemic Wasting Syndrome Reproduced by Co-infection with Korean Isolates of Porcine Circovirus 2 and Porcine Parvovirus. J. Comp. Path. 2003, Vol. 128, 52-59.

2. Park, Choi-Kyu. Kim, Hyun-Soo. Detection of viral pathogens and isolation of porcine circovirus 2 from post weaning multisystemic wasting syndrome-affected piglets. Korean J. Vet. Res. 2004, Vol. 444, 561-569. 


\title{
Field trial comparing two PCV2 vaccines on a farm with late PCV2 infection
}

\author{
Roselyne Fleury ${ }^{1}$ Jessica Thomas ${ }^{1}$ Francois-Xavier Orveillon ${ }^{2}$ \\ 1. Pigalys, Vannes, France; 1. Boehringer Ingelheim, Ingelheim am Rhein, Germany
}

\section{Introduction}

This study took place on a farrow-to-finish farm of 700 sows in France. In Q3 2008, the mortality rate in fattening reached $5.8 \%$ and average daily gain (ADG) over the same period 794 $\mathrm{g} / \mathrm{d}$. Circovac ${ }^{\circledR}$ vaccination was routinely implemented in sows $(2 \mathrm{ml})$ since 2006 and in piglets of four weeks of age $(0.5 \mathrm{ml})$ since September 2007. A screening of the farm in September-October 2008 demonstrated that the PCV2 infection was particularly high at the end of fattening. Necropies revealed typical PMWS lesions in lymphoid tissues which were also positive for PCV2 antigen by IHC. From that time point, due to the continued occurrence of PMWS cases, the veterinarian in agreement with the farmer decided to change the routine PCV2 vaccination in piglets to Ingelvac CircoFLEX ${ }^{\circledR}$. Two months later, a study aiming at comparing the ability of both vaccines in preventing the impact of the PCV2 infection on growth performances and mortality, was implemented.

\section{Material and methods}

The trial was conducted in compliance with the good clinical practice. A total of 1185 4-week-old piglets originating from Cir$\operatorname{covac}^{\circledast}$ vaccinated sows were included over three week groups (WG1, WG2 and WG3) and divided into two treatment groups: CircoFLEX ${ }^{\circledR}$ group $(n=535)$ was injected with Ingelvac CircoFLEX ${ }^{\circledR}$ $(1 \mathrm{ml} \mathrm{IM})$, Circovac $^{\circledast}$ group $(n=548)$ was injected with Circovac $^{\circledR}$ $(0.5 \mathrm{ml} \mathrm{IM})$. A control group ( $\mathrm{n}=102)$ was included to demonstrate presence of PCVD during the trial, control pigs were injected with water for injection ( $1 \mathrm{ml} \mathrm{IM}$ ). Groups were balanced with regard to initial body weight and litter assignment. All animals from the three groups were co-mingled throughout the study ensuring that all animals were under the same housing and infection conditions. Approximately $8 \%$ of the control animals were sampled at 5 time points $(4,10,15,19$ and 22 weeks of age) to identify the time and profile of the PCV2 infection by Real-Time PCR. All study animals were weighed three times $(4,11$ and 23 weeks of age). Weight gain differences between groups were tested using analysis of variance and subsequent t-tests. Mortality between vaccinated groups were compared by Fisher's exact test.

\section{Results}

PCV2 infection was confirmed to occur after 15 weeks of age (study week 11) with a peak at the end of fattening. The presence of PCV2 was further confirmed in necropsies (including $\mathrm{IHC})$.

During the fattening period (11 to 23 weeks of age) when the PCV2 field infection took place, the mortality was significantly reduced in the CircoFLEX ${ }^{\circledR}$ group (1.5\%) when compared to the Circovac $^{\circledR}$ group (3.7\%, $\left.\mathrm{p}=0.0338\right)$. The increase in mortality coincided with the onset of viremia (Figure 1). Over the same period, the CircoFLEX ${ }^{\circledR}$ and Circovac $^{\circledR}$ groups out-performed the control group respectively by $1.75 \mathrm{~kg}(p=0.0640)$ and 1.46 $\mathrm{kg}(\mathrm{p}=0.1226)$. Weight gain between the two treatment groups did not differ significantly ( $p=0.5881)$. When interpreting the differences between the two treatment groups and the control group, it should be taken into account that the control group was primarily included for epidemiological reasons and only comprised 102 pigs.

Figure 1: Cumulative mortality in fattening.

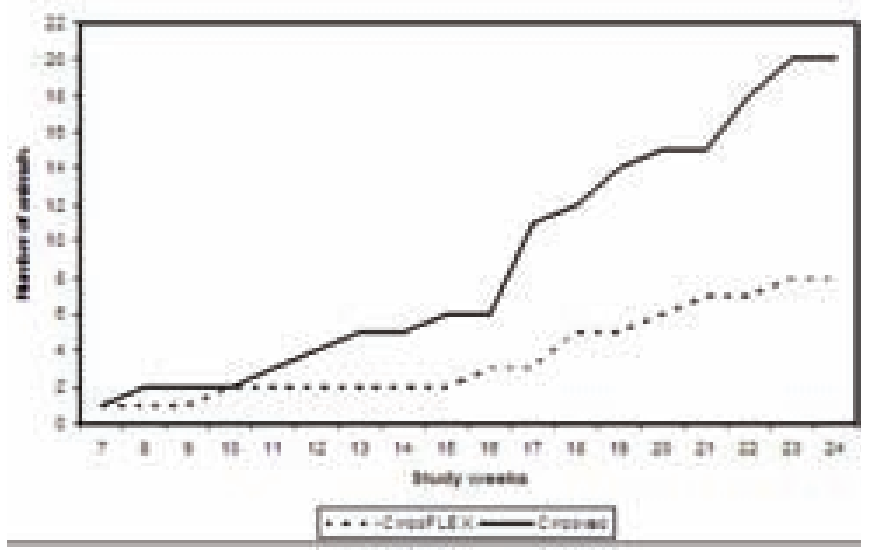

\section{Discussion}

The presence of PCVD on the farm and the late onset of PCV2 infection were confirmed in control animals during the study, demonstrating that sow vaccination does not protect pigs through to slaughter. In addition, Ingelvac CircoFLEX ${ }^{\circledR}$ provided superior protection compared to $\operatorname{Circovac}^{\circledR}$ used in piglets, as shown by a significant reduction in mortality. A significant difference in weight gain between the two treatment groups was not expected as the PCV2 infection occurred late. Furthermore, it is likely that the higher mortality rate in the Circovac $^{\circledR}$ group positively influenced the weight gain by eliminating the poor doers from the calculation. Beside the study results, the superior efficacy of Ingelvac CircoFLEX ${ }^{\circledR}$ is confirmed by the consistently good performance after implementing piglet vaccination with Ingelvac CircoFLEX ${ }^{\circledR}$. Indeed, in Q2 and Q3 2009 when all pigs where vaccinated with Ingelvac CircoFLEX ${ }^{\circledR}$ mortality in fattening was 3.1 and 3.8\%, compared to 4.9 and $5.8 \%$ in Q2 and Q3 2008 when only Circovac ${ }^{\circledR}$ vaccinated pigs where present, reflecting a reduction in mortality of about $2 \%$, very similar to what has been observed in this side-by-side study. 


\title{
P.041
}

\section{Value through vaccination: vaccination against ileitis and PCV2 in a Danish herd}

\author{
Kristian T. Havn² ${ }^{2}$ anne Bak ${ }^{1}$ \\ 1. Boehringer Ingelheim Vetmedica, Copenhagen, Denmark; 2. Svinevet, Haderslev, Denmark
}

\section{Introduction}

Vaccination against either ileitis (1) or PCV2-virus (2) has proven to be economically efficient tools to increase the performance of pigs infected with ileitis or PCV2. Many herds are infected with both organisms, and the question is, whether the economical benefits will hold when using the 2 vaccines in the same herd.

This paper evaluates the benefits of vaccinating against Lawsonia intracellularis and PCV2 in a herd affected by both pathogens.

\section{Materials and Methods}

The study was carried out in a Danish SPF herd that received 1000 weaners every 7th week. At $30 \mathrm{~kg}$ live weight, pigs were moved to fattening units at a different site. Before the vaccination program, the pigs had reduced average daily weight gain (ADWG) and an increase in feed conversion rate (FCR) and mortality. About $10 \%$ of the pigs became pale and lost weight at the end of the finishing period. After blood sampling, a high amount of PCV2-virus was detected by qPCR, and antibodies against Lawsonia intracellularis were found. Vaccination against PCV2 and ileitis started at the same time, with Ingelvac CircoFLEX ${ }^{\circledR}$ (Boehringer Ingelheim) administered i.m. at 2 weeks of age in the sow herd, and Enterisol ${ }^{\otimes}$ Ileitis Vet. (Boehringer Ingelheim) given in the drinking water at 4 weeks of age after arrival to weaning unit. Every 7th week, production data was collected. Comparison of vaccinated and non-vaccinated pigs was done with ANOVA for ADWG, FCR and prescriptions of antibiotics, and with Fishers Exact test for mortality, using $\mathrm{p} \leq 0.05$ as significance level. The economical benefit was calculated using key values from the Danish Pig Producers Organisation (3).

\section{Results}

A statistically significant improvement of the performance of pigs vaccinated with the 2 vaccines was recorded for the 4 parameters (table 1): ADWG, feed conversion, mortality and antibiotic expenses ( $p$-values 0.0130, 0.0010, <0.0001 and 0.0156). The economic value of the improvements exceeded the vaccine cost, with a positive return of investment (ROI) of 1:2.5.

Table 1: Performance of non-vaccinated and double vaccinated finishers (Ileitis and PCV2-vaccine).

\begin{tabular}{|l|c|c|c|c|}
\hline Mean/period & Non-vacc. & Vacc. & Diff. & Value (€/pig) \\
\hline ADWG (g/day $(128$ & 972 & $+44^{*}$ & 0.59 \\
\hline FCR (FE/kg) & 2.85 & 2.72 & $-0.13^{*}$ & 2.43 \\
\hline Mortality (\%) & 3.4 & 1.6 & $-1.79^{*}$ & 1.34 \\
\hline Antibiotics (€/pig) & 2.18 & 1.02 & $-1.15^{*}$ & 1.15 \\
\hline Total value & $5.50 € /$ pig & & \\
\hline *Values with a significant difference $(\mathrm{p} \leq 0.05)$. \\
\hline
\end{tabular}

\section{Discussion}

This study shows that it is economically beneficial to vaccinate against ileitis and PCV2. Every $€$ spend on vaccines is paid back 2.5 times due to an increased ADWG and reductions in FCR, mortality and antibiotic expenses.

Fig. 1: Mortality of finishers before and after vaccination against lleitis and PCV2.

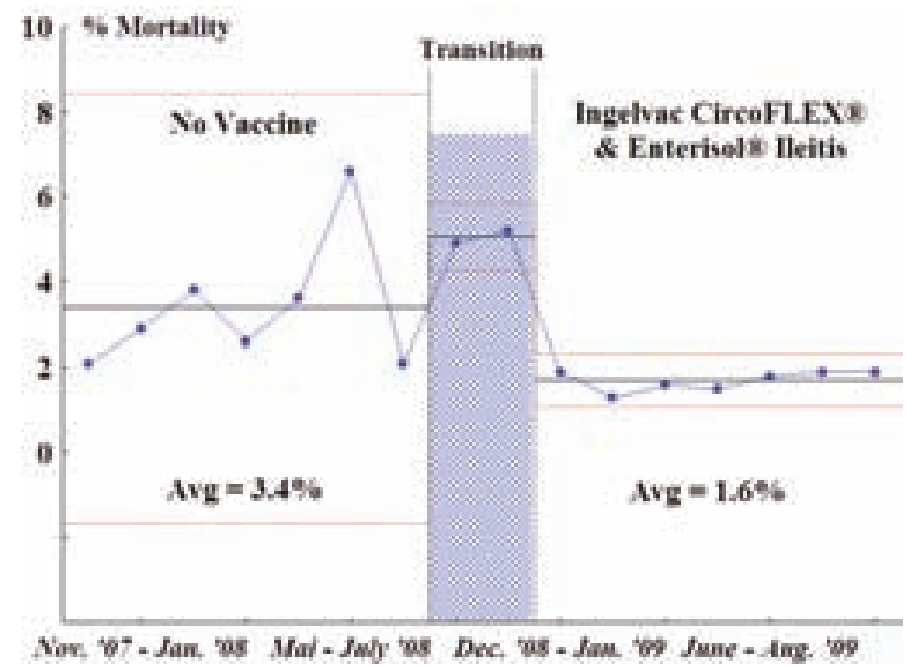

\section{References}

1. Hardge, T. et al. (2004): Pig J. 54, 17 - 34.

2. Cline et al. (2008): Vet. Rec. 163, 737-740.

3. Udesen, F (2007): Note No. 0733, from http://vsp.lf.dk/Publikationer/ Kilder/notater/2007/0733.aspx 


\title{
P.042
}

\section{A Philippine Farm Experience using Ingelvac Circoflex}

\author{
Jonathan Olanday ${ }^{1}$ Rolando Tambago ${ }^{1}$ Marc Bautista $^{2}$ \\ 1. Asturias Farm, Asturias, Cebu, Philippines; 2. Boehringer Ingelheim Philippines Incorporated, Makati City, Philippines
}

\section{Introduction}

Porcine Circovirus 2-associated disease has clearly become a major and complex international problem (1). It is also a common disease in swine farms in the Philippines. This disease can create problems in the farm like increase post-weaning mortality (Post W.M.) and decrease average daily weight gain (ADWG). This study evaluates the benefits of using Ingelvac CircoFLEX on a 1,100 sow farm.

\section{Materials and Methods}

The farm is positive for PCV2, Haemophilus Parasuis, Actinobacillus Pleuropneumoniae, Mycoplasma Hyopneumonia. The farm implemented Ingelvac Circoflex vaccination, $1 \mathrm{ml}$ at day 21 , in December 2008. Weaning day is at day 28-30. The farm records from June 2008 to October 2009 were compiled into a longitudinal study. Except PCV2 vaccination, no other major changes occurred on the farm The monthly data of ADWG and Post W. M. was evaluated through Statistical Process Control (SPC, Statistica v8.0). Data from June - November 2008, December 2008 to April 2009 and May 2009 to October 2009 were grouped into three periods namely Pre-vaccination, Transition and Vaccination respectively. The Transition period was included to ensure that only fully vaccinated groups were compared to completely nonvaccinated ones.

ADWG is evaluated by Simple ANOVA, and adjusted by the Dunnett test. Mortality is evaluated using a Chi-square test. All are tested for an Alpha value equals 0.05 .

\section{Results and Discussion}

The wean-to-slaughter ADWG of the farm during the vaccinated period is 545 grams/day. It has improved by 39 grams/day as compared to the ADWG during the Pre-vaccination period $(p<$ 0.0004 , fig. 1). Mortality in nursery was not significantly different between the two groups ( $4.6 \%$ vs. $5.9 \%, p=0.17$ ) In contrast, the grow-finisher mortality was significantly reduced after introduction of PCV2 vaccination by $50 \%$ from $10.4 \%$ to $5.2 \%$ ( $p<0.004$, fig. 2).
Figure 1. Control chart of growth over the 3 periods.

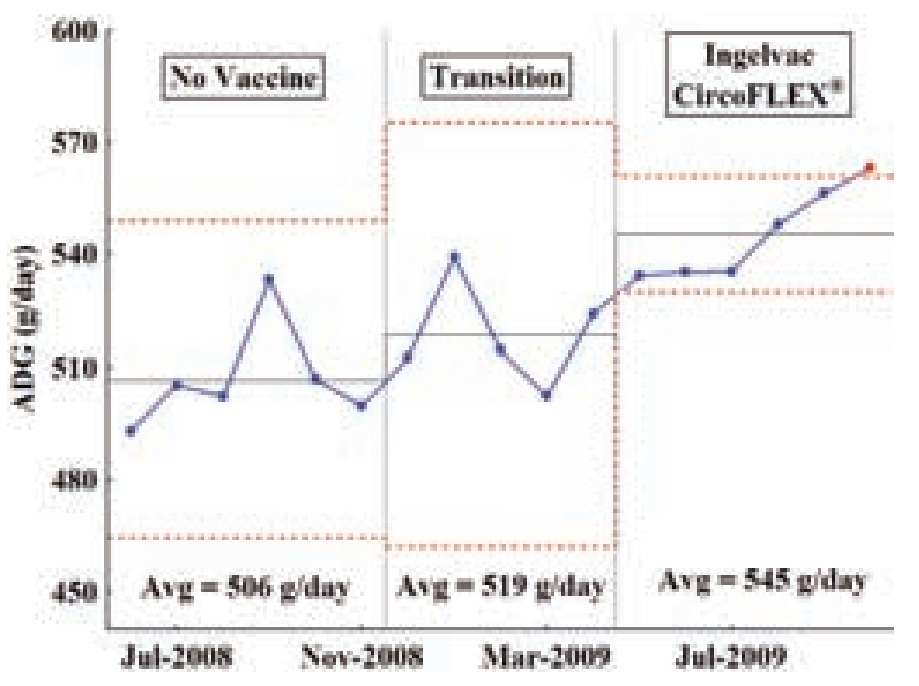

Figure 2. Controls chart for the grow-finisher mortality over the 3 periods.

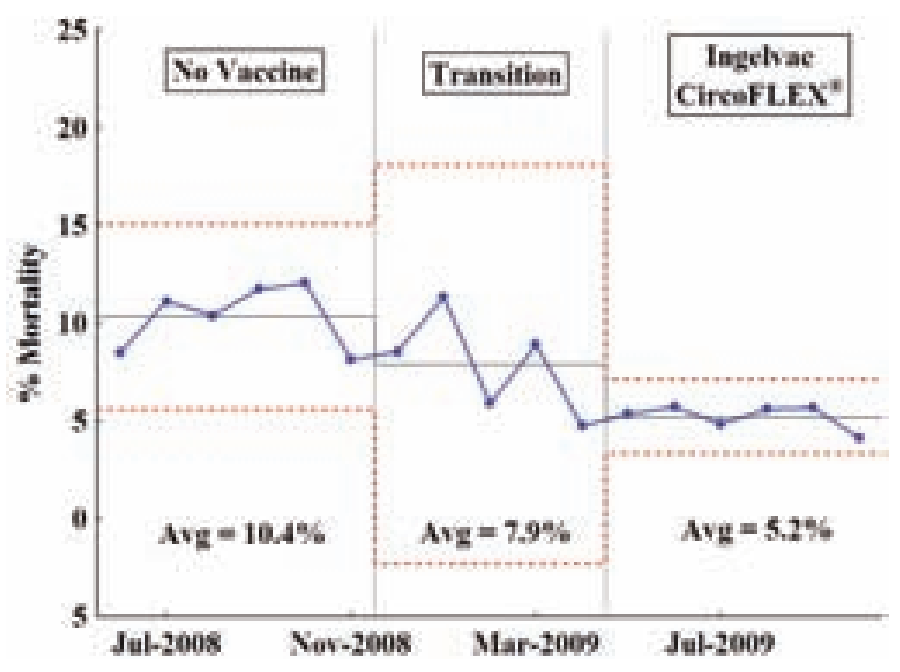

\section{Conclusion}

Introduction of PCV2 piglet vaccination significantly reduced mortality and improved performance on the farm.

\section{References}

1. Harding, JC. (2004). Vet. Microbiol. Feb 4; 98 (2): 131-1355 


\title{
P.043
}

\section{Efficacy of Ingelvac CircoFLEX ${ }^{\circledast}$ in mild forms of PCVD in Thai swine farms}

\author{
Nathaya Duangwhae; Lerdtida Meedacha; Sopon Kongtes; Jitjaroen Channarong \\ Boehringer Ingelheim (Thai), Bangkok, Thailand
}

\section{Introduction}

Recently several PCV2 vaccines have been launched in Thailand. The introduction of PCV2 vaccines has allowed greater control of PCVD. Significant reduction in mortality and improvement in finishing production parameters of swine herds using a single dose PCV2 piglet vaccine, Ingelvac CircoFLEX ${ }^{\circledR}$, were observed throughout Thailand, even though most farms had both PRRSv and PCV2 infection.

The objective of this study was to compare finishing performance parameters in non-vaccinated and Ingelvac CircoFLEX ${ }^{\circledR}$ (Boehringer Ingelheim) vaccinated groups of pigs on two farms, with low mortality in finishing $(<2 \%)$.

\section{Material and method}

Two conventional, finishing pig farms, farm $A$ and $B$, were included in this evaluation. Both farms have used Ingelvac ${ }^{\circledR}$ PRRS MLV and are PRRS stable. The piglets in these farms are serologically negative to PRRS up to 16 weeks of age but seropositive to M. hyopneumoniae and PCV2 virus. Piglets are weaned at 3 weeks of age, raised in the nursery then moved to the finishing site when 8-10 weeks old. This study included, 1,152 non-PCV2 vaccinated pigs (before vaccination) and 1,161 vaccinated pigs (after vaccination). Vaccinated pigs received a single dose $1 \mathrm{ml}$ IM of Ingelvac CircoFLEX ${ }^{\circledR}$ at 21 day of age. Parameters recorded in finishing farms included ADG, total losses, mortality and culling rate, i.e. pigs euthanized before slaughter. The difference in total finishing losses (mortality plus culls) was statistically evaluated using a Chi-square test (Statistica, v.8.0)

\section{Results}

Performance data of the vaccinated and the non-vaccinated pigs are shown in Table1. The ADG of the vaccinated groups was higher than in the non- vaccinated groups, $+56 \mathrm{~g}$ on farm $\mathrm{A}$ and $+81 \mathrm{~g}$ on farm $B$. Total losses were significantly reduced in vaccinated groups on both farms: 1.65 vs $4.51 \%(p=0.002)$ on farm $A$ and 2.69 vs $5.49 \%(p=0.04)$ on farm $B$.
Table1. Performance parameter comparison between vaccinated and non-vaccinated pigs.

\begin{tabular}{|l|c|c|c|c|c|}
\hline & & No. of pigs & Mortality (\%) & Culls (\%) & ADG (g/day) \\
\hline Farm A & $\mathrm{Nx}$ & 732 & 1.78 & 2.73 & 664 \\
\hline & $\mathrm{V} x$ & 721 & 1.25 & 0.40 & 720 \\
\hline & $\mathrm{Diff}$ & & -0.53 & -2.69 & 56 \\
\hline Farm B & $\mathrm{Nx}$ & 420 & 1.19 & 4.3 & 720 \\
\hline & $\mathrm{Vx}$ & 440 & 0.68 & 2.0 & 801 \\
\hline & Diff & & -0.51 & -2.3 & 81 \\
\hline
\end{tabular}

\section{Discussion and conclusion}

Vaccinating piglets with Ingelvac CircoFLEX ${ }^{\circledR}$ in PCV2 infected, PRRS stable herds with low mortality improved finishing performance parameters. This comparison clearly shows the economic benefit of Ingelvac CircoFLEX ${ }^{\circledR}$ under field conditions in face of mild form of PCVAD in finishing period.

\section{Reference}

1. Feldmann L. et al., 2008. Effects of porcine circovirus type2 (PCV2) vaccination on mortality and average daily gain. AASV. p. 317-319.

2. King D., 2008. Benefits of controlling subclinical PCVAD in a large US operation. Boehringer Ingelheim satellite symposium. 24th IPVS. p. 28-31. 


\title{
P.044
}

\section{Field Experience with a subunit PCV2 vaccine in a Chinese Farm with severe PCVD}

\author{
Longtao Yao ${ }^{1}$ Huarong Dai ${ }^{2}$ Brook Fang ${ }^{3}$ Nian Huang ${ }^{3}$ Hanchun Yang $^{4}$ \\ 1. Shanghai Fengxiang Veterinary Center, Shanghai, China; 2. Yuejin Pig Farm, Zhanjiang, China; 3. Boehringer Ingelheim Int'I \\ Trading (Shanghai) Co. Ltd., Beijing, China; 4. China Agricultural University College of Veterinary Medicine, Beijing, China
}

\section{Introduction}

Porcine Circovirus type 2 (PCV2) is widespread and is recognized as the causal agent of post weaning multisystemic wasting syndrome (PMWS) [1] [2]. PMWS was first described in Canada in 1996. Nowadays PMWS and other PCV2 related diseases (PCVD) are present in virtually all pig producing countries [3], causing huge losses to the global swine industry if not effectively controlled. In China PCVD also has a great negative impact on the domestic pig industry. In this study, the efficacy of an imported PCV2 subunit vaccine was investigated in a severe case of PCVD in China.

\section{Materials and Methods}

A one-site production system farm of 1200 sows in Shandong suffered from PCVD. Production performance was severely damaged by the PCV2 infection. High culling and death rates in the nursery and fattening stages were the main causes for economical losses. The farm was positive for PRRSV, PCV2-a, CSFV and Haemophilus parasuis (HPS). The farm started piglet vaccination against PRRS (Ingelvac ${ }^{\circledR}$ PRRS MLV) and HPS (Ingelvac ${ }^{\circledR}$ HP-1) around 7-10 days old from November 2008. However, production performance did not recover to the optimum level. In January 2009, a side-by-side trial was carried out including a total of 800 piglets in two weekly batches. Piglets in the vaccinated group $(n=380)$ received an imported PCV2 subunit vaccine, Ingelvac ${ }^{\oplus}$ CircoFLEX, at 14 days old. The control group comprised 380 non-vaccinated pigs. Average daily weight gain (ADWG), mortality and FCR were recorded in nursery and fattening.

The average daily weight gain (ADWG) was analyzed by t-test, mortality by Chi-square test.

\section{Result}

The results are summarized in Table 1 (nursery performance) and Table 2 (fattening performance). Performance was significantly improved in vaccinated pigs, both in nursery and fattening stages. In nursery mortality was reduced by about $80 \%$, from 22.2 to $4.7 \%$ in vaccinated pigs, whereas ADWG was improved by more than $40 \mathrm{~g} /$ day. Mortality in fattening was reduced by about $70 \%$ from 7.4 to $2.3 \%$ and ADWG increased by about $70 \mathrm{~g} /$ day.
Table 1. Average growth performance (Nursery)

\begin{tabular}{|l|c|c|c|}
\hline & V.G. & C.G. & Diff \\
\hline ADWG (g/day) & 376.5 & 327.9 & $+45.7^{* *}$ \\
\hline Mortality & 4.7 & 22.2 & $-17.5^{* *}$ \\
\hline FCR & 1.89 & 2.25 & -0.36 \\
\hline${ }^{*} p<0.05^{* *} p<0.01$ \\
V.G.: Vaccinated Group C.G.: Control Group \\
\hline
\end{tabular}

Table 2. Average growth performance (Fattening)

\begin{tabular}{|l|c|c|c|}
\hline & V.G. & C.G. & Diff. \\
\hline ADWG (g/day) & 774 & 705 & $+69^{* *}$ \\
\hline Mortality (\%) & 2.3 & 7.4 & $-5.1^{*}$ \\
\hline $\mathrm{FCR}$ & 2.66 & 2.95 & -0.29 \\
\hline${ }^{*} \mathrm{p}<0.05^{* *} \mathrm{p}<0.01$ \\
V.G.: Vaccinated Group C.G.: Control Group \\
\hline
\end{tabular}

\section{Discussion and Conclusion}

In this farm with severe PCVD problems piglet vaccination with a PCV2 subunit vaccine showed excellent efficacy: it effectively controlled the negative impact of the PCV2 infection, significantly reduced PCV2 related mortality, improved the ADWG in nursery and fattening stages, finally stabilized whole herd production. Therefore, it can be concluded that proper use of PCV2 piglet vaccination can effectively control PMWS, PDNS and other PCV2 related diseases, with significant benefits for Chinese pig farms.

\section{References}

[1] Harding JCS., Clark E. G., 1997. Recognizing and diagnosing post weaning multisystemic wasting syndrome (PMWS) [J]. Swine Heal Prod.5 (5) :201-203.

[2] Clark E.G., 1997. Post weaning multisystemic wasting syndrome [A]. Proceedings of the Americans Association of Swine Practitioners[C]. 499-501

[3] Harding JCS, 2009. Porcine circoviral disease-from inception to successful control. 3rd Boehringer Ingelheim Asian PRRSpective Symposium: 28-37. 


\title{
P.045
}

\section{Serological timeline of Circumvent ${ }^{\circ}$ PCV and MycoSilencer ${ }^{\circ}$ ONCE compliance monitoring}

\author{
Jeremy S. Pittman ${ }^{1}$ Brad J. Thacker² John K. Johnson ${ }^{3}$ Sheela Ramamoorthy ${ }^{3}$ \\ 1. Murphy-Brown, LLC, Waverly, VA, USA; 2. Intervet/Schering-Plough Animal Health, Desoto, KS, \\ USA; 3. lowa State University, Veterinary Diagnostic Laboratory, Ames, IA, USA
}

\section{Introduction}

Previously it has been shown that the PCV2 Differential ELISA and Mycoplasma Tween 20 ELISA available at lowa State University, Veterinary Diagnostic Laboratory (ISU-VDL) can be useful tools for vaccination compliance monitoring., ${ }^{1,2}$ Further value could be gained if these tools could be used retrospectively in an investigative manner to determine if groups of sick pigs had not been vaccinated properly. Accordingly, the objectives of this study were to determine the longevity of vaccine induced antibodies and potential development of "background" antibodies.

\section{Materials and Methods}

A total of 150 pigs from 30 litters were used. Within each litter, one pig (control - CON) was vaccinated at weaning ( 3 weeks of age) with single dose PCV2 and Mycoplasma hyopneumoniae (Mhyo) vaccines that do not induce measurable antibodies. The other 4 pigs were vaccinated with Circumvent ${ }^{\circ}$ PCV and MycoSilencer ONCE at weaning and 21 days later (VAC). At 15 weeks of age, $15 \mathrm{CON}$ and $25 \mathrm{VAC}$ pigs were vaccinated with Circumvent ${ }^{\circ} \mathrm{PCV}$ and are designated as RE-CON and RE-VAC, respectively.

All samples were tested at the ISU-VDL by PCV2 Differential ELISA and Mhyo Tween 20 ELISA.3,4 The PCV2 test was modified to measure antibodies to the baculovirus portion of Circumvent ${ }^{\circ} \mathrm{PCV}$. In addition, the BV titer (BV S:P) is reported as an $S: P$ ratio generated by dividing the BV optical density (OD) value by the OD value of serum pool obtained from Circumvent ${ }^{\circ}$ $P C V$ vaccinated, CDCD pigs. The positive/negative cut-offs were 0.250 and 0.240 for the PCV2 and Mhyo ELISAs, respectively.

\section{Results}

The BV S:P results are presented in Table 1. Four weeks after the second Circumvent ${ }^{\circ}$ PCV, $95.8 \%$ of the pigs were positive while none of the controls were positive. Control pigs remained negative throughout the study while the BV titers of vaccinated pigs declined. Re-vaccination greatly boostered BV titers in previously vaccinated pigs and controls showed some titer increase.

The Mhyo titer results are presented in Table 2 . Four weeks after the second vaccination, $85.0 \%$ of vaccinated pigs were positive while none of the controls were positive. The seroconversion rate in vaccinated pigs was $73.3 \%$ and $88.9 \%$ for seropositive and seronegative pigs at 3 weeks of age, respectively. Most vaccinated pigs remained positive while $51.1 \%$ of the control pigs seroconverted.

\section{Discussion}

The data provides field based confirmation of the usefulness of the two ELISAs for monitoring vaccination compliance for both vaccines when pigs are sampled 4 weeks after their second vaccination. For retrospective investigations, interpretation of data is less straightforward based on the decline in BV titers over time and the frequent field exposure of pigs to Mhyo.

Table 1. Baculovirus titer over time

\begin{tabular}{|l|c|c|c|}
\hline Age & Group & BV S:P & No. Pos. \\
\hline 3 (wks) & VAC & 0.012 & $3 / 120$ \\
\hline & CON & 0.012 & $0 / 30$ \\
\hline 10 & VAC & 0.624 & $115 / 120$ \\
\hline & CON & 0.043 & $0 / 30$ \\
\hline 15 & VAC & 0.316 & $59 / 120$ \\
\hline & CON & 0.032 & $0 / 30$ \\
\hline 19 & VAC & 0.190 & $24 / 99$ \\
\hline & CON & 0.023 & $0 / 14$ \\
\hline & RE-VAC & 1.033 & $20 / 20$ \\
\hline & RE-CON & 0.242 & $6 / 14$ \\
\hline
\end{tabular}

Table 2. Mhyo titer over time

\begin{tabular}{|l|c|c|c|}
\hline Age & Group & OD Value & No. Pos. \\
\hline $3(\mathrm{wks})$ & VAC & 0.176 & $31 / 120$ \\
\hline & CON & 0.180 & $10 / 30$ \\
\hline 10 & VAC & 0.480 & $103 / 120$ \\
\hline 15 & CON & 0.027 & $0 / 30$ \\
\hline & VAC & 0.466 & $99 / 120$ \\
\hline 19 & CON & 0.062 & $1 / 30$ \\
\hline & VAC & 0.832 & $115 / 119$ \\
\hline
\end{tabular}

\section{References}

1. Pittman, JS., et al. Proc AASV Ann. Mtg., Dallas, Texas, pp. 207-211, 2009.

2. Schlueter, RH, et al. Proc AASV Ann. Mtg., Kansas City, Missouri, pp. 229-230, 2006.

3. Nawagitgul, P, et al. Clin Diagn Lab Immunol 9:33-40, 2002.

4. Bereiter, M. et al. Vet Micro 25:177-192, 1990. 


\title{
P.046
}

\section{Efficacy of Ingelvac CircoFLEX vaccination on improving swine production performance: a field trial in Northern China}

\author{
Sidang Liu ${ }^{1}$ Aiguo Wang ${ }^{2}{\text { Liande } \text { Zhu }^{2}}^{2}$ \\ 1. Shandong Agricultural University, Shandong, China; 2. Boehringer Ingelheim Ingelheim International Trading (Shanghai) Co. Ltd, Beijing, China
}

\section{Introduction}

PCV2 related diseases (PCVD) have first been described in Canada in 1996, and nowadays are a major threat to the global swine industry. PCVD can have a huge economic impact on farms via increased mortality, reduced growth rate and poorer feed efficiency. Prevalence in China can reach up to 100\%[1].The purpose of this study was to evaluate efficacy of a single-dose PCV2 vaccine (Ingelvac CircoFLEX ${ }^{\circledR}$, Boehringer Ingelheim) on a PCVD affected farm in Northern China.

\section{Materials and Methods}

The study was conducted on a single site, farrow-to-finish farm of 1800 sows in Shandong. The farm was PRRSv positive. Clinical signs on the farm included: Fever, thumping, jaundice, growth retardation, wasting, lethargy can be observed. Herd performance improved when vaccination with Ingelvac ${ }^{\circledR}$ PRRSV MLV was introduced, but did not reach an optimal level and was variable. Further diagnostics confirmed presence of PCV2. Based on that a field study was initiated, with a total of 693 pigs comprising two weekly batches. Half of the pigs $(n=347)$ received a single dose ( $1 \mathrm{ml}$ ) of Ingelvac CircoFLEX ${ }^{\circledR}$ at 3 weeks of age, while the other half $(n=346)$ remained unvaccinated and served as control group. Pigs were weighed individually at weaning, transfer into fattening and at slaughter. Mortality was recorded in nursery and fattening stages. Feed consumption was measured per group to determine FCR (feed conversion ratio). ADG in the two groups were compared with t-test and mortality by Chi-square.

\section{Results}

The results (Table 1 and Table 2 ) indicate significant improvements of vaccination groups in both nursery and fattening stages. During the nursery phase, ADG (average daily gain) in the vaccinated group improved by $+40 \mathrm{~g} / \mathrm{d}$ improvement and mortality was reduced from $13.7 \%$ to $9.2 \%$ reduction; in the fattening phase as well, the vaccinated group showed significant advantages compared to the non-vaccinated group $(+34 \mathrm{~g} / \mathrm{d}$ ADWG, $-9.5 \%$ in mortality).

The high mortality in the nursery during this trial, even in the PCV2 vaccinated groups, can be explained by a PEDV outbreak in the first week group. Mortality in nursery in the first week group was $16.2 \%$ in the vaccinated pigs and $21.3 \%$ in non-vaccinated pigs. Whereas in the week group mortality in vaccinated pigs was only $1.8 \%$ and $5.8 \%$ in the control group.
Table 1. Performance in Nursery

\begin{tabular}{|l|c|c|c|}
\hline & Vac & Control & Diff. \\
\hline Num. of Pigs & 347 & 346 & $/$ \\
\hline ADG (g/day) & 407 & 367 & $+40^{*}$ \\
\hline Mortality (\%) & 9.2 & 13.7 & $-4.4^{*}$ \\
\hline FCR & 1.84 & 2.05 & 0.19 \\
\hline${ }^{*} \mathrm{p}<0.05^{* *} \mathrm{p}<0.01$ &
\end{tabular}

Table 2. Performance in Fattening

\begin{tabular}{|l|c|c|c|}
\hline & Vac & Control & Diff. \\
\hline Num. of Pigs & 314 & 302 & $/$ \\
\hline ADG (g/day) & 694 & 660 & $+34^{*}$ \\
\hline Mortality (\%) & 4.1 & 13.6 & $-9.5^{*}$ \\
\hline FCR & 3.22 & 3.39 & -0.17 \\
\hline${ }^{*} \mathrm{p}<0.0{ }^{* *} \mathrm{p}<0.01$ &
\end{tabular}

\section{Discussion}

Ingelvac CircoFLEX ${ }^{\circledast}$ effectively controlled PCVD on the farm and significantly improved the production performance of the herd. However, the high mortality due to a PEDV outbreak in the nursery indicates, that only comprehensive disease control strategies targeting all major diseases together with good management can result in optimal performance.

\section{References}

[1] Wang Z., Yang H., Guo X., et al (2002) Chinese Journal of Veterinary Medicine, 38(10). 


\title{
P.047
}

\section{Benefits of late vaccination with Ingelvac CircoFLEX ${ }^{\oplus}$ on a Korean grow-out farm}

\author{
Yusik Oh${ }^{1}$ Donhwan Kim ${ }^{1}$ Jina Jung ${ }^{1}$ Seongkyu Kwak ${ }^{2}$ \\ 1. Boehringer Ingelheim Vetmedica Korea Ltd., Seoul, Korea; 2. ChungMiWon, Yangju, Korea
}

\section{Introduction}

Piglet vaccination has been proven to be very successful in controlling Porcine Circovirus Type 2 (PCV2) related diseases. Mostly vaccination is carried out around weaning. In many cases, vaccination has a very positive effect already on nursery mortality and performance 1, and protection last through fininishing until slaughter. However, it has been shown previously that a single dose vaccine, Ingelvac CircoFLEX ${ }^{\circledR}$ can be used successfully at 6 weeks 2 or even as late as 9 weeks 3 of age, if pigs get infected in grow-finish only. In cases where pigs are traded, and are not accessible for vaccination early, it might be necessary to vaccinate piglets at placement into grow-finisher.

This field case describes the benefits of vaccinating early fatteners against PCV2 on a Korean grow-out farm.

\section{Material \& Method}

Two successive weekly batches of pigs, a total of 594 animals, were included in this field observation. One batch of pigs $(n=349)$ was left non-vaccinated, the next batch $(n=245)$ was vaccinated with Ingelvac CircoFLEX, $1 \mathrm{ml}$ i.m., at placement on the grow-out farm. Pigs were placed at 71 days with an average weight of $29.8 \mathrm{~kg}$ (controls) or 72 days and an average weight of $31.3 \mathrm{~kg}$ (vaccinates) on the farm. Vaccinated and non-vaccinated piglets were kept under comparable conditions in two separate buildings on the same site and where sourced from the same piglet producing farm. Pigs seroconvert to PCV2 between 60 and 90 days of age, as determined by Synbiotics ELISA.

Various production parameters, including mortality, ADG and medication costs were recorded for the 2 treatment groups (Table 1).

\section{Results}

Results are summarized in Table 1.

Table 1: Production parameters.

\begin{tabular}{|l|c|c|c|}
\hline & Control & Vaccinates & Diff \\
\hline Pigs ( $\mathrm{n}$ ) & 349 & 245 & \\
\hline Mortality (\%) & 9.6 & 6.5 & -3.1 \\
\hline Light pigs (\%) & 3.94 & 0.81 & -3.13 \\
\hline Medication cost (won*/pig) & 2515 & 1050 & -1465 \\
\hline FCR & 3.14 & 2.98 & -0.16 \\
\hline ADG (g) & 670 & 704 & +34 \\
\hline Days in finisher (days) & 118.5 & 112.7 & -5.8 \\
\hline $\begin{array}{l}\text { Interval between first and last } \\
\text { market day (days) }\end{array}$ & 57 & 37 & -20 \\
\hline Benefit per pigs (won*/pig) & 20,000 & 29,000 & $+9,000$ \\
\hline *1 \$ 1,150 Korean won & \multicolumn{4}{|l}{} \\
\hline
\end{tabular}

\section{Discussion and Conclusion}

All parameters pointed in favor of the vaccinated group. This amounted to a total advantage of 9,000 KRW/pig.

In this farm with a late onset of PCV2 infection, vaccination with Ingelvac CircoFLEX at 10 weeks was still beneficial. However, vaccinating early fatteners is usually a compromise, as often infection occurs in late nursery and optimal performance would only be achieved by vaccinating around weaning.

\section{References}

1. Miyashita, M (2009) Proceedings of the 4th APVS,Tsukuba, Japan, p. 106.

2. Feldman, L, Cline, G, Wilt, V, Diaz, E. (2008). Proceedings of the AASV. Pp. 317-319.

3. Desrosiers, R, Clark, E, Tremblay, D, Tremblay, R, Polson D. (2007) Proceedings of the AASV Pp. 143-145 


\title{
P.048
}

\section{Effect of a single dose of Porcilis PCV at weaning on daily growth on a Danish high health herd with PCVD}

\author{
Peter Astrup $^{1}$ Svend Haugegaard ${ }^{2}$ Anette Rasmussen ${ }^{1}$ Kirsten V. Larsen ${ }^{1}$ \\ 1. Intervet/Schering-Plough Animal Health, Skovlunde, Denmark; 2. Oe-Vet, Horbelev, Denmark
}

\section{Introduction}

Circovirus type 2 (PCV2) is one of the agents responsible for PMWS and PCVD, major causes of losses in pig production. Porcilis PCV has been developed to reduce the losses due to infection with PCV2. Vaccination of piglets with a single $2 \mathrm{ml}$ dose at 3 weeks of age results in both humoral and cellular immunity (1). The purpose of this study was to investigate the efficacy of Porcilis PCV vaccination of piglets in a field case of naturally occurring PCVD.

\section{Materials and method}

A Danish pig farm received 250 piglets every other week from the same sow herd. For the first 8 weeks, the 250 piglets were housed in a separate section containing nine pens, which had previously been cleaned, disinfected, dried and heated. At around $30 \mathrm{~kg}$, the pigs were moved to finishing accommodation operating continuously.

The herd's general health was very high (Danish SPF-declaration: $\mathrm{SPF}+\mathrm{AP} 12$ - meaning free from all serotypes of A pleuropnumoniae (except serotype 12), M hyopneumoniae and PRRS. Three months before the start of the trial, classical PMWS was diagnosed in piglets 4 weeks after introduction onto the farm. When the trial began, the clinical picture had changed, and the disease was classified as PCVD.

Out of a batch of piglets delivered to the farm, 100 pigs were vaccinated with $2 \mathrm{ml}$ of Porcilis PCV on the day of arrival, and 100 pigs were left unvaccinated. These 200 pigs were randomly allocated to pens of 32-34 piglets each, thus mixing vaccinated and unvaccinated pigs in the same pen. This scheme was repeated with the next batch of piglets delivered two weeks later, in a separate section, thus providing, in all, 200 vaccinates and 200 unvaccinated controls.

For each batch, 3 vaccinated and 3 unvaccinated pigs from each pen were bled on days 0 (day of vaccination), 18, 56 and 109. The samples were analyzed by qPCR for PCV2 virus. On days 0, 56 and 109, all the pigs were weighed individually.

Statistical analysis of the ADG was by varians analysis (multifactorial ANOVA, Statgraphics software), and of mortality by the Chi-square test.

\section{Results}

During the trial, serious tail-biting occurred in one pen. Half of the deaths recorded were the result of euthanasia as a sequel to tail-biting and lameness. Apart from this, most cases of lameness and meningitis were cured by appropriate antibiotic therapy. The blood samples showed that the pigs were viraemic from day 56 onwards, throughout the trial period. Vaccinated pigs had lower levels of virus than the unvaccinated controls (Data not shown).
Table 1: Average Daily Gain (ADG) in grams for different growth periods and overall mortality.

\begin{tabular}{|l|c|c|c|}
\hline Period & Porcilis PCV & Control & P-value \\
\hline ADG 0 - 8 weeks (No) & $369(195)$ & $356(192)$ & NS \\
\hline ADG 8 - 16 weeks (No) & $792(186)$ & $753(183)$ & 0.0091 \\
\hline ADG 0 - 16 weeks (No) & $587(186)$ & $561(183)$ & 0.0143 \\
\hline Mortality (all pigs) & $11 / 200$ & $18 / 200$ & NS \\
\hline
\end{tabular}

Figure 1. ADG of different growth classes.

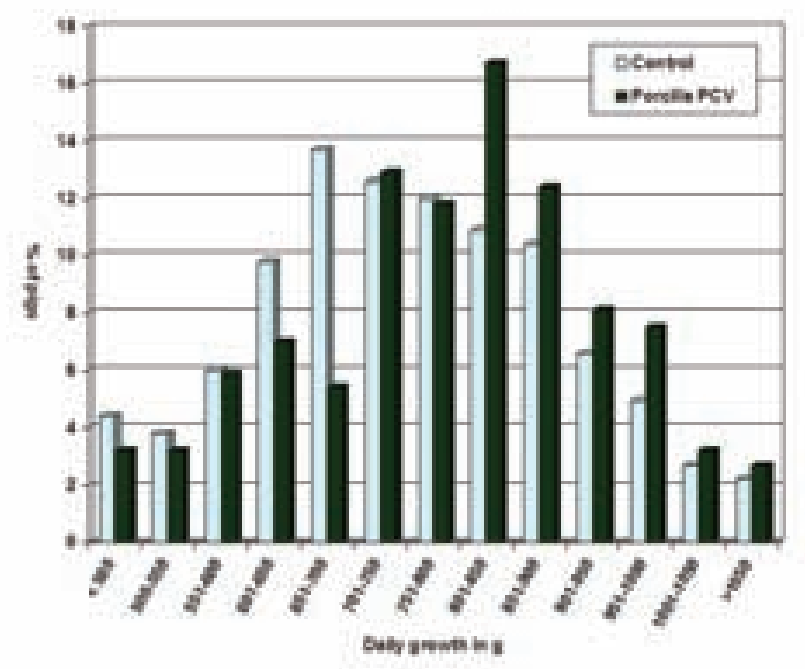

The distribution shows a shift to the right for the pigs vaccinated with Porcilis PCV, indicating the better performance of the vaccinated pigs.

\section{Discussion}

The results showed that, even in a contaminated environment, with vaccinated pigs in direct contact with unvaccinated viraemic pigs, Porcilis PCV vaccination at weaning resulted in a significantly better ADG of $39 \mathrm{~g}$ during the finishing period. This is in accordance with the claim, in the SPC for Porcilis PCV2, that vaccination reduces weight loss due to infection with PCV2 (2). Since no other serious pathogens were present in the farm, the effect is related to vaccination against a 'pure' PCV2 infection.

\section{References}

1: Fort $M$ et al. 2009. Vaccine 27, 4031-7.

2: SPC, EMEA 11/20/2009 


\title{
P.049
}

\section{Safety of Porcilis PCV in pure breed Danish pigs}

\author{
Peter Astrup; Kirsten V. Larsen \\ Intervet/Schering-Plough Animal Health, Skovlunde, Denmark
}

\section{Introduction}

Porcilis ${ }^{\circledR}$ PCV is an inactivated vaccine containing a killed baculovirus vector carrying protective antigen against Porcine Circovirus 2 (PCV2) in Microsol Diluvac Forte ${ }^{\circledR}$ adjuvant. Porcilis ${ }^{\circledR}$ PCV is widely used in the pig industry because circovirus type 2 (PCV2) is considered to be the aetiological agent of postweaning multisystemic wasting syndrome in pigs, a widespread and debilitating condition. Furthermore, it is considered the aetiological agent in porcine circovirus disease (PCVD), known as porcine circovirus associated disease (PCVAD) in the USA.

The data sheets for Porcilis ${ }^{\circledR}$ PCV warn against adverse reactions, which fall into two groups. Firstly, a transient, immediate systemic hypersensitivity-like reaction, and secondly, a transient, painless local swelling, sometimes in the order of several centimetres in diameter. These adverse reactions are to be distinguished from transient, very focal, erythema seen at the site of vaccination (1).

This study reports an investigation into the frequency and characterisation of these two adverse reactions in nucleus pigs from three pure breeds and one cross breed. The investigations were initiated due to speculations from the field, that pure breeds would have a high incidence of local reactions

\section{Materials and Methods}

Four hundred and sixty nine growing pigs between 21 and 40 days of age on two different breeding farms were selected. All were injected with Porcilis ${ }^{\circledR}$ PCV I/M and were observed 2, 15 and 60 minutes and 24 hours after injection for systemic reactions, and at days 1, 8 (or 11 on Farm two), 15 (or 18 on Farm two) and 42 after injection for signs of depression and of local swelling at the injection site. 469 pigs were included: 252 Yorkshire (YY), 119 Duroc (DD), 50 Danish Landrace (LL) and 48 crossbreeds of Yorkshire and Landrace (LY).

\section{Results}

Two of the 469 pigs showed mild, transient systemic side effects, within 15 minutes of injection. Transient, local swellings were observed to begin within twenty four hours after injection and of 469 vaccinated pigs $0.6 \%$ showed reactions on day $1,17.9 \%$ on day $8 / 11,34.4 \%$ on day $15 / 18$ (with the majority of reactions classified as a type B or C) and $0.8 \%$ day 42 (Figure 1 ).

Local swellings occurred significantly more rapidly, and cleared more quickly, in DD pigs. Furthermore, LL, YY and LY groups exhibited significantly higher frequency of swellings than the DD group.

Nine (2\%) of the pigs died during the trial: six from the YY group and 3 from the DD group. Post mortem examination found no evidence that vaccine reactions contributed to the cause of death (data not given)

\section{Discussion}

The purpose of this trial was to investigate the frequency and severity of adverse reactions to Porcilis ${ }^{\circledR}$ PCV vaccine in three different breeds of pigs and cross breeds of these. The reactions to vaccinations seen in this study were as expected from the data sheet advisory, i.e. mild, transient, immediate post vaccination shivering or depression and transient local swellings.

In no cases could treatment or mortality be related to the vaccination with Porcilis PCV. During autopsy of all dead YY-pigs it was judged probable, that deaths were not caused by the vaccination with Porcilis PCV.

Figure 1. The incidence of swellings in all pigs at Day 0, Day 1, Day 8 (and 11), Day 15 (and 18) and Day 42.

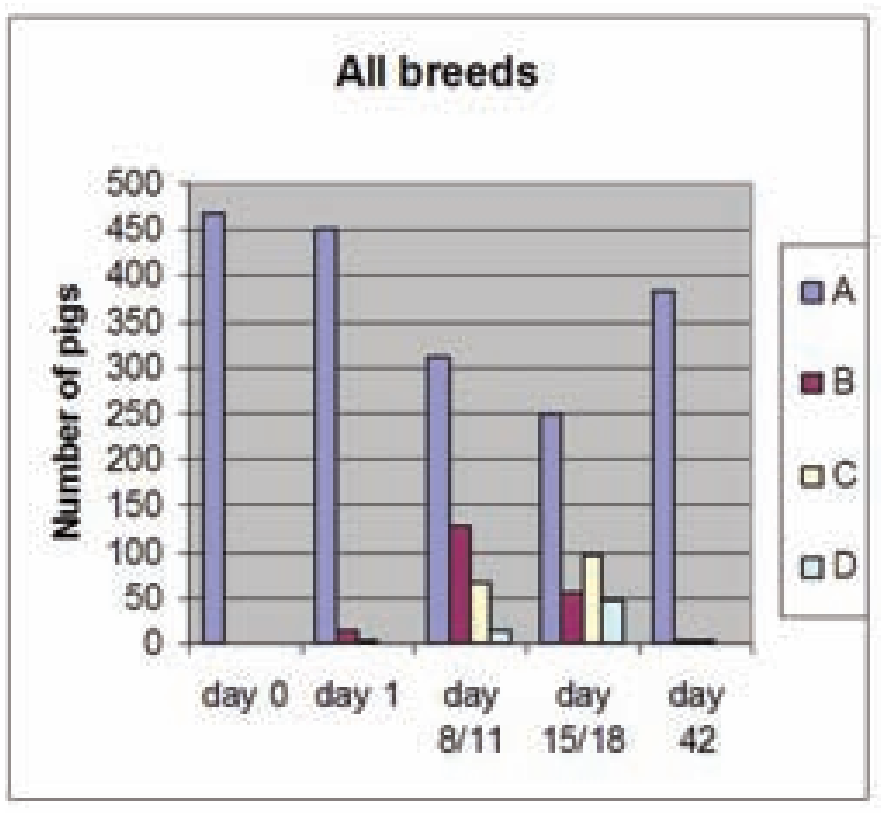

A: no lesions; B: lesions less than $3 \mathrm{~cm}$ diameter; C: lesions between 3 and $8 \mathrm{~cm}$ diameter; D: lesions greater than $8 \mathrm{~cm}$ diameter

\section{Conclusion}

In this study swellings were seen after vaccination, but they were transient and did not negatively impact the vaccinated pigs.

Hypersensitivity reactions were absent except for minor depression and shivering in two pigs.

\section{Reference}

1: EPAR, EMEA, UK. Available at http://www.emea.europa.eu/vetdocs/ PDFs/EPAR/PorcilisPCV. 


\title{
P.050
}

\section{Evaluation of the efficacy of an adjuvanted subunit vaccine (Circumvent ${ }^{\circledR}$ PCV) in the prevention of PMWS under field conditions in Vietnam}

\author{
Alexander A. Eggen ${ }^{1}$ Nguyen T. Toan ${ }^{2}$ Tran T. Dan² Nguyen T. Nam² Le van Huy ${ }^{2}$ \\ Nguyen P. Nam ${ }^{2}$ Vo T. An ${ }^{2}$ Ho T. Nga ${ }^{2}$ Nguyen T. Ninh ${ }^{2}$ Le H. Ngoc ${ }^{2}$ \\ 1. Intervet/Schering-Plough, Boxmeer, Netherlands; 2. Nong Lam University, HCMC, Vietnam
}

\section{Introduction}

PWMS, largely caused by Porcine Circovirus Type 2 , is endemic on pig farms in Vietnam. This study was designed to evaluate the efficacy of Circumvent PCV in protecting pigs on suspected PMWS farms. Circumvent PCV contains a PCV2 viral subunit (ORF2 protein) in X-Solve adjuvant. The antibody response was measured at the time the vaccine was given at 14 or 21 days of age, and again at 42 days.

\section{Materials and Methods}

The study was carried out on two commercial farms (Farms A and B with 400-1000 pigs) in two provinces in Vietnam. Both farms practiced 21-25 day weaning. Pigs were assigned randomly to either a vaccinated or a control group. On Farm A, 396 piglets of 14 days of age from various litters, were divided equally between vaccinated and control groups. On Farm B, 194 piglets of 21 days old, also from various litters were divided equally between vaccinated and controls.

All pigs in the vaccinated group were injected with Circumvent PCV (Intervet Schering-Plough, The Netherlands), on Farm A at 14 and 42 days of age, and on Farm B at 21 and 42 days of age. The trial vaccine was given intramuscularly on one side of the neck, and Mycoplasma vaccine on the other side. The controls did not receive Circumvent PCV. Pigs were observed daily for signs of illness and were weighed at 14, 28 (weaning), 75 and 120 days old on Farm A, and at 21, 84 and 165 days old (slaughter) on Farm B.

Blood samples were collected immediately prior to vaccination on Farm A (Days14 and 42) and at 75 and 168 days of age. On Farm B, blood samples were also taken prior to vaccination (Days 21 and 42) and at 84 and 165 days of age. The sera were tested for antibodies against PCV2 by ELISA (Serelisa ${ }^{\circledR}$, Synbiotics). The result was taken to be positive when $\mathrm{SNc}<0.5$ and titers were calculated from OD at three dilutions of serum, as recommended.

Figure 1. Percentage mortality in vaccinated and control groups on Farms $A$ and $B$

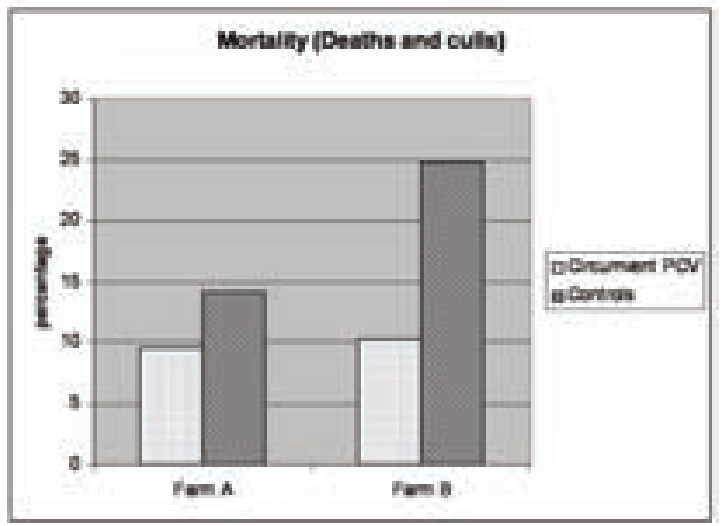

For the statistical analysis, all data were presented as means. Variation between groups was determined by one-way ANOVA analysis and the difference between means by Turkey's multiple comparison test using Minitab Statistical Software 13.20 (USA). In all cases, $\mathrm{P}<0.05$ was considered to be significant.

\section{Results}

On both farms, the vaccinated pigs had better ADG across the age range, and lower mortality, than the controls.

Figure 2. Serological data from Farm A. Vaccinations on days 14 and 42

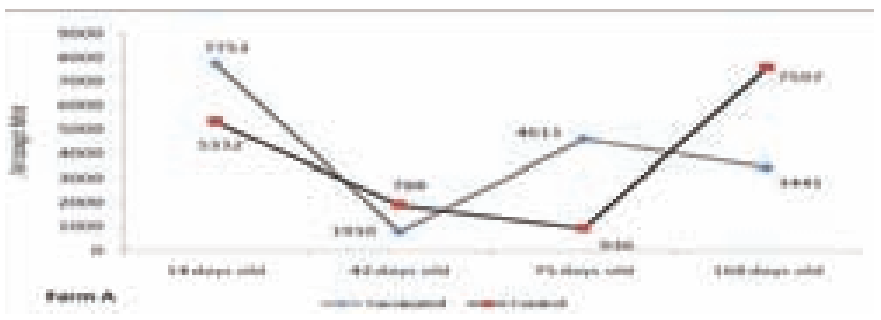

Figure 3. Serological data from Farm B. Vaccinations on days 21 and 42

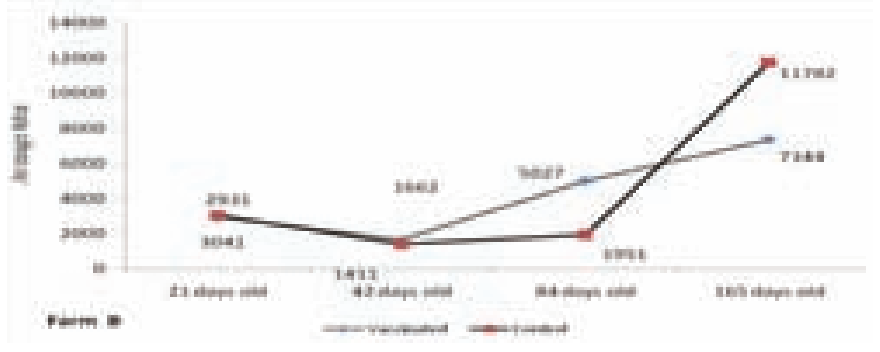

\section{Discussion}

On both farms, vaccination with Circumvent PCV clearly resulted in sero-conversion after the second dose, indicating protection against PCV2 virus (Fort, 2009). This was further evidenced by a better ADG resulting in heavier slaughter weights (2.0\% on Farm $A$ and $3.5 \%$ on Farm $B$ ), lower mortality $(4.5 \%(A)$ and $15 \%(B))$ and a reduced incidence of clinical disease (25\% (A), 12\% (B)) compared to the controls.

Several individual parameters were statistically significantly better in the vaccinated pigs, e.g. post vaccination antibody titers and mortality. More importantly for the pork producer, the overall technical and economic performance improved on both farms.

\section{References}

Fort, Vaccine 2009. 


\title{
P.051
}

\section{Successful PCVD control on a pig farm in Southern China}

\author{
D Xie ${ }^{1}$ Brook Fang ${ }^{2}$ Nian Huang ${ }^{2}$ \\ 1. Guangdong Chengxing Pig Farm, Guangdong, China; 2. Boehringer Ingelheim Int'l Trading (Shanghai) Co. Ltd., Beijing, China
}

\section{Introduction}

Porcine Circovirus type2 (PCV2) is the causative agent of various PCV2 associated diseases, including PMWS, PDNS, PRDC and $P C V 2$ related reproductive failure or enteritis.

A recent epidemiological study revealed severe prevalence of PCV2 infection in China. Positive rates for samples tested for PCV2 in 2002-2003, 2004-2006 and 2008 reached $71 \%, 81.2 \%$ and $100 \%$ respectively [1]. This paper reports a successful case of PCVD control in a 900-sows pig farm suffering from PMWS in Guangdong, a southern province in China.

\section{Materials and Methods}

A one site production 900-sows farm built up in 1994 suffered severe PCVD. The breeding herd is vaccinated against PRRS, PRV, Atrophic rhinitis, CSFV, PPV, JEV and Streptococcus (Str.) suis, while piglets are vaccinated against PRV, CSFV, PRRSV, Haemophilus parasuis and Str. suis. Severe PMWS signs emerged since February 2009. Symptoms in late nursery included growth retardation, wasting, lethargy, anorexia and fever, while in the fattening herd, jaundice, diarrhoea, enlargement of inguinal and mesenteric lymph node, enlarged spleen with infarcts, enlarged kidneys with tan and waxy appearance and petechial haemorrhages and PDNS skin lesions were observed. The average morbidity and mortality ranged between $30-50 \%$ and $25-40 \%$.

In May 2009, the farm started to use Ingelvac CircoFLEX in piglets around 14 days old. In total 40 weekly batches of piglet, 20 before vaccination ( $n=3769)$, 20 vaccinated with Ingelvac CircoFLEX $^{\circledR}(n=3927)$ were included in the evaluation. Diseased pigs (morbidity) as well as dead pigs (mortality) and culls were recorded per batch.

Frequencies of morbidity, mortality and culling were analyzed by Chi-square test.

\section{Results}

Since piglet vaccination with Ingelvac ${ }^{\circledR}$ CircoFLEX, the health status of the vaccinated batches were significantly improved. Morbidity, mortality and culling were significantly reduced in vaccinated pigs, in nursery as well as in fattening (Table1 and Table2). PMWS, PDNS and PCV2 related enteritis was effectively controlled in the farm.

Table 1 Average performance in Nursery (25-75 days old)

\begin{tabular}{|l|c|c|c|}
\hline & \multicolumn{3}{|c|}{ Nursery Herd } \\
\hline & Pre-Vx & Post-Vx & Diff. \\
\hline Pigs ( $\mathrm{n}$ ) & 3769 & 3927 & $/$ \\
\hline Morbidity (\%) & 20.8 & 4.5 & $16.3^{*}$ \\
\hline Mortality and culling rate (\%) & 18.9 & 3.5 & $15.4^{* *}$ \\
\hline${ }^{*} \mathrm{p}<0.05{ }^{* *} \mathrm{p}<0.01$ & \multicolumn{4}{|l}{} \\
\hline
\end{tabular}

Table 2 Average performance in Fattening (76-120 days old)

\begin{tabular}{|l|c|c|c|}
\hline & & Fattening Herd & \\
\hline & Pre-Vx & Post-Vx & Diff. \\
\hline Pigs ( $\mathrm{n})$ & 3057 & 3791 & $/$ \\
\hline Morbidity (\%) & 14.5 & 2.5 & $12.0^{* *}$ \\
\hline Mortality and culling rate (\%) & 11.8 & 1.8 & $10.0^{*}$ \\
\hline${ }^{*} \mathrm{p}<0.05^{* *} \mathrm{p}<0.01$ &
\end{tabular}

\section{Discussion}

This farm was severely affected by PCVD as indicated by a high mortality and culling rate in the nursery and fattening period. PRRSV and CSFV were already controlled through vaccination.

Piglet vaccination with Ingelvac CircoFLEX ${ }^{\circledR}$ successfully controlled PCVD, as demonstrated by a significant reduction in morbidity, mortality and culling rate.

\section{References}

[1] He D.et al (2002) Journal of South Agricultural University, 26(3). 


\title{
P.052
}

\section{The impact of PCV2 sow vaccination on PCVD in grower and finisher pigs}

\author{
Jake Waddilove \\ Eastgate Veterinary Group, Bury St Edmunds, UK
}

\section{Introduction}

Porcine Circovirus Disease (PCVD) has a major impact on pig health and production throughout the world. It affects every category of pig, with its major impact being in the growing herd. Where disease is more established the focus often moves to older pigs. Costs of PCVD in the EU have been estimated at $€ 562$ - 900 million/year (1). Control has been achieved by vaccination of sows \&/or pigs. The benefits of sow vaccination have been shown to extend well beyond the predicted duration of maternally derived antibodies (2). This field study further investigates the benefits of sow vaccination on the progeny of these sows.

\section{Materials and Methods}

The study took place in a 600-sow farrow-to-finish herd housed in a combination of straw based and slatted buildings. Growing pigs were housed in all-in, all-out rooms to $50 \mathrm{~kg}$, and then semi-continuous buildings. The herd had been affected by PCVD for several years. This combined with PRRS and Mycoplasma hyopneumoniae to lead to significant PRDC problems.

Additionally Streptococcus suis meningitis and Haemophilus parasuis problems occurred up to $50 \mathrm{~kg}$. Attempted control had been by vaccinations for PRRS (MLV), M.hyopneumoniae and at times H.parasuis, plus various antimicrobial programmes. Results were of limited success.

In the study sows were vaccinated for PCVD using CIRCOVAC ${ }^{\circledR}$ (MERIAL) in addition to existing vaccines. A primary course given 6 and 3 weeks before farrowing was followed by boosters 3 weeks before farrowing. Progeny from these sows were monitored for pig health, medication, mortality and various slaughter parameters and results compared with previous throughput.

\section{Results}

Progeny from vaccinated sows reached the weaners on 08/05/08 and the finishers on $13 / 08 / 08$. There was a progressive improvement in pig health. Levels of pneumonia and coughing reduced especially in finishers. Streptococcal meningitis in growers dropped to nearly zero, but increased when corrective medication was removed. It reduced again once medication was returned. Clinical evidence of H.parasuis decreased. The costs of medication (in feed, soluble \& injectable) fell from 79p per pig in the 3 months before the pigs came through to $41 p$ in the next 3 months \& then 31 p. Mortality (graph 1) progressively improved except for the temporary increase due to S.suis meningitis.

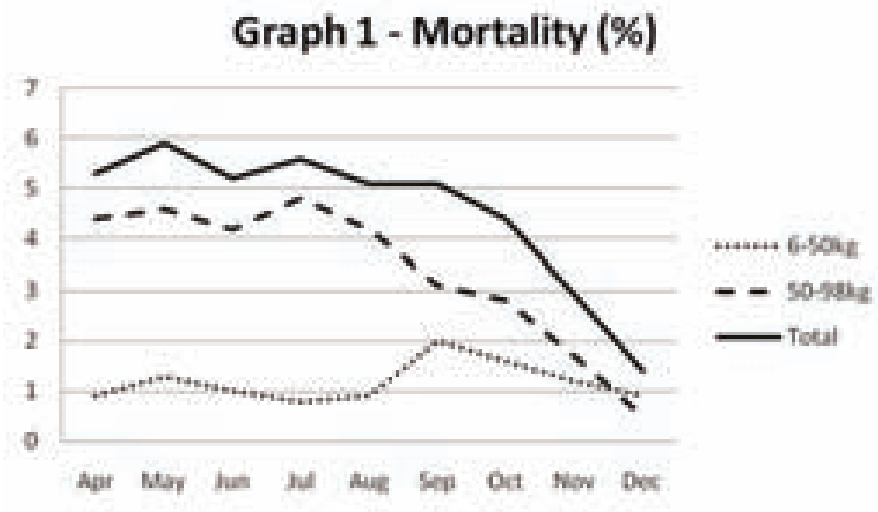

Slaughter house findings (graph 2) showed less pleurisy, pericarditis and peritonitis confirming reduced H.parasuis infection. Growth rates of pigs were not accurately recorded, but days to slaughter fell by approximately 5 days.

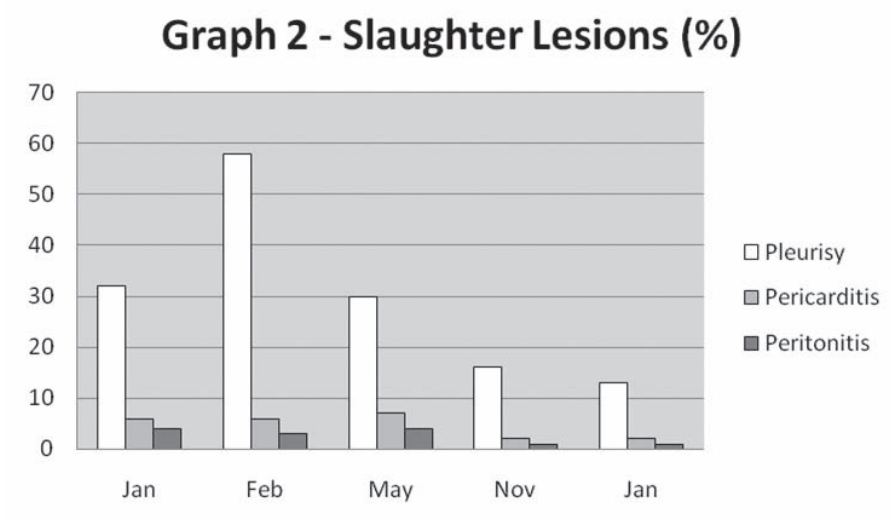

\section{Discussion}

These results show that vaccination of sows using Circovac can have benefits in their progeny though to slaughter. Control of complexed PRDC was improved while reductions in S.suis meningitis and H.parasuis problems suggested that vaccination helped reduce the impact of PCV2 on the immune competence of these pigs. These results further indicate that sow vaccination for PCV2 reduces PCVD in the finishing herd.

\section{References}

1. Tucker, A.M. et al.,(2006) The Pig Journal April 2006

2. Waddilove, $J$ (2008) Proc 20th IPVS p104 


\title{
P.053
}

\section{Prevalence of circovirus (PCV2) in vaccinated and non vaccinated swine's from different Brazilian farms. January 2008 to August 2009}

\author{
Fábio B. Rossi ${ }^{1,2}$ Alberto Yocyitaca Inoue ${ }^{2}$ Gilberto Spricigo ${ }^{2}$ Lissandro Almeida $^{2}$ Masaio Mizuno Ishizuka ${ }^{3}$ \\ 1. Automomous, Campinas, SP, Brazil; 2. Fort Dodge Animal Health, Campinas, Campinas, SP, Brazil; 3. Universidade de São Paulo, São Paulo, Brazil
}

\section{Introduction}

Porcine circovirus type 2 (PCV2) was first described in 1998 and it was associated with a newly observed sporadic wasting disease in weaned pigs in Canada, USA (California), and France (ALLAN and ELLIS, 2000). Actually is known as one of the most important viruses in the global pig industry and the acronym PCVAD is recommended besides PMWS (systemic infection or postwean $\neg$ ing multisystemic disease) and others PCV2-associated diseases as pneumonia, enteritis, reproductive failure, dermatitis and nephropathy syndrome (OPRIESSNIG et al., 2007). Clinical signs and lesions are observed in late nursery (8-10 weeks of age), and finisher pigs (2-3 wks after placement) (BATISTA, 2007; BRUNBORG et al, 2004)

Brazilian isolates of PCV2 were sequenced, analyzed and compared with isolates from other countries and the phylogenetic analysis showed that mostly variants were grouped as PCV2-1 (CIACCI-ZANELLA et al, 2009).

\section{Material and Methods}

Study design: this study included 2090 animals from 93 farms with medium level of biosecurity measures and clinical signs of PCVAD located in 6 states of south, south-east and west-center regions of Brazil (Paraná/PR, Rio Grande do Sul/RS, São Paulo/ SP, Santa Catarina/SC, Mato Grosso do Sul/MS and Minas Gerais/ MG). There were vaccinated 940 animals and 1150 were maintained as control.The SUVAXYN@PCV2 chimeric vaccine was applied intramuscularly in animals aged between 21 and 28 days and serum samples were examined 40,60, 80 and 100 days after vaccination to quantify viral load by TaqMan@ real time PCR assay (BRUNBORG et al, 2004). Statistic procedures were the test for difference of the parameters of two proportions for prevalence's and the Mann Withney test for two median values differences (SIEGEL, 1975) and fixed $a=0,05$.

\section{Results}

There were considered positive all serum with 4500 or more DNA copies of PCV2. Among vaccinated pigs the frequency of positives samples at 40,60,80 and 100 days were respectively, $22,5 \%, 14,7 \%, 23,6 \%$ and $13,3 \%$. Among non vaccinated pigs the frequency of positives samples at 40,60,80 and 100 days were respectively, 36,2\%, 22,5\%, 35,4\% and 58,2\%. The Graphic 1 illustrates the variation of frequencies in vaccinated and non vaccinated and age of serum examination.

\section{Discussion}

In vaccinated and non vaccinated animals, the statistical comparison of frequencies between successive ages (40 with 60 days, 60 with 80 days and 80 with 100 days) revealed a decreasing except at 80 days that could be explained as a result of high challenge due to placement to finishing houses with mixture of animals of different growing rooms. These results permit to do inference about the role of natural immunity among non vaccinated pigs and the efficacy of SUVAXYN ${ }^{\circledR} P C V 2$ in the vaccinated group in farms were de PCV2 is endemic. These results reveal a widespread PCV2 virus in Brazil mainly in areas of important swine industry similarly to mention by OPRIESSNIG et al (2007).

\section{References}

1. BATISTA, L. Porcine Circovirus Associated Diseases (PCVAD) in Canada - Prevalence, Co-Factors, and Risk Factors. Advances in Pork Production, v.18, p. 57, 2007.

2. BRUNBORG, I.M.; MOLDAL, T.; JONASSENA, C.M. Quantitation of porcine circovirus type 2 isolated from serum/plasma andtissue samples of healthy pigs and pigs with postweaning multisystemicwasting syndrome using a TaqMan-based real-time PCR. Journal of Virological Methods, v. 122, p.171-178, 2004.

3. CIACCI-ZANELLA, J.R.; SIMON, N.L.; PINTO L..; VIANCELLI, A,; FERNANDES, L.T.; HAYASHI, M.; DELLAGOSTIN, O.A.; ESTEVES, P.A. Detection of porcine Circovirus type 2 (PCV2-1) and PCV2-2 in Brazilian pig population. Rev Vet Sci, v. 87, n. 1, p. 157-60, 2009.

4. OPRIESSNIG,T.; HALBUR, P.G. Influence of anti-PCV2 passively-acquired antibodies on efficacy of Suvaxyn ${ }^{\circledR} P C V 2$ vaccination in pigs experimentally-infected with PCV2. Proceed. Am. Assoc. Swine Vet. Meeting, Orlando, Florida, USA, p. 299-300, 2007.

5. OPRIESSNIG, T. Current knowledge and future research needed on PCV2 vaccines. Allen D. Leman Swine Conference, p. 63-67, 2008

6. SIEGEL, S. Nonparametric Statistics for the Behavioral Sciences. McGraw-Hill Inc, 350p, 1965. 


\title{
P.054
}

\section{Serological response and influence on virus load in pigs vaccinated with Porcilis PCV}

\author{
Peter Astrup ${ }^{1}$ Svend Haugegaard ${ }^{2}$ Claus B. Larsen ${ }^{3}$ Charlotte K. Hjulsager ${ }^{4}$ Lars E. Larsen 4 \\ 1. Intervet/Schering-Plough Animal Health, Skovlunde, Denmark; 2. Oe-Vet, Horbelev, Denmark; 3. \\ DanVet, Hobro, Denmark; 4. DTU Veterinary Institute, Copenhagen, Denmark
}

\section{Introduction}

Circovirus type 2 (PCV2) is one of the agents responsible for PMWS and PCVD, major causes of losses in pig production. Porcilis PCV has been developed to reduce the losses due to infection with PCV2. Vaccination of piglets with a single $2 \mathrm{ml}$ dose at 3 week of age results in both humoral and cellular immunity (1). The purpose of this study was to investigate the efficacy in controlling viraemia of Porcilis PCV vaccination of piglets in a field case of naturally occurring PCVD.

\section{Materials and Methods}

The trial involved two high-health (SPF + AP12) farms. Farm 1 was a wean to slaughter herd and had vaccinated and unvaccinated pigs in the same pen. Farm 2 was a sow herd which produced 350-400 weaned piglets every third week, which were moved into clean, disinfected, all in/all out accommodation. They had no contact with other pigs until sold at $30 \mathrm{~kg}$ (half of them) or slaughtered at $105 \mathrm{~kg}$. Pigs from two consecutive batches comprised one block and were randomly allocated to a treatment group (2ml Porcilis PCV, 4-6 days before weaning) or an unvaccinated control group. In all, 6 blocks (12 batches) were studied.

In each batch, 5 pigs were blood sampled four times in the growing phase (at treatment time, and at around 6, 12 and 18 weeks after weaning). Samples from the first 4 batches (20 pigs) were analyzed individually by ELISA for antibodies against PCV2. From all 8 batches, virus particles/ml were measured by real time PCR performed on pools of 5 samples from the same batch. (ELISA and PCR to National Veterinary Institute, DTU standards.)

On Farm 1, 6 vaccinated and unvaccinated 6 pigs from the mixed groups were also sampled, at weaning (day 0), days 18, 53 and 109.

\section{Results}

All vaccinated pigs in both farms seroconverted to levels of 1:250 - 1:31250 within the first 6 weeks after vaccination. These levels were slightly declining, anyway all, but one, stayed at at least 1:250 throughout the observation period. Non-vaccinated reached a higher level of serological response

Figure 1: Shows antibody level in individual pigs at farm 1. Light rows are non-vaccinated, dark are vaccinated. 1-6 indicates levels of antibodies from 1:10 through to 1:31,250.

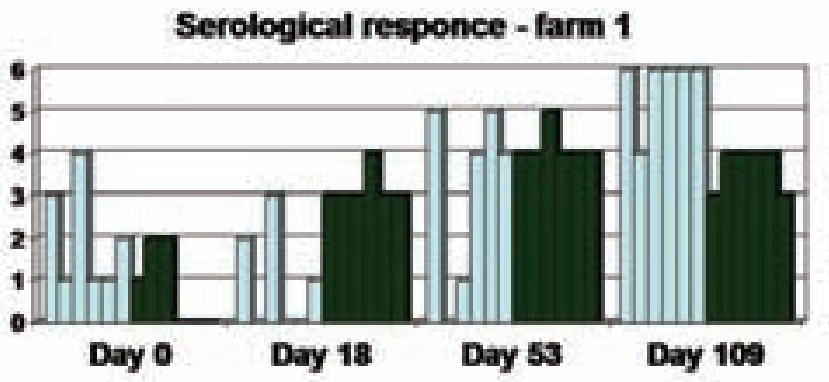

Table 1. Number of PCV2 copies (log(10)) from pools of 5 pigs from individual batches at farm 2. $A-F=$ Blocks of 2 batches. $V=$ Vaccinated, $N=$ Non-vaccinated. Week $=$ Week post weaning.

\begin{tabular}{|l|c|c|c|c|c|c|c|c|c|c|c|c|}
\hline Batch & A & A & B & B & C & C & D & D & E & E & F & F \\
\hline Week & V & N & V & N & V & N & V & N & V & N & V & N \\
\hline 0 & 0 & 0 & 0 & 0 & 0 & 0 & 0 & 0 & 0 & 0 & 0 & 0 \\
\hline 6 & 0 & 0 & 0 & 0 & 0 & 0 & 0 & 0 & 0 & 0 & 0 & 0 \\
\hline 12 & 0 & 4.2 & 0 & 5.2 & 0 & 6.4 & 0 & 5.0 & 0 & 0 & 0 & 6.9 \\
\hline 18 & 0 & 3.7 & 0 & 4.5 & 0 & 5.9 & 0 & 6.1 & 0 & 6.4 & 0 & 6.1 \\
\hline
\end{tabular}

Number of PCV2 copies were significantly lower in vaccinated pigs that were co-mingled with non-vaccinated (farm 1; data not shown) and no copies (above the detection limit of $1 \times 103$ ) were found in vaccinated that were housed separated from nonvaccinated.

\section{Discussion}

Vaccination with Porcilis PCV is shown to elicit an equal and safe immuneresponse in vaccinated pigs. Even though PCV2 virus is shown to circulate in vaccinates at low numbers the measured response seems to be protective against any further stimulation of the immunesystem, indicating that the vaccination have induced an immuneresponse strong enough to avoid the proliferation of virus in infected pigs housed with highly infected littermates.

If pigs are housed only with other vaccinates the vaccine is able to protect pigs from having any development of virus at all.

These results show that the strong cellular and humoral immune response developed by vaccination with one dose of $2 \mathrm{ml}$ of Porcilis PCV in pigs older than 3 weeks, that Fort et al (1) found under laboratory conditions, are highly protective under field conditions.

\section{References}

1. Fort $\mathrm{M}$ et al. 2009. Vaccine $27,4031-4037$ 


\title{
P.055
}

\section{Efficacy of an autogenous vaccine against Porcine Circovirus type 2}

\author{
Vitelio I. Utrera ${ }^{1}$ Susan del Castillo ${ }^{1}$ Andres Boulanger ${ }^{1}$ Jose D. Mogollon ${ }^{4}$ Janett Manzo 5 \\ Elias Sogbe ${ }^{2}$ Natalia Riveros ${ }^{3}$ Manuel Rodriguez ${ }^{5}$ Abel Sequera $^{5}$ \\ 1. Diagnostico y Consultoria Veterinaria CA, Maracay, Venezuela; 2. Universidad Central de Venezuela, Maracay, Venezuela; 3. AABL, Maracay, \\ Venezuela; 4. Universidad Nacional de Colombia, Bogota, Colombia; 5. Empresa de Diagnostico Veterinario CA, Maracay, Venezuela
}

\section{Introduction and Objectives}

Recent studies have shown the effectiveness of vaccines for control of diseases associated with Porcine Circovirus Type II (PCV2). The goal of this study is to demonstrate the efficacy of an autogenous vaccine against PCV2 for the control of Porcine Multisystemic and Wasting Syndrome (PMWS) in a commercial 1000 sow farrow to finish operation in Venezuela.

\section{Materials and Methods}

The study was performed in one pig farm positive to PRRS, Mycoplasma hyopneumoniae, Glasser disease and PMWS. Samples were collected from animals with PMWS clinical signs in order to be evaluated by histopathology and immunoperoxidase assay (1). Tissues from animals identified as positive were used as source of virus for the killed autogenous vaccine.

Seven hundred forty 4 week-old-piglets were randomly selected and divided in two groups:

Group A: 370 piglets were vaccinated with $2 \mathrm{ml}$ of the PCV2 autogenous vaccine at four and six weeks of age;

Group B: 370 piglets were injected with $2 \mathrm{ml}$ of saline solution at 4 and 6 weeks of age.

Both groups were divided in 10 pens with 37 animals per pen, and were fed and managed under the same conditions. A clinical evaluation was assessed to each group weekly. The parameters evaluated were as follows:

Cough index, sneezing index, prevalence of depression, lameness, conjunctivitis, diarrhea, wasting, skin lesions, pig size uniformity in the same pen and mortality rate.

\section{Results}

A summary of the results is shown in Table 1. A higher percentage of wasting pigs, presence of conjunctivitis and sneezing pigs was observed in the control group as compared to that detected in the vaccinated group. On the other hand, a lower mortality rate was observed in the vaccinated group (2.16 vs 5.40 ).

No evidence of diarrhea was observed in any of the groups under study.
Table 1. Comparison of clinical parameters of pigs vaccinated with an autogenous killed PCV2 vaccine against control non vaccinated pigs.

\begin{tabular}{|l|c|c|c|c|}
\hline Parameter & Group A vaccinated & $\%$ & Group B control & $\%$ \\
\hline Cough index & $4^{\mathrm{a}}$ & - & $11^{\mathrm{b}}$ & - \\
\hline Sneeze index & $24^{\mathrm{a}}$ & - & $60^{\mathrm{b}}$ & - \\
\hline Depression & 0 & 0 & 0 & 0 \\
\hline Lameness & 1 & 0.13 & 4 & 1 \\
\hline Conjunctivitis & $5^{\mathrm{a}}$ & 1.33 & $15^{\mathrm{b}}$ & 4.0 \\
\hline Diarrhea & 0 & 0 & 0 & 0 \\
\hline Wasting pigs & $2^{\mathrm{a}}$ & 0.54 & $43^{\mathrm{b}}$ & 11 \\
\hline Skin lesions & $1^{\mathrm{a}}$ & 0.13 & $11^{\mathrm{b}}$ & 2.9 \\
\hline Deaths & $8^{\mathrm{a}}$ & 2.16 & $20^{\mathrm{b}}$ & 5.4 \\
\hline Values with different superscripts differ significantly; $\mathrm{a}, \mathrm{b}: \mathrm{p}<0.05$. & \\
\hline
\end{tabular}

\section{Discussion and Conclusions}

The efficacy of commercial vaccines for the control of PCV2 associated diseases have been previously demonstrated (1). A significant difference in the mortality rate was observed in the vaccinated group as compared with the control non vaccinated group. Even though the weight of the pigs was not evaluated, the proportion of wasting pigs was significantly higher in the control group. These findings suggest the efficacy of the use of an autogenous vaccine for the control of PMWS.

On the other hand, a higher percentage of pigs with conjunctivitis, skin lesions and sneezing were observed in the control non vaccinated group, signs that could be associated with a synergic effect of PCV2 with other infectious agents.

\section{References}

1 Arnold J. et al. (2008) 20th IPVS Durban South Africa. p. 37.

2 Yaeger, M. Diagnosis of PCV2 associated disease a diagnostic pathologist's perspective. American Association of Swine Veterinarians. 2007; 519-524. 


\title{
P.056
}

\section{Evaluation of the safety and and the immune response after different vaccination schedules in sows: preliminary results}

\author{
Eric Pagot ${ }^{2}$ Florian Voisin ${ }^{2}$ Anne Trotel $^{2}$ Alassane Keita $^{2}$ François Joisel $^{1}$ \\ 1. Merial S.A.S., Lyon, France; 2. CTPA Zoopole développement, Ploufragan, France
}

\section{Introduction}

Post-weaning Multisystemic Wasting Syndrome (PMWS), Parvovirosis, Erysipelas, Neonatal Colibacillosis are major diseases in pig farms worldwide. Previous internal trials which tested the effects of simultaneous vaccination of gestating sows/gilts (non-published data) with either PMWS (CIRCOVAC ${ }^{\circledR}$ coded CIR, Merial) and Parvovirosis/Erysipelas (PARVORUVAX ${ }^{\circledR}$ coded PAR, Merial) or CIR and Colibacillosis (NEOCOLIPOR ${ }^{\circledR}$ coded NEO, Merial), both vaccination schemes did not raise safety concerns. This paper reports a study which was conducted in a larger scale under field conditions in France, and evaluates the safety of the two simultaneous vaccination schemes in comparison with the recommended schedules.

\section{Material and Methods}

Overall 181 sows, coming from 2 different commercial farms usually vaccinated with CIR and PAR were included into the study. In each farm, sows of each farrowing batch were randomly assigned to one of three identical treatment groups according to parity: Group 1 (G1): simultaneous vaccination with CIR,PAR 3 weeks before farrowing, and with NEO 2 weeks before farrowing. Group 2 (G2): simultaneous vaccination with CIRC,NEO 2 weeks before farrowing, and with PAR during lactation. Group 3 (G3) was the control group: CIR vaccination 3 weeks before farrowing, NEO 2 weeks before farrowing and PAR during lactation. The study was planned to vaccinate the sows for 2 breeding cycles. Systemic and local site reactions as well as rectal temperature were checked during the first cycle on D0, D0+4H, D1, D2 and D7 after each injection. Statistics (1) used ANOVA (or KruskallWallis test if non-normal data) and Mantel Haenszel chi-square test with $p<0.05$ as level of significance.

\section{Results}

The results for the second cycle are not yet available, only those from the first cycle are shown.

The baseline criteria (parity distribution, median parity and rectal temperature at inclusion) were similar in the three groups. Rectal temperatures were compared within physiological periods. First period (P1) was 3 weeks before farrowing (G1 with CIR,PAR vs $G 3$ with CIR), second period (P2) was 2 weeks before farrowing ( $G 2$ with CIR,NEO vs G1 and G3 with NEO) and third period (P3) was 2 weeks after farrowing (PAR vaccination, G2 vs G3).

The course of rectal temperature is shown in table 1. Depending on the periods, the temperature values were consistently in the normal physiological range for sows: $38.0^{\circ} \mathrm{C}$ to $38.6^{\circ} \mathrm{C}$ before and $38.9^{\circ} \mathrm{C}$ to $39.5^{\circ} \mathrm{C}$ after farrowing.
Table 1: Adjusted mean rectal temperature $\left(\right.$ in $\left.{ }^{\circ} \mathrm{C}\right)$ by group over time

\begin{tabular}{|l|c|c|c|c|c|}
\hline Group & D0 & D0+4h & D1 & D2 & D7 \\
\hline Period 1 -G1 & 38.1 & 38.5 & 38.3 & 38.0 & 38.1 \\
\hline Period 1 -G3 & 38.1 & 38.4 & 38.3 & 38.1 & 38.1 \\
\hline $\begin{array}{l}\text { Period 1 - } \\
\text { Statistics }\end{array}$ & NS & NS & NS & NS & NS \\
\hline Period 2 - G1 & 38.1 & $38.3^{\mathrm{b}}$ & $38.2^{\mathrm{b}}$ & 38.2 & 38.1 \\
\hline Period 2 - G2 & 38.1 & $38.6^{\mathrm{a}}$ & $38.4^{\mathrm{a}}$ & 38.1 & 38.1 \\
\hline Period 2 - G3 & 38.1 & $38.4^{\mathrm{b}}$ & $38.2^{\mathrm{b}}$ & 38.2 & 38.1 \\
\hline $\begin{array}{l}\text { Period 2 - } \\
\text { Statistics }\end{array}$ & NS & p<0.001 & $\mathbf{p}<0.001$ & NS & NS \\
\hline Period 3 -G2 & 39.0 & 39.4 & 39.1 & 39.0 & 38.8 \\
\hline Period 3 - G3 & 39.0 & 39.5 & 39.1 & 39.0 & 38.9 \\
\hline $\begin{array}{l}\text { Period 3 - } \\
\text { Statistics }\end{array}$ & NS & NS & NS & NS & NS \\
\hline
\end{tabular}

${ }_{a, b}$ Different superscripts in the same column mean significantly different values. NS: non significant

For P1 and P3, no significant difference was observed between the rectal temperatures of the three groups. For P2, at D0+4h and at D1, a significant higher temperature was observed in the group 2 compared to the groups 1 and 3. However, it was moderate $\left(0.2^{\circ} \mathrm{C}\right.$ to $0.3^{\circ} \mathrm{C}$ more $)$ and very transient, temperatures being similar in all groups after D1.

At any time point of the study, no significant difference was observed between groups in general condition.

No significant difference was observed between the numbers of piglets born alive per sow of the three groups: respectively 13.3, 14.3 and 13.4 in the groups 1, 2, 3.

No significant difference was observed between groups in the proportion of sows with local reactions after vaccination.

\section{Discussion and Conclusion}

A moderate and transient higher temperature was observed in the group receiving $\mathrm{CIR}$,NEO 2 weeks before farrowing, with no impact on the general condition. Since no adverse reaction was observed in born alive piglet, these results support the safety of the different simultaneous vaccination schedules.

\section{Reference}

1. SYSTAT ${ }^{\oplus} 12$ for WINDOWS ${ }^{\circledR}$, Systat Software Inc., 2007

- CIRCOVAC, NEOCOLIPOR and PARVORUVAX are registered trademarks of Merial in the European Union and elsewhere. 


\title{
P.057
}

\section{Simultaneous vaccination of weaner pigs with SPRINTVAC ${ }^{\circledR}$ and CIRCOVAC $^{\circledR}$ (Merial)}

\author{
H.C. Lim ${ }^{1}$ B.K. Lim² P.Y. Choo ${ }^{3}$ \\ 1. Rhone Ma Malaysia (M) Sdn Bhd, Selangor, Malaysia; 2. Vet Food Agro Diagnostic (M) Sdn Bhd, \\ Selangor, Malaysia; 3. Asia Pacific Special Nutrients (M) Sdn Bhd, Selangor, Malaysia
}

\section{Introduction}

Recommended vaccination schedules for Mycoplasma hyopneumoniae (M. hyo) and porcine cirovirus type 2 (PCV2) for piglets may overlap. The administration of different vaccines simultaneously often gives rise to concerns about safety and efficacy. The present study was conducted to determine the safety and the serological response after simultaneous administration of SPRINTVAC, an oil adjuvanted bacterin vaccine against $M$. hyo and CIRCOVAC, a killed PCV2 vaccine containing Immuneasy ${ }^{\circledR}$, the same adjuvant, in piglets.

\section{Method and material}

A batch of thirty four 4-week old weaners from a farm known to be free of Aujeszky's disease, Porcine Respiratory and Reproductive Syndrome (PRRS) and classical swine fever were randomly allotted to 4 groups (Table 1). When simultaneous vaccination was performed, the vaccines were injected on opposite sites on the neck region. Vaccines were given intramuscularly at 4 weeks of age. All pigs were housed in adjacent pens and managed in same manner throughout the trial period.

Table 1. Group of experimental pigs

\begin{tabular}{|l|c|c|}
\hline Group & Vaccination & N \\
\hline S + C & SPRINTVAC and CIRCOVAC & 10 \\
\hline S & SPRINTVAC only & 8 \\
\hline C & CIRCOVAC only & 8 \\
\hline Control & None & 8 \\
\hline
\end{tabular}

Serums collected weekly from 4 to $10 \mathrm{wks}$ of age and then at 12 and 20 weeks of age were tested for antibodies to M. hyo and PCV2 using the IDEXX test kit, and Synbiotics SERELISA PCV2 test kit respectively. The effects of local site reactions and anorexia were observed for up to 3 weeks after vaccination.

Figure 1: M. hyo antibody serological profiles

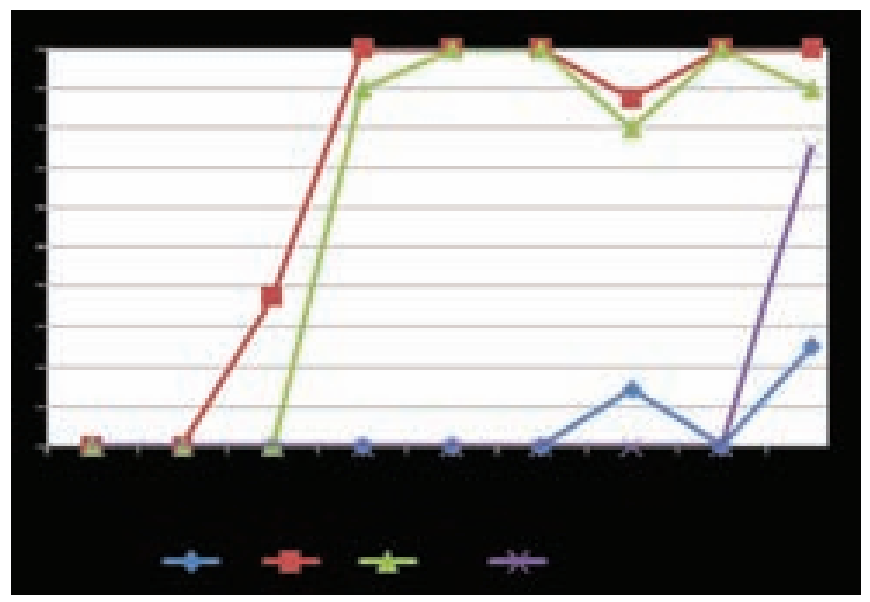

\section{Results and Discussion}

The serological profiles for M. hyo and PCV2 antibodies are shown in Figures 1 and 2.

Three weeks after vaccination, $100 \%$ and $87.5 \%$ of pigs in the $S$ and $\mathrm{C}+\mathrm{S}$ groups were tested positive for $\mathrm{M}$. hyo antibodies and had similar profiles throughout the period of the trial. Pigs in the $\mathrm{C}$ and Control group were negative for $\mathrm{M}$. hyo antibodies until 10-12 weeks of age most likely due to exposure to field M. hyo infection.

All piglets were positive for the presence of maternal antibodies to PCV2 titer at the beginning of the trial. As the pigs aged, reduction in the PCV2 antibody titer was observed in pigs from $\mathrm{S}$ and Control group from 8 weeks of age. In the $C$ and $C+S$ group, PCV2 antibody titers were maintained at high levels up to 20 weeks old when the study was terminated.

There were no adverse local reactions to the vaccinations or anorexia observed post-vaccinations

\section{Conclusion}

In this study, no adverse local reactions or anorexia were observed with the simultaneous vaccination on two different sites intramuscularly in the neck muscles of 4 week-old piglets with SPRINTVAC and CIRCOVAC. The present study showed that simultaneous vaccinations using the vaccines that were tested induced similar serological responses as vaccines used separately.

- SPRINTVAC, CIRCOVAC and Immuneasy are registered trademarks of MERIAL in the United States of America and elsewhere.

Figure 2: PCV2 serological profiles

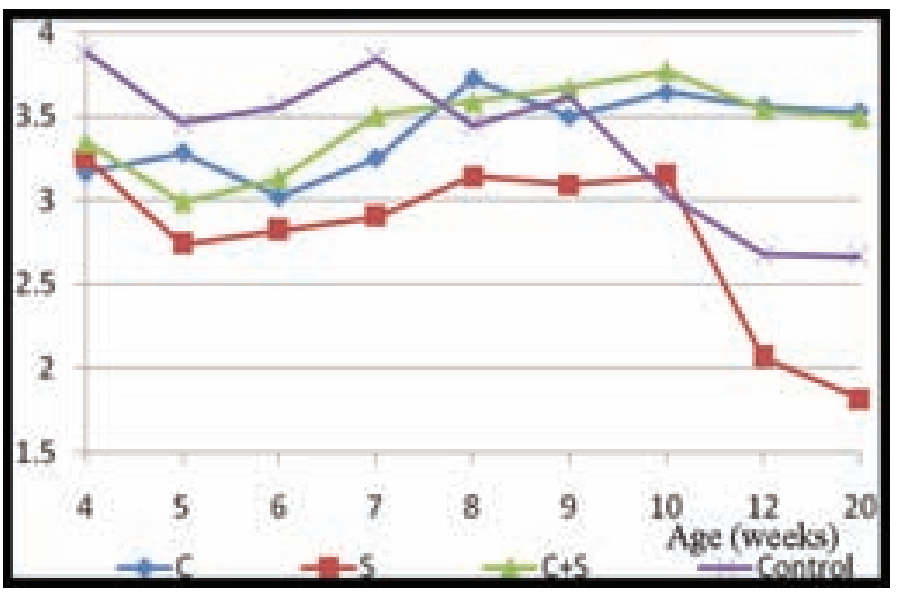




\title{
P.058
}

\section{The impact of PCV2 viremia in a high health Canadian swine herd, a vaccination trial comparing two commercial vaccines}

\author{
Melissa Reindl ${ }^{1}$ Cate Dewey ${ }^{1}$ Kevin Vilaca ${ }^{2}$ Francisco de Grau ${ }^{3}$ Karen Richardson ${ }^{1}$ Zvonimir Poljak ${ }^{1}$ \\ 1. University of Guelph, Guelph, ON, Canada; 2. Maitland Veterinary Professional Corp., \\ Listowel, ON, Canada; 3. Intervet Canada, Kirkland, QC, Canada
}

\section{Introduction}

Subclinical infections with PCV2 are common ${ }^{1,2}$ and can occur in the absence of co-factors or if vaccination does not prevent viral replication ${ }^{3}$. PCV2 viremia produces immune system activation ${ }^{4}$ which causes the redirection of nutrients intended for growth to counteract disease challenge ${ }^{5}$. The objective of this field trial was to compare the productivity of nursery and grower-finisher (G-F) pigs vaccinated with a labeled dose of one of two commercial circovirus vaccines or a placebo. Productivity was measured as average daily gain (ADG), feed disappearance, and mortality.

\section{Materials and Methods}

A total of 2,146 pigs were selected from a PRRS- and $M$. hyopneumoniae-free herd. The pigs were assigned to one of three treatment groups: 1 . One dose (1-D) pigs vaccinated with Circoflex ${ }^{\oplus}$ BIVI $(n=1026) ; 2$. Two dose (2-D) pigs vaccinated with Circumvent ${ }^{\circledR}$ ISPAH ( $\left.n=1020\right)$; and 3. Controls injected with saline $(n=100)$. Pigs were individually weighed at approximately 3,11 and 20 weeks of age as well as prior to slaughter (at least 107 $\mathrm{kg}$ ). Blood samples were taken from a random sample of 122 pigs at approximately 3, 9, 15, 19, and 23 weeks of age and also during the final week of shipping. Viremia was measured using qPCR.

\section{Results}

Throughout the entire G-F phase, the ADG of vaccinated animals outperformed that of unvaccinated controls $(p<0.01)$. Also during this time, controls had a higher mortality (5.1\%) than 1-D (1.7\%) and 2-D (1.6\%) pigs ( $\mathrm{p}<0.05)$.

While the control group had a higher percentage of viremic pigs during the G-F phase compared to the vaccine groups $(p<0.01)$, the 1-D vaccinated pigs were also more likely to be viremic compared to the $2-D$ vaccinated pigs $(p<0.01)$.
In the second half of the G-F phase, when qPCR results indicated the highest natural PCV2 challenge, the ADG of the 1-D pigs began to decrease. From 19 weeks of age to slaughter, the 1-D vaccinates had an ADG that was $42.3 \mathrm{~g} /$ day lower than the 2-D vaccinates $(p<0.01)$ after controlling for starting weight and weaning cohort. In addition, the 1-D vaccinates and 1-D controls had a higher amount of feed disappearance compared to the $2-D$ vaccinates and $2-D$ controls throughout the $G-F$ phase. This means that, although the 1-D pigs were growing at a slower rate, they were potentially eating more feed than the 2-D pigs.

\section{Implications}

Overall, vaccination reduced mortality and increased ADG. However, only the 2-D vaccine was able to control viremia and maximize ADG during the high PCV2 challenge in the finisher phase.

\section{References}

1. Darwich, L.; Segalés, J.; Resendes, A.; Balasch, M.; Plana-Durán, M.; Mateu, E. 2008. Transient correlation between viremia levels and IL-10 expression in pigs subclinically infected with porcine circovirus type 2 (PCV2). Res Vet Sci. 84: 194-198.

2. Krakowka, S.; Ellis, J.; McNeilly, F.; Waldner, C.; Allan, G. 2005. Features of porcine circovirus-2 disease: correlations between lesions, amount and distribution of virus, and clinical outcome. J Vet Diagn Invest. 17: 213-222.

3. Fort, M.; Fernandes, L.T.; Nofrarias, M.; Diaz, I.; Sibila, M.; Pujols, J.; Mateu, E.; Segalés, J. 2009. Development of cell-mediated immunity to porcine circovirus type 2 (PCV2) in caesarean-derived, colostrumdeprived piglets. Vet Immunol and Immunop. 129: 101-107.

4. Kekarainen, T. ; Montoya, M. ; Dominguez, J. ; Mateu, E. and Segalés, J. 2008. Porcine circovirus type 2 (PCV2) viral components immunomodulate recall antigen responses. Vet Immunol and Immunop. 124 : 41-49

5. Colditz, I.G. 2002. Effects of immune system on metabolism: implications for production and disease resistance in livestock Livest Prod Sci. 75(3): 257-268. 


\title{
P.059
}

\section{Reduction of porcine circovirus type 2 load in vaccinated pigs with Circumvent ${ }^{\mathrm{TM}}$ PCV}

\author{
Yeong Hun Kim${ }^{1}$ Myung Hyee Kim² Jeong Hee Han' \\ 1. School of Veterinary Medicine and Institute of Veterinary Science, Kangwon National \\ University, Chuncheon, Korea; 2. Intevet Schering-Plough, Seoul, Korea
}

\section{Introduction}

A new emerging disease termed "post-weaning multisystemic wasting syndrome" (PMWS) was identified and reported in Canada. PMWS manifests as systemic disease characterized by weight loss, a relatively high mortality rate among pigs in the 10-to 17-week-old age group and lymphadenopathy causing immunosuppression. PMWS diagnosis is based on the presence of characteristic histopathological lymphoid lesions and PCV2 within these lesions. Also, the presence in a sample of animals of high PCV2 loads is suggested as indicating the presence of PMWS lesion using real time PCR. Thus, this study was to evaluate whether vaccination against PCV2 can reduce PCV2 load or not.

\section{Materials and Methods}

159 piglets from 3 different farms, Farm A, B, and C located in Hongsung-gun, Choongnam province, Korea, that had a history of PCVD will be included in this study and divided into 2 groups, vaccinated and placebo. Pigs in the vaccination group were given CIRCUMVENTTM PCV (Intevet Schering-Plough) IM in 3 and 6 weeks of age. Control pigs were handled in a similar manner as vaccinated pigs with saline. For quantification of the PCV2 viral load in serum, serum samples were analyzed by TaqMan real time PCR as described previously (A. Olvera et al. 2004)

Table 1. Primer and probe designed for the real time $P C R$

\begin{tabular}{|l|l|}
\hline Oligo & Sequences \\
\hline PCV2F & CCAGGAGGGCGTTGTGACT \\
\hline PCV2R & CGCTACCGTTGGAGAAGGAA \\
\hline PCV2S & AATGGCATCTTCAACACCCGCCTCT \\
\hline
\end{tabular}

\section{Results and Discussion}

The profile of PCV2 load during the course of the study is described in Fig. 1, 2 and 3. All of the farm hadthe presence of high maternal derived antibodies against PCV2. Peak level of PCV2 positive animals were reached when animals were approximately 10-16 weeks of age in placebo-treated group. In vaccinated group, level of positive animal decreased dramatically than placebo-treated group. Animals with high viral loads (106 $\mathrm{PCV} 2$ genome/ml in serum) were observed in the acute phase when is 10 to 16 weeks of age. Compared to the placebo-treated group the proportion of PCV2 positive animals in the vaccinated group was significantly lower $(P=0.0021$, Farm $A ; P<0.0001$, Farm $B ; P<0.0001$, Farm $C)$. Based on the results, vaccination with CIRCUMVENTTM PCV would reduce PCV2 load in serum.
Figures 1-3.

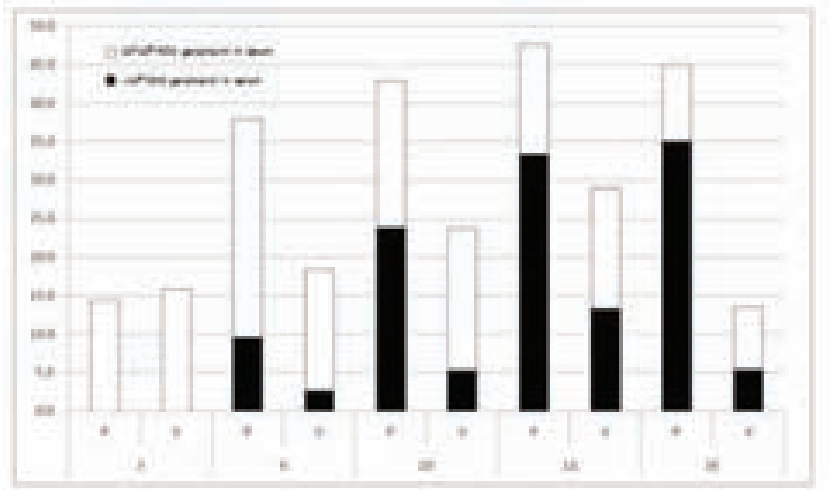

Figur 1 Comparion of the PCV 2 viral load f arm A
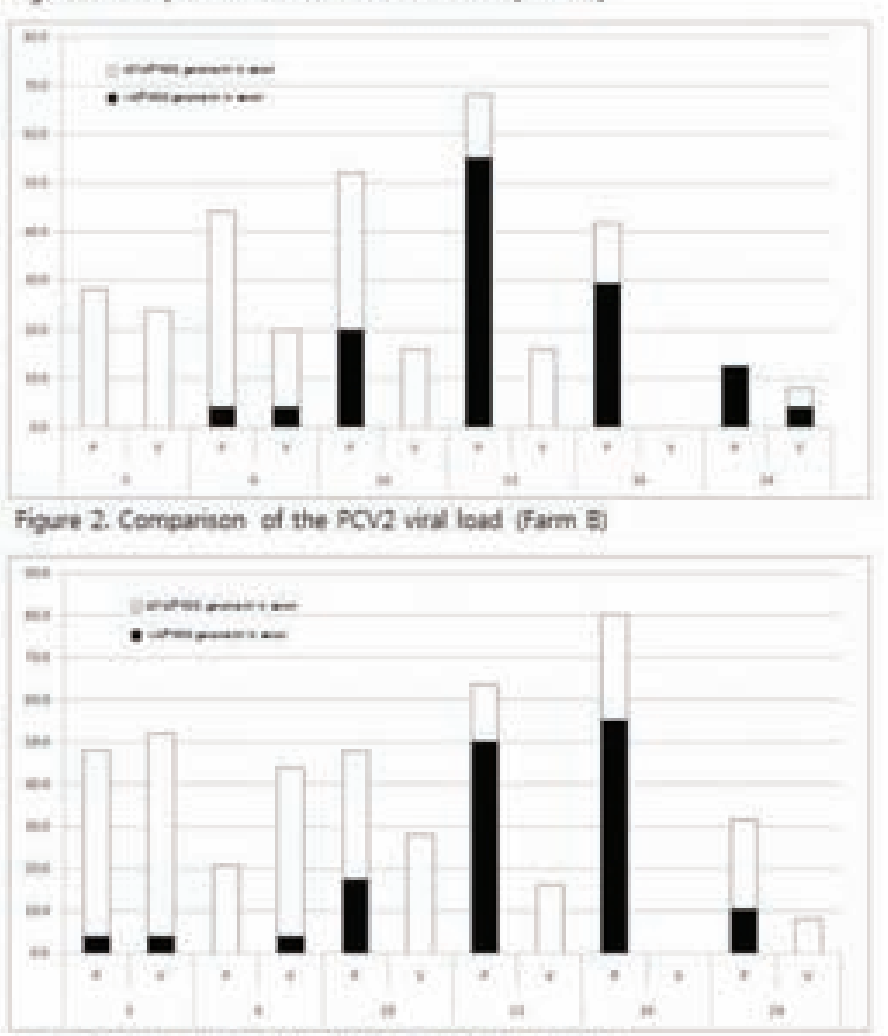

Figure 3. Comearion of the PCV2 veral lead form Co

\section{References}

Olvera A et al. 2004. J Virol Methods. 117: 75-80 


\title{
P.060
}

\section{A field trial on efficacy of DS Circo Pigvac ${ }^{\circledast}$ in South Korea}

\author{
Yeong Hun Kim; Jeong Hee Han \\ School of Veterinary Medicine and Institute of Veterinary Science, Kangwon National University, Chuncheon, Korea
}

\section{Introduction}

PCVAD is now well established as a wasting disease associated with PCV2 and has rapidly become one of the most devastating and economically important concerns in all pig-producing areas of the world. And PCV2 could be an important contributor to PRDC characterized be slow growth, decreased feed efficiency, and increased clinical sign index, such as lethargy, anorexia, fever, cough and dyspnea. In this disease, although the exact pathogenic mechanisms responsible for these diseases are unknown, several studies have suggested that PCV2 infects macrophages and $B$ lymphocytes, inducing apoptosis of the $B$ cells and the damage of lymphoid tissues resulting in extensive lymphocyte depletion. immunosuppression is believed to be a key of PCVAD. The purpose of this study was to evaluate the effects of inactivated PCV2 vaccine in Korean commercial farms that had a history of PCVD.

\section{Materials and Methods}

50 piglets from a farm, located in Choongnam province, Korea, that had a history of PCVD will be included in this study and allocated into 2 groups equally, vaccinated and placebo. Pigs in the vaccination group were given the PCV2 vaccine IM in approximately 3 weeks of age. On the other hands, Control pigs were handled in a similar manner as vaccinated pigs with saline. ADG was obtained by dividing weight gain by the number of days from vaccination to the end of the nursery phase, by number of days from the end of the nursery phase to the entry into the finishing phase. Immune response in serum sample was analyzed using IFA. Mortality rate was calculated for each group. For statistical analysis, student t-test or Wilcoxon Mann-Whitney test was used in order to assess differences with regard to ADG and serology. Fisher's exact test was used to investigate possible differences in mortality.

\section{Results}

Mean ADGs during the overall period for experiment were increased by $19.2 \%$ in vaccinated group. compared with placebo group. Vaccinated group had consistently a significantly higher mean PCV2 antibody titer than placebo group in 10 weeks of age when is the onset of PCV2 viremia. During the period following the vaccination placebo group had an $24.0 \%$ higher mortality rate than it of vaccinated animals.
Table 1. Average daily weight gain (g/day) during different intervals

\begin{tabular}{|l|c|c|c|c|}
\hline Period & Vaccine $\mathbf{( 9 5 \%}$ Cl) & Placebo $\mathbf{( 9 5 \% ~ C l )}$ & Difference & P-value \\
\hline $3-6$ & $209.5(185.1-234.0)$ & $227.6(199.6-255.6)$ & -18.1 & 0.6575 \\
\hline $6-10$ & $458.2(429.8-486.6)$ & $416.8(385.9-447.7)$ & 41.4 & 0.0476 \\
\hline $10-13$ & $685.9(656.0-715.9)$ & $500.3(432.9-567.7)$ & 185.6 & $<0.0001$ \\
\hline $13-16$ & $855.5(818.6-892.4)$ & $716.4(570.8-862.0)$ & 139.1 & 0.0661 \\
\hline $3-16$ & $562.6(538.7-586.3)$ & $469.2(422.7-515.9)$ & 93.4 & 0.0008 \\
\hline
\end{tabular}

Table 2. The mortality rates (\%) of vaccinated group and placebotreated group

\begin{tabular}{|l|c|}
\hline Group & Mortality rate \\
\hline Placebo-treated group (\%) & $24.0^{*}$ \\
\hline Vaccinated group (\%) & 0.0 \\
\hline${ }^{*} \mathrm{P}<0.01$. \\
\hline
\end{tabular}

Figure 1. Comparison of the PCV2 viral load in vaccinated and placebo-treated group.

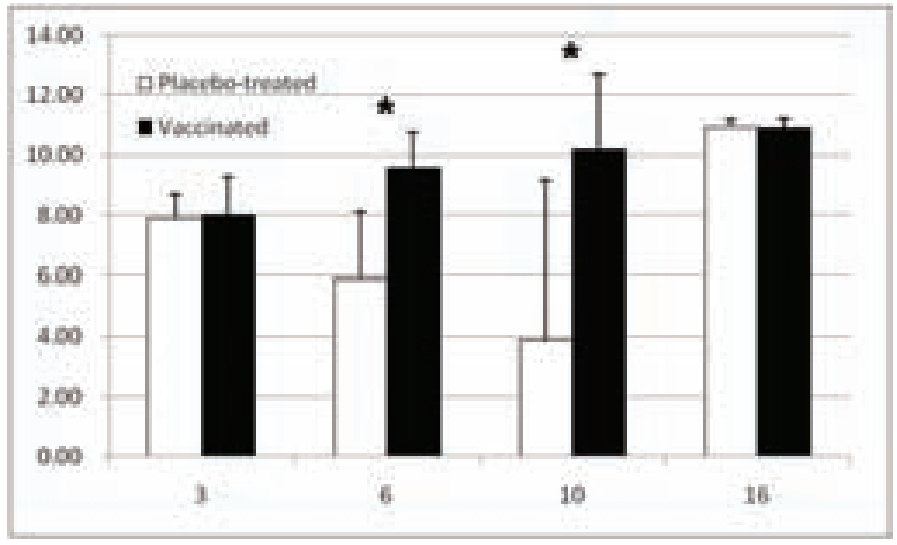

\section{Discussion}

Based on the results, Vaccination against PCV2 would be helpful for improving growth performance and reducing mortality rate as it significantly maintained antibody titers against PCV2 in the point when is the entry of the finishing phase.

\section{Reference}

1. Kixmoller M., et al. 2008. Vaccine, 26, 3443-3451.

2. Horlen KP., et al. 2008. JAVMA, 232, 906-912. 


\title{
P.061
}

\section{Immune response by vaccination with Circumvent ${ }^{\mathrm{TM}} \mathrm{PCV}$}

\author{
Yeong Hun Kim ${ }^{1}$ Myung Hyee Kim² Jeong Hee Han \\ 1. School of Veterinary Medicine and Institute of Veterinary Science, Kangwon National \\ University, Chuncheon, Korea; 2. Intevet Schering-Plough, Seoul, Korea
}

\section{Introduction}

PCVAD is now well established as a wasting disease associated with PCV2 and has rapidly become one of the most devastating and economically important concerns in all pig-producing areas of the world. In this disease, although the exact pathogenic mechanisms responsible for these diseases are unknown, several studies have suggested that PCV2 infects macrophages and B lymphocytes, inducing apoptosis of the B cells and the damage of lymphoid tissues resulting in extensive lymphocyte depletion. immunosuppression is believed to be a key of PCVAD. The purpose of this study was to evaluate the immune response of inactivated PCV2 vaccine in Korean commercial farms.

\section{Materials and Methods}

159 piglets from 3 different farms, Farm A, B, and C located in Hongsung-gun, Choongnam province, Korea, that had a history of PCVD will be included in this study and divided into 2 groups, vaccinated and placebo. Pigs in the vaccination group were given CIRCUMVENTTM PCV (Intevet Schering-Plough) IM in 3 and 6 weeks of age. Control pigs were handled in a similar manner as vaccinated pigs with saline. Immune response in serum sample was analyzed using SERELISA PCV2 Ab Mono Blocking (Synbiotics, Frence) according to the manufacturer's instructions. For statistical analysis, student t-test or Wilcoxon Mann-Whitney test was used in order to assess differences with regard to serology. Statistical analyses were performed using SAS software release 8.2 .

\section{Results}

Maintenance of antibody responses in vaccinated group had been continued with high antibody level until 16 weeks of age in Farm A and 24 weeks of age in Farm B and Farm C by vaccination. However, infectious antibodies in placebo-treated group were confirmed at 13 or 24 weeks of age. Interestingly, at 24 weeks of age, the lower antibody response was observed in vaccinated group than in placebo-treated group.

\section{Discussion}

Analysis of immune response against PCV2 revealed that vaccinated group had the higher immune response more than placebo-treated group at 10 to 16 weeks of age when is the period of acute infection with PCV2. In conclusion, vaccination against PCV2 would have an effect to activate immune response against it.

\section{Reference}

Horlen KP et al. 2008. JAVMA 232(6): 906-912
Figures 1-3.

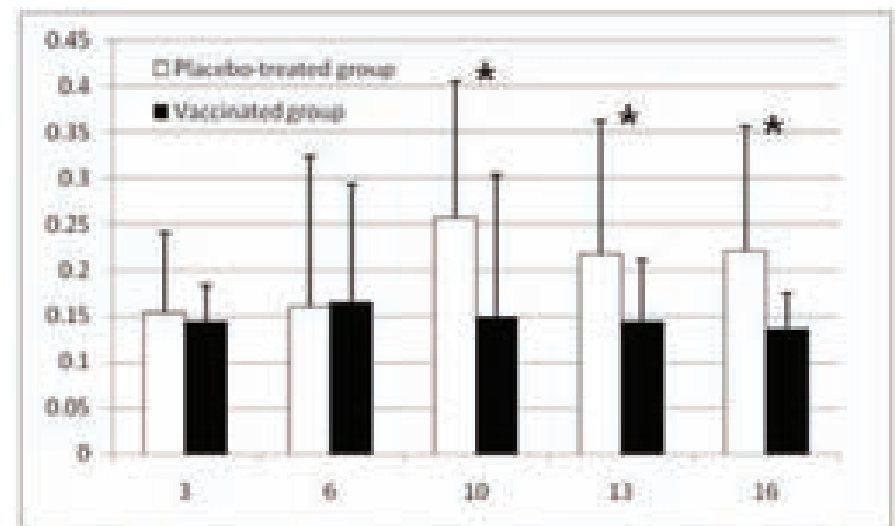

Figure 1. Immune response of vaccinated group and placebo-treated pigs (Farm A)

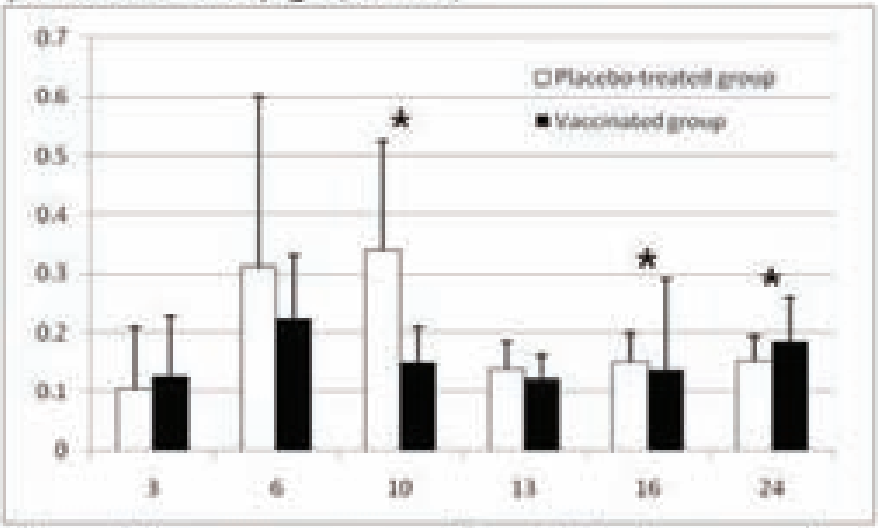

Figure 2. Immune response of vaccinated group and placebo-treated pigs (Fam B)

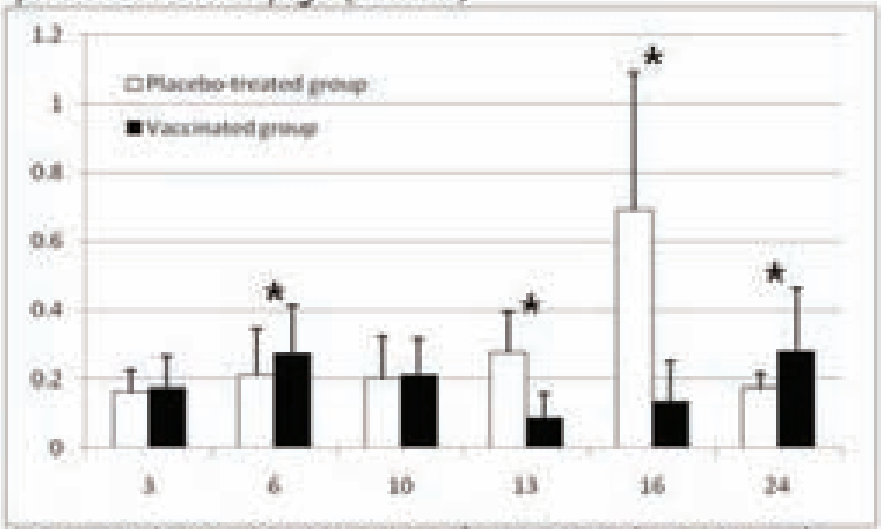

Figure 3. Immune response of vaccinated group and placebo-treated pigs (Farm Q) 


\title{
P.062
}

\section{A field efficacy of growth performance and mortality rate in vaccinated pigs with Circumvent ${ }^{\mathrm{TM}} \mathrm{PCV}$}

\author{
Yeong Hun Kim${ }^{1}$ Myung Hyee Kim² Jeong Hee $\mathrm{Han}^{1}$ \\ 1. School of Veterinary Medicine and Institute of Veterinary Science, Kangwon National \\ University, Chuncheon, Korea; 2. Intevet Schering-Plough, Seoul, Korea
}

\section{Introduction}

Porcine circovirus type 2 has been reported throughout the world since it is first described in Canada in the early 1990s. Recently, these diseases are also called as porcine circovirus associated disease (PCVAD) PCVAD has been reported with increasing frequency in swine herds in Western Europe, North America and eastern Asia. The problem with PCV2 is similar to previous results reported in above country. The introduction of PCV2 vaccines is contributing to reduce the incidence of PMWS and their associated diseases. However, there have been a few investigations demonstrating the efficacy of PCV2 vaccine and no published study on commercial Korean farms. The purpose of this study was to further investigate the effects on growth performance and mortality of vaccination against PCV2 on commercial Korean farms having positivity for PCV2

\section{Materials and Methods}

159 piglets from 3 different farms, Farm A, B, and C located in Hongsung-gun, Choongnam province, Korea, that had a history of PCVD will be included in this study and divided into 2 groups, vaccinated and placebo. Pigs in the vaccination group were given a single dose of the PCV2 vaccine (Intevet ScheringPlough) IM in 3 weeks of age. A second dose of vaccine was administered 3 weeks after the first dose. Control pigs were handled in a similar manner as vaccinated pigs with saline. The individual body weight of all study animals was measured at 3 , $6,9,1316$ and 24 weeks of age. Mortality rate was calculated for each group. For statistical analysis, student t-test or Wilcoxon Mann-Whitney test was used in order to assess differences with regard to ADG. Fisher's exact test was used to investigate possible differences in mortality. Statistical analyses were performed using SAS software release 8.2.

\section{Results}

As seen average daily weight gain, it was considered to be an objective measurable parameter to determine the severity of PCVAD and the effects of vaccination in a large number of animals. Vaccinated groups had significantly improved growth performances more than placebo-treated group in either or both Entry into the finishing phase or/and midpoint of the finishing phase. The total of mortality rate from vaccinated group was significantly lower during the finishing phase, compared with placebo-treated group.

\section{Discussion}

Pigs vaccinated against PCV2 showed more increased ADGs and more reduced mortality rate with statistical differences in the finishing phase compared with placebo-treated group. Thus, vaccination against it can reduce economic losses from PCV2 infection To our knowledge this is the first report of the efficacy of vaccination against PCV2 in Korean field farms.
Tables 1-4.

Table 1. Avenge daily weight gais (g day) during differeat intervale (FarmA).

\begin{tabular}{|c|c|c|c|c|}
\hline Age (wedks) & Vacore gstoc CD & 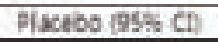 & Difference & Donblut \\
\hline 3.6 & $2182,2061+225.4$ & $1918(1712.212 .4)$ & 264 & 0015 \\
\hline $6-10$ & $19600184-5760$ & 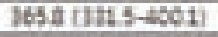 & -92 & 0012 \\
\hline $10-13$ & $674)(6241-7249$ & $6390(5750-70401$ & HA & 000 \\
\hline $1+16$ & 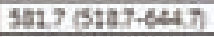 & $4450(1279.925)$ & 1867 & 0000 \\
\hline $3-16$ & $4537(430.8 .476 .6$ & $4100(370.4 .4496)$ & 437 & 0090 \\
\hline
\end{tabular}

Table 2. Average daily weight gaia (g day) during different intervals (Farm B)-

\begin{tabular}{|c|c|c|c|c|}
\hline ge (mvilcs) & Vaccine $9556 \mathrm{CD}$ & Dlacubo $955 \mathrm{CD}$ & Difference & Drialue \\
\hline 26 & 217,4 (196)-2180 & 2149 (204:-27) & -215 & 025 \\
\hline $6-10$ & $4614505-1968$ & $61.5(418+5606)$ & 0.1 & 6001 \\
\hline $10-13$ & $6.416159-6967$ & $5077(5247-6647)$ & $\infty \pi$ & 0310 \\
\hline $12-26$ & 040 (Ded)-9516) & $7296[6345-6046]$ & 1724 & 60001 \\
\hline 1634 & 8926 (341.3.943.9) & 9183 (1321-20056) & 5262 & 0593 \\
\hline 32,24 & $634.4(6596.7092)$ & $6670(2130.7151)$ & 174 & 0310 \\
\hline \multicolumn{5}{|c|}{$\begin{array}{l}\text { Table 3. Average daily weight gaia (g day) during different interval } \\
\text { (Furm C) }\end{array}$} \\
\hline Agen (ventos) & vactire coss CD & DIxabo coss CD & Difference & Dratue \\
\hline 56 & $1942(17342.2150$ & 2276 [1996.2535] & .334 & 0009 \\
\hline 610 & 392905665.4289 & $4130(3 \times 94460)$ & +201 & Q401 \\
\hline 1013 & 359 & $5030(4330.5672)$ & 1269 & 0003 \\
\hline 18 & 979 & 716 & 1615 & 0042 \\
\hline 1654 & 90700 & $20022(3035.10067)$ & 11 & 0619 \\
\hline $3-24$ & $6757(6443.707 .1)$ & 5710 (3156-4345) & 1007 & 0004 \\
\hline
\end{tabular}

Table 4. The mortality mes (\%) of vaceinaed groap and placebotreated group.

\begin{tabular}{ccccc}
\hline & Farm A & Farm B & Fam C & Total \\
\hline Placebo-treated group (5) & 3.8 & 4.0 & $24.0^{*}$ & $11.3^{*}$ \\
\hline Vaccinated group (5) & 2.6 & 0.0 & 0.0 & 1.1 \\
\hline
\end{tabular}

- PCO.05.

\section{References}

1. Horlen KP., et al. 2008. JAVMA, 232, 906-912.

Proceedings of the $21^{\text {st }}$ IPVS Congress, Vancouver, Canada - July 18-21, 2010 


\title{
P.063
}

\section{Reduction of lung lesions in vaccinated pigs with Circumvent ${ }^{\mathrm{TM}}$ PCV}

\author{
Yeong Hun Kim${ }^{1}$ Myung Hyee Kim² Jeong Hee Han' \\ 1. School of Veterinary Medicine and Institute of Veterinary Science, Kangwon National \\ University, Chuncheon, Korea; 2. Intevet Schering-Plough, Seoul, Korea
}

\section{Introduction}

Porcine circovirus type2 (PCV2) is the primary causative agent of porcine circovirus-associated disease (PCVD), which is recently replaced from postweaning multisystemic wasting syndrome (PMWS). PCV2 is required to cause the characteristic lymphoid depletion of PCVAD. Coinfection with several other viral and bacterial pathogens has been shown to cause an increase in incidence and a markedly more severe clinical course of disease. The agent implicated as creating the greatest risk is porcine reproductive and respiratory syndrome virus (PRRSV), porcine parvovirus (PPV), and Mycoplasma hyopneumoniae. Therefore, This study performed to evaluate whether vaccination against PCV2 reduce mycoplasmic lung lesions in pigs or not.

\section{Materials and Methods}

100 piglets from 2 different farms, Farm A and B located in Hongsung-gun, Choongnam province, Korea, that had a history of PCVD will be included in this study and divided into 2 groups, vaccinated and placebo. Pigs in the vaccination group were given CIRCUMVENTTM PCV according to manufacturer's instrument. Control pigs were handled in a similar manner as vaccinated pigs with saline. For lung lesion analysis, at 24 weeks of age, macroscopic lung lesions were estimated based on the amount of lung parenchyma affected by lesions according to Straw et al (1986). Statistical analysis performed with Wilcoxon Mann Whitney test for lung lesion analysis.

\section{Results and Discussion}

In case of farm A, piglets in vaccinated group had a significant lower $(P<0.05)$ mean of lung lesions score $(1.4 \pm 0.7)$ than piglets in placebo-treated group (2.7 \pm 1.3$)$. Vaccine efficacy in reducing lung lesions score was $48 \%$.

In case of farm B, although there was no significant difference in lung lesions between treatment groups, piglets in vaccinated group had lower mean of lung lesions score with placebo-treated group and vaccinated group being 1.8 and 2.5, respectively. Under the conditions described in this study, vaccination against PCV2 in piglets would reduce lung lesions at slaughter.
Figure 1. Lung lesion scoring in vaccinated pigs and placebo-treated pigs at market age (Farm A).

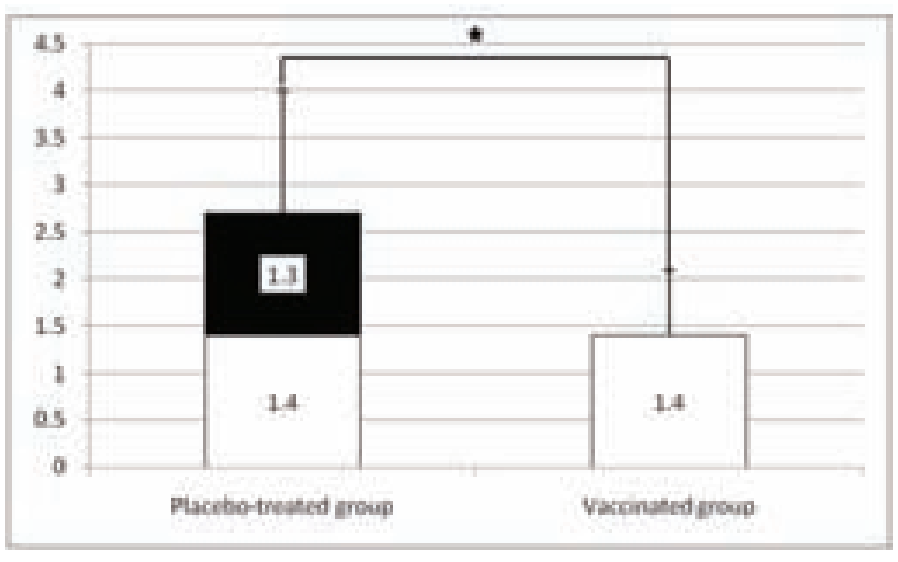

Figure 2. Lung lesion scoring in vaccinated pigs and placebo-treated pigs at market age (Farm B).

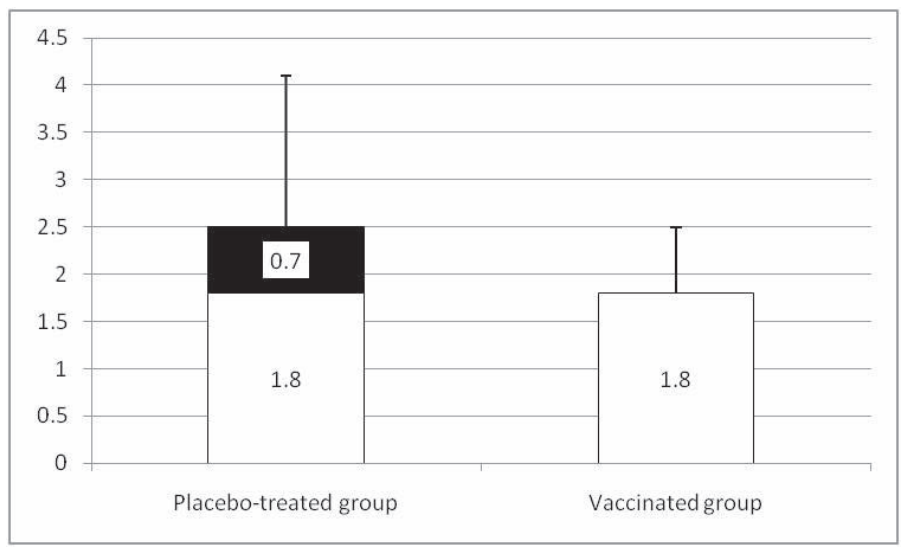

\section{Reference}

1. Opriessnig T et al. 2008. Vet Microbiol, 131, 103-114 


\title{
P.064
}

\section{Detection of Mycoplasma hyopneumoniae from porcine nasal cavity in vaccination against PCV2 in Korea}

\author{
Seung Hyuk Yang; Yeong Hun Kim; Jeong Hee Han \\ School of Veterinary Medicine and Institute of Veterinary Science, Kangwon National University, Chuncheon, Korea
}

\section{Introduction}

Mycoplasma(M) hyopneumoniae, the primary pathogen of enzootic pneumonia, is an integral component of the porcine respiratory disease complex(PRDC). M. hyopneumoniae causes to increase susceptibility to other respiratory disease. The vaccination is easy to control $\mathrm{MH}$ infection. Porcine circovirus type 2(PCV2) is considered to be the causative agent of PMWS. PCV2 infection affects pig's immune system and increases susceptibility of other viruses and bacteria. PCV2 is widely prevalent in pigs with PRDC and should be considered a major respiratory pathogen. The interaction of PCV2 with other respiratory pathogens plays an important role in developing PRDC. The PCV2 vaccination reduces clinical signs of respiratory and the detection rate of other respiratory pathogens in vaccinated pigs. The interactions with different respiratory pathogens, porcine reproductive and respiratory syndrome(PRRS), swine influenza virus(SIV) and Actinobacillus pleuropneumoniae (APP) were studied. It was the purpose of this study to evaluated the detection rate of $M$. hyopneumoniae from nasal swabs in vaccination against PCV2.

\section{Materials and Methods}

A total of 40 3-week-old piglets were allocated into 2 groups and either vaccinated against PCV2(DSPCV2 ${ }^{\circledR}$,Korea) and treated with a placebo. Pigs were inoculated intramuscularly with $2.0 \mathrm{ml}$ either the vaccine or saline at three weeks of age. Blood samples were collected in each pigs at four different points during their growth period: 3, 6, 9, 16 weeks of age. Antibody titers were evaluated by using ELISA Kit(Mycoplasma hyopneumoniae Antibody Test Kit (IDEXX,USA). Intranasal swabs were collected from nine pigs of vaccination groups and eight pigs of placebo group at 3,6, 9, 16 weeks of age. M. hyopneumoniae DNA was extracted by using Accuprep ${ }^{\circledR}$ Genomic DNA extraction kit(Bioneer, Korea) and detected with nest-PCR and conventional PCR. For statistical analysis, student t-test or Wilcoxon Mann-Whitney test was used in order to assess differences with regard to serology. Statistical analyses were performed using SAS software release 8.2.

\section{Results}

The infection of M. hyopneumoniae was demonstrated by ELISA in serum and PCR in nasal swabs. The high antibody titers of M. hyopneumoniae were detected in non-vaccinated group against PCV2 compared to vaccinated group (table 1). M. hyopneumoniae seroconversion was observed at 9 weeks of age in non-vaccinated group against PCV2. M. hyopneumoniae DNA was detected in $37.5 \%$ at 9 weeks of age , $25 \%$ at 16 weeks of age in non-vaccinated group. M. hyopneumoniae DNA was only detected in $11 \%$ at 9 weeks of age in vaccinated groups against PCV2 using nested PCR (table2).

\section{Table 1:}

\begin{tabular}{|l|c|c|c|c|}
\hline Age & $\mathbf{3}$ & $\mathbf{6}$ & $\mathbf{9}$ & $\mathbf{1 6}$ \\
\hline Vaccinated group & $0.132 \pm 0.024^{*}$ & $0.281 \pm 0.139$ & $0.428 \pm 0.082$ & $0.259 \pm 0.159$ \\
\hline Non-vaccinated group & $0.123 \pm 0.021$ & $0.363 \pm 0.110$ & $0.528 \pm 0.176$ & $0.276 \pm 0.133$ \\
\hline P-value & 0.4273 & 0.3578 & 0.1706 & 0.6912 \\
\hline
\end{tabular}

Table 2:

\begin{tabular}{|l|c|c|c|c|c|c|c|c|}
\hline Age & $\mathbf{3}$ & $\mathbf{3}$ & $\mathbf{6}$ & $\mathbf{6}$ & $\mathbf{1 0}$ & $\mathbf{1 0}$ & $\mathbf{1 6}$ & $\mathbf{1 6}$ \\
\hline $\mathrm{PCR}$ & Con & Nested & Con & Nested & Con & Nested & Con & Nested \\
\hline $\begin{array}{l}\text { PCV2 } \\
\text { vaccine }\end{array}$ & $0 / 9(0.0)$ & $0 / 9(0.0)$ & $0 / 9(0.0)$ & $0 / 9(0.0)$ & $0 / 9(0.0)$ & $\begin{array}{c}1 / 9 \\
(11.0)\end{array}$ & $0 / 9(0.0)$ & $0 / 9(0.0)$ \\
\hline Placebo & $0 / 8(0.0)$ & $0 / 8(0.0)$ & $0 / 8(0.0)$ & $0 / 8(0.0)$ & $0 / 8(0.0)$ & $\begin{array}{c}3 / 8 \\
(37.5)\end{array}$ & $0 / 8(0.0)$ & $\begin{array}{c}2 / 8 \\
(25.0)\end{array}$ \\
\hline
\end{tabular}

\section{Discussion}

The infection and antibody titers of $M$. hyopneumoniae decreased in the PCV2 vaccination group. The high titers of PCV2 antibody is intended to decrease antibody of M. hyopneumoniae and decrease of detection of M. hyopneumoniae antigen in nasal swabs. The PCV2 is supposed to infleuence the infection of $M$. hyopneumoniae. In addition, the nest-PCR is effective method to detecting $M$. hyopneumoniae DNA in nasal swabs.

\section{References}

1. Allan GM, Ellis JA. Porcine circoviruses : A review. J Vet Diagn Invest 2000, 12, 3-14.

2. Calsamiglia M, Pijoan C, Trigo A. Application of a nested polymerase chain reaction assay to detect Mycoplasma hyopneumoniae from nasal swabs. J Vet Diagn Invest 1999, 11, 246-252. 


\title{
P.065
}

\section{Efficacy of PCV2 Vaccination on Morbidity under Philippine Field Conditions}

\author{
Edmundo C. Sy ${ }^{2}$ Philip R. Lehrbach¹ Libertad S. Reyes² Civilo Q. Lima ${ }^{3}$ Jose R. Balantakbo ${ }^{4}$ \\ 1. Pfizer Australia, Melbourne, VIC, Australia; 2. Fort Dodge Animal Health Philippines, Inc, Mandaluyong, \\ Philippines; 3. Sta Cruz Farm, Pampanga, Philippines; 4. Reva Farm, Laguna, Philippines
}

\section{Introduction and Objective}

In the Philippines a phylogenetic analysis of the sequences of PCV2, isolated from PMWS affected local farms, allocated isolates into a PCV2 subgroup with Canadian and European isolates (1). Suvaxyn ${ }^{\circledast}$ PCV2 (Fort Dodge Animal Health, USA) is the Chimeric PCV1-2 vaccine containing immunogenic capsid gene of PCV2 cloned into the backbone of the non-pathogenic PCV1. Experimental vaccination with the chimeric PCV1-2 significantly reduced viraemia and decreased the risk of clinical diseases (2). It is on this basis that an efficacy study was performed to measure the difference in morbidity cases between vaccinated and control animals under Philippine field condition.

\section{Material and Methods}

Three farms were selected where PMWS had been positively identified through the analysis of clinical signs, histopathology lesions and a specific PCV2 antigen fluorescent antibody test. At each trial site the pigs were individually ear tagged, numbered and colour coded to indicate the treatment group. Codes were not known to the farms and the identity of the vaccines (Suvaxyn PCV2 or placebo) was blinded when administered.

Individual animals were clinically evaluated on days 0, 21 and 84 post vaccination. The signs evaluated were body condition, presence of respiratory and digestive clinical signs (coughing, dyspnea and diarrhea) and behaviour. Pigs' body condition was recorded by applying a numeric value ranging from 1 to 6 , based on individual evaluation of the pelvis, vertebrae and ribs (3). Presence of coughing, dyspnea, diarrhea in the animals and their behaviour was recorded (4) with a numeric value ranging between 0 and 2. The clinical score (CS) was calculated as:

$\mathrm{CS}=[$ (numeric value of diarrhea + numeric value of behaviour + numeric value of coughing + numeric value of dyspnea divided by 4$)+(6$ - numeric value of physical condition)].

Morbidity, expressed as a percentage of the group was calculated as the number of animals with a CS higher than 0 , divided by the total number of pigs of the clinical study on days 0,21 and 84 of the study.

\section{Result and Discussion}

Morbidities (\%) recorded on each farm are shown in Table 1. These percentages reflect the number of pigs transferred to nursery or hospital pens during the course of the trial.

Table 1. Percent morbidities.

\begin{tabular}{|l|c|c|c|}
\hline Farm Name & Replicates & Vaccinated (Nos.) & Control (Nos.) \\
\hline Farm 1 & 1 & $6.7 \%(8 / 120)$ & $9.2 \%(11 / 120)$ \\
\hline & 2 & $5.0 \%(6 / 120)$ & $8.3 \%(10 / 120)$ \\
\hline & Total & $5.8 \%(14 / 240)$ & $8.8 \%(21 / 240)$ \\
\hline Farm 2 & 1 & $6.7 \%(8 / 120)$ & $13.3 \%(16 / 120)$ \\
\hline & 2 & $4.1 \%(5 / 122)$ & $9.8 \%(12 / 122)$ \\
\hline & Total & $5.4 \%(13 / 242)$ & $11.6 \%(28 / 242$ \\
\hline Farm 3 & 1 & $7.6 \%(9 / 119)$ & $12.5 \%(15 / 120)$ \\
\hline & 2 & $7.6 \%(10 / 130)$ & $14.6 \%(19 / 130)$ \\
\hline & Total & $7.6 \%(19 / 249)$ & $13.6 \%(34 / 250)$ \\
\hline
\end{tabular}

The vaccinated groups had overall significantly reduced incidence of morbidity (one sided $p<0.001$ ). The controls were $1.90(95 \%$ confidence limits $=1.31,2.77)$ times as likely to have morbidity.

These differences were not significant $(p=0.15)$ on piggery Farm 1 , but they were on Farm $2(p=0.01)$ and Farm $3(p=0.02)$.

Thus on the majority of the farms there was a significant reduction in the levels of morbidity in the Suvaxyn PCV2 vaccine group. These results are consistent with an immune response that acts to lessen the impact of the presence of PCV2 on pig growth.

\section{References}

1. Maldonado et al. (2004) J. Vet. Med. Sci. 66: 533-537.

2. Fenaux, M. et al. (2004). J. Virol. 78: 6297-6303.

3. Straw, B.E. et al (1999) In Diseases of Swine (8th edition) p3-18.

4. Kyriakis, C.S. et al. (2002) J. Comp. Pathol. 126: 38-46. 


\title{
P.066
}

\section{The reproduction and production improvements in a South African commercial piggery after introducing whole herd vaccination against PCV2}

\author{
Pieter J. Grimbeek \\ Private Practitioner, Potchefstroom, South Africa
}

\section{Introduction}

A wide range of clinical signs and diseases can be associated with PCV2 infection, including PMWS, PDNS and reproductive failure. While piglet vaccination has been proven to be the most effective tool to control losses attributable to PCV2 infection in grow-finish pigs (1), sow and gilt vaccination might have a positive effect on reproductive performance (2). This is the first report evaluating the benefits of whole herd vaccination (sows, gilts, boars and piglets) with Ingelvac CircoFLEX ${ }^{\circledR}$, a subunit PCV2 vaccine that became available in South Africa mid 2008 under a special import permit.

\section{Materials and Methods}

The piggery is a 4500 sow continuous flow, farrow to finish unit. Most if not all the modern diseases except for PRRS and SIV are present.

During the first quarter of 2008 a nutritional insult resulted in poor on farm performance that was exacerbated by an acute PCV2 challenge with subsequent clinical outbreaks of PMWS and PDNS. PCV2 infection was confirmed by both serology and immunohistochemistry. A Ingelvac CircoFLEX ${ }^{\circledR}$ vaccination program was then implemented in September 2008. All piglets were vaccinated at 18 days of age. As the reproduction parameters were severely challenged and had in fact been under pressure for some time a calculated decision was taken to vaccinate the breeding herd as well. All the gilts, sows and boars were vaccinated once with one $\mathrm{ml}$ of CircoFLEX ${ }^{\circledR}$ intramuscularly, irrespective of their status, and repeated with the same dosage when the sows were 90 days pregnant. All future gilts, when entering the herd as maidens, were vaccinated at 168 days of age and then, if successfully mated, repeated at 90 days of pregnancy.

Farrowing rate, number of piglets born alive and weaned/litter, pre wean mortality for the effect of breeding herd vaccination and post wean mortality for the effect of piglet vaccination were evaluated before and after implementation of PCV2 vaccination with one-way ANOVA, pairwise comparison, using vaccination status as variable factor, and the Dunnett's Test as a post hoc test (Statitistica ${ }^{\circledR}$ v8.0, Statsoft ${ }^{\circledR}$, USA).

\section{Results}

Accurate and comprehensive on farm records are kept depicting all actions and performance levels. Results are summarized in table 1. Figure 1 demonstrates that not only the number of piglets weaned per litter increased, but consistency of production improved as well.
Table 1: Performance parameters before and after implementation of PCV2 vaccination in sows and piglets.

\begin{tabular}{|l|c|c|}
\hline & Before CircoFLEX & After CircoFLEX $^{\odot}$ \\
\hline Farrowing rate & $82^{\mathrm{a}}$ & $88^{\mathrm{b}}$ \\
\hline Born alive & $10.7^{\mathrm{a}}$ & $11.1^{\mathrm{b}}$ \\
\hline Weaned/litter & $9.2^{\mathrm{a}}$ & $9.7^{\mathrm{b}}$ \\
\hline Pre wean mortality & $15.0^{\mathrm{a}}$ & $14.2^{\mathrm{a}}$ \\
\hline Post wean mortality & $6.3^{\mathrm{a}}$ & $3.2^{\mathrm{b}}$ \\
\hline a,b: figures with different superscripts are significantly different $(\mathrm{p}<0.05)$ \\
\hline
\end{tabular}

Figure 1: Monthly records of weaned pigs/litter.

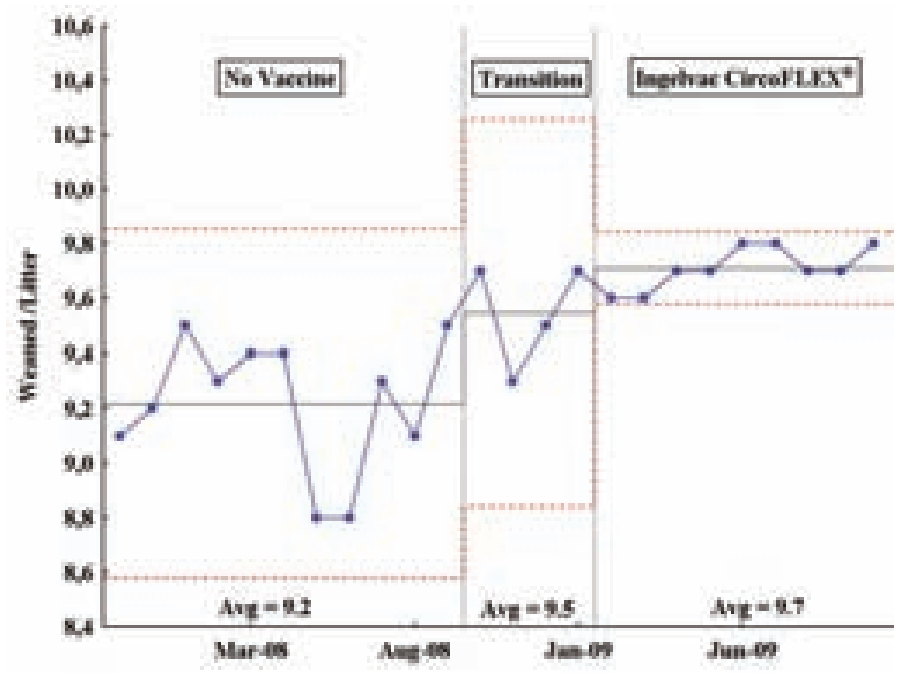

\section{Discussion}

The results of this field observation confirm that PCV2 piglet vaccination is a very effective tool to control PCVD in wean-to-finish pigs. PMWS and PDNS disappeared from the herd and postwean mortality was reduced significantly. Furthermore, in this case the improvement in reproductive performance indicates that vaccinating the breeding herd with Ingelvac CircoFLEX might have additional benefits on top of piglet vaccination.

\section{References}

1. Cardinal, Finishing mortality in a swine production system using different PCV2 vaccination protocols. IPVS 2008 OR.01.34.

2. British Pig Executive PCV2 trial results 26 June 2009. The PigSite July 2009. 


\title{
P.067
}

\section{Effects of PCV2 vaccination on market weights and mortality rates on commercial farms in the Philippines}

\author{
Edmundo C. Sy ${ }^{1}$ Philip R. Lehrbach ${ }^{2}$ Libertad S. Reyes $^{1}$ Civilo Q. Lima ${ }^{3}$ Jose R. Balantakbo ${ }^{4}$ \\ 1. Fort Dodge Animal Health Philippines, Inc, Mandaluyong, Philippines; 2. Pfizer Australia, Melbourne, \\ VIC, Australia; 3. Sta Cruz Farm, Pampanga, Philippines; 4. Reva Farm, Laguna, Philippines
}

\section{Introduction and Objective}

Porcine Circovirus Associated Diseases (PCVAD) is a broad categorization of multi-systemic diseases that can be sub-clinical or cause a severe economic impact due to its ability to cause high mortality and lowered growth performance $(1,2)$. The main causative agent of the disease is Porcine Circovirus Type 2 (PCV2) but other factors are required for clinical signs and lesions to appear (3). In the Philippines a phylogenetic analysis of the sequences of PCV2, isolated from PMWS affected local farms, allocated isolates into a PCV2 subgroup with Canadian and European isolates (4). Suvaxyn ${ }^{\circledR}$ PCV2 (Fort Dodge Animal Health, USA) is the Chimeric PCV1-2 vaccine containing immunogenic capsid gene of PCV2 cloned into the backbone of the nonpathogenic PCV1. Experimental vaccination with the chimeric PCV1-2 significantly reduced viraemia and decreased the risk of clinical diseases (5). This study determined the effects of PCV2 vaccine on market weight and mortality in commercial farms in the Philippines.

\section{Material and Methods}

Three 500 sow level farms in Luzon (the Philippines) were assessed for PCVAD using clinical signs, histopathology lesions and a specific PCV2 antigen fluorescent antibody test. A total of 770 piglets from the three farms were included in the study (Table 1). All piglets in the vaccinated group were given a $2 \mathrm{~mL}$ Suvaxyn PCV2 intramuscular injection at 28 days of age. Harvest weights were taken at 165 days. Mortalities from day 28-150 were recorded.

\section{Results and Discussion}

Table 1. Average weight at harvest and percent mortalities

\begin{tabular}{|l|c|c|c|c|}
\hline Farm & Type & Number of Heads & Average Harvest Weight (kg) & Mortality (\%) \\
\hline Farm 1 & Control & 80 & 69.54 & 14 \\
\hline & Vaccinated & 80 & 74.21 & 8 \\
\hline Farm 2 & Control & 93 & 72 & 16 \\
\hline & Vaccinated & 130 & 78 & 12 \\
\hline Farm 3 & Control & $\begin{array}{c}\text { Previous Average } \\
\text { Performance }\end{array}$ & 87 & 22 \\
\hline & Vaccinated & 387 & 92 & 7.29 \\
\hline
\end{tabular}

All vaccinated groups on the three farms showed a marked improvement in harvest weight and a significant decrease in mortalities compared to controls (Table 1).

The farms also noted that they experienced less respiratory problems and decreased incidence of slow growers in the vaccinated groups.

\section{References}

1. Opriessnig, T. et. al. (2007) J. Vet. Diagn. Invest. 19: 591-615.

2. Segales, J. et. al. (2005) Animal Health Res. Rev. 6: 119-142.

3. Dorr, P.M. et. al. (2007) J. Am. Vet. Med. Assoc. 230: 244-250.

4. Maldonado et al. (2004) J. Vet. Med. Sci. 66: 533-537.

5. Fenaux, M. et al. (2004). J. Virol. 78: 6297-6303. 


\title{
P.068
}

\section{Impact of CIRCOVAC ${ }^{\circledR}$ sow PCV2 vaccination on piglet weaning weight}

\author{
N. Brons ${ }^{1}$ Ricardo Neto $^{2}$ Thaïs Vila $^{3}$ Sophie Longo ${ }^{4}$ François Joisel $^{3}$ \\ 1. Easey Pigs Ltd, Town Farm, Hoxne, Eye, UK; 2. Merial Animal Health Ltd, Woking, UK; 3. Merial S.A.S., Lyon, France; 4. BPC, Labruguière, France
}

\section{Introduction}

Early exposure to PCV2 compromises the ability to respond to infectious agents (viral and bacterial) if the pig is exposed to PCV2 without the presence of protective passive immunity. The objective of this study was to evaluate the effect of CIRCOVAC ${ }^{\circledR}$ sow vaccination on the pre-weaning performance of the pigs.

\section{Material and Methods}

The chosen farm was a 1,270-sow indoor breeding unit farrowing 60 sows per week and weaning off site.

PCVD haven't been previously diagnosed on the progeny of this breeding unit and no PCV2 vaccine was being used before this study.

Dam line gilts were weaned before the weighing and were not included in this study.

The average weaning age before and after was approximately 28 days. Sows started being vaccinated with CIRCOVAC in June 2008 , following the recommended protocol.

To evaluate the impact of PCV2 sow vaccination on piglet weaning weight 1,007 piglets from non-vaccinated sows and 955 pigs born from vaccinated sows were weighed at weaning. The skewness and kurtosis values of the weights at weaning were studied following a normal distribution (at 95\%) and the live weaning weight (LWW) before versus after vaccination was compared using the following statistical tests, Bartlett test, student t-test and Kruskal-Wallis one way analysis of variance.

The distribution of pigs in different weaning weight groups was also evaluated using chi $^{2}$ test.

\section{Results}

The number of total pigs born alive per litter for a period of 35 weeks before and after CIRCOVAC sow vaccination started, was 11.1 and 11.3 , respectively. The number of pigs weaned on average per litter for the same period was 9.5 and 9.4, respectively. This was due to the PRRS problems that affected the sow herd and impacted in the general sow health.

The results of the analysis of the data collected can be seen on table 1. A significant improvement in the average weaning weight after the use of vaccine of $0.93 \mathrm{Kg}$ was showed (student t-test and Kruskal Wallis tests; $\mathrm{p}<0.001$ and $<0.001$ respectively).
Table 1: Evaluation of the weaning weights before and after sow vaccination

\begin{tabular}{|l|c|c|c|}
\hline & $\begin{array}{c}\text { Before vaccination } \\
(\mathbf{n}=\mathbf{1 , 0 0 7 )}\end{array}$ & $\begin{array}{c}\text { After CIRCOVAC vaccina- } \\
\text { tion }(\mathbf{n}=\mathbf{9 5 5})\end{array}$ & Delta \\
\hline Mean & 7.73 & 8.66 & $0.93(\mathrm{p}<0.001)$ \\
\hline Std & 1.486 & 1.424 & \\
\hline
\end{tabular}

The percentage of pigs weighing less than 6, 7 or $8 \mathrm{~kg}$ of LWW can be seen on table 2 .

Table 2: Percentage of pigs weighing less than 6, 7 or $8 \mathrm{~kg}$ of LWW

\begin{tabular}{|l|c|c|}
\hline & $\begin{array}{c}\text { Before Vaccination } \\
(\mathbf{n}=\mathbf{1 , 0 0 7 )}\end{array}$ & $\begin{array}{c}\text { After CIRCOVAC Vaccination } \\
(\mathbf{n = 9 5 5 )}\end{array}$ \\
\hline$\%$ pigs $<6 \mathrm{~kg} \mathrm{LWW}$ & $12.1^{\mathrm{a}}$ & $1.8^{\mathrm{b}}$ \\
\hline$\%$ pigs $<7 \mathrm{~kg} \mathrm{LWW}$ & $33.5^{\mathrm{a}}$ & $11.6^{\mathrm{b}}$ \\
\hline$\%$ pigs $<8 \mathrm{~kg}$ LWW & $56.4^{\mathrm{a}}$ & $32.8^{\mathrm{b}}$ \\
\hline a,b Different superscripts in the same row mean significant difference $\left(\mathrm{Chi}^{2}\right.$ test; $\left.\mathrm{p}<0.001\right)$. \\
\hline
\end{tabular}

\section{Discussion}

Lighter weaning weights and percentage of small pigs are associated with higher mortality and slower growth. Heavier pigs perform better from weaning to slaughter, result of higher daily live weight gains $(1,2)$.

A significant improvement in the mean weaning weight of 0.93 Kg was observed in this study.

Reduction of the number of pigs with a low weaning weight have the advantage of making multi-site systems easier to manage (2) and may also represent a direct financial improvement to the breeding herd as some weaner producers receive premiums based on weaning weights.

In this study, the use of CIRCOVAC resulted in the reduction of the percentage of pigs weighing less than $6 \mathrm{~kg}$ from over $12.1 \%$ to $1.8 \%$. The improvements observed may be attributed to the protection granted by maternally derived immunity against PCV2 challenge, which improves the health of the animals from birth, improving the growth of the animals, resulting in a higher weight at weaning.

\section{References}

1. Main R.G. et al., (2004) J. A. Sc. 82:1499-1507

2. Roberts J., (2000) A. D. Leman S. Conf: 195

${ }^{\circledR}$ CIRCOVAC is a registered trademark of MERIAL S.A.S. in the United States of America and elsewhere. 


\title{
P.069
}

\section{Comparison of $\mathrm{CIRCOVAC}^{\oplus}$ (Merial) and a recently licensed PCV2 weaner vaccine in UK condition}

\author{
Frank Tobin \\ Holmefield Farm Services, Agriculture House, Murton, UK
}

\section{Introduction}

The first cases of PCV2 associated diseases (PCVD) in the UK occurred in 1999. The cost of PCVD to European pig producers was calculated to reach 562-900 M€ per year (1). PCV2 vaccines proved to be an efficient tool to control PCVD.

The first available vaccine, CIRCOVAC $^{\circledR}$ (Merial) (CVAC) was licensed for use in breeding animals but was used to vaccinate piglets at weaning in cases where management factors would compromise the efficacy of sow vaccination. The objective of this study was to assess the impact of a recently licensed piglet vaccine (FVAC) on pig performance compared to the performance achieved using the previous PCV2 vaccine licensed for breeding animals (CVAC).

\section{Material and Methods}

A 700-sow indoor unit was chosen for this study. Pigs were allocated to either group randomly and individually weighed at weaning (W), end of the grower stage (EF) and before slaughter (S). The allocation of pigs was blinded to farm management. Pigs from 2 batches were individually identified and received either $0.5 \mathrm{ml}$ of CVAC or $1 \mathrm{ml}$ of FVAC administered deep intramuscularly at W. 91 pigs were included in the FVAC group and 100 pigs were included in the CVAC group. 20 pigs were bled at W and at the following weightings. The samples were tested by qPCR for quantification of PCV2 genome (minimum detection level $2 \times 103 / \mathrm{ml}$ ) and qELISA for quantification of PCV2 specific antibodies.

Both vaccines were compared on the following parameters: average daily weight gain (ADWG) in post-weaning, ADWG in finishing, ADWG from weaning to slaughter, weight at entry in finishing stage and slaughter weight.

Two-tailed student t-test was used to compare the different parameters of the 2 vaccines for each farm, separately.

\section{Results}

The mean parameters values of CVAC groups are always slightly higher than FVAC groups values in both farm (table 1), but there was no statistically significant difference between the two groups (CVAC and FVAC) for the ADWG. The difference between both vaccines is significant for the slaughter weight parameter only $(p=0.040)$, in farm 1 . Piglets vaccinated with the CVAC presented higher slaughter weight, in average, than the ones vaccinated with the FVAC.
The results of the blood samples can be seen on table 2. Both groups had high titres of antibodies at weaning. All pigs tested were negative at $\mathrm{W}$ and $\mathrm{EF}$ for qPCR, with $23.5 \%$ and $6.7 \%$ positives (of the sampled pigs) at $S$ for FVAC and CVAC respectively, with 1 pig in the group FVAC above 106 PCV2 genome copies.

Table 1: Results of the weighing of each individual pig and the ADWG for farm 1 and 2

\begin{tabular}{|l|l|c|c|c|}
\hline & & CVAC & FVAC & Dif \\
\hline Farm 1 & ADWG wean-slaughter g/day & 741.4 & 728.9 & 12.4 \\
\hline Farm 1 & ADWG finishers g/day & 929.9 & 919.4 & 10.5 \\
\hline Farm 1 & ADWG growers g/day & 560.0 & 547.3 & 12.8 \\
\hline Farm 1 & Weight entry finishing Kg & 48.1 & 47.9 & 0.1 \\
\hline Farm 1 & Slaughter weight Kg & 110.9 & 105.2 & 5.7 \\
\hline Farm 2 & ADWG wean-slaughter g/day & 669.2 & 653.1 & 16.1 \\
\hline Farm 2 & ADWG finishers g/day & 769.6 & 750.3 & 19.2 \\
\hline Farm 2 & ADWG growers g/day & 523.4 & 509.7 & 13.7 \\
\hline Farm 2 & Weight entry finishing Kg & 39.0 & 38.1 & 0.96 \\
\hline Farm 2 & Slaughter weight Kg & 105.3 & 102.6 & 2.7 \\
\hline
\end{tabular}

Table 2: Results of the $q P C R$ and $q E L I S A$ tests at different stages, $q P C R$ at slaughter and qELISA at W, EF and S

\begin{tabular}{|l|c|c|c|c|c|}
\hline & \multicolumn{3}{|c|}{ qPCR } & \multicolumn{2}{c|}{ qELISA } \\
\hline & Positive & $>\mathbf{1 0}^{\mathbf{6}}$ & W & EF & S \\
\hline CVAC & $6.7 \%$ & $0 \%$ & 2686.89 & 3742.63 & 2863.00 \\
\hline FVAC & $23.5 \%$ & $5.9 \%$ & 1569.63 & 1088.95 & 3172.41 \\
\hline
\end{tabular}

\section{Discussion and Conclusion}

Due to the small number of animals involved in this study it was not possible to show a statistical difference, except for slaughter weight in farm 1. Nevertheless average values of ADWG at all stages of production and the slaughter weight parameter are always slightly higher in CVAC vaccinated groups in both farms. In one of the vaccinated groups, FVAC had a slightly higher level of viremia, but this was not associated to disease $(2,3)$.

These results indicate that both vaccines are efficient tools for the control of PCV2, resulting in performance improvement.

\author{
References \\ 1. Tucker D. et al., (2006) P.J. April \\ 2. Maas P. et al., (2009) Proc 1st ESPHM: 61 \\ 3. Holck T. et al., (2009) Leman S. Conf.: 168
}




\title{
P.070
}

\section{Efficacy of $\mathrm{CIRCOVAC}^{\oplus}$ and 2 other PCV2 vaccines in piglets}

\author{
Ronie Pinheiro ${ }^{3}$ Glauber Machado ${ }^{3}$ Edson Bordin ${ }^{2}$ Julie Venet $^{1}$ François Joisel $^{1}$ \\ 1. Merial S.A.S., Lyon, France; 2. Merial Animal Health Ltda., Campinas, SP, Brazil; 3. Integrall \\ - Soluções em Produção Animal Ltda, Patos de Minas, MG, Brazil
}

\section{Introduction}

Porcine circovirus diseases (PCVD) have been described in Brazil since 2000. PCV2 vaccination efficacy with CIRCOVAC $^{\circledR}$ has been extensively confirmed $(1,2)$ so it has been decided not to carry out a trial with negative control.

The objective of this study was to evaluate the effect of CIRCOVAC vaccination over wasting versus 2 other commercial PCV2 vaccines marketed in Brazil, under Brazilian field conditions.

\section{Material and Methods}

The experiment was carried out in a commercial farm with PCVD clinical diagnosis previously established. A total of 1,800 3-weekold piglets were individually weighed and randomly allocated into 3 groups (600 per treatment) vaccinated at weaning:

Treatment 1 (T1): CIRCOVAC, $0.5 \mathrm{ml}$ once

Treatment 2 (T2): Vaccine $\mathrm{X}, 2 \mathrm{ml}$ once

Treatment 3 (T3): Vaccine $Y, 1 \mathrm{ml}$ once

Serology was performed by ELISA using the IT-BAN-028-ELISATest (Nano-Core ${ }^{\circledR}, \mathrm{NJ}$, USA). Three hundred serum samples were collected before vaccination i.e. at 21 days of age and at 36,66 , 96 and 126 days. The experimental unit was the animal for the daily weight gain (DWG), and the pen (40 heads) for the feed conversion evaluation. Animals were fed throughout the experiment with ration according to the Brazilian charts values of poultry and swine (3). Feed consumption per pen was evaluated weekly.

The software package statistical analysis for genetic epidemiology (S.A.G.E.) was used for variance analysis and mean comparison. For mortality rates, $\mathrm{Chi}^{2}$-test and non-parametric tests like Kruskall-Wallis were used.

\section{Results and Discussion}

No difference in weights at weaning was found between groups while piglet vaccination significantly affected the nursery exit weight ( $p$ 0.05). CIRCOVAC vaccination and T3 led to higher weight gains at nursery and slaughter (see Table 1). T1 and T3 showed globally better growth performances than T2 (see Table 1). Performing serologies at different piglet ages, T1 showed higher titres and all treatments showed a significant increase starting at 66 days of age, which led to the hypothesis of wild virus exposition from that day on. In all groups a significant increase in the antibody titres for PCV2 was observed and these were kept high until 126 days (see Figure 2).

Figure 1: Serology results: mean of antibody titres per treatment at different ages

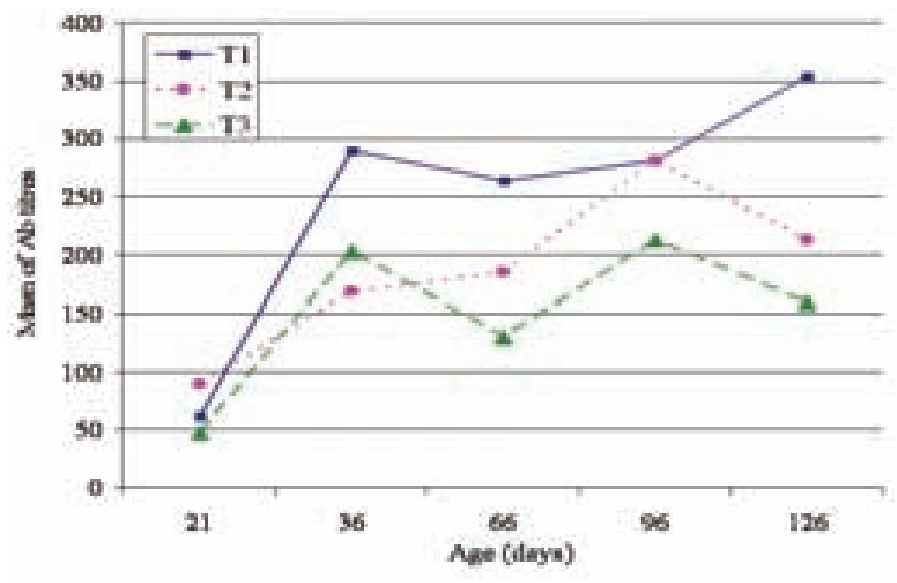

\section{Conclusion}

CIRCOVAC-vaccinated piglets (T1 group) and T3 induced similar weight performance. T1 group showed a stronger seroconversion after vaccination and kept higher ELISA antibody titres up to the end of the experiment compared with the 2 other treatments.

\section{References}

1. Seliger C. et al., (2009) 4th APVS Proceedings, Tsukuba, Japan, p.218

2. Sanchez R. et al., (2009) 4th APVS Proceedings, Tsukuba, Japan, p.231

3. Rostagno, H. S. et al., (2005) Tabelas Brasileiras para Aves e Suínos: Composição de Alimentos e Exigências Nutricionais. Departamento de Zootecnia/UFV, Viçosa

${ }^{-} \mathrm{CIRCOVAC}$ is a registered trademark of Merial in Canada and elsewhere. S.A.G.E. is supported by the Human Genetic Analysis Resource funded by the National Center for Research Resources of the National Institute of Health.

Table 1: Growth performance at nursery stage (until 65 days of age) and globally until the end at 145 days of age and mortality

\begin{tabular}{|c|c|c|c|c|c|c|c|c|c|}
\hline Treatment & $\begin{array}{l}\text { Mortality } \\
\text { rate }\end{array}$ & $\begin{array}{c}\text { Weaning } \\
\text { weight }(\mathrm{kg})\end{array}$ & Nursery exit weight (kg) & \begin{tabular}{|c|}
$\begin{array}{c}\text { Nursery DWG } \\
(\mathrm{kg})\end{array}$ \\
\end{tabular} & $\begin{array}{c}\text { Nursery feed } \\
\text { conversion ratio }\end{array}$ & \begin{tabular}{|c|}
$\begin{array}{c}\text { Final weight } \\
\text { (kg) }\end{array}$ \\
\end{tabular} & $\begin{array}{c}\text { Global DWG } \\
(\mathrm{kg})\end{array}$ & $\begin{array}{c}\text { Global feed con- } \\
\text { version ratio }\end{array}$ & \begin{tabular}{|c|}
$\begin{array}{c}\text { Total consumption } \\
\text { of ration }\end{array}$ \\
\end{tabular} \\
\hline $\mathrm{T} 1$ & $3.16 \%$ & 5.222 & a) 20.829 & a 0.354 & 1.504 & a90.565 & ${ }^{\mathrm{a}} 0.688$ & 2.242 & 191.339 \\
\hline $\mathrm{T} 2$ & $3.33 \%$ & 5.243 & ${ }^{b} 20.306$ & b0.342 & 1.574 & b88.580 & b0.672 & 2.244 & 187.008 \\
\hline T3 & $2.5 \%$ & 5.233 & a 20.905 & ${ }^{\mathrm{a}} 0.356$ & 1.511 & a 90.226 & ${ }^{\mathrm{a}} 0.685$ & 2.270 & 192.934 \\
\hline
\end{tabular}




\title{
Report on the impact of early exposure by PCV2 in a vaccinated flow
}

\author{
Tom Gillespie \\ Renssalear Swine Services, Renssalear, IN, USA
}

\begin{abstract}
Introduction
Porcine circovirus associated diseases (PCVAD) have had a massive negative impact on the global pig industry. ${ }^{1}$ The availability of efficacious commercial vaccines has vastly reduced the clinical signs associated with PCVAD, especially the high mortality rates, wasting and increased frequencies of co-infections with other pathogens. ${ }^{2}$ In this case report we describe the impact of shifting PCV2 infection dynamics in a herd in which PCVAD had previously been well controlled by pig vaccination at five weeks of age.
\end{abstract}

\section{Case History}

This case involves a PRRS naïve and Mycoplasma hyopneumoniae (M. hyo) positive, single site, 1100 sow farrow to finish facility. Pigs are weaned at three weeks of age. Nursery rooms are filled by two weaning events per week. Piglets are vaccinated with Ingelvac CircoFLEX ${ }^{\circledR}$ and Ingelvac MycoFLEX ${ }^{\circledR}$ at five weeks of age. The index nursery room was filled with weanings on August 3rd and August 6th. An increased mortality rate was observed beginning three weeks post weaning. The average mortality for the nursery stage had been $2.51 \%$ but increased to $10.8 \%$ for the index room. The average mortality for the finisher stage had been $1.77 \%$, which increased to $425 \%$ in the room housing pigs from the index nursery room.

The clinical signs in the index nursery room included wasting body condition, respiratory distress, and acute mortality. On September 15th, tissue samples, nasal swabs and serological profiling diagnostics of the nursery pigs were performed. The average pig age for the index room was 60 days. The results from this initial workup showed moderate amounts of PCV2 antigen using IHC staining in the lung and lymphoid tissues confirming a systemic PCV2 infection. Bacterial pneumonia was also present and Streptococcus suis was isolated. M. hyo PCR testing was negative. Thirty gestating sows were then serologically profiled (September 17th) from different breeding weeks and various parities. The sow sera were tested using PCV2 quantitative PCR (qPCR) and all samples were below the detectable level of $<4$ logs/mL serum. Additionally, 60 total serum samples were collected (15 for each age) from randomly selected pigs 21, 31, 41 and 47 days. The growing pig sera were tested using PCV2 qPCR and only one sample in the 47 day of age group had detectable viremia ( $>4$ logs of genetic copies of PCV2 virus $/ \mathrm{mL}$ serum). On September 22nd, a more encompassing tissue collection from pigs 31,41 , and 68 days of age was performed. PCR results indicated PCV2 genetic material presence in pooled tissue samples of all ages. Tissue PCV2 IHC results were negative for PCV2 antigen in the 31 day and 41 day of age tissues but strongly positive in the lung and lymphoid tissue from the 68 days of age group.
Swine influenza, PRRS virus, M. hyo and other respiratory pathogens test results were negative. The laboratory diagnosis was severe interstitial pneumonia associated with PCV2 infection and a mild enterocolitis was also associated with PCV2 infection. PCV2 was detected at high levels in the tissue pools; therefore the diagnosis was confirmed to be PCVAD.

Although reproductive symptoms of PCV2 infection are sometimes hard to detect, and no abnormal reproductive clinical signs were observed, a parity 1 female aborted her developing litter on October 21st, 2009. Six fetuses were collected and submitted for diagnostic testing. Results for PCV2 IHC on heart samples were positive (3+) and multifocal areas of mineralization were present in multiple heart sections.

A diagnostic investigation that included intense profiling of sows in farrowing and their offspring was initiated. The colostrum from 30 sows was collected immediately after farrowing. Twenty-five pigs were selected from different litters, tagged and serially serum profiled at seven days of age, weaning (approximately 20 days of age), and again at 42 days of age. One colostrum sample was positive for PCV2 virus by qPCR, $30 \%$ of the piglets were positive by qPCR at 20 days of age, although there were no positive piglets detected at either 7 or 42 days of age by qPCR.

\section{Discussion and Conclusions}

PCV2 exposure does occur in the adult swine herd, although the results of the infection may be reproductively inapparent. Infection of the adult herd may result in viral transmission from the sow to her offspring. ${ }^{2} \mathrm{~A}$ comprehensive diagnostic program is necessary to understand PCV2 infection dynamics in cases of early clinical symptoms of PCVAD. ${ }^{3}$ Early exposure in this case (prior to planned vaccination) resulted in increased mortality and poor growth performance beginning in the nursery which persisted into the finisher stage. Although mass PCV2 vaccination of the sow herd and moving piglet vaccination earlier (to the time of weaning at 3 weeks of age) was followed by disappearance of clinical signs, additional work is needed to fully understand the risk factors involved in this case.

\section{References}

1. Opriessnig, T. J Vet Diagn Invest. 2007

2. Krakowka, J Vet Diagn Invest. 2007

3. Altherrr. 4th International Symposium on Emerging and Re-emerging pig diseases, 2003 


\title{
P.072
}

\section{Protection of viral shedding after challenge with porcine circovirus type 2 (PCV2) inactivated bivalent vaccine in vaccinated specific pathogen free (SPF) pigs}

\author{
Hyoung Joon Moon; Dae Sub Song; Tae Hoon Oh; Min Joo Yeom; Bo Kyu Kang; Jong Man Kim
}

Research Unit, Green Cross Veterinary Products, Yong In, Korea

\section{Introduction}

Porcine circovirus 2 (PCV2) is non enveloped single stranded DNA virus cause the complicated syndrome called porcine circovirus-associated disease (PCVAD) which is one of the issue in pig industry in the world. To reduce the economic loss from PCVAD, many kind of effective PCV2 vaccines have been developed and used[2]. This study is aimed to evaluate the vaccine efficacy through viral shedding in nasal discharges and feces after challenging to piglets which were vaccinated with inactivated PCV2 vaccine containing both PCV2a and PCV2b strains isolated in Korea.

\section{Materials and Methods}

\section{Experimental design}

The details of the experimental designs were presented in table 1.

\begin{tabular}{|l|l|l|}
\hline Vaccination* $^{*}$ & Challenge $^{\dagger}$ & Heads of pigs $^{\ddagger}$ \\
\hline Twice $^{\S}$ & PCV2 & 4 \\
\hline Non & PCV2 & 2 \\
\hline Non & Non & 1 \\
\hline $\begin{array}{l}\text { *: PCV2 inactivated bivalent vaccine (PCV2a+PCV2b) from Green Cross } \\
\text { veterinary products co. Itd. (GCVP) } \\
\text { t: Field isolates PCV2b, 105.0TCID50/ml, Two weeks after booster. } \\
\text { †: 3-week old SPF pig, §: Two weeks interval, 1ml/dose, IM }\end{array}$ \\
\hline
\end{tabular}

\section{Clinical signs and sampling}

Clinical signs had been observed for 14 days after challenge. In order to measure the titers of released virus, nasal swabs and rectal swabs were collected at the day of challenge, 3, 7, 10, 14, 17, 20, 25 days after challenge.

Virus shedding

Shedding of PCV2 was measured using quantitative SYBR real time PCR in nasal swabs and rectal swabs. The released viral titer was converted to immune fluorescent assay (IFA) titer using standard curve. The viral titers in each group were compared statistically.

\section{Results}

Clinical signs

There were no clinical signs in vaccinated and PCV2 challenged group, however pathognomic signs of PCV2 infection including anorexia, mild loose feces, wasting were shown in some pigs which was challenged without vaccination.

\section{Virus shedding}

Viral shedding in nasal discharges and feces were low and stopped until 14 days after challenge in vaccinated group (vaccinated and challenged). However, the viral shedding in non vaccinated group was high and lasting more than 31 days after challenge in both nasal swabs and rectal swabs.

\section{Discussion}

So PCV2 is transmitted via fecal oral infection that this experiment was focused on viral shedding. The results of this experiment were nearly corresponded with previous study in viral shedding in nasal discharges and feces[1]. Unfortunately, this study did not cover with viremia and serology like in former study. However, as presented in this study this vaccine could efficiently reduce the viral spreading through the nasal discharge and feces. Reduction of the viral shedding could relieve the chance of the transmission of PCV2 to nascent pigs, and prevent the risk of PCVAD in pig heard. To our knowledge, this small experiment is the first report about the efficacy test of PCV2 inactivated vaccine including inactivated whole viruses of both PCV2a and PCV2b strains.

\section{References}

1. Fort, M. et al.,Vaccine, 2008(26) 1063-1071

2. Opriessnig, T. et al, Vaccine, 2009( 27) 1002-1007

Figure 1. Shedding of PCV2 in nasal discharges $(A)$ and rectal swab $(B)$ in PCV2 inactivated bivalent vaccine in pigs. The mean titer in vaccinated group was $10^{0.82} \mathrm{TCID}_{50} / \mathrm{ml}$ and $10^{1.13} \mathrm{TCID}_{50} / \mathrm{ml}$ after challenge in nasal swabs and rectal swabs, respectively.

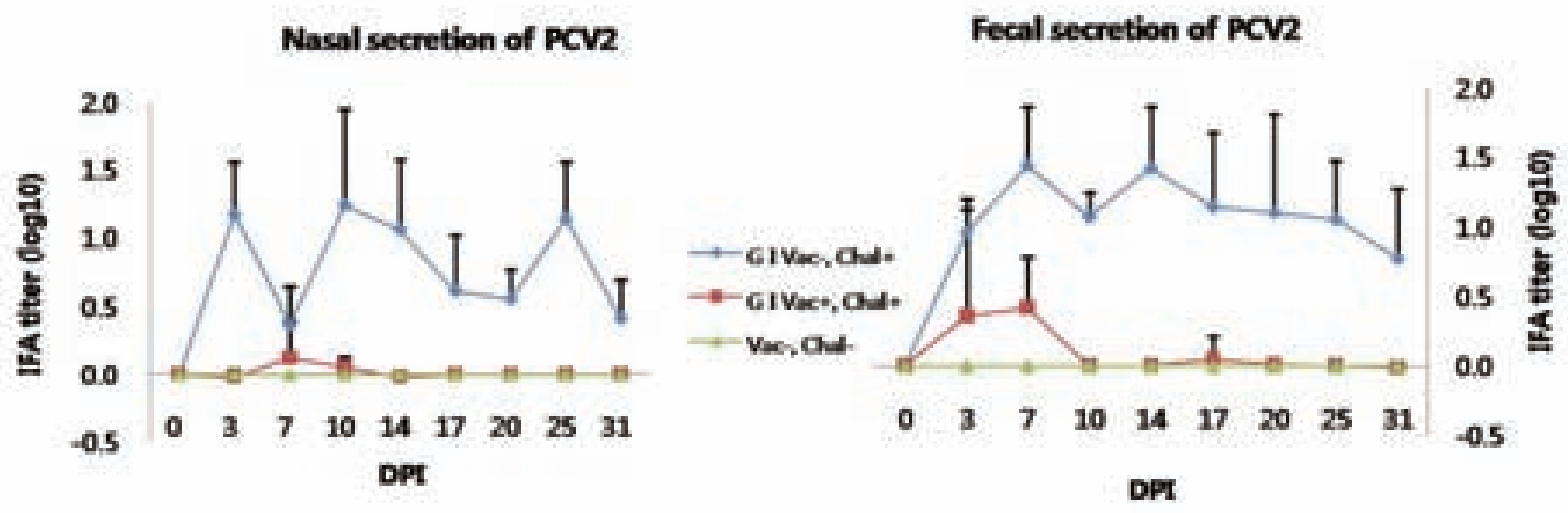




\title{
P.073
}

\section{Seroprofile survey of anti-PCV2 blocking Elisa antibodies of non-vaccinated and CIRCOVAC ${ }^{\circledast}$ vaccinated pigs in PCVD-affected SPF farm}

\author{
Ivan Soukup ${ }^{1}$ Ivan Ps ${ }^{2} i k a l^{2}$ \\ 1. GP Veterinarian, Velvary, Czech Republic; 2. Dyntec Ltd., Terezín, Czech Republic
}

\begin{abstract}
Introduction
Infections of pigs with porcine circovirus 2 (PCV2) is associated with a broad spectrum of clinical conditions recognized as Porcine circovirus diseases (PCVD). Under certain circumstances the illness could be observed in the farm of specific pathogens free status (SPF), where low infection pressure and good standard of management are expected (1). The objective of this field observation was to describe the changes of the health situation in SPF pig farm caused only by PCV2 infection shortly after repopulation and to show an effect of sow vaccination by $\mathrm{CIRCOVAC}^{\circledR}$ on serological profiles in pigs of different age, on clinical changes and production results.
\end{abstract}

\section{Material and Methods}

The farrow-to-finish pig farm was repopulated in the end of year 2005. New health status of the farm was free of PRRSv, M.Hyo, APP, Swine dysentery and P. multocida DNT+. About 1 year after repopulation sporadic health disorder (suddenly died suckings, wasting and icteric postweaners, suddenly died boars) and decrease of production results (total born and weaned piglets/ sow) were observed. Investigation was focused on PCV2 infections using $\mathrm{IHC}$ method in lympho-nodes, quantitative PCR and competitive blocking ELISA (CB-ELISA) to show the dynamics of PCV2 antibodies (seroprofiles) in pigs before and after vaccination by $\mathrm{CIRCOVAC}^{\circledR}$ (batch vaccination and revaccination of pregnant sows and gilts). Blood samples were collected the same day from 5 pigs per group at 4, 8, 12 and 16 weeks of age and from 5 sows according the parity and from 5 gilts $(70 \mathrm{~kg})$. Pigs from each group were bled before initialization of vaccination and at 3 and 6 month after the beginning of vaccination. CB-ELISA uses an HRP-labelled specific anti-PCV2 monoclonal antibody (1C6) as a conjugate which compete with serum antibodies for PCV2 antigen determinants on immobilized PCV2 proteins. Blocking PCV2 antibody titres were measured at three different dilutions $1: 100,1: 1000$ and 1:10000. Any serum sample presenting $\geq 40 \%$ of blocking activity at appropriate dilution was considered positive for the presence of PCV2 antibodies, any sample presenting $<40 \%$ of blocking activity were considered negative. Mean antibody titres and standard deviation in each group were tested using a Student T-test between two groups for each defined age.

\section{Results}

PCVD diagnostic in SPF herd was confirmed by both IHC and quantitative PCR methods according to internationaly recognized procedures (www.pcvd. org). Seroprofiles based on the mean of CB-ELISA PCV2 antibody titres in sows and their progeny are shown in Figure 1.
Figure 1.

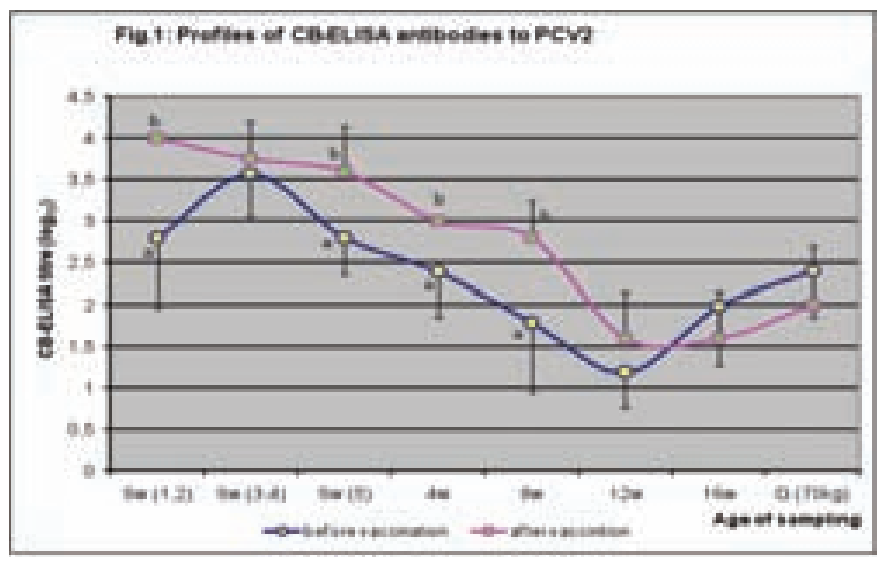

a, b: different superscripts at the same age group mean that PCV2 antibody levels are significantly different.

Dynamics of antibody response to PCV2 infection (round points) in piglets and growing pigs show an initial drop of anti-PCV2 antibody levels to be the lowest at the age of 12 weeks. Further stage of monitoring using CB-ELISA exhibits a sharp seroconversion reaching the peak at the age of 16 and more (70 kg gilts). Similar seroprofile was seen in pigs born to vaccinated (squared points) sows but PCV2 antibody levels were significantly higher at the age 4 and 8 weeks after 3 month since the beginning of CIRCOVAC ${ }^{\circledR}$ application and the active PCV2 antibody increase started at the age of 16 weeks but this increase was gradual. The PCV2 antibody level was significantly higher in the blood of vaccinated sows at $1 .+2$. and $\geq 5$. parity both 3 and 6 month since the beginning of Circovac application.

\section{Discussion and Conclusion}

Except of health status and production parameters improvements, the effect of sow vaccination by $\mathrm{CIRCOVAC}^{\circledR}$ was observed in serological profiles comparable with other authors (2): i) the mean of CB-ELISA PCV2 antibody titres were more homogenous in vaccinated sows of all parity and in their piglets till 8 weeks of age, ii) the active PCV2 antibody increase in pigs born to vaccinated sows occured later in life and it was gradual indicating lower PCV2 infection pressure within the herd.

\section{References}

1. Ladekjaer-Mikkelse, A.-S. et al., (2001a) Vet. Rec. 148, 759-760.

2. Joisel F. et al. (2008) IPVS, Durban, South Africa, p. 15. 


\title{
P.074
}

\section{Comparison of performance before and after the institution of a Circumvent PCV vaccination program}

\author{
Suraphan Boonyawatana ${ }^{1}$ Winai Thongmak² Kridtasak Sang-Gassanee ${ }^{1}$ \\ 1. Intervet (Thailand), Sathorn, Thailand; 2. Live Informatics Company Limited, Moung, Nonthaburi, Thailand
}

\section{Introduction}

Porcine circovirus (PCV) is one of the factors involved in the disease entity known as porcine respiratory disease complex (PRDC). When it was introduced into the country, it provided Thai pig producers with a unique challenge in diagnosis and control.

Current PCV2 vaccination has reduced the problem markedly (Thanawongnuweach, R., 2009). Circumvent PCV was introduced into Thailand in May 2009, and is the vaccine of choice. The aim of this study was to compare performance before and after instituting a vaccination program with Circumvent PCV.

\section{Materials and Methods}

This trial was carried out on a 4,000-sow farrow-to-finish farm, on which pigs were weaned at 24 days of age on average, and kept in the nursery for 3-4 weeks, before being moved to the finishing house where they remained until slaughter at an average 24-25 weeks old. The farm was experiencing a severe problem with PRDC.

Pigs were vaccinated with $2 \mathrm{ml}$ Circumvent PCV 4 and 6 week of age. Pigs were observed for local or systemic reactions following vaccination, culling and mortality rates (\% Loss), average daily weight gain (ADG) and feed conversion ratio (FCR) were all recorded. These performance data were compared with the same parameters before the start of Circumvent PCV vaccination.
Local reactions were observed in $0.8 \%$ of pigs after the first Circumvent PCV dose, and $0.16 \%$ after the second.

The performance data are compared in Table 1.

Table 1: Comparison of performance data before and after the Circumvent PCV program

\begin{tabular}{|l|c|c|c|}
\hline & Before & Circumvent PCV & Diff. \\
\hline$\%$ Loss & 13.41 & 5.68 & 7.73 \\
\hline FCR & 2.44 & 2.40 & -0.04 \\
\hline ADG (g) & 738 & 759 & 21.00 \\
\hline
\end{tabular}

The Circumvent PCV vaccination program led to a lower percentage mortality (and fewer culls), lower FCR and greater ADG when compared with the period prior to the use of the vaccine (Francisco de Grau, A. et al., 2007; Thacker, B. et al., 2008).

\section{References}

1 Thanawongnuweach, R. 2009. Keynote speech1-5 Proceedings of 4th APVS congress, Tsukuba, Japan, p.15-17.

2 Francisco de Grau, A. et al., 2007. Field performance of a conditionally licensed vaccine: Canadian experience. AASV, p.159-161.

3 Thacker, B. et al, 2008. Circumvent PCV vaccine: Performance evaluation and serological studies update. AASV, p.153-156.

\section{Results and Discussion}




\title{
P.075
}

\section{Effect of Piglets PCV2 Vaccination with $\mathrm{CIRCOVAC}^{\oplus}$ in the Farm with Severe Porcine Circovirus Type 2 Disease (PCVD) in Post-Weaning}

\author{
Ivan Soukup ${ }^{1}$ Pavel Dus ${ }^{2} k^{2}$ \\ 1. GP veterinarian, Velvary, Czech Republic; 2. GP veterinarian, C'eská Br 'iza, Czech Republic
}

\section{Introduction}

Porcine multisystemic wasting syndrome (PMWS) is well known and described as caused by porcine circovirus type 2 (PCV2) in post-weaning period of piglet rearing $(1,2)$. The main clinical symptoms are wasting, daily weight gain (DWG) depression, heterogenity in size, high mortality and outbrake of co-infections. CIRCOVAC ${ }^{\circledR}$, an inactivated, adjuvanted vaccine is possible to use successfully in sows in accordance with the leaflet recommendations (3). PCV2 vaccination of piglets may also be an effective way to prevent PMWS.

The objective of this field study was to analyse the effect of CIRCOVAC ${ }^{\circledR}$ piglets vaccination in a farm with clinical symptoms of PMWS and high viral pressure.

\section{Material and Methods}

The studied farm is a conventional 100-sow farrow-to-prefattening farm where clinical signs of PMWS were observed and PCV2 presence was confirmed. Clinical symptomatology, DWG and mortality were recorded in 4 batches and compared to results of the following post-weaner batches under CIRCOVAC $^{\circledR}$ vaccination. The farm was serologicaly ELISA-positive to PRRS, M. Hyo, APP 2,9,11, bacteriologically Str. Suis, H. parasuis, and S. typhymurium were earlier identified.

Sow vaccination schedule: commercial vaccines EC+Clostridium, Ery+Parvo, APP in label recommendation, live PRRS vaccine 14 days after farrowing.

Piglets vaccine schedule: Respisure One (Pficer Animal Health), APP at $9+12$ weeks of age. CIRCOVAC $^{\circledR}$ vaccine $0,5 \mathrm{ml}$, IM at 21 days of age, into neck behind the ear. No new changes of husbandry (Madec's point) had been implemented during the study.

Lab investigations: presence PCV2 in lesions was confirmed by immunohistochemistry (IHC) and qPCR (4,02E7 - 4,19E9/.

Statistical analysis: a Student t-test was used to compare the DWG, and a Chi-square test was used for mortality rate comparison. Statistical analyses were performed with STATGRAPHICS 5.1 (for Windows) and SAS 9.1 (SAS Institute, Cary, NC) software. Statistical significance is based on a two-tailed test of the null hypothesis resulting in $\mathrm{p}$-values of 0.05 or less.

\section{Results}

The production result comparison is shown in table 1. The effect of vaccination was noticed in the first vaccinated batch of postweaners. Absence of wasting, better homogenity in pig size, better vitality, less cases of post-weaning diarhoea and sharp decrease of mortality were observed. Polyserositis were found less frequently during necropsy in the group of vaccinated piglets.

Table 1: Production results comparison of vaccinated and not vaccinated piglets

\begin{tabular}{|l|c|c|c|}
\hline & No PCV2 vaccine & CIRCOVAC & p value \\
\hline Mortality rate in post-weaning(\%) & 13.35 & 3.68 & $<0.001^{1}$ \\
\hline DWG in post-weaning (g) & 359 & 434 & $<0.001^{2}$ \\
\hline Mortality rate in fattening (\%) & 6.46 & 4.32 & $<0.001^{1}$ \\
\hline DWG in fattening (g) & 766 & 752 & $0.369^{2}$ \\
\hline${ }^{1}$ Chi-square test. ${ }^{2}$ Student t-test.
\end{tabular}

\section{Discussion}

Nowadays the use of vaccines is very effective tool in prevention of PCVD. The use of this vaccine in piglets significantly reduced mortality in both sections and dramatically increase DWG in post weaning stage. DWG during fattening showed an important variability due to other cause (checking of different feedstuff formula in last period of fattening due to its price) and the difference between vaccinated and control did not appeared to be significant.

\section{Conclusion}

In this study, very significant and quick effect on DWG and mortality rate was confirmed when using CIRCOVAC $^{\circledR}$ in piglets in age 21 days of age, in the farm with high disease incidence in the postweaning sections where the disesase was most prominent.

\section{References}

1. Thacker E.L. et al.,(1999) Journal of Clinical Microbiology, 37: 620627.

2. Thacker E.L. et al.,(2006) Diseases of Swine. Ames, lowa. Blackwell Publishing. Vol 9, pp706-707. 2006.

3. Delisle C. at al., (2008) 20th IPVS Durban

CIRCOVAC ${ }^{\oplus}$ is a registered trade mark of Merial. 


\title{
P.076
}

\section{Further development of a differential ELISA for PCV2 vaccination compliance}

\author{
Sheela Ramamoorthy ${ }^{3}$ John K. Johnson ${ }^{3}$ Jeremy S. Pittman ${ }^{1}$ Brad J. Thacker ${ }^{2}$ \\ 1. Murphy-Brown, LLC, Waverly, VA, USA; 2. Intervet/Schering-Plough Animal Health, Desoto, KS, \\ USA; 3. Iowa State University, Veterinary Diagnostic Laboratory, Ames, IA, USA
}

\section{Introduction}

Previous work has shown the potential for our PCV2 Differential ELISA as a compliance monitoring test for PCV2 vaccination by measuring antibodies that are uniquely induced by PCV2 vaccination. ${ }^{1}$ This test is based on using a coating antigen (PCV2 ORF2 expressed in baculovirus grown in SF9 insect cells) that is similar to the antigen used in some commercial vaccines plus a coating antigen that lacks the ORF2 expression (referred to as the wild type antigen, WT). ${ }^{2}$ This system has worked well when pigs are tested 3-4 weeks after vaccination with a two dose product (Circumvent ${ }^{\oplus}$ PCV, Intervet Schering Plough Animal Health). However in some operations, non-vaccinated pigs will develop antibody to the WT antigen as they progress through the finishing phase. We presume that this non-specific titer is due to exposure to cross-reacting insect antigens. Our objective was to determine the components of the immune response in vaccinated pigs and if accounting for SF9 antigen cross-reaction could improve test specificity.

\section{Materials and Methods}

Samples from a variety of previous field and laboratory studies that were used to develop the PCV2 Differential ELISA test were tested by ELISA in duplicate using ORF2, WT and SF9 coating antigens. Representative data is reported here. Samples originated from pigs vaccinated with Circumvent PCV at 3 and 6 weeks of age or non-vaccinated control pigs. For the ELISA, appropriate controls including serum from vaccinated and non-vaccinated CDCD pigs were used on each plate. Calculation of the net optical density (OD) due to baculovirus (BV) was done by subtracting the SF9 OD from the WT OD. Results are reported as the group mean average optical density value (ODV). Positive/negative cut-offs for the WT antigen and the BV calculated values were 0.300 and 0.100 , respectively.

\section{Results}

Table 1 presents WT, SF9 and BV results from 3 separate experiments. Sample times were at vaccination and 3 weeks after the second vaccination for PRE and POST, respectively. Table 2 presents $B V$ results from two field studies where the pigs were bled periodically until near market weight. Background BV antibodies were detected in Herd A at 15 and 21 weeks of age but not in Herd B at 14 and 25 weeks of age. BV titers and the percentage of positive animals were similar.

\section{Discussion}

Vaccination with Circumvent PCV induced titers to the ORF2 and WT antigens but not the SF9 antigen, indicating that SF9 antigens may not be a major component in the vaccine or are not sufficiently immunogenic to induce a measurable antibody response. This finding suggests that the vaccine induces antibodies mainly to ORF2 and BV but not SF9 (insect cell) antigens. Overall, the calculation of the BV net OD (WT minus SF9) appears to improve the specificity of the test and may provide a better method for compliance monitoring compared to the WT antigen alone. However, the utility of the BV titer in older pigs is questionable due to the decline in titers observed 6 weeks or more after the second vaccination (both herds) and the development of positive BV titers in non-vaccinated pigs in Herd A.

Table 1: Summary of three experimental studies

\begin{tabular}{|l|c|c|c|c|c|}
\hline & \multicolumn{2}{|c|}{ WT } & SF9 & \multicolumn{2}{c|}{ BV } \\
\hline GRP/TIME & ODV & POS & ODV & ODV & POS \\
\hline VAC/PRE & 0.155 & $1 / 18$ & 0.164 & 0.009 & $0 / 18$ \\
\hline VAC/POST & 0.616 & $35 / 41$ & 0.245 & 0.371 & $38 / 41$ \\
\hline CON/PRE & 0.132 & $0 / 17$ & 0.131 & 0.001 & $0 / 17$ \\
\hline CON/POST & 0.224 & $5 / 23$ & 0.199 & 0.026 & $1 / 23$ \\
\hline
\end{tabular}

Table 2: BV titers over time in two field studies

\begin{tabular}{|l|c|c|c|c|c|}
\hline & AGE & \multicolumn{2}{|c|}{ HERD A } & \multicolumn{2}{c|}{ HERD B } \\
\hline GRP & (WKS) & ODV & POS & ODV & POS \\
\hline VAC & 3 & 0.011 & $0 / 16$ & -0.002 & $0 / 32$ \\
\hline VAC & 6 & 0.120 & $12 / 16$ & 0.057 & $3 / 32$ \\
\hline VAC & 9 & 0.512 & $32 / 32$ & 0.383 & $31 / 32$ \\
\hline VAC & $15 / 14$ & 0.157 & $9 / 16$ & 0.235 & $27 / 32$ \\
\hline VAC & $21 / 25$ & 0.200 & $12 / 16$ & 0.147 & $15 / 30$ \\
\hline CON & 3 & 0.001 & $0 / 16$ & 0.000 & $0 / 6$ \\
\hline CON & 6 & 0.016 & $0 / 16$ & 0.011 & $0 / 6$ \\
\hline CON & 9 & 0.027 & $0 / 16$ & 0.026 & $0 / 6$ \\
\hline CON & $15 / 14$ & 0.120 & $7 / 16$ & 0.062 & $0 / 6$ \\
\hline CON & $21 / 25$ & 0.140 & $12 / 16$ & 0.030 & $0 / 5$ \\
\hline
\end{tabular}

\section{Reference}

1. Pittman, JS., Thacker, B., Johnson, J. Proc AASV Ann. Mtg., Dallas, Texas, pp. 207-211, 2009.

2. Nawagitgul, P, et al. Clin Diagn Lab Immunol 9:33-40, 2002. 


\title{
P.077
}

\section{Evaluation of a commercial ELISA method for measuring PCV2 antibodies}

\author{
Brad J. Thacker $^{1}$ Chinta M. Lamichhane ${ }^{2}$ Cindy Thomson ${ }^{2}$ \\ 1. Intervet/Schering-Plough Animal Health, Desoto, KS, USA; 2. Synbiotics Corporation, College Park, MD, USA
}

\section{Introduction}

Several tests have been developed for measuring antibodies to porcine circovirus Type 2 (PCV2). In the Unites States, the main tests currently in use include an ELISA and an indirect immunofluorescence antibody (IFA) test. These tests are routinely performed in several state/university diagnostic laboratories. Recently, a competitive ELISA based on a three dilution method has been developed and marketed by Synbiotics Corporation. This three dilution method enables calculation of a titer which provides a quantitative evaluation of PCV2 antibody levels. In this report, we present data generated from testing samples of vaccinated and non-vaccinated pigs over time.

\section{Materials and Methods}

The samples originated from several previous studies and had been stored at -20 C for up to 2 years prior to testing. Vaccinations were done per label directions with Circumvent ${ }^{\circ}$ PCV. Pigs were vaccinated at 3 and 6 weeks of age. Sows were vaccinated twice at a 3 week interval. Blood was collected prior to vaccination. Post vaccination sample times are listed in the result tables

The competitive ELISA uses a single well blocking technique. The test is performed in three serum dilutions. Wells are incubated for $1 \mathrm{hr}$ followed by a wash. Peroxidase conjugate PCV-2 Mab is added and incubated for 1 hour. After a second wash, TMB substrate is added and incubated for 30 minutes. Stop solution is added and the optical density is measured. Each value is compared to a linear model using a logistic regression model and interpolation between the three results is correlated to a titer. Group results are expressed as geometric mean titers (GMT).

\section{Results}

Table 1 presents titer data in non-vaccinated pigs from Herd A over time. Maternal antibody levels decline to their lowest level at 9 weeks of age and then seroconversion due to field exposure results in increased titers at 12 weeks of age.

Table 2 presents titer data in vaccinated and control pigs from Herd B over time. In vaccinates, seroconversion started after the first vaccination and the highest titers were observed 3 weeks after the second vaccination. Titers in control pigs indicated that seroconversion due to field exposure occurred later relative to Herd A.

Table 3 presents titer data in sows and pigs from Herd B prior to vaccination and at 3 weeks after their second vaccination. Sow titers prior to vaccination along with relatively high titers after vaccination indicate previous exposure to PCV2. Pig titer responses are consistent with the data presented in Table 2.
Table 1: Titers in six non-vaccinated pigs over time

\begin{tabular}{|c|c|c|c|}
\hline Age (wks) & Titer & Age (wks) & Titer \\
\hline 3 & $1,078.5$ & 12 & $1,011.6$ \\
\hline 6 & 284.0 & 15 & $4,919.4$ \\
\hline 9 & 81.7 & 21 & $5,367.4$ \\
\hline
\end{tabular}

Table 2: Titers in vaccinated and control pigs

\begin{tabular}{|c|c|c|}
\hline Age (wks) & VAC $(\mathbf{n}=\mathbf{8})$ & CON $(\mathbf{n}=\mathbf{6})$ \\
\hline 3 & 383.3 & 257.7 \\
\hline 6 & $1,574.8$ & 49.4 \\
\hline 9 & $6,621.7$ & 88.8 \\
\hline 14 & $5,243.5$ & 179.4 \\
\hline 25 & $1,789.3$ & $1,0815.2$ \\
\hline
\end{tabular}

Table 3: Titers in vaccinated and control sows and pigs (8 per vaccine by age group)

\begin{tabular}{|c|c|c|c|}
\hline Age & Sample & VAC & CON \\
\hline \multirow{2}{*}{ Sow } & Pre & $1,718.6$ & $1,078.2$ \\
\cline { 2 - 4 } & Post & $10,597.1$ & $1,428.7$ \\
\hline \multirow{2}{*}{ Pig } & Pre & 666.4 & 544.5 \\
\cline { 2 - 4 } & Post & $4,770.4$ & 350.2 \\
\hline
\end{tabular}

\section{Discussion}

The PCV2 antibody titers generated with this test were consistent with the vaccination status and age of the pigs. We conclude that this 3 dilution format provides an effective method for measuring PCV2 antibodies.

\section{References}

1. Nawagitgul, P, et al. Clin Diagn Lab Immunol 9:33-40, 2002.

2. Horlen, K, et al. J Am Vet Med Assoc 232:906-912, 2008. 


\title{
P.078
}

\section{A field study to assess the efficacy of the PCV2 sow vaccine Circovac ${ }^{\circledR}$ in a Danish sow herd}

\author{
Charlotte S. Kristensen ${ }^{1}$ Kaj Vestergaard ${ }^{1}$ Mai-Britt F. Nielsen ${ }^{1}$ Leon Lau $^{2} \underline{\text { Poul Bækbo }}^{1}$ \\ 1. Pig Research Centre, Danish Agriculture \& Food Council, Kjellerup, Denmark; 2. Merial, Copenhagen, Denmark
}

\section{Introduction}

PCV2 is the cause of economic losses to the pig producers all over the world. Vaccines against PCV2 are now available and used world wide. The first licensed vaccine on the marked was a killed PCV2 vaccine for use in sows (Circovac ${ }^{\circledast}$, Merial). The objective of this study was to assess the efficacy of Circovac ${ }^{\circledR}$. The study was carried out in three herds - two herds with focus on weight gain and one with focus on mortality. Only results from the latter will be presented here.

\section{Materials and Methods}

The study was designed as a randomised, controlled, and blinded trial comparing the offspring of vaccinated and placebo injected groups of sows. The study was performed in a Danish sow herd where Post Weaning Multisystemic Wasting Syndrome (PMWS) was diagnosed according to the EU definition (www. pcvd.eu).

The study herd was a two-site farrow-to-finish herd accommodating 260 sows and with a wean-to-finish mortality above $8 \%$ at the time of selection of the herd for the trial. The sows and the weaners up to appr. $30 \mathrm{~kg}$ were on one site and the finishers on another site. The herd was infected with $\mathrm{M}$. hyopneumoniae but free from pleuropneumonia and PRRSV.

In each farrowing batch the sows were allocated at random to one of two experimental groups. Group GREEN (165 sows) was vaccinated with $2 \mathrm{ml} \mathrm{Circovac}{ }^{\circledR} 6$ and 3 weeks before expected farrowing. Group WHITE (164 sows) was injected with a placebo (saline) at the same time. Cross fostering of piglets was only allowed within the two sow groups and only for the first 24 hours after farrowing. At farrowing all piglets were given individually numbered ear tags in colours GREEN or WHITE according to the mother sow colour. At weaning the pigs were moved to nursery units and housed in separate pens according to ear tag colour. The pigs were individually weighted three times: at weaning (4 weeks of age; $6.2 \mathrm{~kg}$ ), at transfer to the finishing units (10 weeks of age; $31.5 \mathrm{~kg}$ ) and just before shipment to slaughter (20 weeks of age; $94 \mathrm{~kg}$ ). All dead pigs and the number of individual antibiotic treatments were recorded from weaning to slaughter.

The statistical analysis was done in $\mathrm{SAS}^{\circledR}$, version 9.13 using a logistic regression model for mortality and number of antibiotic treatments (PROC GENMOD) and a linear model (PROC MIXED) for growth (average daily gain (ADG)).

\section{Results}

No side effects after vaccination were observed.

Weaners after vaccinated sows had a significant reduced mortality of $1.1 \%$, an increase in ADG by $19 \mathrm{~g} /$ day and a reduced number of antibiotic treatments compared to pigs after nonvaccinated sows (Table 1).

Table 1. Mortality, ADG, and number of individual antibiotic treatments of weaner pigs (6.2-31.5 kg) after vaccinated and non-vaccinated sows.

\begin{tabular}{|l|c|c|c|c|}
\hline & N & Mortality & ADG $(\mathbf{g} \pm$ SD $)$ & Antibiotic treatments \\
\hline Pigs after vac. sows & 2329 & $1,7 \%$ & $467 \pm 22 \mathrm{~g} /$ day & 0,35 \\
\hline Pigs after non-vac. sows & 2233 & $2,8 \%$ & $448 \pm 21 \mathrm{~g} /$ day & 0,42 \\
\hline P-value & & 0,01 & $<0,0001$ & 0,0008 \\
\hline
\end{tabular}

For finishers the mortality was $0,2 \%$ lower (1.9\% versus $2.1 \%$ ), and the ADG $14 \mathrm{~g}$ higher (910 versus 896 ) for pigs after vaccinated pigs compared to non-vaccinated pigs, but none of these differences were significant ( $P$ : 0.59 and 0.15 , respectively). The number of antibiotic treatments was the same in the two groups of finishers.

\section{Discussion}

As it will be noted, the overall wean-to-finish mortality dropped from above $8 \%$ when the herd was selected to around $5 \%$ when the study took place. This not uncommon when doing clinical trials.

However the vaccine was still able to significantly increase the productivity in the weaner phase of the production, but only marginally in the finishing phase of the production.

The size of effect of the vaccine shown in this study is in good accordance with some preliminary results from a Swedish trial in one herd (1), but in farms with high losses a numerical higher reduction in mortality might be expected as indicated in a Japanese study in one herd (2)

\section{Acknowledgement}

The authors acknowledge the financial support from the European Commission (PCVD, no 513928).

\section{References}

1. Paulsson, M. et al., 2008, Proc.IPVS, OR.01.37

2. Ishikawa, H., et al., 2008 Proc. IPVS, P.01.080 


\title{
P.079
}

\section{Evaluation of the performance of growing pigs after a single dose of Porcilis ${ }^{\circledR}$ PCV}

\author{
Henrique v. Comprido ${ }^{1}$ Francisco Fagundes ${ }^{2}$ João C. Lopes ${ }^{2}$ \\ 1. Agro Pec Valinho, SA, Alcobaça, Portugal; 2. Intervet Shering-Plough, Lisboa, Portugal
}

\section{Introduction}

Nowadays, Porcine Circovirus type 2 (PCV2) is considered to be key to many of the important economic losses in the swine industry, mainly in its relationship with Porcine Circovirus Associated Disease (PCVAD). This syndrome leads to considerable performance failures in weaning and fattening pigs due to increased mortality rates and reduced feed conversion efficiency.

\section{Materials and Methods}

This field study took place on a multi-site 800-sow farm. It involved 192 piglets from 18 litters of a single weaning. The herd was experiencing clinical signs compatible with PCV2 infection. The presence of PCV2 was confirmed by direct quantification of viral load by qPCR.

At vaccination, the litters were weighed and divided into two uniform groups of 96 piglets with an average age of 24 days. All animals with a low birth weight and/or clinical problems were excluded.

Group 1: Vaccinated simultaneously with Porcilis ${ }^{\circledR}$ PCV and Porcilis ${ }^{\circledast} \mathrm{M}$ Hyo (average weight - 7.15 Kg).

Group 2: Vaccinated with Porcilis ${ }^{\circledast} \mathrm{M}$ Hyo (average weight - 7.28 $\mathrm{Kg})$ as controls.

All piglets were monitored for signs of local and systemic reactions in the 14 days following vaccination.

When transferred to the fattening site, the pigs were re-weighed, mortality recorded, and the uniformity of the batches assessed.

During the final stage of the fattening period, randomly selected pigs were blood sampled periodically and tested for PCV2 antibodies by ELISA. At 23 weeks of age, all the animals from both groups were individually weighed.

Statistical analysis was carried out using the T-test.

An economic analysis was undertaken to quantify the return on investment based on current average meat price, financial loss from deaths/culls (60€ per pig) and vaccination cost.

\section{Results}

No abnormal clinical signs or local reactions were observed after vaccination.

As can be seen in Table 1, the vaccinated group had a better ADG and lower mortality/culling rate, which amounted to a benefit over the controls of $4.21 €$ per pig sold. The difference in body weights between the groups $(+4.35 \mathrm{Kg}$ ) was statistically significant $(p=0.0043)$.
Table 1: Technical and economic data

\begin{tabular}{|l|c|c|c|}
\hline Parameter & PCV & Control & Diff \\
\hline Finished weight (Kg) & 97,18 & 92,83 & $4,35(\mathrm{a})$ \\
\hline ADG (g) & 639 & 607 & 32 \\
\hline Total mortality \& culls & $7 \%$ & $8 \%$ & $-1 \%$ \\
\hline Pig Value (euro) & 100,50 & 96,29 & 4,21 \\
\hline
\end{tabular}

Figure 1 shows that the finished weights of the PCV vaccinated group $(\bullet)$ were higher and comprised a more homogeneous distribution than those of the control group $(\boldsymbol{\Delta})$.

Figure 1: Individual weight distribution

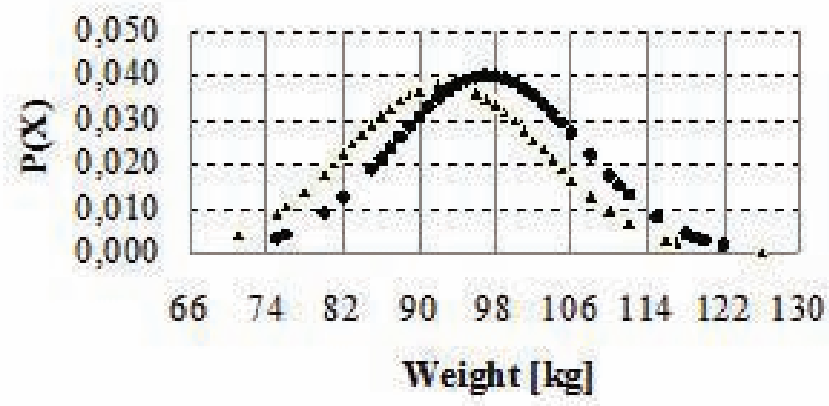

\section{Discussion}

Both technical and economic parameters were superior in the group which received a single dose of Porcilis PCV, with significant difference with respect to end weight.

There was also greater homogeneity in the PCV vaccinated group. This is an important feature for profitable pig production.

The data collected in this study showed that a single dose of Porcilis ${ }^{\circledR}$ PCV is effective in improving the performance of growing pigs.

\section{References}

1. Thacker, $\mathrm{T}$. et al (2008) 18th IPVS, 90.

2. Sato, K. et al (2008) 18th IPVS, 45.

3. Fort, M. et al (2009) Vaccine 27: 4031-4037. 


\title{
P.080
}

\section{Serological response in piglets to PCV2 and Mycoplasma hyopneumoniae vaccines administered separately and simultaneously}

\author{
Juan M. Palacios ${ }^{1}$ Alfredo G. Rendón ${ }^{2}$ \\ 1. Intervet Schering Plough AH, Huixquilucan, MEX, Mexico; 2. Rancho Covadonga, Cuautla Morelos, Mexico
}

\section{Introduction}

The use of vaccines to control diseases associated with PCV2 infection (PCVAD) has increased the number of times piglets need to be handled. Agents often associated with PCV2, such as Mycoplasma hyopneumoniae, have been shown to increase the severity of clinical signs, as well as the damage caused by PCV-21.

The need to avoid the unnecessary handling of piglets while maintaining effective protection has led to the use of vaccines with combined antigens or multiple application systems. The combination of PCV2 and Mh antigens is not available in a commercial vaccine, and the the possibility of combining both agents without affecting individual responses is being evaluated2,3. The aim of this study was to compare the results of applying two different antigens to piglets, either separately or simultaneously.

\section{Materials and Methods}

A weekly production batch of 49 of piglets of three weeks of age were identified individually, and assigned randomly to one of four treatment groups, as follows:

Ta: vaccinated against PCV2 (Circumvent PCV৫); Tb: vaccinated against M.hyopneumoniae (M+Pac@) ; Tc: vaccinated against both PCV2 and M.hyopneumoniae; and Td: Control group injected with $2 \mathrm{ml}$ of sterile diluent. All injections (vaccines and placebo) were administered at 3, 6 and 12 weeks of age. The simultaneous adminstration was given using Dua Vac@ equipment (NJ Phillips Ltd).

The piglets were bled prior to the first dose and again at 6, 13 and 15 weeks of age. The sera were analyzed for antibodies against M.hyopneumoniae using the ELISA test (IDDEX - $s / p<$ 0.4 was taken as the cut-off), and PCV2 by means of an ORF-2 antigen blocking ELISA (Synbiotics $-\mathrm{s} / \mathrm{p} \leq 0.15$ was taken as the cut-off).

The data were analyzed by means of an ANOVA test of two factors (treatment and time) using the SPSS version 15.0 software.

\section{Results}

The following graphs show anti-PCV2 and anti- Mh titers.

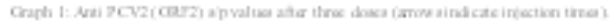

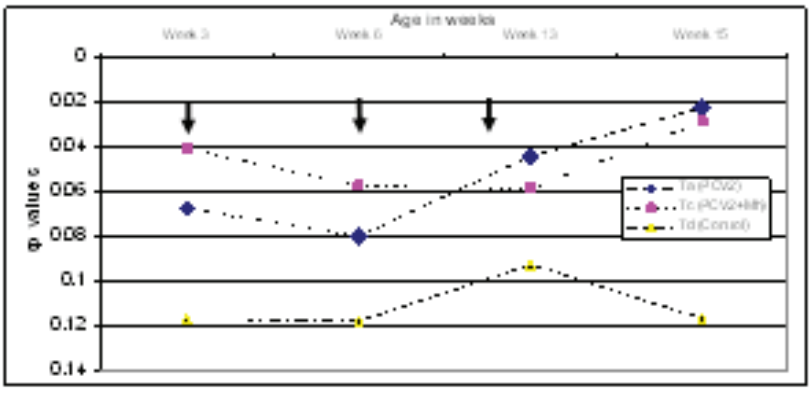

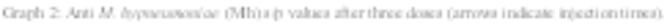

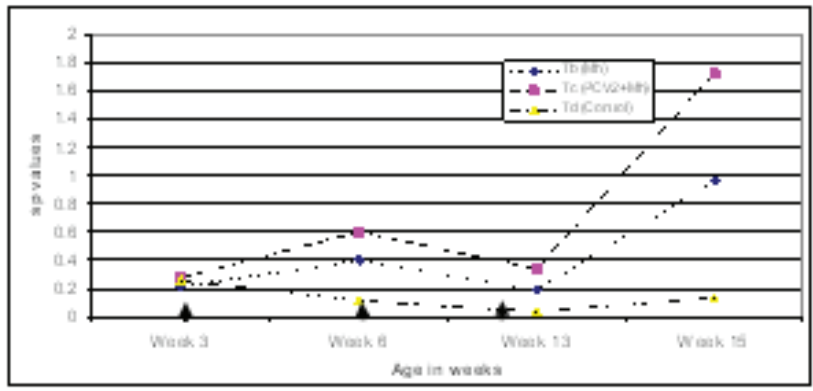

\section{Discussion}

There are no statistically significant differences between the responses to the PCV2(ORF-2) and the M.hyopneumoniae antigens at the recorded times. However, these responses both differ significantly from those of the unvaccinated controls ( $\mathrm{p} \leq$ $0.05)$. The titres against Mycoplasma $s p$ are positive $(s / p \geq 0.4)$ at week 6 and 15 of age.

Several different authors report that the response to the antigens of PCV-2 and Mycoplasma sp do not alter whether the vaccines are given individually or simultaneously. Givng the vaccines at the same time is thus a possible option for the producer, as long as the vaccination regimes are not interefered with by passive immunity (MDA), especially in the case of PCV2 for which the dose is given at weaning (Mycoplasma spp vaccination is given during the first week of life). Both vaccines can be applied simultaneously between 3 and 4 weeks of age, either in individual or combined programs.

\author{
References \\ 1 Opriessnig T. et al. Vet. Pathol. 41 :624-640 (2004) \\ 2 J.Farreres et.al IPVS 2010 \\ 3 A.E. Eggen et.al IPVS 2010
}




\title{
P.081
}

\section{Economic evaluation of a combined vaccination against PCV-2 and Mh on a commercial farm}

\author{
Juan M. Palacios ${ }^{1}$ Florencio Mancilla² Enrique Mariscal ${ }^{3}$ \\ 1. Intervet Schering Plough AH, Huixquilucan, MEX, Mexico; 2. Granja El Volantin, El Grullo, \\ JAL, Mexico; 3. Insumos Veterinarios SA, Guadalajara, JAL, Mexico
}

\section{Introduction}

One of the agents most commonly involved in PRDC is Mycoplasma hyopneumoniae (Mh). Its effects on the defense mechanisms of the respiratory tract mean it is the major cause of economic loss, worldwide. Its co-infection with Porcine Circovirus Type 2 (PCV2) increases the severity of disease, both clinically and pathologically1. Vaccinating piglets against $\mathrm{Mh}$ has been common practice all over the world.

Every vaccination has the potential to be yet another stressor on piglets in the production cycle. Because some farms were experiencing both PCV2 and Mh infections, it was decided to vaccinate piglets simultaneously against PCV2 and Mh2,3. The aim of this trial was to investigate the economic effect of the combined vaccination against PCV2 and Mh on a commercial farm.

\section{Materials and Methods}

The trial was conducted on a 500-sow farm operating a continuous flow system. 60 piglets of 26 days of age were chosen from a weekly production batch. Each piglet was identified individually, and randomly assigned to one of two treatment groups. Group 1 was vaccinated against PCV2 (Circumvent $\odot$ PCV). Group 2 was vaccinated against PCV2 but also against Mycoplasma spp $(\mathrm{M}+\mathrm{Pac} \odot)$. Both groups were vaccinated at 26 and 42 days of age. The simultaneous application of the products was carried out using a double syringe system (Dua Vac@ NJ Phillips Ltd).

All the piglets were weighed at 26,73 and 146 days of age. The average daily weight gain (ADG) was calculated separately for the periods 26 to 73 days and 73 to 146 days of age. Feed intake was measured in each pen for each growing phase, and the total weight for each pen was used to calculate the feed efficiency. All deaths were recorded.

Chart 1. Production and mortality parameters in each group.

\begin{tabular}{|l|c|c|}
\hline Average Daily weight gain ${ }^{1}$ & PCV2 26-40d & Mh+PCV2 26-40d \\
\hline From 26 to 73 days age $(\mathrm{kg})$. & $0.520( \pm 0.0138)$ & $0.554( \pm 0.146)$ \\
\hline From 73 to 146 days age $(\mathrm{kg})$. & $0.706( \pm 0.0184)$ & $0.789( \pm 0.019)$ \\
\hline From 26 to 146 days age $(\mathrm{kg})$. & $0.696( \pm 0.0149)$ & $0.752( \pm 0.015)$ \\
\hline Feed efficiency & & \\
\hline From 26 to 146 days age & 3.02 & 2.46 \\
\hline Mortality & 0 & 0 \\
\hline From 26 to 73 days age (total) & 3 & 0 \\
\hline From 73 to 146 days age (total) & 10 & 0 \\
\hline Total mortality in \% & \multicolumn{2}{|l|}{} \\
\hline${ }^{1}$ Average \pm standard error & \\
\hline
\end{tabular}

The data were analyzed by student t-test, and variance homogeneity was analyzed using the SPSS program version 15.0. The economic analysis was carried out at 146 days of age, before the largest pigs had been selected. The cost of feed and vaccination was deducted from the gross income to determine the gross margin.

\section{Results}

Chart 1 shows the mortality and production parameters for each group. Chart 2 shows the economic analysis for both groups.

\section{Discussion}

The synergistic effect between PCV2 and Mycoplasma sp has been well reported. However, the effect of PCV2 is currently more often associated with the growth and fattening phases, when replication is related to the circulating pathogens. With respect to Mycoplasma sp it is the prevalence around the time of weaning that will determine the overall effect.

From 26 to 73 days when feed-efficiecy is greatest and the piglet tends to gain more weight, the simultaneous vaccination group performed better than the single vaccine group.

Most producers vaccinate against PCV2, but many fail to do so against $M$. hyopneumoniae, and the synergy of the combined infection has a greater impact on herd health. The use of simultaneous vaccination against both organisms will improve production performance.

\author{
References \\ 1 Opriessnig T. et al. Vet. Pathol. $41: 624-640$ (2004) \\ 2 J.Farreres et.al IPVS 2010 \\ 3 A.E. Eggen et.al IPVS 2010
}

Chart 2. Economical analysis in each group

\begin{tabular}{|l|c|c|}
\hline & PCV 26-40d & Mh+PCV 26-40d \\
\hline \# pigs at beginning & 30 & 30 \\
\hline Total mortality (\%) & 10 & 0 \\
\hline Sell kg (bodyweight) & 2270.5 & 2726 \\
\hline Feed cost US\$ & $\$ 1,852.70$ & $\$ 1,843.50$ \\
\hline Mh Vaccine cost US\$ & $\$ 0$ & $\$ 21.92$ \\
\hline PCV2 vaccine cost US\$ & $\$ 59.13$ & $\$ 59.13$ \\
\hline Feed + Vaccines cost US\$ & $\$ 1,911.83$ & $\$ 1,924.55$ \\
\hline Feed efficiency & 3.02 & 2.46 \\
\hline Quality sales distribution & & \\
\hline Premium US\$ & $\$ 1,167.94$ & $\$ 2,770.08$ \\
\hline Lightweights US\$ & $\$ 1,734.96$ & $\$ 867.18$ \\
\hline Total US\$ & $\$ 2,902.90$ & $\$ 3,637.25$ \\
\hline Gross margin & $\$ 991.07$ & $\$ 1,712.70$ \\
\hline Margin per kg. Sold & $\$ 0.44$ & $\$ 0.63$ \\
\hline
\end{tabular}




\title{
P.082
}

\section{Comparison of production performance between pigs vaccinated with a single-dose or a double-dose PCV2 vaccine}

\author{
$\underline{\text { Juan M. Palacios }}{ }^{1}$ Victor Martínez ${ }^{2}$ \\ 1. Intervet Schering Plough AH, Huixquilucan, MEX, Mexico; 2. Granja La Joya, Atlixco, Puebla, Mexico
}

\section{Introduction}

The attention of swine producers all over the world has long been directed against diseases associated with PCV2 (Porcine Circovirus type 2). Their detrimental effect on production been led to various vaccination schemes being considered. Two-dose regimes are aimed at generating a memory response to inactivated antigens, giving rise to a more lasting response, providing protection during both growth and fattening. In spite of the need to handle piglets twice, this system represents both health and economic advantages to justify its use 1.

The aim of this study was to compare the performance of a single-dose vaccine with a double-dose product in four consecutive production cycles.

\section{Materials and Methods}

On a multipurpose farm in Mexico, 3,684 piglets were chosen from four consecutive production runs. Two of these groups $\left(1,887\right.$ piglets) were vaccinated with Suvaxin Respifend ${ }^{\circledR}$ PCV: a single intramuscular dose of $2 \mathrm{ml}$ in the base of the neck at 21 days of age. The other two groups (1,797 piglets) were vaccinated twice with Circumvent ${ }^{\oplus} \mathrm{PCV}$ : two $2 \mathrm{ml}$ doses at 21 and 42 days of age on alternate sides of the neck.

The evaluation was carried out in two phases, from 21 to 86 days of age and from 86 to 156 days of age (finished weight). The averages for each group were taken for the purposes of comparison.

\section{Results}

Total mortality in the single-dose Suvaxin Respifend ${ }^{\circledR}$ PCV group was $6.31 \%$ and in the double-dose Circumvent ${ }^{\oplus} \mathrm{PCV}$ group, $7.16 \%$. Total of culled and runt pigs was $10.64 \%$ and $3.91 \%$ in the single- and double-dose groups respectively. Table 1 shows the feed cost for each treatment group.
Table 1. Total feed intake, efficiency and cost in each group.

\begin{tabular}{|l|c|c|}
\hline Vaccine & Single-dose & Double-dose \\
\hline Total start weight in $\mathrm{kg}$. & $11,606.0$ & $10,694.0$ \\
\hline Total sales weight in $\mathrm{kg}$. & $177,736.0$ & $163,080.0$ \\
\hline Total weight gain in $\mathrm{kg}$. & $166,130.0$ & $152,386.0$ \\
\hline Total feed intake at weaning in kg. & $96,063.1$ & $93,558.0$ \\
\hline Total feed intake at finishing in kg. & $275,144.0$ & $234,026.0$ \\
\hline Total feed intake in $\mathrm{kg}$. & $371,207.1$ & $327,584.0$ \\
\hline Feed efficiency & 2.23 & 2.15 \\
\hline Average feed cost in US\$ by kg. & $\$ 0.29$ & $\$ 0.29$ \\
\hline Total feed cost in US\$ & $\$ 108,811.85$ & $\$ 96,024.62$ \\
\hline
\end{tabular}

Table 2 shows the cost-benefit analysis for each treatment group.

Table 2. Cost benefit analysis in each treatment.

\begin{tabular}{|l|c|c|}
\hline Group & Single dose & Double-dose \\
\hline Feed cost (US\$) & $\$ 108,811.85$ & $\$ 96,024.62$ \\
\hline PCV2 vaccine cost (US\$) & $\$ 2,444.78$ & $\$ 3,017.86$ \\
\hline Subtotal US\$ & $\$ 111,260.63$ & $\$ 99,042.49$ \\
\hline Total kg at sales. & $177,736.0$ & $163,080.00$ \\
\hline Total sales in US\$ & $\$ 257,785.04$ & $\$ 236,528.24$ \\
\hline Gross margin in US\$ & $\$ 146,524.41$ & $\$ 137,485.76$ \\
\hline Gross margin by produced US\$/BW/kg & $\$ 0.82$ & $\$ 0.84$ \\
\hline
\end{tabular}

\section{Discussion}

During the first production phase ( 21 to 86 days of age) the groups behaved alike. The differences between them arose during the second phase ( 86 to 155 days of age). The percentage of culled pigs and runts was critical because of the effect on feed efficiency.

After deducting the cost of the vaccines, the double-dose Circumvent ${ }^{\oplus} \mathrm{PCV}$ group showed a $2.2 \%$ better profit per kg sold than the single-dose Suvaxin Respifend ${ }^{\circledR}$ PCV group. This trial demonstrated that Circumvent ${ }^{\oplus} \mathrm{PCV}$ had a beneficial effect in the older pigs conferring an obvious advantage in terms of the economics of production.

\section{References}

1 Horlen K.P. J.Am.Vet.Med.Asso. 2008;232: 906-912 


\title{
P.083
}

\section{Comparison of the effect on economic performance of two vaccines for the control of diseases associated with porcine circovirus on a commercial farm in Mexico}

\author{
$\underline{\text { Juan M. Palacios }}^{1}$ Fernando Curiel ${ }^{2}$ \\ 1. Intervet Schering Plough AH, Huixquilucan, MEX, Mexico; 2. Granja El Ensueño, Zumpango, MEX, Mexico
}

\section{Introduction}

The use of vaccines in the control of diseases associated with PCV2 (PCVAD) has been shown to offer health and production benefits in farms in Canada, USA and Mexico, and has become common practice. Most of the current products are single-dose vaccines.

Intervet-Schering-Plough has developed a double-dose vaccine, the purpose of which is to reduce the risk of interference from maternally derived antibodies, and to produce a booster response, thus reducing viremia 1 in the growth and fattening phases. The aim of this study was to compare the effect on production and health of a single-dose vaccine (Respifend ${ }^{\circledR}$ PCV-2), with that of a double-dose product (Circumvent ${ }^{\circledast} \mathrm{PCV}$ ).

Materials and Methods On a commercial farm in Mexico, one group of 238 piglets was vaccinated with Respifend ${ }^{\circledR}$ PCV-2 (a single $2 \mathrm{ml}$ dose by intramuscular injection in the neck at 29 days old). Another goup of 309 piglets was vaccinated with Circumvent $^{\circledR}$ PCV ( $2 \mathrm{ml}$ by the same route at 23 and 43 days of age). From each group, 30 piglets were chosen at random, individually ear-tagged, weighed at 99 and 155 days of age and their daily weight gain calculated. Mortality was also recorded. At the end of the cycle, age and weight at sale, and feed efficiency were recorded. The data were analyzed by T-test using SPSS 15.0 software.

\section{Results}

Mortality began in week 6 in both groups, reaching a peak in weeks 7 and 11. The disease affected the alimentary system, and was characterized by progressive wasting. At the necropsy of 3 pigs, Salmonella cholerae suis was isolated from the liver and iliocecal valve. Table 1 shows the mortality results:

Table 1. Mortalityresults in each group

\begin{tabular}{|l|c|c|}
\hline Weaning & Single-dose & Double-dose \\
\hline Total born piglets & 238 & 309 \\
\hline Total weaned piglets & 211 & 272 \\
\hline Total deaths & 27 & 37 \\
\hline Mortality in \% in weaning phase. & $11.3^{\mathrm{a}}$ & $11.9^{\mathrm{a}}$ \\
\hline Growing and fattening & & 272 \\
\hline Pigs entering growingphase & 211 & 252 \\
\hline Total sales pigs & 184 & 20 \\
\hline Total deaths & 27 & $7.3^{\mathrm{b}}$ \\
\hline \% mortality & $12.8^{\mathrm{a}}$ & \\
\hline Different superscript letters inthe same row indicate significant differences \\
\hline
\end{tabular}

The economic analysis utilized different prices at sale for pigs over $80 \mathrm{~kg}$ and those under $80 \mathrm{~kg}$, the latter being considered second class. Feed efficiency was calculated for each group. Table 2 shows the performance data and the gross margin per kg sold for each group.

Table 2. Economic analysis between 2 groups

\begin{tabular}{|l|c|c|}
\hline Group & Single-dose & Double-dose \\
\hline Total weight at start (kg). & 1519.2 & 1915.8 \\
\hline Total sales weight $(\mathrm{kg})$. & 15150.5 & 19554.25 \\
\hline Total weight gain $(\mathrm{kg})$. & 13631.3 & 17638.45 \\
\hline Total feed consumption (kg). & 38167.6 & 48505.7 \\
\hline Feed efficiency & 2.8 & 2.7 \\
\hline Feed cost in US\$ & $\$ 13,111.02$ & $\$ 16,662.28$ \\
\hline Vaccines cost US\$ & $\$ 257.71$ & $\$ 456.79$ \\
\hline Subtotal & $\$ 13,368.73$ & $\$ 17,119.07$ \\
\hline Premium price sales (US\$). & $\$ 20,054.27$ & $\$ 26,766.95$ \\
\hline Second class sales (US\$) & $\$ 2,307.19$ & $\$ 2,315.15$ \\
\hline Subtotal & $\$ 22,361.47$ & $\$ 29,082.1$ \\
\hline Gross margin & $\$ 8,992.74$ & $\$ 11,963.03$ \\
\hline Gross margin per kg sold. & $\$ 0.59$ & $\$ 0.61$ \\
\hline
\end{tabular}

\section{Discussion}

There were significant differences in mortality between the two vaccine systems. In the Circumvent ${ }^{\circledR}$ PCV group, the second dose reinforced the immunity leading to better mortality rates after week 10, in the critical growth and finishing stages.

The inferior performance of the Respifend ${ }^{\circledR}$ PCV-2 group was not only evident in the higher mortality rate, but also in the weights at sale which were lower than the Circumvent ${ }^{\circledR}$ PCV group $(84.52$ and $90.25 \mathrm{~kg}$ respectively). However, the wide variation in the end weights in the Respifend ${ }^{\circledR}$ PCV-2 group led to the trial being abandoned when the first pigs were sold, otherwise the differences would have been even greater.

The pigs vaccinated with Circumvent ${ }^{\circledR}$ PCV had heavier end weights. The other group had more second class pigs and thus poorer feed efficiency. The profit per kg sold is a measure of the better performance of the pigs vaccinated with Circumvent ${ }^{\circledast}$ PCV.

\section{References}

1 Fort M. et al. Vaccine 2008; 26(8): 1063-1071 


\title{
P.084
}

\section{Clinical case: improved sow fecundity after CIRCOVAC $^{\oplus}$ vaccination}

\author{
Guillaume Perreul ${ }^{1}$ Bernard Delahaye ${ }^{2}$ Jean-Bernard Herin ${ }^{4}$ Thaïs Vila $^{3}$ Julie Venet $^{3}$ François Joisel $^{3}$ \\ 1. MERIAL, Lyon, France; 2. Vétérinaire, Thorigne-sur-Due, France; 3. Merial S.A.S., Lyon, France; 4. Merial S.A.S., Ancenis, France
}

\section{Introduction}

Porcine circovirus type 2 (PCV2) is well known to be involved in reproductive disorders. The objective of the present paper is to describe a clinical case in which PCV2 was suspected to be involved and vaccination with $\mathrm{CIRCOVAC}^{\circledR}$ brought an improvement in the reproductive indexes.

\section{Farm/Case Description/Methods and Actions}

Farm: 450-sow 1-week farrowing batch management PRRSnegative operation. Weaning at 24 days; new buildings and good husbandry practices. Al with semen collection on farm.

Case description: poor reproduction indexes for 12 years; high \% of returns to oestrus mainly in parity $4 \& 5$ with fecundity as low as $72 \% ; 1 \%$ abortion and good global productivity: 27.7 .

Differential diagnosis and corrective measures: for the last years, a lot of work has been done to optimize reproduction, mainly in depth review of on farm Al management, full review of the feeding system and full revaccination against Porcine Parvovirus.

PCV2 suspicion: based on big size mummified piglets $>0.5$ per litter, hyperthermia and anorexia at mid-lactation, high prevalence of ear necrosis in 10 of the 7-week-old piglets and pig size heterogeneity in the flow.

PCV2 antibody titration in sows: Serelisa ${ }^{\circledR}$ PCV2 Ab (Synbiotics, Lyon, France).

Vaccination: CIRCOVAC was implemented in sows and gilts as recommended. Boars were vaccinated twice, $2 \mathrm{ml} \mathrm{IM}$.

Fecundity: results were recorded using the batch files collected on farm. Returns to oestrus were recorded on 5-month periods, before and during the vaccine implementation.

Statistical analysis: fecundity indexes were compared using a Yates-corrected Chi2 test.

Savings per avoided return to oestrus was evaluated at $57 €$ according to the reference given by the French Institute for Pig Industry (www.ifip.com) IFIP, Paris, France.

\section{Results}

The sow PCV2 Ab profile before vaccination is shown in Figure 1. Titres are heterogeneous with only $40 \%$ at $4 \log 10$ or more.
Figure 1 : PCV2 Ab titers in sows (ELISA unity log 10) accordig to sow parity.

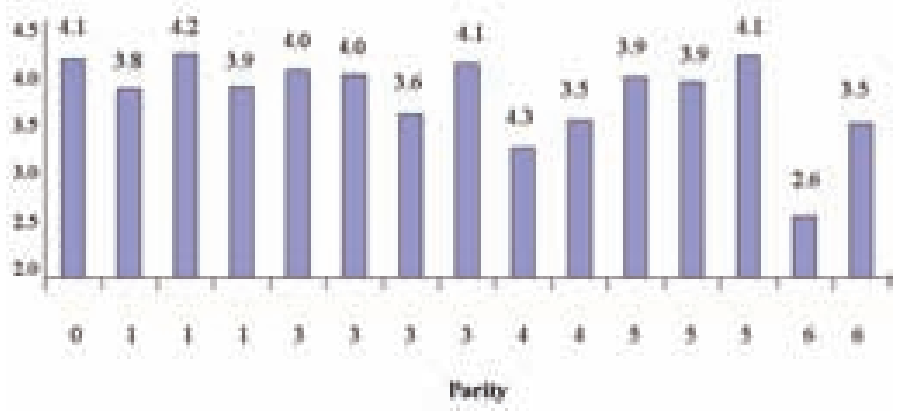

Figure 2 : Month results for fecundity (1st Al).

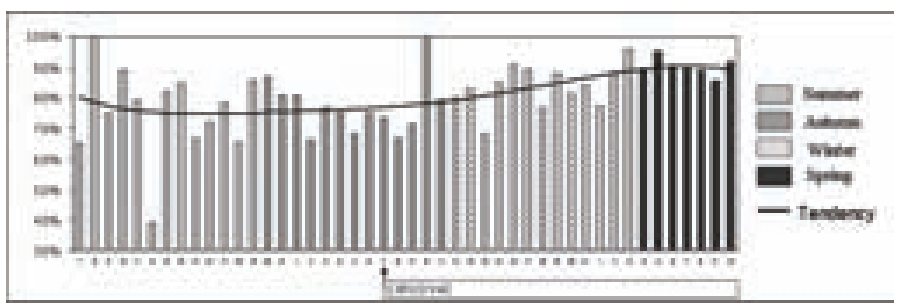

Fecundation after $1^{\text {st }} \mathrm{Al}$ was significantly improved from $76.0 \%$ to $83.5 \%$ after vaccination (Yates-corrected Chi2 test; $p<0.01$ ). Percentage of returns to oestrus before and during the vaccination of the sows with CIRCOVAC significantly dropped down from $21.15 \%$ to $13.51 \%$ respectively.

\section{Discussion}

Heterogeneity of PCV2 ELISA titres showed an inconsistent immune status. A confirmation of the presence of high quantity of PCV2 in late mummies would have been interesting to make sure PCV2 involvement. However, since vaccination of sows with CIRCOVAC, the number of return to oestrus dramatically dropped down. This is coherent with Mateusen et al. (1) findings which showed that embryos were susceptible to PCV2 as soon as they get rid of the zona pelucida. Unfortunately today, no conclusive diagnosis tool does exist for evidencing PCV2 involvment in early embryo death. Only based upon returns to oestrus, CIRCOVAC vaccination brought savings of around $10 €$ per sow per year.

\section{References}

1. Mateusen et al., (2004) Theriogenology, 61: 91-101

${ }^{\circledR} \mathrm{CIRCOVAC}$ is a registered trademark of Merial in the United States of America and elsewhere. 


\title{
P.085
}

\section{Onset of Immunity induced by Porcilis ${ }^{\circledR}$ PCV in MDA positive pigs given at 3 weeks of age by a single dose of $2 \mathrm{ml}$ and challenged with PCV2 virus at 5 weeks of age}

\author{
Alexander A. Eggen ${ }^{1}$ Frank Roerink ${ }^{2}$ Ulrike Schmidt $^{1}$ \\ 1. Intervet/Schering-Plough, Boxmeer, Netherlands; 2. Intervet/Schering-Plough, DeSoto, KS, USA
}

\section{Introduction}

Porcine Circovirus type 2 infection in pigs is regarded as the causative factor for development of Porcine Circovirus Disease (PCVD). PCVD occurs in pigs of different ages. Infection of piglets is associated with low levels of Maternally Derived Antibodies (MDA) and the presence of PCV2 virus. Under field conditions timing of PCV2 virus infection is often unknown. It is important that pigs are protected early and for the whole fattening period (Eggen). Pigs will be vaccinated early in life against PCVD in the presence of varying levels of MDA. The object of this study is to demonstrate efficacy against PCV2 virus of a single dose of $2 \mathrm{ml}$ Porcilis PCV in piglets of 3 weeks of age with MDA. At 5 weeks of age, 2 weeks after vaccination, vaccinated and control animals were challenged with PCV2 virus.

\section{Materials and Methods}

Progeny of SPF sows with PCV2 antibodies were studied. Male piglets were spread across litters to make 2 uniform groups of 10 piglets. At 3 weeks of age, Group 1 was vaccinated with $2 \mathrm{ml}$ of Porcilis PCV and Group 2 with placebo. At 5 weeks of age the pigs were challenged intranasally with $4.5 \log 10$ TCID50 of a heterologous wild type PCV2 challenge virus strain I-12/11. All animals were observed daily for clinical signs and were weighed at critical times.

Three weeks post challenge all animals were necropsied. At post mortem, mesenteric lymphnodes and tonsils were sampled for the determination of PCV2 antigen. Serum samples were taken at vaccination, at challenge, one week later and at necropsy, and were examined for PCV2 antibodies and nucleic acid. Fecal swabs were taken at challenge, one week later and at necropsy, and were examined for PCV2 nucleic acid. Statistical analysis of the serology employed Dunnett's multiple comparison method. Q-PCR data was analyzed by ANOVA and Dunnett using a linear mixed model assuming constant correlation of repeated measurements of a subject.

\section{Results}

All piglets were healthy before and after vaccination. Bodyweight gain did not differ significantly between groups at time of challenge or for the period between challenge and necropsy (see table 1).
Table 1: Bodyweight development $(\mathrm{kg})$

\begin{tabular}{|l|c|c|c|c|}
\hline Weeks of age// wpv* & $\mathbf{5}(\mathbf{2} \mathbf{w p v})$ & $\mathbf{8}(\mathbf{5} \mathbf{w p v})$ & Gain $\mathbf{( k g})$ & ADG (g/day) \\
\hline Group /////////wpc ** & $0 \mathrm{wpc}$ & $3 \mathrm{wpc}$ & & \\
\hline Porcilis PCV & $9.5 \pm 1.6$ & $17.5 \pm 3.5$ & $7.9 \pm 2.1$ & $378 \pm 101$ \\
\hline Control & $9.8 \pm 1.8$ & $17.2 \pm 2.5$ & $7.4 \pm 1.3$ & $351 \pm 61$ \\
\hline * wpv = weeks post vaccination ${ }^{* *} \mathrm{wpc}=$ weeks post challenge \\
\hline
\end{tabular}

Serology (see table 2)

Table 2: Antibody titer development (ELISA log2 values)

\begin{tabular}{|l|c|c|c|c|}
\hline Weeks of age///(wpv) & $\mathbf{3}$ (0 wpv) & $\mathbf{5}$ (2 wpv) & $\mathbf{6}(\mathbf{3} \mathbf{w p v})$ & $\mathbf{8}$ (5 wpv) \\
\hline Group//I/I///wpc & & $0 \mathrm{wpc}$ & $1 \mathrm{wpc}$ & $3 \mathrm{wpc}$ \\
\hline Porcilis PCV & 3.0 & 7.7 & 10.1 & 10.7 \\
\hline Control & 4.1 & 4.0 & 3.3 & 4.3 \\
\hline${ }^{*} \mathrm{wpv}=$ weeks post vaccination ${ }^{* *} \mathrm{wpc}=$ weeks post challenge \\
\hline
\end{tabular}

At challenge, the average PCV2 antibody titer was significantly higher in the vaccinated group $(P<0.0004)$

Q-PCR: At the time of challenge, the amount of viral DNA was negligible in both groups. Three weeks after challenge, the amount of virus in sera of the vaccinated group was significantly lower than the control group $(P<0.05)$. No PCV2 DNA was detected in feces of this group one week after challenge, and in significantly lower amounts at 3 weeks post challenge $(P<0.05)$, the amount of PCV2 nucleic acid in tonsils and mesenteric lymph nodes was significantly lower $(P<0.05)$.

\section{Discussion}

A single $2 \mathrm{ml}$ dose of Porcilis PCV in piglets of 3 weeks of age induced a rapid and strong immunity. Piglets were protected when challenged at 5 weeks of age. This was confirmed by the significant reduction in virus shedding and the PCV2 DNA load in lymphoid organs. The MDA titers in the piglets were purposely low. MDA titers $>5 \log 2$ present at 5 weeks of age can be protective and these MDA influence the take of the challenge virus (Fort, 2009) which will result in no PCV2 load in the controls leading to inconclusive results. This rapid onset of immunity by a single dose in the face of MDA is a feature unique to Porcilis PCV.

\section{References}

Fort; Vaccine 2009.

Eggen; IPVS 2010. 


\title{
P.086
}

\section{Effect of Porcilis ${ }^{\circledR}$ PCV on mortality in finishers in the presence of late severe PCVAD}

\author{
Jesus M. Bollo ${ }^{1}$ Marta Jimenez ${ }^{1}$ Rut Menjon Jesus V. Lopez ${ }^{1}$ Maria T. Tejedor ${ }^{2}$ \\ 1. Intervet Schering Plough, Madrid, Spain; 2. Dept. of Anatomy, Embryology and Genetics \\ Unit of Genetics - Faculty of Veterinary Medicine, Zaragoza, Spain
}

\section{Introduction}

In Spain, porcine circovirus-associated disease (PCVAD) is seen less frequently than in the first few years after the initial reports of the disease. The aim of this study was to assess the efficacy of vaccination with Porcilis ${ }^{\circledast}$ PCV in the presence of late severe PCVAD.

\section{Materials and Methods}

The study was performed on an 800-sow farm operating a closed cycle, three week batch system. Pigs were weaned at 28 days of age, and the farm had facilities to fatten $40 \%$ of its production. The other $60 \%$ were fattened on other farms. The farm was positive for PRRS and Mycoplasma.

In 2008, mortality rates in the fatteners varied between 3 and $5 \%$, and there were approximately $1 \%$ of runts. At the beginning of 2009, there was a notable increase in mortality rates and percentage of runts, reaching a peak of $13.3 \%$ mortality, $16.4 \%$ for deaths and runts together. The clinical presentation began at 13 weeks old, the signs being pallor, general enlargement of lymph nodes, stomach ulcers and a variable number of pigs with porcine dermatitis and nephropathy syndrome (PDNS).

From April onwards, blood samples were taken at 4, 7, 10, 13, 16,19 and 21 weeks of age. Serum was tested for PCV2 IgG and IgM antibodies (Ingezim PCV2 ELISA ${ }^{\circledR}$, Ingenasa), and showed that seroconversion coincided with the start of the clinical signs (Graph 1).

\section{Graph 1: Percentage of samples positive for IgG/M/age}

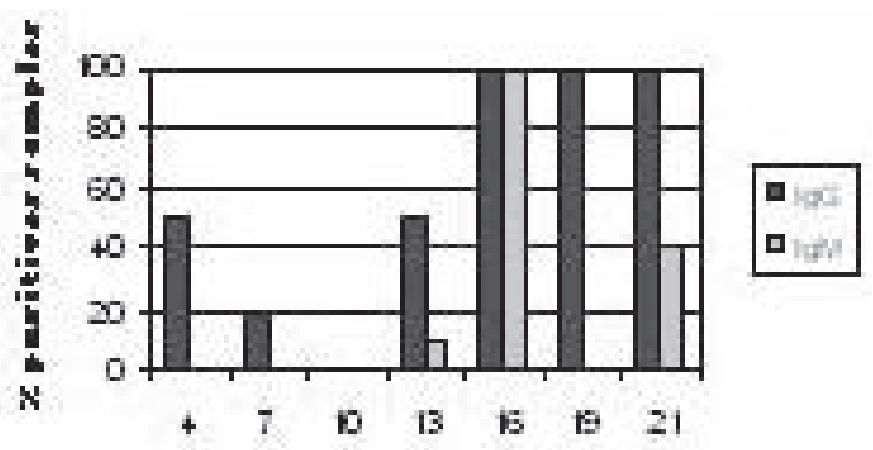

In the light of this, it was decided to vaccinate as many pigs as possible in the shortest possible time. Thus, all batches of pigs at either 45 or 70 days of age were vaccinated with Porcilis ${ }^{\circledR}$ PCV. Thereafter, all pigs were vaccinated at weaning (28 days old). [Data from this latter vaccination regime were incomplete at the time this abstract was submitted.] The results of the following groups were compared:
- Controls: unvaccinated animals (from 10 batches)

- Porcilis PCV (A): a single $2 \mathrm{ml}$ dose of Porcilis ${ }^{\circledR} P C V$ given to pigs 70 days old (note that the disease was probably already present in most of these animals).

- Porcilis PCV (B): a single $2 \mathrm{ml}$ dose of Porcilis ${ }^{\circledR} \mathrm{PCV}$ given to pigs 45 days old.

- The Pearson's chi-square test was used to compare the treatments (percentages), using the SPSS 15.0 software package.

\section{Results}

There was a statistically significant reduction in mortality rates during fattening in both vaccinated groups compared with the unvaccinated controls $(p<0.001)$, the best results being in the pigs vaccinated closer to the recommended schedule (at 3-4 weeks of age). The percentage of runts was also significantly better in the Porcilis PCV (B) group $(p<0.05)$.

Table 1: Production Results

\begin{tabular}{|l|c|c|c|}
\hline & Control & Porcilis PCV (A) & Porcilis PCV(B) \\
\hline No.animals & 7177 & 1196 & 865 \\
\hline \% deaths finishing & 8.7 & $4.9^{* *}$ & $1.6^{* *}$ \\
\hline \% low-weight pigs & 2.1 & $1.6^{\mathrm{NS}}$ & $0.9^{*}$ \\
\hline \% low-weight + deaths & 10.8 & $6.5^{*}$ & $2.5^{* *}$ \\
\hline \multicolumn{2}{|l}{ Porcilis PCV (A) \& (B) groups compared with controls: (NS: $\left.\mathrm{p}>0.05 ;^{*}: p<0.05{ }^{* *}: p<0.001\right)}$. \\
\hline
\end{tabular}

\section{Graph 2: Percentage mortality and runts during finishing}

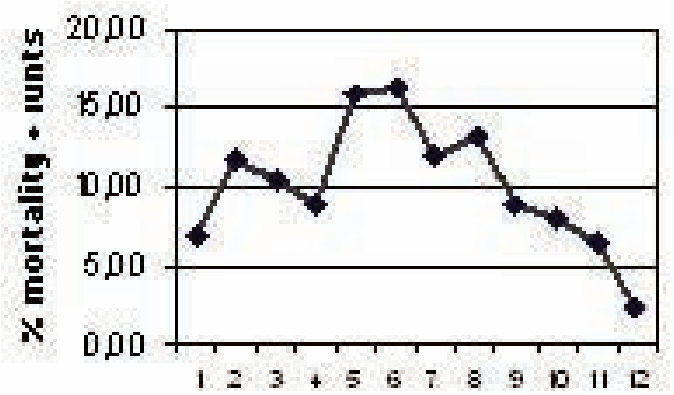

\section{Discussion}

The efficacy of Porcilis ${ }^{\circledR}$ PCV was demonstrated using different administration protocols. Vaccination with a single dose of Porcilis ${ }^{\circledR}$ PCV was found to be very effective in the presence of late severe PCVAD.

Better uniformity was observed in the vaccinated pigs; the lower percentage of runts being one of the reasons. 


\title{
P.087
}

\section{Efficacy of different vaccines against Porcine Circovirus type 2 administered as single shot to 3 week-old piglets with high maternal derived immunity against PCV2}

\author{
Ulrike Schmidt; Melanie Sno; Alexander A. Eggen \\ Intervet/Schering-Plough, Boxmeer, Netherlands
}

\section{Introduction}

Porcine Circovirus type 2 (PCV2) is widespread and has, as major causative agent for development of Porcine Circovirus Disease (PCVD), great economic impact in the swine industry. Infection of sows with PCV2 leads to varying antibody levels in blood and colostrum. High levels of maternal derived antibodies (MDA) can prevent PCV2 infection of piglets during first weeks of life, but may interfere with active immunization of piglets against PCV2 (Palzer,IPVS 2010). In recent years, several vaccines against PCV2 were brought to the market. All piglet vaccines can be given as a single shot vaccination from 2 or 3 weeks of age, when MDA can still be on a high level. Thus, it is essential that this single vaccination at young age is efficacious against possibly high levels of MDA.

The objective of this study was to compare the efficacy of commercially available PCV2 vaccines in piglets with high MDA against PCV2 after a single vaccination at 3 weeks of age.

\section{Materials and Methods}

Fifty piglets from sows with high PCV2 antibody titres (9.8 to $12.5 \log 2$ ELISA units before farrow) were available for this study. Allocation of piglets to five groups of 10 was done across litters the way that average MDA titres per group were comparable. At three weeks of age one of the following PCV2 vaccines was injected:

Group 1 was vaccinated with $2 \mathrm{ml}$ of Porcilis PCV(subunit vaccine), group 2 received $2 \mathrm{ml}$ of a PCV2 vaccine containing inactivated chimeric PCV1-2 virus as antigen, group 3 was injected with $1 \mathrm{ml}$ of a PCV2 subunit vaccine, group 4 received $0.5 \mathrm{ml}$ of an inactivated whole virus vaccine and group 5 was treated with $2 \mathrm{ml}$ of a physiological salt solution and served as negative control group. At 12 weeks of age, all piglets were challenge infected with wild type PCV2 challenge strain I-12/11 by intranasal route.

All animals were observed daily for clinical signs and blood samples were taken on several time points. Three weeks post challenge all animals were necropsied and tonsil, mesenteric lymph node and lung were sampled for determination of PCV2 viral nucleic acid by Quantitative PCR (QPCR). Serology data at time points $>3$ weeks were analysed by Analysis of Covariance (ANCOVA) by time point using the titre at time of vaccination and sow as covariate. Equality of serum antibody titre prior to vaccination was checked by ANOVA. Tukey's multiple comparison method was used to compare treatments. Descriptive statistics were used to summarize QPCR results on organs.

\section{Results}

No clinical signs were observed throughout the study.

Serology: at time of vaccination the piglets had individual PCV2 antibody titers between 9.3 and $15.5 \log 2$ ELISA units, resulting in mean group titres of around $11 \log 2$ ELISA units.
Fig 1: Development of PCV2 antibody titres

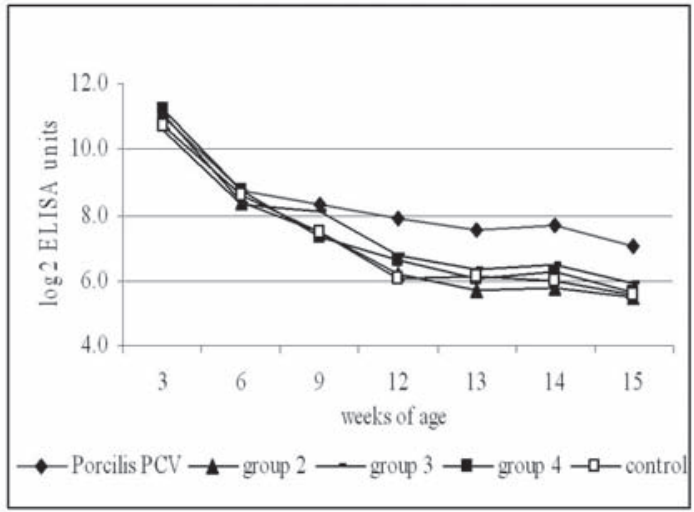

From week 9 post vaccination until the end of the study, average antibody titre of piglets vaccinated with Porcilis PCV was significantly higher than in the other groups (Porcilis PCV/group $2 \mathrm{p}<$ 0.0014 ; group $3 p<0.0155$; group $4 p<0.0037$; control $p<0.0001$ ). No statistically significant difference in PCV2 antibody titres could be found at any time point between other vaccinated groups or unvaccinated controls.

QPCR on organs revealed that piglets vaccinated with Porcilis PCV had the lowest mean load of PCV2 DNA in all tested organs.

Fig. 2: Mean PCV2 nucleic acid load in organs

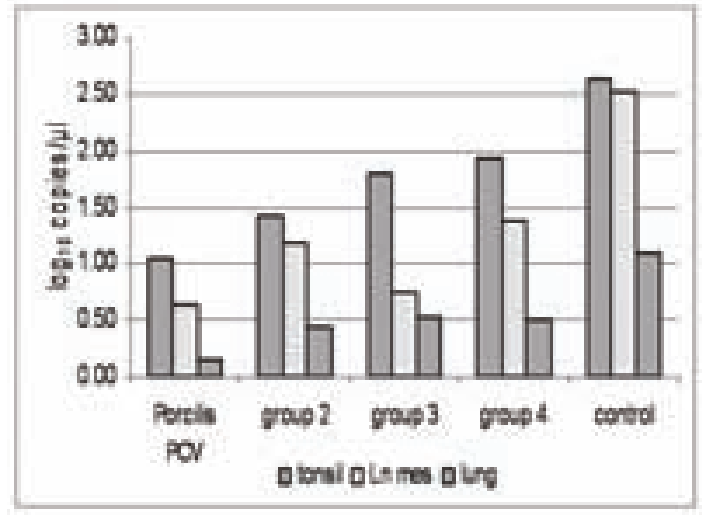

\section{Conclusion}

In this study, a single vaccination with Porcilis PCV induced a significantly higher humoral immune response in piglets with high MDA as compared to other PCV2 vaccines. The excellent protective efficacy of Porcilis PCV was confirmed by the low load of PCV2 DNA in all tested organs upon challenge infection.

\section{Reference}

Palzer, IPVS 2010 


\title{
P.088
}

\section{Evaluation of three commercial PCV2 vaccines in a commercial swine farm}

\author{
Kwang Soo Lyoo $^{1}$ Brian Caldwell ${ }^{2}$ Hyeon Bum Kim ${ }^{1}$ Jerry Torrison ${ }^{1}$ Peter Davies ${ }^{1}$ Han Soo Joo ${ }^{1}$ \\ 1. University of Minnesota, St. Paul, MN, USA; 2. Choice Connection, Mapleton, MN, USA
}

\section{Introduction}

Three commercial PCV2 vaccines have been licensed in the U.S., and all of them are widely used on commercial swine farms. The efficacy of these vaccines is well documented, and all of the vaccines reduced mortality and improved growth performance under field conditions ${ }^{1,2,3}$. However, the 3 vaccines vary in the nature and concentration of the antigen, the type of adjuvant, and the dose of administration. At present, no report is available on the comparative efficacy side by side of the 3 vaccines under conventional field conditions. The purpose of this study was to compare clinical signs, viremia, antibody responses, and average daily gain (ADG) of conventional pigs following vaccination at 3-weeks of age with the respective commercial PCV2 vaccines.

\section{Materials and Methods}

At a commercial farm in Minnesota, 4 pigs of similar body weight were selected from each of 20 litters and ear tagged. Three pigs from each litter were inoculated intramuscularly at 3 weeks of age with PCV2 vaccine A, B, and C, respectively. The fourth pig from each litter remained unvaccinated controls. All 80 pigs were moved into one pen of a wean-to-finish barn together with 520 pigs housed in other pens. The 20 pigs inoculated with vaccine A were revaccinated at 6 weeks of age (3 weeks after the first vaccination) according to the manufacturer's recommendation. All pigs in the barn were monitored daily for clinical abnormalities by the farm manager. Blood samples from each pig were collected at 3 weeks intervals. All pigs were individually weighed at 3 weeks and 24 weeks of age. Antibody responses of each pig were measured by indirect fluorescent antibody (IFA) test. Serum samples were also tested for viremia. The mean antibody titers, viral load, and body weight between the groups were analyzed by student t-test.

\section{Results}

There were no clinical signs observed in any of the pigs in the vaccinated and control groups, although 2 pigs in group B were found dead overnight from unknown causes during the first 3 weeks of the experiment. No medication was used for pigs during the study. ADG for all 4 study groups was calculated for the interval 3 to 24 weeks of age (Table 1). There was no significant difference in the mean body weights among the 4 groups at 3 weeks of age. The ADG of pigs in groups $A(0.81 \mathrm{~kg} \pm 0.03, P$ $<0.016)$ and $B(0.80 \mathrm{~kg} \pm 0.05, P<0.048)$ were significantly higher than ADG of the control group $(0.74 \mathrm{~kg} \pm 0.05)$. The ADG of pigs of the group $C(0.78 \mathrm{~kg} \pm 0.05)$ did not differ significantly from the controls $(P=0.132)$ or the other vaccinated groups $(P>0.173)$

Table 1. Comparison of the mean body weights at 3 and 24 weeks of age and $A D G$ between the pig groups

\begin{tabular}{|c|c|c|c|c|}
\hline \multirow[b]{2}{*}{ Age } & \multicolumn{4}{|c|}{ Group (weight $\mathrm{Kg}$ ) } \\
\hline & A & B & C & Control \\
\hline \multirow[t]{2}{*}{3 weeks } & 7.09 & 7.42 & 7.32 & 7.05 \\
\hline & $n=20$ & $n=19$ & $n=20$ & $n=18$ \\
\hline \multirow[t]{2}{*}{24 weeks } & 126.41 & 126.95 & 123.21 & 117.98 \\
\hline & $n=19$ & $n=16$ & $n=19$ & $n=18$ \\
\hline ADG & $0.81^{\mathrm{a}}$ & $0.80^{\mathrm{a}}$ & 0.78 & $0.74^{\mathrm{a}}$ \\
\hline
\end{tabular}

\section{Discussion}

The present study found differences in growth performance and body weight at marketing age among the study groups. Mean ADG and body weight just before the marketing of all 3 vaccinated groups were numerically higher than those of the control group, although statistically significant differences were observed only for groups $\mathrm{A}$ and $\mathrm{B}$. The differences were concordant with the results for viremia which showed higher levels of viremia in the control at several time points. The study confirms likely production benefits from PCV2 vaccination even in the absence of clinical PCV2 related diseases.

\section{References}

1. Fort M, Sibila M, Allepuz A et al (2008) Vaccine 26: 1063-1071

2. Opriessnig T, Patterson AR, Madson DM et al (2009) Vaccine 27: 1002-1007

3. Urniza A, Segales J, Balasch M et al (2008) Proc IPVS p55 


\title{
P.089
}

\section{Efficacy of Porcilis ${ }^{\circledR}$ PCV in pigs confronted by a severe porcine circovirus infection late in production}

\author{
Elena Vizcaíno ${ }^{2}$ Carlos Piñeiro ${ }^{2}$ Jesus M. Bollo ${ }^{1}$ Marta Jimenez ${ }^{1}$ Rut Menjon ${ }^{1}$ Jesus V. Lopez $^{1}$ \\ 1. Intervet Schering Plough, Madrid, Spain; 2. PigCHAMP Pro Europa S.A., Segovia, Spain
}

\begin{abstract}
Introduction
The aim of the present study was to evaluate the safety, efficacy and economic benefits of vaccination with Porcilis ${ }^{\circledR}$ PCV when pigs are confronted by a severe porcine circovirus infection late in the production cycle.
\end{abstract}

\section{Materials and Methods}

The study was carried out on a closed-cycle 650-sow farm in central Spain, producing pigs in weekly batches. Piglets are weaned at 21 days old, and moved to separate barns for the nursery, growing (10-16 weeks of age) and finishing periods. The farm suffered confirmed PRRS, and also Mycoplasma, for which piglets were vaccinated in their first and third week of age. Clinical signs associated with PCV2 were more evident from 14 weeks of age onwards, that is, mostly during growing and finishing. The average mortality rate (weaning-slaughter) of piglets born in 2008 was $13 \%$, with a peak of $16 \%$. The problem was aggravated by a high percentage of runts (culls).

Prior to vaccination, a serological profile was obtained monitoring the optical density ratios for IgG and IgM (Ingezim PCV Elisa), which demonstrated seroconversion from 15 weeks of age, still evident at 19 weeks.

Initially, all animals between 21 and 70 days of age were vaccinated. Thereafter, pigs were vaccinated 4 days after weaning with a single $2 \mathrm{ml}$ dose of Porcilis ${ }^{\circledR}$ PCV. Mortality rate, percentage of runts, weight at slaughter and days to slaughter were recorded for the following groups: Control Group: unvaccinated. Group A: vaccinated with Porcilis ${ }^{\circledR}$ PCV between 55 and 70 days of age. Group B: vaccinated younger with Porcilis ${ }^{\circledR}$ PCV: slightly more than $2 / 3$ rds of the group at 21 days old (the recommended age according to the leaflet instructions), and the rest ( 4 batches) between 32 and 55 days of age. During the whole study period, no relevant management changes were implemented on the farm.

The different treatments were compared using the Pearsons's chi-square test (proportions) and the Levene test (variances), together with an ANOVA (means), using the SPSS 15.0 software package.

\section{Results}

No local or systemic reactions were observed in the vaccinated animals, regardless of the time of vaccination.

PCV2 was considered to be involved during the growing and finishing periods. The recorded production data are shown in Table 1. For Group B, compared to the controls, the number of culls, and the mortality rate were reduced significantly during all the production phases $(p<0.001)$, leading to greater uniformity at slaughter.
Also a notable improvement in Average Daily Gain (ADG) was discovered later when the ANOVA test was used to compare slaughter weight, with days to slaughter as a covariant - an extra $8.070 \pm 1.800 \mathrm{~kg}$ was estimated for Group B.

Later vaccination, as practised in Group A, also proved effective, though there were fewer benefits than with early vaccination. Compared with the controls, Group A performed statistically significantly better in respect of all recorded parameters, except for mortality during the nursery period and days to slaughter. Overall, Group B performed better than Group A. The combination of Days to Slaughter and Slaughter weight $(\mathrm{kg})$ shows the clear economical advantage of Porcilis PCV vaccination at the recommended age (Group B).

Table 1: Production Results

\begin{tabular}{|c|c|c|c|}
\hline & Control & Porcilis PCV (A) & Porcilis PCV (B) \\
\hline Number of animals & 1719 & 688 & 3254 \\
\hline$\%$ Mortality nursery & 3.49 & $2.18^{\mathrm{NS}}$ & $0.80^{* *}$ \\
\hline$\%$ Mortality growing & 3.14 & $1.45^{*}$ & $0.89^{* *}$ \\
\hline$\%$ Mortality finishing & 6.4 & $1.6^{* *}$ & $1.44^{* *}$ \\
\hline \% Total mortality & 13.0 & $5.73^{* *}$ & $3.10^{* *}$ \\
\hline$\%$ culled pigs & 7.27 & $3.05^{* *}$ & $0.98^{* *}$ \\
\hline$\%$ culled + deaths & 20.27 & $8.42^{* *}$ & $4.12^{* *}$ \\
\hline Days to slaughter & 186.8 & $188^{\text {NS }}$ & $178^{* *}$ \\
\hline Slaughter weight (kg) & 97.86 & 102.32 & 101.71 \\
\hline \multicolumn{4}{|c|}{$\begin{array}{l}\text { In the statistical comparison of Groups A \& B with the Controls - } \\
\text { NS: } p>0.05 ;^{*}: p<0.05 ;^{* *}: p<0.001 \text {. }\end{array}$} \\
\hline
\end{tabular}

An economic model taking account only of (i) the differences in mortality rate and percentage of runts during the growing and fattening periods (but not those the nursery period) (ii) the improvement of days to slaughter, and (iii) the costs of vaccine and labor, yielded financial benefits of vaccinating (Groups A \& B) over not vaccinating (Controls): Late vaccination (Group A): $+10.17 € /$ pig. Earlier vaccination (Group B): $+12.24 € /$ pig. Thus, in both cases there was a good return on investment.

\section{Discussion}

The present study demonstrated that vaccination of pigs with Porcilis ${ }^{\circledast}$ PCV is safe, effective and profitable, even in the face of a severe PCV2 infection, late in the production cycle, contributing to a reduction in production losses caused by PCV disease. Regardless of the age at vaccination with Porcilis PCV, it was shown to be highly profitable, yielding a substantial return on investment. 


\title{
P.090
}

\section{Safety and efficacy of PCV-2 inactivated vaccine in weaned piglets under field conditions in Brazil}

\author{
Raquel L. Santos ${ }^{1}$ Djane Dallanora ${ }^{2}$ Roniê W. Pinheiro ${ }^{2}$ Glauber S. Machado² Zélia I. Lobato Roberto M. Guedes $^{1}$ \\ 1. Universidade Federal de Minas Gerais, Veterinary School, Belo Horizonte, MG, Brazil; 2. \\ Integrall Soluções em Produção Animal, Patos de Minas, MG, Brazil
}

\section{Introduction}

In July 2007, the first commercial vaccine against Porcine Circovirus type 2 (PCV-2) was launched in Brazil. This vaccine consists of inactivated PCV-2 in oil adjuvant, recommended for gilts and sows, protecting the piglets through passive transfer of antibodies (1). Sow vaccination has given good results in farms with PCVAD. However, different serological titration profiles against PCV-2 were observed in farms in Brazil and indicated different exposure and viral circulation. (2). Due to the limited availability of other conventional PCV-2 vaccines in Brazil and the concern regarding the development of the disease in fattening pigs, this vaccine started to be empirically used in weaned piglets. Thus, this field study was conducted in order to evaluate the safety and efficacy of this procedure.

\section{Materials and Methods}

A total of 1140 piglets, males and females, were divided into two groups: vaccinated (single dose, $0.5 \mathrm{ml}$ of vaccine) and control (not vaccinated), blocked by gender. All animals were evaluated from the application of the experimental treatment until market. Safety was evaluated by clinical and body temperature daily examinations in the four days after vaccination. Efficacy was based on comparison of performance (body weight and average daily gain), mortality, antimicrobial usage, diarrhea, coughing and sneezing indexes. Post examination was performed in all dead animals, followed by histopathology and PCV-2 immunohistochemistry evaluation. Lung lesions were evaluated at slaughter. Blood samples were collected at weaning, at the end of nursery and before slaughter and used for lgG serology using IPMA.

\section{Results}

No adverse effects were observed after vaccine application. Vaccinated males had a higher slaughter weight $(2.73 \mathrm{~kg}, \mathrm{p}=0.029)$ and a higher average daily gain $(0.025 \mathrm{~kg}, \mathrm{p}=0.027)$ when compared to control males. In contrast, females had no difference in body weight or average daily again between vaccination and control groups. However, there was a greater final body weight of $1.50 \mathrm{~kg}(p=0.07)$ in vaccinated females compared to the control group. There was no significant difference between groups regarding mortality and culling rates, coughing, sneezing or diarrhea indexes, medication use, pneumonia index, pathological and histopathological lesions, and antibody titration.

\section{Discussion}

Vaccination did not induce any significant adverse effect, as observed by others (3). The results of daily weight gain, slaughter weight and mortality rate in this study agreed with other reports under field conditions $(3 ; 4)$. As there was no reduction in mortality, it was suspected that other conditions were playing an important role.

The absence of statistical difference in the final weight of females $(p=0.07)$ can be explained by the difference in housing conditions compared to males, as the females were continuous in feces contaminated solid floors, were in more densely populated pens and were managed much more often for breeding selection.

PCV2D lesions and PCV2 antigen were observed in both vaccinated and control groups from both genders with no difference among them. If animals had been randomly selected for euthanasia, as was done by Segalés et al. (6), we may have seemed difference between groups.

These data indicate that vaccination against PCV-2 is safe and effective in improving the final weight and the average daily gain in piglets vaccinated under field conditions in Brazil.

\section{References}

(1) Joisel, F. et al. Adv. Pork Production. 19:183-195, 2008.

(2) Lobato, Z. I. P. et al. 5th International Symposium on Emerging and Re-emerging Pig Diseases, 2007, Krakow.

(3) Pejsak, Z. et al. Comp. Immunol Microbiol Infect Dis. (2009) doi:10.1016/j.cimid.2009.09.006.

(4) Fachinger, V. et al. Vaccine. 26:1488-1499, 2008.

(5) Kixmoller, M. et al. Vaccine. 26:3443-3451, 2008.

(6) Segales, J. et al. Vaccine. (2009) doi:10.1016/j.vaccine.2009.09.084. 


\title{
P.091
}

\section{The effect of a single dose of Porcilis $\mathrm{PCV}^{\circledR}$ in piglets at 3 weeks of age on mortality, daily weight gain and carcass weight at slaughter}

\author{
Paolo Martelli ${ }^{1}$ Antonio Caleffi ${ }^{2}$ Stefano Gozio ${ }^{3}$ Alberto E. Cevidalli ${ }^{4}$ Stefano Guazzetti ${ }^{5}$ Paolo Borghetti ${ }^{1}$ \\ 1. Dept. Animal Health - University of Parma, Parma, Italy; 2. Veterinary Practitioner, Mantua, Italy; 3. Intervet/Schering- \\ Plough A.H., Boxmeer, Netherlands; 4. Intervet/Schering-Plough A.H., Milan, Italy; 5. ASL, Reggio Emilia, Italy
}

\section{Introduction}

The control of PCVD is based on management strategies, reduction of co-infections and vaccination. Under field conditions, all commercial PCV2 vaccines lead to reduced mortality and cull rates, and significantly improved weight gain, along with a reduction in co-infections in PMWS-affected herds (Kixmoller et al., 2008; Segalés et al, 2009).

The object of the present study was to investigate the efficacy of a single dose of PCV2 subunit vaccine on two different farms suffering from PCVD by monitoring mortality and culling rates, average daily gain and carcass weight at slaughter.

\section{Materials and Methods}

A double-blinded, randomised and controlled field trial was conducted on two farms with a history of PMWS (Farm 1: 900-sow farrow-to-finish herd; Farm 2: 3-site 1850-sow herd). A total of 818 piglets were included in this study and distributed between two treatment groups.

At inclusion (weaning at $21 \pm 3$ days of age), a total of 408 animals (Group B) received a $2 \mathrm{ml}$ intramuscular dose of Porcilis PCV ${ }^{\circledR}$, a commercial PCV2 capsid-based subunit vaccine expressed in inactivated baculovirus. To act as controls (Group A), 410 pigs received $2 \mathrm{mls}$ of the adjuvant Diluvac Forte ${ }^{\circledR}$, by the same route.

All animals were individually identified, and their weights were recorded at inclusion, and at 12 and 26 weeks of age, and the average daily gain (ADG) calculated. The carcass weights of the pigs from Farm 2 were recorded at slaughter (274 days old). All dead animals (died or were culled) were submitted for postmortem diagnostic investigation, in order to classify them as PMWS-affected (according to the accepted case definition) or not.

To estimate the effect of vaccination on the probability of a pig dying, a mixed effect logistic regression model was constructed to take account of the non-independence of repeated measurement of the same subjects and the effect of the sow (litter effect), using the 'Ime4' package.

\section{Results}

Total losses from PMWS were $9.02 \%$ and $0.2 \%$ in control and vaccinated groups, respectively. For fatal conditions other than PMWS, the proportion of pigs lost were 7.3 and 7.8 in Groups A and $B$, respectively.

The estimated Hazard Ratio for losses related to PMWS in Group $B$ as against Group A was 0.082 (c.i.95\%: 0.030-0.229; p<0.0001).

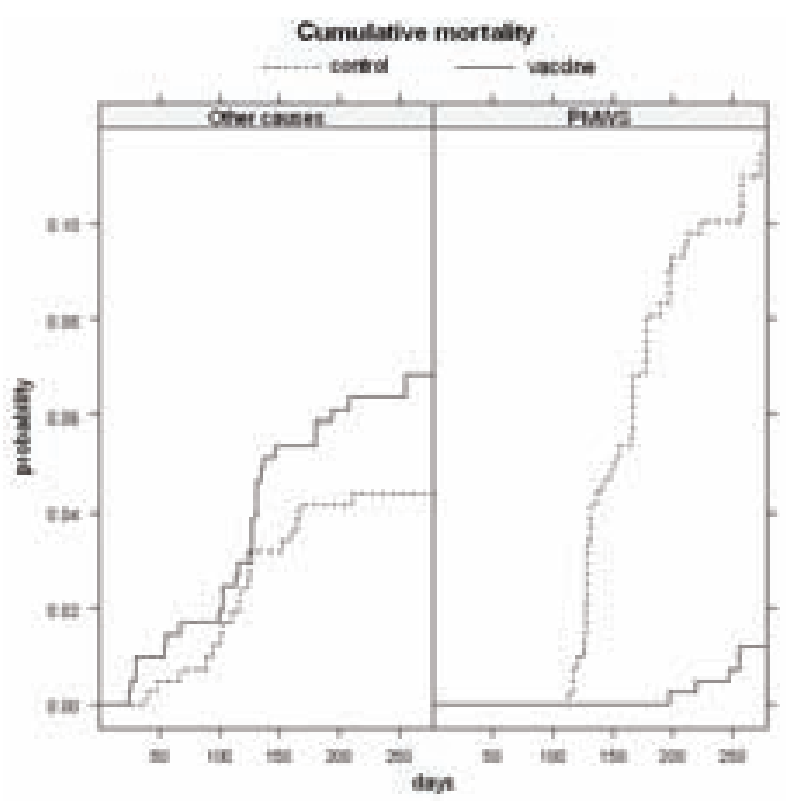

The ADG and carcass weight at slaughter for both groups are shown in Table 1.

\begin{tabular}{|l|c|c|c|c|}
\hline ADG $(\mathbf{g})$ & A & B & Diff. & p value \\
\hline 3-12 weeks of age & 481 & 478 & +3 & $<0.05$ \\
\hline 12-26 weeks of age & 811 & 881 & -70 & $<0.001$ \\
\hline CARCASS $(\mathrm{kg})$ & 140.5 & 145.0 & $+4,5$ & $<0.012$ \\
\hline
\end{tabular}

\section{Discussion}

In this study, the probability of a pig vaccinated with a single dose of Porcilis PCV at 3 weeks of age dying from PMWS was 12 times less than that of an unvaccinated control pig. Moreover, vaccination improved the average daily gain when animals became infected with PCV2.

In addition, the difference in average carcass weight (+4.5 kg in vaccinated) in pigs slaughtered at 274 days of age demonstrated the long term positive effect of Porcilis PCV vaccination on productive performace.

\section{References}

Segalés et al. (2009) Vaccine 27(52), 7313-21.

Kixmoller et al. (2008). Vaccine 26 (27-28), 3443-51. 


\title{
Comparison of the efficacy of Porcilis ${ }^{\circledR}$ PCV and an alternative PCV vaccine under field conditions
}

\author{
Juan L. Ubeda ${ }^{1}$ Rut Menjon² Jesus M. Bollo² Marta Jimenez ${ }^{2}$ Jesus V. Lopez ${ }^{2}$ Javier Rodriguez $^{2}$ \\ 1. Veterinary Practitioner, Zaragoza, Spain; 2. Intervet Schering Plough, Madrid, Spain
}

\section{Introduction}

The aim of the present study was to compare the efficacy of Porcilis ${ }^{\circledR} \mathrm{PCV}$ with another commercial vaccine, under field conditions, with respect to health and production, on a farm with a history of severe losses caused by porcine circovirus-associated disease (PCVAD).

\section{Materials and Methods}

The study was performed on two different sites of a 1000-sow farm in northern Spain. The farm had a history of PCVAD-related problems, with an average $5 \%$ mortality rate in the nursery, a $6-8 \%$ mortality rate in fattening pigs, with $4 \%$ referrable to PCV, and a late clinical picture appearing from 14 weeks of age onwards, and persisting up to 18-20 weeks of age. The farm had tested positive to Mycoplasma and PRRS (unstable and with resurgence at 7, 9 and 16 weeks).

Before the start of the study, PCV2 had been confirmed by clinical signs and gross pathology in diseased pigs, virus isolation from 3 of 4 culled pigs, and clear evidence of seroconversion by 18 weeks of age. After confirmation of the diagnosis, piglets from two weekly batches were selected (a total of 829 piglets) and randomly allocated into 3 groups according to gender, dam and weight, individually identified, and treated at 3 weeks of age.

Group 1: 272 piglets vaccinated with a single $2 \mathrm{ml}$ dose of Porcilis $^{\circledR}$ PCV; Group 2: 276 piglets vaccinated with a single $0.5 \mathrm{ml}$ dose of another commercial vaccine (Vaccine B); Group 3: 281 piglets injected with $2 \mathrm{ml}$ Diluvac Forte ${ }^{\circledR}$ as placebo, acting as controls.

In addition, 60 piglets were selected ( 20 piglets per group), for serological controls at 3, 7, 10, 14, 18, 22 and 25 weeks of age.

After weaning, the pigs were housed in the same barn, with each group in a separate pen. At 10 weeks old, they were moved to the fattening barn, again penned separately in their groups. Temperature and humidity were recorded throughout, as well as individual treatments and mortality. All pigs were weighed at 3 , 10,18 and 25 weeks of age (earliest slaughter date).

Statistical analysis was performed using the General Linear Model (GLM, SPSS 15.0 for Windows).

\section{Results}

At 3 weeks old, all three groups had high levels of MDA with no significant differences between them $(p=0.601)$. At 7 weeks of age, Group 1 had $94.7 \%$ and $84.2 \%$ animals positive for IgG and IgM, respectively, significantly different from the other groups $(p<0.001)$. At 14 weeks, the percentage of pigs positive for lgM increased (see other paper of proceedings of this congress from same aurhors) significantly in Groups 2 and 3, whereas Group 1 remained negative. As there had been no significant differences in average weight $(p=0.429)$ and variance of weights $(p>0.05)$ at the start, all groups were comparable.

During the nursery period no significant differences were found for growth, mortality or treatments $(p>0.05)$.

Table 1:

\begin{tabular}{|l|c|c|c|}
\hline & Control & Vaccine B & Porcilis PCV \\
\hline Kg gained & $70.95 \pm 0.67$ & $72.67 \pm 0.69$ & $74.09 \pm 0.55$ \\
\hline ADG & $651 \pm 0.006$ & $667 \pm 0.006$ & $680 \pm 0.005$ \\
\hline \% Mortality & 4.0 & 4.8 & 1.5 \\
\hline \% Pigs treated/ week & 12.3 & 10.5 & 8.1 \\
\hline \% Total treatments & 40.4 & 33.9 & 25.7 \\
\hline
\end{tabular}

Group 1(Porcilis PCV) was $1.42 \mathrm{~kg}, 13 \mathrm{~g} /$ day better than Group 2 $(p=0.09)$, and $3.14 \mathrm{~kg}, 29 \mathrm{~g} /$ day better than Group 3 ( $p<0.001)$. Group 1 had significantly fewer treatments than the other two groups $(p=0.01)$. Group 1 was significantly better for all parameters than the controls, whereas Group 2 (Vaccine B) performed no better than the controls.

A clinical picture compatible with PCV was found in $41.4 \%$ of dead pigs. Laboratory diagnosis (IHC) confirmed the presence of PCV in none of the Porcilis ${ }^{\circledast}$ PCV group, $3.45 \%$ of the Vaccine B group, and $10.3 \%$ of the control group.

\section{Discussion}

Vaccination of a high percentage of the pigs commingled in the same house probably improved the results of the control group due to a reduced infection pressure, otherwise greater differences would have been expected between groups.

The use of Porcilis ${ }^{\circledR}$ PCV in this situation led to an improvement in all the recorded health and production parameters, not only compared to the control group but also compared to the other commercial vaccine (Vaccine B). In addition to this, the robust seroconversion induced in the presence of maternally derived immunity allows the firm conclusion that Porcilis ${ }^{\circledR} \mathrm{PCV}$ was the better choice of vaccine to protect against a severe, late problem with PCVAD. 


\title{
P.093
}

\section{Safety of Porcilis ${ }^{\circledR}$ PCV in conventional piglets under field conditions}

\author{
Juan Luis Ubeda ${ }^{1}$ Ruth Menjon² Jesus M. Bollo² Marta Jimenez ${ }^{2}$ Jesus V. Lopez ${ }^{2}$ \\ 1. Veterinary Practioner, Zaragoza, Spain; 2. Intervet/Schering-Plough AH, Cantabria, Madrid, Spain
}

\section{Introduction}

Porcine Circovirus Type 2 (PCV2) is involved in the development of several diseases of swine and is undoubtedly of major clinical importance in modern pig production. Vaccination against this pathogen is therefore a very common precaution, usually piglets at weaning.

There are four available vaccines, and as with other products, the main selection criteria used by veterinarians are efficacy and safety. The object of this trial was to compare the safety of piglet vaccination with a subunit vaccine (Porcilis ${ }^{\circledR} P C V$ ) with another PCV vaccine by monitoring local and systemic reactions under field conditions (1).

\section{Materials and Methods}

Porcilis ${ }^{\circledR}$ PCV is a subunit vaccine containing the viral capsular protein coded by the ORF2 of the PCV2 genome in an adjuvant containing dl-a-tocopherol acetate and paraffin (Xsolve). The efficacy of this vaccine has been demonstrated previously (2)

The vaccine was tested on the piglets of a 1000-sow herd in the province of Zaragoza, in north-east Spain, managed on a twophase production system. The piglets suffered a PCV2-related disease from 14 weeks of age.

829 piglets of about 3 weeks of age were randomly assigned to three groups:

Group 1: 272 piglets vaccinated with a $2 \mathrm{ml}$ dose of Porcilis ${ }^{\circledast}$ PCV.

Group 2: 276 piglets vaccinated with $0.5 \mathrm{ml}$ of another PCV vaccine (Vaccine B).

Group 3: 281 piglets injected with 2ml Diluvac Forte, a dla-tocopherol acetate based adjuvant, acting as controls.

The animals were observed for local and systemic effects for 1-2 hours post injection, and at 24 hours, and 7 and 14 days after vaccination.

All the pigs were individually identified, and weighed at 3 weeks old (vaccination time) and 10 weeks old (at the end of the postweaning period) and the average daily gain (ADG) of each group was calculated.

Statistical analysis was performed using the General Linear Model (GLM-SPSS 15.0 for Windows). The means and variances were compared using the ANOVA and Levene tests.

\section{Results}

No local reactions were observed in any of the groups post vaccination. In Group 1, $0.7 \%$ of the piglets showed mild systemic signs that disappeared spontaneously a few minutes after injection.

In Group 2, vomiting was observed in 3.5\% of the piglets about 10 minutes post vaccination. The animals recovered between 5 and 15 minutes later. No systemic signs were observed in Group 3.

There were no significant differences between any of the groups with respect to ADG between 3 and 10 weeks of age. Table 1 summarizes the performance data of the three groups.

Table 1: Performance Results

\begin{tabular}{|l|c|c|c|}
\hline & Group 1 & Group 2 & Group 3 \\
\hline Parameter//Test group & Porcilis PCV & Vaccine B & Control \\
\hline Weight at 3 weeks of age & $5.9 \pm 0.76$ & $5.9 \pm 0.8$ & $5.8 \pm 0.77$ \\
\hline Weight gain (3-10) weeks of age & $12.1 \pm 0.17$ & $12.2 \pm 0.22$ & $12.3 \pm 0.24$ \\
\hline Average Daily Gain & $269 \pm 0.004$ & $273 \pm 0.004$ & $275 \pm 0.004$ \\
\hline
\end{tabular}

There was no significant difference in performance between the three groups ( $p=0$ 307).

At the end of the trial ( 25 weeks of age) Group 1, however showed significant differences for all parameters in comparison to the control group (Group 3), whereas Group 2 (Vaccine B) did not present significant differences vs the control group.

\section{Discussion}

There were no local reactions to the simple vaccination of conventional piglets with Porcilis ${ }^{\circledR}$ PCV. There were a few systemic reactions after vaccination, but they were very moderate and transient.

There was complete equivalence between control and vaccinated piglets as regards growth during the nursery period. According to this study, the vaccination of piglets of 3 weeks of age with Porcilis ${ }^{\circledR}$ PCV has no negative effect on performance, in the few weeks following vaccination.

\section{References}

1. Taneno, A (2008). Proceedings of 20th IPVS Congress, Durban.

2. Ruiz, A. (2008). Proceedings of 20th IPVS Congress, Durban. 


\title{
P.094
}

\section{Economic impact of reduced variation of slaughter weights involving different PCV2 vaccinated groups}

\author{
Dennis DiPietre $^{2}$ Eric Lewandowski $^{3}$ Remy Jagu ${ }^{3}$ Matthias J. Adam $^{1}$ \\ 1. Boehringer Ingelheim Animal Health, Ingelheim am Rhein, Germany; 2. KnowledgeVentures, \\ LLC, Columbia, MO, USA; 3. Boehringer Ingelheim Vetmedica, Pace, France
}

\section{Introduction}

Pig producers selling at the same average weight may have widely different profit outcomes depending on the fraction of production which qualifies for the highest price sections of the buying grid. The main reason for this is variation in slaughter weights. It is not unusual for modern production systems to have a range of $45 \mathrm{~kg}(100 \mathrm{lb})$ live weight between the largest and smallest animals sold at the time of slaughter. Slaughterhouse pricing schemes provide the highest prices in a relatively narrow range of weight and lean percent combinations to incentivize producers to produce and market animals which bring the highest returns to the processing and up-chain sales activities. Pigs falling outside this range are subjected to increasingly severe discounts as they depart farther from the ideal weight and lean parameters1.

It is therefore crucial for pig producers to measure, understand and control the variance of production2. Many factors contribute to variation in final kill weights including natural variation inherent in biological production processes. Insults to the pigs during the production process (such as feed out events, lack of proper ventilation etc.) as well as disease increase the variation of finished weights as each animal varies in its growth and quality response to challenges in the growing environment. Disease is thought to be the most important cause of variation in final slaughter weights. Vaccination measures can affect the standard deviation of the distribution of weights and other production parameters by reducing disease pressure. This paper investigates the economic impact of two vaccination protocols taking into account only the reduction in standard deviation of slaughter weights between the two groups.

\section{Materials and Methods}

A 5,000 head nursery-finish unit in Brittany, France receives weekly, 21-day-old piglets from a unique 900-sow unit. In this side-by-side trial, pigs were randomly assigned at weaning to two treatment groups, $\operatorname{Circovac}^{\circledR}(\mathrm{CV})$ or Ingelvac CircoFLEX ${ }^{\circledR}$ (FLEX). Pigs of both groups were kept in separate pens, but in the same rooms 3. The producer was blind to the treatments and employed his standard selection and marketing procedures to both groups. At slaughter, individual data were gathered for all pigs marketed. While actual average slaughter weights per group were nearly identical, carcass weight standard deviations were significantly different in favor of the FLEX group. 10,000 paired carcass weights and lean percents were randomly drawn from correlated distributions estimated from the actual sales data for each group. The total feed costs were estimated from standard growth and intake curves adjusted to match the historical closeout data of the farm (average feed cost/Mton W-F: $€ 200$; base carcass price: $€ 1.30 / \mathrm{kg}$ ). All observations in each set of sampled observations were priced in the French slaughterhouse grill (matrix) to which the farm sold pigs.

\section{Results}

Standard deviation of weight at slaughter was $16.7 \%$ lower in the FLEX group generating an "as marketed" advantage of $€ 1.85$ / head for the FLEX group.

Table 1: Economic returns over feed/hd for both groups

\begin{tabular}{|l|c|c|c|}
\hline & FLEX Group & CV Group & Difference per head \\
\hline Avg Carcass (kg) & 91.04 & 90.50 & $0.60 \%$ \\
\hline StDev & 6.06 & 7.28 & $-16.7 \%$ \\
\hline Avg price Received & $€ 1.429$ & $€ 1.413$ & $€ 0.016$ \\
\hline Revenue/head & $€ 130.31$ & $€ 127.73$ & $€ 2.58$ \\
\hline Feed cost/head & $€ 59.92$ & $€ 59.18$ & $€ 0.74$ \\
\hline Return over Feed/head & $€ 70.40$ & $€ 68.55$ & $€ 1.85$ \\
\hline
\end{tabular}

\section{Discussion \& Conclusion}

The above differential return takes only into account the reduction in standard deviation of slaughter weights and does not include other production parameters e.g. mortality or growth 3. However pig producers evaluate different strategies based on the most commonly available information, such as the average of production parameters (feed efficiency, etc.) and overlook the hidden economic benefit generated from reduced variation. More understanding of variation at slaughter is critical to capturing more profit, and since slaughter data is for many farms the only availability of individual animal performance data, it is crucial to collect and analyze this important metric. In this trial a differential return of $€ 1.85 / \mathrm{hd}$ was generated by a $16.7 \%$ reduction in standard deviation of slaughter weights for the FLEX group.

\section{References}

1. Tokash M. \& Henry S. (2008) Swine profitability conference, p49-62

2. DiPietre D. (2009) Pork Business Journal, August, p10-15, published by Farms.com

3. Guillaume, J.M., and al. (2009) ESPHM, p52 


\title{
P.095
}

\section{Comparison of the serology of piglets with Porcilis $^{\circledR}$ PCV and an alternative PCV vaccine}

\author{
Juan L. Ubeda ${ }^{1}$ Rut Menjon ${ }^{2}$ Jesus M. Bollo² Marta Jimenez ${ }^{2}$ \\ 1. Veterinary Practitioner, Zaragoza, Spain; 2. Intervet Schering Plough, Madrid, Spain
}

\section{Introduction}

The aim of the study was to compare the development of specific humoral immunity (IgG and IgM) against PCV2 over the whole production cycle of animals vaccinated with two different commercial vaccines and unvaccinated animals, taking account of possible interference with maternally derived antibodies (MDA).

\section{Materials and Methods}

The study was performed on two different sites of a 1000-sow farm in northern Spain. The farm had a history of PCVAD-related problems, with an average 6-8\% mortality during fattening, and a late clinical picture appearing from 14 weeks of age onwards which persisted up to $18-20$ weeks old. Prior to the study, PCV2 was confirmed by clinical signs and gross pathology in diseased animals, isolation from tissues of $4 / 5$ culled piglets, and seroconversion evident at 18 weeks of age.

60 three week old piglets were individually identified and randomly allocated to three groups of 20 animals each, according to dam, sow parity, gender and weight. The pigs were treated at 3 weeks old as follows: Group 1 was vaccinated with Porcilis ${ }^{\circledR}$ PCV (single $2 \mathrm{ml}$ dose); Group 2 with a single $0.5 \mathrm{ml}$ dose of another commercial product, Vaccine B; Group 3, the controls, were injected with $2 \mathrm{ml}$ Diluvac Forte ${ }^{\circledR}$. Blood samples were taken at $3,7,10,14,18,22$ and 25 weeks old. Serum was tested for PCV2 IgG and IgM antibodies (Ingezim PCV2 ELISA ${ }^{\circledR}$, Ingenasa).

\section{Results}

At 3 weeks of age, the animals of all three groups had high levels of MDA, with a high percentage being positive for lgG, but with average optical density (OD) titers showing no significant differences between the groups ( $p=0.601)$. (Group1: 0.7191 \pm 0.1 ; Group 2: $0.853 \pm 0.11$; and Group 3: $0.758 \pm 0.1)$. All the groups were negative for $\lg \mathrm{M}$ at this age.

At 7 weeks old, the piglets of Group 1 had $94.7 \%$ and $84.2 \%$ animals positive for lgG and IgM, respectively, statistically significantly greater than the other groups $(p<0.001)$, and an average $1.36 \pm 0.11$ OD titer compared with that of the other groups which fell below the threshold.

At 10 weeks old, IgM had disappeared from all groups, but Group 1 had $87.5 \%$ animals IgG positive, significantly more than the other groups $(p<0.001)$ which were nearly negative, and remaining so until 25 weeks of age.

At 14 weeks old the percentage of IgM positive animals in Groups 2 and 3 increased significantly, while Group 1 animals remained negative.
Graph 1: Percentage samples positive for lgG

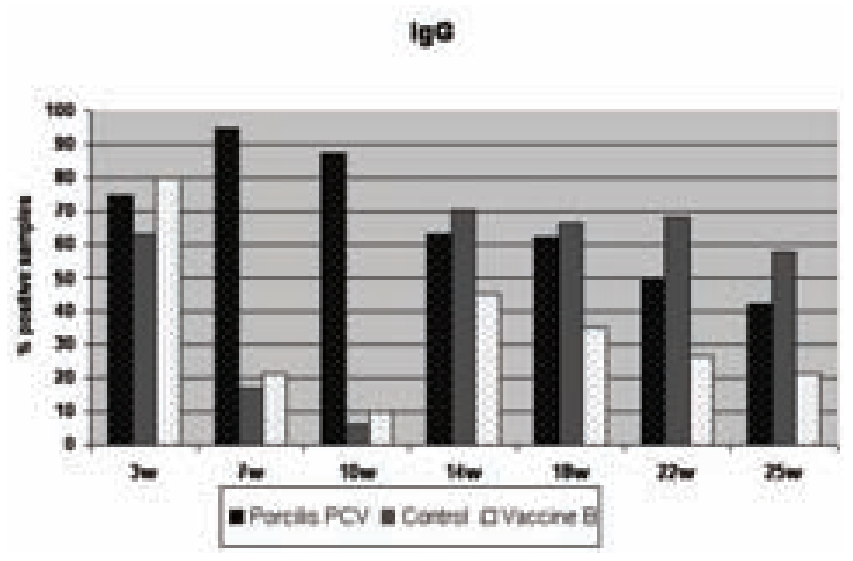

Graph 2: Percentage samples positive for IgM

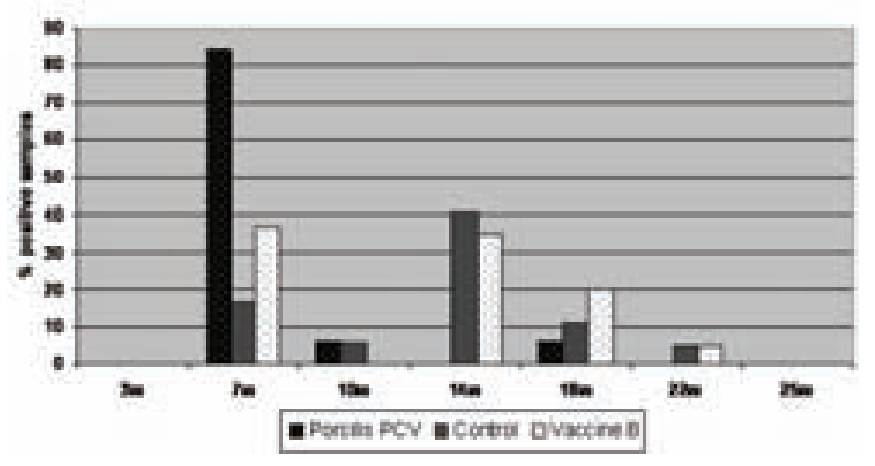

\section{Discussion and Conclusions}

At 3 weeks old, the lgG levels represent the level of MDA, when all piglets were negative for IgM. Unlike the Porcilis ${ }^{\circledR}$ PCV vaccinated group, the Vaccine B group did not seroconvert at 7 or 10 weeks of age, and the control group did not do so until 14 weeks of age, on contact with field virus. This indicates that all previous immune responses had been induced by the vaccine without interference from MDA (1). A high percentage of the Porcilis ${ }^{\circledast}$ $P C V$ vaccinates continued with positive lgG titers until week 25 , whereas the other two groups needed the field virus challenge to induce seroconversion. After challenge, the increased percentage of IgM positive pigs in the Vaccine B and control groups, but not in the Porcilis ${ }^{\circledR}$ PCV group, demonstrates the priming induced by this vaccine.

\section{References}

1. Fort,M. (2009) Vaccine $27,4031-4037 \mathrm{~s}$ 


\title{
P.096
}

\section{Benefits of Porcilis ${ }^{\circledR}$ PCV $^{\circledR}$ PCV vaccination on a farm with late mild PCVAD}

\author{
Juan C. Ezpelata ${ }^{1}$ Alicia Echave ${ }^{1}$ Rut Menjon ${ }^{2}$ Marta Jimenez ${ }^{2}$ Jesus M. Bollo ${ }^{2}$ \\ 1. Grupo AN.Pamplona (Navarra), Pamplona, Spain; 2. Intervet Schering Plough, Madrid, Spain
}

\section{Introduction}

The clinical presentation of porcine circovirosis has modified over recent years, with cases often being less severe, and arising at the end of the production cycle. Nevertheless, the virus still causes significant production losses which it is very important to avoid. The aim of the present study was to assess the benefits of vaccination with Porcilis ${ }^{\circledR}$ PCV against late, subclinical PCVAD.

\section{Materials and Methods}

The study was carried out on a 1500-sow farm in northern Spain, managed in 2 week batches, in a two-phase production system. The farm, which uses LW*LD genetics, finishing with halothanenegative Pietrain, had tested negative for PRRS and Aujeszky's Disease, but positive for Mycoplasma, against which piglets were vaccinated. The average mortality rate had been $3.5 \%$ and culling rate $1.3 \%$.

The clinical presentation of Circovirus was mild and occurred between 20 and 22 weeks of age (the end of fattening period). Prior to this study, PCV2 had been identified by in situ hybridization (in two unthrifty pigs which were culled), and confirmed by high levels of maternally derived antibodies in young animals and seroconversion to PCV2 at 18 weeks of age.

At 3 weeks of age, the pigs were randomly allocated to two equivalent groups of 750 animals each, according to gender and weight. The treatment group received a single $2 \mathrm{ml}$ dose of Porcilis ${ }^{\circledR} \mathrm{PCV}$; the control group was left unvaccinated. In the prefattening period, the groups were kept in separate barns, and during the fattening period they were housed in two twin barns, each holding 750 animals. Handling, feeding and treatment procedures were similar for both groups. Blood samples taken from 10 animals of each group at 5, 7, 10, 14, 18 and 26 weeks of age, were assessed for IgG and IgM (Ingezim PCV Elisa).

\section{Results}

Serology shows that Porcilis ${ }^{\circledR}$ PCV vaccination at 3 weeks old,

Graph 1. IgG/M values. Control group

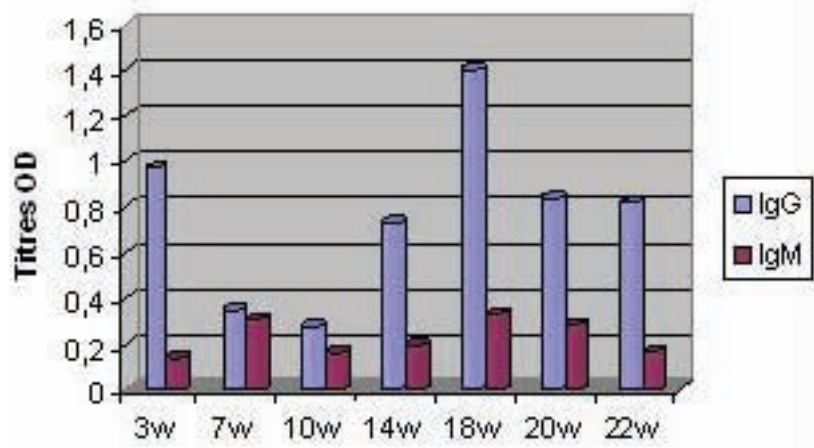

Positive result: $\lg G$ index $>0.461$ and $\lg M$ index $>0.558$ even in the presence of medium-high levels of MDA, led to significant increases in mean IgG and IgM values, which persisted to 14 weeks old, before decreasing. The unvaccinated pigs had lost their IgG titers (MDA) at 7 weeks of age and continued with low levels until seroconversion in week 18 (Graphs 1\&2). This indicates that MDA did not interfere with the vaccine take.

Animals vaccinated with Porcilis ${ }^{\oplus} \mathrm{PCV}$ also showed improvements in various production parameters, including optimal end weight and better carcass yield at slaughter.

Table 1: Production results

\begin{tabular}{|l|c|c|}
\hline & Vaccinated & Controls \\
\hline$\%$ Mortality & 1.73 & 2.40 \\
\hline$\%$ culled pigs due to PCVAD & 0 & 0.93 \\
\hline ADG (grams/day) & 793 & 766 \\
\hline Feed Conversion rate & 2.73 & 2.8 \\
\hline Treatment costs/pig & $1.77 €$ & $2.01 €$ \\
\hline Carcass yield & 81.25 & 80.88 \\
\hline$\%$ Pigs out of range & 3.33 & 6.67 \\
\hline
\end{tabular}

The Return on investment (ROI) by using Porcilis ${ }^{\circledR}$ PCV was $3,19 €$.

\section{Discussion}

Pigs showed clear evidence of seroconversion after Porcilis ${ }^{\circledR} \mathrm{PCV}$ vaccination, even in the presence of high levels of maternally derived antibodies.

Vaccination with Porcilis ${ }^{\circledR} \mathrm{PCV}$ has been proven to be effective even with late onset and mild, subclinical infections, thus contributing to reduced production losses caused by PCV2.
Graph 2. $\lg G / M$ values. Vaccinated group

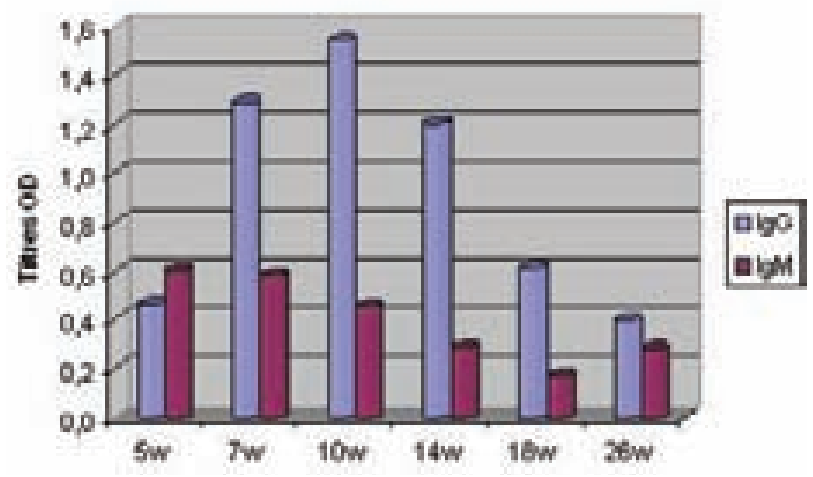

Positive result: $\operatorname{lgG}$ index $>0.455$ and $\lg M$ index $>0.494$ 


\title{
P.097
}

\section{Field trial of Circumvent ${ }^{\mathrm{TM}} \mathrm{PCV}$ in South Korea}

\author{
Yeong H. Kim ${ }^{2}$ Myunghyee Kim ${ }^{3}$ J H. Pi ${ }^{4}$ J H. Han ${ }^{2}$ Alexander A. Eggen ${ }^{1}$ \\ 1. Intervet/Schering-Plough, Boxmeer, Netherlands; 2. School of Veterinary Medicine and Institute of Veterinary Science, Kangwon \\ National University, Chuncheon, Korea; 3. Intevet Schering-Plough Korea, Seoul, Korea; 4. Greeen Farming Corporation, Seoul, Korea
}

\section{Introduction}

Porcine circovirus type2 (PCV2) is the primary causative agent of porcine circovirus-associated disease (PCVAD) PCVAD has been reported with increasing frequency in swine herds. It is difficult to recognize and diagnose because the exact pathogenic mechanisms responsible for the disease are still under review. Nevertheless, immune suppression is considered to be a key factor. Clinical signs of pigs with PDNS include weight loss/ unthriftiness, dyspnea, enlarged lymph nodes, diarrhea, pallor and jaundice, with an increased mortality rate. However, the introduction of PCV2 vaccines is contributing to the reduction of the incidence of PMWS and its associated diseases. The purpose of this study was to further investigate the effects on PCVAD of CIRCUMVENTTM PCV on commercial Korean farms testing positive for PCV2.

\section{Materials and Methods}

159 piglets from 3 different farms with a history of PCVD (Farms $A, B$, and $C$ located in Hongsung-gun, Choongnam province, Korea), were included in this study, and divided into 2 groups, vaccinated and placebo. Piglets in the vaccinated group received CIRCUMVENTTM PCV (Intervet Schering-Plough) IM at 3 and 6 weeks of age. The placebo-treated controls were treated in a similar manner but with saline instead of vaccine.

All the pigs were weighed, blood samples were taken, and nasal and fecal swabs collected at 3, 6, 9, 13, 16 and 24 weeks of age. The mortality was recorded and the rate was calculated for each group. Sera were analyzed to measure the immune response using SERELISA PCV2 Ab Mono Blocking (Synbiotics, France). The PCV2 viral load was quantified by TaqMan real time PCR. Lung lesions were assessed according to Pointon et al (1982) at moment of slaughter.

Statistical analysis was by student t-test. Wilcoxon Mann-Whitney test was used to assess differences with respect to ADG, serology and lung lesions. The ${ }^{\circ} \mathrm{C} 2$-test and Fisher's exact test were used to investigate possible differences in PCV2 viremia and mortality. Statistical analyses were performed using SAS software release 8.2

\section{Results}

Average daily weight gain (ADG) was considered to be an objective measurement by which to determine the severity of PCVAD and the effect of vaccination in a large number of animals. The vaccinated group had significantly better ADG than the placebo group either at entry to the finishing phase or at the midpoint of the finishing phase, or both.

The mortality rate in the vaccinated group was significantly lower during the finishing phase, compared with placebo group.

Pigs on all the farms had high levels of maternally derived antibodies against PCV2. The vaccinal antibody response in the vaccinated group continued at high levels until 16 weeks of age on Farm A and 24 weeks of age on Farms B and C. However, field virus antibodies in the placebo group were confirmed at 13 or 24 weeks of age. Interestingly, at 24 weeks of age, there was a lower antibody response in the vaccinated group than in the placebo group.

In the real time PCR, PCV2-positive animals reached a peak at approximately 10-16 weeks of age in the placebo group. In the vaccinated group, the level of positive animals decreased more dramatically than in the placebo group. Animals with high viral loads (106 PCV2 genome/ml in serum) were observed in the acute phase at 10 to 16 weeks of age. Compared to the placebo group the proportion of PCV2-positive animals in the vaccinated group was significantly lower (FarmA: $P=0.0021$; Farm $B$ : $\mathrm{P}<0.0001$; Farm C: $\mathrm{P}<0.0001$ ).

There were no significant differences between the groups overall in respect of lung lesions, but on Farm $C$, the vaccinated group had significantly lower lung lesion scores than the placebo group.

\section{Discussion}

Based on these results, vaccination with CIRCUMVENTTM PCV was shown to reduce economic losses from PCV2 infection. This appears to be the first report of the efficacy of vaccination against PCV2 on commercial Korean farms.

\section{Reference}

1. Horlen KP., et al. 2008. JAVMA, 232, 906-912. 


\title{
P.098
}

\section{Effect of a single dose of Porcilis ${ }^{\circledR}$ PCV at three weeks of age on mortality caused by Porcine Circovirus type 2}

\author{
Stefan von Rüden ${ }^{1}$ Ralf Werner ${ }^{1}$ Patricia Roesner ${ }^{2}$ Thomas Noé $^{1}$ Alex Eggen ${ }^{3}$ Hans Holtslag $^{3}$ Jos Smeets $^{3}$ \\ 1. Intervet Schering Plough Animal Health, Unterschleissheim, Germany; 2. Tierzucht Nordhausen GmbH Co \\ KG, Nordhausen, Germany; 3. Intervet Schering-Plough Animal Health, Boxmeer, Netherlands
}

\section{Introduction}

Porcine circovirus type 2 (PCV2) is associated with several swine diseases such as PMWS (Post weaning Multisystemic Wasting Syndrome), PDNS (Porcine Dermatitis and Nephropathy Syndrome), and reproductive and intestinal failures.

The object of this study was to demonstrate a reduction in mortality in a herd affected by PCV2 virus circulation, using a single $2 \mathrm{ml}$ dose of Porcilis ${ }^{\circledR}$ PCV (Intervet/Schering-Plough Animal Health), mainly by reducing the number of PDNS cases.

\section{Materials and Methods}

The study was carried out on a large German pig herd $(6,000$ sows and 29,000 finishers) with a high PCV2-related mortality rate during the finishing phase (beginning around the 55th day of finishing). The presence of PCV2 was confirmed by histological and serological examination.

The farm operated three separate production lines. To evaluate the effect of Porcilis ${ }^{\circledR}$ PCV on mortality, the piglets from one of these lines were vaccinated with a single $2 \mathrm{ml}$ dose of Porcilis ${ }^{\circledR}$ PCV before weaning at three weeks of age. On the same day, the piglets were also vaccinated with Porcilis ${ }^{\circledR}$ PRRS intra-dermally with IDAL equipment (Intra Dermal Application of Liquids).

During the nursery and finishing phases, mortality and other disease data were monitored for every batch according to the farm's usual routine. Each nursery unit could accommodate approximately 660 animals and each finishing unit, approximately 550 animals. The data were collected over three periods:

1) The pre-study period: no PCV2 vaccination (March - October 2007)

2) The study period: only batches of piglets from production line 1 were vaccinated with Porcilis ${ }^{\circledR}$ PCV. The piglets from production lines 2 and 3 served as controls (October - December 2007).

3) The post-study period: piglets of all three production lines were vaccinated with Porcilis ${ }^{\circledR}$ PCV (December 2007 - February 2008).

To determine the effect of herd vaccination on the incidence of PDNS, the data from the pre- and post-study periods were compared, the pre-study period serving as historical control. The total mortality data per production line were analyzed for variance (ANOVA) with production line as the independent variable. The PCV2-related mortality data were analyzed using the one-sample T-test.

\section{Results}

During the study period, 43 batches of piglets were included in the trial ( 27 nursery batches and 16 finishing batches). In all, 5,796 vaccinated animals were involved in the batches used for the analysis. The total mortality during the nursery phase was not significantly different (ANOVA: P-value 0.1078) between the vaccinated line $1(0.96 \%)$ and control lines 2 and $3(0.68 \%$ and $1.34 \%$ respectively), and no PCV2-related mortality was recorded.

However, during the finishing phase of the study period, the total mortality was significantly lower (ANOVA; P-value 0.0004) in the vaccinated line $1(1.66 \%)$ than in the unvaccinated line 3 (3.93\%). In production line 3, the PCV2-related mortality was $1.22 \%$, while $0.04 \%$ PCV2-related mortality with respect to PDNS was recorded in the vaccinated line 1 (ANOVA; P-value 0.0012).

A total of 21,703 vaccinated animals were included in the batches used for the historical comparison. This analysis showed that the average total mortality was reduced from $4.77 \%$ in the prestudy period to $1.61 \%$ in the post-study period when all three production lines were vaccinated. The PCV2-related mortality decreased from $2.22 \%$ (pre-study) to $0.04 \%$ (post-study).

\section{Discussion}

The efficacy of single-dose vaccination at 3 weeks of age was clearly demonstrated by the significant reduction in total mortality by more than $50 \%$ during the whole finishing phase: $1.66 \%$ in the vaccinated line against 3.93\% in the controls. The reduction in PCV2-related mortality was even clearer in the comparison between the vaccinated post-study period (1.61\%) and control pre-study period (4.82\%).

It can be concluded that there was a highly significant decrease in PDNS losses in the finishing phase after PCV2 vaccination with Porcilis ${ }^{\circledR}$ PCV. In the control group however, signs related to PDNS were evident in the majority of the pigs which died.

The economic benefits due to a single $2 \mathrm{ml}$ dose of Porcilis ${ }^{\circledR}$ PCV realized in this trial were considerable: $3.22 €$ (study period) and $4.74 €$ (pre-study versus post-study period) per fattening pig. 


\title{
P.099
}

\section{Economic impact of CIRCOVAC $^{\circledast}$ in a PCV2 subclinically infected breeding farm in Switzerland}

\author{
Xaver Sidler ${ }^{1}$ Jeremias Kurmann ${ }^{1}$ Esther Buergi ${ }^{1}$ Enrico Brugnera' ${ }^{1} 2$ Titus Sydler $^{2}$ \\ 1. Division of Farm Animals, University Zurich, Vetsuisse Faculty, Zurich, Switzerland; 2. Institute of \\ Veterinary Pathology, University Zurich, Vetsuisse Faculty, Zurich, Switzerland
}

\section{Introduction}

Postweaning Multisystemic Wasting Syndrome (PMWS) in pigs became worldwide one of the most economical important diseases. In Europe annual losses associated with PMWS amount to 500 to 900 million Euros (Armstrong and Bishop, 2004).

The objective of this study was to investigate the efficacy of the vaccine Circovac ${ }^{\circledR}$ Merial SA Lyon in a small subclinically infected herd under field conditions and to test the effect on reproductive parameters, daily weight gain, days to slaughter, mortality rate and farm economics under Swiss commercial conditions.

\section{Materials and Methods}

This study was conducted in a small breeding herd with 90 sows in 2007. Piglets were sold every week to a regional finisher at the age of approximately ten weeks and with a body weight of 23 to $27 \mathrm{~kg}$. In 2006 only sporadic cases of PMWS had occurred on the breeding herd, but there had been considerable losses in the finishing farm

Randomly sows were chosen either for vaccination or were injected with $\mathrm{Circovac}^{\circledR}$ adjuvans as control group. In the vaccinated group the sows were vaccinated with $2 \mathrm{ml} \mathrm{Circovac}^{\circledR}$ two and four weeks before artificial insemination and in the 12th week of gestation. Parity number, litter weight, number of live and dead piglets, number of mummies, number of piglets weighing below $1 \mathrm{~kg}$, piglet losses during the nursing period and the cause of piglet death were recorded for each sow. The carcass weight was considered to represent $78 \%$ of the live weight at slaughter and was used to calculate the average daily weight gain (AWDG, $\mathrm{g} / \mathrm{d}$ ). A feed price of $0.43 € / \mathrm{kg}$, a feed conversion rate (FCR) of $1.7 \mathrm{~kg}$ feed $/ \mathrm{kg}$ body weight, a price for slaughtered pigs of 2.87 $€ / \mathrm{kg}$ and a price of $3.47 € / \mathrm{kg}$ per piglet at $25 \mathrm{~kg}$ were taken as a basis for the calculation (average Swiss market prices from 2006 - 2008). The vaccination costs were calculated on a base of $7.00 €$ a dose, 2.3 parities/year and $33 \%$ replacements of young sows.

\section{Results}

There were no significant differences of litter size, litter weight, number of live and dead piglets, and number of mummies, number of piglets weighing below $1 \mathrm{~kg}$ and number of lost piglets during the nursing period between offspring of vaccinated and non-vaccinated sows.
Table 1: Average daily weight gain (ADWG)

\begin{tabular}{|l|c|c|}
\hline & Vac. sows & Non-vac. sows \\
\hline ADWG g/d (birth-105kg) & $626 \pm 10^{*}$ & $593 \pm 10$ \\
\hline ADWG g/d (25 -105 kg) & $786 \pm 20^{*}$ & $734 \pm 20$ \\
\hline$\emptyset$ Slaughterage (d) & $168 \pm 2^{*}$ & $177 \pm 2$ \\
\hline
\end{tabular}

However there were significant differences $\left({ }^{*} p \leq 0.05\right)$ in ADWG between offspring of vaccinated and non-vaccinated sows. The feed costs in the vaccinated group were $4.10 € /$ pig lower than in the non-vaccinated group respectively $9254 €$ for all produced piglets in this farm in 2007.

Table 2: Mortality rate before and after vaccination (2007)

\begin{tabular}{|l|c|c|}
\hline & Before vaccination & During vaccination \\
\hline Weaned piglets/sow & 25.3 & 27.3 \\
\hline Weaned piglets/year & 2277 & 2457 \\
\hline Mortality (weaning-25 kg) & $\mathrm{n}=73(3.2 \%)$ & $\mathrm{n}=75(3.0 \%)$ \\
\hline Mortality $(25-105 \mathrm{~kg})$ & $\mathrm{n}=134(6.1 \%)$ & $\mathrm{n}=53(2.2 \%)$ \\
\hline ØSlaughterage $(\mathrm{d})$ & 182 & 168 \\
\hline
\end{tabular}

Moreover, during the vaccination period the mortality rate dropped significantly from $6.1 \%$ (before vaccination) to $2.2 \%$ in the fattening farm and after weaning from $3.2 \%$ to $3.0 \%$ (Table 2 ). The higher mortality rate corresponded to losses of about $25^{`} 664 €$.

\section{Discussion}

The lower mortality rate and feed costs of the piglets of the vaccinated sows were producing an economical benefit about $34^{`} 918 €$ even in a subclinically infected breeding farm. The investment for vaccination in 2007 amounts to $3360 €$. Hence it resulted a Return on Investment of (ROI) on 1: 10.4.

\section{References}

Armstrong G. and Bishop S. (2004). 18th IPVS Congress, Hamburg, Germany, 2004, 809 


\title{
P.100
}

\section{The effect on PCV2 viremia in pigs vaccinated with a single dose of Porcilis $\mathrm{PCV}^{\circledR}$}

\author{
Paolo Martelli ${ }^{1}$ Paolo Bonilauri ${ }^{2}$ Stefano Gozio ${ }^{3}$ Alberto E. Cevidalli ${ }^{4}$ Stefano Guazzetti ${ }^{5}$ Antonio Caleffi ${ }^{6}$ Paolo Borghetti ${ }^{1}$ \\ 1. Dept. Animal Health - University of Parma, Parma, Italy; 2. IZSLER, Reggio Emilia, Italy; 3. Intervet/Schering-Plough A.H., Boxmeer, \\ Netherlands; 4. Intervet/Schering-Plough A.H., Milan, Italy; 5. ASL, Reggio Emilia, Italy; 6. Veterinary Practitioner, Mantua, Italy
}

\section{Introduction}

PCV2 vaccines have been demonstrated to reduce losses (mortality and culling rates), and significantly improve the average daily gain, while reducing the frequency of co-infections. Moreover, they are claimed to reduce virus load and viral-induced lymphoid lesions.

This study was aimed at investigating the effect of a single dose of a PCV2 subunit vaccine administered at weaning, by measuring the proportion of viremic animals and the PCV2 virus load in two herds suffering from PCVD in pigs older than 15 weeks of age.

\section{Materials and Methods}

A double-blinded, randomised and controlled field trial was conducted on two farms with a history of PMWS in pigs older than 15 weeks (Farm 1: 900-sow farrow-to-finish herd and Farm 2: 3-site 1850 sows herd).

A total of 818 piglets (males and females) was enrolled in the study and distributed between two treatment groups. At inclusion (weaning - $21 \pm 3$ days of age), a total of 408 animals (Group B) received a $2 \mathrm{ml}$ intramuscular dose of Porcilis PCV ${ }^{\circledR}$, a commercial PCV2 capsid-based subunit vaccine expressed in inactivated baculovirus. To act as controls (Group A), 410 pigs received $2 \mathrm{mls}$ of the adjuvant Diluvac Forte ${ }^{\circledR}$, by the same route.

On Farm 1, 22 piglets/group were bled at 3 weeks (inclusion / weaning /vaccination), and 4, 5, 6, 7, 9, 12, 15, 16, 17, 18, 19, 20, 22, 26 and 35 weeks of age. On Farm 2, blood samples were collected from 22 animals/group at 3 weeks of age (inclusion / weaning /vaccination), and at 4, 6, 12, 16, 18, 20, 22, 24, 26, and 35 weeks of age.

Real-time quantitative PCR (qPCR) was performed according to Olvera et al. (2004), and the results expressed as the number of PCV2 genome copies/ml of serum.

To estimate the effect of vaccination on the probability of a pig becoming viremic, a mixed effect logistic regression model was constructed to take account of the non-independence of the repeated measurement of the same subjects and the effect of the sow (litter effect) using the " $R$ " software.

\section{Results}

The onset of PCV2 viremia was observed on both farms at 16-17 weeks of age in the control pigs and two weeks later in the vaccinated group. The proportion of PCV2-positive animals in the vaccinated group was statistically significantly lower than that of the controls $(p<0.001)$. Vaccinated pigs, when Q-PCR positive, never had a viral load higher than 106.
Figure 1: Proportion of viremic pigs in vaccinated and control animals in Farm 1 (upper graph) and Farm 2 (lower graph)
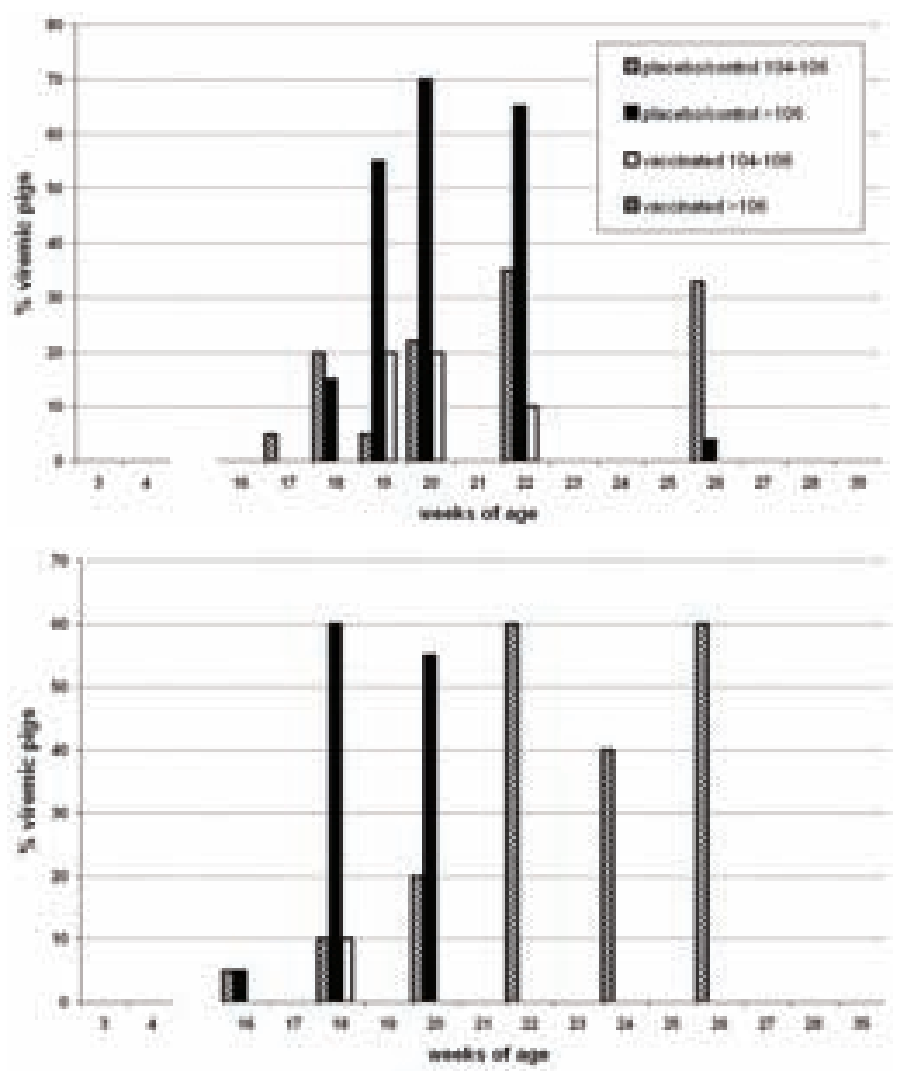

\section{Discussion}

The present study confirms that a single dose of Porcilis $\mathrm{PCV}^{\circledR}$ administered at 3 weeks of age significantly reduces the proportion of viremic pigs and the viral load.

These reductions were associated with an absence of clinical signs, improved daily gain and reduced mortality and are correlated with an increase in the antibody response.

\section{References}

Segalés et al. (2005).Anim Health Res Rev 6, pp. 119-142

Olvera et al. (2004) J Virol Methods 117 (2004), pp. 75-80 


\title{
P.101
}

\section{Immune response to PCV2 in conventional pigs after vaccination with a single dose of Porcilis PCV ${ }^{\circledR}$ and exposure to PCV field infection}

\author{
Paolo Borghetti ${ }^{1}$ Luca Ferrari ${ }^{1}$ Elena De Angelis ${ }^{1}$ Stefano Gozio ${ }^{2}$ Stefano Guazzetti ${ }^{3}$ Paolo Martelli ${ }^{1}$ \\ 1. Dept. Animal Health - University of Parma, Parma, Italy; 2. Intervet/Schering-Plough A.H., Boxmeer, Netherlands; 3. ASL, Reggio Emilia, Italy
}

\section{Introduction}

Under field conditions, PCV2 vaccines improve performance, reducing mortality, clinical signs, the frequency of co-infections, viral load and viral-induced specific lymphoid lesions. Some studies indicate that these effects are related to total and neutralising antibody responses, as well as to cell-mediated immunity (Fort et al., 2009). The present study was aimed at investigating the antibody response and the PCV2 IFN-g secretion (by ELISpot) after vaccination and exposure to a natural infection, under field conditions.

\section{Materials \& Methods}

A double-blinded, randomised and controlled field trial was conducted in a 900 farrow-to-finish sow herd with a history of PMWS in pigs older than 15 weeks. A total of 411 piglets (males and females) were enrolled in the study. At inclusion (weaning $-21 \pm 3$ days of age) 205 pigs (vaccinated group) received a $2 \mathrm{ml}$ intramuscular dose of Porcilis PCV ${ }^{\circledR}$. To act as controls, 206 pigs (placebo/control group) were injected with $2 \mathrm{mls}$ of Diluvac Forte ${ }^{\circledR}$. Thirty randomly selected piglets (20 controls and 10 vaccinated) were bled at 3 (inclusion /weaning /vaccination), and $4,5,6,7,9,12,15,16,17,18,19,20,22,26$ and 35 weeks of age. The sera were tested with a blocking ELISA for the detection of PCV2-specific antibodies in porcine sera, and the titers expressed as log2. The levels of IFN- $\gamma$ secreting cells (SC) in the peripheral blood of pigs were determined according to Martelli et al. (2009). The in vitro recall response was stimulated by the addition of a wild PCV2 strain (I12/11) solution. A mixed effects regression model was fitted to take into account the hyerarchical structure of the observations, using the "nlme" package within the R.

\section{Results}

Vaccination elicited a rapid increase of antibodies with a tripling of titres within 6 weeks, even in pigs with the highest levels of maternally derived antibodies (MDA) at the time of vaccination. In the controls, passively acquired antibodies gradually declined, disappearing altogether by 12 weeks of age. Three weeks postvaccination the number of IFN-g SC peaked with an average of 120 cells. At 16-17 weeks of age, when the PCV2 field infection occurred, the antibody levels increased in both groups. In the controls, a marked IFN-g secretion was detectable (190-240 cells) within 3 weeks of the field infection. Conversely, the infection stimulated only an erratic course of IFN-g SC response in the vaccinated, clinically protected animals.
Figure 1: Plot of geometric mean of ELISA titers in vaccinated and control pigs.

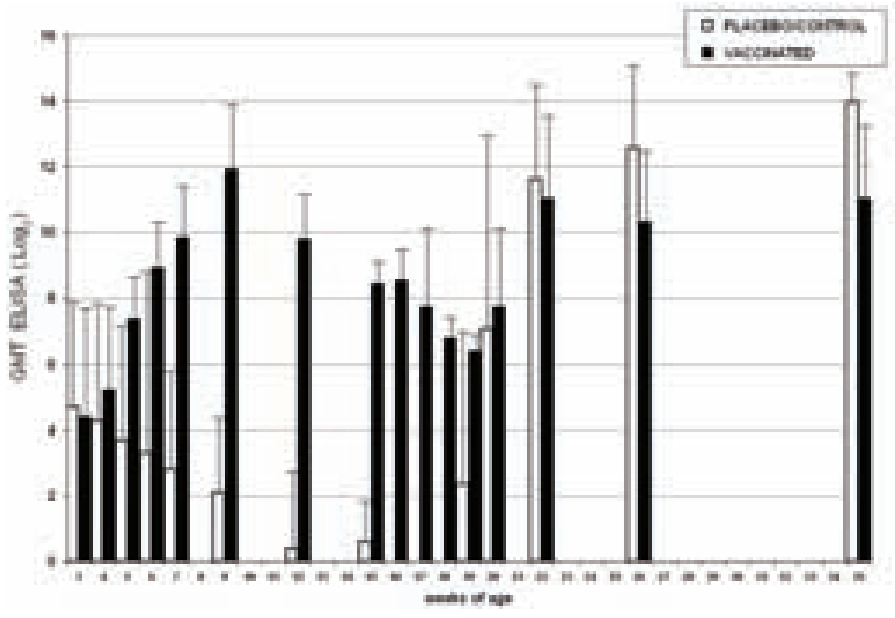

Figure 2: Plot of the number of IFN- $\gamma$ SC/106 PBMC after vaccination and after infection.

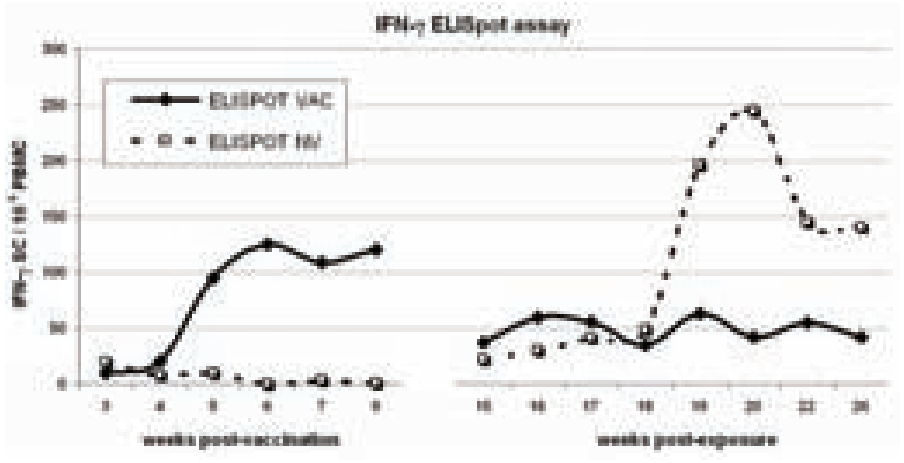

Discussion

After vaccination, there was a rapid and remarkable PCV2-specific IFN-g secretion, as well as a significant increase in antibodies. After infection high levels of antibodies in the vaccinated animals were associated with a reduced or absent PCV2 viremia, and a lack of specific clinical signs. Comparing our results with those of Fort et al. (2009) in experimentally challenged pigs, it could be proposed that the intensity of IFN-g secretion depends on the in vitro stimulation (in this case, whole PCV2), as well as on the type of isolate, viral load and replication in vivo.

\section{References}

Martelli et al. 2009. Vaccine, 27, 3788-3799.

Fort et al., 2009. Vaccine, 27, 4031-4037 


\title{
P.102
}

\section{Serology and safety of the simultaneous use of Porcilis ${ }^{\circledast}$ PCV and $M+\mathrm{PAC}^{\circledast}$ in the field}

\author{
Josep Farreres ${ }^{2}$ Daniel Puig² Rut Menjon¹ Jesus M. Bollo Jesus V. Lopez ${ }^{1}$ Marta Jimenez ${ }^{1}$ \\ 1. Intervet Schering Plough Animal Health, Alcobendas (Madrid), Spain; 2. Cooperativa de Bellcaire, Bellcaire d'Urgell, Lleida, Spain
}

\section{Introduction}

Porcine Circovirus Type 2 (PCV2) and M.hyopneumoniae vaccines are probably the most frequently used in the pig industry all over the world. As both vaccines are very often given at weaning, their simultaneous use would simplify herd management and improve animal welfare. The simultaneous administration of various commercial products has previously been shown to be effective (1). The object of this trial was to demonstrate that the

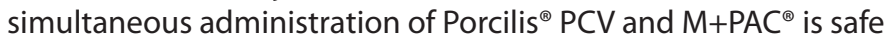
and efficacious in terms of serological response.

\section{Materials and Methods}

The trial was performed on a 250-sow farrow-to-finish herd in north-east Spain, in which piglets had been routinely vaccinated with another PCV2 vaccine at 4 weeks of age, but had not been vaccinated against M.hyopneumoniae.

Porcilis ${ }^{\circledR} \mathrm{PCV}$ is a subunit vaccine containing the viral capsular protein coded by the ORF2 of the PCV2 genome adjuvanted in $\mathrm{X}$-Solve ${ }^{\circledR} . \mathrm{M}+\mathrm{PAC}^{\circledR}$ is an inactivated M.hyopneumoniae vaccine in Emunade $^{\circledR}$, an oil-in-water dual-action adjuvant.

A total of 397 four-week old piglets were allocated to two experimental groups:

Group 1: 197 piglets, vaccinated with a mixture of $2 \mathrm{ml}$ Porcilis ${ }^{\circledR} \mathrm{PCV}$ and $2 \mathrm{ml} \mathrm{M}+\mathrm{PAC}^{\circledR}$ injected in a single site on the left side of the neck.

Group 2: 200 piglets were vaccinated with $2 \mathrm{ml}$ Porcilis ${ }^{\circledR}$ PCV and $2 \mathrm{ml} \mathrm{M}+\mathrm{PAC}^{\circledast}$ in separate sites on either side of the neck.

All animals were identified individually by ear tag, and 10 animals of each group were bled at 4, 7 and 10 weeks of age.

All the pigs were monitored for signs of local or systemic reactions.

The immune response to Porcilis ${ }^{\circledR}$ PCV was evaluated comparing the PCV2 IgG and IgM titers of each group using Ingezim ${ }^{\circledR}$ PCVELISA (Ingenasa, Madrid, Spain).

Levene test was used for the comparison of variances and MannWhitney U-test for the comparison of means.

\section{Results}

No local or systemic reactions were observed in any of the animals of Group 1 (mixed vaccination). One piglet of Group 2 (separate vaccinations) exhibited a transient systemic reaction which rapidly disappeared without any remedial action needed.

Graph 1 shows the PCV2 serology. There were no statistically significant differences between groups either for $\lg M$ or $\lg G$ at any age $(p>0.1)$.

Graph 1. PCV2 IgG and IgM seroconversion

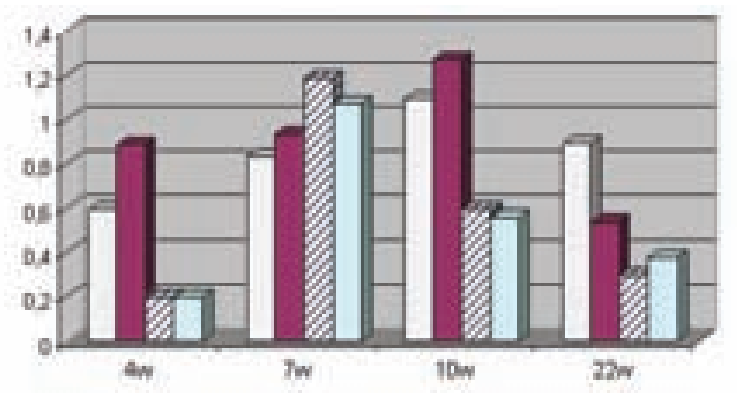

ELISA Ingezim PCV IgG index $>0.520$ and IgM index $>0.66$ - positive result

No clinical signs of PCV2 or M.hyopneumoniae infection were detected in any treatment group.

\section{Discussion}

This trial has demonstrated the compatibility of Porcilis ${ }^{\circledR}$ PCV and $M+P A C^{\circledR}$ in terms of safety and the immune response against PCV2 lgG and IgM, even in the presence of maternally derived antibodies.

Although further studies will be needed to confirm efficacy in the field, these data suggest a way herd vaccination strategies might be simplified and animal welfare improved.

\section{References}

1. Taneno, A (2008). Proc 20th IPVS Durban. 


\title{
P.103
}

\section{Safety and efficacy of the simultaneous administration of Porcilis ${ }^{\circledR}$ PCV and Porcilis ${ }^{\circledR}$ MHyo under field conditions}

\author{
Josep Farreres ${ }^{2}$ Daniel Puig² Rut Menjon¹ Jesus M. Bollo Jesus V. Lopez ${ }^{1}$ Marta Jimenez ${ }^{1}$ \\ 1. Intervet Schering Plough Animal Health, Alcobendas (Madrid), Spain; 2. Cooperativa de Bellcaire, Bellcaire d'Urgell, Lleida, Spain
}

\section{Introduction}

M.hyopneumoniae and PCV2 infections are widespread in pig populations, which are frequently vaccinated against both pathogens. However, as these vaccines are commonly administered at weaning, it is important that they do not interfere with each other. The efficacy of the simultaneous administration of Porcilis ${ }^{\circledR} \mathrm{PCV}$ and Porcilis ${ }^{\circledR} \mathrm{MHyo}$ has been previously demonstrated under laboratory conditions (1). The aim of this study was to confirm the same under field conditions.

\section{Materials and Methods}

The trial was performed in a 250-sow farrow-to-finish herd in north-east Spain, in which piglets had been routinely vaccinated against PCV2 at 4 weeks of age, but not against M.hyopneumoniae.

The trial vaccines were Porcilis ${ }^{\circledR}$ MHyo (a 2-dose inactivated vaccine against $M$.hyopneumoniae with dl-a-tocopherol acetate as adjuvant) and Porcilis ${ }^{\circledR}$ PCV, a subunit vaccine containing the viral capsular protein coded by the ORF2 of the PCV2 genome, adjuvanted in dl- a-tocopherol acetate and paraffin.

All the trial pigs received a $2 \mathrm{ml}$ priming dose of Porcilis ${ }^{\circledR} \mathrm{MHyo}$ at 1 week of age. At 4 weeks old, 515 piglets were allocated to one of two experimental groups:

Group 1: 325 piglets were vaccinated with a mixture of $2 \mathrm{ml}$ Porcilis ${ }^{\circledR} \mathrm{PCV}$ and $2 \mathrm{ml}$ Porcilis ${ }^{\circledR} \mathrm{MHyo}$ injected in a single site on the left of the neck. Group 2: 190 piglets were vaccinated with $2 \mathrm{ml}$ Porcilis ${ }^{\oplus} \mathrm{PCV}$ and $2 \mathrm{ml}$ Porcilis MHyo in separate sites on either side of the neck.

All animals were individually identified by ear tag, and 10 of each group were bled at 4, 7 and 10 weeks of age. All the pigs were examined post mortem, at slaughter.

Safety was assessed by monitoring the piglets for any local or systemic reaction.

All sera were examined for PCV2 IgM and IgG antibodies (Ingezim PCV-ELISA), and the antibody response of the groups were compared.

The efficacy of Porcilis ${ }^{\circledR} \mathrm{MHyo}$ was assessed by scoring the severity (0-5) of any post mortem lung lesions typical of enzootic pneumonia. Lung lesion scores were compared with those observed in contemporary, but unvaccinated, animals from the same farm. For practical reasons not all piglets vaccinated were scored at the slaughterhouse. Statistical analysis was performed using the Levene test, Mann-Whitney U-test and Pearson's chisquare test.

\section{Results}

No local or systemic reactions were observed in any of the animals of Group 1. Five piglets of Group 2 showed a transient systemic reaction that rapidly disappeared without any remedial action needed.

Graph 1 shows the results of PCV2 serology. No significant difference was found in IgM response between the groups, at any age. Although there was a statistically significant difference in $\operatorname{lgG}$ response $(p=0.049)$ at 7 weeks of age, it had disappeared 3 weeks later. No clinical signs of PCV2 infection were detected in any treatment group.

\section{Graph 1. PCV2 IgG and IgM seroconversion}

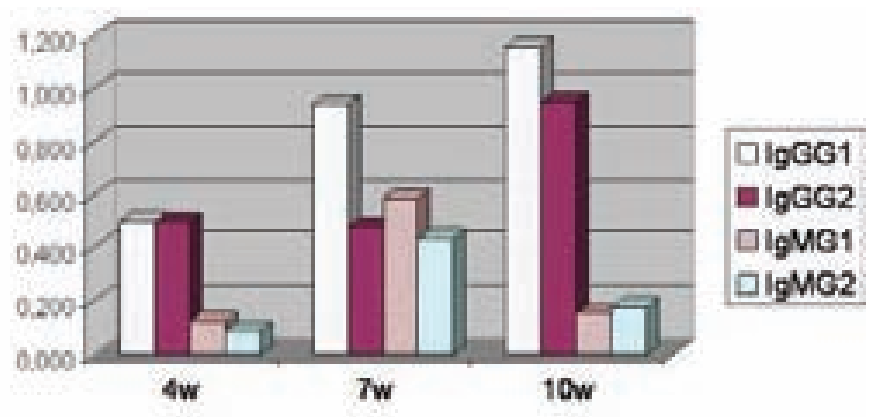

IgG index $>0.460$ and $\lg M$ index $>0.518$ - positive result

Lung lesion scores are summarized in Table 1. No significant differences were found between the two vaccinated groups, but the scores of both groups were significantly statistically different compared to the unvaccinated animals $(p<0.001)$.

Table 1. Lung lesions at the slaughterhouse:

\begin{tabular}{|c|c|c|c|}
\hline Lung Lesions & Unvaccinated & Group 1 (mixed) & Group 2 (separated) \\
\hline $\mathrm{N}^{0}$ animals observed & 254 & 92 & 99 \\
\hline$\%$ affected lungs & $72.57^{a}$ & $43.48^{b}$ & $38.38^{b}$ \\
\hline Average score & $1.11^{\mathrm{c}}$ & $0.66^{d}$ & $0.63^{d}$ \\
\hline
\end{tabular}

\section{Discussion}

The simultaneous use of Porcilis ${ }^{\circledR} \mathrm{PCV}$ and Porcilis ${ }^{\circledR} \mathrm{MHyo}$ has been shown to be safe and efficacious under field conditions, with no statistically significant differences between being mixed and injected in a single site or injected separately in two distinct sites, even in the presence of MDA. These results might be used to simplify herd vaccination strategies and improve animal welfare.

\section{References}

1. Taneno, A (2008). Proc 20th IPVS Durban 


\title{
P.104
}

\section{Efficacy of 3 PCV vaccines in a commercial farm situation}

\author{
John S. Richardson ${ }^{1}$ Mark E. White ${ }^{2}$ \\ 1. Intervet/Schering-Plough UK, Milton-Keynes, UK; 2. Haven Veterinary Group, Hedon, Hull, UK
}

\section{Introduction}

The use of vaccines to control diseases associated with the infection caused by Porcine Circovirus type 2 (PCV-AD) generally has resulted in success. In some farm situations however response to vaccination has been disappointing necessitating a change of PCV vaccine. This study reports such an occurrence.

\section{Background}

The farm has 300 sows, weaning occurs weekly at 4 weeks of age, progeny are finished on site at $100 \mathrm{~kg}$ live weight. Pigs are PRRS and M.hyo positive. In December 2006 wean - finish mortality was $15 \%$ when breeding stock vaccination commenced to control PCV2 infection in piglets; this resulted in wean-finish mortality reducing to $6 \%$ for 7 months to August 2007. Mortality then started to increase to $15 \%$, and whilst previously in pigs at 7-10 weeks of age was now seen later in pigs at 13-14 weeks of age. Piglet PCV vaccination was then instigated using a chimeric PCV1/PCV2 vaccine at 7-8 weeks of age due to concerns about interference from maternally derived antibody (MDA); mortality then decreased to $10 \%$. When sow vaccination had been stopped for more than 6 months, piglets were PCV vaccinated at 3 weeks of age. Mortality then increased to $15 \%$ during early 2009 , and then declined to $10 \%$ in early summer 2009 , before increasing dramatically to $28 \%$ in autumn 2009 despite antibiotic use. PCV-AD was confirmed via post mortem examination, histopathology, virus isolation and immunohistochemistry Porcilis ${ }^{\circledR}$ PCV vaccination at weaning commenced autumn 2009.

\section{Materials and Methods}

Two pigs were bled from each of 10 litters at approximately 3 - 4 weeks of age prior to vaccination with Porcilis ${ }^{\circledR} \mathrm{PCV}$, the same pigs were then sampled later at 8,12 and 15 weeks of age. The serum was analysed to detect antibodies against PCV2 using an ELISA test (Boxmeer R\&D labs). Samples were also q-PCR tested for PCV virus at VLA Weybridge, as were samples from pigs vaccinated with the first chimeric PCV piglet vaccine. Pig mortality was recorded on a monthly basis.

\section{Results}

The following graph shows PCV2 antibody titers at 4 and 8 weeks of age after Porcilis PCV vaccination at 3 weeks of age, on a Log2 scale.

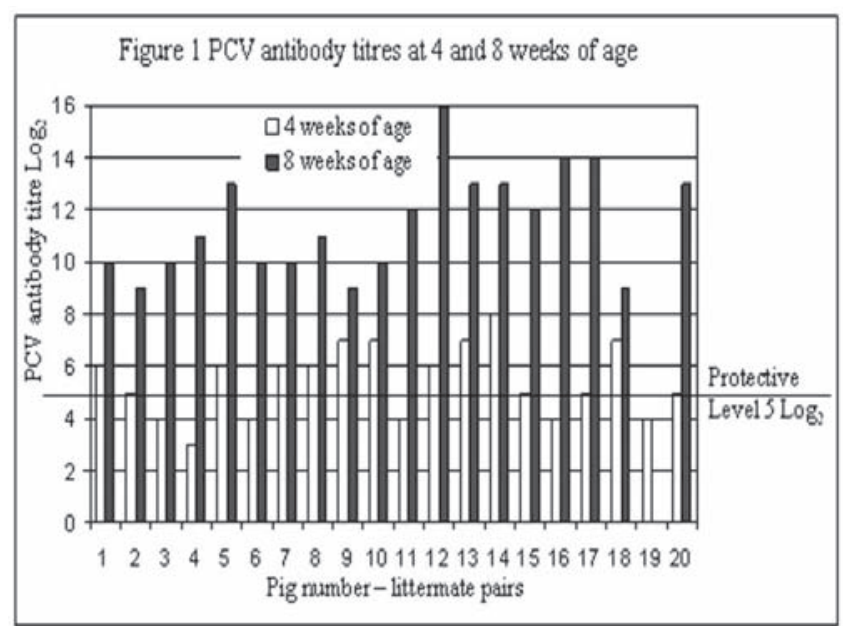

Littermate pairs showed similar PCV2 antibody titres. Response to Porcilis ${ }^{\circledR}$ PCV vaccination in pigs with medium levels of MDA showed a strong positive humoral response.

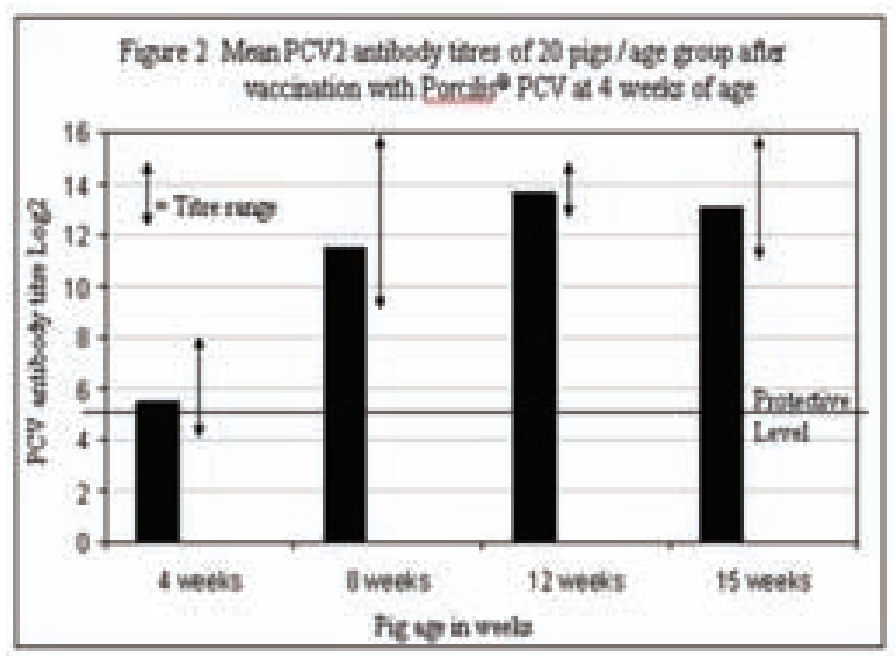

Antibody titres remained particularly high and uniform at 12 and 15 weeks, and all exceed the protective antibody titre level of 5 Log2 (Fort, Vaccine 2009) Significant challenge occured in pigs at 13 weeks of age on transfer to finishing; whilst q-PCR showed that all pigs that had been vaccinated with Porcilis ${ }^{\circledR}$ PCV were PCV virus free at 12 and 15 weeks of age, and clinical signs of PCV-AD were absent in these pigs. Pigs vaccinated with the initial piglet vaccine had $40 \%$ of pigs q-PCR positive for PCV virus associated with clinical signs of PCV-AD. Pig mortality has been reduced from $28 \%$ to $5 \%$ within 6 months, growth rate has increased by $14 \%$ and medication in finishing diets has been discontinued since commencing vaccination using Porcilis ${ }^{\circledR}$ PCV.

\section{References}

Fort, M.Vaccine 2009. 


\title{
P.105
}

\section{Shedding of Infectious Porcine Circovirus 2 (PCV2) Into Colostrum And Milk Of Vaccinated And Non-Vaccinated Naturally Infected Sows}

\author{
Priscilla Gerber; Flávia Garrôcho; Zélia Lobato \\ Universidade Federal de Minas Gerais, Belo Horizonte, MG, Brazil
}

\section{Introduction}

Porcine circovirus type 2 (PCV2) has been associated with swine diseases, collectively referred to as porcine circovirus diseases (PCVD). Epidemiological studies have demonstrated that clinical manifestations of PCVD can be influenced by non-infectious factors such passive immunity and maternal viremia in the peripartum [1]. Recently, the PCV2 DNA was detected in colostrum and milk [4].

The objective of this study was to evaluate the profile of antiPCV2 immunoglobulins and the presence of infectious PCV2 in colostrum and milk of vaccinated and non-vaccinated naturally infected sows.

\section{Materials and Methods}

Forty-one sows from 8 conventional farrow-to-finish herds were sampled, 21 non-vaccinated and 20 vaccinated with CIRCOVAC (Merial) at 30 and 15 days before parturition. Serum samples $(n=41)$ were collected on parturition day. Milk samples $(n=20)$ were collected 10 days post-partum.

Serum and colostrum were tested for total (TA) and neutralising (NA) anti-PCV2 antibodies as described elsewhere [3]. PCV2 isolation in lacteal samples was performed as previously described [2]. PCV2 titre was expressed in TCDI50/50 $\mu$ l. The results of the NA and TA was tested by IPMA and expressed as the average of $\log 2$ titre. Mann-Whitney test was used to analyse the difference in the antibody levels and the viral titres between the vaccinated and non-vaccinated groups. Differences were considered significant when $\mathrm{P}<0.05$.

\section{Results}

Infectious PCV2 was detected in 22/41 (53.6\%) of colostrums samples, and a difference was observed between vaccinated $(7 / 20,35 \%)$ and non-vaccinated sows (15/21, 74\%) $(P=0.02)$. Vaccinated sows excreted a minor amount of infectious PCV2 into colostrum, compared to in the non-vaccinated sows (100.50 \pm 081 vs. $101.63 \pm 1.20 ; P<0.01)$. PCV2 was isolated in 5/20 (25\%) of milk sampled. There was no correlation between the PCV2 titre in colostrum and milk samples or PCV2 titre and the level of anti-PCV2 immunoglobulins.

There was no difference between TA and NA titres in colostrum of vaccinated and non-vaccinated sows, although vaccinated sows had an higher serum NA titre compared to the non-vaccinated sows $(P=0.048)$.

\section{Discussion}

In this study, we observed that PCV2 is shed into milk from vaccinated and non-vaccinated naturally infected sows, even in the presence of high neutralising antibodies titres. Despite the fact that the vaccination of the sows did not prevent the PCV2 shedding in those secretions, it was capable of reducing PCV2 shedding into colostrum.

PCV2 was detected more frequently in samples of colostrum than milk. This difference may be related to PCV2 associated with monocytic lineage cells shedding (Park et al., 2009) that are present in a greater number in the colostrum. The association of PCV2 in the cellular fraction of milk may have an important role in the viral protection against the NA present in those secretions.

The importance of PCV2 shedding into colostrum in the early infection of piglets needs further investigation, especially because herds in which the piglets are infected earlier with PCV2 have a higher risk of developing PCVD. Also, it has previously been shown that sow PCV2 viremia was related to piglet mortality [1]. In conclusion, PCV2 can be shed into milk and colostrum of naturally infected sows even in the presence of high titres of neutralising antibodies. Vaccination of sows can reduce viral shedding in milk secretion, but cannot eliminate it.

\section{Acknowledgment}

This work was funded by Fapemig and CNPq (Brazil).

\section{References}

1. Calsamiglia, $M$, et al. 2007. Sow porcine circovirus type 2 (PCV2) status effect on litter mortality in postweaning multisystemic wasting syndrome (PMWS). Res Vet Sci 82:299-304.

2. Farnham, MW, et al. 2003. Isolation and caracterization of porcine circovirus type-2 from stillborn fetuses. Can J Vet Res, 67(2):108-13.

3. Fort, $M$, et al. 2008. Porcine circovirus type 2 (PCV2) vaccination of conventional pigs prevents viremia against PCV2 isolates of different genotypes and geographic origins. Vaccine 26:1063-71.

4. Park, JS; et al. 2009. Detection of porcine circovirus 2 in mammary and other tissues from experimentally infected sows. J Comp Pathol, 140:208-11. 


\title{
P.106
}

\section{PCV2 associated mummification and stillbirth in gilts}

\author{
Titus Sydler $^{2}$ Enrico Brugnera ${ }^{1,3}$ Monika Engels $^{2}$ Esther Buergi ${ }^{3}$ Xaver Sidler $^{3}$ \\ 1. Institute of Veterinary Pathology, Universitiy of Zurich, Vetsuisse Faculty, Zurich, Switzerland; 2. Institute of Virology, University of \\ Zurich, Vetsuisse Faculty, Zurich, Switzerland; 3. Division of Farm Animals, Universitiy of Zurich, Veterinary Faculty, Zurich, Switzerland
}

\section{Introduction}

Porcine circovirus type 2 (PCV2) is the causative agent for postweaning multisystemic wasting syndrome (PMWS) and is considered to be one of the most important viral pathogens of pigs in many countries. PCV2 has also been associated with additional diseases, referred to as PCV associated diseases (PCVAD) which include PMWS, PDNS, some types of pneumonia, enteritis and also reproductive failures (1). In Switzerland a PMWS epizooty commenced at the end of 2003 (2). However we observed cases of reproductive failures in Swiss sows for the first time in autumn 2008.

\section{Materials and Methods}

In April 2008 gilts originating from a jointly held mating and gestation facility (ringsystem) in 4 farrowing to wean farms showed raised numbers of mummified and stillborn piglets ( $\varnothing 0.6$ dead piglets and 2 mummies per litter whereas the pluriparous sows had $\varnothing 0.3$ stillborn piglets and 0.3 mummies). All gilts had been vaccinated twice against parvovirus and erysipelothrix before insemination.

In August we received tissue fluids to check for PCV2 from 2 mummies from two litters (farm A) which were negative for parvovirus by virological examination. Our quantitative PCR

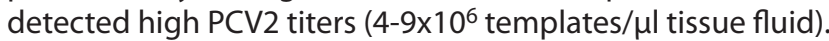
Subsequently we investigated 3 cases with mummies and/or stillborn piglets from 3 other farms (farms B, C, and D) belonging to the same ringsystem. Unfortunately, we did not receive further mummies from the first farm (farm A) but a 3 weeks old dead litter mate and blood of the two sows that produced the mummies mentioned above 3 weeks after gestation. Altogether we investigated 2 mummies ( 16 and $25 \mathrm{~cm}$ ) from farm B, a nonmummified fetus $(23 \mathrm{~cm})$ from farm $C$ and 4 mummified fetuses from farm $D(13,17,22,22 \mathrm{~cm})$ by necropsy, histology, virological methods for parvovirus detection, immunohistochemistry (IHC) for PCV2 (F217 monoclonal antibody (3)) and conventional PCR for PCV2. A quantitative PCR for PCV2 was achieved on the blood samples of the two gilts. We also performed electron microscopy on 2 mummies.

\section{Results}

All fetuses were negative for parvovirus or antibodies against parvovirus. The 3 weeks old littermate from farm A, as well as the non-mummified fetus from farm $\mathrm{C}$ were $\mathrm{IHC}$ negative for PCV2. The fetus showed no inflammatory reaction anywhere and the 3 weeks old littermate had died from an intestinal obstruction (invagination). The tissues of both mummies from farm B and 3 mummies out of 4 from farm $D$ were highly $\mathrm{IHC}$ positive for PCV2. However, the fourth mummy was IHC negative. In some cases the myocard was the most positive organ but also lymphatic tissues (lymph nodes and spleen) as well as liver, lung, kidney and lamina propria of the gut could be very highly positive, sometimes even more so than the heart. An inflammatory reaction could no longer be detected in the mummified tissues, but multifocal distrophic calcification in the myocard was a prominent change in 3 of the 5 PCV2 positive hearts. Interestingly, the electron microscopic preparation of mummified paraffin embedded tissues revealed paracristallinic arrays of icosahedral viral-like particles as described for PCV2 inclusions (4). These were at least still preserved in the liver of one of the investigated mummies. All positive fetuses harbored a PCV2b genotype. Only one of the two gilts mentioned from farm A was slightly viremic 3 weeks after gestation (500 templates/ $\mu$ l blood)

\section{Discussion}

Gilts only had raised numbers of mummies and stillborn piglets. The problem diminished half a year after but only vaccination against PCV2 of all sows finally solved the problem.

Mummies showed always high PCV2 IHC positivity and never moderate or low signals. Myocarditis was not seen due to the poor tissue conservation, however, distrophic calcification in the myocard was prominent. Well preserved viral particles were found by electron microscopy even in these mummified tissues. Interestingly, surviving littermates in affected litters developed normally.

The implication of PCV2 in reproductive failures has still to be estimated in Switzerland.

\section{References}

1) West et al. 1999. J Vet Diagn Invest 11:530-532.

2) Wiederkehr et al. 2004. Vet Microbiol 136: 27-35.

3) McNeilly et al. 2001. Arch Virol 146: 909-922.

4) Crother et al. 2003. J Virol 77: 12026-13041. 


\title{
P.107
}

\section{Retrospective field study of the evolution of growth performances after piglet vaccination against PCV2}

\author{
Pascal Fourchon $^{2}$ Aude Miorcec ${ }^{3}$ Vincent Auvigne ${ }^{1}$ Catherine Belloc ${ }^{3}$ \\ 1. Ekipaj, Pozuelo de Alarcón, Spain; 2. Coopagri Bretagne, Landerneau, France; 3. National College of Veterinary Medicine, Nantes, France
}

\section{Introduction}

In the mid nineties, when PMWS appeared in France, mortality in affected herds was frequently above $10 \%$ during the postweaning period. Fifteen years later, the disease caused by PCV2 looks very different. Post-weaning losses have decreased to normal range in most herds. Few pigs exhibit clinical or postmortem signs linked to PCV2 infection but poor growth rates resulting in high within-batch weight variability are frequent. Consequently many vets advise farmers to vaccinate piglets when growth heterogeneity is high even without clear evidence of PCV2 associated disease. In that context, we wanted to evaluate the impact of piglet vaccination against PCV2 on pig growth in a group of herds.

\section{Materials and Methods}

The study took place between March and August 2009 in 69 farrow-to-finish herds. For each herd we compared data from (i) three batches before and (ii) three batches after the implementation of vaccination. Piglets were vaccinated either with Circoflex ${ }^{\circledR}(1 \mathrm{ml})$, or Circovac ${ }^{\circledR},(0.5 \mathrm{ml})$. The criteria analysed were: Average Daily Gain from birth to slaughter (ADG), mortality rate from $30 \mathrm{~kg}$ to slaughter (MR), lean thickness (M2), percentage of pigs with carcass weight below $80 \mathrm{~kg}(\mathrm{CW}<80)$. For several reasons (quality of recording, batch mixing, other management change during the study) only 23 herds were included in the analysis and some criteria were not available for all herds. MannWithney test was used to compare the three batches before to the three batches after vaccination.

\section{Results}

In nearly half of the herds an increase, or a trend, of growth rate after vaccination was observed. This proportion was the same for both vaccination programs. In these herds, the average increase was $29 \mathrm{~g} /$ day and a positive impact on lean thickness was observed. The proportion of underweight pigs was also improved in six out of 15 herds. Nevertheless we didn't observe a decrease of mortality rate during the fattening period.
Table 1: Evolution of performances after vaccination (number of herds in each category)

\begin{tabular}{lccccc} 
& $\begin{array}{c}\text { Positive } \\
\text { evolution }\end{array}$ & Stable & \multicolumn{2}{c}{$\begin{array}{c}\text { Negative } \\
\text { evolution }\end{array}$} \\
\hline & Yes* & Trend** & & Trend** & Yes * \\
\hline ADG & 7 & 3 & 8 & 0 & 1 \\
\hline $\mathrm{M} 2$ & 6 & 4 & 7 & 2 & 0 \\
\hline $\mathrm{CW}<80$ & 3 & 3 & 8 & 1 & 0 \\
\hline $\mathrm{M} R$ & 0 & 1 & 11 & 1 & 1 \\
\hline$* \mathrm{p}=0.05$ & $* * \mathrm{p}=0.27$ & & &
\end{tabular}

\section{Discussion}

An improvement of technical performances after vaccination was observed only in approximately half of herds. This is probably related with the fact that most often PCV2 associated diseases had not been clearly diagnosed. Our study did not demonstrate an effect of vaccination on mortality rate. This can be linked to some difficulties to collect reliable data on mortality. Additionally, in France, mortality rate associated with PCV2 are currently less important than ten years ago.

Our study has some limitations. It did not compare contemporaneous batches. Consequently results are dependent on other changes in the farm during the study period such as management changes or sanitary events. Nevertheless data obtained here are complementary to those collected in field trials assessing simultaneously several treatments in the same herds. Other limit of our study is the number of herds that were finally included since 46 herds had to be removed because of lack of reliable data.

To conclude, our results suggest that circovirus vaccines can be of economical interest by improving technical performances. However a more accurate diagnosis of PCV2 infection leading to a more relevant vaccine indication could result in a higher success rate of vaccination. 


\title{
P.108
}

\section{Long term field observation using different PCV2 vaccines on a 2000 sow farm in Thailand}

\author{
Pattanapong Karunyasiri ${ }^{1}$ Paitoon Sayvaw ${ }^{1}$ Nathaya Duangwhae ${ }^{2}$ Lerdtida Meedacha ${ }^{2}$ Sopon Kongtes ${ }^{2}$ Jitjaroen Channarong $^{2}$ \\ 1. Karun Farm, Thailand, Thailand; 2. Boehringer Ingelheim (Thai), Bangkok, Thailand
}

\section{Introduction}

Porcine Circovirus type 2 related diseases (PCVD) are a major threat for pig production all around the globe. Fortunately, in recent years PCV2 vaccines became available and piglet vaccination has proven to be very effective in controlling losses associated with PCV2 infection $(1,2)$.

This long term field observation evaluates the performance of fatteners from a 2000 head sow farm using different PCV2 piglet vaccines.

\section{Material and method}

The field observation included in total more than 85,000 pigs from a 2,000 head sow farm in Thailand. Pigs are transferred from the sow farm to the fattening units at about 8 weeks of age. PCV2 piglet vaccination was first introduced in Spring 2007. The first vaccine used was ChoongAng vaccine (produced in Korea), mid 2007 the farm switched to Suvaxyn PCV2 and about a year later Ingelvac CircoFLEX was introduced. Pigs were vaccinated with all vaccines at about 3 weeks of age.

Mortality, culls (pigs euthanized during fattening), and average daily gain (ADG) were recorded per fattening unit. Total loss is the sum of mortality and culls, i.e. the pigs not reaching slaughter age.

The differences between treatment groups in average performance variables (ADG, mortality, culls and total loss) were evaluated using pair-wise Wilcoxon test with a Bonferroni adjustment (SAS System, SAS Inst., Cary, North Carolina, v 8.2).

\section{Results}

Finishing mortality, culls and total loss were significantly reduced in CircoFLEX vaccinated pigs compared to the other two groups, while ADG was significantly improved (table 1).

Table 1. Average performance in fattening units.

\begin{tabular}{|l|c|c|c|}
\hline & Choong Ang & Suvaxyn PCV2 & CircoFLEX \\
\hline Fattening units ( $\mathrm{n})$ & 21 & 79 & 82 \\
\hline Pigs $(\mathrm{n})$ & 9,932 & 37,152 & 38,479 \\
\hline ADG (g/day) & $673^{\mathrm{a}}$ & $679^{\mathrm{a}}$ & $710^{\mathrm{b}}$ \\
\hline Mortality (\%) & $2.64^{\mathrm{a}}$ & $3.13^{\mathrm{a}}$ & $1.37^{\mathrm{b}}$ \\
\hline Culls (\%) & $1.70^{\mathrm{a}}$ & $2.52^{\mathrm{b}}$ & $1.40^{\mathrm{a}}$ \\
\hline Total loss (\%) & $4.35^{\mathrm{a}}$ & $5.65^{\mathrm{a}}$ & $2.77^{\mathrm{b}}$ \\
\hline a,b: Values with different superscripts differ significantly $(\mathrm{p}<0.05 / 3)$ \\
\hline
\end{tabular}

Figure 1 shows that not only average mortality was significantly lower in the CircoFLEX vaccinated units, but that variability in mortality was reduced as well.

Figure 1. Total loss (\%) per fattening unit. $A=$ ChoongAng, $B=$ Suvaxyn PCV2, C=CircoFLEX.

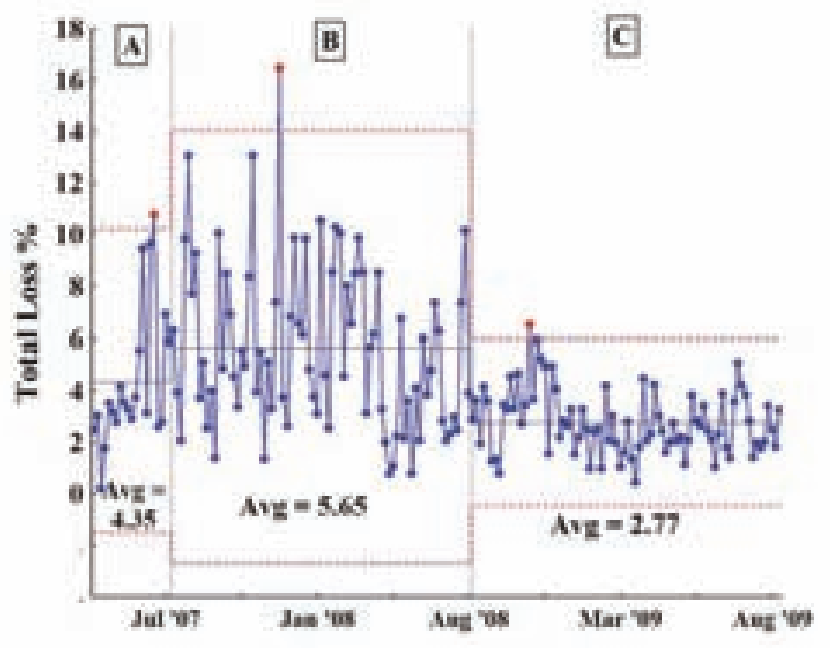

\section{Discussion and conclusion}

PCV2 piglet vaccination has become global routine use (3). The data of this field observation indicates that the choice of vaccine might significantly influence production performance. Ingelvac CircoFLEX vaccinated pigs performed significantly better than pigs vaccinated with two other PCV2 vaccines.

\section{References}

(1) Cline et al (2008) Vet. Record, 163, 737-740.

(2) Desrosiers et al (2009) J Swine Health Prod. 17(3), pp. 148-154.

(3) Hardge (2009) Asian Pork Magazine, Aug/Sep, 28-29. 


\title{
P.109
}

\section{Comparative humoral immunity response to vaccination with Porcilis ${ }^{\circledR}$ PCV, a second commercial vaccine and unvaccinated animals}

\author{
Enric Marco ${ }^{1}$ Jesus M. Bollo ${ }^{2}$ Rut Menjon² Marta Jimenez ${ }^{2}$ \\ 1. Marco y Collell, St Paulí de Nola, Barcelona, Madrid, Spain; 2. Intervet Schering Plough, Madrid, Spain
}

\begin{abstract}
Introduction
Monitoring the correct humoral immune response to vaccination is important when using routine vaccination procedures at early ages (3-4 weeks of age). This can be considered as the first step to assess possible interferences of vaccination with maternally-derived antibodies, and gives an indication about the response that will be obtained in subsequent production phases.
\end{abstract}

\section{Materials and Methods}

The study was performed in a farm housing 1680 sows, located in central Spain. Pigs from this farm showed evident PCVADrelated problems during fattening. The symptoms appeared at first at 13 weeks of life, and the most critical phase of the disease concluded 4 weeks later. Sixty-three animals with a mean age of 24 days ( 2 days before weaning) were selected for the study. The animals were uniquely identified using double ear-tags and were randomized into 3 groups, according to their dam and its parity, and piglet weight and gender, as follows:

- Porcilis ${ }^{\circledR} \mathrm{PCV}$ group, single $2 \mathrm{ml}$ dose, 21 piglets.

- Vaccine A group, single $1 \mathrm{ml}$ dose, 23 piglets.

- Control group, single $2 \mathrm{ml}$ dose of Diluvac Forte ${ }^{\circledR}$ (as a placebo), 19 piglets.

A commercial test was used for the detection of $\lg G$ and $\lg M$ (Ingezim PCV2 ELISA $^{\oplus}$, Ingenasa), comparing the results obtained at 3, 7 and 9 weeks of age. The cut-off thresholds differed for each antibody detection time: from the $1^{\text {st }}$ to the $3^{\text {rd }}$ sampling, the values for IgG were $0,7,0.69$ and 0.65 , respectively and $1.02,0.74$ and 0.71 for $\lg M$, respectively.

Statistical evaluation was performed using the Kruskall - Wallis test, the Pearson's chi-square test, the Fisher's exact test and the Mann-Whitney-U test.

\section{Results}

The first sampling time coincided with the administration of the three treatments, and all the groups presented high levels of maternally-derived immunity, as shown by the high mean optical density ratio (OD) values and the high percentage of animals positive for lgG (Graph 1). Very low or negative values were found for IgM. All the parameters were comparable $(p<0.05)$.

The second sampling at 7 weeks showed a stronger lgG response to vaccination in the Porcilis ${ }^{\circledR} P C V$ group, with significant differences for the percentage of positive animals compared to the other two groups $(p<0.05)$. Vaccine A did not present significant differences vs. the Control group for both parameters $(\mathrm{p}<0.05)$. The Porcilis ${ }^{\circledR} \mathrm{PCV}$ group showed a stronger IgM response (Graph 2), with significant differences when comparing the percentage of positives vs. the Control group $(p<0.05)$ and vs. Vaccine $A(p<0.001)$, with no differences between the Vaccine $A$ and Control groups. The percentage of animals of the Vaccine A group positive for IgM at 4 weeks post vaccination was $0 \%$ At week 9 , a marked increase of the mean OD values was found in the Porcilis ${ }^{\circledR} P C V$ group vs. the other two groups $(p<0.001)$, and for the percentage of positives vs. the other two groups $(p<0.05)$. No significant differences were found for both parameters between the other groups. A strong IgM response occurred in the Control group. Significant differences were found vs. the Porcilis ${ }^{\circledR} P C V$ group $(p<0.05)$ and the Vaccine A group $(p<0.001)$. No significant differences occurred between both vaccinated groups $(p<0.05)$.

Graph 1: \% Samples positive for IgG

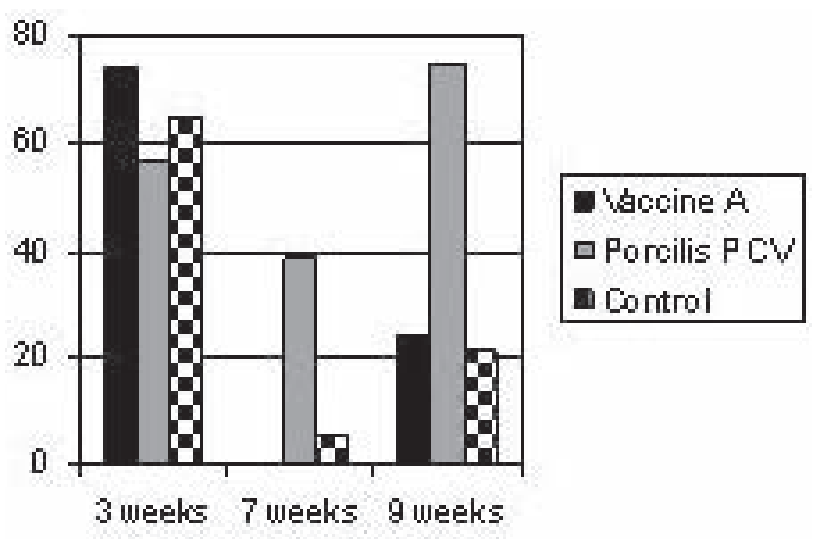

Graph 2: \% Samples positive for IgM

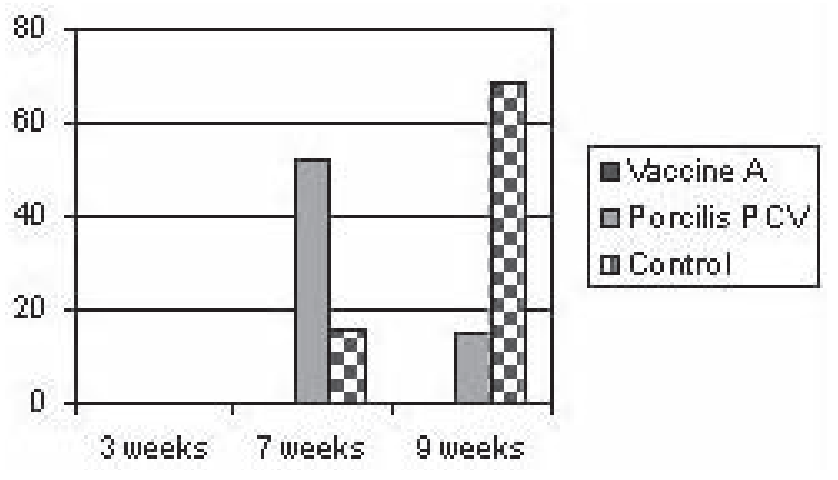

\section{Discussion and Conclusions}

The strong response to the Porcilis ${ }^{\circledR}$ PCV vaccine obtained 4 weeks after vaccination, contrary to the findings for the Vaccine $A$ and Control groups, indicates a correct immunisation of the animals, thus preventing interferences with maternally derived immunity.

Even though the farm had a history of late problems, the increase of IgM values in the Control group at week 7, which was more evident at week 9, probably was due to contact with the field virus. 


\title{
P.110
}

\section{Vaccination with Circovac $^{\oplus}$ in Iberian sows and gilts: effects on insemination results}

\author{
Guillermo Ramis ${ }^{1}$ Angel Garrido ${ }^{2}$ Francisco J. Pallarés ${ }^{3}$ Helena Sanjosé2 Manuel \\ Toledo² Juan J. Queredaํㅣ José M. González² Antonio Muñoz ${ }^{1}$ \\ 1. Universidad de Murcia, Murcia, Spain; 2. Piensos Jiménez S.A. \& Juan Jiménez García S.A.U. , Lorca, Spain; \\ 3. DPTO Anatomía y Anatomía Patológica Comparadas. Universidad De Murcia, Murcia, Spain
}

\section{Introduction}

Porcine Circovirus type 2 associated diseases (PCVD) is the current name for those pathologies directly related to PCV2. Apart from the classical clinical expression formerly called postweaning multisistemic wasting syndrome (PMWS), has been describe a wide range of diseases, including reproductive alterations $(1,2)$. The launch of vaccines against PCV2 has offered a very useful tool to manage the PCVAD, but there is no wide knowledge about the improvement of reproductive performances directly derived of the vaccination $(3,4)$. On the other hand, there is much less knowledge about the effect on Iberian pig breed, a Spanish minority indigenous breed.

The aim of this study was to assess the effect of the vaccination against PCV2 with Circovac $^{\circledast}$ on reproductive performance in Iberian gilts and sows.

\section{Material and Methods}

The experience was carried out in a large unit of Iberian pig production (850 sows) in multiple-sites-production. The breeders were mass vaccinated and revaccinated in a period of 3 weeks. Subsequently, the animals were revaccinated every gestation at least 3 weeks before farrowing expected date. The reproduction is completely based on artificial insemination, and every ejaculate is tested using classical seminal quality tests. Gilts and sows are vaccinated against PRRSv three times per year, alternating modified live and killed vaccines. Boars remained unvaccinated.

The reproductive performance recorded during one year before vaccination and revaccination were used as control.

The analyzed data were: fertile inseminations unfertile inseminations, number of inseminations, return to estrus and type of return to estrus depending on the day returning; early (0-17 dpi), early cyclic (18 to $24 \mathrm{dpi})$, early acyclic (25-30 dpi), later acyclic (31-38 dpi), later cyclic (39-43 dpi) and later ( $>44 \mathrm{dpi})$. A total of 2,741 and 2,897 .inseminations performed in control and vaccinated groups, respectively, were analyzed. All data were analyzed using the SPSS v. 15.0 software (SPPS Inc, USA).

\section{Results}

Data obtained from gilts and sows in both experimental groups are shown in the following table:

\begin{tabular}{|l|c|c|c|}
\hline Group & AMG & FM & NFM \\
\hline Control & & & \\
\hline Gilts & 1.34 & 71.9 & 28.1 \\
\hline Sows & 1.15 & 84.1 & 15.9 \\
\hline Vaccinated & & & 22.6 \\
\hline Gilts & 1.18 & 77.4 & 12.6 \\
\hline Sows & 1.08 & 87.4 & $\mathrm{p}=0.002$ \\
\hline Significance & $\mathrm{p}<0.001$ & $\mathrm{p}=0.002$ & \multicolumn{3}{|l|}{} \\
\hline $\begin{array}{l}\text { Where AMG: average mating per gestation, FM: fertile mating, NFM: non } \\
\text { fertile mating. }\end{array}$
\end{tabular}

There was no difference for the frequency of each return-to-estrus type neither for gilts nor sows $(p=0.47$ and $p=0.45$, respectively). Early and cyclic returns in gilts and sows from both groups were the most frequently types found.

\section{Discussion}

There is controversy regarding the effect of PCV2 on reproductive performance, even when some negative effects have been described by practitioners. This trial shows an improvement in parameters such us average mating per gestation, fertile inseminations and non-fertile inseminations, resulting in reduction of production costs. These findings suggest that the PCV2 could be producing a subclinical effect on reproductive performance and the improvement of health status in sows and gilts derived from PCV2 vaccination could result in these better performances in Iberian pigs reared under intensive conditions.

\section{References}

1. Opriessnig et al. (2007). J Vet Diagn Invest 19:591-615.

2. Guillespie et al. (2009). J Vet Intern Med 23:1151-1163.

3. Madson et al. (2009). Clin Vaccine Immunol 16:830-834.

4. Madson et al. (2009) Theriogenology. 72:747-754. 


\title{
P.111
}

\section{Vaccination with Circovac $^{\circledR}$ in Iberian sows and gilts: effects on farrowing results}

\author{
$\underline{\text { Guillermo Ramis }}^{1}$ Angel Garrido ${ }^{2}$ Francisco J. Pallarés ${ }^{3}$ Helena Sanjosé ${ }^{2}$ Manuel Toledo \\ Juan M. Herrero-Medrano ${ }^{1}$ José M. González $^{2}$ Antonio Muñoz ${ }^{1}$ \\ 1. Universidad de Murcia, Murcia, Spain; 2. Piensos Jiménez S.A. \& Juan Jiménez García S.A.U. , Lorca, Spain; \\ 3. DPTO Anatomía y Anatomía Patológica Comparadas, Universidad De Murcia, Murcia, Spain
}

\section{Introduction}

Porcine Circovirus Diseases (PCVD) is the current name for those pathologies directly related to PCV2. Apart from the classical clinical expression formerly called postweaning multisistemic wasting syndrome, has been describe a wide range of diseases, including reproductive alterations $(1,2)$.

The aim of this study was to assess the effect of the vaccination against PCV2 with Circovac ${ }^{\circledR}$ on farrowing performance in lberian gilts and sows.

\section{Material and Methods}

The experience was carried out in a large unit of Iberian pig production (850 sows) in multiple-sites-production. The breeders were mass vaccinated and revaccinated in a period of 3 weeks. Subsequently, the animals were revaccinated every gestation at least 3 weeks before farrow expected date. The reproduction is completely based on artificial insemination (Al), and every ejacule is tested using classical seminal quality tests. Gilts and sows are vaccinated against PRRSv three times per year, alternating modified live and killed vaccines.

As control the reproductive performance recorded during one year before vaccination and revaccination were used. The analyzed data were: length of gestation (GL), live born piglets (LBP), stillborn piglets (SBP), weaned piglets per litter (WPL) and mortality during suckling (MS). All data were included in a SPSS data base analyzed using the SPSS v. 15.0 software (SPPS Inc, USA) by means of ANOVA. Results from 2,273 and 2,317 farrowings in control and vaccinated group, respectively, were analyzed. In control group 515 and 1,580 farrowings from gilts and sows, respectively, and in vaccinated groups 361 and 1,956 farrows from gilts and sows, respectively, were recorded.

\section{Results}

There was no difference for lactation length ( 22.3 vs 22.6 and 21.5 vs. 22 for sows and gilts, respectively). Data obtained for gilts and sows are shown in the table 1.
Table 1: Reproduction parameters in vaccinated and control groups, for sows and gilts

\begin{tabular}{|l|c|c|c|c|c|c|}
\hline \multirow{2}{*}{ Parameter } & \multicolumn{2}{|c|}{ Control } & \multicolumn{2}{c|}{ Vaccinated } & \multicolumn{2}{c|}{ Significance } \\
\cline { 2 - 7 } & Gilts & Sows & Gilts & Sows & Gilts & Sows \\
\hline GL & 113 & 112.4 & 113.5 & 113.7 & NS & $\mathrm{P}<0.001$ \\
\hline ABP & 6.7 & 7.11 & 6.7 & 7.18 & NS & NS \\
\hline DBP & 0.28 & 0.52 & 0.28 & 0.35 & NS & $P<0.001$ \\
\hline WPL & 6.5 & 6.37 & 6.3 & 6.47 & NS & $P=0.036$ \\
\hline MS & 12.9 & 17.2 & 12.2 & 9.9 & NS & $P<0.001$ \\
\hline $\begin{array}{l}\text { Where GL: gestation length ALV: alive born piglets, SBP: stillborn piglets, WPL: weaned piglets } \\
\text { per litter and MS = mortality during suckling. NS: not significant. }\end{array}$
\end{tabular}

\section{Discussion}

The length of lactation did not influence other parameters. We have found differences for all studied parameters except for ABP in sows, but, there was no difference in gilts. To have a clear idea it should be taken into account that Iberian pigs show a lower reproductive performance than commercial crossbreds. However, there were important advantages derived from the vaccination with Circovac $^{\circledR}$ in sows: the reduction of SBP and mortality during suckling result in a significant increase in weaned piglets per litter. There is also an interesting result: the increase of gestation length in more than one day. This could result in a better vitality and maturity of piglets. The most important difference was mortality during suckling in sows, showing a decrease of more than $57 \%$ (17.2 vs $9.9 \%$ in control and vaccinated group, respectively) which could be a direct result of immunity against PCV2 derived from specific colostral antibodies against the virus, in agree with several previous studies. The lack of effects in gilts group could be explained because gilts are vaccinated only when they are included in the breeder group. So, despite the vaccination every farrow, the sows would have immunity produced by mass vaccinations.

\section{References}

1. Opriessnig et al. (2007). J Vet Diagn Invest 19:591-615.

2. Guillespie et al. (2009). J Vet Intern Med 23:1151-1163. 


\title{
P.112
}

\section{Induction of maternal antibodies and the effect on growth parameters by Circovac ${ }^{\circledR}$ in a field study}

\author{
$\underline{\text { Xaver Sidler }}^{1}$ Jeremias Kurmann ${ }^{1}$ Esther Buergi' Enrico Brugnera² Titus Sydler ${ }^{3}$ \\ 1. Division of Swine Medicine, University Zurich, Vetsuisse Faculty, Zurich, Switzerland; 2. Division of Farm Animals, University Zurich, \\ Vetsuisse Faculty, Zurich, Switzerland; 3. Institute of Veterinary Pathology, University Zurich, Vetsuisse Faculty, Zurich, Switzerland
}

\section{Introduction}

Postweaning Multisystemic Wasting Syndrome (PMWS) in pigs, caused by porcine circovirus type 2 (PCV2), has become one of the most important diseases in swine. Pigs are most commonly affected at 5 to 12 weeks of age. PCV2-specific antibodies can be detected in almost all pigs. In 2008 Circovac $^{\circledR}$, (Merial SAS, Lyon) was introduced in Switzerland. $\operatorname{Circovac}^{\circledR}$ is used to vaccinate pregnant sows to increase the concentration of colostral antibodies.

\section{Materials and Methods}

In this study we used Circovac $^{\circledR}$ in two different farms with a history of naturally occurring PMWS. Herd A was a breeding herd with 90 Yorkshire sows. Piglets were sold to a regional finisher at the age of approximately ten weeks and at a body weight of 22 to $27 \mathrm{~kg}$. Only sporadic cases of PMWS had occurred in herd A since 2006, but there had been considerable losses in the finishing operations. 10 to $15 \%$ of these pigs developed PMWS. Herd $B$ was a breeding herd with 150 Yorkshire $x$ Landrace crossbred sows. Approximately $5-20 \%$ of the pigs had low daily weight gain and profuse untreatable diarrhoea with a herd mortality rate of $5-10 \%$, also indicators to a serious PMWS problem.

Randomly sows were chosen either for vaccination or were injected with Circovac $^{\circledR}$ adjuvans as control group. Sows were vaccinated with $2 \mathrm{ml} \mathrm{Circovac}{ }^{\circledR}$ two and four weeks before artificial insemination and in the 12th week of gestation. $10 \mathrm{ml}$ blood was collected from the jugular vein from of all sows immediately before the first injection, four weeks after the second injection and two weeks after the third injection. 2-5 ml blood was collected from 100 individually tagged piglets at the age of 3, 10, 31, 42 and 63 days p.p. An improved competitive ELISA (SERELISA ${ }^{\oplus}$ PCV2 Ab Mono Blocking Systems (Synbiotics Corporation Europe SAS, Lyon) was used for antibody detection. The carcass weight represents $78 \%$ of the live weight at slaughter and was used to calculate the average daily weight gain (AWDG, g/d).

\section{Results}

Figure 1. In both farms the vaccinated sows showed a significant increase of PCV2 antibody titre from the first to the second vaccination $\left({ }^{* *} p \leq 0.001\right)$

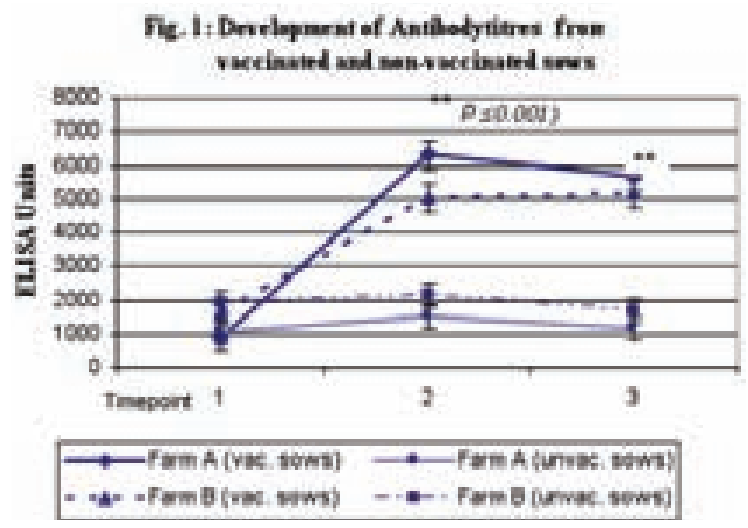

Proceedings of the $21^{\text {st }}$ IPVS Congress, Vancouver, Canada - July 18-21, 2010
Figure 2. Offspring of vaccinated sows had significantly higher antibody titres against PCV2 until day 63 after birth $(* * \leq 0.001)$
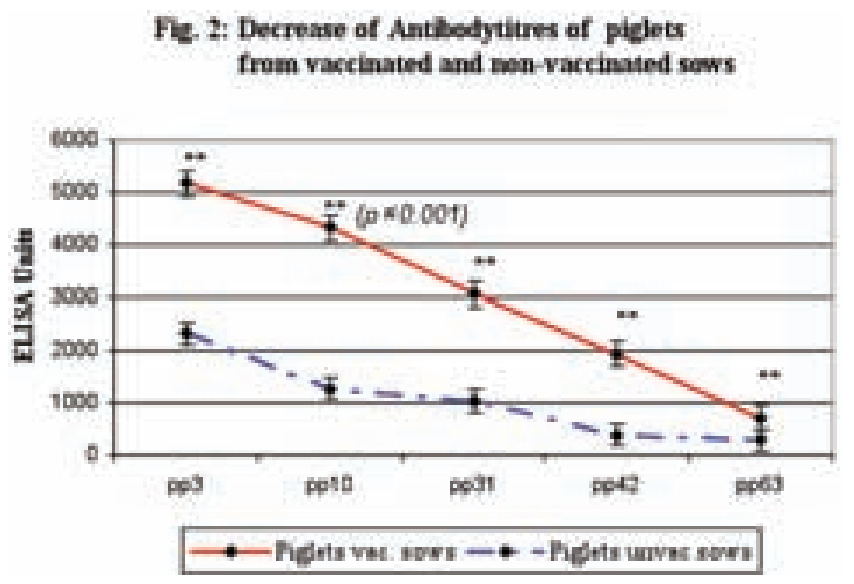

Table 1. Performance parameters for the control group compared to the vaccinated group $\left({ }^{*} p \leq 0.05\right)$

\begin{tabular}{|l|c|c|c|}
\hline & ADWG & ADWG (g/d) & Slaughter \\
\hline & (g/d) & $\mathbf{2 5} \mathbf{- 1 0 5} \mathbf{~ k g}$ & age (d) \\
\hline Control A & 593 & 734 & 177 \\
\hline Circovac A & $626^{*}$ & $785^{*}$ & $168^{*}$ \\
\hline Control B & 565 & 696 & 189 \\
\hline Circovac B & $585^{*}$ & $726^{*}$ & $183^{*}$ \\
\hline
\end{tabular}

\section{Discussion}

We succeeded by vaccination in elevating antibody titres in sows. At day 42 offspring of vaccinated sows had nearly the same antibody titres as piglets from non-vaccinated sows at day $3 \mathrm{pp}$. These higher antibody titres ameliorated growth parameters of piglets in both farms. 


\title{
P.113
}

\section{A field efficacy study with Enterisol ${ }^{\circledast}$ Ileitis and Ingelvac CircoFLEX ${ }^{\circledast}$ in Switzerland}

\author{
Helen Weibel $^{2}$ Titus Sydler $^{3}$ Esther Buergi ${ }^{4}$ Enrico Brugnera ${ }^{4}$ Xaver Sidler ${ }^{1}$ \\ 1. Vetsuisse Faculty, Zurich, Switzerland; 2. Division of Swine Medicine, University Zurich, Vetsuisse Faculty, \\ Zurich, Switzerland; 3. Institute of Veterinary Pathology, University Zurich, Vetsuisse Faculty, Zurich, Switzerland; \\ 4. Division of Farm Animals, University Zurich, Vetsuisse Faculty , Zurich, Switzerland
}

\section{Introduction}

Proliferative enteropathy caused by Lawsonia intracellularis (Li) and porcine circovirus associated diseases (PCVAD) caused by porcine circovirus type 2 (PCV2) are two of the most important swine-diseases in Switzerland. The objective of this study was to investigate the efficacy of the vaccines Enterisol ${ }^{\circledR}$ lleitis against $\mathrm{Li}$ and Ingelvac CircoFLEX ${ }^{\circledR}$ against PCV2 under field conditions and to test whether there is an additive effect on performance parameters when using both vaccines. The absence of Mycoplasma (M.) hyopneumoniae and porcine reproductive and respiratory syndrome virus (PRRSV) in Switzerland allows a proper investigation of the impact of vaccination against these two pathogens.

\section{Materials and Methods}

This study was conducted in a 200 sow farm operating in a weekly rhythm with eight farrowing sows per batch and a finishing site with 800 places. The sites were tested negative for PRRSV, M. hyopneumoniae, Salmonellae, Brachyspira (B.) hyodysenteriae and B. pilosicoli. PCV2 was confirmed by dissection and IHC, Li by faecal PCR and ELISA. In total 1405 piglets were included into the study, 384 vaccinated with Enterisol ${ }^{\circledR}$ Ileitis and Ingelvac CircoFLEX ${ }^{\circledR}$ (EI + CF), 376 with Ingelvac CircoFLEX ${ }^{\circledast}(\mathrm{CF}), 318$ with Enterisol ${ }^{\circledR}$ lleitis (EI) and 327 with oral and parenteral placebo (Control). Because of the unbalanced genetics and the small number of sows per week group, all piglets of two consecutive batches were included into one treatment group. This resulted in 24 week-groups with three repetitions of eight weeks, where the sequence of the treatment groups per repetition was random. Piglets were vaccinated at an average age of 23.5 days. Average Daily Weight Gain (ADWG) was calculated from individual weight measurements at four time points (week 3, 12, 18 and slaughtering) during the entire study. Almost all dead animals were dissected and tested for PCV2 and Li. Blood and faecal samples were taken from at least 10 pre-selected animals per batch at $3,7,12,15$ and 18 weeks of age. Blood samples were tested by qPCR for PCV2 and by ELISA for anti-Li antibodies. Faecal samples were tested by PCR for Li.

\section{Results}

The results from serology and PCR indicate an infection of $\mathrm{Li}$ and PCV2 at the end of the nursery/beginning of fattening (data not shown).

The mortality rate and the ADWG of the fattening period are indicated in table 1.

Table 1: The mortality rate and the ADWG of the fattening period. Different letters $(a / b / c)$ indicate a significant difference $(P \leq 0.05)$.

\begin{tabular}{|l|c|c|c|c|}
\hline Parameter & EI+CF & CF & El & Control \\
\hline Mortality(\%) & $1.1^{\mathrm{a}}$ & $2.5^{\mathrm{a}}$ & $2.3^{\mathrm{a}}$ & $6.3^{\mathrm{a}}$ \\
\hline ADWG (g) & $792^{\mathrm{a}}$ & $772^{\mathrm{b}}$ & $774^{\mathrm{b}}$ & $751^{\mathrm{c}}$ \\
\hline
\end{tabular}

For the control and the El group 18 and 10 death losses were after necropsy diagnosed to be related to PCV2 whereas for the PCV2 vaccinated animals none of the losses were related to PCV2. Li was detected in four dead animals but was never seen as the cause of death.

\section{Discussion}

The observed effects of the mono applications (CF and EN) during the fattening period were comparable with other efficacy studies 1, 2. The data indicates that the concurrent application of Enterisol ${ }^{\circledR}$ Ileitis and Ingelvac CircoFLEX ${ }^{\circledR}$ had additive effects on pig performance parameters. It was also demonstrated that the use of both vaccines was profitable3. The results of this study indicate that after introduction of PCV2 vaccination ileitis vaccination has still a significant impact on performance parameters even when Li is sub-clinically present in a farm.

\section{References}

1. Caspari et al., 2009. Schweiz Arch Tierheilk 151, 31-32

2. Fachinger et al., 2008, Vaccine 26, 1488-1499

3. Weibel et al., IPVS 2010 submitted 


\title{
P.114
}

\section{Economic impact of Enterisol ${ }^{\circledast}$ lleitis and Ingelvac CircoFLEX ${ }^{\circledast}$ in a fattening farm in Switzerland}

\author{
Helen Weibel $^{2}$ Frank Schreiber ${ }^{3}$ Bernd G. Liesner ${ }^{3}$ Xaver Sidler $^{1}$ \\ 1. Vetsuisse Faculty , Zurich, Switzerland; 2. Division of Swine Medicine, University Zurich, Vetsuisse Faculty, \\ Zurich, Switzerland; 3. Boehringer Ingelheim Animal Health GmbH, Ingelheim, Germany
}

\begin{abstract}
Introduction
Proliferative enteropathy caused by Lawsonia intracellularis (Li) and porcine circovirus associated diseases (PCVAD) caused by porcine circovirus type 2 (PCV2) are two of the most important swine-diseases in Switzerland. The objective of this study was to investigate the efficacy of the vaccines Enterisol ${ }^{\circledR}$ Ileitis against Li and Ingelvac CircoFLEX ${ }^{\circledR}$ against PCV2 under field conditions and to test whether there is an additive effect on performance parameters when using both vaccines. This abstract covers the impact of the ileitis and PCV2 vaccination on fattening performance and farm economics under Swiss commercial conditions.
\end{abstract}

\section{Materials and Methods}

This study was conducted in a 200 sow farm operating in a weekly rhythm with eight farrowing sows per batch and a finishing site with 800 places. The farms ware tested negative from porcine reproductive and respiratory syndrome virus (PRRSV), Mycoplasma hyopneumoniae, Salmonellae, Brachyspira (B.) hyodysenteriae and B. pilosicoli. PCV2 was confirmed by necropsy and IHC, Li by faecal PCR and ELISA. In total 1405 piglets were included into the study, 384 vaccinated with Enterisol ${ }^{\circledR}$ Ileitis and Ingelvac CircoFLEX ${ }^{\circledR}$ (EI + CF), 376 with Ingelvac CircoFLEX $^{\circledast}$ (CF), 318 with Enterisol ${ }^{\circledR}$ lleitis (EI) and 327 with oral and parenteral placebo (control). Because of the unbalanced genetics and the small number of sows per week-group, all piglets of two consecutive batches were included into one treatment group. This resulted in 24 week-groups with three repetitions of eight weeks, where the sequence of the treatment groups per repetition was random. Piglets were vaccinated at an average age of 23.5 days. Average Daily Weight Gain (ADWG) was calculated from individual weight measurements at four time points (week 3, 12, 18 and slaughtering) during the entire study. All dead animals were recorded. The economic impact of the fattening farm (week 12 to slaughtering) is expressed by the return on investment (ROI) per year, i.e. the extra profit of $X$ Swiss francs generated per 1 Swiss franc invested. A price for slaughtered pigs of $3.60 \mathrm{sfr} / \mathrm{kg}(2.40 € / \mathrm{kg})$, a price of $4.40 \mathrm{sfr} / \mathrm{kg}$ $(2.93 € / \mathrm{kg}$ ) per piglet at $30 \mathrm{~kg}$ and a price of $6.00 \mathrm{sfr}(4.00 €)$ for both vaccines in total were taken as a basis for the calculation.
The comparison of the control group and the PCV2 vaccinated group is showed in table 1. The pigs vaccinated with Ingelvac CircoFLEX $^{\otimes}$ (CF) had a significant higher ADWG $(+21 \mathrm{~g} / \mathrm{d})$ and a significant lower mortality rate (-3.8\%).

Table 1: Performance parameter and economic impact for the control group compared to the CF group.

\begin{tabular}{|l|c|c|c|}
\hline & Control & CF & $\Delta$ \\
\hline ADWG (g/d) & 751 & 772 & 21 \\
\hline Mortality (\%) & 6.3 & 2.5 & 3.8 \\
\hline ROI & & $3.6: 1$ & \\
\hline
\end{tabular}

Fattening pigs vaccinated with Enterisol ${ }^{\circledR}$ lleitis and Ingelvac CircoFLEX $^{\circledast}(\mathrm{EI}+\mathrm{CF})$ had a significant higher ADWG (+ $\left.20 \mathrm{~g} / \mathrm{d}\right)$ and a lower mortality rate $(-1.4 \%)$ compared to the animals vaccinated with Ingelvac CircoFLEX ${ }^{\circledR}$ (CF). The economic benefit of Enterisol ${ }^{\circledR}$ Ileitis illustrated with the ROI was 2.1:1 (table 2).

Table 2: Performance parameter and economic impact for the CF group compared to the EI +CF group (Enteriso ${ }^{\circledR} / l e i t i s$ group).

\begin{tabular}{|l|c|c|c|}
\hline & CF & El+CF & $\Delta$ \\
\hline ADWG $(\mathrm{g} / \mathrm{d})$ & 772 & 792 & 20 \\
\hline Mortality (\%) & 2.5 & 1.1 & 1.4 \\
\hline ROI & & $2.1: 1$ & \\
\hline
\end{tabular}

\section{Discussion}

The comparison of the ROI of the PCV2 and the additional lleitis vaccination indicates the economic relevance of both vaccinations. On this farm, the effects of the PCV2-vaccine against the clinical PCVAD were a decisive reduction of mortality and an improvement of the ADWG. Despite the fact that the ileitis infection was subclinical vaccination with Enterisol ${ }^{\circledR}$ Ileitis was highly profitable.

\section{References}

1. Weibel et al. IPVS (submitted)

\section{Results}




\title{
P.115
}

\section{Effect of Porcilis PCV one shot vaccination on slaughter weight and mortality in a subclinically infected pig farm}

\author{
Sonja Agten; Stefaan Van Gorp; Bart M. Balis \\ Intervet Schering Plough, Ukkel, Belgium
}

\section{Introduction}

PCV2 infections are ubiquitous among pigs worldwide.

Olvera (2004) and Krakowka (2005) showed that there is a relationship between viral load and the occurrence of disease. High infection levels lead to clinical disease while lower levels induce a subclinical course. The latter may however induce significant economical losses.

The majority of pigs in Belgium are subclinically infected with low levels of PCV2. Even without clear signs of PMWS or PDNS, a subclinical infection with PCV2 often has a negative impact on average daily weight gain, feed conversion, days to slaughter and/or slaughterweight.

The timing of a PCV2 virus infection is often unknown under field conditions. It is important that pigs are protected as early as possible and for the whole of the fattening period (Eggen IPVS 2010).

In the present field study three week old piglets were vaccinated once with Porcilis PCV vaccine. The objective was twofold. It was intended to measure the effect of this Porcilis PCV one shot vaccination on slaughter weight, as well as to evaluate the mortality rate during the growing period, with respect to unvaccinated controls.

\section{Materials and Methods}

A large conventional closed farm with hypothetical subclinical problems of PCV2 was selected.

At 3 weeks of age, group 1 was vaccinated with $2 \mathrm{ml}$ of Porcilis PCV and group 2 was indicated as a control group. Group 1 contained 572 animals and group 2 contained 714 animals. All animals were observed daily for clinical signs and mortality. The body weights were measured at slaughter. Mortality rates between vaccinated and unvaccinated controls were compared and evaluated by the non-parametric test of Kruskal-Wallis. Differences in weights at slaughter were examined using the one-way Anova.

10 serum samples for each were taken at vaccination, at 10 weeks of age, at 14 weeks of age and one week before slaughter. These samples were examined for PCV2 and PRRSv antibodies and nucleic acid of PCV2.

\section{Results}

All piglets were healthy before and after vaccination. Slaughter weights were scored individually for group 1 and 2 and the mean and standard deviation are shown in Table 1. The mortality rate is shown in Table 2.

Table 1: Growth performance and slaughter weight $(\mathrm{kg})$

\begin{tabular}{|c|c|c|}
\hline Group & Porcilis PCV & Control \\
\hline$N^{*}$ & 472 & 647 \\
\hline$B W$ at $10 W(\mathrm{~kg})^{* *}$ & 20.15 & 20.79 \\
\hline $\begin{array}{l}\text { Days to slaughter*** } \\
\text { Average weight at slaughter + } \\
\text { standard deviation }\end{array}$ & $\begin{array}{l}141 \\
\text { a92.1+/- } 10.75\end{array}$ & \begin{tabular}{|l}
142 \\
b90.0 +/- 10.96
\end{tabular} \\
\hline
\end{tabular}

Table 2: Mortality rate of Porcilis PCV vaccinated piglets and control group

\begin{tabular}{|l|l|l|l|l|}
\hline Group & N & Alive & Dead & \% mortality \\
\hline Porcilis PCV & 572 & 560 & 12 & a 2.09 \\
\hline Control & 714 & 681 & 33 & b 4.6 \\
\hline $\mathrm{a}, \mathrm{b} \mathrm{p}<0.05$ & & & \\
\hline
\end{tabular}

The Porcilis PCV-group showed a significant difference of $2.1 \mathrm{~kg}$ in slaughter weight within the same fattening period $(p<0.05)$.

The mortality rate in the Porcilis PCV group was significantly reduced to $2.09 \%$, which is a decrease of $54.5 \%(p<0.05)$.

\section{Discussion}

A single $2 \mathrm{ml}$ dose of Porcilis PCV in piglets of 3 weeks of age, used in subclinical field conditions, led to significantly higher slaughter weights within the same fattening period. This result could justify a vaccination with Porcilis PCV at three weeks of age in subclinically infected farms.

Although, the mortality rate in the control group was reasonably low, it was even 54,5\% lower in the Porcilis PCV vaccinated group.This may indicate that the vaccine was able to significantly reduce mortality, resulting from a subclinical PCV infection.

\section{References}

Olvera, Journ. Vir. Methods, Vol. 117, April 2004, 75-80.

Krakowka, Journal Vet. Diagn. Invest., March 2005, 213-222.

Eggen, IPVS 2010. 


\title{
P.116
}

\section{Effects of Porcilis ${ }^{\circledR}$ PCV vaccination in a significantly large batch of animals with high levels of maternally-derived immunity}

\author{
Miguel A. Jimenez ${ }^{1}$ David Escalada ${ }^{1}$ Ivan Mayor ${ }^{1}$ Rut Menjon ${ }^{2}$ Jesus M. Bollo ${ }^{2}$ Marta Jimenez ${ }^{2}$ \\ 1. Uvesa, Ctra. Zaragoza, TUDELA, Navarra, Spain; 2. Intervet Schering Plough, Madrid, Spain
}

\section{Introduction}

Porcine circovirus infection causes important production losses. Over recent years the disease has been found to occur with an increasingly later onset, with negative effects on the fattening phase of production. Vaccination against this disease, which is usually carried out at an early age, may reduce these losses, but on some farms it will coincide with high maternally-derived antibody (MDA) levels. The aims of the present study were to assess the improvement in production parameters resulting from a Porcilis ${ }^{\circledR} \mathrm{PCV}$ vaccination program and to evaluate the possible interference by maternally-derived immunity.

\section{Materials and Methods}

The study was undertaken on a 2,600-sow farm in North- East Spain (LD x LW, finished with Pietrain), operating a two-phase production system. The herd was positive for M. hyopneumoniae (M. hyo), PRRSv and circovirus. The onset of clinical PCV2 occurred at 17 weeks of age.

Production results were compared of a total of 33,580 piglets which entered phase two between January and September 2009. The animals were housed in different fattening units. The 16,120 pigs entering the units between January and April were not vaccinated with Porcilis ${ }^{\circledR}$ PCV, used as controls. The 17,460 pigs entering the units between May and September were vaccinated against PCV2 (Porcilis ${ }^{\circledR} \mathrm{PCV}$ : single $2 \mathrm{ml}$ dose) at weaning at 3 weeks of age. All the animals in the study were also vaccinated against $M$. hyo ( $\mathrm{M}+\mathrm{PAC}^{\circledR}$ : single $2 \mathrm{ml}$ dose) at 3 weeks of age. The vaccinated group thus received two vaccines at the same time, in separate injections.

Average production indicators for all the animals were recorded over the whole fattening phase (feed conversion rate, ADG, \% mortality, \% runts, treatment cost/pig). A cross-section serological study was also carried out on 10 animals of each group at different ages during the cycle. A commercial test was used to assess PCV2 antibodies in the serum (Ingezim PCV2 ELISA ${ }^{\circledR}$, Ingenasa), for the detection of $\lg G$ and $\lg M$.

\section{Results}

Porcilis ${ }^{\circledR} P C V$ vaccination at 3 weeks of age, even in the presence of high levels of MDA, led to significant increases in mean IgG and IgM values at 10 weeks of age, before they declined. The control group had lost their lgG titers (MDA) at 7 weeks of age and continued with low levels until seroconversion at 17 weeks of age (Graphs 1\&2). This indicates that MDA did not interfere with the immune response to the vaccine.
Graph 1. IgG/M values. Control group

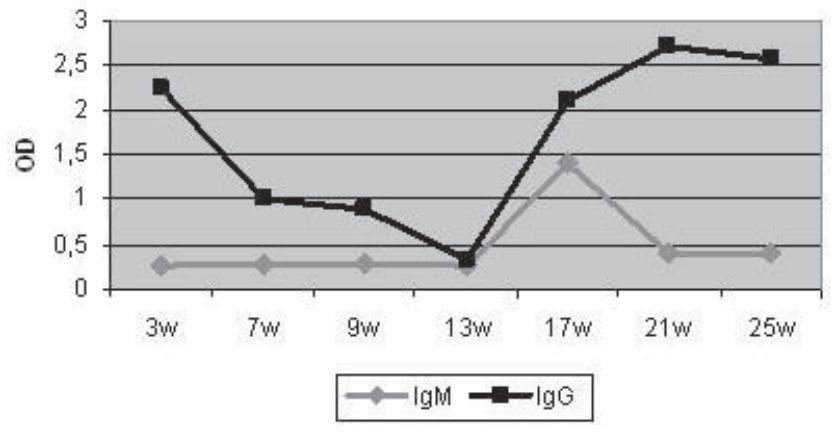

Positive result: $\lg$ G index $>0.827$ and $\lg M$ index $>0.915$

Graph 2. IgG/M values. Vaccinated group

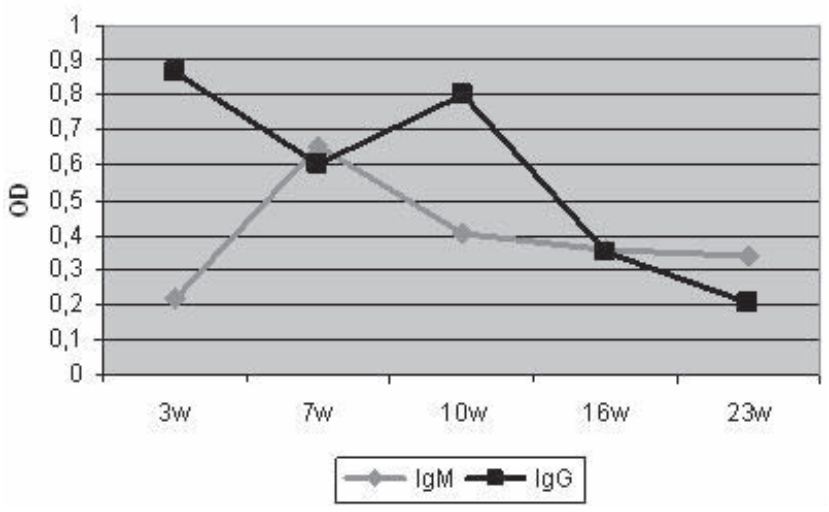

Positive result: IgG index $>0.416$ and $\lg M$ index $>0.520$

Table 1: Production results

\begin{tabular}{|l|c|c|}
\hline & Control group & Vaccinated group \\
\hline No.piglets & 16.120 & 17.460 \\
\hline Feed Conversion & 2.81 & 2.71 \\
\hline ADG & 683 & 702 \\
\hline$\%$ Low-weight pigs & 6.9 & 3 \\
\hline \% Mortality (finishing) & 7.4 & 4.1 \\
\hline Treatments/pig & $2.31 €$ & $1.79 €$ \\
\hline
\end{tabular}

\section{Discusssion}

Vaccination with Porcilis ${ }^{\circledR} \mathrm{PCV}$ at 3 weeks of age, even in the face of high levels of MDA and the occurrence of a late, severe clinical PCV2 infection, improved the production indicators of the farm, leading to significant economic benefits with a $8.8 \mathrm{ROI}$. 


\title{
P.117
}

\section{Vaccination against PCV2 in a herd that had been declared free from PMWS}

\author{
Carl-Johan Ehlorsson² Gunilla Blomqvist ${ }^{1}$ Per Wallgren ${ }^{1}$ \\ 1. Dept of Animal Health and Antimicrobial Strategies, Uppsala, Sweden; 2. Swedish Animal Health Service, Ängelholm, Sweden
}

\section{Introduction}

Combating other diseases and improving management routines has decreased the impact of Post weaning Multisystemic Wasting Syndrome (PMWS) (1). PMWS is associated to porcine circovirus type 2 (PCV2), and the impact of PMWS has been further decreased following introduction of vaccines directed against PCV2 (2). The present study aimed to scrutinise the relevance of vaccinating against PCV2 in a herd previously affected by PMWS, but declared free from clinical disease.

\section{Materials \& Methods}

The study was carried out in a gilt producing herd previously deemed for PMWS, but declared free from PMWS at herd basis since one year. Batches of 24 sows farrowed every third week in previously emptied and cleaned units. Piglets born were weaned at the age of 6 weeks and kept as intact litters in the unit until the age of 12 weeks. At that age barrows were sold, while gilts were transferred to a growing unit with continuous production.

At three weeks of age, 12 litters of one farrowing batch were vaccinated against PCV2 (Ingelvac Circoflex, BI-Vet). The other 12 litters remained unvaccinated. All piglets were weighed at weaning and at 11 weeks of age. The gilts that remained at the herd were also weighed at the age of 21 weeks. Blood from two pigs per litter was collected at 5,8,12 and 16 weeks of age and analysed for presence of serum antibodies to PCV2 with an IPMA technique.

\section{Results}

Figure 1. Log antibody titers to PCV2 in vaccinated $(\diamond)$ and unvaccinated ( $\mathbf{\square})$ pigs.

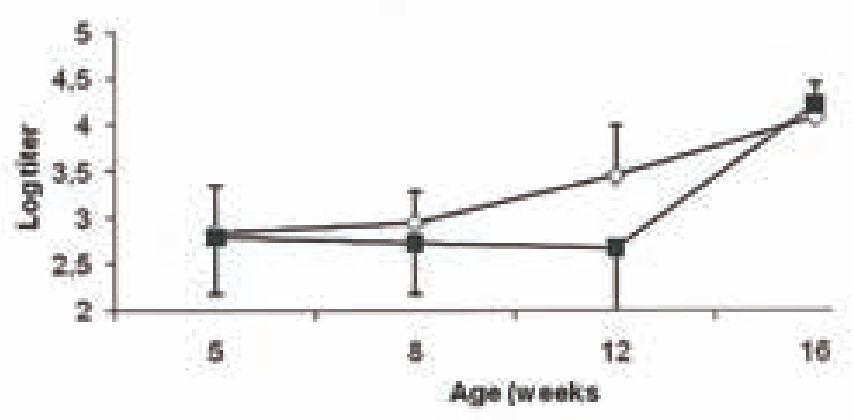

The decreasing levels of serum antibodies to PCV2 from 5 to 12 weeks in the control group indicated a low PCV2-load in the farrowing unit. The vaccinated pigs seroconverted at the age of 12 weeks, while the unvaccinated pigs that seroconverted at 16 weeks of age.
There were no significant differences in mean body weights between the groups at any occasion (Figure 2). However, vaccinated gilts gained significantly more weight from 11 to 21 weeks of age. None of the 48 vaccinated gilts weighed less than $70 \mathrm{~kg}$ at 21 weeks of age. In comparison, 6 out of 54 unvaccinated gilts weighed less than $70 \mathrm{~kg}$ at 21 weeks of age. Out of 53 weaned vaccinated gilts, 48 were still at the herd at the age of 21 weeks (92\%). The corresponding figure for the control group was 54 out of 60 (90\%).

Figure 2. Body weights of pigs vaccinated or not against PCV2 at three weeks of age

\begin{tabular}{lccc}
\hline & Vaccinated & Control & T-test \\
\hline Weaning (n) & 132 & 120 & - \\
\hline Wean (weight, & $11.9 \pm 3.1$ & $12.0 \pm 3.5$ & NS \\
11 w (weight, kg) & $29.4 \pm 5.9$ & $29.7 \pm 7.6$ & NS \\
21 wecks (n) & 48 & 54 & - \\
Pigs <70 kg bw & 0 & 6 & - \\
Mean weight (kg) & $90.4 \pm 11.8$ & $85.5 \pm 14.0$ & $(\mathrm{p}=0.06)$ \\
Gain 11-21 w (kg) & $60.7 \pm 9.0$ & $55.7 \pm 11.9$ & $\mathrm{p}<0.05$ \\
DWG 111-21 & $867 \pm 129$ & $796 \pm 169$ & $\mathrm{p}<0.05$ \\
\hline
\end{tabular}

Statistically significant difference if $\mathrm{p}<0.05$

\section{Discussion \& Conclusions}

We saw no effect of the vaccination in the farrowing unit were the PCV2-load according to the serology was low. Thus, vaccinations against PCV2 with the aim of protecting weaned pigs in such units appear pointless.

However, vaccinated gilts seroconverted earlier when they met PCV2 in the unit with continuous production, indicating a positive effect of the vaccination. They also performed better $(p<0.05)$ from 11 weeks of age, which was achieved by a lower number of poor performing pigs.

The results obtained support theories of improved performance following PCV2-vaccination also in herds without clinical PMWS. However, it ought to be remembered that this herd effectuated continuous production from 12 weeks of age, which coincided with the time when the pigs appeared to meet the infection with PCV2. Therefore, further studies ought to be made in herds with batch wise production to confirm the true relevance of vaccinating young pigs in herds without presence of clinical PMWS. Not the least since a decrease in number of underweighted pigs has a potential to shorten the effective rearing time for the entire batches.

\section{References}

1 Wallgren et al., 2004. Vet. Q. 26:170187.

2. Kixmöller et al., 2008. Vaccine. 26:3443-3451. 


\title{
P.118
}

\section{Vaccination against Porcine Circovirus type $\mathbf{2}$ and Lawsonia intracellullaris in a two- site production system with subclinical manifestations of the microbes}

\author{
Carl-Johan Ehlorsson ${ }^{2}$ Maria Persson ${ }^{1}$ Per Wallgren ${ }^{1}$ \\ 1. Dept of Animal Health and Antimicrobial Strategies, Uppsala, Sweden; 2. Swedish Animal Health Service, Ängelholm, Sweden
}

\section{Introduction}

The superior growth of SPF pigs has raised questions on the impact of subclinical infections on the growth of pigs. Vaccines have recently been registered for the common microbes Porcine Circovirus type 2 (PCV2) and Lawsonia intracellullaris. The present study aimed to scrutinise the relevance of vaccinating piglets against these microbes in a two-site production system with presence of these microbes, but without obvious problems with either of them.

\section{Materials \& Methods}

The study was carried out in a piglet producing herd with 1,000 sows that farrowed in batches of 43 sows every week, and in a fattening herd recruiting full batches from the sow herd at an age of 11 weeks. The herds were free from clinical PMWS and proliferative enteritis, but PCV2 and Lawsonia were demonstrated by serology. All piglets in one farrowing batch were given a unique identity. Piglets in each litter were allotted into three groups. At four weeks of age, group A was vaccinated against PCV2 (Ingelvac Circoflex, Bl-vet), group B against Lawsonia (Enterisol lleitis, BI-Vet), and group $C$ was left as an unvaccinated control. However, gilts had been vaccinated twice against PCV2 (Circovac, Merial), and all sows were revaccinated during pregnancy.

All pigs in the experimental batch were weighted at 4, 10 and 22 weeks of age. Blood was collected from ten pigs per group (from different litters) at 4, 7, 10,13, 16 and 19 weeks of age. Serum was analysed for presence of antibodies to PCV2 by IPMA (1) and Lawsonia by a commercial ELISA (BioScreen, Münster, Germany).

\section{Results}

All piglets received a high maternal protection to PCV2. Piglets vaccinated for PCV2 increased the amount of antibodies to PCV2 at 13 weeks of age and the other groups at 19 weeks of age (Fig. 1). No pig showed any sign of PMWS.

All piglets were seronegative to Lawsonia at 4 weeks of age (Fig 1). At 16 weeks of age, three pigs vaccinated to Lawsonia were seropositive ( $\mathrm{Pl}>34$ ) to Lawsonia compared to one pig in each of the other groups. At 19 weeks of age, three vaccinated pigs were still seropositive compared to 5 or 6 in the other groups. The mean PI-values never differed statistically between groups.

The growth rate was equal for the three groups from 4 to 10 weeks of age (Fig 2). The weight of the pigs was equal for also groups also at the age of 22 weeks.

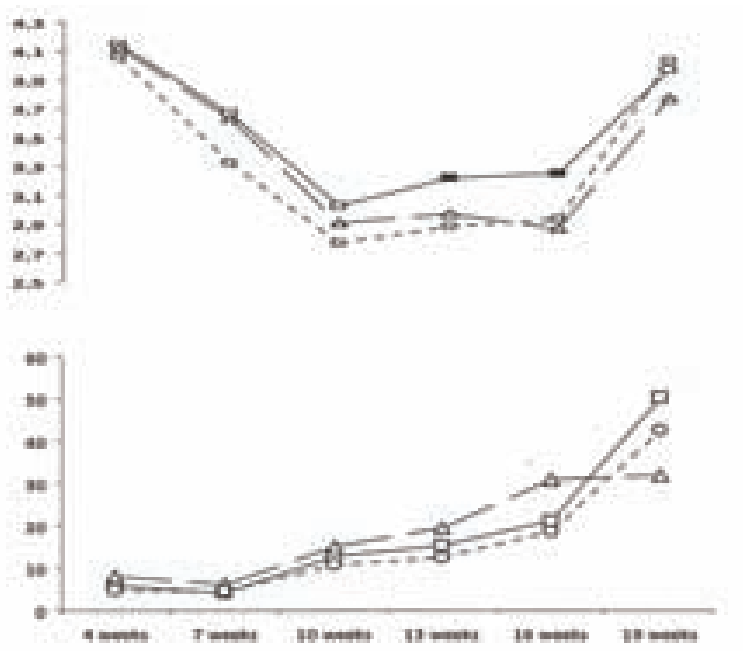

Figur 1. Serum antibodies in pigs vaceinated against PCV2 (D) or Lawsonia ( $\Delta$ ), oe left as controls (o). Different colours within day, pa.05. Above: mean log titres to PCV2. Below: mean Plvalues to Lawsonia

Figure 2

\begin{tabular}{|l|c|c|c|}
\hline Body weights & \multicolumn{3}{|c|}{ Piglets vaccinated } \\
\hline & $\mathbf{P C V} 2$ & Lawsonia & Control \\
\hline Weight $\mathbf{( k g )}$ & $(\mathbf{n = 1 5 2 )}$ & $(\mathbf{n}=\mathbf{1 5 0 )}$ & $(\mathbf{n}=\mathbf{1 4 2})$ \\
\hline 4 weeks & $8.3 \pm 1.9$ & $8.6 \pm 2.2$ & $8.3 \pm 1.9$ \\
\hline 10 weeks & $29.4 \pm 5.4$ & $30.5 \pm 6.4$ & $29.9 \pm 5.7$ \\
\hline 22 weeks & $98.7 \pm 11.0$ & $99.1 \pm 11.8$ & $98.4 \pm 11.8$ \\
\hline
\end{tabular}

\section{Discussion \& Conclusions}

Piglets vaccinated for PCV2 seroconverted between 10 and 13 weeks of age, indicating that PCV2 became activated at that time. However, antibody levels had not increased at 16 weeks in the other groups, indicating a low load of PCV2, presumably achieved by the vaccination of sows and the high amounts of maternal antibodies. Thus unvaccinated pigs seroconverted at a high age when they are less likely to develop PMWS.

Likewise, the serology indicated only a limited load with Lawsonia prior to 16 weeks of age. A protective role of the vaccine may be indicated by somewhat lower PI-values at 19 weeks of age in vaccinated pigs.

We saw no difference in weight gain between the three groups. Thus, vaccinations against PCV2 or Lawsonia with the aim of protecting recently weaned pigs in apparently healthy herds appear pointless, and a true significance of these microbes ought to be proven before expecting any effect in fattening enterprises.

\section{References}

1 Wallgren et al., 2009. Acta Vet Scand. 51:13. 


\title{
P.119
}

\section{Field experiences with Ingelvac CircoFLEX ${ }^{\circledR}$ in mild cases in Spain}

\author{
Jose L. Lorenzo; Marisa Rosas \\ Maporc s.l., San Cristobal de Segovia, Spain
}

\section{Introduction}

PCVD is a worldwide disease and PCV2 is present in nearly all swine herds. Luckily for technicians and producers, in last years, very effective vaccines have been developed and using Circovirus vaccines has been shown to be very profitable (1). Even in cases of mild disease, return of investment of vaccinated animals justify the use of the vaccine (2).

The objective of the present field observation was to evaluate the effect of PCV2 vaccination in mild to moderate cases of $P C V D$ on 3 different farms in Spain.

\section{Materials and Methods}

This field observation included 3 sow farms and 5 fattening units that suffer mild-moderate problems of PCVD. In all cases, peak of signs were observed between 14 and 16 weeks of age. Animals were vaccinated at 3 weeks of age using one shot of $1 \mathrm{ml}$ of Ingelvac CircoFLEX ${ }^{\circledast}$ (Boehringer Ingelheim) following label recommendations. Piglets were moved at 10 weeks of age to the fattening units. Control groups were non vaccinated animals grown in the same building just before vaccinated ones, during 2009. Health status is similar in the three farms: PRRS and Mycoplasma positive (vaccination at 3-4 weeks of age) and APP negative.

Case 1: Farrow-to-finish farm with 550 sows, producing weekly batches of 240 piglets in an AIAO system. Performances in the nursery are really good but animals suffer from respiratory signs and wasting starting at 14 weeks of age.

Case 2: Farrow-to-finish farm with 600 sows, producing weekly batches of 270 piglets. Final Boar is Pietrain. In the nursery there are some problems of Glässer and Streptoccocus suis disease but there are no clinical signs of PCVD in the fattening, only few wasting pigs.

Case 3: Farrow-to-finish farm with 650 sows with genetic nucleus, producing weekly batches of 300 piglets. Final Boar Pietrain. Good performances in nursery and fattening, mild PCVD case with few respiratory problems.

Mortality in controls and vaccinated animals were evaluated using a Chi-square test.

\section{Results}

Case 1: Performance after vaccination on this farm was improved substantially (table 1). Weight gained in the fattening unit was $5,45 \mathrm{~kg}$ more in the vaccinated group spending the same number of days in fattening.

Vaccinated pigs were slaughtered 10 days younger than the control group.
Table 1. Relevant parameters in Case 1

\begin{tabular}{|l|c|c|c|}
\hline & Control & Vaccine & Difference \\
\hline \# piglets tested & 3036 & 950 & \\
\hline Mortality (\%) & $5.4^{\mathrm{a}}$ & $3.1^{\mathrm{b}}$ & -2.35 \\
\hline ADG (g/day) & 708 & 789 & +81 \\
\hline FCR (kg/kg) & 2.576 & 2.374 & -0.202 \\
\hline
\end{tabular}

Case 2: Vaccinated pigs performed much better than the controls (Table 2). Weight gain in the fattening period was $3 \mathrm{~kg}$ more in the vaccinated pigs in 21 days less.

Case 3: The results (Table 2 ) in this case are the mean from 3 different batches in 3 different fattening units.

Table 2. Relevant parameters in Case 2 and Case 3

\begin{tabular}{|l|c|c|c|}
\hline Case 2 & Control & Vaccine & Difference \\
\hline \# piglets tested & 917 & 964 & \\
\hline Mortality (\%) & $4.36^{\mathrm{a}}$ & $2.70^{\mathrm{b}}$ & -1.66 \\
\hline ADG (g/day) & 534 & 756 & +222 \\
\hline FCR (kg/kg) & 2.480 & 2.490 & +0.010 \\
\hline & & & Difference \\
\hline Case 3 & Control & Vaccine & \\
\hline \# piglets tested & 2240 & 2218 & -1.2 \\
\hline Mortality (\%) & $5.0^{\mathrm{a}}$ & $3.8^{\mathrm{b}}$ & +140 \\
\hline ADG (g/day) & 612 & 752 & -0.363 \\
\hline FCR (kg/kg) & 2.817 & 2.454 & \\
\hline a,b. values with a different superscript, differ significantly $(\mathrm{p}<0.05)$ \\
\hline
\end{tabular}

\section{Discussion}

Several authors showed improvements of performance in animals vaccinated with Ingelvac CircoFLEX in different systems around the world (1-6). Data presented in this article confirmed the efficacy in 3 farrow-to-finish farms in Spain. In mild or moderate cases, where mortality is not the main goal, we can appreciate that ADG could be increased by more than 100 grams/day and FCR could be reduced by more than 0,2 .

\section{References}

(1) Cline et al (2008) Veterinary Record, 163, 737-740.

(2) King et al (2008) Proc 20th IPVS Congress, Durban, South Africa, vol. 2, p. 36.

(3) Fachinger et al (2008) Vaccine, 26, 1488-1499.

(4) Ramirez et al (2008) Proc AD. Leman Conference, Rec Res, p.15.

(5) Meedacha et al (2008) Proc 4th APVS Congress, Tsukuba, Japan, p. 106.

(6) Lyoo et al (2008) Proc 20th IVPS Congress, Durban, South Africa, vol. 2, p. 102 


\title{
P.120
}

\section{Effect of vaccination against PCV2 and Lawsonia intracellularis administered concurrently}

\author{
Gonzalo Cano; Joan Sanmartin \\ Optimal Pork Production, S.L., Lleida, Spain
}

\section{Introduction}

Prevention of endemic diseases in farms should be the main objective of the veterinarians in the next years. PCV2 and Lawsonia intracellularis are two of the most widely spread infectious disease in Spain. Both are endemic in all major pig producing countries $(1,2)$. Clinical presentations in farms are variable depending to external factors like nutrition, facilities, co-infections or climatic conditions. Successful experiences with Ileitis vaccination or PCV2 vaccination have been reported in numerous cases. (3-10).

The objective of this study was to evaluate the effect of concurrent PCV2 and Ileitis vaccination administered few days before weaning on performance parameters in fattening.

\section{Materials and Methods}

The study took place in two sow units of the same company, one unit with 4.900 sows and the other one with 2.200. Both units were producing weekly batches and shared gilt source (same quarantine) and did have the same health status: PRRS, APP, and Ileitis positive. Sow and piglet vaccination program were identical and management in both units was very similar.

Performance parameters in the sow farms were good. Piglets were weaned between 21 and 24 days, allocated in nursery until 10 weeks of age and then moved to different fattening units in an all-in/all-out regime (each origin in a separate fattening unit). Identical feed programs and in-feed medications were applied for sows, piglets and pigs.

The before-after study included 18.381 animals divided in 4 groups: non vaccinated, vaccinated against lleitis (Enterisol ${ }^{\circledR}$ lleitis, $2 \mathrm{ml}$ via drench at 17 days of age), vaccinated against PCV2 (Ingelvac CircoFLEX ${ }^{\circledR}, 1 \mathrm{ml}$ IM at 17 days of age) and vaccinated against lleitis+PCV2 (both vaccines administered concurrently at 17 days of age).

All data presented is from different fattening units of the same production system. All batches of all groups were entered simultaneously in fattening units between late November 2008 and late January 2009. Mortality rate was compared between treatment groups using the Chi-square test.

\section{Results}

Performance results show an improvement in each vaccinated group compared to non-vaccinated animals, with, the best results in the group vaccinated with both vaccines (table 1).

In the group vaccinated against lleitis and PCV2 compared to the group with lleitis vaccination only mortality was reduced by $43 \%$ and medication costs by $36 \%$. Comparing the group with concurrent lleitis and PCV2 vaccination with the group vaccinated against PCV2 only, the improvement is obvious in FCR and medication costs.
Table 1: Performance parameters in fattening units comparing 4 groups of vaccination

\begin{tabular}{|l|c|c|c|c|}
\hline & Non vaccinated & Ileitis & PCV2 & Ileitis+PCV2 \\
\hline Num piglets & 5.469 & 5.313 & 3.749 & 3.850 \\
\hline Mortality (\%) & $9.31^{\mathrm{a}}$ & $5.01^{\mathrm{b}}$ & $2.85^{\mathrm{c}}$ & $2.81^{\mathrm{c}}$ \\
\hline Days in fattening & 144 & 138 & 132 & 134 \\
\hline ADG (g/day) & 640 & 672 & 674 & 678 \\
\hline FCR (kg/kg) & 2.880 & 2.710 & 2.770 & 2.700 \\
\hline $\begin{array}{l}\text { Medication costs } \\
(€ / \text { pig) }\end{array}$ & 2.28 & 1.26 & 1.19 & 0.81 \\
\hline $\mathrm{a}, \mathrm{b}, \mathrm{c}:$ : values with a different superscript, differ significantly $(\mathrm{p}<0.05)$ \\
\hline
\end{tabular}

A return of investment of 3,84:1 was calculated for the concurrent ileitis and PCV2 vaccination when compared to the nonvaccinated animals.

\section{Discussion}

The data presented in this study demonstrate that vaccinating pigs concurrently against lleitis and PCV2 improve the fattening performance compared to non-vaccinated animals and animals vaccinated against only one of the diseases. ROI for using both vaccines was 3.84:1 compared to non-vaccinated pigs.

Finally, in this case, both vaccinations were done at the same time, one orally by drench and the other IM. No adverse reaction was observed. This is an example how producers can benefit of doing two treatments together, saving stress to the animals and saving money.

\section{References}

1. Carvajal, A. et al. (2006) Proc IPVS 2006 vol.2, 174

2. Technical Manual Enterisol Ileitis, ver. 3.0.

3. Cano and Sanmartin (2008) Proc IPVS 2008.

4. Lorenzo et al. (2008) IPVS (2008)

5. Cline et al (2008) Veterinary Record, 163, 737-740.

6. King et al (2008) Proc 20th IPVS Congress, Durban, South Africa, vol. 2, p. 36.

7. Fachinger et al (2008) Vaccine, 26, 1488-1499.

8. Ramirez et al (2008) Proc AD. Leman Conference, Rec Res, p.15.

9. Meedacha et al (2008) Proc 4th APVS Congress, Tsukuba, Japan, p. 106.

10. Lyoo et al (2008) Proc 20th IVPS Congress, Durban, South Africa, vol. 2, p. 102. 


\title{
P.121
}

\section{PCV2 vaccination prevented clinical PCVAD and reduced PCV2 viremia and semen virus shedding in boars concurrently infected with PCV2b and Mycoplasma hyopneumoniae}

\author{
Tanja Opriessnig ${ }^{1}$ Darin Madson ${ }^{1}$ Huigang Shen ${ }^{1}$ Susan Brockmeier² Nathan Beach ${ }^{3}$ Xiang-Jin Meng ${ }^{3}$ \\ 1. lowa State University, Ames, IA, USA; 2. National Animal Disease Center, USDA-ARS, Ames, IA, \\ USA; 3. Virginia Polytechnic Institute and State University, Blacksburg, VA, USA
}

\section{Introduction}

It has been determined that porcine circovirus type 2 (PCV2) DNA is shed in semen of naturally and experimentally infected boars (1). Recently, it also has been shown that PCV2 DNA present in semen is infectious in a swine bioassay model (2). However, under experimental conditions the amount of PCV2 shed in semen is low and not sufficient to be transmitted into naïve breeding animals (2). In the growing pig model, Mycoplasma hyopneumoniae infection has shown to potentiate PCV2 replication, PCV2-associated lesions, and disease (3). Under field conditions, young boars that enter boar studs are often exposed to infectious agents (i.e. M. hyopneumoniae) for the first time, are given multiple adjuvanted vaccines, and are exposed to other stressors (mixing, transportation) that are thought to enhance PCV2 replication in growing pigs. The main objective of this study was to determine if the amount of PCV2 shed in semen can be increased in boars experimentally coinfected with M. hyopneumoniae and immune-stimulated via killed parvovirus-leptospira-erysipelothrix (PLE) vaccination. In addition, we wanted to determine if PCV2 vaccination of the boars prior to PCV2 exposure will reduce PCV2 viremia and virus shedding in semen.

\section{Materials and Methods}

Twelve specific-pathogen-free PCV2 and M. hyopneumoniae naïve boars were randomly divided into one of four groups with three animals in each group. Half of the boars were vaccinated against PCV2 35 days before PCV2 challenge using a commercially available inactivated product (Suvaxyn ${ }^{\circledR}$ PCV2; Fort Dodge Animal Health Inc). Fourteen days before and at the day of PCV2 inoculation all 12 boars were vaccinated per label with a commercially available PLE vaccine (FarrowSure ${ }^{\circledR}$, Pfizer Inc.). In addition, 14 days before PCV2 inoculation, 6/12 boars were intranasally inoculated with M. hyopneumoniae. All boars were challenged with PCV2b on Day 0 of the study. Semen and serum samples were collected on a weekly basis and all boars were euthanized 35 days post-PCV2 inoculation. All samples were analyzed for the presence of anti-PCV2 IgG antibodies by ELISA and for the presence and amount of PCV2 DNA by quantitative real-time PCR as described (1).
All vaccinated boars had seroconverted to PCV2 by the time of PCV2 challenge, whereas non-vaccinated boars were seronegative. After M. hyopneumoniae challenge, inoculated boars developed moderate respiratory disease characterized by coughing, respiratory distress, and mucopurulent nasal discharge. After PCV2 challenge, one of three coinfected, non-vaccinated boars became lethargic, lost condition, and died. M. hyopneumoniae infected boars had significantly $(p<0.05)$ higher PCV2 DNA levels in serum compared to boars singularly infected with PCV2. Non-vaccinated boars had significantly $(p<0.05)$ higher PCV2 DNA loads in serum compared to vaccinated boars. Moreover, PCV2 vaccination resulted in significantly $(p<0.05)$ reduced incidence of shedding of PCV2 in semen.

\section{Discussion}

In this study we showed that boars experimentally infected with PCV2 and M. hyopneumoniae can develop clinical manifestation of porcine circovirus associated disease (PCVAD). In addition, PCV2 vaccinated boars did not develop clinical disease and had significantly reduced PCV2 viremia and PCV2 shedding in semen. This information will guide boar stud owners in their decision process when considering PCV2 vaccination.

\section{Acknowledgments}

This works was funded by the lowa Livestock Healthy Initiative. We thank Shayleen Harrison for assistance with the animal work.

\section{References}

1. Madson, D.M. et al. (2008) J. Vet. Diagn. Invest. 20, 725-734.

2. Madson, D.M. et al. (2009) Vet. Res. 40:10.

3. Opriessnig, T. et al. 2004. Vet. Pathol. 41: 624-640.

\section{Results}




\title{
P.122
}

\section{Attenuated vaccine against PCV2 infection by abrogation of ORF3 function}

\author{
Jimmy Kwang \\ Temasek Life Sciences Laboratory, Singapore, Singapore
}

\section{Introduction}

Porcine circovirus 2 (PCV2) is a pathogen causing significant economic impact on swine production. Interestingly, the virus is one of the smallest known viruses with a genome of 1.7 kilobases and has only three identified genes. This virus infection has a high morbidity and leads to severe pathogensis in more than $20 \%$ of the infected pigs. The principle feature of Porcine Circovirus associated Diesease (PCVD) is the compromise of the immune system, marked by lymphadenopathy, lymphocyte depletion and lymphoid organ destruction. The PCV2 infection is usually found with other viral and bacterial co-infections. Our lab identified a novel gene in the PCV2 genome, ORF3, which plays a central role in the apoptosis induced by the virus infection.

\section{Results}

PCV2 open reading frame 3 (ORF3) codes a 105 amino acid protein that causes apoptosis of PCV2 infected cells. In infected cells, the ORF3 causes the accumulation of p53 by interacting with pPirh2 and possibly by disrupting the association of p53 and pPirh2 (J.Virol.81(2007)9560). Mutant PCV2 lacking the expression of ORF3 are infectious and replicate in cells in vitro, but do not cause apoptosis of the infected cells. The ORF3 of PCV2 has been shown to be involved in pathogenesis of the virus in mice model (J. Virol. 80(2006)5065). The pathogenicity of the ORF3 deficient virus is attenuated in the piglets. The mutant virus did not cause any observable disease or perturbation of the lymphocyte count in the inoculated piglets and elicited an efficient immune response. When compared with the wildtype virus infection, the mutant virus infection was characterized by mild viremia and absence of pathological lesions. The lymphoid organ depletion in the ORF3 deficient virus was almost absent as opposed to the wildtype virus infection, which resulted in the characteristic loss of lymphnode architecture. The findings highlight the role of ORF3 in the pathogenesis of PCV2 infection in its host. 


\title{
P.123
}

\section{Piglet vaccination against PCV2 with $\mathrm{CIRCOVAC}^{\circledR}$ (Merial) in 59 German herds: evaluation of the effect on mortality in weaners and fatteners}

\author{
Ariane Schade ${ }^{2}$ Zrinjka Bradaric $^{2}$ Julie Venet $^{1}$ Thaïs Vila$^{1}$ Sophie Longo ${ }^{3}$ François Joisel $^{1}$ \\ 1. Merial S.A.S., Lyon, France; 2. Merial GmbH, Munich, Germany; 3. BPC, Labruguière, France
}

\section{Introduction}

CIRCOVAC ${ }^{\circledR}$ has been registered in mid 2007 in the European Union for the use in sows. Piglet vaccination has been implemented in Germany under special allowance according §17c. Abs.2 TSG since May 2008. Vaccination under special allowance requires collection and report of information on the outcome of the vaccination to authorities. The efficacy of CIRCOVAC sow vaccination in preventing porcine circovirus type 2 (PCV2) infections in piglets and therefore in improving production parameters of their offspring has been extensively proven (1). However in some cases, e.g. piglets from different origins being grouped for fattening, piglet vaccination can be useful (2).

The objective of this study is to evaluate the effect of one-shot vaccination of piglets with $0.5 \mathrm{ml} \mathrm{CIRCOVAC}$ from 2 weeks of age when used in field conditions.

\section{Material and Methods}

Veterinarians taking part in the field study completed questionnaires provided by Merial for vaccine evaluation including questions on production data of the herds before and during CIRCOVAC piglet vaccination.

The analysis was performed in 59 herds from which evaluable information on production data before and during CIRCOVAC piglet vaccination under special allowance was provided. In some herds PCV diseases (PCVD) have been presumed before vaccination as the data showed high mortality and high numbers of wasting animals and animals affected by PDNS. So the vaccination was intended as metaphylactic approach. On other farms vaccination was implemented without presumption of PCVD and with already good performance parameters. Reasons for this could be a preventive approach or the fact that PCV2 piglet vaccination is often requested by swine marketing organisations. Such farms were not excluded from this analysis.

The types of farms are shown in Table 1.

Table 1: Number of present herds by production type

\begin{tabular}{|l|c|c|}
\hline Production type & Number of herds & \% of herds \\
\hline Farrow-to-finish (FF) & 28 & $47.5 \%$ \\
\hline Sows+Weaners (SW) & 23 & $39 \%$ \\
\hline Weaners (W) & 5 & $8.5 \%$ \\
\hline Fatteners (F) & 3 & $5 \%$ \\
\hline Total farms & 59 & $100 \%$ \\
\hline
\end{tabular}

Mortality data and percentage of wasting pigs were obtained from the records on each farm, if they were available. The parameters were analyzed for nursery and fattening period.
Means of each variable were compared between the two different periods of time (before and during vaccination) using Student t-test and/or Kruskal-Wallis test. The level of significance was $5 \%$ for all the tests.

\section{Results}

Mortality rates are significantly decreased after CIRCOVAC vaccination (table 2).

Table 2: Mortality improvement, before versus during vaccination (STD = standard deviation)

\begin{tabular}{|l|c|c|c|l|}
\hline & & Mean (\%) & STD & Decrease of mortality during vaccination \\
\hline $\begin{array}{l}\text { Piglets in } \\
\text { nursery }\end{array}$ & Before & 3.95 & 2.81 & \\
\hline & During & 2.53 & 1.61 & $-1.42 \%(\mathrm{p}=0.016)$ \\
\hline Fattening pigs & Before & 3.35 & 1.91 & \\
\hline & During & 2.21 & 1.17 & $-1.14 \%(\mathrm{p}=0.024)$ \\
\hline
\end{tabular}

Further, the incidence of wasting was lower in both weaners and fatteners after vaccination (-1.6\% in both periods). The difference was close to significance in fatteners $(p=0.060)$.

\section{Conclusion}

CIRCOVAC piglet vaccination induced a distinct improvement with a significant decrease in mortality. This large study demonstrates the positive impact of CIRCOVAC piglet vaccination. For the interpretation of the results, it needs to be taken into account, that farms which vaccinated in a preventive approach were not excluded from the statistics. Therefore it can be hypothesized that restricting the analysis to farms with diagnosed PCV2 problems and reduced performance could lead to even better results.

\section{References}

1. Joisel F. et al., (2007) 5th International Symposium of Emerging and Re-emerging diseases, Krakow, Poland, p.126

2. Vila T. et al., (2009) 40th AASV Proceedings, Dallas, USA, p.231-236

3. Joisel F. et al., (2007) American Association of Swine Veterinarians Proceedings, p.311-314

${ }^{-} \mathrm{CIRCOVAC}$ is a registered trademark of Merial in European Union and elsewhere. 


\title{
P.124
}

\section{Evaluation of the safety of an inactivated porcine circovirus type 2 vaccine $\left(\mathrm{CIRCOVAC}^{\circledR}\right)$ administered to boars: absence of impact on spermatogenesis}

\author{
Thaïs Vila; Valérie Cozette; François Joisel \\ Merial S.A.S., Lyon, France
}

\section{Introduction}

Porcine circovirus type 2 diseases (PCVD) have been reported throughout the world as a major cause of losses in pig herds (1). PCV2 can clearly be associated with some cases of reproductive failure. Furthermore, the presence of infectious PCV2 in semen has been demonstrated $(2,3,4)$. Consequently, PCV2 boar vaccination is commonly used in pig farms. This study was designed to assess the safety of $\mathrm{CIRCOVAC}^{\circledR}$ administered to reproductive boars.

\section{Material and Methods}

The study was performed in a European artificial insemination center. A total of 15 9-month-old boars provided by the same nucleus farm were included in the study and randomized as follows: 5 boars vaccinated twice 3 weeks apart with CIRCOVAC, $2 \mathrm{ml}$ (group 1); 5 boars vaccinated according to the same protocol and treated with paracetamol dose for 3 days since the day before vaccination (group 2); 5 boars non vaccinated but injected with $\mathrm{NaCl} 0.9 \%, 2 \mathrm{ml}$ twice (group 3). Boar semen was sampled once a week from 3 weeks before 1 st vaccination to 8 weeks after 2 nd vaccination. The criteria followed up were: body temperature and semen quality (volume, spermatozoon concentration, motility, abnormality, viability and healthy spermatozoon per ejaculate meaning mobile, with normal mobility and non agglutinated). A one-way analysis of variance (ANOVA) was used to compare temperature results between groups. Semen parameters were compared by a Student t-test after pooling both vaccinated groups 1 and 2 (vacc.) which were similar.

\section{Results and Discussion}

Body temperature was slightly increased after vaccination (4 boars out of 5 were above $39.5^{\circ} \mathrm{C}$ in group 1); this was limited to 1 and 3 boars respectively after 1 st and 2 nd injection in group 2 treated with paracetamol.

Semen quality parameters for each period (before, during and after vaccination) for the vaccinated and control groups are presented in table 1. No group effect was evidenced whatever the period $(p>0.05)$.
Table 1: Semen parameters according to the treatment group (mean value \pm standard deviation)

\begin{tabular}{|l|c|c|c|c|c|c|}
\hline $\begin{array}{l}\text { Semen } \\
\text { parameters }\end{array}$ & \multicolumn{2}{|c|}{$\begin{array}{r}\text { Concentration } \\
\text { (Million/mI) }\end{array}$} & \multicolumn{2}{c|}{$\begin{array}{r}\text { Healthy spz } \\
\text { (Milliard/ejaculate) }\end{array}$} & \multicolumn{2}{c|}{ Viability (\%) } \\
\hline $\begin{array}{l}\text { Groups } \\
\text { (Nb of boars) }\end{array}$ & $\mathbf{V}(10)$ & $\mathbf{C}(5)$ & $\mathbf{V}(10)$ & $\mathbf{C}(5)$ & $\mathbf{V}(10)$ & $\mathbf{C}(5)$ \\
\hline $\begin{array}{l}\text { Before vaccina- } \\
\text { tion (5 weeks) }\end{array}$ & $201.9 \pm 31.2$ & $180.4 \pm 40.8$ & $47 \pm 13.1$ & $45.7 \pm 25.0$ & $85.5 \pm 2.1$ & $86.4 \pm 1.6$ \\
\hline $\begin{array}{l}\text { During vaccina- } \\
\text { tion (4 weeks) }\end{array}$ & $246.5 \pm 62.6$ & $213 \pm 36.4$ & $60.3 \pm 14.1$ & $51.2 \pm 22.4$ & $86.1 \pm 1.8$ & $85.3 \pm 3.1$ \\
\hline $\begin{array}{l}\text { After vaccina- } \\
\text { tion (6 weeks) }\end{array}$ & $201.7 \pm 40.6$ & $191.9 \pm 36.9$ & $\begin{array}{c}59.5 \pm 12.5 \\
(9 \text { boars) }\end{array}$ & $49.5 \pm 18.3$ & $85.1 \pm 2.6$ & $85.1 \pm 2.4$ \\
\hline
\end{tabular}

Only one boar in group 2 had a lower semen concentration during vaccination period. The statistical analysis only evidenced higher values for spermatozoon concentration and viability for vaccinated groups.

Very few impact of CIRCOVAC vaccination was observed in this study on semen quality parameters over a period of 10 weeks after $1^{\text {st }}$ injection.

\section{Conclusion}

CIRCOVAC vaccination showed no impact on spermatogenesis as far as semen parameters of reproductive boars are concerned.

\section{References}

1. Charreyre C. et al., (2006) Proc. Jpn. Pig Vet. Soc., p.49

2. Kim J et al., (2001) J. Virol. Methods, 98(1): 25-31

3. Gava et al., (2008) Pesq. Vet. Braz., 28(1): 70-76

4. Madson et al., (2009) Vet. Res., jan-Feb; 40(1):10

${ }^{\circledR} \mathrm{CIRCOVAC}$ is a registered trademark of Merial in the European Union and elsewhere. 


\title{
P.125
}

\section{IL4, IL5 and IL13 gene expression in porcine PBMC after PCV2 vaccination}

\author{
Juan J. Quereda ${ }^{1}$ Guillermo Ramis ${ }^{1}$ Francisco J. Pallarés ${ }^{2}$ Ludivine Chapat $^{3}$ Anne \\ Goubier $^{3}$ François Joisel $^{3}$ Catherine Charreyre $^{3}$ Antonio Muñoz ${ }^{1}$ \\ 1. Universidad de Murcia, Murcia, Spain; 2. DPTO Anatomía y Anatomía Patológica Comparadas, \\ Universidad De Murcia, Murcia, Spain; 3. Merial S.A.S., Lyon, France
}

\section{Introduction}

Several studies have demonstrated that PCV2 vaccines increase the level of antibodies in the pigs and reduce the severity of the injuries associated to this virus. In the human specie the $\mathrm{TH} 2$ response is mainly regulated by the interleukins (IL) IL4, IL5 and IL13. Sometimes the ELISA technique is not enough sensitive to detect cytokine levels in culture supernatants (1). Nowadays there is no kit for pig IL5 and the ELISA for IL13 is brand new and has never been demonstrated to work with antigen specific response. Quantitative PCR (Q-PCR) is a specific and sensitive technology to quantify the gene expression of mRNA (2). The aim of the present study was to evaluate the $\mathrm{TH} 2$ immune response of PCV2-vaccinated pigs when their peripheral blood mononuclear cells (PBMC) are stimulated with antigenic peptides of the open reading frame 2 (ORF2) of PCV2.

\section{Material and Methods}

Ten SPF 10 weeks old Large White pigs were divided in two groups: vaccinated $(n=5)$ and control (unvaccinated, $n=5$ ). Animals in the first group were vaccinated the day 0 of the experiment with CIRCOVAC $^{\circledR}$ (Merial, France). The day 21 of the experiment a blood sample was obtained and two and a half million PBMC were cultured per well in a total volume of $1 \mathrm{ml}$ complete RPMI under two different conditions: stimulation with PCV2 ORF2 antigenic peptides and a non activation condition where the culture media was composed exclusively by complete RPMI. Gene expression was determined at $24 \mathrm{~h}$ poststimulation. RNA was extracted and retrotranscribed into cDNA. Relative gene expression was calculated using the $2-\Delta \Delta \mathrm{Ct}$ method. Q-PCR for IL4, IL5, IL13 and cyclophilin was developed using published sequences $(3,4)$ in a 7500 real time PCR system (Applied Biosystems, EEUU).

\section{Results}

Major gene expression changes were observed for IL 13 compared to IL4 and IL5 in PBMC. No difference for interleukin gene expression at day 0 between both groups was observed. At day 21 when the cells were stimulated with PCV2 ORF2 peptides a 17 times higher response of IL4 and a 21 times higher synthesis of IL13 was detected in the vaccinated group.

\section{Discussion}

At day 0 of the experiment no specific lymphocytes existed circulating in the blood because no change in IL gene expression was detected after stimulation with PCV2 ORF2 peptides. At day 21 a greater response in the synthesis of IL4 and IL13 was detected in the PCV2 vaccinated group when their PBMC were stimulated with PCV2 ORF2 peptides suggesting that specific lymphocytes have been developed against the vaccine antigens. IL5 gene expression did not show statistical differences between both experimental groups indicating that this IL is low expressed in the pigs as Levast et al., (5) previously demonstrated. Some authors have described that Large White pigs do not express IL4 (6), but the results of the present study showed that gene expression of IL4 took place in the Large White pigs analyzed, suggesting that differences could exist among different Large White genetic lines. Bautista et al., (7) suggested that IL13 could replace IL4 functions in the pig, but our results showed IL13 and IL4 gene expression changes in response to PCV2 ORF2 peptides in the vaccinated group indicating that both IL could be important in the pig $\mathrm{TH} 2$ response.

\section{References}

1. Díaz I \& Mateu E (2005) Vet Immunol Immunopathol 106:107-112.

2. De Boever S, Vangestel C, De Backer T et al., (2008) Vet Immunol Immunopathol 122: 312-317.

3. Dawson HD, Beshah E, Nishi S et al., (2005) Infect Immun 73: 11161128.

4. Flores-Mendoza L, Silva-Campa E, Reséndiz M et al., (2008) Clin Vaccine Immunol 15: 720-725.

5. Levast B, De Monte M, Melo S (2010) Dev Comp Immunol 34:102106.

6. Raymond C \& Wilkie B (2004) Vaccine 22: 1016-1023.

7. Bautista EM, Nfon C, Ferman GS (2007) Vet Immunol Immunopathol 115: 56-57.

${ }^{-} \mathrm{CIRCOVAC}$ is a registered trade mark of Merial in Canada and elsewhere. 


\title{
P.126
}

\section{Swine herd with a prevalent PCV2 subclinical infection: diagnostic investigation results before and after PCV2 vaccination}

\author{
Andrea Luppi ${ }^{1}$ Paolo Bonilauri ${ }^{1}$ Claudio Mazzoni ${ }^{2}$ Rosanna Di Lecce ${ }^{3}$ Michele Dottori ${ }^{1}$ \\ 1. Istituto zooprofilattico sperimentale Lombardia and Emilia Romagna, Reggio Emilia, Italy; 2. Libero \\ professionista, Reggio Emilia, Italy; 3. Dipartimento di Sanità animale, Sezione di Patologia Generale ed \\ Anatomia Patologica, Facoltà di Medicina Veterinaria, Università di Parma, Parma, Italy
}

\section{Introduction}

Porcine circovirus type 2 (PCV2) may cause a variety of diseases (PCVAD - Porcine Circovirus Associated Disease), including postweaning multisystemic wasting syndrome (PMWS) (1). Because subclinical infections are common, diagnostic methods used should be able to perform the PCVAD diagnosis and also evaluate the PCV2 viremia in pigs. In the present longitudinal study the Authors described the use of a diagnostic approach, before and after the PCV2 vaccination, in a herd where PCV2 subclinical infections were prevalent.

\section{Materials and Methods}

The clinical case took place between March and June 2008 in a farrow to finish herd with 170 sows in the Northern Italy. The clinical symptoms of the disease were mainly observed in pigs 12 weeks old and were characterized by loss of appetite, rough hair, paleness, dyspnoea, cough and diarrhoea. Total losses reaching over $7 \%$ in some batches. Before vaccination against PCV2, blood samples and lymph-nodes ( $L n$. inguinales superficiales) were collected from 15 pigs 5, 12 and 19 weeks old (5 pigs for each age) without clinical signs and from 10 pigs 12 weeks old with clinical signs PMWS compatible. Cytology and histology of lymph-nodes were respectively performed on air dried impression smears and on formalin-fixed, paraffinembedded tissue sections. Cytological and histological slides were stained by the May-Grunwald Giemsa and EmatoxilinEosin methods respectively (2). Immunohistochemistry was performed on the same paraffin embedded tissues using a home made monoclonal antibody against PCV2. Real time PCR was performed on blood (sera) samples described above, using primers and probe designed by Olvera et al. (2004). Vaccination against PCV2 was performed using a one-shot commercial vaccine, administered to piglets 3 weeks old. Post vaccination, blood samples and lymph-nodes (Ln. inguinales superficiales) were collected from 15 pigs 5, 12 and 19 weeks old (5 pigs for each age) and submitted to the same investigations described above.

\section{Results}

No microscopic lesions were observed in the lymph-nodes belonging to pigs without clinical signs. No PCV2 viral load was observed on sera of pigs 5 weeks old. Real time PCR logarithmic values (PCV2 genomic copy no./gr.) of sera belonging to pigs 12 and 19 weeks old, without clinical signs, showed moderate (4.7, $5.8,5.9,6.1,6.8)$ and absence or mild viral $\operatorname{load}(0,0,0,5.1,5.1)$ respectively. On 10 pigs with clinical signs PMWS compatible, only 4 animals showed characteristic PMWS microscopic lesions of lymph-nodes and high sera viral load (the real time PCR logarithmic values - PCV2 genomic copy no./gr. were 7.5, 8.3, 9.0, 7.5). The diagnosis was confirmed by immunohistochemistry. The others results of real time PCR on pig sera belonging to this group showed moderate viral load (the real time PCR logarithmic values - PCV2 genomic copy no./gr. were 5.1, 5.6, 5.9, 6.1, 6.9, 7.0). No viral load was observed on 15 pig sera samples collected 2,9 and 16 weeks after PCV2 vaccination.

\section{Discussion}

The field study was conducted to investigate the use of a diagnostic approach, based on cytology and histology of lymph-nodes and sera PCV2 real time PCR, on pigs belonging to an herd showed prevalently a subclinical PCV2 infection. The study was performed before and after the PCV2 vaccination. The results of this study suggested that in herds in which a subclinical PCV2 infection is present, real time PCR on sera seems to represent the better approach to understand the disease development and the subclinical PCV2 infection severity. Two, nine and sixteen weeks after the vaccination, pigs showed no viremia, as well as a significant improvement of productivity performances with a consequent reduction of herd losses $(<3-4 \%)$. This indicates that in absence of obvious clinical PCVAD signs, the virus still induced a detrimental effect on growth and mortality. However, the results of real time PCR on pig sera after vaccination, need to be further investigated and must not be used to evaluate the vaccination efficacy.

\section{References}

1. Krakovka S., Ellis J.,McNeilly F.,Waldner C., Allan G. (2005). J.Vet. Diagn Invest, 17:213-222.

2. Luppi A. (2003). Veterinary Record, 152, (5), 148.

3. Olvera A., Sibila M., Calsamiglia M., Segalés J., Domingo M. (2004). J. Virol.Methods, 117 (1):75-80. 


\title{
P.127
}

\section{Duration of immunity by PCV2 challenge in 17 week-old conventional piglets vaccinated at 3 weeks of age with CIRCOVAC $^{\circledR}$ (Merial)}

\author{
Mathieu Chevalier ${ }^{1}$ Laurent Fischer ${ }^{2}$ François Joisel $^{2}$ \\ 1. Merial CRSV, Saint-Vulbas, France; 2. Merial S.A.S., Lyon, France
}

\section{Introduction}

The aim of this study was to demonstrate, in conventional piglets, the duration of immunity conferred by vaccination with the inactivated adjuvanted PCV2 vaccine CIRCOVAC.

\section{Material and Methods}

Three-week-old piglets were randomly assigned in 2 groups: 15 piglets vaccinated once with CIRCOVAC $0.5 \mathrm{~mL}$, IM and 12 piglets injected with $0.5 \mathrm{~mL}$ of PBS as control. Both groups were kept in isolation and subsequently challenged at 17 weeks of age with an infectious PCV2b field strain. The 2 groups were compared according to body weight evolution and PCV2 viral load in sera and faeces.

\section{Results}

Before challenge, body weights of vaccinated and control animals were similar. After challenge, vaccinated animals were heavier although not significantly (ADWG $p=0.116$ ) than controls: $+2.4 \mathrm{~kg}$ in average at D28 post challenge (Figure 1).

Figure 1: Evolution of average body weight after challenge

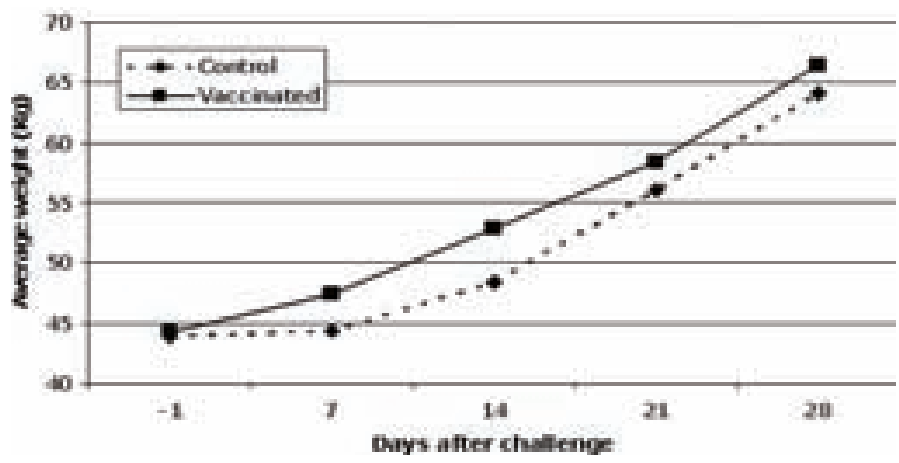

Further, post challenge PCV2 viral DNA load in the sera of vaccinated piglets was significantly reduced as compared to the viral load in the sera of placebo-injected animals (Figure 2a).

The mean viral load during the whole monitoring period as area under the curve (AUC) was significantly lower for vaccinated piglets compared to control animals (Student t-test $\mathrm{p}<0.001$ ) (Figure 2b).

Vaccination also contributed to a significant reduction of post challenge viral excretion in faeces (Figure 2c).

The mean viral load during the whole monitoring period (AUC) was significantly lower for vaccinated piglets compared to control ones (one-tailed student t-test $\mathrm{p}=0.011$ ) (Figure $2 \mathrm{~d}$ ).

\section{Discussion and Conclusion}

All together, these results demonstrated a significant reduction of PCV2 viral load in sera and in faeces, in CIRCOVAC vaccinated animals after PCV2 challenge at 17 weeks of age. A growth improvement ( $+2.4 \mathrm{~kg}$ in 28 days post challenge) in vaccinated animals has been also observed.
Figure 2: a) evolution of $P C V 2$ viral load in the sera during challenge; b) distribution of the AUC PCV2 viral load in sera in control and vaccinated groups; c) evolution of mean PCV2 excretion in faeces; d) distribution of the AUC PCV2 viral excretion in faeces in control and vaccinated groups

a)

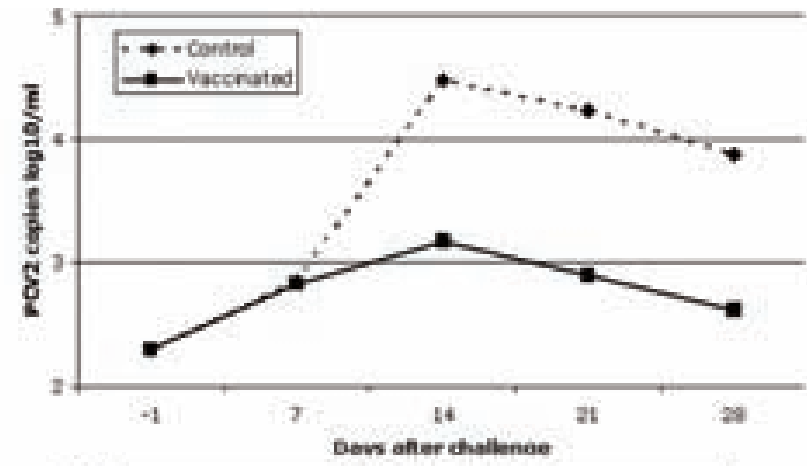

b)

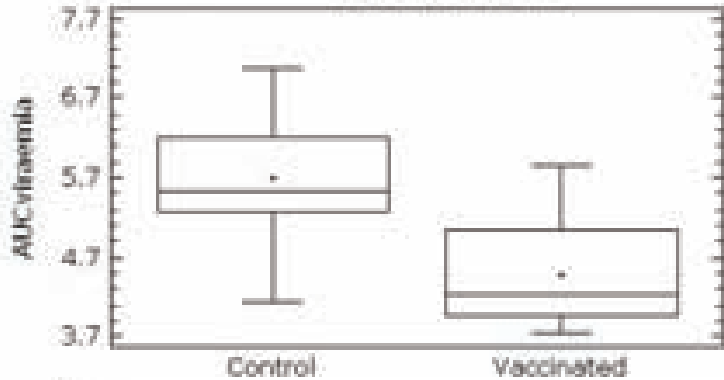

c)

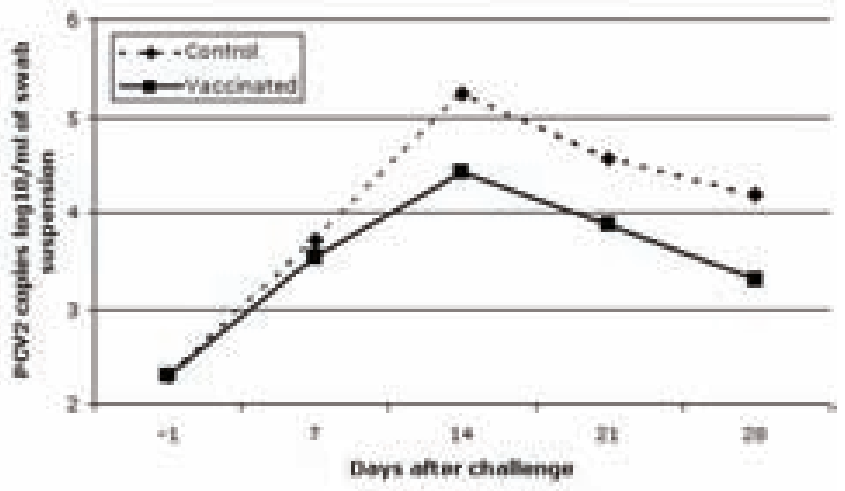

d)

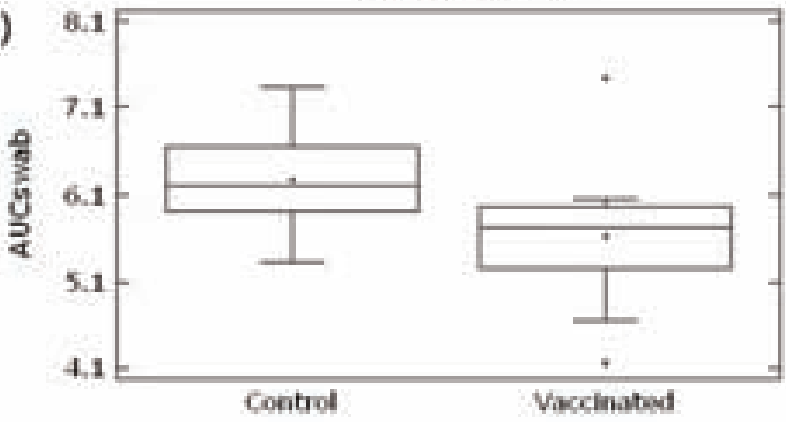

${ }^{\circledR} \mathrm{CIRCOVAC}$ is a registered trademark of Merial in Canada and elsewhere. 


\title{
P.128
}

\section{Beneficial impact of an inactivated PCV2 vaccine (CIRCOVAC ${ }^{\circledast}$ ) under Portuguese conditions: a large scale field study}

\author{
J.M. Lopes Jorge ${ }^{1}$ Thaïs Vila $^{2}$ Sophie Longo ${ }^{3}$ François Joisel $^{2}$ \\ 1. Merial Portuguesa, Lisbon, Portugal; 2. Merial S.A.S., Lyon, France; 3. BPC, Labruguière, France
}

\section{Introduction}

CIRCOVAC has been registered in Europe for sow vaccination since 2007 and has proved efficacy to reduce mortality rate from birth to slaughter (1), improve growth performance (2) and reproductive parameters (3). In an attempt to control PCVD in already born when sow vaccination is started, piglets can be temporarily vaccinated up to the batch of piglets born from completely (2-times vaccinated) sows reaches weaning. This successful method was already described earlier (4).

The objective of this study was to evaluate the effect of this CIRCOVAC vaccination programme under Portuguese conditions.

\section{Material and Methods}

The study was performed in 10 farms located in Azores and Continental Portugal, with clinical PCVD signs after weaning: 8 farrow-to-finish farms and 2 farrow-to-post-weaning units. Farm size ranged from 220 to 1,551 sows, average: 557 sows/farm.

PCV2 sow vaccination was implemented from September 2007 to April 2008 as follows: mass vaccination of all sows, 2 injections of CIRCOVAC ( $2 \mathrm{ml}) 3$ weeks apart, and a booster 15 days before farrowing. At the same time, temporary piglet vaccination was implemented in 8 farms as follows: piglets from weaning to 7 weeks of age were injected with CIRCOVAC once, $0.5 \mathrm{ml}$. All weaned piglets born from non 2-time-vaccinated sows were vaccinated. Then, piglets vaccination was stopped as soon as piglets born from sows vaccinated twice reached weaning. A total of 5,573 sows, 138,017 weaned piglets and 131,925 slaughter pigs were monitored. Effect evaluation was based on post-weaning and fattening mortality rates and on weaned piglets/sow/year that summarizes all aspects of reproduction. A questionnaire was sent to the vets in charge of 33 farms that started CIRCOVAC vaccination between 09/2007 and 04/2008. Global data concerning the different parameters were collected from farm software.

Two similar periods of time were compared for each farm: before the date of first vaccination and during vaccination starting at the date of mass vaccination (duration: 13 to 52 weeks according to available data). Mortality rates were compared using a Kruskal-Wallis test and number of weaned piglets a Student t-test.

\section{Results and Discussion}

Mortality rate in post-weaning decreased in every single farm, as well as the average mortality went significantly down, $p=0.023$ (Table 1). In fattening, completed data were obtained only for the 8 farrow-to-finish farms. Mortality rates decreased in 7 of them. In one farm, mortality that was very low before vaccination $(0.42 \%)$ went up to $0.91 \%$ due to a $\mathrm{S}$. suis outbreak in the period during vaccination. Average decrease of mortality in fattening was not statistically significant due to the small number of farms but the improvement magnitude confirmed what was already seen (1).
The number of weaned piglets/sow/year was improved in 9 out of 10 farms (Table 2). One farm experienced a slight decrease $(-0.19)$ due to a Swine Influenza outbreak during vaccination period. Increase in weaned piglets/sow/year was not found statistically significant but the improvement magnitude confirmed what was already seen (3).

Table 1: Mortality rate before and during vaccination (*STD: standard deviation)

\begin{tabular}{|l|c|c|c|c|c|}
\hline & & $\begin{array}{c}\text { Farms } \\
(\mathbf{n}=)\end{array}$ & Mean (\%) & STD* & Mortamity Decrease \\
\hline Post-weaning & Before & 10 & 5.18 & 3.08 & $2.72 \pm 2.02(p=0.023)$ \\
\hline Post-weaning & During & 10 & 2.46 & 1.10 & $2.72 \pm 2.02(p=0.023)$ \\
\hline Fattening period & Before & 8 & 4.81 & 3.72 & $2.40 \pm 2.03(p=0.208)$ \\
\hline Fattening period & During & 8 & 2.40 & 1.87 & $2.40 \pm 2.03(p=0.208)$ \\
\hline
\end{tabular}

Table 2: Weaned piglets/sow/year before and during vaccination (*STD: standard deviation)

\begin{tabular}{|l|c|c|c|c|}
\hline & Farms $(\mathbf{n}=)$ & Mean & STD* & Improvement \\
\hline Before & 10 & 22.6 & 1.47 & $+1.1 \pm 0.70(\mathrm{p}=0.147)$ \\
\hline During & 10 & 23.7 & 1.83 & $+1.1 \pm 0.70(\mathrm{p}=0.147)$ \\
\hline
\end{tabular}

\section{Conclusion}

The beneficial impact of CIRCOVAC using a vaccination programme including short-term piglet vaccination and long-term sow vaccination has been confirmed in this study. Return on investment must include the decrease of mortality rates and the improvement in reproduction as shown by the increase of weaned piglets/sow/year which was welcomed as an unexpected additional benefit.

\section{References}

1. Pejsak Z. et al. (2009) $4^{\text {th }}$ APVS Tsukuba, Japan, p.232

2. Delisle G. et al. (2009) $4<$ sip >th APVS Tsukuba, Japan, p. 220

3. Joisel F. et al. (2008) $20^{\text {th }}$ IPVS Durban, p.72

4. Vila T. et al. (2009) $4^{\text {th }}$ APVS Tsukuba, Japan, p.233

${ }^{\circledR} \mathrm{CIRCOVAC}$ is a registered trademark of Merial in Canada and elsewhere. 


\title{
P.129
}

\section{Monitoring PCV2 viremia in pigs on Canadian farms after using PCV2 vaccination}

\author{
Francisco de Grau \\ Intervet Canada Corp, Kirkland, QC, Canada
}

\section{Introduction}

Since PCV2 discovery in the early 90's, by Harding1, PCV2 was commonly found in blood samples from Canadian pig farms with PCVAD and on farms where no clinical signs were observed 2. PCVAD occurred sporadically in Ontario and Quebec before 2004 2,3, 4. However, a PCVAD outbreak occurred in these provinces in the winter of 2004 and spread to the rest of Canada in 2005. A new strain of PCV2 (2b) was associated with these outbreaks 5. PCV2 vaccination was introduced in Canada in the spring of 2006. Mortality decreased significantly after vaccination. However, subclinical presentations are still a common problem. The objective of this study was to determine the prevalence of PCV-2 viremia after 3 years of the use of PCV-2 vaccines in Canadian farms.

\section{Materials and Methods}

Twelve swine veterinarians from four veterinary practices and five private companies participated in this study. The data was collected from 23 swine herds from across Canada. All herds were routinely vaccinating with commercial PCV2 vaccines. Practitioners collected 50 pooled blood samples from randomly selected pigs on each farm: 10 pigs at weaning, 10 pigs at the mid nursery stage, 10 pigs at the end of the nursery stage; 10 pigs at the middle of the grower finisher period, and 10 just before slaughter to determine the vaccine program efficacy and viremia control. Blood samples were centrifuged and the sera recovered. Serum samples were sent to the diagnostic lab for PCR testing.

\section{Results}

In total 1260 pigs were sampled. The average herd size was 1580 sows with a range of $170-6000$. Forty-four percent of the farms were farrow-to-finish and 56\% multiple-site production. Twenty-two herds were using a commercial vaccine to control PCV2. Viremia was found on 14 (61\%) herds. According to the practitioner reports, $40 \%$ of these farms reported clinical signs of PCVAD. Ninety percent (9/10) of the farrow-to-finish farms, and $38 \%(5 / 13)$ of the multi-site operations presented PCV2 viremia.

A total of $65 \%(14 / 23)$ farms sampled were PRRV+. Within these farms, PCV2 viremia was found on $71 \%(10 / 14)$. A total of $61 \%$ $(14 / 23)$ of the herds were Mycoplasma positive. Within these mycoplasma positive farms $57 \%(8 / 14)$ had PCV2 viremia.
Thirty-five percent (8/23) of the nurseries, and $48 \%$ grower-finisher $(11 / 23)$ were positive for PCV2 viremia. Eighty percent of all the viremic samples were from grower-finisher barns pigs. More than half of the positive samples $8 / 14$ (57\%) were found to be positive in more than one production phase.

Sixty one percent (14/23) herds reported using the vaccines offlabel. Fifty seven percent of them were positive for PCV2 viremia. $P C V 2$ viremia was found on $57 \%$ percent of the herds where PCVAD signs had been observed.

PCV2a was found on $26 \%(6 / 23)$ of the herds, PCV2b on $39 \%$ $(9 / 23)$ herds, and both strains were found in only one herd. PCV2 was not detected on 7 farms (30\%). Three out of 7 herds negative to PCV2 viremia were also negative for PRRS and Mycoplasma. Only one herd negative to PCV2 viremia was a farrowto-finish operation.

\section{Conclusions and Implications}

The data revealed higher PCV2 viremia in grower-finisher pigs. PCV2 viremia is more prevalent on farms positive for PRRSv and Mycoplasma. PCV2 viremia was found on more than half of the farms where vaccines were used off label.

PCV2a was found on $25 \%$ of the farms ninety percent of these farms were located in Western Canada (Alberta, Manitoba).

This study shows that PCV2 still present in the field even after three years after PCV2 commercial vaccine introduction and use. The Practitioners participated voluntarily and farms were not selected randomly therefore these results may be biased and cannot be extrapolated to all farms in Canada.

\section{References}

Harding, J. Proc WCASP 1996; 21

Cotrell et al., Proc AASV 1999: 389-390

Charbonneau, G. George A. Young Swine Conf 2006: 36-49.

Batista, L Proc AASV 2006; 383-386

Carman et al., AHL Newsletter March 2006. 


\title{
P.130
}

\section{Improvement of reproductive parameters in gilts after $\mathrm{CIRCOVAC}^{\circledR}$ vaccination in a farrow-to-finish farm in France}

\author{
Michel Noirrit ${ }^{2}$ Bernard Fily ${ }^{3}$ Guillaume Perreul ${ }^{4}$ Jean-Bernard Herin ${ }^{3}$ Thaïs Vila $^{1}$ Andrea Delvecchio ${ }^{1}$ Julie Venet $^{1}$ François Joisel $^{1}$ \\ 1. Merial S.A.S., Lyon, France; 2. TOPIGS France, Loperhet, France; 3. Merial S.A.S., Ancenis, France; 4. MERIAL, Lyon, France
}

\section{Introduction}

PCV2 has been associated with reproductive disorders and is regarded as a causative agent of foetal death in swine $(1,2)$.

The objective of the present paper is to report a clinical case in which $\mathrm{CIRCOVAC}^{\circledR}$ vaccination gave an improvement in the reproductive parameter in a farm where PCV2 was suspected to be involved in abortions.

\section{Farm description}

These results come from a 1600-sow farrow-to-finish French farm, having faced reproductive disorders on the long run. Litters coming from non-vaccinated gilts and sows did not show any PMWS clinical signs. This closed farm observes strict 2-month quarantine. The farm productivity was below its potential, and abortions occurred regardless sow parity. But the fertility rate $(>92 \%)$, the number of mummified foetuses $(0.3 /$ litter) and stillborns ( $0.73 /$ litter) consistently remained at a normal level. Piglets used to be weaned at 21 days.

Gilts are raised in a specific unit away from the rest of the other pigs of the farm. The routine vaccination program of the farm use to count a mass-vaccination with PROGRESSIS ${ }^{\circledR}$ against PRRS every $31 / 2$ months, GRIPOVAC ${ }^{\circledR}$ vaccination against swine flu, and vaccination against parvovirus and erysipelas.

\section{Case Description - Diagnosis}

For the last years, lots of changes were carried out to improve the farm reproductive parameters, mostly to reduce the abortion rate.

No other classical abortion infectious causes were identified except Leptospira sp. which were found to be the causative agents of abortions (PCR-positive). Repeated antibiotic metaphylactic treatments were put and kept in place ( 2 to 3 times / year) and a strict control of rodents was implemented.

Despite these measures, abortions up to 80 gestation days still occurred. Thus, PCV2 infection was suspected: at the first attempt, 4 aborted foetuses hearts were found PCV2 negative by PCR. A second analysis was performed 1 year later and large amounts of PCV2 was found by IHC or qPCR in hearts or livers of 8 aborted foetuses from 4 sows.

\section{PCV2 vaccination}

It was decided to carry out a mass-vaccination of all breeding animals with CIRCOVAC. All pens were vaccinated starting with a primo-vaccination of gilts in quarantine followed by a second injection 3 weeks before the insemination.

\section{Results}

Reproductive parameters of gilts are shown in table 1. After CIRCOVAC vaccination, four reproductive parameters were improved although non-significantly: abortions rate, return of oestrus rate, farrowing rate and the weaning to effective service interval at the 1 st weaning.

Table 1: Reproductive parameters before and after CIRCOVAC vaccination ( $p=$ Chi-square test $p$-value)

\begin{tabular}{|l|c|c|c|}
\hline & Non-vaccinated gilts & CIRCOVAC vaccinated gilts & $\mathbf{P}$ \\
\hline No gilts (15 gilts/pens) & 165 & 165 & - \\
\hline \% farrowing & 83.9 & 90.5 & 0.072 \\
\hline No abortions (\%) & $12(7.3 \%)$ & $6(3.6 \%)$ & 0.146 \\
\hline \% return to oestrus & 5.1 & 1.8 & 0.125 \\
\hline $\begin{array}{l}\text { Weaning-to-effective } \\
\text { service interval at 15t } \\
\text { weaning (days) }\end{array}$ & 8.7 & 5.5 & NA \\
\hline No mummified/litter & 0.3 & 0.3 & - \\
\hline No stillborn/litter & 0.8 & 0.8 & - \\
\hline
\end{tabular}

No difference in mummified and stillborn per litter was noticed between vaccinated and non-vaccinated gilts.

\section{Discussion and Conclusion}

Although none of the differences before and after vaccination in gilts seem to be statistically significant, most probably due to the low number of animals, the improvement looked quite spectacular for the farmer. It may be hypothesized that gilts raised in a premise separated from the rest of the farm had minimal contact with PCV2 during their pre-mating life. Then, the suddenly increased viral pressure when they joined back the sow herd may have been the cause of the abortions. At least, a systematic vaccination of the whole herd with CIRCOVAC seemed to have solved the reproductive issue.

\section{References}

1. Pittman J. S., (2008) J Swine Health Prod, 16(3):144-148.

2. Hansen M. S. et al., (2009) Vet Microbiol, doi:10.1016/j. physletb.2003.10.071.

${ }^{-} \mathrm{CIRCOVAC}$ is a registered trademark of Merial in the Canada and elsewhere. PROGRESSIS and GRIPOVAC are registered trademarks of Merial S.A.S. 


\title{
P.131
}

\section{Immediate efficacy of $\mathrm{CIRCOVAC}^{\circledR}$ (Merial) administered to 3 week- old SPF piglets against a challenge at 5 weeks of age}

\author{
Mathieu Chevalier ${ }^{1}$ Laurent Fischer ${ }^{2}$ François Joisel $^{2}$ \\ 1. Merial CRSV, Saint-Vulbas, France; 2. Merial S.A.S., Lyon, France
}

\section{Introduction}

The aim of this study was to demonstrate, in SPF piglets, the onset of immunity conferred by vaccination with the inactivated adjuvanted PCV2 vaccine of Merial: CIRCOVAC ${ }^{\circledR}$.

\section{Material and Methods}

Three-week-old piglets were randomly assigned in 2 groups: 15 piglets vaccinated once with CIRCOVAC $0.5 \mathrm{~mL}$, IM and 15 piglets injected with $0.5 \mathrm{~mL}$ of PBS as control. Both groups were kept in isolation and subsequently challenged at 5 weeks of age with an infectious PCV2b. The 2 groups were compared according to body weight evolution, and PCV2 viral load in sera and faeces.

\section{Results and Discussion}

After challenge, the median RDWG was significantly higher for vaccinated piglets when compared to controls (Mann-Whitney test; $p=0.030$ ) (data not shown), leading to a difference of $1.5 \mathrm{~kg}$ between the average weight of vaccinated and control animals at D42 (Fig. 1).

Figure 1: Evolution of body weights before and after challenge (D14)

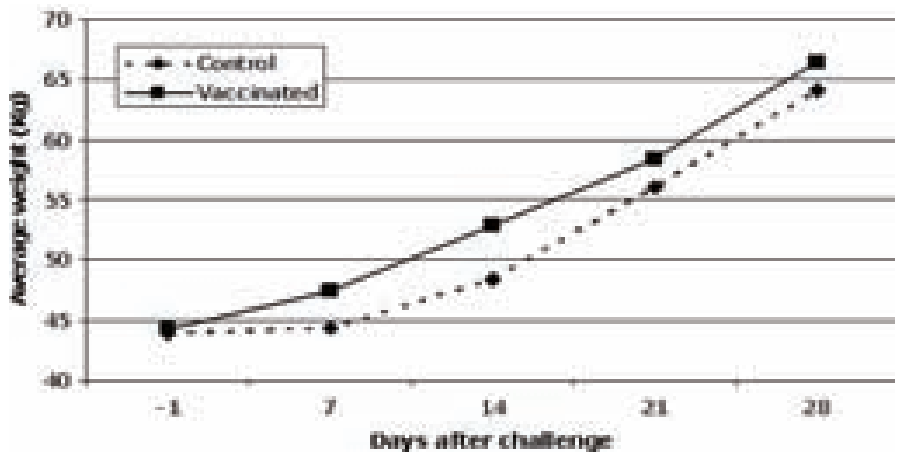

Further, post-challenge PCV2 viral DNA load in the sera of vaccinated piglets was significantly reduced as compared to the viral load in the sera of placebo-vaccinated animals (Fig 2a). Both vaccinated and control piglets had no detectable PCV2 in serum before challenge.

The mean viral load on the whole monitoring period (AUC) was significantly lower for vaccinated piglets compared to control animals (one tailed Student t-test $p=0.003$ ) (Fig. 2b).

Vaccination also contributed to a significant reduction of postchallenge viral excretion in faeces (Fig. 2c). The mean viral load on the whole monitoring period (AUC) was significantly lower for vaccinated piglets compared to control animals (one tailed Student t-test $p=0.004$ ) (Fig. 2d).

\section{Conclusion}

Collectively, these results demonstrated a significant reduction of viral load in blood and faeces of PCV2 and a significant difference of the median RDWG (relative daily weight gain) between vaccinated and controls piglets leading to a difference of +1.5 $\mathrm{kg}$ of body weight in average at the end of the animal phase in CIRCOVAC vaccinated animals against a virulent PCV2 challenge performed only two weeks after vaccination, i.e. at the age of 5 weeks.

${ }^{\circledR} \mathrm{CIRCOVAC}$ is a registered trademark of Merial in Canada and elsewhere.

Figure 2: a) evolution of the mean PCV2 viral load in the sera; $b$ ) distribution of the AUC PCV2 viral load in the sera according to groups; c) evolution of the mean PCV2 viral excretion in faeces; d) distribution of AUCPCV2 viral excretion in the feces according to groups

a)

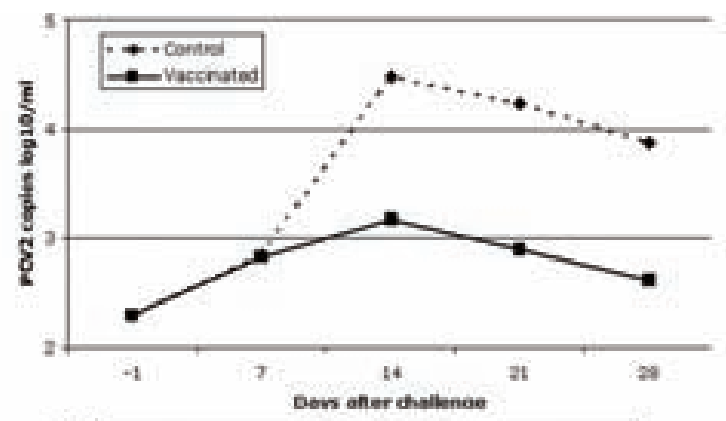

b)

c)
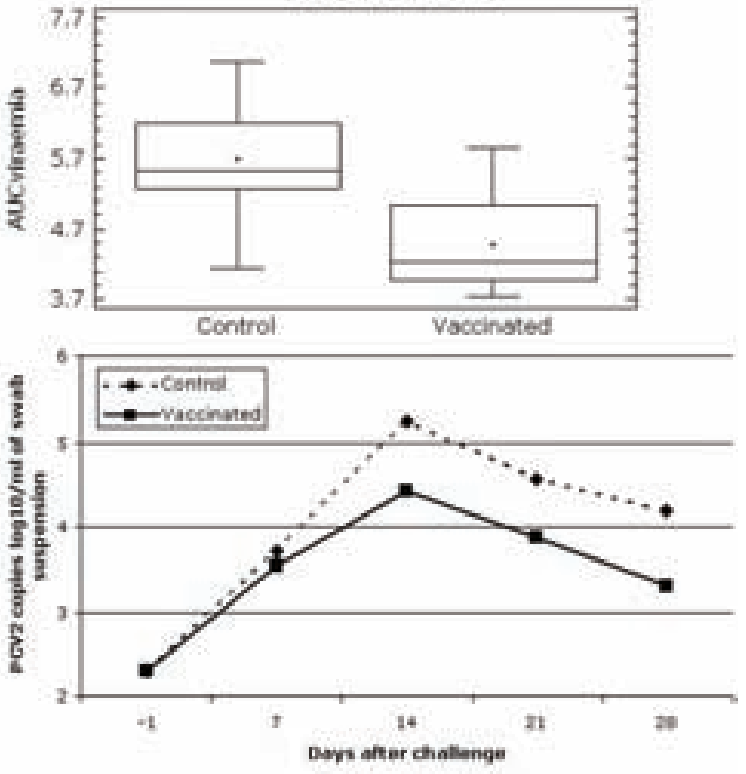

d)

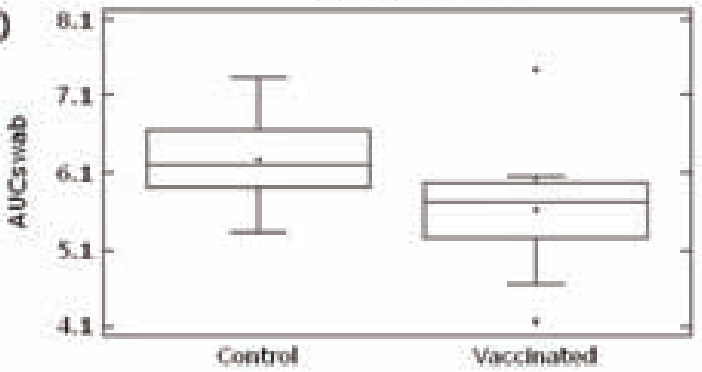




\title{
P.132
}

\section{Clinical and pathologic lesions after simultaneous vaccination with PCV2 and Mycoplasma vaccine in 3 week old piglets}

\author{
Alvaro Ruiz ${ }^{2}$ Juan M. Palacios $^{1}$ \\ 1. Intervet Schering Plough AH, Huixquilucan, MEX, Mexico; 2. Universidad de Concepción, Chillan, Chile
}

\section{Introduction}

The use of simultaneous PCV-2 and Mycoplasma sp vaccines has been a common practice in order to reduce piglet management and stress, both vaccines are used in a similar age at weaning, even there is no a commercial vaccine that combines both antigens the products can be injected each at one neck side or in the same point. The objective of this trial was to evaluate the safety of a simultaneous use of a PCV2 vaccine1 and a Mycoplasma sp bacterin2.

\section{Materials and Methods}

311 piglets, with 3 weeks of age, from a conventional herd were utilized; piglets were vaccinated with $2.0 \mathrm{ml}$ of PCV-2 vaccine and $1.0 \mathrm{ml}$. of Mycoplasma bacterin one day after weaning followed by a second vaccination at six weeks of age. Vaccination was performed using an automatic double syringe (NJ Phillips Ltd) at the same site of the neck (first dose at the right site, second dose 3 weeks alter at the left site). Another group of 26 animals received the vaccines separately (first PCV-2 dose at the right site of the neck and Mycoplasma sp bacterin at the left site) and the second dose 3 weeks later. All animals were observed for two days following each vaccination for changes in general condition (e.g. feed intake) and local reactions at the injection site. Any abnormality or alteration observed during the further test period were recorded. In addition, 5 animals per group were euthanazied and necropsied at 10 weeks of age to perform an histopathological analysis in the injection sites.

1 PCV2 vaccine : Circumvent PCV (Batch no. 213893207).

2 Mycoplasma sp bacterin; M+PAC (Batch no. 05605). Intervet Schering-Plough A.H.

\section{Results and Conclusions}

After vaccination, no systemic or local reactions were observed. The histopathological observations did not reveal any inflammatory or necrotic lesion that could be related to vaccination with PCV-2 and/or Mycoplasma sp bacterin. These field data, demonstrate that concurrent administration of PCV-2 and Mycoplasma $\mathrm{sp}$ bacterin whether using an automatic syringe which injects the two vaccines on the same side of the neck, or injecting them each to one side of the neck, did not induce any systemic or local reaction. In conclusion, concurrent use of PCV-2 with Mycoplasma sp is safe in pigs from 3 weeks of age.

\section{References}

1. J.Farreres et.al IPVS 2010

2. A.E. Eggen et.al IPVS 2010 


\title{
P.133
}

\section{Serological follow-up in SPF piglets vaccinated with CIRCOVAC $^{\circledR}$ (Merial) at 3 weeks of age}

\author{
Mathieu Chevalier ${ }^{1}$ Laurent Fischer ${ }^{2}$ François Joisel $^{2}$ \\ 1. Merial CRSV, Saint-Vulbas, France; 2. Merial S.A.S., Lyon, France
}

\section{Introduction}

The aim of this study was to monitor the serological response in SPF piglets vaccinated with the inactivated adjuvanted PCV2 vaccine CIRCOVAC.

\section{Material and Methods}

A group of 8 piglets was vaccinated with $0.5 \mathrm{ml}$ of CIRCOVAC by intramuscular route at 3 weeks of age, and a group of 3 piglets was left as an unvaccinated control.

The two groups were kept in isolation and compared according to PCV2 anti-ORF2 ELISA and PCV2 seroneutralizing antibody titres.

\section{Results}

Animals from both groups were seronegative for anti-ORF2 PCV2 antibodies measured by ELISA at D0. Control piglets remained seronegative throughout the study (D42). A serological response was clearly observed for piglets belonging to the group vaccinated with CIRCOVAC. All vaccinated piglets had detectable level of anti-ORF2 ELISA antibodies one week after vaccination except one piglet that exhibited detectable level of anti-ORF2 antibodies at D14 and one piglet that showed detectable level of anti-ORF2 at D42 only. Evolution of anti-ORF2 PCV2 antibody titres per group is presented in Figure 1.

Figure 1: Evolution of the mean anti-ORF2 PCV2 antibody titres (ELISA)

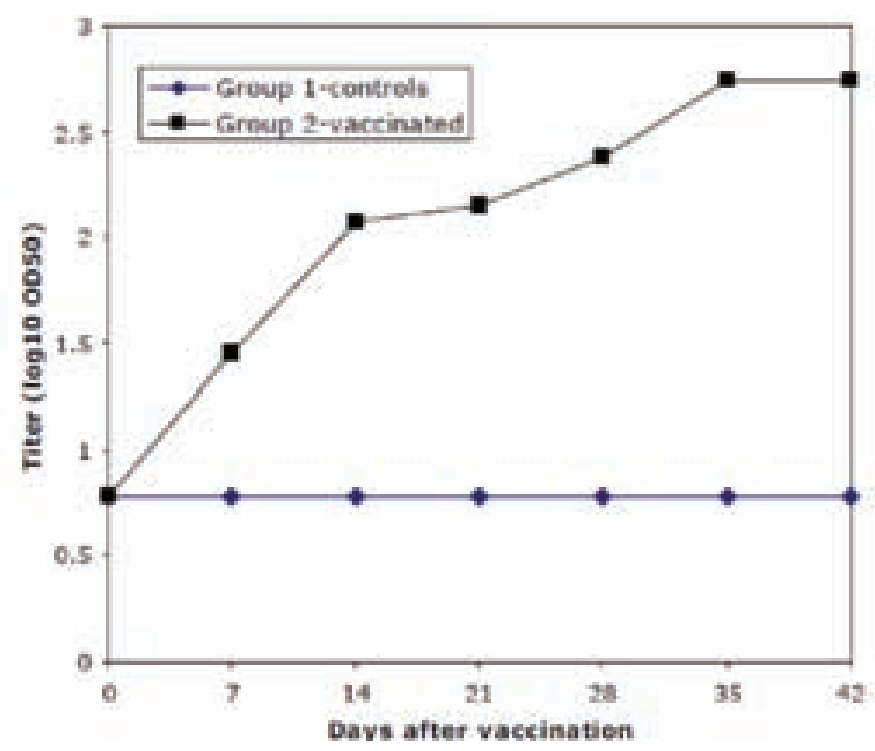

Figure 2: Evolution of the mean anti-PCV2 antibody titres (seroneutralization)

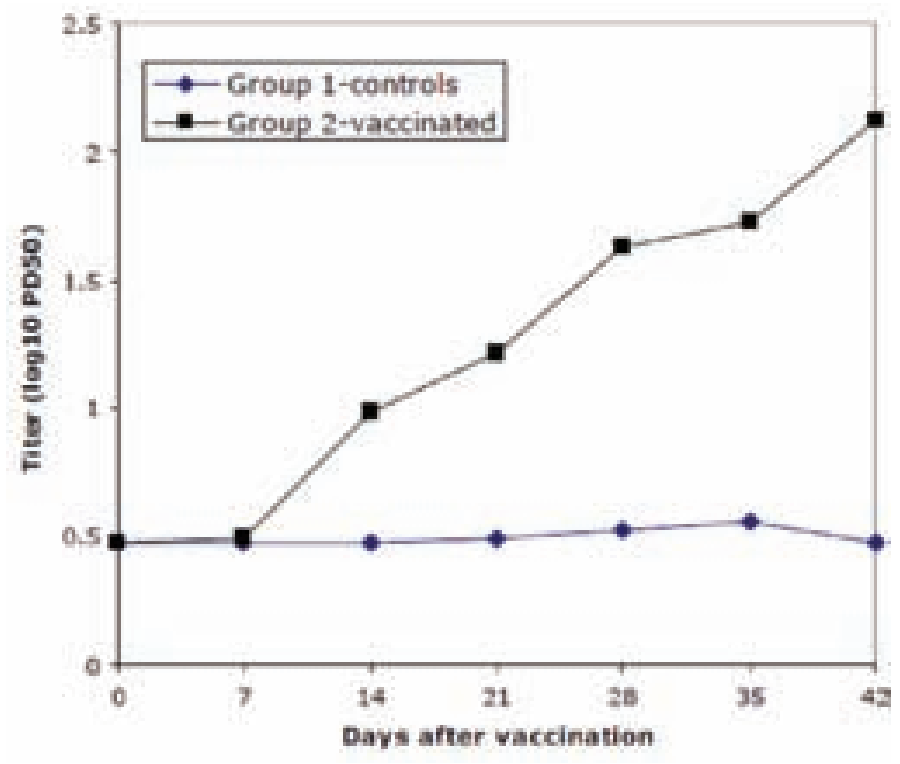

\section{Conclusion}

The seroconversion induced in SPF piglets vaccinated at 3 weeks of age with CIRCOVAC was demonstrated by ELISA and seroneutralization techniques.

${ }^{\circledR}$ CIRCOVAC is a registered trademark of Merial in the Canada and elsewhere. 


\title{
P.134
}

\section{Improved production parameters and profit as a result of PCV2 piglet vaccination in the United Kingdom.}

\author{
Nico Brons ${ }^{2}$ Allison M. Hughes ${ }^{1}$ Matthias J. Adam ${ }^{3}$ \\ 1. Boehringer Ingelheim, Berkshire, UK; 2. Easey Veterinary Services Ltd, Hoxne, UK; 3. Boehringer Ingelheim Ltd, Ingelheim, Germany
}

\section{Introduction}

Porcine Circovirus Disease (PCVD) was first described in the UK in 1997. ${ }^{1}$ The introduction of PCV2 vaccines has allowed greater control of PCVD; a significant reduction in mortality and an improvement in production parameters in herds throughout the UK. ${ }^{2}$ This study looks at the reduction in mortality, improved performance and additional gross margin following the introduction of Ingelvac CircoFLEX ${ }^{\circledR}$ (Boehringer Ingelheim) to a farm in the UK. This farm is part of a 28 breeding unit pyramid. ${ }^{3}$

\section{Materials and Methods}

Routine PCV2 piglet vaccination (a single, $1 \mathrm{ml}$, dose at weaning) was introduced to a 900 sow, outdoor pig unit in East Anglia, England, in May 2008. The farm practiced a 3-week batch cycle and piglets were weaned and transported to one of five all in/ all out, rearing units (wean to slaughter). Pig performance was closely monitored on the rearing units from Nov 2007 to Feb 2009 (before and after vaccine introduction). Data collected included post weaning mortality (PWM), average daily weight gain (ADWG), feed conversion ratio (FCR) and veterinary costs per pig. Eleven batches of pigs ( 11,075 in total) before vaccination and seven batches of pigs (5,851 pigs in total) after vaccination were monitored. Veterinary costs were also compared and these comprised of veterinary visits (routine and emergency) and total antibiotic expenditure per pig. Additional gross margin per pig was calculated on the basis of the average improvements observed after vaccination and the reduction in antibiotic expenditure (assuming a slaughter live weight of 100kg; prices: feed $-£ 170 / t$, weaner $-£ 36 /$ pig \& carcass $-£ 1.23 / \mathrm{kg}$ ).

Data was also collected from the top performing rearing unit (subclinical PCVD farm).

No other significant management, genetic or nutritional changes occurred on these rearing units during that period.

\section{Results}

The average mortality of successive batches of pigs showed a statistically significant decline from $6.4 \%$ to $2.8 \%$ (figure 1 ) due primarily to the disappearance of PDNS and the improved control of respiratory disease. ADWG per pig increased from $669 \mathrm{~g} /$ day to $729 \mathrm{~g} /$ day and FCR reduced from 2.53 to $2.44 \mathrm{~kg} /$ $\mathrm{kg}$. Variation was reduced for all parameters measured. The top performing unit experienced a reduction in mortality of $25 \%$, an increase in ADWG of $36 \mathrm{~g} /$ day and a reduction in FCR of 0.6 after vaccination. In addition, veterinary costs were reduced by $22 \%$ per pig, following PCV2 vaccination.

Based on the average improvement observed, the increased gross margin was calculated at $£ 6.77 /$ pig.

Figure 1: Average batch mortality over time

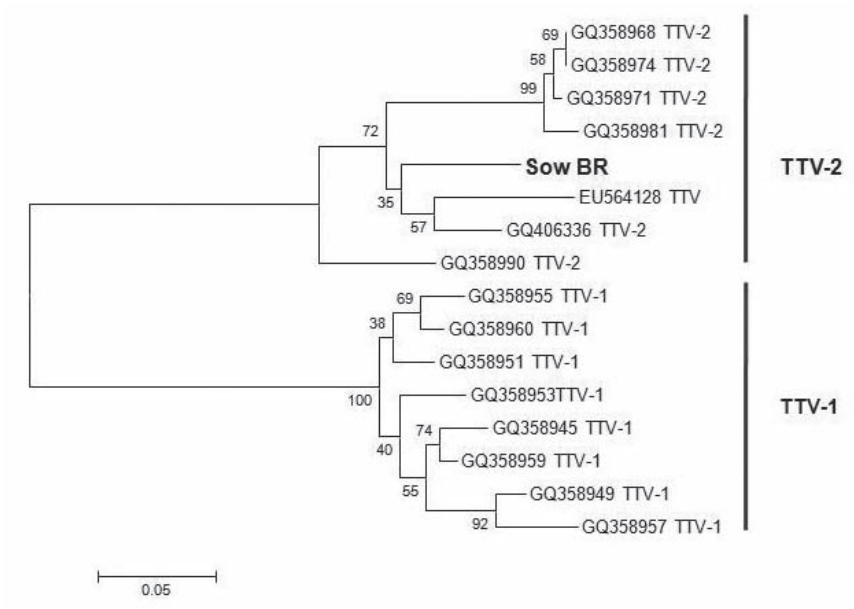

\section{Discussion}

The introduction of PCV2 vaccination to pigs at weaning resulted in a statistically significant reduction in mortality, the disappearance of PDNS, an improvement in ADWG and FCR and an additional gross margin of $£ 6.77$ per pig, from wean to slaughter. In addition, there was a reduction in veterinary costs of $22 \%$ per pig. Substantial improvements in production were also observed in the rearing unit with subclinical PCVD.

\section{References}

1. Kennedy et al. (1998). Vet Record,142,495-496.

2. Burch, D. International Pig Topics. Vol 23, Number 8, 13-15.

3. Brons et al. (2010).IPVS (submitted) 


\title{
P.135
}

\section{Full dose vs half dose of Ingelvac CircoFLEX: field data in Spain}

\author{
Joaquim Bringas \\ Sat Suis, Banyoles, Spain
}

\section{Introduction}

Every day more, PCVD vaccination is becoming a routine in pig production, in some countries, vaccination rates are reaching extremely high percentages, like in US, about 95\% in early 2010. Efficacy and return of investment have been demonstrated in several cases of PCV2 vaccination (1,2).

On the other hand, since 2006, huge economical and financial crisis is affecting pig producers all over the world. Reducing input costs is one of the main objectives, therefore some producers in Spain decided to cut doses of PCV2 vaccines.

The objective of this study is to compare the efficacy of a PCV2 vaccine, Ingelvac CircoFLEX ${ }^{\circledR}$ (Boehringer Ingelheim) at full dose in comparison to half dose in mortality, medication costs and fattening performance.

\section{Materials and Methods}

This study was performed in a farrowing farm with 750 sows. This site one is producing approximately 23 piglets/sow/year, which is not as good as should be due to a high mortality in farrowing period. The herd is PRRS positive, sows are vaccinated against Aujeszky's disease, Parvovirus and porcine Erisypelas. Gilts come from external source and before entering the farm, are vaccinated against Aujeszky's, Parvovirus, Erisypelas and PRRS.

Piglets are weaned between 21 and 24 days and delivered to 3 nursery farms. All piglets are vaccinated against Mycoplasma at 7days of age and against PCV2 at weaning.

Piglets remain in the nursery farm until they reach $20 \mathrm{~kg}$ of live weight, when they are moved to fattening units. In fattening, animals suffered moderate problems of PCVD. Peak of clinical signs were observed between 16 and 17 weeks of age. Before introduction of PCV2 vaccination mortality in fattening averaged at about $6 \%$ with medication costs of more than $3 €$ per pig, animals did not react to antibiotic treatments for secondary infections.

A total of 4.250 animals were included in this before-after study. First, 3 fattening batches (1.700 animals) were vaccinated at half dose $\left(0,5 \mathrm{ml}\right.$ per piglet) of Ingelvac CircoFLEX ${ }^{\circledR}$ off label. After that, 4 batches ( 2.550 animals) were vaccinated at full dose $(1 \mathrm{ml}$ per piglet) following label recommendations.

All data presented is from fattening units. Mortality in both groups were evaluated using a Chi-square test.

\section{Results}

Animals vaccinated with full dose perform better than animals with half dose, (table 1). Weight gained in the fattening unit was $1,8 \mathrm{~kg}$ more in the vaccinated group with full dose spending 2 days more in fattening.

Difference in mortality was highly significant $(p<0,001)$, obtaining a reduction of $56 \%$ in the group vaccinated with full dose in comparison with the results with half dose. Medication costs were reduced by $41 \%$ in the group of full dose.

Table 1. Relevant parameters

\begin{tabular}{|l|c|c|c|}
\hline & Half dose & Full dose & Difference \\
\hline \# piglets tested & 1700 & 2550 & \\
\hline Mortality $(\%)$ & $4.8^{\mathrm{a}}$ & $2.1^{\mathrm{b}}$ & -2.7 \\
\hline Weight gain $(\mathrm{kg})$ & 94.1 & 95.9 & +1.8 \\
\hline $\begin{array}{l}\text { Medication costs } \\
(€ / \text { pig) }\end{array}$ & 2.59 & 1.53 & -1.06 \\
\hline a,b: values with a different superscript, differ significantly $(\mathrm{p}<0,001)$. \\
\hline
\end{tabular}

\section{Discussion}

When comparing data of non vaccinated animals with animals vaccinated at half dose, some improvement was observed, but the clinical situation and mortality was still not satisfactory. Data presented in this field study, demonstrate that using full dose of Ingelvac CircoFLEX ${ }^{\circledR}$ instead of half dose improved the performance and lead to a significant reduction in mortality.

Based on reduction in mortality and medication costs, a yearly return of investment of 5,07:1 for every extra $€$ spent on vaccine was calculated when moving from half to full dose of Ingelvac CircoFLEX.

The results of this study show that using full dose of Ingelvac CircoFLEX (compared to half dose) is improving the health situation on the farm and provides significant economic benefits.

\section{References}

(1) Cline et al (2008) Veterinary Record, 163, 737-740.

(2) King et al (2008) Proc 20th IPVS Congress, Durban, South Africa, vol. 2, p. 36. 


\title{
P.136
}

\section{Evaluation of CIRCOVAC ${ }^{\circledR}$ one shot vaccine applied in three-week-old piglets on production parameters in farms with and without a diagnosis of postweaning multisystemic wasting syndrome (PMWS)}

\author{
Lorenzo Fraile ${ }^{1}$ Llorenç Grau-Roma ${ }^{1}$ Maribel Casas ${ }^{1}$ Patxi Sarasola² Nuria Sinovas ${ }^{2}$ Miquel Nofrarias ${ }^{1}$ Rosa $^{2}$ \\ Lopez-Jimenez ${ }^{1}$ Sergio Lopez-Soria ${ }^{1}$ Marina Sibila ${ }^{1}$ Mathieu Chevalier ${ }^{3}$ Joaquim Segales $^{1}$ \\ 1. Centre de Recerca en Sanitat Anima (CReSA), Bellaterra, Spain; 2. Ondax Scientific HQ, Hondarribia, Spain; 3. Merial, St. Vulbas, France
}

\section{Introduction}

Porcine circovirus type 2 (PCV2) vaccines have demonstrated to be very efficient to control postweaning multisystemic wasting syndrome (PMWS) under experimental and field conditions $(1,2,3)$. Vaccination of sows and gilts increases PCV2 antibody titres in serum or colostrum providing protection of piglets against disease development (4). $\mathrm{CIRCOVAC}^{\circledR}$ is registered in many countries to be used in sows and gilts with the former indication. The objective of the present study was to evaluate the efficacy of the above-mentioned vaccine in farms infected with PCV2 and with or without a PMWS diagnosis.

\section{Material and Methods}

CIRCOVAC ${ }^{\circledR}$ was tested in 17-26 day-old conventional pigs of 2 different Spanish farms. Both farms were selected by their previous recent PMWS history. In each farm, about 300 pigs received one single dose of $0.5 \mathrm{~mL}$ of $\mathrm{CIRCOVAC}^{\circledR}$, while 300 control animals received the same amount of placebo. Also in each farm, 50 pigs from each group were bled and a rectal swab was also taken. PCV2 viral load in serum and rectal swab (real-time PCR) were investigated at 3, 6, 10, 14, 17, 20, 23 weeks of age and before slaughter (S). All animals were weighed and scored for physical condition at 3 and 10 weeks of age and S. Average daily gain (ADG) for the whole postweaning period was calculated. All pigs were necropsied and sampled for a PMWS diagnosis assessment (histopathology and PCV2 in situ hybridization, ISH).

Analysis of variance with Bonferroni multiple comparisons (for normally distributed variables) and Kruskal-Wallis and MannWhitney tests (for non-normally distributed variables) were used to compare ADG, PCV2 DNA loads in sera and faeces between vaccinated and control animals in each sampling time.

\section{Results}

PCV2 infection was demonstrated to occur in both studied farms (measured by qPCR and ISH in tissues). PMWS individual case definition was fulfilled only in farm 1, while no cases accomplishing the diagnostic criteria were found in farm 2. ADG was significantly higher in vaccinated versus control animals in farm 1 and 2. Moreover, PCV2 viral load in serum was significantly lower (1-2 log) in vaccinated compared to control animals in most sampling times after PCV2 exposure in both farms. A similar result was obtained regarding PCV2 viral load in faeces.

\section{Discussion}

Vaccination with $\mathrm{CIRCOVAC}^{\circledR}$ in piglets under field conditions was able to significantly improve production parameters and reduce significantly PCV2 viremia and faecal load. These results agree with other publications using piglet vaccination in PMWS affected farms by means of different vaccine approaches, such as a PCV1-2 chimera vaccine (5) and a subunit vaccine based on PCV2 ORF2 protein (1). The present results support the idea that production parameters are suitable indicators of PCV2 vaccine efficacy.

\section{References}

1. Fachinger et al., 2008. Vaccine 26: 1488-1499.

2. Fort et al., 2008. Vaccine 26: 1063-1071

3. Grau-Roma et al., 2010. Vet J. In press.

4. Opriessnig et al., 2010. Vet Microbiol. In press.

5. Segalés et al, 2009. Vaccine. 27:7313-7321.

CIRCOVAC ${ }^{\circledast}$ is a registered trademark of MERIAL in Canada and elsewhere. 


\title{
P.137
}

\section{Effect of PCV-2 vaccination protocols on late term mortality}

\author{
Imre Biksi ${ }^{1}$ Tamás E. Kis ${ }^{1}$ Zoltan Nemet ${ }^{2}$ Márk Kovács ${ }^{3}$ \\ 1. Szent István University, Üllo", Hungary; 2. SZIU FVSC Large Animal Clinic, Dóra Major, Hungary; 3. Dalmandi Mg. ZRt., Dalmand, Hungary
}

\section{Introduction}

PCV-2 associated diseases and related losses might occur in high health status herds. With the advent of PCV-2 vaccines, these losses can be successfully reduced. In this study we have observed the effects of different vaccination protocols on late term mortality in a gilt development unit of a high health status breeding herd in Hungary.

\section{Materials and Methods}

PCV-2 related problems were detected in the gilt development unit (GDU) of a large breeding complex in Hungary. Pigs were transferred to the GDU at approximately 80 days of age ( $35 \mathrm{~kg}$ bw). PCVAD was manifested mainly in PDNS and gastric ulceration possibly related to systemic illness. The majority of losses occurred between approx. 140-170 days of age (Figure 1.). The herd was free from PRRSV, M. hyopneumoniae, and pathogenic APP strains. No lesions related to these agents were found in necropsy.

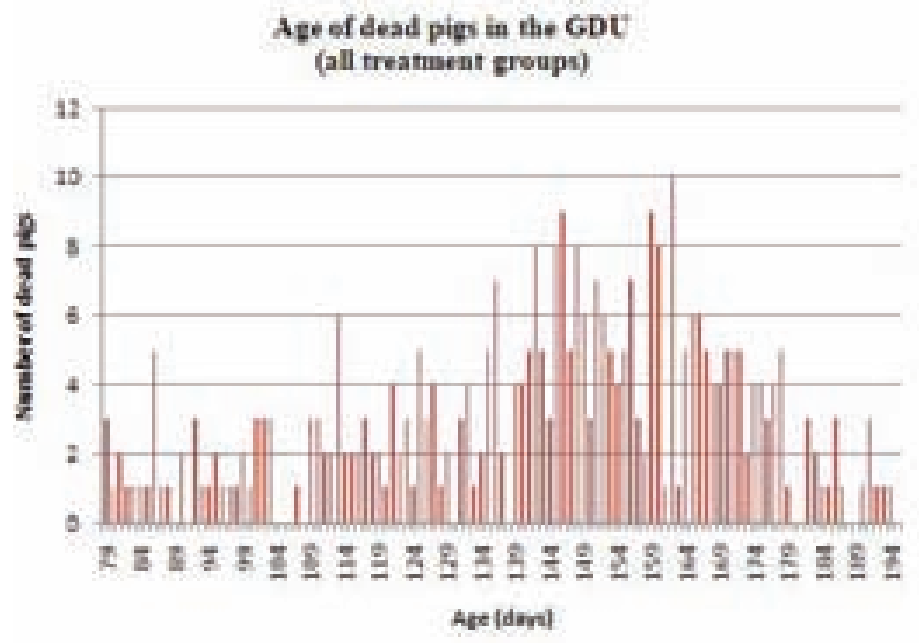

From the beginning of 2009, we have tested two different vaccines and three protocols in order to prevent PCV-2 associated losses in the GDU. We have tested Circovac ${ }^{\circledR}$ (Merial) in 0,5 ml/ pig dose, CircoFLEX ${ }^{\circledR}$ (Boehringer-Ingelheim) in $1 \mathrm{ml} /$ pig dose applied at two different ages, and also observed unvaccinated groups of animals. The sole measure of vaccine efficacy was mortality in the GDU (between 85-190 days of age). Dams of all pigs in this study were vaccinated with Circovac $^{\circledR}$ according to the manufacturer's instructions. All groups entering GDU received the same medication regime against proliferative enteropathy. No major changes in feed composition of feeding technology occurred in the study period. Mortality data were collected for all groups for their entire stay in the GDU. Mean mortality figures were compared by nonparametric statistics (Kruskal-Wallis test with Dunn's multiple comparisons test). Results are summarized in Table 1.

\section{Discussion}

From these mortality data it appears, that on this farm the best protection from PCVAD in the 140-170 days age group was achieved with CircoFLEX $^{\circledR}$ vaccination at approximately 22 days of age. Other vaccination approaches did not decrease total mortality rate compared to unvaccinated controls. It also appears, that vaccination of dams alone or in combination with late vaccination of pigs with $\operatorname{CircoFLEX}^{\circledR}$, or with $\operatorname{Circovac}^{\circledR}$ did not affect total mortality rates. This observation is in line with findings from other studies (Guillaume et al., 2009).

\section{References}

Guillaume, J. M. et al.: Additional benefits of Ingelvac Circoflex ${ }^{\oplus}$ in a Circovac ${ }^{\circledast}$ vaccinated herd. Proc. 1st ESPHM, p. 52., Copenhagen, Denmark, 2009.

Table 1

\begin{tabular}{|l|c|c|c|}
\hline Treatment & Circovac(R) & CircoFLEX(R) & CircoFLEX(R) \\
\hline Age at vaccination, days (median, range) & $39(25-45)$ & $66(65-67)$ & $22(19-27)$ \\
\hline Number of groups & 4 & 4 & 7 \\
\hline Vaccinated animals (sum) & 1666 & 2135 & 3794 \\
\hline Dead animals, sum (median, range) & $99(18.5 ; 18-49)$ & $105(21.5 ; 16-46)$ & $83(12 ; 6-15)$ \\
\hline Mortality \% (median, range) & $5.94^{\mathrm{a}}(5.49 ; 3.32-9.04)$ & $4.92^{\mathrm{a}}(3.97 ; 3.13-8.48)$ & $2.19^{\mathrm{b}}(2.21 ; 1.10-2.76)$ \\
\hline
\end{tabular}




\title{
P.138
}

\section{Immunization program to control PCV2-associated reproductive failure in sows using a commercial vaccines}

\author{
Carlos Martin del Campo ${ }^{2}$ Francisco Aguilar ${ }^{2}$ Salvador Espinoza ${ }^{2}$ Lucia A. Garcia-Camacho ${ }^{1}$ Victor Quintero ${ }^{1}$ \\ 1. FESC-UNAM, Cuautitlán, MEX, Mexico; 2. Propeca SA. de CV., Tepatitlan, JAL, Mexico
}

\section{Introduction}

Porcine circovirus type 2 (PCV2) has been related to reproductive failure (RF) in sows, resulting in infertility, abortions, stillborns, increased non-viable piglets.1,2,7,8 PCV2 detection in stillborn, and newborn piglets, transplacental transmission without participation of other infectious agents and RF experimentally induced have been documented.3,4,5,6,7,8 It has been proved that PCV2 replicates in heart, lung, liver, kidney and lymphoid tissues by intrafetal inoculation. The most consistent neonatal and fetal are non-suppurative myocarditis and encephalitis associated to PCV2 detection by immunohistochemistry and/or in situ hybridization (ISH).8 Regarding vaccination, an improvement of reproductive parameters has been confirmed after vaccination with an inactivated $P C V 2$ vaccine. 2

\section{Material and methods}

The aim of this work was to evaluate an immunization program and its effect on controlling RF in a herd with history of PCV2 associated RF (PCV2-RF). The present study was done in a farm with 2800 sows of central Mexico with reproductive problems such as low parturition rate, late-term abortions (up to $7 \%$ in affected groups), and 3-7\% mummified fetuses. The PCV2 presence was confirmed by diagnosis of non-suppurative myocarditis on histopathology examination and ISH PCV2 detection in aborted fetuses. In addition, the farm has history of positivity to PRRSV, H1N1 influenza virus, and porcine parvovirus. The herd was massive immunized with a commercial vaccine (Baculovirus-expressing PCV2 capside protein). The data prior vaccination and after vaccination of 21 groups were compared as follow: parturition rate (PR), born alive piglet total average (BAT), born alive piglets (BA), stillborns, and mummified fetuses (MF). Results were analyzed by central tendency tests, and media comparison.

\section{Results}

The values from all evaluated parameters are shown in Table 1.
Table 1.

\begin{tabular}{|l|c|c|}
\hline Parameter & Prior vaccination & Post-vaccination \\
\hline PR & $81.31( \pm 3.29)^{*}$ & $6.71( \pm 2.89)$ \\
\hline BAT & $11.3( \pm 0.26)$ & $11.4( \pm 0.24)$ \\
\hline BA & $10.16( \pm 0.20)$ & $10.45( \pm 0.15)$ \\
\hline Stillborns & $6.0( \pm 0.35)$ & $5.45( \pm 0.58)$ \\
\hline MF & $3.65( \pm 0.87)$ & $3.6( \pm 0.56)$ \\
\hline Fertility \% & 89.6 & 93.8 \\
\hline Abortion \% & 1.75 & 1.64 \\
\hline *Data between brackets depict to standard deviations \\
\hline
\end{tabular}

\section{Discussion}

Based on the results, a significant improvement of PR after vaccination (over 5.4\%) was observed. The differences regarding BAT and BA were no significant and minimal. A similar finding was observed in BA and stillborns with no significant variation. The standard deviation of mummified fetuses was open with a tendency to narrow after vaccination. These findings are in agreement with reported data2 which there was an improvement of 2.1 in PR, and marginal improvements of BAT and BA. The latter were higher than our results. Regarding abortion percentage no significant variation was determined as opposed to reported data.

\section{References}

1. Brunborg IM, et al. J Vet Diagn Invest. 2007 Jul;19(4):368-75.

2. Callen, A., Villa,T. Mem. I Cong AMVEP 2008

3. Kim J, et al. Vet Rec. 2004 Oct 16;155(16):489-92.

4. Lyoo KS, et al. J Vet Sci. 2001 Dec;2(3):201-7.

5. Madsen, D.M. et al. 2009, doi;10.1354/vp.08-VP-0234-O-FL

6. Park, JS. et al. J Comp. Path 2005, (132) 139-144.

7. Pensaert, MB, et al. Vet Microbiol.2004 (132)175-183.

8. Pittman, JS. JSHP 2008. (16)144-148. 


\title{
P.139
}

\section{Immunity induced by recombinant adenovirus expressing capsid protein of porcine circovirus $\mathbf{2}$}

\author{
Kyoung Ki Lee; Choi Kyu Park; Seong Hee Kim; Yun Kyung Song; Chae Wun Bae; Chang il Yeo; O Soo Lee \\ National Veterinary Research and Quarantine Service, Anyang, Korea
}

\section{Introduction}

Porcine circovirus associated disease (PCVAD) caused by porcine circovirus type 2 (PCV2) was one of the most important diseases currently threatening global swine industry. To prevent and control this disease, various types of vaccine have been developed and tested for their immunological effects. This study investigated the immune response induced by a recombinant adenovirus (reAdV) expressing the capsid protein, which is involved in the production of protective antibody against PCV2.

\section{Materials and Methods}

The ORF2 gene from field isolate was altered to replace with the codons preferred in mammalian cell for its high-level expression. The replication-defective reAdV was created by homologous recombination in $\mathrm{E}$. coli using human adenovirus serotype 5 DNA deleted the early transcribed E1 and E3 genes. The capsid protein expressed in 293 A cell was identified with immunochemical assay and western blotting. Groups of four female mice were immunized with ten-fold serially diluted reAdV $\left(1 * 10^{7}-1\right.$ * $10^{4} \mathrm{TCID}_{50}$ /mouse) given intramuscularly. Mice were periodically bled under anesthesia by retro-orbital puncture. The antibody titers were detected by IFA test and ELISA.

\section{Results \& Discussion}

The reAdVPCV2cap was produced by homologous recombination of a transfer vector, pAdenoVatorCMV5 and adenovirus DNA, pAdenoVator $\triangle E 1 / E 3$. At 6 days post-transfection in 293 cells, the typical CPE was observed. The titer of purified adenovirus was finally $1 * 10^{9} \mathrm{TCID}_{50} / \mathrm{ml}$. The recombinant capsid protein was expressed in infected 293A cells by investigation of staining with specific MAb (12C48) against capsid protein of PCV2. In addition, bands corresponding to a molecular weight of about $28 \mathrm{kDa}$ were detected in the 293A cell lysate with western blotting.

The immune response induced by reAdVPCV2cap was investigated with IFA test and ELISA. The results of IFA test showed that PCV2-specific antibodies were detected in two mice groups inoculated with $1 * 10^{7}$ and $1 * 10^{6} \mathrm{TCID}_{50}$ /mouse of reAdVPCV2cap, whereas no immune response was found in two mice groups treated with $1 * 10^{5}$ and $1 * 10^{4} \mathrm{TCID}_{50} /$ mouse and control group (Fig. 1). In mice group inoculated with highest titer $\left(1 * 10^{7} \mathrm{TCID}_{50} /\right.$ mouse), the average antibody titers were $1: 128,1: 1024,1: 2436,1: 2048$ and $1: 2436$ at 10, 20, 30, 60, 90 days after immunization, respectively. In mice group inoculated with $1 * 10^{6} \mathrm{TCID}_{50}$ /mouse titer, the average antibody titers showed more than 1:256 at 30 days after immunization. These results indicated that virus titer of at least more than $10^{6} \mathrm{TCID}_{50} /$ mouse was required to induce the hummoral immune response in mice with the reAdVPCV2cap. The results of ELISA analysis also were similar to those of IFA test (Fig. 2).
Fig. 1. Analysis of PCV2 antibody response in mice immunized with reAdVPCV2cap using IFA test. Because no PCV2-specific antibodies $(<1: 16)$ were detected in mice inoculated with $1 * 10^{5}$ and $1 * 10^{4}$ $T C I D_{50}$ /mouse, and PBS, the data were excluded in this figure.

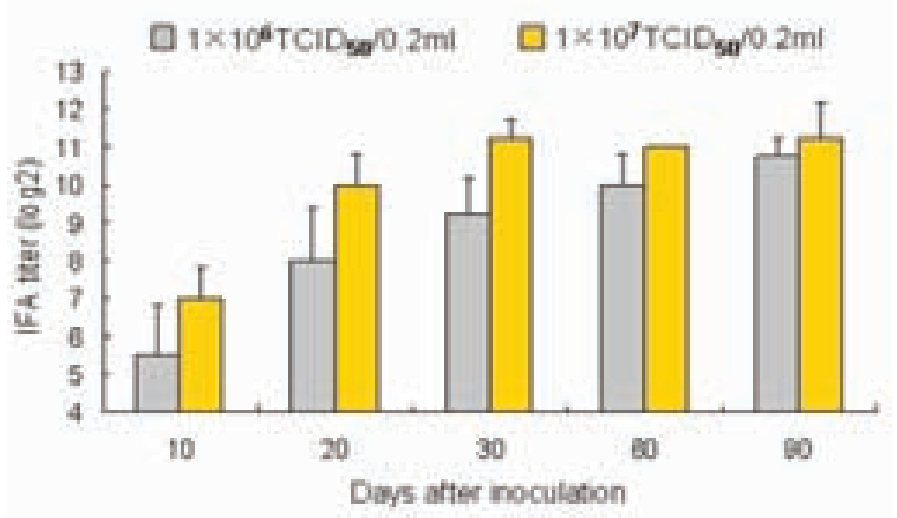

Fig. 2. Analysis of PCV2 antibody response in mice immunized with reAdVPCV2cap using ELISA. Antibody titers of individual mouse $(A)$ and mean titers of each group (B).

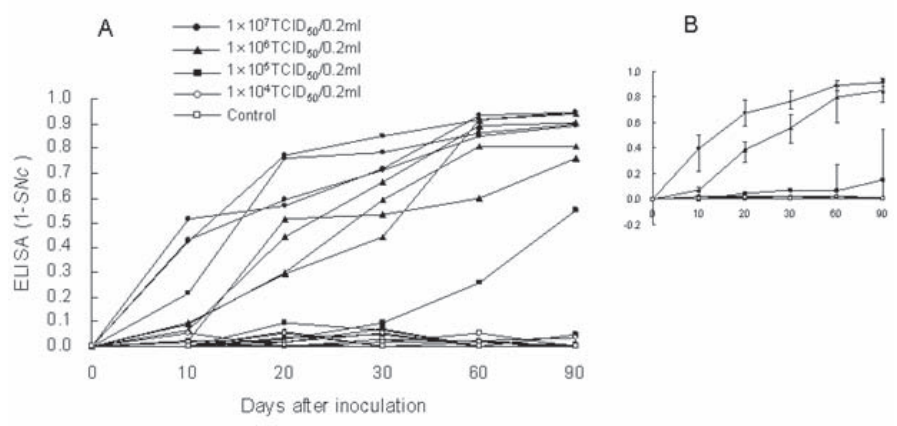

Although titers of antibodies varied in individual mouse, geometric mean titers of each group showed dose dependent. These results show that the reAdV can effectively produce the capsid protein of PCV2 and induce sufficient immune responses.

\section{References}

Nawagitgul P., et al., (2000) J. Gen. Virol. 81, 2281-2287.

Wang X. et al., (2006) Vaccine 24, 3374-3380.

Wesley R.D. et al., (2004) Vaccine 22, 3427-3434. 


\title{
P.140
}

\section{Effects of sugar cane extract and mangerial improvent on PRRSV and PCV2 co-infection}

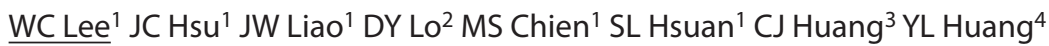 \\ 1. Graduate Institute of Veterinary Pathobiology, National Chung Hsing University, Taichung, Taiwan; 2. Department of Veterinary \\ Medicine, National Chiayi University, Chiyi, Taiwan; 3. Graduate Institute of Veterinary Microbiology, National Chung-Hsing \\ University, Taichung, Taiwan; 4. Animal Health Research Institute, Council of Agriculture Executive Yuan, Taipei, Taiwan
}

\section{Introduction}

Porcine circovirus type 2 associated diseases (PCVAD) has become one of the most concerned diseases in swine industry (4). Though the novel PCV2 vaccines have shown good protection for PCVAD control, implementation of a strict biosecurity and enhancement of host innate immunity are also very important in PCVAD control $(2,3)$. Sugar cane extract (SCE), a natural product, has displayed immunostimulating effects on porcine leukocyte functions (1). Therefore, this study was aimed to evaluate the effects of SCE and managerial improvement on PCV2 and/or PRRSV infection.

\section{Materials and methods}

Experimental design: The study was conducted on two separated experiments. In experiment I, the experimental pigs were weaned at 18 days old and moved into an experimentally controlled house to avoid PRRSV infection. In experiment II, the experimental pigs were weaned and raised at the original farm with PRRSV endemic until 8 weeks old for PRRSV natural exposure and then moved into experimental house. Experimental pigs were grouped and treated as indicated in table 1.

Table 1 Grouping and treatment

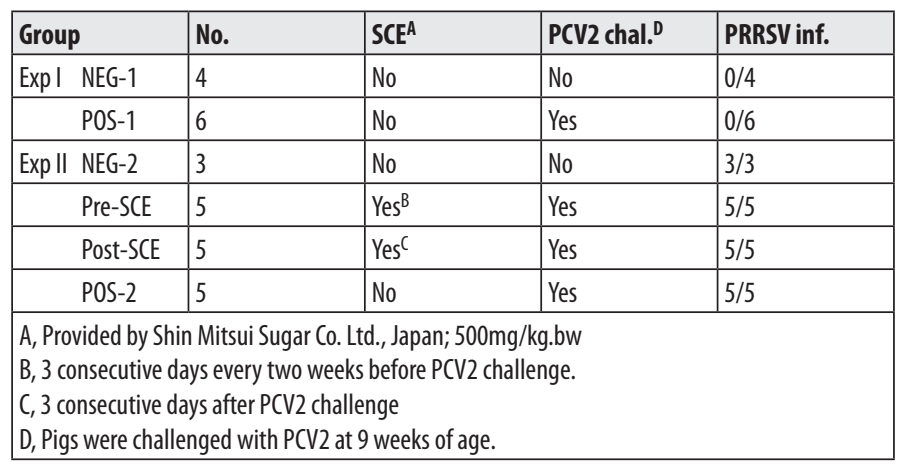

\section{Evaluation:}

1. Clinical record: The body temperatures $(B T)$ were recorded daily and fever was determined by the BT $>40^{\circ} \mathrm{C}$. Body weight was weighted weekly.

2. Pulmonary impairments ( $\mathrm{PI}, \%)$ were scored depending on the area of bronchopneumonia (\%, grossly) and the severity of interstitial pneumonia (\%, microscopically) at the end of experiment, i.e. 4 weeks post PCV2 challenge.

3. PCV2 virus loads were determined by real time PCR.

\section{Results}

Pigs in group POS-1 that weaned early and segregated to the clean house had significantly lower PRRSV infection and $21.5 \%$ lower MDWG after PCV2 challenge. In contrast, pigs in experiment II were all naturally exposed to PRRSV infection. After PCV2 challenge, all pigs were more seriously affected by PRRSV and PCV2 synergistic infection, with a decrease of $44.6 \%$ in MDWG (POS-2). However, SCE administration showed $9.4 \%$ to $20 \%$ improvement on the decreasing injury caused by PRRSV and PCV2 synergistic infections (table 2).

Table 2 Summary of the two experiment Results

\begin{tabular}{|l|c|c|c|c|c|}
\hline Group & MFD $^{A}$ & PI(\%) & PCV2(log10) & MDWG(gm) & Affected(\%) \\
\hline NEG-1 & 0 & 3.6 & - & 0.65 & 0 \\
\hline POS-1 & 2.7 & 17.8 & 10.42 & 0.51 & -21.5 \\
\hline NEG-2 & 0 & 4.3 & - & 0.61 & -6.2 \\
\hline Pre-SCE & 3.0 & 28.5 & 11.03 & 0.49 & -24.6 \\
\hline Post-SCE & 4.0 & 23.7 & 10.98 & 0.42 & -35.2 \\
\hline POS-2 & 7.0 & 34.3 & 11.20 & 0.36 & -44.6 \\
\hline A, MFD: mean fever day; B, pulmonary impairment (\%); C, MDWG: mean daily weight gain \\
\hline \multicolumn{7}{|l}{} \\
\hline
\end{tabular}

\section{Discussion}

Administration of SCE can decrease the injury, but may not avoid the effect of PRRSV and PCV2 synergistic infection. In contrast, early weanling and segregation system can efficiently decrease or control PRRSV infection and also decrease the effect of PRRSV and PCV2 synergistic infections.

\section{References}

1. Lo DY, et al., J Vet Med Sci 67: 591-597, 2005.

2. Madec F, et al., Livest Prod Sci 63: 223-233, 2000.

3. Madec F, et al., Transbound Emerg Dis 55: 273-283, 2008.

4. Rovira A, et al., J Virol 76: 3232-3239, 2002 


\title{
P.141
}

\section{First report of the incidence and distribution of PCV2 virus in Mexico}

\author{
Francisco Robles ${ }^{1}$ Ricardo Angulo² Jean Claude Chevez ${ }^{1}$ Edgar Diaz ${ }^{2}$ \\ 1. Boehringer Ingelheim Vetmedica S.A. de C.V., Guadalajara, JAL, Mexico; 2. Boehringer Ingelheim Inc., St. Joseph, MO, USA
}

\section{Introduction and Objective}

Porcine circovirus type 2 (PCV-2) has been reported as the cause of PMWS and others syndromes $(1,2,3)$. This virus was identified in Mexico during 2001 (4) and different reports show the current presence of PCV-2 in Mexico. Nevertheless, there is not enough information about the prevalence and distribution in Mexico, just some reports of antibodies' presence (5). The main objective of this study was to establish the prevalence of PCV2 in different regions of Mexico using the PCR technique.

\section{Materials and Methods}

The study was conducted in 34 farms located in 10 different regions of Mexico. In 20 of these farms, the pigs showed clinical PMWS, according with the case definition (4). The sampling period was from January to September of 2006, collecting serum $(n=3094)$, organs $(n=122)$ plus lymph node, lung, ileum, and fecal swab samples $(n=437$.) The samples were pooled in accordance with the type of sample. There were 888 pools in total. The conduction of a PCR test amplified a fragment of 506 nucleotides of the ORF2.

\section{Results}

The number of samplings was 89 with 72 samplings equaling $80.89 \%$, testing positive. The analysis showed that 33 of 34 farms, equaling $97.06 \%$, were PCV2 PCR positive.

Table 1. Results of PCV2 analysis for farms and Mexican state

\begin{tabular}{|l|c|c|}
\hline STATE & No. farms analyzed & PCV-2 Status \\
\hline COAHUILA & 1 & NEGATIVE \\
\hline GUANAJUATO & 4 & POSITIVE \\
\hline JALISCO & 4 & POSITIVE \\
\hline MICHOACAN & 1 & POSITIVE \\
\hline MONTERREY & 1 & POSITIVE \\
\hline PUEBLA & 7 & POSITIVE \\
\hline QUERÉTARO & 3 & POSITIVE \\
\hline SONORA & 4 & POSITIVE \\
\hline VERACRUZ & 1 & POSITIVE \\
\hline YUCATÁN & 8 & POSITIVE \\
\hline
\end{tabular}

The analysis for each Mexican state shows that 9 out of 10 states are positive; this represents the $90 \%$ of positives. The only negative state was Coahuila. Nevertheless, for this state only one farm (3 samples) was subject to analysis.
Table 2 shows the results for each type of sample. In this case, the samples showing more findings that are positive were rectal swabs with $56 \%$, and the ones showing fewer positives were semen samples with less than $10 \%$.

Table 2. Results of PCV2 analysis from different samples

\begin{tabular}{|l|c|c|c|c|}
\hline SAMPLE & N0. SAMPLES & N0. POOLS & N0. POSITIVES & $\%$ \\
\hline SERUM & 3094 & 682 & 268 & 39.3 \\
\hline TISSUE & 122 & 110 & 43 & 39 \\
\hline SEMEN & 22 & 11 & 1 & 9.01 \\
\hline RECTAL SWABS & 437 & 85 & 48 & 56.47 \\
\hline
\end{tabular}

\section{Discussion}

PCR is a sensitive diagnostic method widely used for pathogen detection, including PCV2. Since PMWS was described in Canada (1997), PCV2 has been isolated from different samples in Germany, France, Spain, Korea, Japan and other important pig-producing countries (3.) In America, there are reports of the presence of PCV2 in United States, Canada and Brazil, but in Mexico there is only one report of the antibodies' presence and few reported cases have been isolated. Our results are the first report of the incidence and distribution of PCV2 in Mexico's commercial farms (using the PCR method), and show that PCV2 virus is distributed throughout all of Mexico's territory. In this research there was no difference between tissue samples and blood samples. The best samples for diagnostic purposes were found to be rectal swabs (56 per cent positives) and the worst were semen (almost 10 per cent positives). Nevertheless all the different sample types analyzed showed positive results.

The percentage of positives found during this investigation is similar at other reports from around the world.

It is very important to research the sequences of the Mexican PCV2 virus in order to determinate the homology with other PCV2 virus distributed around of the world.

\section{References}

1. Wallgren P. et al. (2004) Veterinary Quartely, 26:170-187.

2. Chae C. (2005) Veterinary Journal, 169: 326-336.

3. Feneaux M. et al. (2000) Clinical Microbiology, 38:2494-2503.

4. Trujano et al. (2001). Vet. Rec. 148: 792.

5. Ramirez-Mendoza H. et al (2007). Res. Vet. Scien. 83: 130-132. 


\title{
P.142
}

\section{Histopathologyc findings of Porcine Multisystemic Wasting Syndrome (PMWS) with non suppurative myocarditis as a cause of sudden death in two pig farms}

\author{
Elias J. Sogbe ${ }^{1}$ Carmen T. Diaz ${ }^{1}$ Jean Cano ${ }^{1}$ Vitelio Utrera' Elias Ascanio ${ }^{1}$ Miguel E. Sogbe ${ }^{1}$ Andres Boulanger ${ }^{2}$ Susan V. Del Castillo ${ }^{1}$
}

1. Universidad Central de Venezuel, Maracay, Venezuela; 2. AABL Veterinaria C.A., Valencia, Venezuela

\section{Introduction}

In recent years Post Weaning Multisystemic Wasting Syndrome (PMWS) has become of significant concern in the swine industry in many countries, particularly Canada, United States of America, Europe, Asia and South America. The syndrome affects pigs mainly between 6 and 14 weeks of age. It is characterized by wasting or unthriftiness, dyspnea, jaundice and enlarged lymph nodes. The syndrome was been associated with myocarditis finding in aborted or stillborn piglets due to PCV2, it was also observed in 8-day-old piglet with PCV2 association (1) The present study was performed in piglets located in 2 porcine farms from Central State of Venezuela (Aragua/Carabobo).

The first description of the syndrome in Venezuela was in 2003 $(4,5)$. Therefore in this report we focus on describe the heart histopathological findings in young pigs naturally infected in two Venezuelan pigs farms with sudden death piglets, both farms were free from classical swine fever, Aujeskys disease, Porcine reproductive respiratory syndrome and encephalo myocarditis

\section{Material and Methods}

The present study was performed in 20 piglets of 6 to 10 weeks of age from 2 pig farms placed at Central States of Venezuela (Aragua/Carabobo).All piglets showed signs of wasting, pallor, dyspnea, diarrhea and enlarged superficial inguinal lymph nodes and has history of sudden and unexplained death.

Each pig was necropsed and tissue samples were collected for the histopathological evaluation, fixed in

Buffered formalin 10\%, processed by rutinary methods, and stained with Hematoxilin-Eosin.

In situ hybridization studies (IHS) were performed on those specimens with histological evidence of PCV2 (heart included) as described by Segalés et al (3)

\section{Results and Discussion}

Clinical aspects: All the evaluated piglets (20) showed signs of wasting, pallor, respiratory distress, diarrhea, and sudden death.

At necropsy there was evidence of pneumonia with non-colapsable lungs as well as inguinal, mesenteric and tracheobronchial lymph nodes enlargement, heart showed focal to locally extensive hemorrhagic areas in myocardium with white spots areas.

The histopathology study showed lymphoid depletion, with loss of follicles architecture in lymph nodes, spleen, and Peyers patches, histiocityc infiltration, basophilic cytoplasmatic inclusions in histiocitic cells, and syncytial cells. These findings described were suggestive of PMWS. (3). The heart study showed focal to locally extensive areas of myocarditis and fibrosis. Other areas showed degeneration of myocardium and infiltration of macrophages and lymphocytes in the heart (Fig.1). In this areas cross striation in cardiomyocytes have disappeared and necroses foci were seen and could explain heart failure and sudden death (Fig 2)

The sample tissues specimen processed by a technique of IHS (Fig.3) revealed the presence of PCV2 in affected lymphoid tissue and heart and confirmed the diagnosis of PMWS.

\section{Conclusions}

The results confirmed the existence of PCV2 associated with nonsuppurative myocarditis as an important cause of sudden heart disease of young piglets in Venezuela

\section{Micrographies}

Fig. 1

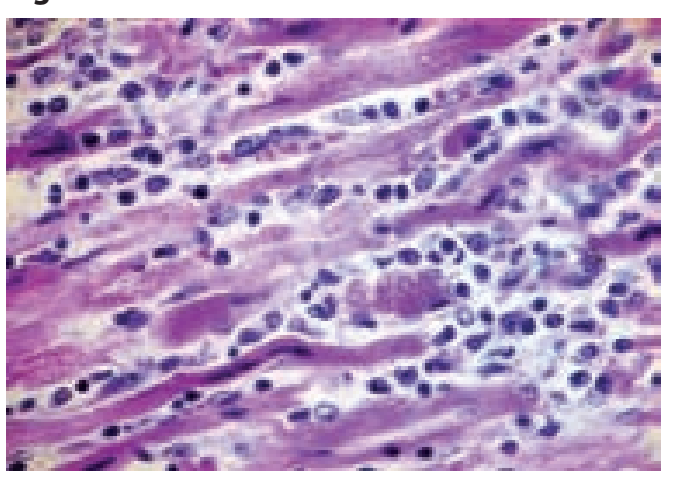

Fig. 2

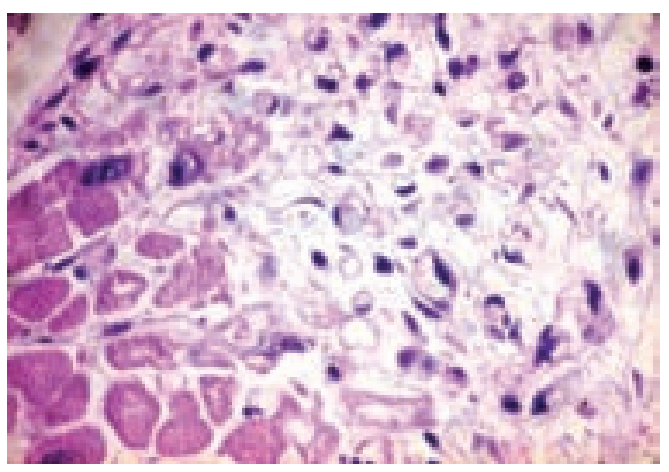

\section{References}

1. Mikami,O. et al (2005) J.Vet.Med.Sci 67(7)735-738

2. Harms, P. et al (2001).Vet.Pathol. 38: 528-539

3. Segales et al (1999) Proc. 17th IPVS congress, 311

4. S ogbe. et al (2003) Rev.Fac.de Ciencias Veterinarias 44: 49-57.

5. Sogbe, et al (2009) Rev.Fac. de Ciencias Veterinarias 50: 33-42. 


\title{
P.143
}

\section{National Level Research on Porcine Circovirus Associated Disease (PCVAD), PCV2 Prevalence and Associations with Herd Level Health and Management Practices}

\author{
Charles A. Haley ${ }^{1}$ Bruce A. Wagner ${ }^{1}$ Sumathy Puvanendiran ${ }^{2}$ Michael Murtaugh ${ }^{2}$ \\ 1. USDA:APHIS:VS:CEAH:NAHMS, Fort Collins, CO, USA; 2. Department of Veterinary \& \\ Biomedical Sciences, University of Minnesota, Saint Paul, MN, USA
}

\section{Introduction}

Porcine circovirus 2 (PCV2) is associated with Postweaning Multisystemic Wasting Syndrome (PMWS) and other Porcine Circovirus Associated Diseases (PCVAD), but also is frequently isolated from healthy swine ${ }^{1,2}$. PCVAD threatened the swine industry in Europe and later North America for about 15 years. In North America in 2006 efficacious PCV2 vaccines was introduced. However, little research to answer basic questions on the disease has been done since 2006.

Molecular biologists warn that this lack of understanding weakens our ability to respond to new outbreaks ${ }^{1}$. We need to increase our understanding of PCVAD should current control strategies fail.

Our objectives were to retrospectively:1. Determine the National Prevalence of PMWS and 2. the National Weaned Pig Mortality from PMWS, 3. Determine the Prevalence of PCV2 (Antibodies and P(R) and 4. Develop National Estimates for the impact of farm and animal level factors on incidence of PMWS.

\section{Materials and Methods}

Questionnaire data and blood samples were collected as part of the NAHMS Swine 2006 study $^{3}$. Questionnaires administered during personal interviews with farm personnel were used to gather data on housing, health management and health status.

Blood samples were collected from up to 35 grower/finisher market pigs (20-32 weeks old) on 185 farms in 16 states. A new ELISA $(n=6,046)$ was used to test for the presence of PCV2 antibodies and a new PCR $(n=4,147)$ was also used to test whether PCV2 DNA was present in the sera of these pigs. In a separate study, these tests' accuracy was determined using Bayesian analysis. For the ELISA, the Sensitivity was up to $90 \%$ while that of the PCR was up to $92 \%$.

\section{Results}

Nationally, approximately one in three sites with weaned market pigs reported PMWS during the 12 months prior to the interview, ranging from $29.7 \%$ of small sites ( $<2,000$ head) to $59.9 \%$ (>4,999 head) of large sites. On sites that reported PCVAD in weaned market pigs, $15.4 \%$ of these pigs were affected and again, larger sites had more pigs affected $(19.8 \% \text { vs. } 7.7 \%)^{3}$.
Out of 6,046 samples $78.8 \%$ were positive for PCV2 antibodies and out of 4,147 samples $82.6 \%$ were positive for PCV2 DNA. Over $80 \%$ of samples tested using both tests were positive for antibodies and DNA. All but two farms had one or more samples positive for PCV2 antibodies and all but one for PCV2 DNA.

We used a weighted logistic regression that generates estimates that can be directly extrapolated back to the population (>93\% of the farms nationally with 100 or more pigs) rather than just the sample it comes from ${ }^{4}$. When farms had one respiratory disease (such as PRRS) the odds of PMWS being present went up almost 14 times compared to when no respiratory disease was present. If there were two or more respiratory diseases the odds went up over 30 times.

\section{Discussion}

Prior to the use of vaccines, more large farms than small farms had PMWS-affecting more animals. PCV2 elicits a humoral immune response yet fails to clear the virus for some unknown reason. Anecdotally, it was believed that PCV2 was ubiquitous but there has not been a study of this magnitude to give more accurate estimates.

Should the vaccines available now begin to fail to control PMWS or more broadly PCVAD, then we must return our efforts to reducing the impact of these respiratory pathogens modeled such as PRRS.

\section{References}

1. Opriessnig T., Meng X. J. and Halbur P. G. PCV type 2 associated disease: update on current terminology, clinical manifestations, pathogenesis, diagnosis, and intervention strategies. J Vet Diagn Investig. 2007: Volume 19, pp. 591-615.

2. Allan, G.M., Ellis J.A. Porcine circoviruses: a review. J Vet Diagn Investig. 2000: Volume 12, pp. 3-14.

3. USDA. 2007. Swine 2006, Part I: Reference of Swine Health and Management Practices in the United States, 2006 USDA:APHIS:VS:CEAH. Fort Collins, $\mathrm{CO}$

4. Dohoo, I., Martin, W., Stryhn, H. Veterinary Epidemiologic Research. Atlantic Veterinary College, Charlottetown, PE, Canada, 2003. 


\title{
P.144
}

\section{Porcine circovirus $\mathbf{2}$ as a causative agent for severe respiratory signs and polyserositis on a Specific Pathogen Free fattening farm}

\author{
Victor Geurts ${ }^{1}$ Chris Schouten ${ }^{2}$ Antoine L.M. Cruijsen ${ }^{1}$ Mark W. de Groot ${ }^{1}$ Mieke P. Vrijenhoek ${ }^{1}$ \\ 1. Intervet Nederland BV, Boxmeer, Netherlands; 2. Veterinarian Practice, Heeswijk-Dinther, Netherlands
}

\section{Introduction}

The clinical expression of PCVAD embraces a complex of signs including those related to the respiratory system. The authors are not aware of any reports relating polyserositis directly to PCV2 infections. Experimental PCV2 infections in Specific Pathogen Free (SPF) pigs has led to severe respiratory signs with high mortality including lung edema, hyperemia of the lungs, icterus, lymphadenopathy and edema of the mesentery (1). In 2009 on 2 locations SPF fattening pigs from one origin suffered from severe respiratory symptoms (dyspnea, coughing), high fever and a high level of mortality in spite of vaccination at 4 and 6 weeks of age against Haemophilus.parasuis (Porcilis ${ }^{\circledR}$ Glässer). Necropsy revealed polyserositis with negative bacteriological results, hyperemia of the lung, and interstitial lung edema. Organs were highly positive for PCV2 by IHC. Paired serological investigation of acutely sick pigs, only revealed seroconversion to PCV2. This strongly suggests that the clinical signs were related to PCV 2 infections in a similar situation as described in experimentally infected SPF pigs by Gauger et al(1). A PCV-vaccination trial was set up in order to help the farm and to explore the relationship between the signs observed and PCV2 infections. After this trial, all new piglets that entered the fattening units were vaccinated.

\section{Materials and Methods}

In a batch of 350 piglets, 197 were vaccinated with a single dose of Porcilis ${ }^{\circledR}$ PCV at 10 weeks of age, on their arrival in the fattening unit. The rest were left unvaccinated. Vaccinated and unvaccinated pigs were housed site by site in three different units. Mortality was recorded and in each trial group serum of 10 pigs was sampled at 10-, 14-, 18- and 22-weeks of age. Sera were tested on antibodies against PCV2 (Synbiotics), PRRS(Idexx), $\mathrm{H}$. parasuis (Biovet), M. hyopneumoniae (Idexx) and the $42 \mathrm{kD}$ outer membrane protein of A. pleuropneumoniae (2). Sera were also tested on PCV2 virusload by qPCR. Average daily intake (ADI), feed conversion rate (FCR) and average daily gain (ADG) during 2 months after the start of the vaccination was compared with the results of not vaccinated pigs during 2 months before the trial.

\section{Results}

All samples were negative against PRRS and M. hyopneumoniae. Also A. pleuropneumoniae titers stayed at low levels in both groups. Some sera of the 10- and 14-week old fatteners were positive against $\mathrm{H}$. parasuis in both groups. These positive results were likely due to the early vaccination beause all 22-week old fatteners became negative.
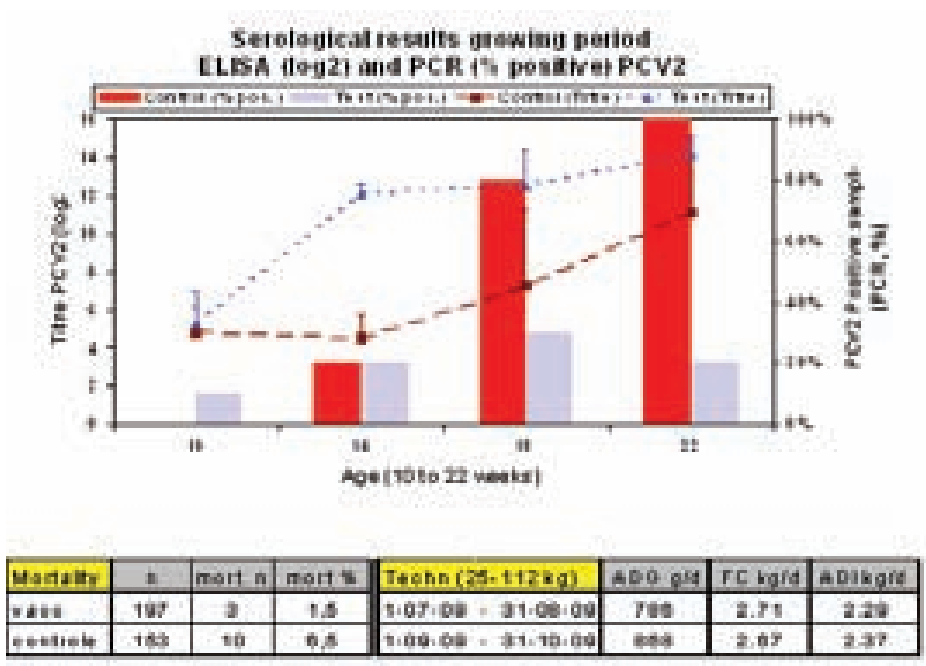

\section{Conclusion and Discussion}

The clinical signs (respiratory distress and polyserositis) on this farm were likely caused by PCV2, no other causative agent was found in the diseased pigs and vaccination against PCV was successful in preventing the disease. Pigs vaccinated with Porcilis ${ }^{\circledast}$ PCV had a reduced mortality, a clear humoral response to vaccination and the amount of PCV2 virus positive pigs was reduced. The historical data show a clear improvement in ADG, $A D I$ and FCR after starting the vaccination. The respiratory signs in this clinical field trial with SPF pigs were similar to those of experimentally infected SPF pigs after PCV 2 challenge (1). The authors are not aware of any reports relating polyserositis to PCV2 infection. A possible explanation for this feature could be a co-infection with Mycoplasma hyorhinis $(3,4)$, an opportunist which is capable of causing polyserositis. Its involvement may be triggered by the immune suppression caused by PCV 2 infection. The practical implication of the results of both this and the Gauger trial is that PCV 2 can be the cause of acute respiratory signs with high fever and high mortality levels.

\section{References}

1. Gauger P.C et al. 2006, Proc. OR.01.28 19th IPVS Copenhagen

2. Kobisch M and JF van den Bosch. 1992, Proc 12th IPVS, The Hague. p. 216

3. Gagnon C.A. et al. 2007,Can.Vet.J. 48 (8) 811-819

4. Lin J.H. et al. 2006, Vet.Microbiol. jun 15:111-6 


\title{
P.145
}

\section{Experimental co-inoculation with porcine circovirus type 2 (PCV2) and Mycoplasma hyopneumoniae in conventional pigs}

\author{
Marina Sibila; Maria Fort; Miquel Nofrarias; Anna Pérez de Rozas; Ivan Galindo-Cardiel; Enric Mateu; Joaquim Segales \\ Centre de Recerca en Sanitat Anima (CReSA), Bellaterra, Spain
}

\begin{abstract}
Introduction
Porcine circovirus type 2 (PCV2) is the etiologic agent of postweaning multisystemic wasting syndrome (PMWS) in pigs. However, PCV2 co-infection with some viral and bacterial agents may potentiate PMWS development (1). Particularly, a previous infection with Mycoplasma hyopneumoniae (M.) has been shown able to trigger PMWS in subsequently PCV2 infected pigs (2). The objective of this work was to study the effect of concurrent PCV2 and $\mathrm{MH}$ co-inoculation in conventional piglets.
\end{abstract}

\section{Material and Methods}

Thirty six 6-week-old male conventional piglets were divided into 4 groups: control ( $n=6$, receiving $2 \mathrm{ml}$ of PBS); PCV2 $(n=6$, intranasally challenged with $1 \mathrm{ml}$ of 105 TCID50 PCV2b per nostril); $\mathrm{MH}$ ( $\mathrm{n}=12$, intratracheally inoculated with $5 \mathrm{ml}$ of $\mathrm{MH}$ 107 CCU on two consecutive days); and PCV2+MH ( $n=12$, same inoculum conditions as above). Clinical signs, rectal temperature and body weight were recorded during the study period. Blood samples, faecal and nasal swabs were collected on $0,7,14$ and $21 \mathrm{dpi}$. At necropsy ( $21 \mathrm{dpi}$ ), tracheal and bronchial swabs were also taken. Extension of cranio-ventral pulmonary consolidation lesions (CVPC) was assessed (3). Moreover, lymphoid tissues and lungs were collected and fixed in 10\% buffered formalin. Sera were examined for PCV2 (total and neutralising (4)) and $\mathrm{MH}$ antibodies. DNA extracted from serum samples, nasal and faecal swabs were analyzed by a PCV2 quantitative PCR (Q-PCR) (5). PCV2 in situ hybridization (ISH) from lymphoid tissues and lung was performed (6). MH DNA from nasal, tracheal and bronchial swabs was analyzed by Q-PCR.

\section{Results}

PCV2 infection remained subclinical although mild PMWS-like microscopic lesions were observed in two PCV2 pigs and in one PCV2+MH animal. PCV2 was detected by ISH in 3 PCV2 and in 4 $\mathrm{PCV} 2+\mathrm{MH}$ challenged pigs. No significant differences on mean body weight and rectal temperature were observed among the 4 groups. Total antibody and NA titres were similar in both PCV2-inoculated groups. Number of PCV2 Q-PCR positive pigs in serum was higher but not statistically significant in all samplings in PCV2+MH than in PCV2 inoculated pigs. No significant differences in viral loads on different sampling days and duration of viremia were detected among PCV2 inoculated groups.
Coughing was observed in 3 piglets from the $\mathrm{MH}$ group and in $6 \mathrm{PCV} 2+\mathrm{MH}$ pigs but it was absent in non-MH inoculated pigs. Proportion of MH Q-PCR positive pigs and mean bacterial DNA load in nasal cavity were similar in $\mathrm{MH}$ and $\mathrm{PCV} 2+\mathrm{MH}$ groups. On the contrary, mean MH DNA load at bronchial and tracheal swabs was higher in the $\mathrm{PCV} 2+\mathrm{MH}$ group compared to the $\mathrm{MH}$ one. No significant differences on percentage of $\mathrm{MH}$ seropositive pigs among $\mathrm{MH}$ inoculated groups were observed. Eight pigs from $\mathrm{MH}$ group and nine from PCV2+MH group showed CVPC. No significant differences in mean lung scoring between $\mathrm{MH}$ group and PCV2+MH group were observed.

\section{Discussion}

In a previous study, $\mathrm{MH}$ inoculation two weeks before PCV2 challenge resulted in an increase of PCV2-associated lesions severity, amount of PCV2 antigen and incidence of PMWS in pigs (2). In the present study, the concurrent inoculation of $\mathrm{MH}$ and PCV2 did not produce that synergic outcome. These divergent results may be explained by the different timing of infection but also by the source of the animals used. In the present study, the inoculated animals were seropositive to $\mathrm{MH}$ - and PCV2 while in the previous one (2) the challenged animals were seronegative against both pathogens. Taking into account that infection with both agents was successful, the present study suggests that timing and initial pig serological status may be the most important issues for the final outcome of the co-infection.

\section{Acknowledgements}

This work is based on data generated in a study commissioned by Intervet/Schering-Plough Animal Health. Part of these results was presented at a satellite symposium hold at the IPVS 2008, Durban (South Africa) organized by this company.
References
1. Gillespie et al., 2009. J.Vet. Intern. Med. 23 (6):1151-63
2. Opriessnig et al. 2004. Vet Pathol. 41:624-640.
3. Goodwin et al. 1973. British Vet J 129(5):456-64.
4. Fort et al. 2007. Vet Microbiol 125:244-255.
5. Olvera et al. 2004. J Virol Methods; 117: 75-80.
6. Rosell et al. 1999. J Comp Pathol; 120: 59-78. 


\title{
P.146
}

\section{High moralities in the nursery, growing and finishing pigs. Risk assessment in Colombian pig herds}

\author{
Carlos A. Diaz ${ }^{2}$ María N. Rodriguez ${ }^{1}$ Gloria Ramirez ${ }^{2}$ Jairo Jaime² Victor Vera² Jose D. Mogollon² \\ 1. Department of Statistics. College of Science. National University of Colombia, Bogotá, Colombia; 2. Department \\ of Animal Health and Production. College of Veterinary Medicine and Animal Science, Bogota, Colombia
}

\section{Introduction}

Porcine Circovirus Associated Diseases (PCVD) have been associated with high mortality rates but PCV2 infection itself hasn't been proved to be a risk factor for develop them $(1,2,3)$. Some management practices, most be triggering the disease once PCV2 infects the pig. The main objective in this study was to determine what kind of swine industry practices and/or production indicators are associated with extremely high mortalities rates in Colombian swine herds.

\section{Materials and Methods}

Sample size was calculated in 70 herds considering a 95\% confidence level, $10 \%$ precision and $50 \%$ prevalence. Herds were randomly selected from 163 farms located in the three major swine rearing areas in Colombia, with 200 or more sows that represent the $74 \%$ of national inventory. An epidemiological survey was applied, and extremely high mortality in the nursery and finishing was defined as mean plus 2 standard deviation of the sample without extreme values when calculating it. 43 serum samples were collected per production cycle and analyzed for PCV2 antibodies (SERELISA ${ }^{\oplus}$ PCV2 SYNBIOTICS). Samples of death animals were collected when present and analyzed for PCVD lesions and PCV2 antigen was identified by Immunohistochemistry (IHQ) within compatible lesions.

Statistical analysis was performed using STATA $9{ }^{\circledR}$. The null hypothesis was that exposure to any of the variables included in the survey do not modifies the risk of developing extremely high mortality rates. Association between high mortalities and exposure variables were assessed through a logistic regression model estimating relative risk adjusting it for variables like clinical disorders or previously diagnosed diseases; backward stepwise regression analysis was performed for each outcome (high mortality in nursery and finishing).

\section{Results}

High positive ELISA reactivity was found in the sow herd (95.3\%) without significant differences between proportions, within parities in the tested animals ( $p>0.05$ ). High positive ELISA reactivity were also found in growing pigs $94,90.7,83.7,89.8$ and $93.6 \%$ for $1,5,10,15$ and 20 weeks of age respectively, being different the proportion of positive animals between animal 5 and 10 week of age $(p=0.009)$ and between animals 10 and 15 weeks of age $(p=0.026)$.
Mean mortality rate in the nursery was estimated in $1.41 \%$ SD 0.74 and $2.2 \%$ in the finishing pigs SD 1.45. The risk to have extremely high mortalities in the nursery was estimated to be 17.5 (IC 95\% 1.8, 168.2; $p=0.01$ ) higher when farms don't use shower baths at the entry to the nursery if the polyserositis (OR 6.1, IC95\% 1.06-35.3; $p=0.43$ ) and low consumption (OR 25.3 IC95\% 2.3 - 274.0; $\mathrm{p}=0.008$ ) effect was controlled in the logistic model; all other variables were dropped off the model because they lost significance when the effect of this three was controlled. All of the variables included in the second model lost significance when the effect of low consumption was controlled in finishing pigs; it was estimated that finishing pigs have a risk 18.8 times higher to develop high mortality if low consumption was reported (IC95\% 1.7 - 203.2; $p=0.016)$. PCVD has been diagnosed and PCV2 has been identified within lesions in some farms but not all result where available at the time of this preliminary report. PCV2 exposure was determined in all farms evaluated and vaccination didn't modify the risk of developing high mortality rates.

\section{Discussion}

PCV2 exposure itself was discarded to be associated with high mortality rates in the nursery and growing to finishing in farms with more than 200 sows in Colombia. Poor management practices could make possible that other swine pathogens, including PCV2, alter pig's health status in such a way that high mortalities appeared. The difference between proportions in positive animals to PCV2 during the nursery and finishing period may be related to high mortalities in certain ages.

\section{References}

1. Dewey C., et al. Postweaning mortality in Manitoba swine. Can J Vet Res. 2006; $70: 161-167$.

2. Vigre H., et al. Spatial and temporal patterns of pig herds diagnosed with postweaning multisystemic wasting syndrome (PMWS) during the first two years of its occurrence in Denmark. Vet Microbio. 2005; 105:17-26.

3. Woobdbine K., et al. Spatiotemporal patterns and risk of the herd break down in pigs with postweaning multisystemic wasting syndrome. Vet Rec. 2007; 160:751-762. 


\title{
P.147
}

\section{Growth depression after severe outbreak of porcine circovirus associated disease (PCVAD) in the finishing unit: case history}

\author{
Piotr Kneblewski; Krzysztof Wilczynski; Pawel Karbowiak
}

Vet-Com, Olsztyn, Poland

\section{Introduction}

The first reports about confirmed PCV2 in Polish swine herds date back to $2000(1,2)$. The aim of the investigation was to determine the impact of outbreak of severe porcine circovirus associated disease (PCVAD) on the performance in the finishing unit.

\section{Materials and Methods}

The investigation was conducted in a fattening farm located in the western part of Poland. The farm consisted of three barns for 2000 finishers with pens for 30-120 animals. Pigs for this study were sourced from a high health farm without clinical signs of PCVAD. They were not vaccinated against PCV2. A total of 1600 pigs were moved to the finisher farm at $40 \mathrm{~kg}$ of weight. The factors which aggravate problems were high temperature during transport and the quality of the feed in the beginning, which caused outbreak of watery, yellowish or grey diarrhea which affected up to $50 \%$ of the animals. After two weeks persistent cough appeared in $30-35 \%$ of pigs and the first cases of death were recorded. Numerous symptoms of PMWS and the first PDNS cases occurred. Weekly mortality increased to 5-10 pigs. Post mortem examination showed cachexia, paleness of the skin, reddish or blackish foci of cutaneous necrosis located mainly in the area of rump, neck and ears, enlargement of inquinal and mesenteric lymph nodes, various inflammation of digestive and respiratory system. Three sick pigs were culled. Their lymph nodes, samples of ileum, cecum, spiral colon and kidneys were analysed microscopically and tested by in situ hybrydysation for PCV2(1,2). Additionally intestine samples were examined by PCR for Lawsonia intracellularis and Brachyspira hyodysenteriae. PMWS typical histopathological lesions and PCV2 DNA were detected in the submitted material. The result for Lawsonia and Brachyspira was negative. After two weeks with many cases of diarrhea and coughing treatment was started with Tiamutin 45\% (tiamulin $8 \mathrm{mg} / \mathrm{kg}$ for 6 days) and then Soludox $50 \%$ (doxycyclin $10 \mathrm{mg} / \mathrm{kg}$ for 6 days) was administered in the drinking water. Such therapy only stopped the progress of disease for two weeks. After this time treatment had to be repeated. Moreover every sick pig was put in hospital pens. All facilities were cleaned and disinfected every day. Additionally all animals in hospital pens (300 pigs at the peak of disease) got
TiaClor (CTC and Tiamulin) and Paracetam(NSAID) in the feed for 21 days. The weight of the pigs was recorded at introduction to the finishing unit and at slaughter. Average daily weight gain (ADWG) was calculated and number of days to slaughter were recorded. Performance of pigs affected with PCVAD was compared with pigs non-affected with PCVAD. Group A consisted of healthy pigs without any clinical symptoms of PCVAD and group $B$ consisted of pigs with PCVAD.

\section{Results}

During the fattening period clinical signs of PCVAD infection occurred in $82 \%$ of the pigs (Group B). Only about $13 \%$ were not affected (Group A). Total mortality rate was $4.8 \%$. The ADWG of pigs with PCVAD (Group B) was reduced compared to non affected (Group A) by $244 \mathrm{~g} /$ day. Number of days to slaughter was extended by 21 days for group B compared to Group A (Table 1).

Table 1. Comparison between two groups of finishers. Group A pigs without any clinical sings of PCVAD and Group B pigs with clinical sings of PCVAD.

\begin{tabular}{|l|c|c|}
\hline & A & B \\
\hline Number of pigs & 206 & 1317 \\
\hline Average weight at the beginning $(\mathrm{kg})$ & 40 & 40 \\
\hline ADWG $(\mathrm{g})$ & 952 & 708 \\
\hline Number of days to slaughter & 75 & 96 \\
\hline
\end{tabular}

\section{Discussion}

Outbreak of PCVAD infection had serious financial consequences, reduction of DWG by $244 \mathrm{~g} /$ day and extension of feeding days by 21 for pigs with PCVAD and the loss was estimated at 35,200 USD or about 27 USD per pig. Had these treatments with antimicrobials, isolation of affected pigs in hospital pens, cleaning and disinfection of pens not been carried out losses would have been higher.

\section{References}

1. Pejsak Z. et al.: Vet Medicine 2001,57, $591-594$

2. Stadejek T. et al.: Vet Medicine 2006, 62, 297,301 


\title{
P.148
}

\section{Effect of co-infection of porcine circovirus type 2 and porcine reproductive and respiratory syndrome virus on porcine alveolar macrophages}

\author{
Fei Song ${ }^{1}$ Qigai He$^{1}$ Weidong Chai ${ }^{1}$ Wentao $\mathrm{LI}^{1}$ Catherine Charreyre ${ }^{2}$ \\ 1. College of Veterinary Medicine, Huazhong Agricultural University, Wuhan, China; 2. Merial Limited, Lyon, France
}

\section{Introduction}

Porcine reproductive and respiratory syndrome virus (PRRSV) and Porcine circovirus type 2 (PCV2) are currently a major concern for the swine industry worldwide. A lot of field surveys have indicated that dual PRRSV and PCV2 infection is common in pigs $(3,4)$. However, whether the immunosupression, in particular in alveolar macrophages, is stronger compared with the singular infection is still unknown.

\section{Materials and Methods}

Porcine alveolar macrophages (PAM) were isolated from lungs of 5-week-old piglets as Chang (2005) description. For subsequent experiments, PAMs were seeded at 24-well plates and infected with PCV2, PRRSV, PCV2+PRRSV (simultaneously inoculation) and PCV2 followed by PRRSV infection at $6 \mathrm{hr}$ later. The uninfected PAMs served as blank control. The supernatant and pellet of infected cells were collected at 0, 12, 24, 48, 60hpi.

We used quantitative real time PCR and indirect immunofluorescence assay (IFA) to analyze the adhesion and replication of PCV2 on PAMS, the cytopathic effect (CPE) and livability of PAMs were monitored and calculated by Typan Blue Staining Cell Viability Assay Kit, and mRNA levels of the cytokines (TNF- $a$, IFN$Y, I L-8)$ in PAMs were detected by semi-quantitative real-time PCR.

\section{Results}

The infection of PAMs with PRRSV neither increased the adhesion nor the replication of PCV2, but the distribution of PCV2 was changed in PAMs. PCV2 could not replicated nor induced CPE in infected PAMs, whereas the co-infected with two viruses could induce the cell death compared to single virus infected group. The expression of TNF- $a, I L-8$ in PAMS induced by coinfected group (different orders) were found to significantly increase and the increase was stronger than singular virus infected groups $(p<0.001)$. However the co-infected group inhibited the expression level of IFN- $\gamma$ compared to virus infected alone $(\mathrm{p}<0.001)$.

\section{Discussion}

The object of our work was to study the effect of PRRSV and PCV2 co-infection on PAMS. The results showed that no replication of PCV2 in PAMs was observed and this phenomenon was not associated with the present of PRRSV or not, PCV2 probably evade the immune systems through low-dose infection. As to the difference of PCV2 distribution between co-infected groups and single virus infection, more evidence is needed to explain this consequence. Besides, the addition of PRRSV to PCV2 caused more severe cell death and increased the expression of TNF- $a, I L-8$ than single virus infection, the results indicated co-infection may cause more serious pulmory pathological lesions. IFN- $\gamma$ is the broad-spectrum anti-virus agent, the stronger inhibition of expression caused by co-infected groups indicated PCV2 and PRRSV co-infection caused more severe immune suppression than infection with PCV2 or PRRSV alone.

\section{Acknowledgement}

The study was granted by Merial Limited.

\section{References}

1. Rossow et al., (1998) Vet. Immunol. Immunopathol. 61, 49-66.

2. Allan et al., (2000) Vet. Diagn. Invest. 12, 3-14.

3. Grau-Roma et al., (2007) Vet. Microbiol. 119, 144-151.

4. Pallares et al., ?2002?Clin. Diagn. Lab. Immunol. 9, 236-242. 


\title{
P.149
}

\section{PDNS was not a homogenous entity in the Swiss PMWS epizooty between 2003 and 2006}

\author{
Sandra Welti ${ }^{2}$ Titus Sydler $^{1}$ Esther Buergi ${ }^{2}$ Enrico Brugnera ${ }^{3,2}$ Xaver Sidler $^{2}$ \\ 1. Institute of Veterinary Pathology, Vetsuisse Faculty, Zurich, Switzerland; 2. Division of Farm Animals, Universitiy of Zurich, Veterinary \\ Faculty, Zurich, Switzerland; 3. Institute of Veterinary Pathology, Universitiy of Zurich, Vetsuisse Faculty, Zurich, Switzerland
}

\section{Introduction}

Porcine circovirus type 2 (PCV2) is the causative agent for postweaning multisystemic wasting syndrome (PMWS) and is considered to be one of the most important viral pathogens of pigs in many countries. PCV2 has also been associated with additional diseases, referred to as PCV associated diseases (PCVAD) which include PMWS, some types of pneumonia, enteritis, reproductive failures and another distinct disease condition, the porcine dermatitis and nephropathy syndrome (PDNS). In Switzerland a PMWS epizooty commenced at the end of 2003 (1). We attempted to reconstruct this epizooty and the retrospectively reevaluated PDNS cases are presented here.

\section{Materials and Methods}

The Institute of Veterinary Pathology, Zürich, is the only laboratory in Switzerland which diagnoses PCV2 infections by immunohistochemistry (IHC) with the monoclonal antibody F217 according to the rules of the sixth framework program (2). A retrospective investigation of all available cases from our archive and the archives of other Swiss laboratories between 2003-2006 were used to elucidate the Swiss PMWS epizooty, which began at the end of 2003. In this material only 14 of 538 declared PCV2 infected animals were IHC negative after reevaluation. 131 cases had a low PCV2 antigen load in the lymphatic tissues and 383 moderate to high. For this communication we selected the cases where PDNS was diagnosed using the "typical" glomerulonephritis as the diagnostic criterion.

\section{Results}

PDNS was diagnosed in 43 out of 538 animals. 21 PDNS cases had only minimal PCV2 antigen in lymphatic tissues and 22 had a moderate to high PCV2 antigen load. The PMWS animals were, as expected, significantly younger than those with PDNS. The PDNS pigs with low PCV2 antigen load in the lymphatic tissues were the same age as those with moderate to high antigen load ( $\varnothing 17$ weeks) but they had significantly higher body weights ( $\varnothing$ $44.1 \mathrm{~kg}, \mathrm{n}=19$ with available data) than those with moderate to severe PCV2 antigen load ( $\varnothing 29.8 \mathrm{~kg}, \mathrm{n}=13$ with available data) (Table 1). The cases with moderate to high PCV2 antigen content also showed depletion and histiocytic infiltrates in lymphatic tissues similar to PMWS.

Not all cases had skin lesions.
Table 1: Mean age and body weight of animals with PMWS and PDNS with low PCV2 content and PDNS with high PCV2 content ( $\left.{ }^{*} P<0.001\right)$

\begin{tabular}{|l|c|c|}
\hline Diagnosis & Age (weeks) & Body weight $(\mathbf{k g})$ \\
\hline PMWS & $10.5 \pm 3.8^{*}(n=167)$ & $14.6 \pm 8.8(n=276)$ \\
\hline PDNS: low PCV2 antigen content & $17.0 \pm 4.2^{*}(n=9)$ & $\mathbf{4 4 . 1} \pm 20.7^{*}(n=19)$ \\
\hline $\begin{array}{l}\text { PDNS: moderate to high PCV2 antigen content } \\
\text { "mixed PDNS-PMW" }\end{array}$ & $17.5 \pm 4.2(n=6)$ & $\mathbf{2 9 . 8} \pm 11.9^{*}(n=13)$ \\
\hline
\end{tabular}

\section{Discussion}

PDNS is suspected to be PCV2 induced (3), however we were not able to demonstrate any PCV2 antigen signal or DNA signal (by insitu hybridisation) within the glomerular lesions. PCV2 antigen signals were primarily in lymphatic tissues. However low PCV2 antigen load in lymphatic tissues is not defined as PCV2 disease because low quantities of PCV2 may be found in healthy animals.

Our set of PDNS cases was clearly divided into two subsets. One comprised PDNS animals with no to scarce PCV2 antigen and good body condition, the other subset suffered from PMWS (with high PCV2 antigen content and lesions in lymphatic tissue) followed by renal failure. We called these cases "mixed PDNSPMWS".

PDNS cases were infrequent in this investigation probably because the easily recognizable typical cases with dermatitis were not submitted for necropsy by the owners.

\section{References \\ 1) Wiederkehr et al. 2004. Vet Microbiol 136: 27-35. \\ 2) McNeilly et al. 2001. Arch Virol 146: 909-922. \\ 3) Wellenberg et al. 2004. Vet Microbiol 99: 203-214}




\title{
P.150
}

\section{Distribution of Porcine Circovirus type 2 (PCV2), Torque teno virus (TTV) and a novel porcine boca-like virus (PBo-likeV) in pigs from postweaning multisystemic wasting syndrome (PMWS)-affected and non-PMWS-affected farms in archival UK samples}

\author{
Michael J. McMenamy ${ }^{1}$ Irene McNair ${ }^{2}$ Catherine Duffy' John McKillen² Bernt Hjertner ${ }^{1}$ Catherine Mulholland² Gordon Allan² \\ 1. Queen's University Belfast, Belfast, UK; 2. Agri-Food and Biosciences Institute Northern Ireland, Belfast, UK
}

\section{Introduction}

Porcine circovirus type 2 (PCV2) is acknowledged as the essential infectious agent of postweaning multisytemic wasting syndrome (PMWS) ${ }^{1}$. However, it has been noted that PMWS may not develop even though a pig has been infected with $P C V 2^{3}$. It is possible that other agents may contribute to the development of PMWS. A possible potentiating co-factor in the development of PMWS by immunologic dysregulation is Torque teno virus genogroup 1 (g1-TTV), even though it is not considered a directly causative agent ${ }^{3}$. Another candidate which may play a role in PMWS development is a recently discovered porcine boca-like virus (PBo-likeV), identified by high-throughput sequencing in lymph nodes taken in a Swedish study from PMWS-affected pigs $^{2}$. Here we investigate the distribution of PCV2, TTV and the new PBo-likeV in archival serum and tissue samples recovered from pigs from 5 UK farms in 2002.

\section{Materials \& Methods}

37 sera samples were collected from pigs on all 5 UK farms, 2 of which were known to be non-PMWS-affected and the other 3 PMWS-affected. 21 pigs from 3 PMWS-affected farms also had tissue samples collected. Tissues included lung, liver, kidney, spleen and lymph nodes from each pig. Viral nucleic acids were extracted from sera and homogenised tissue samples. All sera and tissue samples were analysed by PCR for the presence of PCV2 using ORF2 specific primers ${ }^{1}$. Where possible, resultant amplicons were sequenced to ascertain the PCV2 genotype ${ }^{5}$. The samples were also assessed for the presence of g1-TTV4, g2-TTV and PBo-likeV² using real-time PCR. g2-TTV was detected using a set of primers kindly provided by Dr. Annette Mankertz (Robert Koch-Institut, Berlin, Germany) (data not published).

\section{Results}

Tissue sample PCR results were pooled due to little variation in virus presence from tissue to tissue. The percentage distribution of viruses detected in the samples are detailed in Tables 1 and 2.
Table 1. Distribution of PCV2, TTV \& PBo-likeV in sera from PMWSaffected \& non-PMWS-affected farms (\%).

\begin{tabular}{|l|c|c|c|c|}
\hline Sera Results & PCV2 +VE & g1-TTV +VE & g2-TTV +VE & PBo-likeV +VE \\
\hline PMWS +VE & $22 \%$ & $0 \%$ & $0 \%$ & $0 \%$ \\
\hline PMWS -VE & $61 \%$ & $50 \%$ & $56 \%$ & $0 \%$ \\
\hline
\end{tabular}

Table 2. Distribution of PCV2, TTV \& PBo-likeV in PMWS-affected \& non-PMWS-affected tissues from PMWS-affected farms (\%).

\begin{tabular}{|l|c|c|c|c|}
\hline Tissue Results & PCV2 +VE & g1-TTV +VE & g2-TTV +VE & PBo-likeV +VE \\
\hline PMWS +VE & $100 \%$ & $60 \%$ & $90 \%$ & $100 \%$ \\
\hline PMWS -VE & $100 \%$ & $64 \%$ & $46 \%$ & $82 \%$ \\
\hline
\end{tabular}

\section{Discussion}

The disparity in virus distribution evident in the sera samples could result from the PMWS-affected samples being comprised of sera from the 1st week of life which would limit pig exposure time to these viruses. However, non-PMWS-affected samples also included terminal bleeds at 12 weeks of age. It is apparent from the tissue samples that g2-TTV and PBo-likeV were more prevalent in PMWS-affected pigs. It is also of interest that PCV2 genotype 2a was present in sera samples from non-PMWSaffected farms although genotype $2 \mathrm{~b}$ was exclusively found in tissues from PMWS-affected farms ${ }^{5}$. Further statistical analysis of these results is required to assess if incidence of a virus or specific genotype can be significantly related to disease status.

\section{References}

1. Allan, G.M. et al. 2007. J. Vet. Diag. Inv. 19, 668-73.

2. Blomström, A-L. et al. 2009. Vir. Res. 146, 125-29.

3. Ellis, J.A. et al. 2008. Am. J. Vet. Res. 69, 1608-14.

4. Kerkarainen, T. et al. 2006. J. Gen. Vir. 87, 833-37.

5. Segalés, J. et al. 2008. Vet. Rec. 162, 867-68. 


\title{
P.151
}

\section{Serum endotoxin in pigs affected by wasting syndromes}

\author{
Paolo Candotti; Sara Rota Nodari; Claudia Nassuato \\ IZSLER, Brescia, Italy
}

\section{Introduction}

Postweaning multisystemic wasting syndrome (PMWS) is one of the disease syndromes which have been collectively named porcine circovirus diseases (PCVD). PMWS mainly affects nursery and/or fattening pigs and wasting is considered the most representative clinical sign of this disease. It is considered a multifactorial disease in which other factors in addition to PCV2 are needed, in most cases, to trigger the clinical disease. These factors haven't been clearly identified yet.

The aim of this study was to examine the levels of serum endotoxin in weaning piglets from farms with clinical symptoms of wasting compared to the levels in pigs from healthy farms.

\section{Materials and Methods}

Five (control farms) healthy farms and 13 (case farms) with a wasting syndrome that could be attributed to PMWS were selected for the study. Diagnosis of clinical PMWS and exclusion of other wasting agents was made through anamnesis, clinical findings and anatomo-pathological examination. Ten weaners were blood sampled in each farm (in control farms: animals perfectly healthy; in case farms: animals with a severe wasting syndrome). Blood samples were tested for lypopolisaccarides (LPS) with a Kinetic Cromogenic Assay.

\section{Results}

Mean and standard deviations (SD) of serum LPS (EU/ml) in the different farms are reported in table 1.

Table 1. LPS levels

\begin{tabular}{|c|c|c|}
\hline $\begin{array}{c}\text { case } \\
\text { farms }\end{array}$ & mean & $S D$ \\
\hline 1 & 0,95 & 0,20 \\
\hline 2 & 1,03 & 0,46 \\
\hline 3 & 1,15 & 0,36 \\
\hline 4 & 0,87 & 0,29 \\
\hline 5 & 1,26 & 0,69 \\
\hline 6 & 1,65 & 0,38 \\
\hline 7 & 0,73 & 0,21 \\
\hline 8 & 0,79 & 0,21 \\
\hline 9 & 0,74 & 0,06 \\
\hline 10 & 0,57 & 0,20 \\
\hline 11 & 0,46 & 0,10 \\
\hline 12 & 0,76 & 0,19 \\
\hline 13 & 0,99 & 0,38 \\
\hline
\end{tabular}

In figure 1 the graph of sensitivity-specificity and the cut off value is reported.

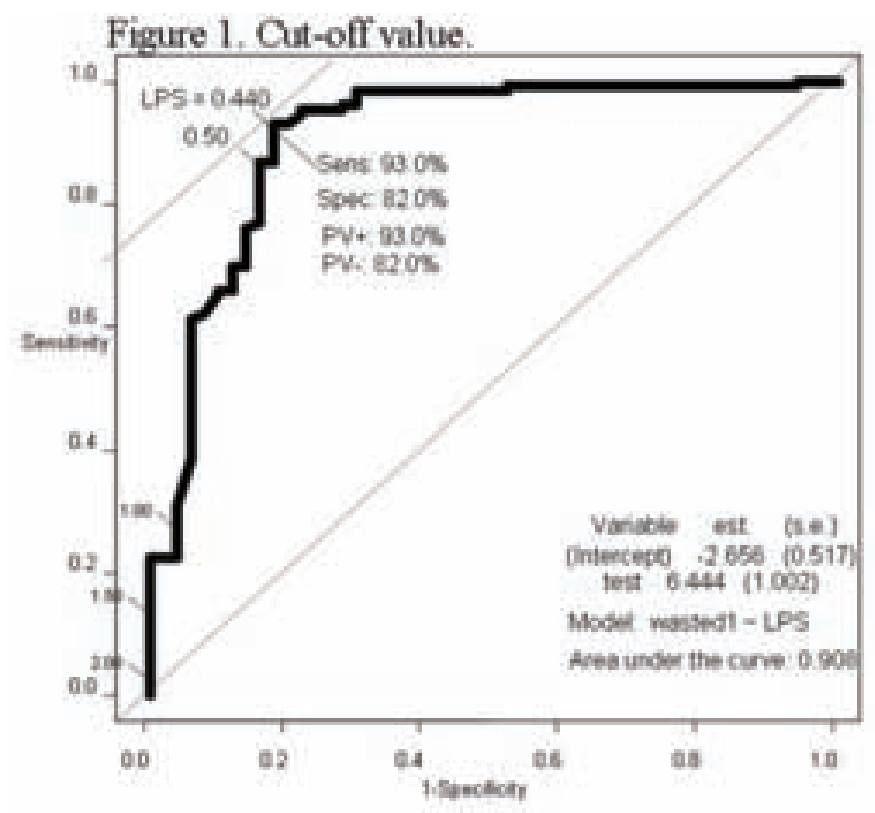

\section{Discussion}

The correlation between the presence of the disease and the level of LPS was statistically significant. The average LPS level in healthy animals was $0.34 \mathrm{EU} / \mathrm{ml}(\mathrm{Cl} 95 \%$ : 0.08-0.59) while in wasted animals $0.92 \mathrm{EU} / \mathrm{ml}$ (Cl 95\%: 0.76-1.08). In presence of wasting, LPS was on average $0.58 \mathrm{EU} / \mathrm{ml}$ higher (Cl 95\%: 0.28-0.88; $\mathrm{p}<0.001$ ). The cut-off value identified for LPS was $0.44 \mathrm{EU} / \mathrm{ml}$.

The limitation of these findings is that PMWS was not confirmed by histopathological examination of lymphoid tissues and detection of PCV2 in damaged tissues. However, these results could be useful in identifying triggering factors in animals affected by wasting syndromes that can't be attributed to specific pathogens and are clinically attributed to PMWS.

\section{References}

CHANG H. W., PANG V. F., CHEN L. J., CHIA M. Y., TSAI Y. C. \& JENG C. R. (2006) Bacterial lipopolysaccharide induces porcine circovirus type 2 replication in swine alveolar macrophages. Veterinary Microbiology 115, 311-319;

OPRIESSNIG T., MENG X. J. \& HALBUR P. G. (2007) Porcine circovirus type 2 associated disease: Update on current terminology, clinical manifestations, pathogenesis, diagnosis, and intervention strategies. Journal of Veterinary Diagnostic Investigation 19, 591. 


\title{
P.152
}

\section{Conventional sows inseminated with artificially PCV2 infected semen: I. In vivo results}

\author{
Giuseppe Sarli ${ }^{1}$ Federico Morandi ${ }^{1}$ S. Panarese ${ }^{1}$ B. Bacci ${ }^{1}$ D. Ferrara ${ }^{1}$ Laura Fusaro $^{1}$ M.L. Bacci ${ }^{1}$ G. Galeati ${ }^{1}$ \\ M. Dottori ${ }^{2}$ P. Bonilauri' ${ }^{2}$ D. Lelli ${ }^{3}$ Giorgio Leotti ${ }^{4}$ Thaïs Vila ${ }^{5}$ François Joisel ${ }^{5}$ F. Ostanello ${ }^{1}$ \\ 1. School of Veterinary Medicine, University of Bologna, Bologna, Italy; 2. IZSLER, Reggio Emilia, \\ Italy; 3. IZSLER, Brescia, Italy; 4. Merial, Milano, Italy; 5. Merial S.A.S., Lyon, France
}

\section{Introduction}

Since 1999, field evidence of transplacental infection by PCV2 and reproductive failure has been reported (1). Several experimental studies were performed: trans-uterine inoculation of fetuses (2), oronasal inoculation of pregnant SPF (3) or conventional (4) sows, intra-uterine inoculation of SPF sows (5). The objective of this study was to evaluate the clinical and pathological consequences of PCV2 infection in conventional pigs by artificially infected semen.

\section{Material and Methods}

Nine prepubertal conventional pigs were randomly divided: 3 controls and 6 infected. Hormonal estrus synchronization was followed by artificial insemination ( $\mathrm{Al}$ ) with a single dose semen added with $10 \mathrm{ml}$ of a PCV2 suspension. After ultrasonography at 29 day post-insemination (DPI), empty sows were euthanized at $30 \mathrm{DPI}$ whilst pregnant ones between 52nd and 56th DPI. Cervix, nasal and rectal swabs, and blood samples were weekly collected from -2 DPI till the end of the experimental period. Serum samples were directly and indirectly tested for PCV2, PRRSV, PPV and ADV. Serum antibody titres were determined by testing serial dilutions of each serum, by competitive $\operatorname{ELISAs}(6,7,8)$. The protocol described by Olvera et al. (9) was employed for PCV2 real time-PCR. Serum progesterone levels were measured by radioimmunoassay (10).

\section{Results}

At the -2 DPI, none pig presented viremia for all tested virus. All animals showed a high anti-PCV2 antibody level at -2 DPI (range 1/1000-1/10000), but only one sow had a lower titre (1/100). Four out of 6 infected sows displayed viremia at 7, 21, 28 and 35 DPI (the subject with the lowest anti-PCV2 level kept showing positive blood results for two successive samplings at 21 and 35 DPI). This latter was also the only sow showing a positive swab (rectal, 35th DPI). The anti-PCV2 antibody proved a decline after 7 DPI followed by a plateau in controls, whereas in infected animals were recorded values higher than controls that declined only after 42 DPI (Figure 1).

No sows showed any signs of estrus after Al. Pregnancies were established in 3 out of the 6 infected sows and in 3 out of the 3 controls (one control sow aborted 3 fetuses at 21 DPI). Serum antibody titres against PPV, PRRSV and ADV (total and anti-gE) decreased between -2 DPI and at the last DPI. Progesterone was absent in all gilts at insemination and in all animals increased after estrus induction but in the three non-pregnant infected animals never raised until the $28 \mathrm{DPI}$.
Figure 1: Mean anti-PCV2 antibody titre in infected (IG) and control groups (CG)

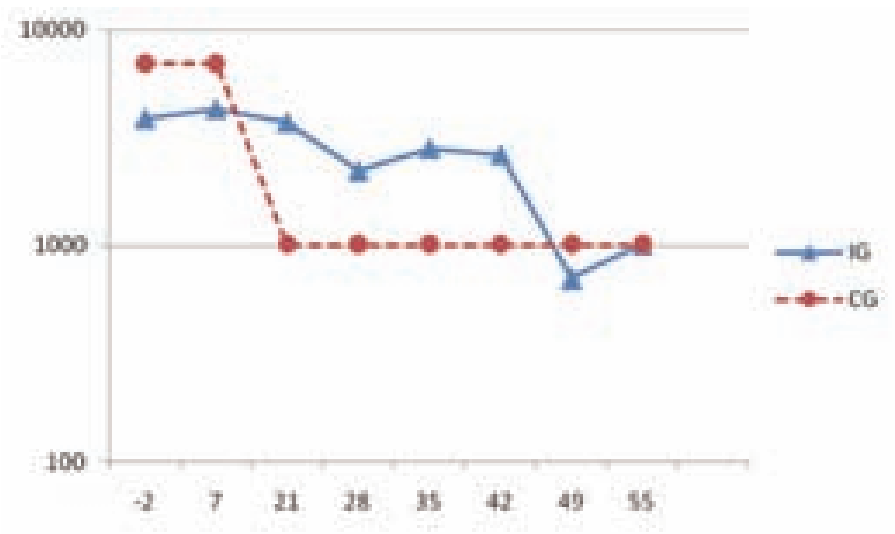

\section{Discussion}

In the present study the assessment of a field situation employing conventional pigs and intrauterine PCV2 exposition was investigated. Viremia was recorded in 4 out of 6 infected animals, besides mean antibody titre higher only in exposed subjects. Three infected sows out of 6 were not pregnant, unlike controls were all pregnant. Moreover, it should be emphasized that only the infected sow with lower PCV2 antibodies titre at -2 DPI showed simultaneously viremia and fecal virus spread. Therefore, i) the PCV2 infection is possible in conventional sows by intrauterine exposition; ii) low antibody titres increase the probability of the infection; iii) PCV2 infection close to insemination reduces the pregnancy rate.

\section{References}

1. West H, et al., 1999. J Vet Diagn Invest 11:530-32

2. Johnson C, et al., 1999. J Vet Diagn Invest 14:507-12

3. Cariolet $R$, et al., 2001. Proc ssDNA viruses of plants, birds, pigs and primates, 128

4. Park JS, 2005. J Comp Pathol. 132:139-44

5. Rose N, et al., 2007. J Comp Pathol 136:133-44

6. Sala G, et al., 2000. Proc. V Int. Congress of the ESVV, 253-54

7. Brocchi E, et al., 1990. Atti SISVET 44, 913-17

8. Cordioli P, et al., 1996. Proc XIV IPVS Congress, 86

9. Olvera A, et al., 2004. J Virol Methods. 117:75-80

10. Tamanini C, et al., 1985. Anim Reprod Sci 9:357-64 


\title{
P.153
}

\section{Occurrence of PCV2 and TTV genotypes from fetus and from nursery and finishing animals}

\author{
Kledna C. P. Reis; Jose L. Santos; Walter V. Guimaraes; Lucas F. Santos; Mayka R. Henriques; Daniel L. Santos \\ Microvet, Vicosa, MG, Brazil
}

\section{Introduction}

Swine with Postweaning Multisystemic Wasting Syndrome (PMWS) under PCR analysis yields both Porcine Circovirus genotypes (PCV2a and PCV2b) as well as association with Torque Teno Virus (TTV), PRRSV, Parvovirus (PPV), Swine Influenza, Coronavirus and also E. coli, Streptococcus suis, M. hyopneumoniae, $\mathrm{H}$. parasuis and $A$. pleuropneumoniae $(1,2,3)$. This work was done to verify the occurrence of PCV2 and TTV genotypes in samples from swine with PMWS and possible other virus and bacteria co-infections.

\section{Material and Methods}

Eighteen samples from swine suspected of PMWS, collected during 2009 in Brazil, were analyzed. Ten samples were from fetus (group I) and eight from nursery/finishing animals (group II). Lymph nodes, lung/heart were investigated by PCR analyses for PCV2a, PCV2b, TTV1, TTV2 and PPV. The DNA extraction and the PCR analyses were done according to Kim et al. (2003). The secondary bacteria infection diagnosis was done by Microvet laboratory (Brazil).

\section{Results}

PCV2b was the most prevalent in both groups. Two samples from group I were positive for PCV2a/PCV2b but this coinfection was not detected in group II. In both groups the most common co-infection was PCV2b/TTV2 followed by PCV2b/PPV. Twenty percent of samples from group I and 50\% from group II had TTV1/TTV2 co-infection (Table 1). In group I, $60 \%$ of the samples were co-infected with one bacterium, mainly, alpha hemolytic E. coli and in group II different species were detected (Table 2).

Table 1. PCR results for 18 samples from fetus and nursering/finishing animals

\begin{tabular}{|l|c|c|}
\hline PCR positive virus & Group I (fetus) & Group II (nursery/finishing \\
\hline PCV2b & $8 / 10(80 \%)$ & $8 / 8(100 \%)$ \\
\hline PCV2a/PCV2b & $2 / 10(20 \%)$ & $0 / 8(0 \%)$ \\
\hline PCV2/PPV & $3 / 10(30 \%)$ & $6 / 8(75 \%)$ \\
\hline PCV2b/TTV1 & $2 / 10(20 \%)$ & $4 / 8(50 \%)$ \\
\hline PCV2b/TTV2 & $9 / 10(60 \%)$ & $7 / 8(87.5 \%)$ \\
\hline TTV1/TTV2 & $2 / 10(20 \%)$ & $4 / 8(50 \%)$ \\
\hline
\end{tabular}

Table 2. Bacterial species associated with PCV2 in 18 samples from fetus and nursering/finishing animals

\begin{tabular}{|l|l|}
\hline Groups & Bacterial secundary agent \\
\hline Group I & E. coli (6/10); S, suis 1/10 \\
\hline Group II & E. coli (2/8);S. suis (2/8); M.hyorhinis (1/8); L. intracellulares (1/8) \\
\hline
\end{tabular}

\section{Discussion}

The viral and bacterial co-infections are common in PMWS $(1,2,3)$. The occurrence of PCV2 and TTV genotype infections could be related to differences in pathogenesis or animal origin. According to Horlen et al (2007) both PCV2a and PCV2b genotypes were found in isolates from PMWS-affected pigs. In this study the presence of PCV2a and PCV2b in group I needs more investigation since they were absent in group II. The PCV2a could be an antecessor in the primary infection or related to abortion or fetus anomalies when in association with PPV and TTV2. The presence of TTV1 and TTV2 in both groups was high, essentially the TTV2. The analysis of infection sequence in animals with PMWS can give interesting clues for the role of the TTV during infection. Taira et al. (2009) using nested PCR found positive results for TTV1 and TTV2 in different organs form PMWS swine. More studies are required to determine if other endogenous or exogenous cofactors are involved in the pathogenesis of PCV2a and PCV2b. In conclusion it can be stated that PCV2b and PCV2b/TTV2 were frequently observed and co-infection with bacteria were common in swine with PMWS.

\section{References}

1. Kim et al. J. Vet. Med. Sci 65: 741-744, 2003

2. Gagnon et al. CVJ 48: 811-819, 2007

3. Fernandes et al. Arq. Bras. Med. Zootec $48: 1-8,2006$

4. Horlen et al. J. Swine Health Prod. 15: 270-278, 2007

5. Taira et al. J. Vet. Mic.139: 347-350, 2009. 


\title{
P.154
}

\section{Association of PCV-2 with porcine respiratory disease complex (PRDC) in field infection from nine swine-producing Brazilian states}

\author{
Juliana Amália F. Nascimento ${ }^{1}$ Fábia S. Campos ${ }^{1}$ Grazielle C. Galinari ${ }^{1}$ Ernane F. Nascimento ${ }^{2}$ \\ Adrienny T. Costa ${ }^{3}$ Marcos B. Heinemann ${ }^{1} \underline{Z e ́ l i a ~ I n e ̂ s ~ P . ~ L o b a t o ~}^{1}$ \\ 1. Laboratório de Pesquisa em Virologia Animal, Escola de Veterinária, UFMG, Belo Horizonte, MG, Brazil; 2. \\ Departamento de Clínica e Cirurgia Veterinárias, Escola de Veterinária, UFMG, Belo Horizonte, MG, Brazil; \\ 3. Instituto de Pesquisas Veterinárias Especializadas Ltda (IPEVE), Belo Horizonte, MG, Brazil
}

\begin{abstract}
Introduction
The porcine circovirus diseases (PCVD) rarely occur as a result of the infection from one infectious agent, like PCV-2, alone. Usually several pathogens work together with the PCV-2 to break down defenses leaving the respiratory tract susceptible to pathogenic organisms. The PCV-2 has been identified in association with lesions in swines with porcine respiratory disease complex (PRDC). The aim of this work was to investigate the presence and distribution of PRDC bacterial coinfections in swines with clinical signals of the PCVD among Brazilian states.
\end{abstract}

\section{Materials and Methods}

Samples from 150 swine lungs with clinical symptoms of PCVD were studied. The samples were originally from field infections collected in 9 different swine-producing Brazilian states and have been previously confirmed to be positive for PCV-2 by PCR. All samples were submitted to microbiological standard tests for bacteria isolation and identification. Histopathological evaluation was carried out in 71 of the 150 samples and microscopic lesions were correlated with presence or absence of associated bacteria.

\section{Results}

Forty two samples (28.0\%) did not present any bacterial agent associated. In 108 (72\%) samples at least one of the following agents was detected: $P$. multocida type A, P. multocida type D, B. bronchiseptica, H. parasuis, A. pleuropneumoniae, S. suis, E. coli. Sixty-seven samples (44.7\%) presented one bacterial agent only, while 41 (27.3\%) had more than one. The bacterial frequencies among the 67 samples were: 18/67 (26.9\%) of S. suis, 7/67 (10.4\%) of A. pleuropneumoniae, 22/67 (32.8\%) of P. multocida type A, $6 / 67$ (9.0\%) of P. multocida type D, 3/67 (4.5\%) of B. bronchiseptica, $7 / 67$ (10.4\%) of H. parasuis and 4/67 (6.0\%) of E. coli. In the 41 samples with more than one of these bacteria, 24 different pathogens combinations were observed. The distribution of the bacteria in the Brazilian states studied showed diversity. The most prevalent bacteria associations were A. pleuropneumoniae and $P$. multocida type $A ; S$. suis and $P$. multocida type $A$; $S$. suis and P. multocida type A and H. parasuis. Minas Gerais state showed the major prevalence of bacterial associations. The pulmonary histopathological lesions observed in samples were grouped as: (i) positive association with bacterial infections (47.89\%), (ii) negative for bacterial association (52.11\%). In the negative group, interstitial pneumonia with thickening of interalveolar walls and inflammatory cells in the alveoli were observed. It was also observed peribronchial and perivascular lymphohistiocytic infiltrates with syncytial cells. In the positive group, pulmonary lesions similar to those from the negative group were observed in 12 samples, while pulmonary lesions with characteristics of bacterial infection were seen in 22 samples.

\section{Discussion}

In the present study we observed a wide dispersion of pulmonary bacteria across different swine-producing farms from nine Brazilian states. Furthermore, the frequence of association between PCV-2 and PRDC bacteria was high, suggesting that the PCV-2 immunossuppression can facilitate these pathogens infection. The results confirm that the respiratory infections are widely spread out among Brazilian herds. Our data are in accordance with previous studies that indicated that pulmonary lesions associated with PRDC had prevalence taxes of the $19.7 \%$ to $75.7 \%$ in swine from different Brazilian states. The high number of different bacterial associations with PCV-2 found reinforce current literature data that demonstrated that the PRDC is a multifactorial disease. It is also important to observe that Minas Gerais is one of the major swine producer states and that its herds are distributed for different states which could explain the dispersion of PCV-2 infection with bacterial associate.

\section{References}

Harms, 2002. J. Swine Health Prod., v. 10, p. 27-30;

Kim et al., 2003. Vet. J., v.166, p.251-256;

Opriessnig et al., 2007. J. Vet. Diag. Invest., v. 19, p. 591-615;

Reis et al., 1992. Arq. Bras. Med. Vet. Zoo., v. 44, p. 407-418;

Silva et al., 2001. In: Congresso Brasileiro de Veterinários Especialistas em Suínos - ABRAVES, Porto Alegre, Rio Grande do Sul, Brasil Anais.... CD Room.;

Thacker and Thanawongnuwech, 2002. Thai J. Vet. Med., v.32, p. 125134. 


\title{
P.155
}

\section{Superficial inguinal lymph node inv various PCVD conditions}

\author{
John Carr \\ Portec Australia, Belmont, WA, Australia
}

\section{Introduction}

An increase in size of the superficial inguinal lymph node is described as a clinical sign of Post-weaning Multisystemic Wasting Syndrome (PMWS). However, examination of the physical properties of the superficial inguinal lymph node indicates that rather than enlargement, the lymph node becomes more prominent as the "flare fat", around the lymph node, disappears with the loss of weight associated with PMWS and other Porcine Circovirus Diseases (PCVD). In Porcine Dermatitis and Nephropathy Syndrome (PDNS), there was a true enlargement of the superficial inguinal lymph node both in terms of length, width and depth.

It is essential, when describing pathology, to avoid terms like "enlargement" without providing physical dimensions.

\section{Materials and Methods}

Normal population: Superficial inguinal lymph nodes were collected from a slaughterhouse or a feed-trial in Western Australia from a variety of live-weight pigs. All the pigs were passed suitable for human consumption or had no gross pathology. The lymph nodes were measured for their length, width and depth. Western Australia was selected because the area is free of many viral pathogens that cause changes in lymph node sizes - particularly Porcine Reproductive and Respiratory Syndrome virus.

Number of animals in each normal group:

10 to $30 \mathrm{~kg}$ live weight: 107 pigs

40 to 60 kg live weight: 312 pigs

90 to $110 \mathrm{~kg}$ live weight 125 pigs

Abnormal population: Superficial inguinal lymph nodes were collected from cases of PMWS, PCVAD and PDNS.

Porcine Circovirus Associated Disease (PCVAD) is defined as pigs from farms where there was a PCVAD score $\geq 1$ but there was no clinical evidence of PMWS on the farm.

\section{Count:PCVAD: 33 pigs; PMWS: 17 pigs; PDNS: 10 pigs}

Approximate age of the pigs in each group and normal weight range for the age group

PCVAD: 8 to 10 weeks

22 to $30 \mathrm{~kg}$

PMWS: 8 to 16 weeks

22 to $65 \mathrm{~kg}$

PDNS: 16 to 20 weeks

65 to $95 \mathrm{~kg}$

\section{Results}

Length of the superficial inguinal lymph node:

\begin{tabular}{|l|c|c|c|}
\hline & Mean $\mathbf{~ m m}$ & Standard deviation & Range $\mathbf{~ m m}$ \\
\hline 10 to $30 \mathrm{~kg}$ & 38 & 7.4 & $20-66$ \\
\hline 40 to $60 \mathrm{~kg}$ & 56 & 8.9 & $34-80$ \\
\hline 90 to $110 \mathrm{~kg}$ & 61 & 9.3 & $22-85$ \\
\hline PCVAD & 32 & 7.4 & $18-50$ \\
\hline PMWS & 44 & 8.7 & $32-63$ \\
\hline PDNS & 90 & 21.7 & $50-110$ \\
\hline
\end{tabular}

The relative length of the superficial inguinal lymph node in the normal pig and various abnormal states

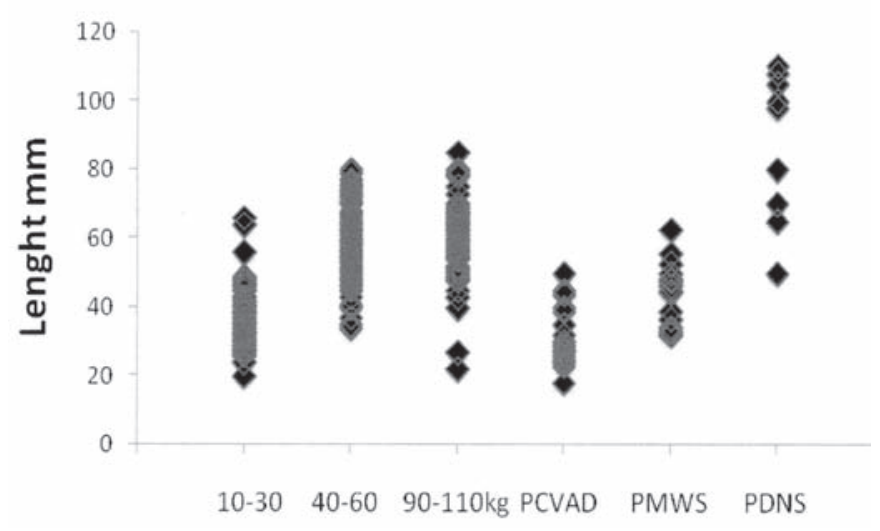

The superficial inguinal lymph node appearance The location is arrowed

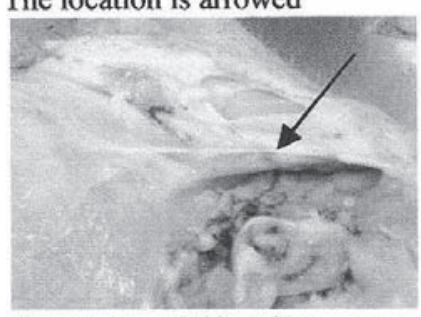

Normal - difficult to see

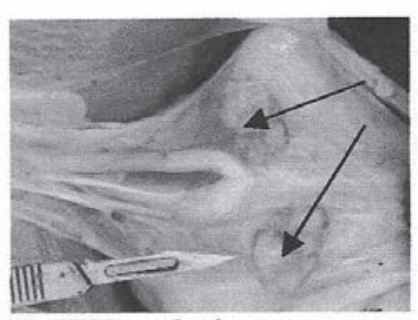

PMWS - obvious

\section{Discussion}

The normal inguinal lymph node increases in size as the pig grows from a mean length of $38 \mathrm{~mm}$ in pigs less than $30 \mathrm{~kg}$ to 61 $\mathrm{mm}$ in the $110 \mathrm{~kg}$ finisher pig

The superficial inguinal lymph node is quite large and is normally virtually invisible; covered by fat.

In cases of cachexia the 'flare fat" disappears around the superficial inguinal lymph node, which then becomes clinically very apparent.

In cases of PCVAD and PMWS the lymph node is not enlarged, when compared to the normal pig. However, it becomes more prominent because the surounding "flare fat" is lost.

The superficial inguinal lymph node is enlarged - in terms of length, breath and depth in cases of PDNS. $P<0.001$. As the tissues of the skin around the perineal region are infected in cases of PDNS, an enlargement of the draining lymph node is to be expected.

Please visit out website: www.portec.com.au 


\title{
P.156
}

\section{Acute Pulmonary Edema (APE), a New Porcine Circovirus Associated Disease??}

\author{
Kara M. Theis ${ }^{1}$ Steve Henry ${ }^{2}$ Lisa Tokach $^{2}$ \\ 1. University of Minnesota, Lino Lakes, MN, USA; 2. Abilene Animal Hospital, Abilene, KS, USA
}

\begin{abstract}
Introduction
We are investigating an on-going case involving porcine circovirus (PCV) and PRRS that has striking clinical differences compared to outbreaks before the advent of circovirus vaccines. Historically clinical circovirus is seen in 8-20 week old pigs with lesions of depleted lymphoid tissue in many organs. Gross pathology is varied and the clinical course is chronic, debilitating and progressive. Implementation of PCV vaccine decreased morbidity and mortality dramatically. In our case the clinical presentation differs with acute death pigs in good body condition. The consistent gross lesion, severe interlobular pulmonary edema, is distinguishing. The affected animals have been vaccinated with a product and protocol that has proven highly effective in the past. This particular animal group is suffering from severe disease while cohort animals, raised on other sites, remain normal.
\end{abstract}

At 21 days of age weaned pigs from two separate PRRS negative breed-to-wean farms move to one of 20 separate growing facilities, either stand-alone nurseries or nursery-finishing sites. Pigs in 9 of the 20 sites remain PRRS negative through their growth period while PRRS infection and seroconversion frequently occurs at 8-10 weeks of age in the other eleven sites. APE associated losses occur in both PRRS positive and PRRS negative populations. The 20 sites are fed by six separate and nonaffiliated feed mills. All grain used has been 2008 grain sources. The four affected sites are fed by three different mills. None of the affected sites are located near one another. All pigs were immunized at four and seven weeks of age with Circumvent PCV vaccine (Intervet Schering-Plough Animal Health) Immunization protocol has remained unchanged since 2007 and has been highly efficacious. Groups affected range from 7-14 weeks of age at onset of clinical signs. In severely affected lots mortality ranged from $13-21 \%$. Predominantly the mortality is acute and associated with APE, however some chronic and debilitated animals developed in certain groups.

\section{Materials and Methods}

Fresh and fixed tissues were collected from seven pigs of seven weeks of age and weighing approximately $35 \mathrm{lbs}$. Three pigs were found dead the morning of necropsy. The four remaining pigs were selected as "clinically normal" controls based on clinical evaluation. None of the other pigs in this four room nursery were coughing. They were clinically normal and were highly active. Pigs were sedated with $1 \mathrm{ml}$ of pentobarbital via jugular venapuncture. Seventy $\mathrm{mls}$ of blood was collected into $10 \mathrm{ml}$ serum separator tubes via an intracardiac stick. Animals were then euthanized with $1.5 \mathrm{mls}$ of pentobarbital and necropsied. Gross pathology, histopathology including IHC for Circovirus, differential and quantitative PCR for PCV and PRRS, IFA antibody titers for PCV, viral genome sequencing and compliance antibody differential sampling with baculovirus antibody testing was conducted.

\section{Results and Discussion}

On the basis of the viral genome sequencing the circovirus and PRRS viruses present are very similar to those historically known to this region and production flow. Compliance antibody evaluations exhibited responses to vaccination and several groups that exhibited disease appeared to have been well vaccinated based on compliance testing. Through IHC, histopatholgy and PCR testing circovirus was also demonstrated as the agent associated with the pulmonary pathology, including necrotizing vasculitis, which is not commonly seen with PCV2. The concentrations of circovirus detected in the tissue and the serum were considered extremely high in most cases with $\mathrm{Ct}<10$ for many tissues and $\mathrm{Ct}<20$ for serum pools.

While interlobular edema is an occasional manifestation of circovirus the pulmonary edema is not. Noncardiogenic pulmonary edema is characterized by diffuse alveolar damage, increased capillary permeability and accumulation of protein rich fluid in the alveoli. The fluid accumulation ultimately results in decreasing oxygenation. This can be caused by either direct alveolar damage i.e. diffuse pulmonary infection or indirectly i.e. severe sepsis resulting from the over expression of the normal inflammatory response. Commonly all of this occurs within 24 hrs manifesting as respiratory failure and acute death $\mathrm{a}$,b.

The consistent lesions of acute death, interlobular edema, necrotizing vasculitis pleural transudate accompanied by very high concentrations of virus in tissues appears clinically to be a new manifestation of PCV2b viremia. Several laboratory tests are still pending.

\section{References}

a. Pernia D.G. Noncardiogenic Pulmonary Edema Emergency Medicine Clinics of North America. 21 (2003) 385-393

b. Straw B.E. et al Porcine Circovirus. Diseases of Swine 9th ed (2006) 299-309 


\title{
P.157
}

\section{Co-infections in PMWS field cases in Italian herds}

\author{
Loris G. Alborali ${ }^{1}$ Pavesi Roberta ${ }^{1}$ Matteo Gradassi ${ }^{1}$ Giuseppe Sarli ${ }^{2}$ Zanoni Mariagrazia ${ }^{1}$ Cristian Salogni $^{1}$ Stefano Giovannini ${ }^{1}$ \\ 1. IZSLER, Brescia, Italy; 2. Facoltà di Medicina Veterinaria Università Bologna, Bologna, Italy
}

\section{Introduction and Objectives}

Porcine Circovirus type 2 (PCV2) is a worldwide endemic viral pathogen of swine, associated with Postweaning Multisystemic Wasting Syndrome (PMWS). PCV2 infection is essential to the development of PMWS, but other co-factors can influence the evolution of PCV2 infection (1). Between these co-factors there are co-pathogens. An enhancement of PCV2 viral load, severity of the lesions and incidence of PMWS have been highlighted when PCV2 co-exists with other swine pathogens like PRRSV or Mycoplasma hyopneumoniae $(2,3)$. Through the diagnostic outcome of investigations, carried out between January 2007 and August 2009 at IZSLER-Brescia, the aim was to determine the prevalence of several viral and bacterial swine pathogens, frequently associated with PCV2 in PMWS cases.

\section{Material and Methods}

193 PMWS affected pigs, from 92 farms, were investigated for other swine pathogens. For diagnosis of PMWS was applied a protocol according to Opriessnig et al., 2007(1). For PRRSV was applied a reaction One-Step RT-PCR multiplex with specific primers, described by Persia et al., 2001(4). For SIV a RT-PCR according to the protocol described by Spackman et al., 2002(5). For Mycoplasma hyopneumoniae a PCR according to the protocol described by Stakenborg et al., 2006(6). For Lawsonia intracellularis a PCR as described by Jone et al., 1993(7). For Haemophilus parasuis was applied a PCR described by Angen et al., 2007(8). Isolation of bacterial agents was performed by routine culture procedures.

\section{Results and Discussion}

The viral and bacterial pathogens identified in PMWS affected pigs are reported in the table below.

The co-pathogens association are shown in Graphic 1.

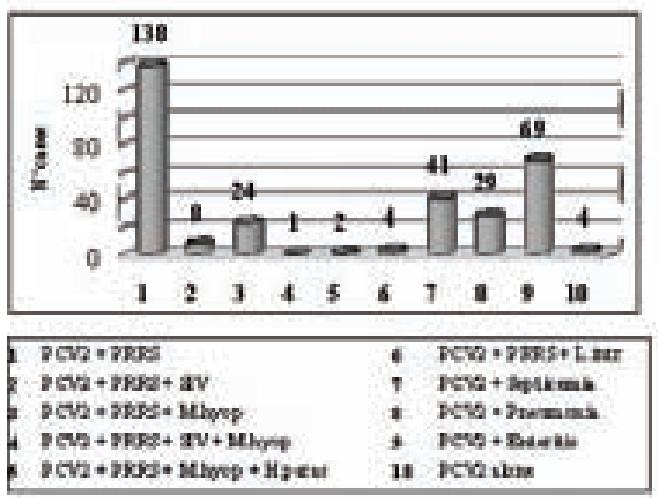

Graptic 1. Association of PCV2 and the cther suine pathogens in P2NWS cuses.
Farms with PMWS problems in which also PRRSV was highlighted were $81 / 92$ (88\%); 68,9\% was previously described in PMWS affected Italian farms (9). PRRSV was detected in $91,2 \%$ of PMWS affected pigs. PRRSV was the pathogens more frequently associated with PCV2 also in field cases in US $(51,9 \%)$ and in Netherlands ( $83 \%)(10,11)$. In case of septicemia and enteritis E.coli was the most frequent bacteria, in pneumonia cases $\mathrm{P}$. multocida . PCV2 alone was highlighted only in the $2,1 \%$ of PMWS cases, which is consistent with the theory according to which other co-factors are required for PMWS development.

\section{References}

1) Opriessnig et al. 2007. J Vet Dia Inv 19, 591-615.

2) Rovina et al. 2002. J Virol 76, 3232-3239.

3) Opriessnig et al. 2004. Vet Path 41, 624-640.

4) Persia et al. 2001. Proc $X$ Intern Symp Vet Lab Diagn 440-441.

5) Spackman et al. 2002. J Clin Micr 40, 3256-3260.

6) Stakenborg et al. 2006. Vet Res Com 30, 239-247.

7) Jone et al. 1993. J Clin Micr 31, 2611-2615.

8) Angen et al. 2007. Vet Micr 119, 266-276.

9) Sarli et al. 2009. Vet Rec 164, 519-523.

10) Pallares et al. 2002. J Vet Diagn Invest $14,515-519$.

11) Wellenberg et al. 2004. Res Vet SC 77, 177-184. 


\title{
P.158
}

\section{A preliminary study of the effects of treating diarrhoeic pigs with oxytetracycline on shedding of Porcine Circovirus Type 2 and Lawsonia intracellularis}

\author{
Patricia K. Holyoake ${ }^{1}$ Charlotte Hjulsager ${ }^{2}$ Lars E. Larsen ${ }^{2}$ Ken S. Pedersen ${ }^{3}$ Markku Johansen ${ }^{4}$ \\ Helle Stege ${ }^{3}$ Beverley Orchard ${ }^{1}$ Marie Stahl² Oystein Angen ${ }^{2}$ Jens P. Nielsen ${ }^{3}$ \\ 1. Wagga Wagga Agricultural Institute, Wagga Wagga, NSW, Australia; 2. National Veterinary Institute, Technical University of Denmark, \\ Copenhagen, Denmark; 3. University of Copenhagen, Copenhagen, Denmark; 4. Danish Pig Research Centre, Copenhagen, Denmark
}

\section{Introduction}

There is pressure to minimize antibiotic use in food-producing animals due to the risks of selecting resistant strains of bacteria. In Denmark, pressure to reduce macrolide antibiotics has resulted in a tendency to increase the use of tetracyclines. ${ }^{1}$ Most antibiotics are used to treat intestinal infections of grower ( $>10$ weeks) pigs, thought to be caused by Lawsonia intracellularis (L. intracellularis) and/or Porcine Circovirus Type 2 (PCV2). Our hypothesis was that individual treatment of diarrhoeic pigs for three days with an oxytetracycline antibiotic would reduce diarrhoea and the faecal shedding of $L$. intracellularis and/or PCV2.

\section{Materials and Methods}

Forty grower pigs were selected on each of six farms (total of 240 pigs) in Denmark for a case-control study. Each pig was eartagged and assessed for faecal consistency (normal, loose, fluid). Approximately half of the pigs had loose or fluid faeces and were classed as "diarrhoeic". Faecal samples were collected from each pig and tested using real-time PCR specific for PCV2 and $L$. intracellularis. ${ }^{2,3}$ Half of the pigs in each group were randomly selected on Day 1 and injected for three consecutive days with oxytetracycline (Engemycin, Intervet). Pigs were re-assessed on Day 4, when repeat faecal samples were collected. The effect of oxytetracycline treatment on faecal PCV2 quantity (negative/ low versus moderate/massive), and $L$. intracellularis bacterial quantity (negative/low versus moderate/massive) on Day 4 were analysed, with diarrhoea status (+/-), PCV2 quantity and L. intracellularis quantity on Day 1 included as fixed effects. All analyses were conducted using generalized linear mixed models, with pen and farm as random effects (Genstat 11 th edition).

\section{Results}

Two farms had no pigs with moderate/massive PCV2 results in faeces. One farm had no pigs with a negative/low faecal PCV2 result. Two farms had pigs with no moderate/massive $L$. intracellularis results. There appeared to be within-pen clustering of PCV2 infection on Day 1, with all or no pigs in some pens being infected. This was also true for L. intracellularis on Day 4. Hence, pen was removed from subsequent analyses involving these variables. Pigs that had diarrhoea on Day 1 and had not been treated with oxytetracycline had $2.6(95 \% \mathrm{Cl} 2,3.2)$ times the odds of having diarrhoea on Day 4 than pigs that were treated $(p<0.05)$. The farms where no pigs had moderate/massive quantities of PCV2 and/or L. intracellularis were excluded from analyses of associations between the two pathogens. Oxytetracycline treatment and PCV2 quantity on Day 1 did not affect PCV2 quantity on Day 4 ( $p>0.05)$. There were no significant associations between the quantities of $L$. intracellularis bacteria on Days 1 and 4 ( $p>0.05$ ). However, treated pigs had 0.076 (95\% Cl 0.03, 0.19 ) times the odds of having moderate/massive quantities of $L$. intracellularis on Day 4 than non-treated pigs.

\section{Discussion}

These preliminary results suggest that oxytetracycline treatment of growing pigs did not affect the quantity of PCV2 shed in faeces. Treating pigs for three consecutive days with injectable oxytetracycline reduced the incidence of diarrhoea and the quantity of $L$. intracellularis shed in faeces, suggesting that this pathogen played a role in causing diarrhoea, and that the treatment was effective.

\section{Acknowledgements}

This project was supported by the University of Sydney, University of Copenhagen, Danish Pig Research Centre, Technical University of Denmark and Australian Pork Ltd. We thank the participating producers and veterinarians for their assistance.

\section{References}

1. Jensen VF. et al. (2008). "Use of antimicrobial agents and occurrence of antimicrobial resistance in bacteria from food animals, foods and humans in Denmark". ISSN 1600-2032

2. Hjulsager CK. et al. (2009). Vet Microbiol. 133:172-178

3. Ståhl M. et al. (2008). Abstract, Advances in qPCR 


\title{
P.159
}

\section{Presence of porcine circovirus type 2, Lawsonia intracellularis and Brachyspira hyodysenteriae in pigs with diarrhoea}

\author{
Anna Szczotka; Jacek Zmudzki; Zygmunt Pejsak; Tomasz Stadejek \\ National Veterinary Research Institute, Pulawy, Poland
}

\section{Introduction}

The aim of the study was to analyze the presence of porcine circovirus type 2 (PCV2) in cases of antibiotic non-responsive diarrhoea and to evaluate the possible role of the virus in development of enteritis in pigs. For differential diagnosis identification of Lawsonia intracellularis (L. intracellularis) and Brachyspira hyodysenteriae (B. hyodysenteriae) was performed.

\section{Materials and Methods}

Internal organs and feces were collected from 76 pigs, 5 - 19 weeks old, from 50 farrow-to-finish farms, PMWS-positive or PMWS-suspected. Sections of lymph nodes and intestines (ileum, caecum and colon) were analyzed for presence of PCV2 DNA by in situ hybridization test (ISH) (2). They were also hematoxilin-eosin (HE) stained for standard histopathological examination. Addtionally, fecal samples were tested for presence of B. hyodysenteriae and L. intracellularis by PCR (3).

\section{Results}

In samples from 37 pigs, 10 - 17 weeks old, large amounts of PCV2 DNA, typical for PMWS, were detected in lymph nodes by ISH. In this group, in samples from 19 pigs, PCV2 was also found in abundant amount in samples of ileum. The remaining 18 pigs, PCV2-positive in lymph nodes, were negative in ileum. In samples from only 1 animal lymph nodes were negative for PCV2 in ISH, but virus was detected in considerble amount in ileum. In HE stained sections of lymph nodes histopathological lesions characteristic for PMWS were identified. Similar lesions were observed in PCV2-positive samples of ileum. Seventy samples of feces were negative in PCR for B. hyodysenteriae and $L$. intracellularis. DNA of $L$. intracellularis was found in feces from 3 pigs and mixed infection caused by both bacteria was detected in 3 animals

\section{Discussion}

According to the obtained results in PMWS-affected pigs similar lesions could be observed both in lymph nodes and in ileum and they correlate with clinical outcome of disease. Also, it was found that presence of PCV2 in ileum could be correlated with diarrhoea in PMWS- free animal, which confirms that the emergence of this virus as an intestinal pathogen may represent a new phenomenon (1). In the animals negative for $\mathrm{B}$. hyodysenteriae and L. intracellularis and PCV2 other causative agents of diarrhoea should be considered. Futher investigation on larger number of samples needs to be performed to confirm the role of PCV2 as an etiological agent of diarrhoea in pigs.

\section{Acknowledgements}

This work was supported by the research grant N N308 075634 from Polish Ministry of Science and Higher Education

\section{References}

1. Jensen T.K. et al. 2008, J. Comp. Path. 135, 176-182

2. Stadejek T. et al. 2006, Medycyna Wet. 62, 297-301

3. Zmudzki J. et al. 2006, Medycyna Wet. 62, 1420-1423 


\title{
P.160
}

\section{Co-infection between porcine circovirus type 1 (PCV1) and genogroups 1 and 2 of porcine torque teno virus (TTV) in fetuses naturally infected by porcine circovirus type 2 (PCV2)}

\author{
Giseli A. Ritterbusch ${ }^{2}$ Camila Sa Rocha ${ }^{3,2}$ Nelson Mores ${ }^{2}$ Neide L. Simon² Eraldo L. Zanella1, \\ ${ }^{4}$ Leonardo Diniz-Mendes ${ }^{5}$ Christian Niel $^{5}$ Janice R. Ciacci-Zanella ${ }^{1}$ \\ 1. Virus and Prion Diseases Research Unit, NADC, ARS, USDA, Ames, IA, USA; 2. Embrapa Swine and Poultry Research Center, Animal Healthy \\ Laboratory, Concordia, SC, Brazil; 3. Agroveterinarian Science Center - CAV - University of Santa Catarina State - UDESC, Lages, SC, Brazil; 4. \\ College of Agronomy and Veterinary Medicine, University of Passo Fundo, Passo Fundo, RS, Brazil; 5. Oswaldo Cruz Institute, Rio de Janeiro, Brazil
}

\section{Introduction}

Porcine circovirus 2 (PCV2) associated diseases (PCVAD) includes reproductive failures (1). PCV2 DNA has been detected by nested-PCR in organs of fetuses naturally infected $(8,5)$. In contrast PCV1 has not been associated with pathologies in pigs. Another DNA circular virus, porcine torque teno virus has also been detected in swine, with at least two genogroups, TTV1 and TTV2 (7). Recent studies have shown a possible role of TTV in increasing clinical signs and severity of lesions caused by PCV2 on piglets (3). The objective of this work is to study the co-infection of both porcine circular DNA viruses, TTV1, TTV2 and PCV1 in organs from fetuses naturally infected by PCV2, aiming to verify the importance of them.

\section{Material and Methods}

Organs from 29 fetuses, previously tested for PCV2, from 11 swine farms, were processed to detect PCV1, TTV1 and TTV2 nucleic acids. Samples of heart, lung, liver, kidney, lymphoid organs and nervous tissues were tested by nested-PCR. Nested$P C R$ reactions were performed using specific primers to detect sequences of PCV1, TTV1 and TTV2 as previously described (3, 4).

\section{Results}

Organs samples of 29 fetuses were tested by nested-PCR and PCV1 DNA was detected in 8 (27.6\%) of them. In addition, TTV1 and TTV2 was detected in 7 (24.1\%) and 24 (82.8\%) fetuses respectively (Figure 1), and viral sequences were found in several tested organs.

\section{Discussion}

These results show the occurrence of TTV1 and TTV2 in analyzed samples, indicating its importance of TTV co-infections with PCV2. In swine, TTV alone has not showed to be pathogenic, but, its role in co-infections with other pathogens has been investigated.

Recent studies showed the first indications of TTV in utero transmission to piglets (6). Other studies claim that TTV is widely spread in Spain and could be associated with PCV2 infections (5). Porcine TTV infections are also widespread in herds of the
USA, Canada, China, Korea and Thailand (9). Multiple infections of novel four U.S prototypes of porcine TTV in a single pig also have reported (10), suggesting that these co-infections are important for disease severity. The co-infection of TTV and PCV2 in piglets from Brazilian herds has also been reported (2). These findings raise the question of the importance of PCV and TTV co-infection in the pathology of PCVAD associated reproductive failures and vertical transmission in swine farms.

Figure 1. Detection of PCV1, TTV1 and TTV2 DNA in organs samples from $P C V 2$ positive fetuses.

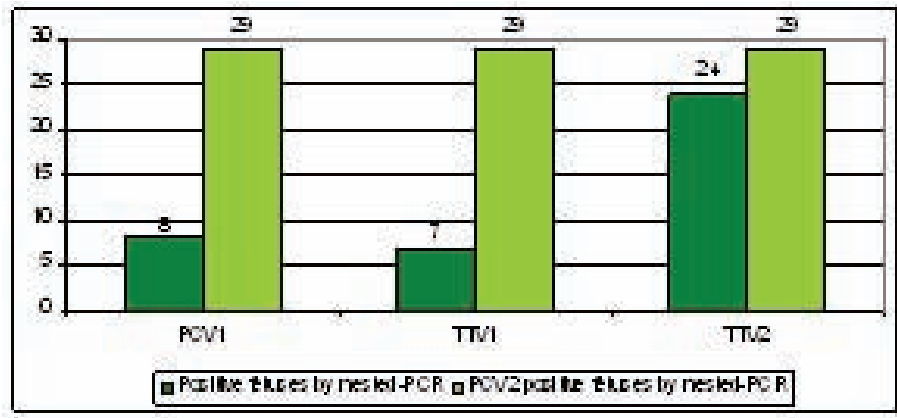

\section{References}

1. Ciacci-Zanella, J.R., et al. 2007. 5th Int. Symp.Emerging and Reemerging Pig Diseases,. Poland. v.1. p.95 - 95.

2. Ciacci-Zanella, J.R., et al. 2009. 28th Annual Meeting American Society for Virology, Vancouver. 1, 335-335.

3. Kekarainen, T. et al. 2006. J. Gen. Virol. 87, 833-837.

4. Kim, J.; et al. 2001. J. Virol. Meth. 98, 25-31.

5. Maldonado, J. et al. 2005. The Veterinary Journal. 169, 454-456.

6. Martínez-Ginó, L. et al. 2009. Theriogenology. 71, 1390-1395.

7. Niel, C. et al. 2005. J. Gen. Virol. 86, 1343-1347.

8. West, K.H. et al. 1999. J. Vet. Diagn Invest. 11, 530-532.

9. McKeown, N.E. et al. 2004. Vet. Microbial. 104: 113-117.

10. Huang, Y.W., 2010. Virology. 396:289-297. 


\title{
P.161
}

\section{Relationship of histopathology and PCV2 detection in fetal and neonatal myocardium from cases of reproductive failure in sows}

\author{
Karina Enriquez; Victor Quintero; Ignacio C. Rangel-Rodriguez; Yolanda Romero; Miguel A. Perez-Razo; Lucia A. Garcia-Camacho
}

FESC-UNAM, Cuautitlán, MEX, Mexico

\section{Introduction}

Porcine Circovirus type 2-associated reproductive failure (PCV2$\mathrm{RF}$ ) is characterized by increased abortions, stillbirths and neonatal mortality. Transplacental trasmission is acknowledged and the most consistent lesion in the fetus of PCV2-RF is a non-suppurative myocarditis with intralesional PCV2 5, 6, 7 PCV2-RF has been described without co-infection with porcine parvovirus (PPV), encephalomyocarditis virus (EMCV) and porcine respiratory and reproductive syndrome virus (PRRSV)1,5 The aim of the present work was to determine PCV2 role in RF development.

\section{Materials and Methods}

In order to assess the role of PCV2 in reproductive failure in Mexico, 107 hearts (predominantly fetal samples) with presumptive diagnosis of PCV-RF were evaluated by standard procedures of histopathology and in-situ hybridization (ISH) using a digoxigenin-labelled PCR- derived probe specific for PCV2. Cases were grouped as follows: Fetuses with myocarditis (Group 1), fetuses without myocarditis (Group 2), stillborns/neonates with myocarditis (Group 3), and stillborns/neonates without myocarditis (Group 4). Among these 107 cases, 50 were selected to perform nested PCR from paraffin-embedded samples. The data were analized by Chi-square test, Cramer coefficient and analysis of variance for categorical data. The later was used to evaluate cardiac lesion degree and PCV2 distribution.

\section{Results}

Microscopically, 48 cases (45\%) exhibited non-suppurative myocarditis, of which $35 / 48$ were PCV2 positive (73\% of cases with myocarditis, $32.7 \%$ overall) and 13 were PCV2 negative ( $27 \%$ of cases with myocarditis, $12.4 \%$ overall). There were 5 (4.67\%) fetal cases PCV2 positive by ISH with no myocarditis. A diagnosis of myocarditis and a positive ISH showed a high relationship $(\mathrm{J} 2<0.005$ y Cramer coefficient $=0.64$ ) revealing that both tests are comparable for disease diagnosis. Twenty out of 107 additional cases, regardless of myocarditis, were detected by PCR, increasing to 55 (51.4\%) the cases positive to PCV2, 42/107 $(38.75 \%)$ with miocarditis and 13 cases ( 9 fetal) without myocar- ditis. In total, 50 fetal cases were PCV2 positive. The myocarditis severity was mild to moderate in groups 3 y 4 , and moderate to severe in fetal tissues. The lesion severity was statistically significant, showing values of $\mathrm{P}=0-04$ y $\mathrm{P}=0-0001$ for $1-3$ and 2-4 groups, respectively. The HIS signals were mild, variable sized and randomly distributed (Figures 4-5), precluding a statistical analysis.

\section{Discussion}

The use of histopathology as screening test is highly recommended but association to PCV2-RF must be confirmed by ISH and/or PCR. Few viral agents produce both non-suppurative myocarditis and RF1,7 Therefore, 33-39\% cases are compatible to PCV2-RF, based on criteria diagnosis, and the vertical transmission was of $46.72 \%$ (50/107 fetal samples) which is higher than previous reports2,3,4,9 Cases with myocarditis but PCV2negative suggest a separate, unknown etiology. Furthermore, no other viruses could be examined since only cardiac tissues were submitted, Therefore, co-infection cannot be entirely excluded. PCV2 relies on cell mitotic activity and DNA polymerase availability7,8,10 Our findings are in agreement, because the lesion severity was age-dependent. The ISH signals suggest low viral load. Thus, real-time PCR is being performed to assess the relationship between lesion severity and viral load.

\section{References}

1. Brunborg IM, et al. J Vet Diagn Invest. 2007 Jul;19(4):368-75.

2. Kim J, et al. Vet Rec. 2004 Oct 16;155(16):489-92.

3. Lyoo KS, et al. J Vet Sci. 2001 Dec;2(3):201-7.

4. Maldonado J, et al. M. Vet. J. 2005, 169:454-456.

5. Mikami O, et al. J Vet Med Sci. 2005 Jul;67(7):735-8.

6. Nielsen J., et al. 2004. Proceedings of the 18th IPVS Congress, Hamburg, Germany Vol I, 14.

7. Pensaert M.B., et al. 2004. Vet. Microbiol. 98, 175-183.

8. Pittman JS., 2008. Reproductive failure associated with porcine Circovirus type 2 in Giles. J Swine Health Prod. 16(3): 144-148.

9. Ritzmann M, et al. 2005. Dtsch Tierarztl Wochenschr 112(9):348-51.

10. Sanchez Jr. R.O., et al. Vet. Microbiol. 2003, 95:15-25 


\title{
P.162
}

\section{Natural infection of porcine circovirus type 2 (PCV2) in fetuses from sows with reproductive failures}

\author{
Giseli A. Ritterbusch ${ }^{2}$ Camila Sa Rocha ${ }^{3}$ Nelson Mores $^{2}$ Neide L. Simon ${ }^{2}$ Janice R. Ciacci-Zanella ${ }^{1}$ \\ 1. Virus and Prion Diseases Research Unit, NADC, ARS, USDA, Ames, IA, USA; 2. Embrapa Swine and Poultry Research Center, Animal Healthy \\ Laboratory, Concordia, SC, Brazil; 3. Agroveterinarian Science Center - CAV - University of Santa Catarina State - UDESC, Lages, SC, Brazil
}

\section{Introduction}

Porcine Circovirus Type 2 (PCV2), etiologic agent of PCVAD or PCV2 associated diseases, can cause reproductive failure in swine $(1,4,5)$. PCV2 has been detected in farms with high occurrence of aborted, mummified and stillborn fetuses, and viral DNA and antigen has been detected by nested-PCR and immunohistochemistry $(\mathrm{IHC})$, respectively, in organs from fetuses naturally infected $(4,6)$. In order to study the pathogenesis of the disease in Brazilian swine herds, the objective of this work is to study the natural infection of PCV2 in organs of fetuses from sows with reproductive failures.

\section{Material and Methods}

Samples from field cases, as aborted fetuses, mummified, stillborn and fragile piglets were collected and processed in order to detect PCV2 DNA and antigens. Samples were collected from 21 farms previously identified with occurrence of reproductive losses, and a total of 169 fetuses were necropsied. Fragments of organs, including heart, lungs, liver, kidney, lymphoid organs and nervous tissues were processed for viral and histopathological diagnostic. For viral isolation, DNA was extracted and nested-PCR reactions were performed using specific primers to detect sequences of PCV2 as previously described (3). For histopathological examination, organs were fixed in $10 \%$ buffered formalin and embedded in paraffin, sectioned at $5 \mu \mathrm{m}$ thick and stained with hematoxylin and eosin for light microscopical examination. Moreover, viral presence was confirmed by IHC, carried out as previously described (2) and performed in tissues that resulted positive by PCR.

\section{Results}

Organs samples of 169 fetuses were tested by nested-PCR and PCV2 DNA was detected in 29 (17.1\%) of them. Viral sequences were found in several tested organs. Microscopic lesions were found in $15.4 \%$ of the samples, mainly on heart and lymphoid organs, with the presence of infiltration of inflammatory cells, syncytial cells and varying degrees of lymphocellular depletion. In addition, IHC was performed and viral antigen was detected in 17 out of 29 positive fetuses (58.6\%).

\section{Discussion}

These results show the occurrence of natural infection of PCV2 in analyzed samples, confirming the importance of PCV2 infection in reproductive failures. Among the analyzed samples, heart was the organ where PCV2 DNA was most frequently detected, following by lymphoid organs and nervous tissues, which agree with results reported by others studies $(5,6)$. Genomic sequencing analyses are underway to classify the different PCV2 isolates. By IHC staining it was observed a variable amount and distribution of viral antigen in tissues, and positive cells were found most commonly on macrophages (Figure 1). These findings raise the question of the importance of PCVAD associated reproductive failures and vertical transmission in swine farms.

Figure 1. Detection of PCV2 by nested-PCR and IHC in organs from fetuses

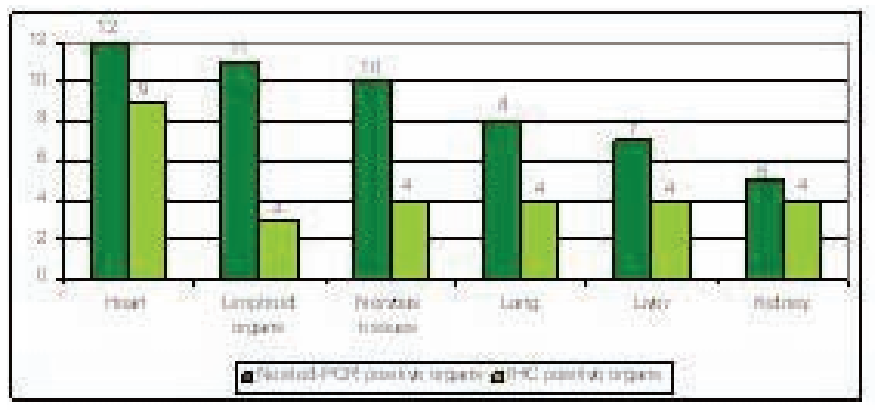

\section{References}

1. Ciacci-Zanella, J.R., et al. 2007. 5th Int. Symp.Emerging and Reemerging Pig Diseases,. Poland. v.1. p.95 - 95.

2. Gava, D. et al. 2008. Pesquisa Veterinária Brasileira. 28, 70-76.

3. Kim, J. et al. 2001. J. Virol. Meth. 98, 25-31.

4. Maldonado, J. et al. 2005. The Veterinary Journal. 169, 454-456.

5. Pensaert, M.B. et al. 2004. Vet. Microbiology. 98, 175-183.

6. West, K.H. et al. 1999. J. Vet. Diagn Invest. 11, 530-532. 


\title{
P.163
}

\section{Distribution of porcine parvovirus type 2 (PPV2, Myanmar erythrovirus) and porcine hokovirus (PHoV) in pigs from post-weaning multisystemic wasting syndrome (PMWS)- affected and non-affected farms in archival samples from UK and Republic of Ireland}

\author{
John McKillen ${ }^{1}$ John P. McKillen ${ }^{1}$ Michael McMenamy² Irene McNair ${ }^{1}$ Gordon Allan ${ }^{1}$ \\ 1. Agri-Food \& Biosciences Institute, Belfast, UK; 2. Department of Veterinary Science, The Queen's University of Belfast, Belfast, UK
}

\section{Introduction}

In 2001 PPV2 $^{1}$, a novel parvovirus related to the genus erythrovirus, family Parvoviridae was detected in swine from Myanmar. $\mathrm{PHoV}^{2}$ is also a member of the family Parvoviridae and along with bovine hokovirus (BHoV) and PARV4 form a distinct cluster within the family Parvoviridae. This has been proposed as a separate genus, Hokovirus. Porcine circovirus type 2 (PCV2) is the causative agent PMWS ${ }^{3}$. PMWS does not however, always occur in PCV2 infected pigs. It is likely that other agents may contribute to the development of PMWS. Here we investigate the distribution of PPV2 and PHoV in archival PMWS-affected and non-affected tissue and serum samples recovered from pigs in the UK and Republic of Ireland from 2000 to 2006.

\section{Materials \& Methods}

44 sera samples were collected from pigs on PMWS-affected UK farms. Also tissues were taken from 64 PMWS-affected pigs and 55 non PMWS-affected pigs, from the UK and Republic of Ireland. Tissues included lung, liver, kidney, spleen and lymph nodes. Viral nucleic acids were extracted from sera and 10\% homogenised tissue samples using Roche MagNA Pure LC. All tissue and sera samples were analysed by real-time QuantiTect SYBR Green I PCR for the presence of PPV2 and PHoV using in-house specific primers designed against deposited GenBank sequences. Between 1 and 4 tissues were tested for each animal. A selection of positive amplicons was cloned into TOPO TA vectors and sequenced using standard protocols. Sequences were compared to GenBank using nucleotide BLAST.

\section{Results}

With one exception all tissues tested positive in any positive animal. Generally PPV2 was present in significantly higher amounts than PHoV as judged by $\mathrm{Ct}$ values, as well as being more prevalent. Both PPV2 and PHoV were more prevalent in tissues of non-PMWS-affected pigs. Sequencing of the amplicons confirmed the specificity of the PCR. No data was available for sera from PMWS -ve farms. Tables 1 and 2 show the \% of animals testing $+v e$ for the two viruses in relation to their PMWS status.
Table 1. \% of PMWS-affected and non-affected pigs with tissues testing PCR +ve for PPV2 and PHoV.

\begin{tabular}{|l|l|l|}
\hline Tissue Results & PPV2 +ve animals & PHoV +ve animals \\
\hline PMWS +ve & $26.6 \%(17 / 64)$ & $4.9 \%(3 / 64)$ \\
\hline PMWS -ve & $54.5 \%(30 / 55)$ & $9.1 \%(5 / 55)$ \\
\hline Total & $39.5 \%(47 / 119)$ & $6.7 \%(8 / 119)$ \\
\hline
\end{tabular}

Table 2. \% distribution of PPV2 \& PHoV in sera from PMWS- affected farms.

\begin{tabular}{|l|l|l|}
\hline Sera Results & PPV2 +ve & PHoV +ve \\
\hline PMWS +ve farms & $18.2 \%(8 / 44)$ & $15.9 \%(7 / 44)$ \\
\hline
\end{tabular}

\section{Discussion}

PHoV was detected in only $6.7 \%$ of tissue samples and $15.9 \%$ of sera samples tested in this study. In Hong Kong $244.4 \%$ of porcine samples tested +ve for PHoV. PPV2 was detected in $39.5 \%$ of the tissues tested and $18.2 \%$ of the sera, as opposed to $10.5 \%$ of tested sera from Myanmar ${ }^{1}$. These preliminary studies suggest that the distribution of these viruses could be widespread and that the extent and geographical range should be further investigated. The significance of these viruses in terms of swine health is not known. Their role as potential pathogens or as co-factors in other disease complexes such as PMWS merits further investigation although higher prevalence in PMWS-affected animals and farms has not been shown in this study.

\section{References}

1. Hijikata, M. et al. 2001. Jpn. J. Infect. Dis. 54, 244-245.

2. Lau, S.K.P. et al. 2008. J. Gen. Virol. 89, 1840-1848.

3. Allan, G.M. et al. 2007. J. Vet. Diag. Inv. 19, 668-73. 


\title{
P.164
}

\section{Reproductive failure associated to PCV2 and its distribution in the Mexican Republic}

\author{
Victor Quintero; Yolanda Romero; Karina Enriquez; Lucia A. Garcia-Camacho \\ FESC-UNAM, Cuautitlán, MEX, Mexico
}

\section{Introduction}

Porcine circovirus type 2 (PCV2) has been associated to reproductive failure (RF) promoting increases of infertility abortions, stillborn, mummifications as well as a marked increase on preweaning mortality. 1,4,5 The most consistent microscopic lesions are non suppurative miocarditis with perivascular and instertitial mononuclear infiltrates, degeneration and necrosis of myocytes and myocardium fibrosis. The association to PCV2-RF is confirmed by reproductive failure to PCV2 is by immunohistochemistry, in situ hybridization (ISH), and PCR in cardiac samples.1,3,5

\section{Material and methods}

The aim of this work was to determine the non-suppurative myocarditis positivity and PCV2 status as well as the locality of origin of cases presented for histopathology diagnosis in the Pathology Department of FES-Cuautitlan, UNAM during the period of 2006-2008. The heart samples were formalin-fixed and paraffin-embedded for routine microscopic evaluation. The PCV2 detection from non-suppurative myocarditis positive cases was done by ISH using a specific digoxigenin-labelled PCR- derived probe.

\section{Results}

A total of 269 heart samples from fetuses and stillborn from 39 cases from different Mexican states were microscopically evaluated. The case distribution regarding origin, myocarditis positivity and PCV2 status is shown in Table 1. Microscopically, 120 cases $(44.6 \%)$ exhibited varying degree of non-suppurative myocarditis, of which 100/120 were PCV2 positive (83.3\%) by $\mathrm{ISH}$. The lesions consisted of perivascular and/or interstitial accumulation of lymphocytes and macrophages, myocardial degeneration, necrosis, and/or fibrosis. Additionally, subepicardial and subendocardial congestion, edema and hemorrhage were observed. Concerning the sample origin, the states with higher case frequency, such as Veracruz and Jalisco, exhibited lower proportion of myocarditis.

\section{Discussion}

Our findings strongly support the role of PCV2 as a cause of RF in Mexican farms since PCV2 was detected throughout the country. In fact, PCV2 was present in the Mexican central states, such Guanajuato, Michoacan and Jalisco where are located the main porcine production areas. Furthermore, the proportion of PCV2 in fetal and neonatal tissues is in agreement of reports elsewhere.2,5 In this study, no other viruses could be examined since the submitted samples were exclusively hearts. Therefore, co-infection cannot be entirely excluded. In fact, the low ratio of myocarditis and/or PCV2 positive cases in states with high case frequency suggests that the cause of abortion must have been different from PCV2. Thus, co-infection with other agents such as PRRSV, porcine parvovirus, Aujezsky virus and porcine rubulavirus must be considered.

Table 1. Relation of Study Cases (Period 2006-2008)

\begin{tabular}{|l|c|c|c|}
\hline STATE & No SAMPLES & MYOCARDITIS (+) & ISH (+) \\
\hline CHIAPAS & 18 & 8 & 8 \\
\hline EDO MEX & 18 & 13 & 9 \\
\hline GUANAJUATO & 17 & 9 & 7 \\
\hline HIDALGO & 10 & 7 & 6 \\
\hline JALISCO & 48 & 18 & 14 \\
\hline MICHOACAN & 38 & 20 & 17 \\
\hline NAYARIT & 2 & 2 & 1 \\
\hline PUEBLA & 2 & 2 & 2 \\
\hline QUERETARO & 8 & 5 & 4 \\
\hline SONORA & 21 & 16 & 13 \\
\hline VERACRUZ & 83 & 18 & 17 \\
\hline YUCATAN & 4 & 2 & 2 \\
\hline TOTAL & 269 & 120 & 100 \\
\hline
\end{tabular}

\section{References}

1. KIM, J., et al. Vet Rec. 2004, (155) 489-492

2. LYOO KS, et al. J Vet Sci. 2001, 2(3):201-7.

3. PARK, J.S., et al. J Comp. Path. 2005, (132) 139-144.

4. PENSAERT, MB, et al. Vet Microbiol. 2004, (132)175-183.

5. PITTMAN, JS. JSHP 2008, (16)144-148.

6. RITZMANN M., et al. Dtsch Tierarztl Wochenschr 2005, 112(9):348-51. 


\title{
P.165
}

\section{Detection of porcine single-stranded DNA viruses in reproductive organs of culled sows}

\author{
Camila Sa Rocha ${ }^{2,3}$ Giseli A. Ritterbusch ${ }^{2,3}$ Nelson Mores ${ }^{3}$ Neide L. Simon ${ }^{3}$ Leonardo \\ Diniz-Mendes ${ }^{4}$ Christian Niel $^{4}$ Janice R. Ciacci-Zanella $^{1}$ \\ 1. Virus and Prion Diseases Research Unit, NADC, ARS, USDA, Ames, IA, USA; 2. Agroveterinarian Science Center- \\ CAV - University of Santa Catarina State - UDESC, Lages, SC, Brazil; 3. Embrapa Swine and Poultry Research Center, \\ Animal Healthy Laboratory, Concordia, SC, Brazil; 4. Oswaldo Cruz Institute, Rio de Janeiro, Brazil
}

\section{Introduction}

Several reasons determine the removal of sows from a swine herd. Sows are culled when they are considered unsuitable for further production and reproductive failure is the predominant cause, ranging between $13-49 \%$ of removals (1). Infectious causes of reproductive failures vary, however many can be due to viral agents (1). Among them the single stranded DNA (ssDNA) viruses play an important role in the co-infections of these diseases. Porcine circovirus 2 or PCV2 (2), porcine parvovirus or PPV (7) and porcine torque teno virus (TTV) with at least two genogroups, TTV1 and TTV2 (3) have been identified in swine fetuses and sows tissues as the cause of infectious reproductive failures. Although a member of the Circoviridae family, PCV1 has not been associated with pathologies in pigs, but it is also an agent to be included in co-infections. Other viral agents, such as porcine reproductive and respiratory virus (PRRSV) can cause significant losses, but are not present in Brazilian swine herds (4). Thus, the objectives of this work is to detect the infection by porcine ssDNA viruses, PCV2, PCV1, PPV, TTV1 and TTV2 in reproductive organs of culled sows, aiming to verify the importance of them in those sites.

\section{Material and Methods}

Reproductive organs from 83 culled sows, from four slaughterhouses of Santa Catarina State, were collected. The reason of their removal was not particularly due to reproductive failures. A total of 71 samples of ovarian follicular fluid (OF) and 83 fragments of ovaries and 83 fragments of uterus were processed for DNA extraction followed by amplification of PCV2, PCV1, PPV, TTV1 and TTV2 nucleic acids. Nested-PCR reactions were performed using specific primers as previously described $(5,6,7)$.

\section{Results}

Organs samples such as ovaries, uterus and ovarian follicular fluids (OF) of 83 culled sows were tested individually by nestedPCR for ssDNA viral amplification of PCV2, PCV1, PPV and TTVs. PCV1 DNA was detected in 14 (16.9\%) of sows organs. Nucleic acids of PCV2 were amplified from 5 (6.0\%) sows. TTV1 and TTV2 were detected in 25 (30.1\%) and 41 (49.3\%) sows respectively. For TTV2 particularly, 27 samples (38\%) tested positive in OF, $19(23 \%)$ in ovaries and $14(17 \%)$ in uterus. Viral sequences of PPV were amplified in 6 (7.2\%) sows. In some cases, the occurrence of co-infections between viral agents in sampled sows was observed.

\section{Discussion}

The present study demonstrates the occurrence of infection of porcine ssDNA viruses in reproductive organs of culled sows. These results show higher prevalence of TTV1 and TTV2 in analyzed samples, indicating its importance, mainly in co-infections with PCV2. TTV2 is more frequently related to PCV2 associated diseases (PCVAD) compared to TTV1 in Spain (77\% prevalence) (5). Other reports indicate that TTV1 viral infection facilitates PCVAD (8). In swine, TTV alone has not showed to be pathogenic, but, its role in co-infections with other pathogens has been investigated. Recent studies showed the first indications of TTV in utero transmission to piglets (3). PPV has been associated with PCV2 and other swine virus such as PRRSV in samples of piglets born from sows with reproductive failure from China and Korea $(9,10)$. These findings raise the question of the importance of these viral infections in the pathology of reproductive failures and vertical transmission in swine.

\section{References}

1. D'Allaire, S. and Drolet, R. 2006. Diseases of Swine. Ch.63, p.10111025.

2. Maldonado, J. et al. 2005. Vet J. 169, 454-456.

3. Martínez-Ginó, L. et al. 2009. Theriogenology 71, 1390-1395.

4. Ciacci-Zanella, J.R. et al. 2004.Ciência Rural 34:449-455.

5. Kekarainen, T. et al. 2006. J. Gen. Virol. 87, 833-837.

6. Kim, J.; et al. 2001. J. Virol. Meth. 98, 25-31.

7. Soucie,J.M. et al., 2000.Transfusion 40:708-711.

8. Ellis et al. 2008 Am J Vet Res. 69:1608-14.

9. Jiang, Y. et al. 2010. Vet J. 183:172-5.

10. Lyoo, K.S. et al. 2001. J Vet Sci. 2:201-7. 


\title{
P.166
}

\section{Diagnostic of postweaning multisystemic wasting syndrome in pigs in Cuba}

\author{
Patricia Dominguez Perez ${ }^{2}$ Humberto Ramirez-Mendoza ${ }^{1}$ Carlos Bulnes Goicochea ${ }^{2}$ Lester \\ Josue Perez ${ }^{2}$ Maria Iriam Percedo ${ }^{2}$ Daine Chong ${ }^{2}$ Jose Francisco Rivera-Benitez ${ }^{1}$ \\ 1. Departamento de Microbiologia E Inmunologia, FMVZ-UNAM, Distrito Federal, DF, Mexico; \\ 2. Centro Nacional de Sanidad Agropecuaria, La Habana, Cuba
}

\section{Introduction}

Porcine circovirus diseases (PCVDs) are nowadays considered as a significant international problem due to increased mortality rates and reduced feed conversion efficiency in weaning and fattening pigs at the worldwide (Segalés et al., 2005). The Postweaning multisystemic wasting syndrome PMWS is economically the most important disease included within this group. This new disease syndrome was firstly identified in Canada in 1991 (Clark, 1997; Harding, 1997) since then, the PWMS has been report in many part of the word (LeCann et al., 1997; Segalés et al., 1997; Kennedy et al., 1998).

\section{Materials and Methods}

Thirty three pigs from five farms were investigated. Clinical signs evaluated included body condition, presence of respiratory and digestive clinical signs (coughing, dyspnea and diarrhoea), behaviour (depression), skin pallor, and enlargement of superficial inguinal lymph nodes. The farms were located in three different provinces of Cuba. Affected pigs were aged 7-15 weeks. Morbidity on the five farms ranged from 10-25\%; mortality was at least $50 \%$ of affected animals on all farms. Samples of tonsil, lung, kidney, spleen, jejunum, ileum, colon, liver and lymph nodes (including superficial inguinal and mesenteric) were collected and fixed by immersion in $10 \%$ neutral buffered formalin. One section was processed for routine histopathology and stained with haematoxylin and eosin. The second tissue section was processed for PCV2 nucleic acid detection by in situ hybridization (ISH). On the other hand the other section was processed for immunohistochemistry. Tissues were evaluated for presence or absence of the PMWS (Segalés et al., 2005) specific lesions.

\section{Results}

The discoveries anatomophatology included the widespread increase of the lymph nodes, especially superficial inguinal and mesenteric, witnesses of whitish diffuse stains in the liver and kidneys and, consolidation of the lobes lung. Histopathological changes were characterised by depletion of lymphocytes in the interfollicular areas and follicular centres. Infiltration by macrophage and multinucleate giant cells were also observed in the lymph nodes.

Porcine circovirus (PCV-2) antigen and nucleic acid were regularly found in lymphoid organs. In lymph nodes, depletion was observed within lymphoid follicles or in the paracortical zones.
PCV-2 antigen was detected in 23 pigs. In all tissues examined was most frequently seen in the cytoplasm of cells. Nuclear labelling was less commonly seen but occasionally appeared to be almost as abundant as cytoplasmic labelling. The smooth muscle trabeculae of the spleen were occasionally labelled. PCV-2 nucleic acid was detected in most of the pigs was found mostly in the cytoplasm of infected cells, and to a lesser extent in the nucleus.

\section{Discussion}

This is the first diagnostic of the PMWS in Cuba, based on all three accepted diagnostic criteria (Segalés et al., 2005) for the case definition of this disease syndrome. The pigs evaluated showed the clinical signs compatible with this illness. Besides, lesions on lymphoid tissues were found. Moreover, the presence of the PCV2 within these lesions in moderate or high amounts of the virus was observed. This study describes the pathological findings in 23 natural cases of PMWS, together with the tissue distribution of PCV antigen and nucleic acid. Pigs were classified as cases of PMWS on the basis of macroscopical and microscopical lesions, and the detection of PCV antigen or nucleic acid (Segalés et al., 1997).

In Cuba, PCV2 infections of the swine herds have been described recently (Pérez et al., 2009). In addition, co-infection PCV2/PPV in several farms of the different geographic region of the country was found (Pérez et al., 2009). On the other hand, classical swine fever is an endemic disease in Cuba. The use of vaccination is an important tool for combat against outbreaks of this disease. Nevertheless, if the vaccinations against classical swine fever virus can act as trigger fact of PMWS will be needed to be explained.

\author{
References \\ Clark, E., 1997. Proc. Western Can. Assoc. Swine Pract. 28, 499-501. \\ Harding, J.C., 1997. Proc. Am. Assoc. Swine Practitioners, p. 503. \\ Kennedy, et al. 1998. Vet. Rec. 142, 495-496. \\ LeCann, et al. P, 1997. Vet. Rec. 141, 660. \\ Pérez, L.J et al., 2009 Res. Vet. Sci. doi:10.1016/j.rvsc.2009.11.014 \\ Segalés, J. et al, 1997. Vet. Rec. 141, 600-601. \\ Segalés, J. et al., 2005. Anim. Health Res.Rev.6, 119-142.
}




\title{
P.167
}

\section{First evidence of porcine circovirus type 2 in pigs from extensive system raising from Goias State, Brazil}

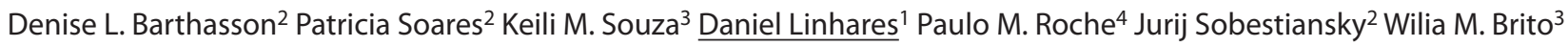 \\ 1. College of Veterinary Medicine, University of Minnesota, Saint Paul, MN, USA; 2. Programa de Pós graduação em \\ Ciência Animal, Escola de Veterinária da Universidade Federal de Goiás/GO, Goiania, GO, Brazil; 3. Laboratório de \\ Virologia Animal, Instituto de Medicina Tropical e Saúde Pública da Universidade Federal de Goiás, Goiania, GO, Brazil; \\ 4. Setor de Virologia, Instituto de Pesquisas Veterinarias Desidério Finamor, RS, Porto Alegre, RS, Brazil
}

\begin{abstract}
Introduction
Porcine circovirus type 2 (PCV2) is ubiquous in swine herds over the world. It is associated with different disease and syndromes in pigs mainly to postweaning multisystemic wasting syndrome (PMWS). In Brazil, PCV2 was first identified in South region,, in 2000 and in other regions later on. All Brazilian' studies were conducted in commercial and intensive raising systems animals (Ciacci-Zanella et al., 2003; Ruiz et al., 2004). In peripheral areas of large cities in Brazil, it is common to create a few pig herds (backyard pigs), for own consumption, under poor hygienic and sanitary conditions and in close contact with others animals species and humans. This swine population is a unique type of extensive raising swine system (ERSS). At our knowledge, no other study was done to verify PCV2 infection in these swine population. The present study was performed to identify PCV2 DNA in clinical specimens of pigs in Goiás State, Brazil ERSS's.
\end{abstract}

\section{Material and Methods}

A total of 200 serum samples from swine of different age groups were collected from 25 ERSS in Goiás state. Tissue samples (lymph nodes, lungs, liver, brain, kidney and spleen) from 5 pigs with growth impairment and 6 pigs with signs of neurological disease were also included. Viral DNA was extracted with standard phenol-chloroform-isoamyl alcohol extraction procedure. Previous described specific primers to ORF2 region of PCV2 [F5'-CGGATATTGTAG TCCTGGTCG-3'] and [R5'-ACTGTCAAGGCTACCACAGTC A-3'] and PCR conditions were used for DNA PCV2 identification (Kim et al., 2001). A fragment of 480pb length was expected.

\section{Results and Discussion}

Out of the serum samples, 57 (28.5\%) were positive. This frequency is lower than that observed in other studies (Casalmiglia et al., 2002; Grau-Roma et al., 2008) although it may represent the occurrence of PCV2 infection in this particular type of swine herd. Seven (63.6\%) of the eleven animals tested showed positivity in at least one tissue sample. Viral DNA was found in inguinal nodes (6/7), lung (1/7) and kidney (1/7). Other authors also found high frequency of identification of PCV2 DNA in inguinal lymph nodes and assert that they are one the main targets for PCV2 replication (Yu et al., 2007). Despite viral DNA had been found in two fragments of pigs with growth impairment it is not enough to ensure the occurrence the syndrome. This also should be associated with clinical signs and pathological findings (Segalés et al., 2003). The results here presented may point out that PCV2 occur in pigs under extensive raising system in Goiás, Brazil.

\section{References}

Calalmiglia et al., 2002 Detection of porcine circovirus types 1 and 2 in serum and tissue samples of pigs with and without postweaning multisystemic wasting syndrome. J. Clin. Microbiol.40(5)1848-1850.

Ciacci-Zanella, J.R., Morés, N. 2003 Circovirose suína. Circular Técnica 37. Concórdia: EMBRAPA/CNPSA, p.1-12.

Grau-Roma, L., et al., 2008. A proposal on porcine circovirus type 2 (PCV2) genotyping and their relation with postweaning multisystemic syndrom (PMWS) occurence. Veterinary Microbiology, 128:23-35.

KIM, J. et al., 2001 Differentiation of porcine circovírus (PCV-1 and PCV-2) in boar semen using multiplex nested polymerase chain reation. Journal of Virological Methods, 98: 25-31.

Ruiz,V.L.A et al 2004 Pesquisa da ocorrência de circovírus suíno e de possíveis co-infecções com parvovírus suíno através da reação em cadeia pela polimerase em oito Estados brasileiros. Arquivo do Instituto Biológico, 71, 484-486.

Segalés,J. et al 2003. How diagnose postweaning multisystemic wasting syndrome, 2003. In: Proceedings of the 4th International Symposium on Emerging and Reemerging Pig Diseases, Rome, Italy, pp $149-150$

Yu, S. et al., 2007 Porcine circovirus type 2 (PCV2) distribution and replication in tissue and immune cells in early infected pigs. Veterinary Immunology and Immunophatology, 115: 261-272. 


\title{
P.168
}

\section{Detection of TTV2 and PCV2 in a high health swine herd located São Paulo State, Brazil}

\author{
Alessandra M. Castro ${ }^{1}$ Cíntia M. Favero ${ }^{1}$ Fernando G. de Castro² Taís Nishikata ${ }^{1}$ \\ Cíntia M. Baldin³ Paulo E. Brandão ${ }^{1}$ Leonardo J. Richtzenhain ${ }^{1}$ \\ 1. Faculdade de Medicina Veterinária e Zootecnia da Universidade de São Paulo, São Paulo, Brazil; 2. Instituto \\ de Zootecnia, Nova Odessa, Brazil; 3. Faculdade de Jaguariúna, Jaguariúna, SP, Brazil
}

\section{Introduction}

Torque Teno virus (TTV) is a non-enveloped, circular, single stranded DNA virus that may infect human, non-primate and domestic species including swine. Two TTV genogroups (TTV1 and TTV2) have been described in pigs. TTV is considered nonpathogenic, but a recent study has shown an increased prevalence of TTV2 in PCV2-associated diseases compared to nonaffected ones (2,3). In Brazil PCV2-associated disease is expected to diminish with the introduction of PCV2 vaccination. However, PCV2-associated disease is still a problem in swine herds. Since the main virus, PRRS, involved in the co-infection of PCV2 to the development of the disease, is absent in Brazil, others agents became important. Nowadays, there are few information about TTV in Brazilian swine herd. Therefore, the aim of this work is to describe, for first time, the detection of co-infections of TTV2 and PCV2 in a high health swine herd.

\section{Materials and Methods}

Twenty and six serum samples were collected from sows from a high health herd located in São Paulo state, Brazil. DNA was extracted from serum using the phenol:chloroform Proteinase $\mathrm{K}$ protocols. TTV DNA was amplified a fragment of $250 \mathrm{bp}$ using a PCR with primers targeting two conserved domains within an untranslated region of the viral genome (4). The detection of PCV-2 was performed by PCR using primers Fa/Ra that amplify a 476 bp fragment (1). One sample was randomly selected and sequenced. Nucleotide sequences were aligned with sequences contained at the GenBank database of the swine TTV and phylogenetic tree constructed.

\section{Results}

TTV2 was detected in 14 (58.3\%) out of the 24 serum samples examined. Among the 14 TTV2 positive samples, five (5/9) was co-infected with PCV2. PCV2 alone was detected in five sow serum samples (Fig. 1). The alignment of the TTV2 sequence obtained in this study showed nucleotide and amino acid identities values of $91.6 \%$ to $82.4 \%$ and 61.0 to $64.6 \%$ with available GenBank sequences of TTV2 and TTV1, respectively. A NeighbourJoining tree was assembled and showed that this sequence had been classified TTV2 group (Fig. 2).

Figure 1: Demonstration of TTV2 and PCV2 positive serum samples collected in a high health herd in São Paulo state, Brazil.

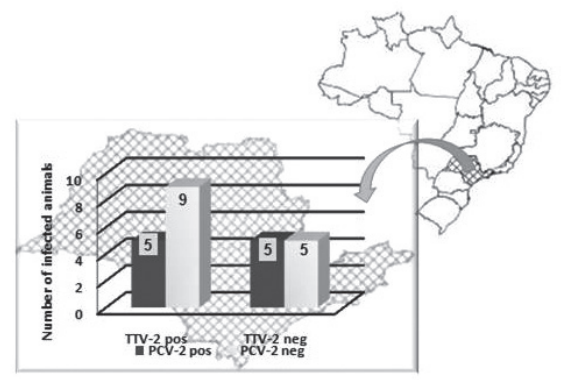

Figure 2: Neighbour-Joining tree of the TTV2 non-coding region Numbers indicates values calculated on 1000 repeats of the alignment with the heuristic method. Sequences are indicated by their GenBank access number.

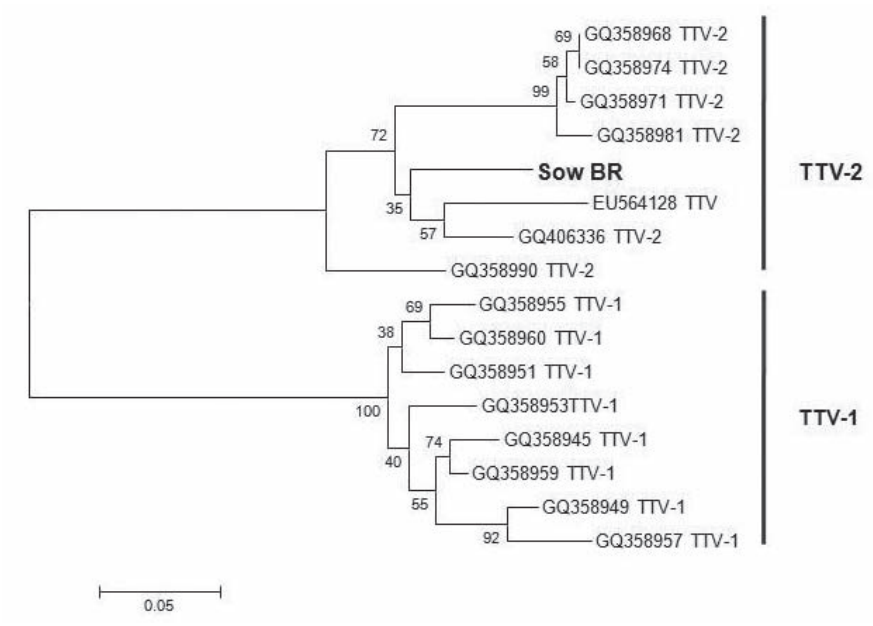

\section{Discussion}

This is the first report of TTV-2 and PCV2 co-infections in Brazilians pigs. The alignment of one sample confirms that the TTV detected belongs to group 2. These results, also, demonstrated that TTV2 is circulating in high health swine herds in Brazil. Previous research showed higher prevalence of TTV2 in PMWSaffected pigs than in healthy ones (2). The involvement of TTV2 in the development of diseases associated with PCV2 is not clear, but the absence of PRRS in Brazil and the low detection of PPV in PCV2-associated disease, make TTV a possible candidate to be involved in disease cases.

\section{References}

1. Castro, et al. (2007). Arch Viro 152 1435-1445.

2. Kekarainen, et al. (2006). J. Gen. Virol. 87, 833-8377.

3. Kekarainen, et al. (2009). Vet. J. 163-168.

4. Segalés, et al. (2009). Vet Micro 134 199-207. 


\title{
P.169
}

\section{Evaluation of the Ct values of non-quantitative real time RT-PCR as a proxy for determining PRRS viral load and its potential for monitoring swine populations, areas and regions}

\author{
Dale Polson; Enrique Mondaca; Kent Doolittle; Greg Hartsook; Eric Vaughn \\ Boehringer Ingelheim Vetmedica, Inc., St Joseph, MO, USA
}

\section{Introduction}

The temporal frequency and dynamic rate with which PRRS virus (PRRSv) transmits within and among swine populations is related to: [1] the inherent infectiousness of a particular PRRS viral isolate, [2] a plethora of environmental factors that influence contact events and rates between infected and susceptible animals, and [3] host animal factors which enable or impede the occurrence of infection events and the resultant magnitude and duration of further transmission risk. Thus, one key component of PRRSv transmission risk within a population would be the magnitude and duration of viral presence occurring within and emanating from infected pigs within a population. In turn, transmission risk among populations would be influenced by the magnitude and duration of viral presence occurring within and emanating from infected populations within areas, regions, flows and networks. The pig and population viral magnitudes and durations will be termed as "viral load". To monitor transmission risk of PRRSv within and among areas, regions, flows and networks - as well as assess the impact of pig, population and area/regional-scale interventions - requires a means with which to reasonably estimate "viral load".

Estimation and monitoring of "viral load" in human medicine has moved beyond the confines of experimental research application into routine operational use, having become a standard method with which to monitor the care of HIV patients and effectiveness of antiretroviral therapies. ${ }^{1}$ As in human medicine, monitoring of PRRS viral load holds potential for routine operational use to monitor the PRRS-related 'care' of populations, areas/regions and networks; and to assess the effectiveness of PRRS control and elimination interventions.

For PRRSv, quantitative RT-PCR (qRT-PCR) is currently used in a relatively limited manner to quantify viral load of experimentally challenged pigs. One factor that has limited broadening the use of qRT-PCR to a more routine operational use is the assay's cost and difficulty with its interpretation. In contrast, the result of a non-quantitative real time RT-PCR assay (rtRT-PCR) is simply reported as a Ct (cycle threshold) value. ${ }^{2}$ Although not technically a quantitative measure, $\mathrm{Ct}$ values have been used as a means with which to quantify a sample's genetic material. ${ }^{3}$

\section{Materials and Methods}

Serum samples from three experimental PRRSv inoculation studies were obtained. Both PRRS qRT-PCR (Lab A) and rtRTPCR Ct (Lab B) testing were done on all samples and the results compared.

\section{Results}

Figure 1: Scatterplot of 599 paired PRRS rtRT-PCR Ct and $q R T-P C R$ Log10 testing results $(R=-0.961, P<0.001)$.

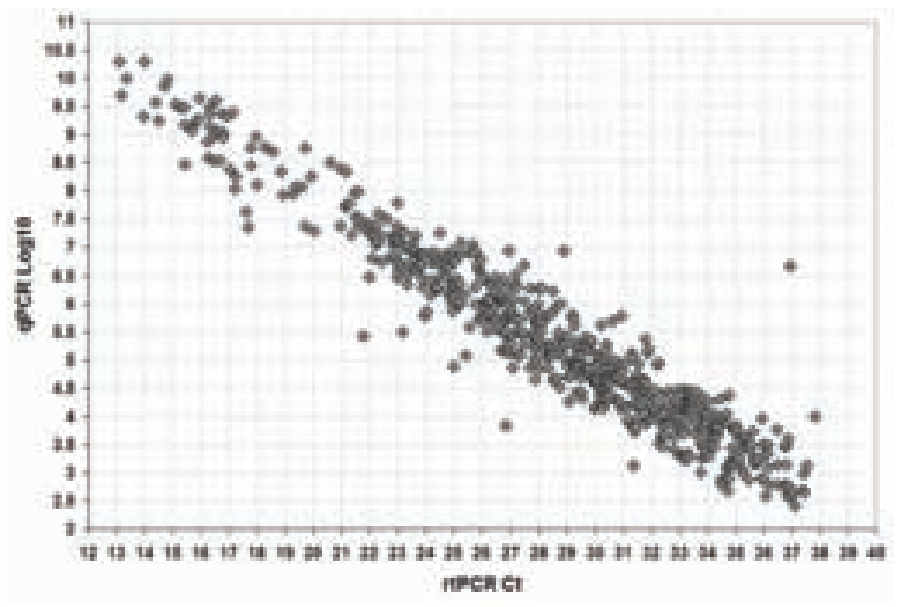

\section{Discussion}

Although not technically a quantitative measure Ct values generated by rtRT-PCR assays for PRRSv detection hold potential for use as a key component for monitoring viral load and tracking progress where PRRS control and elimination are being pursued. Clearly, further evaluation and refinement is needed before rtRT-PCR Ct values are ready for broad application to monitor PRRS viral load within and among pig populations, across areas and networks, and over time. Standardization of methods for quantitative viral load estimation using $\mathrm{Ct}$ values will be needed within testing laboratories over time and among testing laboratories and the various rtRT-PCR assays - including accounting for the influence of PRRS genetic variation and assay component (e.g., primers) detection variation. Further, standardized (as well as inexpensive and convenient) sample procurement methods as well as methods for calculating valid aggregate viral load metrics progressing from animals within sampling, samplings within population, and populations within areas will be required.

\section{References}

1. Malmsten A, et al.; J Med Virology; 76:347-359; 2005

2. http://www.wvdl.wisc.edu/PDF/WVDL.Info.PCR_Ct_Values.pdf

3. Liu W, Saint D; B B Res Comm; 294 (2002) 347-353 


\title{
P.170
}

\section{Comparison of four ELISAs for the detection of antibodies against PRRSV with special respect to false positive outliers}

\author{
Christiane Lang ${ }^{1}$ Andrea Ladinig ${ }^{1}$ Alphonse Minani ${ }^{2}$ Wolfgang Sipos ${ }^{1}$ Friedrich Schmoll ${ }^{3}$ Louis Fischer $^{4}$ Mathias Ritzmann \\ 1. Clinic for Swine, Vienna, Austria; 2. Noack \& Co, Vienna, Austria; 3 \\ . Institute of Veterinary Public Health, University of Veterinary Medicine, Vienna, Austria; 4. Labovet GmbH, Vienna, Austria
}

\section{Introduction}

The points of interest of PRRS control programs are an early detection of disease, a rapid laboratory confirmation and a reliable confirmation of a negative herd status. The most used serological diagnostic tool for PRRS monitoring is the ELISA. A new product, the Ingezim PRRS DR (Ingenasa, Madrid, Spain) was developed, which is able to detect not only lgG but also IgM antibodies, which occur earlier in the course of infection. Furthermore, the high specificity of this test should minimize the risk of false positive results in negative herds. The purpose of this study was to evaluate the performance of four European commercial tests.

\section{Materials and Methods}

In total, 478 pig sera were analysed via four different ELISA test systems (Ingezim PRRS DR (ING-DR) and Ingezim PRRS Universal ${ }^{\circledR}$ (ING-U), Ingenasa, Madrid, Spain, IDEXX HerdCheck ${ }^{\circledR}$ Porcine Reproductive and Respiratory Syndrome Virus Antibody Test Kit (ID), IDEXX Laboratories, Wörrstadt, Germany, Civtest suis PRRS E/S (CIV), Laboratorios HIPRA, S.A., Amer, Spain) according to the manufacturers' specifications. 353 of these serum samples had been collected during routine diagnostic of 148 farms, $16.4 \%$ could be defined as "outliers". An "outlier" was defined as possible false positive sample, which means single samples of randomly tested herds that were positive, while all other analysed samples were negative and showed a S/P (sample/positive control) ratio $<0.4$ in the IDEXX ELISA. 125 sera were collected during a PRRSV challenge.

\section{Results}

The analysis via ING-DR showed a seroprevalence of $67.6 \%$, via ING-U of $56.3 \%$, via ID of $59.2 \%$ and via CIV of $29.9 \%$. The correlations of the different test systems and sample sets were expressed as Spearman's coefficient of correlation $\rho$ (Table 1). When results from the four different tests were compared, it was found that $24.1 \%$ were positive in all four tests and $21.6 \%$ were negative in all tests. $45.1 \%$ showed no corresponding result. $16.1 \%$ of these non-corresponding results were outliers.
Table 1: Spearman's coefficient of correlation $\rho$ of the positive/ negative decisions of the complete sample set (a), outliers (b), sample set excluding the outliers (c) and challenge samples $(d)(*$ level of significance $<0.010$ )

\begin{tabular}{|c|c|c|c|c|}
\hline \multicolumn{2}{|c|}{} & ING-DR & ING-U & ID \\
\hline (a) & ING-U & $0.678^{*}$ & -- & -- \\
\hline (a) & ID & $0.425^{*}$ & $0.513^{*}$ & -- \\
\hline (a) & CIV & $0.414^{*}$ & $0.392^{*}$ & $0.421^{*}$ \\
\hline (b) & ING-U & $0.487^{*}$ & -- & -- \\
\hline (b) & ID & $-0.344^{*}$ & -0.123 & -- \\
\hline (b) & CIV & $0.589^{*}$ & $0.553^{*}$ & -0.019 \\
\hline (c) & ING-U & $0.705^{*}$ & -- & -- \\
\hline (c) & ID & $0.525^{*}$ & $0.608^{*}$ & -- \\
\hline (c) & CIV & $0.390^{*}$ & $0.375^{*}$ & $0.469^{*}$ \\
\hline (d) & ING-U & $0.917^{*}$ & -- & -- \\
\hline (d) & ID & $0.824^{*}$ & $0.869^{*}$ & -- \\
\hline (d) & CIV & $0.444^{*}$ & $0.492^{*}$ & $0.532^{*}$ \\
\hline
\end{tabular}

\section{Discussion}

This study underlines the complexity of PRRSV serology. The analysis of the coefficient of correlation of the complete sample set showed differences between the ELISAs, which were even more distinctive comparing only the outliers. In contrast, the results improved essentially, when outliers were excluded of the calculation. The best correlation could be found evaluating the samples of the challenge, which were defined positive or negative. The ING-DR resulted in the highest seroprevalence because of its ability to detect IgM and IgG, but nevertheless its specificity of $97.3 \%$ should be sufficient to minimize the risk of false positive results. The different answers to different ELISAs could also be related to significant field strain differences, to the phase of the infection or other factors (1).

\section{References}

1. De Wit et al. (2004): Proc. 18th IPVS Hamburg, p. 34. 


\title{
P.171
}

\section{Performance of an immunoenzimatic assay based on the double recognition ELISA for early detection of PRRS virus infection}

\author{
Ivan Díaz ${ }^{1}$ Anna Coll ${ }^{2}$ Ángel Venteo ${ }^{3}$ Belén Rebollo³ Enric Mateu ${ }^{1,2}$ Antonio Sanz ${ }^{3}$ \\ 1. Centre de Recerca en Sanitat Animal (CReSA), Bellaterra, Barcelona, Spain; 2. Departament de Sanitat i Anatomia \\ Animals, Bellaterra, Barcelona, Spain; 3. Inmunología y Genética Aplicada S.A. (INGENASA), Madrid, Spain
}

\section{Introduction}

Routine diagnosis of PRRS is generally done by means of antibodies detection against the virus, most often using indirect or blocking ELISA. With those tests seroconversions are usually detected at +14 post-inoculation $(1,2)$. In other instances, RT-PCR is chosen as diagnostic test for PRRS. RT-PCR usually allows and earlier detection of infected animals compared to serology. Early diagnosis of PRRS is particularly crucial in two circumstances: entry of gilts in PRRS-free farms and testing of boars. INGENASA has recently developed a new ELISA based on the concept of double recognition (DR) of the antigen (3). In the Ingezim PRRS DR (11.PRS.K0) (Ingenasa) for PRRS, plates are coated with the viral protein N. Serum and protein N conjugated to horseradish peroxidase are added to the coated plates in one-step incubation. If PRRS virus (PRRSV)-specific antibodies were bound to the plate, the free binding sites of those antibodies will be able to bind the conjugated N-protein and thus, an increased sensitivity will be obtained after revealing the reaction with a colorimetric substrate. The aim of the present study was to evaluate the performance of the DR for early detection of anti-PRRSV antibodies raised against different genotype-I strains in experimental or field conditions. Comparison with HerdChek 2XR ${ }^{\circledast}$ (Idexx Laboratories) was also performed.

\section{Material and Methods}

ELISAs. Two different ELISAs were used in the present study. In all cases, sera were examined by Ingezim PRRS DR (11.PRS.KO) (INGENASA) and for particular comparisons HerdChek 2XR ${ }^{\circledR}$ (Idexx Laboratories) was used. All tests were used as recommended by the manufacturer. Results were expressed as a ratio of the optical density (OD) of a given sample over the OD of the positive control provided by the test (S/P ratio).

Sera from experimental infections. Sera $(n=38)$ obtained from five experimental infections (days 0 to 28 post-inoculation) of piglets (4 week old) with different PRRSV genotype I strains (S1-S5) were used. Selected strains shared from $90.6 \%$ to $96.0 \%$ of similarity in protein $\mathrm{N}$. In all cases, animals were intranasally inoculated with $\geq 1 \times 10^{5.0} \mathrm{TCID}_{50} / \mathrm{ml}$. All sera were analyzed using DR. Samples for strains S4 and S5 were also examined by the HerdChek ELISA ${ }^{\oplus}$.

Cross-sectional profiling of an endemic farm. A cross-sectional serological profiling was comparatively performed in an endemic farm (pigs of 3, 6, 9, 12, 1518 and 21 weeks of age) using both ELISAs.
Sera from experimental infections. At day $+7 \mathrm{PI}$, DR detected 29/38 sera as positive. Interestingly, for strains S4 and S5 Herd$\mathrm{Chek}^{\circledR}$ did not detect any positive pig by day $+7 \mathrm{PI}$ while DR recognized all animals infected with $\$ 4(8 / 8)$ and one pig in the group infected with S5 (1/8). By day $+14 \mathrm{PI}$, all sera were positive in both HerdChek ${ }^{\circledR}$ and DR tests.

Cross-sectional profiling. Both DR and HerdChek ${ }^{\circledR}$ showed that PRRSV did spread explosively around 9-12 weeks of age as indicated by the seroprofile but DR had an increased sensitivity for detecting low levels of maternal antibodies or early infections as seen in 3-week-old piglets and in the higher proportion of seropositive pigs from week 15 afterwards (Figure 1).

Figure 1. Cross-sectional profiling of a PRRS endemic farm using DRELISA and HerdChek ${ }^{\circledR}$.

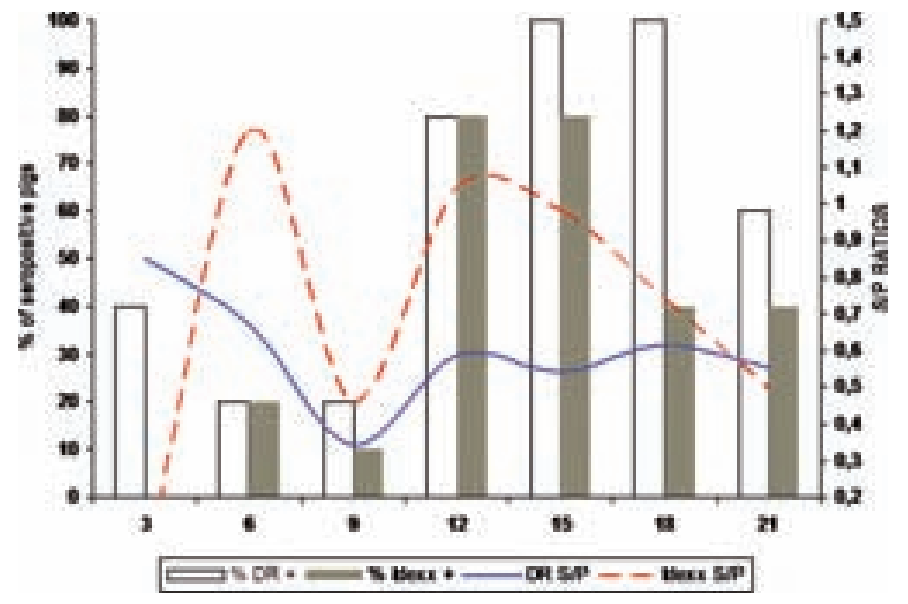

\section{Discussion}

DR-ELISA allowed an earlier detection of experimentally infected pigs (76\% at $7 \mathrm{PI})$. On the basis of the results in field conditions, DR also showed some enhanced sensitivity related to HerdChek $^{\circledast}$. Present results indicate the suitability of the DR-ELISA for early detection of PRRSV infection.

\section{References}

(1) Meier et al. Virology (2003)

(2) Diaz et al. J Gen Virol (2005)

(3) Venteo et al. WALD (2009)

\section{Results}




\title{
P.172
}

\section{Development and characterization of indirect ELISA using heparin-coated PRRSV as an antigen}

\author{
$\underline{\text { Hye-Kwon Kim}}{ }^{1}$ Hyoung-Joon Moon ${ }^{2}$ Seong-Jun Park' Hyun-Ok Keum ${ }^{1}$ Se- \\ Mi Rho' Jae-Yeon Han ${ }^{1}$ Van-Giap Nguyen ${ }^{1}$ Bong-Kyun Park ${ }^{1}$ \\ 1. Department of Veterinary Virology Lab, College of Veterinary Medicine and BK21 Program for Veterinary Science, \\ Seoul National University, Seoul, Korea; 2. Research Unit, Green Cross Veterinary Products, Yongin, Korea
}

\section{Introduction}

Heparan sulfate is known as a receptor for PRRSV attachment (1). The heparin is a soluble form of heparan sulfate. A recent study showed that the use of heparin with virus-like particles as an antigen was effective to reduce non-specific ELISA reaction especially originated from antigenic linearization during coating process in order to detect human papillomavirus-specific antibodies (2). Therefore, this study was performed to estimate the property of the use of heparin with whole viral antigen in PRRSV-specific ELISA.

\section{Materials and Methods}

At first, heparin-binding assay of PRRSV was performed using slightly modified method (two times diltion of whole viral antigen \& 20 times dilution of sera) of previous report about heparin-based ELISA (2).

MARC-145 cell-derived whole viral antigen was mixed with $1 \mathrm{mg} /$ $\mathrm{ml}$ of heparin in PBS in 1:8 ratio and coated on 96well plates (polysorb, Nunc). After fixing with cold ethanol, the plate was blocked with $10 \%$ Horse serum and used as an ELISA reaction. For serum dilution, swine sera was 40 times diluted with a 1:1 mixture of $10 \%$ Horse serum in PBS-T and culture supernatant. After final colorimetric reaction, the O.D values at $450 \mathrm{~nm}$ of viral antigen and cell control coating wells were estimated.

After experimental infection of type I PRRSV, LCS-EU (105 TCID50/ml) and type II PRRSV, CP07-401-9 (105 TCID50/ml), the collected sera until 21 days post challenge (dpc) were tested with commercial ELISA kit (Herdcheck PRRS 2XR, IDEXX) and heparin-ELISA system. The sera were also tested by modified serum neutralizing test (SN test). The heparin-ELISA was tested with not only experimental sera, but also field sera.

The sensitivity and specificity of heparin ELISA were calculated using 310 field swine sera, when IDEXX kit was used as the gold standard. IFA test was also included to confirm true positive or negative in 28 mismatched sera between IDEXX and heparinELISA.

\section{Results}

Heparin-specific PRRSV binding was observed in the heparinPRRSV binding assay with the different concentrations of heparin (fig. 1). In heparin-ELISA system, some unexpected nonspecific reactions found in cell control wells were also reduced in heparin-coating system of this ELISA. (data not shown)

In the experimental infection study, PRRSV-specific IgG began to be detected from 18 to $21 \mathrm{dpc}$ by heparin-ELISA, and from 9 to $10 \mathrm{dpc}$ by IDEXX ELISA. During the study, serum neutralizing ability was not proven by conventional SN test. However, when modified SN test (3) was applied, serum neutralizing ability was detected around 8-9 dpc and 18-21 dpc. In the field sera, IgG detected by heparin ELISA was increased after the viremia of wild type PRRSV.

When IDEXX (2XR) ELISA was regarded as gold standard, the sensitivity and specificity of heparin-ELISA were $89.6 \%$ and 925 $\%$, respectively (0.81 of kappa value). Although $91 \%(10 / 11)$ of false positive in heparin-ELISA was confirmed by IFA test, $60 \%$ $(9 / 15)$ of false negative in heparin ELISA was true negative by IFA test.

Figure 1. ELISA reaction in different concentration of heparin. VC+PS, Viral antigen coating and positive serum; $V C+N S$, Viral antigen coating and negative serum; CC+PS, Cellular antigen coating and positive serum; CC+NS, Cellular antigen coating and negative serum

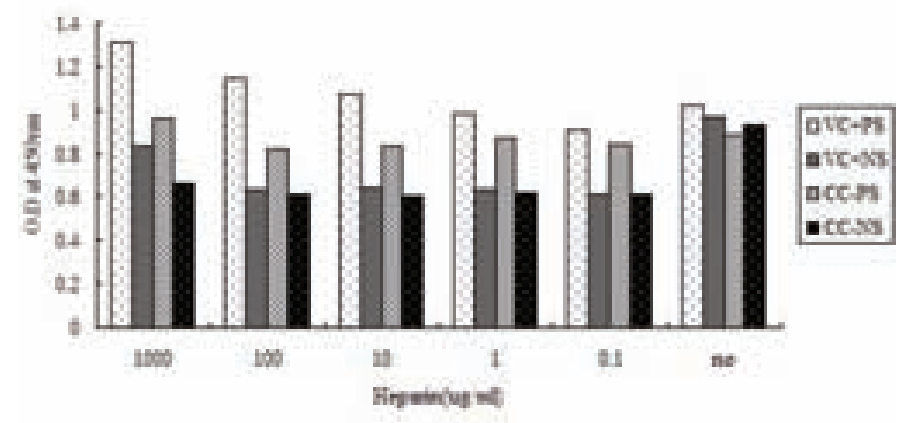

\section{Discussion}

Heparin treatment could be useful for specific detection of lgG specific for PRRSV and theologically protect viral proteins from linearization during the coating process (2). The detection time of IgG for PRRSV by heparin-ELISA was delayed compared to IDEXX ELISA, but correlated with the appearance of neutralizing antibodies that was detected by modified SN test (3). With the fair sensitivity and specificity, the heparin-ELISA was easy to prepare and may provide another approach for PRRSV serology.

\section{Acknowledgement}

This study was supported by a grant (Code\# 20070401034009) from the Biogreen 21 Program, Rural Development Administration, Korea.

\section{References}

1. Delputte and others (2005). J Gen Virol. 86; 1441-5

2. Wang and others (2005). J Gen Virol. 86; 65-73

3. Yoon and others (1994). J Vet Diagn Invest. 6;289-92 


\title{
P.173
}

\section{Rapid Detection of Porcine Reproductive and Respiratory Syndrome Virus by Reverse Transcript Loop-mediated Isothermal Amplification}

\author{
Shangjin Cui \\ Harbin Veterinary Research Institute of CAAS, Harbin, China
}

In order to establish a reverse transcript loop-mediated isothermal amplification method for the rapid detection of porcine reproductive and respiratory syndrome virus, 4 primers specific to 6 regions of $\mathrm{N}$ gene were designed. After amplifying in an isothermal water bath for $1 \mathrm{~h}$, the ladder-like products would be generated with those porcine reproductive and respiratory syndrome virus positive samples while no product was generated with porcine parvovirus, porcine circovirus, classic swine fever virus, pseudorabies virus and swine testis cells. The sensitivity and specificity of this assay were evaluated by comparison with reverse transcript polymerase chain reaction. The detection limit of the assay was shown to be equivalent to $1 \times 100 \sim 1 \times 101$ PRRSV copies/reaction, was about 100 times more sensitive than RT-PCT reaction. The coincidence ratio was showed to be $96.2 \%$. Due to its specificity and simplicity, the assay could be a useful diagnostic tool for the diagnose of PRRSV infection.

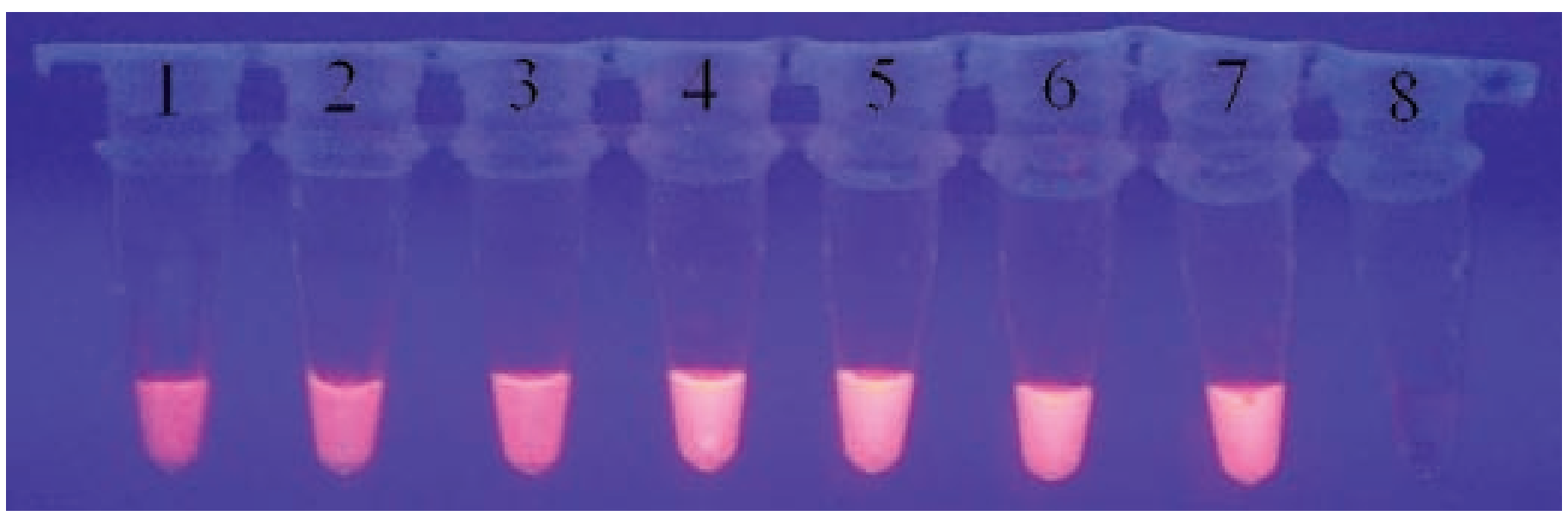




\title{
P.174
}

\section{Multiplex PCR for PRRSV detection and typing}

\author{
Van-Giap Nguyen ${ }^{1}$ Hyoung-Joon Moon ${ }^{2}$ Seong-Jun Park ${ }^{1}$ Hye-Kwon Kim ${ }^{1}$ Hyun- \\ Ok Keum ${ }^{1}$ Se-Mi Rho' Jae-Yeon Han ${ }^{1}$ Bong-Kyun Park ${ }^{1}$ \\ 1. Department of Veterinary Virology Lab, College of Veterinary Medicine and BK21 Program for Veterinary Science, \\ Seoul National University, Seoul, Korea; 2. Research Unit, Green Cross Veterinary Products, Yongin, Korea
}

\section{Introduction}

Since it was first discovered in United States in 1987, PRRS has spread worldwide and become one of the most devastating diseases of swine. The causative agent, PRRSV is divided into two distinct groups: the European (Type 1) and the North American (Type 2). It is well defined about the marked genetic differences between the two genotypes as well as among viruses within each genotype. To identify PRRSV, RT-PCR is one of methods that has been widely applied. However, because of the high degree of sequence variation, the accuracy of RT-PCR might be influenced, especially if mutations are located in the primer or probe binding regions of PCR (3). To overcome those difficulties, previous studies prefered designing primer to amplify part of conserved region of PRRSV genome: ORF1b and ORF7 $(1,5)$.

In this study, a PRRSV detection and typing Multiplex PCR was developed. In our system, the primers were designed to coamplify conserved regions ORF1b and ORF7. In addition, primer set for ORF7 amplicon was type specific that make it possible for differentiation between PRRSV Type 1 and Type 2 .

\section{Materials and Methods}

PRRSV reference strains: Type 1 (105.0 TCID50/ml), Type 2 (104.5TCID50/ml, passage 7 on MARC-145 cell).

Primer design: primers were designed based on PRRSV complete sequence of Type 1 and Type 2 (Genbank: M96262.2, AF046869.1). In details, the following amplicons were amplified: 615bp of ORF1b by CF: IAGGACCATCCTGGGCACCAACA, CR: IIGACGGTTTT CTTTGGGTCCGT; 425bp of Type 1 ORF7 by N26: GCCCTAATTGAATAGGTGAC, FT1: AGAAAAAGAAA

AGTACAGCTCCGAT; 265bp of Type 2 ORF7 by N26/FT2.1: GTGAGCGGCAATTGTGTCTGTCG.

RNA extraction and CDNA synthesis: RNA was extracted using Trizol-Chloroform method. cDNA was synthesized by M-MLV reverse transcriptase kit (Invitrogen, USA) using random hexamers method.

Multiplex PCR reaction: Multiplex was set up according the guidance of previous paper (2) using i-StarMaster mix PCR kit (iNtRON Biotechnology, Korea).

\section{Results}

Specificity and sensitivity of primer set: PCR product was not generated with test RNA virus (TGEV, PEDV) and DW as negative control. In addition, there was no cross reaction of type specific primers. Taken into account with sequencing target bands (data not show), the specificity of primer set was confirmed. By serial 10 fold dilution known titration of PRRSV, the sensitivity of primer set was 102.5TCID50/ml (Type 2), 103.0TCID50/ml (Type 1) and $103.5 \mathrm{TCID} 50 / \mathrm{ml}$ (mixing of Type $1 \& 2$ ).

Sensitivity (Sn) and specificity (Sp) of Multiplex PCR: total 214 field samples (tissue and pool sera originated from different farms) were screening. Comparing to Nested PCR for PRRSV typing (4) (rountine in our lab), 150/214 samples was judged PRRSV true positive. Also, there was no conflict in typing result between Multiplex and Nested PCR. Interestingly, there were 5 tissue samples showed both Type 1 and Type 2 positive. The Sn and Sp of Multiplex PCR was $94.34 \%$ and $90.91 \%$ respectively.

\section{Discussion}

Previous study evaluating RT-PCR method for PRRSV detection (6) concluded that 'the majority of incorrect diagnoses were false-negative results'. One main reason might be the occurrence of mutation that drives a genetic heterogeneity within annealing region of primers. To our knowledge, the probability of false negative will be minimized when more than one conserved regions were co-amplified in PCR. This is partly reflected by the high degree of agreement between new Multiplex PCR and Nested PCR (Kappa value: 0.83). Also, this is the first time Multiplex PCR was set up to co-amplify both conserved regions of PRRSV genome. Slightly high of Sn and Sp might cause by the appropriate primer to the sequence of circulation PRRSV strains. Therefore, more labor is needed in order to get more suitable primer set with recent prevalence PRRSV, Korean strains.

\section{Acknowledgement}

This study was supported by a grant (Code\# 20070401034009) from the Biogreen 21 Program, Rural Development Administration, Korea.

\section{References}

1. Gilbert SA.,et al., (1997).J.Clin.Microbiol.35(1): 264-267

2. Henegariu O. et al., (1997). BioTechniques, 23:504-511

3. Indik S. et al.(2005). Veterinary Microbiology, 107: 171-179

4. Kono Y. et al., (1996). J Vet Med Sci, 58: 941-946.

5. Oleksiewicz MB. et al., (1998). Vet Microbiol, 64: 7-22.

6. Truyen U. et al., (2006). J. Vet. Med. B 53: 68-74. 


\title{
P.175
}

\section{Analysis of ELISA and PCR testing for PRRS in German pig herds}

\author{
Katrin Strutzberg-Minder ${ }^{1}$ Martin Beyerbach² Astrid Tschentscher ${ }^{1}$ Jan Boehmer $^{1}$ Lothar Kreienbrock $^{2}$ Matthias Homuth ${ }^{1}$ \\ 1. IVD GmbH Innovative Veterinary Diagnostics, Hannover, Germany; 2. Department of Biometry, Epidemiology \\ and Information Processing (IBEI), University of Veterinary Hannover, Hannover, Germany
}

\section{Introduction}

Porcine reproductive and respiratory syndrome (PRRS) is one of the most important diseases in pig farms almost worldwide (1). The PRRS virus (PRRSV) causes severe economic losses due to reproductive failures in sows and gilts and respiratory distress in piglets and fattening pigs (1). We analyzed results of diagnostic testing for PRRS in order to obtain current information about PRRS in German pig herds and to determine any associations between different test results, motivation for examination, pig farm type, and the animals' vaccination status.

\section{Material and Methods}

A total of 27,077 serum samples from pigs in Germany were analyzed from October 1, 2007, to September 30, 2009. Antibodies against PRRSV were detected by IDEXX HerdChek* PRRS 2XR Antibody ELISA as described by the manufacturer. Three to five individual serum samples from a single farm were pooled, and these pooled samples $(6,246)$ were also analyzed by PCR based on the ORF7 gene (2). In order to compare the two methods, the individual ELISA results from each pool were averaged to give a pooled ELISA result. From January 1, 2009, data were also collected on herd (type and size, motive for examination, PRRS vaccination status).

\section{Results}

ELISA tests for PRRSV were positive in $525 \%(n=14,213)$ of all individual pig serum samples $(n=27,077)$, whereas PCR tests were positive in $29.0 \%(n=1,816)$ of all pooled samples $(n=6,346)$.

Of the pooled samples from vaccinated pigs ( $n=477), 42.1 \%$ tested positive by both ELISA and PCR, and 9.9\% tested negative by both methods. Only $2.5 \%$ of the serum sample pools from vaccinated pigs tested positive by PCR, but negative by ELISA. Of the serum pools from vaccinated animals, $45.5 \%$ tested positive by ELISA only (and negative by PCR). On the other hand, $31.0 \%$ $(n=52)$ of the pools from unvaccinated pigs $(n=522)$ tested positive by both ELISA and PCR, and $42.7 \%$ tested negative by both methods. Of the pooled samples from unvaccinated pigs, $5.4 \%$ tested positive by PCR but negative by ELISA, while $20.9 \%$ of these samples tested positive by ELISA only (and negative by PCR). (Tab. 1)

Most testing of pig serum samples (55.0\%) was conducted as part of herd monitoring $(n=4,595)$.
Most animals (75.2\% and 66.5\%) from fattening units $(n=427)$ or multipliers ( $n=161$ ), respectively, were unvaccinated, while half $(50 \%)$ of the animals from breeding herds $(n=2,575)$ or nursery units $(\mathrm{n}=423)$ were unvaccinated.

Table 1: ELISA and PCR results of vaccinated and unvaccinated pigs

\begin{tabular}{|l|c|c|c|c|c|}
\hline ELISA results & - & - & + & + & Number of pigs \\
\hline PCR test results & - & + & - & + & tested (100\%) \\
\hline Vaccinated pigs (\%) & 9.9 & 2.5 & 45.5 & 42.1 & 477 \\
\hline Unvaccinated pigs (\%) & 42.7 & 5.4 & 20.9 & 31.0 & 522 \\
\hline
\end{tabular}

Individual ELISA tests were positive in $59.8 \%$ of unvaccinated pigs from fattening units $(n=321)$, whereas these were positive in only about $40 \%$ of unvaccinated pigs from breeding herds ( $n$ $=1277)$, nursery units $(n=217)$ and multipliers $(n=107)$. PCR tests were positive in $36 \%$ of the pools from unvaccinated pigs from breeding herds $(n=275)$ and nursery units $(n=47)$. The most positive $P C R$ results were found in unvaccinated pigs from fattening units: $46.7 \%(n=35$; total $n=75)$.

\section{Discussion}

As expected, we found that vaccination had a very strong effect on the number of positive ELISA tests in pigs. PCR was also affected by the vaccination status of animals, but to a lesser degree. Nevertheless, $57.3 \%$ of the unvaccinated pigs tested positive by ELISA and/or PCR, and thus were shown to be infected with PRRSV.

The vaccination status of the animals depended on the type of pig farm. Most animals (75.2\%) from fattening units were unvaccinated; at least $50 \%$ of these animals were infected with PRRSV. Half (50\%) of the pigs from breeding herds or nursery units were unvaccinated; and at least $36 \%$ of these animals were infected with PRRSV.

Many more interesting associations were discovered in the comparison of test results in regard to vaccination status in combination with herd type and motivation for testing.

\section{References}

1. Zimmermann et al. (2006) in: Straw et al. (eds.), Diseases of Swine, 9thed., 387-417

2. Pesch (2003). Diss. Vet. Fac. University of Leipzig, Leipzig, Germany 


\title{
P.176
}

\section{Analysis of amplification of North America PRRS virus RNA from serial diluted oral fluid samples}

\author{
Ricardo A. Munoz ${ }^{1}$ R Shah ${ }^{1}$ R.R. Rowland ${ }^{2}$ J. El-Attrache ${ }^{1}$ J. Zimmerman ${ }^{3}$ \\ 1. Applied Biosystems, Austin, TX, USA; 2. Kansas State University, Manhattan, KS, USA; 3. lowa State University, Ames, IA, USA
}

\section{Introduction}

Porcine Reproductive and Respiratory Syndrome Virus (PRRSv) surveillance for the purpose of monitoring herd status requires frequent sampling and testing. Less invasive techniques are continuously being explored such as passive blood collection, as well as the use of swabs to collect matrices that contain swine pathogens. Recently, more innovative monitoring techniques and protocols, such as oral fluid collection, have been developed for the swine industry that notably mirror the techniques of pathogen detection employed for human medicine.

The oral fluid is a combination of saliva and "oral mucosa transudations". Saliva is produced by the salivary glands; oral mucosal transudations enter the mouth via the buccal mucosa from the blood capillaries ${ }^{1}$. The purpose of this study was to find a reference performance of sampling protocols in terms of PRRSv North America (NA) strain potential amplification from oral fluid samples after artificial dilution of the original oral fluid samples, using PRRSv negative oral fluid samples. The intention was to mimic what could happened in the field, when just one pig could get infected at the beginning, and other factors, such as the number of negative pigs chewing the rope.

Collection of oral fluids ${ }^{1}$ :

- Use cotton rope, it is more absorbent than synthetic fiber.

- Use $1 / 2$ " rope for nursery and 5/8" for growth and finishers.

- Place the ropes in a clean area, away from water and feed sources.

- Hang the rope at pig shoulder height (do not allow to contact dirt or feces).

- Squeeze the rope inside a plastic bag, avoiding contacting the rope with your exposed hands (to reduce the risk of crosscontaminations).

- Cut one of plastic bag corners and pour into a tube, if there are visible particles, then centrifuge for approximately 10 minutes.

- Maintain cold chain $\left(4\right.$ to $7^{\circ} \mathrm{C}$ ) prior to sample submission

Oral fluids are managed the same as serum samples.

\section{Materials and Methods}

Samples were obtained from a virus challenge study conducted at Kansas State University. In a single experiment, 190 pigs were divided among 12 pens and experimentally infected with 105 TCID50 of a PRRSv 97-7985 strain (NA like virus). Oral fluid samples were collected from each pen and individual animals were bled in intervals over 42 days.

\section{Serial Dilution Experiment:}

Take high titer sample ( $15 \mathrm{cts}$ ) and make a 12 point serial dilution of the sample using negative oral fluid. Dilution series will include 10 fold, 4 fold and 2 fold dilution points. Isolate diluted samples using AM1836 and obtain results using RT-PCR.

This experiment will give us the linear amplification range of and also tell us important dilution information such as, the sample that is a 20 fold dilution of the stock will equate to a sample from one positive pig that has been pooled with 19 negative pigs, assuming they all contribute the same amount of oral fluid to the mix.

\section{Pooling Experiment:}

Take a high titer sample and dilute it in 5, 10 or 20 different negative samples to simulate a high positive pig in a pen with 4,9 or 19 negative pigs. Isolate and run Real-Time PCR to get results. Do the same for a low titer sample to simulate a low positive pig with 4, 9 or 19 negative pigs in a pen. This will give us information about detection limits within pens and how many samples can be tolerated before loss of amplification.

Some pigs may contribute a higher amount of oral fluid compared to others. These experiments assume that the same amount of oral fluid is contributed by each pig

\section{Summary}

The presence of PRRS virus RNA was identified in an early infectious stage utilizing oral fluids as a sample matrix. Real-time PCR can be effectively utilized to amplify PRRS virus RNA obtained from oral fluids samples. Predictably, this type of analysis could provide an enhancement to the routine monitoring and surveillance of populations.

\section{References}

1 Zimmerman, J., Prickett, J. and Johnson, J. Oral Fluids Testing: Science-based applications. Department of Veterinary Diagnostic, College of Veterinary Medicine, lowa State University. Proceedings from the Carlos Pijoan International Symposium, September 2009. University of Minnesota.

For Research Use Only. Not for use in diagnostic procedures. (c) 2009 Life Technologies Corporation. All rights reserved 


\title{
P.177
}

\section{Detection of PRRSV in oral fluid samples: longitudinal study under field conditions}

\author{
Loris G. Alborali; Matteo Gradassi; Pavesi Roberta; Beatrice Boniotti; Claudia Nassuato; \\ Stefano Giovannini; Enrico Giacomini; Silvia Bellini; Maria Pacciarini
}

IZSLER, Brescia, Italy

\section{Introduction and Objectives}

PRRS (Porcine Reproductive and Respiratory Syndrome) control in swine farms is an ambitious challenge that has to be based on different measures and intervention strategies and that, because of the high amount of diagnostic samples required, is often cost prohibitive (1). The selection of oral fluid as diagnostic specimen is likely to be a promising alternative to serum. At present, there are experimental evidences that porcine saliva is a valuable alternative to serum for detection of PRRSV genome $(2,3)$. The main purposes of the present experimental study was to monitor PRRSV infection towards a longitudinal sampling of sera, according to the traditional diagnostic protocols, and match individual serum with collective pen-based oral fluid results.

\section{Material and Methods}

Four site-2 swine commercial farms site in North Italy participated in the study. On each of them, 4 pens of 30 piglets were selected and, at 3, 5, 7 and 9 weeks of age, the following specimen were collected: individual serum samples of 10 ear-tagged pigs/pen and collective oral fluid samples (1 cotton rope/pen). The serum were analysed individually. The presence of PRRSV RNA was shown using a real-time qRT-PCR (Applied Biosystem TaqMan NA/EU PRRSV Kit).

\section{Results}

Comparison showed a good agreement (87,5\%) between PRRSV detection in porcine pen-based oral fluid and individual serum samples, as visualised in Figure 1 and 2.

\section{Figure 1}

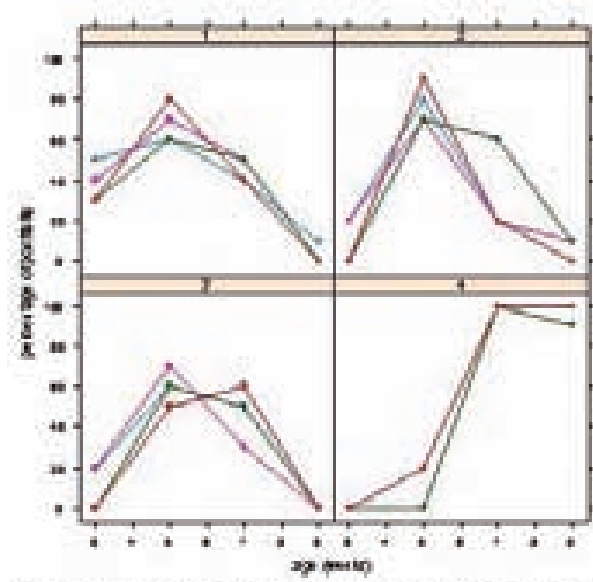

Foure 1 : individual scrum pecentage of PRRSV postivity in tarms 1,2,3and4
Figure 2

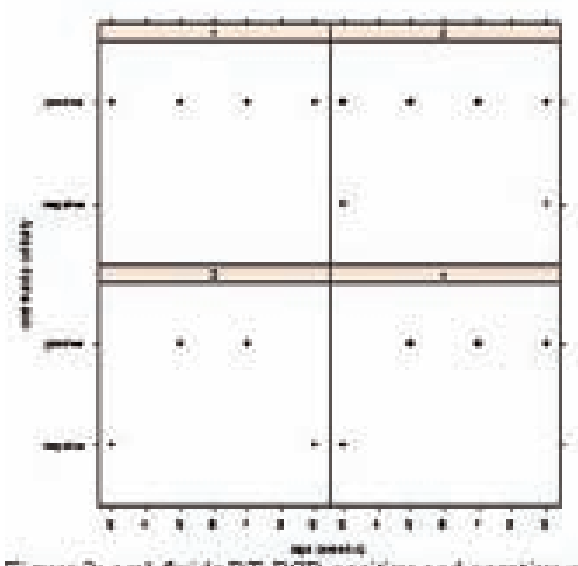

Figure 2: oral fuids RT.PCR positive and nogative resuls in farms $1,2,3$ and 4

Despite oral fluids constantly contained a lower concentration of virus compared to individual positive serum, the level of virus showed a similar temporal course in both specimen.

\section{Discussion}

Our data corroborates that oral fluid samples can be considered a valuable and promising alternative to serum to track PRRSV field infection. Moreover, compared to drawing blood specimen from individual pigs, pen-based saliva offered significant advantages, such as reduced costs, less labour and simplicity. Preliminary data on the diagnostic sensitivity of the quantitative RT-PCR on oral fluids, compared to the gold standard (RT-PCR on individual serum samples), can be accounted as satisfactory. Further analyses are in progress in order to validate saliva qRTPCR at pen level.

\section{References}

1) Neumann E.J. et al., 2005. J. Am. Vet. Med. Assoc. 227: 385-392

2) Pricket J. et al., 2008a. J. Vet. Diagn. Invest. $20: 156-163$.

3) Pricket J. et al., 2008b. J. Swine Health. Prod. $16: 86-91$ 


\title{
P.178
}

\section{Optimization of PRRS PCR for oral fluid testing}

\author{
Apisit Kittawornrat; Wayne Chittick; Wendy Stensland; John R. Prickett; Chong Wang; Erin Strait; K-J Yoon; Jeffrey J. Zimmerman
}

lowa State University, Ames, IA, USA

\section{Introduction}

Oral fluids have been reported to be useful specimens for the detection or diagnosis of infection, 1,2,4 including PRRSV.5,6 Our initial work revealed a low sensitivity for PRRSV PCR on swine oral fluid samples using extraction methods established for serum samples. This sample matrix-related difference has been reported by others, as well.1-3 Thus, oral fluid samples are known to inhibit PCR efficiency.1,3

Preliminary work at the ISU-VDL found that an extended lysis/ processing adaptation to an existing extraction method improved improve test sensitivity (Stensland, unpublished data). In this study, we sought to continue to improve PRRSV PCR for oral fluids. Specifically, we evaluated 5 nucleic acid extraction protocols and assessed the presence of PCR inhibition in swine oral fluids through the use of an internal PCR control.

\section{Materials and Methods}

Oral fluid samples $(n=106)$ were randomly selected from an earlier study in which pigs were inoculated with PRRSV.5 The sample set included 63 pen-based oral fluid samples collected between 7 DPI and $29 \mathrm{DPI}$ and 43 samples from negative control pens. These samples were used to compare 5 extraction protocols based on commercially-available kits using magnetic bead-based extraction (protocols 1 through 4) and spin-column based extraction (protocol 5).

1. Applied Biosystems MagMax ${ }^{\mathrm{TM}}$ Viral RNA Kit - ISU-VDL modified protocol;

2. Applied Biosystems MagMax ${ }^{\mathrm{TM}}$ Viral RNA Kit - High-volume, ISU-VDL modified protocol;

3. Qiagen BioSprint ${ }^{\circledR}$ Vet One-For-All Kit;

4. Applied Biosystems MagMax ${ }^{\mathrm{TM}}$ Total Nucleic Acid Isolation Kit;

\section{Qiagen QIAmp ${ }^{\circledR}$ Viral RNA Mini-Kit.}

PRRSV RNA was detected using Applied Biosystems Ag-Path PRRSV real-time PCR reagents, which contains an internal control for detecting failed reactions. Initially, a standard master mix was prepared according to the instructions from the manufacturer. Later, PCR was re-run using modified master mix with double the amount of enzyme mix (containing both reverse-transcriptase and Taq polymerase enzymes).

\section{Results}

Results indicated significant differences among extraction protocols in the level of detection in PRRS-exposed pen samples, ranging from $17 \%$ to $43 \%$. In general, doubling the PCR enzyme resulted in improvements in detection among all extraction protocols, ranging from $32 \%$ to $56 \%$. The internal control data suggested a differing level of failed reactions among the extraction protocols tested, suggestive of PCR inhibition, although the results were not statistically significant. All protocols had reduced number of failed internal controls when enzymes were doubled.

\section{Discussion}

The oral fluid sample matrix is known to inhibit PCR test process1-3. Cumulatively, our work confirmed this observation and demonstrated that not all extraction methods work equally with the oral fluid matrix.

\section{Acknowledgements}

This study was supported in part through gifts from Applied Biosystems and Qiagen.

\section{References}

1. Amado LA, Villar LM, DePaula VS, et al., 2008. Comparison between serum and saliva for the detection of hepatitis A virus RNA. J Virol Methods 148:74-80.

2. Matto J, Saarela M, Alaluusua S, et al., 1998. Detection of Pophyromonas gingivalis from saliva by PCR by using a simple sample-processing method. J Clin Microbiol Volume:157-160.

3. Oschert AS, Boulter AW, Birnbaum W, et al.: 1994. Inhibitory effect of salivary fluids on PCR: potency and removal. PCR Methods Appl 3:365-368.

4. Pink R, Simek J, Vondrakova J, et al.: 2009. Saliva as a diagnostic medium. Biomed Pap Med Fac Univ Palacky Olomouc Czech Repub 153(2):103-110.

5. Prickett J, Simer R, Christopher-Hennings J, et al., 2008. Detection of PRRSV infection in porcine oral fluid samples: A longitudinal study under experimental conditions. J Vet Diagn Invest 20:156-163.

6. Prickett J, Kim W, Simer R, et al., 2008. Surveillance of commercial growing pigs for PRRSV and PCV2 infections using pen-based oral fluid samples: A pilot study. J Swine Health Prod 16(2):86-91. 


\title{
P.179
}

\section{Brazilian bioassay model to investigate PRRSV on imported swine semen}

\author{
Guido Carlos H. Masson ${ }^{1}$ Ibrahim N. Ibrahim ${ }^{1}$ Luiz Fernando O. Carvalho ${ }^{1}$ Maria Cristina Thomaz Daniel Andaluz ${ }^{1}$ \\ 1. São Paulo State University - UNESP, Jaboticabal, SP, Brazil; 2. Topgen Swine Genetics Farm Araporanga , Jaguariaíva, SP, Brazil
}

\section{Introduction}

Porcine reproductive and respiratory syndrome virus (PRRSV) is a widespread viral disease in the pork industry (Greineir et al., 2000) and causes a potentially devastating disease in swine herds (Wills et al., 2000). Because its potential for rapid spread, significant production and economic losses is considered the most economically important viral disease at swine production. PRRSV has been recorded in most major pig-producing areas throughout the world. However the disease is considerate as an exotic disease in Brazil.

Nowadays, in order to improve the genetics of Brazilian breeding herds, producers imported genetic material (semen and live animals) origination from countries which are recognized by their genetic technology. This trade can lead serious problems for the health status of the piggery because is well know that PRRSV can be shed and transmitted through boar semen (Christopher-Hennings et al., 1996).

The aim of this study was to perform a swine bioassay (in vivo) to test the safety for PRRSV on imported semen from Germany to be used in swine genetics too Topgen Ensure the safety

\section{Materials and Methods}

Six pigs were randomly assigned and kept in flyer-proofed isolations pens. Four piglets were inoculated intraperitoneally which a pool of $2 \mathrm{ml}$ of porcine German imported semen provide from TOPGEN swine Genetics. The other two animals were designed as negative control. Rectal body temperature and PRRS clinical sings (fever, anorexia, lethargy, diarrhea, respiratory symptoms, chemosis, tremors) were done daily from those pigs. Blood samples with EDTA were collected from the sow and each pig at weaning, immediately before PRRSV inoculation an on days 7, 14, 21 post inoculums (PI). Blood samples were analyzed for hematological parameters. At the same day serum samples were collected too in order to test serum-conversion by the PRRS using a commercial Kit - Idexx - Herdcheck PRRS 2XR ELISA. Two piglets were euthanized on day $7 \mathrm{PI}$ and $21 \mathrm{Al}$ at the necropsy room at Laboratório de Sanidade de Suínos at São Paulo State University by electrocution and the necropsy were performed immediately as performed by Ciacci-Zanella et al 2004. At the necropsy, all of the swine were thoroughly examined, blood, serum and organs were collected for histopathology and viral tests.

\section{Results}

For the period of 21 days, nome of the inoculated piglet presented characteristic PRRS symptoms. The blood parameters were normal. At the necropsy, no pathological lesions (macro or microscopic) were found by histopathology. Also the tested pigs were negative to the ELISA test methods.

\section{Discussion}

The goals of this work were achieved. The safety of the semen demonstrates the efficiency of government agencies that oversee the transit of animals and their products through constant monitoring. Swine bioassay is an excellent tool for detecting PRRSV although it is laborious (Christopher-Hennings et al., 1996). The trial period had time for a possible seroconversion that occurs between 14 and 28 days (Wills et al., 2000).

\section{References}

CIACCI-ZANELLA, J. R.; TROMBETTA, C.; VARGAS, I.; da COSTA, D. E. M. 2004 Lack of evidence of porcine reproductive and respiratory syndrome virus (PRRSV) infection in domestic swine in Brazil Ciência Rural, v.34, n.2, p.449-455

CHRISTOPHER-HENNINGS, J.; NELSON, E. A.; BENFIELD, D. A. 1996 Detecting PRRSV in boar semen. Swine Health and Production v.4, n.1, p.37-39.

GREINER, L.L.; STAHLY, T.S.; STABEL, T.J. 2000 Quantitative relationship of systemic virus concentration on growth and immune response in pigs. Journal of Animal Science v.78, p. 2690-2695.

WILLS, R.W.; DOSTER, A.R.; GALEOTA,J.; SUR,J.H.; OSORIO, F.A. 2000 Duration of PRRS Virus Infections and Proportion of Persistently Infected Pigs. Nebraska Swine Reports p.44-46 


\title{
P.180
}

\section{Porcine reproductive and respiratory syndrome (PRRS). Understanding infection dynamics through serumprofiles}

\author{
Isaac R. Ballarà ${ }^{1}$ Sergi Bruguera ${ }^{1}$ David Llopart ${ }^{1}$ Dani Torrents ${ }^{2}$ \\ 1. HIPRA, Technical Services, Amer (Girona), Spain; 2. HIPRA China, Technical Services, Beijing, China
}

\section{Introduction}

Porcine Reproductive Respiratory Syndrome (PRRS) was a problem, is a problem and will be a problem in pig farming. The aim of the vet who is facing this disease is to get a PRRS stabilize population of animals. PRRSV serologic screening results are essential to understand the PRRSV epidemiology in the farm, which is necessary to take the proper decisions in each PRRSV infection scenario. So, in the pig veterinary practice, transversal serum profiles are usually implemented to take most proper decisions in order to control PRRSV.

\section{Materials and Methods}

200 transversal serum profiles from 200 different farms located in Spain, Hungary, Poland, Czech Republic, Slovaquia, Italy and Denmark were analysed. Each serum profile was made from serum samples collected from different pig groups (gilts, sows, piglets and fattening pigs). The sows were grouped together by age (by parities), they were sampled from day of gestation 70 to 100. Piglets and fattening pigs were sampled by weeks of age $(4,7,10,13,16,19,21$ and 24). The sample was blood sera and the amount of samples per group depended on the size of the farm and the estimated infection prevalence. The average number of serum samples per group was 7. Serology analysis was made with ELISA (CIVTESTsuis PRRS/ES Laboratorios Hipra, Amer, España) following the manufacturer instructions. From each monitored farm was noted the productive data, the vaccination program and the clinic situation.

\section{Results}

After testing and analyzing 200 farms, we could determine 3 clear PRRSV infection patterns which let us to define 3 different PRRSV epidemiologic status: 1. Clinically not stable and serologically not stable, (42 farms out of 200) (Graphic 1); 2 . Clinically stable and serologically not stable, (112 out of 200) (Graphic 2); 3. Clinically stable and serologically stable, (36 farms out of 200) (Graphic 3). 10 farms could not be classified in any of these 3 patterns.

\section{Discussion}

Samples results from pigs at 7 weeks of age became essential information. Passive immunity does not reach with high IRPC levels to 7 weeks of age ${ }^{1}$.If we observe high antibody titers at this age we can suspect that the piglets were already viraemic at weaning and for this reason, we can suspect that. So sows were probably farrowing infected PRRSV piglets. In farms where the piglets were vaccinated, we could not determine any pattern, because vaccination serology response could be confused with a serology positive result due to a wild PRRSV infection. However PRRSV sow vaccination does not interfere with this classification, since we observe that the serology response induced by vaccination does not result in IRPC values higher than 100. Recent wild virus infection induces IRPC values higher than 100. We observed that the evolution of PRRSV infection in farms followed these 3 statuses, evolving from one status to the other within 6 to 12 months period. We do not observe differences among the farm status from different countries. We observed that most of the farms that implemented at least vaccination in sows belonged to the second PRRSV infection pattern (Graphic 2). To sum up, according to this classification, swine vet specialists can monitor and take their proper decisions to control PRRSV.

\section{References}

1 Cho JG, Dee SA. Porcine reproductive and respiratory syndrome virus. Theriogenology. 2006 Aug;66(3):655-62 Epub 2006 May 24.

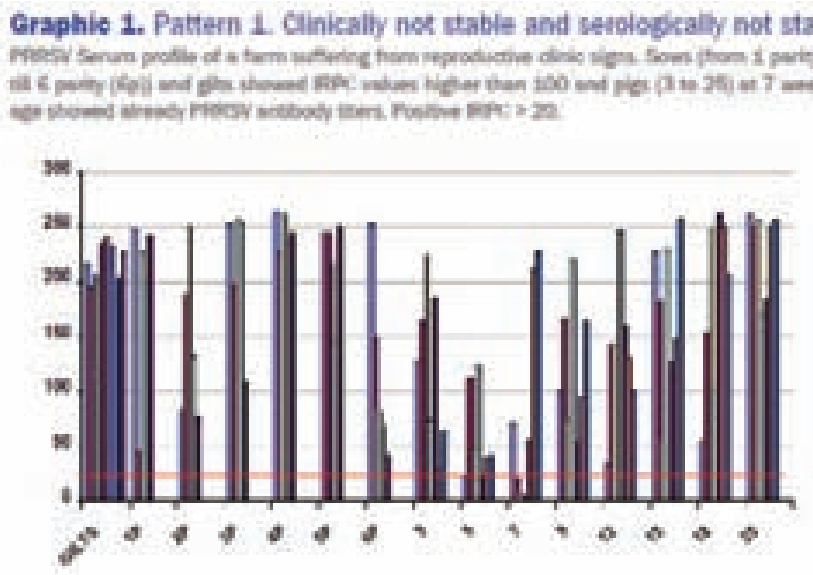

Graphie 2. Pattern 2 Clnically stable and seroboscally not sable.

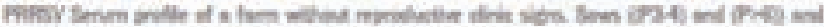

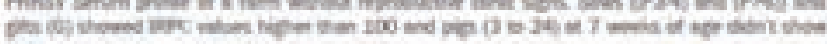

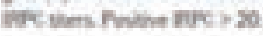

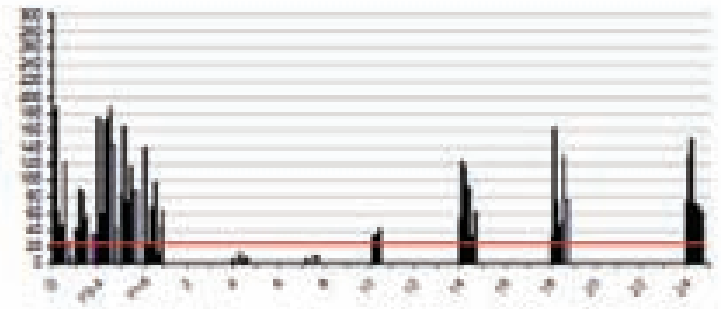

Graphie 3. Patrem 3. Clinically stable and selologically stable

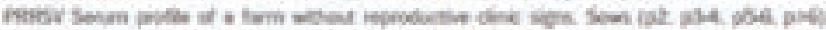

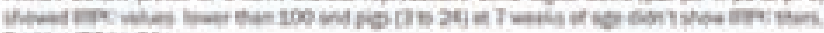

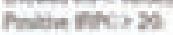

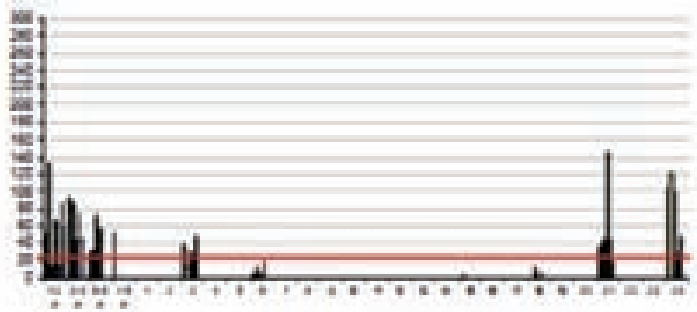




\title{
P.181
}

\section{Porcine reproductive and respiratory syndrome (PRRS) on a large pig farm in Slovenia}

\author{
Zdravko Valencak ${ }^{1}$ Alojz Pertoci ${ }^{2}$ \\ 1. Veterinary Faculty, Ljubljana, Slovenia; 2. Panvita, Veterina, Beltinci, Slovenia
}

\section{Introduction}

PRRS is an economically significant viral disease of swine (1). Slovenia was PRRS free country and later on country with low seroprevalence to PRRS in small pig farms (2). In last years seroprevalence to PRRS increased on small farms and also large pig farms became positive. Vaccination after PRRS outbreak is used control measure (3). In 2008 in one of the large one site pig farm production results and increased mortality leaded to suspision of PRRS. PRRS was confirmed in May 2008 with testing of 152 pig with ELISA, from which 112 were positive to PRRS. Farm started with control measures.

\section{Materials and Methods}

Vaccination programme

- June 2008 all sows on the farm were vaccinated with inactivated vaccine in three weeks.

- August 2008 vaccination with live vaccine. Sows were vaccinated 6 th day after farrowing and 60 th day of pregnancy.

- From July to November piglets were vaccinated with live vaccine at beginig all and after at age of 14 days.

- December 2008 piglets were vaccinated at age of 24 days.

- From may 2009 till October piglets were not vaccinated.

- From August 2009 in sows at 60 th day of gestiation the inactivated vaccine was used.

- October 2009 all sows were vaccinated with live vaccine in 2 days and all piglets were vaccinated at weaning.

- In January 2010 all sows were vaccinated with live vaccine.

Serological control

In year 2008 were tested 101 samples from June to December, and in 2009562 samples with IDEXX PRRS ELISA.

\section{Selected production Results}

Results of 2007, 2008 and 2009 were compared (abortions, farrowing rate, mating rate, weaned piglets per litter, preweaning mortality, postweaning mortality).

\section{Results}

In 2008 only 48 samples (47,5\%) were seropositive. In 2009537 $(95,5 \%)$ sera reacted positive.
Table 1: Comparison of production Results

\begin{tabular}{|l|c|c|c|}
\hline & $\mathbf{2 0 0 7}$ & $\mathbf{2 0 0 8}$ & $\mathbf{2 0 0 9}$ \\
\hline Abortions/month & 14,17 & 43,83 & 66,25 \\
\hline Farrowing rate (\%) & 83,74 & 80,11 & 69,78 \\
\hline Mating rate (\%) & 84,81 & 83,31 & 74,31 \\
\hline Weaned/litter & 9,68 & 8,94 & 8,58 \\
\hline Preweaning mortality (\%) & 12,10 & 8,55 & 20,20 \\
\hline Postweaning mortality (\%) & 4,95 & 5,58 & 7,20 \\
\hline
\end{tabular}

Average number of abortions per month in year 2008 from January to October was 14,8 but increased in November to 153 and in December to 212. In 2009 average number of abortions from January to October was 59,7 monthly, but in November 102 and in December 23.

\section{Discussion}

PRRS reached culmination in November in December 2008 in spite of used programme of vaccination. Success of vaccination in 2009 was not satifactory. In October 2009 the vaccination scheme was changed fundamentaly. With vaccination of all breeding sows in short interval stabilization of herd was expected. Preliminary results shows that number of abortions decreased significantly and number of seropositive animals is improving. Results in January 2010 indicates that vaccine did not achieve long lasting immunity and PRRS virus still circulates in breeding herd and that repeat vaccination of all sows is appropriate measure to improve production results.

\section{References}

1. Neumann EJ, Klebenstein, Johnson CD, Marby JW, Bush EJ, Seitzinger AH, e tal. Am Vet Med Assoc 2005;227:385-92.

2. Valenc ${ }^{2} a k$ Z. Slov Vet Res 2004;41(2):99-101.

3. Gillispie TG, Carroll AL J Swine Health Prod 2003;11(6):291-295. 


\title{
P.182
}

\section{Impact of the Porcine Reproductive and Respiratory Syndrome (PRRS) on reproductive performance in Austrian sow herds}

\author{
Eva-Maria Peinhart ${ }^{1}$ Bettina Exel ${ }^{2}$ Franz Dieber ${ }^{3}$ Wolfgang Sipos $^{1}$ \\ 1. University of Veterinary Medicine Vienna, Vienna, Austria; 2. Artificial Insemination Stud \\ Gleisdorf, Gleisdorf, Austria; 3. Styrian Animal Health Service, Graz, Austria
}

\section{Introduction}

Since the first appearance of porcine reproductive and respiratory syndrome (PRRS) in 1987 in the USA and in the early 1990's in Europe PRRSV has become endemic in most pig producing countries and causes high economic losses (1). PRRSV causes a persistent infection and virus excretion can be detected over several months post infection (2). However, data to substantiate the role of PRRSV on reproductive performance of sows in Austrian pig herds were not available. Due to the different structure of the farms, the data obtained from other countries with much higher concentrated pig herds and higher numbers of animals per herd are not directly comparable to the situation in Austria. So the aim of the study was to evaluate the effect of PRRSV on the reproductive perfomance of Austrian sow herds.

\section{Materials and Methods}

140 Styrian (South-East Austria) production herds were randomly selected. The mean number of sows per herd was 74 animals. The main inclusion criteria were that the farmers had to use the SPon Web soft-ware for reproductive management and to be a member of the Styrian animal health service. On each farm 14 random blood samples were collected. These samples comprised 4 sows, 4 gilts, 4 growers, and 2 boars, if available. All samples were analysed by the IDEXX Herdchek ${ }^{\circledR}$ PRRS Antibody (Ab) test kit.

Then, the farms were divided into three groups: PRRSV-Ab-positive (at least one animal seropositive, no history of vaccination, $n=66)$, PRRSV-Ab-negative $(n=60)$ or vaccinated (EU-strain MLV vaccine, $n=14$ ). Following, reproductive performance data were compared between these three groups.

\section{Results}

No significant difference in the number of live born piglets per sow and year could be found between the PRRSV-Ab-negative ( median=27.4) and the PRRSV-Ab-positive (median=27.0) group. In contrast the vaccinated herds exhibited a significantly $(p<0.05)$ worse productivity with a median of 25.2 live born piglets. However, the PRRSV-Ab-positive herds showed a trend towards greater losses in the suckling period (median=14.6) than PRRSV-Ab-negative (median=13.2) and vaccinated herds (median=11.4).

\section{Discussion}

This study shows that the results from the USA are not directly comparable to the situation in Europe, especially to the one in Austria with its different structure of swine herds. Our data show, that PRRSV-Ab-positive and PRRSV-Ab-negative herds can obtain nearly the same reproductive performance, given PRRSV infection is stable. Interestingly, the number of live born piglets was significantly lower in vaccinated herds. There are several possible explanations for this finding, for example the vaccine does not cover all relevant strains or vaccination regimens were not optimal. However, vaccinated herds achieved better results during the suckling period, which could be a consequence of the initially smaller litter sizes in vaccinated sow herds.

\section{References}

1. Neumann et al. (2005): J. Am. Med. Assoc. 227, 385-392.

2. Straw et al. (eds.): Diseases of swine, 9 th ed., pp. 387-417. 


\title{
P.183
}

\section{Comparison of temporal variation of blood parameters between PRRS naive "5 kg" gilts and PRRSv infected resident piglet population commingled in a nursery}

\author{
Andréanne Caron ${ }^{1}$ Martine Denicourt ${ }^{2}$ Sylvie D'Allaire ${ }^{2}$ Younès Chorfi ${ }^{2}$ Joël Rivest ${ }^{1}$ Christian Klopfenstein $^{1}$ \\ 1. Centre de développement du porc du Québec inc., Québec, QC, Canada; 2. Faculté de \\ médecine vétérinaire, Université de Montréal, St-Hyacinthe, QC, Canada
}

\section{Introduction}

As a method of gilt acclimatization to porcine reproductive and respiratory syndrome virus (PRRSv), many swine producers commingle naive " $5 \mathrm{~kg}$ " replacement gilts (NG) with their PRRS positive resident nursery pigs. Surprisingly, the NG often perform better than the resident piglets (RP). The objective of this study was to describe and compare temporal variation of hematologic, serologic and viremic characteristics between NG and RP.

\section{Materials and Methods}

RP and NG were introduced in an all-in-all-out nursery (d0) at approximately 20 and 40 days of age, respectively. They were transferred to a grow-finishing (GF) unit at d45 of the study. A total of 24 piglets were selected, 12 NG and 12 RP. Blood was collected at d0, d6, d13, d20, d27, d42, d48, d55 and d95. Selected piglets were housed in 6 pens ( 3 for NG and 3 for RP) of 4 piglets in three different rooms of a capacity of 72 piglets each. NG were in separate pens but they had nose-to-nose contact with RP through the fence. All 24 piglets were weighed and scored for clinical signs (1: normal; 2: unthrifty; 3: sick) at each sampling day of nursery. Presence in serum of PRRSv and antibodies against PRRSv were tested by a quantitative RT-PCR (qPCR) and IDEXX-2XR ELISA on all samples. Serum protein electrophoresis ${ }^{1}$ and complete hematologic profile were done on most sampling days. Finally some sera (d6, d13, d27 and d42) were screened for influenza H1N1 and H3N2 antibodies (IHA). Differences between groups of pigs, sampling days and interaction were tested with mixed models ${ }^{2}$.

\section{Results}

Viremia for PRRSv (qPCR positive) was observed in only 2 RP at d0 and in $3 \mathrm{RP}$ at d20. From d42 to d48, most piglets became viremic (PRRSv peak), but no clinical signs were observed on neither group of pigs during this period. All piglets had seroconverted (ELISA) by d95. Clinical signs (mainly dyspnea and unthriftiness) were observed between d 13 and $d 20$ on most piglets and were found to be related to the circulation of H3N2 influenza virus (paired sera). Clinical signs were less severe in NG. However, piglets in both groups had similar nursery growth rates $(A D G=0.487 \mathrm{~kg})$.
Most hematological parameters varied over time in both groups but the dynamics were different between groups. The main variations were observed for cellular immunity and globulins (see table 1).

\begin{tabular}{|c|c|c|c|c|c|}
\hline \multirow{2}{*}{ Parsinecer } & \multirow{2}{*}{ Group } & \multicolumn{2}{|c|}{ H3IT outbreat. } & \multicolumn{2}{|c|}{ PER:yeat. } \\
\hline & & 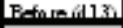 & Afterifid27) & 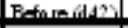 & Aftar (d 55 ) \\
\hline \multirow{2}{*}{$\begin{array}{l}\text { Whats beol celle } \\
10 / \mathrm{L}) \\
\end{array}$} & HG & 163 & $26.6 *$ & 161 & 179 \\
\hline & IP & 203 & $27.6 \cdots$ & $1+5$ & $23 . *$ \\
\hline \multirow{2}{*}{$\begin{array}{l}\text { is pwitel } \\
\text { Wentroflale }(10 / L)\end{array}$} & HG & 6.7 & $143 *$ & 71 & s. 0 \\
\hline & $\mathrm{RP}$ & 109 & $11 \mathrm{~s}$ & 59 & $93 * *$ \\
\hline \multirow{2}{*}{ 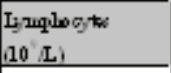 } & HG & 7.6 & 53 & 73 & S.2 \\
\hline & $\mathrm{RP}$ & 7.7 & $12.6 *$ & 71 & $115 *$ \\
\hline \multirow{2}{*}{$\begin{array}{l}\text { Wowastes } \\
10 \text { L) } \\
\end{array}$} & HG & 15 & 19 & 0.4 & $10^{\circ}$ \\
\hline & $\mathrm{EP}$ & 1.6 & 1.4 & $0 S$ & $15 \%$ \\
\hline \multirow{2}{*}{$\begin{array}{l}\text { Golonine } \\
(\gamma \mathrm{L})\end{array}$} & HG & 23 & $312 *$ & 29.2 & $335 *$ \\
\hline & $\mathrm{EP}$ & 1.4 & $26.7^{* *}$ & 222 & $29.4 *$ \\
\hline \multirow{2}{*}{$\begin{array}{l}\text { (- dobtix } \\
\text { (cL) }\end{array}$} & HG & 35 & $39 *$ & $7 S$ & $92^{\circ}$ \\
\hline & $\mathrm{EP}$ & 33 & 3.7 & +3 & $70 * *$ \\
\hline
\end{tabular}

\section{Discussion}

Almost no viral circulation of PRRSv was observed among selected piglets until the end of the nursery period (d42); infection of NG occurred later than expected even though there was nose-to-nose contact with infected piglets. In this study, NG were less affected by the influenza virus than RP and showed different immune responses (cellular and humoral). It could be that NG had a more mature immune system than RP because they were older when introduced in the nursery. Also NG had a higher health status than RP; their immune resources were possibly more readily available to fight pathogens.

\section{References}

1 Chorfi et al. 2004.Vet Clin Pathol. 33:122-127

2 Mixed models, SAS, 2002, SAS version 9.1.3 SAS Inst. Inc. Cary, NC. 


\title{
P.184
}

\section{Time-course of the immune response to Porcine Reproductive and Respiratory Syndrome Virus (PRRSV) in gilts}

\author{
Silvia Dotti ${ }^{1}$ Giampietro Sandri Riccardo Villa ${ }^{1}$ Elisabetta Razzuoli ${ }^{1}$ Enrico Sossi ${ }^{1}$ Massimo Amadori ${ }^{1}$ \\ 1. Istituto Zooprofilattico Sperimentale Lombardia Emilia Romagna, Brescia, Italy; 2. Soc. Agricola Tre Valli, Gruppo Veronesi, Parma, Italy
}

\begin{abstract}
Introduction
The aim of this study was to evaluate the time-course of the immune response to PRRS virus in two PRRSV-positive farms. Gilts came from a PRRS-free herd and the purpose was to house them without causing PRRS outbreaks in the two farms under study. For this reason, two different strategies were adopted: vaccination in farm 1 and direct contact with viremic animals in farm 2. The cell-mediated immune response was evaluated by a PRRSV-specific interferon- $\gamma$ release assay; also, an ELISA assay was performed to detect PRRSV-specific IgA Abs in saliva, the assessment of which could be useful in association with other routine diagnostic tests.
\end{abstract}

\section{Materials and Methods}

Animals. Two PRRS-positive farms, hereunder named 1 and 2 were chosen for the study. Gilts came from a PRRS-free herd, 10 animals from farm 1 and 15 animals from farm 2, respectively. Pigs from herd 1 were primo-vaccinated i.m. with MLV Porsilis ${ }^{\circledR}$ PRRS Intervet at 5 weeks of age, and a second vaccination with KV Progressis ${ }^{\circledR}$ Merial was carried out by the same route on 5-months old animals. In herd 2, animals were housed with viremic pigs until they were 5 months old. In farm 1, blood samples were collected twice: T0, 60 days after MLV vaccination and T1, 60 days after $\mathrm{KV}$ vaccination. In farm 2, blood samples were collected three times: T0 (3- month old animals), T1 (5-months old animals during contact with viremic pigs), T2 (9-months old animals). Moreover, these animals were checked for PRRSVspecific IgA Abs in saliva samples at T1 and T2. Laboratory analyses. Blood samples were collected in tubes with and without lithium-heparin, in order to obtain both serum (for anti-PRRSV IgG, RT-Real Time PCR, anti-SIV Ab) and plasma samples (for IFN- $\gamma$ assay). The IgG Abs ELISA was performed as recommended by the producer (Herdcheck ${ }^{\circledast}$ IDEXX Porcine Reproductive and Respiratory Syndrome Antibody Test Kits); moreover, sera were used in RT-Real Time PCR to detect PRRSV RNA (3) and inhibition of haemoagglutination $(\mathrm{IH})$ for influenza virus type $A / S W / H 1 N 1$, A/SW/H1N2 and A/SW/H3N2 (4). After incubation of whole blood with PRRSV and mock antigens, IFN- $\gamma$ was measured by sandwich ELISA using anti-swine IFN- $\gamma$ capture mAb P2F6 and biotinylated, anti-swine IFN- $\gamma$ mAb MP701B (Thermo Scientific, Rockford, IL). Swine IFN- $\gamma$ was revealed by HRP-conjugated streptavidin, and ortho-phenilenediamine as substrate of the color reaction. Samples were scored positive if the OD corresponding to PRRSV-stimulated whole blood was higher than that of mock-stimulated and unstimulated blood by $20 \mathrm{mOD}$ at least. PRRSV-specific IgA in saliva samples was investigated by an ELISA assay; samples were reacted with sucrose-purified type I PRRSV and mock antigen, respectively. Mucosal Abs were revealed by an anti-swine $\lg A$, HRP-conjugated antiserum and ortho-phenilenediamine. Samples were scored positive if the OD corresponding to PRRSV antigen was higher by $20 \mathrm{mOD}$ at least (background level shown in SPF pigs).

\section{Results}

Animals remained healthy until the end of the study. In farm 1 all pigs, but one, were PRRSV-negative in RT-Real Time PCR at T0 and T1, while they were IgG-positive at both T0 (except 1 animal) and T1; a PRRSV-specific IFN- $\gamma$ response was absent at T0. Instead, there were 4 specific and 2 non-specific responses at T1. These animals also showed a seroconversion to H2N1 SIV. In farm 2, RT-Real Time did not reveal any PRRSV-positive sera; IgG ELISA indicated instead a seroconversion at T1 (except 1 animal), and low Ab titres at T2, too. The IFN- $\gamma$ response was absent at T0; then, 3 PRRSV-specific and 3 non-specific reactions were scored at T1 and T2, respectively. The IgA ELISA assay revealed 10 and 3 positive reactors at $\mathrm{T} 1$ and $\mathrm{T} 2$, respectively, with an inverse frequency ratio to $\mathrm{lg}$ G reactors in serum.

\section{Discussion}

In both farms, the cell-mediated immune response occurred at low intensity as opposed to the $A b$ response. This finding confirms the results by other authors $(1,2)$, i.e. the discrepancy between humoral and CMI response to PRRSV following both vaccination and direct contact with viremic animals. Also, these preliminary data indicate a different time-course between $\lg \mathrm{A}$ and IgG Ab responses, as a possible strategy of PRRSV to escape immune pressure.

\section{References}

1) Charerntantanakul W. et al. 2006 Viral Immun.; 19, 646-661.

2) Mateu E., et al. 2008 Vet. J. 177 (3), 345-51.

3) Revilla F. S. 2005 J.Virol. Methods 126, 21-30.

4) Van Reeth K, et al. (2006) Viral Immunol. 19 (3), 373-82. 


\title{
P.185
}

\section{Serological screening in PRRSV infection}

\author{
Viorel Herman; Corina Pascu; Luminita Costinar; Bogdan Faur; loana Vaduva; Nicolae Catana \\ Faculty of Veterinary Medicine, Timisoara, Romania
}

\section{Introduction}

Porcine Reproductive and Respiratory Syndrome was first described in 1980 in North America, following (from 1990) in Europe. From here PRRS occurs worldwide in most major swineraising countries.

Both domestic and wild boars were the known susceptible species at PRRSV in natural condition.

There are kits to detect specific antibodies against PRRSV in serum samples. The most used kits are: indirect immunofluorescent antibody (IFA), ELISA and serum neutralization.

The formation and decline antibodies kinetic in IFA and ELISA are similar. The ELISA advantages are: rapid execution, low results variation comparing with results interprets by a technician.

\section{Materials and Methods}

For antibodies detection was samplings sows from breeding farm and from pigs from fattening unit that belongs to the anterior mentioned farm. From sows are sampling in the first period of gestation and from fattening pigs at 2, 3 and 5 months of age.

The trials were performed using PRRS IDEXX ELISA (HerdCheck IDEXX Laboratories Switzerland AG.), and xChek (Laboratories IDEXX) software.

\section{Results and discussions}

In the breeding farm were observed more reproductive disorders, and there were serological tested against PRRS.

\section{Figure 1}

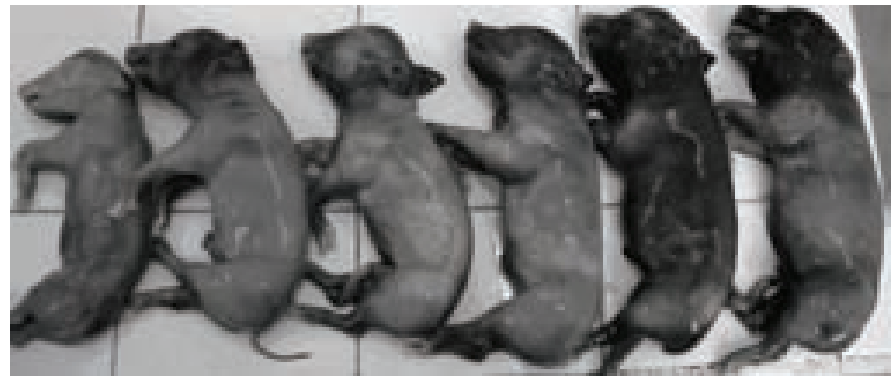

The obtained results are presents in table 1. As it could be observed, in breeding farm and in fattening farm both are present the PRRS infection.

Table 1

\begin{tabular}{|l|c|c|c|c|}
\hline & $\begin{array}{c}\text { Farm 1 - } \\
\text { breeding }\end{array}$ & $\begin{array}{c}\text { Farm 3 - } \\
\text { fattening }\end{array}$ & $\begin{array}{c}\text { Farm 3 - } \\
\text { fattening }\end{array}$ & $\begin{array}{c}\text { Farm 3 - } \\
\text { fattening }\end{array}$ \\
\hline & & 1st sampling & 2nd sampling & 3rd sampling \\
\hline Tolal & 25 & 15 & 20 & 12 \\
\hline Negative samples & 5 & 4 & 6 & 1 \\
\hline Positive samples & 20 & 11 & 14 & 11 \\
\hline Infection \% & 80 & 75 & 70 & 91 \\
\hline
\end{tabular}

In breeding farm, 80\% from samples were positive. In fattening farm, at first sampling 75\% samples were positive, at second sampling, 70\% samples were positive and at third sampling, just after slaughtered, $91 \%$ samples were positive. This proved the seroprevalence rising.

\section{Graphic 1. Dynamic of positive samples in fattening pigs}

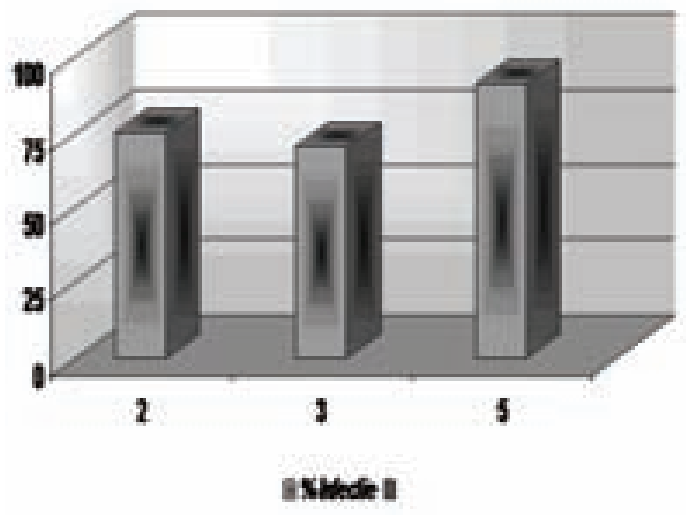

\section{Conclusions}

The breeding farm was positive in a significant proportion (80\%). Also, all three samplings from fattening farm revealed positive reactions, as it follows: $75 \%$ at 2 months old, $70 \%$ at 3 months old and $91 \%$ at 5 months old.

Early identification of PRRS infected herds is essential for epidemiological surveillance and disease control. The herds moving is necessary to be done only between herds with the same immune status, through this disease spreading is limited.

\section{References}

1. BENFIELD, D.A., NELSON, C., STEFFEN, M., ROWLAND, R.R.R. (2000) Proc Annu Meet Am Assoc Swine Pract, pp. 405-408.

2. CA'TANA“" N., POPA, VIRGILIA, HERMAN, V., FODOR, IONICA (2007) -, Lucr. S, t. Med. Vet. Timis, oara, vol. XL, 131-134.

3. MURTAUGH, M.P. (2004) - American Association Of Swine Veterinarians, 359-368.

4. ROTARU, ELENA (2005) - Sindromul tulbura rilor respiratorii $s_{s}$ i de reproduct, ie al porcinelor, În: Boli virotice $s_{s}$ i prionice ale animalelor, sub redact, ia Moga Mânzat R., Ed. Brumar, Timis, oara, 245-261.

5. OIE (2004) - Porcine reproductive and respiratory syndrome. In Manual of Diagnostic Tests and Vaccines for Terrestrial Animals, Part 2, Section 2.6., Chapter 2.6.5. https://www.oie. int/eng/normes/ MMANUAL/A_00099.htm.

6. http://www.idexx.com/view/xhtml/en_us/livestock-poultry/swine/ prrs.jsf (accesat 15.02.2010). 


\title{
P.186
}

\section{Risk factors for the porcine high fever disease in a region of Vietnam}

\author{
Hien T. Le ${ }^{1,2}$ Zvonimir Poljak $^{1}$ Rob Deardon ${ }^{3}$ Cate Dewey ${ }^{1}$ \\ 1. Population Medicine, Guelph, ON, Canada; 2. Nong Lam University, Ho Chi Minh, Vietnam; 3. \\ Department of Mathematics and Statistics, University of Guelph, Guelph, ON, Canada
}

\section{Introduction}

Porcine high fever disease (PHFD) emerged in 2007 as a large and rapidly evolving outbreak affecting most of China, and subsequently, Vietnam (1). The disease was characterized by fever, depression, anorexia, and respiratory distress; with high morbidity. One genotype of the porcine reproductive respiratory syndrome virus (PRRSV) was considered to be the etiological agent, possibly with involvement of other pathogens, because of the disease's unusual characteristics (1). However an interaction between PRRSV and other pathogens was not confirmed. It is important to understand the epidemiological characteristics of PHFD. This study's objective was to evaluate the distribution of, and risk factors for, the occurrence of PHFD in a region of Vietnam which underwent a recent outbreak.

\section{Materials and Methods}

Study area: The area of interest was a district of a province of southern Vietnam that had reported the outbreak of PHFD during 2008. A study area, approximately $10 \times 10 \mathrm{kms}$ in dimension, which contained 5 communes and a total of 37 hamlets, was selected.

Data collection: A survey was conducted in the study area to collect information about the swine health problems during 2008. A group of trained interviewers went to almost all households having pigs with local veterinarians to fill questionnaires, which included 3 sections: general information, clinical signs of disease in pigs, and production factors hypothesized to be risk factors.

Definition of case at household level: Based on clinical signs from the literature $(1,2)$, and opinions of experts and experienced veterinarians in the area, cases were defined as households that experience a pig that died or aborted accompanied by one of the suspected signs, including: high fever (41.5oC), blue ear, respiratory problems in sows, stillbirths, pre- and post weaning death up to $20 \%$, or with mortality over $10 \%$ accompanied by fever and respiratory symptoms in other pigs

Data analyses: Questionnaire data were entered into EpiData 3.1 (EpiData Association, Odense, Denmark). Logistic regression with a random intercept at the hamlet level was used to assess risk factors for PHFD at the household level using Stata 10 (Stata Corporation, Texas, USA).

\section{Results}

Of 955 households with questionnaire data, 33.4\% were classified as cases. Cases occurred over the entire year (2008) with peak frequency in June. In addition to clinical signs defined for cases, agalactia and lameness were observed in sows while trembling and excessive wasting were observed in suckling and nursery pigs. Diarrhea and redness of skin were observed in finishing pigs. The risk factors associated with the occurrence of cases were: higher number of sows and finishing pigs (log 2 transformed), receiving pigs from an external source, and the interaction between using "water green plants" (WGP) as pig feed and owning ducks with/without contact with pigs (duck ct/ duck nct) in the household (Table 1).

Table 1. Factors associated with being a case of PHFD at household level in final model

\begin{tabular}{|l|c|c|c|c|}
\hline Parameter & Coefficient & 95\% Cl (lower) & 95\% Cl (upper) & p value \\
\hline Fixed effect & & & & \\
\hline Intercept & -1.50 & -1.85 & -1.16 & $<0.001$ \\
\hline Log sow & 0.39 & 0.28 & 0.51 & $<0.001$ \\
\hline Log finishing pig & 0.05 & 0.01 & 0.09 & 0.047 \\
\hline WGP* Duck & & & & \\
\hline WGP & 0.04 & -0.34 & 0.43 & $<0.001$ \\
\hline Duck nct & -0.26 & -0.70 & 0.18 & \\
\hline Ducks ct & 0.18 & -0.49 & 0.86 & \\
\hline WGP-duck nct & 0.63 & 0.01 & 1.26 & \\
\hline WGP-duck ct & 1.04 & 0.04 & 2.03 & \\
\hline Receiving pigs & 0.53 & 0.15 & 0.91 & $<0.001$ \\
\hline Random effect & & & & \\
\hline Variance of intercept & 0.07 & 0.02 & 0.32 & 0.020 \\
\hline
\end{tabular}

\section{Discussion}

Number of sows and finishing pigs were used in the model to control for confounders. The final model fitted suggested that the movement of pigs (i.e. receiving nursery pigs form another farm) might be a risk factor with $\mathrm{OR}=1.70$ (1.16-2.48). This factor might have contributed to the observed pattern of disease transmission over the area. The interaction between duck and feeding WGP suggested that potential pathogens originating from a water source, and which further multiplies in ducks, could contribute to occurrence of PHFD.

\section{References}

1. Feng et al., (2008), Emerg. Infect. Dis 14:1774-1776

2. Zimmerman J., et al., (2006), Disease of Swine: $387-418$ 


\title{
P.187
}

\section{Risk assessment of PRRS outbreak in endemic farms according to productivity and health management variables}

\author{
Antonio Callen $^{2}$ I. De Blas ${ }^{3}$ J. Q. Cabanes ${ }^{4}$ J. A. Munoz François Joisel ${ }^{1}$ \\ 1. Merial S.A.S., Lyon, France; 2. Merial Laboratorios S.A., Barcelona, Spain; 3. Veterinary Faculty, \\ Universidad de Zaragoza, Zaragoza, Spain; 4. Agrocesa, Valladolid, Spain
}

\section{Introduction}

Porcine reproductive and respiratory syndrome (PRRS) is a devastating disease in intensive pig production and is an endemic disease in most farms in the world, but triggering factors for outbreaks should be studied for each scenario.

The objective of this study was to assess the factors linked with PRRS outbreak presentation in field conditions.

\section{Material and Methods}

Forty-three Spanish farms, belonging to the same company and with a census between 100 and 5,250 sows and gilts (mean \pm SD: $643 \pm 1,012$ ) were surveyed during a minimum period of 3 years (and a maximum of 10 years) (total of 397 farm-years) in a prospective observational study.

Twelve variables related to farm facilities, stocking management, health status and strategies were studied.

Four different strategies for PRRS control were used in these farms: S1: PRRS-Positive Gilts, Live Vaccine and No Infection with Piglets; S2: PRRS-Positive Gilts, Live Vaccine and Infection with Piglets; S3: PRRS-Positive Gilts, Killed Vaccine and Infection with Piglets; and S4: PRRS-Negative Gilts, Killed Vaccine and Infection with Piglets. Live vaccine was AMERVAC ${ }^{\circledR}$-PRRS (HIPRA) and killed vaccine was PROGRESSIS ${ }^{\circledR}$ (MERIAL). They were applied according to producer specifications. A commercial indirectELISA test was used to confirm PRRS diagnosis (Ingezim PRRS K1, Ingenasa).

In this study, a PRRS outbreak was defined as the sudden occurrence of reproductive problems associated to an abrupt increase of seroprevalence to this disease. Comparison of outbreak frequencies was performed with Likelihood Ratio test (for more than 2 frequencies) or Fisher's exact test (for 2 frequencies). Relative Risk and 95\% confidence interval were calculated for significant associated variables. Error a was established at 0.05 . All statistical analyses were carried out with SPSS $^{\circledR} 15.0$ for WINDOWS ${ }^{\circledR}$.

\section{Results and Discussion}

During the study period only 7 PRRS acute outbreak were recorded. The only variable significantly associated with outbreak risk was the stock variation (see Table 1), so an increase of census led to a higher probability of PRRS outbreak occurrence (40\%) versus a probability of $1.3 \%$ when no changes in census; that corresponded to a significant Relative Risk of 31.2 (95\%Cl: 7.8 - 124.3). It means, that there is 312 times more risk for PRRS outbreak to occur in farms that undergo a relevant increase of population size, than in farms with constant census.

Other variables had not been associated with PRRS outbreak (Table 1). In this study, we didn't find any significant impact of the type of vaccine used (live or killed) on the outbreak risk.
However several variables could be related to outbreak risk, but the very low number of outbreaks did not allow us to get significant results (i.e. existence of infected piglets in post-weaning, use of outsource gilts and presence of in-farm Al station).

Table 1: Relationship between productivity \& health management variables and PRRS acute outbreak

\begin{tabular}{|c|c|c|c|c|}
\hline Variable & Category & $\mathbf{N}$ & Outbreak & $\mathbf{p}$ \\
\hline \multirow[t]{2}{*}{ Fattening unit } & Absence & 328 & $1.8 \%$ & $>0.999^{2}$ \\
\hline & Presence & 69 & $1.4 \%$ & \\
\hline \multirow[t]{2}{*}{ Post-weaning unit } & Absence & 10 & $0.0 \%$ & $>0.999^{2}$ \\
\hline & Presence & 387 & $1.8 \%$ & \\
\hline \multirow[t]{2}{*}{ In-farm Al station } & Absence & 377 & $1.9 \%$ & $>0.999^{2}$ \\
\hline & Presence & 20 & $0.0 \%$ & \\
\hline \multirow[t]{2}{*}{ In-farm gilts rearing } & No & 358 & $1.7 \%$ & $0.518^{2}$ \\
\hline & Yes & 39 & $2.6 \%$ & \\
\hline \multirow[t]{3}{*}{ Stock variation } & Decrease & 2 & $0.0 \%$ & $<0.001^{1}$ \\
\hline & No changes & 390 & $1.3 \%$ & \\
\hline & Increase & 5 & $40.0 \%$ & \\
\hline \multirow[t]{2}{*}{ Gilt source } & Out & 363 & $1.9 \%$ & $>0.999^{2}$ \\
\hline & $\ln$ & 34 & $0.0 \%$ & \\
\hline \multirow[t]{2}{*}{ PRRS status of gilts } & Negative & 178 & $2.2 \%$ & $0.705^{2}$ \\
\hline & Positive & 219 & $1.4 \%$ & \\
\hline \multirow[t]{2}{*}{ Gilt infection with piglets } & No & 69 & $1.4 \%$ & $>0.999^{2}$ \\
\hline & Yes & 328 & $1.8 \%$ & \\
\hline \multirow[t]{2}{*}{ Type of vaccine for gilts } & Live & 164 & $1.2 \%$ & $0.705^{2}$ \\
\hline & Killed & 233 & $2.1 \%$ & \\
\hline \multirow[t]{2}{*}{ Killed vaccine use } & Only gilts & 357 & $1.4 \%$ & $0.150^{2}$ \\
\hline & All breeders & 40 & $5.0 \%$ & \\
\hline \multirow[t]{2}{*}{$\begin{array}{l}\text { Infected piglets in post- } \\
\text { weaning }\end{array}$} & No & 26 & $0.0 \%$ & $0.289^{2}$ \\
\hline & Yes & 46 & $8.7 \%$ & \\
\hline \multirow[t]{4}{*}{ Health strategy for gilts } & S1 & 67 & $1.5 \%$ & $0.896^{1}$ \\
\hline & S2 & 96 & $1.0 \%$ & \\
\hline & S3 & 54 & $1.9 \%$ & \\
\hline & S4 & 177 & $2.3 \%$ & \\
\hline \multicolumn{2}{|l|}{ Total } & 397 & $1.8 \%$ & \\
\hline
\end{tabular}

\section{Conclusion}

Increase of the population size is a critical risk factor for acute outbreak in farms with endemic PRRS independently of implemented health strategies.

${ }^{\oplus}$ PROGRESSIS is a registered trademark of Merial in France and elsewhere. 


\title{
P.188
}

\section{Correlation between genetic, temporal and geographic distances of porcine reproductive and respiratory syndrome virus (PRRSv) strains isolated in swine herds from a high density area in Quebec, Canada}

\author{
Marie-Ève Lambert ${ }^{1}$ Zvonimir Poljak $^{2}$ Julie Arsenault ${ }^{1}$ Sylvie D'Allaire ${ }^{2}$ \\ 1. University of Montreal, Faculty of Veterinary Medicine, St. Hyacinthe, QC, Canada; \\ 2. University of Guelph, Ontario Veterinary College, Guelph, ON, Canada
}

\section{Introduction}

The transmission of PRRSv between herds can occur over long distance by the introduction of infected animal or semen (1), but also over short distances by mechanisms such as aerosols or various mechanical/biological vectors (2). Short distance processes of transmission could be largely enhanced in high density production area, complicating PRRS control at a herd level. PRRSv strains sharing a high level of homology are suggestive of a common source of infection, and thus can be used to explore the temporal and geographical spread of the virus. The objective of our study was to determine the correlation between genetic, temporal and geographical distances of PRRSv strains in a high density area of swine production.

\section{Materials and Method}

As a part of a larger study on the transmission and control of PRRS, a census of production sites was conducted in 10 municipalities located in a high density area (Monteregie) between February 2005 and June 2007. PRRS herd status was determined by ELISA and qRT-PCR and when PCR positive, sequencing of ORF5 was performed. For each production site, geographical coordinates were obtained using GPS at the closest building from public road. A pairwise alignment of all ORF5 sequences obtained was generated in Bionumerics software and genetic distances were calculated from nucleotides. Euclidian and temporal distances were calculated in SAS (SAS Institute 1989). Mantel test procedure was computed in R software to examine the correlation among genetic, geographical and temporal distances. P-values were computed based on 999 permutations.

\section{Results}

PRRS herd status was determined on 191 of the 200 participating sites, of which 176 were positive for PRRSv. One sequence was identified for 132 production sites, which were attended by 25 different veterinarians. Independent and integrated producers (7 production systems) accounted for $61 \%$ and $39 \%$ of the total. Sequences were identified on farrowing /farrow-tofinish and on weaner/finisher sites in a proportion of $58 \%$ and $42 \%$, respectively. Table 1 shows descriptive results of pairwise distances included in the distance matrices used to compute the Mantel test. The bivariate Mantel test revealed a significant association between genetic and temporal distances $\left(r_{M}=0.06\right.$ $p=0.04$ ). No significant relationship was found between geographical distance and either genetic $(p=0.68)$ or temporal $(p=0.60)$ distances.
Table 1: Descriptive results of pairwise distances between production sites included in the continuous distance matrices $(n=8646)$

\begin{tabular}{|l|c|c|c|}
\hline Distance variable & mean & sd & min - max \\
\hline Euclidean (m) & 14957 & 8458 & $43-45726$ \\
\hline Sampling time (day) & 214 & 159 & $0-852$ \\
\hline Genetic (\%) & 12 & 3 & $0-19$ \\
\hline
\end{tabular}

\section{Discussion}

The correlation observed between genetic and temporal distances revealed that sequences isolated far away in time showed more genetic diversity, suggesting possible genetic evolution of the virus during the study period. The absence of correlation between genetic and geographic distances as previously reported (3) suggests that many processes could be involved in the between-herd transmission of the virus at both short and long distances. Further analyses will be performed to take into account other factors such as corporate organization membership.

\section{References}

1. Mousing et al. 1997. Vet Microbiol 55:323-328.

2. Otake et al. 2002. J Swine Health Prod 10: 59-65.

3. Goldberg et al. 2000. J Gen Virol 81: 171-179. 


\title{
P.189
}

\section{Risk factors for introduction and spread of PRRSV and PCV2 in pig farms in Korea}

\author{
Oun Kyong Moon \\ National Veterinary Research \& Quarantine Service, Anyang, Korea
}

\section{Introduction}

Although Porcine Reproductive and Respiratory Syndrome (PRRS) and Porcine Circovirus Disease (PCVAD) induce severe economical losses in domestic pig farms, most research are concentrated on the diagnosis of the causative agent and there is a need for more studies on epidemiological analysis of risk factors related to the transmission of these diseases. In this study, we conducted a survey to identify risks factors for PRRS and PCV2 infection in pig farms in Korea.

\section{Materials and Methods}

Survey was conducted from July 2008 to December 2009 through on-farm visits and use of questionnaires which were designed based on risk factors identified during previous epidemiological investigations conducted for Foot-and-Mouth Disease and Classical Swine Fever. Also, swab samples were collected from various key areas and equipments in the participating farms and tested to identify the degree of PRRS and PCV2 contamination during each season in 2009. Samples were tested using RT-PCR and nested PCR.

\section{Results}

A total of 58 pig farms in 9 provinces were surveyed. In our survey, the percentage of farms that disinfected the farm entrance, pig house entrance, inside of the pig house and farm surroundings were $36 \%, 26 \%, 22 \%$ and $16 \%$, respectively. For tools, equipment and feces removal vehicles, disinfection was conducted in $93 \%, 93 \%$ and $69 \%$ of the farms, respectively when entering the farms. In contrast, disinfection was conducted in only $42 \%, 38 \%$ and $42 \%$ of the farms, respectively when leaving the farms. For refrigerators used to store veterinary drugs, $29 \%$ of the farms responded that they conducted disinfection.

Samples were collected from pig farms during spring, summer, autumn and winter, which were totals of $6,14,24$ and 21 farms, respectively. The percentage of farms where PRRSV and PCV2 were detected were $83.33 \%$ and $10.00 \%$, respectively during spring, $3.35 \%$ and $16.32 \%$, respectively during summer, $19.62 \%$ and $38.06 \%$, respectively during autumn, and $39.94 \%$ and $54.45 \%$, respectively during winter. In particular, investigation of refrigerators in 6 pig farms during each season showed PRRSV and PCV2 contamination in 100\% and 33.3\% of the farms, respectively during spring, $0 \%$ and $16.7 \%$, respectively during summer, $0 \%$ and $66.7 \%$, respectively during autumn, and $0 \%$ and $80.0 \%$, respectively during winter. Viruses were consistently detected from shoes, vehicles or various tools and equipment such as wheel barrows and shovels used in the farms, although there were slight variations depending on the season. Also, there was persistent detection of viruses from the floor of pig houses.

\section{Discussion}

This study demonstrated that pathogens can be transmitted during the movement of people or equipment associated or used in pig farms, and the rates of detection of these two viruses were different according to the season. Refrigerators could become an important source of diseases as viruses in refrigerators would be less influenced by the difference in the season. On a positive note, the rates of detection of viruses were lower on the secondary tests after the farm had been educated, than on the primary investigation. The method used in this study including sample collection and epidemiological investigation can be applied to the standard surveillance for porcine diseases conducted in Korea. Widespread use of this method in the porcine industry would result in a more scientifically sound surveillance allowing for more effective disease control measures to be implemented.

\section{References}

1. Pinto $\mathrm{CJ}$ et al. Biosecurity practices on intensive pig production system in Chile. Prev Vet Med 2003, 59, 139-145.

2. Horst $\mathrm{HS}$ et al. Risks and economic consequences of introducing classical swine fever into The Netherlands by feeding swill to swine. Rev Sci Tech 1997 Apr;16(1): 207-14. 


\title{
P.190
}

\section{Spatiotemporal distribution of the dominant PRRSV genotype in Ontario}

\author{
Thomas Rosendal; Cate E. Dewey; Robert M. Friendship; Sarah K. Wootton; Young Beth; Zvonimir Poljak \\ University of Guelph, Guelph, ON, Canada
}

\begin{abstract}
Introduction
Modeling the spatiotemporal distribution of porcine reproductive and respiratory syndrome virus (PRRSV) is important in the pursuit to understand how PRRS spreads in a population of pig herds. The distribution in space and time of PRRSV describes part of a set of connections between herds. The objectives of this study are to search for trends and clusters in space and time of a common genotype of PRRSV in Ontario and to assess the importance of ownership structure in of PRRSV genotypes in Ontario.
\end{abstract}

\section{Methods}

Herds were eligible for inclusion in the study if a PRRSV positive diagnostic sample was identified at the Animal health Laboratory at the University of Guelph between Sept 2004 and Aug 2007. Herd managers who were willing to participate were interviewed to determine herd ownership. The ORF5 gene of the PRRSV positive sample was sequenced and the RFLP type according the University of Minnesota method was determined. Spatial and temporal autocorrelation and clustering of the most common type, Minnesota RFLP type 1-18-4, were investigated using the space-time k-function and the SaTScan Bernoulli model. The distribution in space and time was modeled using the generalized additive model (GAM). The effect of ownership on the space-time distribution was tested using fixed effects in the GAM for the owners with the largest number of members.

\section{Results}

The k-function showed that autocorrelation was present in PRRSV type 1-18-4 up to a distance of $6 \mathrm{~km}$ and 45 days as well as a distance of $12 \mathrm{~km}$ and 15 days. A cluster of cases was found in the eastern part of the study period lasting 4 months from Nov/2005 - Feb/2006 ( $P=0.046)$. A trend in the spatial distribution of type 1-18-4 was found using the GAM $(P=0.001)$ and no significant temporal trend was found $(P=0.09)$. An association was found between ownership of herds and the presence of type 1_18_4 ( $\mathrm{P}=0.005)$. When ownership was added to the space-time model the shape of the spatial trend did not change. Two owners had $\mathrm{OR}=7.3(\mathrm{P}=0.004)$ and $\mathrm{OR}=6.7(\mathrm{P}=0.02)$ of having type 1-18-4, where small owners, those with less than 6 herds in the study, were at reduced odds, compared to large ownerships, of having type 1_18_4 (OR=0.12, $\mathrm{P}<0.001)$.

\section{Discussion}

The autocorrelation in cases of 1-18-4 indicates that herds up to $6 \mathrm{~km}$ from a case herd are more likely to be cases if tested within 45 days of each other but also out to as far as $12 \mathrm{~km}$ if they are tested within 15 days of each other. Type 1-18-4 was also not uniformly distributed in Ontario during the study period (Fig 1a). Although, some of the variation can be explained by the ownership structure, a spatial trend remains after accounting for ownership (Fig 1b). The shape of the spatial trend did not change after accounting for ownership; this is an indication that the spatial effect and the effect of ownership are not confounded, meaning that the spatial effect is not explained by ownership. Further investigation is needed to shed light on the origin of this trend. The spatial pattern could be a result of similarities of breeding stock, animal or semen suppliers between herds or be an indication of transmission between herds. The variables describing herd suppliers could not be adequately accounted for using these methods and future work will address these connections.

Figure 1: GAM of the spatial distribution of RFLP type 1-18-4. The ellipse indicates the location of the significant cluster of cases. The top figure (a) is the smoothed distribution of type 1-18-4 without accounting for ownership. The bottom figure $(b)$ is the predicted distribution of RFLP type 1-18-4 for the owner with $O R=7.3$
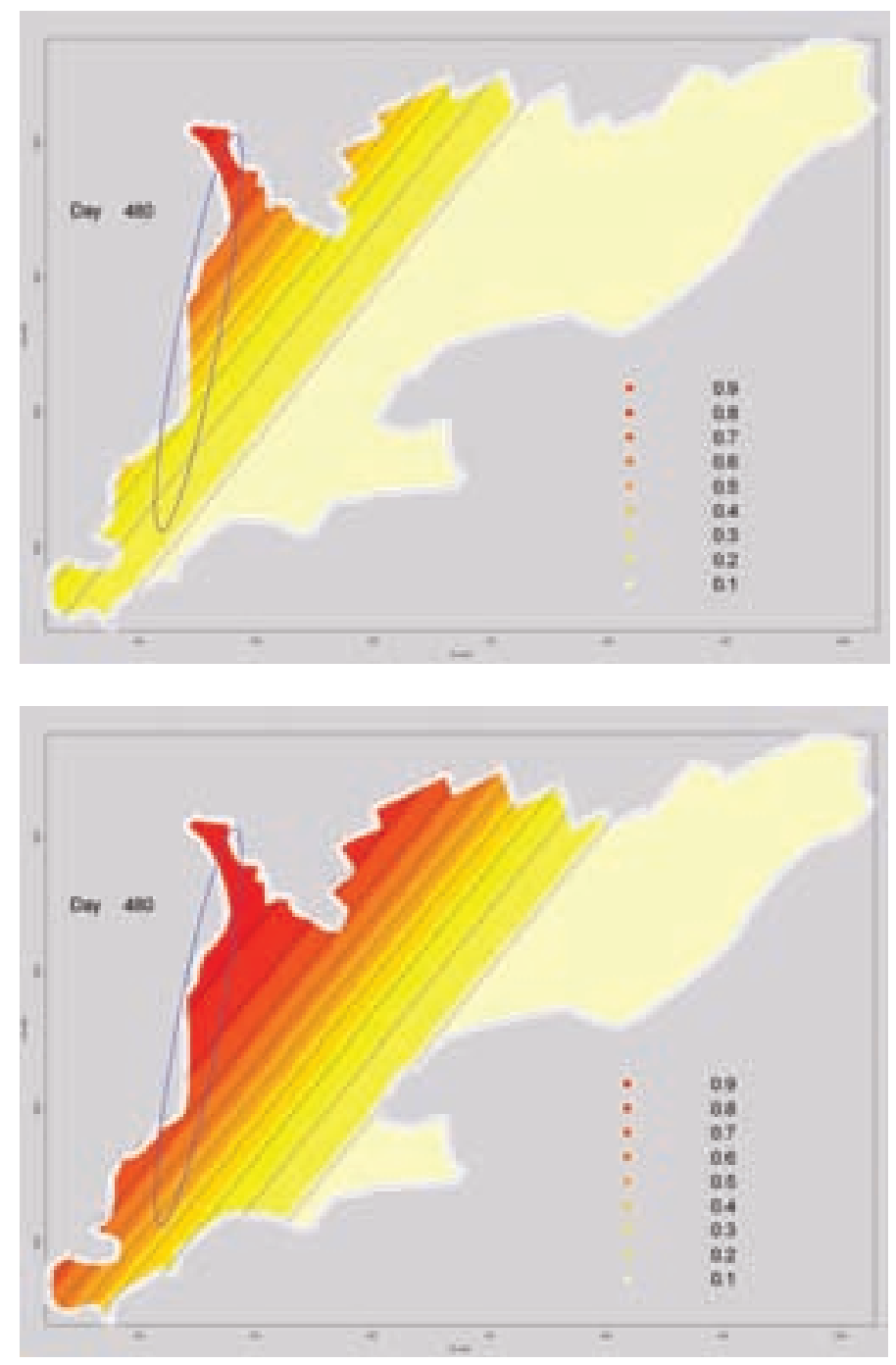


\title{
P.191
}

\section{Association between genetic similarity of the PRRSV ORF5 sequence and the similarity in clinical signs of PRRS in Ontario swine herds}

\author{
Thomas Rosendal; Cate E. Dewey; Robert M. Friendship; Sarah K. Wootton; Young Beth; Zvonimir Poljak
}

University of Guelph, Guelph, ON, Canada

\section{Introduction}

Porcine reproductive and respiratory syndrome (PRRS) is a clinically variable disease. Clinical signs that are recognized by the producer include abortion and anorexia in sows, stillborn and mummified piglets, dyspnea, respiratory disease and increased mortality in all ages of pigs (1). Isolates of PRRSV are genetically variable in the ORF5 gene. The association between clinical disease and the ORF5 gene have been infrequently investigated under field conditions (2). The objectives of the present study were to measure the association between RFLP type and clinical signs of PRRS in the herd and to assess the association between clinical similarity between herds and the similarity of PRRSV isolates from the herds.

\section{Materials and Methods}

Herds were eligible for the study if a PRRSV PCR test result from the Animal Health Laboratory at the University of Guelph was found between Sept 2004 and Aug 2007. Herd managers were surveyed about clinical signs of PRRS in the herd and ORF5 of the PRRSV isolate was sequenced. The RFLP type of the virus was determined and the genetic similarly of all virus pairs was calculated. The similarity of each clinical sign was determined to be either similar or dissimilar for all pairs of herds.

The association between RFLP type and clinical disease was tested by regressing each clinical sign on the RFLP types using logistic regression ( $P<0.05$ was significant). The association between sequence similarity and clinical similarity was tested by the Mantel test and the generalized additive model where the outcome was similarity of a given clinical sign and the independent variable was the similarity of the ORF5 gene.

\section{Results}

RFLP type 1-0-4 was associated with increased probability of abortion, sows off-feed, stillbirths, weak-born pigs, sow/boar mortality, and preweaning mortality. RFLP type 1-0-2, was associated with clinical disease in the farrowing phase of production and type 184 was significantly associated with increased abortions.

The GAM models indicated a significant association between PRRSV sequence similarity and similarity of both abortions and stillbirths and that the chance of similarity of these clinical signs was significantly increased when sequences were $>92 \%$ similar (Fig 1). Including vaccine like viruses in the analyses caused similarly model to decline at the upper end of homology.
Figure 1: GAM models for the association between virus similarity abortion similarity and stillbirth similarity.

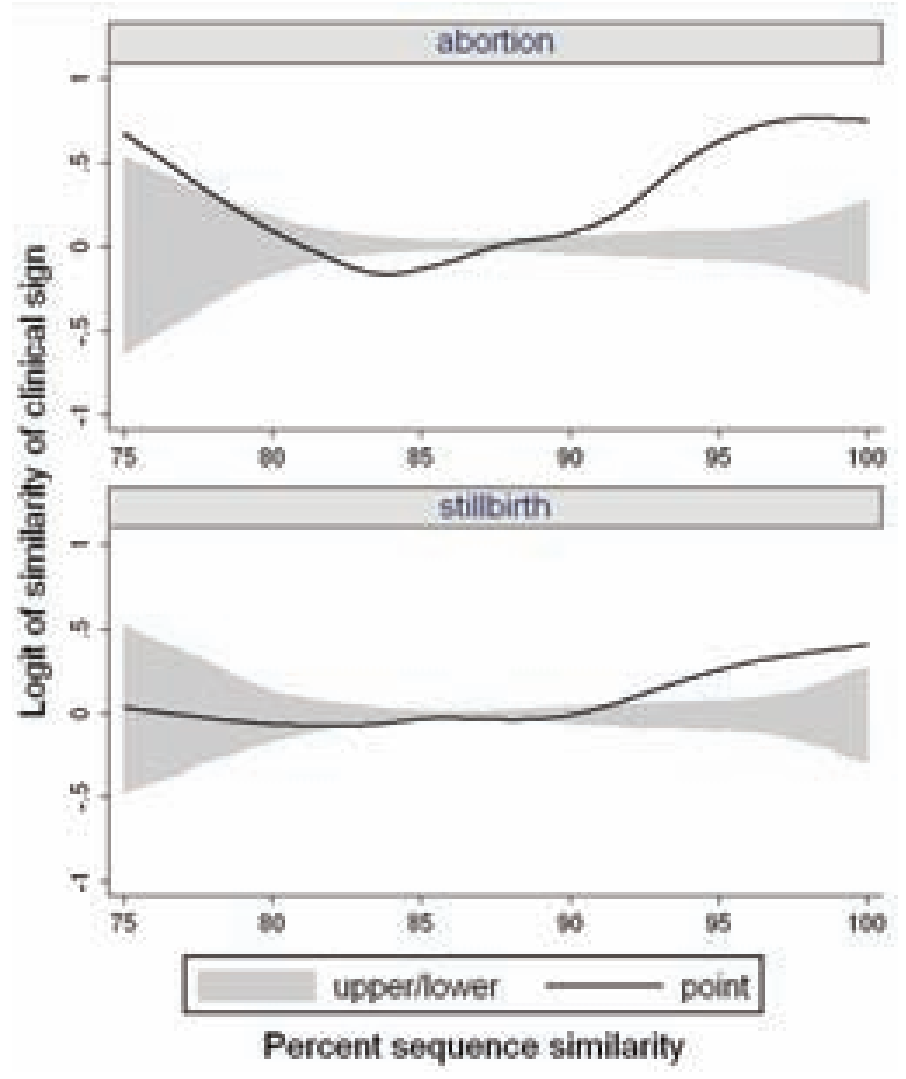

\section{Discussion}

These results indicate that variation in clinical signs of PRRS is caused in part by variation in the PRRSV. For abortions and stillbirths, PRRSV isolates of $>92 \%$ homology of the ORF5 gene are associated with similar clinical signs. This is in contrast to the benchmark where viruses of $<98 \%$ ORF 5 sequence similarity are considered different (3). Presence of vaccine type virus in a population can have an impact on the interpretation of PRRSV epidemiology. Removal of vaccine type observations before analysis may be necessary to interpret studies such as this because vaccine isolates are genetically similar but are not expected to be associated with similar clinical signs of disease.

\section{References}

1. Zimmerman J, et al. Diseases of Swine. 2006;387-417.

2. Goldberg TL, et al. Prev Vet Med 2000;43:293-302.

3. Larochelle R, et al. Virus Res 2003;96:3-14. 


\title{
P.192
}

\section{Clinical and virological characterization of European-type PRRSV isolates of high and low virulence}

\author{
Francisco Javier Martínez-Lobo; Francisco Díez-Fuertes; Carlo García-Artiga; Isabel Simarro; José María Castro; Cinta Prieto \\ Facultad de Veterinaria Universidad Complutense de Madrid, Madrid, Spain
}

\section{Introduction}

Porcine Reproductive and Respiratory Syndrome (PRRS) is a swine disease produced by PRRS virus (PRRSV), which is antigenically and genetically heterogeneous (1). PRRSV variability extends also to pathogenicity and highly and moderately virulent isolates have been described within the American genotype (2). The infection by highly virulent isolates produces severe clinical signs, significant gross lung lesions and high levels of viremia $(2,3)$. In a previous study, we characterized the pathogenicity of seven European-type isolates including one isolate which virulence was comparable to that described for American type isolates (4). The aim of this study was to compare the pathogenic properties of this isolate with that exhibited by a European type isolate of remarkable low virulence.

\section{Materials and Methods}

Two European-type isolates were used in this study. Additionally a modified live vaccine strain, Ingelvac PRRS, was used as an attenuated control. European isolates were propagated in porcine alveolar macrophages (PAM) and the fifth passage was used for pig inoculation. Vaccine strain was used directly from the bottle. For pig inoculation, a total of 45 3-week old piglets seronegative to PRRSV were divided into 3 groups. On Day 0 of the experiment piglets of groups $A, B$ and $C$ were inoculated intranasally (IN) with 5 x 105 TCID50 of highly virulent isolate, low virulent isolate and vaccine strain, respectively. All pigs were examined daily for clinical signs and rectal temperatures were recorded until the end of the experiment. Biological samples, including serum, nasal and rectal swabs were collected every three days. Five piglets per group were sacrificed on days 7, 14 and 21 postinfection (p.i.) and different tissue samples were collected. At necropsy macroscopic lung lesions were evaluated. Determination of viremia and organic distribution were carried out by virus isolation as previously described (5) on cultures of PAM in the case of field isolates and MARC 145 in the case of vaccine strain. Clinical and viremic results for each pig were converted to an approximate area under the curve (AUC) and a complete statistical study was carried out to estimate differences between groups. Finally, a cluster analysis of clinical and virological parameters was performed.

\section{Results}

After inoculation, severe clinical signs were observed in pigs ex- posed to highly virulent isolate, leading to a AUC values of 5.13, 8.0 and 0.4 on the first, second and third week p.i. On the contrary, pigs infected with low virulent isolate and vaccine strain remained clinically normal throughout the experiment, reflected in AUC values that never exceeded 0.5. Seemingly, fever was only recorded in pigs exposed to highly virulent isolate, namely from day 2 p.i. to day 10 p.i. Consistently with the clinical score macroscopic lung lesions were more severe in pigs inoculated with the highly virulent isolate.

Looking at the virological parameters, viremia was more intense with statistically significant differences in those pigs inoculate with highly virulent isolate. Additionally, highly virulent virus was isolated more frequently from tissue samples than low virulent or vaccine strain, being viral load also higher in the former group. Finally, remarkably low virulent isolate was never detected in any swab, indicating a very low shedding rate.

The apathogenic phenotype of the low virulent isolate was confirmed by the grouping of pigs of this group with those infected with the vaccine strain in the cluster analysis.

\section{Discussion}

This is the first description of the existence of highly pathogenic and apathogenic PRRSV isolates within the European type. The ultimate causes of the differences in pathogenicity between these two isolates were not elucidated in our study. However, there are strong indications that the replication rate of the isolates and the organic distribution in the pigs upon inoculation can determine their virulence.

\section{Acknowledgements}

This study was supported by grant CSD-2006-00007 (Consolider Ingenio 2010).

\section{References}

1. Meng, XJ. 2000. Vet Microbiol, 74:309-329

2. Halbur, PG. et al. 1996. J vet Diagn Invest, 8:11-20

3. Jhonson, W. et al. 2004. Vet Imm Immunopath 102: 233-247

4. Martínez-Lobo et al. Proceedings IPVS 2008.

5. Scortti, M. et al. 2005. Vet J, 172:506-514 


\title{
P.193
}

\section{Genetic variation and pathogenicity of the recently isolated PRRSV in Korea}

\author{
Hyun Jang; Ji-Youl Jung; Sung-Su Park; Seong-cheol Moon \\ Komipharm International Co. LTD, Gyeonggi-do, Korea
}

\section{Introduction}

The porcine reproductive and respiratory syndrome virus (PRRSV) is the causative agent of porcine disease, which has respiratory failure in neonate and abortion in sow. The PRRS is one of the most serious endemic diseases to swine producing countries and heavy economic burden due to the high mortality rate in infected pig. PRRSV is a positive sense single stranded RNA virus of the family Arteriviridae. The genome of PRRSV consist nine ORFs. Among nine ORFs, ORF5 and Nsp2 gene show the highest genetic variations. Especially ORF5 gene encodes GP5 major surface protein, which is major inducer of neutralizing antibodies in vivo. GP5 protein is presumed to interact with sialoadhesin. Recently isolated PRRSV show different glycosylation pattern in the GP5 protein and is also related with pathogenicity of PRRSV. The aims of this study are analyze genetic variation in the ORF5 gene and its relation to pathogenicity of recently isolated PRRSV in Korea

\section{Materials and Methods}

PRRSV isolated lung tissue samples from pig, which was show typical clinical signs of PRRSV infection in the field. Homogenate lung tissue clarified by centrifugation and supernatant filtered using $0.22 \mu \mathrm{m}$ membrane filter and then inoculate into monolayer forming Marc145 cell. 3 to 5 days after inoculation, virus harvested and quantified virus concentration. GP5 protein gene amplified using PCR method and sequenced and then analyzed and compared with previous reported GP5 gene sequence. PRRS negative pigs were inoculated intratrchealy with isolated PRRSV (Komi-NA263, Komi-NA316 each virus titer adjusted 105.0 $\mathrm{TCID} 50 / \mathrm{ml}$ ) for pathogenicity test. After challenge clinical signs, body temperature and virus shedding observed every day and autopsy was performed for examination of pathological lesions.

\section{Results}

Two field isolated PRRSV Komi-NA263 and Komi-NA316 ORF5 gene sequence show $94 \%$ and $87 \%$ respectively. But both strains was show identical pathogenicity, such as high fever, virus shedding through nasal and respiratory distress. Grossly there was pulmonary edema and multifocal red spots in the lung. Enlargement of spleen and lymph nodes were also observed. Viral antigens in the lung also detected by immunohistochemical staining. GP5 amino acid sequence at 34 and 35 of the both strains was asparagine and serine respectively. This positions were major glycosylation site at ectodomain of the GP5 protein.

\section{Discussion}

The major envelope protein GP5 is the most variable structural protein in PRRSV. In this study virulence of the PRRSV was related to specific amino acid sequence change by the accumulation of mutations rather than overall genetic variations

\section{Reference}

1. Tjeerd G. Kimman*, Lisette A. Cornelissen, Rob J. Moormann, Johanna M.J. Rebel,Norbert Stockhofe-Zurwieden. 2009. Challenges for porcine reproductive and respiratory syndrome virus (PRRSV) vaccinology. Vaccine. 27, 3704-3718. 


\title{
P.194
}

\section{PRRS strain diversity in a European pig production area 14 years after the primary infection}

\author{
Benoît Gouvars ${ }^{1}$ Vincent Auvigne $^{2}$ Tomasz Stadejek $^{3}$ Eric Sellal ${ }^{4}$ \\ 1. UGPVB, Rennes, France; 2. EKIPAJ, Pozuelo de Alarcon (Madrid), Spain; 3. NVRI, Pulawy, Poland; 4. LSI, Lissieu, France
}

\section{Introduction}

The porcine reproductive and respiratory syndrome (PRRS) virus is widely distributed over the various pig production areas worldwide.

The diversity of the American PRRS strains has been well documented. That of the European strains has been described only recently $(1,2)$. The aim of this study was to describe the diversity of the PRRS strains present in a European pig production area that was contaminated in 1991 and has around 7000 pig farms.

\section{Materials and Methods}

The study carried out used the blood samples of piglets $(9,12$ and 15 weeks of age) of breeder-fattener farms, taken over a period of 4 months (December 2004 to March 2005). The farms were known to be infected and had or had not observed signs that could be attributed to PRRS (in breeding animals and growing pigs). After screening the serum samples using a PCR kit allowing the detection of European and US strains of the PRRS Virus (Adiavet ${ }^{\circledR}$ PRRS), amplification and sequencing of ORF7 and ORF5 genes were carried out for each farm (one sample per farm). Sequence alignment and analysis was carried out using CLUSTALW (1.82), Lasergene (DNAStar) and PHYLIP 3.67 program packages. For the comparative analysis, selected sequences representing the full range of European genotype diversity were used.

\section{Results}

In total 51 ORF7 and 46 ORF5 were obtained. All sequences obtained were of the European genotype. The identity with the Lelystad strain was 91 to $100 \%$ for ORF7 and 82 to $98 \%$ for ORF5. These sequences were also highly similar to the FR/IV4A strain, isolated 14 years earlier at the time of the introduction of PRRSV in the region (Fig. 1).

No clusters were found in association with the clinical signs observed or with the geographical proximity of the farms.

\section{Discussion}

The results of this study underline the very high genetic identity, both between the isolated strains and compared to the Lelystad strain. This finding is rather unique considering the genetic diversity in many European countries $(3,4)$, where the heterogeneity of the strains is much higher. In the region studied, the initial outbreak occurred in 1991 with a strain closely related to the Lelystad strain (5). It can be concluded that the great majority of the farms in this region were infected at the time of the original outbreak or later but with the strains originating from the original introduction. Only in 4 farms the strains were found to be unrelated. Since 1991, no new strains have been introduced and the original strains seem to have undergone a 'natural drift'.

\section{References}

1. Stadejek, T .et al.(2002). J.Gen.Virol. 83, 186-1873

2. Mateu, E. et al.(2003). J.Gen.Virol. 84, 529-534

3. Stadejek, T. et al. (2006). J. Gen. Virol. 87, 1835-1841

4. Cay, AB.et al.(2006). Proc.19th IPVS Vol.2, 9

5. Albina E. et al. (1998). Vet. Res. 54, 1.

\section{Acknowledgements}

Intervet and Merial are acknowledged for their financial support.

Figure 1. Phylogenetic tree based on the ORF5 sequence of the strains studied.

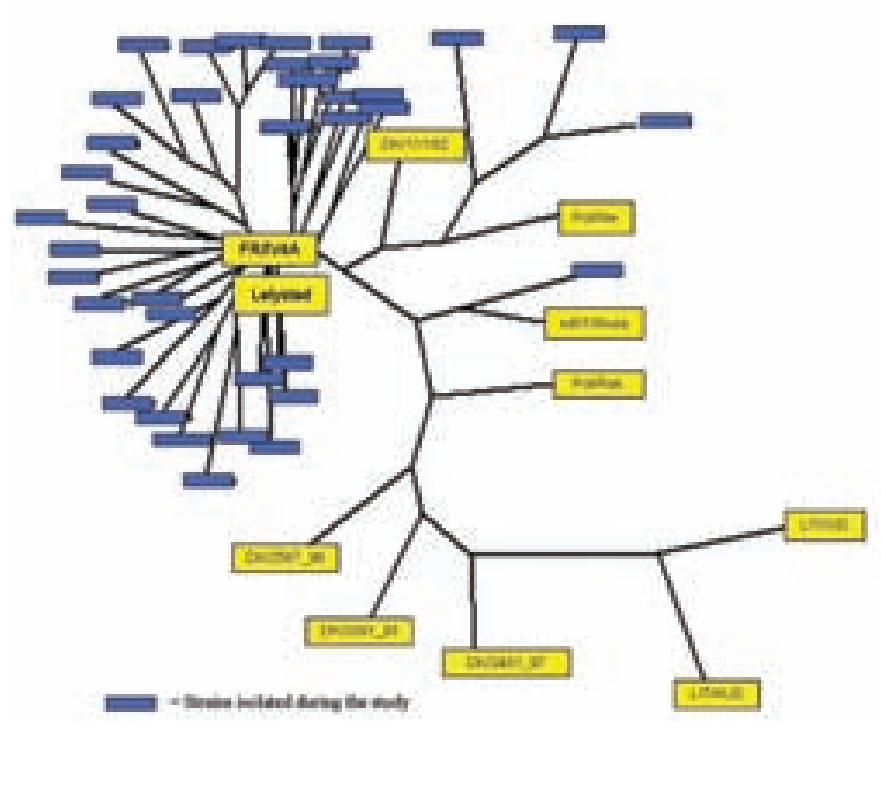




\title{
P.195
}

\section{Genetic diversity and evolution of Porcine Reproductive and Respiratory Syndrome virus in a large sequence data set from Quebec swine herds}

\author{
Benjamin Delisle; Carl A. Gagnon; Sylvie D'Allaire \\ Universite de Montreal, St-Hyacinthe, QC, Canada
}

\begin{abstract}
Introduction
Porcine reproductive and respiratory syndrome virus (PRRSv) is known to be genetically highly variable; knowledge of this diversity within a geographical region may help to understand the epidemiology of the disease as well as the evolutionary drift in GP5 amino acid sequences. The purposes of our study were to classify the genetic diversity of PRRS in subgenotype clusters for various strains affecting herds in the Province of Quebec, and also to evaluate amino acid (a.a.) variation in vaccine-like strains found in the field.
\end{abstract}

\section{Materials and Methods}

As part of a PRRS surveillance project, samples were submitted by veterinarians over an 11-year period. Samples originated either from herds 1) experiencing reproductive or respiratory problems, 2) monitoring regularly their PRRS status, or 3) participating in a research project on risk factors for PRRS (see abstract by Lambert et al. in this IPVS proceeding). A total of 1345 ORF 5 sequences from 659 production sites were obtained. RT-PCR and sequencing were done to obtain ORF5 gene. A dendrogram was made by phylogenetic analysis with the Neighbor-Joining algorithm in the Bionumerics software. Two different subsets were constructed and analyzed by phylogenetic alignment and selective evolutionary pressure by non-synonymous to synonymous rate, $d \mathrm{~N}-d \mathrm{~S}$ (SNAP web utility and PAML 4.3)

\section{Results}

The first and largest dataset consisted of 520 sequences obtained after excluding redundant sequences, e.g. those with more than $98 \%$ of homology. Following a clustering analysis, 28 different groups were obtained. Half of the clusters were primarily composed of cases submitted in the earlier part of the surveillance project (2004-2006), whereas some others mainly included more recent cases (2007-2009), suggesting temporal variations in strain proliferation and consequently, its evolution. Some geographical clustering could also be observed. Amino acid non-synonymous substitution was generally high in the GP5 peptide signal region but also in two hypervariable regions (HVR) located between aminos 32-35 and 57-59 for all clusters. Very few amino substitutions were observed in the principal neutralizing epitope (PNE) between aminos 36-52, which is flanked by both HVR.
The second dataset extracted from the database focused on PRRS vaccine and vaccine-like strains. A total of 159 PRRS MLVlike and 46 PRRS ATP-like deduced a.a. sequences were obtained. A comparison of these strains found in the field with both vaccines and their parental strains (VR2332 and JA142) showed that a single amino, F101, was conserved when compared to actual and past wild-type field strains. Also, separate comparison of both vaccine-like field strains collected revealed that MLV-like had undergone very little a.a. variations in the last 2/3 of the GP5 amino acid sequence, as compared to ATP-like strains which had undergone more variation thru synonymous mutations (negative selection) and some non-synonymous mutations (positive selection). Positive selective pressure was statistically significant ([1-P] value was $\geq 98.5 \%$ ) for 7 a.a. sites in MLV-like $(3,13,32-34$, 58 and 151) and 5 a.a. sites in ATP-like strains (32, 57-59 and 102).

\section{Discussion}

The interpretation of low or high PRRS subgenotype viral spread through time in affected regions can possibly help in predicting trends in emerging strain clusters. Even though some clusters could be attributed to temporal evolution or geographical area, the proportion or importance of each of these factors will have to be estimated. Further analysis of these clusters by SNAP showed PNE region to be relatively well conserved among clusters (1). This might indicate molecular constraints in possible viral receptor recognition site as seen for other RNA viruses $(2,3)$.

The mutation found on almost all vaccine and vaccine-like strains can help distinguish field strains from vaccine derived field strains, therefore fulfilling a need for a typing tool, this is particularly important considering possible reversion to virulence by these vaccine like-strains (4). Analysis of these clusters by SNAP showed slightly different patterns of evolution. Position of sites under selective pressure might indicate a link between PNE (conserved region) and adjacent HVR. The factors implicated in selective pressure and evolution of vaccine derived strains remain to be clarified.

\section{References}

1. Hu et al. 2009, Arch. Virol. 154: 391-398.

2. Ostrowski et al. 2002, J. Virol. 76: 4241-4250.

3. Ruigrok and Gerlier 2007, PNAS 104: 20639-20640.

4. Storgaard et al. 1999, Arch. Virol. 144: 2389-2401. 


\title{
P.196
}

\section{Genetic analysis and typing of PRRSV isolates in ORF5 and ORF7 originating from pig farms in Central Europe}

\author{
Stefan Vilcek; Anna Jackova; Michaela Vlasakova; Valeria Leskova \\ University of Veterinary Medicine and Pharmacy, Kosice, Slovak Republic
}

\begin{abstract}
Introduction
Porcine reproductive and respiratory syndrome virus (PRRSV) is emerging pathogen causing serious clinical problems in pig farms around world. The viral genome consisting positive-single strand RNA is evolutionary diverse. PRRSV exists in two genotypes: European (EU) genotype, type 1 (PRRSV-1) and North American (NA) genotype, type 2 (PRRSV-2). Based on nucleotide sequence variability EU genotype is genetically divided (Stadejek et al., 2006) forming three subtypes (Stadejek et al., 2008). The aim of this work was to contribute to genetic characterization of PRRSV isolates collected in Central Europe, namely in Slovakia, The Czech Republic and Austria by their analysis in ORF5 and ORF7 regions.
\end{abstract}

\section{Material and Methods}

Ten viral samples (serum, lymph nodes, and lung) from pig farms in Slovakia, 10 viruses from The Czech Republic and 10 viral samples from Austria were collected in the period of last 5 years from clinically suspicious animals showing respiratory problems. The total RNA was extracted using Trizol reagent (Invitrogen) according to the manufacturer's manual. The cDNA was prepared using gene specific primer and SuperScript reverse transcriptase (Invitrogen). ORF5 and ORF7 fragments were prepared by a nested RT-PCR using outer and inner primers according to Oleksiewicz et al. (1998) and Drew et al. (1997). Purified PCR products were sequenced using automatic sequencer ABI PRISM. Partail ORF5 sequences (432 nt) and entire ORF7 were assembled using SeqMan. The alignment was carried out and percentage of nucleotide identity were calculated using MegAlign (DNASTAR).

\section{Results}

All 30 PRRSV strains from Slovakia, The Czech Republic and Austria were typed in both genomic regions, e.g. ORF5 and ORF7, as EU genotype (PRRSV-1), subtype 1. No geographic distribution of PRRSV isolates were observed in the phylogenetic trees. However, the grouping of viruses according to farms, especially with isolates from Slovakia was observed. Percentage of nucleotide sequence identity in ORF5 between Slovakian strains varied in the range $83.6-100 \%$, Czech strains $-82.6-100 \%$ and
Austrian strains $-84.5-98.1 \%$. Similar values were obtained when sequences were compared to the European genotype 1 prototype strain Lelystadt: 85.4-89.1 \%, 85.0-98.6 \%, 89.4-98.6\%. Corresponding values in ORF7 were as follows: $87.3-100 \%$, 87.6$100 \%$ and $91.5-100 \%$, to Lelystadt strain: $90.7-93.3 \%$, 89.4-100 $\%$ and $94.6-98.7 \%$.

When compared amino acid sequence (aa) for entire ORF7 region, in all strains analyzed the size of this region was the same - 128 aa. However, the polymorphism in ORF7 size reaching 132 aa in two isolates from a pig farm in Slovakia was observed. The size was not the result of insertion/deletion of amino acids but longer ORF7 at C-terminus.

\section{Discussion}

Our work contributes to information on genetic characterization of PRRSV isolates in pig farms of Central Europe. The isolates belong to EU genotype, as it is prevalent in other western European strains (Fosberg et al., 2002) independently if they were analyzed in ORF5 or ORF7 genomic regions. On the other hand our results revealed new and the first time observed polymorphism on ORF7 size since two isolates had ORF7 size 132 aa, differing from standard size - 128 aa for PRRSV-1, subtype 1 isolates. Recently it has been suggested that the ORF7 size 128 aa can be used as characteristic parameter for typing of EU genotype (PRRSV-1), subtype 1 strains (Stadejek et al., 2008). The results of our work indicate that two strains from Slovakia failed this criterion. Reason for ORF7 size polymorphism is not clear at present.

\section{Acknowledgements}

This work was supported by project APVV 20-019605, VEGA 1/0389/08 and INFEKTZOON - Center of Excellence for infectious diseases of animals and zoonoses financed by EC.

\section{References}

Drew, T.W. et al.: Vet. Microbiol., 55, 209, 1997

Fosberg, R. et al.: Virology 299, 38, 2002.

Oleksiewicz, M.B. et al.: Vet. Microbiol., 64, 7, 1998.

Stadejek, T. Et al..: J. Gen. Virol., 87, 1835, 2006.

Stadejek, T. Et al.: Arch. Virol., 153, 1479, 2008. 


\title{
P.197
}

\section{Genetic diversity of European genotype PRRSV in Central and Eastern Europe - An update}

\author{
Tomasz Stadejek ${ }^{1}$ Gyula Balka² Katarzyna Chabros ${ }^{1}$ Zoran Lipej ${ }^{3}$ Milicevic Vesna ${ }^{4}$ Dinko Novosel ${ }^{3}$ Arunas Stankevicius $^{5}$ \\ 1. National Veterinary Research Institute, Pulawy, Poland; 2. Szent Istvan University, Budapest, Hungary; 3. Croatian Veterinary Institute, \\ Zagreb, Croatia; 4. Scientific Institute of Veterinary Medicine, Belgrade, Serbia; 5. Lithuanian Veterinary Academy, Kaunas, Lithuania
}

\section{Introduction}

A high degree of variability exists within European genotype of porcine reproductive and respiratory syndrome virus (EUPRRSV). The EU-PRRSV strains can be grouped into several subtypes based on the sequence of the ORF7 gene coding for the capsid protein (2). While in majority of Europe relatively similar strains circulate, having ORF7 387 nucleotides long (subtype 1), the East European countries like Latvia, Lithuania, Belarus, Ukraine and Russian Federation harbor extremely diverse strains with ORF7 size from 375 to 393 nucleotides that belong to multiple genetic subtypes $(2,3)$. Exploring variability of the ORF7 is of major importance because it can influence the sensitivity and specificity of the PCR and serological diagnostic methods (1, 2). Here we determined the ORF7 sequences of PRRSV from recently obtained samples from Latvia, Lithuania, Belarus, Ukraine, Russia, Poland, Hungary, Croatia and Serbia.

\section{Materials and Methods}

Samples of serum, aborted fetuses or lungs were submitted for diagnosis to the authors' laboratories. Total RNA was extracted, complete ORF7 sequences were amplified and sequenced with different methods used in the laboratories. The sequences were assembled and analysed using programs from the Lasergene program package, (DNASTAR) and the CLC Sequence Viewer (CLC Bio). As a reference, a set of 69 ORF7 sequences representing the full range of EU-PRRSV diversity was used.

\section{Results}

A total of 28 complete ORF7 sequences representing field PRRSV strains from, Latvia (1), Lithuania (3), Belarus (3), Ukraine (1), Poland (5), Hungary (8), Croatia (4) and Serbia (3) were obtained and all of them were classified to the European genotype. Their analysis showed that Polish, Hungarian, Croatian and Serbian strains were closely related and had ORF7 consisted of 387 nucleotides characteristic for subtype 1 strains. On the other hand, the new Latvian, Lithuanian, Belarusian and Ukrainian ORF7 sequences were distantly related to those from abovementioned countries and exhibited extensive but expected size polymorphism $(375,378$ or 387 nucleotides) as it was shown before $(2,3)$.

\section{Discussion}

This collaborative report provides another evidence for already well documented extreme diversity of EU-PRRSV. The position of the most diverse strains in the countries formerly being part of the Soviet Union (Latvia, Lithuania, Belarus, Ukraine and Russia) indicates that the EU-PRRSV could have emerged there. Possibly only a single variant of the genotype diffused westward giving the origin to the presently globally spread subtype 1 . The oldest serological evidence of PRRSV in Europe comes from East Germany from 1986. The first PRRS outbreak in Europe occurred also in Germany at the time of the country's re-unification in 1989. These two pieces of evidence suggest that the source of virus for the first outbreak could be in East Germany. PRRS was recognized in Central Europe a few years later than in West Europe and several anecdotal reports pointed that the infection occurred after importation of pigs or contaminated semen from West Europe. However, it cannot be excluded that before the European epidemic PRRSV was present not only in East Germany but also in other countries of Central Europe. In summary, the presented data confirm that the strains from Central and Western Europe are closely related and have common origin while the strains from East Europe are much more diverse.

\section{References}

1. Karniychuk et al.: The current antigenic heterogeneity between PRRSV strains can influence the reliability of serological surveys. Proc. 2009 International PRRS Symposium, Chicago, p. 74.

2. Stadejek et al. Definition of subtypes in the European genotype of porcine reproductive and respiratory syndrome virus: nucleocapsid characteristics and geographical distribution in Europe. Arch Virol 153, 1479-1488, 2008.

3. Stankevicius A. et al.: Exceptional diversity of PRRSV strains in Latvia and Lithuania. Proc. 5th International Symposium on Emerging and Re-emerging Pig Diseases. PMWS PRRS Swine influenza and associated diseases. Krakow, 2007, p. 178. 


\title{
P.198
}

\section{Broad cross protection against PRRS induced by chimeric viruses containing mixed structural gene fragments from distinct heterologous PRRS viruses}

\author{
Dong Sun ${ }^{1}$ Wonil Kim ${ }^{2}$ Yongil Cho ${ }^{1}$ Sangho $\mathrm{Cha}^{3}$ Eunjin Choi $^{3}$ Kyoung-Jin Yoon $^{1}$ \\ 1. lowa State University, Ames, IA, USA; 2. Chonbuk National University, Jeonju, Korea; 3. \\ National Veterinary Research and Quarantine Service, Seoul, Korea
}

\section{Introduction}

PRRS virus (PRRSV) is one of the economically significant pathogens in the swine industry throughout the world. Vaccines can be an intervention tool, but a high degree of genetic variation among PRRSVs makes the protection induced by a monovalent vaccine limited to its homologous virus strains, impeding effective prevention of PRRS. Thus vaccines that can produce broad protection against heterologous viruses are highly desired for better control of the disease.

PRRSV GP5 is known to play a major role in the induction of antiPRRSV neutralizing antibody. Yet, our recent study has demonstrated that GP3 and M also significantly contribute to crossneutralization between different PRRSVs.1 Furthermore, GP3 was more critical than GP5 or M in overall virus neutralization against a PRRSV strain whose GP5 is highly glycosylated. Therefore, it was hypothesized that a chimeric virus of 2 distinctive PRRSVs can confer better cross-protection against those viruses if necessary genes from the 2 viruses are put together in a structured manner based on their prime role in virus neutralization.

\section{Materials and Methods}

To test the hypothesis, 3 chimeric viruses designated as JAP5, JAP56 and JAP2-6, respectively, were generated from the VR2332 infectious cDNA clone by replacing its ORF5, ORFs 5 and 6 or ORFs 2- 6 with that/those of the JA142 strain that is genetically and antigenically distinct from the VR2332 strain. A total of 114, 3-week-old pigs were divided into 6 groups and each group was inoculated with one of the chimeric viruses, VR2332, JA142, or sham inoculum. At 44 days post inoculation (dpi), 8 pigs each within each group were randomly selected, housed separately and challenged intranasally with VR2332, JA142, or sham inoculum to determine if protective immunity was conferred by inoculation of the chimeric viruses. All pigs were bled periodically until $72 \mathrm{dpi}$ and tested for viremia and antibody response. Half of the pigs in each room were necropsied at 14 days after the challenge and the remaining at 28 days for pathological evaluation.
To further assess the validity of chimeric virus strategy, 12 additional chimeric viruses randomly combining ORFs 2-4 and ORFs 5-6 of four antigenically and genetically distinct PRRSVs (1648$02,17198-2$, MN184 and SDSU73) were constructed using the VR2332-backbone infectious clone. Antisera against each donor virus were generated in 3-week-old pigs which were euthanized at $45 \mathrm{dpi}$. Neutralizing activity of each serum against each of the chimeric viruses was tested in vitro by a two-way fluorescentfoci neutralization assay.

\section{Results}

JAP56 was well neutralized in vitro by antisera raised against each of the donor strains whereas each donor strain was neutralized only by the homologous antisera. In pigs, the prior inoculation with JAP5 or JAP56 effectively protected the pigs from the challenge with VR2332 while the pigs inoculated with JAP56 or JAP2-6 demonstrated excellent protection against JA142 infection, suggesting that the JAP56 chimeric virus may be used as a vaccine candidate to induce broad cross-protection against both VR2332 and JA142.

As observed with the VR2332 and JA142 chimeric viruses, antisera generated against other donor viruses neutralized each of the chimeric viruses containing ORFs 2 to 4 from one strain and ORFs 5 and 6 from another as effectively as the homologous donor virus, while little cross neutralization was observed among the donor viruses.

\section{Discussion}

These observations suggest that the strategy of constructing chimeric viruses by combining structural genes from distinct heterologous strains in an organized manner can provide broad protection and can be widely applicable as a novel vaccine platform for PRRSV.

\section{References}

1. Kim W-I, Yoon K-J. 2008. Molecular assessment of the role of envelope-associated structural proteins in cross neutralization among different PRRS viruses. Virus Genes 37:380-391. 


\title{
P.199
}

\section{Lack of correlation between genetic and antigenic classification of European- type porcine reproductive and respiratory syndrome virus isolates}

\author{
Francisco Javier Martínez-Lobo; Francisco Díez-Fuertes; Lidia Hornillos-Gumiel; Isabel Simarro; José María Castro; Cinta Prieto \\ Facultad de Veterinaria Universidad Complutense de Madrid, Madrid, Spain
}

\section{Introduction}

One of the main characteristics is its great variability. The extensive heterogeneity observed at the genomic level is likely to cause wide antigenic differences among the diverse PRRSV isolates and, in fact, significant differences have been reported between different isolates (1). However, current knowledge of antigenic variability of PRRSV is rather limited compared to the existing information on genomic diversity. Consequently, the main objective of the present study was to characterize European-type porcine reproductive and respiratory syndrome virus isolates by means of cross-neutralization assays, studying the possibility of the existence of serogroups, and to verify whether genotypic relationship reflect antigenic associations between PRRSV isolates.

\section{Materials and Methods}

Twenty nine PRRSV isolates were used in this study, of which one belonged to genotype II and was isolated in Denmark and 28 belonged to the European genotype. Of these 17 were Spanish isolates, 5 originated from Western Europe, 4 from Italy and 2 from Poland and Czech Republic, respectively. These isolates were adapted to replication in MARC-145. ORF5 of all isolates were amplified and sequenced and a phylogenetic tree was constructed. Monoespecific antisera against these isolates were produced in 6-month-old PRRSV seronegative pigs and used in seroneutralization (SN) assays performed as described by Yoon et al (2). Homologous SN titers were adjusted to a titer of 1:128 either by dilution or concentration by antibodies precipitation using ammonium sulphate. All sera were used in cross neutralization assays, and heterologous titers were used to estimate antigenic similarity coefficients $(R)$ that were used to construct a dendogram (3).

\section{Results}

The dendrogram obtained shows that the PRRSV isolates are antigenically diverse and are segregated into a considerable number of groups. When we use $R$ values $\geq 25$, which have been previously associated with significant antigenic differences (3), 19 different groups are defined, of which only 5 comprise more than one isolate. The comparison of antigenic and genomic dendograms indicates that there is no correlation between antigenic and genomic clustering of PRRSV isolates. Analysis of ORF5 and particularly of neutralizing epitope sequences or potential $\mathrm{N}$-glycosilation sites is not predictive of antigenic similarity.

\section{Discussion}

The results of our study show that antigenic diversity among European isolates is significant and that no clear antigenic groups can be defined. Additionally the comparison of the antigenic and genomic dendograms indicated a lack of correlation between antigenic and genetic classification of PRRSV isolates.

\section{References}

1. Meng, XJ. 2000. Vet Microbiol, 74:309-329

2. Yoon et al. 1994. J Vet Diagn Invest, 6:289-292

3. Becher, P. et al. 2003. Virology, 311:96-104

Figure 1. Antigenic dendogram of PRRSV isolates

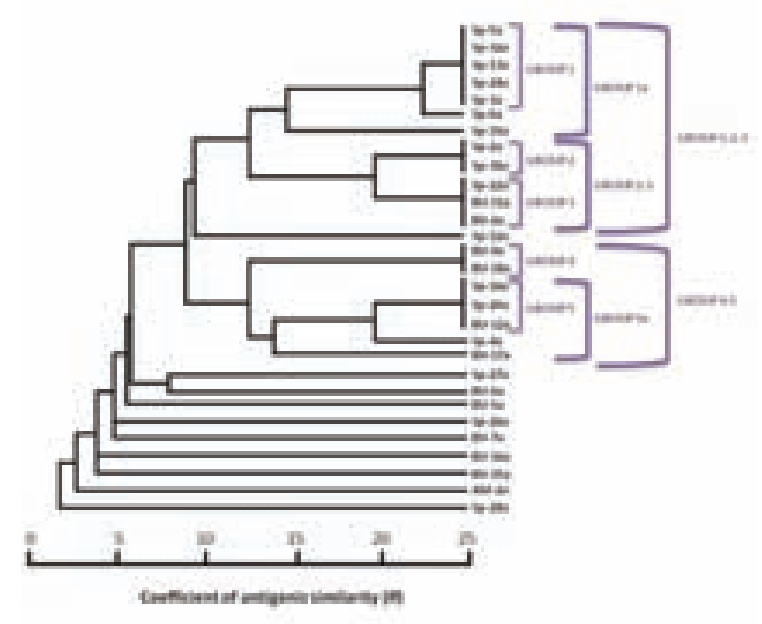

Figure 2. Genomic dendogram of PRRSV isolates

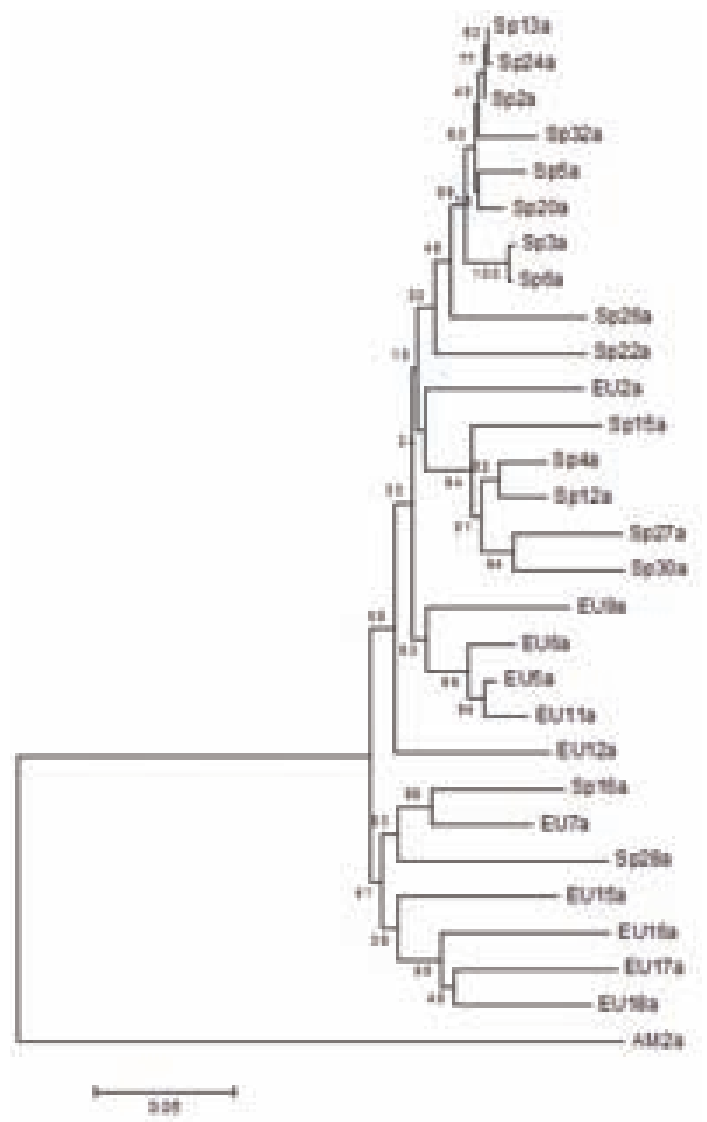




\title{
P.200
}

\section{Median infection dose (ID50) of PRRSV isolate MN-184 for young pigs via aerosol exposure}

\author{
Tim D. Cutler ${ }^{1}$ Apisit Kittawornrat ${ }^{1}$ Steven J. Hoff² Chong Wang ${ }^{2}$ Jeffery J. Zimmerman ${ }^{3}$ \\ 1. lowa State University, Ames, IA, USA; 2. lowa State University, Department of Statistics, Ames, IA, USA; 3. \\ lowa State University, Dept. of Vet Diagnostic and Production Animal Medicine, Ames, IA, USA
}

\section{Introduction}

The objective of this research was to quantify the likelihood of PRRSV transmission via aerosols as a function of exposure dose. This data is critical to understanding of airborne transmission of PRRSV between pigs, within herds, and betwe en herds.

\section{Materials and methods}

The study used PRRSV isolate MN-184 (kindly provided by Dr. Scott Dee, UMN). All procedures were approved by the lowa State University IACUC committee (\#9-07-6429-S). All pigs were confirmed PRRSV negative prior to commencement of the experiment and were housed in HEPA-filtered isolation units throughout the experiment to avoid inadvertent transmission of pathogens.

The study was conducted in 10 replicates, 10 pigs per replicate, with pigs randomly assigned to treatment. One negative control pig and one positive control pig were included in each replicate. The negative control pig was used to validate biosecurity procedures, i.e., was present in the laboratory during the aerosol exposure treatments, was housed in the same room as pigs exposed to PRRSV aerosols, and was monitored throughout the observation period. The positive control pig was inoculated intramuscularly with $10 \mathrm{ml}$ of fluid from the last air sample in each run to prove that infectious, airborne PRRSV was in the air to which pigs were exposed throughout the experiment.

Figure 1: Cartoon depiction of aerosol exposure. [A] Nebulizer with PRRSV, [B] Impinger for sampling cloud, [C] Aerosol reservoir, [D] Spirometer

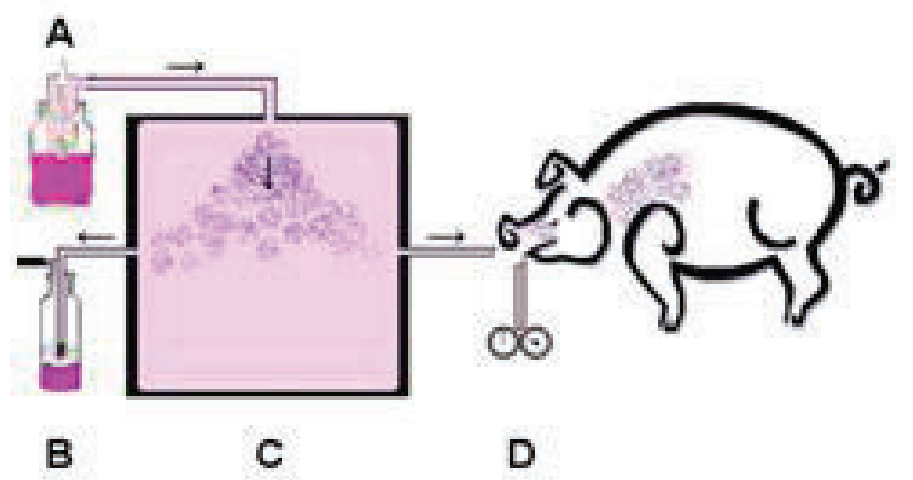

To conduct the experiment (Figure 1), PRRSV MN-184 was aerosolized [A] into a dynamic aerosol toroid [C]. Pigs to be exposed to the PRRSV aerosol were anesthetized, fitted with a canine surgical mask attached to a pediatric spirometer [D]. Each pig respired 10 liters of virus aerosol. Air samples [B] were collected before and after each pig were used to estimate the exposure dose. Serum samples collected 5 and 10 days post-exposure (DPE) were tested for the presence of PRRSV to determine whether exposure resulted in infection. The dose-response curve for exposure to airborne PRRSV was derived from the proportion of pigs infected by dose.

\section{Results}

Three replicates were disqualified due to failure to meet quality criteria; therefore, the infectious dose 50 (ID50) estimate was based on 7 replicates. Initial analysis showed that the infective dose 50 (ID50) of MN-184 under the parameters of this study (pig body size and age, exposure dose and time) was $<1$ x 101 TCID50.

\section{Discussion}

Under comparable conditions, the ID50 estimate we report for MN-184 is lower than the ID50 estimate for isolate VR-2332 (Hermann et al., 2009). The data suggest that the ID50 for airborne PRRSV may vary among isolates and supports reports of transmission of PRRSV via aerosol (Dee et al., 2005).

\section{Acknowledgements}

This project was funded in part through Pork Checkoff funds distributed through the National Pork Board (Des Moines, lowa).

This study was supported in part by the PRRS CAP, USDA-NIFA Award 2008-55620-19132.

\section{References}

Hermann JR, Muñoz-Zanzi, Zimmerman JJ. 2009. A method to provide improved dose-response estimates for airborne pathogens in animals: An example using PRRSV. Vet Micro 133:297-302.

Dee SA., Deen J., Jacobson L, Rossow KD., Mahlum C., Pijoan C. 2005. Laboratory model to evaluate the role of aerosols in the transport of porcine reproductive and respiratory syndrome virus. Vet Record 156:501-504 


\title{
P.201
}

\section{Experimental study of the effect of the B1 fumonisin and the PRRS virus: injuries histopathologíc lesions in pig kidney and identification of the of the PRRS virus}

\author{
Susana E. Mendoza_Elvira ${ }^{1}$ Carolina Moreno-Ramos ${ }^{1}$ Abel Ciprian-Carrasco ${ }^{1}$ David Trujillo-Ceballos \\ Ernesto MorenoMartinez ${ }^{1}$ Eliseo Hernández-Baumgarten ${ }^{1}$ Rosalia Melendez-Perez ${ }^{1}$ Horacio Lara- \\ Puente ${ }^{2}$ Francisco Quezada² Ruben Echeveste ${ }^{2}$ Jorge Tortora-Perez ${ }^{1}$ Isabelle P. Oswald ${ }^{3}$ \\ 1. FESC-UNAM, Cuautitlán, MEX, Mexico; 2. Laboratorios Avi-Mex, S A de C V, Iztapalapa, Mexico; 3. Pharmacology \\ and Toxicology Laboratory, UR 66, National Institute of Agronomic Research INRA, Toulouse, France
}

\section{Introduction}

The FAO (1999) estimates that at least $25 \%$ of world grain and seed production is contaminated by fungi and their mycotoxins, and are considered as one of the greatest risks that affect human and animal health (1). The fumonisins have been associated with certain diseases in animals such as the leucoencefalomalacia in equines (ELEM) and pig lung edema (PLE) (3). The porcine reproductive and respiratory syndrome (vPRRS) has economically impacted the national and international swine industry, for 20 years

\section{Material and Methods}

The reference Strain ATCC 2332 of the PRRS virus was cultivated in MA-104 African Green monkey kidney cells, and the pigs were challenged with 104 TCFF50 /ml. The FB1 used was a standard (SIGMA), in $5 \mathrm{mg}$ and $10 \mathrm{mg}$ vials with $98 \%$ purity. A stock solution was prepared at a concentration of $87 \mathrm{ppm}$ in distilled water. The FB1 was administered to weaned pigs in 12ppm ( $\mathrm{mg} / \mathrm{kg}$ liveweight) by the oral route by means of a probe.

Experimental animals: Twenty five recently weaned pigs 22-36 Days of age, with a weight of 4.17 to $7.6 \mathrm{~kg}$, from a vPRRS free farm were used. The animals were distributed into 5 groups; each group consisting of five pigs Group A: negative Control. Group B: Treated with FB1 12 ppm on day 0 (beginning of the experiment). Group C: inoculated with PRRSv on day 8. Group D: inoculated with vPRRS on day 0 and simultaneously intoxicated with FB1 12ppm. day 0 day 0 . Group E: intoxicated with FB1 $12 \mathrm{ppm}$ on day 0 and inoculated with vPRRS on day 8 . Blood was collected in vacuum tubes containing EDTA on days $0,8,16$ and 18 , for processing by the nested RT-PCR technique to identify the PRRSv. For the histopathological study samples of kidney were collected and fixed in $10 \%$ buffered formalin for further processing (4) and finally stained with hematoxylin and eosin.

\section{Results}

The nested RT-PCR was negative for groups A and B. For groups C, D and $E$ which were inoculated with PRRSv were positive. Histopathology in Group A was without evi9dent pathological changes. The kidney damage was observed in groups B, D and $\mathrm{E}$, and was characterized by basophililic cytoplasm in the cells of the tubules, suggestive of immature cells in the process of repair, on these tubules in presented polyploidy and in most of the observed lesions the tubules did not showed light (aberrant repair, non functional). These are located next to the glomerulus and areas of straight interlobulillar tubules, so probably correspond to the proximal convoluted tubules. In most cases, it was observed mononuclear cell infiltration in association with these damaged nephrons. At the glomerular level very cellular and swollen glomeruli were observed, suggesting proliferative glomerulitis. We also observed in lesser quantities retracted, smaller atrophied glomeruli. In some sections the interstitial cortical area showed mononuclear infiltration, but rarely the medullary level.

\section{Discussion}

The kidney lesions observed are suggestive of toxic processes, apparently in proximal convoluted tubules, that can relate to the presence of FB1, of which there are no previous reports in pigs, only in rodents (5), so we suggest conducting trials to evaluate these effects. Glomerulonephritis and the PRRSv can lead to a hipoproteínemia and to the development of edema. In Group C, where only PRRSv was applied, we also noted kidney injuries, it was noteworthy since the presence of these alterations in this pathology is not common. It has been reported kidney lesions associated with infection of the PRRSv, showing inflammatory infiltration observed in renal cortex and Medulla, as well as renal vascular changes (2).

\section{Conclusions}

It would be desirable to focus an investigation that concentrates in assessment of renal damage in pigs by FB1

\section{We thank}

Ing. Draucin Celis for his technical support.

\section{Bibliography}

1. Bullerman,L.B.(1996). Adv.Exp.Med. Biol.392:27-38.

2. Cooper,V.L. et al.(1997). J.Vet.Diagn.Invest. 9:198-201.

3. Harrison, L.R.et al.(1990).J.Vet.Diagn.Invest. 2:217-221.

4. Valladares, J.C.C., Salinas,V. (2007). Manual de Proc. de laboratorio. N.L., México.

5. Ryley, R.T. and Voss, K.A.( 2006). Toxicological Sciences. 92(1): 335345 


\title{
P.202
}

\section{Useful markers to study extrinsic and intrinsic pathways of apoptosis in porcine paraffin-embedded tissues}

\author{
$\underline{\text { Inmaculada Barranco }}{ }^{1}$ Jaime Gómez-Laguna Irene M. Rodríguez-Gómez ${ }^{1}$ Francisco J. \\ Salguero ${ }^{2}$ Francisco J. Pallarés ${ }^{3}$ Antonio Bernabé ${ }^{3}$ Librado Carrasco $^{1}$ \\ 1. Departamento de Anatomía y Anatomía Patológica Comparadas, Universidad de Córdoba, Córdoba, Spain; 2. Veterinary Laboratories Agency, \\ New Haw, Addlestone, Surrey, UK; 3. Departamento de Anatomía y Anatomía Patológica Comparadas, Universidad de Murcia, Murcia, Spain
}

\section{Introduction}

Apoptosis is a strictly regulated mechanism of cell death that involves a complex network of biochemical pathways. Whether a cell undergoes apoptosis or not depends on a delicate balance of anti- and pro-apoptotic stimuli (1). This phenomenon can be induced by two differents pathways: intrinsic and extrinsic pathways $(2,3)$. The main aim of this study was to determine the ideal fixative and antigen retrieval method in porcine paraffin embedded tissues for the immunohistochemical detection of apoptosis mediators, from both extrinsic and intrinsic pathways.

\section{Materials and Methods}

To carry out this study we used 5 week old pigs from a previous experiment (4) in which the animals were inoculated with a European PRRS virus strain. From this previous experiment we selected animals that were caspase 3-positive and TUNELpositive. At the necropsy samples from the lung, tonsil and retropharyngeal lymph node were collected and fixed in different fixatives: $10 \%$ neutral buffered formalin, Bouin's solution and Zinc salts solution. Four $\mu \mathrm{m}$ sections were performed and 6 primary antibodies were used for the immunohistochemical study: caspase 3, caspase 8, caspase 9, bcl-2, Fas, iNOS; and a kit for TUNEL technique. Different antigen retrieval methods were carried out: no pretreatment, Tween 20, Citrate microwave and Proteinase K. All the samples were evaluated determining the intensity of the positive reaction as well as the background. In addition, the number of cells per $\mathrm{mm} 2$ or structure (lymphoid follicle, paracortex, or medulla) and the main cells immunolabelled against each antibody were examined

\section{Results}

The best fixative and antigen retrieval method for the antibodies against caspase 8 and Fas (extrinsic pathway of apoptosis) were Zinc salt fixative and Citrate microwave unmasking technique. To determine the intrinsic pathway the ideal fixative and antigen retrieval method for each antibody was as follows: for caspase 9 , fixation in Zinc salt solution and antigen retrieval with Tween 20; for $\mathrm{BCl}-2$ and iNOS the optimal immunolabelling was observed in $10 \%$ neutral buffered formalin fixed samples and with Proteinase $\mathrm{K}$ and Citrate microwave as antigen retrieval methods, respectively.

The main cells immunolabelled against each antibody were macrophages and lymphocytes from paracortical areas (T-cell areas) of lymphoid organs and from the alveolar septa of the lung. Occasional immunolabelled cells were also observed in the lymphoid follicles (B-cell areas) of the retropharyngeal lymph node and tonsil and in the alveoli of the lung. Few neutrophils showed positive immunostaining against caspase 9 and iNOS antibodies.

\section{Discussion}

Our results show useful new tools which may be used to study apoptosis phenomena and to deep in the knowledge of the pathogenesis of several porcine diseases.

\section{References}

1. Costers et al. (2008) Arch Virol 153:1453-1465.

2. Roy et al. (2000). J. Exp. Med. 192, F21-F26.

3. Mitchell and Cotran (2007). Basic Pathology, 8th Edit., pp. 19-22.

4. Gómez-Laguna et al., 2009. J Comp Pathol 


\title{
P.203
}

\section{PRRSV is the most frequently diagnosed respiratory pathogen in Austrian pigs}

\author{
Sabine Elicker ${ }^{2}$ Louis Fischer $^{3}$ Nina Scherer ${ }^{1}$ Elisabeth Mayrhofer ${ }^{1}$ Franz Schilcher ${ }^{1}$ Herbert Weissenböck ${ }^{1}$ Wolfgang Sipos $^{1}$ \\ 1. University of Veterinary Medicine Vienna, Vienna, Austria; 2. Veterinary Practice Entenfellner, Stössing, Austria; 3. LaboVet GmbH, Vienna, Austria
}

\section{Introduction}

Respiratory diseases cause important economic losses in industrialized pig production. As respiratory diseases are mainly caused by multiple infections, these are referred to as porcine respiratory disease complex (PRDC). Viral pathogens, especially the Porcine Reproductive and Respiratory Syndrome Virus (PRRSV) and the Porcine Circovirus Type 2 (PCV2), are important players within the pathology of PRDC (1-3). Therefore an exact etiological diagnosis is of great importance for effective management of respiratory diseases.

\section{Materials and Methods}

A total of 677 pigs (494 weaned piglets, 151 fattening pigs, 11 gilts, 21 sows), which had been submitted to the clinic for swine of the University of Veterinary Medicine Vienna for diagnostic reasons due to respiratory distress in the course of three years, was sacrificed and post mortem analysed by means of standard bacteriological analyses and serological measurements using commercially available ELISA kits.

\section{Results}

Most frequently, PRRSV could be identified as etiological agent in weaned piglets ( $34.7 \%$ ) as well as in fattening and breeding pigs (50.5\%). A. pleuropneumoniae was isolated out of 21.3 $\%$ of weaners and $31.1 \%$ of pigs over $30 \mathrm{~kg} \mathrm{BM}$. In weaners, a-hemolysing streptococci could be isolated out of $20.6 \%$ and P. multocida out of $16.6 \%$ of cases, whereas in fattening and breeding pigs the corresponding percentages were 17.4 and 26.6. The seroprevalence of SIV-antibodies was approximately the same in all age groups (14.4-14.7\%). Also for $\mathrm{H}$. parasuis no marked differences (weaners: $11.6 \%$, fattening and breeding pigs: $12.6 \%$ ) could be found. A. pyogenes, B. bronchiseptica, and mycoplasmae were isolated at even lower percentages.
The most frequent lung lesions were interstitial pneumonias (weaners: $79.1 \%$, fattening and breeding pigs: $68.9 \%$ ), followed by fibrinous-purulent broncho-pneumonias and hyperemic districts in weaners ( $25.9 \%$ each). In fattening and breeding pigs emphysemas could be diagnosed quite often (38.3\%). These could be found to a lesser degree in weaners (21.1\%). Other pathologic lung lesions, such as purulent catarrhalic pneumonias, fibrinous pleuropneumonias, and pleuritides could be diagnosed less frequently.

\section{Discussion}

The results highlight the drawbacks of nowadays routine diagnosis as only in $43 \%$ of investigated pigs one or more specific pathogen(s) could be identified. This could be the consequence of an antibiotic treatment prior to submission to the clinic, or the missing of a more intense diagnostic search for viral pathogens, such as PCV2. The high incidence of viral diseases can also be delineated from the high incidence of interstitial pneumonias. Interestingly, the comparison of pathogen incidences in the two age groups (weaners on the one hand and fattening and breeding pigs on the other hand) clearly shows very similar distributions. Out of these reasons, increasing attention should be paid to (I) more sophisticated diagnostic procedures and (II) proper vaccination and management strategies against viral respiratory pathogens.

\section{References}

1. Harms et al. (2002): J Swine Health and Prod 10, 27-30.

2. Elicker et al. (2009): Wien Tierarztl Monat 96, 246-252.

3. Elicker et al. (2009): Tierärztl Umschau 64, 484-489. 


\title{
P.204
}

\section{Targeted sampling for enhancing the detection of PRRS virus infection in suckling piglets}

\author{
Jean P. Cano ${ }^{1}$ Scott Dee ${ }^{2}$ Albert Rovira² Claudia M. Zanzi² Sukumarannair Anil ${ }^{2}$ Robert Morrison² \\ 1. Boehringer Ingelheim Vetmedica, Inc., St Joseph, MO, USA; 2. College of Veterinary Medicine, University of Minnesota, St Paul, MN, USA
}

\section{Introduction}

An important challenge for swine practitioners is to determine whether porcine reproductive and respiratory syndrome virus (PRRSv) transmission is occurring in the sow herd. A "traditional monitoring protocol" used in North America is to sample 20-30 piglets at weaning, pooling the samples in groups of 5, and testing them by PCR. ${ }^{1,2}$ However, if the prevalence is very low, the probability of detecting infected piglets with this protocol is also very low. "Targeted sampling" has been used to increase the likelihood of detecting low prevalence diseases in animal populations. ${ }^{3,4}$ This strategy focuses sampling on high-risk individuals in which specific risk factors exist. It was hypothesized that pigs with specific attributes were more likely to be infected. The objective of this study was to identify those factors and their distribution across infected and non-infected piglets and litters.

\section{Materials and Methods}

A 1500 sow farrow-to-wean farm originally populated as PRRSvnaïve became infected. The owners and the veterinarian decided to inject serum containing the herd specific PRRSv isolate to all the sows in an attempt to hasten the spread of the virus. This decision provided an opportunity to conduct the proposed study. To estimate the prevalence of PRRSv infected litters and piglets, blood was collected from a random sample of 38 litters at birth and again at weaning. All piglets in selected litters were sampled. Two farrowing groups were selected as being representative of epidemic (4 weeks post-inoculation / PI) and endemic infection (12 wks PI). Serum samples were randomly pooled in groups of 5 ( $1 \mathrm{~mL}$ of serum per piglet) and tested by RT-PCR. All samples in a positive pool were tested individually. Clinical signs and production parameters were examined by the same investigator every sampling day. Litter attributes included size, weight, gilt-origin litter and diarrhea. Piglet attributes were gender, weight, light piglet, diarrhea, paleness, fuzziness and dome head. The association between attributes of the piglets or litters and the detection of PRRSv was screened by univariate and multivariate logistic regression analyses (SAS/STAT 9.1).

\section{Results}

During the study, 133 PCR-positive results were obtained from 1,507 piglets at birth and weaning. In the univariate logistic regression analysis, litter indicators including, light average litter weight and gilt litters were associated with the detection of PRRSv in at least one of the sampling times $(P \leq 0.1)$. Significant piglet indicators included being a barrow, having a dome shape head and having a fuzzy hair coat $(P \leq 0.1)$. The odds for a barrow sampled from light litters to be detected as PCR-positive was 6.86 ( $\mathrm{Cl}: 2.4$ - 19.6) times higher than for the rest of the piglets sampled at weaning 12 wks PI (Table 1).

Table 1. Multivariate logistic regression analysis for attributes associated with PRRSv detection 12 wks PI

\begin{tabular}{|l|c|c|}
\hline Attribute & Birth $(\mathbf{n = 4 2 1 )}$ & Wean (n=379) \\
\hline Litter level & & $\mathrm{NI}$ \\
\hline Litter size & $77(1.5-1000)^{*}$ & $\mathrm{NI}$ \\
\hline Gilt litter (1=gilt) & $0.4(0.01-33.3)$ & $4.1(0.6-25)$ \\
\hline Light litter & $\mathrm{NR}$ & $\mathrm{NR}$ \\
\hline Presence of diarrhea & & \\
\hline Individual level & $\mathrm{NI}$ & $5.4(2.6-11.1)^{*}$ \\
\hline Gender (barrow=1) & $\mathrm{NI}$ & $\mathrm{NI}$ \\
\hline Weight (kg) & $\mathrm{NI}$ & $\mathrm{NI}$ \\
\hline Light piglet & $\mathrm{NR}$ & $\mathrm{NR}$ \\
\hline Presence of diarrhea & $\mathrm{NR}$ & $\mathrm{NR}$ \\
\hline Paleness & $4.7(2.7-8.3)^{*}$ & $\mathrm{NR}$ \\
\hline Fuzziness & $0.73(0.4-1.4)$ & $\mathrm{NR}$ \\
\hline Dome shaped head & \multicolumn{2}{|c|}{} \\
\hline $\begin{array}{l}\text { Values are 0dds ratios }\left(95 \%(\mathrm{Cl})^{*}, 0 \mathrm{R}(\mathrm{P}<0.05) .\right. \\
\text { NR=not reported, } \mathrm{NI}=\text { not included }(\mathrm{P}>0.1 \text { univariate })\end{array}$ \\
\hline
\end{tabular}

\section{Discussion}

Alternative sampling protocols should consider targeted sampling of piglets with attributes associated with infection. In our study, sampling barrows from light litters at weaning increased the probability of detecting PRRSv by PCR. However, further investigation is required to validate attribute associations and to improve monitoring programs.

\section{References}

1. Spronk, G. Proc Leman Conf 2006: 126-128.

2. Dudley, S. Proc AASV Conf 2006: 351-355.

3. Diherr, M. Prev Vet Med 2001:(51)3-16.

4. Tournpanich S. Am J Vet Res 2006:(67-5)821-828. 


\title{
P.205
}

\section{Porcine Reproductive and Respiratory Syndrome Virus (PRRSV) in Northern Taiwan swine herds}

\author{
Cheng-Yao Yang ${ }^{1}$ Ming-Tang Chiou ${ }^{2}$ Pei-Ling Wang ${ }^{1}$ Ping-Cheng Yang ${ }^{1}$ \\ 1. Animal Technology Institute Taiwan, Miaoli, Taiwan; 2. Department of Veterinary Medicine, \\ National Pingtung University of Science and Technology, Pingtung, Taiwan
}

\section{Introduction}

Porcine reproductive and respiratory syndrome virus (PRRSV) causes severe respiratory diseases in nursery and growingfinishing pigs and has a major role in porcine respiratory disease complex (PRDC). Outbreaks of PRRS in Taiwan swine herds between July 1991 and June 1993 incurred significant economic loss to the local pig industry (1). Following Vietnam, the Philippines was the second country experience a serious outbreak of the disease. Philippine pig herds were infected with highly pathogenic PRRSV in 2008, which has a $98 \%$ sequence similarity with the Chinese type and is characterized by high fever and pathogenic clinical syndrome (4). The growing commodity trade between Taiwan and Southeast Asian countries has increased the risk of importing PRRSV into Taiwan. This study collected serum samples from northern Taiwan pig farms to test for PRRSV antibody. Oral-nasal swabs were collected from sero-positive pig farms and PRRSV was detected by molecular diagnostic method. The data revealed the distribution of European, North American or China strain of PRRSV in Northern Taiwan.

\section{Materials and Methods}

The epidemiological survey of PRRSV was performed using nationwide stratified random sampling method. The sample size of 15 pigs per herd was tested that is intended to detect PRRSV at $95 \%$ confidence interval with a $20 \%$ infection rate. The serum samples were tested for PRRSV antibodies using a commercially available enzyme-linked immunosorbent assay (ELISA) kit (IDEXX PRRS Ab test kit), and a ratio higher than 0.4 was considered seropositive for PRRSV (2). Three farms were selected from each county that was seropositive for PRRSV, and 10-15 porcine oral-nasal swabs were collected from each farm for testing by reverse transcription and polymerase chain reaction (RT-PCR) to determine whether PRRSV was European type (EU) (3), North American type (NA) (3) or China type (CN)(4). All PCR products were sequenced and compared with PRRSV sequences obtained from Genbank.

\section{Results}

The epidemiological survey conducted in 2009 estimated the herd prevalence of PRRSV in northern Taiwan. Sixty-five randomly selected herds and 953 blood samples were examined in this study. The overall PRRSV herd prevalence was $80 \%$ (52/65) and sero-prevalence was 62\% (595/953). The within-herd prevalence in the fifty-two sero-positive herds was $100 \%$ in twenty-four (37\%) herds, between $50 \%$ and $100 \%$ in sixteen $(25 \%)$ herds, and less than $50 \%$ in twelve herds. From each of twelve selected farms, 12-15 oral-nasal swabs were collected from nursing and growing-finishing pigs. Only one swab sample was positive for PRRSV NA type by RT-PCR. Sequence analysis of PCR products showed that the PRRSV was $91 \%$ homologous to VR-2332 (NA stain). No EU or CN type was detected in the study.

\section{Discussion}

Analysis of 953 blood samples taken from sixty-five northern Taiwan pig herds revealed a 62\% sero-prevalence of PRRSV antibody, which was slightly lower than the $64-86 \%$ prevalence reported in a 1996 investigation (5). None of the sampled swine herds had received PRRSV vaccine within the previous year. The $80 \%$ herd prevalence of PRRSV could be considered the actual field PRRSV infection rate. Only one PRRS vaccine (Ingel$\mathrm{vac}^{\circledR}$ PRRS MLV) has been used in the field in Taiwan recently. Ingelvac ${ }^{\oplus}$ PRRS MLV is a modified live vaccine based on VR-2332 strain. However, sequence analysis demonstrated a substantial difference between VR-2332 strain and PRRSV detected from swab in swine herds, indicating that the status of PRRSV in swine herds had changed in Taiwan. Fortunately, no CN-type PRRSV was found. However, continuous tracking of PRRSV surveillance is essential.

\section{References}

1. Chung WB et al. Can J Vet Res. 61: 292-298, 1997.

2. Wasilk A et al. J CLIN MICROBIOL 42:4453-61, 2004.

3. Huang TS et al. Exp Rep NIAH .36: 23-28, 2000.

4. Feng Youjun et al. Emerging infectious diseases 14: 1774-1776, 2008.

5. Huang TS et al. Exp Rep TAHRI. 32: 9-16, 1996. 


\title{
P.206
}

\section{Oral fluid diagnostic samples can be collected from individually-penned adult swine}

\author{
Jeremy Johnson ${ }^{1}$ Mark Engle ${ }^{2}$ Apisit Kittawornrat ${ }^{1}$ John R. Prickett ${ }^{1}$ Trevor Schwartz $^{1}$ Dan \\ Whitney ${ }^{1}$ Chris Olsen ${ }^{1}$ Kent Schwartz ${ }^{1}$ Chong Wang ${ }^{1}$ Jeffrey J. Zimmerman ${ }^{1}$ \\ 1. lowa State University, Ames, IA, USA; 2. PIC North America, Hendersonville, TN, USA
}

\section{Introduction}

Cumulatively, the literature supports the implementation of oral fluid-based diagnostics as a method to monitor infectious diseases in swine populations: (1) oral fluid samples are quickly and easily collected; (2) detectable levels of pathogen-specific $\lg \mathrm{A}$, IgM, and IgG antibodies are present in oral fluids; (3) a variety of infectious agents are shed in oral fluid, including highly significant pathogens of production animals, e.g., FMDV, CSFV, PRRSV (Prickett et al., 2008a), and PCV2 (Prickett et al., 2008b). Previous experiments used pen-based oral fluids to monitor PRRSV and/ or PCV2 infections in wean-to-finish populations (Prickett et al., $2008 a, b)$. The objective of this study was to determine if oral fluids could be collected from individually-housed boars.

\section{Materials and Methods}

The study was conducted using boars owned by PIC North America (Hendersonville, TN USA). Housing, procedures, and protocols were approved and supervised by the PIC USA Health Assurance and Welfare department. Three trials of 24 boars (6 months to 3.6 years of age) were conducted. Trial 1 used only select (young) boars; Trials 2 and 3 included both select and cull boars. Samples were collected for 8 days using 5/8", 3-strand, $100 \%$ cotton rope was cut into 22 "lengths. One end was untwisted, soaked overnight in a flavoring solution (unsweetened apple juice with $50 \%$ (v/v) sucrose) and air-dried. Ropes were placed in brackets, threaded through the pipe, and knotted to prevent slipping. Boars had access to the ropes for 20 minutes. To recover the sample, a 6 " segment of the "boar end" of the rope was cut, inserted, and sealed into a re-sealable plastic bag. Oral fluid was extracted by passing the plastic bag, with the rope inside, through a hand wringer, allowing the fluid to accumulate in a bottom corner of the bag. The bag was then cut and the contents decanted into a $50 \mathrm{ml}$ tube.

\section{Results}

Among 576 attempted oral fluid samples (8 days $x 24$ boars $\mathrm{x}$ 3 trials), 524 (91\%) samples were collected. Among a potential total of 192 potential samples in each trial, 188 (98\%) samples were collected in Trial 1, 155 (81\%) in Trial 2, and 181 (94\%) in Trial 3. The rate of successful collection initially increased during the observation period and then stabilized. Analysis of these data showed that trial $(p=0.0339)$ and observation day $(p<$ 0.001 ) were statistically significant sources of variation, but boar age $(p=0.9525)$ was not.

The average volume of oral fluid collected per boar across all trials was $17.9 \mathrm{ml}$ (range: 1 to $39 \mathrm{ml}$ ). Analysis of the sample volume data showed that day $(p<0.0001)$, age $(p=0.0127)$ and in the interaction of trial $x$ sampling day $(p<0.001)$ were statistically significant sources of variation.

\section{Conclusions and Discussion}

Oral fluid samples can be collected from individually-housed adult animals. Improvements in the success rate through the use of attractants or flavorings needs to be evaluated. As opposed to pen-based oral fluid testing, oral fluid samples from individual animals provide the opportunity to evaluate individual responses to infection under experimental and/or field conditions. This approach could offer significant improvement in the ease, timeliness, and cost of disease surveillance.

\section{Acknowledgements}

This work was supported in part by the USDA PRRS CAP (USDANIFA Award 2008-55620-19132).

\section{References}

Prickett J, Simer R, Christopher-Hennings J, et al. 2008a. Detection of PRRSV in Porcine oral fluid samples: A longitudinal study under experimental conditions. JVDI 20:156-163.

Prickett J, Simer R, Yoon K-J, et al. 2008b. Oral-fluid samples for surveillance of commercial growing pigs for porcine reproductive and respiratory syndrome virus and porcine circovirus type 2 infections. J Swine Health Prod 16(2):86-91. 


\title{
P.207
}

\section{Seroprevalence of Porcine Reproductive and Respiratory Syndrome (PRRS) in wean to finish pig farms}

\author{
Luminita Costinar ${ }^{1}$ Viorel Herman ${ }^{1}$ Corina Pascu ${ }^{2}$ Bogdan Faur ${ }^{1}$ Ioana Vaduva ${ }^{1}$ Anca Surpat ${ }^{1}$ Sorina leremie ${ }^{1}$ \\ 1. Faculty of Veterinary Medicine Timisoara, Timisoara, Romania; 2. USAMVB Timisoara, Timisoara, Romania
}

\section{Introduction}

PRRS is probably the most important swine disease of the last half-century. PRRS has been observed in the majority of European countries. In Romania, the disease has been continuously spreading in the pig population since it was first diagnosed by Stanuica and col. 1998. The PRRS virus is an enveloped RNA virus in the genus Arterivirus, classified in the virus family Arteriviridae and produce reproductive disorders in breedings farms and respiratory disorders in finishing farms.

An important feature of the PRRS virus is its ability to persist in long-term carrier pigs, greater than 200 days. The aim of the present study was to a cross-sectional study on 6 fattening farms to estimate the seroprevalence of antibodies directed against PRRSV in 4 week-old pigs, 12-13 week-old pigs and 20-27 weekold pigs.

\section{Materials and Methods}

There were tested 262 blood samples from 6 finishing farms at different times. Samples were analyzed for the presence of antibodies against PRRS by PRRS IDEXX ELISA (HerdChek ${ }^{\circledR}$ PRRS 2X, IDEXX, Switzerland). The presence or absence of antibody to PRRS in the sample is determined by relating the value of the unknown to the positive control mean by calculating the sample to positive (S/P). An S/P $\geq 0.4$ was considered seopositive. Statistical analysis was performed using the XChek IDEXX (vers. 3.3 2003). Studied herds were unvaccinated for PRRS.

\section{Results}

Serology is an essential part of a PRRS diagnostic and control program. ELISA antibodies are not detectable until 9-10 days post infection and persist for longer periods, at least 5-6 months. The seroprevalence of PRRSV in studied farms was 51,5\% (135 samples positive from 262 tested).

The seroprevalence of PRRSV in 4, 12-13 and 20-27 weeks-old pigs' varied between all the three samplings for each farm. In the farm 1 and 2 seroprevalence of PRRSV in the younger pigs ( 4 week-old pigs) was significantly higher suggesting the presence of maternal antibodies, and high prevalence in the other category (12-13 and 20-27 weeks-old) show that PRRS infection or reinfection taken place in fattening units.

A high seroprevalence in 12-13 week-old pigs and 20-27 weekold pigs to indicate an active immune response to PRRSV, this fact it could be observed in farms 3, 4 and 6, showing a recent infection. Persistence of infection may be associated with large herds in pig-dense regions with repeated reintroduction.
Table 1

\begin{tabular}{|l|c|c|c|}
\hline Farms & 1st sampling & 2nd sampling & 3st sampling \\
\hline & \multicolumn{3}{|c|}{ No. sample positive /No. sample tested } \\
\hline 1 & $10 / 10$ & $6 / 10$ & $10 / 10$ \\
\hline 2 & $10 / 10$ & $5 / 10$ & $10 / 10$ \\
\hline 3 & $14 / 40$ & $13 / 20$ & $21 / 28$ \\
\hline 4 & $7 / 19$ & $5 / 15$ & $10 / 10$ \\
\hline 5 & $0 / 10$ & $2 / 11$ & $2 / 19$ \\
\hline 6 & $0 / 10$ & $1 / 10$ & $9 / 10$ \\
\hline Total positive samples/ & \multicolumn{3}{|c|}{} \\
Total samples tested & \multicolumn{3}{|l}{$135 / 262$} \\
\hline
\end{tabular}

In farm 5 the infection percent was lower than the other studied farms, that means the herd became free of infection or the animals was recently infected and is undergoing seroconversion.

\section{Discussion}

We conclude that most farms pigs wean to finish are seropositive for PRRSV. they indicate that the prevalence of PRRSV antibody in the swine populations tested was relatively high. Method ELISA may be helpful in confirming the seropositivity and to indicate high levels of antibody in recent infections. An accurate diagnosis is essential to confirm presence PRRSV and may serologic profile of pigs in the various stages of production. The lack of an antibody response in recently weaned pigs indicated the absence of PRRSV infection in the breeding and farrowing populations.

\section{References}

1. Albina, E., Madec, F., Cariolet, R. et. al. 1994, Vet. Rec., 134, 567-573

2. Doo-Sung, C., Chanhee, C., Yong-Soon, L., 1997, J. Vet. Diagn. Invest. 9:434-436

3. Nodelijk, G., van Leengoed, L.A.M.G., Schoevers, E.J. et. al. 1997, Veterinary Microbiology, 21-31

4. Stanuica, D., Panca, C., Sarca, M., Caraivan, I., Draghici, D. 2000, Studies and researches in veterinary medicine, $8,1-13$ 


\title{
P.208
}

\section{Monitoring of PRRS control protocol in two farrow-to-finish herds during three years in high risk area}

\author{
Patrick Gambade ${ }^{1}$ Arlette Laval ${ }^{2}$ Luc Mieli $^{4}$ Martial Rigaut ${ }^{3}$ \\ 1. Selas Univet Santé Elevage, Loudéac, France; 2. ONIRIS, Nantes, France; 3. Intervet/Schering- \\ Plough AH, Beaucouzé, France; 4. LDA 22, Ploufragan, France
}

\section{Introduction}

In France, PRRS mass vaccination with a modified live Vaccine $(\mathrm{MLV})$, Porcilis ${ }^{\circledR}$ PRRS, Intervet / Schering-Plough, have been already described $(1,2)$.

This study presents a three years monitoring PRRS control program used in two farrow-to-finish herds in PRRS high risk area.

\section{Material and Methods}

The trial was performed in two farrow-to-finish herds: Herd A (180 sows) known infected with PRRS virus since 1994; herd B (350 sows) since 2002.

From June 2005, a vaccination scheme was established as follows: Gilts: one injection at arrival in quarantine. Sows and boars: "mass vaccination" three times a year. Piglets: one injection between 5 and 6 weeks of age.

Serological monitoring data from February 2005 to December 2006 showed that PRRSv was under control since November 2005 (1). Monitoring was extended from 2007 to 2009 (3 years) in two ways:

- Serological monitoring: every 3 or 4 weeks, ELISA individual serology on sera sample (IDEXX Herdcheck PRRS 2XR) were done on a minimum of 10 non-vaccinated piglets. Each piglet, was identified by an ear tag. So a new cohort of ten piglets was chosen every six months and was followed from 70 to 140 days old.

- Virological monitoring: every 3 weeks, PCR tests (ADIAVET PRRS = RT-PCR EU and US strains qualitative test) done on 2 pools of three sera taken on 140 days old vaccinated piglets. All sera were analyzed by LDA 22 (laboratory, Ploufragan, France).

\section{Results}

In total, from January 2007 to December 2009, 630 non-vaccinated growing pigs were controlled by serology, and 585 vaccinated pigs by PCR. No positive results linked with PRRSv infection were observed (Table 1 and Table 2). The only serological positive results (farm B, first semester of 2007) were linked to indirect contacts of non-vaccinated piglets with vaccinated ones. In table 1: ${ }^{*} 2$ positive sera, tested with other kit (AFSSA antigen): 1 negative, 1 inconclusive and negative by $\mathrm{PCR} ;{ }^{* *} 13$ positive sera, also positive by PCR, but $100 \%$ homology with the vaccine strain by PRRSv ORF7 sequencing.

\begin{tabular}{|c|c|c|c|}
\hline \multirow{2}{*}{$\begin{array}{c}\text { Famm } \\
\text { year }\end{array}$} & \multicolumn{3}{|c|}{ Number of coutrols (sera) } \\
\cline { 2 - 4 } & Total & Nceative & Positive \\
\hline A 2007 & $9(90)$ & $9(90)$ & $0(0)$ \\
A 2008 & $6(60)$ & $6(60)$ & $0(0)$ \\
A 2009 & $7(70)$ & $7(70)$ & $0(0)$ \\
\hline B 2007 & $13(130)$ & $8(115)$ & $2(2)^{*}$ and 3(13) ** \\
B 2008 & $14(140)$ & $14(140)$ & $0(0)$ \\
B 2009 & $14(140)$ & $14(140)$ & $0(0)$ \\
\hline A + B & $\mathbf{6 3 ( 6 3 0 )}$ & $58(615)$ & $5(15)$ \\
\hline
\end{tabular}

Table 2: Vaccinated pigs virological Results

\begin{tabular}{|r|c|c|c|}
\hline \multirow{2}{*}{ Famn / year } & \multicolumn{3}{|c|}{ Number of coutrols (sera) } \\
\cline { 2 - 4 } & Total & Negative & Positive \\
\hline A 2007 & $14(90)$ & $14(90)$ & $0(0)$ \\
A 2008 & $17(153)$ & $17(153)$ & $0(0)$ \\
A 2009 & $14(126)$ & $14(126)$ & $0(0)$ \\
\hline B 2007 & $13(78)$ & $13(78)$ & $0(0)$ \\
B 2008 & $10(60)$ & $10(60)$ & $0(0)$ \\
B 2009 & $13(78)$ & $13(78)$ & $0(0)$ \\
\hline Total A + B & $81(585)$ & $81(585)$ & $0(0)$ \\
\hline
\end{tabular}

\section{Discussion and Conclusion}

Regular controls showed no circulation of PRRSv "wild" or vaccine strain during 3 years. Since 2007, the sensibility of serological monitoring was increased: to avoid contact with vaccinated piglets, the non-vaccinated monitored piglets were split with other non-vaccinated piglets ( 80 piglets in total). In case of PRRSv infection, virus multiplication would probably be more important according to the high number of animals without vaccine protection. This field data confirm efficiency of Porcilis ${ }^{\circledR}$ PRRS in reducing significantly viral excretion after infection, and to control virus shedding within these two farms located in high risky area. Even if, we can't claim eradication, results show that it might be a mean to start or even to achieve eradication, especially in geographic area where infection prevalence is low.

\section{Références}

1. Gambade $P$ et al. 2007. 5th ISERPD Proc., Krakow, 205

2. Lebret A. et al. 2006. 19th IPVS Proc., Copenhagen, vol. 2, 75.

Table 1: Non-vaccinated piglets serological Results 


\title{
P.209
}

\section{Feasibility of using FTA cards to transport samples for PRRSV testing}

\author{
Daniel Linhares ${ }^{1}$ Albert Rovira ${ }^{2}$ Montserrat Torremorrell ${ }^{1}$ \\ 1. College of Veterinary Medicine, University of Minnesota, Saint Paul, MN, USA; 2. Veterinary Diag \\ Lab, College of Veterinary Medicine. University of Minnesota, Saint Paul, MN, USA
}

\begin{abstract}
Introduction
Porcine reproductive and respiratory syndrome (PRRS) is a devastating disease caused by PRRS virus (PRRSV). Farmers and veterinarians require having access to state of the art readily available diagnostics in order to respond rapidly to disease treats. FTA cards consist of a cellulose-based matrix paper containing chemicals that lyse the cells in the sample while preserving the nucleic acids and are available for the safe transfer of diagnostic samples. Because these chemicals render bacteria and viruses inactive, samples embedded in FTA cards are not infectious. Samples in FTA cards can be shipped and stored at room temperature because the nucleic acids are protected from degradation and clinical samples can be stored in a safe nonbiohazard way suitable for molecular diagnostics. Data from a previous study has shown that PRRSV is detectable by RT-PCR from FTA cards (Inoue, 2000). However this study failed to validate the methods to properly assess the diagnostic sensitivity of RT-PCR in samples embedded in FTA cards.
\end{abstract}

The objective of this study was to evaluate the analytical sensitivity (limit of detection) and stability of FTA cards containing serum and oral fluid samples spiked with PRRSV under controlled laboratory conditions.

\section{Materials and Methods}

Porcine sera and oral fluid samples were spiked with a concentration of $10 \wedge 5$ TCID50 of PRRSV. Samples were diluted in 10-fold dilutions (from $10 \wedge 5$ to $10 \wedge-2$ TCID50/ml) and used to inoculate FTA cards. Each sample from each dilution was inoculated in duplicate in 2 FTA cards and let dry at room temperature overnight. One FTA card embedded sample was tested directly at $24 \mathrm{~h}$ post inoculation. The other card was kept at room temperature and tested after 2 weeks. Oral fluids were evaluated directly and following a pre-processed protocol which consisted of treatment with a lysis solution before the extraction protocol (Prickett et al. 2008).

To elute the RNA from the FTA card, the RNA Rapid Extraction Solution (Ambion-Applied Biosystems) was used as recommended by the manufacturer. RNA was then purified and concentrated using high throughput magnetic bead-based technology (Ambion-Applied biosystems). The RT-PCR protocol used was a real time TaqMan-based multiplex protocol that detects both North American and European Strains of PRRSV (NAEU PRRSV) (Dee et al. 2009).

\section{Results and Discussion}

Results in table 1 indicate the minimum concentration of PRRSV detected by RT-PCR in samples embedded in FTA cards. Under the conditions of this study, the analytical sensitivity of PRRSV detection by RT-PCR from serum embedded-in FTA cards was reduced 10 times compared to detecting the virus directly from serum. However, the virus could still be detected at a very low concentration in FTA cards (10^1 TCID50). Moreover, there was no loss of sensitivity when comparing detection on FTA cards at $24 \mathrm{~h}$ or 14 days of storage

Table 1. PRRSV minimum concentration (in TCID50/ml) detected by RT-PCR.

\begin{tabular}{|l|c|c|c|}
\hline & Direct & FTA 24h & FTA 2 wk \\
\hline Serum & $10 \wedge 0$ & $10 \wedge 1$ & $10 \wedge 1$ \\
\hline Oral fluids & neg & $10 \wedge 3$ & $10 \wedge 5$ \\
\hline $\begin{array}{l}\text { Oral fluids (pre- } \\
\text { processed) }\end{array}$ & $10 \wedge 0$ & NA & NA \\
\hline
\end{tabular}

Sensitivity of oral fluids in pre-processed samples was comparable to that of serum but was poor when testing was done directly.

In summary, this study provides preliminary data that indicate that the use of FTA cards is a sensitive tool to store and submit pig serum and saliva samples for PRRSV molecular diagnostics. Further validation is in process to evaluate the suitability to transport samples under field conditions.

\section{Acknowledgments}

This work has been partially funded by the National Pork Board. The authors would like to thank Michele Leiferman and Yin Jiang from the University of Minnesota Veterinary Diagnostic Laboratory for their excellent laboratory work. We also thank Dr. Enrique Mondaca from Boehringer Ingelheim for his ongoing support for this project.

\section{References}

Inoue R et al. (2007). J Virol Methods 141(1):102-6.

Dee $S$, et al (2009). Vet Res 40:39.

Prickett JR, et al. 2008. 51st AAVLD Annual Conf.Greensboro-NC,Oct 22-27,2008. 


\title{
P.210
}

\section{Modified live PRRS virus vaccination as a key component in nursery pig performance improvement}

\author{
Gordon Spronk ${ }^{1}$ Tom Wetzell ${ }^{2}$ Reid Philips ${ }^{2}$ \\ 1. Boehringer Ingelheim Vetmedica, Inc., Pipestone, MN, USA; 2. Boehringer Ingelheim Vetmedica, St Joseph, MO, USA
}

\section{Introduction}

Two sow farms had experienced continual PRRS instability (large periodic increases in abortion rates) due to multiple PRRS virus isolate introductions over the past several years. The highest mortality rates in grower pigs occurred in the first 8 weeks postweaning with mean mortality rates in excess of $6 \%$ in nursery 1 flow for the previous 24 months and $12 \%$ during the previous 12 months in nursery 2 flow. The objective of this study was to determine if stabilization of the sow herd along with vaccinating pigs pre-weaning (5-10 days of age) with Ingelvac ${ }^{\circledR}$ PRRS ATP could reduce mortality in the first 8 weeks post-weaning.

\section{Materials and Methods}

The study was conducted in two 3,250 sow farrow to wean farms located in lowa and Minnesota.

The breeding herd PRRS stabilization program followed a "Load/ Close/Homogenize" approach. A 200 day supply of gilts was entered into the farms which were then closed to new animal entries for a minimum of 200 days. All breeding animals including developing gilts were mass vaccinated twice 30 days apart with Ingelvac ${ }^{\circledR}$ PRRS ATP (Boehringer Ingelheim Vetmedica, Inc., St Joseph, MO). Both herds practiced industry standard MCREBEL methods with no piglet cross-fostering after 24 hours of age for at least 30 days.

Piglets were vaccinated at 5-10 days of age with the same vaccine. On most continuous flow sites, mass vaccination of all pigs occurred twice 30 days apart when the first PRRS vaccinated weaned pigs entered the site. PRRS PCR testing was conducted every 2-4 weeks on 20 poor quality pigs aged 5 days and younger pooled in groups of 5. "Sow herd stability" was defined as negative results on two consecutive PRRS PCR tests on these pooled piglet sera. PRRS PCR testing of 15 pigs ( 3 pools of 5 from sick pens) from each group at 4-8 weeks post weaning was done and PCR positives were sequenced. An in-process intervention analysis was done using statistical process control methods on mortality for the first 8 weeks post weaning.

\section{Results}

Pig mortality rates during the first 8 weeks post-weaning were significantly reduced from $6.4 \%$ to $2.84 \%$ and $12.6 \%$ to $3.0 \%$ from Farms 1 and 2 respectively.
Figure 1. Farm 1 pig mortality rates the first 8 weeks post-weaning before/after intervention.

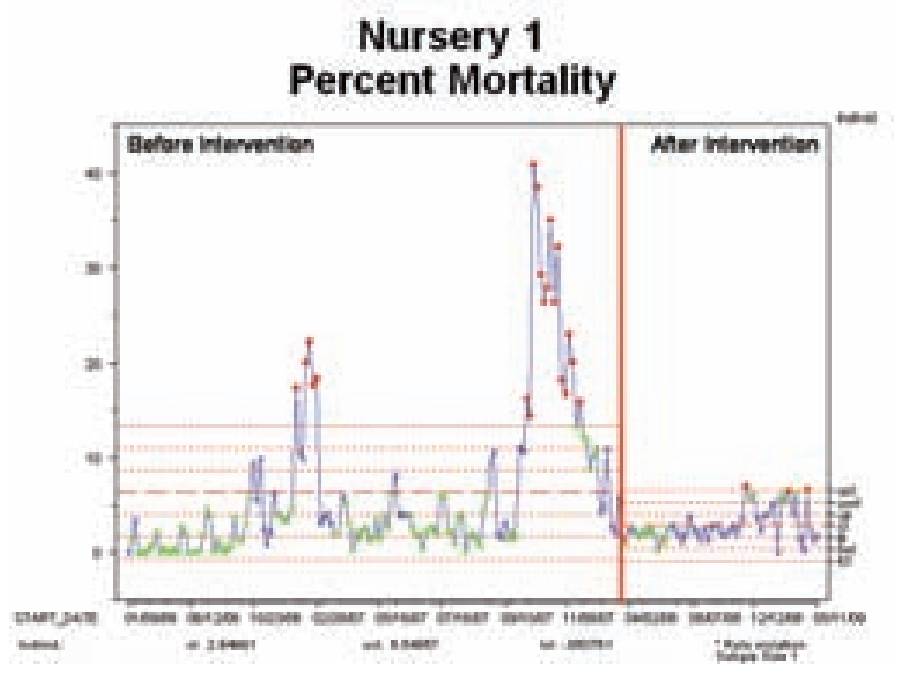

Figure 2. Farm 2 pig mortality rates the first 8 weeks post-weaning before/after intervention.

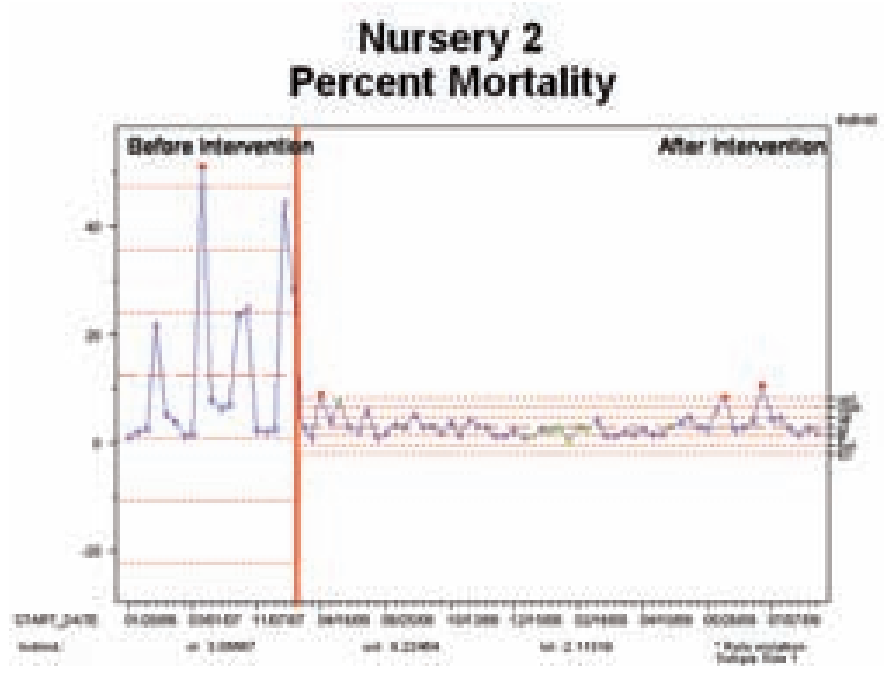

\section{Discussion}

The sow herd stabilization program along with vaccination of pigs at 5-10 days of age has proven to be very effective in reducing mortality during the first 8 weeks post weaning. This occurred in spite of wild-type virus, including highly virulent isolates 1-18-2 and 1-8-4, persisting on or being laterally introduced to twelve of the nursery sites. 


\title{
P.211
}

\section{Efficacy of Ingelvac PRRS MLV on HP PRRS in a Chinese Pig Farm}

\author{
Zhuogui Ao $^{1}$ Aiwu Huang ${ }^{2}$ Nian Huang $^{3}$ \\ 1. Shanwei Baoshan Pig Farm, Guandong, China; 2. Guangdong Import \& Export Trade Company, \\ Guangdong, China; 3. Boehringer Ingelheim Int'I Trading (Shanghai) Co. Ltd., Beijing, China
}

\section{Introduction}

Highly Pathogenic Porcine Reproductive and Respiratory Disease (HP PRRS), which caused great economic losses in Chinese hog industry, induces reproductive failure in sows and high culling rate in fatteners(1). To prevent viral disease like PRRS, vaccination is the optimum choice (2). The present study evaluated the effect of Ingelvac ${ }^{\circledR}$ PRRS MLV in a pig farm in Guangdong Province, China.

\section{Materials and Methods}

The 700-sow farm in Guangdong was built years ago with poor ventilation. Killed PRRS vaccine had been used before March 2006 but performance kept low. PCR test results confirmed PRRS positive before PRRS MLV vaccination.

From March 2006, mass vaccination program for breeders with Ingelvac ${ }^{\circledR}$ PRRS MLV started: 3 times per year in sows and twice per year in boars. 5 month later, in August 2006, piglets started to be inoculated with Ingelvac ${ }^{\circledR}$ PRRS MLV 1 dose/pig at 14 days of age. Replacement gilts were vaccinated twice before mating, with one month interval.

\section{Results}

Table 1. Reproductive performance during 2005-2008

\begin{tabular}{|l|c|c|c|c|}
\hline & $\mathbf{2 0 0 5}$ & $\mathbf{2 0 0 6}$ & $\mathbf{2 0 0 7}$ & $\mathbf{2 0 0 8}$ \\
\hline Conception rate(\%) & 95.24 & 94.88 & 96.09 & 94.42 \\
\hline Farrowing rate (\%) & 88.90 & 88.55 & 88.41 & 86.02 \\
\hline Reheats (sows) & 75 & 80 & 61 & 81 \\
\hline Abortion (litters) & 24 & 26 & 23 & 12 \\
\hline Pseudopregnancy (sows) & 19 & 20 & 19 & 16 \\
\hline Culled sows & 56 & 50 & 73 & 56 \\
\hline Piglets/litter & 10.44 & 10.85 & 11.02 & 10.9 \\
\hline Piglets born alive/litter & 9.5 & 9.73 & 9.91 & 9.94 \\
\hline Farrowing interval (days) & 151.7 & 150.4 & 150 & 151.3 \\
\hline Weak piglets (\%) & 0.01 & 1.67 & 1.53 & 1.39 \\
\hline Deformity (\%) & 5.01 & 4.18 & 4.39 & 3.81 \\
\hline Stillborn (\%) & 2.65 & 3.71 & 3.17 & 2.85 \\
\hline Mummies (\%) & 1.27 & 0.76 & 1.00 & 0.83 \\
\hline Pre-weaning mortality (\%) & 3.92 & 4.48 & 4.17 & 3.68 \\
\hline
\end{tabular}

* In 2008, sow feed had been contaminated by mycotoxin for 2 months, causing poor reproductive performance.
Figure 1. Survival rate of commercial pigs after PRRS KV and MLV vaccination

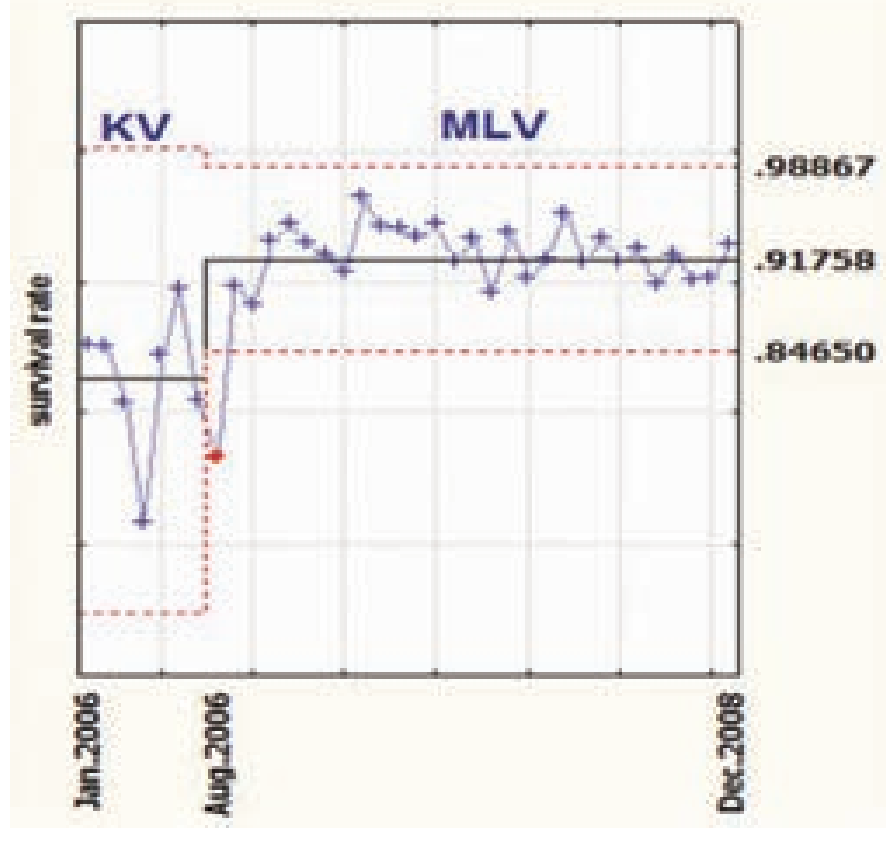

Great increase is seen in average fattener survival rate by $9.23 \%$ since Aug. 2006(figure 1). The other parameters remained stable even despite a Mycotoxin problem in 2008.

\section{Discussion}

In this study, PRRS MLV vaccination improved sow reproductive performance in conception rate, abortion, pseudopregnancy, live and weak born, more than KV (table 1). Much more pigs were marketed after vaccination changed from KV to MLV (figure 1). Feed free of mycotoxin is an important contributor to stable reproductive performance (2).

In conclusion, Ingelvac ${ }^{\circledast}$ PRRS MLV vaccination program and good feed and health management are essential to achieve good performance in PRRS positive farms.

\section{References}

1. Tong GZ et al. Highly Virulent porcine reproductive and respiratory syndrome virus emerged in China. Transbound Emerg Dis. 2008 May;55(3-4):152-64.

2. Zimmerman J, et al. Porcine Reproductive and Respiratory Syndrome, Disease of Swine 9th edition:387-418 


\title{
P.212
}

\section{Successful control of Porcine High Fever Disease in a Chinese 1200 sow farm: a case report}

\author{
GR Lin ${ }^{1}$ Shuhe Fang ${ }^{2}$ Nian Huang ${ }^{3}$ \\ 1. Swine Practioner, Guangdong, China; 2. Boehringer Ingelheim Shanghai Pharmaceuticals, Co. Ltd, \\ Beijing, China; 3. Boehringer Ingelheim Int'l Trading (Shanghai) Co. Ltd., Beijing, China
}

\section{Introduction}

Porcine High Fever Disease(PHFD), the primary pathogen of which is highly virulent porcine reproductive and respiratory syndrome virus (HP PRRSV), causes huge economic losses because of high morbidity and mortality in all infected Chinese pig farms(1). An outbreak of HP PRRS in a 1200 sow pig farm started in April of 2008 in Guangdong province, and it was controlled by Ingelvac ${ }^{\circledR}$ PRRS MLV mass vaccination one month later.

\section{Materials and methods}

This pig farm was built in 1990,, with two units of 600 sows each, continuous flow management. Two-hundred newly purchased gilts were introduced into the breeding herd without acclimation in Jan.2007. PHFD broke out with evident clinical signs from Apr. 20th, whereby all animals seemed to be affected with following clinical signs: Anorexia, lethargy, dyspnea, hyperthermia and discolouration of the skin. On April 25th, abortion, preterm birth and stillbirth started. Until Apr. 30th, 80 sows aborted and 120 sows were culled.

On 26th, 40 blood samples and 5 tissue samples from the abortion embryo and stillbirth were sent to Guangdong swine disease diagnosis center for antibody and antigen test.

On May 1, all no-sign breeders, healthy suckling piglets were vaccinated with Ingelvac ${ }^{\circledR}$ PRRS MLV. Then breeders were given second shot 4 weeks later. Replacement gilts were vaccinated twice at a 4 -week interval before mating. Piglets were given one shot at 2 weeks of age. Antibiotics were administered in water or feed against bacterial infection at same time.

\section{Results}

Table 1. Lab diagnosis results before Ingelvac PRRS MLV vaccination

\begin{tabular}{|c|c|c|c|}
\hline \multicolumn{2}{|c|}{ PRRS Antibody Test } & \multicolumn{2}{c|}{ HP PRRSv PCR test } \\
\hline samples & positive & samples & positive \\
\hline 40 & 20 & 5 & 5 \\
\hline
\end{tabular}

On May 10, sows started returning to normal appetite. Abortion reduced to 20 litters and no death was observed following start of vaccination. All pigs recovered in July. Reproductive performance of all sows in each quarter was getting better and better after vaccination, and even better than that before PHFD outbreak.

Table 2. Average reproductive performance each quarter in 2008

\begin{tabular}{|l|c|c|c|c|}
\hline & Jan-Mar & Apr-Jun/Apr & Jul-Sept & Oct-Dec \\
\hline Farrowing (\%) & 81 & $70 / 14$ & 83 & 85 \\
\hline Abortion (\%) & 4 & $21 / 72$ & 3 & 3 \\
\hline Pigs born alive/litter & 9.2 & $7 / 4.5$ & 9.6 & 9.5 \\
\hline Stillbirth (\%) & 8.8 & $20 / 35$ & 6.7 & 5.8 \\
\hline suckling interval (\%) & 93 & $85 / 70$ & 93 & 94 \\
\hline Nursery survival (\%) & 95 & $88 / 65$ & 93 & 96 \\
\hline sows culled & 93 & $214 / 150$ & 94 & 90 \\
\hline sows died & 8 & $20 / 18$ & 0 & 0 \\
\hline
\end{tabular}

${ }^{*}$ PHFD broke out on Apr.20. Ingelvac ${ }^{\circledR}$ PRRS MLV intervention started from May 1.

\section{Discussion}

Analyzing the data recorded in 2008, we found that Ingelvac ${ }^{\circledR}$ PRRS MLV improved the reproductive performance by two times of vaccination in 4 weeks. One shot of Ingelvac ${ }^{\circledR}$ PRRS MLV to piglets obviously reduced the mortality and morbidity in nursery. Ingelvac ${ }^{\circledast}$ PRRS MLV provided cross-protection against HP PRRS in the farm. In conclusion, Ingelvac ${ }^{\circledR}$ PRRS MLV is a useful tool to control and prevent PHFD.

\section{References}

1. Zhou YJ, Hao XF, et.al, Highly virulent porcine reproductive and respiratory syndrome virus emerged in China. Transbound Emerg Dis. 2008 May;55(3-4):152-64. 


\title{
P.213
}

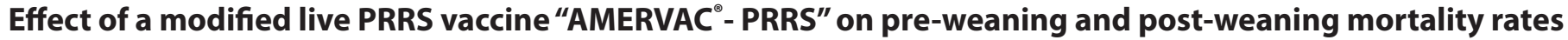

\author{
Michael E. Quilitis $^{2} \underline{\text { Sergi D. Bruguera }}^{1}$ Redentor A. Manuel ${ }^{2}$ Maria I. Torres ${ }^{2}$ Dani G. Torrents ${ }^{3}$ David V. Llopart $^{1}$ \\ 1. HIPRA, Technical Services, Amer, Spain; 2. HIPRA Philippines, INC. Technical Services, \\ Manila, Philippines; 3. HIPRA China, Technical Services, Beijing, China
}

\section{Introduction}

In previous studies in Asia, in spite of the increasing new PRRSV strain diversity ${ }^{3}$, vaccination programs with a live PRRS European (EU) strain vaccine could reduce the mortality in piglets ${ }^{1}$ and in growing-finishing pigs $^{2}$. The objective of this study was to monitor the effect in pre-weaning and post-weaning mortality rates of a modified live virus PRRS EU strain vaccine (AMERVAC ${ }^{\bullet}$-PRRS) program in sows and piglets.

\section{Materials and methods}

A 4000 sow level farm located north of Manila, Philippines, had a complaint of high pre-weaning (weaning at 4 weeks of age) and post-weaning (4-10 weeks of age) pig mortalities. PRRSV diagnosis was confirmed with the use of a commercial serology ELISA kit (CIVTEST ${ }^{\mathrm{TM}}$ suisPRRS E/S, HIPRA) an early serum conversion at 6-7 weeks of age. Gestating sows farrowed infected piglets which infected other litter mates before weaning time. So after veterinary advice, two mass vaccinations with a live PRRSV vaccine (AMERVAC ${ }^{\circ}$-PRRS) in breeders within 4 weeks interval were implemented in November 2007. At the same moment sows were administered with the second mass vaccination (December 2007), piglet vaccination started. Every piglet at 25 days of age was vaccinated intramuscularly with one shot of the same live PRRSV vaccine. Pre-weaning and post-weaning pig mortality rates were compared between batches of pigs 6 months before and 6 months after the implementation of the second mass vaccination in sows (December 2007).

\section{Results}

Mortality rates were significantly decreased $(\mathrm{P}<0.001 ; \mathrm{t}$ test) after the vaccine program implementation (Table 1). Pre-weaning and post-weaning mortality rates decreased $75 \%$ and $80 \%$ respectively.

Table.1. Monthly mean (Mean \pm SD) pre-weaning (Pre) and postweaning (Post) pig mortality 6 months before and 6 months after PRRSV vaccine implementation.

\begin{tabular}{|l|c|c|}
\hline Treatment & \% Pre. Mortality & \% Post. Mortality \\
\hline Before vaccination & $20.0 \pm 3.27$ & $9.9 \pm 1.83$ \\
\hline After vaccination & $5.06 \pm 1.48^{*}$ & $1.8 \pm 1.16^{*}$ \\
\hline *Results were significantly different, $\mathrm{P}<0.001$. \\
\hline
\end{tabular}

\section{Discussion}

We can observe that as in previous field trials ${ }^{4,5}$, a mass vaccination in breeders with a live PRRSV vaccine resulted in a sow protected population, which farrowed healthier new born piglets and consequently induced a decrease of pre-weaning and post-weaning mortality rates. Vaccinated pigs at 3 weeks of age showed a lower post-weaning mortality rates that may be
Graphic 1. Monthly mean (Mean \pm SD) pre-weaning (Pre) and postweaning (Post) pig mortality 6 months before and 6 months after PRRSV vaccine implementation.

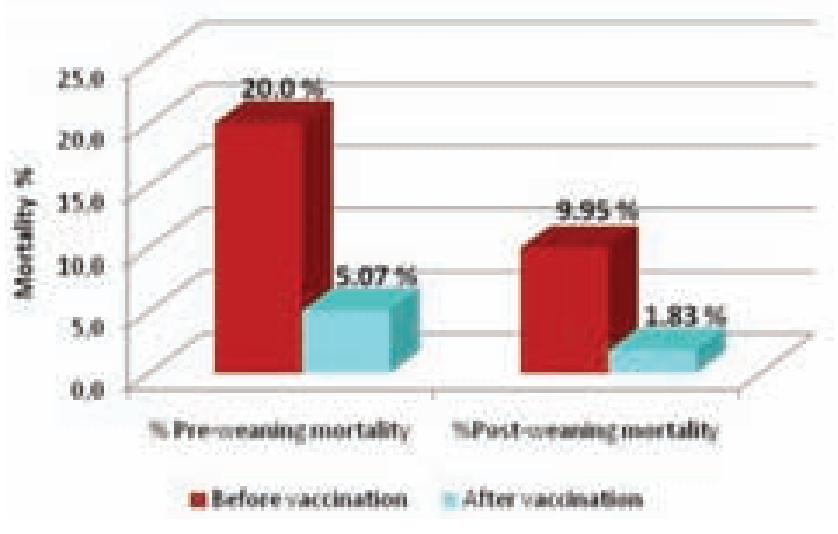

maintained during fattening period as in Guerzón, V. et al, 2008. In spite of the increasing diversity of PRRSV strains described worldwide ${ }^{3}$, in this field experience in Asia, a PRRSV vaccine, available in the field for more than 10 years (AMERVAC ${ }^{\oplus}$-PRRS) was able to induce enough protection to reduce the negative impact of a PRRSV infection. Actual PRRSV strain classification according to genetic sequence of the ORF 5 RNA fragment of the PRRSV genome is not useful to forecast cross protection ${ }^{6}$. Previous studies confirmed that distant PRRSV strains according to this ORF 5 sequence classification, contrary to what was expected, can present clear cross protection 7 . Therefore, further studies are needed to disclose either viral structures or immune path ways or genetic markers that may determine cross protection between PRRSV strains.

\section{Conclusion}

Within these field conditions a vaccination program in sows and piglets with an EU MLV PRRSV vaccine (AMERVAC ${ }^{\bullet}$-PRRS) reduced pre-weaning and post-weaning pig mortalities caused by a PRRSV infection.

\section{References}

1. Bell, D et al. 2007. Proceedings of the 3rd Congress of the Asian Pig veterinary Society (APVS) Wuhan; China. 2007. pag 135.

2. Guerzon, V. et al. 2008. Proc. IPVS 2008.P01.118.

3. Hu, H. et al. 2009. Arch Virol. 2009;154(3):391-8. Epub 2009 Jan 30.

4. Kim, S., Lange, S. (2005) Proc. 2nd APVS p. 111.

5. Maala et al. 2006. Proc. IPVS 2006 p. 39.

6. Prieto, C. Et al. 2008. Vet J. 2008 Mar;175(3):356-63.Epub 2007 Jun 8

7. Scortti. M. Et al. 2006. Theriogenology 66 (2006) 1884-93. 


\title{
P.214
}

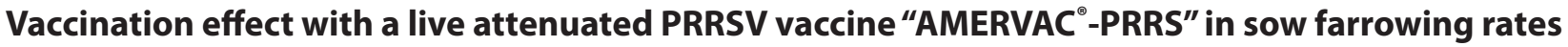

\author{
$\underline{\text { Michael E. Quilitis }}^{2} \underline{\text { Sergi D. Bruguera }}^{1}$ Ruel C. Macaraig² Maria I. Torres ${ }^{2}$ Dani G. Torrents ${ }^{3}$ David V. Llopart $^{1}$ \\ 1. HIPRA. Technical Services, Amer, Spain; 2. HIPRA Philippines, INC. Technical Services, \\ Manila, Philippines; 3. HIPRA China. Technical Services, Beijing, China
}

\section{Introduction}

Porcine Reproductive and Respiratory Syndrome Virus (PRRSV) is one of the primary precursors of economic losses ${ }^{2}$ in pig production. Acute impact costs range from \$236-502 per sow per year ${ }^{3}$. Clinical signs include decreasing farrowing rates, abortions, stillborns and increase of respiratory clinic problems in pigs.

The objective of this field experience is to assess the effect of a live PRRSV European (EU) vaccine (AMERVAC ${ }^{\circ}$-PRRS) in the monthly farrowing rates in a farm endemically infected by PRRSV.

\section{Materials and methods}

A 2500 sow farm with a 2 site production system that is located in the southern Philippines had a complaint of unstable and variable farrowing rates. PRRSV diagnosis was based on Polymerase chain reaction technique $3(P C R)$ from lungs and lymph nodes from stillbirths and 1 day old weak born piglets. A PRRSV vaccination program was implemented in sows at October 2007, using a modified live PRRSV European (EU) vaccine (AMERVAC ${ }^{\oplus P R R S)}$. Every batch of sows was vaccinated with one dose at 60 days of pregnancy and another dose 7 days after each farrowing. Farrowing rates from April 2007 until December 2009 were monitored. Farrowing rates from April to October 2007 were compared with farrowing rates from November 2007 till December 2009 (unpaired t test).

\section{Results}

Variable and unstable farrowing rates were monitored from April to October 2007. The monthly farrowing rates after vaccine implementation became less variable and were significantly higher (Table 1) along the whole monitored period than in previous unvaccinated sows.

Table 1. Monthly mean (Mean $\pm S D$ ) sow farrowing rates before and after PRRSV vaccine program implementation in sows.

\begin{tabular}{|l|c|}
\hline Groups & \% Farrowing rate \\
\hline Before vaccination & $75.7 \pm 7.08^{*}$ \\
\hline After vaccination & $82.3 \pm 3.58^{*}$ \\
\hline *Results were significantly different, $\mathrm{P}<0.001$. \\
\hline
\end{tabular}

Graphic.1. Monthly sow farrowing rates evolution from April 2007 to December 2009. Discontinuous line marks the beginning of the vaccine program.

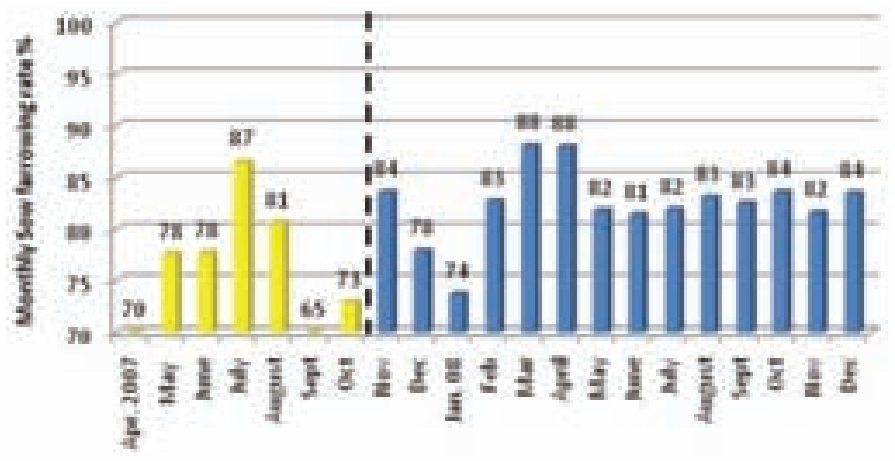

\section{Discussion}

The monthly farrowing rates seem to get stabilized around 3-4 months after the beginning of the vaccine program implementation (graphic.1.). This slow recovery may be related with the necessary time to administer at least one vaccine dose to all the sows in the farm. Contrary to this experience, mass PRRSV vaccination programs in sows are more usually applied ${ }^{1}$. Mass live PRRSV vaccination may build up faster vaccine immune response in the whole sow population just with the first dose. Nevertheless in the case of PRRSV infection the time needed to come back to normality after PRRSV infection depends also on some other factors, which are not directly related to vaccines or vaccination programs.

\section{Conclusion}

The PRRSV vaccination program implemented within these field conditions achieved to improve and stabilize sow monthly farrowing rates along the whole experiment.

\section{References}

1. Bell, D et al. 2007. Proceedings of the 3rd Congress of the Asian Pig veterinary Society (APVS) Wuhan; China. 2007.pag 135.

2. Desrosiers, R. 2006 Proceedings of the North Carolina Health Hogs Seminar.

3. Yoon KJ. 2007 Proceedings of the Asian Pig Veterinary Society. 


\title{
P.215
}

\section{Effect of a PRRSV (Porcine Reproductive and Respiratory Syndrome Virus) vaccination program in sows with a live attenuated PRRSV vaccine, in a Spanish farm, which implemented first parity sows segregation}

\author{
Pedro U. Sánchez ${ }^{3}$ Ramón G. Riquelme ${ }^{1}$ Sergi D. Bruguera ${ }^{1}$ Pere P. Riera ${ }^{1}$ Dani G. Torrents ${ }^{2}$ David V. Llopart \\ 1. HIPRA. Technical Services, Amer, Spain; 2. HIPRA China. Technical Services, Beijing, China; \\ 3. Piensos Garla S.A. Technical Director, Huercal Overa (Almeria), Spain
}

\section{Introduction}

Sows infected by Porcine Reproductive and Respiratory Syndrome Virus (PRRSV) reduce farrowing rates and farrow infected piglets that will suffer higher pre-weaning and post-weaning mortality rates. The objective of this field experience was to assess the effect of a PRRSV vaccination protocol in multiparous (MS) and first pregnancy sows (FPS) in a PRRSV infected farm.

\section{Materials and methods}

The study is carried out in a 4000 sow farm located in South East Spain, endemically infected with a PRRSV. This is a farm that rears pigs only till $20 \mathrm{Kg}$ live weight (8-9 weeks of age). Gilts (G) and FPS are segregated and they remain separated from MS. From June 2008 return to oestrus \% (RTO\%) increased either in MS or FPS. Besides this, born alive piglets per litter (TAL) and weaned piglets per litter (TWL) decreased. From August to October 2008 post-weaning (from 3 to 8-9 weeks of age) mortality \% (PM\%) increased dramatically in pigs from both FPS and MS. PRRSV infection diagnosis was confirmed with the use of a commercial serology ELISA kit (CIVTEST ${ }^{\mathrm{T}}{ }^{\mathrm{s}}$ suisPRRS E/S, HIPRA) in sows and PCR PRRSV detection from stillbirths and dead pigs. In September and again in October 2008, a mass vaccination was implemented in all MS, FPS and $\mathrm{G}$ with a live PRRSV vaccine (AMERVAC PRRS). Afterwards a mass vaccination with AMERVAC ${ }^{\oplus}$-PRRS was implemented regularly every 4 months in MS and FPS. Every new batch of $\mathrm{G}$ was vaccinated twice with AMERVAC ${ }^{\circ}$-PRRS one moth apart before the first mating. In this field experience we monitored monthly RTO\%, TAL, TWL and PM\%.

\section{Results}

TWL improved in MS and in FPS but the improvement was more remarkable in FPS (Graph.2). RTO\% (Graph.1) decreased constantly from the first mass vaccination program in MS. RTO\% from FPS decreased every month but in February 09 RTO\% of FPS increased again till 28,2\%. After this increase all the MS and FPS were vaccinated again (defined in material and methods) and the next month RTO\% in FPS was reduced back to normality. Pigs in post-weaning period from non-yet vaccinated MS and FPS suffered a peak of mortality in September and October 08 (Graph.3). Weaned pigs from vaccinated sows may be healthier and PM\% decreased markedly from November 2008 till the end of the study.

\section{Discussion}

The decreasing RTO\% and the clear increasing trend of TWL in pigs from MS and FPS indicate, as in previous experiences ${ }^{1,2}$, that the immune response induced by the vaccine may build up a protection in sows that reduced the birth of infected piglets ${ }^{2}$. This effect probably reduced also the PM\%, especially in batches of weaned piglets from FPS (Graph.3). According to these results FPS and their progeny seemed to suffer more severely during a PRRSV infection. This observation occurs frequently in the practice and recalls the need to submit new replacement gilts to proper acclimatisation procedures against PRRSV, previous to be introduced in an endemically PRRSV infected farm.

\section{Conclusion}

Within these field conditions, a vaccination program with AMERVAC ${ }^{\circ}$-PRRS in sows induced an immune response that reduced markedly the negative impact of a wild PRRSV infection in sows and their progeny.

\section{References}

1. Bell, D et al. (2007) Proceedings of the 3rd Congress of the Asian Pig veterinary Society (APVS) Wuhan; China. 2007. pag 135

2. Scortti, M. et al. 2006. Theriogenology. 2006 Nov; 66(8):1884-93. Epub 2006 Jun 27.

Graph 1. Return to oestrus \% in MS (RTO\% MS) and FPS (RTO \% FPS).

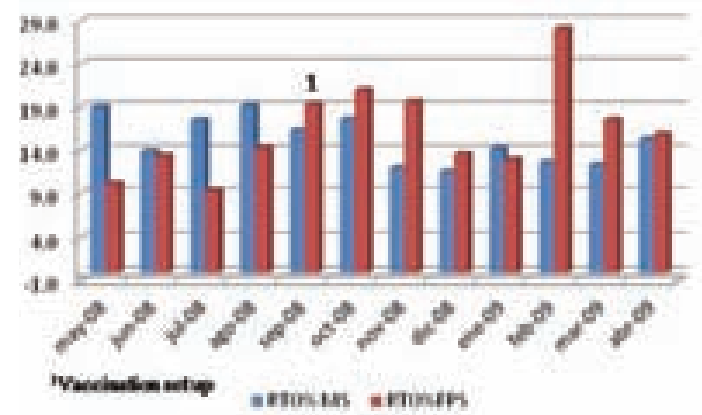

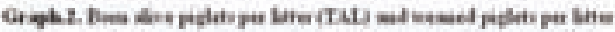

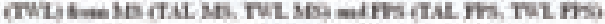

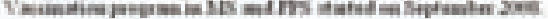

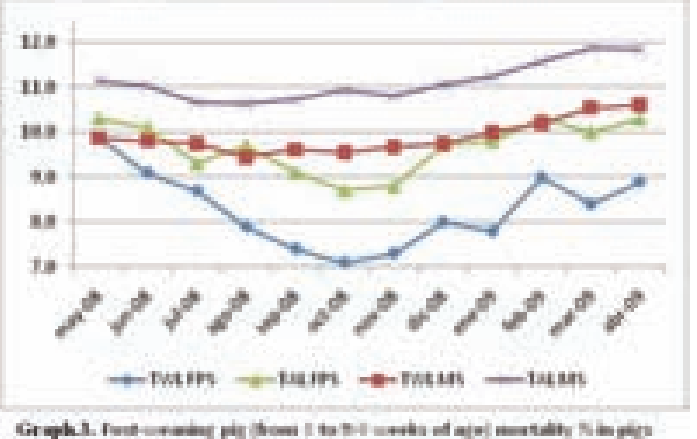

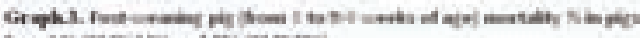

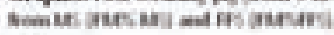

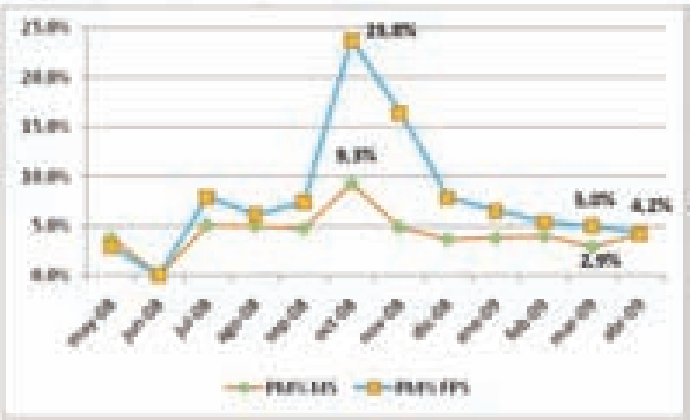




\title{
P.216
}

\section{Evaluation of a porcine reproductive respiratory syndrome virus (PRRSV) vaccination program with a modified live PRRSV Vaccine during 3 consecutive years in a Polish farm}

\author{
Isaac R. Ballarà ${ }^{2}$ Sergi Bruguera ${ }^{2}$ Robert Panek $^{1}$ David Llopart $^{2}$ \\ 1. HIPRA Polska, Warsaw, Poland; 2. HIPRA, Technical Services, Amer, Spain
}

\section{Introduction}

Porcine reproductive and respiratory syndrome is an economically significant disease. Polish farms have suffered the two forms of this syndrome either from reproductive or respiratory clinic episodes ${ }^{1}$. Modified live vaccines (MLV), in several field and experimental cases, have induced enough protection to control the negative clinic signs of PRRSV infection ${ }^{1}$. The objective of this study is to evaluate the efficacy of a PRRSV vaccination program, in sows and pigs, with a modified live European (EU) PRRSV vaccine (AMERVAC ${ }^{\circledR}$-PRRS Laboratorios HIPRA S.A.) along 3 consecutive years.

\section{Materials and Methods}

This study was performed in a one site farrow to finish farm with 2000 reproductive sows, located in Swidwin, Poland. We evaluated the PRRSV situation through several transversal serumprofiles (CIVTEST ${ }^{\mathrm{TM}}$ suisPRRS E/S ELISA). The first serumprofile was performed in November 2005, in this moment the farm were suffering high pre-weaning and post-weaning mortality, and acute reproductive failure in sows. The second serumprofile was performed in October 2006, and the last one in October 2007. The reproductive parameters in sows, pre-weaning and post-weaning pig performance parameters were monitored from November 2005 to October 2007. On December 2005 sows were vaccinated with a mass vaccination with a MLV vaccine (AMERVAC ${ }^{\circledR}$-PRRS). One month after the mass vaccination sows were vaccinated with the following regular vaccination program: Gilts: One vaccine dose at 22 weeks of age and a second dose at 25 weeks of age in gilts. Sows: They were vaccinated in each gestation at 56 days of pregnancy, and once again in lactation period at 6 days after farrow. Piglet vaccination didn't start till March 2006; piglets were vaccinated with one dose of AMERVAC ${ }^{\circledR}$-PRRS at 26-28 days of age.

\section{Results}

After the implementation of the vaccination the farm became stabilized clinically and serologically, with a relevant reduction every year of \% piglet mortality (Graphic 1 and Table 2), \% mummified piglets, \% stillbirth, \% weak born piglets and the number of pigs in hospital pens.

Moreover a significant increase of average piglet weight at birth and at 10 weeks of age was recorded (Table 2). Sows also increase clearly the farrowing rates during the three year period (Table 1).

\section{Table 1.}

\begin{tabular}{|l|c|c|c|}
\hline SOWS & $\mathbf{2 0 0 5}$ & $\mathbf{2 0 0 6}$ & $\mathbf{2 0 0 7}$ \\
\hline $\begin{array}{l}\text { \% return to } \\
\text { oestrus }\end{array}$ & 26,2 & 20,4 & 18,4 \\
\hline$\%$ farrowing rate & 68,4 & 75,2 & 78 \\
\hline
\end{tabular}

Table 2

\begin{tabular}{|l|c|c|c|}
\hline PIGS & $\mathbf{2 0 0 5}$ & $\mathbf{2 0 0 6}$ & $\mathbf{2 0 0 7}$ \\
\hline Total born & 10,1 & 10,9 & 11 \\
\hline Total born alive & 9,41 & 10,3 & 10,5 \\
\hline Mummified piglets & 0,2 & 0,1 & 00,5 \\
\hline Stillbirth & 0,7 & 0,6 & 0,5 \\
\hline \% Weak born piglets & 14 & 10 & 10 \\
\hline$\%$ pre-weaning mortality & 13,5 & 10,4 & 8,9 \\
\hline$\%$ post-weaning mortality & 10,8 & 6,2 & 4,3 \\
\hline \% piglets treated & 18,6 & 12,1 & 9,4 \\
\hline Number of pigs in hospital pens & 40 & 25 & 18 \\
\hline Average piglet born weight & 1,32 & 1,44 & 1,48 \\
\hline Average pig weight at 10 weeks & 23,8 & 24,9 & 26,2 \\
\hline
\end{tabular}

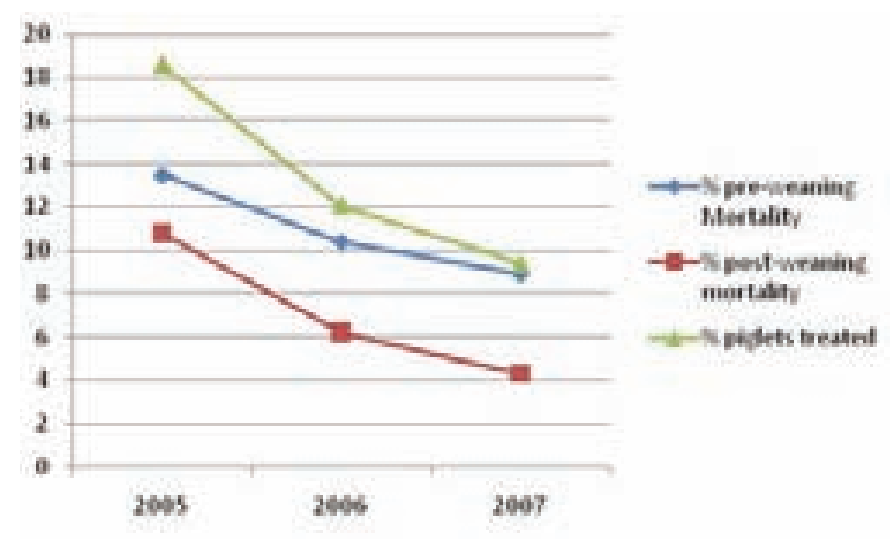

\section{Discussion}

MLV vaccine efficacy in pigs and in sows has been also described previously. In spite of these positive long lasting results, PRRSV control depends also on other important factors rather than vaccines or vaccination programs as proper gilt replacement policies or strict biosafety measures. Regardless the increasing diversity of PRRSV strains described recently in Poland ${ }^{2}$, a PRRSV vaccine, available in different European and Asian countries for several years (AMERVAC ${ }^{\circledR}$-PRRS) was able to induce enough cross protection to reduce the negative impact of a PRRSV infection.

\section{Conclusion}

We concluded that the use of a MLV vaccination program in sows and piglets during the whole studied period resulted in the control of the negative effects of a PRRSV infection and improved sows and piglets performance.

\section{References}

1. Kim, S., Lange, S. (2005) Proc. 2nd APVS, Manila, Philippines. p.111.

2. Stadejek T, et al. 2006. The Journal of General Virology $2006 \mathrm{Jul} ; 87(\mathrm{Pt}$ 7):1835-41. 


\title{
P.217
}

\section{Viability of PRRS (Porcine Reproductive and Respiratory Syndrome) vaccine virus VP046-BIS (AMERVAC - PRRS) when it was mixed with an inactivated Haemophilus parasuis vaccine (HIPRASUIS ${ }^{\diamond}$-GLASSER)}

\author{
Sergi Bruguera $^{3}$ Reina Alemany ${ }^{2}$ Daniel Torrents ${ }^{1}$ \\ 1. HIPRA China, Technical Services, Beijing, China; 2. HIPRA, Research \& Development, Amer \\ (Girona), Spain; 3. HIPRA, Technical Services, Amer (Girona), Spain
}

\section{Introduction}

In Asia, nowadays, pig producers need to protect their pigs and sows against several pathogens. Many vaccination programs have to be implemented in specific close periods during the pigs' life either whether it is in sows during the gestating or lactating period or whether it is during the first weeks of life in pigs. Then the possibility of reducing or administering vaccines simultaneously can potentially improve either the pig welfare status or the labour efficiency. Therefore, the objective of this trial is to find out if the attenuated PRRS vaccine virus VP046-BIS (AMERVAC ${ }^{\circ}$-PRRS) can keep alive when it is reconstituted with an inactivated Haemophilus parasuis vaccine (HIPRASUIS ${ }^{\circ}$-GLÄSSER) as a previous step to try in vivo the actual effect of these combined vaccines.

\section{Material and methods}

Material: 50 dose freeze-dried tablets of AMERVAC ${ }^{\circledR}$-PRRS vaccine (attenuated PRRS virus), 50 dose of inactivated Haemophilus parasuis vaccine HIPRASUIS ${ }^{\circledR}$-GLÄSSER, $100 \mathrm{ml}$ aqueous solvent vials and CLON8 cell line.

Methods: Two tablets of AMERVAC ${ }^{\circ}$-PRRS were reconstituted with HIPRASUIS -GLÄSSER and with aqueous solvent respectively. After 0, 2, 4 and 6 hours post reconstitution the virus was titred in CLON8 cell line.

\section{Results}

Table 1. Results of titres per dose of PRRS vaccine virus VP046-BIS. Vaccine virus titres VP046-BIS per dose (2ml) (TCID 50 dose). Regarding by CPE (Cytopathic Effect).

\begin{tabular}{|c|c|c|}
\hline $\begin{array}{c}\text { Hours at standard Temperature } 2^{\circ}{ }^{\circ} \mathrm{C} \\
\text { after reconstitution }\end{array}$ & AQUEOUS SOLVENT & $\begin{array}{c}\text { HIPRASUIS } \\
\text { GLASSER }\end{array}$ \\
\hline 0 & $10^{4.4}$ & $10^{4.5}$ \\
\hline 2 & $10^{4.3}$ & $10^{4.4}$ \\
\hline 4 & $10^{4.2}$ & $10^{4.2}$ \\
\hline${ }^{*} 6$ & ${ }^{*} 10^{4.1}$ & ${ }^{*} 10^{4.1}$ \\
\hline
\end{tabular}

* PRRS vaccine virus VP046-BIS (AMERVAC -PRRS) kept viable and with a dosage titre value equivalent or higher of $10^{3.5} \mathrm{TCID}_{50}$ (minimum necessary effective concentration as product manufacturer recommendation) at 6 hours after reconstitution with the inactivated Haemophilus parasuis vaccine HIPARSUIS ${ }^{\circ}$ GLÄSSER (100ml). The same results were obtained when mixed with the usual prescribed aqueous solvent $(100 \mathrm{ml})$.

\section{Discussion}

This in vitro trail experience may be also applicable in vivo as in Roof et al 2000. In Roof et al 2000, there was no interference between vaccine antigens either when a live PRRSV vaccine was reconstituted with a multi-antigen inactivated vaccine and administered in pigs.

\section{Conclusion}

HIPRASUIS $^{\circ}$-GLÄSSER vaccine allows the viability of the attenuated PRRS vaccine virus VP046-BIS (AMERVAC ${ }^{\circ}$-PRRS) even 6 hours after the reconstitution. Further studies in vivo are required to discard any vaccine incompatibility.

\section{References}

Roof, M.B. Burkhart, K. Schlesinger, K. Hayes, P. 2000. The 16th International Pig Veterinary Society Congress, Melbourne, Australia, 17-20 Sept. 2000. 


\title{
P.218
}

\section{In vitro evaluation of the impact of a needleless device on Ingelvac ${ }^{\bullet}$ PRRS ATP vaccine virus viability}

\author{
Enrique Mondaca ${ }^{1}$ Albert Rovira ${ }^{2}$ Dale Polson ${ }^{1}$ \\ 1. Boehringer Ingelheim Vetmedica, Inc., St Joseph, MO, USA; 2. College of Veterinary Medicine, University of Minnesota, St Paul, MN, USA
}

\section{Introduction}

Needle-free injection devices deliver product by pressure directly into subcutaneous or muscular tissue layers. This technology has been used in human and veterinary medicine since mid 1900 's, offering some advantages over conventional medicine delivery methods: elimination of broken needles, consistent vaccine delivery, elimination of accidental worker needle sticks, elimination of needle disposal, and less pain and stress ${ }^{1}$. It also has an significant potential as a tool when population-based injectable interventions are suitable for large livestock systems such as area/regional disease control programs. Some of their disadvantages include the relatively large initial financial investment in equipment and the need for technical training. Another potential disadvantage is the effect of the device's high pressure on the integrity of viral particles when applying modified live vaccines.

The aim of this study was to determine if the needle-less injector AcuShot $^{\mathrm{TM}}$ (AcuShot, Inc., Winnipeg, CA) affects the viability of Ingelvac ${ }^{\oplus}$ PRRS ATP (Boehringer Ingelheim Vetmedica, Inc., St. Joseph, MO) vaccine virus by comparing samples obtained directly from the bottle of vaccine to samples obtained through 10 of the AcuShot ${ }^{\mathrm{TM}}$ devices.

\section{Materials and Methods}

$\mathrm{A} \mathrm{TCID}_{50}$ assay was run at the Virology Laboratory of the University of Minnesota on samples of Ingelvac ${ }^{\circledR}$ PRRS ATP vaccine virus obtained through: AcuShot ${ }^{\mathrm{TM}}$ devices, conventional needlesyringe, and pipetted directly from the vaccine bottle (controls). Thirty five samples were taken from each of the 12 treatments in study: AcuShot ${ }^{\mathrm{TM}}$ devices (10), conventional needle-syringe (1), and pipetted directly from the vaccine bottle (control, 1). A sample size of 35 was determined applying Minitab ${ }^{\circledR}$ software version 15.1.0.0 (Minitab Inc., State College, PA), to detect a maximum difference between means of $0.3 \log _{10}$ or about half a recommended dose of vaccine at $\mathrm{P}<0.05$, and power $>80 \%$.

The statistical analysis was run with Minitab applying ANOVA and Tukey's test for comparison of means.

\section{Results}

$\mathrm{TCID}_{50}$ mean values $\left(\log _{10}\right)$ did not differ significantly between any of the eleven treatments (ten Acushot devices and one needle/syringe) and the Control (samples pippeted directly from the bottle). The $95 \%$ confidence interval for all eleven treatments included zero, indicating no difference at $\mathrm{P}<0.05$ from the Control (Figure 1).
Figure 1.95\% confidence intervals for the differences in mean $T C I D_{50}$ values from Controls.

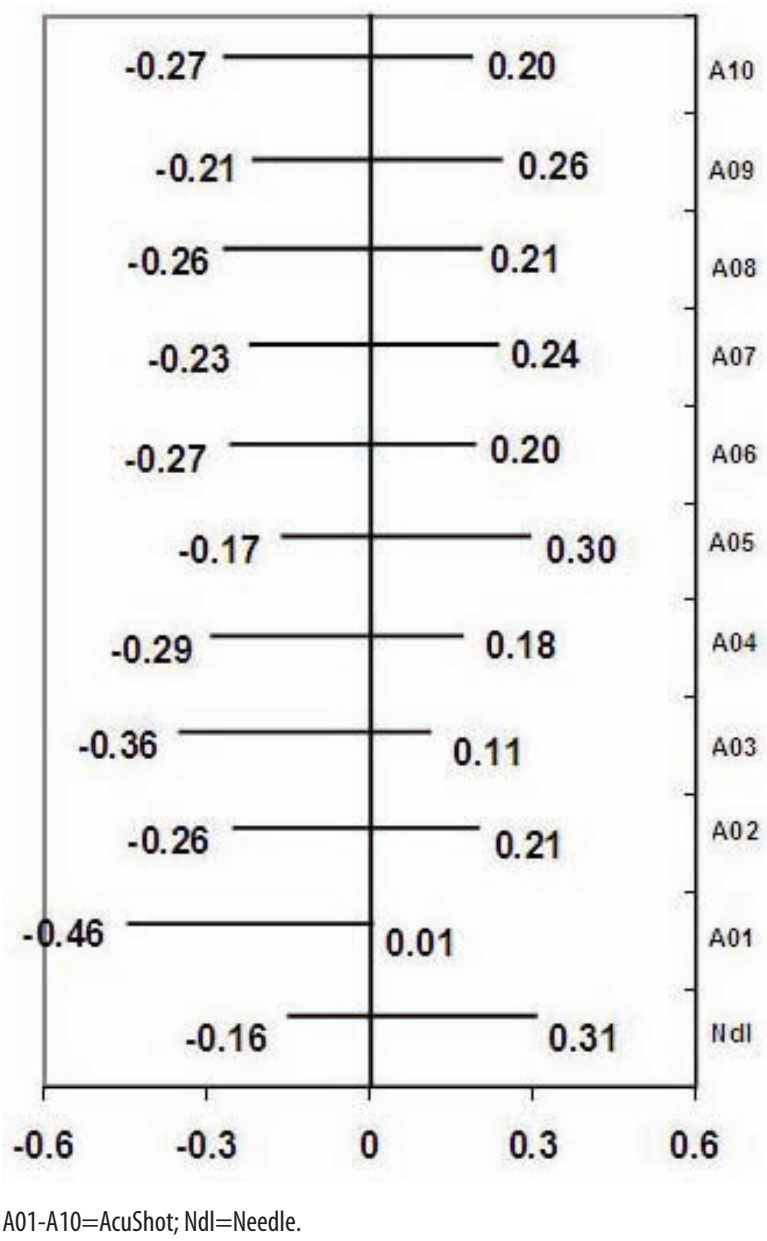

\section{Discussion}

The results of this study indicate that the viability of Ingelvac ${ }^{\circ}$ PRRS ATP vaccine is not affected when applied through AcuShot $^{\mathrm{TM}}$ needleless devices. Further evaluation of needleless devices under field conditions is warranted to ensure their suitability for use in swine production.

\section{References}

1. Chase, C. JSHAP 2008, 16(5):254-261. 


\title{
P.219
}

\section{Effect of a PRRS modified live vaccine (AMERVAC - PRRS) on mortality rates in a pig farm in Venezuela}

\author{
Carlos Rojo ${ }^{4}$ Gustavo Garcia ${ }^{3}$ Daniel Torrents ${ }^{1}$ Sergi Bruguera $^{2}$ \\ 1. HIPRA China, Technical Services, Beijing, China; 2. HIPRA, Technical Services, Amer (Girona), Spain; 3. \\ Orograinca, Turmero (Aragua), Venezuela; 4. Agrosuplidores, fl., Valencia, Venezuela
}

\section{Introduction}

Porcine Reproductive and Respiratory Syndrome (PRRS) is an economically important disease in pig production, mainly due to losses related to reproductive failure in sows and high mortality rates in commercial pigs from lactation to growing- finishing phase. In Venezuela, PRRS virus (PRRSV) was first identified in $1996^{1}$ and nowadays is and endemic pathogen ${ }^{2}$ causing big impact on the productive parameters of swine farms in Venezuela ${ }^{3}$. The objective of this study was to monitor the effect of a live European (EU) PRRSV vaccine (AMERVAC ${ }^{\circledR}$-PRRS) program on mortality rates in pigs at 3 different periods: pre-weaning, postweaning and growing-finishing period.

\section{Materials and methods}

An 800 sows farm located in Venezuela, had a complaint of high pig mortalities. PRRSV diagnosis was confirmed with PRRSV antibody detection in serum samples with a serology ELISA kit (HerdChek PRRS 2XR, IDEXX) in sows and pigs at early ages. After veterinary advice, in October and November 2008 within a 4 weeks interval, two mass vaccinations with a live PRRSV vaccine (AMERVAC ${ }^{\circ}$-PRRS) were implemented in breeders. From January 2009 onwards, every piglet at weaning was vaccinated intramuscularly with one shot of the same live PRRSV vaccine. Mortality rate was compared between batches of pigs 6 months before and 8 months after the implementation of the second mass vaccination in sows (November 2008).

\section{Results}

Mortality rates were significantly decreased $(\mathrm{P}<0.05$ or $\mathrm{P}<0.001$; unpaired $t$ test) after the vaccine program implementation (Table 1). Pre-weaning, post-weaning and growing-finishing mortality rates decreased $45 \%, 46 \%$ and $34 \%$ respectively.

Table 1. Monthly (mean $\pm S D$ ) pre-weaning (Pre), post-weaning (Post) and growing-finishing (G-F) pig mortality rates (\%) 6 months before and 8 months after PRRS vaccine implementation (November 2008).

\begin{tabular}{|l|c|c|}
\hline Period & Before vaccination & After vaccination \\
\hline$\%$ Pre & $15.5 \pm 1.1$ & $8.6 \pm 1.4^{* *}$ \\
\hline$\%$ Post & $4.8 \pm 0.3$ & $2.6 \pm 0.8^{* *}$ \\
\hline$\%$ G-F & $8.9 \pm 0.2$ & $5.9 \pm 2.8^{*}$ \\
\hline $\begin{array}{l}\text { * Results were significantly different, } \mathrm{P}<0.05 \\
\text { ** Results were significantly different } \mathrm{P}<0.001\end{array}$ \\
\hline
\end{tabular}

Figure 1.\% Pig mortality evolution from July 2008 till August 2008. Mass vaccination in breeders $\left({ }^{*}\right)$ set up on October 2008. Piglet vaccination at weaning set up on January 2009.

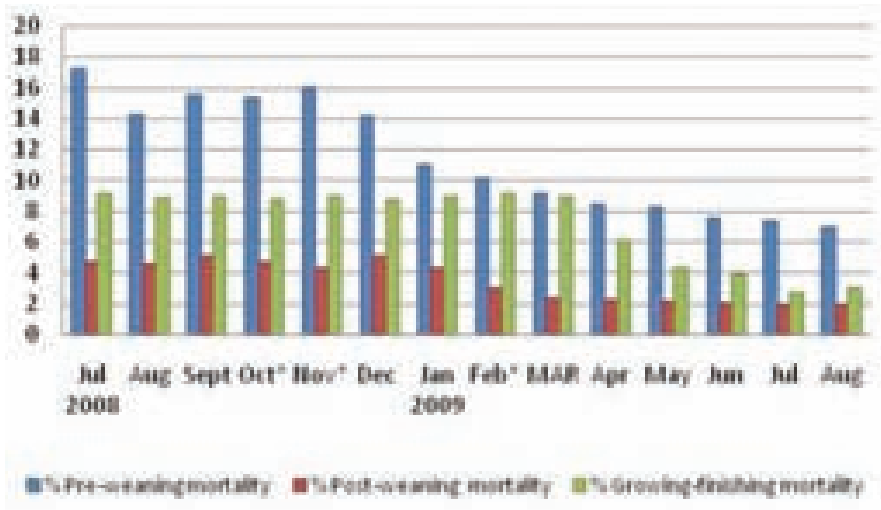

\section{Discussion}

Within these field conditions, we can observe that as in previous field trials ${ }^{4,5,6}$, a live PRRSV vaccine program resulted in a sow protected population, which farrowed healthier new born piglets that may respond properly to a intramuscularly live PRRSV vaccine dose and consequently induced a decrease of mortality rates during the whole growing period. In spite of the increasing diversity of PRRSV strains described worldwide ${ }^{7}$, in this field experience in Venezuela, (AMERVAC ${ }^{\circledast}$-PRRS) was able to induce enough protection to reduce the negative impact of a PRRSV infection.

\section{Conclusion}

Within these field conditions a vaccination program in sows and piglets with an EU MLV PRRSV vaccine (AMERVAC ${ }^{\circ}$-PRRS) reduced pig mortalities caused by a wild PRRSV infection in a pig farm in South America.

\section{References}

1. Diaz C. et al., 1999. Rev. Cient. FCV-LUZ, 9:215-22.

2. Cano JP. et al., Proc. IPVS 2002 p. 241.

3. Cano JP. et al., 2004. Rev. Fac. Cs. Vets.-UCV, 45:19-29.

4. Kim, S., Lange, S. Proc. 2nd APVS 2005 p. 111.

5. Maala et al. Proc. IPVS 2006 p. 39.

6. Quilitis M. et al., Proc APVS 2009 p 270.

7. Prieto, C. et al. 2008. Vet J. 2008 Mar;175(3):356-63. Epub 2007. 


\title{
Efficacy of AMERVAC ${ }^{\circ}$-PRRS vaccine on sow reproductive performance in a PRRSV (Porcine Reproductive and Respiratory Syndrome Virus) infected pig farm in Malaysia
}

\author{
Hang C. Lim² Ban K. Lim² Pow Y. Choo² Sergi D. Bruguera' ${ }^{1}$ \\ 1. HIPRA. Technical Services, Amer, Spain; 2. Vet Food Agro Diagnostic (M) Sdn Bhd, Selangor, Malaysia
}

\section{Introduction}

PRRSV is a common pathogen causing respiratory and reproductive clinic signs in Malaysian pig farms. PRRS vaccination in sows with AMERVAC ${ }^{\circ}$-PRRS has been used in pig farms in different countries and the benefits have shown to be encouraging (Quilitis ${ }^{2}$ et al. 2009; Rodriguez ${ }^{3}$ et al. 2009). The objective of this trial is to monitor the effect of AMERVAC ${ }^{\circ}$-PRRS vaccination on sows' reproductive performance in an infected PRRSV farm and to determine whether there is any adverse reaction observed in pregnant sows post vaccination.

\section{Materials and methods}

A 1800 sow commercial farrow-finish pig farm located in Selangor state, Malaysia, was diagnosed with a PRRSV infection by means of the PRRSV antibody detection from serum samples with an ELISA kit test (Herdcheck 2XR, Idexx) and by PRRSV detection with a PCR technique (data not shown). Serology results indicated the presence of active PRRSV infection in the sows' population. On veterinary prescription, all breeders (sows and gilts) were vaccinated with a live European PRRS vaccine (AMERVAC ${ }^{\circ}$-PRRS) on September 2008 and boosted with the same vaccine 1 month later. Sows were then vaccinated regularly once every 4 months with AMERVAC ${ }^{\ominus}$-PRRS. The farm is using Pig Champ ${ }^{\circledast}$ recording system to monitor production performance of the farm. Reproductive performance data for a period of 5 months before and after AMERVAC ${ }^{\varpi}$-PRRS vaccination was collected and analyzed. The parameters and results observed are stated in Table 1.

\section{Results and Discussion}

There were no adverse reactions observed in sows after any of the vaccine inoculations. Reproductive parameters improved significantly after the vaccine program was implemented. A significantly improvement $(P<0.05)$ in farrowing rate $(+6 \%)$ and the average piglets born alive per litter $(+0.54)$ was observed. The Return to estrus rate and average pigs weaned per sow per litter were improved but the results were not significantly different 5 months after vaccine implementation. These results confirmed previous positive field experiences in Asia after the use of a live European PRRSV (genotype-I) vaccine in sows ${ }^{1,2}$.
Table.1. Sows' reproductive performance before and after vaccination with AMERVAC ${ }^{\circledR}-P R R S$.

\begin{tabular}{|l|c|c|c|}
\hline Parameters & Before & After & Difference \\
\hline $\begin{array}{l}\text { \% Sows on estrus within 7 days } \\
\text { after weaning }\end{array}$ & 83.52 & 88.03 & $+4.51^{*}$ \\
\hline \% Sows Return to estrus & 24.12 & 18.23 & $-5.89 \%$ \\
\hline Farrowing rate \% & 67.45 & 73.45 & $+6 \%^{*}$ \\
\hline $\begin{array}{l}\text { Average piglets born alive per } \\
\text { litter }\end{array}$ & 9.83 & 10.38 & $+0.54^{*}$ \\
\hline $\begin{array}{l}\text { Average piglets weaned per sow } \\
\text { per litter }\end{array}$ & 8.14 & 8.70 & +0.56 \\
\hline${ }^{*} P<0.05$ & & \\
\hline
\end{tabular}

\section{Conclusions}

Vaccination in lactating and gestating sows with AMERVAC ${ }^{\circ}-$ PRRS did not induce any adverse effect and improved reproductive performance in an endemic PRRSV infected farm in Malaysia.

\section{References}

1. Bell, D et al. 2007. Proceedings of the 3rd Congress of the Asian Pig veterinary Society (APVS) Wuhan; China. 2007. pag 135.

2. Quilitis, M, et al. 2009. Proceeding of the 4th Congress of Asian Pig Veterinary Society (APVS) Tsukuba, Japan. 2009, pg 271

3. Rodriguez, l et al. 2009. Proceeding of the 4th Congress of Asian Pig Veterinary Society (APVS) Tsukuba, Japan. 2009, pg 275 


\title{
P.221
}

\section{Protection efficacy of hyperimmune serum and vaccines from homologous and heterologous PRRS virus to challenge with highly pathogenic Chinese-type PRRSV}

\author{
Sergey A. Kukushkin; Alexander E. Fomin; Taufik Z. Baybikov; Elena P. Baborenko \\ FGl Federal Centre for Animal Health, Vladimir, Russian Federation
}

\section{Introduction}

Epidemic of highly virulent PRRS caused by atypical virus of North American (NA) genotype was registered in China and Vietnam in 2006-2007 (Tian et al., 2007; To et al., 2007). In August 2007 the similar virus - "Irkutsky 2007" strain - was isolated in the Irkutskaya Oblast of Russia, where the first outbreak of new atypical PRRS outside South-East Asia was registered (Kukushkin et al., 2008). The purpose of our study was to compare resistance of pigs to challenge with highly pathogenic Chinese-type PRRS virus after seroimmunization and vaccination with commercial vaccines from European, North American PRRSV genotypes and a vaccine from a homologous strain.

\section{Materials and Methods}

Twenty-six 4-month-old PRRS-seronegative piglets were divided into five groups: Group 1 (6 piglets) was single immunized with a commercial live vaccine from North American PRRSV genotype; Group 2 (5 piglets) was twice immunized with an inactivated vaccine from a homologous PRRSV “Irkutsky 2007" strain; Group 3 (4 piglets) was twice immunized with a commercial inactivated vaccine from European PRRSV genotype; Group 4 (8 piglets) was immunized with hyperimmune (positive) serum to a homologous PRRSV "Irkutsky 2007" strain at a dose of $1 \mathrm{ml} / \mathrm{kg}$ before challenge; Group 5 (3 piglets) was a non-immunized control. After immunization all piglets were intranasally challenged with a highly pathogenic Chinese-type PRRS virus "Irkutsky 2007" strain. Blood sera collected before and 16 days after inoculation were tested for PRRSV antibodies using ELISA (IDEXX, Westbrook, ME, USA). All animals were slaughtered on day 16 after challenge for examination of lung lesions score according to Halbur et al. (1995).

\section{Results}

Hyperthermia was registered in all groups. The most lasting hyperthermia was observed in Groups 3 and 5 (12-15 days, maximum $41.7^{\circ} \mathrm{C}$ ), hyperthermia of medium duration was recorded in Groups 2 and 4 (11-12 days, maximum $\left.41.8-41.9^{\circ} \mathrm{C}\right)$, the shortest hyperthermia was in Group 1 (7 days, maximum 40.7$41.0^{\circ} \mathrm{C}$ ). Disease clinical signs and their duration between groups were analogous to the manifestation of hyperthermia. Less pronounced clinical signs were present in the Group immunized with a commercial live vaccine.
The highest level of lung lesions was in Groups 5 and 3, the lowest level was in the Group immunized with a commercial live vaccine from $\mathrm{N}$. American genotype (Table 1).

\begin{tabular}{|l|c|c|c|c|}
\hline Group & $\begin{array}{c}\text { Number } \\
\text { of piglets }\end{array}$ & \multicolumn{2}{|c|}{$\begin{array}{c}\text { Mean antibody level in } \\
\text { ELISA IDEXX, s/p }\end{array}$} & $\begin{array}{c}\text { Average lung } \\
\text { lesions, } \%\end{array}$ \\
\hline $\begin{array}{l}\text { Live vaccine from NA } \\
\text { genotype }\end{array}$ & 6 & $0.85 \pm 0.65$ & $1.25 \pm 0.58$ & $0.38 \pm 0.72$ \\
\hline $\begin{array}{l}\text { Inactivated vaccine } \\
\text { from a homologous } \\
\text { strain }\end{array}$ & 5 & $1.51 \pm 0.99$ & $2.35 \pm 0.23$ & $12.03 \pm 12.80$ \\
\hline $\begin{array}{l}\text { Inactivated vaccine } \\
\text { from European } \\
\text { genotype }\end{array}$ & 4 & $2.70 \pm 0.08$ & $2.97 \pm 0.73$ & $28.69 \pm 17.63$ \\
\hline $\begin{array}{l}\text { Hyperimmune serum } \\
\text { to a homologous strain }\end{array}$ & 8 & $0.02 \pm 0.03$ & $1.42 \pm 0.53$ & $7.13 \pm 11.86$ \\
\hline $\begin{array}{l}\text { Non-immunized } \\
\text { control }\end{array}$ & 3 & $0.00 \pm 0.00$ & $1.35 \pm 0.43$ & $39.67 \pm 31.63$ \\
\hline
\end{tabular}

\section{Discussion and Conclusion}

Testing results showed that the best protection of piglets against challenge with highly virulent PRRS virus was provided by a commercial live vaccine from N. American genotype. Inactivated vaccine and hyperimmune serum from a homologous strain provided middle protection of lungs but did not prevent the appearance of clinical signs.

\section{References}

Halbur, P.G., et al., 1995. Vet. Pathol. 32: 648-660.

Kukushkin, S.A. et al., 2008. In: Proc. 2008 International PRRS Symposium, Chicago, Illinois, p. 23.

Tian, K. et al., 2007. PLoS ONE. 2(6), e526.

To, L.T. et al., 2007. In: Proc. 2007 International PRRS Symposium, Chicago, Illinois, p. 74. 


\title{
P.222
}

\section{PRRSV (type I) challenge in vaccinated and non-vaccinated pigs}

\author{
Andrea Ladinig ${ }^{1}$ Gyula Balka² Herbert Weissenboeck ${ }^{3}$ Armin Saalmueller ${ }^{4}$ Wilhelm \\ Gerner $^{4}$ Tobias Kaeser ${ }^{4}$ Miklós Rusvai ${ }^{2}$ Mathias Ritzmann ${ }^{1}$ \\ 1. Clinic for Swine, University of Veterinary Medicine Vienna, Vienna, Austria; 2. Department of Pathology and Forensic Veterinary Medicine, \\ Faculty of Veterinary Science, Szent István University, Budapest, Hungary; 3. Institute of Pathology and Forensic Veterinary Medicine, University \\ of Veterinary Medicine Vienna, Vienna, Austria; 4. Institute of Immunology, University of Veterinary Medicine Vienna, Vienna, Austria
}

\section{Introduction}

Recently it has been demonstrated that significant differences can be observed among Type 1 PRRSV isolates (1). Previous studies demonstrated that genetic diversity within European genotype may affect the efficacy of currently available vaccines (2).

The aim of the present study was to investigate the pathogenicity of a recently isolated virulent PRRSV Type I, subtype 1 field strain by evaluating the clinical outcome of the infection as well as the pathological lung lesions in vaccinated and non-vaccinated growing pigs.

\section{Materials and Methods}

Thirty-six 3-week-old PRRSV negative pigs were randomly assigned to three treatment groups with 12 pigs each. Group 3 was vaccinated with a commercial live PRRSV Type 1 vaccine (DV strain). Five weeks after vaccination pigs of groups 2 and 3 were challenged with $2.2 * 10^{5} \mathrm{TCID}_{50}$ of a Type 1 , subtype 1 virulent PRRSV field isolate. The challenge isolate and the vaccine strain shared $89 \%$ nucleotide and $91 \%$ amino acid identity on ORF5 (GP5) and $95 \%$ nucleotide and $96 \%$ amino acid identity on ORF7 (N). Group 1 served as a negative control. During the course of the study, viraemia, serology, clinical signs, rectal temperature and average daily weight gain were monitored. Animals were euthanized on $10 \mathrm{DPI}(\mathrm{n}=6$ per group) and 21 DPI ( $n=6$ per group). Severity of macroscopic and microscopic lung lesions was scored (0-3). The microscopical evaluation was performed as a blinded analysis.

\section{Results}

All group 3 animals became viremic following vaccination and all group 2 animals became viremic following challenge. Seroconversion was observed within one week after onset of PRRSV viraemia. Following challenge on SD 35 , a significant $(p \leq 0,05)$ increase in number of animals with fever $\left(\geq 40,5^{\circ} \mathrm{C}\right)$ occurred in the vaccinated group in comparison to the control group between SD 39-44, while a significant increase was observed in the challenged group between SD 42-44. Coughing was observed in more than $60 \%$ of animals in group 2 and 3 but in less than $10 \%$ of group 1 animals. Within the first 10 days following challenge, group 1 animals showed a significant higher body weight gain $(6.8 \mathrm{~kg})$ than group $2(3.9 \mathrm{~kg}, \mathrm{p}<0.001)$ and group $3(5.0$ $\mathrm{kg}, \mathrm{p}=0.004$ ) animals. Average daily weight gain of all 3 groups before and after challenge is presented in Figure 1.
Concerning lung lesions, significant differences (macroscopic lesions: $p \leq 0.0285$, microscopic lesions: $p \leq 0.0001$ ) were observed between challenged animals (group 2 and group 3 ) and the negative controls (group 1). In contrast to that, summarized lung score values of vaccinated (group 3 ) and non-vaccinated pigs (group 2) were not significantly different (macroscopic lesions $p$ $\geq 0.0607$, microscopic lesions: $p=0.7144$ ).

Figure 1: Average daily weight gain before and after challenge.

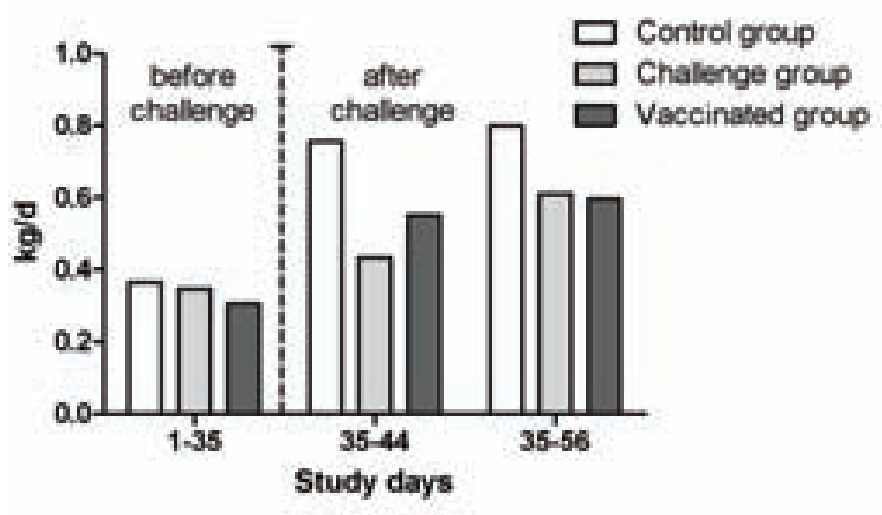

\section{Discussion}

The data demonstrate that a recent PRRS Type 1 field strain is able to induce clinical signs and macroscopic and microscopic lung lesions upon experimental challenge of growing pigs. The fact that no significant differences were observed between vaccinated and non-vaccinated challenged pigs is in line with other reports indicating that available live modified PRRS vaccines do not always provide complete protection against all types of currently circulating PRRS strains (2).

\section{References}

1. Stadejek T. et al. 2008. Arch. Virol. 153; 1479-1488.

2. Labarque G. et al. 2004. Vaccine 22; 4183-4190. 


\title{
P.223
}

\section{Comparaison of immune response between PRRS vaccination using PROGRESSIS ${ }^{\circledR}$ an inactivated vaccine or a combination of inactivated and a modified live vaccine}

\author{
M. Gimeno ${ }^{2,3}{ }^{3}$ Diaz $^{3}$ L. DARWICH ${ }^{3}$ Enrique Mateu $^{2,3}$ Antonio Callen ${ }^{4}$ Julie Venet $^{1}$ François Joisel ${ }^{1}$ \\ 1. Merial S.A.S., Lyon, France; 2. Dept. Sanitat I Anatomia Animals, Bellaterra, Spain; 3. Centre de Recerca \\ en Sanitat Animal (CRESA), Barcelona, Spain; 4. Merial Laboratorios S.A., Barcelona, Spain
}

\section{Introduction}

Successful control of PRRS often involves strategies for immunizing gilts and maintaining immunity in sows. The choice of a given strategy depends on the safety/efficacy balance. Objections about using a live PRRSV strain in gestating sows - except in the case of a severe PRRS outbreak- may coexist in the same farm with the use of live PRRSV. In Spain, another approach was tested in which gilts are firstly immunized by using live virus and then booster vaccination of sows is performed using PROGRES$\mathrm{SIS}^{\circledR}$. This may avoid the use of live PRRSV in gestating sows. The present report examines the efficacy of such approach.

\section{Material and Methods}

Thirty-two high health pigs free from all major pig diseases were selected at weaning in a PRRSV-free farm. Animals were confirmed to be free of PRRSV by ELISA (HERDCHEK 2XR, IDEXX Laboratories) ear-tagged and let to acclimatize for two weeks. At six weeks of age, piglets were randomly allocated in four separated groups of eight animals: A, B, C and D and vaccinated with an adjuvanted modified-live PRRSV vaccine (MLV) or a placebo. Three months later, piglets were PROGRESSIS-revaccinated (once or twice) or with a MLV as shown in Table 1. At 6.5 months of age, all pigs were intranasally challenged with $1 \times 10^{6} \mathrm{TCID}_{50}(2$ $\mathrm{ml}$ ) of a Lelystad-like PRRSV strain and were followed for 21 days. Humoral response was evaluated by ELISA and the frequency of PRRSV-specific IFN- $\gamma$ cells in blood was measured by ELISPOT as reported before (1). After challenge viremia was examined by RT-PCR at days $0,+3,+7,+10,+14,+21$. Statistical analysis was done using StatsDirect v2.7.5 by means of the Kruskal-Wallis test with multiple comparisons performed by the Connover-Inman test.

Table 1: Design of the experiment

\begin{tabular}{|l|c|c|c|c|c|}
\hline Group/Months of age & $\mathbf{1 . 5}$ & $\mathbf{4 . 5}$ & $\mathbf{5 . 5}$ & $\mathbf{6 . 5}$ & $\mathbf{7 . 5}$ \\
\hline $\mathrm{A}$ & $\mathrm{MLV}$ & $\mathrm{KV}$ & $\mathrm{Pl}$. & $\mathrm{Ch}$. & End \\
\hline $\mathrm{B}$ & $\mathrm{MLV}$ & $\mathrm{MLV}$ & $\mathrm{Pl}$. & $\mathrm{Ch}$. & End \\
\hline $\mathrm{C}$ & $\mathrm{MLV}$ & $\mathrm{KV}$ & $\mathrm{KV}$ & $\mathrm{Ch}$. & End \\
\hline $\mathrm{D}$ & $\mathrm{PI}$. & $\mathrm{Pl}$. & $\mathrm{Pl}$ & $\mathrm{Ch}$. & End \\
\hline $\mathrm{KV}=$ PROGRESSIS; PI.=Placebo; Ch. = Challenge
\end{tabular}

\section{Results}

No reaction after vaccination was observed, showing safety of these treatments. Initial vaccination with the MLV produced seroconversion and raised the PRRSV-specific cell-mediated response (Table 2 and Figure 1). No differences were seen in the humoral response but the use of PROGRESSIS as a booster antigen was equal or better than the use of a repeated MLV administration (group B) in terms of maintaining the PRRSV-specific IFN$\gamma$ response. Thus, at the time of challenge, the IFN- $\gamma$ response of group $C$ was higher $(p<0.05)$ than the IFN- $\gamma$ frequencies in groups B and D. After challenge, the higher booster in IFN- $\gamma$ was seen in group $C$ as well. By the end of the experiment, group $A$ showed the higher IFN- $\gamma$ frequencies compared to other groups $(p<0.05)$. RT-PCR results showed that all unvaccinated became viremic after challenge while in groups $A, B$ and $C$ only one or two pigs became infected by day $3 \mathrm{PI}$ (data not shown).

Table 2: Serological evolution (ELISA) of pigs (S/P ratios)

\begin{tabular}{|l|c|c|c|c|c|c|c|}
\hline Group/Months of age & $\mathbf{1 . 5}$ & $\mathbf{2 . 5}$ & $\mathbf{3 . 5}$ & $\mathbf{4 . 5}$ & $\mathbf{5 . 5}$ & $\mathbf{6 . 5}$ & End \\
\hline $\mathrm{A}$ & 0.05 & 1.2 & 2.9 & 2.8 & 2.8 & 2.7 & 2.5 \\
\hline $\mathrm{B}$ & 0.04 & 1.1 & 2.4 & 2.0 & 2.5 & 1.9 & 2.3 \\
\hline $\mathrm{C}$ & 0.07 & 1.2 & 2.8 & 1.9 & 2.7 & 2.4 & 2.2 \\
\hline $\mathrm{D}$ & 0.07 & 0.1 & 0.1 & 0.1 & 0.1 & 0.1 & 2.1 \\
\hline
\end{tabular}

Figure 1: IFN- $\gamma$ response (ELISPOT) after vaccination

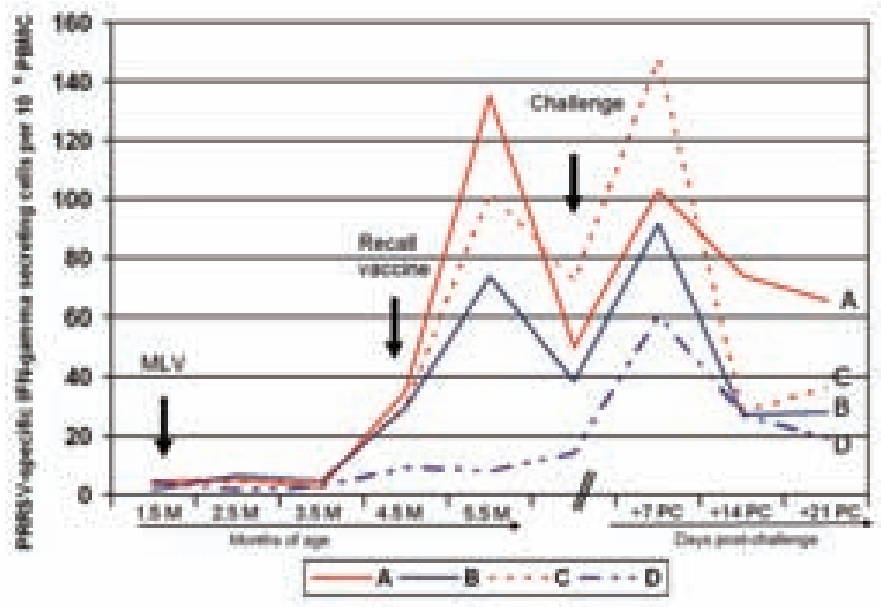

\section{Conclusion}

The present results show that the use of PROGRESSIS or MLV vaccine or combination of both gave similar results.

\section{References}

1. Diaz et al., (2005) J Gen Virol, 86:1943-1951.

${ }^{\oplus P R O G R E S S I S ~ i s ~ a ~ r e g i s t e r e d ~ t r a d e m a r k ~ o f ~ M e r i a l ~ S . A . S ., ~ F r a n c e . ~}$ 


\title{
P.224
}

\section{Clinical protection provided by AMERVAC ${ }^{\bullet}$ - PRRS against heterologous challenge with a highly virulent Asian (Genotype - II) strain}

\author{
Merce Roca ${ }^{1}$ Sergi Bruguera ${ }^{1}$ Mariona Gimeno ${ }^{2,}{ }^{3}$ Ivan Diaz ${ }^{2}$ Ivan Galindo ${ }^{2,3}$ Joaquim Segales $^{2,3}$ Laila $^{2}$ \\ Darwich $^{2,3}$ Enric Mateu ${ }^{2,3}$ Eva Martinez $^{1}$ David Llopart ${ }^{1}$ Jaime Maldonado ${ }^{1}$ Ricard March ${ }^{1}$ \\ 1. HIPRA, Amer (Girona), Spain; 2. Centre de Recerca en Sanitat Animal (CReSA), Bellaterra (Barcelona), \\ Spain; 3. Departament de Sanitat i Anatomia Animals (UAB), Bellaterra (Barcelona), Spain
}

\section{Introduction}

The emergence of highly virulent PRRS virus (PRRSV) strains in China in $2006<$ sup $<1,2$ was a milestone in the epidemiology of PRRS in Asia. Nowadays, genotype II strains of different virulence coexist with genotype-I strains ${ }^{3,4}$ in this continent. At present, AMERVAC ${ }^{\circ}$-PRRS (a genotype-I vaccine) is licensed and marketed in many countries of Asia and, therefore, contact of vaccinated herds with highly virulent genotype-Il strains can be expected. The present study was aimed to explore the safety and potential efficacy of AMERVAC ${ }^{\circledR}$-PRRS against highly virulent genotype-II strain.

\section{Material and Methods}

Twenty-one 4-week-old, PRRSV-naïve pigs were randomly divided into three groups ( $G 1, n=8 ; G 2$ n=8; and $G 3, n=5)$ and housed separately in biosafety level 3 facilities at CReSA. Pigs in G1 were immunized intramuscularly with AMERVAC -PRRS $(2 \mathrm{ml}$ of $10^{4,6} \mathrm{TCID}_{50} / \mathrm{ml}$ ). G2 and $\mathrm{G} 3$ were kept as unvaccinated groups. At day 32 post-vaccination, $\mathrm{G} 1$ and $\mathrm{G} 2$ pigs were intranasally inoculated with $2 \mathrm{ml}$ of $\left(1 \times 10^{5} \mathrm{TCID}_{50} / \mathrm{ml}\right)$ of the Asian highly virulent isolate PRRSV21 ( $99 \%$ similar in ORF5 to other highly virulent isolates of Asia). Pigs in G3 remained as uninoculated controls. For the following 21 days, pigs were clinically examined (C) and rectal temperatures $(\mathrm{T})$ were taken. Blood samples were taken on days, $0,3,7,10,14$ and 21 post-challenge (PC). RT-PCR was used to assess the virological outcome of the challenge. Pigs were euthanized and necropsied on day 21 PC. Statistics were done using StatsDirect 2.7.5 software.

\section{Results}

Vaccination did not produce any adverse or side effect. All inoculated pigs became infected as determined by RT-PCR and developed fever but differences were noticed among groups (Figure 1). Thus, G1 pigs suffered less days of fever $(p<0.05)$ than $\mathrm{G} 2$ ones did. Also, naïve (G2) pigs developed evident respiratory signs from day 5 PC that often persisted until day 18 PC. Two G2 pigs died (25\%). In G1 pigs, signs were milder, of shorter duration and affected less number of pigs. At necropsy, lesions were more frequent and more severe in $\mathrm{G} 2$ pigs compared to $\mathrm{G} 1$ (Table 1). Findings compatible with bacterial complications (i.e. polyserositis and bronchopneumonia) were more common in G2.
Figure 1. Clinical signs and body temperatures.

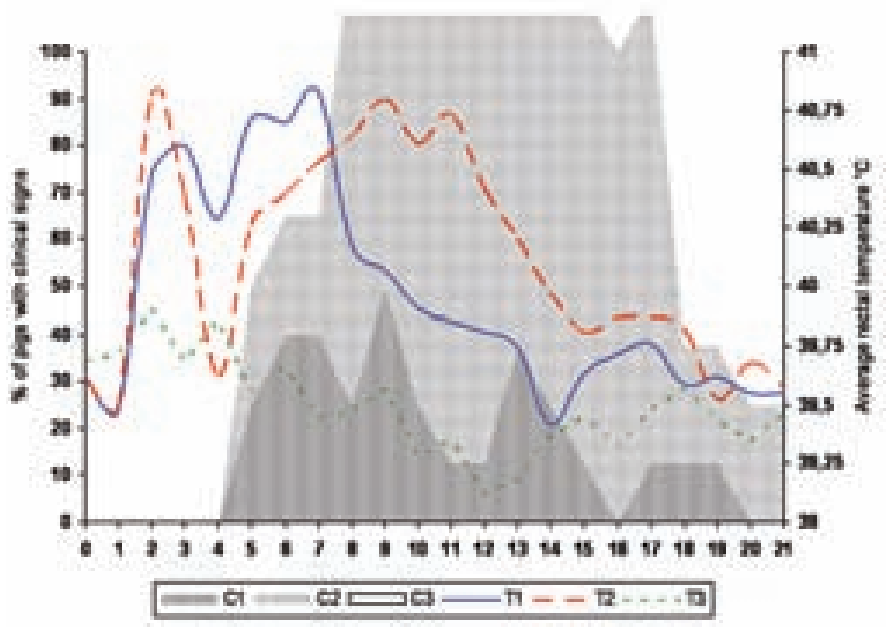

C1, C2, C3 (shadowed areas): \% of pigs with clinical signs in groups G1, G2, G3. T1, T2, T3 (lines): average rectal temperatures in groups $\mathrm{G1}, \mathrm{G} 2, \mathrm{G} 3$

Table 1. Main findings in necropsies of G1, G2 and G3 pigs.

\begin{tabular}{|l|c|c|c|}
\hline Lesion & G1 & G2 & G3 \\
\hline Average pneumonia score (1-4) & 1.0 & 2.2 & 0.0 \\
\hline Catarrhal bronchopneumonia & $0 / 8$ & $4 / 8$ & $0 / 5$ \\
\hline Interstitial nephritis & $1 / 8$ & $5 / 8$ & $0 / 5$ \\
\hline Gall bladder oedema & $0 / 8$ & $3 / 8$ & $0 / 5$ \\
\hline Fibrinous polyserositis & $2 / 8$ & $5 / 8$ & $0 / 5$ \\
\hline Arthritis & $0 / 8$ & $3 / 8$ & $0 / 5$ \\
\hline
\end{tabular}

\section{Discussion}

Vaccinated animals had clinical protection against the virulent challenge in spite of the different genotypes of the vaccine and the challenge isolate. This observation also suggests the existence of conserved epitopes among PRRSV genotypes.

\section{References}

1. Tian et al. 2007. PLoS ONE, 2: e526

2. Li et al. 2007. Vet J, 174:577-584.

3. Nam et al. 2009. Arch Virol, 154: 629-638.

4. Amonsin et al. 2009. Virol J, 16: 143. 


\title{
P.225
}

\section{In-vivo evaluation of Ingelvac ${ }^{\circledR}$ PRRS MLV through the AcuShot ${ }^{\mathrm{TM}}$ needle-free injector}

\author{
Reid Philips; Fred Sick; Enrique Mondaca; Dale Polson \\ Boehringer Ingelheim Vetmedica, Inc., St Joseph, MO, USA
}

\section{Introduction}

Needle-free injection devices deliver product by pressure directly into subcutaneous or muscular tissue layers. This technology has been used in human and veterinary medicine since the mid 1900 's, offering some advantages over conventional vaccine or medicine delivery methods: elimination of broken needles, consistent product delivery, elimination of accidental worker needle sticks, elimination of needle disposal, and less pain and stress. ${ }^{1}$ It also has significant potential as a tool when population-based injectable interventions are suitable for large livestock systems such as area/regional disease control programs. Some of their disadvantages include the relatively large initial financial investment in equipment and the need for technical training. Another potential disadvantage is the effect of the device's high pressure on the integrity of viral particles when applying modified live vaccines.

The aim of this study was to compare the post-vaccination seroresponse to Ingelvac ${ }^{\bullet}$ PRRS MLV (Boehringer Ingelheim Vetmedica, Inc., St. Joseph, MO) in pigs vaccinated with either the traditional syringe and needle technology, or AcuShot ${ }^{\mathrm{TM}}$ (AcuShot, Inc., Winnipeg, CA) needleless approach.

\section{Materials and Methods}

Forty 4-week old PRRS virus and M. hyopneumoniae negative/ naïve piglets were randomly allocated into two treatment groups: Treatment Group 1 (AcuShot) and Treatment Group 2 (Needle), both with a sample size of 20 pigs. Each pig in each treatment group was vaccinated I.M. with a $2 \mathrm{~mL}$ dose of Ingelvac ${ }^{\circ}$ PRRS MLV per label recommendations. Blood samples were collected at days $0,7,14,28,42$ and 56 for assessment by PRRS ELISA, PCR and quantitative PCR (qPCR).

The statistical analysis was run with Minitab ${ }^{\oplus}$ software version 15.1.0.0 (Minitab Inc., State College, PA), applying Mann-Whitney and Two Proportions tests.

\section{Results}

No significant difference was found at $\alpha=0.05$ between treatments for viral load as determined by qPCR (Table 1).

Table 1. Quantitative PCR mean values $\left(\log _{1} 0\right)$ post-vaccination. Different superscripts within a column indicate significant differences at $P<0.05$.

\begin{tabular}{|l|c|c|c|c|c|}
\hline & Day7 & Day14 & Day28 & Day42 & Day56 \\
\hline AcuShot & $5.61^{\mathrm{a}}$ & $5.79^{\mathrm{a}}$ & $4.09^{\mathrm{a}}$ & $3.97^{\mathrm{a}}$ & $3.21^{\mathrm{a}}$ \\
\hline Needle & $5.45^{\mathrm{a}}$ & $5.77^{\mathrm{a}}$ & $4.71^{\mathrm{a}}$ & $3.91^{\mathrm{a}}$ & $3.48^{\mathrm{a}}$ \\
\hline
\end{tabular}

No significant differences at $\mathrm{a}=0.05$ were detected between treatments for PRRS ELISA S/P ratios or percent PCR positive samples post-vaccination (Figure 1).

Post-vaccination viremia was detected by PCR at day 7, and was still present in serum in $20-26 \%$ of pigs at day 56 post-vaccination. Post-vaccination ELISA positive samples were detected from day 14 to the end of the study at day 56 (Figure 1).

Figure 1. Percent of PPRSV PCR (+) samples and ELISA S/P post-vaccination mean values per treatment ( $\mathrm{AcS}=$ AcuShot; $N d l=$ Needle).

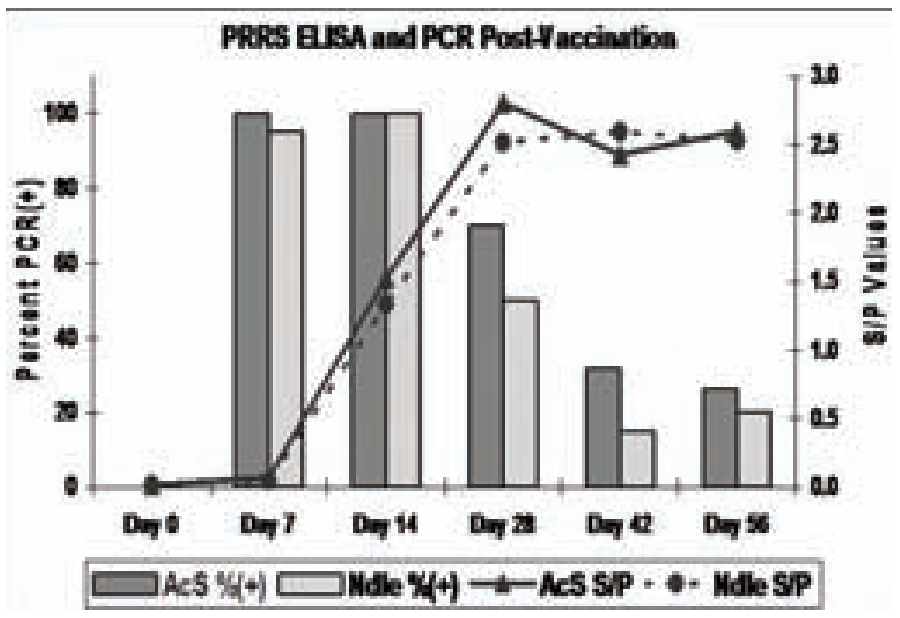

\section{Discussion}

Under the conditions of this study, no significant differences were found between AcuShot and traditional syringe and needle in the Ingelvac ${ }^{\circ}$ PRRS MLV post-vaccination seroresponses as measured by ELISA, PCR and qPCR.

Further evaluation of needleless devices under field conditions is warranted to ensure their suitability for use in swine production.

\section{References}

1. Chase, C. JSHAP 2008, 16(5):254-261. 


\title{
P.226
}

\section{Evaluation of a needle-free injection system (AcuShot ${ }^{\mathrm{Tm}}$ ) for reduction of hematogenous transmission of PRRS virus}

\author{
Seth Baker ${ }^{1}$ Enrique Mondaca² Dale Polson² Scott Dee ${ }^{3}$ \\ 1. University of Minnesota, St Paul, MN, USA; 2. Boehringer Ingelheim Vetmedica, Inc., St Joseph, MO, \\ USA; 3. College of Veterinary Medicine, University of Minnesota, St Paul, MN, USA
}

\section{Introduction}

Needle-free injection devices deliver product by pressure directly into subcutaneous or muscular tissue layers. This technology offers some advantages over conventional vaccine or medicine delivery methods: elimination of broken needles, consistent vaccine delivery, elimination of accidental worker needle sticks, elimination of needle disposal, and less pain and stress ${ }^{1}$. Since it has been demonstrated that hematogenous transmission of porcine reproductive and respiratory syndrome virus (PRRSv) can occur from infected pigs to susceptible pigs via repeated use of the same needle ${ }^{2}$, another potential advantage of needle-free technology is the reduction of hematogenous transmission of PRRSv.

The aim of this study was to evaluate the ability of the AcuShot ${ }^{\mathrm{TM}}$ needle-less injection system (AcuShot, Inc., Winnipeg, CA) to reduce or eliminate the hematogenous transmission of PRRSv.

\section{Materials and Methods}

Four replicates of this study were conducted, each involving 22 four-week-old PRRSv naïve pigs. Each replicate consisted of 5 groups, and each group was housed in a separate room in an isolation facility at the University of Minnesota. Group \#1 (PRRSv source population) consisted of 10 pigs which were intranasally inoculated on day 0 with $2 \mathrm{~mL}$ of the MN-184 PRRSv isolate at a concentration of $104 \mathrm{TCID}_{50} / \mathrm{ml}$. Group \#2 AcuShot, Group \#3 conventional needle or positive control, Group \#4 shaminoculated, and Group \#5 negative control, each consisted of three pigs. All pigs in Group 4 (the sham-inoculated group) were intranasally inoculated with $2 \mathrm{~mL}$ of virus-free media. On days 5, 6 and 7 post-inoculation (PI) of Group \#1, pigs from groups $\# 1$, \#2 and \#3 were vaccinated with a killed Mycoplasma hyopneumoniae (M. hyo) bacterin (Ingelvac MycoFLEX ${ }^{\circledR}$, Boehringer Ingelheim Vetmedica, Inc., St. Joseph, MO). The same AcuShot device and needle/syringe used to initially M. hyo-vaccinate the PRRSv source pigs (group \#1) on opposite sides of the neck were used to subsequently M. hyo-vaccinate pigs in groups \#2 and \#3, respectively. Serum samples were collected from all pigs on days $0,2,5,7,12,19$ and 22 PI for PRRS PCR, and PRRS ELISA at days 0 and 22. The statistical analysis was run with Minitab software, applying Fisher's exact test.

\section{Results}

At arrival (day 0), all pigs tested negative by PRRS ELISA and PCR. All Group 1 pigs tested PRRS PCR positive at days 2 and 5 PI. All 12 pigs (4 reps of 3 pigs) injected with the conventional needle/ syringe became PRRS PCR-positive at days 12 and 19 PI, while 4 out of 12 pigs injected by AcuShot tested PRRS PCR-positive by day $19 \mathrm{PI}$. The proportion of PRRS PCR-positive animals was significantly lower in the AcuShot group compared with the needle/syringe group ( $\mathrm{P} \leq 0.05$, Table 1$)$. On day $22 \mathrm{PI}$, the final day of the study, all PRRSv source pigs tested PRRS ELISA-positive, as did the 12 animals injected with conventional needle/ syringe. Three of 12 animals treated using AcuShot and none of the negative controls or sham-inoculated pigs tested ELISA positive on day $22 \mathrm{PI}$.

Table 1. Proportion of PRRS PCR-positive pigs per treatment by testing day.

\begin{tabular}{|l|c|c|c|c|c|}
\hline Group & & D 7 & D 12 & D 19 & D 22 \\
\hline 3 & Needle & $9 / 12^{\mathrm{b}}$ & $12 / 12^{\mathrm{b}}$ & $12 / 12^{\mathrm{b}}$ & NT \\
\hline 2 & AcuShot & $0 / 12^{\mathrm{a}}$ & $2 / 12^{\mathrm{a}}$ & $4 / 12^{\mathrm{a}}$ & $3 / 12^{\mathrm{a}}$ \\
\hline 4 & Sham-Inoc. & NT & NT & NT & $0 / 12^{\mathrm{a}}$ \\
\hline 5 & Neg Control & NT & NT & NT & $0 / 12^{\mathrm{a}}$ \\
\hline $\begin{array}{l}\text { NT=No samples taken. Different superscripts within columns indicate significant differ- } \\
\text { ences at P } \leq 0.05 .\end{array}$
\end{tabular}

\section{Discussion}

The results of this study indicate that hematogenous transmission of PRRSv occurs from infected pigs to susceptible pigs via repeated use of the same needle and that needle-free systems reduce, but do not eliminate, the hematogenous transmission of PRRSv. Further research is needed to identify the mechanisms of PRRSv transmission by needle-free devices. Further evaluation of needleless devices under field conditions is warranted to ensure their suitability for use in swine production.

\section{References}

1. Chase, C. JSHAP 2008, 16(5):254-261.

2. Otake, S. VetRec 2002, 150(4):114-115. 


\title{
P.227
}

\section{Effect in a Vietnamese farm of a live attenuated genotype-I PRRSV (Porcine Respiratory Reproductive Syndrome Virus) vaccine in sows that were previously vaccinated regularly with a live attenuated genotype-II PRRSV vaccine}

\author{
Chung Kim³ Bui T. Dung² Sergi D. Bruguera' ${ }^{1}$ \\ 1. HIPRA. Technical Services, Amer, Spain; 2. HIPRA Malaysia Sdn. Bhd., Selangor, Malaysia; 3. Kim \\ Long Livestock Producer and Feed Processing Pte. Ltd. Co, Binh Duong, Vietnam
}

\section{Introduction}

In the last years pig Vietnamese farms have suffered the infection of very virulent PRRSV ${ }^{1}$ strains, which were responsible of important economic loses. The use of live PRRSV vaccines either of the genotype-I (European) or the genotype-II (American) family virus has been spread in Vietnam and other Asian countries in order to reduce PRRSV negative effects. Some Vietnamese pig farms, which were endemically infected by PRRSV, have been already for more than 4 years vaccinating their sows with different family type vaccines. The objective of this field study is to compare reproductive sow performance data of a pig farm in Vietnam, in which sows and gilts were vaccinated regularly during two different periods of time with live PRRSV vaccines of different genotype family.

\section{Material and Methods}

The study was carried out in a one site production pig farm with 3000 sows, which is located in Binh Duong province, Vietnam. In 2004 PRRSV was diagnosed and a vaccination program with a live genotype-II virus vaccine started. A vaccine dose was regularly inoculated in gilts at 80 days of age and sows were vaccinated once in each pregnancy on the $4^{\text {th }}$ week of gestation. At the end of 2007 the live genotype-II PRRSV vaccine was replaced by a live genotype-I PRRSV vaccine. This new genotype-I vaccine is still used in the farm today (January 2010). The vaccination protocol implemented with the new vaccine was the same like it was with the genotype-II PRRSV vaccine. We have compared the average sow reproductive performance data between the last 2 years implementation period of both the live genotype-II PRRSV vaccine (from 2006 to 2007) and the live genotype-I PRRSV vaccine (from 2008 to 2009).

\section{Results}

In spite of the already long time stable reproductive results with the use of a live genotype-II PRRSV vaccine in sows we observed that reproductive parameters were improved during the next 2 years period, in which a live genotype-I PRRSV vaccine was used (Table 1). The only studied parameter that did not improve significantly was the total number of born piglets per litter.
Table 1. Monthly average reproductive performance results during 2 years periods, from year 2006 to 2007 (use of live genotype-II PRRSV vaccine) and from year 2008 to 2009 (use of live genotype-I PRRSV vaccine).

\begin{tabular}{|l|c|c|}
\hline & Genotype-II vaccine & Genotype-I vaccine \\
\hline Farrowing rate \% & $77.60 \pm 4.59$ & ${ }^{*} 80.60 \pm 4.08$ \\
\hline Born piglets /litter & $10.99 \pm 0.30$ & $11.00 \pm 0.51$ \\
\hline Weaned piglets/litter & $10.27 \pm 0.34$ & ${ }^{*} 10.54 \pm 0.52$ \\
\hline$\%$ Litter scatter ${ }^{1}$ & $13.24 \pm 2.70$ & ${ }^{*} 11.03 \pm 3.47$ \\
\hline $\begin{array}{l}\text { *Significantly different ( } \mathrm{P}<0.05 \text {, unpaired t test). }{ }^{1} \text { Litter scatter }<8 \text { born } \\
\text { piglets. }\end{array}$ \\
\hline
\end{tabular}

\section{Discussion and conclusion}

The use of a live genotype-I PRRSV vaccine improved results in a farm endemically infected with PRRSV, in which sows had been vaccinated with genotype-II PRRSV vaccine. Different degree of efficacy was also previously observed ${ }^{2}$ with the use of 2 different live PRRSV vaccines in experimental conditions against a pathogenic PRRSV challenge in sows at 90 days of pregnancy. In this experience, lower \% of new born viraemic piglets resulted also in a lower $\%$ of pre-weaning piglet mortality. So the vaccine that could reduce better vertical transmission from sows to foetus was also the one giving as a result healthier born litters with lower mortality. This field experience is also indicating that other virus epitopes ${ }^{3}$ or other immune response factors, common in both genotype PRRSV families, could be related to vaccine protection features.

\section{Conclusion}

A genotype-I PRRSV vaccine within these field conditions seemed to induce better degree of efficacy against a PRRSV infection in sows, when used in an endemically PRRSV infected farm in Vietnam.

\section{References}

1. Feng, Y, et I. 2008. Emerg.Infect.dis, 2008,vol 14, Num 11, pg.1774-6.

2. Scortti M, et al. 2006. Theriogenology 66 (2006),pg.1884-93.

3. Díaz l, et al.Vaccine. 2009 Sept 18;27(41):5603-11. 


\title{
P.228
}

\section{Construction of attenuated porcine reproductive and respiratory viruses with a marker of Nsp2-coding region deletion}

\author{
Aihua Fang; Xin Guo; Xinna Ge; Hanchun Yang \\ China Agricultural University, Beijing, China
}

\section{Introduction}

Porcine reproductive and respiratory syndrome (PRRS) is one of the most economically important diseases for the pig industry worldwide. Non-structural protein 2 (Nsp2) of PRRSV is the most variable region that is able to tolerate natural and artificial amino acid deletion $(1,2,3,4)$. In the present study, we generated two candidates of PRRSV vaccine with a marker of Nsp2coding region deletion using the reverse genetic operation method, based on the attenuated PRRSV strain HB-1/3.9M which can provide sufficient protection for pigs against the Chinese highly pathogenic PRRSV

\section{Materials and Methods}

Cells and viruses. MARC- 145 cells were used for propagation of PRRSV and transfection of full-length cDNA clones. An attenuated PRRSV strain (HB-1/3.9M) was used for the study.

Construction of full-length cDNA clones HB-1/3.9M. The construction strategy of full-length cDNA clones for the virus was basically performed according to the methods previously described (1). The full-length cDNA clone plasmid was designated pWSK-HB1-WT.

Deletion mutagenesis of Nsp2-coding region of PRRSV HB$1 / 3.9 \mathrm{M}$. A plasmid $p E B-A$ possessing the first 3585 nucleotides of HB-1/3.9M was constructed, which contains partial Nsp2. To delete HB-1/3.9M Nsp2-coding region with 270 nt (Nsp2 1627 1896) and 528nt (Nsp2 $\Delta$ 1627-2154) respectively, overlapping extension PCR was performed on pEB-A. The PCR product was gel-purified, digested with proper enzymes, and cloned into $p E B-A$, cut with the same enzymes. After confirmation by sequencing, mutated fragment $A$ insert into the infectious clone backbone to construct the final full-length deletion clone designated as pWSK-HB1-D90 and pWSK-HB1-D176, respectively.

Recovery and identification of the viruses. The viruses were rescued from each full-length cDNA clone according to the method previously described (3). The cytopathic effect (CPE) was daily observed. The rescued viruses were identified by immunofluorescence assay (IFA) using monoclonal antibody for $\mathrm{N}$ protein of PRRSV. Meanwhile, the genetic markers were detected to differentiate the rescued viruses and parental viruses. Growth characterization of the rescued viruses was determined.

\section{Results}

Viable viruses could be rescued from the full-length cDNA clones. A typical PRRSV CPE could be observed at the first passage for the all full-length cDNA clones. The three rescued viruses designated as RVHB1-WT, RVHB1-D90 (90-aa deletion) and rVHB1-D176 (176-aa deletion) were identified by IFA, genetic markers check and partial Nsp2 sequencing (data not shown).
Growth characteristics of the rescued viruses. The rescued viruses could be passaged stably on MARC-145 cells with higher viral titres. All the three rescued viruses displayed the similar replication rate like their parental virus (Fig. 1). After 20 passages, the deletions in Nsp2 of rVHB1-D90 and rVHB1-D176 were examined by RT-PCR and followed by sequencing, indicating that the deletions stably exist.

Figure 1. Growth curve of the rescued viruses on MARC-145 cells.

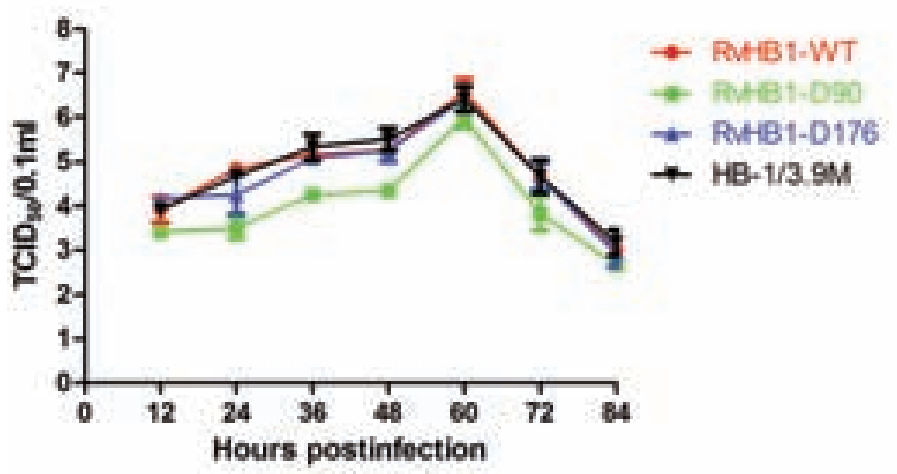

\section{Discussion}

In recent years, the highly pathogenic PRRSV was pandemic in China and brought huge losses for swine production. Therefore, developing an attenuated vaccine with a marker is important for prevention and control of the virus. We have attenuated a strain of Chinese PRRSV HB-1/3.9M that presented satisfactory immune efficacy against highly pathogenic PRRSV infection. In the present study, we obtained two viruses (RVHB1-D90 and RVHB1-D176) on the basis of HB-1/3.9M by using infectious cDNA clones, which could be propagated stably in vitro. These viruses contain a deletion within the Nsp2-encoding region, respectively, suggesting that they are potential to be used as the candidates of PRRSV vaccine with a marker. Their immunogenicities and methods for detection of antibody to the deleted region of Nsp2 for differentiating vaccinated pigs from infected pigs by field virus are still under investigation.

\footnotetext{
References

1. Ran, Z. G. et al., (2008). Arch. Virol. 153:899-907.

2. Fang, Y. et al., (2006). J. Virol. 80:11447-11455.

3. Zhou, L. et al., (2009). J. Virol. 83:5156-5167.

4. Han, J. et al., (2006). Virus Res. 122:175-182.
} 


\title{
P.229
}

\section{Efficacy of an attenuated live PRRS vaccine against a severe PRRS outbreak in sows and piglets}

\author{
Antonio Palomo ${ }^{1}$ Jose M. Garcia' Jesus M. Bollo² Ruth Menjon² Marta Jimenez ${ }^{2}$ Jesus V. Lopez ${ }^{2}$ \\ 1. SETNA NUTRICION-INVIVO NSA, Madrid, Spain; 2. Intervet/Schering-Plough AH, Cantabria, Madrid, Spain
}

\section{Introduction}

Porcine Reproductive and Respiratory Syndrome (PRRS) is considered to be one of the most important causes of loss for the swine industry worldwide. Several techniques have been proposed not only to reduce the economic impact of this disease, but also to minimize PRRS virus circulation. Among them, vaccination with attenuated vaccines seems to be one of the most effective.

This paper describes field experience with a vaccine based on an attenuated strain (Porcilis ${ }^{\circledR}$ PRRS), used in a sow herd with active PRRS virus circulation.

\section{Materials and Methods}

This study was performed in a closed cycle herd of about 500 sows in the province of Toledo, in central Spain. Before the introduction of Porcilis ${ }^{\circledR}$ PRRS, replacement stock was vaccinated twice with an inactivated vaccine with a 3 week interval, during the quarantine period. This measure alone appeared unable to prevent new PRRSv circulation in the herd. This was evidenced by (between May and June 2009) more abortions, and weak born piglets and increasing mortality in lactating sows and weaned piglets associated with high levels of antibodies against PRRSv in the sows (Antibody test kit - IDEXX Laboratories Inc), and by the detection of PRRSv in piglets of 4 and 5 weeks of age (PCR and inmunoperoxidase test).

The ORF- 5 of the PRRSv strain involved in this disease was sequenced as $91.4 \%$ homologous with the Lelystad strain.

Porcilis ${ }^{\circledR}$ PRRS (Intervet International BV, Boxmeer, NL) is an attenuated live vaccine, based on a European strain of PRRS virus identified as DV strain.

In July 2009, the whole herd was vaccinated twice with Porcilis ${ }^{\circledR}$ PRRS with a three week interval between doses. After October 2009 , sows were vaccinated according to a '5/50' scheme (5 days into lactation and 50 days into pregnancy). To complement these measures, no replacement stock were introduced for 5 months, and the M. hyo and PCV2 vaccination of piglets ceased.

Statistical analysis was performed using the General Linear Model (GLM-SPSS 15.0 for Windows) and Pearson's chi-square test was used for comparisons of means.

\section{Results}

Table 1 and Graphs 1 and 2 summarize the development of the main parameters recorded.
Table 1. The main parameters recorded over time

\begin{tabular}{|l|c|c|c|c|}
\hline & $\%$ & $\%$ & $\%$ Mortality & $\%$ Mortality \\
\hline & abortions & Still born & Lactation & Nursery \\
\hline Jan to April & $0.27^{* *}$ & $1.85^{* *}$ & 16.98 NS & $2.9^{* *}$ \\
\hline May to Aug & 3.15 & 2.4 & 17.03 & 10.6 \\
\hline Sept to Dec & $1.6^{*}$ & $1.95^{* *}$ & 15.83 NS & $4.5^{* *}$ \\
\hline \multicolumn{5}{|l}{ Comparing the first and third periods with the second: NS: $\left.p>0.05 ;{ }^{*}: p<0.05 ;{ }^{* *}: p<0.001\right)}$. \\
\hline
\end{tabular}

\section{Graph 1. Abortion rate over time}

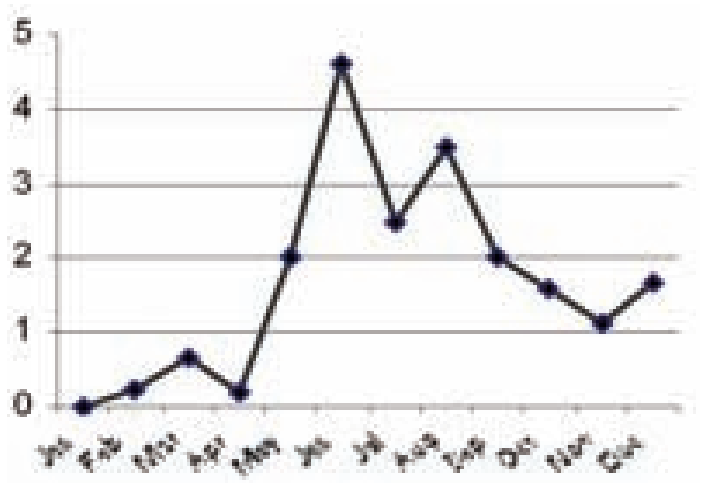

After vaccination, the number of abortions and stillbirths and the mortality rate were clearly reduced during the lactation and post-weaning periods. In September, piglets of 9 weeks of age were found to be seronegative for PRRS, indicating that the improvements were related to the changing pattern of PRRSv circulation.

Graph 2. Mortality rate in weaned piglets over time

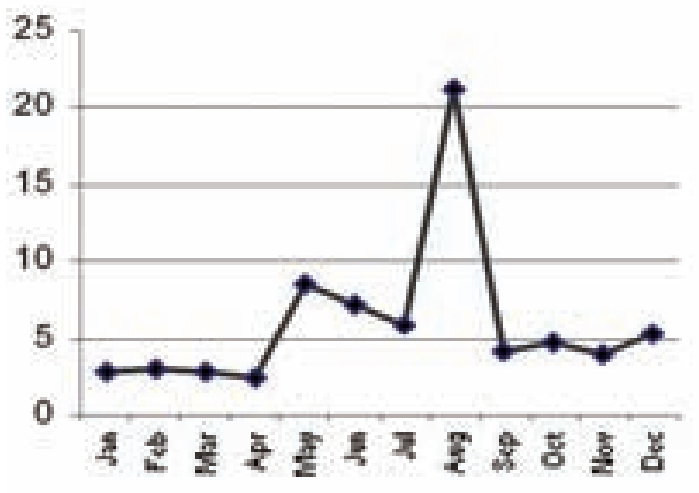

\section{Conclusion}

Vaccination with Porcilis ${ }^{\circledR}$ PRRS is an effective means of controlling PRRS clinically, as well as from an epidemiological viewpoint. 


\title{
P.230
}

\section{Case control and management of virulent PRRS in a commercial farm in the Philippines}

\author{
Angel Antonio C. Manabat \\ PIC Philippines, Ortigas, Pasig City, Philippines
}

\section{Introduction}

For years, active PRRS infections in local swine farms have resulted in large economic losses for farmers because of poor breeding performances, high morbidities and medication costs and mortalities. In 2008, there were reports that PRRS strains similar to China were found in selected Philippine farms 2, which could have further increased the maginutde of this disease problem.

There are many approaches in stabilising herds with PRRS, however for farms located in areas with high density pig population, a good vaccination program seems to be the main and possibly the only option to take1.We report here a case of a farm slowly getting back to profitability after months and possibly years of frustrating management of their PRRS problem.

\section{Case description}

An $800+$ sow level farrow to finish operation located in a farm estate in Bulacan Province Philippines have been experiencing the effects of an active PRRS infection. Mortalities in the pre-weaning and post weaning have been in alarming levels for months, and there was a reported 15 abortions per month observed. Farrowing rates have stayed in the 50 to $65 \%$ rates for sometime. Other pig farms are located in the same farm estate, one even located just a few meters away, many reporting their own problems in dealing with a respiratory and reproductive problems in their herds. The farm has had many control and management measures implemented, strict adherence to all-in all-out loading, breeding audits, farm personnel trainings, strategic medication programs and was already on a breeder mass vaccination and piglet vaccination program for PRRS Modified Live Vaccine (MLV) using a European strain vaccine. However, performances stayed at unsatisfactory levels.On September 2008, the farm decided to implement a shift to another PRRS vaccine (Ingelvac PRRS MLV ${ }^{\circledR}$, Boehringer Ingelheim). They mass vaccinated the breeding herd and placed the piglet vaccination on day 21 , improvements in piglet mortalities would be their major evaluation factor.

\section{Results}

A few months after the shift of PRRS vaccine used, the farm has reported a significant decrease in mortalities of piglets in both the pre-weaning and post weaning stages (figure 1).
Figure 1. Statistical Process Chart Data of Mortality showing the period of vaccinating with the EU strain vaccine as against Ingelvac PRRS MLV

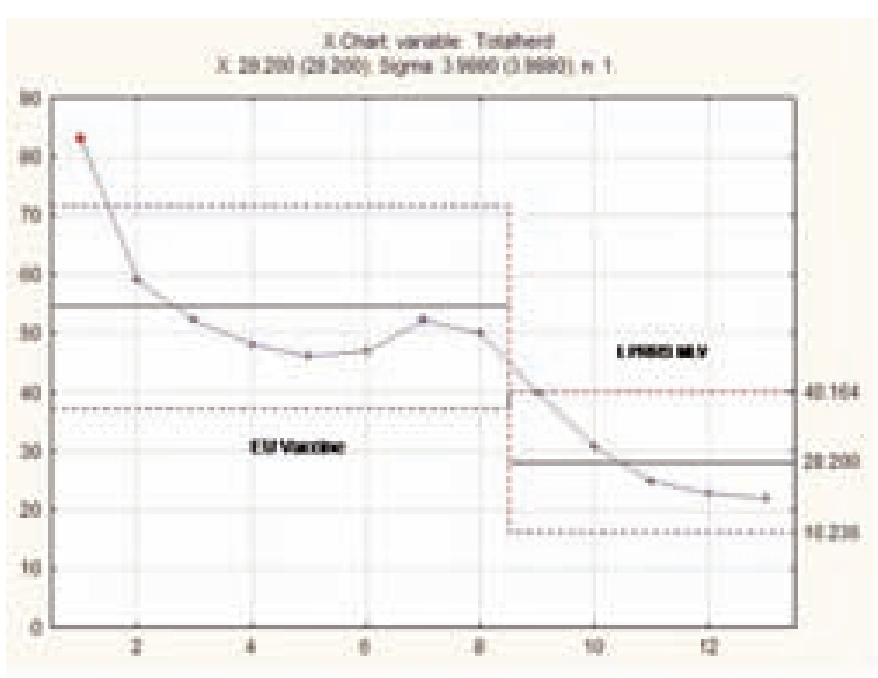

Farm also reported a reduction in abortion cases by half after initiating the program in September and partly due to this, farrowing rates are now improving and already reaching the $80 \%$ level.

\section{Conclusion}

Despite dramatic improvements, the farm is still a case-control in progress, vaccinating with Ingelvac PRRS MLV just barely 6 months as of data availability. Inspite of a virulent PRRS that hit the herd, which wiped out the piglets before weaning, the use of a VR2332-strain MLV showed that it can bring back the performance particularly livability to more acceptable levels.

\section{References}

1. Ballesteros, C. (2007). The 1st Asian PRRSpective, Shanghai, China. Pp. 52-54.

2. Philippine College of Swine Practitioners (2008). An Atypical PRRS Field Primer, p. 7. 


\title{
P.231
}

\section{Comparison of serological and virological analysis for infection patterns of porcine reproductive and respiratory syndrome virus to establish a farm level control strategy}

\author{
Choi-Kyu Park; Seong-Hee Kim; K-K Lee; Y-K Song; O-S Lee \\ National Veterinary Research \& Quarantine Service, Anyangcity, Korea
}

\section{Introduction}

Porcine reproductive and respiratory syndrome virus (PRRSV) has plagued pig populations worldwide causing severe economical impacts. In order to establish effective strategies for prevention of PRRS, infection patterns on the herd level were primarily evaluated. In the present study, therefore, serological and virological analyses were conducted in 20 pig farms suffering from PRRS.

\section{Materials and Methods}

A total of 20 pig farms were selected that they were a 150-250sow, three-site multiplier herd. The blood samples were taken from sow, piglet, 40, 70, 100, 130 days-old pig groups. A commercial kit for PRRS (HerdCheck; IDEXX Laboratories) was used for serological testing according to the manufacturer's instruction. A reverse transcriptase-PCR (RT-PCR) was used to amplify open reading frame 7(ORF7) of PRRSV as previously described by Lyoo(1998).

\section{Results}

Seroprevalence levels in each farm were grouped into 3 patterns: SN (Stable sow groups/Not infected piglet groups), SI (Stable sow groups and Infected piglet groups), and UI (Unstable sow groups and Infected piglet groups). The rate of each serological pattern were 15\% $(n=3), 10 \%(n=2)$, and 75\% $(n=15)$, respectively. In addition, the pattern analysis was extended to virological monitoring on the same farms that further included suckling pig groups. As a result, the infection pattern was classified into 4 categories: SNI (Stable sow groups/Not infected suclker groups/Infected piglet groups), SIl (Stable sow groups/ Infected suckler groups/Infected piglet groups), UNI (Unstable sow groups/Not infected suckler groups/Infected piglet groups), and UII (Unstable sow groups/Infected suckler groups/Infected piglet groups). The rate of each viroprevalence were estimated at $50 \%(n=10), 30 \%(n=6), 10 \%(n=2)$, and $10 \%(n=2)$, respectively. PRRSV viroprevalence results of suckling pig groups revealed that 8 farms were considered the virus positive. In 2 farms among these farms, PRRSV appears to be transmitted vertically to suckling piglets from their sows. In contrast, piglet-to-piglet horizontal transmission of PRRSV seems to occur in sucking herds of the remaining farms. Thus, this virological analysis on suckling piglets will provide useful information to understand PRRSV transmission routes during the suckling period and to improve a PRRS control program.

\section{Discussion}

Our seroprevalence and viroprevalence data found that infection patterns between sow and piglet groups are not always coincident in the same farm. Remarkably, 15 farms belonging to the UI seroprevalence pattern showed four distinct viroprevalence patterns (SNI; 7, SII; 4, UNI; 2 and UII; 2). Among these farms, 11 farms with unstable seprevalence sow groups were further identified as the stable viroprevalence pattern. These results indicated that despite the absence of typical seroconversion, PRRSV infection was detected in several farms, implying the limitation of serological analysis. Taken together, our data strongly suggested that both seroprevalence and viroprevalence should be determined in parallel so that a PRRS control strategy could be efficiently developed on a farm level.

\section{References}

Dee, S. and Joo, H.S. (1997) Vet. Microbiol. 55, 347-353

Le Potier et al. (1997) Vet. Microbiol. 55, 355-360

Lyoo, Y.S. et al.(1998) Korean J. Vet. Res. 38, 77-83

Nodelijk, G et al. (1997) Vet. Microbiol. 56, 21-32

Yoon, S.H. et al. (2008) Arch .Virol. 153, 627-635 


\title{
P.232
}

\section{Evaluation of the efficacy of PRRSV modified live vaccine in one-day old piglets}

\author{
Sayoko Ishizeki ${ }^{1}$ Hiromichi Iswhikawa ${ }^{1}$ Hiroshi Iseki ${ }^{2}$ Michihiro Takagi ${ }^{2}$ \\ 1. Summit Veterinary Services, Ltd., Kiryu-city, Japan; 2. National Institute of Animal Health, Tsukuba-city, Japan
}

\section{Introduction}

Porcine reproductive and respiratory syndrome virus (PRRSV) causes major economic losses in the pig industry. It has been proven that the modified-live vaccine (MLV) is one of the options to control PRRSV. The objective of this study was to evaluate the efficacy of using PRRSV-MLV in one day-old piglets to provide them with protective immunity in their nursery-growing stages.

\section{Materials and Methods}

The study was conducted in a conventional farm in Gunma, Japan ( 2 site system, 3,000 sows). A total of 25,000 pigs were born between February 2008 and June 2008, and the piglets received a half dose of PRRSV-MLV intramuscularly at 1-day of age. A total of 18 piglets were randomly bled prior to vaccination, at 28, 35 and 42 days of age. Sera were tested for PRRSV by ELISA and PCR. Samples that were PCR-positive were sequenced to determine the homogeneity of ORF-5 region of the viral nucleic acids. Additionally, tonsil, lymph nodes, lung, liver, spleen, intestines, kidney and brain were collected from one pig that died at 52 days of age. All the tissue samples were examined by histopathology. The same set of tissue samples was also collected from a 38-day old pig that was euthanized. All the tissue samples were tested for PRRSV by PCR. Production performance data including nursery mortality and average daily gain (ADG) were obtained, and compared with the data from the batches that were born prior to the vaccine implementation in a previous year (non-vaccinated controls). Statistical analysis using T-test was conducted to determine whether there was significant difference between vaccinated piglets and non-vaccinated piglets.

\section{Results}

Prior to vaccination, no piglets showed viremia or any other clinical signs. The vaccinated pigs showed typical PRRS clinical symptoms in suckling and nursery periods. The sera and tissue samples from the vaccinated pigs at 28,35 and 42 days of age showed viremia for PRRSV(11/18). (Table 1) ORF-5 sequencing revealed that 10 out of 11 PCR-positive samples were $99.2-99.8 \%$ homologous to MLV PRRSV strain, while 1 out of 11 was found to be a wild strain of PRRSV (87.7\% homologous to PRRSVMLV strain ). Typical PRRS-like histopathological lesions were found from the tissues collected from the dead pig at 52-day old. PRRSV nucleic acid was detected by PCR from the lymph nodes collected from the euthanized pig at 38-day old. Nursery mortality and ADG were significantly worse in vaccinated piglets compared to non vaccinated controls.

\begin{tabular}{|l|c|c|c|}
\hline sample ID & days of age & $\begin{array}{c}\text { RT-PCR for } \\
\text { sequence }\end{array}$ & $\begin{array}{c}\text { homologous to } \\
\text { PRRSV-MLV strain }\end{array}$ \\
\hline P129-1 & 28 & + & $99.5 \%$ \\
\hline P129-2 & 28 & + & $99.7 \%$ \\
\hline P129-3 & 28 & - & \\
\hline P129-4 & 28 & - & \\
\hline P129-5 & 28 & + & $87.7 \%$ \\
\hline P129-6 & 28 & + & $99.8 \%$ \\
\hline P129-7 & 28 & + & $99.7 \%$ \\
\hline P129-8 & 35 & + & $99.7 \%$ \\
\hline P129-9 & 35 & + & $99.5 \%$ \\
\hline P129-10 & 35 & + & $99.2 \%$ \\
\hline P129-11 & 35 & + & $99.3 \%$ \\
\hline P129-12 & 35 & - & \\
\hline P129-13 & 42 & + & $99.7 \%$ \\
\hline P129-14 & 42 & - & \\
\hline P129-15 & 42 & - & \\
\hline P129-16 & 42 & + & \\
\hline P129-17 & 42 & - & \\
\hline P129-18 & 42 & - & \\
\hline
\end{tabular}

\section{Discussion}

Under the conditions of this study, it is concluded that vaccination of 1-day old piglets with PRRSV-MLV vaccine has negate effects on clinical signs, viremia, as well as production performance including nursery mortality and ADG. 


\title{
P.233
}

\section{Effect of vaccination using AMERVAC ${ }^{\oplus}$-PRRS on different production parameters in three Philippine commercial swine farms}

\author{
Renato T. Policarpio ${ }^{1}$ Michael Felipe E. Quilitis ${ }^{3}$ Sergi D. Bruguera ${ }^{2}$ Ma. Immaculada T. Torres ${ }^{4}$ David V. Llopart $^{2}$ \\ 1. Philippine College of Swine Practitioners, Quezon City, Philippines; 2. Hipra Swine Technical Department, \\ Amer, Girona, Spain; 3. Swine Technical Services, Hipra Philippines, Muntinlupa, Philippines; 4. Laboratory \\ and Technical Manager - DIAGNOS, Hipra Philippines, Muntinlupa City, Philippines
}

\section{Introduction}

Porcine Reproductive and Respiratory Syndrome Virus (PRRSV) has affected many farms in Asia including the Philippines. Clinical signs observed include abortions, pre-mature births, inconsistent farrowing rates and high mortality in sucklings, weanlings and growing-finishing pigs. As previously described, vaccination using a live PRRS vaccine (AMERVAC ${ }^{\circledR}$-PRRS) has positive effects on different production parameters as farrowing rates ${ }^{1,2}$ and mortality in growing pigs ${ }^{4}$. In this study, the objective is to assess the effect of continuous vaccination using a Modified Live (MLV) PRRS vaccine (AMERVAC ${ }^{\circledR}-$ PRRS) in different production parameters in three Philippine swine farms.

\section{Materials and Methods}

Three Philippine commercial farms with sow level ranging from 1300 to 3000 , one site production system are involved in this study. PRRSV diagnosis was carried out with PCR ${ }^{3}$ and an ELISA serology technique (CIVTEST ${ }^{\mathrm{TM}}$ suis PRRS E/S) from necropsy findings and serum samples.

Farm A: 1800 sows, one site production system located in south of Manila. The farm had complaint of abortion in sows and high mortalities in growing pigs in June 2008. Two mass vaccinations in sows with 4 weeks interval were implemented in July 2008 with AMERVAC ${ }^{\circledR}$-PRRS. Pigs from 18 to 70 days of age were also vaccinated once at the same time. Next batches of piglets were vaccinated once intramuscularly at 21-28 days of age.

Farm B: 3000 sows, one site production system located north of Manila had abortion storms in July 2008. High mortality in pre-weaning, weanling and growing-finishing pigs was also observed simultaneous with reproductive failure in sows. Two mass vaccinations with 4 weeks interval were implemented in August 2008. Pigs from 18 to 70 days of age were also vaccinated once at the same time. Next batches of piglets were vaccinated once intramuscularly at 21-28 days of age.

Farm C: 1300 sows, one site production system located south of Manila. It had complaint of abortions, erratic farrowing rate, and high mortality in growing pigs. Same vaccination program as in the farms A and B was implemented starting September 2008.

Average monthly Farrowing rate, Pre-weaning, Post-weaning and Growing-Finishing mortalities for the period of three years were obtained and summarized in the Table 1.

\section{Results}

All the parameters monitored within this study improved considerably in farms A, B and C during 2009. In 2009 all the sows and piglets had been already vaccinated. Reproductive performance stability was observed from the 3rd month after the beginning of the vaccination program until the end of 2009.

Table 1. Mean production data from three Philippine commercial swine farms. PRRSV vaccination program started in July 2008 in Farm A, in August 2008 in Farm B and in September 2008 in Farm C.

\begin{tabular}{|l|c|c|c|}
\hline Production Parameters & $\mathbf{2 0 0 7}$ & $\mathbf{2 0 0 8}$ & $\mathbf{2 0 0 9}$ \\
\hline Average Farrowing rate \% & $77.64 \pm 7.02$ & $68.85 \pm 4.63$ & $80.00 \pm 4.63$ \\
\hline Pre-weaning Mortality \% & $12.65 \pm 4.75$ & $17.62 \pm 8.14$ & $7.18 \pm 4.70$ \\
\hline Nursery Mortality \% & $4.86 \pm 4.44$ & $13.92 \pm 16.22$ & $4.90 \pm 6.43$ \\
\hline Growing-Finishing Mortality\% & $5.20 \pm 1.12$ & $9.95 \pm 6.04$ & $4.83 \pm 2.55$ \\
\hline
\end{tabular}

\section{Discussion}

Based on these specific results, the implementation of PRRS vaccination (AMERVAC PRRS ${ }^{\circledR}$ ) in sows resulted in stabilization of breeding performance. It has subsequent positive effect in reducing pre-weaning mortality since vaccination lessens the negative effects of PRRS field virus. Vaccination in piglets reduced substantially the mortality in growing-finishing stages.

\section{Conclusion}

Based on the data presented, regular vaccination program with AMERVAC ${ }^{\circledR}$-PRRS in sows and pigs improved production performances and prevented the negative impact of a field PRRSV infection.

\section{References}

1. Bell, D et al. 2007. Proceedings of the 3rd Congress of the Asian Pig Veterinary Society (APVS) Wuhan; China. 2007. pag 135.

2. Scortti, M. et al. 2006. Theriogenology. 2006 Nov; 66(8):1884-93. Epub 2006 Jun 27.

3. Martínez E, et al. Res Vet Sci. 2007 Dec 1(Epub ahead of print).

4. Guerzon, V. et al. 2008. Proc. IPVS 2008. P01.118. 


\title{
P.234
}

\section{PRRS vaccine serological response of a live PRRSV vaccine (AMERVAC ${ }^{\circledR}$-PRRS) in pigs when the diluents is a Haemophilus parasuis bacterin (HIPRASUIS ${ }^{\circledR}$-GLASSER)}

\author{
Michael Felipe E. Quilitis ${ }^{1}$ Ma. Immaculada T. Torres² Sergi D. Bruguera ${ }^{3}$ David V. \\ Llopart $^{3}$ Criselda T. Bautista ${ }^{2}$ Zoilo M. Lapus ${ }^{4}$ Redentor A. Manuel ${ }^{1}$ \\ 1. Swine Technical Services, Hipra Philippines, Muntinlupa, Philippines; 2. DIAGNOS, Hipra Philippines, Muntinlupa City, Philippines; 3. \\ Hipra Swine Technical Department, Amer, Girona, Spain; 4. Fellow, Philippine College of Swine Practitioners, Quezon City, Philippines
}

\section{Introduction}

Combined administration of vaccines may be practical and convenient; however it may not be always possible. Haemophilus parasuis vaccine is most usually inoculated in pigs at 7 and 21-28 days of age. Vaccination at 21-28 days of age with a live PRRSV vaccine is common practice in PRRSV infected farms. A previous study ${ }^{1}$ showed that PRRS vaccine virus of AMERVAC ${ }^{\circledR}$-PRRS vaccine virus (VP046-BIS) could survive when the solvent used was a Haemophilus parasuis bacterin (HIPRASUIS ${ }^{\circledR}-$ GLASSER). In this study, the objective is to determine if PRRSV vaccine (AMERVAC ${ }^{\circledR}$-PRRS) serological response in piglets when PRRSV vaccine is used in pigs after reconstitution with a Haemophilus parasuis bacterin in a commercial pig farm.

\section{Materials and Methods}

1007 day-old piglets (Day 0) were randomly selected and allocated in two groups.

Group A: (Hps + Amervac PRRS) 50 apparently 7 day-old piglets were allocated separately and ear notched. This group was vaccinated with $2 \mathrm{ml}$ dose of a Haemophilus parasuis bacterin (HIPRASUIS ${ }^{\circledR}$-GLASSER) at 7 days of age. The same group of animals was vaccinated intramuscularly in the same point of injection, in the long neck muscle, with the PRRSV vaccine (AMERVAC ${ }^{\circledR}$-PRRS), using the Haemophilus parasuis bacterin vaccine (HIPRASUIS ${ }^{\circledR}$-GLASSER) as diluents at 21 days of age. 15 piglets out of 50 were randomly selected for serial bleeding done every 2 weeks.

Group B: (Hps and Amervac PRRS) 50 apparently 7 day-old piglets were allocated separately and ear notched. This group was vaccinated with $2 \mathrm{ml}$ dose of a Haemophilus parasuis bacterin (HIPRASUIS ${ }^{\circledR}$-GLASSER) at 7 days of age. The same group of animals was vaccinated intramuscularly at 21 days of age with a PRRSV vaccine (AMERVAC ${ }^{\circledR}$-PRRS) and a Haemophilus parasuis bacterin (HIPRASUIS ${ }^{\circledR}$-GLASSER) in separate long neck muscles. 15 piglets out of 50 would be randomly selected for serial bleeding done every 2 weeks. Serum samples were taken at day 0 , 14, 28 and 42 and analyzed with a PRRSV Elisa kit (CIVTEST ${ }^{\mathrm{TM}}$ suis PRRS E/S).

\section{Results}

Serological response to vaccination has no significant difference in the two groups during the course of the trial. Similarly, no significant difference was observed in terms of percentage of positive serum samples.
Figure 1. Individual antibody levels in each group using (CIVTEST ${ }^{\mathrm{TM}}$ suis PRRS E/S). Hps and Amervac (Group B); Hps + Amervac (Group A).

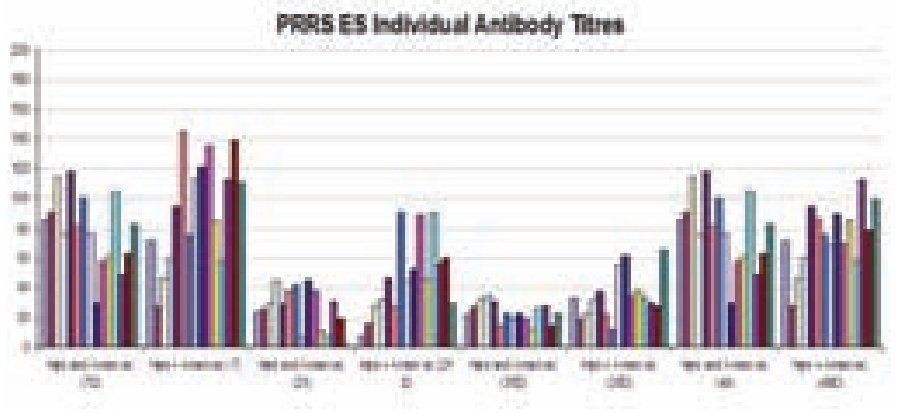

\section{Discussion}

Under field conditions, simultaneous vaccination of a live PRRSV vaccine (AMERVAC ${ }^{\circledR}-$ PRRS) and a Hemophilus parasuis can reduce stress to the animals and maximize the time for farm personnel in the implementation of vaccination. Previous study ${ }^{2}$ also concluded that simultaneous vaccination with the live PRRSV vaccine and another vaccine did not influence PRRS serological response in pigs. In another in vivo ${ }^{3}$ experience there was no interference with live PRRSV vaccine when reconstituted with a multi-antigen inactivated vaccine and administered in pigs.

\section{Conclusion}

There were no differences observed on the serological PRRSV response in pigs when AMERVAC ${ }^{\circledR}$-PRRS vaccine was inoculated separately or combined at the same inoculation point with HIPRASUIS $^{\circledR}$-GLASSER vaccine. This knowledge may have practical field implications. Further immune response studies should be done to discard any negative immune response interferences between vaccines.

\section{References}

1. Bruguera et al. 2009. Proceedings of the 4th Congress Of Asian Pig Veterinary Society; in Tsukuba, Japan. WS2-P-069, page 273.

2. Talactac, M et al 2008. Proceed. of the 20th IPVS Congress, Durban, South Africa. P06.016, page 414.

3. Roof, M et al. 2000. Proc. of The 16th IPVS Congress, Melbourne, Australia, 17-20 Sept. 2000. 


\title{
P.235
}

\section{Serological response to PRRS vaccination using a mycoplasma bacterin (MYPRAVAC ${ }^{\circledR}$ SUIS) as diluents for a live attenuated PRRSV vaccine "AMERVAC ${ }^{\circledR}$ PRRS" $^{-1}$}

\author{
Michael Felipe E. Quilitis ${ }^{1}{ }^{\text {Zoilo M. Lapus }}{ }^{2}$ Sergi D. Bruguera ${ }^{4}$ Ma. Immaculada T. \\ Torres $^{5}$ Criselda T. Bautista ${ }^{3}$ Redentor A. Manuel ${ }^{1}$ David V. Llopart ${ }^{4}$ \\ 1. Swine Technical Services, Hipra Philippines, Muntinlupa, Philippines; 2. Fellow, Philippine College of Swine Practitioners, \\ Quezon City, Philippines; 3. DIAGNOS, Hipra Philippines, Muntinlupa City, Philippines; 4. Hipra Swine Technical Department, \\ Amer, Girona, Spain; 5. Laboratory and Technical Manager - DIAGNOS, Hipra Philippines, Muntinlupa City, Philippines
}

\section{Introduction}

With the advent of combination vaccines, conveniences in the administration and the efficacy under field condition have been the primary concerns. Since the recommended vaccination program for Mycoplasma hyopneumoniae is at 7 and 21 days and for PRRSV is 21 days, then the second dose of Mycoplasma can be given simultaneous with PRRRSV vaccine. Previous study has demonstrated that PRRSV vaccine can survive under laboratory condition using a Mycoplasma bacterin as the diluents ${ }^{1}$. In this study, the objective is to determine if PRRSV vaccine (AMERVAC ${ }^{\circledR}$-PRRS) could elicit serological response using a mycoplasma bacterin (MYPRAVAC ${ }^{\circledR}$ SUIS) as the diluents under field conditions.

\section{Materials and Methods}

1007 day-old piglets were randomly selected and allocated in two groups.

Group A. (Mypravac suis + Amervac PRRS) 50 apparently 7 dayold piglets allocated and ear notched. This group was vaccinated with $2 \mathrm{ml}$ dose of a Mycoplasma hyopneumoniae bacterin (MYPRAVAC $^{\circledR}$ SUIS) at 7 days of age. The same group of animals was vaccinated with the combination of PRRSV vaccine (AMERVAC ${ }^{\circledR}$ PRRS) and Mycoplasma hyopneumoniae bacterin (MYPRAVAC ${ }^{\circledR}$ SUIS) at 21 days of age. 15 piglets out 50 were randomly selected for serial bleeding done every 2 weeks.

Group B. (Mypravac suis and Amervac PRRS) 50 apparently 7 dayold piglets allocated and ear notched. This group was vaccinated with $2 \mathrm{ml}$ dose of a Mycoplasma hyopneumoniae bacterin (MYPRAVAC $^{\circledR}$ SUIS) at 7 days of age. The same group of animals was vaccinated with a PRRSV vaccine (AMERVAC ${ }^{\circledR}$-PRRS) and Mycoplasma hyopneumoniae bacterin (MYPRAVAC ${ }^{\circledR}$ SUIS) in separate injection sites at 21 days of age. 15 piglets out 50 would be randomly selected for serial bleeding done every 2 weeks.

Blood samples were taken at day 0, 14, 28 and 42 of the experiment.

\section{Results}

Vaccination with the combination and separate injection has no significant difference in terms of serological response.

\section{Discussion}

Under field conditions, simultaneous vaccination of MLV PRRSV vaccine and Mypravac suis has been proven valid because it can reduce stress to the animals and can reduce cost. In this study, the combination of a MLV PRRSV vaccine (AMERVAC ${ }^{\circledR}$-PRRS) and a Mycoplasma bacterin (MYPRAVAC ${ }^{\circledR}$ SUIS) can be useful tool to control the two diseases.

\section{Conclusion}

With the use of the two vaccination protocols, no difference was observed serologically in the two groups of animals. We can therefore recommend that the two vaccines (MYPRAVAC ${ }^{\circledR}$ SUIS and $A M E R V A C^{\oplus}$-PRRS) can be used separately or in combination without reducing their efficacy.

\section{References}

1. Bruguera et al. 2009. Proccedings of Asian Pig Veterinary Society. Tsukuba, Japan

Figure 1. Individual antibody levels in each group using (CIVTEST ${ }^{\mathrm{TM}}$ suis PRRS E/S). Mypravac suis + Amervac PRRS (Group A); Mypravac suis and Amervac PRRS (Group A).

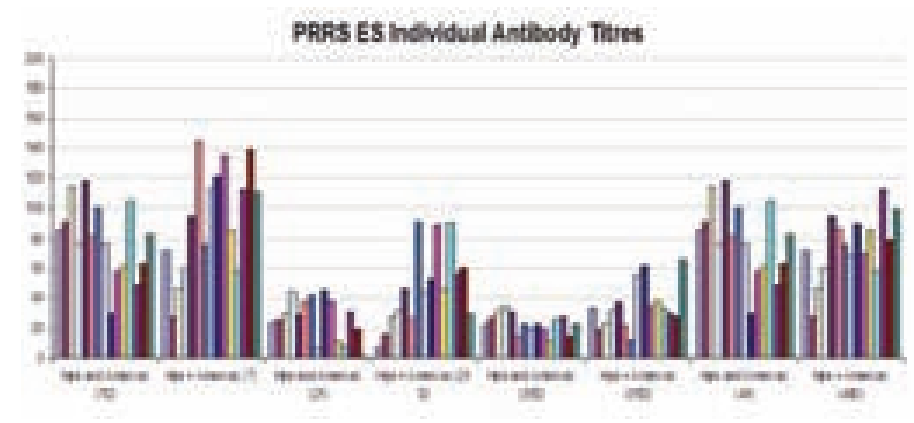

Figure 2. Percent positive animals in each group in different stages of experiment using (CIVTESTTMPRRS). Mypravac suis + Amervac PRRS(Group B); Mypravac suis and Amervac (Group B).

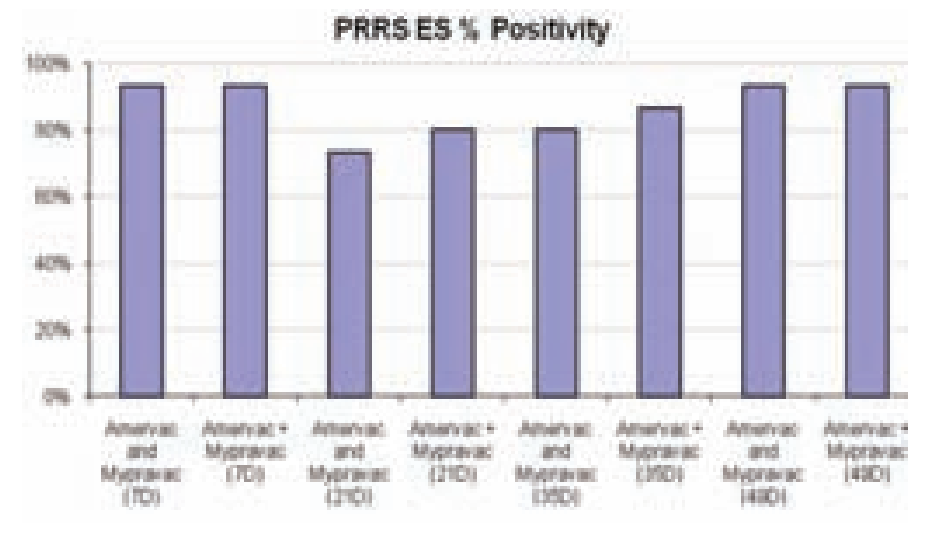




\title{
P.236
}

\section{Antibody titer against porcine reproductive and respiratory syndrome virus (PRRSV) after modified live PRRSV vaccination}

\author{
Em-on Olanratmanee; Roongroje Thanawongnuwech; Annop Kunavongkrit; Padet Tummaruk
}

Chulalongkorn University, Bangkok, Thailand

\section{Introduction}

Porcine reproductive and respiratory syndrome virus (PRRSV) can be detected in the lymphoid tissue of pig up to 8-9 months post infection (1). During this period, the virus can be replicated continuously at a low level and transmitted to susceptible animals via direct contact. In the PRRSV endemic herds, the present of PRRSV subpopulations (susceptible pigs) may lead to reoccurrence of the disease in the herds. Herd closure, gilts acclimatization and mass exposure have been recommended to eliminate the subpopulations (2). The use of vaccination to immunize the pigs has been evaluated, in most cases, at individual level. However, only few studies on the immune response of PRRSV in the infected herd have been evaluated. Earlier study has demonstrated that vaccination of the entire herd (mass vaccination) could reduce persistence and duration of shedding even though the wild type of virus was not eliminated from the pigs (3). However, the successful results are varied among the herds and a limited information on the immune response of PRRS modified live virus (MLV) vaccine is available in pregnant gilts and sows. The present study aims to evaluate the humoral immune response of gilts/sows after mass vaccination of PRRS MLV vaccine in a PRRSV infected herd in Thailand.

\section{Materials and Methods}

The present study was conducted between May and September, 2009 in a 1,200-sow inventory swine herd in Thailand, which produced their own replacement gilts. The herd has never been vaccinated the gilts and sows with PRRSV vaccine. An occurrence of abortion in sows was observed in January 2009 and an American strain of PRRSV was detected in serum of aborted sows using reverse transcription polymerase chain reaction (RT-PCR). Gilts/ sows were vaccinated against PRRS MLV vaccine (Ingelvac ${ }^{\circledR}$ PRRSTM MLV, Boehringer-Ingelheim, Vetmedica Inc., St. Josept, Missouri, USA) 2 doses 3 weeks apart and booster at 14 week and 3 months. Blood samples were collected from replacement gilts and pregnant gilt/sow (36 samples) one day prior to first vaccination (week 0 ) and subsequently at 2, 5, 9, 12 and 18 weeks after vaccinations in the same animals. The sera were obtained for viral detection using RT-PCR technique (pooled serum) and analyzing antibodies against PRRSV using ELISA (HerdChek ${ }^{\circledR}$ PRRS virus antibody test kit 2XR, IDEXX Laboratories, Inc., USA). The percentage of sero-positive gilt/sow was compared using logistic regression. The differences of means of the serum sample/positive control (S/P) ratio were compared using pair t-test. $\mathrm{P}<0.05$ was considered as statistically significant.

\section{Results and Discussion}

It was found that no viral shedding was detected during 2 to 18 weeks post vaccination (Table 1). Before vaccination, $11.1 \%$ (4/36) PRRSV susceptible pigs were observed. The percentage of PRRSV subpopulation was reduced to $6.1 \%$ within 18 week post vaccination $(P=0.401)$. The $S / P$ ratio was slightly increased two weeks post vaccination and significantly decreased at 5 weeks post vaccination (Table 1).

Table 1. The S/P ratio (means \pm S.E.M.), the percentage of sero-positive gilts and sows and the viral detection by weeks post vaccination

\begin{tabular}{cccl}
\hline weeks & S/P ratio & \% positive & RT-PCR \\
\hline 0 & $1.61 \pm 0.19^{\mathrm{ab}}$ & $88.89^{\mathrm{a}}$ & Negative \\
2 & $1.88 \pm 0.16^{\mathrm{a}}$ & $94.44^{\mathrm{a}}$ & Negative \\
5 & $1.47 \pm 0.16^{\mathrm{b}}$ & $86.11^{\mathrm{a}}$ & Negative \\
9 & $1.32 \pm 0.15^{\mathrm{b}}$ & $88.89^{\mathrm{a}}$ & Negative \\
12 & $1.46 \pm 0.17^{\mathrm{b}}$ & $85.29^{\mathrm{a}}$ & Negative \\
18 & $1.23 \pm 0.07^{\mathrm{b}}$ & $93.94^{\mathrm{a}}$ & Negative \\
\hline All & $1.50 \pm 0.06$ & 89.57 & Negative \\
\hline
\end{tabular}

a,b different letters differ significantly $(P<0.05)$

The present study demonstrated that mass vaccination of PRRSV MLV in pregnant gilts and sows did not cause viral shedding during 2-18 weeks post vaccination and might possibly minimize the number of PRRS subpopulations in the PRRSV infected herds. However, the shedding of PRRSV during 0-2 weeks post vaccination and reproductive performance of pregnant gilts/ sows should be evaluated further.

\section{References}

1. Wills et al., 2003. J. Clin. Microbiol. 41: 58-62.

2. Cano et al., 2007. Vaccine 25: 4382-4391.

3. Cano et al., 2007. Am. J. Vet. Res. 68: 565-571. 


\title{
P.237
}

\section{Vaccination effect of a live attenuated PRRSV vaccine "AMERVAC ${ }^{\circledR}$ PRRS" in sow farrowing rates: the story continues}

\author{
$\underline{\text { Michael Felipe E. Quilitis }}{ }^{1} \underline{\text { Sergi D. Bruquera }}^{2}$ Ma. Immaculada T. Torres ${ }^{3}$ Ruel C. Macaraig ${ }^{1}$ Dani G. Torrents ${ }^{4}$ \\ Catherine C. Ceniza ${ }^{5}$ Jaspearl T. Ontanillas ${ }^{5}$ Karen Anne A. Pakingan ${ }^{5}$ David V. Llopart ${ }^{2}$ \\ 1. Swine Technical Services, Hipra Philippines, Muntinlupa, Philippines; 2. Hipra Swine Technical Department, Amer, \\ Girona, Spain; 3. Laboratory and Technical Manager - DIAGNOS, Hipra Philippines, Muntinlupa City, Philippines; \\ 4. Hipra China, Technical Services, Beijing, China; 5. Farm Veterinarian, GFI, Cebu, Philippines
}

\section{Introduction}

Porcine Reproductive and Respiratory Syndrome Virus (PRRSV) is one of the primary precursors of economic losses ${ }^{2}$ in pig production. Only in Japan the annual cost of the disease in 2008 was close to 280 million USD<sup4. Clinical signs include decreasing farrowing rates, abortions, stillborns and increase of respiratory clinic problems in pigs. The objective of this field experience is to assess the effect of continuous vaccination using a live PRRSV European (EU) vaccine (AMERVAC ${ }^{\circledR}$ PRRS) in the monthly farrowing rates in a farm endemically infected by PRRSV.

\section{Materials and Methods}

A 2000 sow farm with a 2 site production system that is located in the southern Philippines had a complaint of unstable and variable farrowing rates. PRRSV diagnosis was based on Polymerase chain reaction technique 3 (PCR) from lungs and lymph nodes from stillbirths and 1 day old weak born piglets. A PRRSV vaccination program was implemented in sows at October 2007, using a modified live PRRSV European (EU) vaccine (AMERVAC ${ }^{\oplus}$ PRRS). Every batch of sows was vaccinated with one dose at 60 days of pregnancy and another dose 7 days after each farrowing. Farrowing rates from April to October 2007 were compared with farrowing rates from November 2007 until December 2009 (unpaired t test).

\section{Results}

Variable and unstable farrowing rates were monitored from April to October 2007. The monthly farrowing rates became less variable and were significantly higher during the 25 months period after the implementation of the PRRSV vaccination program.

Table 1. Monthly mean (Mean $\pm S D$ ) sow farrowing rates (December 2009) before and after PRRSV vaccine program implementation in sows.

\begin{tabular}{|l|c|}
\hline Groups & \% Farrowing Rate \\
\hline Before Vaccination & $75.6 \pm 7.10^{*}$ \\
\hline After Vaccination & $82.55 \pm 2.69^{*}$ \\
\hline *Results were significantly different, $\mathrm{P}<0.001$ \\
\hline
\end{tabular}

Graphic 1. Monthly sow farrowing rates evolution from April 2007 to December 2009. Red arrrow marks the beginning of the vaccination program.

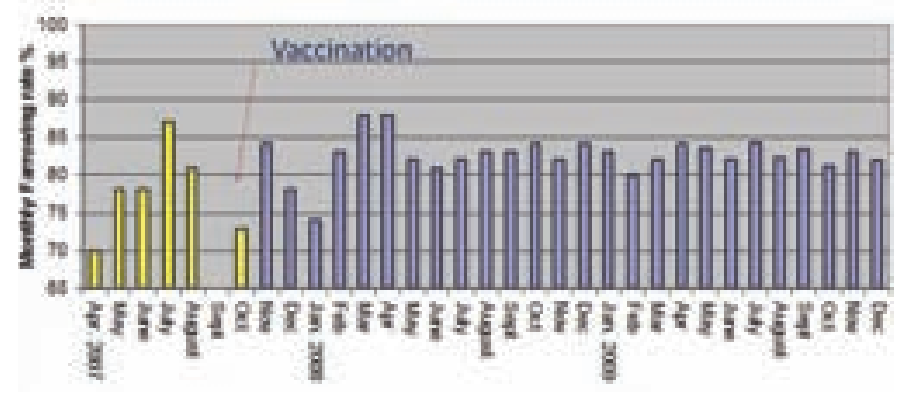

\section{Discussion}

These results match with previous field cases in Asia where PRRSV vaccination prevented against negative effects of a PRRSV infection in sows ${ }^{1}$. PRRSV regular vaccination after two years seemed to keep a degree of protection in sows that protects them again new reproductive clinic signs outbreaks due to the field PRRSV in the farm and may reduce the economic losses provoked by unstable reproductive performance.

\section{Conclusion}

The continuous long term implementation of a PRRSV vaccination program in sows with AMERVAC ${ }^{\circledR}$-PRRS was effective and improved the monthly farrowing rates in a PRRSV infected farm.

\section{References}

1. Bell, D et al. 2007. Proceedings of the 3rd Congress of the Asian Pig veterinary Society (APVS) Wuhan; China. 2007. pag 135.

2. Desrosiers, R. 2006 Proceedings of the North Carolina Health Hogs Seminar.

3. Yoon KJ. 2007 Proceedings of the Asian Pig Veterinary Society.

4. Dr. Hiromichi Ishikawa, Vice-President of APVS 2009 in Tsukuba, Japan. 2009. Proceedings of the 4th Congress of Asian Pig Veterinary Society. Keynote speech 1-4. pag 12. 


\title{
P.238
}

\section{Efficacy of an experimental inactivated PRRSV vaccine based on a recently isolated field strain}

\author{
Marc F. Geldhof ${ }^{1}$ Merijn Vanhee ${ }^{1}$ Jan Van Doorsselaere ${ }^{2}$ Hans J. Nauwynck $^{1}$ \\ 1. Laboratory of Virology, Faculty of Veterinary Medicine, Ghent University, Merelbeke, Belgium; 2. Department of Health \\ Care and Biotechnology, KATHO Catholic University College of South-West Flanders, Roeselare, Belgium
}

\begin{abstract}
Introduction
Porcine reproductive and respiratory syndrome virus (PRRSV) infection is characterized by reproductive failure in sows (1) and is associated with respiratory problems in pigs of all ages (2). The vaccines that are available give variable results regarding the efficacy against genetically diverse field strains. At present, it is generally accepted that there is a need for new safe and effective vaccines that easily can be adapted to prevalent PRRSV strains. Recently, it was demonstrated that by the use of a new procedure, an inactivated PRRSV vaccine can be developed that induces virus-neutralizing antibodies and offers partial protection upon homologous challenge (3). The aim of this study was to compare the efficacy of a homologous experimental inactivated PRRSV vaccine, with the efficacy of a heterologous (Lelystad virus) experimental inactivated PRRSV vaccine and commercially available, inactivated and attenuated vaccines upon challenge.
\end{abstract}

\section{Materials and Methods}

The following PRRSV vaccines were included in this study: a vaccine based on a circulating PRRSV strain $07 \mathrm{~V} 063$ that was isolated from foetuses during reproductive failure in a vaccinated herd and inactivated using the procedure described in Delrue et al. (4); a heterologous experimental inactivated PRRSV vaccine based on Lelystad Virus (3); a commercial attenuated European virus vaccine; a commercial attenuated American virus vaccine and a commercial inactivated European virus vaccine. Piglets from a PRRSV-free farm were vaccinated twice at 5 and 9 weeks of age with the inactivated vaccines. The attenuated vaccines were given at 7 weeks of age. One group of pigs was kept unvaccinated and served as control group. All animals were challenged intranasally with $10^{6.0} \mathrm{TCID}_{50}$ of the virulent virus field strain 07V063 at the age of 13 weeks. Sera were collected by jugular venipuncture at $0,1,2,3,4,5,6$ and 7 weeks after (primo) vaccination and at $0,1,3,5,7,10,14,21,28$ and 35 days post challenge. Virus-specific and virus-neutralizing antibodies were determined by an IPMA- and SN-test, respectively. Virus titers in serum were determined by virus titration on pulmonary alveolar macrophages.

\section{Results}

It was shown that all vaccines, except the commercial inactivated vaccine, induced virus-specific antibodies after vaccination and only the two inactivated experimental vaccines induced 07V063-specific neutralizing antibodies after vaccination. Upon challenge, viremia was observed for 4 weeks in the control group and the groups that were vaccinated with the commercial inactivated and the experimental heterologous inactivated vaccine. Vaccination with the attenuated vaccines reduced the duration of viremia with one week. The virological protection upon challenge was the best in the group receiving the experimental homologous inactivated $07 \mathrm{~V} 063$ vaccine (Fig. 1.).

Fig. 1. Viremia in all groups in function of time post challenge.

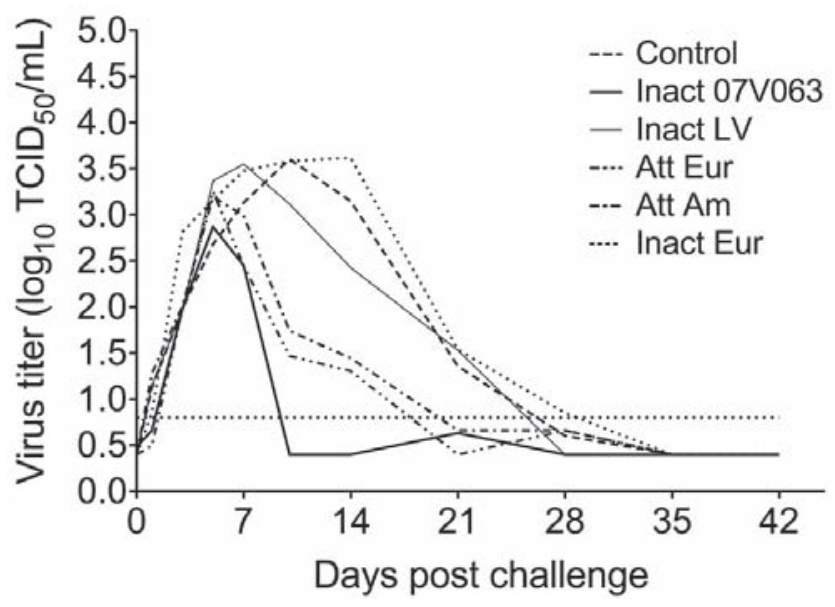

\section{Discussion}

The genetic diversity of PRRSV can cause problems with the efficacy of the current available vaccines that are all based on one virus strain. In general, the advice is to use a vaccine strain that is homologous to the present PRRSV-isolate in the herd. A farmspecific vaccine, based on the circulating PRRSV field strain can be a solution on (vaccinated) farms with severe PRRSV-related problems. Future work will be done to investigate the efficacy of this kind of inactivated vaccine in the field.

\section{Acknowledgements}

This work was funded by the Federal Public Service Health, Food chain safety and Environment (RT - Theme 7 - Acron. PORRS) and the European Union (Seventh Framework Programme; Project No. 245141; coordinated by Dr. H.J. Nauwynck).

\section{References}

(1) Christianson et al. (1992). Am J Vet Res. 53, 485-488

(2) Van Gucht et al. (2004). Vet Immunol Immunopathol, 102, 165-178

(3) Vanhee et al. (2009). Vet Res. 40:63

(4) Delrue et al. (2009). Vet Res. 40:63 


\title{
P.239
}

\section{Vaccination of pigs with replicon particles expressing various PRRSV open reading frames}

\author{
Mark Mogler $^{1,2}$ Ryan Vander Veen ${ }^{1,2}$ Brandon Russell1, 2 DL Hank Harris ${ }^{1,2}$ \\ 1. lowa State University, Ames, IA, USA; 2. Harrisvaccines Inc, Ames, IA, USA
}

\section{Introduction}

Porcine reproductive and respiratory syndrome (PRRS) virus causes abortion, anorexia and pneumonia. Due to the prevalence of the virus and the ineffectiveness of current countermeasures, it has become one of the most economically significant threats to the swine industry. Current immunization strategies rely on autogenous killed-virus vaccine, modified-live virus vaccine, or deliberate exposure to virulent virus. Each method has drawbacks, such as: ineffective cross-protection, shedding of virus to penmates and piglets, and lack of serological differentiation between infected and vaccinated animals (DIVA). New vaccination strategies are needed to achieve the goals of local and regional elimination of PRRS virus.

One such method involves the use of replication-incompetent, alphavirus-derived, single-cycle viral replicon particle (RP) vaccines. These vaccines consist of a viral particle derived from Venezuelan equine encephalitis (VEE) virus containing a modified RNA genome that does not include the VEE structural genes. The lack of vector-encoded structural genes renders the vector unable to form a new particle once it enters the host cell. This feature adds to the safety of the vaccine and helps the vector overcome host anti-vector immunity.

Our group has evaluated RP vaccine candidates expressing open reading frame (ORF) 3, 4, 5, and 6 in a young-pig heterologous challenge model.

\section{Materials and Methods}

Replicon plasmids containing the PRRS virus genes of interest were created and used to generate RP vaccines for the study as previously described ${ }^{1,2}$. Briefly, in vitro transcribed replicon RNA and helper RNAs were electroporated into Vero cells, which were then incubated for 18 hours. After incubation, packaged RP were harvested by affinity chromatography and formulated in phosphate buffered saline with $5 \%$ sucrose and $1 \%$ swine serum. Safety tests were performed to ensure that no replication-competent virus existed in the batch of RP. Aliquots of RP were frozen at $-80 \mathrm{C}$ until use in the study. On the day of each vaccination, RP were thawed and diluted to the desired dosage, then administered by intramuscular injection in the neck. Each formulated vaccine batch was titrated on Vero cells and quantified using an indirect fluorescent antibody assay.
Forty piglets were obtained at three weeks of age from a herd serologically and clinically negative for PRRS virus, swine influenza virus, and porcine circovirus 2. Pigs were randomized into four groups of ten pigs each. Blood samples were collected at intervals throughout the study. At four weeks of age, pigs were vaccinated with the appropriate RP vaccine, and booster immunization occurred at seven weeks of age. At ten weeks of age, pigs were challenged intranasally with $2 \times 10^{5} \mathrm{TCID}_{50}$ PRRS virus. Study termination and necropsy occurred at twelve weeks of age. Serum samples from throughout the study were used to determine the magnitude of antibody and cell-mediated responses, as well as post-challenge viremia. Gross and microscopic pathology scores were determined by a pathologist blinded to the treatment groups.

Vaccine groups for the study were as follows: 1)RP expressing PRRS virus ORFs 3 and 4; 2)RP expressing PRRS virus ORFs 5 and 6; 3)RP expressing PRRS virus ORFs 3, 4, 5 and 6; 4) Placebo.

\section{Results and Discussion}

Prior to challenge, the majority of vaccinated pigs had a specific antibody response to the RP-expressed antigen in their vaccine, as determined by Western blot. Prior to challenge, no pigs had a positive anti-nucleocapsid antibody response, as determined by the IDEXX Herd-Chek ELISA at the ISU Diagnostic Laboratory.

An interferon-gamma ELISPOT assay was used to determine the magnitude of cell-mediated immunity on the day of challenge. These results indicated that vaccinates receiving RPs expressing ORFs 5 and 6 (with or without ORFs 3 and 4) had significantly higher antigen-specific cell stimulation than placebo. The lack of seroconversion to the nucleocapsid antigen among vaccinates illustrates the DIVA capabilities of the RP vaccines. The induction of specific cell-mediated and antibody-mediated immune responses following vaccination with RP vaccines demonstrates their potential to overcome the limitations of existing vaccine technology.

Additional results will be reported at the meeting.

\section{Acknowledgments}

The authors would like to thank AlphaVax Inc. (Research Triangle Park, NC) for assistance constructing the replicon plasmids.

\footnotetext{
References

1. Hooper JW, et al. Vaccine 2009 (28)2:494-511.

2. Kamrud Kl, et al. Virology 2007 360(2):376-87.
} 


\title{
Failure in protection conferred by recombinant Aujeszky's disease viruses expressing non-apoptotic fragments of PRRSV GP5 protein in pigs
}

\author{
Francisco Díez-Fuertes; Cinta Prieto; Francisco Javier Martínez-Lobo; Isabel Simarro; Lidia Hornillos-Gumiel; José María Castro
}

Facultad de Veterinaria Universidad Complutense de Madrid, Madrid, Spain

\section{Introduction}

In the last years, porcine reproductive and respiratory syndrome virus (PRRSV) envelope glycoprotein GP5 has been a leading target for the design of new generation vaccines that solve safety problems of live attenuated vaccines and the limited efficacy of inactivated vaccines against PRRS. In a previous study, our research group identified and characterized different regions in the GP5 implicated in apoptotic processes (1). Taking advantage of this knowledge, EC1, a recombinant Aujeszky's disease virus (rADV) which express a GP5 fragment deleting the first 127 amino acids of the N-terminus of the original GP5, and EA4, a rADV which express a chimera fusing 32-69 amino acids fragment to the last 74 amino acids fragment of the original GP5, were generated (2). Therefore, the aim of the present study was to test the immunoprotective properties of EC1 and EA4 by immunization of PRRS-negative pigs.

\section{Materials and Methods}

The experimental design included eighteen six-month old PRRS seronegative pigs. Five pigs of group A were immunized with 106,0 TCID50 of the Spanish virulent strain Sp-3 (positive controls). Groups B and C, both of five animals, were immunized intramuscularly with 106,5 TCID50 of EA4 or EC1, respectively. Three pigs of group $D$ were not immunized but challenged (negative controls). The pigs were immunized on days 0, 21 and 42 of the experiment. On day 70, all animals were challenged intranasally with $5 \times 106,0$ TCID50 of the Spanish virulent field strain Sp-5. Twenty one days post-challenge all animals were euthanized and necropsied. Blood samples and oral, rectal and nasal swabs were collected on immunization days, on challenge day and every three days after challenge. Different tissue samples were collected from all necropsied animals. All samples were tested for PRRSV detection by viral isolation. Specific antibodies production against PRRSV was assayed by ELISA (CIVTEST SUIS PRRS E/S), Western-blot and virus neutralization assay. $A$ T-lymphocyte proliferation assay and an ELISPOT assay (3) were also carried out on days 42, 70 and 90 to quantify the development of cell mediated immune response.

\section{Results}

None of the vaccinated pigs showed any obvious clinical signs before challenge. After challenge, viremic animals were observed among rADV vaccinated pigs through all the experiment (Table 1). Moreover, PRRSV was found in at least one of the tissue samples collected at necropsy from each pig (except in one pig of group C) and in nasal, oral and rectal swabs collected during the first 15 days after challenge.
Table 1. Viremia results after challenge

\begin{tabular}{|c|c|c|c|c|c|c|c|c|}
\hline Group & Chall. & $3 \mathrm{dpc}$ & 6dpc & 9dpc & $12 \mathrm{dpc}$ & 15dpc & 18dpc & 21dpc \\
\hline A & $0 / 5^{\mathrm{a}}$ & $0 / 5$ & $0 / 5$ & $0 / 5$ & $0 / 5$ & $0 / 5$ & $0 / 5$ & $0 / 5$ \\
\hline$B$ & $0 / 5$ & $5 / 5^{*}$ & $5 / 5^{*}$ & $5 / 5^{*}$ & $5 / 5^{*}$ & $5 / 5^{*}$ & $2 / 5$ & $3 / 5$ \\
\hline$C$ & $0 / 5$ & $5 / 5^{*}$ & $4 / 5^{*}$ & $5 / 5^{*}$ & $4 / 5^{*}$ & $4 / 5^{*}$ & $3 / 5$ & $1 / 5$ \\
\hline$D$ & $0 / 3$ & $3 / 3^{*}$ & $2 / 3^{*}$ & $3 / 3^{*}$ & $3 / 3^{*}$ & $3 / 3^{*}$ & $2 / 3$ & $2 / 3$ \\
\hline
\end{tabular}

Detectable ELISA antibodies against PRRSV were found in one pig of group $C$ at day 42 and in one animal of each rADV vaccinated group on day 70. However, specific antibodies production in groups $B$ and $C$ could not be demonstrated by Western-blot or virus neutralizing assay. T-lymphocyte proliferation measurement did not show any statistically significant difference between groups but a statistically significant difference between groups B and D $(p<0.05)$ was found by ELISPOT on day 70.

\section{Discussion}

Enhanced expression levels obtained in vitro after GP5 apoptotic region deletion encouraged the study of the potential immunoprotective properties of EC1 and EA4 in pigs. However, both experimental vaccines failed to provide protection after challenge as demonstrate by virus isolation from experimental samples taken along the study. On the other hand, pigs immunized with a different PRRSV isolate were fully protected. As no significant differences in cell mediated immune response between rADVs vaccinated pigs and positive controls were found, protection failure elicited by rADVs could be related with the incapacity to observe neutralizing antibodies production. Although a neutralizing epitope has been described in the ectodomain of GP5, it is possible that conformational changes in the recombinant protein expressed by EA4 have prevented the recognition of this epitope by the immunized pigs. In the same way, EC1 has not this neutralizing epitope so the failure in protection conferred by rADV could be completely related with the absence or modification of this epitope.

\section{References}

1. Fernández et al.(2002). Virus Res., 83(1-2):103-18.

2. Díez-Fuertes et al. (2008). IPVS Congress.

3. Díaz et al. (2005). J Gen Virol., 86(Pt 7):1943-51. 


\title{
Immunization of pigs with PRRSV GP5 subunit vaccine produces a severe enhancement of clinical disease after challenge
}

\author{
Cinta Prieto; Francisco Javier Martínez-Lobo; Francisco Díez-Fuertes; Patricia Aguilar-Calvo; Isabel Simarro; José María Castro
}

Facultad de Veterinaria Universidad Complutense de Madrid, Madrid, Spain

\section{Introduction}

In the last years our research group has studied extensively the variability of PRRSV and obtained evidence of the existence of isolates with a greater ability to induce neutralizing antibodies capable to cross-react with different strains than others (Martínez-Lobo et al., 2008). These results have encouraged the development of a subunit vaccine expressing the GP5 of one of the pluripotent isolates described. On the other hand, no adverse effects have ever been observed after challenge of animals immunized with recombinant PRRSV vaccines and for this reason, the aim of the present communication is to report the exacerbation of disease observed after challenge with a virulent PRRSV isolate in a group of pigs that had been previously immunized with a PRRSV GP5 subunit vaccine produced in E. coli.

\section{Materials and Methods}

Eighteen six-month old PRRSV seronegative pigs were divided into three groups. Six pigs of group A were immunized twice with $600 \mu \mathrm{g}$ of rGP5 on day -42 and with $300 \mu \mathrm{g}$ of rGP5 on day -21 . Six pigs of group B were challenged but not vaccinated and six pigs of group $C$ were not vaccinated and not challenged. Three weeks after immunization, pigs of group A and B were inoculated intranasally with 105 TCID50 of PRRSV strain 5710. Clinical signs, rectal temperature and food intake were evaluated for each pig every day after vaccination and challenge. Blood samples and nasal culture swabs were collected in vaccination days and at various time points post-vaccination and post-challenge. All pigs were euthanized 18 days after challenge and different tissue samples were collected at necropsy. Every collected sample was assayed by virus isolation and titration. Specific antibodies production against PRRSV was evaluated by ELISA (CIVTEST SUIS PRRS E/S) and virus neutralization assay. Tissues samples were collected for routine histopathological examination.

\section{Results}

No adverse reactions to immunizations were noted in pigs but after challenge, moderate to severe clinical signs were observed in pigs of groups $A$ and $B$. These clinical signs were more severe in pigs vaccinated with rGP5 than in pigs of group B. Furthermore, significant lower daily body weight gain and higher mean temperatures were found in pigs of group A. Pigs of both challenged groups exhibited high viremia titers through all the experiment and no statistically significant differences were encountered at any time. PRRSV was detected at least in one of the tissue samples collected from each pig of groups A and B. On the day of challenge, only two pigs on group $A$ had seroconverted by ELISA and none of them had neutralizing antibodies. After challenge an increase in antibody titre was observed in pigs of group A but no statistically significant differences were observed between groups $A$ and $B$ in the neutralization assays. Pigs of groups $A$ and $B$ showed mild moderate to gross lesions, including areas of congestion in the lung and moderate enlargement and congestion of some lymph nodes. Microscopically, moderate subacute interstitial pneumonia was recorded in animals of group $A$.

\section{Discussion}

Results of the present study demonstrate that immunization of growing pigs with a recombinant fusion GP5 protein of PRRSV not only failed to provide protection, but appeared to result in an exacerbation of the acute disease produced by challenge with a virulent isolates of the virus. Exacerbation of disease can be mediated by different mechanisms, including an antibodydependent enhancement (ADE) of infection (3) that facilitates viral uptaking and efficiency of replication in target cells. However, the exact mechanisms of in vivo vaccine-induced enhancement observed remain uncertain since no association could be established between clinical severity and non-neutralizing antibody titers or viral burden in serum samples or tissues collected at necropsy.

\section{References}

1. Martínez-Lobo et al. (2008). IPVS Congress.

2. Yoon et al. (1996). Viral Immunology 9, 51-63 


\title{
P.242
}

\section{Elimination of PRRS with herd closure on small pig farm in Slovenia}

\author{
Marina Stukelj; Zdravko Valencak \\ Institute for Health Care of Pigs, Ljubljana, Slovenia
}

\section{Introduction}

Porcine reproductive and respiratory syndrome (PRRS) has plagued the swine industry for the past two decades and is considered the most economically devastating disease of modern swine production (1). A variety of strategies has been described for PRRS eradication; total depopulation/ repopulation, partial depopulation, segregated early weaning, test and removal, mass vaccination with unidirectional pig flow and herd closure (2).

PRRS elimination protocols by the herd closure method have been developed from the knowledge that infected animals will recover from the infection and will eliminate the PRRS virus from the body's tissue when interruption of the introduction of susceptible animals takes place. With herd closure the introduction of gilts is temporally stopped to allow the older sow population to produce antibodies and to eliminate the virus from the herd. It is known and actually happens quite often in Europe that the PRRS infection can sporadically disappear from small swine population $(2,3)$. Herd closure is financially advantageous over depopulation because there is no required downtime, sows are not slaughtered, and there is no clean-up cost (1).

PRRS prevalence in Slovenia was according annual serological monitoring low before 2004. After joining to EU PRRS situation, worsen due to free trade of pigs. The aim of the study was to prove the effectiveness of herd closure in selected conditions on small pig farm on PRRS.

\section{Materials and Methods}

\section{Farm}

The study was conducted on one site pig farm with 50 sows and 3 boars, free of Classical swine fever and Aujeszky disease. Actual epizootiological data and increased mortality rate lead to suspicion of PRRS.

Sampling

- Nine blood samples (2 fatteners, 2 gilts, 2 sows, 3 boars) were taken after suspicion of PRRS.

- Three weeks later 15 blood samples (6 weaners, 5 fatteners, 4 gilts) were taken.

- After two months blood was taken from all sows (50) and boars (3).
All serum samples were tested with IDEXX PRRS ELISA (HerdChek, IDEXX LaboratoriesWestbrook, Maine, USA). Results were expressed in S/P (sample: positive) ratios.

Records of weaner succumbs and culls

Number of succumbed and culled weaners was recorded weekly.

Closure of the farm

After confirmation of PRRS, farm was closed (no introduction of gilts and boars on the farm) for at least 3 months.

\section{Results}

First sampling confirmed PRRS. S/P ratios were high (more than 2) in 8 samples. Second sampling showed high titers (14 samples). Third sampling showed uniformly low S/P (less than 1) in all samples. Number of succumbs and culls at the beginning was $77(16 \%)$ and decreased to $18(6,25 \%)$ after last sampling.

\section{Discussion}

The effect of herd closure was evident by serological testing and with decreased number of succumbed and culled weaners. In future, we expect that farm will become PRRS negative if new PRRS virus will not be introduced on the farm. Strict biosecurity procedures and isolation area for all replacement pigs are imperative for farm to keep its health status.

\section{References}

(1) Schaefer N, et al. 2007. J Swine Health Prod 15(3):152-155.

(2) Torremorell M, et al. 2002.Advan Pork Indus 13:169-176.

(3) Freese WR, et al. 1994. J Swine Health Prod 2(1):13-15. 


\title{
P.243
}

\section{Porcine Reproductive and Respiratory Syndrome regional elimination in Minnesota}

\author{
Robert B. Morrison; Cesar A. Corzo; Scott Dee; Montserrat Torremorell; Peter Davies \\ University of Minnesota, St Paul, MN, USA
}

\begin{abstract}
Introduction
Porcine reproductive and respiratory syndrome (PRRS) has become one of the most important diseases in swine production around the world. PRRS was first diagnosed in the United States in 1987.1 The disease is manifested by reduced reproductive performance, poor growth, decreased feed efficiency and increased mortality. Recently, the cost of this disease in North America has been estimated to cost US\$ 560 million per year in the United States.2 Veterinarians and producers have been able to eliminate the from farms, however, some herds become reinfected and aerosol3 transmission is often suspected. Therefore, a regional elimination approach may increase the odds of success of eliminating pathogens among swine herds.
\end{abstract}

\section{Materials and Methods}

In 2004, local producers in Stevens county a county located in west central Minnesota proposed to bring PRRS herd elimination to the next level and start a regional elimination project. This county has many breeding-stock herds with high biosecurity and health standards. The first step was to contact all producers in the county to obtain their farm location as well as a basic description of the type of pigs being raised. Locations were plotted on a map using ArcGIS 9.3 (ESRI, Redlands, CA). The second step was to obtain producer consent for testing and sharing of PRRS results. The third step was to have all herds tested through serology and PCR. The PRRS virus in infected herds would be further tested to characterize the strain present in the herd. Each location was classified as positive, negative or unknown based on expert opinion of the local veterinarian or by diagnostic tests such as serology and PCR. Infected breeding herds would start a control program called herd closure to stop transmission within the herd. Sites with growing pigs eliminated the virus through depopulation or strict movement of pigs, personnel and equipment. Quarterly meetings were held to share results, validate information and provide scientific information with regards to the latest PRRS findings.

\section{Results and Discussion}

A total of 89 farm locations, one truck cleaning facility, one truck service facility and one hog-buying station were plotted on the map. Of the 89 locations, there are 19 with sows, eight nursery sites, 40 finishing sites, two boar studs, two boar stud isolation units, two $4 \mathrm{H}$ finishing sites, 13 empty sites and 3 sites that have been abandoned. In 2004 there were 31 locations that were thought to have positive pigs. By 2006, the number of positive locations had decreased to 15 and in 2008 there were four positive locations. Presently, there is only one positive location that is under elimination planning (Table 1). A total of 18 PRRS isolates have been sequenced. Three sow sites eliminated the virus by serum inoculation and herd closure, two sow sites eliminated the virus through depop/repop and the remaining sites which were nursery/finishing sites have eliminated the virus through depop/repop. The project has been expanded to include six surrounding counties. To date, 85 additional locations have been identified, of which three are positive, 18 are in the process of status definition and the remaining sites are negative. Due to the low density of these additional six surrounding counties and the low prevalence of positive herds, the project has been expanded once again to northern Minnesota where farm location and testing has already started. To date, an additional 140 locations have been identified of which 15 are positive, 30 are negative and the remaining are in the process of status definition. These results indicate that PRRS can be eliminated from a region.

Table 1. Number of positive, negative and unknown locations in Stevens County and Northern Minnesota.

\begin{tabular}{|l|c|c|c|c|}
\hline PRRS Status & $\begin{array}{c}\text { Stevens } \\
\text { County 2004 }\end{array}$ & $\begin{array}{c}\text { Stevens } \\
\text { County 2008 }\end{array}$ & $\begin{array}{c}\text { Stevens } \\
\text { County 2009 }\end{array}$ & $\begin{array}{c}\text { Northern } \\
\text { Minnesota }\end{array}$ \\
\hline Positive & 31 & 4 & 1 & 19 \\
\hline Negative & 19 & 85 & 88 & 154 \\
\hline Unknown & 37 & 0 & 0 & 141 \\
\hline Total & 87 & 89 & 89 & 314 \\
\hline
\end{tabular}

\section{Acknowledgements}

Authors would like to acknowledge E Mondaca, S Wayne, local veterinarians and producers. Funding was provided by NPB, USDA, AASV, Boehringer Ingelheim Vetmedica and MN Pork Board.
References
1. Benfield D. et al. (1992). Diseases of Swine. 756-762.
2. Neumann E. et al. (2005). JAVMA. 3:385-392.
3. Dee S. et al. (2009). Vet Res. 40:39. 


\title{
P.244
}

\section{Benefits of using Pulmotil Premix ${ }^{\circledast}$ in swine production infected by PRRSV}

\author{
Annick Saives ${ }^{1}$ Nathalie Gautier $^{2}$ \\ 1. Elanco Europe, Brussels, Belgium; 2. Selas Veterinaire de la Hunaudaye, Lamballe, France
}

\section{Introduction}

Respiratory disease is one of the most important diseases in swine production, with major economic consequences. Porcine Respiratory and Reproductive Syndrome Virus (PRRSV) is one of the main agents. Treatments are mostly used when an outbreak appears, especially during the fattening phase. The present study aimed to evaluate the effects of a treatment with Pulmotil Premix $^{\mathrm{TM}}$ in a farm with history of PRRSV, before the outbreak in fattening pigs.

\section{Materials and Methods}

The swine facility is a farrow to finishing farm of 220 sows having a history of PRRSV symptoms at the end of post-weaning and at the beginning of fattening. PRRSV was diagnosed by laboratory analysis (Histology, March 2006).

Pulmotil Premix ${ }^{\mathrm{TM}}$ medication was implemented starting in March 2006 a dosage of $5 \mathrm{~kg} / \mathrm{T}(200 \mathrm{ppm})$ in feed from 12 to $20 \mathrm{~kg}$ of AW. The objective was to intervene before clinical symptoms appear, to improve the immunity of animals and to decrease the virus circulation, replication and virus expression. It was demonstrated before that tilmicosin has an effect in decreasing in vivo viral replication (1). Nothing else was changed during the follow-up period.

A monitoring technique was set up by comparing a time period before treatment (from 04/01/05 to 03/31/6), a transition period where all the pigs had not yet been treated (from 04/01/06 to $03 / 31 / 07$ ) and a final period where all pigs received the treatment (from 04/01/07 to 03/31/08).

A 4th treatment period was evaluated (from 04/01/08 to $07 / 31 / 09)$, since the producer decided to continue the treatment to see if the improvement would continue.

To evaluate the economic interest of using Pulmotil ${ }^{\mathrm{TM}}$ we referred to the latest estimates published by the French Pig Institute (IFIP) (2)

\section{Results}

Table 1 presents the technical criteria per time period used and Table 2 shows the improvement in each criterion and its economic impact per pig.

\section{Discussion and Conclusion}

This study confirms the advantage of using Pulmotil Premix ${ }^{\mathrm{TM}}$ for the treatment of respiratory diseases even within a viral context. The cost of treatment, being estimated at $1.39 €$ per $25 \mathrm{~kg}$ of feed consumed per pig, shows the return on investment using Pulmotil is therefore at $12.28 / 1.39=8.8$

\section{References}

1. Benfield, DA et al. (2002). Proc. AASV, p87-91.

2. IFIP (2008) Résultats Porcs Bretagne
Table 1: Technical criteria per period

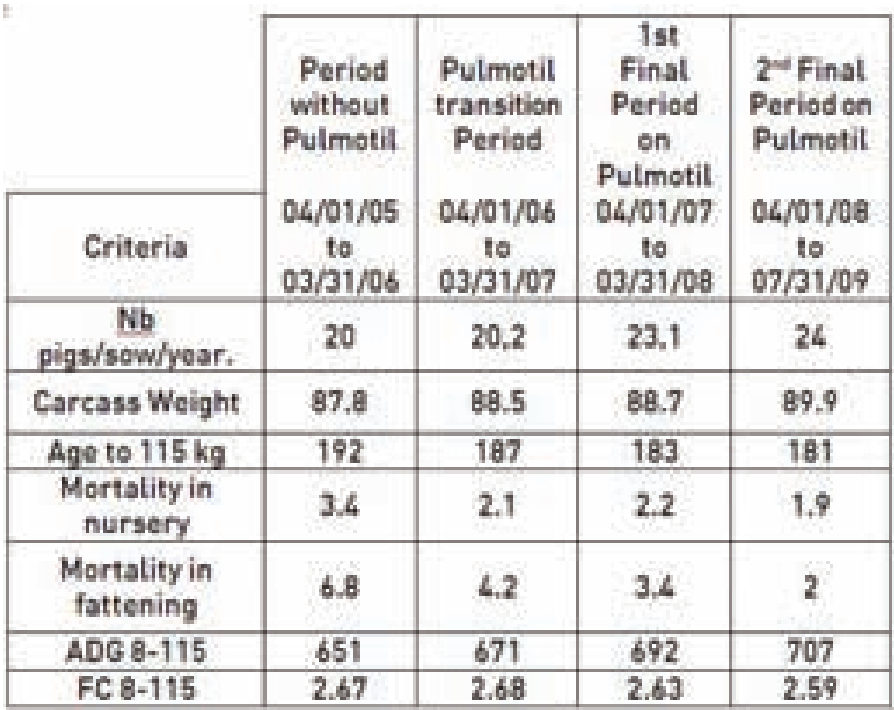

Table 2: Gain for each criterion with Pulmotil treatment

\begin{tabular}{|c|c|c|}
\hline Criteria & $\begin{array}{c}\text { Improvement between } \\
\text { last period and period } \\
\text { without trentment }\end{array}$ & $\begin{array}{c}\text { Economie } \\
\text { benefit }\end{array}$ \\
\hline Cateass weight & $2.1 \mathrm{~kg}$ & $2.66 €$ \\
$\begin{array}{c}\text { Age to } 115 \mathrm{~kg} \\
\text { Mortality in } \\
\text { nursery }\end{array}$ & 11 days & $1.76 €$ \\
$\begin{array}{c}\text { Mortality in } \\
\text { fattening }\end{array}$ & $1.5 \%$ & $0.9 €$ \\
IC 8-115 & $4.8 \%$ & $4.32 €$ \\
\hline
\end{tabular}




\title{
P.245
}

\section{In vitro efficiency of a disinfectant (Virusnip ${ }^{T M}$ ) on CSFV, PRV and PRRSV}

\author{
Napawan Bunpapong ${ }^{1}$ Sasiwimon Talummuk ${ }^{2}$ Kai Sievert ${ }^{3}$ Penchan Chaiyanate ${ }^{2}$ Roongroje Thanawongnuwech $^{1}$ \\ 1. Chulalongkorn University, Bangkok, Thailand; 2. Novartis (Thailand) Ltd, Bangkok, Thailand; 3. Novartis Animal Health, Basel, Switzerland
}

\section{Introduction}

Classical swine fever virus (CSFV), pseudorabies virus (PRV) and porcine reproductive and respiratory syndrome virus (PRRSV) are economically significant pathogens in major swine raising countries including Thailand. Vaccination, quarantine regulation and management strategies have been practiced as prevention and control strategies to improve the animal herd health status (1). Biosecurity using disinfectants is able to break the disease cycle by reducing the pathogen load in the environment. Disinfectants can be classified into various groups such as phenol, chlorine-releasing, acid, alkalis etc (2). The objective of this study is to evaluate the in vitro efficacy of Virusnip (Novartis Animal Health) against the major swine pathogens (CSFV, PRV and PRRSV) in Thailand.

\section{Materials and Methods}

Disinfectant: Virusnip, a commercial oxidizing disinfectant composed of Potassium monopersulfate. Viruses and cells: CSFV (ALD) and PRV (Tongsong) were prepared in SK6 cell line $(2,3)$. PRRSV (01NP1) was prepared in MARC-145 with 5\% fetal bovine serum (FBS) in growth medium incubated in $5 \% \mathrm{CO} 2$ at $37^{\circ} \mathrm{C}$ for 48 hours (5).

Virucidal activity: Virusnip was prepared in a $2 X$ concentration and mixed with the equal volume of the virus stock to yield a final concentration of 1:100, 1:200 and 1:400. The mixtures were incubated at room temperature for 30 seconds and 5 minutes then were filtrated through SephadexTM column and titrated in a 10-fold dilution manner. The mixtures were inoculated into a 96 well microtiter plates then incubated in $5 \% \mathrm{CO} 2$ at $37^{\circ} \mathrm{C}$ for 1 hour. Maintenance medium with $2 \%$ FCS was added and incubated in $5 \% \mathrm{CO} 2$ at $37^{\circ} \mathrm{C}$ for 48 hours. Indirect immunoperoxidase monolayer assay (IPMA) was performed for CSFV and PRRSV antigen demonstration to confirm the infections. An evidence of cytopathic effect (CPE) was observed to determine the PRV-infected cell compared with the control. Virus titer was calculated by Reed and Muench method (4). The titers were expressed as TCID50/ml.

\section{Results and Discussion}

The efficacy of Virusnip against CSFV, PRV and PRRSV is shown in Table 1. CSFV was completely killed at all dilutions tested within 30 seconds. However, PRV remained alive at all dilutions within 30 seconds but was completely killed at all dilutions by 5 minutes. PRRSV was completely killed at all dilutions within 30 seconds. CSFV, PRV and PRRSV are enveloped swine viruses. These swine viruses may be highly resistant in the suitable environment but are easily destroyed by lipophilic agent and very sensitive to acid or alkalis. Virusnip is able to kill CSFV, PRV and PRRSV at different concentrations depending on the viral susceptibility. CSFV and PRRSV appeared to be more susceptible to Virusnip than PRV. Virusnip can be considered as an alternative disinfectant in the swine farms.

Table 1: In vitro efficacy of Virusnip on virus titers at different times and concentrations.

\begin{tabular}{|c|c|c|c|}
\hline \multirow{3}{*}{ Virus } & \multirow{2}{*}{$\begin{array}{c}\text { Concentration } \\
\text { of Virusnip }\end{array}$} & \multicolumn{2}{|c|}{$\begin{array}{c}\text { Titers after contacted } \\
\text { with Virusni (logl0) }\end{array}$} \\
\cline { 2 - 4 } & & $\mathbf{3 0} \mathbf{~ s e c}$ & $\mathbf{5}$ min \\
\hline \multirow{4}{*}{ CSFV } & $1: 100$ & 0 & 0 \\
\cline { 2 - 4 } & $1: 200$ & 0 & 0 \\
\cline { 2 - 4 } & $1: 400$ & 0 & 0 \\
\cline { 2 - 4 } & Control & 4.5 & 4.5 \\
\hline \multirow{4}{*}{ PRV } & $1: 100$ & 4 & 0 \\
\cline { 2 - 4 } & $1: 200$ & 3.5 & 0 \\
\cline { 2 - 4 } & $1: 400$ & 2.5 & 0 \\
\cline { 2 - 4 } & Control & 6.5 & 6.5 \\
\hline \multirow{4}{*}{ PRRSV } & $1: 100$ & 0 & 0 \\
\cline { 2 - 4 } & $1: 200$ & 0 & 0 \\
\cline { 2 - 4 } & $1: 400$ & 0 & 0 \\
\cline { 2 - 4 } & Control & 5.5 & 5.5 \\
\hline
\end{tabular}

\section{References}

1. Hooft van Iddekinge et al., 1996. Vaccine 14(1):6-12.

2. Linton et al., 1987. Blackwell scientific, 167 p.

3. Mendoza et al., 2007. Vaccine 25(41):7120-7124.

4. Reed and Muench, 1983. Am J Hygiene 27:493-497.

5. Thanawongnuwech et al., 1998. Vet Pathol 35:988-406. 


\title{
P.246
}

\section{Antiviral activity of Tilmicosin vs. European and North American genotypes of Porcine Reproductive and Respiratory Syndrome Virus (PRRSV)}

\author{
Yijun Du ${ }^{1}$ Dongwan Yoo ${ }^{1}$ Gail Scherba ${ }^{1}$ Marie Anne Paradis ${ }^{2}$ Tom Shryock $^{3}$ \\ 1. University of Illinois at Urbana-Champaign, Urbana, IL, USA; 2. Elanco Canada, Montreal, QC, Canada; 3. Elanco Animal Health, Greenfield, USA
}

\section{Introduction}

Porcine reproductive and respiratory syndrome (PRRS) has spread to most pig-producing countries and has continued to evolve. Thus, vaccination for PRRS has been less effective than anticipated and the PRRS-associated economic losses continue. Since a satisfactory vaccine is currently absent, antiviral chemotherapy is a worthwhile consideration for PRRS. Tilmicosin is a macrolide antibiotic, which has been developed and marketed under the brand name Pulmotil ${ }^{\circledR}$ as a premix and Pulmotil $A C^{\circledR}$ as an aqueous concentrate for swine respiratory disease. Tilmicosin has previously been shown to have antiviral effects on PRRSV in pigs, with improved clinical scores, feed consumption and gain, and a reduction in mortality and abortion rate in treated vs. untreated sows $(1,2,3)$. Tilmicosin accumulates in macrophages at high concentrations (4), and since PRRSV preferably infects alveolar macrophages as the primary target cells, the inhibitory effect of tilmicosin in macrophages may explain the reduced clinical severity in vivo as observed in the tilmicosin-fed pigs. In the present study, the antiviral effects of tilmicosin were examined for both North American and European genotypes of PRRSV in porcine alveolar macrophages (PAMs), and the mechanism of action has been studied.

\section{Materials and Methods}

Cells and viruses: PAMs were grown in RPMI1640 with 10\% FBS. PA8 strain (5) was used as a representative virus for North American genotype PRRSV, and Lelystad virus $(L V, 6)$ was used as the European genotype PRRSV.

Determination for antiviral activity: PAMs were seeded in 96-well plates in a volume of $100 \mu \mathrm{l}$ per well. Cells were treated with tilmicosin, and $24 \mathrm{~h}$ later, infected with 100 TCID50 of PA8 or LV in the presence of tilmicosin. Supernatants were collected and titrated by micro-titration infectivity assays $18 \mathrm{~h}$ post-infection. For real-time RT-PCR, viral RNA was extracted from cell culture supernatants and subjected to quantitative reverse transcription (RT)-PCR.

\section{Results}

Fig. 1. Antiviral activities of tilmicosin on the North American genotype $(A, B)$ and European genotype PRRSV $(C, D)$, as determined by virus titration $(A, C)$ and quantitative $R T-P C R$ for viral genomic $R N A(B, D)$. Tilmicosin concentrations are indicated on the $X$-axis. Virus titers and relative amounts of RNA are indicated on the $Y$-axis. Star $\left(^{*}\right)$ denotes a statistically significant difference $(p<0.05)$. Each concentration was tested in triplicate, and the experiment was repeated twice.
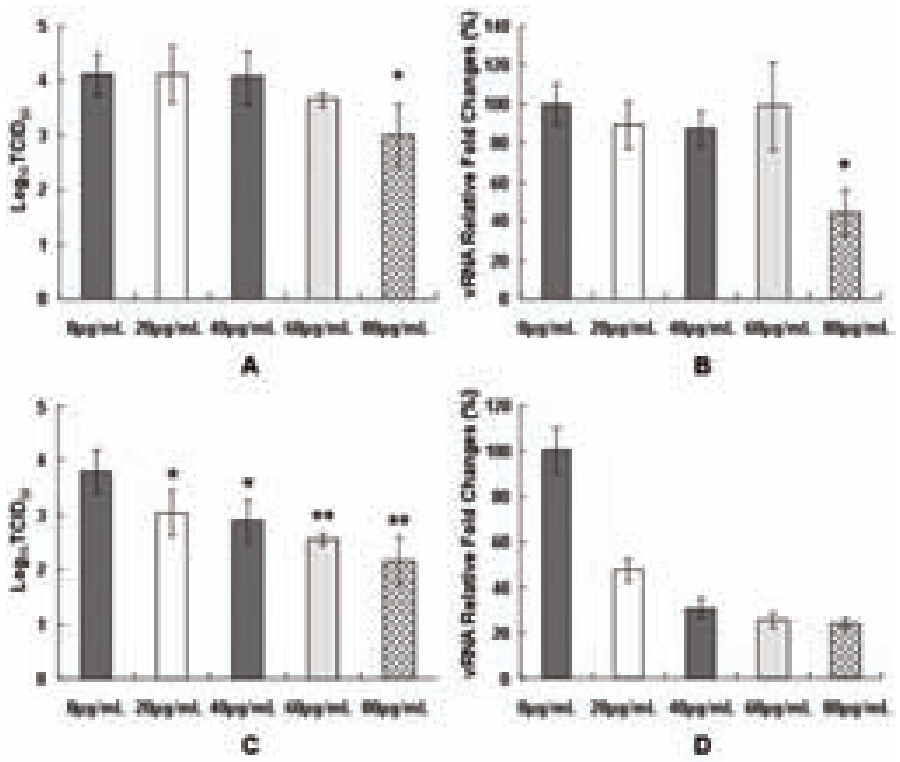

\section{Discussion}

The results show that tilmicosin inhibited replication of both genotypes of PRRSV, but was more potent vs. the European genotype.

\section{Conclusion}

The data suggests that tilmicosin may be used as a preventative measure for pigs infected with either the European or North American PRRSV genotype.

\section{References}

1. Batista et al., 2009, Proc. 23rd Am. Assoc. Swine Vet. 173-177.

2. Benfield et al., 2002, Proc. 16th Am. Assoc. Swine Vet. 87-91.

3. Misener et al., 2006, Proc. 19th IPVS Congr. p13.

4. Scorneaux B and T. Shryock, 1998. J. vet Pharmacol, Ther. 21: 257268.

5. Wootton et al., 2000, Arch. Virol. 145: 2297-2323.

6. Meulenberg et al., 1993, Virology 192: 62-72. 


\section{P.247}

\section{Development of a certification procedure for a PRRS-unsuspected status of Al-Stations - a project of the swine health services}

Hendrik Nienhoff ${ }^{1}$ Sylvia Baier ${ }^{1}$ Tom Duinhof $^{2}$ Sabine Eger ${ }^{3}$ Juergen Harlizius ${ }^{4}$ Ernst Lohner $^{5}$ Hermann Niemeyer ${ }^{6}$ Thomas $^{2}$ Barmettler ${ }^{7}$ Jean Schoos ${ }^{8}$ Helga Vergara ${ }^{9}$ Gottfried Schoder ${ }^{10}$ Frederic Vangoenweghe ${ }^{11}$ Uta Wettlaufer-Zimmer ${ }^{12}$

1. Swine Health Service, Chamber of Agriculture Lower Saxony, Oldenburg, Germany; 2. Gezondheidsdienst voor Dieren, Deventer, Netherlands; 3. SGD TSK Thueringen, Bad Langensalza, Germany; 4. SGD Chamber of Agriculture, Bonn, Germany; 5. SGD TSK BW, Stuttgart, Germany; 6. SGD TGD Bayern, Grub, Germany; 7. SUISAG, SGD, Bern, Switzerland; 8. Convis, Luxembourg, Luxembourg; 9. SGD TSK Sachsen, Dresden, Germany; 10. SGD TGD, Linz, Austria; 11. DGZ, Torhout, Belgium; 12. SGD Landesuntersuchungsamt Rheinland-Pfalz, Koblenz, Germany

\section{Introduction}

PRRS has a high economic impact on swine production worldwide (4). Eradication of the disease on a herd basis is described by different groups $(1,3,5)$. Every breeding organisation has its own strategy to clarify the being unsuspected status for PRRS of their farms, esp. Al-stations. The schemes, diagnostic tests and sample numbers can not be compared at this moment.

\section{Materials and Methods}

A PRRS working group of the swine health services of Germany, Austria, Switzerland, Luxembourg, Belgium and the Netherlands has developed a standard procedure of certification for a PRRSunsuspicious status of Al-stations.

\section{Auditing process:}

1. Requirements:

- Agreement with the local Swine Health Service

- Compliance with the biosecurity standards

- Clinical unsuspected for PRRS

- Unsuspected for PRRS by Serology and PCR

- Purchase livestock from unsuspected farms

- Quarantine

- No vaccination

2. Biosecurity check

- Biosecurity check according to a specially developed checklist by a Swine Health Service Veterinarian

3. Status survey

- Al-stations: serological survey of all boars on PRRS alternative $12 x$ / year $1 / 12$ of the herd, or $4 x /$ year $1 / 4$ of the herd or $2 x$ / year $1 / 2$ of the herd.

- All aquired boars 3x negative (PCR and serological)

- Minimum time between two surveys 4 weeks maximum 6 months.

4. Ongoing investigations

- Minimum 30 samples / epidemiological unit per 1/2 year (serological) (2)

- 2 visits per year by a Swine Health Service Veterinarian or/ and official government veterinarian (guideline EU 90/429)
5. Clarification of "false" positive Results

- Resampling of the positive animal within two weeks along with at least 10 contact animals (PCR and serological investigation)

- Again positive animals gets removed from the station

6. Certification:

- Biosecurity-check positive

- Status survey all samples negative

- Ongoing investigations all negative or clarified

- The certificate is valid for the last 1/2 year

\section{Results}

So far $15 \mathrm{Al}$-stations have joined the program.

\section{Discussion}

The above procedure makes comparison between Al stations possible.by using same standards for biosecurity, numbers of samples, test procedures and frequencies of farm visits. Hence, this contributes towards a non-governmental approach to control PRRS.

\section{References}

1. Baker, R.B.: 37th Ann. Meeting Am. Assoc. Swine Vet., Toronto:339-405 (2006)

2. Cannon R.M., R.T. Roe: Australian Bureau of Animal Health (1982)

3. Große Beilage, E: Tierärztl. Prax. 34 (G):249-55 (2006)

4. Neumann, E.J., J.B Kliebstein., C.D Johnson., J.W Mabry., E.J. Bush, A.H. Seitzinger, A.L. Green, J.J. Zimmermann: JAVMA 227:385-392. (2005)

5. Schröder, C., S. Bremerich: Tierärztl. Umsch. 58:532-536 (2003)

6. Vangroenweghe, F., Miry, C., Castryk, F. Proc. 19th IPVS, Durban SA, OR.01.73 (2008) 


\title{
P.248
}

\section{Phylogenetic analysis of Dutch PRRSV strains}

\author{
Gerard J. Wellenberg ${ }^{1}$ Toine Cruijsen ${ }^{2}$ \\ 1. Animal Health Service, Deventer, Netherlands; 2. Intervet/Shering-Plough Animal Health, Boxmeer, Netherlands
}

\section{Introduction}

Since the introduction of porcine reproductive and respiratory syndrome virus (PRRSV) in Europe at the beginning of the 90ths, the characterisation of different virus strains demonstrated significant genetic differences between isolated virus strains. It became clear that genetic differences were no longer observed between American and European strains (1), but also within European PRRSV strains. The aim of this study was to characterise the ORF5 sequence of Dutch PRRSV strains and compare them with other EU and US strains.

Figure 1: Parsimony tree using 200 bootstrap simulations, based on EU- and US-type PRRSV ORF5 sequences.

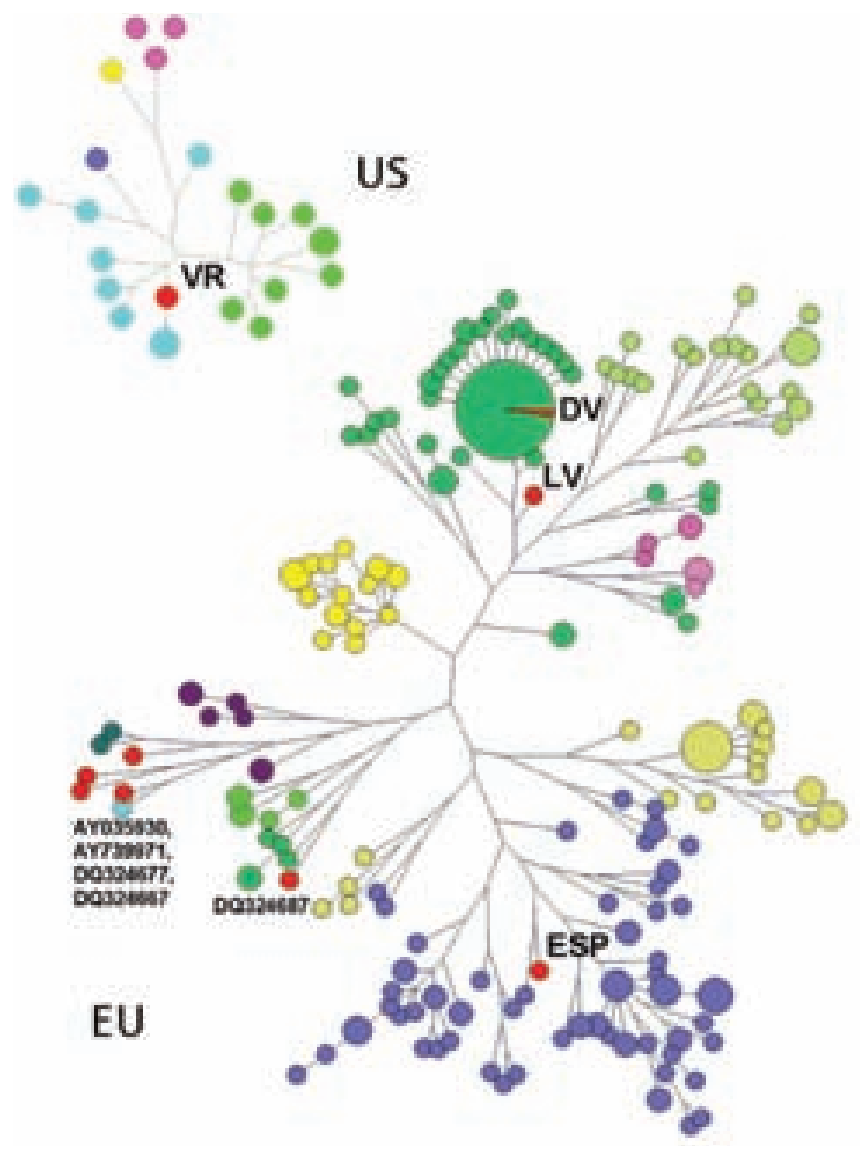

\section{Materials and Methods}

Dutch PRRSV strains ( $n=284)$ were selected from PRRSV-positive samples during 2004-2009. Tissue samples or serum samples were originated from aborted fetuses or weak born piglets, and sera were also obtained from diseased and healthy (vaccinated) pigs. ORF5 sequence alignments and construction of parsimony trees were performed using the BioNumerics package (Applied Maths NV, Sint-Martens-Latem, Belgium).

\section{Results}

Two separate parsimony trees (Figure 1) were constructed based on the EU- of US-type PRRSV ORF5 sequences. The EU-type tree was constructed based on 262 field strains, using the EU-type vaccine strain (DV), Lelystad virus (LV; M96262), Italian strains (AY035930 and AY739971), Belarus strains (DQ324677 and DQ324687), Lithuanian (DQ324667) and the Spanish strain (ESP; DQ345755) as reference strains.

For the US-type PRRSV, 22 field strains (7.7\%) were compared with the US reference strain VR-2332 (VR). Thirty strains (10.6\%) were very likely EU modified live vaccine strains (>99\% homology (NT) with DV) and all these 30 strains were isolated from DV vaccinated pigs (within 4 weeks post-vaccination).

Clear genetic differences were detected in the remaining PRRSV strains $(81.7 \%)$ with homologies ranging from 83 to $100 \%$ compared to the DV vaccine strain (DV) (Figure 2). The average in homology was $90 \%$ in 2005, and it decreased to $88 \%$ in 2007 and $86 \%$ in 2009.

Figure 2: Percentages of Dutch EU-type PRRSV strains, expressed as percentages of homology to DV-ORF5 sequence, within the period 2005-2009.

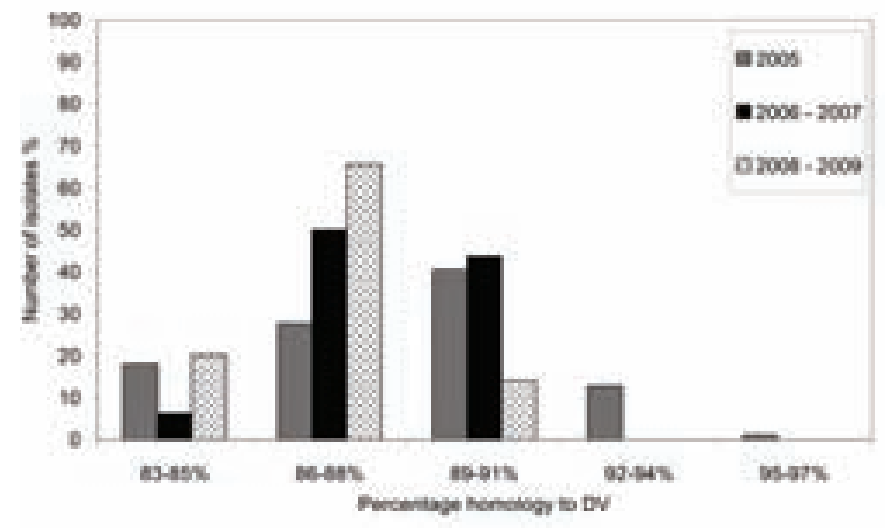

\section{Discussion}

The investigated ORF5 from Dutch EU-type PRRSV field strains, detected in the period 2004 - 2009, are on average $88 \%$ homologous to DV, and LV was not found. Compared to the DV vaccine strain, the homology of ORF5 sequences of EU-type PRRSV field strains might be decreased since 2005.

The spread of the DV strain appears to be very limited as it was found in DV-vaccinated animals (1-4 weeks pv).

Whether new branches, e.g. ESP-branches, appear in the PRRSV parsimony tree in the Netherlands, or whether this is a European phenomenon, has to be investigated.

\section{Reference}

Anderyev, V.G. et al. (1997). Arch. Virol. 142, 993-1001 


\title{
P.249
}

\section{Outbreak and fade out of Porcine Reproductive and Respiratory Syndrome virus in a Dutch SPF-herd: role of vaccination and strict hygienic procedures}

\author{
Manon Houben ${ }^{1}$ John van der Wielen ${ }^{2}$ Toine Cruijsen $^{3}$ Leo van Leengoed ${ }^{4}$ \\ 1. PorQ BV, Breugel, Netherlands; 2. Veterinary Centre The Overlaet, OSS, Netherlands; \\ 3. Intervet/shering Plough, Boxmeer, Netherlands; 4. Veterinary Faculty, Utrecht, Netherlands
}

\begin{abstract}
This paper describes a newly started SPF-herd getting infected by an EU-strain of Porcine Reproductive and Respiratory Syndrome virus (PRRSv).The consecutive eradication strategy was based upon both vaccination and hygienic measurements to prevent the infection from becoming endemic and to produce PRRSv negative pigs again. Fade out of infection was extensively monitored by stratified cohort sampling. A cohort consists of all piglets born in 1 farrowing compartment. Nine months after the initial outbreak no PRRS infected pigs were traced anymore.
\end{abstract}

\section{Introduction}

PRRSv is widely spread among Dutch sow herds. There is an increased demand for PRRS negative- breeding gilts and grower pigs. PRRSv-free herds risk introduction of virulent field virus strains. Strategies to cope with PRRS may vary between herds and depend on estimated introduction risk and the ability to control endemic infections. Preferably, this strategy should lead to eradication of the field infection from the herd. This study demonstrates how vaccination and well-defined internal biosecurity are successfully combined.

\section{Material and Methods}

Herd: A 350 SPF-sow herd was started with caesarian derived piglets from various breeding-herds and combined with gilts form existing SPF-herds. Selected sows with a high genetic index produced the piglets for this new herd (1).

Monitoring: The herd was repeatedly (3 times/year) tested for PRRSv-antibodies (30 randomly selected pigs). In addition, all produced pigs that were sold for reproduction were tested. At August 2006, 10 months after the herd was started, 3 boars out a group of 16 were tested positive for PRRSv (Idexx Elisa). The oldest pigs at the farm then aged 9-10 months. All pigs from the herd were bled and tested for presence of PRRSv antibodies. Thirty-one out of 421 pigs were tested positive and culled. Three weeks later 380 pigs were retested and 28 animals tested positive. Positive tests, reconfirmed by IFA, indicated the herd being infected by an eu-type PRRS-v. Approximately 8 weeks after PRRSv was diagnosed all pigs of the herd were vaccinated with Porcillis PRRS ${ }^{\oplus}$ (EU-live vaccine, Intervet/Shering-Plough) by IDAL (intradermal applicator of liquids) in an attempt to prevent further spread of the infection. Needle-free injection was used to avoid pig-to-pig transmission by needle.

Farm management and hygiene protocol: Stringent hygienic procedures were implemented: -the farrowing departments were strictly isolated by blocked corridors. -clothes were changed between farrowing compartments (max.10 sows per compartment), as well as surgical gloves, masks and hairnets. From each litter pooled blood samples of 4 piglets were tested by PCR to ensure absence of viremia. When negative by PCR, all piglets were tested serologically at 6 weeks and 10 weeks of age to ensure absence of PRRS. Only those cohorts of piglets that were tested negative for PRRS were allowed to be moved to the grower compartments.

\section{Results}

Initial introduction of PRRSv in this SPF-herd, did not coincide with clinical signs. The infection was detected by serology in August 2006 ( 3 out of 16 boars). 3 weeks later $7 \%$ of the pigs in the herd tested serologically positive. After culling the positive pigs again 7 $\%$ of the remaining pigs tested positive by Idexx 3 weeks later.

Two months later (October 2006), when the first piglets were born, characteristic reproductive signs of PRRS were seen, like high numbers of still born piglets, mummies, weakly born piglets and high preweaning mortality. Thereafter these signs gradually declined. The course of infection was extensively monitored by blood sampling of the newborn piglets by PCR.

From February 2007 on most of the cohorts were PCR negative. From May on, sera from 6 and 10 weeks old pigs, tested negative for PRRS.

\section{Conclusion and Discussion}

The vaccination and well-defined internal biosecurity were successfully combined. In our strategy we assume that vaccination reduces vertical transmission of PRRSv and shortens the infectious period of infected pigs. Together with compartmenting of the herd hygienic procedures will lower the risk for susceptible pigs of becoming infected (3). By combining vaccination and hygienic procedures the infection faded out, resulting in the production of PRRSv-negative pigs.

Although introduction of PRRSv in a naive population may not induce clinical signs, the infection may continue to spread within the herd. Especially when no pregnant sows are present, a PRRSv infection may stay unapparent. Only during end-term pregnancy the virus will infect the placenta that leads to mummification, fetal death and weakly born piglets. Viremic born piglets will further spread the infection through the herd. As a consequence the infection may become endemic in weaned piglet groups (2).

In our study we prevented further spread of the infection by vaccination, internal biosecurity procedures, and the removal of viremic born piglets. The relative effect of these contributing factors cannot be calculated, and will need further research.

Our data proved that this approach was successful in eradicating PRRSv from the offspring of a breeding-herd.

\section{References}

1.Groenland G.J.et al,, IPVS 2008; OR.01.75

2. Nodelijk G, et al., Epidemiol Infect. 2000 124(1):173-82.

3. Pitkin A, et al, Can. J. Vet. Res. 73(4):298-302 


\title{
P.250
}

\author{
Antiviral effect of dietary alminosilicate supplement against porcine reproductive \\ and respiratory syndrome virus in experimentally infected pigs \\ Bock-Gie Jung ${ }^{1}$ Jae-Hyung Ko II-Yung Yang ${ }^{1}$ Yeon-Kwon Jung ${ }^{2}$ Bong-Joo Lee $^{1}$ \\ 1. College of Veterinary Medicine, Chonnam National University, Gwanju, Korea; 2. Seobong Biobestech Co., Ltd, Seoul, Korea
}

\section{Introduction}

Porcine reproductive and respiratory syndrome (PRRS), caused by PRRSV, is characterized by respiratory problems in growing and finishing pigs and reproductive failure in sows, with late term abortion, stillbirth and increased preweaning mortality. ${ }^{1}$ Our previous study has demonstrated that dietary aluminosilicate $\left(\mathrm{Al}_{2} \mathrm{SiO}_{5}\right)$ supplement (DAS) enhances immune activity in mice and reinforces clearance of porcine circovirus type 2 in experimentally infected pigs. ${ }^{2}$ The aim of the present study was to demonstrate the antiviral effects of the DAS against PRRSV in experimentally infected pigs.

\section{Materials and Methods}

\section{Animals and diets}

Conventional 6-week-old pigs were used to demonstrate antiviral effect of DAS against experimental PRRSV infection. All animals were confirmed to be free of PRRSV, as determined by a commercial ELISA kit (IDEXX Laboratories, Westbrook, ME, USA). They were randomized into two groups of three pigs each. The control group received a commercial, nutritionally complete, swine feed. The experiment group received the same swine feed supplemented with $0.3 \%$ (w/w) DAS.

\section{Virus titration and histological analysis}

All pigs were acclimatized to the particular diet for 2 weeks before experimental virus infection. Five milliliters of the viral culture $\left(1 * 10^{7} \mathrm{pfu} / \mathrm{ml}\right)$ was inoculated intranasally in each nostril of each pig. All pigs were sacrificed at 12 day post-infection (DPI). Lymphoid tissues and lung were collected from each animal. Titration of PRRSV in each sample was performed by plaque assay. Histological analysis was performed as previously descisribed. ${ }^{2}$

\section{Results}

\section{Virus quantity in various tissues}

Virus titer in bronchial lymph node, tonsil, thymus and lung of the $0.3 \%$ DAS feeding group was significantly decreased compared with those of the control group at $12 \mathrm{DPI}(\mathrm{P}<0.05)$ (Fig. 1).

Fig. 1. Virus quantity in bronchial lymph node (BLN), inguinal lymph node (ILN), tonsil (TON), thymus (THY), mesenteric lymph node (MLN), spleen (SPN) and lung (LUN) at $12 \mathrm{DPI}$.

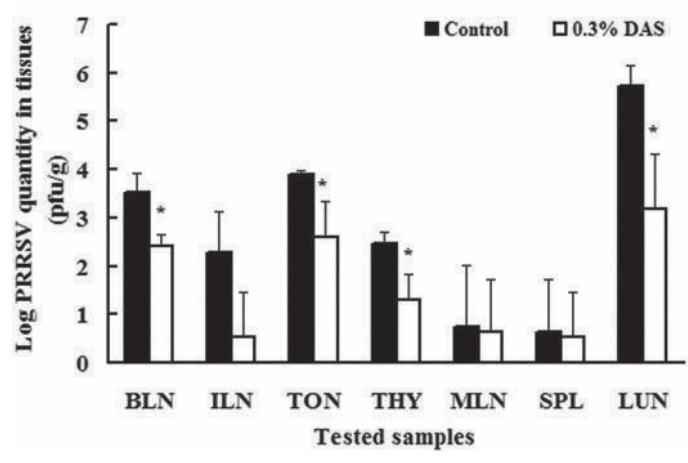

\section{Histological analysis}

Experimentally PRRSV infected pigs showed interstitial pneumonia. These lesions in the $0.3 \%$ DAS feeding group were milder (Fig. 2b) compared with in the control group (Fig. 2a). Additionally, the $0.3 \%$ DAS feeding group shown less severe lymphoid depletion in thymus (Fig. 2d) compared with the control group (Fig. 2c).

Fig. 2. Histological features of lung $(a, b)$ and thymus $(c, d)$ in experimental PRRSV-infected pigs.

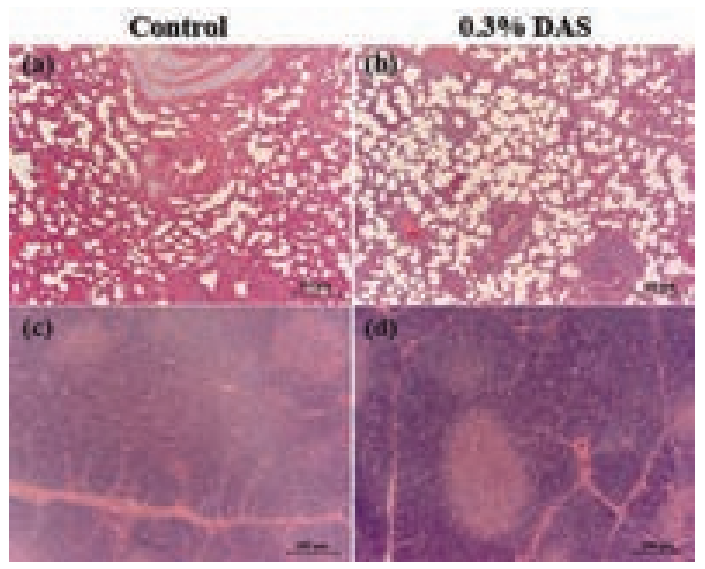

\section{Discussion}

In the present study, titer of PRRSV in almost tested lymphoid tissues and lung of $0.3 \%$ DAS feeding group was significantly decreased compared with those of the control group at 12 DPI. These results are similar to those of previous our study that $0.3 \%$ DAS reinforces clearance of porcine circovirus type 2 in experimentally infected pigs. ${ }^{2}$ Previous studies have reported that $\mathrm{Al}_{2} \mathrm{SiO}_{5}$ act as nonspecific immunostimulators similar to superantigens. ${ }^{3}$ In addition, our previous study has also demonstrated that DAS enhances immune activity in mice. ${ }^{2}$ Therefore, antiviral effect of DAS may relate to nonspecific immunostimulatory effects of $\mathrm{Al}_{2} \mathrm{SiO}_{5}$. Taken together, these findings suggest that DAS have an antiviral effect against PRRSV in experimentally infected pigs.

\section{References}

1. Mateu E, Diaz I. Vet. J. 2008; 177: 345-351.

2. Jung BG, Toan NT, Cho SJ, Ko JH, Jung YK, Lee BJ. Vet. Microbiol. In Press [Epub].

3. Aikoh T, Tomokuni A, Matsukii T, Hyodoh F, Ueki H, Otsuki T, Ueki A. Int. J. Oncol. 1988; 12: 1355-1359. 


\title{
Control of PRRS outbreak in a naïve herd using killed local US-strain PRRSv vaccine: A case report
}

\author{
Pariwat Poolperm; Kitcha Urairong; Preeyaphan Udomprasert; Worawidh Wajjwalku \\ Kasetsart University, Nakornpathom, Thailand
}

\section{Introduction}

Porcine reproductive and respiratory syndrome (PRRS), caused by PRRS virus (PRRSv), is the most costly disease currently affecting pig industry worldwide. PRRS outbreak seriously caused high losses in reproductive system, including highly increase in abortion, mummified fetuses, stillbirth, weak piglets born and sow death. For a naïve herd, these losses are drastically high and need more intensive action to control and stop losses. Vaccine is one effective tool in controlling the disease. Commercial vaccines are available worldwide. Killed PRRSv vaccine was chosen due to the regulation of the company. However, killed PRRSv US-strain vaccine is not commercialized in Thailand. Therefore, a homemade killed vaccine using local US strain (KU-killed-PRRS) was developed at the faculty of veterinary medicine, Kasetsart University, Thailand.

The objective of this study was to assess the efficacy of the KU-killed-PRRS vaccine in controlling PRRS outbreak in a naïve nucleus herd.

\section{Materials and Methods}

A 2,100 sows nucleus herd, had been free from PRRS for 6 years, was serologically positive for PRRS antibody using ELISA (HerdChek 2XR Ab, IDEXX Lab., Westbrook, MA, USA) in March 2009. The virus was determined as US strain using RT-PCR. Area veterinary consultants decided to use a vaccine, developed in a research project funded in part by National Research Council of Thailand, using local PRRSv US-strain (KU-killed-PRRS). The vaccine contains at least 106.5 TCID50/dose in oil-in-water adjuvant.

Mass vaccination was applied to all sows twice using KU-killedPRRS vaccine, 4 weeks apart. Then vaccine was programmed at week 11 and 14 of gestation. In piglets, vaccine was programmed at 2 and 6 weeks of age to enhance antibody level for protecting early infection during suckling period.

Serological profiles and RT-PCR were monitored on monthly basis. Sow reproductive performance, percentage of preweaning and nursery losses were recorded.

\section{Results}

Sow reproductive and lactational performance parameters were shown in table 1. Serological profiles and RT-PCR results were positive in sows, suckling and nursery pigs. However, serological and RT-PCR results for suckling and nursery pigs turned to be negative in August, 6 months after vaccine was applied. During the outbreak, there was no increasing of losses in nursery pigs, however, the ADG was dropped about 26\% during Apr-Jun 2009 (ADG $460 \mathrm{~g} / \mathrm{d}$ to $340 \mathrm{~g} / \mathrm{d}$ ).

Table 1. Represents sow reproductive and lactational performance during Feb-Aug 2009. (sb=\%stillbirth, $m m=\%$ mummies, pwm=\%preweaning mortality, Iwg=average daily litter weight gain $(\mathrm{kg} / \mathrm{d}), a b=$ number of aborted sows, day=average gestation length at abortion)

\begin{tabular}{|l|c|c|c|c|c|c|c|}
\hline & Feb & Mar & Apr & May & Jun & Jul & Aug \\
\hline $\mathrm{sb}$ & 6.1 & 15.6 & 14.7 & 8.9 & 7.5 & 6.6 & 4.6 \\
\hline $\mathrm{mm}$ & 7.9 & 22.3 & 36.1 & 44.1 & 19.0 & 12.8 & 14.9 \\
\hline $\mathrm{pwm}$ & 18.0 & 34.2 & 49.6 & 21.6 & 16.3 & 12.5 & 9.1 \\
\hline $\mathrm{Iwg}$ & 1.97 & 1.14 & 0.7 & 1.32 & 1.44 & 2.04 & 2.34 \\
\hline $\mathrm{ab}$ & 21 & 52 & 33 & 33 & 27 & 12 & 13 \\
\hline day & 35.8 & 77.1 & 89.7 & 80.5 & 61.4 & 57.9 & 78.8 \\
\hline
\end{tabular}

\section{Discussion}

The first PRRS outbreak in a naïve nucleus herd caused severe abortion in all parity sows, as high as $10 \%$. The most detrimental effects are during lactation period, including, high percentage of stillbirth, mummies and weak piglets consequently increase preweaning mortality. Application of homemade killed local PRRSv US-strain vaccine could stop losses in an outbreak herd within two months and could produce PRRS free-infection for weaned and nursery pigs within 6 months. Off-site nursery is a recommended strategy to achieve PRRS free herd in the future. 


\title{
P.252
}

\section{Elimination of PRRS virus infection in a boar stud by vaccination and replacement}

\author{
Michael Alt; Friedrich Delbeck \\ Chambre of Agriculture, Oldenburg, Germany
}

\section{Introduction}

As PRRS imposes high costs on pig production elimina-tion of PRRSV is the best response to the disease (1). Transmission of the virus also by airosols and insects is possible and re-infections are frequent so that eradication must be conducted on a regional basis. Although the risk of virus transmission by semen from infected boars is low compared to other factors (2), negative herds should only be supplied from negative boar studs. If re-infection occurs, PRRSV should be eliminated immediately to prevent transmission to other farms. Successful eradications by vaccination with live vaccines have been reported from breeding herds (3), whereas for boars killed vaccines should be preferred because no adverse effects on semen quality nor shedding of vaccine virus were reported (4). A combined testing of blood samples by PCR and serological methods has been shown to be the safest method for monitoring boar studs (5).

\section{Material and Methods}

A boar stud consisting of two parts was re-infected after a period of several years being negative. The infection was limited to one part consisting of 200 boars. In order to prevent the transmission to the other part that is situated only $150 \mathrm{~m}$ apart housing 100 boars, strict bio-security measures were implemented including elimination of arthropods and rodents as well as separation of personal and feeding supply. For monitoring samples were taken every month and tested using a RT-PCR (blood and semen) and the IDEXX ${ }^{\oplus}$ Herd check ELISA $2 X$ and X3. All young boars ( $60 \%$ replacement/a) absolved a triple testing within 6 weeks in separate isolation farms situated at least $5 \mathrm{~km}$ apart. Two months after infection all boars of the infected stud 2 were vaccinated applying a killed vaccine (PROGRESSIS ${ }^{\oplus}$, Merial). The vaccination of this herd is repeated three times at an interval of three months. Young boars are vaccinated before introduction as well. Semen quality is controlled permanently.

\section{Results}

Vaccinated boars of stud 2 revealed no adverse effects concerning general behaviour or semen quality. All boars in stud 1 remained free of infection until now. Whereas virus material could be detected by the PCR in blood samples only at the onset of infection, no PRRSV in semen occurred at any time. Initially high levels of PRRSV antibodies declined on a relatively low and stable level. All of the tested animals with the exception of two developed antibodies after vaccination.
Figure 1: ELISA Antibodies of boars in stud 2 after infection and vaccination with PROGRESSIS ${ }^{\circledR}$

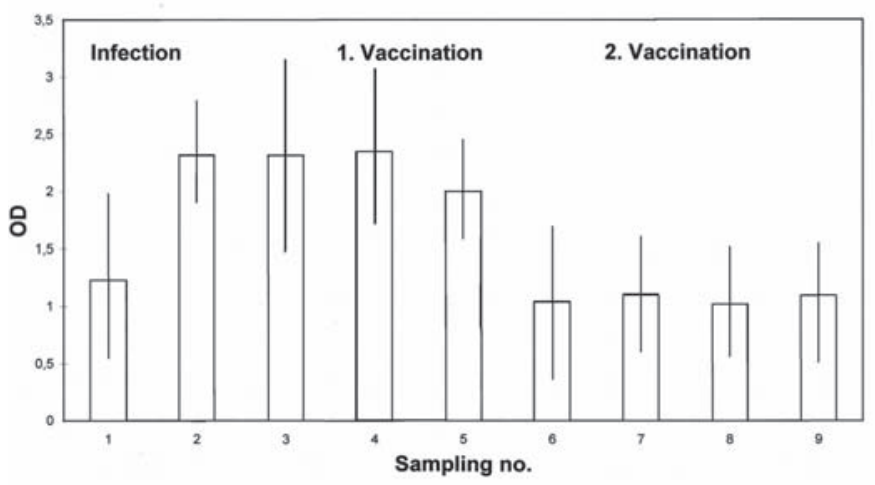

Table 1: PCR and ELISA Results in boar stud 1 (free) and boar stud 2 (infected and vaccinated)

\begin{tabular}{|l|c|c|c|c|c|}
\hline & & Total & RT PCR blood & $\begin{array}{c}\text { RT PCR } \\
\text { Semen }\end{array}$ & ELISA serum \\
\hline & No. samplings & No. sampless & pos/neg & pos/neg & pos/neg \\
\hline Boar stud 1 & 9 & 202 & $0 / 86$ & $0 / 10$ & $0 / 106$ \\
\hline Boar stud 2 & 9 & 304 & $10 / 140$ & $0 / 24$ & $94 / 36$ \\
\hline
\end{tabular}

\section{Discussion}

No adverse effects of semen quality have been found following the vaccination. The spreading of the virus in stud 2 and transmission of virus to stud 1 could be prevented. The vaccination antibodies declined. Newly introduced boars remained free of detectable virus material. The antibody level is expected to decline after the end of the vaccination period. These preliminary results promise that elimination of the PRRSV can be achieved without depopulation.

\section{References}

1. Zimmermann, J. (2006) Proc.19th IPVS, Copenhagen, 1:18-25

2. Torremorell, M. et al. (2004) Proc. 18th IPVS, Hamburg, 1:103

3. Gillespie, T.G. et al. (2003), J Swine Health Prod 11(6), 291-295

4. Papatsiros, V. et al. (2006) Proc. 19th IPVS, Copenhagen, $2: 46$

5. Rovira, A. et al. (2006) Proc. 19th IPVS, Copenhagen $1: 70$ 


\title{
P.253
}

\section{Eradication of PRRSV: an approach using mass vaccination and high standard internal biosecurity without herd closure and partial depopulation}

\author{
Geertjan van Groenland ${ }^{1}$ Tom F. Duinhof ${ }^{2}$ Paul J.M. Franssen ${ }^{2}$ \\ 1. TOPIGS S\&D, Helvoirt, Netherlands; 2. GD Deventer, Deventer, Netherlands
}

\section{Introduction}

For the eradication of PRRS from infected farms methods as herd-closure, partial or total depopulation including strict biosecurity (1), and the use of vaccination (2) have proved to be effective. However, the costs of these methods, caused by loss of production and genetic material, question if other methods can be effective. Aim of this paper is to describe an attempt to eradicate PRRSV using all herd-vaccination combined with high standard internal hygiene and biosecurity procedures, without the use of (partial) depopulation. This approach was used on a breeding farm holding all sows, weaners, rearing and testing of breeding stock under one roof.

\section{Materials en Methods}

A breeding farm with 500 sows and 2000 young breeding stock, that started in August 2006 on a new location with SPF PRRSVfree gilts, was infected early 2007. March 2007 the diagnoses of an infection with an EU-type PRRS-virus strain was confirmed by PCR and serology. Sows were vaccinated 4 times, all other pigs (piglets included) were vaccinated twice, starting from an age of 14 days, with an interval of 2 weeks. The used vaccine was Porcilis PRRS (Intervet/Schering Plough) administered using Intra-dermal application (IDAL-technique), to avoid the use of needles. After the initial mass vaccination, the sows were vaccinated by 60 (gest.) - 6 (lact.) scheme, the piglets at 3 and 5 weeks of age. Vaccination of piglets was ended 6 months after the outbreak. For biosecurity four separate internal units were created with hygiene-barriers between them: a. sows and replacement gilts; b. farrowing rooms; $c$. weaned piglets; $d$. rearing testing stock. The hygiene-barriers included: changing of coveralls and boots, disinfection of hands and separated materials, including those for individual treatments. Unilateral flow and strict all in all out were standard procedures. The used sampling scheme was: a. monthly sampling of 20 non vaccinated weaners of 9 weeks of age (tested with Idexx ELISA Herdcheck PRRS 2XR), b. weekly sampling of 4 piglets per each newborn litter, samples pooled for rt-PCR-testing, c. all sold breeding pigs at individual level.

\section{Results}

In table 1 the results of testing are provided. In total 4 litters, 4 weaners and three 6 months old breeding pigs were found positive (Table 1).

\section{Discussion}

Although more than $99 \%$ of the tested animals became seronegative (weaners at 6 months and rearing stock at 12 months after the outbreak) the occurrence of a few infected pigs indicates that eradication of PRRSV was not fully successful. Reasons for finding positive test-results could not be determined. Unfortunately sequen-cing of the EU-virus strain found in a breeding pig in September 2008 was not possible due to technical reasons. Thus it could not be determined if a new strain had entered the farm, and virus spread from persistently infected sows could not be ruled out. Possible reasons for new virus circulation could be: internal influx of gilts into the sowherd started again after 3 in stead of 6 months, and the fact that not all sows were culled which could account for a low level of virus circulation. In spite of these facts the used vaccination-protocol and the high level of internal biosecurity have contributed to a rapid decline in percentage of infected animals.

Unfortunately the effect of the used approach could not be evaluated totally, because the farm got infected with a new PRRS-virus strain in December 2008, 96\% homologous to the US-MLV-vaccine strain VR2332. Possible routes of this new virus introduction were considered to be: a) introduction of virus in incoming air either from neighbouring farms (500 m distance),b) from a slurry-container, c) non compliance to biosecurity protocols for humans d) open doors when loading pigs for transport. Next to reconsidering biosecurity protocols, the farmer now considers the use of air filtration to prevent virus introductions in future.

\section{References}

1. Cho, J.G et al.; Porcine reproductive and respiratory syndrome virus; Theriogenology 66 (2006) 655-662

2. Voglmayer, T et al.; PRRSV eradication in a closed breeding unit without interruption of production by means of application of a MLV vaccine and herd closure; Tierärztl Prax 2006; 34: 241-8

Table 1: Results of sampling

\begin{tabular}{|l|c|c|c|}
\hline Age group & Sampling period & $\begin{array}{c}\text { Number of } \\
\text { bloodsamples/ Test }\end{array}$ & $\begin{array}{c}\text { Pos } \\
\text { samples }\end{array}$ \\
\hline Litters & $10-2007 / 07-2008$ & $406 /$ PCR (pool) & $4^{\mathrm{a}}$ \\
\hline & & & \\
\hline Weaners 9 wks old & $10-2007 / 08-2008$ & $319 /$ ELISA & $4^{\mathrm{b}}$ \\
\hline & & & \\
\hline & $06-2008 / 08-2008$ & $36 /$ PCR & 0 \\
\hline & & & \\
\hline Rearing Stock & $02-2008 / 10-2008$ & $305 /$ ELISA & $3^{c}$ \\
\hline & & & \\
\hline & & $60 /$ PCR & 0 \\
\hline
\end{tabular}

a Dec 2007 and March 2008 bMarch 2008, 'May and Sept 2008; in September one 6 months old breeding pig was found virus-positive (in the lung lymphnode, sequence of this virus could not be confirmed). 


\title{
P.254
}

\section{Potential positive effect of commercial spray-dried porcine plasma on pigs challenged with PRRS virus}

\author{
Ivan Díaz ${ }^{1}$ Cristina Lorca ${ }^{1}$ Ivan Galindo ${ }^{1}$ Joy Campbell ${ }^{2}$ Inmaculada Barranco ${ }^{3}$ Liudmila Kuzemtseva $^{1}$ \\ Irene M. Rodríguez-Gómez ${ }^{3}$ Joe Crenshaw $^{2}$ Louis Russell ${ }^{2}$ Javier Polo ${ }^{4}$ Joan Pujols $^{1}$ \\ 1. Centre de Recerca en Sanitat Animal (CReSA), Bellaterra, Barcelona, Spain; 2. APC, Ankeny, IA, USA; 3. \\ Dept. Anatomía y Patología Comparada, Córdoba, Spain; 4. APC Europe, Granollers, Spain
}

\section{Introduction}

Spray-dried porcine plasma (SDPP) is used as a feed ingredient in diets of weanling pigs to provide beneficial effects on productive parameters. Some epidemiological studies have described a significant association of dietary SDPP with lower post weaning mortality ${ }^{1}$ and improved productive records in sows from a PRRS unstable farm ${ }^{2}$. According to these studies the positive effect of SDPP could be related to a modulation of the inflammatory immune responses. The aim of the present study was to determine the effect of SDPP as a supplement in the diet on the outcome of experimental PRRS virus (PRRSV) infection.

\section{Material and Methods}

Experimental design. 55 three-weeks-old piglets obtained from a PRRSV-free farm were randomly distributed in three groups: pigs in I and II were intranasally inoculated with PRRSV $\left(10^{6}\right.$ $\mathrm{TCID}_{50} / \mathrm{ml}$ ) and group III was kept as a control. Group I received a supplemented diet (8\% SDPP, AP-820P from APC Europe, SA) from four days before challenge to 28 days post-inoculation (PI) and drinking water supplemented with $2 \%$ spray-dried porcine serum until 7 days PI.

Clinical follow-up and sample taking. Individual body temperatures were measured daily (from 0 to $14 \mathrm{PI}$ ). Sera samples were taken from 0 to 28 days PI and analyzed for PRRSV viremia by nested RT-PCR (nPCR) ${ }^{3}$. The presence of antibodies to PRRS was checked by a commercial ELISA kit (HerdChek ${ }^{\circledR}$ PRRS 2XR, Idexx).

Histopathology. Serial examination of tissues was performed after humane killing of pigs at days 0, 3, 7, 14 and $28 \mathrm{PI}$. Severity of microscopic interstitial pneumonic lesions was semi-quantified following a previously reported scoring system ${ }^{4}$.

Immunohistochemistry (IHC). IFN- $\gamma$, IL-1 and IL-10 were measured from apical and intermediate lobes of lungs by IHC as described before ${ }^{5}$.

Statistics. Statistical analysis was performed with StatsDirect 2.7.5

\section{Results}

Clinical follow-up. Temperatures of both infected groups showed a biphasic pattern of two peaks (day $4 \mathrm{PI}$ and day 8-12 PI) of fever $\left(\geq 40^{\circ} \mathrm{C}\right)$. All infected groups seroconverted at day $14 \mathrm{PI}$ and were positive by $\mathrm{nPCR}$ until $21 \mathrm{PI}$. Five of six pigs in group II and only two of five in group I were viraemic at day $28 \mathrm{PI}(p=0.07)$.

Histopathology. Sum of interstitial pneumonia scores from day 3 to $28 \mathrm{PI}$ showed significant differences among groups $(\mathrm{II}=129>$ $\mathrm{I}=94>\mathrm{III}=25 ; \mathrm{p}<0.05$ ).

IHC. Interestingly, higher frequency of cells expressing IFN- $\gamma$ and IL-1 (day $3 \mathrm{PI}$ and days 3, 7 and $14 \mathrm{PI}$, respectively) were detected in apical and intermediate lobes for group I $(p<0.05)$ (Figure 1). No significant differences were observed for IL-10.
Figure 1. Mean of cells by $\mathrm{mm}^{2}$ expressing IFN- $\gamma$ and IL-1 in apical and intermediate lobes.
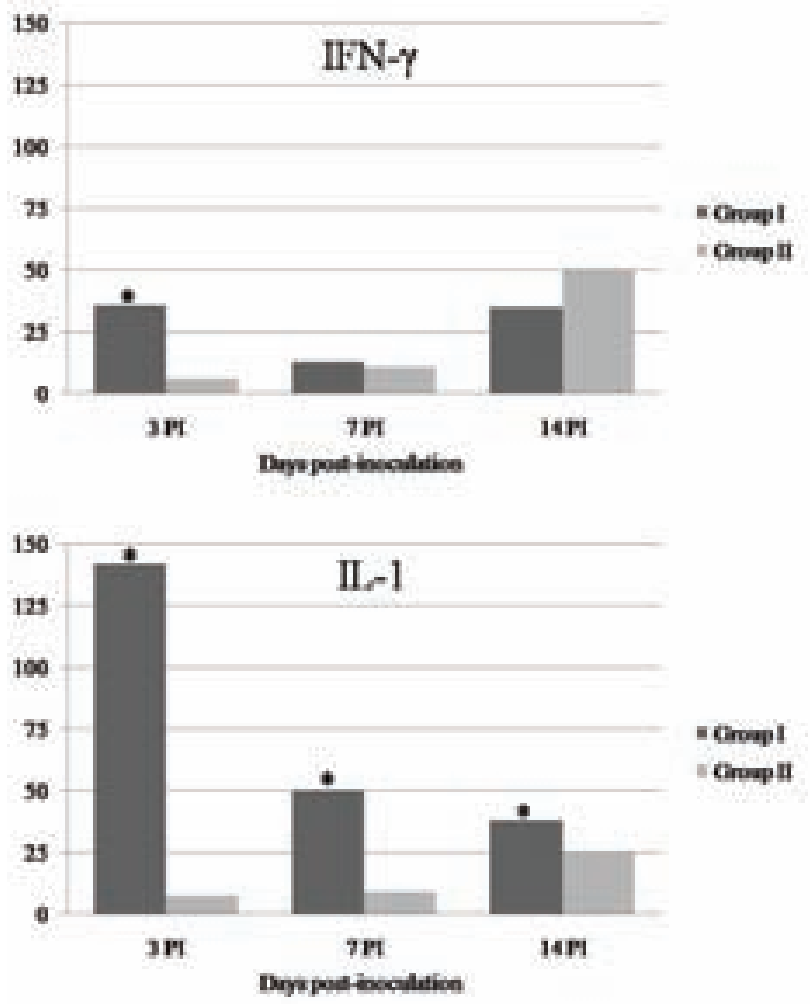

${ }^{*}$ Group I $>$ Group II $(p<0.05)$

\section{Discussion}

After the PRRSV inoculation, a significant lower interstitial pneumonia was found in the group receiving SDPP $(I)(p<0.05)$. Besides, this group also showed less nPCR positive pigs at day $28 \mathrm{PI}(p=0.07)$. Both facts suggest that pigs fed SDPP may have an earlier virus clearance compared to non-SDPP challenged group (II).

The increase of cytokine expression (IFN- $\gamma$ and IL-1) in lungs of group I points to a Th1 enhancement that might be related with the data discussed above.

Results of the present report indicate that SDPP used in the diet may have a potential positive effect during a PRRS challenge.

\section{References}

1 Dewey et al. (2006) Can J Vet Res. 70:161-167

2 Campbell et al. (2006) AASV Proc. 139-142

3 Diaz et al. (2005) J Gen Virol. 1943-1951

4 Opriessnig et al. (2004) Vet Pathol. 624-640

5 Gómez-Laguna et al. (2010) J Comp Pathol 51-60 


\title{
P.255
}

\section{Comparative analysis of different strategies for PRRS control}

\author{
I. De Blas ${ }^{1} \underline{\text { Antonio Callen }}^{2}$ J. Q. Cabanes ${ }^{3}$ J. A. Munoz ${ }^{3}$ François Joisel ${ }^{4}$ \\ 1. Veterinary Faculty, Universidad de Zaragoza, Zaragoza, Spain; 2. Merial Laboratorios S.A., \\ Barcelona, Spain; 3. Agrocesa, Valladolid, Spain; 4. Merial S.A.S., Lyon, France
}

\section{Introduction}

PRRS is a devastating disease for intensive pig production. Seroprevalence studies show that it is an endemic disease in most farms. Several control measures are currently used at farm level with variable results (1).

The objective of this study was to assess, under field conditions, the effectiveness of different health management strategies for PRRS control.

\section{Material and Methods}

Forty-three Spanish farms belonging to the same company and with a census between 100 and 5,250 sows and gilts (mean \pm SD: $643 \pm 1,012$ ), were surveyed during a minimum period of 3 years (maximum of 10 years) (total of 397 farm-years) in a prospective observational study.

Four different strategies for PRSS control were used in these farms: S1: PRRS-Positive Gilts, Live Vaccine and No Infection with Piglets; S2: PRRS-Positive Gilts, Live Vaccine and back passage (infection) with viremic piglets; S3: PRRS-Positive Gilts, Killed Vaccine and Infection with Piglets; and S4: PRRS-Negative Gilts, Killed Vaccine and Infection with Piglets.

Live vaccine (AMERVAC ${ }^{\circledR}$-PRRS (Hipra, Spain)) and killed vaccine (PROGRESSIS ${ }^{\circledR}$ (MERIAL)) were applied according to producer specifications in gilts in quarantine, except in some farms where adult sows were also vaccinated with PROGRESSIS.

In this study, a PRRS outbreak was defined as the sudden occurrence of reproductive problems associated to an abrupt increase of seroprevalence to this disease.

A commercial indirect-ELISA test was used for PRRS diagnosis (Ingezim PRRS K1, Ingenasa). Seroprevalences were calculated every year from min. 20 samples (max. 370) depending on farm size $(48.3 \pm 35.1)$.

Comparison of average year seroprevalences was performed with ANOVA or Student t-test (normality was previously checked with Kolmorogov-Smirnov test). Error a was established at 0.05. Statistical analyses were performed with SPSS ${ }^{\circledR} 15.0$ (WINDOWS ${ }^{\oplus}$.

Comparison between outbreak prevalence was tested with classical percentage comparison.

\section{Results}

Using live vaccine and introducing PRRS-positive gilts (S1, starting point) gave us an average seroprevalence of 52\% (minimum 11\%). Further infection of gilts with positive piglets (S2) produced a significant increment of seroprevalence $(71.9 \%$, minimum 28.6\%). A change from live to killed vaccine (S3) led to a significant decrease of seroprevalence $(63.4 \%$, minimum $2 \%)$. Finally, introducing PRRS-negative gilts (S4) led to a further reduction of seroprevalence $(47.4 \%$, with some farms becoming totally seronegative) in spite of the infection of gilts with piglets (Table 1).
However, some farms under S4 strategy still showed high seroprevalence as a probable result of PRRS virus circulation. Only a very low number of outbreaks occurred whatever the strategy (Table 2).

Table 1: Average seroprevalences depending on control strategy

\begin{tabular}{|l|c|c|c|c|}
\hline & & Seroprevalence (\%) & & \\
\hline Strategy & $\mathrm{N}$ & Mean \pm SD $^{*}$ & Min & Max \\
\hline Strategy 1 & 67 & $52.0 \pm 26.6^{\mathrm{a}}$ & 11.0 & 100 \\
\hline Strategy 2 & 96 & $71.9 \pm 17.8^{\mathrm{b}}$ & 28.6 & 100 \\
\hline Strategy 3 & 54 & $63.4 \pm 24.3^{\mathrm{c}}$ & 2.0 & 100 \\
\hline Strategy 4 & 177 & $27.5 \pm 30.2^{\mathrm{d}}$ & 0.0 & 100 \\
\hline Total & $\mathbf{3 9 4}$ & $\mathbf{4 7 . 4 \pm 3 2 . 4}$ & $\mathbf{0 . 0}$ & $\mathbf{1 0 0}$ \\
\hline
\end{tabular}

*ANOVA p-value $<0.001$ (different superscripts indicate significant differences among seroprevalences applying Duncan's post-hoc test)

Table 2: Relationship between seroprevalence and PRRS outbreak prevalence during the subsequent year

\begin{tabular}{|l|c|c|c|c|c|}
\hline $\begin{array}{l}\text { Use of a killed } \\
\text { vaccine }\end{array}$ & $\begin{array}{c}\text { PRRS } \\
\text { outbreak }\end{array}$ & & & $\begin{array}{c}\text { Seroprevalence } \\
(\%)\end{array}$ & \\
\hline & & $\mathrm{N}$ & $\%$ & Mean \pm SD & $\mathrm{p}^{*}$ \\
\hline Only gilts & No & 351 & $1.71^{\mathrm{a}}$ & $45.1 \pm 31.8$ & \\
\hline & Yes & 6 & & $16.4 \pm 13.8$ & 0.003 \\
\hline All breeders & No & 39 & $2.56^{\mathrm{a}}$ & $69.6 \pm 28.6$ & \\
\hline & Yes & 1 & & 95.0 & 0.386 \\
\hline Total & No & $\mathbf{3 9 0}$ & $\mathbf{1 . 7 9}$ & $\mathbf{4 7 . 6 \pm 3 2 . 3}$ & \\
\hline \multicolumn{7}{|c|}{ Yes } & $\mathbf{7}$ & & $\mathbf{2 7 . 6 \pm 3 2 . 3}$ & $\mathbf{0 . 1 0 6}$ \\
\hline * Student t-test p-value; ${ }^{\text {a }}$ non significantly different \\
\hline
\end{tabular}

\section{Discussion and Conclusion}

It is well known that ELISA antibodies are more of a proof that pigs have been in contact with the virus than related to protection (2) and that ELISA antibody response to PROGRESSIS vaccination are variable (3). The use of killed vaccine in seronegative gilts with infected piglets did lead to a significant reduction of PRRS-seroprevalence in most farms due to no new virus brought through the incoming gilts and through the control of existing infection. This was confirmed by the low overall number of outbreaks occurring during the study. The PRRS status reached in most of the farms could eventually let us consider an eradication program.

\section{References}

1. Zimmermann F. et al., (2003) PRRS Compendium. National Pork Board. Des Moines, IA, USA

2. Murtaugh MP et al., (2002) Viral Immonol 15, 533-547

3. Mieli L. et al., (2002) 17th IPVS, Ames, lowa, USA, p. 363

${ }^{\oplus}$ PROGRESSIS is a registered trademark of Merial in France and elsewhere. 


\title{
P.256
}

\section{Isolation of a swine influenza type A virus from pigs during a reproductive outbreak in Mexico}

\author{
Pedro J. Pradal-Roa ${ }^{1}$ Ivan Sánchez-Betancourt ${ }^{1}$ Mario Velasco-J² Antonio Morilla-González ${ }^{3}$ \\ 1. Universidad Nacional Autónoma de México (UNAM), Facultad de Medicina Veterinaria y Zootecnia, Departamento de Producción Animal: \\ Cerdos, Ciudad, CHIH, Mexico; 2. National University of Mexico, Mexico, DF, Mexico; 3. CENID-Microbiologia, INIFAP, Mexico, DF, Mexico
}

\section{Introduction}

Swine influenza virus (SIV) causes outbreaks of pneumonia and respiratory disease of short course with high fever and morbidity and mortality of less than $1 \%$. Clinical signs generally are of respiratory type however it has also been reported as a cause of reproductive disease $(1,2,3)$ and it is a public health concern. In this paper the isolation in Mexico of an influenza type A virus from pigs during a reproductive outbreak is described.

\section{Materials and Methods}

The outbreak took place in a 250 sow herd which produces only weaners, located in the State of Mexico and considered free of PRRSv and Porcine Rubulavirus. Antibodies against PRRSv, influenza virus $\left(\mathrm{H}_{1} \mathrm{~N}_{1}, \mathrm{H}_{3} \mathrm{~N}_{2}\right)$ were measured by ELISA (IDEXX), for Parvovirus and Porcine Rubulavirus haemagglutination inhibition test $(\mathrm{HI})$ was used. Tissues from dead newborns and semen from males were inoculated in PK15 and Vero cell line and in chicken embryos for virus isolation. Primers against the $\mathrm{M}$ gen (Matrix) were used for RT-PCR. From the isolated virus an inactivated vaccine was prepared with $1 \times 10^{5} 50$ IDCE and Freud adjuvant.

\section{Results}

Herd fertility decreased at the end of 2007 (figure 1), and born death and mummies increased. Mortality during the suckling period increased from 3\% to $8 \%$ and piglets showed nervous and respiratory signs. An increment of repeating sows with irregular cycles, abortions and sudden dead were recorded. None of the males had apparent testicular abnormalities but some had azoospermia and others agglutination, low viability or death spermatozoa. Serology of sows and piglets was negative for Porcine Rubulavirus and PRRSv, and few sows had antibodies to parvovirus at low titers. Therefore these viruses were not circulating in the herd. Antibodies against $\mathrm{H}_{1} \mathrm{~N}_{1}$ and $\mathrm{H}_{3} \mathrm{~N}_{2}$ SIV were detected only in 4 th to 5 th parity sows indicating that these types of influenza virus infected the herd in 2006. A haemagglutinating virus was isolated from lung tissue and semen. The isolated virus was positive to type A influenza virus by RT-PCR and was inhibited weakly with an specific anti $\mathrm{H}_{1} \mathrm{~N}_{1}$ sera, but not with $\mathrm{H}_{3} \mathrm{~N}_{2}$. By means of an $\mathrm{HI}$ test using the isolated virus, all parity sows ( 1 to 5 ) had antibodies indicating that the new virus was infecting them. The inactivated vaccine was applied to gestating sows and males at the end of 2008 and fertility returned to normal values by mid 2009.

\section{Discussion}

The clinical presentation described has not been reported globally and it is very important to mention that it can only be diagnosed through molecular biology tests such as RT-PCR or Real-Time PCR, once the complete viral genome has been fully sequenced. The typification and characterization of the virus is on its way, however, the search for new viral subtypes is needed because at the moment the reproductive clinical impact associated with the influenza virus still unknown; since, generally reproductive disorders are associated with other viruses such as Aujeszky, Parvovirus, Circovirus or PRRS, that means that we are identifying the influenza virus as directly responsible for the reproductive failure. It was concluded that a type $A$ influenza virus, different to $\mathrm{H}_{1}$ or $\mathrm{H}_{3}$ subtypes, was involved in the reproductive outbreak described.

\section{References}

1. Olsen, C. W., et al. (2006). Swine Influenza. In Diseases of Swine (9th edition). Straw, B. E., et al. eds. Blackwell Publishing, Ames, lowa, USA. 2(28): 469-482.

2. Stephano, H. A. (2004). Proceedings XXXIX AMVEC National Congress (Mexico). 107-114.

3. Woods, G. T. and Mansfield, M. E. (1974). Res. Commun. Chem. Pathol. Pharmacol. 10: 629-632.

\section{Percentage of fertility (2007-2009)}

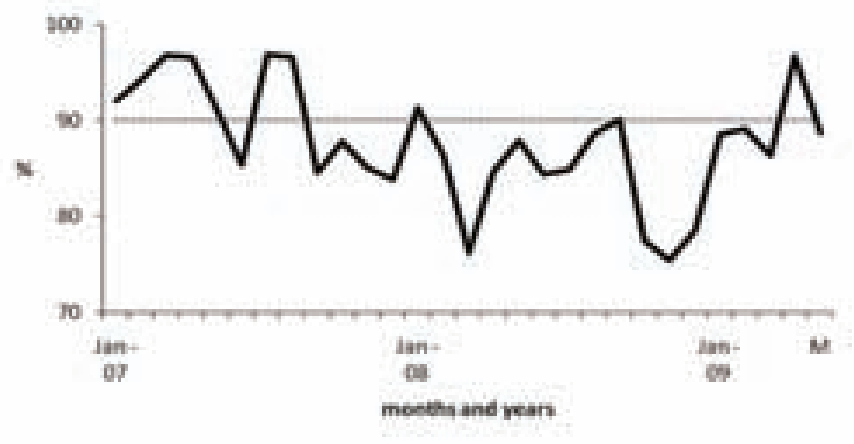




\title{
P.257
}

\section{An Outbreak of Influenza Virus H3N2 Subtype in a farm in Argentina}

\author{
Javier A. Cappuccio ${ }^{1}$ Ariel Pereda ${ }^{2}$ ucas Insarralde ${ }^{1}$ María A. Quiroga ${ }^{1}$ Mariana Machuca María I. \\ Craig $^{2}$ Agustina Rimondi ${ }^{2}$ Lindomar Pena ${ }^{3}$ Jorge Mendez ${ }^{3}$ Daniel Perez ${ }^{3}$ Carlos J. Perfumo \\ 1. Cátedra de Patología Especial, Facultad de Ciencias Veterinarias, Universidad Nacional de La Plata, La Plata, Argentina; 2. Instituto de \\ Virología, CICVyA. INTA Castelar, Castelar, Argentina; 3. Department of Veterinary Medicine, University of Maryland, Blacksburg, MA, USA
}

\section{Introduction}

In South America, reports about swine influenza virus (SIV) are scarce. Serological surveys have revealed that SIV subtypes $\mathrm{H} 3 \mathrm{~N} 2$ and $\mathrm{H} 1 \mathrm{~N} 1$ are currently in circulation in Venezuela, Colombia, Brazil and Argentina (1). However, as neither isolation nor sequence has been performed, subtype, genotype and source of virus remains unknown. In this work we describe the epidemiology, clinical outcome, pathology and the techniques applied for isolation, subtype and sequence of influenza A virus outbreak in Argentina.

\section{Materials and Methods}

The disease appeared at the nursery barns of 3 site farrow-to-finish farm with its own replacement gilts, located in Buenos Aires province. Each barn had an average of 3,000 pigs with a total pig population at risk of more than 21,000 . The clinical signs such as cough, dysnea, off of feed and fever affected 1,2\% of 40 days-old pigs and rapidly elevated up to $40 \%$ in pigs of $48-55$ days-old and almost disappeared at 83 days. The cycle of infection persisted for 8 weeks. Average weight of pigs was recorded by barns. Ten pigs were submitted for post-mortem studies. Lung samples were processed for histopathology and immunohistochemistry (IHC) against NP of influenza A viruses (2). Nine bronchial swabs and lungs samples from 45 to 50 days-old pigs with pneumonic lesions were processed by real time RT-PCR directed to the matrix (M) gene (3). Positive samples by rRT-PCR were processed for virus isolation in MDCK cells. The genome segments were amplified by RT-PCR and directly sequenced with the BigDye terminator kit (Applied Biosystems ${ }^{T M}$, Foster City, CA, USA). Partial sequences of HA, NP, NA, M1, NS, and PB2, PB1 and PA were obtained. Genomic information was derived from overlapping sequences covered by forward and reverse primers. At least two independent RT-PCR reactions were produced for each gene and used for sequencing. Nucleotide BLAST analysis was initially used to identify the most closely related influenza $A$ virus gene for each respective segment.

\section{Results}

Overall average daily gain, feed intake and mortality records were not affected during the outbreak. However, in 9 postmortem examined pigs, body condition was under the target weight. All pigs had craneoventral plum colored consolidate areas. In 2 pigs, distinctive lobular consolidation like "chess- board" distribution was observed. In 6/10 severe necrotizing bronchiolitis was a characteristic microscopic finding. Small and medium sized bronchioles were plugged with neutrophils, cellular debris and proteinaceous exudates. IHC showed distinctive positive reaction only in the bronchial or bronchiolar epithelia that showed minimal changes. Seven samples were rRT-PCR positive to influenza A. Further, rRT-PCR procedure demonstrated H3N2 subtype. The partial sequence of the eight genome segments revealed a high nucleotide identity with wholly human origin influenza A H3N2.

\section{Discussion}

Since 1970, infection of pigs with H3N2 viruses of human origin have been reported in Asia and Europe, but only after 1984 it was associated with clinical outbreaks (4). Currently, double and triple reassortant $\mathrm{H} 3 \mathrm{~N} 2$ viruses are the predominant in Europe and in North America respectively (5). However, wholly human influenza viruses are some times isolated from pigs without sustained circulation. The origin of this isolate and its prevalence in Argentinean farms are yet unknown. From an epidemiological, clinical and pathological point of view the reported outbreak was in agreement with those described for endemic SIV. However cycle of infection and clinical signs persisted longer than those reported (5). The age-dependent susceptibility of pigs may reflect a higher innate immune response to the virus in older pigs (6) or the decline of maternally acquired immunity. This is the first report of SIV H3N2 isolated and genotyped from a clinical case in Argentina and South America.

\section{References}

1 Piñeyro P et al. Proceedings 5th Int. Symp Emerg Re-emerg. Pig Dis. 24-27 June 2007. Krakow, Poland. 252.

2 Vincent LL et al. J Vet Diag Invest. 9:191-95, 1997

3 Spackman ES et al. JClin Microbiol. 40:3256-60. 2002

4 Brown IH. Trends in Emerging Viral Infection of Swine. Blackwell Publishing, lowa, USA. 2002

5 Olsen CW et al. In Diseases of Swine, 9th Edition BE Straw et al. Blackwell Publishing, lowa, USA. 2006

6 Richt JA et al. J Clin Microbiol. 41: 3198-3205, 2003

This work was supported by grants from ANPCyT OC/AR PICT 2005-33987 and P.E. AESA1573 INTA, NIAID-NIH Contract No.HHSN266200700010C 


\title{
P.258
}

\section{Existence of reassortant H1N2 swine influenza viruses isolated in eastern part of Thailand}

\author{
Donruethai Sreta Metta Makhanon² Suparlark Nuntawan Na Ayudhya' \\ Nataya Charoenvisal ${ }^{1}$ Pravina Kitikoon ${ }^{1}$ Roongroje Thanawongnuwech ${ }^{1}$ \\ 1. Chulalongkorn University, Bangkok, Thailand; 2. Novartis (Thailand) Ltd, Bangkok, Thailand
}

\section{Introduction}

Swine influenza virus (SIV) subtypes H1N1, H1N2 and H3N2 are well established in the pig population worldwide. Since 1999, the H1N2 subtype has emerged and caused abortion storms in pregnant sows in the U.S. swine farms (1). In Thailand, H1N2 SIV was first isolated in the non-clinical swine farms in Saraburi province located in the central part in 2005 (2). It was not until 2009 that we found the H1N2 SIV associated with severe respiratory distress in two pig farms in the eastern part of Thailand. The objective of this study was to characterize the major envelope glycoprotein genes, $\mathrm{HA}$ and NA of the re-emerging Thai H1N2 SIV.

\section{Materials and Methods}

Two separate pig farms in Chacheongsoa province located in the eastern part of Thailand, faced with respiratory problems in the nursery to early grower pigs (4-12 wk). The morbidity rate was over $50 \%$ and mortality rate was approximately $10-20 \%$. Fresh 6 lung tissues were submitted to the Chulalongkorn UniversityVeterinary Diagnostic Laboratory. Lungs were diagnosed of PRRSV, PCV2 and SIV by PCR. SIV subtyping and characterization of $\mathrm{HA}$ and NA gene were performed (1).

Both SIV isolates designated as A/Swine/Thailand/CU-K4/09 (H1N2) and A/Swine/Thailand/CU-CHL2/09 (H1N2) were characterized.

\section{Results}

The nucleotide homology of the HA and NA genes of the two current $\mathrm{H} 1 \mathrm{~N} 2$ virus compared to the first Thai swine $\mathrm{H} 1 \mathrm{~N} 2$ isolated in 2005 (H1 N2/2005) are 87.5-88.2\% and 82.3-82.6\%, respectively. HA genes of both 2009 isolates belonged to classic swine lineage similar to the $\mathrm{H} 1 \mathrm{~N} 2 / 2005$ but located in different clusters. The NA gene of both $2009 \mathrm{H} 1$ N2 belonged to the human-like SIV similar to the H1N2/2005 but also located in different clusters.

\section{Discussion}

Previous data indicated that the Thai $\mathrm{H} 1 \mathrm{~N} 1$ and $\mathrm{H} 3 \mathrm{~N} 2$ subtypes were separated into 2 clusters ( $a$ and b) (2). The Thai H1N2 SIV reported in 2005 contained a combination of H1N1 (H1-classical swine $(\mathrm{Cl}) \mathrm{b}$ ) and $\mathrm{H} 3 \mathrm{~N} 2(\mathrm{~N} 2-$ human $(\mathrm{H}) \mathrm{b}$ ). The origin of the N2 of both $2009 \mathrm{H} 1 \mathrm{~N} 2$ viruses in this study appeared to be similar to the human-like in cluster a $(\mathrm{Ha})$ closely related to the Thai human influenza viruses. Characterization of the internal genes is pending. No pathogenesis or epidemiological data was done with the $2005 \mathrm{H} 1 \mathrm{~N} 2$ SIV.
Fig. 1: Phylograms of HA gene (A) and NA gene (B) of SIV H1N2.Both 2009 H1N2 isolates were associated with the severe respiratory signs in pigs. This study demonstrates the presence and on-going diversity of the H1N2 SIV in the Thai swine population. The study also highlights the importance of SIV as a contributor to PRDC which remains as a major problem in the Thai pig industry
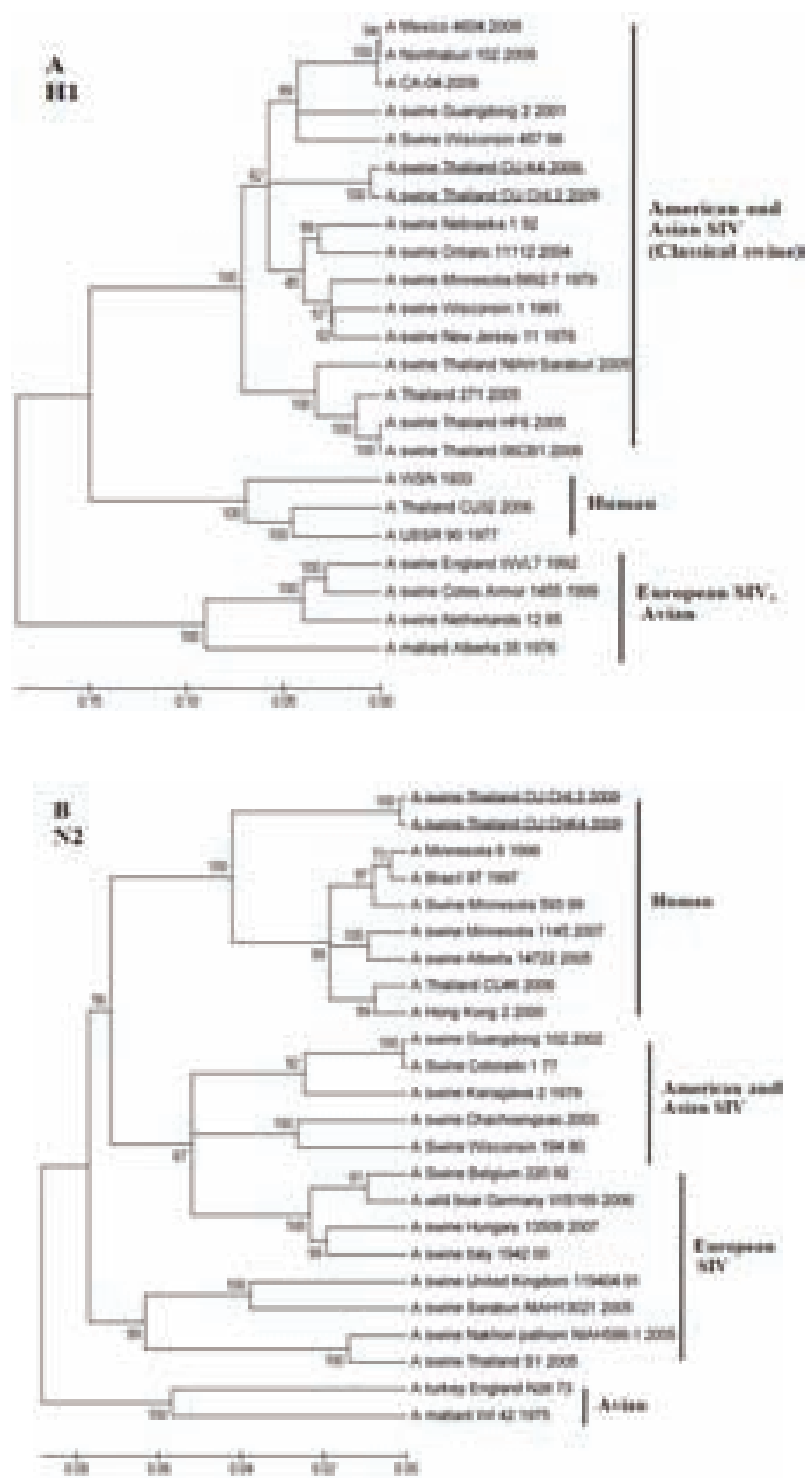

\section{References}

1. Karasin et al. 2002. J. Clin. Microbiol. 40 (3):1073-1079.

2. Takemae et al. 2008. Influenza Other. Respi. Viruses. 2(5):181-189. 


\title{
P.259
}

\section{Seroprevalence of H1N1, H3N2 and H1N2 influenza virus in 29 pig farms in France in 2009}

\author{
E. Foni ${ }^{1}$ M. Bublot ${ }^{2}$ C. Chiapponi ${ }^{1}$ Jean-Bernard Herin ${ }^{3}$ François Joisel ${ }^{2}$ Guillaume Perreul ${ }^{2}$ I. Zanni $^{1} \underline{\text { Thaïs Vila }}^{2}$ \\ 1. Istituto Zooprofilattico della Lombardia e dell'Emilia Romagna, Parma, Italy; 2. Merial S.A.S., Lyon, France; 3. Merial S.A.S., Ancenis, France
}

\section{Introduction}

Swine influenza viruses (SIVs) represented by three different subtypes, H1N1, H3N2 and H1N2 are now co-circulating in European countries (1). In France, according to recent virological and serological surveillance studies, $\mathrm{H} 1 \mathrm{~N} 1$ and $\mathrm{H} 1 \mathrm{~N} 2$ are the major subtypes and no or very few $\mathrm{H} 3 \mathrm{~N} 2$ viruses have been evidenced $(2,3)$.

The objective of this survey was to evaluate the seroprevalence of SIVs in farms affected with acute respiratory clinical signs in France.

\section{Material and Methods}

Experimental design: 29 farms ( 2 farrowing units, 17 farrowto-finish and 10 finishing herds) located mostly in Brittany and western part of France were investigated. These farms had been affected with acute respiratory clinical signs at least 3 weeks before sampling. Four farms used to vaccinate the sows against H1N1 and H3N2 strains; in these specific cases, only H1N2 serological results were considered on sow sera. Farms were sampled from January to June 2009 as follow: 10 sows of different parity in farrowing units, 10 sows and 10 fattening pigs before slaughter in farrow-to-finish farms and 10 pigs before slaughter in fattening farms. In one farm, where fattening pigs showed fever, nasal swabs were collected for virus isolation. All sera and swabs were sent to IZLER laboratory at Parma, Italy, to be submitted to haemagglutination inhibition tests (HI) (4) and to virus isolation. The strains used for the $\mathrm{HI}$ test were $\mathrm{H} 1 \mathrm{~N} 1 \mathrm{sw} /$ Belgium/1/98, H1N2 sw/Scotland/41440/94, H1N2 sw/France/134240/09 (strain isolated on nasal swabs from one farm in Brittany during the study) and H3N2 sw/Gent/84. The results were analyzed according to the following rules: samples were considered positive for one specific subtype if the titre is $\geq 1 / 20$; one farm was classified as positive against one given subtype if at least 2 sera showed an $\mathrm{HI}$ titre $\geq 1 / 20$ against this subtype. Risks of misinterpretation due to cross-reactivity between $\mathrm{H} 1 \mathrm{~N} 2$ and $\mathrm{H} 1 \mathrm{~N} 1$ strains were decreased by considering negative for $\mathrm{H} 1 \mathrm{~N} 2$ a serum that showed higher $(3 \log 2) \mathrm{HI}$ titre against H1N1.

\section{Results and discussion}

The serological results are presented in table 1. Globally, $96.6 \%$ of farms tested were positive for at least one SIV subtype. The use in the $\mathrm{HI}$ test of the strain $\mathrm{H} 1 \mathrm{~N} 2$ sw/France/13240/09, isolated in Brittany, increased the sensitivity of the $\mathrm{HI}$ test, as showed in the table 2. This observation confirms the importance to use local strains in HI tests in SIV surveillance programme. Some farms were infected with both strains H1N1 and H1N2: this represents $38 \%$ of tested farms. One farm not vaccinated against flu was found to show positive sera against $\mathrm{H} 3 \mathrm{~N} 2$, and another one was doubtful. H3N2 subtype infection has not been shown since many years in pigs in Brittany, the possibility that cross reactions could have been occurred must be considered.
Table 1: positive serological results by $\mathrm{HI}$ tests (\%)

\begin{tabular}{|l|c|c|}
\hline Tested strain & $\mathbf{N}^{\circ}$ farms tested & \% positive farms \\
\hline H1N1 sw/Belgium/1/98 & 29 & 72.4 \\
\hline H1N2 sw/Scotland/41440/94 & 29 & 13.8 \\
\hline H1N2 sw/France/134240/09 & 29 & 58.6 \\
\hline H3N2 sw/Gent/84 & 29 & $3.4^{*}$ \\
\hline $\begin{array}{l}\text { *1 farm only was found positiveTable 2: H1N2 results in one farm: comparison of positive samples } \\
\text { by strain used for the HI test }\end{array}$
\end{tabular}

Table 2:

\begin{tabular}{|l|c|c|c|c|c|c|}
\hline Titre & $<\mathbf{2 0}$ & $\mathbf{2 0}$ & $\mathbf{4 0}$ & $\mathbf{8 0}$ & $\mathbf{1 6 0}$ & Total \\
\hline $\begin{array}{l}\text { H1N2 sw/Scotland } \\
\text { /41440/94 }\end{array}$ & 20 & 0 & 0 & 0 & 0 & 20 \\
\hline H1N2 sw/France /134240/09 & 4 & 1 & 7 & 3 & 5 & 20 \\
\hline
\end{tabular}

\section{Conclusion}

This serological survey confirms the major role of SIVs in swine respiratory disorders in French farms. SIV of H1N1 and H1N2 subtype are enzootic in swine producing region of Brittany, particularly about $50 \%$ of tested farms were found to be infected by $\mathrm{H} 1 \mathrm{~N} 2$ subtype when tested with a strain representative of the strains circulating in the region.

\section{Acknowledgements}

The authors wish to thank all practitioners who participated in this survey.

\section{References}

1. Kuntz-Simon et al., (2009) Zoonoses and Public Health, 56, 31 0-325

2. Kyriakis et al., (2009) Zoonoses and Public Health

3. Madec et al., (2004) Proc. 18th IPVS Hamburg, Vol.1p.5

4. Van Reeth et al., (2008) Influenza and Other Respiratory Viruses, 2,99-105 


\title{
Surveillance for swine influenza in Polish herds over 10 years period
}

\author{
Iwona Markowska-Daniel; Andrzej Kowalczyk; Zygmunt Pejsak \\ National Veterinary Research Institute, Pulawy, Poland
}

\section{Introduction}

Swine influenza (SI) remains one of the most important and worldwide prevalent viral respiratory diseases in pig population, causing economic losses in swine industry. It is also considered an important factor in public health concern. Therefore the regular monitoring of the epidemiological situation as well as rapid and sensitive detection of IV in clinical specimens is of great necessity.

The surveillance program for swine influenza was initiated in Poland at the end of $90 \mathrm{~s}$. The aim of this paper is to present both, the current epidemiological situation concerning SI in Poland and its retrospective analysis over 10 years period.

\section{Materials and Methods}

Serological survey. In total 33001 sera were tested, including 30829 pig sera taken in slaughter houses located in all 16 districts of Poland; 1477 blood samples taken from heart or pleural cavity of wild boars, hunted in 11 districts; and 695 human sera taken from volunteers (554 from producers or animal caretakers and 141 from veterinarians). The samples were tested according to the standard procedure in $\mathrm{HI}$ test against the reference viruses representing 3 subtypes ( $\mathrm{H} 1 \mathrm{~N} 1$ - A/sw/Belgium /1/98, H3N2 - A/ sw/Flanders/1/98, H1N2 - A/sw/Gent/ 7625/99). All necessary reference sera, virus and $\mathrm{RBC}$ controls were included in each test. The sample was considered positive when the antibody titer was $\geq 1: 20$.

Virological survey. In total 421 samples were tested, including: 312 nasal swabs, 49 lung tissue samples and 60 blood samples. The biological material was taken from pigs demonstrating influenza-like symptoms, raised in 41 farms located in 12 districts. Additionally 4 aborted foetuses were taken from the sows demonstrating influenza symptoms. Virological investigations include: virus isolation in 10 days old embryonated SPF chicken eggs and tissue culture, detection of viral RNA by RT-PCR, molecular subtyping in mPCRs, and phylogenetic analysis.

\section{Results}

In serological investigation of the pig sera it was clearly demonstrated that H1N1 subtype is dominated in Poland. In 2009 the seroconversion reached $14 \%$ of tested sera. The percentage of animals possessing antibodies against the mentioned subtype varied over the time, with the range from 5 to $14.4 \%$. Within last five years we observed about 2 fold increase of seroconversion against $\mathrm{H} 1 \mathrm{~N} 1$. We also evidenced that the H1N1 subtype circulate at the higher frequency in the West part of Poland, in pig densely area where the pig production is better developed. The survey for H1N2 subtype performed in 2009 demonstrated $9.4 \%$ of seropositive samples. It also varied over the time, ranging from 2.4 to $9.4 \%$, with about 2 -fold increase within last 5 years. The seroconversion to H3N2 antigen in 2009 was detected in $10.9 \%$ of tested sera. The significant increase (about 5 fold) of antibodies against H3N2 subtype was evidenced within last 5 years. In contrast to $\mathrm{H} 1 \mathrm{~N} 1, \mathrm{H} 3 \mathrm{~N} 2$ subtype circulate at the higher frequency in the East part of the country, where the small private farms are mainly located and pig production is less developed. The situation among wild boars was similar to that observed among pigs but the prevalence of antibodies was significantly smaller. Interestingly the highest occurrence of antibodies against the tested SIV strains was evidenced in human. In 2009 seroprevalence for H1N1, H1N2 and H3N2 antigens reached $45.4 \%, 34 \%$ and $62.4 \%$, respectively. Over the time it ranged from 9.4 to $45.4 \%$ in case of $\mathrm{H} 1 \mathrm{~N} 1$; from 14.3 to $37.5 \%$ for H1N2 and from 62.4 to $88.5 \%$ for H3N2 subtype.

Using conventional virology method 45 SIV isolates were obtained. RNA of SIV was detected in 127 (30\%) samples (49\% of tested lung and $33 \%$ of tested nasal swabs), originated from $68.3 \%$ farms. In molecular subtyping $93 \%$ of isolates were determined as $\mathrm{H} 1 \mathrm{~N} 1$ subtype, $4.4 \%$ as H3N2 subtype and $2 \%$ as $\mathrm{H} 1 \mathrm{~N} 2$.

$\mathrm{HA}$ gene of Polish isolates have been derived from contemporary strains of "avian-like" SIV. M1 gene may have originated from avian influenza viruses. H3N2 strain was located in swine cluster, in the main prevalent European group of $\mathrm{H} 3 \mathrm{~N} 2$ isolates (A/ PortChalmers/1/73-like lineage).

\section{Discussion}

Due to the fact that infections of pigs with $\mathrm{H} 1 \mathrm{~N} 1$ virus were described in most European countries intensively producing pig we can state that epidemiological situation of Polish pig herds concerning $\mathrm{SI}$ is similar to that observed in European countries but the prevalence of virus specific antibodies is smaller and is entirely different from that observed in USA (66.3\%-100\%) and Asia (45\%).
References
1. Brown I. (2000) Vet. Microbiol. 74, 29-46.
2. deJong J.C. et al. (2007) J Virol. 81, 4315-4322
3. Kowalczyk A. et al. (2009) Bull.Vet.Inst. 53, 319-325.
4. Markowska-Daniel I. et al. (2005) Med.Wet. 61, 669-672. 


\title{
Serological study of $\mathrm{H} 1$ and $\mathrm{H} 3$ swine influenza viruses from pigs in the high pig population provinces in Thailand
}

\author{
Donruethai Sreta ${ }^{1}$ Roongtham Kedkovid' Supatta Jittimanee ${ }^{1}$ Nantasak Musiksilp² \\ Ranida Tuanudom ${ }^{1}$ Roongroje Thanawongnuwech ${ }^{1}$ Pravina Kitikoon $^{1}$ \\ 1. Faculty of Veterinary Science, Chulalongkorn University, Bangkok, Thailand; 2. Veterinary Research and \\ Development Center (Western region), Ratchaburi, Thailand
}

\section{Introduction and objective}

Swine influenza virus (SIV) causes an acute respiratory disease in pigs that occasionally can be transmitted to human. It is one of the major viral pathogen that contributes to porcine respiratory disease complex (PRDC) (1). In Thailand H1N1, H3N2 and H1N2 SIV has been reported in the pig population. SIV-infected pigs shed virus for a short period. Therefore serological tests have been used as an indirect method to study SIV epidemiology in the Thai swine population. However the most updated SIV seroprevalence data was reported since 2005 (2). Previous genetic data characterized Thai SIV H1 subtype into classical swine lineage which is subgrouped into two clusters; classical swine cluster a (Cla) and b (Clb). Thai $\mathrm{H} 1 \mathrm{Cla}$ and $\mathrm{Clb}$ were thought to have derived from a hypothetical Classical swine H1N1 common ancestor in 1981 and 1990, respectively. The Thai SIV H3 subtype belonged to human lineage and can be subgrouped into two clusters; human cluster a $(\mathrm{Ha})$ and b $(\mathrm{Hb})$. Thai $\mathrm{H} 3 \mathrm{Ha}$ SIVs were closely related to Thai human H3N2 virus isolated in 1997 while Thai H3 Hb SIVs were closely related to human-like H3N2 virus circulating in the early 1970s (3). This study, we provide an update data

of the SIV seroprevalence based on both clusters of $\mathrm{H} 1$ and $\mathrm{H} 3$ SIV in pigs from the high pig population provinces in Thailand during June 2008 to May 2009. Materials and methodDuring June 2008 to 2009, 850 pig sera were collected from pigs of various age in the four provinces that have the highest density of pigs in Thailand. Those provinces include, Ratchaburi, NakhonPathom, Chonburi and Chachoengsao. A standard hemagglutination-inhibition $(\mathrm{HI})$ assay was used to evaluate the antibody titer against Thai $\mathrm{H} 1$ and $\mathrm{H} 3 \mathrm{SIV}$. $\mathrm{HI}$ test was performed, according to $\mathrm{WHO}$ (4). All sera subjected to test with $\mathrm{H} 3$ virus were treated with receptor-destroying enzyme (RDE) and adsorbed with $50 \%$ chicken red blood (RBC) cells to remove non-specific inhibitors of agglutination and natural serum agglutinins. Sera subjected to test with $\mathrm{H} 1$ SIV were absorbed with $50 \%$ RBC without RDE treatment. Positive $\mathrm{SI}$ was considered when $\mathrm{HI}$ titer was $\geq 40$. Virus antigen used for $\mathrm{HI}$ tests were $\mathrm{H} 1 \mathrm{~N} 1 \mathrm{Cla}$ and $\mathrm{Clb}$ and $\mathrm{H} 3 \mathrm{~N} 2 \mathrm{Ha}$ and $\mathrm{Hb}$ (3).

\section{Results and discussion}

Table 1 shows the percentage of pigs with positive antibody to $\mathrm{H} 1$ and $\mathrm{H} 3 \mathrm{SIV}$ in each province. It was demonstrated that pigs in all provinces were antibody positive to both $\mathrm{H} 1$ and $\mathrm{H} 3$ SIV. However, overall the percentage of positive pigs to $\mathrm{H} 3 \mathrm{Ha}$ was significantly higher than $\mathrm{H} 3 \mathrm{Hb}(\mathrm{p}<0.01)$. There were geographical differences of seropositive pigs to different clusters of $\mathrm{H} 3$.

Pigs that were seropositive to $\mathrm{H} 3 \mathrm{Ha}$ in Nakorn-Pathom was significantly higher than Chonburi and Chachoengsao $(p<0.01)$. The percentage of seropositive pigs to H1N1 SIV was indifferent between each province. However, percentage of seropositive pigs to $\mathrm{H} 1 \mathrm{Clb}$ was significantly higher than $\mathrm{Cla}(\mathrm{p}<0.01)$. The findings indicated that Thai SIV currently circulating in Thai pig populations are diverse antigenically. The data highlights the importance of updating SIV test antigen used in serological test for diagnostic purposes.

Table 1 Seropositive pigs to H1 and H3 SIV by HI test

Table 1 Seropositive pigs to $\mathrm{HI}$ and $\mathrm{H} 3 \mathrm{STV}$ by $\mathrm{HI}$ test

\begin{tabular}{|c|c|c|c|c|}
\hline \multirow{2}{*}{ Province } & \multicolumn{4}{|c|}{ \% seropositive of HI test } \\
\hline & H1 (C'A) & $\underset{\text { (Clb) }}{\mathrm{HI}}$ & $\begin{array}{c}\text { H3 } \\
\text { (Ha) }\end{array}$ & $\begin{array}{c}\text { H3 } \\
\text { (Hb) }\end{array}$ \\
\hline Rasclabun & $\begin{array}{c}16.2 \\
(25 / 117)\end{array}$ & $\begin{array}{c}44.5 \\
(101 / 227)\end{array}$ & $\begin{array}{c}83.3^{\circ} \\
(110132)\end{array}$ & $\begin{array}{c}13.8 \\
(19 / 138)\end{array}$ \\
\hline Nakbon-Pathom & $\begin{array}{c}15.9 \\
(33 / 207)\end{array}$ & $\begin{array}{c}44.6 \\
(103 / 231)\end{array}$ & $\begin{array}{c}95.2 \\
(158166)\end{array}$ & $\begin{array}{c}18.9 \\
(24127)\end{array}$ \\
\hline Chonburi & $\begin{array}{c}9.9 \\
(881)\end{array}$ & $\begin{array}{c}56.3 \\
(40 / 71)\end{array}$ & $\begin{array}{c}69.1^{\circ} \\
(3855)\end{array}$ & $\begin{array}{c}6.5 \\
(4.62)\end{array}$ \\
\hline Chachoerigase & $\begin{array}{c}21.9 \\
(61 / 278)\end{array}$ & $\begin{array}{c}56.9 \\
(173 / 304)\end{array}$ & $\begin{array}{l}79.805 \\
(67 / 84)\end{array}$ & $\begin{array}{c}24.1 \\
(1458)\end{array}$ \\
\hline Total & $\begin{array}{c}18.6 \\
(127 / 683)\end{array}$ & $\begin{array}{c}50.1 \\
(417,833)\end{array}$ & $\begin{array}{c}85.4 \\
(373 / 437)\end{array}$ & $\begin{array}{c}15.8 \\
(61 / 385)\end{array}$ \\
\hline
\end{tabular}

\section{References}

1. Easterday, B.C. and Van Reeth, K. 1999. Swine influenza. In Straw et al(eds), Diseases of Swine, 8th ed, lowa State Press, Ames, p. 277290.

2. Damrongwatanapokin et al. 2006. The 19th IPVS Congress. Proceeding, Copenhagen, Denmark. 2:09-16.

3. Takemae et al. 2008. Genetic diversity of swine influenza viruses isolated from pigs during 2000 to 2005 in Thailand. Influenza Other. Respi. Viruses. 2(5):181-189.

4. Webster et al, 2002. WHO Manual on Animal Influenza Diagnosis and Surveillance. World Health Organization, 15-67. 


\title{
P.262
}

\section{Development of Swine Influenza Virus Nucleic Acid Purification Workflow for Surveillance}

\author{
Angela M. Burrell; Weiwei Xu; Quoc Hoang; Rohan Shah; Manohar Furtado
}

Life Technologies, Austin, TX, USA

Swine Influenza Virus (SIV) is a (-) ssRNA, enveloped, Influenza $A$ virus in the family Orthomyxoviridae. It is a continuously evolving virus that causes swine respiratory disease with fever, reduced activity and food intake, conjunctivitis, sneezing and coughing and nasal discharge. Respiratory disease can be caused by multiple SIV genotypes continuously undergoing changes by genetic drift and shift. SIV genotype is determined by the antigenic characteristics of two surface glycoproteins (hemagglutinin and neuraminidase). The three main genotypes currently present in the swine population are $\mathrm{H} 1 \mathrm{~N} 1, \mathrm{H} 1 \mathrm{~N} 2$, and $\mathrm{H} 3 \mathrm{~N} 2$. Monitoring/surveillance and genetic characterization of SIV in swine herds are critical for successful to control of the disease and are essential for selection of vaccine treatment and novel vaccine development.

The following data were filed to support a Product License Application filed with the USDA, Center for Veterinary Biologics.

Applied Biosystems developed an integrated workflow consisting of high throughput nucleic acid purification, and $\operatorname{TaqMan}^{\circledR}$ RT-PCR for amplification and genotyping of Swine Influenza. Nucleic acid is purified using the MagMAXTM Nucleic Isolation Kit and purified viral RNA is detected using the VetMAXTM - Gold SIV Detection Kit. The SIV-A TaqMan assay targets multiple genetic regions of the Influenza A genome decreasing the likelihood of false negatives due to viral mutation. The assay is multiplexed with an internal control RNA, Xeno ${ }^{\text {TM }}$ RNA, to monitor nucleic acid purification efficiency, detect the presence of reaction inhibitors, and determine assay reagents functionality. The analytical amplification limit is 50 copies of SIV-A transcript RNA per 25ul TaqMan PCR.
The performance of the SIV-A TaqMan reagents was compared to a published SIV screening protocol (Richt, et. al. 2004). Nucleic acid from an SIV vaccine (Novartis PneumoSTARTM vaccine) was purified using the MagMAXTM -96 Viral RNA Isolation Kit. The purified nucleic acid was then serially diluted to extinction and tested using both the VetMAXTM SIV screening reagents and the published assay protocol. SIV Cts using the VetMAXTM - Gold SIV Detection Kit were 4-5Cts earlier than the published protocol and detection sensitivity was approximately 64-fold better. To ensure the specificity of the SIV screening reagents, RNA from field samples containing other common porcine pathogens (PCV2, M.Hyopneumoniae, PRRSV) were purified using MagMAXTM -96 Viral RNA Isolation Kit and tested with the VetMAX ${ }^{\mathrm{TM}}$ SIV Detection Kit $(n=3)$. No false positives were detected using the VetMAX-Gold SIV Detection Kit

The performance of the workflow was evaluated using field samples $(n=60)$ of known SIV status and diverse reference SIV genotypes. RNA was isolated from swine nasal swabs, tonsil and lung tissue homogenates, virus, and cell lysate supernatant using MagMAXTM -96 Viral RNA Isolation Kit. The purified RNA was utilized for TaqMan RT-PCR employing the SIV screening reagents on the Applied Biosystems 7500 Fast Real-Time PCR System. The SIV screening results showed $100 \%$ concordance with secondary laboratory PCR results. These results as well as the superior analytical sensitivity demonstrate that this method provides an economical and rapid solution for SIV identification. 


\title{
P.263
}

\section{Identification of Swine Influenza A Virus by RT-PCR}

\author{
Jae-Yeon Han ${ }^{1}$ Hyoung-Joon Moon ${ }^{2}$ Seong-Jun Park' Hye-Kwon Kim¹ Hyun-Ok Keum ${ }^{1}$ \\ Se-Mi Rho' Van-Giap Nguyen ${ }^{1}$ Bong-Kyun Park ${ }^{1}$ \\ 1. Department of Veterinary Virology Lab, College of Veterinary Medicine and BK21 Program for Veterinary Science, \\ Seoul National University, Seoul, Korea; 2. Research unit, Green Cross Veterinary Products, Yongin, Korea
}

\section{Introduction}

Swine influenza is an acute respiratory disease caused by influenza A viruses . Influenza A viruses cause respiratory disease in humans. There are many sorts of assays to detect SIV. Specially, The M gene is used commonly as PCR target for screening, as well as the NP whose sequences are highly conserved. The aim of this study was to report the prevalence of swine influenza virus by targeting matrix gene which is highly conserved among Influenza A.

\section{Materials and Methods}

Virus isolation

A total of samples, all tissues including lung were submitted from the swine farms in South Korea from July 2009 to January 2010. About $20 \%$ suspension of lung tissue homogenate was prepared in PBS. Then, they were centrifuged at 3000rpm for 10 min., the supernatants were used for virus isolation and RNA extraction.

\section{Viral RNA extraction}

Viral RNA was extracted from virus suspension using Trizol LS .750ul of Trizol LS was mixed with 250ul of homogenized samples. 200ul of Chloroform was added and the suspension was centrifuged at $12000 \mathrm{rpm}$ for $15 \mathrm{~min}$. The RNA-containing aqueous phase was

precipitated with an equal volume (350ul) of isopropanol, maintained for $10 \mathrm{~min}$ at room temperature, centrifuge at $12000 \mathrm{rpm}$ for $15 \mathrm{~min}$. The RNA pallet was washed by $75 \%$ ethanol, centrifuged at $12000 \mathrm{rpm}$ for $10 \mathrm{~min}$. Dried over $15 \mathrm{~min}$, resuspended by 30 ul of DEPC-DW.

\section{RT PCR}

Table1. Primers sequences for $m$ gene SIV RT-PCR.

\begin{tabular}{|l|c|c|}
\hline specificity & primer & sequence 5' $^{\prime} \mathbf{3}^{\prime}$ \\
\hline matrix gene & $M R$ & ATGCTAAAGACAAGACCAA \\
\hline & $M F$ & TATAYCAGGCCATCAA \\
\hline
\end{tabular}

The 2 ul of template, 2 ul of primer mix( 1 ul each), $16 u$ of i-star master sol(intron). The 32 cycles of denaturing at $94^{\circ} \mathrm{C}$ for $5 \mathrm{~min}$, annealing at $53^{\circ} \mathrm{C}$ for $30 \mathrm{~s}$ and extension at $72^{\circ} \mathrm{C}$ for $7 \mathrm{~min}$.

The PCR amplified products were analyzed by $1 \%$ agarose gel electrophoresis.

\section{Results}

The sizes of amplified products, determined by agarose gel electrophoresis were 280bp.Table2. Number of influenza virus isolates, 2009.7-2010.1

\begin{tabular}{|l|c|c|}
\hline DATE & $\begin{array}{c}\text { N0.of RT-PCR } \\
\text { positive samples }\end{array}$ & $\begin{array}{c}\text { No. of RT-PCR positive samples/ } \\
\text { No. of samples(\%) }\end{array}$ \\
\hline 09, July & 5 & $5 / 49(10 \%)$ \\
\hline Aug & 0 & $0 / 36(0 \%)$ \\
\hline Sep & 16 & $16 / 63(25 \%)$ \\
\hline 0ct & 8 & $8 / 58(13 \%)$ \\
\hline Nov & 15 & $15 / 51(29 \%)$ \\
\hline Dec & 1 & $1 / 40(2 \%)$ \\
\hline 10, Jan & 1 & $1 / 31(3 \%)$ \\
\hline
\end{tabular}

Of the 328 samples, 46 samples proved positive for matrix gene of influenza A virus.

\section{Discussion}

From July 2009 to January 2010, we collected tissue samples and placed them in PBS. In field specimens selected at random from Korean farms, the influenza virus was detected in 46 of 328 samples. As shown (table2), we could know the prevalence of Influenza A viruses. Influenza A was peaked from September to November which would be the change of seasons. The percentage of positive samples for Influenza A declined sharply for winter from November. The winter seanson would not be a multiplication period of Influenza A viruses. Acknowledgement

This study was supported by a grant (Code\# 20070401034009) from the Biogreen 21 Program, Rural Development Administration, Korea.

\section{References}

C.S.Lee,Kang, B.K,D.H.et al,One step multiplex RTPCR for detection and subtyping of siv $\mathrm{H} 1, \mathrm{H} 3, \mathrm{~N} 1, \mathrm{~N} 2$ viruses in clinical samples using a DPO system, Journal of Virological Methods,2008

Alessio Lorusso, Kay S.Faaberg.et al, One step real-time RT-PCR for pandemic influenza A virus(H1N1)2009 matrix gene detection in swine samples, Journal of Virological Methods,2008 


\title{
P.264
}

\section{Swine influenza virus detection and identification by the use of an integral diagnosis program}

\author{
Pedro J. Pradal-Roa; Ivan Sánchez-Betancourt; Rolando Beltran-Figueroa; Maria E. Trujillo-Ortega; \\ Roberto Martinez-Rodriguez; Marco A. Herradora-Lozano \\ Universidad Nacional Autónoma de México (UNAM), Facultad de Medicina Veterinaria y Zootecnia, \\ Departamento de Producción Animal: Cerdos, Ciudad, $\mathrm{CHIH}$, Mexico
}

\section{Introduction}

Swine influenza virus (SIV) is an RNA virus that belongs to the Orthomixoviridae family. There are different subtypes characterized for their surface glycoprotein's a hemagglutinin and a neuraminidase. The main viral subtypes affecting pigs are $\mathrm{H}_{1} \mathrm{~N}_{1}$ and $\mathrm{H}_{3} \mathrm{~N}_{2}$ (2). The SIV is associated as a primary agent of the porcine respiratory complex and currently pigs have been identified as intermediaries because in their respiratory tract they have epithelial cells of human and avian viruses. For this reason the diagnosis is important to understand the dynamics of infection by a serological profile and by molecular diagnostic tools, identify specific viral RNA to implement prevention, control and treatment strategies.

\section{Materials and Methods}

A longitudinal study was carried out for the diagnosis of $\mathrm{H}_{1} \mathrm{~N}_{1}$ and $\mathrm{H}_{3} \mathrm{~N}_{2}$ SIV subtypes. A total of 111 serum samples and nasal swabs were collected from pigs of 4, 8, 12, 16, 20, 24 weeks of age. Serologic diagnosis was made by Hemoagglutination inhibition $(\mathrm{HI})$ test with a 1:80 cutting point, multiple RT-PCR technique (1) was applied for the molecular diagnosis from sera and nasal swabs. Titters from IH were transformed to a logarithmic base to apply statistical analysis using variance test between treatments $(P<0.05)$. The mean difference was determined by Tukey test and Ji-square was used to evaluate the differences in the percentage of positive and negative animals per treatment.

\section{Results}

Serology showed $100 \%$ prevalence for the $\mathrm{H}_{1} \mathrm{~N}_{1}$ subtype in 4 week old pigs. In week 8 decreased to $73 \%$ for week 12 increased to $94 \%$ and for weeks 16,20 and 24 a $100 \%$ of samples were positive (Figures 1-2). For subtype $\mathrm{H}_{3} \mathrm{~N}_{2} 75 \%$ of seropositives were recorded in week 4, 36\% for week 8 and $83 \%$ for week 12 (Fig. 2). Significant statistical differences $(P<0.05)$ for the $H_{1} N_{1}$ subtype were found between samples from week 8 and weeks 4, 16 and 24; for subtype $\mathrm{H}_{3} \mathrm{~N}_{2}$ significant differences were found in week 8 when compared to 4, 16, 20 and 24 weeks, however, samples from week 8 did not showed differences with the 12 week samples. RT-PCR identification of viral nucleic acid for the $\mathrm{H}_{1} \mathrm{~N}_{1}$ subtype was $1.8 \%$ from serum and $6.3 \%$ in nasal swabs, for the $\mathrm{H}_{3} \mathrm{~N}_{2}$ subtype $5.4 \%$ was identified in nasal swabs where statistical differences were significant $(\mathrm{P}<0.05)$. All serum samples were negative for subtype $\mathrm{H}_{3} \mathrm{~N}_{2}$.
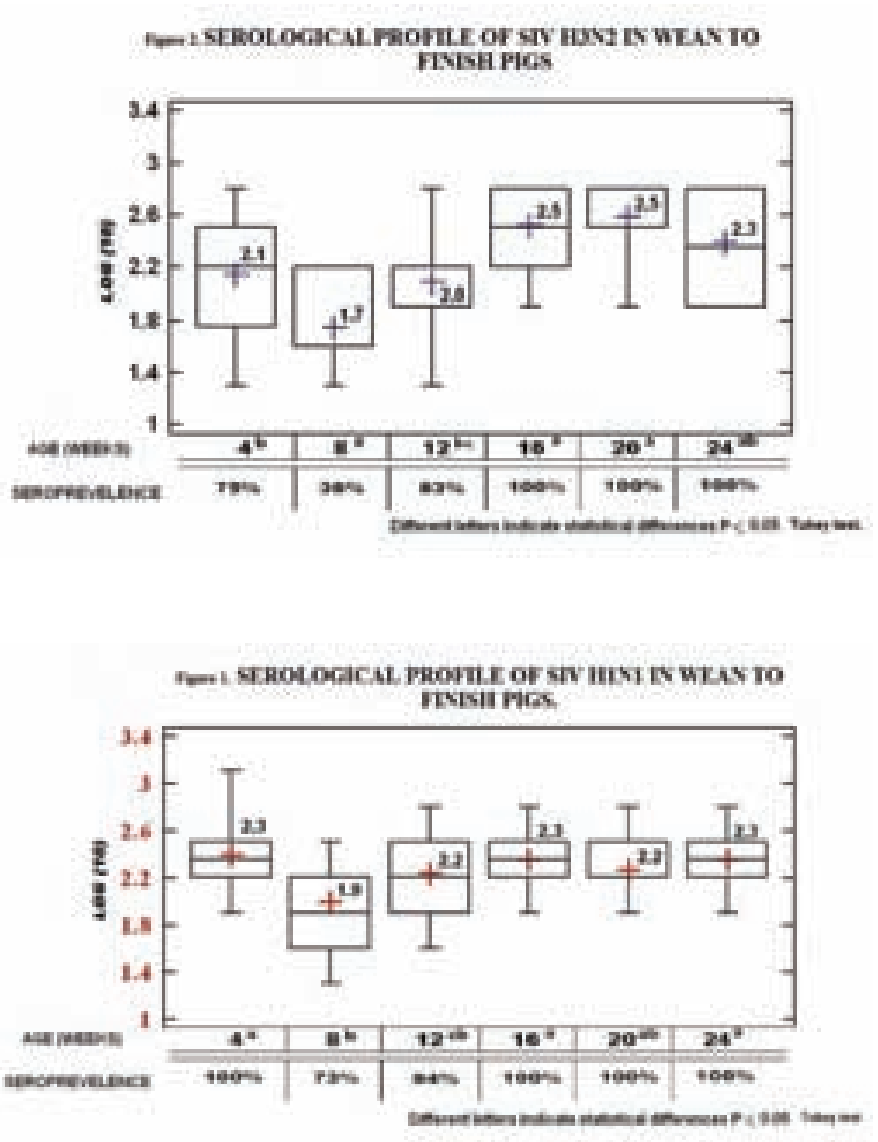

\section{Discussion}

The co-existence of diagnostic techniques allows us identifying with a high sensitivity and a very timely manner any subtype of SIV present in a sample. Through the integration of diagnosis we are able to establish the dynamics of viral infection within the farm and allows us to identify the group of animals that carry the virus (PCR), as well as those animals that have already been affected by the virus and that are negative to PCR, but have antibodies $(\mathrm{HI})$; both panoramas show us the dynamics of infection associated with the clinical signs, allowing us to understand the process of the disease in the herd. With this information you can intervene promptly to control and prevent the infection process.s

\section{Reference}

1. Beltran, F. R., et al. (2007). Memorias del XLII Congreso Nacional de AMVEC. 190.

2. Olsen, C. W., et al. (2006). Swine Influenza. In Diseases of Swine (9th edition). Straw, B. E., et al. eds. Blackwell Publishing, Ames, lowa, USA. 2(28): 469-482. 


\title{
P.265
}

\section{Influence of various adjuvants on antibody avidity and relative ratios of IgG2 to IgG1 (Th1 to Th2) in pigs after booster immunizations with SIV vaccines}

\author{
Boh C. Lin; Karen K. Brown; Jack D. McGonigle; Leann M. Siedlik \\ MVP Laboratories, Inc., Omaha, NE, USA
}

\section{Introduction}

Since no single adjuvant is universally applicable for all antigens, it is critical to include different types of adjuvant systems in an experimental vaccine for evaluation. In this study, an inactivated commercial swine influenza A (SIV) vaccine was used to evaluate the influence of five adjuvant systems on antibody avidity and the relative ratios of $\operatorname{lgG} 2$ to $\lg G 1$ (Th1 to Th2) in young pigs after booster immunizations.

\section{Materials and Methods}

Thirty six 21 day old pigs were assigned to six groups. Experimental vaccines were prepared by mixing the manufacturer's recommended amount of SIV antigens with one of five adjuvants supplied by MVP Laboratories, Inc. Pigs of group 1 through group 5 were given vaccines containing 20\% Emulsigen ${ }^{\circledR}-\mathrm{D}$, $12 \%$ Emulsigen ${ }^{\circledast} / 4 \%$ Rehydragel, 20\% Emulsigen ${ }^{\circledR}-\mathrm{BCL}, 20 \%$ Polygen $^{\mathrm{TM}}$, and an adjuvant supplied by the manufacturer of the commercial SIV vaccine respectively. Pigs of group 6 were given PBS only. On day 0 , all of the vaccinated pigs were given a $2 \mathrm{ml}$ dose intramuscularly. On day 21, all pigs were given a second dose. All pigs were bled on day 0 , day 21 , and day 42 . The hemagglutination inhibition ( $\mathrm{HI}$ ) test was conducted by the Veterinary Diagnostic Laboratory at lowa State Univesity. The antibody avidity of each pig serum was measured using a thiocyanate elution ELISA as described (1). IgG1 and IgG2 were determined in pig sera by ELISA as described (2).

\section{Results and Discussion}

Influence of adjuvant on relative ratios of $\lg G 2$ to $\lg G 1$ in pigs: Polygen ${ }^{\mathrm{TM}}$ induced the highest H1N1-specific and H3N2-specific IgG2 response (Table 1). This signals a Th1 response that leads to cell-mediated immunity. Furesz, et al, demonstrated that a high anti-hemolysin IgG2:IgG1 ratio correlated with hemolysin neutralization antibody titers and reduced lung lesion scores in A. pleuropneumoniae infected pigs (3). Emulsigen ${ }^{\circledR}-D$ and Emulsigen ${ }^{\circledast} /$ Rehydragel induced the highest levels of H1N1-specific and H3N2-specific lgG1. This indicates a Th2 response that leads to humoral immunity which is of importance in SIV infections.

Influence of adjuvant on antibody avidity: There is a significant difference between the SIV-specific antibody avidity indices (GMAI) of the five adjuvant groups with Emulsigen ${ }^{\circledR}-D$ producing the highest results (Table 2). It has been reported that higher serum avidity indices indicate more effective bactericidal activity of the microphages (2). This study indicates that the measurement of ratios of $\lg G 2$ to $\operatorname{lgG} 1$ and GMAl are useful as supplementary methods for predicting the properties of each test adjuvant in terms of their tendency to direct cellular (Th1) or humoral (Th2) immune response and antibody functional activity. These findings suggest that Th1 or Th2 responses in pigs are determined by both the adjuvant and its co-injected antigen.
Table 1. Adjuvant effects on relative ratios of $\mathrm{H} 1 \mathrm{~N} 1$-specific $\lg \mathrm{G} 2$ to $\lg G 1$ and H3N2-specific lgG2 to $\lg G 1$.

\begin{tabular}{|l|c|c|c|c|c|c|}
\hline \multirow{2}{*}{$\begin{array}{l}\text { Vaccine adju- } \\
\text { vant }\end{array}$} & \multicolumn{3}{|c|}{ H1N1-specific } & \multicolumn{3}{c|}{ H3N2-specific } \\
\cline { 2 - 7 } & $\begin{array}{l}\text { IgG2:IgG1 } \\
\text { ratio }\end{array}$ & HI titer & $\begin{array}{c}\text { Response } \\
\text { profile }\end{array}$ & $\begin{array}{c}\text { IgG2:IgG1 } \\
\text { ratio }\end{array}$ & HI titer & $\begin{array}{c}\text { Response } \\
\text { profile }\end{array}$ \\
\hline Emulsigen ${ }^{\oplus}$-D & 0.81 & 1973 & Th2 & 0.74 & 2133 & Th2 \\
\hline $\begin{array}{l}\text { Emulsigen } \\
\text { Rehydragel }\end{array}$ & 0.73 & 347 & Th2 & 0.81 & 200 & Th2 \\
\hline Emulsigen $^{\oplus}$-BCL & 1.09 & 987 & Th1 & 0.92 & 480 & Th2 \\
\hline Polygen $^{\text {TM }}$ & 1.24 & 147 & Th1 & 1.05 & 67 & Th1 \\
\hline Control adjuvant $^{*}$ & 1.00 & 427 & Th1 & 0.81 & 280 & Th2 \\
\hline${ }^{*}{ }^{2}$ Control adjuvant was obtained from the commercial vaccine used in this study. \\
\hline
\end{tabular}

Table 2. Adjuvant effects on the geometric mean avidity indices (GMAI) of anti-SIVIgG antibodies in pigs after booster immunization.

\begin{tabular}{|c|c|c|c|}
\hline & Adjuvant & GMAI (Range) & $\begin{array}{c}\text { Statistical difference } \\
\text { between Adjuvant (p } \\
\text { value) }\end{array}$ \\
\hline 1. & Emulsigen $^{\circledast}-D$ & $1.360(1.200-1.400)$ & \\
\hline 2. & Emulsigen ${ }^{\circledast} /$ Rehydragel & $1.100(0.900-1.400)$ & $\mathrm{p}=0.0101(1 \text { vs } 2)^{*}$ \\
\hline 3. & Emulsigen ${ }^{\circledR}-\mathrm{BCL}$ & $0.970(0.400-1.400)$ & $p=0.0331(1 \text { vs } 3)^{*}$ \\
\hline 4. & Polygen $^{\mathrm{TM}}$ & $0.800(0.600-1.000)$ & $\mathrm{p}=0.0001(1 \text { vs } 4)^{*}$ \\
\hline 5. & Control adjuvant & $1.000(0.900-1.100)$ & $p=0.0101(1 \text { vs } 5)^{*}$ \\
\hline
\end{tabular}

\section{References}

1. Pullen, G. R., Fitzgerald, and C. S. Hosking. 1986. Antibody avidity determination by ELISA using thiocyanate elution. J. Immunol Methods. 86(1):83-87.

2. Vermont, C. L., van Dijken, H. H., van Limpt, C. J. P., de Groot, R., van Alphen, L., and G. P. J. M. van den Dobbelsteen. 2002. Antibody avidity and immunoglobulin $\mathrm{G}$ isotype distribution following immunization with a monovalent Meningococcal B outer membrane vesicle vaccine. Infect. Immun. 70:584-590.

3. Furesz, S. E., Wilkie, B. N., Mallard, B. A., Rosendal, S., and J. I. Maclnnes. 1998. Anti-haemolysin lgG1 to IgG2 ratios correlate with haemolysin neutralization titres and lung lesion scores in Actinobacillus pleuropneumoniae infected pigs. Vaccine. 16 (20):1971-1975. 


\title{
P.266
}

\section{Efficient protection against heterologous H1N2 infection using a trivalent swine flu vaccine}

\author{
Michael Schlegel ${ }^{1}$ Andi Krumbholz ${ }^{2}$ Roland Zell ${ }^{2}$ Hans-Joachim Selbitz ${ }^{1} \underline{\text { Ralf Dürrwald }}^{1}$ \\ 1. IDT Biologika GmbH, Dessau-Roßlau, Germany; 2. Universitätsklinikum Jena, Institut für Virologie und Antivirale Therapie, Jena, Germany
}

\section{Introduction}

In the course of several reassortment events the novel swine influenza A virus subtype H1N2 emerged in Europe in the 1990s (1). Its lacking cross-reactivity to $\mathrm{H} 1 \mathrm{~N} 1+\mathrm{H} 3 \mathrm{~N} 2$ vaccines required the development of a new trivalent swine flu vaccine (2).

\section{Materials and Methods}

Three strains of subtypes $\mathrm{H} 1 \mathrm{~N} 1, \mathrm{H} 3 \mathrm{~N} 2$, and $\mathrm{H} 1 \mathrm{~N} 2$ were selected as vaccine strains. Viruses were grown in cell culture and inactivated. A substance from the carboxyvinylpolymers group was used as an adjuvant. Experimental batches were blended and investigated in laboratory and clinical trials. A major focus of investigation was the proof of efficacy against heterologous $\mathrm{H} 1 \mathrm{~N} 2$ field strains in experimental infection trials. Five challenge experiments were conducted on a total of 158 pigs. Three recently isolated field isolates of subtype $\mathrm{H} 1 \mathrm{~N} 2$ were used for the experiments. The strains were sequenced and analysed phylogenetically. Pigs were vaccinated twice. For proof of efficacy an aerosol method was used in which high doses of field viruses are sprayed by a generator. For comparison an additional intratracheal infection was done in one trial.

\section{Results}

Vaccinated pigs displayed neutralizing and hemagglutination inhibiting antibodies as early as 7 days after the second vaccination.

Vaccinated animals had almost no clinical symptoms after infection, whereas unvaccinated animals exhibited fever, dyspnoea and sleepiness. The viral load in the lungs was significantly lower in vaccinated pigs compared to unvaccinated animals. Lung lesions typical for influenza were observed in the apical parts of medial and cranial lung lobes of the control animals, whereas vaccinated pigs showed no lesions. Moreover, histological investigations revealed a higher degree of inflammation in the unvaccinated pigs.Fig. 1: Viral lung load in egg infectious dose 50 (EID50), clinical score and rectal temperatures of vaccinated and unvaccinated pigs after experimental infection with H1N2 virus; Statistics: Mann-Whithney U test $(p=$ statistical probability, $\leq 0.05$ significant, $\leq 0.01$ highly significant, $\leq 0.001$ very highly significant); Clinical score: 1, enhanced abdominal breathing, 2, strong abdominal breathing, 3 , severe breathing covering the whole body; Vaccinated, vaccinated pigs ( $2 \times 2.0 \mathrm{ml}$ i.m ); Control, unvaccinated pigs ( $n=15$ pigs in each group) a) aerosol infection with strain Kitzen/IDT6142/07 (H1N2), arithmetic mean

\begin{tabular}{|l|c|c|c|}
\hline & Vaccinated & Control & Statistics, $\mathbf{p}$ \\
\hline EID50/g, day 1 & 3388 & 38538 & 0.003 \\
\hline Clinical score, day 1 & 0.07 & 2.47 & 0.000 \\
\hline Temperature, ${ }^{\circ}$ C, day 1 & 39.65 & 41.07 & 0.000 \\
\hline EID50/g, day 3 & 471 & 38857 & 0.003 \\
\hline Clinical score, day 3 & 0 & 0.93 & 0.004 \\
\hline Temperature, ${ }^{\circ}$ C, day 3 & 39.67 & 40.56 & 0.004 \\
\hline
\end{tabular}

b) intratracheal infection with strain Kitzen/IDT6142/07 (H1N2), arithmetic mean

\begin{tabular}{|l|c|c|c|}
\hline & Vaccinated & Control & Statistics, $\mathbf{p}$ \\
\hline EID50/g, day 1 & 525 & 12600 & 0.021 \\
\hline Clinical score, day 1 & 0.4 & 1.53 & 0.016 \\
\hline Temperature, ${ }^{\circ}$ C, day 1 & 39.93 & 40.15 & 0.436 \\
\hline EID50/g, day 3 & 5 & 38857 & 0.001 \\
\hline Clinical score, day 3 & 0.29 & 1.71 & 0.007 \\
\hline Temperature, $^{\circ}$ C, day 3 & 39.7 & 40.19 & 0.026 \\
\hline
\end{tabular}

\section{Discussion}

Vaccinated pigs were protected against all three $\mathrm{H} 1 \mathrm{~N} 2$ field strains used in a number of experimental infection trials. The very high efficacy of the vaccine against different strains of European $\mathrm{H} 1 \mathrm{~N} 2$ viruses has been demonstrated.

\section{References}

1 Brown IH, Harris PA, McCauley JW, Alexander DJ (1998) Multiple genetic reassortment of avian and human influenza $A$ viruses in European pigs, resulting in the emergence of an $\mathrm{H} 1 \mathrm{~N} 2$ virus of novel genotype. J. Gen. Virol. 79: 2947-2955

2 Van Reeth K, Van Gucht S, Pensaert M (2003) Investigations of the efficacy of European $\mathrm{H} 1 \mathrm{~N} 1$ - and H3N2-based swine influenza vaccines against the novel H1N2 subtype. Vet. Rec. 153: 9-13 


\title{
P.267
}

\section{Field safety of the administration of a new swine influenza virus vaccine Respiporc $^{\circledR}$ Flu3/Gripovac ${ }^{\circledR} 3$ in pregnant sows and gilts}

\author{
Michael Schlegel ${ }^{2}$ Ralf Dürrwald ${ }^{2}$ Lothar Kreienbrock $^{3}$ Thaïs Vila $^{1}$ Hans-Joachim Selbitz $^{2}$ \\ 1. Merial S.A.S., Lyon, France; 2. IDT Biologika GmbH, Dessau-Rosslau, Germany; \\ 3. Tierärztliche Hochschule Hannover, Hannover, Germany
}

\section{Introduction}

The objective of these studies was to confirm the safety of a new swine influenza vaccine including the 3 swine influenza $A$ virus subtypes, H1N1, H3N2 and H1N2: RESPIPORC ${ }^{\circledR}$ FLU3/GRIPOVAC ${ }^{\circledR} 3$ administered during gestation to sows and gilts in the field.

\section{Material and Methods}

A total of 107 sows and gilts from several farrow-to-finish conventional farms were used at different stages of pregnancy.

Experimental design

\begin{tabular}{|l|c|c|c|c|}
\hline & Sows T1 & Sows T2 & Sows T3 & Gilts \\
\hline Total sows in farm & 206 & 530 & 200 & 530 \\
\hline Vaccinated sows & $\mathrm{n}=12$ & $\mathrm{n}=14$ & $\mathrm{n}=12$ & $\mathrm{n}=17$ \\
\hline Control sows & $\mathrm{n}=12$ & $\mathrm{n}=16$ & $\mathrm{n}=11$ & $\mathrm{n}=13$ \\
\hline
\end{tabular}

Vaccination schedule

\begin{tabular}{|l|c|c|}
\hline Group & 1st inj./period in the repro. cycle & 2nd injection \\
\hline T1 sows & D0 $=$ Gestation 1st to 38th day & D21 \\
\hline T2 sows & D0 $=$ Gestation 39th to 78th day & D21 \\
\hline T3 sows & D0 $=$ Gestation 76th to 114 day & D21 \\
\hline Gilts & D0 $=5$ weeks before insemination & 2nd inj. D21; 3rd inj. D133 \\
\hline
\end{tabular}

Safety criteria were rectal body temperature, local reactions i.e. skin colour, swelling, abscess, and systemic reactions i.e. behaviour, respiration, digestion, skin, coughing, others. Clinical status was monitored every day throughout the study. Clinical observation: T1, T2 and T3 sow temperature was measured on D0, D0+4h, D1, D2, D21, D21+4h, D22 and D23. In gilts it was measured on D0, D0+4h, D1, D2, D21, D21+4h, D22, D23, D133, D133+4h, D134 and D135.

The local reactions have been monitored at the same time points and on D106 in T1 sows, on D100 in T2 sows, on D64 in T3 sows and on D177 in gilts, i.e. the last day of the study in each case. Statistical analyses: A Student T test was used to compare the absence of difference between temperatures. The level of significance was $5 \%$.

\section{Results}

Neither relevant local nor systemic reactions were noticed. The temperature was significantly non-different both in vaccinated and control groups.
Figure 1: Temperature in sows
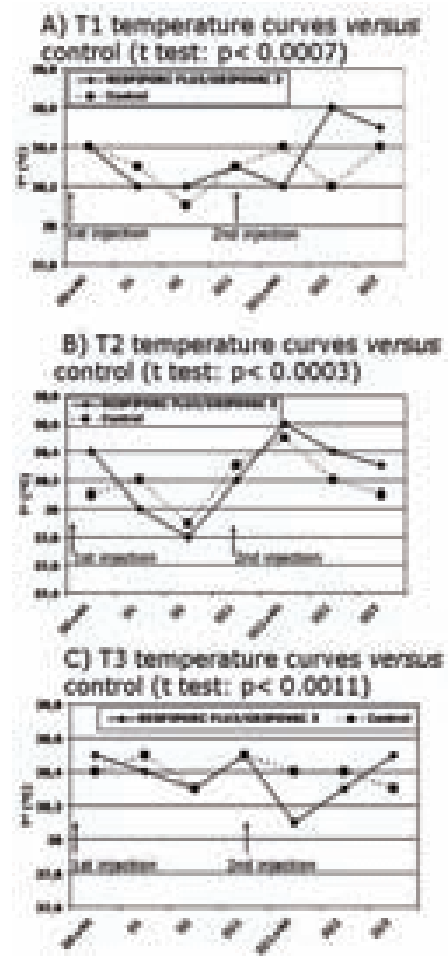

Figure 2: Temperature in gilts

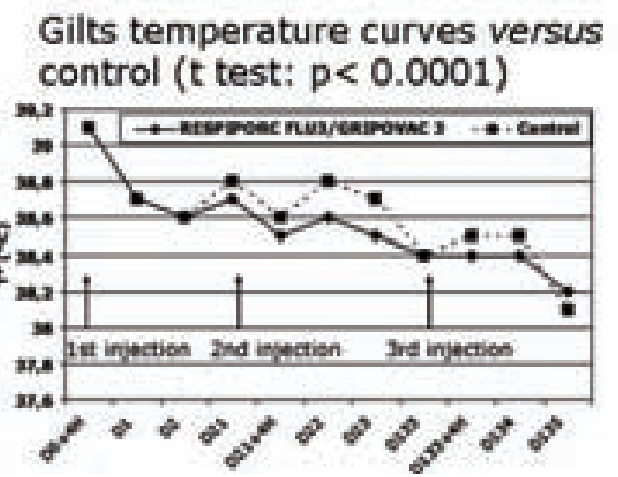

\section{Conclusion}

The use of RESPIPORC FLU3/GRIPOVAC 3 is perfectly safe in pregnant sows and gilts, any time during gestation.

${ }^{\circledR}$ RESPIPORC is a registered trademark of IDT. ${ }^{\circledR}$ GRIPOVAC is a registered trademark of MERIAL S.A.S. 


\title{
P.268
}

\section{Duration of clinical protection after vaccination with Respiporc ${ }^{\circledR}$ Flu3/Gripovac ${ }^{\otimes} 3$}

\author{
Michael Schlegel ${ }^{1}$ Hans-Joachim Selbitz ${ }^{1}$ Thaïs Vila² Ralf Dürrwald ${ }^{1}$ \\ 1. IDT Biologika GmbH, Dessau-Rosslau, Germany; 2. Merial S.A.S., Lyon, France
}

\section{Introduction}

This paper summarizes several studies aiming at showing the duration of clinical protection after vaccination of piglets with a new swine influenza vaccine (RESPIPORC FLU3/GRIPOVAC 3) including the FLUAVsw subtypes H1N1, H3N2 and H1N2.

\section{Material and Methods}

Before vaccination, piglets were free of antibodies against H1N1, H1N2 and H3N2. The studies were blinded, placebo controlled and randomised. Challenge at more than 4 months after basic immunisation was done by aerosol nebulisation with heterologous strains from Central Europe isolated from pigs affected with severe respiratory illness as described in table 1.

Table 1: Study parameters

\begin{tabular}{|l|c|c|c|}
\hline Subtype & H1N1 & H1N2 & H3N2 \\
\hline No of pigs & 26 & 30 & 30 \\
\hline Vaccinated & 13 & 15 & 15 \\
\hline Control & 13 & 15 & 15 \\
\hline Aerosol challenge & $\begin{array}{l}10^{6.5} \mathrm{TCID}_{50} / \mathrm{ml} 148 \text { days } \\
\text { after 2nd vaccination }\end{array}$ & $\begin{array}{l}10^{6.75} \mathrm{TCID}_{50} / \mathrm{ml} 126 \\
\text { days after 2nd vac- } \\
\text { cination }\end{array}$ & $\begin{array}{l}10^{7.5} \mathrm{TCID}_{50} / \mathrm{ml} 126 \text { days } \\
\text { after 2nd vaccination }\end{array}$ \\
\hline
\end{tabular}

Vaccination schedule: vaccination after 56 days of age $2 \mathrm{ml}$, twice 3 weeks apart. Control piglets were injected with $\mathrm{NaCl}$ $0.9 \%$.Criteria of data collection: Temperature was measured twice a day. Dyspnoea was scored from 1 to 3: 1= enhanced abdominal breathing; $2=$ severe abdominal breathing; $3=$ strong breathing covering the whole body.

\section{Results}

After challenge 4 months after vaccination, flu-associated clinical signs as high temperature or dyspnoea were significantly lower in vaccinated piglets than in control piglets (Figure 1 and table 2).
Figure 1: Temperatures recorded after challenge for the 3 FLUAVsw subtypes: A)H1N1, B)H1N2, C)H3N2

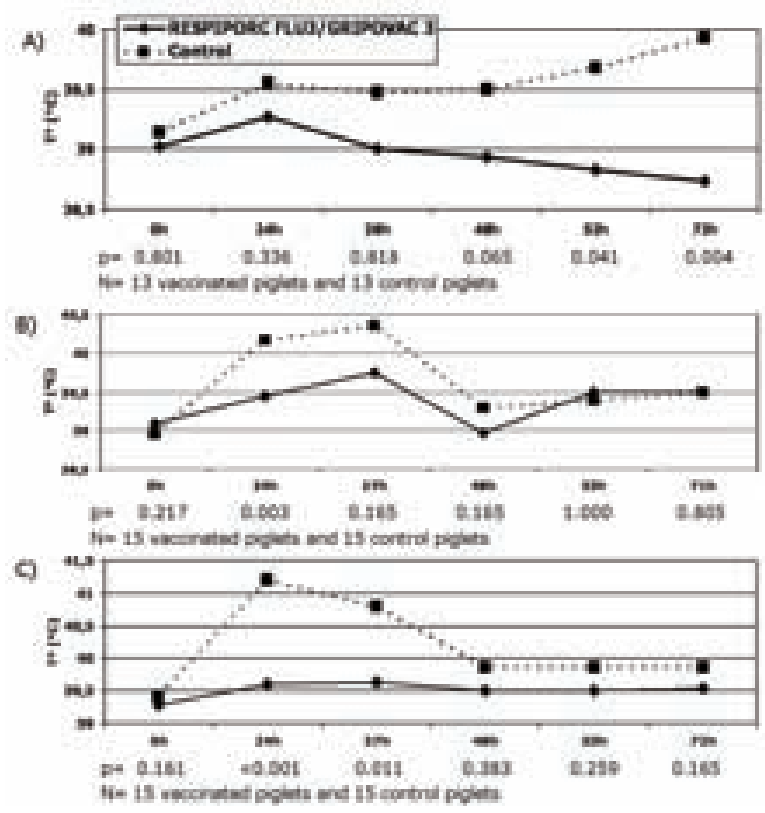

Table 2: Dyspnoea scores after challenges H1N1, H1N2, H3N2

\begin{tabular}{|l|c|c|c|c|c|}
\hline Challenge H1N1 & $\mathbf{2 4 h}$ & $\mathbf{2 8 h}$ & $\mathbf{4 5 h}$ & $\mathbf{7 3 h}$ & - \\
\hline $\begin{array}{l}\text { RESPIPORC FLU3 / } \\
\text { GRIPOVAC 3 }\end{array}$ & 0.29 & 0.13 & 0 & 0 & - \\
\hline Control & 1.46 & 1.75 & 1.08 & 0.5 & - \\
\hline $\mathrm{p}$ & 0.002 & 0.002 & 0.065 & 0.394 & - \\
\hline Challenge H1N2 & $24 \mathrm{~h}$ & $27 \mathrm{~h}$ & $48 \mathrm{~h}$ & $53 \mathrm{~h}$ & $71 \mathrm{~h}$ \\
\hline $\begin{array}{l}\text { RESPIPORC FLU3 / } \\
\text { GRIPOVAC3 }\end{array}$ & 0.48 & 0.36 & 0 & 0.07 & 0 \\
\hline Control & 1.15 & 1.36 & 0.82 & 0.29 & 0 \\
\hline $\mathrm{p}$ & 0.002 & 0.017 & 0.001 & 0.165 & 1.000 \\
\hline Challenge H3N2 & $24 \mathrm{~h}$ & $27 \mathrm{~h}$ & $48 \mathrm{~h}$ & $53 \mathrm{~h}$ & $72 \mathrm{~h}$ \\
\hline $\begin{array}{l}\text { RESPIPORC FLU3/ } \\
\text { GRIPOVAC 3 }\end{array}$ & 0.4 & 0.14 & 0.29 & 0.18 & 0.21 \\
\hline Control & 2.33 & 2.64 & 2 & 1.64 & 1.5 \\
\hline$p$ & $<0.001$ & 0.001 & 0.004 & 0.017 & 0.017 \\
\hline
\end{tabular}

\section{Conclusion}

Piglets vaccinated with RESPIPORC FLU3/ GRIPOVAC 3 after 8 weeks of age were well clinically protected against $\mathrm{H} 1 \mathrm{~N} 1, \mathrm{H} 1 \mathrm{~N} 2$ and $\mathrm{H} 3 \mathrm{~N} 2$ subtype challenges up to 4 months after the 2 nd injection of the vaccination.

${ }^{\circledR}$ RESPIPORC is a registered trademark of IDT. GRIPOVAC is a registered trademark of MERIAL S.A.S. 


\title{
P.269
}

\section{Effect of ibuprofen, aspirin or saline water medications on pigs challenged with swine influenza virus (SIV)}

\author{
Peggy Anne Hawkins ${ }^{1}$ Nathan Winkelman² Michael Strobel ${ }^{1}$ Enrique Mondaca ${ }^{1}$ \\ 1. Veterinary Provisions, Inc., Northfield, MN, USA; 2. Swine Services Unlimited Inc., Rice, MN, USA
}

\section{Introduction}

Swine influenza virus (SIV) commonly infects growing pigs. Antibiotics are of no use against viral infections. However, antiinflammatory products such as ibuprofen and aspirin are often proportioned to pigs through the water. The objective of this study was to evaluate the anti-inflammatory action of ibuprofen (IB) or aspirin (SS) (sodium salicylate) or saline (SA) in pigs challenged with SIV. The ibuprofen significantly reduced fever as compared to aspirin or saline. Additionally, the use of ibuprofen during a field SIV outbreak that reduced death loss and signs of disease is covered in the discussion.

\section{Materials and Methods}

Sixty-three SIV-negative, M. hyopneumoniae-negative and PRRSV negative crossbred pigs were randomly assigned to 3 treatments blocked by gender and weight and placed in 9 pens at 7 pigs per pen. Pens were randomized into treatments. Pigs were acclimated for 1 week and then challenged with SIV (H1N1 sub-type, Asw lowa 239:2004) provided by U of Minnesota. Treatments were dosed according to label directions. IB $160 z$ $(473 \mathrm{ml})$ per gallon stock solution ( 6 $\mathrm{mg} / \mathrm{lb})$. SS $80 \mathrm{z}(236 \mathrm{ml})$ per gallon stock solution (4mg/lb). SA $12 \mathrm{oz}(354 \mathrm{ml})$ per gallon stock solution. Stock solution should then be metered at 1:128. Twenty-four (24) hours after inoculation, treatments were added to the water and remained in the water for 5 complete days. Individual measures of clinical observations, weight, rectal temperature, nasal swabs, blood samples and lung lesions scores were recorded. Water and feed intakes were measure by pen (1).

\section{Results}

The SIV challenge was successful. On day three, 61 of 63 pigs had virus by nasal swab PCR or virus isolation. Virus was not detected in $2 \mathrm{SA}$ pigs. For this study, $103.5^{\circ} \mathrm{F}$ or less was considered normal rectal temperature. Across treatments, pigs started with a low- normal rectal temperature at inoculation $\left(103.1^{\circ} \mathrm{F}\right)$. 24-hours later all treatments spiked a fever (104.9 F). One day after treatment began the pigs in the IB group had a significantly lower rectal temperature averaging $103.2^{\circ} \mathrm{F}$ compared to the SS $\left(103.6^{\circ} \mathrm{F}\right)$ or SA $\left(103.7^{\circ} \mathrm{F}\right)$ groups. On the 5 th day of the study, the SS group spiked a fever. The cause was unknown (Fig. 1). ${ }^{* * i m-}$ age $1^{* *}$ There were no differences between treatments in clinical observations, feed intake, water intake or weight gain. The IB group did have higher lung lesion scores on treatment day 10 as compared to SA or SS. Wilcoxon rank sum test was used for analysis. A $P$ value of $<0.05$ was considered significant.

\section{Discussion}

Sodium salicylate (SS) was chosen over acetylsalicylic acid (ASA) as a result of work done by Patterson et al. showing that measureable plasma concentrations of SS and not ASA were found when administered at $4.5 \mathrm{mg} / \mathrm{lb}$ body weight (2). In this study treatments were added 24 hours after inoculation to simulate a field outbreak in which a producer would notice that pigs are sick and then would start treatment the next day. As a result of having the IB in the water, rectal temperatures were reduced to normal levels. Saline control and aspirin did not affect the rectal temperature. The number of pigs in this study limited the ability to see other significant differences. In a related field case, a 1000 head barn lost 17 pigs in an outbreak of SIV. The herd veterinarian put IB in the water for 5 days in 3 subsequent 1000 head barns and lost only 2 pigs to SIV. The cost of treatment with IB was about $\$ 0.75$ per pig. Diagnosing SIV quickly is key to getting IB in the water fast enough to stop/reduce death loss and morbidity.

\section{References}

1. Winkelman N, Hawkins PA, Strobel M, Mondaca E, Effect of ibuprofen water medication on pigs challenged with SIV, and a field case study, AASV Proceedings, Omaha, NE. 2010 (in press)

2. Patterson AR, Karriker LA, Apley MD, Imerman PM, Plasma concentrations of sodium salicylate in nursery pigs treated orally, JSHAP. 2007; Vol 15, No 3(146-151) 


\title{
P.270
}

\section{Duration of clinical protection after vaccination of pigs at more than 96 days of age with Respiporc ${ }^{\circledR}$ Flu3/Gripovac ${ }^{\circledR} 3$}

\author{
Michael Schlegel ${ }^{1}$ Hans-Joachim Selbitz ${ }^{1}$ Thaïs Vila $^{2}$ Ralf Dürrwald ${ }^{1}$ \\ 1. IDT Biologika GmbH, Dessau-Rosslau, Germany; 2. Merial S.A.S., Lyon, France
}

\section{Introduction}

This paper summarizes 3 vaccination studies aiming at showing the duration of clinical protection of RESPIPORC FLU3/GRIPOVAC 3 vaccination in pigs vaccinated from 96 days of age against the 3 swine influenza virus (FLUAVsw) subtypes: H1N1, H3N2 and H1N2.

\section{Material and Methods}

Piglets were obtained from farms free of infections with influenza viruses. The study was blinded, placebo controlled and randomised. A challenge was performed at more than 6 months after basic immunisation by aerosol nebulisation with heterologous strains from Central Europe isolated from pigs affected with severe respiratory illness as described in table 1.

Table 1: Study parameters

\begin{tabular}{|l|c|c|c|}
\hline Subtype & \multicolumn{1}{|c|}{ H1N1 } & \multicolumn{1}{c|}{ H1N2 } & \multicolumn{1}{c|}{ H3N2 } \\
\hline No of pigs & 40 & 40 & 40 \\
\hline Vaccinated & 20 & 20 & 20 \\
\hline Control & 20 & 20 & 20 \\
\hline $\begin{array}{l}\text { Aerosol chal- } \\
\text { lenge }\end{array}$ & $\begin{array}{l}10^{6.25} \mathrm{TCID}_{50} / \mathrm{ml} \\
211 \text { days after } \\
\text { vacc. }\end{array}$ & $\begin{array}{l}10^{6.5} \mathrm{TCID}_{50} / \mathrm{ml} \\
211 \text { days after } \\
\text { vacc. }\end{array}$ & $\begin{array}{l}10^{8.75} \mathrm{TCI}_{50} / \mathrm{ml} \\
209 \text { days after } \\
\text { vacc. }\end{array}$ \\
\hline & & & \\
\hline
\end{tabular}

Vaccination schedule: pigs were vaccinated from 96 days of age (including gilts) $2 \mathrm{ml}$, twice 3 weeks apart. Control pigs were injected with $\mathrm{NaCl} 0.9 \%$.

Criteria of data collection: Temperature was measured twice a day. Dyspnoea was scored from 1 to 3: $1=$ enhanced abdominal breathing; $2=$ severe abdominal breathing; $3=$ strong breathing covering the whole body.

\section{Results and Discussion}

Temperatures were found significantly lower at several time points during the observation period in pigs vaccinated at more than 96 days of age and challenged with H1N2 and H3N2 subtypes 6 months after vaccination compared to control pigs (figure 1).Regarding respiratory signs evaluated by dyspnoea scoring, pigs vaccinated with RESPIPORC FLU3/GRIPOVAC 3 were clinically protected.
Figure 1: $T^{\circ}$ after challenge A)H1N1, B)H1N2, C)H3N2 in pigs vaccinated at more than 96 days of age

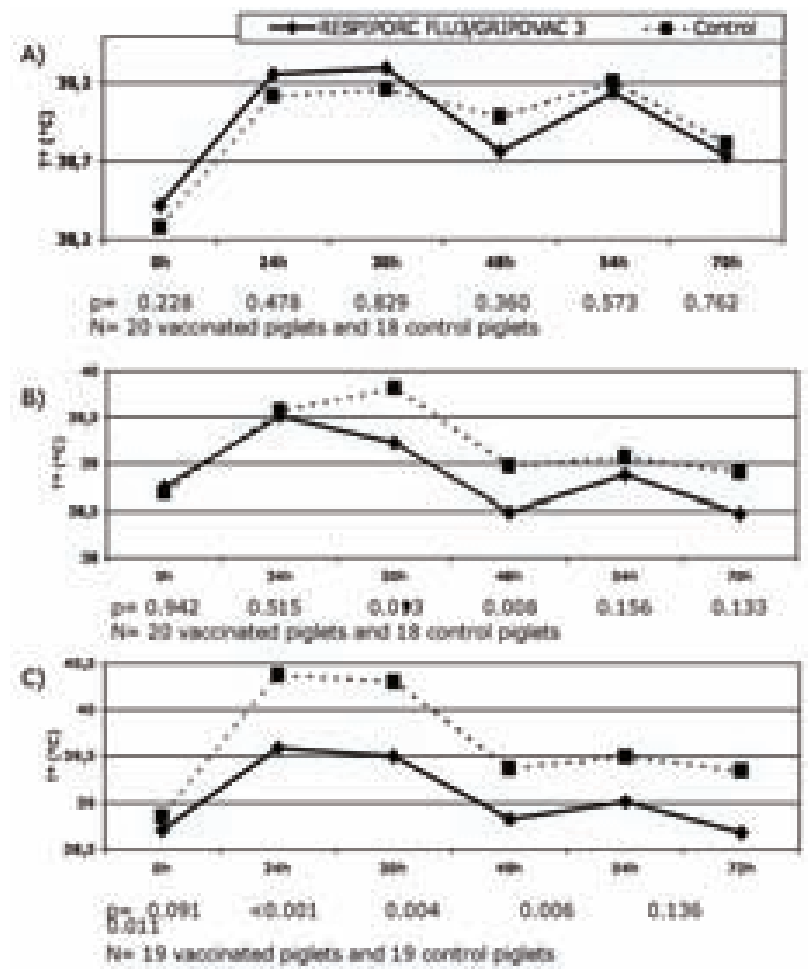

Table 2: Dyspnoea scores

\begin{tabular}{|l|c|c|c|c|c|}
\hline Challenge H1N1 & $\mathbf{2 4 h}$ & $\mathbf{3 0 h}$ & $\mathbf{4 8 h}$ & $\mathbf{5 4 h}$ & $\mathbf{7 0 h}$ \\
\hline RESPIPORC FLU3/GRIPOVAC3 & 0.88 & 0.5 & 0 & 0.1 & 0 \\
\hline Control & 1.67 & 0.81 & 0.19 & 0.31 & 0 \\
\hline $\mathrm{p}$ & 0.005 & 0.360 & 0.408 & 0.460 & 1.000 \\
\hline Challenge H1N2 & $24 \mathrm{~h}$ & $30 \mathrm{~h}$ & $48 \mathrm{~h}$ & $54 \mathrm{~h}$ & $70 \mathrm{~h}$ \\
\hline RESPIPORC FLU3/GRIPOVAC3 & 1.35 & 1.1 & 0.2 & 0.2 & 0 \\
\hline Control & 1.86 & 1.5 & 1.22 & 0.5 & 0.28 \\
\hline $\mathrm{p}$ & 0.228 & 0.356 & 0.002 & 0.243 & 0.243 \\
\hline Challenge H3N2 & $24 \mathrm{~h}$ & $30 \mathrm{~h}$ & $49 \mathrm{~h}$ & $54 \mathrm{~h}$ & $72 \mathrm{~h}$ \\
\hline RESPIPORC FLU3/GRIPOVAC3 & 0.47 & 0 & 0.22 & 0.06 & 0 \\
\hline Control & 2.08 & 1.78 & 1.39 & 1.22 & 0.56 \\
\hline $\mathrm{p}$ & 0.002 & $<0.001$ & $<0.001$ & 0.001 & 0.113 \\
\hline
\end{tabular}

\section{Conclusion}

Pigs vaccinated with RESPIPORC FLU3/GRIPOVAC 3 from 96 days of age were protected against the 3 FLUAVsw subtypes H1N1, $\mathrm{H} 3 \mathrm{~N} 2$ and $\mathrm{H} 1 \mathrm{~N} 2$ up to 6 months after the 2 nd vaccination.

${ }^{\circledR}$ RESPIPORC is a registered trademark of IDT. GRIPOVAC is a registered trademark of MERIAL S.A.S. 


\title{
P.271
}

\section{Development of a trivalent swine flu vaccine based on a safe adjuvant}

\author{
$\underline{\text { Ralf Dürrwald }}{ }^{1}$ Michael Schlegel ${ }^{1}$ Hans-Joachim Selbitz ${ }^{1}$ Andi Krumbholz ${ }^{2}$ Roland Zell ${ }^{2}$ \\ 1. IDT Biologika GmbH, Dessau-Roßlau, Germany; 2. Universitätsklinikum Jena, Institut für Virologie und Antivirale Therapie, Jena, Germany
}

\section{Introduction}

The introduction via reassortment of a human $\mathrm{H} 1$ hemagglutinin into the European swine influenza $A$ virus population that lacks cross-reactivity to avian and classical porcine $\mathrm{H} 1 \mathrm{~N} 1$ viruses has remarkably changed the otherwise epidemiologically very stable situation of swine flu $(1,2)$. This stimulated the development of a new trivalent swine flu vaccine that provides protection against all three subtypes, including the new subtype H1N2, and improves safety through the use of a non-pyrogenic and non-virucidal adjuvant.

\section{Materials and Methods}

A surveillance program for swine influenza in Germany was initiated which gave an overview on the epidemiological situation and led to the isolation of numerous viruses of all three subtypes. Three virus strains of subtypes H1N1, H3N2, and H1N2 originally isolated in regions with dense swine populations were chosen as vaccine strains. A very efficient cell culture production procedure was developed. Viruses were produced and inactivated by ethylenimine. Experimental and production batches were blended. A substance from the carboxyvinylpolymers group was used as an adjuvant. Dose titration studies and safety and efficacy investigations were performed. The vaccination scheme comprises a twofold administration of $2.0 \mathrm{ml}$ of the vaccine 21 days apart intramuscularly. An aerosol-mediated challenge procedure was applied to investigate efficacy of the vaccine. A batch potency test was established which measures the geometric mean of neutralizing antibodies in guinea pigs. Clinical trials were carried out.

\section{Results}

It was shown by phylogenetic analysis of the hemagglutinin and neuraminidase genes of the vaccine strains that all three strains fit well with currently circulating swine flu viruses throughout Europe. The production in cell culture provides virus harvests with titers up to $10^{8.5} \mathrm{TCID}_{50} / \mathrm{ml}$ within a short time. The viruses are inactivated rapidly by ethylenimine without influencing the content of antigen. The range of the immunostimulating activity of the adjuvant was shown to be very broad and the final adjuvant blending was chosen midway through. The optimal antigen content was determined in dose titration experiments. None of the vaccinated animals had elevated body temperatures after administration of the vaccine, not even in the critical phase of the first 24 hours after vaccination in which mineral-oil adjuvanted vaccines cause severe fever reactions up to $42^{\circ} \mathrm{C}$. Vaccinated pigs display neutralizing and hemagglutination inhibiting antibodies as soon as 7 days after the second vaccination which is the onset of immunity. For proof of efficacy an aerosol method was developed in which field viruses are sprayed by a generator. Vaccinated animals had no clinical symptoms or only slight ones after infection, whereas unvaccinated animals exhibited fever, dyspnoea and sleepiness, accompanied by varying degrees of loss of appetite. The viral load in the lungs was significantly lower in vaccinated pigs compared to unvaccinated animals. The duration of immunity is age-dependent and lasts up to 6 months in older pigs after two vaccination shots. A third vaccination stabilizes the immune response and prolongs immunity. The batch potency was determined. The vaccine induces at least $10.22,10.53$ and 12.34 GMNU (Geometric Mean of Neutralizing Units $\log _{2}$ ) in guinea pigs against the $\mathrm{H} 1 \mathrm{~N} 1, \mathrm{H} 3 \mathrm{~N} 2$, and $\mathrm{H} 1 \mathrm{~N} 2$ strains respectively.

\section{Discussion}

A new trivalent swine flu vaccine has been developed in response to recent epidemiological events in the European swine influenza A virus population and an increased sensitivity of pigs to vaccinations. The high safety of the vaccine and its very high efficacy against each of the three subtypes currently circulating in swine has been demonstrated in a number of safety and infection trials. The vaccine went through a central registration procedure and was approved in 2010.

\section{References}

1 Brown IH, Harris PA, McCauley JW, Alexander DJ (1998) Multiple genetic reassortment of avian and human influenza $A$ viruses in European pigs, resulting in the emergence of an $\mathrm{H} 1 \mathrm{~N} 2$ virus of novel genotype. J. Gen. Virol. 79: 2947-2955

2 Van Reeth K, Van Gucht S, Pensaert M (2003) Investigations of the efficacy of European $\mathrm{H} 1 \mathrm{~N} 1$ - and H3N2-based swine influenza vaccines against the novel H1N2 subtype. Vet. Rec. 153: 9-13. 


\section{P.272}

\section{Serological response to Respiporc ${ }^{\circledast}$ Flu3/Gripovac ${ }^{\circledast} 3$ in pregnant sows against $H 1 N 1, H 1 N 2$ and $H 3 N 2$ influenza viruses}

Ralf Dürrwald ${ }^{1}$ Michael Schlegel ${ }^{1}$ Lothar Kreienbrock ${ }^{2}$ Thaïs Vila $^{3}$ Hans-Joachim Selbitz $^{1}$

1. IDT Biologika GmbH, Dessau-Rosslau, Germany; 2. Tierärztliche Hochschule Hannover, Hannover, Germany; 3. Merial S.A.S., Lyon, France

\section{Introduction}

The prevalence of swine influenza viruses may be high with $60 \%$ of farms affected (1). Previous studies showed an increase in the prevalence of the H1N2 subtype (2).

The objective of these studies was to show the serological responses in pregnant sows after vaccination of sows with a new swine flu vaccine, RESPIPORC FLU3/GRIPOVAC 3 including the 3 subtypes, H1N1, H3N2 and H1N2.

\section{Material and Methods}

For this blinded controlled trial, 77 swine flu-antibody-free or with very low antibody titres sows from several farrow-to-finish conventional farms were used at different stages of pregnancy (Table 1).

Sows were kept in the farm of origin.

Table 1: Experimental design

\begin{tabular}{|l|c|c|c|}
\hline & Sows T1 & Sows T2 & Sows T3 \\
\hline Total sows in farm & 206 & 530 & 200 \\
\hline Vaccinated sows & 12 & 14 & 12 \\
\hline Control sows & 12 & 16 & 11 \\
\hline
\end{tabular}

$\mathrm{T} 1=1 \mathrm{st}$ third of pregnancy. $\mathrm{T} 1=2 \mathrm{nd}$ third of pregnancy. $\mathrm{T} 3=3 \mathrm{rd}$ third of pregnancy.Antibodies were titrated using a HI test (Haemagglutination Inhibition) on blood samples involving homologous vaccine strains.

Table 2: Vaccination schedule and lab investigations

\begin{tabular}{|c|c|c|c|c|c|}
\hline T1: study day & DO & D21 & D28 & D78 & D106 \\
\hline Vaccination & $\mathrm{X}$ & $x$ & & & \\
\hline Blood sampling & $\mathrm{X}$ & & $X$ & & $x$ \\
\hline T2: study day & Do & $\mathrm{D} 21$ & $\mathrm{D} 28$ & D72 & D100 \\
\hline Vaccination & $x$ & $x$ & & & \\
\hline Blood sampling & $\mathrm{X}$ & & $\mathrm{X}$ & & $x$ \\
\hline T2: study day & Do & $\mathrm{D} 21$ & $\mathrm{D} 28$ & D35 & D64 \\
\hline Vaccination & $x$ & $x$ & & & \\
\hline Blood sampling & $x$ & & $x$ & & $x$ \\
\hline \multicolumn{6}{|c|}{$\mathrm{T1}: \mathrm{D} 0=$ from day 1 to day 38 of pregnancy } \\
\hline \multicolumn{6}{|c|}{$\mathrm{T} 2: \mathrm{D} 0=$ from day 39 to day 78 of pregnancy } \\
\hline \multicolumn{6}{|c|}{$\begin{array}{l}\text { T3: D0=from day } 76 \text { to day } 114 \text { of pregnancyStatistical analyses: Fisher's exact test was used to } \\
\text { compare the titres. Significance: } p<0.01 \text {. }\end{array}$} \\
\hline
\end{tabular}

\section{Results}

Mean antibody titres after vaccination versus control are shown in Figure 1: in the 1st third, only H1N2 titres are significantly different. They are all significantly different in the 2 nd third. And both $\mathrm{H} 1 \mathrm{~N} 2$ and $\mathrm{H} 3 \mathrm{~N} 2$ titres are significantly different in the 3rd third.

Figure 1 a,b,c: Mean Hl antibody titres against the different subtypes at different pregnancy stages in sows

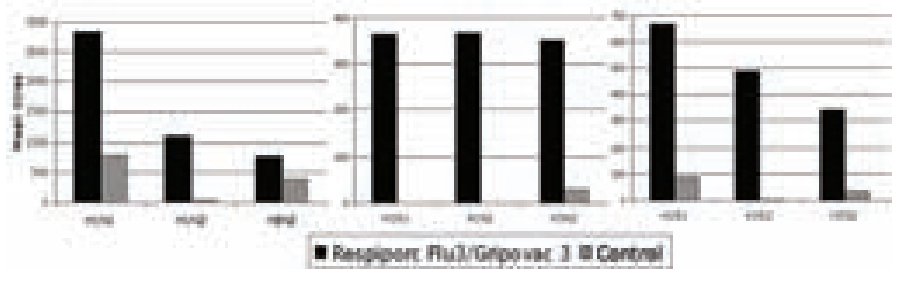

No difference was noticed between vaccinated and control sows in all groups for performance parameters such as:

- Live born piglets

- Still born piglets

- Weaned piglets

- Suckling piglets loss

\section{Conclusion}

These studies show this vaccine ability to induce a significant seroconversion in pregnant sows against the subtypes $\mathrm{H} 1 \mathrm{~N} 1$, $\mathrm{H} 3 \mathrm{~N} 2$ and $\mathrm{H} 1 \mathrm{~N} 2$ of swine influenza A virus.

\section{References}

1. Madec et al., (2004) Journées de la Recherche Porcine, 36, 353-358.

2. Van Reeth K et al., (2003) Influenza and other Respiratory Viruses DOI: 10.1111/j.1750-2659.2008.0043. ${ }^{\circledR}$ RESPIPORC is a registered trademark of IDT. ${ }^{\oplus}$ GRIPOVAC is a registered trademark of MERIAL S.A.S. 


\title{
P.273
}

\section{Safety of the administration of a new swine influenza vaccine: Respiporc $^{\circledast}$ Flu3/Gripovac ${ }^{\circledast} 3$ in sows during lactation}

\author{
Olaf Lüder ${ }^{1}$ Guntram Hagemann ${ }^{1}$ Michael Schlegel ${ }^{1}$ Hans-Joachim Selbitz ${ }^{1}$ Lothar Kreienbrock $^{2}$ Thaïs Vila $^{3} \underline{\text { Ralf Dürrwald }}^{1}$ \\ 1. IDT Biologika GmbH, Dessau-Rosslau, Germany; 2. Tierärztliche Hochschule Hannover, Hannover, Germany; 3. Merial S.A.S., Lyon, France
}

\section{Introduction}

The objective of this study was to evidence the safety of the vaccination with a new swine influenza vaccine RESPIPORC ${ }^{\circledast}$ FLU3/ GRIPOVAC ${ }^{\circledR} 3$ including $\mathrm{H} 1 \mathrm{~N} 1, \mathrm{H} 3 \mathrm{~N} 2$ and $\mathrm{H} 1 \mathrm{~N} 2$ in sows during the lactation.

\section{Material and Methods}

Sows: a total of 13 conventional lactating sows, 7 vaccinated and 6 control, were used in this trial.

Vaccination schedule: the 7 vaccinated sows were injected with a double dose of RESPIPORC FLU3/GRIPOVAC 3 just after farrowing (D0) and a single dose of vaccine 3 weeks after. The 6 control sows were injected with $\mathrm{NaCl} 0.9 \%$ at the same time of the vaccinated sows.

Temperature monitoring: in vaccinated and control sows the temperature was measured on D0, D2, D4, D14, D18, D20, D21, D22 and D23. In gilts it was measured on D0, D0+4h, D1, D2, D21+2h, D21+8h, D22, D24 and D28.

Clinical observation: adverse clinical signs were monitored every day throughout the study until Day 35. Safety criteria were lactation performance (the day of farrowing (D0) and the day of weaning (D35): number and weight of weaned piglets) rectal body temperature, food consumption, local reactions, i.e. skin colour, swelling, abscess, and systemic reactions i.e. behaviour, respiration, digestion, skin, coughing, and other possible signs.

Statistical analyses: permutation tests (for non-parametric Behrens Fisher problem) for equality were used in the study.

\section{Results}

Temperatures in vaccinated sows were not significantly different from temperature in control groups (Figure 1). No statistical difference was found between the 2 groups compared regarding neither reproductive parameters (Table 1) nor in the performance of their litter (Figure 2).

Neither local reactions nor systemic reactions were noticed at any time during the observation period.

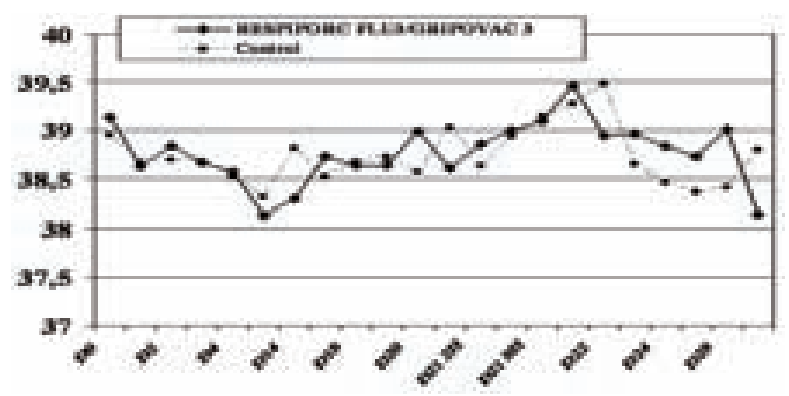

Figure 1: temperature curves of vaccinated sows versus control

Table 1: lactation performance in control and vaccinated sows

\begin{tabular}{|l|c|c|}
\hline Group & RESPIPORC FLU3/GRIPOVAC3 & Control \\
\hline Viable piglets per sow at farrowing & 11.3 & 11.8 \\
\hline Weaned piglets per sow D35 & 10.7 & 11.0 \\
\hline Losses per sow at weaning & 0.6 & 0.8 \\
\hline
\end{tabular}

Figure 2: weight gains of piglets born from vaccinated and control sows

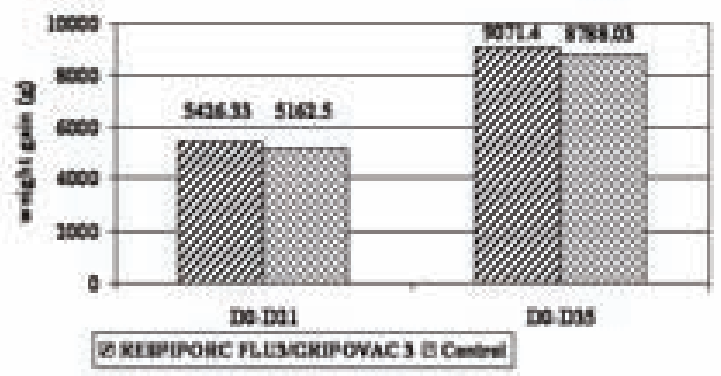

\section{Conclusion}

The administration of RESPIPORC FLU3/GRIPOVAC 3 in sows is safe during lactation. ${ }^{\circledR}$ RESPIPORC is a registered trademark of IDT.

${ }^{\circledR}$ GRIPOVAC is a registered trademark of MERIAL S.A.S. 


\title{
P.274
}

\section{Cross-protection of Respiporc ${ }^{\circledR}$ Flu3/Gripovac ${ }^{\circledR} 3$ against swine flu: challenge trials with two recent European H1N1 strains}

\author{
$\underline{\text { Ralf Dürrwald }}{ }^{1}$ Michael Schlegel ${ }^{1}$ Hans-Joachim Selbitz ${ }^{1}$ Thaïs Vila $^{2}$ \\ 1. IDT Biologika GmbH, Dessau-Rosslau, Germany; 2. Merial S.A.S., Lyon, France
}

\section{Introduction}

Swine influenza viruses of three different subtypes, H1N1, H3N2 and $\mathrm{H} 1 \mathrm{~N} 2$ are now co-circulating in European countries (1). The commercial vaccine based on $\mathrm{H} 1 \mathrm{~N} 1$ and H3N2 strains doesn't protect against $\mathrm{H} 1 \mathrm{~N} 2$ infection (2). A new swine influenza vaccine RESPIPORC ${ }^{\circledR}$ FLU3/ GRIPOVAC 3 adapted to European epidemiology contains $\mathrm{H} 1 \mathrm{~N} 1, \mathrm{H} 3 \mathrm{~N} 2$ and $\mathrm{H} 1 \mathrm{~N} 2$ recently isolated strains. The objective of this study was to evidence the crossprotection of this vaccine against recent $\mathrm{H} 1 \mathrm{~N} 1$ strains isolated in France.

\section{Material and Methods}

H1N1 strains from two different genotypes of swine influenza viruses isolated in Brittany (France) were used in this study: $A$ / sw/Cotes d'Armor/0190/06 (reassortant H1N1=RA) and A/sw/ Morbihan/0070/05 (avian-like H1N1=AL) (3). The antigenic character of these strains was investigated by cross-neutralisation with specific hyper immune sera of pigs (Kuntz-Simon et al., in preparation). They were kindly provided by G. Kuntz-Simon, AFSSA-LERAPP, F-Ploufragan. Pig vaccination: All 49 experimental pigs were seronegative against $\mathrm{H} 1 \mathrm{~N} 1, \mathrm{H} 1 \mathrm{~N} 2$ and $\mathrm{H} 3 \mathrm{~N} 2$ prior to vaccination. Half of the pigs $(\mathrm{N}=24)$ were vaccinated with $2 \mathrm{ml}$ RESPIPORC FLU3/GRIPOVAC 3, IM twice, 3 weeks apart. The other half was injected with a placebo.Challenge: One week after 2 nd administration of $2.0 \mathrm{ml}$ of the vaccine, an aerosol challenge validated in previous studies was conducted.Viral load in lung tissue homogenates was quantified by egg culture and expressed in number of egg infection dose $50 \%$ (EID50).

\section{Results and Discussion}

The challenge strain RA did not induce clinical signs of the disease (neither dyspnoea nor fever). Vaccinated pigs had significantly lower viral lung load than not vaccinated pigs both 1 and 3 days after challenge.

Figure 1: Mean viral load (EID50 in $10 \mathrm{mg}$ lung tissue) after aerosol challenge with strain $R A$

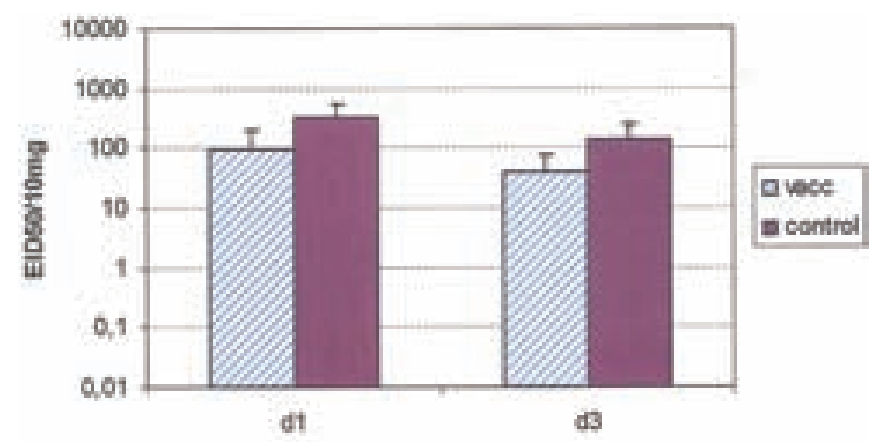

Day 1: $p=0.026$; Day 3: $p=0.021$ The challenge strain $A L$ did induce only mild clinical signs of disease (mild dyspnoea but no fever), consequently no difference was observed between treatment groups.

Vaccinated pigs had a highly significantly lower viral lung load than not vaccinated pigs (no virus in the lungs of the vaccinated pigs) both 1 and 3 days after challenge.

Figure 2: Mean viral load (EID50 in $10 \mathrm{mg}$ lung tissue) after aerosol challenge with strain $A L$

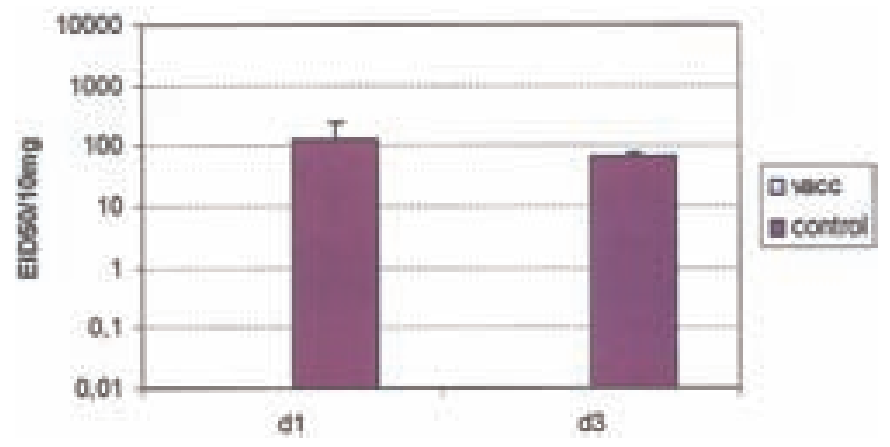

Day 1 and Day 3: $p=0.008$

\section{Conclusion}

These experiments with heterologous swine influenza viruses challenge clearly show the cross-protection of the vaccine strains of RESPIPORC FLU3/ GRIPOVAC 3 with recent H1N1 Swine Influenza A field strains from Europe.

\section{References}

1. Kuntz-Simon G. et al., (2009) Zoonoses and Public Health, 56, 310325

2. Van Reeth K. et al., (2003) Veterinary Record, 153,9-13

3. Franck N. et al., (2007) Proc. 5th Int. Symp. on Emerging and Reemerging Diseases in pigs, Krakow, Poland, p. $250^{\circ}$ RESPIPORC is a registered trademark of IDT. GRIPOVAC is a registered trademark of MERIAL S.A.S. 


\title{
P.275
}

\section{Cross-protection of Respiporc ${ }^{\circledR}$ Flu3/Gripovac ${ }^{\circledR} 33$ against swine flu: challenge trials with two recent European H1N2 strains}

\author{
Ralf Dürrwald ${ }^{1}$ Michael Schlegel ${ }^{1}$ Hans-Joachim Selbitz ${ }^{1}$ Thaïs Vila $^{2}$ \\ 1. IDT Biologika GmbH, Dessau-Rosslau, Germany; 2. Merial S.A.S., Lyon, France
}

\section{Introduction}

Swine influenza viruses of three different subtypes, H1N1, H3N2 and $\mathrm{H} 1 \mathrm{~N} 2$ are now co-circulating in European countries (1). The commercial vaccine based on H1N1 and H3N2 strains doesn't protect against $\mathrm{H} 1 \mathrm{~N} 2$ infection (2). A new swine influenza vaccine RESPIPORC ${ }^{\circledR}$ FLU3/ GRIPOVAC ${ }^{\circledR} 3$ adapted to European epidemiology contains $\mathrm{H} 1 \mathrm{~N} 1, \mathrm{H} 3 \mathrm{~N} 2$ and $\mathrm{H} 1 \mathrm{~N} 2$ recently isolated strains.

The objective of this study was to evidence the cross-protection of the vaccine against two H1N2 French strains.

\section{Material and Methods}

Two strains of the H1N2 subtype isolated in Brittany were used in this study: A/sw/Cotes d'Armor/0214/06 (genotype $1 \mathrm{H} 1 \mathrm{N2}=\mathrm{G} 1$ ) \& A/sw/Cotes d'Armor/0113/06 (genotype 2 $H 1 N 2=G 2)(3)$. These strains are heterologous from the vaccine strain and belong antigenetically to the human-like H1N2 subtype as confirmed by cross-neutralisation (Kuntz-Simon et al., in preparation). They were kindly provided by G. Kuntz-Simon, AFSSA-LERAPP, F-Ploufragan.Vaccination and challenge G1: All pigs $(\mathrm{N}=28)$ were seronegative against $\mathrm{H} 1 \mathrm{~N} 1, \mathrm{H} 1 \mathrm{~N} 2$ and $\mathrm{H} 3 \mathrm{~N} 2$ prior to vaccination. Half of the pigs were vaccinated twice 3 weeks apart with $2 \mathrm{ml}$ of RESPIPORC FLU3/GRIPOVAC 3. One week after 2 nd administration of the vaccine, an aerosol challenge was performed.Vaccination and challenge G2: All pigs $(\mathrm{N}=20)$ were seronegative against $\mathrm{H} 1 \mathrm{~N} 1, \mathrm{H} 1 \mathrm{~N} 2$ and $\mathrm{H} 3 \mathrm{~N} 2$ prior to vaccination. Half of the pigs were vaccinated with $2 \mathrm{ml}$ of RESPIPORC FLU3/GRIPOVAC 3. One week after 2nd administration of the vaccine, an aerosol challenge was performed.Viral load in lung tissue homogenates was quantified 1 and 3 days ( 11 and d3) after challenge by egg culture and expressed in number of egg infection dose 50\% (EID50).

\section{Results}

The challenge with strains $G 1$ and $G 2$ induced typical clinical signs of influenza, i.e. dyspnoea and fever in control pigs. Vaccinated pigs were protected against fever and other clinical signs (data not shown). Vaccinated pigs had very significantly lower viral lung load than non-vaccinated pigs (Figures 1 and 2).
Figure 1: Mean viral load (EID50 in $10 \mathrm{mg}$ lung tissue) after aerosol challenge with strain G1 H1N2 (d1 and d3: $p=0.001$ )

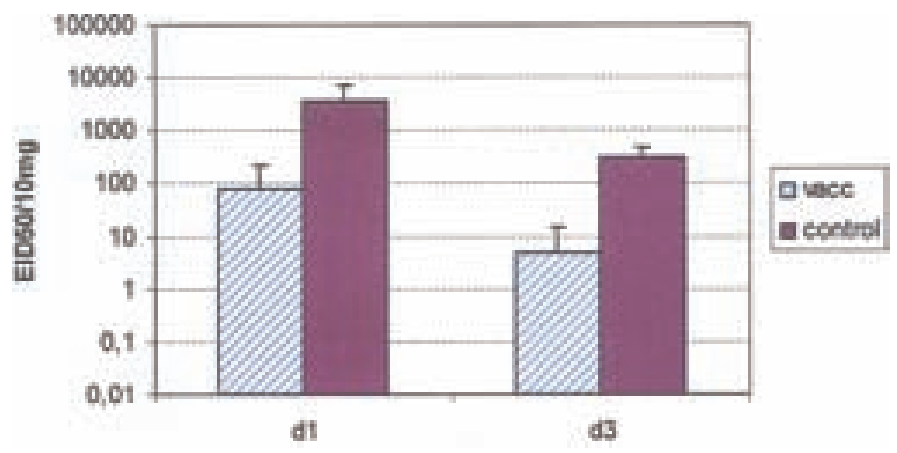

Figure 2: Mean viral load (EID50 in $10 \mathrm{mg}$ lung tissue) after aerosol challenge with strain G2 H1N2 (d1: $p=0.016 ; d 3: p=0.032$ )

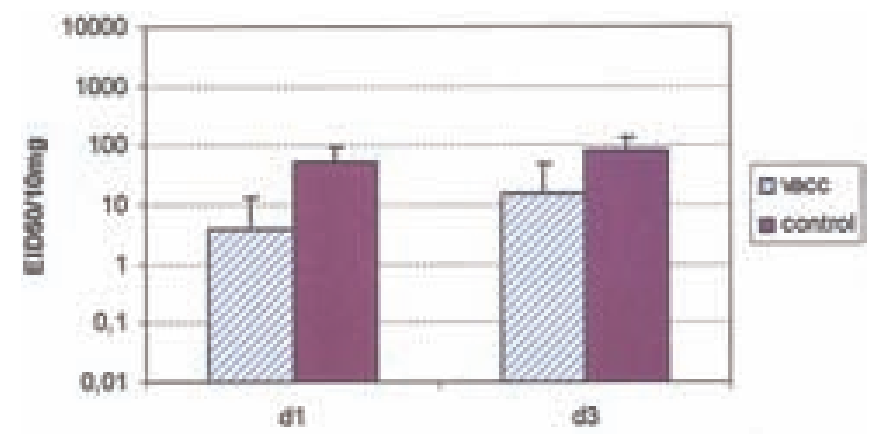

\section{Conclusion}

Clinical and viral cross-protection of pigs vaccinated with RESPIPORC FLU3/ GRIPOVAC 3 with recent Swine Influenza A Virus $\mathrm{H} 1 \mathrm{~N} 2$ strains in Europe has been confirmed in these challenge studies.

\section{References}

1. Kuntz-Simon G. et al., (2009) Zoonoses and Public Health, 56, 310325

2. Van Reeth K. et al., (2003) Veterinary Record, 153, 9-13

3. Franck N., Quéguiner S., Gorin S., Eveno E., Fablet C., Madec F. and Kuntz-Simon G., (2007) Proceedings of the 5th International Symposium on Emerging and Re-emerging Diseases in Pigs, Krakow, Poland, p. $250^{\circ}$ RESPIPORC is a registered trademark of IDT. GRIPOVAC is a registered trademark of Merial S.A.S. 


\title{
P.276
}

\section{Protective immune responses following vaccination of a consensus H1N1 Swine influenza DNA vaccine using electroporation in pigs}

\author{
Douglas R. Kern ${ }^{1}$ Douglas R. Kern ${ }^{1}$ Amir S. Khan² \\ 1. VGX Animal Health, The Woodlands, TX, USA; 2. VGX Pharmaceuticals Inc, The Woodlands, TX, USA
}

Over the last two decades, DNA vaccines have become a potent means of providing immune responses or protective immunity against viruses, bacteria and parasites. DNA vaccines comprised of plasmid DNA encoding proteins from pathogens, allergens, and tumors, are being evaluated as prophylactic vaccines and therapeutic treatments for infectious diseases, allergies and cancer. The use of DNA as a means of vaccination offers advantage in protective efficacy, cross-strain applicability, development speed with lower manufacturing cost over conventional vaccines. DNA vaccines are known to be particularly effective in inducing antibody responses which are critical to fighting infections.

Recently, the USDA reported there was limited cross-reactivity against the new $2009 \mathrm{~A} / \mathrm{H} 1 \mathrm{~N} 1$ virus in pig herds vaccinated with existing swine influenza vaccines.

This presentation describes a study in which pigs were immunized with consensus H1N1 influenza DNA vaccine candidate on day 0 , day 14 , and day 28 . Sera collected on day 28 and day 42 , after two or three doses of vaccine, respectively, showed hemagglutination inhibition $(\mathrm{HI})$ titers above the protection threshold in $100 \%$ of the vaccinated animals against a swine $\mathrm{H} 1 \mathrm{~N} 1$ virus previously isolated from pigs (Swine 1999). In addition the immune sera were tested for responses against a virus isolated from the current circulating strain of swine origin influenza A/ H1N1 (Swine A/Mexico/InDRE4487/2009). Four of the six pigs immunized with the $\mathrm{H} 1 \mathrm{~N} 1$ vaccine developed $\mathrm{HI}$ titers exceeding the 1:40 level commonly associated with protective immunity (see Table 1 ).

These findings have been verified in other animal species. In a previous study, we demonstrated that mice immunized with SynCon ${ }^{\mathrm{TM}} \mathrm{H} 1 \mathrm{~N} 1$ DNA vaccine provided $100 \%$ protection in a lethal challenge study against an unmatched $\mathrm{H} 1 \mathrm{~N} 1$ virus that caused the 1918 Spanish flu, which killed over 40 million people worldwide. In addition, ferrets immunized with similar SynCon ${ }^{\mathrm{TM}}$ universal flu vaccine combinations were $100 \%$ protected against death and sickness in a challenge with the A/H1N1 Mexico/ InDRE/4487/2009 pandemic swine-origin influenza.
VGX Animal Health's novel SynCon ${ }^{\mathrm{TM}}$ technology enables the design of DNA-based vaccines with the potential to protect against unmatched sub-types and strains of pathogens. Using a designer approach, VGX Animal Health can formulate these DNA plasmids together to rapidly develop a universal influenza vaccine potentially targeting all important strains. The resulting vaccine could target seasonal as well as pandemic-potential influenza strains such as avian influenza and swine flu, which has already been designated pandemic status. Significantly, being based on a common set of antigens derived from a broad range of flu strains, such a universal vaccine would have the potential to provide greater protection against evolving, unmatched flu strains in multiple species.

Table 1 HI Titers to Swine 1999 and Mexico '09 (MX09)

\begin{tabular}{|l|c|c|c|c|c|}
\hline Animal \# & Treated & $\begin{array}{c}\text { HI (Swine } \\
\text { 1999) wk 10 }\end{array}$ & $\begin{array}{c}\text { HI (MX09) } \\
\text { wk 6 }\end{array}$ & $\begin{array}{c}\text { HI (MX09) } \\
\text { wk 10 }\end{array}$ & $\begin{array}{c}\text { HI (MX09) } \\
\text { wk 14 }\end{array}$ \\
\hline 107 & Yes & 160 & $<20$ & $<20$ & $<20$ \\
\hline 119 & Yes & 160 & 20 & 40 & 40 \\
\hline 124 & Yes & 80 & $<20$ & $<20$ & $<20$ \\
\hline 131 & Yes & 160 & 40 & 80 & 80 \\
\hline 140 & Yes & 160 & 20 & 40 & 40 \\
\hline 158 & Yes & 320 & 20 & 40 & 40 \\
\hline 116 & No & 10 & $<20$ & $<20$ & $<20$ \\
\hline 114 & No & 10 & $<20$ & $<20$ & $<20$ \\
\hline 137 & No & 10 & $<20$ & $<20$ & $<20$ \\
\hline
\end{tabular}

HI titers against Swine 1999 and Mexico 2009 for pigs immunized with Swine H1N1 concensus DNA vaccine, Day 28 - 2 weeks post 2nd immunization; Day 56 - 2 weeks post 3rd immunization and Day 70 - 4 weeks post 3rd immunization 


\title{
Pandemic (H1N1) 2009 influenza a infection in pigs in Northern Ireland
}

\author{
Jesus Borobia Belsué \\ MOSSVET, Portadown, UK
}

\section{Introduction}

In October 2009 a 120 sow unit experienced an increase in abdominal breathing, coughing and inappetance in the breeding herd and sucking piglets. The breeding herd and weaners are 05 miles away from the growing/finishing accommodation.

Clinical signs started 4 days before vet visit.

A week earlier, 6 year old farmer's daughter had a "cold". All family members -except the farmer-contracted this illness.

Changeable weather conditions in the area two weeks earlier.

\section{Materials and Methods}

Clinical investigation consisted in a farm visit (27 Oct 2009). Rectal temperature was taken from affected animals. Two dead 4 week old piglets from the same litter were post-mortemed and their lungs submitted to the laboratory (AFBI, Belfast, Northern Ireland) with 5 clotted blood samples of affected sows.

Two more farm visits were carried out ( 3 \& 10 Nov 2009). More clotted blood samples were collected.

\section{Results}

During the first farm visit, 5 lactating sows, 7 dry sows, 2 sows in service room and one boar had abdominal breathing, barking cough, inappetance and prostration. Piglets of affected lactating sows had abdominal breathing but good appetite. Five 4 week old piglets of a litter of 11 died. No fertility problems detected.

Rectal temperature ranged from 40 to $41.5^{\circ} \mathrm{C}$.

Growing/finishing accommodation did not have clinical signs.

Post-mortem examination of the lungs detected $30-40 \%$ lung consolidation of cranioventral lobes with interstitial pneumonia. Bronchiomediastinal lymph nodes were congested.

Histological changes of the bronchiole showed desquamation and necrosis of the respiratory epithelium, along with marked lymphocytic infiltration of the lamina propia and submucosa. Furthermore, there was perivascular lymphocyte infiltration in the lung.

Virology came back positive to FluMatrix RTPCR.

Serology came back negative to Aujeszky, PRRS and H1N1 Belg/79. However, one sow was positive to PRCV and all were positive to H1N1 Eng/92. Serological progression is shown in Table 1.
Table 1. Serological results for H1N1 Eng/92 from clotted bloods

\begin{tabular}{|c|c|c|c|}
\hline ANIMAL ID & $\mathbf{2 7} \mathbf{0 c t} \mathbf{2 0 0 9}$ & $\mathbf{3}$ Nov $\mathbf{2 0 0 9}$ & $\mathbf{1 0}$ Nov 2009 \\
\hline 373 & $1 / 8$ & $1 / 256$ & $1 / 128$ \\
\hline 375 & $1 / 16$ & $1 / 64$ & $1 / 128$ \\
\hline 377 & $1 / 32$ & $1 / 64$ & NT \\
\hline 355 & $\mathrm{IS}$ & $1 / 16$ & $1 / 32$ \\
\hline 348 & $1 / 16$ & $1 / 256$ & $1 / 128$ \\
\hline 343 & $\mathrm{NT}$ & $1 / 16$ & $1 / 64$ \\
\hline \multirow{3}{*}{ IS= Insufficient Sample NT= Not Tested } \\
\end{tabular}

\section{Discussion}

SIV (Swine Influenza Virus) is an accute, infectious, respiratory disease caused by influenza A viruses. It causes a sudden onset, coughing, dyspnea, fever, prostration, followed by a rapid recovery.

Northern Ireland has a high intensity of Pandemic (H1N1) 2009 in human population (ECDPC, 2009).

Weather conditions were favorable for the spread of the virus during that period of time.

Likely humans infected pigs and/or airborne in this case. Mortality was low. Taylor (1995) \& Easterday et al. (1999) reported $<1 \%$ mortality unless intercurrent infections and/or pigs very young.

PRCV did not appear to exacerbate infection experimentally (Lanza et al., 1992; Taylor, 1995).

SIV morbidity is reported near $100 \%$ (Taylor, 1995; Easterday et al., 1999). It was, however, $60 \%$ in this case. This could be related to the pathogenicity of the virus and/or prompt treatment by nursing care of warm environment, minimising stress and medicating the sow's diet.

No reproductive, farrowing and neonatal problems were reported in this case, contrary to Taylor, 1995; Muirhead et al., 1997; Easterday et al., 1999 and Jackson et al. 2007).

Viral isolation and/or specific antibodies detection is necessary for diagnosis (Easterday et al., 1999).

Serum samples were positive to SI/A/Swine/England/92(H1N1) with rising titres the following weeks. Viruses with the same $\mathrm{H}$ (haemagglutinin) cross react with antibodies to related strains (Brown, 2002).

PCR in lung confirmed Pandemic (H1N1) 2009.

Bacteriology was not performed because the laboratory had issues about hazards to staff.

SIV is not a Notifiable Disease (WHO, GB and rest of EU). However, Influenza A viruses are Notifiable Disease in Northern Ireland (The Disease of Animals (Northern Ireland) Order 1981). Some authorities can over-react in this situation and there is some concern of the possible consequences on pig trade and consumption since first signs in humans appeared in Mexico (April 2009). 


\title{
P.278
}

\section{Outbreak of 2009 pandemic influenza A H1N1 in Korean pigs}

\author{
Seonghee Kim; C-I Yeo; C-W Bae; Y-K Song; K-K Lee; O-S Lee; Choi-Kyu Park \\ National Veterinary Research \& Quarantine Service, Anyangcity, Korea
}

\section{Introduction}

Pigs could play the role of intermediate host as a "mixing vessel" which could give rise to an outbreak of new variants through the process of genetic reassortment event because their tracheal cells contain both human and avian influenza virus receptors. That is why continuous monitoring of influenza virus in pigs is significant in terms of public health. To this end, National Veterinary Research \& Quarantine Service (NVRQS) in South Korea, starting the 2 nd quarter of 2009 , launched a nationwide disease prevention program and embarked on monitoring of $\mathrm{pH} 1 \mathrm{~N} 1$ in pig farms throughout the nation. In the case of commercial pig farms, 708 farms, 1,057 farms and 1,060 farms were monitored in the 2nd, 3rd and 4th quarter of 2009, respectively. Meanwhile, with respect to pig breeding farms, 198 farms were monitored in the 4th quarter of the year, since influenza viruses typically have high prevalence during winter season.

\section{Materials and Methods}

For test sample, seventeen nasal swab samples per one pig farm were collected from the pigs showing respiratory symptom among weaners, growers and finishers. The samples were inoculated into each embryonated chicken egg and MadinDarby canine kidney (MDCK) cells. Total RNA was extracted, using RNeasy ${ }^{\circledR}$ Mini Kit (Qiagen), from the samples whose hemagglutination titers were higher than 2 times. Identification of influenza virus among the extracted total RNA was run on a Roche LightCycler 2.0 using artus $^{\circledR}$ Infl./H1 LC/RG RT-PCR kit (Qiagen). Final confirmation of the $\mathrm{pH} 1 \mathrm{~N} 1$ virus were done through sequence analysis of hemagglutine (HA), neuraminidase (NA) and matrix $(\mathrm{M})$ region using the universal primers.

\section{Results}

As a result, while the $\mathrm{pH} 1 \mathrm{~N} 1$ virus was not found at the tests conducted during the 2 nd and 3rd quarters of 2009, it was detected from 17 farms during the period from the 4th week of November to December of the year. While most of pH1N1-positive farms were tested negative at the tests conducted a week after notification of the virus infection to them, one pig-breeding farm still remained positive to the virus. After a week of suspension, additional antigen test was conducted for the pig-breeding farm. As the test did not detect any antigen, the farm was freed from the movement restriction. This fact indicates that, in the case of large-scale pig-breeding farms where biosecurity is relatively well managed at each breeding stage, the transmission speed of influenza virus is relatively slow, causing a continuous circulating virus infection to antibody-negative pigs.

The phylogenetic analyses on $\mathrm{HA}$ and NA gene of pH1N1 isolated in South Korea found that they were similar to the $\mathrm{pH} 1 \mathrm{~N} 1$ which is currently prevalent among humans. With the existing $\mathrm{pH} 1 \mathrm{~N} 1$ virus isolated from pigs in Canada, $\mathrm{HA}$ and NA nucleotide homology of $98.3-99 \%$ and $98.5-99.8 \%$ were observed, respectively, with Korean isolates. Furthermore, HA and NA genes of Korean isolates showed homology of $98.7-100 \%$ and $98.5-100 \%$, respectively, among themselves.

\section{Discussion}

Movement restrictions had been imposed on pH1N1-confirmed pig farms for three weeks, during which antigen tests were conducted at the interval of one week and the restrictions were lifted for the farms that had been tested negative. For the pigs that were to be shipped out to market, the shipment, even during the restriction period, was allowed to each individual pig, if tested negative for the $\mathrm{pH} 1 \mathrm{~N} 1$ virus.

All things taken together, it is highly likely that the outbreak of $\mathrm{pH} 1 \mathrm{~N} 1$ in South Korea was caused by the transmission from infected humans to pigs as with other countries, given following factors: first, the outbreak of pH1N1 in pigs was reported after the first week of November 2009 when the rate of Influenza-like Illness of Koreans was at its peak of $50 \%$. Second, any epidemical relevance indicating that the $\mathrm{pH} 1 \mathrm{~N} 1$ was transmitted by the movement of pigs of infected pig farms was not found. Even though an analysis on the full genome sequencing data of the $\mathrm{pH} 1 \mathrm{~N} 1$ isolated in Korea assures that so far there has not been any outbreak of new variant through genetic reassortment (data not shown), we can not rule out the possibility of emergence of those variants in the near future. Thus, continuous surveillance on the pig farms has been conducted to detect the emergence of any variants in South Korea, and the use of vaccine for pig against the $\mathrm{pH} 1 \mathrm{~N} 1$ which is at its final stage of development is being carefully considered.

\section{References}

BROWN, I.H. (2001) Int. Congr. Ser. 1219, 173-178

GARTEN, R.J. et al. (2009) Science 325, 197-201

HOFFMANN, E. et al. (2001) Arch. Virol. 146, 227 


\title{
P.279
}

\section{Pandemic influenza virus (A/ H1N1) outbreak in pig farm in north of Italy}

\author{
Loris G. Alborali; Matteo Gradassi; Mariagrazia Zanoni; Anna Moreno Martin; Anna \\ Catelli; Cristian Salogni; Enrica Sozzi; Emanuela Foni; Paolo Cordioli
}

\author{
IZSLER, Brescia, Italy
}

\section{Introduction and Objectives}

The swine influenza virus (SIV) frequently causes acute respiratory disease in pigs worldwide and is an important pathogen in the porcine respiratory disease complex (PRDC). SIV is one of the "door opener" and frequently is associated with viral (PRRSV,PCV2), bacterial respiratory pathogens and M.hyopneumoniae.(1). H1N1, H3N2 and H1N2 are the most common influenza subtypes isolated in Italian pig farms (2) In 2009 a novel (H1N1)v influenza A virus caused annual worldwide epidemics. In Italy, in order to examine the emergence of the new pandemic virus and confirm the recent reports about human to animal transmission $(3,4)$, all swine type A influenza positive samples were tested for $(\mathrm{H} 1 \mathrm{~N} 1) \mathrm{v}$. The first $\mathrm{H} 1 \mathrm{~N} 1$ pandemic outbreak in sow farm in Italy is described in this paper.

\section{Material and methods}

On November 2009, in a Northern Italy farm clinical signs in sows and piglets were observed in farrowing units. The farm was a 1250 sows site with outside growing gilts- and weaning-units. Weekly clinical investigations were reported and performance data were collected to evaluate and quantify losses. Nine dead piglets and four nasal swabs from sows were submitted to the diagnostic laboratory. Lung homogenates were tested. Clinical specimens were screened for the presence of influenza A viruses by real time RT-PCR (6). Positive samples were further tested for the $(\mathrm{H} 1 \mathrm{~N} 1) v$ by real time RT-PCR (rtRT-PCR), according to the CDC procedure (7). All samples were also cultured for the most common bacterial swine respiratory pathogens and tested for PRRSV, PCV2 and M. hyopneumoniae by using RT-PCR, multiplex PCR and PCR assays respectively $(8,9,10)$.

Sera were collected in sows and piglets both when clinical signs were observed and 28 days P.I.. Specimens were examined by NPA-ELISA and HI tests swine antigen A/H1N1,A/H3N2,A/ H1N2 and pandemic A/H1N1.

\section{Results}

Figure 1: Total born, born alive and weaned piglets in 2008, 2009, November - January 2008/09 and 2009/10

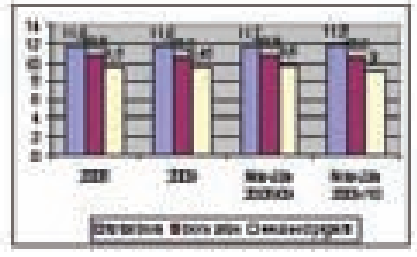

Figure 2: Sow mortality and abortions \% in 2008, 2009, November - January 2008/09 and 2009/10

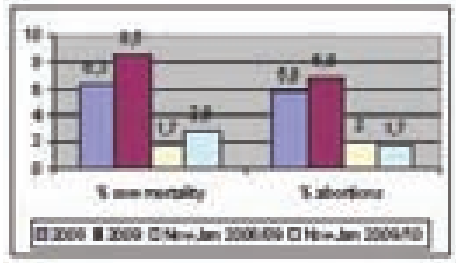

Sows presented fever, depression, anorexia and agalactia, while 5-10 days piglets diarrhoea and weight loss. The morbidity in sows was approximately $30-50 \%$ and the performance sow and piglets data are reported in Figure 1 and 2.

Gross lesions were catarrhal enteritis in 8 piglets and pneumonia with a purple area of consolidation in the apical lobes in one piglet. The latter and one of the two pools of 4 piglets were positive for type A influenza (H1N1)v assay. Only PRRSV European strain was detected from the first pool, whereas other samples resulted negative for the tested pathogens.

Sow sera collected at 28 days P.I. were positive by NPA-ELISA and $\mathrm{HI}$ tests pandemic $\mathrm{A} / \mathrm{H} 1 \mathrm{~N} 1$.

\section{Discussion}

The main clinical signs were depression and agalactia in sows during the first week after farrowing. Different weights were observed in litters and piglets showed wasting and dehydration. The loss interested litters and were quantified in increased mortality in farrowing units, performance reduction in sows consisting in enhanced mortality and fertility rate reduction.

\section{References}

1) Gradassi M. Et al 2009 Atti SIPAS 2009 - XXXV 229-241.

2) Luppi A. Et al..2007 Atti SIPAS , XXXIII, 231-242.

3) Centers for Disease Control and Prevention. Swine influenza $A$ (H1N1) 2009. MMWR. 2009; 58:400-2

4) Hofshagen M. Et al. 2009. Euro Surveill. 14(45):pii 19406.

5) OIE World Animal Health Information Spackman E. Et al.2002 J Clin Microbiol 40(9):3256-60.

6) Centers for Disease Control and Prevention, CDC 2009). Calsamiglia M et al 1999 J Vet Diagn Invest.11: 246-251.

7) Ouardani M. Et al $1999 \mathrm{~J}$ Clin Microbiol. 37(12): 3917-3924.

8) Suárez P.et al.1994 Arch Virol 135: 89-99. 


\title{
P.280
}

\section{Pigmeat price dynamics: effects of Influenza A H1N1 in Mexico?}

\author{
Ernesto Bobadilla ${ }^{1}$ Angelica Espinoza ${ }^{1}$ Maria Trujillo ${ }^{2}$ Giovanna Penuelas ${ }^{1}$ Francisco E. Martinez Castañeda $^{1}$ \\ 1. Universidad Autonoma del Estado de Mexico, Toluca, MEX, Mexico; 2. Universidad Nacional Autonoma de Mexico, Mexico, DF, Mexico
}

\section{Introduction}

Several factors can modify price fluctuations in different magnitude and time. Mexico as all world, experimented a terrible human disease in middle 2009. At the beginning of the disease it was named swine disease, hog fever, and other names that contributed someway to increase fear for these products. Price fluctuations are a discipline that combines historical dynamics and prospective analysis. The aim of these study was to evaluate 2009 pigmeat price fluctuations and identify the effect of $A$ H1N1 Influenza disease.

\section{Materials and Methods}

2009 daily pigmeat, hog prices and slaughter were analyzed. Data were collected from Secretaria de Economía. A multiplicative components model with a component separation was used. $Y=T^{*} S^{*} C^{*}$ l, where $Y=$ variable; $T=T e n d e n c y ; S=S e a s o n a l i t y$; $\mathrm{C}=$ Fluctuations and; I irregular movements.

\section{Results}

Both live hog and pigmeat price showed fluctuations around $10 \%$ in one year, percentage that destabilized the sector. Live price fluctuation was $12.49 \%$ and began a negative dynamic from April 20th to May 31. Pigmeat fluctuation was $11.87 \%$ all year but from April 12th to June 10th the variation was $10.70 \%$ (Fig 1). Figure 2 showed 2 different negatives tendencies, the first one from March 31 to April 9th and the second from April 17 th to June $3 r d$.

\section{Discussion}

Price fluctuation more than $10 \%$, can provoked wrong signals both consumer and producer at different time. When a Pandemic A H1N1 Influenza occurred, live hog price dropped and fluctuated more than $10 \%$, and it happens in a short period of time, 41 days. Mexican Pigmeat cycle is 42 month long (Martínez et al, 2009). That means, the sector needs at least 21 months in order to recover the bad fluctuation or vise versa. The effects that were observed during the Pandemic correspond with the negative live price fluctuation. Some way it had affected Mexican hog production but it surly will have effect when the market it selves recovered or adjust the fluctuation regular movements. Unfortunately, this market arrangements represents the lost of many producers, and the federal policies implemented weren't made to protect or stimulated pigmeat and Mexican hog sector.

Pigmeat fluctuation ran with the same tendency, but the time registered wasn't 41 days like live price, it was 10 days more, i.e. 51 days long, almost 2 months. Once price bottom it never recovered, the price average maintained below the no fluctuation value, i.e. CERO.
Figure 1. Live hog and Pigmeat price fluctuations in Mexico. 2009.

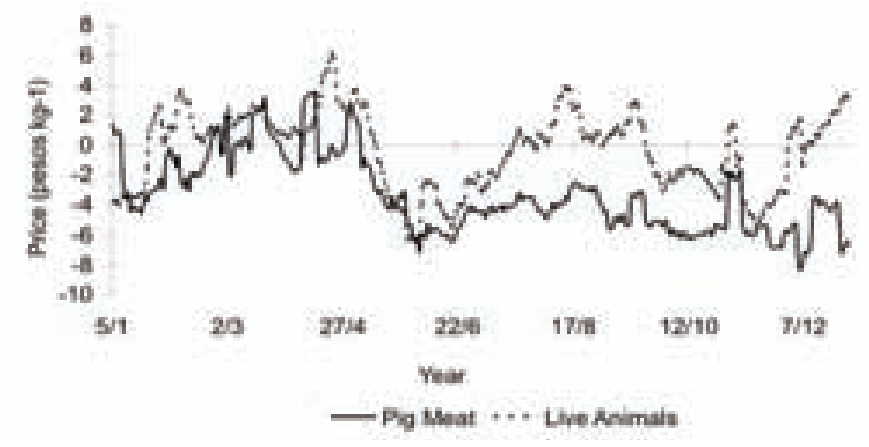

Slaughter registered two negatives periods, the first one is "regular" performance, because it coincidence with the Traditional Mexican "Seaman Santa" (Eastern break).

Figure 2. Slaughter variations in Mexico. 2009.

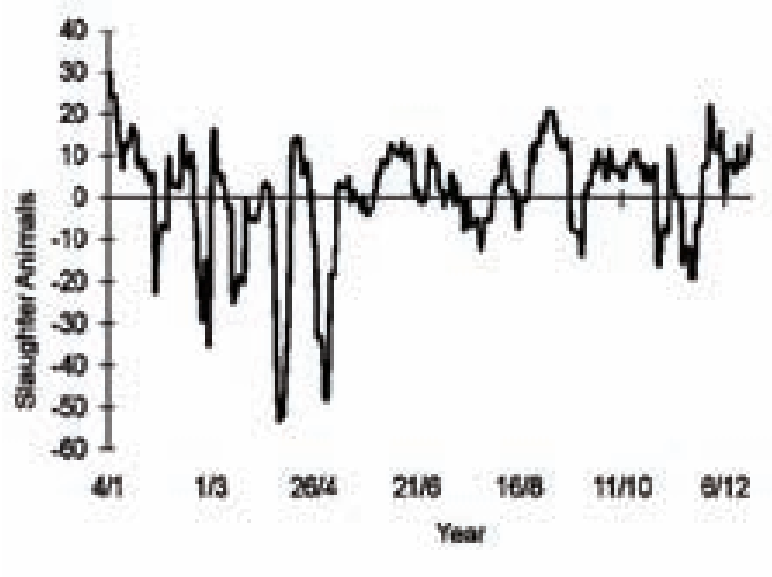

\section{References}

Martínez et al., 2009. AMVEC Proceedings. 


\title{
P.281
}

\section{Lung Histopathology from Naturally Infected Pigs with Pandemic H1N1 2009}

\author{
Javier A. Cappuccio ${ }^{1}$ Ariel Pereda ${ }^{2}$ María A. Quiroga ${ }^{1}$ Mariana Machuca María I. Craig ${ }^{2}$ Agustina Rimondi $^{2}$ Lucas Insarralde $^{1}$ \\ Santiago Capalvo ${ }^{3}$ Adriana Risso ${ }^{4}$ Sergio Samus ${ }^{5}$ Marcela Testa ${ }^{5}$ Ramón Sanguinetti ${ }^{6}$ Carlos J. Perfumo ${ }^{1}$ \\ 1. Cátedra de Patología Especial, Facultad de Ciencias Veterinarias, Universidad Nacional de La Plata, La Plata, Argentina; \\ 2. Instituto de Virología, CICVyA. INTA Castelar, Castelar, Argentina; 3. Bioter S.A, Pilar, Argentina; \\ 4. Private practitioner, Venado Tuerto, Argentina; 5. LADIPREVET, La Plata, Argentina; 6. SENASA, Buenos Aires, Argentina
}

\section{Introduction}

Swine influenza virus (SIV) is a primary pathogen of the respiratory tract of pig (1). The target cells of SIV are the epithelia lining the entire respiratory tract. However, a major pathological significance are those located in the small airways (2). Young pigs are fully susceptible to the swine origin pandemic $\mathrm{H} 1 \mathrm{~N} 1$ 2009 influenza A as it was reported in several countries including Argentina. Experimental infections with this novel virus induced mild clinical signs, however, virus shedding and lung lesions were similar to infection with SIV (3). The present work was aimed to review the histopathology (HP) and immunohistochemistry (IHC) features of lung lesions of naturally infected pigs with the pandemic H1N1 2009 influenza A.

\section{Materials and Methods}

A total of 21 slide lung samples belonged from 9 naturally infected pigs were examined. Cases were selected by gross lesions and rRT-PCR positive. For each, HP, and IHC studies were done. Morphometric study of changes at level of bronchi, bronchiole, alveoli, blood vessels and connective tissue was performed according with Richt et al (2). For statistical analysis, ANOVA test were used and, post hoc Fisher's LSD analysis was applied when differences were detected.

\section{Results}

The histopathological hallmark was a severe necrotizing bronchiolitis. Small and medium sized bronchioles were plugged with neutrophils, necrotic epithelial cell and mucous exudates. Affected airways were denuded or lined by flat epithelium. In few of them focal areas of metaplasia were seen. Bronchi and large bronchiole showed only minor changes. Alveolar inflammation was distributed in a lobulillar or lobar fashion. In them, two coexisting patterns of response were observed in the same sample. The first, infiltrative non-exudative reaction with the presence of neutrophils and few macrophages within the alveoli or in the alveolar wall. The second was serofibrinous exudates into the alveoli with inflammatory cells such as macrophages and neutrophils, hyaline membranes, capillary thrombosis and in few cases, focal necrosis. In 2 cases, lesions resembled infection by A. pleuropneumoniae. The ANOVA analysis showed significant statistical differences among structure affected $(p=0.0000)$. Bronchiolar lesions were statistically higher than others structures $(p<0.05)$, followed by those seen in the alveoli and bronchi. Marked IHC labelling was observed in the severely denuded epithelial cells of bronchioles, pneumocytes and alveolar macrophages of affected or normal alveoli.

\section{Discussion}

The spectrum of pathological lesions in pigs appears to vary little among SIV subtypes (4). Epithelial cells necrosis of bronchioles, airway plugging with desquamate cells, neutrophils infiltration and mucus are the features of SIV infection (1,2). Changes such as hyaline membranes, capillary thrombosis and alveolar necrosis observed in the study are not commonly reported in SIV infection as has been in human influenza (5). These differences could be associated with the lack of immune response against this "new" subtype. Neutrophils were the common inflammatory cells seen in the airways and alveoli. It may be the results of high level of cytokines secreted by the infected bronchiolar epithelial cells and macrophages which in turn is related with the virus load on these cells (4). In the examined samples, the higher bronchiolar scores were correlated with the more marked immunolabeling. However, secondary bacterial infection can not be excluded. More extensive studies are needed to evaluate the consistence of the above described lesions of the pandemic H1N1 2009.

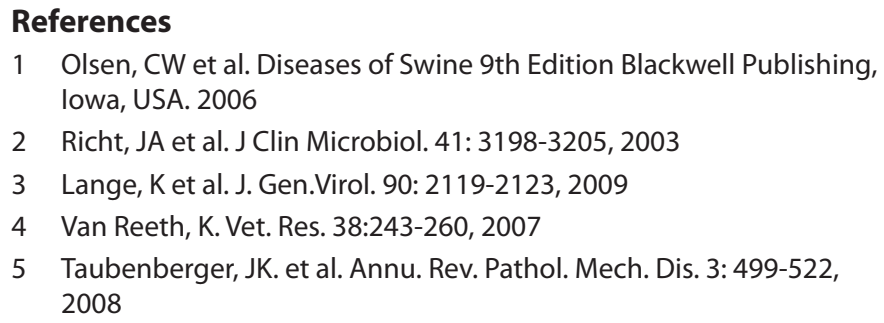

This work was supported by grants from ANPCyT OC/AR PICT 2005-33987 and P.E. AESA1573 INTA, NIAID-NIH Contract No.HHSN266200700010C 


\title{
P.282
}

\section{Lung pathology in slaughtered pigs from Norwegian herds naturally infected with pandemic influenza A (H1N1) 2009 virus}

\author{
Mette Valheim $^{1}$ Hans Gamlem $^{1}$ Britt Gjerset ${ }^{1}$ Lars Erik Larsen$^{2}$ Bjørn Lium ${ }^{1}$ \\ 1. National Veterinary Institute, Oslo, Norway; 2. National Veterinary Institute, Technical Institute of Denmark, Copenhagen, Denmark
}

\section{Introduction}

The Norwegian swine population has been considered free from influenza A virus infection documented trough a serological surveillance program running since 1997 (1). The swine population is also documented free from PRRS-virus and Mycoplasma hyopneumonia $(1,2)$. The first human cases of the pandemic influenza A (H1N1) 2009 virus infection in Norway were detected in early May 2009. The first porcine case was detected in October, and human to pig transmission was suspected (3). This paper describes the lung lesions in slaughtered fattening pigs from herds naturally infected with pandemic influenza A (H1N1) 2009 virus.

\section{Materials and Methods}

The slaughtered fattening pigs originated from three herds. The lungs from pigs from herd 1 and 2 had been selected for examination because "new" types of lung lesions were observed at the meat inspection. From these herds, lungs from 9 and 10 pigs, respectively, were examined. Herd 3 had been tested positive for pandemic influenza A (H1N1) 2009 virus two weeks before the pigs were slaughtered, and 10 lungs were submitted for examination. From each examined lung one swab from trachea and one from a bronchus were submitted for virus detection by real-time PCR (4). One sample from the right cranial lung lobe was submitted for bacteriological examination. The gross pathological lung lesions were recorded. One sample from trachea and nine samples from different lung lobes were fixed in $10 \%$ buffered formalin for $24 \mathrm{~h}$ and then transferred to $70 \%$ ethanol. The samples were routinely processed, and 3-4 $\mu \mathrm{m}$ sections were cut and stained with hematoxilin and eosin. Immunohistochemical examinations using monoclonal antibodies to detect influenza $A$ virus (5) and PCV2 in the lung tissues were performed.

\section{Results}

In herd 1 and 2 the pandemic influenza $A(H 1 N 1) 2009$ virus was detected in 17 of 18 examined lungs. In herd 3 influenza virus was not detected in the five examined lungs. The lungs from the three herds showed a multifocal, lobular pattern of atelectasis and dark red discoloration. The lesions were most pronounced in the cranioventral parts of the lungs. The areas with atelectasis were slightly firm compared to the surrounding tissue. In the bronchi there were varying amounts of mucus to mucopurulent exudate. The tracheobronchial lymph nodes were slightly to moderately enlarged. Histological examination of the lungs from pigs from herd 1 and 2 showed comparable lesions but also revealed variation between animals and within the lungs. The main findings in the affected lobules were atelectasis and moderate to intense infiltration of leukocytes in the mucosa of the small bronchi and bronchioles. Attenuation of bronchial and bronchiolar epithelial lining was seen, but acute epithelial necrosis was seldom identified. Large peribronchiolar and perivascular infiltrates of lymphocytes, and in some pigs, also lymphoid follicles were observed. There were increased numbers of leukocytes in the interalveolar septa, and varying numbers of granulocytes, lymphocytes and few macrophages were seen in the partial collapsed alveoli. In the lungs from pigs from herd 3 the major findings were atelectasis, prominent peribronchial and peribronchiolar lymphoid follicles and thickened interalveolar septa. The bacteriological examination showed sparse to moderate growth of different Streptococcus species or nonpathogenic bacteria from the majority of the lungs. Influenza A virus was detected by immunohistochemial examination in the bronchiolar epithelium, in cellular debris in bronchiolar lumen or in scattered cells in the interalveolar septa in 5 of 9 pigs in herd 1 and in 9 of 10 pigs in herd 2. PCV2 virus was not detected by immunohistochemical examination of the lung lesions.

\section{Discussion}

The pandemic influenza A (H1N1) 2009 virus infection induced significant lung pathology in the pigs from the three herds. The primary locations for lesions seemed to be small bronchi and bronchioles. In herd 1 and 2 the duration of the infection may have varied between 5-10 days prior to slaughter, and the lesions in most lungs were significantly different from the lungs from the pigs in herd 3 slaughtered 18 days after the detection of the virus in the herd. The lesions observed in the lungs were similar to the lesions described for "classical swine influenza".

\section{References}

1. Lium B et al. Annual report 2007. Oslo: National Veterinary Institute; 2008. p. 99-102.

2. Lium B et al. 2010. 21st IPVS Congress,Vancouver p?

3. Hofshagen M et al. Euro Surveillance 2009, 14 (45):pii=19406.

4. Statens Seruminstitutt Institutt, Denmark, unpublished

5. Löndt BZ. (2008). J. A. Pat. 37, 619-627. 


\title{
P.283
}

\section{Development of a one-step RT-PCR assay for detection and differentiation between swine influenza H1N1 virus and pandemic H1N1 2009 virus in Thai swine herds}

\author{
Aunyaratana Thontiravong; Rachod Tantilertcharoen; Ranida Tuanudom; Donruethai Sreta; Alongkorn \\ Amonsin; Roongroje Thanawongnuwech; Kanisak Oraveerakul; Pravina Kitikoon
}

Faculty of Veterinary Science, Chulalongkorn University, Bangkok, Thailand

\section{Introduction and objective}

A recently emerged influenza A H1N1 virus ( $\mathrm{pH} 1 \mathrm{~N} 1)$ spread globally from human-to-human causing the first pandemic of this century (1). The $\mathrm{pH} 1 \mathrm{~N} 1$ originated from the reassortment of swine influenza virus (SIV) with human, avian, classical swine and Eurasian swine lineages (2). Since then reports of reverse zoonotic cases in avian species and pigs have been documented in the field (3). These events raise concern of possible reassortment between the $\mathrm{pH} 1 \mathrm{~N} 1$ and other SIVs that may cause a new virus with zoonotic potential. Rapid detection and differentiation of the $\mathrm{pH} 1 \mathrm{~N} 1$ virus from swine $\mathrm{H} 1 \mathrm{~N} 1$ influenza virus (sH1N1) is essential for prompt preparedness for such events. Since the genome of pH1N1 is of swine origin, it is difficult to differentiate $\mathrm{pH} 1 \mathrm{~N} 1$ from all sH1N1 currently circulating in pigs worldwide. Fortunately, the origin of PB1 gene in the Thai and European sH1N1 isolates are mainly avian-like while that in $\mathrm{pH} 1 \mathrm{~N} 1$ is human-like (4). In this study, a conventional one-step reverse transcriptase (RT)-PCR assay targeting PB1 gene was developed for detection and differentiation of $\mathrm{pH} 1 \mathrm{~N} 1$ from other $\mathrm{SH} 1 \mathrm{~N} 1$ viruses. Additional RT-PCR assay targeting HA and NA genes was also developed and modified for subtyping $\mathrm{H} 1$ of classical swine and $\mathrm{N} 1$ of European swine viruses.

\section{Materials and Methods}

The two pairs of primers, PB1-P and PB1-S, specific for pH1N1 and $\mathrm{sH} 1 \mathrm{~N} 1$ viruses, respectively were designed based on PB1 gene. Additional $\mathrm{H} 1$ and N1 primers (N1 modified from (5)) were designed for subtyping H1N1 viruses from all species. The primers are shown in Table 1. All primers used in this study run with the same condition allowing detection of different genes simultaneously. The conditions are as follow: reverse transcription at $48^{\circ} \mathrm{C}$ for $45 \mathrm{~min}$, initial denaturation at $94^{\circ} \mathrm{C}$ for $3 \mathrm{~min}$ and 40 cycles of denaturation at $94^{\circ} \mathrm{C}$ for $30 \mathrm{sec}$, anneal at $55^{\circ} \mathrm{C}$ for 30 sec and extension at $72^{\circ} \mathrm{C}$ for $1 \mathrm{~min}$, followed by final extension at $72^{\circ} \mathrm{C}$ for $10 \mathrm{~min}$. The sensitivity of this assay was tested. The specificity of the assay was determined by testing with $\mathrm{pH} 1 \mathrm{~N} 1$, SIV subtype $\mathrm{H} 1 \mathrm{~N} 1, \mathrm{H} 1 \mathrm{~N} 2$ and $\mathrm{H} 3 \mathrm{~N} 2$, avian influenza $\mathrm{H} 5 \mathrm{~N} 1$ virus, seasonal human influenza H1N1 (huH1N1) virus and other swine respiratory viral pathogens.

\section{Results and Discussion}

The results showed that the RT-PCR assay can be applied to detect and differentiate $\mathrm{pH} 1 \mathrm{~N} 1$ virus from other SIVs, including sH1N1 viruses and huH1N1 virus (Fig 1). This novel assay was sensitive and can detect 0.001 and $0.01 \mathrm{TCID} 50 / \mathrm{ml}$ of $\mathrm{pH} 1 \mathrm{~N} 1$ and sH1N1 viruses, respectively. They showed $100 \%$ specificity and did not amplify genetic material from other subtypes of SIV and other swine respiratory viral pathogens (data not shown). Overall, the results clearly indicated that the novel one-step RT-PCR assay has high sensitivity and specificity to detect $\mathrm{pH} 1 \mathrm{~N} 1$ virus. The assay can discriminate $\mathrm{pH} 1 \mathrm{~N} 1$ from huH1N1 virus, Thai
sH1N1 and possibly European H1N1 SIVs since the PB1 gene is of the same avian-like origin. Moreover, the RT-PCR assay targeting $\mathrm{H} 1$ and $\mathrm{N} 1$ genes can be used to subtype the virus using the same PCR condition which allows rapid and simultaneous detection and differentiation of the influenza A viruses. In summary, this cost-effective assay can be used for rapid screening of current circulating SIV isolates for detecting pH1N1 in the swine population.

Table1. Primers used in this study

\begin{tabular}{|l|c|c|c|c|}
\hline Name & Virus target & Gene & Sequence & $\begin{array}{c}\text { Product size } \\
\text { (bp) }\end{array}$ \\
\hline PB1-P-1038F & $\begin{array}{c}\text { Pandemic (H1N1) } \\
2009\end{array}$ & PB1 & CGAGTGGTTCAGAAACATCC & 408 \\
\hline PB1-P-1446R & $\begin{array}{c}\text { Pandemic (H1N1) } \\
2009\end{array}$ & PB1 & $\begin{array}{c}\text { CACTAACTTGCAGGTC- } \\
\text { CTGTAG }\end{array}$ & \\
\hline PB1-S-1036F & Swine (H1N1) & PB1 & $\begin{array}{c}\text { CCTGAATGGTTCAGAAAT- } \\
\text { GTTTT }\end{array}$ & 410 \\
\hline PB1-S-1446R & Swine (H1N1) & PB1 & $\begin{array}{c}\text { AACCAGTTTGCAGGTC- } \\
\text { CTATAA }\end{array}$ & 706 \\
\hline H1-231F & All influenza (H1N1) & HA1 & $\begin{array}{c}\text { GCATTTGGGTAAAT- } \\
\text { GTAACATTGC }\end{array}$ & \\
\hline H1-937R & All influenza (H1N1) & HA1 & $\begin{array}{c}\text { GAAATGGGAGGCTGGT- } \\
\text { GTTTAT }\end{array}$ & \\
\hline N1-F849 & All influenza (H1N1) & NA1 & GAGGAATGTTCCTGTTACCC & 309 \\
\hline N1-R1158 & All influenza (H1N1) & NA1 & CATCCATTAGGATCCCAATCA & \\
\hline
\end{tabular}

Fig1. Detection, differentiation and subtyping of influenza $A$ (H1N1) viruses by RT-PCR assay. Lane 1-6: PB1-P primer specific to $p H 1 N 1$ virus. Lane 8-13: H1 primer for subtyping all H1 subtype. Lane M: 100-bp ladder.

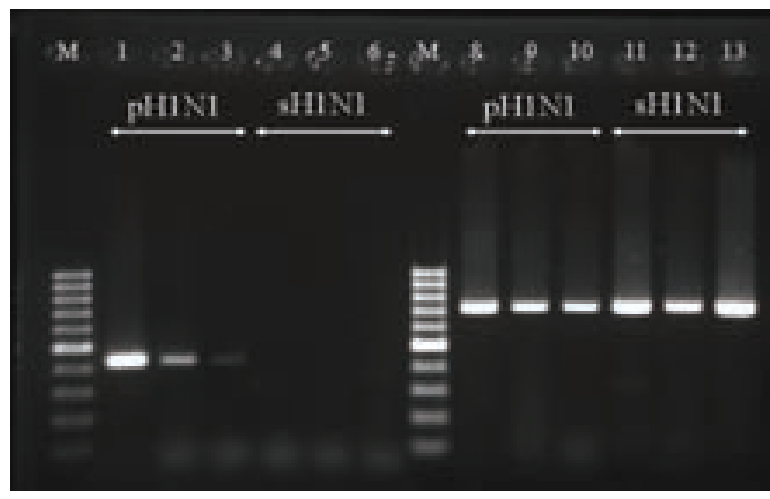

\section{References}

1. WHO, 2010. http://www.who.int//csr/don/2010_01_15/

2. Kingsford et al., 2009. PLoS ONE 4:6402.

3. Weingartl et al., 2009. J Virol 16.

4. Neumann et al., 2009. Nature 459: 931-939.

5. Chutinimitkul et al., 2008. Virus Res 132:122-131. 


\title{
P.284
}

\section{Analysis of cross reactivity of porcine sera in the haemagglutination inhibition assay using swine H1N1 or the new pandemic H1N1 influenza virus}

\author{
Gerard E. Martín-Valls ${ }^{1}$ Meritxell Simon-Grifé ${ }^{1}$ Núria Busquets ${ }^{1}$ Ivan Díaz ${ }^{1}$ Margarita Martín² Jordi Casal ${ }^{2,1}$ Enric Mateu $^{2,1}$ \\ 1. Centre de Recerca en Sanitat Animal (CReSA), UAB-IRTA, Campus de la Universitat Autònoma de Barcelona, Bellaterra, Barcelona, Spain; \\ 2. Departament de Sanitat i d'Anatomia Animals. Universitat Autònoma de Barcelona, Bellaterra, Barcelona, Spain
}

\section{Introduction}

In April 2009, a new influenza A/H1N1 strain circulating in the human population was reported in Mexico. The new H1N1 virus was a triple swine/human/avian reassortant that afterwards spread pandemically. Fortunately, the virulence of the pandemic H1N1 (panH1N1) seems to be low but this episode made clear the need for a better surveillance of influenza A viruses infection. Swine influenza is most often caused by subtypes $A / H 1 N 1$, $\mathrm{A} / \mathrm{H} 1 \mathrm{~N} 2$ and $\mathrm{A} / \mathrm{H} 3 \mathrm{~N} 2$. Hemagglutination inhibition test $(\mathrm{HI})$ still is the serological assay of reference and it is assumed to be subtype-specific ${ }^{1}$. The main objective of the present work was to assess to what extent the use of different strains of a same subtype may affect the outcome of $\mathrm{HI}$ when examining swine serum samples.

\section{Material and methods}

Ninety-four serum samples were selected from a sera bank (2007-2009) corresponding to a cross-sectional study of swine influenza in Spain where sera had been tested in $\mathrm{HI}$ using an European avian-like A/swine/Neth/Best/96 strain (NLH1N1)(GD laboratories, Deventer, Netherlands). The group of selected sera included negative and positive anti-NeH1N1 sera $(\leq 1: 320)$ that were re-tested in $\mathrm{HI}$ using the Spanish avian-like H1N1 A/swine/ Spain /53207/2004 strain (ESH1N1) and the novel pandemic H1N1 (A/Catalonia/S2169/2009) strain (panH1N1) isolated from a human sample. Similarity of the predicted amino acid sequences of $\mathrm{H} 1$ were $89.3 \%$ between NLH1N1 and ESH1N1; 75.1\% between NLH1N1 and panH1N1 and 71 5\% between ESH1N1 and panH1N1. Sera were also examined using $A / H 1 N 2$ and $A / H 3 N 2$ (A/swine /Gent/7625/99 and A/swine/Neth/St.Oedenrode/96) (GD laboratories, Deventer, Netherlands) strains. Statistical analysis was done with Statdirect 2.7.5.

\section{Results}

Figure 1 show the comparative distribution of $\mathrm{HI}$ titres using the different H1N1 strains. Comparison of results of sera analyzed with NLH1N1 or ESH1N1 showed that both strains produced fairly similar titres $\left(\mathrm{r}=0.79 ; \mathrm{Cl}_{95 \%}: 0.70-0.85\right)$. In contrast, regression of log-transformed titres for NLH1N1 or ESH1N1 and panH1N1 was non significant, suggesting that antibodies towards panH1N1 did not cross-react with other H1N1. No cross-reactivity between none of the $\mathrm{A} / \mathrm{H} 1 \mathrm{~N} 1$ and either $\mathrm{H} 1 \mathrm{~N} 2$ or $\mathrm{H} 3 \mathrm{~N} 2$ was observed (data not shown). Interestingly, in 4 farms animals seropositive $(\geq 1: 40)$ against panH1N1 were found. In one of those farms (analysed in the first quarter of 2009) 8 animals were positive (Table 1); some of them reacted with other swine influenza strains.
Figure 1. Distribution $\mathrm{HI}$ titres against three different $\mathrm{H} 1$ influenza $A$ strains: a) ESH1N1 vs. NLH1N1; b) ESH1N1 vs. panH1N1 and, c) NLH1N1 vs. panH1N1

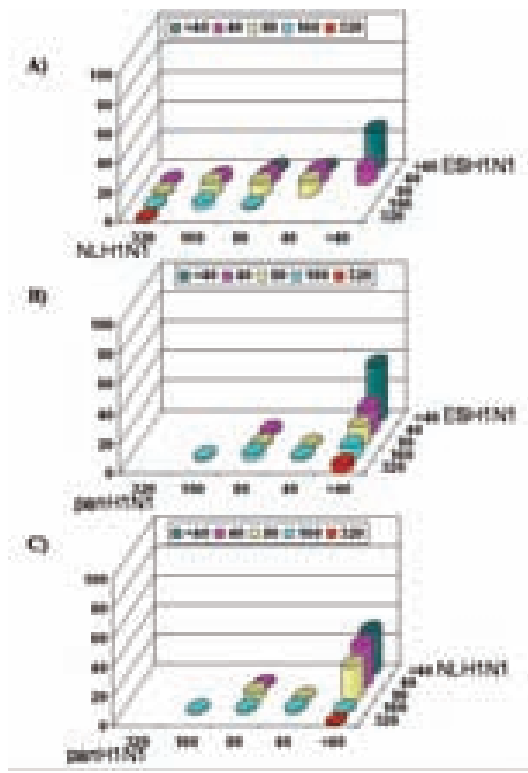

Table 1. Results by HI with different A/influenza strains of the 8 pigs seropositive to panH1N1.

\begin{tabular}{|c|c|c|c|c|c|}
\hline Pig & panH1 & NLH1 & ESH1 & H1N2 & H3N2 \\
\hline 1 & $1: 160$ & $1: 160$ & $1: 160$ & $1: 80$ & Neg. \\
\hline 2 & $1: 80$ & $1: 80$ & $1: 80$ & Neg. & Neg. \\
\hline 3 & $1: 80$ & $1: 80$ & $1: 80$ & $1: 80$ & $1: 160$ \\
\hline 4 & $1: 80$ & $1: 80$ & $1: 160$ & Neg. & Neg. \\
\hline 5 & $1: 80$ & $1: 40$ & $1: 160$ & Neg. & $1: 80$ \\
\hline 6 & $1: 80$ & $1: 80$ & $1: 40$ & Neg. & $1: 640$ \\
\hline 7 & $1: 80$ & $1: 160$ & $1: 160$ & $1: 80$ & $1: 160$ \\
\hline 8 & $1: 80$ & $1: 80$ & $1: 40$ & Neg. & $1: 320$ \\
\hline
\end{tabular}

\section{Discussion}

The present results indicate that panH1N1 has little or no crossreactivity in terms of $\mathrm{HI}$ with other contemporary swine $\mathrm{H} 1 \mathrm{~N} 1$ viruses. However, the results also showed that, most probably, $\mathrm{H} 1$ viruses cross-reacting with the pan $\mathrm{H} 1 \mathrm{~N} 1$ strain already existed before or at the same time of the emergence of that new pandemic strain. Considering the limited virulence of current panH1N1 it can not be discarded that similar or cross-reacting $\mathrm{H} 1 \mathrm{~N} 1$ virus circulated completely unnoticed in pig herds. Also, raise the question of how to interpret the $\mathrm{HI}$ results obtained with a given influenza strain.

\section{References}

1. Van Reeth, K. et al. Viral Inmunology. 19;373-382 (2006). 


\title{
P.285
}

\section{Detection of Pandemic H1N1 2009 in swine herds}

\author{
Yeun-Kyung Shin; Sorah Yoon; Soon-Seek Yoon \\ National Veterinary Research and Quarantine Service, Anyang, Korea
}

\begin{abstract}
Introduction
Pandemic H1N1 2009 was first reported in human population in April, 2009 and on the same month, pig infection was also reported in Alberta, Canada. With the increasing number of human death toll and with more cases of swine infections all around the world, Korean government enlisted Pandemic H1N1 2009 in notifiable animal diseases under the Act of ACT ON THE PREVENTION OF CONTAGIOUS ANIMAL DISEASES on August 21, 2009 and started monitoring of Pandemic H1N1 2009 in pig nerds. Accordingly, the necessity of rapid and reliable detection method of swine influenza virus or antibodies in pig herds for a number of heads on a regular basis was increased. Here we present one-step M gene multiplex RT-PCR system which detect Pandemic H1N1 2009 from swine samples, one-step HA1/3 subtyping multiplex RT-PCR system and one-step NA1/2 subtyping multiplex RT-PCR system from swine influenza viruses.
\end{abstract}

\section{Materials and Methods}

Viruses

A human pandemic flu virus (A/Korea/01/2009 (H1N1), isolated in Korea, a pandemic flu virus (A/sw/Korea/J2/2009) isolated from pig, 10 various subtypes of endemic swine influenza viruses, and Reference influenza viruses (from HA subtype 1 to 16) were used. Virus titres were measured by Hemagglutinin assay or Reed-Munch method using MDCK cells.Oligonucleotides design

The matrix (M) genome sequences of Pandemic H1N1 2009 influenza virus (A/California/04/2009), endemic swine influenza viruses and avian influenza $A$ viruses were retrieved from the GenBank database and analyzed to select sites which is specific for pandemic flu. Primers were also selected for the subtyping of $\mathrm{HA} 1 / \mathrm{HA} 3$ or NA1/NA2 among endemic swine influenza viruses.

\section{Results}

One-step multiplex RT-PCR assay was performed with the RNA extracted from diluted pandemic flu virus (titre of stock: $1 \times 106$ $\mathrm{TCID} 50 / \mathrm{ml}$ ) at $10-1$ to $10-8$. A positive amplification could be visualized up to 10-5-6dilution, which meant the lowest concentration with positive results was 1-10 TCID50/ml. One-step multiplex RT-PCR assay was also performed with RNA standard prepared to know the exact sensitivity as copy number. The minimum copy number of matrix gene RNA detectable with our system was approximately 101-2 copies of RNA.
Specificity of the system was tested using various endemic swine influenza viruses and reference viruses with different $\mathrm{HA}$ subtype. All were negative for the pandemic H1N1 2009 virus specific bands. Bands for influenza A common M gene were only visible.

One-step HA1/HA3 subtyping multiplex RT-PCR system and onestep NA1/NA2 subtyping multiplex RT-PCR system were also tested for the specificity and sensitivity with a panel of influenza A viruses. The limit of detection was $101 \mathrm{TCID} 50 / \mathrm{ml}$ for HA1/HA3 subtyping, and 101-2TCID50/ml for NA1/NA2 subtyping.

\section{Discussion}

Pig can be infected with avian, human or swine influenza A viruses, hence, is well known as a mixing vessel. With the advent of swine origin Pandemic H1N1 2009, the importance of national or international level of monitoring of swine influenza viruses is well appreciated. It is important to develop or update the virus or antibody detection method from pigs to know the exact situation of influenza virus infection. It is necessary to have a reliable and highly sensitive swine flu detection method from pig herds since the viruses are cleared rather quickly from pigs. Monitoring of swine flu on regular basis will contribute public health and pork industry at the same time. Our system will be a good tool for monitoring of Pandemic H1N1 2009 in swine herds on a regular basis.

\section{References}

Lorusso et al. Journal of Virological Methods. 2009

Pabbaraju et al. Journal of Clinical Microbiology. Vol.47. No.11. 34543460 


\title{
P.286
}

\section{Development of inactivated pig vaccine for Pandemic H1N1 2009}

\author{
Jae-Young Song ${ }^{1}$ Yeun-Kyung Shin ${ }^{1}$ Young-Ki Choi ${ }^{2}$ \\ 1. National Veterinary Research and Quarantine Service, Anyang, Korea; 2. College of medicne and medical research, \\ Choongbook National University, Cheongju, Korea
}

\section{Introduction}

First case of pig infection of Pandemic H1N1 2009 was reported in Canada in April, 2009 and aftermath, there were additional cases in more than 10 countries all around the world. Korean government practiced active monitoring of Pandemic H1N1 2009 from May 2009. Approximately $10 \%$ of the fattening farms, which meant $10 \%$ of the total fattening farms and included 700 farms, all breeding farms and all Artificial Insemination centers (200 locations) were monitored. 18 farms in different places all around the country were found to have infected pigs. Since pigs were well known as a mixing vessel of influenza viruses, it was necessary to develop vaccines which can prevent pigs from being infected or producing reassorted pandemic flu viruses.

\section{Materials and Methods}

\section{Generation of vaccine candidate viruses}

A/PR8/34 H1N1 influenza A virus, which is a backbone virus of seasonal human influenza vaccine and Pandemic H1N1 2009 virus, A/sw/Korea/SCJ01/2009, isolated from pigs in Korea were co-infected in 9-10 day old embryonated SPF eggs to generate reassorted vaccine candidate virus(CL A/swine/Korea /SCJ01/09 $x$ PR8). Allantoic fluid was harvested and plaque assay was performed to select reassorted virus with 6 internal genes from PR8 virus and with 2 surface glycoprotein (NA and $\mathrm{HA}$ ) genes from pandemic flu virus. Immunogenicity in mice and pigs

Immunogenicity of recombinant virus in mouse and in pigs was tested using concentrated viruses with $2 \%$ alum. Mice were immunized with $3.5 \mathrm{ug}$ inactivated vaccine two times with two weeks interval. Pigs were immunized with 7.5ug. Sera were collected 2 weeks after each immunization and Hemagglutination inhibition ( $\mathrm{HI}$ ) tests were performed.Virus challenge and protection test

Two groups of pigs were immunized with different type of vaccines two times. Group I was immunized with endemic swine influenza H1N1 vaccine and Group II were immunized with pandemic flu vaccine candidate. Two weeks after the last immunization, both groups were challenged with $1 \mathrm{ml}$ pandemic $\mathrm{H} 1 \mathrm{~N} 12009$ virus in intranasal route at $1 \times 105 \mathrm{TCID} 50 / \mathrm{ml}$. For transmission studies, new group of pigs were housed aerosol contact with immunized and challenged groups a day after infection. Nasal swabs were collected p.i. 2, 4, 5, 6, 7, and 8 days from all animals to examine the ability of the vaccine to inhibit virus replication.

\section{Results}

Immunogenicity in mice and pigs

$\mathrm{HI}$ titer of one time immunized mice were 40-80. When immunized two times, the titers were 160-320. In the case of pigs, $\mathrm{HI}$ titer of one time immunized pigs were 40-80, and 160-320 with two immunizations. Virus challenge and protection test

Both of the vaccine and challenged group started to shed virus 2 days p.i. Much higher titer of virus was detected from pigs immunized with endemic swine influenza virus vaccine longer time. No virus was detected from contact group with pandemic flu vaccine immunized group while virus was detected from contact group with endemic vaccine.

\section{Discussion}

It is well known that vaccination for influenza A virus would be a good way to control transmission of viruses and overcome illness in humans. Vaccination of pigs will add up one more advantage: inhibitions of possible generation of reassorted influenza $A$ viruses by combination of endemic influenza $A$ viruses and Pandemic H1N1 2009. Continuous monitoring of influenza $A$ viruses circulating among pigs and application of right vaccination for the current strains will benefit both public health and pork industry.

\section{References}

Brookers et al, Veterinary Record, June 132009

Smith et al, Nature, June, 2009

Reeth et al, Vet Res 38, 243-260, 2007 


\title{
Spreading of Actinobacillus pleuropneumoniae in the pig farm. A field case
}

\author{
Kazimierz Tarasiuk ${ }^{1}$ Marcin Bzdawka² \\ 1. PIC Poland, Warsaw, Poland; 2. Rogóźno Farm, Łasin, Poland
}

\section{Introduction}

Actinobacillus pleuropneumoniae (App) is the etiologic agent of swine pleuropneumonia, a highly infectious, often fatal disease. The main route of spread is by direct contact from pig to pig or by aerosol within short distances. Although some authors $(1,2)$ reported that airborne transmission between closely located pig units is possible but rare. The objective of this study was to follow up the spreading of App serotype 2 infection within a small pig herd.

\section{Materials and Methods}

A high-health multiplying farrow to finish herd (240 sows) was infected with App serotype 2 in August 2009. The herd was free of the major diseases including PRRS, EP and Actinobacillus pleuropneumonia. The farm consisted of two buildings $40 \mathrm{~m}$ from each other with no direct connection established between them. One building is devoted to grow-finish pigs managed AIAO by room and the other one is populated with sows and weaning pigs up to 7 weeks of age. The grow-finish building consisted of 4 rooms split, each containing 2 pens with 45 pigs in each. The infection entered into the building in one of the outer rooms which was separated physically from the other part with a solid wall. Once the first symptoms of the infection emerged in pigs (temperature $41.5^{\circ} \mathrm{C}$, coughing, lack of appetite) the facility was locked and the possibility to get in from outside was only used. All the animals in this room immediately were treated with tetracycline in the water for 7 consecutive days. Immediately extra biosecurity conditions (different stockperson, separate shower facility, clothes and boots) were introduced into the farm. After the clinical signs declined and antibiotic withdrawn, all the animals from this room were slaughtered. Slaughtercheck was performed and tonsils from 15 randomly selected pigs with lung lesions were taken for PCR. The affected lung tissues were submitted to the laboratory for App culture. The empty pens were first fumigated with $1 \%$ Virkon and left for 5 days. The floor was soaked with a glutaryl aldehyde based disinfectant and left until completely dried. After 5 days the entire facility was cleaned, washed, disinfected, dried and left for 30 days before new pigs were introduced. The pigs from the other 3 rooms were observed clinically and were given tetracycline in the water for 7 consecutive days as prevention. From every pen 15 randomly selected pigs were bled for serology. During the next 8 weeks all the animals from the first building were gradually slaughtered. Tonsils from 5 randomly selected pigs / pen were taken for PCR. In the meantime many precautions were undertaken to protect the sow building from contamination. Ten sentinels naïve for App were introduced into the breeding sector of the farm allowing them to have contact nose to nose with the local pigs. All sentinels were tested serologically twice: 1 and 2 months after introduction. After two months from infection the blood from 45 randomly selected sows was taken for serology. For detection of antibodies against App serotype 2, a LC-LPS ELISA was used (Swine-Check App, Biovet, Canada).

\section{Results}

App serotype 2 infection was confirmed by culture from lung lesions of pigs from the outer perimeter room. Pigs from this facility were sick with a typical clinical signs of pleuropneumonia. Slaughtercheck revealed typical lesions of App infection (abscess-like nodules present in the diaphragmatic lobes) in 35 of 52 pigs. Two hundred and seventy pigs from the other three rooms gradually slaughtered did not show any lung lesions. The serology carried out in randomly selected pigs from every room revealed that all the results were negative for App serotype 2. In total 90 pigs were bled for serological investigations. No clinical signs of respiratory problems were observed in sows and weaning pigs as well as sentinel animals. Serological investigations performed on 45 randomly selected sows shown all of them to be negative for App serotype 2. Tonsils of 15 pigs from the infected room were tested PCR positive. Tonsils of pigs from the other three rooms of the building were tested PCR negative. Sentinel pigs were tested serologically negative for App serotype 2 either 4 or 8 weeks upon introduction.

\section{Discussion}

Results obtained clearly show that App infects naïve pigs if there is direct contact with contaminated material. In this particular case the source of infection there was very likely the stockman (fomites) attending the loading process. Only the pigs in the outer perimeter room became infected with App serotype 2, of course the loaded animals get infected as well. It is worth noting that infection did not spread across the entire building. The swift actions undertaken upon the first signs of infection appeared allowed to protect pigs from the rest of the rooms of this building and other building from the App infection. This confirms previous observations that airborn transmission of App is possible but limited (1, 2).

\section{References}

1. Desrosiers R. 2004. Proc Am Ass Swine Vet 9-37.

2. Kristensen CS et al. 2004. Vet. Microbiol 98:243-249. 


\title{
P.288
}

\section{Serotype determination of a collection of Actinobacillus pleuropneumoniae isolates from Argentina}

\author{
Fernando A. Bessone ${ }^{1}$ María de la Luz Ducommun ${ }^{1}$ Gustavo C. Zielinski ${ }^{1}$ Javier E. Sarradell ${ }^{2}$ \\ 1. Instituto Nacional Tecnologia Agropecuaria, Marcos Juárez-Córdoba, Argentina; 2. FCV-Univ. Nac. de Rosario, Casilda-Sta Fé, Argentina
}

\section{Introduction}

Porcine pleuropneumoniae is a worldwide spread disease responsible for severe economic loss to pig producers because of mortality, lower weight gains and increased production costs. Its aetiological agent, Actinobacillus pleuropneumoniae (App), has 2 biotypes (I and II) dependent and non-dependent for "in vitro" growth of nicotinamideadeninedinucleotide (NAD), respectively. The species has been classsified into serotypes 1-15, according to composition of surface cell walls antigens (LPS). This is important for immunity and protection, since most of the serotypes do not cross-react immunologically, having different pathogenic potential. So that, knowledgment on relative prevalence of different serotypes in a particular producing area is important for establishing preventive control strategies. The objective of this work was to classify a collection of argentinean isolates of App into serotype groups, based on PCR amplification of selected sequences belonging to the omIA and apx genes.

\section{Material and Methods}

Forty eight App cultures isolated from clinical cases of pleuropneumoniae in swine farms from different locations of the country were utilized. The strains were cultured and biochemically classified by conventional bacteriology laboratory works and stored lyophilized. For serotype classification by PCR, bacterial ADN was extracted by a commercial kit (Fermentas ${ }^{\mathrm{TM}}$ ) and ran using primers and conditions reported previously (1).

\section{Results}

Serotype group classification and percentages can be observed in the following table 1. In figure 1 can be visualized the geographical location within Argentina where the strains were isolated.

\section{Table 1.}

\begin{tabular}{|c|c|c|}
\hline Serotypes & $\mathbf{n}$ isolations & $\%$ \\
\hline $1 / 9 / 11$ & 26 & 54 \\
\hline $2 / 8$ & 0 & 0 \\
\hline $3 / 6$ & 15 & 31 \\
\hline 4 & 0 & 0 \\
\hline 5 & 0 & 0 \\
\hline 7 & 7 & 15 \\
\hline 10 & 0 & 0 \\
\hline 12 & 0 & 0 \\
\hline 15 & 0 & 0 \\
\hline Total & 48 & 100 \\
\hline
\end{tabular}

Figure 1. Spatial distribution of isolations in Argentina.

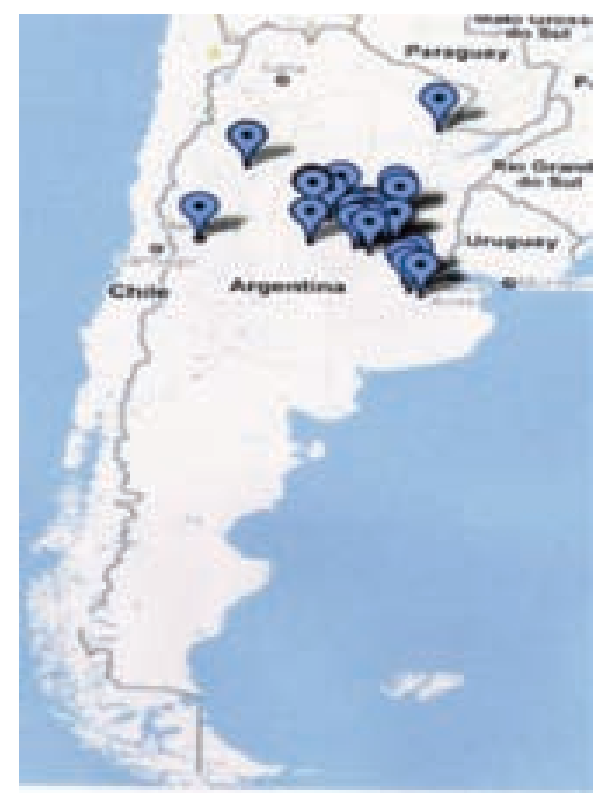

\section{Discussion}

It can be observed that the most frequent group of serotypes detected is 1-9-11, since the test does not discriminate among these particular serotypes. It was expected that serotype 1 was the most prevalent since was described as it long ago (2). However, a relative high frequency of serotypes 3-6 was detected, which is not surprising since existance of serotype 3 was previously described (3). This is an important finding, since no conventional vaccines containing App serotypes 3-6-8 exist in the argentine market. Serotype 7 also showed a relatively high prevalence within this collection, which is not surprising either, since it was previously isolated from a clinical case of pleuropneumoniae (4).

In this work, strains from the most important producer areas from Argentina were studied, such as showed in figure 1. In the future a higher number of isolates should be processed, so that a more complete map of App serotype distribution in Argentina will be obtained. This information will allow to improve control and preventive strategies for this disease.

\section{References}

1. Gram T., Ahrens P., Andreasen M. (2000) Vet. Microbiol. 75, 43-57.

2. Perfumo C.J. (1981) Rev. de Med. Vet. 62,89-108.

3. Di Cola G., Zielinski G. (2004) unpublished.

4. Moredo F., (1999) Memorias. Congreso Chileno de Microbiología. Valdivia, Chile, pp12. 


\title{
P.289
}

\section{Animals infected with Actinobacillus pleuropneumoniae serotype 15 develop antibodies that cross-react with serotypes 3, 6 and 8 by LC-LPS ELISA}

\author{
Marcelo Gottschalk ${ }^{2}$ Sonia Lacouture ${ }^{1}$ Donald Tremblay ${ }^{1}$ Josée Harel${ }^{2}$ \\ 1. Université de Montréal, St-Hyacinthe, QC, Canada; \\ 2. Faculté de médecine vétérinaire, GREMIP and CRIP, Université de Montréal, St-Hyacinthe, QC, Canada
}

\section{Introduction}

Swine pleuropneumonia caused by Actinobacillus pleuropneumoniae (App) is a contagious disease that may produce important economic losses. Clinical signs of acute disease are dyspnea, coughing, anorexia, depression, fever and sometimes vomiting. In the absence of treatment, the disease can progress very rapidly and death can occur within a few hours. Chronic infections are characterized by coughing and pleuritis in lungs. There are 15 serotypes of App described so far. Serotype 15, the last described serotype, was suggested as being present only in Australia ${ }^{1}$. In this country, it seems to produce important losses in the swine industry. However, strains have later been isolated in USA, Canada and Argentina ${ }^{2}$ and, more recently, in Japan ${ }^{3}$, causing sporadic disease. In the present study, we analyzed sera from naturally infected animals in Australia, as well as, sera from experimentally infected animals with the reference strain HS-143 and with North American strains. In addition, we studied the genetic diversity of within USA App serotype 15 strains.

\section{Materials and Methods}

Strain and isolates: The reference strain of App serotype 15 (HS-143) was kindly supplied by Prof. J. Frey, Switzerland. Other reference strains came from our own collection. A total of 22 isolates recovered from diseased animals originated from the University of Minnesota Veterinary Diagnostic Laboratory. These isolates were sent to our diagnostic laboratory for serological characterization.

Antigenic and genotypic characterization: Serotyping was carried out by coagglutination, immunodifussion and indirect hemagglutination tests. Hyperimmune serum against strain HS-143 was produced in rabbits. Toxine profile was performed by PCR. Genotyping was carried out by ERIC- PCR and Random Amplified Polymorphic DNA (RAPD).

Field and experimentally infected swine sera: Field serum samples from two Australian herds clinically infected with serotype 15 strains (kindly supplied by Dr. Patricia Spicer) as well as sera from six experimentally infected pigs with strain HS-143 were tested by LC-LPS ELISA against serotypes 1 to $12^{4}$. A LPS antigen for serotype 15 was produced as described before ${ }^{4}$ using the reference strain. Selective immunoblot tests, using different antigens and sera, were also performed.

\section{Results}

- Serotyping: All field isolated presented a strong and clear reaction with anti-serotype 15 rabbit serum. Most strains presented also cross-reactions with serotypes 3, 6 and/or 8 with most techniques used. Some isolates presented crossreactions with serotypes 7 or 12 .

- Genotyping: A relative degree of heterogeneity, with at least 7 different clusters, was observed.

- Toxine profile: apxIVA, apxIBD, apxIICA, apxIIICA and apxIIBD(production of ApxIV, ApxII and ApxIII).

- Serology:

\begin{tabular}{|c|c|c|c|c|c|c|}
\hline & \multicolumn{7}{|c|}{ LC-LPS ELISA WITH ANTIGENS } \\
\hline $\begin{array}{c}\text { Sera from } \\
\text { infected animals }\end{array}$ & App 15 & App 3 & App 6 & App 8 & App 7 & App 12 \\
\hline App 15 & +++ & +++ & +++ & +++ & - & - \\
\hline App 3 & +++ & +++ & +++ & +++ & - & - \\
\hline App 6 & +++ & +++ & +++ & +++ & - & - \\
\hline App 8 & +++ & +++ & +++ & +++ & - & - \\
\hline App 7 & - & - & - & - & +++ & - \\
\hline App 12 & - & - & - & - & - & +++ \\
\hline
\end{tabular}

- Most field sera presented a clear positive reaction with antigens from serotypes 3, 6, 8 and 15. Common LPS epitopes in these serotypes was confirmed by immunoblot.

\section{Discussion}

- App serotype 15 infection is relatively common in North America.

- LPS from App serotype 15 is antigenically related to that from serotypes 3, 6 and 8, as previously suggested by chemical analysis ${ }^{5}$.

- A slight cross-reactivity at the capsular level is observed in serotying, which also can be explained by previous capsular structure analysis ${ }^{5}$.

- Strains presented in USA are not clonal .

- Serologic diagnosis of herds infected by these strains can be done by the LC-LPS ELISA test using either homologous or serotype 3,6 or 8 antigen.

\section{References}

1. Blackall et al. Vet. Microbiol. 2002. 84:47-52.

2. Gottschalk, M. Proc. 38th AASV. 2007. 381-84.

3. Koyama et al. J. Vet. Med. Sci. 2007, 69:961-64.

4 Gottschalk et al.Vet. Microbiol. 1994. 42: 91-104.

5 Perry et al. Biochem. Cell Biol. 2004. 83:61-9. 


\title{
P.290
}

\section{An atypical biotype I Actinobacillus pleuropneumoniae serotype 13 is present in North America}

\author{
Marcelo Gottschalk 1 Sonia Lacouture ${ }^{2}$ Oystein Angen ${ }^{3}$ Branko Kokotovic $^{3}$ \\ 1. Faculté de médecine vétérinaire, GREMIP and CRIP, Université de Montréal, St-Hyacinthe, QC, Canada; 2. Université de Montréal, \\ St-Hyacinthe, QC, Canada; 3. National Veterinary Institute, Technical University of Denmark, Copenhagen, Denmark
}

\section{Introduction}

Swine pleuropneumonia caused by Actinobacillus pleuropneumoniae (App) is a contagious disease that may produce important economic losses ${ }^{1}$. Clinical signs of acute disease are dyspnea, coughing, anorexia, depression, fever and sometimes vomiting. In the absence of treatment, the disease can progress very rapidly and death can occur within a few hours. Chronic infections are characterized by coughing and pleuritis in lungs. There are two biotypes of App differentiated on the basis of their requirement for nicotinamide adenine dinucleotide (NAD). Biotype 1 strains require NAD, whereas biotype 2 strains can synthesise NAD in the presence of specific pyridine nucleotides or their precursors ${ }^{1}$. There are 13 serotypes of biotype I (serotypes 1 to 12 and 15), and 2 serotypes of biotype II (serotypes 13 and 14), based on surface polysaccharide antigens. Some strains belonging to serotypes such as 2, 4, 7 and 9, mainly isolated in Europe, have been described as being either biotype I or biotype II. So far, in North America, serotypes 1 to 3, 4 (Canada), 5 to 8, 10, 12 and 15 have been isolated ${ }^{2}$. We report here for the first time the isolation of serotype 13 strains in USA and Canada. However, these strains are NAD-dependent and antigenically different from the reference strain ${ }^{3}$.

\section{Materials and Methods}

Isolates: Two isolates (003-16 and 15816), from a Canadian and an USA herds, respectively, were characterized. Both isolates were recovered from animals presenting clinical signs and lesions compatible with swine pleuropneumonia. Both strains were shown to be a typical biotype I (NAD-dependant) App. Both strains were shown to be App by two species-specific PCR.

Strain characterization: Serotyping was carried out by coagglutination (CoAg), immunodifussion (ID) and indirect hemagglutination tests (IHA). Toxine profile was performed by PCR. Genotyping was carried out by Amplified Fragment Length Polymorphism (AFLP) 4 .

Field and experimentally infected swine sera: Field serum samples from herds infected with strains $003-16$ or 15816 as well as sera from three experimentally infected pigs (with strain 003-16) were tested by LC-LPS ELISA against serotypes 1 to 15 and against the homologous strains. Selective immunoblot tests using different antigens and sera were also performed.

\section{Results}

- Serotyping:

\begin{tabular}{|c|c|c|c|}
\hline & \multicolumn{3}{|c|}{ Antiserum against $^{\mathrm{a}}$} \\
\hline Strain Used & Serotype 13 & Serotype 10 & 0ther serotypes \\
\hline Reference serotype 13 & $++^{\mathrm{b}}$ & - & - \\
\hline $003-16$ & $++^{\mathrm{b}}$ & $++^{\mathrm{c}}$ & - \\
\hline 15816 & $++^{\mathrm{b}}$ & $++/+^{\mathrm{d}}$ & $+/$ e $^{\mathrm{b}}$ \\
\hline
\end{tabular}

aSera from 6 different rabbits from 2 laboratories; bBy all techniques used; 'By ID only (LPS-

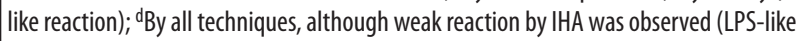
reaction by ID); ' Light cross-reactions with serotypes 5, 6 and 8 .

- Sera from either naturally or experimentally infected animals presented a positive reaction using LC-LPS ELISA when either the homologous (high values) or serotype 10 (intermediate values) LPS antigen was used. Negative results were obtained with LPS from the reference strain of serotype 13. Immunoblot tests clearly indicated a reaction with sera from experimentally infected animals with serotype 10 LPS.

- Toxine profile: apx ICA; apx IBD (ApxI production).

- Genotyping: Both strains clustered together with the same profile, clearly different from the serotype 13 reference strain.

\section{Discussion}

- Although slight differences, both Canadian and USA strain are antigenically similar.

- These strains are different from the reference strain: a) biotype I; b) LPS similar to serotype 10 App; c) Apx I toxin production; d) a different genetic cluster.

- These strains probably possess a capsular type of serotype 13 and a LPS of serotype 10.

- Serologic diagnosis of herds infected by these strains should be done by the LC-LPS ELISA test using the homologous (preferable) or serotype 10 antigen.

\section{References}

1. Gottschalk \& Taylor. A. pleuropneumoniae. Diseases of Swine. 2006. lowa University Press. 563-76.

2. Gottschalk, M. Proc. 38th Annual meeting AASV. 2007. 381-84.

3. Nielsen et al. Vet Microbiol. 1997; 54: 35-46.

4. Kokotovic \& Angen. J. Clin. Microbiol. 2007, 45:3921-9. 


\title{
Profiles of Actinobacillus pleuropneumoniae serotypes in Dutch slaughter pigs
}

\author{
Gerard J. Wellenberg ${ }^{1} \varnothing y s t e i n$ Angen ${ }^{1}$ Eric. van Esch² Anja Rothkamp ${ }^{1}$ Frits Bouwkamp ${ }^{1}$ Tom Duinhof ${ }^{1}$ \\ 1. Animal Health Service, Deventer, Netherlands; 2. National Veterinary Institute, Copenhagen, Denmark
}

\section{Introduction}

Although Actinobacillus pleuropneumoniae (App) infections have been evidenced in The Netherlands, the actual distribution of the different App serotypes in Dutch pigs is unknown and so, it is not clear which types of App should be tested for App control or eradication programmes Therefore, a study was performed to get;

1. more insights in circulating App serotypes in Dutch herds with unknown history of $A p p$ by performing a serological survey in slaughter pigs and

2. by characterisation of App strains isolated from clinical cases.

\section{Materials \& Methods}

500 serum samples from slaughter pigs, originated from 100 ad random chosen herds ( $n=5$ per herd), were analysed for the presence of App antibodies using 7 different ELISA's. These ELISA's were able to detect antibodies directed against lipopolysaccharides (LPS) antigens of App, specific to one or a group of serotypes 1 - 12 (Biovet, Canada). In addition, 24 serum samples collected from a swine herd, positive by Apx-IV Idexx ELISA but without App related clinical signs, were analysed using the LPS antigens ELISA's. The Bayesian True Prevalence model was used for calculating the App antibody prevalences in Dutch slaughter pigs.

Within the period February - December 2008, 63 App strains were isolated from clinically affected swine after necropsy. The App strains were obtained from other herds than the herds described above. App strains were serotyped by standard coagglutination assay using a panel of type-specific anti-sera against all 15 serotypes (National Veterinary Institute, Denmark). If necessary the serotype of the App strains was further characterised by the immunodiffusion method and/or serotype specific PCR's. The Apx-toxin profiles were determined by PCR.

\section{Results}

Table 1 presents the results of serotype specific ELISA's, including the calculated App seroprevalences in Dutch slaughter pigs. In 142 (28.4\%) of the 500 examined slaughter pigs, no positive reactions were recorded in any of the 7 App antibody ELISA's. In at least $44 \%$ of the examined herds positive reactions were recorded in more than one of the 7 ELISA's.
Table 1: Results ( $n=500$ sera) of the App serotype or serogroup specific ELISA's, and the calculated prevalences with $95 \%$ confidence intervals (CI).

\begin{tabular}{|c|c|c|}
\hline $\begin{array}{c}\text { Serotype(s) } \\
\text { / serogroup }\end{array}$ & $\begin{array}{c}\text { Positive sera } \\
\text { (n) }\end{array}$ & $\begin{array}{c}\text { Prevalences } \\
(\%)[95 \%-C l]\end{array}$ \\
\hline LPS 1,9, 11 & 33 & $3.6[0.2-8.2]$ \\
\hline LPS2 & 180 & $29.1[14.7-38.9]$ \\
\hline LPS 3, 6, 8 & 105 & $19.4[12.0-24.6]$ \\
\hline LPS 4,7 & 106 & $17.1[9.6-23.5]$ \\
\hline LPS 5a, 5b & 12 & $0.4[0.0-1.5]$ \\
\hline LPS 10 & 5 & $0.3[0.0-1.1]$ \\
\hline LPS 12 & 146 & $21.6[14.0-28.6]$ \\
\hline
\end{tabular}

In the 24 swine sera of one Apx-IV ELISA positive herd, antibodies directed against App serotypes 3, 6, $8(n=15), 4,7(n=3)$ and serotype $12(\mathrm{n}=5)$ were detected.

Sixty (95\%) of the $63 \mathrm{App}$ isolates were characterised as serotype 2 (70\%) or 9 (25\%: Table 2). The Apx-toxine profiles of 61 (97\%) of the 63 examined App isolates were similar to one of the serotypes that were detected too by serotyping and/or App typespecific PCR.

Table 2: Results of the serotyping of the App strains and the Apx-toxine profiles ( $n=63)$.

\begin{tabular}{|c|c|c|}
\hline No of isolates $(\mathbf{n})$ & Serotype & Apx-profile \\
\hline $44(70 \%$ & 2 & $2,4,6,8$ \\
\hline $16(25 \%)$ & 9 & $1,5,9,11$ \\
\hline $1(2 \%)$ & $\mathrm{NT}$ & 7,12 \\
\hline $1(2 \%)$ & $9 / 11$ & $1,5,9,11$ \\
\hline $1(2 \%)$ & $13^{*}$ & 7,12 \\
\hline \multicolumn{2}{|l|}{} \\
\hline
\end{tabular}

\section{Discussion}

This study shows that Dutch slaughter pigs have:

- Rather high App antibody prevalences against serotypes $2,3 / 6 / 8,4 / 7$ and 12 , and low antibody prevalences against serotypes 1/9/11, $5 \mathrm{a} / 5 \mathrm{~b}$ and 10 .

- Herds could be positive for antibodies against more than one App serotype

- Antibodies against App serotypes 3/6/8, 4/7 and serotype 12 were found in one herd without clinical signs related to $A p p$ infections

- The serological profile of App serotypes in slaughter pigs differs from the App serotype profile found in clinically affected pigs. In $95 \%$ of these pigs, originated from other herds, App serotypes 2 and 9 were found. 


\title{
P.292
}

\section{A case control study of on-farm risk factors for acute pleuropneumonia in fatteners}

\author{
Nils Holmgren ${ }^{2}$ Nils Lundeheim ${ }^{1}$ Sivert Johansson ${ }^{2}$ Leif Göransson ${ }^{1}$ Gösta Gustafsson ${ }^{4}$ Johan Ehlorsson ${ }^{3}$ \\ 1. Swedish University of Agric. Sciences, Uppsala, Sweden; 2. Swedish Animal Health Service, Skara, Sweden; \\ 3. Swedish Animal Health Service, Ängelholm, Sweden; 4. Swedish University of Agric. Sciences, Alnarp, Sweden
}

\section{Introduction}

Actinobacillus pleuropneumoniae causes pleuropneumonia (PP) in swine throughout the world and can cause severe economic losses (3). Acute outbreaks often appear unpredictable at the end of the fattening period (1). On average $0.6 \%$ of slaughterpigs in Sweden show acute PP, but the variation between herds is substantial 0-60\% (5). Limited options are available to prevent the disease. Improvements in barn climate have not been evaluated as preventive measures. Experiences made by practitioners indicate that feeding may influence on PP. Therefore, the aim of the present study was to look for climatologic and nutritional risk factors for PP.

\section{Material and Methods}

The trial was conducted as a case-control study in 25 barns with documented high, and in 25 barns with low prevalence of PP at slaughter. Averages by quarter of PP-prevalence $(n=8)$ before, during and after the trial were in case-barns $4.6 \%(\mathrm{SD}=0.2 \%)$ and in control-barns $0.2 \%(\mathrm{SD}=0.1 \%)$. Case- and control-barns were matched for slaughter plants, and season at visit (April - Sept).

Measurements of climate and environmental factors were performed in a standardised way by an engineer specialised on climate in pig houses. Measurements taken at herd visits (pig age 20-24w) were: max. ventilation rate; air inlet capacity; underpressure (Dwyer type Mark II); air velocity in the resting zones at different ventilation rates (0-100\%); balance between exhaust and inlet air; heat balance; sectioning between barns; air contamination; functioning of climate regulation equipments; pen hygiene; concentrations of $\mathrm{CO}_{2}$ and $\mathrm{NH}_{3}$. The release (amount) of $\mathrm{NH}_{3}$ in relation to animal density (CR) was calculated (4). Composition of feeds and feed allowances during the fattening period was recorded at farm visits. The statistical analyses were performed by Students t-test and logistic regression, using the SAS software (PROC TTEST; PROC GENMOD; PROC GLIMMIX).

\section{Results}

On average, PP barns had more deficiencies in air inlet and in air outlet capacities compared to control barns. These differences were however not significant. There was a tendency for better control of air in- and outlet in control herds $(p=0.07)$.

Analysed differences between case-control barns are presented in Table 1. In total, 9 possible risk factors differed significantly between the two group of barns. When including all these risk factors as explanatory variables in the logistic regression, 3 of these factors had a $p$-value $=<0.15$ (Table 2).
Table 1. Estimated difference (t-test) of risk factors related to barn climate and feeding

\begin{tabular}{lccc}
\hline Risk factor & $\begin{array}{c}\text { Case } \\
\text { barns }\end{array}$ & $\begin{array}{c}\text { Contr. } \\
\text { barns }\end{array}$ & p-value \\
\hline No.fatteners in herd & 1938 & 888 & $<0.001$ \\
No. barns f. fatteners & 5.3 & 2.9 & $<0.01$ \\
Farrow to finish, \% & 72 & 96 & $<0.05$ \\
NH3, RRm/h & 1165 & 1022 & $<0.05$ \\
NH3-release* & 21.1 & 14.1 & $<0.12$ \\
Bad pen hygiene, \% & 56 & 20 & $<0.01$ \\
Grain, \% of DM & 56.9 & 74.3 & $<0.05$ \\
Alternative feeds, no. & 2.5 & 1.3 & $<0.001$ \\
Energy, MJ day 7-63 & 1551 & 1472 & $<0.05$ \\
\hline * Long-term measurements of CR, average 13h
\end{tabular}

\begin{tabular}{|c|c|c|c|c|}
\hline $\begin{array}{l}\text { Independent } \\
\text { riskfactors }\end{array}$ & Coef & $\begin{array}{l}\text { Conf. } \\
\text { intery. }\end{array}$ & OR & $\begin{array}{l}\text { P- } \\
\text { value }\end{array}$ \\
\hline $\begin{array}{l}\text { No of } \\
\text { fatteners in herd } \\
\text { Baseline } 360-800 \\
820-6000\end{array}$ & 2.77 & $0.5-5.1$ & 16.0 & 0.02 \\
\hline $\begin{array}{l}\text { Energy MJ, day 7-63 } \\
\text { Baseline } 1217-1445 \\
1450-1681\end{array}$ & 1.73 & $-0.1-3.5$ & 5.6 & 0.06 \\
\hline $\begin{array}{l}\text { Alternative feeds } \\
\text { No } \\
\text { Yes }\end{array}$ & 1.31 & $-0.5-3.1$ & 3,7 & 0.15 \\
\hline
\end{tabular}

\section{Discussion}

Climate in barns had unexpected low impact on the occurrence of PP. However, a more intense feeding plan (+1MJ/pig/d) and use of many alternative feed-stuffs had negative impact on pen hygiene. In the final model there were tendencies for alternative feeds and intense feeding to be risk factors for PP. If this is due to an increased metabolic stress in fast growing pigs is unknown and has to be elucidated by further investigations. The number of fatteners in herds had a strong impact on the occurrence of PP. The same risk factor has also been identified for pleurisy in slaughtered pigs (2).

\section{References}

1. Bergström G 2004. Proc.18th IPVS Congr. 563-575

2. Beskow $P$ et al. 2008. Swedish Vet Journal 12;11-18

3. Gottschalk M, Taylor D 2006. Dis. of Swine 563-575

4. Gustafsson G et al. Proc AgEng2000, paper 00-AP-023

5. Swedish Animal Health Service 2008, Postbox 30167 , S-10425 Stockholm 


\title{
P.293
}

\section{Acute-phase responses following repeated infections with Actinobacillus pleuropneumoniae in the presence and absence of medical treatments}

\author{
Marie Sjölund $^{1,2}$ Caroline Fossum² Per Wallgren ${ }^{1,2}$ \\ 1. SVA National Veterinary Institute, Uppsala, Sweden; 2. SLU Swedish University of Agricultural Sciences, Uppsala, Sweden
}

\section{Introduction}

Acute-phase proteins mirror the magnitudes of primary Actinobacillus pleuropneumoniae (APP) infections (1) which are influenced by early initiated antimicrobial treatments, and also correlated to the efficacies of such treatments (2). However, little is known about the acute-phase response following re-infections, especially how it is influenced by responses following previous treatments. Therefore we studied the acute-phase response after a re-challenge with APP in pigs treated with drugs of different efficacies following the first exposure.

\section{Materials and Methods}

30 nine week old SPF-pigs were allocated to 5 groups and housed in separate rooms with a high level of biosecurity. After acclimatisation, 4 groups were inoculated intranasally with $10^{11}$ CFU of APP serotype 2 (APP2). At the onset of clinical symptoms, 20 hours pi, the groups were either treated with enrofloxacin, tetracycline or penicillin. One group served as an inoculated control and did not receive any treatment and one group served as an un-inoculated control group.

On day 28, all 5 groups were challenged with $10^{11}$ CFU of APP2. No treatments were carried out this time (3).

Blood was collected once daily following inoculations and thereafter once or twice weekly (Fig 1). Haptoglobin and Serum amyloid A (SAA) in serum were analysed using commercial assay kits (PHASETMRANGE, Tridelta), according to the manufacturer's instructions.

\section{Results}

Results regarding clinical signs, antibody responses and postmortem findings are well documented and published (3). Acute phase responses mirrored serology and clinical manifestations well (3). Following the first inoculation, both haptoglobin and SAA-levels increased in all inoculated pigs while no changes were observed in the un-inoculated pigs (Fig. 1). Acute-phase responses were not observed in any of the previously inoculated groups except the enrofloxacin treated group following the re-challenge. In contrast, increased levels were recorded in the previously un-inoculated control group and in the enrofloxacin treated group (Fig. 1).

\section{Discussion}

Early medication with enrofloxacin has proven very effective in treating acute actinobacillosis, preventing seroconversion (4). However, these pigs were unprotected upon re-challenge, despite the initiation of an acute-phase response after the primary exposure to APP2 (3). This implies that early treatments with very effective antimicrobials prevent any immunological memory to develop. Consequently, an acute-phase response appears following re-challenge of such animals.
Figure 1. Mean serum concentrations of haptoglobin (a) and SAA (b) following inoculation with A. Pleuropneumoniae serotype 2 on days 0 and 28. Two groups served as controls, one was inoculated on both day 0 and 28 ( $\square)$, and one only on day 28 (). Three groups received antimicrobial treatments for 3 consecutive days after the first inoculation: $(\diamond)$ enrofloxacin, $(0)$ tetracycline and $(\triangle)$ penicillin.

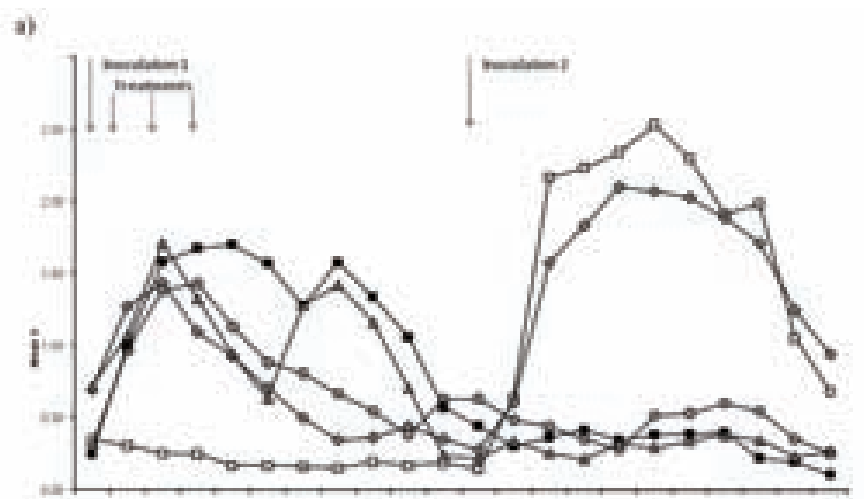

)

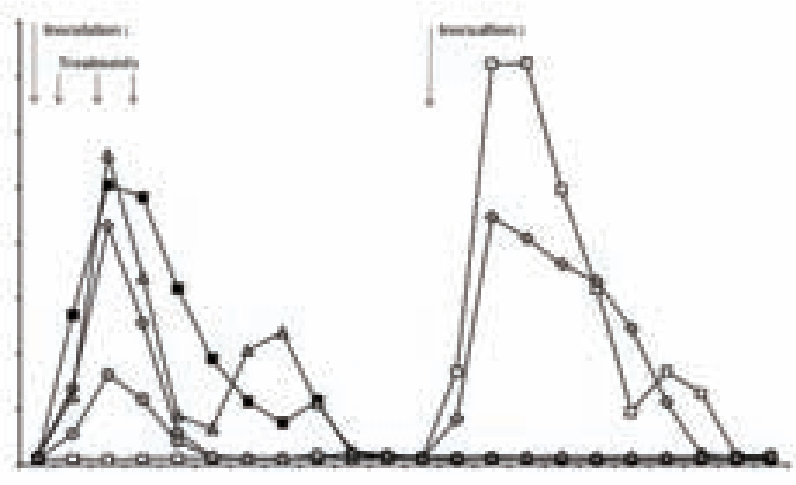

Early treatment with tetracycline was clinically effective, but pigs seroconverted to APP2 as did pigs in the other inoculated groups (3). The seropositive pigs remained unaffected and did not express any acute-phase responses following the re-challenge with APP2. Thus, both haptoglobin and SAA appear to be sensitive indicators of ongoing infections with APP.

\section{References}

1. Heegard et al., 1998, Comp. Biochem. Physiol., 119B(2), 365-373

2. Hultén et al., 2003, Vet. Microbiol., 95(1-2), 75-89

3. Sjölund et al., 2009, Vet. Rec., 164, 550-555

4. Wallgren et al., 1999, J. Vet. Med. B., 47, 727-738 


\title{
P.294
}

\section{Innate immune parameters in the respiratory tract after infection with Actinobacillus pleuropneumoniae}

\author{
Isabel Hennig-Pauka ${ }^{1}$ Doris Hoeltig ${ }^{1}$ Ruediger Koch² Hagen Gasse² Frank Blecha ${ }^{3}$ Karl-Heinz Waldmann Gerald-F. Gerlach ${ }^{4}$ \\ 1. Clinic for Swine and Small Ruminants, Hannover, Germany; 2. Department of Anatomy, University of Veterinary Medicine, Hannover, Germany; \\ 3. Department of Anatomy and Physiology, College of Veterinary Medicine, Kansas State University, Kansas City, USA;
}

4. IVD GmbH, Hannover, Germany

\section{Introduction}

The severity of respiratory tract disease is essentially determined by the quality of immediately activated reactions of the innate immune system as the first line of defense, which is also responsible for naturally occuring disease resistance in breeding lines (1). Understanding local innate mucosal immune responses of the upper and lower respiratory tract will be the basis for the development of new control strategies against disease. The tissue distribution of the antibacterial peptid PR-39 which has been found to be a local respiratory disease marker in BALF has been observed as an example for the constitutive defense readiness of the upper and lower respiratory tract. In addition systemic disease markers as acute phase proteins are involved also in local immune defense mechanisms in the lung (2); they have been examined in this study in healthy pigs; and in pigs infected with Actinobacillus pleuropneumoniae (A.pp).

\section{Materials and Methods}

The immunhistochemical examination of tissue samples was performed in three healthy mock-infected pigs and six pigs infected with different serotypes of A.pp in the acute $(n=2)$ and chronic $(n=4)$ stage of infection. Subsequently samples from the Concha nasalis, Tonsilla pharyngea, Trachea, Lung (Lobus cranialis, Lobus caudalis as well as from altered lung tissue at other locations) and the Lymphonodi tracheobronchales craniales were sampled. For the demonstration of PR-39, sections were stained with a monoclonal mouse anti-PR-39-antibody and a secondary peroxidase-coupled polyclonal goat-anti-mouse antibody. The determination of acute phase proteins Haptoglobin ( $\mathrm{Hp})$, C-reactive protein (CRP), pig major acute phase protein (pig MAP), serum amyloid A (SAA) and the LPS binding protein (LBP) in BALF and serum was performed prior to and at days 4 and 20 after aerosol infection with an A.pp serotype 7 strain AP76.

\section{Results}

PR-39 was detected in all tissue samples of healthy and pneumonic pigs. In healthy lung tissue a homogenous distribution of the peptide was found while a high increase of positive cells could be detected in pneumonic areas (Fig. 1a and b).

$\mathrm{Hp}, \mathrm{CRP}$ and pigMAP were significantly increased in BALF and serum at day 4 after infection, while HP concentrations in BALF further increased to day 21 after infection (Fig 2). SAA was only increased in serum at day 4 after infection, while LBP concentrations showed no significant changes.
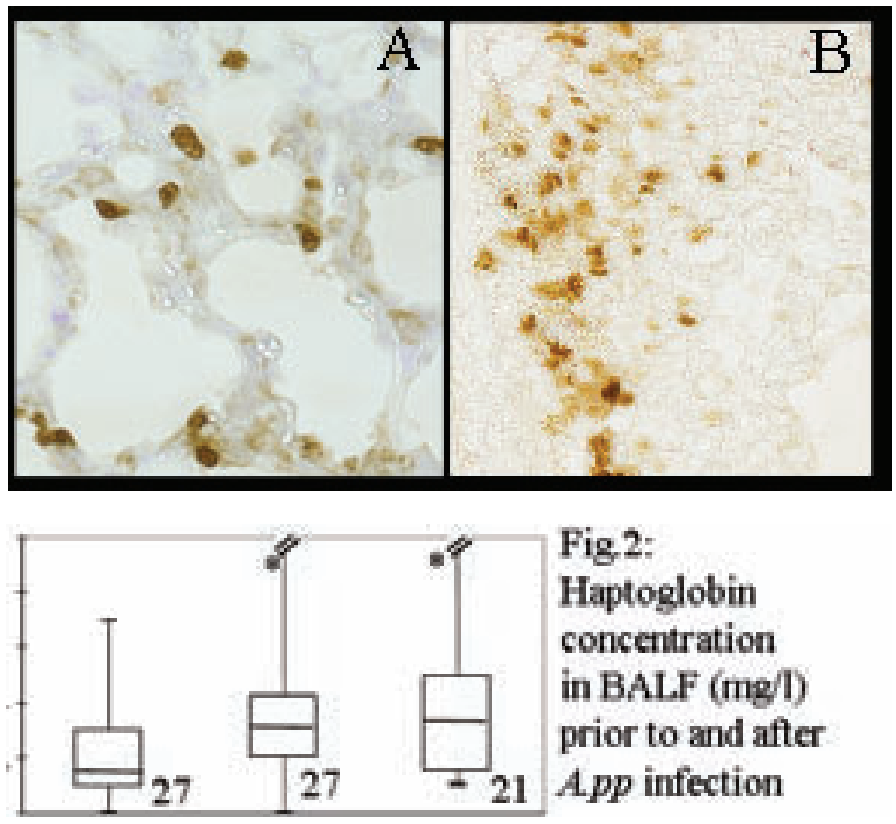

\section{Discussion}

According to the tissue and cell distribution of PR-39 not only neutrophils but also other cells might be responsible for PR-39 expression or uptake. An increase of Haptoglobin in BALF supports the hypothesis of a local production in lung cells, so that it can be considered also as a respiratory disease marker reflecting lung tissue alterations. Day 4 after infection might have been too late to detect early changes in the LBP concentration as a proximate effector molecule of the innate immune system.

\section{References}

1. Hoeltig, D; Hennig-Pauka, I; Thies, K; Rehm, T; Beyerbach, M; Gerlach, GF and Waldmann KH (2009): A novel Respiratory Health Score supports a role of acute lunge damage and pig breed in the course of an Actinobacillus pleuropneumoniae infection. BMC Vet Res, 5: 14

2. Hiss, S; Willbrenning, GS; Suntz, M; Reinacher, M; Sauerwein, H (2008): Immunohistochemical localization of haptoglobin in porcine lungs. Anat Histol Embryol, 37:196-9. 


\title{
P.295
}

\section{Actinobacillus pleuropneumoniae exotoxin Apxl induces expression of proinflammatory cytokines in porcine alveolar macrophages}

\author{
Zeng-Weng Chen ${ }^{1,4}$ Maw-Sheng Chien ${ }^{1}$ Chi-Ming Wu ${ }^{1}$ Nai-Yun Chang ${ }^{1}$ Cheng-Chung Lin \\ Ter-Hsin Chen ${ }^{1}$ Chih-Ming Liao ${ }^{2}$ Joey Yu ${ }^{3}$ Shih-Ling Hsuan ${ }^{1}$ \\ 1. Graduate Institute of Veterinary Pathobiology, College of Veterinary Medicine, National Chung Hsing University, Taichung, Taiwan; \\ 2. Animal Health Division, Bayer Taiwan Co., Ltd,, Taipei, Taiwan; 3. Animal Health Division, Bayer Taiwan Co., Ltd,, Taichung, Taiwan; \\ 4. Graduate Institute of Microbiology and Public Health, College of Veterinary Medicine, National Chung Hsing University, Taichung, Taiwan
}

\section{Introduction}

Actinobacillus pleuropneumoniae (AP) causes fibrinous, hemorrhagic, necrotizing pleuropneumonia in all ages of pigs. The Actinobacillus pleuropneumoniae exotoxins (Apx) are one of the major virulence factors of AP. Previous studies had revealed that pigs endotracheally inoculated with live AP bacteria resulted in the expression of proinflammatory cytokines in lungs, including IL-1, IL-8 and TNF- $a$; however, what types of cells and bacterial components involved in this process remained unidentified (1, 2). Therefore, the objective of this study was to examine whether AP serotype 10-derived exotoxin, Apxl, caused proinflammatory cytokines expression in porcine alveolar macrophages (PAMs), one of the first line defense cells in lung.

\section{Materials and Methods}

PAMs were obtained from 3- to 6-week-old healthy piglets through lavage. Apxl was harvested from the culture supernatant of AP serotype 10. For experiments, PAMs were treated with $0.5-1 \mathrm{CU} / \mathrm{ml}$ or heat-inactivated $A p x I(\triangle A p x l)$ in the presence of polymyxin B for 2-4 h. The mRNA and protein levels of cytokine expression were determined by real-time reversetranscriptase PCR (RT-PCR) and quantitative ELISA (Quantikine ${ }^{\circledR}$, R\&D system), respectively. Proinflammatory cytokines examined included IL-1 $\beta$, IL-8 and TNF- $a$.

\section{Results}

To examine the protein expression level of cytokines under treatment with APP serotype 10 derived Apxl, cytokines IL-1 $\beta$, IL-8 and TNF- a were determined by quantitative ELISA. Significant elevation of IL- $1 \beta$, IL-8 and TNF- a were found in culture supernatants of PAMs at $4 \mathrm{~h}$ after stimulation with Apxl (image1). Levels of these cytokines in $\triangle$ Apxl-treated group did not differ significantly form untreated control group, suggesting that the induction of cytokines was attributable to the bioactivity of Apxl.

To further examine the transcription levels of IL-1 $\beta$, IL-8 and TNFa genes expression, PAMs treated with Apxl were subjected to RTPCR analysis. The results indicated that Apxl induced significantly higher mRNA levels of IL-1 $\beta$, IL-8 and TNF- $\alpha$ in PAMs than that in $\triangle A p x l$ or control group (image2). These data are consistent to the findings in quantitative ELISA and indicate that AP-derived ApxI induces IL-1 $\beta$, IL-8 and TNF- $a$ expression in PAMs.

\section{Discussion}

Overproduction of proinflammatory cytokines has been implicated in the AP-induced lung damage (1). In this study, we demonstrated that Apxl, in addition to its hemolytic and cytotoxic activities, induces the expression of IL-1, IL- 8 and TNF- $\alpha$ in PAMs that might have a role in the pathogenesis of AP.

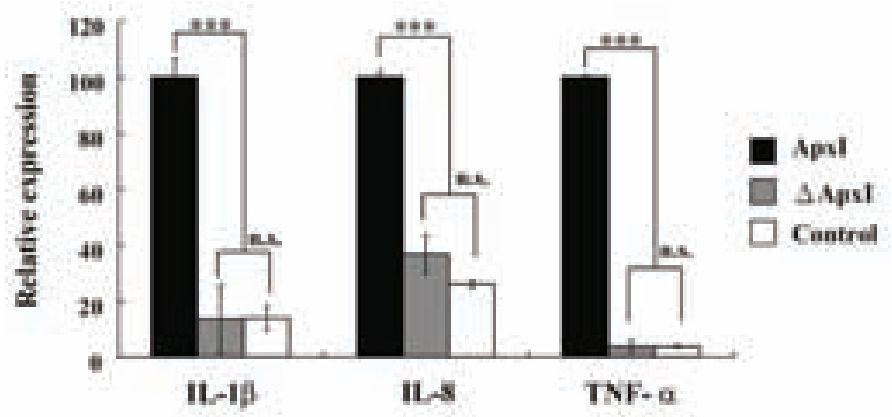

Apxl induced IL-1, IL-8, and TNF- a secretion from PAMs. PAMs were incubated with $1 \mathrm{CU} / \mathrm{ml}$ of Apxl or heat-inactivated $\mathrm{Apxl}(\triangle A p x \mathrm{l})$ for $4 \mathrm{~h}$. Protein levels of cytokines in culture supernatants were determined by quantitative ELISA. ${ }^{* * *} p<0.001$, n.S., no significance.

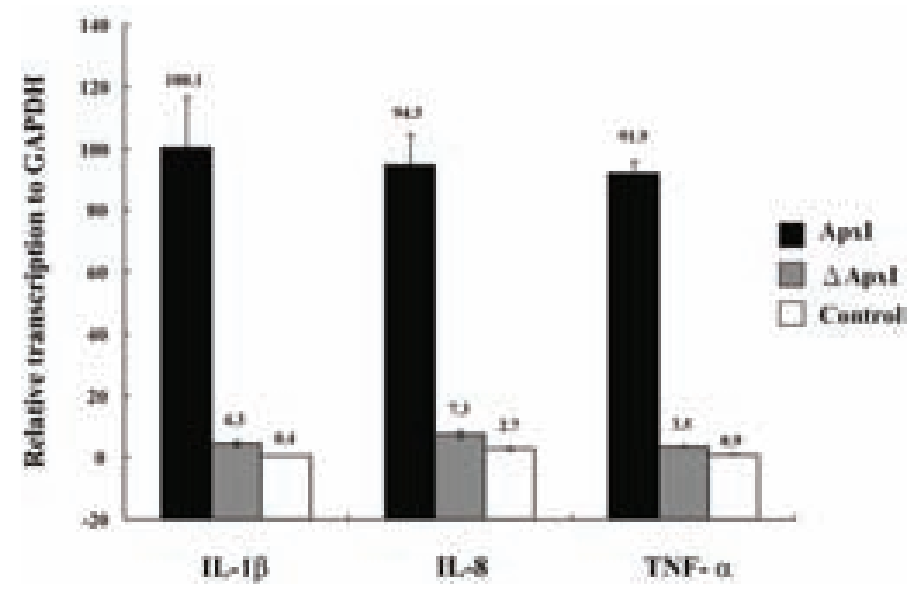

Apxl induced gene expression of IL-1, IL-8, and TNF- $a$ in PAMs. PAMs were incubated with $0.5 \mathrm{CU} / \mathrm{ml}$ of Apxl or heat-inactivated ApxI ( $\triangle A p x I)$ for $2 h$ and subjected to RT-PCR analysis.

\section{References}

1. Baarsch et al., 1995. Infect. Immun. 63:3587-94

2. Baarsch et al., 2000. Am. J. Vet Res. 61:684-90 


\title{
P.296
}

\section{Identification of genetic modulators for susceptibility of pigs to an infection with Actinobacillus pleuropneumoniae}

\author{
Inga Probst ${ }^{1}$ Doris Hoeltig ${ }^{2}$ FUGATO consortium RePoRI ${ }^{2}$ \\ 1. Medizinische Hochschule Hannover, Hannover, Germany; 2. University of Veterinary Medicine Hanover, Foundation, Hannover, Germany
}

\section{Introduction}

Respiratory tract infections in pigs pose a severe economic problem to farmers due to immense costs for antibiotic treatments and increased mortality rate among piglets. Vaccines developed so far only alleviate clinical symptoms but do not prevent colonisation by the pathogens. Therefore there is a strong interest in the identification of genes which modulate the susceptibility of pigs to respiratory tract infections. This work was performed under the auspices of the project FUGATO-IRAS and the ongoing project RePoRI. The aim of the project is the development of genetic markers to predict resistance. By means of these markers a pre-selection of animals with reduced susceptibility to respiratory tract infections shall be enabled in breeding programs.

\section{Materials and Methods}

Highly informative microsatellite markers have been developed for 35 candidate genes. These genes belong either to the innate immune system (e.g. toll-like receptors) or are supposed to be directly related to an infection with $A$. pleuropneumoniae. For identification of suitable microsatellite motifs within or in proximity of the target gene genomic porcine sequences available in the NCBI database or shotgun genomic sequences from porcine BAC clones have been used. Genotyping was carried out using standardized PCR-techniques with subsequent direct-blotting and chemiluminescence detection of the products. By means of these markers two different objectives were pursued. The first aim was the investigation of the current gene pool in different pig populations in Germany. The second aim was the comparative genotyping of pigs from four different breeding lines with a mild or severe course of disease after infection with $A$. pleuropneumoniae in order to identify loci which modulate the susceptibility of the animals to respiratory tract infections. For the diversity study 74 samples from rare and / or old pig breeds and 69 samples from a consumer study (meat samples bought in supermarkets and butcheries in 30 cities all over Germany) were taken. For the association study 100 animals with defined phenotype after infection have been genotyped (infection model standardized by the Tierärztliche Hochschule Hannover).

\section{Results}

The diversity study revealed large differences of the allele distribution among the examined cohorts. The largest allele diversity was observed in the consumer study gene pool whereas the allele pool in the tested breeding lines showed less variation. Unique alleles were only observed in the wild boar population whereas the gene pools of the examined breeding lines represented parts of the consumer study gene pool at each marker locus.

The analysis of the association study data revealed statistically significant differences concerning the genotype distribution of the mildly and severely infected pigs at 7 marker loci mainly belonging to the interferon receptor and tumor necrosis factor receptor superfamilies.

\section{Discussion}

The next step will be the comparative sequencing of homozygous carriers of risk or resistance alleles respectively at the pre-selected loci to enable the identification of SNPs. With these data a haplotype analysis will be performed to minimize the genomic region responsible for the association.

\section{References}

Herold C, Becker T. Genetic association with FAMHAP: a major program update. Bioinformatics 25(1): 134-136 (2009)

Hoeltig D, Hennig-Pauka I, Thies K, Rehm T, Beyerbach M, StrutzbergMinder K, Gerlach GF, Waldmann KH; FUGATO-consortium IRAS. A novel Respiratory Health Score (RHS) supports a role of acute lung damage and pig breed in the course of an Actinobacillus pleuropneumoniae infection. BMC Veterinary Research 21(5): 14 (2009)

Mekus F, Dörk T, Deufel T, Morral N, Tümmler B. Analysis of microsatellites by direct blotting electrophoresis and chemiluminescence detection. Electrophoresis 16(10): 1886-8 (1995) 


\title{
P.297
}

\section{Involvement of Mitogen-Activated Protein Kinases p38 and JNK in Actinobacillus pleuropneumoniae exotoxin ApxI-Induced apoptosis in porcine alveolar macrophages}

\author{
Chi-Ming Wu ${ }^{1}$ Maw-Sheng Chien ${ }^{1}$ Nai-Yun Chang ${ }^{1}$ Zeng-Weng Chen ${ }^{1}$ Chih-Ming Liao ${ }^{2}$ \\ Joey $\mathrm{Yu}^{3}$ Jiunn-Wang Liao ${ }^{1}$ Wei-Cheng Lee ${ }^{1}$ Shih-Ling Hsuan ${ }^{1}$ \\ 1. Graduate Institute of Veterinary Pathobiology, Taichung, Taiwan; 2. Animal Health Division, Bayer Taiwan Co., Ltd, Taipei, Taiwan; \\ 3. Animal Health Division, Bayer Taiwan Co., Ltd,, Taichung, Taiwan
}

\section{Introduction}

Actinobacillus pleuropneumoniae (AP) is the causative agent of fibrinous, necrotic and hemorrhagic pleuropneumoniae in swine. Actinobacillus pleuropneumoniae exotoxins (Apx) are one of the major virulence factors of AP (2). Our previous study has demonstrated that native Apxl at low concentration causes apoptosis in porcine alveolar macrophages (PAMs), one of the first line defense cells in lungs (1). However, the molecular mechanisms underlying Apxl-induced apoptosis are not well understood. Therefore, the aim of this study was to elucidate the signaling pathways mediating Apxl-induced apoptosis in PAMs with focuses on the effect of Apxl on mitogen-activated protein kinase (MAPK) and the role of MAPK in Apxl-induced apoptosis of PAMs.

\section{Materials and Methods}

PAMs were obtained from 3-6-week-old healthy piglets through lavage. The exotoxin Apxl of A. pleuropneumoniae serotype 10 was prepared from bacterial culture supernatant. For experiments, PAMs were treated with 0.5-1 CU/ml Apxl in the presence of polymyxin B for 0-6 h. The levels of active, phosphorylated MAPKs p38 and JNK were determined by Western blot analysis. PAMs were pretreated with a p38 inhibitor (SB203580) or JNK inhibitor (SP600125) for $1 \mathrm{~h}$, followed by Apxl stimulation, Hoechst dye staining, and scored for apoptosis.

\section{Results}

To evaluate the potential role of MAPKs in Apxl effect, the phosphorylated p38 and JNK were assessed by Western analysis for the indicated time periods. The levels of phospho-p38 increased substantially and reached a plateau between 0.5 and $2 \mathrm{~h}$ post Apxl stimulation (Fig. 1A). The phospho-JNK isoforms were observed at $1 \mathrm{~h}$ post-treatment with Apxl and persisted to $4 \mathrm{~h}$ (Fig. 1B).

To further determine the involvement of p38 and JNK in Apxlinduced apoptosis, selective inhibitors was used to pre-treat PAMs prior to stimulation of Apxl and scored for apoptosis. The results indicated that addition of p38 inhibitor and JNK inhibitor caused $\mathrm{a} \sim 45 \%$ and $\sim 35 \%$ reduction in Apxl-induced apoptosis, respectively ( $p<0.05$; Fig. 2). Co-administration of SB203580 and SP600125 prevented PAMs from Apxl-induced apoptosis almost completely $(p<0.001)$. These results demonstrated that $p 38$ and JNK both contributed to apoptosis of PAMs in response to Apxl.

\section{Discussion}

In this study, it was demonstrated that both p38 and JNK were activated in PAMs stimulated with Apxl. Blocking activities of these two kinases rescued PAMs from apoptosis by Apxl. These findings suggest a crucial role of MAPK signaling in Apxl-mediated apoptosis.
Figure 1. Apxl activated MAPKs p38 (A) and JNK (B) in PAMs. PAMs were treated with $1 \mathrm{CU} / \mathrm{ml}$ of Apxl or left untreated for 0 to $6 \mathrm{~h}$ and subjected to Western blot analysis for phosphorylated $p 38$ ( $p-p 38)$ and JNK ( $p-J N K)$. Beta-actin served as a loading control.

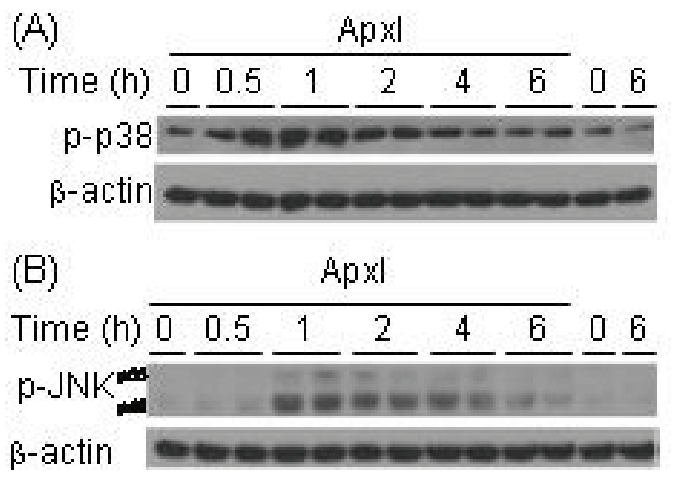

Figure 2. $p 38$ and JNK inhibitors attenuated Apxl-induced apoptosis in PAMs. PAMs pre-treated with $20 \mu M$ of $p 38$ inhibitor SB203580 (SB), JNK inhibitor SP600125 (SP), or both were stimulated with Apxl for $6 \mathrm{~h}$, followed by apoptosis scoring. ${ }^{*} p<0.05 ;{ }^{* * *} p<0.001$. V, vehicle DMSO used to dissolve inhibitors.

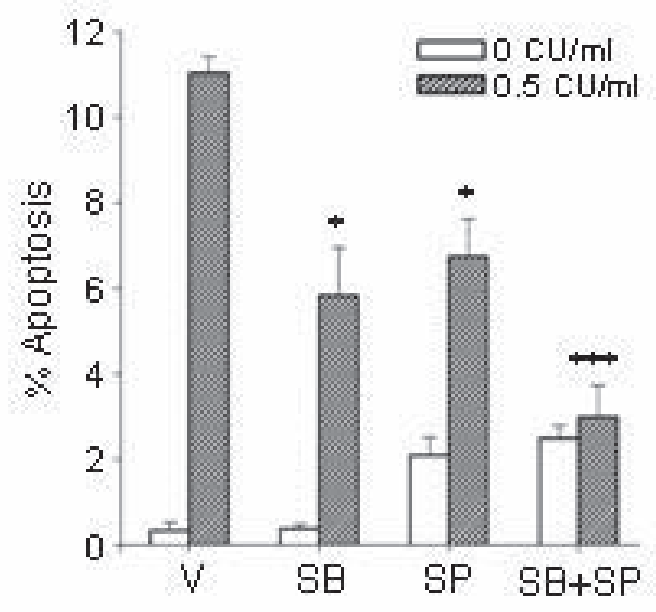

\section{References}

1. Chien et al., 2009. Vet. Microbiol. 135:327-33.

2. Frey J., 1995. Trends Microbiol. 3:257-61. 


\title{
P.298
}

\section{Characterization of apxIVA of Actinobacillus pleuropneumoniae Korean isolate}

\author{
Min-kyoung Shin; Seung-Bin Cha; Won-Jung Lee; Ji-Hyun Sung; Yu-Mi Roh ; Han-Sang Yoo \\ Department of Infectious diseases, College of Veterinary Medicine, Seoul National University, Seoul, Korea
}

\section{Introduction}

Actinobacillus pleuropneumoniae (APP) causes a highly contagious pleuropneumonia in pigs with major economic losses in pig farming worldwide. The four major RTX toxins Apxl, Apxll, ApxIII and ApxIVA are secreted by the different serotypes in various combinations. Compared to Apxl, Apxll and ApxIII, ApxIV is produced by all serotypes of APP, highly specific to APP and expressed only in vivo but not in vitro conditions. Based on the information in GenBank, apxIVA gene was sequenced after PCR amplification from A. pleuropneumoniae serotype 2 Korean isolate. ApxIVA of Korean isolate was analyzed and characterized using determined amino acid sequences after sequencing of the gene.

\section{Materials and Methods}

Primer sequences of apxIVA were designed according to the reference strain (GenBank assession No. AF021919). Primer walking method was performed for nucleotide sequences of unknown region of ApxIVA Korean strain. Extended PCR amplification and sequencing except apxIVA were performed to confirm sequences. ApxIVA of Korean isolate was determined amino acid sequences. Analyzed sequences of apxIVA were compared with the reference strains. In addition, the phylogenetic analysis was performed based on nucleotide and amino acid sequences compared with 5 different APP reference strains (GenBank assession No. AF021919, AF030511, CP000569, GQ332268 and CP000687) using MEGA 4.0 program. The regions of similarity between Korean isolate and the reference strains is represented by a dotplot. The percent identity was performed based on nucleotide sequences and amino acid sequences compared with reference strains using MegAlign 5.0 DNASTAR. The determined amino acid sequences were analyzed to characterize bioinformatics of ApxIVA Korean isolate using ProtScale, emboss polydot and Hphob. / Kyte \& Doolittle program.

\section{Results}

The full length nucleotide size of apxIVA was 5,856bps and confirmed by nucleotide sequencing. ApxIVA of Korean isolate was determined amino acid sequences with a predicted molecular mass of 218,124.77 Da and a theoretical pl of 4.76. ApxIVA Korean isolate gene is preceded immediately upstream by a ORF1. Upstream of ORF1, there is a stem loop structure. This stem loop structure is preceded the C-terminal end of mrp gene. LacZ gene is present the $\mathrm{C}$-terminal of apxIVA. Stem loop structure is located between C-terminal of ApxIVA and LacZ. ApxIVA of Korea isolate was closely related to 5 different reference strains, showing 96.4, 96.1, 96.4, 96.8 and $98.4 \%$ nucleotide homology. ApxIVA Korea isolate showed 100, 95.2, 95.3, 96.2 and 98.2\% homology compared with China(CP000569), Switzerland(AF030511), Canada, China(GQ332268) and Switzerland(AF021919) in amino acid level. Conserved region and repeats are easy to see in ApxIVA Korean isolate by dotplot program. The C-terminal part of ApxIVA Korean isolate contains 30 glycinerich nonapeptides with the consensus sequence L/V-X-G-G-X-G-N/D-D-X. The hydrop- athy plot of ApxIVA Korean isolate was shown by Hphob. / Kyte \& Doolittle program. This analysis revealved a hydrophobic region at the $\mathrm{N}$-terminus and a hydrophilicity in general region.

\section{Discussion}

The amplified apxIVA Korean isolate from A. pleuropneumoniae serotype 2 was confirmed and analyzed by sequencing. Nucleotide and amino acid sequences of whole apxIVA of Korea isolate were similar to the reference strain. Conserved region and repeats are shown in ApxIVA Korean isolate by dotplot program. There are 30 glycinerich nonapeptides with the consensus sequence in the C-terminal part of ApxIVA Korean isolate. The hydropathy plot of ApxIVA Korean isolate revealved a hydrophobic region at the $\mathrm{N}$-terminus and a hydrophilicity in general region.

\section{Acknowledgements}

This work was supported by a grant (20080401034048) from BioGreen 21 Program, RDA, Research Institute of Veterinary Science, BK 21 for Veterinary Science, Seoul National University, Korea.

\section{References}

1. A. Dreyfus. et al. Veterinary Microbiology (2004) 227-238.

2 Hongliang Huang. et al. Molecualar Immunology (2006) 2130-2134.

3. Alain Schaller. et al. Microbiology (1999), 145, 2105-2116. 


\title{
P.299
}

\section{Efficacy of Protective Immune Response for Surface expressed Actinobacillus pleuropneumoniae ApxIIA in Saccharomyces cerevisiae in pig}

\author{
Min-kyoung Shin ${ }^{1}$ Myung Hwan Jung ${ }^{1}$ Seung-Bin Cha ${ }^{1}$ Won-Jung Lee ${ }^{1}$ Yu-Mi Roh ${ }^{1}$ \\ Jung-Mi Kim² Ji-Eun Cho ${ }^{3}$ Dae-Gun Choi ${ }^{3}$ Dae-Hyuk Kim² Han-Sang Yoo ${ }^{1}$ \\ 1. Department of Infectious diseases, College of Veterinary Medicine, Seoul National University, Seoul, Korea; \\ 2. Research Center of Bioactive Materials, Chonbuk National University, Jeonju, Korea; 3. CTCBIO INC. , Hwasung, Korea
}

\begin{abstract}
Introduction
Saccharomyces cerevisiae has been used as a tracer for the oral application of vaccines and drugs because it is relatively stable, nonpathogenic and noninvasive in the gut compared with the other biodegradable vehicles. Oral vaccination of Saccharomyces cerevisiae expressing full-genes of ApxIIA was previously reported and capable of inducing antigen-specific immune responses in murine model. In this study, S. cerevisiae was engineered to anchor the neutralizing epitope of ApxIIA on the outer layer of the cell. Neutralizing epitope of ApxIIA was derived from Actinobacillus pleuropneumoniae serotype 2 Korean isolate. In vitro experiments using porcine alveolar macrophages, surface displayed ApxIIA in S. cerevisiae had effective immune-stimulating activity. Oral vaccination of surfaced ApxIIA in S. cerevisiae was investigated in piglets by comparing the systemic and local immune responses and clinical signs and pathologic lesions after challenge with $A$. pleuropneumoniae serotype 5 Korean isolate.
\end{abstract}

\section{Materials and Methods}

Porcine alveolar macrophages were obtained from 8-week-old healthy piglets through lavage and isolated using previously described procedures (Iglesias et al., 1989). The cells were dispensed at a concentration of $1 \times 106$ cells/ml in 24 well microplates. The cells were stimulated with $4 \times 105$ cells $/ \mathrm{ml}$ of vector control- and surface-displayed ApxIIA in S. cerevisiae and $1 \mu \mathrm{g} /$ $\mathrm{ml}$ of LPS. After stimulation, the concentrations of secreted nitric oxide (NO), TNF- $a$ and IL- 6 were measured with ELISA (R\&D SYSTEMS).

Three-week-old piglets were used in this study. Each experimental group consisted of 5 pigs was deginated into four groups; non-treated group, vector control group, oral vaccinated groups with surface-displayed ApxIIA in S. cerevisiae. This was followed by three-time administrations with gradually increasing amount of the vaccines with 1 week intervals. After last boosting, pigs were challenged by intra-tracheal and intra-nasal injection with A. pleuropneumoniae serotype 5 Korean isolate. ApxIIA-specific immune responses were measured in sera, nasal wash and fecal samples by ELISA. Protectivity was investigated by comparing clinical signs, pathological lesions and histopathologic analysis after challenge with A. pleuropneumoniae serotype 5 Korean isolate.

\section{Results}

The amount of secreted NO, TNF- $a$ and IL- 6 in the supernatant of porcine alveolar macrophages were measured after stimulation with vector control- and surface-displayed ApxIIA in S. cerevisiae, and LPS. While IL- 6 and NO were not measured, the production of TNF- $a$ from porcine alveolar macrophages increased time-dependently after exposure.
Pigs immunized orally with surfaced-ApxIIA in S. cerevisiae maintained a higher ApxIIA-specific IgG response than the nontreated and vector control group in serum. ApxIIA-specific IgA titers of the vaccinated groups were a little higher than the nonvaccinated groups in serum and fecal sample. After challenge with A. pleuropneumoniae serotype 5 Korean isolate, scores of clinical signs and pathological lesions of vaccinated pigs were lower than those of the control group. Survival rate was high in the vaccinated group.

\section{Discussion}

The secretion of TNF- a was evaluated as a parameter of macrophages stimulation. Higher secretion of TNF- a was showed in the supernatant of macrophages exposed to surface-displayed ApxIIA in S. cerevisiae. Porcine alveolar macrophages did not produce any NO after stimulation. Present result lends support to the theory that porcine macrophage are not able to produce NO in vitro. Although the secretion of IL- 6 was not almost induced after $24 \mathrm{~h}$ of stimulation, supplementary experiment needs such as arrangement of experiment schedule and the amount of stimulants for confirmation of this conclusion.

Pigs immunized orally with surface-displayed ApxllA showed ApxllA-specific immune response in serum and nasal wash. Although ApxllA-specific IgA was not significantly increased, protectivity was significantly different in the vaccinated group in the challenge. These results suggested that the feeding surfacedisplayed ApxIIA in S. cerevisiae might be an effective vaccination strategy. Based on these results, more effective oral vaccination strategy should be established through further study.

\section{Acknowledgements}

This study was supported by ARPC (107034-03-3-CG000), BioGreen 21 Program (20080401034048), BK21 for Veterinary Science and Research Institute of Veterinary Science, Seoul National University, Republic of Korea.

\section{References}

1. Sung Jae Shin. et al. FEMS Immunol. Med. Microbiol. 43. (2005) 155-164.

2. Kyung-yeol Lee. et al. FEMS immunol Med Microbiol. 48(2006) 381-389.

3. Petra Zelnickova. et al. Nitric Oxide. 19 (2008) 36-41.

4. Maw-sheng Chien. et al. Vet. Microbiol. 135 (2009) 327-333. 


\title{
P.300
}

\section{Biofilm formation in field isolates of Actinobacillus pleuropneumoniae}

\author{
Corina Pascu; Luminita Costinar; Viorel Herman
}

Faculty of Veterinary Medicine, Timisoara, Romania

\section{Introduction}

Actinobacillus pleuropneumoniae is the causative agent of swine pleuropneumonia, a highly contagious and often fatal respiratory disease that affects pigs worldwide.

Biofilms play a key role in the pathogenesis of many bacterial infections. As a first step toward understanding the mechanism of A. pleuropneumoniae biofilm formation and the role it may play in the pathogenesis of swine pleuropneumonia, we sought to develop methods to observe the biofilm phenotype in the laboratory, and to determine the prevalence of biofilm formation among various $A$. pleuropneumoniae field isolates from Romania. In this report we show that $A$. pleuropneumoniae forms biofilms in glass tubes. This phenotype is stable when A. pleuropneumoniae is cultured on agar but is rapidly lost when cells are cultured in broth.

\section{Material and Method}

All field isolates (30 strains) were from the lungs of pigs with swine pleuropneumonia. All isolates were tested for gram stain reaction and requirement for NAD. Bacteria were grown on $\mathrm{BHI}$ agar and broth or in Mueller Hinton broth. The media were supplemented with $0.1 \%$ of NAD. Cultures were incubated in air at $37^{\circ} \mathrm{C}$ for 24 hours.

\section{Results and Discussions}

Strains formed thick biofilms at the air-liquid interface when cultured in glass tubes with agitation. The biofilm formation phenotype was maintained indefinitely when cultures were passaged on agar but was lost after one or two passages in broth.

Our findings indicate that biofilm formation is a prevalent phenotype among A.pleuropneumoniae field isolates, and that this phenotype may have been previously overlooked because of its tendency to be lost upon subculturing in broth. Biofilm formation may have relevance to the colonization, pathogenesis and transmission of this bacterium.

\section{Conclusions}

Considering the ability of A.pleuropneumoniae strains to produce biofilm on the walls of test tubes in which they had been cultivated in $\mathrm{BHI}$ broth, as a defining indicator of adesin production linked pathogenity, there were found differences among the strains we isolated, only $56 \%$ of our strains had the ability to produce micro-colonies adherent to the tube walls, although all strains were hemolytic.

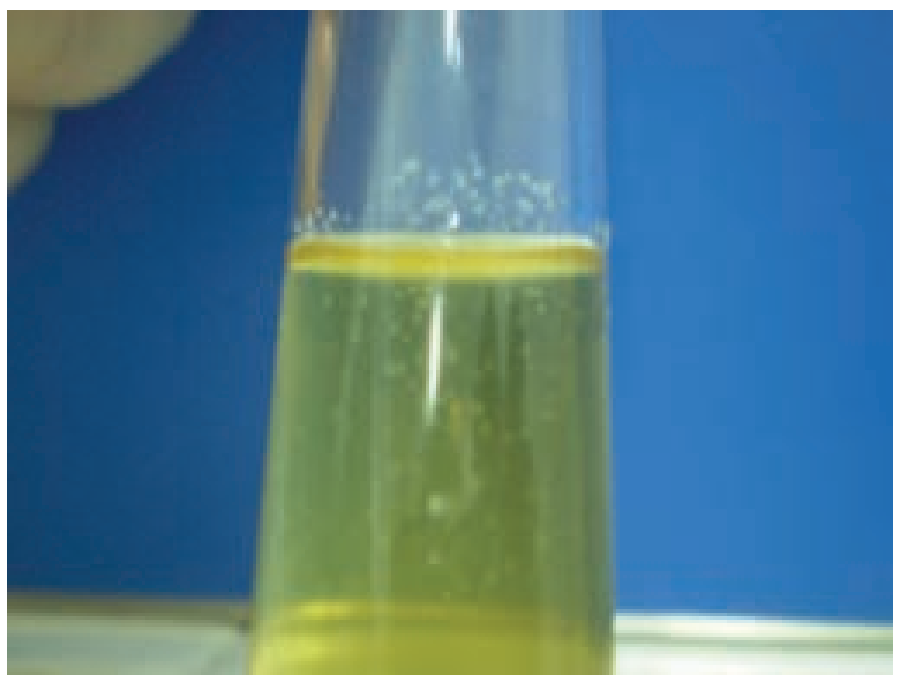

\section{References}

1. Dalai, B., Zhou, R., Wan, Y., Kang, M., Li, L., Li, T., Zhang, S., Chen, H. (2009), Microbial Pathogenesis, 46, 128-134

2. Kaplan, J.B., Mulks, Martha - (2005), Vet. Microbiol., 108, 1-2, 89-94

3. Kaplan, J.B., Velliyagounder, K., Ragunath, C., Rohde, H., Mack, D., Knobloch, J.K.-M., Ramasubbu, N. - (2004), J. of Bacteriol., 186, 24, 8213-8220

4. Li, L. , Zhou, R., Li, T., Kang, M., Wan, Y., Xu, Z., Chen, H. - (2008), Microbial Pathogenesis, 45, 192-200 


\title{
Loop-mediated isothermal amplification for detection of Actinobacillus pleuropneumoniae
}

\author{
Yang Wang; Pin Chen; Yang Chen; Hui Liu; Qigai He \\ State Key Laboratory of Agricultural Microbiology, Huazhong Agricultural University, Wuhan, China
}

\section{Introduction}

Porcine contagious pleuropneumonia is a severe infection caused by Actinobacillus pleuropneumoniae (APP). Timely implementation of effective antimicrobial treatment depends on rapid diagnosis. The apxIVA gene was showed highly specificity in Actinobacillus pleuropneumoniae and does not exist in other species that closed to A.pleuropneumoniae. But not all the strains of A.pleuropneumoniae have high virulent, The strains that product APXI toxin were high virulent strains. Loop-mediated isothermal amplification (LAMP) method was a novel nucleic detection method with high specificity and sensitivity. Using the LAMP method targeting the apxIVA gene and apxl gene, we could rapidly and correctly detect A.pleuropneumoniae infection and the high virulent strains of A.pleuropneumoniae within 1 hour which is more rapid than conventional PCR.

\section{Material \& Methods}

LAMP was carried out in $25 \mu \mathrm{L}$ reactions containing $1.6 \mu \mathrm{M}$ of each inner primer (FIP and BIP), $0.2 \mu \mathrm{M}$ of each outer primer (F3 and B3), $0.4 \mu \mathrm{M}$ of each loop primer (LF and LB), 1.4 $\mu \mathrm{M}$ deoxynucleoside triphosphates, and $1 \mathrm{M}$ betaine (Sigma). Each LAMP reaction also included $20 \mathrm{mM}$ Tris- $\mathrm{HCl}(\mathrm{pH} 8.8), 10 \mathrm{mM} \mathrm{KCl}, 10$ $\mathrm{mM}(\mathrm{NH} 4) 2 \mathrm{SO} 4,6 \mathrm{mM} \mathrm{MgSO} 4,0.1 \%$ Tween 20, $8 \mathrm{U}$ of Bst DNA polymerase large fragment (New England Biolabs), and $1 \mu \mathrm{L}$ of template DNA. The mixture was incubated at $63^{\circ} \mathrm{C}$ for $30 \mathrm{~min}$ in a water bath and heated at $80^{\circ} \mathrm{C}$ for an additional $10 \mathrm{~min}$ to terminate the reaction.

\section{Results}

The LAMP assay amplified the target gene within 30 minutes, and required only a laboratory water bath for the reaction to occur. The apxIVA LAMP and apxI LAMP respectively successful amplified all serovars of $A$. pleuropneumoniae and serovars of $1,5,9,10,11$ which were high virulent strains of $A$. pleuropneumoniae. A total of 100 lung samples were collected from 100 pigs with apparent respiratory problems. The LAMP and PCR methods were also compared in field trials. Eighteen lung tissue samples tested positive for A. pleuropneumoniae by both apxIVA-nested PCR and apxIVA-LAMP methods. We also use apxI PCR and apxI LAMP for the 18 samples. Six samples were test positive by both apxI PCR and apxI LAMP. The PCR and LAMP have a great agreement.

\section{Discussions \& Conclusions}

The LAMP is a novel nucleic detection method with high specificity and sensitivity. In our sensitivity test, we found that the sensitivity of LAMP assay is about 10 times more than that of conventional PCR. In the field trail, ApxIVA-LAMP picked up 10 additional positive samples that were missed by nested-PCR $(p<0.0001$,chi-square test). Based on the apxIVA gene and apxl gene, LAMP assay could detection of $A$. pleuropneumoniae infection and high virulent strains of $A$. pleuropneumoniae with 1 hour which is more rapid than conventional PCR. So the LAMP method is a alternative method for detection of $A$. pleuropneumoniae.

\section{References}

Notomi, T., et al., 2000, Loop-mediated isothermal amplification of DNA. Nucleic Acids Res. 28, e63. 


\title{
P.302
}

\section{Detection of Actinobacillus pleuropneumoniae DNA by quantitative real time PCR}

\author{
T.J. Tobias $^{1}$ A. Bouma ${ }^{1}$ A.J.J.M. Daemen ${ }^{1}$ D. Klinkenberg ${ }^{1}$ J.A. Stegeman ${ }^{1}$ J.A. Wagenaar ${ }^{2}$ B. Duim ${ }^{2}$ \\ 1. Utrecht University, Utrecht, Netherlands; \\ 2. Utrecht University, Faculty of Veterinary Medicine, Dep. Infectious Diseases and Immunology, Utrecht, Netherlands
}

\section{Introduction}

Quantitative detection of Actinobacillus pleuropneumoniae (App) in live pigs is usually done by laborious and relatively nonspecific selective bacterial examination techniques (SBE). PCR techniques for identification of App, described so far, are used for qualitative rather than quantitative detection. In this study we describe the design, development and validation of a quantitative real time PCR ( $q P C R$ ) for the detection of apxIVA DNA, using previously described primers and a newly designed probe.

\section{Material and Methods}

BLAST homology search was performed in development of a probe sequence. A real time PCR protocol was developed for use in a IQ5 PCR machine. Commercially available hot start mix was combined with primers earlier described (APXIVANEST1-F and APXIVANEST1-R1) and a newly designed Taqman probe (with a 5' FAM fluorescent group and at the $3^{\prime}$ end a BHQ group), dissolved in TE-buffer. DNA from pure App cultures and samples from pigs infected with App were isolated according to Instagene ${ }^{\mathrm{TM}}$ Matrix protocol2. Negative and process controls of DNA isolation and qPCR reaction were included to detect possible contamination. App species specific reactions were determined by testing DNA from pure cultures of 15 reference App serotypes, several field isolates of App and other Pasteurellaceae and bacterial swine pathogens. The analytical detection limit was determined with DNA from a 10-fold serial dilution of App serotype 1536 in saline, ranging from $1-5.0 \times 106 \mathrm{CFU} / \mathrm{ml}$. The influence of target clinical material was tested in spiking experiments using the same App1536 dilution series and added to tonsil scrapings. Intra- and inter-assay reproducibility was determined by testing duplet dilution series in triplets on different plates $(n=6)$. For validation with samples from pigs, material from live pigs was obtained from $\mathrm{S}$. suis and App experimentally infected pigs using cotton swabs for samples from the ventral nasal meatus and toothbrushes for tonsil scrapings. Correlation of quantitative detection between SBE on CGVA plates3 and PCR was studied in samples from App infected pigs. For DNA quantification a standard dilution series was included in each PCR run. The statistical analyses were performed using statistical software package $R$ version 2.10.1. (The R Foundation for Statistical Computing). Linear models with threshold cycle as outcome and the log of the bacterial cell concentration, assay and spike and their interactions as explanatory variables. Triplets within assay were analyzed separately as a random effect. The AIC criterion was used to select the best model. Linear regression models described the correlation between SBE and qPCR results in samples from App infected pigs.

\section{Results}

The qPCR product was 377 base pairs. In non App species 26/26 $P C R$ reactions were negative and in App reference serotypes 15/15 PCR reactions tested positive. The analytical detection limit was 1-5 CFU/reaction. Analysis of linearity showed that the regression coefficient of the slope $=-3.30(\mathrm{Cl}-3.36--3.25)$ and the intercept $=42.77(\mathrm{Cl} 42.5-43.0)$, with $\mathrm{R} 2=0.99$, corresponding to a PCR efficiency of $100 \%$ (CI $98.4-103.1 \%)$. Statistical analysis showed no significant intra-assay variability. An effect of interassay variability is observed on the intercept, showing the need to incorporate reference samples in each new assay. Small but non-significant inhibitory effects were found for testing spiked DNA samples. Freezing $\left(-20^{\circ} \mathrm{C}\right)$ of spiked samples before DNA isolation showed to be essential to prevent PCR inhibition. Optimization and standardization of protocols have to be performed to minimize inter assay variability. Results from the correlation between qPCR and quantitative SBE of tonsil scrapings of App infected animals will be presented.

\section{Discussion}

The apxIVA qPCR could be a valuable alternative for quantitative SBE for the detection of App in samples from pigs. Strict compliance to sample handling and DNA isolation protocols is a prerequisite for reproducibility. Future experiments have to provide more data on optimal sampling sites in live pigs to provide an appropriate estimate of the App infection status of a pig. Sensitivity and specificity of the qPCR have to be determined in field validation experiments before implementation as a diagnostic assay can be considered.

Intervet-Schering Plough, Boxmeer, the Netherlands and Central Veterinary Institute, Lelystad, the Netherlands are greatly acknowledged for providing bacterial strains.

\section{References}

1. Schaller, A et.al., 2001, Vet. Microb. 79., 1, 47

2. Frey J, 2003, in: Meth. in molecular biology, 216,87

3. Velthuis AG et.al., 2003, Prev. Vet. Med. 60, 1, 53 


\title{
P.303
}

\section{Identification of strain specific marker genes for Actinobacillus pleuropneumoniae infection in pig}

\author{
Mario Drungowski ${ }^{1}$ Doris Höltig ${ }^{2}$ FUGATO consortium RePoRI ${ }^{2}$
}

1. ATLAS Biolabs GmbH, Berlin, Germany; 3. University of Veterinary Medicine Hannover, Hannover, Germany

\section{Introduction}

Bacterial respiratory tract infections cause severe losses to the pig industry in the European Union and worldwide. Genetic traits associated with porcine host resistance have not been identified to date. The aim of this project is the identification of strain specific genetic markers, putatively associated with resistance to infection caused by the bacterial respiratory tract pathogen Actinobacillus pleuropneumoniae (APP).

\section{Materials and Methods}

For the identification of candidate genes a well established aerosol infection model with APP was used. Pigs of three different strains (German Landrace, Pietrain and Hampshire) were infected, clinically monitored during the stages of infection and probed on day 4 (acute phase) post infection.

The samples were used for transcriptome analysis using the Affymetrix Gene Chip Porcine Genome Array enabling a genome wide analysis of porcine gene expression.

\section{Results}

In the course of the analysis we performed an intensive re-annotation of Affymetrix probe sets since the incomplete sequence of the pig genome still poses a challenge in genome wide data interpretation. We then applied analysis of variance (ANOVA) in order to identify potential strain specific marker genes. The genes identified as being strongly regulated post infection show important differences in the pig strains indicating strain-specific responses to APP lung infection. The candidates were further functionally characterised with enrichment analysis in order to identify affected molecular pathways. Here, we identified predominantly pathway components of immune response related mechanisms. 


\title{
P.304
}

\section{Improvement of APP vaccine through adjuvant selection}

\author{
Sébastien Deville ${ }^{1}$ Laurent Dupuis ${ }^{1}$ Ghislaine lonkoff ${ }^{1}$ François Bertrand ${ }^{1}$ Dmitri Biryuchenkov ${ }^{2}$ \\ Vladimir Rusaleev² Sergey Kukushkin² Anatoli Frolovtseva² Taufic Baybikov² Vladimir Borisov² E. Belik ${ }^{2}$ \\ 1. SEPPIC, Puteaux, France; 2. FGI Federal Centre for Animal Health, Yur'evets, Russia
}

\section{Introduction}

Actinobacillus pleuropneumoniae (APP) is a gram negative bacterium responsible of severe respiratory disease in growing and in finisher pig. This disease is causing significant loss to farmers particularly when finisher pigs are involved. Improvement of existing vaccine can be safety and/or efficacy improvements but also to provide longer lasting protection allowing single vaccine procedure for a lifetime pig protection. Our studies demonstrate the ability of various adjuvants from Montanide ${ }^{\mathrm{TM}}$ range to improve the both safety and efficacy profile of pig APP vaccines.

\section{Materials and Methods}

Experimental vaccines: All experimental vaccine formulations were done by simple dilution of the antigenic media in the adjuvants under a gentle stirring. Various Adjuvants technologies were studied: oil in water emulsified formula (Montanide ${ }^{\mathrm{TM}}$ ISA 15 A VG), Polymeric adjuvant (Montanide ${ }^{\mathrm{TM}}$ Gel 01) and several nanoparticles based formula (Montanide ${ }^{\mathrm{TM}}$ IMS range). Two trials were performed to assess the safety and efficacy of experimental vaccines.

Trial 1: Montanide ${ }^{T M}$ Gel, Montanide ${ }^{T M}$ ISA 15 A VG, Montanide ${ }^{T M}$ IMS 1335 VG, Montanide ${ }^{\text {TM }} 2215$ VG and Montanide ${ }^{\text {TM }}$ IMS 251 CVG. A commercially available vaccine was used as positive control (oil in water emulsified adjuvant) as well as an aluminum salts based formula. The antigen media injected in saline buffer was the negative control. APP seronegative (PCR and ELISA-test) pigs weighting 10 to $15 \mathrm{~kg}$ were vaccinated intramuscularly in the neck twice with $1 \mathrm{ml}$ dose, 28 days apart. Groups of 7 pigs were used except for the negative control group where 9 animals were inserted.

Trial 2: Montanide ${ }^{\mathrm{TM}}$ Gel, Montanide ${ }^{\mathrm{TM}}$ ISA 15 A VG and Montanide $^{\text {TM }}$ IMS 1335 VG. The adjuvant ratio was reduced compared to trial one. APP seronegative pigs weighting 40 to $45 \mathrm{~kg}$ were vaccinated with $1 \mathrm{ml}$ intramuscularly in the neck and injection site dissected 14 days after injection. Groups of 4 pigs were used.

Data collected: Rectal temperature and behavior of the animals were followed during 3 days after each injection. Loins were dissected at the slaughterhouse in order to look for intramuscular local reactions. An internal scale was created allowing the scoring of observed local reactions. Blood samplings were performed before injection, 14 and 28 days after vaccine delivery. ELISA test on individual sera were performed at each sampling date to detect antigen specific lgG antibodies induced after vaccine injection. An infectious challenge was performed 38 days after first injection to assess the protection induced by the vaccines. Lung scoring was used to assess the level of protection in surviving pigs.

\section{Results and Discussion}

Trial 1: Pyrogenic reactions observed after vaccine delivery were insignificant whatever the vaccine (less than $1^{\circ} \mathrm{C}$ ). Nevertheless, pigs showed a transient anorexia during 2 days after each injection. Two kind of local reactions were at the injection site observed: fibrosis and sterile abscess. Montanide ${ }^{\mathrm{TM}}$ IMS $251 \mathrm{C}$ VG and aluminum based formula induced large local reactions while other formulation presented a low occurrence or even no local reaction. When comparing the ELISA results no significant differences could be observed in between groups whatever the sampling date. On the opposite, the challenge demonstrated that only two formulations were able to protect at $100 \%$ the animals: those formulas were based on Montanide ${ }^{\mathrm{TM}}$ IMS $1335 \mathrm{VG}$ and ISA 15 AVG.

Trial 2: Pyrogenic reactions observed after vaccine delivery were unmodified compared to trial 1. Injection site dissection showed that as early as 14 days post injection, the muscle presented a normal aspect at dissection for Montanide ${ }^{\mathrm{TM}}$ ISA 15 A VG and IMS 1335 VG group while small granulomatous fibrosis was observed for the Montanide ${ }^{\mathrm{TM}}$ Gel based formulation.

Montanide ${ }^{\mathrm{TM}}$ based formulations showed different behavior; best results were obtained with Montanide ${ }^{\mathrm{TM}}$ IMS $1335 \mathrm{VG}$ and ISA 15 A VG reaching a $100 \%$ of protection without any impact on the animal welfare or meat quality. Surprisingly, compared to previously published data, the Gel based formulation induced local reactions in the injected muscle. These findings highlight the crucial importance of the antigenic media composition upon the vaccine performance.

\section{Conclusion}

Our study allowed identifying promising vaccine candidate compared to the existing vaccine available for pig farmers. The efficacy performances of our experimental formulations were identical to marketed vaccine while the local and general reactions were reduced these improvements of animal welfare also means a reduced risk of carcass blemish linked to vaccine adverse effect. Field experiments upon larger number of animal will now allow assessing the benefits of the new vaccine in terms of long term protection. 


\title{
P.305
}

\section{Sensitivity of Actinobacillus pleuropneumoniae biovars to three cephalosporins}

\author{
Jaime Maldonado $^{1}$ Laura Valls$^{1}$ Susana Colomer ${ }^{1}$ Marta Fernandez ${ }^{2}$ Jesús Navas ${ }^{2}$ \\ 1. Laboratorios Hipra, Amer, Spain; 2. Facultad de Medicina, Universidad de Cantabria, Santander, Spain
}

\section{Introduction}

Swine pleuropneumonia (SP) caused by Actinobacillus pleuropneumoniae is traditionally controlled through antibiotic treatment. However, antibiotic resistance against widely used drugs like $\beta$-lactamics and tetracyclines has emerged (1). Currently, three generations of cephalosporins are recommended for the empirical treatment of SP. However, little is known about the quantitative activity of these compounds against A. pleuropneumoniae biovar 1 [nicotinamide adenine dinucleotide (NAD) dependent] and even less against biovar 2 (NAD-independent) strains, which are prevalent in some regions (2). We aimed to compare the in vitro activity of cephalexin, ceftiofur and cefquinome against clinical isolates of A. pleuropneumoniae, representing most prevalent serovars in biovars 1 and 2 .

\section{Materials and Methods}

Minimum Inhibitory Concentration (MIC) determinations were performed by the macrodilution broth method with twentynine A. pleuropneumoniae clinical isolates belonging to biovar $1(n=19)$ serovars 2, 4, 5, 9 and 11 , and biovar $2(n=10)$ serovars 2 , 7 and NT (non-typable). Isolates (2002-2008) came from pneumonic lungs of pigs housed in fattening units located in seven Spanish provinces.

Bacterial inoculums ( 5 x105 UFC/ml) were grown on Veterinary Fastidious Medium broth supplemented with $0.025 \%$ of NAD at $37{ }^{\circ} \mathrm{C}, 5 \% \mathrm{CO} 2$ for $24-48 \mathrm{~h}$ (3). The MIC value of each drug tested corresponded to the lowest concentration of the compound that completely inhibited the visible growth of the tested isolate under the established conditions. The cut-off for resistance of A. pleuropneumoniae to cephalexin and ceftiofur/cefquinome were MICs $\geq 32 \mu \mathrm{g} / \mathrm{ml}$ and $\geq 8 \mu \mathrm{g} / \mathrm{ml}$, respectively.

\section{Results and Discussion}

Overall, cephalexin was the drug against which a higher number of isolates showed resistance and susceptible ones exhibited higher MIC values (always $\geq 1 \mu \mathrm{g} / \mathrm{ml}$ ) (Table 1 ).

Although only one strain was resistant to ceftiofur and cefquinome, when CMI values were compared at the strain level for these two compounds (data not shown) lower MICs were obtained in 15 isolates susceptible to ceftiofur, while cefquinome showed higher activity only against two isolates. The remaining 12 isolates that were susceptible to both drugs showed identical CMIs.
Figure 1. CMI values for 29 A. pleuropneumoniae clinical isolates. Shadow areas correspond to the range of drug dilutions tested and the number of isolates in each CMI; dotted lines indicate the cut-off for resistance.

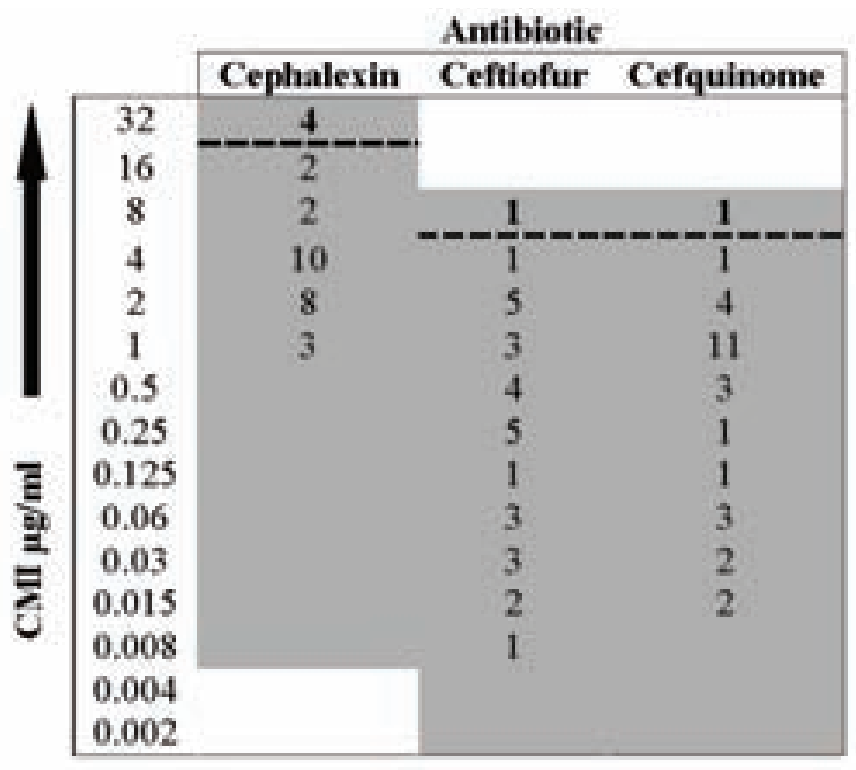

We tested few samples in each biovar to establish differences in sensitivity between them in a reliable way. However, we observed a trend towards a higher average MIC values for biovar 1 isolates (data not shown). Ongoing work is focused on testing more isolates, particularly of the biovar 2 that seems to emerge as an important swine pathogen in some areas.

All together, these findings indicate that ceftiofur is the most active antibiotic against $A$. pleuropneumoniae compared to cephalexin and cefquinome.

\section{References}

1. Aarestrup FM, et al. 2008. Anim Health Res Rev. 9:135-48.

2. Maldonado J, et al. 2009. J Vet Diagn Invest. 21:854-57.

3. Clinical and Laboratory Standards Institute. Documents M31-A y M31-A2. 


\section{P.306}

\section{Antimicrobial susceptibilities of Canadian isolates of Actinobacillus pleuropneumoniae}

Marie Archambault; Julien Gouré; Josée Harel; Mario Jacques

Université de Montréal, St-Hyacinthe, QC, Canada

\section{Introduction}

Actinobacillus pleuropneumoniae is the causative agent of porcine pleuropneumonia, a severe and highly contagious respiratory disease responsible for economic losses in the swine industry worldwide. To date, 15 serotypes have been described based on capsular antigens $(1,2)$. Although antimicrobial resistance in A. pleuropneumoniae has been recently reported in Europe (3-5) and in Japan (6), the current situation in Canada is unknown. The aim of the present study was to determine the antimicrobial susceptibility patterns of 43 strains of $A$. pleuropneumoniae isolated in Canada.

\section{Materials and Methods}

Bacterial isolates and growth conditions. A total of 43 isolates were recovered from swine herds in Saskatchewan, Ontario and Québec (Table 1). Isolates were cultivated overnight on chocolate agar plates supplemented with PolyVitex at $37^{\circ} \mathrm{C}$ with $5 \% \mathrm{CO}_{2}$.

Table 1. Distribution of the 43 clinical isolates by serotypes and provinces.

\begin{tabular}{|l|c|c|c|c|c|c|c|}
\hline & $\mathbf{1}$ & $\mathbf{5 a}$ & $\mathbf{5 b}$ & $\mathbf{3 ( 8 )}$ & $\mathbf{7}$ & $\mathbf{1 2}$ & $\mathbf{1 5}$ \\
\hline Québec $^{1}$ & 1 & 3 & 2 & 1 & 5 & - & - \\
\hline Ontaro $^{2}$ & 4 & - & 4 & - & 4 & - & - \\
\hline Saskatchewan $^{3}$ & 2 & 3 & 4 & - & 3 & 1 & 6 \\
\hline
\end{tabular}

Antimicrobial susceptibilities. The antimicrobial susceptibilities of the isolates were determined by the micro-dilution method using the commercial BOPO1F Sensititre 96-well microtitre plates (Trek Diagnostic Systems Inc., UK) in Veterinary Fastidious Medium in accordance with the guidelines provided by the Clinical and Laboratory Standards Institute (CLSI, M31-A3). The plates were incubated for $20-24 \mathrm{~h}$ at $35^{\circ} \mathrm{C}$ in $5 \% \mathrm{CO}_{2}$. The minimal inhibitory concentration (MIC) value was considered to correspond to the first dilution at which no bacterial growth was detectable. The MICs that inhibited 50\% ( $\mathrm{MIC}_{50}$ ) and 90\% $\left(\mathrm{MIC}_{90}\right)$ of the isolates were also recorded. Resistance breakpoints were used according to the CLSI document M31-A3.

PCR detection of resistance genes. PCR amplification of resistance genes was performed using Taq DNA polymerase and specific oligonucleotide primers.

\section{Results and Discussion}

All isolates were susceptible to ceftiofur, florfenicol, tilmicosin and trimethoprim/sulfamethoxazole (Table 2). The mean \% of resistance to penicillin and ampicillin was $16 \%$ based on the presence of the gene $b / a_{\mathrm{ROB}-1}$ since there are no specific breakpoints for A. pleuropneumoniae for these drugs. We observed a high level of resistance to chlortetracycline (88.4\%) and oxytetracycline (90.7\%). The strains showing resistance to tetracycline antimicrobials contained at least one of the following tet genes: tet $B$, tet $O$, tet $C$, or $t e t H$. The high level of resistance to tetracyclines is similar to what was previously reported in Spain (3). A low level of resistance was observed toward tiamulin (7\%). Five isolates showed multi-resistance to $\beta$-lactam, aminoglycoside and tetracycline antimicrobials.

Table 2 . MICs obtained for the 43 clinical isolates.

\begin{tabular}{|l|c|c|c|}
\hline Antimicrobial & MIC $_{50}$ & MIC $_{90}$ & \% of resistance \\
\hline Ampicillin & 0.25 & $>8$ & 16.3 \\
\hline Ceftiofur & $\leq 0.25$ & 0.5 & 0 \\
\hline Chlortetracycline & 4 & $>4$ & 88.4 \\
\hline Clindamycin & $>1$ & $>1$ & $\mathrm{nc}$ \\
\hline Danofloxacin & $\leq 0.06$ & 0.25 & $\mathrm{nc}$ \\
\hline Enrofloxacin & $\leq 0.06$ & 0.25 & $\mathrm{nc}$ \\
\hline Erythromycin & $>2$ & $>2$ & $\mathrm{nc}$ \\
\hline Florfenicol & 0.25 & 0.25 & 0 \\
\hline Gentamicin & $>4$ & $>4$ & $\mathrm{nc}$ \\
\hline Neomycin & $>16$ & $>16$ & $\mathrm{nc}$ \\
\hline Oxytetracycline & $>4$ & $>4$ & 90.7 \\
\hline Penicillin & 0.5 & $>4$ & 16.3 \\
\hline Spectinomycin & $>32$ & $>32$ & $\mathrm{nc}$ \\
\hline Sulfachloropyridazine & 64 & $>128$ & $\mathrm{na}$ \\
\hline Sulfadimethoxine & $>128$ & $>128$ & $\mathrm{na}$ \\
\hline Sulfatiazole & 128 & $>128$ & $\mathrm{na}$ \\
\hline Tiamulin & 16 & 16 & 7 \\
\hline Tilmicosin & 8 & 8 & 0 \\
\hline Trimethoprim/Sulfa & $\leq 0.25$ & $\leq 0.25$ & 0 \\
\hline $\begin{array}{l}\text { nc, no CLSI resistance breakpoints available for these drugs for A. pleuropneumoniae; na, not } \\
\text { available since the breakpoint is higher that the highest concentration tested in the range. }\end{array}$ \\
\hline
\end{tabular}

\section{References}

1. Blackall et al. 2002, Vet Microbiol $84: 47$.

2. Dubreuil et al. 2000, Anim Health Res Rev $1: 73$.

3. Gutierrez-Martin et al. 2006, Vet Microbio $115: 218$.

4. Hendriksen et al. 2008, Acta Vet Scand $50: 19$.

5. Matter et al. 2007, Vet Microbiol $122: 146$.

6. Morioka et al. 2008. J Vet Med Sci $70: 1261$. 


\title{
P.307
}

\section{Actinobacillus pleuropneumoniae (APP) Elimination with injectable Enrofloxicin and early weaning}

\author{
Hans Rotto ${ }^{1}$ John Swart ${ }^{2}$ \\ 1. IAS LLC Veterinary Clinic, Ames, IA, USA; 2. Exemplar Genetics, Sioux Center, IA, USA
}

\section{Introduction}

Actinobacillus pleuropneumoniae (APP) is an economically significant pathogen in the swine industry.(1) The control of APP associated disease through feed medication and delivery can be difficult for some herds. The trial group was a 75 head sow herd farrow to finish operation composed of APP positive gilts, sows, and boars. The objective of this report was to eliminate APP serotype 3, 5 and 7 from the off-spring of an APP positive sow herd using only injectable antibiotics and early weaning. The APP negative off-spring would then be used to establish a new herd.

\section{Materials and Methods}

The APP elimination medication plan is as follows:

The first step was a whole herd (sows, gilts, boars and growing pigs on site) injection with Baytril at $1 \mathrm{cc} / 29.4 \mathrm{lbs}$. (7.5 mg/kg). The whole herd injection with Baytril was repeated five days later. Sows and gilts were injected again using the same dose two days prior to their projected farrow date throughout the duration of the elimination program. Piglets were given Naxcel (ceftiofur) per label dose at birth in combination with iron. Piglets were given $0.5 \mathrm{cc}$ of Baytril at eight days of age and again at weaning. All piglets were weaned off-site by fourteen days of age into clean environments with limited risk for APP exposure. The first two nursery diets had Denagard / CTC in the ration post weaning. The feed was non-medicated after the first two rations.

The APP diagnostic test plan was as follows:

The first diagnostic tests were a serological examination of the herd prior to purchase. Following purchase, tonsil scrapings were pooled in groups of three and submitted for APP PCR testing to establish the current prevalence of positive pigs within the population. This same testing method was used for APP PCR testing of medicated early weaned pigs post-weaning. At maturity gilts and boars were serologically tested using the Multi-APP screen to monitor for potential APP infection and/or spread.(2)

\section{Results}

Before implementation, the initial screening of the herd using APP serotype testing showed positive pigs for APP 3, 5 and 7. Post purchase $24 / 26$ pools of sows, boars and gilts were positive for APP by PCR. Two of five tonsillar pools from growing pigs, (6 to 14 weeks of age) were also positive. Approximately 30 days after the second whole herd medication treatment, nine random tonsil scrapings were pooled in groups of three from the resident herd and were negative by PCR. The first medicated early weaned pigs were sampled at approximately five weeks post wean and were individually negative by APP PCR and APP culture on tonsillar scrapings. Three tonsils from adult breeding stock were submitted and were negative by APP PCR and for APP culture. Later, eight pools of tonsil scrapings from growing pigs were submitted for APP PCR and were negative. Five months after the first pigs were weaned, serology was used to screen the oldest gilts. One positive test to the Multi-APP screen was found. Roommates were tested and 4/14 were serologically positive. The positive gilts were found to be positive for APP serotype 5 on further testing. PCR testing of ten pooled tonsil scrapings were negative in growing pigs. Due to the lack of spread between pen-mates and limited positive gilts on serology and APP PCR, it was determined that all breeding age gilts would be screened using the Multi-APP test. Any suspects or positives were not used for the re-population of the new herd. Serological testing of the gilts at six to eight months of age using the Multi-APP test determined that 4/61 gilts would not be used. No antibodies have been detected in recent tests of gilts at 6 to 7 months of age.

\section{Discussion}

APP negative gilts and boars were produced from the positive herd to establish an APP serotype 3, 5 and 7 free sow herd. Gilts have remained negative by PCR and culture of tonsillar scrapings for APP. The Multi-APP screening test detected some antibodies suggesting exposure in few individual gilts; however, there was no evidence of APP spread in pens, rooms or barns. On previous APP elimination trials Multi-APP serological screening has shown cross reactivity with other APP serotypes.(3) Test results indicate the herd has eliminated the APP 3, 5 and 7 from retained gilts and boars. The use of injectable Enrofloxicin and early weaning was successful in limiting the vertical spread of APP.

\section{References}

1. Taylor, D. Diseases of Swine 8th Ed. 1999. 343-354.

2. Gottschalck, M. , Actinobacillus pleuropneumoniae serotypes, pathogenicity and virulence. 2007 AASV Proceedings 381-384.

3. Rotto H., Claborn, J. Actinobacillus pleuropneumoniae case study elimination and diagnostic test decisions. 2009 AASV Proceedings 435-437. 


\title{
P.308
}

\section{Efficacy of COGLAPIX ${ }^{\circledR}$ in susceptible piglets against a challenge with Actinobacillus pleuropneumoniae; evaluation of post-mortem findings, clinical data and immune response}

\author{
Miklos Tenk ${ }^{1}$ Georges Országh ${ }^{1}$ János Benyeda ${ }^{2}$ Imre Biksi $^{3}$ Tamás Kis $^{4}$ István Alapi ${ }^{1}$ \\ József Herczeg ${ }^{1}$ Jérôme Thevenon ${ }^{1}$ Bálint Kupcsulik¹ Botond Siklódi ${ }^{1}$ Benaouda Kadra1 \\ 1. CEVA-Phylaxia Veterinary Biologicals Co. Ltd., Budapest, Hungary; 2. Prophyl Immunolab, Mohács, Hungary; \\ 3. Szent István University, Faculty of Veterinary Sciences, Budapest, Hungary; 4. SCG Bt., Budapest, Hungary
}

\section{Introduction}

Actinobacillus pleuropneumoniae is a major pathogen of pigs causing porcine pleuropneumonia, which generates significant economical losses for the swine industry. Among other virulence factors, the APX toxins belonging to RTX toxin family play a significant role in the pathogenesis of A. pleuropneumoniae. The combination of bacterins and inactivated APX toxins have been shown to be cross-serotype protective. The bacterin-toxoid vaccine, Coglapix ${ }^{\circledR}$, using the optimal combination of somatic antigens together with Apx toxoids (Apxl, ApxII \& ApxIII) has been developed to provide effective protection against infection caused by various serotypes of A. pleuropneumoniae.

\section{Materials and Methods}

Coglapix ${ }^{\circledast}$ and a competitor vaccine was injected to 9-week-old A. pleuropneumoniae sero-negative piglets 2 times 3 weeks apart, using the recommended dose of the vaccines. A group of non vaccinated animals was also included. Each group consisted of 8 animals. Seven weeks after the 2 nd vaccination, the animals were challenged intranasally with a broth culture of a virulent serotype- 2 A. pleuropneumoniae strain in a dose of $5 * 106 \mathrm{col}-$ ony forming units per animal. Serum samples were taken on day (D) 0, D21, D35, D69 and D76. The animals' immune response was followed with Swinecheck APP 2, Swinecheck APP 1, 9, 11 (Biovet, Canada) and Apxl, ApxII and ApxIII in-house developed monoclonal-antibody based ELISAs. After the challenge (D69), the animals were monitored daily for the presence of general and respiratory symptoms. The rectal temperatures were also measured on a daily basis from D69 to D76. The body weight gain was recorded between D69 and D76. After 1 week, (D76) the animals were slaughtered and necropsied. The lung changes were scored according to EP:1360. The App bacteria were recovered from the lungs using suitable culture methods. The microscopic lung lesions were observed in paraffin-embedded, haematoxylin-eosin stained sections.

\section{Results}

In this study, both Coglapix ${ }^{\circledR}$ and the Competitor vaccine showed similar protection in terms of preventing from lung lesions.

The cumulative results of clinical and pathological observations are presented in Table 1.
Table 1. Average clinical and pathological scores of the nonvaccinated controls and the animals vaccinated with Coglapix and the Competitor vaccine

\begin{tabular}{|l|c|c|c|}
\hline & Coglapix $^{\circledast}$ & Competitor & Control \\
\hline No. of pigs & 8 & 8 & 8 \\
\hline Clinical score $(\mathrm{m} \pm \mathrm{sd})$ & $2.0 \pm 2.0$ & $3.4 \pm 2.9$ & $6.8 \pm 10.7$ \\
\hline Lung score $(\mathrm{m} \pm \mathrm{sd})$ & $2.6 \pm 3.1$ & $3.9 \pm 3.6$ & $5.9 \pm 8.9$ \\
\hline Histo-path. score $(\mathrm{m})$ & 4.6 & 5.5 & 5.4 \\
\hline
\end{tabular}

The serological titers before vaccination have proven the seronegativity by all of the 5 ELISA systems used.

The negative control group remained sero-negative until the date of challenge.

Both vaccines induced high titers already with first vaccination, which was boosted by the second one.

In general, the Competitor vaccine induced higher antibody titers against somatic antigens. Overall, the Competitor induced higher anti-Apxl titers, the anti-Apxll titers were similar whereas Coglapix ${ }^{\circledast}$ induced higher anti-Apx III titers.

The bacteriological culture results showed higher recovery rate from the Competitor-vaccinated group then the Coglapix ${ }_{-}$vaccinated one, with a 2.25 times higher odds ratio.

\section{Discussion}

Coglapix ${ }^{\circledast}$ vaccine has proven to have similar protective features as the Competitor in this challenge. Further studies needed involving more challenge strains to prove the broad-range protective efficacy of the vaccine, which has already been shown under field conditions

\section{References}

1. Ramjeet M. et al (2008). Anim Health Res Rev. 9:25-45.

2. European Pharmacopoeia, 01/2008:1360.

3. Horváth et al (2008) 20th IPVS, Poster presentation. 


\title{
P.309
}

\section{Clinical field trial of COGLAPIX ${ }^{\circledR}$ (App vaccine) in pigs in The Philippines}

\author{
Romeo Sanchez² Eric Brunier ${ }^{3}$ Georges Országh ${ }^{1}$ Miklos Tenk Benaouda Kadra1 Jérôme Thevenon ${ }^{1}$ \\ 1. CEVA-Phylaxia Veterinary Biologicals Co. Ltd., Budapest, Hungary; 2. College of Veterinary Medicine, University of the Philippines, \\ Los Banos, Philippines; 3. CEVA Animal Health Asia Pacific, Malaysia, Selangor, Malaysia
}

\section{Introduction}

A new Actinobacillus pleuropneumoniae (App) vaccine has been developed. The formulation of this new App vaccine was optimized to obtain a good balance between safety and efficacy.

The vaccine was thoroughly evaluated in field conditions, in pigs in The Philippines, using competitor vaccines for the comparison.

\section{Materials and Methods}

Coglapix ${ }^{\circledast}$ contains inactivated, native Apx antigens (Apxl, Apxll \& ApxIII) and somatic antigens of App. The vaccine is adjuvanted with aluminium hydroxide gel. It is administered by IM route $(2$ $\mathrm{ml} / \mathrm{pig}$ ) and two injections are given at 6 and 9 weeks of age.

This trial was carried out in The Philippines, in one farrow-to-finish farm, positive for App. Altogether, 300 pigs were allocated in 5 treatment groups ( 1 control and 4 vaccinated groups, Table 1.).

Table 1: Vaccines used in the clinical field trial

\begin{tabular}{|c|c|}
\hline Group 1 & Bacterin vaccine serotypes 1,2,3,4,5,7 \\
\hline Group 2 & Toxoid and 0MP vaccine \\
\hline Group 3 & Coglapix \\
\hline Group 4 & Control group \\
\hline Group 5 & Toxoid and 0MP vaccine \\
\hline
\end{tabular}

The vaccines were applied at $6 \& 9$ weeks of age. Precise clinical observations were performed on 15 pigs / group: general reactions and rectal temperatures. The bodyweights of all pigs were measured regularly ( 5 times) and feed conversion rate (FCR) was also calculated.

The seropositivity for App was tested before vaccination on 15 sows and 15 marketed hogs (5-6 months of age), using IDEXX CHEKIT APP-ApxIV ELISA kit.

From each group, 20 pigs were examined at slaughter where lung lesions were recorded using a scoring system of a maximum of $28 /$ lung (4/lobe).

\section{Results}

Out of the 15 sampled sows 12 (80\%), whereas from the 15 sampled hogs 5 (33\%) was seropositive.

The average rectal temperature increase values by vaccine group are presented in Table 2.
Table 2: Summarized safety results of the field trial

\begin{tabular}{|c|c|c|c|c|}
\hline $\begin{array}{c}\text { Group (n=60 } \\
\text { / group) }\end{array}$ & $\begin{array}{c}\text { Vaccine } \\
\text { treatment }\end{array}$ & $\begin{array}{c}\text { Temp. increase } \\
\left.\text { (in }{ }^{\circ} \text { @ @ v1 \& v2) }\right)\end{array}$ & $\begin{array}{c}\text { Post-vaccinal } \\
\text { reactions }\end{array}$ & Comments \\
\hline 1 & $\begin{array}{c}\text { s1,2,3,4,5,7 } \\
\text { bacterin }\end{array}$ & $+0.7+0.2$ & None & $/$ \\
\hline 2 & Toxoid \& 0MP & $+1.2+1.5$ & $2 / 15$ @ V1 \& V2 & $\begin{array}{c}\text { Dyspnoea, depression, } \\
\text { vomiting \& skin } \\
\text { discoloration }\end{array}$ \\
\hline 3 & Coglapix ${ }^{\circledR}$ & $+0.7+0.2$ & None & $/$ \\
\hline 4 & Saline control & -0.10 .0 & None & $/$ \\
\hline 5 & Toxoid \& 0MP & $+1.4+1.1$ & $1 / 15$ @ V1 & $\begin{array}{c}\text { Hyper-salivation \& } \\
\text { incoordination }\end{array}$ \\
\hline
\end{tabular}

No post-vaccinal adverse reactions were observed in Coglapix ${ }^{\circledast}$ vaccinated piglets unlike in the piglets from groups 2 and 5 .

Table 3: Summarized efficacy results of the field trial

\begin{tabular}{|c|c|c|c|c|}
\hline $\begin{array}{c}\text { Group (n=60 } \\
\text { / group) }\end{array}$ & $\begin{array}{c}\text { Vaccine } \\
\text { treatment }\end{array}$ & $\begin{array}{c}\text { ADG (g/d) } \\
\text { weaning-slaughter }\end{array}$ & $\begin{array}{c}\text { FCR } \\
\text { (60d-market) }\end{array}$ & $\begin{array}{c}\text { Lung score } \\
\text { average (mean) }\end{array}$ \\
\hline 1 & $\begin{array}{c}\text { s1,2,3,4,5,7 } \\
\text { bacterin }\end{array}$ & $513^{\text {a }}$ & 3.24 & 5.95 \\
\hline 2 & Toxoid \& 0MP & $506^{\mathrm{a}}$ & 3.26 & 5.85 \\
\hline 3 & Coglapix $^{\circledR}$ & $526^{\mathrm{b}}$ & 3.15 & 4.05 \\
\hline 4 & Saline control & $500^{\mathrm{a}}$ & 3.36 & 7.45 \\
\hline 5 & Toxoid \& 0MP & $517^{\mathrm{a}}$ & 3.21 & 5.35 \\
\hline
\end{tabular}

Coglapix ${ }^{\circledR}$ vaccinated pigs have shown a better productivity performance than the other groups.

Coglapix ${ }^{\circledR}$ vaccinated pigs have had the lowest average lung lesion score at slaughter.

\section{Discussion}

In this trial, Coglapix ${ }^{\circledR}$ has shown an improved safety with no adverse reactions in minimum age piglets as well as the best trend of economical parameters.

\section{References}

1. Frey J. et al. (1993), J. Clin. Microbiol. 139, 1723-1728.

2. Sanchez R. et al. (2009), APVS, Tsukuba, Japan, 425.

3. European Pharmacopoeia, 01/2008:1360. 


\title{
P.310
}

\section{Clinical safety studies of COGLAPIX ${ }^{\oplus}$ (App vaccine) in minimum age susceptible piglets in laboratory conditions}

\author{
Georges Országh ${ }^{1}$ József Herczeg ${ }^{1}$ János Benyeda ${ }^{2}$ Miklos Tenk ${ }^{1}$ Botond Siklódi ${ }^{1}$ \\ Bálint Kupcsulik Benaouda Kadra' Jérôme Thevenon ${ }^{1}$ Roman Krejči ${ }^{3}$ \\ 1. CEVA-Phylaxia Veterinary Biologicals Co. Ltd., Budapest, Hungary; 2. Prophyl Immunolab, Mohács, Hungary; \\ 3. CEVA Santé Animale, Libourne, France
}

\section{Introduction}

A new Actinobacillus pleuropneumoniae (App) vaccine has been developed. The formulation of this new App vaccine was optimized to obtain a good balance between safety and efficacy.

The vaccine safety of a dose and of an overdose was thoroughly evaluated in susceptible piglets in laboratory conditions, in Hungary.

\section{Materials and Methods}

Coglapix ${ }^{\circledast}$ contains inactivated, native Apx antigens (Apxl, Apxll $\&$ ApxIII) and somatic antigens of App. The vaccine is adjuvanted with aluminium hydroxide gel. It is administered by the IM route (standard dose: $2 \mathrm{ml} / \mathrm{pig}$ ) and two injections are given from the age of 6 weeks to protect piglets as early as possible for the duration of the fattening period.

Safety of a maximum LPS content Coglapix ${ }^{\circledR}$ batch was evaluated at the youngest age ( 6 weeks) recommended in susceptible piglets as presented in table I. The serological status of the animals was tested with Swinecheck APP 2, Swinecheck APP 1, 9, 11 (Biovet) and the CHEKIT APP-ApxIV (IDEXX) ELISA kits. Every pigs were observed for general and local reactions for 14 days after each injection. The rectal temperature was measured 3 days before vaccination, 2, 4, 6 hours after each injection and on the 4 consecutive days. Performance measurements were recorded (weight gain, appetite). The injection sites of each pig was palpated and examined at post-mortem, 2 weeks after the last injection (D28).

\section{Results}

Table 1: Summarized serological status of piglets before vaccination

\begin{tabular}{|l|c|c|c|c|}
\hline Animal category & $\begin{array}{c}\text { Sample } \\
\text { No. }\end{array}$ & $\begin{array}{c}\text { App 1,9, 11 } \\
\text { positive }\end{array}$ & $\begin{array}{c}\text { App 2 positive } \\
\text { (\%) }\end{array}$ & $\begin{array}{c}\text { Apx IV positive } \\
\text { (\%) }\end{array}$ \\
\hline Piglets (5-6 weeks of age) & 44 & $0(0 \%)$ & $0(0 \%)$ & $1(0.4 \%)$ \\
\hline
\end{tabular}

Table 2: Summary results of the safety studies performed in laboratory conditions

\begin{tabular}{|c|c|c|c|c|c|}
\hline Treatment & $\begin{array}{l}\text { Pig } \\
\text { No. }\end{array}$ & $\begin{array}{c}\text { Mean max. temp. } \\
\text { increase oC }( \pm \mathrm{sd}) \\
\text { V1@D0 }\end{array}$ & $\begin{array}{c}\text { Mean max. temp. } \\
\text { increase oC }( \pm \mathrm{sd}) \\
\text { V2@D14 }\end{array}$ & $\begin{array}{l}\text { AWG* kg } \\
\text { (D0-D14 } \\
\text { period) }\end{array}$ & $\begin{array}{l}\text { AWG* kg } \\
\text { (D14-D28 } \\
\text { period) }\end{array}$ \\
\hline $\begin{array}{l}\text { Coglapix }^{\circledast} \\
\text { One dose } 2 \mathrm{ml}\end{array}$ & 13 & $1.0( \pm 0.5)$ & $0.2( \pm 0.6)$ & 8.51 & 11.35 \\
\hline $\begin{array}{l}\text { Coglapix }^{\circledast} \\
\text { Overdose } 4 \text { ml } \\
\text { D0 } 2 \text { ml D14 }\end{array}$ & 12 & $1.5( \pm 0.7)$ & $1.1( \pm 0.5)$ & 9.42 & 9.15 \\
\hline Control & 12 & $0.3( \pm 0.5)$ & $0.5( \pm 0.4)$ & 9.84 & 9.43 \\
\hline
\end{tabular}

In laboratory conditions, the maximum rectal temperature increase after vaccination with Coglapix ${ }^{\circledast}$ was not higher than $1.5^{\circ} \mathrm{C}$ as required by the EU Ph even after the overdose administration.

No general adverse reactions were observed after the administration of Coglapix ${ }^{\circledast}$ in both standard and overdose administrations.

\section{Discussion}

Coglapix ${ }^{\circledast}$ has shown a good safety even with a maximum LPS content batch in laboratory conditions.

\section{References}

1. Frey J. et al. (1993) J. Clin. Microbiol. 139, 1723-1728.

2. European Pharmacopoeia, 01/2008:1360.

${ }^{\circledR}$ COGLAPIX is a registered trademark of CEVA SANTE ANIMALE. 


\title{
P.311
}

\section{Study on preventive effects of garlic on pigs experimentally infected with Actinobacillus pleuropneumoniae serotype 2}

\author{
Norbert Stockhofe-Zurwieden ${ }^{1}$ Henk J. Wisselink ${ }^{1}$ Monique F. Mul² Petra M. Becker ${ }^{2}$ Carola van der Peet-Schwering ${ }^{2}$ \\ 1. Central Veterinary Institute of Wageningen UR, Lelystad, Netherlands; 2. Wageningen UR Livestock Research, Lelystad, Netherlands
}

\section{Introduction}

Actinobacillus pleuropneumoniae (App) can induce severe (per) acute pleuropneumonia, but certain serotypes as serotype 2 are more known to cause chronic disease resulting in pleuritis or focal pleuropneumonia, often without overt signs of clinical disease (1).

Garlic is alleged to have antimicrobial properties (2) and antimicrobial in vitro effects on App from garlic-derived volatile allyl methyl sulfide (AMS) has been shown (3). In this study we wanted to investigate the possible preventive effect of garlic on the occurrence of clinical disease and pathology of a mild course of an experimental infection with App in pigs.

\section{Materials and Methods}

In two different studies pigs of about 7 weeks old from a herd free of App were used. Pigs were fed with a commercial freezedried garlic powder (Enteroguard ${ }^{\mathrm{TM}}$ garlic). Garlic was added to the diet in increasing concentrations during 6 days with finally $5 \%$ or $10 \%$ at the day of challenge (Table 1 ).

Table 1: Design experimental infections

\begin{tabular}{|c|c|c|c|c|}
\hline Study & $\begin{array}{c}\text { Pigs [n] } \\
\text { control/garlic }\end{array}$ & $\begin{array}{c}\text { Garlic concentration } \\
\text { in feed [w/w] }\end{array}$ & $\begin{array}{c}\text { Challenge dose in aerosol } \\
\text { inoculum (CFU/ml) }\end{array}$ & $\begin{array}{c}\text { Post infection } \\
\text { phase (days) }\end{array}$ \\
\hline la & $5 / 5$ & $10 \%$ & $10^{7}$ & 4 \\
\hline Ib & $5 / 5$ & $10 \%$ & $10^{8}$ & 4 \\
\hline II & $15 / 15$ & $5 \%$ & $10^{9}$ & 4 \\
\hline \multicolumn{5}{|l}{ a Garlic concentration in feed at day of inoculation. } \\
\hline
\end{tabular}

During the aerosol inoculation three pigs were housed per run for 20 (Study I) or 30 minutes (Study II) in a $0.6 \mathrm{~m}^{3}$ stainless steel chamber. Aerosols of App serotype 2 reference strain 17399 were introduced with an Aeroneb ${ }^{\text {TM }}$ Pro micropump nebulizer (Aerogen Ltd., Ireland) from $5 \mathrm{ml}$ inocula containing $10^{7}, 10^{8}$ or $10^{9} \mathrm{CFU} / \mathrm{ml}$ (Table 1). During each inoculation run viability of bacteria was controlled by passing air from the aerosol chamber through an Anderson stage sampler. During the post infection phase of 4 days, pigs were monitored for occurrence of clinical signs, fever and changes in WBC. After euthanizing, pigs were examined patholocially and bacteriologically. In study II the relative lung weights were determined, comparisons were made between the treatment groups by using the Wilcoxson-MannWhitney two sample rank sum test.

\section{Results}

In the first study no clinical signs of an App infection were observed, fever was observed in 2 pigs from the garlic fed group and in 3 pigs of the control group. In 4 of 10 pigs of the control group and in 1 of 10 pigs in the garlic fed group small focal (pleuro)pneumonia was found at necropsy. In the second study one pig of the control group died 24 hours after inoculation due to a severe pleuropneumonia. No differences were found in clinical signs between the two treatment groups. At necropsy small focal lung lesions were found in 7 of 15 pigs in the control group and in 5 of 15 pigs of the garlic fed group. The relative lung weight was increased in the control group compared to the garlic fed group $(p=0,06)$.

Table 2: Results of in vivo studies in pigs to preventive effects of garlic

\begin{tabular}{|c|c|c|c|c|c|c|c|}
\hline Study & $\begin{array}{c}\text { Fed with } \\
\text { garlic }\end{array}$ & $\begin{array}{l}\text { Clinical signs } \\
\text { of disease (\%) }\end{array}$ & $\begin{array}{c}\text { Fever } \\
\text { index }(\%)^{\mathrm{a}}\end{array}$ & $\begin{array}{l}\text { Leucocyte } \\
\text { index }(\%)^{b}\end{array}$ & $\begin{array}{c}\text { Rel. lung } \\
\text { weigth (\%) }\end{array}$ & $\begin{array}{l}\text { Path. } \\
\text { [n] }\end{array}$ & $\begin{array}{l}\text { Bact. } \\
{[n]^{d}}\end{array}$ \\
\hline \multirow{2}{*}{ la } & Yes & 0 & 10 & 7 & ND & 0 & 0 \\
\hline & No & 0 & 5 & 13 & ND & 2 & 2 \\
\hline \multirow{2}{*}{$\mathrm{lb}$} & Yes & 0 & 5 & 0 & ND & 1 & 2 \\
\hline & No & 0 & 10 & 20 & ND & 2 & 2 \\
\hline \multirow[t]{2}{*}{ I․ } & Yes & 18 & 0 & 17 & $1.13 \pm 0.10$ & 5 & 5 \\
\hline & No & 17 & 4 & 23 & $1.21 \pm 0.15$ & 7 & 7 \\
\hline
\end{tabular}

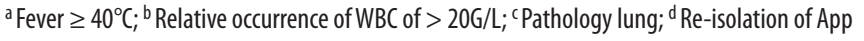
by culture

\section{Discussion}

A mild clinical course of infection was introduced in this aerosol challenge model of App. No clear differences in clinical signs were observed between the groups. A trend for an effect of garlic on pathology findings was observed.

It has to be emphasized, that the used concentrations of Entergoguard $^{\mathrm{TM}}$ garlic $5 \%$ to $10 \%$ far exceeded the concentrations applied in practice of $1 \%$.

\section{References}

1. Kristensen et al., 2004, Vet. Microbiol. 98:243-249

2. Lissiman, E., 2009, Cochrane database of systematic reviews. CD 006206

3. Becker et al., 2010 personal communication 


\title{
P.312
}

\section{Study of some Actinobacillus pleuropneumoniae strains' susceptibility at antimicrobials}

\author{
Corina Pascu; Luminita Costinar; Viorel Herman; loana Vaduva; Bogdan Faur \\ Faculty of Veterinary Medicine, Timisoara, Romania
}

\section{Introduction}

The sensitivity of A.pleuropneumoniae to antibiotics and chemotherapics we studied showed a various and unstable behavior of this species, very different in time and place, dependent on the area and time of isolation, reason why the antibiogram represents an indispensable tool before a treatment is established in pig pleuropneumonia.

The goal of our research was to determine actual sensibility to antimicrobials of A. pleuropneumoniae strains isolated from farms situated in the west part of country, used NCCLS methodology.

\section{Materials and Methods}

The A. pleuropneumoniae strains studied were isolate from animal with clinical symptoms of pig pleuropneumonia from wean to finish and fattening farms.

All 40 strains were identified according well knowing methodology. After completion of the susceptibility testing of the test strains and the quality control strain, we record the obtained results using zone-diameter in millimetres and categorize each of the tested strains as either "resistant" (R), "intermediate" (I) or "susceptible" (S) against each tested antimicrobial agent.

\section{Results and Discussions}

In all the 40 strains we examined in this study, the best results we found with fluorphenicol, tulathromycine, penicillin and tilmicozin. To be noticed that 3 of these belong to a newer class of antibiotics and only $66.6 \%$ of the strains were very sensitive to tilmicozin and $87.5 \%$ were very sensitive to fluorphenicol. Toward lincozamide (lincomycin), some blactamine (amoxicillin), aminoglycosides (gentamicin, streptomycin) the strains we studied had a moderate sensitivity, while they had a noticeable resistance toward amoxiklav, ampicillin, oxitetraciclin, enrofloxacin and cephalosporins, fact that urge us to contraindicate these antibiotics and chemotherapics (enrofloxacin) in the absence of an antibiogram which shows their sensitivity.

To be noticed that $20-30$ years ago seemed an unquestionable truth the fact that all A.pleuropneumoniae strains are sensitive to penicillin, including the strains isolated in this area of the country, while nowadays $30 \%$ of the strains isolated by us are resistant to penicillin and it seems that is because of the excessive usage of this antibiotic in time. In this matter our results are similar to those of other authors.

\section{Conclusions}

Our studies show that an excessive usage of some antibiotics and chemotherapics in order to prevent or treat pig pleuropneumonia, especially using medicated food may have negative results, as the selection of resistant strains which in time may replace the sensitive strains to a certain antibiotic or chemotherapic that is used at random, without knowing the sensitivity of germs from the population.

Also it is worth mentioning that changes of sensitivity toward some antibiotics - tetraciclins and chemotherapics - quinolons were revealed during the present investigation, comparing the results obtained on strains isolated from the same population at an interval of about 6-7 months in which the antibiotics were administered continuously.

\section{References}

1. Euzeby, J.P. - (2002), Dictionnaire de bacteriologie veterinaire www. bacterio.cict.fr/bacdico/garde.htlm

2. Gutiérrez, C.B., Barbosa, R., Tascon, R.I., Costa, I., Riera, P., Ferri Rodríguez, E.F. - (1995), Vet. Rec. 137, 62-64

3. Gutiérrez, C.B., Piriz, S., Vadillo, S., Rodriguez, E.F. - (1993), Am. J. Vet. Res. 54, 546-550

4. Holt, J.G., Krieg, N.R., Sneath, P.H.A., Staley, J.T., Williams, S.T. - (1994), IX-th Edition Bergey's Manual of Determinative Bacteriology. Williams \& Wilkins

5. Pascu, Corina. - (2008), Etiological and immunoprofilactic researches in pig pleuropneumonia. PhD thesis. Faculty of Veterinary Medicine Timisoara, Romania

6. Straw, Barbara., Zimmerman, J.J., Sylvie, D'allaire, Taylor, D.J. - (2006), Diseases of Swine. 9th edition, Blackwell Publishing, Ames lowa, USA

7. *** (2002), Performance Standards for Antimicrobial Disk and Dilution Susceptibility Tests for Bacteria Isolated from Animals; Aproved Standard - second edition. NCCLS document M31-A2 


\title{
P.313
}

\section{Intradermal vaccination by low dosage of antigen, Actinobacillus pleuropneumoniae model}

\author{
Jan Bernardy \\ University of Veterinary and Pharmaceutical Sciences, Brno, Czech Republic
}

\section{Introduction}

The objective of the presented study was to describe different dynamics of humoral immune reply to intradermally (ID) administered gradually diluted antigen and find out the lowest dose of antigen, administered ID, able to invoke measurable humoral immune reply.

ID vaccination is known to be a very practical method of vaccination especially in pigs, able to save a lot of antigen and could increase the number of available doses of vaccine (1), easy to mass administer and without residual changes and/or abscedation in the meet of food animals, and transmission of infectious diseases (2).

An experimental vaccine against APP was used and gradually diluted. The lowest dose of antigen APP was not disclosed. In concentration 3.104, decided as very low, the IgG and IgM reply was measured in levels comparable to higher concentrations of APP antigen.

\section{Material and Methods}

Seven groups of SPF piglets were used $(n=6)$ at age of 6 weeks of life. Experimental vaccine, based on commercial toxoid vaccine Suivac APP ID (Dyntec, CZ) was used and gradually diluted. Group 1 was vaccinated by full ID dose of vaccine, containing 6.0 ug of equal mixture of APX toxoids, OMP and LPS. Groups 2 to 5 were vaccinated and revaccinated three weeks later by gradually diluted antigen in the vaccine $-3 x, 9 x, 27 x$ and $81 x$. Group 6 was vaccinated by standard IM dose of Suivac APP IM (positive control, containing the same amount of antigen as ID dose), and group 7 was negative control group. Piglets were blooded weekly for 6 weeks after prime vaccination. Specific antibodies to APP APX were determined in blood serum using indirect isotype-specific ELISA procedure. Statistical software Statistica 9.0 was used for evaluation of the data The results of serological examinations were expressed as arithmetic mean values of optical densities obtained by ELISA test. Significance of between-group changes detected by ELISA were assessed with Kruskal-Wallis nonparametric test.

\section{Results and Discussion}

The very low dose of antigen ( $27 x$ dilution) administered by intradermal route induced comparable immune reply either in fast immune response of $\lg M$, either of more permanent lgG antibody response. All dilutions of antigen, used in the trial, involved measurable and mostly strong immune reply. The lowest dose of APX antigen was not disclosed. In concentration $1: 81$, expected as very low, the IgM (data not shown) and lgG response (Figure 1) was measured in levels still comparable to higher concentrations of APP antigen.

Intradermal administration of vaccines was found not only involving measurable humoral protection (lgG and $\lg M$ ) after administration of highly diluted dose, it was very effective in promoting a cytotoxic T cell response (data not shown). Attempts to dissect the mode of action of some intradermal administered vaccines by cytometric flow analysis of lymphocyte populations from the spleen, lung, liver and thymus revealed an effect of route of vaccine delivery upon the composition of specific lymphocyte subsets in these various organ compartments (3). In some cases lower dose of antigen in ID vaccination dose seems to be more effective in compare to standard IM dosage(4). The very low concentration, 1:81 dilutions, seemed to be too low to stimulate any replyafter single administration of very low dose of antigen, but booster vaccination of the same thin dose involved measurable lgG and IgM response.

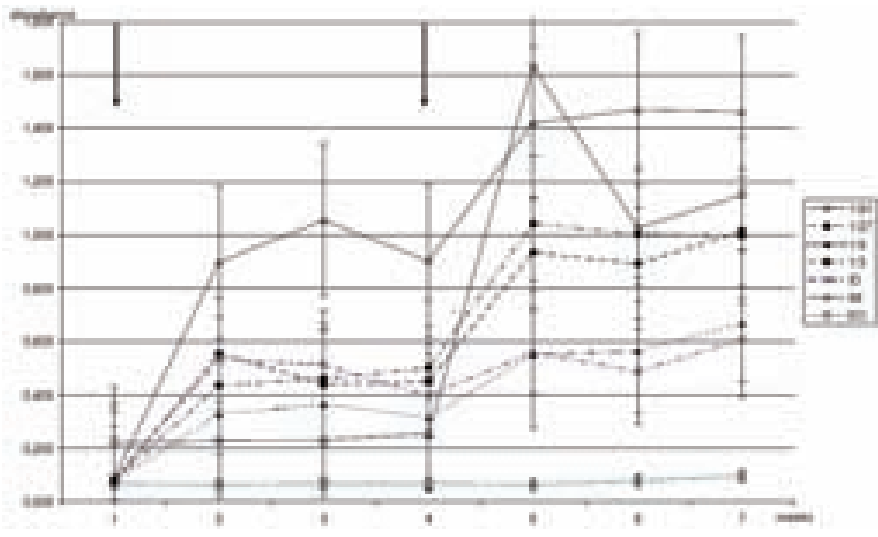

\section{Reference List}

1. Belshe R.B.,et al., N Engl J Med 2004; 351:2286.

2. Mitragotri S., Nat Rev Immunol 2005; 5:905.

3. Chin J. et al., J Biotechnol 1996; 44:13.

4. Bernardy J, et al., Vaccine $2008 ; 26: 6368$. 


\title{
P.314
}

\section{Cost Mycoplasma hyopneumoniae outbreak in Brazil}

\author{
Tatyane P. Sales ${ }^{2}$ Patricia Soares ${ }^{3}$ Wilia M. Brito ${ }^{4}$ Daniel Linhares ${ }^{1}$ Marcos L. Lucena ${ }^{5}$ Paulo H. Curado ${ }^{2}$ Jurij Sobestiansky $^{3}$ \\ 1. College of Veterinary Medicine, University of Minnesota, Saint Paul, MN, USA; 2. Escola de Veterinaria - Universidade Federal de Goias, \\ Goiania, GO, Brazil; 3. Programa de Pós graduação em Ciência Animal, Escola de Veterinária da Universidade Federal de Goiás/GO, \\ Goiania, GO, Brazil; 4. Laboratório de Virologia Animal, Instituto de Medicina Tropical e Saúde Pública da Universidade Federal de Goiás, \\ Goiania, GO, Brazil; 5. Nutrial, Goiania, GO, Brazil
}

\section{Introduction}

The modern pig industry is managed by practices geared for maximum production efficiency. Strategies to reduce the impact of diseases and to promote welfare of animals are essential to ensure business survival. Mycoplasma hyopneumoniae (Mh) is the most important respiratory pathogen in the Brazilian pig industry. It impairs production efficiency and increases production costs. Better understanding of these costs associated with Mh outbreaks will assist Veterinarians and producers to come up with cost-effective biosecurity and health assurance programs, aiming to avoid or at least to minimize potential losses associated with outbreaks.

Considering the economic impact of Mh on pig industry worldwide and understanding that controlling important diseases is cost-effective, the objective of this work was to estimate the economic losses of a Brazilian farm that experienced an outbreak of enzootic pneumonia, disease caused by Mh.

\section{Materials and Methods}

This study was conducted from September to December 2009 in a 170 sows farrow-to-finish herd, located in the State of Goias, Brazil. This farm was free of $\mathrm{Mh}$ based on absence of clinical signs and routine serology monitoring. The clinical signs were first recognized on August, when diagnosis was established by clinical examination, gross pathology and histopathology. The analysis and economic evaluation were based on the mean change of death rates of farrowing, nursery and growing/finishing pigs, as well as the expenses of additional drugs, vaccines and feed intake. The periods used for comparison were (1) the average from April to July, when the farm was serologically free of $\mathrm{Mh}$ and (2) the average from September to December, period post infection.

\section{Results and Discussion}

The death rate of farrowing piglets rose from $7.2 \%$ to $12.37 \%$. At nursery, the change was from $1.33 \%$ to $25.17 \%$ and at growingfinishing period, the death rate jumped from $2.9 \%$ to $5.85 \%$. The mortality rates before the outbreak were in accordance with the expected losses for Brazilian pig farms as suggested by Sobestiansky et al (2007).
The phase that had the higher spike in mortality was the nursery, what was expected and can be explained by the fact that piglets probably got infected at the farrowing barns by their dams. The increase of mortality in preweaning piglets was higher than usual in a Mh outbreak and can be explained by the fact that the average weaning age of this farm was 30 days of age. We believe that interaction of other pathogens such as Circovirus and Pasteurella with Mh infection have contributed to the increase of mortality rates.

The expenses from September to December 09 with Mh-vaccines, anti Mh-drugs and with ration that was fed to animals that died with Mh clinical signs was computed U\$33,600. When we consider that these extra animals that died after the outbreak could have been sold to slaughter at weight of $95 \mathrm{~kg}$, after discounting the cost with feeding, the lost value was US\$15,800. Combining both costs, the total loss with this outbreak from September to December was US\$ 49,400.

\section{Conclusion}

The high economic losses associated with an outbreak of enzootic pneumonia, in the form of deaths and cost of therapy, corroborates the need for establishing effective preventive measures. Biosecurity protocols can prevent the entry of pathogens especially by the introduction of positive animals in the herd and control of fomites and transit of vehicles, since these pose potential risk of disease transmission.

\section{Referencias}

1. Piffer, I. A. \& Brito, J. R. F. Descrição de um modelo para avaliação e quantifiação de lesões pulmonares de suínos e formulação de um índice para classificação dos rebanhos. Concódia, SC, EmbrapaCNPSA, 1991. 12p.

2. Sobestiansky, J. \& Barcellos D. Doença dos suínos. Goiânia, Cânone Editorial, 2007, 768p. 


\title{
P.315
}

\section{Colonization, serologic and parity state to Mycoplasma hyopneumoniae in vaccinated sows and their piglets}

\author{
Pablo J. Tamiozzo; Alicia I. Carranza; Bibiana R. Pelliza; Carlos Goñi; Arnaldo Ambrogi \\ Universidad Nacional de Rio Cuarto, Rio Cuarto, Cordoba, Argentina
}

\section{Introduction}

Mycoplasma hyopneumoniae (Mh) is the etiologic agent to Enzootic Pneumonia (EP). The transmission can occur horizontally from infected pigs to naïve pigs or vertically from sow to its piglets (1). It has been suggested that sow-to-piglet transmission may be influenced by sow parity (2-4) but the results are controversial. Due the fact sow vaccination did not affect piglets` colonization (5) and the different results about serologic and colonization status of sow and piglets according to parity distribution the objective of this study was to determinate colonization, serologic and parity status on Mh multiple-vaccinated sows and their piglets.

\section{Materials and Methods}

A 4100-sow, multiple-site herd was selected to carry out this study. Mh vaccination was used in sows, gilts and boars (at least with five doses of vaccines against Mh ([RespiSureONE, Pfizer, Argentina]). This vaccine scheme had been adopted since 3 years before. A longitudinal study was performed. Eighty-two sows were selected (being 15\% of the weekly farrows).All parturition distribution was considered in each sampling time (1st-9th). From each sow two piglets were randomly selected; (one hundred and sixty-four piglets). None of these piglets were cross-fostered. Blood samples for ELISA (HerdChek ${ }^{\circledast}$ Mycoplasma hyopneumoniae, IDEXX, Maine, USA) and nasal swabs for nPCR (6) were taken in the sows, at 0 day of gestation, at 8 weeks pre-farrowing and 2-5 days post-farrowing, and on the piglets at 2 weeks old. In order to study the effect of parity number, sows were divided into the following groups: A- low parity number (1-2), B- intermediate parity number (3-4), C-high parity number (5-6) and D-Very high parity number (7 or higher). The median Optical Density (OD) values of sera were calculated to compare seropositive sows and piglets according to different parity distribution and to compare seropositive sows at different sampling time.

\section{Results}

The results are shown in tables 1 and 2 .

Table 2. Median and range of Optical density (OD) according to parity distribution in sows and their piglets.

\begin{tabular}{|c|c|c|c|c|c|c|}
\hline \multirow{2}{*}{ Group } & \multicolumn{3}{|c|}{ SOWS OD } & \multicolumn{3}{c|}{ PIGLETS OD } \\
\cline { 2 - 7 } & $\mathbf{n}$ & Median & Range & $\mathbf{n}$ & Median & Range \\
\hline A & 28 & 0.783 & $0.482-1.218$ & 51 & 0.774 & $0.264-1.373$ \\
\hline B & 24 & 0.890 & $0.353-1.212$ & 45 & 0.840 & $0.235-1.412$ \\
\hline C & 15 & 0.986 & $0.585-1.292$ & 33 & 0.858 & $0.253-1.455$ \\
\hline D & 10 & 1.101 & $0.748-1.329$ & 19 & 1.021 & $0.403-1.405$ \\
\hline
\end{tabular}

\section{Discussion}

The percentages of seropositive sows were higher than the obtained in previous studies $(6,5)$. However, seronegative sows were found. This, may be explained by the fact that vaccine efficacy is not $100 \%$, in addition to the existence of subpopulations of susceptible animals (7), and/or different responses to vaccination (8). Through different sampling time, the proportion of seronegative sows in group B was greater than the others groups, these result are according to previous studies $(2,4)$ and would indicate that young sow (Group B) are more likely to transmit Mh to their piglets. The highest level of nPCR positives was found at 8 weeks pre-farrow and in this sample the OD value (from seropositives sows only) was lowest. That suggests that while protective antibodies decreased, exists a major shedding of Mh due to the decline in vaccine antibodies (9) or the protective antibodies are transferred from the blood to the udder in agreement with previous studies (10). At post-farrowing sampling time, the OD values increase according to parity number of sows (and their piglets). This suggests that the increased maternal antibodies concentration is not completely due to vaccination but also the repeated exposure of sows to Mh under field condition.

Table 1. Median OD at different sampling time and positives/sampled (and percentage) of ELISA and nPCR at different sampling time taking into account parity distribution.

\begin{tabular}{|c|c|c|c|c|c|c|c|c|c|c|c|c|c|c|c|c|}
\hline \multirow{3}{*}{$\frac{\text { GROUP }}{A}$} & \multicolumn{4}{|c|}{ ZERO DAY OF GESTATION } & \multicolumn{4}{|c|}{8 WEEKS PRE-FARROW } & \multicolumn{4}{|c|}{ 2-5 DAYS POST-FARROW } & \multicolumn{4}{|c|}{2 WEEK OLD PIGLETS } \\
\hline & \multicolumn{2}{|c|}{ ELISA } & \multicolumn{2}{|c|}{$\mathrm{nPCR}$} & \multicolumn{2}{|c|}{ ELISA } & \multicolumn{2}{|c|}{$\mathrm{nPCR}$} & \multicolumn{2}{|c|}{ ELISA } & \multicolumn{2}{|c|}{ nPCR } & \multicolumn{2}{|c|}{ ELISA } & \multicolumn{2}{|c|}{$\mathrm{nPCR}$} \\
\hline & $27 / 28$ & (96.4) & $6 / 28$ & (21.4) & $28 / 28$ & $(100)$ & $10 / 28$ & (35.7) & $28 / 28$ & $(100)$ & $11 / 29$ & $(37.9)$ & $53 / 57$ & $(92.9)$ & $13 / 57$ & $(22.8)$ \\
\hline B & $24 / 25$ & (96) & $9 / 25$ & (36) & $24 / 25$ & (96) & $10 / 25$ & (40) & $24 / 26$ & (92.3) & $2 / 25$ & (8) & $43 / 50$ & (86) & $11 / 51$ & $(21.5)$ \\
\hline C & $16 / 16$ & $(100)$ & $3 / 16$ & (18.7) & $17 / 17$ & $(100)$ & $4 / 17$ & (23.5) & $15 / 15$ & $(100)$ & $3 / 15$ & (20) & $33 / 34$ & $(97)$ & $3 / 34$ & (8.8) \\
\hline D & $10 / 10$ & $(100)$ & $2 / 9$ & (22.2) & $10 / 10$ & $(100)$ & $7 / 8$ & $(87.5)$ & $10 / 10$ & $(100)$ & $3 / 9$ & (15) & $19 / 21$ & $(90.4)$ & $3 / 21$ & (14.1) \\
\hline TOTAL & $77 / 79$ & (97.4) & $20 / 78$ & (26.6) & $79 / 80$ & (98.7) & $31 / 80$ & (38.7) & $77 / 79$ & (97.4) & $19 / 78$ & $(24.3)$ & $148 / 162$ & (91.3) & $30 / 163$ & (18.4) \\
\hline
\end{tabular}

\section{References}

1. Thacker E. 2006. Mycoplasmal diseases. In Diseases of Swine, 9th ed. Blackwell Publishing Ltd., Oxford, UK; 701-717.

2. Goodwin, R. 1965. Vet. Rec. 77 (14) 383-387.

3. Calsamiglia and Pijoan. 2000. Vet. Rec. 146, 530-532.

4. Fano, E. et al. 2006. 19 th IPVS Congress O.11-03. p. 96
5. Sibila et al.2007.Vet. Microbiol. 127 (1-2), 165-170.

6. Ruiz A, et al. 2003. J. Swine Health and Prod. 11 (3), 131-135.

7. Dee S, et al. 1996. J. Swine Health and Prod. 4 (3), 147-149.

8. Thacker E. 1998. J. Swine Heath and Prod. 6 (3), 107-112.

9. Maes, D. 1999. Vaccine 17. 1024-1034

10. Wallgren 1998. Vet microbiol.1998. 60(2-4), 193-205 


\title{
P.316
}

\section{Retrospective study on Mycoplasma hyopneumoniae infections in suckling and nursery pigs}

\author{
Heiko Nathues $^{1}$ Rene Kubiak$^{2}$ Regina Tegeler $^{1}$ Elisabeth Grosse Beilage $^{1}$ \\ 1. Field Station for Epidemiology in Bakum, University of Veterinary Medicine Hannover, Bakum, Germany; \\ 2. Boehringer Ingelheim Pharma GmbH \& Co KG, Ingelheim am Rhein, Germany
}

\section{Introduction}

Mycoplasma hyopneumoniae, the causative agent of porcine enzootic pneumonia, is predominantly transmitted through nose to nose contact between animals and more infrequently by aerosol, maintaining horizontal spread of the agent from infected to naïve pigs of the same age, or via vertical transmission from sows to their offspring (2). If the latter occurs frequently, it is suggested that vaccination of suckling piglets during their first days of life may assist M. hyopneumoniae control (1). However, only a few studies have surveyed the infection of suckling and nursery pigs under field conditions and the results are inconsistent. The main objective of our study was to evaluate the occurrence of $M$. hyopneumoniae infections in suckling and nursery pigs originating from a broad range of farms located in a region with intensive pig production and a high pig density.

\section{Material and Methods}

A retrospective analysis was applied to data obtained by routine diagnostics over two consecutive years from a laboratory working as specified in the ISO/IEC 17025:2005. Herd parameters were amended on the basis of enquiries with the herd-attending veterinarians. A correlation between the detection of $M$. hyopneumoniae in lung tissue on one hand and the vaccination history of the herd, the lung and pleura lesions examined during necropsy, and the detection of other bacterial and viral respiratory pathogens on the other hand was tested during further data analysis. The list of parameters was divided into suitable subsets and each individual parameter was considered initially by conducting a Chi-Square analysis to examine the independence of the respective parameter levels and the occurrence of a M. hyopneumoniae infection. A linear logistic regression model was then used to examine the relationship between the binary response of $M$. hyopneumoniae detection and a set of explanatory variables which were defined by all infectious agents that were found to be significant $(\mathrm{P}<0.05)$ in the individual considerations. The log odds function (LOGIT) was used as a link function.

\section{Results}

The occurrence of $M$. hyopneumoniae infections in young pigs was surveyed on 1,122 datasets, where either suckling or nursery pigs were examined for $M$. hyopneumoniae in lung tissue. Findings were correlated with lung lesions, detection of other respiratory pathogens, vaccination history and parameters describing the herd of origin. The prevalence of $M$. hyopneumoniae in lung tissue from suckling pigs ( $n=201$ ) was $2.0 \%$ and, thus, significantly lower than in lungs from nursery pigs $(n=921)$, which was $9.3 \%$. Previous use of antimicrobials and vital status of the pigs when delivered for necropsy did not influence the detection of $M$. hyopneumoniae infection. The presence of PRRSV-EU genotype, Pasteurella multocida, Haemophilus parasuis, Mycoplasma hyorhinis or Streptococcus suis was correlated with a higher probability of also finding $M$. hyopneumoniae. Positivity for H. parasuis, M. hyorhinis and S. suis strongly indicated the presence of $M$. hyopneumoniae. Neither vaccination (yes/no), the time of first/second application nor type of vaccine (one-shot vs. two-shot) influenced the detection of $M$. hyopneumoniae. Correlation between "type of herd" and presence of M. hyopneumoniae was statistically insignificant and no effect of farrowing rhythm could be confirmed.

\section{Discussion}

The model of a retrospective study is, on one hand, lacking in the possibility of standardisation in sampling, e.g. number and age of animals, and, moreover, the possibility to further examine the samples. On the other hand, the analysis of datasets from routine diagnostics can provide representative data from the field, if inclusion and exclusion criteria are well chosen to rule out a selection bias (3). For the present analysis 1,122 dataset entries from a nationwide operating laboratory were included, representing $27.5 \%$ of all examined pigs over a period of two entire years. This study corroborates the infrequent occurrence of $M$. hyopneumoniae infection in suckling and nursery pigs under field conditions. It also underlines the low prevalence and rules out any significant impact of vaccination on the frequency and time of the infection.

\footnotetext{
References

1. Martelli, P., et al. (2006) J.Vet. Med. B 53:229-233

2. Thacker, E. (2006) Diseases of Swine. pp. 701-717

3. Thrusfield, M. (1986) Epidemiology. pp. 167-175
} 


\title{
P.317
}

\section{Cloning and expression of pyruvate dehydrogenase E1-a subunit from Mycoplasma hyopneumoniae in Escherichia coli}

\author{
Chung-Nan Weng \\ Animal Technology Institute Taiwan, Taipei, Taiwan
}

\section{Introduction}

Mycoplasma hyopneumoniae is the causative agent of swine enzootic pneumonia (SEP), one of the most important chronic respiratory diseases that affects swine production worldwide. The pathogen results in considerable economic losses through retarded growth, poor food conversion and increased susceptibility of pigs to infections by other organisms. Prevention of SEP may be achieved by improving hygiene and management in pig farms. In addition, vaccination is an effective strategy for control and prevention of SEP [1]. Commercial M. hyopneumoniae vaccines were prepared from killed bacteria (bacterins). However, mass production of $\mathrm{M}$. hyopneumoniae vaccine employing the complicated cultivation method is expensive and time consuming. The use of specific immunogenic proteins as subunit vaccines produced by recombinant DNA technology may be a preferable alternative to the use of killed bacteria. In our previous research, 19 immunogenic proteins of M. hyopneumoniae PRIT-5 were identified using an immunoproteomic approach [2]. These immunogenic proteins are potential subunit vaccine candidates. In order to evaluate the immunogenic proteins as subunit vaccines, cloning and expression of genes encoding immunogenic proteins in heterologous expression systems is needed. In this report, we describe the cloning and expression of a gene encoding immunogenic pyruvate dehydrogenase E1- a subunit (PdhA) from M. hyopneumoniae PRIT-5 in Escherichia coli.

\section{Materials and Methods}

The pyruvate dehydrogenase E1- a subunit gene (pdhA) was amplified by polymerase chain reaction (PCR) from $M$. hyopneumoniae PRIT-5 genomic DNA template followed by TA-cloning. In order to replace mycoplasma -specific TGA (Trp) codons in the cloned pdhA gene, which did not allow its full length expression in E. coli, by the universal TGG codon, the overlap extension-PCR method was used. The silent mutated pdhA was cloned into pET29a-D. The resulting plasmid, pET-DPdhA, was transformed into E. coli BL21 (DE3) for expression of PdhA. Protein expression was induced with $1 \mathrm{mM}$ isopropyl- $\beta$-D-thiogalactopyranoside. The cell pellet of E. coli was collected and analyzed by SDS-PAGE and western blot. Recombinant PdhA prodced by the E. coli expression system was purified from cell pellet by immobilized metal-ion affinity chromatography (IMAC).

\section{Results}

The pdhA gene was cloned by PCR. DNA sequence analysis showed that the pdhA consists of an open reading frame of 1,125 bp coding for a 374-amino acid residues and contains 8 TGA codons. The deduced amino acid sequence of $M$. hyopneumoniae PRIT-5 PdhA showed high degree identity (99\%) compared with PdhA from other strains including J, 232 and 7448. In order to correctly express the PdhA in E. coli, all the TGA codons carried by pdhA were mutated in TGG. The mutated pdhA was further cloned into E. coli expression vector and expressed in E. coli as a recombinant fusion protein containing a hexahistidine $\mathrm{N}$-terminal sequence and $\lambda$ capsid protein $D$ in insoluble form. Recombinant PdhA could be purified from E. coli by IMAC (Figure 1).

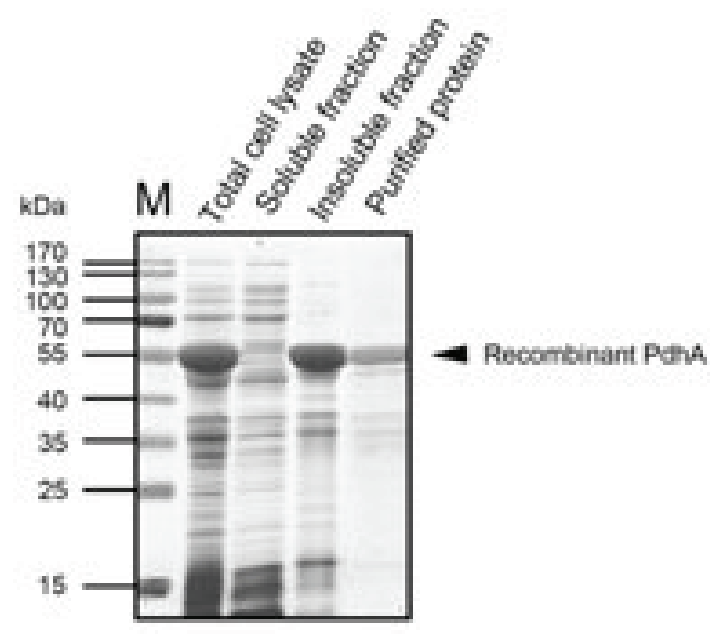

\section{Discussion}

In this study, we reported the successful cloning, expression and purification of pyruvate dehydrogenase E1- a subunit from $M$. hyopneumoniae PRIT-5. The purified recombinant PdhA will be further used for the purpose of immunobiological studies.

\section{References}

1. Maes, D., Segales, J., Meyns, T., Sibila, M., et al. 2008. Control of Mycoplasma hyopneumoniae infections in pigs. Vet. Microbiol. 126: 297-309.

2. Lin, J.H., Wang, J.P., Hsieh, M.W., and Weng, C.N. Research on the major antigen and its application of swine mycoplasma by reverse vaccinology. The 4th Academic Congress of Asian Organization for Mycoplasmology, P. 41. Taipei, Taiwan, Nov 2-3, 2009. 


\title{
P.318
}

\section{Reduction in pulmonary lesions in pigs vaccinated with a novel live temperature sensitive M. hyopneumoniae vaccine compared to pigs vaccinated with an inactivated vaccine}

\author{
Horacio Lara-Puente ${ }^{1}$ Francisco Quezada-Monroy ${ }^{1}$ Ruben Echeveste-Garcia de Alba ${ }^{1}$ \\ Bernardo Lozano-Dubernard ${ }^{1}$ Ernesto Soto-Priante ${ }^{1}$ David Sarfati-Mizrahi ${ }^{1}$ Abel Ciprian-Carrasco ${ }^{2}$ Joaquin Delgadillo-Alvarez $^{3}$ \\ Rima Youil ${ }^{4}$ David Tinworth ${ }^{4}$ Susana Mendoza-Elvira ${ }^{2}$ Yolanda Moreno-Morales ${ }^{3}$ Youssef Abs-EL Osta ${ }^{4}$ \\ 1. Laboratorio Avi-Mex, S. A. de C. V., Mexico City, DF, Mexico; 2. FES-C UNAM, Cuautitlan, MEX, Mexico; \\ 3. CENASA/SENASICA, Tecamac, MEX, Mexico; 4. Bioproperties Pty Ltd, Victoria, VIC, Australia
}

\section{Introduction}

Enzootic pneumonia (EP), also called mycoplasmal pneumonia, is recognized as one of the most economically significant swine respiratory diseases. The etiological agent of this disease $M$. hyopneumoniae (Mh) attaches to the ciliated epithelial lining of the respiratory tract, resulting in impairment of the mucociliary mechanisms, predisposing to secondary bacterial infections. Vaxsafe ${ }^{\circledR}$ MHP is a live temperature sensitive Mh strain that is being developed by Bioproperties Pty Ltd (Australia) as the first live vaccine against EP.

\section{Materials and Methods}

Forty (40) SPF pigs 3 weeks of age were used in this study. All pigs were ear tagged with an individual number, weighed and assigned to 4 study groups each containing 10 pigs housed under the same conditions in negative pressure isolation rooms. Pigs were acclimatized for 3 days before treatment.

Group 1 was the Negative control. Pigs within this group were administered vaccine diluent. Group 2 was vaccinated with Vaxsafe ${ }^{\circledR}$ MHP. Pigs within group 3 were vaccinated with an inactivated commercial vaccine. Group 4 was the positive control. Vaccination day was designated as "days post vaccination-zero" (DPV-0). Vaccination with Vaxsafe ${ }^{\circledR}$ MHP was performed by intranasal spray and the commercial vaccine was administered by intramuscular injection. Vaccinated pigs were allowed to develop immunity over a period of 22 days, and subsequently they were challenged on two occasions with live culture of Mh IOWA strain 194 administered by aerosol at a dose of $\sim 106.0$ $\mathrm{ccu} / \mathrm{mL} / 45$ minutes in a chamber specifically designed for pigs. On the first round pigs were challenged three times at 4 days apart ie. DPV 22, 27 and 32. All groups were then subjected to a second round of challenge with a similar live culture of $\mathrm{Mh}$ administered on 3 consecutive days (DPV-84, -85 and -86). Postmortems were performed 19 days after the second round of challenge (DPV-105). A number of parameters were employed in order to achieve the objectives of this study including assessment of clinical signs, nasal swabs (nPCR) at post-mortem, gross lung lesions typical of $\mathrm{Mh}$ infection were recorded on lung lesion diagrams employing the planimetric method.

\section{Results}

The planimetric scoring method was used to determine the average percentage area of affected lung for each group. Small lesions (unrelated to Mh as demonstrated by $\mathrm{nPCR}$ results) were found in the negative control group. Pigs in the positive control group showed lesions affecting an average of $10.98 \%$ of the lung (Table 1).

A signficant reduction in the severity of lung lesions (1.34\%) was observed in the pigs vaccinated with Vaxsafe ${ }^{\circledR} \mathrm{MHP}$. By comparison a higher lesion score $(6.93 \%)$ was detected in the pigs vaccinated with the commercial inactivated vaccine. There were significant differences $(P \leq 0.05)$ in the severity of lung lesions between groups vaccinated with Vaxsafe ${ }^{\circledR}$ MHP and the positive control group. However, there was no significant difference $(P \geq 0.05)$ between group vaccinated with a commercial vaccine and the positive control group.

Table 1: Incidence and severity of lung lesions.

\begin{tabular}{|l|c|c|}
\hline Group & $\begin{array}{c}\text { Incidence of lung lesions } \\
\text { (as affected lungs \%) }\end{array}$ & $\begin{array}{c}\text { Severity of lung lesions (as } \\
\text { surface of lung affected) }\end{array}$ \\
\hline Negative control & 57.14 & 1.7 \\
\hline Vaxsafe ${ }^{\oplus H P}$ & 75 & 1.34 \\
\hline Commercial vaccine & 100 & 6.93 \\
\hline Positive Control & 100 & 10.98 \\
\hline
\end{tabular}

No significant difference in the incidence of lung lesions was observed between the Vaxsafe ${ }^{\circledR}$ MHP and the commercial inactivated vaccine groups.

ELISA tests performed on the two vaccinated groups showed $78 \%$ positive for the inactivated vaccine but only $12.5 \%$ positive for the live Vaxsafe ${ }^{\circledR}$ MHP vaccine.

Conclusions:Following challenge, Vaxsafe ${ }^{\circledR}$ MHP provided a significantly higher level of protection against severity of lung lesions compared with an inactivated commercial vaccine. This study also showed that ELISA (commercial) assay is not a good indicator for protection provided by this live vaccine.

\section{Bibliography}

Lara, J.H. et al. Experimental infection with Mycoplasma hyopneumoniae in SPF pigs using an aerosol chamber. 20th International Pig Veterinary Society Congress. June 22-26, Durban, South Africa, (2008). 


\title{
P.319
}

\section{Comparison of daily weight gain in pigs vaccinated with a temperature sensitive Mycoplasma hyopneumoniae vaccine or an inactivated commercial vaccine}

\author{
Ruben Echeveste-Garcia de Alba ${ }^{1}$ Francisco Quezada-Monroy ${ }^{1}$ Bernardo Lozano-Dubernard ${ }^{1}$ David Sarfati-Mizrahi ${ }^{1}$ \\ Ernesto Soto-Priante ${ }^{1}$ Rima Youil ${ }^{2}$ Youssef Abs-EL Osta ${ }^{2}$ David Tinworth ${ }^{2}$ Abel Ciprian-Carrasco ${ }^{3}$ \\ Susana Mendoza-Elvira ${ }^{3}$ Joaquin Delgadillo-Alvarez ${ }^{4}$ Yolanda Moreno-Morales $^{4} \underline{\text { Horacio Lara-Puente }}^{1}$ \\ 1. Laboratorio Avi-Mex, S. A. de C. V., Mexico City, DF, Mexico; 2. Bioproperties Pty Ltd, Victoria, VIC, Australia; \\ 3. FES-C UNAM, Cuautitlan, MEX, Mexico; 4. CENASA/SENASICA, Tecamac, MEX, Mexico
}

\section{Introduction}

Enzootic pneumonia (EP) of swine, also called mycoplasmal pneumonia, is recognized as one of the most economically significant swine respiratory diseases. The etiological agent of this disease $M$. hyopneumoniae $(\mathrm{Mh})$ attaches to the ciliated epithelial lining of the respiratory tract, resulting in impairment of the mucociliary mechanisms, predisposing to secondary bacterial infections. Pigs affected with the disease show poor conversion and thus low weight gain. Vaxsafe ${ }^{\circledR}$ MHP is a temperature sensitive Mh strain that is being developed by Bioproperties Pty Ltd (Australia) as the first live vaccine against EP.

\section{Materials and Methods}

Forty (40) SPF pigs 3 weeks of age were used in this study. All pigs were ear tagged with an individual number, weighed and assigned to 4 study groups each containing 10 pigs. All groups were housed under the same conditions in negative pressure isolation rooms. Pigs were acclimatized for 3 days before treatment.

Group 1 was the Negative control. Pigs within this group were administered vaccine diluent. Group 2 was vaccinated with Vaxsafe ${ }^{\circledR}$ MHP. Pigs within group 3 were vaccinated with a commercial vaccine. Group 4 was the positive control. Vaccination day was designated as "days post vaccination-zero" (DPV-0). Vaccination with Vaxsafe ${ }^{\circledR}$ MHP was performed by intranasal spray and the commercial vaccine was administered by intramuscular injection. Vaccinated pigs were allowed to develop immunity over a period of 22 days, and subsequently they were challenged on two occasions with live culture of Mh. IOWA strain $194 \mathrm{admin}-$ istered by aerosol at a dose of $\sim 106.0 \mathrm{ccu} / \mathrm{mL} / 45$ minutes in a chamber specifically designed for pigs. On the first round pigs were challenged three times at 4 days apart. DPV 22, 27 and 32. All groups were then subjected to a second round of challenge with a similar live culture of $\mathrm{Mh}$ administered on 3 consecutive days (ie. DPV 84, 85 and 86). Post-mortems were performed 19 days after the second round of challenge (DPV-105). A number of parameters were examined in order to achieve the objectives of this study including assessment of growth performance.

\section{Results}

Vaccinated groups showed increased weight gain compared to the positive control group. However, analysis of variance for weight gain between the positive control group and the vaccinated groups indicated that the increase was significant $(P \leq 0.05)$ only for Vaxsafe ${ }^{\circledR}$ MHP and the negative control group (see Table 1)
Table 1 Weight values

\begin{tabular}{|l|c|c|c|}
\hline Group & $\begin{array}{c}\text { Total weight } \\
\text { at 105 DPV } \\
(\mathbf{k g})\end{array}$ & $\begin{array}{c}\text { Differential weight at } \\
\text { 105 DPV compared to } \\
\text { positive control (kg) }\end{array}$ & $\begin{array}{c}\text { Differential weight at 105 } \\
\text { DPV compared to nega- } \\
\text { tive control group (kg) }\end{array}$ \\
\hline Negative control & 54.8 & $9.5(0.001)$ & $0.0(\mathrm{NA})$ \\
\hline Vaxsafe ${ }^{\circledast}$ MHP & 52.74 & $8.5(0.004)$ & $-2.0(0.160)$ \\
\hline Commercial vaccine & 50.9 & $5.6(0.315)$ & $-3.9(0.463)$ \\
\hline Positive control & 45.2 & $0.0(\mathrm{NA})$ & $-9.5(0.001)$ \\
\hline P values in parenthesis & \\
\hline
\end{tabular}

No significant difference $(P \geq 0.05)$ in weight loss was observed between the vaccinated groups and the negative control group. Only the positive control group showed a significant $(P \leq 0.05)$ weight loss due to challenge (Table 1).

\section{Conclusions}

Vaxsafe ${ }^{\circledR}$ MHP showed a significant difference with respect to weight gain in comparison to the commercial vaccine. An average increase in weight of $7.5 \mathrm{~kg}$ was observed for the group vaccinated with Vaxsafe ${ }^{\circledR}$ MHP compared to the positive control. The group vaccinated with a commercial vaccine on the other hand did not show any significant difference in weight gain when compared to the positive control group. Compared to the negative control, the group vaccinated with Vaxsafe ${ }^{\circledR}$ MHP lost only $2 \mathrm{~kg}$ while the group vaccinated with a commercial vaccine lost $3.9 \mathrm{~kg}$ following challenge

\section{Bibliography}

Lara, J.H. et al. Experimental infection with Mycoplasma hyopneumoniae in SPF pigs using an aerosol chamber. 20th International Pig Veterinary Society Congress. June 22-26, Durban, South Africa, (2008). 


\title{
Vaxsafe ${ }^{\circledR}$ MHP: A new temperature sensitive live vaccine against Mycoplasma hyopneumoniae - a histological perspective and efficacy at various doses
}

\author{
$\underline{\text { Horacio Lara-Puente }}^{1}$ Ruben Echeveste-Garcia de Alba ${ }^{1}$ Francisco Quezada-Monroy ${ }^{1}$ Bernardo Lozano-Dubernard ${ }^{1}$ \\ David Sarfati-Mizrahi ${ }^{1}$ Ernesto Soto-Priante ${ }^{1}$ Abel Ciprian-Carrasco ${ }^{2}$ Joaquin Delgadillo-Alvarez $^{3}$ Rima Youil $^{4}$ \\ David Tinworth ${ }^{4}$ Susana Mendoza-Elvira ${ }^{2}$ Yolanda Moreno-Morales ${ }^{3}$ Youssef Abs-EL Osta 4 \\ 1. Laboratorio Avimex, Mexico City, DF, Mexico; 2. FES-C UNAM, Cuautitlan, MEX, Mexico; 3. CENASA, Tecamac, MEX, Mexico; \\ 4. Bioproperties Pty Ltd, Victoria, VIC, Australia
}

\section{Introduction}

M. hyopneumoniae ( $\mathrm{Mh}$ ) is the etiological agent of enzootic pneumonia (EP). The organism attaches to the ciliated epithelial lining of the respiratory tract, resulting in impairment of the mucociliary mechanisms, predisposing the lungs to secondary bacterial infections. Vaxsafe ${ }^{\circledR}$ MHP a temperature sensitive Mh strain that is being developed by Bioproperties Pty Ltd (australia) as the first live vaccine against EP.

\section{Materials and Methods}

Forty (40) SPF pigs 3 weeks of age were used in this study. Group 1 was the Negative control. Pigs within this group were administered vaccine diluent. Groups 2, 3, 4 and 5 were vaccinated with Vaxsafe ${ }^{\circledR}$ MHP at 103, 104, 105 and 106 ccu per dose, respectively.Group 6 ws vaccinated with a commercial inactivated vaccine. Group 7 was the positive control. All the details concerning the vaccination and challenge processes are included in other papers of the series. Representative lung samples were collected and fixed in $10 \%$ buffered formalin. Routine paraffin inclusion and HE stains were performed.

\section{Results}

Histopathological examination of lesions showed typical bronchopneumonia with mononuclear and polimorphonuclear cell infiltrate and BALT hyperplasia. A clear correlation was observed between the vaccine dose administered and the severity of the lesions. Increasing doses of vaccine resulted in milder manifestation of lesions with the highest vaccine dose resulting in a severity score of $1.34 \%$ (Table 1). The lowest dose of Vaxsafe ${ }^{\circledR}$ MHP resulted in a severity score of $4.38 \%$. However, this will still lower than the severity of lung lesions observed for the inactivated vaccine $(6.93 \%)$ which was delivered at the commercial dose. Negative control showed lung lesions unrelated to Mh infection as shown by $\mathrm{nPCR}$ test results.
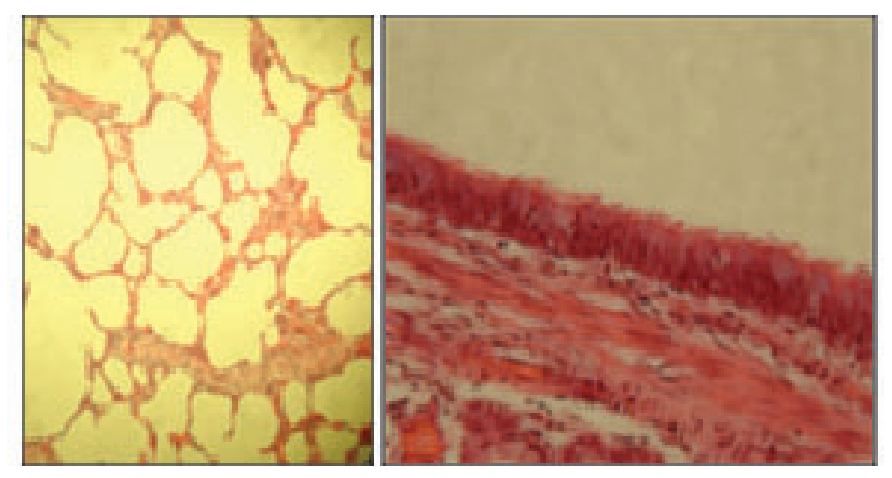

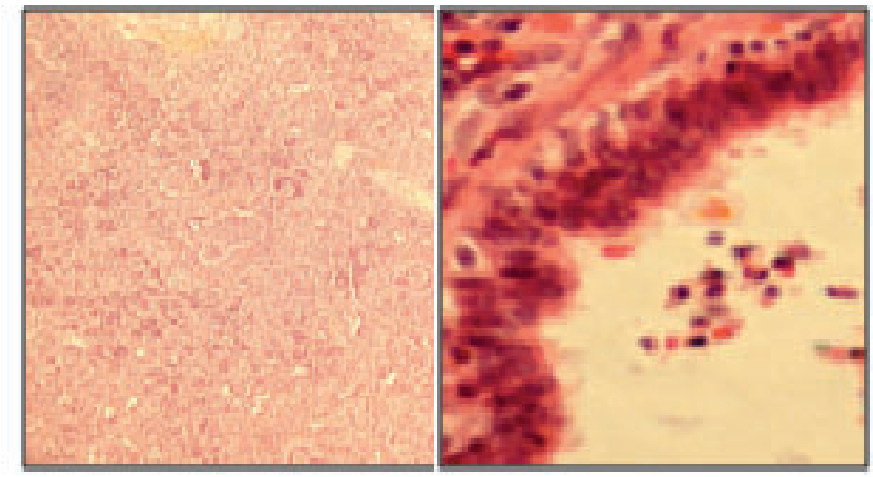

Figure 3 shows bronchopneumonia with abundant mononuclear (MN) and polimorphonuclear (PMN) cell infiltrate. Bronchus associated Lymphoid Tissue (BALT) and other lung structures are difficult to differentiate due to infiltrate severity. Figure $\mathbf{4}$ shows the epithelial cells of bronchus still displaying some cilia but PMN infiltrate is already present.

Table 1: Incidence and severity of lung lesions

\begin{tabular}{|l|c|c|}
\hline Group & Incidence of lesions (\%) & Severity of lesions (\%) \\
\hline Negative control & 57.14 & 1.70 \\
\hline Vaxsafe $^{\circledast}$ MHP $10^{3}$ & 87.50 & 4.38 \\
\hline Vaxsafe $^{\circledast}$ MHP $10^{4}$ & 90 & 3.46 \\
\hline Vaxsafe $^{\circledast}$ MHP $10^{5}$ & 75 & 1.91 \\
\hline Vaxsafe $^{\circledast}$ MHP $10^{6}$ & 100 & 1.34 \\
\hline Commercial vaccine $^{5}$ & 100 & 6.93 \\
\hline Positive control & 100 & 10.98 \\
\hline
\end{tabular}

\section{Conclusions}

The use of Vaxsafe ${ }^{\circledR}$ MHP resulted in a notable reduction of severity of lung lesions (explained as percentage of lung area affected), but not in the absence of lesions. This is in direct relation to the vaccine dosage.

\section{Bibliography}

Lara, J.H. et al. Experimental infection with Mycoplasma hyopneumoniae in SPF pigs using an aerosol chamber. 20th International Pig Veterinary Society Congress. June 22-26, Durban, South Africa, (2008).

Figure 1 Normal lung structures.

Figure 2 Normal epihelial cells with cillia. 


\title{
P.321
}

\section{Simultaneous vaccination of pigs with SPRINTVAC $^{\oplus}$ and PESTIFFA ${ }^{\oplus}$}

\author{
H.C. Lim ${ }^{1}$ B.K. Lim² P.Y. Choo ${ }^{3} \underline{\text { Henry Too }}^{4}$ \\ 1. Rhone Ma Malaysia (M) Sdn Bhd, Selangor, Malaysia; 2. Vet Food Agro Diagnostic (M) Sdn Bhd, Selangor, Malaysia; \\ 3. Asia Pacific Special Nutrients (M) Sdn Bhd, Selangor, Malaysia; 4. MERIAL Asia Pte. Ltd, Selangor, Malaysia
}

\section{Introduction}

The administration of different vaccines simultaneously often gives rise to concerns about safety and efficacy. A previous study on the simultaneous use of MERIAL vaccines in sows showed no adverse effects on safety and similar immune responses (1). However, there has been no study on the simultaneous use of mycoplasma vaccines with swine fever vaccines in piglets. The present study was conducted to evaluate the safety and serological response induced by the two vaccines, SPRINTVAC ${ }^{\circledR}$ and PESTIFFA ${ }^{\oplus}$ (MERIAL) used for the control of swine enzootic pneumonia (SEP) and classical swine fever (CSF) respectively following simultaneous administration to piglets.

\section{Material and Methods}

Twenty four 4-week old weaners from farm known to be free of Aujeszky's disease, Porcine Respiratory and Reproductive Syndrome (PRRS) and CSF were randomly allotted to 3 groups (Table 1). i.e. vaccinated intramuscularly (IM) in the neck with SPRINTVAC and PESTIFFA on the same day (Group S), vaccinated IM with PESTIFFA 5 days after SPRINTVAC (Group NS), and not vaccinated (Control group). When simultaneous vaccination was performed, the vaccines were injected on opposite sites on the neck region. All pigs were housed in adjacent pens and managed in same manner throughout the trial period.

Table 1: Groups of experimental pigs

\begin{tabular}{|c|c|c|}
\hline Group & Vaccination & N \\
\hline S (simultaneous) & SPRINTVAC and PESTIFFA on the same day at 4 weeks of age & 8 \\
\hline NS (non simultaneous) & PESTIFFA vaccinated 5 days after SPRINTVAC vaccination & 8 \\
\hline Control & None & 8 \\
\hline
\end{tabular}

Weekly serum samples from 4 to $10 \mathrm{wks}$ of age and at 12 and 20 weeks of age were tested for antibodies to M. hyo and CSF using IDEXX test kit, and Hipra CIV swine fever test kit respectively. The effects of local site reactions and anorexia were observed for up to 3 weeks after vaccination.

\section{Results and Discussion}

The serological profiles for mycoplasma and swine fever antibodies are shown in Figures 1 and 2.

Seven (87.5\%) of pigs from Group S seroconverted to PESTIFFA 2 weeks post vaccination. Only $25 \%$ of pigs from NS group seroconverted at three weeks post vaccination. CSF antibodies were detected in all pigs at 4 weeks (Group S) and 5 weeks (Group NS) post vaccination.
Figure 1: Swine fever antibody serological profiles

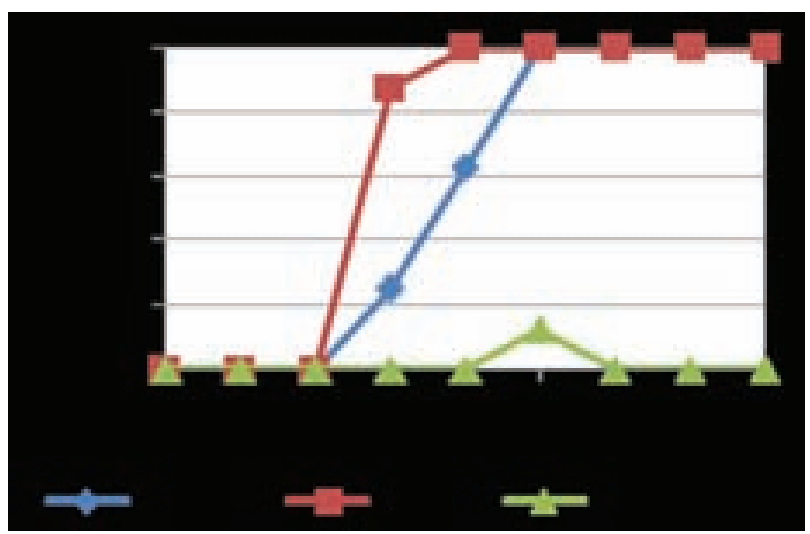

Figure 2: M. hyo antibody serological profiles

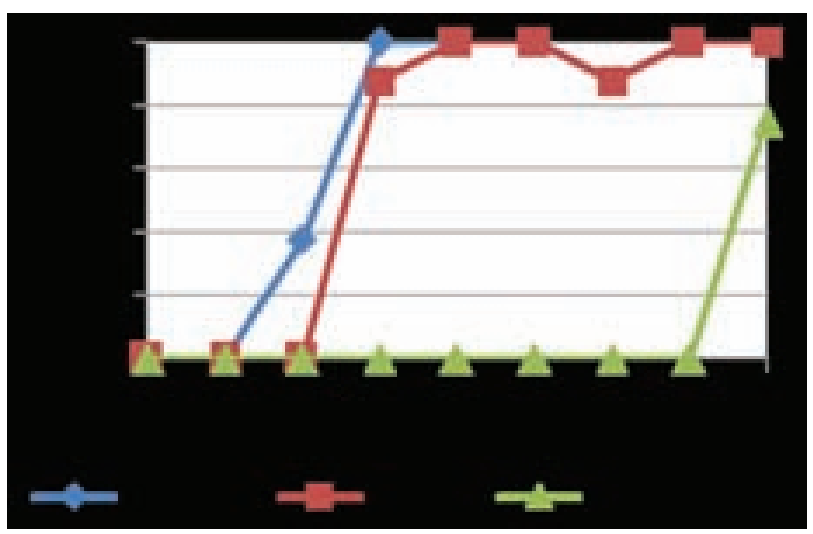

Three (37.5\%) of the pigs from NS group seroconverted two weeks following SPRINTVAC vaccination while all but one $(87.5 \%)$ of the pigs from $S$ group seroconverted 3 weeks post vaccination. Seroconversion rate for both groups at 5 weeks post vaccination remain above $90 \%$ until the age of 20 weeks old. Seventy-five per cent of pigs from control group were seroconverted at the age of 20 weeks old. This is ascribed to field challenge of Mycoplasma hyopneumoniae.

There were no adverse local reactions to the vaccinations or anorexia observed post-vaccinations

\section{Conclusion}

In this study, no adverse local reactions or anorexia were observed with the simultaneous vaccination SPRINTVAC and PESTIFFA. The serological responses were similar in both simultaneous vaccination and non simultaneous group.

\section{References}

1. Cruz et al., (2007). Proc. 3rd Congress APVS. p. 459-461

-SPRINTVAC is a registered trademark of MERIAL in the United States of America and elsewhere. PESTIFFA is a registered trademark of MERIAL S.A.S., France. 


\title{
Safety and Efficacy of a novel live vaccine for Mycoplasma hyopneumoniae: Vaxsafe ${ }^{\circledR}$ MHP
}

\author{
Youssef Abs EL-Osta; David Tinworth; Rima Youil \\ Bioproperties Pty Ltd, Bundoora, VIC, Australia
}

\begin{abstract}
Introduction
Vaxsafe ${ }^{\circledR}$ MHP is a live temperature sensitive vaccine developed by Bioproperties Pty Ltd for the protection against Mycoplasma hyopneumoniae (Mh) infection in pigs. Vaxsafe ${ }^{\circledR}$ MHP has been developed in two formats: wet frozen (WF) and freeze dried (FD). A number of in vivo studies have been completed which assessed safety, efficacy and colonization of Vaxsafe ${ }^{\circledR}$ MHP in target animals following intra-nasal administration. In this paper two regulatory based studies will be presented.
\end{abstract}

\section{Materials and Methods}

Study 1: Six-week-old healthy Mh free commercial pigs were used in this study. A dose of $109.3 \mathrm{ccu} / \mathrm{mL}$ of Vaxsafe ${ }^{\circledR}$ MHP (WF) was tested for safety. The regulatory guidelines for live vaccines stipulate that safety of a 10 times (10X) overdose should be tested. The dose of 109.3 ccu represents a significant overdose of the vaccine surpassing the established 10X overdose by 1000 fold. This study also investigated efficacy of Vaxsafe ${ }^{\circledR}$ MHP (WF) at doses of 105.7, 106.9 and $108.0 \mathrm{ccu} / \mathrm{mL}$. Positive and negative control groups were included. At 22 days post vaccination (DPV), all groups except the negative control group were challenged by intra-nasal spray with Mh wild type strain.

Study 2: Three-week-old healthy Mh free commercial pigs were used in this study. A dose of $105.0 \mathrm{ccu} / \mathrm{mL}$ of both the WF and FD formats of Vaxsafe ${ }^{\circledR}$ MHP were tested for efficacy. A group of pigs given a commercial inactivated vaccine as well as positive and negative control groups were also included in this study. Groups vaccinated on DPV- 0 as well as the positive control group, were challenged by intra-nasal spray on two occasions (DPV-24 and -67) with Mh wild type strain. Post mortems were conducts at DPV-102. Safety of Vaxsafe ${ }^{\circledR}$ MHP (FD) was also investigated at a dose of $108 \mathrm{ccu} / \mathrm{mL}$. This dose was at least 10-fold higher than the expected maximum release titre (MaxRT) of the vaccine.

\section{Results \& Discussion}

Study 1: The safety profile indicated that despite the high overdose, Vaxsafe ${ }^{\circledR}$ MHP did not result in any deaths. Analysis of data revealed that no significant difference in body temperature and weight gain existed between the vaccinated (safety group) and negative control group. At post-mortem, 2/10 pigs had a small lung lesion (not significant $(P>0.05)$ compared with the negative group). Also, the lesions did not translate to any adverse effect on weight gain nor on any clinical signs. At post mortem 4/10 pigs in the unvaccinated, challenged control group presented with significant lung lesions ( $30 \%$ of the lung). No pigs in either the 105.7 or the $106.9 \mathrm{ccu} /$ dose vaccinated groups showed any lesions. In the $108.0 \mathrm{ccu} /$ dose vaccinated group, 1/10 pigs presented with a minor lung lesion ( $7 \%$ of the lung). PCR analysis of nasal swabs taken at DPV-21 prior to challenge showed presence of live Vaxsafe ${ }^{\circledR}$ MHP in $60 \%$ to $70 \%$ of pigs from each of the vaccinated groups. Tracheal colonization was investigated by taking swabs from the upper, middle and lower regions of the trachea at post-mortem (DPV-59). Swabs were only collected from the safety (unchallenged) group. Therefore, Mh detected would be due to Vaxsafe ${ }^{\circledR}$ MHP. Results obtained from PCR analysis showed that Vaxsafe ${ }^{\circledR}$ MHP was present in the trachea of $60 \%$ of pigs in this group. This indicates that Vaxsafe ${ }^{\circledR}$ MHP is capable of colonizing the respiratory passages of vaccinated pigs for at least 8 weeks post vaccination.

Study 2: The unvaccinated challenged group presented with lung lesions in $100 \%$ of pigs with a lung lesion score (planimetric method) of $9.2 \%$. At a dose of 105.0 ccu lung lesions were detected in $30 \%$ (WF) and $10 \%$ (FD) of vaccinated pigs postchallenge with average lesion scores of $0.7 \%$ and $1.7 \%$ respectively. Presence of lung lesions in the commercial inactivated vaccine group was detected in $60 \%$ of pigs with an average lesion score of $8.5 \%$. A direct comparison between the commercial inactivated vaccine group and the $105.0 \mathrm{ccu} /$ dose Vaxsafe ${ }^{\circledR}$ MHP group showed a significant reduction in both the incidence of lung lesions (60\% to $10 \%)$ and in the severity of lung lesions $(8.5 \%$ to $1.7 \%)$, in the Vaxsafe ${ }^{\circledR}$ MHP vaccinated group. The $105 \mathrm{ccu} / \mathrm{mL}$ dose of Vaxsafe ${ }^{\circledR}$ MHP (FD) provided a protective index of $90 \%$ (incidence) and $81 \%$ (severity) of lung lesions. The commercial inactivated vaccine provided a protective index of $40 \%$ (incidence) and only $8 \%$ (severity) of lung lesions. All indices were calculated in comparison to the positive control group.

Administration of an overdose of Vaxsafe ${ }^{\circledR}$ MHP did not result in pig mortalities, any adverse clinical signs, nor any significant increase in the incidence or severity of lung lesions compared to the negative control group. A comparative study with a commercial inactivated vaccine showed Vaxsafe ${ }^{\circledR}$ MHP to offer a higher level of protection following challenge. Overall, these two studies indicate that Vaxsafe ${ }^{\circledR}$ MHP to be a safe and efficacious live vaccine against $M$. hyopneumoniae infection. 


\title{
P.323
}

\section{Efficacy of SPRINTVAC ${ }^{\circledR}$ (MERIAL) vaccination in piglets affected by swine enzootic pneumonia, Mycoplasma hyopneumoniae, in Brazilian field conditions}

\author{
Edson Bordin ${ }^{1}$ Ronie Pinheiro ${ }^{2}$ Flavio Barcelos ${ }^{1}$ \\ 1. Merial Animal Health Ltda., Campinas, SP, Brazil; 2. Integrall - Soluções em Produção Animal Ltda, Patos de Minas, MG, Brazil
}

\section{Introduction}

Mycoplasma hyopneumoniae is considered one of the most important agents associated with chronic diseases in swine herds, and the primary agent of swine enzootic pneumonia (Meyns et al., 2006). Moreno et al. (1999) found a prevalence of $59.9 \%$ of farms affected by Mycoplasma hyopneumoniae in several Brazilian states.

The objective of this study was to evaluate the efficacy of vaccination of piglets with the vaccine SPRINTVAC ${ }^{\circledR}$ (Merial) compared with the vaccine RESPISURE ONE ${ }^{\circledR}$ (Pfizer Animal Health), combined with strategic medication in pulses, by measuring the weight gain, prevalence and extent of lesions in the respiratory tract, rates of occurrence of coughs and mortality rate.

\section{Material and Methods}

The experiment was conducted in a growing and finishing unit of a commercial farm, with a system of all in/all out. A total of 32 pens with about 60 animals (i.e. 1,980 animals) were randomly divided into two groups:

T1: 16 pens vaccinated with SPRINTVAC at 21 days of age.

T2: 16 pens vaccinated with RESPISURE ONE at 21 days of age.

Pulses with strategic medication occurred at the same time and same intensity for all groups.

The cough index was determined throughout the experimental period, according to standard, scientifically validated methodology (Mores et al., 1999).

At slaughter 120 animals of each treatment were submitted to a lung lesion evaluation according to the methodology of Piffer \& Brito (1991).

The software package statistical analysis for genetic epidemiology (S.A.G.E.) was used for variance analysis and mean comparison. $\mathrm{Chi}^{2}$-test and non-parametric tests like Kruskal-Wallis were used.

\section{Results}

As shown in table 1 no significant difference in the weight output termination ( $p>0.05$ ) and for GPD phases analyzed. Table 1 also presents the prevalence of lesions of pneumonia, in addition to objective index to assess the extent of these lesions (IPP Index Pneumonia) and no significant difference was observed between both groups (Chi²-test; p>0.05).

No significant difference was found in mortality rates for the entire experimental period: $2.6 \%$ for animals vaccinated with SPRINTVAC and $2.85 \%$ for those with RESPISURE ONE ( $p>0.05$ ).

As shown in table 2 related to the rate of cough there was no significant difference between treatments (Chi²-test; $\mathrm{p}>0.05$ ).
Table 1: Performance (23 to 149 days) and evaluation of lung lesions at slaughter

\begin{tabular}{|c|c|c|c|c|c|c|}
\hline \multirow{2}{*}{ Treatment } & \multicolumn{2}{|c|}{$\begin{array}{c}\text { Performance } \\
\text { (23 to 149 days) }\end{array}$} & \multicolumn{4}{|c|}{ Evaluation of lung lesions at slaughter } \\
\cline { 2 - 7 } & P 149 days & DWG & Number of lungs & No-lesion & Lesion & (IPP) \\
\hline $\mathrm{T} 1$ & $101.130^{\mathrm{a}}$ & $0.746^{\mathrm{a}}$ & 126 & $11(8.7 \%)$ & $115(91.3 \%)$ & 1.26 \\
\hline $\mathrm{T} 2$ & $101.361^{\mathrm{a}}$ & $0.748^{\mathrm{a}}$ & 116 & $13(11.2 \%)$ & $103(88.8$ & 1.20 \\
\hline \begin{tabular}{l}
$\mathrm{P}=149$ days weight at 149 days of age \\
DWG = daily weight gain throughout the experiment \\
Same letters in the same column indicate the absence of statistical difference between values \\
\hline
\end{tabular}
\end{tabular}

Table 2: Index of coughing during the experiment

\begin{tabular}{|c|c|}
\hline Phase-treatment & Cough index \\
\hline Nursery - T1 & 0.011 \\
\hline Nursery - T2 & 0.034 \\
\hline Growing - T1 & 0.226 \\
\hline Growing - T2 & 0.181 \\
\hline Finishing - T1 & 1.105 \\
\hline Finishing - T2 & 0.970 \\
\hline
\end{tabular}

\section{Conclusion}

In the conditions of this experiment, the vaccination of piglets at weaning with SPRINTVAC and RESPISURE ONE showed no difference in performance (final weight and DWG), slaughter lung lesions, mortality and number of antibiotic treatments (not shown).

\section{References}

1. Meyns D.M. et al., (2006), Vaccine 24 : 7081-7086

2. Moreno A. et al., (1999), IX ABRAVES : 161-162

3. Piffer l. et al., (1991) Embrapa suínos e aves 23

4. Morés N. et al., (1999) Embrapa, CT 242

- $S P R I N T V A C$ is a registered trademark of MERIAL in the United States of America and elsewhere.

S.A.G.E. is supported by the Human Genetic Analysis Resource funded by the National Center for Research Resources of the National Institute of Health. 


\title{
P.324
}

\section{Safety evaluation of SPRINTVAC ${ }^{\otimes}$ by measure of piglet body temperature in a commercial farm in Brazil}

\author{
J. Sarubbi ${ }^{1,5} \underline{\text { Edson Bordin }}^{2}$ D. Jorge De Moura ${ }^{6}$ F. Oliveira ${ }^{3}$ Andrea Delvecchio ${ }^{4}$ François Joisel $^{4}$ \\ 1. FEAGRI UNICAMP, Campinas, SP, Brazil; 2. Merial Animal Health Ltda., Campinas, SP, Brazil; 3. MULTIPLAVET, Campinas, SP, Brazil; \\ 4. Merial S.A.S., Lyon, France; 5. UFSM, Palmeiras das Missões, Brazil; 6. UNICAMP, Sao Paulo, Brazil
}

\section{Introduction}

Mycoplasma hyopneumoniae (M. hyo) is considered to be one of the most economically important pathogens in the swine industry. The effectiveness of vaccination in reducing the economic impact of M. hyo has been shown previously $(1,2)$.

The objective of this study was to evaluate the safety of SPRINTVAC ${ }^{\circledR}$ vaccination in piglets by the measure of piglet body surface temperature compared to body surface temperature of piglets treated with another current vaccination protocol.

\section{Material and Methods}

A total of 140 piglets from a 400-sow farrow-to-finish herd located in Brazil were used in this study. Piglets were randomly divided into 2 treatment groups: group T1 vaccinated with one dose of SPRINTVAC ( $2 \mathrm{~mL}, I \mathrm{M})$, group T2 vaccinated with one dose of Suvaxyn ${ }^{\circledR}$ Respifend MH (Pfizer Animal Health).

Piglet body surface temperature of piglets was measured with an infrared thermographic camera Testo ${ }^{\circledR}$ (figure 1).

Figure 1: Infrared thermografic camera used to verify the distribution of temperature on piglet skin

All piglets were photographed at 5 timepoints: before vaccination, $30 \mathrm{~min}, 45 \mathrm{~min}, 24 \mathrm{~h}$ and $48 \mathrm{~h}$ after vaccination. Acquired images were processed and analyzed using the IRTesto ${ }^{\circledR}$ software to calculate average surface temperature.

To compare piglets from both treatments, a polygon was drawn to cover the regions of their neck, flank and hind legs. From these regions, the average surface skin temperature of the left profile of each piglet was extracted. The average temperatures were compared by Tukey's test, after verification of normality of the data distribution.

\section{Results}

Thirty minutes after vaccination, the average skin temperature of T1 animals was the same as the temperature measured before vaccination. The average temperature of $\mathrm{T} 2$ animals increased by $1.6^{\circ} \mathrm{C}$. Forty-five min after vaccination, the average temperature of $\mathrm{T} 1$ animals increased by $0.8^{\circ} \mathrm{C}$ compared to the average temperature found before vaccination but stays $0.4^{\circ} \mathrm{C}$ lower than in $\mathrm{T} 2$ group. Twenty-four hours after vaccination, the average skin temperature of $\mathrm{T} 1$ animals decreased by $13^{\circ} \mathrm{C}$ compared the previous measurement. Piglets of $\mathrm{T} 2$ showed a decrease of a similar value $\left(1.4^{\circ} \mathrm{C}\right)$. At this point, animals from $\mathrm{T} 2 \mathrm{kept}$ their average temperature $\left(03^{\circ} \mathrm{C}\right)$ a little higher than $\mathrm{T} 1$ animals. Forty-eight hours post-vaccination, piglets in both treatment groups showed an increase in their average temperature, being the highest increase in the animals from $\mathrm{T} 2\left(0.9^{\circ} \mathrm{C}\right.$ against $0.5^{\circ} \mathrm{C}$ from $\left.\mathrm{T} 1\right)$.

At all time points, pigs from group $\mathrm{T} 2$ showed a numerically higher average temperature than in group T1, although, statistical differences were found only at 30 minutes and $48 \mathrm{~h}$ postvaccination (Table 1).

Table 1: comparison of average surface temperatures of piglets from $T 1$ and $T 2$

\begin{tabular}{|c|c|c|c|c|}
\hline \multirow{2}{*}{$\begin{array}{l}\text { Moment from } \\
\text { vaccination }\end{array}$} & \multicolumn{2}{|c|}{ T1 } & \multicolumn{2}{|l|}{$\mathrm{T2}$} \\
\hline & $\begin{array}{l}\text { Average surface } \\
\text { temperature }\left({ }^{\circ} \mathrm{C}\right)\end{array}$ & STD & $\begin{array}{l}\text { Average surface } \\
\text { temperature }\left({ }^{\circ} \mathrm{C}\right)\end{array}$ & STD \\
\hline Before & 34.7 & $(+0.6) \mathrm{a}$ & 34.6 & $(+0.7) \mathrm{a}$ \\
\hline 30 min after & 34.7 & $(+1.0) \mathrm{a}$ & 36.2 & $(+0.8) b$ \\
\hline 45 min after & 35.5 & $(+0.6) \mathrm{a}$ & 35.9 & $(+0.6) \mathrm{a}$ \\
\hline $24 \mathrm{~h}$ after & 34.2 & $(+1.2) a$ & 34.5 & $(+1.0) \mathrm{a}$ \\
\hline 48h after & 34.7 & $(+0.9) \mathrm{b}$ & 35.4 & $(+1.1) \mathrm{a}$ \\
\hline
\end{tabular}

\section{Discussion and Conclusion}

Measure of piglet body surface temperature with an infrared thermographic camera seems to be a good tool limitating labour and pig stress. For a more precise evaluation, it would be necessary that the piglets would be under an efficient climatization system, that more piglets would be studied and during more days.

It is believed that SPRINTVAC adjuvant gives a smoother response compared to the other studied vaccine, explaining the behavior of the lower surface temperature of the piglets vaccinated with this bacterin These results confirm the safety of SPRINTVAC vaccine.

\section{References}

1. Kyriakis S C et al., (2001) Journal Veterinary Medicine, Nov;48 (9): 675-684

2. Maes D et al., (1999) Vaccine Mar 5; 17(9-10):1024-1034

-SPRINTVAC is a registered trademark of Merial in the United States of America and elsewhere. 


\title{
P.325
}

\section{Efficacy of a combined vaccination schedule with RHINIFFA ${ }^{\otimes} T$ in sows and SPRINTVAC ${ }^{\circledR}$ in piglets versus another commercial protocol}

\author{
J. Sarubbi ${ }^{1} \underline{E d s o n}$ Bordin $^{2}$ D. Jorge De Moura ${ }^{3}$ F. Oliveira ${ }^{4}$ Andrea Delvecchio ${ }^{5}$ François Joisel ${ }^{5}$ \\ 1. FEAGRI UNICAMP, Campinas, SP, Brazil; 2. Merial Animal Health Ltda., Campinas, SP, Brazil; 3. UNICAMP, Sao Paulo, Brazil; \\ 4. MULTIPLAVET, Campinas, SP, Brazil; 5. Merial S.A.S., Lyon, France
}

\section{Introduction}

Atrophic rhinitis (AR) and enzootic pneumonia (EP) are two diseases affecting swine herds worldwide.

The vaccines against (EP) provide protection to swine by the reduction of lesions and improvement of the productivity indexes, although they do prevent neither the colonization by mycoplasma nor the establishment of carrier swine (1). AR in swine is characterized by a deformation of snouts and a turbinate atrophy (2). Vaccination of sows is among the effective strategies used for the prevention and control of AR in piglets (3).

The objective of this study was to evaluate a combined vaccination schedule with RHINIFFA ${ }^{\circledR}$ T in sows followed by SPRINTVAC ${ }^{\circledR}$ in piglets, compared to another currently applied protocol.

\section{Material and Methods}

Farm: A 400-sow farrow-to-finish herd located in Brazil and selected on its previous historic of lung lesion condemnations was used in this study.

Experimental design: 3 replicates of 120 commercial pigs, housed in 3 separate pens, were used in the study. Each repetition was divided into 2 treatment groups. In the first group (T1) piglets born from sows vaccinated with 2 doses of RHINIFFA T (MERIAL, 2mL, IM 6 to 8 weeks before farrowing and the 2 nd dose was performed at 2 to 3 weeks before farrowing) received 1 dose of SPRINTVAC (MERIAL - 2mL, IM, at 3 weeks of age) In the second group (T2) piglets born from sows vaccinated with RINIPRAVAC DT ${ }^{\oplus}(2 \mathrm{~mL}, \mathrm{IM}, 1$ st dose at 6 to 8 weeks and the 2 nd dose at 2 to 3 weeks both before farrowing) were vaccinated with 2 doses of MYPRAVAC Suis ${ }^{\circledR}(2 \mathrm{~mL}, \mathrm{IM}, 1$ dose at 7 to 10 days and the second dose at 28 days of age).

Efficacy parameters: Pneumonia Index (PI) and Progressive Atrophic Rhinitis Index (ARI) were used to evaluate the vaccines protocols (4). PI is the numeric representation of the severity level of the pneumonic lesion incidence in examined animals, while ARI is based on the weighted average score of changes and lesions in nasal turbinates. The assessment of lung lesions and nasal turbinates was done by visual appreciation.

Statistical methods: Chi-square Yates-corrected test for concordance of lung and turbinate lesions between the two treatment groups was performed.

\section{Results and Discussion}

The prevalence of lung lesions was numerically found slightly higher in $\mathrm{T} 2$ animals (Table 1 ).

Table 1: Prevalence of lung lesions, nasal turbinate lesions, PI and ARI (averages).

\begin{tabular}{|l|c|c|c|}
\hline \multirow{2}{*}{} & \multicolumn{2}{|c|}{ Treatments } & \multirow{2}{*}{ p } \\
\cline { 2 - 3 } & T1 & T2 & \\
\hline Prevalence of lung lesions (\%) & 39.9 & 44.9 & 0.369 \\
\hline Pneumonia Index (PI) & 0.44 & 0.57 & ND \\
\hline Prevalence of nasal turbines lesions (atrophy) (\%) & 42.1 & 57.9 & 0.117 \\
\hline Atrophic Rhinitis Index (ARI) & 0.52 & 0.82 & ND \\
\hline ND: not done. & \multicolumn{3}{|l|}{} \\
\hline
\end{tabular}

PI and ARI average scores were 0.13 and 0.30 lower in the T1 animals than in the T2 animal, respectively. It is usually admitted that $\mathrm{PI}$ scores are indicative of $\mathrm{PI}<0.56$ : herds pneumonia free; $0.56<\mathrm{PI}<0.89$ : Pneumonia is not a threat; $\mathrm{PI}>0.90$ : there is a significant pneumonia incidence (4).

All repetitions show an about $16 \%$ higher prevalence of lesions in the nasal turbinates in T2 animals (close to a statistical trend).

It is interesting to note that in the T2 animals, some batches showed purulent lesions in the nasal turbinates.

\section{Conclusion}

Numerical differences between the results of the two protocols were not found statistically significant. Considering the lung lesion and nasal turbinate prevalence, in this study, T1 treatment provided a good protection against the two diseases.

\section{References}

1. Hsuan S.-L. et al., (2009) Vaccine, 27 : 2923-29

2. Conceição F. R. and Dellagostin O. A., (2006) Cienc. Rural 36(3):10341042

3. Kavanagh N. T., (1994) Vet. Rec. 134:218219

4. Piffer I. and Brito J., (1991) Concordia, SC. EMBRAPA-CPNSA, 12 p. ه SPRINTVAC and RHINIFFA T are registered trademarks of MERIAL.

- MYPRAVAC Suis and RINIPRAVAC DT are registered trademarks of HIPRA. 


\title{
P.326
}

\section{Comparative efficacy of single dose $\mathrm{M}+\mathrm{PAC}^{\circledR}$ vaccination schedule and another single dose vaccine in a high $M$. hyopneumoniae infection pressure environment}

\author{
Enric Marco ${ }^{1}$ Joan Escobet ${ }^{2}$ Miquel Collell ${ }^{2}$ Jesus M. Bollo $^{3}$ Rut Menjon ${ }^{3}$ Marta Jimenez ${ }^{3}$ Jesus V. Lopez $^{3}$ \\ 1. Marco y Collell, St Paulí de Nola, 6. 08004 Barcelona (Spain), Madrid, Spain; 2. Marco y Collell, St Paulí de Nola, \\ 6. 08004 Barcelona (Spain), Barcelona, Spain; 3. Intervet Schering Plough. c/ Cantabria 2, Edificio Amura 28.108 Alcobendas, Madrid, Spain
}

\section{Introduction}

The objective of the study was to evaluate the safety and efficacy of a single dose $\mathrm{M}+\mathrm{PAC}^{\circledR}$ vaccination schedule compared with another single dose vaccine for prevention of $M$. hyopneumoniae (M. hyo) in a high infection pressure environment, to assess the real efficacy of both products.

\section{Materials and Methods}

The study was performed in a farm with 450 sows, using 3 week batches, which did not vaccinate for $\mathrm{M}$. hyo before the study and presented a moderate level of pulmonary affection due to $\mathrm{M}$. hyo: average score 0.91 , using a 0-5 scoring system (1).

The piglets were divided into 4 groups, and the first 3 ones were randomized according to the sow and piglet gender and weight (weights were comparable at the start of the study $(p<0.05)$, Table 1:

a) $\mathrm{M}+\mathrm{PAC}^{\circledR}$ (Intervet Schering-Plough), single $2 \mathrm{~mL}$ dose given at 3 weeks of age (121 animals).

b) Vaccine $A$, single dose vaccine, $2 \mathrm{~mL}$ given at 3 weeks of age (122 animals).

c) Control, $2 \mathrm{~mL}$ saline given at 3 weeks of age (236 animals).

d) Control A, not included in the study, with no individual animal identification, used to increase the environmental infectious pressure (250 animals).

The 4 groups were commingled in the same pens, keeping the same ratio of each group both during growing and finishing, so that the 2 vaccinated groups represented only $35 \%$ of the animals in each pen.

Statistical evaluation was performed using analysis of variance, together with the Levene test, Pearson's chi-square test, Fisher's exact test and Mann-Whitney-U test.

\section{Results}

Table 1

\begin{tabular}{|l|c|c|c|}
\hline & Control & Vaccine A & M+PAC \\
\hline Weaning weight $(\mathrm{kg})$ & 6.49 & 6.61 & 6.74 \\
\hline Initial fattening weight $(\mathrm{kg})$ & 20.83 & 21.01 & 21.21 \\
\hline
\end{tabular}

No statistical significant differences were found for the initial fattening weight (55 days of age), which indicated the safety of $M+P A C$.

Table 2

\begin{tabular}{|l|c|c|c|}
\hline & Control & Vaccine $A$ & M+PAC \\
\hline ADG fattening $(\mathrm{g} /$ day) & 663 & 690 & 705 \\
\hline End fattening weight $(\mathrm{kg})$ & 87.83 & 90.68 & 92.45 \\
\hline
\end{tabular}

Concerning ADG during fattening (Table 2), the animals of the $\mathrm{M}+\mathrm{PAC}^{\circledR}$ group presented higher growths than the control group ( $+42 \mathrm{~g} /$ day, $\mathrm{p}=0.02$ ), and than the Vaccine A group (+16 g/ day, $p=0.658)$. On the contrary, Vaccine $A$ did not present a significant statistical difference with the Control group $(p=0.656)$.

The $M+P A C^{\circledR}$ group showed statistically significant higher average end of fattening weights (157 days of age) than the Control group ( $+4.62 \mathrm{~kg} . \mathrm{p}=0.027)$, and than the Vaccine A group (+1.77 kg. $p=0.659)$. Vaccine $A$ did not attain significant statistical differences vs the Control group ( $p=025)$.

The analysis of variance of both the ADG during fattening and the average end of fattening weights indicated better results of the group vaccinated with $\mathrm{M}+\mathrm{PAC}^{\circledR}$ compared to the Control group ( $p=0.002$ and $p=0.011$, respectively) but vaccine $A$ did not present a statistical significant difference vs the non-vaccinated animals $(\mathrm{p}=0.050)$.

No differences concerning mortality were found among the groups $(p=0,835)$.

Table 3. Study of lesions at the slaughterhouse

\begin{tabular}{|c|c|c|c|}
\hline Pulmonary lesions & Control & Vaccine $A$ & $M+P A C$ \\
\hline$\%$ affected lungs & $53.5^{\mathrm{a}}$ & $38.8^{\mathrm{b}}$ & $32.2^{c}$ \\
\hline$\%$ lungs with highest score lesions & $2.5^{\mathrm{a}}$ & $2.5^{\mathrm{a}}$ & $0^{\mathrm{a}}$ \\
\hline Average score & $0.86^{\mathrm{a}}$ & $0.51^{b}$ & $0.37^{c}$ \\
\hline \% lungs with pleuritis & $6.4^{\mathrm{a}}$ & $10.6^{\mathrm{a}}$ & $1.1^{\mathrm{b}}$ \\
\hline
\end{tabular}

$\mathrm{M}+\mathrm{PAC}^{\circledast}$ produced the highest reduction of pulmonary lesions ascribed to M.hyo and pleuritis.

When grouping together the grade $0-3$ scores and the grade 4-5 ones (highest score lesions), the average ADG was 709 and 492g/ day, respectively $(p=0.012)$, with the ADG during fattening relating with the severity of the pulmonary lesions which were found.

\section{Discussion}

Animals vaccinated with $\mathrm{M}+\mathrm{PAC}^{\circledR}$ showed the highest reduction of pulmonary lesions, and both a higher ADG during fattening and end of fattening weight compared to the Control group, not occurring in the group vaccinated with Vaccine $A$.

Analysis of variance showed that the animals vaccinated with $\mathrm{M}+\mathrm{PAC}^{\circledast}$ presented a more uniform growth and end weight than the Control group, which was not found for the Vaccine A group.

\section{References}

1. Bollo J.M. et al (2008). Proc.20th. IPVS Congress. Vol 1, 104 


\title{
P.327
}

\section{Efficacy of Suvaxyn ${ }^{\circledR}$ MH-One under field conditions}

\author{
Florence Pillet ${ }^{3}$ Mathieu Couteau ${ }^{1}$ Olivier Roy ${ }^{3}$ Paolo Doncecchi ${ }^{2}$ Patrick Bourguignon ${ }^{4}$ Geoffrey Labarque $^{2}$ \\ 1. Fort Dodge Animal Health, Tours, France; 2. Pfizer Animal Health, Paris, France; 3. Cebiphar, Fondettes, France; 4. Bocavet, Cerizay, France
}

\section{Introduction}

Respiratory disease is the most important pathology in modern swine husbandry in Europe. Mycoplasma hyopneumoniae (M.hyo) is a key player in the porcine respiratory disease complex (PRDC), a multi-factorial disease syndrome caused by several bacteria, viruses, management and husbandry factors. To control the clinical expression of this syndrome and its negative impact on pigs' performance, vaccination with $M$. hyo vaccines is a wide-spread practice in European swine farms. Vaccines against M.hyo are by far the most widely used swine vaccines in Europe.

Suvaxyn ${ }^{\circledast}$ MH-One (Fort Dodge Animal Health) is a one-shot vaccine against $M$. hyo to be administered by intramuscular injection in piglets starting from 3 weeks of age. The efficacy of this vaccine has been confirmed under experimental conditions (1). The present study aimed to confirm the efficacy of this vaccine under field conditions by comparing its performance with another commercially available one-shot Mycoplasma vaccine.

\section{Materials and Methods}

The study was conducted in a closed breeding-fattening herd in France. Before the start of the study, piglets were vaccinated against M.hyo with a commercially available one-shot vaccine at 35 days of age (i.e. one week after weaning). Two consecutive batches with a three weeks interval were included in this study. The piglets of each of the two batches were divided into two groups: one group ( $\mathrm{n}=182$ in total) was vaccinated with Suvaxy $^{\circledR} \mathrm{MH}$-One at 5 weeks of age and the other group $(n=170$ in total) was vaccinated at the same age with another commercially available one-shot Mycoplasma vaccine (designated vaccine A), following the manufacturers' instructions. The piglets did not receive any other vaccine throughout the study.

The efficacy of both vaccines was evaluated using clinical parameters [morbidity (signs of respiratory disease), mortality, number of treatments if required], growth performance parameters [average daily weight gain (ADWG), feed conversion ratio (FCR)] and the results of post-mortem lung examinations at the slaughterhouse. Lung lesions were scored from 0 to 4 per lung lobe. A final score was obtained by adding the scores given to each lung lobe (between 0 and 28) (2). Pleurisy lesions were scored from 0 to 2. The mean lung lesion scores and the percentage of animals having lung lesions were statistically compared using a one-way analysis of variance (ANOVA) and a Chi2 test, respectively.

\section{Results}

The results of the field study are summarized in Table 1. No signs of respiratory disease were observed throughout the study. The mortality was the same in both groups (3 pigs in both vaccinated groups).
There were no differences in the growth performance parameters ADWG and FCR between both groups.

There were no differences in the mean lung lesion scores between both groups ( 0.7 for the Suvaxyn ${ }^{\circledast} \mathrm{MH}$-One group and 0.9 for the vaccine $A$ group) $(P>0.05)$. The percentage of animals with lung lesions (i.e. animals having a score of $>0$ ) was somewhat lower in the Suvaxyn ${ }^{\circledast} \mathrm{MH}$-One group (23.5\%) when compared to the vaccine A group (26.9\%). However, this difference was not significant $(P>0.05)$. Five animals presented pleurisy at slaughter: 2 in the Suvaxyn ${ }^{\circledR} \mathrm{MH}$-One group and 3 in the vaccine A group.

Table 1. Clinical observations, growth performances and post-mortem lung lesions following vaccination with one-shot Mycoplasma vaccines in the field.

\begin{tabular}{|l|c|c|c|c|}
\hline \multirow{2}{*}{} & \multicolumn{2}{|c|}{ Suvaxyn ${ }^{\oplus}$ MH-One } & \multicolumn{2}{c|}{ Vaccine A } \\
\cline { 2 - 5 } & Batch 1 & Batch 2 & Batch 1 & Batch 2 \\
\hline No. of pigs & 99 & 83 & 87 & 83 \\
\hline Mortality rate & $0 \%$ & $3.6 \%$ & $1.1 \%$ & $2.4 \%$ \\
\hline ADWG (g/day) & 764.3 & 740.5 & 761.8 & 749.2 \\
\hline FCR & 2.33 & 2.32 & 2.35 & 2.30 \\
\hline Mean lung lesion score & 0.4 & 1.3 & 0.3 & 1.6 \\
\hline \% of pigs with lung lesions & 15.6 & 36.8 & 18.1 & 38.7 \\
\hline ADWG: average daily weight gain, FCR: food conversion ratio \\
\hline
\end{tabular}

\section{Discussion}

As no respiratory disease occurred throughout the study, the efficacy of Suvaxyn ${ }^{\circledR} \mathrm{MH}-$ One could only be evaluated using growth performance parameters and the results of the postmortem lung examinations at slaughter. The analysis of these parameters did not reveal any differences between the Suvaxyn ${ }^{\circledR}$ $\mathrm{MH}-O n e-v a c c i n a t e d$ pigs and the pigs vaccinated with the competitor vaccine. The percentage of pigs presenting lung lesions and pleurisy was somewhat lower in the Suvaxyn ${ }^{\circledR} \mathrm{MH}$-One group, but these differences were not statistically significant.

\section{References}

1. Thacker E., et al, 2004. Proc.18th IPVS Congress, Hamburg, Germany, $1,383$.

2. Madec F., Kobisch M., 1982. Journées de la Recherche Porcine en France, 14, 405-412. 


\section{Clinical evaluation of intradermal vaccination against porcine Enzootic Pneumonia (Mycoplasma hyopneumoniae)}

Panagiotis D. Tassis ${ }^{1}$ Eleni D. Tzika ${ }^{1}$ Vassilios G. Papatsiros ${ }^{2}$ Tom Nell $^{3}$ Costas Alexopoulos $^{5}$ Dominiek Maes ${ }^{4}$ Spiros C. Kyriakis ${ }^{6}$

1. Clinic of Farm Animal Medicine, Faculty of Veterinary Medicine, Aristotle University of Thessaloniki, Thessaloniki, Greece; 2. Clinic of Medicine, School of Veterinary Medicine, University of Thessaly, Karditsa, Greece; 3. Clinical Trial Unit, Intervet International, Boxmeer, Netherlands; 4. Department of Obstetrics, Reproduction and Herd Health, Faculty of Veterinary Medicine, University of Ghent, Merelbeke, Belgium;

5. Deceased author, Thessaloniki, Greece; 6. Foundation of Biomedical Research, Academy of Athens, Athens, Greece

\section{Introduction}

Mycoplasma hyopneumoniae (M. hyo) is the etiologic agent of mycoplasma pneumonia. The term enzootic pneumonia (EP) describes the epidemiological pattern of the disease in combination with other etiological agents or lesions involved, (high morbidity including clinical respiratory signs such as prolonged non-productive coughing and areas of lung adjacent to normal tissue) $(1,2)$. The aim of this study was to determine the efficacy of a single dose of an aqueous inactivated $M$. hyo vaccine against EP, on the basis of reduction of frequency and severity of lung lesions and improvement of growth performance. The study was financed by Intervet SPAH through the Research Committee of Aristotle University of Thessaloniki.

\section{Materials and Methods}

The study was carried out in a commercial farrow-to-finish pig farm, (900 sows under production) with confirmed M. hyo infection. The intradermal vaccination was performed with an inactivated aqueous vaccine containing $M$. hyo strain 11 inactivated $M$. hyo cells with a light paraffin oil and dl-a-tocopheryl acetate based adjuvant (Porcilis ${ }^{\circledR}$ M. Hyo one shot IDAL, Intervet SPAH, Boxmeer, The Netherlands). A total of 700 healthy suckling piglets at the age of $28 \pm 3$ days were ear tagged and randomly assigned to one of two experimental groups: 346 piglets in treatment group (IDAL) which received a single vaccination at 4 weeks of age (start of the trial period), and 354 in control (C) group which received a placebo (dl- a-tocopherol-acetate based adjuvating

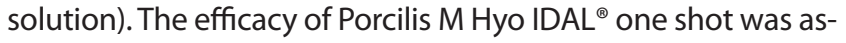
sessed on the basis of the reduction of lung lesions and improvement of the average daily weight gain in the vaccinated piglets as compared to the controls. Pleuritis lesions, medication and mortality were evaluated as secondary parameters of efficacy. The statistical evaluation was performed with $\mathrm{SAS}^{\circledR}$ version 9.1 .

\section{Results and Discussion}

The severity of lung lesions and pleuritis at slaughter were reduced significantly in the IDAL group (Table 1) as compared to the controls.

The ADG during the finishing period and the ADG over the total period were significantly better in the IDAL vaccinated pigs than in the controls (Table 2). The improvement in the IDAL group was $27 \mathrm{~g} /$ day during the finishing phase and $23 \mathrm{~g} /$ day over the total period.
No difference in medication was observed in the nursery, but during the finishing phase significantly less (chi-square test: $p<0.001$ ) IDAL vaccinated pigs were treated (5.1\%) than controls (15.5\%). Three quarters of all treatments during the finishing phase were given for the control of respiratory signs. Also the mortality was significantly lower (Chi-square test: $p=0.0208$ ) in the IDAL vaccinated pigs (4.7\%) as compared to the control group (9.0\%).

It is concluded that a single intradermal vaccination of piglets with Porcilis ${ }^{\circledR} \mathrm{M}$ Hyo IDAL at the age of around 4 weeks is effective against Enzootic Pneumonia in a farrow-to-finish swine unit infected with $M$. hyo. The prevalence and severity of lung was improved and the performance was improved.

\begin{tabular}{|l|c|c|}
\hline Table 1 & \multicolumn{2}{|c|}{ Trial group } \\
\hline & \multicolumn{2}{|c|}{ Lung Lesion Score } \\
\hline & IDAL & Control \\
\hline No lesions & $58^{\mathrm{a}}$ & $17^{\mathrm{b}}$ \\
\hline Score $>0$ & 267 & 293 \\
\hline Mean \pm SD & $7.03 \pm 6.9^{c}$ & $13.13 \pm 7.3^{\mathrm{d}}$ \\
\hline & \multicolumn{2}{|c|}{ Pleuritis Score } \\
\hline No lesions & $240^{c}$ & $124^{\mathrm{d}}$ \\
\hline Spots & 67 & 142 \\
\hline Larger & 17 & 44 \\
\hline $\begin{array}{l}|c| \\
\text { ab: Chi-square test: } p<0.001 \\
\text { cd: Rank Sum test: } p<0.001\end{array}$ \\
\hline
\end{tabular}

\begin{tabular}{|l|c|c|}
\hline Table 2 & \multicolumn{2}{|c|}{ Trial group } \\
\hline Period & IDAL & Control \\
\hline & ADG & ADG \\
\hline Nursery & $473 \pm 85$ & $461 \pm 88$ \\
\hline Finishing & $666 \pm 35^{\mathrm{a}}$ & $640 \pm 37^{\mathrm{b}}$ \\
\hline Total & $623 \pm 33^{\mathrm{a}}$ & $600 \pm 33^{\mathrm{b}}$ \\
\hline Ab: Ancova: $p<0.001$ \\
\hline
\end{tabular}

\section{References}

1. Thacker, E.L., 2006. Diseases of Swine. lowa State University Press, Ames, pp. 701-717.

2. Sibila M. et. al. 2007. Vet Microb 122: 97-107. 


\title{
P.329
}

\section{Evaluation of peripheral lymphocytes after Weaning and vaccination for Mycoplasma hyopneumoniae}

\author{
Glen W. Almond ${ }^{1}$ Andrew Kick ${ }^{1}$ Martin Liebstein ${ }^{1}$ Patricia A. Routh ${ }^{1}$ Mark J. Hammer ${ }^{2}$ \\ 1. College of Vet Med, North Carolina St Univ, Raleigh, NC, USA; 2. Novartis Animal Health, Greensboro, NC, USA
}

\section{Introduction}

An early report indicated that vaccination against Mycoplasma hypopneumoniae (MHYO) or pathogen challenge failed to induce a nonspecific change in peripheral lymphocyte populations; however, interferon- $\gamma$ (INF- $\gamma$ ) secreting cells were important to the protective response (1). Overall, a local mucosal humoral and a systemic cell mediated immune response are important to controlling mycoplasmal pneumonia in pigs (2). The porcine immune system is unique in the expression of CD4+CD8+ lymphocytes, which may be involved in the immune response to MHYO. Therefore, the primary aim of the study was to investigate immune cell populations following intramuscular injection with an one-dose MHYO vaccine and novel adjuvant.

\section{Materials and Methods}

The animal phase of the study was conducted at the Swine Teaching Unit, College of Veterinary Medicine. The unit has a high health status and approximately 40 sows. Based on previous serology, animals seroconverted to MHYO two months after placement in the finishing barn. Pigs ( $n=24$ barrows) were selected at weaning ( $\approx 3$ wks of age) and ear tagged for identification. Eight pigs served as controls and 16 pigs were vaccinated with MYHO bacterin (PneumoSTAR ${ }^{\mathrm{TM}}$ Myco) at placement in the nursery. Blood samples were collected immediately before injection, and 3, 7, 14, 30, and 60 days after injection. Whole blood was used for complete blood counts ( $C B C^{\prime}$ 's) and preparation for the flow cytometry analysis. In addition, serum was collected to assess the humoral response to the vaccine or natural infection. Lymphocytes evaluated with flow cytometry included CD3+, CD4+, CD8+, CD4+CD8+, and CD21+ cells. Also, CD25+ cells, and cells expressing lgG and/or IgA were evaluated, but not included in the results. The statistical analyses used an analysis of variance for repeated measures and Tukey's test to compare means.

\section{Results}

Following vaccination, there were no apparent adverse effects in any of the pigs. The CBC's did not differ between the groups within day; however, total WBC, neutrophils, and lymphocytes increased $(\mathrm{P}<.05)$ from day 0 to day $30(7.2+5$ to $16.5+.7 \times 103 /$ ul, $3.1+.3$ to $6+.2 \times 103 /$ ul, $3.3+.3$ to $9.3+.7 \times 103 /$ ul, respectively). An initial increase in CD4+ (Fig 1), CD3+ and CD21+ cells occurred from day 0 to day 3 in both groups of pigs. The number of CD8+ and CD4+CD8+ lymphocytes increased during the initial 3 days in the control group, but not in the vaccinated pigs (Fig 1). The SP ratios for MHYO remained at 0 for control pigs, whereas the titers increased to 1.4 at Day 30 in vaccinated pigs.
Fig 1. Changes in CD8+ (top), $C D 4+C D 8+$ (middle) and CD4+ cells in pigs after MHYO vaccination (solid lines) or sham treatment (dashed lines). ( ${ }^{*}$ Within day, values differed $P<.05$ )
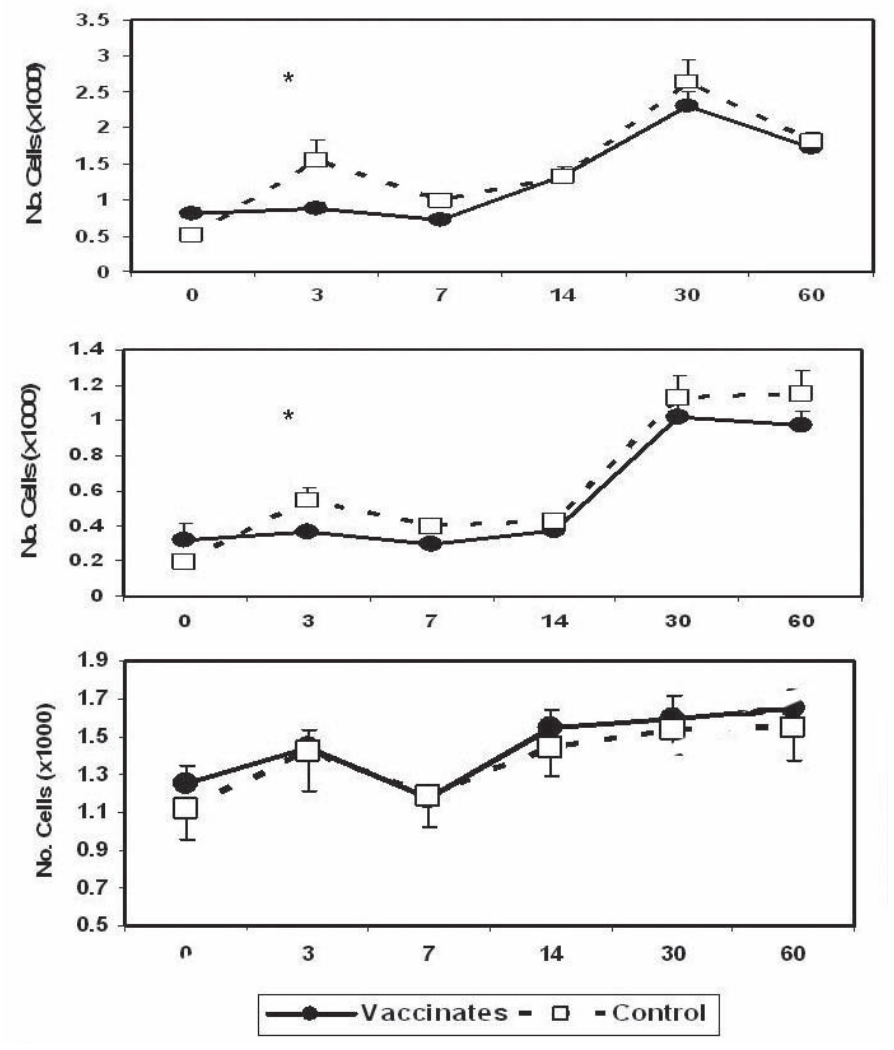

\section{Discussion}

It was expected that the total WBC counts increased with age of the pigs. The increase in the three lymphocyte populations likely reflects antigenic challenge resulting from the changes in diet and environment. The lack of change in the CD8+ and CD4+CD8+ lymphocytes in vaccinated pigs was surprising; however, it could be partially attributed to lymphocyte trapping in the lymph nodes and lymphatic system, and thus the number of these cells failed to change in the peripheral circulation. The differences between immune cells were observed shortly after vaccination (weaning) and prior to the increased ELISA titers. It is likely that the antigenic challenges associated with weaning confounded the response to vaccination.

\section{References}

1. Thanawongnuweck R, et al. 2004. Clin \& Diag Lab Immunol 11:901908.

2. Thacker E, et al. 2000. Am J Vet Res 61:1384-1389. 


\title{
P.330
}

\section{Effect of vaccination against Mycoplasma hyopneumoniae on its transmission under field conditions}

\author{
Iris Villarreal; Dominiek Maes; Katleen Vranckx; Frank Pasmans; Freddy Haesebrouck
}

Faculty of Veterinary Medicine, Ghent University, Merelbeke, Belgium

\begin{abstract}
Introduction
Mycoplasma hyopneumoniae (Mh) is the primary agent of enzootic pneumonia (EP), a contagious respiratory disease with major economical impact for the pig industry. Vaccination of piglets is widely practiced as a control strategy for this disease. However, the currently available vaccines do not induce a complete protection against colonization and transmission (2). Virulence differences (3) and protein variability (1) between Mh strains may indicate that vaccination efficacy could vary depending on the strain. The aim of this study was to assess the effect of vaccination with a commercial bacterin vaccine against low and highly virulent Mh strains at 4 and 8 weeks post infection (PI).
\end{abstract}

\section{Materials and Methods}

Ninety, 3 week-old, cross-bred (Rattlerow Seghers), Mh and PRRSV free, weaned piglets were moved to the experimental facilities of the Faculty of Veterinary Medicine, Ghent University, Belgium. After one week acclimatization, they were randomly allocated to 10 different groups housed in absolute filters (HEPA U15) to avoid spread of the pathogen between groups. Forty piglets were vaccinated $(v)$ with a one-shot vaccine (Stellamune One, Pfizer), 40 were left unvaccinated (nv) and 10 were assigned to the negative nv and non-challenged (NC) control group. The experimental design is summarized in Table 1. One low (LV) and one highly (HV) virulent Mh strain were used for experimental infection. After anesthesia, the pigs were inoculated intratracheally with $7 \times 107 \mathrm{CCU}$ of $\mathrm{Mh}$ in $7 \mathrm{ml}$ inoculum or with $7 \mathrm{ml}$ of sterile culture medium. Half of the piglets were euthanized at $4 \mathrm{w}$ $\mathrm{PI}$ and the other half at $8 \mathrm{w}$ PI. The severity of coughing was assessed daily using a respiratory disease score (RDS) (values from 0 to 6). At necropsy, macroscopic lung lesions were quantified using the lung lesion score diagram of Hannan et al. (1982), ranging from 0 to 35. Additionally, lung tissue samples were taken from each pig to assess the severity of histopathological lesions (score from 0 to 5). Nasal swabs and bronchoalveolar lavage fluid (BALF) were collected after euthanasia and analyzed using nPCR to confirm presence of Mh.

\section{Results}

At 4w PI, the vHV group showed significantly milder respiratory symptoms and significantly lower macroscopic and microscopic lesion scores than the nvHV group $(P<0.05)$. The vLV group showed no significant differences for RDS, macroscopic and microscopic lesions scores, compared to the nvLV group. At $8 \mathrm{w} \mathrm{PI}$, both vHV and vLV groups had significantly lower RDS scores in comparison to the nvHV and nvLV groups, respectively $(\mathrm{P}<0.05)$. Only the vHV group showed significantly lower macroscopic lesions in relation to the nvHV group. Results are summarized in Table 2. As expected, all pigs, except for the nvNC group, were positive by $\mathrm{nPCR}$ on BALF and nasal swabs.
Table 1: Results of the nested PCR on bronchoalveolar lavage fluid (BALF) in the vaccinated (V) and non-vaccinated (NV) groups at 3 and 9 of age and $R n$ values during nursery.

\begin{tabular}{|l|c|c|c|}
\hline \multirow{2}{*}{$\begin{array}{l}\text { Pigs age (weeks) at } \\
\text { sampling }\end{array}$} & \multicolumn{2}{|c|}{ Number (\%) of Mh $\mathbf{n P C R}$ positive pigs on BALF } & \multirow{2}{*}{ P-value } \\
\cline { 2 - 3 } & $\mathbf{V}$ & $\mathbf{N V}$ & \\
\hline 3 (weaning) & $5 / 36(14)$ & $13 / 36(36)$ & $\geq 0.05$ \\
\hline 9 (end of nursery) & $11 / 36(31)$ & $23 / 36(64)$ & $\leq 0.01$ \\
\hline & 6 new infected & $\mathbf{1 0}$ new infected & \\
\hline Rn & 0.71 & 0.56 & $\geq 0.05$ \\
\hline
\end{tabular}

\section{Discussion and conclusions}

The results confirm the difference in virulence between the two strains (3), when measured during a period of either 4 or 8 weeks $\mathrm{PI}$. Vaccination significantly reduced the severity of disease in the vHV group at 4 or 8 weeks PI for most parameters. Vaccination was also beneficial in the LV groups, but the effects were less pronounced and depended on the measured parameter. Further research is warranted to assess the precise effect of vaccination on individual strains under field conditions, since combined infections might lead to more severe clinical disease in a pig herd (4).

\section{References}

1. Calus et al., 2007. Vet. Mic. 120: 284-291

2. Meyns et al., 2004. Prev Vet Med 66: 265-275

3. Vicca et al., 2003. Vet Mic. 97:177-190

4. Villarreal et al., 2009. Vaccine 27:1875-9

\section{Acknowledgements}

The study was financially supported by IWT project number 050642. Special thanks to Hanne Vereecke for all the help, effort and work dedicated to the project. 


\title{
Efficacy of Econor ${ }^{\circledast}$ (valnemulin) for the treatment of mycoplasma pneumonia of swine under field conditions in Japan
}

\author{
Nobuo Nakanishi' ${ }^{2}$ Koji Ishii ${ }^{1}$ Hiroshi Otaki ${ }^{1}$ Ulrich Klein ${ }^{3}$ \\ 1. Novartis Animal Health K.K., Tokyo, Japan; 2. Kyodoken Institute,Co.,Ltd, Kyoto, Japan; 3. Novartis Animal Health Inc, Basel, Switzerland
}

\section{Introduction}

Mycoplasma pneumonia of swine (MPS) caused by Mycoplasma hyopneumoniae $(\mathrm{Mhp})$ is one of major respiratory diseases in pigs all over the world. The susceptibility of Mhp field isolates from Japan against valnemulin, the active ingredient of Econor ${ }^{\circledR}$, has been confirmed recently (1). The objective of this study was to evaluate the clinical efficacy of Econor ${ }^{\circledR} 1 \%$ premix for pigs affected by MPS under field conditions in Japan as part of routine post-approval regulatory requirements.

\section{Materials and Methods}

This study was conducted from October 2006 to November 2007 at two different farms in central part of Japan (Aichi Prefecture). Two farms were historically known as MPS-positive farms. Prior to the study, two pig farms were randomly selected for necropsy to confirm the presence of typical macroscopic MPS legion in the lung and isolation of Mhp. Isolation of M. hyorhinis, Actinobacillus pleuropneumoniae and Pasturella multocida was also confirmed. In total 105 crossbred pigs ( 80 to 100 days of age; 45 pigs at Farm A, 60 pigs at Farm B) were randomly allocated to the following three groups; negative control (T1), Econor ${ }^{\circledR} 50$ ppm (T2) and Econor ${ }^{\circledR}$ 200 ppm (T3). Econor ${ }^{\circledast}$ was medicated in-feed for 7 consecutive days in accordance to the label in Japan. Body weight was measured at the start of study (Day 0), at the end of the treatment (Day 7) as well as 7 and 14 days after the end of the treatment (Day 14 and 21) to determine the weight gain. Twelve pigs (two pigs per group in each farm) were randomly selected at Day 9, two days after the end of treatment, for necropsy, and lungs were carefully examined to score the macroscopic lesion (score 0-4, $0=$ no lesion to $4=$ severe). Also, the area of MPS lesion in the lung was carefully measured to calculate the MPS-lesion ratio (\%) in a whole lung surface. In addition, Mhp, M. hyorhinis, A. pleuropneumoniae and $P$. multocida were isolated when found.

\section{Results and Discussion}

The weight gain of pigs in the two Econor groups were significantly higher than the gain of control group during Day 0-7 (T3: $6.0 \mathrm{~kg}$ vs. T1: $4.4 \mathrm{~kg}$ ) and Day 7-14 (T2 and T3: $5.6 \mathrm{~kg}$ and $6.0 \mathrm{~kg}$ vs. T1: $4.4 \mathrm{~kg}$ ) (Table 1). With regard to the lung lesion score and MPS-lesion ratio, there were no significant differences among the groups, although there was a trend that values for those at Econor groups were smaller than those of the control group (Table 1). At the necropsy before study onset, Mhp was isolated from all four pigs, and M. hyorhinis, A. pleuropneumoniae and P. multocida were also isolated from some pigs due to secondary infection, which is not unusual under field conditions (Table 2). At Day 9, the control group had similar frequency and distribution of bacteria concerned, whereas Econor ${ }^{\circledR}$ groups had tendency to reduce those bacteria from the level of Day 0 . In the case of $M h p$, reduction of isolation in three groups was dose dependent. It has to be noted that M. hyopneumoniae, A. pleuropneumoniae and $P$. multocida were not found and isolated from the lungs of pigs treated with Econor $^{\circledast} 200$ ppm for 7 consecutive days (Table 2).

\section{Conclusion}

In a MPS-positive farm, Econor ${ }^{\circledast}$ has proven its clinical efficacy based on the improvement of body weight gain, the reduction of the bacterial load $(\mathrm{Mhp})$ in the lung and the reduction of the MPS-lesion ratio.

Table 1. Average weight gain and MPS-lung lesion

\begin{tabular}{|l|c|c|c|c|}
\hline \multirow{2}{*}{ Pre } & \multirow{2}{*}{ M. hyopneumoniae } & Day9 & Day9 & Day9 \\
\cline { 3 - 5 } & & T1 & T2 & T3 \\
\hline M. hyorhinis & 100 & 100 & 25 & 0 \\
\hline A. pleuropneumoniae & 50 & 50 & 25 & 25 \\
\hline P. multocida & 50 & 25 & 0 & 0 \\
\hline${ }^{*} \mathrm{p}<0.05$ & 25 & 25 & 0 \\
\hline
\end{tabular}

Table 2. The ratio (\%) of bacteria isolation from the lung lesions

\begin{tabular}{|l|c|c|c|c|}
\hline & & T1 & T2 & T3 \\
\hline Average weight gain(kg) & Day 0-7 & $4.4 \pm 2.5$ & $5.7 \pm 3.0$ & $6.0 \pm 2.4^{*}$ \\
\hline Average weight gain(kg) & Day 7-14 & $4.4 \pm 1.7$ & $5.6 \pm 2.1^{*}$ & $6.0 \pm 2.1^{*}$ \\
\hline Average weight gain(kg) & Day 14-28 & $8.4 \pm 5.4$ & $7.8 \pm 4.0$ & $9.7 \pm 5.0$ \\
\hline Average weight gain(kg) & Day 0-28 & $17.6 \pm 7.5$ & $19.4 \pm 8.0$ & $21.7 \pm 8.3$ \\
\hline MPS-lung lesion & Score & 3.5 & 1.8 & 1.8 \\
\hline MPS-lung lesion & Av. lesion ratio (\%) & 37.5 & 20.6 & 16.7 \\
\hline
\end{tabular}

\section{References}

1. Kobayashi H. et al. 2008. Proceedings 20th IPVS Congress, Durban, Vol II, p 187. 


\title{
P.332
}

\section{In vitro susceptibility of Porcine Mycoplasmas to antimicrobial agents during 2008-2009}

\author{
Pacharee Thongkamkoon ${ }^{1}$ Nuvee Prapasarakul2 Metta Makhanon² Sasiwimon Talummuk ${ }^{3}$ Ulrich Klein $^{4}$ \\ 1. National Institute of Animal Health, Department of Livestock Development, Thailand, Bangkok, Thailand; \\ 2. Dept. of Veterinary Microbiology, Faculty of Veterinary Science, Chulalongkorn University, Bangkok, Thailand; \\ 3. Novartis (Thailand) Limited, Bangkok, Thailand; 4. Novartis Animal Health Inc., Basel, Switzerland
}

\section{Introduction}

Porcine Mycoplasma including Mycoplasma hyopneumoniae $(\mathrm{MH})$ and $\mathrm{M}$. hyorhinis (MHR) concurrently cause respiratory problems as well as polyserositis/arthritis and lameness from fattening to breeder. Antigenic variation of the bacteria can lead to variation of the immune response and acquired resistance can cause failure in disease treatment and control. MIC (minimum inhibitory concentration) data are a trustworthy tool for the evaluation of antibiotics and their effect against Mycoplasma pathogens $(1,2,4,6,7)$. The objective of this study was to determine the MIC of seven antimicrobials: tiamulin, valnemulin (pleuromutilins), lincomycin (lincosamides), tylosin (macrolids), enrofloxacin (fluoroquinolones), doxycycline (tetracycline), florfenicol - against Thai porcine Mycoplasma strains isolated during 2008-2009.

\section{Materials and Methods}

Twenty field isolates of MH and MHR, were collected in 2008/2009 from lungs, heart, brain or joints of pigs with lameness and pneumonia. Mycoplasmal identification was done by PCR detection. Mycoplasmal isolates were diluted to a concentration of $5 \times 105$ $\mathrm{CFU} / \mathrm{ml}$ and were incubated at $35^{\circ} \mathrm{C}$ for 2 hours. MICs were carried out using microdilution protocol based on CLSI guidelines. The concentration of antimicrobials were serially two-fold diluted, from $0.006-12.5 \mu \mathrm{g} / \mathrm{ml}$ for doxycycline, florfenicol, lincomycin, tiamulin, valnemulin and tylosin, and from $0.024-50 \mu \mathrm{g} / \mathrm{ml}$ for enrofloxacin. Mycoplasma growth was confirmed by media indicator and microscopic observation. The lowest concentrations of each antimicrobial showing inhibition of visible color change were interpreted as the MIC values at 5th PI for MHR and 7th PI for MH.

\section{Results}

The MIC values of the tested antimicrobials are shown in Tables 1 and 2 .

\section{Discussion}

Low MIC90 values indicate high susceptibility of MH strains to valnemulin, tiamulin and tylosin. In contrast, high Tylosin MIC90 values show low sensitivity of MHR strains. Resistance to enrofloxacin might be caused by the widely use in piglets to control E.coli. (7) MIC90 of florfenicol against MH and MHR was detected in a resistant range (breakpoint $<2 \mu \mathrm{g} / \mathrm{ml}$ ) and was higher than in previous studies $(1,2,5,8)$. Increase of MIC values might be induced from the other antimicrobials that result in mutation at the 50 s ribosomal subunit of bacteria (3). Valnemulin showed the lowest MIC values against all tested Mycoplasmas and is considered as the most effective antibiotic in this study. Tiamulin inhibited MHR and MH at MICs lower than 0.1 $\mu \mathrm{g} / \mathrm{ml}$ - no trend of MIC increase was found. In conclusion, the Pleuromutilin antibiotics show the lowest MICs and are considered to be the most efficacious therapeutics for the control of Mycoplasmas in Thai pig farms.
Table $1 \mathrm{MIC}(\mu \mathrm{g} / \mathrm{ml})$ values of 7 antimicrobial agents against Thai M.hyopneumoniae strains in 2008 and 2009

\begin{tabular}{|l|c|c|c|c|c|c|}
\hline \multirow{2}{*}{ Drugs } & \multicolumn{5}{|c|}{ M.hyopneumoniae(n2008=10; $\mathbf{n 2 0 0 9 = 1 0 )}$} \\
\cline { 2 - 7 } & \multicolumn{2}{|c|}{ MIC range } & \multicolumn{2}{c|}{ MIC50 } & \multicolumn{2}{c|}{ MIC90 } \\
\cline { 2 - 7 } & 2008 & 2009 & 2008 & 2009 & 2008 & 2009 \\
\hline Enrofloxacin & $0.048-6.25$ & $\begin{array}{c}<0.024- \\
6.25\end{array}$ & 3.12 & 1.56 & 6.25 & 6.25 \\
\hline Doxycycline & $3.12-6.25$ & $1.56-6.25$ & 3.12 & 3.12 & 6.25 & 6.25 \\
\hline Lincomycin & $0.19-0.39$ & $0.19-0.78$ & 0.19 & 0.19 & 0.39 & 0.78 \\
\hline Tiamulin & $0.048-0.097$ & $0.048-0.19$ & 0.048 & 0.048 & 0.048 & 0.097 \\
\hline Tylosin & $0.097-0.19$ & $0.048-0.19$ & 0.19 & 0.097 & 0.19 & 0.097 \\
\hline Valnemulin & $<0.006$ & $<0.006$ & $<0.006$ & $<0.006$ & $<0.006$ & $<0.006$ \\
\hline Florfenicol & $1.56-3.12$ & $1.56-3.12$ & 1.56 & 1.56 & 1.56 & 3.12 \\
\hline
\end{tabular}

Table 2 MIC $(\mu \mathrm{g} / \mathrm{ml})$ values of 7 antimicrobial agents against Thai M.hyorhinis strains in 2008 and 2009

\begin{tabular}{|l|c|c|c|c|c|c|}
\hline \multirow{2}{*}{ Drugs } & \multicolumn{5}{|c|}{ M.hyorhinis(n2008=11; n2009=9) } \\
\cline { 2 - 7 } & \multicolumn{2}{|c}{ MIC range } & \multicolumn{2}{c|}{ MIC50 } & \multicolumn{2}{c|}{ MIC90 } \\
\cline { 2 - 7 } & 2008 & 2009 & 2008 & 2009 & 2008 & 2009 \\
\hline Enrofloxacin & $0.78-25$ & $0.78-50$ & 1.56 & 3.12 & 3.12 & 50 \\
\hline Doxycycline & $0.78-3.12$ & $0.39-1.56$ & 0.78 & 1.56 & 3.12 & 1.56 \\
\hline Lincomycin & $0.39-1.56$ & $0.78-3.12$ & 0.78 & 1.56 & 0.78 & 3.12 \\
\hline Tiamulin & $0.048-0.097$ & $0.048-0.097$ & 0.048 & 0.097 & 0.097 & 0.097 \\
\hline Tylosin & $0.078-3.12$ & $1.56-6.25$ & 3.12 & 3.12 & 3.12 & 6.25 \\
\hline Valnemulin & $<0.006$ & $<0.006$ & $<0.006$ & $<0.006$ & $<0.006$ & $<0.006$ \\
\hline Florfenicol & $1.56-3.12$ & $1.56-3.12$ & 3.12 & 3.12 & 3.12 & 3.12 \\
\hline
\end{tabular}

\section{References}

1. Kobayashi, et al. (1996). Antimicrob. Agents Chemother.. 40(4): 10301032.

2 Kobayashi, et al. (2005). J. Vet. Med. Sci. 67(8):795-800.

3. Long, et al. (2006). Antimicrob. Agents Chemother. 50(7):2500-2505.

4. Senterfit, L.B. (1983). Methods in Mycoplasmology. Vol. 2, U.S.A. pp. 397-401.

5. Shin, et al. (2005) Vet.Microbiol.106:73-77.

6. Stipkovits, et al. (2004). Proceedings of the 18th IPVS congress. 2:518.

7. Ter Laak, et al. (1991). Antimicrob. Agents Chemother. 35(2): 228-233.

8. Vicca, et al.(2004) Antimicrob.Agents Chemother. 48(11):4470-4472. 


\title{
P.333
}

\section{Efficacy of Denagard ${ }^{\circledR} 10$ (tiamulin hydrogen fumerate) in-feed antibiotic in a Mycoplasma hyopneumoniae infection model study}

\author{
J. M. Hammer; Stephen King; Elizabeth Roberts; Linda Roycroft
}

Novartis Animal Health, US, Greensboro, NC, USA

\section{Introduction}

Mycoplasma hyopneumoniae (M. hyo) is a highly prevalent insidious respiratory disease in pigs. Although mortality is low morbidity can be high. It is a component of enzootic pneumonia and is also considered an initiator in secondary bacterial pneumonia of pigs which can cause increased mortality and decreased performance with associated higher production costs. Controlling $M$. hyo infections in pig herds should improve respiratory health and performance in growing pigs leading to lower production costs.

\section{Objective}

To evaluate Denagard ${ }^{\circledR} 10$ in-feed antibiotic at 137.5 ppm or 165 ppm for a 14 day treatment period to control (M. hyoin an induced infection model.

\section{Materials and Methods}

Approximately 8 week old pigs ( 11 to $36 \mathrm{~kg}$ ) serologically negative for M. hyowere endotracheal challenged with a M. hyohomogenate containing at least 109 organisms per $\mathrm{mL}$. Once a minimum of four animals on a given day showed $5 \%$ or greater lung involvement through necropsy confirmation, the treatment phase was initiated. Pigs were allocated to one of three treatment groups (Control 0 ppm Denagard, 137.5 ppm Denagard for 14 days, or 165 ppm Denagard for 14 days). The pen was the experimental unit. The primary variable 'total lung lesion percentage' was transformed and analyzed using an analysis of variance with 'Treatment' as the classification variable. Secondary clinical variables (depression score, respiratory score, and cough score), overall treatment success, production parameters (average daily gain, feed intake, efficiency), and mortality were also determined and statistically analyzed.

\section{Results}

The primary variable for efficacy determination of Denagard was the total lung lesion percentage at necropsy 72 hours post cessation of the Denagard application. Statistically significant differences in favor of the Denagard treatment groups were noted in total lung lesion percentage when compared to the control (Table 1).

Table 1. Summary Statistics for Percent Total Lung Lesion

\begin{tabular}{|l|c|c|c|}
\hline Treatments & N (pens) & Mean & SD \\
\hline Denagard 137.5 ppm & 8 & $16.02^{\mathrm{a}}$ & 5.26 \\
\hline Denagard $165 \mathrm{ppm}$ & 8 & $11.76^{\mathrm{a}}$ & 2.97 \\
\hline Control Group (0 ppm) & 8 & 22.35 & 6.93 \\
\hline a statistical difference from control $(\mathrm{p}<0.05)$ & \\
\hline
\end{tabular}

As coughing is a primary clinical sign associated with $M$. hyo infection, the number of pigs coughing was further examined by time of day (Figure 1). Cough in the morning was differential between treatment groups with lower rate of increase in the Denagard groups over the trial period.
Figure 1. Regression analysis of the number of pigs coughing in the morning (AM) for each treatment group over the study period (Day 0 to Day 16)

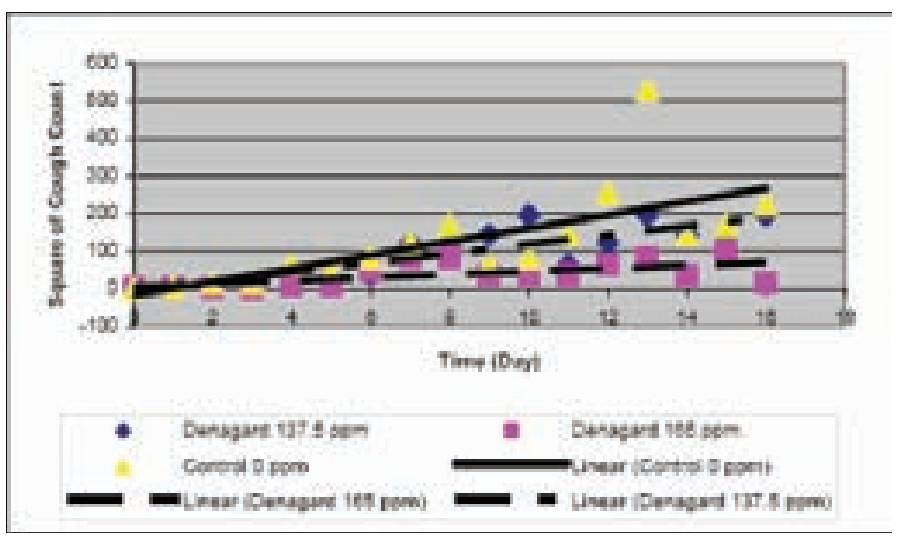

Control vs Denagard $137.5 \mathrm{ppm} \mathrm{p}=0.0087$, Control vs Denagard 165 ppm $\mathrm{p}=0.0106$, Denagard 165 ppm vs Denagard 137 ppm $\mathrm{p}=0.0004$

No statistically significant differences were observed for any of the secondary variables or production parameters, though the production parameters were numerically better in the Denagard treated animals.

\section{Discussion}

Denagard when administered as an in-feed antibiotic at 137.5 and 165 ppm for 14 days provides control of $M$. hyo infection based on the reduction in lung lesion percentage at 72 hours after in-feed antibiotic removal. Pigs that received Denagard had a lower rate of increase in the number of coughs in the morning over time when compared to control animals. The Denagard 165 ppm group showed a lower rate of increase in coughing in the morning than the Denagard 137 ppm group.

\section{Conclusion}

Denagard 10 (tiamulin in-feed antibiotic) at a dose of at least $137.5 \mathrm{ppm}$ reduced rates of coughing which is a positive clinical benefit in pigs infected with M. hyo. Feeding Denagard 10 at either level significantly decreases lung lesions resulting in improved lung health. 


\title{
Mycoplasma hyopneumoniae elimination in a 3800 sow, multi-site system
}

\author{
Jerome O. Geiger ${ }^{1}$ Doug Groth ${ }^{2}$ \\ 1. PIC-NA, Hendersonville, TN, USA; 2. Carthage Veterinary Service, Carthage, IL, USA
}

\section{Introduction}

Mycoplasma hyopneumoniae (Mh) was successfully eliminated from a 3800 sow multi-site system. Several elements were considered important to the successful elimination: stabilization of the sow herd's immunity, segregation of all nursery/finishing animals, strategic use of antibiotics in the sow herd and neonates, complete depopulation of the nursery/finisher facilities, one-directional pig flow, and strict control of people traffic.

\section{Materials and Methods}

A 3800 sow multi-site production system was stocked and operating $>5$ years as Mh negative based on monthly serology, clinical signs and post-mortem examinations. The system was also negative for Porcine Reproductive and Respiratory Syndrome (PRRS). Breeding, gestation and farrowing occurred on Site 1. A gilt development unit (GDU) was also contained in Site 1. Except for the internal replacement gilts in the GDU, the weaned pig flow rotated around three facilities, 18-60 miles from the sow herd.

In Jan.08, clinical signs indicated a change of health status in the sow herd. Mh was diagnosed by Dako ELISA serology and histopathology in the sow herd and followed normal flows through the nursery/finisher system. All animals in the internal GDU and sow herd were infected by natural exposure. The breeding herd and GDU were vaccinated against $\mathrm{Mh}(\mathrm{M}+\mathrm{PAC}$, Intervet) in late Jan.08 and again in Feb.08.

In Oct.08, efforts began to eliminate Mh, starting with depopulation of the GDU. The entire reproductive herd was vaccinated against Mh in Dec.08 (M+PAC, Intervet) and again Jan.09 (RespiSure, Pfizer). During Jan.09, the breeding herd received Lincomycin (Lincocin, Pfizer) through the water system and piglets received a weight-appropriate dose of injectable tulathromycin (Draxxin, Pfizer, $2 \mathrm{mg} / \mathrm{kg}$ ) at birth and 11 days later. During Jan.09, weaning occurred at $>18$ days of age (average weaning age was 20.6 days). By Feb.09, all females in Site 1 breeding herd were $>11$ months of age.

Starting in Feb.09, naïve replacement gilts entered the GDU and weaned pigs flowed into depopulated facilities. Each site of the post-weaning facilities were empty $>2$ weeks, though individual rooms were empty much longer.

Starting one month after the first negative replacement gilts (sentinels) entered Site 1, random sentinels were serologically tested monthly. Random pigs were tested monthly in the downstream flow as well, allowing for maternal antibody degradation. Mh IDEXX ELISA was used as the screening test. Mh Dako ELISA was used as the differential/confirmatory test on any positive IDEXX samples.

\section{Results}

Although sows infected naturally still exist in the breeding herd, shedding of Mh has apparently not occurred, based on continued monthly serologic sampling of sentinel animals and pig flow after the nursery depopulations. The absence of clinical signs and lack of Mh lesions on slaughter checks and routine necropsies (including Mh cultures, PCRs and histopathology) support this position. At the time of writing, serologic testing of sow herd sentinels at Site 1 has been Mh negative 12 months (>350 samples) and the off-site finishers have provided $\mathrm{Mh}$ negative slaughter-aged pigs six months (>100 samples). Tissues from $>25$ animals have been submitted for complete Mh analysis. Three slaughter checks from two finisher sites ( $n=90$ pigs total) have shown no Mh lesions.

\section{Discussion}

In this case, Mh was eliminated with the following key factors:

- Mh vaccination of the entire sow herd shortly after initial exposure and at end of elimination.

- Continuous matings and farrowings throughout elimination process.

- Youngest Mh positive animals were $>11$ months of age and in their second parity before introduction of Mh naïve replacements and weaning of Mh (suspected) negative piglet flow.

- Lincomycin in water medication to breeding herd at end of elimination at Site 1.

- Tulathromycin injection of piglets at birth and 11 days later.

- Depopulation of nursery and finisher facilities before receiving $\mathrm{Mh}$ negative pigs. 


\title{
P.335
}

\section{Antimicrobial susceptibilities and molecular typing of Canadian isolates of Mycoplasma hyopneumoniae}

\author{
Marie Archambault ${ }^{1}$ Audrey Charlebois ${ }^{1}$ Pierre Hélie ${ }^{1}$ Marcelo Gottschalk ${ }^{1}$ Carl A. Gagnon ${ }^{1}$ Corinne Marois² Marylène Kobisch ${ }^{2}$ \\ 1. Université de Montréal, Saint-Hyacinthe, QC, Canada; 2. AFSSA, Ploufragan, France
}

\section{Introduction}

Mycoplasma hyopneumoniae is present in the majority of swine herds around the world. It is the causative agent of porcine enzootic pneumonia. Its importance is also linked to its ability to increase the severity of infections caused by viruses $(1,2)$, as well as bacteria (3). However, little is known about this microorganism in Canadian herds. The aim of this study was to evaluate the genetic diversity of M. hyopneumoniae and to determine their antimicrobial susceptibilities.

\section{Materials and Methods}

Bacterial isolates, growth conditions and PCR. A total of 160 swine lungs with lesions suggestive of enzootic pneumonia were recovered from two slaughterhouses. They were cultured in Friis medium and tested by PCR or real-time PCR for M. hyopneumoniae and M. hyorhinis (4) as well as PRRSV (Tetracore, USA), influenza and PCV2 viruses (5). Samples were also cultured for other commonly encountered swine pathogenic bacteria.

Antimicrobial susceptibilities and resistance genes. Antimicrobial susceptibilities were determined by minimum inhibitory concentration (MIC) for 18 antibiotics using the Sensititre ${ }^{\circledR}$ plates (\#BOPO6F, Nova Century Scientific, Ontario, Canada) as described previously (6) without the use of M. hyorhinis antibodies. PCRs were performed for previously described antimicrobial resistance genes tetM and ermB, and point mutations in L4, L22 and 235 rRNA genes were investigated.

MLVA and RFLP. PCR-RFLP analysis, primers and conditions for locus P97-RR1 and locus P97-RR2 were used as previously described (7). Primers and conditions for locus 1 and 2 were provided as a personal communication from C. Marois, Agence française de sécurité sanitaire des aliments (AFSSA), France.

Cluster and data analysis. Digital images of PCR-RFLP gels and MLVA profiles were imported in the BioNumerics software (Applied Maths, USA) The Student " $t$ " test was performed to evaluate significance $(p<0.05$; SAS software v.9.1).

\section{Results and Discussion}

Ninety percent of the samples were positive for M. hyopneumoniae (Real-time PCR) while $5.6 \%$ were positive only for $M$. hyorhinis (PCR). Within the 25 selected M. hyopneumoniae positive lungs (culture or real-time PCR), 10 were in co-infection with P. multocida, 12 with S. suis, 9 with PCV2 and 2 with the PRRS virus. MLVA and PCR-RFLP analyses of M. hyopneumoniae showed a high diversity among field isolates. However, there seem to be more homogeneity within the same herd. The MLVA analysis also demonstrated that almost half of the field isolates had less than $55 \%$ homology with the vaccine and reference strains used in this study. The absence of amplification of one locus (locus 1) of M. hyopneumoniae was significantly associated with a reduced concentration of bacteria and a lower severity of the lung lesions. All M. hyopneumoniae isolates had low to intermediate MICs against the antimicrobial tested (Table 1). Cultures with intermediate MICs for tetracyclines, macrolides and lincosamides were tested for the presence of previously described resistance genes tetM, ermB and L4, L22 and 23S rRNA point mutations. None of these genes were found but one point mutation, G2057A, was identified. This mutation is responsible for the intrinsic resistance of $M$. hyopneumoniae against 14-membered macrolides. These results indicate that there is no acquired antimicrobial resistance within these isolates.

\section{References}

1. Opriessnig, T. 2004. Vet Pathol 41:624-40.

2. Thacker, E.L., et al. 1999. J Clin Microbiol 37(3):620-7.

3. Ciprian, A. et al. 1988. Can J Vet Res 52:434-8.

4. Stakenborg, T., et al. 2006. Vet Res Commun 30:239-47

5. Gagnon, CA et al. 2008. J Vet Diagn Invest. 20(5): 545-58.

6. Wu, C.C. et al. 1997. Swine Health and Prod 5:227-230.

8. Stakenborg, T., et al. 2006. J Microbiol Methods 66:263-75

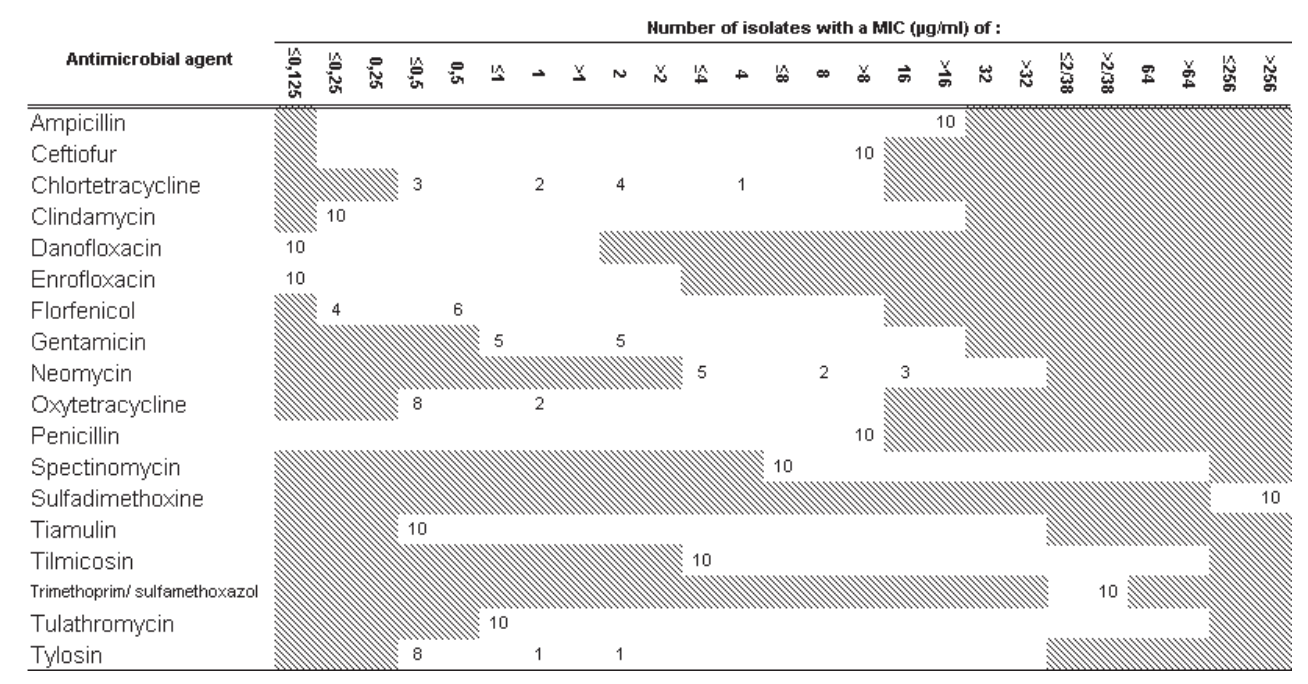

Proceedings of the 21 $1^{\text {st }}$ IPVS Congress, Vancouver, Canada - July 18-21, 2010 


\title{
P.336
}

\section{Mycoplasma eradication using Draxxin ${ }^{\circledast}$ (Tulathromycin), Lincomix 44 Premix ${ }^{\circledast}$ (Lincomycin), and Respisure ${ }^{\circledR}$ in a 2750 sow isowean facility}

\author{
Timothy P. Snider \\ Snider Veterinary Services, Steinbach, MB, Canada
}

\section{Introduction}

Mycoplasma hyopneumoniae is a primary contributor to the Porcine Respiratory Disease Complex (PRDC). (1) Its impact on the cost of finisher pig production makes eradication a serious alternative for profitable production. The possibility of producing effectively negative pigs following any one of several different eradication procedures has been well documented.(2)

Mycoplasma eradication procedures commonly involve steps that have a negative impact on efficient breeding herd productivity. Examples include: 1)restrictions on replacement gilt flows to allow for Mycoplasma exposure and cessation of shedding, and 2)periods with no farrowing or ultra early weaning to prevent the infection of and shedding from piglets. (3) This paper describes a Mycoplasma eradication program that avoids these traditional pitfalls while rapidly achieving negative piglet production utilizing sow herd treatment with injectable Draxxin ${ }^{\oplus}$, feed grade Lincomix $44^{\circledR}$, and Respisure ${ }^{\circledR}$ vaccine. The Draxxin ${ }^{\circledR}$ application to sows and piglets was a modification of the protocol described by Yeske(4). The duration of Draxxin ${ }^{\circledR}$ application to piglets was extended in order to address the potential that recently exposed animals may continue to shed the organism for up to eight months. (5)

\section{Materials and Methods}

A Mycoplasma outbreak was confirmed in one of two naïve sow herds that combined their piglet production to produce marketable groups of weaned piglets. The outbreak occurred following the introduction of infected replacement breeding stock.

At the time of the outbreak, these herds were about to change from commercial weaned piglet sales to retained ownership into several large continuous flow wean to finish facilities. These wean to finish facilities were well isolated from other pig production and had a high likelihood of maintaining a Mycoplasma negative status.

The eradication protocol included the following steps:

- Herd wide vaccination with Respisure ${ }^{\circledR}$ at the onset of the outbreak and just prior to eradication;

- Herd wide Lincomix $44^{\oplus}$ treatment $(220 \mathrm{mg} / \mathrm{kg}$ ) for 3 weeks prior to initial Draxxin ${ }^{\circledR}$ treatment and for 5 weeks following initial Draxxin ${ }^{\circledR}$ treatment;

- Sow Draxxin ${ }^{\circledR}$ doses (2 $5 \mathrm{mg} / \mathrm{kg}$ ) estimated the day prior to mass treatment using flank taping;

- All breeding stock (ie sows and boars) and piglets were treated with Draxxin ${ }^{\circledast}$ on Day $1 \& 2$ of the procedure and again on Day 15\&16 (14 day interval) if they were still present on the farm;

- For 5 months following the first Draxxin ${ }^{\circledR}$ treatment, all piglets received an injection with Draxxin ${ }^{\circledR}$ as soon as possible after birth and again at 12-14 days of age;
- Piglets were weaned at 18 - 21 days. No piglets were held on the farm beyond 24 days of age.

- All weaned piglets received Respisure ${ }^{\circledR}$ vaccination in the nursery for 5 months following the eradication procedure.

Mycoplasma naïve gilts entered the herd immediately following the second breeding herd treatment. Monitoring occurred using serology in Mycoplasma negative sentinel gilts. Post mortem sampling and the monitoring of clinical signs for Mycoplasma were also utilized.

\section{Results}

Piglets from the treated herd have been comingled with naïve piglets from another sow herd for 8 months. No Mycoplasma has been detected clinically or through other diagnostic methods.

\section{Discussion}

Although the cost of treating the complete sow herd was significant, the shareholders immediately recognized the value of rapidly achieving Mycoplasma negative piglet production to the profitability of the wean to finish system. Following the sow Draxxin ${ }^{\circledR}$ protocol, there continued to be a concern of shedding of Mycoplasma from parity 1 sows that entered the herd late in the process. The cost of a Mycoplasma outbreak in the large finisher operations would have been significant. With the assumption that Mycoplasma negative piglets can be produced using Draxxin $^{\circledR}$ therapy in the event of shedding from their dams, all piglets were treated with Draxxin ${ }^{\circledR}$ for a period of 8 months after the last breeding animal was estimated to have been infected.
References
1. Dee (1996) Swine Health Prod 4, p. 147-149
2. Yeske (2007) AASV Proceedings, p. $367-370$
3. Schneider (2006) Allen D Leman Conference Proceedings, p. 82 - 86
4. Yeske (2008) IPVS Proceedings, p110
5. Pieters (2007) AASV Proceedings, p $9-10$ 


\title{
P.337
}

\section{The Norwegian program for eradication of Mycoplasma hyopneumoniae is successfully completed}

\author{
Bjørn Lium ${ }^{1,2}$ Anne Jørgensen² Mona Gjestvang ${ }^{2}$ \\ 1. National Veterinary Institute, Oslo, Norway; 2. Animalia, Oslo, Norway
}

\section{Introduction}

In the early 1990s The Norwegian Pig Health Service conducted successful eradication programs for $M$. hyopneumoniae in several pig herds based on age-segregation and medication (1). Preliminary serological surveys indicated that about $15 \%$ of the pig herds in Norway were infected with M. hyopneumoniae. Based on this knowledge it was decided to initialize a program to systematically reduce the occurrence of, and from year 2000, to totally eliminate M. hyopneumoniae-infection in the Norwegian pig population.

\section{Materials and Methods}

Organization. The nation-wide eradication program was decided in a co-operation between the pig breeders' and the slaughterhouse industry in 2000, and the Norwegian Pig Health Service was appointed to lead the operation. The program was based on voluntary participation, with some financial support from the pork industry and an agricultural tax fund. The pig producers' economic benefit was a higher market price on live pigs in addition to an improved economic production yield on farm.

Classification of herds. The nucleus and multiplier herds were classified as free from $M$. hyopneumoniae if all of respectively 60 and 40 blood or colostrum samples were negative for antibodies against $M$. hyopneumoniae. For conventional herds the initial classification was based on blood or colostrum samples from at least 20 pigs (sows or slaughtered pig). Herds with 1 or 2 positive samples were retested. To maintain status as free from M. hyopneumoniae, the nucleus and multiplier herds are tested annually (at least 10 pigs per herd). The conventional herds were also tested annually in the first years of the program. The surveillance of these herds is now based on clinical signs and control of lung lesions at slaughter. Herds with respiratory symptoms are tested serologically.

Laboratory methods. The serum and colostrum samples have been analysed for the presence of antibodies to M. hyopneumoniaeby a monoclonal blocking ELISA (DAKO ${ }^{\circledR}$, Denmark), which has a very high sensitivity and specificity (2).

Eradication programs. Most of the infected breeding and breeding to finishing herds performed an eradication program based on a combination of age-segregation and medication (1). Finishing herds and some breeding herds were depopulated and restarted with pigs free from $M$. hyopneumoniae, and some herds gave up the pig production.

\section{Results}

During the years 1994 to 2009 samples from 138635 pigs from 3247 different herds were tested for antibodies against $M$. hyopneumoniae, 5538 (4\%) of the samples and 389 (12\%) of the herds were declared as positive. The prevalence of positive herds differed from 5.8 in the South region to $19.2 \%$ in South-West region, Table 1. In 2009 all 9612 pigs tested from 839 herds were negative for antibodies against $M$. hyopneumoniae.

Table 1. Screening for M. hyopneumoniae antibodies in Norwegian pigs during the years 1994 to 2009.

\begin{tabular}{|l|c|c|c|}
\hline Region of Norway & Herds tested $\mathbf{n}$ & Herds positive $\mathbf{n}(\%)$ & Last positive test (year) \\
\hline North & 165 & $11(6.7)$ & 2002 \\
\hline Mid & 649 & $67(10.3)$ & 2005 \\
\hline North-West & 288 & $20(6.9)$ & 2007 \\
\hline South-West & 1013 & $195(19.2)$ & 2008 \\
\hline South & 291 & $17(5.8)$ & 2007 \\
\hline East & 841 & $79(9.4)$ & 2006 \\
\hline All Norway & 3247 & $389(12.0)$ & 2008 \\
\hline
\end{tabular}

\section{Discussion}

A fundamental decision in our project was to start with establishing freedom from $M$. hyopneumoniae in all the nucleus and multiplier herds. With exception of the South-West region, which has a high density of pig farms, re-infections with $M$. hyopneumoniae has not been a problem in Norway. This may be explained by many small farms, long distances between farms, hilly landscape and a well-organised market for fatteners and gilts. In areas with a dense pig population we experienced that a strict regionalization of the eradication is crucial to succeed. Similar programs for eradication of $M$. hyopneumoniae have been implemented in Switzerland and Finland $(3,4)$. It is roughly estimated that freedom from $M$. hyopneumoniae means a benefit of 1-2.5 million euros per year for the Norwegian pig industry with a total of 1.5 million pigs slaughtered per year.

\section{References}

1. Lium B, et al. 1992. Proc. 12th IPVS Congress, The Hague: 300.

2. Sørensen V, et al. 1997. Vet. Microb. 54: 23-34.

3. Stärk KD, et al. 2007. Rev. Sci. Tech. 26(3): 595-606.

4. Ala-Risku V et al. 2004. Proc. 18th IPVS Congress, Hamburg: 1: 237 


\title{
P.338
}

\section{Serotype distribution of A. pleuropneumoniae and H. parasuis in the Czech Republic}

\author{
Linda Czanderlova; Marek Zizlavsky; Dita Kellnerova; Marcela Gambova; Svetlana Odehnalova; Jiri Odehnal
}

\author{
Sevaron Counselling, Brno, Czech Republic
}

\section{Introduction}

Actinobacillus pleuropneumoniae and Haemophilus parasuis cause health problems in pigs with a worldwide economic impact on industrialized pig production. Precise serotype identification is important for studying the epidemiology of these diseases, because the virulence and the prevalence of specific serotypes vary with geographic location (1). The knowledge of the predominant serotypes will facilitate the control of these diseases by vaccination, as strains can be then formulated in vaccines that will contain antigens homologous to those prevalent in the field.

\section{Materials and Methods}

The study conducted in the year 2008 and 2009 included 203 strains of Actinobacillus pleuropneumoniae and 53 strains of Haemophilus parasuis. All strains were isolated from the lungs of pigs showing clinical symptoms of respiratory diseases. A. pleuropneumoniae and $\mathrm{H}$. parasuis were cultivated on chocolate agar based on Müller Hinton agar with $10 \%$ of ovine blood. The isolates of $\mathrm{A}$. pleuropneumoniae and $\mathrm{H}$. parasuis were serotyped by a standard coagglutination assay. The accuracy of the test was confirmed using reference strains for each serotype. Serotyping of $\mathrm{H}$. parasuis was carried out by the Veterinary Research Institute, Brno, Czech Republic.

\section{Results}

Table 1 shows the proportion of A. pleuropneumoniae isolates detected and typed in this study. Serotype 9, identified in 129 strains, i.e. in $63.5 \%$ of all examined strains, was found to have the highest prevalence. Serotype 2 was detected quite frequently (51 strains, $25.1 \%$ ), serotype 11 was less frequent (13 strains, $6.4 \%)$. Serotype 7 was identified in 5 strains, all detections of this serotype were recorded in the year 2009. The isolation of serotypes 3 and non-typable (NT) was sporadic. Other serotypes of A. pleuropneumoniae were not recorded.

Results of serotyping of $\mathrm{H}$. parasuis are presented in Table 2. Serotype 4 was found to have the highest prevalence followed by serotypes 5 and non-typable strains. Serotypes 1, 2, 12 and 13 were detected less frequently. The isolation of serotypes 3 , 7 and 9 was sporadic. Other serotypes of $\mathrm{H}$. parasuis were not recorded.
Table 1 Serotyping of Actinobacillus pleuropneumoniae

\begin{tabular}{|c|c|c|c|c|}
\hline & $\mathbf{2 0 0 8}$ & $\mathbf{2 0 0 9}$ & Total & \\
\hline Serotype & $\mathbf{N}^{\circ}$ positive & $\mathbf{N}^{\circ}$ positive & $\mathbf{N}^{\circ}$ positive & \% positive \\
\hline 2 & 38 & 13 & 51 & 25.1 \\
\hline 3 & 0 & 2 & 2 & 1.0 \\
\hline 7 & 0 & 5 & 5 & 2.5 \\
\hline 9 & 61 & 68 & 129 & 63.5 \\
\hline 11 & 10 & 3 & 13 & 6.4 \\
\hline NT & 2 & 1 & 3 & 1.5 \\
\hline & & & & \\
\hline
\end{tabular}

Table 2 Serotyping of Haemophilus parasuis

\begin{tabular}{|c|c|c|c|c|}
\hline & $\mathbf{2 0 0 8}$ & $\mathbf{2 0 0 9}$ & Total & \\
\hline Serotype & Nopositive & Nopositive $^{\circ}$ & Nopositive & \% positive \\
\hline 1 & 3 & 3 & 6 & 11.3 \\
\hline 2 & 3 & 1 & 4 & 7.5 \\
\hline 3 & 1 & 0 & 1 & 1.9 \\
\hline 4 & 10 & 3 & 13 & 24.5 \\
\hline 5 & 7 & 3 & 10 & 18.9 \\
\hline 7 & 0 & 1 & 1 & 1.9 \\
\hline 9 & 0 & 1 & 1 & 1.9 \\
\hline 12 & 3 & 0 & 3 & 5.7 \\
\hline 13 & 1 & 3 & 4 & 7.5 \\
\hline NT & 5 & 5 & 10 & 18.9 \\
\hline & & & & \\
\hline
\end{tabular}

\section{Discussion}

In the territory of the Czech Republic, the highly pathogenic serotype 9 is a dominant serotype of A. pleuropneumoniae followed by frequently isolated serotype 2 . This finding is in harmony with preceding studies performed in the Czech Republic (2). In case of $\mathrm{H}$. parasuis serotypes the situation is more varied. Nine different serotypes and ten non-typable strains were recorded. This finding results in the need of careful laboratory diagnostics in pig production in case of vaccination against $\mathrm{H}$. parasuis. Owing to the fact that the vaccination is effective against homologous strains, but ineffective against heterogenous strains, autogenous vaccines containing strains isolated in a given herd prevail. But autogenous vaccines must be updated frequently to cover the entire spectrum of strains occurring in a given herd.

\section{References}

1. Mittal K.R. Vet Microbiol, 32, 1992:135-148.

2. Kucerova Z. at al. Vet.Med.-Czech, 50, 2005:355-360. 


\section{P.339}

\section{Pericarditis by Mycoplasma hyorhinis}

Lucas F. Santos; Kledna C. . Reis; Joao Paulo S. Castro; Daniel L. Santos; Mayka R. Henriques; Walter V. Guimaraes; Jose L. Santos

Microvet, Vicosa, MG, Brazil

\section{Introduction}

Mycoplasma hyorhinis is a common agent found in the respiratory tract of pigs, and it can cause polyserositis when become systemic, especially in nursery and grower pigs (Rovira, 2009). Fibrinous pericarditis can be one of the clinical signs found at necropsy. (Buttenschøn et al.1997). The fibrinous pericarditis has been linked to various agents such as Haemophilus parasuis, Pasteurella multocida, Actinobacillus pleuropneumoniae, Actinobacillus suis and Streptococcus suis (Mores, 2007). However, there are cases where you do not get the isolation of bacteria. Based on this aspect, we conducted a study to verify the presence of $M$. hyorhinis in pericarditis cases where the bacterial isolation was negative.

\section{Materials and Methods}

We evaluated 40 cases of pericarditis from pigs with age ranging from 20 to 106 days, negative for bacterial isolation performed by Microvet laboratory. The DNA genomic samples were extracted using the kit PureLink Genomic DNA Mini Kit (Invitrogen) and polymerase chain reaction (PCR) was performed according to Assunção et al. 2005.

\section{Results}

From the 40 samples studied, $67.5 \%$ were positive for $\mathrm{M}$. hyorhinis, 7.5\% for Mycoplasma hyopneumoniae, 7.5\% for $\mathrm{M}$. hyorhinis and $\mathrm{M}$. hyopneumoniae, and $17,5 \%$ negative for both agents (Graphic 1).

\section{Discussion}

It has been observed in recent years a considerable increase in detection of Mycoplasma hyorhinis in polyserositis cases, however always associated with another bacterial agent or associated with PCV-2 causing a severe respiratory syndrome (Santos et al.2008). Some authors consider this pathogen as the secondary agent of infection. This study showed that the $M$. hyorhinis can act as primary agent in some cases, mainly of fibrinous pericarditis. Another aspect to consider is in related to PCR technique, which proves to be very sensitive, rapid and less costly for diagnosis of this agent.

\section{Reference}

[1] Assunção, P. et al., The occurrence of Mycoplasma in the lungs of swine in Gran Canaria (Spain). Veterinary Research Communication. 2005, 29(6): 453-462.

[2] Buttenschon J, et al. Microbiology and pathology of fibrinous pericarditis in Danish slaughter pigs. Zentralblatt fur Veterinarmedizin. 1997, 44: 271-280.

[3] Mores, N. In: Sobestiansky, J.; Barcellos, D. (Org.). Doenças dos suínos. Goiänia, Go: Grafica Editora Pallotti. 2007, 609-610.

[4] Rovira, A. Review of Mycoplama hyorhinis. In: Proceeding of Allen D. Leman Swine Conference. Saint Paul. 2009, 87-88.

[5] Santos, D.L. et al. PCV2 and Mycoplasma hyorhinis coinfection in swine In: Proceeding of the 20th IPVS Congress. Durban. 2008, 2:100.

Graphic 1: Results of PCR for and M. hyorhinis M. hyopneumoniae in pericarditis from pigs

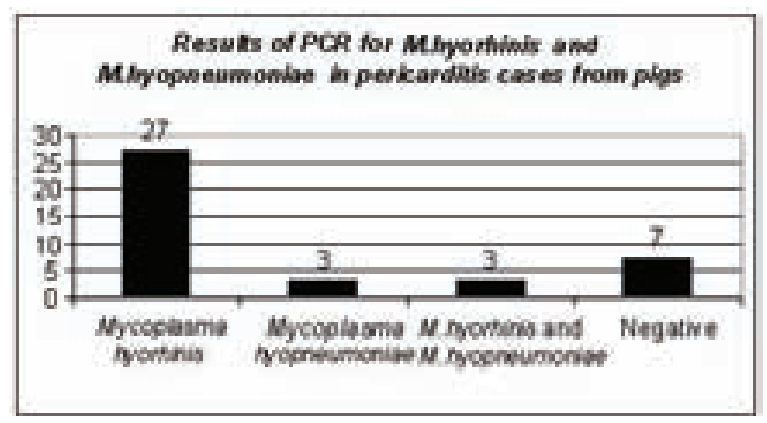




\title{
Prevalence of lesions of pleuritis at slaughterhouse
}

\author{
Jesus M. Bollo ${ }^{1}$ Rut Menjon ${ }^{1}$ Marta Jimenez ${ }^{1}$ Jesus V. Lopez ${ }^{1}$ Maria L. Perez-Brena Maria T. Tejedor ${ }^{2}$ \\ 1. Intervet Schering Plough. c/ Cantabria 2, Edificio Amura 28.108 Alcobendas, Madrid, Spain; 2. Dept. of Anatomy, \\ Embryology and Genetics Unit of Genetics. Faculty of Veterinary Medicine, Zaragoza, Spain
}

\section{Introduction}

Pleuritis is a frequent finding in dead pigs whether they have died on farm or been killed in the slaughterhouse. The lesions are the result of infection by various respiratory pathogens, leading directly to economic losses, often price reduction or carcase condemnation. Thus it is important to investigate the prevalence of pleuritis.

\section{Materials and Methods}

The study was undertaken in Spanish slaughterhouses from 2000 to 2010, examining 145,456 lungs from 1,085 slaughterhouse batches, originating from 451 farms.

Only the presence or absence of pleuritic lesions was recorded, without noting their position within the pulmonary lobes or whether in visceral or parietal pleura.

The aetiology of the lesions was not investigated, but it was suspected that the most probable pathogens were $A$. pleuropneumoniae, P. multocida and H. parasuis.

Statistical analyses were carried out by using the SPSS 15.0 software package. ANOVA was used for the comparison of means.

\section{Results}

The mean percentage of lungs with pleuritic lesions was $14.10 \%$ ( \pm 0.34$)$. Graph 1 is a histogram fitted to a normal curve to show the distribution of the percentage prevalence of pleuritic lesions in the different slaughterhouse batches assessed.

Graph 1: Distribution of percentage of pleuritis in the assessed slaughterhouse batches

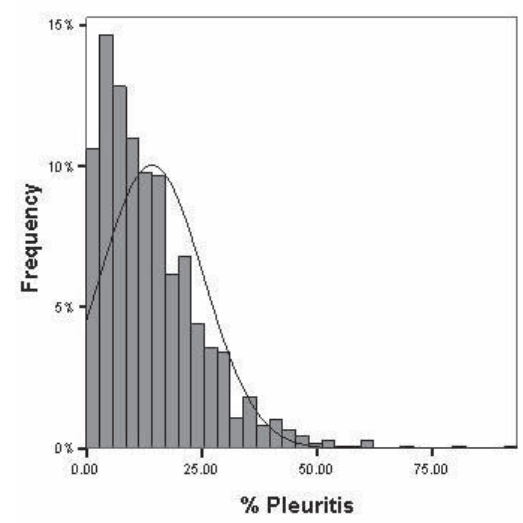

For the $15 \%$ of batches with the lowest rate of pleuritis

( $\leq 3.84 \% ; 162$ batches), the mean percentage of lesions was $2.06 \% \pm 0.09$. For the $15 \%$ of batches with the highest rate of lesions ( $\geq 25.00 \% ; 163$ batches), the mean value was $34.52 \% \pm .81$.
Table 1 shows \% pleuritis over the 11 years of the study.

Table 1: Mean \% of pleuritis by year

\begin{tabular}{|c|c|c|}
\hline YEAR & No. of batches & Mean \% of pleuritis \\
\hline 2000 & 43 & $9.81 \pm 1.21$ \\
\hline 2001 & 20 & $13.77 \pm 2.22$ \\
\hline 2002 & 16 & $15.48 \pm 2.78$ \\
\hline 2003 & 114 & $12.74 \pm 1.03$ \\
\hline 2004 & 220 & $17.93 \pm 0.74$ \\
\hline 2005 & 178 & $15.44 \pm 0.87$ \\
\hline 2006 & 218 & $13.86 \pm 0.69$ \\
\hline 2007 & 160 & $12.32 \pm 1.01$ \\
\hline 2008 & 73 & $11.97 \pm 1.31$ \\
\hline 2009 & 37 & $8.11 \pm 1.20$ \\
\hline 2010 & 6 & $7.36 \pm 2.33$ \\
\hline
\end{tabular}

Significant differences $(p<0.05)$ were found between the year 2004 and the years 2000, 03, 06, 07, 08 and 09, and also when comparing the year 2005 with the year 2009.

When the results were grouped for the 316 batches of pigs which had been fattened during the winter months (December 1 to March 31) for all the years, and compared with the results obtained during the rest of the year (769 batches), the mean prevalence of pleuritic lesions was $15.11 \%$ and $13.69 \%$ respectively.

There were no statistically significant differences between these means, though the $p$ value was close to the limit of significance $(F=3.556 \mathrm{NS} ; \mathrm{p}=0.06)$.

In the periods 2003-2005 and 2007-2009 there was an evident increase in the incidence of pleuritis during the coldest months, but this tendency was not repeated in any of the other years studied, which may explain the results obtained.

\section{Discussion}

Lesions of pleuritis are a common finding at the slaughterhouse. Important differences emerged between years in the extent of the lesions present, indicating a need to evaluate further the severity of the lesions found (1) and to diagnose the cause of the pleuritis when there is a high prevalence, in order to be able to implement effective control measures in herd and disease management.

In previous studies, the Slaughterhouse Pleurisy Evaluation System (SPES) and Actinobacillosis Pleuropneumoniae-Index (APPI) have been shown to be valuable tools in assessing pleuritis $(1,2)$.

\section{References \\ 1) Dottori M. et al (2008). Proc.20th. IPVS Congress \\ 2) Meyns T. et al (2008) Proc.20th. IPVS Congress}




\title{
Case report: Can a combination of PRRSv and Mycoplasma hyorhinis cause Enzootic Pneumonia-like lung lesions?
}

\author{
Mark Roozen; John B. Tasker; Oliver Duran \\ ECO Animal Health Ltd, London, UK
}

\section{Introduction}

Mycoplasma hyopneumoniae is the primary pathogen associated with Enzootic Pneumonia in pigs, and causes characteristic lesions. We report a case in which similar lesions were associated with respiratory disease during a PRRS virus outbreak. These observations were recorded during a trial comparing the use of a macrolide, tylvalosin (TVN), with a combination of tylosin and doxycycline for control of respiratory disease ${ }^{1}$.

\section{Material and Methods}

A 3,500 finisher herd in the most densely populated pig area in The Netherlands had a history of PPE, PRRSv and multifactorial respiratory disease. PCR assay including multiplex PCR and Real time PCR for M. hyopneumoniae was carried out by the IVD Lab on endotracheal Bronchi-Alveolar Lavage Fluid (BALF). BALF samples were taken on arrival of the finishers at 10 weeks of age. ELISA (HerdChek* Mycoplasma hyopneumoniae Antibody and PRRS 2XR-Antibody Testkit, IDEXX) was used when the pigs were 17 weeks of age. Lung scoring was carried out during two slaughterhouse examinations when the pigs were $\sim 24$ weeks of age.

\section{Results}

\section{Diagnostic tests results}

At 10 weeks of age all samples were positive for $M$. hyorhinis and PRRSv, but negative for PRCV, M. hyopneumoniae, SIV, PCMV and $P C V-2$. At 17 weeks of age there were no clear positives for $M$. hyopneumoniae (9 negative, 1 doubtful).

\section{Macroscopic lesions at slaughterhouse}

Lungs (177 pigs in the first batch, 131 pigs in the second batch) were scored as healthy, minor (10-50\% of the lung tissue affected) or extensive (over $50 \%$ of lung tissue affected) with lesions of Enzootic Pneumonia (dark red/purple consolidation on the apical, cardiac and intermediate lobes). The number of lungs with lesions varied between the 2 batches, extensive lesions were present in 13/177 (7\%) and 13/131 (10\%). Minor lesions were present in 17/177 (10\%) and 21/131 (16\%). This resulted in $17 \%$ and $26 \%$ of the total number of animals with macroscopic lesions of catarrhal bronchopneumonia in the two batches. The incidence of pleuritis was very low (2-3\%) whereas $10-15 \% 2$ is typical. The incidence of pericarditis was $2-3 \%$, and for abscessation $0-2 \%$.
Figure 1. Slaughterhouse results

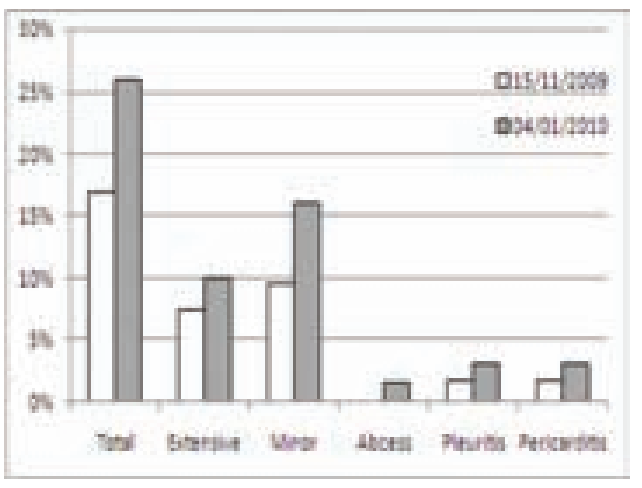

\section{Discussion and conclusions}

These results suggest that $M$. hyopneumoniae did not play a part in the respiratory disease in this herd. M. hyopneumoniae was not found in BALF by PCR assay and the serum ELISA was also negative. $M$. hyorhinis has been shown in challenge studies to produce pneumonia ${ }^{3}$ with histological lesions similar to infection with M. hyopneumoniae ${ }^{4}$. PRRSv infection has been shown to damage lung tissue, permitting colonisation of the serous membrane by $M$. hyorhinis ${ }^{5}$.

These diagnostic tests are frequently used in the field. However, as the sensitivity of the ELISA for $M$. hyopneumoniae is relatively low and macroscopic lesions are non-specific a combination of diagnostic methods is advised ${ }^{3}$. BALF is recognized as a valuable diagnostic method for porcine respiratory disease, but the isolation of pathogens is not sufficient to diagnose the cause of respiratory disease on the farm ${ }^{6}$.

The diagnosis of M. hyopneumoniae, the pathogenesis of Enzootic Pneumonia and the role of $M$. hyorhinis in EP is not clear and further research is needed.

\section{References}

1. ECO report pending

2. De Vries T (2007) Pers. comm.

3. Thacker E (2006) In Diseases of Swine 9th ed. B. Straw et al, 705-709

4. Gois` J et al (1971) J Comp Vet Path 71: 401-10

5. Palzer A et al (2009) Proc. 1st ESPCM Kopenhagen, 23

6. Hennig-Pauka et al (2007) J Vet Med Series A 54: 431-440. 


\title{
P.342
}

\section{Swine respiratory diseases in Aragon (Spain): economic, pathology and microbiological study during the year 2008}

\author{
Juan Grandia ${ }^{1}$ Ana Cristina Berges ${ }^{1}$ María Victoria Falceto ${ }^{2}$ \\ 1. Agrotest Control. S.L., Zaragoza, Spain; 2. Faculty of Veterinary Medicine, Zaragoza, Spain
}

\section{Objective}

The objective was to evaluate the prevalence and economic impact of swine respiratory pathology in the region of Aragón (Spain).

The swine sector contributes greatly to the Spanish economy, since it has an annual production of more than 4,000 million euros/year. Spain is the second and fourth european and world swine producer respectively. Aragón contributes $18.60 \%$ to the Spanish pig census. Aragón has 411861 sows and 1884649 feedlot hogs (1). An important part of the economic losses of the Spanish pig sector are due to respiratory disease, leading to worsening conversion rates, loses of daily average gain, prolonged stays in the pig farm and increased mortality. (2)

\section{Materials and Methods}

The development of this investigation has been based on three sections:

1) Study of 52 surveys answered by Aragón veterinarians responsible for 216,085 sows and $2.039,255$ hogs. In the survey farm's productive data were asked: \% mortality due to respiratory problems, treatment costs and vaccination, $\%$ of delayed pigs following respiratory disease, seizures at slaughterhouse, conversion rates and veterinarian's time dedicated to respiratory problems. The data was processed in a computer simulator of costs and sales (SIP Consultors Model).

2) Pulmonary post-mortem inspection of 7,014 lungs of pigs sacrificed throughout the year 2008 was reported. An evaluation of the lung was made to identify areas of pneumonia. A fast scale was used which allowed to obtain between 0 to 5 points per lung. The scores were assigned according to the following criteria:

0 : Absence of pulmonary injuries

I: Injuries just in the apical pulmonary lobes

II: Small injuries in other pulmonary lobes

III: Injuries in three pulmonary lobes.

IV: Injuries in the intermediate lobe in addition to others.

V: Presence of injuries in all the lobes.

The presence of pleurisy and/or pericardial diseases and other injuries were also documented.

3) Microbiological culture in 616 samples and detection of viral antigens by indirect inmunoperoxidase in 470 samples.

\section{Results}

After survey analysis, an economic estimate was obtained. A negative average of $13.72 €$ was found by each pig that goes to the slaughter house.

After pulmonary inspection at the slaughterhouse, it was verified that during summer and winter, $32 \%$ and $43.70 \%$ respectively, of the animals had healthy lungs (0). The pulmonary injuries were small (I) in 45.89\% during summer and $62.54 \%$ during winter. The most serious pulmonary injuries (II) were $55 \%$ during summer and $14.75 \%$ during winter.

Table 1: Isolated bacteriological agents.

\begin{tabular}{|l|c|}
\hline Isolated bacteriological agent & \% cases \\
\hline Actinobacillus pleuropneumoniae spp. & 5,43 \\
\hline Bordetella bronchiseptica & 9,49 \\
\hline Escherichia coli & 4,58 \\
\hline Haemophilus parasuis & 6,1 \\
\hline Pasteurella spp. & $\mathbf{1 6 , 4 4}$ \\
\hline Staphilococcus. coag. negative & 4,58 \\
\hline Streptococcus spp. & $\mathbf{3 1 , 5 2}$ \\
\hline Others & $\mathbf{2 , 8 8}$ \\
\hline Isolated bacteriological agent & 18,98 \\
\hline
\end{tabular}

Table 2: Percentage of viral antigens detected.

\begin{tabular}{|l|c|}
\hline Detected antígen & $\%$ \\
\hline Aujeszky gE & 0,41 \\
\hline Coronav. resp. & 0 \\
\hline Influenza & 4,13 \\
\hline M.hyopneumoniae & 71,07 \\
\hline M.hyorhinis & 2,89 \\
\hline PCV-2 & 1,24 \\
\hline PRRS & 20,25 \\
\hline
\end{tabular}

\section{Discussion}

The animals that have more pulmonary injuries are those coming from small capacity farms.

The low level of Aujeszky GE antigens indicates the effort made to eradicate this disease in Aragón.

\section{Acknowledgments}

This research was supported by the Asociación de Veterinarios de Porcino de Aragón (AVPA). (www.avparagon.com)

The authors thank the farm groups and laboratories that participated in this study.

\section{References}

1. Pig Census in April of 2008. General subdivision of Statistic. Government of Spain.

2. P. Retamal. "Epidemiologia of the respiratory diseases of the pig". TECNOVET: Year 7 n³, December 2001 


\section{Association between TTV genogroups 1 and 2 and PCV2 as well as various respiratory pathogens - a retrospective study}

\section{Christiane Lang ${ }^{1}$ Heidi Söllner ${ }^{1}$ Andrea Barz ${ }^{1}$ Andrea Ladinig ${ }^{1}$ Rebecca Langhoff ${ }^{1}$ Herbert Weissenböck ${ }^{2}$} Tuija Kekarainen 3 Joaquim Segalés 3 Mathias Ritzmann¹

1. Clinic for Swine, Vienna, Austria; 2. Institute of Pathology and Forensic Veterinary Medicine, University of Veterinary Medicine Vienna, Vienna, Austria; 3. Centre de Recerca en Sanitat Animal (CReSA), UAB-IRTA, Campus de la Universitat Autònoma de Barcelona, Barcelona, Spain

\section{Introduction}

Torque teno viruses (TTV) are viruses discovered in humans and several domestic and wild animal species including swine (1). The virus belongs to the floating genus Anellovirus. Two TTV genogroups are identified in domestic pig as well as in wild boar $(2,3)$. Association between TTV and PMWS affected pigs has already been determined (4). In humans, also respiratory diseases linked to TTV have been described (5). In the present study a possible association between TTV and PCV-2 and respiratory pathogens was investigated.

\section{Materials and Methods}

A total of 253 sera collected in 2008 were tested by conventional PCR (6) for TTV genogroups 1 and 2. Retrospectively, results of former diagnostic findings of those samples were evaluated and possible association to TTV was investigated (Table 1). Furthermore, the association between the final diagnosis PRDC and TTV as well as PCV2 was evaluated. The diagnosis PRDC was based on patho-morphological findings (pneumonia, bronchitis, pleurisy). The percentage of corresponding results, the Pearson's coefficient of correlation and the level of significance $(p)$ were calculated. P-values less than or equal 0.05 were considered significant.

Table 1: Pathogens, methods and number of analyzed samples

\begin{tabular}{|l|c|c|}
\hline TTV1/TTV2 & PCR & $\mathbf{2 5 3}$ \\
\hline PCV2 & In situ hybridization & 136 \\
\hline PCV2-IgM & ELISA & 70 \\
\hline Mycoplasma (M.) hyopneumoniae & Bacteriology & 43 \\
\hline Pasteurella (P.) multocida & Bacteriology & 145 \\
\hline Haemophilus (H.) parasuis & Bacteriology & 145 \\
\hline Actinobacillus pleuropneumoniae (APP) & Bacteriology & 145 \\
\hline
\end{tabular}

\section{Results}

Association between TTV and PCV2 is shown in table 2. There was no correlation between TTVs and P. multocida or APP. A negative correlation between both TTVs and $M$. hyopneumoniae ( $p>0.05)$ as well as $H$. parasuis $(p=0.041)$ was found. Significant correlation was found between TTV2 and the final diagnosis PRDC $(p=0.000)$, whereas there was no association between TTV1 or PCV2 (ISH) and PRDC.

Table 2: Associations of TTV with PCV2

\begin{tabular}{|l|c|c|c|c|c|}
\hline & & TTV1 & TTV1 & TTV2 & TTV2 \\
\hline & & neg. & pos. & neg. & pos. \\
\hline PCV2 ISH & neg. & $48.5 \%$ & $22.8 \%$ & $44.9 \%$ & $26.5 \%$ \\
\hline PCV2 ISH & pos. & $15.4 \%$ & $13.2 \%$ & $5.2 \%$ & $23.5 \%$ \\
\hline & Pearson & 0.145 & 0.145 & 0.368 & 0.368 \\
\hline & $\mathbf{p}$ & 0.092 & 0.092 & $0.000^{*}$ & $0.000^{*}$ \\
\hline PCV2 IgM & neg. & $47.1 \%$ & $14.3 \%$ & $34.3 \%$ & $27.1 \%$ \\
\hline PCV2 IgM & pos. & $20.0 \%$ & $18.6 \%$ & $12.9 \%$ & $25.7 \%$ \\
\hline & Pearson & 0.258 & 0.258 & 0.219 & 0.219 \\
\hline & $\mathbf{p}$ & $0.031^{*}$ & $0.031^{*}$ & 0.068 & 0.068 \\
\hline
\end{tabular}

\section{Discussion}

TTV in swine may have clinical relevance as a cofactor in multifactorial diseases. Association between TTV and PMWS affected pigs (caused by PCV2) has already been described. Also in the present study a significant correlation between PCV2 IgM-Ab and TTV1 as well as PCV2 virus and TTV2 was detected. IgG-Ab were not included into the evaluation because they could have originated from vaccination, whereas IgM-Ab only rarely appear in context with a vaccination. No correlation between other respiratory agents and TTVs could be found, but an association between PRDC and TTV could be determined. To what extent TTV has to be considered as a cofactor for the development of respiratory diseases in swine still needs to be investigated.

\section{References}

1. Kekarainen and Segalés (2009): Vet. J. 180, 163-168.

2. Niel et al. (2005): J. Gen. Virol. 86, 1343-1347.

3. Martinez et al. (2006): Vet. Microbiol. 118, 223-229.

4. Kekarainen et al. (2006): J. Gen. Virol. 87, 833-837.

5. Maggi et al. (2003): J. Virol. 77, 2418-2425.

6. Segalés et al. (2009): Vet. Microbiol. 134, 199-207. 


\title{
P.344
}

\section{Pleurisy: characterisation of associated pneumonia and viral infections (PCV2, PRRSV, SIV)}

\author{
Henrike J. Jager ${ }^{5}$ TJ McKinley ${ }^{1}$ James L. Wood ${ }^{1}$ Gareth P. Pearce ${ }^{1}$ Susanna M. Williamson² \\ Nicholas Woodger ${ }^{2}$ Benjamin Strugnell ${ }^{3}$ Stanley H. Done ${ }^{3}$ Henrike Habernoll ${ }^{4}$ Alexander W. Tucker $^{1}$ \\ 1. University of Cambridge, Cambridge, UK; 2. Veterinary Laboratories Agency, Bury St Edmunds, UK; \\ 3. Veterinary Laboratories Agency, Thirsk, UK; 4. BQP Ltd, Stradbrooke, UK; 5. Garth Partnership, Beeford, UK
}

\section{Introduction}

Pleurisy is recognized as an important aspect of respiratory disease. There are insufficient data on the extent of the relationship between pneumonia and pleurisy but it undoubtedly exacerbates the economic losses of respiratory disease due to consequent required carcase trimming at slaughter. A number of studies have identified risk factors associated with pleurisy (environmental (1), bacterial (2), and recently viral (3)). This study explored the relationships between histological types of pneumonia and active or resolved pleurisy and investigated the role of viral infections (PRRS, PCV2, and influenza) in pleurisy.

\section{Materials and Methods}

A total of 79 pigs were selected for necropsy across 12 UK finisher units that reported a consistent pleurisy prevalence of $>10 \%$ at slaughter. Pigs were selected on the basis of clinical respiratory signs and included the youngest pigs showing respiratory signs (such as dyspnoea and/or coughing). Pigs on $3 / 12$ units were vaccinated against PRRSV (18/79 pigs in study). On 10/12 units pigs were vaccinated against PCV2 (63/79 pigs) and PCV2 associated disease (PCVAD) was not reported as a clinical problem on any units. Each pig was allocated a pleurisy status based on a combination of gross and histopathological findings (no pleurisy (NP), active pleurisy (AP), resolved pleurisy (RP)). Histopathological evaluation permitted each pig to be categorised as having no pneumonia (NPn), bronchopneumonia (BPn), broncho-interstitial pneumonia (BIPn), or interstitial pneumonia (IPn). Virology investigations in each pig comprised PCR on blood for PRRSV, histopathology and immunohistochemistry on lung for PCV2-associated pneumonia, and on inguinal and ileocaecocolic lymph node for PCV2-associated lymphoid changes, and virus isolation on pooled tonsil, trachea and lung for swine influenza virus (SIV).

\section{Results}

Of the 79 pigs, 23 had no detectable pleurisy (age range 5-21wks), 25 showed active pleurisy (5-21wks) and 31 showed resolved pleurisy (10-21wks). AP and RP were found on all 12 units. Histopathology revealed the following findings: NPn -8 cases, BPn - 21 cases, BIPn - 45 cases, IPn - 5 cases. Twenty four of the 79 pigs had a PRRS viraemia, 11 had PCV2-associated pneumonia, and 13 had PCV2-associated pathology in lymph node. Analysis of the relationship between pleurisy and pneumonia revealed statistically significant associations ( $p=0.0006)$. For the no pleurisy group, pneumonia types were broadly distributed across NPn (30\%), BPn (39\%), BIPn (17\%) and IPn (13\%). However, for the AP group there was a marked increase in the proportion of cases with broncho-interstitial pneumonia (NPn $0 \%, \mathrm{BPn}-28 \%, \mathrm{BIPn}-64 \%, \mathrm{IPn}-8 \%$ ). This increased prevalence of BIPn was even more marked in the RP group (NPn - 3\%, BPn - 16\%, BIPn - 81\%, IPn - 0\%).Associations were found between pleurisy and infection by PRRSV and PCV2. For PRRSV viraemia, $\mathrm{NP}=4 \mathrm{pos} / 23(17 \%), \mathrm{AP}=9 \mathrm{pos} / 25$ (36\%), RP = 11 pos/31 (35\%); $\mathrm{p}=0.03)$. For $\mathrm{PCV}$-associated pneumonia, $\mathrm{NP}=0 \mathrm{pos} / 23(0 \%), A P$ $=7 \mathrm{pos} / 25(28 \%), \mathrm{RP}=4 \mathrm{pos} / 31(13 \%) ; \mathrm{p}=0.02)$. For $\mathrm{PCV} 2$ detection in lymph node, NP=0 pos/23 (0\%), AP=7 pos/25 (28\%), RP=6 pos/31 (19\%); $p=0.02)$. SIV was found in 1 pig.

\section{Discussion}

This study focused only on pigs from production units with consistently high pleurisy prevalence. Within these units we found that pigs affected by pleurisy were more likely to be affected by broncho-interstitial pneumonia than pigs on the same units without pleurisy. Furthermore, we found associations between pleurisy and PRRSV viraemia and pleurisy and PCV2-associated pneumonia or PCV2 pathology in lymph nodes. The majority of units undertook vaccination against PCV2-associated disease (PCVAD) and none reported clinically active PCVAD. These findings highlight the value of reviewing procedures to maximise PCV2 and PRRSV vaccine-induced protection as part of a strategy to control pleurisy.

\section{References}

1. Cleveland-Neilsen et al, 2002 PrevVetMed 55:121-135

2. Jirawattanapong et al, 2010 ResVetSci 88:11-15

3. Wellenberg et al., 2010 Vet Micro (in press). 


\title{
P.345
}

\section{Serum acute phase proteins as biomarkers of pleuritis and cranio- ventral pulmonary consolidation in slaughter-aged pigs}

\author{
$\underline{\text { Lorenzo Fraile }}^{1}$ Yolanda Saco ${ }^{2}$ Merce Giménez² Ana Alegre ${ }^{1}$ Rosa López-Jimenez ${ }^{1}$ Marti Cortey ${ }^{1}$ Joaquim Segalés $^{1}$ Anna Bassols ${ }^{2}$ \\ 1. CReSA, Bellaterra, Spain; 2. Departament de Bioquimica i Biologia Molecular, Barcelona, Spain
}

\section{Introduction}

Pleuritis and cranio-ventral pulmonary consolidation (CVPC) are the most frequent pathological findings in pig lungs at slaughter (3). Several environmental factors and infectious agents have been associated with the occurrence of lung lesions in swine. Mycoplasma hyopneumoniae and Actinobacillus pleuropneumoniae are considered to be the most important primary bacterial respiratory pathogens (6). On the other hand, the acute phase protein (APP) response plays an important role in lung infection (5). Therefore, the purpose of this study was to investigate the relationship between the existence of lung lesions in pigs at slaughter and the concentration of three APPs, haptoglobin (Hp), pig-major acute protein (Pig-MAP) and C-reactive protein (CRP).

\section{Material and Methods}

Twenty-four farms were used for the present study. Pleuritis lesions were assessed based on the Slaughterhouse Pleurisy Evaluation System (SPES score). An A. pleuropneumoniae index (APPI) was defined to provide information on the prevalence and severity of pleuritis lesions as previously described (1). Farms were classified as "pleuritis negative $(\mathrm{P}-)$ or positive $(\mathrm{P}+)^{\prime}$ " if they had low or high APPI values, respectively. Moreover, farms were classified also as "CVPC negative (M-) or positive $(\mathrm{M}+)^{\prime}$ " if the percentage of lungs with these lesions at farm level were lower or higher than $60 \%$, respectively. CVPC lesions were evaluated using a previously described method (2). Selected farms were divided into four combined groups ( $\mathrm{P}-\mathrm{M}-, \mathrm{P}-\mathrm{M}+, \mathrm{P}+\mathrm{M}-, \mathrm{P}+\mathrm{M}+$ ), including 6 farms per category. Blood from 20 randomly selected pigs from each farm was collected and selected APPs determined in serum. Hp was quantified by a spectrophotometric method (haemoglobin binding assay). Pig-MAP levels were assessed with an ELISA kit (PigCHAMP ProEuropa, Segovia, Spain). Finally, CRP was determined using a commercial immunoturbidimetric method (Olympus System Reagent, OSR 6147).

A non-parametric test (Mann-Whitney) was used to evaluate the effect of pleuritis or CVPC lesions on the concentration of APPs. Finally, a Receiver Operating Characteristic (ROC) analysis was carried out to determine the capacity of discrimination of APPs in farms classified as $\mathrm{P}$ - or $\mathrm{P}+$ or $\mathrm{M}$ - or $\mathrm{M}+$ and when the four groups were taken into account (P-M-, $\mathrm{P}-\mathrm{M}+, \mathrm{P}+\mathrm{M}-, \mathrm{P}+\mathrm{M}+)$.

\section{Results}

All APPs concentrations were significantly higher for $\mathrm{M}+$ farms than for $\mathrm{M}$ - ones. However, only $\mathrm{Hp}$ and Pig-MAP showed significantly higher values for $\mathrm{P}+$ farms than for $\mathrm{P}$ - ones $(\mathrm{p}<0.05)$. Pig-MAP was the most sensitive indicator since it was able to clearly discriminate between $\mathrm{P}-/ \mathrm{P}+$ and $\mathrm{M}-/ \mathrm{M}+$ groups $(\mathrm{p}<0.001$ in both cases). Hp was an excellent marker for pleuritis and good for CVPC lesions. CRP was able to discriminate for CVPC lesions but not for pleuritis. ROC analysis showed that Pig-MAP was the best biomarker, with $67.8 \%$ sensitivity and $78.8 \%$ specificity to discriminate between $\mathrm{P}-\mathrm{M}-$ and $\mathrm{P}+\mathrm{M}+$ farms.

\section{Discussion}

There is little information in the literature about the relationship between serum APP and the degree of lung lesions of pigs at slaughter (4). The present study shows that both Pig-MAP and $\mathrm{Hp}$ can be used as unspecific markers for the presence of pleuritis and/or CVPC lesions at slaughter. None of the tested APPs was able to discriminate both types of lesions; such result is not surprising, taking into account to their unspecific role in the inflammatory response. It is very important, however, to note that pigs involved in the present study did not show any clinical sign of disease at slaughter, and lesions were identified in a retrospective evaluation by abattoir surveillance.

\section{References}

1. Fraile, 2009. Vet Journal. Ahead of print

2. Madec, 1982. J Rech Porc en France 14, 405-412.

3. Martinez, 2007. Vet Journal 174:160-164.

4. Pallares, 2008, Vet Journal 177:369-373.

5. Petersen, 2004. Veterinary research 35: 163-187.

6. Sorensen, 2006. Diseases of the respiratory system. Diseases of Swine. 9th edition 


\title{
P.346
}

\section{Potential use of long pentraxin 3 (PTX3) as a biomarker in pigs}

\author{
Elisa Crisci ${ }^{2}$ Lorenzo $_{\text {Fraile }}^{2,1}$ Sonia Valentino $^{3}$ Meritxell Simon-Grifé ${ }^{2}$ Gerard E. Martín-Valls ${ }^{2}$ \\ Tufária Mussá ${ }^{2}$ Barbara Bottazzi ${ }^{3}$ Alberto Mantovani ${ }^{3}$ Maria Montoya2, 1 \\ 1. Institut de Recerca i Tecnologia Agroalimentàries (IRTA), Barcelona, Spain; 2. Centre de Recerca en Sanitat Animal (CReSA), \\ UAB-IRTA, Campus de la Universitat Autònoma de Barcelona, Bellaterra, Spain; 3. Istituto Clinico Humanitas, Rozzano, Italy
}

\section{Introduction}

Long pentraxin 3 (PTX3) is a conserved pattern-recognition molecule and a host-defense-related component of the humoral innate immune system. PTX3 is produced in a variety of cell types and tissues, most notably dendritic cells (DC) and macrophages, in response to Toll-like receptor engagement and inflammatory cytokines. PTX3 regulates the complement cascade, facilitates the pathogen recognition via cellular receptors (opsonisation) and plays a regulatory role during inflammation. Moreover, PTX3 is related to female fertility in human and mice $(1,2,4)$.

PTX3 has an antiviral role in early host defence against influenza infections (5) and it might be a useful biomarker of acute lung injury (4) in mice. No information on PTX3 role in pigs is available at the moment.

The aim of our study was to investigate the role of PTX3 as a biomarker in vivo and in vitro in pigs.

\section{Material and Methods}

For in vitro study, porcine bone marrow-derived DC were generated and infected with a H3N2 swine influenza virus (A/Swine) Spain/80598-LP1/2007).

For in vivo study, sixteen conventional pig farms with history of pleuritis lesions and cranio-ventral lung consolidation were used (3). Blood from 20 randomly selected pigs from each farm was collected and selected acute phase proteins were determined in serum. Hp was quantified by a spectrophotometric method (haemoglobin binding assay). Pig-MAP levels were assessed with an ELISA kit (PigCHAMP ProEuropa, Segovia, Spain). Finally, CRP was determined using a commercial immunoturbidimetric method (Olympus System Reagent, OSR 6147). Moreover, sera from 48 conventional sows from parity one to eight showing high swine influenza virus (SIV) antibody titres were also used.

PTX3 concentration in sera and culture supernatant was determinate by sandwich ELISA (2C3 and 6B11 antibodies) as previously described (8).

Statistical analysis was performed using SPSS 15.0 software (SPSS Inc., Chicago, IL, USA).

A linear regression between different variables was carried out and a Kruskal-Wallis test was used to compare PTX3 serum levels between different sow parities. All this statistical analysis was performed using SPSS 15.0 software (SPSS Inc., Chicago, IL, USA).

\section{Results}

The in vitro study showed that porcine myeloid bone marrowderived DC produced PTX3 after infection with porcine influenza virus H3N2.

The in vivo results have shown that PTX3 serum levels are correlated neither with $\mathrm{Hp}, \mathrm{CRP}$ or Pig-MAP nor with lung lesions score in conventional swine farms. PTX3 concentration in SIV antibody positive sera of studied sows increased with the parity number showing statistical tendency between the youngest and the oldest sows ( $p=0.07$ ). Moreover, there was no statistical correlation between SIV antibody titres and PTX3 amount in serum.

\section{Discussion}

PTX3 is produced by myeloid DC in human and mice and the present study confirmed in vitro production on porcine myeloid DC.

In vivo results have shown that PTX3 is not a good biomarker for chronic lung lesion status in swine farms when compared with $\mathrm{Hp}$ and Pig-MAP (7). However, PTX3 concentration in SIV antibody positive sera correlated with a greater exposure to infections, indicating that PTX3 might be used as a biomarker under certain conditions. Further studies should be performed to elucidate its possible practical application.

This is the first analysis of PTX3 role in pigs and future studies will determine its contribution in porcine innate immunity.

\section{References}

1. Bottazzi, 2010. Annu. Rev. Immunol. 28: 157-83

2. Bottazzi, 2009. Immunol. Rev. Jan; 227: 9-18

3. Fraile, 2009 Vet Journal. Ahead of print

4. Garlanda, 2005, Annu. Rev. Immunol. 23: 337-66

5. He, 2009. Intensive Care Med. Nov 18

6. Reading, 2008. J Immunol. Mar; 180: 3391-8.

7. Saco, 2010. Res Vet Sci submitted

8. Salio, 2008. Circulation 117: 1055-1064 


\title{
P.347
}

\section{Development of lung macrophage subpopulations in potbellied minipigs after weaning}

\author{
Marco A. Vega-López; Angel Balam-May; Carmen Ramírez-Estudillo; Gloria Lazo \\ CINVESTAV-IPN, Dept. Infectomics and Molecular Pathogenesis, México, DF, Mexico
}

\section{Introduction}

Respiratory diseases are one of the main problems in human and animal health. Several models to study the respiratory immune system has been used, but humans and pigs share the unique feature of a non-constitutive bronchus-associated lymphoid tissue (BALT) (1-2). In spite of its importance, no studies on lung macrophage (Mf) development have been carried out. We examined the development of Mf in blood, bronchoalveolar lavage (BAL) and lung parenchyma (LP) of pigs after weaning by flow cytometry.

\section{Material and Methods}

Five 5 weeks-old weaned pigs (WP), five 3 months-old ( $3 \mathrm{~m}$ ) and four 5 years-old (adults) SPF potbellied mini pigs from the animal unit of CINVESTAV (UPEAL), compiling with local and federal regulations of animal care and with no vaccination protocols running, were used. PBMC were obtained by Ficoll/Isopaque gradient, BAL cells were obtained by tracheobroncheal lavage with PBS and lung parenchyma cells (LP) were obtained after enzymatic digestion of fresh tissue. Mouse monoclonal antibodies to pig CD172a (IgG1 -MIL-2-), CD14 (IgG2b -74-22-15-) and MHCII (IgG2a -MSA-3-) were used with fluorochrome conjugated goat anti-mouse secondary antibodies (IgG1-PE, IgG2b-APC, IgG2aFITC). The cells were run in a FACScalibur (B-D) flow cytometer. Relative and absolute numbers of positive cells were calculated and the results were analyzed by ANOVA.

\section{Results}

The proportion (\%) of CD14+MHC-II+ Mf was higher in all tissues of WP (WP >adults=3m) (Fig 1A). CD14+CD172a+cells were higher in $3 \mathrm{~m}$ PBMC $(3 \mathrm{~m}>W P)$, and LP of adults (Adults $>W P=3 \mathrm{~m})$ (Fig 1B). CD14+ double positive (DP) cells were higher in all tissues of $3 \mathrm{~m}$ and adults ( $3 \mathrm{~m}=$ adults $>$ WP) (Fig 1C) and CD14+ double negative (DN) cells were higher in WP PBMC (WP $>3 \mathrm{~m})$ and adult LP (Adults $>3 \mathrm{~m}$ ) (Fig 1D). Absolute numbers of CD14+MHC-II+ were higher in PBMC (WP $>3 \mathrm{~m}), \mathrm{BAL}$ and LP of WP (WP $>3 \mathrm{~m}=$ adults, Fig 1E). CD14+CD172a+ were higher in PBMC of $3 \mathrm{~m}$ pigs ( $3 \mathrm{~m}>\mathrm{WP}$, Fig 1F) only. CD14+DP cells were higher in $3 \mathrm{~m} \mathrm{BAL}(3 \mathrm{~m}>\mathrm{WP})$ and $\mathrm{LP}(3 \mathrm{~m}>\mathrm{WP}=$ adults, Fig $1 \mathrm{G})$ and CD14+DN cells were higher in WP PBMC (WP $>3 \mathrm{~m}=$ adults) and LP (WP>3m, Fig 1H).

\section{Discussion}

Mf subpopulations in the lung undergone important changes in proportion and number with age. At least four different CD14+ subpopulations were identified. MHC-II+ and CD172a+ cells may have different roles since they tend to inversely change with age (Fig 1A, E \& B, F). DP cells tend to increase with age (Fig 1C, G) in contrast with DN cells (Fig $1 \mathrm{D}, \mathrm{H}$ ). MHC-II+ may correspond to activated Mf, CD172a+ is a SIRP molecule, related to activation control (3-5). DP cells may be related to fully differentiated Mf and DN to scavenger non-activated cells. Their role in lung protection remain to be elucidated. These data also show evidence of important differences among tissues.

Figure 1. Relative $(A-D)$ and absolute $(E-H)$ numbers of $C D 14+$ macrophage subpopulations in $P B M C$, bronchoalveolar lavage (BAL) and lung parenchyma (LP) of weaned (WP), 3 months-old $(3 \mathrm{~m})$ and 5 years-old (adults) SPF potbellied mini pigs ( $n=5 /$ group). Bars show the mean plus SEM. ANOVA test. ${ }^{*} P<0.05,{ }^{* *} P<0.01,{ }^{* *} P<0.001$.

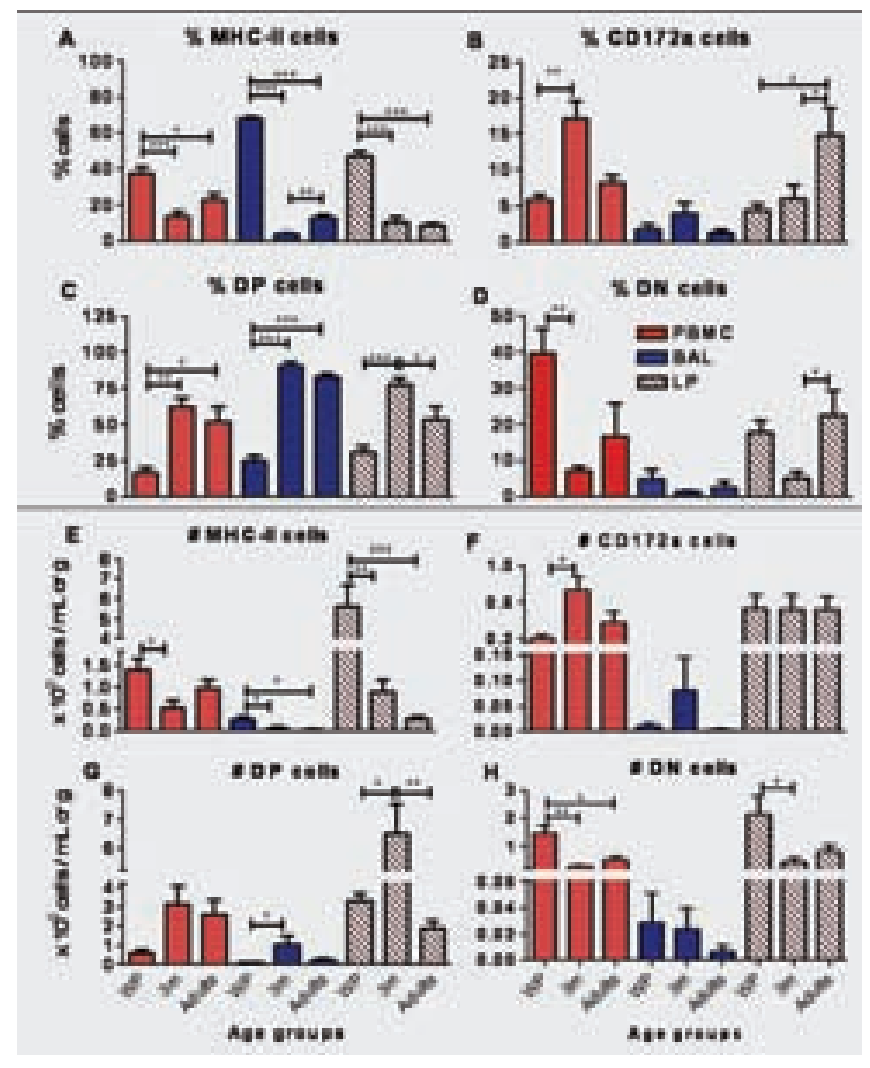

\section{Acknowledgments}

To MVD Manuel Flores (UPEAL-CINVESTAV) for technical assistance. Project partially supported by CONACyT (60941) and Instituto de Ciencia y Tecnología del D.F.

\section{References}

1. Hiller AS, et al., Scand J Immunol. 1998. 47(2):159-62.

2. Pabst R, et al., Am J Respir Cell Mol Biol. 1990. 3(2):131-5.

3. Adams S, et al., J Immunol. 1998. 161(4):1853-9.

4. Cagiola M, et al., Vet Immunol Immunopathol. 2006. 112(3-4):31620.

5. Pollanen P, et al., J Reprod Fertil. 1988. 82(2):437-45. 


\title{
P.348
}

\section{Interaction between Mycoplasma hyopneumoniae and fumonisin B1 toxin in the porcine respiratory tract}

\author{
Roland Pósa ${ }^{2}$ Melinda Kovács ${ }^{2}$ Judit Szabó-Fodor ${ }^{2}$ József Mondok² Péter Bogner ${ }^{3}$ Imre Repa $^{3}$ Tibor Magyar $^{1}$ \\ 1. Veterinary Medical Research Institute, Budapest, Hungary; 2. Kaposvár University, Faculty of Animal Science, Kaposvár, Hungary; \\ 3. Institute of Diagnostic Imaging and Radiation Oncology, Kaposvár, Hungary
}

\section{Introduction}

Respiratory disease is one of the most important health concerns for swine production. The term porcine respiratory disease complex (PRDC) refers to a condition in which an interaction between various pathogens and inappropriate environmental conditions lead to severe respiratory disorders (1).In this study we examined the possible synergy between $\mathrm{M}$. hyopneumoniae (Mh) and fumonisin B1 (FB1) toxin in the lung of pigs. Computed tomography (CT) proved useful for imaging pneumonia (3). CT was applied to follow up the pathological events in the lung.

\section{Materials and Methods}

Twenty-eight 3-day-old pigs, obtained from a high health-status herd, were used in the study. The arrival of the pigs was day 0 of the study. After an early weaning, the piglets were artificially reared receiving milk-replacer until day 16 and then appropriate solid feed until the termination of the experiment (day 58). Four groups of 7 pigs were formed:

Group 1: uninfected untreated controls

Group 2: uninfected - treated with FB1-toxin

Group 3: infected with Mh - untreated

Group 4: infected with Mh - treated with FB1-toxinGroup 3 and 4 were infected with Mh (strain Mp 496) on day 30. The inoculum was prepared by suspending $1.5 \mathrm{~g}$ Mh containing lung tissue in $13.5 \mathrm{ml}$ phosphate buffered saline ( $\mathrm{pH}$ 7.2). Pigs were inoculated intra-tracheally with $0.5 \mathrm{ml}$ of the suspension containing 109 colour-changing units per ml. Groups 2 and 4 were fed with FB1contaminated feed $(10 \mathrm{mg} / \mathrm{kg})$ from day 16 until termination. The toxin was produced as described (2).CT images of the whole lung were acquired at days 16, 30, 44 and 58 using a SIEMENS Somatom Emotion 6 multislice CT scanner. At termination, the pigs were humanly killed and lung lesions were evaluated post mortem (4). CT images were analysed by Medical Image Processing V1.0 software. Affected were compared with and non-affected areas.

\section{Results}

From day 37 the infected animals showed clinical signs (coughing, laboured breathing and huskiness). CT examination did not reveal lesions in the negative control pigs (group 1) and the FB1-treated group (group 2) suggesting that the toxin did not induce macroscopic lesion at this dose level. In the Mh infected groups (groups 3 and 4) lung lesions were found in all animals on day 44. The most pronounced lesions were observed in the cranial, middle, and the accessorial lung lobes and in the front of the caudal lobe. The lung lesions appeared first around the small respiratory airway passages, later whole lobules and lobes became affected. Necropsy did not reveal lung lesion in the negative control pigs (group 1). In group 2, mild interstitial oedema was observed in four animals. In the Mh infected groups (group 3 and 4) we found acute catarrhal pneumonia in all animals. The macroscopic lung lesions were more pronounced in group 4 than in group 3. Significant differences were detected between non-infected and infected groups by CT evaluation

\section{Discussion}

The Mh infection produced lung lesions in young piglets that were increased by treatment with FB1 toxin. The only lethal case and the highest rate of lung lesions were found in group 4 that received $\mathrm{FB} 1$-toxin treatment beside the bacterial infection indicating that the FB1-toxin increased the severity of pneumonia caused by the bacterial pathogen. These findings confirm the idea of PRDC that various factors may act in harmony to produce pneumonia. Our results also indicate that CT can be applied for studying the pathological conditions in the lower respiratory tract of swine.

\section{References}

1. Brockmeier, S. et al (2002) ISBN 1-55581-244-9, 231

2. Fodor, J. et al (2006) Acta Agr Kaposvar 10:277-284

3. Pósa, R. et al (2008) Proc 20th IPVSC, OR.05.11.

4. Straw, B.E. et (1989) JAVM 195:1702-1706 


\section{P.349}

\section{Virulence genes and PFGE types of Pasteurella multocida field isolates from pigs in South Korea}

Aeran Kim; Ji Youn Lee; Chun Tae Lim; Kichan Lee; Suk Chan Jung

National Veterinary Research \& Quarantine Service, Anyang, Korea

\section{Introduction}

Pasteurella multocida is widely recognized as the causative agent of progressive atrophic rhinitis and hemorrhagic septicemia in swine and is of considerable economic importance to the pig rearing industry through-out the world. The present study reports the frequency, features and molecular epidemiology of P. multocida isolates from diseased and finished pigs.•

\section{Materials and Methods}

A total of 140 P. multocida isolates was collected from 751 cases of diseased and finished pigs during 2007-2009 in South Korea. The isolates were analyzed by PCR for the capsular type (A, B, D, $E, F)$ and the presence of virulence genes. The 4 virulence-associated genes were detected with varying frequency. Genotyping of the isolates were performed by Pulsed field gel electrophoresis (PFGE)

\section{Results}

The most prevalent capsular type among P. multocida isolates was capA (54.1\%), followed by capD (45.9\%) from diseased pigs. However, from finished pigs, the prevalent of capA $(97.5 \%)$ was higher than capD (2.5\%). A higher percentage of toxA was detected among diseased pigs (12.5\%) compared with strains isolated from finished pigs (3.8\%). Among clinical isolates hgbB and tpbA was more frequently identified in strains isolated from diseased pig $(95.8 \%, 54.2 \%)$ whereas $78.8 \%$ and $18.8 \%$ of the finished pig isolates harboured this gene. However, pfh A gene detected more frequently from finished pigs (50.0\%) than diseased pigs (16.7\%). The PFGE patterns of the isolates revealed five different genotypes.

\section{Discussion}

These data enforce further virulence and epidemiological studies, examining the properties of porcine P. multocida strains as well as factors of the porcine hosts themselves, which might be involved in disease susceptibility.

\section{References}

1. Bethe A, Wieler LH, Selbitz HJ, Ewers C., Genetic diversity of porcine Pasteurella multocida strains from the respiratory tract of healthy and diseased swine. Vet Microbiol. 2009 Oct 20;139(1-2):97-105.

2. Ewers C, Lübke-Becker A, Bethe A, Kiebling S, Filter M, Wieler LH., Virulence genotype of Pasteurella multocida strains isolated from different hosts with various disease status. Vet Microbiol. 2006 May 31;114(3-4):304-17.

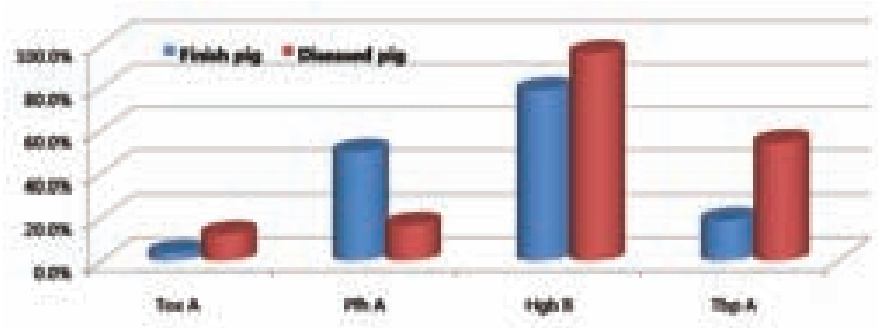

Flgure 1. Detectlon rates of virulence-assoclated genes. 


\title{
P.350
}

\section{FUGATO RePoRI: Development of a powerful tool for the analysis of bacterial lung infections in pigs}

\author{
Martin Selke ${ }^{3}$ Doris Hoeltig ${ }^{1}$ Gerald F. Gerlach ${ }^{2}$ Karl-Heinz Waldmann ${ }^{1}$ Fugato Consortium IRAS ${ }^{1}$ Fugato consortium RePoRI ${ }^{1}$ \\ 1. Clinic for Swine and Small Ruminants, Forensic Medicine and Ambulatory Service; \\ University of Veterinary Medicine Hanover, Foundation, Hannover, Germany; 2. IVD GmbH, Hannover, Germany; \\ 3. Institute of Microbiology, Department of Infectious Diseases, University of Veterinary Medicine Hanover, Foundation, Hannover, Germany
}

\section{Introduction}

Lung infections have a great impact on the pig breeding and fattening industries worldwide. Economical losses due to stunted growth of piglets or massive need of antibiotic treatment of infected herds along with the oftentimes missing alternative of vaccination strategies encouraged a change in strategy toward a breeding programme focusing on lung health. Therefore the FUGATO-IRAS project and the follow-up project RePoRI work on the identification of genetic markers responsible for an increased resistance towards bacterial lung infection by the use of the infection model Actinobacillus pleuropneumoniae (App).

\section{Materials and Methods}

Within IRAS 106 pigs of 4 breeding lines (German Landrace, Piétrain, Hampshire, Large White) were infected. The aerosol infection was performed using App serotype 7 strain AP76. Sampling (BALF, blood) and phenotyping were carried out twice (day 4 and day 20 post infection [p.inf.]). Pigs were euthanized immediately after the sampling, lung lesions were scored during necropsy by the Lung Lesions Score according to Hannan et al., and lung tissue was taken. For additional phenotyping a Respiratory Health Score (1) was used. Acute phase proteins were determined (blood, BALF), glycoproteins (BALF) were analyzed via differential two-dimensional gel electrophoresis (2D-DIGE) and RNA expression was investigated using the Affymetrix GeneChip ${ }^{\circledast}$ porcine Genome Array. In addition, a consumer study investigating the allele diversity within German pork was realized and SNPs for candidate genes, derived from known human genes, were detected and characterized.

As tool for the analyses of the host-pathogen-interaction a versatile, multi-species microarray based on the CustomArrayTM platform of Affymetrix ${ }^{\circledast}$ was developed. Using extensive in silico analyses using BLAST and MUMMER (2) followed by hard and soft pruning, the resulting APP76PIG Chip contains the complete genome of 3 serotypes of App, the Mycoplasma hyopneumoniae strain 232 as well as the 1.500 most promising probes of the Affymetrix GeneChip ${ }^{\circledR}$ porcine Genome Array. Each gene is represented by 8 to $1125 \mathrm{mer}$ probes as match and mismatch probe sets to subtract unspecific binding of labeled cDNA.

\section{Results}

Pair wise whole-genome comparisons of our infection model strain (App serovar 7) with App serovars 3 and 5 using the Maximal Unique Matching subsequence decomposition program MUMMER (Vers.3.0) delivered a number of serovar specific loci for each of the three genomes with 82 open reading frames (ORF) in serovar 3 and 68 ORF in serovar 5 which were not also present in serovar 7. Using BLAST and the Affymetrix ${ }^{\circledR}$ pruning software we condensed this list to 28 (serovar 3) and 49 ORF (serovar 5), respectively, which were unambiguous serovar specific and added these probes to the APP76PIG array. Now we are able to analyze the expression of three App serovars which differ in their clinical severity in pigs.

Next we added all OST of Mycoplasma hyopneumoniae strain 232, another important lung pathogen. This enables us to evaluate our App derived results with another bacterial infection model.

In a final step 1.500 probes of the porcine Genome Array representing the most promising potential genetic markers of FUGATO IRAS were added to the APP76PIG array.

\section{Discussion}

With this powerful tool for expression analyses we are able to not only get a detailed picture of the bacterial expression of App but also, simultaneously, of the direct response of the porcine host, based on our previous results from FUGATO IRAS. Moreover, using e.g. field samples of acute infections in pigs we should be able to compare these results with two additional serovars of App. Also, we can now get an impression detached from our chosen infection model by analyzing pigs infected with Mycoplasma hyopneumoniae and thereby ensure that our genetic markers are in fact lung specific and not App specific markers.

\section{References}

(1) Hoeltig, D et al. (2009): A novel Respiratory Health Score supports a role of acute lunge damage and pig breed in the course of an Actinobacillus pleuropneumoniae infection. BMC Vet Res, 5: 14

(2) Stefan K. et al. (2004): Versatile and open software for comparing large genomes. Genome Biology, 5:R12 


\title{
P.351
}

\section{Herd based computerized analysis for swine respiratory diseases using ELISA}

\author{
Kim Woo-Chang ${ }^{1}$ Joo Hoo-Don ${ }^{1}$ Kim Sung-Hee ${ }^{2}$ Lee Kyung-Kee ${ }^{2}$ Kim Baek-Sop ${ }^{3}$ Pak Sonil ${ }^{4}$ Park Choi-Kyu $^{2}$ \\ 1. Biotechnology Laboratory, JENO Biotech Inc., Chuncheon, Korea; 2. National Veterinary Research Institute (NVRQS), Anyang, Korea; \\ 3. Hallym University, Chuncheon, Korea; 4. Vet College, Kangwon National University, Chuncheon, Korea
}

\section{Introduction}

The chronic respiratory diseases cause serious economic loss in the pig farms for a long time. Especially, PRRSV, PCV2, MH (Mycoplasma hyopneumoniae) were main causative pathogens in respiratory diseases. Moreover, PMA (Pasturella multocida), APP (Actinobacilus pleuropneumoniae), HP (Hemophillus parasuis) were also complex infective agents in chronic respiratory diseases in pig farms. It was difficult to diagnose infection or immune situation of herds. Most of diagnosis results were supplied as positive rates, individual antibody titers. These kinds of data mostly not useful in herd control for respiratory diseases. We need herd based overall statistical data to get information for profiling of farm including immune status and infection ages.

\section{Materials and Methods}

Antibody tests: The several commercial ELISA kits were used for testing antibody level of PRRSV, PCV2, MH, APP, PMA and HP (JENO Biotech Inc. Korea). For PRRSV, IDEXX 2XR was also used for testing.

Serum samples: 46 serum samples per herd according to age groups were collected from 200 or more farms in Korea. The age groups were divided with 7 groups by gilt, sow, suckling (20 days), weaner (40 days), early grower (70 days), late grower (100 days) and finisher (130 days). Serum samples were collected from 4, 10, $12,5,5,5$ and 5 heads by each age group respectively.

Statistical analysis: The several data including positive rate, mean SP value and CV\% (Percentage Coefficient variation) were analyzed by age group of each herd. Mean SP value and CV\% were used to determine the antibody level and variation of pig herds. We determine the sero-conversion age group by comparing SP value and $\mathrm{CV} \%$ of each age group.

Software: Widows XP based standalone software was designed and used to analyze the ELISA results. It gets OD values from each ELISA and then transforms the data to report for herds.

\section{Results}

Total about 200 farms or above were tested and analyzed. Most of farm showed dramatic sero-conversion in specified age groups. Generally, high-level maternal antibody was detected in sucking group and drop down the level on weaner or early grower group. Then it showed increasing antibody again on early or late grower group regardless vaccinated or not.
We can determine simply sero-converted age, mean antibody level and variation as figure 1. Most pathogens were positive by PCR or RT-PCR on before 2-3 weeks of sero-converted age (Data not shown). It means sero-converted age determined by mean $\mathrm{SP}$ is important parameter in herd. Most infected herds showed higher SP value and CV\% than maternal antibody and increased continuously after sero-conversion. It means cyclic infection and unstable immunity of herds was suspected. From the report based on seroconversion, mean SP value and CV\% of each age group, disease and immunity information could be supplied to field veterinarian. It was consider as very effective to control respiratory diseases.

Fig.1. The reporting data example by PCV2 ELISA results by age group. (line: mean SP value, bar: CV\%)

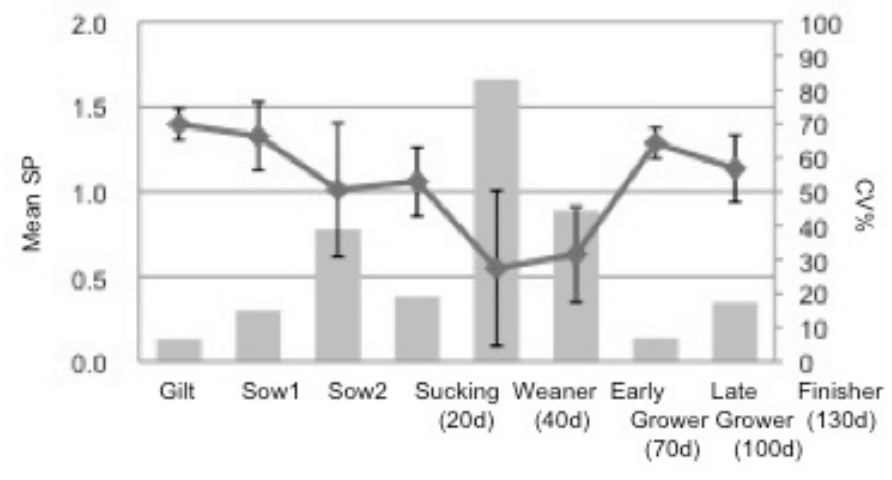

\section{Discussion}

The respiratory diseases have many differences from the other pig diseases. We didn't have ideal tool to evaluate immune and infection status. This novel model of computerized statistical approach can be applied to control swine respiratory diseases.

\section{References}

1. Park Choi-kyu. et al. Survey on vaccination management of commercial pig farms in Korea. Kor J Vet Pub Health. 31(3) 2007. 257258.

2. Park, Choi-Kyu, et al. Infection patterns of porcine reproductive and respiratory syndrome virus by serological analysis on a farm level. Kor J Vet Sci. 2008. 67-73. 


\title{
P.352
}

\section{Identification of Mycoplasma flocculare by Multiplex PCR}

\author{
$\underline{B o g d a n}$ Faur $^{1}$ Virgilia Popa $^{2}$ Viorel Herman ${ }^{1}$ Corina Pascu ${ }^{1}$ Luminita Costinar $^{1}$ loana Vaduva ${ }^{1}$ \\ 1. Faculty of Veterinary Medicine Timis, oara, Timis, oara, Romania; 2. National Society Pasteur Institut, Bucuresti, Romania
}

\section{Introduction}

Enzootic pneumonia (EP) is a chronic respiratory disease that causes considerable economic losses in the pig industry worldwide. Mycoplasma hyopneumoniae (M.hyopneumoniae) is the aetiological agent of enzootic pneumonia in pigs (Assuncao and all).

Mycoplasma flocculare (M.flocculare) was, and is still consider by majority of researchers a lack of patogenicity species. Anyway, some researchers (Armstrong and Friis) isolated this Mycoplasma species from lungs with characteristic lesions, which could sustain the hypothesis in which M.flocculare has a patogenic potential, generate symptoms and gross lesions like M.hyopneumoniae.

\section{Materials and Methods}

In September-October 2009 period, in more pig farms from the west side of Romania were reported serious respiratory disorders, a low rate of average daily weight gain and low feed conversion efficiency. Breeding farms were vaccinated against M.hyopneumoniae. The serological tests revealed that farms with respiratory problems were positive at PRRS virus.

There were investigated a number of lungs with lesions. From younger pings (10-12 week-old of age) from 8 lungs were made cultivations on PPLO broth and agar supplemented with horse serum. All cultures were incubated for $3-4$ weeks at $37^{\circ} \mathrm{C}$. We can isolate only a strain which was subcultures in 3 tubes notes $A, B$, and $C$. Tube A contain the strain after 5 days of incubation, tube $B$ contain the strain after 7 days of incubation, and tube $C$ contain the strain incubated 7 days in PPLO broth supplemented with horse serum $5 \%$.

This strain was identified by Multiplex PCR in Laboratory of Molecular Biology at Pasteur Institute Bucharest. DNA extraction was made with QiAamp DNA mini kit (Qiagen,).

Amplification was performed using illustraTMPuReTaqTM ReadyTo-GoTM PCR Beads (GE Healthcare 27-9557-01 UK).

\section{Results}

The isolated strain was identified as Mycoplasma spp. based on cultural and morphologic aspects.

At Multiplex PCR result for genomic detection/ M.hyopneumoniae, $M$. hyorhinis and/or M.floculare typing was positive for M.flocculare and negative for the other two Mycoplasma species.

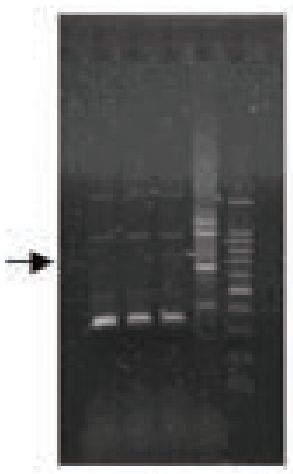

Gel-electrophoresis postPCR: line 1: tube $\mathrm{A}$, line 2: tube $\mathrm{B}$, line 3 : tube C, line 4: multiplex positive martor - M.hyorhinis ATCC-27717 with amplicon by $1129 \mathrm{pb}$, Mflocculare ATCC-27716 with amplicon by 754 $\mathrm{pb}$ and. Mhyopneumoniae with amplicon by $648 \mathrm{pb}$, line 5. DNA standard: $100 \mathrm{pb}$ DNA Ladder (Promega).

All samples were generated unspecific amplicons for M.hyopneumoniae. It is recorded the amplicon by 754 pb specific for M.flocculare only in one tube, in which was PPLO broth supplemented with horse serum. For the other two we consider that in these tubes was insufficient DNA for testing.

\section{Conclusions}

Besides all unspecific amplicons for M.hyopneumoniae for all three samples, $754 \mathrm{pb}$ specific amplicon for M.flocculare was record only in a sample, cultivated in PPLO broth supplemented with horse serum $5 \%$.

Isolation of M.flocculare from specific enzootic pneumonia lesions could sustain the hypothesis of patogenicity of this species, but more searching is demanding.

\section{References}

1. Assuncao, P., De La Fe, C., Kokotovic, B., Gonzalez, O., Poveda, J.B., 2005. The Occurrence of Mycoplasmas in the Lungs of Swine in Gran Canaria (Spain). Veterinary Research Communications, 29, 453-462.

2. Stakenborg, T., Vicca, J., Butaye, P., Imberechts, H., Peeters, J., De Kruif, A., Haesebrouck, Maes, D., 2006. A Multiplex PCR to Identify Porcine Mycoplasmas Present in Broth Cultures. Veterinary Research Communications, 30, 239-247.

3. Holko, I., Urbanova, J, Holkova, T., Kmet, V., 2004. Diagnostics of main bacterial agents of porcine respiratory diseases complex (PRDC) using PCR detection of Mycoplasma hyopneumoniae. Vet. Med. Czech, 49, 35-41.

4. Armstrong, C.H., Friis, N.F., 1981. Isolation of Mycoplasma flocculare from swine in the United States. American Journal of Veterinary Research, 42(6), 1030-2. 


\title{
P.353
}

\section{Visual-only evaluation of lung lesions as an alternative to palpation at necropsy}

\author{
$\underline{\text { Angela Baysinger }}^{1}$ Dale Polson ${ }^{1}$ Reid Philips ${ }^{1}$ Micah Taylor $^{2}$ Greg Hartsook ${ }^{1}$ Kelly Lechtenberg ${ }^{3}$ \\ 1. Boehringer Ingelheim Vetmedica, Inc., St Joseph, MO, USA; 2. liowa State University, Ames, IA, USA; \\ 3. Midwest Veterinary Services, Oakland, NE, USA
}

\section{Introduction}

As swine practitioners become increasingly involved in on-farm research trials, increase their practice geographic areas, and work around stringent biosecurity requirements, their ability to get to farms to perform hands-on necropsies becomes more difficult. We evaluated a potential alternative to hands-on evaluation of lung lesions in order to address these challenges. The objective of this study was to evaluate the inter-observer and inter-method reliability of visually scoring lungs for Mycoplasma hyopneumoniae lesions from digital images and from visual scoring of actual lungs with the benefit of palpation at necropsy.

\section{Materials and Methods}

This study evaluated lungs from 344 pigs in a M. hyopneumoniae challenge study. ${ }^{1}$ The lungs were from pigs in five treatment groups; one strict control, one non-vaccinated and challenged, and three $M$. hyopneumoniae vaccinated and challenged groups. The pigs were euthanized and necropsied 28 days post $M$. hyopneumoniae intra-tracheal challenge. Removed lungs were observed and palpated by two veterinarians who independently scored each lobe as a percentage of the area affected by M. hyopneumoniae (necropsy evaluation). M. hyopneumoniae-induced lesions were defined as dark-red-to-purple consolidated areas. Observations and digital images for each lung were completed within 15 minutes of being removed from the pig. The digital images were later presented to the same observers, who visually evaluated and scored the lungs as described above, without the benefit of palpation (image evaluation). Lung scores were weighted by the approximate volume each lobe contributes to total lung volume to derive a value for total percent lung affected, as previously described. ${ }^{2}$ A linear regression model was used to determine the correlation of necropsy versus image evaluation as well as inter-observer reliability for both methods.

\section{Results}

The mean score across both observers for image evaluation was $8.21 \%$, while the mean score for necropsy evaluation was $6.21 \%$. Means for image evaluation were $10.32 \%$ and $5.89 \%$ and for necropsy evaluation were $7.64 \%$ and $4.77 \%$ for observer 1 and 2, respectively. The linear regression showed a good fit of the data for all three parameters, including image versus necropsy (adjusted $R 2=0.76, p<0.001$ ), image between observers (adjusted $\mathrm{R} 2=0.67, \mathrm{p}<0.001$ ), and necropsy between observers (adjusted $\mathrm{R} 2=0.84, \mathrm{p}<0.001)$. The correlation coefficients were $0.873,0.819$, and 0.921 , respectively, suggesting very good correlation.

\section{Discussion}

There was a high correlation between the image and necropsy evaluation and between the observers for both methods. There did appear to be a better correlation between observers when scoring at necropsy with the benefit of palpation, suggesting that palpation remains a more consistent method of evaluating lung lesions. However, image evaluation appears to be a potential alternative to necropsy evaluation. Practitioners that are unable to necropsy pigs because of biosecurity or time restrictions now have another tool to evaluate the respiratory health of their client's pigs. Practitioners may also utilize this tool to send images to experienced lung scorers to assist in their onfarm research trials. Overall, visual evaluation with the benefit of palpation is still the preferred method.

\section{References}

1. Baysinger, A., Polson, D., Edler, R., Phillips, R., Diaz, E. 2008. Comparison of 1 and 2 dose vaccine regimes using three currently licensed Mycoplasma hyopneumoniae vaccines in the pig model. Proc Allen D. Leman Conference Suppl:25.

2. PigMON(R) slaughter inspection procedures manual. USDA National Animal Health Monitoring System and the University of Minnesota Swine Group. June 1992. 


\title{
P.354
}

\section{Assessment of four sampling methods to detect Mycoplasma hyopneumoniae in live pigs}

\author{
Christelle Fablet; Corinne Marois; Virginie Dorenlor; Florent Eono; Eric Eveno; \\ Typhaine Poezevara; Marylène Kobisch; François Madec; Nicolas Rose \\ AFSSA-Site de Ploufragan, Ploufragan, France
}

\section{Introduction}

Mycoplasma hyopneumoniae (Mhp) is the primary aetiological agent of enzootic pneumonia in pigs and in association with bacteria and viruses is also involved in the Porcine Respiratory Disease Complex (PRDC) (1). The availability of accurate, rapid and easy to perform diagnostic tools is necessary for epidemiological and control purposes. For practical reasons, under field conditions, the nasal cavities are the most frequently chosen sampling sites to assess Mhp contamination of live pigs. However, in experimental trials, tracheal and tracheo-bronchial washings are the most efficient samples for detecting $\operatorname{Mhp}(2 ; 3)$. The aim of the study was to assess the abilities of four sampling methods to detect Mhp by nested-PCR on live pigs in a field context.

\section{Materials and Methods}

The study was carried out on a herd chronically affected by respiratory disorders. A sample of 60 pigs was constituted by a random selection from a batch of finishing pigs. Each pig was restrained and submitted to 4 samplings: oral-pharyngeal brushing, tracheo-bronchial swabbing, tracheo-bronchial washing and nasal swabbing. Mhp DNA was identified by modified nestedPCR (4). The sensitivity and specificity of each sampling method were estimated using a Bayesian analysis framework (5). Parameters distributions were based on previous external data. Since samples taken from SPF pigs gave negative results, a deterministic constraint was used for the specificities of all sampling methods which was taken as equal to one. The sensitivity of the parameter estimation to the choice of priors was assessed by comparing 3 models incorporating different sets of prior distributions ranging from vague priors (M1) to more informative ones (M3). Model convergence was assessed using the Raftery and Lewis tests and the Gelman-Rubin diagnosis. The models were compared on the basis of the deviance information criterion, the number of parameters estimated in the model and of the Bayesian p-value. The models were run using the freeware program WinBUGS (6).

\section{Results}

Mhp was detected by nasal swabbing, oral-pharyngeal brushes, tracheo-bronchial washing and tracheo-bronchial swabbing in $13.3 \%, 40.0 \%, 53.3 \%$ and $60.0 \%$ of the pigs respectively. Whatever the model, nasal swabbing had the lowest sensitivity and tracheo-bronchial swabbing the highest with mean sensitivities of $19 \%$ and $74 \%$, respectively (Table 1 ).

\section{Discussion}

Since the infection status of the pigs tested under these conditions was unknown, and no gold standard is available, the sensitivities of the sampling methods were analyzed using a Bayesian approach. To the best of our knowledge, this is the first field study to use such an approach to evaluate four sampling methods for assessing Mhp infection in live pigs. The results of the present study indicate that tracheo-bronchial swabbing and washing, in combination with nested-PCR assay, were the most sensitive sampling methods for Mhp detection in naturally infected live pigs, the sensitivity of the tracheo-bronchial swabbing being slightly higher. As far as practical aspects are concerned, swabbing the tracheo-bronchial area with a sterile catheter is almost as convenient as obtaining nasal swabs under field conditions and only requires adding a gag to the sampling equipment. Tracheo-bronchial swabbing ensures a gain in diagnostic accuracy, being 3.5 times more sensitive than the nasal swabs commonly used in pig farms.

\section{References}

1. Sibila et al. (2009). Vet. J. 181, 221-231.

2. Kurth et al. (2002). J. Vet. Diagn. Invest. 14, 463-469.

3. Marois et al. (2007). Vet. Microbiol. 120, 96-104.

4. Calsamiglia et al. (1999). J. Vet. Diagn. Invest. 11, 246-251.

5. Berkvens et al. (2006). Epidemiology 17, 145-153.

6. Spiegelhalter et al. (1996). Medical Research Council Biostatistics Unit, Cambridge, UK.

Table 1: Mean and $95 \%$ Credibility Interval of posterior distributions of the sensitivity of the four sampling methods of Mhp detection by nested$P C R$, according to the 3 models with different prior distributions (60 pigs sampled, specificity $=1$ for all models and sampling methods)

\begin{tabular}{lcccc}
\hline Model* & $\begin{array}{c}\text { Nasal } \\
\text { Swabbing }\end{array}$ & $\begin{array}{c}\text { Oro-pharyngeal } \\
\text { Brushing }\end{array}$ & $\begin{array}{c}\text { Tracheo-bronchial } \\
\text { Washing }\end{array}$ & $\begin{array}{c}\text { Tracheo-bronchial } \\
\text { Swabbing }\end{array}$ \\
\hline M1 & 0.19 & 0.51 & 0.66 & 0.72 \\
M2 & $(0.09-0.31)$ & $(0.35-0.67)$ & $(0.49-0.81)$ & $(0.55-0.86)$ \\
& 0.19 & 0.53 & 0.68 & 0.74 \\
M3 & $(0.09-0.32)$ & $(0.38-0.68)$ & $(0.53-0.82)$ & $(0.59-0.86)$ \\
& 0.25 & 0.53 & 0.62 & 0.73 \\
& $(0.14-0.37)$ & $(0.39-0.65)$ & $(0.51-0.73)$ & $(0.59-0.84)$ \\
\hline
\end{tabular}

$* \mathrm{M} 1$ : vague priors on sensitivities, specificity of the 4 sampling methods $=1$, M2: mildly informative priors on the prevalence, specificity of the 4 sampling methods-1, M3: mildly informative priors on the prevalence and parameters to estimate, specificity of the 4 sampling methods=1 


\title{
P.355
}

\section{Surveillance using oral fluid samples - PRRSV, SIV, PCV2, and TTV field data}

\author{
$\underline{\text { Apisit Kittawornrat }}{ }^{1}$ John R. Prickett ${ }^{1}$ Rodger Main ${ }^{1}$ John Johnson ${ }^{1}$ Roman Pogranichniy ${ }^{2}$ Amber Clark ${ }^{2}$ Jeffrey J. Zimmerman ${ }^{1}$ \\ 1. lowa State University, Ames, IA, USA; 2. Purdue University, West Lafayette, IN, USA
}

\section{Introduction}

Oral fluid specimens have been used extensively in human diagnostic medicine, but only recently has the concept been investigated in production animals (Prickett and Zimmerman, 2010). The objective of this research was to evaluate the use of oral fluid samples for monitoring PRRSV, SIV, PCV2, and TTV in commercial pig populations.

\section{Materials and Methods}

Experimental design: Oral fluid samples were collected from one wean-to-finish barn on each of 10 sites across the Midwest, USA. At each site (i.e., barn), oral fluid samples were collected from the same 6 pens at 2-week intervals from placement ( 3 weeks of age) to slaughter. Oral fluid samples were collected by farm personnel after a brief training session with the investigators. Thereafter, samples were assayed for PRRSV, SIV, PCV2, and TTV by PCR-based assays. Sample Collection: Standard operating procedures were developed to ensure the collection of uniform, clean, and clearly identified samples. Investigators demonstrated the first two collections and trained site personnel in the procedure. Once trained, site managers were responsible for the timely collection and submission of samples. Sample collection "kits" containing all of the required materials were assembled and delivered to each site prior to the commencement of the study. Each kit included a pre-cut length of cotton rope, scissors, poly-boots, screw cap tubes, permanent markers, diagnostic submission forms, ice packs, zip-lock bags, and postage-paid addressed shipping containers. Upon arrival to the lowa State University Veterinary Diagnostic Laboratory, oral fluid samples were centrifuged at $9000 \times \mathrm{g}$ for $10 \mathrm{~min}$. Prior to testing, all samples were completely randomized and re-labeled.

\section{Results}

Data indicated that oral fluid samples could be used to monitor the circulation of the targeted pathogens (Figure 1). Temporal patterns of pathogen detection suggests that oral fluid could be used to routinely suveil for pathogens of interest in swine populations.

\section{Discussion}

Oral fluid sampling offers a cost effective solution to infectious disease surveillance in populations of growing swine and offers a new tool for studying the disease causality, ecology, and economics of emerging (TTV) and endemic (SIV, PRRSV, PCV2) viruses in commercial swine populations.

\section{References}

Prickett JR, Zimmerman JJ. 2010. The development of oral fluid-based diagnostics and applications in veterinary medicine. Animal Health Research Reviews (in press)

\section{Acknowledgements}

This project was funded in part through Pork Checkoff funds distributed through the National Pork Board (Des Moines, lowa), the PRRS CAP, USDA-NIFA Award 2008-55620-19132, and Murphy-Brown, LLC, and Pfizer Animal Health.

Figure 1. Cumulative $P C R$ results for the 10 studied populations by pathogen and sampling point.

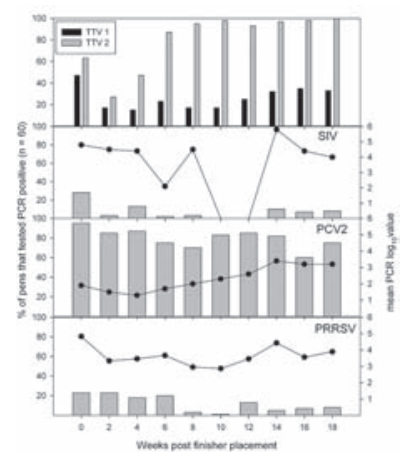




\title{
P.356
}

\section{Computertomographic investigation on porcine pleuropneumonia}

\author{
Carsten Brauer ${ }^{1}$ Doris Hoeltig ${ }^{1}$ Gerald F. Gerlach ${ }^{2}$ Karl-Heinz Waldmann ${ }^{1}$ \\ 1. Clinic for Swine and Small Ruminants, Forensic Medicine and Ambulatory Service; University of Veterinary Medicine Hanover, \\ Foundation, Hannover, Germany; 2. IVD GmbH, Hannover, Germany
}

\section{Introduction}

The aim of this study was to investigate the correlation between computertomographic (CT) and digital x-ray diagnosis, clinical as well as pathomorphological findings during necropsy in pigs experimentally infected with Actinobacillus (A.) pleuropneumoniae. This investigation should lead to the development of a CT scoring system which might allow an objective interpretation of CT images of the pig lung and therefore could be used as a tool for an early stage diagnosis of lung alterations in pigs.

\section{Materials and Methods}

29 German Landrace pigs (age: 7 weeks, averaged weight: $13,7 \mathrm{~kg}$ ) coming from an A. pleuropneumoniae free herd were infected. The pigs were randomized upon weight and sex and divided into four groups. For the infection A. pleuropneumoniae serotype 7 strain AP76 and two deletion mutants of different virulence were used in an established aerosol infection model (1). One group was mok infected and used as control.

Diagnostic imaging was done twice, on days 7 and 21 post infection (p.inf.); the clinical monitoring of the pigs was performed daily. All imaging procedures were carried out under anesthesia ( $2 \mathrm{mg} / \mathrm{kg}$ Azaperon im., 20mg/kg Ketamine im.). The pigs were euthanized on day 21 p.inf., and a necropsy with pathomorphological examination of the lung was done.

For the radiographic examination 3 images were taken (2 laterolateral, 1 dorsoventral; film-focus distance: $150 \mathrm{~cm} ; 80 \mathrm{kV}$ and shutter priority [Precimat, Fa. Picker Int., München, Germany]). For the computertomographic examination a scanogram and transversal slices ( $7 \mathrm{~mm}$ thickness) of the whole thorax using a single slice scanner of the 3rd generation (Tomoscan M, Fa. Philips, Hamburg, Germany) were performed. All acquired pictures were set in lung window (exposure settings: $120 \mathrm{kV}, 40 \mathrm{~mA}$ ). For interpretation of the CT images every single slice was related to its position (precardiac, cardiac, postcardiac) in the scanogram and divided into four quadrants.

For the quantification of results of the clinical, the radiographic and the pathomorphological examination three different established scoring systems were used $(2,3)$.

\section{Results}

During evaluation of the lung alterations visible in the $\mathrm{CT}$ lung window mainly ground-glass opacities and consolidations could be found. The density of the consolidations was $>-150$ Hounsfield Units. The results of the CT examination are shown in Table 1. The results of the different examination methods used as established controls are shown in Table 2.
Table 1: Results of the computertomographic analysis.

\begin{tabular}{|l|c|c|c|c|}
\hline & \multicolumn{4}{|c|}{$\begin{array}{c}\text { uumber of animals with affected parts of the lung } \\
\text { (precardiac, cardiac, postcardiac); } \mathrm{n}=22\end{array}$} \\
\hline & $n 0$ & 1 part & 2 parts & 3parts \\
\hline day 7 p.inf. & 4 & 5 & 7 & 6 \\
\hline day 21 p.inf.* & 5 & 4 & 7 & 4 \\
\hline *2 animals euthanized between day 7 p.inf. and day 21 p.inf. \\
\hline
\end{tabular}

Table 2: Results of the clinical, radiographic and pathomorphological examination.

\begin{tabular}{|l|c|c|c|c|}
\hline & \multicolumn{4}{|c|}{ Number of animals with pleuropneumonia; $\mathrm{n}=22$} \\
\hline Examination method & $n 0$ & mild & moderate & severe \\
\hline Clinical, day 7 p.inf. & 3 & 19 & 0 & 0 \\
\hline Radiographic, day 7 p.inf. & 4 & 11 & 5 & 2 \\
\hline Clinical, day 21 p.inf. ${ }^{*}$ & 15 & 5 & 0 & 0 \\
\hline Radiographic, day 21 p.inf. ${ }^{*}$ & 3 & 13 & 4 & 0 \\
\hline Necropsy, day 21 p.inf. & 6 & 6 & 6 & 4 \\
\hline${ }^{*}$ 2 animals euthanized between day 7 p.inf. and day 21 p.inf. \\
\hline
\end{tabular}

\section{Discussion}

Regarding the comparison of the computertomographic examination of the lung and the already established examination methods, the CT imaging is considered as a diagnostic tool of similar informative value. Therefore the development of a CT scoring system appears feasible and is the next step to be done. After finishing this development a second statistically approved assessment is necessary before application of the scoring system as a diagnostic tool.

\section{References}

1. Maas, et al., 2006. Vaccine, 24: 7226-7237

2. Hoeltig et al., 2009. BMC Veterinary Research 5:14.

3. Hannan et al., 1982. Res.Vet. Sci. 33, 76-88. 


\title{
P.357
}

\section{Medical infrared imaging of the porcine thorax for diagnosis of lung pathologies}

\author{
Carsten Siewert; Doris Hoeltig; Carsten Brauer; Hermann Seifert; Isabel Hennig-Pauka \\ University of Veterenary Medicine, Hannover, Germany
}

\section{Introduction}

Respiratory diseases in pigs affecting pork production worlwide have an impact on consumer protection, live stock trade and animal welfare. The early, non-invasive detection of lung disorders is a valuable tool for the maintanance of respiratory health in pig herds. A promising approach might be the quantification of the skin surface temperature of the thoracic cavity, which is mainly determined by the heat exchange between skin and environment, metabolic activity, blood circulation and anatomical structures beneath the skin. Zapoudrina, et al., (2008) demonstrated as part of a human medical study the good reproducibility of temperature distribution patterns in the central body area of 16 test subjects [1].

\section{Materials and Methods}

Thoracic imaging and examination of 17 pigs (German Landrace, age: 11 weeks) was performed before and after aerosol infection with Actinobacillus pleuropneumoniae (A.pp.) by passive infrared thermography for the recording of infrared (IR) images. After necropsy pathomorphological changes in the lung were evaluated according to a lung lesion score [2].

An infrared thermographic camera operating in the wavelength range from 8 to $12 \mu \mathrm{m}$ was used (IR Flex Cam R2). The IR camera used a microbolometer array as the detector. The IR image matrix of the camera was $160 \times 120$ pixels, which are interpolated internally to $320 \times 240$ pixels. The thermal sensitivity is, according to the manufacturer's specifications, $70 \mathrm{mK}$ with reference to a temperature of the measured object of $30^{\circ} \mathrm{C}$. For the pigs' skin an emissivity of 0.95 was assumed. Air temperature and humidity as well as body core temperature as well as heart and breathing frequencies were recorded.

Medical imaging (IR and X-ray)

a) The recorded thoracic IR images were evaluated by means of the image viewer software IRBIS3plus, (colour temperature coding: combined rainbow colour and black/white-scale, narrow temperature range over $\left.13^{\circ} \mathrm{C}\right)$.

b) Computed tomography (CT) images: CT images were scanned using the system Tomoscan M/EG Compact (Philips Medical Systems)- a single slice scanner of 3rd generation. Scans were performed at $7 \mathrm{~mm}$ slice thickness. All acquired pictures are set in lung window. Exposure settings: 120 kV, 40 $\mathrm{mA}$.

\section{Results}

IR images were suitable to visualize the location of the diaphragm (Fig. 1a,c yellow line) and abnormal warm regions of the thorax which correlate to inflammatory alterations shown in CT images.

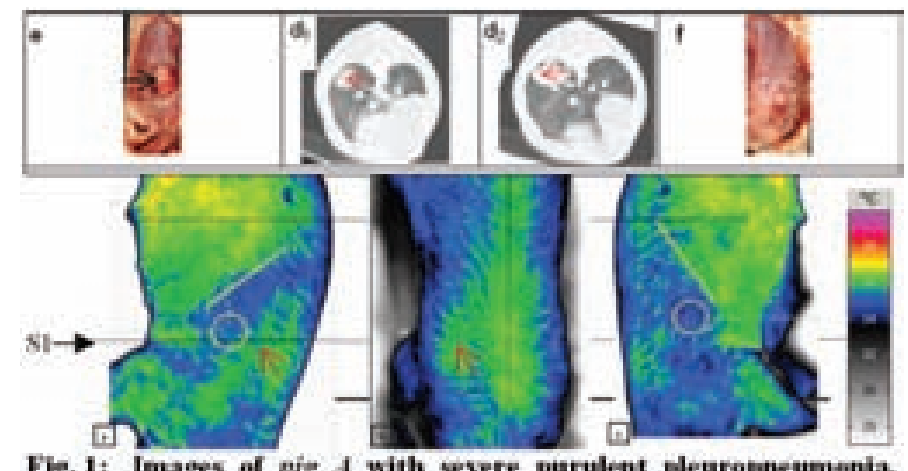

Fie 1: Images of pig $A$ with severe purulent pleuropmeumenia. a,bs: IR-images, $T_{A}=17 \mathrm{C}$, pulse. $79 \mathrm{~mm}$, bending frequency. $36 \mathrm{~min}, d_{13}:$ CT-imase slices coufirmung poeumonat arcis in the nght candal pulmoary lobe, es: Necropsy finilings Anows indicate bue Isaions cansed by $A$. phemopuremontrav. Yellow circles indicale iegona of normal ventilition less warm than altered regons.

\section{Discussion}

The anatomical localization of inflammatory processes leading to reduced ventilation in the outer lung regions can be determined under certain conditions (IR-image Fig. 1a). Pathological findings (Fig. 1e) are visualized in the corresponding CT-images (Fig. 1d). The opposite region of the thorax is less warm (Fig. 1c, yellow circle) due to normal ventilation in the lung. The cool air breathed-in probably acts as a contrast agent, thus distinct anatomical structures become visible (diaphragm, Fig. 1a,c, yellow line). It can be assumed, that pathological changes in the peripherical (outer) lung regions characterized by reduced ventilation can be recognised non-invasively using passive IR thermography. A new generation of IR thermography and image processing might be a practical tool for the diagnostic of many cases of pneumonia in pigs.

\section{References}

[1] Zapoudrina N, Varmavuo V, et al. (2008) Reproducibility of infrared thermography measurements in healthy individuals, Physiol. Meas. $29515-524$

[2] Hannon PC, Bhogal BS, Fish JP: (1982) Tylosin tartrate and tiamultilin effects on experimental piglet pneumonia induced with pig lung homogenate containing mycoplasmas, bacteria and viruses, Res. Vet. Sci. 33: 76-88 


\title{
P.358
}

\section{Evaluation of DRAXXIN ${ }^{\circledR}$ Injectable Solution for the control of swine respiratory disease}

\author{
Michael Senn; Robert Nutsch; Merlyn Lucas \\ Pfizer Animal Health, New York, NY, USA
}

\begin{abstract}
Introduction
Swine respiratory disease (SRD) remains an important cause of disease and economic loss for swine producers worldwide. DRAXXIN ${ }^{\circledR}$ Injectable Solution (10\% tulathromycin, Pfizer Animal Health) is one of the newest anti-infectives for treating SRD. It is rapidly released and absorbed from the injection site and extensively distributed, so the product acts quickly against respiratory disease, and provides an extended clinical duration of activity in pigs of at least nine days after a single dose1. The clinical efficacy of DRAXXIN ${ }^{\circledast}$ as a single IM injection for the control of naturally occurring swine respiratory disease was evaluated in a multilocation study.
\end{abstract}

\section{Materials and Methods}

The study was conducted at 5 sites in North America 2 The animals at all locations were mixed sex commercial feeder pigs purchased from farms that had previously experienced swine respiratory disease. Pigs weighed between 9.3 and 44.8 kilograms at enrollment. All pigs were individually identified. (SRD) was defined as a pig that had a respiratory score of $>2$ , and a depression score of $>2$ (both a scale of 0 to 3 , where 0 is normal), and a rectal temperature of $>104^{\circ} \mathrm{F}$. On study day 0 , the day that a pen had at least $15 \%$ of the pigs meeting the case definition for SRD, all individual pigs in the pen were randomly enrolled in one of two treatment groups. Treatment 1 (T1), Saline, intramuscular, administered once, or Treatment 2 (T2) DRAXXIN ${ }^{\circledR}$ Injectable Solution (tulathromycin), $2.5 \mathrm{mg} / \mathrm{kg}$, intramuscular, administered once. Each study location enrolled 2 pens with 30 to 40 pigs per pen. No concomitant medication of enrolled animals with systemic antimicrobial or anti-inflammatory agents was permitted during the study. Animals that exhibited clinical signs of disease other than SRD were removed from the study. Treatment groups were commingled within each pen, with the individual pig being the experimental unit. General health observations were performed twice a day on enrolled animals from Study Day 0 through Study Day 6, and the morning of Study Day 7. All study animals remaining on Study Day 7 were clinically evaluated for respiratory scores, depression scores, and rectal temperatures. On Study Day 7, all remaining enrolled animals were euthanized and necropsied to aid in characterizing the swine respiratory disease. An animal was classified as a treatment success if on Study Day 7; it was alive, had both a respiratory score and a depression score $<1$, and a rectal temperature of $<104^{\circ} \mathrm{F}$. Failure to meet any one of these criteria classified the animal as a treatment failure. Secondary clinical endpoints assessed were mortality and lung lesion scores.

This protocol was reviewed by the Pfizer Animal Health Ethical Review Board and the Pfizer Animal Health IACUC committee and is in compliance with their recommendations.

\section{Results}

Four hundred fifty five (455) animals were enrolled in the study; 228 in T1 (Saline) and 227 in T2 (DRAXXIN ${ }^{\circledR}$ ). The number of animals classified as a treatment success was significantly higher, $p<0.05$, for the animals administered DRAXXIN ${ }^{\circledR}(59.2 \%)$ vs animals administered saline (41 2\%). The number of mortalities found dead plus euthanized due to SRD was higher for the saline-treated animals $(11 / 227,4.8 \%)$ than the tulathromycintreated animals $(4 / 226,1.8 \%)$, but not significantly different, $p$ $=0.0676$. The back-transformed least squares mean percentage of total lungs with lesions was significantly higher, $p=0.0096$, for animals administered saline, $(13.1 \%)$ vs animals administered DRAXXIN $^{\circledR},(6.6 \%)$.

\section{Discussion}

The results confirm the value of using DRAXXIN ${ }^{\circledR}$ for the control of swine respiratory disease associated with Actinobacillus pleuropneumoniae, Pasteurella multocida and Mycoplasma hyopneumoniae. Treating groups when a percentage of the population is exhibiting signs of swine respiratory disease will help veterinarians to better manage respiratory disease outbreaks. Veterinarians often rely on water medi $\neg$ cation for its ease of administration.3 Problems associated with water medication include variability in flow rates and inadequate consumption by ill pigs. 3 Variation in drinker flow rates and problems associated with dilution and delivery of antimicrobials create an important challenge for farm personnel. 4 DRAXXIN ${ }^{\circledR}$ provides high and long-lasting concentrations in the lung tissues providing for a complete treatment of individual pigs exhibiting swine respiratory disease, and excellent efficacy treating and controlling swine respiratory disease, ensuring instant compliance and fast long-lasting therapy, while reducing the need for extra labor and less handling of sick animals.

\section{References}

1. Waag, TA, Bradford, JR, Lucas, MJ, et al. J Swine Health Prod 2008; 16(3):126-130.

2. Data on file, Study Report. No. 113C-60-07-279, Pfizer Inc.

3. Nemechek, M.S., Dorr, P., Scheidt, A., Gebreyes, W., Baynes, R., Almond, G. 2008. American Association of Swine Veterinarians Proceedings: 55-56.

4. Dorr, P.M., Nemechek, M.S., Scheidt, A.B., Baynes, R.E., Gebreyes, W.A., Almond, G.W. JAVMA, Vol 235, No. 3, August 1, 2009. 


\title{
P.359
}

\section{Health and performance improvements in pigs treated with Draxxin ${ }^{\circledR}$ Injectable Solution (tulathromycin) for swine respiratory disease (SRD)}

\author{
John C. Johnson; Thayer C. Hoover \\ Outcomes Research Pfizer Animal Health, Spirit Lake, IA, USA
}

\begin{abstract}
Introduction
Draxxin ${ }^{\circledR}$ Injectable Solution is approved in the USA for singledose therapy of swine respiratory disease (SRD) caused by Actinobacillus pleuropneumoniae, Pasteurella multocida, Bordetella bronchiseptica, Haemophilus parasuis, and Mycoplasma hyopneumoniae, and for the control of SRD caused by A. pleuropneumoniae, P. multocida and M. hyopneumoniae. Two studies were conducted, with the first in a natural outbreak of SRD, comparing Draxxin to untreated pigs (saline), and pigs medicated using conventional therapy (penicillin G procaine). Variables included treatment response and weight gain. The second study compared Draxxin to untreated controls against induced SRD using a live M. hyopneumoniae challenge (MHYO). This study tracked performance (gain, feed efficiency) as well as clinical response. Study protocols were reviewed by the Pfizer Animal Health Ethical Review Board, Kalamazoo MI to ensure that animal use was consistent with sound scientific practices and ethical considerations.
\end{abstract}

\section{Materials and Methods}

Study 1.1 One hundred seventy pigs (170) from two farms positive for various bacterial and viral respiratory pathogens were commingled with 200 single-source Isowean pigs of similar age and weight. Pigs $(\mathrm{N}=370)$ were housed in a conventional finisher equipped with an AutoSort food court providing feed and electronically capturing individual pig weights. Pigs with SRD ( $\mathrm{n}$ $=135 ; 36 \%$ morbidity) were randomly assigned in a 1:2:2 ratio to one of three treatments: saline (negative control), penicillin $\mathrm{G}$ procaine (IM; $5 \mathrm{~mL} / 45 \mathrm{~kg} /$ day for two [2] consecutive days), or Draxxin (IM; $1 \mathrm{~mL} / 41$ kg, once). Pigs were observed daily, and clinically re-evaluated and weighed seven and 56-days after treatment.

Study 2. 2 Two hundred (200) weaned pigs, sero-negative for MHYO were challenged intra-tracheally once daily for three consecutive days with a live culture of the organism. Ninety-six (96) pigs meeting SRD-criteria were enrolled and assigned to 24 pens according to a randomized block design. Each pen (the experimental unit) contained pigs treated with saline or Draxxin. Pig weights and feed consumption were measured at 10,20,30, 45, and 60 days post-treatment; response to therapy was evaluated 10-days post-treatment.

\section{Results}

Study 1. Compared to untreated pigs, Draxxin-treated pigs had a lower mortality rate the first week post-treatment and a significantly $(P=0.0360)$ lower mortality rate over the study. Further, pigs treated with Draxxin had a significantly $(P=0.0409)$ higher mean gain $(3.7 \mathrm{~kg}[8.3 \mathrm{lb}])$ compared to controls (2.8 kg [6.2 lb]) one week post-treatment. Mean weight gain in pigs treated with Draxxin was $4.5 \mathrm{~kg}$ (10 lb) over pigs treated with penicillin $(P=0.0597)$ and $7.2 \mathrm{~kg}(16 \mathrm{lb})$ over controls $(P=0.0268)$, when adjusted to the 75th percentile of mean weight at enrollment. Draxxin-treated pigs were 1.6 times less likely fail to respond to treatment than pigs treated twice with penicillin; and 1.85 times less likely to fail than pigs that were untreated. The risk of treatment failure was reduced in the Draxxin group 36\% compared to conventional therapy, and reduced $46 \%$ compared to controls. In field settings similar to this study, six pigs (6) treated with Draxxin would be projected as the number needed to treat to reduce or prevent one treatment failure using conventional therapy (penicillin $\mathrm{G}$ procaine).

Study 2. Pigs with SRD, treated with Draxxin had a significantly ( $P$ $=0.0121$ ) improved average daily gain ranging from $0.74-0.90$ $\mathrm{kg} / \mathrm{hd} /$ day $(1.63-1.99 \mathrm{lb})$ over controls ranging from $0.61-0.80$ $\mathrm{kg} / \mathrm{hd} /$ day (1.35 - $1.77 \mathrm{lb})$. Feed consumption was higher in the Draxxin group with a corresponding improvement in Feed:Gain (1.26 kg feed/1.0 kg gain [2.78 lb/1.0 lb) compared to controls (1.62 kg [3.58 lb]; P = 0.1032). Treated pigs had a lower mortality rate ( $8.7 \%$ vs. $4.2 \%)$. Evaluated at 10 -days, the proportion of Draxxin-treated pigs "normal" for attitude/depression and respiratory character was $11 \%$ and $24 \%$ higher compared to untreated cohorts, respectively.

\section{Discussion}

Pigs treated with Draxxin for SRD under field conditions, or in an experimentally induced $M$. hyopneumoniae challenge, responded with one treatment. This reduces antimicrobial use as well as animal stress and handling using multiple day therapy regimens. One-time Draxxin treatment improved both health and performance in both studies. These results support that Draxxin is an effective health management tool for reducing sickness and death loss due to SRD, a cost effective alternative to conventional treatment[s] requiring multiple day administration, and may be an important aid to improve performance and profitability in swine operations affected with respiratory disease.

\section{References}

1. Data on file, Pfizer Inc. Study 1123R-60-07-283.

2. Data on file, Pfizer Inc. Study 1121R-60-07-292. 


\title{
Evaluation of two in feed medication therapeutic programs for the control of swine respiratory disease in nursery
}

\author{
$\underline{\text { Paolo Ferro }}^{1}$ Claudio Mandelli ${ }^{2}$ \\ 1. Elanco Animal Health, div. of Eli Lilly Italia, Sesto Fiorentino (FI), Italy; 2. G.I.ma. S.p.A., Rubiera (RE), Italy
}

\section{Introduction}

Many studies showed that the initial prevalence of pathogens within the group plays a central role in the severity of the diseases and also that early interventions can reduce this prevalence(1). The objective of this study was to evaluate two different in feed medication therapeutic programs at weaning for the control of swine respiratory disease in nursery.

\section{Materials and Methods}

The study was performed on the nursery sector of a multisite farm in the north of Italy. After the confirmation of respiratory disease two groups of around 170 pigs / group were evaluated from weaning (age: 21 days) to the end of the nursery period (age: 67 days). Fifty pigs per group were individually ear tagged, numbered and weighed at weaning and at the end of the nursery period. The pigs were divided in the two groups according to the type of medication included in their prestarter feed:

- Pulmotil ${ }^{\mathrm{TM}}$ Group: Tilmicosin (Pulmotil ${ }^{\mathrm{TM}}$ ) 400 ppm,

- Control Group: Sulfadimetossin 1400 ppm + CTC 560 ppm

(Polisulfan $^{\mathrm{TM}}$ ).

The treatment period was the same (21 days after weaning) for both groups.

The parameters controlled and recorded were:

- Number of pigs / group,

- Average weight (kg) at weaning,

- Average weight (kg) at weaning of individually weighed pigs,

- Number of deaths + culls / group,

- Average weight $(\mathrm{kg})$ at the exit from the nursery sector,

- Average weight $(\mathrm{kg})$ at the exit from the nursery sector of individually weighed pigs.

Statistical analysis: means individual weights were compared between group by ANOVA one-way analysis when Bartlett's test assess the homogeneity of variances and by Kruskal-Wallis test when homogeneity of variances was not demonstrate with Intercooled Stata 7.0 software. The significant level for this evaluation was $p=0.01$.

\section{Results and discussion:}

The general collected results are summarized in table 1. At the exit from nursery the means weight of individually weighed animals was significantly higher in the Pulmotil ${ }^{\mathrm{TM}}$ group $(\mathrm{P})$ than in the Control group (C) (29.48 kg vs $25.92 \mathrm{~kg}$, Anova test F1,60 $\mathrm{p}<0.01$, Table 1 and Graphic 1$)$.
Table 1. Results.

\begin{tabular}{|l|c|c|c|}
\hline & P & C & Diff. \\
\hline Pigs Number & 175 & 164 & 11 \\
\hline Days on nursery sector & 46 & 46 & $/$ \\
\hline Average weight $(\mathrm{kg})$ at weaning & 7.47 & 7.58 & -0.11 \\
\hline $\begin{array}{l}\text { Average weight }(\mathrm{kg}) \text { at weaning of } \\
\text { individually weighed pigs }\end{array}$ & 7.63 & 7.52 & 0.11 \\
\hline $\begin{array}{l}\text { Average weight }(\mathrm{kg}) \text { at the exit from } \\
\text { nursery sector }\end{array}$ & 29.15 & 26.65 & 2.50 \\
\hline $\begin{array}{l}\text { Average weight }(\mathrm{kg}) \text { at the exit } \\
\text { from nursery sector of individually } \\
\text { weighed pigs }\end{array}$ & $29.48^{*}$ & 25.92 & 3.57 \\
\hline ADG (g/d) & 470 & 410 & 70 \\
\hline Deaths + Culls $(\mathrm{n})$ & 3 & 3 & $/$ \\
\hline${ }^{*} \mathrm{p}<0.01$ & \multicolumn{3}{|l}{} \\
\hline
\end{tabular}

Graphic 1. Weight distribution of individually weighed pigs at the end of the nursery period.

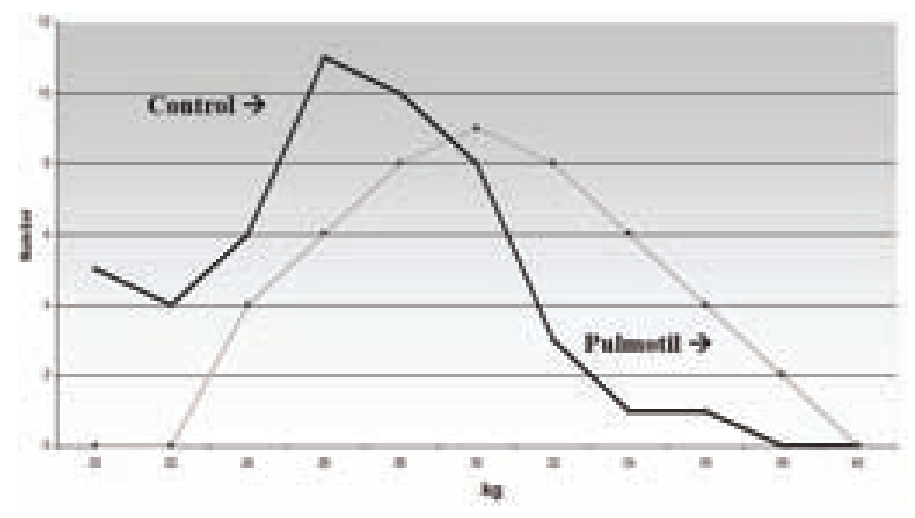

\section{Conclusions}

These results confirm that Pulmotil ${ }^{\mathrm{TM}}$ increases the animal health, with better growing performances from weaning to the end of the nursery period. As reported on other studies(2), the better weight at this age may improve the performances in the fattening and increase the number of Full Value Pigs ${ }^{\mathrm{TM}}$ that arrive at the slaughterhouse.

\section{References:}

(1) Harker J.W. et al., 2006; AASV proceedings, 127-130.

(2) Ferro P. et al., 2008; IPVS proceedings, 373. 


\title{
P.361
}

\section{Comparison of a soluble treatment with doxicicline or Pulmotil AC during a respiratory outbreak}

\author{
$\underline{\text { Xavier de Paz }}{ }^{2}$ Jose L. Lorenzo Miguel Abad² Susana Lapuente ${ }^{2}$ \\ 1. Maporc, Segovia, Spain; 2. Elanco Animal Health, Madrid, Spain
}

\section{Introduction}

Respiratory disease causes great economic losses in pig production (1) and usually involves several pathogens, causing a significant and quick increase in mortality and also affecting growth and feed efficiency of the animals (2). Soluble treatments are mostly used when an outbreak appears, especially during the fattening phase (3). The present study aimed to evaluate the effects of a soluble treatment with PulmotilAC ${ }^{\mathrm{TM}}$ during a respiratory outbreak in fattening pigs.

\section{Materials and Methods}

Two groups of pigs were included in the study $(C, n=998$ and $P$, $\mathrm{n}=876$ ). When pigs showed respiratory signs (around $100 \mathrm{~d}$ of age) and several pathogens were found (PRRSV, M.hyo and App), the pigs were treated either with Pulmotil AC at $17 \mathrm{mg} / \mathrm{kg} \mathrm{BW}$ (P) or Doxicicline at $10 \mathrm{mg} / \mathrm{kg}(\mathrm{C})$ in water for 5 days. Before the treatments started, the mortality rates were $1.0 \%$ and $1.3 \%$ for the control and the Pulmotil group respectively. During the 5 days of treatment the mortality rates were $0.6 \%$ and $0.5 \%$ for the Control and Pulmotil group. The animals were monitored until the end of the fattening period. The variables collected and analyzed were mortality, average daily gain, feed conversion rate, and percentage of lightweight pigs at the end of the period. Also, a cost analysis calculation was done with the production results.

\section{Results and Discussion}

The treatment with PulmotilAC at the time of the disease outbreak decreased the mortality from $10.6 \%$ to $4.8 \%$. Also, a difference in pigs sold as lightweights was detected (fig. 1), therefore the percentage of FVP ${ }^{\mathrm{TM}}$ was also impacted $\mathrm{C}=67.3 \% ; \mathrm{P}=88 \%$.

Figure 1. Mortality and lightweight pigs

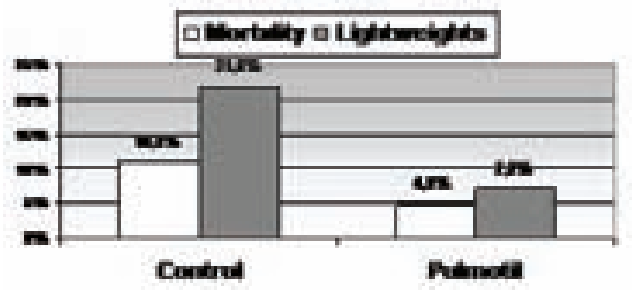

There was also a difference in average daily gain and a decrease of the FCR (table 1). This was highly affected because the weights of dead pigs ( $C=4456 \mathrm{~kg}$ vs $P=1801 \mathrm{~kg}$ ) were not used for the FCR calculation but the feed consumed was not excluded.
Table 1. Results during the fattening period.

\begin{tabular}{|l|c|c|}
\hline & Control & Pulmotil \\
\hline No Pigs & 998 & 876 \\
\hline Weight in $(\mathrm{kg})$ & 31.01 & 27.98 \\
\hline Weight out $(\mathrm{kg})$ & 88.1 & 95.7 \\
\hline Days to market & 183 & 181 \\
\hline ADG $(\mathrm{g})$ & 493 & 597 \\
\hline FCR & 3.09 & 2.52 \\
\hline
\end{tabular}

Table 2 includes the cost analysis performed; the numbers have been standardized for equal number of pigs to start. The main differences between both groups were basically: feeding costs (due to the great difference in FCR and mortality), pig cost (due to the mortality rate improvement), and fixed costs (due to the improvement in ADG). As a consequence, the economic benefit of using a soluble treatment with Pulmotil AC instead of Doxicicline during a respiratory outbreak in the growing-finishing barn was $16.03 € /$ pig sold.

Table 2. Total costs of production

\begin{tabular}{|l|c|c|}
\hline & Control & Pulmotil \\
\hline Feed, $€ /$ pig & 55.34 & 43.83 \\
\hline Pig, $€ /$ pig & 61.31 & 57.93 \\
\hline Fix costs, $€ /$ pig & 12.77 & 9.96 \\
\hline Other, $€ /$ pig & 2.22 & 3.88 \\
\hline TOTAL, $€ /$ pig & 131.63 & 115.60 \\
\hline
\end{tabular}

\section{Discussion}

When a respiratory outbreak appears during the growing-finishing period, the animals treated with Pulmotil AC show an effective response. In this case the benefits were not only clinical but also in performance parameters and economic results. The pigs treated with Pulmotil in water improved the ADG, FCR and mortality rates. In addition, more Full Value Pigs ${ }^{\mathrm{TM}}$ were produced.

\section{References}

1. Lapuente $S$ et al (2004). Pulmotil efficacy on the control of PRDC. Proc 18th IPVS - Vol 1 p.37.

2. Bown P (2004). Effects of Disease on Pig Performance-A Review. NPA(UK) - http://www.npauk.net

3. Marco E. (2009). Comparación entre medicaciones en pienso o en agua en caso de enfermedad. www.3tres3.com. 


\title{
P.362
}

\section{Early treatment with Pulmotil ${ }^{\mathrm{TM}}$ in piglets vaccinated against PCV- II. Effects on the performance of the piglets}

\author{
$\underline{\text { Xavier de Paz }}{ }^{1}$ Albert Vidal ${ }^{2}$ Jordi Bonet ${ }^{2}$ Hernan Torrelles ${ }^{2}$ Miguel Abad ${ }^{1}$ Susana Lapuente ${ }^{1}$
}

1. Elanco Animal Health, Madrid, Spain; 2. Vall-Companys, Lleida, Spain

\section{Introduction}

The presence of respiratory disease increases medication costs but also causes a loss of performance (ADG and FCR) since energy and protein is diverted towards fighting disease (1). The standard use of Pulmotil ${ }^{\mathrm{TM}}$ at weaning to prevent respiratory disease was evaluated to see the effects in piglets vaccinated against PCV-II.

\section{Materials and Methods}

A comparison study was performed with two different in-feed treatments for 3 weeks after weaning: a positive control, (C) with $300 \mathrm{ppm}$ of amoxicillin against 400ppm of Pulmotil (P). In total, 144021 -day old piglets $(C=721$ and $P=719)$ were individually tagged and weighed at weaning, at the end of the treatments (day 20), and at the end of the study (day 37). In addition, the feed also included 120ppm of colistin and 2500ppm of $\mathrm{ZnO}$. After the first 3 weeks, all the piglets received the same in-feed treatment with amoxicillin at 300ppm, colistin at $120 \mathrm{ppm}$ and $\mathrm{ZnO}$ at 2500ppm. Also, all the piglets were vaccinated against PCV-Il with Ingelvac CircoFLEX ${ }^{\mathrm{TM}}$ (Boehringer Ingelheim). Performance and medication administration was evaluated. The data came from 2 consecutive replicas and were analyzed considering the variance (proc GLM of SAS). The treatments were balanced by sex and initial distribution was homogenous for both groups in both replicas, $p=0.9114$ (NS).

\section{Results and Discussion}

The mortality rate in the Pulmotil group was slightly higher than in the Control group ( $C=2.99 \%$ vs $P=3.71 \% ; p=0.4522)$. The number of injections was not different except for replica 1 the Pulmotil group received less treatments than the control $(16.9 \%$ vs $12.5 \%$; $p<0.001$ ). The initial liveweight (LW) of the piglets (figure1) was not different between both treatments $(p=0.4901)$

Figure 1. Average $L W(\mathrm{~kg})$ at day 0.

As can be seen in figures 2 and 3, the Pulmotil piglets were heavier at day 20 and 37 for both replicas $(p<0.05)$.

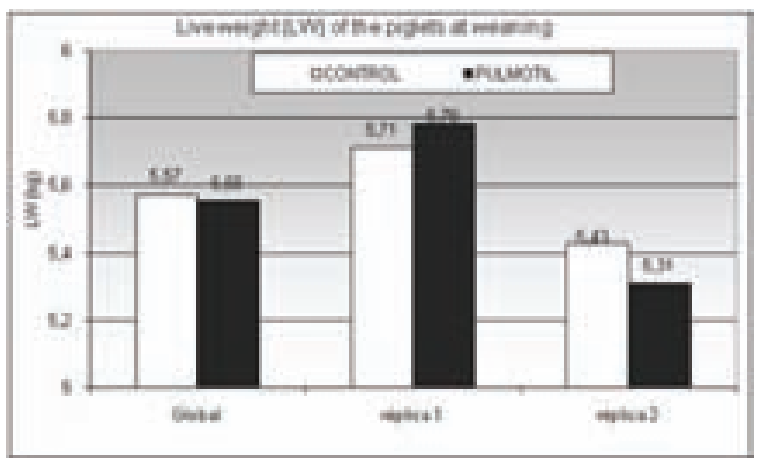

Figure 2. Average LW (kg) at day 20 and 37.

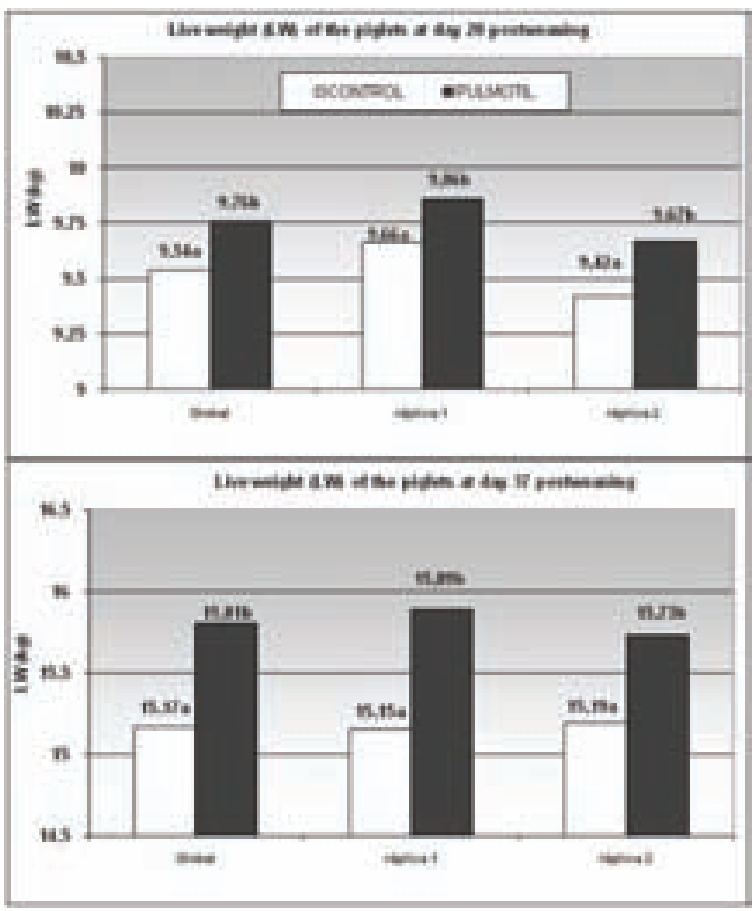

The ADG of the piglets was higher in the Pulmotil group for all the periods of the study.

Table 1: $A D G$ and FCR for the different periods.

\begin{tabular}{|l|c|c|}
\hline & Control & Pulmotil \\
\hline$A D G, g$ (day 0-20) & $199^{\mathrm{a}}$ & $211^{\mathrm{b}}$ \\
\hline$A D G, g$ (day 20-37) & $331^{\mathrm{a}}$ & $355^{\mathrm{b}}$ \\
\hline$A D G, g$ (day 0-37) & $259^{\mathrm{a}}$ & $277^{\mathrm{b}}$ \\
\hline FCR (day 0-20) & 1.20 & $1.14_{(\mathrm{p}=0.1513)}$ \\
\hline \multicolumn{3}{|l}{} \\
\hline
\end{tabular}

\section{Conclusions}

The inclusion of Pulmotil during the first three weeks after weaning improves animal performance even in farms in which vaccination is being carried out against PCV-II:

- The pigs in the Pulmotil group grew more during the nursery and their final weight was greater $(p<0.001)$ increasing the Full Value Pigs ${ }^{\mathrm{TM}}$ in the fattening unit.

- This has been consistently seen in other studies (2).

\section{References}

1. Dial G, et al. Costs, consequences and control of endemic diseases. London Swine Conference 2002.

2. Harker JW. et al. The impact of Pulmotil feeding in the nursery on finishing performance of at-risk pigs. AASV 2006. 


\title{
P.363
}

\section{Early treatment with Pulmotil ${ }^{\mathrm{TM}}$ in piglets vaccinated against PCV-II. Effects on the weight distribution at the end of the nursery period}

\author{
$\underline{\text { Xavier de Paz }}{ }^{1}$ Albert Vidal ${ }^{2}$ Jordi Bonet ${ }^{2}$ Hernan Torrelles ${ }^{2}$ Miguel Abad ${ }^{1}$ Susana Lapuente ${ }^{1}$ \\ 1. Elanco Animal Health, Madrid, Spain; 2. Vall-Companys, Lleida, Spain
}

\section{Introduction}

The presence of respiratory disease increases mortality and medication costs but also causes a loss of performance (ADG and FCR) (1). In this study, the use of Pulmotil ${ }^{\mathrm{TM}}$ after weaning was evaluated to see the effects on weight distribution of the piglets and on the percentage of lightweights at the end of the nursery.

\section{Materials and Methods}

A comparison study was performed with two different in-feed treatments for 3 weeks after weaning: a positive control, (C) with $300 \mathrm{ppm}$ of amoxicillin against 400ppm of Pulmotil (P). In total, 144021 -day old piglets $(C=721$ and $P=719)$ were individually tagged and weighed at weaning, at the end of the treatments (day 20), and at the end of the study (day 37). The rest of the treatments were the same for both groups, including a vaccination against PCV-II with Ingelvac CircoFLEX ${ }^{\mathrm{TM}}$ (Boehringer Ingelheim). At each weighing time, four different quartiles were defined in order to have $25 \%$ of the total population was in each class (Light, Median1, Median2 and Heavy). The data came from 2 consecutive replicas and was analyzed with an F-test. The percentage of animals in each category depending on treatment was analysed by an association chi-square test.

\section{Results and Discussion}

At day0, the proportion of pigs in each weight class was the same for both treatments $(p=0.9114)$; however, the treatment with Pulmotil reduced the proportion of "lightweights" at day 37 $(C=27.86 \%, P=21.79 \%, p<0.01)$.

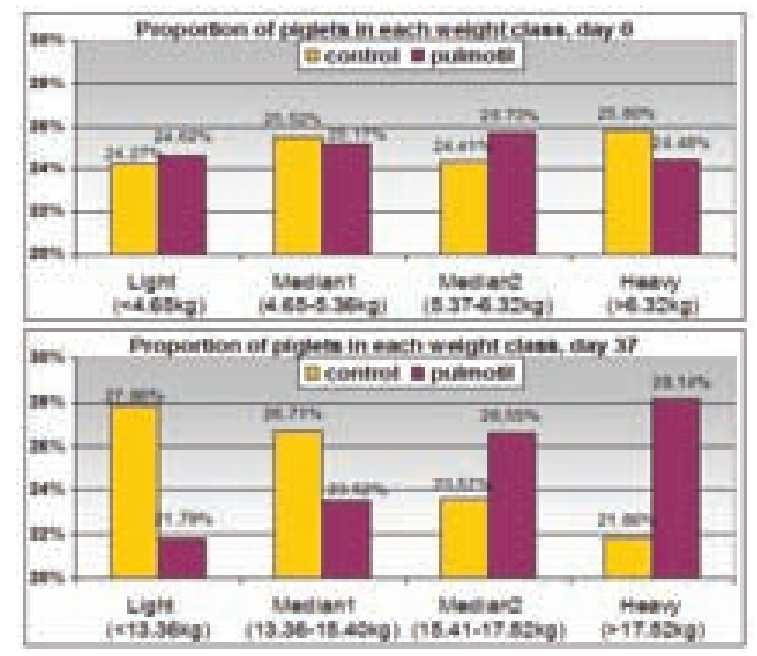

Table 1 represents the odds ratios of all the piglets for both groups. The main effects in the Pulmotil group were:

- The "Light" piglets at day 0 are more likely to be "Median 2" at the end of the study $(p=0.05)$.

- The "Median 1" piglets at day 0 are more likely to be "Heavy" at the end of study (day 37) $(p=0.02)$.

- The pigs classified as "Median 2" at day 0 are more likely to be "Heavy" at day 37 ( $p=0.05)$.

Table 1. Percentage of pigs in each class on day37 depending on the class at day 0 and treatment received

\begin{tabular}{|c|c|c|c|c|}
\hline $\begin{array}{l}\text { Cass on } \\
\text { Day } 0\end{array}$ & $\begin{array}{c}\text { Clavo on } \\
\text { dav } 37\end{array}$ & $\begin{array}{c}\text { Coarral } \\
\text { doet }\end{array}$ & $\begin{array}{c}\text { Pohnetil } \\
\text { [5er }\end{array}$ & $\begin{array}{l}\text { Odes } \\
\text { matie? }\end{array}$ \\
\hline \multirow{5}{*}{ Lesht } & bigla & 76,20 & 11.2 & 1.29 \\
\hline & Mrdan! & 5.41 & 6.42 & $0 . t$ \\
\hline & Medan? & 2.22 & a.63 & A.54* \\
\hline & Heavy & 0.85 & 1.11 & 0.4 \\
\hline & Drat & t.11 & 1.25 & 0.35 \\
\hline \multirow{5}{*}{ Medan 1} & tegh & 9.13 & 5.25 & $1.88^{4}$ \\
\hline & Madion: & 3.18 & 107 & 108 \\
\hline & Medan 2 & 5.55 & 2.09 & 0.85 \\
\hline & Hiavy & 0.97 & 2.64 & as. \\
\hline & Drad & 0.97 & 1.11 & 017 \\
\hline \multirow{5}{*}{ Medan 2} & Lights & 2.22 & 27 & 079 \\
\hline & Median I & 3. 32 & 6.82 & 124 \\
\hline & Modin 2 & 791 & 7,07 & 100 \\
\hline & Heavy & 3,27 & 2,5 & abst \\
\hline & Dead & 0 요 & 2.2 & 157 \\
\hline \multirow{5}{*}{ Heavg } & bigin & 2.67 & 2.17 & 201 \\
\hline & Madiml & 4.02 & $1.7 \%$ & 2,974 \\
\hline & $\operatorname{Mindan} 2$ & 6. & 4.54 & 1.84 \\
\hline & Hexyy & 14.15 & 15.58 & 0.15 \\
\hline & Drad & 0.16 & 249 & 0.17 \\
\hline
\end{tabular}

${ }^{1}$ Ratio of relative risk; a value higher than 1 indicates higher risk for piglets in the Control group. (* indicates $\mathrm{p}<0.05$ )

\section{Conclusions}

In piglets vaccinated PCV-II, the use of Pulmotil after weaning to prevent respiratory disease in early age still reduced the percentage of lightweights and provided more Full Value Pigs ${ }^{\mathrm{TM}}$. The opposite effect was seen for the PCV-II vaccinate piglets that received a control treatment.

These effects are consistent with other studies (2).

\section{References}

1. Dial G, et al. Rademacher C, Wiseman B, Roker J, Freking B. Costs, consequences and control of endemic diseases. London Swine Conference 2002.

2. Harker JW. et al. The impact of Pulmotil feeding in the nursery on finishing performance of at-risk pigs. AASV 2006. 


\title{
P.364
}

\section{Metaphylaxis with tulathromycin (Draxxin ) and therapeutic use of ceftiofur (Naxcel ${ }^{\bullet}$ ) against Swine Respiratory Disease and Polyserositis Complex in pigs: comparison with the use of in-feed Amoxycillin}

\author{
Ian A. Nanjiani ${ }^{1}$ Joaquin Morales $^{2}$ Carlos Piñeiro ${ }^{2}$ Pascale Sierra $^{3}$ JensChristian E. Jensen ${ }^{5,4}$ \\ 1. Pfizer VMRD, Sandwich, UK; 2. PigCHAMP Pro Europa SA, Segovia, Spain; 3. TVIVERITAS France, Gerstheim, \\ France; 4. Pfizer Animal Health EUAfME, Paris, France; 5. Orion Pharma Animal Health, Nivå, Denmark
}

\section{Introduction}

Swine respiratory disease (SRD) and polyserositis (PS) have substantial economic and welfare impacts on the European Swine industry. Disease control is generally achieved by environmental control, vaccination and antibiotic treatment. This GCPV compliant study compared two post-weaning metaphylaxis programmes: 1 - SRD metaphylaxis with tulathromycin $(2.5 \mathrm{mg} /$ kg Draxxin , Pfizer Ltd), followed by systemic treatment of SRD or PS clinical cases with ceftiofur injection $\left(5 \mathrm{mg} / \mathrm{kg} \mathrm{Naxcel}{ }^{\circ}\right.$, Pfizer Ltd); 2 - SRD metaphylaxis with oral amoxycillin (400 ppm in-feed) followed by systemic treatment of SRD or PS clinical cases with long acting amoxycillin by injection (15 mg/ $\mathrm{kg}) ; 3$ - A sentinel group of animals (receiving no metaphylaxis treatment) included to confirm disease; clinical cases in this group were treated with long acting amoxycillin injection $(15 \mathrm{mg} / \mathrm{kg})$. The study was conducted to demonstrate the value of responsible, targeted antimicrobial use in disease control under field conditions.

\section{Materials and Methods}

Four-hundred piglets from two farms with a history of SRD \& PS in the nursery period entered the study on the day following weaning (the first day of metaphylaxis treatment). During the nursery period (approximately 41 days) piglets were group housed in 40 pens, each of 10 animals.

Animals were penned in four rooms and pens randomly assigned to one of the three groups in an 18:18:4 ratio ensuring a balance of treatments within each airspace.

Animals were observed daily for signs of abnormal health; veterinarians conducting clinical examinations were unaware of the treatment group attributed to each pig. Animals were assessed for SRD and PS using pre-defined criteria (a combination of clinical signs and pyrexia of $>40^{\circ} \mathrm{C}$ ). Animals were weighed at the beginning and end of the nursery phase, and feed intake measured on a pen basis.

\section{Results}

SRD and PS were observed and bacterial involvement confirmed by isolation from clinical cases (Pasteurella multocida and Streptococcus suis). The combined tulathromycin and ceftiofur programme significantly reduced the incidence of SRD, number of antimicrobial treatment days, number of SRD or PS relapses and significantly improved the feed conversion ratio when compared to the amoxycillin metaphylaxis/therapy group (1.66 vs. 1.83 respectively, $P=0.02$ ). A tendency towards reduced mortality was observed in T1 compared to T2 (least squares mean of pen values $0.67 \%$ vs. $4.30 \%$ respectively, $P=0.071$ )
Table 1: Incidence of Swine Respiratory Disease (SRD) and Polyserositis (PS) (number and \%)

\begin{tabular}{|c|c|c|c|c|c|}
\hline \multirow{2}{*}{$\begin{array}{l}\text { Group (number } \\
\text { of pigs) }\end{array}$} & \multicolumn{2}{|c|}{ Number of SRD/PS cases ${ }^{1,3}$} & \multicolumn{3}{|c|}{ Incidence of SRD/PS $(\%)^{2,3}$} \\
\hline & SRD & PS & TOTAL & SRD & PS \\
\hline T1 (180) & 23 & 5 & 6.91 & 6.07 & 0.55 \\
\hline T2 (180) & 76 & 9 & 35.76 & 32.63 & 1.76 \\
\hline $\mathrm{T} 3(40)$ & 19 & 0 & 49.06 & 49.56 & 0.02 \\
\hline T1 vs. T2 P-value & N/A & N/A & 0.0005 & 0.0006 & 0.25 \\
\hline \multicolumn{6}{|c|}{${ }^{1}$ Some animals had both conditions concurrently; mortalities included } \\
\hline \multicolumn{6}{|c|}{$\begin{array}{l}2 \text { Incidence \% calculated using Least Squares Means to reduce distortions produced by pen } \\
\text { effects }\end{array}$} \\
\hline \multicolumn{6}{|c|}{${ }^{3}$ Statistical analyses conducted using the pen as the experimental unit (not the pig) } \\
\hline
\end{tabular}

\section{Discussion}

In-feed antibiotics are widely used for metaphylaxis due to their ease of administration and low cost. Potential interactions with other ration components, difficulties insuring drug homogeneity in feed and the inappetence of sick animals can result in individual animals being underdosed, compromising efficacy. The use of injectable antimicrobial products for metaphylaxis \& therapy allow accurate, targeted dosing of the individual animal, particularly when the product chosen has a sufficiently long duration of activity to cover the period of expected disease and a suitable spectrum of activity. In the particular circumstances of this study, based on the drug and piglet (20-25kg live-weight) prices current in Spain at the time (making no allowance for labour costs), this disease management protocol provided a return of approximately $€ 1.50$ per pig compared to that used in group T02.

\section{References}

Nanjiani et al 2009; Obiettivi e Documenti Veterinari 5, 35 - 43 


\title{
P.365
}

\section{A treatment protocol designed to control Mycoplasma hyorhinis infection in a commercial herd points out to a potential association with Streptococcus suis}

\author{
Charles Surprenant ${ }^{1}$ Marcelo Gottschalk ${ }^{2}$ \\ 1. F. Ménard Inc., Ange-Gardien, QC, Canada; 2. Université de Montréal, Saint-Hyacinthe, QC, Canada
}

\section{Introduction}

Mycoplasma hyorhinis was named and characterized by Switzer et al. in $1955^{1}$. Since then, this microorganism has been associated with different clinical conditions in pigs: pneumonia ${ }^{2}$, arthritis $^{3}$, otitis ${ }^{4}$, eustachitis ${ }^{5}$. Over the past 5 years, commercial swine nurseries, negative for Mycoplasma hyopneumoniae and belonging to the same pyramid, experienced clinical losses caused by $M$. hyorhinis and/or Streptococcus suis. A treatment protocol was designed to reduce respiratory problems associated with M. hyorhinis infection. Materials and Method

Herd description: Since 2002, a multisite commercial pig flow has experienced clinical signs of respiratory and S. suis disease at the nursery level. The 1800 crossbred sow unit is negative for PRRSV. Each week, 800 5-kg piglets averaging 18 days of age are transferred to a 3 barn nursery site. Each barn is filled up over a period of 10 days and completely emptied 40 days after the first pigs arrive. The sow herd is $M$. hyopneumoniae positive, however sows have been vaccinated at 3 weeks prior to farrowing for over 10 years in order to stabilize the herd. Piglets are vaccinated against $M$. hyopneumoniae at 3 and 6 weeks of age.

Clinical Signs: Shortly after arrival, some piglets started to exhibit a dry cough and sneezing. These clinical signs became more severe and prevalent over time with some pigs developing pneumonia and/or a clinical syndrome associated with S. suis (meningitis, sudden death). Pig performance was adversely affected. Laboratory tests were always negative for $M$. hyopneumoniae (Table 1). However, M. hyorhinis and S. suis were found repeatedly in different samples.

Treatment protocol: After concluding that M. hyorhinis was the primary cause of the respiratory signs, a novel treatment protocol was implemented to control $M$. hyorhinis. All the piglets were injected once at weaning with $2.5 \mathrm{mg} / \mathrm{kg}$ of tulathromycin $\left(\right.$ Draxxin $^{\circledast}$ ) starting on February 3, 2009. No other changes were made.

Statistical analysis: Although the implementation of the new treatment protocol was not designed as a clinical trial, a Wilcoxon test was done using the median values of different parameters to compare results for 9 pre-treatment pig lots versus 10 post-treatment lots.

\section{Results}

Shortly after the implementation of this new procedure, coughing, sneezing and respiratory signs almost disappeared. Posttreatment results show an improvement in feed conversion and average daily gain. The overall viability of the post-treatment lots was significantly increased $(P=0.02)$. A reduction in the number of Penicillin $V$ water treatments, the number of days on Penicillin V treatment and, surprisingly, in the frequency and severity of S. suis episodes was observed (Table 2).
Table 1. Laboratory tests

\begin{tabular}{|l|c|c|c|c|c|c|c|}
\hline & & PCR test & PCR test & PCR test & PCR test & $\begin{array}{c}\text { Bacterial } \\
\text { culture }\end{array}$ & $\begin{array}{c}\text { Bacterial } \\
\text { culture }\end{array}$ \\
\hline Date & Tissues & $\begin{array}{c}\text { M. } \\
\text { hyorhi. }\end{array}$ & $\begin{array}{c}\text { M. hyo- } \\
\text { pneu. }\end{array}$ & H1N1 & H3N2 & $\begin{array}{c}\text { S. suis } \\
\text { (serovar) }\end{array}$ & $\begin{array}{c}\text { 0ther } \\
\text { (serovar) }\end{array}$ \\
\hline $07 / 06 / 07$ & Heart & ND & ND & ND & ND & $+(1 / 2)$ & - \\
\hline $01 / 14 / 08$ & Lung & - & - & - & - & $+(1 / 2)$ & - \\
\hline $02 / 18 / 08$ & Lung & + & - & - & - & - & 0ther Strep. \\
\hline $05 / 12 / 08$ & Lung & - & - & + & - & ND & ND \\
\hline $06 / 02 / 08$ & Lung & + & - & - & + & ND & ND \\
\hline $09 / 22 / 08$ & Lung & + & - & - & - & $+(1 / 2)$ & ND \\
\hline $10 / 06 / 08$ & Lung & + & - & - & - & ND & ND \\
\hline $10 / 09 / 08$ & Nasal & + & - & - & - & ND & ND \\
\hline $11 / 24 / 08$ & Lung & + & - & - & - & $+(1 / 2)$ & ND \\
\hline $02 / 02 / 09$ & Lung & + & - & - & - & $+(33)$ & H. parasuis (7) \\
\hline $09 / x \times 09$ & Lung & + & ND & ND & ND & ND & ND \\
\hline${ }^{*}$ by bacterial culture \\
\hline
\end{tabular}

Table 2. Performance results

\begin{tabular}{|l|c|c|c|c|c|c|c|}
\hline $\begin{array}{l}\text { Number } \\
\text { of pigs }\end{array}$ & $\begin{array}{c}\text { Viability } \\
\text { (\%) }\end{array}$ & FC & $\begin{array}{c}\text { ADG (g/ } \\
\text { day) }\end{array}$ & $\begin{array}{c}\text { S. suis* } \\
\text { (dead/1000 } \\
\text { pigs) }\end{array}$ & $\begin{array}{c}\text { Mean } \\
\text { number of } \\
\text { Pen V tx }\end{array}$ & $\begin{array}{c}\text { Mean num- } \\
\text { ber of days } \\
\text { on Pen V tx }\end{array}$ \\
\hline Before & 17149 & $97.8^{\mathrm{a}}$ & 1.53 & 427 & 8.5 & 3.5 & 11.0 \\
\hline Draxxin & 17609 & $98.8^{\mathrm{b}}$ & 1.46 & 429 & 3.3 & 2.3 & 7.3 \\
\hline
\end{tabular}

\section{Discussion}

The injection of Draxxin ${ }^{\circledR}$ markedly improved the health status of these nurseries. M. hyorhinis was identified as the primary agent and because tulathromycin is effective against $M$. hyorhinis and not against $S$. suis, it is believed that the improvement observed in pig performance was associated with the action of tulathromycin on M. hyorhinis. A decrease in S. suis incidence was also observed. Can M. hyorhinis be a predisposing factor in the development of clinical $S$. suis infection like some irritants ${ }^{6}$ ? Further work is needed to answer.

\section{References}

1. W.P. Switzer et al., Am J Vet Res, 16:540(year?).

2. K Kawashima et al., J Comp Pathol, 114:315(1996).

3. M Kobisch et al., Rev Sci Tech, 15:1569 (1996).

4. T Morita et al., Vet Pathol, 32:107 (1995).

5. T Morita et al.., Vet Pathol. 36:174 (1999).

6. F Pallares et al., Can J Vet Res, 67: 225(2003). 


\title{
P.366
}

\section{Elimination of PRCV and H3N2 swine influenza virus from a production network using a 4-week batch farrowing system}

\author{
Manon St-Hilaire ${ }^{1}$ Desrosiers Robert $^{2}$ \\ 1. Génétiporc Inc, St-Jean-Chrysostome, QC, Canada; 2. Boehringer Ingelheim, St-Hyacinthe, QC, Canada
}

\section{Introduction}

Porcine respiratory coronavirus (PRCV) is a pathogen of low economic impact. Swine influenza virus (SIV) can produce losses but is also often present on farms without significant clinical signs of the disease. Nevertheless, the presence of these two pathogens can, for certain farms with a high health status, constitute an obstacle to exportation of breeding animals. This paper describes a production network using a 4-week batch farrowing system where both viruses were eliminated.

\section{Materials and Methods}

Two high health sow herds ( $A$ and $B$ ) of 1200 sows each produced weaned piglets that went to a common nursery site, and from there to three different finishing sites. Replacement gilts and boars used in herds $A$ and $B$ came from the three finishing sites. In June 2006 herd A became infected with PRCV, and within a month pigs on all sites became infected as well. The biosecurity level on these sites is very high and to date the source of initial contamination has not been found. In January 2007, following the introduction of replacement animals in herd B, coughing was observed and determined to be caused by SI H3N2. Within a month, presence of the virus was detected in all sites. In January 2008 the owner elected to switch to a 4-week batch farrowing system for various reasons that included the possibility to more easily get rid of both viruses. An autogenous H3N2 vaccine was obtained for the vaccination of gilts (vaccinated twice in isolation and kept there for 8 weeks) and sows (vaccinated twice three weeks apart). Starting three to four weeks later sows were also vaccinated before farrowing in an attempt to maximize colostrum antibody content. Following that program, gilts were introduced in the two sow herds every four weeks. Circulation of the viruses within the herds was monitored using an IDEXX ELISA for PRCV and an IHA test, containing a H3N2 Quebec strain, for SI. Both pigs at the end of the finishing period and sentinels introduced in the two sow herds were tested.

\section{Results}

After infection of the sow herds, both PRCV and SIV circulated in finishing pigs. The first farrowings of the 4-week batch farrowing system occurred in June 2008 (batch 1). All 14 batches produced and tested so far, except batches 8 and 9, have remained negative to PRCV. The two infected batches are thought to have been infected at the finishing site from an indirect and undetermined cause. For SI the first two batches were infected, but all others have remained negative since then. Negative sentinels introduced in September 2008 in herd B, but not in herd A, seroconverted to PRCV.

Those introduced in June 2009 did not. In October 2008 sentinels introduced in Herd B seroconverted to $\mathrm{SI} \mathrm{H3N2,} \mathrm{but} \mathrm{those}$ introduced in February 2009 have remained negative.

\section{Discussion}

Both PRCV and SIV are considered to be organisms that are usually shed for only short periods of time following infection. The 4-week batch farrowing system allows a sow herd to be without suckling piglets on site every four weeks. If the herd is temporarily closed to introduction of susceptible gilts and/or boars, this system should theoretically increase the chances of being able to get rid of pathogens, particularly those that are not shed for long periods of time following infection. This is because for a while there are no susceptible animals on the farm to maintain circulation of the organism.

In this study it was possible to get rid of both PRCV and SIV from two different sow herds, the nursery site and the three finishing sites and this occurred following the implementation of the 4-week batch farrowing system. It is not sure if elimination of these two viruses was due mainly, partially or not at all to the use of that production system. In fact in the case of SIV an autogenous vaccine was used and may have contributed to the results obtained. Furthermore there are situations where SIV was eliminated from swine farms without doing anything, or by delaying the introduction of gilts to every two months.1,2 This being said, the results obtained in this study as well as the theoretical advantages this system confers suggest, at the very least, that further studies should be conducted to determine to what extent it could improve the chances of eliminating some of the pathogens that have to be dealt with in swine production.

\section{References}

1. Desrosiers R, Boutin R, Broes A. Persistence of antibodies after natural infection with swine influenza virus and epidemiology of the infection in a herd previously considered influenza-negative. JSHAP, 2004, 12:78-81.

2. Torremorell M, Juarez A, Chavez E, Yescas J, Doporto JM. Procedures and economics of swine influenza virus elimination in a three-site production herd. Proc IPVS, 2008, Vol 2, 23. 


\title{
P.367
}

\section{Palatability of Tilmovet $250 \mathrm{mg} / \mathrm{ml}$ Concentrate for Oral Solution compared to a reference formulation containing tilmicosin}

\author{
Alain Kanora; Henk Lecluyse; Koen De Gussem
}

Huvepharma NV, Antwerp, Belgium

\section{Introduction}

Tilmicosin is a semi-synthetic macrolide antibiotic obtained from tylosin which is affecting bacterial protein. Macrolides have a proven benefit as treatment for a wide range of infectious diseases, with an increased interest for respiratory infections. The objective of this study was to compare the palatability of Tilmovet $^{\circ} 250 \mathrm{mg} / \mathrm{ml}$ Concentrate for Oral Solution with that of a reference formulation containing tilmicosin and to evaluate the impact on pig water consumption.

\section{Materials and methods}

On a Belgian fattening unit containing two open compartments, 11 pens were selected, each containing 14 pigs with an average weight of $+60 \mathrm{~kg}$. Two test groups of about 150 pigs each were identified. Compartments had own dosing equipment installed at $1 \%$. The water uptake of each group was monitored during 5 days, split into 2 phases: 2 days of no medication (negative, control) and 3 days with medicated drinking water (Tilmovet ${ }^{\oplus}$ or a tilmicosin reference formulation). The inclusion rate of tilmicosin was calculated based on the registered dose of $16 \mathrm{mg} / \mathrm{kg}$ bodyweight and the actual water intake of the pigs. Each day, a fresh solution was prepared and administered.

\section{Results}

During the first phase of the trial period, temperatures ranged from $26^{\circ} \mathrm{C}$ to $30^{\circ} \mathrm{C}$ and for the animals receiving unmedicated drinking water in this phase, a daily water consumption was recorded ranging from 57 to $67 \mathrm{ml} / \mathrm{kg}$ bodyweight and both groups had very similar drinking water intake levels. In the second trial phase, in which the animals received medicated drinking water for 3 days, the registered temperatures ranged between $20^{\circ} \mathrm{C}$ and $23^{\circ} \mathrm{C}$. During this phase, the Tilmovet group had a somewhat higher daily water consumption intake level (53-68 $\mathrm{ml} / \mathrm{kg}$ bodyweight, i.e. 3.18-3.81 liter/day/pig) than the group receiving the medicated drinking water with the tilmicosin reference formulation (49-60 ml/kg bodyweight, i.e. 2.473.05 liter/day/pig) (Figure 1). In terms of dosing, the pigs in the Tilmovet group consistently reached higher dose levels than the tilmicosin reference group. The registered minimum dose of $16 \mathrm{mg} / \mathrm{kg}$ bodyweight was not reached in the tilmicosin reference group on any of the trial days, whereas in the Tilmovet group, mostly higher dose levels were attained (Figure 2).
Figure 1. Daily drinking water consumption

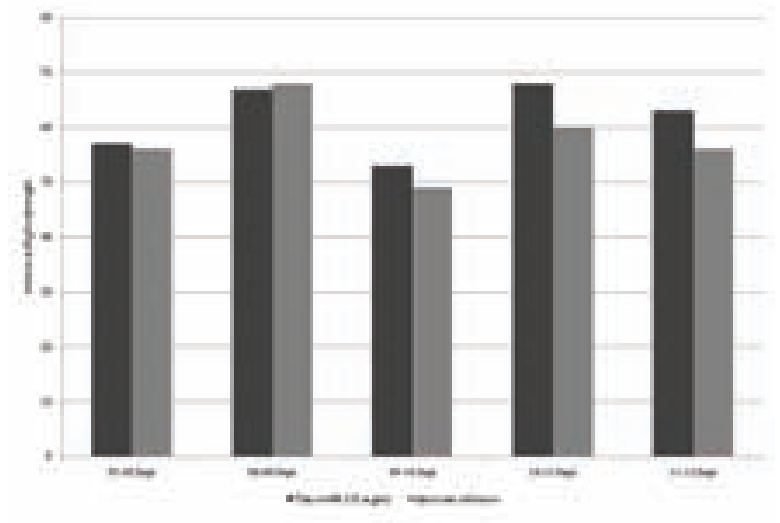

Figure 2. Tilmicosin dose intake levels ( $\mathrm{mg} / \mathrm{kg}$ bodyweight) vs registered dose of $16 \mathrm{mg} / \mathrm{kg} \mathrm{BW}$

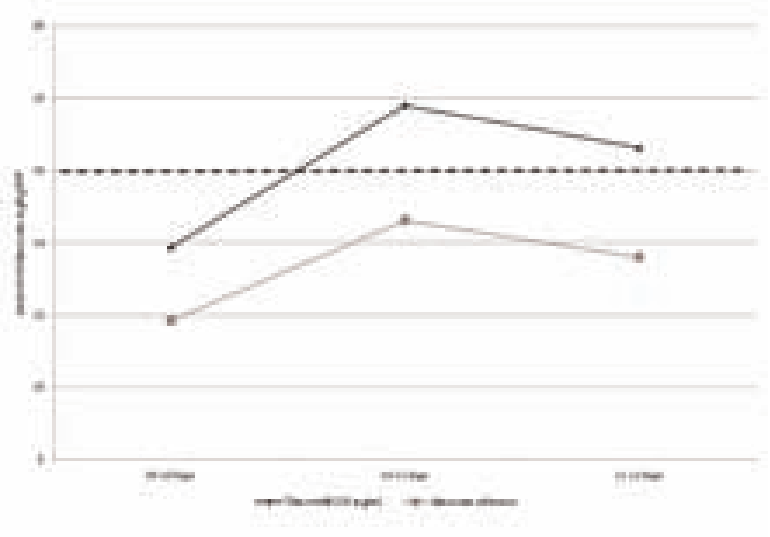

\section{Discussion and conclusion}

It can be concluded from this study that the overall daily drinking water consumption is not influenced by Tilmovet $250 \mathrm{mg} /$ $\mathrm{ml}$ Concentrate for Oral Solution and that the calculated concentration of tilmicosin is fully compliant with the registered dose of $16 \mathrm{mg} / \mathrm{kg}$ bodyweight. 


\title{
P.368
}

\section{Treating weaned piglets with tilmicosin oral solution to prevent circulation of viral infection in a closed sow herd}

\author{
Jan Van Steelant ${ }^{1}$ Lieven Tanghe $^{2}$ Henk Lecluyse ${ }^{2}$ Alain Kanora $^{2}$ \\ 1. Voeders Depré, Vaart-Noord 11, Beernem, Belgium; 2. Huvepharma NV, Antwerp, Belgium
}

\section{Introduction}

The PRRS virus is one of the most important pathogens in the pig breeding business. In the field, co-infections with multiple bacterial respiratory pathogens are commonly found, making effective treatment more difficult. The objective of this trial was to use tilmicosin drinking water medication as an effective tool next to vaccination with a life vaccine to prevent PRRSV replication. This was done in a previously vaccinated, yet positively diagnosed sow herd.

\section{Materials and Methods}

A Belgian closed sow herd ( $n=350)$, suffering from early parturition (on average 2 days early) and having received multiple PRRSV vaccination (3 times in the previous year) after PRRSV was diagnosed with RT-PCR strain (in combination with influenza $\mathrm{H} 3 \mathrm{~N} 2, \mathrm{H} 1 \mathrm{~N} 1$ and H1N2) still had unusually high prevalence of abortions. Change in feed and feed supplier did not bring the expected result either. Blood samples were taken and through vertical serology the diagnoses of PRRSV circulating was reconfirmed (Figure 1).

Figure 1. S/P ratio

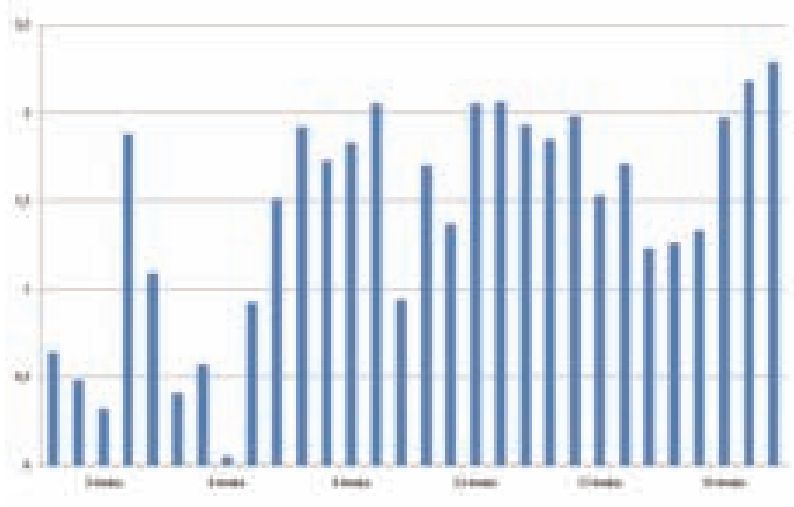

Instead of re-vaccinating the herd (piglets) with the same vaccine, the vet decided to go for an alternative treatment with tilmicosin to lower the infection pressure in the farrowing house and battery. Four days after weaning, the piglets (each weighing $+6 \mathrm{~kg}$ ) were treated with tilmicosin (Tilmovet $250 \mathrm{mg} / \mathrm{ml}$ Oral Solution - Huvepharma) for 3 to 5 days at a dose of 0.5 liter of tilmicosin per 1,000 liter. Mortality rates were then monitored and evaluated.

\section{Results}

Mortality rates dropped considerably in both the farrowing house and in the group of weaned piglets, respectively $-2.30 \%$ and $-1.40 \%$ (Figure 2).

Figure 2. Mortality rates (\%), farrowing house and group of weaned piglets

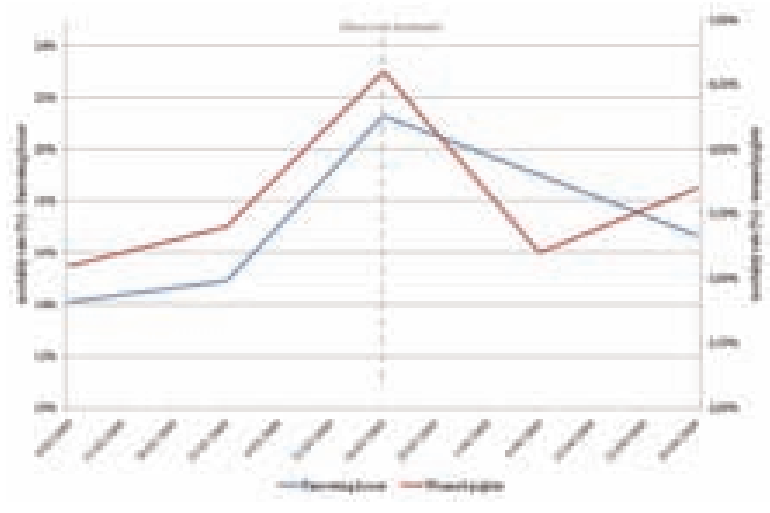

\section{Discussion}

The 3-5 days treatment with tilmicosin oral medicated drinking water has successfully controlled the circulating viral infection of PRRSV in both farrowing house and within the group of weaned piglets. This was assessed by means of the piglet mortality rate which had dropped quite considerably.

\section{References}

1. Campi, T.W. et al. (2009), Pulmotil type A medicated article for the control of swine respiratory disease, AASV 2009, p. 179-181.

2. Froejk, G.F. (2006), Elimination of clinical symptoms, improvement in performance and reduction of serological titers in a conventional herd with PRDC by moving pigs offsite and treatment with tilmicosin, IPVS 2006, Vol. 2, p. 312.

3. Misener, M. et al. (2006), Preliminary evaluation of clinical effects and cost-effectiveness of in-feed Pulmotil (tilmicosin) and serum inoculation in an outbreak of PRRS, IPVS 2006, Vol. 2, p. 13. 


\title{
P.369
}

\section{Economic benefits from the eradication of enzootic pneumonia and PRRS from a new 800-gilt herd}

\author{
David G. Burch ${ }^{1}$ Nigel J. Woolfenden ${ }^{2}$ \\ 1. Octagon Services Ltd, Old Windsor, Berkshire, UK; 2. Bishopton Veterinary Group, Ripon, N. Yorkshire, HG42QR, UK
}

\section{Introduction}

In 2008, due to a period of high feed prices and poor meat prices, it was decided to kill out one of three approx. 800 sow herds (Herd 1), which was badly affected with chronic porcine circovirus type 2 (PCV2), porcine reproductive and respiratory syndrome virus (PRRSV), enzootic pneumonia (EP) and Actinobacillus pleuropneumoniae ST3. Herd 2, which was also positive for PRRS, EP \& PCV2 but had a good reproductive performance and low post-weaning to slaughter mortality, was to be the source of gilts for Herd 1. It was decided to try to eliminate PRRS and EP through vaccination and medication prior to farrowing. Elimination of PRRS had been described (1) using gilt pool management and infection stabilization and Mycoplasma hyopneumoniae (Mhp) elimination by the use of tiamulin and chlortetracycline in combination (2).

\section{Materials and Methods}

Approximately, 50 cross-bred (LRxLWXLW) gilts/week were weaned at 4 weeks of age into straw-based accommodation over a 21 week period from the start of 2008. The young pigs were vaccinated with a killed mycoplasma vaccine (Ingelvac ${ }^{\oplus}$ $\mathrm{M} \mathrm{Hyo}$ ) at 3 weeks of age and again at 8 weeks of age. A PCV2 vaccine (Ingelvac ${ }^{\oplus}$ Circoflex) was given also at 3 weeks of age. A live PRRS vaccine (Porcilis ${ }^{\circledR}$ PRRS) was given at 8 weeks of age. Following the closure of Herd 1 and the removal of all pigs at the beginning of June, the premises were thoroughly cleaned and disinfected. Three weeks later (21st June) the gilts were moved over in batches of 300 from Herd 2. Matings were started in the older gilts on the 11th of October and they were given Porcilis PRRS and a parvovirus and erysipelas vaccine (Porcilis ${ }^{\circledR}$ Ery + Parvo) 3 weeks before service. To ensure the required uniform immunity to PRRS, the whole gilt herd was vaccinated on 24th November with Porcilis PRRS (live), an inactivated PRRS vaccine (Progressis ${ }^{\circledR}$ ) on the 15 th of December and a final Porcilis PRRS (live) on the 5th of January, 2009. Medicated feed was also started on the 5th of January, 2009 for 6 weeks and containing sufficient tiamulin (Denagard ${ }^{\circledR}$ Premix) at 500ppm and chlortetracycline (Aurogran ${ }^{\circledR}$ Premix) 1500ppm to give 5 and $15 \mathrm{mgs}$ of active/ $\mathrm{kg}$ bodyweight respectively. The first farrowings commenced on the 3rd of February. The younger gilts ( $<300$ days of age) and boars were also injected with tiamulin (Denagard 200 injectable) at $15 \mathrm{mg} / \mathrm{kg}$ bodyweight, 3 weeks into the in-feed medication period.

Blood samples were taken from 60 pigs (8-22 weeks old) on July 14th and examined by ELISA tests for PRRSV (LSI Hipra) and Mhp (DAKO blocking). In December, an acute outbreak of coughing occurred in Herd 1 and a further 30 samples from 16-20 week old pigs were taken. A number of lungs at slaughter were also examined for EP lesions \& pleurisy in July (240) and December (115).

\section{Results}

Table 1. Blood test results for PRRSV and Mhp.

\begin{tabular}{|c|c|c|c|}
\hline Blood test & No of samples & PRRSV +ve & Mhp +ve \\
\hline July 2009 & 60 & 0 & 0 \\
\hline Dec 2009 & 30 & $15(50 \%)$ & $0(0 \%)$ \\
\hline
\end{tabular}

Table 2. Comparative production figures before and after PRRSV and EP eradication programme in Herd 1

\begin{tabular}{|l|c|c|c|}
\hline & Herd 1 & Herd 1 & Herd 2 \\
\hline & Pre-prog 2007 & Post-prog 2009 & Original herd 2009 \\
\hline No born alive & 11.9 & 13.4 & 12.0 \\
\hline Mort pre-wean & $9.8 \%$ & $8.5 \%$ & $7.5 \%$ \\
\hline Mort post-wean & $11.4 \%$ & $7.6 \%$ & $3.5 \%$ \\
\hline FCE & 2.59 & 2.46 & - \\
\hline
\end{tabular}

All of the lungs examined were free from EP lesions. Pleurisy had fallen from $>30 \%$ in 2007 to $<6 \%$ in 2009.

\section{Discussion}

The EP part of the programme appears to have been very successful for over a year according to blood and lung lesion results. There appears to have been a breakdown in PRRSV in December, although the herd remained free clinically for over 10 months, suggesting the programme had been effective originally. Contaminated transport was the suspected cause. Number of pigs born alive/litter was increased by 1.5. Mortality was reduced in all stages of production by $5.1 \%$ but from post-weaning to slaughter by $3.8 \%$ or an extra 730 pigs sold resulting in a $£ 25,500$ margin over feed. FCE was reduced by 0.13 , which equated to savings of $£ 34,944$, and a combined total of $£ 60,444$. PRRS and EP medication costs were $£ 11,970$, so the med cost/benefit ratio was 1:5.

\author{
References \\ 1 Dee, S. et al (1994) SHAP, 3, 2, 64-69 \\ 2. Baekbo, P. et al (1994) Proc. 13th IPVS Congress, p135
}




\title{
P.370
}

\section{Use of Tilmovet $250 \mathrm{mg} / \mathrm{ml}$ Concentrate for Oral Solution in prefarrowing sows - a Danish field trial}

\author{
Niels H. Rough ${ }^{1}$ Alain Kanora $^{2}$ Koen De Gussem² \\ 1. Samsvet, Tingvej 1, Samsoe, Denmark; 2. Huvepharma NV, Antwerp, Belgium
}

\section{Introduction}

Macrolides have been intensely studied in the past with regards to their effect on pig production parameters. A Danish sow farm suffered from an outbreak of Mycoplasma hyopneumoniae including pregnant sows. To minimize the infection pressure in the farrowing house, all pregnant sows were given tilmicosin (Tilmovet $^{\bullet}$ - Huvepharma) ${ }^{1,2}$. The objective of this field trial was to control the Mycoplasma circulation in piglets by using tilmicosin in prefarrowing sows.

\section{Materials and Methods}

The Danish sow farm ( $n=680$, races $L Y$ and Zig Zag cross) is located on the island Samsø. Every week, there are 29 farrowings (average of 6 months prior to current trial) and all gilts are produced on farm. Historical data on the number of piglets being born, weaned, pig weight and piglet mortality were available. During an 8 week period (1 October- 23 November), all sows received an individual treatment of tilmicosin (Tilmovet ${ }^{\circ}$ ) of 12 $\mathrm{ml}$ per sow for 5 consecutive days prefarrowing. First pigs from treated sows were weaned around 28 days ( 28 October) and the last around 16 December. Weaning age was 28 days. Data on average piglet weight at time of weaning (data representing 2970 pigs weaned) were then compared to historical data relative to 6,186 pigs weaned (period of 17 weeks) and to data from 690 pigs weaned during the two weeks post treatment period. Secondly, piglet mortality data from the treated group were compared to the pre-trial historical data.

\section{Results}

In terms of average weight, the historical data showed an average weight per pig of $6.61 \mathrm{~kg}$, whereas the piglets from the Tilmovet treated group had an average weight of $6.81 \mathrm{~kg}$, i.e. an increase of $2.92 \%$ (Table 1). During the post trial period of 2 weeks which was considered, the average weight per pig decreased again to $6.55 \mathrm{~kg}$, being a decrease of $3.78 \%$ compared to the treated group.

Table 1. Avg. piglet weight, historical vs treatment

\begin{tabular}{|l|c|c|c|}
\hline Data relative to period & $\begin{array}{c}\text { Number of pigs } \\
\text { weaned }\end{array}$ & $\begin{array}{c}\text { Weight of pigs } \\
\text { weaned }\end{array}$ & $\begin{array}{c}\text { Avg. weight per } \\
\text { pig (kg) }\end{array}$ \\
\hline Before tilmicosin & 6,186 & 40,920 & 6.61 \\
\hline Tilmicosin treatment & 2,970 & 20,220 & 6.81 \\
\hline Post trial (2 wk) & 690 & 4,520 & 6.55 \\
\hline
\end{tabular}

Secondly, the historical mortality data (over a period of 5 weeks) were compared to the mortality rate relative to the Tilmovet ${ }^{\oplus}$ treated groups. Taking into account the start and ending of the treatment, the first weaned pigs from these groups were in week 44 and the last ones in week 51. A clear tendency in mortality decrease in the treated groups can be noticed (Figure 1). The average mortality rate prior to this study was $16 \%$, whereas the average mortality rate of the treated groups was $13.67 \%$.
Figure 1: Post-weaning mortality rate (\% of dead piglets)

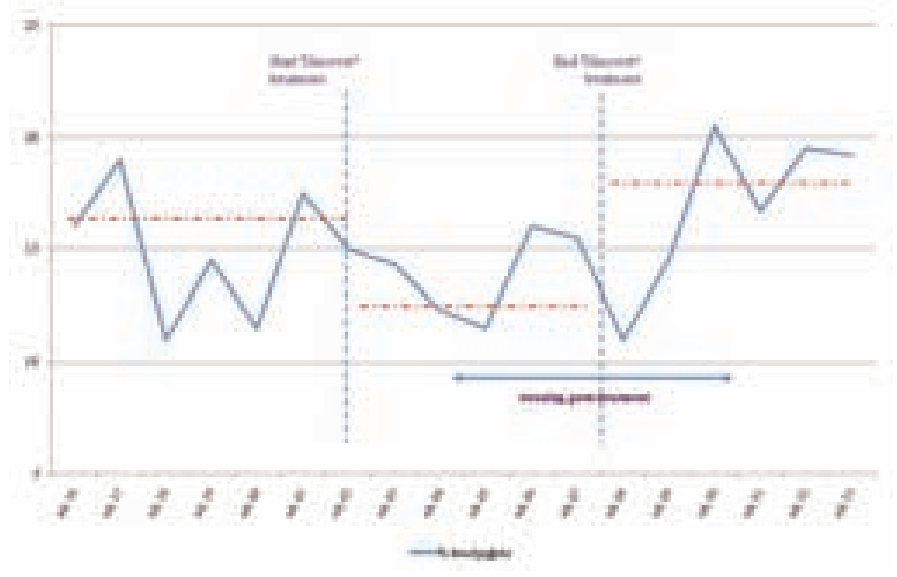

\section{Discussion}

Historical data showed that untreated sows had piglets of inferior weaning weight. This observation clearly confirmed the ability of tilmicosin to control the disease pressure. Secondly, it demonstrated the indirect effect on the average piglet weight of Tilmovet treated sows when treated in the prefarrowing stage. Consequently, the average mortality rate of treated sows showed a tendency to be lower (in comparison to historical data).

\section{References}

1. Roedbro, T. et al. (2002), Elimination of mycoplasma hyopneumoniae and actinobacillus pleuropneumoniae by tilmicosin, IPVS Congress 2002, Proceed. Vol. 1, p. 147.

2. Vraa-Andersen, L. and Gram, S. (2004), How to eliminate APP, IPVS Congress 2004, Proceed. Vol. 2, p. 834. 


\title{
P.371
}

\section{Efficacy of florfenicol (Nuflor $\mathbf{4 0} \mathrm{mg} / \mathrm{g}$ Premix for swine) for the control of porcine respiratory disease complex in an Iberian pig farm}

\author{
Pablo Arevalo ${ }^{1}$ Antonio Palomo ${ }^{2}$ Jesus M. Bollo ${ }^{3}$ Ruth Menjon ${ }^{3}$ Marta Jimenez ${ }^{3}$ Jesus V. Lopez ${ }^{3}$ \\ 1. Granja Iberica Alcudia, S. Lu., Ciudad Real, Spain; 2. SETNA NUTRICION-INVIVO NSA, Madrid, Spain; \\ 3. Intervet/Schering-Plough AH, Cantabria, Madrid, SpainObjective
}

The object of the study was to compare safety and efficacy of florfenicol (Nuflor $40 \mathrm{mg} / \mathrm{g}$ Premix for Swine) with that of Tilmicosin for prevention of porcine respiratory disease complex (PRDC).

\section{Materials and Methods.}

Study was performed on a farm with 3,000 lberian sows crossbred with Duroc and Iberian boars in a closed production cycle. The farm had a history of PRDC with clinical and/or laboratory diagnosis of P. multocida, M. hyopneumoniae and S. suis.

A total of 604 piglets from two consecutive weanings were included in the study (302 per group). Animals from both groups were randomized according to their weaning group, age, weight and gender.

The treatments to be compared were mixed with pre-starter feed as follows:

80 ppm florfenicol (Nuflor 40 mg/g Premix for Swine, 2 kg/Tm) during 14 days.

200 ppm tilmicosin (1 kg/Tm) during 14 days.

The mean age at weaning was 27 days, and the study lasted 42 days, so piglets were 69 days old at end of study.

The pen was the experimental unit when weighing the animals (25, occasionally 26 pigs per pen). Pigs were weighed on day 0 (at weaning, the start of medicated feed intake), on day 14 (at end of medicated feed intake) and on day 42 (at end of starter feed intake and entry to pre-fattening barn) and average daily gain (ADG) calculated. Feed intake was recorded and feed conversion ratios (FCR) calculated.

The statistical study was performed using ANOVA, Pearson's chisquare test and Fisher's exact test.

\section{Results}

Both treatments proved safe; no adverse effects were observed. The average weights of both groups at start of the study were comparable $(p=0.677)$.

Table 1: Mean weights $(\mathrm{kg})$

\begin{tabular}{|l|c|c|c|}
\hline & Day 0 & Day 14 & Day 42 \\
\hline Florfenicol & 7.46 & 10.85 & 23.15 \\
\hline Tilmicosin & 7.55 & 10.44 & 21.7 \\
\hline
\end{tabular}

The florfenicol group performed statistically significantly better for average weight on day $14(p=0.001)$ and on day $42(p<0.01)$ (Table 1).
Better growth rates and weights were found in the florfenicol group, both during pre-starter and starter periods, and these differences were statistically significant in all cases (Table 2).

Table 2: Weight gains and growths per study period

\begin{tabular}{|l|c|c|c|c|c|c|}
\hline & $\begin{array}{c}\text { Weight } \\
(\mathbf{k g})\end{array}$ & $\begin{array}{c}\text { Weight } \\
(\mathbf{k g})\end{array}$ & $\begin{array}{c}\text { Weight } \\
(\mathbf{k g})\end{array}$ & ADG & ADG & ADG \\
\hline Period (Days) & $0-14$ & $14-42$ & $0-42$ & $0-14$ & $14-42$ & $0-42$ \\
\hline Florfenicol & $3.39^{\mathrm{a}}$ & $12.30^{\mathrm{c}}$ & $15.69^{\mathrm{e}}$ & $242^{\mathrm{a}}$ & $440^{\mathrm{c}}$ & $374^{\mathrm{e}}$ \\
\hline Tilmicosin & $2.89^{\mathrm{b}}$ & $11.26^{\mathrm{d}}$ & $14.15^{\mathrm{f}}$ & $207^{\mathrm{b}}$ & $403^{\mathrm{d}}$ & $337^{\mathrm{f}}$ \\
\hline $\mathrm{a} \mathrm{b}(\mathrm{p}=0.001), \mathrm{cd}(\mathrm{p}=0.002)$ ef $(\mathrm{p}<0.01)$ \\
\hline
\end{tabular}

The florfenicol group had a lower mortality rate (1.7\%) than the tilmicosin group (4\%) though this was not statistically significant. However, significantly fewer pigs in the florfenicol group $(9.6 \%)$ received injectable treatment than in the tilmicosin group (14.9\%) $(p=0.031)$.

Table 3: Production data

\begin{tabular}{|l|c|c|}
\hline & Florfenicol & Tilmicosin \\
\hline Feed intake $(\mathrm{g})$ & 583 & 579 \\
\hline FCR & 1.56 & 1.72 \\
\hline
\end{tabular}

Table 3 indicates a 9\% improvement in FCR of the florfenicol group over the tilmicosin group ( $160 \mathrm{~g}$ less feed per kilogram gained). This represents an extra intake of 2.26 kilograms of feed in the tilmicosin group during this period.

\section{Conclusions}

It can be concluded that florfenicol (Nuflor 40mg/g Premix for Swine) in pre-starter feed for prevention of PRDC on an Iberian pig farm was safe.

Also,compared to treatment with tilmicosin at recommended inclusion rate, florfenicol led to better mortality rate and FCR, and statistically significantly better ADG, end weight, and \%age animals receiving injectable treatment.

The in-feed use of florfenicol can be recommended as effective in reducing the effects of PRDC (1).

\section{References}

1. Palomo A. et al. 2nd Symposium Veterinary Sciences. Zaragoza (Spain) 2008, pag 37. 


\title{
P.372
}

\section{Draxxin metaphylaxis against Swine Respiratory Disease (SRD) in suckling piglets: benefits on performance and morbidity}

\author{
Ian A. Nanjiani ${ }^{1}$ Joaquin Morales $^{2}$ Carlos Piñeiro ${ }^{2}$ Pascale Sierra $^{3}$ JensChristian E. Jensen 4 \\ 1. Pfizer VMRD, Sandwich, UK; 2. PigCHAMP Pro Europa SA, Segovia, Spain; 3. TVIVERITAS France, Gerstheim, France; \\ 4. Orion Pharma Animal Health, Nivå, Denmark
}

\section{Introduction}

Swine Respiratory Disease (SRD) is a complex multifactorial disease which has substantial economic and welfare impacts on the swine industry, particularly when present in young animals. Disease control is generally achieved by environmental control, vaccination and antibiotic treatment. This GCPV compliant study was conducted to investigate the effects of a metaphylactic treatment administered early to piglets at risk of SRD during their first week of life on the disease and their subsequent growth when compared to a conventional "intervention" approach.

\section{Materials and Methods}

One hundred and twenty litters containing 1170 Large White $x$ Landrace piglets were included in fives batches on two commercial farms sites in Spain with history of respiratory disease in young piglets.

Litters were randomised within airspace to either: T01 - metaphylactically treated with tulathromycin $(2.5 \mathrm{mg} / \mathrm{kg}$ Draxxin , Pfizer Ltd), at 3 days of age on Day 0 (60 litters, 582 piglets); or T02 - control group (60 litters, 588 piglets) individuals treated therapeutically with amoxycillin injection when showing signs of SRD. Animals were observed daily for signs of abnormal health; veterinarians conducting clinical examinations were unaware of the treatment group attributed to each pig. All piglets were weighed on Day 0 prior to treatment and on the day of weaning (Day 24 or Day 25). Any piglet (T01 and T02) diagnosed with SRD during the observation period was treated with amoxicillin long acting injectable at the recommended dose rate of 15 $\mathrm{mg} / \mathrm{kg}$ repeated at a 48-hours interval if necessary.

The primary variable for Draxxin metaphylaxis effectiveness was the average daily weight gain between Day 0 and weaning (ADG). Secondary variables included the homogeneity of weaning body weight per litter, the percentage of pigs with SRD during the observation period, and the number of days and injections for treatment for SRD.

\section{Results}

Clinical signs of respiratory disease were observed in all study batches, though they were generally mild in character. Pasteurella multocida, Haemophilus parasuis and Streptococcus suis were isolated (nasal or lung swabs taken from pigs with SRD), and serological evidence of Actinobacillus pleuropneumoniae was present on both sites.
Table 1. Homogeneity of final body weight per litter, weaning weights, ADG \& Proportion requiring therapy

\begin{tabular}{|l|c|c|c|c|c|c|}
\hline \multirow{2}{*}{ Group (No. of pigs) } & \multicolumn{2}{|c|}{ Homogeneity1 } & \multicolumn{2}{c|}{ Body Weight } & \multicolumn{2}{|c|}{ ADG (g/day) } \\
\cline { 2 - 5 } & BW Day 0 & $\begin{array}{c}\text { BW Day } \\
\mathbf{2 5}\end{array}$ & $\begin{array}{c}\text { Initial } \\
\text { Weight } \\
\text { (kg) }\end{array}$ & $\begin{array}{c}\text { Weaning } \\
\text { Weight } \\
\text { (kg) }\end{array}$ & \multicolumn{2}{|c|}{$\begin{array}{c}\text { \% needing SRD } \\
\text { therapy }\end{array}$} \\
\hline T01 Draxxin (582) & 82.04 & 80.68 & 2.286 & 8.092 & 237.3 & 3.09 \\
\hline T02 Control (588) & 82.19 & 78.76 & 2.365 & 7.906 & 229.5 & 4.59 \\
\hline SEM & 0.636 & 1.227 & 0.079 & 0.326 & 15.67 & 0.795 \\
\hline P-value & 0.86 & 0.07 & 0.30 & 0.0627 & 0.0604 & 0.0825 \\
\hline
\end{tabular}

${ }^{1} 100$ - Coefficient of Variation of bodyweight (higher values reflect greater homogeneity)

\section{Discussion}

The use of injectable antibiotics for metaphylaxis allows the effective treatment of an at-risk group by ensuring accurate, targeted dosing of the individual animal. This study is consistent with recent studies in showing respiratory problems early in current swine production systems. In this study, a tulathromycin metaphylaxis approach during a mild disease outbreak produced a tendency to better weight gain and higher bodyweight at weaning, more homogeneous litter weights at weaning and less need for subsequent antibiotic treatment in the metaphylactically treated group when compared to a conventional "intervention" approach.

\section{References}

Sibila, M. et al. Veterinary Microbiology, 2007, 121: 352-356.

Moorkamp, L. et al. Transboundary and Emerging Diseases 2009, 56 (1-2): 54-56.

Morales, J., Piñeiro, C \& Sierra, P.. Anaporc, November 2009: 21-26. 


\title{
P.373
}

\section{On-farm comparison of the efficacy and profitability of Draxxin ${ }^{\circledast}$ vs. medicated- feed for the prevention of Swine Respiratory Diseases on weaners}

\author{
Cyrille Malherbe $^{2}$ Audrey Dedeurwaerdere ${ }^{1}$ Arlette Laval $^{3}$ \\ 1. Pfizer Animal Health, Paris, France; 2. Cabinet Vétérinaire Gâtines, Valençay, France; 3. ONIRIS National Veterinary School, Nantes, France
}

\section{Introduction}

The possible transmission of most respiratory pathogens and the heavy consequences of early SRD clinic on pigs' performances make the weaning period the most critical moment for controlling SRD. This can be achieved using in-feed medication, a practical and often satisfactory way of preventing SRD on weaners. An alternative way is the use of single-shot, long-acting injectable antibiotics. In Europe Draxxin ${ }^{\circledR}$ (tulathromycin 100mg/ $\mathrm{mL}$ ) is labeled for the treatment and prevention of SRD associated with Pasteurella multocida, Actinobacillus pleuropneumoniae Mycoplasma hyopneumoniae and Haemophilus parasuis.

This study compared on-farm results of a single shot of Draxxin ${ }^{\circledast}$ at weaning to the normal farm practice using a tilmicosine medicated starter. Focus here was on clinical efficacy and profitability at batch level, while a second trial investigated further clinical results at individual level.

\section{Materials and Methods}

Herd history The farrow-to-finish herd, located in western France, showed a high SRD pressure: PRRS, PCV2 and Mhyo positive, plus history of App. Clinical outbreaks were under control via in-feed medication on weaners and occasional oral treatments on growers. Decreased performance and increased heterogeneity were reported in the post weaning units. Due to lack of palatability, the two-week administration of medicated starter (300ppm tilmicosine) was delayed one week from weaning (21 to 28 days of age).

Longitudinal study Five consecutive batches were included at 2-weeks interval of 21 days-old piglets between April and August 2009. The first two batches $(n=666)$ followed the normal farm practice (NF group). The other batches $(n=938)$ received a single IM injection of tulathromycine at $2.5 \mathrm{mg} / \mathrm{kg}$ on weaning day instead of the in-feed medication (Draxxin group). No other parameter changed.

Data Mortality was registered at batch level during the 7.5 weeks of post-weaning period. Average daily weight gain (ADG) and feed consumption ratio (FCR) were recorded at pen level from 21 to 42 days of age. These data were analysed with a T-test for independent populations. The ROI was calculated based on the 2008 French National references for farrow-to-finish farms and translated with Osiris ${ }^{\circledR}$ software (Pfizer). Extra-labor and treatment costs were registered on-farm.

\section{Results}

Performance data The mortality rate over the weaning period significantly decreased by $1.2 \%$ in the Draxxin group. ADG and FCR showed no significant differences.
Table I Comparison of the mortality rate, ADG and FCR [means (interquartile range)]

\begin{tabular}{|l|c|c|c|}
\hline & Mortality (\%) & ADG (g/d) & FCR \\
\hline NF group & $1.95^{\mathrm{a}}$ & $321.6(70)$ & 1.46 \\
\hline Draxxin group & $0.75^{\mathrm{b}}$ & $298.2(40)$ & 1.28 \\
\hline $\mathrm{a}, \mathrm{b}: \mathrm{p}$-value $<0.05$ & & \\
\hline
\end{tabular}

Return On Investment analysis In this case, the dispenser injected 250 piglets per hour. Variations of performance results only between the groups added to $1.52 €$ gain/pig. Overall profitability was 1.74€/pig for Draxxin group.

Table II Variation of result and benefit per ROI data

\begin{tabular}{|l|c|c|}
\hline & $\begin{array}{c}\text { Difference NF/Draxxin } \\
\text { groups }\end{array}$ & $\begin{array}{c}\text { Gain/pig for Draxxin } \\
\text { compared to NF group }\end{array}$ \\
\hline Mortality & $-1.2 \%$ & $+0.82 €$ \\
\hline ADG & $-23.4 \mathrm{~g} / \mathrm{d}$ & $-0.66 €$ \\
\hline FCR & -0.18 & $+1.36 €$ \\
\hline Labor cost & & $-0.08 €$ \\
\hline Treatment cost & & $+0.30 €$ \\
\hline
\end{tabular}

\section{Discussion}

Although not a randomised study, this investigation included multiple batches and management was consistent between batches, so reliable conclusions can be drawn on the efficacy of the products. During this observation period the use of Draxxin ${ }^{\circledast}$ at weaning proved better clinical and economical results than normal farm in-feed medication. ADG was less variable and the feed-conversion efficiency was numerically higher in the Draxxin-treated batches.

A possible explanation for these findings may reside in treatment compliance. Given the individual variations of feed intake (linked with observed lack of palatability), especially on young weaners of 21-days of age in case of high SRD pressure, a proportion of piglets may not receive the calculated dose of oral medication. Thus it would lead to a fraction of poor-performing and unhealthy piglets, diminishing the general performances of the batch. This bias disappears with single-shot injectables, guarantying full compliance and superior overall efficacy of the treatment.

\section{References}

Malherbe $C$., thèse vétérinaire 2010, submitted

Nanjiani et al 2009; Obiettivi e Documenti Veterinari 5, 35 - 43

Morales, J., Piñeiro, C \& Sierra, P.. Anaporc, November 2009: 21-26. 


\title{
P.374
}

\section{On-farm comparison of the clinical efficacy of Draxxin ${ }^{\circledR}$ vs. oxytetracycline medicated- feed for the prevention of Swine Respiratory Diseases in at-risk weaners}

\author{
Cyrille Malherbe $^{2}$ Audrey Dedeurwaerdere ${ }^{1}$ Arlette Laval $^{3}$ \\ 1. Pfizer Animal Health, Paris, France; 2. Cabinet Vétérinaire Gâtines, Valençay, France; 3. ONIRIS National Veterinary School, Nantes, France
}

\section{Introduction}

The possible transmission of most respiratory pathogens and the heavy consequences of early SRD disease on performance make the weaning period a critical time for controlling SRD. This is often achieved using in-feed medication, a practical and often satisfactory way of preventing SRD on weaners. An alternative way is the use of single-shot, long-acting injectable antibiotics. In Europe Draxxin ${ }^{\circledR}$ (tulathromycin $100 \mathrm{mg} / \mathrm{mL}$ ) is licensed for the treatment and prevention of SRD associated with Pasteurella multocida, Actinobacillus pleuropneumoniae Mycoplasma hyopneumoniae and Haemophilus parasuis.

This study compared the efficacy of a single shot of Draxxin ${ }^{\circledR}$ at weaning to the normal farm practice using a medicated starter, in two different herds in normal farm conditions. The first part of the trial (vs. tilmicosin, separate poster) addressed clinical efficacy and profitability at batch level. In this second herd clinical results were investigated further at individual level as well.

\section{Materials and Methods}

Herd history The farrow-to-finish herd was located in Normandy, France and was positive for SIV, Mhyo and (historically) App. The farm was reported to be PRRS and PCV2 negative. Regular outbreaks of clinical respiratory disease occurred in the postweaning units, with high morbidity, individual weight loss and heterogeneity commonly reported. Normal farm practice scheduled preventive treatment for 3 weeks at weaning (medicated starter, oxytetracycline, $1000 \mathrm{ppm}$ ) then additional oral treatment (20mg OTC/kg BW x 4 to 5 days) for therapy when needed.

Longitudinal study Six consecutive batches of 21 days-old piglets were included at 1-week intervals between July and August 2009. Three batches $(n=944)$ followed the normal farm practice (NF group). The other batches $(n=1155)$ received a single IM injection of tulathromycine at $2.5 \mathrm{mg} / \mathrm{kg}$ at weaning (Draxxin group) instead of the starter medication. No other parameter changed. In one batch of each group 120 piglets from 10 litters were chosen at random by the dispenser and identified for individual observations.

Data Mortality \& additional oral treatment were recorded at batch level during the 7.5 weeks of post-weaning. Clinical examinations and weighings were recorded once a week up to 21 days after weaning. Quantitative data were analysed with a T-test for independent populations, qualitative ones with Pearsonx test.

\section{Results}

Mortality rate decreased from $1.68 \%$ to $0.65 \%(p=0.021)$ and therapeutic oral treatment from 3.71 to 1.42 days/pig $(p=0.007)$ in favor of the Draxxin group. Mean pen ADG reflected the mean individual ADG recorded per 1-week period, significantly superior in the Draxxin group.
Table 1: Development over time of respiratory signs and ADG

\begin{tabular}{|c|c|c|c|c|c|c|}
\hline & \multicolumn{2}{|c|}{$\begin{array}{l}\text { Mean coughing } \\
/ 2^{\prime} / 100 \text { pigs }\end{array}$} & \multicolumn{2}{|c|}{$\begin{array}{l}\text { Nb of pigs with } \\
\text { dyspnoea (ind**) }\end{array}$} & \multicolumn{2}{|c|}{$\begin{array}{c}\text { Mean ADG } \\
(\mathrm{g} / \mathrm{d} / \mathrm{pig}, \text { ind })\end{array}$} \\
\hline & NF group & $\begin{array}{l}\text { Draxxin } \\
\text { group }\end{array}$ & NF group & $\begin{array}{c}\text { Draxxin } \\
\text { group }\end{array}$ & NF group & $\begin{array}{l}\text { Draxxin } \\
\text { group }\end{array}$ \\
\hline DO & $0.97^{*}$ & $0.10^{*}$ & $2^{*}$ & $3^{*}$ & & \\
\hline D7 & $0.32^{*}$ & $0.22^{*}$ & 16 & 5 & 73.5 & 157.6 \\
\hline D14 & 4.05 & 0.08 & 22 & 8 & 190.5 & 279.6 \\
\hline D21 & 13.77 & 0.98 & 30 & 16 & 358.8 & 441.9 \\
\hline & $p<0.025$ & & $p<0.025$ & & $p<0.0001$ & \\
\hline
\end{tabular}

\section{Discussion}

Although not a randomised study, this investigation included multiple batches and management was consistent between batches, so reliable conclusions can be drawn on the clinical efficacy of the preventive treatments. Dramatic difference in individual weight gains in the first days after weaning while no visible clinical sign (at individual or group level) was observed, supports the hypothesis of an early viral challenge, then of secondary respiratory infections noticeable at piglet level 7 days after weaning, and at group level after 2 weeks.

The difference in clinical efficacy between the two preventive treatments may then reside in compliance. Indeed, early challenged piglets, even if not visibly sick, won't eat their share. Added to the individual variations of feed intake, especially on newly weaned piglets of 21 days of age, one can assume that a proportion of piglets would not receive the calculated dose of oral medication. This would lead to a fraction of unhealthy and poor-performing piglets, diminishing the general performances of the batch - and increasing the risk of SRD transmission. This bias disappears with single-shot injectable antibiotics, guarantying full compliance and superior overall efficacy of the preventive treatment.

\section{References}

Malherbe $C$., thèse vétérinaire, submitted.

Nanjiani et al 2009; Obiettivi e Documenti Veterinari 5,35-43

Morales, J., Piñeiro, C \& Sierra, P.. Anaporc, November 2009: 21-26. 


\title{
P.375
}

\section{Pulmotil ${ }^{\otimes}$ (Tilmocosin) in feed reduces clinical disease associated with PRDC resulting in increased nursery performance}

\author{
Chanunda Raphanaphraiwan ${ }^{2}$ Ampol Chayomchai ${ }^{2}$ Thitima Tripipat $^{1}$ Dachrit Nilubol ${ }^{1}$ \\ 1. Faculty of Veterinary Science, Chulalongkorn University, Bangkok, Thailand; \\ 2. Elanco Animal Health A Division of Eli Lilly Asia, Inc. Thailand Branch, Bangkok, Thailand
}

\section{Introduction}

Several reports suggest that the severity of PRDC associated clinical diseases reduced when Pulmoti ${ }^{\otimes}$ (Tilmicosin, Elanco Animal Health, Thailand) was used1, 2. The objectives of the study were to investigate the efficacy of Pulmotil ${ }^{\circledR}$ in feed as an early intervention regimen in the reduction of PRDC associated clinical disease and to observe the antibody response against PRRSV as measured by ELISA (Idexx, USA) in pigs administered Pulmoti ${ }^{\circledast}$ in feed.

\section{Materials and Methods}

PRRSV status of the experimental herd was considered stable/ inactive in which clinical diseases associated with PRRSV infection and PRDC were observed in the nursery period. 266 barrows were randomly selected based on the stratification of weight and ear-tattooed. Pigs were allocated into 2 groups (Control and Pulmotil ${ }^{\circledR}$ groups. Pigs were weaned at 18-21 days of age into a wean-to-finish facility located few kilometers away from the sow herd. Upon arrival,each group was housed in 2 pens. Pigs in control group $(n=140)$ were medicated in feed with Amoxycillin, Tiamulin and Colistin at the dosages of 200, 100 and 80 ppm, respectively. Pigs in Pulmotil ${ }^{\circledR}$ group $(n=126)$ were medicated in feed for 3 consecutive weeks with Pulmotil ${ }^{\circledR}$ and Colistin at the dosages of 200 and 150 ppm, respectively. All pigs were weighed individually at weaning and 8 weeks of age. Feed consumption was monitored daily. Average daily gain (ADG) and feed conversion ratio (FCR) were calculated. Death and cull were recorded. Ten same pigs were followed serologically for antibody response against PRRSV as measured by ELISA (Idexx, USA) at weaning and 8 weeks of age. At 8 weeks of age, 5 pigs were randomly selected from each group and necropsied to assess the pneumonic lung lesion. Bacterial culture was performed in all lungs. Bronchial alveolar lavage fluid (BAL) samples were collected and assayed for the presence of PRRSV by PCR. The study was done in duplicate.

\section{Results}

Nursery performance including antibody response as measured by ELISA is displayed in Table 1. Pigs on Pulmotil ${ }^{\circledR}$ in feed had ADG and \% CV of age at 8 weeks of age slightly better than pigs in the control group and the difference was not statistically significant.

\begin{tabular}{|l|c|c|}
\hline \multicolumn{1}{|c|}{ Parameters } & & \\
& Control & Pulmotil \\
& & \\
\hline Average Weight at 3 weeks $(\mathrm{Kg})$ & 6.15 & 6.14 \\
\hline Average Weight at 8 weeks $(\mathrm{Kg})$ & 21.3 & 22.9 \\
\hline Average weight Gain $(\mathrm{Kg})$ & 15.45 & 16.76 \\
\hline ADG (Kg/day) & 0.51 & 0.51 \\
\hline FCR & 1.2 & 1.2 \\
\hline$\%$ CV of Weight at 8 weeks & 19.25 & 13.76 \\
\hline Average S/P ratio at 3 weeks & 0.63 & 0.54 \\
\hline Average S/P ratio at 8 weeks & 0.67 & 0.12 \\
\hline
\end{tabular}

Bacterial Secondary bacterial infection was found in the control pigs and none was detected in the Pulmotil group. PCR results indicated that all BAL samples of the control pigs were positive for PRRSV, while that of Pulmotil ${ }^{\circledR}$ pigs were negative. In addition, most of the control pigs increased in their antibody response against PRRSV. The S/P ratio of the Pulmotil ${ }^{\circledR}$ pigs, however, decreased and was below the cut-off level (0.4)

\section{Discussion}

The results of the study suggested that the administration of Pulmotil in feed reduced the severity of the clinical disease associated with PRRSV and PRDC resulting in increased nursery performance.
References
1. Benfield, D.A. 2002 AASV: 87 - 91
2. Lehe, K. 2003 AASV: $129-130$ 


\title{
P.376
}

\section{Porcine respiratory disease complex, from diagnosis to efficient prevention: the role of infection dynamics and the quality of vaccination}

\author{
Henri Guilmoto \\ Selas Vétérinaire de la Hunaudaye, Lamballe, France
}

\section{Introduction}

The practitioner needs perseverance and critical self-appraisal. This is illustrated by this case report which starts with a complaint of a drop in carcass weight (CW) and mortality in spite of piglets PRRS vaccination.

\section{Materials and methods}

The case concerns a 350 sow breeding-finishing farm, 28 days weaning once a week, all in / all out post-weaning and finishing, dynamic depression ventilation, in a low pig density area. Piglets are weighed at weaning and at the end of post-weaning. The farm is known to be infected by the influenza virus, M. hyop, PRRSV, $H$. parasuis and P. multocida. It is free from APP. The respiratory disease control plan includes vaccinations of the breeding stock against rhinitis, influenza, and PRRS (live). This last is also used in piglets at 7 weeks of age. There is no Mh vaccination and no antibiotic prevention after the nursery feed.

June 05: PRDC, with the "18-week wall" and CW 6 kg less than before. Observations at the slaughterhouse and the absence of vaccination lead to the diagnosis of enzootic pneumonia. Mh vaccination was set up, and PRRS vaccination maintained. Situation did not improve. Flu-like episodes continued, and lab analysis indicated the presence of SI, PCV2, PRRS and Mh.

Dec. 06: Follow-up of 12 sentinel piglets showed a late circulation of Mh and an early circulation of PRRSV. Necropsy and histology confirmed Mh infection. Antibiotic prevention was set up during 22 weeks, while PRRS vaccination was suspended. In parallel, three further elements were examined (Jan.-Apr. 2007): (a) colostrum analysis showed an absence of antibodies against atrophic rhinitis despite sow vaccination, (b) an analysis of weight at the start of finishing showed a sudden drop of over $3 \mathrm{~kg}$ in 2005. In the same batches, a drop in carcass weight was observed, (c) PRRSV PCR in sentinel showed early circulation (6-10 wks).

April 2007. The following action plan was up:

(a) Continuation of vaccination against Mh (PORCILIS M HYO at 1 and 4 weeks of age),

(b)earlier vaccination against PRRS (PORCILIS PRRS at 4 instead of 7 weeks of age).

\section{Results}

After adjusting the timing and quality of vaccination, a clear improvement was observed: improvement of mortality rates and lung scores (Figure 1), increase of body weight at the start and end of the finishing period (Figure 2). Taken over 6 years, data show that, after an important drop in performance between 04 and 06, all indicators of 08 and 09 were better than in 04 (Table $1)$.
Figure 1: Losses and lung scores (Nov. 06 to Oct. 08)

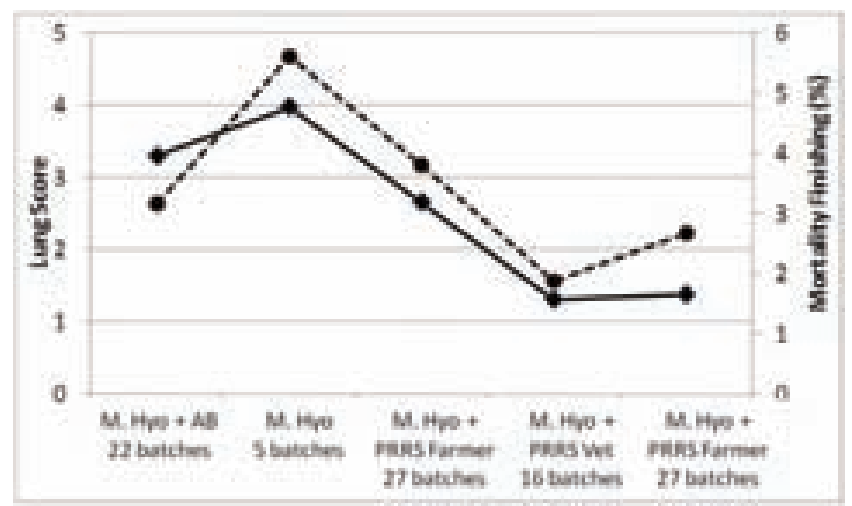

Figure 2: Body weights evolution (Nov. 06 to Oct. 08)

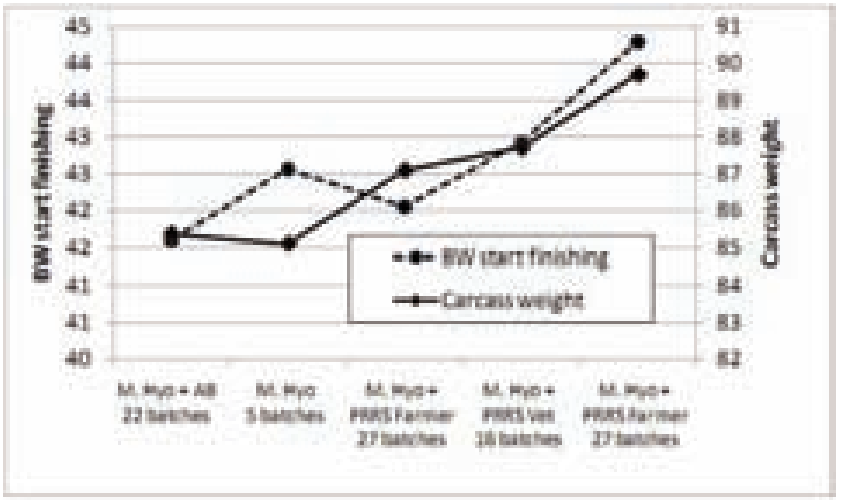

Table 1: Evolution of results over 6 years

\begin{tabular}{|l|c|c|c|c|c|c|}
\hline & $\mathbf{0 4}$ & $\mathbf{0 5}$ & $\mathbf{0 6}$ & $\mathbf{0 7}$ & $\mathbf{0 8}$ & $\mathbf{0 9}$ \\
\hline BW start finish & 42.6 & 39.0 & 40.3 & 41.5 & 43.4 & 43.5 \\
\hline Mortality finish & 6.4 & 7.4 & 6.7 & 3.0 & 2.1 & 2.5 \\
\hline Carcass wt & 86.9 & 84.7 & 84.2 & 85.6 & 88.4 & 89.8 \\
\hline FCR & 2.93 & 3.04 & 3.16 & 2.90 & 2.85 & 2.8 \\
\hline
\end{tabular}

\section{Conclusion}

- Consider the impact of postweaning on finishing.

- Check quality of vaccination

- Record technical and used them as a diagnostic tool.

- Use diag. lab and observations in the slaughterhouse.

\section{Acknowledgements}

The author would like to thank the farmer for his trust and Intervet Schering-Plough $\mathrm{AH}$ for its support. 


\title{
P.377
}

\section{Field observation of the effects on the performance of weaned piglets of Pulmotil ${ }^{\mathrm{TM}}$ in large farms in Spain}

\author{
$\underline{\text { Xavier de Paz }}{ }^{1}$ Susana Lapuente ${ }^{1}$ Miguel Abad ${ }^{1}$ Joan Sanmartin² Gonzalo Cano ${ }^{2}$ \\ 1. Elanco Animal Health, Madrid, Spain; 2. Optimal Pork Production, Lleida, Spain
}

\section{Introduction}

The presence of respiratory disease increases medication costs but also causes a loss of performance (ADG and FCR) since energy and protein is diverted towards fighting disease (1).

The objective of the present field observations was to evaluate the effects of a standard medication with Pulmotil ${ }^{T M}$ at weaning to prevent respiratory disease in two large farms in Spain.

\section{Materials and Methods}

Two different in-feed treatment programs were compared after weaning: a control group $(C)$ received a prestarter feed with 120ppm of colistin and 3200ppm of $\mathrm{ZnO}$ and a pulmotil group $(P)$ that received a prestarter feed which included 400ppm of Pulmotil besides 120ppm of colistin and 3200ppm of ZnO. Every piglet received $5 \mathrm{~kg}$ of this prestarter feed. Also, all the piglets were vaccinated against M.hyo at one week of age. The data of these field observations came from 18015 weaned piglets coming from 2 different sow farms during 10 consecutive weeks.

Farm 1: 2480 sow-farm where piglets are weaned at 21 days and moved to nursery facilities in another location for approximately 6 weeks.

Farm 2: 4865 sow-farm where piglets are weaned at 21 days and moved to nursery facilities in another location for approximately 6 weeks.Data was analyzed using the GLM procedure of SAS System. The model includes the treatment effect and batch was the experimental unit. No statistical differences were found neither for weight or age at start of trial so no covariate was included. Mortality was analysed using the logistic regression with treatment as fixed effect.

\section{Results and Discussion}

The global results are summarized in table 1.

Table 1. Production parameters observed in the two nursery farms

\begin{tabular}{|l|c|c|c|c|c|c|}
\hline & $\begin{array}{c}\text { Piglets in } \\
(\mathbf{n})\end{array}$ & $\begin{array}{c}\text { Piglets } \\
\text { out (n) }\end{array}$ & $\begin{array}{c}\text { Mortality } \\
(\%)\end{array}$ & $\begin{array}{c}\text { Initial LW } \\
(\mathbf{k g})\end{array}$ & $\begin{array}{c}\text { Final LW } \\
(\mathbf{k g})\end{array}$ & ADG (g) \\
\hline Control & 9032 & 8931 & 1.12 & 6.10 & 18.01 & 304.39 \\
\hline Pulmotil & 8983 & 8916 & 0.75 & 6.02 & 19.06 & 333.32 \\
\hline sig. (p=) & & & 0.0098 & 0.52 & $<0.0001$ & $<0.0001$ \\
\hline
\end{tabular}

The initial liveweight (LW) of the piglets was not different between both treatments $(p=0.52)$ but at the end of the nursery period ( 60.2 days of life) the piglets in the Pulmotil group were heavier (18.01 kg vs $19.06 \mathrm{~kg} ; \mathrm{p}<0.0001)$.

The ADG of the piglets was higher in the Pulmotil group $(304 \mathrm{~g} / \mathrm{d}$ vs $333 \mathrm{~g} / \mathrm{d} ; \mathrm{p}<0.001)$. The mortality rate in the Pulmotil group was slightly lower than in the Control group ( $C=1.12 \%$ vs $\mathrm{P}=0.75 \%)$.

The treatment with Pulmotil affect significantly $(p=0.0098)$ the mortality of piglets. Also, the odds ratio for mortality was 1.505 (1.10-2.0 Confidence interval at 95\%) so Pulmotil piglets had less probability to die.

\section{Conclusions}

The inclusion of Pulmotil in the prestarter feed of piglets improves animal performance even in farms with good health status (around 1\% mortality rate). As seen in other studies (2), the piglets in the Pulmotil group were heavier at the end of the nursery (1.05kg more at 60.2 days of life). Therefore, the piglets in the Pulmotil group grew more which enables them to increase the Full Value Pigs ${ }^{T M}$ in the fattening unit.

\section{References}

1. Dial G, et al. Costs, consequences and control of endemic diseases. London Swine Conference 2002.

2. Harker JW. et al. The impact of Pulmotil feeding in the nursery on finishing performance of at-risk pigs. AASV 2006. 


\title{
P.378
}

\section{Relationship between lung lesions and haptoglobin serum concentration in slaughter pigs}

\author{
Christelle Fablet; Virginie Dorenlor; Florent Eono; Eric Eveno; Jean-Pierre Jolly; Gaëtan Marquier; Eric Boilletot; François Madec \\ AFSSA-Site de Ploufragan, Ploufragan, France
}

\section{Introduction}

During the first step of the immune defence, the concentrations of a group of plasmatic proteins called the Acute Phase Proteins (APPs) change. These APP seem to be sensitive and accurate biomarkers of pig health. Therefore quantification of their concentrations in plasma or serum of pigs could provide valuable diagnostic information for prognosis and monitoring disease [1]. In intensive production systems, respiratory disorders are considered as the most serious problem affecting finishing pigs worldwide and represent one of the major health issues [2]. The aim of the study was to assess the relationship between macroscopic lung lesions and serum concentration of one APP: haptoglobin ( $\mathrm{Hp})$ in slaughter pigs.

\section{Materials and Methods}

A sub sample of 70 pig farms was selected from a sample of 125 French farrow-to-finish herds included in an analytical survey designed to identify risk factors for Porcine Respiratory Disease Complex. The 70 farms were selected according to their pneumonia mean score, assessed on a sample of 30 slaughtered pigs:

- mean score $\leq 1,7,37$ herds (mild pneumonia)

- mean score $\geq 5,5,33$ herds (severe pneumonia)

For each herd, at the slaughterhouse, individual blood samples were obtained at exsanguination from a sample of 10 finishing pigs selected at random. For every pig the lungs were collected on the slaughter line and pneumonia, pleuritis and abscesses were recorded. As regard enzootic pneumonia, the lungs were scored [3]. In a second step, pigs were classified into 4 groups according to the extent of pneumonia (0:no lesion, $1 \leq$ score $\leq 4$; $5 \leq$ score $\leq 8$; score $\geq 9$ ). The serum level of $\mathrm{Hp}$ was measured by a spectrophotometric method using a commercial assay kit (Tridelta Development, Greystones, Ireland). Differences in serum $\mathrm{Hp}$ concentrations between healthy pigs vs those showing pleuritis or abcesses were assessed using Mann and Whitney test whereas Kruskal-Wallis test was used for pneumonia $(p<0.05 ;$ SAS Inc.).

\section{Results}

Among the 700 selected pigs, the correspondence between blood and lung sampling was achieved for 517 animals. The levels of $\mathrm{Hp}$ were not significantly different between pigs showing or not either pleuritis or lung abscesses $(p>0.05$,
Table 1. Mean serum concentration of $\mathrm{Hp}$ was associated with the severity of enzootic pneumonia $(p<0.05$,

\begin{tabular}{|c|c|c|c|c|}
\hline \multirow[b]{2}{*}{ 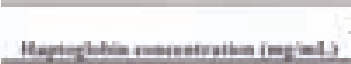 } & \multicolumn{2}{|c|}{ Pratith } & \multicolumn{2}{|c|}{ Alomen: } \\
\hline & Aneres & Proutser & Alouser & hours: \\
\hline Minien & 8 & "1" & 7 & 8.10 \\
\hline Manines & (4) & 3.71 & 06 & $1 \mathrm{~m}$ \\
\hline Mess & 100 & 1.99 & 1,49 & 1.7 \\
\hline$=$ & 111 & 1.12 & in & $1 \pi$ \\
\hline s. & 414 & 31 & 111 & 4 \\
\hline
\end{tabular}

Table 2.

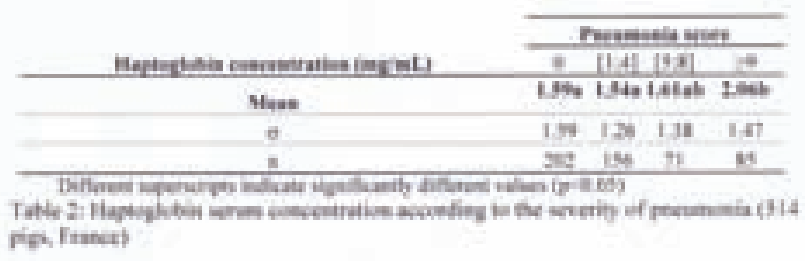

\section{Discussion}

The results of our study indicated that serum Hp concentration in slaughter pigs was higher in case of extended pneumonia whereas no relationships between $\mathrm{Hp}$ levels and pleuritis or lung abscesses were found. To the best of our knowledge, this is the first report on $\mathrm{Hp}$ levels measured in a large sample of commercial pig farms in France. These findings are partially consistent with those of Amory et al., [4] and Pallares et al., [5]. Nevertheless, infectious conditions other than respiratory ones or injury like lameness, diarrhoea, tail or ear biting may induce elevated levels of $\mathrm{Hp}$ [6]. As these conditions were not examined in the present study, it is likely that these kind of diseases have occurred in some pigs and may have induced an Acute Phase Response. This may be one explanation of the relative "high" mean value of Hp observed in the group of pneumonia free pigs when compared to values encountered in commercial healthy finishing pigs. This is in line with previous literature conclusions telling that $\mathrm{Hp}$ should be considered as a general biomarker rather than a specific respiratory infection indicator.

\section{References}

1. Eckersall, P. (2000). Rev. Med. Vet., 151, 577-584.

2. Sorensen, V. et al. (2006). Diseases of Swine, 9th edition, Straw, B. et al., Editors. 149-177.

3. Madec, F. and Kobisch, M. (1982). JRP, 14, 405-412.

4. Amory, J.R. et al. (2007). Res. Vet. Sci., 83, 428-435.

5. Pallares, F.J. et al. (2008). Vet. J., 177, 369-373.

6. Fablet, C. and Madec, F. (2009). Rev. Med. Vet., 160, 28-43. 


\title{
P.379
}

\section{Impact of porcine respiratory disease complex on the efficacy of classical swine fever vaccination}

\author{
Ming T. Chiou ${ }^{1}$ Cheng Y. Yang ${ }^{2}$ Tsung C. Chang ${ }^{1}$ Ming L. Hsieh ${ }^{1}$ \\ 1. National Pingtung University of Science and Technology, Pingtung, Taiwan; \\ 2. Animal Technology Institute Taiwan, Chunan, Taiwan
}

\section{Introduction}

Porcine respiratory disease complex (PRDC), an endemic disease caused by co-infection with several respiratory pathogens, typically adversely affects pig weaning and growth, leading to considerable economic losses worldwide. Porcine circovirus type 2 (PCV2) and porcine reproductive and respiratory syndrome virus (PRRSV) are the most common pathogens causing PRDC; these pathogens cause severe respiratory disorders and interfere with pig immune function. Classical swine fever (CSF) is a highly contagious disease. In many countries vaccination is the main strategy for protection against CSF. Notably, CSF vaccine is generally administered after weaning. Therefore, determining whether the CSF vaccine efficacy is influenced by PRDC in pigs is warranted.

\section{Materials and Methods}

Based on routine pathogen screening and polymerase chain reaction (PCR) analysis results, this study selected 2 farrow-tofinish pig herds with a history of PRDC infection for more than 3 years. Thirty 4-week-old weaned piglets were selected randomly from each herd and ear tagged. Half of the pigs from each herd were housed in the same barn in independent pens with nontested weaned pigs (group A). The remaining tested pigs were transferred into barrier-separated animal rooms after weaning (group B). All tested pigs were provided the same feed. Food and water were provided ad libitum. Both groups were vaccinated with 1 dose of Lapinized China (LPC) strain CSF virus at 6 and 9 weeks of age. Blood samples were collected from each pig at $4,6,9,12$, and 15 weeks. Clinical signs were recorded during regular visits. Competitive enzyme-linked immunosorbent assay (ELISA) tests were performed to identify CSF-specific antibodies. All study protocols were approved by the Animal Care and Use Committee of National Pingtung University of Science and Technology. Data were analyzed using the Student t-test. Values of $P$ $<0.05$ were considered statistically significant.

\section{Results}

Group A pigs from both herds presented marked clinical signs of respiratory disorder and had a high mortality rate $(4 / 15 ; 26.7$ $\%)$ during the test period. The cause of death for both herds was diagnosed as PRDC, except for the case of 1 pig in herd B. However, pigs from group $B$ in both herds did not show remarkable clinical signs and only 1 pig died due to acute bacterial infection. The serologic profiles of pigs from both herds were extremely similar. Figure 1 shows the serological examination results of herd A pigs. The antibody titers of pigs in both groups were similar at different time points; the titer decreased gradually from weaning to 9 weeks of age, increased markedly after vaccination, and reached a maximum in 15-week-old pigs. The CSFV-specific antibody titer in group A and B pigs did not differ significantly at all tested time points.

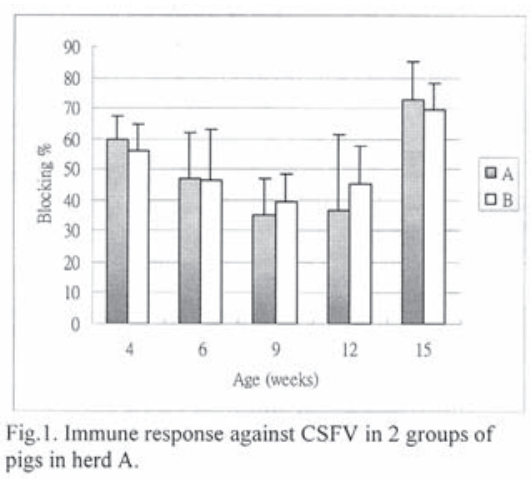

\section{Discussion}

This study housed 2 groups of pigs at different levels in pathogens concentration. The pigs in group A had a higher mortality rate and clearer clinical signs of PRDC compared with pigs in group B. The CSFV-specific antibody titer in 4- to 15-week-old pigs did not differ between the two groups. Unlike previous reports suggesting that some pathogens may alter pig immune function, this study shows that pathogenic agents have no or little effect on the efficacy of CSF vaccination in PRDC-affected pigs.

\section{References}

1. Suradhat S. et al. Vaccine. 24:2634-42, 2006.

2. Chiou MT. et al. The 19 th international pig veterinary society congress. P. 79, 2006. 


\title{
P.380
}

\section{Porcilis AR-T: uniformity of titers optimizing passive protection against Atrophic Rhinitis}

\author{
Marc Martens ${ }^{1}$ Alex Eggen ${ }^{2}$ \\ 1. Veterinarian, Nistelrode, Netherlands; 2. Veterinarian, Malden, Netherlands
}

\section{Introduction}

The infection in piglets that results in Progressive Atrophic Rhinitis (PAR) takes place in the first weeks of life. Sorensen (1990) reported the relationship between high titers against Pasteurella Multocida Toxin (PMT) and the absence of turbinate atrophy. In the swine industry, uniformity of batches is important at all stages of production. To ensure this level of consistency throughout the production system, all piglets need to obtain the same high level of protection.

\section{Materials and Methods}

Trial 1 Breeding stock was classified according to their titer against PMT. (Ridremont, B. et al IPVS 2006). Serological profiles were undertaken on 135 farms, selected by veterinarians, with a history of persistent minor or major PAR problems in spite of vaccination with Porcilis AR-T. Anti-PMT titers were assessed and the animals were grouped according to titer and age. Farms were classified according to the percentage of animals in the different qualitative groups. Advice on the correct vaccination scheme was given to farmers and some farms were checked again, 6 or 10 months later, to determine whether the quality of the serological profile had improved. The following classification was used on both occasions. Excellent: when $80 \%$ of both age groups had a high titer; Good: when $50 \%$ of both age groups had a high titer; Average-1: when $30-80 \%$ in the old sows, and up to $30 \%$ in the young sows had a high titer; Average- 2 : when $30-60 \%$ in the young sows and up to $20 \%$ in the old sows had high titers; Insufficient: when less than $20 \%$ in both age groups had a high titer.

Trial 2 Gilts were vaccinated with either Porcilis AR-T or a competitor vaccine (Supat Suwanna et al IPVS 2008) and the resulting titers were measured. Both vaccines contain PMT and inactivated Bordetella bronchiseptica cells. Gilts of 120 days of age were randomly allocated to one of two groups of 12 and vaccinated with one or other vaccine in accordance with the manufacturer's instructions and recommended vaccination program. The animals were grouped according to their titer measured on D42, 2 weeks after the second dose.

\section{Results}

Trial 1: In figure 1, there is clearly a wide variation amongst breeding stock in their titers against PMT, under field conditions, despite vaccination. Figure 2 shows that the situation can be improved by adopting better vaccination schemes.

Trial 2: Figure 3 shows the distribution of the gilts vaccinated with either Porcilis AR-T or the competitor product over the different $\log 2$ groups.
Figure 1: Percentage of young ( $P 1$ and $P 2)$ and old sows ( $P \geq 3$ ) classified according to their titer (for classification criteria, see above)

Figure 2: Percentage of farms classified according to their titer distribution. Arrows indicate improvement after either adjusting the vaccination scheme or improving on vaccination compliance

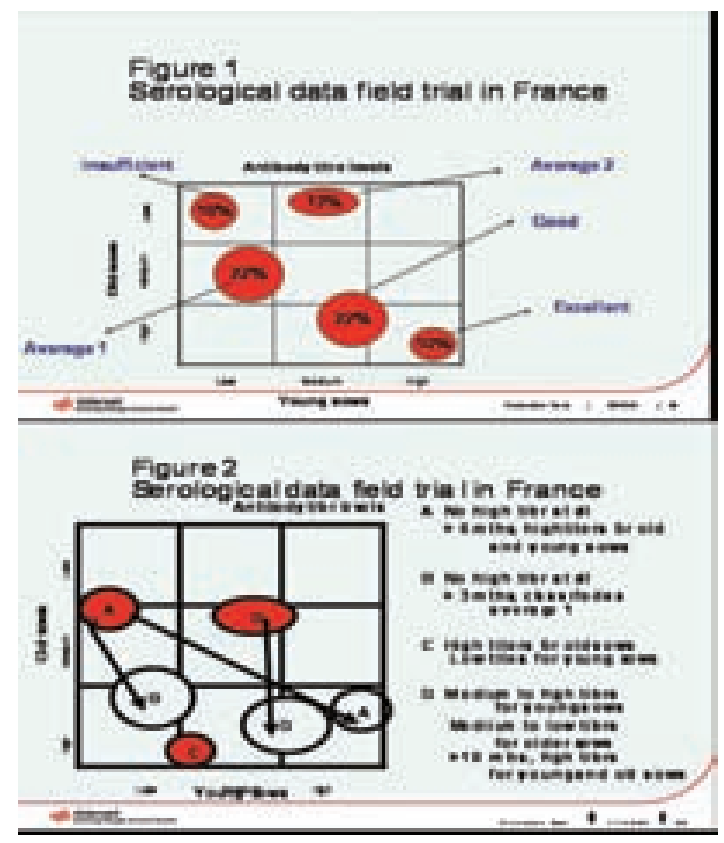

Figure 3: PMT titers of the two vaccine groups

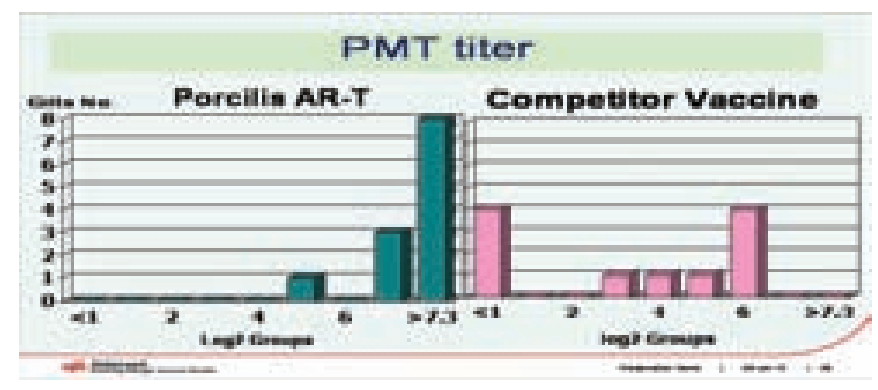

\section{Discussion}

The Trial 1data show improvement after an updated vaccination schedule with more farms achieving uniformly high titers across the different age groups (See farms $A$ and D). Trial 2 shows that vaccines differ in their ability to induce homogeneous titers in sows. The combination of Porcilis AR-T achieving optimal titers and the correct vaccination schedule led to the best results.

\section{References}

Sorensen et al in proc. IPVS 1990 Lausanne p 57.

Ridremont et al in proc. IPVS 2004 Hamburg p 452. 


\title{
The prevalence of DNT and BfrZ genes in Bordetella bronchiseptica
}

\author{
Iwona Markowska-Daniel; Katarzyna Stepniewska; Kinga Urbaniak; Zygmunt Pejsak
}

National Veterinary Research Institute, Pulawy, Poland

\section{Introduction}

Bordetella bronchiseptica (Bbr) is worldwide spread pathogen of pigs' respiratory tract. When infecting the pig alone it may cause mild form of atrophic rhinitis (AR) called NPAR, while invading the respiratory tract in cooperation with Pasteurella multocida $(\mathrm{Pm})$ it may cause progressive changes characteristic for PAR.

Among different factors influencing the pathogenic properties of Bbr dermonecrotoxin (DNT) possessing cytotoxic properties as well as the factor involved in iron acquisition and transport, necessary for Bbr replication and survival, are the most important $(1,2,4)$.

The aim of the study was the detection of the exogenous ferric siderophore receptor gene - BfrZ gene and DNT gene in Bbr strains existing in nasal cavities of healthy and diseased pigs and estimation the correlation between the presence of both genes in Bbr.

\section{Materials and Methods}

Samples. The prevalence of BfrZ gene was checked in 280 DNA samples in total. From this number 150 DNA samples were obtained directly from nasal swabs of pigs, raised in 19 farms, located in 10 provinces. Because symptoms of PAR were observed in some of the farms all DNA samples extracted directly from the swabs were previously tested by PCR with the primers specific for DNT gene of both, Pm and Bbr. 99 DNA samples were obtained from Bbr cultures on G20G agar base in which the presence of DNT gene was previously confirmed. The remaining 31 samples were obtained from Bbr cultures negative in PCR specific for DNT.

PCRs. DNA was extracted using Genomic DNA Prep Plus Kit (A\&A Biotechnology). Detection of DNT in Pm based on Register's procedure with some ours modifications (5). Detection of DNT and BfrZ in Bbr based on PCR protocols elaborated by ourselves (3). The predicted sizes of amplified DNA were $501 \mathrm{bp}, 224 \mathrm{bp}$ and $368 \mathrm{bp}$, for DNT Pm, DNT Bbr and BfrZ, respectively. The PCRs were performed in $25 \mu \mathrm{l}$ mixture containing 10xPCR Gold Buffer, $25 \mathrm{mM} \mathrm{MgCl} 2$ solution, polymerase AmpliTaq Gold 5U/mL, $10 \mathrm{mM}$ dNTPs, $20 \mathrm{pM}$ of each primer and water free from DNAse and RNase.

\section{Results}

One hundred fifty (100\%) DNA samples extracted directly from nasal swabs were positive in PCR test for detection of DNT Bbr. From this number 59 samples (39.3\%) possessed BfrZ gene.

The presence of DNT Pm was evidenced in 23 out of 150 (15.3\%) tested samples. 13 (8.6\%) of them possessed also BfrZ gene. In 10 remaining samples lack of BrfZ gene was evidenced.

Among $99 \mathrm{Bbr}$ isolates possessing DNT gene BfrZ gene was found in 51 samples (51.5\%). The lowest number of positive results was obtained in case of Bbr strains which did not possess DNT gene. From 31 tested strains only in 5 (16.2\%) the BfrZ gene was detected.

\section{Discussion}

Bordetellaceae have wide range of ferric siderophore receptors genes but BfrZ gene, which was used in our study, is present only in Bbr genome so PCR test for its detection can be used as species specific assay.

The results obtained in our study demonstrate that over half of analysed strains (51.5\%) have both, ferric siderophore receptor and dermonecrotoxin genes. Additionally we evidenced that BfrZ gene is present in both types of Bbr strains - those with, as well as those without DNT gene. However the highest number of positive results in PCR with primers specific to BfrZ gene was obtained in Bbr strains possessing DNT gene. Lower percentage of positive results in PCR test for BfrZ gene detection were obtained when PCR was performed with DNA extracted directly from nasal swabs. The lowest number of positive results was received in the group of Bbr strains, which don't present DNT gene.

Moreover, the correlation between the presence of BfrZ and DNT genes, especially in the case of bacterial culture, was evidenced. There is no doubt that among Bbr strains which don't possess BfrZ gene there is high probability of occurrence of DNT gene.

\author{
References \\ 1. Brickman T.J. et al. Biomet.,2007, 20, 303-322 \\ 2. Brockmeier S.L. et al. Infec. Immun.,2002, 70, 481-490 \\ 3. Markowska-Daniel I. et al. Med.Wet., 2010, 1, 45-50 \\ 4. Murphy E.R. et al. Infec. Immun., 2002, 70, 5390-5403 \\ 5. Register K.B. et al. Vet. Mictrobiol., 2006, 117, 201-210.
}

* Paper prepared within Project No N308 322833 


\title{
P.382
}

\section{Ecology of Lawsonia intracellularis infection across rearing systems in Australia}

\author{
Patricia K. Holyoake ${ }^{1}$ Peter Thomson ${ }^{2}$ David Hamilton ${ }^{3}$ Alison Collins ${ }^{4}$ \\ 1. Wagga Wagga Agricultural Institute, Wagga Wagga, NSW, Australia; 2. University of Sydney, Camden, NSW, Australia; 3. South Australian \\ Research and Development Institute, Adelaide, SA, Australia; 4. Elizabeth Macarthur Agricultural Institute, Menangle, NSW, Australia
}

\section{Introduction}

In Australia, increasing numbers of pigs are housed on bedding (straw or rice hulls) in custom-built sheds (ecoshelters, hoops) instead of on concrete (partial or full slats) in 'traditional' sheds. ${ }^{1}$ A potential disadvantage compared with traditional sheds having under-floor effluent drains, is that the deep litter may only be cleaned out when the pigs are removed, giving the pigs ready access to their faeces for the entire period of their stay in the shed. Proliferative enteropathy (PE) is an enteric disease of pigs caused by the intracellular bacterium Lawsonia intracellularis (L. intracellularis). Its major effects include poor growth and diarrhoea or sudden death and dysentery. ${ }^{2}$ This project was designed to investigate the ecology of L. intracellularis herd infection of pigs raised in ecoshelters on deep litter bedding, compared with those raised in 'traditional' pig housing (concrete-based).

\section{Materials and Methods}

Pigs (Large White $x$ Landrace) were reared on 6 different 2-site (weaner/grow-out) commercial farms. The study was repeated on 2 of the sites. Pigs were transferred at 8-10 weeks of age to grow-out farms (4 traditional, 4 ecoshelters). A group of 65 pigs per farm was identified and blood samples collected at transfer to the grow-out farm, and at 16 and 22-24 weeks of age. Sera were tested using an ELISA according to the manufacturer's instructions (BioScreen ELISA, Synbiotics, France). Factors affecting a pig being test-positive were analysed using logistic regression (Generalised Linear Mixed Model, GenStat Release 12). Housing (traditional/ecoshelter) and age of pig (weaner, grower, finisher) were included in the model as fixed effects, and farm as a random effect. There was no interaction between housing and age of pig. The positive predictive value and negative predictive value of the ELISA were used to estimate seroprevalence and standard errors.

\section{Results}

There was no effect of housing type of test-positive status ( $\mathrm{F}=$ $0.00 ; \mathrm{df}=1,14 ; \mathrm{P}=1.00$ ). Mean seroprevalence was identical in pigs housed in traditional (36.3\%, SE 19.8\%) and ecoshelter (36.3\%, SE 26.6\%). There was a strong effect of age, with testpositive status/seroprevalence increasing steadily with age $(\mathrm{F}=$ 16.3; $\mathrm{df}=2,14 ; \mathrm{P}=<0.001$ (Table 1).
Table 1: Model-based test-positive results with standard errors, expressed as percentages from 8 groups of 65 pigs/group housed on 6 farms for L. intracellularis-specific antibodies.

\begin{tabular}{|c|c|c|c|c|}
\hline Age & Test +(\%) & SE(\%) & Prevalence(\%) & SE(\%) \\
\hline Weaner & 1.9 & 1.9 & 4.4 & 2.8 \\
\hline Grower & 65.7 & 12.9 & 65.4 & 17.9 \\
\hline Finisher & 81.1 & 9 & 80.2 & 12.4 \\
\hline
\end{tabular}

\section{Discussion}

There were substantial increases in the seroprevalence of $L$. intracellularis-specific antibodies with increasing age of pigs. This appeared to be unrelated to the use of bedding at the fattening stage i.e. under the conditions of this study, deep litter systems did not appear to pose a greater risk to pigs of infection compared with traditional concrete-based systems.

\section{References}

1. Kruger I. et al. (2006). Primefact 68, New South Wales Department of Primary Industries

2. Love R. et al. (1977) Vet Rec;100:65-68.

\section{Acknowledgements}

Collaborating producers are warmly thanked for their support. Financial and academic support for this project was provided by the Australian Research Council, Boehringer Ingelheim, University of Sydney, Australian Pork Ltd and NSW Department of Industry and Investment. Special thanks to Jocelyn Gonsalves for her technical work in running the ELISA. 


\title{
P.383
}

\section{Pig-associated Lawsonia intracellularis in various stages of on-farm Dipteroid fly species}

\author{
Steven McOrist ${ }^{1}$ Connie J. Gebhart ${ }^{2}$ Ruth Blunt $^{1}$ \\ 1. University of Nottingham, Sutton Bonington, UK; 2. University of Minnesota, St Paul, MN, USA
}

\section{Introduction}

Insects on pig farms can cause loss in various ways, including a potential mechanical or biological vector ability to spread disease within and between farms. This may conceivably include re-infection of eradication or start-up project farms by insects travelling from adjacent farms when infected with the agent in question. Proliferative enteropathy (PE, or ileitis) is one of the most ubiquitous of pig infections worldwide, but there is little work on possible vectors, such as insects. Most infections in pigs are gained by pigs contacting infected faeces or faecal-contaminated materials. Other studies have indicated that flies and some floor-dwelling insects, such as cockroaches and beetles, may be capable of carrying other agents such as PRRS, pseudorabies (Aujesky's disease), Salmonella and E coli for significant periods on commercial pig and chicken farms. Increases in farm bedding usage will presumably also increase potential infected vectors. This study aimed to address if insects on pig farms could be infected with Lawsonia intracellularis and whether this was associated with specific insect types.

\section{Materials and Methods}

On 15 farm study sites within England and Ireland, invertebrate communities were regularly trap-collected, counted and sorted into genus and species groups. The fly (Diptera) community was generally dominated by Musca domestica (house fly, $n=14$ farms), but also included Muscina stabulans (false stable fly, $n=9$ ), Ophyra spp (garbage fly, $n=10$ ), Drosophila (fruit flies, $n=8$ ), Stomoxys calcitrans (stable fly, $\mathrm{n}=2$ ) and Syrphidae containing Eristalis species (hover flies, $n=2$ ); Blatta orientalis cockroaches were noted on 2 farms. Total DNA was extracted from at least ten 12-week-old pig feces on each of the 15 farms and also from available adult, pupae and larvae samples of each Dipteroid group. Each DNA sample was tested for the presence of Lawsonia intracellularis DNA by specific PCR. A wide selection of Lawsonia-positive PCR products and controls, were tested by the variable number tandem repeat isolate typing (VNTR) method. This method distinguishes finely among isolates in intergenic Lawsonia DNA zones and so distinguishes between isolates from different farm systems. This VNTR testing was conducted by blinded technicians who did not know the origin of each DNA sample.

\section{Results}

Lawsonia-specific PCR products were routinely detected in weaner-grower pig faeces on 13/15 farms. On these farms, 12 were positive for numerous Musca domestica; total DNA extracted from the Musca collected on 5 farms where this was the dominant fly type, was also positive for Lawsonia DNA PCR reactions. All sets of adults, pupae and larvae of the Syrphidae hover fly Eristalis sp, collected from the only two farms positive for this fly, also had positive Lawsonia DNA reactions. Fruit flies were not found to be positive. Sub-typing of the Lawsonia DNA obtained from pigs and from insects by variable number tandem repeat analysis indicated that the same VNTR isolate type occurred within the positive farm pigs and the dominant pig-associated dipteroid fly stages on the same farm.

\section{Discussion}

This study indicates that the Dipteroid flies associated with pigs and pig bedding feed troughs and flooring, such as Musca domestica and Eristalis spp had the most likely potential to carry and transmit Lawsonia intracellularis. In contrast, Drosophila fruit flies, which do not have significant pig or pig faeces interactions on-farm were not found to be positive for Lawsonia. This suggests that a program aimed at reducing infection levels or re-infection with this agent should include not only Enterisol ileitis ${ }^{\mathrm{TM}}$ vaccination and appropriate pig medication, such as pleuromutilins or macrolides, but also anti-insect population control measures, targeted at those insects in association with pigs, particularly in summer months when fly populations peak. The infection of Dipteroid fly insects in different stages of growth also indicates that some transmission between farms may be possible, including to nearby farms in start-up situations, by infected adult flies. We are continuing these studies with experimental challenge exposure studies in Musca flies. 


\title{
P.384
}

\section{Increased variation in pig weight and reduced feed intake in pigs sub-clinically affected with proliferative enteropathy}

\author{
Alison M. Collins ${ }^{1}$ Shayne Fell ${ }^{1}$ Jolien van Straaten ${ }^{2}$ Nadine Bolsius $^{2}$ Lindsay Penrose $^{3}$ \\ 1. Elizabeth Macarthur Agricultural Institute, Narellan, NSW, Australia; 2. University of Utrecht, Utrecht, Netherlands; \\ 3. Orange Agricultural Institute, Orange, NSW, Australia
}

\section{Introduction}

Proliferative enteropathy (PE) varies in its clinical presentation, ranging from acute intestinal haemorrhage and sudden death of pigs, to diarrhoea and weight loss, or sub-clinical disease, where histological lesions of PE occur in the absence of diarrhoea?. Clinical PE is a costly disease because it reduces weight gains and feed conversion efficiency (FCE) in pigs by as much as $50 \%$ and increases variation in growth ${ }^{2}$. The impact of sub-clinical $P E$ is difficult to estimate because many producers are unaware that sub-clinical PE is present in their herd. The aim of this study was to quantify the impact of sub-clinical PE on feed intake and live-weight gain.

\section{Materials and Methods}

Thirty six male hybrid weaner pigs (Large White $x$ Landrace) were randomly allocated at $13.8 \pm 1.0 \mathrm{~kg}$ into 2 treatments: pigs infected with L.intracellularis, and a cohort of uninfected pigs. Pigs were housed in individual pens, with strict quarantine between treatments (separate rooms). Serology and faecal PCR monitoring of pigs prior to challenge indicated that all pigs were naïve to L.intracellularis. At 9 weeks of age, one group of 18 pigs was orally challenged with $5.9 \times 10^{9}$ viable L.intracellularis extracted from a PE-affected mucosa, and the control group was inoculated with phosphate buffered saline. The consistency of pig's faeces was scored daily as either normal or diarrhoeic. Blood collected at 0, 28 and 38 days post inoculation (pi) was tested for L.intracellularis- specific IgG using an indirect fluorescent antibody test (IFAT). Faeces collected twice per week from 0 to 35 days pi was tested for L.intracellularis DNA by a conventional PCR. Feed intake and pig weights were measured weekly but changes were analysed over 3 periods: pre-infection (day -14 to day 0 ), early infection ( 0 to 21 days pi) and late infection (21-42 days pi).

\section{Results}

L.intracellularis infection was demonstrated by faecal PCR and IFAT between 14 and 38 days pi in all pigs challenged with L.intracellularis. The majority of infected pigs (>80\%) were sub-clinically affected, but L.intracellularis infected pigs had a significantly higher probability of diarrhoea than control pigs between day 22 and 26 pi. L.intracellularis infection did not significantly reduce pig weights or weight gains, but did significantly increase variation in pig weight in the early infection period, as seen in the error variance of random effect models predicting pig weight as a function of infection period. When L.intracellularis infected pigs started to recover, their rate of weight increase was significantly greater $(P<0.05)$ in the late infection period than in the early infection period, unlike for the uninfected pigs. L.intracellularis infection significantly decreased feed intake over the early infection period $(P=0.017)$, but not in the pre-infection or late infection periods $(P=0.668$ and $P=0.416$ respectively). Pig weight was a strong covariate for feed intake in each period $(P<0.001)$.

\section{Discussion}

This study highlighted the reduced feed intake and the large variation in weight in a group of pigs sub-clinically affected with PE. While animal genetics plays a significant role in weight gain, it alone can not account for the observed variation in pig weights with sub-clinical PE. It is likely that differences in the host's immune status or intestinal microflora may explain the variation in weight between infected pigs. Control of L.intracellularis infections in herds with sub-clinical PE can minimize production losses and improve profitability for pig producers.

\section{Acknowledgments}

The authors acknowledge the support of the Australian Research Council, Boehringer Ingelheim, and the University of Sydney.

\section{References}

1. Lawson, G.H.K et al. (2000). J.Comp.Path. 122, 77-100.

2. Gogolowski, R.P. et al. (1991). Aust.Vet.J. 68, 406-408 


\title{
P.385
}

\section{Prevalence of Lawsonia intracellularis infection in grower-finisher pigs in Thailand during 2008-2009}

\author{
Chanunda Raphanaphraiwan; Yongyot Sriyong; Ampol Chayomchai \\ Elanco Animal Health A Division of Eli Lilly Asia Inc. Thailand Branch, Nonthaburi, Thailand
}

\section{Introduction}

Proliferative enteropathy (PE) or ileitis is an important enteric disease in swine industry worldwide. It is caused by an obligate intracellular bacterium, Lawsonia intracellularis (LI). In general, the specific immunoglobulin $\mathrm{G}(\mathrm{lgG})$ against $\mathrm{LI}$ infection is usually detectable 2-3 weeks after infection. This IgG response can be found in serum for upto 12 weeks after exposing to the bacteria, depending on the form of ileitis and the severity of the disease (1).

This IgG response can be used as a marker for screening on the status of LI infection in the herd. Several serological tests have been developed and available for use. IleiTEST ${ }^{\circledR}$ is an indirect fluorescent antibody test that are commercialy available. In Thailand, we have little information about the dynamic and the prevalence of $\mathrm{LI}$ infection in grower-finisher pigs. The objective of this study was to estimate the prevalence of $L I$ infection in grower-finisher herds in Thailand using IleiTEST ${ }^{\circledR}$.

\section{Materials and Methods}

This study was conducted in 40 commercial grower-finisher swine herds located in Northern, Southern, Western, Eastern, Middle and Northeast part of Thailand during 2008-2009. Eight blood samples were randomly collected from each age of pigs as cross-sectional at 4, 8, 12, 16 and 20 weeks of age. A total of 1,600 blood samples were submitted to the diagnostic laboratory at Faculty of Veterinary Medicine, Kasetsart University, Kamphaengsaen campus. All serum samples were measured for serum IgG antibody against LI infection by using IleiTEST ${ }^{\circledR}$, which was provided by Elanco Animal Health.

\section{Results and Discussion}

The specific antibodies against LI infection were found in 36 of 40 (90\%) grower-finisher herds in Thailand. In those positive herds, it shows that at least one-time point of pigs in the production system was exposed to the bacteria. Eighty percents of herd were positive with the IleiTEST $^{\circledR}$ at 4 weeks of ages (Figure 1). It is more likely that the positive results may be due to the passive antibody from sow to their piglets. Guedes et al (2002) found that passive immunity can be found in piglets up to 5 weeks of age (2). If this is the case, it indicated that most of the breeding herds in Thailand have the natural infection of LI.

The peak of herd prevalence was $85 \%$ when pigs were 12 weeks of age. Unfortunately, the prevalence of $\mathrm{LI}$ infection in growerfinisher herd decreased to $62.5 \%$ at 20 weeks of ages.
Figure 1. Herd prevalence of $L I$ infection at each time points from 40 herds of grower-finisher pigs in Thailand

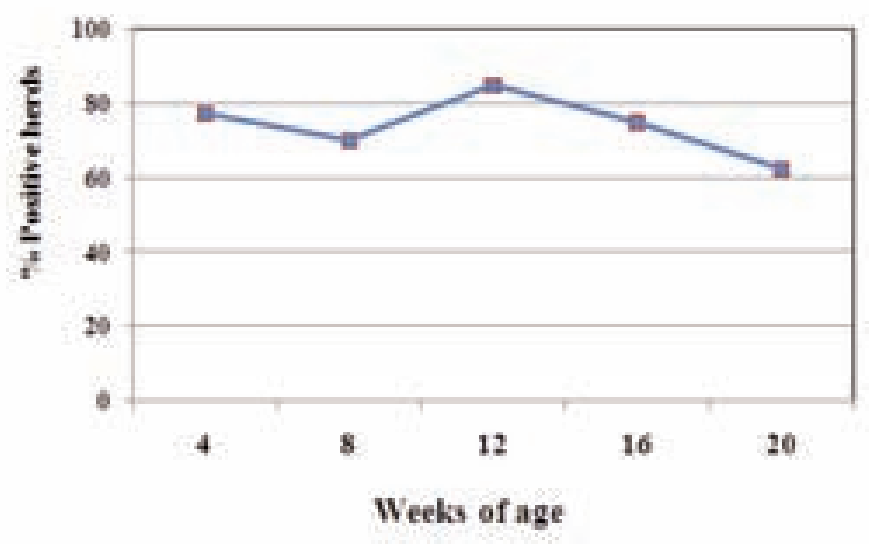

Figure 2. The average of percent of positive samples from 40 herds at each time point

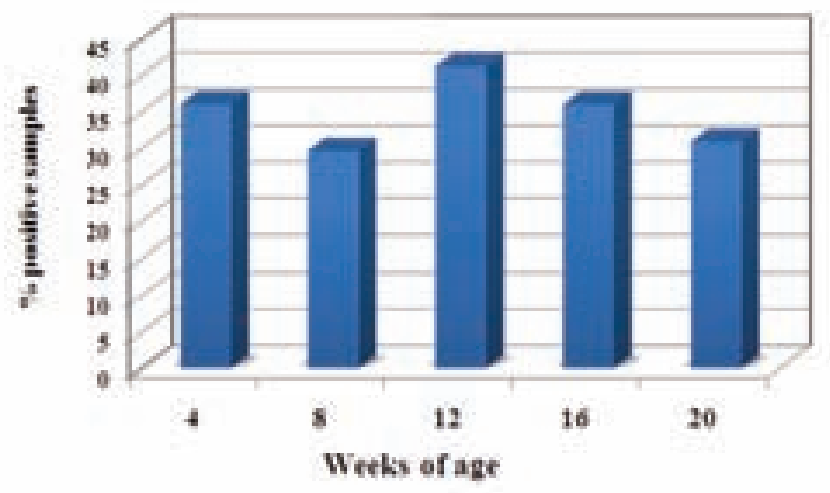

Overall, the dynamic of LI infection in grower-finisher herd in Thailand was summarized in Figure 2. The results from this investigation suggested that the prevalence of $\mathrm{LI}$ infection is more likely increasing during 8-12 weeks of age of pigs. Therefore, several strategies for treatment and control of LI infection might need to be developed and applied during that period.

\section{References}

1. Gebhart CJ. 2008. AASV.493-497.

2. Guedes RMC et al. 2002. CJVR. 258-263.

\section{Acknowledgements}

The data in this study were analyzed and reviewed by the assistance from Dr. Pariwat Poolperm and Dr. Suphot Wattanaphansak. 


\title{
P.386
}

\section{Granulomatous enteritis and regional lymphoadenitis associated with Lawsonia intracellularis infection}

\author{
Javier A. Cappuccioํㅜ Mariana Machuca Adrián Barragán ${ }^{1}$ Lucas Insarralde $^{1}$ \\ María A. Quiroga' Jose L. Cancer² Silvio Arrospide² Carlos J. Perfumo ${ }^{1}$ \\ 1. Cátedra de Patología Especial, Facultad de Ciencias Veterinarias, Universidad Nacional de La Plata, La Plata, Argentina; \\ 2. Private practitioner, Roque Perez, Argentina
}

\section{Introduction}

Lawsonia intracellularis (L. intracellularis) is an obligate intracellular bacterium causing porcine proliferative enteropathy (PPE), an enteric disease condition affecting growing and finishing pigs. The ileal mucosa is thickened due to proliferation of immature cryptal enterocytes (3). Porcine circovirus type 2 associated diseases (PCV-AD) include systemic or localized infection. The later recognized: PCV-associated pneumonia, enteritis, reproductive disorders and dermatitis nephropathy syndrome (4). Main histological lesions consist of lymphocyte depletion together with histiocytic and multinucleate giant cell infiltration in the lymphoid organs and, in a wide range of tissues with the presence in some cases of inclusion bodies (4).

The aim of the present study was to report a case of granulomatous enteritis (GE) and regional granulomatous lymphadenitis associated with L. intracellularis infection in growing and marked pigs.

\section{Materials and Methods}

A total of 310 pigs ( 23.3 weeks-old) with an average of $113 \mathrm{kbw}$ were examined in 2 slaughter inspections. lleum samples, taken at $10 \mathrm{~cm}$ of the ileocecal junction and mesenteric lymph nodes were collected from 46 randomly selected pigs. Samples were fixed in $10 \%$ buffered formalin for histopathological (HP) and immunohistochemical (IHC) studies against PCV-2 and L. intracellularis. HP results of intestine were evaluated for statistical association by Chi square test.

\section{Results}

Characteristic histopathological lesions of PPE such as enterocyte hyperplasia with the absence of goblet cells were observed at the level of cryptal epithelia. In the lamina propia and Peyer's patches lesions consisted of infiltration of histiocytes, multinucleate giant and epitheloid cells. In the regional mesenteric lymph-node lesions were similar to the above described with a diagnosis of granulomatous lymphoadenitis. There was statistical significance association between PPE and granulomatous lesions $(p=0.0029)$. By IHC, strong immunolabeled of L. intracellularis antigen was detected in 34 samples of ileum (73.9\%) and in 26 samples of mesenteric lymph-nodes (56.5\%). Whereas, slight PCV-2 antigens was observed in 1 ileum (2.2\%) and 3 lymph-nodes (6.5\%) samples. These samples also showed a strong immunostaining against $L$. intracellularis. All samples were negative for Mycobacterium spp or fungi by PAS and Ziehl Neelsen stains.

\section{Discussion}

Previously studies reported the identification of L. intracellularis associated with granulomatous enteritis $(2,5)$. However, few samples were available and also PCV-2 antigen was identified (2, 5). In this study results showed statistical significant association between PPE and GE lesions. Further, immunostaining against L. intracellularis was strongly positive in multinucleated giant cells and histiocytes. In addition, PCV-2 antigens were not found in Peyer's patches with granulomatous lesions and only a few positive cells were observed in the lamina propria. Lesions induced by L. intracellularis are restricted to enterocytes, but ocassionally epithelial cells and bacterium were found in mesenteric lymph nodes $(5,6)$. In the study, granulomatous lesion in lymph nodes was also associated with strong immunostaining of L. intracellularis in macrophages, histiocytes and giant cells. According with the pathogenesis of the granulomatous inflammation (1), the above results might suggest that $L$. intracellularis may induce $a$ granulomatous response, in absence of PCV-2 infection, when resolution of lesions and disappearance of the bacterium not take place. In this context, the granulomatous enteritis and lymphoadenitis could be considered as a part of PPE complex.

\section{References}

1. Ackermann MR. (2007). In: Pathologic Basis of Veterinary Disease, 4th Edit, MD McGavin, et. al Eds., Mosby Inc, St. Louis, pp. 153-192

2 Jensen TK, et. al.. (2006). J. Comp Pathol, 135, 176-183 2006

3. Mc Orist S, Gebhart CJ. (2006). Diseases of Swine. Blackwell Publishing, Oxford, pp. 727-738

4. Opriessing T, Meng XJ, Halbur P. (2007). J. Vet. Diag. Invest., 19, 591615

5. Segalés J, et. al. (2001). Vet. Pathol., 38, 343-346

6. Smith DG, Lawson GH. (2001). Vet. Microbiol. 82, 331-345.

This work was supported by grants from ANPCyT OC/AR PICT 200533987 


\title{
Characteristics of the cellular response to pathogenic action of Lawsonia intracellularis in the small intestine of pigs with diarrhea
}

\author{
Ismael M. Dolso; Judhit Bertone; Gabriel Di Cola; Silvia Romanini; Raúl Yaciuk \\ Universidad Nacional de Río Cuarto, Río Cuarto, Argentina
}

\section{Introduction}

Lawsonia intracellularis (Li), curved bacillus Gram (-), intracellular, does not grow on conventional media in vitro in the absence of enterocytes, their presence in the body produces a humoral response variable and short-term (1). Therefore, the study of cellular response is truly meaningful to characterize and understand the pathogenesis, which has not yet been deepened. Affects several animal species, mainly in pigs, producing porcine proliferative enteritis (PPE) causing stunting and increased mortality. The objective of this study is to determine the response characteristics of cells involved in the lesions of the small intestine (ID) of pigs who were diagnosed by immunoperoxidase technique (IPX) and PCR mucosa, the presence of Li.

\section{Materials and Methods}

Se trabajó sobre 20 establecimientos confinados pertenecientes a 7 de las provincias con mayor producción de cerdos de la República Argentina. Fueron muestreados los ileon de un total de 60 cerdos con diarrea. Por cada animal se tomaron dos muestras, una a $50 \mathrm{~cm}$ por delante de la válvula-ileocecal y otra a 10 $\mathrm{cm}$ por delante de la misma, las cuales se procesaron para histopatología con H/E. Work was confined about 20 stores belonging to 7 provinces with higher production of pigs in Argentina. The ileum were sampled a total of 60 pigs with diarrhea. For each animal were taken two samples, one at $50 \mathrm{~cm}$ anterior to the ileocecal valve, and another $10 \mathrm{~cm}$ in front of it, which were processed for histopathology with $\mathrm{H}$ / E. We searched for lesions compatible with EPP as villus atrophy, enlarged and branched crypts, hyperplasia of immature enterocytes in the crypts and absent or fewer goblet cells. The samples processed by IPX were considered positive when there was a brown color reaction in the luminal epithelium and criptal and special attention was paid to the mononuclear inflammatory response in lamina propria and submucosa. In addition, samples were processed to determine the Li Ag by PCR mucosa.

\section{Results}

Five of the 60 pigs sampled were positive for IPX. Three of them showed hyperplastic reaction in the epithelium of the crypts elongated and branched in macrophages, activated macrophages reticular appearance and giant cells in the lamina propria. The other two animals showed no reaction in the deep epithelium, as these despite being enlarged but showed goblet cell hyperplasia. Was observed in these ID staining in a few macrophages and giant cells of the lamina propria and in some macrophages in the submucosa. Furthermore, in all the samples had large numbers of immature lymphocytes and plasma cells. In the epithelia of villi and crypts increased the number of intraepithelial lymphocytes. These 5 pigs were also positive with PCR mucosa (PCR F).

\begin{tabular}{|c|c|c|c|}
\hline Portion of ileum & HE & IPX+ & PCRM+ \\
\hline 50 & $S / L(n=1)$ & $0 / 1$ & $0 / 1$ \\
\hline 50 & $C / L(n=60)$ & $5 / 60$ & $4 / 58$ \\
\hline 10 & $S / L(n=0)$ & $0 / 0$ & $0 / 0$ \\
\hline 10 & $C / L(n=60)$ & $5 / 60$ & $4 / 58$ \\
\hline
\end{tabular}

\section{Discussion}

It is noteworthy that the pathogenic action of Li can be determined by the histopathological lesions and the technique of IPX. However, the cellular response that has deserved special attention because it is an infiltration and / or histiocytic proliferation and hyperplasia of intraepithelial lymphocytes that remain present even when the epithelial lesions have disappeared, as indicated in animals with hyperplasia goblet cells (2). It would be important to deepen the studies of the pathogenic mechanisms of Li mainly related to apoptosis, and cellular response it generates, through the marking of different cell types acting. Thus differentiating it from other agents such as circovirus and / or Yersinia that produce similar lesions and granulomatous responses (3). Moreover, these results related to the pathogenesis would allow us to begin to explore possible associations with Li circovirus in clinical presentation of PMWS as seen in the caseloads of our pathology and histopathology.

\section{References:}

1. J. Comp. Path. 122:77, 2000.

2. J. Comp. Path. 115(1):35, 1996.

3. J. Comp. Path. 135:176, 2006. 


\title{
P.388
}

\section{Detection of $L$. intracellularis, B. hyodysenteriae and B. pilosicoli in German wild boars}

\author{
Gerald Reiner; Melina Winkelmann; Sonja Hillen; Hermann Willems \\ Department of Veterinary Clinical Sciences, Justus-Liebig-University, Giessen, Germany
}

\section{Introduction}

Wild boars are recognized as a potential reservoir of pathogenic microorganisms for domestic pigs. Brachyspira hyodysenteriae ( $B$. h.) and B. pilosicoli (B. p.) are important swine pathogens causing swine dysentery and porcine intestinal spirochetosis, respectively. Because of difficulties in culturing Brachyspira species and due to the obligate intracellular parasitism of $L$. intracellularis (L. i.) (Ileitis), diagnostics of these bacteria primarily relies on the detection of genomic sequences. Small amounts of these bacteria can often be isolated from subclinical cases. Few data exist about the occurence of Brachyspira and Lawsonia spp. in wild boar populations. Infections with $L$. i. have been demonstrated in single wild boars from Spain (Segalés et al. 2001), and Brasil (Zlotowski et al. 2008; commercial herd). Jacobson et al. (2005) did not find Brachyspira spp. or L. i. in Swedish wild boar samples. L. i. was detected in $29.6 \%$ and $9.1 \%$ of free ranging and enclosed wild boars from Czech Republic (Tomanova et al. 2002; Dezorzova-Tomanova et al. 2006). Nyree et al. (2009) found $18.9 \%, 8.1 \%$ and $0.45 \%$ of 222 Australian feral pigs infected with L. i., B. h. and B. p. The aim of the present study was to determine the prevalence for the three species in German wild boars.

\section{Materials and Methods}

Samples from ileal mucosa of 165 wild boars were collected from 19 hunting grounds situated in fourteen of the 16 federal states of Germany between November 2005 and March 2006. The town states Hamburg and Bremen were not sampled. Wild boars were gathered after the hunting at central places. The wild boars were on average 12 months ( 1 to 84 months) old, with an average carcass weight of $34 \mathrm{~kg}$ (6 to $110 \mathrm{~kg}$ ). Samples were taken half an hour to 2 hours after death with fresh, sterile disposable gloves and scalpels, and stored at $-20^{\circ} \mathrm{C}$ until further usage. DNA was extracted from $25-50 \mathrm{mg}$ of tissue sample using the Qiagen Viral RNA Mini Kit (Qiagen, Hilden, Germany). Differentiating primers and probes for conventional and real-time PCR targeted the nox gene of Brachyspira spp. and the aspA gene of L. i. Real-time PCR was performed with $2.5 \mu$ l of the isolated DNA on a 7300 Real Time PCR System (Applied Biosystems, Germany). The analytical sensitivity for the real-time PCR assay, expressed as limit of detection (LOD) was 14, 26, and below 10 DNA copies for B. p., B. h., and L. i., respectively. The analytical sensitivity for the conventional PCR assay was below 10 DNA copies for all three species.

\section{Results}

None of the intestines sampled did show any gross pathological signs of enteritis. Pathological histology was not available. The overall prevalence for L. i., B. h. and B. p. was $20.6 \%, 2.4 \%$ and $12.1 \%$, respectively. None of the three agents was detected in $68.5 \%$ of the wild boars; $17.0 \%, 1.2 \%$ and $9.7 \%$ were monoinfected with $L$. i., B. $h$., and B. p., respectively; $2.4 \%$ and $1.2 \%$ were mixed infections with $L$. i./B. p. and $L$. i./B. $h$., respectively. $84.2 \%$ of the hunting grounds were found positive for at least one of the three agents; L. i., B. h. and B. p. were detected in 15.8\%, $0.0 \%$ and $10.5 \%$ of the hunting grounds as monoinfections; $36.8 \%$ and $21.1 \%$ of the hunting grounds were positive with L. i./B. $p$. and $L$. i./B. h., respectively. Numbers of bacteria per sample were consistently low, and none of the positive samples was above the quantitation limit of 100 DNA copies per PCR reaction.

\section{Discussion}

This is the first time that $B$. $h$. was detected in genuinewild boars. Detection rates substantiate a wide distribution of the three agents in wild boar populations. Prevalence of $L$. i. corresponds well with those reported for the Czech Republic and for feral pigs in Australia. The occurrence of B. h. is rather low. Missing of gross pathological lesions and the low number of bacteria per sample argue for a commensal role. Further research is needed to get insight into the epidemiological impact of these results.

\section{References}

Dezorzova-Tomanova et al. 2006. Vet. Microbiol. 134, 294-299.

Jacobson et al. 2005. J. Vet. Med. B. 52, 386-391.

Segalés et al. 2001. Vet. Pathol. 38, 343-346.

Tomanova et al. 2002. Vet. Rec. 151, 765-767.

Zlotowski et al. 2008. Ciencia Rural, Santa Maria 38, 2540-2544. 


\title{
P.389
}

\section{Mixed infections with $L$. intracellularis, B. hyodysenteriae, and B. pilosicoli in faecal field samples from German pig herds, simultaneously detected and quantified by realtime multiplex PCR}

\author{
Gerald Reiner $^{1}$ Marion Kixmöller ${ }^{2}$ Hermann Willems ${ }^{1}$ \\ 1. Department of Veterinary Clinical Sciences, Justus-Liebig-University, Giessen, Germany; 2. Novartis Animal Health, München, Germany
}

\section{Introduction}

Brachyspira (B.) hyodysenteriae and B. pilosicoli are important swine pathogens causing swine dysentery and porcine intestinal spirochaetosis, respectively. Because of difficulties in culturing Brachyspira species and due to the obligate intracellular parasitism of $L$. intracellularis diagnosis of these bacteria primarily relies on the detection of genomic sequences. There is a close relation between the faecal load of $B$. hyodysenteriae and $L$. intracellularis and the clinical outcome of the infection $(1,2)$. Small amounts of these bacteria can often be isolated from subclinical cases and the positive result of a conventional PCR is often misinterpreted and does not lead to the causal diagnosis. Thus, to provide information from a clinical point of view, it is advisable to detect and quantify the putative agent of disease. We have developed a multiplex real-time PCR (mrtPCR) assay for the simultaneous detection and quantitation of $L$. intracellularis, $B$. hyodysenteriae and $B$. pilosicoli from faecal samples. The mrtPCR was applied to study qualitative and quantitative distribution of the three agents in commercial herds from Germany, in order to gain information on prevalences, bacterial loads, and the occurrence of mixed infections.

\section{Materials and Methods}

A total of 1176 individual faecal samples from 95 herds (including 33.000 sows, 64.000 piglets and 145.000 fattening pigs) were sent in by 48 practitioners in the course of a diagnostic study. All samples were accompanied by a detailed questionnaire. For DNA extraction $300 \mathrm{mg}$ of faeces were thoroughly mixed and applied to a Quiagen extraction kit. Finally, DNA aliquots extracted from $12.5 \mathrm{mg}$ faeces were used for mrtPCR. Primers and probes for real-time PCR were designed with the Primer Express software (Applied Biosystems) targeting the nox gene of Brachyspira and the aspA gene of $L$. intracellularis. Gene sequences were retrieved from the Genbank database. Real-time PCR was performed on a 7300 Real Time PCR System (Applied Biosystems, Germany). The limit of detection was below $1 \times 103$ cells $/ g$ of faeces for all three species.

\section{Results}

From the individual samples, $12.7,8.3$, and 3.2 percent were positive for $L$. intracellularis, B. hyodysenteriae and B. pilosicoli, respectively. The relation of mono- to mixed infections per sample was $70.2 \% / 29.8 \%$

Regarding herds, 48.4, 24.2, and 31.6\% were positive with $L$. intracellularis, B. hyodysenteriae, and B. pilosicoli, respectively. $68.4 \%$ of the herds were infected with at least two of the three organisms. $60.1 \%, 82.6 \%$, and $73.3 \%$ of infections with L. intracellularis, B. hyodysenteriae, and B. pilosicoli, respectively, were mixed infections. Medians for the log number of cells/g of faeces (with 5\% and 95\% percentiles in brackets) were 3.3 (2.2-7.2), 5.9 (2.9-7.5), and 3.2 (2.1-5.8) for L. intracellularis, B. hyodysenteriae, and $B$. pilosicoli, respectively. Incidence and severity of diarrhea were correlated with the quantity of $B$. hyodysenteriae and $B$. pilosicoli, but not with that of $L$. intracellularis. Haemorrhagic diarrhea was associated with the presence of $B$. hyodysenteriae and was most severe with mixed infections of the two Brachyspira species. Of the positive faecal samples, $60 \%, 60 \%$, and $80 \%$ did not correspond with the tentative clinical diagnosis of ileitis, dysentery, and both diseases, respectively.

\section{Discussion}

In conclusion, the three economically important pathogens have been shown to have a wide distribution among herds. The overlapping clinical signs, the relatively high incidence of mixed infections and the occurrence of B. pilosicoli in herds can lead to significant misinterpretations in the field, underlining the impact of a fast and reliable molecular diagnosis. The introduced mrtPCR can be a relevant tool in the diagnosis and prognosis of infections with L. intracellularis, B. hyodysenteriae, and B. pilosicoli.

\author{
References \\ 1. Davis et al. 2005. Curr. Microbiol. 50,285-291. \\ 2. Guedes et al. 2003. Vet. Rec. 153,432-433.
}




\title{
P.390
}

\section{Demonstration of Lawsonia intracellularis in necrotic ileitis cases by immunohistochemistry}

\author{
Dinko Novosel $^{1}$ Zoran Lipej $^{1}$ Željko Grabarević $^{2}$ \\ 1. Croatian Veterinary Institute, Zagreb, Croatia; 2. Veterinary Faculty, Zagreb, Croatia
}

\section{Introduction}

Proliferative enteropathy (PE) caused by Lawsonia intracellularis is well known common intestinal and one of most economically important disease. There are several different syndromes of PE porcine intestinal adenomatosis, hemorrhagic enteropathy and necrotic enteritis [1]. Immunohistochemistry (IHC) continue to be golden standard as diagnostic tool [2]. Most typical histopathological lesions is absence of goblet cells and little evidence of infiltration of inflammatory cells, which is not common [3]. The aim of this study was to detect and describe morphological findings possible presence of PE syndromes in Croatian pig production units.

\section{Material and Methods}

From three different fattening farms was reported onset dying without clinical symptoms or with greyish diarrhoea shortly before death. Totally, 11 animals were necropsied and after completely post-mortem examination three samples of ileum, before ileocecal valve were collected in $10 \%$ buffered formalin. Tissue was routinely processed until paraffin blocks. Stained with H\&E and for immunohistochemical detection Lawsonia intracellularis is used anti Lawsonia intracellularis (Euroclone ${ }^{\circledR}$ ) monoclonal antibodies according previously described protocol [4].

\section{Results}

In ileum of 5 animals strong Lawsonia intracellularis signal in enterocytes were detected by IHC (Fig. 1.). Histopathological lesions in all 5 positive animals were uniform in distribution. The mucosa is thickened by necrosis of apex of villi. The strong inflammatory reaction with lymphocytes and globular leucocytes between villi was clearly seen (Fig. 2). In crypts were still vital immature epithelial cells. The most prominent findings were absence of goblet cells.

\section{Discussion}

The present study describes the ileum lesions associated with Lawsonia intracellularis infection in fattening pigs. In our cases most prominent histopathological findings were necrosis of apex of villi and absence of goblet cells. A strong inflammatory reaction with lymphocytes and globular leucocytes was seen which is in contrast with previous studies which reported very mild inflammatory infiltration [3]

According to histopathological findings and positive IHC signal in enetrocytes of swine with necrotic ileitis are fulfilled key criteria which confirmed the first objective findings of Lawsonia intracellularis as PE causative agent in Croatia. As far as we know this is the first report about necrotic ileitis in pig population in Croatia. Nevertheless, infection with Lawsonia intracellularis caused considerable economic losses in pig production during many years ago.
Figure 1. Positive signal in enterocytes. IHC anti-Lawsonia intracellularis, $40 x$

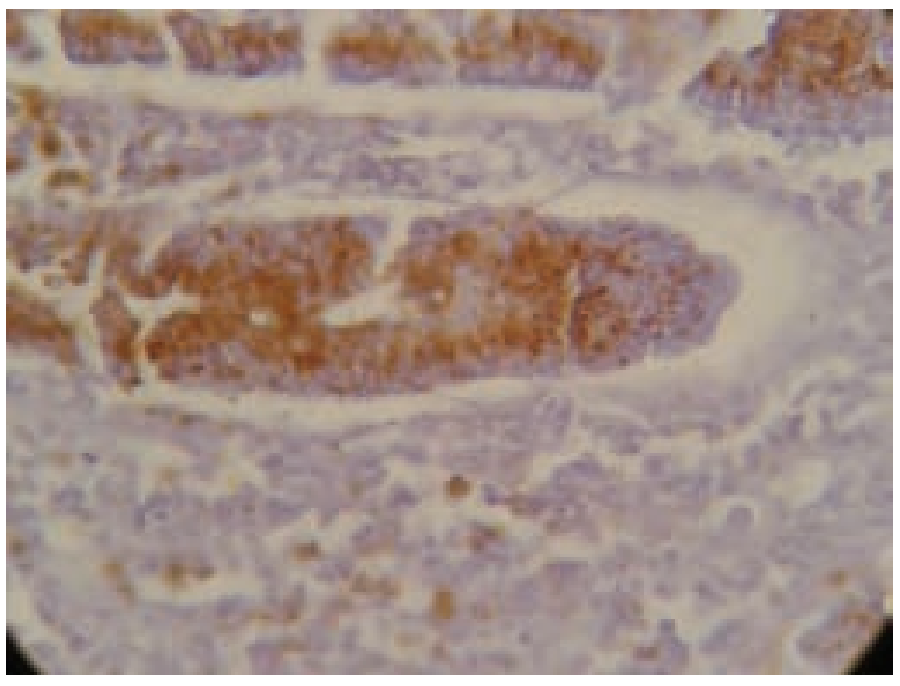

Figure 2. Strong inflammatory reaction and absence of goblet cells. $H \& E, 20 x$

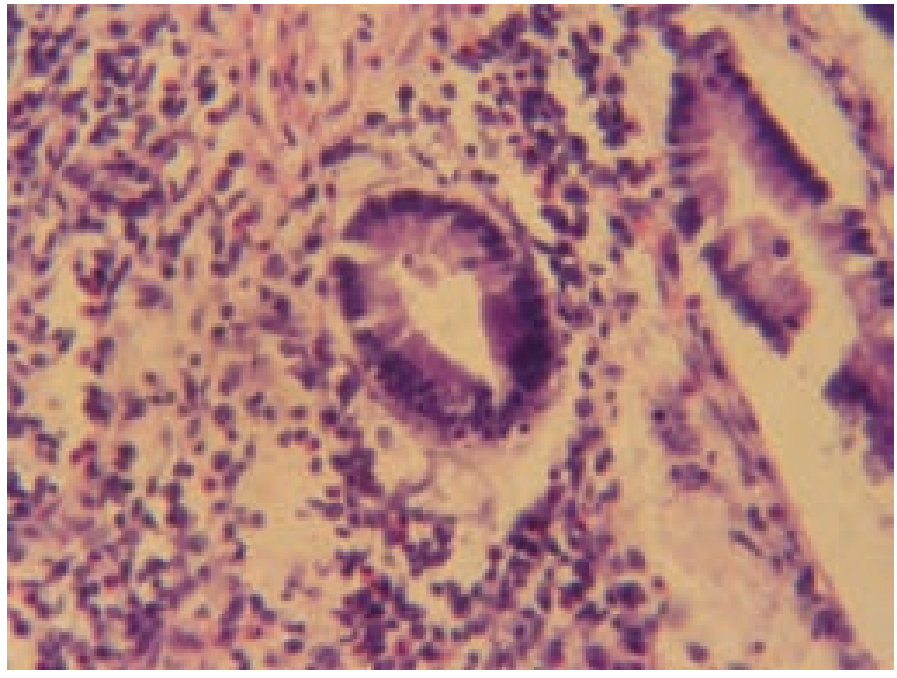

\section{References}

1. McOrist, S. and C.J. Gebhart (1999): in Disease of Swine 8th edn.

2. Kroll, J.J., et al. (2005): Anim Health Res Rev,. 6: p. 173-97.

3. Rowland, A. and G.H. Lawson (1992): in Disease of Swine 7th edn, B. Straw, et al., Editors. lowa State University Press. p. 590-596.

4. McOrist, S., et al. (1987): Vet Rec,. 121: p. 421-2. 


\title{
Prevalence of faecal Lawsonia intracellularis shedding in piglets: A European field study using Lawsonia FIRSTtest ${ }^{\circledR}$
}

\author{
Annick Saives $^{2}$ Vincent Auvigne $^{1}$ Susana Lapuente ${ }^{1}$ \\ 1. EKIPAJ, Madrid, Spain; 2. Elanco Europe, Brussels, Belgium
}

\section{Introduction}

Lawsonia intracellularis (LI) is a major bacterial pathogen in pigs, responsible for ileitis (Proliferative Enteropathy), a digestive disease that greatly reduces the animal's performance in the growing and finishing stage. The pathogen is endemic worldwide. The level of its excretion is variable and is linked to the severity of clinical signs (1). It is therefore important to gain a better knowledge of the epidemiology of the excretion of Lawsonia, for a better, targeted preventive and therapeutic approach.

\section{Materials and Methods}

Lawsonia FIRSTtest ${ }^{\circledast}$ is a new ELISA diagnostic tool designed to detect $\mathrm{LI}$ in faeces (2). A qualitative result is obtained by comparison to a positive control by indication of a color change to blue. By validation testing comparing to PCR, it is determined that if more than 3 out of 10 samples are positives, the batch is considered as shedding LI with a prevalence of at least $40 \%$ (3). For most herds, a sample size of ten is adequate to determine if a farm is positive or negative.

The study was carried out in 2008 and 2009 in 12 European countries. 7362 growing pigs of 531 farms were included. On 138 farms, two or more age groups were sampled. The average number of fecal samples per age and per farm was 10.8 . Age (weeks of life) was available for 624 out of the 684 sampled batches. When more than one age group was sampled, the effects of age and herd were studied, using the Wilcoxon Match-Pairs Signed Ranks Test and Spearman's rank correlation, respectively.

\section{Results}

The overall individual and batch prevalence was 55\% and $82 \%$, respectively. The intra-batch prevalence (image. 1) shows a bimodal distribution with one subpopulation around the 50 $\%$-prevalence mark and another subgroup with a prevalence of $90 \%$ or more.

The herd effect was confirmed in this study: if pigs of 16 weeks and older were positive, pigs from the same herd in age groups 11-13 weeks and 14-16 weeks tended to be positive as well. The age effect was also confirmed: pigs aged 11-13 weeks shed significantly less bacteria that those aged 14-16 weeks or 16 weeks and older (Tab 1).
Fig. 1: Distribution of the intra-batch prevalence

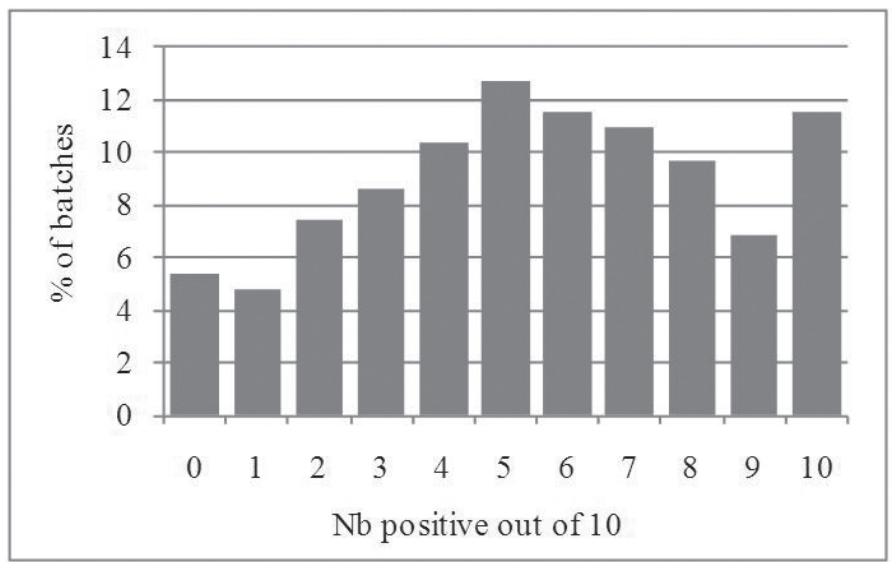

Table 1: Age pattern and age effect in herds with at least 2 sampled batches

\begin{tabular}{crrrrrc}
\cline { 2 - 6 } & \multicolumn{3}{c}{ Categories of age } & \multicolumn{2}{c}{ p level } \\
\hline $\begin{array}{c}\text { nb of } \\
\text { herds }\end{array}$ & $3-10$ & $11-13$ & $14-16$ & $>16$ & $\begin{array}{c}\text { Age } \\
\text { pattern }\end{array}$ & $\begin{array}{c}\text { Herd } \\
\text { effect }\end{array}$ \\
\hline 37 & & & $49 \%$ & $55 \%$ & 0.18 & $\mathbf{0 . 0 0 2}$ \\
\hline 32 & & $\mathbf{4 3 \%}$ & $\mathbf{5 6 \%}$ & & $\mathbf{0 . 0 2}$ & 0.09 \\
\hline 30 & $45 \%$ & & $54 \%$ & & 0.43 & 0.24 \\
\hline 25 & & $\mathbf{5 1} \%$ & & $\mathbf{6 3 \%}$ & $\mathbf{0 . 0 5}$ & $\mathbf{0 . 0 3}$ \\
\hline 21 & $43 \%$ & $56 \%$ & & & 0.74 & 0.83 \\
\hline
\end{tabular}

\section{Discussion}

This study confirms the high prevalence of LI on pig farms (4). However, shedding is not homogeneous, as shown by the herd and age effect. Shedding should therefore be evaluated herd level by measuring shedding in 2 different ages in the same farm. Sampling should be sufficient to assess the intra-batch prevalence and to adjust the preventive and therapeutic approach. Sampling should be standardised to allow for a comparison of the results between farms.

\section{References}

1. Paradis, M. et al. Proceeding AASV (2005).

2. Gebhart, C. Elanco internal report (2008).

3. Green, L. Epidemiologic report. Elanco internal report (2008)

3. Strachan, W. et al. Proc. 20th IPVS (2008).2, 294 


\title{
P.392
}

\section{A longitudinal study comparing four diagnostic tests for Lawsonia intracellularis}

\author{
Annick Saives ${ }^{1}$ Vincent Auvigne $^{2}$ Xavier De Paz Solanes ${ }^{1}$ Susana Lapuente ${ }^{1}$ \\ 1. Elanco Europe, Brussels, Belgium; 2. EKIPAJ, Madrid, Spain
}

\section{Introduction}

Lawsonia intracellularis (LI) is a major bacterial pathogen in pigs, and is endemic worldwide. An accurate diagnostic is necessary for a targeted preventive and therapeutic approach. Available tests either measure the immune response or the faecal shedding. The aim of the study was to compare the results of four of them.

\section{Materials and Methods}

The study was carried out between Dec. 2008 and Apr. 2009, on a 300-sow breeding farm, with an all-in-all-out management from weaning to slaughter. At weaning (21 days), 20 piglets were randomly selected and individually double-tagged. Samples (blood and faeces) were taken every two weeks between 3 and 21 weeks of age. The serological status was determined at the LDG lab (Barcelona, Spain), using an IFA test (Ileitest, Elanco) and ELISA test (Bioscreen) for LI. Faecal shedding was determined at the CReSA laboratory (Barcelona, Spain) using in-house PCR and at Labopat (Segovia, Spain) using FIRSTtest ${ }^{\oplus}$. FIRSTtest $^{\oplus}$ is a new antigen ELISA which uses magnetic beads technology. All results were qualitative. Doubtful ELISA results were considered positive.

\section{Results}

All piglets remained in good clinical condition and could be followed up during the entire study period. All methods confirmed that the farm was contaminated by LI, although there was a difference in the percentage of positive animals and the evolution of the infection depending to the tests. Faecal LI shedding was detected by the FIRSTtest ${ }^{\circledR}$ from 5 weeks of age. Between weeks 7 and 19, nearly all piglets were positive, although shedding seemed to decrease by 21 weeks of age. PCR detected the first excreting piglets by week 11, and shedding only became widespread ( $>40 \%$ of positives) at 17 and 19 weeks of life. Four piglets were never found positive for PCR. ELISA only detected a few positive animals between 3 and 7 weeks of age, probably animals with remaining maternal antibodies. This was followed by a massive seroconversion from 13 weeks of age. Between 13 and 21 weeks of age, the ELISA negative piglets were not systematically the same for all time points. The percentage of animals positive for IFA evolved little during the study period (minimum 30\%, maximum 50\%). However, two periods can be distinguished: between 3 and 9 weeks of age, there is a large variation between IFA test results of individual animals, from one time point to another. In contrast, between 9 and 21 weeks, there is little evolution in the IFA status of the individuals: positive animals remain positive and negatives remain negative.
Fig. 1: Comparative results of the four tests

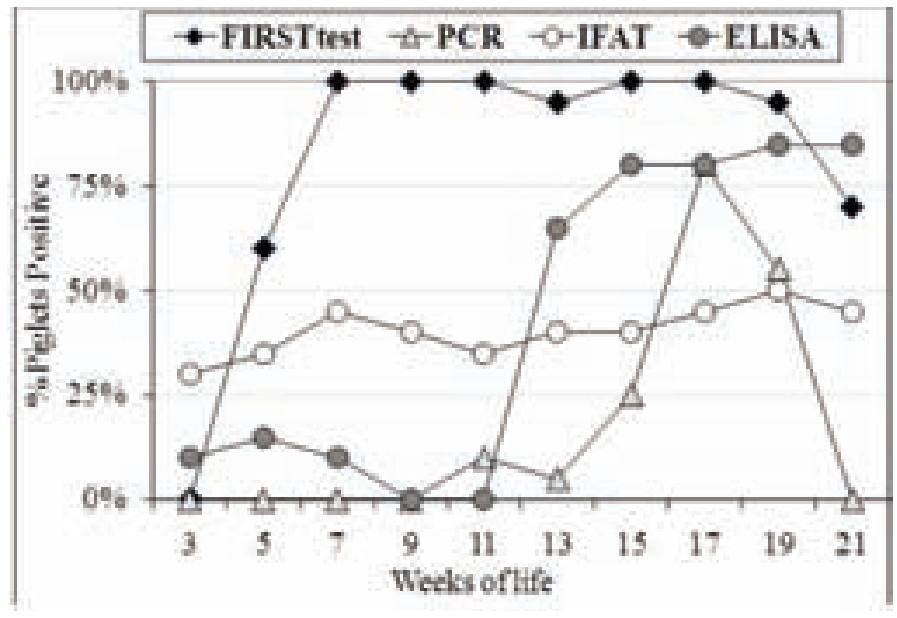

\section{Discussion}

In the absence of a gold standard, this study has not allowed to reach a direct estimation of the sensitivity and specificity of tests. Furthermore, different biological parameters were used for the tests (immune response vs. faecal shedding). Nevertheless, it can be concluded that, under the study conditions, ELISA allows better than IFA to determine the time of seroconversion. The results obtained by PCR is consistent with another longitudinal study where PCR+ faeces were observed before ELISA seroconversion (1). This inconsistency might be due to the presence of inhibiting factors in the faeces or to intermittent shedding. Finally, the FIRSTtest ${ }^{\circledR}$ seems a highly sensitive technique since shedding is detected as early as 5 weeks of age.

These results might aid the veterinary practitioner in the choice of test to be used in the field.

\section{References}

1. Jordan D.M. et al. Vet. Microbiol (2004).104, 83-90 


\title{
P.393
}

\section{Sub-clinical Ileitis: use of a diagnostic tool, Lawsonia FIRSTtest, to understand the excretion of sows and piglets and the impact of Tylan treatments on sows}

\author{
Annick Saives \\ Elanco Europe, Brussels, Belgium
}

\section{Introduction}

Lawsonia intracellularis (LI) is a major bacterial pathogen in pigs, responsible for ileitis (Proliferative Enteropathy), a digestive disease that greatly reduces the animal's performance in the growing and finishing stage. The pathogen is endemic worldwide. The level of its excretion is variable and is linked to the severity of clinical signs (1). The aim of this study is to control the level of shedding of sows and their piglets in 5 farms presenting signs of subclinical ileitis and to understand if a Tylan treatment on sows could decrease the shedding.

\section{Materials and Methods}

Lawsonia FIRSTtest ${ }^{\oplus}$ is a new ELISA diagnostic tool designed to detect $\mathrm{LI}$ in faeces (2). A qualitative result is obtained by comparison to a positive control by indication of a color change to blue. By validation testing comparing to PCR, it is determined that if more than 3 out of 10 samples are positives, the batch is considered as shedding LI with a prevalence of at least $40 \%$ (3). For most herds, a sample size of ten is adequate to determine if a farm is positive or negative.

Five farrow-to-finish herds with same gilt source demonstrating sub-clinical ileitis at the beginning of the finishing period, were selected. Baseline level of LI shedding in production sows and piglets from these sows after weaning (aged between 5 and 7 weeks) was measured before treatment. Sample sizes were determined based on sow farm size and estimated prevalence. A Tylan premix treatment at $5 \mathrm{mg} / \mathrm{kg}$ for 15 days was administered to the animals at the time of placing the sows before farrowing. Testing was repeated in sows and piglets to determine effect due to Tylan administration to sows.

Percentages of shedding per animal category, before and after treatment, were analyzed by using the Mantel Haenszel Chisquare test adjusted on farms (EPIINFO 3.5.1).

\section{Results}

The results of shedding levels for the 5 farms are presented in tables 1 and 2 by animal category, before and after treatment. Farms were pooled for analysis. Results are listed in Table 1, and shown graphically in Figure 1. The inclusion of Tylan in the sow ration for 15 days initiated prior to farrowing resulted in decreased prevalence of $\mathrm{LI}$ as indicated by fecal shedding in both sows and piglets.
Table 1: \% of shedding level of sows and piglets

\begin{tabular}{c|c|c|c|c|c} 
& $\begin{array}{c}\text { Sows before } \\
\text { treatment }\end{array}$ & \multicolumn{2}{c|}{$\begin{array}{c}\text { Sows after } \\
\text { treatment }\end{array}$} & $\mathrm{P}$ \\
\hline & $\begin{array}{c}\text { No. of } \\
\text { samples }\end{array}$ & $\%+$ & $\begin{array}{c}\text { No. of } \\
\text { samples }\end{array}$ & $\%++$ & \\
\hline Total & 104 & 71.2 & 79 & 39.2 & $\begin{array}{c}\mathrm{S} \\
\text { (P-0.0001) }\end{array}$ \\
\hline & $\begin{array}{c}\text { Piglets before } \\
\text { treatment }\end{array}$ & $\begin{array}{c}\text { Piglets after } \\
\text { treatment }\end{array}$ & $\mathrm{P}$ \\
\hline & $\begin{array}{c}\text { No. of } \\
\text { samples }\end{array}$ & $\%+$ & $\begin{array}{c}\text { No, of } \\
\text { samples }\end{array}$ & $\%+$ & \\
\hline Total & 50 & 76 & 60 & 43.3 & $\mathrm{~S}(0.0003)$ \\
\hline
\end{tabular}

Figure 1: $\%$ of shedding before and after Tylan treatment for each category of animal

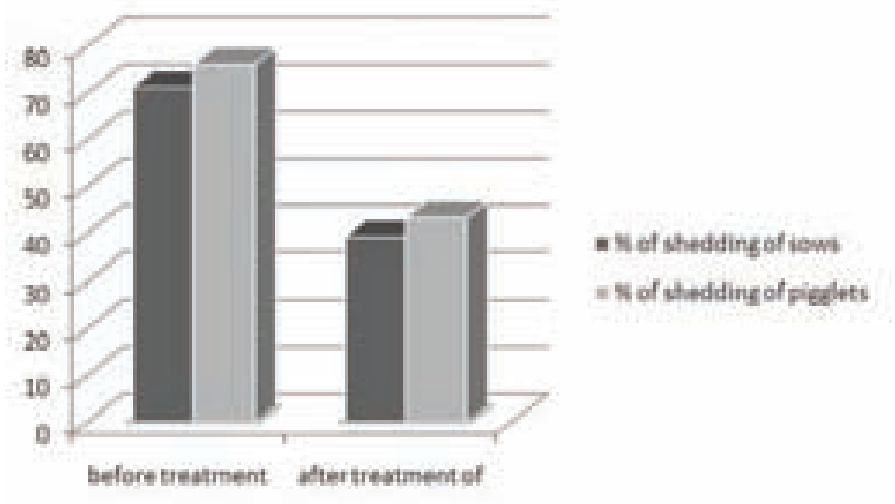

\section{Discussion and Conclusion}

This study demonstrates that in herds with sub- clinical ileitis, the shedding of sows is an important factor and could contribute to the contamination of their young. The Tylan treatment of sows allowed a significant reduction of the shedding level from 71.2 to $39.2 \%$. The study showed that the shedding level also decreases in the young of the treated sows, without using a treatment, from 76 to $43.3 \%$.

Lawsonia FIRSTtest ${ }^{\circledast}$ is an interesting tool in the understanding of Lawsonia shedding in herds with sub clinical ileitis and may allow a better understanding of bacterium circulation and its impact on farming.

\section{References}

1. Paradis, M. et al. Proceeding AASV (2005).

2. Gebhart, C. (2008) Internal Elanco report..

3. Green,L. (2008) Internal Elanco report.. 


\title{
P.394
}

\section{The importance of the choice of gold standard and target population in the evaluation of diagnostic methods, such as serology, for the detection of antibodies to Lawsonia intracellularis in pigs.}

\author{
Magdalena Jacobson ${ }^{1}$ Per Wallgren² Ann Nordengrahn³ Malik Merza ${ }^{3}$ Ulf Emanuelson \\ 1. Dept. of Clinical Sciences, SLU, Uppsala, Sweden; 2. National Veterinary Institute, Uppsala, Sweden; 3. Svanova Biotech, Uppsala, Sweden
}

\section{Introduction}

Diagnosis of Lawsonia intracellularis in live animals is based on the demonstration of microbial DNA in fecal samples, or the demonstration of $L$. intracellularis-specific antibodies in sera. The diagnostic sensitivity (DSn) and specificity (DSp), used to assess the performance of a new method, are commonly evaluated by analysis of reference samples from animals with a known infectious status and compared to analysis by a gold standard. However, these reference samples may not reflect the actual population for which the methods are intended and a true gold standard is not always available. The aim of the present study was to compare the results obtained by a blocking ELISA to an IFAT in the detection of serum antibodies to L. intracellularis.

\section{Materials and Methods}

200 pigs from 25 herds were included. Sera obtained from three previous studies were stored at $-80^{\circ} \mathrm{C}$. The pigs in study (1) and (2) were 8-12 weeks old. Study (1) included 54 pigs from 9 herds with poor performance and diarrhoea in growers, and 12 pigs from 4 herds with good performance and no diarrhoea. In (2), 110 pigs from 11 herds with diarrhoea and poor performance were selected. In (3), 24 six-week-old pigs from an SPF herd free from $L$. intracellularis, were included.

Sera were analysed by two serological methods, an IFAT (4) and a blocking ELISA (5) using PI 35 as cutoff value. The two methods were compared using pigs from study (1) and (2). The DSn and DSp were determined by a 2 * 2 table using either the IFAT or the ELISA as gold standard. Further, samples from ten pigs confirmed to be infected with $L$. intracellularis by nested PCR on faeces, and the 24 pigs from herd (3), were used as reference samples and similarly compared.

\section{Results}

As analyzed by the blocking ELISA, 32 of 66 samples from population (1) and 30 of 110 samples from (2) were judged as positive. As analyzed by the IFAT, 47 samples from (1) and 28 samples from (2) were judged as positive. All samples from confirmed cases of $L$. intracellularis were serologically positive and all samples from population (3) were deemed as negative by both methods.
Table 1. The diagnostic sensitivity and specificity of the blocking ELISA, using the IFAT as gold standard and applied on pigs from population (1) and/or (2).

\begin{tabular}{|c|c|c|}
\hline & \multicolumn{2}{|c|}{ ELISA } \\
\hline Population & DSn & DSp \\
\hline$(1)$ & 0.64 & 0.89 \\
\hline$(2)$ & 0,79 & 0.92 \\
\hline (1) and (2) & 0.69 & 0.91 \\
\hline
\end{tabular}

Table 2. The diagnostic sensitivity and specificity of the IFAT, using the blocking ELISA as gold standard and applied on pigs from population (1) and/or (2).

\begin{tabular}{|c|c|c|}
\hline & \multicolumn{2}{|c|}{ IFAT } \\
\hline Population & DSn & DSp \\
\hline$(1)$ & 0.94 & 0.50 \\
\hline$(2)$ & 0.73 & 0.94 \\
\hline (1) and (2) & 0.84 & 0.82 \\
\hline
\end{tabular}

Analysing reference samples from known L. intracellularis-positive and -negative animals, the DSn and DSp was $100 \%$ for both methods.

\section{Discussion}

Experimentally, L. intracellularis-specific antibodies are detected 2 weeks after challenge, but the time point of infection and the actual infectious status are usually unknown in clinical samples. Further, estimating the diagnostic sensitivity and specificity by a $2 * 2$ table requires the use of a true gold standard with DSn and DSp close to 1.00. However, none of the tests applied in this study can be considered to be appropriate for that purpose. Hence, in the present study the diagnostic sensitivity and specificity varied depending on the gold standard and on the population. In the absence of a true gold standard, Bayesian modelling techniques may be used to validate samples obtained from the target population.

\section{References}

1. Jacobson et al., 2003. Res Vet Sci 74:163-169.

2. Jacobson et al., 2006. Proc. 19th IPVS, pp. 181.

3. Wallgren et al., 1999. J. Vet. Med. B, 249-260.

4. Knittel et al., 1998. Am J Vet Res 59:722-726.

5. Keller et al., 2004. Proc. 18th IPVS, pp. 293. 


\title{
P.395
}

\section{Susceptibility of different mouse strains to Lawsonia intracellularis infection using intestinal mucosa homogenate and pure culture}

\author{
Aline M. Viott ${ }^{1}$ Fabio A. Vannucci ${ }^{1}$ Juliana S. Oliveira ${ }^{1}$ Mirella C. Costa ${ }^{1}$ Connie J. Gebhart ${ }^{2}$ Roberto M. Guedes $^{1}$ \\ 1. Universidade Federal de Minas Gerais, Veterinary School, Belo Horizonte, MG, Brazil; \\ 2. Department of Veterinary and Biomedical Sciences, University of Minnesota, Saint Paul, MN, USA
}

\section{Introduction}

The susceptibility of laboratory mice to Lawsonia intracellularis infection has been demonstrated by experimental infection 1 ; however, the occurrence of proliferative enteropathy (PE) lesions varies among different mouse strains2. Moreover, Murakata et al.2 showed that there are differences with respect to the establishment of PE lesions in mice according to the type of inoculum used. The authors demonstrated that mice inoculated with intestinal mucosa homogenate extracted from PE-affected rabbits had more severe lesions than those inoculated with pig intestinal homogenate. The purposes of this study were to describe experimental infections of L. intracellularis in four different mouse strains using intestinal mucosa homogenate $(\mathrm{MH})$ extracted from $\mathrm{PE}$-affected pigs and pure culture $(\mathrm{PC})$ of the bacteria as inoculum and to evaluate fecal shedding of $L$. intracellularis in these animals.

\section{Materials and Methods}

Forty 3-to-4-week-old male mice of four different strains (BALB/C, C57BL/6, DB/A2 and Swiss) were used. On D0, 16 animals of each strain were inoculated with $0.5 \mathrm{ml}$ of $\mathrm{L}$. intracellularis $\mathrm{PC}(7.75$ $x$ 107)3, 16 with $0.5 \mathrm{ml}$ of $\mathrm{MH}(8.9 \times 108) 3$ and eight with sham inocula using an intragastric gavage. Four and two mice from the infected and control groups, respectively, were euthanised on D7, D14, D21 and D28. Intestinal samples from the mice were analyzed by histology and immunohistochemistry (IHQ)4. A pool of fecal samples was collected from each cage weekly and tested using a nested PCR assay 5 for $L$. intracellularis. Fisher exact test was used for the statistical analyses.

\section{Results}

No diarrhea or other clinical signs were observed in these mice. Macroscopically, DB-A mice inoculated with PC had thickening of the cecum and ileum wall at 14D. According to the exact Fisher test, the histological lesions and IHQ were more severe in mice inoculated with $\mathrm{PC}$ than in those inoculated with $\mathrm{MH}$. Strains DB-A and Swiss had more intense lesions and IHQ label than the other strains. Results of fecal PCR were reflected the severity of the histologic lesions. Swiss mice inoculated with PC shed L. intracellularis on D7 and D14; those inoculated with $\mathrm{MH}$ shed on D14 only. BALB/C mice inoculated with either inoculum were positive by PCR only on D14. C57BL/6 mice inoculated with PC shed the bacteria on D7 and D14 while those inoculated with $\mathrm{MH}$ were negative throughout the experiment. $\mathrm{PC}$ and $\mathrm{MH}$ inoculated DB-A mice were positive by PCR on D7, D14 and D21. All control mice were negative in the analyses performed.

\section{Discussion}

Gross and microscopic lesions typical of L. intracellularis infection were successfully reproduced in the mice in this study. Our results demonstrate a significant variation of lesion severity and of $L$. intracellularis infection among the four mice strains studied. DB-A and Swiss strains were the most susceptible to PE lesions, while the lesions in BALB/C and C-57BL/ 6 mice were mild. These differences in susceptibility to infectious agents have been previously known, but the genetic basis for these differences remains to be determined2. Typical major lesions and bacterial shedding occurred in mice strains on D14, similar to what is described in pigs6. DB-A and Swiss developed more severe PE lesions and shed more bacteria, especially when inoculated with PC. This information can be useful for the establishment of an experimental model for $L$. intracellularis infection in mice.

\section{References}

1. Inf. Immun. 2000, 68(12): 6737-6743.

2. J. Comp. Pathol. 2007, 139: 8-15.

3. Vet. Microbiol. 2003, 93: 159-166.

4. J. Vet. Diag. Invest. 2003, 15(5): p. 438-446.

5. J. Clin. Microbiol. 1993, 31: 2611-2615.

6. Vet. Pathol. 2003, 40:421-432. 


\title{
P.396
}

\section{Quantitative assessment of Lawsonia intracellularis in feces by real-time PCR}

\author{
Ken S. Pedersen ${ }^{1}$ Klaus Pedersen ${ }^{1}$ Marie Ståhl${ }^{2} \varnothing$ ystein Angen ${ }^{2}$ Charlotte Hjulsager ${ }^{2}$ Lars E. Larsen $^{2}$ Helle Stege $^{1}$ Jens $^{\text {P. Nielsen }}{ }^{1}$
}

1. University of Copenhagen, Frederiksberg, Denmark; 2. National Veterinary Institute, Technical University of Denmark, Frederiksberg, Denmark

\section{Introduction}

Conventional PCR tests designed for detection of Lawsonia intracellularis (LI) in feces may detect infections with or without effect on clinical disease and production parameters. Quantification of fecal excretion may therefore serve as a method to distinguish between clinically and economically significant and insignificant infections. Recently a quantitative real-time PCR (q-PCR) for detection of LI has been developed (1). Repeatability is an important test characteristic for quantitative diagnostic tests. Q-PCR repeatability can be influenced by features inherent to the q-PCR assay, homogeneity of fecal samples and variation in level of excretion for the same pig within the same day. The objective of the current study was to investigate variation in fecal excretion of LI detected by q-PCR within one day. Variation in fecal dry matter was also determined for the same pigs and will be reported elsewhere in the proceedings.

\section{Materials and Methods}

A cross-sectional field study with follow-up was designed. A Danish farrow- to finish herd with a history of LI was selected at convenience. A batch of approximately 8 weeks old pigs having an outbreak of diarrhea was selected. The pigs had not received antibiotic treatment within the last 4 weeks and treatment of the current outbreak was not initiated before the fecal samples were collected. Thirty pigs were selected at convenience. Fecal samples were collected at 9am, $11 \mathrm{am}, 1 \mathrm{pm}, 3 \mathrm{pm}$ and $5 \mathrm{pm}$ from the rectum of each pig. The pigs were identified with two identical ear tags. At each time point the pigs were examined in the same chronological order. All fecal samples were transported to DTU-VET, stored at $40 \mathrm{C}$ and further processed the following day. All fecal samples were subjected to q-PCR testing designed for detection of $\mathrm{LI}$ (1). Fecal dry matter content (DM\%) was determined by the microwave method (2). DM\% of the fecal samples from the first time point was used to stratify the pigs into non-diarrheic (DM\%>18.8), semi-diarrheic (18.8 $\geq \mathrm{DM} \%>13.1)$ or diarrheic (DM\% $\leq 13.1)$ in the statistical analysis (3). The data was analyzed in a linear regression model with a repeat measurement structure using the mixed procedure in SAS, version 9.1.

\section{Results}

A total of 29 pigs and 144 observations were included in the analysis. Twenty-eight pigs had detectable levels of $\mathrm{LI}$ in feces at all time points. One pig was negative at one time point. Decreasing DM\% was significant associated with an increasing excretion of $\mathrm{LI}(\mathrm{p}<0.001)$. A significant effect of time on mean fecal excretion of LI was observed for non-diarrheic $(p<0.02)$, semi-diarrheic ( $p$ $<0.01)$ and diarrheic $(p<0.01)$ pigs, table 1 . In all three groups the mean fecal excretion of $\mathrm{LI}$ was lowest at $1 \mathrm{pm}$.The maximum individual change in fecal $\mathrm{LI}$ excretion between any time points was below one log unit for 25 of the pigs. The most extreme change observed was 1.09 log units.
Table 1. Mean copies of Lawsonia intracellularis (Log10/gram feces), in fecal samples from 29 pigs examined 5 times within one day $(n=144$ fecal samples)

\begin{tabular}{|l|c|c|c|c|c|}
\hline & \multicolumn{5}{|c|}{ Time point } \\
\hline & $\mathbf{9 a m}$ & $\mathbf{1 1} \mathbf{a m}$ & $\mathbf{1 p m}$ & $\mathbf{3 p m}$ & $\mathbf{5} \mathbf{p m}$ \\
\hline Non-diarrheic (DM\% $>18.8)(\mathrm{n}=7$ pigs) & $4.3 \mathrm{a}$ & $4.2 \mathrm{a}$ & $3.9 \mathrm{~b}$ & $4.3 \mathrm{a}$ & $4.1 \mathrm{ab}$ \\
\hline Semi-diarrheic $(18.8 \geq \mathrm{DM} \%>13.1)(\mathrm{n}=9$ pigs) & $5.3 \mathrm{a}$ & $5.1 \mathrm{ac}$ & $4.8 \mathrm{~b}$ & $5.0 \mathrm{c}$ & $4.9 \mathrm{bd}$ \\
\hline Diarrheic (DM\% $\leq 13.1)(\mathrm{n}=13$ pigs) & $5.6 \mathrm{a}$ & $5.4 \mathrm{a}$ & $5.2 \mathrm{~b}$ & $5.6 \mathrm{a}$ & $5.4 \mathrm{ab}$ \\
\hline Time points with different subscripts within rows are significant different $(\mathrm{p}<0.05)$. \\
\hline
\end{tabular}

\section{Discussion}

The increasing excretion of $\mathrm{LI}$ in pigs with decreasing fecal DM\% indicates that quantification of LI can be applied to distinguish between significant and insignificant infections. The mean fecal Ll excretion changed significantly during the examination day. However, the change was small within each group of pigs and may not affect the potential of q-PCR as a group level test. At the individual level the change of more than one log unit in $14 \%$ of the pigs may indicate that within day variation is more important for application of q-PCR as a diagnostic test at the individual level. The lowest level of excretion was detected at time $1 \mathrm{pm}$ for all 3 groups. This implies that the other 4 time points better represents the mean fecal excretion of LI. This should be taken into consideration during collection and interpretation of q-PCR test results for detection of $\mathrm{LI}$.

\section{References}

1. Ståhl, M. et al., Abstract, Advances in qPCR 2008.

2. Pedersen, K.S. et al., 2009. Proc 1st ESPHM: page 66.

3. Pedersen, K.S. et al., 2009. Proc 1st ESPHM: page 67

\section{Acknowledgements}

This project was supported by Böhringer Ingelheim. 


\title{
P.397
}

\section{Identification of Lawsonia intracellularis in sections of intestines by immunohistochemistry}

\author{
Anna Szczotka; Jacek Zmudzki; Zygmunt Pejsak; Tomasz Stadejek \\ National Veterinary Research Institute, Pulawy, Poland
}

\section{Introduction}

Proliferative enteropathy, caused by Lawsonia intracellularis (L. intracellularis), is one of the major diseases contributing to economic losses in intensive swine production worldwide. The recommended methods for diagnosis of the disease are polymerase chain reaction (PCR) or immunohistochemistry (1, 2 ). The aim of the study was to develop immunohistochemistry (IHC) for L. intracellularis detection and to compare this method with PCR.

\section{Materials and Methods}

Field samples used in the study comprised of intestines (ileum, caecum and colon) and feces from 21 pigs with clinical symptoms of proliferative enteropathy, originating from 11 medium and large swine units. They were analyzed by PCR (5) for the presence of $\mathrm{L}$. intracellularis. Also, intestines were fixed in $10 \%$ buffered formalin, embeded in paraffin, cut and tested in (IHC), according to avidin-biotin complex method.

Sections of intestines positive for L. intracellularis, kindly provided by dr. T.K. Jensen (Danish Institute for Food and Veterinary Research, Copenhagen, Denmark) and dr. Dinko Novosel (Croatian Veterinary Institute, Zagreb) were used as positive controls. Sections of intestines from clinically healthy pigs, negative for $\mathrm{L}$. intracellularis by PCR (5), served as negative controls. All the sections were additionally hematoxylin-eosin (HE) stained.

\section{Results}

DNA of L. intracellularis was found by PCR in samples of intestines and in the corresponding feces from 16 pigs from 9 farms. $\mathrm{L}$. intracellularis antigen was detected by $\mathrm{IHC}$ in all positive controls and in sections from 9 diseased pigs, representing 7 farms. In the HE-stained sections of positive controls and IHC-positive field samples enlarged and branched crypts with proliferating immature epithelial cells were found, which are characteristic lesions of proliferative enteropathy (4). The remaining 5 sections were negative in $\mathrm{IHC}$ and presented no lesions.
Image: IHC. Immunolabelling within the apical cytoplasm of hypertrophic enterocytes, resulting in specifically stained rims around the crypt lumina. x 200

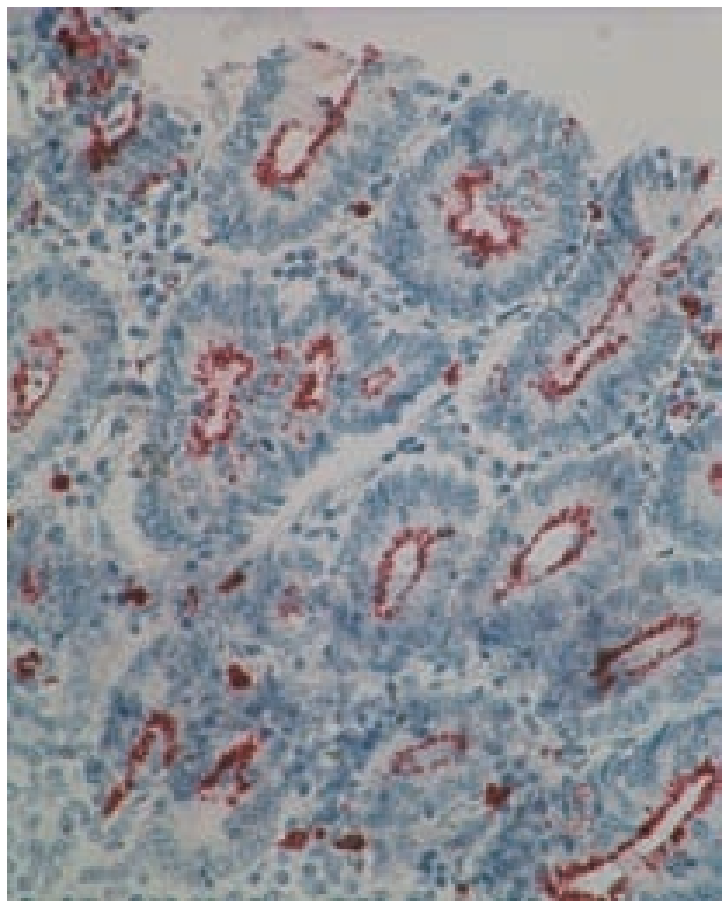

\section{Discussion}

The results of this study showed that $L$. intracellularis antigen could be detected in formalin-fixed, paraffin-embedded tissues of pigs using monoclonal antibodies. The IHC test showed high specificity but lower sensitivity than PCR. Most probably the samples with no obvious lesions characteristic for proliferative enteropathy, negative in IHC and positive in PCR most likely originated from chronic cases of disease or final stages of recovery, where bacteria were present in a small number or already absent in the tissue (3).

\section{References}

1. Guedes R.M.C., Gebhart C.J., Winkelman N.L., Makie-Nuss R.A.C., Marsteller T.A. et al. (2002). Canadian Journal of Veterinary Research, 66, 99-107.

2. Huerta B., Arenas A., Carrasco L., Maldonado A., Tarradas C. et al. (2003). Journal of Comparative Pathology, 129, 179-185.

3. Ladinig A., Sommerfeld-Stur I., Weissenbock H. (2009). J. Comp. Path. 140, 140-148.

4. Lawson G.H.K., Gebhart C.J. (2000). Journal of Comparative Pathology, 122, 77-100.

5. Zmudzki J. et al. 2006, Medycyna Wet., 62 (12) 1420-1423

\section{Acknowledgements}

This work was supported by the research grant N N308 075634 from Polish Ministry of Science and Higher Education 


\title{
P.398
}

\section{Development of SYBR green base quantitative-PCR for quantification of Lawsonia intracellularis}

\author{
Suphot Wattanaphansak ${ }^{1}$ Janet Anderson ${ }^{2}$ Connie Gebhart ${ }^{2}$ Randall Singer $^{2}$ \\ 1. Chulalongkorn University, Pathumwan, Bangkok, Thailand; 2. Veterinary and Biomedical Sciences, University of Minnesota, St. Paul, MN, USA
}

\section{Introduction}

Lawsonia intracellularis (LI) is an obligately intracellular organism and is the causative agent of proliferative enteropathy (PE) in pigs and other animals. In many studies, the estimation of bacterial quantity or level of LI infection in samples (pig feces, intestinal tissue, gut mucosal homogenate, and pure culture) is required. However, we lack the assays for accurate quantification of LI in those samples. Previously, estimates of LI loads were obtained using a cumbersome immunostaining procedure with specific antibody for LI (1). The objective of this study was to develop a quantitative PCR (qPCR) using SYBR green for quantification of $\mathrm{LI}$ in feces and bacterial pure culture.

\section{Materials and Methods}

The LI strain PHE/MN1-00 was used for all steps in the development and optimization of the qPCR. Specific primers were designed using the aspA gene as a target. The size of PCR product is $162 \mathrm{bp}$. Serial 10-fold dilutions of pure culture samples and several sets of spiked feces in the range of 108 to $101 \mathrm{LI} / \mathrm{ml}$ and $\mathrm{LI} / \mathrm{g}$ of feces, respectively, were tested for qPCR accuracy and the limit of quantification. The specificity of the primers was tested against 16 swine enteric bacteria using qPCR and conventional PCR. DNA of LI from pure culture bacteria and from fecal samples was extracted using the DNeasy ${ }^{\circledR}$ Blood and Tissue Kit and the QIAamp ${ }^{\circledast}$ DNA Stool Mini kit, respectively. qPCR was conducted using the Brilliant ${ }^{\circledR} \|$ SYBR $^{\circledR}$ Green Master Mix and $5 \mu$ l of DNA template in a final reaction volume of $25 \mu \mathrm{l}$.The qPCR amplification and quantification were performed using an Mx3000P RealTime PCR System. The standard curve was constructed using the DNA of LI pure culture in which the final bacterial concentration was determined using a direct count method (2) before DNA extraction. Several qPCR parameters were tested: the limits of detection and quantification, the linear range, and the inter- and intra-assay repeatability.

\section{Results and Discussion}

When detected from pure culture, the limit of detection and quantification of $\mathrm{LI}$ was $5.1 \times 101 \mathrm{LI} / \mathrm{ml}$ while the lower limit of the linear range of the assay was $5.1 \times 102 \mathrm{LI} / \mathrm{ml}$. In spiked feces, the limit of detection of $\mathrm{LI}$ was $2.55 \times 101 \mathrm{LI} / \mathrm{g}$ while the limit of quantification was $2.55 \times 103 \mathrm{LI} / \mathrm{g}$. A concentration between $2.55 \times 104$ and $2.55 \times 103 \mathrm{LI} / \mathrm{g}$ was the lower limit of the linear range. No cross-reactivity of qPCR was found when the assay was tested using the DNA extracted from 16 species of swine enteric bacteria. Coefficients of variation of inter- and intra-assay reapeatability was lower than $10 \%$. The dissociation curve of the aspA amplicon (Figure 1) showed a single melting temperature (Tm) peak at 79oC. In one of five spiked fecal samples, a nonspecific peak could be found (Figure 2, right peak) but only at low concentration of 104 to $101 \mathrm{LI} / \mathrm{g}$. This peak of incorrect Tm appeared only after cycle 36.
Figure 1. Dissociation curve analysis of LI pure culture.

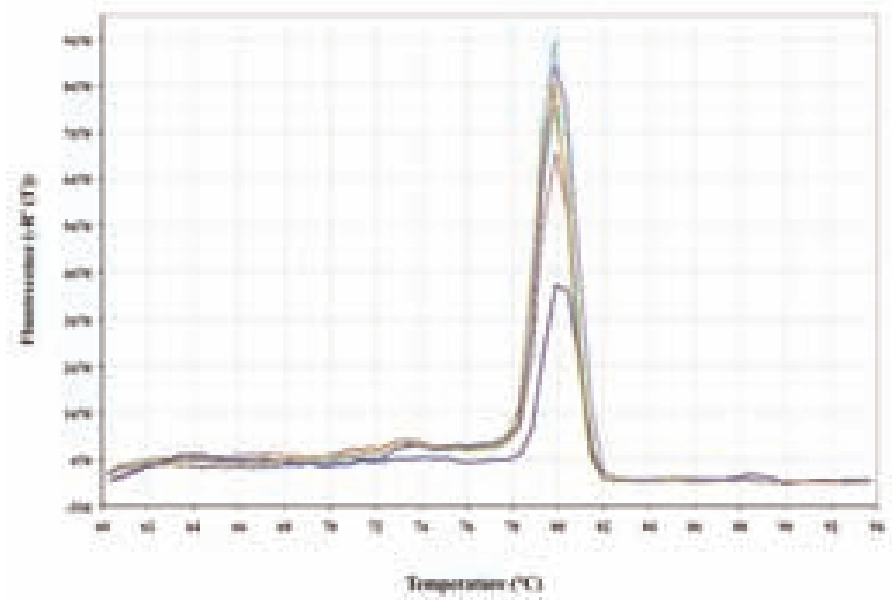

Figure 2. Dissociation curve analysis of spiked feces.

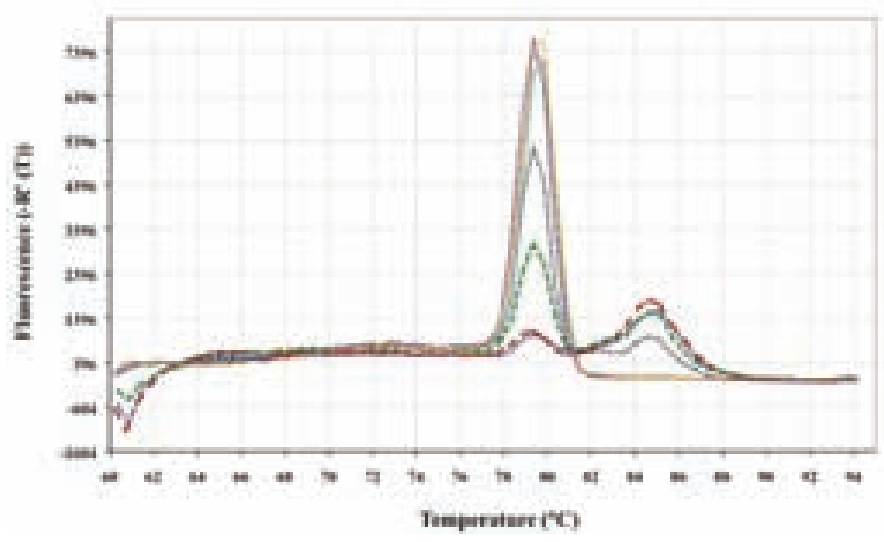

The results obtained from this assay are sensitive, specific and precise for the detection and quantification of $\mathrm{L}$. intracellularis in samples. This new qPCR assay will be helpful in future disease transmission and epidemiology studies. We will use this assay to determine the dynamics and quantity of LI excreted post-infection through feces after experimental and natural infections.

\section{References}

1. Guedes RM and Gebhart CJ. 2003. Vet. Microbiol. 93.159-166.

2. Wattanaphansak et al. 2009. Vet. Microbiol.134. 305-10. 


\title{
P.399
}

\section{Efficacy of enterisol ileitis in a Chinese pig farm}

\author{
Qinzhang Lu \\ Preventive Veterinary Lab, College of Animal Science and Veterinary Medicine, Guangxi University, Naning, China
}

\section{Introduction}

lleitis, caused by Lawsonia intracellularis (LI), is a widespread enteric disease in swine. It leads to reduced average daily gain (ADG), poor feed conversion in nursery and fattening and high variation of body weight at slaughter. McOrist (1) reported that 90 $\%$ of pig farms in the world were LI infected. Most of the herds are only subclinically affected by ileitis meaning that the animals do not show any clinical signs but reduction and variability in growth. Wang et al. (2) tested 2,700 blood samples collected from 54 Chinese pig farms with a blocking ELISA for anti-LI-antibodies. In their survey $70 \%$ of the investigated farms were positive already at the end of nursery period. Adam (3) estimated that the economical impact of clinical and subclinical ileitis in Europe is in average 4.70 and 3.47 Euro respectively per pig.

\section{Materials and Methods}

The trial was carried out in a Chinese pig farm in Guangxi Zhuang Autonomous Region from 2nd of March to 25th of June 2008. Animals were assigned randomly into 2 treatment groups: the vaccinated group (Group V) and the non-vaccinated group (Group Nv). There were 489 pigs in Group V in replicating subgroups of 141, 142 and 206 pigs which were all vaccinated with Enterisol $^{\circledR}$ Ileitis at 14 days of age. There were 489 pigs in Group $\mathrm{Nv}$, which consisted of 3 replicating subgroups as well, with 140, 141, and 208 pigs, respectively. Both Group V \& Group Nv were housed in the same barn, provided with the same feed and health management. An infection of the trial animals with LI was conformed by clinical signs and serological testing.

\section{Results}

ADG of the vaccinated animals was $18.9 \mathrm{~g}$ higher and FCR was 0.09 lower compared to the non-vaccinated animals.

Overall mortality was lower in the vaccinated animals by $0.82 \%$.

An additional 46 Yuan/pig profit was generated by vaccination, including:

1. Feed consumption decreased by $2.16 \mathrm{~kg}$ ( $\approx 4$ Yuan)/V pig;

2. Final weight increased by $2.14 \mathrm{~kg}(\approx 28$ Yuan)/V pig;

3. Mortality and culling rates decreased, saving about 14.6 Yuan/V pig
Table 1. Effects of Enteriso/ ${ }^{\otimes} / /$ eitis on performance data

\begin{tabular}{|l|c|c|c|}
\hline & Group V & Group Nv & Difference \\
\hline pigs included (N) & 489 & 489 & \\
\hline mortality (n/\%) & $16 / 3.27$ & $20 / 4.09$ & $-4 /-0.82$ \\
\hline pig with diarrhea (n/\%) & $145 / 29.7$ & $160 / 32.7$ & $-15 /-3.07$ \\
\hline average birth weight (kg) & 1,40 & 1.43 & -0.03 \\
\hline average post-weaning weight (kg) & 6.42 & 6.48 & -0.06 \\
\hline average final weight (kg) & 58.28 & 56.14 & 2.14 \\
\hline trial duration (d) & 115 & 115 & 0 \\
\hline ADG (g) & 494.61 & 475.74 & 18.87 \\
\hline feed consumption (kg) & 107.98 & 110.14 & -2.16 \\
\hline FCR (wean to growing) & 2.08 & 2.31 & -0.23 \\
\hline
\end{tabular}

\section{Discussion}

Throughout the trial, animals of Group V showed a better growth performance than those of Group Nv. Findings in the present trial demonstrate that Enterisol ${ }^{\circledR}$ Ileitis can effectively protect pigs from clinical ileitis in Chinese farms. Moreover, vaccination can improve the production performance, as indicated by ADG, FCR and mortality, leading to a higher profit margin. In conclusion, Enterisol ${ }^{\circledR}$ lleitis is a powerful tool to control ileitis and boost profitability of pig farms.

\section{References}

1. McOrist, S. et al. (2003): Can J Vet Res 67(3): 232-234.

2. Wang, J. et al (2006): Proc. 19th IPVS Congress Vol. 2: 190.

3. Adam, M. (2008) Proc. 20th IPVS Congress 2008. 


\title{
P.400
}

\section{Influence of lleitis vaccination on gross margin by improved uniformity}

\author{
Hanne Bak; Poul Henning S. Rathkjen \\ Boehringer Ingelheim AS, Copenhagen, Denmark
}

\section{Introduction}

The vaccine Enterisol ${ }^{\circledR}$ Ileitis Vet. effectively controls ileitis caused by Lawsonia intracellularis (LI). In a pan-european survey, the gross margin (GM) increased 3-6€ per pig after vaccination, when benefits of increased average daily weight gain (ADWG), reduced feed conversion rate (FCR) and losses were valued (1). On top, the vaccine increases uniformity of pigs (2), and this parameter was not included in the calculations. As a rule of thumb for acceptable uniformity, the producers organisation in Denmark estimates, that every pig in a batch should have reached the minimal required weight (70 kg carcase weight), when half of the batch has been shipped for slaughter. Each kg a pig weighs below this minimal weight at this cut-off day, the GM per pig decreases by $0,53 €$ because of extra costs in production or depreciation of carcass value (3). Hence, a thorough calculation of economical benefits should not only include values of ADWG, FCR and losses, but also value arising from increased uniformity. In the present study, losses in GM caused by lack of uniformity were calculated for vaccinated and non-vaccinated pigs by calculation of their weight at the cut-off day, when $50 \%$ of the pigs had been slaughtered.

\section{Materials and Methods}

The study contained 9494 pigs from 3 Danish slaughter pig herds. From each herd, 16 batches of pigs were included, and pigs from every second batch (batch 1, 3, 5, 7, 9, 11, 13 and 15) were vaccinated against LI with Enterisol ${ }^{\circledR}$ Ileitis Vet. (Boehringer Ingelheim). Remaining batches were non-vaccinated controls. In herd 1 and 2, the vaccine was given in drinking water 1-5 days after weaning, and in herd 3, vaccine was administered orally by drench before weaning. Infection with LI was confirmed by laboratory diagnosis on intestines. The date of weaning was recorded, and individual data for slaughter date and carcase weight were obtained from the slaughterhouse. The number of days to slaughter and ADWG was calculated for each pig. The carcase weight of pigs at the cut-off day (when $50 \%$ of the pigs had been slaughtered) was calculated backwards from the cut-off day using actual carcase weight and assuming an ADWG of $850 \mathrm{~g} /$ day from the cut-off day until the actual day of slaughter. To put a value on (lack of) uniformity, the difference $(\Delta)$ between calculated carcase weight at the cut-off day and $70 \mathrm{~kg}$ was calculated for each pig. Lost uniformity GM was valued per pig as $\Delta \mathrm{kg} \times 0.53 €$. Comparison of vaccinated pigs and controls was made with Students t-test for ADWG and with Kruskal-Wallis test for lost uniformity GM (significance level $p \leq 0.05$ ).
Table 1: Performance of pigs from weaning to slaughter.

\begin{tabular}{|l|l|c|c|c|}
\hline Herd & & $\mathbf{1}$ & $\mathbf{2}$ & $\mathbf{3}$ \\
\hline \# pigs & $\mathrm{Vx}$ & 2083 & 1414 & 2436 \\
\hline & $\mathrm{Cx}$ & 1388 & 1129 & 1044 \\
\hline ADWG wean to slaughter (g/day) & $\mathrm{Vx}$ & 713 & 752 & 705 \\
\hline & $\mathrm{Cx}$ & 667 & 718 & 678 \\
\hline & $\mathrm{Vx}-\mathrm{Cx}$ & $46^{*}$ & $34^{*}$ & $27^{*}$ \\
\hline Uniformity GM lost $(€)$ & $\mathrm{Vx}$ & 0.19 & 0.17 & 0.40 \\
\hline & $\mathrm{Cx}$ & 0.78 & 0.59 & 1.29 \\
\hline & $\mathrm{Vx}-\mathrm{Cx}$ & $-0.59^{*}$ & $-0.52^{*}$ & $-0.89^{*}$ \\
\hline${ }^{*}=$ Statistically significant difference $(\mathrm{p}<0.05)$. & & \\
\hline
\end{tabular}

\section{Results}

In all 3 herds, vaccination gave a significant improvement in ADWG (table 1). The improvement varied from 27 to $46 \mathrm{~g} /$ day, comparable to results from other trials (1). The reduction in lost uniformity GM was between 0.52 and $0.89 € /$ pig. Fig. 1 shows the improved uniformity of the vaccinated pigs in herd 1, where only $8 \%$ of vaccinated pigs were below $70 \mathrm{~kg}$ carcase weight at the cut-off day, compared to $21 \%$ of non-vaccinated animals.

Figure 1: Lost uniformity GM from pigs in herd 1.

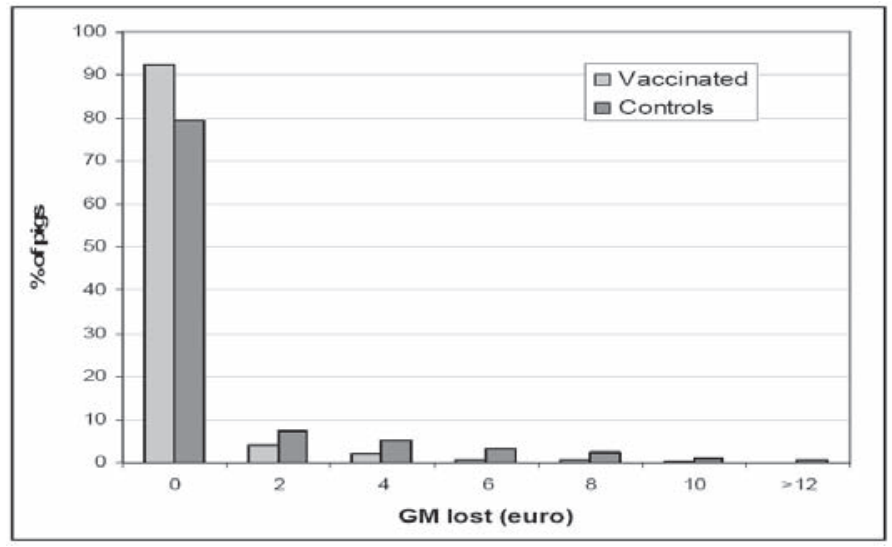

\section{Discussion and Conclusion}

Vaccination with Enterisol ${ }^{\circledR}$ lleitis Vet. significantly reduces losses in GM caused by low uniformity of pigs. To demonstrate full economical benefits of vaccination against LI, value of improved uniformity GM should be added to more traditional benefits, such as improved ADWG, FCR and mortality.

\section{References}

1. Adam M(2008): IPVS proc., Durban, SA,157.

2. Hardge T et al. (2004): Pig J. 54, 17 - 34.

3. Udesen F (2009): Personal communication. 


\title{
P.401
}

\section{Vaccination against Lawsonia intracellularis in two Danish farms}

\author{
Markku Johansen ${ }^{1}$ Poul Baekbo' MaiBritt F. Nielsen² \\ 1. Pig Research Centre; Danish Agricultural and Food Council, Kjellerup, Denmark; \\ 2. Pig Research Centre; Danish Agricultural and Food Council, Copenhagen, Denmark
}

\section{Introduction}

Lawsonia intracellularis (L.i.) is the causative agent of porcine proliferative enteropathy (PPE). PPE is an economically important enteric disease, causing diarrhea and reduced weight gain in weaners and grower-finishers (1). Lawsonia is present in most pig producing countries. In a Danish study, L.i was detected in 93.7 $\%$ of the tested herds (2). Together with management, antibiotics have been used to control the disease. However, the use of antibiotics can cause an increased level of resistant bacteria, and this might cause public concern about the production method in the industry. Vaccination is a consumer-friendly alternative, and the objective of this study was to evaluate the impact of vaccination with Enterisol ${ }^{\circledast}$ Ileitis Vet. on the average daily weight gain in nurseries and finisher barns in two Danish pig farms.

\section{Materials and Methods}

This study was conducted as a randomized blinded clinical trial with parallel treatment and a placebo group in two pig farms with clinical PPE problems. The time of infection was established by serological profiles using an ELISA (3) to detect antibodies against L.i. From both herds, two to four unthrifty pigs were submitted to a diagnostic laboratory to confirm the PPE diagnosis by immunohistochemistry (4). The pigs were vaccinated orally by drench one to two weeks before weaning; half of the litters in each batch of pigs being vaccinated and the other half treated with placebo. In each of the two herds, approx. 250 vaccinated pigs and approx. 250 placebo pigs were ear tagged individually and weighed at vaccination, at the end of the nursery period and at the end of the fattening period. Statistical analysis was performed by linear regression in SAS Proc Mixed with average daily gain (ADG) as the dependent variable, start weight, herd and observation period as covariates and batch as the random effect. Light pigs were arbitrarily defined as pigs weighing less than $67.5 \mathrm{~kg}$ live weight at the end of the fattening period. The study was performed in cooperation with Boehringer Ingelheim and supported by grants from the EU and Danish Ministry for Food, Agriculture and Fisheries.

Table 1. Weight of pigs vaccinated against L.i. and pigs treated with placebo

\begin{tabular}{|l|c|c|c|}
\hline & N & Placebo & Vaccine \\
\hline Start weight, $\mathrm{kg}$ & 987 & $7.8(1.8)^{\mathrm{a}}$ & $7.3(1.7)$ \\
\hline End nursery weight, $\mathrm{kg}$ & 815 & $29.5(7.6)$ & $31.7(7.3)$ \\
\hline End fattening weight, $\mathrm{kg}$ & 607 & $85.6(13.5)$ & $87.6(11.7)$ \\
\hline a Standard Deviation
\end{tabular}

\section{Results}

The mean weight measurements of the pigs are shown in table 1. In the nurseries, the vaccinated pigs grew significantly faster in both herds. In herd 2, the vaccinated pigs also grew significantly faster in the observation period $7-90 \mathrm{~kg}$. The results are shown in Table 2.

Table 2. Results from analysis of vaccination with Enteriso/ ${ }^{\circledR} /$ leitis Vet. on Average Daily Gain (g/day) in two herds

\begin{tabular}{|l|c|c|c|c|}
\hline & Placebo & Vaccine & Difference & P value \\
\hline 7-30 kg & & & & \\
\hline Herd 1 & 399 & 418 & +19 & 0.05 \\
\hline Herd 2 & 494 & 525 & +31 & 0.002 \\
\hline 7-90 kg & & & & \\
\hline Herd 1 & 691 & 686 & -5 & 0.63 \\
\hline Herd 2 & 631 & 665 & +34 & 0.005 \\
\hline
\end{tabular}

Figure 1 shows the distribution of the weight of vaccinated and placebo pigs at the end of fattening in herd 2. The fraction of light pigs was lower in the vaccinated group of pigs. In this group $6 \%$ had a weight below $67.5 \mathrm{~kg}$, while $17 \%$ of placebo treated pigs weighed less than $67.5 \mathrm{~kg}$.

Figure 1. Distribution of weight measurements of placebo and vaccinated pias at the end of fattening in herd 2.

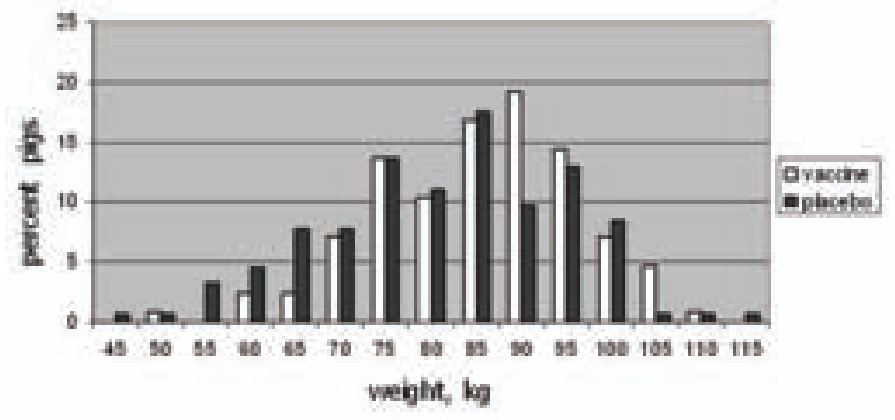

\section{References}

1. McOrist, S. et al, (2006) In Dis. of Swine 9th Ed. p 727-737

2. Stege, H.; et al. (2000).. Prev Vet Med, 46:279-292

3. Boesen, HT, et al. (2005). Vet. Microbiol. Vol. 109, 105-112

4. Jensen, T. K (2006).J. Comp. Path.,Vol. 135,176-182 


\title{
P.402
}

\section{Attempt to eradicate Lawsonia intracellularis during establishment of a new breeding herd by combined strategic medication with tiamulin (Denagard ${ }^{\oplus}$ ) and cleaning/disinfection}

\author{
Marion Kixmoeller ${ }^{1}$ Magdalena Kmiec ${ }^{2}$ Josef Szancer $^{3}$ \\ 1. Novartis Animal Health, Munich, Germany; 2. Risum-Lindholm Practice, Risum-Lindholm, Germany; \\ 3. Novartis Animal Health, Copenhagen, Denmark
}

\section{Introduction}

Lawsonia intracellularis (L.i.) is widely distributed among pigs in Germany and seems to play a dominant role in sows (1). The aim of this study was to eliminate L.i. from a breeding farm by medication with tiamulin (Denagard ${ }^{\circledR} 10 \%$ oral powder) combined with cleaning and disinfection.

\section{Materials and Methods}

The eradication program was carried out in a breeding farm with 2500 sows with production of $30 \mathrm{~kg}$ pigs. Six weeks before the program started the farm was completely depopulated, cleaned and disinfected and routine rodent control was carried out. Barns were repopulated with 2500 gilts aged $20-24$ weeks (60 - $120 \mathrm{~kg}$ ) delivered in 5 consecutive batches (April to September 2008). The delivery multiplier herd was infected with L.i.. Periodic outbreaks of PE (Proliferative Enteropathy) were observed and confirmed by post mortem investigations and L.i. was detected in faeces by polymerase chain reaction (PCR). Before arrival, 10 of 11 faeces samples from purchased gilts were positive for L.i.. The medication program was carried out in a quarantine stable. After each batch received the medication, the gilts moved to a new facility $9 \mathrm{~km}$ away.

\section{Medication}

To reduce shedding of L.i. and to minimize the risk of contaminating the quarantine area, the gilts were given a dosage of $8 \mathrm{mg} /$ $\mathrm{kg}$ bodyweight/day (bw/d) of tiamulin (Denagard) orally three days before being transported from the farm of origin to the new facility. After arriving at the quarantine stable, the gilts were fed a dosage with $8 \mathrm{mg} / \mathrm{kg}$ bw/day of tiamulin (Denagard $10 \%$ oral powder) for another 28 days. The dosage was calculated based on the heaviest gilt of approximately $120 \mathrm{~kg}$. Tiamulin was given via wet feed twice a day. $4 \mathrm{mg} / \mathrm{kg}$ bw/d once in the morning and $4 \mathrm{mg} / \mathrm{kg}$ bw/d after $8-10$ hours.

\section{Cleaning/Disinfection}

Faeces was removed from the pens twice daily after each feeding. Twice a week the whole pen was disinfected after cleaning with a disinfectant (Venno Vet 1 super).

\section{Monitoring}

Since the eradication program was conducted in October 2008 the herd has been clinically monitored twice a month for 15 months. 50 faecal samples from progeny weighing 20 - 60 $\mathrm{kg}$ were examined for L.i. by PCR and 56 blood samples from progeny (40-100 kg) were tested for specific antibodies for L.i. by ELISA test (2). Pigs showing the clinical symptoms of diarrhea were submitted to gross pathology for further laboratory examination.

\section{Results}

There have been no clinical signs of PE since completing the eradication program. As shown in the Table, all 50 faeces samples tested negative for L.i., but 2 blood samples were positive at the cut-off point which could be considered positive.

Table: Monitoring for L.i. in faeces and blood of the offspring (weaners and gilts) by PCR and ELISA after eradication program was carried out

\begin{tabular}{|c|c|c|}
\hline Date & $\begin{array}{c}\text { Number of positive/negative } \\
\text { faeces samples tested by PCR }\end{array}$ & $\begin{array}{c}\text { Number of positive/negative } \\
\text { blood samples tested by ELISA }\end{array}$ \\
\hline Nov. 2009 & $0 / 50$ & $1 / 30$ \\
\hline Dec. 2009 & - & $1 / 26$ \\
\hline
\end{tabular}

Pigs with clinical symptoms which were submitted to laboratory examination were tested negative for L.i.. Furthermore, there were no lesions characteristic for PE.

\section{Discussion}

This field trial indicates that L.i. may be successfully eradicated from a breeding herd by the presented procedure of medication with tiamulin combined with cleaning/disinfection. The two very low reactions for L.i. antibodies in blood are difficult to explain. It cannot be ruled out entirely that these two blood samples on the cut-off point could have been false positives since all data are against any signs of PE and in large parts blood samples as well all faeces samples tested negative for L.i.. Further investigation both in faeces by PCR and by serology for L.i. will be carried out in the near future to ascertain the herd is free from L.i.. The results will be presented.

\section{References}

1. Herbst, W. et al Berl Munch Tierarztl Wochenschr 2004, 117, 493-498.

2. Boesen, H.T. et al. Veterinary Microbiology 2005, 109, 105-112. 


\title{
P.403
}

\section{Denagard $^{\circledast}$ (tiamulin) treatment in a Lawsonia intracellularis pure culture seeder pig challenge study}

\author{
Dana C. Beckler $^{2}$ J. M. Hammer ${ }^{1}$ Connie Gebhart ${ }^{3}$ Linda Roycroft $^{1}$ \\ 1. Novartis Animal Health, US, Greensboro, NC, USA; 2. Gut Bugs, Inc., Fergus Fall, MN, Uruguay; 3. University of Minnesota, St Paul, MN, USA
}

\section{Introduction}

Proliferative enteropathy (PE) causes considerable economic losses to the swine industry worldwide. ${ }^{1}$ Judicious use of Denagard ${ }^{\circledR}$ has been shown to be an effective means of reducing economic loss due to PE. ${ }^{2}$ A pure culture seeder pig model was designed to show transmission of Lawsonia intracellularis ( $\mathrm{Li}$ ) infection from $\mathrm{Li}$ challenged pigs in a population to susceptible pen mates. This model mimics the paradigm for natural transmission of the disease. ${ }^{3}$ The objectives of this study were to document the spread of $\mathrm{Li}$ infection from a seeder pig to multiple susceptible pen mates, document levels of shedding in seeder and susceptible pigs, and evaluate the effect of placing Denagard $^{\circledR}$ at 38.5 ppm ( $35 \mathrm{~g} /$ ton) when a shedding threshold of $30 \%$ in susceptible pig was reached.

\section{Materials and Methods}

Li was isolated from a field case and cultured under conditions previously described ${ }^{4}$ and used as the challenge material for the seeder pigs. Sixty pigs were randomly placed into one of six pens. All pens were separated by an empty pen or a solid partition to prevent fecal oral contact between pens. The seeder pig to be challenged was designated as the pig closest to the median pig weight of each pen. Each seeder was challenged with $1.6 \times 10^{10} \mathrm{Li}$ via esophageal tube. Pigs were weighed and blood samples were collected once a week. Weekly blood samples were analyzed by the immunoperoxidase monolayer assay (IPMA) for the detection of circulating Li antibodies. Fecal consistency, abdominal appearance, and demeanor scores were recorded three times a week. Fecal samples were analyzed by quantitative polymerase chain reaction (qPCR) to determine the amount of $L i$ being shed per gram of feces. Denagard ${ }^{\circledR}$ at $38.5 \mathrm{ppm}$ ( $35 \mathrm{~g} /$ ton) was placed in three (treatment T2) of the six pens when $30 \%$ of the sentinel pigs were shedding $L i$ as detected by PCR. Three of the six pens remained untreated (treatment T1) throughout the trial. Pen was used as the experimental unit in all analyses.

\section{Results}

Fecal shedding of $L i$ from 3 of the seeder pigs peaked on 21 days post-challenge, the other 3 seeder pigs peaked on day 11, 18 , and 32 . The highest amount of $L i$ shed by a seeder pig was $8.62 \times 10^{8}$ bacteria/gram feces on day 11 post-challenge. The highest amount of $L i$ shed by a susceptible pigs was $1.52 \times 10^{9}$ bacteria/gram feces on day 32 post-exposure to a seeder pig. There was a significant difference in the percent of IPMA and qPCR positive pigs between Denagard ${ }^{\circledR}$ treated (T2) and control (T1) pens (Fig.1). There was a significant difference in mean fecal consistency and mean abdominal appearance scores between T1 and T2 (Fig. 2). Numerically, T2 when compared to T1 had a lower percent of days with abnormal (score $>0$ ) fecal consistency scores (3.91\% vs. 8.44\%, respectively) and abnormal (score $>0$ ) abdominal appearance scores $(17.08 \%$ vs $38.89 \%$, respectively).
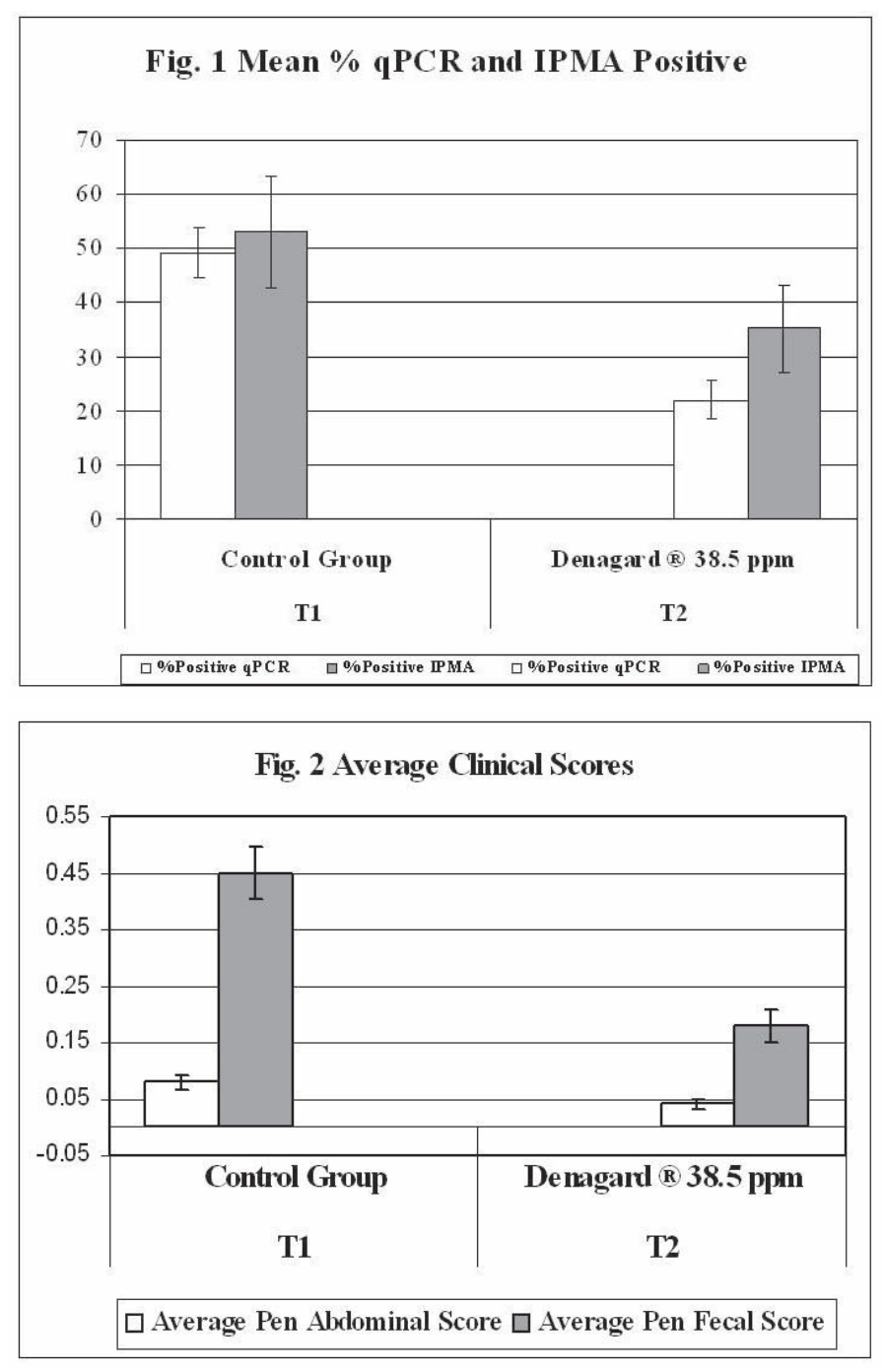

\section{Discussion}

Spread of $L i$ and levels of fecal shedding were documented in this pure culture seeder pig model. Direct challenged seeder pigs shed lower quantity of $L i$ than susceptible contact pigs when shedding was detected. Use of Denagard at 38.5 ppm (35 $\mathrm{g} /$ ton) in the model significantly decreased the clinical effects of $\mathrm{PE}$ in the susceptible pigs. Fewer Denagard ${ }^{\circledR}$-treated pigs tested positive by PCR or serology.

\section{References}

1. Lawson and Gebhart 2000 J Comp Pathol. 122:77-100

2. Walter et al. 2001 J. Swine Health Prod. 9:109-115

3. Jordan et al. 2004 Vet Microbiol. 104:83-90

4. Guedes and Gebhart 2003 Vet Microbiol. 19:159-166 


\title{
P.404
}

\section{Long term benefits of Ileitis vaccination in a $\mathbf{5 0 0 0}$ sow herd in Spain}

\author{
Joan Sanmartin; Gonzalo Cano \\ Optimal Pork Production, S.L., Lleida, Spain
}

\section{Introduction}

Ileitis is an infectious endemic disease present in most of the Spanish farms (1) and all over the world. Multisite production systems have not been able to control the infection and the clinical signs of the disease or to eliminate its negative economical impact in the fattening period.

Enterisol ${ }^{\circledR}$ Ileitis is the unique oral ileitis vaccine for controlling the negative impact of the disease. The objective of this study was to evaluate the effect of ileitis vaccination in a 5000 sow system one year after introducing the vaccine.

\section{Materials and Methods}

The study took place in a three site production system with a 5000 sow farrowing site, 16,000 places in the nursery and 32,000 fattening places. The system produced weekly batches of 2000 piglets. Some of the batches went to external fattening sites.

At the beginning of the trial the performance of the sow herd and in the nursery were above the average level in Spain. Nevertheless the management was not satisfied with the performance achieved during fattening period especially concerning growth, mortality and cull rate. From 10-12 weeks of age onwards until end of fattening, animals had chronic diarrhoea and some animals showed clinical signs typical for Porcine hemorrhagic enteritis. Before ileitis vaccination was implemented Lawsonia intracellularis infection was confirmed and the time of infection was determined by serology and PCR of fecal samples.

In May 2007 ileitis vaccination with Enterisol ${ }^{\circledR}$ |leits was implemented and piglets were vaccinated via the drinking water in troughs between 3rd and 4th week of live, just after weaning. Later due to logistic reasons in farm, the animals were vaccinated by drench.

The study compares 6 batches of animals before implementation of ileitis vaccination (12,048 pigs) with 15 batches of vaccinated animals (29.221 pig as Enterisol ${ }^{\circledR}$ Ileitis group). All batches were kept under similar husbandry conditions. All data presented is from fattening period and dates in the SPC charts are starting dates of each batch in fattening.

Performance of both treatment groups was compared by SPC (Statistical Process Control), using Statistica ${ }^{\circledR}$ Version 8.0, Statsoft. Return of Investment was calculated using pork and feed average prices of the dates that animals were slaughtered.

\section{Results}

SPC analysis revealed a significant increase in ADG in favour of the vaccinated animals (+70g, fig. 1). The FCR was $0.4 \mathrm{~kg} / \mathrm{kg}$ lower in the vaccinated animals compared to the non-vaccinated (fig. 2).
Figure 1. SPC chart of fattening Average Daily Gain (g/day).

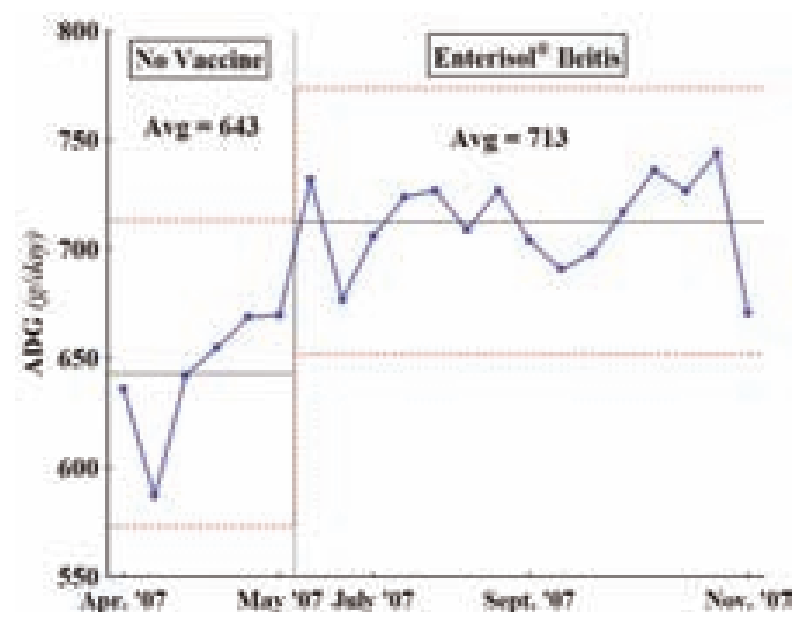

Figure 2. SPC chart of fattening Feed Conversion Ratio ( $\mathrm{kg} / \mathrm{kg})$.

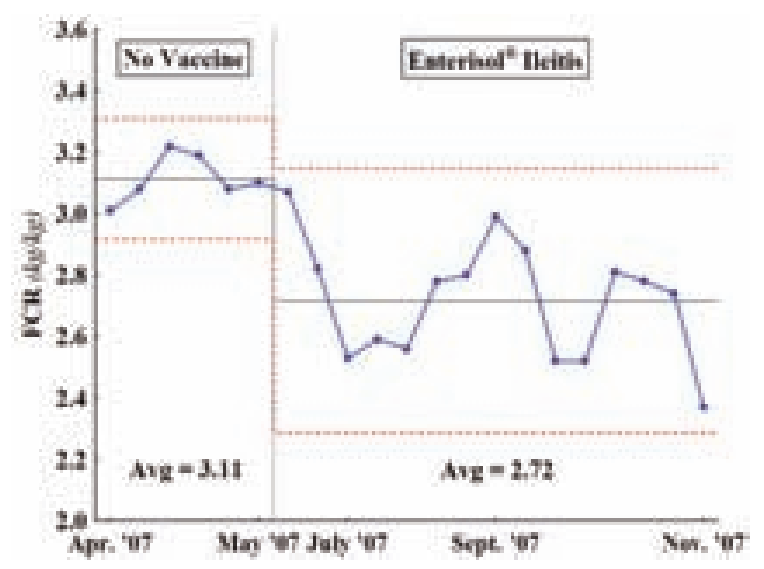

The mortality rate was $1.3 \%$ lower after implementation of ileitis vaccination (6.55 \% in the control group and $5.28 \%$ in the vaccinated animals).

Finally, the cost of medication for enteric problems in the fattening period was dramatically reduced by $89 \%$ by cutting out two routine antibiotic treatments that were established in the farm before the start of the study. These improvements in performance resulted in a Return of investment $8.6: 1$ per pig (including the vaccination cost).

\section{Discussion}

In the present study Enterisol ${ }^{\circledR}$ lleitis vaccination improved significantly the performance during fattening and was highly profitable. Fattening period is a critical point from an economical point of view and all the tools to improve its efficiency must be considered.

\section{References}

1. Carvajal, A. et al. (2006) Proc IPVS 2006 vol.2,174

2. Technical Manual Enterisol lleitis ver 3.0 


\title{
P.405
}

\section{Evaluation of the in feed use of Larch sawdust in growing pigs for treatment and prophylaxis of proliferative enteropathies}

\author{
Eleni D. Tzika ${ }^{1}$ Vassilios G. Papatsiros ${ }^{2}$ Panagiotis D. Tassis ${ }^{1}$ Dimitrios Koylialis ${ }^{1}$ Spiros Kritas ${ }^{3}$ Costas Alexopoulos $^{4}$ Spiros C. Kyriakis ${ }^{5}$ \\ 1. Clinic of Farm Animal Medicine, Faculty of Veterinary Medicine, Aristotle University of Thessaloniki, Thessaloniki, Greece; \\ 2. Clinic of Medicine, School of Veterinary Medicine, University of Thessaly, Karditsa, Greece; 3. Laboratory of Microbiology and Infectious Diseases, \\ Faculty of Veterinary Medicine, Aristotle University of Thessaloniki, Thessaloniki, Greece; 4. Deceased author, Thessaloniki, Greece;
}

5. Foundation of Biomedical Research, Academy of Athens, Athens, Greece

\section{Introduction}

Proliferative enteropathies (PE), also known as ileitis, are a group of acute and chronic conditions of widely differing clinical signs but with a common underlying pathological change visible at necropsy; a thickening of the mucosa of the small intestine and the colon. The primary causative agent is the obligate intracellular bacterium Lawsonia intracellularis. Ileitis is a common disease which affects pigs maintained under various management systems worldwide (1). The aim of this field study was the assesment of the possible antimicrobial activity of Larch sawdust based on the reduction of proliferative enteropathies prevalence in growing pigs. This trial was part of the SAFEWASTES specific targeted research project financed by the European Union (6th Framework Project).

\section{Materials and Methods}

This study was carried out on a commercial pig farm in northern Greece with a capacity of 250 sows under production. The farm was selected due to previous history of PE occurrence. 120 healthy of the same genetic background growing pigs at 8 weeks of age were used. A randomized block design was used with 3 treatments and 4 replicates (pens of 10 pigs each with equal sex ratio) per treatment. A day before allocation, all pigs were weighed and monitored for diarrhea. They were allocated to different groups, in order to achieve homogeneity in average weight, sex and diarrhea signs at the beginning of the trial.

The first group, designated as negative control group received feed formulated with $99 \%$ basic diet (grower ratio of wheat/ barley/soya mix with no antimicrobial agents or performance enhancers) and $1 \%$ corn starch from trial day 0 until the end of the trial ( 6 weeks later). The piglets of the second group received feed formulated with $99 \%$ basic diet and 1\% larch sawdust, while the third group was offered feed formulated with $97.5 \%$ basic diet and $2.5 \%$ larch sawdust. Productivity parameters and diarrhea score (DS) were recorded daily, while blood samples from 10 pigs/group at days 0, 14, 28 and 42 were collected for the detection of $\mathrm{L}$. intracellularis antibodies.

\section{Results and Discussion}

The in-feed use of larch did not show a clear positive antimicrobial effect against L. intracellularis infection in growing pigs, although the 3rd group (larch 2.5\%) had less positive blood samples at 14 th week relatively to the other 2 groups $(p=0.114)$ (graph 1). However, for the overall trial period, productivity parameters and DS of larch-fed pigs were improved relatively to the negative controls (tables 1 and 2).
Graph 1: Serological results of L. intracellularis antibodies in blood samples (Number of positive samples)

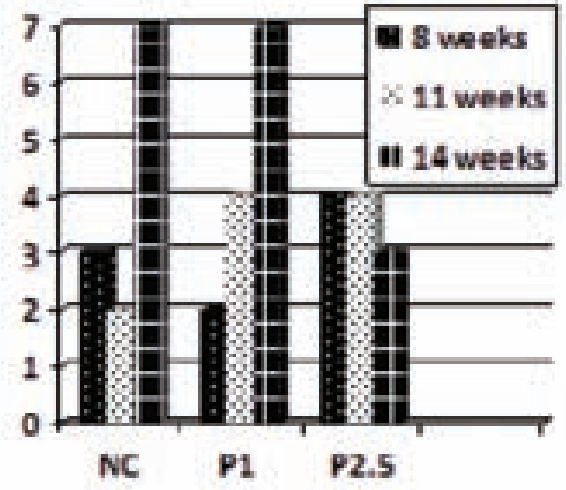

Table 1: Average Daily Weight Gain (kg), Average Daily Feed Intake $(\mathrm{kg})$ and Feed Conversion Ratio (Mean \pm SD)

\begin{tabular}{|l|c|c|c|}
\hline Groups & ADWG & ADFI & FCR \\
\hline Negative control & $0.589 \pm 0.068(n=39)$ & $1.323 \pm 0.023(n=4)$ & $2.24 \pm 0.03(n=4)$ \\
\hline Larch $(1 \%)$ & $0.604 \pm 0.094(n=39)$ & $1.340 \pm 0.014(n=4)$ & $2.22 \pm 0.07(n=4)$ \\
\hline Larch $(2.5 \%)$ & $0.607 \pm 0.084(n=40)$ & $1.334 \pm 0.010(n=4)$ & $2.20 \pm 0.02(n=4)$ \\
\hline
\end{tabular}

Table 2: Diarrhoea score based on pen calculation for the total trial period (Mean \pm SD)

\begin{tabular}{|l|c|}
\hline Groups & Diarrhea score $(\mathrm{n}=24)$ \\
\hline Negative control & $0.52 \pm 0.18$ \\
\hline Larch (1\%) & $0.44 \pm 0.16$ \\
\hline Larch $(2.5 \%)$ & $0.42 \pm 0.18$ \\
\hline
\end{tabular}

\section{References}

1. McOrist S and Gebhart CJ (2006) In Diseases of Swine. 9th edn. Eds Straw BE, Zimmerman JJ, D'Allaire, Taylor DJ pp. 727-737. Blackwell Sciences, London, UK 


\title{
P.406
}

\section{Longitudinal field case study on the impact of ileitis vaccination on performance}

\author{
Kerstin Klien ${ }^{2}$ Bernd Grosse Liesner $^{3}$ Ricarda Deitmer $^{1}$ \\ 1. Boehringer Ingelheim Vetmedica GmbH, Ingelheim am Rhein, Germany; 2. Practitioner, Gribow, Germany; \\ 3. Boehringer Ingelheim Animal Health GmbH, Ingelheim am Rhein, Germany
}

\section{Introduction}

It is widely demonstrated that vaccination controls the effects of Lawsonia intracellularis (Li) infections allowing improved performance (growth, feed efficiency, uniformity etc.) and generating higher profitability 1,2 . However any established practice is challenged from time to time to save costs. This longitudinal case study describes changes in production parameters and therefore the economic impact of the implementation and termination of ileitis vaccination .

\section{Materials and Methods}

The study took place in a farrow-to-finish farm of 1,600 sows and 10,000 fattening places in Germany. All pigs were housed under identical conditions. Parameters monitored were recorded per batch. ADG (average daily gain), FCR (feed conversion rate), mortality, and amount of antibiotics used against enteric diseases ( $A B$ use) in the finishing unit were monitored by SPC methodology (Statistical Process Control) since 2004 3. ADG, FCR and antibiotic use were evaluated by one-way ANOVA, pairwise comparison and adjusted by Bonferroni approach. Mortality is evaluated using a Chi-square test. All tests were performed with the software Statistica ${ }^{\circledR}$ v8.0, Statsoft ${ }^{\oplus}$. Two time periods were considered for evaluation of the ileitis vaccination impacts. During these two periods no other changes than implementation and withdrawal of ileitis vaccination were undertaken.

Implementation: August 2004 to March 2005 and March 2005 to November 2007 representing 95,134 animals. Sows were mass vaccinated against PRRSV every 4 month. Pigs were vaccinated against PRRSV (14 days of age) as well as against Mycoplasma hyopneumoniae ( 21 days of age). The producer added a third piglet vaccine, Enterisol ${ }^{\circledR}$ Ileitis in March 2005 (vaccination by drench, 14 days of age).

Withdrawal: February 2008 to April 2008 and April 2008 to September 2008, representing 16,848 animals. Enterisol ${ }^{\circledR}$ Ileitis was taken out of the vaccination program by April 2008.

The batches between these two periods were not taken into account since other changes were implemented in the vaccination protocol: vaccination with Ingelvac CircoFLEX ${ }^{\circledR}$ against PCV2 ( 21 days of age) was started by December 2007, and by end of February 2008 vaccination against PRRSV was terminated as the flow became PRRSV negative. The overall performance improved by $10 \%$ during this time.

\section{Results}

The improvement in performance following implementation of ileitis vaccination was similar to the decrease following withdrawal (table 1).

Table 1: Average performances and changes observed in ileitis vaccinated and non-vaccinated pigs

\begin{tabular}{|c|c|c|c|c|}
\hline & \multicolumn{2}{|c|}{ Implementation } & \multicolumn{2}{|c|}{ Withdrawal } \\
\hline Vaccines & M.hyo, PRRS & M.hyo, PRRS, Ileits & PCV2, M.hyo, lleitis & PCV2, M.hyo \\
\hline ADG g/day & 738 & $771^{*}$ & 811 & $781^{*}$ \\
\hline Diff. & & +33 & & -30 \\
\hline FCR & 3.16 & $3.06^{*}$ & 2.80 & $2.97^{*}$ \\
\hline Diff. & & -0.10 & & +0.17 \\
\hline Mortality \% & 4.12 & $3.04^{*}$ & 3.15 & 3.47 \\
\hline Diff. & & -1.08 & & +0.32 \\
\hline$A B$ use $\mathrm{kg} /$ group & 2.23 & $0.91^{*}$ & 0 & $2.32^{*}$ \\
\hline Diff & & -1.32 & & +2.32 \\
\hline
\end{tabular}

\section{Discussion}

The decline in performance after withdrawal of ileitis vaccination mirrors the gain observed three years earlier. Also the antibiotic use was reduced after implementation and increased again after withdrawal of ileitis vaccination. This indicates the consistent effect of the ileitis vaccination on performance parameters. The results indicate as well that losses in performance due to Lawsonia infections in non-vaccinated animals could not be prevented by treatment with antibiotics. Furthermore the decline in the second period indicates the potential additive effect of ileitis vaccination on top of PCV2 vaccination.

\section{References}

1. Scholz A.M. et al (2008). Pig journal 61: 26-35

2. Adam M. et al (2008). Leman conference v. 35 sup.: 36

3. Deitmer R. et al (2009). Praktischer Tierarzt 90; 4, 346-355 


\title{
P.407
}

\section{Reduction of growth rate variation for finishing pigs vaccinated with Enterisol ${ }^{\circledR}$ ileitis oral vaccine in a sub-clinically infected Australian pig farm}

\author{
Geoffrey P. Pollock ${ }^{1}$ Greg V. Marr² \\ 1. Boehringer-Ingelheim Animal Health, North Ryde, NSW, Australia; 2. IAS Management Services, Kingaroy, QLD, Australia
}

\section{Introduction}

Porcine Proliferative Enteropathy (PPE or lleitis) is due to the infection of Lawsonia intracellularis. It is a major factor affecting intestinal health of growing pigs. The economic impact of ileitis has been reported in Australia to be AUD\$15.00/sow/year and AUD\$141.00/sow/year with the non-haemorrhagic and hemorrhagic PPE respectively 1 . Sub-clinical ileitis was observed as uneven growth rates and longer time taken to reach market weights. Enterisol ${ }^{\circledR}$ |leitis is a modified live oral vaccine that offers lifelong immunity to grower pigs. The objective of this study was to document the efficacy of Enterisol ${ }^{\circledR}$ lleitis in a herd with subclinical lleitis.

\section{Materials and Methods}

A field evaluation was conducted in a 700 sow commercial piggery in Queensland. The presence of Lawsonia was confirmed by ELISA test. Seroconversion was found to be in late finisher at around 17 to 18 weeks of age. Clinical signs were not observed. However some variation in growth rate was observed regularly by the management. Two batches of vaccinated animals ( $V x)$ were compared to two batches of non-vaccinated animals (CX). Pigs were vaccinated with Enterisol lleitis at 14 days of age. Pigs were identified by batch with ear tags. Pigs were housed by batch in separate rooms. Growth rates from weaning until 21 weeks of age for 477 animals

( 2 batches) were calculated and compared to 544 non-vaccinated animals ( 2 batches). An average weaning weight was collected for each batch of the control groups $(6.46 \mathrm{Kg}, 6.30 \mathrm{Kg})$ and vaccinated groups (6.54 and $6.51 \mathrm{~kg}$ ). Pigs were weighed at 21 weeks of age in groups.

The farm used in-feed antibiotics against Lawsonia intracellularis infections in both treatment groups; OTC at 200ppm from 3 to 8 weeks of age, Olaquindox at 50ppm from 12 to 14 weeks of age, and Tylosin at 100 ppm from 18 to 20 weeks of age. No changes to in-feed medication or farm management were made during the trial. Data was analysed with Statistica ${ }^{\circledR}$ V 8.0.

\section{Results}

Mean Growth rate of vaccinated animals from weaning until 21 weeks of age was $726 \mathrm{~g}$ per day versus Non-vaccinates $678 \mathrm{~g}$ per day an improvement of $48 \mathrm{~g}$ per day. A frequency analysis of the data indicates that 61.5 percent of non-vaccinated animals grew at growth rates under 700 grams per day. In comparison, only $22 \%$ of vaccinated animals grew under 700 grams per day $(p<0.000001)$.
Figure 1: Distribution of wean to 21 week average daily weight gain in vaccinated and non-vaccinated animlas

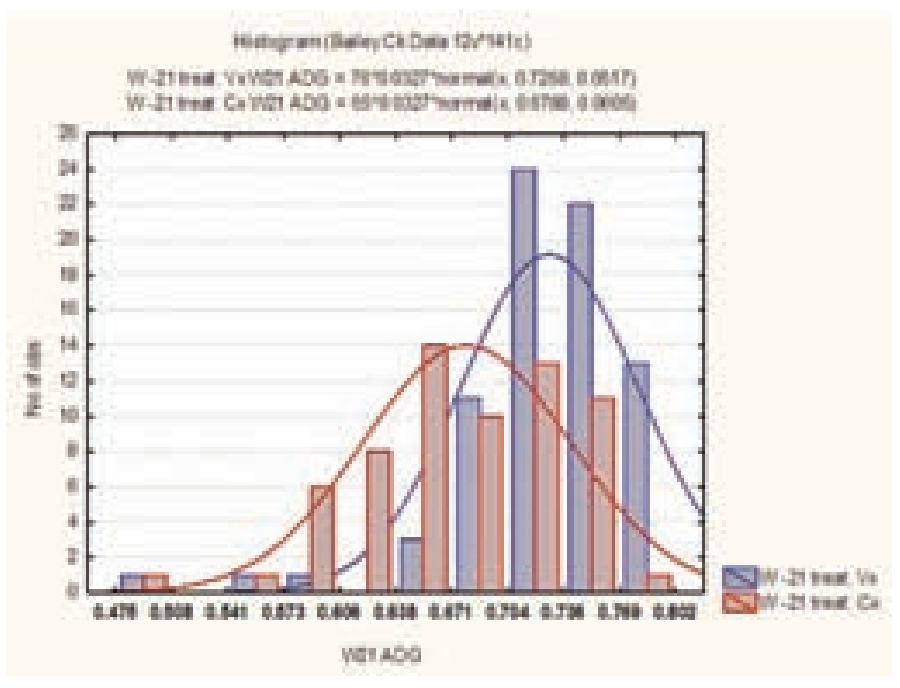

\section{Discussion}

Vaccination with a live oral vaccine improved growth rate in finishing pigs and variation significantly. The implications of this are that pigs will be turned off more quickly, reducing feed costs and improving profitability. A reduction of in-feed medications specifically targeting would also offer further cost savings.

Despite the use of in feed antibiotics, the long lasting protection though vaccination against Lawsonia intracellularis significantly improved performance. Vaccination improved growth rates and variation which resulted in cost savings through reduced to days to market.

\section{References}

1. Holyoake P.K, Mullan B.P., and Cutler, R.S. (1996) Australian Veterinary Journal; 73: pp. 89-92 


\title{
P.408
}

\section{Tylvalosin (Aivlosin ${ }^{\circledR}$ ) water medication for control of porcine proliferative enteropathy (PPE): a field trial carried out in Denmark}

\author{
Jens Sørensen ${ }^{2}$ Klaus Hellmann ${ }^{3}$ Isabel Radeloff ${ }^{3}$ John B. Tasker ${ }^{1}$ \\ 1. ECO Animal Health Ltd, London, UK; 2. Odder Veterinary Clinic, Odder, Denmark; 3. Klifovet AG, Munich, Germany
}

\section{Introduction}

Porcine proliferative enteropathy (PPE) is a ubiquitous and commercially important enteric disease of pigs, caused by the obligate intracellular bacterium Lawsonia intracellularis ${ }^{1,2}$. This is the first report of water medication with tylvalosin to control PPE on a commercial pig farm.

\section{Material and Methods}

This GCP-compliant trial was conducted in Denmark. Tylvalosin (TVN, Aivlosin $625 \mathrm{mg} / \mathrm{g}$ Granules) was administered in drinking water at $5 \mathrm{mg}$ TVN/kg bodyweight for 5 days and compared to water-soluble tylosin (Tylan ${ }^{\mathrm{TM}}$, Elanco). Tylosin is approved in Denmark at a dose rate of $5-10 \mathrm{mg} / \mathrm{kg}$ bodyweight for 5 days. In this study the highest approved dose of tylosin was used namely $10 \mathrm{mg} / \mathrm{kg}$.

309 pigs, mean weight $11.5 \mathrm{~kg}$, were allocated to two groups (TVN 155, tylosin 154 pigs).

Within each group response to treatment was assessed (treatment claim). The ability of each product to prevent PPE developing in clinically normal pen-mates was also assessed (prevention, or metaphylaxis claim).

A diagnosis of PPE was made before the trial using clinical history, necropsy and faecal PCR. On the final day of treatment (D5 of the study) $54 / 78$ (69\%) faecal samples taken from pigs with positive faecal scores were PCR-positive for L. intracellularis.

Medication started when $27 \%$ of all the pigs (85/309) had a faecal score of $\geq 2$ (diarrhoea). These pigs were included in the treatment claim (TVN 46, tylosin 39 pigs) At this point 155 pigs had faecal scores of zero and were assessed for PPE metaphylaxis (TVN 72, tylosin 83 pigs). 69 pigs failed to meet the inclusion criteria for efficacy assessment but were treated and observed for adverse reactions.

The primary efficacy variable for the treatment claim was faecal score, and for the metaphylaxis claim, the \% of pigs that developed a faecal score of $\geq 2$ during the study. L. intracellularis shedding, average daily liveweight gain, condition, demeanour, abdominal appearance and mortality were measured in both groups.

\section{Results}

A clear clinical response was observed in the treatment groups. Mean faecal score was reduced from 2.13 to 0.24 in the TVN group, and from 2.08 to 0.25 in the tylosin group (89\% and $88 \%$ reduction respectively). The clinical scores of the pigs in the TVN group were equivalent to those in the tylosin-treated group throughout the study.

Treatment was followed by a significant reduction in the proportion of PCR-positive results in samples taken from pigs in the treatment groups (Table 1).

Table 1: Shedding of L. intracellularis during the trial (\% PCR-positive)

\begin{tabular}{|c|c|c|}
\hline Days post-treatment & Tylvalosin & Tylosin \\
\hline D5 & $29 / 44(65.9 \%)$ & $25 / 34(73.5 \%)$ \\
\hline D21 & $3 / 38(7.9 \%)$ & $4 / 36(11.1 \%)$ \\
\hline \% reduction & $88 \%$ & $85 \%$ \\
\hline
\end{tabular}

In the metaphylaxis group, pre-treatment clinical scores suggested that pigs had sub-clinical disease. Both products were very effective in preventing the development of diarrhoea during the trial, with only one animal in each group developing a faecal score of $\geq 1$.

No pigs died during the study and no adverse reactions were observed.

\section{Discussion}

In this trial $5 \mathrm{mg}$ tylvalosin/kg bodyweight given in drinking water for 5 days was effective for treatment of PPE. This dose also prevented development of disease in pigs exposed to challenge from infected pen-mates. Medication was followed by significantly reduced shedding of $L$. intracellularis.

In conclusion, tylvalosin given at half the dose of tylosin but for the same treatment period produced the same clinical effect. Such a reduction in antimicrobial use is an important objective in many European countries.

\section{References}

1. Lawson GHK et al (1993). J. Clin. Microbiol. 31: 1136-1142.

2. McOrist S \& Gebhart CJ (2006) In Diseases of Swine 9th Edition. 


\title{
P.409
}

\section{Growth rate performance and uniformity improvements for finishing pigs vaccinated with Enterisol ${ }^{\circledR}$ Ileitis oral vaccine in an Australian pig farm}

\author{
Geoffrey P. Pollock ${ }^{1}$ Greg V. Marr² \\ 1. Boehringer-Ingelheim Animal Health, North Ryde, NSW, Australia; 2. IAS Management Services, Kingaroy, QLD, Australia
}

\section{Introduction}

Porcine Proliferative Enteropathy (PPE or lleitis) is due to the infection of Lawsonia intracellularis. It is a major factor affecting intestinal health of growing pigs. The economic impact of ileitis has been reported in Australia to be AUD\$15.00/sow/year and AUD\$141.00/sow/year with the non-haemorrhagic and hemorrhagic PPE respectively 1 . The aim of this study was to compare the growth performance of vaccinated animals with non-vaccinated animals in a commercial environment.

\section{Materials and Methods}

ELISA testing for serum antibodies prior to the experiment confirmed infection with Lawsonia intracellularis at about 11 to 12 weeks of age. Pigs were showing clinical signs of diarrhoea and poor growth at 14 to 15 weeks of age. All animals were weaned to a separate site and data was collected from all male animals. The farm used in-feed antibiotics against Lawsonia intracellularis infections in both treatment groups. Olaquindox at $25 \mathrm{ppm}$ from 12 to 14 weeks of age, OTC at 200ppm from 15 to 17 weeks of age and Tylosin at 50ppm from 18 to 19 weeks of age. No changes to in-feed medication or farm management were made during the trial.

Females were not weighed as they were sent to other grow-out units for the grower-finisher stage. Pigs were housed in conventional housing until 7 weeks of age, straw based shelters from 7 to 15 weeks of age. At 15 weeks pigs were moved into conventional grower accommodation until slaughter. All pigs had liveweight measured at 15 and 19 weeks of age in groups of $4-6$ animals per weight group. Pigs were housed by batch in separate sheds. Growth rates of 900 animals ( 2 batches) vaccinated at 14 days of age were calculated and compared to 1,425 non-vaccinated animals ( 3 batches). Growth rates presented are calculated from mean liveweight of the group at weaning until the 19 week liveweight. An average weaning weight was collected for each batch of the control groups (6.45, 6.42 and 7.16 $\mathrm{kg}$ ) and vaccinated groups (6.41and $6.71 \mathrm{~kg}$ ). Data was analysed with Statistica ${ }^{\circledR}$ V 8.0.

\section{Results}

Vaccinated pigs were significantly heavier at both 15 weeks and 19 weeks of age (Table 1: $\mathrm{P}<0.001$ ) with liveweight improvements of $3.19 \mathrm{~kg}$ and $3.33 \mathrm{~kg}$ respectively. Liveweight variability as measured by standard deviation was also lower for the vaccinated animals at both ages. Wean to 19 week growth rate was significantly improved by $30 \mathrm{~g} /$ day in vaccinated pigs (Table 1: $p<0.0005)$.

Table 1: Liveweight at 15 and 19 weeks of age and wean to 19 week average daily weight gain in vaccinated and non-vaccinated animals

\begin{tabular}{|l|c|c|c|}
\hline & Control & Vaccinated & P-value \\
\hline No. of pigs & 1,425 & 900 & \\
\hline liveweight 15 wks (kg) & 44.73 & 47.92 & 0.0002 \\
\hline SD & 6.51 & 5.83 & \\
\hline liveweight 19 wks (kg) & 71.13 & 74.43 & 0.0008 \\
\hline SD & 10.82 & 7.19 & \\
\hline Wean to 19 wk ADG (g/day) & 589 & 619 & 0.001 \\
\hline
\end{tabular}

\section{Discussion}

The study indicates that despite the use of in feed antibiotics, the lifelong protection though vaccination against Lawsonia intracellularis significantly improved performance. Vaccination improved growth rates and variation which resulted in cost savings through reduced to days to market.

\section{References}

1. Holyoake P.K, Mullan B.P., and Cutler, R.S. (1996) Australian Veterinary Journal; 73: pp. 89-92 


\title{
P.410
}

\section{Additional benefit of a ileitis and PCV2 vaccination in a two-fold vaccinated pig flow}

\author{
Kerstin Klien ${ }^{1}$ Matthias Adam² Ricarda Deitmer ${ }^{3}$ \\ 1. Practitioner, Gribow, Germany; 2. Boehringer Ingelheim Animal Health GmbH, Ingelheim am Rhein, Germany; \\ 3. Boehringer Ingelheim Vetmedica $\mathrm{GmbH}$, Ingelheim am Rhein, Germany
}

\section{Introduction}

Prophylactic measures e.g. vaccination programs are of high importance in order to control disease. In the present case the effect of ileitis and PCV2 vaccination on finisher performance was examined in a pig flow that was already vaccinated against PRRSV and Mycoplasma hyopneumoniae (M. hyo).

\section{Materials and Methods}

The study took place in a farrow-to-finish farm of 1,600 sows and 10,000 fattening places in Germany. All pigs were housed under similar conditions. During the entire evaluation period neither the genetics of the pigs nor the management were changed. Since 2004 ADG (average daily gain), FCR (feed conversion rate), mortality, and amount of antibiotics used against enteric diseases ( $A B$ use) were monitored batchwise by SPC methodology (Statistical Process Control) for the finishing unit. ADG, FCR and antibiotic use were evaluated by one-way ANOVA, pairwise comparison and adjusted by Bonferroni approach. Mortality was evaluated using a Chi-square test. All tests were performed with the software Statistica ${ }^{\circledR}$ v8.0, Statsoft ${ }^{\circledR}$. During period I of the study (August 2004 - March 2005, 32 batches, 16,032 pigs included) sows were mass vaccinated against PRRSV (Ingelvac ${ }^{\circledR}$ PRRS MLV) every 4 month. Pigs were vaccinated against PRRSV (Ingelvac ${ }^{\circledR}$ PRRS MLV, 14 days of age) as well as against M. hyo ( 21 days of age, $\mathrm{M}+\mathrm{Pac}^{\circledR}$ ). At the beginning of period II (March 2005 - November 2007, 152 batches, 7,7731 piglets) a third piglet vaccine, Enterisol ${ }^{\otimes}$ Ileitis (vaccination by drench, 14 days of age) was added. At the beginning of period III (10 batches, 4,932 pigs included) PCV2 vaccination (Ingelvac CircoFLEX ${ }^{\circledR}$, same time but different injection sites as $M$. hyo vaccination) was implemented as a fourth vaccine.

\section{Results}

Following introduction of ileitis vaccination in 2005 significant improvements were observed in ADG (by $33 \mathrm{~g} /$ day), FCR (by $0.1 \mathrm{~kg} / \mathrm{kg}$ ) and mortality rate (by $1.08 \%$ ). At the same time, the amount of oral antibiotics used decreased by $59 \%$ to 0.9 kg per fattening group. In 2007 vaccination against PCV2 was implemented in addition to the three other vaccines generating further significant improvements: ADG (+42 g/day) and FCR $(-0.25 \mathrm{~kg} / \mathrm{kg})$.
Table 1: Average performances of pigs over the three periods of time

\begin{tabular}{|l|c|c|c|}
\hline Period & $\mathrm{I}$ & II & III \\
\hline No. of batches & 32 & 152 & 10 \\
\hline Vaccines & M.hyo, PRRSV & M.hyo, PRRSV, lleitis & M.hyo, PRRSV, Ileitis, PCV2 \\
\hline ADG g/day & $738^{\mathrm{a}}$ & $771^{\mathrm{b}}$ & $813^{\mathrm{b}}$ \\
\hline FCR & $3.16^{\mathrm{a}}$ & $3.06^{\mathrm{b}}$ & $2.82^{\mathrm{c}}$ \\
\hline Mortality \% & $4.12^{\mathrm{a}}$ & $3.04^{\mathrm{b}}$ & $3.47^{\mathrm{b}}$ \\
\hline AB use kg/group & $2.23^{\mathrm{a}}$ & $0.91^{\mathrm{b}}$ & $0^{\mathrm{b}}$ \\
\hline \multicolumn{4}{|l|}{ Different superscripts indicate a significant difference with a p-value $\leq 0.05}$. \\
\hline
\end{tabular}

The mortality rate in the observation period III was numerically higher than in period II. The breakdown of causes of losses in the herd in suspected PCV2-related as well as non-PCV2 related cases confirmed however that the increased losses were not attributed to PCV2. In period II (no PCV2 vaccination) every 4th loss was considered to be caused by PCV2. In the case of the PCV 2 vaccinated animals only one pig from a total of 4932 pigs in period III could be attributed to a PCV2 infection.

\section{Discussion}

The present work provides an example how additional significant improvements in performance are made possible after implementation of a third and fourth piglet vaccination into an already established vaccination scheme. The two additional vaccines Enterisol ${ }^{\circledR}$ Ileitis and Ingelvac CircoFLEX ${ }^{\circledR}$ also increased profitability by generating an overall additional revenue of $€ 11.48 /$ pig1.

\section{References}

1. Deitmer R. et al (2009). Praktischer Tierarzt 90; 4, 346-355 


\title{
P.411
}

\section{Field Evaluation of the effect of Aivlosin ${ }^{\circledR}$ (Tylvalosin) for the control of Porcine Proliferative Enteropathy using two dosing regimens}

\author{
Vladan Miljkovic ${ }^{1}$ Andrea Ladinig ${ }^{1}$ Oliver Duran² Mathias Ritzmann¹ \\ 1. Clinic for Swine, University of Veterinary Medicine Vienna, Vienna, Austria; 2. Eco Animal Health, London, UK
}

\section{Introduction}

Porcine proliferative enteropathy (PPE) caused by Lawsonia intracellularis $(\mathrm{Li})$ is one of the most important diseases economically in the swine industry worldwide (1). Control of the disease can be achieved by the use of antibiotics given either parenterally, in feed or in water. The aim of this field trial was to evaluate the efficacy of an in feed formulation of tylvalosin (TVN, Aivlosin ${ }^{\circ}$ $42.5 \mathrm{mg} / \mathrm{g}$ Premix, Eco Animal Health) in two different dosage regimens at reducing the production losses associated with infection with $L i$.

\section{Materials and Methods}

The field trial was conducted on a 1000 sow commercial gilt producing farm in Austria. Piglets became naturally infected with $L i$ from 5 weeks of age as confirmed by clinical signs (diarrhea, uneven growth), serology and fecal PCR for $L i$. In total, 782 piglets, about 5 weeks old, were randomly assigned to three groups. The pigs of each group were housed in 8 different pens in the same room. Group $1(n=262)$ served as the untreated control group. Pigs in group $2(n=252)$ were given feed containing 42.5ppm TVN daily for 14 days. Group $3(n=268)$ pigs were given feed containing $85 \mathrm{ppm}$ TVN daily for 10 days. These inclusion rates were equivalent to dose rates of 2.125 and 4.25 $\mathrm{mg}$ TVN/kg bodyweight for group 2 and group 3 pigs respectively. TVN treatment was started at 5 weeks of age. All pigs were ear tagged and individually weighed 3 times: at the beginning of the trial (at 5 weeks of age), at 13 weeks of age (end of nursery period) and at 24 weeks of age (before slaughter). Data on feed intake by pen was available during the nursery phase only. For statistical analysis the pen and the pig were used as the experimental unit. Pig uniformity was assessed from the coefficients of variation of individual bodyweight by using $F$ test.

\section{Results}

Diarrhea was not detected in the treated pigs.

The body weight (BW) at 13 weeks of age and the average daily gain (ADG) over the nursery period (G1vs G3 $p=0.0023$; G1 vs $G 2 p=0.044$ ) were significantly higher in both TVN treated groups compared to the control when analyzing the data by pen. As the BW at 5 weeks of age was higher in group 3 the initial body weight was included as a covariate in the analysis. The Feed Conversion Ratio (FCR) was significantly better during the nursery phase for pigs in group 3 vs. group 1 ( $2.06 \pm 0.04$ vs. $2.26 \pm 0.04 ; p=0.0174)$. Individual pig analysis by analysis of variance showed an improvement in BW and ADG for both treated groups when compared with untreated control (see table 1). At 24 weeks of age pigs in group 3 were significantly more uniform than the control pigs (G1 vs. G3 Ratio 1.19; $p=0.0046$ ).
Table 1. Bodyweight (BW) and average daily gain ( $A D G)$. Least square mean \pm standard error. G1: Control; G2: TVN (2.125 mg/kg); G3: TVN $(4.25 \mathrm{mg} / \mathrm{kg})$

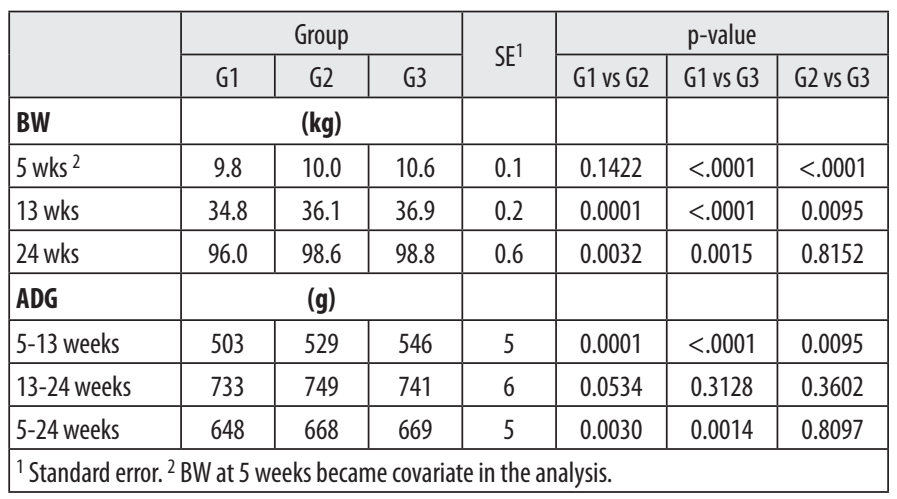

\section{Discussion}

Tylvalosin has previously been proven to be effective against infection with $L i$ using two different dosage regimens $(2,3)$. In this trial the comparison of TVN at $2.125 \mathrm{mg} / \mathrm{kg}$ body weight for 14 days and $4.25 \mathrm{mg} / \mathrm{kg}$ for 10 days with a negative control confirmed the effective reduction of production losses caused by $L i$. Pigs that received the higher dose were more uniform in weight at the time of slaughter. Analysis of the individual pig data between treatments confirms that bodyweight at slaughter and the ADG during the whole trial period was better with both dosage regimens when compared to the control and not significantly different from each other. It appears that one well timed treatment with Aivlosin ${ }^{\circledR}$ can result in a reduction of productivity losses due to PPE.

\section{References}

1. McOrist S. 2005. Vet. J. 170, 8-9.

2. Guedes R.M.C. et al. 2009. Vet. Rec. 165, 342-45.

3. Pommier P. et al. 2008. Rev. Med. Vet. 159, 579-82. 


\title{
P.412
}

\section{Denagard $^{\circledast}$ (tiamulin) liquid concentrate dose titration in a Lawsonia intracellularis Mucosal Homogenate lleitis challenge study - performance response}

\author{
Nathan Winkelman ${ }^{1}$ Mark Hammer ${ }^{2}$ Linda Roycroft ${ }^{2}$ Ulrich Klein ${ }^{3}$ Connie Gebhart ${ }^{4}$ \\ 1. Swine Services Unlimited Inc, Rice, MN, USA; 2. Novartis Animal Health, Greensboro, NC, USA; \\ 3. Novartis Animal Health Inc., Basel, Switzerland; 4. University of Minnesota, St. Paul, MN, USA
}

\section{Introduction}

Clinical and subclinical ileitis (PPE) is ubiquitous in the global swine industry. Both forms have an economical impact on a swine unit's profitability. A Lawsonia intracellularis (Li) disease challenge model can reproduce both forms of the disease'. Denagard ${ }^{\circledR}$ administered in the water at 60 ppm is very effective in minimizing the negative impact of PPE on growth performance $^{2}$. There is no information on the efficacy of higher doses of tiamulin (LC) to possibly eliminate PPE carriers during the course of treatment or after the treatment is withdrawn. The objective was to compare three treatment dose levels of tiamulin LC in pigs given a high dose Li mucosal homogenate challenge.

\section{Materials and Methods}

One hundred and fifty five-week-old PPE naïve crossbred pigs were randomized by weight and gender into five treatment groups of 30 pigs per group ( 6 pens with 5 pigs/pen): T1 was a strict negative control group (unchallenged, no medication), T2 was a positive control group (Li challenged, no medication), $\mathrm{T} 3, \mathrm{~T} 4$, and T5 were all Li challenged groups receiving 60, 120, and 180ppm Denagard respectively. All pigs were individually infected by intraoesophageal gavage with $2.0 \times 10^{9} \mathrm{Li}$ organisms per pig on Day 0. Treatment with tiamulin water medication was initiated on Day 7 (DM = first day of medication) when $20 \%$ of pigs and $60 \%$ of the pens had a minimum fecal score of 1 (semi-solid feces). Treatment continued to Day $12(\mathrm{DM}+5)$ when all pigs returned to non-medicated water for the remainder of the study. All surviving pigs were euthanized on Day $27(\mathrm{DM}+20)$ post Li challenge. Gross pathological PPE lesion scores were recorded for each pig on Day 27. Pigs were individually weighed and feed consumption per pen was recorded on Day - 1 (acclimation start), $\mathrm{DM}, \mathrm{DM}+5, \mathrm{DM}+10, \mathrm{DM}+15$ and $\mathrm{DM}+20$ for individual average daily gain (ADG), pen Feed Conversion Ratio (FCR -feed/ gain) and Average Daily Feed Intake (ADFI) calculations. Pen was the experimental unit in all analyses.

\section{Results - Performance Variables}

Body weights were significantly higher in all treatment groups compared to the positive control from $\mathrm{DM}+10$ to $\mathrm{DM}+20$. Overall Average Daily Gain: (ADG from (DM to DM+20) was significantly lower for the positive control group compared to all other groups and significantly higher for the negative control compared to all groups. There were significantly higher ADFI and improved FCR in all three treatment groups compared to the positive Li challenged control groups. Gross Pathology: Significantly lower PPE gross lesion scores in the ileum, jejunum, and colon in all treatments compared to the T2 challenged controls were found. There were also significantly lower ileal PPE scores in the Denagard $120 \mathrm{ppm}$ and Denagard $180 \mathrm{ppm}$ compared to the Denagard $60 \mathrm{ppm}$ treatment groups. The lesion length was significantly longer in the $\mathrm{T} 2$ challenged controls compared to

\begin{tabular}{|c|c|c|c|c|c|}
\hline \multicolumn{6}{|c|}{ Perternance (DM o DM } \\
\hline & $\pi$ & $\tau 2$ & 73 & 74 & TS \\
\hline Treotment & $\begin{array}{c}\text { Neg } \\
\text { Coetrat }\end{array}$ & $\underset{\text { Controt }}{\mathrm{L}}$ & $\underset{p+\infty}{\infty}$ & $120 \mathrm{Fgm}$ & $190 \mathrm{ygm}$ \\
\hline Bodyty act & 2736 & 192 & 253 & 25.4 & 253 \\
\hline NDO DOS & $394 \mathrm{c}$ & $313^{\circ}$ & $531^{b}$ & $531^{\circ}$ & $531^{\circ}$ \\
\hline ADI OCO & $945^{5}$ & $640^{\circ}$ & sub & 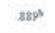 & sast \\
\hline FCR & $160^{\circ}$ & $207 *$ & $167^{\circ}$ & $16 \%$ & 167 \\
\hline \multicolumn{6}{|l|}{ store } \\
\hline theun & $0.99^{\circ}$ & $215^{\circ}$ & $0.8 \%$ & $0.08 \%$ & 025 \\
\hline \multirow[t]{2}{*}{ Jejueran } & $000^{\circ}$ & $186^{\circ}$ & 0.30 & $0.00^{\circ}$ & $0.00^{\circ}$ \\
\hline & $0.00 \%$ & $0.28^{\circ}$ & $0.07^{6}$ & 0.005 & $0.00^{\circ}$ \\
\hline thasalght & w* & $n+4$ & 100 & 0.52 & 194 \\
\hline
\end{tabular}

all other treatment groups. The T1 non-challenged control group remained negative throughout the study as evidenced by no fecal PCR positives and lack of Li IPMA titers (not shown).

\section{Discussion}

There were no differences between the three tiamulin LC treatments in performance variables. The results show excellent efficacy at the 60 ppm dose even when pigs were challenged with a high $\left(10^{9}\right)$ Li mucosal homogenate in which PPE mortality occurred in the challenged controls. Ileum PPE scores were significantly better in the 120 ppm and 180ppm groups compared to the 60 ppm group. No other group differences in pathological scores were identified.

\section{References}

1. Winkelman, N., et.al, "Various doses of Lawsonia intracellularis in a Mucosal Homogenate Challenge Model" Proc 17th IPVS Congress Ames, lowa 211 vol 22002

2. Walter, D. et.al. (2001) Treatment and Control of PPE Using different tiamulin Delivery Methods. J. Swine Health and Prod 2001; 9(3):109115 


\title{
P.413
}

\section{Denagard $^{\circledR}$ (tiamulin) liquid concentrate dose titration in a Lawsonia intracellularis Mucosal Homogenate lleitis challenge study - clinical response}

\author{
Nathan Winkelman ${ }^{1}$ Mark Hammer ${ }^{2}$ Linda Roycroft ${ }^{2}$ Ulrich Klein ${ }^{3}$ Connie Gebhart ${ }^{4}$ \\ 1. Swine Services Unlimited Inc, Rice, MN, USA; 2. Novartis Animal Health, Greensboro, NC, USA; \\ 3. Novartis Animal Health Inc., Basel, Switzerland; 4. University of Minnesota, St. Paul, MN, USA
}

\section{Introduction}

Clinical and subclinical ileitis (PPE) is ubiquitous in the global swine industry. Both forms have a negative economical impact on a swine unit's profitability. A Lawsonia intracellularis (Li) disease challenge model can reproduce both forms of the disease ${ }^{1}$. Denagard $^{\circledR}$ administered in the water at 60 ppm is very effective in minimizing the negative impact of PPE on growth performance $^{2}$. There is no information on the efficacy of higher doses of tiamulin (LC) to possibly eliminate PPE carriers during the course of treatment or after the treatment is withdrawn. The objective of this trial was to compare three treatment dose levels of tiamulin LC and control pigs given a high dose Li mucosal homogenate challenge. The clinical data is shown in this abstract.

\section{Materials and Methods}

One hundred and fifty five-week-old PPE naïve cross-bred pigs were randomized by weight and gender into five treatment groups of 30 pigs per group ( 6 pens with 5 pig/pen): T1 was a strict negative control group (unchallenged, no medications), $\mathrm{T} 2$ was a positive control group (Li challenged, no medication), T3, T4, and T5 were all Li challenged groups receiving 60, 120, and 180ppm Denagard respectively. All pigs were individually infected by intraoesophageal gavage with $2.0 \times 10^{9}$ per pig Li organisms on Day 0. Treatment with tiamulin water medication was initiated on Day 7 (DM = first day of medication) when $20 \%$ of pigs and $60 \%$ of the pens had a minimum fecal score of 1 (semi-solid feces). Treatment continued to Day $12(\mathrm{DM}+5)$ when all pigs returned to non-medicated water for the remainder of the study. All surviving pigs were euthanized on Day $27(\mathrm{DM}+20)$ post Li challenge. Parameters recorded daily were mortality, abdominal appearance score (AAS), pig demeanor score (DS), and fecal score (FS). Gross PPE lesion scores were recorded for each pig on Day 27. Pigs were individually weighed and feed consumption per pen was recorded on Day -1 (acclimation start), $\mathrm{DM}, \mathrm{DM}+5, \mathrm{DM}+10, \mathrm{DM}+15$ and $\mathrm{DM}+20$ for individual average daily gain (ADG), pen Feed Conversion Ratio (FCR) and Average Daily Feed Intake (ADFI) calculations. In addition, fecal samples were collected on all pigs on days $\mathrm{DM}, \mathrm{DM}+5, \mathrm{DM}+10, \mathrm{DM}+15$, and $\mathrm{DM}+20$ for Li polymerase chain reaction (PCR) analysis. Li immunoperoxidase monolayer assay (IPMA) serum samples were collected from two pigs/pen on Day -1 and DM+20. Pen was experimental unit for all analysis.

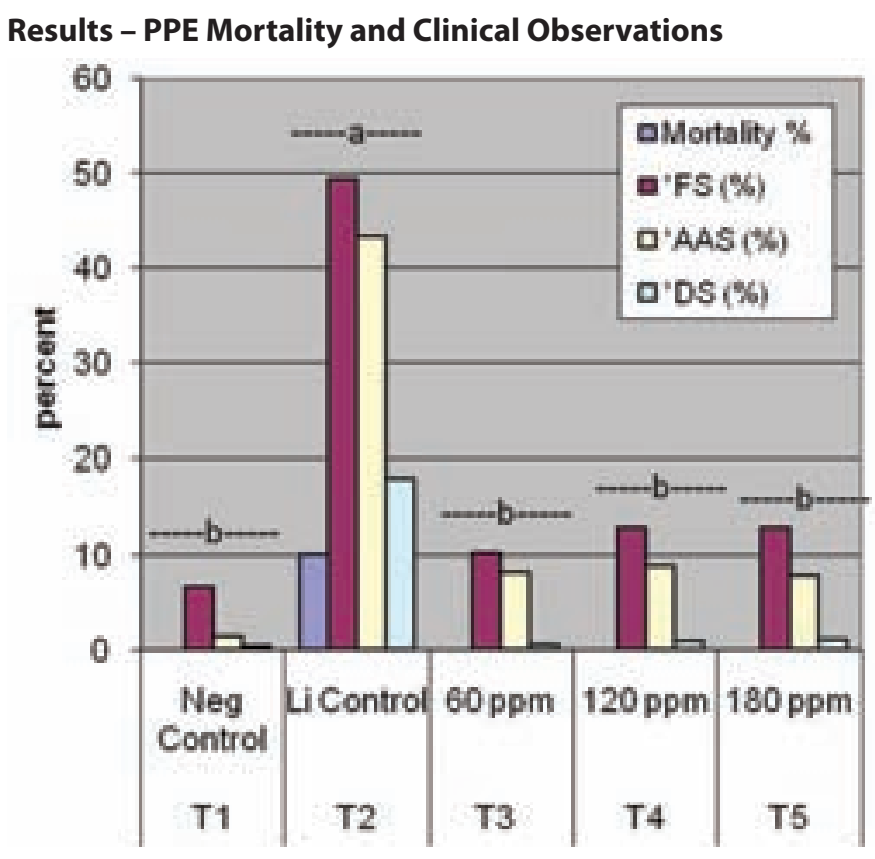

$a, b$ different letters between treatments are sign. different $\mathrm{p}<0.05$ *Abnormal Score $\%=\%$ of pig-days with abn. score per pen.

Mortality: There were three pigs which died due to PPE, all in the T2 challenge control group. Fecal Score: There was a significantly higher average pen fecal score for the positive control group when compared to all other groups on all days after day DM+1. Abdominal Appearance Score: There was significantly higher average AAS for the positive control group when compared to all other groups starting on $\mathrm{DM}+7$ and continuing each day through DM+20. Demeanor Score: There was a significantly higher average pen demeanor score for the positive control group compared to all other treatments from DM+10 through $\mathrm{DM}+20$.

\section{Discussion}

There were no differences between the three tiamulin LC treatments in clinical measures. The results show excellent efficacy even at the 60 ppm dose when pigs were challenged with a high $\left(10^{9}\right)$ Li mucosal homogenate in which mortality occurred in the challenged controls.

\section{References}

1. Winkelman, N., et.al, "Various doses of Lawsonia intracellularis in a Mucosal Homogenate Challenge Model". Proc 17th IPVS Congress, Ames, lowa 211 vol 22002.

2. Walter, D. et.al. (2001) Treatment and Control of PPE Using different tiamulin Delivery Methods. J. Swine Heath and Prod 2001:9 (3): 109115. 


\title{
P.414
}

\section{Efficacy of Aivlosin ${ }^{\circledast}$ Water Soluble Granules for the control of porcine proliferative enteropathy in pigs challenged with Lawsonia intracellularis}

\author{
Elizabeth M. Abbott ${ }^{2}$ Mark J. Gnozzio ${ }^{3}$ Rickie J. Domangue ${ }^{4}$ Nate Winkelman ${ }^{5}$ Chris D. Eliopoulos ${ }^{3}$ Sharon A. Bauer Jeff B. Wilson ${ }^{1}$ \\ 1. Novometrix Research Inc, Moffat, Canada; 2. Eco Animal Health, London, UK; 3. Eco LLC, Princeton, NJ, USA; 4. Statistical Consulting \\ Services, Broadway, VA, USA; 5. Swine Services Unlimited, , Canada; 6. Novometrix Research Inc., Guelph, ON, Canada
}

\section{Introduction}

Porcine proliferative enteropathy (PPE), or ileitis, caused by the obligate intracellular bacterium Lawsonia intracellularis ( $\mathrm{Li})$, is an important enteric disease of swine ${ }^{1}$. In vitro studies have demonstrated that tylvalosin accumulates rapidly in high concentrations inside intestinal epithelial cells ${ }^{2}$, and has proven efficacy in the control of PPE when administered in-feed ${ }^{3-5}$. The objective of this study was to evaluate the efficacy of tylvalosin administered in drinking water for the treatment of PPE in weaner pigs.

\section{Materials and Methods}

In this multi-centre study with three separate sites, 144 five week-old weaned pigs at each site (Rice, Minnesota; Harrisonburg, Virginia; Burford, Ontario) were randomly assigned to one of three treatment groups: Challenged - Untreated, Challenged - Treated (50 ppm Aivlosin in drinking water) and Challenged - Treated (75 ppm Aivlosin in drinking water). Pigs were orally challenged with Li from a field case of PPE on Day 0. Drinking water was medicated with Aivlosin ${ }^{\circledR}$ Water Soluble Granules (ECO Animal Health, London, UK) for five consecutive days and treatment began when $\sim 15 \%$ of pigs had semi-solid feces. Body weights and feed consumption were measured throughout the study. Twenty-two days after the start of treatment fecal samples were collected for PCR analysis and all surviving animals were euthanized. At necropsy, the intestinal tract was scored for gross lesions consistent with PPE and ileal samples were obtained for immunohistochemistry (IHC) analysis. Statistical analyses were conducted using analysis of variance and contingency table methods.

\section{Results and Discussion}

During the study, $14.9 \%$ of the untreated pigs died due to PPE infection. There was no mortality due to PPE in either of the treated groups, and this difference was significant $(P<0.01)$ when compared with the untreated group. Pigs in both treated groups had significantly improved mean pen gross lesion scores $(P<0.03)$, and pen production parameters (Table 1 ), compared to the non-medicated group. Significantly decreased frequencies of Li positive fecal PCR and ileal IHC samples were found in the treated groups compared to the untreated group (Table 1).
Table 1. Mean pen average daily gain (ADG) and feed efficiency (gain/ intake) and proportion of pigs testing positive for Li by fecal PCR and IHC at study termination.

\begin{tabular}{|l|c|c|c|}
\hline & \multicolumn{3}{|c|}{ Treatment Groups } \\
\hline & No medication & Aivlosin 50 ppm & Aivlosin 75 ppm \\
\hline ADG (kg/pig/day) & $0.31^{\mathrm{a}}$ & $0.48^{\mathrm{b}}$ & $0.49^{\mathrm{b}}$ \\
\hline Feed efficiency (gain/intake) & $0.41^{\mathrm{a}}$ & $0.54^{\mathrm{b}}$ & $0.56^{\mathrm{b}}$ \\
\hline$\%$ positive PCR & $73^{\mathrm{a}}$ & $50^{\mathrm{b}}$ & $52^{\mathrm{b}}$ \\
\hline \% positive IHC & $91^{\mathrm{a}}$ & $46^{\mathrm{b}}$ & $39^{\mathrm{b}}$ \\
\hline ab Rows with no common letters are significantly different at $\mathrm{p}<0.03$ \\
\hline
\end{tabular}

The decreased frequency of positive fecal PCR samples collected at the end of study indicates that fecal shedding of Li was reduced due to treatment when compared to the untreated pigs. Appetite may be depressed as a result of Li infection. Therefore administration of Aivlosin ${ }^{\circledR}$ in drinking water can be advantageous as pigs can receive adequate uptake of the drug. Water medication also allows treatment to be initiated promptly once a diagnosis has been confirmed and the relatively low dose and short treatment duration is consistent with prudent use of antibiotics ${ }^{6}$. This study demonstrates that tylvalosin administered in drinking water is effective in reducing mortality, intestinal lesions, fecal shedding of Li and growth suppression that are associated with PPE in pigs orally challenged with Li.

\section{References}

1. McOrist S, Gebhart CJ. Proliferative enteropathies. In: Straw et al., eds. Diseases of Swine. 9th ed.Oxford, UK. Blackwell Publishing. 2006.

2. Stuart $A D$, et al. Intra-cellular accumulation and trans-epithelial transport of aivlosin, tylosin and tilmicosin. Pig J. 2007. 60:26-35.

3. Guedes RMC, et al. Use of tylvalosin-medicated feed to control porcine proliferative enteropathy. Veterinary Record. 2009. 165:342-345

4. Pommier P, et al. Comparison of Tylvalosin with Tylosin for the control of subclinical ileitis in swine. Revue de Medecine Veterinaire. 2008. 159(11):579-582.

5. Tasker JB, et al. Use of Aivlosin in feed for control of ileitis in USA and Europe. Proc IPVS Cong. Hamburg, Germany. 2004 (1):256.

6. Holyoake PM, et al. Identifying obstacles to reducing the use of antibiotics to control porcine proliferative enteropathy. Aust Vet J. 2009. 87(1\&2):33-34. 


\title{
P.415
}

\section{Efficacy of Aivlosin ${ }^{\circledR}$ Type A Medicated Article for the control of porcine proliferative enteropathy in pigs challenged with Lawsonia intracellularis}

\author{
Elizabeth M. Abbott ${ }^{2}$ Mark J. Gnozzio ${ }^{3}$ Rickie J. Domangue ${ }^{4}$ Nate Winkelman ${ }^{5}$ Chris D. Eliopoulos ${ }^{3}$ Sharon A. Bauer ${ }^{6}$ Jeff B. Wilson ${ }^{1}$ \\ 1. Novometrix Research Inc, Moffat, Canada; 2. Eco Animal Health, London, UK; 3. Eco LLC, Princeton, NJ, USA; 4. Statistical Consulting \\ Services, Broadway, VA, USA; 5. Swine Services Unlimited, Rice, USA; 6. Novometrix Research Inc., Guelph, ON, Canada
}

\section{Introduction}

Porcine proliferative enteropathy (PPE), or ileitis, is a significant enteric disease in swine. The obligate intracellular bacterium Lawsonia intracellularis (Li) is the etiologic agent, and the disease is characterized by a thickening on the mucosa of the small intestine and colon, evident at necropsy' ${ }^{1}$. Tylvalosin, a macrolide antimicrobial, has demonstrated efficacy in the control of PPE ${ }^{2-4}$. The objective of this multi-site study was to evaluate the efficacy of tylvalosin administered in feed for the control of PPE in weaner pigs challenged with Li.

\section{Materials and Methods}

Two studies were conducted at separate sites in North America. 144 four-week-old weaned pigs in each study were randomly assigned to one of two groups: treated (42.5 ppm tylvalosin in feed) or untreated ( $0 \mathrm{ppm})$. Pigs were orally challenged with Li on Day 0 . Treatment began when $\sim 15 \%$ of pigs had semi-solid feces and continued for 14 days. Pigs were scored daily for abdominal appearance, demeanor and fecal consistency. Body weights and feed consumption were measured throughout the study. Animals were euthanized 21 days after the start of treatment. At necropsy, the intestinal tract was scored for gross lesions consistent with PPE and ileal samples were collected for immunohistochemistry analysis. Data from the two study sites were analyzed separately using analysis of variance and contingency table methods.

\section{Results and Discussion}

Cumulative mortality due to PPE was lower in the treated pigs across the study sites, with statistically significant $(P<0.03$, Table 1) differences observed at one of the two sites.

Table 1. Proportion of pigs that died due to PPE, mean proportion of pens with abnormal fecal scores and mean pen lesion scores for the treatment groups across both sites.

\begin{tabular}{|l|c|c|c|c|}
\hline Study Site & \multicolumn{2}{|c|}{ Rice, MN } & \multicolumn{2}{c|}{ Burford, ON } \\
\hline Treatment Group & $0 \mathrm{ppm}$ & Aivlosin $42.5 \mathrm{ppm}$ & $0 \mathrm{ppm}$ & Aivlosin $42.5 \mathrm{ppm}$ \\
\hline PPE Mortality (\%) & 1.4 & 0.0 & $15.5^{\mathrm{a}}$ & $4.2^{\mathrm{b}}$ \\
\hline Pen fecal score (\%) & $51.4^{\mathrm{a}}$ & $14.7^{\mathrm{b}}$ & $67.7^{\mathrm{a}}$ & $39.9^{\mathrm{b}}$ \\
\hline Pen lesion score & $0.61^{\mathrm{a}}$ & $0.29^{\mathrm{b}}$ & $0.89^{\mathrm{a}}$ & $0.67^{\mathrm{b}}$ \\
\hline
\end{tabular}

Pigs in the treated groups at both sites had significantly improved mean pen gross lesion scores $(P<0.03)$ and mean pen fecal scores $(P<0.001)$ compared with the untreated pigs (Table 1). Pen feed efficiency was significantly increased $(P<0.05)$ in the treated pigs compared with the untreated (Table 2). Mean pen average daily gain was numerically greater in treated pigs and this difference was significant $(P<0.001$, Table 2$)$ at one study site.

Table 2. Mean pen average daily gain (ADG) and mean pen feed efficiency (gain/intake) at 21 days after start of treatment.

\begin{tabular}{|l|c|c|c|c|}
\hline Study Site & \multicolumn{2}{|c|}{ Rice, MN } & \multicolumn{2}{c|}{ Burford, 0N } \\
\hline Treatment Group & $0 \mathrm{ppm}$ & Aivlosin 42.5 ppm & $0 \mathrm{ppm}$ & Aivlosin 42.5 ppm \\
\hline ADG (kg/pig/day) & 0.61 & 0.64 & $0.18^{\mathrm{a}}$ & $0.34^{\mathrm{b}}$ \\
\hline Feed efficiency (gain/intake) & $0.50^{\mathrm{a}}$ & $0.53^{\mathrm{b}}$ & $0.24^{\mathrm{a}}$ & $0.40^{\mathrm{b}}$ \\
\hline ab Rows at each site with different letters are significantly different at $\mathrm{p}<0.05$ \\
\hline
\end{tabular}

Treatment with Aivlosin ${ }^{\circledast}$ Premix for 14 consecutive days in Li challenged pigs was associated with significantly lower gross PPE lesion scores in the treated groups when compared with untreated animals. Mortality due to PPE was decreased in the treated pigs compared with the untreated group. The treated groups showed improved performance in production parameters such as ADG and feed efficiency.

Consistent with previous studies ${ }^{2-4}$, this multi-site study demonstrates that Aivlosin ${ }^{\circledR}$ Premix is effective in reducing mortality, clinical signs, intestinal lesions, and growth suppression that are associated with PPE in weaner pigs orally challenged with Li.

\section{References}

1. McOrist, S., Gebhart, C.J. Proliferative enteropathies. In: Straw BE, et al., eds. Diseases of Swine. 9th ed Oxford, UK. Blackwell Publishing. 2006.

2. Guedes RMC, et al. Use of tylvalosin-medicated feed to control porcine proliferative enteropathy. Veterinary Record. 2009. 165:342-345

3. Pommier $\mathrm{P}$, et al. Comparison of Tylvalosin with Tylosin for the control of subclinical ileitis in swine. Revue de Medecine Veterinaire. 2008. 159(11):579-582.

4. Tasker JB, et al. Use of Aivlosin in feed for control of ileitis in USA and Europe. Proc IPVS Cong. Hamburg, Germany. 2004. (1):256. 


\title{
P.416
}

\section{The prevalence of swine enteropathogens in Brazilian grow/finisher}

\author{
Aline M. Viott ${ }^{1}$ Andrey P. Lage ${ }^{1}$ Eduardo C. Cruz Jr. ${ }^{1}$ Daiane D. Spessatto ${ }^{2}$ Roberto M. Guedes ${ }^{1}$ \\ 1. Universidade Federal de Minas Gerais, Veterinary School, Belo Horizonte, MG, Brazil; 2. Universidade Federal do Paraná, Palotina, PR, Brazil
}

\section{Introduction}

Brazil is the fourth largest pig producer in the World, behind China, the European Union and United States. The state of Minas Gerais is the fourth largest producer of pork in Brazil, and the growth of pig production in the state has been increasing year after year. As a result, it is necessary to accurately detect and identify porcine pathogens in order to devise proper treatments and prevention programs1. Enteric infections are among the most frequent problems in pig production, being responsible for significant losses. The purpose of this study was to determine the prevalence of the enteric pathogens and the occurrence of mixed infections in Brazilian growing/finishing pigs in herds with diarrhea or a history of diarrhea in the state of Minas Gerais, Brazil.

\section{Materials and Methods}

Forty-six farrow-to-finish herds; 31 with $101-500$ sows and 15 with $>500$ sows; with diarrhea or a history of diarrhea were randomly selected from four different geographical regions of the state of Minas Gerais, Brazil [south and southwest (SSW), metropolitan area of "Belo Horizonte" (MBH), "zona da mata" (ZM) and Triangle mining and high "Paranaiba" (TMHP)]. Randomly were collected feces from rectum for approximately 10 animals of each property between 60 and 140 days old. Multiplex PCR was used for the diagnostic of L. intracellularis, B. hyodysenteriae and B. pilosicoli2 and for enterotoxigenic E. coli3. For the detection of Trichuris suis eggs in the swine faeces, the faecal samples were individually examined by flotation technique (Willis-Mollay). Salmonella was isolated according Waltman, 20004. For statistical analyses Fischer's exact test was used.

\section{Results}

A total of 512 faecal samples were examined. The analysis by the Fisher exact test showed no significant association between the pathogen and the size of the farm. The only subspecies of Salmonella spp. found, potentially pathogenic for pigs, was Salmonella enterica subspecies Typhimurium. The results are demonstrated in Table 1. The mainly mixed infection observed was Salmonella Typhimurium associated with L. intracellularis present in 5 herds (10,8\%). T. suis eggs and B. hyodysenteriae were not diagnosed. The B pilosicoli was observed in two farms with mixing infection cases. The most prevalent enterotoxigenic E. coli virotype was StaP-Stb-F18.
Table 1. Distribution of prevalence according to herd size and pathogen.

\begin{tabular}{|l|c|c|c|c|c|c|}
\hline & \multicolumn{7}{|c|}{ Herds } \\
\hline Pathogen & \multicolumn{2}{|c|}{$101-500$} & \multicolumn{2}{|c|}{500} & Total & Prev. \\
\hline & No & $\%$ & No & $\%$ & No & $\%$ \\
\hline E. coli enteroxigenic & 2 & 4.3 & 3 & 6.5 & 5 & 10.8 \\
\hline L. intracellularis & 5 & 10.8 & 4 & 8.7 & 9 & 19.5 \\
\hline S. Typhimurium & 1 & 2.2 & 2 & 4.3 & 3 & 6.5 \\
\hline Mixed infection & 10 & 21.7 & 4 & 8.7 & 14 & 30.4 \\
\hline
\end{tabular}

\section{Discussion}

The prevalence of herds only infected by L. intracellularis was $19.5 \%$. This result is in agreement with previously reports from other countries, where the prevalence of $L$. intracellularis varied between $15 \%$ and $93.7 \% 5$. The differences among prevalence indexes observed may be due to regional variations, different swine breeding systems, antibiotics use and population sampled. The prevalence of mixing infections was high in our work when compared with European data5. The overall prevalence for Salmonella Typhimurium alone (6.5\%) was elevated mainly when we associate this bacterium to the cases of mixing infection. The presence of enterotoxigenic E. coli in grow/finishing pigs it is not clear. It seems that this pathogen may alter the ecological gut flora, which would favor some pathogenic bacteria6.

\section{References}

1. Brazilian Journal of Microbiology. 2003, 34: 225-229.

2. Letters in Applied Microbiology. 2006, 42: 284-288.

3. Arquivo Brasileiro de Medicina Veterinária e Zootecnia. 2007, 59: 1117-1123.

4. Salmonella in Domestic Animals, 2 ed. 2000, 355-372.

5. The Veterinary Record. 1998, 142: 235-239

6. Research in Veterinary Science 74, 163-169

\section{Acknowlegdment}

Fundação de Amparo a Pesquisa do Estado de Minas Gerias - FAPEMIG. 


\title{
P.417
}

\section{Rodent dispersal between piggeries following total pig depopulation as part of a swine dysentery eradication program}

\author{
Alison M. Collins ${ }^{1}$ Anil Manavalan ${ }^{2}$ Jocelyn Gonsalves ${ }^{1}$ Shayne Fell ${ }^{1}$ \\ 1. Elizabeth Macarthur Agricultural Institute, Narellan, NSW, Australia; 2. University of Wollongong, Wollongong, NSW, Australia
}

\section{Introduction}

Rodents have been identified as potential vectors for a number of bacterial pathogens of pigs, including Lawsonia intracellularis ${ }^{1}$, Brachyspira pilosicoli, and B. hyodysenteriae ${ }^{2}$. Monitoring the dispersal of rodents between piggeries in relatively close proximity may be important in assessing the risk of transmission of these pathogens to naïve pigs, especially following disease eradication programs. The aim of this study was to use microsatellite markers to demonstrate the genetic diversity between rats trapped at two separate pig farms and therefore rodent dispersal.

\section{Materials and Methods}

Twenty-eight black rats (Rattus rattus) were caught in snap traps over a three month period in two pig units separated by one kilometre (sites $A$ and $B$ ). During this period, pigs had been removed from site $A$ as part of a swine dysentery eradication program. Both pig farms were surrounded by natural boundaries to rodent dispersal (roads and rivers) but alternate food sources were available at neighbouring pig farms ( 4 and $9 \mathrm{~km}$ away). Genomic rat DNA was extracted from the rat's ears using a QIAGEN tissue kit (DNeasy). Microsatellite markers were amplified from the rat DNA as previously described ${ }^{3-4}$ using 8 sets of PCR primers (D5Rat83, D8Rat28, D11Rat56, D16Rat81, D18Rat75, Rr54, Rr107 and Rr114). PCR products were typed with an $A B I$ genetic analyzer using the GeneMapper v3.7 software (Applied Biosystem). The genetic relatedness of rats was analyzed with STRUCTURE software ${ }^{5}$ trialing the number of populations $(k=1-5)$.

Faecal samples were collected from pigs at site B at the completion of rat trapping. DNA extracted from pig faeces and rat intestines were tested for the presence of L.intracellularis, B.hyodysenteriae and B.pilosicoli by conventional PCR.

\section{Results}

All of the microsatellite loci except D8Rat28 were polymorphic in our rat populations. A total of 27 alleles were detected across the 8 microsatellite loci in population A versus 26 alleles in population B. However, $66 \%$ of alleles were common in both populations and $44 \%$ were different between populations $A$ and $B$. The genotypes of the 28 rats analyzed was best explained by one population (maximum log likelihood reached a plateau at $\mathrm{k}=1$ ).

L.intracellularis DNA was detected in the faeces of the majority of the pigs sampled, but was not detected in the small intestine of any rats. B.hyodysenteriae and B.pilosicoli DNA were not detected in any of the porcine faecal samples or rat intestinal samples.

\section{Discussion}

The genotype of rats trapped at the two units was similar, suggesting that rats were able to interbreed, and could travel more than $1 \mathrm{~km}$. This was significantly further than previously thought ${ }^{6}$, and may have been due to the scarcity of food at site A during the swine dysentery eradication program. While rats are unlikely to travel far from pig farms where food and shelter are provided, pig depopulation as part of a disease eradication program may provide the stimulus for dispersal. Although we didn't find porcine enteric pathogens in rats trapped at these pig farms, previous reports have found L.intracellularis and B.hyodysenteriae in rats trapped on pig farms ${ }^{1,2}$. If rodents can travel more than $1 \mathrm{~km}$ between pig farms, disease eradication programs may be more effective if performed on a regional basis.

\section{References}

1. Friedman, M et al. (2009). Lett. Appl. Micro. 47, 117-121.

2. Hampson, DJ et al. (1991). Aust.Vet.J. 68,308.

3. Abdelkrim, J. et al. (2005). Molecular Ecology 14, 2923-31.

4. oiseau, A. et al. (2008). Molecular Ecology Resources. 8, 916-918.

5. Pritchard, JK et al., (2000). Genetics 155, 945-959.

6. Leung, LKP and Clark, NM. (2005). Intnl. Biodeteriation Biodegradation $55,77-84$ 


\title{
P.418
}

\section{Population structure of Brachyspira hyodysenteriae from commercial pigs in Spain}

\author{
Jesús Osorio ${ }^{1}$ Ana Carvajal ${ }^{1}$ Álvaro Hidalgo ${ }^{1}$ Héctor Argüello ${ }^{1}$ German Naharro ${ }^{1}$ Pedro Rubio ${ }^{1}$ David J. Hampson ${ }^{2}$ \\ 1. University of León, León, Spain; 2. Murdoch University, Perth, WA, Australia
}

\section{Introduction and Objectives}

The research group at León University (Spain) is working on different topics associated with swine dysentery (SD), a severe mucohaemorrhagic diarrhoeal disease that primarily affects pigs during the growing-finishing period caused by $B$. hyodysenteriae. We have found that more than $30 \%$ of Spanish farms and $12 \%$ of faecal specimens testing positive for B. hyodysenteriae (1). However, it is necessary to obtain information about the population structure of $B$. hyodysenteriae in Spain. A multilocus sequence typing (MLST) scheme was used as a tool for investigating the diversity of Spanish isolates of B. hyodysenteriae (2).

\section{Material and Methods}

In total, 50 Spanish and 1 Portuguese isolates of $B$. hyodysenteriae from 47 farms spread all around the country were included in the analysis. All isolates were recovered from pigs affected with SD between 2001-2007 and came from the collection held at the University of León. Isolates were obtained from the most important pig production regions of our country. Data from strain reference B204 were obtained from PubMLST (http://pubmlst.org) and included in the study. Seven MLST loci (2) were used and the MLST results were visualised by constructing a dendrogram using the unweighted pair-group method for arithmetic means (UPGMA) in START2 (3).

\section{Results}

Spanish isolates were allocated to 9 sequence types (STs), of which 7 were new with an allele frequency ranging from 2 to 8 per locus. The predominat ST1 included 21 Spanish isolates from 18 farms located in 8 different autonomous regions distributed from the North to the South of the country. STs 2, 8 and 10 each also included several isolates from different regions. ST8 is widespread as well whilst ST2 appeared in the northen regions of the country. 4 singletons were identified (4, 5, 6 and 9), one of them represented by the Portuguese isolate (H32). We confirmed than $B$. hyodysenteriae got a moderate heterogeneity as previously described for Australian isolates (2). Based on the number of isolates the population had an Index of Association value of 0.786 and 0.990 based on the number of STs. Significant linkage disequilibrium was found in both analysis ( $P=0,000 ; P=0.001)$. For 5 Spanish farms where multiple isolates were available, 2 of the isolates belonged to different STs (1 and 8). The eBURST analysis (data not shown) indicated ST1 and ST8 do not belong to the same clonal complex pointing out that 2 isolates might be present in the same farm at the same time. When we combined our 51 isolates to the population as a whole (2) we found 3 monophyletic clusters each including 3 STs.
Fig. 1 Dendrogram showing the relationships of 9 sequence types (STS) obtained from $51 \mathrm{~B}$. hyodysenteriae isolates. Reference strain B204 was also included.

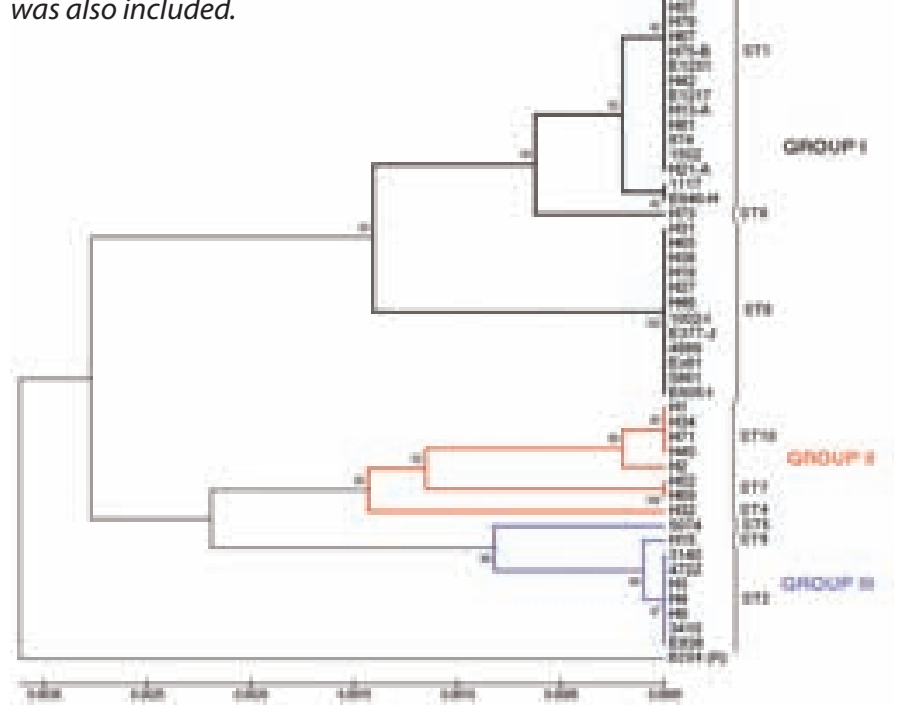

Fig. 2 Map representing the autonomous regions of Spain. Regions where farms were located are coloured and the number of isolates/STs are indicated.

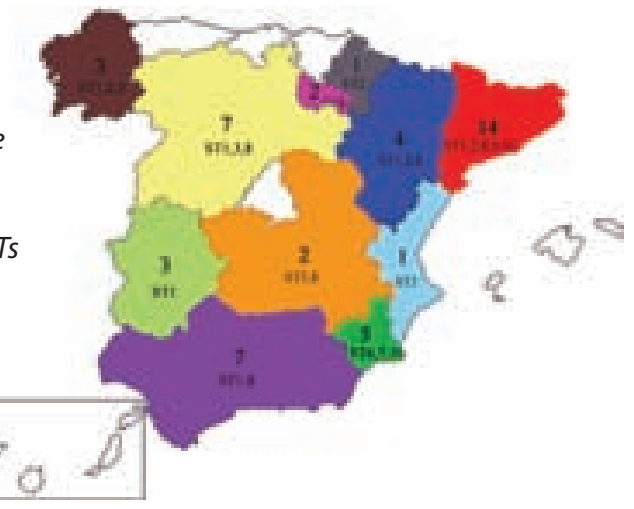

\section{Discussion}

This study has gained an insight into the population structure of $B$. hyodysenteriae using a reliable method which provides useful molecular epidemiological data to investigate the diversity of $B$. hyodysenteriae isolates, and allows the easy comparison of results between laboratories. The bacterial population was found to be clonal with evidence of likely transmision of strains between farms all around the country. Three major lineages of descent have been detected in Spain.

\section{Acknowledgements}

This study was an international collaboration between the University of León, Spain, and Murdoch University, Australia.

\section{References}

1. Carvajal et al., 2006. Vet Rec, 700-701.

2. La et al., 2009. Vet Microbiology, 138: 330-338.

3. Jolley et al., 2001. Bioinformatics, 17: 1230-1231. 


\title{
P.419
}

\section{ELISA using meat juice to detect herds with swine dysentery}

\author{
Yong Song ${ }^{1}$ Barbara Frey $^{2}$ David J. Hampson ${ }^{1}$ \\ 1. School of Veterinary and Biomedical Science, Murdoch University, Perth, WA, Australia; 2. Consistent Pork, Perth, WA, Australia
}

\section{Introduction}

Swine dysentery (SD) is a mucohaemorrhagic colitis resulting from infection of the large intestine with the anaerobic spirochaete Brachyspira hyodysenteriae. Successful disease control programmes rely on accurate, cost-effective and timely detection of infected animals and herds. Recently an enzyme-linked immunosorbent assay (ELISA) using recombinant Bhlp29.7, an outer membrane protein of the spirochaete, has been shown to have potential to be used at the herd level as a routine serological surveillance test (1). The aim of the current study was to determine whether the ELISA could be used with muscle fluid from pigs at slaughter to detect evidence of $B$. hyodysenteriae infection in herds with SD.

\section{Materials and Methods}

Pigs from two healthy herds, and three with a recent history of $\mathrm{SD}$, were sampled. Disease in the infected herds was routinely controlled using in-feed medication. Diaphragm muscle from 50 finisher pigs in each herd was collected at slaughter. A piece of muscle was excised and placed into the mouth of a sterile plastic funnel set over a sterile bottle. This was covered with plastic film, held at $-20^{\circ} \mathrm{C}$ overnight, and after thawing the passively-released muscle fluid ("meat juice") was collected. The samples were diluted 1:20 in PBS and were tested in two ELISAs for specific IgG and IgM against recombinant Bhlp29.7, as previously described (1). The optical density (OD) was measured at $450 \mathrm{~nm}$ on a microplate reader. For across-plate standardization, a positive control consisting of a meat juice sample collected from a pig that previously had recovered from SD, was added in triplicate to each plate. Calibrated ODs were calculated according to the following formula: (OD value of test sample - OD value of blank control)/average of (OD value of control sample - OD value of blank control). Cut-off values were calculated as the mean plus three standard deviations of the readings from the samples from the two negative herds. Herds with any pigs with readings over that value were considered to be infected

\section{Results}

The diagnostic threshold values for the assays were set at ODs of $0.68 \pm 0.13$ for IgG and $0.64 \pm 0.12$ for IgM. Using these criteria, the two negative herds were recorded as negative in both assays. Single serum samples from each of the three infected herds exceeded these cut-off values in the two ELISAs. In each of these herds, different serum samples werer positive in the lgG and IgM ELISAs.

\section{Discussion}

Meat-juice ELISAs have been used in a number of sero-epidemiological studies and in surveillance for specific microbial diseases of pigs (eg 2). Taking muscle samples at the abattoir avoids on-farm biosecurity risks, and generally muscle samples are easier to collect at the abattoir than are blood samples. Although serological ELISAs previously have been developed for detecting SD at the herd level, this is the first report where meat juice samples have been used as a source of antibodies. Under the test conditions, both the IgG and IgM ELISAs were specific and sensitive as herd tests. The IgM ELISA may detect more recent infection than the IgG ELISA.

Although Bhlp29.7 worked well as a plate-coating antigen in the current study, a recent report that the gene encoding Bhlp29.7 could not be detected by PCR in 33 (45\%) recent German isolates of $B$. hyodysenteriae is of concern (3). Consequently, further work is required to identify additional specific $B$. hyodysenteriae antigens that can be used in meat juice (and serum) ELISAs.

In conclusion, meat juice samples from pigs in herds with SD contain IgG and IgM antibodies that react with $B$. hyodysenteriae antigens. As such, testing meat juice samples is a convenient way for screening herds for SD. Such testing could be integrated into other meat juice ELISA screening, for example for Salmonella infections.

This study was supported by a grant from the Australian Cooperative Research Centre for an Internationally Competitive Pork Industry ("the Pork CRC").

\section{References}

1. La T. et al. (2009). Vet. Microbiol. 133, 98-104.

2. Nielsen B. et al. (1998) J Vet Diag Invest 10, 158-163.

3. Barth S. et al. (2009) Proc 5th Int Conf Colonic Infect Animals and Humans. León, Spain p31. 


\title{
P.420
}

\section{$\mathrm{N}$-terminal amino acid sequencing of $22 \mathrm{kDa}$ protein in Brachyspira hyodysenteriae reacting strongly with convalescent antibody of pig affected with swine dysentery}

\author{
Yoshikazu Adachi; Susumu Ochiai \\ School of Agriculture, Ibaraki University, Ami-machi, Japan
}

\section{Objectives}

Presence of $22 \mathrm{kDa}$ has been discovered by using convalescent antibodies after recovering from swine dysentery. The antigen has been confirmed among 11 serogroups and 17kDa antigen as well. However, we have never been studying on amino acid sequencing of the $22 \mathrm{kDa}$ protein and gene cloning. In this paper, we attempted amino acid sequencing of the protein.

\section{Materials and Methods}

ATCC27164, ATCC31212, B169, A1, WA6, Q16, NSW1, ATCC49887, and ATCC29796 were used in this test. SDS-PAGE was carried out according to Ochiai et al.(2000). 2D-PAGE were carried out as previously described(2000).Amino acid sequencing was carried out for the comparison of the amino acid sequence among serogroups.

\section{Results and Discussion}

The 22 and 17kDa proteins of Brachyspira hyodysenteriae which reacted with convalescent antibody have been analyzed by a peptide sequencer after 2 dimension polyacrylamide electrophoresis. The results demonstrated the N-terminal amino acid arrangement was consistent among ATCC27164, ATCC31212, and B169 but it did not get any results by western blotting technique after SDS-PAGE. The amino acid arrangement was Met-Thr Val-Thr-Phe-Gln-Gly-Ala-The-Gln. These results indicated that $22 \mathrm{kDa}$ protein was species specific antigen of Brachyspira hyodysenteriae. Furthermore, the $22 \mathrm{kDa}$ protein has been found in every isolate of 11 serogroups of Brachyspira hyodysenteriae, they also reacted strongly with the infected piglet sera and they was also digested with poteinase $\mathrm{K}$. Therefore, $22 \mathrm{kDa}$ protein was real protein and it also may play a role of the protection against swine dysentery. It may be like $16 \mathrm{kDa}$ antigen as described by Sellwood(1995)( 1 ).

\section{References}

1. Sellwood,R. et al.(1995) Expression of SmpA outer membrane lipoprotein of Serpulina hyodysenteriae strain P18A in vivo. Vet. Microbiol., 44, 25-35.

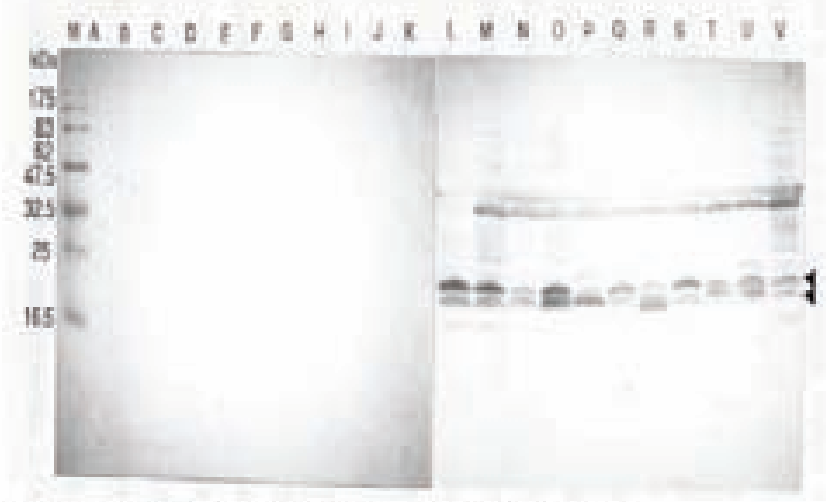

Fig 1 the presence af 22 and 17 Da preteins ofcams B hyodysenteriaeciems reacting with convalescent senum in pig with swine dysentery Lefl plate is proternase K-digested samples and noht plate is non-digested samples $A$ to $K$ and $L$ to $V$ show serogroups $A$. B. C, D. E, F, J, K, C, L and I Aerow show the 22 and $17 \mathrm{kDa}$ proteiss 


\title{
P.421
}

\section{A retrospective comparison of the specificity of culture and PCR technique for the detection of Brachyspira hyodysenteriae and Brachyspira pilosicoli}

\author{
Christina L. Planz ${ }^{2,1}$ Judith Rohde ${ }^{3}$ Elisabeth Grosse Beilage ${ }^{1}$ Heiko Nathues ${ }^{1}$ \\ 1. Field Station for Epidemiology, University of Veterinary Medicine Hannover, Bakum, Germany; 2. IBEI - University of Veterinary Medicine \\ Hannover, Hannover, Germany; 3. Institute for Microbiology, University of Veterinary Medicine Hannover, Hannover, Germany
}

\section{Introduction}

Brachyspira hyodysenteriae, the etiological agent of swine dysentery, and less commonly Brachyspira pilosicoli, the infectious agent of spirochaetal diarrhoea, are frequent causes of colitis and diarrhoea in grower and finisher pigs. Especially swine dysentery causes considerable economic loss for pig farmers due to reduced weight gain, mortality and cost-intensive treatment, eradication and preventive measures. To get the disease under control, a quick and reliable laboratory diagnosis is a prerequisite. Up to date, culture is a very common since highly sensitive method, with the disadvantage of being extraordinarily time-consuming and therefore leading to delayed diagnosis with all its negative consequences. The use of PCR as a very rapid method could solve the problem, given a similar sensitivity and specificity to culture. However, veterinarians and farmers fear false positive results from PCR including all consequences for trading of piglets and gilts, and, therefore, sometimes prefer the cultural isolation. To evaluate the accuracy of PCR under field conditions, PCR applied in routine diagnostics was compared to culture for its level of agreement.

\section{Material and Methods}

Data obtained from routine diagnostics between Jan 2006 and Dec 2009 in North-Western Germany was analysed retrospectively: Results from faecal samples or anal swabs from pigs that were submitted to the Field Station for Epidemiology for the purpose of detecting enteric pathogens were extracted from a SQL database. Datasets were further filtered for those where samples had been examined for the presence of Brachyspira species by both methods PCR and culture. The multiplex PCR assay applied to the samples detects specific genome fragments of Brachyspira hyodysenteriae and Brachyspira pilosicoli and additionally fragments of Lawsonia intracellularis (Nathues et al. 2007). For cultural testing, identical samples were examined on Brachyspira selectiveand Columbia Blood Agar incubated anaerobically at 42 degree Celsius for at least 6 days. Differentiation of Brachyspira species was based on biochemical reactions and nox-RFLP. For statistical analysis of the data $\mathrm{SAS}^{\circledR}$, version 9.1., was used to calculate the K-value as measure of concordance.

\section{Results}

During four consecutive years (2006-2009), 6,228 samples were tested for the presence of specific genome fragments of $B$. hyodysenteriae and B. pilosicoli by PCR. Overall, 184 samples were tested by both PCR and culture since the veterinarians and/or farmers did not rely on PCR alone. In 61 samples, unidentifiable Brachyspira spp. or others than B. hyodysenteriae /B. pilosicoli were detected culturally. These cases were excluded from further analysis to maintain exact comparability. Of the remaining 123 samples, 44 were positive for $B$. hyodysenteriae in PCR compared to 55 in culture, resulting in detection rates of $35.8 \%$ (PCR) versus $44.7 \%$ (culture). Twelve samples identified as positive culturally were negative in PCR whereas one sample negative in culture resulted positive in PCR. The level of agreement is $80.1 \%$. Only in one sample B. pilosicoli was found (detection rate of 0.8 $\%)$, this being positive in PCR and culture, resulting in an agreement of $100 \%$. The unweighted $\mathrm{k}$-value is 0.78 , which is $96 \%$ of the maximum possible $\mathrm{k}$-value of 0.82 .

Table 1: Results for Brachyspira hyodysenteriae and Brachyspira pilosicoli from culture and $P C R$

\begin{tabular}{|l|c|c|c|}
\hline & $\begin{array}{c}\text { PCR / } \\
\text { negative }\end{array}$ & $\begin{array}{c}\text { PCR / B. hyodysenteriae } \\
\text { positive }\end{array}$ & $\begin{array}{c}\text { PCR / B. pilosicoli } \\
\text { positive }\end{array}$ \\
\hline Culture / negative & 66 & 1 & 0 \\
\hline $\begin{array}{l}\text { Culture / B. hyodysenteriae } \\
\text { positive }\end{array}$ & 12 & 43 & 0 \\
\hline Culture / B. pilosicoli positive & 0 & 0 & 1 \\
\hline
\end{tabular}

\section{Discussion}

This retrospective analysis on a subset of data obtained by routine diagnostic indicates a substantial to almost perfect agreement between results from PCR and culture to detect $B$. hyodysenteriae and B. pilosicoli. As mentioned, culture was preferred in terms of suspicion of better specificity than PCR in 58 submissions comprising 123 samples. However, results show that specificity of both methods were equal. Contrary, the PCR is lacking sensitivity when compared to culture, which might be caused by low concentration of the target in some samples or by inhibition of the PCR. Nonetheless, the PCR is a suitable alternative to cultural methods with comparable specificity for the diagnosis of $B$. hyodysenteriae and B. pilosicoli, with the added advantage of more rapid results.

\section{References}

Nathues et al. (2007); J Vet Med A; 54: 532-538 


\title{
P.422
}

\section{Antimicrobial susceptibility of Brachyspira hyodysenteriae isolates from cases of swine dysentery in Italy recovered by different sampling procedures}

\author{
Chiara F. Magistrali; Lucilla Cucco; Nicoletta D’Avino; Michele Tentellini; Giovanni Pezzotti \\ Istituto Zooprofilattico Sperimentale Umbria e Marche, Perugia (PG), Italy
}

\section{Introduction}

Swine dysentery (SD) by Brachyspira hyodysenteriae is still a common disease and a major cause of production losses in Italian pig industry. Control of SD is based upon the use of antimicrobials, but few antibiotics are licensed for treatment; moreover, multi-resistant strains were recorded in Europe, Italy included. The aim of this work was to assess antimicrobial susceptibility of B. hyodysenteriae isolates from pigs in Italy. For this purpose, B. hyodysenteriae isolates were collected from culture collections of diagnostic laboratories (passive sampling) and from pigs affected by SD (active sampling). All the isolates collected were tested for antimicrobial susceptibility using the broth dilution method.

\section{Materials and Methods}

Isolates for antimicrobial testing were recovered using two different procedures: passive and active sampling.

Passive sampling: $B$. hyodysenteriae isolates $(n=27)$ were taken from bacterial collections in diagnostic laboratories.

Active sampling: samples were collected from clinical cases of $\mathrm{SD}$, following a standard sampling procedure, and a questionnaire was filled in. Thirty-nine herds were sampled, $28 \%$ of them were farrow to weaning, 33\% finishing and 39\% farrow to finish herds. The average number of sows/herd was 600 , while in finishing herds the number of finishers was 2550/year on average. Regarding treatment, $54 \%$ of the herds used pleuromutilins either for medication purposes in the groups of pigs sampled. On each farm, animals showing symptoms suggestive of SD, or living in the same box of symptomatic pigs, were sampled. At least 9 fecal samples were taken from each herd, cultured anaerobically on TSA-BJ medium at $41,5^{\circ} \mathrm{C}$ for five days. Suspected colonies were isolated on Blood agar and typed by PCR. Thirty isolates of $B$. hyodysenteriae from active sampling and 27 from passive sampling were tested as described below.

Broth Dilution Method: was performed according to Rohde, 2004 , using a standardized MIC panel for Brachypira spp. (VetMIC TM Brachy ver 2, SVA, Uppsala, Sweden). The concentrations ranged from $8 \mu \mathrm{g} / \mathrm{ml}$ to $0.063 \mu \mathrm{g} / \mathrm{ml}$ (tiamulin), $4 \mu \mathrm{g} / \mathrm{ml}-0.031$ $\mu \mathrm{g} / \mathrm{ml}$ (valnemulin), $64 \mu \mathrm{g} / \mathrm{ml}-0.5 \mu \mathrm{g} / \mathrm{ml}$ (lincomycin), $128 \mu \mathrm{g} /$ $\mathrm{ml}-2 \mu \mathrm{g} / \mathrm{ml}$ (tylosin). The interpretation of MIC values for both strain collections was based upon MIC-breakpoint values reported by Ronne \& Szancer, as follows (1990).Tiamulin; $\leq 1 \mu \mathrm{g} / \mathrm{ml}$ : sensitive $(\mathrm{S})_{;}>1-\leq 4 \mu \mathrm{g} / \mathrm{ml}$ : intermediate $(\mathrm{l}) ;>4 \mu \mathrm{g} / \mathrm{ml}$ : resistant (R). Valnemulin: $\leq 1 \mu \mathrm{g} / \mathrm{ml}: \mathrm{S} ;>1-2 \mu \mathrm{g} / \mathrm{ml}$ : l; $\geq 4 \mu \mathrm{g} / \mathrm{ml}: \mathrm{R}$. Lincomycin: $\leq 4 \mu \mathrm{g} / \mathrm{ml}: \mathrm{S} ;>4-\leq 32 \mu \mathrm{g} / \mathrm{ml}: \mathrm{l} ; \geq 32 \mu \mathrm{g} / \mathrm{ml}$ : R. Tylosin: $\leq 1 \mu \mathrm{g} /$ $\mathrm{ml}: \mathrm{S} ;>1-\leq 4 \mu \mathrm{g} / \mathrm{ml}: \mathrm{l} ;>4 \mu \mathrm{g} / \mathrm{ml}: \mathrm{R}$.

\section{Results}

MIC values of tested antimicrobial agents for B. hyodysenteriae isolates from active and passive samplings are shown in Table 1 and 2 .

Table 1: $A B$ MIC values for $B$. hyodysenteriae isolates from active sampling $(n=30)$.

\begin{tabular}{|l|c|c|c|c|c|c|c|c|c|c|c|c|c|}
\hline & 0.031 & 0.063 & 0.125 & 0.25 & 0.5 & 1 & 2 & 4 & 8 & 16 & 32 & 64 & 128 \\
\hline Tiamulin & & 2 & & 6 & 7 & 11 & 4 & & & & & & \\
\hline Valnemulin & 3 & 4 & 2 & 1 & 3 & 8 & 9 & & & & & & \\
\hline Lincomycin & & & & & & & & 1 & 5 & 4 & 6 & 14 & \\
\hline Tylosin & & & & & & & & & & & & 1 & 29 \\
\hline
\end{tabular}

Table 2: $A B$ MIC values for $B$. hyodysenteriae isolates from passive sampling $(n=27)$.

\begin{tabular}{|l|c|c|c|c|c|c|c|c|c|c|c|c|c|}
\hline & 0.031 & 0.063 & 0.125 & 0.25 & 0.5 & 1 & 2 & 4 & 8 & 16 & 32 & 64 & 128 \\
\hline Tiamulin & & 1 & 2 & 6 & 2 & 5 & 5 & 1 & 5 & & & & \\
\hline Valnemulin & 3 & 1 & 2 & 1 & 6 & 1 & 5 & 8 & & & & & \\
\hline Lincomycin & & & & & & 1 & 1 & 6 & 6 & 4 & 2 & 7 & \\
\hline Tylosin & & & & & & & 2 & & & & & 1 & 24 \\
\hline
\end{tabular}

\section{Discussion}

B. hyodysenteriae isolates from both active and passive samplings were resistant to tylosin, confirming results of previous studies reported, while most of them resulted in intermediate results to lincomycin. Regarding pleuro-mutilins, a different picture was found by active and passive samplings: no tiamulin or valnemulin resistant isolate from active sampling was found. The presence of multi-resistant strains in Italy was confirmed by passive sampling. Identified MIC differences recorded for passive vs. active sampling may be due to a selection of isolates originating from farms with recurrent SD problems. Those samples are sent to diagnostic laboratories. The sensitivity to pleuromutilins of B.hyodysenteriae strains is good in the majority of farms in Italy. Emergence and spreading of multi-resistant strains must be kept under control by implementation of appropriate dosing regimes, biosecurity measures and continuous monitoring of the isolates.

\section{References}

1. Rohde, J. (2004) Vet. Microbiol. 102: 25-32.

2. Ronne \& Szancer (1990) 11th IPVS Proceedings: 1126 


\title{
P.423
}

\section{In vitro susceptibility of Brachyspira hyodysenteriae to a tiamulin-doxycycline combination}

\author{
Philip Vyt ${ }^{1}$ Koen De Gussem² Alain Kanora² \\ 1. Medic Lab, Diagnostic Laborotory, Zonnestraat 3, Aalst, Belgium; 2. Huvepharma NV, Antwerp, Belgium
}

\section{Introduction}

Swine dysentery is a serious hazard on swine health in affected farms ${ }^{1}$. The increasing resistance of the bacterium Brachyspira hyodysenteriae against pleuromutilins complicates treatment options on several farms ${ }^{2}$. Since elimination protocols are mainly based on the use of tiamulin or valnemulin, eliminating the disease on farms with a resistant strain becomes problematic. One option could be a higher efficiency of tiamulin. In literature, a synergistic effect of tiamulin and tetracylines is reported, more specifically on respiratory pathogens in poultry and swine ${ }^{3,4}$. Therefore, the aim of the present study was to examine whether a combination of tiamulin and doxycycline resulted in in vitro susceptibility of $B$. hyodysenteriae at lower tiamulin concentrations.

\section{Materials and Methods}

Susceptibility of 26 Brachyspira hyodysenteriae isolates was determined using the agar dilution technique ${ }^{2}$. Minimal inhibitory concentrations (MIC) were determined against tiamulin (range $0.03-16 \mu \mathrm{g} / \mathrm{ml}$ ), doxycycline (range $0.06-16 \mu \mathrm{g} / \mathrm{ml}$ ) and against tiamulin (range $0.03-16 \mu \mathrm{g} / \mathrm{ml}$ ) supplemented with respectively $16,2,1$ and $0.5 \mu \mathrm{g} / \mathrm{ml}$ doxycycline. MIC was determined as the lowest concentration where growth was inhibited.

\section{Results}

Table 1 represents the MIC values for the 26 isolates tested. Nine strains showed decreased susceptibility to tiamulin (MIC> $1 \mu \mathrm{g}$ / $\mathrm{ml}$ ), eleven isolates had decreased susceptibility to doxycycline $(>4 \mu \mathrm{g} / \mathrm{ml}$ ) and six to both antibiotics.

Table 1. MIC $(\mu \mathrm{g} / \mathrm{ml})$ of Brachyspira hyodysenteriae for tiamulin (T), doxycycline $(D)$ and their combination

\begin{tabular}{|l|c|c|c|c|}
\hline Molecule & $\mathbf{n}$ & range & MIC $_{50}$ & MIC $_{90}$ \\
\hline Tiamulin & 26 & $<0.03->16$ & 0.250 & 8 \\
\hline Doxycycline & 26 & $<0.06-8$ & 1 & 8 \\
\hline T+D $16 \mu \mathrm{g} / \mathrm{ml}$ & 12 & $<0.03-<0.03$ & $<0.03$ & $<0.03$ \\
\hline T+D $2 \mu \mathrm{g} / \mathrm{ml}$ & 26 & $<0.03->16$ & $<0.03$ & 8 \\
\hline T+D $1 \mu \mathrm{g} / \mathrm{ml}$ & 24 & $<0.03->16$ & $<0.03$ & 8 \\
\hline T+D $0.5 \mu \mathrm{g} / \mathrm{ml}$ & 24 & $<0.03->16$ & $<0.03$ & 8 \\
\hline
\end{tabular}

Considering the combination of both antibiotics, growth was inhibited in all isolates when the concentration of doxycycline in the agar exceeded the MIC of the isolate, independent of the MIC for tiamulin. In case the MIC for doxyclin was higher than the agar concentration, a lower MIC for tiamulin when combined with doxycycline was noted in 33 to $68 \%$ of the isolates (Table 2). When considering the strains with decreased susceptibility to tiamulin, two strains (22\%) had a decreased MIC for tiamulin in combination with doxycycline. A difference of at least two dilutions was considered valid when comparing MICs for tiamulin with or without doxycycline.
Table 2. Number of B. hyodysenteriae isolates where the MIC for tiamulin $(T)$ was decreased when combined with doxycycline (D).

\begin{tabular}{|l|c|c|c|}
\hline & T+D $2 \boldsymbol{\mu g} / \mathbf{m l}$ & T+D $\mathbf{\mu} \mathbf{g} / \mathbf{m l}$ & T+D 0.5 $\mathbf{~ g} / \mathbf{m l}$ \\
\hline MIC D $\geq$ agar conc. (n) & 15 & 16 & 18 \\
\hline MIC T+D $<$ MIC T (n) & 5 & 11 & 7 \\
\hline MIC T+D $<$ MIC T (\%) & 33 & 68 & 38 \\
\hline
\end{tabular}

\section{Discussion}

In the present study the in vitro susceptibility of B. hyodysenteriae isolates to tiamulin was compared with and without doxycycline. There was no difference in $\mathrm{MIC}_{50}$ or $\mathrm{MIC}_{90}$ when all isolates were considered. When looking at the isolates with higher MIC than the concentration in the agar, these in vitro data demonstrate a higher susceptibility to tiamulin in the presence of 0.5 to $2 \mu \mathrm{g} / \mathrm{ml}$ doxycycline for one third of the isolates. From the strains with decreased susceptibility to tiamulin, two were in vitro susceptible when tiamulin was combined with doxycycline.

Further examination is necessary to confirm this combined effect of tiamulin and doxycycline on Brachyspira hyodysenteriae. Based on these limited in vitro data, a positive effect of combining both antimicrobials in the treatment of swine dysentery may be expected for some strains which can be helpful in treatment protocols.

\section{References}

1. Vyt, P. et al (2009), Flem. Vet. J., 76, 124.

2. Vyt, P., Hommez, J. (2006), Flem. Vet. J., 75: 279.

3. Caudron, C. et al (2009), Proc. 8th Journées de la Recherché Avicole, 385.

4. Burch, D. et al (1986), Vet. Rec., 119, 5: 108. 


\title{
P.424
}

\section{Efficacy of Econor ${ }^{\circledR}$ (Valnemulin) for the treatment of swine dysentery under field conditions in Japan}

\author{
Nobuo Nakanishi $^{2}$ Koji Ishii $^{1}$ Hiroshi Otaki ${ }^{1}$ Ulrich Klein ${ }^{3}$ \\ 1. Novartis Animal Health K.K., Tokyo, Japan; 2. Kyodoken Institute,Co.,Ltd, Kyoto, Japan; 3. Novartis Animal Health Inc, Basel, Switzerland
}

\section{Introduction}

Swine dysentery (SD) caused by Brachyspira hyodysenteriae (Bh) is one of the common and important enteric diseases worldwide. The objective of this study was to evaluate the clinical efficacy of Econor $1 \%$ premix in-feed medication against SD under field conditions in Japan as part of routine post-approval regulatory requirements.

\section{Materials and Methods}

Sixty six SD-infected crossbreed pigs were enrolled from three geographically different farms in Japan (Aichi, Kyoto and Ehime) from April, 2008 to April, 2009 (62 fattening pigs at age of 90 to 160 days and 4 sows). SD was confirmed by fecal microscopy and isolation of Bh. Other infections including parasites, Salmonella spp, Campylobacter spp, Clostridium perfringens and rotavirus were excluded based on additional tests. MICs of valnemulin (VAL), tylosin (TS), lincomycin (LCN) and tiamulin (TML) were evaluated using $46 \mathrm{Bh}$ strains. The pigs were treated in-feed with Econor at 30 ppm $(n=33)$ or 100 ppm $(n=33)$ for 7 consecutive days in accordance to the label in Japan. No control group was established. Combined clinical scores or CC ( $\min 0, \max$ 12) representing the fecal consistency (score $0-3,0=$ normal to $3=$ severe), pig demeanor (0-2), appetite (0-3), skin condition (0-2) and emaciation (0-2) was recorded daily (Day 0 to Day 9) and 7 days after end of the medication (Day 14) to evaluate possible relapses of SD. Fecal swabs were collected from all pigs at 2 days after treatment (Day 9) to investigate the presence of Bh. Based on the individual CC at Day 0 (CCD0) and Day 9 (CCD9), the individual percentage of improvement (IC) as primary efficacy endpoint was calculated as $100 \% *(C C D 0-C C D 9) / C C D 0$, followed by categorizing each pig into one of four categories; $(A)$ marked improvement (85$100 \%)$, (B) good improvement (70-85\%), (C) moderate improvement (50-70\%) and (D) little improvement $(<50 \%)$.

\section{Results and Discussion}

Both treated groups showed quick recovery from clinical signs with reduction of CC with more than $90 \%$ (Figure 1, Table 1). All pigs showed marketed or good improvement by treating with Econor ${ }^{\circ}$, and $75.8 \%$ of pigs with lower dose (30 ppm) and $87.9 \%$ of pigs with higher dose (100 ppm) were evaluated as marketed improvement at Day 9, which was 2 days after end of the medication (Table 2). None of the treated pigs was relapsed at Day 14. Bh was not detected from faecal samples at Day 9. Bh isolates $(n=46)$ showed high susceptibility against VAL and TML, and MIC of TS and LCM were both above $12.5 \mu \mathrm{g} / \mathrm{ml}$ (Table 3).

\section{Conclusion}

The results demonstrate the superb clinical efficacy of Econor at $30 \mathrm{ppm}$ and $100 \mathrm{ppm}$ in-feed medication against SD. The results of MIC were in alignment with earlier published data $(1,2)$.

Figure 1. Average combined clinical scores during the study from Day 0 to Day 9

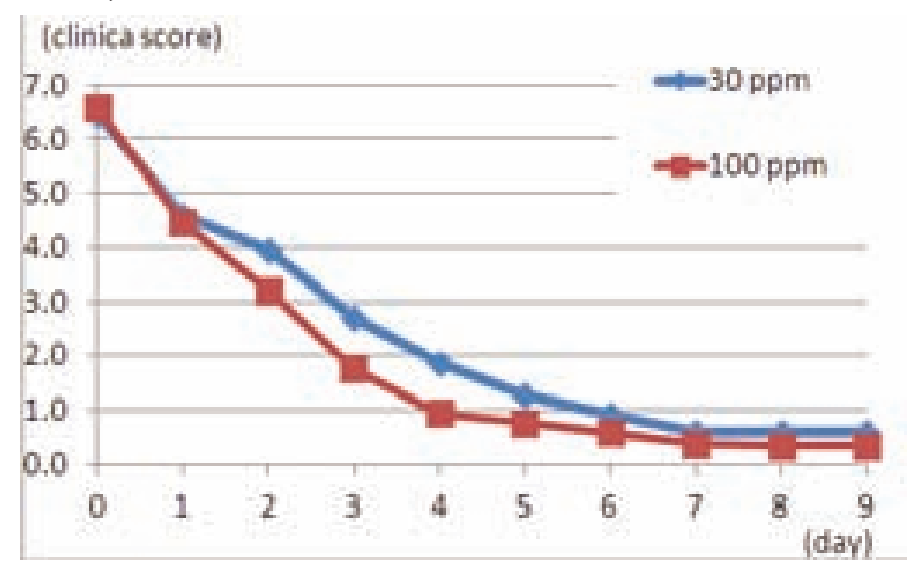

Table 1. Combined clinical scores and IC at Day 9

\begin{tabular}{|c|c|c|c|}
\hline & & $\mathbf{3 0 p p m}$ & $\mathbf{1 0 0 p p m}$ \\
\hline Clinical score & average score & 17.1 & 12.7 \\
\hline Clinical score & \% improvement & 92.2 & 95.5 \\
\hline
\end{tabular}

Table 2. Distribution of IC at Day 9

\begin{tabular}{|c|c|c|c|c|}
\hline \multirow{2}{*}{} & \multicolumn{4}{|c|}{ Number of pigs in four improvement categories } \\
\cline { 2 - 5 } & (A) & (B) & (C) & (D) \\
\hline $\mathbf{3 0} \mathbf{p p m}$ & 25 & 8 & 0 & 0 \\
\hline $\mathbf{1 0 0 p p m}$ & 29 & 4 & 0 & 0 \\
\hline
\end{tabular}

Table 3. MIC tested of antibodies for Bh $(\mu \mathrm{g} / \mathrm{ml})$

\begin{tabular}{|c|c|c|c|}
\hline & MIC Range & MIC $_{50}$ & MIC $_{90}$ \\
\hline VAL & $\leq 0.00625 \sim 0.0125$ & $\leq 0.00625$ & 0.0125 \\
\hline TS & $12.5 \sim>100$ & 100 & $>100$ \\
\hline LCM & $12.5 \sim 25$ & 12.5 & 25 \\
\hline TML & $\leq 0.00625 \sim 0.10$ & $\leq 0.00625$ & 0.1 \\
\hline
\end{tabular}

\section{References}

1. Adachi Y. et al. 2008. Proceedings 20th IPVS Congress, Durban, Vol II, p 239.

2. Karlsson M. et al 2002. J. Vet. Microbiol. 84: 123-133. 


\title{
P.425
}

\section{Effectiveness of two antibiotics against Brachyspira hyodysenteriae: a field study}

\author{
Alicia I. Carranza; Sergio O. Bautista; Natalia V. Illanes; Pablo J. Tamiozzo; Julián Parada; Carlos Goñi; Arnaldo Ambrogi
}

Universidad Nacional de Rio Cuarto, Rio Cuarto, Córdoba, Argentina

\section{Introduction}

Brachyspira hyodysenteriae (B. hyo) is the etiological agent of Swine Dysentery (SD), a disease responsible of high economic losses in the swine production. The objective of the present study was to compare the macrolide antibiotic Tylvalosin (Aivlosin ${ }^{\circledR}$ ECO Animal Health) with tiamulin by evaluating production parameters, presence of the agent and SD clinical signs.

\section{Materials and Methods}

A longitudinal study was carried out in a 500-sows, farrow-tofinish herd with antecedents of SD. All the pigs (250) weaning at 1, 3 and 5 and at 2,4 and 6 weeks constituted the groups GA (Tylvalosin) and GT (Tiamulin) respectively. Pigs from week 7 and 8 were used as control group (CG). The medication, 50 ppm of Tylvalosin and 100 ppm of Tiamulin, was given in the feed in 3 periods of time, 21 to 42,73 to 83 and 100 to 110 days of age. Five pigs per group were euthanized at 42 and 83 days old for necropsies and sampling for bacteriology and histopathology. All the pigs were weighed at weaning and at 42 days and were sent to slaughter at $105 \mathrm{~kg}$ bodyweight. Average daily gain $(A D G)$ and feed conversion rate (FCR) were determined. The percentage of pigs with diarrhoea was recorded at $42,60,83$, 100 and 150 days of age. The percentage of unthrifty pigs and mortality was also recorded. At slaughter, pigs from the three groups were evaluated as per those euthanized earlier.

Colon macroscopic lesions were recorded as follow: 1) Normal, 2) Oedema, congestion and mucus, 3) Oedema, congestion and red-brown mucus. For histopathology: 1) Normal, 2) Hyperplasia of crypts and calceiform cells and/or presence of abscess, 3) Necrosis. The 187 colon samples were inoculated in blood agar plate with antibiotics and in anaerobic atmosphere for 6 days. Samples for DNA extraction for nox-specific PCR (1) to genera determination were taken with the aid of sterile filter paper when spirochete growth was detected. Duplex PCR for B. hyodysenteriae and B. pilosicoli differentiation (2) was performed from the positive nox-specific PCR. Three strains were processed by biochemical assay.

\section{Results}

Tables 1 and 2 show the results of the different determinations. The purified strains were identified as negative indol B. hyo. None of the samples were positive to B. pilosicoli.
Table 1. Production parameters for different treatments

\begin{tabular}{|l|l|c|c|c|}
\hline \multicolumn{2}{|l|}{ PARAMETER } & GA & GT & GC \\
\hline 21 to 70 Days & ADG & 0.316 & 0.335 & 0.333 \\
\hline & FCR & 2.13 & 1.82 & 1.84 \\
\hline & \% Unthrifty & 6 & 11.3 & 4 \\
\hline & \% Mortality & 2.1 & 1.7 & 3 \\
\hline & \% Diarrhoea & 0.66 & 1.33 & - \\
\hline 70 to finish & ADG & 0.809 & 0.757 & 0.727 \\
\hline & FCR & 2.15 & 2.19 & 2.25 \\
\hline & \% Mortality & 3.9 & 4.0 & 3.1 \\
\hline & \% Diarrhoea & 4.0 & 7.3 & - \\
\hline
\end{tabular}

Table 2. Gross and microscopic lesions and PCR at different sampling times (positive/number samples)

\begin{tabular}{|l|c|c|c|c|c|c|c|c|c|}
\hline Samples & Group & \multicolumn{2}{|c|}{ PCR } & \multicolumn{3}{|c|}{ Macro } & \multicolumn{3}{c|}{ Micro } \\
\hline & & B. spp & B. hyo & 1 & 2 & 3 & 1 & 2 & 3 \\
\hline Day 42 & GA & $0 / 15$ & $0 / 0$ & 14 & 1 & 0 & 15 & 0 & 0 \\
\hline & GT & $1 / 15$ & $0 / 1$ & 14 & 1 & 0 & 15 & 0 & 0 \\
\hline Day 83 & GA & $2 / 15$ & $1 / 2$ & 11 & 1 & 3 & 10 & 5 & 0 \\
\hline & GT & $5 / 15$ & $5 / 5$ & 5 & 1 & 9 & 5 & 9 & 1 \\
\hline Slaughter & GA & $6 / 50$ & $4 / 6$ & 39 & 10 & 0 & 42 & 6 & 1 \\
\hline & GT & $17 / 48$ & $15 / 17$ & 30 & 20 & 4 & 36 & 16 & 2 \\
\hline & GC & $15 / 29$ & $12 / 15$ & 15 & 10 & 4 & 18 & 9 & 2 \\
\hline
\end{tabular}

\section{Discussion}

The presence of diarrhoea, the pathologic lesions and the isolation of B. hyo indicated that the animals were affected of SD. The GA group presented fewer animals with clinical signs as well as less organism isolation and pathological lesions. This would explain the difference in ADG (52 and $82 \mathrm{gr}$ ) and the reduced days to slaughter (5 to 10 days) compared with the GT and GC respectively. The reason of the better activity of Tylvalosin in this field trial could be a higher susceptibility of the brachyspira strain to this drug compared to Tiamulin taken into account the extensive use of the latter in Argentina for many years and particularly on the study farm. A higher Tylvalosin dose rate should be recommended for similar situations since SD was also observed on the GA group.

\section{References}

1. Rhode, J. et al. 2002. J. Clin. Microbiol. 40 (7), 2598-2600.

2. La, T. et al. 2003. J. Clin. Microbiol. 41 (7): 3372-3375. 


\title{
P.426
}

\section{Eradication of swine dysentery in an outdoor breeding herd and its production pyramid}

\author{
David G. Burch ${ }^{1}$ Mark J. Howells ${ }^{2}$ \\ 1. Octagon Services Ltd, Old Windsor, Berkshire, UK; 2. H.M.J. \& C.M. Howells, Veterinary Surgeon, Easingwold, UK
}

\section{Introduction}

The use of tiamulin (Denagard ${ }^{\circledR}$ - Novartis Animal Health Inc.) for the eradication of swine dysentery caused by Brachyspira hyodysenteriae in outdoor herds has been described (1). Herd eradication is usually planned to eliminate the infection from a chronically infected herd. The chance of success is greatest when high temperatures and dryness prevail (summertime) and at a time when the breeding herd is being moved to a new uncontaminated pasture; both will naturally assist the decontamination process. B. hyodysenteriae has been shown to survive in faeces and slurry for up to 60 days in cold conditions (2).

Diarrhoea was first noticed in a 1,500 sow outdoor breeding herd in mid-September 2008; the diagnosis was confirmed by FAT, and cultures from faeces samples submitted by the private veterinarian in the course of a routine visit two weeks later. The MICs for two of the isolates against tiamulin hydrogen fumarate were 0.031 and $0.125 \mu \mathrm{g} / \mathrm{ml}$ respectively. It was thought that the infection had originated from a nearby farm $(3 \mathrm{~km})$ which was known to be infected.

The affected unit formed part of a 10,000 sow breeder/nursery/ finisher operation comprising two separate production pyramids. Progeny were routinely co-mingled at weaning with weaners from three other unaffected breeding units. This enabled the thirteen nurseries and 23 finisher sites in the pyramid to be filled quickly; stores moved to finisher sites at about $40 \mathrm{~kg}$ and pig flow was truly "all in/all out" by unit. After consultation, an eradication programme commenced just as reports of diarrhoea were reported in some of the nurseries, which had been recently filled. At this stage there were no reports of dysentery in the finishers.

\section{Materials and Methods}

Medication programme

Breeders

The breeding herd was medicated with high levels of tiamulin (Denagard) at $8 \mathrm{mg} / \mathrm{kg}$ bodyweight for 28 days, achieved by incorporation at $800 \mathrm{ppm}$ in the dry sow ration and 500ppm in the lactating sow diet. Resolution of clinical signs would trigger a reduction to $5 \mathrm{mg} / \mathrm{kg}$ bwt for a further 28 days' in feed medication.

\section{Nursery pigs}

Five nurseries had been recently supplied from the breeding unit and all feed on these units was medicated with tiamulin at $10 \mathrm{mg} / \mathrm{kg}$ bwt (200ppm). A thorough cleaning and disinfection programme was introduced between batches.

\section{Finishers}

Despite the absence of clinical signs, all the finisher pigs in the pyramid, except those selecting for slaughter, were medicated in feed with $10 \mathrm{mg} / \mathrm{kg}$ bwt (200ppm) tiamulin for 4 weeks. Subsequent breakdowns on finisher sites led to the belief that infection had been present for longer than initially thought. A full cycle of in-feed medication (tiamulin at $10 \mathrm{mg} / \mathrm{kg}$ bwt (200ppm) for 4 weeks, reducing to $5 \mathrm{mg} / \mathrm{kg}$ (100ppm) for 4 weeks) successfully eliminated the infection from all the finisher sites.

The in-feed medication regime was complimented by parenteral and water medication with tiamulin (Denagard) in consultation with the private veterinarian to ensure rapid treatment of affected individuals.

\section{Management}

Pig flow was adjusted to separate the affected breeding unit and its progeny from all other pigs. Biosecurity procedures were reviewed and standard operating procedures tightened; risk assessments were undertaken and transport logistics were scrutinised and adjusted accordingly. Staff were advised of the situation and movement between farms was limited and controlled. Clothing changes and boots were made available on every site for visitors and disinfectant foot baths were introduced.

\section{Results and Discussion}

Sows health improved rapidly and clinical signs resolved within a week of instigating the medication programme. October was a mild and dry month, but November was cold and wet. The clinical response was excellent on all nursery and finisher sites.

The pyramid has remained free of dysentery for over 12 months since the end of medication. Progeny from the affected unit had been considered to be the poorest in the company, but have subsequently outperformed all others (data available).

\section{References}

1. Wilkinson, J.D. (1988) Pig Journal, 21, 177-184

2. Chia, S.P. \& Taylor, D.J. (1978) Proc. 5th IPVS Congress, KB57 


\title{
P.427
}

\section{Swine dysentery eradication by strategic medication without depopulation}

\author{
Johan Kamp ${ }^{1}$ Rita Schuttert-Wilps ${ }^{1}$ Susan Kars-Hendriksen ${ }^{2}$ \\ 1. De Oosthof, Dierenkliniek Hellendoorn, Hellendoorn, Netherlands; 2. Novartis Animal Health, Breda, Netherlands
}

\section{Introduction}

Swine dysentery (SD) has a major negative effect on the productivity performance. The objective of this study was to eradicate Brachyspira hyodysenteriae from a multiplier herd by medication with tiamulin (Denagard ${ }^{\circledR} 45 \%$ WSG) without partial depopulation.

\section{Materials and Methods}

The 910 sow multiplier herd was chronically infected by Brachyspira Hyodysenteriae over the past years. This resulted in recurrent outbreaks of dysentery in the summer and autumn of 2006. The weaners (8-25 kg) were therefore continuously medicated and the finishers at different sites were underperforming. All sows and weaners were housed at one site. The diagnosis was made based on clinical symptoms, post-mortem findings and confirmed by detection of B.hyodysenteriae in fecal samples of finishers and sows by culture and PCR.

The eradication program consisted of the medication of breeding animals, suckling piglets and weaners combined with thorough cleaning and disinfection of the stables. It was financially impossible to carry out a total or partial depopulation.

Prior to the start of the program, biosecurity was improved, effective rodent control was started, no visitors were allowed and no new animals were introduced. All staff was involved in establishing a cleaning and disinfection protocol.

To investigate if the eradication could be performed without feed medication, solubility and palatability of Denagard $45 \%$ in drinking water for gestation sows was tested. Most gestation sows showed a sufficient water intake with 650 gram Denagard $45 \% / 1000$ I drinking water. So treatment was only by drinking water and injection. All sows and weaners were medicated with Denagard $45 \% 9 \mathrm{mg} / \mathrm{kg}$ bodyweight in drinking water for 14 days, and then the dosage was reduced to $4.5 \mathrm{mg} / \mathrm{kg} \mathrm{BW}$ for the next 14 days. This was followed by another 28 days medication with $225 \mathrm{mg} / \mathrm{kg}$ BW. Suckling piglets were treated with tiamulin injections $10 \mathrm{mg} / \mathrm{kg} \mathrm{BW}$ on day 1, 7 and 14 after the start of medication. All animals with reduced appetite were injected with tiamulin $10 \mathrm{mg} / \mathrm{kg} \mathrm{BW}$. During the program every day all manure was removed. Every 3 days all places possibly contaminated with manure were cleaned and disinfected (Virocid). After finishing the program the herd was monitored on the presence of B. hyodysenteriae.

\section{Results}

The eradication program was completed in the beginning of 2007. A few days after starting the medication there were no clinical signs of dysentery anymore. Every month the farm was monitored for clinical symptoms. Routine use of antimicrobials against enteric diseases was no longer required.

Fecal samples of sows, piglets and finishers were screened post-eradication for Brachyspira by PCR regularly (Jan 2008-June 2009) and were all negative.

The reproduction performances improved (see table 1) and so did the growth of the piglets and finishers. After the eradication program the herd performance significantly improved.

Table 1. Comparison of technical results before and after SD eradication

\begin{tabular}{|l|c|c|}
\hline & $\mathbf{2 0 0 6}$ & $\mathbf{2 0 0 8}$ \\
\hline No. of sows & 929 & 1034 \\
\hline Farrowing rate & 84.2 & 89.3 \\
\hline Live born piglets per litter & 11.62 & 12.64 \\
\hline Weaned piglets/sow/year & 21.89 & 24.39 \\
\hline
\end{tabular}

\section{Discussion}

There are many closed herds for which it will be very costly to perform an eradication strategy with partial or total depopulation. Successful eradication of B. hyodysenteriae without any depopulation is also a reasonable option as indicated by the results. Big advantage is that the pig flow is stable, which at the end translates into normal revenues for the farm. If biosecurity is kept at a very high level it is possible to keep the farm free of SD. All biosecurity measurements like cleaning and disinfection to prevent re-infection from the environment are as important as the medication strategy.

Eradication of diseases fits the current approach of decreasing the use of antibiotics in the Netherlands.

The study results confirm previously published data on SD eradication with strategic medication combined with intensive cleaning and disinfection. In the project described here no partial depopulation was performed. 


\title{
P.428
}

\section{The drug-susceptibility of Japanese isolates of Brachyspira hyodysenteriae in 2008}

\author{
Yoshikazu Adachi; Hiromi Nakajima; Akihiro Misawa
}

School of Agriculture, Ibaraki University, Ami-machi, Japan

\section{Introduction}

Brachyspira(B.) hyodysenteriae isolated from slaughtered pigs in Ibaraki Prefecture in Japan ,2008. The MIC values of B.hyodysenteriae isolates to 7 antibacteral agents have been investigated and were compared with those of previous results (1).

\section{Materials and Methods}

Eighteen pig isolates of $B$. hyodysenteriae have been identified by PCR using NOX primers and sequencing with primers $3 F\left(5^{\prime}-G G-\right.$ CGATGCGTCTTAAGC-3') and 500R(5'-AATCCGAGCAACGTTTG-3') (2). Indole production test was used for identification. The drugsusceptibility test was performed as previously described (3) and carbadox, valunemulin, lincomycin, tylosin, tiamulin, penicillin $\mathrm{G}$, and metronidazol were used.

\section{Results and Discussion}

The isolates with over $99.7 \%$ homology to B.hyodysenteriae ATCC27164 have been identified as B.hyodysenteriae and 18 isolates were B.hyodysenteriae. As shown in Table 1, carbadox and metronidazole were very effective and MICs were $0.01>$ and $0.4>$, respectively but carbadox was prohibited to use in pig herds. Valunemulin was effective to the isolates and MIC values were slightly higher than those of previous results (1).

Penicillin $\mathrm{G}$ was also effective except for one isolate with MIC of 100 and 17 isolates were less than MIC of 0.4 . MIC values of tiamulin were ranging from 13 to 0.1 and MIC values of 9 isolates were less than MIC of 0.4. On the other hand, MIC values of tylosin were ranging from 100 to 3.1 and MICs of tylosin were $\geq 100$ in most isolates. MIC values of lincomycin were ranging from 100 to 0.2 . The isolates have acquired the resistance to tylosin and lincomycin and the values of MICs were similar to the previous values(1), while those isolates were still susceptible to valnemulin and tiamulin and the values were slightly higher than previous results(1).

\begin{tabular}{|l|c|c|}
\hline Drugs & \multicolumn{2}{|c|}{ MICs $(\mu \mathrm{g} / \mathrm{ml})$} \\
\hline Penicillin G & Range & For $\geq 90 \%$ of the isolates \\
\hline Valunemulin & $100-0.2$ & $\leq 0.4$ \\
\hline Lincomycin & $6.3-0.1$ & $\leq 6.3$ \\
\hline Tiamulin & $100-0.2$ & $\leq 25$ \\
\hline Tylosin & $13-0.1$ & $\leq 6.3$ \\
\hline Metronidazol & $100-3.1$ & $\geq 3.1$ \\
\hline Carbadox & $1.6-0.1$ & $\leq 0.4$ \\
\hline
\end{tabular}

\section{References}

1. Adachi Y, et al. 2008. Proc. Cong. Int.Pig. Vet. Soc 20:239

2. Okojima K. and Adachi Y. 2007.Int.Conf.Colonic Spiro. Infect. Anim. Human.4:7

3. Uezato et al. 2004..J.Vet.Med.Sci.,66,307-309 


\title{
P.429
}

\section{Comparison between two different methods for antimicrobial susceptibility testing of Brachyspira hyodysenteriae}

\author{
Chiara F. Magistrali ${ }^{1}$ Lucilla Cucco ${ }^{1}$ Gloria D’Angelo ${ }^{1}$ Yuri Gherpelli² Paolo Bonilauri2 Giuseppe Merialdi ${ }^{3}$ \\ 1. Istituto Zooprofilattico Sperimentale Umbria e Marche, Perugia, Italy; 2. Istituto Zooprofilattico Sperimantale della Lombardia e dell'Emilia \\ - Romagna, Reggio Emilia, Italy; 3. Istituto Zooprofilattico Sperimentale della Lombardia e dell'Emilia - Romagna, Bologna, Italy
}

\section{Introduction}

Antimicrobials are the most relevant tool to control swine dysentery caused by Brachyspira hyodysenteriae in pig herds. Pleuromutilins (tiamulin and valnemulin) and lincomycin are the most important agents used for this purpose, nevertheless, multi-resistant B. hyodysenteriae isolates have been reported in many countries, Italy included. Currently no standard antimicrobial susceptibility testing method is internationally established. Agar dilution technique and broth dilution test methods are available. The aim of this work was to compare the two methods used for antimicrobial susceptibility testing of $B$. hyodysenteriae isolates in two different Italian laboratories.

\section{Materials and Methods}

Isolates: twenty B. hyodysenteriae isolates from clinical cases of Swine Dysentery in Italian pig herds were used during the study. The isolates were tested independently and blindly by the two different laboratories using the methods described below.

Broth Dilution Method: the broth dilution method was performed according to published literature (Rohde, 2004). Briefly, approximately $10^{6}$ of each isolate in $0,5 \mathrm{ml}$ of $\mathrm{BHI}$ broth supplemented with calf fetal serum was added to each well of a MIC panel for Brachypira spp. (VetMIC TM Brachy ver 2, SVA, Uppsala, Sweden), and incubated on a shaker for 4 days in an anaerobic atmosphere (Genbox, Biomerieux, Lyon, France) at $37^{\circ} \mathrm{C}$. Reference control strains were used as controls ( B. hyodysenteriae B78, ATCC 27164). The concentration tested were from $8 \mu \mathrm{g} / \mathrm{ml}$ to $0.063 \mu \mathrm{g} /$ $\mathrm{ml}$ for tiamulin, from $4 \mu \mathrm{g} / \mathrm{ml}$ to $0.031 \mu \mathrm{g} / \mathrm{ml}$ for valnemulin and from $64 \mu \mathrm{g} / \mathrm{ml}$ to $0.5 \mu \mathrm{g} / \mathrm{ml}$ for lincomycin.

Quick-MIC agar dilution method: a simplified agar dilution method using only antibiotic break point values was applied according to Ronne and Szancer (1990).

The interpretation of MIC values was based upon MIC-breakpoint values reported by Ronne \& Szancer, as follows (1990). Tiamulin; $\leq 1 \mu \mathrm{g} / \mathrm{ml}$ : sensitive (S); $>1-\leq 4 \mu \mathrm{g} / \mathrm{ml}$ : intermediate (I); $>4 \mu \mathrm{g} / \mathrm{ml}$ : resistant (R). Valnemulin: $\leq 1 \mu \mathrm{g} / \mathrm{ml}: S_{;}>1-2 \mu \mathrm{g} / \mathrm{ml}$ : l; $\geq 4 \mu \mathrm{g} / \mathrm{ml}: \mathrm{R}$. Lincomycin: $\leq 4 \mu \mathrm{g} / \mathrm{ml}: \mathrm{S} ;>4-\leq 32 \mu \mathrm{g} / \mathrm{ml}: \mathrm{l} ; \geq 32 \mu \mathrm{g} /$ $\mathrm{ml}:$ R. Tylosin: $\leq 1 \mu \mathrm{g} / \mathrm{ml}: \mathrm{S} ;>1-\leq 4 \mu \mathrm{g} / \mathrm{ml}:$ l; $>4 \mu \mathrm{g} / \mathrm{ml}: \mathrm{R}$.

\section{Results}

The results are shown in Table 1 and 2. By broth dilution , 4 isolates were classified resistant to tiamulin, 6 to valnemulin and 7 to lincomycin.
Table 1: MIC values of $B$. hyodysenteriae isolates by agar diffusion technique $(n=20)$.

\begin{tabular}{|l|c|c|c|c|c|}
\hline $\mathbf{\mu g} / \mathbf{m l}$ & $<\mathbf{1}(\mathbf{S})$ & $\mathbf{1 - 4}(\mathbf{I})$ & $>\mathbf{4}(\mathbf{R})$ & $\mathbf{4 - 3 6}(\mathbf{I})$ & $<36(\mathbf{R})$ \\
\hline Tiamulin & 5 & 10 & 5 & & \\
\hline Valnemulin & 7 & 6 & 7 & & \\
\hline Lincomycin & & & & 12 & 8 \\
\hline
\end{tabular}

Table 2: MIC values of $B$. hyodysenteriae isolates by broth dilution method $(n=20)$.

\begin{tabular}{|l|c|c|c|c|c|c|c|c|c|c|c|c|}
\hline $\boldsymbol{\mu g} / \mathrm{ml}$ & .031 & .063 & .125 & .25 & .5 & $\mathbf{1}$ & $\mathbf{2}$ & $\mathbf{4}$ & $\mathbf{8}$ & $\mathbf{1 6}$ & $\mathbf{3 2}$ & $\mathbf{6 4}$ \\
\hline Tiamulin & & 1 & 2 & 5 & 2 & 3 & 1 & 1 & 4 & & & \\
\hline Valnemulin & 2 & 1 & 2 & 1 & 4 & 1 & 3 & 6 & & & & \\
\hline Lincomycin & & & & & & & 1 & 6 & 2 & 3 & 1 & 7 \\
\hline
\end{tabular}

For tiamulin, 8 strains were classified as intermediate by agar dilution, and sensitive by broth dilution, for valnemulin 5 and for lincomycin 7. The isolates classified intermediate for tiamulin by agar diffusion and sensitive by broth dilution showed MIC values above $0,125 \mu \mathrm{g} / \mathrm{ml}$ in the broth dilution test . No isolate classified as sensitive by one of the two methods resulted resistant by the other one.

\section{Discussion}

The need of standardization and quality control for antimicrobial susceptibility testing of Brachyspira spp. has been repeatedly pointed out. Broth method has been reported to give MICs lower by one dilution step in literature. The trial results confirm lower MIC values for the tested isolates by using broth dilution method compared to agar diffusion technique. Using the MIC breakpoints thresholds, some isolates classified as intermediate to pleuromutilins and lincomycin by agar dilution technique were classified as sensitive by broth dilution test. Nevertheless, no isolates were classified as resistant by agar dilution technique and sensitive by broth dilution test or vice versa. In conclusion, classification of isolates in different susceptibility groups using the two test methods can occur, in particular for those showing intermediate values.

\section{References}

Rohde, J. (2004) Vet. Microbiol. 102: 25-32.

Ronne\& Szancer (1990) 11th IPVS Proceedings: 1126. 


\title{
P.430
}

\section{Evolution of recent antimicrobial sensitivity data of Brachyspira hyodysenteriae in Belgium}

\author{
Frédéric A. Vangroenweghe; Evelyne De Graef; Mia Vanrobaeys; Cora Miry \\ DGZ-Vlaanderen, Torhout, Belgium
}

\section{Introduction}

Evolution of antimicrobial resistance (AMR) of Brachyspira hyodysenteriae, especially to pleuromutilins, should be monitored regularly to update the actual treatment possibilities in case of clinical disease or eradication strategies. A study, using strains isolated in 2003, on the link between AMR and clinical effect of treatment to $B$. hyodysenteriae infections revealed that only $13 \%$ of the strains tested were susceptible to lincomycin and just 4\% to tylosin (1). Since 2006, recent data are yearly available on B. hyodysenteriae AMR to tiamulin and valnemulin in Belgium.

\section{Materials and Methods}

Strains of B. hyodysenteriae were isolated from routine diagnostic samples during 2006-2008-2009 and immediately after isolation, antimicrobial susceptibility testing was performed. Besides the field isolates, available reference Brachyspira strains were also tested (2). Susceptibility testing was performed as previously described $(1,2)$. The MIC was recorded as the lowest concentration at which no distinct hemolysis was seen in the spot.

\section{Results}

The reference strain of $\mathrm{B}$. hyodysenteriae (B78) was more susceptible to valnemulin $(\mathrm{MIC}<0.03)$ than to tiamulin $(\mathrm{MIC}=0.06)$. Results of the field isolates of the different $B$. hyodysenteriae strains ( $n=40$ strains per year) are given in Figure 1 for tiamulin and valnemulin. Minimal inhibitory concentrations for 50\% (MIC50) and $90 \%$ (MIC90) of the strains tested are also given in Table 1.

Table 1. Minimal inhibitory concentrations of tiamulin and valnemulin required to inhibit 50 and $90 \%$ (expressed in $\mu \mathrm{g} / \mathrm{ml}$ ) of $B$. hyodysenteriae strains isolated in Belgium in 2006-2008-2009

\begin{tabular}{|c|c|c|c|c|}
\hline \multirow{2}{*}{} & \multicolumn{2}{|c|}{ Tiamulin } & \multicolumn{2}{c|}{ Valnemulin } \\
\cline { 2 - 5 } & MIC 50 & MIC 90 & MIC 50 & MIC 90 \\
\hline 2006 & 0.25 & 2 & 0.03 & 0.50 \\
\hline 2008 & 0.50 & 8 & 0.12 & 8 \\
\hline 2009 & $>8$ & $>8$ & 8 & $>8$ \\
\hline
\end{tabular}

\section{Discussion and Conclusions}

In comparison with previous studies (1), MIC50 and MIC90 were significantly increased for $\mathrm{B}$. hyodysenteriae. Considering that isolates with MIC $<1 \mu \mathrm{g} / \mathrm{ml}$ should be regarded as not responding to therapy in vivo, more than $50 \%$ of all isolates obtained in 2009 should be considered resistant to both tiamulin and valnemulin (1).
Figure 1. Percentage of strains with their respective minimal inhibitory concentrations of tiamulin (A) and valnemulin (B) for B. hyodysenteriae strains isolated in Belgium in 2006 (red) -2008 (green) -2009 (purple)

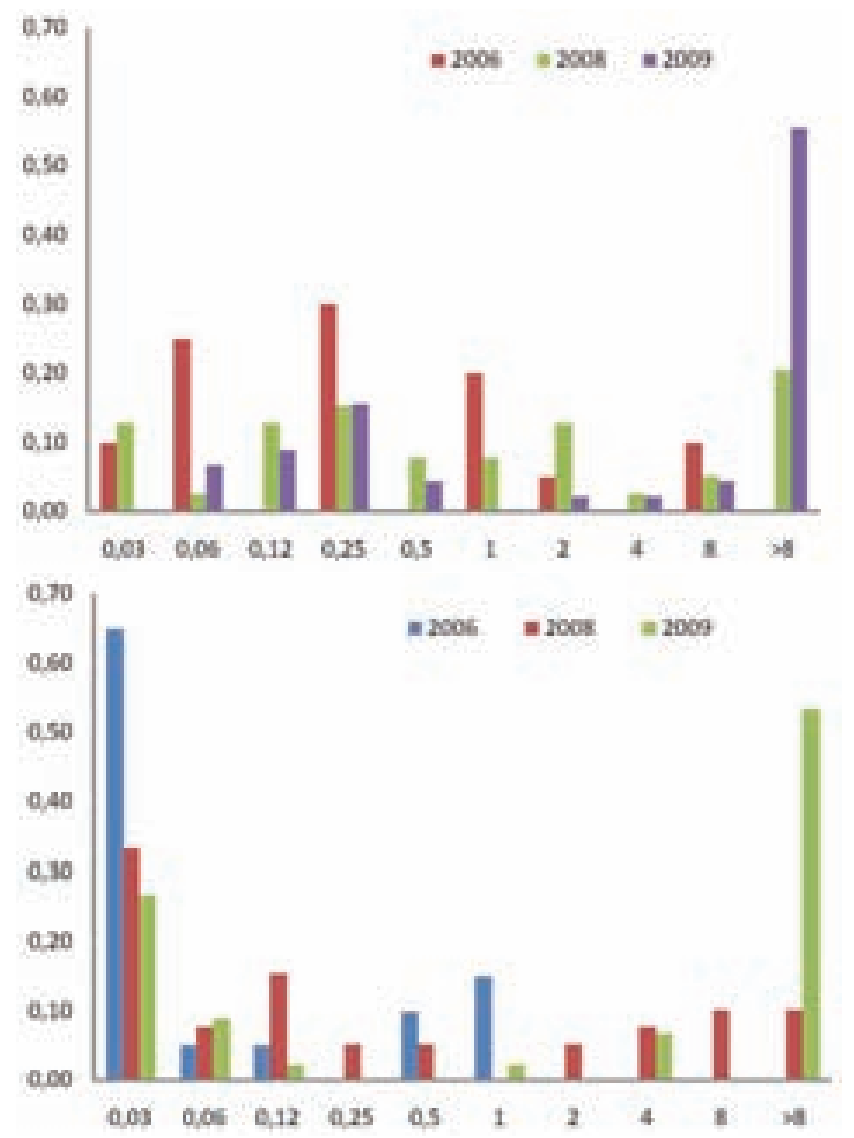

For valnemulin, the number of resistant strains ( $n=27)$ significantly increased last year and became nearly as high as for tiamulin $(n=29)$. The fact that an increasing number of strains reaches MIC-values beyond the highest concentrations ( MIC $>8 \mu \mathrm{g} / \mathrm{ml}$ ) tested for both pleuromutilins is a serious concern for the Belgian pig industry. Especially in relation to eradication protocols, further research focussing at potential alternative control measures is necessary. Farms should be aware to further improve their external en internal biosecurity measures to prevent introduction of new $B$. hyodysenteriae strains in the farm.

In conclusion, antimicrobial resistance to pleuromutilins has evolved towards a higher prevalence of resistance and higher MIC50 and MIC90 values as compared to previous data.

\section{References}

1. Vyt P., Hommez J. 2006. Antimicrobial susceptibility of Brachyspira hyodysenteriae isolates compared with the clinical effect of treatment. Vlaams Diergeneeskundig Tijdschrift 75, 279-285.

2. Hommez J., Devriese L.A., Castryck F., Miry C., Lein A., Haesebrouck F. 1998. Susceptibility of different Serpulina species in pigs to antimicrobial agents. Vlaams Diergeneeskundig Tijdschrift 67, 32-35. 


\title{
P.431
}

\section{Case report: therapeutical effect of Vetmulin ${ }^{\circledR}$ premix on a clinical outbreak of Brachyspira murdochii in a fattening herd in Belgium}

\author{
Frédéric A. Vangroenweghe ${ }^{1}$ Evelyne De Graef ${ }^{1}$ Lieven Claerhout $^{2}$ Henk Lecluyse $^{3}$ Alain Kanora ${ }^{3}$ \\ 1. DGZ-Vlaanderen, Torhout, Belgium; 2. DAP Curavet, Roeselare, Belgium; 3. Huvepharma NV, Antwerpen, Belgium
}

\section{Introduction}

Spirochaetes belong taxonomically to the order Spirochaetes, which contains several genera such as Brachyspira and Leptospira. The intestinal spirochaetes are included in the genus Brachyspira, which contains at present seven species, of which five are known to be present in pigs: B. hyodysenteriae, B. pilosicoli, B. intermedia, B. murdochii and B. innocens. Porcine diarrheal disease in fatteners is mainly caused by $B$. hyodysenteriae and B. pilosicoli. In Belgium, B. hyodysenteriae has been diagnosed in $16 \%$ of the fecal samples $(n=2264)$ analyzed through PCR during the last 3 years (1). In bacteriological culture, B. hyodysenteriae was present in $26 \%$ of the analyzed fecal samples (2). Besides the major pathogenic B. hyodysenteriae, $B$. murdochii also occurs in $9 \%$ of the fecal samples analyzed through PCR and in 5\% of the bacteriological cultures for Brachyspira species. Brachyspira murdochii is generally considered as a minor pathogen (3). The present case report focuses on clinical outbreak caused by B. murdochii in a fattening herd in Belgium.

\section{Materials and Methods}

A fattening herd of 600 fattening places went through a period of clinical diarrhea in the group of $60-70 \mathrm{~kg}$. The piglets all originated from one single sow farm. Clinically speaking the diarrhea consisted of flat feces with a slimy aspect without bloody contents. All fattening pigs were strongly contaminated with fecal material. Fecal samples of clinically affected pigs were collected for diagnostic purposes. The samples were cultured through standard anaerobic bacteriological Brachyspira culture and colonies were phenotypically identified to the species level.

\section{Results}

Bacteriological culture of Brachyspira resulted in the identification of B. murdochii in pure culture. As no other relevant bacterial species could be isolated from the fecal samples, it was considered to be the agent causing clinical diarrhea in the fattening herd. Following the bacteriological diagnosis, antimicrobial therapy with tiamulin (Vetmulin ${ }^{\circledR}$ premix; Huvepharma) at a dose of $10 \mathrm{mg}$ active substance per kg live weight was started. The antimicrobial was administered through the feed for at least 7 days. Following the therapy, clinical recovery and normal fecal consistency could be observed after 5 days of treatment with Vetmulin ${ }^{\circledast}$. After the treatment, clinical signs disappeared in the treated group. However, identical clinical symptoms were observed in other untreated fattening herds with piglets originating from the very same farm. In these fattening herds, similar therapy was introduced and resulted in identical therapeutical effect on clinical symptoms.

\section{Discussion}

The present case report clearly demonstrates that although B. murdochii is generally considered as a minor pathogen without specific clinical symptoms, occasionally clinical outbreaks can occur. The absence of bloody contents in the feces could indicate that $\mathrm{B}$. hyodysenteriae was not the etiological agent, although clinical signs of B. hyodysenteriae in Belgium are not always associated with bloody feces. The importance of rapid and accurate diagnostics in order to install an efficient therapeutic plan is herewith demonstrated. The good therapeutic response of $\mathrm{B}$. murdochii to the administration of tiamulin preparations is in accordance with several data on antimicrobial susceptibility, showing no significant resistance to tiamulin $(4,5)$.

In conclusion, the present case report clearly shows that $\mathrm{B}$. murdochii is not as apathogenic as generally accepted. A clear relation with other piglets originating from the same farm could be observed, which indicates a possible contamination during the suckling or post-weaning period.

\section{References}

1. Goyvaerts, E. 2010. Analytical results of PCR-laboratory, DGZ 2009. Personal communication.

2. Fevery, T. 2010. Analytical results of bacteriological culture laboratory, DGZ 2009. Personal communication.

3. Råsbäck, T. et al. 2005. Assessment of diagnostics and antimicrobial susceptibility testing of Brachyspira species using a ring test, Vet. Microbiol., 109, 229-243.

4. Vangroenweghe, F. et al. 2008. Recent antimicrobial sensitivity data of Brachyspira sp. in Belgium. Proc. 20th IPVS Congres, Durban, South-Afrika.

5. Hommez, J. et al. 1998. Susceptibility of different Serpulina species in pigs to antimicrobial agents, Flem. Vet. J., 67, 32-35. 


\title{
P.432
}

\section{Minimum inhibitory concentration of tiamulin, valnemulin and tylosine for Brachyspira hyodysenteriae, Brachyspira pilosicoli and Brachyspira spp. isolated from pig farms in México}

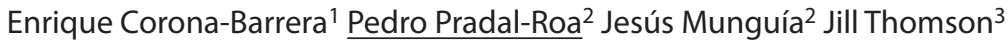 \\ 1. CIR, Biomedicas, Mérida, YUC, Mexico; 2. Depto. Prod. Animal Cerdos., Fac Med Vet Zoot-UNAM. Ciudad Universitaria, México, DF, Mexico; \\ 3. Vet Science Division-Scottish Agricultural College, Edinburgh, UK
}

\section{Introduction}

The pathologies caused by the intestinal spirochaetes (IS) Brachyspira hyodysenteriae-swine dysentery and Brachyspira pilosicoli-porcine colonic spirochaetosis have been documented in pig production sector of many countries. These enteropathogens have been identified in México (2). Values for minimum inhibitory concentration (MIC) of the antimicrobials tiamulin, valnemulin and tylosin have already been reported $(1,4)$. The aim of this work was to determine MIC values of the antimicrobials above for Brachyspira specimens isolated from pigs in México.

\section{Material and Methods}

A total of 19 Brachyspira specimens, B. pilosicoli $(n=6)$, Weakly $\beta$-haemolytic IS (WBHIS) indol -ve (presumably B. pilosicoli, $\mathrm{n}=11)$ and $B$. hyodysenteriae $(n=2)$ were used to determine MIC values of tiamulin (Novartis $A H$ ), valnemulin (Novartis $A H$ ) and tylosin (USP ref. std) using agar dilution method. Propagation of spirochaetes was done on blood agar plates (Columbia agar, Oxoid, UK $+8.0 \%$ horse blood) incubated for 3 days under anaerobic conditions using GasPak (AnaeroGen, Oxoid, UK) in jar. The harvest of those plates was used to make a working bacterial suspension adjusted to 1.0 MacFarland standard. Concentrations from 128 to $0.0125 \mu \mathrm{g} / \mathrm{ml}$ of the antimicrobials were obtained by dilution from mother concentrations of 10,000 mg/l. Wilkins Chalgren (WC) [Anaerobe agar, (Oxoid UK) $+8.0 \%$ horse blood] plates containing the antimicrobial dilutions were prepared. Volumes of $10.0 \mu \mathrm{l}$ of the bacterial suspension of each Brachyspira specimen and 2 replicates were inoculated on WC plates. Control plates (no antimicrobial) were also inoculated for viability of Brachyspira cells. WC plates were incubated at $38{ }^{\circ} \mathrm{C}$ under anaerobic conditions in jars for $48 \mathrm{hrs}$. MIC assays were repeated at least once.

\section{Results}

MIC values $>4.0 \mu \mathrm{g} / \mathrm{ml}$ are considered as resistant. The MIC values obtained for the antimicrobials of study are shown below.

MIC values:

B. pilo $(\mathrm{n}=6)$ Tia $0.025-0.5 \mu \mathrm{g} / \mathrm{ml}$ Val 0.025-0.25 $\mu \mathrm{g} / \mathrm{ml}$ Tyl 2.0-16 $\mu \mathrm{g} / \mathrm{ml}$

WBHIS $(\mathrm{n}=11)$ Tia $0.5-4.0 \mu \mathrm{g} / \mathrm{ml}$ Val 0.01-0.5 $\mu \mathrm{g} / \mathrm{ml}$ Tyl -

B. hyo $(\mathrm{n}=2)$ Tia $0.025-0.1 \mu \mathrm{g} / \mathrm{ml}$ Val 0.025-0.1 $\mu \mathrm{g} / \mathrm{ml}$ Tyl 2.0-128 $\mu \mathrm{g} / \mathrm{ml}$

Tiamulin MIC values at the level of $4.0 \mu \mathrm{g} / \mathrm{ml}$ were observed on isolates of Brachyspira recovered from the same farm. Control inocula developed the Brachyspira characteristic growth showing that the assay conditions were appropriate and the purity of those cultures was confirmed on Gram stained smear preparations.

\section{Discussion}

The MIC values obtained for tiamulin and valnemulin in the present study are among those reported on previous studies $(3,5)$. In the present study a MIC value at the level of $4.0 \mu \mathrm{g} /$ $\mathrm{ml}$ for tiamulin was obtained (that should be interpreted as a resistance value). Tiamulin resistance values have been reported by Czech Republic authors who have tested Brachyspira isolates $(n=202)$ for a 10 year period (1). However in another MIC study using B. pilosicoli isolates obtained since 1996 no resistance was observed (5). The current study is the first one on MIC values of tiamulin, valnemulin and tylosin for Brachyspira. The tylosin high MIC values (up to 16 for B. pilo $\mu \mathrm{g} / \mathrm{ml}$ and up to $128 \mu \mathrm{g} / \mathrm{ml}$ for $B$. hyo) found in this study show resistance, $A$ Brachyspira feature already reported elsewhere (1) and is consistent with results from other authors among the Brachyspira international group as discussed on the last biannual meeting. Performing MIC assays on WC agar implies to adapting cultures on this medium which takes time making the testing more costly.

\section{Conclusion}

The MIC values of tiamulin were in some cases at the level of $4.0 \mu \mathrm{g} / \mathrm{ml}$, this is at the limit of susceptibility-resistance. The MIC values of valnemulin were low $(<0.5 \mu \mathrm{g} / \mathrm{ml})$ showing that Brachyspira is far from being resistant. The technical information presented in this study gives confidence to the use of these anticmicrobials, particularly valnemulin for treatment of Brachyspira infections.

\section{Acknowledgements}

Novartis Animal Health provided the funds for this study.

\section{References}

1. Cizek et al., (2007). IV Int Conf Intest Spiroch., 16.

2. Corona-Barrera et al., (2008). XLIII AMVEC., 161.

3. Hidalgo et al., (2007). IV Int. Conf Intest Spiroch, 20.

4. Pejsak et al., (2002). 17th IPVS., 361.

5. Ripley et al., (2004). 18th IPVS-Novartis., 1-4. 


\title{
P.433
}

\section{Activity of propyl propyl thiosulfinate and propyl propyl thiosulfonate against Brachyspira hyodysenteriae field isolates}

\author{
Álvaro Hidalgo; Jesús Osorio; Héctor Argüello; Ana Carvajal; Pedro Rubio \\ University of León, León, Spain
}

\section{Introduction}

Brachyspira hyodysenteriae is the causative agent of swine dysentery (SD), one of the most important gastrointestinal disorders in pigs. Treatment and control of SD involve mainly the use of antimicrobial as no commercial vaccines are available. However, field isolates of $B$. hyodysenteriae with decreased susceptibility to one or more of the drugs commonly used for the treatment of SD have been reported $(1,2)$. These isolates represent a threat to the pig industry.

The antimicrobial properties of plants of genus Allium have been known for ages. Accordingly, the effectiveness of some garlic substances (Allium sativum) against some human gastroenteric bacteria have been reported previously $(3,4)$. However, knowledge on antimicrobial activity of these substances against animal pathogens is scarce.

This study aims to evaluate the potential use of two compounds of natural origin obtained from plants of genus Allium, propyl propyl thiosulfinate (PTS) and propyl propyl thiosulfonate (PTSO), to assist in the treatment and control of SD.

\section{Materials and Methods}

Bacterial strains and growth conditions. Forty seven isolates of $B$. hyodysenteriae from the bacterial collection held at the Animal Health Department at the University of León, Spain, were used in this study. Bacterial isolates had been recovered from different farms distributed across Spain between 2001 and 2009. Thawed isolates were grown on fastidious anaerobe agar supplemented with $5 \%$ horse blood in an anaerobic atmosphere (10\% hydrogen, $10 \%$ carbon dioxide and $80 \%$ nitrogen) at $39^{\circ} \mathrm{C}$.

Table 1. $M I C_{50}$ and $M I C_{90}(\mu \mathrm{g} / \mathrm{ml})$ of PTS, PTSO and PTS+PTSO for 47 Spanish field isolates of $B$. hyodysenteriae.

\begin{tabular}{|c|c|c|c|}
\hline & PTS & PTSO & PTS + PTSO \\
\hline MIC $_{50}$ & 25 & 200 & 50 \\
\hline MIC $_{90}$ & 100 & $>400$ & 100 \\
\hline Range & $12.5-100$ & $\leq 6.25->400$ & $\leq 6.25-200$ \\
\hline
\end{tabular}

Susceptibility panel and broth dilution procedure. A susceptibility testing panel was designed using twofold serial dilutions of PTS, PTSO and a combination of both called PTS+PTSO (17.5\% PTS + 82.5\% PTSO) [DMC Research Center SL, Granada, Spain] ranging from 6.25 to $400 \mu \mathrm{g} / \mathrm{ml}$. For this purpose, 48-well plates were used, following the broth dilution method described by Karlsson et al. (1, 2). Growth control wells were included in each plate. MIC was determined as the lowest concentration of Allium spp. derivatives that prevented visible growth. Absence of contamination was checked by phase contrast microscopy.

\section{Results and Discussion}

The results and MIC distributions of the susceptibility test are shown in Table 1 and Figure 1, respectively.

Figure 1. Distribution of MIC of PTS, PTSO and PTS+PTSO for 47 Spanish field isolates of $B$. hyodysenteriae.

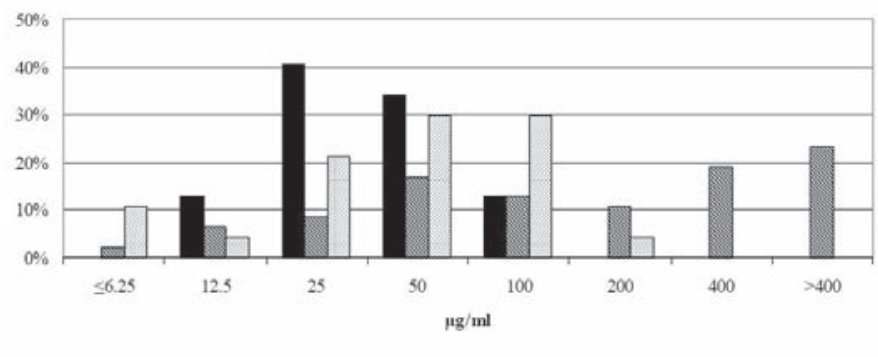

ㅁ PTS $\mathbf{0}$ PTSO $\square$ PTS + PTSO

Although susceptibility of Spanish B. hyodysenteriae field isolates to PTS and PTSO were markedly different, MIC distributions indicated that growth of this bacterium was affected by the use of Allium spp. derivatives on their own or combined. PTS presented lower $\mathrm{MIC}_{50}$ and $\mathrm{MIC}_{90}$ than PTSO, showing a higher antimicrobial activity against this spirochaete. Accordingly, PTS was able to prevent the growth of all isolates at the tested range of concentrations, while PTSO enabled the growth of more than $20 \%$ of the isolates at $400 \mu \mathrm{g} / \mathrm{ml}$. The combination of both substances retained the PTS antimicrobial effect in spite of decreasing its concentration to $17.5 \%$.

The results of the in vitro susceptibility testing suggest that clinical trials should be performed to evaluate the use of PTS and PTSO in the diet of pigs in order to treating and controlling SD.

\section{References}

1. Karlsson et al., 2002. Vet Microbiol, 84:123-133.

2. Karlsson et al., 2003. J Clin Microbiol, 41:2596-604.

3. O'Gara et al., 2000. Appl Environ Microbiol, 66:2269-2273

4. Ross et al., 2001. Appl Environ Microbiol, 67(1):475-480 


\title{
P.434
}

\section{Detection of a novel Brachyspira species associated with haemorrhagic and necrotizing colitis}

\author{
John Harding ${ }^{1}$ Manuel Chirino-Trejo ${ }^{1}$ Champika Fernando ${ }^{1}$ Mark Jacobson ${ }^{2} Z^{2}$ enon Forster ${ }^{3} J_{a n e t}$ E. Hill ${ }^{1}$ \\ 1. University of Saskatchewan, Saskatoon, SK, Canada; 2. Warman Veterinary Services, Warman, SK, Canada; \\ 3. Big Sky Farms Inc, Humboldt, SK, Canada
}

\section{Introduction}

Several Brachyspira species cause disease in grow-finish pigs, notably B. hyodysenteriae and B. pilosicoli which cause colitis and typhlitis. B. innocens, B.intermedia and B. murdochii are generally considered non-pathogenic. Described herein are case submissions from 2 unrelated farms experiencing a mucohaemorrhagic colitis associated with a novel Brachyspira species.

Farm D

Fresh and fixed tissues from 3 grow-finish pigs exhibiting bloody diarrhea were submitted from a single source nursery-finisher barn (Table 1). Histologic examination confirmed a necrotizing catarrhal colitis. Brachyspira sp. was not isolated by bacterial culture from any case. The affected pigs were PCV2-vaccinated and had been pulse medicated with $33 \mathrm{~g} / \mathrm{T}$ tylosine ( 1 week of 3 ) from 9 to 15 weeks of age. The incidence of bloody diarrhea in 11 to 24 week old pigs was infrequent. Total annualized growfinish mortality was 3.5 to $4.5 \%$.

Farm $\mathrm{H}$

Four submissions of various types were made from grow-finish pigs exhibiting bloody diarrhea in a single source finisher barn (Table 1). Histologic examination confirmed a mucohaemorrhagic and suppurative colitis. Affected pigs were 13 to 16 weeks old, and were PCV2 and erysipelas vaccinated. Diets were medicated with $44 \mathrm{~g} / \mathrm{T}$ lincomycin from 10 to 16 weeks of age, and with 110 $\mathrm{g} / \mathrm{T}$ lincomycin for 1 week when significant diarrhea was noted in a group, which included all groups during the submission period. The total mortality rate attributable to bloody diarrhea was estimated to be $1 \%$ to $2 \%$.

PCR and sequence analysis (colon)

Species-specific PCR assays targeting $B$. hyodysenteriae and pilosicoli were negative for all samples. PCR primers designed to amplify a region of the NADH oxidase 1 gene (nox1) of Brachyspira spp. ${ }^{1}$ yielded a fragment of the expected size and direct sequencing of the nox 1 amplicons yielded identical sequences from all samples. The nox 1 sequence detected had a maximum of $92 \%$ identity to any previously characterized species. Phylogenetic analysis of relevant sequences (Figure 1) shows that the case sequence is distinct from other species which exhibit very little intraspecific diversity, and that its distance from other species is typical of interspecific distances for the genus Brachyspira.
Table 1. Diagnostics of pigs with bloody diarrhea and colitis unrelated to B. hyodysenteriae and B. pilosicoli

\begin{tabular}{|c|c|c|c|}
\hline Farm Case & Sample Type ${ }^{1}$ & Colon Culture $^{2}$ & PCR Results ${ }^{3}$ \\
\hline \multicolumn{4}{|l|}{ Farm D } \\
\hline 17757 & $\mathrm{Fr}, \mathrm{Fx}$ & $E, L, C$ & Bh \& Bp: Neg \\
\hline 22958 & $\mathrm{Fr}, \mathrm{Fc}$ & $\mathrm{Neg}$ & Bh \& Bp: Neg \\
\hline 28573 & $\mathrm{Fr}, \mathrm{Fx}$ & $E$ & Bnox: Pos \\
\hline \multicolumn{4}{|l|}{ Farm H } \\
\hline 26620 & $\mathrm{Fc}$ & $\mathrm{S}, \mathrm{A}, \mathrm{E}$ & Bh \& Bp: Neg \\
\hline 27021 & $\mathrm{Fc}$ & $\mathrm{Neg}$ & Bnox: Pos \\
\hline 28403 & $\mathrm{Fx}, \mathrm{Fc}$ & $\mathrm{S}, \mathrm{E}$ & All Neg \\
\hline \multirow[t]{2}{*}{30446} & Car & B & Bh \& Bp: Neg \\
\hline & & & Bnox: Pos \\
\hline \multicolumn{4}{|c|}{$\begin{array}{l}\text { Legend: } \\
{ }^{1} \mathrm{Fr}=\text { fresh tissue, } \mathrm{Fx}=\text { formalin fixed tissue, } \mathrm{Fc}=\text { feces } C \text { ar=carcass } \\
{ }^{2} \mathrm{~L}=\text { Lactobacillus spp., } \mathrm{C}=\text { Campylobacter spp., } \mathrm{E}=E \text {. coli, } \mathrm{S}=\text { Streptococcus spp., } \mathrm{A}=\text { Acinetobacter } \\
\text { spp., } \mathrm{B}=\text { Brachyspira spp. } \\
{ }^{3} \text { Bnox=Brachyspira spp. } \mathrm{nox} 1 ; \mathrm{Bh}=B \text {. hyodysenteriae, } \mathrm{Bp}=B \text {. pilosicoli }\end{array}$} \\
\hline
\end{tabular}

Figure 1. Phylogenetic tree of $810 b p$ nox 1 gene sequence from colitis cases and published reference sequences.

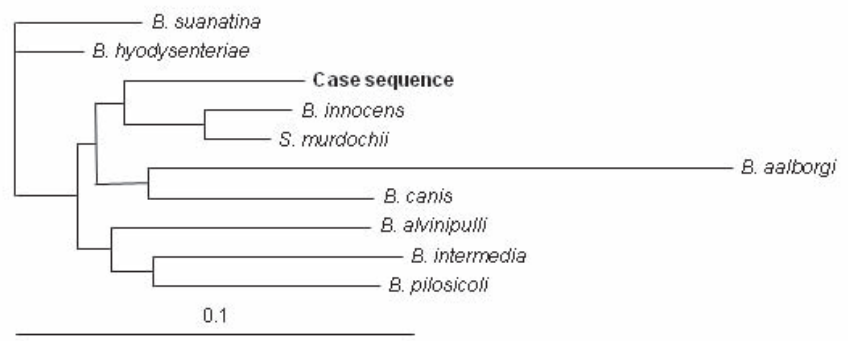

\section{Discussion}

Our results indicate a putatively novel Brachyspira spp. associated with haemorrhagic colitis and diarrhea in grow-finish pigs in the absence of other known pathogens including $B$. hyodysenteriae and $B$. pilosicoli. More studies are planned to determine its causality.

\section{References}

1. Rhode, J et al (2002) J Clin Microbiol. 40(7), 2598-2600 


\title{
P.435
}

\section{Effects of different diets on pigs with naturally-acquired Brachyspira pilosicoli infection}

\author{
Jill R. Thomson; Brian P. Murray; Lorraine E. Henderson; Joan Dick; Colin Morgan \\ Scottish Agricultural College, Edinburgh, UK
}

\section{Introduction}

The impact of diet on porcine colonic spirochaetal diseases has been demonstrated in a number of studies. Experimentally, Brachyspira pilosicoli-infected pigs developed milder disease when fed cooked rice as compared with wheat based diets $(1,2)$, attributed to reduced colonic fermentation and sparse, dry colonic content in rice-fed pigs. Commercial pig grower rations in the UK are mostly wheat based, and those with higher non-starch polysaccharide content can be associated with nonspecific colitis (3). Whether such diets promote or enhance the impact of infectious forms of colitis is poorly understood. This study compared the effects of different diets on pigs with porcine colonic spirochaetosis (PCS).

\section{Materials and Methods}

Pigs were acquired from six units with outbreaks of B.pilosicoli infection for a series of farm-specific studies. For each trial, twelve diarrhoeic pigs and four normal pigs (controls) were obtained from the farm in question and housed in individual pens for the duration of the study. All pigs were growers (weights between $20-40 \mathrm{~kg}$ at start). During acclimatisation, all pigs remained on the farm's ration and were tested for enteropathogens, faecal $\%$ dry matter (\%DM) and intestinal retention time (IRT). On Day 0, six affected and two control pigs were changed to the test ration while the other cohort of 6 affected and 2 control pigs remained on the farm's ration. The test ration was comprised of barley, soya, fishmeal with vitamin and mineral balancer formulated to the same nutritional specification, and pelleted at the same mill as the farm's ration. Faecal \%DM and brachyspira cultures were carried out 3 times weekly and IRT measurement on days 6 and 12. Mean IRT was measured by feeding known numbers of polythene beads followed by 4-hourly faecal collections. Pigs were weighed on days 0 and 14 . On day 15 all pigs were euthanased for pathological examination and bacteriology.

\section{Results}

B.pilosicoli was isolated from all diarrhoeic pigs during the course of the trials, but not from normal pigs. During acclimatisation, all affected pigs had typical clinical signs of PCS, with faecal \%DM values ranging between $16-23 \%$. In all trials, changing to the test ration gave a noted improvement in clinical appearance and an increase in faecal \%DM from day 4. Significant differences in mean IRT between the farm's ration and the test ration were recorded for all trials $(p<0.001)$ (Table 1$)$. In normal pigs, the test ration gave an average increase in IRT of 4.8 hours as compared with the farms' ration. In pigs with PCS, the difference was more marked, giving an average increase in IRT of 6.6 hours in pigs on the test ration. A significant positive correlation was recorded between mean IRT and growth rate in pigs with PCS. On postmortem examination all affected pigs were confirmed to have lesions of PCS. A histopathological scoring system for colonic lesions showed significantly higher mean scores (i.e. worse colitis) for pigs on the farms' ration as compared with test ration.
Table 1. Mean intestinal retention time in pigs with or without porcine colonic spirochaetosis (PCS) from 6 farms; comparison of farms' ration versus test ration on day 12 of trials. $n=$ number of pigs from each farm

\begin{tabular}{|c|c|c|c|c|}
\hline \multirow{2}{*}{ Farm } & \multicolumn{4}{|c|}{ Mean intestinal retention time } \\
\cline { 2 - 5 } & \multicolumn{2}{|c|}{ Farms' rations } & \multicolumn{2}{c|}{ Test rations } \\
\cline { 2 - 5 } & Normal $\mathrm{n}=2$ & $\mathrm{PCS} \mathrm{n}=6$ & Normal $\mathrm{n}=2$ & $\mathrm{PCS} \mathrm{n}=6$ \\
\hline 1 & 24.1 & 20.3 & 30.7 & 27.8 \\
\hline 2 & 25.0 & 18.9 & 32.5 & 26.2 \\
\hline 3 & 26.6 & 21.8 & 33.0 & 28.5 \\
\hline 4 & 29.4 & 22.3 & 31.9 & 29.3 \\
\hline 5 & 24.4 & 19.8 & 27.5 & 25.2 \\
\hline 6 & 27.0 & 21.5 & 29.8 & 27.2 \\
\hline Average & 26.1 & 20.8 & 30.9 & 27.4 \\
\hline
\end{tabular}

\section{Discussion}

The improvement in the clinical parameters and IRT in pigs with PCS when fed barley-based ration as compared with wheatbased rations suggested that the dietary constituents had direct effects on enteric physiological processes and disease expression. In view of the matched dietary specifications, the increased growth rates achieved by PCS-affected pigs that were fed the barley-based ration could be attributable to an increase in IRT and to a reduction in both the severity of diarrhoea and the colonic inflammatory lesions.

\section{References}

1. Hampson D.J. et al. 2000. Vet. Microbiol. 73: 75-84

2. Lindecrona R.H. et al. 2004. Vet Rec. 154:264-267

3. Chase-Topping M. et al. 2007. Vet J. 173: 353-360 


\title{
P.436
}

\section{Spirochaetes isolated from pigs in Spain}

\author{
Jesús Osorio; Ana Carvajal; Álvaro Hidalgo; Héctor Argüello; Sonia Álvarez; German Naharro; Pedro Rubio \\ University of León, León, Spain
}

\section{Introduction and Objectives}

The research group at León University (Spain) is working on different topics associated with Brachyspira species. The bacterial genus Brachyspira consists of several species of intestinal spirochetes with the capability of colonizing a broad spectrum of hosts. B. hyodysenteriae and B. pilosicoli have been commonly detected in pigs suffering gastrointestinal disorders in Spain (1). However, there is no information about other species within the Brachyspira genus recovered from swine faeces. The purpose of this study is to give an overview of the different non-pathogenic Brachyspira species that we have detected in pigs from Spain.

\section{Material and Methods}

A total of 67 Spanish field isolates of Brachyspira spp. from 54 farms were included in the analysis. All isolates were weakly $\beta$-haemolytic, recovered from pigs between 2006-2009 and came from the collection held at the University of León. None of the isolates had been designed into the $B$. hyodysenteriae/ $B$. pilosicoli species by culture and PCR detection routinely used in our laboratory $(2,3)$. A partial sequence of NADH oxidase (nox) gene was used for the identification of the isolates. Sequence data for well-known isolates and reference strains retrieved from Genbank were also included in the study. Sequence and sequencing analysis was performed according to previously described procedures (4). The genetic relationships between strains were visualised by constructing a dendrogram using the 'maximun composite likehood' model and the neighbour-joining tree in MEGA version 4 (www.megasoftware.net) (5).

\section{Results}

All the isolates used were pure cultures and PCR confirmed as different from $B$. hyodysenteriae and B. pilosicoli. Information of partial nox sequences allocated most of the isolates to the three non-pathogenic species B. murdochii (31), B. innocens (26) and $B$. intermedia (7). The three remaining isolates could not be included into any of the Brachyspira species which naturally infect pigs. Isolates $\mathrm{H} 29$ and $\mathrm{H} 51$ were recovered from Iberian pigs belonging to 2 different herds. The analized nox sequence of isolates $\mathrm{H} 29$ and $\mathrm{H} 51$ got 63 nucleotide substitutions (7.4\%) with regard to the sequence of the $B$. hyodysenteriae isolates. Nox gene information confirmed previous classifications and showed larger heterogeneity in B. intermedia isolates than in the other species. None of the isolates were included in the strongly $\beta$ haemolytic species $B$. hyodysenteriae and "B. suanatina" even though we have check that several of the weakly haemolytic isolates gave weak positive bands to $B$. hyodysenteriae PCR used for diagnostic purposes, mainly $B$. intermedia isolates. We have found $B$. murdochii and $B$. innocens much more prevalent that $B$. pilosicoli and B. intermedia in swine herds in Spain.
Fig. 1. Dendrogram based on partial nox DNA sequences from isolates belonging to all currently described Brachyspira species which colonize pigs.

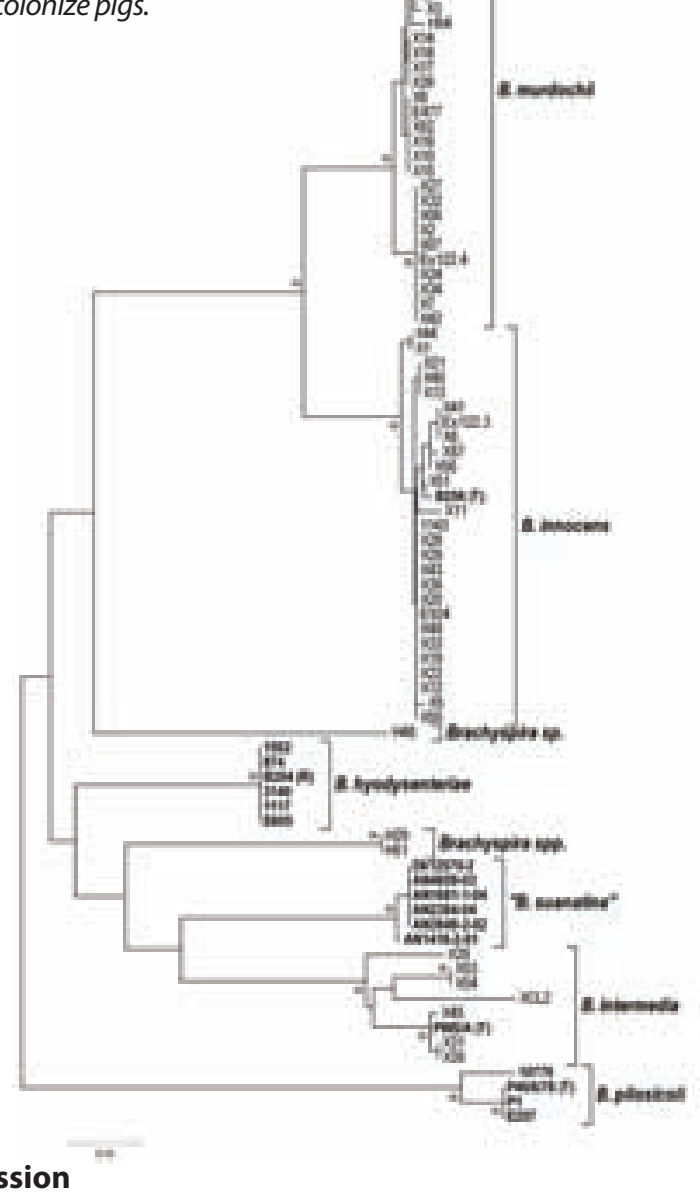

\section{Discussion}

The isolates were identified as B. murdochii (46.3\%), B. innocens (38.9\%) and B. intermedia (10.4\%). Three of the isolates (4.4\%) could not be included into any of the Brachyspira species which infect pigs. Therefore, in this study on weakly haemolytic intestinal spirochaetes in Spanish pigs, we have found new groups of intestinal spirochetes which differ from previous descriptions and could possibly be proposed as new Brachyspira species. More isolates should be idenfied in order to confirm this finding. The present study adds knowledge to the biodiversity and evolution of Brachyspira swine species in Spain.
References
1. Carvajal et al., 2006. Vet Rec, 700-701.
2. Leser et al., 1997. Mol Cell Probes, 11: 363-72.
3. Muniappa et al., 1997. J Vet Diagn Invest, 9: 165-171.
4. Rhode et al., 2002. J Clin Microbiology, 2598-2600.
5. Tamura et al., 2007. Mol Biol Evolution, 24: 1596-1599. 


\title{
P.437
}

\section{A field experience using oregano essential oil in six swine herds with hemorrhagic bowel syndrome}

\author{
$\underline{\text { Robert W. Evelsizer }}^{1}$ Darryl Sanden ${ }^{2}$ Martha A. Mellencamp ${ }^{1}$ \\ 1. Ralco Nutrition, Inc., Marshall, MN, USA; 2. PIGS Inc., Fairmont, MN, USA
}

\section{Introduction}

Hemorrhagic bowel syndrome (HBS) is a condition of finishing pigs between 2 to 6 months of age that is known to cause sporadic, sudden mortality. It has been theorized that intestinal volvulus may account for most, if not all, of what has been described as HBS (1). Some suggest that excessive gas production in the caudal intestine is the underlying cause of HBS. Post mortem observations include a pale carcass with abdominal distension and gas distended intestines that are markedly reddened and filled with blood-stained watery fluid. The jejunum is thin walled. The stomach is filled with feed. In a recent paper, the name "Porcine Intestinal Distension Syndrome" (PIDS) was proposed to more accurately reflect the nature and cause of this condition (2). Investigation of a condition known as "whey bloat", which appears to be identical to HBS or PIDS, has demonstrated an increase in abdominal pressure sufficient to cause impairment of venous return through the mesenteric veins (3). This results in massive pooling of blood in the intestines and ultimately leads to multiple organ failure and sudden death. Actual volvulus is not necessary for HBS and is absent in a majority of cases. Reduction of HBS mortality with in-feed administration of antibiotics has been reported (1). This paper describes a field investigation in which oregano essential oil (OEO) was applied either in the water or feed in six herds that were experiencing HBS. Results showed significant reduction in death loss on these farms.

\section{Materials and Methods}

An experienced grow-finish field supervisor (D.S.) who oversaw 60,000 finishing pigs per week had commonly encountered HBS on several of his farms. Factors associated with HBS on these farms related to feed intake patterns. These included genetics, weather, diet changes, out of feed events, feeder type, and water intake. He had taken several approaches to resolving this problem, including increasing feed particle size, reducing out of feed events, water chlorination, and antibiotics. After noticing that a farm feeding an OEO product had a lower rate of HBS, he began to implement OEO in either feed or water on his other farms in an attempt to reduce HBS.

\section{Results and Discussion}

A summary of recorded deaths from six farms before and after OEO use is shown in Table 1. No changes in management or how deaths were recorded were made. OEO in the water or feed was associated with an $87.5 \%$ reduction of HBS mortality. Odds ratio analysis (4) showed that OEO-treated pigs were 8.5 times more likely to survive than untreated pigs ( $5 \% \mathrm{Cl}$ : 5.45 to 11.96$)$. On one farm, OEO was fed in one barn, while the other barn served as the "control". In the OEO barn, death loss from HBS dropped to very low levels, while the control barn continued to experience the same number of HBS deaths. OEO has been shown to inhibit many species of bacteria, including Clostridium perfringens (5). It is theorized that OEO may reduce gut fermentation due to alteration of intestinal microflora, resulting in a reduction in observed HBS.

Table 1. Recorded deaths from HBS with and without oregano essential oil (OEO).

\begin{tabular}{|l|c|c|}
\hline Parameter & No OEO & With OEO \\
\hline Total number of pigs & 14,090 & 17,923 \\
\hline Deaths listed as HBS (\%) & $182(1.29)$ & $29(0.16)$ \\
\hline
\end{tabular}

\section{References}

1. Straw, B., C. Dewey, J. Kober, et al. 2002. Factors associated with death due to hemorrhagic bowel syndrome in two large commercial swine farms. J Swine Health Prod 10:75-79.

2. Buddle, J. and D. Twomey. 2002. The 'Porcine Intestinal Distension Syndrome.' The Pig J 50:68-82.

3. Thomson J, W. Miller, et al. 2007. Pressure-related abdominal changes in pigs with 'whey bloat'- a case report. The Pig J 59:152-159.

4. Ramirez, A. 2008. Use of odds ratios as a field diagnostic tool. Proc 16th Ann Swine Dis Conf for Swine Pract.

5. Si W., X. Ni, J. Gong, et al. 2008. Antimicrobial activity of essential oils and structurally related synthetic food additives towards Clostridium perfringens. J Appl Microbiol 106:213-220. 


\title{
P.438
}

\section{Investigation into the cause of hemorrhagic bowel syndrome in grower pigs on commercial farms in South Africa}

\author{
$\underline{\text { Annemarie Labuscagne }}^{1}$ B. T. Spencer ${ }^{2}$ J. A. Picard ${ }^{2}$ M. C. Williams ${ }^{2}$ \\ 1. Charles Street Veterinary Consultancy, Pretoria, South Africa; 2. Department of Production Animal Studies, \\ Faculty of Veterinary Science, University of Pretoria, Pretoria, South Africa
}

\section{Introduction}

Over the last five years generally excellent pig farmers in South Africa, with optimal management systems, have reported to consulting veterinarians that some of their grower pigs die acutely without prodromal signs, especially during summer. Most of these pigs appear to die from what can be described clinically and pathologically as hemorrhagic bowel syndrome (HBS). It tends to affect the best grown pigs close to market age, making it an expensive disease. Because of this, it was decided to investigate the syndrome in more detail.

\section{Experimental Design}

37 Necropsies were done by pig veterinarians. Only those carcasses that had marked intestinal congestion, with red contents, were selected for sampling: For histopathology a $1 \mathrm{~cm}$ thick slice of intestine was collected from the ileum $(30 \mathrm{~cm}$ proximal to the ileo-caecal valve), mid section of jejunum and from the duodenum (10 cm distal from the stomach) for histopathology. For bacteriology a $6 \mathrm{~cm}$ piece of ileum, taken just cranial to the histopathology sample, was removed. Once the intestinal contents had been removed, a mucosal scraping was made. The slide was stained using the Gram's staining method. Another mucosal scraping was made by rubbing the ileal mucosa with a cottontipped swab and cultured aerobically as well as anaerobically. Histology sections were made using standard methods and the sections were evaluated for the presence of lesions.

\section{Results}

The average age of the sampled pigs was 16 weeks and the range was from $9-22$ weeks of age. Mortalities tended to occur in the early hours of the morning. At necropsy the skin was usually pale, without signs of trauma. Bacteriology cultures only revealed $C$. perfringens in a heavy, almost pure culture, where it was cultured from 12 (40\%) of the samples. Histological lesions characteristic for enterotoxigenic Clostridium infection were found. Large, Gram-positive bacilli were sometimes abundant in sections and mucosal smears of the intestine. The lesions included necrosis, haemorrhage and congestion

\section{Discussion}

The discrepancy between the histological observation of Clostridia-like bacteria and culture of $C$. perfringens was disappointing but not unexpected. This anaerobic bacterium can be overgrown by commensal bacteria. Furthermore, prolonged sampling times after death, and suboptimal preservation methods, may have negatively impacted on the viability of the bacteria.

The most likely cause of HBS in this study is Clostridium perfringens. Modern genetics has resulted in a commercial pig with improved average daily gain (ADG), feed conversion ratio (FCR) and leaner meat but perhaps because of this they are more predisposed to metabolically-induced diseases such as HBS. Farms included in this study had growth rates above the national average. The pigs are probably pushed to their physiological limits from an early stage. These pigs are intolerant to mistakes in management or feeding2, especially when rations are high in carbohydrates (typical South African rations), which under certain circumstances can lead to the proliferation of $C$. perfringens 11 . The extremely high ambient temperatures experienced in summer on all of these farms can lead to a decreased appetite during the day, with a compensatory increase in feeding at night. This could lead to over engorgement, with the delivery of increased amounts of intestinal carbohydrate and protein. This, in turn, could lead to enteric microflora proliferation, which is supported by the fact that most of the deaths are discovered early in the morning, with mortalities probably occurring early (04h00) in the morning. The above hypothesis might explain why HBS is more prevalent in summer.

\section{Conclusions}

Clostridium perfringens most likely plays a major role in heamorrhagic bowel syndrome (HBS) in South African piggeries during the hot summer months in rapidly growing pigs.

\section{References}

1. Brumm, M.C., Richert, B.T., Marchant Forde, J.N., Marchant Forde, R. 2004. Out-of-feed events in grow-finish pigs: causes \& consequences. Proceedings of the 45th George A. Young Swine Health \& Management Conferece. p. 6-15.

2. Pejsak, Z., 2007, Krwotoczny zespół jelitowy u świń, Zycie Weterynaryjne, 398 - 340. Translated by Beata Leszczynska. 


\title{
P.439
}

\section{Case report of Gastric Ulcers in grower pigs affected with persistent Porcine Respiratory disease complex (PRDC)}

\author{
Sasiwimon Talummuk; Rapeepat Kunalintip; Metta Makhanon
}

Novartis (Thailand) Limited, Bangkok, Thailand

\section{Introduction}

Gastric ulceration is an important disease in pigs. It is a direct or indirect cause of pigs death. The gastric ulcer can affect pigs at any ages but the highest rate of ulceration occurs is in 3-6 months of age. Lesion of gastric ulcer is sited at pars oesophagia. There are a lot of risk factors including some infectious agents such as PCV2 and Helicobacter -pylori-like-bacteria and non-infectious causes such as stress, management, and nutrition(1, 3 ). However, there are also other unknown causes. Clinical signs are depended on the causes of disease and varied from no clinical sign to sudden death. Gastric ulcer is indirect cause of mortality in case of PRDC disease due to anorexia and stress from some pathogens including PCV2 (4). If the stomach is empty more than 24 hours, gastric ulcer can develop. This report is the case of severe death caused by gastric ulcer in a Thai pig farm and discuss how to handle the case successfully.

\section{Materials and Methods}

Farm history: the multiple unit fattening pig farm with conventional housing (909 grower pigs per house) and commercial pellet feed is used. Herd heath status is positive to PRRSV and PCV2. There is no Mycoplasmal vaccination. Pigs showed respiratory and diarrhoeal signs one week after weaning with almost $10 \%$ mobility. The grower pigs show more severe clinical signs including fever $\left(38^{\circ}-39^{\circ} \mathrm{C}\right)$, nasal discharge, lethargy, labored abdominal-breathing, severe coughing, pale, feed intake drop to $0.6 \mathrm{~kg}$ per days, and diarrhoea (Fig1). Highest morbidity (100\%) and mortality (12\%) is at 14 weeks old. Culling rate of the weak pigs is $27.93 \%$. Total loss is $39.93 \%$.

Necropsy finding: Post mortem two 14 wk old pigs. The lesion were pale skin, cranioventral pneumonia, total lung score approximately 50-60\%(Fig 2), diffuse blood in stomach(Fig 3), gastric ulcer score 3 (Fig 4) (2,5), flabby heart, pale kidney, no feed content in gastrointestinal organ, severe colitis and ulceration. Screening test by directed smear was positive to Balantidium coli.

Laboratory diagnosis: lung, blood content and large intestine are collected for bacterial culture. Pool organs of lung, lymph node, kidney, and spleen are collected for PRRS and PCV2 identification by PCR.

Diffentrial diagnosis: PRDC, Helicobacter-pylori-like-bacteria, Clostridium perfringens, and Walfarin poisoning.

Treatment: All pigs were individually injected with Denagard ${ }^{\circledR}$ (tiamulin) $20 \%$ injection at $10 \mathrm{mg} / \mathrm{Kg}$ body weight for 3 days and water medication with Denagard ${ }^{\circledR}$ (tiamulin) $45 \%$ at $10 \mathrm{mg} /$ $\mathrm{Kg}$ body weight for 5 days.
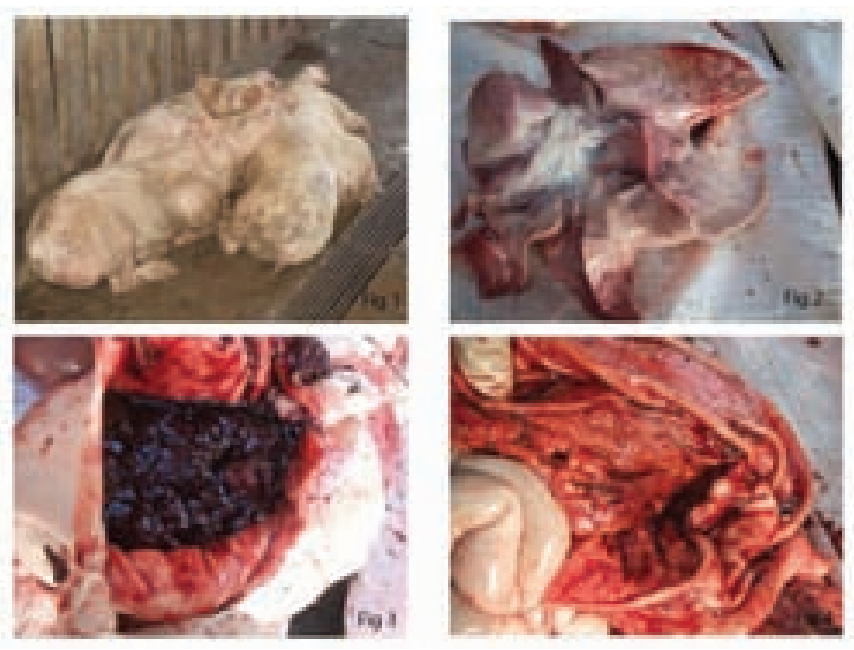

\section{Results}

The organisms isolated from lung lesions are Mycoplasma hyorhinis, Pasturella multocida and Streptococcus suis. Alpha Hemolytis streptococcus is isolated from large intestine. PRRS and PCV2 are positive by PCR. Helicobacter-like bacteria can be cultured from blood content in stomach. Clostridium perfringens is not found. There is no Walfarin used in this farm. Clinical signs improve slightly after treatment at late time of infection.

\section{Discussion}

According to this case, PRDC can induce severe of gastric ulceration and cause of high loss due to the mortality, morbidity and culling rate $(1,3)$. Route of medical administration and early treatment are the key factor for the successful treatment. Therefore, water medication and injection is more effective route when feed intake drop(4).

\section{References}

1. Correa, et al. 2008. Gastric ulcers in pigs affected with postweaning multisystemic wasting syndrome. Pesq.Vet.Bras.28(12):601-605.

2. Embaye, et al. 1990. Histopathology of oesphagogastric lesions in pigs. J.Comp.Pathol,103:253-264

3. Friendship, R.M. 2006. Gastric ulcers. In: Disease of Swine 9thEd. B.E Straw., J. Zimmerman, S. D'Allaire and D.J. Taylor, (ed). Blackwell Publishing. Ames. lowa. 891-899.

4. Harm et al. 2001.Experimental reproductive of severe disease in CD/ $C D$ pigs concurrently infected with type 2 Porcine circovirus and Porcine reproductive and respiratory syndrome virus. Vet Pathol. 38:528-39.

5. Pig Heath Monitoring Service.1989. A guide to abattoir procedures. Department of Primary Industries. Queensland. 


\title{
Clinical, pathological and laboratorial investigations of a severe outbreak of acute ulcers in pigs
}

\author{
$\underline{\text { Nuria Llanes }}{ }^{1}$ Carmen Alonso $^{1}$ Joaquim Segales ${ }^{1,2}$ \\ 1. CReSA, Bellaterra, Spain; 2. Departament de Sanitat i Anatomia Animals, Bellaterra, Spain
}

\section{Introduction}

Gastric ulcers in pigs have been extensively studied (1). Annual mortality due to gastric ulcers is estimated to be between 0.5 and $0.75 \%$. Therefore, its economical weight on the pork industry is high. The specific causes of gastric ulceration are not well known, but a number of factors have been pointed as risky (1). A severe case of acute gastric ulcers affecting several farms from an integration company located in a high density pig rearing area in North-Eastern Spain allowed the study of potential risk factors associated with such condition.

\section{Materials and Methods}

An estimated number of 5000 pigs died because of acute gastric ulcers during a 4-month period. Pigs corresponded to a 40.000sow company that produce around 900.000 finishing pigs per year. It was known that other pig producing companies in the area had also a similar problem. The majority of affected pigs weighted between 30 and $40 \mathrm{~kg}$ and no previous clinical signs were found prior the gastric ulceration outbreaks. Most affected farms were in good health conditions before the appearance of the problem.

Acute gastric ulceration with massive haemorrhage appeared in $90 \%$ of the dead animals. Mesenteric oedema, hepatomegaly and soft, orange-yellowish livers were found also at necropsy in $80 \%$ of the deaths. Mortality rate rapidly increased from $0.5-$ $5 \%$ (normal mortality values in fatteners) to $30-40 \%$ in most severely affected farms.

A number of laboratorial analyses were performed, including necropsy and histopathology (with in situ hybridization to detect Porcine circovirus type 2 (PCV2), haemogram, hepatic enzyme determination, measurement of vitamin $\mathrm{E}$ and selenium in blood and feed, mycotoxins in livers and raw feed material, heavy metals in liver and perirenal fat, and a general toxicological analysis on feed, raw feed material and livers.

In the meantime, in order to counteract the problem, the feed particle size was increased in size (from 700 to $800 \mu \mathrm{m}$ ). Concomitantly, a dramatic change in the diet formulation was performed: reduction of wheat content from 45 to $25 \%$, a source of fibre was added (soybean hulls at $2 \%$ ), sodium bicarbonate was added at a dose of $10 \mathrm{~kg} / \mathrm{ton}$ and levels of vitamin $\mathrm{E}$ and selenium were increased from 10 and 0.2 to 40 and $0.5 \mathrm{ppm}$, respectively. Feed were served in mash form in most affected farms. Moreover, vitamin E and selenium were also given in pigs as injection. Finally, ranitidine (inhibitor of gastric acid production) was added in the water of some farms.

\section{Results}

Clinical response to farm actions: Injection of vitamin $\mathrm{E}$ and selenium had a positive response in some of the farms.

Pathology: Most consistent lesion, besides gastric ulceration, was massive hepatic centrolobular necrosis and or liver plate disruption with massive loss of hepatocytes, suggesting either a chronic intoxication or hepatosis dietetica. PCV2 was not detected in tissues of affected animals.

Haemogram and biochemical determinations: Most significant results were severe anaemia and increased levels of hepatic enzymes in serum

Vitamin $\mathrm{E}$ and selenium determination: Low levels of vitamin $\mathrm{E}$ $(<0.5 \mu \mathrm{g} / \mathrm{ml})$ and selenium $(0.78 \mu \mathrm{g} / \mathrm{ml})$ were found (2).

Other analyses: No significant mycotoxins levels or heavy metals were detected in analysed samples. Toxicological analysis was indicative of peroxidation in the feed.

\section{Discussion}

Clinical response and performed analyses pointed to a deficiency in vitamin E and selenium. However, it is very likely that other factors were involved in the triggering of the problem due to the erratic appearance of the cases. Peroxidation and free radicals on the feed may have accounted as major predisposing factors for the clinical situation observed.

\section{References}

1. Friendship R. 2006. Diseases of Swine. pp891-899.

2. Schwartz KJ, 2009 Leman Pre-conference workshop. 


\title{
P.441
}

\section{Occurrence of gastric ulcers in Swedish pigs}

\author{
Katarina Karlsson; Malin Cerne \\ Swedish Animal Health Service, Falkenberg, Sweden
}

\section{Introduction}

Gastric ulceration is common in pigs throughout the world. Increased incidence of gastric ulcers has paralleled intensive husbandry practices $(2,5)$. The syndrome may have an economic impact because of increased mortality rate $(2,4)$ and decreased weight gain $(1,6)$. It is also an issue of animal welfare as gastric ulcers may cause pain.

Abattoir surveys in different countries demonstrate the prevalences of lesions of the pars oesophagea ranging from 32 - $99 \%$ (2). In several surveys possible risk factors have also been investigated. There is no published survey describing the prevalence of gastric ulcers among pigs in Sweden.

\section{Materials and Methods}

Our study was carried out during one week in September 2007, at the abattoir of Kristianstad in the south of Sweden. A total of 19360 finishing pigs and 436 sows were slaughtered during the same period. Out of these we examined a total of 3858 stomachs, randomly selected, mostly from finishing pigs. The stomachs were examined directly after being cut open and emptied.

Alterations of the pars oesophagea were graded according to Dansk Svineproduktiońs index of ulceration (3). The categories of alterations were; category 1 healthy stomachs, category 2 stomachs with parakeratosis, category 3 stomachs with both parakeratosis and small erosions, category 4 extended erosions, ulcers and scares, category 5 strictures of various severities and category 6 bleeding ulcers.

\section{Results}

Out of 3858 stomachs, 1893 (49.1\%) were normal without any alterations of the pars oesophagea. 1260 (32.6\%) were judged as category 2 and $258(6.7 \%)$ as category 3 . We found 379 (9.8 $\%)$ in category 4 and 57 (1.5\%) in category 5 . Finally 11 stomachs had bleeding ulcers $(0.3 \%)$.

Fg 1. Prevalences of gastric ulcers of dithrent de grees in 3858 plgs eramined at slaughter

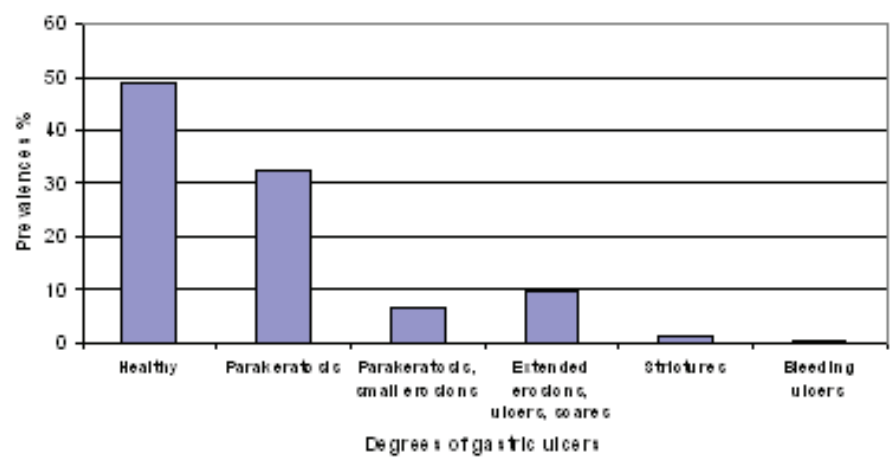

\section{Discussion}

Ulceration of the oesophageal region of the stomach is a common and well known condition of finishing pigs throughout the world. In our study we found about $49 \%$ of the examined stomachs to be normal and without any alterations of the pars oesophagea but almost $51 \%$ had some kind of alterations. Most of the alterations found were mild and are not considered to have any impact on the performance of pigs (2). Approximately $12 \%$ of the examined stomachs had serious alterations like extended ulcers, scars, strictures and in some cases bleeding ulcers. Stomachs with similar contents and similar alterations clustered, which indicates that they belonged to the same farms.

\section{References}

1. Ayles et al., Swine Health and Production, 1996; 4:211-216.

2. Friendship et al., Diseases of swine, 2006; 891-899.

3. Madsen et al., Manual - Maveforandringer hos polte og søer. www. dansksvineproduktion.dk

4. Melnichouk., Can Vet J, 2002; 43:223-225

5. O’Brien, Diseases of swine, 1992; 680-691.

6. Smith et al., IPVS, 1996; 693 


\title{
P.442
}

\section{Gastric ulceration in $\mathbf{3 0}$ fattening herds in Sweden}

\author{
Malin Cerne; Katarina Karlsson \\ Swedish Animal Health Service, Falkenberg, Sweden
}

\section{Introduction}

Ulceration of the oesophageal region of the stomach is a common condition of finishing pigs throughout the world. Increased incidence of gastric ulcers has paralleled intensive husbandry practices $(2,5)$.

Many abattoir surveys from different countries have shown various prevalences of gastric ulcers. In several surveys possible risk factors have also been investigated.

Well known risk factors for gastric ulcers in pigs include reducing particle size of the feed and pelleting, as does withholding feed for 24 hours $(1,6)$. Other factors that have been associated with the development of gastric ulcers in pigs are infection with helicobacter, infection with PCV2 (7) and treatment with NSAID. It also appears that access to straw may compensate for several of the risk factors.

In a pre-studie of the occurrence of gastric ulcers in Swedish Pigs, we found that stomachs with similar contents and similar alterations clustered, which indicates that they belonged to the same farms (3).

\section{Materials and Methods}

Our study was carried out during one week in September 2007, at the abattoir of Kristianstad. We examined 460 stomachs from 30 randomly selected farms. The stomachs were examined directly after being cut open and emptied.

The alterations of the pars oesophagea were graded according to Dansk Svineprouktions index of ulceration (4). We recorded six different categories of alterations. Categories 1, 2 and 3 were healthy stomachs, stomachs with parakeratosis and stomachs with mild erosions in pars oesophagea respectively. These alterations are not considered to affect performance or wellbeing in pigs (2). Categories 4, 5 and 6 were stomachs with severe erosions, ulcers, scars and strictures. These alterations are considered to affect the pigs in a negative way.

\section{Results}

405 stomachs were judged as category 1, 2 or 3.

55 stomachs were judged as category 4, 5 or 6 .

$70 \%$ of the stomachs judged as category 4, 5 or 6 originated from 4 of the 30 examined farms (no 6, 10, 28 and 30), fig 1.
Fig 1. Distribution of stomachs with severe alterations among examined herds

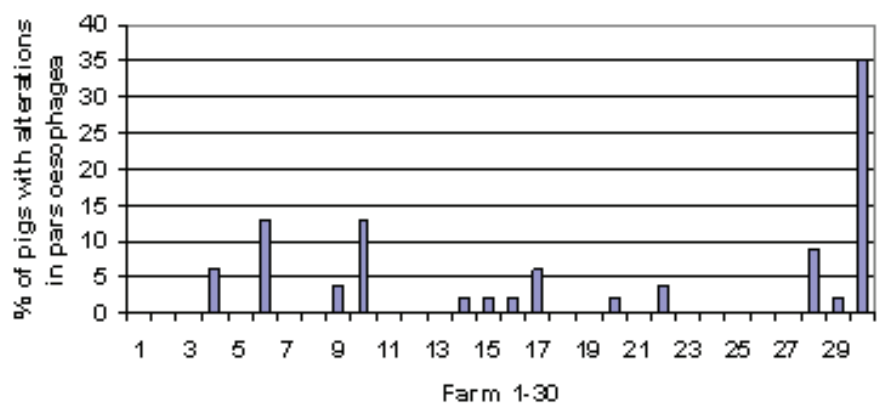

\section{Discussion}

As several of the risk factors have something to do with feed, feeding or housing it is not surprising that pigs from the same farm have the same alterations in pars oesophagea.

In an Australian survey from 2002 one found differences in prevalences between farms, but also between regions/states (6). The regions had different kinds of climate and different conditions for crops. There is probably a difference between regions in Sweden. The abattoir in Kristianstad serves the southern part of Sweden. In this area it is common to feed pigs with surplus products from the food industry, such as products from bakeries, dairies, distilleries, crisps- and chocolate producers. It has not been investigated what impact these products have on the prevalences of gastric ulcers.

Gastric ulceration has been reported frequently in PMWS-affected pigs, although no direct connection between PCV2 infection and this lesion has been established. At the time of our survey PMWS was widespread in the southern part of Sweden.

\section{References}

1. Bäckström et al., Am J Vet Res. 1981; 42:538-543.

2. Friendship, Diseases of swine, 2006. p. 891-899.

3. Karlsson et al., IPVS, Vancouver, 2010

4. Madsen et al., Manual - Maveforandringer hos polte og søer. www.dansksvineproduktion.dk

5. O'Brien et al., Diseases of swine, 1992. p. 680-691.

6. Robertson et al., Pre Vet Med. 2002; 53:292-303.

7. Segalés et al., Meeting of Amer Assoc of Swine Vet; 2006, www.pcvd.org 


\section{P.443}

\section{Esophagogastric ulcer: differences in hematological values comparing depending on non-glandular mucosa lesional stage}

Guillermo Ramis ${ }^{1}$ Serafín Gómez ${ }^{2}$ Juan J. Quereda ${ }^{1}$ José J. Cerón ${ }^{3}$ Francisco J. Pallarés²

François Joise ${ }^{4}$ Catherine Charreyre ${ }^{4}$ Sophie Longo ${ }^{4,5}$ Antonio Muñoz ${ }^{1}$

1. Universidad de Murcia, Murcia, Spain; 2. DPTO Anatomía y Anatomía Patológica Comparadas, Universidad De Murcia, Murcia, Spain; 3. Hospital Clinico Veterinario, Universidad de Murcia, Murcia, Spain; 4. Merial S.A.S., Lyon, France; 5. BPC, Labruguière, France

\section{Introduction}

Esophagogastric ulcer is a widespread disease affecting pigs of different ages, especially to finishers. The ethiology of the disease is multifactorial, influencing nutritional, genetic, pathologic and management factors on its development and severity. The pathogeny has not been completely understood yet (1). The aim of this study was to assess the hematological and blood biochemical values in animals showing different esophagogastric lesional stages.

\section{Material and Methods}

Eighty commercial pigs 1, 3, 6, 9, 12, 15, 18 and 21-week old ( $n=5$ each group), were randomly selected. The animals were humanely euthanized and a macroscopical evaluation of non glandularis mucosa lesional stage was carefully done. Blood samples were taken on EDTA and dry tubes to recover whole blood and serum from each pig.

A complete hematological study was performed including white blood cells count (WBC), red blood cells (RBC), hemoglobin $(\mathrm{HG})$, hematocrite (HT), mean corpuscular volume (MCV), mean corpuscular hemoglobin concentration (MCHC), platelets count (PTL), red blood cells volume distribution (RDW-SD), mean platelets volume (MPV), and total proteins (TP), using an Automatic analyzer SYSMEX F-800 (Japan)

The macroscopical lesional stage results were included in two categories for statistical analysis: apparently normal (AN), and abnormal (ABN). The abnormal category included all the stomachs showing some kind of alteration, including pre-ulcerous changes as parakeratosis and erosions.

Data were analyzed by means of Student t-test comparation using SPSS v. 15.

\section{Results}

Twenty stomachs were macoscopically classified as normal, while 60 stomachs showed some kind of non glandularis mucosa alteration. There were significant differences between groups for RBC, MCHC, RDW-SD and MPV. The values for every parameter are shown in the following table:
Table 1.

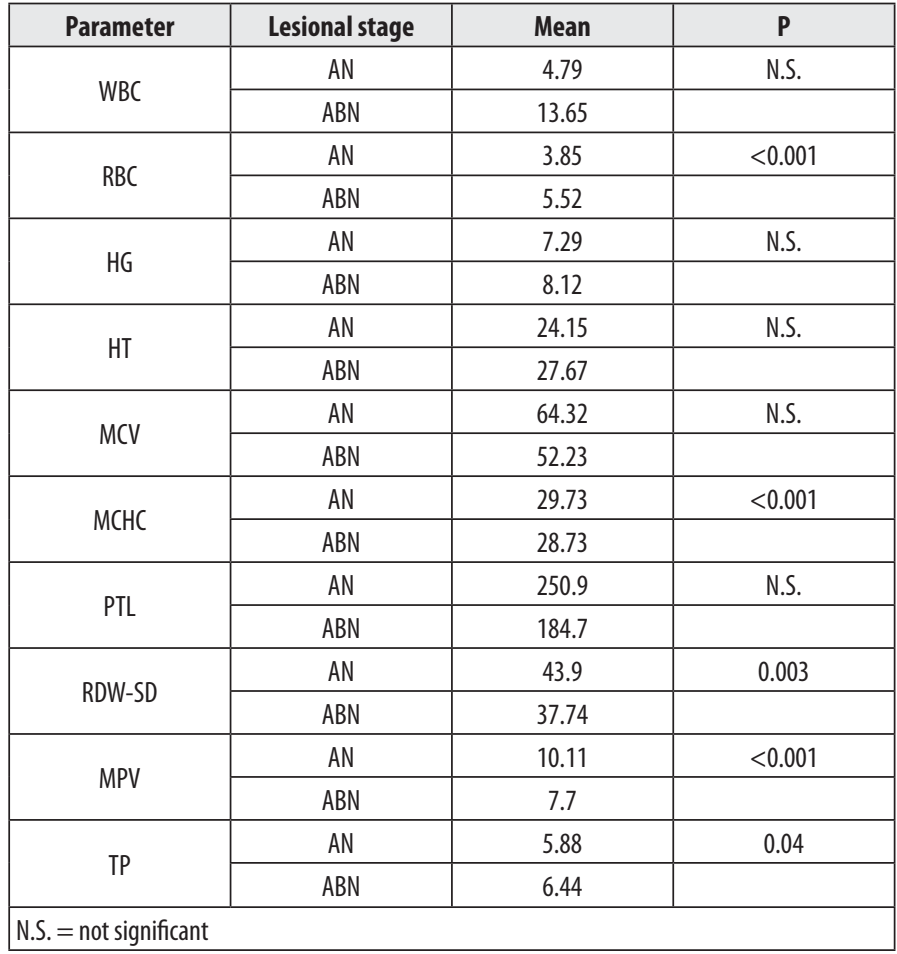

\section{Discussion}

Some changes in hematological values such us decreased RBC or MCV had been previously described (2). We have found significant differences in some hematological values comparing groups, but most of them (RBC, MCV, MCH and RDW-SD) are into the different reference intervals published $(3,4)$. The MPV is the only value under references values in abnormal stomachs, and could be related to increased coagulation derived from slow continuous blood loss, suggested also by reduced (even not significant) MCHC. The reduced MPV indicates a quick replacement of platelets.

\section{References}

1. Ramis G. 2002. PhD Thesis. Universidad de Murcia, Spain.

2. Curtin T. M et al. 1963. J. Am. Vet. Med. Assoc. 143: 854-860

3. Swenson and Reece, 1993. Duke's physiology of animal domestics. 11 th ed. Cornell University Press.

4. Thorn CE. 2000. Normal hemathology of the pig. Shalm's veterinary hematology. 


\title{
P.444
}

\section{Esophagogastric ulcer: differences in blood biochemical values comparing depending on non-glandular mucosa lesional stage}

\author{
Guillermo Ramis ${ }^{1}$ Serafín Gómez² Juan J. Quereda ${ }^{1}$ José J. Cerón ${ }^{3}$ Francisco J. Pallarés² \\ François Joisel ${ }^{4}$ Catherine Charreyre ${ }^{4}$ Sophie Longo ${ }^{4}$ Antonio Muñoz ${ }^{1}$ \\ 1. Universidad de Murcia, Murcia, Spain; 2. DPTO Anatomía y Anatomía Patológica Comparadas. Universidad De Murcia, Murcia, Spain; \\ 3. Hospital Clinico Veterinario, Universidad de Murcia, Murcia, Spain; 4. Merial S.A.S., Lyon, France
}

\section{Introduction}

Esophagogastric ulcer is a widespread disease affecting pigs of different ages, especially to finishers. The ethiology of the disease is multifactorial, influencing nutritional, genetic, pathologic and management factors on its development and severity. The pathogeny has not been completely understood yet (1). The aim of this study was to assess the hematological and blood biochemical values in animals showing different esophagogastric lesional stages.

\section{Material and Methods}

Eighty commercial pigs 1, 3, 6, 9, 12, 15, 18 and 21-week old ( $n=5$ each group), were randomly selected. The animals were humanely euthanized and a macroscopical evaluation of non glandularis mucosa lesional stage was carefully done. Blood samples were taken on EDTA and dry tubes to recover whole blood and serum from each pig.

A complete blood biochemical study was performed including glucose, urea, cholesterol, triglycerides, creatinin, Gammaglutamil transferase (GGT), phosphorus, alanine aminotransferase (ALT), Alkaline Phosphatase (ALP), Aspartate amiontranferase (AST), creatine kinase (CK), albumin, bilirrubin, and iron by means of a COBAS MIRA PLUS analyzer (ABX Diagnostics, France)

The macroscopical lesional stage results were included in two categories for statistical analysis: apparently normal (AN), and abnormal (ABN). The abnormal category included all the stomachs showing some kind of alteration, including pre-ulcerous changes as parakeratosis and erosions.

Data were analyzed by means of Student t-test comparation using SPSS v. 15.

\section{Results}

Twenty stomachs were macoscopically classified as normal, while 60 stomachs showed some kind of nonglandularis mucosa alteration. There were significant differences between groups for cholesterol, creatinine, GGT, ALP, ALT, albumin, bilirrubin and iron. The values for those significant different parameter are shown in the following table:
Table 1.

\begin{tabular}{|c|c|c|c|}
\hline Parameter & Lesional stage & Mean & $\mathbf{P}$ \\
\hline \multirow{2}{*}{ Cholesterol } & AN & 158.6 & $<0.001$ \\
\hline & ABN & 104.8 & \\
\hline \multirow{2}{*}{ Cratinine } & AN & 1.34 & 0.016 \\
\hline & ABN & 1.62 & \\
\hline \multirow{2}{*}{ GGT } & AN & 42.65 & 0,001 \\
\hline & ABN & 88.39 & \\
\hline \multirow{2}{*}{ ALP } & AN & 811.18 & $<0.001$ \\
\hline & ABN & 318.96 & \\
\hline \multirow{2}{*}{ ALT } & AN & 62.21 & $<0.001$ \\
\hline & ABN & 39.57 & \\
\hline \multirow{2}{*}{ Albumin } & AN & 2.88 & $<0.001$ \\
\hline & $A B N$ & 3.56 & \\
\hline \multirow{2}{*}{ Bilirrubin } & AN & 1.09 & $<0.001$ \\
\hline & ABN & 0.58 & \\
\hline \multirow{2}{*}{ Iron } & AN & 354.64 & $<0.001$ \\
\hline & ABN & 69.12 & \\
\hline
\end{tabular}

\section{Discussion}

Even when significant differences in some hematological values comparing groups have been found, all of them are into reference values $(2,3)$ except for ALP and iron. It should take into account that some biochemical values can vary over the life of the animals and we have analyzed animals from 1st to 21 st weeks of life. The iron value is much lower in abnormal group compared with normal group, and this parameter could be related to chronic loss of blood. Another hypothesis would be a reduced iron content derived from feed deficiencies for this metal. But, interestingly, has been previously recorded how the deficiency in nutritional iron results in a significant acid gastric secretion, reducing the lesional stage of the animals (4). So, in this case the lower iron value in lesionated animals should be related to iron losses. The iron seric value could be a help to diagnosis in cases of esophagogastric alteration.

\section{References}

1. Ramis G. 2002. PhD Thesis. Universidad de Murcia, Spain.

2. Swenson and Reece, 1993. Duke's physiology of animal domestics. 11 th ed. Cornell University Press.

3. Thorn CE. 2000. Normal hemathology of the pig. Shalm's veterinary hemathology.

4. Hannan, J. 1971. Vet. Rec. 88: 181-190 


\title{
P.445
}

\author{
Neonatal Diarrhea in Danish pig farms - a questionnaire among veterinarians \\ $\underline{\text { Peter Astrup }}^{1}$ Kirsten V. Larsen ${ }^{1}$ Sven E. Jorsal ${ }^{2}$ Lars E. Larsen ${ }^{2}$ \\ 1. Intervet/Schering-Plough Animal Health, Skovlunde, Denmark; 2. DTU Veterinarinstitutet, Copenhagen, Denmark
}

\section{Introduction}

During the recent years neonatal diarrhea of unknown etiology seems to have occurred in many sow herds. The disease can usually not be effectively controlled by known vaccination or treatment strategies (1). The aim of this investigation was to systematize the perception of the character of the disease among Danish veterinarians.

\section{Material and Method}

A questionnaire, considering different management factors, clinical symptoms and laboratory as well as autopsy findings was developed. Two technicians were trained in interviewing veterinarians with the questionnaire as basis. Interviews were made from March through August 2009. Sixty-four veterinarians specialized in pig production were interviewed about their perception, without further documentation, of neonatal diarrhea in sow herds.

\section{Results}

Sixty-one of 64 veterinarians knew of this disease in one or more of their herds. The veterinarians interviewed were estimated to represent more than $50 \%$ of the Danish pig production.

Based on the questions the following answers were given (brackets indicate the percentage of veterinarians approving the statement in question).

- Neonatal diarrhea has increased during the recent years (95\%)

- In affected herds the prevalence of litters with clinical signs is between 30 and 70\% (66\%).

- The piglet incidence in the litter is 70-100 \% (61\%).

- There is no relation between litter size and clinical signs (79\%).

- Mortality in the farrowing unit is increased with 0-5\%-point (56\%), but can increase to more than 20\%-point above normal.

- Diarrhea is yellow (100 \%) and watery (99\%) in some cases foamy and pasty (15\%) and more seldom green colored (10\%).

- Onset of clinical signs is from immidiately after birth with maximum frequency 2-3 days after birth, where after frequency decrease and diarrhea disappears 7-10 days after birth (table 1).

- Autopsy findings are non-specific: Flaccid dilated intestines (93\%) and hyperemic intestines with watery content (48\%).

- At laboratory examinations Clostridium perfringens type $A$ (85\%), Non-hemolytic E. coli (82\%), Clostridium difficile (16\%) and a few hemolytic E. coli or rotavirus have been found. Clostridium perfringens type $\mathrm{C}$ has not been found.

- There is no obvious relation between use of sow vaccinations and clinical signs.

- No obvious relation between management and clinical signs (54\%).

- Health status (95\%), hygiene in farrowing unit (65\%), hygiene in dry sow facilities (85\%) and purchase of breeding stock (85\%) has no impact on clinical signs

- Low age (gilts) increase the risk of clinical signs (73\%).

- Poor feeding with colostrum is a possible or certain cause of clinical signs (74\%).

- Quality or source of feed can not be related to increased incidence $(87 \%)$. Wet feed (31\%), high protein in sow feed $(34 \%)$ or toxins in feed (11\%) might pose a risk. A few vets point at rapeseed as a specific risk factor.

Table 1. Number of vets out of 61 that see diarrhea on a specific day after birth.

\begin{tabular}{|c|c|c|c|c|c|c|c|c|}
\hline Day & $\mathbf{0}$ & $\mathbf{1}$ & $\mathbf{2}$ & $\mathbf{3}$ & $\mathbf{4}$ & $\mathbf{5}$ & $\mathbf{6}$ & $\mathbf{2 7}$ \\
\hline No & 30 & 47 & 53 & 51 & 45 & 33 & 23 & 12 \\
\hline
\end{tabular}

\section{Discussion and Conclusion}

Based on the questionnaire the disease can best be described as a new type of diarrhea with increased incidence in Danish pig production. It is a watery to creamy diarrhea with non-characteristic pathological findings and without detection of a certain etiological agent. Nevertheless, Non-hemolytic E. coli and Clostridium perfringens type $A$ are isolated with high frequency.

The morbidity is very high and the mortality low. Especially litters after gilts and young sows are at risk of being affected.

These findings are in accordance with reports from other parts of the world with intensive pig production and underline the need for further investigations to clarify the nature of the disease.

\section{Reference}

1. Svensmark B. Proceedings of the 1st ESPHM, Copenhagen, Denmark, 2009. 


\title{
P.446
}

\section{Farrowing progress (chronopart) and enzootic neonatal diarrhoea: observational study in nine commercial herds}

\author{
Jean-Noel Sialelli² Fabien Vautrin ${ }^{2}$ Yannick Lautrou ${ }^{3}$ Isabelle Oswald ${ }^{4}$ Nathalie Quiniou ${ }^{5}$ Guy-Pierre Martineau $^{1}$ \\ 1. National Veterinary School, Toulouse, France; 2. Farm'apro, Lamballe, France; 3. ESA Angers, Angers, France; \\ 4. INRA Toulouse, Toulouse, France; 5. IFIP, LeRheu, France
}

\section{Introduction}

In some area of France, almost $20 \%$ of the farms are concerned with enzootic neonatal diarrhoea (END) (Sialelli et al., 2009). Onfarm investigations conclude that many different bacteria such as Clostridium perfringens type A, Clostridium difficile, Enterococcus durans and others may be isolated but not consistently (Gin, 2008). Our hypothesis is that END origin at the sow level. The aim of this study was to describe the parturition in herds with END in comparison of herds without END.

\section{Material and Methods}

Selection of herds: among 100 herds from the same Coop (COOPERL-Arc Atlantique, Brittany, France), nine commercial herds were retained according to presence or absence of END but also on the level of productivity, hygiene, management and stockmanship: 4 herds were regularly affected by END and 5 herds without any history of diarrhea. The farrowing has been followed in 26 sows (Table 1 ).

Measures: chronoparts (individual time at birth of littermates), piglets' weight at birth and at $24 \mathrm{~h}$ of age (in order to estimate colostrum production) and colostral data (lgG) were obtained.

Table 1: Herds and sows selected according to presence or absence of END (Enzootic Neonatal Diarrhea)

\begin{tabular}{|l|c|c|c|}
\hline Herds & $\begin{array}{c}\text { Control } \\
\mathrm{N}=5\end{array}$ & \multicolumn{2}{|c|}{$\begin{array}{c}\text { Affected by END } \\
\mathrm{N}=4\end{array}$} \\
\hline END status of sows & $\mathrm{No}$ & $\mathrm{No}$ & Yes \\
\# sows & 33 & 36 & 17 \\
Mean piglet's interval & 11.6 & 14.8 & 18.1 \\
\% bom $>3$ hrs & 7.1 & 19.7 & 26 \\
Total born & 14.9 & 14.9 & 14.0 \\
Born alive & 13.9 & 13.7 & 13.3 \\
Farrowing (min) & 158 & 197 & 213 \\
\hline
\end{tabular}

\section{Results and Discussion}

Only data on farrowing duration is reported. Data on IgG in colostrum and piglets' sera has been previously reported (Sialelli et al., 2009).

Parities 1 and 2 sows presented more risk to develop piglets'END $(O R=3.6, p<0.05)$. Duration of farrowing was longer in affected sows in affected herds than in unaffected ones. In affected herds, the duration of farrowing of unaffected sows was intermediate is between unaffected sows and sows from control herds (Table 1).

Pattern of farrowing is different according to the size of the litter (Figure 1).
Figure 1: Chronopart from sows in affected herds and control herds. In affected herds, sows are divided in unaffected sows and affected sows

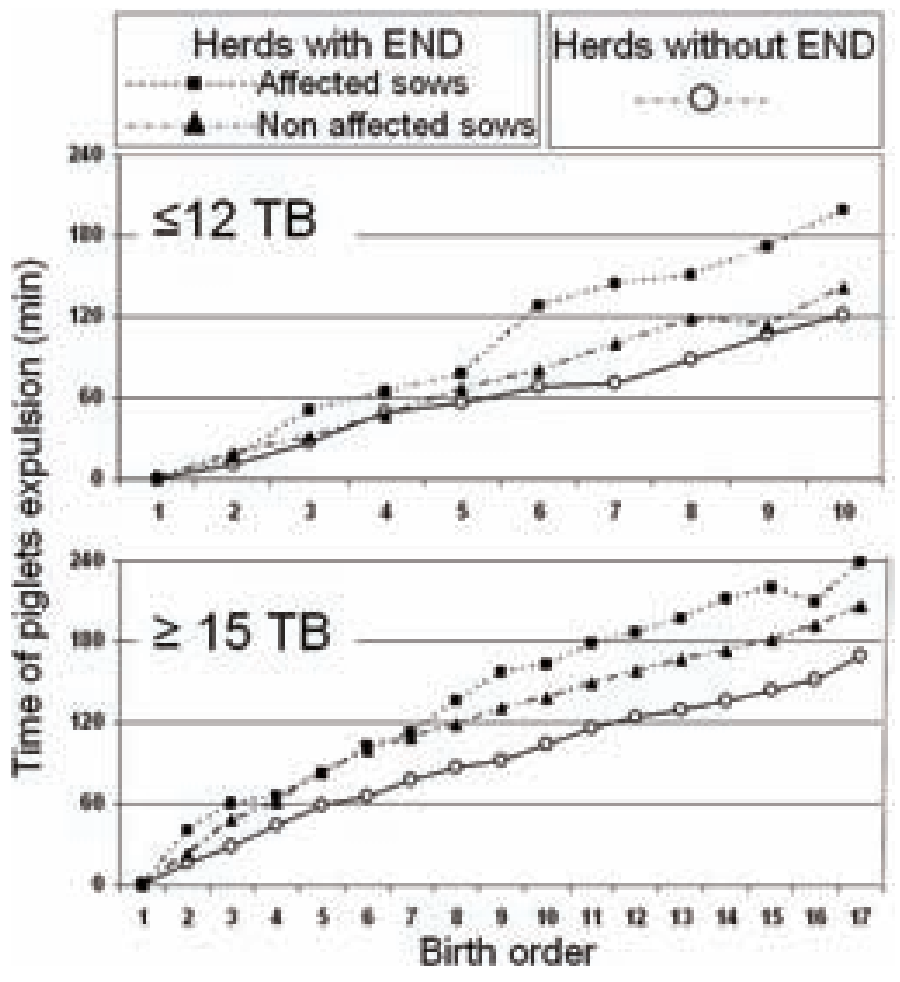

In litter $\leq 12$ total born piglets, birth rhythm is similar for the beginning of farrowing whatever the status of the sows (END pos or neg) or herds (control vs affected). However, there is a difference after birth of the 5th piglet (Figure 1). In large litter ( $\geq 15$ TB), birth rhythm is always upper in sows from control negative herds. In affected herds, a significant difference appears after the birth of the 8th piglet.

\section{Conclusion}

END is probably a consequence of a problem at the sow level. Investigations are under evaluation to approach farrowing in herds affected by long farrowing (Sialelli et al., 2010).

\section{References}

Gin T. Veterinary Thesis. Toulouse Veterinary School. 2008, pp72

Sialelli J-N et al., Journées Rech Porc. France. 2009 ; 41 : 167-172

Sialelli J-N et al., Proceedings IPVS 2010 


\title{
P.447
}

\section{Symbiotic action on piglet digestive tract-Ajigsaw for practitionners}

\author{
Frederic Pelenc $^{1}$ Guy Pierre Martineau ${ }^{2}$ \\ 1. Original Process, Lille, France; 2. Ecole Nationale Veterinaire, Toulouse, France
}

\section{Introduction}

At weaning, the digestive flora of piglets is unstable and is considered as a key factor of enteric disease. Alternative solutions to antibiotics or other products such as $\mathrm{ZnO}$ are proposed and give effective and repetitive results.

Symbiotic (prebiotic and probiotic) are shown to be one of these solutions. To have the widest comprehensive of this approach, the practitioner has to mix physiology, bacteriology, pathology, immunology, etc. It is why we propose to summarize the control of enteric flora at weaning by a jigsaw with 7 different pieces.

Control of enteric flora at weaning: the jigsaw

The seven pieces are illustrated in Figure 1.

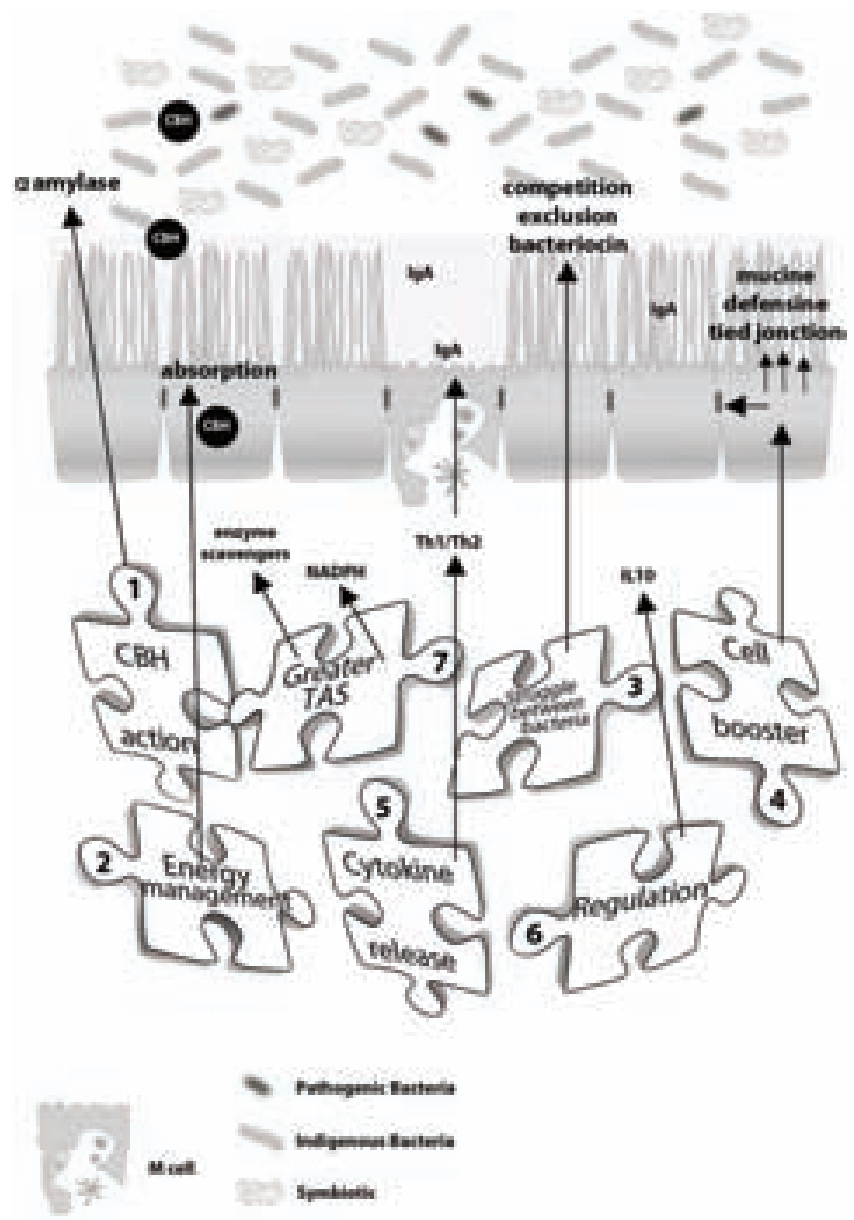

exukaticantion

Piece 1: CBH action.

Due to the manufacturing technique, some specific Lactic Acid Bacteria1 are adapted to starch medium and bring a lot of a-amylases. Lactic acid produced improves pancreas secretion and impact on assimilation of carbohydrate chain $(\mathrm{CBH})$ metabolism.
Piece 2: Enterocyte energy management.

Better balance of digestive flora which decrease basal energy metabolism needed and impact (improve) on FCR.

Piece 3: Struggle between bacteria.

The competition / exclusion effect and environmental changes impact on natural defense at the mucus level.

Piece 4: Cell booster.

Stimulation of cell mucine and defensine production and reinforcement of tied-junctions impact on natural defense at the cell level.

Piece 5: Cytokine release.

Interaction with intestinal immune system modulating cytokine pro-inflammatory synthesis, increasing APC (Antigen Presenting Cell) activity, stimulating cytokine IL-12 synthesis (linked to Th1 and Th2 balance), improving IgA production and finally impact on gut immune system at the lamina propria level.

Piece 6: Regulation.

Regulation of oral tolerance, key of intestinal homeostasis, with improvement of cytokine IL10 secretion impact on gut immune system at the lamina propria level.

Piece 7: Greater TAS (Total Antioxidant Status)

Improving glucose assimilation, it improves NADPH regeneration with anti-oxidant function, added to the anti-oxidant enzyme and scavengers brought by the Symbiotic impact on TAS (decrease deleterious effect of oxidative stress as catabolism).

\section{Discussion}

According to recent results, we showed that it is worth to use symbiotic to get repetitive effectiveness than only prebiotic or probiotic. Although the interaction between Symbiotic and digestive tract is complex, we are able to analyze via the proposed jigsaw.

Using symbiotic 1 as feed additive in trials, zootechnical effects has been statistically observed (FCR: Pelenc 2009; ADG: Criqui 2003), as well as immune response (Borghetti 2009). During studies on other species we systematically showed a better total anti-oxidant status (Laude 2008).

\section{Conclusion}

We can claim that the Symbiotic is acting on different goals on the digestive tract.

\section{References}

1. The Symbiotic used in our trial is stemmed from a natural technological industrial process which causes a fermentation of germinated barley with 8 strengths of Lactic Acid Bacteria (LAB): Micronil ${ }^{\circledR}$ 


\title{
P.448
}

\section{Evaluation of a microwave procedure for fecal dry matter determination}

\author{
Ken S. Pedersen; Klaus Pedersen; Helle Stege; Jens P. Nielsen \\ University of Copenhagen, Frederiksberg, Denmark
}

\section{Introduction}

Previously studies have reported variable intra- and interobserver agreement for assessment of fecal consistency $(1,2)$. Diarrhea is characterized by defecation of feces which contain decreased dry matter content. Therefore assessment of fecal dry matter is an objective measure of diarrhea. A microwave procedure has recently been described (3). The objective of the current study was to evaluate repeatability, robustness and agreement with freeze-drying for microwave determination of dry matter content in feces from weaners and growers.

\section{Materials and Methods}

A normal household microwave oven with a maximum effect of 700 Watt was used in the study. Fecal samples were dried to constant weight using the procedure previously described (3). Fecal dry matter content was calculated as: Dry matter = (weight of container and dried feces - weight of container) / weight of wet feces.

Three different studies were conducted. Study 1: Intra- and inter-serial variation were evaluated in a fractional three-way factorial design. The 3 factors were main fecal sample, operator and replica. Fecal subsamples (observational unit) were obtained from 4 main fecal samples with different consistencies. The analyses were repeated by 2 persons (operators). From each main fecal sample 8 fecal subsamples were heated together (replica). Coefficient of variation (CV) for fecal dry matter content was calculated. Data were analyzed by a linear mixed model with main fecal samples and weight of wet feces included as fixed effects. Operator and replicas were included as random effects. Study 2: Effect of weight of wet feces was evaluated. Two fecal samples representing 2 different fecal consistency scores were prepared. A total of 80 subsamples representing 5 different weights categories of wet feces were prepared. The categories were 0.5 gram, 1.0 gram, 1.5 gram, 2 gram and 3 gram. From each consistency score 8 subsamples from each weight category were heated together. All samples were evaluated by the same person. Data were analyzed by linear regression.

Study 3: Agreement between microwaves and freeze-drying was evaluated. Eighty fecal samples representing different fecal consistencies were collected in a herd. Two subsamples from each fecal sample were prepared and fecal dry matter was determined by microwaves and freeze-drying. A Band-Altman plot was constructed and Lin's concordance correlation coefficient for agreement was calculated. Trends of agreement in relation to increasing dry matter content were further evaluated by linear regression. All statistical analyses were performed in STATA/IC 11.0.

\section{Results}

For the intra- and inter-serial variation an overall CV of 0.03 was determined. Replica and operator accounted for $12 \%$ of the variation while $88 \%$ of the variation was with-in replicas. Confidence limits (95\%) for any future fecal subsample examined by any operator in any replica was calculated to be $+/-0.85 \%$ dry matter.

Weighing off 05 gram of wet feces gave significant $(p<0.001)$ different dry matter content than the other weight categories of wet feces. The other weight categories gave the same dry matter content.

Lin's concordance correlation coefficient for agreement between microwaves and freeze-drying was 0.94. On average the dry matter values was $1.7 \%$ higher in freeze dried samples compared to samples prepared by the microwave procedure. The difference was increasing by $0.18 \%$ dry matter $(p<0.001)$ for each $1 \%$ increase in fecal dry matter of the examined sample.

\section{Discussion}

The observed repeatability for the microwave procedure was considered acceptable for determination of fecal dry matter. The variation distribution implies that homogenization of the original fecal samples before analysis is important and that the variation due to the microwave procedure it self and different operators can be neglected. The microwave method gave consistent results using 1 to 3 gram of wet feces for the determination. This implies that a minimum of 1 gram of wet feces should be used. The microwave procedure showed a high level of agreement with freeze-drying, although slightly higher dry matter values were obtained using freeze-drying. Neither method can be regarded as gold standard, but the current study show that similar results can be obtained with both methods. The increasing difference between the two methods with increasing dry matter content indicate that the results of the current study may only be valid for feces collected from weaners and growers. Feces from older pigs may contain a higher level of fecal dry matter which may influence the results.

In conclusion: The microwave procedure offers a fast, repeatable, robust and accurate method for determination of fecal dry matter.

\section{References}

1. Pedersen, K.S. et al., 2009. Proc 1st ESPHM: page 64.

2. Pedersen, K.S. et al., 2009. Proc 1st ESPHM: page 65.

3. Pedersen, K.S. et al., 2009. Proc 1st ESPHM: page 66. 


\title{
P.449
}

\section{Diurnal variation of fecal dry matter in pigs}

\author{
Ken S. Pedersen; Klaus Pedersen; Helle Stege; Jens P. Nielsen \\ University of Copenhagen, Frederiksberg, Denmark
}

\section{Introduction}

Classification of pigs as having diarrhea or not is important for results and interpretation of research in enteric diseases. Recently an objective procedure for determination of fecal dry matter (DM\%) based on microwave drying has been described(1). Diurnal fecal dry matter variation may influenza the outcome of clinical examinations and the results of research.

The objective of the current field study was to investigate the variation in fecal dry matter and diarrhea classification with-in one day for pigs with and without diarrhea. The quantitative excretion of Lawsonia intracellularis from the same pigs was also determined and will be reported elsewhere in these proceedings.

\section{Materials and Methods}

A cross-sectional study with follow-up was designed. A Danish farrow- to finish herd with a history of L. intracellularis was selected at convenience. Pigs were selected from a batch of 350 pigs with an approximately age of 8 weeks. Within the batch, 20 pigs with diarrhea and 10 pigs without diarrhea were selected at convenience. Fecal samples were collected five times (9am, 11am, 1 pm, 3pm and 5pm) from the rectum of each pig. Defecation was triggered by rectal digital manipulation if necessary. The fecal samples were at all times kept chilled. Fecal dry matter content was determined for all fecal samples 24 hours after collection by the microwave method as described previously(1). The examined pigs were re-classified into non-diarrheic (DM\% $\geq 18.8)$, semi-diarrheic (18.8>DM\% $\geq 13.1)$ or diarrheic $(\mathrm{DM} \%<13.1)$ based on $\mathrm{DM} \%(2)$ of the fecal sample from the first time point.

The data was analyzed in a linear regression model (LogDM\% as outcome, time points as independent variables) with a repeat measurement structure using the mixed procedure in SAS, version 9.1.

\section{Results}

A total of 29 pigs and 144 observations were included in the analysis. Seven pigs were classified as non-diarrheic, 9 were semi-diarrheic and 13 were diarrheic at the first time point. A significant change in mean DM\% between time points was observed for both non-diarrheic $(p=0.01)$, semi-diarrheic $(p=$ $0.01)$ and diarrheic pigs $(p=0.02)$. The combined analysis of all pigs showed a significant $(p<0.01)$ lower mean DM\% at the last time point $(5 \mathrm{pm}-7 \mathrm{pm})$ compared to the other time points.

Only one initial non-diarrheic pig stayed non-diarrheic all day. The other 6 initially non-diarrheic pigs changed classification once or twice during the day. Five of the initial semi-diarrheic pigs stayed diarrheic all day. The other 4 pigs changed from diarrheic to non-diarrheic and back. Eleven initial diarrheic pigs stayed diarrheic all day while 2 pigs changed to non-diarrheic and back, table 1.
Table 1. Diurnal variation in classification of diarrhea for individual pigs

\begin{tabular}{|l|l|c|c|c|c|c|}
\hline & & \multicolumn{5}{|c|}{ Time point } \\
\hline Status at first time point (9am) & Status at later time & 9am & $\mathbf{1 1 a m}$ & $\mathbf{1 p m}$ & $\mathbf{3 p m}$ & $\mathbf{5 p m}$ \\
\hline Non-diarrheic (DM\%>18.8) & Non-diarrhea & 7 & 6 & 3 & 4 & 3 \\
\hline & Diarrhea & 0 & 1 & 4 & 3 & 4 \\
\hline Semi-diarrheic (18.8 $\geq$ DM\% $>$ 13.1) $)$ & Non-diarrhea & 0 & 4 & 2 & 1 & 0 \\
\hline & Diarrhea & 9 & 5 & 7 & 8 & 9 \\
\hline Diarrheic (DM\% $\leq 13.1)$ & Non-diarrhea & 0 & 0 & 1 & 2 & 0 \\
\hline & Diarrhea & 13 & 13 & 12 & 11 & 13 \\
\hline
\end{tabular}

\section{Discussion}

The results of the current study shows that fecal dry matter content can change during the day and that time of day is important for classification of pigs as diarrheic or not. This indicates that examination of pigs at the same time of the day may be important in research studies. It does also have implications for monitoring of diarrhea at both individual and herd level in veterinary practice. Stress caused by several times of handling and fixation in a period of 10 hours may have contributed to the observed decrease in mean fecal dry matter content during the day. A larger sample of pigs is needed to determine whether a true diurnal variation in fecal dry matter exists. In conclusion, the current study supports the existence of a within day variation in fecal dry matter.

\section{References}

1. Pedersen, K.S. et al., 2009. Proc 1st ESPHM: page 66.

2. Pedersen, K.S. et al., 2009. Proc 1st ESPHM: page 67 


\title{
P.450
}

\section{Intestinal pathogens in acute diarrhea in weaners: A case control study in 20 Danish herds}

\author{
Ken S. Pedersen ${ }^{1} \varnothing y s t e i n$ Angen ${ }^{2}$ Sven E. Jorsal ${ }^{2}$ Helle Stege ${ }^{1}$ Jens P. Nielsen ${ }^{1}$ \\ 1. University of Copenhagen, Frederiksberg, Denmark; 2. National Veterinary Institute, Technical University of Denmark, Frederiksberg, Denmark
}

\section{Introduction}

Recurring therapeutic batch medication of acute outbreaks of diarrhea in weaners is a common cause of antibiotic consumption in Danish pig production. The objective of the current study was to investigate the prevalence of selected pathogens in outbreaks of acute treatment indicated diarrhea in pigs between 10 and 70 days post weaning. Results of clinical and pathological investigations are reported elsewhere in these proceedings.

\section{Materials and Methods}

A case control study was conducted. Herds were selected by multistage sampling. All herds serviced by six specialized swine veterinarians from the same vet practice at Zealand and fulfilling the inclusion criteria were selected. The criteria were recurring therapeutic use of in-feed or in-water medication for diarrhea at room level in pigs between 10 and 70 days post weaning. Only herds representing modern intensive production systems were selected. One outbreak of acute diarrhea was investigated in each herd. All herds were visited the day following notification from the farmer/veterinarian of an acute treatment requiring outbreak of diarrhea and the farmer was not allowed to medicate before the pigs were examined. If the pigs had received antibiotic batch medication within the last 7 days of the examination day, the outbreak was excluded from the study. A sample of 80 pigs in each herd was selected by systematic random sampling among all pigs in the nursery room where the outbreak of acute diarrhea occurred. The selected pigs were subjected to a clinical examination and fecal samples were collected. Among the examined pigs a simple random sample of 8 pigs with diarrhea and 8 pigs without diarrhea was selected and euthanized. Intestinal content was examined for rotavirus by an ELISA method and Escherichia coli, Brachyspira spp. and Salmonella spp by standard culturing methods. Dry matter (DM\%) was determined for the fecal samples. DM\% $\leq 18.8$ was considered as diarrhea (1) and was used (if necessary) to reclassify the pigs as diarrheic (cases) or non-diarrheic (controls) in the statistical analysis. Chi-sq test was applied for unconditional testing of different pathogen prevalence in pigs with and without diarrhea.

\section{Results}

Twenty-four non-diarrheic and 12 diarrheic pigs (determined at clinical examination) were reclassified according to the DM\% determination. Eight pigs were excluded from analysis. Results are displayed in Table 1. Infection with one or more of the listed pathogens was demonstrated in $76 \%$ of the pigs with diarrhea and $69 \%$ of the pigs without diarrhea. There were no significant ( $p>0.05$ ) differences between the prevalence of pathogens found in pigs with and without diarrhea Salmonella spp., B. hyodysenteriae and rotavirus were not detected.
Table 1. Prevalence of selected intestinal pathogens* in 20 herds with outbreaks of acute diarrhea with indication of treatment 10 to 70 days post weaning ( $n=312$ pigs)

\begin{tabular}{|l|c|c|}
\hline & $\begin{array}{c}\text { Pigs with diarrhea } \\
(\mathbf{D M} \% \leq \mathbf{1 8 . 8})(\mathbf{n = 1 6 6})\end{array}$ & $\begin{array}{c}\text { Pigs without diarrhea } \\
(\mathbf{D M} \% \mathbf{> 1 8 . 8})(\mathbf{n = 1 4 6})\end{array}$ \\
\hline Small intestinal content: & & \\
\hline Hemolytic E. coli & & $3 \%$ \\
\hline Pure culture & $11 \%$ & $7 \%$ \\
\hline Dominant in mixed culture & & \\
\hline Non-hemolytic E. coli & $7 \%$ & $6 \%$ \\
\hline Pure culture & $32 \%$ & $37 \%$ \\
\hline Dominant in mixed culture & & \\
\hline Large intestinal content: & $7 \%$ & $5 \%$ \\
\hline B. intermedia & $29 \%$ & $3 \%$ \\
\hline B. innocens, B. murdochii & $7 \%$ & \\
\hline B. pilosicoli & & \\
\hline * Demonstrated by bacteriological culturing & \\
\hline
\end{tabular}

\section{Discussion}

The non-significant differences in the prevalence of pathogens found in pigs with and without diarrhea could imply that the detected infections were not the cause of diarrhea. It may also imply that the non-diarrheic pigs were in the incubation phase of diarrhea development or that some pigs were able to resist the infections without having diarrhea. In the latter case the results imply that it does not matter whether samples for microbiological examinations are collected from pigs with or without diarrhea. However, a quantitative evaluation of the pathogens present in the two groups might tell a different story. Further conclusion awaits the remaining microbiological examinations by quantitative real-time PCR for detection of PCV2, Lawsonia intracellularis, B. pilosicoli and E. coli.

\section{References}

1. Pedersen, K.S. et al., 2009. Proc 1st ESPHM: page 67. 


\title{
P.451
}

\section{Frequency of enteropathogens in diarrheic and non-diarrheic neonatal piglets}

\author{
Eduardo C. Cruz Jr. ${ }^{1}$ Felipe M. Salvarani ${ }^{1}$ Isabella S. Alberafo ${ }^{1}$ Rodrigo O. Silva ${ }^{1}$ Francisco F. Lobato ${ }^{1}$ Edel F. Stancioli ${ }^{2}$ Roberto M. Guedes ${ }^{1}$ \\ 1. Universidade Federal de Minas Gerais, Veterinary School, Belo Horizonte, MG, Brazil; \\ 2. Institute of Biological Sciences, Universidade Federal de Minas Gerais, Belo Horizonte, MG, Brazil
}

\section{Introduction}

Enteric diseases represent the most important cause of losses in neonatal piglets due to antimicrobial use, labor, reduced weaning weight and mortality (1). The most important agents involved with enteric disorders up to 7 days of age are enterotoxigenic Escherichia coli (ETEC), Clostridium perfringens types A and C, Clostridium difficile, Rotavirus and Isospora suis. There is little information regarding the frequency of these agents in Brazilian swine production systems. As a result, the aim of this study was to establish the frequency of enteropathogens in diarrheic and non-diarrheic neonatal piglets.

\section{Material and Methods}

Sixty 1 to 7-day-old piglets from 15 different swine farms ( 2 diarrheic and 2 non-diarrheic piglets, age matched, per farm) were euthanized and evaluated. A fresh stool sample was taken from each animal and submitted to different analysis, such as: $\mathrm{E}$. coli isolation and typification using PCR multiplex for virulence factors (2); Rotavirus detection using poliacrylamide gel electrophoresis (PAGE); C. difficile toxins detection using an ELISA kit; C. perfringens isolation and typification (type A or C) based on a multiplex PCR; and a flotation fecal test for detection of I. suis oocists. Jejunum, ileum, colon and cecum segments were collected and formalin fixed for histopathological evaluation. This project was approved by the ethics committee for animal experimentation of the Universidade Federal de Minas Gerais.

\section{Results}

C. perfringens type A was the most frequent agent detected, being present in $43.3 \%$ of the diarrheic piglets and in $83.3 \%$ of the non-diarrheic ones. However, for all $C$. perfringens type $A$, $76.9 \%$ of the diarrheic animals were $\beta 2$ gene positive, while $64 \%$ of the non-diarrheic animals had the $\beta 2$ gene. No $C$. perfringens type $C$ was detected. $C$. difficile was the second most frequently detected agent, present in $23.3 \%$ and $10 \%$ of diarrheic and non-diarrheic piglets. Rotavirus and E. coli were the third most important enteropathogens (Table 1).

Eight piglets out of 10-ELISA positive for C. difficile had significant neutrophilic colitis. Three of four Rotavirus PAGE positive animals had necrosis of the tip of villi. Only one animal had cocobacilar bacterial colonies adhered to enterocytes compatible to ETEC. No histologic lesion was attributed to C. perfringens type A even though there were some positive $\beta 2$ results.
Table1. Diagnostics of each piglet group

\begin{tabular}{|l|c|c|}
\hline & Diarrheic piglets & Non-diarrheic piglets \\
\hline C. difficile & $23.3 \%(7 / 30)$ & $10 \%(3 / 30)$ \\
\hline C. perfringens type A B2 & $33.3 \%(10 / 30)$ & $53.3 \%(16 / 30)$ \\
\hline C. pefringens type C & $0 \%(0 / 30)$ & $0 \%(0 / 30)$ \\
\hline Enterotoxigenic E. coli & $10 \%(3 / 30)$ & $3.3 \%(1 / 30)$ \\
\hline Isospora suis & $0 \%(0 / 30)$ & $3.3 \%(1 / 30)$ \\
\hline Rotarvirus & $13.3 \%(4 / 30)$ & $0 \%(0 / 30)$ \\
\hline
\end{tabular}

\section{Discussion}

Our results were similar to YAEGER (3) who found $47 \%$ and $48 \%$ for $C$. perfringens type $A$ and $C$. difficile, respectively, in young piglets with diarrhea. However, one should be careful as $C$. perfringens type $A$ and $C$. difficile are part of the normal flora and were also detected in non-diarrheic piglets. As a result, there is no easy diagnosis and the results should be interpreted with cautious. Histopathological findings should also be considered. Rotavirus was not highly prevalent but was present in a significant number and should not be forgotten. ETEC frequency was low, as demonstrated in different studies (3). It is important to emphasize the relevance of performing the detection of virulence factors in order to diagnose relevant ETEC strains.

\section{References}

1. Songer,J.G.;Uzal,F.A. Clostridial enteric infections in pig. J. Vet. Diagn. Invest., v.17, p. 528-536, 2005.

2. Macêdo,N.R., et al.Detection of pathogenic strains by multiplex PCR and antimicrobial sensitivity of Escherichia coli isolated from piglets. Brazilian Journal of Veterinary and Animal Sciences.

3. Yaeger, M.J. Prospective and retrospective studies on Clostridium perfringens type A enteritis in neonatal swine. American Associate of Swine Veterinarians, p.101-103, 2007.

\section{Acknowledgments}

This work was supported by FAPEMIG. We want to thank INTEGRALL for helping during the sample collection. 


\title{
P.452
}

\section{Clinical and laboratory investigations in 10 French pig herds dealing with enzootic neonatal diarrhea}

\author{
Thomas Gin ${ }^{1}$ Jean Le Guennec ${ }^{2}$ Hervé Morvan³ Guy-Pierre Martineau \\ 1. National veterinary school, Toulouse, France; 2. Labofarm, Loudeac, France; 3. LDA22, Ploufragan, France
}

\section{Introduction}

Although non fatal, enzootic neonatal diarrhea (END) is still a problem in many French swine high producing herds[1]. Dealing with this issue, veterinarians have to perform a diagnosis in order to well implement the treatment and the prevention. In this paper, we focus on the diagnosis approach of END.

\section{Materials and Methods}

The study was conducted in 10 French farrow-to-finish pig farms (see Materials and Methods in: Gin et al., 2010). In order to identify the onset of diarrhea, piglets were watched 3 times a day and during 7 consecutive days. Four piglets from 2 different litters, having diarrhea for less than 12 hours, were euthanized and on-farm necropsied. Microbiological analyses were performed on samples from the small and large intestine, kept at $5^{\circ} \mathrm{C}$ after the necropsy. Histological examinations were performed on samples (kept in formaline 10\%) from duodenum, jejunum, ileum and helicoidal part of the colon. Microbiological and histological analyses were performed by LDA22 (Ploufragan, Côtes d'Armor, France) or by Labofarm (Loudéac, Côtes d'Armor, France), depending on the location of the farm.

\section{Results}

Although herds were selected for END, 2 farms remained diarrhea free during the week of investigation. This is an illustration of END variability between different batches within the same herd.

Clinical investigation highlights that END is an issue in very good herds (average of 11.4 piglets weaned per sow per litter) where producers and veterinarians are implementing a lot of interventions: zootechnical interventions (cross-fostering, change of the processing date...) and medical interventions (antibiotics, symbiotics, autogenous vaccines,...). However, nothing seems to be working and, sometimes, it is getting worse.

Necropsies highlight prevalence of mesocolon edema observed in $6 / 8$ herds ( $17 / 27$ piglets) but with different etiological diagnosis. Therefore, there is no link between a specific pathogen (like C. difficile3) and mesocolonic edema.

On 8 etiological herd diagnosis, there were 2 cases of Enterococcus durans, one case of $C$. difficile, one case of both, $C$. difficile and $E$. durans, 3 cases of $C$. perfringens type $A$, one case of rotaviral diarrhea and none with E. coli.

\section{Discussion}

In human medicine, especially in hospital, daily interventions can quickly spread a pathogen like C. difficile for example[4]. Recently, it was shown that $C$. difficile is a concern in intensive care units for cats and dogs5. In a given herd, a farrowing room could be compared to an hospital. The producer is like a nurse: he/she is going from litters to litters, moving animals and performing some treatments. During all these interventions (zootechnical and medical), the producer can easily spread a "pathogen". This is called a nosocomial disease and it may be a "new" risk factor of END.
Moreover, always in human medicine, antibiotics can induce diarrhea and Antibiotic-Associated Diarrhea 6 starts to be better understood (Figure). This is called a iatrogenic disease and it may also be another "new" risk factor of END.

Finally, Enterococcus durans diarrhea occurs mainly in litters from parity 1 and 2 sows, 3 to 5 days after birth. Before having a very watery diarrhea, piglets are often vomiting. Usually, the whole litter is having scour and death occurs quickly if an appropriate antibiotic treatment (such as florfenicol or amoxicillin) is not implemented. Acute enteritis with a small intestine full of water is observed during necropsies. Histological analyses reveal gram positive cocci bind to the jejunum and ileum epithelium.

To conclude, this field study shows that neonatal diarrhea is changing with "new" pathogens (E. durans) and, beside other new identified risk factor[1], other risk factors such as nosocomial and/or iatrogenic diseases have to be considered.

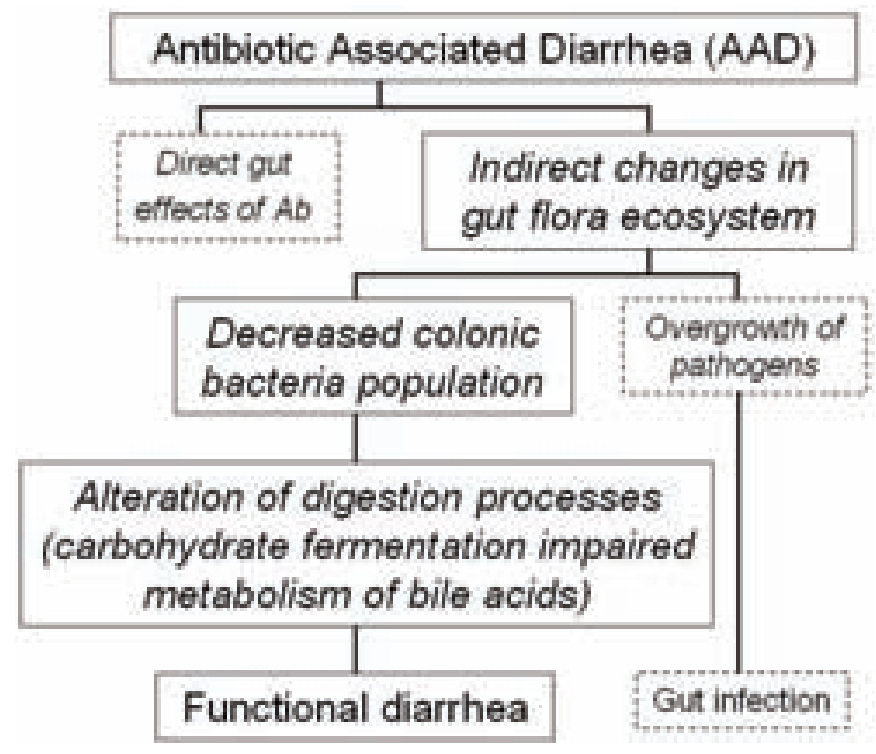

References

1. Sialelli et al. Proceed. Journées Recherche Porcine. 2009; 41 : 167-172.

2. Gin et al. Proceed. IPVS 2010.

3. Yaeger et al. Journal of Veterinary Diagnotic Investigation. 2007;52-59.

4 Nolan et al. Gut. 1987;1467-1473.

5. Clooten et al. Veterinary Microbiology. 2008;209-214.

6. Beaugerie et al. Best Practice and Research Clinical Gastroenterology. 2004;337-352. 


\title{
P.453
}

\section{Diagnostic of post-weaning diarrhoea on the farm level}

\author{
Albert Sundrum; Amke Goebel; Christina Werner \\ University of Kassel, Witzenhausen, Germany
}

\section{Introduction}

Post-weaning diarrhoea (PWD) is a multi-factorial disease. Clinical symptoms are often linked to a com-bination of different factors such as low feed intake after weaning, low hygiene, low age at weaning, low piglet live weight at weaning, and a high number of piglets per pen (Madec et al., 1998). Associated with the myriad of stressors to which the piglets are sub-jected in the weaning process, are marked changes to the digestive and absorptive capacity. Therefore, intake of creep feed and diet composition can have severe impacts on the occurrence of PWD (Callesen et al., 2007) too. Although organically reared piglets are, in general, six weeks of age at weaning, and are kept with a high space allowance, post-weaning diar-rhoea is a severe problem in organic pig production (Bonde \& Sörensen, 2006; Sundrum et al., 2010). Due to restrictions in the use of antibiotics in organic livestock production, there is a need to focus primarily on the implementation of preventive measures. When health problems are recognised, it is of high importance to make an accurate diagnosis and iden-tify the farm-specific causes to enable the elimina-tion of relevant risk factors. The objective of this study was to assess, qualitatively, the possible causes of weaning diarrhoea in an on-farm approach on those organic farms which have previously reported about PWD problems.

\section{Material and Methods}

Case studies were performed on six organic sow herds in Germany, affected with PWD problems. Clinical examinations and data on critical control points (CCPs) were assessed on the farms in an interval of 6 months. On the first visit a comprehensive interview was carried out with the herd manager gathering information on performance level, mortality and morbidity rates, hygiene management, feeding re-gime, and medicine usage. Piglets were housed in stables with outdoor runs. Weaning age averaged 44.8 days. Feed was purchased from an independent feed mill, creep feed and post-weaning diets were analysed for crude nutrient content.

Faeces were collected on each farm via swabs from five clinical healthy and five piglets suffering from postweaning diarrhoea, respectively. Faeces were analysed microbiologically for content of haemolytic E. coli. Differential analysis includes salmonellosis, swine dysentery, porcine proliferative enteropathy and porcine colonic spirochetosis.

\section{Preliminary Results}

The second visits on the farms have just started so that currently only preliminary results have been obtained.

The examination of the CCPs showed that all farms showed more or less severe deficits in the hygiene management and in the nutrient regime, although varying in their details considerably between the farms. Laboratory analyses provided proof for the presence of different strains of haemolytic E. coli, Brachyspira pilosicoli and Lawsonia intracellularis, independent of the health status of the piglets.

After interpretation of all data, farm specific measures have been recommended to the farmers. Effects of the implementation of measures will be assessed on the second farm visit.

\section{Implications}

Effective clinical management includes the iden-tification of risk factors and the implementation of changes aimed at reducing the incidence of PWD. Further investigations should be directed towards the impact of hygiene management and feeding regime on piglet susceptibility to GIT disorders and enteric infections. The development of diagnostic tools to be used on the farm level should be enforced to enable appropriate and promptly counteractive measures.

\section{References}

Bonde, M., Sörensen, J.T., 2006. Animal health and welfare in organic European pig production. Joint Organic Congress, Odense, Denmark, May 30-31, 2006, p. 562-563.

Callesen, J., Halas, S. Thorup, F., Bach Knudsen, K.E., Kim, J.C., Mullan, B.P., Hampson, D.J., Wilson, R.H., Pluske, J.R., 2007. The effect of weaning age, diet composition, and categorisation of creep feed intake by piglets on diarrhoea and performance after weaning. Livestock Science 108, 120-123.

Madec, F., Bridoux, N., Bounaix, S., Jestin, A., 1998. Measurement of digestive disorders in the piglet at weaning and related risk factors. Prev. Vet. Med. 35, 53-72.

Sundrum A., Goebel A., Bochicchio D., Bonde M., Bourgoin A., Dietze K., Dippel S., Hegelund L., Leeb T., Lindgren K., Prunier A., Wiberg S., 2010. Health status in organic pig herds in Europe. Proc. of the IVPS conference, July 18-21, 2010, Vancouver, Brit-ish Columbia, Canada. 


\title{
P.454
}

\section{Is pathomorphology sufficient for diagnosis of enteritic infections?}

\author{
$\underline{\text { Sabine Elicker }}^{2}$ Daniela Philadelphy ${ }^{1}$ Franz Schilcher ${ }^{1}$ Louis Fischer ${ }^{3}$ Herbert Weissenböck ${ }^{1}$ Wolfgang Sipos ${ }^{1}$ \\ 1. University of Veterinary Medicine Vienna, Vienna, Austria; 2. Veterinary Practice Entenfellner, Stössing, Austria; 3. LaboVet GmbH, Vienna, Austria
}

\section{Introduction}

Enteritides continue to be amongst the most important factors that limit the profitability and efficiency in pig industry. For this reason exact diagnosis is needed in order to avoid great economic losses [1, 2]. Therefore the aim of this retrospective study was to look for possible relations between diarrhea-causing pathogens and pathological findings of the gastrointestinal tract of swine suffering from enteritis to possibly provide a useful tool for diagnosis in the field.

\section{Materials and Methods}

Results of the clinical, pathomorphological, and bacteriological examinations from 444 pigs (29 suckling and 314 weaned piglets, 88 fatteners, and 13 sows) all suffering from diarrhea, which were submitted to the Clinic for Swine at the University of Veterinary Medicine Vienna over a period of three years, were analysed with pathological findings only being taken into account in cases of monoinfection. Pathological findings were classified as serous-catarrhalic enteritis, hemorrhagic-necrotizing enteritis, fibrinous enteritis, ulcerative enteritis, thickening of the ileal or colonic mucosa, mesenterial edema, lymphoplasmacytic enteritis, lymphocyte depletion of the MALT, and crypt abscesses.

\section{Results}

Infections with one or more bacterial pathogens could be detected in only $72.1 \%$ of investigated pigs. However, no causative pathogen was found in the remaining $27.9 \%$. Most frequently, E. coli could be isolated (59.0\%), followed by infections with L. intracellularis (10.4\%), Cl. perfringens (9.7\%), Brachyspira spp. (5.0\%) and Salmonella spp. (1.4\%). Monoinfections with one of these pathogens were determined in 122 animals. Pathomorphologically, in most cases (63.9\%) a lymphoplasmacytic enteritis could be diagnosed irrespective of the pathogen. A reliable hint for a mutual occurence of a specific pathogen and a corresponding pathomorphological finding was only seen in infections with L. intracellularis. In the majority of these cases $(87.5 \%)$ the ileum exhibited a thickening of the mucosa.

\section{Discussion}

The only correlation between a specific pathogen and a pathomorphological finding could be demonstrated for L. intracellularis-infections. Therefore, it can be concluded that all other bacterial pathogens causing enteritic infections in pigs do not go hand in hand with specific lesions.

In a large proportion of cases no causative pathogen could be isolated. When taking into account that in most cases a lymphoplasmacytic enteritis could be diagnosed, an infection by some viral pathogen is conclusive. As a consequence these results may give a hint towards PCV2 being a possibly important pathogen also in cases of diarrhea. However, we did not prove this assumption by further molecular diagnostic techniques.

\section{References}

1. Straw et al. (eds., 2006): Diseases of swine, 9th ed., pp. 37-55.

2. Elicker et al. (2010): Tierarztl. Umschau 65, 111-114. 


\title{
P.455
}

\section{Clinical case of diarrhea with E. coli F4 in late fattening pigs: is it relevant?}

\author{
Philippe Leneveu ${ }^{1}$ Marianne Bertrand ${ }^{2}$ Alassane Keita ${ }^{3}$ Hervé Morvan ${ }^{4}$ Roland Foret $^{5}$ Catherine Belloc $^{6}$ \\ 1. ISPAIA Zoopole Développement, Ploufragan, France; 2. Coopagri, Landerneau, France; 3. CTPA Zoopole Développement, Ploufragan, France; \\ 4. LDA 22, Ploufragan, France; 5. CCPA, Janzé, France; 6. ENVN, Nantes, France
}

\section{Introduction}

Aetiology of Grey Scour Syndrom (GSS) is a matter of question in France. Lawsonia intracellularis ( $\mathrm{LI}$ ) and Brachyspira sp (Br) have been isolated from faeces of pigs with GSS in some cases but not in the majority of concerned farms (1). However, the seroprevalence of $\mathrm{LI}$ is as high as in the others countries (2). Escherichia coli is the most common pathogen found in GSS but its role as a causative agent is questionable. This paper discuss a case of late colibacillosis.

\section{Materials and Methods}

A field investigation was carried out in a selected farrow to finish farm ( 200 sows) concerned by GSS and heterogeneity. An outbreak of diarrhoea usually occurred during post weaning (8 weeks of age) followed by GSS until 12 weeks. A batch of 308 pigs was monitored from weaning to slaughter. Several clinical evaluations were conducted, with sampling of faeces and blood. If we observed an increase in GSS intensity or otherwise at the end of the follow up (145 days), it was planned to euthanize 9 pigs at laboratory: 3 with clinical signs of diarrhoea or doubtful body condition, 3 lean pigs (Ip) and 3 control pigs (without diarrhoea). Digestibility was evaluated by analyzing colonic content: flora, digestibility of organic material (DO, \%) evaluated by the use of indigestible part of the feed as a marker and volatile fatty acids (Vfa, g/l).

\section{Results}

Two main outbreaks of liquid diarrhoea with general clinical signs occurred at 48 and 97 days of age respectively. For the first one, 3 different E. coli were found on 3 necropsied pigs. Only non typable $\mathrm{E}$. coli has been identified for the second one (sickest pigs were under treatment at sampling time). At each herd examination some pens were concerned by diarrhoea (evident or doubtful situation) with a maximum of $50 \%$ of the pens at 77 days. As GSS was mild, pigs were euthanized at 145 days (Table 1). Diarrheic pigs didn't seem to be sick but E. coli F4 was found attaching the small intestine on the two heaviest.

This farm was positive for $\mathrm{LI}$ and this batch had seroconverted between 109 days (1+/16 pigs. ELISA) and 145 days (6+/16). Necropsy of lean pigs didn't give relevant information: peritonitis / no lesion / pneumonia and pleuritis respectively. No histological lesion of PCV2 was found on any pig.

Digestibility of organic mater wasn't depreciated on these diarrheic pigs but their fermentation in colon was low (Vfa).
Table 1: Clinical and analytical observations for the 9 necropsied pigs (145 days).

\begin{tabular}{|c|c|c|c|c|c|c|c|c|c|}
\hline & $\begin{array}{c}\text { Control } \\
\text { pigs }\end{array}$ & $\begin{array}{c}\text { Control } \\
\text { pigs }\end{array}$ & $\begin{array}{c}\text { Control } \\
\text { pigs }\end{array}$ & $\begin{array}{c}\text { Lean } \\
\text { pigs }\end{array}$ & $\begin{array}{l}\text { Lean } \\
\text { pigs }\end{array}$ & $\begin{array}{l}\text { Lean } \\
\text { pigs }\end{array}$ & $\begin{array}{l}\text { Diar- } \\
\text { rheic }\end{array}$ & $\begin{array}{l}\text { Diar- } \\
\text { rheic }\end{array}$ & $\begin{array}{l}\text { Diar- } \\
\text { rheic }\end{array}$ \\
\hline & $A$ & $B$ & $C$ & $D$ & $\mathrm{E}$ & $F$ & $G$ & $\mathrm{H}$ & 1 \\
\hline As & ws & ws & ws & Ip & Ip & Ip & ws & ws & ws \\
\hline W & 85 & 77 & 92 & 57 & 47 & 53 & 103 & 93 & 81 \\
\hline CS & - & - & - & - & - & - & $D$ & D & D \\
\hline Ec & $F^{*}$ & $\mathrm{Nt}$ & F4 & $\mathrm{Nt}$ & - & - & F4 & F4 & F4 \\
\hline LI & - & - & + & - & - & - & - & - & + \\
\hline $\mathrm{Br}$ & - & - & $+^{*}$ & - & - & - & - & - & - \\
\hline DO & 75 & 80 & 84 & 79 & 85 & 80 & 83 & 77 & 85 \\
\hline Vfa & 7 & 7 & 6 & 8 & 9 & 6 & 5 & 1 & 4 \\
\hline
\end{tabular}

\section{Discussion}

Normal digestibility on these diarrheic pigs is relevant with their weight and aspect. Hence, due to the lack of other clinical signs and their optimal weight, we can wonder on the real impact of this late colibacillosis. Moreover, the batch had not been treated and nothing happened. We can also wonder if the diarrhoea is due to the colibacillosis, to the lack of fermentation in the gut or the both. This case suggests then to minimize the importance of E. coli isolated on fattening pigs not showing global clinical signs (situation different from postweaning diarrhoea). Therefore one hypothesis could be a mix of different profiles for GSS in France: profile of economic importance when due to LI for example; profile of more doubtfully economical importance when associated with E. coli only.

Authors thank Boehringer Ingelheim for its financial support in this field investigation.

\section{References}

1. Leneveu et al., IPVS 2008, 1, 135

2. Hardge et al., IPVS 2006, 1, 77 


\title{
P.456
}

\section{Prevalence of enterotoxigenic Escherichia coli (ETEC) virulence genes isolated from scouring piglets in Taiwan}

\author{
YF Sun; MH Liao; MC Lee; SP Chen \\ ATIT, Miaoli, Taiwan
}

\section{Introduction}

Enterotoxigenic Escherichia coli (ETEC) are associated with diarrhea in neonatal and post-weaning piglets, and these infections constitute an important problem relating to farrowing in modern intensified piggery management. The strains of ETEC associated with intestinal colonization in piglets resulting in hypersecretory diarrhoea express F4, F5, F6, F17, F18 and F41 fimbriae and/or STa, STb and LT enterotoxins. Most studies of the prevalence of fimbrial adhesins and enterotoxins of porcine ETEC isolates have been conducted, until recently, by the use of polymerase chain reaction (PCR). Previous studies using enterotoxin genes cloned into a nontoxigenic fimbriated strain have suggested that both LT and STb play an important role in dehydrating diarrheal disease in piglets $<1$ week old and also enhances bacterial colonization of the intestine. Little is known about fimbriae associated with piglet ETEC-induced scours in Taiwan. Therefore, our aim was to investigate the prevalence of toxin and fimbrial genes among E. coli isolated from diarrheic piglets obtained over a 3-year period (2007-2009) in Taiwan.

\section{Materials and Methods}

Between 2007 and 2009, a total of 426 E. coli strains were isolated from piglets with diarrhea at suckling and weaning periods on 12 pig farms in Taiwan. After incubation at $37^{\circ} \mathrm{C}$ for $18 \mathrm{hr}$ on MacConkey's and Blood agar plates, single colonies were selected and identified as E. coli. The identification of $\beta$-hemolysin in all of E. coli strains were conducted visually after overnight growth on SBA. The detection of fimbrial and enterotoxin genes was performed by PCR. E. coli reference strains were used as positive control for each virulence factor detected by PCR, and a non-pathogenic commensal E. coli strain was used as a negative control. PCR for the identification of fimbrial (F4, F5, F6, F41 and F18) and enterotoxin (STa, STb and LT) genes was carried out as previously described (Do et al., 2005). Amplification procedures were carried out in a thermal cycler (TaKaRa, PCR Thermal Cycler Dice). Reactions were incubated at $94^{\circ} \mathrm{C}$ for $3 \mathrm{~min}$ for $1 \mathrm{cycle}$, then $94^{\circ} \mathrm{C}$ for $30 \mathrm{sec}$, the melting temperature for $30 \mathrm{sec}$ and $72^{\circ} \mathrm{C}$ for $90 \mathrm{sec}$ for $35 \mathrm{cycles}$, followed by a final incubation $72^{\circ} \mathrm{C}$ for $3 \mathrm{~min}$. The PCR products were analyzed by electrophoresis on $1.5 \%$ agarose gel, and the amplicons were visualized under ultraviolet light after ethidium bromide staining.

\section{Results}

A total of $426 \mathrm{E}$. coli isolates were screened for those genes by PCR. The prevalence of pathogenic ETEC among E. coli strains from field isolates was $114(26.8 \%)$ strains in the present study. Of these 114 isolates, 103 (90.4\%) carried at least one ETEC virulent gene and $44(38.6 \%)$ carried genes for the fimbrial adhesins. Percentages of E. coli strains carrying each virulence factors were as follows: F4 (6.14\%), F5 (8.78\%), F6 (13.16\%), F18 (10.53\%), STa (84.2\%), STb (85.09\%), LT (43.86\%). We assessed whether the presence of enterotoxin genes (STa, STb, LT and Stx2) could be correlated with the presence of fimbrial genes (Table 1). STa/STb enterotoxin genes were co-expressed in F5, F6 and F18 of the ETEC strains. $\mathrm{STa} / \mathrm{STb} / \mathrm{LT}$ were found to co-express with F4 and F41genes. STa enterotoxin was found to co-express with F6 and F18.

Table 1. Fimbrial and enterotoxin genes detected in 112 ETEC in 12 pig farms.

\begin{tabular}{|l|c|c|c|c|c|c|}
\hline Enterotoxin genes & F4 & F5 & F6 & F18 & F41 & ND \\
\hline STa/STb/LT & 4 & & & & 1 & 42 \\
\hline STa/STb & & 10 & 13 & 6 & & 2 \\
\hline STb/LT & 3 & & & & & \\
\hline STa/LT & & & & & & \\
\hline STb & & & & & & 3 \\
\hline STa & & & 2 & 4 & & 12 \\
\hline LT & & & & & & \\
\hline ND & & & & & \\
\hline${ }^{*}$ ND: non-detected. & & & & & \\
\hline
\end{tabular}

\section{Discussion}

The present study was conducted to provide baseline information on the significance of specific ETEC serogroups and pathotypes associated with diarrhoea in piglets. These results suggest $\mathrm{Sta} / \mathrm{STb}$ was the most prevalent pathotype in diarrheal piglets, which is different from the findings in USA (Zhang et al., 2007). A highly prevalent toxin in USA is STb (72.6\%). Different patterns of fimbrial gene expression were detected in ETEC from different pig farms in Taiwan. Therefore, vaccination and vaccine development based on fimbrial adhesion antigens needs to consider the spectrum of these antigens detected in pig farms in Taiwan.

\section{References}

Dubreuil, J.D., 2008. FEMS Microbiology Letters 278, 137-145.

Do et al., 2005. Aust Vet J 83, 293-299.

Zhang et al., 2007. Vet Microbiol 123:145-152 


\title{
P.457
}

\section{Prevalence of virulence genes of Escherichia coli isolated from diarrheic pigs in Korea}

\author{
Byeong Y. Jung; Jae W. Byun; Ha Y. Kim; Dong H. Shin; Hyoung S. Kim; O S. Lee \\ National Veterinary Research \& Quarantine Service, Anyang, Korea
}

\section{Introduction}

Escherichia coli strains such as enterotoxigenic E. coli (ETEC), and shiga toxin-producing E. coli (STEC) are associated with diarrhea and edema disease in pigs. Adherence factors (F4, F5, F6, F18, F51, adhesion involved in diffuse adherence [AIDA]-I, and porcine attaching and effacing-associated factor [paa]), heat-stable enterotoxins (STa and STb), heat-labile enterotoxin (LT), and enteroaggregative $\mathrm{E}$. coli heat-stable enterotoxin 1 (EAST1) have been known to be virulence factors of ETEC (1). Shiga toxin $2 e$ (Stx2e) produced by STEC plays an important role in the pathogenesis of edema disease in postweaned pigs. The objective of this study was to determine the prevalence of virulence genes among E. coli isolated from pigs with diarrhea in Korea.

\section{Materials and Methods}

One hundred thirty five E. coli were isolated from diarrheic pigs on different farms in 2009 in Korea. Pigs were divided with four different age groups, suckling ( $\leq 3$ weeks of age, $n=65$ ), postweaning ( $>3$ weeks to $<10$ weeks, $n=40)$, growing $(\geq 10$ weeks to $<17$ weeks, $n=22)$, and finishing ( $\geq 17$ weeks, $n=8)$, respectively. After incubation at $37^{\circ} \mathrm{C}$ for $18 \mathrm{hr}$ on MacConkey agar plate, a single colony was pick up and identified as $\mathrm{E}$. coli by VITEK 2 -Compact system (bioMerieux, France). $\beta$-Hemolysis was defined as a zone of complete erythrocyte lysis surrounding a bacterial colony observed after an overnight grown at $37^{\circ} \mathrm{C}$ on a blood agar plate. The isolates were grown overnight in tryptic soy broth and stored at $-80^{\circ} \mathrm{C}$ after the addition of $10 \%$ glycerol until further use.

For PCR test, 12 sets of oligonucleotide primers (Bioneer, Korea) used to detect the genes encoding for the following virulence factors: adherence factors (F4, F5, F6, F18, F41, AIDA-I, and paa) and enterotoxins (STa, STb, LT, Stx2e, and EAST1). The PCR assay for detection of virulence factors of the isolates was carried out as described previously (2).

\section{Results}

Of 135 E. coli isolates, 78 (57.8\%) were shown to be positive for the genes encoding more than one of the virulence factors. Forty eight isolates (35.6\%) carried both adherence factors and enterotoxins. A presence of diverse patterns of genetic profiles in virulence was observed, and the most prevalent pathotype was EAST1 only $(n=15,11.1 \%)$, followed by EAST1/paa $(n=11$, $8.1 \%)$, and paa only $(n=7,5.2 \%)$, respectively.
The prevalent pathotypes were determined by age. F4, LT, EAST1, and paa pathotypes were detected all age groups of pigs, whereas F5 was not detected in this study. F4 was the most prevalent in the postweaning pigs (22 5\%) compared with the suckling (13.8\%), finishing (12.5\%), and growing (9.1\%), respectively $(\mathrm{P}>0.05)$.

Almost all the virulence genes tested (F4, F6, F18, LT, STa, Stx2e, EAST1, and paa) were more prevalent in $\beta$-hemolytic E. coli than in non-hemolytic E. coli $(\mathrm{P}<0.05)$. On the other hand, prevalence of STb and AIDA-I in this study was higher in non-hemolytic E. coli than $\beta$-hemolytic E. coli, but this difference was not significant.

\section{Discussion}

The isolates showed the diverse patterns of virulence genes with LT/EAST1/F4 ( $n=5)$, followed by EAST1/F4 ( $n=3)$, EAST1/F4/ paa $(n=3)$, STa/F6/F18/paa $(n=3)$, and EAST1/STb/AIDA-I $(n=3)$, respectively. The isolates of $\mathrm{E}$. coli that harbor genes for adherence factors or enterotoxins are classified as ETEC, and pathogenic ETEC that cause colibacillosis must have both of them (3). The EAST1 gene was reported commonly found in E. coli isolated from diarrheic pigs. Frydendahl (4) reported that $65.8 \%$ of the isolates carried the EAST1 gene, however, E. coli isolated from pigs without diarrhea also carried this EAST1 gene (5). On the other hand, paa gene was demonstrated to be necessary for development of lesion caused by EPEC strains (6). However, paa gene was detected nearly even in isolates from diarrheic and non-diarrheic pigs, suggesting that the paa gene may not be a virulence factor in diarrhea (5).

In this study, we found that EAST1 was most prevalent in diarrheic pigs in Korea, followed by paa, and F4, respectively. And also, we found that almost all the virulence factors tested in this study were more prevalent in $\beta$-hemolytic E. coli than in nonhemolytic E. coli.

\section{References}

1. Bertschinger \& Fairbrother. Disease of Swine, p 431-468, 1999

2. Zhang et al. Vet Microbiol, 123: 145-152, 2007

3. Leclere et al. Res Microbiol, 158: 97-104, 2007

4. Frydendahl K. Vet Microbiol, 85: 169-182, 2002

5. Ngeleka et al. J Vet Diag Invest, 15: 242-252, 2003

6. Batisson et al. Infect Immun, 71: 4516-4525, 1996 


\section{P.458}

\section{Seroprevalence of F4+ and F18+ Escherichia coli in Cuban young sows}

Pedro Yoelvys de la Fe Rodriguez ${ }^{1,2}$ Emanuele Del Fava ${ }^{3}$ Jose Cortiñas Abrahantes ${ }^{3}$ Ziv Shkedy ${ }^{3}$ Luis O. Maroto Martin ${ }^{1}$ Eduardo Cruz Muñoz ${ }^{1}$ Luc Duchateau ${ }^{4}$ Eric Cox ${ }^{2}$ Annelies Coddens ${ }^{2}$ Bruno M. Goddeeris 2,5

1. Departamento de Medicina Veterinaria y Zootecnia, F. de Ciencias Agropecuarias, Universidad Central "Marta Abreu" de Las Villas, Santa Clara, Cuba; 2. Laboratory of Veterinary Immunology, Faculty of Veterinary Medicine, Ghent University, Merelbeke, Belgium; 3. Center for Statistics, Hasselt University, Diepenbeek, Belgium; 4. Department of Physiology and Biometrics, Faculty of Veterinary Medicine, Ghent University, Merelbeke, Belgium;

5. Department of Biosystems, Division Gene Technology, Faculty of Bioscience Engineering, Katholieke Universiteit Leuven, Leuven, Belgium

\section{Introduction}

In Cuba, gastroenteric disorders are the cause of the $31 \%$ and $37 \%$ of mortality in suckling and weaned piglets respectively. Taking decisions when an outbreak starts is difficult as specific diagnose of enteric pathogens is depressed (Cabrera and García, 2009). The present survey has the aim of contributing to the knowledge of the colibacillosis epidemiology in Cuba, specifically for $\mathrm{F}^{+}$and $\mathrm{F} 18^{+}$Escherichia coli.

\section{Materials and Methods}

1044 sera samples were randomly collected from gilts (6-8 months old) in 34 piggeries spread in the three main Cuban regions. The complement inactivated serum was treated with kaolin and tested for anti-F4 and anti-F18 lg at the fix dilution of $1 / 15$ in duplicates. Both fimbriae were purified by mechanical sharing protocol using the strains IMM01 (F4ac-LT-STb+) and 107/86 (F18ab-SLTIIv ${ }^{+}$). For F4 it was followed an indirect double antibody ELISA and for F18 a direct one. For the data analysis, that included the optical density values (OD), was fitted a mixture modeling in R (package "flexmix")(Leisch, 2004).

\section{Results}

F4 and F18 are highly prevalent in Cuba. A three components model was fitted for F4 as represented by the 3 thinner curves (Fig 1), while the thicker is the estimated mixture model. The cutoff points were given by the curves intersection: between weak and moderately positives was 0.775 , while between moderately and highly positives was 1.343 . Fig 2 shows the description of farms (finest cluster division) depending on the OD of serum samples, different letters mean different geographical areas. Piggeries were clearly distributed in each component showing a different epidemiologic situation. For F18 OD, only a one component model was found and the Box plot description was homogeneous.

\section{Discussion}

Considering a no previous vaccination against $\mathrm{F} 4$, the distribution of F4 OD in three components with the $14 \%$ and $42 \%$ classified as hard and moderately positive respectively, means that in Cuba is a high prevalence or association of $\mathrm{F}^{+}$E. coli in diarrhea episodes. From 1997 to 2001, Lazo et al., (2005) clinically reported a $43.8 \%$ of morbidity and $29.3 \%$ of lethality due to swine enteric colibacillosis in V. Clara, Cuba. Verdonck et al., (2002) mentioned that the slower immune response following a $\mathrm{F} 18^{+}$VTEC infection and the resulting longer excretion increases it's spreading; it can justify in part the F18 OD distribution, but $\mathrm{F} 18^{+} \mathrm{ETEC}$ strains have to be considered. In a later study

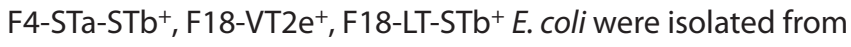
diarrheic piglets in a Cuban province (de la Fé, P., Butaye, P., Goddeeris, B., Cox, E., manuscript under preparation).

\section{Conclusions}

$\mathrm{F}^{+}$and $\mathrm{F} 18^{+} E$. coli are widespread in Cuban piggeries as proved by serological survey and mixture modeling. A more effective diarrhea prevention and control is needed.

Fig 1: Histogram for the F4 mixture model.

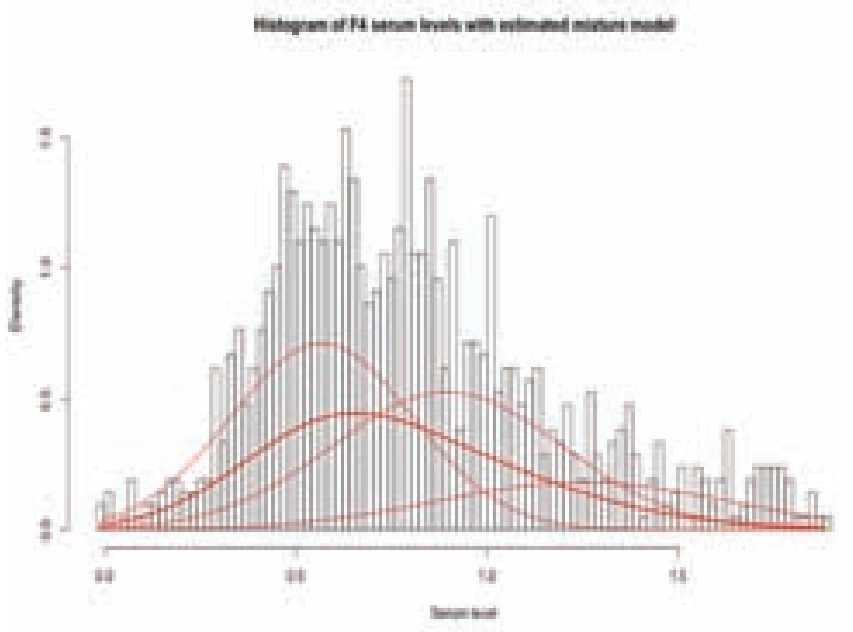

Fig 2: Box plot for F4 OD values grouped by piggeries.

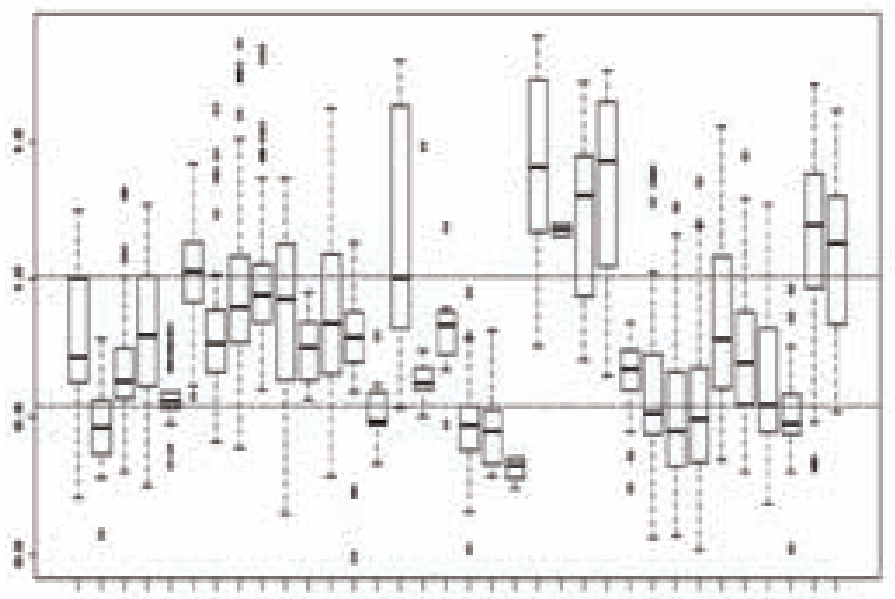

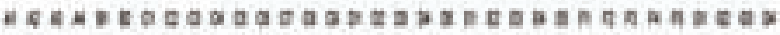

\section{References}

Cabrera and García, 2009. Bol Técnico Porcino. 11, 11-2. Leisch, 2004. J.

Statistical Software (JSS). 11, 1-18.

Lazo et al., 2005. Rev. Salud Anim. 27, 129-32.

Verdonck, 2002. Vaccine. 20, 2995-3004. 


\title{
P.459
}

\section{Influence of different housing systems and feeding regime on E.coli problems of post-weaning piglets in Central Vietnam}

\author{
Nguyen Quang Linh; Hoang Nghia Duyet; Nguyen Thi Thuy \\ Hue University of Agriculture and Forestry, Hue City, Vietnam
}

\begin{abstract}
Introduction
Post-weaning diarrhoea (PWD) is a significant gastrointestinal disease in pigs. It is considered a multifactorial disease associated with proliferation of enterotoxigenic Escherichia coli in the intestinal tract of affected pigs. The aim of this study was to analyse risk factors related to the occurrence of post-weaning diarrhoea on Vietnam piglet producing farms. In Central Vietnam, many pig farms have the problems for piglets after weaning periods, there is no vaccine for the prevention and control, it was inpact on their farm'income and decrease pig production in Vietnam.
\end{abstract}

\section{Materials and Methods}

The data of a follow-up study of 72 conventional piglet producing farms was used in the case-control study. The selection of the 36 PWD case and 36 control piglets. All of piglets were raised on 6 pig farms that was based on the use of antimicrobials for treating diarrhoea in weaned pigs and the answers related to the occurrence of diarrhoea after weaning in the questionnaire. Four intermediate farms were excluded from the statistical analysis. Altogether 36 factors related to herd characteristics, weaner pig management and pig health were studied. The median number of sows was the same breed and feeding situation on the case and the control farms, respectively. The significances of the univariable associations between the explanatory variables and the outcome variable were tested, and in the multivariate analysis linear models were applied.

\section{Results}

An decreased risk of PWD was associated with the regimen of feeding levels, twice a day and feed restriction after weaning $(P$ $=0.05$; compared to feeding three or more meals a day or the use of ad libitum feeding) and with a rotation housing system of sows on the farm $(P=0.01$; risk decreasing with increasing housing systems for sows removed from farrowing pens). Temperature and huminity control was associated with a decreased risk of PWD ( $P=0.03$; compared to manual temperature and huminity control).

\section{Discussion}

Temperature and huminity for resistance to pathogenic bacteria that is a global threat and therefore increasing attention is being paid to the prudent use of feeding control[1]. Gastrointestinal diseases of growing pigs, which are economically important for pig production worldwide [2] and enteric bacterial infections. The total of feeding levels in pigs and that gastrointestinal diseases were the most common indications for prescriptions in this age group[1]. PWD is considered to be a multifactorial factors[2]. Recently weaned pigs are variously predisposed to enteric disorders. Newly weaned pigs are stressed by nutritional, psychological, environmental and physiological factors [3]. At weaning the feed is changed from milk to a weaner diet, piglets are separated from their sow and often moved from the farrowing pen and mixed with unfamiliar pigs. Weaned pigs also lose passive intestinal immunity provided by antibodies in sow's milk. After weaning there are alterations in stable in farrowing pens until 2 weeks after weaning. During the first two weeks after weaning, pathogenic Escherichia coli plays a significant role in the etiology of PWD [1,2], although infection with pathogenic E. coli does not unequivocally lead to the development of diarrhoea in weaned pigs. housing systems with pathogenic $E$. coli strains are likely sources of infection for weaned pigs, but the infection can also be acquired before weaning. The susceptibility for diarrhoea after weaning has been associated with management related factors such as low feed intake during the first week after weaning, excessive feed intake, low number of meals, the hygiene and management level, a low weaning weight and age, moderate cold stress, draught, texture of feed, number of feeder spaces per pen and vaccinating gestating sows against $E$. coli. A sastification of temperature and humility in the housing during first two weeks after weaning with a decreased risk of PWD. This study was done to provide information on risk factors related to the occurrence of PWD on weaning piglet producing farms.

\section{References}

1. Ball RO, Aherne FX: Effect of diet complexity and feed restriction on the incidence and severity of diarrhea in early-weaned pigs. Can J Anim Sci 1982, 62:907-913.

2. Ball RO, Aherne FX: Influence of dietary nutrient density, level of feed intake and weaning age on young pigs.

2. Apparent nutrient digestibility and incidence and severity of diarrhea. Can J Anim Sci 1987, 67:1105-1115.

3. Rantzer D, Svendsen J, Westrom B: Effects of a strategic feed restriction on pig performance and health during the post-weaning period. Acta Agric Scand Sect A, Animal Sci 1996, 46:219-226. 


\section{Comparison of the pathotypes and virotypes of pathogenic Escherichia coli in diseased pigs in Vietnam and Quebec, Canada}

Thuy N. Do ${ }^{1}$ Darren J. Trott ${ }^{2}$ Éric Nadeau ${ }^{3}$ Clarisse Desautels ${ }^{4}$ John M. Fairbrother ${ }^{4}$

1. National Institute of Veterinary Research, Hanoi, Vietnam; 2. The University of Adelaide, Adelaide, SA, Australia; 3. Prevtec Microbia Inc., Saint-Hyacinthe, QC, Canada; 4. Reference laboratory for E. coli, Faculté de médecine vétérinaire, Université de Montréal, Montréal, QC, Canada

\section{Introduction}

Diseases caused by E. coli have been a recognised problem for as long as pigs have been raised. Different categories of $E$. coli have been associated with diarrhoeal disease in animals worldwide with Enterotoxigenic E. coli (ETEC) and Shiga toxin-producing $E$. coli (STEC) being the most common (DebRoy \& Maddox, 2001). The objective of this study was to compare the distribution of virotypes of pathogenic $E$. coli isolates from diseased pigs in two geographically distinct countries, Vietnam and Canada.

\section{Materials and Methods}

E. coli isolates obtained from diseased pigs (diarrhea, sudden death or edema disease) in 2007 and submitted to the NIVR in Vietnam or the FMV in Québec, Canada, were initially tested by PCR for the presence of defining genes of ETEC and STEC pathotypes. They were then virotyped by colony hybridisation (Ngeleka et al., 2002) for detection of the presence of 20 virulence genes most commonly found in animal pathogenic $E$. coli.

Table 1: Virotypes of ETEC-STEC isolates

\begin{tabular}{|l|c|c|}
\hline \multicolumn{2}{|c|}{ ETEC-STEC pathotype } \\
\hline Virotype & \multicolumn{2}{|c|}{ Number of isolates } \\
\hline ETEC-STEC F18 & Vietnam & Quebec \\
\hline LT:STa:Stx2:Paa:F18 & 13 & 0 \\
\hline STa:STb:Stx2:AIDA:F18 & 4 & 0 \\
\hline STa:STb:Stx2:Paa:AIDA:F18 & 3 & 0 \\
\hline 0ther & 6 & 0 \\
\hline Total ETEC-STEC F18 & $\mathbf{2 6}$ & $\mathbf{0}$ \\
\hline 0ther & $\mathbf{1}$ & $\mathbf{4}$ \\
\hline
\end{tabular}

Table 2: Virotypes of STEC isolates

\begin{tabular}{|l|c|c|}
\hline \multicolumn{2}{|c|}{ STEC pathotype } \\
\hline & \multicolumn{2}{|c|}{ Number of isolates } \\
\hline Virotype & Vietnam & Quebec \\
\hline STEC F18 & & 2 \\
\hline Stx2:Paa:AIDA:F18 & 4 & 1 \\
\hline Stx2:AIDA:F18 & 4 & 8 \\
\hline Stx2:EAST1:AIDA:F18 & 0 & 3 \\
\hline 0ther & 2 & $\mathbf{1 4}$ \\
\hline Total STEC F18 & $\mathbf{1 0}$ & $\mathbf{9}$ \\
\hline 0ther & $\mathbf{4}$ & \\
\hline
\end{tabular}

Table 3: Virotype of ETEC isolates

\begin{tabular}{|l|c|c|}
\hline \multicolumn{2}{|c|}{ ETEC pathotype } \\
\hline & \multicolumn{2}{|c|}{ Number of strains } \\
\hline Virotype & Vietnam & Québec \\
\hline ETEC F4 & & 5 \\
\hline LT:STa:STb:EAST1:Paa:F4 125 & 12 & 0 \\
\hline STa:STb:EAST1:F4 & 8 & 14 \\
\hline LT:STb:EAST1:F4 & 3 & 19 \\
\hline LT:STb:EAST1:Paa:F4 & 2 & 8 \\
\hline Other & 2 & $\mathbf{4 6}$ \\
\hline Total ETEC F4 & $\mathbf{2 7}$ & 0 \\
\hline ETEC F18 & & 2 \\
\hline STa:STb:AIDA:F18 & 3 & 1 \\
\hline LT:STa:STb:EAST1:F18 & 0 & $\mathbf{3}$ \\
\hline 0ther & 3 & $\mathbf{6 3}$ \\
\hline Total ETEC F18 & $\mathbf{6}$ & $\mathbf{6 3}$ \\
\hline ETEC F5-F41 & & $\mathbf{1 3}$ \\
\hline STa:Paa:F5 & 17 & 0 \\
\hline Other & $\mathbf{1 7}$ & \\
\hline Total ETEC F5-F41 & $\mathbf{2 0}$ & \\
\hline 0ther & 13 & \\
\hline LT:STa:STb:EAST1:Paa & & \\
\hline Other & & \\
\hline Total & & \\
\hline
\end{tabular}

\section{Discussion}

Our results demonstrate that the most important pathotypes associated with diarrhea, ETEC F4, ETEC F18, and ETEC F5, and edema disease, STEC F18, are present in both countries. On the other hand, typing using additional virulence genes permitted further discrimination of the isolates, which demonstrated that distinct virotypes predominate in each country. Interestingly, the ETEC-STEC F18 pathotype, was very frequently observed in Vietnam, but was not identified in isolates from Quebec. Thus, virotyping is a valuable tool for monitoring the geographical distribution of virotypes worldwide and the emergence of new pathogenic variants. We are presently setting up a database for the global surveillance of animal pathogenic E. coli.

\section{References}

DebRoy, C. \& Maddox, C. W. (2001). Animal Health Research Reviews 2, 129-140.

Ngeleka M, Brereton L, Brown G, Fairbrother JM. 2002. Avian Dis 46:143152. 


\title{
P.461
}

\section{Identification of virulence genes of E. Coli in diarrheic pigs in the preweaning and postweaning period by simple and duplex PCR assay}

\author{
Jorge R. Lopez \\ Universidad Nal. Autonoma de Mexico, México, DF, Mexico
}

\section{Introduction}

Diarrhea in pigs from the nursery and post-weaning are mainly of infectious origin and cause economic losses of great magnitude. Strains of enterotoxigenic E. coli (ETEC) and E. coli that produce Shiga toxins (STEC) cause this disease. The virulence factors such as fimbriae and toxins produced by this bacterium are involved in the establishment of the disease. Fimbriae and most common toxins produced by E. coli are F4, F5, F6, F18, F41, STa, STb, LT and STx. In the present study, the aim was to identify virulence genes of E. coli associated with porcine diarrhea using PCR.

\section{Material and Methods}

One hundred and sixty six fecal samples obtained from pigs with diarrhea in five Mexican farms, shows that 113 belong to pigs with diarrhea and 53 to pigs without diarrhea. E. coli strains were isolated in Eosin Methylene Blue (EMB) solid media and MacConkey subsequently underwent biochemical tests. The extraction of DNA from the isolates was done according to Zhang (5). PCR assay simple and duplex using eight specific oligonucleotides as primers were used for the identification of the eight virulence genes (LT, STa, STb, Stx1, Stx2, F18, F41 and F4). The conditions of the PCR's used were in accordance to what has already been reported, with some modifications (1, 2, 3,4). Amplification products were identified by agarose gels and stained with $2 \%$ ethidium bromide. Reference strains were used as positive and negative controls for each one of the genes.

\section{Results}

One hundred and fifty samples corresponded to E. coli, where 110 (73.33\%) were positive for one or more virulence factors of ETEC and EHEC, of which 30 (52.7\%) were from nursing piglets and 41 of weaned pigs. Strains from piglets without diarrhea, 39 $(84.8 \%)$ had one of the genes studied (Table 1).

\section{Discussion}

STa was the toxin detected in $31 \%$, consistent with studies by Thuy N., Do et al., Cicuta M.E. et al. and Chen X et al. $(6,7)$ The F41 fimbriae gene was found in more than 59\% unlike other countries where F41 is detected in less than 3\%. This is of great importance, since it is a new finding and allows us to consider that the strains of E. coli isolated from pigs in Mexico, exhibit different virulence gene profile from those reported by other countries (Table 2). There were 19 combinations; the most frequent was STa/F41 at $11.3 \%$. This work is of great importance, since it is one of the first made in Mexico on the detection of virulence genes of E. coli in nursing and weaned pigs, using this technique suggests that both the fimbriae and toxins are important for the presentation of diarrhea in nursing and weaned pigs. For this reason, our work in a second step will allow us to develop a rapid and accurate diagnostic method of this enteric disease related to E. coli infections.
Table 1. Positive strains of E. coli in piglets with and without diarrhea.

\begin{tabular}{|c|c|c|}
\hline Pigs & Isolates of E. Coli & $\begin{array}{c}\text { Percentage of positive } \\
\text { strains of E. Coli }\end{array}$ \\
\hline Nursery & 57 & 52.63 \\
\hline Weaning & 47 & 87.23 \\
\hline Without diarrhea & 46 & 84.78 \\
\hline Total & 150 & 73.33 \\
\hline
\end{tabular}

Table 2. Percentage of virulence factors in strains of E. coli.

\begin{tabular}{|c|c|}
\hline Genes Identified & Presence of genes in E. Coli strains (\%) \\
\hline F41 & 59 \\
\hline STa & 31 \\
\hline STb & 18 \\
\hline LT, F18 & 8 \\
\hline STx2, F4 & $\leq 6$ \\
\hline STx1 & 0 \\
\hline
\end{tabular}

\section{References}

1. Imberechts, H., Bertschinger, H. U., Nagy, B., Deprez, P., Pohl, P. 1997. Adv Exp Med Biol. 412: 175-183.

2. Osek, J., Gallien, P., Truszczynski, M., Protz, D. 1999. Comp Inmmunol Microbiol Infect Dis. 22(3): 163-174.

3. Ojeniyi, B., Ahrens, P., Meyling, A. 1994. Zentralbl Veterinarmed B. 41(1): 49-59.

4. Vu-Khac, H., Holoda, E., Pilipcinec, E., et al. 2006. Vet Res. 20; 2: 10.

5. Zhang, W., Zhao, M., Ruesch, L., Omot, A. and D. Francis. 2007. Veterinary Microbiology 123: 145-152.

6. Do T, Phu, Huyen X, Tuan X, Quy N, Steve J, et al. 2006. Journal of medical Microbiology 55: 93-99.

7. Cicuta M. E, Parma A. E, Viñas M. R, Sanz M.E, Boeheringer S. I, et al. 1999-2000. Rev. Vet 10/11, 1y2. 


\title{
P.462
}

\section{Comparing PCR-methods diagnosing fimbriae and toxins with serotyping of $E$. coli}

\author{
Sigbrit Mattsson; Lennart Melin; Per Wallgren \\ Dept of Animal Health and Antimicrobial Strategies, Uppsala, Sweden
}

\section{Introduction}

Serotyping of $E$. coli from piglets with diarrhoea gives a historical view of disease patterns, but is laborious as there are more than 170 O-serotypes of $E$. coli (1). The panels used must for practical reasons be limited, and despite continuous updating around $50 \%$ of the samples often turn out as O?. As the most essential for clinicians are to achieve knowledge of whether the strains are potentially pathogenic or not (2) diagnosing presence of adhesion fimbriae and enterotoxin production instead of serotyping is tempting. Thus, PCR-methods have replaced serotyping in many laboratories.

Despite change of method, still only around $50 \%$ of the samples in Sweden were diagnosed as potentially pathogenic. When these PCR-positive isolates were serotyped, in general only $\mathrm{O} 149$ and 0147 were diagnosed. Thus, it appeared that some information received by serotyping had been lost. Therefore the aim of this work was to serotype $E$. coli isolates previously diagnosed by PCR.

Fig 1. Serotypes vs toxins and adhesion diagnosed by $P C R$.

\begin{tabular}{|c|c|c|c|c|c|c|c|}
\hline Serobype & $(n)$ & $L T$ & & STa: & $S T b$ & $\sqrt{12 e}$ & $=$ \\
\hline 098 & 5 & - & & 1 & $\cdot$ & 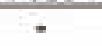 & 4 \\
\hline 0115 & 1 & - & & - & 1 & - & 0 \\
\hline 0138 & 3 & - & & 1 & 3 & - & 1 \\
\hline 0139 & 6 & 1 & & - & 1 & - & 5 \\
\hline 0141 & 23 & - & & 4 & 2 & - & 17 \\
\hline 0147 & 23 & $\therefore$ & & 13 & 15 & 1 & 6 \\
\hline O149 & 50 & 49 & & 10 & 49 & - & 0 \\
\hline 0157 & 6 & - & & - & 1 & - & 5 \\
\hline $8,9,45,179$ & 9 & - & & - & . & - & 9 \\
\hline O? & 180 & 1 & & 11 & 23 & - & 154 \\
\hline TOTAL & 306 & 51 & & 40 & 95 & 1 & 201 \\
\hline Serotype & $n$ & F4 & F5 & F6 & FI8 & F4I & - \\
\hline 098 & 5 & $\cdot$ & 1 & $\cdot$ & - & 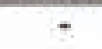 & 4 \\
\hline 0115 & 1 & - & $\cdot$ & - & $=$ & - & 1 \\
\hline 0138 & 3 & - & - & - & 2 & - & 1 \\
\hline 0139 & 6 & 1 & - & 1 & - & - & 4 \\
\hline 0141 & 23 & - & 4 & - & - & - & 19 \\
\hline 0147 & 23 & $=$ & 1 & - & 15 & - & 7 \\
\hline 0149 & 50 & 49 & - & 2 & 2 & - & 1 \\
\hline 0157 & 6 & - & - & - & - & - & 6 \\
\hline $8,9,45,179$ & 9 & : & $B$ & - & : & - & 9 \\
\hline O? & 180 & 3 & 2 & - & 6 & 4 & 154 \\
\hline TOTAL & 306 & & 51 & 40 & 95 & 1 & 292 \\
\hline
\end{tabular}

\section{Materials \& Methods}

E. coli from pig herds with diarrhoea during 2009 were analysed for presence of toxins (LT, STa, STb, VT2e) and adhesion fimbriae (F4, F5, F6, F18, F41) by multiplex PCR systems. All isolates were thereafter serotyped with a panel of 16 different O-groups, previously confirmed to be the most common in Swedish pigs with diarrhoea caused by E. coli. Also, the MIC-values to 12 different antimicrobial substances were analysed (3).
Fig 2. Resistance to 12 substances in 306 E. coli strains.

\begin{tabular}{lccllcc}
\hline & \multicolumn{2}{c}{ Resistance } & & \multicolumn{2}{c}{ Resistance } \\
\cline { 2 - 3 } \cline { 6 - 7 } Substance & n & $\%$ & & Substance & n & $\%$ \\
\hline Sulfa & 77 & 25 & & Ciproflox & 18 & 6 \\
Streptomyc & 71 & 23 & & Kanamyc & 15 & 5 \\
Ampicil & 61 & 20 & & Tetracyc & 9 & 3 \\
Trimetho & 57 & 19 & & Gentam & 3 & $I$ \\
Chloramfen & 24 & 8 & & Cefotax & 0 & 0 \\
Nalidixic ac & 18 & 6 & & Florfen & 0 & 0 \\
\hline
\end{tabular}

Fig 3, Antimicrobial resistance and properties of $E$. coll

\begin{tabular}{lccccccc}
\hline & & \multicolumn{1}{c}{ Resistant to substances (12 tested) } \\
\cline { 3 - 8 } & (n) & None & 1 & 2 & 3 & $4-5$ & $6-7$ \\
\hline O-posPCR+ & 149 & 83 & 3 & 11 & 7 & 7 & 9 \\
On \& PCR- & 157 & 113 & 19 & 8 & 12 & 33 & 1 \\
\hline TOTAL & 306 & 196 & 22 & 19 & 19 & 40 & 10 \\
TOTAL & $\%$ & 64 & 7 & 6 & 6 & 13 & 3 \\
\hline
\end{tabular}

\section{Results and Discussion}

As seen in the Fig 1 \& 2, serotyping identified 126 out of the 306 samples scrutinised (41\%). The PCRs identified 110 isolates as potentially pathogenic (36\%). Generally these strains belonged to serotypes $\mathrm{O} 149$ or O147. Serotypes O8, O9, O45 and OX179 were never identified by the PCR systems. Together PCR and serotyping identified 149 strains as potentially pathogenic (52\%).

The resistance pattern was favourable (3) and fairly equal for different categories of $E$. coli (Figs 2 \& 3). More than $91 \%$ of the strains were sensitive to 8 of the 12 substances tested. Sixteen strains (5\%) were resistant to 4 substances or more.

In conclusion, $\mathrm{PCR}$ will diagnose some $E$. coli strains that express common pathogenic factors. However, some potentially pathogenic serotypes may remain undetected. Serotyping is still a diagnostic tool to use as a complement to PCR in herds with diarrhoeic problems.

\section{References}

1. Ørskov \& Ørskov (1984) Serotyping of E. coli. In: Methods in Microbiology. Vol.14, 43-112.

2. Gyles (1992) E. coli cytotoxins and enterotoxins. Can. J. Microbiol. 38, 734-746.

3. www.sva.se Search for SVARM. 


\title{
P.463
}

\section{Vaccination with Coliprotec ${ }^{\circledast}$ vaccine for the prevention of post-weaning diarrhea associated with F4(K88)-positive enterotoxigenic Escherichia coli (ETEC)}

\author{
Eric Nadeau $^{1}$ Danielle Tremblay' ${ }^{1}$ John M. Fairbrother ${ }^{2}$ \\ 1. Prevtec Microbia, Saint-Hyacinthe, QC, Canada; 2. University of Montreal, Saint-Hyacinthe, QC, Canada
}

\section{Introduction}

ETEC harboring F4 (K88) fimbriae are recognized as the major cause of post-weaning diarrhea (PWD) (1). A genetically inherited receptor for F4 (F4R) located in the villous brush border facilitates binding of ETEC-F4 to intestinal villi leading to colonization and disease. Antimicrobials are frequently used on farms to treat PWD but emergence of resistance has been observed for causative strains (2). The objective of this controlled challenge study was to evaluate the efficacy of a single oral dose of Coliprotec $^{\oplus}$ (Prevtec microbia Inc.), an avirulent live E. coli vaccine, to prevent PWD caused by ETEC-F4 in situations of low and high infectious pressure.

\section{Materials and Methods}

The study consisted of 2 trials, each including a group vaccinated with Coliprotec ${ }^{\circ}$ and a group receiving sterile water as a placebo. Trial A (low infectious pressure) consisted of 20 pigs per group with 5 and 8 RF4-positive pigs for the vaccinated and placebo groups, respectively. Trial B (high infectious pressure) consisted of 20 and 21 pigs, with 18 and 20 RF4-positive pigs, for the vaccinated and placebo groups, respectively. For both trials, weaned pigs of $18 \pm 1$ days of age were introduced into isolation facilities on day 0 . At day 1 , pigs received an oral dose of the Coliprotec ${ }^{\circ}$ vaccine using a syringe, as per label instructions, or placebo. On day 8, all pigs were challenged via gavage with the virulent ETEC-F4 strain ECL 8559. The challenge inocula contained $5 \times 10^{8}$ and $1 \times 10^{9} \mathrm{CFU}$ per pig for the trials $A$ and $B$, respectively. Necropsies were carried out on day 11. Efficacy parameters included ileal colonization of the challenge strain (day 11), fecal excretion of the challenge strain, accumulation of fluid in the intestines (day 11; scored from 0 for normal to 3 for presence of more liquid or totally liquid), diarrhea (scored from 0 for normal to 4 for totally liquid), and daily weight gain (DWG).

\section{Results}

Trial A: The prevalence of pigs excreting the challenge strain at days 9 and 11 was significantly lower $(p<0.0001)$ for vaccinates ( 0 and $26 \%$ ) than for placebo pigs ( $100 \%$ for both days). The colonization level of the challenge strain in the ileum was also lower $(p<0.0001)$ for vaccinates $\left(4.0 \times 10^{6} \mathrm{CFU} / \mathrm{g}\right)$ than for placebo pigs $\left(1.4 \times 10^{8} \mathrm{CFU} / \mathrm{g}\right)$. Severity of diarrhea and accumulation of fluid in the intestine was significantly lower for vaccinates than for the placebo group (Table 1). Finally, DWG was significantly higher after challenge for vaccinates than placebo pigs (Table 1).
Table 1: Severity of diarrhea and intestinal fluid ${ }^{1}$ and DWG ${ }^{2}$

\begin{tabular}{|l|l|c|c|}
\hline Parameter & Group $^{3}$ & Trial A & Trial B \\
\hline Diarrhea & Vaccinates & $1.5 \mathrm{a}$ & $2.0 \mathrm{a}$ \\
\hline & Placebo & $3.5 \mathrm{~b}$ & $5.0 \mathrm{~b}$ \\
\hline Intestinal fluid & Vaccinates & $2.0 \mathrm{a}$ & $3.0 \mathrm{a}$ \\
\hline & Placebo & $6.5 \mathrm{~b}$ & $10.0 \mathrm{~b}$ \\
\hline DWG & Vaccinates & $410 \mathrm{a}$ & $400 \mathrm{a}$ \\
\hline & Placebo & $308 \mathrm{~b}$ & $44 \mathrm{~b}$ \\
\hline
\end{tabular}

${ }^{1}$ Median of cumulative scores for diarrhea ( 3 days post-challenge) or for content in the jejunum, ileum, colon, caecum, and rectum. ${ }^{2}$ Mean daily weight gain (gram). ${ }^{3}$ Groups included all pigs (RF4-positive and RF4-negative). a,b values in a row for a parameter with different superscripts differ significantly $(\mathrm{p}<0.05)$.

Trial B: All pigs of both vaccinated and placebo groups excreted the challenge strain on days 9 to 11 . However, vaccination significantly reduced ( $p<0.0003$ for each day) the level of excreted challenge bacteria in feces by 1.4, 1.7 and 2.5 LOG for days 9, 10 and 11 , respectively. The colonization level of the challenge strain in the ileum was also lower $(p<0.0001)$ for vaccinates $(8.5 x$ $\left.10^{4} \mathrm{CFU} / \mathrm{g}\right)$ than for placebo pigs $\left(4.1 \times 10^{7} \mathrm{CFU} / \mathrm{g}\right)$. As observed in trial A, severity of diarrhea, accumulation of fluid in the intestines and weight loss were significantly lower for vaccinates than for placebo pigs (Table 1).

\section{Discussion and Conclusion}

This controlled challenge study demonstrate the efficacy of an oral single dose of Coliprotec ${ }^{\circ}$ to significantly reduce intestinal colonization, excretion, accumulation of fluid in the intestines and diarrhea when the overall infectious pressure is either low or high. In both situations, vaccination with Coliprotec ${ }^{\bullet}$ induced a significant protection after virulent ETEC-F4 challenge in $18 \pm 1$ day-old weaned pigs resulting in normal growth rates after challenge compared to the rapid weight loss observed for unvaccinated pigs.

\section{References}

1. Fairbrother J. M. et al. (2005). Anim Health Res Rev. 6: 17-39.

2. Maynard C. et al. (2003). Antimicrob Agents Chemother. 47: 32143221. 


\title{
P.464
}

\section{Control of neonatal diarrhea with commercial vaccines}

\author{
SeungYoon Lee; PilSoo Jeong \\ Hanbyol Farm Tech, Gyeonggi-do, Namyangju-city, Korea
}

\section{Introduction}

Neonatal diarrhea causes poor weaning weight and high preweaning mortality. Recently, some farms have had this problem quite a long time. We tried to control piglet diarrhea with different commercial vaccine at two different large herds.

\section{Materials and Methods}

Two herds have around 1,500 sows each were selected for this trial. A herd has had piglet diarrhea problems for about one month(Fig1). B herd has had this problem for about one year(Fig1). Laboratory results of each herd show multiple bacterial and viral infections(Table1). Three commercial vaccines were inoculated to pregnant sows in this trial(Table2,3).

Fig 1. Clinical signs of piglet diarrhea (Top-A herd, Bottom-B herd)
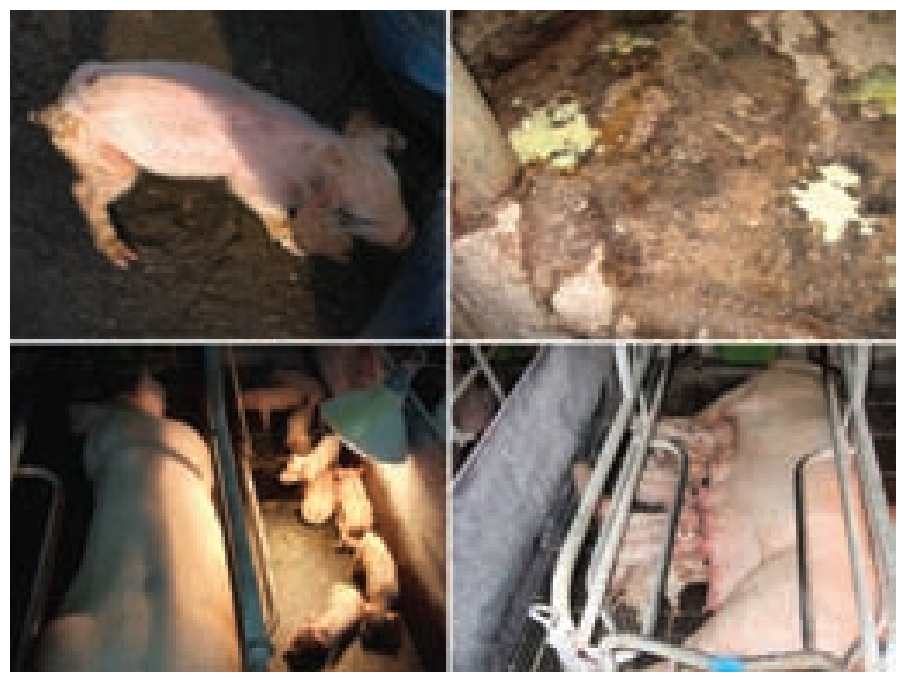

Table 1. Lab. Results for feces of piglets

\begin{tabular}{|c|c|c|c|c|c|c|}
\hline Herd & E.coli & Sal & Clo & TGE & PED & Rota \\
\hline A Herd & $0 / 4$ & $0 / 4$ & $2 / 4$ & $0 / 3$ & $0 / 3$ & $3 / 3$ \\
\hline B Herd & $3 / 3$ (K88) & $0 / 3$ & $3 / 3$ & $0 / 10$ & $3 / 10$ & $9 / 9$ \\
\hline \multicolumn{6}{|c|}{ E.coli is hemolytic E.coli, Sal is Salmonella, Clo is Clostridium } \\
\hline
\end{tabular}

Table 2. Commercial vaccines used for these trials

\begin{tabular}{|c|l|}
\hline Vaccine & Contents \\
\hline A & $\begin{array}{l}\text { Modified live TGE, Rotavirus(G\&A), E.coli pilus antigens (K88,K99,F41,987P), } \\
\text { Clostridium perfringens toxoid }\end{array}$ \\
\hline B & Modified live TGE and Rotavirus \\
\hline C & E.coli bacterin, Clostridium perfringens beta toxin \\
\hline
\end{tabular}

Table 3. Vaccine programs

\begin{tabular}{|l|c|c|c|c|c|}
\hline Herd & \multicolumn{3}{|c|}{ A herd } & \multicolumn{2}{c|}{ B herd } \\
\hline Vaccine & A & B & None & A & C \\
\hline Program & Two shot & Two shot & none & One shot & Two shot \\
\hline
\end{tabular}

\section{Results (see below)}

Fig 2. Diarrhea rate $\%$, average weight of weaners and average number of wean piglets per sow of A herd (weaning age is around 26 days).

Fig 3. Diarrhea rate $\%$ of $B$ herd.

Fig 4. Parity effect on diarrhea rate $\%$ of $B$ herd.

\section{Discussion}

Neonatal diarrhea causes poor survival rate before weaning and weaning weight. Most of neonatal diarrhea in Korea caused by multiple agents including Rotavirus, Porcine epidemic virus(PED), Clostridium and pathogenic E. coli. Vaccine A contain multiple viral and bacterial antigen except PED virus and shows better results for controlling neonatal diarrhea in these two herds(Fig2,3). Weaning weight increased by $0.4 \mathrm{~kg}$ per a head. Low parity sows shows neonatal diarrhea more often than high parity sows(Fig4).

\section{Reference}

See product label for directions for use and other information.
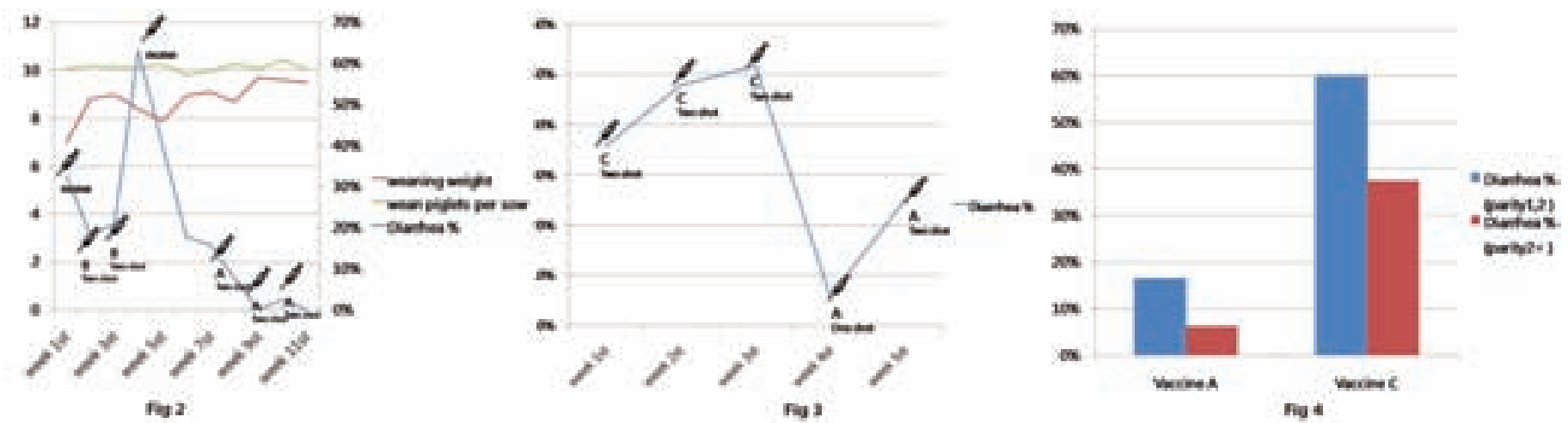


\title{
P.465
}

\section{Effects of Tilmicosin and Apramycin on proliferation of enterotoxemic Escherichia coli and release/production of shiga toxin $2 e$}

\author{
Masuo Sueyoshi; Tomoko Kuroda; Ryoko Uemura; Hiroshi Nagatomo \\ Department of Veterinary Hygiene, Faculty of Agriculture, University of Miyazaki, Miyazaki, Japan
}

\section{Introduction}

The fatal accidents of piglets resulting from treatment of edema disease with antibiotics have made vets to hesitate the use of antibiotics to this disease. Various efforts have been made in the field for the treatment of edema disease, but no control or treatment methods has been established. We reported that the susceptibilities of the isolated Enterotoxemic E. coli (ETEEC) had diminished in regard to antimicrobials[1]. Furthermore, we found that more shiga toxin (Stx) 2e accumulated in the bacterial cells than was released into supernatant[2]. Therefore, evaluation of the direct effect of antibiotics to ETEEC in an experimental system will give useful information for selecting appropriate antibiotic in the field. In this study, in vitro effects of Tilmicosin (TMS) and Apramycin (APM) on proliferation of ETEEC and release/production of Stx were investigated.

\section{Materials and Methods}

The strains used for this study were ETEEC MVH 269 (O 139 serotype, stx2e+, fedA+, LT-, ST-, beta-hemolytic+) isolated from the pigs with E. coli enterotoxemia. As test antibiotics, TMS, APM, Ampicillin (ABPC) and Enrofloxacin (ERFX) were used. For examination of MIC, agar plate dilution method was used, according to CLSI (2002). The organism was inoculated into BHIB (Difco, USA). The medicated BHIB was prepared at the final concentrations of ${ }^{*} 1$ and ${ }^{*} 50$ of MIC. Non-medicated BHIB was used as the control. The organism suspension was inoculated into each substance or control BHIB with final bacterial concentration of approximately $8.0 \log 10 \mathrm{cfu} / \mathrm{mL}$ and cultured in the shaking incubator for $4 \mathrm{hrs}$ at $37 \mathrm{C}$. Then, each BHIB was measured for the number of viable colibacilli. BHIB diluted properly with PBS was smeared to TSA (Difco). For the preparation of released Stx2e, after centrifugation (8,000rpm, $10 \mathrm{~min}$ ) of BHIB, supernatant was filtered with a 0.20 um filter. For the preparation of produced Stx2e, after ultrasonic fragmentation in ice, BHIB was centrifuged and supernatant was filtered. Vero cell sheets were cultured on 96 well microplate. After incubation for $72 \mathrm{hrs}$ with 5\%CO2 at 37C, CD50 (50\%Cytotoxicity Dose) was determined.

\section{Results}

MICs of ETEEC MVH269 for TMS, APM ABPC and ERFX were 64ug/ $\mathrm{mL}, 4 \mathrm{ug} / \mathrm{mL}, 256 \mathrm{ug} / \mathrm{mL}$ and $<0.125 \mathrm{ug} / \mathrm{mL}$, respectively.

In the TMS medication, the number of viable colibacilli significantly decreased at x50 MIC compared to the control. No significant differences were observed in released Stx2e at both $\mathrm{x} 1$ and x50 MIC, whereas produced Stx2e decreased dosedependently (Fig. 1a, 1b). In the APM medication, the number of viable colibacilli decreased at 50x MIC (Fig. 2). The amount of released /produced Stx decreased compared to the control (Fig. $2 a, 2 b)$. On the other hands, in the ABPC and ERFX medication, the number of viable colibacilli decreased significantly at $1 \times$ MIC and 50x MIC. However, in the ABPC, the amount of released Stx markedly increased compared to control.

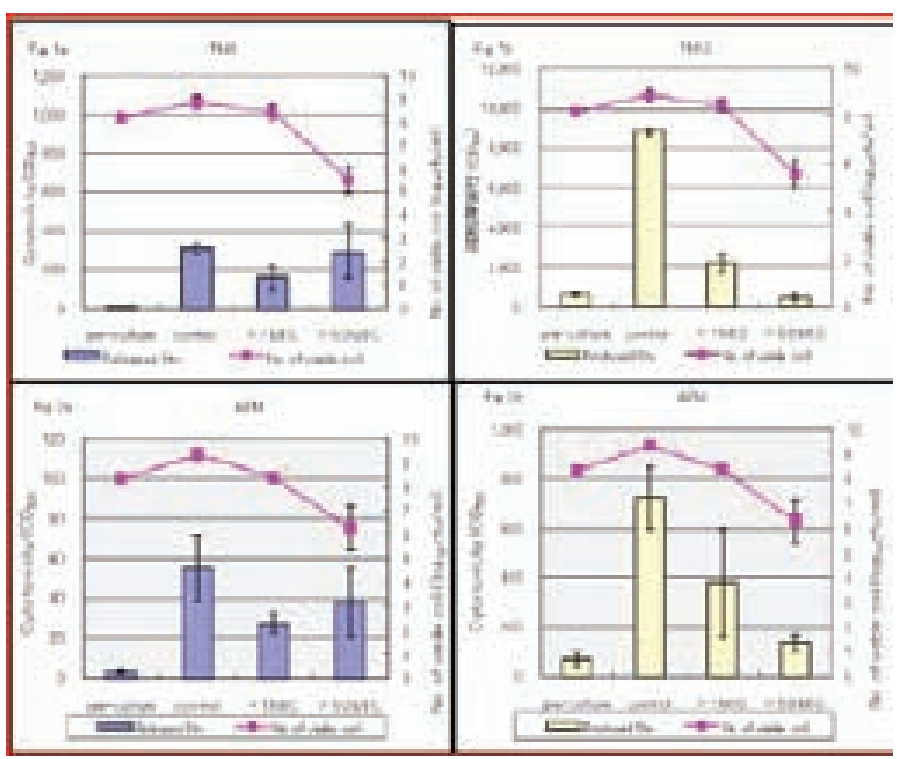

\section{Discussion}

ETEEC (MVH269) strain tested was not susceptible to ABPC, highly susceptible to ERFX and APM, and moderately susceptible to TMS. The reduction rate of viable colibacilli of TMS and APM were comparable to ABPC or ERFX, but the reduction rate of produced Stx2e was higher, suggesting the possibility that TMS and APM inhibited Stx2e production.

\section{References}

1. Uemura R., Sueyoshi M. et al., Microbiol. Immnol., 47: 57-61(2003).

2. Uemura R., Sueyoshi M. et al., J. Vet. Med. Sci, 66: 899-903(2004). 


\title{
P.466
}

\section{Comparison of Porcilis ${ }^{\circledR}$ Coli $6 \mathrm{C}$ with another vaccine by measuring maternally derived antibody levels of $E$. coli virulence factors in piglets}

\author{
$\underline{\text { Rut Menjon Ruiz }}{ }^{1}$ Antonio Ubiergo ${ }^{2}$ Teresa Abizanda² Jorge Sistac ${ }^{2}$ Jesus M. Bollo $^{1}$ Marta Jimenez ${ }^{1}$ Jesus V. Lopez $^{1}$ \\ 1. Intervet/Schering-Plough A.H., Alcobendas, Spain; 2. Piensos Mazana, Capella, Spain
}

\section{Introduction}

Enterotoxigenic E.coli associated with neonatal diarrhea may posses one or more F4(K88), F5(K99), F6(987p) and F41 fimbriae, with K88 having the highest prevalence (1). Adequate immunization of sows is fundamental for providing piglets with effective protection against this disease via the colostrum(2).

The object of the study was to compare the levels of specific antibodies against the main E.coli virulence factors in piglets from sows vaccinated with Porcilis ${ }^{\circledR}$ Coli $6 \mathrm{C}$ and another commercial vaccine with the same indication (vaccine $B$ )

\section{Materials and Methods}

The study was performed during 2009 in two phases on a breeding farm with 2400 sows in northern Spain.

Thirty sows from different production cycles were selected together with their piglets: 20 primiparous sows and 10 multiparous (same parities per group). These were divided into two groups (Porcilis coli 6C and Vaccine B). The vaccines were administered at 70 and 90 days of pregnancy.

After farrowing, 3 piglets were randomly selected from each litter $(n=90)$. Blood samples were taken at 7 days of age to measure the level of maternally derived antibody (MDA) specific for the main E.coli virulence factors, acquired via the colostrum . A specific ELISA test (internal ISPAH test) was used, which detects titers against K88ab, K88ac, K99, 987P antigens in serum or colostrum. Titers are expressed in $\log _{2}$ titers. Titers lower than $6 \log _{2}$ are considered to be negative.

Statistical analysis was performed using the General Linear Model (GLM-SPSS 15.0 for Windows), the Mann-Whitney U-test. The Pearson's chi-square test was used for comparisons of means, and the Levene test for comparison of variances.

\section{Results}

The results of the serum sample assays showed significant differences in the mean antibody titers of both groups.

Table 1: Mean $\log _{2}$ antibody titers $( \pm S D)$

\begin{tabular}{|l|c|c|c|c|}
\hline & K88ab & K88ac & K99 & 987p \\
\hline Porcilis $^{\oplus}$ Coli 6C & $9.38^{\mathrm{a}} \pm 0.22$ & $9.95^{\mathrm{c}} \pm 0.22$ & $8.42^{\mathrm{a}} \pm 0.21$ & $8.63^{\mathrm{a}} \pm 0.16$ \\
\hline Vaccine B & $7.84^{\mathrm{b}} \pm 0.32$ & $8.89^{\mathrm{d}} \pm 0.34$ & $8.26^{\mathrm{a}} \pm 0.25$ & $8.56^{\mathrm{a}} \pm 0.19$ \\
\hline
\end{tabular}

$a, b, c, d$ : values with different superscripts in the same columns represent statistically significant differences: ${ }^{a, b}: p<0.001, c, d: p<0.05$.

The distribution of the mean antibody titers for all the virulence factors studied was more uniform in piglets from the Porcilis ${ }^{\circledR}$ Coli $6 \mathrm{C}$ group $(\mathrm{p}<0,01)$, only the titers against K88ab in primiparous sows failed to show a statistically significant difference (Graph 1,Graph 2).
Graph 1: Distribution of $A b$ titers in multiparous sows

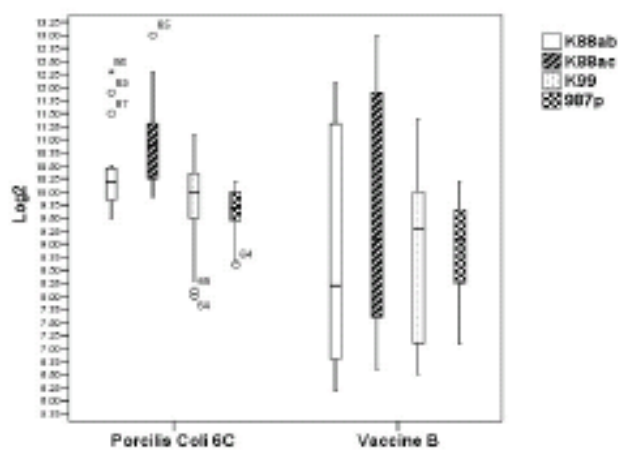

Graph 2: Distribution of Ab titers in primiparous sows

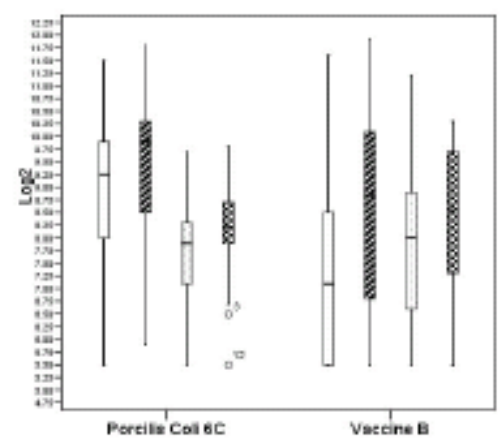

When comparing animals with negative titers for K88ab and K88ac antigens, a higher percentage of negative titers was found in the group of piglets from sows vaccinated with Vaccine $B(p<0,05)($ Graph 3)

\section{Graph 3: \%age piglets with $<6.00$ Ab titers}

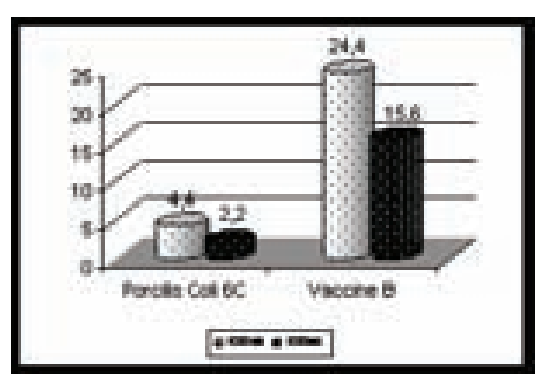

\section{Discussion}

Porcilis ${ }^{\circledR}$ Coli 6 C induced both higher MDA against the most prevalent E.coli virulence factors, and higher titers against the main virulence factor (K88) than another vaccine with the same indication. Consequently, Porcilis ${ }^{\circledR}$ Coli $6 \mathrm{C}$ confers stronger protection against the main antigen involved in neonatal diarrhea in piglets.

\section{References}

1. Henriques, M.R. et al (2008). Proc.20th. IPVS Congress

2. Fairbrother,J.M 1999.In:Straw,B. et al. Diseases of swine 


\title{
P.467
}

\section{Vaccination with SUIVAC EDT ${ }^{\circledR}$ against oedema disease - a field study}

\author{
Anne Jørgensen² Magne Haugum ${ }^{1}$ Hilde M. Juul ${ }^{1}$ Anja Kristoffersen ${ }^{1}$ Bjarne Bergsjø $\varnothing^{1}$ Bente Fredriksen ${ }^{2} \underline{B j ø r n ~ L i u m}^{1}$ \\ 1. National Veterinary Institute, Oslo, Norway; 2. Animalia, Oslo, Norway
}

\section{Introduction}

Oedema disease in pigs is usually caused by infection with F18positive and verotoxin 2e producing hemolytic E. coli O 138, O 139 or 0141 . In some herds this infection may cause considerable economical losses due to high mortality in pigs during the first 1-4 weeks after weaning. This field trial was performed to assess the efficacy and safety of a commercial vaccine against oedema disease in weaned pigs.

\section{Materials and Methods}

Herds

Two conventional herds where high mortality due to oedema disease in weaned pigs had been a persistent problem for some years were selected to participate in this trial. The diagnosis oedema disease was confirmed by autopsy and bacteriology positive for haemolytic E.Coli O 138, O 139 or O 141.

Herd 1: integrated production with 22 week batches with 56 sows per batch.

Herd 2: piglet production with 3 week batches with 48 sows per batch.

\section{Study design}

A double blinded randomized block design study, with herd and litter as block factors, was performed in 2008-2009. A total of 1980 piglets were vaccinated at 2 and 4 weeks of age. Within each litter, the piglets were randomized to get either Suivac EDT ( $n=981)$ or placebo (phosphate buffered saline) $(n=999)$. Weaning took place a minimum of three days after last vaccination. Clinical symptoms and adverse reactions were recorded from weaning to $30 \mathrm{~kg}$. Post mortem examination was performed on all piglets that died or were euthanized during the study period. Only pigs with characteristic pathological lesions, and from which haemolytic E. coli O 138, 139 or O 141 were isolated, were diagnosed as "dead due to oedema disease".

\section{Statistical analysis}

To evaluate the association between the probabilities of getting oedema disease and placebo contra real vaccination, a mixed effect logistic regression model taking into account the effect of herd and litter was used. The analysis was done in R 2.9.1 (1), library Ime4 (2).

\section{Results}

A total of 82 pigs ( $4.1 \%$ ) died during the study period. Haemolytic E. coli O139 was identified in 54 pigs with pathological lesions indicating oedema disease. Another 9 pigs had pathological changes consistent with oedema disease but negative bacteriology. Nineteen pigs died of other reasons. Table 1 shows the distribution of confirmed cases of oedema disease per herd and treatment
Table 1. Distribution of piglets $n(\%)$ that died of oedema disease.

\begin{tabular}{|c|c|c|c|}
\hline & Placebo & Vaccine & Total \\
\hline Herd 1 & $37 / 740(5.0)$ & $13 / 733(1.8)$ & $50 / 1473(3.4)$ \\
\hline Herd 2 & $2 / 241(0.8)$ & $2 / 266(0.8)$ & $4 / 507(0.8)$ \\
\hline Total & $39 / 981(4.0=$ & $15 / 999(1.5)$ & $54 / 1980(2.7)$ \\
\hline
\end{tabular}

The odds ratio for getting oedema disease was 0.33 for vaccinated animals $(p=0.002)$. When litter was used as random effect, herd did not contribute with further explanation in the model. The Suivac EDT vaccine was safe and did not produce marked adverse reactions.

\section{Discussion}

In this field trial the prevalence of postweaning mortality due to oedema disease was markedly reduced in pigs vaccinated with Suivac EDT compared to pigs in the placebo group. The results indicate that vaccination may be a good alternative to high doses of zink oxide as food additive or treatment with antiserum to prevent oedema disease in weaned pigs. A higher beneficial effect of vaccination with VT2e-toxoid against oedema disease has previously been documented in a Danish study (3)

\section{Acknowledgements}

We would like to thank the veterinarians Håvard Okkenhaug, Eli Maria Stenklev, Øyvind Skei and Regina Boch for practical work in the herds.

\section{References}

1. R Development Core Team (2009). ISBN 3-900051-07-0, http://www.R-project.org.

2. Douglas Bates and Martin Maechler (2009). http://CRAN.R-project.org/package=Ime4

3. Johansen $M$ et al. 1997. Can J Vet Res; 61: 280-285 


\title{
P.468
}

\section{Comparison of the antimicrobial resistance of pathogenic escherichia coli in diseased pigs in Vietnam and Quebec, Canada}

\author{
Thuy N. Do ${ }^{1}$ Darren J. Trott ${ }^{2}$ Éric Nadeau ${ }^{3}$ Clarisse Desautels ${ }^{4}$ John M. Fairbrother ${ }^{4}$ \\ 1. National Institute of Veterinary Research, Hanoi, Vietnam; 2. The University of Adelaide, Adelaide, SA, Australia; 3. Prevtec Microbia Inc., \\ Saint-Hyacinthe, QC, Canada; 4. Reference laboratory for E. coli, Faculté de médecine vétérinaire, Université de Montréal, Montréal, QC, Canada
}

\section{Introduction}

Escherichia coli is an important cause of a wide range of diseases in production animals and humans worldwide, including pigs. In certain cases, animals are a reservoir of infection for humans, either by direct contact, contamination of the environment, or via contaminated food. The objective of this study was to compare the distribution of antimicrobial resistance of pathogenic E. coli isolates from diseased pigs in two geographically distinct countries, Vietnam and Canada, with differing antimicrobial use.

\section{Materials and Methods}

E. coli isolates obtained from diseased pigs (diarrhea, sudden death or edema disease) in 2007 and submitted to the NIVR in Vietnam or the FMV in Québec, Canada, were initially tested by PCR for the presence of defining genes of ETEC and STEC pathotypes. They were then virotyped by colony hybridisation (Ngeleka et al., 2002) for detection of the presence of 20 virulence genes most commonly found in animal pathogenic $E$. coli. The isolates were examined by the disk diffusion test to identify antimicrobial resistance.

\section{Results}

Table 1: Prevalence of antimicrobial resistance in pathogenic E. coli isolates

\begin{tabular}{|l|c|c|}
\hline Antimicrobial & \multicolumn{2}{|c|}{ \% of resistance } \\
\hline & Canada $(\mathrm{n}=124)$ & Vietnam $(\mathrm{n}=50)$ \\
\hline Ceftiofur & 5 & 0 \\
\hline Ampicillin & 67 & 42 \\
\hline Apramycin & 12 & 0 \\
\hline Spectinomycin & 46 & 4 \\
\hline Gentamycin & 7 & 14 \\
\hline Enrofloxacin & 0 & 20 \\
\hline Florfenicol & 12 & 32 \\
\hline Trim-Sulfa & 48 & 82 \\
\hline Neomycin & 23 & 18 \\
\hline Tetracycline & 95 & 92 \\
\hline
\end{tabular}

Figure 1: Prevalence of antimicrobial resistance in porcine pathogenic E. coli isolates from Vietnam and Canada

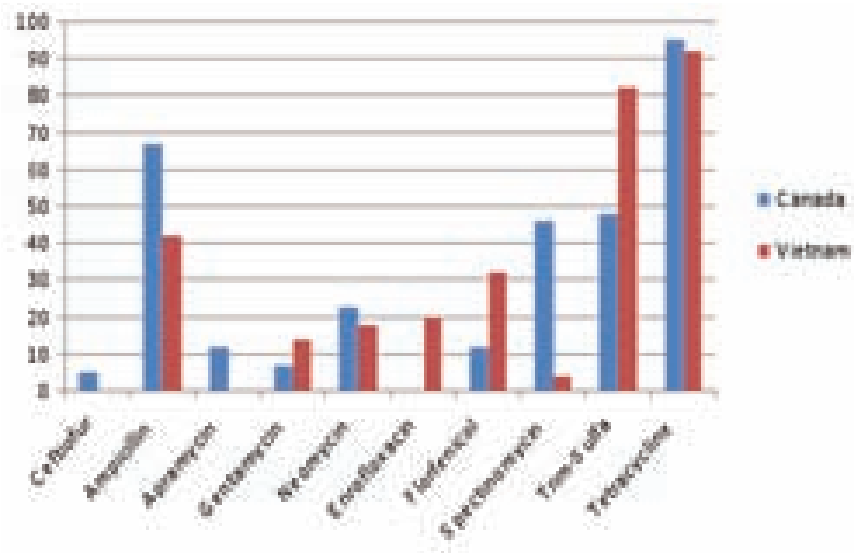

\section{Discussion}

The antimicrobial resistance profiles generated can be considered to be representative of each geographical region. Some interesting similarities and differences were demonstrated between the two data sets. Overall, very high rates of resistance were found to tetracycline in isolates from both countries, Canada and Vietnam (95 and 92\%, respectively). Interestingly, all the Vietnamese isolates were sensitive to ceftiofur and apramycin, whereas no resistance was recorded for enrofloxacin with the Canadian isolates. The similarities and differences in antimicrobial resistance between the two countries could be attributed to variation in veterinary antimicrobial regulation and usage between Canada and Vietnam. This was definitely the case for tetracycline, ampicillin, and sulfa drug use in both countries, and ceftiofur and apramycin use in Canada, and enrofloxacin use in Vietnam. Frequent usage of agents in the past, such as chloramphenicol in Vietnam and nitrofurantoin in Canada also was associated with resistance. Other factors may include the distribution of serotypes and pathotypes between the two countries, and differences in farrowing shed management.

\section{References}

Ngeleka M, Brereton L, Brown G, Fairbrother JM. 2002. Avian Dis 46:143152. 


\title{
P.469
}

\section{In vitro immune stimulating activities of Escherichia coli fimbriae encapsulated with thiolated Eudragit}

\author{
Wonjung Lee; Seungbin Cha; minkyoung Shin; Yumi Roh ; Myunghwan Jung; Hansang Yoo \\ Seoul National University, Seoul, Korea
}

\section{Introduction}

Vaccination has been one of the most effective ways of preventing disease. Oral delivery of vaccine induces both humoral and cellular responses against mucosal infections. Thiolated eudragit as delivery system have been known to exhibit $\mathrm{pH}$ dependent, permeation enhancing, enzyme inhibitory and mucoadhesive properties to improve the peroral absorption efficiencies.

Intestinal infections with Escherichia coli are an important cause of diarrhea and mortality in pigs. Especially enterotoxigenic $\mathrm{E}$. coli (ETEC) infections immediately after birth and weaning are responsible for significant economical losses due to diarrhea, growth retardation, and mortality. The infections are mainly caused by the fimbiral colonization factor F4 (K88) and F18 (F107). In this study, Eudragit-cysteine conjugate were prepared and chacterized for the fimbriae of E. coli.

Immune stimulating activity of F4 and F18-loaded thiolated Eudragit was checked in murine macrophage cell-line.

\section{Materials and Methods}

F4ac and F18ab and ac were detected by PCR amplification from E. coli strains in Korea as described by Lee et al. The fimbria proteins were purified as described by Wim et al. Briefly, the selected E. coli strain were cultured in cultured in tryptic soy broth at $37^{\circ} \mathrm{C}$ overnight with shaking. The bacteria were harvested and suspended with PBS. F18 fimbriae were purified by heat shock $\left(60^{\circ} \mathrm{C}\right.$ for $\left.20 \mathrm{~min}\right)$ and $\mathrm{F} 4$ fimbriae by homogenizing the bacterial suspension using an Ultra Turrax at 24,000 rpm for $15 \mathrm{~min}$. It was centrifuged at 8,500 rpm for $20 \mathrm{~min}$ and then 10,000 rpm for 40 $\mathrm{min}$. The solubilized fimbriae were precipitated with $20 \%(\mathrm{w} / \mathrm{v})$ ammonium sulphate.

Thiolated Eudragit were prepared according to the procedure previously developed by Quan et al. Raw 264.7 was cultured in 12 well plates containing $2.2 \times 105$ cells per well with DMEM supplemented with $10 \% \mathrm{FBS}$ at $37{ }^{\circ} \mathrm{C}$ under $5 \% \mathrm{CO} 2$. After a $18 \mathrm{~h}$ incubation, they were stimulated with $1 \mathrm{ug} / \mathrm{ml}$ of each fimbria protein loaded TEMs. LPS $(1 \mathrm{mg} / \mathrm{ml})$ and unloaded TEMs were used as positive and negative controls, respectively. After stimulation, secreted concentrations of TNF- $a$ and IL- 6 were measured by ELISA method.

\section{Results}

Average particle sized of TEMs(thiolated Eudragit), F4 loaded TEMs and F18 loaded TEMs are $4.2 \pm 0.75 \mu \mathrm{m}, 4.7 \pm 0.50 \mu \mathrm{m}$ and $4.5 \pm 037 \mu \mathrm{m}$, respectively. The morphologies of TEMs, F4 and F18-loaded TEMs were observed as spherical shapes. The particle sizes of TEMs became bigger after loading of fimbria proteins into TEMs. Production of TNF- a from raw 264.7 cells was increased in a time-dependent manner after exposure to F18-loaded TEMs, F4-loaded TEMs, TEMs and LPS. Secretion of IL-6 from raw 264.7 cells was increased in a time-dependent manner after exposure to F18-loaded TEMs, F4-loaded TEMs and LPS.

\section{Discussion}

The size of microspheres is quite important to target vaccines for uptake into the mucosal associated lymphoid tissues (MALTs) of the gut. Also, the majority of the microspheres below $10 \mathrm{~mm}$ in diameter were needed to be transported through the efferent lymphotics within macrophages. Therefore, it will be expected that F4-loaded TEMs and F18-loaded TEMs will be effective for delivery. Production of TNF- $a$ and IL-6 in F4-loaded TEMs and F18-loaded TEMs were higher than production in only F4 and F18 proteins. Compared to F4 protein, F18 protein and F18loaded TEMs produced more TNF- $\alpha$ and IL-6. Therefore, F4 and F18-loaded TEMs could be considered as a candidate for oral vaccine of enterotoxigenic E. coli.

\section{Acknowledgements}

This study was supported by a grant (20070401034009) from Biogreen21 program RDA, Korea, BK21 program for Veterinary Science, and Research Institute of Veterinary Science, Seoul National University, Korea.

\section{References}

1. Wim Van den Broeck et al., Receptor-specific binding of purified F4 to isolated villi. Veterinary Microbiology 68 (1999) 255 263.

2. Ji-Shan Quan et al., pH-sensitive and mucoadhesive thiolated Eudragit-coated chitosan microspheres, International Journal of Pharmaceutics 359 (2008) 205-210 


\title{
P.470
}

\section{Efficacy of the booster dose of SUISENG ${ }^{\circledR}$ : a novel friendly adjuvanted vaccine against neonatal colibacilosis and clostridiosis}

\author{
Nicolas G. Liaño; Xavier Gibert; Mercè Roca; Marta Sitjá
}

HIPRA, Girona, Spain

\section{Introduction}

This work shows the results of one study where the safety and the efficacy of SUISENG ${ }^{\bullet}$ (a vaccine against the Neonatal Piglet Colibacillosis, $C$. perfringens and $C$. novyi enterotoxicosis) were assessed. Applying the basic vaccination and the Revaccination to pregnant sows, the efficacy was evaluated by means the serological response, the transfer of maternal immunity and the clinical signs in challenged piglets with two pathogenic strains of $E$. coli.

\section{Materials and Methods}

SUISENG ${ }^{\circledR}$ was used to vaccinate pregnant gilts. The basic vaccination scheme consists of two doses: the first dose at approximately 6 weeks before farrowing and a second dose at approximately 3 weeks before farrowing. Revaccination on next subsequent gestation, administering one dose 3 weeks before the expected date of farrowing.

The piglets from the second parturition were challenged using two heterologous pathogenic strains to induce neonatal colibacillosis.

Table 1: Studies design

\begin{tabular}{|l|c|c|}
\hline & Study $\mathbf{1}$ & Study 2 \\
\hline No Sows Vaccinated & 5 Suiseng & 5 Suiseng \\
\hline & 5 placebo & 5 placebo \\
\hline No piglets challenged & 39 Suiseng & 56 Suiseng \\
\hline & 52 Placebo & 44 Placebo \\
\hline Challenge & Strain 1 & Strain 2 \\
\hline
\end{tabular}

\section{Results}

\section{Serological response in sows and transfer of maternal immunity}

Next is shown the IgG profile against the F4ac in the sow sera after the immunization. The rest of valences included in the vaccine followed a similar profile. (See figure 1.a)

The figure 1.b shows the presence of antibodies against each valence in the colostrum/milk samples of the sows. The serology of the piglets sera demonstrated the presence of specific antibodies against all the valences.

Figure 1.a and 1.b.Serological response in sows and presence of

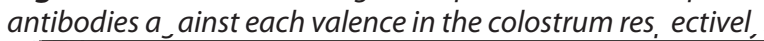

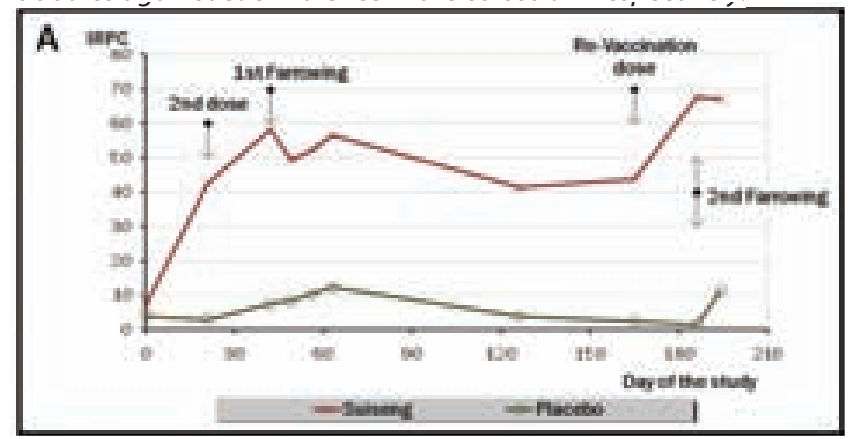

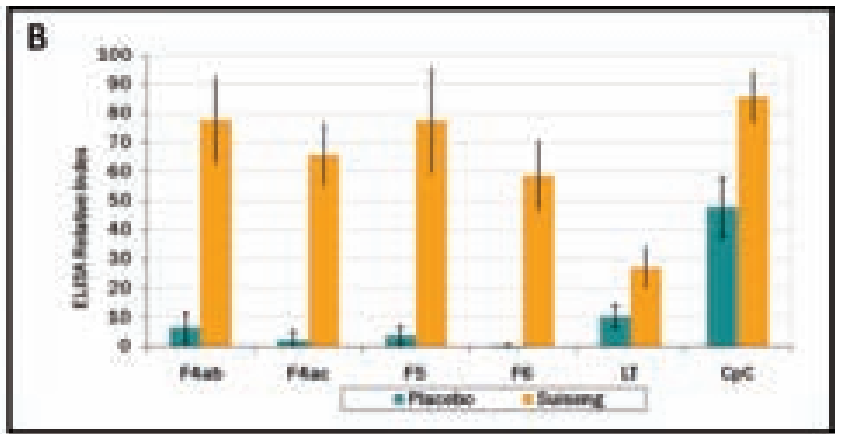

Clinical sign of colibacillosis

The clinical signs after the challenge were evaluated by means a score record during 8 days. Next (figure 2.a) are the results of this score, significant differences between treatments for both challenges were found ( $p>0,05)$.

In the same way, the mortality rates of each group are shown below (see figure 2.b). Significant differences between the Suiseng and the Placebo were demonstrated for the two challenges.

Figures 2.a and 2.b. Clinical signs and mortality rates of each group respectively.

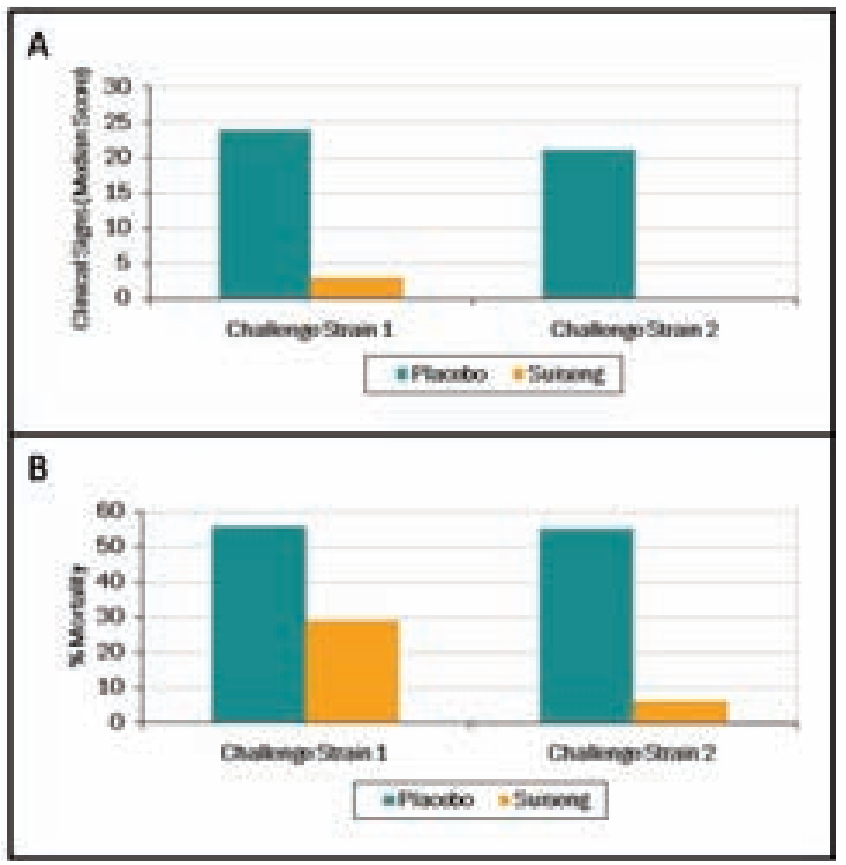

\section{Discussion and conclusion}

The results of this study demonstrated the efficacy of SUISENG ${ }^{\circledR}$ after a booster dose against the experimental infection with enterotoxigenic E. coli. The vaccination reduced significantly the clinical signs and the mortality of the piglets suffering from colibacillosis. 


\title{
P.471
}

\section{Increased antimicrobial resistance for colistin of haemolytic Escherichia coli strains from clinical cases in Belgium using the E-test}

\author{
Frédéric A. Vangroenweghe; Evelyne De Graef; Cora Miry; Frans Castryck \\ DGZ-Vlaanderen, Torhout, Belgium
}

\section{Introduction}

The last few years, acquired resistance to colistin in Escherichia coli (E. coli) has been described quite frequently. It has been shown that the standard agar dilution test is not a reliable method for the detection of colistin resistance in several bacterial species. Therefore, the need for a reliable test to check colistin susceptibility of pathogenic E. coli strains in routine diagnostics was rather high. A practical study, comparing 4 different antimicrobial susceptibility tests revealed that the E-test was the most reliable alternative agar-based colistin susceptibility testing method for use in E. coli strains (1). The objective of the present study is to evaluate the evolution of results obtained following the introduction of the E-test in the diagnostic laboratory procedure.

\section{Materials and Methods}

All samples from the content of the small intestine -positive for hemolytic E. coli- were tested using the E-test (AB Biodisk) with direct reading of the minimal inhibitory concentration (MIC) and expressed in $\mu \mathrm{g} / \mathrm{ml}$. The interpretation towards susceptibility or resistance was performed according to Table 1.

Table 1. Interpretation of antimicrobial susceptibility testing to colistin using the E-test

\begin{tabular}{|c|c|}
\hline Interpretation & $\boldsymbol{\mu g} / \mathbf{m l}$ \\
\hline susceptible & $<4$ \\
\hline intermediately susceptible & $4-16$ \\
\hline resistant & $>16$ \\
\hline
\end{tabular}

\section{Results}

The distribution of antimicrobial susceptibility testing of the (haemolytic) E. coli strains isolated from diagnostic bacteriology of samples from small intestines in the period 2006-2009 are presented in Figure 1. Overall, an evolution from 8\% intermediately susceptible strains to colistin in 2006 to $28 \%$ in 2009 could be observed. On average, 150 strains of haemolytic E. coli were tested in routine diagnostics yearly.

\section{Discussion and Conclusions}

Colistin is used worldwide as an oral antimicrobial agent for the prevention and treatment of neonatal and weaning-associated E. coli infections in piglets.

Considering its extensive use and the fact that antimicrobial formulations are often underdosed (2), it is not surprising to find an increasing colistin resistance in veterinary E. coli strains (3).
Figure 1. Distribution of antimicrobial susceptibility results with the E-test obtained from (haemolytic) E. coli strains isolated in diagnostic bacteriology from 2006 till 2009. S: susceptible, I: intermediate, $R$ : resistant

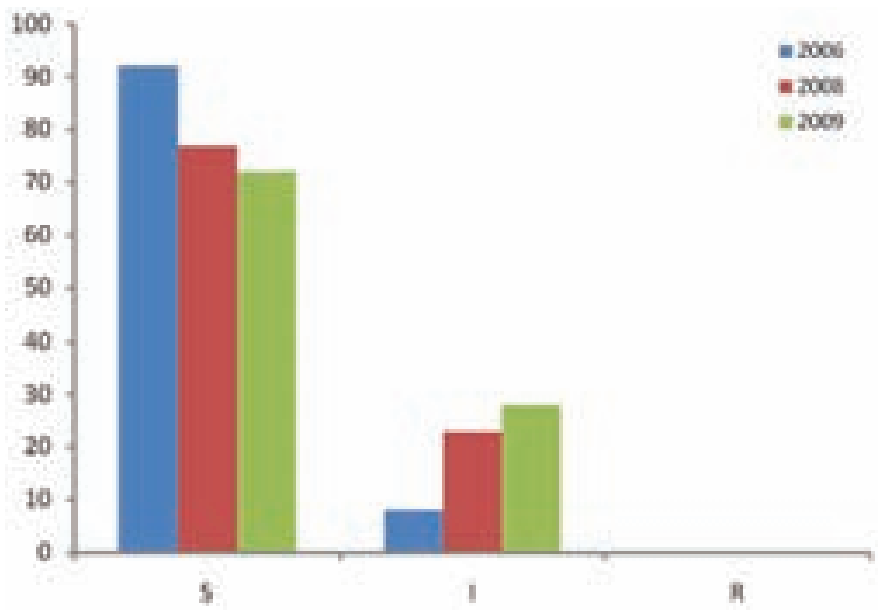

Following introduction of a more reliable antimicrobial susceptibility testing method, using the E-test, the occurrence of a high percentage intermediately susceptible E. coli strains could be observed in Belgium. This is in accordance with the information provided by veterinary practitioners concerning the decreasing efficacy of colistin in the treatment and prevention of E. coli infections, especially after weaning. Monitoring of the further evolution of antimicrobial susceptibility will be necessary in order to guarantee treatment success when applying colistin in antimicrobial formulations for piglets post-weaning.

\section{Acknowledgements}

The financial support of Veepeiler-varken in the development and introduction of the E-test into the routine diagnostic procedure is greatly acknowledged.

\section{References}

1. Boyen F., Vangroenweghe F., Butaye P., De Graef E., Castryck F., Heylen P., Vanrobaeys M., Haesebrouck F. 2010. Disk prediffusion is a reliable method for testing colistin susceptibility in porcine E. coli strains. Veterinary Microbiology, accepted.

2. Timmerman T., Dewulf J., Catry B., Feyen B., Opsomer G., de Kruif A., Maes D. 2006. Quantification and evaluation of antimicrobial-drug use in group treatment for fattening pigs in Belgium. Preventive Veterinary Medicine, 74: 251-263.

3. Harada K., Asai T., Kojima A., Oda C., Ishihara K., Takahashi T. 2005. Antimicrobial susceptibility of pathogenic Escherichia coli isolated from sick cattle and pigs in Japan. Journal of Veterinary Medical Science, 67: 999-1003. 


\title{
P.472
}

\section{Efficacy of SUISENG ${ }^{\circledR}$ : a novel friendly adjuvanted vaccine against neonatal colibacilosis and clostridiosis}

\author{
Nicolas G. Liaño; Xavier Gibert; Marta Sitjá; Daniel Torrents \\ HIPRA, Girona, Spain
}

\section{Introduction}

This work shows the results of two studies where the safety and the efficacy of SUISENG ${ }^{\circledR}$ (a vaccine against the Neonatal Piglet Colibacillosis, C. perfringens and C. novyi enterotoxicosis) were assessed. Applying the basic vaccination scheme to pregnant sows, the efficacy was evaluated by means the serological response, the transfer of maternal immunity and the clinical signs in challenged piglets with two pathogenic strains of $E$. coli.

\section{Materials and Methods}

Suiseng was used to vaccinate pregnant gilts applying the the basic vaccination scheme consists of two doses: the first dose at approximately 6 weeks before farrowing and a second dose at approximately 3 weeks before farrowing.

The piglets of the sows were challenged using two heterologous pathogenic strains to induce neonatal colibacillosis. (See table 1)

Table 1. Studies design

\begin{tabular}{|l|c|c|}
\hline & Study 1 & Study $\mathbf{2}$ \\
\hline № sows vaccinated & 5 Suiseng & 5 Suiseng \\
\hline № sows vaccinated & 5 Placebo & 5 Placebo \\
\hline № piglets challenged & 39 Suiseng & 59 Suiseng \\
\hline № piglets challenged & 52 Placebo & 44 Placebo \\
\hline Challenge & Strain 1 & Strain 2 \\
\hline
\end{tabular}

\section{Results}

Serological response in sows and transfer of maternal immunity

Two IgG serological profiles were observed. For the F4ab, F4ac, F5, LT and CpC valences high antibody titres were obtained after the first dose of the vaccine. In the case of F6 and CnB valences, two doses of the vaccine were needed to achieve high antibody titres. (See figure 1.a)

The presence of specific antibodies was demonstrated in the colostrum/milk samples of the sows. The transfer to their offspring was confirmed with the serology of the piglet sera. (See figure 1.b)

Figure $\mathbf{1}(\mathbf{a}-\mathbf{b})$. Shows the serological response in sows and the present of specific antibodies in the colostrum of the sows.

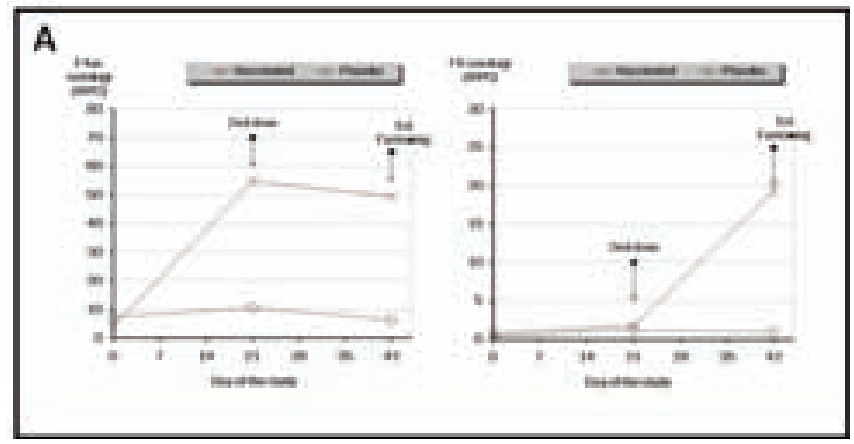

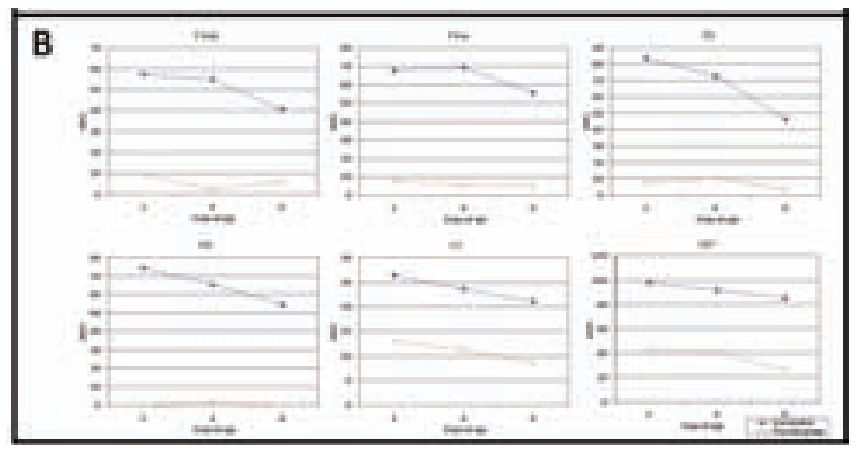

Clinical sign of colibacillosis

The clinical signs after the challenge were evaluated by means a score record during 8 days, as well as, the mortality rate (\%) of each group. Next are the results of this score and percentages, significant differences between treatments were found in all the studies. (See figure 2).

Figure $\mathbf{2}$ (a-b). Shows the clinical signs scoring and the mortality rate of each group respectively.

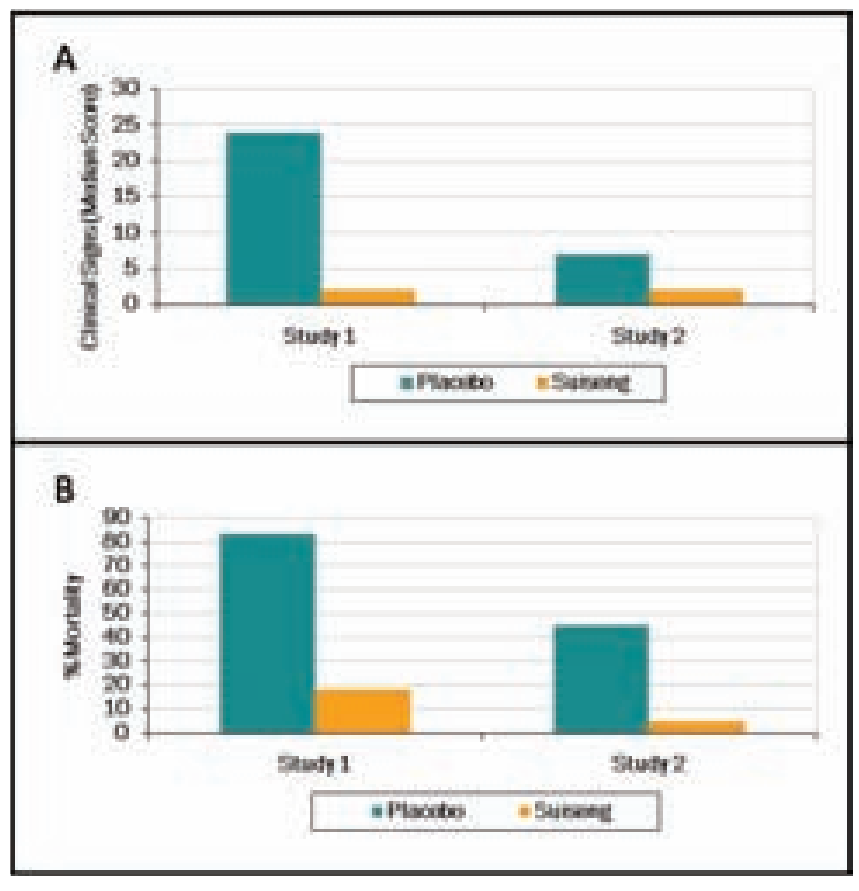

\section{Discussion and conclusion}

The results of the two studies demonstrated the efficacy of Suiseng (applying the basic vaccination scheme) against the experimental infection with enterotoxigenic $E$. coli. The vaccination reduced significantly the clinical signs and the mortality of the piglets suffering from collibacilosis. 


\title{
P.473
}

\section{The effect of Saccharomyces cerevisiae supplement on surviving and resistance in piglets before weaning}

\author{
Jan Bernardy; Dana Kumprechtova \\ University of Veterinary and Pharmaceutical Sciences, Brno, Czech Republic
}

\section{Introduction}

Addition of Saccharomyces cerevisiae (SC) to the prestarter premix may provide early stimulation of the immune system of the piglets before weaning. Cell walls of SC contains betaglucans, an immunomodulator that can enhance the innate immune system providing protection against bacterial (1) and viral infections (2). Mechanisms of action include activation of macrophages, neutrophils, and NK cells, along with B and T lymphocytes and increased phagocytosis and cytokine production in macro-phages (3). Our previous results show better ability of piglets stimulated by SC to reduce mortality and enterotoxigenic E.coli strains (ETEC) in faeces of pre-weaning piglets. The objection of the study was not only confirm our previous results(4), but also closer understanding of interaction between SC and ETEC and its impact to the resistance to the diarrhoea and chance of survival of piglets before weaning.

\section{Material and Methods}

Farrow to finish farm with 500 sows was used for the study, separated into multiple outlaying sections. Sows from two farrowing sections were included into the trial, randomly allocated into the groups giving birth concurrently, 16 sows each. There were 156 piglets live born in one group (avg 9.75 per sow) and 192 in another group (avg 12.00 per sow). The first group (SC group) of breeding sows and piglets received standard feed and prestarter premix, supplemented with SC culture at 1000 ppm (Actisaf Sc47, Lesaffre Feed Ad-ditives, France) each. The second group of sows and piglets (C group) was fed by the same feed without SC supplementation.

Piglets and sows were rectally swabbed - sows before and after the farrowing and piglets every week until the weaning at 28 days of age, cultivated and haemolytic strains of E.coli were further examined to the pathogenic factors. Symptoms of diarrhea and mortality were checked, as well as prestarter consumption at daily basis; piglets were weighed weekly and ADG was calculated. The data were evaluated by non-parametric ANOVA Kruskal-Wallis test and visualized by Statistica v.9 software.

\section{Results and Discussion}

Number of enteropathogenic E.coli in SC group significantly decreased in compare to Controls in the second and third week $(p=0.030$ and 0.044 respectively). The SC dietary supplementation reduced significantly number of ETEC colonies at $2 \mathrm{nd}$ and 3rd week about 8 and $14 \%(p=.03$ and .044$)$ respectively (week three - Figure 1), followed by lower or nil diarrhea symptoms and lower mortality, reduced about 1.03 piglet per litter $(p=0.04)$ in $\mathrm{SC}$ group (Table 1). The major part of the response seems to be caused by changes in intestinal microbiology, including enhanced numbers of favourable microorganisms and decreased numbers of potentially pathogenic bacteria (E.coli, etc.) together with higher fermentation along the intestines. Some investigations have been made with varying diet composition or supplementing with different substances of animal or plant origin.
However, positive effects on the gut have often been more variable and sometimes inconsistent (5). However, measurable effects of these factors on intestinal physiology and muco-sal immunology are not clear or at least difficult to interpret, our results declare measurable impact on number of ETEC strains and lower pre-weaning mortality in the group fed by diet enriched with SC.

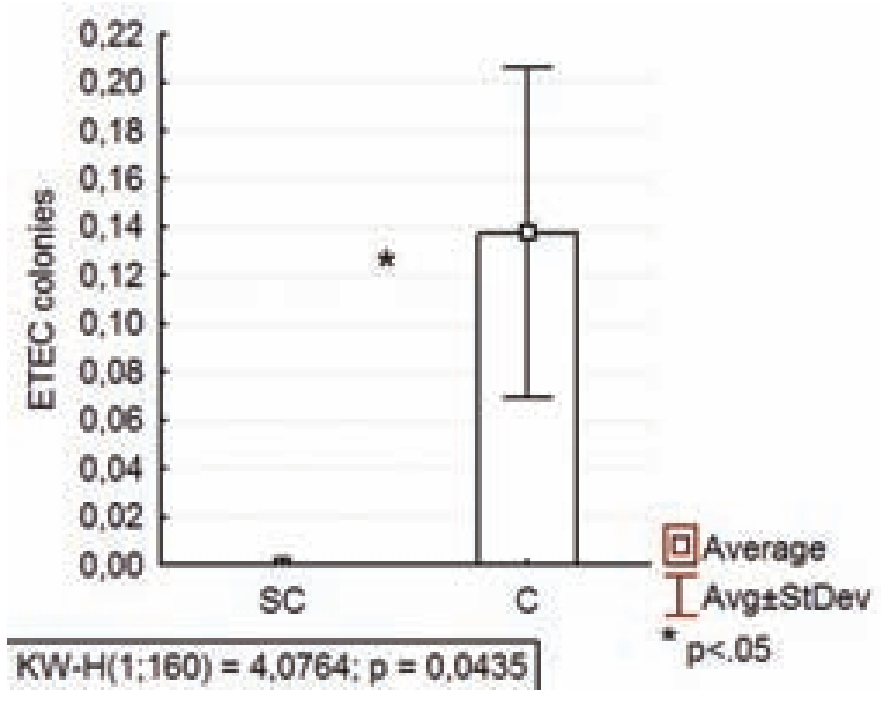

\begin{tabular}{|l|c|c|c|c|}
\hline & \multicolumn{2}{|c|}{$\mathbf{S C}(\mathbf{n}=\mathbf{1 6}$ litters) } & \multicolumn{2}{c|}{$\mathbf{C}(\mathbf{n}=\mathbf{1 6}$ litters $)$} \\
\hline & Average & StDev & Average & StDev \\
\hline Live born (n) & 9.75 & 3.21 & 12.00 & 2.76 \\
\hline Mortality (n) & 1.67 & 1.12 & 2.70 & 1.25 \\
\hline Weaning age (d) & 25.56 & 1.59 & 25.75 & 1.48 \\
\hline
\end{tabular}

\section{Reference}

1. Onderdonk $A B$, et al. Infect Immun 1992; 60:1642.

2. Rouhier P, et al. Phytochemistry $1995 ; 39: 57$.

3. Seljelid RL, et al. Scan J Immunol 1987; 25:55.

4. Bernardy J, et al. Proceedings IPVS 2008; 2:521.

5. Lalles JP et al. Livestock Science 2007; 108:82. 


\section{P.474}

\section{Evaluation of the in feed use of pumpkin for treatment and prophylaxis of escherichia coli postweaning diarrhoea}

Eleni D. Tzika' ${ }^{1}$ Panagiotis D. Tassis ${ }^{1}$ Vassilios G. Papatsiros ${ }^{2}$ Dimitrios Koylialis $^{1}$ Evanthia Petridoy ${ }^{3}$ Costas Alexopoulos $^{4}$ Spiros C. Kyriakis $^{5}$

1. Clinic of Farm Animal Medicine, Faculty of Veterinary Medicine, Aristotle University of Thessaloniki, Thessaloniki, Greece;

2. Clinic of Medicine, School of Veterinary Medicine, University of Thessaly, Karditsa, Greece; 3. Laboratory of Microbiology and Infectious Diseases, Faculty of Veterinary Medicine, Aristotle University of Thessaloniki, Thessaloniki, Greece; 4. Deceased author, Thessaloniki, Greece;

5. Foundation of Biomedical Research, Academy of Athens, Athens, Greece

\section{Introduction}

Postweaning diarrhea due to Escherichia coli (PWD) is a transmissible diarrhoea mediated by enterotoxins and observed mainly observes after weaning, causing major losses in weaned piglets worldwide (1). The aim of this field study was the assessment of possible antimicrobial activity of Pumpkin based on the reduction of E. coli postweaning diarrhoea prevalence in weaned piglets. This trial was part of the SAFEWASTES specific targeted research project financed by the European Union (Framework Project 6).

\section{Materials and Methods}

This study was carried out on a commercial pig farm in northern Greece with a capacity of 250 sows under production. The farm was selected due to previous history of E. coli PWD occurrence. Under a randomized block design 150 healthy and genetically similar weaned piglets at 4 weeks of age were divided into 3 groups (5pens of 10 piglets each with equal sex ratio/group. The negative control group (NC), received feed formulated with $99 \%$ basic diet and $1 \%$ corn starch from trial day 0 (weaning day) until the end of the trial after 28 days. The second group received feed formulated with $99 \%$ basic diet and $1 \%$ pumpkin (P1), while the third received feed formulated with $97.5 \%$ basic diet and $2.5 \%$ pumpkin (P2.5) for the same time period. Average daily weight gain (ADWG), average daily feed intake (ADFI), feed conversion ratio (FCR) and pen diarrhea score (DS) were recorded. Additionally, faecal samples were collected at day 0,14 and 28 of the trial for $\mathrm{E}$.coli shedding quantitative analysis (strains K88, K99, F18, F41, 987P) and Salmonella spp and Clostridium spp presence.

\section{Results and Discussion}

The mean DS for the total trial period did not differ significantly between the 3 treatment groups, although there was a slight improvement in P1 and P2.5 groups (graph 1). Faecal analysis did not reveal the presence of Salmonella spp or Clostridium spp. Referring to E. coli shedding the P1 and P2.5 groups showed a better (not significantly) performance (graphs $2 a, b, c)$.

Graph 1: Daily DS per pen during the total trial period

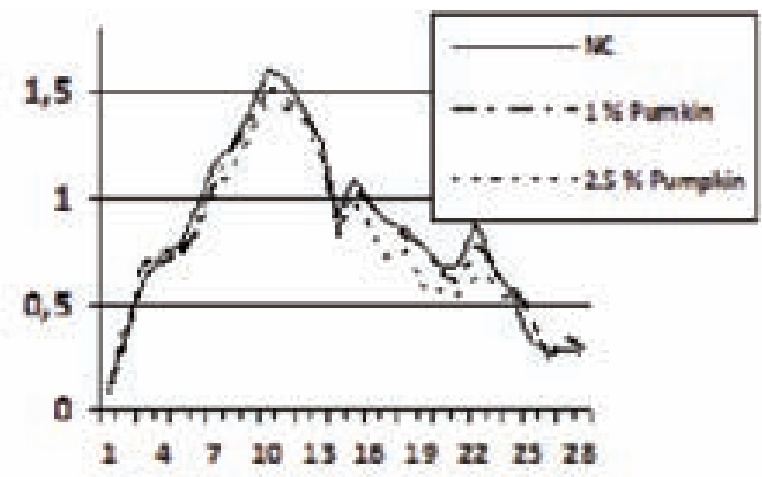

Graph 2 (a,b,c): Quantitive analysis of E. coli shedding $\left(1=<10^{7}\right.$, $2=10^{7}-9 \times 10^{7}, 3=10^{8}-9 \times 10^{8}, 4=10^{9}-9 \times 10^{9}, 5=10^{10}-9 \times 10^{10}(\mathrm{fu} / \mathrm{grR})$ at 0 , 14th and 8th day of trial

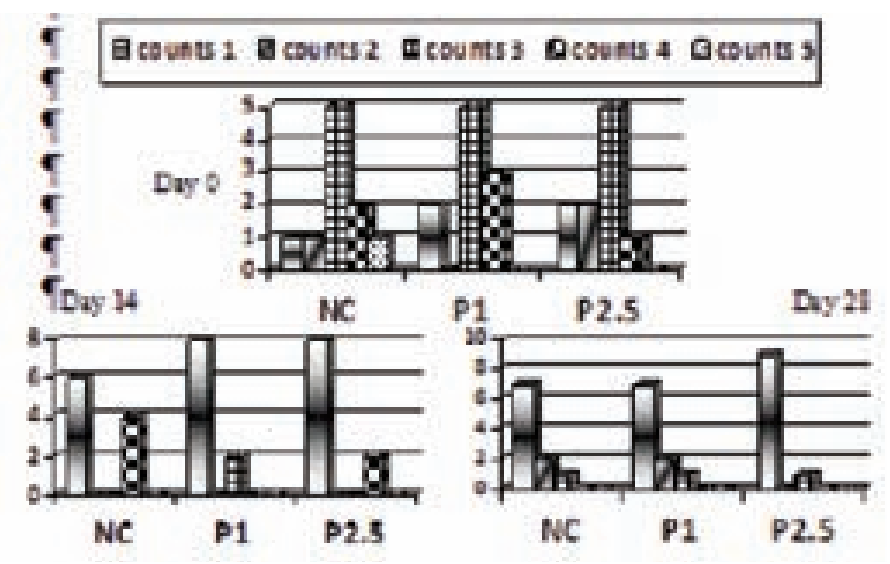

Table 1: ADWG (kg), ADFI (kg), FCR (Mean \pm SD)

\begin{tabular}{|c|c|c|c|}
\hline Groups & ADWG & ADFI & FCR \\
\hline NC & $0.357 \pm 0.069(n=44)$ & $0.522 \pm 0.029(n=5)$ & $1.46 \pm 0.06(n=5)$ \\
\hline P1 & $0.360 \pm 0.061(n=48)$ & $0.527 \pm 0.021(n=5)$ & $1.48 \pm 0.05(n=5)$ \\
\hline P2.5 & $0.362 \pm 0.062(n=47)$ & $0.523 \pm 0.028(n=5)$ & $1.45 \pm 0.12(n=5)$ \\
\hline
\end{tabular}

The results of the trial showed that pumpkin at $1 \%$ and $2.5 \%$ inclusion rates did not show a clear positive antimicrobial effect against E. coli infection in weaners. However, in P2.5 group scores of almost all the recorded parameters were better than the negative controls.

\section{References}

1. Faibrothe, J.M. and Gyles, C.L. (2006). Diseases of Swine. 9th edn. Eds Straw, B. E., Zimmerman, J.J, D’Allaire, and Taylor, D.J. pp. 649-662. Blackwell Sciences, London, UK. 


\title{
P.475
}

\section{Oregano essential oil improves sow and piglet performance - a summary of three field investigations}

\author{
Martha A. Mellencamp ${ }^{1}$ Robert W. Evelsizer $^{1}$ Ron Dvorak $^{1}$ Rafael Cabrera ${ }^{1} \mathrm{Jim}_{\text {Hedges }}{ }^{1}$ \\ Denise Mouat ${ }^{2}$ David Cadogan ${ }^{3}$ Malcolm Mottram³ \\ 1. Ralco Nutrition, Inc., Marshall, MN, USA; 2. Halls Farms, Millmerran, QLD, Australia; 3. Feedworks, Romsey, VIC, Australia
}

\section{Introduction}

Oregano essential oil (OEO) is a phytogenic feed additive with a spicy, aromatic flavor that stimulates appetite and promotes feed intake. OEO is being studied because of its well documented anti-bacterial, anti-fungal and anti-oxidant activities and for its ability to support a healthy gut (1). University of Minnesota research (2) showed that inclusion of OEO in sow gestation and lactation diets increased number of piglets born alive, increased litter birth weight and improved average daily gain of piglets. The authors attributed higher piglet growth to the higher quality colostrum (increased lgG and immune cells) produced from sows supplemented with OEO. This report summarizes three field investigations of the effects of OEO on sow and litter performance. In trials $1 \& 2$, OEO was fed in gestation and lactation diets. In trial 3, OEO was given in water for $10 \mathrm{~d}$ at farrowing. Results showed that feed intake, reproductive performance and litter health were significantly improved for OEO-fed sows compared with controls.

\section{Materials and Methods}

In trials 1\&2, OEO or control diet were fed during gestation and lactation (Table 1). Feed intake and reproduction traits were used to assess sow performance. Litter health and performance were assessed by preweaning mortality, litter gain, and incidence of scours. In trial 3, OEO was delivered in water for $3 d$ prior and 7d after farrowing, which allowed us to compare litter health only. Statistical analysis (Chi-square, ANOVA Proc Mixed, SAS) and odds ratios (3) were performed for each study.

Table 1. Three trials to study the effects of OEO on sow and litter health.

\begin{tabular}{|c|c|c|c|c|c|}
\hline Trial & Location & \multicolumn{2}{|c|}{ Number } & \multicolumn{2}{c|}{ OE0 inclusion rate } \\
\hline & & $0 E 0$ & Control & Gestation & Lactation \\
\hline 1 & N. Carolina & 192 & 199 & $440^{\mathrm{a}}$ & $650^{\mathrm{a}}$ \\
\hline 2 & Australia & 162 & 146 & $250^{\mathrm{a}}$ & $250^{\mathrm{a}}$ \\
\hline 3 & Illinois & 48 & 48 & none & $47^{\mathrm{b}}$ \\
\hline \multicolumn{3}{|l}{} \\
aGrams/metric tonne; ${ }^{b}$ Milliliters/liter of stock solution metered at 1:128.
\end{tabular}

\section{Results}

In trial 1, lactation feed intake was increased for OEO-fed sows compared with control sows $(\mathrm{P}<0.001)$ (4). OEO-sows weaned an additional 0.6 pigs/litter $(\mathrm{P}<0.001)$, and pre-weaning mortality was $3.3 \%$ lower than controls: $11.1 \%$ and $14.4 \%$, respectively $(\mathrm{P}=0.06)$. OEO-fed sows had fewer litters affected by Isospora suis scours $(P=0.001)$, and litter medications were reduced $(P=0.027)$. Odds ratios showed that litters from control sows were 22 times more likely to scour than those from OEO-sows ( $95 \% \mathrm{Cl}: 1.42$ to 3.3). In trial 2 , OEO-fed sows weaned an additional 0.38 pigs/litter $(P=0.026)$, and litters were healthier, as shown by reduced scours $(\mathrm{P}=0.008)$. Litters from control sows were 3.5 times more likely to scour than those from OEO-fed sows $(95 \% \mathrm{Cl}, 158$ to 7.80$)$. Trial 3 was conducted on a farm with lab-confirmed neonatal scours caused by Clostridium perfringens type A. Results showed that OEO-sows weaned 0.74 additional pigs/litter than control sows $(\mathrm{P}<0.001)$. Pre-weaning mortality was reduced from $16.0 \%$ for control sows to $6.4 \%$ for OEO-sows $(\mathrm{P}<0.001)$. Odds ratios showed that litters from OEO-sows were 2.8 times more likely to survive than control litters $(95 \% \mathrm{Cl}, 2.09$ to 3.76$)$. Financial analysis showed that return on investment ranged from 3.9:1 to 9.7:1.

\section{Discussion}

These three studies demonstrated that dietary OEO improved sow reproductive performance and delivered better litter health. Litters from OEO-fed sows had reduced preweaning mortality, increased litter weights, and lower incidence of scours than litters from control sows. OEO litters were healthier and more robust than control litters. These results may be attributed to the anti-bacterial and anti-oxidant activity of OEO, which improves sow gut health and colostrum immunity, leading to healthier piglets (2). OEO is an economical way to deliver more full value pigs at weaning.

\section{References}

1. Si, W, et al. J Appl Microbiol. 100:296, 2006.

2 Arizo-Nieto C, et al. J Anim Sci. 84:(Suppl.2):48, 2006.

3. Ramirez, A. Proc 16th Ann Swine Dis Conf Swine Pract. 1:50-54, 2008.

4. Cabrera R, et al. Proc AASV. 1:93, 2008. 


\title{
P.476
}

\section{Effect of benzoic acid and a probiotic containing Bacillus cereus var Toyoi on growth performance and diarrhoea in weaning piglets}

\author{
$\underline{\text { Vassilios G. Papatsiros }}{ }^{2}$ Panagiotis D. Tassis ${ }^{1}$ Eleni D. Tzika ${ }^{1}$ Dimitrios S. Papaioannoy² \\ Evanthia Petridoy ${ }^{3}$ Costas Alexopoulos ${ }^{4}$ Spiros C. Kyriakis ${ }^{5}$ \\ 1. Clinic of Farm Animal Medicine, Faculty of Veterinary Medicine, Aristotle University of Thessaloniki, Thessaloniki, Greece; \\ 2. Clinic of Medicine, School of Veterinary Medicine, University of Thessaly, Karditsa, Greece; 3. Laboratory of Microbiology and Infectious Diseases, \\ Faculty of Veterinary Medicine, Aristotle University of Thessaloniki, Thessaloniki, Greece; 4. Deceased author, Thessaloniki, Greece;
}

5. Foundation of Biomedical Research, Academy of Athens, Athens, Greece

\section{Introduction}

The use of probiotics containing Bacillus cereus var Toyoi in weaners, finishers, sows and their litters has positive effects on their health and performance (1), as well as on the reduction of incidence and severity of PWDS (2). Recently, it was also noticed that Benzoic Acid improved piglets' performance (3). The purpose of this study was to assess the efficacy of a probiotic containing Bacillus cereus var Toyoi spores (Toyocerin ${ }^{\circledR}$ ) and Benzoic Acid (VevoVitall ${ }^{\circledR}$ ) on health and growth performance parameters in weaning pigs, against negative controls. The study was financed by Rubinum SA through the Research Committee of Aristotle University of Thessaloniki.

\section{Materials and Methods}

The study was carried out on a commercial farrow-to-finish pig farm in Greece (420 sows under production). The farm had a history of PWDS due to E. coli and it was free of Brachyspira hyodysenteriae \& pilosicoli, Salmonella spp., Cryptosporidium spp., rotavirus and coronaviruses.

The animals of each weekly batch were allocated into 4 experimental groups $[8$ pens $\times 10$ piglets $/$ pen $=80$ piglets $/$ group $\times 4$ groups=320 piglets]. The trial groups were as follows: (a) NC: No treatment. (b) TOYO group: Same feed as in the controls plus Toyocerin ${ }^{\circledR}$ at a dose of $1 \times 10^{9} \mathrm{~B}$. cereus var Toyoi spores / kg feed, (c) BA group: Same feed as in the controls plus Vevovital $^{\circledR}$ at a dose of $5 \mathrm{~kg} / \mathrm{kg}$ feed (5000 ppm benzoic acid) and (d) TOYO+BA group: Same feed as in the controls plus Toyocerin ${ }^{\circledR}$ at a dose of $1 \times 10^{9} \mathrm{~B}$. cereus var Toyoi spores and Vevovital ${ }^{\circledR}$ at a dose of $5 \mathrm{~g} / \mathrm{kg}$ feed.

Morbidity, mortality and growth performance data including body weight (BW), average daily gain (ADG), average daily feed intake (ADFI) and average feed conversion ratio (FCR) were recorded at weaning day and then every 7 days (until 35th day) and for the total trial period. Diarrhoea score (DS) and daily DS/ pen was calculated on litter basis after a daily monitoring. On the day of weaning, 4 piglets/pen were randomly selected for faecal sampling. Samples were subjected to microbiological examination for the determination of E. coli counts, as well as for the presence of ETEC strains.

\section{Results and Discussion}

Morbidity rates were significantly decreased in TOYO and TOYO+BA groups compared to $\mathrm{NC}(\mathrm{P}<0.05)$, but there were no significant differences in mortality rates. The mean DS for the total trial period is given in Table 1. E. coli ETEC strains presence and counts $\left(x 10^{6}\right)$ in faecal samples are shown in Table 2.

\begin{tabular}{|c|c|c|c|c|}
\hline \multirow[t]{2}{*}{ TABLE 1} & \multicolumn{4}{|c|}{ Experimental groups } \\
\hline & NC & TOYO & $\mathrm{BA}$ & TOYO+BA \\
\hline Diarrhea score & $0.322 \pm 0.050^{\mathrm{a}}$ & $0.096 \pm 0.021^{b c}$ & $0,132 \pm 0.037^{b}$ & $0.064 \pm 064^{c}$ \\
\hline \multicolumn{5}{|l|}{ TABLE 2} \\
\hline \multicolumn{5}{|l|}{\begin{tabular}{|l|} 
ETEC strains \\
\end{tabular}} \\
\hline Day 0 & $3.1 \%$ & $0.0 \%$ & $3.1 \%$ & $3.1 \%$ \\
\hline Day 14 & $6.9 \%$ & $3.2 \%$ & $3.4 \%$ & $0.0 \%$ \\
\hline Day 28 & $3.4 \%$ & $0.0 \%$ & $0.0 \%$ & $0.0 \%$ \\
\hline \multicolumn{5}{|c|}{ E. coli counts $\left(x 1^{6}{ }^{6}\right)$} \\
\hline Day 0 & $9.09 \pm 19.45$ & $11.48 \pm 24.71$ & $11.24 \pm 35.09$ & $14.03 \pm 26.83$ \\
\hline Day 14 & $56.19 \pm 79.98^{\mathrm{a}}$ & $14.73 \pm 30.02^{b}$ & $36.72 \pm 27.99^{\mathrm{ab}}$ & $15.20 \pm 20.50^{b}$ \\
\hline Day 28 & $23.33 \pm 51.44$ & $10.34 \pm 18.71$ & $17.24 \pm 5.94$ & $10.18 \pm 18.57$ \\
\hline
\end{tabular}

The mean BW of piglets increased significantly in the 3 treatment groups compared to NC group $(P<0.05)$. There were no significant differences in ADFI between all groups, except a reduction in $B A$ group at the total period of day 0 - day 35 after weaning. The ADG and FCR were significantly higher in all medicated groups compared to the NC group $(\mathrm{P}<0.05)$. In conclusion, the addition of Toyocerin ${ }^{\circledR}$ or Vevovital ${ }^{\circledR}$ in weaners' feed improved their health and growth performance compared to the negative controls. However, the most beneficial effects were obtained in the combination of Bacillus cereus var Toyoi spores with benzoic acid.

\section{References}

1. Stamati, S. et al. 2006. Int J Probiot. Prebiot (1): 33-40

2. Kyriakis, S.C. et al., 2003. Asian Australas J Anim Sci 16 (9): 1326-1331.

3. Torrallardona, D. et al., 2007. Liv Sci, 108 (1): 210-213 


\title{
P.477
}

\section{The use of acidificants and probiotics in piglets ration and its effect on their performance after weaning}

\author{
José Cristani ${ }^{1}$ Maria C. Thomaz ${ }^{2}$ Sandra D. Traverso ${ }^{1}$ André Thaler Neto ${ }^{1}$ Juliana Lazaroto ${ }^{1}$ Daniela Miyasaka ${ }^{3}$ \\ 1. UDESC, Lages, SC, Brazil; 2. UNESP, Jaboticabal, SP, Brazil; 3. Ouro Fino Agronegocios Ltda, Ribeirão Preto, Brazil
}

\section{Introduction}

Probiotics are used on animal feeding with the aim of to increase meet, milk, eggs yield. Its utilization has been widely investigated, mainly during the last years, because of the forbiddance of any antibiotics as a growth promoter among all countries in the world (1). This study aimed to evaluate the efficiency of acidificants and probiotics as growth promoters in piglets. They were fed from 21 to 63 days of age to have investigate the performance or percentage of piglets delivered to units of growth and termination.

\section{Materials and Methods}

A total of 630 piglets (castrated males and females) from a commercial herd were weaned with 21 days of age, weighing in average $6.3 \mathrm{Kg}$. The randomized block design was used, being the pen with 14 pigltes the experimental unit, with 5 treatments performed in 9 replications and density being $0.33 \mathrm{~m} 32 / \mathrm{pig}$. Piglets received water and ration ad libitum. Treatments were as following: 1 - Negative Control - Basal Diet (DB); 2- Antibiotic - DB + 20ppm doxiciclin; 3- Acidificant - DB + acidificant (4Kg/ ton of ration from day 21 to 49 , and $2 \mathrm{~kg} /$ ton of ration from day 50 to 63); 4- Probiotic - DB + probiotc (Saccharomyces cerevisiae var. boulardii - $100 \mathrm{~g} /$ ton of ration); 5 - Probiotic + Acidifiant - DB + associations of treatments 3 and 4 . The acidificant used was a blend of organic acids composed of $10 \%$ citric acid, $10 \%$ fumaric acid, $10 \%$ phosphoric acid, $30 \%$ benzoic acid and $40 \%$ of vehicle. The daily feed intake (CDR), gain of weight (GPD) and food conversion (CA) as well as the percentage of piglets delivered were evaluated in three periods: 1 (days 21 to 35), 2 (days 21 to 49) and 3 (days 21 to 63). The percentage of piglets delivered was calculated based on the relation between piglets weighing $18 \mathrm{Kg}$ on day 63 and the total number of piglets used in the experiment

\section{Results and Discussion}

The CDR, GPD and CA for animals from the different experimental groups are showed on table 1 . On period 1 only CDR was not influenced by treatments $(P<0.05)$, being the highest GPD and CA, observed in piglets that were fed with antibiotic containing ration. The same group presented, on periods 2 and 3 the highest $(P<0.05)$ CDR and best GPD and CA in comparison to the others. The same way, the percentage of piglets to the units of growth and termination (Table 1) was influenced $(P<0.05)$ by the treatments, being the group fed with antibiotics the one that showed the best performance. The low percentage of piglets delivered impairs the realization of efficient cleaning and disinfection, and dirty pens result in an increase of negative effects caused by infectious agents that are present. Animals maintained in such conditions tend to present sub-clinical or opportunist diseases, what might increase the rate of waste (refugo?) and increase the lot yield causing economic losses due to extra expenses with feeding, medicines and labor.

\section{Conclusion}

Animals fed using antibiotics as growth promoters after weaning obtained the best performance if compared to animals fed using acidificants and probiotics.

\section{References}

1 RUTZ, F.; LIMA, G.J.M.M. In: CONGRESSO BRASILEIRO DE VETERINÁRIOS ESPECIALISTASEM SUÍNOS, 10, Anais... Porto Alegre: ABRAVES 2001, CD-ROM.

Table 1. Averages observed for CDR, GPD, CA, coeficients of variation (CV), probability values $(P)$ and \% of piglets delivered, according to treatments and experimental periods

\begin{tabular}{|c|c|c|c|c|c|c|c|c|c|c|}
\hline Trat & & Period 1 & & & Period 2 & & & Period 3 & & $\%$ of piglets delivered \\
\hline & CDR & GPD & $C A$ & $C D R$ & GDP & $C A$ & $C D R$ & GDP & $C A$ & \\
\hline 1 & 0,195 & $0,081^{b}$ & $2,536^{\mathrm{a} b}$ & $0,353^{a b}$ & $0,196^{b}$ & $1,804^{b}$ & $0,493^{\mathrm{ab}}$ & $0,287^{b}$ & $1,725^{b}$ & $51,2^{b}$ \\
\hline 2 & 0,206 & $0,124^{\mathrm{a}}$ & $1,754^{\mathrm{a}}$ & $0,391^{\mathrm{ab}}$ & $0,246^{a}$ & $1,605^{\mathrm{a}}$ & $0,549^{a}$ & $0,351^{\mathrm{a}}$ & $1,523^{a}$ & $75,4^{\mathrm{a}}$ \\
\hline 3 & 0,212 & $0,097^{\mathrm{ab}}$ & $2,219^{\mathrm{ab}}$ & $0,398^{a}$ & $0,211^{b}$ & $1,861^{b}$ & $0,527^{\mathrm{ab}}$ & $0,294^{b}$ & $1,798^{b}$ & $52,8^{b}$ \\
\hline 4 & 0,212 & $0,083^{b}$ & $2,669^{b}$ & $0,373^{\mathrm{a}}$ & $0,192^{b}$ & $1,946^{b}$ & $0,502^{\mathrm{ab}}$ & $0,270^{b}$ & $1,865^{b}$ & $51,7^{b}$ \\
\hline 5 & 0,202 & $0,096^{a b}$ & $2,418^{a b}$ & $0,350^{b}$ & $0,188^{b}$ & $1,857^{b}$ & $0,487^{b}$ & $0,262^{b}$ & $1,862^{b}$ & $43,6^{b}$ \\
\hline $\mathrm{cV} \%$ & 2,28 & 8,00 & 7,13 & 9,93 & 10,97 & 8,09 & 9,19 & 11,07 & 8,50 & \\
\hline$P$ & 0,56 & 0,01 & 0,04 & 0,03 & $<0,001$ & 0,0008 & 0,05 & $<0,0001$ & 0,0009 & \\
\hline
\end{tabular}




\title{
P.478
}

\section{The influence of acidificants and probiotics in the feed of post weaned piglets and their effect on diarrhea and total and fecal coliforms}

\author{
José Cristani ${ }^{1}$ Maria C. Thomaz ${ }^{2}$ Sandra D. Traverso ${ }^{1}$ André Thaler Neto ${ }^{1}$ Daniela Miyasaka ${ }^{3} J_{u l i a n a}$ Lazaroto $^{1}$ \\ 1. UDESC, Lages, SC, Brazil; 2. UNESP, Jaboticabal, SP, Brazil; 3. Ouro Fino Agronegocios Ltda, Ribeirão Preto, Brazil
}

\section{Introdução}

To avoid weaning adverse effects and at the same time to work as growth promoters, sub-therapeutic levels of antibiotics are added to piglets feed and this might cause bacterial resistance to antibiotics (1). To avoid this issue probiotics and organic acids have been used as an alternative to the use of antobiotics. This study aimed to evaluate the efficiency of alternative additives, as growth promoters, instead of the use of antibiotics. Its effects on the occurrence of diarrhea and total and fecal coliforms were evaluated through the administration in piglets ration from days 21 to 63 of age

\section{Material and Methods}

A total of 630 piglets from a commercial herd were weaned with 21 days of age, weighing in average $6.3 \mathrm{Kg}$. The randomized block design was used, being the pen with 14 pigltes the experimental unit, with 5 treatments performed in 9 replications and density being $0.33 \mathrm{~m} 2 /$ pig. Piglets received water and ration ad libitum.

Treatments were as following:

1- Negative Control - Basal Diet (DB);

2- Antibiotic-DB + 20ppm doxiciclin;

3- Acidificant - DB + acidificant (4Kg/ton of ration from day 21 to 49 , and $2 \mathrm{~kg} /$ ton of ration from day 50 to 63 );

4- Probiotic - DB + probiotc (Saccharomyces cerevisiae var. boulardii - $100 \mathrm{~g} /$ ton of ration);

5- Probiotic + Acidifiant - DB + associations of treatments 3 and 4.

The acidificant used was a blend of organic acids composed of $10 \%$ citric acid, $10 \%$ fumaric acid, $10 \%$ phosphoric acid, $30 \%$ benzoic acid and $40 \%$ of vehicle. The occurrence of diarrhea was daily evaluated during the first 15 days post-weaning, according to the scores: 0: firm stool, with normal consistency; 1: less consistent stool; 2: loose stool; 3 liquid stools. Diarrheal stool were considered for scores 2 and 3 (2). On days 10 and 20 post-weaning, 3 piglets per treatment were slaughtered and a portion from jejunum was aseptically collected refrigerated and sent to the laboratory for total and fecal coliforms counting (3).

\section{Results and Discussion:}

Table 1 shows data regarding the incidence of diarrhea for total and partial post-weaning periods. For the total period, from day 1 to 15 post-weaning the lowest incidence of diarrhea $(P<0.05)$ was observed for piglets that received ration added of antibiotics. Table 2 shows data regarding the amount of fecal and total coliforms between days 10 and 20 post-weaning. An increase of fecal and total coliforms was observed for animals from all treatments on day 10 , which was followed by a reduction on day 20 post-weaning. These results coincide with the incidence of diarrhea that has started on day 4 post-weaning, increasing until day 10 and then reduced until day 15 being practically controlled at that point.
Table 1. Incidence of diarrhea on piglets during partial and total periods, according to the experimental treatments

\begin{tabular}{|l|c|c|c|c|}
\hline \multirow{2}{*}{$\begin{array}{l}\text { Experimental } \\
\text { treatments }\end{array}$} & \multicolumn{4}{|c|}{ Days post-weaning } \\
\cline { 2 - 5 } & $\mathbf{1} \mathbf{a} \mathbf{5}$ & $\mathbf{6}$ a $\mathbf{1 0}$ & $\mathbf{1 1}$ a $\mathbf{1 5}$ & $\mathbf{1}$ a 15 \\
\hline Cn & $13,0^{\mathrm{a}}$ & $30,0^{\mathrm{a}}$ & $23,0^{\mathrm{a}}$ & $22,0^{\mathrm{a}}$ \\
\hline Ant & $7,0^{\mathrm{c}}$ & $11,0^{\mathrm{d}}$ & $20,0^{\mathrm{b}}$ & $12,0^{\mathrm{d}}$ \\
\hline Acid & $8,0^{\mathrm{bc}}$ & $17,0^{\mathrm{c}}$ & $22,0^{\mathrm{ab}}$ & $16,0^{\mathrm{c}}$ \\
\hline Prob & $10,0^{\mathrm{ab}}$ & $22,0^{\mathrm{b}}$ & $23,0^{\mathrm{ab}}$ & $18,0^{\mathrm{b}}$ \\
\hline Prob + ACid & $11,0^{\mathrm{ab}}$ & $27,0^{\mathrm{ab}}$ & $25,0^{\mathrm{a}}$ & $21,0^{\mathrm{a}}$ \\
\hline a,b Same letter in the same line are not different according to F-test $(\mathrm{P}>0.05)$ \\
\hline
\end{tabular}

Table 2. The Most probable number (NMP) for total and fecal coliforms (log CFU/g) in piglets between 10 and 20 days post-weaning, according to the different experimental diets

\begin{tabular}{|c|c|c|c|c|}
\hline & \multicolumn{4}{|c|}{ NMP, Log UFC/g } \\
\hline & \multicolumn{2}{|c|}{ Total Coliforms } & \multicolumn{2}{|c|}{ Fecal coliforms } \\
\hline & \multicolumn{4}{|c|}{ Days post weaning } \\
\hline Treatment & 10 & 20 & 10 & 20 \\
\hline $\mathrm{Cn}$ & 7,00 & 5,09 & 5,92 & 1,25 \\
\hline Ant & 6,49 & 2,84 & 6,49 & 0,73 \\
\hline Acid & 5,09 & 2,60 & 5,09 & 0,00 \\
\hline Prob & 7,00 & 1,75 & 6,16 & 0,87 \\
\hline Prob+acid & 6,71 & 2,60 & 5,11 & 0,42 \\
\hline Media & $6,46^{\mathrm{a}}$ & $2,98^{b}$ & $5,75^{\mathrm{a}}$ & $0,65^{h}$ \\
\hline CV\% & \multicolumn{2}{|c|}{44,90} & \multicolumn{2}{|c|}{51,44} \\
\hline Treatment Effect & \multicolumn{2}{|c|}{0,49} & \multicolumn{2}{|c|}{0,68} \\
\hline Effect of age & \multicolumn{2}{|c|}{0,0002} & \multicolumn{2}{|c|}{$<0,0001$} \\
\hline
\end{tabular}

\section{Conclusion}

In commercial swine herds with high health challenge, the use of alternative products is not able to prevent post-weaning diarrhea in an appropriate

\section{Reference}

1) RUTZ, F.; LIMA, G.J.M.M. In: Anais. Porto Alegre: ABRAVES 2001, CDROM

2) MORÉS, N.; MARQUES, L.L.J.; SOBESTIANSKY, J.; et al.. Pesq.Vet.Bras. v.10, n.3/4, p.85-88, 1990.

3) SCHIMIDT, V.; GOTTARDI, C.P.T.; SANTOS, M. A. A.; et al. Ars Veterinária, v.18, n.3, p.1-12, 2002 


\title{
P.479
}

\section{Epidemiologic survey for Clostridium difficile in an integrated swine operation}

\author{
Roger B. Harvey ${ }^{1}$ Keri N. Norman² Morgan Scott ${ }^{3}$ Michael E. Hume ${ }^{1}$ Kathleen Andrews ${ }^{1}$ Bo Norby ${ }^{2}$ Mack Scanlan ${ }^{2}$ \\ 1. FFSRU, ARS, U.S. Dept. Agriculture, College Station, TX, USA; 2. Texas A\&M University, College Station, TX, USA; \\ 3. Kansas State University, Manhattan, KS, USA
}

\section{Introduction}

Since 2003, the incidence and severity of disease associated with toxigenic Clostridium difficile (Cd) have increased in hospitals in North America $(1,2)$. These increases have coincided with the emergence of a new, highly virulent, and more resistant epidemic strain (PFGE NAP1, Toxinotype III). The origins of this strain have yet to be determined. Various strains of $\mathrm{Cd}$, including NAP1, can be isolated from food animals and retail meat (3-5); however, the predominant strain from food animals is NAP7 (3). Because $\mathrm{Cd}$ has been isolated from food animals and meat, it has been proposed that $\mathrm{Cd}$ may be transmitted via food sources $(3-5,6)$. The objective of the present study is to compare the prevalence of $\mathrm{Cd}$ in multi-age production groups within a Texas integrated swine operation.

\section{Materials and Methods}

Composite fecal samples ( $n=2936$, representing approximately 10 pigs/sample) were collected monthly for 36 months (20042006) from asymptomatic, clinically healthy, multiple-age swine from 12 separate farms at 3 geographical locations in Texas. Cultivation of $\mathrm{Cd}$ was accomplished by alcohol shock and enrichment techniques described for feces (3). PCR was used to characterize $C d$ for toxin $A \& B$ genes, tcdC gene deletion, toxinotype, binary toxin gene, and pulsed field gel electrophoresis (PFGE) genotype (7). Cd isolates were tested for susceptibility to 11 antibiotics.

\section{Results}

In total, $252 \mathrm{Cd}$ were isolated: 175 from farrowing, 37 from grower/finisher, 14 from nursery, and 26 from breeding animals. Of the isolates, 66 were PFGE pattern NAP7, 173 variant pattern (with $91 \%$ similarity to NAP7), and 4 other patterns. There were 236 Toxinotype V, 7 Toxinotype V-like, 7 Toxinotype XI, and 2 of unknown toxinotype; 250 isolates had the 39 bp deletion for the tcdC gene; 252 isolates were positive for binary toxin; and 238 were Toxin $A+/ B+, 7$ were $A-/ B+$, and 7 were $A-/ B-$. All isolates were sensitive to amoxicillin/clavulanic acid, piperacillin/ tazobactam, and vancomycin; and the majority were sensitive to chloramphenicol, metronidazole, and tetracycline. The most prevalent resistance observed was for cefoxitin, imipenem, and ciprofloxacin. Intermediate resistance was seen for clindamycin and ampicillin.

\section{Discussion}

The majority of swine isolates came from the farrowing barn and this agrees with others in that young animals are more prone to be colonized with $\mathrm{Cd}$ than older ones $(3,7,8)$. The predominant genotype among our isolates was PFGE pattern NAP7 and Toxinotype $\mathrm{V}$, and this agrees with that reported for pig and calf isolates $(6,8)$. Although the predominant strain in our study has been recovered from humans (6), none of our isolates were of the human epidemic strain (NAP1, Toxinotype III). Antibiotic sensitivities were consistent with those reported in an earlier study (7). Our isolates appear to have less resistance than observed for human clinical strains (9). On the basis of our study, we conclude that young animals have a higher prevalence of $\mathrm{Cd}$ than older swine. If $\mathrm{Cd}$ is indeed a potential food-associated organism, the lower carriage rate of $\mathrm{Cd}$ in market-age pigs in our study would suggest a reduced transmission risk to the food chain.

\section{References}

1. McDonald L, et al. 2005. N Engl J Med 353:2433-41.

2. CDC. 2008. MMWR 57:340-3.

3. Rodriguez-Palacios A, et al. 2006. Emerg Infect Dis 12:1730-6.

4. Songer J, et al. 2009. Emerg Infect Dis 15:819-21.

5. Rodriguez-Palacios A, et al. 2007. Emerg Infect Dis 13:485-7.

6. Jhung $M$, et al. 2008. Emerg Infect Dis 14:1039-45.

7. Norman K, et al. 2009. Anaerobe 15:256-60.

8. Keel K, et al. 2007. J Clin Microbiol 45:1963-4

9. Loo V, et al. 2005. N Engl J Med 353:2442-9. 


\section{P.480}

\section{Development of a sandwich ELISA for C. difficile toxin A for in-process control of protective antigen production in vaccine development}

Botond Siklódi; Imre Tóth; József Sipos; Ágnes Horváth; Benaouda Kadra

Ceva-Phylaxia, Budapest, Hungary

\section{Introduction}

Clostridium difficile is among the 5 most important pathogens causing diarrhea in swine (Songer 2006). Vaccination is a promising measure in fighting the agent (Aboudola 2003, Ghose 2007). A reliable analytical tool for controlling the protective antigen(s) in production process is of paramount importance in vaccine development and production. We have developed a sandwichtype ELISA for quantitative detection of C. difficile toxin A (TcdA) using the same monoclonal antibody $(\mathrm{mAb})$ as capture and detector reagents. With this method we are able to quantitatively detect TcdA in the form of bioactive toxin as well as in the form of toxoid.

\section{Materials and Methods}

Capture antibody: TcdA-specific mAb produced by the hybridoma PCG-4 (ATCC access No: HB-8712). Detector antibody: biotin-labeled mAb PCG-4. Conjugate: Extravidine-HRPO (Sigma E2886).

ELISA procedure: Each incubation step except with TMB substrate is performed in a thermo-regulated shaker followed by 4 washing cycles. Nunc Maxisorp ELISA plate is coated with capture mAb diluted 1:1000 in carbonate buffer ( $\mathrm{pH} 9.6)$ for 60 $\mathrm{min}$ at $37^{\circ} \mathrm{C}$. Standards and samples are incubated for $60 \mathrm{~min}$ at $37^{\circ} \mathrm{C}$. Detector mAb is diluted 1:500 in PBS supplemented with $0.05 \%$ Tween-20 and $05 \%$ milk powder, and incubated for 60 min at $37^{\circ} \mathrm{C}$. Conjugate is used in 1:2000 dilution in the same buffer as above, and incubated for $30 \mathrm{~min}$ at $37^{\circ} \mathrm{C}$. TMB substrate in $0.1 \mathrm{M}$ acetate buffer ( $\mathrm{pH} 5.5$ ) is incubated for $30 \mathrm{~min}$ at $\mathrm{RT}$ in dark, and color reaction is stopped by $2 \mathrm{~N}$ sulphuric acid.

\section{Results}

Quantitation limit of the validated ELISA method determined by an eight-point standard curve (Graph 1 ) is $10 \mathrm{ng} / \mathrm{ml}$. No cross-reaction has been found with toxin B (TcdB). Intra- and interassay variation is within $10 \%$.

\section{Graph 1}

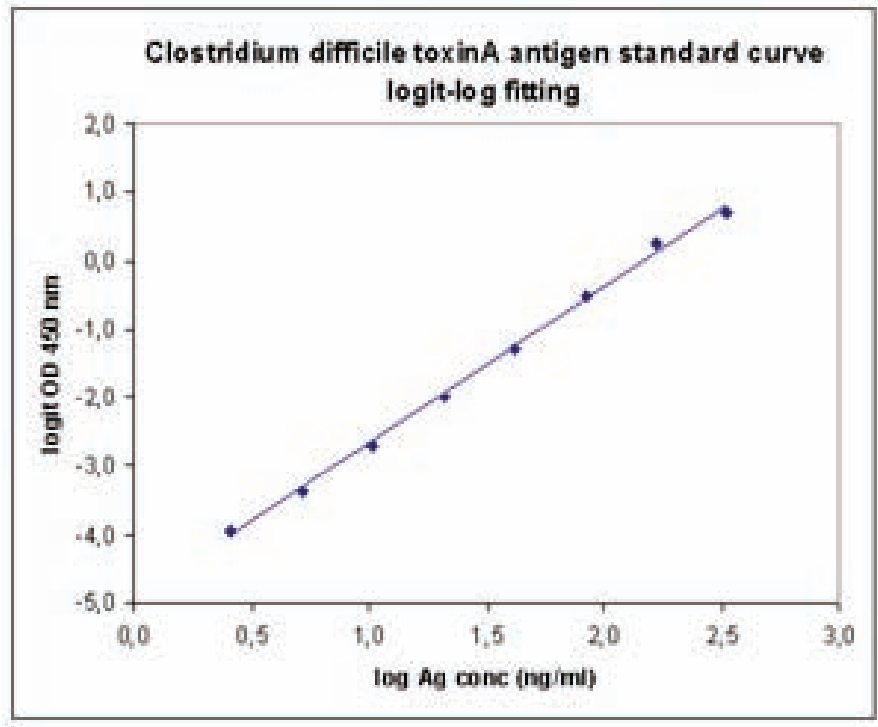

\section{Discussion}

This ELISA is a reliable and convenient method for monitoring the optimization process of $C$. difficile fermentation aiming at the most economical production of the protective antigen TcdA in vaccine development. The method is also used for the quantitative detection of toxoid as long as the epitope conformation is preserved after formol inactivation of the bioactive toxin.

\section{References}

Aboudola, S. et al. 2003: Clostridium difficile vaccine and serum immunoglobulin $\mathrm{G}$ antibody response to toxin A. Infect Immunity, $71,1608-10$

Songer, J.G., Anderson, M.A., 2006. Clostridium difficile: an important pathogen of food animals. Anaerobe 12, 1-4

Ghose, C. et al. 2007: Transcutaneous immunization with Clostridium difficile toxoid $A$ induces systemic and mucosal immune responses and toxin A-neutralizing antibodies in mice. Infect Immunity, 75, 2826-32 


\title{
P.481
}

\section{Dietary supplementation of $\mathrm{BMD}^{\circledR}$ in sow diets enhances nursing piglet immunoglobulin status}

\author{
Daniel A. Nelson' ${ }^{1}$ Samuel K. Baidoo² \\ 1. Alpharma Animal Health, East Bethel, MN, USA; 2. University of Minnesota, Waseca, MN, USA
}

\section{Introduction}

$\mathrm{BMD}^{\bullet}$ (bacitracin methylene disalicylate, Alpharma Inc., Bridgewater, $\mathrm{NJ}$ ) is approved by the FDA in the USA for the control of clostridial enteritis in suckling piglets caused by Clostridium spp. susceptible to bacitracin when fed to sows at $275 \mathrm{ppm}$ ( $250 \mathrm{~g} /$ ton) starting 2 weeks prior to farrowing and throughout lactation. Thirteen previous studies ${ }^{1,2}$ have documented an improvement in pig weaning weights in those pigs nursing sows consuming BMD. This experiment was conducted to determine the effect of BMD supplementation in sow diets from day 99 of gestation and throughout lactation on the immunoglobulin status of sows and piglets and sow milk nutrient composition.

\section{Materials and Methods}

Crossbred sows (Topigs, Landrace X Yorkshire; $n=16$; parities $=2.4 \pm 0.28$ ) were randomly assigned to one of two dietary treatments. Diet 1 was a non-medicated corn-soybean meal control diet and Diet 2 was Diet 1 supplemented with 275 ppm BMD. Sows were fed their respective diets from day 99 of gestation through weaning. Piglet blood samples (1 pig/litter/period) and sow blood and milk samples were taken on day 0,9 , and 18 of lactation.

\section{Results and Discussion}

No differences were observed between treatments for sow milk crude protein, fat, ash or sow milk immunoglobulin status (Table 1). The average sow serum immunoglobulin status was not affected by treatment (Table 2). The average IgA content of piglet serum was not affected by treatment. However, the overall average lgG status in the serum of piglets nursing sows consuming $B M D$ was significantly $(P=0.04)$ enhanced and was more than twice that of pigs nursing Control sows (Table 2). Because there is virtually no placental immunoglobulin transfer in pigs, the higher lgG serum content of pigs nursing sows consuming BMD had to be derived from the IgG of colostrum. The IgG concentration of colostrum was numerically greater in BMD-fed sows. In conclusion, BMD supplementation did not affect sow milk nutrient composition nor sow milk or serum immunoglobulin status but did enhance the immunoglobulin status of piglets. The greater serum IgG concentrations of piglets nursing sows consuming BMD may contribute to the heavier pig weaning weights reported in previous research.
Table 1. Average sow milk nutrient and immunoglobulin concentration.

\begin{tabular}{|l|c|c|}
\hline & 275 ppm BMD & Control \\
\hline Sow milk crude protein, \% & 6.31 & 6.22 \\
\hline Sow milk fat, \% & 8.06 & 8.19 \\
\hline Sow milk ash, \% & 0.75 & 0.72 \\
\hline Sow milk $\mathrm{lgA}, \mathrm{mg} / \mathrm{ml}$ & 2.38 & 2.15 \\
\hline Sow milk $\mathrm{lgG}, \mathrm{mg} / \mathrm{ml}$ & 0.12 & 0.10 \\
\hline
\end{tabular}

Table 2. Average sow and piglet serum immunoglobulin concentration.

\begin{tabular}{|l|c|c|}
\hline & 275 ppm BMD & Control \\
\hline Sow serum $\lg \mathrm{A}, \mathrm{mg} / \mathrm{ml}$ & 0.26 & 0.34 \\
\hline Sow serum $\operatorname{lgG}, \mathrm{mg} / \mathrm{ml}$ & 0.48 & 0.59 \\
\hline Piglet serum $\operatorname{lgA}, \mathrm{mg} / \mathrm{ml}$ & 0.94 & 0.90 \\
\hline Piglet serum $\operatorname{lgG}, \mathrm{mg} / \mathrm{ml}^{\mathrm{a}}$ & 0.58 & 0.27 \\
\hline aP=0.04 & \\
\hline
\end{tabular}

\section{References}

1. Wolff T. (2005). Proc AASV, 101-106

2. Turner et al. (2007) J. Anim Sci. 85 (Supp 1):578 


\title{
P.482
}

\section{Antimicrobial susceptibility of Clostridium perfringens type $A$ isolated from neonatal pigs with and without diarrhea in Minas Gerais, Brazil}

\author{
Felipe M. Salvarani; Roberto Maurício C. Guedes; Isabella S. Albefaro; Eduardo C. Cruz Júnior; \\ Rodrigo Otávio S. Silva; Prhiscylla S. Pires; Guilherme G. Alves; Francisco Carlos F. Lobato \\ Escola de Veterinária da Universidade Federal de Minas Gerais, Belo Horizonte, MG, Brazil
}

\section{Introduction}

Clostridium perfringens a spore-forming anaerobic bacilli, Grampositive, and strictly-to-oxygen-tolerant, is widely distributed in the environment and in the intestinal tracts of domestic animals . It has been recognized as one of the most important causes of diarrhea in neonatal pigs. $C$. perfringens types $A$ and $C$ and $C$. difficile are the main pathogenic clostridia in swine (5). Antibiotics recommended for the treatment of $C$. perfringens-associated diarrhea include amoxicillin, tylosin, and tetracycline, but there are few data about this microorganism susceptibility to these substances, in Brazil. The aim of this study was to determine the in vitro susceptibility of $C$. perfringens type $A$ to seven relevant antimicrobials for swine production.

\section{Materials and Methods}

Fifty C. perfringens type $A$ isolates, each obtained from different piglets ( 25 diarrheic and 25 non diarrheic) were evaluated. All strains were obtained in swine herds in Minas Gerais, Brazil. The following antibiotics, manufactured for analytical purpose, were tested: amoxicillin, ceftiofur, florfenicol, josamycin, lincomycin, tetracycline, and tylosin. Minimum inhibitory concentrations (MICs) were determined on Brucella's agar plates containing doubling of the antibiotics, from 0.25 to $256.0 \mu \mathrm{g} / \mathrm{L}$, and with $5 \%$ of sheep blood, vitamin $\mathrm{K}(1 \mu \mathrm{g} / \mathrm{ml})$, and hemim $(5 \mu \mathrm{g} / \mathrm{ml})$. All strains were tested twice and, for negative control, Bacteroides fragillis (ATCC25285) was included in every test plate. All MICs values were determined using the National Committee for Clinical Laboratory Standards (NCCLS) (3).

\section{Results}

MICs values of each antibiotic for $C$. perfringens type $A$ are summarized in Table 1. All isolates were susceptible to amoxicillin and ceftiofur. Forty four samples (88\%) were considered susceptible to tylosin and only $6 \%$ of the strains were resistant. Thirty eight strains (76\%) were susceptible to florfenicol but 10 (20\%) were considered resistant. In contrast, 42 (84\%) and 36 (72\%) of the C. perfringens type A were resistant to tetracycline and lincomycin, respectively.

Table 1. MIC distribution of amoxicillin, ceftiofur, florfenicol, josamycin, lincomycin, tetracycline and tylosin for fifty swine Clostridium perfringens type $A$ isolates.

\begin{tabular}{|c|c|c|c|c|c|c|c|c|c|c|c|c|c|c|}
\hline \multirow[t]{2}{*}{ Antimicrobial } & \multicolumn{14}{|c|}{ Number of C. perfringens isolates with values $(\mu \mathrm{g} / \mathrm{ml})$} \\
\hline & 0,25 & 0,5 & 1 & 2 & 4 & 8 & 16 & 32 & 64 & 128 & 256 & $>256$ & MIC50 & MIC90 \\
\hline Amoxicillin & 5 & 31 & 9 & 2 & 2 & 1 & 0 & 0 & 0 & 0 & 0 & 0 & 0,5 & 1 \\
\hline Ceftiofur & 21 & 9 & 2 & 4 & 6 & 8 & 0 & 0 & 0 & 0 & 0 & 0 & 0,5 & 8 \\
\hline Florfenicol & 0 & 1 & 2 & 24 & 9 & 2 & 2 & 2 & 8 & 0 & 0 & 0 & 2 & 64 \\
\hline Josamycin & 0 & 0 & 0 & 2 & 3 & 16 & 5 & 0 & 1 & 0 & 5 & 16 & 16 & $>256$ \\
\hline Lincomycin & 0 & 0 & 0 & 7 & 7 & 8 & 3 & 0 & 0 & 0 & 6 & 19 & 16 & $>256$ \\
\hline Tetracycline & 0 & 0 & 0 & 4 & 0 & 4 & 21 & 2 & 0 & 4 & 15 & 0 & 16 & 256 \\
\hline Tylosin & 14 & 22 & 5 & 2 & 1 & 0 & 3 & 3 & 0 & 0 & 0 & 0 & 0,5 & 16 \\
\hline
\end{tabular}

\section{Discussion}

Several studies using different C. perfringens samples also demonstrated too high-level resistant (MIC > 16 $\mu \mathrm{g} / \mathrm{ml}$ ) to tetracycline, an antimicrobial commonly recommended for treatment of $C$. perfringens-associated diarrhea in animals including piglets. Important to Clostridium species can carry tetracycline resistance genes $(2,4,6)$. Most $C$. perfringens strains from chickens and turkeys used by WATKINS et al (6) were considered resistant to lincomycin with high MICs (> $8 \mu \mathrm{g} / \mathrm{ml})$. However the present study showed that amoxicillin, ceftiofur, florfenicol, and tylosin have a good effect against $C$. perfringens strains, as described elsewhere $(1,4,6,7)$. These results emphasis the importance of conscious use of antimicrobials and the relevance of hygiene and vaccination as preventive measures.

\section{References}

1. Citron, D.M. et al. In vitro activities of ramoplanin, teicoplanin, vancomycin, linezolid, bacitracin and four others antimicrobials against intestinal anaerobic bacteria. Antimic. Ag. Chemot., v.47, p.2334-2338, 2003.

2. Kather, E.J. et al. Determination of the prevalence of antimicrobial resistance genes in canine C. perfringens isolates. Vet. Microb., v. 113, p.97-101, 2006.

3. NCCLS. Methods for antimicrobial susceptibility testing of anaerobic bactereia. Approved standard, 6th ed. M11-A6. National Committee for Clinical Laboratory Standards, Wayne. Pennsylvania, 2004.

4. Silva, R.O.S. et al. Antimicrobial susceptibility of C. perfringens strains isolated from broiler chicken. Braz. J. Microb., v.40, p.262-264, 2009.

5. Songer, J.G. and Uzal, F.A. Clostridial enteric infections in pig. J. Vet. Diagn. Invest., v.17, p. 528-536, 2005.

6. Watkins, K.L. et al. In vitro antimicrobial susceptibility of Clostridium perfringens from commercial turkey and broiler chicken origin. Vet. Microb., v. 54, p.195-200, 1997.

7. Tansuphasiri, U.; et al. Antimicrobial reistance among C. perfringens isolated from various sources in Thailand. South. Asian J. Trop. Med. Public Health, v.36, p. 954-961, 2006.

\section{Acknowledgments}

This work was supported by CNPq, Fapemig and FEPMVZ. 


\title{
P.483
}

\section{Implementing the 5-week $B M D^{\circledR}$ feeding program produces heavier pigs at weaning than feeding BMD only during lactation}

\author{
Daniel A. Nelson ${ }^{1}$ Roy A. Schultz ${ }^{2}$ \\ 1. Alpharma Animal Health, East Bethel, MN, USA; 2. Alpharma Animal Health, Avoca, IA, USA
}

\section{Introduction}

$\mathrm{BMD}^{\circledR}$ (bacitracin methylene disalicylate, Alpharma, Inc, Bridgewater, NJ) is approved by the FDA in the USA for the prevention of clostridial enteritis in suckling piglets caused by Clostridium spp. susceptible to bacitracin when fed to sows starting 2 weeks prior to farrowing and throughout lactation. Thirteen previous studies $^{1,2}$ have documented the benefits of feeding BMD during this 5-week period, including heavier piglet and litter weaning weights and reductions in piglet mortality and sow lactation weight loss. The 14-day pre-farrow BMD feeding period is sometimes perceived as too difficult to implement. Therefore, two separate studies conducted by large swine integrators and reported herein evaluated the benefits of feeding 275 ppm $(250 \mathrm{~g}$ BMD/ton) only in lactation or starting 2 weeks prior to farrowing and throughout lactation (5-week).

\section{Material and Methods}

In the first study a total of 106 sows were allotted to treatment (non-medicated or BMD in lactation) via parity and line. BMD was added to the lactation diets of test sows to provide 275 ppm. BMD feeding began when sows were moved into farrowing rooms (an average of 2 days pre-farrow) and continued until weaning. Total number liveborn and stillborn pigs/sow, mortality/litter, number of piglets weaned/sow, individual live pig birth weight within $24 \mathrm{hrs}$ post-farrowing, and individual weaned pig weight were recorded. The second study contained 3 treatments: 1) Non-medicated 2) BMD only in lactation 3) BMD fed 2 weeks prefarrow and throughout lactation. A total of 568 sows of common genetic base were blocked by location within farrowing room and balanced by parity to treatment. Similar gestation and lactation diets were fed to all treatment groups with the exception that BMD was added to supply 275 ppm for the treatment groups. Sows were individually weighed before farrowing and at weaning. Entire litters were weighed at birth, after fostering and at weaning. In lactation, sows were given adlibitum access to feed.

\section{Results and Discussion}

In Study 1, there were no differences between treatments in the total number of pigs born, the number of pigs born alive, the number of pigs that were stillborn, the number of mummies, the number of pigs weaned nor the number of pigs that died/litter. Weaning weights of pigs nursing sows consuming BMD were significantly $(P=0.02)$ greater (6.0 vs $5.8 \mathrm{~kg} / \mathrm{pig})$. In Study 2 , net sow weight post-farrowing, post-weaning, and net sow weight loss in lactation were not different among treatments. The number of pigs started and weaned/sow, and pre-weaning mortality were not different among treatments. Adjusted 22-day piglet weaning weights were significantly $(P<0.05)$ greater $(+0.3 \mathrm{~kg} /$ pig) for pigs nursing sows consuming BMD for the 5-week period compared to pigs nursing Control sows (6.7 vs $6.4 \mathrm{~kg} / \mathrm{pig}$ respectively). The adjusted 22- day piglet weaning weights of pigs nursing sows fed BMD only in lactation were intermediate (6.5 $\mathrm{kg} / \mathrm{pig}$ ) and not significantly different from pigs nursing Control sows or pigs nursing sows consuming BMD for the 5-week period. This is a logical observation as the two-week prefarrow BMD feeding period begins to reduce clostridial shedding before sows enter and "seed" the farrowing crate. In both studies, BMD increased pig weaning weight by $0.2 \mathrm{~kg} / \mathrm{pig}$ when used only in lactation, and by $0.3 \mathrm{~kg} / \mathrm{pig}$ when use for 2 weeks prefarrow and throughout lactation in Study 2 (Figure 1). In the presence of Clostridium perfringens type $A$ and $C$, dietary supplementation of sow feed with 275 ppm BMD will improve the daily gain and weaning weights of nursing pigs. Implementing the 5-week BMD feeding program produces heavier pigs at weaning than feeding BMD only during lactation.

\section{Figure 1. Study 1 and 2 Piglet Weaning Weight Advantage/Pig for BMD over Non-Medicated}

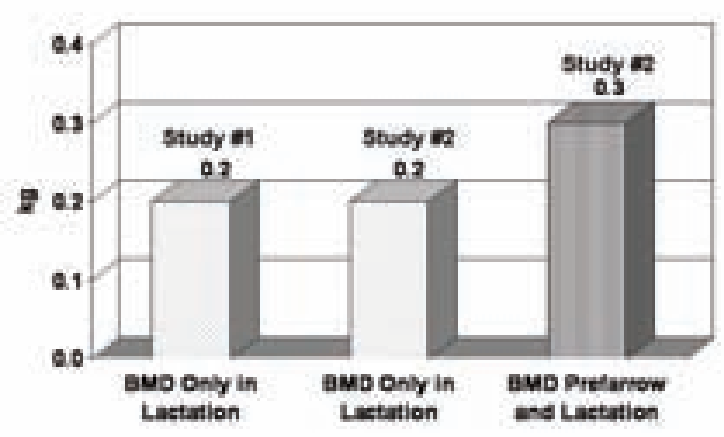

References

1. Wolff T. (2005). Proc AASV, 101-106

2. Turner et al. (2007) J. Anim Sci. 85 (Supp 1):578 


\title{
P.484
}

\section{In vitro susceptibility of porcine $C$. perfringens Type A isolates to tylosin in Czech Republic}

\author{
Martina Masaříková; Jiří Smola \\ Veterinary and Pharmaceutical Sciences Brno, Brno, Czech Republic
}

\section{Introduction and objectives}

Enteritis caused by the Clostridium perfringens Type A range among the most important piglets diseases in the Czech Republic. The causes underlying the spread of the infection are still unclear. Our hypotheses suppose a relation with the absence of the previously used antibiotic growth promoters, and a possible fall in sensitivity, alternatively an acquired resistance of the virulent isolates Clostridium perfringens to the antibiotics frequently used in therapy in pig herds. The aim of the study was to establish the MIC values of tylosin and to prove the emergence of resistance in the porcine Clostridium perfringens, isolated from piglets with enteritis.

\section{Material and Methods}

The Clostridium perfringens isolates (280) were acquired in 20042007 via cultivation of rectal swabs of piglets aged to 7 days with diarrhoea from 83 different pig herds. The identification of Clostridium perfringens was based on the demonstration of the species-specific gene (cpa) by PCR, another molecular genetic examination was performed to differentiate between Types $A$ and $C$ of Clostridium perfringens (1). Isolates of Clostridium perfringens Type A were selected as the basic set for further testing. The MICs of the tylosin (E. Lilly \& Co., Elanco) were established using the agar dilution method according to CLSI M11-A5 (2001), the interpretative criteria adopted from Prescott (2).

\section{Results}

In the year 2004, the largest group were susceptible isolates $(43,9 \%)$, followed with $38,2 \%$ intermediate isolates, at least $(17,9 \%)$ were resistant isolates of Clostridium perfringens Type A. Next year get to significant increasing $(84,5 \%)$ of susceptible isolates at the expense of isolates intermediate susceptible $(3,4$ $\%)$ and resistant (12,1\%). In 2006 still predominated susceptible isolates (56,9\%), nevertheless others categories increased to approximately $20 \%$. In 2007, the largest group were continuously susceptible isolates $(66,7 \%)$, but resistant isolates increased too $(25,9 \%)$, see Graph 1 . The assessment of the dynamics of the susceptibility and resistance of porcine Clostridium perfringens Type $A$ isolates from the $\mathrm{MIC}_{90}$ point of view shows resistance level to tylosin with the range from 8 to $16 \mathrm{mg.l}^{-1}$, see Table 1.

\section{Categories of $C$. perfinigens type $A$ isolates}

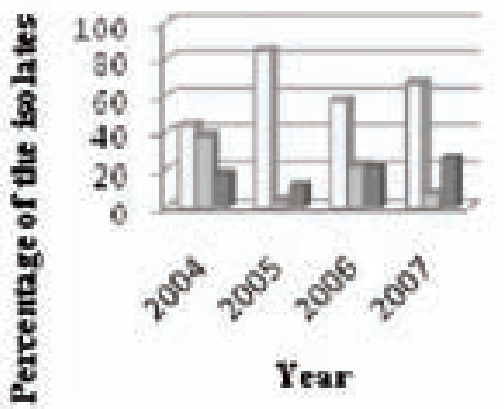

\begin{tabular}{|c|c|c|c|c|}
\hline Year & $\mathbf{n}$ & MIC $_{50}$ & MIC $_{90}$ (mg. $^{-1}$ & MIC distribution $_{\text {(mg. }^{-1}}$ \\
\hline 2004 & 123 & 1 & 8 & $0,125-\geq 16$ \\
\hline 2005 & 58 & 0,5 & 8 & $0,25-\geq 16$ \\
\hline 2006 & 72 & 0,5 & 16 & $0,125-\geq 16$ \\
\hline 2007 & 27 & 0,5 & 16 & $0,25-\geq 16$ \\
\hline
\end{tabular}

\section{Discussion}

Because of a lack of data on the susceptibility of Clostridium perfringens Type A isolates of porcine origin, we had to compare our results with the isolates obtained from poultry and dogs. For instance in the USA was reported a few times lower $\mathrm{MIC}_{90}$ of tylosin (2 $\mathrm{mg.}^{-1}$ ) in chickens Clostridium perfringens isolates obtained from necrotic enteritis cases (3). $\mathrm{MIC}_{90}$ of tylosin in Clostridium perfringens isolated from clinical cases of enteritis in dogs in USA was also lower, concretely $1 \mathrm{mg}^{-1}(4) . \mathrm{MIC}_{90}$ of tylosin higher than those ascertained in our study have been published only in USA in Clostridium perfringens isolated from turkeys with necrotic enteritis (256 $\mathrm{mg.l}^{-1}$ ) (3). MICs of tylosin calculated in Belgium for poultry isolates of Clostridium perfringens ranged from 0.03 $\mu \mathrm{g} \cdot \mathrm{ml}-1$ to $1 \mu \mathrm{g} \cdot \mathrm{ml}^{-1}$, (5), while MIC values of our porcine isolates of Clostridium perfringens Type $A$ in same year ranged from 0.125 mg. $\mathrm{I}^{-1}$ to $>16$ mg. $\mathrm{I}^{-1}$.

\section{Conclusions}

Obtained $\mathrm{MIC}_{90}$ values (16 mg. $\mathrm{I}^{-1}$ ) of tylosin in isolates from newborn piglets are showing acquired resistance which is probably a result of frequent feed incorporation of the drug in swine. Furthermore acquired resistance had enabled to this subpopulation of Clostridium perfringens to take selective advantage in intestinal microflora of gilts and saws first in new born piglets.

\section{Acknowledgement}

The financial resources for the study were provided by the grant NAZV QH 1278.

\section{References}

1. Buogo, C. et al. (1995). J Vet Med 42: 51-58.

2. Prescott, J.F. (2000). In: Antimicrobial therapy: 229-262.

3. Watkins, K.L. et al. (1996). Vet Microbiol 54: 195-200.

4. Marks, S.L. and Kather, E.J. (2003). Vet Microbiol 94: 39-45.

5. Martel, A. et al. (2004). Avian Pathol 33: 3-7. 


\title{
P.485
}

\section{Clostridiosis in the farrowing room with an atypical onset: a clinical case}

\author{
Claudio Mazzoni ${ }^{1}$ Valeria Raffi ${ }^{1}$ Carlo Rosignoli² Paolo Bonilauri ${ }^{2}$ Brunella Spaggiari² Daniela Gelmetti ${ }^{2}$ \\ Lucia Gibelli2 Giulia Maioli2 Silvia Faccini² Michele Dottori² Francesco Tonon ${ }^{3}$ Andrea Luppi ${ }^{2}$ \\ 1. Libero professionista, Reggio Emilia, Italy; 2. Istituto Zooprofilattico Sperimentale della Lombardia ed Emilia Romagna-Brescia, Brescia, Italy; \\ 3. Libero professionista, Conegliano Veneto, Italy
}

\section{Introduction}

Clostridia are large, gram-positive, spore forming rods. Clostridium perfringens type $\mathrm{A}$ and $\mathrm{C}$ are the most important species, producing toxins responsible of enteritis in pigs. Infection is transmitted from piglet to piglet and the sows are the most important source of infection (4). Antibiotics are used for therapy, in particular $\beta$-lactam antibiotics (2) and tylosin (3).

In this work the Authors describe a clostridial enteric disease in several suckling piglets (one-day old) occurred in a herd in the Northern Italy and characterized by an atypical onset.

\section{Materials and Methods}

The clinical case took place between December 2008 and January 2009 with an increasing mortality rate in suckling piglets. Fourteen suckling piglets, belonging to a pig herd in Northern Italy and showing a syndrome characterized by death 12 hours after the onset of clinical signs, were submitted for necropsy to the Istituto Zooprofilattico Sperimentale Lombardia and Emilia Romagna, Laboratory of Reggio Emilia.

The intestinal contents and several organs were taken for microbiological cultures and histological examination.

Histology was performed on formalin-fixed paraffin-embedded tissue sections stained by Ematoxilin-Eosin and Gram methods. Genes for toxins $\alpha$ and $\beta 2$ were detected, using a multiplex polymerase chain reaction method, from C.perfrigens isolated (see results) (2).

Toxicological investigations on sow feed were carried out to evaluate the presence of zearalenon, aflatoxins B1, B2, G1 and $\mathrm{G} 2$, ochratoxin A and fumonisin B1 and B2.

\section{Results}

Clinical signs observed 24 hours after the birth in the suckling piglets, were characterized by jaundice, lethargy and diarrhoea. The death occurred during the 12 hours after the onset of clinical signs. Gross lesions were characterized by necrosis of small intestine mucosa and liver degeneration with foci of parenchymal necrosis. Later, during the evolution of the clinical case, the dead piglets showed haemorrhagic-diarrhoea, intestinal hyperemia and mesenteric oedema, especially in jejunum and ileum.

C.perfrigens was isolated from the small intestine of several piglets ( $1 \times 10^{7}$ ufc of C.perfrigens from each gram of intestinal content). Genes for toxins $\alpha$ and $\beta 2$ were detected and the C.perfrigens isolated was genotyped as type A. Histological examination of samples collected during the necropsy showed vacuolar liver degeneration, necrotic enteritis and multiple gram-positive, spore-forming bacterial colonies in the lumen. The results of the toxicological investigations on sow feed showed values under the legislative about maximally allowed.

A drug-treatment against C.perfrigens, using a long acting macrolid on piglets in the first 12 hours after the birth, led to a complete solution of the syndrome.

\section{Discussion}

In this work the Authors described a syndrome characterized by death, 12 hours after the onset of clinical signs in the suckling piglets. The syndrome did not appear to be related to dietary or any other specific management factor. The clinical signs of the disease, the gross lesions observed, the results of laboratory investigations and response to therapy suggested a diagnosis of clostridiosis due to a beta2 toxin-producing C.perfrigens type A strain.

\section{References}

1. Nigrelli A.D. (2009). Atti XXXV Meeting annuale Sipas, pp. 134-141.

2. Rosignoli C., Faccini S., Franzini G., Costa A., Nardi M., Nigrelli A.D. (2004). VI Congresso Nazionale S.I.Di.L.V. - Abano Terme 10-12 Novembre 2004, pp 205-206.

3. Songer J.G. and Uzal F.A. (2005). J. Vet. Diagn. Invest. 17: 528-536.

4. Tayler D.J. (2003). Disease of swine. pp. 51-60. 


\title{
P.486
}

\section{B. licheniformis for C. perfringens control in sows}

\author{
$\underline{\text { Robert Lantz }}{ }^{4}$ Inge Knap ${ }^{1}$ Douglas Cook ${ }^{2}$ William Minton ${ }^{3}$ \\ 1. Chr. Hansen, Hoersholm, Denmark; 2. Akey, Lewisburg, OH, USA; \\ 3. Four Star Veterinary Services, Chickasaw, OH, USA; 4. Chr. Hansen, Milwaukee, WI, USA
}

\section{Introduction}

Clostridium perfringens related scours is a form of diarrhea that affects young pigs and has been reported worldwide. The objective of this study was to determine the effectiveness of Bacillus licheniformis (Chr Hansen strain) in reducing the shedding of Clostridium perfringens in sows and the resulting Clostridium perfringens related scours in baby pigs.

\section{Materials and Methods}

The trial was conducted in the fall of 2009 in a 5,000 sow commercial farrow to wean operation using PIC genetics and Akey nutrition. (Lewisburg, Ohio, USA) The sow farm had 2 separate gestation barns, and 1 farrowing building comprised of 16 rooms of 64 farrowing crates each. The gestation barns had separate dedicated feed lines, 2 separate feed systems run through the farrowing facilities.

The entire herd was split into one of two groups. The groups of gestating sows were placed either on a standard control diet or on a standard diet containing B. licheniformis $(1.6 \mathrm{E}+6 \mathrm{cfu} / \mathrm{gram}$ of feed). The sows were maintained on these diets throughout gestation. Once the sows entered the farrowing facilities, the groups maintained their integrity with the control sows being placed on a control farrowing and lactation diet. The sows that had received $B$. licheniformis in gestation continued to receive B. licheniformis $(1.6 \mathrm{E}+6 \mathrm{cfu} / \mathrm{gram}$ of feed) in the farrowing and lactation diet. Both the control diet and B. licheniformis diets where identical with the exception of the B. licheniformis being added to the treatment diet.

A total of 12 sows from each of the two groups were randomly selected for fecal sample collection Fecal samples were taken directly from the rectum of the sow and analyzed at the lowa State University Veterinary Diagnostic Laboratory (Ames, lowa, USA) to determine $C$. perfringens type and count. Samples were collected in gestation (approximately 38 days pre-farrowing and before the treatment group was placed on B. licheniformis), as sows were moved to farrowing (average 4.5 days pre farrow) and then again in lactation (average 2.5 days post farrow). In addition, feed samples where collected throughout the trial to determine the presence and level of $\mathrm{B}$. licheniformis in the feed. At approximately 15 days of age, fecal samples were collected from at least 1 pig from each sow (both control and treated) to determine the presence of $\mathrm{B}$. licheniformis in the pigs.

All samples were stored at $-34^{\circ} \mathrm{C}$ until shipment to the ISU VDL. Samples were shipped overnight, on dry ice, in insulted containers and placed at $-70^{\circ} \mathrm{C}$ upon arrival at the ISU VDL. Samples were thawed at room temperature; each sample was well mixed and immediately diluted for the standard plate count procedure (Quinn et al. 1994). Any isolates with typical colony morphology and double zone of hemolysis were gram stained for confirmation as large gram positive rods. These were recorded as $C$. perfringens. Twenty four of the isolates were submitted for
Genotyping by the "Multiplex polymerase chain reaction assay for genotyping C. perfringens (Meer and Songer 1997).

\section{Results}

In gestation - treated sows (B. licheniformis) started with 5.6 times more clostridium type $A$ than the control group (7.7E+5 vs 1.4E+5). NS

In farrowing - treated sows had 1.3 times more clostridium $(7.8 \mathrm{E}+5$ vs. $6.0 \mathrm{E}+5)$. Treated sows increased their count from gestation at a rate that was $97 \%$ slower than that of the control group.

$\mathrm{P} \leq 0.05$

In lactation - the treated sows had $49 \%$ the level of clostridium as the control sows $(4.6 \mathrm{E}+6$ vs. $9.2 \mathrm{E}+6)$.

The treated sows increased in clostridium counts from Farrowing to lactation at a rate that was $55 \%$ slower than that of the control. NS

The rate of increase in clostridium in the fecal samples from gestation to lactation grew in the treated group at a rate that was $57 \%$ slower than that of that in the control group. $\mathrm{P} \leq 0.05$

All sampled pigs in the treated group had detectable B. licheniformis in their faeces compared to the control group pigs which had no detectable B. licheniformis.

\section{Discussion}

In summary the results show that $\mathrm{B}$. licheniformis has the ability to reduce shedding of $C$. perfringens in sows which could lead to a reduction in C. perfringens related scours in baby pigs. In addition, when fed to sows in lactation, B. licheniformis can be found in the faeces of pigs nursing treated sows.

\section{References}

Quinn, P. J., Carter, M. E., Markey, B. and Carter, G. R. 1994. Clinical Veterinary Microbiology. Mosby Wolfe Publisher.

Meer, R.R. and Songer, G. 1997. Multiplex polymerase chain reaction assay for genotyping Clostridium perfringens. AJVR: 58(7) p702-705. 


\title{
P.487
}

\section{In Vitro Susceptibility of Tiamulin in Czech Isolates of Clostridium perfringens Type A}

\author{
Martina Masaříková; Jiří Smola \\ Veterinary and Pharmaceutical Sciences Brno, Brno, Czech Republic
}

\section{Introduction and objectives}

Neonatal enteritis in suckling piglets caused by Clostridium perfringens Type $\mathrm{A}$ is an important factor influencing economic efficiency of pig operations in the Czech Republic. Because Type A Clostridium perfringens is commonly present in swine intestinal content, it often becomes the unintentional target of peroral antimicrobial at the time of therapeutic interventions. One of antimicrobial substances that is very often used in pig herds to control swine dysentery and therefore could, in our opinion, influence susceptibility in Clostridium perfringens, is tiamulin. The aim of the study was to determine the minimal inhibitory concentration (MIC) of tiamulin in Clostridium perfringens Type A isolates from suckling piglets with diarrhoea between 2001 and 2004.

\section{Material and Methods}

The set tested for susceptibility and resistance to tiamulin consisted of 213 Clostridium perfringens Type $A$ isolates obtained from cases of diarrhoea in piglets under 7 days of age. The samples were taken at 83 different farms. The tiamulin (Novartis AG) MICs were determined using the agar dilution method with blood Wilkins-Chalgren agar in accordance with CLSI recommendations (1). As no interpretative criteria for the evaluation of MICs of Clostridium perfringens isolates to tiamulin have been defined yet, we have adopted criteria intended for porcine isolates of Brachyspira hyodysenteriae (2).

\section{Results}

2001: MICs to tiamulin obtained in 30 selected Clostridium perfringens Type A isolates ranged from 2 to $>32 \mathrm{mg} . \mathrm{l}^{-1}$. Susceptibility and intermediate susceptibility were demonstrated in 14 $(46.7 \%)$ of the isolates, 2 (6. $6 \%)$ isolates were resistant.

2002: MICs in 30 Clostridium perfringens Type $A$ isolates ranged

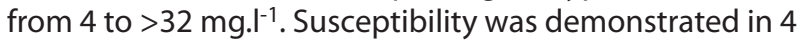
isolates (13.3\%), intermediate susceptibility in $9(30.0 \%)$ and resistance in 17 (56.7\%) isolates.

2003: MICs range in 30 isolates was the same as in the year 2002. Susceptibility and resistance was demonstrated in $6(20.0 \%)$ isolates, intermediate susceptibility in $18(60.0 \%)$ isolates.

2004: Susceptibility was found in 36 (29.2\%) isolates, resistance in 5 (4.1\%) isolates. The MIC values ascertained in most of the isolates $82(966.7 \%)$ were those corresponding to intermediate susceptibility; see Graph 1 and Table 1.

\section{Discussion}

Regardless of situation when tiamulin isn't registered for treatment of clostridial enteritis in piglets, were $\mathrm{MIC}_{90}$ surprisingly high (16 - >32 $\mathrm{mg.l}^{-1}$ ) in monitored period, in comparison with data obtained in Canada ( $\mathrm{MIC}_{90} 1 \mathrm{mg}^{-\mathrm{I}^{-1}}$ ) (3). We believe that this unfavourable development was connected with intensive medication of pigs with tiamulin in breeding herds affected with swine dysentery (SD), because a gradual growth in tiamulin MICs was also observed in Brachyspira hyodysenteriae isolates in the same time (4). Also the decrease in MICs of tiamulin in subsequent years is probably due to the decrease in the use of tiamulin, because of clinical resistance of Brachyspira hyodysenteriae at the period of treatment.

\section{Conclusions}

Based on the results of our study, we may assume a close relationship between bacteria forming part of intestinal microflora with respect to the establishment and development of resistance to antimicrobials frequently applied in feed or in water. Even though tiamulin has not been employed in the therapy of enteric infections, isolates keep unexpected high value of resistance. Possible explanation for findings sowing increasing MICs can consists in frequent use of tiamulin in the therapy of swine dysentery. Furthermore this findings corresponds with decreased sensitivity of Brachyspira hyodysenteriae isolates in the same time.

\begin{tabular}{|c|c|c|c|c|}
\hline & $\begin{array}{c}\mathbf{2 0 0 1} \\
(\mathbf{n}=\mathbf{3 0})\end{array}$ & $\begin{array}{c}\mathbf{2 0 0 2} \\
(\mathbf{n}=\mathbf{3 0})\end{array}$ & $\begin{array}{c}\mathbf{2 0 0 3} \\
(\mathbf{n}=\mathbf{3 0})\end{array}$ & $\begin{array}{c}\mathbf{2 0 0 4} \\
(\mathbf{n}=\mathbf{1 2 3})\end{array}$ \\
\hline $\mathrm{MIC}_{50}\left(\mathrm{mg} . \mathrm{I}^{-1}\right)$ & 8 & 32 & 16 & 8 \\
\hline $\mathrm{MIC}_{90}\left(\mathrm{mg} . \mathrm{I}^{-1}\right)$ & 16 & $>32$ & 32 & 16 \\
\hline$M I \mathrm{Cs}^{-}$range $\left(\mathrm{mg} . \mathrm{I}^{-1}\right)$ & $2->32$ & $4->32$ & $4->32$ & $<0,5-32$ \\
\hline
\end{tabular}

\section{Categories of $C$. perfinguens type $A$ isolates}

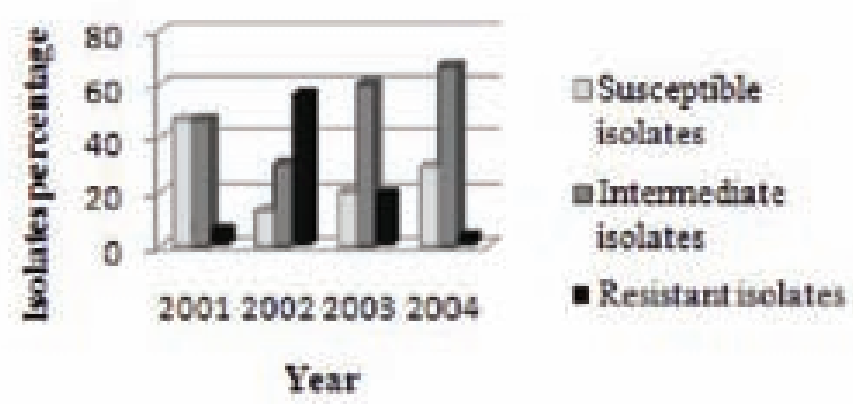

\section{Acknowledgement}

The financial resources for the study were provided by the grant NAZV QH 1278.

\section{References}

1. CLSI, (2001). Approved standard M11-A5.

2. Rønne, H. and Szancer, J. (1990). In: Proceedings of the 11th IPVS, 1126.

3. Prescott, J.F. (2000). In: Antimicrobial therapy: 229-262.

4. Lobová, D. (2004). J Med Microbiol 53: 287-291. 


\title{
P.488
}

\section{Treatment with diclazuril 1,5\% in a farm swine with history of isospora suis: effect on the daily weight, diarrhea and mortality, from birth-weaning}

\author{
Ismael M. Dolso; Gabriel Di Cola; Mercedes Vazquez; Silvia Romanini; Judhit Bertone
}

Universidad Nacional de Río Cuarto, Río Cuarto, Argentina

\section{Introduction}

Isospora suis is the causative agent of the swine neonatal coccidiosis (1). The disease usually occurs during the second week of life and is characterized by the development of diarrhea, dehydration and loss of body condition $(2,3,4)$. The study aimed to assess the effectiveness of the diclazuril on a pig farm with a history of Isospora suis and its effect on the daily gain weight, diarrhea and mortality from birth to weaning.

\section{Materials and Methods}

The experience was on a farm in 500 mothers, located south of the province of Córdoba, Argentina, with a history of Isospora since 2007. During three weeks of deliveries are weekly formed two groups of piglets, selected at random, belonging to 23 mothers each group. A total of 517 piglets at $256(49.51 \%)$ is given by mouth, $1 \mathrm{~cm} 3$ of saline (group Control C). To 261 (50,49 $\%$ ) is given $2 \mathrm{~cm} 31.5 \%$, (Group Treaty D) Diclazuril . Both treatments were on the second day of life. All animals were heavy individually to the $1 \mathrm{st}$, 7th, 14th and weaning at 19 days. Recorded the number of animals presented diarrhea and those who died with or without diarrhea.. All data were analysed and processed by the statistical programme SPPS.

\section{Results}

See the results in Table 1 and 2.

\section{Discussion}

Table 1 shows that animals treated with Diclazuril $1.5 \%$ on the second day of life, obtained a better daily weight, while Table 2 noted that it was effective to prevent the occurrence of diarrhea caused by Isospora suis, as well to reduce mortality.

\section{References}

1- Lindsay, D. S., et al.1992. Compendium on Continuing Education for the Practicing Veterinarian (14), $698-702$.

2- Christensen, J.P.B.; et al. 1994. Sheding of oocyst in piglets experimentally infected with Isospora suis. Acta Vet. Scand. 35 (2): 165172.

3- Lindsay, D.S.; et al. (1984). Prevalence of oocyst of Isospora suis and Eimeria spp. From sows on farms with ans without a history of neonatal coccidiosis. Javma. 185 (4): 419-421.

4- Vazquez M; et al (2000). Dynamics of Isospora suis infection in pigs raisers at open air. InVet 2: 333-337.
Table 1: Results of the study phase

\begin{tabular}{|l|c|c|}
\hline & Control & Diclazuril \\
\hline Births Live & 256 & 261 \\
\hline Births Dead & 9 & 9 \\
\hline Deliveries & 23 & 23 \\
\hline Births Live/sow & 11,13 & 11,34 \\
\hline Births Dead /sow & 0.39 & 0.39 \\
\hline Births Totals /sow & 11.52 & 11.73 \\
\hline Weaned & 233 & 248 \\
\hline Average & 10,13 & 10,78 \\
\hline Slaughtered & 6 & 6 \\
\hline Dead & 17 & 7 \\
\hline \% Mortality & 6,64 & 2.68 \\
\hline Quantity animals with diarrhea & 13 & 2 \\
\hline Weight Average at birth $(\mathrm{kg})$ & 1,3474 & 1.29 \\
\hline Weight Average 7 days $(\mathrm{kg})$ & 2,226 & 2,295 \\
\hline Weight Average 14 days ((kg) & 3,7546 & 3,8819 \\
\hline Weight Average to weaning (kg) & 5,0126 & 5,3145 \\
\hline Gain daily average (kg) & 0,1957 & $0,2133 *$ \\
\hline Minimum daily gain $(\mathrm{kg})$ & 0.10 & 0.09 \\
\hline Maximum Daily gain $(\mathrm{kg})$ & 0.33 & 0.32 \\
\hline 0ffset Standat & 0,03647 & 0,04726 \\
\hline Variance & 0.001 & 0.002 \\
\hline * $\mathrm{p}<0.001$ & & \\
\hline
\end{tabular}

Table 2

\begin{tabular}{|l|c|c|}
\hline \multicolumn{1}{|c|}{ Group } & Control & Diclazuril \\
\hline Weaned & 233 & 248 \\
\hline Average & 10,13 & 10,78 \\
\hline Quantity animals with diarrhea & 13 & $2^{*}$ \\
\hline Quantity of dead pigs & 17 & 7 \\
\hline$\%$ Mortality & 6,64 & $2,68^{*}$ \\
\hline${ }^{*} \mathrm{P}<0.005$ & \multicolumn{3}{|l}{} \\
\hline
\end{tabular}




\title{
P.489
}

\section{Effect of diclazuril $1.5 \%$ on the uniformity of the litter to the weaning in a farm with Isospora suis antecedents}

\author{
Ismael M. Dolso; Gabriel Di Cola; Mercedes Vazquez; Judhit Bertone; Silvia Romanini \\ Universidad Nacional de Río Cuarto, Río Cuarto, Argentina
}

\section{Introduction}

Isosporosis in piglets, caused by coccidium Isospora suis, is recognised as a disease having a significanrt negative impact on the pig industry, not as a cause of high mortality, but by reducing productivity and eroding the economic returns to pig producers (1). Piglet coccidiosis has been associated with descreased nutrient absorption, impaired immunocompetence, incresead susceptibility to secundary bacterial infeccion, uneven weigt gains and uneven litters at weaning $(2,3)$.

The training target was to evaluate the effect of Diclazuril $1.5 \%$, in a pig farm with antecedents of Isospora suis on the uniformity of the litter to the weaning.

\section{Materials and Methods}

The experience was realised in a farm of 500 mothers, located to the south of the province of Cordoba (Argentina), with antecedents of Isospora from year 2007. During three weeks of childbirths two groups of pigs, selected at random, pertaining to 23 mothers were satisfied weekly each group. Of a total of 517 pigs, to $256(49,51 \%)$ it was administered to them by oral route, $1 \mathrm{~cm} 3$ of saline solution, (Group Control C). To 261 (50.49\%) 2cm3 of Diclazuril $1.5 \%$ was administered to them, (Treated Group D). Both treatments were realised to the second day of life. All the animal were heavy in individual form to the day of birth, and the 19 days, age of weaning. All the data were analyzed and process by statistical program SPPS.

\section{Results}

Table 1 shows the animal birth weight distribution.

Table 1: Distribution the weight at birth.

\begin{tabular}{|l|c|c|}
\hline The birth weight & Control & Diclazuril \\
\hline Grams & $\%$ & $\%$ \\
\hline$<800$ & 3,13 & 2,68 \\
\hline $801-1000$ & 9,38 & 11,49 \\
\hline $1001-1200$ & 16,41 & 21,07 \\
\hline $1201-1400$ & 28,52 & 33,72 \\
\hline $1401-1600$ & 23,83 & 19,16 \\
\hline $1601-1800$ & 12,50 & 9,20 \\
\hline$>1801$ & 6,25 & 2,68 \\
\hline N & 256 & 261 \\
\hline Average Kg & $1,347^{*}$ & 1,290 \\
\hline Minimun Kg & 0,52 & 0,65 \\
\hline Maximum Kg & 2,26 & 1,95 \\
\hline Desv. typically & 0,29339 & 0,25074 \\
\hline Variance & 0,086 & 0,063 \\
\hline
\end{tabular}

The Group C, 71,09\% of animals were over 1200 grams, while in Group D, $64,75 \%$ of the animals only achieved this weight to its time, the difference between the average weight at birth, were 138 grams heavier Group C (* $p<0,01)$.

In Table 2 shows the animals at weaning weight distribution.

Table 2: Distribution the weights of the animals at weaning.

\begin{tabular}{|l|c|c|}
\hline Weight to the weaning & Control & Diclazuril \\
\hline Grams & $\%$ & $\%$ \\
\hline$<3000$ & 3,86 & 2,42 \\
\hline $2001-400$ & 10,73 & 10,48 \\
\hline $4001-4500$ & 11,16 & 11,29 \\
\hline $4501-5000$ & 19,31 & 12,10 \\
\hline $5001-5500$ & 28,76 & 16,53 \\
\hline $5501-6000$ & 15,45 & 18,95 \\
\hline$>6000$ & 10,73 & 28,23 \\
\hline $\mathrm{N}$ & 233 & 248 \\
\hline Average $\mathrm{Kg}$ & 5,012 & $5,314 *$ \\
\hline Minimun $\mathrm{Kg}$ & 2,44 & 2,38 \\
\hline Maximum Kg & 8,03 & 8,36 \\
\hline Desv. typically & 0,93753 & 1,12225 \\
\hline Variance & 0,879 & 1,259 \\
\hline
\end{tabular}

The Group C, 26,18\% of animals were in excess of 5, $501 \mathrm{~kg}$ While in Group D, $63,71 \%$ of the animals they achieved this weight to its time, the difference between the average weights at weaning, were 302 grams heavier Group D (* $p<)(0.01)$.

\section{Conclusion}

One demonstrated that the animal of group D, that although they were lighter when being born, when applying Diclazuril $1.5 \%$ to the second day of life, were but heavy to the weaning and the litter but uniform it was compared with the group nontreated.

\section{References}

1 Torres, J.2004. Proceedings 18 th IPVS Congress, Hamburg, Germany. p. 243.

2 Böhne, l; et al. 2006. Proceedings of the 1 st International Bayer Pig Symposium. Copenhagen. p 92.

3 Mundt, H; et al. 2001. Control of coccidiosis, in Pig Progess. P 9-11. 


\title{
P.490
}

\section{Age, not infection dose, determines the outcome of Isospora suis infections in suckling piglets}

\author{
Hans-Christian Mundt ${ }^{1}$ Hanna L. Worliczek ${ }^{2}$ Soelen Siemers ${ }^{2}$ Anja Joachim ${ }^{2}$ \\ 1. Bayer Animal Health GmbH, Monheim, Germany; 2. Institute of Parasitology and Zoology, \\ Department of Pathobiology, University of Veterinary Medicine, Vienna, Austria
}

\section{Introduction}

In the field, clinical presentations of neonatal isosporosis range from subclinical infection with oocyst excretion to excessive watery diarrhea with exsiccosis and unthriftiness within the same litter. Several publications on a small set of animals indicate that age is more important for the clinical outcome of isosporosis than the infection dose (Meyer et al., 1999; Martineau and de Castillo, 2000; Mundt et al., 2003). In this evaluation a larger data set from experimental studies under highly standardized conditions was analysed retrospectively.

\section{Materials and Methods}

Data from 13 trials involving 124 suckling piglets experimentally infected with Isospora suis were evaluated for the effects of infection dose and age at infection on the clinical and parasitological outcome of infection in four different models. Infections were carried out with single oral inoculations of different numbers of sporulated oocysts of $I$. suis per animal at different ages (days of life, d.o.l.): model 1: 1000 oocysts on the 1st d.o.l (9 piglets from 3 litters); model 2: 1000 oocysts on the 4th d.o.l (25 piglets from 11 litters); model 3: 1500 oocysts on the 4th d.o.l. (40 piglets from 20 litters); model 4: 10,000 oocysts on the 4th d.o.I (50 animals from 10 litters). Weights were determined on the day of birth and in weekly intervals. Faecal consistency and quantitative oocysts excretion were evaluated for 2 weeks starting 4 days after infection (d.p.i.) as described (Mundt et al., 2006).

\section{Results}

Piglets showed typical weight depression in the second week of life during the acute phase of infection. This effect was most noticeable in model 1 (infection on the first d.o.l.) where animals only gained $2.08 \mathrm{x}$ their birth weight until the $22 \mathrm{nd}$ d.o.l., compared to 2.31 (model 2), 2.52 (model 3) and 2.38 (model 4) $x$ the birth weight in the other groups.
This correlated with the occurrence of watery diarrhoea which was found in $37 \%$ of the samples in the acute phase (4-11 d.p.i.) in model 2 but only $12-20 \%$ of the samples in the other models. Oocyst excretion prevalences showed the earliest peak 5 d.p.i. in the group with the highest dose and the highest peak (100\% for 2 days) in the group infected on the first day of life. The average excretion days 5-11 d.p.i. were $41.3 \%$ (mean OPG: 9189 ) in model 1, 30.0\% (mean OPG: 8545 ) in model 2, $39.5 \%$ in model 3 (mean OPG: 3143), and 75.2 \% (mean OPG: 26 841) in model 4.

Median oocyst excretion peaked earlier in the models with higher infection doses but reached the highest values in model 1 (early infection).

\section{Discussion}

As in previous studies, this cross-sectional analysis of a larger number of animals confirms the influence of age on the outcome of isosporosis in suckling piglets, stressing the need to control the infection at an early life phase.

\section{References}

Martineau GP, del Castillo J (2000). Epidemiological, clinical and control investigations on field porcine coccidiosis: clinical, epidemiological and parasitological paradigms? Parasitol. Res. 86: 834-7

Meyer C, Joachim A, Daugschies A (1999). Occurrence of Isospora suis in larger piglet production units and on specialized piglet rearing farms.Vet. Parasitol. 82: 277 - 284

Mundt, HC, Joachim A, Daugschies A, Zimmermann M (2003): Population biology studies on Isospora suis in piglets. Parasitol. Res. 90, Suppl. 158-159.

Mundt, HC, Joachim A, Becka M, Daugschies A (2006): Isospora suis: an experimental model for mammalian intestinal coccidiosis. Parasitol. Res. 98, 167-175 


\title{
P.491
}

\section{Impact of piglet coccidiosis on the gut health and economic benefits of the prevention with toltrazuril}

\author{
Abdulkerim Deniz; Veerle Hamaekers \\ Bayer Animal Health, Monheim, Germany
}

\begin{abstract}
Introduction
Piglet coccidiosis is a parasitic disease mostly affecting young and suckling piglets in the farrowing pens. The responsible agent is Isospora suis which is very reproductive protozoa with a prepatent period of 4- 6 days. It causes pasty yellowish diarrhoea, but it can also occur subclinically without any sing of disease. Isoporosis is prevalent around $74 \%$ in German, 76\% in Belgium, 79\% in Greek and Austrian, 90\% in Polish, 56\% in French (1), 75\% in Japanese (2) and $70 \%$ in Canadian pig farms (3). Coccidiosis in suckling piglet is of economic importance due to retarded growth $(4,5)$.
\end{abstract}

\section{Impact of isosporosis on gut health}

I. suis may cause fibrinous enteritis which mainly affects the middle and posterior part of the jejunum; villous necrosis and atrophy are frequently described (6). Serious damages on the villious and gut mucosa due to coccidiosis in pigs were already detected by different scientists $(6,7)$. Coccidiosis can run concomitantly with Clostridium perfringens, E. coli, Rota virus, Adeno virus and salmonellosis (8). Primarily coccidiosis is also predisposing factor for the contaminant diseases. Typically the disease causes significant weight gain decrease at weaning and also its impact can result in prolonged production period (slaughter age) up to 9 additional days and in high postweaning $\operatorname{FCR}(9,10)$. This may be seen as primary reason why the piglets have frequently bacterial infections during the course of coccidiosis (10). A mixed infection of Clostridium perfringens and I. suis caused a mortality rate of $12,1 \%$ in the first week of life and $16,2 \%$ up to weaning in Germany (8). This mortality rate due to mixed infection was reduced by $71 \%$ in the first week of life and $64 \%$ up to weaning by preventative treatment of 3 days old pigs with toltrazuril $5 \%$ suspension (Baycox). Prevention from the primary destructive affect of I. suis on the gut health with early toltrazuril treatment resulted in significantly reduced mortality. Similarly in an experimental study (11) the same results were reproduced. Earlier coccidiosis episode predisposed pigs for postweaning Lawsonia infections which has been detected serologically in untreated pigs aged 21 to 105 days and resulted in higher FCR (10). However, not all treatment options give optimal results as reported by Mundt et al. 2007 (7). Sulphadimidine, diclazuril and toltrazuril ( $20 \mathrm{mg} / \mathrm{kg}$ one shot) were used to control isosporosis in suckling piglets, but none of the treatments other than toltrazuril were able to protect gut mucosa and to prevent from weight loss.

\section{Economic benefits of the prevention}

Even in subclinical coccidiosis the weight gain of pigs can be affected significantly (5). Under subclinical coccidiosis pressure the preventative treatment with toltrazuril (Baycox 5\%) resulted in $€ 0,23$ net profit per piglet at weaning. Similarly, Scala et al. 2009 (4) also stated that the early preventative treatment with toltrazuril in italian pig farm provides better returns $(€ 10,64)$ than sulphonamide and trimetoprim combination $(€ 8,57)$ and untreated control group $(€ 9,72)$ based on the weight at weaning day 28. Not doing preventative measurements against

Proceedings of the $21^{\text {st }}$ IPVS Congress, Vancouver, Canada - July 18-21, 2010 coccidiosis has prolonged the feeding time by around 12 days (9). This statement has also been supported by McOrist et al. 2009 (10). The FCR of toltrazuril treated pigs between ages 21 to 105 was $10 \%$ significantly less than untreated pigs. Based on the increase of weaning weight (647 g) and including handling and treatment cost, Sepulveda et al. in 2008 (12) calculated a surplus of $€ 1,09$ for the troltrazuril treated pigs at weaning. The increase of weight gain up to weaning via toltrazuril prevention is presented in figure 1. Prevention from coccidiosis at early ages can also reduce the frequency of antibiotic treatments up to weaning by around $88 \%$ (13), this can be explained by the improvement of gut health due maintenance of gut mucosa integrity and low diarrhoea incidence in those pigs treated with toltrazuril.

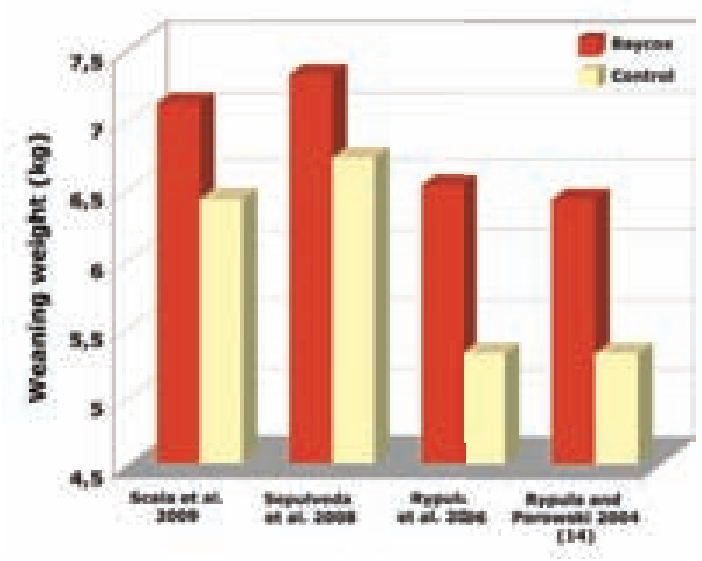

\section{Conclusion}

Isosporosis in suckling piglets has life long affects and should not be underestimated because it has economic impacts on production parameters and can cause loss of profit in pig rearing.

\section{References}

1 Torres A (2004): IPVS, June 27-July1st, Hamburg, Germany.

2 Matsuba et al. (2009): APVS, October 26-28, Tsukuba-Japan.

3 Aliaga-Leyton et al. (2008): AAVP, July 19-22, New Orleans, US.

4 Scala et al. (2009): Veterinary Parasitology, 163, 362-365.

5 Maes et al. (2007): The Veterinary Journal, 173: 197-199.

6 Koudela and Kucerova (2000): Parasitology Research 86, 861-863.

7 Mundt et al. (2007): Parasitol Res, 100:401-411.

8 Westphal et al. (2007): Tierärztliche Umschau 62, 682 - 689.

9 Rypula et al. (2006): IPVS, July 16-19, Cophenhagen, Denmark.

10 McOrist et al. (2009): WAAVP, Calgary, Canada, August 8 - 14.

11 Mundt et al. (2009): WAAVP, Calgary, Canada, August 8 - 14.

12 Sepulveda et al. (2008): Albeitar, ublicações Ciência e Vida, Odivelas, Portugal, Nov./Dez., 6, 136-138.

13 Driesen et al. (1995): Aust Vet J. 1995 Apr, 72(4):139-41.

14 Rypula and Porowski (2004): IPVS, June 27-July 1st, Hamburg, Germany. 


\title{
P.492
}

\section{Evaluation of efficacy of oral toltrazuril (Baycox $5 \%{ }^{\circledR}$ ) for the improvement of post-weaning gut health in pigs in Romania}

\author{
Steven McOrist ${ }^{1}$ Daniel Spiru² Marion Ocak ${ }^{3}$ Abdulkerim Deniz ${ }^{4}$ \\ 1. University of Nottingham, Sutton Bonington, UK; 2. SMF Veterinary Services, Timisoara, Romania; 3. MD Research, Munich, Germany; \\ 4. Bayer Health Care, Monnheim, Germany
}

\section{Introduction}

Isospora suis disease often occurs around the second week of life, through to the normal pig weaning age of 3-weeks-old. It is characterized by yellowish pasty diarrhoea for one to several days. Oocysts can survive outside the pig, in the farrowing area, for many months. The time for intervention in piglets with a control strategy is critical; use of a preventive drug usually take place at 3 to 4 days of age so that it can kill appropriate stages of the parasite within newly infected piglets. In the European Union, the symmetrical triazintrione toltrazuril is the only coccidiocidal registered (since 2002) specifically for the control of porcine neonatal coccidiosis in a single oral dose format of $20 \mathrm{mg}$ per $\mathrm{kg}$ bodyweight. Clinical studies of toltrazuril after weaning have not been comprehensive - pigs are only considered to show limited compensatory growth. The exact reasons for this are not clear, but could include intestinal maturity and enzymatic activity and susceptibility to further post-weaning infectious agents. The aim of this on-farm study was to evaluate the efficacy of toltrazuril (Baycox ${ }^{\circledR} 5 \%$ oral suspension) for the improvement of gut health in a large-scale grower-finisher pig herd.

\section{Methods}

Four-day-old matched piglets either received toltrazuril (Green, $\mathrm{n}=2,870$ ) or placebo (Blue, $\mathrm{n}=2,812$ ) via blinded colour-coded medications. The subsequent diarrhoea scores, growth performance, gut health and entero-pathogen load in each group was monitored by blinded observations, feed and pig weighing, faecal PCR tests for Brachyspira and Lawsonia and PCV2, culture for coliforms and Clostridia, as well as Lawsonia immunofluorescence assay serology. Observations were made daily and measurements collected weekly from day 21 up to day 150 in the grower-finisher stage of farming.

\section{Results}

There was a significant drop in oocyst count in piglets around weaning in the Baycox ${ }^{\mathrm{TM}}$ treated group $(p<0.01)$. There was markedly improved feed conversion in treated weaner-finisher pigs (2.41 cf. 2.63). Weight gains were consistently and significantly better through to day 150 , see chart. Lawsonia serology results indicated a late (between day 84 and 105) and moderate onfarm exposure, which was more severe in non-treated pigs.

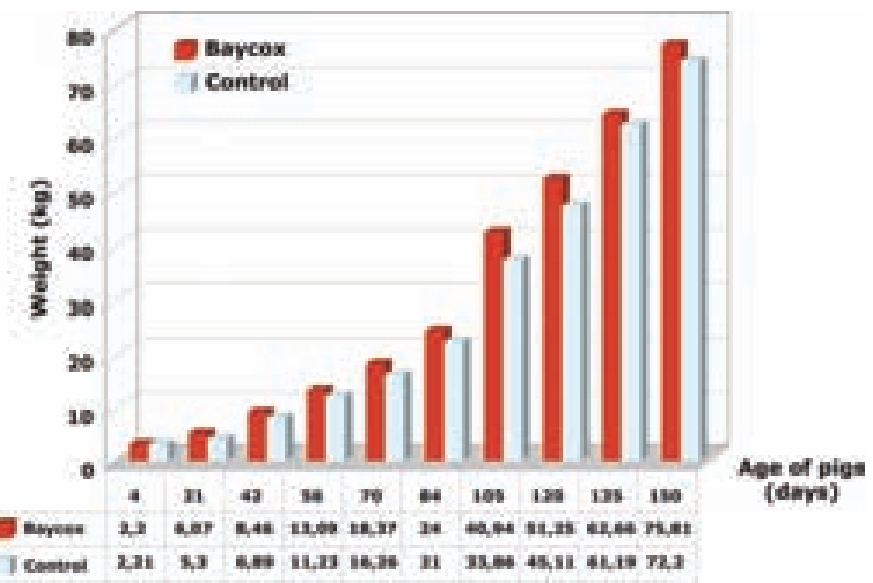

\section{Discussion}

These results confirm the protective effect of toltrazuril (Baycox ${ }^{\top M}$ ) for coccidiosis prior to weaning and demonstrate specific beneficial effects on gut health and feed conversion after weaning. We also found that post-weaning enteric diseases including ileitis were more severe in pigs untreated for coccidiosis.

We therefore suggest there are two main reasons why the lifetime effects of small weaning weight and peri-weaning infections of coccidia are so important and detrimental. First, there is an innate anatomical issue in that smaller, gut-damaged piglets being weaned with a limited supply of mature villi units, will grow more slowly and second, there is the likelihood that pre-weaning infections can carry forward into an increased number of post-weaning infections such as ETEC, Lawsonia and Brachyspira.

In the past 5 years, novel and effective preventive programs for Lawsonia and Isospora been introduced, related to an oral live attenuated vaccine and the oral anti-coccidial medication toltrazuril $\left(\right.$ Baycox $\left.{ }^{\mathrm{TM}}\right)$, respectively. Unlike coccidiosis in chickens, no major vaccines have been identified for coccidiosis in pigs. 


\title{
P.493
}

\section{Prevalence of Coccidia in piglets in Poland in years 2003-2009}

\author{
Jacek Karamon; Zygmunt Pejsak \\ Nat. Vet. Res. Inst in Pulawy, Pulawy, Poland
}

\section{Introduction}

Coccidiosis caused by Isospora suis and Eimeria spp. is one of the common diarrhoeal diseases of piglets, occurring in all countries with intensive swine production. The first clinical symptoms of this disease appear between 9 and 15 days of piglet life. The faeces are pasty at the beginning, and what is typical diarrhoeic piglets do not respond to antibiotics. The diagnosis is usually made by detecting of oocysts in faeces of the affected pigs. The reports about prevalence of piglet coccidiosis caused by I. suis demonstrate a high farm prevalence of the disease $(45 \%$-to 90\%).

The objective of this study was to summarize results of routine diagnosis for piglet coccidiosis performed in our laboratory during the period from $2003-2009$.

\section{Material and Methods}

The study was carried out between 2003 and 2009, in 14 out of 16 Polish provinces. Faecal samples were taken from 2-3 weeks piglets from 3 types of farms: large ( $>100$ sows), medium ( 25 to 100 sows) and small (<25 sows). In mentioned farms diarrhoea of suckling piglets at the age 8-15 days were observed in all of the examined herds, the antibiotic treatment of sick piglets gave non satisfactory results. Overall, 966 litters of suckling piglets from 132 farms and 273 mother sows, during mentioned above period, were examined. Faecal samples of 5 to 28-day-old piglets were collected directly from the rectum. Samples were taken from 3 to 5 piglets per litter and pooled. 5-10\% of litters from each farm were investigated. The faeces were analyzed with the flotation method. The saturated $\mathrm{NaCl}$ solution sugar enriched was used. The samples were examined at 100x-400x magnification. Samples were mixed with $2.5 \%$ potassium dichromate solution and stored on Petri dishes at $250 \mathrm{C}$ in order to induce the sporulation if unsporulated oocysts were present. The species of coccidia were defined based on oocysts morphology and sporulation time.

\section{Results and Discussion}

Overall, I. suis infection was found in 286 litters of suckling piglets $(29.6 \%)$ from 88 farms $(66.7 \%)$. The coccidia of Eimeria genus (E. debliecki, E. polita, E. suis) were detected in only 23 litters (2.4\%) from 13 farms (9.8\%).

Comparison of the results from different years indicated that percentage of positive farms in different periods was similar. In any period percentage of I. suis did not exceed 74\% (2005) and never was lower than 58\% (2008).
Tab. 1. Prevalence of Coccidia in suckling piglets on different types of Polish farms

\begin{tabular}{|l|c|c|c|c|}
\hline $\begin{array}{l}\text { Types of farms } \\
\text { (no. of sows on } \\
\text { farm) }\end{array}$ & \multicolumn{2}{|c|}{$\begin{array}{c}\text { \% of farms infected } \\
\text { with coccidia }\end{array}$} & \multicolumn{2}{c|}{$\begin{array}{c}\text { \% of litters infected } \\
\text { with coccidia }\end{array}$} \\
\hline & I. suis & Eimeria & I. suis & Eimeria \\
\hline Large $(>100)$ & 91.1 & 6.3 & 33.3 & 1.1 \\
\hline Medium $(25-100)$ & 51.2 & 4.9 & 19.1 & 2.0 \\
\hline Small $(<25)$ & 27.3 & 54.5 & 13.2 & 28.9 \\
\hline Total & 66.7 & 9.8 & 29.6 & 2.4 \\
\hline
\end{tabular}

As shown in Tab. 1. in the large farms I. suis infection was detected the most often ( $91,1 \%$ of farms, $33.3 \%$ of litters), whereas in the small farms frequency of this infection was found to be twice as rare. The situation with Eimeria spp. was just the opposite - for the large and medium sized farms Eimeria spp. oocysts were very uncommon, while on the small farms these infections were found in as many as $54.5 \%$ of farms, $28.9 \%$ litters. Similar results confirming a higher occurrence of $I$. suis in large farms than in smaller ones were obtained in Nordic countries (1).

The examination of faecal samples collected from sows (mothers of examined piglets), yielded the following results: I. suis oocysts were found in 19 sows (7\%) and Eimeria spp. (E. debliecki. E. polita, E. perminuta, E. suis, E. scabra) in 18 sows (6.6\%). From 73 sows delivering I. suis infected piglets just in 12 sows (16.4\%) I. suis oocysts and in as little as 4 (5.4\%) Eimeria spp. oocysts were found. In the remaining 56 sows (78\%) no cases of coccidian infections were detected. Small number of sows excreting coccidian oocysts and a lack of correlation between the presence of oocysts in the sows, and the I. suis infected piglets were demonstrated also by other authors $(2,3)$

Study concerning coccidiosis in piglets carried out during last 7 years proved that I. suis and Eimeria spp. were quite common in piglets $2-3$ weeks of age in all sizes of farms. It might be also stated that those intracellular parasitic species play an important role as a cause of diarrhoea in suckling piglets. The level of coccidia infections seems to be stable during evaluated period.

\section{References}

1. Larsen, K., (1996). Dan. Vet. Tijdsskr. 79, 387-392.

2. Farkas (2004) IPVS Congress 314

3. Nilsson (1984) Nordisk. Vet. 36, 103-110. 


\title{
P.494
}

\section{Prevalence of isosporidial infection in piglets with clininical signs of enteropathy}

\author{
Bozidar Savic; Ivan Pavlovic; Vojin Ivetic; Milenko Zutic; Branislav Kureljusic \\ Scientific Veterinary Institute of Serbia, Belgrade, Serbia
}

\section{Introduction}

The disease caused by coccidia Isospora suis has been described as an important cause of suckling piglet diarrhea and reported from all types of farrowing facilities and under all types of management systems $(2,3)$. The developmental stages of Isospora suis occur both inside the host animal and outside, in the external environment. The target organ of this parasite is the small intestine, where it undergoes development within the mucosal tissue. Isospora suis can infect pigs of any age, but only causes clinical signs in piglets, usually those between the ages of 7 and 14 days. Older pigs act as carriers. The sow plays a minor or nonexistent role in the transmission of Isospora suis.

Depending on the rate of infection, the disease complexes can range from sub clinical infections to infections that include severe intestinal problems with severe lesions in the gastrointestinal tract that can result in high mortality and/or a negative impact on the factors of production $(2,4)$. At same time, those parasites play important role at complex of neonatal entheropathy of piglets, spread together with other infectious agents (1, 3).

In this study we aimed to investigate about presence of Isospora suis prevalence in farm-farrowing conditions.

\section{Materials and Methods}

During 2007-2009 a total of 428 pigs, 2 days -21 weeks old, were exanimate in the laboratory of Scientific Veterinary Institute of Serbia. All of the examined pigs were sings of diarrhea. After routine necropsy, to parasitological examination we used faecal swab, gut contents and a mucous scrape of gut which we examined with Sheather's sugar flotation and in direct smears stained with Gimsa strain and Ziehl-Neelsen stain and examined by use of light microscopy. In addition section of jejunum, ileum and spiral colon from each pig were fixed in natural buffered $10 \%$ formal in, embedded in paraffin, sectioned at 6 micrometer, stained with hematoxylin and eosin and examined by use of the light microscopy. At same time we examined 979 samples of faeces originated from various product categories (fating pigs, sows, board) a coprological examination was done within 24 hours after sampling using flotation method in Sheather's sugar solution.

\section{Results and Discussion}

The occurrence of Isospora suis was found to be highest in piglets at the age of 7-15days. Isosporosis prevalence in piglets was highest on the second week of piglet age (32.8\%) with the highest prevalence value on day 13 of piglet age (44.3\%) In infected piglets the presence of $\mathrm{I}$. suis was detected most frequently in connection with watery diarrheas (37.4\%) and least frequently in piglets with shaped faeces (15.0\%). In piglet with watery diarrheas only very weak isosporous infections were usually found, together with other enteropathogenic agents of bacterial or viral etiology contributed to the origin of diarrheas (like E.coli, C.perfrigens tip C rotaviruses, and etc.) in parallel infections in piglets to a large extent.

Reports on the presence, prevalence and epidemiology of Isospora suis have come from virtually every country in the world (4). Studies conducted in most countries have shown a high prevalence of the disease on farms (18-85\%). Results of our examination are at similar range Reports on the highest occurrence of Isospora suis in relation to piglet age vary. The highest prevalence of isosporosis was reported most frequently at the piglet age of two in some cases also third week of life. During our examination most prevalence are find at 13 days of piglet age. In our study we found sig $\neg$ nificant correlation between isosporosis and wa-tery diarrhoeas because the highest isosporosis prevalence was demonstrated in piglets with watery diarrhoeas.

We found out total isosporosis prevalence $27.4 \%$ mostly in a litter housing system; these re $\neg$ sults document that in our conditions there are not any significant differences in the prevalence and time of occurrence of Isospora suis between differ-nent housing systems and time periods compared to the results obtained in a litterless system of piglet housing.

\section{Acknowledgments}

This work was partly funded by Serbian Ministry of Science and Technological Development, project number TP: 20110.

\section{References}

1. Pavlovic',I. et al. (1995)Mac.Vet.Rev.24,69-72

2. Pavlovic',l. et al. (2004) Vet.Glasnik 58 (3-4), 561-565

3. Pavlovic',I. et al. (2008) Proc.20th IPVS Congress Durban, South Africa, 345

4. Mundt H.-C., Daugschies A. (2004): Procc.18th Congress IVS Hamburg, 11-14. 


\title{
P.495
}

\section{Impact of the pharmaceutical quality of different toltrazuril suspension formulations on the control of Isospora suis infections in piglets}

\author{
$\underline{\text { Hans-Christian Mundt }}{ }^{1}$ Soelen Siemers ${ }^{1}$ Iris Heep ${ }^{1}$ Daniel Gondol ${ }^{1}$ Klaudia Lusky² Abdulkerim Deniz $^{1}$ \\ 1. Bayer Animal Health GmbH, Monheim, Germany; 2. IVPT, Bernau, Germany
}

\section{Introduction}

Piglet coccidiosis due to infection with Isospora suis is controlled by, among other measures, a single oral treatment with Baycox ${ }^{\circledR}$ $5 \%$ suspension in the first few days of life. The product was invented and developed specifically for this purpose. Almost two decades of experience and expertise in epidemiology and control demonstrate that both coccidial infections and infections due to $C$. perfringens on problem farms are reliably controlled (Mundt et al., 2007; Mundt and Daugschies, 2007; Mundt et al., 2008). Generic formulations containing toltrazuril have appeared in some countries since 2002. Their pharmaceutical parameters were analyzed and compared with the reference product (approved specification of the pioneer formulation). The impact on practical clinical use is discussed.

\section{Materials and Methods}

A total of 108 generic formulations and/or different batches were analyzed for their chemical, physical and microbiological properties. Investigations were carried out with HPLC (quantity of toltrazuril, impurities and by-products, preservatives), GC, analysis of color, density, sedimentation behavior, redispersibility, particle size (microscopy and measurement of particles and crystals), pH, microbiological status. In total, 10 to 15 analyses were conducted per sample. All methods were compliant with FDA, GMP and 21 CRF Part 11 requirements. Stability testing was performed at various temperatures in compliance with the most recent VICH guidelines in a GMP and cGMP environment. The clinical impact was additionally assessed in pharmacokinetic studies carried out by way of example with some formulations in piglets (comparison with the pioneer formulation).

\section{Results}

All the formulations tested were found to deviate from the specification for the pioneer formulation. There were differences in terms of the active ingredient (impurities, content of methyltoltrazuril etc.), type and content of preservatives, particle size and distribution (some particles up to $300 \mu \mathrm{m}$, Figure 1), sedi- mentation behavior, redispersibility among others. The differences were confirmed by the pharmacokinetic behavior of the products (Cmax, AUC).

Figure 1: Particle size of toltrazuril in pioneer (left; $<10 \mu \mathrm{m}$ ) and generic (right) formulation
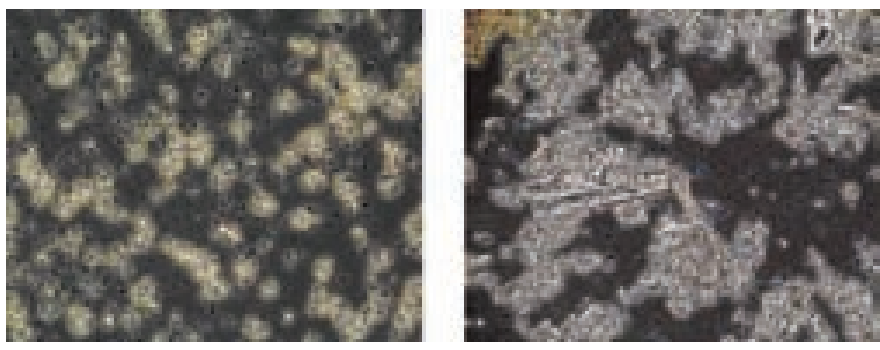

\section{Discussion}

The studies showed differences, some of them considerable, between the pharmaceutical quality of the generic formulations tested and Baycox ${ }^{\circledR}$. Their impact in terms of bioavailability, bioequivalence, efficacy, safety/tolerability, withdrawal time, convenience and cost-benefit is discussed.

\section{References}

Mundt HC, Mundt-Wüstenberg S, Daugschies A, Joachim A (2007): Efficacy of various anticoccidials against experimental porcine neonatal isosporosis. Parasitology Research 100, 401-411

Mundt HC, Daugschies A (2007): Current understanding of the epidemiology of intestinal coccidiosis in mamma-lian livestock. In: M. Fürll (ed.) Leipzig: Production Diseases In: Farm Animals, Merkur Druck und Kopier-Zentrum GmbH, Leipzig, 90-107:

ISBN 978-3-934178-90-8

Mundt HC, Krüger M, Westphal B, Mengel H, Krüger MU, Dittmar K, Kuhnert Y, Daugschies A (2008): Investigations into the synergic effect of postnatal experimental infection with Isospora suis and natural infection with Clostridium perfringens $\beta 2$ for the development of necrotic enteritis in piglets. 20th

Congress of the IPVS, June 22-25, Durban, South Africa. 


\title{
P.496
}

\section{Prevalence of Isospora suis in the South of Belgium}

\author{
Martine Laitat ${ }^{1}$ Veronica lanosi ${ }^{1}$ Valery Delleur ${ }^{1}$ Frédéric Farnir ${ }^{1}$ Bertrand Losson ${ }^{1}$ Dominique Gevaert ${ }^{2}$ Claude Saegerman $^{1}$ \\ 1. University of Liège, Liège, Belgium; 2. Bayer SA-NV, Animal Health Division, Diegem, Belgium
}

\section{Introduction}

Isospora suis is the causal agent of coccidiosis, a frequent digestive disease usually noted in 5 to 14 days old piglets. The disease is characterized by diarrhoea and growth retardation but no (or a few) mortality unless after secondary bacterial infection. It has a strong economical impact for the farmer and even more if the aetiology remains unknown, causing loss of time and money using antibiotics that will be ineffective as the pathogen is a parasite. The objective of this work was to determine the prevalence of Isospora suis and the incidence of litters affected in pig farms of the South of Belgium - Wallonia. Epidemiological data were collected in each farm and compared (Table 1) to those reported in a previous study (Leten and al., 2002) performed in the North of Belgium - Flanders.

\section{Materials and Methods}

Faeces of three to five 10-18 day old piglets per litter were collected in 58 at random selected pig farms where 10 litters were available and no toltrazuril treatment (Baycox ${ }^{\circledR} 50 \mathrm{mg} / \mathrm{ml}$, Bayer) was administered. Processing of faeces (1 $\mathrm{g}$ per sample) was performed using $7 \mathrm{ml}$ of a saturated $\mathrm{NaCl}$ solution with $500 \mathrm{~g}$ added glucose per litre (Henriksen and Christensen, 1992). An optical microscope $10 \times 40$ was used to detect oocysts. A positive diagnosis for a farm (F) or for a litter $(L)$ was recorded when at least one (sporulated or not) oocyst was present.

Possible relation between clinical signs (diarrhoea), cleaning and disinfection of the farrowing crate and the presence of I. suis or the percentage of infected litters were tested using ${ }^{\circ} \mathrm{C} 2$ test.

\section{Results}

Table 1 presents and compares results (NS not significant) obtained in Wallonia and previously in Flanders (Leten and al., 2002).

Table 1

\begin{tabular}{|l|c|c|}
\hline & Flanders & Wallonia \\
\hline Year of faeces sampling & 2000 & $2006-2007$ \\
\hline Number of farms & 30 & 58 \\
\hline Herd size (n sows) & $100-450$ & $20-250(\tilde{n}=90)$ \\
\hline Positivity criteria & sporulated & sporulated or not \\
\hline Method of diagnostic & Telemann & modified Henriksen \\
\hline Positive farms $\mathrm{F}(\%)$ & 80 & 69 \\
\hline Positive litters L/F (\%) & 33 & 37 \\
\hline Relation diarrhoea-F or $\mathrm{L}$ & NS & NS \\
\hline Cleaning crate effect on F or $\mathrm{L}$ & NS & NS \\
\hline Disinfecting crate effect on $\mathrm{F}$ & NS & NS \\
\hline Disinfecting crate effect on $\mathrm{L}$ & p $<0.005$ & NS \\
\hline
\end{tabular}

\section{Discussion}

Isospora suis has been shown to be a common parasite in 10-18 day old piglets in the South of Belgium. Diarrhoea was not significantly correlated with the presence of the coccidia. Perhaps the not quantitative method of diagnostic used and the high frequency of diarrhoea - also due to other pathogens - in suckling piglets could explained this result. Cleaning and disinfecting the farrowing crate had no significant effect on the occurrence of the infection. These results confirm that if farm hygiene measures are important to control the infection pressure, they only reduce it. Whatever the differences between pig production and studies from both the North and South part of Belgium, similar results were obtained.

\section{Acknowledgements}

Authors thank farmers and their veterinarians who made this study possible.

\section{References}

Henriksen S.A., Christensen J.P. Vet. Rec. 1992, 131, 443-444.

Leten J., Smets K., Claerebout E., Mundt H.C., Heesen H., Vercruysse J.

Vlaams Diergeneeskundig Tijdschrift, 2002, 71, 63-67.

Visa number of the study: VI VET 06/01/19/02. *Both authors brought an equivalent contribution to the study. 


\title{
P.497
}

\section{Efficacy of Virusnip ${ }^{\circledR}$ disinfectant and Quixalud ${ }^{\circledast}$ supplement against Balantidium spp. following the treatment of sows in a pig farm in Thailand}

\author{
Woraporn Sukhumavasi ${ }^{1}$ Manop Muangyai ${ }^{1}$ Padet Tummaruk¹ Metta Makhanon² Sasiwimon Talummuk ${ }^{2}$ Kai Sievert $^{3}$ \\ 1. Dept. of Obstetrics, Gynaecology and Reproduction, Faculty of Veterinary Science, Chulalongkorn University, Bangkok, Thailand; \\ 2. Technical Service, Novartis (Thailand) Ltd., Bangkok, Thailand; 3. Global Technical Service, Novartis, Basel, Switzerland
}

\section{Introduction}

Balantidium spp. is a ciliated binucleate protozoan parasite infecting the large intestine of mammals including pigs, primates and humans. Representative, species of Balantidium in mammals are B. suis, B. coli and B. caviae. Balantidiasis appears to be problematic mostly of developing countries in pig industry and zoonosis $(1,6)$. Previous report of $B$. coli prevalence in Thailand was $37.3 \%$ in fattening pig farms (4). In the case of preexisting pathogenic bacterial intestinal infection, Balantidium aggravates the ulcerative colitis lesion due to its hyarulonidase activity. The ciliated motile trophozoite and dormant cyst are two stages responsible for the pathogenesis and transmission, respectively. The cyst is environmentally resistant and capable of infecting the host due to its thick wall hence protecting it from desiccation and other environmental stress (5). The transmission is direct so the pigs become infected either from their sows or through contaminated water or coprophagy (6). To prevent the disease from spreading, farm sanitation and hygienic measures are important factor to control Balantidium apart from treating diarrhea pigs with antiprotozoal drug. Recent reports showed the in vitro activity of Virusnip ${ }^{\circledR}$ disinfectant to disrupt B. coli cyst wall (3) and the efficacy of Quixalud ${ }^{\circledR}$ for the treatment of diarrhea in fattening pigs caused by B. coli (2). The objective of this study is to test the activity of Virusnip ${ }^{\circledR}$, a disinfectant composed of potassium monopersulphate (PMP), sodium dichloroisocyanurate (SDIC), sulphamic acid, water softener and surfactant, and Quixalud ${ }^{\circledast}(60 \%$ Halquinol), a combination of hydroxy quinoline derivatives, in reducing Balantidium spp. transmission from the sows to the nursery pigs in a pig farm as an environmental control and an antiprotozoal activity, respectively.

\section{Materials and Methods}

The experiments were conducted in 4 groups of 30 pregnant sows composed of control (non-treated), Virusnip ${ }^{\circledR}$ treatment, Quixalud $^{\circledR}$ supplement in feed, and Virusnip ${ }^{\circledR} /$ Quixalud $^{\circledR}$ combination. Virusnip ${ }^{\circledR}$ was diluted with water to the working solution at 1:200 and 1:1000 in which it was used for disinfecting the farrowing pens (a week before bringing in the full-term sows) and pregnant sows (on the due date of farrowing), respectively. 180 ppm of Quixalud ${ }^{\circledR}$ was supplemented in feed of sows between 1 week pre- and post-farrowing period. The fecal samples from the control and treated sows at $1 \mathrm{wk}$ post-farrowing and their weaning piglets at $4 \mathrm{wk}$ and $8 \mathrm{wk}$-old were collected and qualitatively examined for Balantidium cyst by the direct fecal smear technique. The statistical analysis was carried out by using the Chi-square test.

\section{Results and Discussion}

After the Virusnip ${ }^{\circledR}$ treatment, $63.0 \%$ of the sows were found positive for Balantidium cysts. Interestingly, the percentage of Balantidium-positive samples from the 4 wk-old pigs born from this group was greatly reduced to $6.7 \%(p<0.0001)$ and there was a slight increase to $9.7 \%$ after following them up until $8 \mathrm{wk}$ of age. However, there was no significant difference between the last two groups ( $p>0.05$ ). For the Quixalud ${ }^{\circledR}$-treated group, the percentage of Balantidium cyst were $60.0 \%, 10.0 \%$ and $0 \%$ in sows, 4 wk-old pigs and 8 wk-old pigs, respectively. There were significant differences in reduction of this protozoa between sows and both groups of their offsprings $(p<0.0001)$. For the Virusnip $^{\circledR}$ and Quixalud ${ }^{\circledR}$ combination group, the percentage of cyst from sows and 4 wk-old pigs were decreased from $34.6 \%$ to $6.5 \%(p=0.0075)$. However, we found the increased percentage at 8 wk-old group up to $61.3 \%$. The latter results need to be further evaluated other causative agents that could play a role in potentiating this findings. This study is the first to show the promising application of Virusnip ${ }^{\circledR}$ disinfectant and Quixalud ${ }^{\circledR}$ to reduce the level of Balantidium transferring from sows to nursery pigs in the farm.

\section{References}

1. Anargyrou, K et.al. 2003. Am. J. Hematol. 73:180-183.

2. Makhanon M, et. al. 2008. Proceeding 20th IPVS. 461.

3. Makhanon M, et. al. 2009. Proceeding 4th APVS. Oct. 26-28: 375.

4. Muangyai M and Bhodigen SE. 1980. Thai J.Vet.Med. 10(3):139-153.

5. Rees, CW. 1927. Science. 61:89-91.

6. Schulster, FL and Ramirez-Avila L. 2008. Clin. Micro. Rev. Oct. 2008: 626-638. 


\title{
P.498
}

\section{Efficacy of Virusnip ${ }^{\circledR}$ disinfectant and Quixalud ${ }^{\circledR}$ supplement against Balantidium spp. in a nursery pig farm in Thailand}

\author{
Woraporn Sukhumavasi ${ }^{1}$ Manop Muangyai ${ }^{1}$ Padet Tummaruk² Metta Makhanon ${ }^{3}$ Sasiwimon Talummuk ${ }^{3}$ Kai Sievert $^{4}$ \\ 1. Parasitology Unit, Dept. of Pathology, Faculty of Veterinary Science, Chulalongkorn University, Bangkok, Thailand; \\ 2. Dept. of Obstetrics, Gynaecology and Reproduction, Faculty of Veterinary Science, Chulalongkorn University, Bangkok, Thailand; \\ 3. Technical Service, Novartis (Thailand) Ltd., Bangkok, Thailand; 4. Global Technical Service, Novartis, Basel, Switzerland
}

\section{Introduction}

Balantidiasis is a disease caused by the ciliated parasitic protozoan Balantidium spp. The large intestine of several mammalian hosts including pigs serves as its niche $(6,2)$. Although Balantidium infection is usually asymptomatic, the trophozoite can invade the epithelium during the concurrent pathogenic bacteria infection hence worsening the disease severity of ulcerative colitis resulting in diarrhea $(7,2)$. Pigs acquire the cysts, an infective stage, from the contaminated water or food or from their sows via fecal-oral route (6). In Thailand, the prevalence was $37.3 \%$ in fattening pig farms and increased with age correlating to the study in Oklahoma $(5,8)$. Since the Balantidium cyst wall is thick thus protecting it from the environmental stress, the measures for control this pathogen are recommended composed of sanitation, hygiene and farm management to prevent the pigs from succumbing to the infection by this secondary invader (1). Based on the recent findings, Virusnip ${ }^{\circledR}$ disinfectant appeared to be effective in disrupting B. coli cyst wall in vitro (4). Also, another report showed the anti-protozoal activity of Quixalud ${ }^{\circledR}$ in treating diarrhea in fattening pigs caused by B. coli infection (3). The aim of this study is to test the activity of Virusnip ${ }^{\circledR}$ (Potassium monopersulphate) disinfectant and Quixalud ${ }^{\circledR}$ (60\% Halquinol), a combination of hydroxy quinoline derivatives, in reducing the environmental contamination and infection of Balantidium spp. in the nursery pigs, respectively.

\section{Materials and Methods}

The experiments were conducted in 4 groups of 30 nursery pigs composed of non-treated control, Virusnip ${ }^{\circledR}$ treatment, Quixalud $^{\circledR}$ supplement in feed, and Virusnip ${ }^{\circledast} /$ Quixalud ${ }^{\circledR}$ combination. Virusnip ${ }^{\circledR}$ was diluted with water to the working solution at 1:200 and used for disinfecting the pens of nursery pigs three times a week starting from 5 wk old until 7 wk of age. 300 ppm of Quixalud ${ }^{\circledR}$ was supplemented in feed during the period of $5 \mathrm{wk}$ to $7 \mathrm{wk}$ old. The control group was treated as the routine cleaning management and feeding. The fecal samples were collected before starting the experiment at $5 \mathrm{wk}$ old and at the end of the treatment, 7 wk old. Direct fecal smear technique was used for qualitative examination for Balantidium cyst. The statistical analysis was performed by using the Chi-square test.

\section{Results}

Before the treatment, the percentage of Balantidium-positive pigs were similar in every group in which the percentage of control group, Quixalud ${ }^{\circledR}$-treated group, Virusnip ${ }^{\circledR}$-treated group

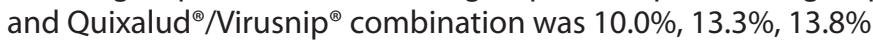
and $16.7 \%$, respectively ( $p>0.05$ ). After the period of $2 \mathrm{wk}$-experiment, the percentage of cyst-positive pigs in control group was increased twice (20\%) compared to the $5 \mathrm{wk}$ old control group whereas the slight reduction of positive sow percentage was found in Quixalud ${ }^{\circledR}$-treated and Quixalud ${ }^{\circledR} /$ Virusnip $^{\circledR}$ com- $^{-}$ bination group (10.0\% and 6.7\%, respectively) ( $p>0.05)$. For the Virusnip ${ }^{\circledR}$ treatment group, there was an insignificant increase from $13.8 \%$ to $19 \%$ ( $p>0.05)$.

\section{Discussion}

Without the treatment, the control group revealed the increase in number of Balantidium-infected nursery pigs within 2 weeks correlating to the previous findings in that the older pigs harbored higher loads of parasites $(5,8)$. In addition, there was a tendency in reduction of number of infected pigs after treatment with Quixalud $^{\circledR}$ or Quixalud ${ }^{\circledR} /$ Virusnip $^{\circledR}$ despite the nonsignificant differences. This study provides a possible application of both Quixalud ${ }^{\circledR}$ and Virusnip ${ }^{\circledR}$ in control Balantidium in swine farm by reducing the environmental contamination, infection as well as improving the farm biosecurity.

\section{References}

1. Hampton, J, et.al. 2006. EcoHealth. 3:103-108.

2. Kaufmann, J. 1996. Parasitic infections of domestic animals: a diagnostic manual. p.295-296.

3. Makhanon M, et. al. 2008. Proceeding 20th IPVS. 461.

4. Makhanon M, et. al. 2009. Proceeding 4th APVS. Oct. 26-28: 375.

5. Muangyai M and Bhodigen SE. 1980. Thai J.Vet.Med. 10(3):139-153.

6. Schulster, FL and Ramirez-Avila L. 2008. Clin. Micro. Rev. Oct. 2008: 626-638.

7. Skotarczak, B. 1997. Acta Parasitol. 42:230-233.

8. Morris, RG, et. al. 1984. Am. J. Vet. Res. 45:2421-2423. 


\title{
P.499
}

\section{Modified technique for collecting and processing fecal material for diagnosing intestinal parasites in swine}

\author{
Jeremy S. Pittman ${ }^{1}$ Brad J. Thacker ${ }^{2}$ Gene Shepherd ${ }^{3}$ Gil H. Myers ${ }^{4}$ \\ 1. Murphy-Brown, LLC, Waverly, VA, USA; 2. Intervet/Schering-Plough Animal Health, Desoto, KS, USA; \\ 3. Oklahoma State University, College of Veterinary Medicine, Stillwater, OK, USA; 4. Myers Parasitology Services, Magnolia, KY, USA
}

\begin{abstract}
Introduction
Intestinal parasites of swine are still a concern in modern swine production and cause ill thrift, reduced growth rates, decreased feed efficiency, respiratory distress and condemnations of livers at harvest. ${ }^{1}$ Fecal flotation methods for diagnosing intestinal parasites and monitoring effectiveness of deworming programs are commonly used in many areas of veterinary medicine and are easily implemented in any clinic or laboratory. ${ }^{2}$ Monitoring of swine populations for the presence of intestinal parasites using the Modified Wisconsin Sugar Centrifugal-Flotation Method is the most sensitive and accurate method for detecting parasite eggs. ${ }^{3}$ The authors herein describe and discuss the "Waverly-Modified" method of collection and processing of fecal samples for the detection of intestinal parasites in swine.
\end{abstract}

\section{Materials and Methods}

For sampling a breeding herd, a 95 (\% confidence)/10 (minimum $\%$ prevalence) sampling scheme was used and 30 samples were appropriate for most sow herds. Sows were sampled post-farrowing, which has been shown in other species to be associated with a periparturient rise in egg counts. ${ }^{4} \mathrm{~A}$ fresh $2 \mathrm{cc}$ fecal core sample was taken from each sow, using a 10cc syringe cut at the needle hub end. A new syringe was used for each sample because of the potential high numbers of eggs in an individual sample and the "sticky" nature of parasite eggs which could cause cross sample contamination.

For growing pigs, a composite sample was prepared where one fresh 1cc sample was taken from five separate fecal piles, totalling $5 \mathrm{cc}$, within a single pen. The number of composites collected per barn was based on the capacity of the barn; 10 composites from barns with up to 800 animals and 12 from barns with 800 to 1,200 animals. For barns above 1,200 animals, an additional composite was collected per 80 head up to a maximum of 24 . By pen, the composite numbers provided a 95/22 sampling scheme; by animal, a 95/6 scheme.

Each core sample was then discharged into a labelled $50 \mathrm{ml}$ conical centrifuge tube. This tube acts as the mixing container for the first laboratory step, thereby reducing additional steps and equipment. The $50 \mathrm{ml}$ tubes are returned to the laboratory, and the following steps are performed: 1) a concentrated sugar solution ( $0.45 \mathrm{~kg}$ in $354 \mathrm{ml}$ water) is added to the fecal sample directly into the $50 \mathrm{ml}$ tubes; $15 \mathrm{ml}$ for sows, $20 \mathrm{ml}$ for composites; 2 ) the fecal-sugar mixture is homogenized by shaking vigorously or using a wooden tongue depressor; 3 ) the fecal-sugar mixture is strained through a tea strainer into a 150 $\mathrm{ml}$ waxed paper cup; 4) the remaining liquid is pressed through the strainer using a wooden tongue depressor; 5) the strained contents are transferred into a $15 \mathrm{ml}$ conical centrifuge tube; 6 ) the tube is centrifuged for 5 minutes at 1,000 rpm; 7) the tube is placed in a test tube rack; 8 ) sugar solution is again added to the tube until a slight meniscus forms; 9) a $22 \times 22 \mathrm{~mm}$ cover slip is placed on top and allowed to sit in place for 5 minutes; and 10) the cover slip is then placed on a microscope slide. Slides are read at 40X total magnification on a binocular microscope with a movable stage. Starting at one corner of the cover slip, move in a vertical plane, counting any intestinal parasite eggs seen. When the end of the field is reached, move the field horizontally until in a new vertical plane. Continue this method for 5 total planes, allowing evaluation of the entire cover slip.

\section{Results and Discussion}

The protocol provided clean, easy to read cover slips for the accurate detection of Ascaris suum and Trichuris suis eggs in over 3,000 samples. The modifications were made by the authors to standardize sample volume, collection methods, minimize excess fecal material and consolidate steps to allow for faster processing as part of a large scale intestinal parasite survey from which the results are reported in two accompanying papers. The above modified method should allow any veterinarian or veterinary clinic to perform in-house diagnostic testing for intestinal parasites.

\section{References}

1. Stewart, TB and Hoyt PG. Internal Parasites. In: Straw BE, Zimmerman JJ, D'Allaire S, Taylor DJ, eds. Diseases of Swine. 9th ed. Ames, lowa: Blackwell Publishing; 2006:901-914.

2. Bowman, DD. Fecal Examination. In: Bowman, DD, ed. Georgis' Parasitology for Veterinarians. 7th ed. Philadelphia, Pennsylvania: W.B. Saunders Company; 1999:285-294.

3. Dryden MW, Payne PA, Ridley R, Smith V. Vet. Ther. 2005;6:15-28, 2005.

4. Courtney $\mathrm{CH}$, Parker CF, McClure KE, Herd RP. Int J Parasitol. 14:377$381,1984$. 


\title{
P.500
}

\section{Prevalence of internal parasites in a production system: Part I - Sows}

\author{
Jeremy S. Pittman ${ }^{1}$ Gene Shepherd ${ }^{2}$ Brad J. Thacker ${ }^{3}$ Gil H. Myers ${ }^{4}$ Charles J. Francisco ${ }^{3}$ \\ 1. Murphy-Brown, LLC, Waverly, VA, USA; 2. Oklahoma State University, College of Veterinary Medicine, Stillwater, OK, USA; \\ 3. Intervet/Schering-Plough Animal Health, Desoto, KS, USA; 4. Myers Parasitology Services, Magnolia, KY, USA
}

\section{Introduction}

Historically, internal parasites have been a source of economic loss for the swine industry, estimated at $\$ 155$ million annually. ${ }^{1}$ The 2006 NAHMS survey indicated that $74.7 \%$ of breeding sites regularly gave anthelmintics. ${ }^{2}$ However, internal parasitism is a topic of little discussion or concern in many modern swine production systems. The overall prevalence of internal parasitism has likely declined with the advent of indoor rearing of swine and the application of more effective anthelmintics in parasite control programs. However, once a barn or premise is contaminated with the eggs of the key internal parasites of swine, namely Ascaris suum (Large Roundworm) and Trichuris suis (Swine Whipworm), there is continuous risk for reduced performance due to parasitism. The objective of this study was to investigate the prevalence of internal parasites in a large integrated production system.

\section{Materials and Methods}

A total of 1,197 individual sow fecal samples, from 40 sow farms within a common ownership and management structure, representing 44,050 breeding animals were collected and processed. In addition to sample collection, a questionnaire was completed for each sow farm to capture historical parasite status, current health and production parameters and facility design features to assess potential risk factors that might influence parasite burden. Sow parity and lactation age were collected for each individual sow sample. Data was analyzed by either linear or logistic regression depending on the parameter evaluated. All fecal samples were processed using a modification of the Wisconsin Sugar Centrifugation flotation technique. Each sample was read and scored based on presence and total number of parasite eggs detected ( $0=$ no eggs; $1=<10$ eggs per coverslip; $2=10-49$ eggs; or $3>50$ eggs). The presence of any eggs was considered positive and a farm with at least one positive sample was considered positive.

\section{Results}

Ascaris suum was the only parasite detected. Overall, $25 \%$ of sow farms were positive, representing 12,000 breeding animals. Additional positive results by a selected subset of farm type, farm size and facility design are reported in Table 1. However, statistical analysis found no risk factors related to farm parasite status and overall sow farm health and production levels were not related to farm parasite status.

\section{Discussion}

The prevalence of intestinal parasites within this system was higher than anticipated prior to performing the survey. The system had quit deworming sows prior to farrowing approximately 18 months prior to this survey based on not observing any adult worms and economic considerations. Selective treatment of positive sow herds along with implementing a continuous monitoring program is planned for this production system in the future.

Table 1. Prevalence of intestinal parasites by farm, farm type, farm size and parity

\begin{tabular}{|l|c|c|c|}
\hline & No. Farms & No. Positive & \% Pos \\
\hline Total & 40 & 10 & 25.0 \\
\hline Farm Type* & & & \\
\hline FTF & 30 & 7 & 23.4 \\
\hline FTFd & 6 & 2 & 33.3 \\
\hline FTW & 4 & 1 & 25.0 \\
\hline Farm Size (sows) & & & \\
\hline$\leq 1000$ & 33 & 8 & 24.2 \\
\hline$>1000$ & 7 & 2 & 28.6 \\
\hline Individual Animals By Parity & & & \\
\hline Gilts & 192 & 5 & 2.6 \\
\hline $1-2$ & 340 & 8 & 2.4 \\
\hline $3-4$ & 308 & 8 & 2.6 \\
\hline $5-7$ & 285 & 1 & 0.4 \\
\hline $8+$ & 42 & 2 & 4.8 \\
\hline Unknown & 30 & 3 & 10.0 \\
\hline *Farm defined as: Farrow-to-finish (FTF), farrow-to-feeder pig (FTFd) or farrow-to-wean (FTW). \\
\hline
\end{tabular}

\section{References}

1. Stewart TB, Hale OM. 1988. J Anim Sci 66:1548-1554.

2. USDA:APHIS:VS. Part II: Reference of Swine Health and Health Management Practices in the U.S. 2006. National Animal Health Monitoring System (NAHMS); 2007 Aug. N471.1107. 


\title{
P.501
}

\section{Prevalence of internal parasites in a production system: Part II - Finishing pigs}

\author{
Jeremy S. Pittman ${ }^{1}$ Gene Shepherd ${ }^{2}$ Brad J. Thacker ${ }^{3}$ Gil H. Myers ${ }^{4}$ Charles J. Francisco ${ }^{3}$ \\ 1. Murphy-Brown, LLC, Waverly, VA, USA; 2. Oklahoma State University, College of Veterinary Medicine, Stillwater, OK, USA; \\ 3. Intervet/Schering-Plough Animal Health, Desoto, KS, USA; 4. Myers Parasitology Services, Magnolia, KY, USA
}

\section{Introduction}

Historically, internal parasites have been a source of economic loss for the swine industry, estimated at $\$ 155$ million annually. ${ }^{1}$ The 2006 NAHMS survey indicated that $30.6 \%$ of grow-finish sites regularly administered anthelmintics. ${ }^{2}$ The overall prevalence of internal parasitism has likely declined with the advent of indoor rearing of swine and the application of more effective anthelmintics in parasite control programs. However, once a barn or premise is contaminated with the eggs of the key internal parasites of swine, namely Ascaris suum (Large Roundworm) and Trichuris suis (Swine Whipworm), there is continuous risk for reduced performance due to parasitism. The objective of this study was to investigate the prevalence of internal parasites in a large integrated production system, while also taking into consideration the facility design and production parameters of the various farms.

\section{Materials and Methods}

A total of 1,697 composite fecal samples were collected, processed and analyzed from finishing pigs. A composite contained a pool of 5 separate fecal samples from within the same pen. The total number of composites per barn ranged from 10 to 24 , depending on the capacity of the barn. The 1,697 finishing composite samples represent 153 barns (33.2\% of barns) and 91 finishing sites (100\% of sites). The finishing pig samples represent 124,003 finishing spaces by barn and 367,758 spaces by sites. In addition to sample collection, a questionnaire was completed for each finishing barn sampled to capture historical parasite status, current health and production parameters and facility design features to assess potential risk factors that might influence parasite burden. Data was analyzed by either linear or logistic regression depending on the parameters evaluated. All fecal samples were processed using a modification of the Wisconsin Sugar Centrifugation flotation technique. Each sample was read and scored based on presence and total number of parasite eggs detected ( $0=$ no eggs; $1=<10$ eggs per coverslip; 2 = 10-49 eggs; or $3>50$ eggs). The presence of any number of eggs was considered positive and a barn with at least one positive sample was considered positive.

\section{Results}

Ascaris suum was the predominant parasite detected, while Trichuris suis was found in one finishing site. Overall $31.7 \%$ of barns and $38.1 \%$ of sites were positive, representing 37,911 and 90,072 finishing spaces, respectively. Results by selected risk factors are shown in Table 1. Statistical analysis indicated that the presence of intestinal parasites was mainly influenced by floor type, waste handling system and previous detection.
Table 1. Prevalence of intestinal parasites by farm and facility type for finishing sites.

\begin{tabular}{|l|c|c|c|}
\hline & No. Farms/Barns & No. Positive & \% Pos \\
\hline Total & & & \\
\hline Sites & 91 & 35 & 38.5 \\
\hline Barns & 153 & 51 & 33.3 \\
\hline By Farm Type (Sites) & & & \\
\hline Farr-to-Fin & 30 & 3 & 10.0 \\
\hline Finish only & 61 & 32 & 52.5 \\
\hline By Floor Type (Barn) & & & \\
\hline Total Slats & 114 & 25 & 21.9 \\
\hline Partial Slats & 16 & 7 & 43.8 \\
\hline Solid Floor & 23 & 19 & 82.6 \\
\hline By Waste Handling System (Barn) & & & \\
\hline Flush Under & 86 & 11 & 12.8 \\
\hline Open Flush & 22 & 18 & 81.8 \\
\hline Pull Plug & 45 & 22 & 47.7 \\
\hline
\end{tabular}

\section{Discussion}

In summary the prevalence of intestinal parasites within this system was high, especially in the finishing sites. The source of infestation appeared to be the facility itself and not the sow herd of origin. Facility factors and evidence of previous infestation were strongly associated with a site's parasite status.

\section{References}

1. Stewart TB, Hale OM. J Anim Sci. 1988;66:1548-1554.

2. USDA:APHIS:VS. Part II: Reference of Swine Health and Health Management Practices in the United States, 2006. Fort Collins, CO: National Animal Health Monitoring System (NAHMS); 2007 Aug. N471.1107. 


\title{
P.502
}

\section{Gastrointestinal nematodes in Swiss pig farms - an explorative overview}

\author{
Linus Eichhorn ${ }^{1}$ W. Zimmermann² Bruno Gottstein ${ }^{3}$ Caroline F. Frey ${ }^{3}$ Marcus G. Doherr ${ }^{4}$ Friederike Zeeh $^{1}$ \\ 1. Vetsuisse Faculty Bern, Swine Clinic, Bern, Switzerland; 2. Vetsuisse Faculty, Dept. of Clinicial Veterinary Medicine, Swine Clinic, Bern, \\ Switzerland; 3. Institute for Parasitology, Bern, Switzerland; 4. Department of Clinical Research and VPH, Bern, Switzerland
}

\section{Objective}

Porcine gastrointestinal (Gl) nematodes such as Ascaris (A.) suum, Trichuris (T.) suis, gastrointestinal strongyles, Strongyloides ransomi and others occur worldwide and can significantly influence feed efficiency, daily weight gain, feed intake and the health status of the individual pigs as well as of the whole herd. In most Swiss finishing pig farms, deworming is not routinely performed. Recent prevalence estimates for intestinal parasites are lacking. The aims of our study were thus to exploratively assess prevalence data of porcine GI nematodes in Swiss pig farms and to identify potential risk factors for intestinal endoparasitosis.

\section{Materials and Methods}

The study included 110 farms: 90 conventional and 20 outdoor farms distributed all over Switzerland. A total of 2762 (conventional) and 291 (outdoor) samples was taken.

Rectal fecal samples were collected only in one weight group per farm during the farm visit. Four groups were formed: weaned with 4 weeks-40 kg (A), 41-60 kg (B), 61-80 kg (C) and $>80 \mathrm{~kg}$ (D). Distribution of herds was nearly equal between groups.

Fecal specimens were analyzed in pools of 5 samples for the presence of parasites by a combined sedimentation and flotation method using a $44 \%$ Zinc-Chloride solution. If a pool was positive, every single specimen out of that pool was individually retested. Farms were declared positive if one or more individual fecal samples were positive. A questionnaire was completed during a farm visit to identify potential risk factors. Statistics were performed using a multiple logistic regression model with $\mathrm{p}<0.05$ and $\mathrm{Cl}$ of $95 \%$ (NCSS 2007).

\section{Results}

The number of collected samples ranged from 6 to 37 in outdoor housing farms and from 4 to 58 in conventional farms.

T. suis was the most prevalent $\mathrm{GI}$ nematode in conventional farms, followed by A. suum and gastrointestinal strongyles. Only one farm was positive for Strongyloides sp. (Tab. 1).

All parasites in conventional farms were found most frequently in group (D). Prevalence of $T$. suis and $A$. suum inclined continuously from group (A) to group (D). T. suis was common in group (A) whereas $A$. suum was very rarely present in this group (Tab. 2).

In outdoor farms, T. suis was the most common Gl nematode found, followed by A. suum, gastrointestinal strongyles and Strongyloides ransomi. In addition Metastrongylus sp. eggs were detected in pigs from 6 outdoor housing farms.
"Lack of visitor's clothing", "silage" and "no rodent control" could be identified as risk factors for a farm being positive for Gl nematodes. "Lack of visitors clothing", "dirty boots and farmer's clothing" and "no rodent and insect control" could be identified as risk factors for an infection with $T$. suis.

Table 1: Percentage of outdoor and conventional herds infected with Gl nematodes and Metastrongylus sp.. Multiple infections occurred. "-": parasite negative.

\begin{tabular}{|l|c|c|c|c|c|}
\hline & T. suis & A. suum & $\begin{array}{c}\text { GI } \\
\text { strongyles }\end{array}$ & Strongyloides sp. & Metastr. sp. \\
\hline Outdoor & $60 \%$ & $35 \%$ & $20 \%$ & $5 \%$ & $30 \%$ \\
\hline Conventional & $32.2 \%$ & $13.3 \%$ & $3.3 \%$ & $1.1 \%$ & - \\
\hline
\end{tabular}

Table 2: Percentage of infected conventional herds in different age (weight) groups. "-": parasite negative.

\begin{tabular}{|l|c|c|c|c|c|}
\hline & T. suis & A. suum & $\begin{array}{c}\text { GI } \\
\text { strongyles }\end{array}$ & Strongyloides sp. & Metastr. sp. \\
\hline$<40 \mathrm{~kg}$ & $20 \%$ & $3 \%$ & - & $3 \%$ & - \\
\hline $41-60 \mathrm{~kg}$ & $30 \%$ & $15 \%$ & - & - & - \\
\hline $61-80 \mathrm{~kg}$ & $40 \%$ & $15 \%$ & - & - & - \\
\hline$>80 \mathrm{~kg}$ & $45 \%$ & $25 \%$ & $15 \%$ & - & - \\
\hline
\end{tabular}

\section{Discussion}

The study showed that GI nematodes are frequently found in outdoor and in conventional farms in Switzerland. Pigs from outdoor farms showed a higher worm burden than pigs from indoor housing. This can be explained by the higher exposition of outdoor pigs to a possibly contaminated environment. Furthermore, T. suis seems to be the most common Gl parasite in Switzerland. Its presence in conventional herds in all age groups indicates it being a problem already in breeding as well as in fattening farms. On the other hand, the low prevalence of $A$. suum in age group $(A)$ indicates it being a problem only in fattening herds .

Metastrongylus sp. infections were demonstrated for the first time in Swiss commercial outdoor pigs. Wild boars are most likely to be the source of infection. All risk factors are related to general farm management. 


\title{
P.503
}

\section{Endoparasite infections and hygiene management in organic fattening herds}

\author{
Albert Sundrum; Christina Werner \\ University of Kassel, Witzenhausen, Germany
}

\section{Introduction}

Studies in different European countries have revealed that infections with endoparasites are one of the most important health problems in swine herds (Roepstorff et al., 1999; Eijck \& Borgsteede, 2005). In organic pig farming, the regulation (EC) No 889/2008 includes several specifications which are expected to have severe impacts on parasite infection, i.e. straw bedding, access to outdoor run, addition of roughage to the daily feed ration and no prophylactic anthelmintic medication. Organic pig farms are also characterised by heterogeneous management practices and a lack in control measures (Ebke \& Sundrum, 2004; Werner et al., 2009) providing a non-assessable risk for helminth egg contamination in the environment. The aim of the study was to assess the prevalence of endoparasite infections in fattening pigs on organic swine herds and to assess effectiveness and efficiency of management measures with respect to the reduction of endoparasite infections.

\section{Materials and Methods}

The study was performed in 17 organic swine herds in Germany. An interview with the farmer was performed using a standardised questionnaire, containing closed and open-ended questions on anthelmintic treatment, cleaning and disinfectant measures, documentation of production and management data and diagnostic measures on farm-level. Furthermore, 10 faecal samples per farm, taken twice during spring and autumn, were collected from fatteners. They were examined within $12-24 \mathrm{~h}$ after collection for nematode egg counts per gram of faeces (EPG) and coccidian oocysts using the McMaster technique. A total number of 326 fatteners were sampled. Data were categorised to evaluate the relationship between hygiene management and parasite infection.

\section{Results}

Strongylid infections were detected in $58.8 \%$ of the herds with a mean within-farm prevalence of $30.3 \%$. Ascaris (A.) suum and Trichuris suis eggs were detected in 21 and $9.8 \%$ of faecal samples of fatteners, respectively. Coccidia infections were diagnosed in $88 \%$ of the farms. Pigs were treated with anthelmintics (mainly flubendazol) on all farms. The hygiene management showed a high variation between the farms. Only half of the farmers performed wet cleaning in their fattening units $(n=8)$, whereas two farmers did not clean the stables at all. 15 of the total number of 17 farmers made no use of chemical disinfectant measures versus endoparasites. However, no egg-shedding of A. suum and strongyles was detected in three of these 15 herds. Four additional herds were free of strongyles.

\section{Discussion}

Results show that farms varied to a high degree with respect to the prevalence of endoparasite infection and the implementation of hygiene measures. In contrast to expectancy, the prevalence rate of Ascaris suum infection was on a low level in comparison to other studies (Carstensen et al., 2002; Eijck \& Borgsteede, 2005).

No correlation was found between hygienic procedures and the occurrence of parasite infection, confirming previous results from Roepstorff et al. (1999). Thus, those farms who use comprehensive hygiene measures are not necessarily gaining the best results with respect to parasite infection. It is concluded that farm specific control and feedback mechanism are required to minimize both endoparasite infections and efforts to control them.

\section{References}

Carstensen, L., Vaarst, M., Roepstorff, A., 2002. Helminth infections in Danish Organic swine herds. Vet. Parasitol. 106, 253-264.

Ebke, M., Sundrum, A. (2004): Problems and challenges with the certification of organic pigs. Proc. of the 2nd SAFO Workshop; Witzenhausen, p. 193-198.

Eijck, I.A., Borgsteede, F.H., 2005. A survey of gastrointestinal pig parasites on free-range, organic and conventional pig farms in the Netherlands. Vet. Res. Commun. 29, 407-414.

Roepstorff, A., Nilsson, O., O'Callaghan, J., Oksanen, A., Gjerde, B., Richter, S.H., Ortenberg, E.Ö., Christensson, D., Nansen, P., Eriksen, L., Medley, G.F., 1999. Intestinal parasites in swine in the Nordic countries: multilevel modelling of Ascaris suum infections in relation to production factors. Parasitology 119, 521-534.

Werner, C., Dietze, K., Sundrum, A., 2008. Implementation of animal health plans in organic sow herds in Germany. Landbauforschung vTI agriculture and forestry research 58, 271-281. 


\title{
P.504
}

\section{Routine deworming of pregnant sows in group housing systems}

\author{
Jan Jourquin; Jos Jacobs; Lieve Goossens; Leo Van Leemput \\ Janssen Animal Health, Beerse, Belgium
}

\section{Introduction}

Group housing of pregnant sows is becoming more and more the norm in modern pig production. Although clearly beneficial in view of animal welfare, this poses specific challenges towards day-to-day practicalities.

It not only involves the stressful event of grouping which has to be addressed to avoid a decrease in reproductive performance (1), but it makes it also much more difficult to correctly administer routine treatments to the sows once they are grouped. Injection treatments become 'rodeo-like', dangerous and stressful events for both animal and man. For in-feed medication it becomes more difficult to get the dose correct as sows of different parity, condition and gestation length have different nutritional needs and it implies specific medicated feed deliveries posing additional logistic problems. Top dressing is not possible with feeding stations and becomes more difficult if sows are not individually confined during meals. This leaves the drinking water as the most convenient and least stressful medium to administer routine medication, such as deworming.

Solubenol ${ }^{\circledast}$ is a flubendazole based anthelmintic for drinking water application that recently was granted an authorization for use in pregnant sows. Flubendazole is a broad spectrum anthelmintic for pigs used for strategic deworming programs. In a target animal safety study (2), Solubenol ${ }^{\circledR}$ showed to be safe to the pregnant sow when given at multiple doses and occasions before, during and after pregnancy, indicating the absence of the risk for overdosing.

This study was performed to evaluate its safety and convenience as a routine dewormer at the therapeutic dose, in a large group of sows at different stages of pregnancy.

\section{Materials and Methods}

Four consecutive batches, each of approximately 110 sows of mixed parities and different housing system were either treated with $5 \mathrm{mg}$ flubendazole per kg body weight spread over 5 consecutive days or left untreated. Sows were treated through the drinking water system in the group housed animals or individually dosed on the wet feed using a drenching gun in the individual pen housing (Table 1).

Clinical observations of the sows and their piglets, monitoring of the litter size (total, live or stillborn and mummies) and viability (mortality up to weaning and piglets weaned) were the evaluation criteria for the safety of the treatment.
Table 1: Study design

\begin{tabular}{|c|c|c|c|}
\hline Batch & Stage gestation & Number of litters (Solubenol ${ }^{\otimes} /$ Control) $^{\text {) }}$ & Application route \\
\hline 1 & late & $62 / 48$ & wet feed \\
\hline 2 & late & $56 / 47$ & water \\
\hline 3 & mid & $50 / 65$ & wet feed \\
\hline 4 & early & $70 / 45$ & wet feed \\
\hline
\end{tabular}

\section{Results}

In total, 238 sows treated with Solubenol ${ }^{\circledR}$ and 205 control sows were included. No abnormal clinical observations were present resulting from the treatment of the pregnant sows. No statistical significant differences in the way of administration or litter size were present between both groups (total born 14.2 versus 14.4), although overall, somewhat more live born piglets and less stillborn piglets were present in the Solubenol ${ }^{\circledR}$ group (Table 2).

Mortality up to weaning, as well as the number of piglets weaned was comparable between groups.

Furthermore, there was no influence of the sow parity.

Table 2: Averaged production parameters

\begin{tabular}{|c|c|c|}
\hline & Solubenol $^{\circledR}$ & Control $^{-1}$ \\
\hline live born & 13.1 & 12.7 \\
\hline stillborn & 0.8 & 1.3 \\
\hline mummies & 0.3 & 0.4 \\
\hline mortality & 1.5 & 1.6 \\
\hline weaned & 11.2 & 11.0 \\
\hline
\end{tabular}

\section{Discussion}

From the results it is clear that the Solubenol ${ }^{\circledR}$ treatment, as a routine dewormer, is safe for use in every stage of the pregnancy. No differences in any of the parameters were detected between the results in the drinking water or wet feed treatment and the control. This renders both larger batch treatments as well as targeted individual treatments very convenient without causing additional stress to the sows.

\section{References}

1. J. Jourquin et al., Proceedings 20th IPVS Congress, Durban, p 215

2. P. Richez et al., J. vet. Pharmacol. Therap. 32 (Suppl. 1) p 131 


\title{
P.505
}

\section{Deworming via the drinking water system with flubendazole, both safe and efficacious}

\author{
Jos Jacobs; Jan Jourquin; Lieve Goossens; Leo Van Leemput \\ Janssen Animal Heatlh, Beerse, Belgium
}

\section{Introduction}

In pig industry, disease prevention has become a pillar for good veterinary practice. Biosecurity, housing and ventilation, vaccination and routine deworming all add up to successfully achieve that goal.

Routine treatment by injections are very labor intensive and an important stress factor for both pigs and caretaker. Vaccinations are usually done at a young age when piglets are still manageable. Routine deworming however is done in older pigs which make injections troublesome. In-feed medication is a good alternative but in many cases, there are logistical and legal constraints. This leaves the drinking water as the most convenient and least stressful medium to deworm. More and more farms are equipped with a separate medicated drinking water system and dosing pumps are no longer exceptions. This offers great flexibility and the possibility of targeted treatments by the stockman. Solubenol ${ }^{\circledR}$ is a safe and efficacious flubendazole based anthelmintic for drinking water application for pigs of all ages. It is authorised at a total dose of $5 \mathrm{mg}$ flubendazole per $\mathrm{kg}$ body weight (BW) to be administered over a 5-day period for the control of the economic most important Ascaris suum infections. Unlike levamisole, that has a narrow safety margin.

In view of reducing labor pressure, an alternative dosing schedule of the same total flubendazole dose, but administered over only 2 days was evaluated. This included two efficacy studies, a residue depletion and a target animal safety study.

\section{Materials and Methods}

The first efficacy study was run under controlled laboratory conditions (UK). It included pigs with a confirmed, naturally acquired Ascaris suum infection. They were divided in two randomised groups: one treated with Solubenol ${ }^{\circledR}$ at $25 \mathrm{mg}$ flubendazole per $\mathrm{kg}$ BW on two consecutive days, the other group was left untreated. Efficacy was based on reduction in worm counts and faecal egg counts (EPG), one week after the last day of treatment.

In the second efficacy study (France) the same groups were composed but under field conditions.

In the residue depletion study, concentrations of parent flubendazole and its major metabolite were determined in edible tissues over a period of 7 days after the last administration.

The target animal safety study looked at changes in clinical, biochemical and hematological parameters after a treatment with 1, 3 and 5 times the $2.5 \mathrm{mg}$ per $\mathrm{kg}$ BW dose over a 3-fold treatment duration.

\section{Results}

The results (Table 1) indicated that the treatment was fully effective.

Table 1: Efficacy study UK

\begin{tabular}{|c|c|c|c|c|}
\hline Group & Worm count & Effectiveness (\%) & EPG & Reduction (\%) \\
\hline Control $^{11}$ & 11 & 945.3 & \\
\hline Solubenol $^{\circledR}$ & 0 & 100 & 0.9 & 99.9 \\
\hline p-value & & 0.0022 & & 0.0152 \\
\hline
\end{tabular}

The second efficacy study is ongoing at this moment.

One day after treatment, major total residues were found in liver and kidney; relatively low residues were quantifiable in muscle and skin/fat. Residues declined rapidly in liver and kidney and were not quantifiable in muscle and skin/fat after 3 days. The concentrations determined in the edible tissues after the 2-day treatment did not compromise the withdrawal time of 4 days as also established for the 5-day treatment.

The target animal safety study, conducted at 2.5, 7.5 and 12.5 $\mathrm{mg} / \mathrm{kg}$ BW/day for 6 days did not result in any abnormal clinical observations and demonstrated no relevant changes in none of the parameters measured. This indicates that this treatment schedule has a very large safety margin. No palatability issues were noted in any of the studies using a higher day concentration of Solubenol ${ }^{\circledR}$ in the drinking water.

\section{Discussion}

From the results it is clear that a Solubenol ${ }^{\circledR}$ treatment, administered over two days while keeping the total flubendazole dose at $5 \mathrm{mg}$ per $\mathrm{kg} \mathrm{BW}$, is effective, safe and does not imply a different withdrawal period. This additional dosing schedule offers more flexibility in treatment duration. Accidental overdosing if animals drink excessively or when different age groups are treated at the same time, can be avoided with flubendazole which has a very high LD50. Therefore, Solubenol ${ }^{\circledR}$ is the safer choice for routine deworming of fatteners. 


\section{P.506}

\section{Spike glycoprotein gene-based phylogenetic analysis of recent transmissible gastroenteritis virus isolates in Korea}

Seong-Jun Park' Hyoung-Joon Moon ${ }^{1}$ Hye-Kwon Kim¹ Hyun-Ok Keum ${ }^{1}$ Se-Mi Rho' Jae-Yeon Han' Van-Giap Nguyen ${ }^{1}$ Bong-Kyun Park

1. Department of Veterinary Virology Lab, College of Veterinary Medicine and BK21 Program for Veterinary Science, Seoul National University, Seoul, Korea; 2. Research unit, Green Cross Veterinary Products, Yongin, Korea

\section{Introduction}

Transmissible gastroenteritis virus (TGEV) has caused a devastating enteric disease with acute diarrhea, vomiting, weight loss, dehydration and high mortality and resulting in severe economical losses to the affected farms [1]. The spike glycoprotein stimulates induction of neutralizing antibodies in the host. Moreover, the spike glycoprotein has been recognized as not only a tropism but also a virulence determinant [2]. The purpose of the present study was to investigate the genetic diversity among the Korean TGEV field isolates and was to find out more prevalent TGEVs in Korea, according to sequence and spike glycoprotein gene-based phylogenetic analyses with Korean and non-Korean TGEV and PRCV reference strains.

\section{Materials and Methods}

A total of 10 porcine samples (from 5 different farms) including feces or intestinal contents had been taken from young piglets showing watery diarrhea, dehydration and high mortality. Five pairs of sense and anti-sense primers were designed based on the reference TGEV sequences and used to generate full spike glycoprotein gene of TGEV. The full spike glycoprotein gene of TGEV was amplified by RT-PCR, gel extracted and directly sequenced. Nucleotide sequence of full spike glycoprotein gene was determined and phylogenetic tree was then generated using an alignment of spike glycoprotein gene nucleotide sequences with reference TGEV and PRCV strains by applying neighbor-joining method in the MEGA 3.1 program. To assess the relative support for each clade, bootstrap values were calculated from 1000 replicate analyses.

\section{Results}

Nucleotide sequences of the full spike glycoprotein genes of 10 Korean TGEV isolates were determined and compared with one another, as well as with reference TGEV and PRCV strains. By phylogenetic analysis, all compared twenty-two viruses, including 10 Korean TGEV isolates, 11 TGEV and 1 PRCV reference strains, certainly fell into two groups at the nucleotide sequence level. One group comprised KT2, KT3, KT4, HKT2, 133, MIL65AME, BRI70-FS, PUR46-MAD, NEB72, TOY56, HOL87 and 8 Korean TGEV isolates. Another group consisted of TFI83 and 2 Korean TGEV isolates.
Figure 1. Phylogenetic tree generated on the basis of nucleotide sequences of full spike glycoprotein genes of 10 Korean TGEV isolates with reference TGEV and PRCV strains.

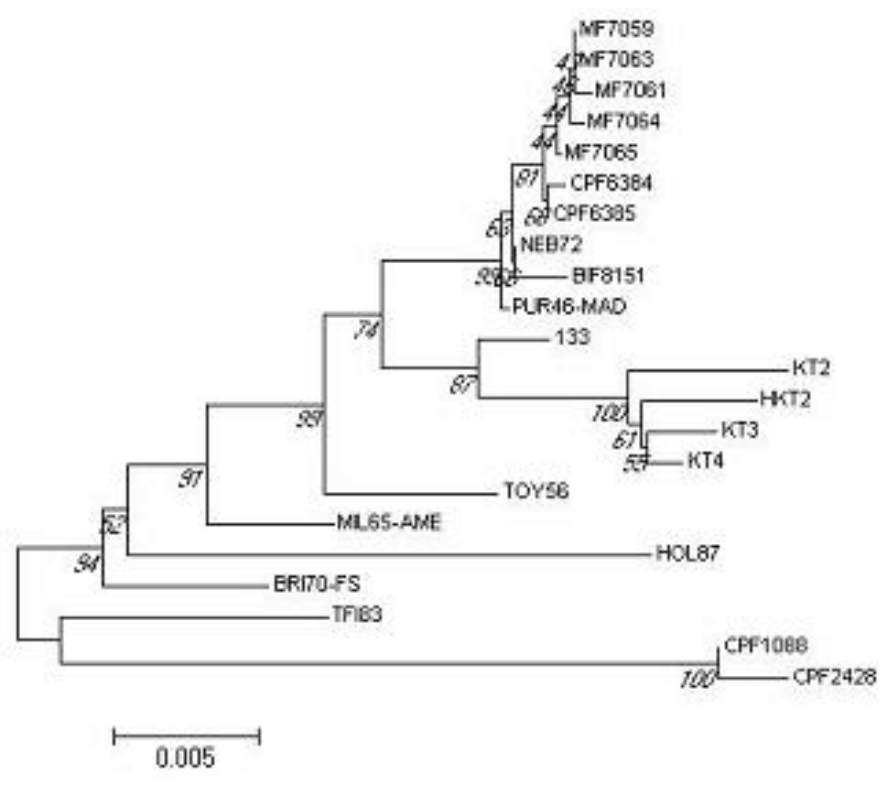

\section{Discussion}

By spike glycoprotein gene-based phylogenetic analysis, all Korean TGEV isolates were divided into two groups. It was suggested that recent, many Korean TGEV field isolates are closely related to the group including KT2, KT3, KT4, HKT2, 133, MIL65AME, BRI70-FS, PUR46-MAD, NEB72, TOY56 and HOL87 rather than to the group including TFI83. It is notable that recently more prevalent TGEVs in Korea are especially close to the NEB72, respiratory Purdue type TGEV.

\section{References}

1. Saif and Sestak (2006). Diseases of Swine; 489-516

2. Sanchez and others (1999). J. Virol. 73; 7607-7618

\section{Acknowledgements}

This work was supported by a grant (Code \#20070401034009) from BioGreen 21 Program, Rural Development Administration, Republic of Korea. 


\title{
P.507
}

\section{Novel vaccine against swine transmissible gastroenteritis using Salmonella choleraesuis as a live vector}

\author{
Weidong Chai; Qigai He; Fei Song; Kun Zhang; Lili Xu \\ College of Veterinary Medicine, Huazhong Agricultural University, Wuhan, China
}

\section{Introduction}

Swine transmissible gastroenteritis(TGE) is a highly contagious enteric disease in piglets causing high mortality rate of up to $100 \%$ and resulting in severe economical losses to the affected farms. The currently commercial available vaccines could provide only partial protection, and this emphasizes the importance of developing a new vaccine to prevent this disease. The mucosal immunity is required for protection. Salmonella has been proved as good vector that stimulates mucosal immunity. How to enhance expression of main antigen is a crucial strategy. Site $A$ and site $D$ in the spike protein $(S)$ of transmissible gastroenteritis virus are the main inducer of neutralizing antibodies while Site $A$ is a major B cell epitope. Peptide N321, which is located on the nucleoprotein $(\mathrm{N})$, defines a functional T helper epitope eliciting $T$ cells capable of collaborating with B cells specific for different proteins of TGEV(1).

\section{Material and Methods}

Antigen site A, site D and antigen site N321 of TGEV were combined by two Linker ((GGGGS)4 as a fusion peptide (SLN). By using Sall and Xhol site, two copies of SLN (2SLN) were constructed. Non-resistance expressing plasmids pYA-SLN and pYA-2SLN were constructed, respectively. The two plasmids were electrotransformated into Salmonella choleraesuis C500 competent cells to construct the recombinant Salmonella strains C500 (pYA-SLN) and C500 (pYA-2SLN) respectively. We evaluated the immunogenicity and protective capacity of the recombinant Salmonella strains through a vaccination and challenge trial in mice. Furthermore, field trail of the recombinant vaccine was applied in a farm which was being affected by TGE which was confirmed by our in-house RT-PCR in Jan. 2009 and the mortality rate was around $60 \%$.

\section{Result}

We successfully constructed the recombinant Salmonella choleraesuis strains (500 containing plasmid pYA-SLN) and pYA$2 S L N$, respectively, in which the gene integrated could be highly expressed. Both of the recombinant Salmonella strain induced the immunized mice a high titer of serum lgG and gut mucus secreted IgA against the antigens of Salmonella and SLN-protein. A higher titer of $\lg G$ and $\lg A$ could be induced in mice vaccinated with recombinant salmonella choleraesuis containing PYA-2SLN than that containing PYA-SLN, indicating that a peptide antigen displaying repeating copies of SLN is a more potent inducer of TGEV-neutralizing antibodies than SLN alone. Furthermore, IgG subclasses, among which the lgG2a titer is higher than IgG1, were induced in mice immunized by Salmonella choleraesuis harboring PYA-2SLN. The slgA could also be detected in mice four week after vaccination and peak at six week. The primary result of field trail showed that by oral administration, no new cases were observed, and about $99 \%$ piglets recovered from diarrhea between 48-72 hours after oral administration of the new vaccine.

\section{Discussion}

Due to the mucosal immunity is important factor in the protective mechanism of swine TGE which inactivated vaccine always lack. In this work, we used Salmonellas choleraesuis as a live vaccine to express main antigen protein of TGEV. The three eiptopes domains were combinaed, cloned and integrated the genes into non-resistance expressing plasmid, non-resistance recombinant Salmonella strains were successfully constructed and efficiently expressing the fragments integrated in. Furthermore, we evaluated the immunogenicity and protective capacity of the recombinant Salmonella strains using a vaccination and challenge trial both in mice and in piglets. These results indicated that the recombinant $\mathrm{C} 500$ (pYA-2SLN) vaccine showed the potential live vaccine against Swine transmissible gastroenteritis

\section{Acknowledgement}

This work was granted by project from Ministry of Science and Technology of China (nycytx-009 and nyhyzx07-034). 


\title{
P.508
}

\section{Genetic diversity of porcine rotaviruses in selected Korean pig farms, 2009}

\author{
Shienyoung Kang ${ }^{1}$ Yunkyung Song ${ }^{1}, 2$ Seonghee Kim² Kyoungki Lee ${ }^{2}$ Changil Yeo $^{2}$ Chaewun Bae $^{2}$ Osoo Lee $^{2}$ Choikyu Park $^{2}$ \\ 1. Chungbuk National University, Cheongju, Korea; 2. National Veterinary Research and Quarantine Service, Chungang-ro, Anyang, Korea
}

\section{Introduction}

Rotaviruses(RV) are important causes of diarrheal disease in animals and humans and a common cause of gastroenteritis in sucking and postweaning pigs (1). Based on viral protein(VP) 6 , major component of the inner capsid, RV are divided into seven distinct groups, A to G. Among them, group (G) A, B, C and E RV have been detected in pigs. GARV are the most commonly associated with diarrhea in pigs (1). GCRV were first detected in pigs in 1980 and have been identified as the causes of diarrhea in suckling and newly weaned piglets. In Korea, GARV and GCRV have been detected in pigs with diarrhea (2). GARV are classified into $G$ and $P$ types based on the specificity of two outer capsid protein VP7(glycoprotein) and VP4(protease-sensitive protein), respectively (1). The examination of the diversity and prevalence of $\mathrm{G}$ and $\mathrm{P}$ types is important to survey the contemporary circulating strains.

\section{Materials and Methods}

Eighty nine intestinal or fecal specimens were obtained from 42 pig farms in Korea between January and July 2009. All specimens were examined by reverse transcription-polymerase chain reaction(RT-PCR) using group-specific primers for detection of GARV, GBRV and GCRV $(2,3)$. Positive samples for GARV were subjected to $G$ genotyping by RT-PCR using type-specific primers (4). The RT-PCR products of VP6 of GCRV and VP7 amplicons from samples positive for G9 RV were sequenced and analyzed.

\section{Results}

Of 89 samples from 42 pig farms, 40 from 22 pig farms and 40 from 21 pig farms were positive for GARV and GCRV, respectively. No samples showed positive for GBRV. Twenty-three out of 40 GARV-positive samples were genotyped by RT-PCR. The $G$ genotypes of 23 samples were determined as follows; three samples were $G 3$, six were $G 5$, nine were $G 9$, three were $G 3+G 5$, one was $\mathrm{G} 3+\mathrm{G} 9$ and another one was $\mathrm{G} 3+\mathrm{G} 5+\mathrm{G} 9$. The identity of the partial nucleotide(nt) sequence of the VP6 gene from 20 porcine GCRV detected in this study was $87-99 \%$ and that of the amino acid(aa) was $92-100 \%$. By the phylogenetic analysis of the partial VP6 gene of 20 GCRV detected in this study and reference strains of human, bovine and porcine GCRV, it was found that 19 Korean GCRV belonged to the porcine lineage and one didn't belong any porcine, bovine and human lineages. By the phylogenetic analysis of VP7 gene of G9 RV, four amplicons were shown to belong to lineage $\mathrm{VI}$ and sublineage $\mathrm{b}(\mathrm{VIb})$ and two amplicons were VIc according to the novel nomenclature for $\mathrm{G} 9$ RV (6 lineages with 11 sublineages) (5).

\section{Discussion}

This study indicated that GCRV(50.3\%) and GARV(52.3\%) were widespread in Korean pig farms. Genetic analysis of partial VP6 gene of GCRV showed that most GCRV belonged to the porcine lineage. However, relatively short nucleotide sequences of GCRV VP6 gene were analyzed due to the direct sequencing of detected amplicons. For further detailed genetic sequence analysis, the comparison of the extended or whole nucleotide sequence of GCRV VP6 gene will be necessary. The determination of G genotypes showed that G3, G5 and G9 types were prevalent in Korean pig farms examined in this study. To define the more detailed diversity of circulating RV, $\mathrm{P}$ typing is absolutely needed. Phylogenetic analysis of VP7 gene of G9 RV indicated that seven isolates were close to the porcine G9 RV rather than human G9 RV. For further study, it is necessary to isolate the circulating porcine RV and characterize them genetically and serologically and develop the vaccine by the selection of the most prevalent and effective strains.

\section{References}

1. Yuan L, Stevenson G, Saif LJ. 2006. Rotavirus and Reovirus. In Diseases of Swine. 9th Edition. Blackwell Publishing. Ames, lowa. p435-454.

2. Jeong YJ, Park SI, Hosmillo M, Shin DJ, Chun YH, Kim HJ, Kwon HJ, Kang SY, Woo SK, Park SJ, Kim GY, Kang MI, Cho KO. 2009. Vet Microbiol 138: 217-224

3. Elschner M, Prudlo J, Hotzel H, Otto P, Sachse K. 2002. J Vet Med B 49: 77-81

4. Gouvea V, Allen JR, Glass RI, Fang ZY, Bremont M,Cohen J, McCrae MA, Saif LJ, Sinarachatanant P, Caul EO. 1991. J Clin Microbiol 29: 519-523

5. Martella V, Pratelli A, Greco G, Tempesta M, Ferrari M, Losio MN, Buonavoglia C. 2001. Clin Diagn Lab Immunol 8: 129-132 


\title{
P.509
}

\section{Prevalence of rotavirus and coronavirus in piglet production herds in the Czech Republic}

\author{
Linda Czanderlova; Marek Zizlavsky; Dita Kellnerova; Marcela Gambova; Svetlana Odehnalova; Jiri Odehnal
}

Sevaron Counselling, Brno, Czech Republic

\section{Introduction}

Pre-weaning diarrhoea in pigs is a complex problem involving a large number of causative agents. Multiple enteric infections can occur, often in association with non-infectious factors. Viruses can play an important role by modifying the intestinal mucosa and thus predisposing secondary infections (1). Group A rotaviruses have been detected most frequently in swine. Rotaviruses and TGE and PED coronaviruses are important swine pathogens in nearly all countries with an intensive pig production. The significance of rotavirus and coronavirus infections is frequently underestimated in practice and these viruses are not often included in the differential diagnostics of diarrhoeal diseases in suckling piglets.

The aim of this study is to report the data of identification of rotavirus group $\mathrm{A}$ and TGE a PED coronaviruses in suckling piglets with diarrhoea in the Czech Republic.

\section{Materials and Methods}

The study included in total 98 pool samples of faeces taken from 53 pig production herds in the territory of the Czech Republic in the year 2008 and 2009. The samples were taken from suckling piglets with clinical manifestations of diarrhoea at the age from 2 to 28 days. All samples were tested for the presence of rotavirus group A, TGE coronavirus and PED coronavirus. Commercial immunochromatographic tests Rota Ag test kit and TGE/PED Ag test kit (Bionote) were used to prove these viruses in laboratory.

\section{Results}

Of 98 faeces samples included in the study, the presence of rotaviruses and/or coronaviruses was detected in 66 samples (67.3\%). The capture of rotaviruses and coronaviruses in the year 2008 and 2009 is presented in Table 1. TGE coronavirus was diagnosed most frequently (51 positive laboratory diagnoses). PED coronavirus was detected in 41 cases, rotavirus was diagnosed less frequently (30 positive laboratory diagnoses). Monoinfections were detected only in 25 cases ( $37.8 \%$ of all positive laboratory diagnoses), mixed infections with various combinations of rotaviruses and coronaviruses were diagnosed in the remaining cases (see Table 2). The combination of TGE and PED coronaviruses was found to have the highest prevalence, followed by TGE monoinfection and combined infection of rotavirus, TGE and PED coronavirus.
Table 1. Capture of rotavirus group $A$ and coronaviruses in the year 2008 and 2009

\begin{tabular}{|c|c|c|c|c|}
\hline & $\mathbf{2 0 0 8}$ & $\mathbf{2 0 0 9}$ & Total & \% positive \\
\hline Rotavirus & 12 & 18 & 30 & 30.6 \\
\hline TGE & 26 & 25 & 51 & 52.0 \\
\hline PED & 18 & 23 & 41 & 41.8 \\
\hline
\end{tabular}

Table 2. Prevalence of single infections and multiple infections caused by rotavirus group $A$ and coronaviruses

\begin{tabular}{|c|c|c|}
\hline & N'positive & \% positive \\
\hline Rotavirus+TGE+PED & 15 & 22.7 \\
\hline Rotavirus+PED & 6 & 9.1 \\
\hline Rotavirus+TGE & 3 & 4.5 \\
\hline TGE+PED & 17 & 25.8 \\
\hline Rotavirus & 6 & 9.1 \\
\hline TGE & 16 & 24.2 \\
\hline PED & 3 & 4.5 \\
\hline
\end{tabular}

\section{Discussion}

The acquired results indicate that the problem of viral diseases of digestive system in suckling piglets is still topical in the Czech Republic. These results and observations from farm audits suggest that environmental conditions and husbandry practices (poor disinfection) may be predisposing suckling piglets to viral intestinal infection. Although the infections related to rotaviruses and coronaviruses have an enzootic course in pig herds in the Czech Republic, they lead to atrophy and degeneration of enterocytes and aggravate the course of intercurrent infections (e.g. C. perfringens type A, C. difficile, ETEC).

When solving these viral infections in practice, vaccination of breeding stock sows before delivery, either by a commercial vaccine containing ETEC and the rotavirus group $A$, or by an autogenous vaccine with ETEC, rotavirus group $A$ and TGE and PED coronaviruses proved good.

\section{References}

1. Johnson M.W. at al. Veterinary Medicine, 1992 April:382-386. 


\title{
P.510
}

\section{Interspecies transmission of goat rotavirus to swine}

\author{
Min-A Hwang; Jung-Ah Lee; Seung-Yong Park; Chang-Sun Song; In-Soo Choi; Joong-Bok Lee
}

Konkuk University College of Veterinary Medicine, Seoul, Korea

\section{Introduction}

Rotavirus $A$, the major cause of gastroenteritis in children and animals, is interspecies pathogen. The notion of interspecies transmission between porcine and human(1), porcine and equine(2), and porcine and bovine(3) was reported. Transmissibility between porcine and goat was not reported up to date. An attempt was carried out to support the evidence that rotavirus $A$ isolated from different species may infect others by demonstrating that goat rotavirus may infect pigs.

\section{Material and Methods}

Inoculum was $1 \mathrm{ml}$ of 104 TCID50 caprine rotavirus, GRV G3P5[3], that was isolated from fecal specimen of 3-month-old goat(4). GRV was orally administered to the 3-day-old piglets which were raised with replacement milk after ceserian section (GRV $n=6$, mock $n=3)$. For six days, clinical sign was monitored by scoring diarrhea level ( $0=$ normal, $1=$ mild, $2=$ moderate, $3=$ severe). In order to confirm fecal shedding and to see if GRV is disseminated to other organs besides conventional infection site of rotavirus, fecal samples were sampled daily and then ileum, liver, brain and lung tissue were collected by the end of experiment period. NSP4 gene of rotavirus was amplified by nested PCR to confirm viral infection. In addition, mRNAs from spleens were collected to profile mRNAs for innate immunity and pro-inflammatory cytokines. Quantitative analysis using reverse transcription realtime PCR using SYBR ${ }^{\oplus}$ green reagent was conducted..

\section{Result}

Mild diarrhea began at day 1 post inoculation (PID 1) and got worse day by day till PID 3. The severe diarrhea peaked at PID 4 and reached flat for 24 hours. The PCR detection rate of PID 4 and 5 were significantly higher than the others (Fig 1).

Fig. 1. The mean diarrhea score (line) and density of $P C R$ products (bar).

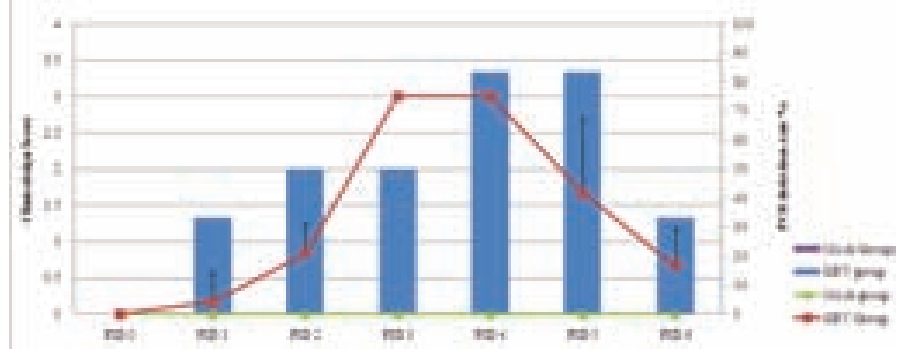

As shown in Table 1, PCR products of GRV appeared at $50 \%$ of ileum, liver, lung, and brain, respectively. It is interesting that one of infected piglet did not shed, but spread virus to all of the tested tissue.
Table 1. Detection of rotavirus RNA in varying organs of GRV-infected piglet

\begin{tabular}{|l|c|c|c|c|}
\hline \multirow{2}{*}{} & \multicolumn{4}{|c|}{ No.positive/No.tested (\%) } \\
\cline { 2 - 5 } & Ileum & Liver & Lung & Brain \\
\hline GRV & $3 / 6(50)$ & $3 / 6(50)$ & $3 / 6(50)$ & $3 / 6(50)$ \\
\hline mock & $0 / 3(0)$ & $0 / 3(0)$ & $0 / 3(0)$ & $0 / 3(0)$ \\
\hline
\end{tabular}

In GRV infected piglet, mRNAs of both type I and II interferon (IFN- $a, \gamma$, respectively) were elevated in parallel with increase of mRNAs for IFN -inducible antiviral molecules, OAS and PKR. mRNAs of TNF- $a$ and IL-4 increased, while those of IL-6, 8, 10 decreased slightly (Fig. 2).

Fig 2. Profiling innate immunity and pro-inflammatory cytokine mRNAs in spleen.

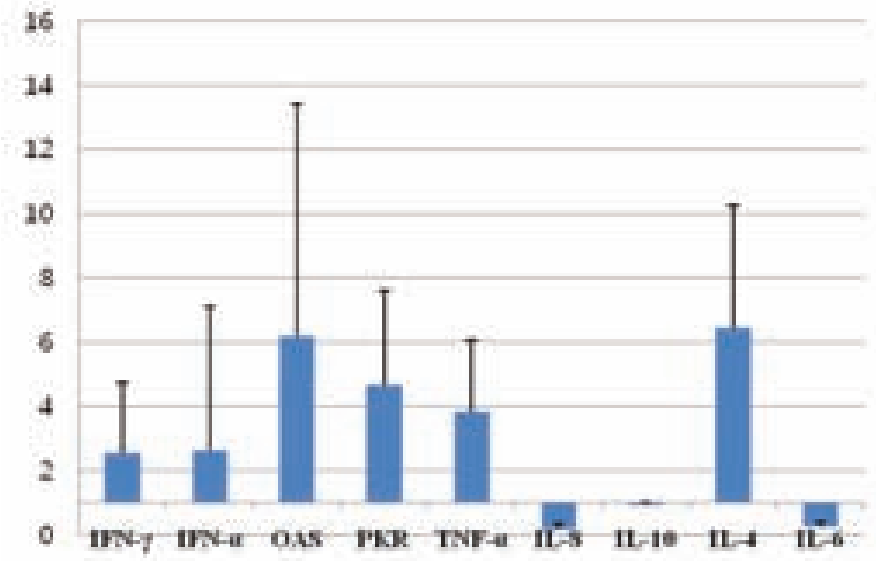

\section{Discussion}

This study directly support the possibility of interspecies transmission. Cytokine analysis also showed that GRV strain may evoke innate immunity to provide antiviral activity. In an attempt to control co-circulation of rotavirus infection in animal and/or human population, to understand mechanism by which transmission of interspecies occurs is important.

\section{References}

1. Ciarlet M. et al., (2001), Virus Gene 22,5-20

2. Gouvea V. et al.,(1994), J Clin Microbiol 32,1338-1340

3. Lee J.B. et al.,(2003), Arch Virol 148,643-657

4. Racz M.L. et al., (2001), J Clin Microbiol 38,2443-2446 


\title{
P.511
}

\section{Development and validation of a quantitative PCR for the detection of Actinobacillus suis}

\author{
Maria J. Clavijo; Simone Oliveira \\ University of Minnesota, Saint Paul, MN, USA
}

\section{Introduction}

Actinobacillus suis is an emerging pathogen considered to be a new threat for swine production. The isolation of this pathogen from tissues submitted to the University of Minnesota Veterinary Diagnostic Laboratory (MVDL) increased considerably in the past three years. In 2006, a 16s rRNA gel-based PCR was developed and since then requests for $A$. suis PCR testing have raised significantly. ${ }^{1}$ This tool has been highly employed in protocols attempting to eradicate $A$. suis. However, complete elimination of the pathogen has not been achieved. 2,3 Limitations of this gelbased PCR include the non-specific amplification of $A$. equuli and the lack of bacterial quantification. Therefore, a more sensitive and specific quantitative diagnostic tool is needed to improve eradication protocols. The objective of this study was to develop and validate a sensitive and specific quantitative PCR assay for the detection of Actinobacillus suis in clinical samples.

\section{Materials and Methods}

Forward and Reverse primers and a TaqMan probe specific for the amplification and detection of an $A$. suis housekeeping gene involved in thiophene oxidation (Thdf) were designed using the Primer 3 Software. Quantitative PCR conditions were optimized utilizing an ATCC A. suis reference strain. This strain was also utilized to evaluate the analytical sensitivity of the quantitative PCR by testing 10-fold dilutions of extracted DNA. The analytical specificity was evaluated by testing 29 unrelated bacterial species frequently isolated from swine in addition to $A$. equuli, which generated non-specific amplifications with the gel-based $16 \mathrm{~S}$ rRNA PCR. The stability of the targeted gene sequence among $A$. suis field isolates was evaluated by testing 70 different isolates representing 12 genotypes circulating among U.S swine herds.

\section{Results}

The newly developed quantitative PCR detected a minimum of $3.5 \times 101 \mathrm{CFU} / \mathrm{ml}$, compared to $7.2 \times 102 \mathrm{CFU} / \mathrm{ml}$ detected by the $16 \mathrm{~S}$ rRNA gel-based PCR test. The Thdf quantitative PCR was highly specific, detecting exclusively $A$. suis isolates. There was no non-specific amplification of $A$. equuli DNA, as previous observed with the gel-based PCR. The new quantitative PCR amplified the Thdf gene from all 70 A. suis field isolates tested, indicating that this housekeeping gene is highly conserved among different strains circulating in U.S. swine herds.

\section{Discussion}

We have developed a more sensitive and specific quantitative PCR for detection of $A$. suis in clinical samples. This new test will allow swine veterinarians to perform a more comprehensive comparison of different control and eradication protocols. All attempts to eradicate $A$. suis from endemically infected populations have failed so far. In most of these cases, $A$. suis was not detected at weaning following intensive antibiotic treatments, but was detected at the end of the nursery using the gel-based $P C R$. The new quantitative PCR, which is more sensitive and specific than the previous gel-based PCR, will allow a better characterization of prevalence at weaning. Quantification of $A$. suis in tonsil swabs will also provide a more detailed characterization of treatment effect, and will allow swine veterinarians to evaluate the cost-benefit of each protocol. We expect the new $A$. suis quantitative PCR to be an important aid in $A$. suis control and eradication.

\section{References}

Oliveira S., Gayle R., Tomaszewski J., Collins J. (2007) Development of a duplex PCR for the simultaneous detection of Actinobacillus suis and Actinobacillus pleuropneumoniae in clinical samples from swine. Proceedings of the 50th American Association of Veterinary Laboratory Diagnosticians Annual Meeting, Reno, p. 135.

Geiger J., Engle M., Oliveira S. (2007) Actinobacillus suis eradication: is it possible? Proceedings of the Swine Disease Eradication Center Annual Meeting., Minneapolis, MN p. 14.

Miller S.P., Stanford, S, Oliveira, S. (2008) Case study - Actinobacillus suis: elimination or reduction? Proceedings of the 2008 Carlos Pijoan International Symposium: New solutions to old problems. In: Allen Leman Swine Conference Proceedings, saint Paul MN, p. 29-32. 


\title{
P.512
}

\section{Preliminary study of Actinobacillus suis colonization in upper and lower respiratory tract in naturally infected animals}

\author{
Marina Sibila; Eva Huerta; Llorenç Grau-Roma; Virginia Aragón; Joaquim Segales \\ Centre de Recerca en Sanitat Anima (CReSA), Bellaterra, Spain
}

\section{Introduction}

Actinobacillus suis (A. suis) is considered an early colonizer of the upper respiratory tract, which may also cause fatal cases of septicaemia in young piglets. A. suis infection has been also described in adult animals with fever, inappetence and erypselalike skin lesions (1). However, in high-health farms this pathogen has been linked as well to different pathological conditions including arthithis, pneumonia, enteritis, meningitis, abortion and endocarditis in pigs at different ages (2). Besides, A. suis infection can cause very similar lesions to those of Actinobacillus pleuropneumoniae (App). In fact, both pathogens share some virulent factors including Apx toxins, urease, and iron-regulated outer membrane proteins. Moreover, distinction of A. suis isolates from App biotype II ones can be difficult if it is not confirmed biochemically or by molecular biology techniques. This high similarity between both pathogens may sometimes lead to erroneous diagnosis (2).

Little information on the A. suis prevalence in animals of different ages and clinical conditions is nowadays available. Therefore, the goal of the present study was to gain insight into A. suis prevalence in upper and lower respiratory tract samples from pigs with different clinical conditions.

\section{Material and Methods}

Forty seven pigs were included in this study. From these, 34 pigs (from 6 to 18 weeks of age) were submitted for necropsy to the Pathology Diagnostic Veterinary Service at the Veterinary School of Barcelona (Spain) with different clinical signs (mainly, growth retardation, digestive and respiratory problems). The remaining pigs were 136 -week-old healthy pigs. The following samples were collected from each animal: nasal swab (NS), tonsillar swab (TS), tonsillar brush (TB), tonsil tissue (T), bronchial swab (BS) and lung (L). TB was not taken in 9 of the 47 animals. Lung was always taken from the accessory lobe, independently on the presence or absence of lesion.

DNA from swabs and tissues was extracted using commercial kits and processed according to the manufacturer's instructions. In order to differentiate between A. suis and App, samples were tested with two PCRs. One to detect the Apx IV gene (3), present exclusively in all App serotypes, and another to detect the Apx Il gene (4), present in A. suis and in most of App serotypes. The Apx IV PCR was done in a singleplex mode in all samples but when ambiguous results were obtained the nested PCR was performed (5).

\section{Results}

Apx II and IV toxin genes were detected in a number of animals showing different clinical signs. Positivity was found in samples from the upper (NS, TS, TB and T), but not in those from lower respiratory tract (BS and $\mathrm{L}$ ). On the other hand, all tested samples from healthy animals were PCR negative to both toxins.

Apx II was more frequently detected (15 out of 47 [32\%]) than Apx IV (9 out of 47 [19\%]). In both cases, the number of PCR positive samples per animal varied from one (mainly TS) up to 3 (TS, TB and T or NS). From the 47 tested animals, 31 (66\%) were negative to both Apx II and IV PCRs in all the tested samples. Nine (19\%) out of 47 pigs were PCR positive to both Apx II and IV at least in one sample. Finally, $6(13 \%)$ animals were positive only to Apx II PCR while only 1 (2\%) was positive exclusively to Apx IV.

\section{Discussion}

Detection of Apx II in absence of Apx IV amplification in some samples would indicate $A$. suis infection in such animals. These preliminary results suggest that $A$. suis detection in upper respiratory tract by means of direct PCR is feasible and relatively frequent in animals of different ages and showing different clinical conditions. The best sample to be tested by direct PCR resulted to be the TS or TB.

The concomitant detection of both toxins in some animals together with the existence of an animal in which only Apx IV was found, indicated the presence of different App serotypes in the samples.

More studies will be useful to assess the role and prevalence of A. suis in porcine respiratory pathologies.

\section{References}

1. Taylor D.J. Disease of Swine, 8th Edition. lowa State University press. Ames lowa. p 624-627

2. Oliveira S. 2007, AASVp 371-376

3. Schaller et. al. 2001. Vet. Microbiol 79, 47-62.

4. rey et. al. 1996. Schweiz Arch Tierheilkd 138, 121-124.

5. McInnes et al., 2008. Can. J. Vet. Res. 72(3): 242-8. 


\title{
P.513
}

\section{Detection of haemophilus parasuis requires strategic diagnostic}

\author{
Rose-Leah Austin-Busse ${ }^{1}$ Andrea Ladinig ${ }^{2}$ Joachim Spergser ${ }^{3}$ Mathias Ritzmann ${ }^{2}$ Karl Heinritzi ${ }^{1}$ Andreas Palzer $^{1}$ \\ 1. Clinic for Swine, Veterinary Faculty Ludwig-Maximilians University Munich, Munich, Germany; 2. Clinic for Swine, Veterinary \\ University Vienna, Vienna, Austria; 3. Institute of Bacteriology, Mycology and Hygiene Veterinary University, Vienna, Austria
}

\section{Introduction}

Haemophilus parasuis (HPS) is the etiological agent of porcine polyserositis and arthritis (Glässer's disease). Despite the increased prevalance and severity of Glässer's disease in recent years (1), diagnosis of Glässer's disease is still a challenging task. Not only is the isolation and cultivation of the bacteria tedious due to its fastidious nature, but routine diagnostic methods are hampered by the heterogenic nature of HPS and its commensal status in the upper respiratory tract of healthy pigs (2).

The aim of this study was to evaluate the detection of HPS by $P C R$ and bacterial culture $(B C)$ in different materials taken from weaned piglets that were experimentally infected with HPS serovar 5.

\section{Materials and Methods}

36 piglets, approximately 3 weeks old, were obtained from a commercial farrowing farm which had no previous history of porcine reproductive and respiratory syndrome virus (PRRSV). Also, the animals were Mycoplasma hyorhinis PCR negative and HPS serologically negative. Piglets were housed in two separate units in a biosafety level 3 facility. After an acclimatization period of 17 days, 27 piglets were infected intratracheally with 5 $x 10^{8}$ colony-forming units (CFU) of HPS serovar 5 . The remaining 9 animals served as control animals and received phosphatebuffered saline (PBS) instead. The study was terminated 15 days after infection. Dry swabs of the serosal surfaces (pleura, pericardium, and peritoneum) and the brain, synovia and cerebrospinal fluid (CF), and joint capsule were taken for PCR (3) analysis from each animal either at study termination or at removal. Furthermore, swabs from the serosal surface were investigated for bacterial examination.

\section{Results}

Results of the PCR and BC of the infected pigs are illustrated in Tab. 1. All sample materials from the control animals were negative by PCR analysis and BC. A total of 12 infected pigs died or were euthanized due to humane reasons within 10 days after infection. HPS was more frequently isolated in samples from these animals than animals that died at study termination. The sample material and method with the highest HPS detection was the PCR analysis of serosal swabs. HPS genome was detected by PCR in the joint capsule of 5 animals, however, the synovia fluid from these animals were HPS negative. Similarly, the synovia samples from four animals were PCR positive but the corresponding joint capsules from these animals were negative. Also, HPS genome was more frequently detected in the cerebrospinal fluid than the brain swab by PCR testing. At study termination, HPS genome was detected predominantly in the synovia fluid; all other sample materials were negative by PCR analysis and BC.
Table 1: Results of PCR and bacteriological examination of samples from pigs infected with HPS serovar 5

\begin{tabular}{|c|c|c|c|c|c|c|c|}
\hline $\begin{array}{c}\text { Day of } \\
\text { study } \\
\text { exit }\end{array}$ & $\begin{array}{c}\text { No. of } \\
\text { pigs }\end{array}$ & $\begin{array}{c}\text { Serosal swab- } \\
\text { bacterial } \\
\text { culture }\end{array}$ & $\begin{array}{c}\text { Serosal } \\
\text { swab- } \\
\text { PCR }\end{array}$ & $\begin{array}{c}\text { Brain } \\
\text { swab- } \\
\text { PCR }\end{array}$ & CF-PCR & $\begin{array}{c}\text { Synovia } \\
\text { fluid }\end{array}$ & $\begin{array}{c}\text { Joint } \\
\text { fluid- } \\
\text { PCR }\end{array}$ \\
\hline 2 & 3 & $3 / 3$ & $3 / 3$ & $2 / 3$ & $2 / 3$ & $0 / 3$ & $2 / 3$ \\
\hline 3 & 3 & $3 / 3$ & $3 / 3$ & $0 / 3$ & $2 / 3$ & $0 / 3$ & $0 / 3$ \\
\hline 4 & 3 & $3 / 1$ & $3 / 3$ & $0 / 3$ & $2 / 3$ & $0 / 3$ & $0 / 3$ \\
\hline 6 & 1 & $1 / 2$ & $1 / 1$ & $1 / 1$ & $1 / 1$ & $0 / 1$ & $1 / 2$ \\
\hline 10 & 2 & $0 / 15$ & $2 / 2$ & $0 / 2$ & $0 / 2$ & $0 / 2$ & $1 / 2$ \\
\hline 15 & 15 & $0 / 15$ & $0 / 15$ & $0 / 15$ & $0 / 15$ & $4 / 15$ & $1 / 15$ \\
\hline
\end{tabular}

\section{Discussion and Conclusion}

In the current experiment, 12 pigs died within 10 days after infection. This study confirms the findings of Kielstein and Rapp-Gabrielson (4) who classified serovar 5 as very pathogenic, capable of eliciting death within 4 days. Although this study was conducted with a relatively small number of animals, differences in HPS detection were found, particularly after comparing PCR and culture results between animals that died acutely or at study termination. Turni et al. (2) noted that sample material tends to yield differing results depending on if samples were obtained after a subacute, acute or chronic infection.

Based on the results from this study, conclusive HPS detection is best achieved by examining samples from various sites using both PCR and BC. Furthermore, HPS detection is more successful in sample material from animals which are in the acute phase of infection. In this study, HPS genome was only detected in the synovia fluid when animals were chronically infected. Also, PCR testing of CF was more successful at detecting HPS genome than a brain swab. Further investigations are necessary to confirm these results.

\section{References}

1. De la Fuente et al. (2009) Res. Vet. Sci. 86: 230-234

2. Turni C. and Blackall P. (2007) Aust. Vet. J. 85: 177-184

3. Angen O. et al. (2006) Vet. Micro. 119: 266-276

4. Kielstein P. and Rapp-Gabrielson V. (1992) J. Clin. Micro. 30: 862-865 


\title{
P.514
}

\section{Rapid identification of Haemophilus parasuis by MALDI-TOF Mass Spectometry}

\author{
Marie Helene Bayon-Auboyer; Marie Laure Malcoste; Rosine Danguy-des-Déserts; Herve Morvan
}

LDA22, Ploufragan, France

\section{Introduction}

Matrix-assisted laser desorption ionisation-time of fly mass spectrometry (Maldi-TOF-MS) in combination with dedicated analysis software is a reliable method for the identification of microoorganisms (1).

The analysis software incorporates functionalities for the processing of mass spectra as well as for identification.

Identification of bacteria is achieved by comparison of generated mass spectra with reference spectra stored in a ready to use data base which contains the characteristic spectra information of species and subspecies. However, Haemophilus parasuis spectrum was not present.

We present here, the generation of a new reference pattern for $H$. parasuis and its use for a rapid identification of $H$. parasuis strains in a clinical approach as an alternative to time-consuming and expensive routine methods.

\section{Materials and Methods}

The bacterial strains used to evaluate the $H$. parasuis reference mass spectra were recovered from clinical diagnosis. They were identified using traditional biochemical tests (4) and PCR (3). These bacterials strains were H. parasuis (29), A. pleuropneumoniae (1), Actinobacillus taxon C (1), Actinobacillus minor (6), B. bronchiseptica (1) and P. multocida (1).

Bacterial strains were grown over night on different media and under diverse culture conditions according to the microorganisms.

To established a reference mass spectrum for $H$. parasuis, the well known and identify reference strain CIP 105098 was used (CIP : Pasteur Institute collection). A good average was achieved by the measurement of 24 spectra of the reference strain. The software automatically generated the peak lists from the whole set of the 24 spectra and extracted the typical peaks which were present in all the spectra to established the $H$. parasuis reference spectrum. This spectrum was added to the library.

Direct "cell smear", a simple method applicable to many bacteria, was used. Small amounts of biological material (single colony) were smeared directly as a thin film onto a Maldi target. One microliter of chemical matrix was deposited and allowed to dry down.

The samples were analysed in a Bruker Microflex (LT) Maldi-Tof Mass Spectrometer.

The MALDI BioTyper ${ }^{\mathrm{TM}}$ software was used to process raw spectra. Identification of the bacterial strains was performed by comparison with the library of reference spectra from Bruker and our $\mathrm{H}$. parasuis reference spectrum. A matching score based on identified masses and their intensity correlation was generated and used for ranking of results.

\section{Results}

Traditional identification of $H$. parasuis is based on colony morphology, Gram staining, NAD requirement and biochemical testing (4). However, those techniques remain time consuming and labor intensive procedures. H. parasuis identification strain from clinical samples processing needs, at least, 3 days.

Starting from a few biological material, mass spectra could be observed for each bacterial isolate tested. Results were known in a few minutes

In total, Maldi Tof MS identified 29 of the 29 isolates H. parasuis (100\%), concordant with traditional bacterial methods and PCR. The remaining tested bacteria were identified at the species or genus level, but not as $H$. parasuis. Since the validation of the $H$. parasuis reference spectrum, numerous strains have been identified using this new method.

\section{Discussion}

These results show that it's easy to create a new reference mass spectrum for $H$. parasuis which is nowadays used for routine and rapid identification in our laboratory. Now, H. parasuis is identified within $24 \mathrm{~h}$ rather than the $72 \mathrm{~h}$ or more needed for traditional bacteriological methods. Furthermore, samples preparation and analysis do not exceed traditional methods cost.

Thus, Maldi Tof MS offers a good alternative to traditional laboratory methods in veterinary and environmental diagnostics (2). The speed and minimal sample preparation make it well suitable for routine use. Prerequisite is the establishment of high quality spectra libraries.

A further field of application is the potential of lineage typing, analysis of taxonomic relationships.

\section{References}

1. Fenselau, C. et al. 2001. Characterization of intact microorganisms by maldi mass spectrometry. Mass Spectrom. Rev. 20 : 157-171.

2. Holland R. et al. 1996. Rapid identification of intact whole bacteria based on spectral patterns using matrix-assisted laser desorption/ ionization with time-of-flight mass spectrometry" Rapid Commun Mass Spectrom. $10: 1227-32$.

3. Oliveira S. et al. 2001. development of a PCR test to diagnose Haemophilus parasuis infections. J. Vet. Diagn. Invest. $13: 495-501$.

4. Rapp-Gabrielson V.J., Diseases of swine 8th Editions, Ames, loxa: lowa State University Press. 1999, 474-479 


\title{
P.515
}

\section{Serotyping of Haemophilus parasuis isolates from German pigs}

\author{
Katrin Strutzberg-Minder ${ }^{1}$ Jan Boehmer ${ }^{1}$ David Goldstein ${ }^{1}$ Herve Le Galludec ${ }^{2}$ Matthias Homuth ${ }^{1}$ \\ 1. IVD GmbH Innovative Veterinary Diagnostics, Hannover, Germany; 2. Fort Dodge Animal Health, GM Naarden, Netherlands
}

\section{Introduction}

Haemophilus parasuis is the cause of Glässer's disease (porcine polyserositis and arthritis) and other clinical disorders in pigs, but it can also be isolated from the upper respiratory tracts of healthy pigs.

Fifteen serotypes of Hps, defined by an immunodiffusion or agar gel precipitation test with heat-stable antigens, have been described by (1), and it has been shown that there is. an association between serotype and the degree of virulence (2). A new indirect hemagglutination (IHA) test for serotyping of Hps isolates has also been developed and evaluated (3). The latest published data on the prevalence of Hps serotypes in Germany are from 1998 (4)

\section{Material and Methods}

The IHA test was performed according to the method of (3). Antisera against all 15 serotype-specific reference strains were prepared as described (1) with some modifications.

A total of 96 strains isolated from German pig herds with clinical problems were typed by IHA, most of them with the objective of making a herd-specific vaccine. No precise data were available on the source tissue of the isolates and on clinical symptoms.

\section{Results}

The most prevalent Hps serotype was $1(n=18)$, followed by serotypes $2(n=16), 13(n=13), 4(n=12)$, and $5(n=9)$ (Fig. 1). Less prevalent were the serotypes $12(n=5), 7(n=4), 9(n$ $=2), 11(n=1)$ and $14(n=1)$. No Hps isolate was identified as serotype $3,6,8,10$ or 15 . In all, 15 of the Hps isolates showed no reaction with any of the 15 serotype-specific antisera and were thus not typeable (s. Fig. 1: n.t.).

Figure 1: Frequency distribution of serotypes of Hps isolates from German pig herds. Striped columns represent serotypes associated with death, checkered columns, those with a moderate to severe degree of virulence, and completely filled columns, those associated with no lesions; source: (2)

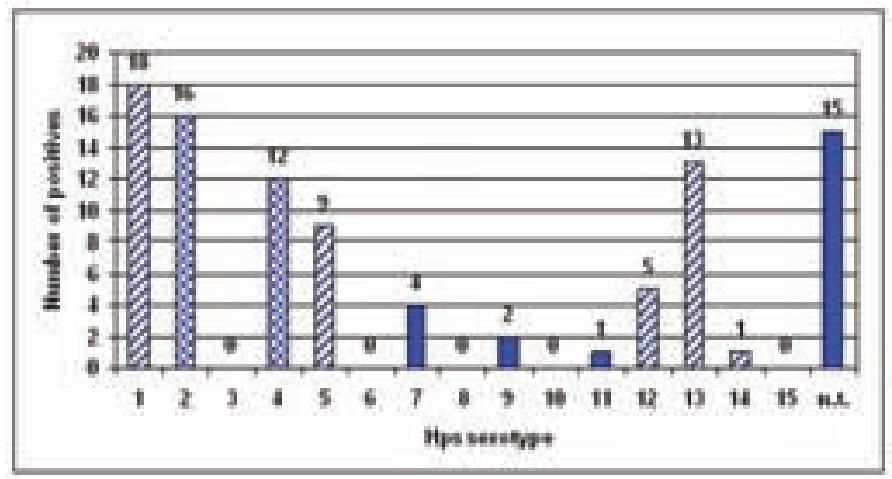

\section{Discussion}

The most frequent serotypes 1, 2, 13, 4 and 5 (73.8\%) are associated with a moderate to severe degree of virulence (2). Our data support the assumption that these isolates were involved in the clinical problems of the herds. The IHA test for serotyping Hps isolates is thus a useful tool for evaluating whether an isolate can be expected to have a clinical impact.

The IHA test was originally developed as a new method for serotyping of Hps and was evaluated by determining the prevalence Hps in North America. More than $90 \%$ of the field strains of Hps were found to be typeable by IHA, including those exhibiting cross-reactivity in immunodiffusion which fails to identify approximately $30 \%$ of field isolates of $\mathrm{Hps}(3)$. In our study, 15 (15.6\%) field isolates could not be identified with any serotype, although $84.4 \%$ of the isolates were typeable. Since genotyping is not strictly correlated with serotyping and virulence is associated with serotype, there is still a need for antigen-based typing. Furthermore, despite great progress in genotyping techniques, antigenic characterization of prevalent strains of Hps is still essential for the development of effective vaccines and serodiagnostic tests and for epidemiological analysis.

\section{References}

1. Morozumi and Nicolet (1986). J. Clin. Microbiol. 23:1022-1025

2. Kielstein and Rapp-Gabrielson (1992). J. Clin. Microbiol. 30:862-865

3. Tadjine et al. (2004). J. Clin. Microbiol. 24:839-840

4. Kielstein and Wuthe (1998). TU 53:250-258

5. Rapp-Gabrielson et al. (2006). in: Straw et al. (eds.), Diseases of Swine, 9thed., 681-690 


\title{
P.516
}

\section{A Modified Multi-Locus Sequence Typing (MLST) Scheme for Haemophilus parasuis}

\author{
Karen B. Register; Michael A. Mullins; Darrell O. Bayles \\ USDA/ARS/National Animal Disease Center, Ames, IA, USA
}

\section{Introduction}

Haemophilus parasuis is the etiologic agent of Glässer's disease and pneumonia in swine. Phenotypic classification systems are of assistance in epidemiologic studies but molecular methods provide numerous distinct advantages. An MLST scheme proposed by others appears promising (4). However, recently acquired genome data $(3,5)$ reveal the primers used, based on sequences of other bacteria, are not optimal for $H$. parasuis. Furthermore, several different PCR mixes and cycling programs are required for amplification of all targets. Here we report modifications to enhance and simplify the original method, thereby facilitating data acquisition and increasing accuracy. The modified MLST was applied to a group of 35 diverse $H$. parasuis isolates and the results compared with those obtained using the original method.

\section{Materials and Methods}

Thirty-five genetically distinct strains of $H$. parasuis, including the 15 serovar reference strains, were evaluated by the original (4) and modified MLST methods. Amplicon sequences were trimmed, concatenated and used to construct Neighbor-Joining trees as reported (2). Target-specific alignments used to guide primer redesign included genome sequences of four $\mathrm{H}$. parasuis strains (3,5). Vector NTI Advance was used for sequence editing and analysis and to assist in primer design.

\section{Results and Discussion}

Alignments of genome sequences from 7 target genes, atpD, infB, $m d h$, rpoB, 6pgd, g3pd and $f r d B$, were examined for variability and quality of match within the original primer-binding regions (see Fig. 1 for an example). Ten of the 14 originally recommended primers (4) were redesigned to eliminate mismatches and avoid regions of high sequence heterogeneity.

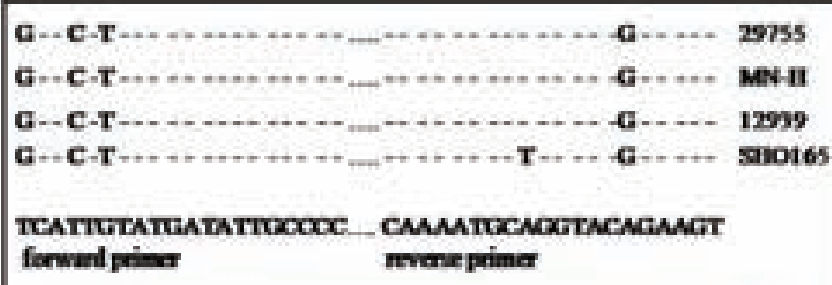

Fig. 1. DNA sequence from the mdh gene of the $H$. parasuis strain indicated on the right as compared to the MLST primers originally recommended. Base mismatches with the primers are indicatod.

To simplify the method for end users, we standardized the Tm of all primers and identified a universal PCR mix and set of cycling conditions that resulted in robust and reproducible amplification of all targets.
The number and percentage of variable positions within the dataset obtained using our modifications (240 of 3154 positions; $7.6 \%$ ) is slightly less than for the original method (408 of 3806 positions; $10.7 \%$ ) but the grouping of strains in major clusters is nearly identical (Fig. 2). Analysis of $H$. parasuis genomes indicates some MLST targets are in close proximity along the chromosome (e.g., 6pgd and frdB are separated by $\sim 10 \mathrm{~Kb}$ ), in opposition to general guidelines for MLST (1). Nonetheless, the discriminatory power of the scheme proposed here suggests it is a highly useful phylogenetic and epidemiologic tool.

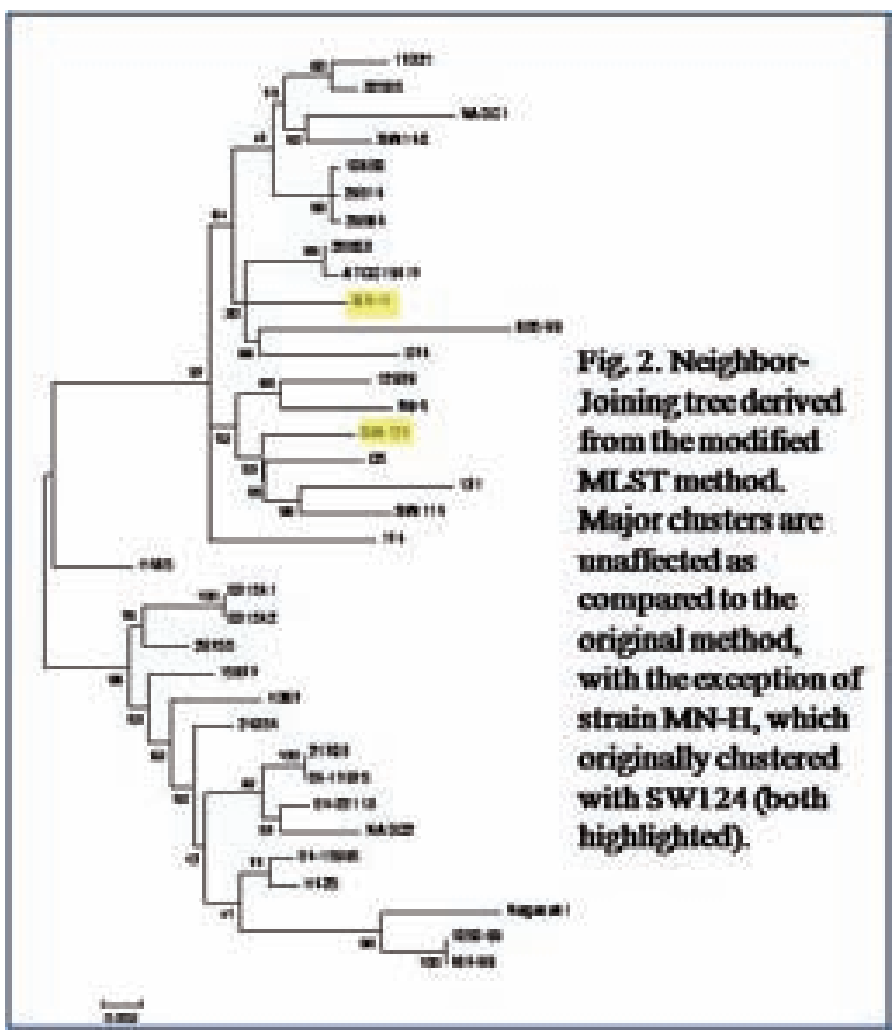

\section{References}

1. Maiden, MC. 2006. Ann Rev Microbiol 60:561-88.

2. Mullins, MA et al. 2009. J Bacteriol 191:5988-6002

3. Mullins, MA, Bayles, DO, Register, KB. unpublished

4. Olvera, A et al. 2006. Microbiol 152:3683-3690.

5. Yue, M et al. 2008. J Bacteriol 191:1359-60. 


\title{
P.517
}

\section{Phylogenetic Analysis of Dutch and Belgian Haemophilus Parasuis Isolates Using ERIC-PCR}

\author{
Remco Dijkman ${ }^{1}$ Gerard J. Wellenberg ${ }^{1}$ Tom Duinhof $^{1}$ Harold M.J.F. van der Heijden ${ }^{1}$ \\ Refke Peerboom ${ }^{1}$ Alex Olvera ${ }^{2}$ Cora Miry ${ }^{3}$ Anja Rothkamp ${ }^{1}$ Eric. van Esch ${ }^{1}$ \\ 1. Animal Health Service, Deventer, Netherlands; 2. Centre de Recerca en Sanitat Animal (CReSA), Barcelona, Spain; \\ 3. Animal Health Care Flanders, Torhout, Belgium
}

\section{Introduction}

Haemophilus parasuis (H. parasuis), a ubiquitous bacterium in the upper respiratory tract of swine, is the causative agent of Glässer's disease. To date, more than $15 \mathrm{H}$. parasuis serotypes have been described and isolates with the same serotype can be very heterogeneous in virulence (1).

Several attempts have been made to improve the differentiation of field isolates both on phenotypic and genotypic level. In this study, the Enterobacterial Repetitive Intergenic Consensus-PCR (ERIC-PCR) for fingerprinting of field isolates has been used to identify separate lineages in Dutch and Belgian H. parasuis isolates. ERIC-PCR lineages were compared with serotyping results.

\section{Materials and Methods}

From October 2007 until September 2009, 89 H. parasuis isolates have been collected from pigs which were presented at the Dutch Animal Health Service for postmortem examinations. Typical growth on chocolate (+NAD) agar plates, satellite growth without hemolysis on blood agar plates using Staphylococcus aureus nurse strain and a negative CAMP test were used for the identification of $\mathrm{H}$. parasuis. Ten $\mathrm{H}$. parasuis reference strains and 11 Belgian field isolates were examined too. The serotypes were determined at the IVD GmbH (Gesellschaft für Innovative Veterinärdiagnostik, Germany) using type-specific antisera. ERIC-PCR was performed as described by Rafiee et al (2). PCR products were visualized on a $2 \%$ agarose gel. Gel images were analyzed using the BioNumerics software package (Applied Maths NV, Sint-Martens-Latem, Belgium).

\section{Results}

Serotyping revealed in general the serotypes $1(n=14), 2(n=12)$, $4(n=29)$ and $13(n=18)$, whereas serotype 5 was serotyped 8 times.

ERIC-PCR gel images of the 100 isolates were analyzed by the BioNumerics software. Similarities were determined using Pearson correlation and clustered using the UPGMA algorithm (Figure 1). ERIC-PCR revealed a very heterogeneous pattern. No clear correlations between serotypes and ERIC-PCR lineages were found.
Figure 1: Dendrogram based on H. parasuis ERIC-PCR results constructed using Pearson correlation and the UPGMA algorithm.

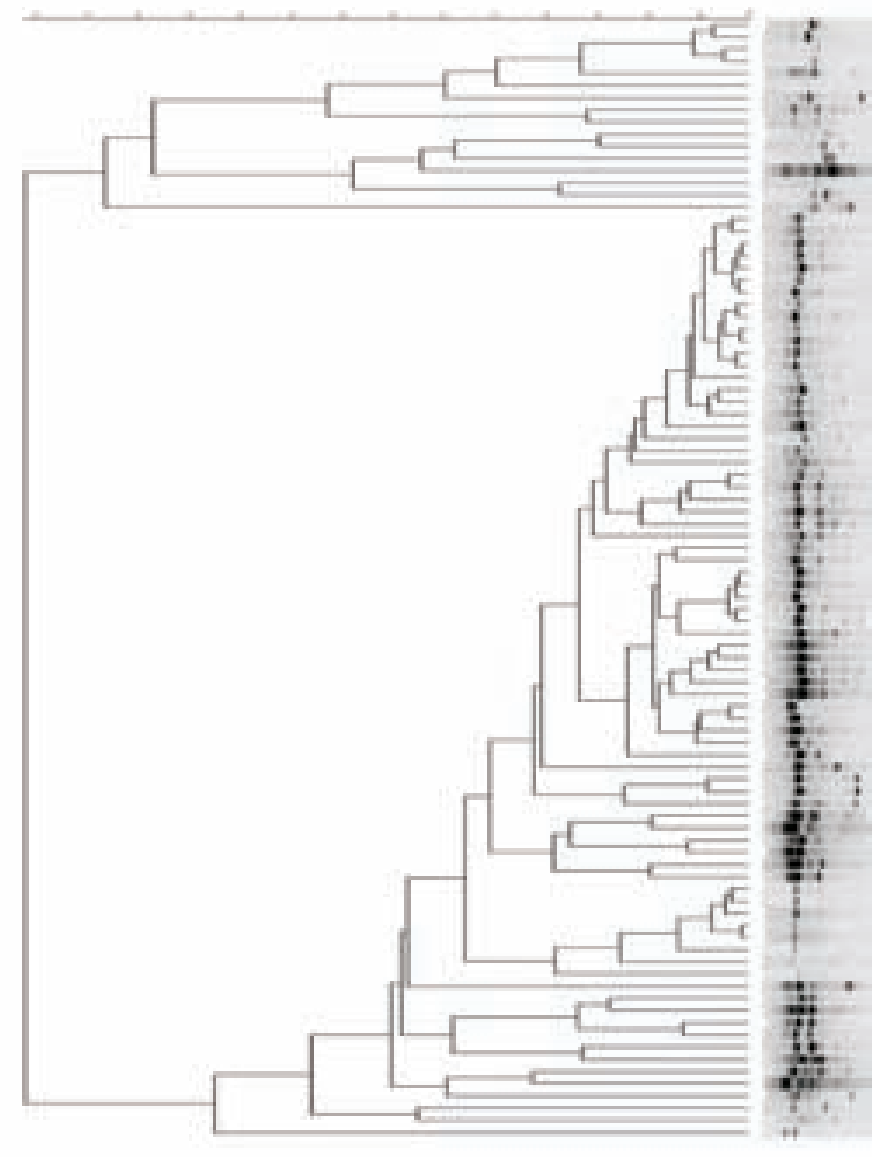

\section{Discussion}

Different $H$. parasuis serotypes and ERIC-PCR lineages were found in Dutch and Belgian isolates. The overall correlation between serotype and ERIC-PCR analyses was low. Isolates with different ERIC-PCR patterns were found within the same serotype. Other techniques and further analyses of these isolates in correlation with clinical and pathological findings will be used to identify more virulent isolates and to separate these from low virulent isolates.

\section{References}

1. Kielstein, P., and Rapp-Gabrielson, V.J. (1992). J. Clin. Microbiol. 30 : 862-865

2. Rafiee, M. et al (2000). Austr. Vet J. 78(12):846-849. 


\title{
P.518
}

\section{Phylogenetic Analysis of Dutch and Belgian Haemophilus Parasuis Isolates Using HSP60 Sequence Typing}

\author{
Gerard J. Wellenberg ${ }^{1}$ Remco Dijkman ${ }^{1}$ Tom Duinhof $^{1}$ Harold M.J.F. van der Heijden ${ }^{1}$ \\ Refke Peerboom ${ }^{1}$ Alex Olvera ${ }^{2}$ Cora Miry ${ }^{3}$ Anja Rothkamp ${ }^{1}$ Eric. van Esch ${ }^{1}$ \\ 1. Animal Health Service, Deventer, Netherlands; 2. Centre de Recerca en Sanitat Animal (CReSA), Barcelona, Spain; \\ 3. Animal Health Care Flanders, Torhout, Belgium
}

\section{Introduction}

Haemophilus parasuis (H. parasuis) is the etiological agent of Glässer's disease. Glässer's disease is characterized by serofibrinous and fibrinopurulent polyserositis, arthritis, meningitis which can also lead to acute death, especially in piglets. H. parasuis can also be isolated from lungs.

At this moment, 15 different serotypes are described (1). Certain serotypes may be associated with virulence but the link between serotype and virulence is often inconclusive. Several attempts have been made to improve the differentiation of field isolates both on a phenotypic and a genotypic level. Partial sequencing of the heat shock protein 60 gene ( $h s p 60$ gene) proved to be a reliable marker for epidemiological studies of $H$. parasuis and showed that the analysis of its sequence is superior to other fingerprinting techniques (2). The objective of this study was to identify lineages of Dutch and Belgian H. parasuis isolates. These isolates, including $10 \mathrm{H}$. parasuis reference strains, were subjected to serotyping and $h s p 60$ sequence analysis.

\section{Materials and Methods}

From October 2007 until September 2009, 134 H. parasuis isolates were collected from pigs which were submitted to the Dutch Animal Health Services for postmortem examinations. Typical growth on chocolate (+NAD) agar plates, satellite growth without hemolysis on blood agar plates using Staphylococcus aureus nurse strain and a negative CAMP test were used for the identification of $H$. parasuis. Ten reference strains and 11 Belgian field isolates were examined too.

The serotypes were determined at the IVD GmbH (Gesellschaft für Innovative Veterinärdiagnostik, Germany) using type-specific antisera. The $h s p 60$ universal PCR was performed as described by Goh et al. (3). PCR products were sequenced and sequence data was aligned and analyzed using the BioNumerics software package (Applied Maths NV, Sint-Martens-Latem, Belgium).

\section{Results}

Serotyping revealed in general the serotypes $1(n=14), 2(n=12), 4$ $(n=29)$ and $13(n=18)$, whereas serotype 5 was serotyped 8 times.

Hsp60 partial sequences (498bp) of 145 isolates were aligned, and parsimony trees (using 200 bootstrap simulations) were constructed (Figure 1). Variations were detected at 116 nucleotide positions and pair-wise similarities using Pearson correlation ranged from 86 to $100 \%$. In total, 56 different sequence types (STs) were detected. Using $99 \%$ sequence similarity, as threshold for group separation, 4 separate clusters (I, II, II and IV) could be distinguished. When comparing STs with serotyping data no strong correlation was observed, except for a possible correlation between cluster I and serotype 5 .
Figure 1: Parsimony tree using 200 bootstrap simulations, based on hsp60</em sequence analysis.

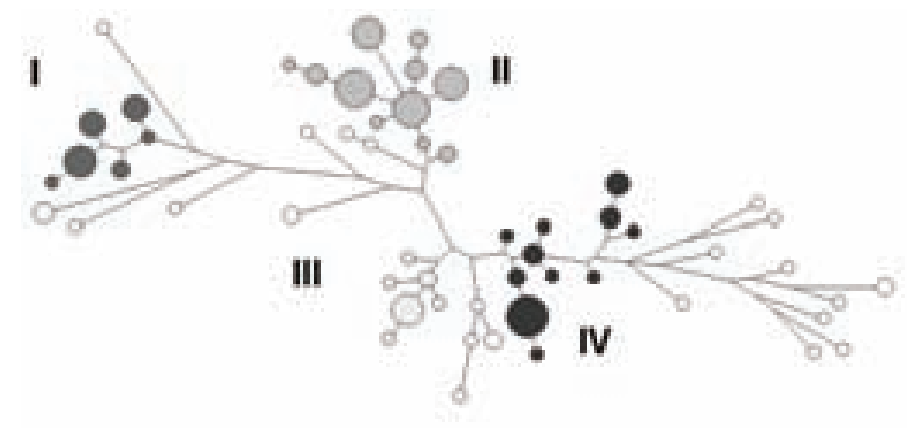

\section{Discussion}

Different $H$. parasuis serotypes and hsp60 lineages were found after analysis of the $145 \mathrm{H}$. parasuis isolates. The overall correlation between the two methods, serotyping and $h s p 60$ sequence analysis, was low, except for serotype 5 . A possible association between cluster I and $\mathrm{H}$. parasuis serotype 5 and/or higher virulence will be examined furthermore based on the recorded clinical symptoms and pathological lesions.

\section{References}

1. Kielstein, P., and Rapp-Gabrielson, V.J. (1992). J. Clin. Microbiol. 30 : 862-865

2. Olvera, A., et al (2006). Appl Environ Microbiol. 72(6):3984-3992.

3. Goh, S., et al (1996). J. Clin. Microbiol. $34: 818-823$ 


\title{
P.519
}

\section{Identification of a novel immunogenic and species-specific protein in Haemophilus parasuis}

\author{
Nubia R. Macedo; Simone Oliveira
}

University of Minnesota, Saint Paul, MN, USA

\section{Introduction}

Haemophilus parasuis continues to be one of the most important causes of nursery mortality in modern swine production. One of the main challenges in $H$. parasuis control is the lack of a reliable serological test to characterize the development of antibodies in sows and piglets. This information is critical to understand herd immunity and to identify the best timing for vaccination. In this study we have identified a highly immunogenic and speciesspecific protein in $\mathrm{H}$. parasuis. This protein is a strong candidate for the development of a $\mathrm{H}$. parasuis-specific ELISA test and of a universal subunit vaccine. ${ }^{1,2}$

\section{Material and Methods}

Twenty pigs involved in an $H$. parasuis outbreak were selected based on clinical signs (fever > $105 \mathrm{~F}$, respiratory distress, lameness, CNS signs) for necropsy and $H$. parasuis isolation $(n=10)$ or for antibiotic treatment to produce convalescent sera $(n=10)$. Whole cell proteins obtained from $H$. parasuis isolates recovered from diseased pigs were probed with convalescent serum using Western blot. An immunodominant protein observed in all $\mathrm{H}$. parasuis outbreak strains and 13 reference strains was identified and sequenced. Specificity of the highly immunogenic protein was tested by doing Western blot analysis using whole cell proteins obtained from 10 additional bacterial species commonly isolated from swine tissues, including Actinobacillus porcinus, $A$. indolicus, A. minor, A. pleuropneumoniae, A. suis, Streptococcus suis, Bordetella bronchiseptica, Pasteurella multocida, Escherichia coli, and Salmonella choleraesuis. Pre-colostrum serum obtained from a 1 day-old piglet was used as a negative control in all Western blots.

\section{Relevant results and discussion}

The convalescent sera obtained from pigs that survived the $H$. parasuis outbreak recognized a wide range (27 kDa - $77 \mathrm{kDa}$ ) of whole cell proteins in both outbreak and reference strains. One single band with an estimated molecular weight of 52 kDa was clearly immunodominant and it was observed in all $H$. parasuis strains tested. This protein was not identified in any of the unrelated bacterial species, indicating that it was specifically expressed by $H$. parasuis. Pre-colostrum swine serum also failed to detect this protein in all isolates tested. The $52 \mathrm{kDa}$ immunodominant protein was identified by sequencing as an oligopeptide permease $A B C$ transporter membrane protein in $H$. parasuis (OppA). This protein has now been cloned and expressed in E. coli (Figure 1). We have identified a novel immunogenic and species-specific protein in Haemophilus parasuis. This protein will now be utilized to develop a $H$. parasuis-specific ELISA test and will be evaluated as a potential universal subunit vaccine.
Figure 1 - Haemophilus parasuis OppA protein expressed in Escherichia coli (arrow).

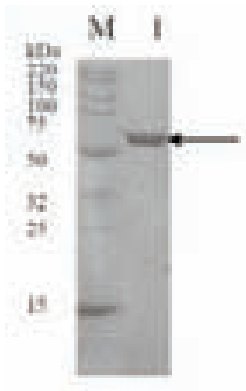

\section{References}

1. Higgins C.F. and Hardie M.M. J. bacteriol 155(3):1434-1438

2. Tanabe M, Atkins HS, Harland DN, et al. Infect Immun. 2006 Jun;74(6):3687-91.

\section{Acknowledgements}

This project was partially funded by the Minnesota Pork Board and Newport Laboratories. 


\title{
P.520
}

\section{Simultaneous infection by serotypes 4 and 12 of Haemophilus parasuis in a swine herd: a clinical case}

\author{
Andrea Luppi ${ }^{1}$ Giuseppe lodice $^{2}$ Luigi Franchi $^{3}$ Paolo Bonilauri ${ }^{1}$ Giuseppe Merialdi $^{4}$ Marianna Merenda ${ }^{5}$ Michele Dottori $^{1}$ \\ 1. Ilstituto Zooprofilattico Sperimentale della Lombardia ed Emilia Romagna, Sezione di Reggio Emilia, Reggio Emilia, Italy; \\ 2. Libero professionista, Reggio Emilia, Italy; 3. Medico Veterinario, Gruppo Progeo Mangimi, Reggio Emilia, Italy; \\ 4. Istituto Zooprofilattico Sperimentale della Lombardia ed Emilia Romagna, Sezione di Bologna, Bologna, Italy; \\ 5. Istituto Zooprofilattico Sperimentale della Lombardia ed Emilia Romagna, Sezione di Parma, Parma, Italy
}

The clinical expression of Haemophilus parasuis (HPS) in pigs is well described since many years, in the form of polyserositis and arthritis (Glässer's disease) (4). Of the 15 known serovars, strains belonging to serovars 1,5,10,12,13,14 have been shown to be highly virulent, serovars 2,4 and 15 moderately virulent, serovar 8 mild virulent while serovars 3,6,7,9 and 11 avirulent (3). The purpose of this paper is to report a clinical case where 2 different pathogenic serotypes of HPS were isolated.

\section{Materials and Methods}

This clinical case took place between April and May 2009. The farm was a farrow-to-feed unit with 500 sows. The piglets were vaccinated against PCV2 and Mycoplasma hyopneumoniae at 3 and 5 weeks respectively. The clinical symptoms of disease started at 7 weeks of age and the mortality rate increased at 12 weeks of age reaching over $10 \%$ in some batches. The symptoms observed were: loss of appetite, swollen joints, lameness, dyspnoea, cough, sometimes nervous signs and sudden death. Seven dead piglets were submitted for necropsy to the Istituto Zooprofilattico Sperimentale Lombardia and Emilia Romagna (IZSLER), laboratory of Reggio Emilia, following routinary methods. From dead piglets several samples of affected organs were collected. Bacteriological cultures were performed using Gassner agar, nutrient agar and blood agar added whith nicotinnamide adenine dinucleotide (NAD). H.parasuis PCR was performed from pathological samples (fibrinous lesions in the lung and pericardium) collected and stored at $-20^{\circ} \mathrm{C}$ (4). From six live piglets in the affected age groups, blood samples were collected and examined for antibodies against PRRSV (indirect ELISA IDEXX), against PCV2 (competitive ELISA IZSLER-Brescia) and for the presence of PCV2 and PRRSV using a rt-PCR and a real time PCR respectively $(2,5)$. The strains of $H$.parasuis isolated were serotyped using the agar gel immunodiffusion test and by indirect haemoagglutination (1). Phenotypic methods were used to identify other bacteria isolated.

\section{Results}

The gross lesions observed were: arthritis in the carpal and tarsal joints with fibrinous content, fibrino-purulent pneumonia, pericarditis and peritonitis with fibrin trends over the abdominal organs in four pigs. Two of these showed also pleuropneumonia. Catarrhal bronchopneumonia without other lesions was observed in two pigs, while no lesions were observed in one pig. Two serovars of H.parasuis were isolated from diseased pigs. Two strains isolated from pigs with systemic disease (polyserositis) were serotyped as serovar 4, while another strain isolated from a pig with bronchopneumonia was serotyped as serovar 12 . Two strains of Actinobacillus pleuropneumoniae biovar 2 serovar 7 and biovar 1 serovar 9 were isolated from the two lungs with pleuropnemonia respectively. PCR results confirmed bacteriological findings and also demonstrate the presence of H.parasuis in two cases of polyserositis in which this pathogen was not isolated by culture. All sera samples showed antibodies against PRRSV (S/P values ranging between 1 and 2,5) and also antibodies against PCV2. Positive PRRSV rt-PCR (European strain) was observed in a pool of three sera, while all sera resulted PCV2 real time PCR negative.

\section{Discussion}

The results agreed with the classification of Kielstein and RappGabrielson (1992), in which the serovar 12 has been shown to be highly virulent (generally isolated from pigs without polyserositis), while serovar 4 moderately virulent (generally isolated from pigs with polyserositis). However, the connection between serovar isolated and gross lesions observed can be influenced by several factors (host factors, vaccinations and other bacterial and viral diseases). In this work H.parasuis coexisted with other pathogens like A.pleuropneumoniae and PRRSV. The results of this work showed the importance of isolates serotyping, mainly if the vaccination is used for the disease protection.

\section{References}

1. Blackall P.J., Rapp-Gabrielson V.J., Hampson D.J. (1996). Aust. Vet. J. 73:93-95.

2. Bonilauri P. et al. (2003). 4th Int. Symp. on Em. and Re-emerging Pig Diseases- Roma June 29th July 2nd, 2003, pp.98-99.

3. Kielstein P., Rapp-Gabrielson V.J. (1992). J. clin. microbiol. 30, 826865.

4. Oliveira S. and Pijoan C. (2004). Veterinary microbiology 99, 1-12.

5. Olvera A., Sibila M., Calsamiglia M., Segalés J., Domingo M. J. Virol. Methods (2004); 117 (1):75-80.

5. Olvera A., Sibila M., Calsamiglia M., Segalés J., Domingo M. J. Virol. Methods (2004); 117 (1):75-80. 


\section{P.521}

\section{Characterization of field isolates of Haemophilus parasuis from Colombian swine farms}

Paula C. Manrique Ramirez ${ }^{1}$ Jose D. Mogollon ${ }^{2}$ Ricardo J. Piñeros Duque ${ }^{3}$ Ivonne I. Hernández ${ }^{3}$ María A. Rincon Monroy ${ }^{3}$

1. Pontificia Universidad Javeriana, Bogota, Colombia; 2. Universidad Nacional de Colombia, Bogota, Colombia;

3. Instituto Colombiano Agropecuario ICA, Bogota, Colombia

\section{Introduction}

Haemophilus parasuis ( $\mathrm{Hp}$ ) is an important commensal opportunistic respiratory pathogen of swine. These organisms can potentially invade the host and cause systemic infection characterized by fibrinous poliserositis, arthritis and meningitis. $\mathrm{Hp}$ usually in our country affects young pigs after 7 weeks of age in the nursery. Some serovars of Hp seem to be more virulent than other, but so far no true virulence factors have been identified.

A tentative diagnosis can often be made on the basis of history, clinical signs and gross lesion observed during necropsy. Isolation and culture of this microorganism is considered the gold standard. To control this disease the characterization of the strains associated with systemic disease is required. The goals of this study were (i) to isolate Hps from intensive swine affected herds (ii) to apply the ERIC - PCR technique to detect the genetic diversity among the isolated strains.

\section{Materials and Methods}

Samples were collected from eleven commercial farrow -to-finish pig farms located in three different raising regions. The farms had a previous history of Glässer's disease outbreak in nursery piglets.

Clinical samples (joints, pericardium, pleural and abdominal fluid swabs, lungs and spleen) were examined by isolation. Routine biochemical tests to identify $H$. parasuis were performed, although final identification was achieved by PCR [1,2]. Additionally, to characterize the Haemophilus parasuis isolates ERIC-PCR was done as described. $[3,4]$.

\section{Results and Discussion}

Haemophilus parasuis was recovered in pure culture from sick piglets (joint, pericardium, pleural cavity, abdominal fluid and from some lungs). The DNA patterns (fingerprints) of the Hps strains were distributed in four clusters, indicated heterogeneity between herds (Figure1). Additionally, the isolates obtained from the respiratory tract didn't differ from those collected from systemic sites. The Hp strains obtained were not serotyped because of the lack of this type of reagents.

The isolates F-R2 and F-S3 had the same ERIC-PCR profile, because they were the only two strains isolated from the same herd, even through they were collected in different dates. Despite of these preliminary results the information obtained with this procedure may be useful to understand the epidemiology in the Colombian swine farms and to suggest control measures using autogenous vaccines.
Figure 1. ERIC-PCR fingerprints analysis. C+: positive control; FS: FarmSystemic sites; FR: Farm-Respiratory sites.
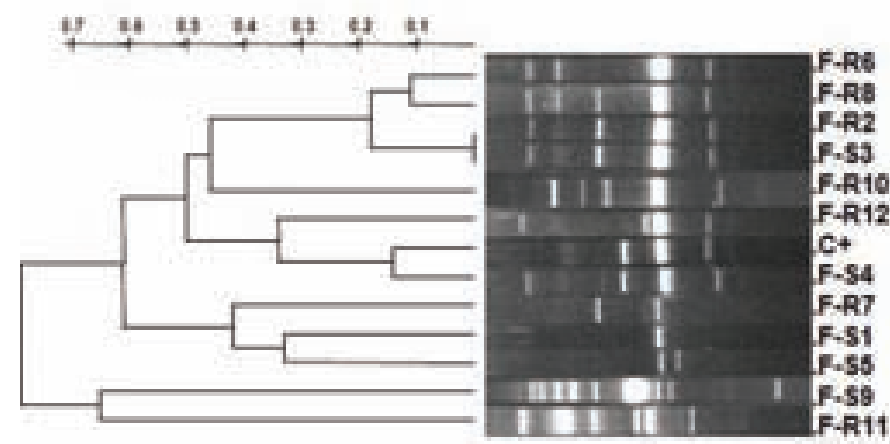

\section{References}

1. Oliveira, S. et al. (2001). J. Vet. Diagn. Invest, 13 (6): p. 495-501.

2. Angen, Ø. et al (2007). Vet. Microbiol. 119(2-4): p.266-276.

3. Oliveira, S. et al (2003). Am. J. Vet. Res. 64:p.435-442.

4. Olvera A, et al (2006). Appl. Environ. Microbiol. (72):p.3984-3992. 


\title{
P.522
}

\author{
The serological titer course of Haemophilus parasuis in a coinfection \\ with Mycoplasma hyorhinis, PRRSV and PCV2 \\ Andreas Palzer $^{1}$ Katharina Kolb ${ }^{2}$ Mathias Ritzmann ${ }^{3}$ Christiane Lang $^{3}$ Teija M. Stricker ${ }^{3}$ \\ Susanne Zoels ${ }^{2}$ Katrin Strutzberg-Minder ${ }^{4}$ Karl Heinritzi ${ }^{2}$ \\ 1. Tierarztpraxis Scheidegg, Scheidegg, Germany; 2. Clinic for Swine of the LMU Munich, Oberschleissheim, Germany; \\ 3. Clinic for Swine of the VUW, Vienna, Austria; 4. Innovative Veterinary Diagnostics Laboratory, Hannover, Germany
}

\section{Introduction}

Clinical and diagnostic evidence have suggested that porcine respiratory diseases are caused by an association of several viral and bacterial infections. The clinical symptoms like fever, a slower growth and a worse feed conversion lead to increasing economic losses. The aim of this study was to describe the serological and temporally course of a $\mathrm{H}$. parasuis infection on three farms. Furthermore the influence of other agents, like PRRSV, $\mathrm{PCV} 2$ and $\mathrm{M}$. hyorhinis onto the course of $\mathrm{H}$. parasuis infection was proved.

\section{Material and Methods}

The study was conducted on three breeding farms and their finishing units. Clinical examination (based on a score system for body condition, gastrointestinal tract, respiratory tract and lameness) and blood sampling were performed in two-week intervals between $1 \mathrm{st}$ and $11 \mathrm{th}$. and in four-week intervals between 14th and 22 nd week of life. Also the sows were sampled in the 1 st week after farrowing. The samples were analysed via ELISA for antibodies against $\mathrm{H}$. parasuis, M. hyorhinis, PCV2 and PRRSV.

\section{Results}

A significant correlation between the antibodies of the sows and their piglets was found for all agents in the 1st week of life. In the 1 st week of life, identical antibody values for $\mathrm{H}$. parasuis were measured in the sows and piglets. The PRRSV and PCV2 antibody values of the piglets were even higher at this time. The decrease of antibody values in the following week of life depended on the specific agent and the basic antibody value of the piglet in the 1 st week of life. If in the first week of life the antibody value was very high, the decrease was prolonged. In the three farms no uniform seroconversion time for the four examined agents could be determined. The highest antibody values could be detected in one pig during the 14 th to 22 nd week of life:

On farm $125 \%$ of the pigs got seropositive for all four agents and $75 \%$ for three agents (H. parasuis, PRRSV, PCV2). Farm 2 was PRRSV negative and there $52 \%$ of the pigs got seropositive for all three, $29 \%$ for two $(5 \% \mathrm{H}$. parasuis and PCV2, 24\% M. hyorhinis and PCV2) and 19\% for only one (PCV2) agent. On farm $355 \%$ of the pigs became seropositive for all four agents, 35\% for three (M. hyorhinis, PRRSV, PCV2) and 10\% for two (PRRSVand PCV2) of the tested agents.

On farm 2 the H. parasuis antibody values increased between the 7th and the 9th week of life and on farm 1 and 3 in week 11. The first seropositive pigs were detected on all farms until the 14th week of life. The $H$. parasuis seroprevalence on farm 1 was $90 \%$ in the 18 th and $100 \%$ in the 22 nd week of life. At the same time the seroprevalence for $\mathrm{H}$. parasuis on farm 2 was $50 \%$ and on farm $345 \%$.
Farm 1 had the highest score of lameness in the 14th week of life. The M. hyorhinis antibody titer increased from the 9 th until the 22 nd week of life.

On farm 1 the PCV2 antibody titer increased between 18th and 22 nd week of life, on farm 2 between 9th and 18th, and on farm 3 between 11th and 18th week of life. At the end of the finishing period antibodies against PCV2 could be detected in all study pigs.

On farm 1 the PRRSV antibody values increased between the 18 th and the 22 nd week of life until $100 \%$ of the study pigs were seropositive and on farm 3 between week 11 and 22 .

\section{Discussion}

The present study demonstrates the difficulty of the interpretation of serological profiles and indicates that an appropriate interpretation of the serological profile can only be done when the clinical signs are also taken into consideration. A specific serological profile on every single farm for each relevant agent should be provided.

The time point of infection with the four examined agents was different on the three farms, but the study showed that the incidence of multiple infections increased during the finishing period. On farm 2 and 3 a simultaneous seroconversion time in the 9 th and 11 th week of life was detected for $\mathrm{H}$. parasuis, $\mathrm{M}$. hyorhinis and PCV2. The association between $\mathrm{H}$. parasuis and PCV2 in the 18th week of life was significant, which is an evidence for a simultaneous infection time with those two agents on the respective farms. Also on farm 1 a simultaneous seroconversion for PRRSV and PCV2 in the 18th week of life could be observed, but the association was not significant.

Interaction between $\mathrm{H}$. parasuis, M. hyorhinis and PCV2 is possible and between PRRSV and PCV2 even more likely. High antibody values in sows are the basic requirement for a good passive immunity of the piglets in the first weeks of life and the maternal immunity against $\mathrm{H}$. parasuis, PRRSV and PCV2 could be improved by sow vaccination. 


\title{
P.523
}

\section{Efficacy of Porcilis ${ }^{\circledR}$ Glässer vaccination of sows to control H.parasuis serotype 15 infection in young piglets}

\author{
Albert Finestra ${ }^{2}$ Rut Menjon ${ }^{1}$ Jesus M. Bollo ${ }^{1}$ Jesus V. Lopez ${ }^{1}$ Marta Jimenez ${ }^{1}$ \\ 1. Intervet Schering Plough Animal Health, Alcobendas (Madrid), Spain; 2. Veterinary Practitioner, Les Borges Blanques, Lleida, Spain
}

\section{Introduction}

For many years, Glässer's disease has been considered of only minor importance, mainly seen at the end of the weaning or the beginning of the finishing periods. More recently, its incidence has increased, and the disease is frequently observed during the first weeks of life.

The efficacy of commercial vaccines has been controversial: some have proved very successful (1), while others have appeared to be lacking. Various causes have been proposed, such as inappropriate vaccination program, lack of cross-protection between serotypes and deficient immune response (2). The aim of this study was to show that sow vaccination with Porcilis ${ }^{\circledR}$ Glässer is an effective tool in the control of H.parasuis infection in young piglets, even against a heterologous challenge. Porcilis ${ }^{\circledast}$ Glässer is a vaccine based on an inactivated whole cell concentrate of Haemophilus parasuis serotype 5, strain 4800, mixed with dl- a-tocopherol acetate as adjuvant.

\section{Materials and Methods}

This study was performed in an 1100-sow herd, managed on a three site production system in the north-east of Spain. From January 2009, a high incidence of arthritis had been observed in young piglets. It was first seen in piglets of 10 days old and, although no mortality was recorded during the suckling period, clinical signs increased at the start of weaning, leading to $>7 \%$ mortality. The presence of $\mathrm{H}$. parasuis was confirmed from the results of bacteriological examinations of synovial fluid and lungs of affected but untreated piglets. An indirect hemagglutination test revealed the pathogen to be H.parasuis serotype 15.

The piglets were treated with $0.2 \mathrm{ml}$ long-acting ceftiofur at 5 days of age, and then vaccinated at 3 weeks of age with a polyvalent inactivated vaccine available on the Spanish market, which included a H.parasuis bacterin, and some management improvements were implemented.

Clinical signs were still being observed in May 2009, and weaning mortality remained above $6 \%$. Consequently, the treatment regime was abandoned completely in favour of vaccinating the sows with two doses of Porcilis ${ }^{\circledR}$ Glässer, between 7 and 6 weeks, and again between 3 and 2 weeks, before farrowing.

Clinical signs and mortality were recorded over a 5 month period for litters born from vaccinated sows, and were compared with the same parameters prior to the Porcilis ${ }^{\circledR}$ Glässer vaccination program. Statistical analysis was performed using the Pearson's chi-square test and Fisher's exact test.

\section{Results}

No negative effects were observed on the health status of the vaccinated sows or their progeny. The clinical signs of Glässer's disease were clearly reduced in both sucking pigs and weaners. In sucking pigs incidence of arthritis moved from 15\% to $1 \%$ in piglets born from vaccinated sows. There was a statistically significant reduction in mortality rates $(p<0.001)$ after weaning (Table 1).

Table 1. Mean monthly mortality during weaning period of piglets born to vaccinated and unvaccinated sows.

\begin{tabular}{l}
\begin{tabular}{|l|c|c|c|c|c|c|}
\hline Unvaccinated & Jan & Feb & Mar & Apr & May & Mean \\
\hline Mortality (\%) & 4.8 & 7.6 & 3.5 & 6 & 6.2 & $5.62^{\mathrm{a}}$ \\
\hline Vaccinated & Jun & July & Aug & Sept & Oct & Mean \\
\hline Mortality (\%) & 2.5 & 2.4 & $3.8^{*}$ & $4^{*}$ & 2.4 & $3.02^{\mathrm{a}}$ \\
\hline
\end{tabular} \\
* Litters affected by an E.coli infection \\
a, b: values with different superscripts represent statistically significant differences ( $<<0.01)$. \\
\hline
\end{tabular}

\section{Discussion}

This study showed that the vaccination of sows with Porcilis ${ }^{\circledast}$ Glässer is a safe and efficacious treatment which can prevent Glässer's disease in young piglets.

These data obtained from field conditions confirmed previous laboratory results demonstrating that sow vaccination with Porcilis ${ }^{\circledR}$ Glässer increased H.parasuis colostral antibody levels, and that the transmission of these MDA could protect piglets up to 6 weeks of age (3).

The efficacy of Porcilis ${ }^{\circledR}$ Glässer has been shown previously against homologous and heterologous serotypes 1, 5, 12, 13 and 14 (4). The study reported here proves that Porcilis ${ }^{\circledR}$ Glässer can also provide effective immunity against a heterologous challenge with H.parasuis serotype 15 .

\section{References}

1. Geurts, V et al (2006) Proceedings of the 19th IPVS Congress, Copenhagen, Denmark, $\cdot$ Vol 1

2. López, J.V. (2005). Anaporc.

3. Müller C et al (2004). Proceedings of the 18th IPVS Congress, Hamburg, Germany, Vol 1

4. Bak, $\mathrm{H}$ et al (2002) Veterinary Record, 151: 502-505. 


\title{
P.524
}

\section{Assessment of the antimicrobial in vitro sensitivity for Streptococcus suis in porcine farms from Venezuela}

\author{
Susan V. Del Castillo ${ }^{1}$ Andres Boulanger ${ }^{2}$ Carlos Antillano ${ }^{2}$ Janett Manzo ${ }^{3}$ Manuel Rodriguez ${ }^{2}$ \\ Abel Sequera ${ }^{3}$ Renny Blanco ${ }^{2}$ Elias Sogbe ${ }^{1}$ Vitelio Utrera ${ }^{1}$ Carmen Diaz ${ }^{1}$ \\ 1. Universidad Central de Venezuela, Maracay, Venezuela; 2. Diagnostico y Consultoria Veterinaria CA, Maracay, AR, Venezuela; \\ 3. Empresa de Diagnostico Veterinario CA, Maracay, Venezuela
}

\section{Introduction and Objectives}

The goal of our study was to evaluate the in vitro sensitivity pattern for Streptococcus suis strains isolated from pigs showing neurological clinical signs in porcine farms from Venezuela

\section{Materials and Methods}

A total of 9 strains of Streptococcus suis were isolated from brain tissue samples from 41 suspected cases coming from farms with histories of neurological signs in 5 to 12 week-old piglets between January 2005 and December 2008. For the purpose of this study, the piglet heads were transported to our laboratory were they were dissected under sterile conditions and samples were collected in selective media (blood agar) and then incubated in a microaerofilic environment at 37 Celsius for 48 hours. Colonies were identified considering their morphology and biochemical properties (Quinn, P.J. et al en 1994). An antibiogram from the isolated strains was done through the method of Kirby-Bauer (NCCLS, 1990, MA-A4). The antibiotics assessed in this study were enrofloxacin, norfloxacin, gentamicin, neomicin, sulfamethoxazole-trimethoprim, ceftiofur, amoxicillin, florfenicol, tetracyclin, lincomicin and penicillin. The interpretation of the antibiogram was performed according to the NCCLS (National Committee for clinical Laboratory Standards) standards (2).

\section{Results}

The Streptococcus suis strains isolated in this study had the highest percentage of resistance to lincomicin, gentamicin, tetracyclin, neomicin, florfenicol and sulfa-trimethoprim and to a lesser degree to enrofloxacin and amoxicilin. One hundred percent (100\%) of the strains assessed were sensitive to Penicillin and Ceftiofur. Likewise, it was observed that $100 \%$ of the evaluated strains had moderate sensitivity to norfloxacin. Finally, $100 \%$ of the strains in this study were resistant to lincomicin. The results are summarized in table 1.

Table 1. Percentage of sensitivity to antibiotics of Streptococcus suis strains isolated from January 2005 to December 2008.

\begin{tabular}{|l|c|c|c|}
\hline Antibiotic & Sensitive & Resistant & Intermedium \\
\hline Enrofloxacin & 33,3 & 11,1 & 55,6 \\
\hline Norfloxacin & 0 & 0 & 100 \\
\hline Gentamicin & 11,1 & 88,9 & 0 \\
\hline Neomicin & 11,1 & 88,9 & 0 \\
\hline Sulfa-Trimethoprim & 33,3 & 56,6 & 0 \\
\hline Ceftiofur & 100 & 0 & 0 \\
\hline Amoxicillin & 55,6 & 44,4 & 0 \\
\hline Florfenicol & 11,1 & 77,8 & 11,1 \\
\hline Tetracycline & 11,1 & 88,9 & 0 \\
\hline Lincomicin & 0 & 100 & 0 \\
\hline Penicillin & 100 & 0 & 0 \\
\hline
\end{tabular}

\section{Discussion and Conclusions}

In our study, a high percentage of Streptococcus suis strains were resistant to Sulfa-Trimethoprim. Our results differ from those published by Tarradas M.C. in 1994 (3) who suggested the use of this drug as an alternative for Streptococcus suis control. Likewise, our results show a high percentage (44.4\%) of resistance to Amoxicicillin which also differs from other studies (Ristow, et al., 2008) (4) which have shown less than $1 \%$ resistance to this drug. Finally, we observed a high proportion of strains resistant to lincomicin, tetracyclin, gentamycin, sulfa-trimethoprim and sensitive to Penicillin as was shown in previous studies in Venezuela (Pineda, et al; 1996) (5). Based on our in vitro data, penicillin and ceftiofur appear to be the antibiotics of choice against Streptococcus suis infections in these farms; however, most previous studies show the highest susceptibility of this bacteria to amoxicillin when compared to penicillin. During the last years there has been wide spread use of amoxicillin in pre-initiation and initiation food in Venezuela which could explain an increase in resistant strains to this antibiotic. During this study, a total of 41 cases were assessed during a 4 year period and only in 9 of these, strains of Streptococcus suis were isolated. The low number of isolated strains is probably the consequence of the wide spread use of antibiotics in the food in farms in our country, which is a limitation for our study.

\section{References}

1. Gottschalk, Marcelo. Porcine Streptococcus suis strains as potential sources of infection in humans: an underdiagnosed problem in North America?. Journal of Swine Health and Production. 2004; 12:4:197-199.

2. Performance Standard for Antimicrobial Disk and Dilution Susceptibility Tests for Bacteria Isolated from Animals. 1999. NCCLS. M31-A, V. 19. N. 1.

3. Tarradas, M., et al. Susceptibility of Streptococcus suis to various antimicrobial agents. Zentralblatt für Veterinarmedizin, Reihe B 41, 685-8. 1994.

4. Ristow, L., et al. In vitro sensitivity evaluation of Streptococcus suis strains. 20th International Pig Veterinary Society Congress. Durban, South Africa. 2008.

5. Pineda, Y., et al. Aislamiento de cepas de Streptococcus suis y su susceptibilidad in vitro a los agentes antimicrobianos. Veterinaria Tropical 21 (2): 155-165. 1996. 


\title{
P.525
}

\section{Maternal immunity to Streptococcus dysgalactiae subsp. equisimilis and incidence of lameness in piglets}

\author{
Mate Zoric; Maria Persson; Per Wallgren \\ National Veterinary Institute, Uppsala, Sweden
}

\section{Introduction}

Lameness is one of the most important animal welfare issues in suckling piglets and about every $10^{\text {th }}$ piglet could be expected to be treated with antimicrobials because of lameness before being weaned (1). Despite this, lameness is only diagnosed in about every second litter, and the treatment incidence varies from one to all piglets in affected litters. Consequently, an effect of the sow could be expected. Streptococcus dysgalactiae subsp. equisimilis (SE) is commonly associated to arthritis in piglets and the aim of this study was to scrutinise the maternal protection to that microbe.

\section{Materials \& Methods}

Three indirect ELISA-systems detecting antibodies to SE were designed by using sonicated field strains of SE isolated from piglets diagnosed with arthritis in three herds. The three ELISA systems were used to define the levels of serum antibodies to SE in three day old piglets from these herds in a cross-over model. Each serum was diluted 1/100 in PBS-T and analysed with respect to presence of antibodies to SE by all three ELISA systems.In addition, colostrum from sows that had been collected from sows in the three herds at the day of farrowing were analysed for presence of antibodies to SE in whey diluted 1/50 in PBS-T employing the same ELISA systems.Finally, the amounts of antibodies to SE in colostral whey of the 93 sows in herd $C$ were related to the incidence of lameness in the offspring. Also parity number and litter size were compared to the incidence of lameness in the offspring.

Figure 1. Mean absorbance values of serum antibodies and whey antibodies to SE in 3 day old pigs and from sows at the day of farrowing from 3 different herds detected by 3 different antigens preparations.

\begin{tabular}{|c|c|c|c|}
\hline & Ag, herd $A$ & Ag, herd B & Ag, herd C \\
\hline Senuscialdir & $n=32$ & $\mathrm{n}=111$ & $\mathrm{n}=284$ \\
\hline Herd A & $0.58 \pm 0.19$ & $0.59 \pm 0.25$ & $0.76 \pm 0.29$ \\
\hline Hend B & $0.54 \pm 0.18$ & $0.40 \pm 0.17$ & $0.44 \pm 0.16$ \\
\hline Hend C & $0.72 \pm 0,40$ & $0.59 \pm 0.39$ & $0.61 \pm 0.40$ \\
\hline Caleitnalutag & $n=6$ & $n=37$ & $\mathbf{n}=95$ \\
\hline Herd A & $1.76 \pm 0.23$ & $0.95 \pm 0.26$ & $1.08 \pm 0.28$ \\
\hline Herd B & $1.30 \pm 0.38$ & $0.99 \pm 0.35$ & $1.18 \pm 0.49$ \\
\hline Hend C & $1.46 \pm 0.80$ & $1.11 \pm 0.73$ & $1.21 \pm 0.74$ \\
\hline
\end{tabular}

\section{Results}

As seen in Figure 1, the amounts of serum antibodies varied somewhat due to herd and to antigen used in the tests. However, the optical density values were fairly constant in samples regardless of antigen used. In contrast, the levels of antibodies in colostral whey clearly differed between herds, but was again fairly nondependent of antigen preparation used.The standard deviation of the OD-values in colostral whey indicated a large variation between sows, and the levels of antibodies to SE, as well as litter size and party number were compared with the incidence of lameness in the offspring (Fig. 2).

Figure 2. Lameness in the offspring to 93 sows in herd $C$, related to the amount of antibodies to SE in colostral whey, and to litter size and parity number of the sows.

\begin{tabular}{|c|c|c|c|c|}
\hline & \multirow[b]{2}{*}{$\mathrm{A}$} & \multicolumn{3}{|c|}{ Fof laters with lame pukts } \\
\hline & & Niver & ar kast 1 lame & $>1$ lume \\
\hline \multicolumn{5}{|l|}{ DDintex } \\
\hline$<09$ & 37 & 67.6 & 324 & 13.5 \\
\hline $0.9-1.9$ & 43 & 65.1 & 34.9 & 9.3 \\
\hline$>20$ & 13 & 923 & 7.7 & 0 \\
\hline \multicolumn{5}{|c|}{ Bimgreveler } \\
\hline $1^{\prime \prime}$ and $2^{\prime \prime}$ & 26 & Mos & 192 & 0 \\
\hline $3^{4} 65^{4}$ & 40 & 65.0 & 35.0 & 15.0 \\
\hline 6 or mare & 27 & 66.7 & 333 & 11.1 \\
\hline \multicolumn{5}{|l|}{ Litirestor } \\
\hline 11 oe leses & 48 & 75.0 & 250 & 42 \\
\hline 12 ot mare & 45 & 644 & 356 & 15.5 \\
\hline
\end{tabular}

\section{Discussion \& Conclusions}

The results obtained indicate that an indirect ELISA-system detecting antibodies to SE based on a field isolate from one herd could be used also in other herds. However, mean OD-values in piglets appear to be fairly equal between herds, and if the target is to identify piglets at risk each individual sample must be assessed individually.We therefore paid attention to the levels of antibodies in colostral whey, with the aim of identifying litters at risk. Indeed, lameness were more rarely diagnosed in sows with high levels of colostral antibodies to $S E$ ( $p=0.06$ to 0.08 ). However, also litter size and parity numbers influenced the incidence of lameness (1), and it is striking that the only litter with 6 piglets treated for lameness included 16 piglets and emanated from a sow with low levels of antibodies to SE (OD-value 0.70).The results obtained indicate that high levels of colostral antibodies to SE can have a potential to decrease the incidence of lameness in piglets, and this ought to be further scrutinised.

\section{Reference}

1. Zoric et al., 2003. Vet Rec. 153:323-328. 


\title{
P.526
}

\section{A highly virulent Streptococcus suis serotype 33 strain as a cause of acute sepsis in pigs}

\author{
Marcelo Gottschalk ${ }^{1}$ Charles Surprenant ${ }^{2}$ Marie-Pier Lecours ${ }^{3}$ Marycruz Dominguez Punaro 3 Sonia Lacouture ${ }^{3}$ \\ 1. Faculté de médecine vétérinaire, GREMIP and CRIP, Université de Montréal, St-Hyacinthe, QC, Canada; \\ 2. F. Ménard Inc., Ange Gardien, QC, Canada; 3. Université de Montréal, St-Hyacinthe, QC, Canada
}

\section{Introduction}

Streptococcus suis is one of the most important swine pathogens worldwide. It can cause septicemia, meningitis and other infections ${ }^{1}$. There are 35 serotypes described, with serotype 2 considered as the most virulent and the most frequent type isolated from diseased pigs in Eurasia. However, its prevalence in North America is lower, and some other serotypes are also implicated in clinical disease ${ }^{2,3}$. S. suis, especially serotype 2 , has been pointed out as an emerging zoonotic agent. Recently, many streptococcal toxic shock like-syndrome (STSS) human cases with high mortality have been described ${ }^{1}$. This syndrome is characterized by a short incubation time, an exaggerated inflammatory response with massive organ failure and mortality. Serotype 33 is not a common serotype, and it represents less than $1 \%$ of S. suis strains recovered from diseased animals in Canada ${ }^{2}$. In the present study, we report an atypical strain of S. suis serotype 33 responsible for a STSS cases in pigs.

\section{Materials and Methods}

Herd: The affected herd is a multisite production system, with a sow herd of 1000 animals. Weaning is carried out at 18 days of age. Nurseries are operated All in All out per building. The herd is actively infected with PRRSV.

Health problems in nursery: Acute infections of PRRSV in the sow herd lead to abortion problems and weak piglets at weaning. During the first 2 weeks post weaning (PW), piglets presented different respiratory and intestinal clinical signs, accompanied by typical cases of septicemia and meningitis caused by S. suis. Response to antibiotic treatment (penicillin, amoxicillin or ceftiofur) was good. During weeks 3 to 6 PW, the clinical picture changed, with a relatively high proportion of animals presenting typical signs of a STSS, such as sudden death with septic shock, multi-organ failure, ventral edema with weak response to antibiotic treatments.

Laboratory methods: S. suis isolation/serotyping were carried out as described ${ }^{2}$. In some experiments (comparison purposes), the reference strain of $S$. suis serotype 33 and a well characterized virulent strain of serotype 2 were used. Cytokine measurements in sera were performed by ELISA. Genotyping was performed by ERIC-PCR. Virulence was evaluated using a well standardized mouse model, with a dose of $5 \times 10^{7}$ C.F.U./mouse ${ }^{4}$.

\section{Results}

Bacteriology: Two different serotypes of $S$. suis were present: a) a serotype 4, usually isolated from meningitis cases (1-2 weeks PW); b) a serotype 33, responsible for most STSS cases (weeks 3-6 PW). Both strains were equally susceptible to the antibiotic used.

Cytokine levels in swine sera: Sera from STSS caused by serotype 33 affected animals presented in general higher levels of IL-6, IL-12 and IL-8 than healthy animals.

Genotyping: A single clone of serotype 33 was responsible for the STSS cases in the farm. The strain was genetically different from the reference strain.

Figure1. Virulence in a mouse model of infection

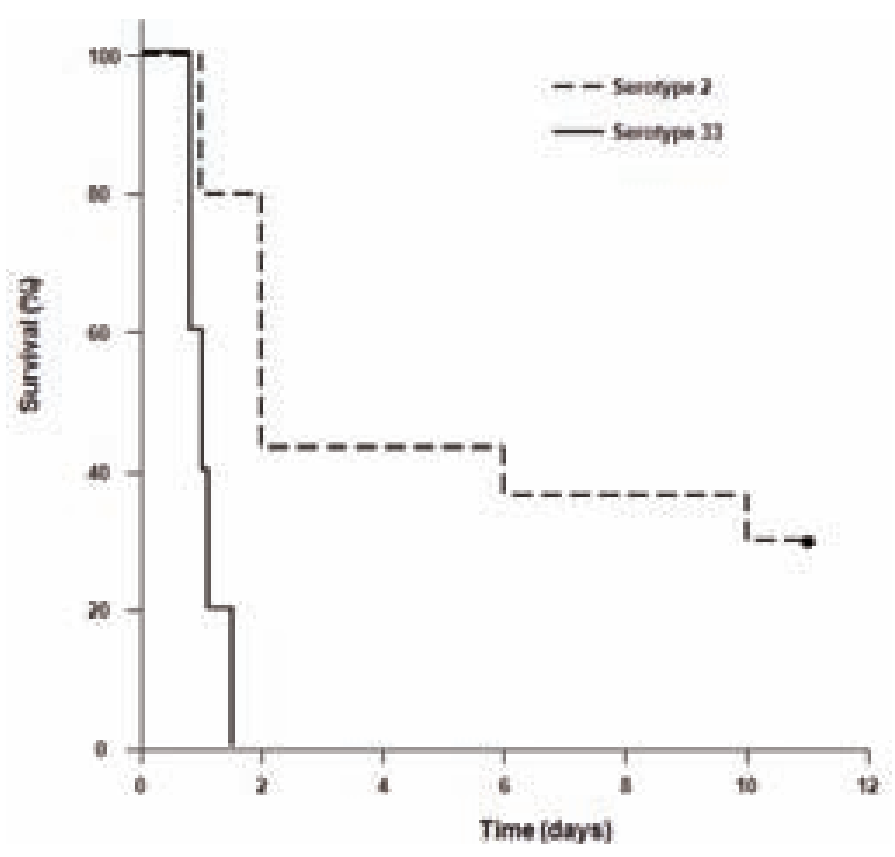

\section{Discussion}

- S. suis is able to induce a STSS in pigs, similar to that observed in humans in Asia, with high concentrations of pro-inflammatory cytokines in serum of affected animals.

- An atypical highly virulent strain of serotype 33 (genotypically different from the reference strain) was responsible for such cases, which hardly respond to antibiotic treatment.

- Serotypes different from serotype 2 may cause important disease in North American herds, especially when an active PRRSV infection is present.

\section{References}

1. Gottschalk et al. Anim Health Res Rev. 2007;8:29-45.

2. Messier et al. Can J Vet Res. 2008;49:461-2.

3. Fittipaldi et al. Vet Microbiol. 2009; 139:310-7.

4. Dominguez-Punaro et al. J Immunol. 2007;179:1842-54. 


\title{
P.527
}

\section{Capsular polysaccharide modulates Streptococcus suis interactions with swine dendritic cells}

\author{
Marcelo Gottschalk ${ }^{1}$ Marie-Pier Lecours ${ }^{2}$ Mariela Segura ${ }^{2}$ Mathieu Houde ${ }^{2}$ Charles Surprenant ${ }^{3}$ Tufaria Mussa $^{4}$ Maria Montoya $^{4}$
}

1. Faculté de médecine vétérinaire, GREMIP and CRIP, Université de Montréal, St-Hyacinthe, QC, Canada; 2. Université de Montréal, St-Hyacinthe, QC, Canada; 3. F. Ménard Inc., Ange Gardien, QC, Canada; 4. CReSA, Universitat Autonoma de Barcelona, Bellaterra, Barcelona, Spain

\section{Introduction}

Streptococcus suis is a major swine pathogen and a zoonotic agent causing mainly meningitis and septicemia. Serotype 2 is considered the most virulent and the most frequently type isolated from both pigs and humans ${ }^{1}$. Mechanisms involved in the host innate and adaptive immune responses toward $S$. suis as well as the mechanisms used by $S$. suis to subvert these responses are unknown. The capsular polysaccharide (CPS) is the only proven critical virulence factor ${ }^{1}$. Dendritic cells are powerful antigen presenting cells that initiate the immune response against pathogens, and interactions between DCs and pathogens can strongly influence the outcome of a disease. DCs capture and process the antigens, and then migrate to the adjacent lymphoid organs where they activate T cells. In the present study, we used porcine bone marrow-derived DCs (bmDCs) to investigate the capacity of S. suis to interact with DCs and to induce their maturation and activation.

\section{Materials and Mathods}

Bacterial strains: S. suis serotype 2 virulent strain 31533, originally isolated from a case of porcine meningitis, and its isogenic CPS-negative mutant B218 were used .

Generation of bmDCs: Bone marrow was removed from femurs of 7 week-old SPF piglets. Cells were obtained and characterized by morphology and appropriate receptors by FACS ${ }^{2}$.

Phagocytosis assay and intracellular survival: Bacteria were either not opsonized or pre-opsonized with complete normal serum. Phagocytosis was left to proceed for different incubation times before antibiotics were added into the wells for $1 \mathrm{~h}$ to kill extracellular bacteria. Cells were then washed and lysed to determine viable intracellular bacteria. The intracellular survival of $S$. suis was performed in a similar manner, but antibiotics were added for a period times up to $6 \mathrm{~h}$. Cells were processed as described above.

Microscopy analysis: The interactions between S. suis and bmDCs were analyzed by scanning electron microscopy (SEM). Internalization of S. suis by bmDCs was confirmed by confocal microscopy.

Cytokine measurement and expression of co-stimulatory molecules: Levels of IL-6, IL-8, IL-12 and TNF- $a$ in cell culture supernatants following $S$. suis stimulation were measured by ELISA. The expression of MHC-II and CD80/86 were analyzed by FACS.

\section{Results}

In vitro interactions: S. suis interacts with bmDCs. This interaction is modulated by the CPS, as shown by SEM.

Phagocytosis: The CPS interferes with the uptake of S. suis by bmDCs (Fig.1). Once internalized, both strains are equally destroyed.

Fig 1: Interactions between S. suis strain 31533 and its non-encapsulated mutant with bmDCs as observed by confocal microscopy. White arrows show bacteria.
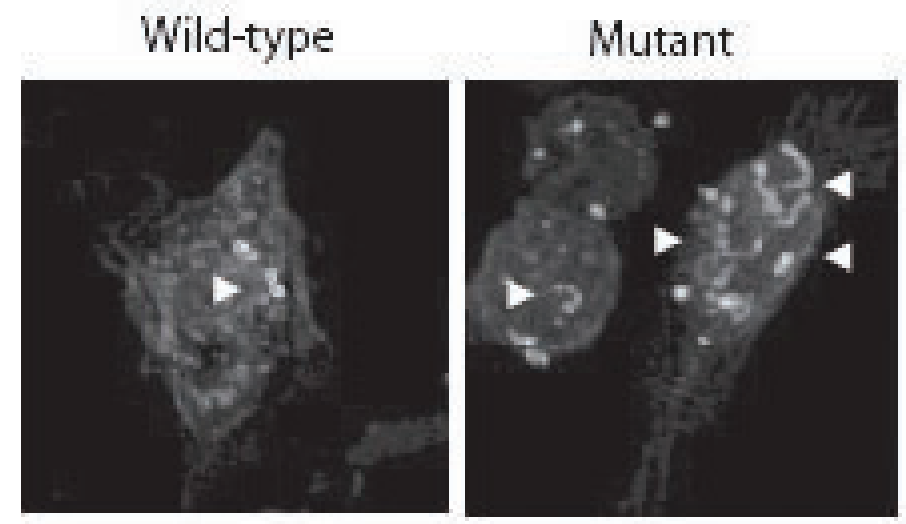

Cytokines and co-stimulatory molecules: S. suis triggers the release of IL-6, IL-8, IL-12 and TNF- $a$ as well as the expression of MHC-II and CD80/86 by bmDCs. However, the CPS-negative mutant induced higher levels of cytokine production and costimulatory molecule expression by bmDCs than the wild-type strain.

\section{Discussion}

- S. suis interacts with bmDCs, which results in an activation of these cells.

- The CPS highly interferes with S. suis internalization by and activation of bmDCs, as previously shown with other professional phagocytes ${ }^{1}$.

- This reduced level of antigen uptake/activation due to CPS might lead to a reduced host immune response.

\section{References}

1. Gottschalk et al. Anim Health Res Rev. 2007;8:29-43.

2. Kekarainen et al. Vet. Immunol. Immunopathol. 2009; 124: 41-9. 


\title{
P.528
}

\section{Distribution of Streptococcus suis capsular types isolated from diseased pigs in 2008 and 2009 in Canada}

\author{
Marcelo Gottschalk ${ }^{1}$ Sonia Lacouture ${ }^{1}$ Laetitia Bonifait $^{2}$ Daniel Grenier $^{2}$ \\ 1. Faculté de médecine vétérinaire, GREMIP and CRIP, Université de Montréal, St-Hyacinthe, QC, Canada; \\ 2. Faculté de médecine dentaire, Université Laval, Québec, QC, Canada
}

\section{Introduction}

Streptococcus suis is an important pathogen of swine causing meningitis, sudden death, septicemia, endocarditis, and pneumonia ${ }^{1}$. The distribution of different serotypes varies depending on the geographical origin of the strains ${ }^{1}$. As a reference laboratory, we routinely perform serotyping of S. suis isolates recovered in Québec (and sometimes in other provinces of Canada) from tissue samples of diseased pigs. Exceptionally, samples from other animal species are also received. Serotype determination remains a valuable tool used by veterinary practitioners and diagnosticians, to understand the epidemiology of a particular outbreak or to increase the possibility of success of a vaccination program within a herd. The present communication presents the distribution of the serotypes isolated in 2008 and 2009 in Canada.

\section{Materials and Methods}

Isolates: Animals from which these isolates have been obtained presented a variety of clinical signs, as for example, nervous signs, sudden death, arthritis, and/or respiratory problems. All but two S. suis strains were recovered from pigs. Although it is not possible to determine whether $S$. suis was the sole causative agent of the pathological condition, it was isolated in pure culture or in a significant number to be considered involved in the pathology observed. One strain was isolated from milk from a caw presenting mastitis and one strain was isolated from a human case of meningitis.

Laboratory analysis: Serotyping was carried out by using the coagglutination test with reference antisera. All reagents for serotyping are prepared in our laboratory according to a described procedure ${ }^{2}$. Serotype 2 strains were tested for the proposed virulence markers muramidase-released protein (MRP), extracellular factor (EF) and suilysin (Sly) by PCR and/or red blood cell lysis and/or immunoblotting ${ }^{2}$. Non-typable (NT) or autoagglutinating (AA) isolates were confirmed to belong to the Streptococcus suis species using a well described specific PCR ${ }^{3}$. Representative NT and $A A$ isolates were tested for the presence of a capsule using electron microscopy and polycationic ferritin labelling.

\section{Results}

Distribution of capsular types of S. suis in 2008 and 2009.

\begin{tabular}{|c|c|c|c|c|c|}
\hline \multicolumn{3}{|c|}{ Year 2008} & \multicolumn{3}{|c|}{ Year 2009} \\
\hline $\begin{array}{c}\text { Capsular } \\
\text { type }\end{array}$ & $\begin{array}{c}\text { Number of } \\
\text { isolates }\end{array}$ & $\begin{array}{c}\% \text { of total } \\
\text { isolates }\end{array}$ & $\begin{array}{c}\text { Capsular } \\
\text { type }\end{array}$ & $\begin{array}{c}\text { Number of } \\
\text { isolates }\end{array}$ & $\begin{array}{c}\% \text { of total } \\
\text { isolates }\end{array}$ \\
\hline 3 & 43 & 13.8 & 3 & 33 & 12.0 \\
\hline 2 & 40 & 12.9 & 2 & 29 & 10.6 \\
\hline $1 / 2$ & 32 & 10.3 & 8 & 20 & 7.3 \\
\hline 8 & 27 & 8.7 & 22 & 18 & 6.6 \\
\hline 4 & 15 & 4.8 & $1 / 2$ & 17 & 6.2 \\
\hline 7 & 14 & 4.5 & 33 & 11 & 4.0 \\
\hline 22 & 13 & 4.2 & 4 & 10 & 3.6 \\
\hline 5 & 10 & 3.2 & 5 & 10 & 3.6 \\
\hline 9 & 6 & 1.9 & 7 & 9 & 3.3 \\
\hline 21 & 6 & 1.9 & 16 & 6 & 2.2 \\
\hline NT/AA & 56 & 18.0 & $\mathrm{NT} / \mathrm{AA}^{\mathrm{a}}$ & 61 & 22.3 \\
\hline Others & 49 & 15.8 & Others & 50 & 18.2 \\
\hline
\end{tabular}

- Most NT/AA isolates (including a bovine isolate) were confirmed to be $S$. suis by a species-specific PCR.

- Three representative NT/AA isolates were shown to be nonencapsulated by electron microscopy.

- The human strain was shown to be a serotype 14.

- Most serotype 2 strains are MRP+/-, EF- and Sly-.

\section{Discussion}

- The distribution of the most common serotypes in 2008/2009 is comparable with that from previous years4.

- The prevalence of serotype 2 is much lower than that from European countries1.

- As most North American strains, serotype 2 isolates in 2008/2009 do not carry European virulence markers MRP, EF and Sly1.

- The first serotype 14 isolate recovered from human in North America was identified.

- Most NT/AA isolates recovered from diseased pigs were shown to belong to the $\mathrm{S}$. suis species.

- Many of these NT/AA isolates are non-encapsulated, indicating that they are probably not "new serotypes": it is possible that they belong to known serotypes before they lost the capacity to express capsule.

\section{References}

1. Gottschalk et al. Anim Health Res Rev. 2007;8:29-45.

2. Fittipaldi et al. Vet Microbiol. 2009;139:310-7.

3. Okwumabua et al. FEMS Microbiol Lett. 2003; 218:79-84.

4. Messier et al. Can J Vet Res. 2008;49:461-2. 


\title{
P.529
}

\section{Preliminary report on the characterization of pathological lesions and serotype of Streptococcus suis infection in Serbia}

\author{
Bozidar Savic; Oliver Radanovic; Milenko Zutic; Ivan Pavlovic; Vojin Ivetic \\ Scientific Veterinary Institute of Serbia, Belgrade, Serbia
}

\section{Introduction}

Streptococcal disease is a worldwide problem in the swine industry. Among Streptococci, serogroups which are considered pathogenic in pigs belonged to Lancefield group D, E, C and occasionally other groups. In most parts of the world, Streptococcus suis (S. suis) is the predominant agent of Streptococcal infection, which is associated with a variety of diseases including meningitis, septicaemia, arthritis, endocarditis and pneumonia (1). Thirty-three serotypes (types 1-31, 33, and 1/2) have been described based on capsular polysaccharides,polysaccharides, with serotype 2 being most commonly associated with diseases in pigs and humans, and also the most frequently reported serotype worldwide $(2,3)$. The objectives of this study were to determine the anatomic distribution and pathologic conditions associated with $\mathrm{S}$. suis infection and prevalence of $\mathrm{S}$. suis strains isolated in pigs from Serbia.

\section{Materials and Methods}

The pigs were from 15 conventional farrow-to-finish pig farms which were submitted to the Pathology Department of the Veterinary Institute during 2008 - 2009. Over 600 pigs of different ages were necropsied including: 337 suckling piglets, 179 nursing piglets, 107 grower/finisher pigs and 4 aborted foetuses. The samples for bacteriology were obtain from corresponding lesions and different tissues including: joint, brain, heart, lung abscesses and blood as well as 39 samples of milk, 27 vaginal swabs from clinical cases of mastitis and endometritis and 38 tonsils swabs from slaughtered pigs. The samples were cultured on Columbia blood agar base and base for blood agar (M 144- Hi Media) containing 5\% defibrinated ovine blood. The plates were incubated at $370 \mathrm{C}$ for 48 hours. Bacterial isolates were identified by biochemical tests and commercial kits (Api Strep.) and for Lancefield classifications, Slidex Strepto Kit (Biomerieux ref. 58 810)). Serotyping of S. suis was carried out by a coagglutination test, using commercial specific sera against serotype 1-10 (Statens Seruminstitut, Copenhagen, Denmark).

\section{Results}

Pathomorphological finding in suckling, nursring and grower/ finisher pigs and clinical conditions in sows associated with $\mathrm{S}$. suis infections as well as total number of examined and number of positive isolates of $\mathrm{S}$. suis according to site of isolation and clinical material were presented in table 1. Distribution of serotypes of S. suis isolated from corresponding lesions and clinical material obtained from sows were presented in table 2 .

\section{Discussion}

Overall, meningoencephalitis, septicemia and arthritis, otitis interna with encephalitis endocarditis and periarticular abscesses and pneumonia, appeared to be most frequent pathomorphological finding in suckling, nursing and grower/finisher pigs respectively and mastitis and endometritis are the most fre-
Table 1 Pathomorphological findings, clinical conditions, a total number of examined and number of positive isolates of $S$ suis.

\begin{tabular}{|l|l|c|c|c|}
\hline Category & Pathmorphological findings & No of exam. & No of pos. & \% of pos. \\
\hline \multirow{4}{*}{$\begin{array}{l}\text { Suckling } \\
\text { piglets }\end{array}$} & Mono./poly arthritis & 84 & 23 & 27.3 \\
\cline { 2 - 5 } & Str. septicemia & 30 & 11 & 37 \\
\cline { 2 - 5 } & Meningoencephalitis & 43 & 27 & 63 \\
\cline { 2 - 5 } & Endocarditis & 65 & 14 & 21.5 \\
\hline \multirow{4}{*}{$\begin{array}{l}\text { Nursering } \\
\text { piglets }\end{array}$} & Meningoencephalitis & 31 & 5 & 16.1 \\
\cline { 2 - 5 } & Pneumonia & 57 & 7 & 12.2 \\
\cline { 2 - 5 } & Otitis interna & 10 & 4 & 40 \\
\cline { 2 - 5 } & Periarticular abscesses & 37 & 17 & 46 \\
\hline \multirow{3}{*}{$\begin{array}{l}\text { Grower/ } \\
\text { Finisher }\end{array}$} & Pneumonia & 39 & 4 & 10.5 \\
\cline { 2 - 5 } & Tonsils/swabs & 38 & 11 & 28.9 \\
\cline { 2 - 5 } & Clinical conditions & & & \\
\hline \multirow{4}{*}{ Sows } & Endometritis/vaginal swabs & 27 & 3 & 11.1 \\
\cline { 2 - 5 } & Mastitis/milk & 67 & 9 & 13.4 \\
\cline { 2 - 5 } & Abortion/tissue of fetuses & 4 & 1 & 25 \\
\hline \multirow{2}{*}{ Total } & & & 532 & 136 \\
\hline \multirow{2}{*}{} & & & & \\
\hline
\end{tabular}

Table 2 Distribution of S. suis serotypes

\begin{tabular}{|c|c|c|c|c|c|c|c|c|}
\hline$\%$ & type 2 & type 1/2 & type 1 & type 4 & type 7 & type 9 & type 5 & type 3 \\
\hline $\begin{array}{c}\text { Streptococcus } \\
\text { suis }\end{array}$ & 47 & 17 & 13 & 7 & 6 & 5 & 4 & 3 \\
\hline
\end{tabular}

quent clinical conditions in sows associated with streptococcal infections of pigs from Serbia. These findings are in concordance with described lesions in swine from other European countries (4), however, encephalitis and periarticular abscesses are most frequent pathological conditions in suckling and nursing piglets and seems to be main lesions of Streptococcal infections in Serbia in 2008 and 2009.

From the distribution of S. suis strains in corresponding lesions, serotype 2 was the most frequently isolated strain, which is at this time main causal agent of Streptococcal diseases in Serbia. In addition, serotype $1 / 2$ was also highly prevalent, and some generally acknowledgment invasive serotypes in European countries such as 9, 3 and $7(2,4)$ were much less encountered in our study.

\section{Acknowledgments}

This work was funded by Serbian Ministry of Science and Technological Development, project number TP: 20110.

\section{References}

1. Sanford, S.E., Higgins, R. (1992) In: Diseases of Swine. lowa State University Press, Ames, pp. 588-590.

2. Wisselink, H.J. et al. (2000) Vet. Microbiol. 74, 237-248.

3. Hill, J.E. et al. (2005) Vet. Microbiol. 107, 63-69.

4. Aarestrup, F.M. et al. (1998) Vet. Microbiol. 60, 59-66 


\title{
P.530
}

\section{Case study on a project to eradicate Streptococcus suis serotype 2 from a breeding herd}

\author{
Grace Webster ${ }^{2}$ Karen Degenstein ${ }^{3}$ David Woolfall ${ }^{4}$ Brian Hunt $^{5}$ Jennifer Thacker $^{1}$ Jill R. Thomson $^{1}$ \\ 1. Scottish Agricultural College, Edinburgh, UK; 2. Meadows Veterinary Centre, Old Meldrum, UK; 3. Royal (Dick) School of Veterinary \\ Studies, Edinburgh, UK; 4. Vion Food Scotland Ltd, Banff, UK; 5. Veterinary Laboratories Agency, Bury St Edmunds, UK
}

\section{Introduction}

Streptococcus suis serotype 2 infection in pig herds has significant health, economic and zoonotic implications $(1,2,3)$ and therefore, disease freedom is essential for specific pathogen-free breeding pyramids. Starting new herds with caesarian-derived piglets is recognized to be the most successful method of achieving disease freedom but it is costly, slow and labourintensive. Disease eradication from established herds has been attempted by antimicrobial treatment (4) and medicated-earlyweaning programmes (5). However, the success rate has been generally poor, and attributed to subclinical carriage of infection in tonsil crypts resulting in failure to detect and eliminate carriers (6). This paper describes a programme that aimed to eliminate $S$. suis serotype 2 from a nucleus herd through assiduous testing of pigs and removal of carriers, medication, vaccination and herd relocation.

\section{Materials and Methods}

The herd: A 400-sow herd with valuable genetics, in an isolated location and area of low pig density.

Strategy: Initially all sows were tested twice and any positives were culled, remaining sows were vaccinated twice (interval 4 weeks) with Porcilis Strep suis (Intervet-Schering Plough Ltd), then all received in-feed medication with potentiated penicillin for 6 weeks and were moved to a clean unit. However the herd continued to have evidence of S. suis serotype 2 infection, therefore the plan was amended, to create a clean, pure, nucleus herd from the progeny. All piglets received the above vaccine at 14 and 28 days of age and moved to 4 separate sites at weaning. All pigs in all batches were tested 4 times at a maximum of 6 week intervals from 10 weeks of age onwards, and any batch with a positive result was culled in its entirety. From the batches that were negative after the first 2 tests gilts were selected and moved into isolation for 8 weeks and were then moved to a further clean site to start the new herd.

Testing: Initially this was by culture and immuno-fluorescent antibody test on tonsil swabs and vaginal swabs from individual sows, and tonsil swabs from piglets. Testing then changed to PCR (7) on tonsil swabs and scrapings from growing pigs. Tonsil swabs and scrapings were collected from the palatine tonsils, applying as much pressure to the tonsils as possible. Mucus samples collected by scraper were transferred to swabs before submission to the laboratory.

\section{Results}

10 of the 400 sows were culled due to being positive on initial testing. Of the remaining 390 sows that moved to the clean site 4 were culled following subsequent testing. 9 batches of weaners in groups of 80-100 were tested and of those 4 groups were culled as positive. 5 batches comprising a total of 450 pigs were negative on two occasions. The selected gilts (approx 180) tested negative for a further two successive tests and were retained to form the new herd. These gilts have been bred and are due to farrow in the coming weeks. To date they have remained disease-free.

\section{Discussion}

Repeating the same testing schedule on the first crop of piglets should provide an indication of the initial success of the programme. However, long term monitoring for both the agent and any clinical signs of infection will be needed before concluding whether the methods used were genuinely successful. If successful, the key factors are considered to be effective sampling and testing methods that allow detection and elimination of pigs that remain carriers despite vaccination and medication. Use of the tonsil scrapers allow more pressure to be exerted on the tonsil tissue and better expression of mucus from the tonsil crypts. The change to PCR testing detected some positive animals that had previously tested negative by swabbing the tonsil surface and hence, aided the elimination of carriers. Establishing a practical and successful means of eradicating S. suis serotype 2 from breeding herds without total depopulation or using caesarian-derived methods of piglet retrieval will be a valuable resource for companies undertaking radical health improvement programmes in the future.

\section{References}

1. Staats, J.J.et al.1997. Vet. Res. Com. 21:381-407.

2. Tucker, A.W. 2006. Pig J. 57:178-191.

3. Strachan, W.D. 2005. Pig J. 56:202-213.

4. Strachan, W.D. et al. 2003. Pig J. 51:177-183.

5. Amass, S.F.et al.1996. J. Vet Diag Invest. 8:64-67.

6. Taylor, D.J. 2006. Pig Diseases 8th Ed. St Edmundsbury Press Ltd, Suffolk.

7. Wisselink, H.J. et al. Vet Microbiol, 67, 143-157. 


\title{
P.531
}

\section{Development of immunohistochemistry for detection of Brucella suis type- 2 in formalin-fixed tissues from pigs}

\author{
Zeljko Mihaljevic; Dinko Novosel; Zoran Lipej \\ Croatian Veterinary Institute, Zagreb, Croatia
}

\section{Introduction}

The aim of this study is to develop an immunohistochemical method for detection Brucella suis type 2 and to compare this method with PCR detection and bacteriological isolation.

\section{Material and Methods}

Testis samples obtained from 10 naturally infected pigs that were PCR and bacteriologically positive to Brucella suis type 2 were analyzed by immunohistochemistry (IHC). Demonstration of Brucella suis type 2 in formalin-fixed, parafin-embedded tissues was performed using polyclonal antibodies and positive samples kindly provided by Dr. Silvio Spicic (Croatian Veterinary Institute, Zagreb, Croatia). Testis tissue from clinically healthy pigs and PCR and bacteriologically negative tissue samples served as negative controls. Briefly, $5 \mu \mathrm{m}$ cuts were placed on silanized slides, section was deparafinized in xilen and rehydrated through different grades of ethanol. Endogenous peroxidase was blocked with $3 \% \mathrm{H} 202$ in $0.5 \mathrm{M} / 0.15 \mathrm{M}$ Tris/ $\mathrm{NaCl}$ (TBS). Slides were pretreated with Proteinase $\mathrm{K}$ (Dako ${ }^{\circledR}$ ) $3 \mathrm{~min}$. on room temperature according manufacture instruction. Non-specific reaction was blocked with incubation 1 hour in 5\% normal goat serum. Primary antibody was diluted $1 / 500$ in $2 \%$ normal goat serum in TBS and incubated over night at $+4^{\circ} \mathrm{C}$. Anti-mouse secondary antibody marked with labeling polymer (Dako Envision) was incubated according with manufacture instruction. For visualization is used DAB chromogen, slides were counterstained with Mayer's hematoxylin and then were dehydrated and mounted with DPX.

\section{Results}

In testis of PCR and bacteriologically positive pigs Brucella suis type 2 was found in all diseased animals. In their testis samples large amounts of Brucella suis type 2 antigen were concentrated in the cells of the inflammatory foci. All samples from healthy animals were IHC negative.
Figure 1. IHC detection of Brucella suis type 2 antigen, Mayer's hematoxylin counterstain. $40 x$.

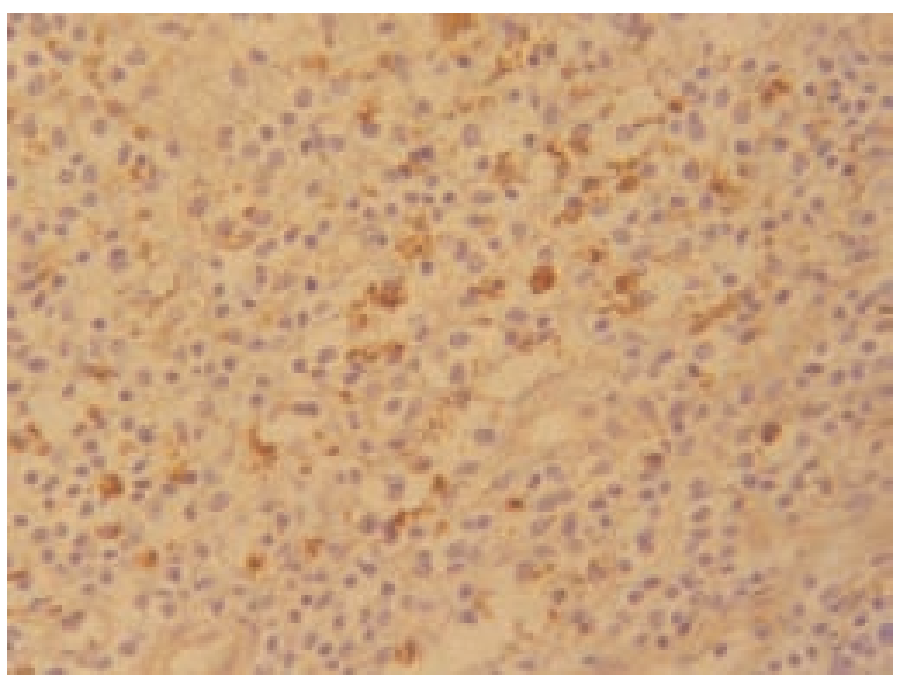

\section{Discussion}

An optimized IHC protocol was developed for the detection of Brucella suis type 2 in formalin-fixed, paraffin-embedded tissues and its results were compared with those determined by PCR and bacteriological isolation. The results obtained show that the use of IHC test is a reliable detection method. In the present study observed infection pattern is consistent with similar studies. When only formalin-fixed tissues are available, IHC would be useful diagnostic methods for the detection of Brucella suis type 2. The high specificity of $\mathrm{IHC}$ test for Brucella suis type 2 could be used in experimental studies of disease in pigs.

\section{References}

Bergagna et al., 2009. J WildI Dis 45, 1178-1181.

Cvetnic et al., 2003. Acta Vet Hung 51, 465-473.

Cvetnic et al., 2005. Vet Rec 156, 584-585.

Delrue et al., 2004. FEMS Microbiol Lett 231, 1-12.

Foster, R.A., Ladds, P. W., 2007. Male genital system. In: Maxie, M.G. (Ed.), Pathology of Domestic Animals. Elsevier, pp. 585-587.

Fretin et al., 2008. Vet Microbiol 131, 376-385.

Perez et al., 1998. Vet Diagn Invest 10, 17-21.

Thirlwall et al., 2008. Vet Res Commun 32, 209-213. 


\title{
P.532
}

\section{Effect of Erysipelothrix rhusiopathiae bacterial cell lysate on immunogenecity of swine erysipelas killed vaccine}

\author{
Hyun Jang; Kyung-sil Kim; Min-su Park; Min-su Park
}

Komipharm International Co. LTD, Gyeonggi-do, Korea

\section{Introduction}

Swine erysipelas may occur as an acute septicemic disease or chronic disease typically characterized by endocarditis and polyarthritis. Previous research shows the protective antibodies were elected against $64-66 \mathrm{kDa}$ and $40 \mathrm{kDa}$ protein of the membrane component in E. rhusiopathiae. Mice immunized with the purified 64-66 kDa proteins from E. rhusiopathiae were protected against challenge by the virulent strain. The $66-64 \mathrm{kDa}$ protein appears to be most immunogenic when complexed with glycolipid. This may be due to an adjuvant effect of polysaccharide antigens. Therefore cell homogenate of E. rhusiopathiae was very important component for effective vaccine development. In this study the effect of E. rhusiopathiae bacterial cell homogenate to the efficacy of the swine erysipelas killed vaccine was tested by challenge test using highly virulent E. rhusiopathiae T2 strain and also we will determined which was the major antigen in cell homogenate using western blotting methods.

\section{Materials and Methods}

The bacterial strain E. rhusiopathiae SE9 was used in this experiment. The initial culture was grown on solid $\mathrm{BHI}$ agar media containing $5 \%$ sheep blood. Bacterial cell number was expanded in the flask culture using semi defined and complex media. Measuring the optical density at $600 \mathrm{~nm}$ monitored bacterial cell growth. The concentrated bacterial cells were homogenized using high-pressure homogenizer and cell breakage determined by measuring viable cell count. Trial vaccines were made with inactivated cell homogenate, whole bacterial cell and mixture of the inactivated cell homogenate and whole cell bacterial cell. Immunoblotting, SDS-PAGE and ELISA assay were performed following general methods. Efficacy test of trial vaccines was performed by mouse challenge test. using E. rhusiopathiae T2 strain.

\section{Results}

Antibodies to a $66-64 \mathrm{kDa}$ and $34 \mathrm{kDa}$ proteins released from the cell surface have been shown to be involved in protective immunity. The efficacy of the vaccine using the bacterial cell homogenate was better than vaccine using the bacterial whole cell. $66-64 \mathrm{kDa}$ and $34 \mathrm{kDa}$ proteins on the E. rhusiopathiae SE9 are major antigen of the immune response in mouse

\section{Discussion}

The results of this study provide important information for the development of safe and efficacious vaccine development. 66-64kDa and $34 \mathrm{kDa}$ proteins on the E. rhusiopathiae can act as an important antigen of the vaccine protectivity. In addition, 66-64kDa and $34 \mathrm{kDa}$ proteins can be used as a protein partner for glycolipid conjugate vaccine production.

\section{References}

1. Groschup, M.H., Cussler, K., Weiss, R. and Timoney, J.F., 1991. Characterization of a protective protein antigen of Erysipelothrix rhusiopathiae. Epidemiol. Infect., 107: 637-649

2. Lachmann, P.G. and Deicher, H., 1986. Solubilization and characterization of surface antigenic components of Erysipelothrix rhusiopathiae T28. Infect. Immun., 52:818-22. 


\title{
P.533
}

\section{Analysis of the surface protective antigen (Spa) patterns of Erysipelothrix spp. isolates in tissues, environmental samples and vaccine strains obtained from vaccinated farms experiencing acute erysipelas outbreaks in the Midwest}

\author{
Joseph Bender; Huigang Shen; Tanja Opriessnig \\ lowa State University, Ames, IA, USA
}

\section{Introduction}

Erysipelothrix spp. isolates are divided into four groups ( $E$. rhusiopathiae, E. tonsillarum, E. sp. strain 1, E. sp. strain 2) and 28 serotypes ( $1 \mathrm{a}, 1 \mathrm{~b}, 2-26, \mathrm{~N})(6)$ Diagnostic investigations of U.S. field cases have indicated that E. rhusiopathiae serovar $1 \mathrm{a}$ is typically the cause of acute septicemic erysipelas and serovar 2 is most common in subacute and chronic cases of swine erysipelas. All other known serovars are thought to have low levels of virulence for swine (6). Currently available commercial attenuated-live vaccines against swine erysipelas are based on serovar 1a (3). Swine practitioners often rely on serotyping results to determine if a vaccine will be cross-protective. Recently, the surface protective antigen (Spa) protein of Erysipelothrix spp. was characterized and shown to be highly immunogenic (2). Moreover, it was found that Erysipelothrix spp. isolates typically harbor one of three known Spa proteins (Spa A, Spa B, Spa C). There appears to be cross-protection between isolates within the same Spa type but not between Spa types (4). The objective of this study was to characterize the Spa types present in Erysipelothrix spp. isolates involved in suspected vaccine breaks.

\section{Materials and Methods}

Eighteen tissue samples and 126 environmental samples were collected from six farms in the Midwest experiencing erysipelas outbreaks in vaccinated animals. In addition, autogenous and commercially available modified-live erysipelas vaccines that were used on the farms were included in the analysis. Tissue specimens were also included from cases submitted to the lowa State University Diagnostic Laboratory where swine erysipelas was suspected (1). Enrichment broth-based isolation was used for detection of Erysipelothrix spp. (1). Further characterization was done by serotyping of the isolates (1). In addition, a novel multiplex real-time PCR method for simultaneous detection of Spa $A, B 1, B 2$, and $C$ was used which was previously validated using 28 Erysipelothrix spp. reference strains (5).

\section{Results}

Erysipelothrix spp was isolated from tissue samples obtained from all six farms which were investigated and included serovars 1a, 2, 10, and 21. Environmental samples were positive for Erysipelothrix spp on each farm; however in two farms the serovar identified from the tissues did not correlate with serovars found in environmental samples. Spa PCR results on 109 selected tissue and environmental isolates indicated that isolates identified as E. rhusiopathiae serovar 1a, 2, 6, 9, 10, 11, and 21 all were positive for Spa A, while isolates identified as E. tonsillarum serovars 7 and 10 were negative for Spa A, B1, B2, and C. E. sp. strain 1 (serovar 13) was also identified on one of the six farms (negative for all Spa A, B1, B2, and C). All vaccines tested were found to be E. rhusiopathiae serovar $1 \mathrm{a}$ and were positive for Spa A.

\section{Discussion}

Previous investigations have shown Spa proteins to be highly immunogenic as antibodies are formed against these cell surface proteins $(2,4)$. Current literature reports that each Erysipelothrix spp serovar is specific to a particular Spa protein based on analysis of reference strains (4). Results of this study indicate that variability exists between Spa proteins and serovars, this conclusion is supported by the fact that the U.S. field isolates investigated during this study differed in Spa type when compared to the Spa types reported for the reference strains. Currently available attenuated live vaccines in the US are based on E. rhusiopathiae serovar 1a, and contain Spa A. Studies have shown that there is cross protection within the same Spa type; however, minimal cross protection exists between Spa types. In the current study, the Spa type indentified in the vaccines strains was not always identical to the Spa type identified in the tissue and environmental samples. Further investigation is warranted to explore the cross protection provided between Spa types under field conditions.

\section{References}

1. Bender, J.S. et al. (2009) J. Vet. Diagn. Invest. 21:863-868.

2. Makino, S. et al (1998) Microb. Pathog. 25:101-109.

3. Opriessnig, T. et al. (2004) J. Vet. Diagn. Invest. 16:101-107.

4. To, H. and Nagai, S. (2007) Clin. Vac. Immunol. 14:813-820.

5. Shen, H. et al. (2010) Submitted for publication.

6. Wood, R.L. (1999) In: Diseases of Swine, 8th: pp. 419-430. lowa State University Press, Ames, IA. 


\title{
P.534
}

\section{Post serological monitoring of a live vaccine implementation swine erusipela in piglets on a farm in Puebla State in Mexico}

\author{
Oscar Huerta Alva; E. V. Vivanco; Y. L. Santos; L. Valencia \\ Laboratorios Sanfer SA de CV, Mexico, DF, Mexico
}

Erysipelas is a contagious infectious disease of pigs caused by the bacterium Erisipelothrix rhusiopathiae. The Swine Erysipelas is an actual disease, as reported Schwarts (AASV 2002) with the increase of cases at the University of lowa, with economic impact in the herd losses may include loss of death (1\%) - \$ $2.50 \mathrm{dls} /$ hembra ; treatment cost $\$ 1 /$ hembra; abortion (4\%) - \$ $5 \mathrm{dls} /$ hembra for a total of $\$ 10 \mathrm{dls} /$ hembra. Also in the growth stage - complete the death loss (3\%) - \$2.50dls; treatment cost $\$ 1 \mathrm{dls}$; retarded (3\%) - $\$ 1.25 \mathrm{dls}$ for a total of $\$ 4.75 \mathrm{dls} /$ cerdo the market. The cost of an outbreak can be high, and this does not include effects on carcass quality, or forfeitures. In 2008, in traces of Sonora, Queretaro and Mexico City for the confiscation of channels because of erysipelas (personal communication) has been frequent.

Sierra (2006) reports that Erysipelas is the second most isolated bacteria in the presence of PRRS in farms of Guanajuato, Jalisco, Michoacan and Perea (2006) reports that $90 \%$ of animals tested in the State of Mexico are positive Erysipelas, Huerta (2006-2008) reported $80 \%$ incidence of seropositivity in Sonora. According to remarks by Dr. Calderon (personal communication) $75 \%$ of the farms of La Piedad and Tepatitlán have subclinical Erysipelas.

\section{Materials and Methods}

The aim of this study was to monitor the serological to 5 piglets before and after application of a live vaccine and see if through the Elisa test (kit for the detection and / or quantification of specific antibodies Erisipelotrix rhusiopathiae INGENASA pig serum) we identified a serological response at 30 and 60 days after vaccination. The piglets are from a farm of 1,100 stomachs located in the State of Puebla, stable PRRS virus and by late last year introduced Erysipelas problems and proceeded to vaccinate the herd and piglets savanna around week 7 of age . 5 piglets were selected at random, were identified individually and they took blood samples at day 1 (before vaccination) and 30 and 60 days after vaccination, sera were sent to Diagnostic Laboratory IASA, obtaining the following results.

\begin{tabular}{|c|c|c|c|c|c|}
\hline \# piglets & $\mathbf{1}$ & $\mathbf{2}$ & $\mathbf{3}$ & $\mathbf{4}$ & $\mathbf{5}$ \\
\hline 1st blood test & 0.098 & 0.095 & 0.078 & 0.101 & 0.088 \\
\hline 2nd blood test & 0.095 & 0.100 & 0.130 & 0.088 & 0.084 \\
\hline 3th blood test & 0.111 & 0.113 & 1.0135 & 0.119 & 0.100 \\
\hline
\end{tabular}

Graphically this is the result.

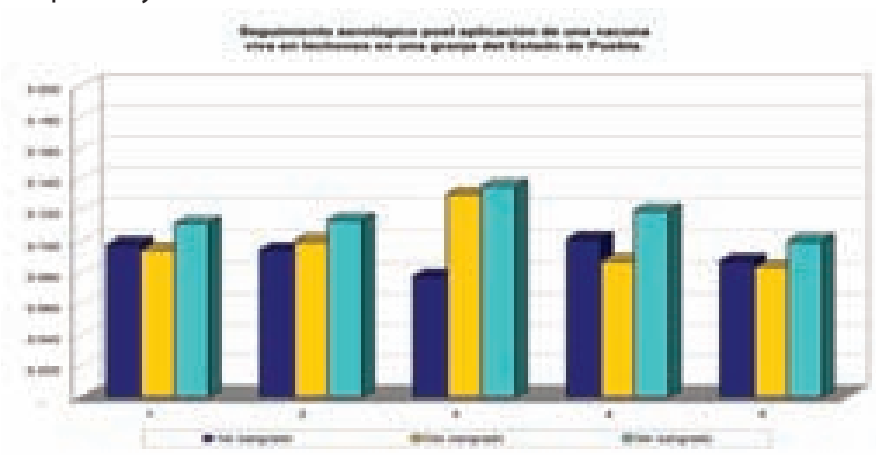

\section{Discussion}

3 / 5 piglets showed a decrease in the immune response during the 2 nd bleeding and 5 / 5 piglets have a greater serological response and uniform prior to vaccination. We believe that using the Elisa test for the detection and / or quantification of specific antibodies in swine serum Erisipelotrix rhusiopathiae (INGENASA) can be used to measure the response post vaccination, but this work must be replicated again to set specific parameters post-vaccination response, since this kit is designed to identify negative animals, suspicious and positive in the presence of erysipelas in the field, which we know will change the immune response towards each individual and the degree of infection present. For the third bleeding, we can see that there is a similar response in 5 cases. It is noteworthy that farm animals had clinical symptoms of Erysipelas and after vaccination this extint.

\section{References}

1. Perea, CML, et al. Determinación de anticuerpos contra Erisipelothrix rhusiopathiae.en sueros de cerdos utilizando una prueba sencilla de aglutinación., Congreso AMVEC, 2006.

2. Huerta, AOF; Montaño MB, Reporte de casos de Erisipela porcina en el Estado de Sonora, Congreso AMVEC, 2006

3. Huerta, AOF; Montaño MB, Reporte de casos de Erisipela porcina en el Estado de Sonora, IPVS, 2008.

4. Schwartz, K.J.,2002, Erysipelas: an old disease returns to the swine industry or something NEW?, American Association of Swine Veterinarians.

5. Sierra, N., Multi etiologías complejas de interacción entre PRRS y otros patógenos. Congreso AMVEC, 2006.

5. Wood, R.L., 1999, Erysipelas. In Diseases of Swine 8th Ed, ISU press, Ames lowa. 


\title{
P.535
}

\section{Usability of quantification of interferon-gamma production after in vitro stimulation with Mycobacterium-specific antigen in diagnosis of mycobacterial infection in pigs: a limited field study}

\author{
Martin Faldyna; Eduard Gopfert; Hana Kudlackova; Marija Kaevska; Iva Slana; Ivo Pavlik \\ Veterinary Research Institute, Brno, Czech Republic
}

\section{Introduction and Objectives}

At present, there is no doubt that Th1-type immune response is crucial for the control of mycobacterial infections [1,2]. Interferon-gamma (IFN- $\gamma$ ) is a key cytokine produced by Th1 lymphocytes. It plays a role in modulation of immune response associated with activation of macrophages [3]. Th1-type immune response is also associated with the 4th type of hypersensitivity. This fact has been used in intravital diagnosis of mycobacterial infections using intradermal administration of a specific antigen.

In the 1990, Wood and coworkers showed that quantification of IFN- $\gamma$ produced by lymphocytes after in vitro stimulation with a specific antigen is a useful tool for detection of tuberculosis in cattle $[4,5]$. A similar test is used also in human medicine [6].

At the 20th IPVS Congress, we showed that IFN- $\gamma$ release detection after in vitro stimulation could be used in pigs experimentally infected with M. avium subsp. avium and hominissuis (MAA and $M A H$ ), with the latter having weaker production [7]. The aim of the present study was to ascertain whether the test is useful for individual diagnosis of naturally infected pigs.

\section{Materials and Methods}

Sixty-one clinically healthy pigs, aged 4-5 months, utilized in feeding experiments at the Veterinary Research Institute were used in the study.

One month before slaughter, samples of heparinised blood were taken from the pigs. The samples were in vitro stimulated for 20 hours with M. avium - specific antigen. Produced IFN- $\gamma$ was quantified using available ELISA kit (BioSource International, Inc., USA) in accordance with manufacturer's recommendations.

Simultaneously, intradermal administration of antigen designed for such a type of test (Avitubal, Bioveta na Hane, Czech Republic, containing 28000 IU of M. avium antigen) was performed. Based on manufacturer's recommendation, local lesions were evaluated 48 hours after administration.

In a slaughterhouse, carcasses were inspected and tuberculous lesions were evaluated by microbiological methods (Ziehl-Neelsen staining, culture examinations, qPCR).

\section{Results}

No animals were positive to intradermal tests, i.e. no local reaction at the site of injection exceeded $2 \mathrm{~cm}$ in diameter 48 hours after administration.

Lymphocytes of 3 animals produced detectable amounts of IFN- $\gamma$, i.e. more than $18 \mathrm{pg} / \mathrm{ml}$. Two of these 3 animals were bacteriologicaly proven to be infected with $\mathrm{MAH}$. On the other hand, one pig infected with $\mathrm{M}$. xenopi was not able to produce a detectable amount of IFN- $\gamma$.

\section{Conclusions}

Our results indicated that detection of IFN- $\gamma$ production after in vitro stimulation with Mycobacterium-specific antigen could be used in diagnosis of mycobacterial infections in naturally infected pigs. It should be noted, however, that much lower amounts of IFN- $\gamma$ were produced by naturally infected pigs than by experimentally infected ones [7].

\section{References}

1. Flesch, I.E., Kaufmann, S.H.: Role of cytokines in tuberculosis. Immunobiology. (1993) 189:316-339.

2. Kaufmann, S.H., Flesch, I.: The role of T cell-macrophage interactions in tuberculosis, Springer Semin. Immunopathol. (1988) 10:337-358.

3. Murray, H.W. Interferon-gamma, the activated macrophage, and host defense against microbial challenge. Ann. Intern. Med. (1988) 108: 595-608.

4. Rothel, J.S. et al.: A sandwich enzyme immunoassay for bovine interferon-gamma and its use for the detection of tuberculosis in cattle. Aust. Vet. J. (1990) 67: 134-137.

5. Wood, P.R. et al.: Field comparison of the interferon-gamma assay and the intradermal tuberculin test for the diagnosis of bovine tuberculosis. Aust. Vet. J. (1991) 68: 286-290.

6. Mori, T.: Usefulness of interferon-gamma release assays for diagnosing TB infection and problems with these assays. J. Infect. Chemother. (2009) 15:143-155.

7. Faldyna, M. et al.: Antigen-specific immune response of piglets to experimental infection with Mycobacterium avium ssp. avium and Mycobacterium avium ssp. hominissuis. 20th International Pig Veterinary Society Congress, Durban, South Africa, Poster proceedings, p.312, ISBN 978-0-620-41364-0.

The study was supported by the Ministry of Agriculture of the Czech Republic (MZe 0002716202) and Ministry of Education, Youth and Sports of the Czech Republic (CZ.1.05/2.1.00/01.0006 AdmireVet). 


\title{
P.536
}

\section{Testing immunogenicity of Mycoplasma hyosynoviae vaccine candidates; Induction of antibodies and IFNY response}

\author{
Klara T. Lauritsen; Ulla Riber; Jens Nielsen; Jeanne T. Jakobsen; Gregers Jungersen \\ National veterinary Institute, Technical University of Denmark, Copenhagen, Denmark
}

\section{Introduction}

Mycoplasma hyosynoviae (Mhs) is a significant cause of acute arthritis in swine above 10 weeks of age. No effective prophylaxis is available. Therefore attempts are made to develop a vaccine candidate with potential to protect against arthritis caused by Mhs. First step is screening of the immunogenic effect of Mhs antigen in combination with various adjuvants. Earlier studies of the infection have shown that antibodies can provide a certain degree of protection against the infection (Lauritsen et al., 2006), but a cell-mediated immune response has also been shown to play a significant role in protection (Lauritsen, 2005).

\section{Materials and Methods}

The four vaccine candidates tested, were based on the same formalin fixed Mhs, but combined with various EMULSIGEN oil-in-water adjuvant formulations (MVP Laboratories). One dose contained $300 \mu \mathrm{g}$ antigen in aqueous solution and adjuvant was added to $20 \%$ of the total volume. Eleven pigs at 12 -weeks of age (one litter, from Mycoplasma free herd) were administered the primary vaccination i.m. (EMULSIGEN-D: 3 pigs, EMULSIGEN: 2 pigs, EMULSIGEN-BCL: 3 pigs and EMULSIGEN-P: 3 pigs). Revaccination was carried out with identical boosters after three weeks. Another three weeks later the pigs were killed.

Mhs-specific lgG in serum was measured by ELISA (Nielsen et al. 2005). IFN $\gamma$ measurements were included as an indicator of the ability of the vaccines to induce a cellular immune response. Mhs specific IFN- $\gamma$ response was measured by ELISA (Riber \& Jungersen 2007) in supernatants from stimulated heparinstabilised blood.

\section{Results and Discussion}

When measuring Mhs-specific lgG in serum, all pigs seroconverted from day 24 to 28 after first vaccination (i.e. day 3 and 7 after boost). Plateau was reached 7 to 14 days after boost, OD values ranging from 0.5 to 1.75. Measurement of IFN $\gamma$ in supernatants from whole blood cultures stimulated with Mhs antigen showed that all groups had pigs that reacted to vaccination with a very high IFN $\gamma$ response. All pigs had IFN $\gamma$ measurements reaching beyond $500 \mathrm{pg} / \mathrm{ml}$, in some pigs beyond $4000 \mathrm{pg} / \mathrm{ml}$ (Figure 1). The negative control samples (PBS stimulated samples) did not exceed an IFN $\gamma$ level of $102 \mathrm{pg} / \mathrm{ml}$. However, no group of pigs had consistently higher IFN $\gamma$ level than the others.
Figure 1. Mycoplasma hyosynoviae-specific IFN- $\gamma$ response. Type of vaccine used is indicated for each pig; Square; EMULSIGEN-D, Triangle; EMULSIGEN-BCL, Stippled line; EMULSIGEN, Line; EMULSIGEN-P.

Earlier attempts to develop a vaccine against Mhs have focused

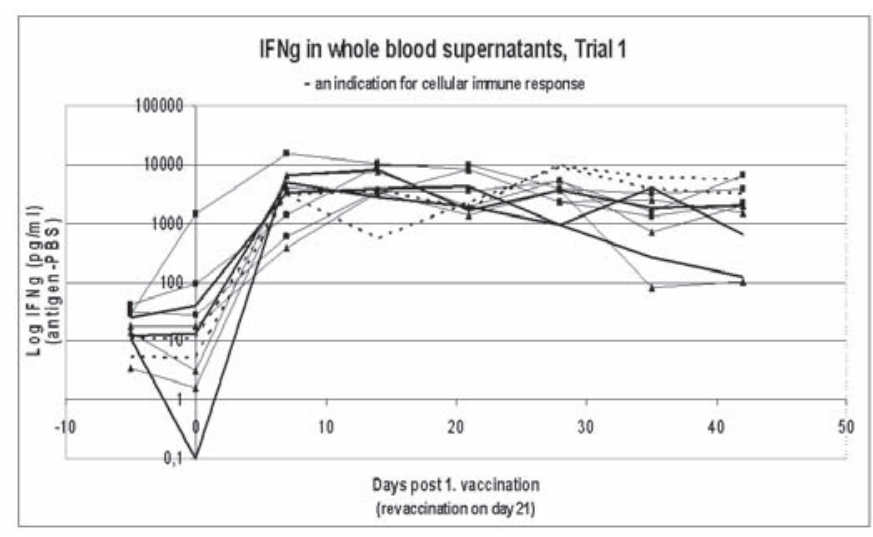

on the serological response (Ross, 1978; Feenstra et al., 1994). Our experiment, however, focuses on the use of adjuvants that are reported to favour cell mediated immune response, while still giving rise to an antibody response. The investigation is a part of a larger initial screening for vaccine candidates against Mhs. Thus more candidates are to be tested, and finally a promising candidate, with ability to induce both serological end cell-mediated immune response, will be tested in challenge experiments.

\section{References}

Lauritsen 2005. ph.d. thesis. The Royal Veterinary Agricultural University, Copenhagen, Denmark

Lauritsen et al. 2006. Proc. 4th International Veterinary Vaccines and Diagnostics Conference, Oslo, Norway. 119

Nielsen et al. 2005. Vet. Microbiology 111: 41-50.

Riber \& Jungersen 2007. Vet Immun: Immunopath. 116: 13-25

Feenstra et al. 1994. Proceedings of the 10th International Congress of the International Organization for Mycoplasmology. Bordeaux, France. pp. 306-307

Ross, R.F., 1978. Zentralblatt Für Bakteriologie, Parasitenkunde, Infektionskrankheiten Und Hygiene, Erste Abteilung Originale 241A, 246. 


\title{
P.537
}

\section{Test of vaccine against M.hyosynoviae; Induction of specific B-cell memory}

\author{
Klara T. Lauritsen ${ }^{1}$ Josephine S. Rasmussen ${ }^{1}$ Jeanne T. Jakobsen ${ }^{1}$ Ulla Riber ${ }^{1}$ \\ Elisabeth O. Nielsen² Poul Bækbo² Sardar Ahmad ${ }^{1}$ Gregers Jungersen ${ }^{1}$ \\ 1. National Veterinary Institute, Technical University of Denmark, Copenhagen, Denmark; 2. Danish Pig Production, Copenhagen, Denmark
}

\section{Introduction}

Mycoplasma hyosynoviae (Mhs) causes arthritis in swine. Since an effective immunoprophylaxis against the disease is not available, the aim of this project was to attempt to develop one.

\section{Materials and Methods}

Twenty-six Mhs-free cross-bred pigs (3 litters) from an infected herd were isolated from $31 / 2$ weeks of age. A vaccine containing formalin inactivated Mhs (strain M60, $300 \mu \mathrm{g} /$ dose) with EMULSIGEN-BCL ${ }^{\bullet}$ (MVP Laboratories) as adjuvant was administered to 13 pigs at 7 and 10 weeks of age. 13 placebo pigs received adjuvant in saline. Intranasal challenge infection with Mhs was given 3 weeks after boost. 14-21 days after this, the pigs were autopsied.

Mhs-specific lgG in serum was measured by ELISA (Nielsen et al. 2005). IFN $\gamma$ measurements were included as an indicator of the ability of the vaccines to induce a Mhs-specific cellular immune response. Mhs specific IFN- $\gamma$ response was measured by ELISA (Riber \& Jungersen 2007) in supernatants from stimulated heparin-stabilised blood.

\section{Results and Discussion}

Three pigs in the placebo group and one in the vaccine group developed lameness after inoculation. Eight vaccinated and 10 placebo pigs had pathological changes in joints at autopsy. Primarily mild changes were seen. One day before inoculation a significantly higher level of IFN $\gamma$ was measured in supernatants of antigen stimulated full blood in the vaccinated group compared to the placebo group. On day 6 after inoculation the placebo group had a significantly higher IFN $\gamma$ level when compared to the vaccinated group. Compared to placebo pigs, all vaccinated pigs had a more potent response in Mhs specific lgG in serum after challenge, with a more rapid onset and higher levels reached before autopsy (Fig 1). The latter results show that the vaccinated pigs have been primed to mount a more rapid specific antibody response when encountering the infection. Presence of different levels of maternally derived antibodies in the three litters did not seem to interfere with this priming effect (Figure 1).
Figure 1: $M$. hyosynoviae specific IgG measured in serum of two of the litters during the experimental period. Stippled line; vaccinated pigs. Line; Placebo pigs. Note that level of maternally transferred antibodies varied.
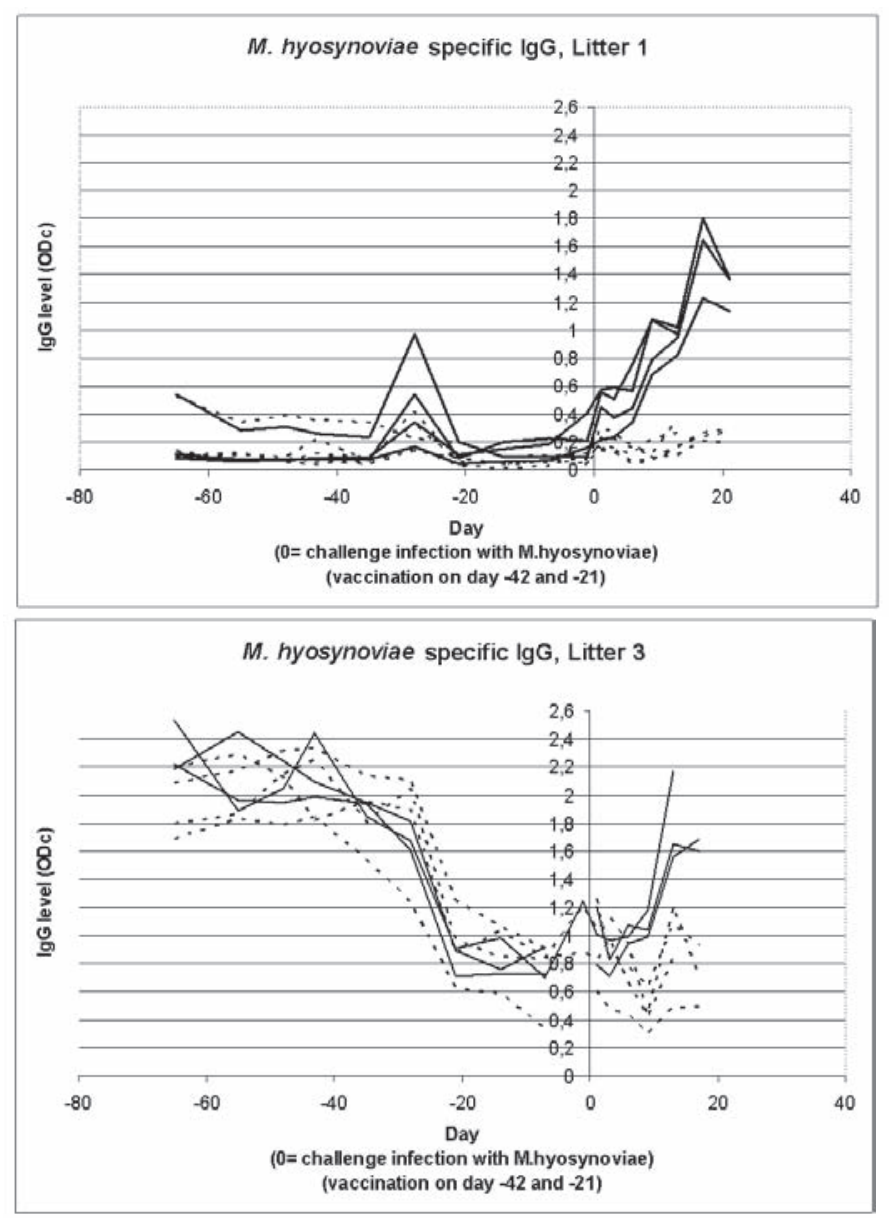

In order to be able to conclude anything on the protective effect of the vaccine against clinical disease, larger experimental groups are needed.

\section{References}

Nielsen et al. 2005. Vet. Microbiology 111:41-50.

Riber \& Jungersen 2007. Vet Immun: Immunopath. 116: 13-25 


\title{
P.538
}

\section{Novel vaccine against Mycoplasma hyosynoviae: The immunogenic effect of iscom-based vaccines in swine}

\author{
$\underline{\text { Klara T. Lauritsen }}{ }^{1}$ Annette V. Heydenreich ${ }^{2}$ Ulla Riber $^{1}$ Jeanne T. Jakobsen ${ }^{1}$ Nikolai Kirkby ${ }^{2}$ Gregers Jungersen ${ }^{1}$ \\ 1. National veterinary Institute, Technical University of Denmark, Copenhagen, Denmark; \\ 2. Rigshospitalet, Dept. for Clinical Microbiology, Copenhagen, Denmark
}

\section{Introduction}

Arthritis in swine is frequently caused by Mycoplasma hyosynoviae (Mhs). For the development of an effective vaccine, we investigated the immunogenic effect of three vaccine preparations with the ISCOM adjuvant Posintro ${ }^{\mathrm{TM}}$ from Nordic Vaccine.

\section{Materials and Methods}

Vaccine A consisted of formalin fixed whole-cells Mhs $(300 \mu \mathrm{g} /$ dose) mixed with Posintro. Vaccine B consisted of Deoxycholate extracted lipoproteins from Mhs organisms (DOC-antigen, 300 $\mu \mathrm{g} /$ dose) in Posintro and finally Vaccine $C$ consisted of DOC antigen $(50 \mu \mathrm{g} /$ dose $)$ in Posintro. Each vaccine-group contained three pigs. Vaccinations (i.m.) were performed at 12 and 15 weeks of age. Three weeks after the last dose the pigs were autopsied. The presence of specific lgG in serum was measured during the experiment. Furthermore secretion of the cytokines IFN $\gamma$ and IL-12p40 by stimulated blood cells was measured. IFN $\gamma$, was measured in supernatants of whole-blood cultured with Mhs-antigen whereas IL-12p40 was measured in supernatants from stimulated peripheral blood mononuclear cells. The latter two measures were included to describe the cell-mediated immune response $(\mathrm{CMI})$.

\section{Results and Discussion}

Vaccine $B$ induced a high level of specific serum IgG in all animals 1 week after boost. Vaccine $C$ gave a variable response after boost, with two pigs seroconverting, while no response was seen by vaccine A. All pigs secreted IFN $\gamma$ after primary vaccination followed by an increased production after booster vaccination (Fig 1). The $C M I$ response was highest with vaccine $B$ when compared to responses induced by vaccine $A$ and $C$ at one $(\mathrm{P}<0.01)$ and two $(\mathrm{P}<0.001)$ weeks after boost. IL-12p40 levels in the supernatants corresponded with the IFN $\gamma$ responses. At autopsy only vague signs of tissue damage could be seen, and only in pigs that received $\mathrm{A}$ and $\mathrm{C}$.
Figure 1: Mhs specific IFN- $\gamma$ response of the individual pigs after vaccination. Triangle; vaccine A, Square; vaccine B, Line; vaccine $C$

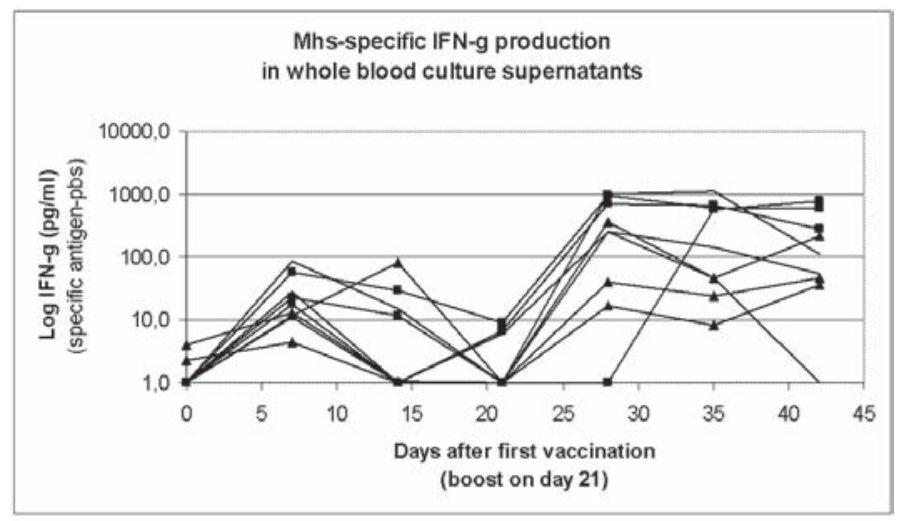

Clearly, Posintro ${ }^{\mathrm{TM}}$ Mhs-vaccines possess the potency to raise a high level of both a cellular and a humoral immune response. Whether the induced immune response prevents clinical disease after infection should be measured in a challenge study. 


\title{
P.539
}

\section{Detection of Mycoplasma suis in healthy pig populations}

\author{
Erin Strait; Nyssa Levy; Cassandra Roe; Alejandro Ramirez \\ lowa State University, Ames, IA, USA
}

\section{Introduction}

Mycoplasma suis (formerly Eperythrozoon suis) is a blood-borne bacterial pathogen and causative agent of immune-mediated hemolytic anemia in pigs. Once infected, animals become lifelong carriers, causing a variety of signs that have been reported to include reproductive failure, hypoglycemia, general unthriftiness, increased susceptibility to secondary infections, and even acute death ${ }^{1}$. Good diagnostics for this agent are currently lacking, and because of this, the true prevalence and significance of this disease is unknown. This organism is not able to be cultured in the laboratory, and until recently, diagnosis of $M$. suis was primarily by acridine-orange staining of red blood cells; however, recent studies have shown this method to have limited sensitivity $^{2}$. Recently, an intracellular strain of $M$. suis was identified that was unable to be detected by this staining method. An ELISA test has been described, but is not commercially available. This highlights the need for a rapid and sensitive test such as realtime PCR. A real-time PCR assay targeting the msg1 gene has been recently described, but can only be performed on a single instrument that is not widely used, thus limiting its application ${ }^{3}$. The main objective of this study was to develop a real-time PCR based on the msg1 assay that could be performed on a wide range of instruments and facilitate diagnosis of $M$. suis. In addition, a sampling of normal pig populations of various ages was performed to begin to assess the prevalence of this organism in US swine herds.

\section{Materials and Methods}

Blood samples were collected in EDTA and/or heparin tubes from apparently healthy pigs in 4 age groups, each from different herds: 3 -day old piglets $(n=13), 12-18$ week old pigs $(n=17)$, 6-24 month old boars $(n=23)$ and sows at time of slaughter $(n=42)$. Additionally, meat juices $(n=50)$ were obtained from muscle samples taken at the time of slaughter at 10 unique sites in 3 different states and processed at the lowa State University Veterinary Diagnostic Laboratory- Meats Laboratory. DNA was extracted by spin column (Qiagen QiAmp DNA mini kit) and/or magnetic bead-based protocol (Ambion Mag-Max kit).

\section{Results}

We determined that extracting blood samples by the spin column method is the most sensitive protocol for positive detection of M. suis by real-time PCR. Positive samples were found within each sample group at various rates between 2-28.6\%, except for the 3-day old piglets which were all negative. EDTAblood samples from PCR-positive $(n=10)$ and PCR-negative $(n=15)$ pigs were submitted for complete blood count analysis. While not statistically significant due to low sample number, a trend for lower clinical blood values in PCR-positive samples was observed (Table 1).

Table 1. Average clinical blood values from selected $M$. suis $P C R$ positive or negative blood samples.

\begin{tabular}{|c|c|c|c|}
\hline PCR result & RBC\# (x10^6/ul) & [Hemoglobin] (gm/dl) & Hematocrit (\%) \\
\hline positive & $6.03+/-$ SD 0.44 & $11.02+/$-SD 0.77 & $33.3+/-$ SD 2.77 \\
\hline negative & $6.20+/$-SD 0.44 & $12.2+/$-SD 0.66 & $35.8+/$-SD 2.38 \\
\hline
\end{tabular}

\section{Discussion}

Results of this study suggest that the presence and impact of $M$. suis in otherwise healthy pigs may be currently underappreciated. Further testing of both apparently healthy and clinicallyaffected animals will be necessary to determine the true prevalence and significance $M$. suis-induced disease in today's swine industry.

\section{References}

1. Hoezle LE. 2008. Vet. Microbiol. 130, 215-226.

2. Groebel K, et al. 2008. 77:2, 576-584.

3. Hoezle LE, et al. 2007. J. Microbiol Methods. 70:2, 346-354. 


\title{
Detection of African swine fever virus (ASFV) DNA in several cell-types from formalin-fixed paraffin-embedded infected tissues using a new in situ hybridisation (ISH) protocol
}

\author{
Maria Ballester ${ }^{1}$ Ivan Galindo-Cardiel ${ }^{1}$ Carmina Gallardo ${ }^{2}$ Jordi M. Argilaguet ${ }^{1}$ \\ Joaquim Segales ${ }^{1}$ Javier M. Rodriguez ${ }^{3,4}$ Fernando Rodriguez ${ }^{1}$ \\ 1. Centre de Recerca en Sanitat Anima (CReSA), Bellaterra, Spain; 2. Centro de Investigación en Sanidad Animal, Valdeolmos, Spain; \\ 3. Centro de Biología Molecular "Severo Ochoa" (CSIC-UAM), Universidad Autónoma de Madrid, Madrid, Spain; \\ 4. Centro Nacional de Microbiología, Instituto de Salud Carlos III, Madrid, Spain
}

\section{Introduction}

African swine fever (ASF) is a highly contagious hemorrhagic disease of domestic pigs. The disease was reported for the first time in Kenya in the 1920s, and actually remains endemic in Sardinia and in East and Southern Africa, producing important economical losses in the pig industry (1). The recent outbreak in Georgia last 2007, and its extension to some Russian counties, has increased the chances for the ASFV to be extended to European and Asian countries. Due to the absence of vaccines available against this disease, ASF control is based on an efficient diagnostic and the slaughter of infected animals.

The aim of the present work was to develop an optimized in situ hybridization (ISH) protocol to detect ASF virus genome in formalin-fixed paraffin-embedded tissues.

\section{Material and Methods}

Animal samples. Tissue samples including liver, spleen, kidney, lung, and gastro-hepatic lymph node were obtained from experimentally infected animals using Ba71L and E75L virulent ASFV isolates.

ISH method. Three different digoxigenin (DIG) labeled probe approaches were used: a pool of DIG-oligonucleotide probes complementary to ASFV sequences, a $18.5 \mathrm{~kb}$ Sall fragment (SD probe) of the genomic Ba71V DNA, and the total E75 viral DNA (CG probe). Two different proteolytic treatments were also tested: proteinase $\mathrm{K}$ and pepsine digestion.

Combined ISH and immunohistochemistry (IHC): in order to verify the presence of ASFV DNA in the nucleus of lymphocyticlike cells, a double ISH-IHC protocol was performed in different tissues. T-lymphocytes were first identified by IHC using a polyclonal anti-human CD3 antibody (Dako). ISH was next performed using the CG probe after proteinase K digestion.

\section{Results}

Positive specific ASFV signal was obtained in most of ASFV-infected tissues tested either using SD or CG probes. Nonetheless, no signal was detected using the DIG-oligonucleotide probes.

While the SD probe was able to detect the presence of ASFV DNA only in Ba71L infected tissues, the CG probe hybridized with both E75L and Ba71L infected tissues. Proteinase K treatment resulted more efficient than pepsin digestion, yielding a better tissue integrity and a lower background.
Regarding ISH staining distribution, intranuclear and perinuclear positive signal was mainly found in cells of the monocytemacrophage cell lineage. Positive staining was also found in other cell types, including endothelial cells, hepatocytes, neutrophils, and T-lymphocytes (double staining performed).

\section{Discussion}

In the present study, a new optimised protocol to detect ASFV genome in formalin-fixed paraffin-embedded tissues has been developed. The viral distribution described in this study is in accordance with that previously described (2). However, this is the first time that intranuclear location of the AFSV DNA has been described in different cell types, such as macrophages and T-lymphocytes, from ASFV-infected tissues. This surprising result might be the in vivo reflection of an early step of intranuclear ASFV replication, as it has been previously described after the in-vitro infection of cell lines with the virus $(3,4)$.

Presented results indicated the utility of this ISH protocol to study ASFV pathogenesis and open new avenues to be used in the future as a diagnostic tool.

\section{Acknowledgements}

This work was funded by the Spanish Ministry of Science and Innovation (projects Consolider -"Porcivir" CDS2006-00007 and AGL200766441-C03-01/GAN).

\section{References}

1. Dixon et al (2008) Animal viruses: molecular biology, chapter 10.

2. Oura et al (1998) J Virol Methods 72, 205-17

3. Tabarés and Sánchez-Botija (1979) Arch Virol 61, 49-59.

4. García-Beato et al (1992) Virology 188, 637-49. 


\title{
African swine fever threats: are we ready?
}

\author{
C. Gallardo ${ }^{1}$ A. Soler ${ }^{1}$ A. Simón ${ }^{1}$ E. Martín ${ }^{1}$ R. Martín ${ }^{1}$ V. Pelayo ${ }^{1}$ JM. Sánchez-Vizcaíno² E. Okoth ${ }^{3}$ \\ R. Bishop ${ }^{3}$ MA. Sánchez ${ }^{1}$ G. de Mi ${ }^{4}$ E. Couacy-Hymann ${ }^{5}$ M. Arias ${ }^{1}$ \\ 1. Community Reference Laboratory for African Swine Fever, CISA-INIA, Madrid, Spain; 2. OIE World Reference Laboratory for African Swine Fever, \\ Veterinary Faculty, UCM, Madrid, Spain; 3. International Livestock Research Institute, Nairobi, Kenya; 4. Istituto Zooprofilattico Sperimentale dell \\ "Umbria e delle Marche", Perugia, Italy; 5. Ministère de la Production Animale et des Ressources Halieutiques -LANADA-LCPA, Abidjan, Ivory Coast
}

African swine fever (ASF) is one of the most complex and lethal disease of swine. The disease transmission involves domestic pigs, wildboars, warthogs and bushpigs. ASFV usually induces unapparent infection in a variety African wildboar population. Soft ticks (Ornithodoros spp) act as reservoir and biological transmission vectors.

ASF is endemic in the majority of sub-Saharan Africa. Within EU, it is confined to Sardinia. On June 2007, ASF was notified in the Caucasus region, in Georgia. It was associated to a new isolate, related to p72 genotype II, circulating in East Africa. Since then, ASF progressed to neighbouring countries Armenia, Azerbaijan and Russian Federation, reaching the near border with Ukraine, and, on October 2009, jumping to St Petersburg in north-western Russia, in the Baltic Sea. Current situation of ASF threatens the EU countries, Eastern Europe, the Black Sea basin countries and - in the worst case scenario - central Asia and even China, which has the largest pig population in the world.

There is no vaccine available and control strategies are based on rapid laboratory diagnosis. But, are we ready to combat with ASF? Would current diagnostic techniques be sensitive enough taking into consideration the new circulating viruses?

From 2000 to date, reliable, specific, and fast PCR tools have been developed and validated for virus detection. They have been shown to be highly sensitive for the detection of the new current circulating isolates in Europe and Africa.

However, serological surveillance studies performed in East Africa among 2004-2009, have shown low seroprevalence with, in contrast, a high incidence of virus in domestic pigs. This could be related to genome variability of antigenic ASF proteins in Eastern African isolates, the more variable and genotypically distant. To what extent current serological diagnostic techniques might be missing some of these new variants?
To study these questions, different ASFV were selected on the basis of genome variability criteria and date collection. Particular emphasis was placed to those belonging to $p 72$ genotype $X$, the most variable, and $p 72$ genotype II, current European circulating genotype. After successfully adapted to grow in COS cells, new soluble cytoplasmic antigens were used in indirect ELISA tests for initial standardization. To evaluate the capability and competence of these new tests with regards to the formal ASF serological tests, a comparative study is carrying out using a wide panel of serum comprising: i) experimental sera from "in vivo" experiments with East Africa and Caucasus ASFV circulating isolates ii) field sera from East-West Africa and Sardinia from 2004 to 2009. The preliminary results using the new $p 72$ genotype $X-$ based antigen indicate correlation to those using OIE serological tests and INGEZIM PPA.K3 Compac ${ }^{\circledast}$. It's important to highlight the results achieved analysing negative serum samples from "apparently healthy" virus-positive pigs from East Africa. The capability of new others ASFV antigens from Africa and Caucasus to be used in ASF serological tests is under evaluation.

Theses results together with those obtained from in vivo experiments at CISA-INIA with recent isolates from East Africa and Caucasus regions, could be the first approach demonstrating the capability of the formal diagnostic techniques to perform a serological diagnosis with high sensitivity, specificity and confidence, adapted to current epidemiological situations.

\section{Acknowledgments}

Work at INIA was supported by Community reference laboratory for ASF (UE-1 LR PPA/03) and by the European project ASFRISK project (Grant Agreement) KBB211691 


\title{
P.542
}

\section{The role of sylvatic cycle involving wild pigs, ticks and domestic pigs in the epidemiology of African swine fever in Kenya}

\author{
C. Gallardo ${ }^{1}$ V. Pelayo ${ }^{1}$ E. Okoth ${ }^{2}$ M. Arias ${ }^{1}$ E. Martín ${ }^{1}$ A. Simón ${ }^{1}$ R. Nieto ${ }^{1}$ M. del Pozo ${ }^{1}$ C. Pérez ${ }^{1}$ R.P. Bishop ${ }^{2}$ \\ 1. CommunityReference Laboratory for ASF. Centro de Investigación en Sanidad Animal, INIA, Madrid, Spain; \\ 2. International Livestock Research Institute, Nairobi, Kenya
}

\section{Introduction}

African swine fever (ASF) is a lethal, hemorrhagic disease of domestic pigs for which animal slaughter and area quarantine are the only methods of control. Since first description of ASF in Kenya in 1921, the disease was maintained in African until the initial export in 1957 to Lisbon spreading between 1957 to 1990 throughout Europe and America. Currently ASF is present in Sardinia, in Caucasus Republics and in most than twenty SubSaharan Africa countries, where ASFV can infect hosts through either a domestic cycle or a sylvatic cycle involving warthogs and bushpigs as well as ticks of the genus Ornithodoros.

In 2006, ASF broke out among commercial pigs in Kenya-Uganda border and spread in 2007 to Central Kenya around the city of Nairobi, after an apparent absence from the entire country since 2001. The outbreaks were traced to movements of domestic pigs and were not linked to warthogs or bushpigs. However, Kenya has had several outbreaks caused by regular reemergence of ASFV since its first description in 1921. These outbreaks could be result primarily from direct contact between infected domestic pigs with tick-involvement. In addition, experimental evidence suggests that pigs can exceptionally become infected from eating infected warthog tissues playing an important role in the transmission of the disease in the country. We report the first extensive study to investigate the role of sylvatic cycle involving warthogs, O. porcinus porcinus ticks and domestic pigs in the epidemiology of ASF in Kenya.

\section{Materials and Methods}

Samples from 83 ASF asymptomatic domestic pigs, 51 warthogs and a total of 1,576 Ornithodorus porcinus porcinus ticks from 26 independent warthog's burrow were collected from area of interface between domestic and wild pigs in Central Kenya, where ASF had been reported. Their infection status with ASFV was determined using combined $p 72$-based PCRs, restriction analyses and virus isolation. In addition, sera from wild suids and domestic pigs were tested for anti-ASF antibodies using OIE serological prescribed methods. Different ASFV isolates were selected for genotyping purposes using a combination of full length $p 54$-gene and p32-gene sequencing [1, 2], partial p72-gene sequencing [3], and analysis of tetrameric amino acid repeat regions within the variable region of the $B 602 \mathrm{~L}$ gene (CVR) [4].

\section{Results}

The presence of ASFV was determined in 62 of 285 (22\%) 0. porcinus pools, 3 of 51 (3.22\%) warthogs serum samples and 41 of 83 (49\%) domestic pigs serum samples. Interestingly, no seropositive response was found in any domestic pig in contrast with the warthog's results where all of them were seropositives. Twenty O. porcinus, twelve domestic pigs and three warthog ASFV isolates were selected for genotyping. Phylogenetic analysis indicated that the ASFV isolates obtained in this study from ticks and healthy domestic pigs clustered in a sylvatic cycle P72 genotype $X$, whose range encompasses viruses from Rwanda, Burundi, Tanzania and Kenya, whereas ASFV isolates collected from warthogs were placed into p72 genotype IX related to recent domestic pig outbreaks occurred in Kenya in 2007.

\section{Discussion}

The detection and characterization of ASFV in warthogs, ticks and domestic pigs in this study verifies and expand on the first report of the relevance of vertebrate and invertebrate hosts in the transmission and maintenance of ASF following disease outbreaks in Kenya. Together these results signal the possible establishment of domestic pig- warthog and domestic pig-tick cycle transmission playing an important role in the maintenance and possible reemergence of future outbreaks of the disease in Kenya. This highlights the complex epidemiological situation in Eastern African countries which complicate control of the disease in these regions and emphasize the requirement for improved regulation and monitoring of domestic pig as well as a most effectiveness control by preventing contact between domestic pigs and wild suids and their ticks.

\section{References}

[1] Gallardo et al (2009). Virus Genes Feb;38(1):155-70

[2] R.J Rowlands (2009) Emerg Infect Dis. December; 14(12): 18701874.

[3] Bastos A.D.S. et al., (2003). Arch Virol 148 (4), 693-706I

[4] Nix et al., (2006). Arch Virology Dec; 151(12):2475-94.

\section{Acknowledgments}

Work at INIA was supported by Community reference laboratory for ASF (UE-1 LR PPA/03) and by the European project ASFRISK project (Grant Agreement) KBB211691 


\title{
P.543
}

\section{Immunogenicity of commercial vaccines for the control of Porcine Rubulavirus causing Blue Eye Disease}

\author{
Francisco Quezada-Monroy; Ruben Echeveste-Garcia de Alba; Bernardo Lozano-Dubernard; \\ David Sarfati-Mizrahi; Ernesto Soto-Priante; Horacio Lara-Puente \\ Laboratorio Avi-Mex, S.A de C. V., Mexico City, DF, Mexico
}

\section{Introduction}

Porcine Rubulavirus is the causal agent of Blue Eye Disease (BED). This is an endemic disease of the central area of Mexico, been the only country reporting the disease (Stephano et al, 1981). The objective of this study was to analyze the capacity of commercial vaccines to stimulate the pig immune system to produce neutralizing antibodies.

\section{Material and Method}

3 weeks-old SPF piglets were divided in 2 groups. The first group with a commercial inactivated vaccine $A(n=16)$ and the second group with a commercial inactivated vaccine $B(n=16)$. Both groups were vaccinated at days 0 and 14 of the experiment with a dose of $2.0 \mathrm{~mL}$. Blood samples were taken on days 0, 14, 21 and 28 post vaccinations (PV). Antibody detection was done by means of Virus Neutralization (VN) test, with two-fold dilutions in a 12 wells plaque. A positive result was considered when the sample reached a titer equal or higher than 1:16 (Ramirez et al, 1996).

\section{Results}

Twelve animals died due to causes not related to the trial. Group A started with 13 pigs and group B with 7.

For vaccine $A$, sero-conversion was first detected on day $14 \mathrm{PV}$ (76.92\%), and by day 28 PV all animals were positive, as shown in graph 1.

For vaccine $\mathrm{B}$, sero-conversion was first detected on day $28 \mathrm{PV}$ (28.57\%), as shown in graph 1.

\section{Discussion and Conclusions}

Vaccine A showed a better capacity to stimulate the immune system of SPF pigs (antibody level was 1:128 at day 14, reaching 1:2048 on day 28). Vaccine B showed a lower capacity to stimulate the immune system of SPF pigs (at day 28 antibody level varied from 1:16 to 1:256). Statistical differences were detected among both groups at days 14,21 and 28 using a $T$ test $(P=0.05)$.

Serological response differences found for both commercial inactivated vaccines can be due to several factors such as the seed used, viral titer before inactivation, quantity and quality of antigen, inactivation process, production process and type of adjuvant, among others.
Graph1. Comparative neutralizing antibody levels induced by 2 applications of two commercial inactivated Blue Eye Disease vaccines.

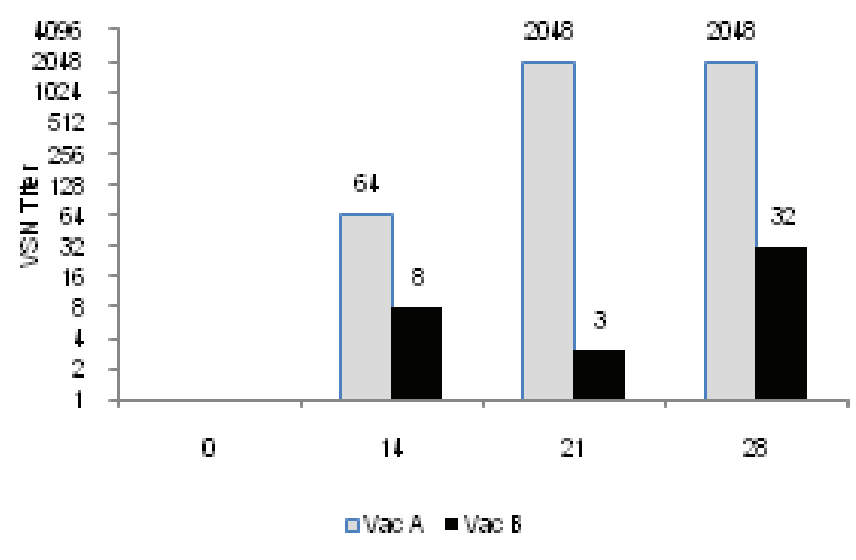

\section{Bibliography}

Ramirez M.H. et al, Vet. Mex. 27(3):257- 259 (1996).

Stephano H.A. et al, XVII AMVEC. Pp. 46-47 (1981). 


\title{
P.544
}

\section{Cross serological reactions obtained in SPF pigs inoculated with different Rubulavirus isolates (Blue Eye Disease)}

\author{
Horacio Lara-Puente; Ruben Echeveste-Garcia de Alba; Bernardo Lozano-Dubernard; \\ David Sarfati-Mizrahi; Ernesto Soto-Priante; Francisco Quezada-Monroy
}

Laboratorio Avi-Mex, S. A. de C. V., Mexico City, DF, Mexico

\section{Introduction}

A controversy in Veterinary Medicine in reference to the existence of cross reaction or cross immunity exists, the fact that this phenomenon can affect the vaccination and diagnose processes. A classical example is Newcastle disease virus, any vaccine strain protects against any pathogenic isolate, independently of the homology percentage and is equally employed in laboratory tests. In pigs PRRS virus (1), which can or can't protect against different isolates when employed as a vaccine. In a negative sense we find Bovine Viral Diarrhea (BVD) and Classical Swine Fever (CSF)(2), since the presence of BVD antibodies can yield a false positive result for CSF. The objective of this study was to acknowledge cross reactions that exists between antibody levels detected in serum of challenged animals and confronted with isolates of homologous and heterologous Blue Eye Disease (BED) isolates.

\section{Material and Method}

Nine SPF 8 weeks old pigs were employed distributed as follows: Group A ( $n=3)$, Group B $(n=3)$ and Group C $(n=3)$. The animals were challenged with isolates of BED virus, from 1999 (virus A), 2000 (virus B) and 2006 (virus C). Challenge dose was $10^{5.0}$ TCID $50 \% / \mathrm{mL}$ administered $1.0 \mathrm{~mL} / \mathrm{IM}$ and $1.0 \mathrm{~mL} / \mathrm{IN}$. Blood samples were taken from 0 days post challenge $(\mathrm{dpc})$ and up to $36 \mathrm{dpc}$, with intervals of 4 days. Virus sero neutralization test (VSN) was employed with serial double dilutions starting at 1:2 and up to 1:4096; a positive result was considered with a titer of 1:16. For such test all 3 virus ( $A, B$ and $C$ ) were employed as test antigens $\mathrm{Ag}$ ) for the virus employed in the challenge (An antigen homologous to each challenge isolate) and also a 4th virus isolated in 1992 (virus D) (heterologous antigen for all the challenge isolates).

\section{Results}

At days 0 and 4 post challenge (PC) all animals remained negative. At day 8 PC group $A$ became positive to $A g A$ and $D$ but remained negative to $B$ and $C$; Group $B$ became positive to $A g A, B$ and $D$ but negative to $C$; Group $C$ became positive to $A g A$ and $D$ but negative to $B$ and $C$. At days 12 and 16 animals form group $A$ were negative to antigen $C$ but positive to $A, B$ and groups $A$ and $\mathrm{C}$, became positive to all $4 \mathrm{Ag}$ used with mid to low titers. From day 20 to 36 all animals became positive to all four Ag with mid to high titers (see table 1). It was noticeable that challenge virus $\mathrm{C}$ was less antigenic than the other 2 towards the 4 tested $\mathrm{Ag}$. It is also noticeable that using $\mathrm{Ag} \mathrm{D}$ a higher titer of antibodies was reached, even in comparison with homologous reactions.
Table 1. Average serological results at different days PC using VSN tests.

\begin{tabular}{|c|c|c|c|c|c|}
\hline Sampling & Challenge & Ag A & Ag B & Ag C & Ag D \\
\hline 3 DPC & Virus A & $1: 20$ & $1: 12$ & $1: 5$ & $1: 34$ \\
\hline 3 DPC & Virus B & $1: 24$ & $1: 20$ & $1: 6$ & $1: 32$ \\
\hline 3 DPC & Virus C & $1: 17.33$ & $1: 14$ & $1: 5.33$ & $1: 28$ \\
\hline 16 DPC & Virus A & $1: 144$ & $1: 32$ & $1: 4$ & $1: 64$ \\
\hline 16 DPC & Virus B & $1: 256$ & $1: 128$ & $1: 128$ & $1: 512$ \\
\hline 16 DPC & Virus C & $1: 96$ & $1: 96$ & $1: 64$ & $1: 192$ \\
\hline 24 DPC & Virus A & $1: 256$ & $1: 256$ & $1: 256$ & $1: 512$ \\
\hline 24 DPC & Virus B & $1: 256$ & $1: 126$ & $1: 128$ & $1: 512$ \\
\hline 24 DPC & Virus C & $1: 128$ & $1: 128$ & $1: 64$ & $1: 512$ \\
\hline 36 DPC & Virus A & $1: 256$ & $1: 256$ & $1: 128$ & $1: 512$ \\
\hline 36 DPC & Virus B & $1: 128$ & $1: 256$ & $1: 128$ & $1: 256$ \\
\hline 36 DPC & Virus C & $1: 128$ & $1: 64$ & $1: 64$ & $1: 512$ \\
\hline
\end{tabular}

\section{Discussion}

Cross reaction is given in function to characteristic or antigenic distance between the virus that produces immunity and the employed virus, for the case, in VSN. For this study the cross reaction was from low to negative intensity at 8 days PC, the antibodies of the 3 challenged groups being able to detect A and D antigens but in low titers, 1:20 and 1:30 respectively. As days PC passed the antibody levels became mid to high, antibodies from all 3 challenged groups identified all $4 \mathrm{Ag}$. C antibodies were unable to detect homologous virus in comparation to $A$ and $B \mathrm{Ag}$ at 8 days $P C$. Antibodies had higher affinity to $A$ and $D$ from day $8 \mathrm{PC}$ and posterior samplings. The cross reaction can be linked to the viral capacity to stimulate high antibody titers as observed with $\mathrm{Ag} \mathrm{C}$, the less antigenic.

\section{Bibliography}

1. http://www.exopol.com/general/circulares/231.html

2 http://www.senasica.sagarpa.gob.mx/web/ropuestas_web/221204/ salud_animal/Fiebre Porcina Clasica/Manual sobre FPCFAO.doc 


\title{
P.545
}

\section{Potency of a Blue Eye Disease commercial vaccine against 3 different viral challenges (Lung lesions)}

\author{
Horacio Lara-Puente; Bernardo Lozano-Dubernard; David Sarfati-Mizrahi; Ernesto Soto-Priante; \\ Francisco Quezada-Monroy; Ruben Echeveste-Garcia de Alba
}

Laboratorio Avi-Mex, S. A. de C. V., Mexico City, DF, Mexico

\section{Introduction}

Blue Eye Disease (BED) was observed in the 80's in farms around La Piedad, Michoacan, Mexico. Affecting pigs of different ages. Lung damage caused by the virus is of capital importance since the loss of pulmonary capacity repercutes in productive parameters and days to market, impacts medication costs and pigs become diffusers of the disease in the farm and the geographical zone (ISU 2006 and Hernandez et al 2004). The objective was to determine the lung lesion percentage produced by 3 different isolates of BED and to evaluate the protective response of a vaccine by reduction of lung lesions against these 3 genetically different viruses.

\section{Material and Method}

Virus: 3 isolates collected in Mexico between 1980 and 2007. Animals: 28 SPF pigs between 5 and 6 weeks of age distributed in 7 groups, 4 piglets each, Negative Control (NC), Positive control Challenged with A virus (PCCAV), Positive Control challenged with $B$ virus (PCCBV), Positive Control Challenged with $C$ virus (PCCCV), Vaccinated Challenged with $A$ virus (VCAV), Vaccinated challenged with B virus (VCBV) and Vaccinated Challenged with $C$ virus (VCCV). All groups were maintained with strict biosecurity measures, with daily monitoring of rectal temperature and clinical signs. Blood samples were drawn at day 0 and repeated every 7 days. On day 0 pigs were vaccinated with $2.0 \mathrm{~mL} / \mathrm{IM}$ of a commercial vaccine against BED, repeating at day 17. Challenge was performed 15 days post vaccination (pv) using 3 viral isolates ( $A, B$, and $C$ ) with a genetic homology of $100 \%, 99.7 \%$ and $97.6 \%$ respectively, using $1.0 \mathrm{~mL} / \mathrm{IM}$ and 1.0 $\mathrm{mL} / \mathrm{IN}$ at $10^{5.0} \mathrm{CCID} 50 \% \mathrm{~mL}$. At day $18 \mathrm{pc}$ all animals were sacrificed and necropsied, lung lesions were evaluated and blood was collected. Lung lesions were valorated by the planimetry method described by Ciprian et al (1988). An ANOVA test and Tukeýs test was done to the collected data.

\section{Results}

Lung lesions on pig groups PCCAV, PCCBV and PCCCV reached an average of $6.9 \%, 8.0 \%$ and $2.2 \%$ respectively. On groups VCAV, VCBV and VCCV this average lowered to $1.5 \%, 3.6 \%$ and $0.3 \%$ that is a reduction of a least $55 \%$ (Table 1, Graph 1).
Table 1. Lung Lesion Average.

\begin{tabular}{|c|c|c|c|c|}
\hline $\begin{array}{c}\text { Challenge } \\
\text { virus }\end{array}$ & $\begin{array}{c}\text { Negative } \\
\text { control }\end{array}$ & Challenged & $\begin{array}{c}\text { Vaccinated } \\
\text { Challenge }\end{array}$ & $\begin{array}{c}\text { Reduction of } \\
\text { lesion }\end{array}$ \\
\hline A & 0 & 6.9 & 1.5 & 78.3 \\
\hline B & 0 & 8.0 & 3.6 & 55.0 \\
\hline C & 0 & 2.2 & 0.3 & 86.4 \\
\hline Average & $0 \mathrm{a}$ & $5.7 \mathrm{~b}$ & $1.8 \mathrm{c}$ & 73.2 \\
\hline \multicolumn{5}{|l}{ Different literals indicate statistical differences $(\mathrm{P} \leq 0.05)}$. \\
\hline
\end{tabular}

Graph 1. Percentage of Lung Lesions

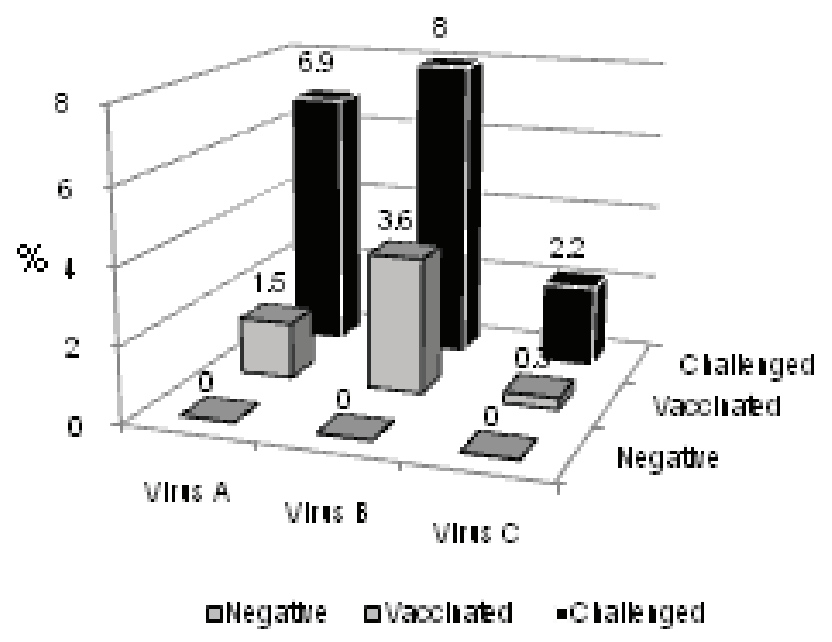

\section{Discussion/Conclusions}

The commercial BED vaccine clearly showed effectively lung lesions reduction and protected independently to genetic differences of the challenge virus. No statistical difference was found between lesion reduction and viral genotype employed in challenge, however it was found between challenged pigs and vaccinated challenged pigs $(P \leq 0.05)$.

\section{Bibliography}

Porcine Rubulavirus infection lowa State University, Institute for International Cooperation in animal biologics. (2006).

Hernández J., et al. Vet Mex (35) 1. (2004).

Ciprián A., et al. Can. J. Vet. Res., 52: 434 438. (1988). 


\title{
P.546
}

\section{Clinical response in SPF pigs challenged with isolates of Porcine Rubulavirus (Blue Eye Disease)}

\author{
Ruben Echeveste-Garcia de Alba; Bernardo Lozano-Dubernard; David Sarfati-Mizrahi; Ernesto Soto-Priante; \\ Francisco Quezada-Monroy; Horacio Lara-Puente \\ Laboratorio Avi-Mex, S. A. de C. V., Mexico City, DF, Mexico
}

\section{Introduction}

Porcine Rubulavirus affect swine and produces nervous signs and high mortality in 2 to 5 days-old piglets. It also produces reproductive failures. Affected sows increase the wean to service and repetitions rate, it also increases the number of stillbirths and mummies; in males, libido and semen quality are affected due to a permanent damage to testicular structures. In affected animals, regardless to their age, corneal opacity (blue eye) can be observed around 30 days post-infection in about $10 \%$ of the herd. The most important macroscopic lesion observed in pigs of all ages is pneumonia (consolidation zones) affecting the ventral region of the cranial lobes. Such clinical sign depends on the age, immunological status, production system and concomitant infections present (Stephano et al, 2002). The objective of this study was to acknowledge the behavior of 3 Porcine Rubulavirus isolates in relation to days with fever and percentage of macroscopic lung lesions in order to correlate fever with lung damage.

\section{Material and Method}

Eight weeks old SPF pigs (Avifarma, SA de CV, Mexico) were divided in 3 groups and challenged with different BED isolates. Group A ( $n=3)$ challenged with isolate A (1999); Group B $(n=3)$ challenged with isolate B (2000), and Group C $(n=3)$ challenged with isolate $C$ (2006). Challenge with any of all isolates was done with a $10^{5.0}$ TCID50\% administered by intramuscular and intranasal routes ( $1 \mathrm{~mL}$ each). Rectal temperature and clinical signs were monitored daily. At day 18 post challenge (PC), one pig from each group was humanely sacrificed in order to evaluate lung lesions. At the end of the experiment on day 36 PC, all pigs were humanely euthanized and lung lesions were evaluated. Lung lesions score was performed according to the planimetry method described by Ciprian et al (1988). The correlation between fever and lung lesion was analyzed.

\section{Results}

Among the 3 viral isolates employed, virus $B$ showed the highest number of days with fever (24) and also the highest percentage of lung lesion (8\%), compared with the other 2 viruses employed (see graph 1), which indicates a direct correlation among fever and lung damage. Pigs also had a fever peak at day 3-5 PC in all 3 groups.

\section{Discussion}

A direct correlation between days with fever and lung lesions was detected, the higher the numbers of days with with fever the higher the percentage of lesions. Pigs had a higher immune response towards virus $A$ and $B$ than to $C$. This is coincident with field observations regarding clinical signs. Apparently signology and clinical lesions differ towards what was described in the initial reports of the disease in 1980. The lung damage and days with fever induced by Rubulavirus or any other pathogen has a lot of impact in pigs, due to the loss of lung capacity and appetite loss, which in time repercutes in productive parameters as daily gain, alimentary conversion and in consequence days to market, and also negatively impacts medication costs in order to control secondary pathogens.

\section{Graph 1. Average of lung lesions and days with fever}

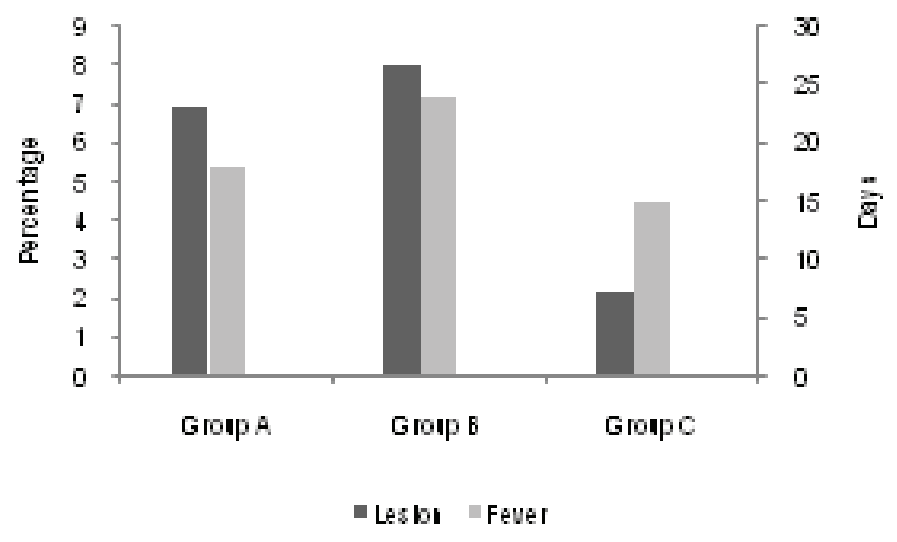

\section{Bibliography}

Stephano A. et al. Trends in emerging viral infections of swine $p 47$ (2002).

Ciprian A. et al, Can J. Vet. Res. 52:434-438.(1988). 


\title{
Impact in days with fever after the employment of a commercial Blue Eye Disease vaccine against 3 viral challenges
}

\author{
Horacio Lara-Puente; Bernardo Lozano-Dubernard; David Sarfati-Mizrahi; Ernesto Soto-Priante;
}

Francisco Quezada-Monroy; Ruben Echeveste-Garcia de Alba

Laboratorio Avi-Mex, S. A. de C. V., Mexico City, DF, Mexico

\section{Introduction}

Blue Eye Disease (BED), was originally described in Mexico in the 80 's. Besides the lesions produced by the viral replication it is important to consider fever as a generator of productive losses since an animal during this process eats very little or nothing at all, situation that repercutes in its productive efficiency and also converts them into easy targets for other pathogens (Hernández, et al, 2004). The objective of this research was to evaluate the impact of the use of a BED commercial vaccine in days with fever in animals challenged with 3 genetically different BEDv isolates.

\section{Material and Method}

Virus: 3 BED isolates, obtained during 1999 - 2006 (Quezada, et al, 2008). Animals: Twenty eight SPF, 5 - 6 weeks old pigs, distributed in 7 groups of 4 pigs each: (NC) Negative Control, Positive control Challenged with A virus (CA), Positive Control challenged with $B$ virus (CB), Positive Control Challenged with $C$ virus (CC), Vaccinated Challenged with A virus (VCAV), Vaccinated challenged with B virus (VCBV) and Vaccinated Challenged with $C$ virus (VCCV). All groups were maintained in strict biosecurity measures, with daily monitoring of rectal temperature and clinical signs. For temperature monitoring infrared thermometers (Dottori UR 100), were employed, reading the temperature on the neck area posterior to the ear of the pigs. In order to determine presence or absence of fever the average daily temperature of $\mathrm{NC}$ was employed, we determined a group average and Standard deviation (SD) was calculated, we added twice this value to the obtained average, all data above this rank was considered as fever. On day 0 they were vaccinated with $2.0 \mathrm{~mL} /$ IM of a commercial BED vaccine, repeating it 17 after; challenge was done 15 after revaccination, using 3 different isolates (A, B and $C$ ) with a genetic homology of $100,99.7$ and $97.6 \%$ respectively, using $1.0 \mathrm{~mL} / \mathrm{IM}$ and $1.0 \mathrm{~mL} / \mathrm{IN}$ at $10^{5.0} \mathrm{TCID}_{50} \% / \mathrm{ml}$. At day 36 post challenge all animals were sacrificed, and necropsied.

\section{Results}

None of the NC group had fever or clinical signs at all. The per se effect of the vaccination on the pigs was minimal and was detected as fever 1 days PV that spontaneously receded without any other effect. On vaccinated/challenged groups with isolates A, B or C (VCAV, VCBV and VCCV) between 1 and 3 days with fever were detected (Table 1), on non vaccinated/challenged groups (CA, CB and CC) had between 15 to 24 days with fever (Graph 1)
Table 1. Days with fever

\begin{tabular}{|c|c|c|c|}
\hline Virus & Negative Control & Challenged & Vaccinated \\
\hline A & 0 & 18 & 1 \\
\hline B & 0 & 24 & 3 \\
\hline C & 0 & 15 & 3 \\
\hline
\end{tabular}

Graph 1. Days with fever, challenged animals and vaccinated/ challenged animals.

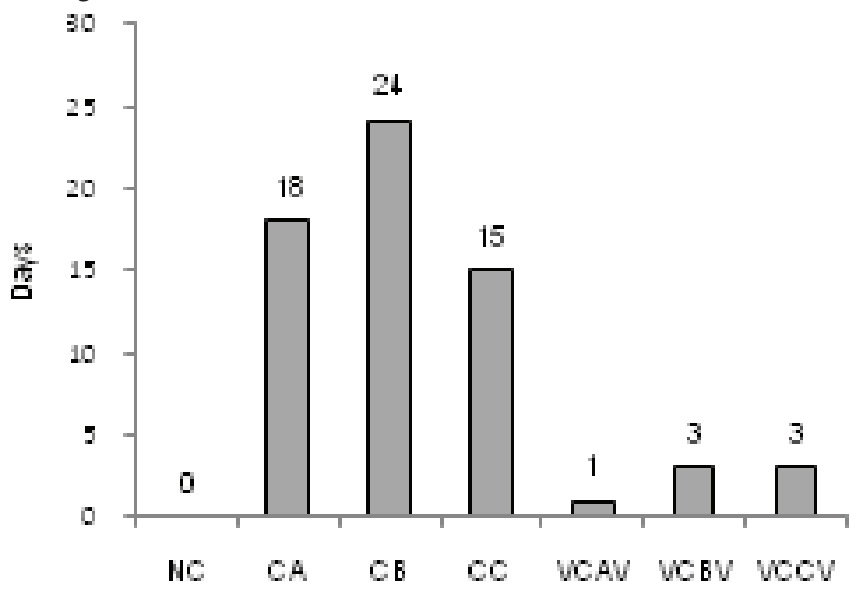

\section{Conclusions}

Clinical manifestations of viral infection on SPF pigs were less severe than expected. It is probable that secondary infections in commercial pigs and other unfavorable environmental farm conditions become a factor in the clinical onset of the disease along with the pigs age. The decrease of the disease's impact in the field by employing a commercial vaccine is shown not only in the adverse effects of the infection but with a reduction of at least $80 \%$ of days with fever after challenge, which in turns manifests as a considerable improvement in the herd.

\section{Bibliography}

Hernández J. et al, Vet. Mex. (35) p1 2004.

Quezada M. F. et al, Memorias AMVEC Querétaro, 2008.

\section{Non cited Bibliography}

Porcine Rubulavirus Infection, lowa State University, Institute for international cooperation in animal biologics. 2006. 


\title{
P.548
}

\section{Hemmaglutination inhibition test using different porcine Rubulavirus Isolates (Blue Eye Disease)}

\author{
Francisco Quezada-Monroy ${ }^{1}$ Efrain Verde-Rojo ${ }^{2}$ Ruben Echeveste-Garcia de Alba ${ }^{1}$ Bernardo Lozano-Dubernard ${ }^{1}$ \\ Ernesto Soto-Priante ${ }^{1}$ David Sarfati-Mizrahi ${ }^{1} \underline{\text { Horacio Lara-Puente }}^{1}$ \\ 1. Laboratorio Avi-Mex, S. A. de C. V., Mexico City, DF, Mexico; 2. Diagnosticos Clinicos Veterinarios, Mexico City, DF, Mexico
}

\section{Introduction}

Currently the diagnostic for Porcine Rubulavirus by antibody detection is done through different serologic tests such as Immunoperoxidase, ELISA, Virus Sero Neutralization (VSN) and Hemmaglutination Inhibition $(\mathrm{HI})$ test. $\mathrm{HI}$ has been widely employed for Blue Eye Disease (BED) diagnostics and seroepidemiology due to its easiness of implementation and that the results are repeatable, reproducible, and of low cost, when compared to other tests (Gonzalez, 2002). The objective of this study was to compare the results obtained by $\mathrm{HI}$ tests using 2 different isolates of Porcine Rubulavirus.

\section{Materials and Methods}

Samples: 144 field sera.

Virus: two different isolates of different origin were employed.

Hemmaglutination Inhibition test $(\mathrm{HI})$ : All samples were inactivated at $56^{\circ} \mathrm{C}$ for 30 minutes. Then adsorbed as follows: $100 \mu \mathrm{l}$ of serum, $50 \mu \mathrm{l}$ kaolin and $50 \mu \mathrm{l}$ of $5 \%$ bovine erythrocytes resting all night. Once adsorbed, double serum dilutions were employed from 1:4 and up to 1:512, $8 \mathrm{HAU}$ were added incubating 30 minutes at room temperature, once finished bovine erythrocytes at $0.5 \%$ were added. Sample was considered positive when they showed a titer equal or higher to 1:16 (Ramirez, 1996).

\section{Results}

Using Virus A we detected 91 positive and 53 negatives sera; virus $B$ detected 85 positive and 59 negative sera (Table 1). It was noticeable that in $80.56 \%$ of the samples there were no differences in the dilutions or only one difference, with 2 differences $13.19 \%$ and with 3 or more $6.25 \%$ (Table 2). To know if there were differences among results, a T student test was performed and did not found statistical differences among the results employing both viruses $(p=0.01)$
Table 1. HI test results with different isolates.

\begin{tabular}{|c|c|c|}
\hline Virus & Positives & Negatives \\
\hline A & $63.19 \%$ & $36.81 \%$ \\
\hline B & $59.03 \%$ & $40.97 \%$ \\
\hline
\end{tabular}

Table 2. Differences in the number of dilutions among sera.

\begin{tabular}{|c|c|}
\hline Differences & Serum Samples \\
\hline 0 & $56(38.89 \%)$ \\
\hline 1 & $60(41.67 \%)$ \\
\hline 2 & $19(13.19 \%)$ \\
\hline 3 & $7(4.86 \%)$ \\
\hline 4 & $1(0.69 \%)$ \\
\hline 5 & $1(0.69 \%)$ \\
\hline
\end{tabular}

\section{Discussion}

In this study there were samples which did not completely agreed in their results using both viruses, $6.25 \%$ of the samples had 3 or more dilution differences, this may be due to the percentage of false positive and false negative inherent to the $\mathrm{HI}$ test (Hernandez, 1992).

We did not found any statistical difference employing both isolates at the moment of the report of the results of the HI test, It has been said that result differences may exist in the test results using different viruses (Sanchez, 2004), but our results agree with the theory that there are no antigenic variations among the different isolates of Porcine Rubulavirus.

\section{Bibliography}

González V.D. Trends in emerging viral infections of swine. lowa State Press Pp. 55-57 (2002).

Ramírez MH. Vet. Mex. 27(3):257-259 (1996).

Hernández JP. Vet. Mex. 23(3):217-22 (1992).

Sánchez BJI. PanVet (2004). 


\title{
P.549
}

\section{Real time PCR diagnosis of Blue Eye disease}

\author{
Alvarado I. Arcelia ${ }^{1}$ Cuevas R. Sandra ${ }^{1}$ Colmenares V. German ${ }^{1}$ A. A. Blormstron ${ }^{1,2}$ Garcia F. Arturo ${ }^{1}$ Mejia S. Pedro ${ }^{1}$ M. M. Berg ${ }^{2}$
}

1. INFAP-CENID-Microbiologia Animal, México, DF, Mexico; 2. Upsala Sweden University, Suecia, Sweden

\section{Introduction}

Currently, Blue Eye Disease represents a barrier for the exportation of swine; it is a disease that affects pigs at different ages, although 2 to 15 day old piglets are the most susceptible. Most notable symptoms are changes in behavior, nervous alterations, respiratory failure, and reproductive alterations in dams and sires and in some cases corneal opacity of one or both eyes.

The objective of this study was the development of a sensitive and specific enough diagnostic method that could to detect pigs persistently infected with the porcine Rubulavirus (PoRVLPMV) that is the causal agent of this disease.

\section{Materials and Methods}

This method was developed and standardized for the detection of the gene fragment that codifies for the porcine Rubulavirus phosphoprotein, in tissue and blood samples. To read it, the assay was adapted to a Light Cycler 1.5 system. For standardizing this technique a plasmid developed in the University of Agriculture of Sweden was used, which is formed by a 1384 base pairs of gene fragment that codifies for the PoRV-LPMV phosphoprotein. Serial decimal dilutions were performed with this plasmid to establish the sensitivity of the assay and then the assay was tested on samples from naturally and experimentally infected animals in order to validate it.

\section{Results}

Results obtained showed an assay sensitivity of 10 copies for detecting the positive plasmid control. Assay conditions were established according to the protocol corresponding to the Light Cycler TaqMan Master, whereby the presence of porcine Rubulavirus was established. The assay has $1.98 \%$ efficiency according to the Light Cycler Instrument 1.5 equipment system.

\section{Discussion and Conclusions}

Real time PCR technique is efficient for detecting with high sensitivity animals that carry the porcine Rubulavirus since it is possible to carry out the diagnosis with up to a minimum of 10 phosphoprotein gene copies. Technology developed may be transferrable to national diagnostic laboratories.

\section{References}

Duvigneau JC, Hartl RT, Groiss S, Gemeiner M. (2005). J of Immunological Methods 306: 16-27.

Belák, S. And Thorén. (2001) Expert Rev Mol Diagn. 1, 434-443.

Berg M, Sundqvist A, Moreno-Lopez J, Linne T. (1991)..J Gen Virol. 72 :1045-50.

Hjertner B, Linne T, Moreno-Lopez J.(1997). Acta Vet Scand. 38(3):213-24.

McNeilly F, Walker I, Allan GM, Foster JC, Linne T, Merza M, Hernandez P, Kennedy S, Adair B.(1997). J Vet Diagn Invest. 1997 Jan;9(1):3-9. 


\title{
P.550
}

\section{Persistence of Porcine Rubulavirus (PoRV) in experimentally infected boars}

\author{
Jose Francisco Rivera-Benitez ${ }^{1}$ Rebeca Martinez-Bautista ${ }^{1}$ Adelfa Del Carmen García-Contreras ${ }^{2}$ \\ Julio Roberto Reyes-Leyva ${ }^{3}$ Jesus Hernandez Lopez ${ }^{4}$ Humberto Ramirez-Mendoza ${ }^{1}$ \\ 1. Departamento de Microbiologia E Inmunologia, FMVZ-UNAM, Distrito Federal, DF, Mexico; 2. DCBS, DPAA, UAM-X, Distrito Federal, DF, Mexico; \\ 3. CIBIOR, IMSS, Puebla, COAH, Mexico; 4. CIAD, Sonora, SON, Mexico
}

\section{Introduction}

Porcine rubulavirus (PoRV) is the etiological agent of blue eye disease (BED) of pigs that has been endemic in Mexico (Kirkland \& Stephano, 2006). The clinical signs are age-dependent. Orchitis, epididymitis and decrease of seminal quality are presented in boars, eventually leading to infertility (RamirezMendoza et al., 1997; Solís et al., 2007). Viral RNA persistence has been documented in the central nervous system, lymph nodes and reproductive tract (Wiman et al., 1998; Cuevas et al., 2009) but not detected in semen and viral isolation not is record in persistent infection status in boars. The aim of the present work was to detect persistent PoRV in semen and reproductive tract of experimentally infected boars.

\section{Materials and Methods}

Nine 12-month-old York * Landrace boars were obtained from a PoRV free farm. Boars were inoculated by intranasal instillation with $5 \mathrm{ml} 105$ TCID50/ml of strain PAC-3 Jalisco/1992 (RamirezMendoza et al., 1997) and kept for 142 day post inoculation (dpi). Serum and semen samples were collected twice per week. Antibody response was evaluated by haemagglutination inhibition $(\mathrm{HI})$ and virus neutralization (VN) test. Boars were killed at 10, 16 and 20 weeks post inoculation (pi) and testicle and epididymis were collected. For PoRV RNA detection in serum and semen samples, RT-PCR was performed and amplified $375 \mathrm{bp}$ of $\mathrm{N}$ gene. For viral isolation, semen and reproductive tissues were used and cultured in MDCK cell line in order to be stained by an indirect immunofluorescence assay.

Figure 1. Antibody response from PoRV infected boars.

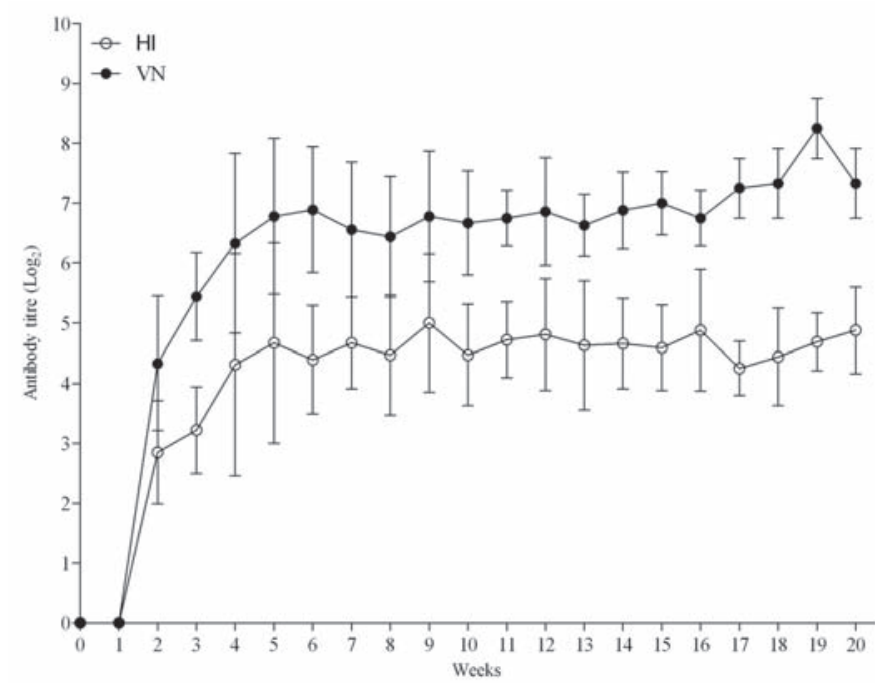

\section{Results}

PoRV infection caused clinical signs in day 5 pi, testicular swelling and pathological changes in epididimys were observed as well as the spermatic concentration that was significantly decreased. At necropsy three boars showed testicular and epididymal lesions. All boars seroconvert, $\mathrm{HI}$ and $\mathrm{VN}$ antibodies were detected in the second week pi, showing a high titer until the end of the experiment (Fig. 1). Viral isolation and PoRV RNA results showed in table 1.

Table 1. Detection of PoRV and viral RNA in samples collected from boars experimentally infected.

\begin{tabular}{|c|c|c|c|c|}
\hline $\begin{array}{c}\text { Number of positive } \\
\text { boars }\end{array}$ & Sample & DPI & Viral isolation & RT-PCR \\
\hline $3 / 9$ & Serum & $2-30$ & & + \\
\hline $9 / 9$ & Semen & & + & + \\
\hline $1 / 9$ & Serum & $31-64^{*}$ & & + \\
\hline $4 / 9$ & Semen & & + & + \\
\hline $1 / 9$ & Testicle & & + & \\
\hline $1 / 9$ & Epididymis & & + & \\
\hline $0 / 9$ & Serum & $65-110 \dagger$ & & - \\
\hline $1 / 8$ & Semen & & - & + \\
\hline $2 / 8$ & Testicle & & + & \\
\hline $2 / 8$ & Epididymis & & + & \\
\hline $0 / 4$ & Serum & $110-142 \dagger$ & & - \\
\hline $1 / 4$ & Semen & & - & + \\
\hline $1 / 4$ & Testicle & & + & \\
\hline $2 / 4$ & Epididymis & & + & \\
\hline *Tissues from one boar killed. Tissues from four boars killed. & \\
\hline \multicolumn{5}{|l|}{} \\
\hline
\end{tabular}

\section{Discussion}

The nine boars exhibited testicular changes and negative effects in seminal quality. Antibody response in persistent status was demonstrated. These results were consistent with previous experiments (Ramirez-Mendoza et al., 1997; Hernandez et al., 1998; Cuevas et al., 2009). Viral isolation is a novel report in persistent status, though other researchers only detected viral RNA in epididymis in long time of infection and PoRV in semen in acute infection (Solís et al., 2007; Cuevas et al., 2009). Thus, these results confirm that PoRV RNA persists in semen and the virus is present in genital organs during prolonged periods of infection.

\section{References}

Cuevas, J. S., et al., 2009. Vet. Immunol. Immunopathol. 127:148-152. Hernández, J., et al., 1998. Vet. Immunol. Immunopathol. 64: 367-381. Kirkland P. D. \& Stephano A. 2006. Diseases of swine. 9th Ed. Ramírez-Mendoza, H. et al., 1997. J. Comp. Path. 117:237-252. Solís, M., et al., 2007. Res. Vet. Sci. 83: 403-409.

Wiman, A. C., et al., 1998. J. Neurovirol. 4: 545-52. 


\title{
P.551
}

\section{Assay of humoral immunity to porcine rubulavirus (PoRV) experimental infection}

\author{
Jose Francisco Rivera-Benitez ${ }^{1}$ Rebeca Martinez-Bautista ${ }^{1}$ Ana Cecilia Escobar-Lopez ${ }^{1}$ Adelfa Del Carmen García-Contreras ${ }^{2}$ \\ Jesus Hernandez Lopez ${ }^{4}$ Julio Roberto Reyes-Leyva ${ }^{3}$ Humberto Ramirez-Mendoza ${ }^{1}$ \\ 1. Departamento de Microbiologia E Inmunologia, FMVZ-UNAM, Distrito Federal, DF, Mexico; 2. DCBS, DPAA, UAM-X, Distrito Federal, DF, Mexico; \\ 3. CIBIOR, IMSS, Puebla, COAH, Mexico; 4. CIAD, Sonora, SON, Mexico
}

\section{Introduction}

Blue eye disease in pigs is an emerging and endemic disease in México. The causal agent is porcine rubulavirus a paramyxovirus isolated for first time in La Piedad, Michoacan (Kirkland \& Stephano, 2006). Humoral immune response in clinic phase has been identified by conventional test, such haemagglutination inhibition (HI), virus neutralization (VN) and indirect fluorescent antibodies (IFA) (McNeilly et al., 1997; Hernández et al., 1998) and persistent stage of infection with ELISA specific HN antibodies (Cuevas et al., 2009). Quantification of antibody response in persistent infection has not been investigated. The objective of this study was evaluated the humoral immune response in clinical and persistent experimental PoRV infection.

\section{Materials and Methods}

202 serum samples were collected from pigs experimental infected ( 24 sera preinfection and 178 posinfection), analyzing two weeks pre and 20 pi.

Haemagglutination inhibition $(\mathrm{HI})$ and virus neutralization (VN) test were carried according to previous standard procedures (Ramírez-Mendoza et al., 1996; Hernández et al., 1998) with next modifications for $\mathrm{HI}, 8$ and 16 haemagglutination units were used with $1: 8$ and 0 cut point, respectively. For VN $0.5 \%$ chicken and bovine erythrocytes were used for measurement haemagglutination limit, all assays were performed in MDCK line cells. Immunoperoxidase monolayer assay was performed in MDCK cell line infected with PoRV at $0.01 \mathrm{MOI}$. Indirect fluorescent antibodies test was developed in same cell line, goat anti swine lgG conjugated with fluorescein isothiocyanate was used. All sera were placed in double serial dilutions. The results were analyzed with SPSS 10.0 statistical package.

\section{Results}

The results showed in figures. For the IHA test shows no difference in the two combinations. In SN differences were evaluated only three weeks, obtaining a lower antibody titter to observe cytophatic effect. The IPMA test was similar to the HI, showing differences only evaluated in one week. For the IFA test results are similar to the VN, except evaluated in two weeks, where the neutralizing antibody titter was lower.
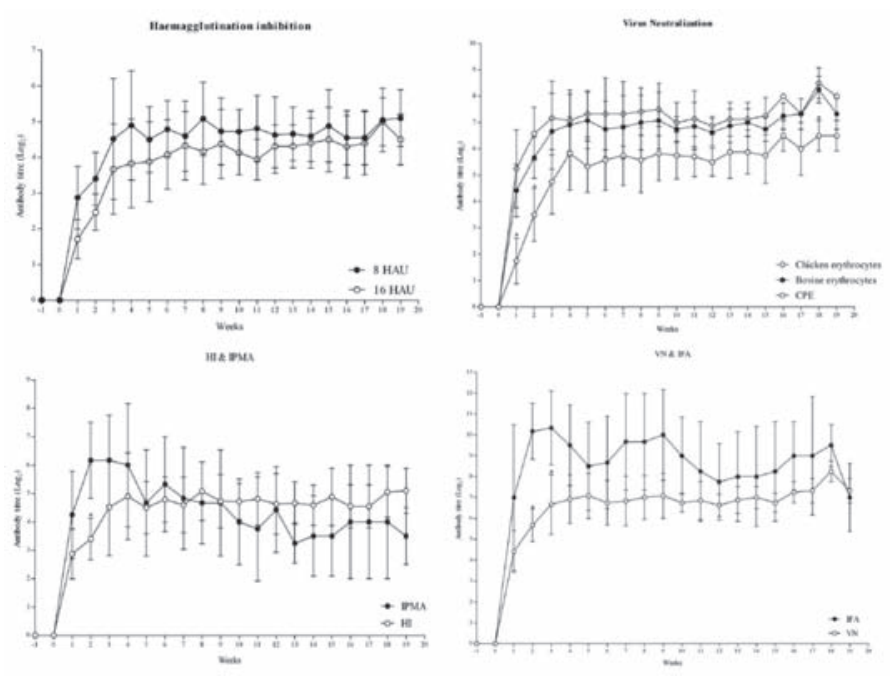

\section{Discussion}

The humoral immune response begins in the first week postinfection and it maintained high titters for prolonged periods in the persistent phase of infection. Cuevas et al. (2009), it has been reported that animals retaining seropositivity during the persistent phase of infection, but was not quantified with standard methods. The results correspond to previously report by Hernández et al. (1998), which analyzed the humoral immune response by $\mathrm{HI}$ and $\mathrm{VN}$ in the clinical phase of disease. The results of the IPMA and IFA demonstrate the utility of fixed and inactivated antigens in the development of serological diagnostic tests.

\section{References}

Cuevas, J. S., et al., 2009. Vet. Immunol. Immunopathol. 127:148-152. Hernández, J., et al., 1998. Vet. Immunol. Immunopath. 64: 367-381. Kirkland P. D. \& Stephano A. 2006. Diseases of swine. 9th Ed. McNeilly et al., 1997. J Vet Diagn Invest 9:3-9. Ramírez et al., 1996. Vet Méx. 27:257-259. 


\title{
P.552
}

\section{Glycoprotein E2 of classical swine fever virus expressed in yeast protects swine from challenge infection}

\author{
Guang-Jan Lin ${ }^{1}$ Maw-Sheng Chien ${ }^{2}$ Ting-Yu Liu ${ }^{1}$ Zeng-Weng Chen ${ }^{1}$ Chih-Chiang Cheng ${ }^{2}$ Ming-Chung Deng ${ }^{3}$ Chienjin Huang $^{1}$
}

1. Graduate Institute of Microbiology and Public Health, College of Veterinary Medicine, National Chung Hsing University, Taichung, Taiwan; 2. Graduate Institute of Veterinary Pathobiology, College of Veterinary Medicine, National Chung Hsing University, Taichung, Taiwan; 3. Hog Cholera Division, Animal Health Research Institute, Council of Agriculture, Executive Yuan, Tansui, Taipei, Taiwan

\section{Introduction}

Classical swine fever (CSF) is an economically important swine disease worldwide. The glycoprotein E2 of classical swine fever virus (CSFV) is capable of inducing the production of neutralizing antibodies in the host (1). A CSF subunit marker vaccine has been developed which contains the $\mathrm{E} 2$ produced in insect cell by the recombinant baculovirus expression system (2). Recently, we have constructed a recombinant E2 protein using the yeast Pichia pastoris secreted expression system, and the yeast-expressed E2 protein (yE2) is able to induce a protective immune response against CSFV infection (3). The aim of this study was to determine the optimal dose of $y E 2$ vaccine for conferring full protection against CSF.

\section{Materials and Methods}

Twelve 6-week-old specified pathogen-free (SPF) piglets were randomly allotted to three $\mathrm{yE} 2$ vaccine groups and a control group. Concentrated secreted product from the recombinant E2-expressing yeast (yE2) was used for vaccine preparation without further purification steps. Each pig was immunized at 6- and 9-week-old respectively intramuscularly in the pig's neck with 2 $\mathrm{ml}$ vaccine containing 200,300, or $400 \mu \mathrm{g}$ yE2 (yE2/200, yE2/300, and $y E 2 / 400$ vaccine groups respectively) or normal saline (control group) in a 1: 1 water-in-oil emulsion with the adjuvant IMS 1113 (SEPPIC). The pigs at 12-week-old were challenged intramuscularly via the neck with a $1 * 10^{5} \mathrm{TCID}_{50}$ dose of virulent CSFV strain. Serum samples were tested for their neutralizing activities by serum neutralization test. Neutralizing titers were expressed as the reciprocal of the highest dilution that cause complete neutralization.

\section{Results}

All yE2 vaccine groups including yE2/200, yE2/300, and yE2/400 mounted anamnestic response after booster vaccination with the average neutralizing antibody titers of 64, 928, and 2683 respectively. Neutralizing antibody titers of yE2 vaccine groups increased dramatically after challenge infection (Fig. 1)

The control pigs developed an acute febrile response after challenge infection and had to be euthanized because of severe clinical symptoms by the post-infection day 10 . None of the $y E 2 / 300$ and yE2/400 pigs became febrile (Fig. 2). All yE2-vaccinate pigs survived after challenge without any apparent clinical symptoms and post-mortem evaluation at 14 days post challenge revealed no evidence of CSFV infection.

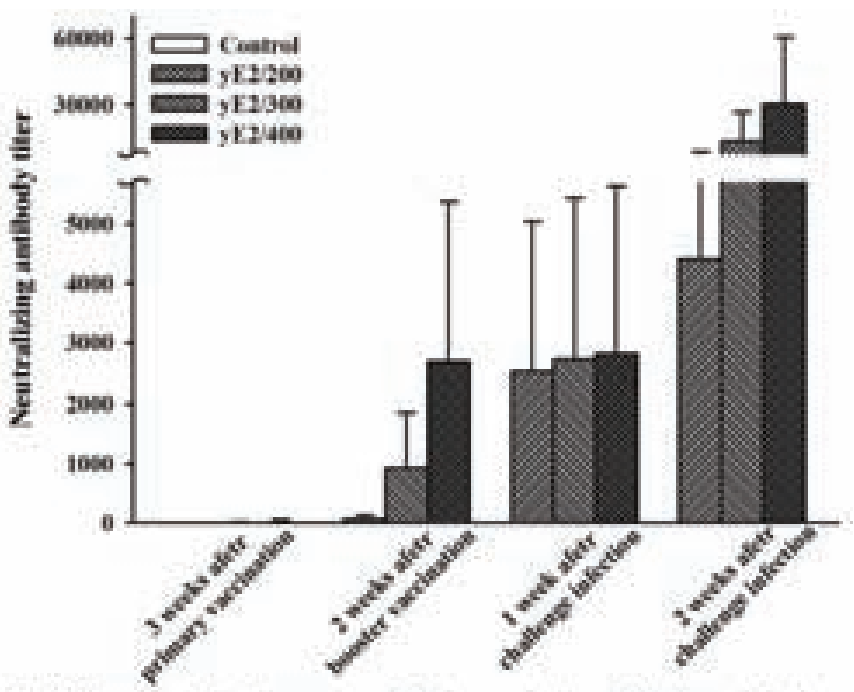

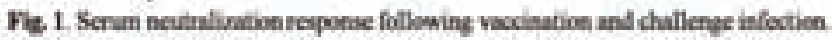

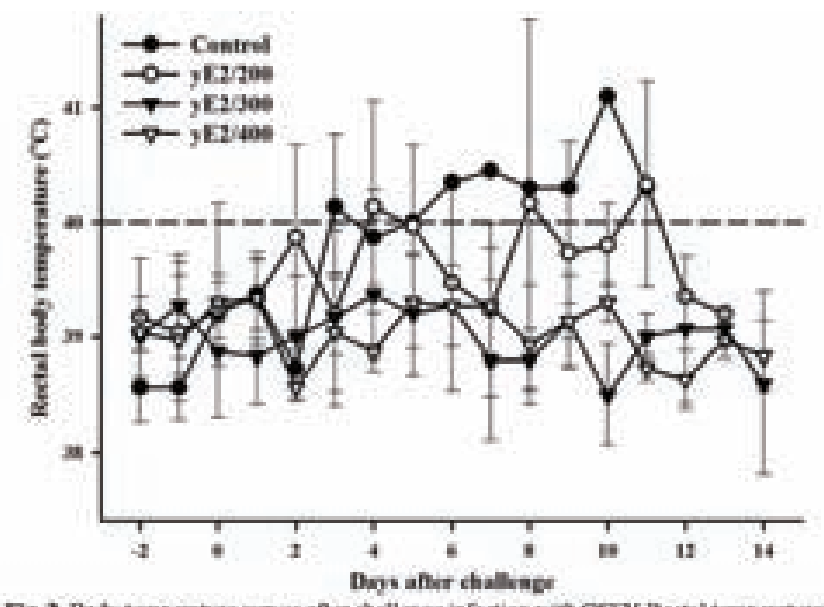

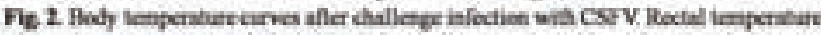

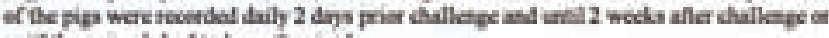
urfl the animalahad so be cubarind.

\section{Discussion}

All of three doses of $y E 2$ vaccine could induce protective immune response against CSFV infection. The optimal dose of yE2 was recommended to be $300 \mu \mathrm{g}$ of total secreted protein. The E2 constituted $5-10 \%$ of total secreted protein $(300 \mu \mathrm{g})$ which is equivalent to $15-30 \mu \mathrm{g}$ of E2 in one dose of $\mathrm{yE2}$ vaccine. Yeastexpressed E2 recombinant protein (yE2) represents an alternative subunit E2 marker vaccine candidate with advantages of easy manipulation and low cost.

\section{References}

1. Bouma et al., 2000. Vaccine 18: 1374-1381.

2. Moormann et al., 2000. Vet. Microbiol. 73: 209-219.

3. Lin et al., 2009. Vet. Microbiol. 139: 369-374. 


\title{
P.553
}

\section{Gross and histopathological scoring systems to evaluate Classical Swine Fever virus infections}

\author{
Francisco J. Salguero; Helen E. Everett; Alejandro Nunez; Trevor Drew; Helen Crooke
}

VLA-Weybridge, Addlestone, UK

\section{Introduction}

Classical swine fever (CSF) virus infections cause large economic losses in the pork industry and have profound social and welfare consequences. CSF has been reported in many countries throughout America, Asia and Europe in the recent years. Control strategies are based on stamping out as well as vaccination. Clinical and pathological diagnoses have proven to be very important in the early steps of an outbreak. However, many clinical signs and gross lesions cannot be differentiated from other porcine diseases. The characterization of CSF virus infections by different isolates is key to identify the common and unique features associated with infection. Few clinical and very few and basic post mortem gross scoring systems have been applied to these characterization experiments. In this paper we describe two detailed scoring systems, gross and histopathological, and their use in experimental infections with two genetically diverse CSF virus strains.

\section{Material and Methods}

Two different animal experiments were carried out. Six 10 weekold pigs were inoculated by the intranasal route with $10^{5} \mathrm{TCID}_{50}$ of the corresponding isolate (UK2000/7.1 and CBR/93) and housed with 3 animals used as "in contact" sentinels, while 2 animals were housed separately as non inoculated negative controls. Clinical signs were monitored daily and animals were euthanized at 18-21 dpi. Post mortem examination was carried out following standard procedures and any observed lesion was recorded. 21 parameters were used for the gross pathology scoring system: body condition, skin and subcutis, tonsils, fluid in body cavities, spleen, kidney, ileum (and ileocaecal valve), caecum and colon, brain and meninges, respiratory system, eight lymph nodes (precrural, inguinal superficial, mandibular, lateral retropharyngeal, medial retropharyngeal, prescapular, ventral cervical superficial and ileocaecal), urinary bladder, thymus and conjunctiva. Scores from 0 (normal) to 3 (severe) were given to observed lesions for each parameter (e.g. hemorrhages, thymic atrophy, etc.)

Samples from tonsils, spleen, kidney, ileocaecal valve, colon, encephalon, lung, thymus, pancreas, liver, bone marrow and the eight lymph nodes were fixed in buffered formalin and embedded in paraffin wax. $4 \mu \mathrm{m}$ sections were stained with H\&E for histopathological examination. 33 parameters were used for the histopathological scoring system and the severity of lesions was also recorded from 0 (no lesion) to 3 (severe). Data from both scoring systems were analysed by Kruskal-Wallis non-parametric mean comparison test and differences were considered significant for $\mathrm{P}<0.05\left(^{*}\right)$.

\section{Results}

Results are summarized in figure 1 (A, B; gross pathology) (C, D; histopathology). Boxes represents percentile 25 to 75; whiskers represents maximum and minimum values; lines represent medians. No statistical differences were found between UK2000/7.1 and CBR/93 inoculated or UK2000/7.1 and CBR/93 in contact animals. Significant differences were observed between the 3 groups (inoculated, in-contact and control) within the same experiment.

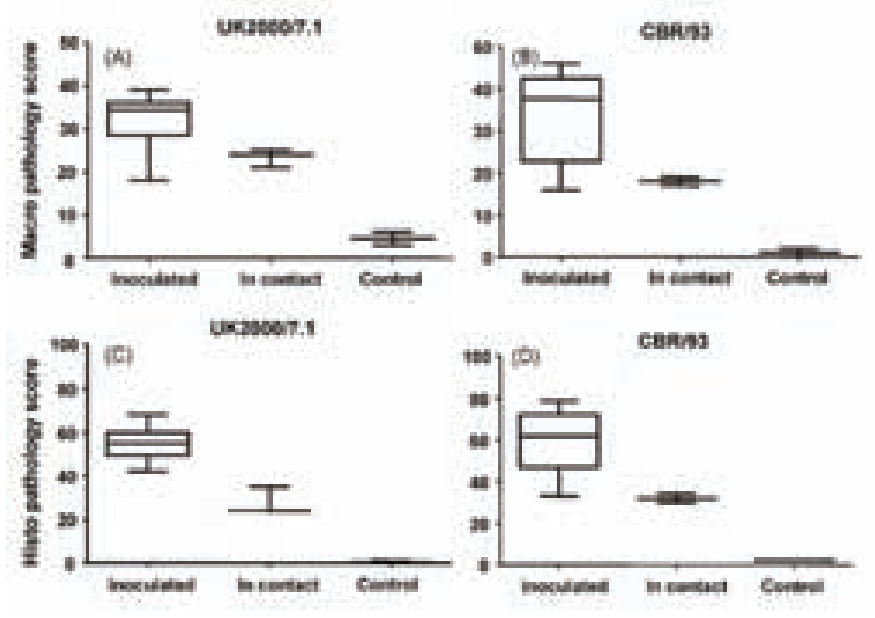

\section{Discussion}

The extended gross and histopathological scoring systems described here have proven to be a valuable tool for systematic comparison and assessment of pathological findings related to CSF infection.

\section{References}

1. Everett et al., Veterinary Microbiolgy, in press. doi:10.1016/j.vetmic.2009.09.039

2. Floegel-Niesmann et al., Veterinary Microbiology 139, 165-169 (2009)

3. Mittelholzer et al., Veterinary Microbiology, 74, 293-308 (2000)

4. S.Parchariyanon et al., Thai Vet. Med Assoc 52, 2544 (2001).

This work has been funded by Defra project SE0778 and FP7-EU NADIR (228394) 


\title{
P.554
}

\section{IFN-gamma immunodetection in tissues from pigs vaccinated (C-strain) and challenged with Classical swine fever virus (UK 2000 7.1)}

\author{
Francisco J. Salguero; Helen E. Everett; Simon P. Graham; Felicity Haines; Olubukola Sosan;
} Helen Johns; Alejandro Nunez; Trevor Drew; Helen Crooke

VLA-Weybridge, Addlestone, UK

\section{Introduction}

Classical Swine Fever (CSF) is a viral haemorrhagic disease caused by Pestivirus within the Flaviviridae family. The disease is characterized by haemorrhages and lymphopenia, and the main target cell for the virus is the monocyte-macrophage. The attenuated C-strain is an effective vaccine, widely used in many countries but its use within the EU has been hindered by limitations in differentiating vaccinated from infected animals by serology. To investigate mechanisms behind the effective protection afforded by this vaccine, we have studied the immunohistochemical detection of IFN-gamma within lymphoid tissues of pigs vaccinated and challenged with CSF virus.

\section{Material and Methods}

Groups of six, ten-week old pigs were vaccinated $(V)$ with the Reimser C-strain vaccine by intramuscular route 5,3 or 1 day/s prior to intranasal challenge (Ch) with UK2000/7.1 CSF virus strain. Four unvaccinated pigs were also challenged to control for infection $(C+)$. In all cases, challenged animals were re-introduced to unvaccinated, "in-contact" (IC) animals twenty-four hours post-challenge. Animals were monitored twice daily and blood samples were taken to assess viremia (RT-PCR). Animals were euthanized and gross and histopathological scores were calculated as described (see poster presented by this group). At necropsy, medial retropharyngeal lymph node, tonsil and spleen were also collected and fixed with Bouin's solution for immunohistochemical detection of IFNgamma (R\&D Systems Inc.) by means of a modified $A B C$ technique.

\section{Results}

Unvaccinated animals developed clinical signs, viraemia and transmitted the virus to "in contact" animals within 14 days of inoculation. Animals vaccinated 5 days prior to challenge were completely protected. Five of the 6 animals vaccinated 3 days prior to challenge had only few clinical signs and pathological changes while half of the animals vaccinated 1 day prior to challenge were protected to some extent. Results of gross pathology and histopathology are summarized in Figures $1 \& 2$ respectively.

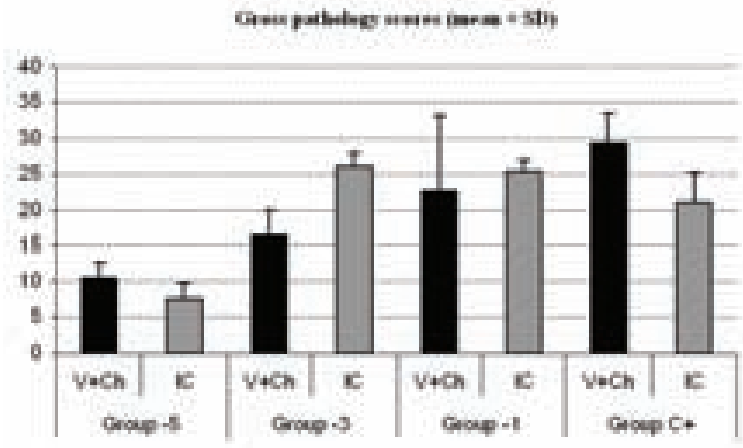

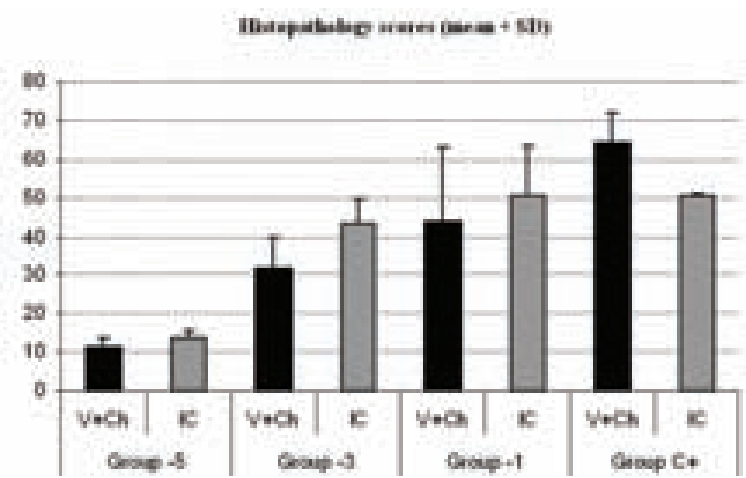

Results for IHC detection are summarised in Table 1 (-, less than $5 ;+, 5-25 ;++, 26-50 ;+++,>50$ cells per sq. $\mathrm{mm}$ )

\begin{tabular}{|l|c|c|c|c|c|c|c|c|c|}
\hline & $\begin{array}{c}-5 \text { V+ } \\
\text { Ch }\end{array}$ & $-\mathbf{5 ~ I C ~}$ & $\begin{array}{c}-3 \\
\text { V+Ch }\end{array}$ & $-\mathbf{3}$ IC & $\begin{array}{c}-\mathbf{1} \\
\text { V+Ch }\end{array}$ & -1 IC & C+ Ch & C+ IC & C- \\
\hline Lymph node & & & & & & & & & \\
\hline - Lymphoid follicles & + & - & - & - & - & - & - & - & - \\
\hline - Medulla & +++ & + & + & - & + & + & + & - & - \\
\hline Tonsil & & & & & & & & & \\
\hline - Lymphoid follicles & + & - & - & - & - & - & - & - & - \\
\hline - Interfollicular & +++ & + & + & + & + & + & + & + & - \\
\hline Spleen & & & & & & & & & \\
\hline - Red pulp & ++ & + & - & - & - & - & + & - & - \\
\hline - White pulp & + & - & - & - & - & - & - & - & - \\
\hline
\end{tabular}

Immunohistochemical detection of IFNgamma displayed high levels in animals vaccinated 5 days prior to challenge, mostly in lymph node medulla, interfollicular areas of the tonsils and splenic red pulp. IFNgamma-expressing cells were reduced in numbers in all the other groups. The level of IFNgamma expression in animals showing some degree of protection was higher than those not protected within the group vaccinate 1 day prior challenge.

\section{Discussion}

C-strain vaccination provides good protection against CSF virus infection by 5 days post vaccination and this protection is correlated with IFNgamma expression in lymphoid tissues. These results support the hypothesis that the presence of high levels of IFNgamma secreting cells provide a cell mediated mechanisms that are able to control CSF virus infection.

\section{References}

1. van Oirschot. Veterinary Microbiology, 96, 367-384 (2003)

2. Everett et al., Veterinary Microbiology, in press. doi:10.1016/j.vetmic.2009.09.039

3. Graham et al., Veterinary Microbiology, in press. doi:10.1016/j.vetmic.2009.09.040

This work has been funded by Defra project SE0778 and FP7-EU NADIR (228394) 


\title{
P.555
}

\section{Comparison of PCR methods for detection of classical swine fever virus and other pestiviruses}

\author{
Katarzyna Podgórska; Katarzyna Kamieniecka; Zygmunt Pejsak; Tomasz Stadejek \\ National Veterinary Research Institute, Pulawy, Poland
}

\section{Introduction}

Classical swine fever virus (CSFV) is an etiological agent of an economically important notifiable pig disease - classical swine fever (CSF). Other viruses belonging to the same Pestivirus genus, border disease virus (BDV) and bovine viral diarrhoea virus (BVDV) types 1 and 2 are also able to infect pigs, sometimes causing lesions resembling infection with a low-virulent CSFV strain (1). Therefore, discrimination of these infections is important from epidemiological point of view.

The aim of the study was to compare the usefulness of PCR methods for CSFV detection and differentiation from other pestiviruses.

\section{Materials and Methods}

Viruses. Strains representing all species within Pestivirus genus were propagated in cell cultures and included into the study: CSFV (strain Alfort/187 of genotype 1 and field strain 1795/94 of genotype 2), BVDV 1 (NADL), BVDV 2 (Short) and BDV (Moredun).

RNA extraction and reverse transcription. RNA was extracted using Total RNA Prep Plus kit (A\&A Biotechnology) and eluted in $100 \mu \mathrm{l}$ of RNase-free water. Next, cDNA was synthesized using MMLV reverse transcriptase as described elsewhere (4). Tenfold dilutions of CDNA's were aliquoted and stored at $-70^{\circ} \mathrm{C}$.

PCR methods. Two Real-Time methods and conventional onetube nested RT-PCR were applied for specific detection of CSFV. TaqMan I method was performed according to previously described protocol (2). In TaqMan II assay csfv 6 and csfv 7 primers designed by Uttenthal et al. (7) and CSFV specific probe (Stadejek et al., unpublished) were used. Nested PCR was based on Katz et al. (3) with later modifications (5) including immobilization of the inner primers pair in tube caps.

Additionally two pan-pestivirus specific methods, Real-Time SYBR Green I (6) and gel-based one-tube nested RT-PCR (8) with further modifications (5) were included into the study.

All PCR reactions were performed in triplicates. Real-Time PCR reactions were run on Stratagene Mx3005P system using Quantitect PCR Probe or SYBR Green I kits (Qiagen). CSFV-specific nested RT-PCR amplified fragment of genome encoding E2/NS2. All other assays were designed based on 5'UTR genome region.

\section{Results}

The highest sensitivity in detection of CSFV was observed using TaqMan I method. Similar results were obtained by CSFV-specific nested RT-PCR. These methods allowed detection of the virus in examined samples containing 101 and 102 TCID50/ml of Alfort/178 respectively.

Both pan-pestivirus specific methods were less sensitive in detection of CSFV. The virus was detected in samples containing 105 and 103 TCID50/ml using SYBR Green I and nested PCR method respectively. Only the nested RT-PCR method detected BDV strain. Moreover, the sensitivity of this test for BVDV detection was 2 or 4 logs higher (depending on the strain) compared to SYBR Green I method.

\section{Discussion}

In this study sensitivity and specificity of 3 methods specific for CSFV and 2 methods specific for all persiviruses was compared. The results confirmed that both, conventional and Real-Time PCR methods, are useful for detection of CSFV and discriminating from other pestiviruses. However, the compared methods differed in sensitivity to detect CSFV. The Reak-Time PCR method of Hoffmann et al (2) and conventional PCR of Stadejek and Pejsak (5) which employed primers described by Katz et al. (3) proved to be the most sensitive.

\section{Acknowledgements}

The study was funded by a grant No N N308 293537 from the Polish Ministry of Science and Higher Education.

\section{References}

1. Deregt D, et al. (2006). J Virol Methods 136: 17-23

2. Hoffmann B, et al. (2005) J Virol Methods 130: 36-44

3. Katz JB, et al. (1993) J Clin Microbiol 31:565-568

4. Podgórska K, Stadejek T, (2010) Acta Vet Hung 58 (in press)

5. Stadejek T, Pejsak Z (2000) Med Wet 56: 73-136

6. Stadejek T, et al. (2006) Med Wet 62: 121-240

7. Uttenthal A, et al. (2003) Vet Microbiol 92: 197-212

8. Vilcek S, et al. DJ (1994) Arch Virol 136: 309-323 


\title{
P.556
}

\section{Research of Porcine Parvovirus (PPV) in organs and stomach fluid of stillbirth, mummified and aborted fetuses and phylogenetic analysis of PPV isolated from fetal tissue}

\author{
Camila Sa Rocha ${ }^{2}$ Neide L. Simon ${ }^{3}$ Giseli A. Ritterbusch ${ }^{3,2}$ Aline Viancelli ${ }^{3}$ Nelson Mores ${ }^{3}$ \\ Armando L. Amaral ${ }^{3}$ Paulo A. Esteves ${ }^{3}$ Janice R. Ciacci-Zanella ${ }^{1,4}$ \\ 1. Virus and Prion Diseases Research Unit, NADC, ARS, USDA, Ames, IA, USA; 2. Agroveterinarian Science Center - CAV - \\ University of Santa Catarina State - UDESC, Lages, SC, Brazil; 3. Embrapa Swine and Poultry Research Center, \\ Animal Health Laboratory, Concordia, SC, Brazil; 4. Labex-USA, EMBRAPA, Brasilia, DF, Brazil
}

\section{Introduction}

Porcine Parvovirus (PPV) was described for the first time in the 60 's (1) and until today it is known as the main infectious agent associated with swine reproductive failures (2). In Brazil, the porcine parvovirus infection is diagnosed as the cause of reproductive problems for at least 20 years (3). The fetal infection can occur through the placenta or from fetuses to fetuses (4) thus, those tissues are very important source of contamination $(4,5)$. Because it is an endemic disease in several countries, including Brazil, and recently another viral agents have been implicated as the cause of reproductive problems the purpose of this study was to investigate the presence and distribution of PPV in tissues and in stomach liquid of stillbirth, mummified and aborted fetuses from 27 swine farms with reproductive losses. In addition to that, the phylogenetic analysis of PPV isolated from fetal tissues was also performed.

\section{Material and Methods}

A total of 230 stillbirths, mummified and aborted fetuses were collected and necropsied from 27 selected farms. The farms of this study were located in the Southern States of Brazil and were chosen based on overall reproductive failure rates (6). Organs (heart, lung, liver, spleen, thymus, kidney, brain, cerebellum, spinal cord, submandibular lymph nodes, inguinal lymph nodes and mesenteric lymph nodes) from all 230 fetuses and the stomach fluid of 109 of those fetuses were collected. DNA was extracted by proteinase $\mathrm{K}$ method (7) and further amplified by nested-PCR using specific primers from the PPV nonstructural protein gene, NS1 (8). The positive samples were sequenced by the Big dye terminator method and phylogenetically analyzed.

\section{Results and Discussion}

PPV DNA was found in organs of six (2.6\%) out of 230 necropsied fetuses by nested-PCR. The positive organs were cerebellum (50\%), spinal cord $(50 \%)$, brain $(33.3 \%)$, lungs (33.3\%), spleen $(33.3 \%)$, inguinal lymph nodes (33.3\%), mesenteric lymph nodes (33.3\%), heart (16.7\%), liver (16.7\%) and kidney (16.7\%). Stomach fluid from the 109 fetuses were also analyzed by nested-PCR and the PPV DNA was detected in three (2.8\%) of them. Those findings confirm the results obtained by other studies about the most frequently infected organ, as heart and lung (9) and, together with others factors can help the understanding of virus pathogenesis in fetuses. This study indicated a low frequency of the infection by PPV on the studied farms, although the disease is considered endemic in Brazilian swine herds. Thus, vaccination and management measures may be playing an important role controlling PPV infection in Brazil. Sequencing and phylogenetic analysis showed that PPV samples isolated from fetuses had 97 to $100 \%$ identity with classical PPV. These samples were grouped with isolates from China (ZJ-EU790642), South Korea
(VR-1-AY390557), United Kingdom (CHALLENGE-AY684866) and Germany (IDT-AY684872) (Figure 1), indicating that similar isolates are present in the various countries.

Figure 1. Phylogenetic tree of samples sequenced in this study (24LM).

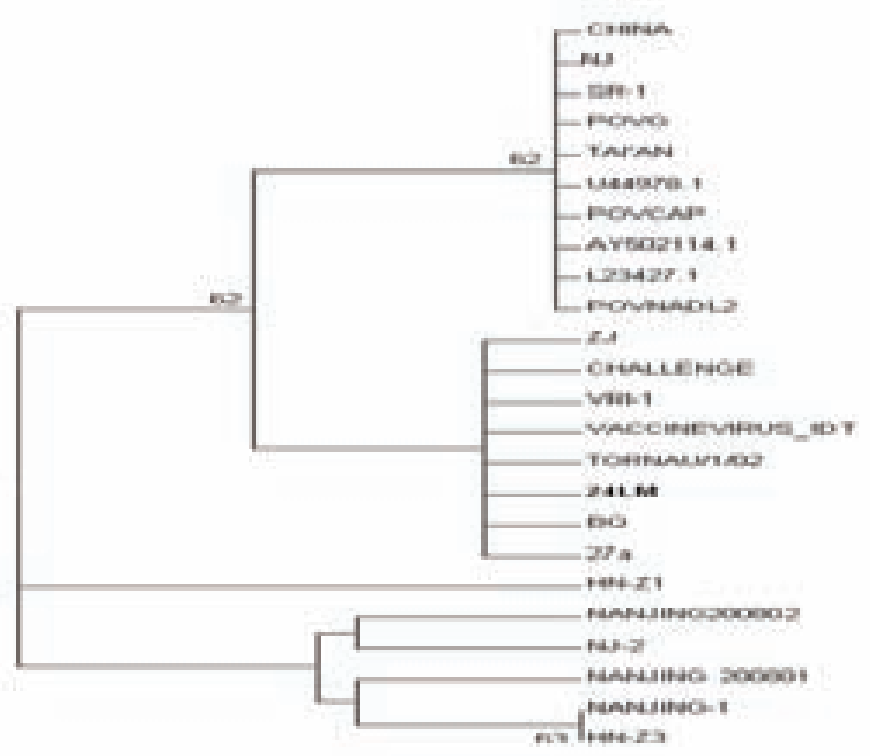

\section{References}

1. Cartwright S.F. \& Huck, R.A. 1967. Veterinary Record. 81: 196-197

2. Mengeling W.L. 1972. American Journal of Veterinary Research. 33: 2239-2248

3. Gouvêia A.M.G. et al., 1984. Pesquisa Veterinária Brasileira. 4: 17-22;

4. Roehe P.M. etal.,2007.Doenças de Suínos, pp.286-293;

5. Moraes M.P. \& Costa P.R.S. 2007. Virologia Veterinária. pp.377-396;

6. Almond, G.W., et al, 2006. Diseases of Swine. Ch..6, p.113-147.

7. Sambrook, J. et al., 1989. Molecular cloning: A laboratory manual. 2.Ed;

8. Soucie, J. M. et al., 2000. Transfusion. 40: 708-711.

9. Wolf et al., 2008. Genetics and molecular research. 7: 2: 509-517 


\title{
P.557
}

\section{Safety evaluation of different combined Parvovirosis/Erysipelas vaccines}

\author{
Alberto Morillo ${ }^{2}$ Antonio Callen ${ }^{1} \mathrm{G}$. Cano ${ }^{2}$ \\ 1. Merial Laboratorios S.A., Barcelona, Spain; 2. Tests \& Trials, Lérida, Spain
}

\section{Introduction}

The aim of this study was to confirm previous results (1), to include new vaccines in the comparison and to study the impact if any on feed intake.

\section{Material and Methods}

The trial was a multicentric, randomised and positive controlled GCP study in three farrow-to-finish Spanish farms. Following a simultaneous 4-weeks batch farrowing, each sow of one farrowing batch was randomly assigned to one of 3 treatment groups (PARVORUVAX ${ }^{\circledR}$, and 2 other different Parvovirosis and Erysipelas (P/E) vaccines coded VA and VB) according to parity. In total, 196 sows were included in the general study, and 95 of them in the part of the trial involving feed intake. Sows received a booster vaccination 1 week before weaning, being checked for rectal temperature and for swelling at the injection site before and after injection (at Day 0, Day $0+6$ hours, Day 1 and Day 2 after injection). Daily feed intake (from Day -2 to Day 2) was determined only in one of the farms using dried feeding. For this purpose the experiment in this farm was repeated in two subsequent batches. Site reactions were classified in 2 categories: absent or inflammation. The statistical procedures used for rectal temperature and feed intake was the variance analysis implemented by the MIXED procedure of SAS ${ }^{\circledR}$. The statistical model included the sow, group, site, day and their interactions and local site reactions were analysed using a logistic regression implemented by the LOGISTIC procedure of SAS.

\section{Results}

At $6 \mathrm{~h}$ post-injection a significant lower temperature was observed in the PARVORUVAX group ( $P$ group) compared to vaccine $V A,-0.33^{\circ} \mathrm{C}(\mathrm{P}<0.0001)$, and a close to significant difference, $-0.16^{\circ} \mathrm{C}(\mathrm{P}=0.05)$ with vaccine VB. Significantly, less local site reactions at Day $0+6 h$ were observed $(p<0.01)$ in the P group $(14.9 \%)$ compared to the VA (55.8\%) and VB (51.0\%) groups. This difference persisted till the end of the experiment, at D2 (table 1).

Table 1: safety parameters values per group

\begin{tabular}{|c|c|c|c|c|c|c|c|c|}
\hline Group & $\begin{array}{c}\text { No } \\
\text { Sows }\end{array}$ & $\begin{array}{c}\text { T Day } \\
\mathbf{0}^{*}\end{array}$ & $\begin{array}{c}\text { T Day } \\
\mathbf{0 + 6 h}\end{array}$ & $\begin{array}{c}\text { T Day } \\
\mathbf{1}\end{array}$ & $\begin{array}{c}\text { T Day } \\
\mathbf{2}\end{array}$ & $\begin{array}{c}\text { LSR Day } \\
\mathbf{0 + 6 h}\end{array}$ & $\begin{array}{c}\text { LSR } \\
\text { Day } \mathbf{1}\end{array}$ & $\begin{array}{c}\text { LSR } \\
\text { Day 2 }\end{array}$ \\
\hline P & 64 & $38.59^{\mathrm{a}}$ & $38.88^{\mathrm{a}}$ & $38.68^{\mathrm{a}}$ & $38.76^{\mathrm{a}}$ & $14.9^{\mathrm{a}}$ & $10.6^{\mathrm{a}}$ & $0.0^{\mathrm{a}}$ \\
\hline VA & 65 & $38.55^{\mathrm{a}}$ & $39.21^{\mathrm{b}}$ & $38.89^{\mathrm{b}}$ & $38.84^{\mathrm{a}}$ & $55.8^{\mathrm{b}}$ & $53.9^{\mathrm{b}}$ & $26.9^{\mathrm{b}}$ \\
\hline VB & 67 & $38.53^{\mathrm{a}}$ & $39.04^{\mathrm{b}}$ & $38.79^{\mathrm{a}, \mathrm{b}}$ & $38.85^{\mathrm{a}}$ & $51.0^{\mathrm{b}}$ & $53.1^{\mathrm{b}}$ & $44.9^{\mathrm{b}}$ \\
\hline \\
a,b values in the same column with different superscripts were significantly different $(\mathrm{p}<0.05)$. \\
* Temperature on Day 0 was used to covariate the rest of temperature analysis \\
\hline
\end{tabular}

Feed intake was influenced by the type of vaccine used. As shown in Figure 1, sows vaccinated with vaccine VA and VB ate on the day of vaccination, 0.18 and $0.14 \mathrm{~kg}$ less than sows in $P$ group $(\mathrm{P}<0.05$ and $\mathrm{P}=0.05$ respectively).
Figure 1: Time course of the feed intake ( $\mathrm{kg} / \mathrm{sow} /$ day) by group for sows receiving different Parvovirosis-Erysipelas inactivated vaccines

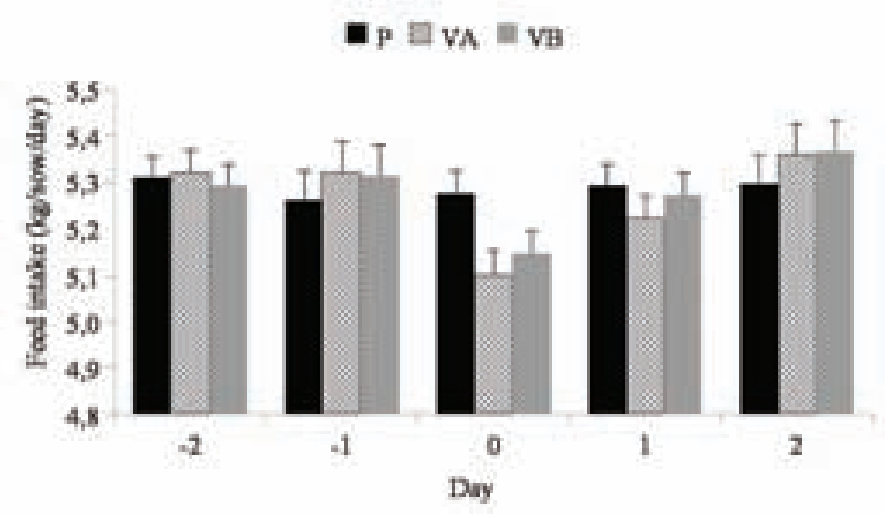

\section{Discussion and Conclusion}

These results confirm that the use of some Parvovirosis/Erysipelas vaccines may have a safety concern as it can have welfare and production negative consequences in practice. Our results not only confirm the safety of PARVORUVAX compared to the vaccine VB, included in a former study, but also to vaccine VA, not analyzed previously. With our experimental design we have been able not only to reproduce the effects on rectal temperature and site reactions but also to proof an impact of these products in feed intake witnessing a general reaction. While hyperthermia and local reactions have an obvious impact in the animal wellbeing, a transient reduction of feed intake during lactation could have further consequences such as reduction of milk production and consequently pre-weaning growth retardation in the litter.

\section{References}

1. Keïta A. et al., (2006) Proc. 19th IPVS Congress, Copenhagen, Denmark, Vol. 2, 159.

ه PARVORUVAX is a registered trademark of Merial and SAS is a registered trademark of SAS Institute Inc, in the United States of America. 


\title{
P.558
}

\section{A genome characterization of Korean porcine Parvovirus}

\author{
Hye-Young Jeoung; Dong-Jun An; Wooseog Jeong; Byoung-Han Kim \\ National Veterinary Research \& Quarantine Service, Korea, Korea
}

\section{Introduction}

Porcine parvovirus (PPV) is classified in the genus Parvovirus, in subfamily Parvovirinae and the family Parvoviridae. The large ORF1 and the small ORF3 encode for the non-structural proteins NS1, NS2 and NS3. The ORF2 encodes the structural proteins VP1, VP2 and VP3. VP1 and VP2 are a result of alternative spliced RNAs, giving VP1 a specific N terminus of 150 aa. VP3 is generated by proteolytic cleavage of VP2. Recently, PPV has gained importance as an agent able to potentiate the effects of porcine circovirus type 2 (PCV2) infection in the clinical course of postweaning multisystemic wasting syndrome, a significant economical disease worldwide.We researched two strains in this study that PVK1 and SK2 strain isolated from abortion fetus of sows in 1980 and 2008, respectively. According to molecular analysis of the two Korean strains and previous studied VRI-1 strain, we were to compare a genome characterization and virulence among other countries strains.

\section{Material and Methods}

Two Korean PPV sequences and 20 sequences from PPVs from around the world obtained from GenBank were aligned using the Clustal X 1.83 sequence alignment program. Nucleotide and amino acid sequence identities among the PPV strains were calculated using BIOEDIT 7.053. The phylogenetic tree was created by neighbor-joining method using MEGA 4.0 software and bootstrap analyses were conducted using 1000 replicates. The recombination among PPV strains was analyzed by the recombination detection program (RDP).

\section{Results}

Of six amino acid position sites within VP1/VP2 proteins, Korean SK2 strain was different only one position (T365I) and VRI-1 strain was just two positions (G528D and Q533H) comparison with Kresse strain whereas PVK1 strain was only two positions (T195S and R715K) comparison with NADL-2 strain. The sequences of NADL-2, POVNADL-2, POVG, SR-1, China and POVCAP in this region were the longest among the strains, and contained two identical 127-bp repeat units whereas strains 15a, 21a, 27a, $106 \mathrm{~b}$, Kresse and Tornau contained only one complete 127-bp unit (Table 1). Strains SK2 and PVK1 lacked 75 bases from 74 to 148 bases but VRI-1 strain cann't analyzed due to data none. Phylogenetic tree analysis of VP2 protein of 22 PPV strains using a Neighbor joining (NJ) method in MEGA 4.0 software didn't divide clearly as the attenuated and moderate and highly virulent groups, but three Korean strains showed grouping similarly on tree topology.

\section{Discussion}

The genotypic grouping in this study could also be associated with neither clinical presentation nor geographical origin of the samples, whereas three Korean strains showed grouping similarly on tree topology. In our judgment of three genome analysis (mutation amino acid of VP1/VP2 protein, a 127-bp repeat of ORF right and phyogenetic tree), three Korean strains are presumed to include a moderate virulence. On the other hand, The genomes among all strains by in GENECONV, SiSCAN, BootScan, MaxChi, and 3Seq methods in RDP program analysis weren't determined a possiblie breakpoints of the two recombinants which are the major and minor parent strains. On accession of no breakpoint may be a very high homology among worldwide PPV strains as well as Korean strains.

In conclusion, two Korean strains have one or two difference of six amino acid position sites within VP1/VP2 protein with Kresse strain and on a $127 \mathrm{nt}$ repeat after the right ORF encoding the VP1/VP2, strains SK2 and PVK1 lacked 75 bases. Three Korean strains on the phylogenetic tree analysis for VP2 gene were grouped similarly lineage. Korean PPV strains are presumed to include a moderate virulence.

\section{References}

1. Ellis, J. et al (2004) Vet Microbiol 94, 159-163.

2. Kim, B.H. et al (2004) J Virol Bacteriology 34, 201-212.

3. Shangjin, S. et al (2009) Virus Reseach 140, 209-215. 


\title{
P.559
}

\section{Comparison of two attenuated PRV vaccines in Aujeszky's Disease control}

\author{
Liande Zhu; Shumin Chen; Aiguo Wang \\ Boehringer Ingelheim International Trading (Shanghai) Co. Ltd, Beijing, China
}

\section{Introduction}

Aujeszky's Disease (AD) or Porcine Pseudorabies (PR), which had been eradicated successfully in many countries of North- America and Europe(1), is still a widely spread viral disease in China (2). The use of a gene-altered vaccine makes it possible to identify infected pigs from vaccination population. Attenuated live marker vaccines are routinely used for a combined vaccinationeradication program for PR eradication. Several kinds of marker vaccines, home-made or imported, are approved for PR control in China (2). In this study, efficacy of 2 imported marker vaccines was compared by $\mathrm{gE}$-antibody test.

\section{Materials and Methods}

A North Chinese pig company (3,500 sows) has 2 farms with 2,000 sows in Farm 1 and 1,500 sows in Farm 2. There is 2 km between the two farms. Both farms implement continuous pig flow, all-in-all-out management. All sows are from same genetic farm and kept in same housing, feed and health management systems.

Both farms used home-made attenuated PRV vaccine. After 2 years' vaccination, more than $95 \%$ breeding animals were still $\mathrm{gE}$ positive, with poor reproductive and growth performance.

In early March 2008, both farms decided to replace the local vaccine with imported PRV vaccines. Farm 1 switched to vaccine $B$ (Ingelvac ${ }^{\circledR}$ Aujeszky MLV, Bartha K-61 strain, with water adjuvant). Sows and boars were vaccinated 4 times per year. In piglets, an intranasal vaccination was given at 1 to 3 days of age, and a second was given intramuscularly at 8 weeks of age. Farm 2 changed to vaccine $\mathrm{M}$ (another imported attenuated PRV vaccine, Bartha strain, with oil-in-water adjuvant). Sows and boars were vaccinated 4 times per year. In piglets, a first intramuscular shot was given at 8 weeks of age, and a second at 12 weeks of age.

Blood samples from boars, sows, 40-55-day old piglets and 130-150-day old fatteners, were collected from both farms in July-Oct. 2008 and May 2009. gE antibody were detected with IDEXX PRV-gE ELISA kit.

\section{Results}

Fig. 1 showed that the seropositive rate of field strain decreased more by Vaccine B in farm 1 than Vaccine $M$ in farm 2. In 5 months, fatteners in farm 1 became $g E$ antibody negative and that in farm 2 were not.

\section{Discussion}

Piglets born to sows infected with wild type virus should acquire $\mathrm{gE}$ positive colostral antibody. We reckoned it was colostral antibody that caused piglet $\mathrm{gE}$ positive, and fatteners were negative while $\mathrm{gE}$ antibody disappeared and no wild virus infected the group pigs in farm 1. In farm 2, the fatteners were gE positive which indicated wild virus infected the pigs earlier. vaccination by local replication and development of mucosal immunity in the presence of passively acquired colostral immunity. During acute infection, intranasal vaccine virus colonizers the trigeminal ganglia, and prevents infection and reduces latent infection (3) Two applications of intranasal and intramuscular vaccinations of Vaccine $B$ ensure much better protection than two intramuscular vaccinations of Vaccine M. Vaccine $B$ was more effective than Vaccine $M$ in preventing latent infection.

Figure 1. Seropositive rates of both farms in July, Oct. 2008 and May 2009
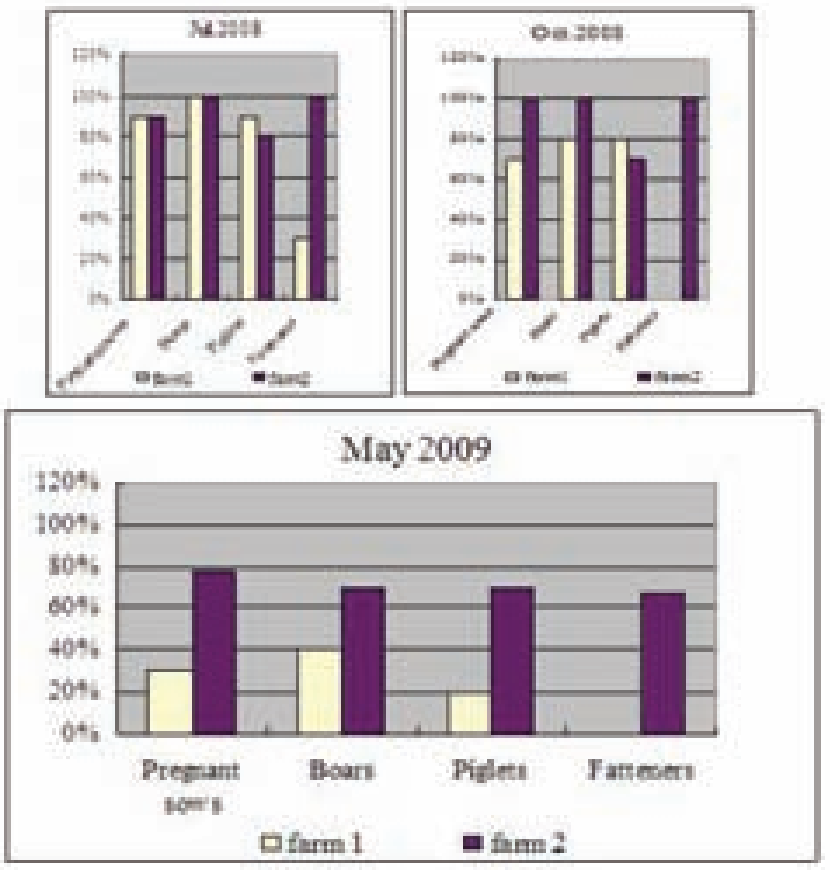

The eradication of PR can be most successfully achieved by the slaughter of all the seropositive animals, strict control of pig movement. However, this is not cost-effective. For most Chinese farmers, the optimal method of eradication is to implement combined vaccination-eradication program (1).

In this study, Vaccine B (Ingelvac ${ }^{\circledR}$ Aujeszky MLV) was proved a powerful tool for PR control and eradication.

\section{Reference}

1. Pajsak Z.K, et al. Aujeszky's Disease, Disease of Swine, 9th edition:419-434

2. Torrents $D$, et al. Pseudorabies (Aujeszky's virus disease) vaccination protocols in China. IPVS2008 poster:3

3. Mengeling $W L$, et al. Effect of various vaccination procedures on shedding, latency, and reactivation of attenuated and virulent pseudorabies virus in swine. Am J Vet Res 53:2164-2173

Intranasal vaccination is more effective than intramuscular 


\title{
Viability of Aujeszky disease virus vaccine virus BARTHA K61 (AUSKIPRA ${ }^{\circledR}-$ GN) when it was mixed with an inactivated Haemophilus parasuis vaccine (HIPRASUIS ${ }^{\circledR}$-GLASSER)
}

\author{
Sergi Bruguera $^{3}$ Reina Alemany ${ }^{2}$ Daniel Torrents ${ }^{1}$ \\ 1. HIPRA China, Technical Services, Beijing, China; 2. HIPRA, Research \& Development, Amer (Girona), Spain; \\ 3. HIPRA, Technical Services, Amer (Girona), Spain
}

\section{Introduction}

In Asia, nowadays, pig producers need to protect their pigs and sows against several pathogens. Vaccination programs to prevent Aujeszky disease virus and Glässer disease (Haemophilus parasuis) contemplate sow and gilt vaccination. The combination of both vaccines implemented simultaneously in the same inoculation point, saving two intramuscular injections, can potentially improve both the pig welfare status and the labour efficiency. Therefore, the objective of this trial is to find out if the attenuated gE deleted Aujeszky virus Bartha K61 (AUSKIPRA ${ }^{{ }_{-}}$ GN) can keep alive when it is reconstituted with a inactivated Haemophilus parasuis vaccine (HIPRASUIS ${ }^{\circledR}$-GLÄSSER) as a previous step to try in vivo the actual effect of these combined vaccines.

\section{Material and Methods}

Material

50 dose freeze dried tablets of AUSKIPRA ${ }^{\circledR}$-GN vaccine (attenuated gE deleted Aujeszky virus), 50 dose of inactivated Haemophilus parasuis vaccine HIPRASUIS ${ }^{\circledR}$-GLÄSSER, $100 \mathrm{ml}$ aqueous solvent vials and PK15 cell line.

Methods

Two tablets of AUSKIPRA ${ }^{\oplus}-G N$ were reconstituted with HIPRASUIS ${ }^{\circledR}$-GLÄSSER and with aqueous solvent respectively. After 0, 2, 4 and 6 hours post reconstitution the virus was titred in PK15 cell line.

\section{Results}

Table 1. Results of titres per dose of vaccine virus BARTHA K61. Vaccine virus titres BARTHA K61 per dose (2mI) (TCID50/dose). Reading by CPE (Cytopathic Effect).

\begin{tabular}{|c|c|c|}
\hline $\begin{array}{l}\text { Hours at standard Temperature } \\
25^{\circ} \mathrm{C} \text { after reconstitution }\end{array}$ & AQUEOUS SOLVENT & HIPRASUIS ${ }^{\oplus}$-GLASSER \\
\hline 0 & $10^{6.2}$ & $10^{6.2}$ \\
\hline 2 & $10^{6}$ & $10^{6}$ \\
\hline 4 & $10^{5.9}$ & $10^{6}$ \\
\hline$* 6$ & $* 10^{5.9}$ & $* 10^{5.9}$ \\
\hline
\end{tabular}

* BARTHA K61 vaccine virus Bartha K61 (AUSKIPRA ${ }^{\circledR}-G N$ ) kept viable and with a dosage titre value equivalent or higher of $10^{5.5} \mathrm{TCID}_{50}$ (minimum necessary effective concentration as product manufacturer recommendation) at 6 hours after reconstitution with the inactivated Haemophilus parasuis vaccine HIPARSUIS ${ }^{\circledR}$-GLÄSSER $(100 \mathrm{ml})$. The same results were obtained when mixed with the usual prescribed aqueous solvent $(100 \mathrm{ml})$.

\section{Conclusions}

HIPRASUIS $^{\circledR}$-GLÄSSER vaccine allows the viability of the $g E$ deleted Bartha K61 vaccine virus (AUSKIPRA ${ }^{\circledR}-\mathrm{GN}$ ) even 6 hours after the reconstitution. Further studies in vivo are required to discard any vaccine incompatibility. 


\title{
P.561
}

\section{Pseudorabies virus partial gE protein production for immunochromatographic strip test}

\author{
Suparlark Nuntawan Na Ayudhya ${ }^{1}$ Roongtham Kedkovid ${ }^{1}$ Kampon Kaeoket $^{2}$ Roongroje Thanawongnuwech $^{1}$ \\ 1. Chulalongkorn University, Bangkok, Thailand; 2. Mahidol University, Bangkok, Thailand
}

\section{Introduction}

Pseudorabies virus (PRV) is a cause of an important infectious disease in the pig industry known as Aujeszky's disease. The disease is lethal in piglets; however, in older pigs, the disease is not lethal characterized by severe depression, anorexia, pyrexia, ataxia and pneumonia. In pregnant sows, the infection may result in abortion1. PRV eradication program is usually based on the use of the marker vaccine deleted in the non-essential glycoprotein $\mathrm{E}(\mathrm{gE})$ gene2. The vaccinated pigs can be differentiated from the natural infected pigs by detection of anti-gE antibody, commonly done by the competitive ELISA. However, current ELISA assays have some limitations such as multi-step and timeconsuming procedures, requirement of an ELISA reader and other equipments and subsequent calculations to interpret the results. The immunochromatographic strip test is a new diagnostic technique which has several advantages over the commercial ELISA, including less time-comsuming (immediate results) and no requirement for skilled technicians or expensive equipments. Thus, this assay has a potential to be used as a field test. The objective of this study was to produce the recombinant $\mathrm{gE}$ protein for the immunochromatographic strip test.

\section{Materials and Methods}

The pseudorabies virus DNA was extracted and used for PCR to produce a partial $\mathrm{gE}$ fragment (coded for the major epitope domain of PRV gE gene, nucleotide position 156-714). The purified PCR product was cloned into the PGEM ${ }^{\oplus}$-T-easy vector (Promega, USA.) and then cut with EcoRI. The DNA fragment was inserted downstream to the TAC promoter of the expression vector pGEX $5 x-3$, resulted in $g E$ pGEX $5 x-3$. The recombinant plasmid was transformed into the E. coli Rosetta (DE3) pLysS strain (Novagen, USA.). For expression, the E. coli carrying recombinant plasmid was 1:50 (v/v) diluted into 1 liter of LB medium (with $100 \mu \mathrm{g} / \mathrm{ml}$ ampicillin and $34 \mu \mathrm{g} / \mathrm{ml}$ chloramphenical) and incubated at 37 oC for $3 \mathrm{~h}$. Then, the protein synthesis was induced by isopropy 1 - $\beta$-D-thiogalactopyranoside (IPTG, $0.1 \mathrm{mM}$ final conc.) and the bacteria were further incubated at $37^{\circ} \mathrm{C}$ for $4 \mathrm{~h}$. The bacteria were harvested and then disrupted by sonication. The size and the specificity of the recombinant protein were examined using SDS-PAGE and western immunoblotting, respectively.

\section{Results}

The expressed products were determined by SDS-PAGE revealing the extra protein band of approximately $38 \mathrm{kDa}$ molecular weight (Figure 1). The ability of the expressed product to bind with anti-gE antibody was further confirmed by the western immunoblotting analysis Figure 1, lane 3) using the IDEXX ELISA gE positive pig serum as a primary antibody.
Figure 1. SDS-PAGE and western immunoblotting analysis of the recombinant protein.

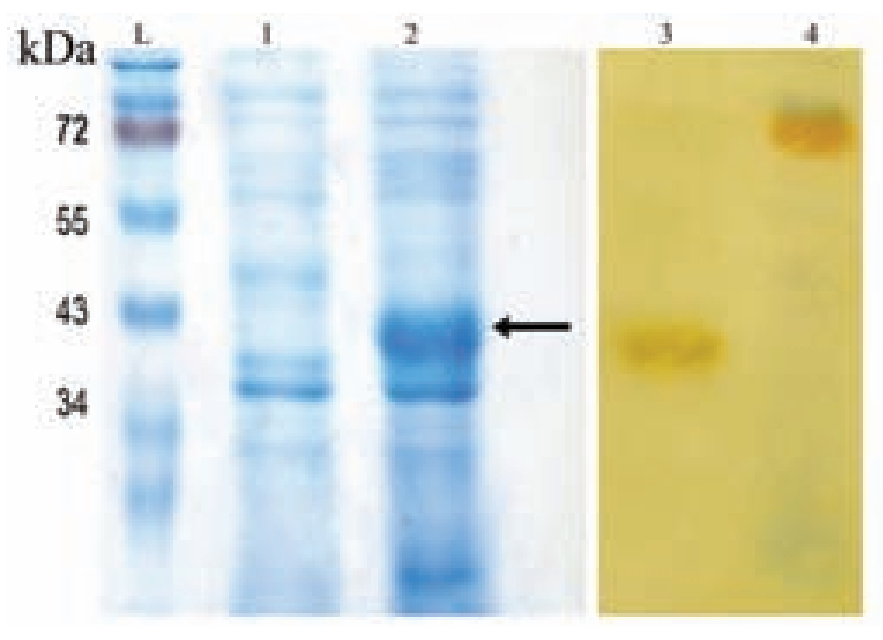

Expression of gE pGEX $5 x-3$ in E. coli showed an extra band by the SDS-PAGE (lane 2, 38 kDa, arrow) compared with non induced E. coli (lane 1). The ability to bind with anti-PRV antibody was showed by western immunoblotting analysis (lane 3 ). $L=$ protein marker.

\section{Discussion}

The recombinant $\mathrm{gE}$ protein could be expressed in the $\mathrm{E}$. coli system and this recombinant protein was able to stain with the IDEXX ELISA gE positive pig serum demonstrating its specificity. This recombinant protein will be used for further study such as the checker-board titration and testing for the sensitivity and specificity to ensure the efficacy of the developing immunochromatographic strip test.

\section{Acknowledgements}

The authors are thankful to the Chulalongkorn University-Veterinary Diagnostic Laboratory for providing PRV, pig serum and laboratory equipments. This study was funded by the National Science and Technology Development Agency (NSTDA).

\section{References}

Baskerville, A., 1981, New Zealand Vet. J. 29, 183-185.

Mettenleiter, T.C., 1994, Acta Veterinaria Hungarica 42, 153-177. 


\title{
P.562
}

\section{Minimum protective dose efficacy test of Auphyl ${ }^{\circledR}$ Plus (deleted mutant live pseudorabies vaccine) in susceptible piglets}

\author{
József Herczeg $^{1}$ Tamás Szalai $^{1}$ Erzsébet Sombor ${ }^{1}$ Georges Országh ${ }^{1}$ Jean deFoucauld ${ }^{1}$ \\ Jérôme Thevenon ${ }^{1}$ Zsófia Benyeda ${ }^{2}$ János Benyeda $^{2}$ Roman Krejčí ${ }^{3}$ \\ 1. CEVA-Phylaxia, Budapest, Hungary; 2. Prophyl Immunolab, Mohács, Hungary; 3. CEVA Sante Animale, Libourne, France
}

\section{Introduction}

Aujeszky's disease (AD or pseudo-rabies) is one of the most important swine diseases of the developing word. The disease has effects on both reproductive and fattening performance of the herds. Live, gene-deletant vaccine strains formulated with oil adjuvants, are considered the most efficient tools (by DIVA) for $A D$ eradication programs in the European Union. AD eradication still remains a heavy task in Eastern-Europe and in Asia. Virus strain of Auphyl ${ }^{\circledR}$ Plus vaccine is a $\mathrm{gE}$ ( $\mathrm{gl}$ ) deletant, UL21 mutant and $\mathrm{gC}$ positive $\mathrm{AD}$ virus (ADV), which is a recombinant derivate of the Bartha's strain (K/61).

Material and Methods: Minimum Protective Dose (MPD) trial was dedicated to confirm the choice of vaccine dose of Auphyl ${ }^{\circledR}$ Plus in ADV sero-negative 8 week (w) old pigs.

Study design: Four vaccine dose-groups and a control group were formed using 5 piglets in each. Pigs in Group 1 (G1) received one shot commercial dose (6.1 log10 TCID50). Animals of G2, G3 and G4 received 1/10-1/1000 (5.1-3.1 log10 TCID50) doses. The challenge (ch) was performed 1 month after the intramuscular vaccination. The ch-virus, strain AyV-NIA-3 was applied intra nasally in $2 \mathrm{ml} /$ nostril. The re-titrated ch-dose was 6.7log10/mI TCID50.

Clinical parameters: 1 ) symptoms of $A D, 2)$ rectal temperature, 3) body weight, 4) ch-virus re-isolation. Post-challenge (pch) observations were conducted till the 7th day (1) \{European Pharmacopoea\}.

\section{Results}

1. Two control pigs died on the 5 th or 7 th pch days (pchD). Central neural symptoms were shown in all controls and none in vaccinates. Total cumulative respiratory symptom scores were between 8 and 10 in G1-G4 and 29 in G5.

2. Rectal temperature increase of the controls was higher than in any of the vaccinates, but the difference was not significant. See figure 1.

Figure 1: The group-mean rectal temperature $\left(C^{\circ}\right)$ of the pigs before and after the challenge

3.

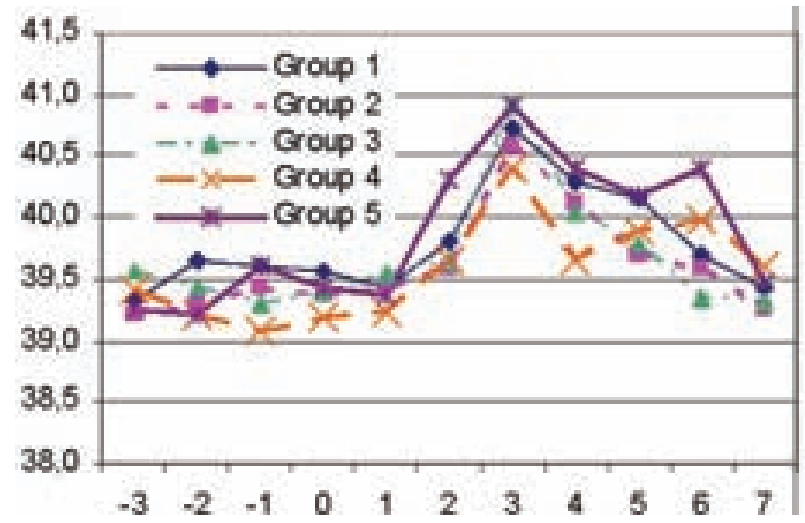

Daily body weight gain was almost equal $(0.4-0.3 \mathrm{~kg})$ between pchD0 and pchD7 in G1, G2 and G3. In G4 it was 0.1 kg. Controls showed $-0.4 \mathrm{~kg}$ daily body weight loss. See table 1 .

Table 1: Summarized efficacy results post-challenge

\begin{tabular}{|l|c|c|c|}
\hline Group (vaccine) & No. Death / Total & ADWG D0-pchD7 & $\begin{array}{c}\text { Ch-virus re-isolation } \\
\text { (log10TCID50/ mI) @ } \\
\text { pchD4 }\end{array}$ \\
\hline 1 (Auphyl Plus) & $0 / 5$ & 0.3 & 1.9 \\
\hline $2(1: 10)$ & $0 / 5$ & 0.3 & 2.6 \\
\hline $3(1: 100)$ & $0 / 5$ & 0.4 & 3.2 \\
\hline $4(1: 1000)$ & $0 / 5$ & 0.1 & 4.0 \\
\hline 5 (controls) & $2 / 5$ & -0.4 & ND \\
\hline
\end{tabular}

Among the vaccinates, the less protected pigs were those animals which received $1 / 1000$ dose of the vaccine.

4. Ch-virus re-isolation results (log10TCID50/ $\mathrm{ml}$ ) on pchD4 showed significant differences between the treatment groups: average geometrical mean in $\mathrm{G} 1=1.9 \log 10$, $\mathrm{G} 2=2.6 \log 10, \mathrm{G} 3=3.2 \log 10, \mathrm{G} 4=4.0 \log 10$.

\section{Discussion}

Auphyl ${ }^{\circledast}$ Plus vaccine was protective against death and central neural symptoms even in 1/1000 dose (3.1 log10TCID50/ml). $1 / 100$ is the lowest dose which is protective against the significant daily body weight loss in $8 \mathrm{w}$ old pigs.

Apply DIVA system. Due to these features the vaccine is recommended to perform AD eradication programs.

\section{References}

1. European Pharmacopoeia 01/2008:0745 corrected 6.0: Aujeszky's disease vaccine (live) for pigs for parenteral administration

2. Robbins A.K. et al., J. Virol. (1989), 63, 250-258.

3. Van Oirschot J.T. et al., J. gen. Virol. (1986). 67, 1179-1182.

4. Van Oirschot J.T. et al., Vet. Record (1987) 121, 305-306

${ }^{\circledR A U P H Y L ~ P L U S ~ i s ~ a ~ r e g i s t e r e d ~ t r a d e m a r k ~ o f ~ C E V A ~ S A N T E ~ A N I M A L E . ~}$ 


\title{
P.563
}

\section{Eradication of PRV in Chinese farm with Ingelvac ${ }^{\circledR}$ Aujeszky MLV}

\author{
David Xu; Liande Zhu \\ Boehringer Ingelheim International Trading (Shanghai) Co. Ltd, Beijing, China
}

The objective of this project was to eradicate PRV (Pseudorabies Virus) form a 1500 sows farm by combining vccination with Ingelvac ${ }^{\circledR}$ Aujeszky MLV and positive animals elimination acccording to serology diagnosis. It is a one-site production system farm located in Jiangshu province China, suffered a severe loss due to PRV outbreak in 2003. Production performance after the outbreak could not reach back to normal level.

\section{Materials and Methods}

Pre eradication: Before eradication, 100 samples were randomly collected in each section for serology (HerdChek ${ }^{\circledR}$ PRV gB and HerdChek $^{\circledR *}$ Anti-PRV gl(gE) from IDEXX)to analyze the wild PRV infection in the farm. The results showed an over $50 \%$ positive rate in all herd (Table1).

Vaccinated program: Breeders: After first massive vaccination, a booster vaccination was implemented in a month, then with regular vaccination every 4 months. Piglets: intranasal vaccination at 1 day old, with another intramuscular booster vaccination on 65 days old. Gilts: vaccinated twice before mating.

Management: Strict management according to Madec's 20 was implemented in this farm at the mean time.

Measurement: New herds were isolated for 30 days before introducing to farm with a serology and PCR test against PRV (positive results would be tested twice for confirmation). In the first 3 years blood samples were randomly collected from all herds of the farm to monitor the eradication effect ( $N=1,282$ samples). In the 2nd year after the eradication implementation, all PRV-gE serological positive boars were eliminated; in the 4th year all PRV positive breeding swine (PRV-gE antibody and PCR tested) were eliminated; in the 5th year, randomly sampling from the whole herd for serology test to evaluate the eradication efficacy.

\section{Results}

Before eradication, the average abortion rate of the sows was above $30 \%$. Intractable diarrhea and severe respiratory problems were prevalent in the nursery and fattening herds. The clinical signs were reduced gradually since the eradication and after 1 year, the whole herds were stabilized.

Serology surveillance was strictly implemented at the same time as vaccination began. During this period, seropositive animals were eliminated. Positive rate of the serology test decreased consistently and steadily every year. In the 2 nd year and 4th year after eradication, 5 boars and 13 pigs were eliminated. Serology results showed the success since the 5 th year (Table1). The all negative results indicated the success of the eradication. Since then, the farm stopped PRV vaccination in sows and piglets.
Table1. Positive rate of PRV-gE ELISA test Before/After Eradication

\begin{tabular}{|l|c|c|c|c|}
\hline & Sows & Suckling & Nursery & Fattening \\
\hline Pre eradication & $50 \%(50 / 100)$ & $705(84 / 120)$ & $60 \%(66 / 110)$ & $50 \%(50 / 100)$ \\
\hline $\begin{array}{l}\text { 1st year post } \\
\text { eradication }\end{array}$ & $18 \%(20 / 111)$ & $24 \%(25 / 104)$ & $20 \%(30 / 150)$ & $18 \%(15 / 83)$ \\
\hline $\begin{array}{l}\text { 2nd year post } \\
\text { eradication }\end{array}$ & $4 \%(5 / 125)$ & $7 \%(8 / 114)$ & $6 \%(6 / 100)$ & $5 \%(4 / 80)$ \\
\hline $\begin{array}{l}\text { 3rd year post } \\
\text { eradication }\end{array}$ & $2 \%(2 / 100)$ & $4 \%(5 / 125)$ & $3 \%(3 / 100)$ & $2 \%(2 / 90)$ \\
\hline $\begin{array}{l}\text { 4th year post } \\
\text { eradication }\end{array}$ & $0(0 / 100)$ & $0(0 / 115)$ & $0(0 / 90)$ & $0(0 / 100)$ \\
\hline $\begin{array}{l}\text { 5th year post } \\
\text { eradication }\end{array}$ & $0(0 / 100)$ & $0(0 / 110)$ & $0(0 / 100)$ & $0(0 / 110)$ \\
\hline
\end{tabular}

\section{Discussion}

This farm is now considered to be PRV negative. Vaccination with proved to be effective for the eradication of PRV in the field in this case.

\section{References}

1. Pensaert M, Labarque $\mathrm{G}$, Favoreel $\mathrm{H}$, et al. Aujeszky's disease vaccination and differentiation of vaccinated from infected pigs. Dev Biol (Basel) [J], 2004, 119: 243-254.

2. Vannier P, Strugar S, Pansart J F, et al. Progress in eradication and control programmes against Aujeszky's disease (pseudo rabies) in $\mathrm{F}$ rance[J]. Vet Res, 2000, 31: 151-152. 


\title{
P.564
}

\section{Safety of overdose, safety on pregnancy and progeny of Auphyl ${ }^{\circledR}$ Plus (deleted mutant live pseudorabies vaccine) vaccine on swine}

\author{
$\underline{\text { József Herczeg }}^{1}$ Zsófia Benyeda $^{3}$ Tamás Szalai $^{1}$ Diana Krassóvári ${ }^{1}$ Georges Országh ${ }^{1}$ Jérôme Thevenon ${ }^{1}$ \\ Jean deFoucauld ${ }^{1}$ János Benyeda ${ }^{3}$ Roman Krejčí ${ }^{2}$ \\ 1. CEVA-Phylaxia, Budapest, Hungary; 2. CEVA Sante Animale, Libourne, France; 3. Prophyl Immunolab, Mohács, Hungary
}

\section{Introduction}

Aujeszky's disease (AD or pseudo-rabies, PRV) is one of the most important swine diseases of the developing word. The disease has effects on both reproductive and fattening performance of the herds. Several inactivated or attenuated live, oil adjuvated vaccines were developed against $A D$. Effect of oil adjuvant raises questions of general and local safety of the vaccine. Following topics are presented: i) safety of overdose of a $\mathrm{gE}$ (gl) deletant, UL21 mutant and gC positive virus strain MNC+ of Auphyl-plus vaccine strain and its solvent after the reconstitution, ii) safety of the vaccine on pregnancy and progeny.

i) Overdose safety test was conducted on two batches of the vaccine to prove its general and local safety.

\section{Material and Methods}

Three groups of 8 week old pigs were formed with 7 animals in each. Parameters of general and local tolerance: 1 ) rectal temperature, 2) body weight gain, 3) appetite, 4) colour of reactive zone at the vaccination site, 5 ) diameter of the reactive zone.

\section{Results}

1) Febrile effect of the vaccine batches was moderate $\left(2.1^{\circ} \mathrm{C}\right.$ and $2.4^{\circ} \mathrm{C}$ ) in two pigs for 1 day, only. Temperature decreased by $0.7-1.0^{\circ} \mathrm{C}$ for the next day. The individual temperature increase values in each treatment group were calculated in comparison with the individual base line values. This parameter ranged between $0.6^{\circ} \mathrm{C}$ and $2.4^{\circ} \mathrm{C}$. For another parameter, the group-mean increase values, non-significant difference $(p=0.1462)$ was found by ANOVA between of the treatment groups as the treatment stress has also caused temperature increase in the control group ranging between $0.5-1.3 \mathrm{C}^{\circ} .2$ ) No remarkable break or decline and non-significant difference appeared in the body weight gain tendency of the vaccinated groups $(P=0.99$ on $D 7$ and $P=0.93$ on D14). 3) None of pigs showed remarkable feed refusal during the observation period. 4\&5) None of animals showed colourisation reaction at the injection site after the vaccination and no any swollen local reactions appeared.

\section{Conclusion}

Neither immediate general nor local lesions with intolerance attributable to the vaccine were seen. Mild fever reaction lasting more than 24 hours occurred in case of two animals only. Nonsignificant body weight gain difference between the treatment groups appeared.

ii) Safety on pregnancy and progeny test was conducted to show the reproductive performance of sows and vitality of progeny piglets after use Auphyl-plus vaccination regime in the mothers.

\section{Material and Methods}

14 gilts were vaccinated and 8 received placebo at 8 weeks of age with repetition 1 month apart. 4 pregnant gilts received a third vaccination 3 weeks before the farrowing and 4 pregnant gilts were kept for controls. Commercial dose of the vaccine was used in $2 \mathrm{ml}$ containing $6.1 \log 10$ TCID50 ADV. Test parameters of overdose safety were used and completed with monitoring of reproductive performance (6), progeny vitality (7) and body weight of piglets (8).

\section{Results}

1) No fever appeared in any of the treatment groups. 2) All animals performed normal daily body weight gain in the rearing period enabling them to be inseminated after their heating period. 3) No remarkable feed refusal was recoded. 4-5) One gilt showed red spot 1 hour after the first vaccination. This local reaction disappeared by the next day. No other local reaction was recorded. 6) Same litter size was delivered by both treatment groups of the sows (43-43 piglets). $95.3 \%$ and $97.7 \%$ life born, 7\%-7\% weak, 4.7\%-2.3\% still-born progenies were recorded, born from vaccinated and control sows, respectively. 7) Vitality of piglets was assessed by scoring of any clinical signs. Piglets of the vaccinated sows performed score value 21 while controls 18 within the 1 week observation period. 8) Body weight of piglets at birth and on PpD21: this parameter didn't show a significant difference between the groups $(P=0.13$ and $P=0.46)$. Mothers were also included in the calculation as they can affect the body weight of the new born piglets.

\section{Conclusion}

No immediate post vaccination reactions and no adverse effect on the feeding behaviour were recorded. Very mild and temporary local post-vaccination reaction, rarely appeared, mild and transient fever, normal body weight gain tendency, and good reproductive performance of vaccinated gilts were seen. Assessment of the litters of both groups showed good tolerance of vaccination from the progenies of vaccinated sows was proven. No adverse effect on vitality, normal body weight performance results of piglets originating from the vaccinates were recorded.

${ }^{\oplus A U P H Y L ~ P L U S ~ i s ~ a ~ r e g i s t e r e d ~ t r a d e m a r k ~ o f ~ C E V A ~ S A N T E ~ A N I M A L E . ~}$ 


\title{
P.565
}

\section{Study on seropositivity for Pseudorabies virus in swine farms in Pinerolo District: correlation with management and geographic factors}

\author{
Giuseppe Martano; Giorgio Burzio \\ ASI TO3, Vigone, Italy
}

\section{Introduction}

The aim of this study is to evaluate PRV seropositivity in Pinerolo district and to underline the most probable risk factors about the seropositivity for Aujeszky's disease.

We collected data on potential risk factors for infectious disease in 41 pig farms in Asl To3, Pinerolo district, Northwestern Italy. UTM geographic coordinates were recorded for each farm and entered in a geographic information system ( GIS). Herds size ranged from 15 to 11.050 animal. Results of 6564 tests effected in the course of 613 herd's visit have been compared in geographic areas of Asl To3, from 1997 to 2009. The number of PRV+ herds has clearly decreased from 1997, even though areas with higher risk exist.

\section{Material and Methods}

Aujeszky's disease is a viral disease due to porcine herpesvirus-1 which belongs to the Alphaherpesvirinae subfamily, herpesviridae family.

In April 1997, a programme to eliminate Aujeszky's disease virus infection from the swine herds in Italy was initiaded. The programme was based on intensive compulsory vaccination, with gE-deleted vaccines of all pigs. From May 1997 to December 2009 we collected 6564 serum samples from pigs and they were tested for antibodies to pseudorabies virus (PRV) by the enzyme-linked immunosorbent assay (ELISA test). Every farms was tested at least one time for year. Also we collected data about management and biosicurity level in 41 farms. The data were deloped by Epi-info 6. UTM geographic coordinates were recorded for each farm and entered in a geographic information system ( GIS).

\section{Results}

Analysis of spatial arrangement shows that the farms PRV+ are closer together compared to all the farms. Proper application of the vaccination plan and observance of basic principles of biosecurity to ward off the risk of infection.

Movement of infected pigs is considered the most likely route of Aujeszky's disease route in PRV-free herds.

Farrow to growing farms were the first to become PRV free. A high pig density in the area and presence of finishing pigs in farm was associated with a increase in odds of a herd being seropositive.
Figure 1. Seropositivity prevalence PRV in the partecipating herds.

\begin{tabular}{|c|c|c|c|c|c|c|}
\hline $\mathbf{1 9 9 7}$ & $\mathbf{1 9 9 8}$ & $\mathbf{1 9 9 9}$ & $\mathbf{2 0 0 0}$ & $\mathbf{2 0 0 1}$ & $\mathbf{2 0 0 2}$ & $\mathbf{2 0 0 3}$ \\
\hline $84 \%$ & $77 \%$ & $56 \%$ & $58 \%$ & $24 \%$ & $19 \%$ & $21 \%$ \\
\hline $\mathbf{2 0 0 4}$ & $\mathbf{2 0 0 5}$ & $\mathbf{2 0 0 6}$ & $\mathbf{2 0 0 7}$ & $\mathbf{2 0 0 8}$ & $\mathbf{2 0 0 9}$ & \\
\hline $12 \%$ & $15 \%$ & $18 \%$ & $12 \%$ & $8 \%$ & $2 \%$ & \\
\hline
\end{tabular}

In 1997 the seropositivity herd 's prevalence was 84\%, in 2009 was $2 \%$.

Figure 2. Seropositivity prevalence in single tested pigs.

\begin{tabular}{|c|c|c|c|c|c|c|}
\hline $\mathbf{1 9 9 7}$ & $\mathbf{1 9 9 8}$ & $\mathbf{1 9 9 9}$ & $\mathbf{2 0 0 0}$ & $\mathbf{2 0 0 1}$ & $\mathbf{2 0 0 2}$ & $\mathbf{2 0 0 3}$ \\
\hline $38 \%$ & $30 \%$ & $19 \%$ & $18 \%$ & $16 \%$ & $18 \%$ & $19 \%$ \\
\hline $\mathbf{2 0 0 4}$ & $\mathbf{2 0 0 5}$ & $\mathbf{2 0 0 6}$ & $\mathbf{2 0 0 7}$ & $\mathbf{2 0 0 8}$ & $\mathbf{2 0 0 9}$ & \\
\hline $10 \%$ & $\mathbf{7} \%$ & $6 \%$ & $4 \%$ & $4 \%$ & $1 \%$ & \\
\hline
\end{tabular}

The seropositivity prevalence in tested pigs decreased from 38 $\%$ in 1997 to $1 \%$ in 2009.

\section{Discussion}

The results show that if a correct vaccination and satisfactory biosecurity level applied, the risk of viral circulation decreased rapidly in few years.

\section{References}

Tamba, Calabrese, Finelli, Cordioli (2002): "Risk factors for Aujeszky's disease seropositivity of swine herds of a region of northern Italy". Preventive Veterinary Medicine, 54.

Leontides, Eward, Mortensen, Willeberg (1995): “Factors associated with seroprevalence of Aujeszky's 


\title{
P.566
}

\section{Evaluation on seropositivity and antibody titre for Aujeszky's Disease in fattening and farrow to feeder pig herds}

\author{
Giuseppe Martano; Igor Ferenaz \\ ASI TO3, Vigone, Italy
}

\section{Introduction}

In North-West Italy control and eradication for Aujeszky's disease represents a target still to be reached. The aim of this work is to obtaine an evaluation for seropositivity prevalence Aujeszky's disease in farrow to feeder and fattening herds. The antibodies titres has been evaluated on pigs in fattening herds. The examination has been carried on 64 herds in the period 2007-2008.

\section{Materials and Methods}

In Italy, Aujeszky's disease has been a subject of a mandatory eradication program since 1997. The vaccination program prescribes three inoculation/year for the sow and three inoculation for the fattening pigs( the first at 60-90 days, the second 30 days later and the third at 180 days).We used a gE-delete dead vaccine in the farrow to feeder herds and a $\mathrm{gE}$ - delete live vaccine in fattening herds. In our district, the pigs were slaughtered at 270 days old. From January 2007 to December 2008 a survey was conducted in Pinerolo District, North-west Italy. Sixty four randomly selected farms was tested to evaluate the provincial pseudorabies virus ( PRV) herd seroprevalence. The sample included 34 farrow to feeder herds and 30 fattening herds. The farms were randomly selected. Blood sample collection for each farm was calculated so as to detect disease with an estimated prevalence of $20 \%$ and at a confidence limit of $95 \%$. 2117 pigs were tested during 152 inspections and they were tested for antibodies to pseudorabies virus (PRV) by the EnzymeLinked Immunosorbent Assay (ELISA test). Antibodies against PRV ( glycoprotein gB and glycoprotein gl ) were examined.

\section{Results}

Seropositivity prevalence in farrow to feeder herds was $6,1 \%$ and in fattening herds was $13,5 \%$.

The seropositive pigs prevalence was 1,21\% in farrow to feeder herds and 5,34 \% in fattening herds.

In seronegative fattening herds a significant percentage of pigs with low antibodies titres ( glycoprotein $\mathrm{gB}$ ) has been observed.

\begin{tabular}{|l|c|}
\hline negative samples & $25,1 \%$ \\
\hline positive samples 1:8 & $9,5 \%$ \\
\hline positive samples 1:16 & $8,3 \%$ \\
\hline positive samples 1:32 & $28,4 \%$ \\
\hline positive samples 1:64 & $5,6 \%$ \\
\hline positive samples 1:128 & $17,1 \%$ \\
\hline positive samples 1:256 & $1,2 \%$ \\
\hline positive samples $\geq 256$ & $4,8 \%$ \\
\hline
\end{tabular}

\section{Discussion}

The target is to reduce seroprevalence in swine herds stopping the entry of the virus through latently infected pigs.

The current report shows that in seronegative fattening herds a significant percentage of pigs with low antibodies titres has been observed. This indicates that the herd immune status of the farms was insufficient to prevent a field virus infection. Probably the vaccination in some fattening herds is carried out not correctly. Fattening herds could became an important reservoir for pseudorabies virus.

It is necessary to use a correct vaccination program with management improvements and rigorous biosecurity measures.

\section{References}

Alborali L., Guadagnini ., Accini A.., Zanoni M., Salogni., Cordioli., (2007)“ Sieroprevalenza della malattia di Aujeszky in suini pesanti macellati in pianura Padana". Atti SIPAS, Modena, 283-287.

Martini M., Drigo M., Dalla Pozza M., Ferrari G., Sona B., (2003) “ A study of the progress of the Aujeszky's disease control programme in Italy using survival analysis".Journal Veterinary Medicine B50, 191-195.

Bolla C., (2005) " Monitoraggio della Malattia di Aujeszky, applicazione in campo e prospettive". Atti SIPAS, Mantova,315-319. 


\title{
P.567
}

\section{Duration of immunity of Auphyl ${ }^{\oplus}$ Plus (deleted mutant live pseudorabies vaccine) in progeny of vaccinated sows}

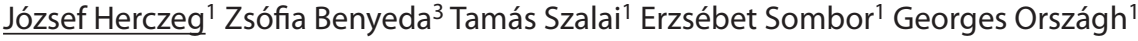 \\ Jérôme Thevenon ${ }^{1}$ Jean deFoucauld ${ }^{1}$ János Benyeda ${ }^{3}$ Roman Krejči ${ }^{2}$ \\ 1. CEVA-Phylaxia, Budapest, Hungary; 2. CEVA Santé Animale, Libourne, France; 3. Prophyl Immunolab, Mohács, Hungary
}

\section{Introduction}

Aujeszky's disease (AD or pseudo-rabies) is one of the most important swine diseases of the developing word. The disease has effects on both reproductive and fattening performance of the herds. Live, gene-deleted vaccine strains formulated with oil adjuvants, are considered the most efficient tools (by DIVA) for AD eradication programs in the European Union. AD eradication still remains a heavy task in Eastern-Europe and in Asia. Virus strain of Auphyl ${ }^{\circledR}$ Plus vaccine is a gE-deleted, UL21 mutant and gC positive $A D$ virus (ADV), which is a molecularly modified derivate of the Bartha's strain (K/61).

\section{Materials and Methods}

Duration of Immunity in Progeny: DOI by maternally derived antibodies (MDA) in the progenies of vaccinated sows was tested by challenging 4- and 6-w-old piglets.

Study design: 4 sows were vaccinated 3 times at 2, 3 months of age and 3 ws before farrowing. Their progenies ( 16 piglets) had no vaccination but were sero-positive due to the MAD transfer. Each of 8 piglets were challenged at $4(\mathrm{G} 2)$ or $6(\mathrm{G} 1)$ ws of age. 8 piglets from the progenies (G3) of non-vaccinated sows were involved as ch-controls at 6 ws of age.

Challenge circumstances and clinical parameters were the same as detailed in the MPD trial.

\section{Results}

1. All control piglets (G3) died within 7 pchDs. All piglets of $G 2$ (4-ws-old) were protected but 2 out of 8 piglets from the G1 (6-ws-old) died.

2. The ANOVA didn't show any significant difference between rectal temperatures of the groups ( $p>0.05)$. Note: the rectal temperature decrease in Group 2 is a virtual one as pre-moribund or moribund animals showed lower rectal temperature a day or two days after their fever peak. All pigs from this group died one or two days after that their rectal temperatures declined.
Figure 1: The group-mean rectal temperature $\left(C^{\circ}\right)$

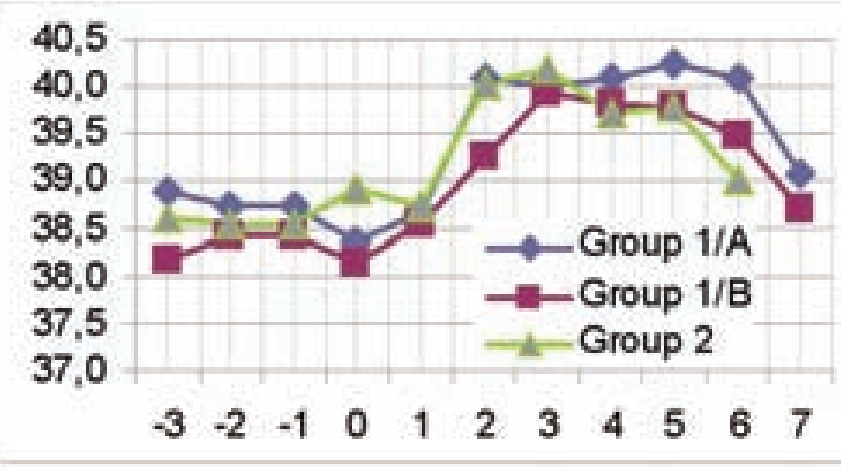

3. Mortality and post-ch average daily body weight (DBW) and the weekly body weight gain (WBWG): are shown in Table 1.

\begin{tabular}{|l|c|c|c|c|}
\hline Table 1. & $\begin{array}{c}\text { No. Death / } \\
\text { Total }\end{array}$ & $\begin{array}{c}\text { DBW at } \\
\text { chD0 }\end{array}$ & $\begin{array}{c}\text { DBW at } \\
\text { PchD7 }\end{array}$ & $\begin{array}{c}\text { WBWG between } \\
\text { chD0 and PchD7 }\end{array}$ \\
\hline Group 1/A (ch at 6 w) & $2 / 8$ & 10.14 & 11.24 & 0.16 \\
\hline Group 1/B (ch at 4 w) & $0 / 8$ & 4.91 & 5.71 & 0.11 \\
\hline Group 2, controls (ch at 6 w) & $8 / 8$ & 9.04 & 7.33 & -0.25 \\
\hline
\end{tabular}

4. There was no significant difference between MDA protected and control piglets in the excretion of the ch-virus on pchD4 and pchD7.

\section{Discussion}

Auphyl ${ }^{\circledR}$ Plus vaccine was efficient to protect swine against death, nervous symptoms and body weight loss caused by Aujeszky's disease. The vaccine used by the prescribed application regime, significantly decreased the virus shedding.

\section{References}

1. European Pharmacopoeia 01/2008:0745 corrected 6.0: Aujeszky's disease vaccine (live) for pigs for parenteral administration

2. Robbins A.K. et al., J. Virol. (1989), 63, No. 1, 250-258.

3. Van Oirschot J.T. et al., J. gen. Virol. (1986). 67, 1179-1182.

4. Van Oirschot J.T. et al., Vet. Record (1987) 121, 305-306

${ }^{\circledR}$ AUPHYL PLUS is a registered trademark of CEVA SANTE ANIMALE. 


\title{
P.568
}

\section{Duration of immunity of Auphyl ${ }^{\circledR}$ Plus (deleted mutant live pseudorabies vaccine) in fattening pigs}

\author{
József Herczeg ${ }^{1}$ Tamás Szalai $^{1}$ Zsófia Benyeda ${ }^{3}$ Diana Krassóvári ${ }^{1}$ Georges Országh ${ }^{1}$ \\ Jérôme Thevenon ${ }^{1}$ Judit Szucs ${ }^{3}$ János Benyeda ${ }^{3}$ Roman Krejčí ${ }^{2}$ \\ 1. CEVA-Phylaxia, Budapest, Hungary; 2. CEVA Santé Animale, Libourne, France; 3. Prophyl Immunolab, Mohács, Hungary
}

\section{Introduction}

Aujeszky's disease (AD or pseudo-rabies) is one of the most important swine diseases of the developing word. The disease has effects on both reproductive and fattening performance of the herds. Live, gene-deleted vaccine strains formulated with oil adjuvants, are considered the most efficient tools (by DIVA) for $A D$ eradication programs in the European Union. AD eradication still remains a heavy task in Eastern-Europe and in Asia. Virus strain of Auphyl ${ }^{\circledR}$ Plus vaccine is a gE-deleted, UL21 mutant and $\mathrm{gC}$ positive AD virus (ADV), which is a molecularly modified derived of the Bartha's strain (K/61). Duration of Immunity (DOI) in Fatteners test was conducted to show the level of protection in 6 month old pigs.

Material and Methods: Study design: 8 pigs received primo-vaccination (G1) at 2 month-of-age and it was boosted one month later. 4 sero-negative, non- vaccinated controls were involved (G2) in the study, with same age. Challenge was performed at 6 months-of-age, 3 months after the last vaccination.

Challenge circumstances and clinical parameters were the same as detailed in the MPD trial.

\section{Results}

1. No death occurred in control group within 7-day observation period. Central nervous symptoms were observed in each of the controls but not in any of the vaccinates. Total cumula-

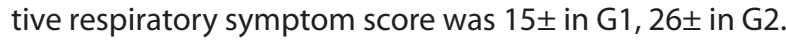

2. Rectal temperatures of controls (G2) were significantly higher than in the vaccinates from pchD3 till pchD7 ( $p$-value $=0.01$ ).

Figure 1: Rectal temperature results

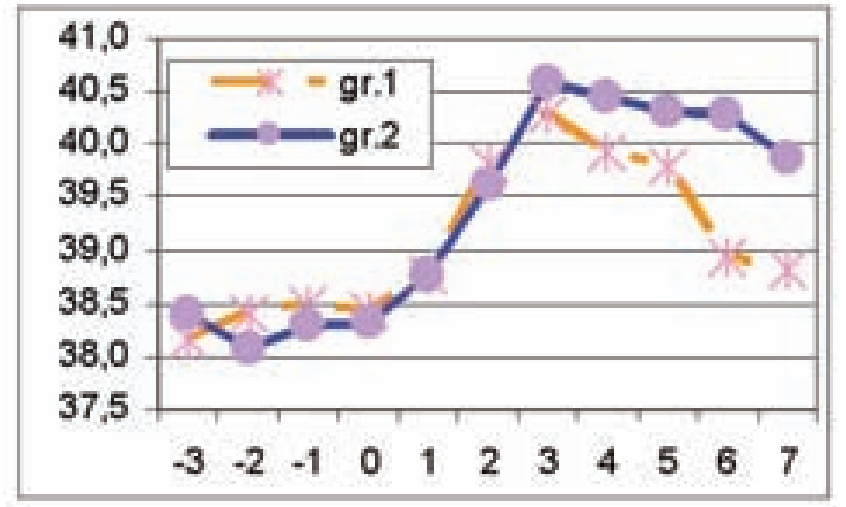

3. Post-ch DBWG in vaccinated group was $+0.34 \mathrm{~kg}$ versus -1.14 $\mathrm{kg}$ in controls: Difference $1.48 . \mathrm{Kg}$ fulfilling the requirement of the (1) European Pharmacopoeia.
Table 1: Group mean body weight (GMBW) kg) of treatment groups and calculated daily body weight gains (DBWG)

\begin{tabular}{|c|c|c|c|c|}
\hline Table 1. & chD0 & PchD7 & GMBW: chD0 - PchD7 & DBWG (group average values) \\
\hline Group 1. & 126.3 & 128.7 & 2.4 & $2.4 / 7=0.34$ \\
\hline Group 2. & 119.7 & 111.7 & -8.0 & $-8 / 7=-1.14$ \\
\hline
\end{tabular}

4. The ch-virus re-isolation results ( $\log 10 \mathrm{TCID} 50 / \mathrm{ml}$ ) of $\mathrm{G} 2$ were significantly higher (5.2 on pchD4 and 3.3 on pchD7), than in G1 (3.8 on pchD4 and 0 on pchD7).

\section{Discussion}

The 4 month duration of immunity of vaccine Auphyl ${ }^{\circledR}$ Plus was proven. It was protective against death and central nervous symptoms of $A D$. The vaccine diminished the fever response in challenged animals. The vaccine was highly protective against the body weigh loss and significantly decreased the ch-virus shedding.

\section{References}

1. European Pharmacopoeia 01/2008:0745 corrected 6.0: Aujeszky's disease vaccine (live) for pigs for parenteral administration

2. Robbins A.K. et al., J. Virol. (1989), 63, No. 1, 250-258.

3. Van Oirschot J.T. et al., J. gen. Virol. (1986). 67, 1179-1182.

4. Van Oirschot J.T. et al., Vet. Record (1987) 121, 305-306

${ }^{\oplus} \mathrm{AUPHYL}$ PLUS is a registered trademark of CEVA SANTE ANIMALE. 


\title{
P.569
}

\section{Pathogenesis and transmission of feral swine pseudorabies virus isolates}

\author{
Eraldo L. Zanella ${ }^{1}{ }^{2}$ Kelly M. Lager ${ }^{1}$ Laura C. Miller $^{1}$ Sabrina L. Swenson ${ }^{3}$ Troy T. Bigelow ${ }^{4}$ Marcus E. Kehrli, Jr ${ }^{1}$ \\ 1. National Animal Disease Center-USDA-ARS, Ames, IA, USA; 2. College of Agronomy and Veterinary Medicine - University of Passo Fundo, \\ Passo Fundo, RS, Brazil; 3. National Veterinary Services Laboratories, USDA, Veterinary Services, Ames, IA, USA; \\ 4. National Center for Animal Health Programs, USDA, Veterinary Services, Des Moines, IA, USA
}

\section{Introduction}

Aujesky's Disease or pseudorabies, is one of the oldest recognized swine diseases. It is caused by pseudorabies virus (PRV), an alpha-herpesvirus that can induce respiratory disease, reproductive failure, and affect the central nervous system. PRV vaccines, in conjunction with serologic tests, have been used to eradicate the virus from swine herds and even from entire countries. One weakness in PRV control and eradication programs is the indigenous PRV infection of feral swine. This wild-life reservoir is responsible for frequent infection of domestic swine.1 A better understanding of this source of infection may lead to improved PRV control programs. This report summarizes studies investigating the pathogenicity and transmissibility of feral swine PRV isolates from the USA.

\section{Materials and Methods}

Seventy-three four-week-old pigs were randomly allocated to one of nine treatment groups, each housed in a separate isolation room. Pigs in each group received either a low dose (LD about $\left.10^{3} \mathrm{CCID}_{50}\right)$ or high dose (HD - about $\left.10^{6} \mathrm{CCID}_{50}\right)$ intranasal challenge of virus: Group 1 ( $n=7)$ LD feral swine isolate FS268; Group 2 ( $n=7)$ HD FS268; Group 3 ( $n=7)$ LD feral swine isolate C3R Ossabaw Is; Group 4 (n=7) HD C3R Ossabaw Is; Group 5 $(n=7)$ LD domestic swine isolate ISUVDL4892; Group $6(n=7)$ HD ISUVDL4892; Group $7(n=10)$ LD panther isolate of a feral swine virus FP117-05; Group 8 ( $n=10)$ HD FP117-05; Group $9(n=11)$ controls that received three $\mathrm{ml}$ of cell culture media. Nasal swabs (NS) were collected on days $0,2,4,7,9,11,14,21$, and at necropsy 28 days-post-inoculation (dpi) at which time blood, lung lavage and tissues were collected for virus isolation and detection of viral DNA by real-time PCR.2

On 2 dpi, ten direct contact pigs (DC) were comingled with the pigs inoculated with HD C3R Ossabaw Is isolate, and five DC pigs were comingled with the pigs inoculated with HD FP117-05. After five days of contact the inoculated pigs were moved to different rooms and each group of DC pigs was maintained for about 16 weeks.

\section{Results}

Clinical signs: Mean body temperature spiked 2 dpi in all PRV inoculated groups and returned to normal by 5-7 dpi. For each virus pair the HD pigs were more affected than LD pigs. By $1 \mathrm{dpi}$ some pigs were lethargic and at 2 dpi respiratory distress (sneezing, dyspnea, dysphonia (altered squeal (laryngeal edema)), congested nasal passages, and dog sitting) was observed. Transient anorexia was detected in most pigs. Central nervous system disease was observed in some pigs (ataxia, opisthotonos, paddling, and seizure). Most pigs from the feral swine isolate groups recovered; however, six out of seven group 6 pigs were euthanized or died in the first $7 \mathrm{dpi}$. In group 4, two pigs and in group 1 , one pig were euthanized in the first $8 \mathrm{dpi}$. Clinical signs in DC pigs exposed to C3R Ossabaw Is isolate were mild. In contrast, all DC FP117-05 exposed pigs had moderate to severe respiratory disease, but each pig did recover.

Virology: Virus was isolated from NS from 2-14 dpi with most positives between 2-9 dpi, and more frequently from the HD than LD challenge groups. Virus was isolated infrequently from 28 dpi tissues.

Serology: Based on a commercially available ELISA, all PRVinoculated pigs seroconverted by $14 \mathrm{dpi}$. Likewise, all DC pigs seroconverted by 14 days post contact and were seropositive throughout the duration of the study, about four months.

\section{Discussion}

Collectively, results from these studies indicate natural infection of domestic swine with feral swine PRV isolates should be detected either through detection of virus during the acute phase of the infection, or through detection of antibody in the convalescent phase of the infection. This conclusion supports many current control programs that screen sera for PRV-specific antibody, and may test tissues for virus by real-time PCR.

\section{Acknowledgements}

We thank Dr. Romero from the University of Florida for the feral swine isolate FS268, Dr. Stallknecht from the Southeastern Cooperative Wildlife Disease Study for the feral swine isolate C3R Ossabaw Is and the panther isolate FP117-05, and Dr. Yoon from lowa State University for the domestic swine isolate ISUVDL4892. Funding was provided by USDA Veterinary Services and Agricultural Research Service. Eraldo Zanella is a recipient of a scholarship from $\mathrm{CNPq} /$ Brazil.

\section{References:}

1. Wyckoff, AC, et al., 2009. J Wildlife Dis, 45;422-429.

2. Ma, W, et al., 2008. J Vet Diagn Invest, 20:440-447. 


\title{
P.570
}

\section{Isolation and in vivo and in vitro characterization of the first Chinese porcine teschovirus strain (PTV-8)}

\author{
Shangjin Cui \\ Harbin Veterinary Research Institute of CAAS, Harbin, China
}

Investigations were made to identify the causal agent of a case of acute diarrhea, respiratory distress and death in a swine farm in the Jilin Province, northern China. Only porcine teschovirus (PTV) was isolated from samples of organs and identified as PTV-8. Presence of virus which was designated Jilin/2003, was confirmed by the production of a specific cytopathic effect on susceptible cells and by the results from IPMA assay, polymerase chain reaction, and electron microscopy. Other main pathogenic agents causing diarrhea, respiratory distress and death (e.g., porcine rotavirus, transmissible gastroenteritis virus of swine, Porcine epidemic diarrhea virus, classical swine fever virus, pseudorabies virus, porcine circovirus, porcine reproductive and respiratory syndrome virus, Japanese encephalitis virus, mycoplasma, leptospira, Streptococcus, Listeria, and Brucella species) were eliminated as possible causal agents because they were not consistently associated with the disease of the pigs. The PTV-8 strain was adapted to growth in swine primary kidney (PK-15) cells and in a swine testicular cell line (ST cells). When inoculated into healthy pigs, PTV-8 Jilin/2003 caused the same symptoms as observed in the affected herd. We conclude that PTV-8 Jilin/2003 was the causal agent of this disease. To the best of our knowledge, few reports so far indicate that PTV strains infect swine in China.
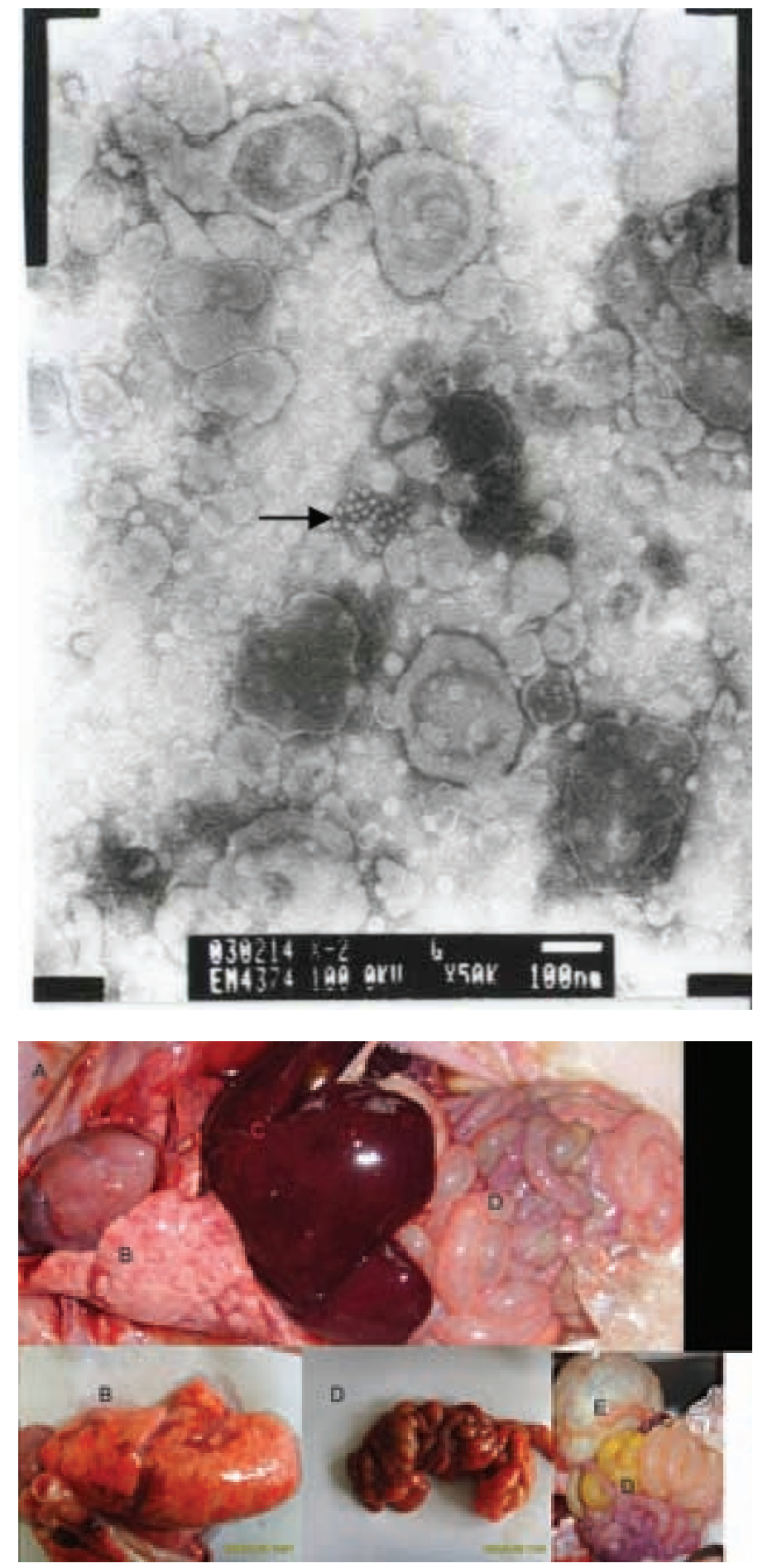


\title{
Teschen Talfan Disease - contagious swine paralysis - a case report
}

\author{
$\underline{\text { Katja Brase }}^{1}$ Eva Ursula Peitzmeier ${ }^{2}$ \\ 1. Landwirtschaftskammer Niedersachsen, Oldenburg, Germany; 2. Praxis, Meente 24, Hille, Germany
}

\section{Introduction}

The enterovirus encephalomyelitis of pigs (porcine enteroviral Polioenzephalomyelitis) rarely occurs in German pig population. The Tescher Disease, caused by porcine Teschovirus (PTV) Type 1 hasńt been reported in Germany for over 20 years. It is a human analogue of the spinal polio disease, which mainly is caused by Porcine Teschovirus (PTV) strains. Porcine Teschovirus enters the body by oral route, reproduces in the gastro- intestinal organs and will be excreted by feces for up to 8 weeks. The majority of infected animals will not show any symptoms but will excrete the virus with the feces for weeks. If neurovirulent strains of the virus reproduce and spread in the brain, general illness with paralysis of different degree can be the result. The animal's awareness of the environment will not be influenced, sensitivity of the skin stays intact. An improvement of symptoms after a few days can be considered the rule. Usually an immunity to the virus within the contained group of animals will be established within 6 weeks. If tonic-clonic muscle spasms, nystagmus and other paralytic symptoms appear during the course of the disease, morbidity and mortality will be high due to dyspnoea and cardial insufficiency. Hardly any information about therapy of this disease can be found in literature. Diagnosis via PCR and serological determination of the type of virus will decide about the obligation to report, as is the case with PTV type 1. Only the virus extraction from the CNS can be considered proof, the antigen itself has to be traced. The large number of different Teschovirus makes determination of types difficult. For clinical considerations, proof of the species Teschovirus can be considered sufficient. In differential diagnosis, ESP and AD will have to be considered.

\section{Material and Methods}

The descripted farm has 160 sows in a closed system. Jung sows come from different farms in denmark with a yearly replacement of $35 \%$. Piglets are weaned after 28 days of nursing. First clinical signs can be observed 7 to 10 days later in flatdeck. About $10 \%$ of a group show high degree paralysis of the limbs. Animals are aware of their environment, food and water intake appear to be normal. They show a swaying gait with dropping down in front while the hind quarters remain upright. Sensitivity of body surface stays intact. In almost all affected animals edema of the eye lids is prominent. In some bad cases, animals are lying on the side with walking motion oft he legs, show opisthotonus, nystagmus and tonic-clonic spasms. About $4 \%$ of affected animals die within 12 hours after onset of symptoms. The others recover within a few days. None of these animals show long term signs of paralysis. Treatment with cortisone und vitamines $B$ did not change any of the clinical symptoms. In this farm is, however, no reliable inventory of immunity. There remain clinical symptoms in the piglets in flatdecks.

\section{Results}

By autopsy and following microbiological examination, porcine Enterovirus type 8,9 and 10 could be proven in organs and large intestine as well as PTV virus in the large intestine. Infection by ESP and AD could be excluded in blood samples. Cerebrum, cerebellum and brain stem showed low grade edema. The Bioscreen Laboratory in Münster examined organ matter for PTV virus (before: porcine enterovirus type 1 to 7 and 11) and porcine enterovirus type 8,9 and 10.The examination was performed via multiplex PCR. A type determination of the different PTV virus types is not possible in German laboratories. Thus it could not be proven if the isolated virus is PTV type 1.

\section{Discussion}

By definition of the government from 12.08.2005 the Tescher disease has to be proven by virus breeding and Type 1-identifiation. Additionally, histological proof of non-purulent polioenzephalomyelitis is needed. A durable immunity against the virus probably could not be established because too many strains were present in the contained group. A possible reason might be the acquisition of young sows from different sources. There are no cross- immunities, so there will always be susceptible animals supporting virus reproduction. As enterovirus is spread by the feces of sick animals, controlled access to the feces of older sows would be desirable to reach quick contamination of every animal. By this measure, maternal protection of piglets should be optimized, so a durable immunity can be established. It is important to keep access to the feces on a moderate level, since enterovirus may not only course neurological symptoms but also abortions.

Group- specific vaccines cannot be produced at this point of time.

\section{References}

Lehrbuch der Schweinekrankheiten, Karl-Heinz Waldmann, Michael Wendt, 4 Auflage, Parey Verlag 2004;

Case definitions of the Federal Ministry for Consumer Protection, Food and Agriculture, dated 12.08.2005

Annual Report 2008 of the Tierseuchenkasse Baden Wurttemberg 


\title{
P.572
}

\section{The distribution and genotyping of porcine teschovirus in Taiwan}

\author{
Yeou-Liang Lin; Yu-Wen Huang; Susan Yun Fan Lin; Tien-Shine Huang; Ming-Hwa Jong ; Wei-Ming Chang; Chin-Cheng Huang
}

Animal Health Research Institute, Taipei County, Taiwan

\section{Introduction}

Porcine teschovirus (PTV), previously named porcine enterovirus 1 , causes disease in pigs (1). Based on serology more than $70 \%$ of the swine population in Taiwan were positive. The percentage of detected lesion samples in Taiwan from which PTV was isolated was higher than that of other swine viral pathogens (PRRSV etc).

The aim of this study was to assess the number of PTV serotypes, and their distribution, in Taiwan.

\section{Material and Methods}

A total of 525 swine fecal samples were collected from 5 pig farms of each county and city in Taiwan. Each fecal sample was mixed in MEM solution and a 10\% mixture filtered through a filter membrane with a pore size of $0.22 \mathrm{um}$. The filtrate was mixed with growth media (MEM) and incubated with PK 15 cells at $370 \mathrm{C}$ in $3.5 \% \mathrm{CO} 2$, for 3 days. Culture fluid was analysed for the presence of PTV by RT-PCR.

The VP1 gene fragment of PTV was amplified in a reverse transcription-polymerase chain reaction (RT-PCR) using extracted PTV viral RNA and PTV-specific primers. The PTV VP1 amplimers were then purified by gel filtration and sequenced. Using DNAstar software, phylogenetic analysis was carried out using the PTV VP1 sequences and sequence data obtained from web sources (4).

\section{Results}

A total of 58 samples positive for PTV, out of a total of 525 , from 21 counties and cities in Taiwan, were obtained by virus isolation and RT-PCR. The phylogenetic analysis of the sequences of 58 PTV isolates in Taiwan and 20 reference PTV strains covering 11 serotypes of PTV showed that all the isolated PTV strains had sequence homologies similar to 10 different serotypes of PTV (the exception of serotype 5 ). The 10 serotypes were distributed randomly around Taiwan (figure 1).

\section{Discussion}

In this study, 58 fecal samples out of 525 were positive for PTV. Infected pigs did not show any clinical symptoms, consistant with the findings that PTVs specifically infect pigs and are shed in pig feces (5), and that PTV infections are most frequently asymptomatic $(3,6)$. Previous workers have shown that specific PTV sequences equate to the serotypes 1-11 (2). The results of the phylogenetic tree analysis indicated that the 58 isolated PTVs, were aligned among 10 different serotypes (PTV1-PTV4 and PTV6-PTV11) and these serotypes were distributed randomly around Taiwan. Many of the larger pig farms located in the prefectures within middle and southern Taiwan (TCP, NTP etc) had animals shedding more than 1 isolate serotype. However, there was only one serotype in some counties. We concluded that at least 10 different serotypes of PTV existed in Taiwan now. The distribution of these serotypes was not restricted geographically.
Figure 1. The distribution of 10 different serotypes PTV isolates in Taiwan. Full circle indicates the serotype that isolate of each county or city was classified. U means unclassify

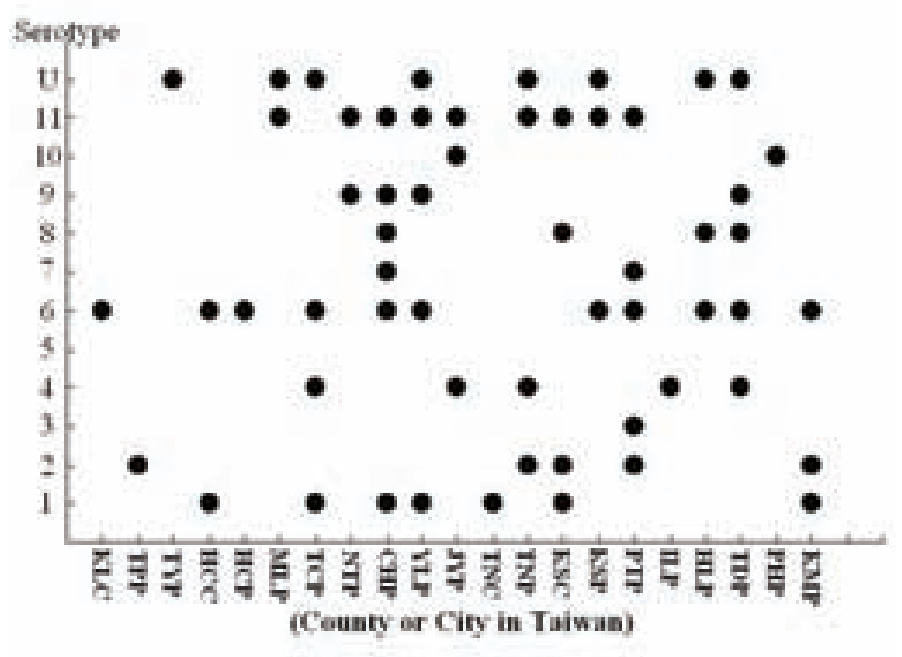

\section{References}

1. King, A. M. Q. et al. (2000). 7th Rep. ICTV. Academic Press, New York, N.Y. p. 657-673.

2. Zell, R.et al. (2001). J. Virol. 75, 1620-1631.

3. Forman, A. J. et al. (1982). Aust.Vet. J. 58: 136-142.

4. http://www.ncbi.nlm.nih.gov/nucleotide/

5. Jim'enez-Clavero M. A. (2003). Appl. Environ. Microbiol. 69(10), 6311-6315.

6. Kaku, Y. et al. (2001). J. Gen. Virol. 82:417-424. 


\title{
P.573
}

\section{Impact of Torque teno virus (TTV) on porcine dendritic cell function and activity}

\author{
Laura Martínez-Guinó ${ }^{1}$ Kenneth C. McCullough ${ }^{2}$ Artur Summerfield ${ }^{2}$ Joaquim Segales $^{1}$ Tuija Kekarainen $^{1}$ \\ 1. Centre de Recerca en Sanitat Anima (CReSA), Bellaterra, Spain; 2. Institute of Virology and Immunoprophylaxis (IVI), Mittelhäusern, Switzerland
}

\section{Introduction}

Torque teno virus (TTV) is a small, non-enveloped single stranded circular DNA virus classified in the floating genus Anellovirus, which infects several vertebrates including human and swine. Two genetically distinct genogroups have been described in swine, genogroup 1 (TTV1) and genogroup 2 (TTV2). TTV is ubiquitous and considered non-pathogenic but it has been linked to several diseases or pathological conditions in humans and, lately, in swine (1). To date, information related with the immunological events behind TTV infection is not available. Several viruses are known to modulate dendritic cell (DC) function. One example is porcine circovirus type 2 (PCV2), related genetically and structurally to TTV. It has been reported that PCV2 DNA is a potent modulator of DC and mediated inhibition of IFN- a production by plasmacytoid DC (pDC) (2). In the present work, the role of viral DNA from two genetically distinct swine TTV genogroups was analyzed for its potential interaction and modulation of DCs activity.

\section{Material and Methods}

PCV2 and TTV genomes were isolated by digestion from plasmids containing double-stranded DNA forms of the complete viral genomes. Peripheral blood mononuclear cells (PBMCs) were isolated by Ficoll-Paque differential centrifugation. pDCs were then enriched by magnetic antibody cell sorting (MACS). Enriched pDCs were incubated with decreasing amounts of PCV2, TTV1 or TTV2 DNA prior to stimulation with CpG-ODN. Supernatants from activated cultures were tested for IFN- a production by ELISA.

\section{Results}

Preliminary results showed that TTV1 and TTV2 viral DNA mediated inhibition of IFN- a production by CpG-ODN induced pDCs. The effect was dose dependent (Figure 1). TTV DNA was compared with DNA from PCV2, and it was observed that the inhibitory capacity of TTV1 was similar than the one observed for PCV2 and different from that of TTV2 (Figure 2).
Figure 1. IFN-a production by $p D C$ s in the presence of linear PCV2, TTV1 and TTV2 DNAs.

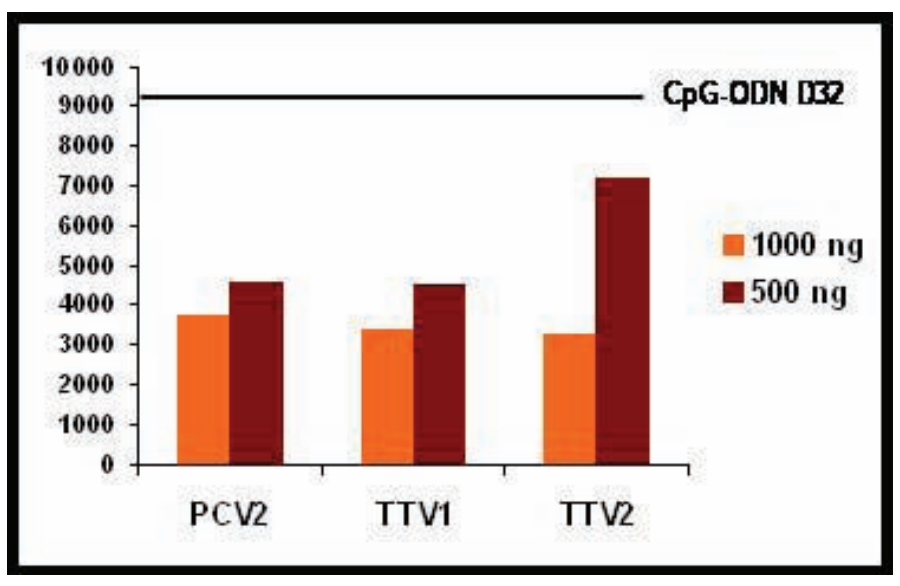

Figure 2. Percentage of IFN- a inhibition in the presence of liner PCV2, TTV1 and TTV2 DNAs, relative to levels obtained with CPG-ODN alone.

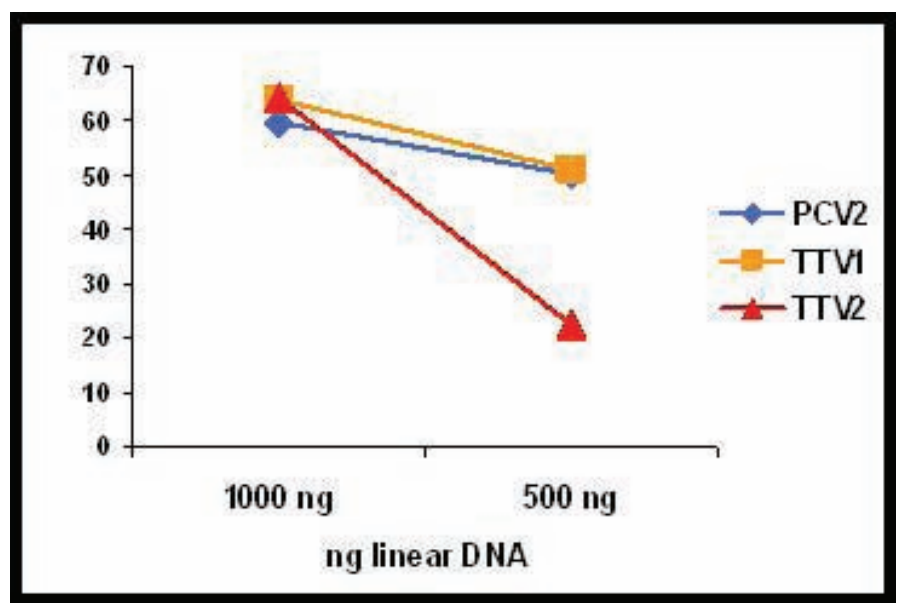

\section{Discussion}

TTV viral DNA was found to interfere in the ability of swine pDC to produce IFN- $a$ in response to known CpG-ODN stimuli (2). This study also reconfirms the effect or PCV2 DNA (2). The immunomodulatory effect observed for these viruses could potentially interfere in the development of efficient host innate and adaptative immune responses.

\section{Acknowledgements}

This work was funded by grants AGL2006-02778 /GAN, TRT2006-00018 and CSD2006-00007 from Spanish government. T. Kekarainen was supported by Spanish Government, Ramón y Cajal program.

\section{References}

1. Kekarainen and Segales (2009) Vet. J. 180, 163-168

2. Vincent et al. (2006) Immunology $115,388-398$ 


\title{
Torque teno sus virus 2 (TTV2) in situ hybridization: from Durban 2008 to Vancouver 2010
}

\author{
Mario Aramouni ${ }^{1}$ Tuija Kekarainen ${ }^{1}$ Maria Ballester ${ }^{1}$ Gerard E. Martin-Valls ${ }^{1}$ Joaquim Segalés ${ }^{1,2}$ \\ 1. CReSA/UAB, Bellaterra (Barcelona), Spain; 2. Departament de Sanitat i Anatomia Animals, Universitat Autònoma de Barcelona, Bellaterra, Spain
}

\section{Introduction}

Torque teno virus (TTV) is a non-enveloped, circular, single stranded DNA virus that may infect human, non-primate and domestic species including swine. Two specific TTV genogroups (TTV1 and TTV2) have been described in pigs. Even though the infection in pigs is considered non-pathogenic, TTVs have been somewhat linked with porcine circovirus diseases like postweaning multisystemic wasting syndrome (PMWS) and a porcine dermatitis and nephropathy syndrome (PDNS)-like syndrome $(1,2,3)$.

Two years ago, at the IPVS 2008 in Durban (South Africa), an in situ hybridization (ISH) method was described for the detection of TTV2 in formalin-fixed, paraffin embedded tissues (4). The study was performed on tissues of healthy, PMWS, and PDNS affected animals. Major conclusion of such study was: "TTV2 amount, location and cell type labelled resembled substantially those of PCV2 in PMWS affected animals. These findings raise the question on the potential role of TTV2, in co-infection with PCV2, in the pathogenesis of PMWS". During the development of the technique, a number of positive and negative controls were used to validate the technique. Once presented as an oral communication in the IPVS 2008, further validation studies were carried out. However, by the authors' surprise, different validation results were obtained from the initial ones and investigating in depth it was concluded that the oligonucleotide probe to detect TTV2 was also detecting PCV2. The parallelism between TTV2 and PCV2 amount was explained by the probe cross-reactivity, and presented results were discarded. Therefore, in view of these previous regrettable results, the authors further insight in the development of a new, specific ISH and fluorescent ISH (FISH) method to detect TTV DNA in TTV-DNA transfected cells and formalin-fixed, paraffin embedded pig tissues.

\section{Materials and Methods}

Cells and tissues. PK-15 cells were transfected with $0.5 \mu \mathrm{g} /$ well (six-well plate) of cloned TTV2 genome and fixed on coverslips. Formalin-fixed, paraffin-embedded tissues (lymph node, lung, kidney and liver) from healthy and PMWS pigs were tested with a TTV semi-quantitative PCR (data not shown) and tissues with the highest amounts of virus were selected for ISH and FISH.

ISH and FISH. To prepare TTV2 specific probe for hybridizations, total TTV2 viral DNA was labelled with digoxigenin (DIG), previous digestion with Xholl and Smal. Negative controls and specificity test of the TTV probe were: non transfected, pGFP (plasmid-green fluorescent protein) transfected, and PCV2 transfected PK-15 cells. The detection was made by an anti-DIG antibody and developed with NBT/BCIP as chromogen. The FISH method was performed using Cy3 labelled anti-DIG-antibody. In tissues, to enhance the signal, TSA (tyramine signal amplication) system was used. ISH and FISH methods were based on previously described protocols $(5,6)$ respectively.

\section{Results}

Both techniques, ISH and FISH, displayed positive cytoplasmic cell labelling using TTV2 probe on TTV2 transfected cells, while no labelling was observed in any of the negative controls including PCV2 transfected cells. Once a specific positive signal was obtained in the transfected cells, ISH and FISH methodologies were applied on formalin-fixed, paraffin-embedded tissues carrying TTV2 (detected by PCR). No specific labelling was found in any of the tested tissues.

\section{Discussion}

ISH and FISH were successfully developed to detect TTV2 nucleic acid in PK-15 transfected cells. In contrast, no specific ISH and FISH positive signal was found in formalin-fixed, paraffin embedded tissues from TTV2 infected pigs. These tissues were positive by PCR; considering the lower sensitivity of ISH techniques versus PCR, it is likely that the TTV2 DNA amounts in the tissues were below detection limit of ISH. However, we can still not rule out if formalin fixation and paraffin embedding alters the detection by ISH or FISH. Nowadays, experiments are being done to determine the detection limits of the technique.

\section{Acknowledgements}

This work was funded by the grants AGL2006-02778/GAN, TRT200600018 and CSD2006-00007 from Spanish government. M. Ballester and T. Kekarainen were supported by the Spanish Government, Juan de la Cierva and Ramón y Cajal programs, respectively.

\section{References}

1. Ellis et al (2008) Am. J. Vet. Res. 69, 1608-1614

2. Krakowka et al (2008) Am. J. Vet. Res. 69, 1615-1622

3. Kekarainen et al (2006) J Gen Virol 87, 833-7.

4. Martin-Valls (2008) IPVS oral proceedings OR.01.96

5. Rosell et al. (1999) J Comp Pathol 120, 59-78.

6. Ballester et al (2998) J Cell Biochem 105, 262-70 


\title{
P.575
}

\section{Genetic variability and phylogeny of swine Torque teno virus 1 (TTV1) and 2 (TTV2) based on complete genomes}

\author{
Martí Cortey ${ }^{1}$ Lisa Macera ${ }^{1,2}$ Joaquim Segales $^{1}$ Tuija Kekarainen $^{1}$ \\ 1. Centre de Recerca en Sanitat Anima (CReSA), Bellaterra, Spain; 2. Retrovirus Centre and Virology \\ Section, Department of Experimental Pathology, University of Pisa, Pisa, Italy
}

\section{Introduction}

Torque teno viruses (TTVs) are circular, single-stranded DNA viruses classified in the floating genus Anellovirus. They are currently considered non-pathogenic although they have been lately linked to several diseases in human and swine (1-3). The aim of this study was to determine the level of genetic variability in full-length genomes of swine TTVs, study the types and effects of mutations and to determine phylogenetic relationships with all known swine TTV genomes. For these reason 13 novel full-length genomes of swine TTVs were obtained from Europe and analysed together with 10 published genomes.

\section{Material and Methods}

Sera samples used in the study were collected during 2005-2007 and stored in the CReSA sera collection. Full-length genomes of TTV1 and TTV2 were amplified with a proofreading DNA polymerase and sequenced by DNA walking.

\section{Results and Discussion}

TTV genomes presented an untranslated region and three potential ORFs. Conserved regions like Arginin rich $\mathrm{N}$-terminus and rolling-circle replication domains were found in all swine TTV genomes. TTV1 genomes showed a high amount of variable positions compared with TTV2 (46.3\% vs $23.9 \%)$, that were differently distributed along the genome: lower in the UTR $(13.2 \%$ for TTV1 and $11.4 \%$ for TTV2), and higher in the translated region (59.8\% for TTV1 and $29.7 \%$ for TTV2). Low variability in the UTR region is most likely due to its importance in replication and transcription (4). The pattern of substitutions among ORFs was also different, more skewed for ORF1 and more evenly distributed for ORF2 and ORF3. TTV ORF1 seems to be affected by stronger levels of purifying selection compared with ORF2 and ORF3, most likely caused by the function of the protein products encoded by every ORF (5). TTV1 and TTV2 should be considered as species confirming the classification proposed (6). The phylogenetic reconstructions indicate that TTV1 is divided into three types. One of them (1c) is being reported here for the first time (Fig.1). Also, a certain kind of geographic structure among phylogenetic types may exist. Quite the opposite, the proposed subtypes for TTV2 clustered mixed and its definition was unclear.
Figure 1. Neighbor-Joining tree based on the percentage identity among 23 swine TTV genomes.

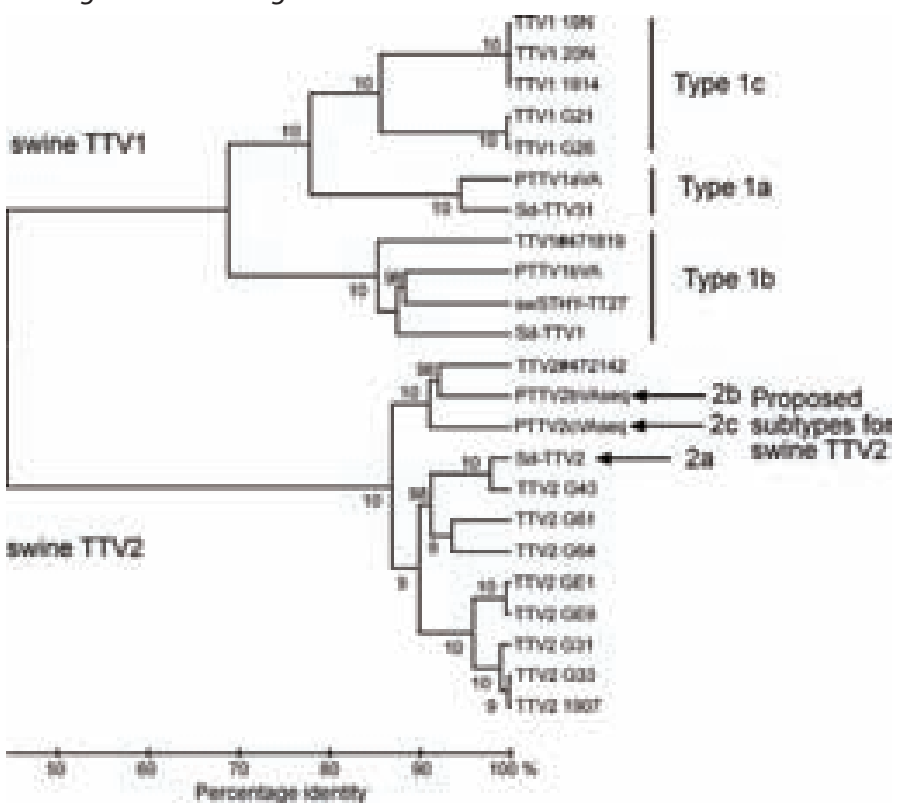

\section{Aknowledgements}

This work was funded by the grants AGL2006-02778/GAN, TRT200600018 and CSD2006-00007 from the Spanish government. M. Cortey holds a Beatriu de Pinós grant from the Government of Catalonia. T. Kekarainen was supported by the Spanish Government, Ramón y Cajal program.

\section{References}

1. Ellis et al., 2008. Am J Vet Res, 69, 1608-1614.

2. Kekarainen et al., 2006. J Gen Virol 87, 833-837

3. Krakowka \& Ellis, 2008. Am J Vet Res, 69, 1623-1629.

4. Mankertz et al., 2004. Vet Micro 98, 81-88.

5. de Villiers and zur Hausen, 2009. TTV Viruses. The still elusive Human Pathogens. Springer Verlag, Heidelberg Germany.

6. Huang et al., 2010. Virology 396, 289-297. 


\title{
P.576
}

\section{Rolling circle amplification to detect anelloviruses in pig serum samples}

\author{
Lisa Macera ${ }^{1}$ Martí Cortey ${ }^{1}$ Fabrizio Maggi ${ }^{2}$ Joaquim Segales $^{1}$ Tuija Kekarainen $^{1}$ \\ 1. Centre de Recerca en Sanitat Anima (CReSA), Bellaterra, Spain; 2. Retrovirus Centre and Virology Section, \\ Department of Experimental Pathology, University of Pisa, I-56127, Pisa, Italy
}

\section{Introduction}

Anelloviruses are vertebrate infecting single-stranded circular DNA viruses which have been recently linked to swine diseases (1). In swine two genetically distinct Anellovirus genogroups are described, namely Torque teno virus 1 (TTV1) and 2 (TTV2) (2). It is probable that novel swine genogroups exist as shown in the human TTV counterpart. Rolling-circle amplification (RCA) is a novel technique used to amplify circular DNA templates using random hexamers, yielding 109 or more copies of a circular sequence in about an hour (3). The potential of this technique has been shown to be increased when combining it with SequenceIndependent Single Primer Amplification (SISPA) (4). The aim of the present study was to apply the combined RCA-SISPA approach and optimize a novel RCA method using Anellovirusspecific primers (Anello-RCA) to discover new members of the genus Anellovirus in pigs. Also, efficiencies of the two techniques were compared.

\section{Materials and Methods}

A TTV2 positive swine serum sample (assayed by nested $P C R$ ) was selected to test the RCA-SISPA technique. Standard RCA involves the prolonged elongation of random primers bound to a circular DNA template by Phi29 DNA polymerase. The RCA products, that are tandem repeat copies of the viral complete genome, were used for the SISPA method, based on the use of endonuclease restriction of target sequences. These products were separated on agarose gel and different length products were ligated to specific linkers followed by PCR amplification and sequencing.

Short Anellovirus-specific primers were designed corresponding to a highly conserved region among all full-lenght Anellovirus genomes from several species available in the GenBank. AnelloRCA was optimized using short Anello-specific primers (6 nt) using swine sera known to contain TTVs. Furthermore, AnelloRCA was applied using swine sera and plasma samples negative for TTVs. The resulting products from Anello-RCA were used as template in inverse-PCR with Anello-specific PCR primers (12 and $15 \mathrm{nt}$ in length). PCR products purified from agarose gel were sequenced.

\section{Results}

Typically, the combined RCA-SISPA method resulted in several fragments of various lengths (Figure 1A). After sequencing 66 fragments, 3 of them were positive for TTV2 demonstrating that the RCA-SISPA is applicable to detect TTV in swine sera. Anello-RCA approach resulted in fragments between 2 and $3 \mathrm{~kb}$ of length (Figure 1B); sequencing of 12 fragments yielded 5 as positive for TTV1 and 1 for TTV2.
Fig.1. Digestion products obtained using RCA-SISPA approach (A). Amplification patterns from inverse-PCR on Anello-RCA product ( $B)$.
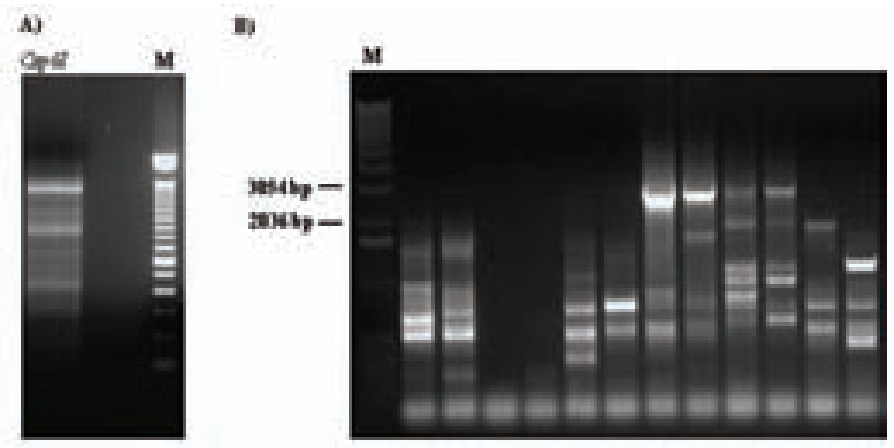

\section{Discussion}

Both described methods are applicable for Anellovirus detection in pig samples. The RCA-SISPA is more tedious than Anello-RCA due to the high number of different size fragments to be analysed. Anello-RCA technique resulted in larger and fewer fragments for analysis and is, therefore, more straightforward and less laborious. However, Anello-RCA detects only Anelloviruses while RCA-SISPA could detect any circular DNA virus present in a sample. Since primers used in Anello-RCA are located in the highly conserved region, this technique could be applicable for Anellovirus detection in other species as well.

\section{References}

1. Ellis et al. (2008) Am J Vet Res 69:1608-1614.

2. Kekarainen and Segalés (2009) Vet J. 180(2):163-168.

3. Niel et al. (2005) J Gen Virol 86:1343-1347.

4. Biagini et al. (2007) J Gen Virol 88:2696-2701.

\section{Acknowledgements}

This work was funded by the grants AGL2006-02778/GAN, TRT200600018 and CSD2006-00007 from Spanish Government. Tuija Kekarainen was supported by the Spanish Government, Ramón y Cajal program. 


\title{
P.577
}

\section{Torque teno virus tissue distribution by a semi-quantitative PCR method}

\author{
Mario Aramouni; Joaquim Segalés; Martí Cortey; Tuija Kekarainen
}

CReSA/UAB, Bellaterra (Barcelona), Spain

\section{Introduction}

Torque teno viruses (TTVs) are small, non-enveloped viruses with a circular single-stranded DNA genome, belonging to the family Anelloviridae, genus lotatorquevirus. TTVs are widely distributed and may infect human, non-primate and domestic species including pigs. In swine, two genetically distinct genogroups have been identified so far, TTV1 and TTV2 (1). The aim of the present study was to study the distribution and age-relation of swine TTV1 and TTV2 in tissues. Viral DNA obtained from tissue extractions were tested by a semi-quantitative PCR for the presence of both TTVs.

\section{Materials and Methods}

Thirty healthy animals corresponding to six different age-groups (second and last third of gestation, 5 days and 5, 15 and 24 weeks of age), 5 pigs per group, were sampled for 9 different tissues: brain, lung, mediastinal lymph nodes, heart, liver, spleen, kidney and bone marrow. Presence of TTV1 and TTV2 was tested with a semi-quantitative PCR, amplifying the virus in three different dilutions of extracted DNA ( 90 ng, 9 ng and $0.9 \mathrm{ng}$ ) at 43 cycles (optimal cycling for detecting the lowest standard concentration of 101 molecules). Negative samples at 43 cycles were tested at 50 cycles to enhance the detection sensitivity and ensure the negativity of the sample. The degree of TTV1 or TTV2 positivity was graded into highly positive (4), medium (3), low (2), very low (1), and negative (0). Contingency tables (Chi-square) were used to compare prevalence and grades of TTV1 and TTV2 in different samples at the different studied ages and in the different tissues. Statistical significance level was set at $\mathrm{p}<0.05$.

\section{Results}

No significant differences were found between genogroup prevalence in the same tissue at any age, while the prevalence increased with the age reaching $100 \%$ in all the organs by 5 weeks of age onwards. The first three age groups had negative to low levels of virus, and the last three groups, medium to high levels. Negative tissues were only found in foetuses and 5 day-old pigs. The only TTV1 PCR negative organs in some pigs were kidney, mediastinal lymph node, brain and heart. For TTV2, those negative tissues were brain and heart, mediastinal lymph node in some pigs.

\section{Discussion}

Both TTV genogroups followed similar infection dynamics in all the tested tissues, increasing progressively in prevalence and virus load over the time. The highest prevalence (100\%) was reached at 5 weeks of age, and the highest loads of virus in the different tissues were seen in the oldest animals (15 to 24 weeks of age). Those results are in agreement with an earlier study reporting increasing prevalence in sera in a longitudinal analysis (4). No animals were found to be negative to TTV, even foetuses, supporting the role of vertical transmission of TTV $(2,3)$. In conclusion, the present study indicates that swine TTV1 and
TTV2 can be found in tissues like brain, lung, mediastinal lymph nodes, heart, liver, spleen, kidney, mesenteric lymph nodes and bone marrow. Both swine TTV genogroups were present in high levels in most if not all adult animals while viral negative tissues were only found in 5 week-old pigs or foetuses.

Figure 1. Mean grade of TTV1 (black bars) and TTV2 (grey bars) in different age groups for some tissues. (IIG: 2 nd third of gestation, IIIG: last third of gestation, 5D: 5 days, 5W: 5 weeks, 15W: 15 weeks, 24W: 24 weeks). See definition of grading in Materials and Methods.

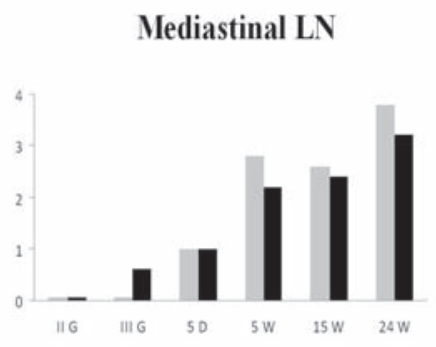

Liver

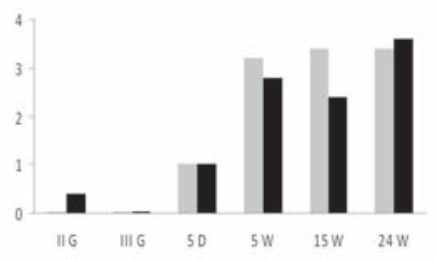

Lung
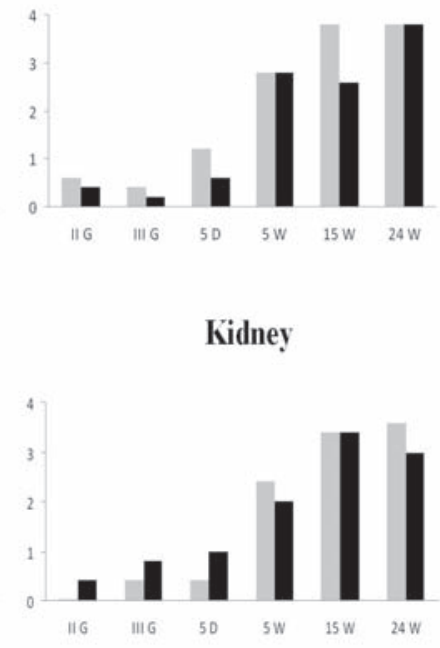

\section{Acknowledgements}

This work was funded by the grants AGL2006-02778/GAN, TRT200600018 and CSD2006-00007 from Spanish Government. Tuija Kekarainen was supported by the Spanish Government, Ramón y Cajal program.

\section{References}

1. Kekarainen and Segalés. 2009. Vet J. 180, 163-168

2. Martínez-Guinó et al. 2009, Theriogenology. 71, 1390-1395.

3. Pozzuto et al, 2009, Vet. Microbiol. 137:375-379.

4. Sibila et al. 2009. Vet. Microbiol. 139, 213-218. 


\title{
P.578
}

\section{Retrospective study on swine Torque Teno virus genogroups 1 and 2 and porcine circovirus type 2 coinfection in Italian pig sera (from 1990 to 2009)}

\author{
Marcello Bresaola ${ }^{1}$ Tina Lombardo² Riccardo Villa² Enrico Sossi² ${ }^{\text {Maura Ferrari }}{ }^{2}$ \\ 1. University of Parma (Italy), Parma, Italy; 2. Istituto Zooprofilattico Sperimentale della Lombardia e dell'Emilia Romagna, Brescia, Italy
}

\section{Introduction}

Torque Teno virus (TTV) is a non-enveloped, circular, singlestranded virus that has been detected in humans and animals, including pigs (1). Two swine TTV genogroups (TTV1 and TTV2) have been described (2) and the ubiquitous nature of this infection suggest that TTV have been infecting pigs for a long period of time. Moreover, recent studies suggest that swTTV could play an aetiological role in pig diseases, in particular PMWS (3). The main objective of this retrospective study is to asses whether there was a statistically significant association between swTTV and PCV2 infection.

\section{Materials and Methods}

Ninety five pig sera sampled between 1990 and 2009 were used in this study (no more information about these sera was available). A maximum of 10 pig sera per year were analysed. As much as possible, were analysed two year periods at the time (Table 1). Samples were stored at $-80^{\circ} \mathrm{C}$ until analysed. Sera were tested to detect swTTV1 and swTTV2 using specific PCR methods. Moreover, some PCR products from both swTTV genogroups were sequenced and phylogenetically analysed

\section{Results}

Both swTTV genogroups were found in pig sera from the very first year examined (Table 1).

Taking into account the whole period under study, 73 out of 95 animals $(76,84 \%)$ were infected with one or the other genogroup of swTTV, while 27 out of 95 pigs $(28,42 \%)$ were co-infected with both genogroups. swTTV genogroup 1 (54 out of 95, 56,84\%) was more prevalent than genogroup 2 (46 out of $95,48,42 \%$ ). Moreover, 41 out of 95 animals $(43,16 \%)$ were also infected with PCV2 (Table 1). Moreover, 20 out of 95 animals (21,05\%) were co-infected with swTTV1 and PCV2, while 27 out of 95 animals (28,42\%) were co-infected with swTTV2 and PCV2 (Table 2).

Phylogenetic analyses indicated no relationship between the date of virus detection and the cluster in the distance trees.

\begin{tabular}{|c|c|c|c|c|c|c|}
\hline year & $\mathbf{n}$ & $\begin{array}{c}\text { TTV1 or } \\
\text { TTV2 }\end{array}$ & $\begin{array}{c}\text { TTV1 and } \\
\text { TTV2 }\end{array}$ & TTV1 & TTV2 & PCV2 \\
\hline 1990 & 10 & 4 & 1 & 3 & 2 & 0 \\
\hline 1992 & 10 & 8 & 1 & 1 & 8 & 8 \\
\hline 1996 & 5 & 1 & 0 & 0 & 1 & 1 \\
\hline 1998 & 10 & 10 & 7 & 10 & 7 & 1 \\
\hline 2000 & 10 & 10 & 5 & 8 & 7 & 5 \\
\hline 2002 & 10 & 6 & 0 & 6 & 0 & 1 \\
\hline 2006 & 10 & 6 & 0 & 5 & 1 & 1 \\
\hline 2007 & 10 & 8 & 3 & 5 & 6 & 7 \\
\hline 2008 & 10 & 10 & 7 & 8 & 9 & 9 \\
\hline 2009 & 10 & 10 & 3 & 8 & 5 & 8 \\
\hline tot & 95 & 73 & 27 & 54 & 46 & 41 \\
\hline$\%$ & 76,84 & 28,42 & 56,84 & 48,42 & 43,16 \\
\hline
\end{tabular}

\section{Discussion}

This study indicates that TTV genogroups have been circulating al least since 1990 in the Italian pig population.

Moreover, these results indicate a statistically significant association between swTTV2 and PCV2 infection, but not between swTTV1 and PCV2 (Fisher's exact test with $\mathrm{p}<0.05$ ).

Phylogenetic analyses show no significant changes in the proportion of viral genogroups over time.

\section{References}

1. Taira O. et al (2009) Veterinary Microbiology 139, 347-350

2. Segalés J. et al (2009) Veterinary Microbiology 134, 199-207

3. Kekarainen. et al (2006) The Journal of General Virology 87, 833-837 


\title{
P.579
}

\section{Detection of TTV-2 by PCR in feces samples from different pig herds in São Paulo State, Brazil}

\author{
Cíntia M. Favero ${ }^{1}$ Alessandra M. M. G. de Castro ${ }^{1}$ Cíntia M. Baldin² Taís Nishikata $^{1}$ \\ Simone Miyashiro' Paulo E. Brandão ${ }^{1}$ Leonardo J. Richtzenhain ${ }^{1}$
}

1. Faculdade de Medicina Veterinária e zootecnia-Universidades de São Paulo, São Paulo, Brazil; 2. Faculdade de Jaguariúna, Jaguariúna, SP, Brazil

\section{Introduction}

Torque teno virus is a small non enveloped virus with circular single strand DNA genome classified within the Anellovirus. Two distinct TTV genogroup were identified circulating among domestic pig population, TTV-1 and TTV21. TTV have been detected by PCR in lungs, lymph nodes, tonsil, ileum, serum, marrow bone, liver and kidney2,3,4. Swine TTV has been detected in serum, plasma, nasal swab, rectal swab and feces, indicating fecal-oral transmission as the most significant way of spreading5,6. The aim of this work was to detect TTV-2 DNA in fecal samples from pig herds in São Paulo state, Brazil

\section{Materials and Methods}

Ninety seven fecal samples were collected from two different phases (nursery and growing) between 2009 and 2010 in three different swine herds from São Paulo state, Brazil. The samples were frozen, thawed three times and DNA extraction was carried out by phenol:chloroform:proteinase K protocol. TTV2 amplification was performed according to previous procedure described 7.

\section{Results}

Twenty six from a total of 97 fecal samples from three different pig herds were positive for TTV-2 (27\%).The number of positive samples within each pig herd (1, 2 and 3$)$ was $11 / 42(26 \%)$, $13 / 40$ (33 \%) and 2/13 (13\%), respectively (Fig. 1). Considering breeding phase (nursery and growing) we found a interesting data, since the number of positive animals were 10/68 (15\%) and $16 / 29$ (55\%) for nursery and growing, respectively (Fig. 2)

\section{Discussion}

The presence of TTV-2 in three commercial pig herds from São Paulo state, Brazil was investigated and the proportions of infected animals shedding virus were $27 \%$. A study carried out on Canada with commercial swine herd showed a higher proportion of infected animals (60.3\%). According to the results obtained in recent researches, the proportion may vary due the phase of breeding. A higher percentage of positive animals for TTV-2 was seen in growing (55\%) than in nursery (15\%). This finding are agreement with the results published 6 where $<20 \%$ of the rectal swabs were positive for TTV in pigs ranging from 1 to 15 weeks. In conclusion our results contribute for the understanding of the dynamic excretion of the TTV. This is the first description of excretion of TTV2 on feces in different commercial swine herds from São Paulo state, Brazil.
Figure 1: Number of positive and negative TTV-2 in different pig herds

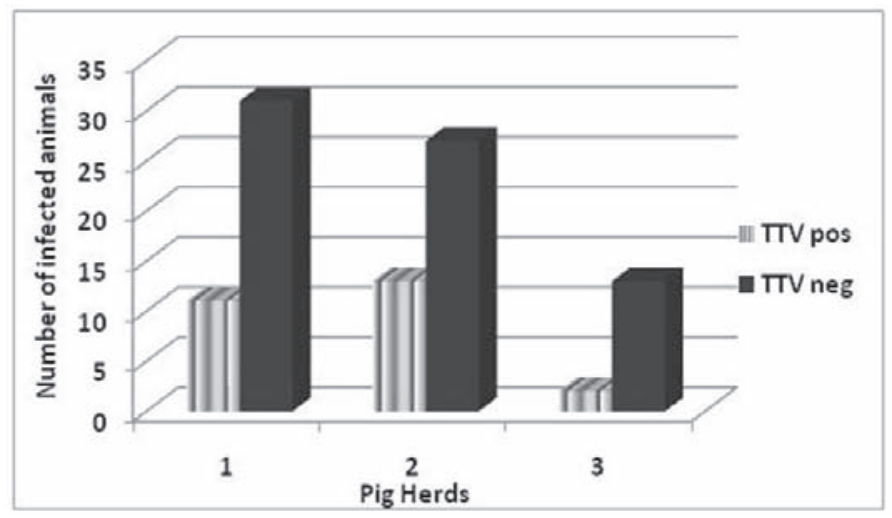

Figure 2: Percentage of positive animals according to breeding phase

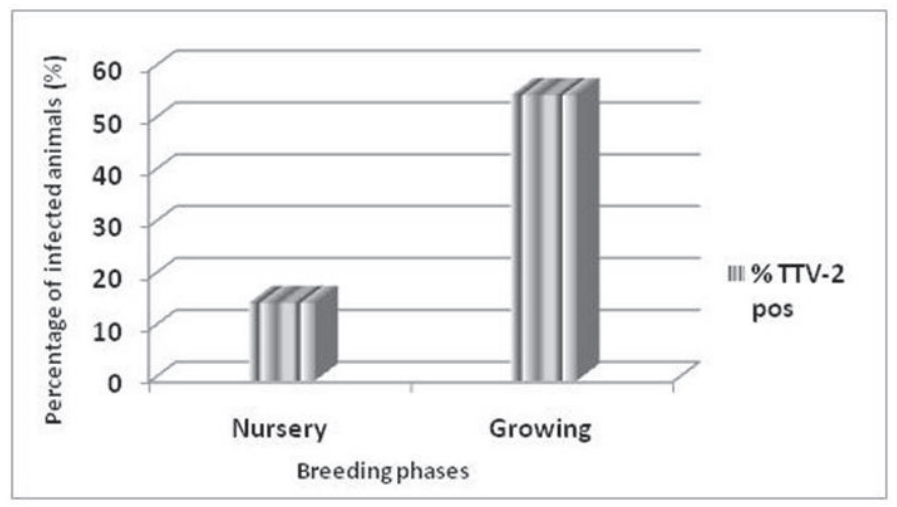

\section{References}

1. Niel, C. et al. (2005). J. Gen. Virol. 86, 1343-1347

2. Bigarré, L. et al. (2005). J. Gen. Virol. 86, 631-635

3. Martínez, L. et al. (2006). Vet Micro:118, 223-229

4. Kekarainen, T. et al. (2009). J. Gen Virol, 90, 648-653.

5. Brassard, J. et al. (2008). Vet Microbiol 126, 271-276

6. Sibila, M. et al. (2009). Vet Microbiol 139, 213-218.

7. Kekarainen, T. et al. (2009). J. Gen Virol, 90, 648-653. 


\title{
P.580
}

\section{Prevalence and age distribution of porcine TT viruses}

\author{
Vladimir Celer; Veronika Jarosova \\ University of Veterinary and Pharmaceutical Sciences, Brno, Czech Republic
}

\section{Introduction}

Torque teno sus virus 1 (TTSV1) and Torque teno virus sus 2 (TTSV2) belong to the genus lotatorquevirus within the family Anelloviridae (Carstens, 2010).

The role of swine TT viruses in the pathogenesis of swine diseases is currently being investigated. Although it seems that TTSV infection is not directly responsible for any disease, the possibility that they play a role in different co-infections still remains. In a recently published study a significantly higher prevalence of swine TT viruses was found in sera from PMWS affected animals compared to non-affected animals. The main goal of this work was to estimate the prevalence of porcine TT viruses in the Czech Republic in different age categories of pigs.

\section{Materials and Methods}

Samples

Two hundred twelve pig blood samples from pigs from 27 swine herds were tested to obtain an overview of the presence of TTSV in the Czech swine population. Samples originated from animals of different ages (newborn piglets $n=66$, weaned pigs (4-12 weeks) $n=127$, gilts $n=11$, sows $n=8$ ) and with different health status with regard to PMWS infections. All specimens used were fresh or stored frozen before DNA extraction.

\section{PCR}

The sequence of primers for two rounds of amplification was based on primers already published by Martinez et al. 2006.

\section{Results}

The results of snPCR testing performed on the panel of 212 samples are shown in Table 1. TTSV1 was detected in 91 samples. TTSV2 was found in 99 samples. In 66 samples both TTS viruses were detected together. The prevalence of individual TT viruses in the Czech Republic could be estimated at 42.9\% (TTSV1) and 46.7\% (TTSV2). The average prevalence of TTS viruses is thus $44.8 \%$.
The age distribution of both genogroups is shown in Table 1. TT viruses were not distributed equally in all age categories. Although the prevalence of TTSV1 and TTSV2 was determined to be $52.7 \%$ and $60.6 \%$ respectively in piglets at weaning, the percentage increased to $90.9 \%$ (TTSV1) and 63.6\% (TTSV2) in gilts and 75\% (TTSV1) and $87.5 \%$ (TTSV2) in sows.

Both viruses were detected in newborn piglets (1-2 days post parturition). In 59 samples from a group of newborn piglets TTSV was detected in 16 cases, which suggest possible transplacental transmission.

\begin{tabular}{|l|c|c|c|c|}
\hline & TTSV1 (\%) & TTSV2 (\%) & Dual infection & Total \\
\hline Newborn pigs & $8(12.12)$ & $8(12.12)$ & $4(6)$ & 66 \\
\hline Weaned pigs & $67(52.75)$ & $77(60.6)$ & $50(39.37)$ & 127 \\
\hline gilts & $10(90.9)$ & $7(63.6)$ & $6(54.5)$ & 11 \\
\hline sows & $6(75)$ & $7(87.5)$ & $6(75)$ & 8 \\
\hline
\end{tabular}

\section{References}

Carstens, E. B., 2010. Ratification vote on taxonomic proposals to the International Committee on Taxonomy of Viruses (2009). Archives of Virology 155, 133-146.

Martinez, L., Kekarainen, T., Sibila, M., Ruiz-Fons, F., Vidal, D., Gortazar, C., Segales, J., 2006. Torque teno virus (TTV) is highly prevalent in the European wild boar (Sus scrofa). Veterinary Microbiology 118 (3-4), 223-229.

This work was supported by the Grant agency of Ministery of youth and education project no. ME08108 and Grant Agency of the Czech Republic project no. 524/09/0673. 


\title{
Detection of Torque teno virus (TTV-2) by PCR in tissue samples from Brazilian swine population
}

\author{
Cíntia M. Favero ${ }^{1}$ Alessandra M. M. G. de Castro ${ }^{1}$ Cíntia M. Baldin² Taís Nishikata ${ }^{1}$ \\ Sueli Taniwaki Paulo E. Brandão' Leonardo J. Richtzenhain ${ }^{1}$ \\ 1. Faculdade de Medicina Veterinária e zootecnia-Universidades de São Paulo, São Paulo, Brazil; 2. Faculdade de Jaguariúna, Jaguariúna, SP, Brazil
}

\section{Introduction}

Torque teno virus is circularized single stranded DNA viruses, classified belonging to genus Anellovirus. Cattle, sheep, pigs, cats, dogs and chickens are described be infected with speciesspecific TTV 2,3. Two distinct TTV genogroup were identified circulating among domestic pig population, TTV-13 and TTV24. TTV have been detected by PCR in lungs, lymph nodes, tonsil, ileum, serum, marrow bone, liver and kidney ${ }^{1,7,8}$. Prevalence varying between $24 \%$ and $100 \%$ in different parts of the world are related ${ }^{1,5,6}$. The aims of this study were to standardizing a PCR and detect TTV-2 in samples from swine collected between 2002 and 2003 in five Brazilians states.

\section{Materials and Methods}

The partial sequence of 250 bases of the non-coding region of swine TTV was amplified ${ }^{8}$ and inserted into pTZ57R/T (InsTAclone - Fermentas ${ }^{\circledR}$ ), according to the manufacturer's instructions. Cloned DNA was selected and plasmid DNA quantified with a NanoDrop 1000 (Thermo Scientific). Sensitivity of the PCR technique was performed with ten-fold dilutions of recombinant plasmid (10-1-10-10). Threshold detection was 25 copies of virus DNA. Eighteen samples of tissue from five different Brazilian states (SP, GO, SC, MS and PR) collected between 2002 and 2003 were homogenized by Stomacher 80 (Seward=Lab System) in $20 \%(\mathrm{v} / \mathrm{w}$ ) TE buffer. DNA extraction was carried out by phenol: chloroform: proteinase $\mathrm{K}$ protocol and the TTV2 amplification was performed according to previous procedure described ${ }^{8}$.

\section{Results}

TTV-2 was detected in 12 (67 \%) out 18 tissue samples (67\%). Among the 12 TTV-2 positive samples, seven (7/7) belong to the fattening, three (3/6) to the growing and four (4/7) to the nursery pigs (Fig. 1). According to Brazilian states, the following distribution were observed: four belong to SP (4/4), one to MS $(1 / 1)$, three to GO (3/4) and four to SC (4/8). The unique sample from PR was negative for TTV-2 (Fig. 2)

\section{Discussion}

This study represents the first report of TTV-2 in swine tissues from different Brazilian states. The results found in this work, that is, $67 \%$ of positivity for TTV-2, are in agreement with the high percentage of this genogroup observed in pigs herd from Spain (over $70 \%)^{5}$. However, the high percentage of positive animals in the fattening pigs was not observed in Italy, which related a higher percentage on TTV-2 positive animals in weaners $(57.4 \%)$ than fatteners $(22.9 \%)^{6}$.

Thus we conclude the TTV-2 is spread at least in four Brazilian states since 2002 and here to for there is no association with clinical disease. Future prospects about sites of viral replication, viral pathogenesis and TTV-2 relation to co-infection with TTV-1 and PCV are necessary.
Figure 1: Distribution of TTV-2 positive animals among nursery growing and finish pigs.

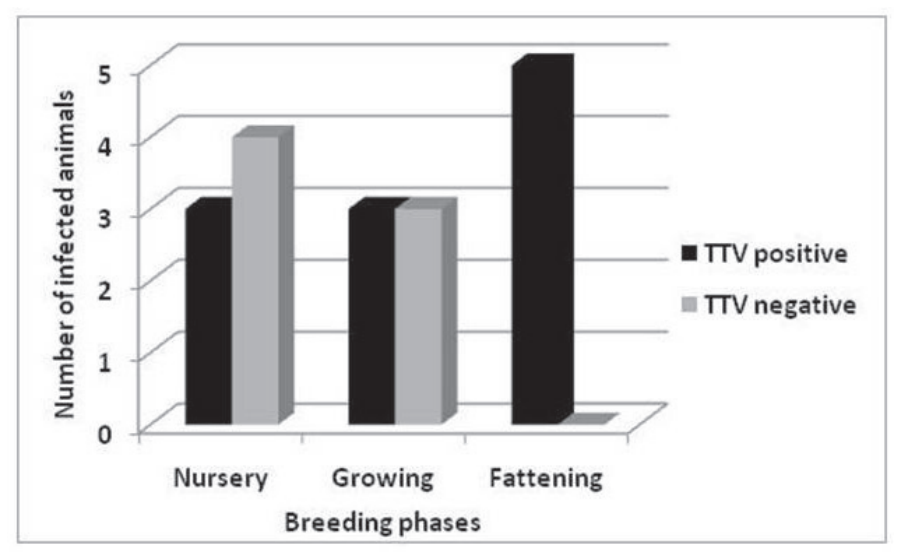

Figure 2: Number of infected TTV-2 animals according to Brazilian states.

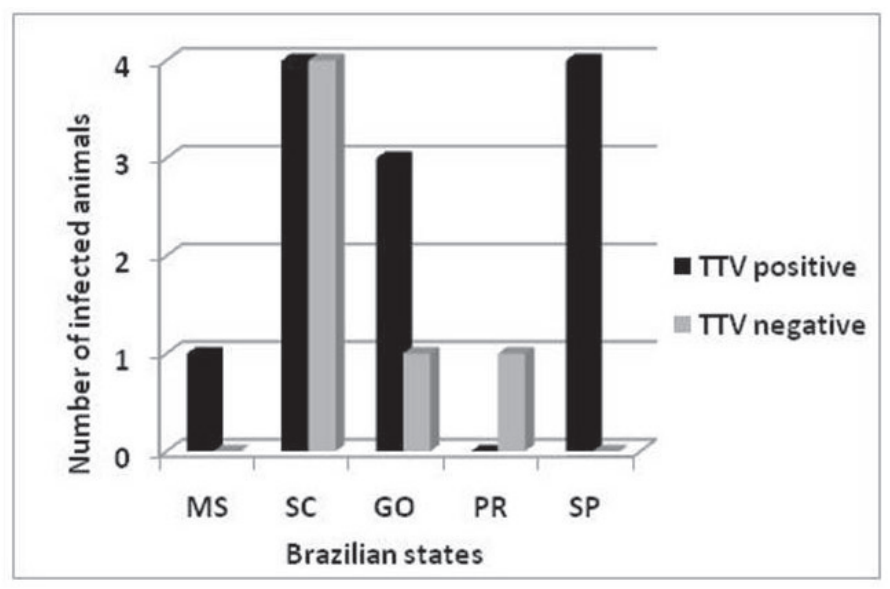

\section{References}

1. Bigarré, L. et al. (2005). J. Gen. Virol. 86, 631-635

2. Leary, T. P, et al. (1999). J. Gen. Virol. 80, 2115-2120

3. Okamoto et al. (2002). J. Gen. Virol., 83, 1291-1297

4. Niel, C. et al. (2005). J. Gen. Virol. 86, 1343-1347

5. Kekarainen, T. et al. (2006). J. Gen. Virol. 87, 833-8377.

6. Martelli, F. et al. (2006). J.Vet. Med, 53, 234-238.

7. Martínez, L. et al. (2006). Vet Micro:118, 223-229

8. Kekarainen, T. et al. (2009). J. Gen Virol, 90, 648-653.

9. Pozzuto T. et al. (2009)Vet Micro137; 375-379. 


\title{
P.582
}

\section{First report of TTV-2 DNA detection in samples from sows with reproductive failure in Brazil}

\author{
Cíntia M. Favero ${ }^{1}$ Alessandra M. M. G. de Castro ${ }^{1}$ Cíntia M. Baldin² Taís Nishikata ${ }^{1}$ \\ Tânia A. Coutinho' Andrea M. Moreno ${ }^{1}$ Paulo E. Brandão ${ }^{1}$ Leonardo J. Richtzenhain ${ }^{1}$ \\ 1. Faculdade de Medicina Veterinária e zootecnia-Universidades de São Paulo, São Paulo, Brazil; 2. Faculdade de Jaguariúna, Jaguariúna, SP, Brazil
}

\section{Introduction}

Torque teno viruses (TTVs) are small, non-enveloped, singlestranded, negative sense, circular DNA viruses, belonging to the genus Anellovirus. Swine are infected with the two identified genotypes TTV-1 and TTV-21. Transmission route of TTV in pig population are mainly fecal-oral ${ }^{2}$ but there is evidence of vertical transmition. Recently, it has been verified that colostrum is an important source of TTV infection on piglets ${ }^{3}$. Also, a high prevalence of TTV genogroups has been detected in boar semen ${ }^{4}$ and in utero infection was repoted as a commum event on multiparous sows 5 . The aim of this study was the detection TTV-2 DNA in abortion, stillborn and mummified fetuses.

\section{Materials and Methods}

Pool of tissues (heart, lungs, liver, kidney, and spleen) from fourty two fetuses were collected in different pig farms located in São Paulo and Paraná state in Brazil. Samples were segregated in three fetal stages: abortion, stillborn and mummified. The samples were homogenized by Stomacher 80 (Seward=Lab System) in 20\% (v/w) TE buffer. DNA extraction was carried out by phenol: chloroform proteinase $\mathrm{K}$ protocol and the TTV2 amplification were carried out according to previous procedure described $^{6}$.

\section{Results}

Fourteen of 42 tissue samples from different animals were positive for TTV-2. Within the fetal stages, 6/8 (75 \%) abortion, 8/25 (32\%) stillborn and none mummified fetuses were PCR TTV-2 positive (Figure 1and 2). From all six abortion positive fetuses for TTV-2, a high detection was obtained in fetuses samples from sows in the last third of the gestational 4/6 (67\%) than on first third of gestational 2/6 (33\%) period

\section{Discussion}

This is the first report of TTV-2 infection in fetal stages in Brazil. PCR positive for TTV-1, 15 out of the 30 (50\%) and for TTV-2, 2 out of the $30(7 \%)$ in serum from stillborn fetuses have already described $^{3}$. However, our results showed a higher number of positive samples in stillborn tissues (30\%) probably due to the kind of the sample. Serum had higher virus quantities than tissues, on other hand TTV-2 is easily found in tissues ${ }^{7}$. The lack of positive samples for mummified fetus must be due the difficulty to separate suitable tissue. An interesting result was the higher prevalence of TTV-2 in aborted fetuses (75\%) occurring in the last third of the gestational period than in the first. Further studies are necessary to confirm this hypothesisis, but it is possible that TTV infection affects the development of fetuses and it can be responsible for mummified, stillborn and weak piglets ${ }^{5}$.
Figure 1: Number of infected animals for TTV-2 according to fetal stages.

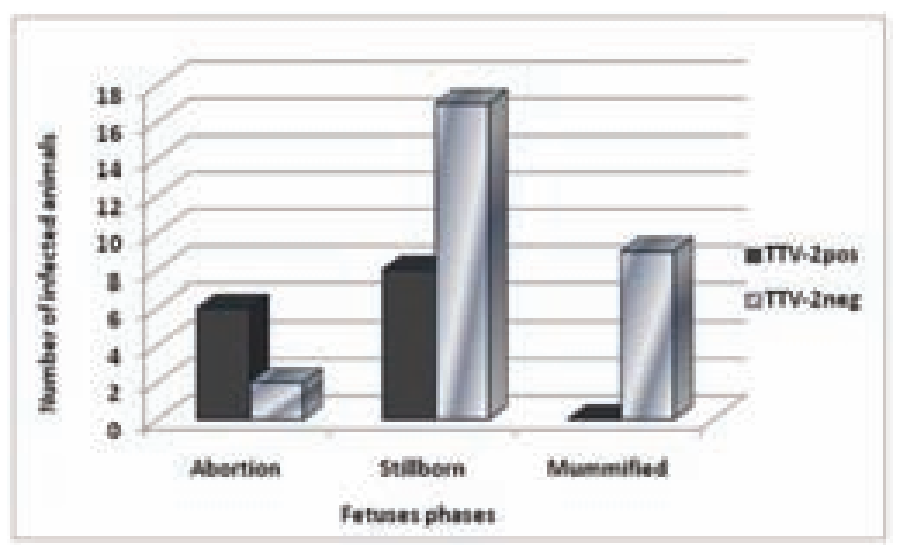

Figure 2: Percentage of infected animals for TTV-2 according to fetal stages.

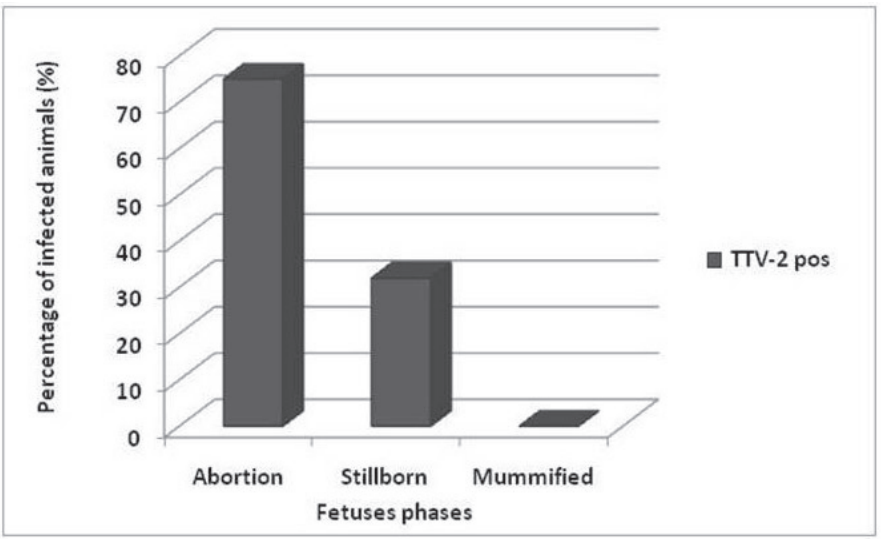

\section{References:}

1. Niel, C. et al. (2005). J. Gen. Virol. 86, 1343-1347

2. Brassard, J. et al. (2008). Vet Microbiol 126; 271-276.

3. Martínez-Guinó, L. et al. (2009). Theriogenology doi:10.1016/j. theriogenology.2009.01.010.

4. Kekarainen, T. et al. (2007). Theriogenology 68, 966-971.

5. Pozzuto, T. et al. (2009). Vet Microbiol 137, 375-379.

6. Kekarainen, T. et al. (2009). J. Gen Virol, 90, 648-653.

7. Krakowa, S. et al. (2008). Proc of the International Pig Veterinary Society Congress, Durban South Africa. 


\title{
P.583
}

\section{Inclusion Body Rhinitis and Porcine Cytomegalovirus in pigs in Denmark}

\author{
Peter Astrup ${ }^{1} \underline{\text { Uffe Nielsen }}^{2}$ \\ 1. Intervet/Schering-Plough Animal Health, Skovlunde, Denmark; 2. LVK, Hobro, Denmark
}

\section{Introduction}

Porcine cytomegalovirus (PCMV) was discovered in 1955 as the causative agent of inclusion body rhinitis (IBR) of pigs, and is closely related to human herpesviruses. Cytomegalovirus is associated with cytomegalic cells and large intranuclear inclusion bod-ies. In pigs, the turbinates and the respiratory tract are major sites of infection, and clinical signs may be fetal death, and rhinitis, pneumonia, reduced weight gain and death in piglets (1).

It is stated that the herd prevalence in Europe is greater than $90 \%$, and that within herds, as many as $98 \%$ of pigs may be seropositive. PCMV can be transmitted horizontally or vertically, and in com-mercial farms the infection is believed to take place perinatally or early postnatally (2).

There has been only one Danish study in fattening pigs to investigate the prevalence of PCMV. The re-sult was $43 \%$ PCR positive and $24 \%$ PCR positive in control and case groups, respectively (3). This paper describes the first case of IBR diagnosed in suckling pigs in Denmark.

\section{Materials and Methods}

The herd studied is a high health herd, which had been declared infected with APP type 6 and 12, M hyopneumoniae and PRRS genotype US. The 440 sows and pregnant gilts are fed and reared conventionally. Piglets are weaned at $7 \mathrm{~kg}$ and sold, after the nursery period, at $30 \mathrm{~kg}$. Replacement gilts are selected from the nursery and reared on farm. The sows are routinely vaccinated against Glässers disease, type C enteritis, E coli, PPV and erysipelas.

The herd was investigated according to usual consul-tative clinical procedures and submissions to The Pig Disease Laboratory, Danish Pig Producers, Kjellerup, Denmark.

\section{Results}

In the farrowing unit, widespread sneezing and coughing was heard amongst the piglets. Pigs from 2 weeks old to weaning age showed nasal discharge, ill-thrift and dyspnea. Stenosis of the turbinates was audible on expiration. The litters were relatively small with fewer than 13 live born pigs per litter, but the mortality rate was as low as $10 \%$.

The symptoms remained prominent for 2 weeks after weaning, but the pigs recovered clinically thereafter. The estimated mortality rate in the nursery period was only $1 \%$.
Five pigs of 18 days of age were submitted for post mortem examination. They were in normal body condition, and most pigs exhibited macroscopically catarrhal bronchopneumonia, peritoneal edema, hepatic congestion and petecchial bleeding in the kidneys. Bacteriological examination of the liver, kidney and lungs revealed no specific pathogens, and nose swabs were negative for $B$ bronchiseptica and $P$ multocida toxin. Lungs tested negative for Mycoplasma spp. by cultivation, and negative for SIV, M hyopneumoniae, PCV2 and PRRSV by PCR. Nasal swabs tested negative for PRRS, but positive for PCMV by PCR. Finally, histological examination of nasal epithelium revealed mononuclear infiltration in the lamina pro-pria and enlargement of the mucus gland epithelium cells with basophilic inclusion bodies.

\section{Discussion}

The histological findings are considered pathognomonic, and the symptoms in general are in accordance with previous descriptions of infection with PCMV in pigs (1). Thus the proper diagnosis of the herd's respiratory disease problems is IBR caused by infection with PCMV. Based on the clinical description, a number of Danish herds must be suspected of suffering from IBR.

No specific treatment or vaccination against PCMV is available. In order to control the infection, batch farrowing, all in/all out management and McRebel procedures are all recommended. Replacement gilts should be naturally immunized before mating, and secondary infections in general should be controlled by appropriate vaccination.

\section{References}

1. Guedes MIMC. PhD thesis, College of Veterinary Medicine, University of Minnesota, USA, 2003: 20 - 36.

2. Yoon K-J, Edington N, Diseases of swine. 9th ed. Ames, lowa, USA. 2006: 323-329.

3. Sif-Hansen M, DVHS 30. april 2009, Kolding, Danmark. Available at http://www.dvhs.dk, accessed December 15, 2009. 


\title{
P.584
}

\section{Congenital tremors of suckling pigs in Jeju, Korea}

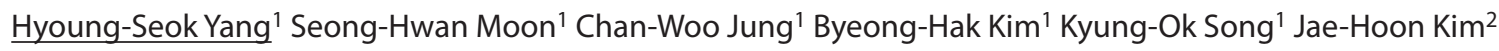

1. Jeju Veterinary Research Institute, Jeju, Korea; 2. College of Veterinary Medicine, Jeju National University, Jeju-si, Korea

\section{Introduction}

Congenital tremor (CT) is a congenital abnormality caused by hypomyelination or demyelination of the brain and spinal cord. Unlike in splaylegged pigs where the deficit in myelin formation specifically affects the motor system, with CT there is an overall reduction of myelin in the spinal cord (Lamar et al., 1975). CT in pigs is associated with myelin deficiency and may be caused by genetic abnormalities (Harding et al., 1975), or in utero infection with classical swine fever virus (CSFV, Harding et al., 1966) or Aujeszky's disease virus (ADV, Maré et al., 1974). In the recent investigations, porcine circovirus infection has been detected in tissues of CT pigs (Stevenson et al., 2001).

\section{Materials and Methods}

January 2010 in Jeju, Korea, 1 day-old suckling pigs showed acute nervous signs including paddling, tremors, and clonic muscular contraction. Two suckling pigs were necropsied in the diagnostic lab of Jeju Veterinary Research Institute. After gross examination, brain, spinal cord, and internal organs were fixed in $10 \%$ neutral buffered formalin, processed routinely and embedded in paraffin wax. Sections $(4 \mu \mathrm{m})$ were cut and stained with Hematoxylin and Eosin (H\&E) and Luxol Fast Blue (LFB) method for histological examination. For bacterial culture, the swabs from the cerebrums were inoculated on sheep blood and MacConkey agar and aerobically incubated for $48 \mathrm{hr}$ at $37^{\circ} \mathrm{C}$. Polymerase chain reaction (PCR) for porcine circovirus type II (PCV-2), CSFV, ADV, Japanese encephalitis virus (JEV), and porcine reproductive and respiratory syndrome virus (PRRSV) were carried out on brains and spinal cords.

\section{Results}

Grossly, there were no lesions in cerebrums, cerebellums and spinal cords of suckling pigs. Histo-pathologically, there were no significant lesions in brains. However marked hypomyelination was observed in the spinal cord of pigs. Hypomyelination is most evident and reduction in cross-sectional area is greatest in the white matter of spinal cord. In bacterial examination, pathogenic bacterial colonies were not observed on blood and MacConkey agars. PCR analysis of brain and spinal cord resulted in amplification of PRRSV-specific nucleotide sequence, but not in PCV-2, CSFV, ADV and JEV.

\section{Discussion}

Based on the clinical and histo-pathological finding and LFB staining, this case was diagnosed as CT. Hypomyelination was predominant lesion in the white matter of spinal cords of two suckling pigs. PRRSV nucleic acid was detected in the brain and spinal cord. Therefore PRRSV should be considered as a possible cause of CT in suckling pigs.

\section{References}

Harding JDJ et al. 1966. Vet Rec 79:388-390.

Harding JDJ et al. 1973. Vet Rec 92:527-529.

Lamar CH et al. 1975. Vet Pathol 12:1-5.

Maré CJ et al. 1974. J Am Vet Med Assoc 164:309-310.

Stevenson GW et al. 2001. J Vet Diagn Invest 13:57-62. 


\section{P.585}

\section{Detection and Phylogenetic analysis of Porcine encephalomyelitis virus in South Korea}

$\underline{\text { Se-Mi Rho }}{ }^{1}$ Hyoung-Joon Moon ${ }^{2}$ Seong-Jun Park ${ }^{1}$ Hye-Kwon Kim¹ Hyun-Ok Keum¹ Jae-Yeon Han ${ }^{1}$ Van-Giap Nguyen ${ }^{1}$ Bong-Kyun Park ${ }^{1}$

1. Department of Veterinary Virology Lab, College of Veterinary Medicine and BK21 Program for Veterinary Science, Seoul National University, Seoul, Korea; 2. Research unit, Green Cross Veterinary Products, Yongin, Korea

\section{Introduction}

Porcine encephalomyelitis virus (PHEV), which belongs to the order Nidovirales, family Coronaviridae, genus Coronavirus, was first isolated in Canada in 1962. PHEV infection is known to be widespread in worldwide and usually remains subclinical, because pigs are protected by colostral antibodies and subsequently they develop age-related resistance against PHEV. However when PHEV-seronegative piglets (less than 3 weeks old) are introduced to this virus, the mortality reaches almost $100 \%$. This is significant for gnotobiotic pig farms or SPF pig farms [1] where the pigs do not receive colostral antibodies. Also, even in not one of those specialized farms, nonimmune subpopulation might be existed in a large gilt pool and they might act as potential sources of PHEV to induce clinical syndromes [2]. There are two types of clinical syndrome, the vomiting and wasting disease (VWD), and the encephalomyelitis form. There are not many isolates of PHEV which are registered in GenBank so our final goal is to isolate new Korean strain of PHEV. To achieve this final goal, we preferentially performed PHEV screening test from pig tissue samples and sequencing of specific gene, $\mathrm{N}$ gene, to compare with each other and existing strains.

\section{Materials and Methods}

From 2009 Nov. to 2010 Jan., a total of 90 swine tissue samples including solid organs and brains were tested for PHEV. The age range of these pigs was from suckling to finisher. They had respiratory, enteric or neurologic sings but some were unknown. Viral RNA of each samples were extracted from $10 \%$ homogenates and their CDNA were synthesized using specific external reverse primer of N (nucleocapsid protein) gene. The primers for RT-PCR and nested PCR of PHEV were based on the nucleotide sequences of nucleocapsid gene of the HEV-JT06 strain and designed as follows: NF(external forward primer) CAGGAGGGACTGTTGTACCC, NR (external reverse primer) CCCAACAAACAATGCA CTGT, N2F (internal forward primer) TGCACCA GGAGTCCCAT CTA, N2R (internal reverse primer) GCCAAGTTTTGC CAGAACAA. The size of amplified product was $472 \mathrm{bp}$. The final PCR products were sequenced and aligned to evaluate the relationship with others using the Clustal $X$ (1.83) multiple sequence alignment program and the phylogenetic tree was constructed by MEGA (4.1).

\section{Results}

Among 90 samples, 9 showed positive results for PHEV after performing nested PCR. The sequence results of these PCR products were confirmed PHEV by BLAST, showing 97-98\% nucleotide identity. These sequences of the 9 samples were aligned with three reference strains, JT06, VW572 and IAF-404, and the sequence identities of the samples were the highest with IAF404. The 9 samples had their unique nucleotides compared to the reference JT06 strain in the region between 586 and 622. The phylogenetic tree was drawn below.

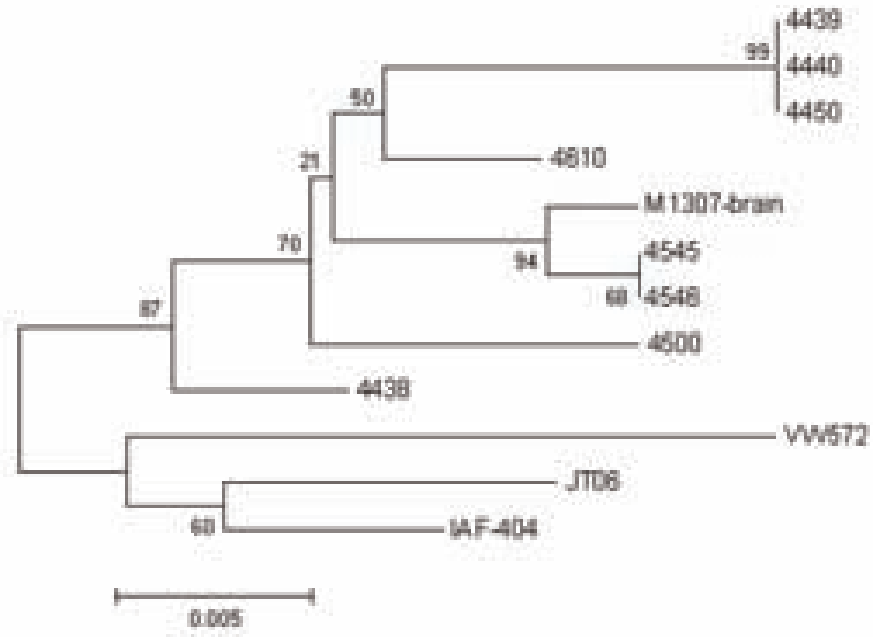

\section{Discussion}

This study showed that PHEV is actually present in some of the farms in South Korea. Even though the positive samples established a new group, there were not so much variations compared to existing PHEV strains. Since the use of gnotobiotic and SPF pigs for xenotransplantation and transformation is common these days, this virus is not negligible when handling PHEV-seronegative pigs.

\section{References}

1. Sekigochi Y, J. Vet. Med. Sci. 66(4): 367-372, 2004

2. Quiroga M. A, Emerg. Infect. Dis. 14(3): 484-486, 2008

\section{Acknowledgement}

This study was supported by a grant (Code \#20070401 034009) from BioGreen 21Program, Rural Development Administration, Korea. 


\title{
P.586
}

\section{Seroprevalence of Bovine Viral Diarrhea virus in finisher hogs on Ontario swine herds}

\author{
Terri L. O'Sullivan ${ }^{1}$ Robert Friendship ${ }^{1}$ David Pearl ${ }^{1}$ Bev McEwen ${ }^{2}$ Susy Carmen ${ }^{3}$ Cate Dewey ${ }^{1}$ \\ 1. University of Guelph, Guelph, ON, Canada; 2. University of Guelph Animal Health Laboratory, Guelph, ON, Canada; \\ 3. University of Guelph Diagnostic Virology Laboratory, Guelph, ON, Canada
}

\section{Introduction}

The genus pestivirus contains four species currently recognised by the International Committee on Taxonomy of Viruses namely, Classical Swine Fever virus (CSFV or hog cholera virus), Bovine Viral Diarrhea virus Type 1 (BVDV-1) and Type 2 (BVDV-2), and Border Disease virus (BDV) of sheep. BVDV under natural conditions mainly infects cattle and sheep; however, the virus has been associated with disease in goats and swine as well (1). Herd level prevalence of BVDV (in CSVF-free countries) has been reported anywhere from $2-43 \%$ with cattle implicated as the most common source of infection of BVDV in pigs (1). Pestivirus infections in animals can lead to fertility problems, diarrhea, immuno-suppression, thrombocytopenia, and central nervous system disease. In pregnant animals, transplacental infection can lead to abortion, stillbirth, foetal malformation, and persistent infection of offspring (1).

Interest in the prevalence and clinical significance of pestivirus' in swine herds has recently been highlighted with the documentation of a neurologic and reproductive disease outbreak associated with a novel pestivirus in the USA (2) and a disease outbreak in Australia (3). Such novel strains of pestivirus have yet to be identified in Ontario swine herds and the current prevalence of BVDV in Ontario swine herds is unknown. The objectives of this study were to determine the prevalence of neutralizing antibodies to BVDV type- 1 and type- 2 in Ontario swine herds and to determine if the presence of cattle on the same premise was a risk factor for seropositivity.

\section{Methods}

Five hundred samples of stored finisher pig sera (10 samples per herd) were tested. The stored samples were from 50 swine herds that participated in a swine sentinel herd program at the University of Guelph in 2005 (4). The Ontario sentinel herds were distributed across the province and the program collected herd level information and blood samples from each herd. The samples were tested by virus neutralization (VN) for BVDV-type 1 and BVDV-type 2 by the Animal Health Laboratory at the University of Guelph. The degree of contact with cattle for each herd was compiled from the sentinel project herd records.

\section{Results}

All 500 samples from, the 50 herds, tested negative (titres $<1: 2$ ) for antibody to BVDV-type 1 and BVDV-type 2. Of the 47 herds with information available on the presence of cattle, cattle were present on $27.6 \%$ of the farms. On the remaining $72.3 \%$ of the farms, only pigs were present.

\section{Discussion}

The results indicate that BVDV did not appear to be present in Ontario swine herds in 2005 regardless of the presence of cattle on the same premise. These findings correspond to other findings where the seroprevalence of ruminant pestiviruses in swine was found to be low $(1,5)$. The Ontario swine industry is experiencing a rapid decline in the presence of mixed farming practices and has moved almost exclusively to species specialization of farms, a phenomenon witnessed in many parts of the world today. It has been speculated that this trend in farming practices has resulted in the decline of BVDV in swine worldwide as supported by this study (1).

\section{References}

1. Le Potier M, et al. Classical Swine Fever and Other Pestiviruses. In: Straw B, et al., eds. Diseases of Swine. 9th edition. Ames, lowa: Blackwell Pub. 2006:309-311.

2. Pogranichniy RM, et al. Vet Microbiol. 2008; Sep131(1-2):35-46.

3. Kirkland PD, et al. Virus Research. 2007;129:26-34.

4. Poljak Z, et al. Can J. Vet. Res. 2008;72:303-310.

5. Loeffen W, et al., Vet Microbiol. 2009; May 12;136(3-4):240-5. 


\title{
P.587
}

\section{Diagnosis of a Swinepox virus infection in a German swine production unit}

\author{
Friedrich Schmoll ${ }^{1,4}$ Susanne Richter ${ }^{2}$ Julia Jäger ${ }^{1}$ Louis Fischer ${ }^{3}$ Tatjana Sattler $^{1}$ Petra Winter ${ }^{2}$ \\ 1. Large Animal Clinic for Internal Medicine, Leipzig, Germany; 2. AGES-Institute for Veterinary Disease Control, Mödling, Austria; \\ 3. LaboVet GmbH, Vienna, Austria; 4. Institute for Veterinary Public Health, VetmedUni Vienna, Vienna, Austria
}

\begin{abstract}
Introduction
Poxvirus infections of pigs are either caused by Vaccinia virus and Cowpox virus (Orthopox) or by Swinepox virus (Suipox, SwP). SwP is a severe disease in young pigs [1]. Adult pigs generally develop a mild, self-limiting form of the disease. Morbidity can be up to $100 \%$ in pigs up to 4 months of age. Transmission by insects is less common; horizontal transmission between pigs may occur. Small skin injuries may facilitate the entry for Swinepox virus (SwPV). Macroscopic examination of skin lesions often leads to an insecure diagnosis. However, other methods that allow for a rapid and accurate diagnostic are appreciated. This paper describes the diagnostic procedure including electron microscopic analysis and a novel SwPV-specific PCR.
\end{abstract}

\section{Materials and Methods}

Between Nov. 2008 and March 2009 piglets in a German two side production unit showed clinical signs of red skin lesions first at maculae stages, following by papula-, pustule and finally crust stages. The lesions were equally distributed over the entire body surface of the nursery piglets. Nearly $50 \%$ of the piglets were affected. First symptoms were seen 1-2 weeks after relocation into the nursery. First macroscopical diagnosis was SwPV-infection.

For PCR, scraped skin tissues from crusts as well as epidermal biopsies were collected. SwPV specific PCR primers (for: $5^{\prime}-\mathrm{CC}$ GAGGAGTATAGACGTGTTATCG-3', rev: 5'-AGGAATCCGTAGATACAGCCGA-3') were designed (PrimerQuest ${ }^{S M}$, Integrated DNA-Technologies) based on published DNA-sequences of the thymidinkinase-gene (TK-gene) of the SwPV (GenBank: Acc. Nr. AF410153, M64000 and M59931). Orthopoxvirus-DNA was included to exclude orthopox infections. PCR products were sequenced and aligned to confirm identity of SwPV.

Electron microscopy (TEM) was performed by negative staining of sample suspensions including enrichment with ultracentrifugation. Moreover, epidermal biopsies embedded in Epon were prepared for tissue sectioning. Ultrathin sections stained with UA were analysed in a TEM (Zeiss 906,80kv).

\section{Results}

SwPV was confirmed by PCR and sequencing of the TK-gene; PCR for Orthopox was negative. All amplified products of the TK-gene were $473 \mathrm{bp}$ long and did show $100 \%$ homology to published sequences (Acc.Nr. AF410153, M64000 and M59931).

Histology revealed marked ballooning cytoplasmatic degeneration and central nuclear cleaning of epidermal cells of the stratum spinosum. As revealed by TEM, infected epithelial cells were greatly distended by huge cytoplasmic lipid A-type inclusion bodies, filled with mature virions and uniformly granular, electron dense material. Intracellular mature virions within inclusions had gained additional outer coats formed by this granular material. Intracellular virus particles found in A-type inclusions have the typical structure of poxviruses; extracellular virus par- ticles often exhibited a second outer virus membrane. Immature virions in B-type inclusions (= virus factories) developed by formation of dense foci of viroplasm were surrounded by crescent shaped, phospholipid membranes later closing to circular membranes. Like in other poxviruses [2,3], virus crescents reveal no continuity between cellular intermediate compartment (IC), though proteins from IC are of the same origin. Like in other poxviruses, the transport of immature virions and crescents within the cell seem to be in accordance with filaments seen in contact with virus stages. Early A-type inclusion formation involves the attachment of intracellular mature virions to small lipid globules.

\section{Discussion}

PCR, sequence analysis and electron microscopic analysis helped to confirm an infection with SwPV in the above mentioned herd. Electron microscopy confirms the practitioner's first diagnosis. The descibed PCR-assay enables a rapid and an accurate diagnosis of SwPV, that my help to review management practices and the implementment of control measures and treatments.

\section{References}

1. Delhon et al. 2006. In: Diseases of swine 9th Ed. 483-387.

2. Weli et al. Med Electron Microsc 2004;37:225-235.

3. Hollinshead et al. J Virol 1999;73: 1503-1517. 


\title{
P.588
}

\section{Detection of porcine endogenous retrovirus (PERV) A/C recombinants in U.S. pigs by quantitative real-time RT-PCR}

\author{
Shayleen Harrison ${ }^{1}$ Rodney Baker ${ }^{1}$ Narinder Pal ${ }^{1}$ Linda Scobie $^{2}$ A Tucker ${ }^{3}$ Tanja Opriessnig $^{1}$ \\ 1. lowa State University, Ames, IA, USA; 2. Glasgow Caledonian University, Glasgow, UK; 3. University of Cambridge, Cambridge, UK
}

\section{Introduction}

Retroviruses are RNA viruses that exist in two main groups: endogenous or exogenous retroviruses. Pigs carry porcine endogenous retroviruses (PERV) in their genome (1) and three PERV subtypes have been identified to date, PERV-A, B, and C. PERV-A and PERV-B are present in genomes of all pigs. In contrast, PERV-C is absent from many pigs and this virus with its host range mostly restricted to porcine cells is not infectious to human cells (2). More recently, the possible existence of exogenous retrovirus; a recombinant between PERV A and PERV $C$ (PERV A/C), as opposed to endogenous retroviruses in pigs was proposed $(3,4)$. The objective of this study was to determine if there is a relationship between retroviremia and clinical disease in pigs by using real-time PCR methods to detect the different PERV types on serum samples obtained from clinically healthy and clinically affected 3 to 25 week old pigs.

\section{Material and Methods}

A total of 369 serum samples were collected from 204 pigs from three commercial swine operations in the U.S. during 2006 and 2007. Farm 1 consisted of a total of 84 pigs with subclinical porcine circovirus associated disease (PCVAD). Farm 2 samples were obtained from 60 three-week-old pigs. Thirty of the 60 pigs were clinically healthy and the remaining 30 pigs suffered from weight loss and diarrhea associated with Salmonella typhimurium and $\mathrm{E}$. coli. Samples collected on Farm 3 were obtained from 60 25-weekold pigs with a recent PCVAD outbreak resulting in $30 \%$ mortality. As with Farm 2, at the time of blood collection, 30 pigs were clinically healthy and 30 showed clinical signs consistent with lethargy, decreased weight gain or weight loss and respiratory disease. The presence of PRRSV, PCV2, and respiratory disease was confirmed by routine diagnostics. The RNA of all samples was extracted and all samples were tested by three different real-time PCR assays: A general PERV-ABC real-time RT-PCR, a real-time RT-PCR specific for PERV-C, and a quantitative real-time RT-PCR specific for PERV-A/C recombinants. Pairwise t-tests were used for data analysis with a level of significance of $<0.05$.

\section{Results}

While all 369 samples were found to be positive for PERV-ABC RNA, PERV-C RNA and PERV-A/C RNA were detected in $24.1 \%$ $(89 / 369)$ and $18.7 \%(69 / 369)$ of the serum samples respectively. Twenty percent $(43 / 215)$ of the samples collected during the nursery stages (3-9 weeks of age) were found to be positive for PERV-A/C RNA versus $16.9 \%$ (26/154) of the samples collected during the grow-finish stages of production (12-25 weeks of age). When clinically affected pigs were compared to unaffected pigs, $25.0 \%$ (15/60) of the samples from affected pigs were found to be positive for PERV-A/C RNA, whereas in clinically healthy pigs $8.3 \%$ (5/60) of the samples were found to be PERV$\mathrm{A} / \mathrm{C}$ positive. Interestingly, PERV A/C was also identified in the same pigs on more than one consecutive bleeding time.

\section{Discussion}

In this study, it was determined that $100 \%$ of the investigated pigs were positive for PERV-ABC as expected. PERV-C and PERV$A / C$ were found in varying percentages $(24.1 \%$ versus $18.7 \%)$ on all farms and production stages. Finally, when we compared clinically healthy and diseased pigs, in one of the two investigated farms, evidence was found that clinically affected pigs had a higher incidence of PERV A/C. Since the current study was limited to selected farms further studies using sample sets from pigs with different clinical expressions are warranted.

\section{References}

1. Langford, G.A. et al. (2001) Transplantation 72:1996-2000.

2. Takeuchi, Y. et al. (1998) J. Virol. 72, 9986-9991.

3. Wood, J.C. et al. (2004) J. Virol. 78, 2494-2501.

4. Scobie, L. et al (2004) J. Virol. 78, 2502-2509. 


\title{
P.589
}

\section{Development of a real-time PCR assay for the detection of novel swine boca-like virus DNA (SwBoV)}

\author{
Catherine Duffy ${ }^{1}$ Julie McClintock ${ }^{2}$ Michael McMenamy ${ }^{1}$ Irene McNair ${ }^{2}$ John McKillen² Michael Welsh² Gordon M. Allan ${ }^{1}$
}

1. Queen's University Belfast, Belfast, UK; 2. AFBI, Belfast, UK

\section{Introduction}

Two antigenically distinct swine boca-like viruses (SwBoV) have been isolated from pigs in Northern Ireland. The viruses were recovered from a small intestinal tissue and faecal specimen from two pigs on different PMWS-affected farms by virus isolation in primary cell cultures. Although both samples exhibited a CPE, PCR testing for a range of known porcine viruses failed to identify either agent. DNA sequencing and molecular characterization subsequently established that both isolates share genetic homology and possible genomic arrangement with parvoviruses BPV-1, CMV and HBV. The isolates were designated as SwBoV1 and SwBoV2. The discovery of a novel porcine bocalike virus (PBo-likeV) in PMWS-affected pigs in Sweden has also recently been reported ${ }^{1}$. Here we describe the development of a real-time PCR assay for the detection of SwBoV DNA.

\section{Materials and Methods}

Primers were designed against conserved nucleotide sequences of SwBoV1 and SwBoV2. Real-time PCR was carried out on a MJ Research Opticon 2 instrument using SybrGreen PCR master mix (Qiagen, West Sussex, UK) in $25 \mu$ reaction volumes containing $2 \mu \mathrm{l}$ of template. Assay optimisation involved determination of optimal $\mathrm{Ta}_{1} \mathrm{Mg}^{2+}$ and primer concentrations. To determine assay sensitivity, serial dilutions of quantified amplicons were prepared for each isolate. Assay specificity was assessed against a number of cell lines, porcine and non-porcine DNA viruses. A 2.8Kb representative amplicon, derived from a UK sample, served as PBo-likeV positive control DNA. All nucleic acid extractions were performed using the MagNA Pure LC Total nucleic acid extraction kit (Roche, Burgess Hill, UK).

\section{Results}

PCR optimisation resulted in an assay designed to work at a Ta of $52^{\circ} \mathrm{C}$ and $0.5 \mu \mathrm{M}$ primer concentration. Amplification was achieved using 40 cycles of $95^{\circ} \mathrm{C}$ for $30 \mathrm{sec}, 52^{\circ} \mathrm{C}$ for $30 \mathrm{sec}, 72^{\circ} \mathrm{C}$ for $30 \mathrm{sec}$ followed by a melt-curve between $60-95^{\circ} \mathrm{C}$. Amplicons of $313-316 \mathrm{bp}, \mathrm{Tm}$ of approximately $79^{\circ} \mathrm{C}$ and $78^{\circ} \mathrm{C}$ and detection limits of $2 \times 10^{1}$ and $2 \times 10^{2}$ copies/ $\mu$ l of PCR product were determined for SwBoV1 and SwBoV2, respectively. The specificity of the assay was also confirmed against a range of cell lines and DNA viruses.
Fig. 1 Fluorescence $v$ cycle no. for SwBoV PCR assay run against lymphoid tissue from field samples.

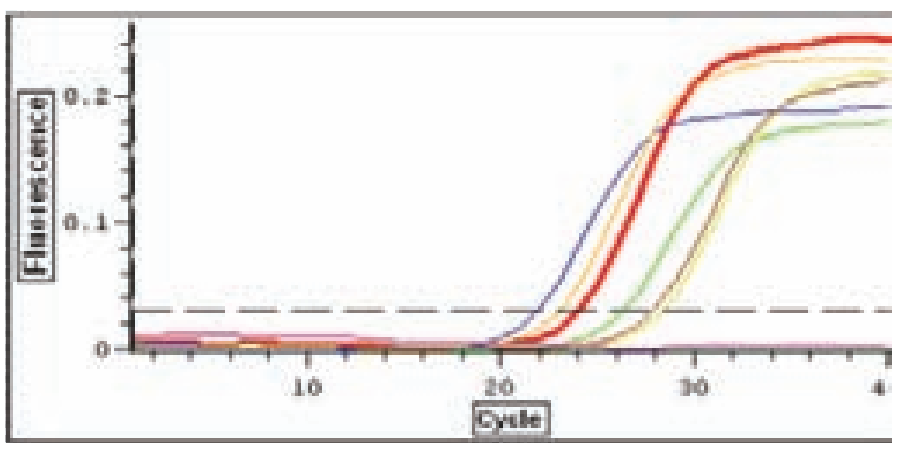

The assay was applied to a number of archived field specimens. Fig. 1 shows the results of positive samples, with Tm ranging from $78.1-79.5^{\circ} \mathrm{C}$. Sequence analysis of two positive field samples established that they shared $>90 \%$ nucleotide homology with SwBoV1 and SwBoV2 within the 900bp region analysed. Nucleotide database searches (BLASTn) reported that the amplicons most closely resemble HBV, CMV and BPV-1. DNA sequencing analysis of the remaining positive samples is on-going.

\section{Discussion}

New and re-emerging pathogens continue to pose a risk to both human and animal health. Bocavirus infection is already associated with a number of disorders in other species ${ }^{2}$. The discovery of a SwBoV therefore warrants further investigation. The results suggest that this PCR assay may offer a sensitive method for the detection of SwBoV DNA. The development of a SwBoV-specific $P C R$ assay provides a means to investigate the prevalence, clinico-pathological significance, epidemiology and genetic diversity of SwBoV within swine populations.

\section{References}

1. Blomström, A., et al. (2009) Virus Res. 146, 125-129.

2. Manteufel, J. and Truyen, U., (2008) Intervirology 51, 328-334. 


\title{
P.590
}

\section{Distal ulna osteochondrosis in fattening pigs}

\author{
Friederike Zeeh ${ }^{1}$ A. Gutzwiller ${ }^{2}$ W. Zimmermann ${ }^{1}$ P. Kircher ${ }^{3}$ H. Stich ${ }^{4}$ P. Schawalder ${ }^{5}$
}

1. Vetsuisse Faculty Bern, Swine Clinic, Bern, Switzerland; 2. Agroscope Liebefeld-Posieux, Posieux, Switzerland; 3. Vetsuisse Faculty Zurich, S ection of Diagnostic Imaging, Zurich, Switzerland; 4. Klinik für Zahnerhaltung, Präventiv- und Kinderzahnmedizin der Medizinischen Fakultät, Universität Bern, Bern, Switzerland; 5. Vetsuisse Faculty Bern, Division of Surgery, Orthopedics and Stomatology, Bern, Switzerland

\section{Introduction}

Osteochondrosis (OC) is a major predisposing factor to lameness, impairing growth performance and animal welfare. It is defined as generalized disturbance of the enchondral ossification at predilection sites such as the articular cartilage and metaphyseal growth plates. Little is known about the pathogenesis, and diverging views regarding the causes of the disease exist. Most reports focus on the articular lesions. In a feeding trial the hypothesis was tested that ad libitum feeding of a diet containing excessive amounts of calcium ( $\mathrm{Ca}$ ) and protein combined with a high physical activity, i.e. risk factors for the development of canine panostitis ${ }^{1}$, cause osteochondrosis in growing pigs.

\section{Materials and Methods}

From 25 to $105 \mathrm{~kg}$ body weight (BW), four groups of five Large White pigs received either restricted amounts of feed containing the recommended nutrient level ("R") or an enriched diet (" $E$ ": $\mathrm{Ca}+30 \%$, crude protein $+20 \%$, $2000 \mathrm{IU} / \mathrm{kg}$ vitamin D) ad libitum and were either individually housed in small (S) pens or group housed in large $(L)$ pens (Table 1$)$.

Table 1: Feeding and housing of the pigs

\begin{tabular}{|l|c|c|c|c|}
\hline Group & RS & RL & ES & EL \\
\hline Pigs $(\mathrm{n})$ & 5 & 5 & 5 & 5 \\
\hline Feeding & restricted & restricted & ad lib & ad lib \\
\hline Pen & small & large & small & large \\
\hline
\end{tabular}

Every week, each pig was weighted and clinically monitored, in particular for lameness, leg deformities and accessory bursae. After slaughtering, one forelimb per pig was radiographed in lateral and antero-posterior position. Findings at the distal ulna growth plate (DUGP) were recorded.

Two radiologically normal and four altered legs were histologically evaluated using light microscopy. For this, bones were sawed in $5 \mathrm{~mm}$ slices in the area of the DUGP and fixed in $70 \%$ alcohol. Bone sections were embedded in acryl and stained with Fuchsin/Acetic-Light Green.

\section{Results}

The ad libitum intake of feed $\mathrm{E}$ as well as housing in small pens significantly increased the daily weight gain (ES 841g, EL 757g, RS 716g, RL $693 \mathrm{~g}$ ).

Limping or stiff walking were observed in 7 pigs from 16 weeks of age onwards but were always slight or moderate and disappeared within one or two weeks without therapy. Bowed legs (17 pigs) and accessory bursae or callosities (20 pigs) were frequent and occurred in all 4 groups.

Radiographs showed OC lesions as radiolucent, widened and irregular growth plates and/or subchondral sclerosis in the distal ulna of 14 pigs. Four of the 14 pigs with radiologic changes had a history of lameness.

Histological findings ranged from normal growth plates to severe lesions in the distal ulna. Irregular width, abnormal ossification or mineralization, and separation in the area of the cartilage breakdown zone were observed. In one case, separation extended to the metaphyseal bone (Fig. 1). On the rims of the clefts, reparation processes occurred.

Fig. 1: Separation along the distal ulna growth plate (DUGP), extending in the metaphysis $(M)$ of the distal ulna, 1.6x.

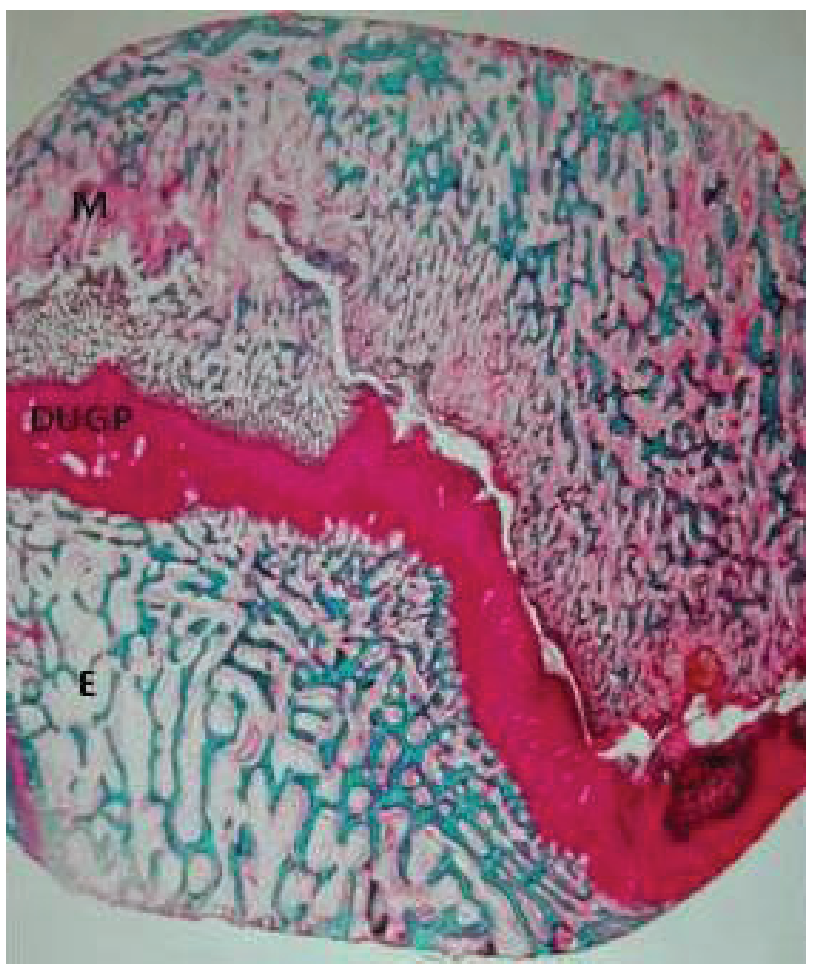

\section{Discussion}

Severe lameness could not be provoked by the study design. Nevertheless, radiological and histological investigation revealed OC lesions in all 4 study groups. The separation in the distal ulna epiphysis (fissure) has been described in literature ${ }^{2}$, but recent reports are lacking. Thus, we want to draw attention to this histological finding as part of the OC features.

\section{References}

1. Schawalder , P. et al. 2002 : Die Panostitis beim Hund - [...] Part I and II. Schweiz Arch Tierheilk; 144/3: 115-130 and 144/4:163-173.

2. Reiland, S. 1978: Morphology of osteochondrosis and sequelae in pigs. Acta Radiol Suppl. 358: 45-90. 


\title{
P.591
}

\section{Microbial etiology of infectious arthritis in nursing piglets}

\author{
Marie Sjölund ${ }^{1,3}$ Maria Lindberg² Ulrika Grönlund-Andersson ${ }^{1}$ \\ 1. SVA Statens Veterinärmedicinska Anstalt, Uppsala, Sweden; 2. Swedish Animal Health Services, Uppsala, Sweden; \\ 3. SLU Swedish University of Agricultural Sciences, Uppsala, Sweden
}

\section{Introduction}

Infectious arthritis is one of the most common causes of antimicrobial treatment of nursing piglets with approximately $10 \%$ of the piglets receiving antimicrobial treatment because of lameness $(1,2)$. Affected piglets mostly receive treatment during the first three weeks of life. At present, there are few studies on the prevalence of pathogens isolated from affected joints of piglets with clinical signs of arthritis.

As infectious arthritis causes both suffering of the individual animal and economic losses due to reduced growth rate, increased mortality, costs for treatments and increased labor, it is most important to initiate treatments as soon as possible with the most appropriate antimicrobial to minimize both losses and other negative effects. Therefore, the aim of this study was to, on a national level, obtain a basis for an optimal treatment regime.

\section{Materials and Methods}

From each herd with $\geq 100$ sows ( $n \approx 400$ ), one nursing piglet ( 0 to 35 days of age) with clinical signs of infectious arthritis such as lameness, fever and swollen joints were identified by the regional Animal Health Service veterinarian on a regular visit. The piglets were euthanized with an intra peritoneal injection of barbiturates ( $\geq 1 \mathrm{ml} / \mathrm{kg}$ body weight) and then frozen at $-20^{\circ} \mathrm{C}$ before being sent to SVA for necropsy.

At necropsy, two affected joints per piglet were sampled for bacterial cultivation including Mycoplasma sp., and Haemophilus sp. Antimicrobial susceptibility was determined by broth microdilution using VetMIC ${ }^{\mathrm{TM}}$ (SVA, Uppsala). Epidemiological cut-off values according to EUCAST were used to determine if the isolates were resistant or not (www.eucast.org). Isolates of Staphylococcus (S.) hyicus were in addition examined for penicillinase production by the "clover-leaf" method.

\section{Results}

The preliminary results from 36 herds, represented by 36 piglets and 72 joints, demonstrated bacterial isolation from 39 of 72 sampled joints. The dominant finding was Streptococcus dysgalactiae subsp. (S.) equisimilis isolated from 23 joints, followed by Escherichia (E.) coli isolated from 5 joints and then S. hyicus isolated from 4 joints. Mycoplasma sp. and Haemophilus sp. could not be isolated from any of the 72 sampled joints. From seven joints, the bacterial species have not yet been identified.
In 10 piglets, the same bacterial species could be isolated from both joints sampled. S. equisimilis was isolated from both joints in eight piglets and $E$. coli in two piglets. In nine of 36 piglets, bacterial cultivation was negative from both joints.

All streptococci were susceptible to penicillin. Three of four S. hyicus isolates were penicillinase producers. However, all S. hyicus and E. coli isolates were susceptible to trimethoprim.

\section{Discussion}

The isolation of the same bacterial species from both of the sampled joints in 10 piglets indicates a hematogenic spread of the infection which is in accordance with previous findings (3). In four of the nine piglets where no bacteria could be isolated, lameness was most probably caused by trauma. The other five piglets had gross pathological lesions indicative of arthritis.

This study shows that treatments with penicillin would be successful in 22 of 25 piglets, provided that treatments were initiated early on during the course of infection. However, as three S. hyicus were penicillinase producers, and three E. coli isolates were resistant to ampicillin, some cases of treatment failure using penicillin could be expected. Thus, given the results, trimethoprim-sulphonamides should be considered as the second hand choice after penicillin.

\section{References}

1. Zoric et al. 2003, Vet Rec 13;153(11):323-8

2. Zoric et al. 2004, J Vet Med B, 51(6):278-84

3. Zoric et al. 2009, Acta Vet Scand, 51:23 (1-9) 


\title{
P.592
}

\section{Hind limbs paralysis outbreak in a pig herd. A case report}

\author{
Eduardo J. Kwiecien ${ }^{1}$ Carolina Gutierrez ${ }^{2}$ Evelyn Cadenas ${ }^{2}$ \\ 1. EKM Servicios Veterinarios, Maracay, Venezuela; 2. Carval Venezuela S.A., Valencia, Venezuela
}

\section{Introduction}

Locomotory disturbances in swine are a significant and commun problem in pigherds 1,2,3. Nutritional, bacterial and viruses infection are the main factors related to paralysis of hind legs in young pigs4,5. Even though the incidence of pathology is low6, no field reports have been done so far. The aim of this study is to describe an outbreak of hind limbs paralysis in an pig farm in Venezuela.

\section{Materials and Methods}

The study was carry on in a 600 sows farrow to finish herd in the Cojedes State of Venezuela. A historical morbility records showed and incidence in 5 to 10 pigs with hind legs recumbence or paralysis in the last three months before to this study. An interview with the owner and stockmanship in the farrowing and weaner areas was scheduled to assess the key factors intervening in the cases. Data of numbers of pigs suffering this condition, mortality attributed to hind quarters paralysis was taken to determine the prevalence and incidences. Necropsy was performed in pigs and samples of limph nodes and spinal cord were taken for bacteriological and histopathological study.

\section{Results}

Interview results and management procedures in tha farrowing area showed failures during piglets processing after farrow. Findings necropsy showed an intervertebral abscess at L4-L5. Nodular proliferative abscess were presented in the spinal cord canal (Fig. 1, ). Degenerative changes were manifest grossly as narrowing of the intervertebral space and conversion of the nucleus pulposus to a red dark, granular mass (Fig. 2). A mix of mononuclear and neutrophils infiltration in spinal cord on microscopic view was observed.

Changes in proccesing piglets and an antimicrobial medication inyectable and in the weaner feed reduced the incidence of this pathology.
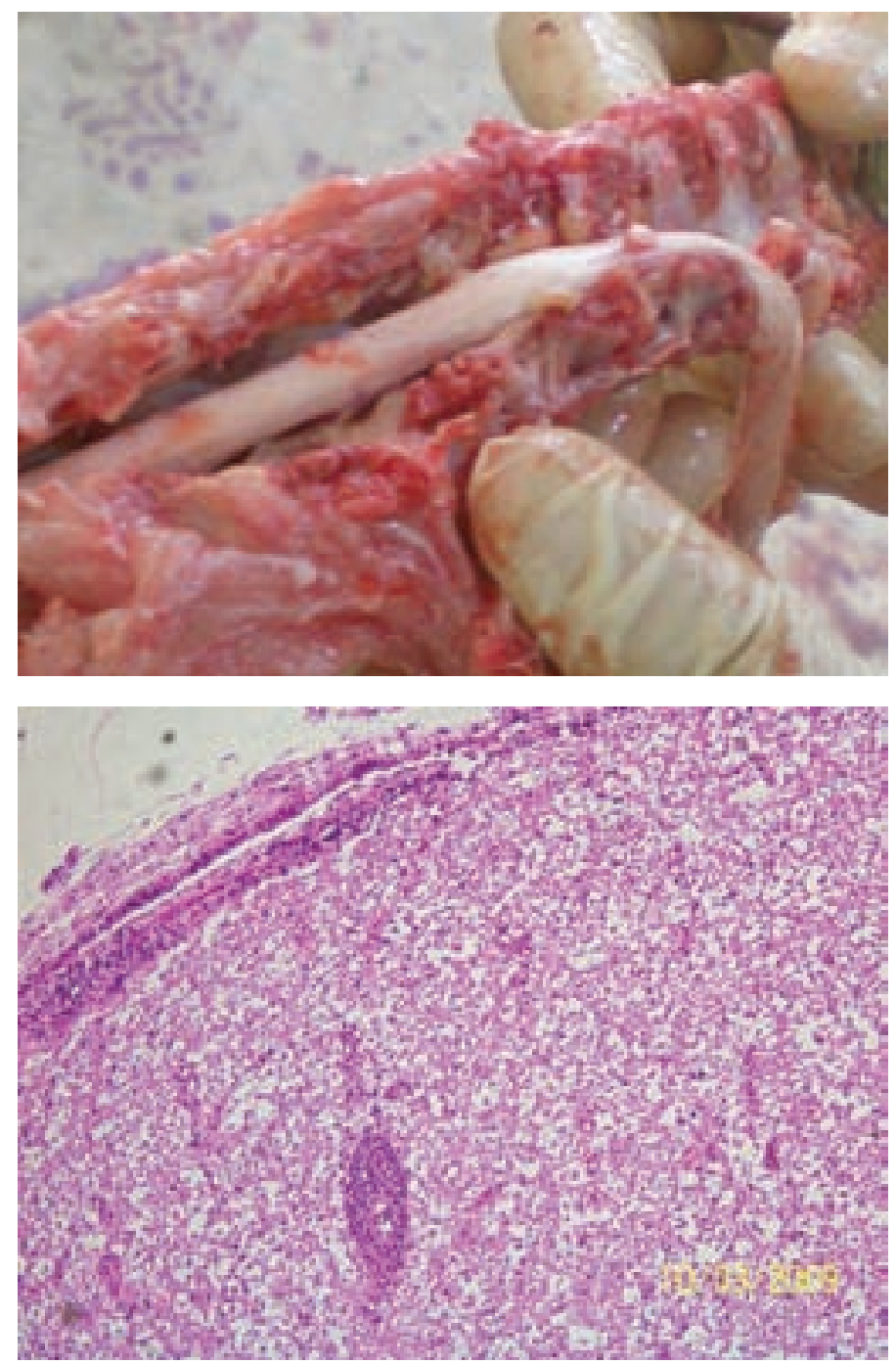


\title{
P.593
}

\section{Etiology of the ear necrosis syndrome - investigation of bacteria, fungi and mycotoxins in feeding stuff}

\author{
Christiane Lang ${ }^{1}$ Thomas Voglmayr $^{2}$ Franz Waxenecker ${ }^{3}$ Ursula Hofstetter $^{3}$ Herbert Weissenböck ${ }^{4}$ \\ Mario Ogris 5 Georg Bruns ${ }^{6}$ Mathias Ritzmann ${ }^{1}$ \\ 1. Clinic for Swine, Vienna, Austria; 2. Traunkreis VetClinic, Ried im Traunkreis, Austria; 3. Biomin GmbH, Herzogenburg, Austria; \\ 4. Institute of Pathology and Forensic Veterinary Medicine, University of Veterinary Medicine Vienna, Vienna, Austria; \\ 5. Intervet GesmbH, Vienna, Austria; 6. Tieraerztliche Klinik Duemmerland, Steinfeld, Germany
}

\section{Introduction}

During the summer months lesions of the so-called ear necrosis syndrome can be observed frequently in weaners during the raising period. The stages range from mild epidermal changes to severe ulcers and necrosis (1). In the past, contaminants of feeding stuff have been discussed to be responsible for the development of ear necrosis, but the exact etiology of this disease could not be determined. Aim of this study was the evaluation of bacteria, fungi and different mycotoxins in feeding stuff of pigs with ear necrosis syndrome.

\section{Materials and Methods}

A total of 15 farms were included in the study. Feeding stuff of piglets with clinical signs of the ear necrosis syndrome in an initial stage was collected 3 times, once during summer time, when the symptoms occurred for the $1^{\text {st }}$ time, once in winter after storage and once again in summer. The investigated parameters are summarized in table 1.

Table 1: Contaminants in feeding stuff

\begin{tabular}{|l|l|}
\hline Bacteria & Aerobe mesophile bacteria \\
\hline Fungi & Mould fungi, Aspergillus spp., Fusarium spp., Penicillium spp. \\
\hline Mycotoxins & T2 toxin, HT2 toxin, Diacetoxyscirpenol \\
\hline Mycotoxins & Deoxynivalenol (DON), Acetyldeoxynivalenol, Nivalenol, Zearalenone (ZON) \\
\hline Mycotoxins & Ergosin, Ergotamin, Ergocornin, Ergocryptin, Ergocristin \\
\hline
\end{tabular}

\section{Results}

Aerobe mesophile bacteria could be detected in a concentration between $1.0 \times 10^{4}$ to $3.0 \times 10^{7}$. Concentrations up to $10^{6}$ are of no clinical relevance. $34.4 \%$ of the samples had results higher than $10^{6}$. Aspergillus spp., Fusarium spp. and Penicillium spp. were determined in a concentration of $10^{3}$, which is not harmful to pigs. Only 1 sample exceeded a Penicillium and 2 samples a Fusarium amount of $10^{4}$. Concerning the mycotoxin concentrations, T2 toxin, HT2 toxin, Diacetoxyscirpenol, Acetyldeoxynivalenol and Nivalenol could not be detected. Table 2 shows the results of the other toxins.
Table 2: Mycotoxin concentrations in $\mathrm{mg} / \mathrm{kg}$

\begin{tabular}{|l|c|c|c|}
\hline Toxin & Max & MV & SD \\
\hline DON & 1.243 & 0.253 & 0.246 \\
\hline ZON & 0.126 & 0.041 & 0.036 \\
\hline Ergosin & 0.486 & 0.043 & 0.111 \\
\hline Ergotamin & 0.241 & 0.025 & 0.053 \\
\hline Ergocornin & 0.015 & 0.001 & 0.003 \\
\hline Ergocryptin & 0.822 & 0.106 & 0.227 \\
\hline Ergocristin & 2.015 & 0.372 & 0.583 \\
\hline \multicolumn{4}{|l|}{ Max = maximum, MV = mean value, SD = standard deviation } \\
\hline
\end{tabular}

\section{Discussion}

Especially mycotoxins can cause ear necrosis. A good quality of feeding stuff can be an essential prophylactic tool. The general hygienic status of the feeds tested in the present study was moderate, which could be an initiating factor for the development of several diseases. Increased mycotoxin concentrations could also be detected. Whereas DON and ZON amounts were mostly tolerable, the detected alkaloid concentrations were unexpectedly high. If those concentrations are sufficient for the development of ear necrosis still needs to be investigated.

\section{Acknowledgment}

This study was supported by the Dres. Jutta und Georg-Bruns-Stiftung.

\section{References}

Mirt (1999): Vet. Rec. 144, 92-96. 


\title{
P.594
}

\section{Etiology of the ear necrosis syndrome - data collection through questionnaires}

\author{
Christiane Lang ${ }^{1}$ Rosmarie Pyrek ${ }^{1}$ Thomas Voglmayr ${ }^{2}$ Franz Waxenecker ${ }^{3}$ Ursula Hofstetter ${ }^{3}$ Georg Bruns $^{4}$ Mathias Ritzmann $^{1}$ \\ 1. Clinic for Swine, Vienna, Austria; 2. Traunkreis VetClinic, Ried im Traunkreis, Austria; 3. Biomin GmbH, Herzogenburg, Austria; \\ 4. Tieraerztliche Klinik Duemmerland, Steinfeld, Germany
}

\section{Introduction}

During the summer months lesions of the so-called ear necrosis syndrome can be observed frequently in weaners during the raising period. The stages range from mild epidermal changes to severe ulcers and necrosis (1). In the past, various pathogens have been discussed to be responsible for the development of ear necrosis, but the exact etiology of this disease could not be determined. Aim of this study was the data collection through questionnaires in farms with pigs with ear necrosis syndrome.

\section{Materials and Methods}

Questionnaires were sent to Austrian and German veterinarians. The parameters of interest were kind of necrosis (tip of the ear $(T)$, margin of the ear (M) or flank (F) necrosis) stadium of disease, severity, percentage of affected pigs, age and weight of pigs, additional symptoms, feeding, husbandry, positive experience with any treatment or prophylaxis, previous illness as well as occurrence of cannibalism.

\section{Results}

In total, 54 questionnaires were returned to the clinic for swine. $88.7 \%$ of them declared to see the ear necrosis syndrome often and $94 \%$ estimated ear necrosis to be a considerable problem in swine production. $90.7 \%$ reported to have problems with necrosis at the tip of the ear, $57.4 \%$ with necrosis at the margin of the ear, and only $14.8 \%$ with flank necrosis. Percentage of affected pigs, as well as age and weight of pigs are presented in table 1. Most of the animals, independent of the kind of necrosis, were in a chronic stadium of disease, there were only a few acute cases. The severity of disease was estimated as follows: flank necrosis: mild symptoms, both kinds of ear necrosis: moderate symptoms. In about half of the farms the pigs showed wasting, $32.1 \%$ had diarrhea. Concerning feeding, only one veterinarian reported a low quality of feeding stuff. $22.6 \%$ associated the occurrence of ear necrosis with a change in the feeding strategy. Only $5.6 \%$ observed a reduced feed intake. Related to husbandry the main problem was the pig density. All other parameters as temperature, cleaning, disinfection were not considered to be problematic. $72.2 \%$ reported positive experiences with different treatment or prophylactic measures (68.4\%: antibiotics, $2.6 \%$ : vaccination, $2.6 \%$ : management improvement, $28.2 \%$ : combination of different measures). $22.2 \%$ of the farms reported any illness previous to the occurrence of ear or flank necrosis (41.7 \%: bacterial infection (gram negative), $25.0 \%$ : bacterial infection (gram positive), $8.3 \%$ : viral infection, $25.0 \%$ : multifactorial infection). Cannibalism was considered to be the reason for the occurrence of ear necrosis in $51.0 \%$ of the cases.
Table 1: Statistical evaluation of collected data

\begin{tabular}{|l|c|c|c|c|c|}
\hline Parameter & Kind & Min & Max & MV & SD \\
\hline Pigs affected (\%) & T & 2 & 100 & 38.8 & 28.5 \\
\hline Pigs affected (\%) & M & 2 & 100 & 34.3 & 27.7 \\
\hline Pigs affected (\%) & $\mathbf{F}$ & 2 & 40 & 10.6 & 13.4 \\
\hline Age (weeks) & $\mathbf{T}$ & 5.5 & 15.0 & 7.9 & 1.7 \\
\hline Age (weeks) & $\mathbf{M}$ & 5.5 & 15.0 & 8.2 & 1.8 \\
\hline Age (weeks) & $\mathbf{F}$ & 10.0 & 15.0 & 11.0 & 2.2 \\
\hline Weight (kg) & $\mathbf{T}$ & 12.0 & 30.0 & 18.1 & 4.8 \\
\hline Weight (kg) & $\mathbf{M}$ & 10.0 & 27.5 & 17.7 & 4.3 \\
\hline Weight (kg) & $\mathbf{F}$ & 17.5 & 42.5 & 28.0 & 9.4 \\
\hline Min = Minimum, Max = Maximum, MV = Mean value, SD = Standard deviation \\
\hline
\end{tabular}

\section{Discussion}

The present study showed that ear and flank necrosis is of concern to swine practitioners. If ear necrosis appears, about one third of the pigs are affected. The economic losses increase because of concomitant symptoms as diarrhea and wasting. Not only infectious diseases seem to be relevant; management, feeding and husbandry are also significant factors. The results of this evaluation pointed out clearly that ear and flank necrosis are mainly caused multifactorial as shown in other studies $(2,3)$.

\section{Acknowledgment}

This study was supported by the Dres. Jutta und Georg-Bruns-Stiftung.

\section{References}

1. Mirt (1999): Vet. Rec. 144, 92-96.

2. Richardson et al. (1984): Vet. Pathol. 21, 152-157.

3. Osweiler (2006): Diseases of swine, 915-930. 


\title{
P.595
}

\section{The occurrence of fungi and mycotoxins in swine feed in Korea}

\author{
Wen Jin; Yeong Hun Kim; Jeong Hee Han \\ Kangwon National University, Chunchuan, Korea
}

\section{Introduction}

Fusarium spp. are recognized as a source of common mycotoxins, and storage fungi include the genera Aspergillus and Penicillium, which account for several mycotoxins important in swine production. A few of significant mycotoxins include aflatoxin and Ochratoxin A belonged to Aspergillus spp, ochratoxin, dexynivalenol, zearalenone, fumonisin and T-2 toxin belonged to Fusarium spp. Aflatoxin and ochratoxin mycotoxins, as well as Fusarium, have been shown to be the destruction of the immune system and immunosuppressive. This study was to survey about fungi and mycotoxins occurrence in Swine Feed of different regions in Korea [1].

\section{Materials and Method}

A total of 202 feed samples were evaluated for survey of Fungi and Mycotoxins. Also all samples were classified by growth period, regions. Culture processes of samples were performed by potato dextrose agar. Then microscopic examination was performed after staining process. The photomicrographs captured by OPTIMAS 6.1 were referred to mycology online and CAB International by E-mail. Simultaneity, the samples collected were examined by ELISA technique after processing. AgraQuant ${ }^{\circledR}$ Mycotoxin Test Kit was used to estimate mycotoxin ELISA titre.

\section{Results}

In morphologic diagnosis process, 3 types of fungi, Aspergillus spp.,Fusarium spp. and Rhizopus spp. were identified by microscopic examination. Aspergillus spp., Fusarium spp. and Rhizopus spp. were observed in 97, 25 and 19 samples, respectively. The rests of 61 samples were unidentified. By analyzed samples from various growth period, belonged to Aspergillus spp., in growing, fattening, pregnant, and farrowing were 17, 32, 27 and 21, respectively. In Fusarium spp., were 2, 12, 9 and 2 samples, in Rhizopus spp., were 1, 11, 4 and 3 samples, respectively. By regions, belonged to Aspergillus spp., in Gangwon-do, Gyeongsang-do, Chungcheong-do, Gyeonggi-do and Jeolla-do were 37, 21, 19, 11 and 9 samples, respectively. In Fusarium spp., were 4, 5, 3, 7 and 6 samples, in Rhizopus spp., were 3, 5, 2, 3 and 6 samples, respectively. By feeder type, 30 samples were dry and humid-typed feeder and 41 samples were dry-typed feeder. In dry and humid-typed feeder, Aspergillus spp., Fusarium spp. and Rhizopus spp, were observed in 12, 5 and 6 samples. Whereas, in dry-typed feeder, those were observed in 9, 4 and 4 samples, respectively. By ELISA analyze of mycotoxins, incidences of contaminated samples were $75.24 \%$ for aflatoxin, $5.94 \%$ for ochratoxin A, $47.02 \%$ for zearalenone, $68.31 \%$ for fumonisin, not detected for T-2 and $78.91 \%$ for deoxynivalenol, respectively. The mean value of contaminated samples were $7.67 \pm 1.86 \mu \mathrm{g} / \mathrm{kg}$ for aflatoxin, $5.10 \pm 2.87 \mu \mathrm{g} / \mathrm{kg}$ for ochratoxin A, $128.32 \pm 99.04 \mu \mathrm{g} /$ $\mathrm{kg}$ for zearalenone, $880.00 \pm 800.00 \mu \mathrm{g} / \mathrm{kg}$ for fumonisin, not detected for T-2 and $510.00 \pm 210.00 \mu \mathrm{g} / \mathrm{kg}$ for deoxynivalenol. Eleven samples were against MEL regulation with those contaminated at a concentration of over 10ppb of aflatoxin. Among them, 6 samples had mixed contamination with zearlenone, fumonisin and deoxynivalenol.

\section{Conclusions}

The most of the fungi identified from swine feed were Aspergillus spp. By growth period, any fungi were not observed in feed of weaned pig. In rest period, 3 types of fungi were similarly observed. But generally, a number of fungi were observed in feed of fattening pig. By province, the most of fungi were observed in Gangwon-do, Gyeongsang-do and Chungcheong-do. Eleven samples exceed maximum exposure limits regulation on aflatoxin, although T-2 has not been detected in our samples. Detection rates of humid-typed feeder were shown higher than those of dry-typed feeder. Based on the results, the development of mycotoxins might be associated with environment. Therefore, the management of feed would consider important to provide against the damage by mycotoxins, as environment and climate are responsible for the development of it.

\section{References}

1. Peadar $G$, Lawlor and P. Brendan Lynch. Mycotoxins in pig feeds 2: clinical aspects. Irish veterinary journal. 54(4), 172 176 


\title{
P.596
}

\section{Biochemical parameters in blood from pigs experimentally intoxicated with aflatoxin B1 and Fumonisina B1}

\author{
Susana E. Mendoza_Elvira ${ }^{1}$ Abel Ciprian-Carrasco ${ }^{1}$ Carolina Moreno-Ramos ${ }^{1}$ Eliseo Hernández- \\ Baumgarten ${ }^{1}$ Ernesto MorenoMartinez ${ }^{1}$ Francisco Quezada² Jorge Tortora-Perez ${ }^{1}$ Rosalia Melendez- \\ Perez ${ }^{1}$ Horacio Lara-Puente ${ }^{2}$ Isabelle P. Oswald ${ }^{3}$ Patricia Perez ${ }^{1}$ Sara Valdes-Martinez ${ }^{1}$ \\ 1. FESC-UNAM, Cuautitlán, MEX, Mexico; 2. Laboratorios Avi-Mex, S A de C V, Iztapalapa, Mexico; 3. Pharmacology \\ and Toxicology Laboratory, UR 66, National Institute of Agronomic Research INRA, Toulouse, France
}

\section{Introducción}

The mycotoxins are secondary metabolites produced by fungi, of which the aflatoxin B1 (AFB1) and Fumonisin B1 (FB1) are important due to their toxicity and carcinogenicity. Aflatoxin affects negatively growth, feed conversion and consumption in pigs. The most affected organs are: the liver, and kidney. Fumonisin on the other hand causes pulmonary edema $(1,2,3)$.

\section{Material y Methods}

The FB1 used was a standard (SIGMA), in $5 \mathrm{mg}$ and $10 \mathrm{mg}$ vials with $98 \%$ purity. A stock solution was prepared at a concentration of $87 \mathrm{ppm}$ in distilled water. The FB1 was administered to weaned pigs in $12 \mathrm{ppm}$ ( $\mathrm{mg} / \mathrm{kg}$ live-weight) by the oral route by means of a probe. For AFB1 we used a Sigma standard in presentation $5 \mathrm{mg}$ per vial with a $98 \%$ purity. A stock solution was prepared in benzene-acetonitrile. The administration of the AFB1 to weaned pigs was at a dose of $8 \mathrm{ppb}$ ( $\mu \mathrm{g} / \mathrm{kg}$ live-weight) by mouth using a probe. The experimental animals were 16 pigs 21 to 36 days of age of either sex, with an average weight of 7 $\mathrm{kg}$. The animals were distributed randomly into 4 groups, each group was composed of 4 pigs, with the following distribution: negative Group A: Control.; Group B: intoxicated with FB1. Group C intoxicated with 8ppb of AFB1. Group D: intoxicated with 12ppm FB1and 8ppb.with AFB1.

The studied variables were body weight, the serum concentration of total proteins, albumin, the enzyme concentration of AST, ( $\gamma$-glutamic tranferase) GGT, direct, indirect and total Bilirrubins. The data were evaluated by ANOVA with a $95 \%$ significance level, using the statistical programme Statgraphics Plus version 5.0 .

\section{Resultados}

The results of the measurement of daily weight gain were: 0.22 kg for Group A, 0.043 kg for Group B, 0.074 kg for Group C and finally $0.002 \mathrm{~kg}$ for Group D. Evaluation of AST and GGT serum, results showed an increased activity in groups $B, C$ and $D$. Protein levels showed no significant changes. Levels of albumin showed a slight decrease in the group $B$. The bilirulubins were increased in groups $B, C$ and $D$, and the effect was more marked in Group B.

\section{Discusión}

The daily weight gain showed significant differences between group $A$ and $D$, with values of $0.22 \mathrm{~kg}$ and $0.002 \mathrm{~kg}$ respectively. These results differ from those reported by Zomborszky et al 4., where they cite that the differences in body weight gain was not statistically significant at the end of the experiment. AST levels in groups, B, C and D showed increased values that is consistent with Dilkin, P. et al, 5 . The GGT levels reported in literature are very diverse, but all showed an increase in intoxication by mycotoxins. The decrease in protein levels was notorious in group $B$ compared with the other groups. The variability of values is due to the concentration of mycotoxins, time of exposure and age of the animals (6). The decrease in levels of serum albumin was more pronounced than total proteins. Albumin has a plasma half-life of 21 days, so that as a measurement of liver function is not very pronounced until three weeks of intoxication (6) that the liver failure is triggered. The decline of bilirubin was most significant in Group B, this show that the FB1 affects the liver

\section{Conclusions.}

The assessment of biochemical parameters shows sensitivity and effectiveness to detect liver damage using small doses of mycotoxins.

\section{Referencias bibliográficas.}

1. D’Mello et al.,1999. Animal Feed Science and technology: 80: 183205.

2. Guzmán D., Peña J.J.2005. Revista latinoamericana de Microbiología Vol. 47 Julio-Diciembre

3. Gordon S.S. et al.2000. J. Agic. Food Chemical:48: 1860-1864.

4. Zomborszky et al., 2000. J. Vet. Med. B 47, 277-286.

5. Dilkin, P. et al, (2003). Food and chemical toxicology 41: 1345-1353.

6. Cardinali, P. D, Dvorkin. A.M., 2005. Bases Fisiológicas de la práctica médica, 13a Ed.Medica panamericana Buenos Aires pp. 533-552. 


\title{
Salinomycin and tiamulin toxicosis in Finish pigs
}

\author{
Kialane C. de Almeida ${ }^{1}$ Michelle d. Gabardo ${ }^{1}$ José Cristani $^{1}$ Joselito Daniluz $^{2}$ Sandra D. Traverso ${ }^{1}$
}

1. UDESC, Lages, SC, Brazil; 2. COOPERCAMPOS, Campos Novos, SC, Brazil

\section{Introduction}

The Salinomycin ionophore is an antibiotic used as anticoccidial in poultry and as growth promoters in cattle, sheep and pigs (1). The tiamulin is used in treatments for respiratory and enteric complications (2) These, when used alone in appropriate doses, do not cause damage to animals, but ionophores are myotoxic in excessive doses (3). Poisonings occur because of mixing error of the drug with the feed (4), dosage error (5), use on non-target species (6), or normal doses associated with drugs that maximizing its effects, such as chloramphenicol and tiamulin . (7). The tiamulin acts by reducing the elimination of the ionophore, leading to intoxication (8). Clinical signs are characterized by vomiting, inapetency and locomotor disorders (9). The work aims at reporting an outbreak of natural poisoning by salinomycin associated tiamulin in pigs in Brazil.

\section{Materials and Methods}

Epidemiological data were obtained with the vet about the animals. Two pigs were euthanized for necropsy. Samples of viscera, brain, heart and skeletal muscles were collected, fixed in 10\% formalin, processed routinely for histological examination (10).

\section{Results}

A batch of 940 finishing pigs were fed with diets containing 30 ppm of salinomycin. The pigs received tiamulin at a dose of 110 ppm in-feed for eight days, for control of respiratory diseases. In the first day after the end of treatment, one animal was found slumped. On the next day two animals showed coordination disorders, stiff gait, reluctance to move, muscular trembling, depression, emaciation and recumbence. These animals were euthanized for necropsy, which presented slight pallor of the muscles of the leg, Histological examination showed, marked extensive hyaline necrosis of skeletal myofibers with mild to moderate infiltration of macrophages. The lesions were more intense in longissima, diaphragm and masseter muscles. The heart had mild multifocal infiltration of macrophages, loss of myofibers and areas with regeneration. The feed of the entire batch was replaced. On the following days, several animals showed locomotor disturbances and after the grace period of medicines, all animals were sent for emergency slaughter.

\section{Discussion}

Epidemiological data, clinical and lesion sign observed and reported are consistent with toxic myositis.and are similar to those described in ionophore poisoning in pigs (9). In this outbreak the doses of salynomicin and tiamulin were in accordance with the theurapeutic dosage for age and species when used alone, indicating that tiamulin probably interfered in the excretion of salinomycin. Concerning pigs in Brazil, the poisoning ionophore should be distinguished from Vit.E - Se deficiency, where the muscle injuries are usually accompanied by cardiac and hepatic lesions similar to those observed in mulberry heart disease and hepatosis dietetica (11); Aeschinomene indica poisoning (12), where locomotor difficulties are secondary to specific neurological lesions and Cassia occidentalis (13) poisoning which can be distinguished through the epidemiological data of the supply of ionophore to animals or through the evidence of the plant mixed in the diet.

\section{References}

1. Barragry T.B. (1994) Lea and Febiger, Philadelphia, 607-615.

2. Ballarini, G. (1996) Rivista di Zootecnia e Veterinária, 24(2):3-14.

3. Novilla M.N. (1992) Vet. Hum. Toxicol. 34:66-70.

4. Ganter M., Kieckhofer H.M. e Kucza A. (1995) Hora Veterinária 15(85):12-16.

5. Rollinson J., Taylor F.G.R. \& Chesney J.N. (1987) Vet. Rec. 121:126-128.

6. Griffiths G.L., Hiller P. e Sutherland R.J. (1989) Aust. Vet. J. 66(10):326329.

7. Radostitis, O. M. (2002) Clínica Veterinária, 9 ed.

8. Witkamp R.F et. al. (1995) Drug Metabol. Dispos. 23(5): p. 542-547.

9. Dost G. (1980). Forsch. Sonderheft 37 Kongressband.

10. Prophet et. al. (1994) Armed Forces Institute of Pathology. American Registry of Pathology;

11. Sobestiansky J. e Barcellos D. (2007) Doenças dos Suínos. 770p.

12. Oliveira F. N. et. al. (2005) Pesq. Vet. Bras. 25(3) p.135-142

13. Martins E. et. al. (1986) Pesq. Vet. Bras. 6(2) p. 35 -38 


\title{
P.598
}

\section{Changes of interleukin-10 mRNA expression are predictive for 9-day survival of pigs in an emergency preservation and resuscitation model}

\author{
Wolfgang Sipos $^{1}$ Catharina Duvigneau ${ }^{1}$ Wolfgang Weihs ${ }^{2}$ Fritz Sterz $^{2}$ Alexandra Graf ${ }^{2}$ Romana Hartl ${ }^{1}$ \\ Keywan Bayegan² Andreas Janata² Danica Krizanac ${ }^{2}$ Wilhelm Behringer ${ }^{2}$ \\ 1. University of Veterinary Medicine Vienna, Vienna, Austria; 2. Medical University of Vienna, Vienna, Austria
}

\section{Introduction}

Outcome after prolonged normovolemic cardiac arrest is poor. The technique of evaluating the neurologic state and the overall performance category in pigs resuscitated after cardiac arrest has already been optimized (1). Emergency preservation and resuscitation (EPR), which is the induction of hypothermia during the arrest, followed by hypothermic stasis during transport to resuscitation with cardiopulmonary bypass (CPB), might be a new concept for patients with prolonged cardiac arrest. We have shown previously that induction of deep cerebral hypothermia via ice cold saline aortic flush during cardiac arrest just before resuscitation improved neurologic outcome in pigs (2). To avoid fluid overload, we further developed a cardiopulmonary bypass cooling system (CPBCS) for rapid induction of hypothermia (3). We hypothesized that changes in cytokine expression during EPR could be predictive of resuscitability and 9-day survival.

\section{Materials and Methods}

21 Large White sows (31-38 kg BM) were subjected to $15 \mathrm{~min}$ of untreated ventricular fibrillation, followed by cooling with a CPBCS for 1,3 , or 5 min achieving brain temperatures (Tbr) of 30.4 $\pm 1.6^{\circ} \mathrm{C}, 24.2 \pm 4.6^{\circ} \mathrm{C}$, and $18.8 \pm 4.0^{\circ} \mathrm{C}$, respectively. After $40 \mathrm{~min}$ of rewarming with $\mathrm{CPB}$, pigs were defibrillated and kept at $\mathrm{Tbr}$ of $34.5^{\circ} \mathrm{C}$ for $20 \mathrm{hrs}$, survival was for 9 days. Plasma samples were analysed for interleukin (IL)-6, tumor necrosis factor-a (TNF-a), and IL-10 levels by ELISA. Total RNA out of PBMCs was analyzed by real-time PCR for IL-1, IL-2, IL-4, IL-10, TNF-a, interferon- $\gamma$, iNOS, and heme oxygenase-1 (HO-1) gene expressions.

\section{Results}

11 out of 21 pigs survived to day 9 . In the plasma of all animals, a remarkable increase in proinflammatory cytokine levels could be observed following cardiac arrest and resuscitation. However, no significant differences in cytokine expressions could be found as a consequence of different intra-arrest hypothermia levels. On the other hand, IL-10 was identified as prognostic marker with decreasing mRNA levels during cardiac arrest in 9-day surviving animals but increasing ones in the non-survivors.

\section{Discussion}

The systemic changes in the postresuscitation syndrome are mainly characterized by a proinflammatory state similar to the systemic inflammatory response syndrome (SIRS) and thus termed a sepsis-like syndrome (4). Interestingly, SIRS is not only characterized by elevated inflammatory cytokine levels including IL-1, IL-6, and TNF-a, but also may be the consequence of a more profound dysregulation of the immune system, which is inferred from elevated IL-10 titers (5). Our study confirmed that pigs upregulate markers of systemic inflammation following cardiac arrest, intra-arrest hypothermia, and resuscitation, thus resembling the postresuscitation syndrome. The slight increase of IL-10 mRNA in non-survivors may account for a change in the functional properties of white blood cells. These findings hopefully will lead to improved intervention methods for the benefit of survival rate after restoration of spontaneous circulation following cardiac arrest.

\section{References}

1. Sipos et al. (2008): Wien Tierarztl Monat 95, 28-38.

2. Janata et al. (2007): Crit Care Med 35, 569-572.

3. Weihs et al. (2009): Resuscitation, in press.

4. Adrie et al. (2004): Curr Opin Crit Care 10, 208-212.

5. Frink et al. (2009): Scand J Trauma Resusc Emerg Med 27, 49

This study was supported by a grant from the "Bürgermeisterfonds der Stadt Wien", Project No: AP07075BGM. 


\title{
P.599
}

\section{Chemotherapy induces partial remission of mast cell leukemia in a minipig}

\author{
$\underline{\text { Wolfgang Sipos }}^{1}$ Johannes Hirschberger $^{2}$ Wolfram Breuer ${ }^{2}$ Isabella Zenker ${ }^{2}$ Sabine Elicker ${ }^{3}$ \\ 1. University of Veterinary Medicine Vienna, Vienna, Austria; 2. Ludwig-Maximilians University Munich, Munich, Germany; \\ 3. Veterinary Practice Entenfellner, Stössing, Austria
}

\begin{abstract}
Introduction
Mast cell tumors are classified as cutaneous or systemic. Cutaneous mast cell tumors represent a comparably frequent malignancy in dogs and cats, but only single reports about cutaneous mast cell tumors in pigs and only one referring to a case of porcine mast cell leukemia (1) can be found in the literature. In the following, a chemotherapy in a porcine leukemia patient is presented for the first time.
\end{abstract}

\section{Case Report}

This report describes a chemotherapeutic approach of treating mast cell leukemia in a three-year old, male-castrated Göttingen miniature pig weighing $27 \mathrm{~kg}$. The animal had been submitted to the clinic due to a mildly depressed behaviour lasting for a month and a mild anemia. Hematologic analysis (Cell-Dyn 3500 ${ }^{\circledR}$; Abbott) including a modified Wright stain led to the diagnosis of a mast cell leukemia. Three days after the diagnosis of mast cell leukemia, chemotherapy was started based on a protocol applied in dogs suffering from cutaneous mast cell tumors (2). Induction therapy comprised weekly IV infusions of $2 \mathrm{mg} / \mathrm{m} 2$ vinblastine sulfate for four weeks. Concomitantly, $2 \mathrm{mg} / \mathrm{kg}$ prednisolone were given daily per os. IV infusions were performed under general anaesthesia achieved with IM application of 20 $\mathrm{mg} / \mathrm{kg}$ ketamine and $2 \mathrm{mg} / \mathrm{kg}$ azaperone. Combined chemotherapy led to a reduction of total leukocyte count from $30.6 \mathrm{G} / \mathrm{l}$ to $25.3 \mathrm{G} / \mathrm{I}$ and a reduction of mast cell count from $18.05 \mathrm{G} / \mathrm{l}$ to $10.57 \mathrm{G} / \mathrm{l}$. Animal's behaviour became undisturbed again and no adverse side effects could be observed. For the next two weeks, no vinblastine was given. During this time, mast cell number increased to $35.34 \mathrm{G} / \mathrm{l}$. As a consequence, weekly vinblastine applications were restarted and prednisolone was replaced by oral $50 \mathrm{mg}$ cyclophosphamide boli supplemented by oral 20 mg furosemide boli, both given every second day. Furosemide administration aimed at achieving an accelerated clearance of cyclophosphamide metabolites and at abrogating the establishment of a hemorrhagic cystitis. Again, no adverse side effects could be recorded, but mast cell number continued to increase and peaked at $55.59 \mathrm{G} / \mathrm{l}$ one month thereafter. The multiagent protocol was applied for 6 weeks. Then vinblastine was given in a two-week interval. After 17 treatment rounds corresponding to an overall treatment period of half a year, overall behaviour became depressed again and feed consumption was decreased. At that point, total white blood cell number had increased to $83.7 \mathrm{G} / \mathrm{l}$ with a mast cell count of $33.48 \mathrm{G} / \mathrm{l}$. At the same time, red blood cell count had dropped markedly. Therefore, the owner decided to stop treatment and to euthanize the animal.

At necropsy a reduced body condition, an anemic state, a splenomegaly, and gastric ulcers were evident. Histologically, an infiltration of inner organs as well as bone marrow by neoplastic cells, which stained multifocally positive for CD117 and more diffusely positive for chloroacetate esterase, could be found.

\section{Discussion}

Minipigs, which are kept as pets, are increasingly cared for at a higher medical standard as conventional pigs (3). Unfortunately, there is a lack of suitable treatment regimens of systemic mast cell tumors. Therefore, humans suffering from mast cell leukemia are treated mainly by corticosteroids and antihistamines. Prognosis for human mast cell leukemia is poor. Also in dogs systemic mast cell tumors are bad responders to chemotherapeutic interventions (4). In the presented case, the initial response to a vinblastine-prednisolone combination protocol was quite satisfying with mast cell number decreasing during the first four weeks of chemotherapy. Due to the following relapse another cytotoxic agent, cyclophosphamide, was included, but was unsuccessful in averting tumor progression.

\section{References}

1. Bean-Knudsen et al. (1989): Vet Pathol 26, 90-92.

2. Davies et al. (2004): J Am Anim Hosp Ass 40, 124-130.

3. Sipos et al. (2007): J Vet Med A 54, 504-511.

4. Thamm and Vail (2001): Small Animal Clinical Oncology, 1st ed., pp. 261-282. 


\title{
P.600
}

\section{Improved neurologic deficit score for pigs in a cardiac arrest model}

\author{
Katharina Haas ${ }^{1}$ Michael Holzer ${ }^{2}$ Alexandra Graf ${ }^{2}$ Fritz Sterz ${ }^{2}$ Wolfgang Weihs ${ }^{2}$ Andreas Janata ${ }^{2}$ Wilhelm Behringer $^{2}$ Wolfgang Sipos $^{1}$ \\ 1. University of Veterinary Medicine Vienna, Vienna, Austria; 2. Medical University of Vienna, Vienna, Austria
}

\section{Introduction}

Cardiac arrest is unique in that when the heart stops, many otherwise healthy organs suffer injury, particularly the brain, the myocardium, and the endothelium. Therefore the goal of resuscitation is to restore victims to full health, including good neurological outcome. Resuscitation research studies the pathophysiology of acute terminal states and clinical death. Acute and short-term experiments in animals help to better understand mechanisms of dying and to evaluate the short-time effect of new resuscitation protocols. To evaluate long-term benefit of potential new treatments on neurologic recovery in resuscitation research, clinically realistic long-term outcome studies including evaluation of neurologic and histologic brain damage in animals high on the phylogenetic scale are necessary.

Recently, our group published a novel and reliable score for evaluation of neurologic outcome in pigs after cardiac arrest.1 The neurologic deficit score (NDS) consists of four equally weighted sections: mental status, breathing pattern, cranial nerve reflexes, and motor and peripheral sensory functions. Aim of this study was to re-evaluate and optimize the neurologic examination procedure in order to make it even more observerindependent by elucidating and then topping off the critical points of the NDS.

\section{Materials and Methods}

16 female Large White pigs (32 kg in the mean) were put into ventricular fibrillation cardiac arrest of 15 min duration followed by cooling to achieve different degrees of preservative hypothermia for $40 \mathrm{~min}$. After resuscitation (ROSC: restoration of spontaneous circulation), controlled ventilation was for $24 \mathrm{~h}$, and intensive care for 3 days. Neurologic evaluation was done independently by two observers, blinded to the experiments, at $72 \mathrm{~h}$ and 9 days post ROSC. The critical points of the NDS as published by Sipos et al. (2008), namely the evaluation of the mental state and, interestingly, the breathing pattern, were optimized.

\section{Results}

A significant difference in the NDS score between hypothermia groups was found (f-test $p=0.0127$ ). No significant difference in the NDS score was found between the two observers. The ICC (intra-class correlation coefficient) determined using analysis of variance components for the NDS score was $20.7 \%$ indicating that $20.7 \%$ of the total variability can be explained by the variability between the pigs. The largest contribution to the total variability came from the variability between time points (intervisit $\mathrm{SD}=68.22$ ). Furthermore, a large contribution came from the variability between hypothermia groups (inter-treatment $S D=47.15)$. The large variation over days and treatments also explained the small ICC. Practically no contribution to the total variability came from the variability between the observers (interrater SD $<0.001$ ), which indicates a good repeatability of the score between observers.

\section{Discussion}

The evaluation of a pig's mental state is very tricky under special situations, as for sure is the immediate post-no-flow time, as pigs are, by their nature, psychically very autonomous animals. However, the optimized NDS showed to be even more examinerindependent than the already published one, as also the mental state as well as the breathing pattern were now judged the same by both investigators in the majority of cases. At the same time, also the published overall performance category (OPC) score (1) was optimized (data not shown). The OPC score gave nearly 100 $\%$ identical results by both investigators and may be even more appropriate than the NDS in specific clinical settings.

\section{References:}

1. Sipos et al. (2008): Wien. Tierarztl. Monat. 95, 28-38. 


\title{
P.601
}

\section{Ovariectomy and calcium shortage have no obvious effect on bone metabolism in adult sows}

\author{
Wolfgang Sipos ${ }^{1}$ Martina Rauner ${ }^{2}$ Peter Pietschmann ${ }^{3}$ \\ 1. University of Veterinary Medicine Vienna, Vienna, Austria; 2. Carl Gustav Carus Universitätsklinikum Dresden, Dresden, Germany; \\ 3. Medical University of Vienna, Vienna, Austria
}

\section{Introduction}

For a long time, osteoporosis was ascribed nearly exclusively to an increased activity of osteoclasts, but now it has been recognized, that also depressed osteoblastogenesis contributes to osteoporosis. Recently, osteoimmunology has emerged as a central area of interest as peripheral blood cells, cytokines, and other soluble immunomodulators exert their impact on a series of osteopathologies including osteoporosis (1). Thus, osteoporosis has been recognized to involve more than simply a skeletal system with decreased bone mineral density. Consequently, new therapeutic strategies need to be developed. To date, the majority of basic osteologic experiments has been performed using rodents, which have a lot of advantages in terms of laboratory management, but also large animal models might be beneficial for osteoporosis research due to biomechanical issues.

Multiparous sows - in contrast to nulliparous sows - being fed a standard diet containing $1.5 \%$ calcium exhibited significantly increased plasma PTH and calcitriol levels at 8 months post ovariectomy and alkaline phosphatase levels at 12 months post ovariectomy. No significant changes concerning bone chemistry and histomorphometry could be observed (2). On the other hand, there have already been successful efforts to establish an osteoporosis minipig model (3). Ovariectomy in 4 months old minipigs resulted in a $6 \%$ decrease in bone mineral density (BMD), $15 \%$ in bone volume, and $13 \%$ in trabecular number, and an increase of $15 \%$ in trabecular separation, whereas ovariectomy in combination with a mild nutritive calcium shortage $(0.75 \% \mathrm{Ca} 2+)$ led to a $10 \%$ reduction in vertebral BMD and significant increases in final erosion depth and vertebral marrow star volume. These data implicate, that an ovariectomy alone is sufficient to induce an osteoporotic phenotype in (mini)pigs, which can be even more pronounced by restricting calcium supply.

\section{Materials and Methods}

32 Large White sows aged 33.5 months in the mean were included and distributed equally to four groups:
1. sham-ovariectomy, $0.75 \% \mathrm{Ca} 2+$
2. sham-ovariectomy, $0.3 \% \mathrm{Ca} 2+$
3. ovariectomy, $0.75 \% \mathrm{Ca} 2+$
4. ovariectomy, $0.3 \% \mathrm{Ca} 2+$

Blood samples were collected by venipuncture every 2 months. The experiment was terminated after 10 months. Serum levels of following bone metabolism markers were determined by commercially available ELISA kits: receptor activator of nuclear factor$\mathrm{KB}$ ligand, osteoprotegerin, calcitriol, $\mathrm{PTH}$, bone specific alkaline phosphatase, osteocalcin, CICP, pyridinoline, and crosslaps.

\section{Results}

Analyses of indicated marker kinetics revealed no meaningful trends in general or differences between the four groups and thus gave no evidence of any ovariectomy- or calcium shortageinduced change in bone metabolism.

\section{Discussion}

Interestingly, ovariectomy and calcium shortage alone as well as a combination of these two did not lead to any effect on bone metabolism as delineated from the analysed marker kinetics. This is quite surprising, as other authors (2) found increasing PTH, calcitriol, and BAP plasma levels in sows of the same age group as the ones we investigated, although those were only ovariectomized but fed a standard diet. On the other hand, most investigators analysing the effects of ovariectomy and/or calcium shortage used minipigs or growing pigs, thus making a comparison with the data obtained from our study difficult.

\section{References}

1. Rauner et al. (2007): Int Arch Allergy Immunol 143, 31-48.

2. Scholz-Ahrens et al. (1996): Z Ernährungswiss 35, 13-21.

3. Mosekilde et al. (1993): J Bone Miner Res 8, 1311-1321.

This study was supported by a grant from the Austrian Wissenschaftsfonds, Project No: P20337-B13. 


\title{
P.602
}

\section{Cytokine modulation of yeast derivatives investigated on a lipopolysaccharid-activated monocyte-macrophage cell line}

\author{
Anja Ganner; Christian Stoiber; Manuela Pelz-Studlar; Simone Schaumberger; Gerd Schatzmayr \\ Biomin Research Center, Tulln, Austria
}

\section{Introduction}

Cytokines are signaling molecules which play an important role in cellular communication. Modulation of cytokine secretion may offer novel approaches in the prevention and treatment of a variety of diseases in human and veterinary medicine. TNFalpha, IL-1, IL-6 are important cytokines which are secreted by immune cells such as macrophages after endotoxin (LPS) exposure and are involved in endotoxic shock which can cause severe economic losses in swine production. Therapeutical approaches in veterinary field aim to minimize or to prevent inflammatory parameters triggered by endotoxins. Counteraction of proinflammatory cytokines with feed additives such as yeast derivates might be an interesting approach.

Yeast derived beta-glucan fractions are described in the literature to interact with cytokines.

Aim of the present study was to investigate the effect of different yeast derivatives such as beta-glucans, whole yeast, yeast extract and yeast nucleotides on nitric oxygen (NO) production, pro-and anti-inflammatory cytokine levels after LPS stimulation of the murine monocyte-macrophage cell line J774A.1.

\section{Materials and Methods}

Monocyte-macrophage cell line:

J774A. 1 is a BALB/C mouse derived monocyte-macrophage cell line isolated from a tumour. Cells were cultured in tissue culture flasks at $37^{\circ} \mathrm{C}$ in DMEM complete growth medium supplemented with $10 \%$ fetal bovine serum. Subcultures were prepared by scraping adherent cells 1 to 2 times per week and re-seeded 1:4 into fresh media.

Cell culture assay:

J774A.1 cell number and viability were assessed by trypan blue dye exclusion on a Neubauer hematocytometer. For the experiment, cells were seeded at a concentration of 106 cells/ well in a 24-well tissue culture plate. Cells were incubated for 48 hours with LPS of E. coli 0127:B8 and the yeast derivative, which was either yeast derived beta-glucan, yeast nucleotide, yeast extract or whole yeast $(0.1 \%, 0.02 \%, 0.01 \%$ solutions in phosphate buffered saline). Produced nitric oxygen was determined after 48 hours of incubation in the supernatant of the cultures using a Griess reagent which provides a colorimetric reaction. Pro-inflammatory cytokines IL-1, IL- 6, TNF-alpha and the antiinflammatory cytokine IL-10 were detected with ELISA assay (R\&D Systems).

\section{Results}

Yeast extract and yeast nucleotides inhibited LPS induced NOproduction between 20 and 50\%; beta-glucan fraction up to $80 \%$ depending on the used concentration. Whole yeast did not affect LPS induced NO-production. IL- 6 was inhibited between 20 and $40 \%$ by beta-glucan, by yeast nucleotide around $20 \%$. Whole yeast did not inhibit IL-6 on a notable level. IL-1 was inhibited to $70 \%$ by a yeast nucleotide product, between 40 and $100 \%$ by beta-glucan and to $50 \%$ by whole yeast. TNF-alpha was inhibited around $40 \%$ by nucleotides, beta-glucans and also by whole yeast. All yeast derivatives tested induced IL-10 production.

\section{Discussion}

The present results revalue that yeast derivatives inhibited LPS induced pro-inflammatory cytokines and elevate anti-inflammatory IL-10 levels. However, the mechanism behind, the mode of action is not yet understood. A fully chemical characterization concerning mannan-and glucan content and nucleotide profile might be illuminating.

In conclusion, yeast derivatives which interfered in this in vitro study with IL-1, IL-6, TNF-alpha and IL-10 might be an interesting approach in the control and prevention of endotoxic shock in swine production and this might impact positively on animal health. However, it is too early to estimate to what extent these expectations are going to be fulfilled in vivo. In any case they justify further multidisciplinary research in this field.

\section{References}

Olson et al. (1996); Infection and Immunity 64(9): 3548-3554

Bedirli et al. (2007); Shock 27(4): 397-401 


\title{
P.603
}

\section{Isoimmune thrombocytopenic purpura in newborn piglets: a case report}

\author{
Elena Galletti ${ }^{1}$ Giuseppe Merialdi $^{1}$ Andrea Antonelli² Elisa Brini ${ }^{3}$ Laura Fusaro $^{3}$ \\ Giuseppe Sarli Maria C. Fontana ${ }^{1}$ Brunella Spaggiari ${ }^{1}$ Paolo Martelli4 \\ 1. Istituto Zooprofilattico Sperimentale della Lombardia e dell'Emilia Romagna, Bologna, Italy; 2. Veterinary Practitioner, Ravenna, Italy; \\ 3. Veterinary Clinic Department, University of Bologna, Bologna, Italy; 4. Department of Animal Health, University of Parma, Parma, Italy
}

\section{Introduction}

Isoimmune thrombocytopenic purpura (ITP), firstly described by Stomorken et al. in 1963, is a sporadic disease affecting newborn piglets, mainly from secondiparous or multiparous sows. Large White and Landrace are the most affected breeds. ITP is an immune-mediated disease subsequent to colostral transfer antiplatelet (PLTs) antibodies produced by sow against the antigens originating from the boar semen (Saunders and Kinch, 1968). Multiple haemorrhages develop in piglets when platelets are lower than 30.000-50.000/ $\mu$ l, leading to death the whole litter or a part of it. The Authors describe a case of ITP occurred in a farrow to finish pig herd.

\section{Materials and Methods}

An hemorrhagic syndrome occurred in 28 litters of a farrow to finish pig herd between May 2008 and Jan 2009. Only one of the sows was primiparous. Sows had been inseminated during 3 days using mixed semen from different boars. During the first week of age seventeen percent of the piglets showed haemorrhagic diathesis, characterised by multiple haemorrhages, spontaneous pethechiae and diffuse ecchymoses. The death rate ranged from $0 \%$ to $50 \%$. Six piglets were submitted to necropsy, histological bacteriological, virological and toxicological examinations. Furthermore, 6 piglets with haemorrhagic syndrome and 3 coetaneous healthy piglets from the same farrowing batch were submitted to complete blood cell count (BCC).

\section{Results}

Hemorrhagic lesions characterised by multiple haemorrhages, spontaneous pethechiae and ecchymoses were detected in piglet skin, subcutaneous tissue, inner organs and mucous/serous membranes. The most affected sites were head, abdomen, groin and soles. Kidneys and lymph nodes presented disseminated hemorrhages and hemorrhagic suffusions were evident in mesocolon and epicardium. Histology confirmed the presence of multivisceral interstitial hemorrhages, extramedullary hematopoiesis and interstitial pneumonia. Bacteriological, virological and toxicological examinations provided negative outcomes. $\mathrm{CBC}$ results are presented in the following table.
Table 1.

\begin{tabular}{|l|c|c|}
\hline & ITP (mean) & Healthy (mean) \\
\hline Hemoglobin (Hb) gr \% & 5,5 & 9,3 \\
\hline Hematocrit (HCT) \% & 17,5 & 28,2 \\
\hline RBCs/mm3 & 2.525 .000 & 4.390 .000 \\
\hline PLTs /mm3 & 33.557 & 241.133 \\
\hline Leukocytes/mm3 & 6.750 & $4.833,3$ \\
\hline MCV fL & 69,9 & 64,3 \\
\hline MCHC gr \% & 32,2 & 33,1 \\
\hline MCH pgr & 22,6 & 21,3 \\
\hline RDW \% & 22,1 & 19,2 \\
\hline NRBC/100 WBC & 13,2 & 1,7 \\
\hline Reticulocytes \% & 12,2 & 2,35 \\
\hline Lymphocytes $/ \mathrm{mm} 3$ & 2.358 & 1.692 \\
\hline Neutrophils/mm3 & 3.225 & 2.867 \\
\hline Eosinophils/mm3 & 113,05 & 120 \\
\hline
\end{tabular}

\section{Discussion}

On the basis of laboratory results ITP was diagnosed. BCC showed a marked decrease in PLTs (absence of PLT aggregation), $\mathrm{Hb}, \mathrm{HCT}$ and RBCs in affected animals. Moreover, a pattern of rigenerative anemia and reticulocytosis was detected. For differential diagnosis, infectious and toxic diseases were ruled out. Combining all data a diagnosis of an immuno-mediated disease was suggested. The marked thrombocytopenia and the absence of direct or indirect signs of erythrocytolisis allowed to rule out the haemolytic disease of newborn pigs (Taylor 2008). From Jan 2009 the insemination practice was modified using the semen from one boar only for each sow. Consequently, one boar was associated with the occurrence of the diseases in offspring and it was culled.

\section{References}

Saunders C.N., Kinch D.A. (1968) J. Comp. Path. 78. 513-525. Stormorken H. et al. (1963) Nature. 198. 1116-1117. Taylor D.J. (2006) “Pig diseases" 8 ed. Cambridge. Burlington Press. 


\title{
P.604
}

\section{Agents isolated in pericarditis cases from pigs in the nursery, growing and finishing in Brazil}

\author{
Jose L. Santos; Lucas F. Santos; Daniel L. Santos; Hellen P. Pasqualon; Walter V. Guimaraes
}

Microvet, Vicosa, MG, Brazil

\section{Introduction}

Pericarditis in pigs can be caused by various pathogens, such as Haemophilus parasuis, Pasteurella multocida, A. pleuropneumoniae, A. suis, Streptococcus suis and Mycoplasma sp (Mores, 2007). Pericarditis is the inflammation of the parietal and visceral surfaces of the pericardial cavity. May occur independent of other lesions, but is often observed in association with a pleurisy. Among the various forms of pericarditis, fibrinous pericarditis is more commonly found. It's characterized by the presence of a serous fluid and marked fibrin deposition on the surfaces of the pericardium and epicardium, leading to heart failure (Jones et al., 2000). This study aims to identify the mainly agents involved in fibrinous pericarditis in pigs in the nursery, growing and finishing.

\section{Materials and Methods}

We evaluated 129 cases of fibrinous pericarditis in animals with ages raging from 20 to 106 days. Swabs were collected from pericarditis, then added $3 \mathrm{ml}$ PBS and plated on blood sheep agar and blood sheep agar with staff nurse. The plates were incubated a at $37^{\circ} \mathrm{C}$ for 24 to 48 hours. And were performed the morphology identication of the colonies and biochemical tests for confirmation of the agent. And serology for $\mathrm{H}$. parasuis and $\mathrm{S}$. suis were performed in Microvet laboratory.

\section{Results}

We obtained a result quite varied among the several agents. The mainly agent observed was the $\mathrm{H}$. parasuis (Hps) 38/129, distributed as: non serotypable (14/129), serotype 5 (10/129), serotype
4 (4/129), serotype 1 (3/129), serotype 2 (3/129), serotype 12 $(2 / 129)$ and serotype 14 (2/129). Other agents were observed such as: P. multocida type A (Past A) 20/129 and P. multocida type D (Past D) 12/129. S. suis 8/129, distributed as: serotype 2 (4/129), serotype 3 (2/129), serotype 7 (1/129) and serotype 8 (1/129). A. pleuropneumoniae (APP) 4/129, A. suis (3/129). Some co-infection were observed between APP and the Past A (1/129), Hps serotype 12 and the Past D (1/129), Hps non serotype and Past D (1/129) and S. suis serotype 2 and Past D (1/129). And 40 animals had were negative for bacterial isolation (Graphic 1).

\section{Discussion}

This study allows us to conclude that the onset of pericarditis is actually connected to various agents as described by Mores, in 2007. However, it is noteworthy that the $\mathrm{H}$. parasuis has a great contribution to pericarditis. The serotype 5 is closely associated to conditions of Glasser's disease in Brazil (Santos et al. 1998), and was found in most reported cases of pericarditis caused by this agent, like $\mathrm{H}$. parasuis non serotypable. Other agents such P. multocida A and D, can also has great importance in the development of fibrinous pericarditis.

\section{References}

1. Jones, T. C. et al. Patologia Veterinária. 2000, 1005-12.

2. Mores, N. In: Sobestiansky, J.; Barcellos, D. Doenças dos suínos. Gráfica Editora Pallotti. 2007, 609-10.

3. Santos, J.L. et al. The frequency of Haemophilus parasuis serovars in Brazil. Proceedings of the IPVS Congre ss. 1998, 15: 278.

Graphic 1: Agents involved in pericarditis from pigs in Brazil

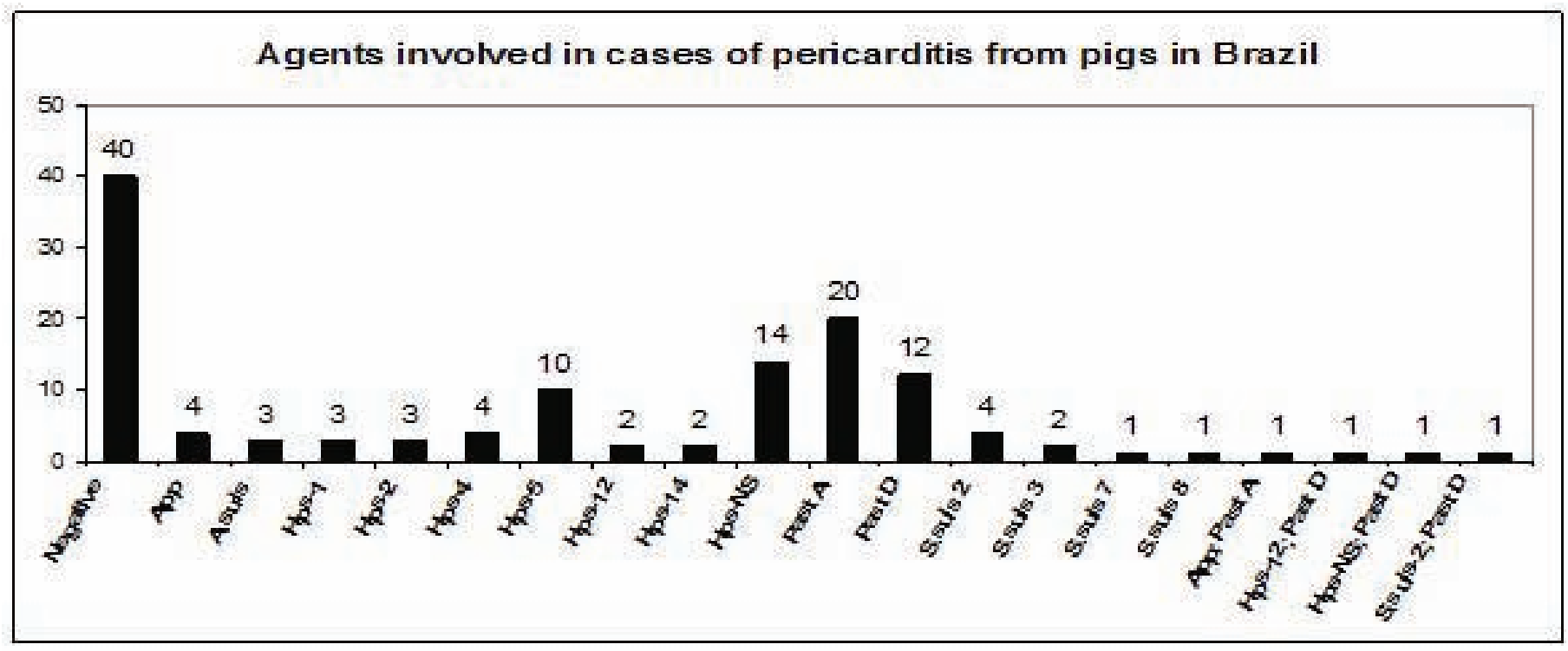




\title{
P.605
}

\section{Positional and functional genomics to resolve genetic disease resistance in swine}

\author{
Gerald Reiner \\ Department of Veterinary Clinical Sciences, Justus-Liebig-University, Giessen, Germany
}

\section{Introduction}

This review deals with genetic disease resistance in pigs as a prospective opportunity to improve swine production. Even under favourable terms of housing and management, infectious diseases are wide-spread, and have to be ranked among the major sources for production losses, decreased production efficiency and suffering in international pig production. Thus, breeding for disease resistance might be the prophylactic measure of first choice. Increased resistance to a certain pathogen or even a group of pathogens would reduce infectious pressure, followed by a decreased incidence of disease. Using disease resistant breeds is already of considerable importance in some livestock species. Examples with highest significance are the resistance to coccidiosis and Mareks disease in fowl, to trypanosomiasis and ticks in cattle, to mastitis in dairy cattle, and to gastro-intestinal nematodes in sheep. In pigs, however, examples of disease resistance in commercial breeding programs are sparse. The prime example is resistance to fimbriated F18 Escherichia coli, the cause of postweaning diarrhoea and edema disease. It is an exceptional case of single-gene control of disease resistance. Other examples include the breeding for improved immune responsiveness, and resistance to fimbriated F4 Escherichia coli (neonatal diarrhoea). Today worldwide efforts are concentrating on marker development and genomic selection strategies to improve genetic disease resistance. In spite of the currently limited commercial application in swine, a wide range of genetic variation in disease resistance has been observed regarding different bacterial, viral and parasitic diseases. The problem is to recognize breeding animals with favourable or unfavourable gene variants. Their identification is impeded by highly variable and influential farm-specific environmental effects (e.g. load of pathogens, immunity, housing, feeding and management conditions), the polygenic mode of inheritance in most of the resistance traits, the limited availability of animal models, and the limited knowledge on pathogenicity of several porcine diseases. Improved knowledge of the porcine genome combined with the tools and technologies developed in the context of the genome projects are creating new opportunities to dissect the genetic control of complex traits, including host responses to infection. Thus, one alternative might be to identify the gene variants responsible based on informative families tested under defined environmental conditions. Upon the development of a genetic marker for disease resistance, breeding stock could then be chosen by marker-assisted selection independently from environmental factors. The aim of our studies is to develop such animal models and to describe disease resistance on a molecular basis. Our models deal with the Pseudorabies virus (PrV; Reiner et al., 2002), Sarcocystis miescheriana (S.m.; Reiner et al., 2007), Salmonella typhimurium, PRRSV (Reiner et al., 2010), and $A$. pleuropneumoniae (APP).

\section{Materials and Methods}

Potential host-pathogen models are studied based on accurate infection models with purebred breeds or lines. Functional genome analysis with DNA microarray techniques gives first hints on candidate genes. They are combined with positional genome analysis (QTL[quantitative trait loci] analysis) to define candidate genes worth looking for single nucleotide polymorphisms (SNPs) that might be useful as markers in future selection.

\section{Results and Discussion}

Our results show marked differences in resistance to PrV, S.m., PRRSV and APP. Moderate to high heritabilities point towards favorable and unfavorable gene variants. QTL analysis has identified the chromosomal regions involved, and some candidate genes have been suggested for further research. In spite of the polygenic character of disease resistance, our results verify that also single outstanding loci, suitable for selection are involved. We have further provided evidence for common mechanisms of resistance in different disease models. This is of high significance for future breeding programs, because it implies that selection towards the resistance to a specific pathogen might also increase resistance against other pathogens. Although there is still a long way from the model to the marker, our results and those of others give hope for applicable functional markers in the near future.

\section{References}

Reiner et al. 2002. J. Gen. Virol. 83, 167-172.

Reiner et al. 2007. Genomics 89, 638-646.

Reiner et al. 2010. J. Anim. Breed. Genet., in press. 


\title{
P.606
}

\section{Unfolding the genetic architecture of clinical-chemical and haematological traits in swine}

\author{
Gerald Reiner; Hermann Willems \\ Department of Veterinary Clinical Sciences, Justus-Liebig-University, Giessen, Germany
}

\section{Introduction}

Clinical-chemical and haematological traits are essential parameters for veterinary practice to evaluate the health status of individuals and herds. A major concern with the interpretation of haematological values is their pronounced variability caused to a great extent by non-systematic factors. Differences in clinicalchemical and haematological traits within breeds and populations provide evidence for genetic factors as primary cause, but little is known about the genetic architecture underlying these traits, especially in swine. Involvement of additive genetic variance of some of these traits argue for the existence of favourable and unfavourable gene variants that are responsible for phenotypic variation. Identification of these variants might improve the diagnostic use of such traits in the future. It might also provide a measure to advance general health of pig populations by selection. Linkage mapping using dense genetic maps is a straightforward approach to locate genes that control inherited diseases. The effects of gene loci contributing to this phenotypic variation were considered undetectable until the introduction of quantitative trait loci (QTL). QTL mapping may be a valuable first step towards the identification of genes and causal polymorphisms responsible for variability of clinical-chemical and haematological traits. The aim of the present study was to detect and evaluate QTL for such traits in swine, and to define potential candidate genes.

\section{Materials and Methods}

The experiments were based on a Pietrain/Meishan F2 family. 139 F2 pigs were consecutively included into the study. All pigs were clinically healthy at the start of the experiment and free of internal and external parasites. In the course of eleven study periods, groups of five to eleven pigs were housed in tiled boxes of $16 \mathrm{~m} 2$ on straw beddings and fed ad libitum with a customary cereal diet (energy content: $11.5 \mathrm{MJ} / \mathrm{kg}$ ) without food additives throughout the whole period of observation. Blood samples were taken at four time points, representing baseline values (day 0 , directly before challenge with Sarcocystis miescheriana), acute (day 14 post infection [p.i.]), sublinical (day 28 p.i.) and chronic (day 42 p.i.) stage of Sarcocystosis. Further details on clinical and clinical-chemical patterns of Sarcocystosis have been described elsewhere (Reiner et al., 2006). A broad aspect of clinicalchemical and haematological traits was analysed by standard procedures. QTL analysis was based on 101 informative microsatellite markers using the web-based application "QTL express". For more details see Reiner et al. (2007).

\section{Results}

54, 38, 27, and 38 QTL for clinical-chemical, erythroid, blood gas, and leukocyte traits have been mapped, respectively. 15, 9, 5, and 9 QTL, respectively were genome-wide significant, the remaining showed chromosome-wide significance. 30, 40, 42, and 45 QTL were associated with different traits during basal stage, acute, subclinical and chronic disease, respectively. Many of the QTL involved were only detected under the burden of the model disease on the metabolism. A variety of candidate genes for different traits has been defined based on their physiology and chromosomal location (e.g. erythropoietin and erythropoietin-receptor: red blood cells; GOT1: aspartate-aminotransferase; and many others). SNPs identified within such candidate genes significantly improved the effects of the QTL and allowed to retrospectively detect QTL effects even on basal traits that had not been visible before. Also the confidence intervals for the QTL were significantly reduced.

\section{Discussion}

Our study confirms that clinical-chemical and haematological traits in swine are influenced by multiple genes, partly representing reasonable effects on their traits. The data represent a significant first step to define candidate genes and to identify the associated polymorphisms. Identification of the molecular basis of this variation has the potential to improve future diagnostics. As individuals with sound values of crucial clinicalchemical and haematological traits should need a higher burden to reach pathological thresholds, breeding animals with favourable gene variants might be selected for higher robustness of individuals and herds in the future.

\section{References}

Reiner et al. 2006. Vet. Parasitol. 145, 2-10.

Reiner et al. 2007. Genomics 89, 638-646. 


\title{
P.607
}

\section{Development of genetic markers for the resistance against infections of the respiratory tract of swine (RePoRI)}

\author{
Gerald Reiner; Natalie Bertsch; Hermann Willems; The RePoRI Consortium \\ Department of Veterinary Clinical Sciences, Justus-Liebig-University, Giessen, Germany
}

\section{Introduction}

Animal health is one of the major concerns in production economics, animal welfare, and food safety. Genetic predispositions of different breeds to infection is well documented for all major domestic species and for many diseases. RePoRI, which is processed in a network of six co-operating research facilities, is a follow-up project of IRAS (Infection and resistance in the respiratory tract of swine) which is granted by the Federal Ministry of Education and Research of Germany. In the scope of the RePoRI project following major tasks are to be conducted: 1) breeding of a segregating F2 family from two pig breeds differing in susceptibility to Actinobacillus (A.) pleuropneumoniae, 2) aerogen exposure to the pathogen with clinical, phenotypical, and pathological-anatomical characterization of animals, 3) genotyping of the F0, F1, and F2 population, 4) expression analysis of the lymph nodes and/or lungs in the most extreme quartils, 5) QTL analysis and identification of putative positional candidate genes, 6) eQTL analysis, 7) identification and characterization of functional and pseudo-functional SNPs, and 8) modelling of host-pathogen interactions and developing of hypotheses accounting for host resistance.

The task of the present study is to provide information about positional candidate genes derived from QTL and eQTL analysis. To achieve this, the following objectives had to be established: i) a panel of informative microsatellite markers with a distance no more than $15 \mathrm{cM}$, ii) multiplex PCR conditions for marker amplification, and iii) genotyping of the complete F2 pig families. Additionally, informative microsatellite markers should be positioned on the physical pig map.

\section{Materials and Methods}

The F0 family consisted of two (relative) resistant boars and 6 susceptible dams which were mated to generate a F1 family with a total of 21 animals. Rearing and infection of the segregating F2 population is in progress. Primers to amplify microsatellites were taken from the USDA list (www.marc.usda.gov/ genome/swine/swine.html) and were synthesized (biomers,
Germany) with forward primers labelled with DY-780 or DY-680. In a first PCR markers were tested for informativity with DNA extracted from blood of the 2 resistant boars and 6 susceptible dams. All PCR reactions were performed in a total volume of 10 $\mu \mathrm{l}$, consisting of $5 \mu \mathrm{l}$ of 2 x Multiplex Mastermix (Qiagen, Germany), $2.5 \mu \mathrm{l}$ of primermix (final concentration [f.c.] $0.4 \mu \mathrm{M}$ ) and $2.5 \mu \mathrm{l}$ of template DNA (50 ng). PCR products were run on $6 \%$ polyacrylamide gels and analyzed with 1DScanEX (Scanalytics). Only PCR conditions from markers with $100 \%$ informativity were optimized. Finally, multiplex PCRs were established combining primers with identical or nearly identical annealing temperatures using the same PCR setup as described above. Only primer concentrations had to be optimized so as to generate bands with equal or nearly equal intensity.

Multiplex PCR reactions were run with three different touchdown protocols each consisting of 23 cycles. Denaturation $\left(95^{\circ} \mathrm{C}\right.$, $30 \mathrm{sec}$ ) and extension $\left(72^{\circ} \mathrm{C}, 30 \mathrm{sec}\right)$ steps were identical for all three protocols, only annealing temperatures varied. The first 8 cycles had annealing temperatures of 70,65 , and $63^{\circ} \mathrm{C}$, the following 8 cycles 68,60 , and $59^{\circ} \mathrm{C}$ and the last 7 cycles 66,55 , and $55^{\circ} \mathrm{C}$, respectively with annealing times of $90 \mathrm{sec}$.

\section{Results and Discussion}

A total number of 434 microsatellites were tested for informativity, of which unexpectedly only $188(43.3 \%)$ proved to be informative. The average distance of the microsatellite markers was 14.5 cM. Nevertheless, a few gaps with distances greater than $15 \mathrm{cM}$ could not be closed because either the microsatellite markers were not informative or there were no further microsatellites available in the corresponding maps for the respective chromosomal region. 174 microsatellite markers were combined in 50 multiplex reactions with 3 to 4 markers per reaction and were tested with animals from the F0 and F1 generation. In conclusion, a panel of informative microsatellite markers has been established for the genotyping of F2 pigs from the ongoing challenge with $A$. pleuropneumoniae, in order to find markers for the resistance to this important respiratory disease in swine. 


\title{
P.608
}

\section{Mapping QTL for innate immune response in a Duroc x Pietrain resource population}

\author{
Muhammad J. Uddin; Mehmet U. Cinar; Dawit Tesfaye; Ernst Tholen; Chirawath Phatsara; Christian Looft; Karl Schellander \\ Institute of Animal Science, Bonn, Germany
}

\section{Introduction}

Cytokines and Toll-like receptors are basic components of the innate immune system. Immune responses are reported to be under the genetic control. QTL mapping and candidate gene studies have been widely used to investigate genetic variation, to identify genes and chromosomal regions contributing to immune response. Therefore, this research was conducted to identify chromosomal regions controlling the variability in cytokines (IL-2, IL-10 and IFN- $\gamma$ ) and Toll-like receptors (TLR-2 and TLR-9) production by QTL analysis.

\section{Materials and Methods}

A total of 334 Duroc x Pietrain F2 animals used for QTL analysis was genotyped by 82 genetic markers. Blood was collected from pigs at 52,83 and 115 days of age and serum was separated for phenotyping. IL-2, IL-10 and IFN- $\gamma$ were measured by sandwichELISA (Invitrogen). TLR-2 and TLR-9 were measured by in house developed indirect-ELISA (CosmoBio). Descriptive statistics were performed by using SAS (vs. 9.2) whereas QTL Express (Seaton et al., 2002) was used for both single and two-QTL analysis.

\section{Results}

The level of IL-10 and IFN $\gamma$ were increased over the time from 52 to 115 days of age. The variations of production were found significant between 52 and 83 days for IFN- $\gamma(P \leq 0.05)$ and IL-2 $(P \leq 0.001)$. In case of IL-2, the differences between 83 and 115 days, and between 52 and 115 days were also significant $(P \leq 0.001)$. In all time points, TLR-9 responses were higher than that of TLR-2. TLRs production variation between 52 and 83 days, and between 83 and 115 days were significant $(P \leq 0.001)$. Th1 type cytokines (IL-2 and IFN- $\gamma$ ) were 5 - 6 times lower than that of Th2 type cytokine (IL-10). Date of sampling, month of birth and year of birth were found to have significant effect on immune traits. Parity, litter and sex had also effect on these innate immune parameters at some age points. A total of 67 single QTL were detected by QTL Express on almost all porcine autosomes, of which 38 for cytokines (IFN- $\gamma$, IL-2 and IL-10) and 29 for TLRs (TLR-2 and TLR-9). All the QTL were significant at 5\% chromosome-wide level, of which 12 and 52 QTL were significant at $1 \%$ and $5 \%$ genome-wide level, respectively. A total of 38 QTL were detected for cytokines, of which 12, 9 and 17 QTL influenced IFN- $\gamma$, IL-2 and IL-10, respectively. A total of 29 QTL regions influencing Toll-like receptors were identified, of which six for TLR-2 and 23 for TLR-9. Besides single QTL, ten QTL were identified by two-QTL model of which three were in coupling phase and two were in repulsion phase. Most of the QTL were identified on SSC $3,5,11,12$ and 17. IFN- $\gamma, I L-10$ and TLR-9 are found to be controlled by the chromosomal regions of their location but all innate immune traits are influenced by multiple chromosomal regions implying multiple gene action.

\section{Discussion}

Most of our QTL are supported by the presence of genes which play immunologically important roles. TOLLIP, TRAF6, IRAK4 and CASP8 are found to be influencing TLRs production whereas IL3, IL5, IL13, IL 10, IFN- $\gamma$ and BPI are influencing cytokines in this study. The QTL knowledge of cytokines and TLRs may contribute towards a more complete understanding of the innate immune system in terms of whole animal physiology. Furthermore, these findings will be helpful to research in these QTL regions to identify new cytokines and their utilization in marker-assisted selection to improve the innate immune response and disease resistance in pigs.

Table: Summary of significant QTL for innate immune traits using QTL Express under two-QTL model.

\begin{tabular}{|c|c|c|c|c|c|c|c|}
\hline SSC & Trait & Position (cM) & Position (cM) & F-value & $F$-value & Sig a & $\mathrm{Sig}^{\mathrm{a}}$ \\
\hline & & QTLA & QTLB & 2vs1 & $2 \mathrm{vs} 0$ & $A$ & B \\
\hline 5 & TLR9 D-83 & 78 & 161 & 5.9 & 5 & *** & * \\
\hline 14 & TLR9 D83 & 35 & 142 & 5.9 & 4.6 & *** & * \\
\hline 18 & TLR9 D115-83 & 103 & 0 & 6.4 & 6.7 & ** & *** \\
\hline 18 & TLR9 D83 & 7 & 100 & 6.4 & 4.7 & *** & * \\
\hline 18 & IL10 D115 & 20 & 104 & 5.2 & 4.6 & *** & ** \\
\hline \multicolumn{8}{|c|}{$\begin{array}{l}\text { asignificant threshold of the F-value determines if the QTL reached the significance level under } 2 \\
\text { vs } 0 \text { QTL ( } 2 \text { degrees of freedom), or } 2 \text { vs } 1 \text { QTL ( } 1 \text { degree of freedom); with * }{ }^{*} \text { chromosome-wide } P \\
<0.05 \text {; ** genome-wide } P<0.05 \text {; *** genome-wide } P<0.01\end{array}$} \\
\hline
\end{tabular}

\section{References}

Seaton G, Haley CS, Knott SA, Kearsey M, Visscher PM: QTL Express: mapping quantitative trait loci in simple and complex pedigrees. Bioinformatics 2002, 18(2):339-340 


\title{
P.609
}

\section{The comparison of the base alignments of D-loop region in the mitochondrial DNA of Ryukyu wild boars with those of the others}

\author{
Yoshikazu Adachi; Chihiro Nagahama \\ School of Agriculture, Ibaraki University, Ami-machi, Japan
}

\section{Objective}

Small type wild boars and large types live in Japan and Ryukyu wild boars are small type. The range of Ryukyu wild boars is not in Taiwan, China, and the other east Asia. They were in Vietnam and were different from those in Taiwan, China, and other east Asia. Our aim is to analysis the genetic diversity of Ryukyu wild boars.

\section{Materials and Methods}

Samples of Ryukyu wild boars have been collected in Ishigaki Island, Nago City and Kadena Cho in Okinawa Island while 9 samples of wild boars in Honshu island e.g. Large type, were collected in Yamaguchi, Ibaraki and Miyagi Prefectures.

DNA extract, PCR and sequencing: Template DNA extracted from the root of the hair was used for PCR and the primers consisting of ; Forward 5'-GGAGACTAACTCCGCCATCA-3' and Reverse 5'-TGTCCCGTAACCATTGACTG-3' were used. Sequencing has been performed using BigDye Terminator V3.1 Cycle Sequence kit by ABI PRISM 3100 sequencer. PCR-RFLP has been studying on analysis of Hinf I digested PCR product.

\section{Results and Discussion}

The base alignments of $D$-loop region in the mitochondrial DNA of Ryukyu wild boars and others have been analyzed. Especially, the base alignment of Ryukyu wild boar was consistent among the Ryukyu wild boar, and the homology between the Ryukyu wild boar and Japanese wild boar was $98 \%$ while a cluster unlike a Japanese wild boar on the phylogenetic tree based on D-loop was drawn. Ryukyu is a province name of Okinawa Prefecture in Japan and located on southern islands. On the other hand, the presence of a cross breed pig called Inobuta in the wild boar which was captured in Okinawa Prefecture was confirmed and we perform attempted to perform PCR-RFLP using PCR products by Hinf I digestion of a D-loop part fragment with Hinf I site. The results, demonstrated the distinguishment of Ryukyu wild boar from other wild boars, Inobuta and a pig completely (Fig.1).
Figure 1 The profiles of Hinf I digested D-loop fragment with 227bp between Ryukyu wild boars and the other wild boar and pigs.

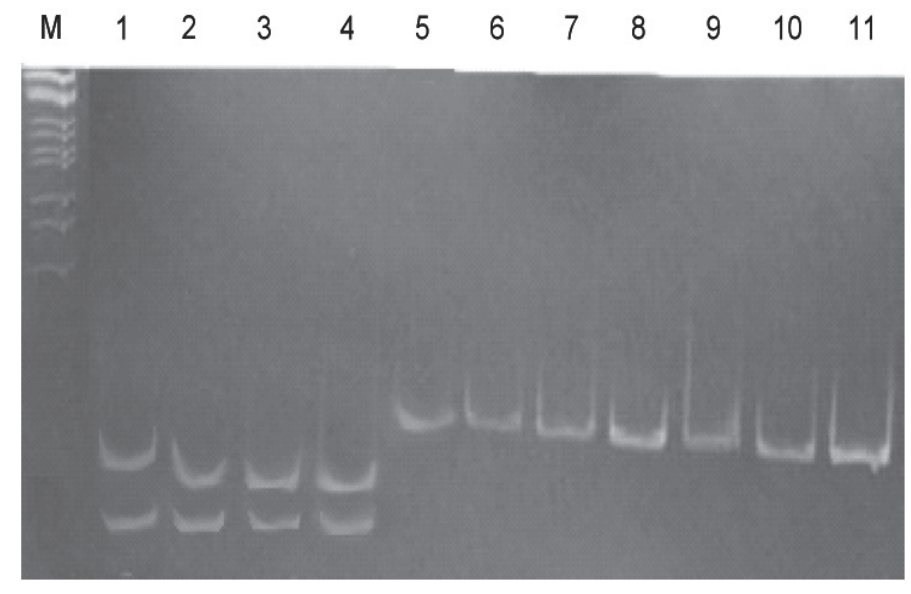

1 4, Ryukyu wild boars; 5 11, Other wild boars including Inobuta. 


\title{
P.610
}

\section{Single-Nucleotide Polymorphisms in collagenous lectins and other innate immune genes associated with common infectious diseases of pigs}

\author{
$\underline{B r a n d o n}$ N. Lillie $^{1}$ E. James Squires ${ }^{3}$ Natalie D. Keirstead ${ }^{2}$ Gordon E. VanderVoort ${ }^{3}$ Mohsen Jafarikia ${ }^{4}$ Brian Sullivan $^{4}$ M. Anthony Hayes ${ }^{1}$ \\ 1. Dept. of Pathobiology, OVC, University of Guelph, Guelph, ON, Canada; 2. Ross University of Veterinary Medicine, Basseterre, Saint Kitts and Nevis; \\ 3. Dept. of Animal and Poultry Science, University of Guelph, Guelph, ON, Canada; 4. Canadian Centre for Swine Improvement, Inc., Ottawa, ON, Canada
}

\section{Introduction}

Pigs vary widely in their susceptibility to many common viral and bacterial pathogens, both between breeds and between different litters on the same farm, suggesting there may be a genetic basis for the variation. If so, genetic selection for higher innate disease resistance should be possible. We have been identifying genetic defects in innate immune genes involved in innate resistance to infectious disease. Previously we identified porcine mannan-binding lectins (MBLs) and ficolins, two collagenous lectins, as bacteria-binding proteins in porcine plasma [1,2]. Within the genes encoding the two porcine MBLs we identified single-nucleotide poly-morphisms (SNPs) similar to those found in the human MBL gene that predispose humans to infections $[3,4,5]$. Some SNPs alter the structure and function of the protein while others decrease the amount of the protein produced. Earlier studies suggest these SNPs are more frequent in pigs having common infectious diseases $[6,7]$. This study looked for associations between these SNPs and SNPs found in other collagenous lectin genes with animals having common diseases and pathogens, as well as the effects of these SNPs on production. For comparison, we included known or newly identified SNPs in other porcine innate immune genes.

\section{Materials and Methods}

DNA was collected from 1324 healthy Duroc, Landrace or Yorkshire/Large White pigs from production facilities in Canada and the United States and 461 diseased pigs submitted to the Animal Health Laboratory (Guelph, ON). Pigs were genotyped for a panel of 27 innate immune SNPs by Sequenom MassARRAY (Analytical Genetics Technology Centre, Toronto, ON). Gross necropsy and histological evaluation were used to classify the pigs into disease groups, with pathogen grouping based on bacteriology and virology tests as requested by the case pathologist. Genotype frequencies (\% of animals possessing the mutant allele) were compared between healthy pigs and pigs in disease and pathogen groups. Associations between SNPs and an array of production parameters were also examined (birth weight, days to $100 \mathrm{~kg}$, litter size, backfat, carcass lean yield, mortality etc ).

\section{Results}

Of the 27 SNPs examined, three SNPs in three different collagenous lectin genes were most consistently associated with the disease and pathogen groups. In the MBL2 gene, encoding $\mathrm{MBL}-\mathrm{C}$, the A allele at position -1081 in the promoter region was approximately twice as common in all disease groups (healthy: $16.6 \%$, diseased: $30.3-37.5 \%)$. The same allele was more frequent in 4 of 6 bacterial groups and in pigs with PRRSV identified (pathogens: 36.9-44.1\%). Similarly a miscoding SNP in the surfactant protein $\mathrm{A}$ gene was more frequent in all disease groups (healthy: $45.8 \%$, diseased: $66.0-75.4 \%$ ) as well as 4 of 6 bacterial groups and PRRSV and PCV-2 positive groups (pathogens: 72.083.0\%). The third collagenous lectin SNP was in the MBL1 gene, encoding MBL-A. The T allele at position 273 was more frequent in all disease groups except serositis (healthy: $31.4 \%$, diseased: $50.5-54.7 \%$ ), as well as in 4 of 6 bacterial groups and the PRRSV group (pathogens: $54.2-66.7 \%)$. In the other innate immune genes, the C(96)T SNP in galectin-4 (8 of 14 disease and pathogen groups) and the C(1919)T SNP in Toll-like Receptor 5 (7 of 14 disease and pathogen groups) were significantly more frequent most often. Significant associations between SNP frequency and production parameters were rare.

\section{Discussion}

Out of 27 SNPs, five were significantly associated with most of the common diseases and pathogens of swine examined. The identification of several mutations consistently more frequent in pigs with infectious diseases, but without significant negative effects on production, suggests that genetic selection for disease resistance based on some innate immune genotypes might be possible. Further work to examine genetic defects in the entire set of genes involved in innate disease resistance is required. Innate immune genotypes that reduce the severity of infectious diseases might facilitate marker-based selection, decrease production costs, improve animal welfare, and reduce antimicrobial use in pork production.
References
1. Lillie BN, et al. Dev Comp Immunol 2006; 30:954-65.
2. Brooks AS, et al. Dev Comp Immunol 2003; 27:835-44.
3. Ip WK, et al. Immunol Rev 2009; 230:9-21.
4. Garred P. Biochem Soc Trans 2008; 36:1461-6.
5. Gupta K, et al. Indian J Med Res 2008; 127:431-40.
6. Lillie BN, et al. Dev Comp Immunol 2007; 31:830-46.
7. Lillie BN, et al. Immunogenetics 2006; 58:983-93. 


\title{
P.611
}

\section{Traceability and genetic evaluation in order to improve the swine products quality}

\author{
$\underline{\text { Sam Balasch }^{1}}{ }^{\text {Joan Tibau }}{ }^{2}$ Joaquim Soler ${ }^{2}$ Marina Gispert ${ }^{2}$ Maria Angels Oliver ${ }^{2}$ Agnès Juvanteny ${ }^{1}$ Glòria Cucurull ${ }^{1}$ Paula Grassi ${ }^{1}$ \\ 1. Alea! Technological Centre of the Animal Life, Masies de Roda, Spain; \\ 2. Institute for Food and Agricultural Research and Technology - IRTA, Monells (Girona), Spain
}

\section{Introduction}

The purpose of the Project is the development and implementation of a traceability system along the production process, starting in the focus of reproductive sows and finishing when the product arrives at the slaughterhouse as loin and ham. This traceability will allow us to evaluate the genetics provided by Gepork in a productive and a meat quality scale.

Total traceability will allow us to monitor the treatments that animals would have received along their productive lives and will allow us to obtain meat products free of antibiotic.

Finally, a customers' survey will be done in order to discover the customers' priorities referring to the gene of porcine stress.

\section{Material and Methods}

**Selection of 150 hybrid sows LDxLW and 45 boars, assigned for coverage.

**Specific software and devices (electronic eartags and reading terminals for the farms).

- Identification by electronic eartags of all the animals taking part in the Project.

- Coverages in batches of 25 sows; with an interval of 6 weeks.

- Monitoring of many processes: gestation, birth, breastfeeding and weaning (feeding, weight, treatments, etc.).

- Extraction of a biological sample of each animal.

- Monitoring of different indicators: daily growth, feeding daily consumption, corporal composition, etc. in 3 genders: male, female and immunocastrated male pigs.

- Evaluation of the productive results of the descendants of the boars used in each one of the coverages of the 6 batches.

- Monitoring of some indicators in the slaughterhouse (individual weight before the slaughtering, carcass pork flesh weight, fat thickness, etc.) and monitoring of the traceability in the slaughterhouse referring to loin and ham.

- Slaughtering in controlled conditions at $110 \mathrm{Kgs}$., including:

1. Analysis before slaughtering (alive animal weight, interpretation of back fat and loin).

2. Analysis in the slaughterhouse (carcass pork flesh weight, back fat thickness -G34FOM- and muscle -MFOM-, pH, etc.).

3. Analysis of carcass pork flesh quality in the butchering room ( $\mathrm{pH}$ and $\mathrm{CE}$ in loin and ham -pHuLT, pHuSM, CEuLT and CEuSM-, color, veined, muscle area in loin, etc.).

- Evaluation of the incidence of the porcine stress gene by means of the crossbreed of boars of the three genetics (NN, $\mathrm{Nn}$ and $\mathrm{nn}$ ) with sows negative to the stress gene.

- Elaboration of a consumers' survey about ham and fresh loin, addressed to 200 consumers.

\section{Results}

We got $100 \%$ of traceability in controlled conditions in the slaughterhouse and $97.01 \%$ of traceability in the commercial slaughterhouse.

We discovered that genetics and gender affect the carcass pork flesh (\% loin and fat), as the descendants of NN animals have more fat thickness and less \% loin that the descendants of $n n$ genetics.

Referring to gender, female pigs have better values than entire male pigs (best carcass performance, \% loin, fat thickness and muscle thickness -MFOM-). The immunocastrated male pigs have a bigger alive weight, but carcass performance is worse than females' carcass performance, they are less conformed and MFOM values are just sufficient.

\section{Conclusions and Discussion}

First of all, we verified that it is possible to ensure the traceability of the evaluated animals from the farm until the slaughterhouse.

Referring to carcass quality, the descendants of $\mathrm{Nn}$ boars can be placed between the descendants of $\mathrm{NN}$ and $\mathrm{nn}$, but referring to meat quality values, the results obtained are better, overall in the NN boars that are descendants of the crossbreed of NNxNP, thanks to the analysis of Microsatellite Moleculars Markers (MMM).

Referring to conformation, the descendants of nn boars are more conformed than the descendants of NN boars. 


\title{
P.612
}

\section{Extraction of DNA using a technique pork non-invasive sampling hair}

\author{
Eva Dolores ${ }^{1}$ Reyna Rojas ${ }^{2}$ Jose Herrera ${ }^{2}$ Adelfa Garcia ${ }^{3}$ Clemente Lemus ${ }^{4}$ Jhanina Garbay ${ }^{1}$ Giovanna Penuelas $^{1}$ \\ 1. UAEM, Toluca, MEX, Mexico; 2. Colegio de Postgraduados, Montecillos, MEX, Mexico; 3. Universidad Autonoma \\ Metropolitana Xoxhimilco, Mexico, DF, Mexico; 4. Universidad Autonoma de Nayarit, Nayarit, Mexico
}

\section{Introduction}

There are several methods of extracting DNA, some fast, efficient and useful in the isolation of high-quality DNA extraction kits such as commercial. However, most of them are expensive and serve a small number of samples, since they have to standardize, thus arises the need for DNA extraction methodologies fast, simple and low cost allowing genomic DNA obtained good quality, free of secondary compounds, proteins and polyphenols, which inhibit DNA The Mexican regulations restricts the mobilization of semen and blood plasma within and between different regions of Mexico as a biosecurity measure, so the objective was to standardize a technique of extraction and purification of DNA from hair, non-invasive technique which allows evaluation of animals from different sources without being stressed at the time of sampling.

\section{Materials and Methods}

Different farms were sampled in the country, pig hair was collected from pulling back sharply with the thumb, index and middle, removing the hair bulb of about 50 hairs per animal. We obtained the DNA extraction and purification from pig hair for being a non-invasive sample. The methodology developed was based on a combination of various pre-established protocols and modified by reference to the proposal by Sambrook et al. (1994), which was to establish the extraction protocol in which they conducted a first lysis step through the detergent SDS and subsequently for total DNA purification method was applied to phenol-chloroform-isoamyl alcohol-enabling removal products degradation, which preserves the DNA for longer periods, finally washed with alcohol to eliminate waste and verify the quality of DNA in agarose gel $1 \%$.

\section{Results}

The extraction method was efficient resulting standardized DNA integrity of a good quantity and quality, a technique that allows for non-invasive samples such as hair, thus avoiding sampling techniques that involve the capture or stress on animals. In this research, the electrophoresis gels show the specificity of the DNA extraction technique hair of pigs used in this study (Fig. 1). The extraction of DNA from hair was standardized despite being listed as a protocol difficult from the standpoint of DNA extraction methods, results revealed that it is highly reproducible and reliable "almost perfect", in the form PCR amplification, all samples were amplified, these tests were conducted with the objective of finding a methodology to obtain samples without any restrictions.
Fig. 1. Agarose gel 1\%; (M) $1 \mathrm{~kb}$ marker; (1-7) DNA hair from pigs.

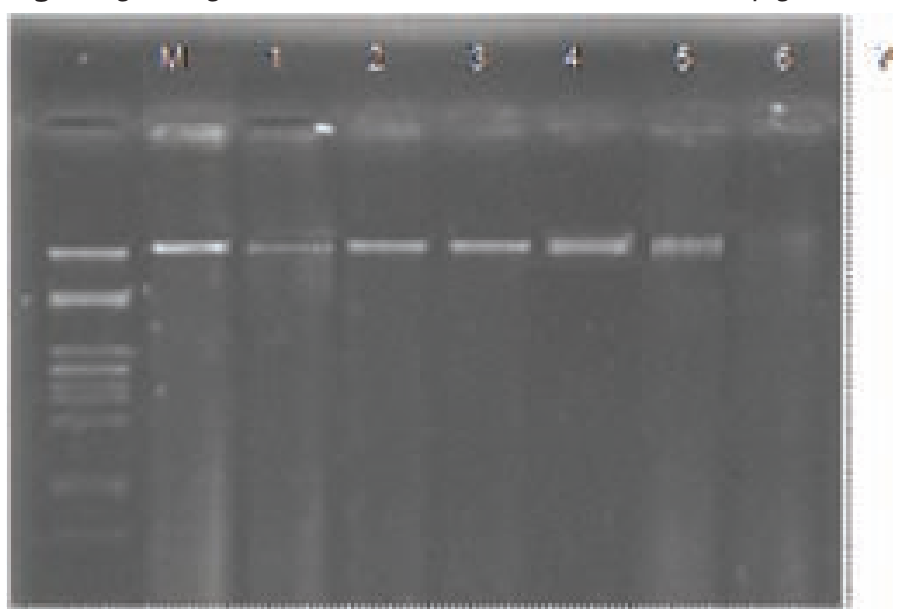

\section{Discussion}

This standardized extraction method, will allow for non-invasive samples such as hair and although they usually do not recommend this type of sample for extraction, were successful in securing a high quality genomic DNA, quantity and purity, Moller y Brinkmann (1994). Although it took a long period of incubation and careful series of steps to follow when handling, they showed a high specificity of the technique (100\%) and that often gets lost during the processing of samples, attributed perhaps a shortage of nucleated cells containing the hairs, located in the bulge or the inhibitory effect of soluble melanins generated during the DNA extraction process. This option opened doors to sample farms which hardly can be entered for biosecurity

\section{References}

Moller A. and B. Brinkmann. 1994. The STR approach. Adv. in For. Haem. 5: 336-338.

Sambrook J., F. E. Fritsch y T. Maniatis. 1994. Molecular Cloning. A laboratory manual. Cold Spring Harbor Laboratory Press. New York. E. U. A. 


\title{
P.613
}

\section{Innate immunity and management in two different herds}

\author{
Livia Moscati ${ }^{1}$ Marco Sensi ${ }^{1}$ Lorenzo Battistacci $^{1}$ Massimo Amadori ${ }^{2}$ \\ 1. Istituo Zooprofilattico Sperimentale Umbria e Marche, Perugia, Italy; 2. Istituto \\ Zooprofilattico Sperimentale Lombardia Emilia Romagna, Brescia, Italy
}

\section{Introduction}

Various environmental effects may adversely bear on welfare of farmed pigs under intensive farming conditions, which may cause chronic rather than acute stress as a long-term effect of modern husbandry techniques. These may in fact overtax the animals' coping ability and force them to long-lasting homeostatic control actions.

\section{Materials and Methods}

This investigation was carried out in winter on pigs from Danish genealogy book in two fattening farms. Farm 1 consisted of two pens housing weaned and growing-finishing pigs. In this herd there were frequent arrivals of animals of various origins, so that no all-in-all-out housing scheme was applied during the trial. A chronic respiratory syndrome by Actinobacillus pleuropneumoniae had been present for a long time with high morbidity and mortality. Herd 1 was compared with herd 2, located in the same area and consisting of two fattening pens. In this latter farm pigs were born in the same breeding unit, moved to herd 2 at a weight of some $30 \mathrm{Kg}$ and further reared up to a slaughter weight of $160-170 \mathrm{Kg}$. An all-in-all-out housing scheme was routinely applied. The overall clinical conditions of pigs were satisfactory. The time-course of clinical immunology and clinical chemistry parameters was evaluated in the two herds with such a different sanitary and management status. Blood samples from 15 randomly chosen animals of the same age were collected in herds 1 and 2 at monthly intervals in vacuum tubes without anti-coagulant. I The following parameters were investigated: haemolytic complement activity $(\mathrm{CH} 50 / 150 \mu \mathrm{l})$ as previously described by Barta (1), serum lysozyme (SL) (3), serum bactericidal activity (SBA) (2), haptoglobin (Hp) by a commercial kit (Tridelta Development Ltd).) zinc ( $Z n$ ) and iron (Fe) were assessed by a Konelab 2001 biochemical analyzer. Resultes were submitted to a statistical examination was performed by ANOVA.

\section{Results}

Results are shown in Tables 1 and 2. Animals of the herd 1 showed at time 0 a significantly higher serum haptoglobin concentration $(P<0.01)$ about two weeks after a respiratory syndrome and a trend to high serum levels was evidenced at later samplings, too. Reduced plasma levels of iron and zinc were always evidenced in herd 1, as a possible indicator of a steady-state inflammatory condition, the greatest differences being revealed at time $3(p<0.001)$. Interestingly, animals of both "problem" and healthy herd showed low SBA and high lysozyme concentrations in later samplings (Table 2) as a possible outcome of difficult adaptation during the growing phase.

Table 1. Results of the samplings $\pm d s$

\begin{tabular}{|l|c|c|c|c|}
\hline & T.0 herd 2 & T.0 herd 1 & T.1 herd 2 & T.1 herd 1 \\
\hline SBA \% & $40 \pm 32$ & $27.1 \pm 14^{*}$ & $25 \pm 14$ & $36 \pm 27$ \\
\hline CH50/150 $\mu \mathrm{l}$ & $37 \pm 26$ & $53 \pm 14$ & $50 \pm 5$ & $55 \pm 11$ \\
\hline $\mathrm{SL} \mu \mathrm{g} / \mathrm{ml}$ & $5 \pm 1$ & $5 \pm 2$ & $7 \pm 2$ & $8 \pm 5$ \\
\hline $\mathrm{Hp} \mathrm{mg} / \mathrm{ml}$ & $0.7 \pm 0.2$ & $1.7 \pm 1.2^{* *}$ & $0.8 \pm 0.3$ & $0.7 \pm 0.2$ \\
\hline $\mathrm{Zn} \mu \mathrm{g} / \mathrm{dl}$ & $76 \pm 24$ & $59 \pm 28$ & $76 \pm 18$ & $17 \pm 6^{*}$ \\
\hline $\mathrm{Fe} \mu \mathrm{mol} / \mathrm{L}$ & $19 \pm 7$ & $17 \pm 5$ & $20 \pm 8$ & $68 \pm 8$ \\
\hline
\end{tabular}

Table 2. Results of the samplings $\pm d s$

\begin{tabular}{|l|c|c|c|c|}
\hline & T.2 herd 2 & T.2 herd 1 & T.3 herd 2 & T.3 herd 1 \\
\hline SBA \% & $25 \pm 10$ & $37 \pm 24$ & $26 \pm 11$ & $13 \pm 11^{* *}$ \\
\hline $\mathrm{CH} 50 / 150 \mu \mathrm{l}$ & $62 \pm 0.2$ & $61 \pm 2$ & $47 \pm 11$ & $56 \pm 11$ \\
\hline $\mathrm{SL} \mu \mathrm{g} / \mathrm{ml}$ & $6 \pm 1$ & $3 \pm 1^{*}$ & $2 \pm 0.4$ & $5 \pm 2^{* *}$ \\
\hline $\mathrm{Hp} \mathrm{mg} / \mathrm{ml}$ & $1.3 \pm 0.7$ & $2.3 \pm 1.9$ & $1.9 \pm 2$ & $2,5 \pm 1,9$ \\
\hline $\mathrm{Zn} \mu \mathrm{g} / \mathrm{dl}$ & $80 \pm 13$ & $64 \pm 27$ & $121 \pm 22$ & $81 \pm 33^{* *}$ \\
\hline $\mathrm{Fe} \mu \mathrm{mol} / \mathrm{L}$ & $25 \pm 5$ & $19 \pm 7^{*}$ & $32 \pm 6$ & $25 \pm 8^{* *}$ \\
\hline
\end{tabular}

* Significant difference $(p<0.05){ }^{* *}$ Highly significant difference $(p<0.01)$

\section{Discussion}

Our results indicate that animal health and welfare in pigs can be monitored by combining clinical, environmental and innate immunity investigations, as a multi-disciplinary approach to animals' environmental adaptation. Most important, the important role of immunological parameters for evaluation of welfare conditions confirms that the effector mechanisms in the immune response are remarkably similar for both infectious and non-infectious stressors.

\section{References}

1. Barta V., Barta O., (1993) Testing of Haemolytic Complement and its components. In: Barta O., (ed.) Vet. Cl. Imm. Lab., Bar-Lab, Blacksburg, USA

2. Dorm W. et al. (1980). Arch. Exper. Vet. Med. 34,635-650

3. Osserman E.F et al. (1966). J. Exp. Med. 124, 921- 952 


\title{
P.614
}

\section{The interferon-alpha response in piglets at weaning}

\author{
Elisabetta Razzuoli; Riccardo Villa; Enrico Sossi; Silvia Dotti; Massimo Amadori \\ Istituto Zooprofilattico Sperimentale Lombardia Emilia Romagna, Brescia, Italy
}

\section{Introduction}

Our interest into the functions of type I interferon (IFN) under health conditions was stimulated by established models of physiological IFN response (1) and constitutive expression in lymphoid and non-lymphoid tissues in the absence of any specific inducer (2), as well as from clinical studies documenting serious inflammatory disorders in humans with a defective type I IFN response. In the veterinary field, pigs under intensive farming conditions are an interesting model for such studies, since an IFN-a response following stressing events like weaning and truck transportation has been known for a long time (3). In the present study we investigated the IFN-a response in pigs weaned at different ages.

\section{Materials and Methods}

The study was carried out in May-June 2008 on 40 Landrace $x$ Large White, randomly selected piglets of four litters. Group 1 (2 litters, 20 piglets) was weaned at 28 days of age, and group 2 ( 2 litters, 20 piglets) at 22 days of age. All piglets were given the same starter feed containing amoxicillin (400 ppm), colistin (120 ppm) and zinc oxide (2000 ppm) over the first 10 days after weaning. Pigs were clinically inspected and blood samples in vacuum tubes without anti-coagulant were collected at days 0 , $+6,+12$ with respect to weaning, in order to evaluate the most common viral infections and IFN-a production. Viral infections were investigated by PCR and antibody assays whereas IFN- $a$ production was tested by a cpe inhibition assay on MDBK cells with Vesicular Stomatitis Virus (VSV) (4). Other blood samples were collected in vacuum tubes with sodium heparin in order to assess intracellular porcine IFN-alpha and the expression of porcine IFN-alpha genes in peripheral blood mononuclear cells (PBMC). IFN-alpha in PBMC was investigated by flow cytometry, and gene expression by RT-Real Time PCR on 9 animals of group 2 according to Cheng (5).

\section{Results}

All pigs remained healthy during the whole period of observation. In particular, there was no detectable clinical difference between the two groups under satisfactory hygiene and welfare conditions. There was no evidence of infection by the usual viral agents of swine (PRRSV, PCV2, SIV, PRV) in the first 2 weeks after weaning (PCR and antibody assays). The sera of all piglets in group 2 and of 7 animals only in group 1 were IFN-a positive at day 6 after weaning. This result was confirmed by a neutralization assay of IFN-a on MDBK cells by a monoclonal antibody to porcine IFN-alpha1.

A small percentage of PBMC from several piglets at day +6 were also positive in a flow cytometry assay for intracellular porcine IFN alpha.4 and 10 animals in groups 1 and 2, respectively, were positive, and the prevalence of IFN alpha-positive PBMC showed no evident correlation with that of the sera.

The results of RT-Real Time PCR showed at day 0 the expression of the IFN alpha5 and 6 genes in 7 out of 9 piglets and of IFN alpha12, 1, 7, 11 and 2 genes in fewer pigs. All the above genes were not expressed at day +6 , and expressed again at day +12 .

\section{Discussion/Conclusions}

Our results are in agreement with previous reports about constitutive expression and secretion of IFN-alpha; in particular, the need for an IFN-a response to the weaning stress was shown to be much higher in 3, compared with 4-weeks old piglets. Transcriptional control on gene expression in PBMC coincided with peak serum IFN-a titres. These findings highlight the importance of type I IFNs as homeostatic agents in the response to environmental, non-infectious stressors.

\section{Acknowledgements}

This study was supported by the Italian Ministry of Health, grant PRC2005002.

\section{References}

1. Bocci V et al. 1985. Lymphokine Res, 4: 151-158.

2. Soos JM et al 2003. In: Thomson AW, Lotze MT eds. The cytokine handbook, vol. 1, London: Academic Press, 549-566.

3. Wattrang E et al. 1998. J Vet Med B, 45: 7-17.

4. Meager A 1987 In: Clemens MJ, Morris AG, Gearing, AJH, eds. Lymphokines and interferons, a practical approach, Oxford: IRL Press Limited, 129-147.

5. Cheng et al 2007. J. Interferon Cytokine Res, 27: 579-587. 


\title{
P.615
}

\section{Peripheral T lymphocyte changes in neonatal pigs}

\author{
Glen W. Almond ${ }^{1}$ Laetitia Bourdieu ${ }^{2}$ Guy-Pierre Martineau ${ }^{2}$ Martin Liebstein ${ }^{1}$ \\ 1. College of Vet Med, North Carolina St University, Raleigh, NC, USA; 2. Ecole Nationale Vétérinaire de Toulouse, Toulouse, France
}

\section{Introduction}

Previous studies characterized lymphocyte development and Ig production in fetal pigs $(1,2)$. The porcine immune system is well developed prior to birth, but prenatal factors influence immune function in suckling pigs (3). Furthermore, in utero transmission of pathogens also altered piglet peripheral and tissue $T$ lymphocytes (4). In contrast to controlled experiments under ideal conditions, our understanding of cell-mediated immunity (CMI) is limited for newborn pigs in commercial farms. Therefore, the primary goal of this study was to characterize peripheral $\mathrm{T}$ lymphocytes in neonatal pigs on a conventional sow farm.

\section{Materials and Methods}

The animal phase of the study was conducted on a PRRSV-stable sow herd. Four sows (parity 1, 2, 3, and 6) were identified prior to farrowing. Farrowing was closely supervised and birth order of the piglets was recorded. Whole blood samples were collected from 12 piglets per sow prior to suckling (day 0), $24 \mathrm{hr}$ after suckling (day 1) and at 7 days of age. Piglets were weighed at the time of each blood collection. Complete blood counts were performed on each sample. Samples were prepared for flow cytometry and the number and percent of CD3+, CD4+ and CD8+ lymphocytes were determined. Spearman rank correlations were used to assess associations among piglet body weights, birth order and immune cell types.

\section{Results}

Piglet body weight (BW) did not differ between day 0 (1.3+.05 $\mathrm{kg})$ and day $1(1.4+.05 \mathrm{~kg})$, but increased $(P<.05)$ at day 7 $(2.4+.08 \mathrm{~kg})$. Total lymphocyte counts increased with age; however, the percentage of lymphocytes and neutrophils did not change with age. The number of CD3+, CD4+ and CD8+ lymphocytes increased with age (Fig 2). In contrast, the percentage of CD4+ cells decreased from day 0 to day 7.

Prior to suckling, the $\%$ and number of $C D 8+$ cells were positively correlated with birth order $(r=0.47, P<.001$ and $r=0.28, P<.05$, respectively) and the percent and number of CD3+ cells were positively correlated with body weight $(r=0.32, P<.02$ and $r=0.48$, $\mathrm{P}<.001$, respectively). For the three days of blood samples, there was a positive correlation among the number of CD4+ and CD8+ cells with body weight and day.
Figure 1. Changes (mean+SEM) in number (top) and percentage (lower figure) of $C D 3+, C D 4+$ and $C D 8+$ lymphocytes in neonatal pigs.

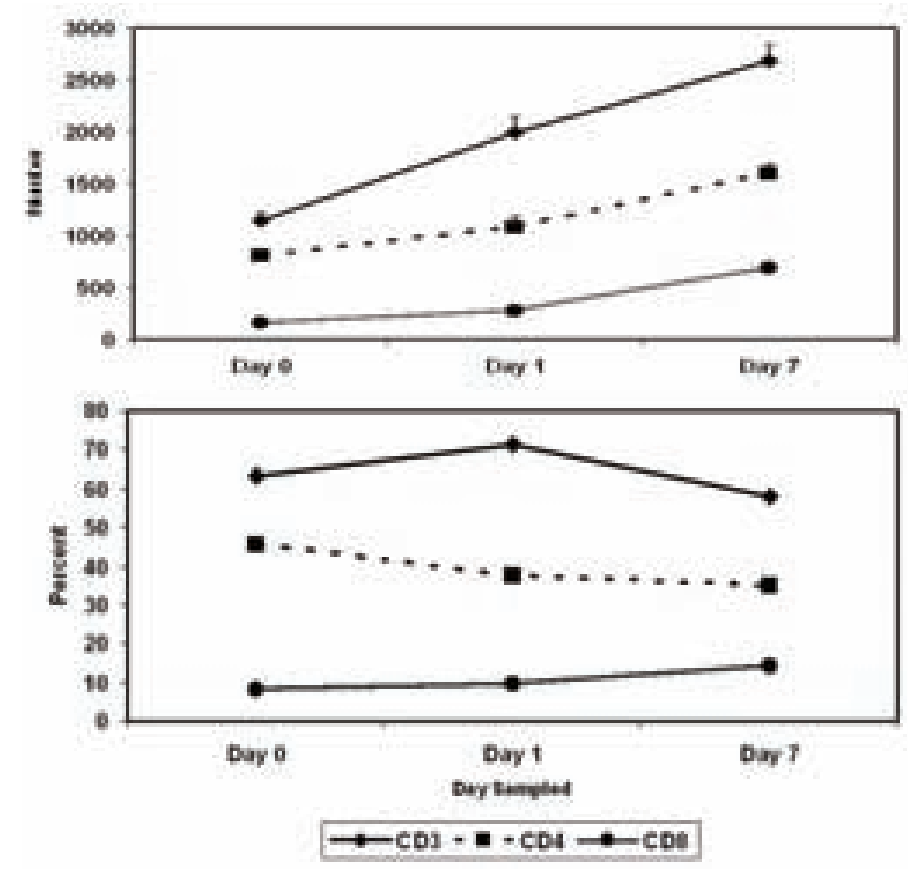

\section{Discussion}

The percentages and numbers of CD4+ and CD8+ lymphocytes agreed with previous studies of PRRSV-free pigs $(3,4)$. It is expected that the number of CD4+ cells decrease while the number of CD8+ cells continue to increase at weaning or shortly thereafter. The CD4+ TH1 and TH2 subsets bind to macrophages and antigen-specific B cells, respectively, and thus play roles in CMI. The CD8+ lymphocytes (cytotoxic T cells) are programmed to become effector cells and become more critical to immunity as the pigs mature. As shown in previous studies $(1,2)$, it is evident that newborn pigs in commercial farms are "armed" with the appropriate cells for CMI. However, our results showed that lighter piglets have lower immune cell numbers. These pigs will require additional care and perhaps, they are more susceptible to infections.

\section{References}

1. Butler, JE et al. 2009. Vet Immunol \& Immunopath 128:147-170.

2. Sinkora, M et al. 2005. Vet Immunol \& Immunopath 108:177-184.

3. Tuchscherer, M et al. 2002. Vet Immunol \& Immunopath 86:195-203.

4. Feng, WH et al. 2002. Virology 302:363-372. 


\title{
P.616
}

\section{Disease Resistance Assay for Animals (DRAA) positively associates T-cell responses with average daily gain (ADG)}

\author{
John Harding ${ }^{1}$ Crissie Auckland ${ }^{1}$ Saba Siddiqi² Caroline Billard ${ }^{2}$ Jenny Phipps ${ }^{2}$ Michel Phipps $^{2}$ \\ 1. University of Saskatchewan, Saskatoon, SK, Canada; 2. Metadis Inc., Ottawa, ON, Canada
}

\section{Introduction}

Genetic differences in antibody and cell-mediated immunity have been described, and swine selection programs based on immune responsiveness have been proposed as a means of improving health status and growth performance ${ }^{1}$. The objective of this study was to evaluate the relationship between growth performance and an individual pigs' general immune competence assessed using a novel mitogen-induced lymphocyte proliferation assay (Disease Resistance Assay for Animals; DRAA). These results are part of a larger study evaluating relationships among general immune competence, post-natal performance, and PCV2 risk.

\section{Materials and Methods}

This study was performed in a 600 sow high health farrowfinish farm using 45 PIC Camborough sows blocked by parity. Two male and 2 female piglets, representing small and large birth weight, were selected from each litter at birth and monitored until 15 weeks of age. After weaning at 3 weeks of age, piglets were reared in conventional all-in-all-out nursery and grow-finish rooms and fed commercial diets typical of western Canada. Individual weights were obtained at birth, 3, 9 and 15 weeks of age. The average daily gain (ADG) for each period was calculated. From each piglet, $5 \mathrm{ml}$ heparinized blood was collected at 3,9 and 15 weeks of age. DRAA: The individual's general immune competence was assessed using the DRAA (Metadis Inc.). Lymphocytes were isolated by density gradient centrifugation using 'Lymphoprep', then proliferative responses to a panel of mitogens were assessed as 6 replica of $10^{4}$ cell aliquots. The mitogens in the panel were chosen in order to mimic a wide array of pathogenic stimuli and each was representative of a specific T-cell molecular signaling pathway and genetic signature. Since some of the elicited receptors respond to multiple mitogens, multivariate-based mathematical analysis was used to eliminate overlaps, leading to a measurable response along 3 fundamental T-cell pathways on which the blastogenic responses relied. For each pathway a TABI score was calculated reflecting the strength of an individual's expected immune response to a pathogen. TABI 1 encompasses general $\mathrm{CD}^{+}$ $\mathrm{T}$-cell responses. $\mathrm{TABI} 2$ reflects the ratio of given $\mathrm{T}$-cell receptors specific to each individual animal. $\mathrm{TABI} 3$ represents the nature of $\mathrm{CD}^{+}{ }^{+}$subsets and the clonal expansion of B lymphoblasts present in the peripheral blood. Statistical analysis: Generalized linear mixed models (STATA v9, StataCorp, College Station, Tx) were developed to assess potential relationships between the week $15 \mathrm{TABI}(1,2,3)$ scores and ADG. Two ADG periods (weeks 9-15 and 0-15) were considered. The TABI 1, 2 and 3 scores measured at week 15 and biologically relevant cofactors that potentially impacted ADG including gender, birth weight and farrowing week were retained in the final model if $P<0.05$.

\section{Results}

Mean birth weight was $1.4 \pm 0.3 \mathrm{~kg}$. Mean body weight at 15 weeks of age was $62.5 \pm 9.0 \mathrm{~kg}$. Mean ADG 9-15 was 0.819 \pm 0.18 and mean ADG 0-15 was $0.581 \pm 0.01 \mathrm{~kg} /$ day. The week $15 \mathrm{TABI}$ 1 scores ranged from -1.37 to 3.54 ; TABI 2 scores ranged from -2.16 to 3.1; and TABI3 scores ranges from -1.99 to 1.75. The TABI 1 score was significant and positively associated with ADG 9-15 $\left(r^{2}=0.43 ;<\wedge \mathrm{em}>\mathrm{P}<0.001\right)$ and ADG 1-15 $\left(r^{2}=0.46 ; P<0.001\right)$ (Table 1). TABI 2 and TABI 3 scores were not associated with ADG during either period, nor was gender.

Table 1. Relationship between TABI 1 (wk 15) score and average daily gain from weeks 9-15 and weeks 0-15.

\begin{tabular}{|l|c|c|c|}
\hline & Beta (kg/d) & $95 \%$ Cl & $P$ value \\
\hline ADG 9-15 & & & \\
\hline TABI 1_wk15 & 0.046 & 0.006 to 0.086 & 0.024 \\
\hline Birth wt (kg) & 0.141 & 0.061 to 0.221 & 0.001 \\
\hline ADG 0-15 & & & \\
\hline TABI 1_wk15 & 0.026 & 0.007 to 0.045 & 0.007 \\
\hline Birth wt (kg) & 0.124 & 0.086 to 0.161 & $<0.001$ \\
\hline
\end{tabular}

\section{Discussion}

Many factors, including health, potentially impact the growth performance in finisher pigs. This study demonstrates that a vigorous and positive $T$ cell response, as quantified by the DRAA, is associated with enhanced growth rate. For each 1 point increase in the TABI 1 score, ADG 9-15 and 0-15 increased 46 and 26 grams/day respectively, or about $4-5 \%$. Of the 3 fundamental T-cell pathways evaluated, only the general $\mathrm{CD}^{+}$response pathway was significantly associated with growth. Surprisingly, ADG was not associated with gender in this study.

\section{References}

1. Wilkie B et al (1999) Vet Immunol Immunopathol. 72:231-235. 


\title{
P.617
}

\section{Microbiological analysis of swine tonsils collected from carcasses at slaughter}

\author{
Terri L. O'Sullivan ${ }^{1}$ Robert Friendship ${ }^{1}$ Cate Dewey ${ }^{1}$ Tim Blackwell ${ }^{2}$ David Pearl ${ }^{1}$ Susy Carmen ${ }^{3}$ Bev McEwen $^{4}$ Durda Slavic $^{5}$ \\ 1. University of Guelph, Guelph, ON, Canada; 2. Ontario Ministry of Agriculture Food and Rural Affairs, Fergus, ON, Canada; \\ 3. University of Guelph Diagnostic Virology Laboratory, Guelph, ON, Canada; \\ 4. University of Guelph Animal Health Laboratory, Guelph, ON, Canada; \\ 5. University of Guelph Bacteriology Laboratory, Guelph, ON, Canada
}

\section{Introduction}

The difficulty of sampling swine tonsils ante-mortem makes collection at the time of slaughter an obvious alternative for disease monitoring purposes (1). The funnelling of large number of animals during the slaughter process also provides an opportunity to sample a wide range of animals and animal sources on one premise. The objectives of this 7-month study were to determine the prevalence of porcine pathogens of the tonsil of the soft palate at slaughter, to determine if sampling normal or abnormal hog carcasses provides different microbiological profiles, and to determine if the slaughter plant provides a feasible sampling frame and environment for detecting and monitoring important pathogens in tonsils that have health implications for swine and humans.

\section{Materials and Methods}

Twenty (20) tonsil samples were collected every week for 20 consecutive weeks, June to December 2008, from swine carcasses at a federally inspected abattoir in southern Ontario, Canada. The sampling was performed by experienced abattoir staff during normal slaughter operations. Microbiological analyses of the tonsils were conducted by the Animal Health Laboratory (AHL) at the University of Guelph. Guelph, Ontario. Testing included bacteriological culture and identification, real-time PCR testing for PPRS virus and IHC for Porcine Circovirus-2 (PCV-2). The relationship between finding a pathogen from pigs on the hold rail (abnormal carcasses) vs. normal carcasses was examined by logistic regression. A generalized mixed model using farm as a random intercept was utilized to determine whether there was any clustering at the farm level (2).

\section{Results}

A total of 395 samples were collected of which 180 were tonsils from normal carcasses and 215 from carcasses that were on the hold rail. 264 different farms were represented and no clustering occurred at the farm level. Most commonly isolated bacterial pathogens included: S. suis (53.7\%), A. pyogenes (29.9\%), P. multocida (27.3\%), and S. porcinus (19.5\%). PRRS virus and PCV-2 were identified in $22.0 \%$ and $11.9 \%$ of the samples respectively. Tonsils had 2 times greater odds ( $\mathrm{OR}=2.16, \mathrm{Cl} 1.44-3.24)$ of being positive for $\mathrm{S}$. suis if sampled from the holdrail vs. normal carcasses. Similarly, tonsils had an $8(\mathrm{OR}=8.79, \mathrm{Cl} 4.24-18.23)$ and 7 (OR=7.51, $\mathrm{Cl}$ 2.89-19.54) times greater odds of being positive for S. porcinus and Staph hyicus respectively, if collected from the hold rail vs. normal carcasses. However, tonsils that were positive for PRRSV or PCV-2 were no more likely to be from the hold rail than from the normal carcasses. The odds were 2 times greater for tonsils that were PRRSV-positive for being positive for S. equisimilis ( $\mathrm{OR}=2.15, \mathrm{Cl}$ 1.22-3.27). However, being PRRSV positive did not increase the odds of the sample being positive for any other bacteria or PCV-2. Tonsils that were positive for PVC-2 had a 2 times higher odds of being positive for S. porcinus (OR= $2.15, \mathrm{Cl} 1.10$ - 4.22). However, being positive for PCV-2 did not increase the odds of a sample being positive for any other bacteria or PRRSV. Two samples $(0.5 \%)$ were positive for Salmonella typhimurium and then further typed. One bacterium was Phage Type 104a and the other was Copenhagen Phage Type UT1. Bio and serotyping were undertaken for all of the Yersinia enterocolitica positive (1.8\%) samples. Bio-serotype 4/0:3 was the most common type found in 5 out of 7 of the positive samples (71.4\%) The remaining 2 positive samples were bio-serotype 2/0:5, 27.

\section{Discussion}

The sampling frame and sampling method proved to be an efficacious way to collect swine tonsil tissue. Accurate tissue recovery occurred (99.7\%), the sampling protocol was not technically challenging, and plant production was minimally affected during the sampling periods. Tissue collection during the slaughter process was a superior method of tonsil tissue collection compared to reports of ante mortem techniques where only $48.9 \%$ of samples were correctly obtained by tonsil biopsy methods (1). Interestingly, tonsils that were positive for PRRSV or PCV-2 were not more likely to be positive for zoonotic pathogens or other swine pathogens with major production limiting implications.

\section{References:}

1. Bierk MD, Dee SA, Rossow KD, Collins JE, Guedes MI, Molitor TW. Experiences with tonsil biopsy as an antemortem diagnostic test for detecting porcine reproductive and respiratory syndrome virus infection in breeding swine. J Swine Health Prod. 2000;8:279-282.

2. StataCorp. 2007. Stata Statistical Software: Release 10. College Station, TX: StataCorp LP. 


\title{
P.618
}

\section{Surveillance using oral fluid samples - PRRSV and PCV2 experimental data}

\author{
John R. Prickett ${ }^{1}$ Tanja Opriessnig ${ }^{1}$ John Johnson ${ }^{1}$ Suzanne Stone ${ }^{2}$ Jeffrey J. Zimmerman ${ }^{1}$ Apisit Kittawornrat $^{1}$ \\ 1. lowa State University, Ames, IA, USA; 2. University of Minnesota, St. Paul , MN, USA
}

\section{Introduction}

Oral fluid specimens have been used extensively in human diagnostic medicine, but only recently has the concept been investigated in the production animal setting (Prickett and Zimmerman, 2010). Two studies were conducted under experimental conditions. The objective of the first study was to determine whether porcine reproductive and respiratory syndrome virus (PRRSV) and/or specific anti-PRRSV antibodies were present in oral fluids. The second study evaluated the detection of porcine circovirus type 2 (PCV2) and anti-PCV2 antibody in oral fluids.

\section{Materials and methods}

Experiment 1 (PRRSV): The onset, level, and duration of PRRSV and anti-PRRSV antibodies in serum and oral fluids was evaluated in 3 age groups of pigs (4, 8, and 12 weeks of age). Each age group consisted of 16 pigs (12 PRRSV-inoculated, 4 negative controls) housed in pens of 4 pigs each. Pigs were inoculated IM on DPI 0 with $2 \mathrm{ml}$ of inoculum containing 1 x 101.7 TCID50 of PRRSV per ml. After PRRSV inoculation, serum, buccal swabs, and oral fluids were collected at regular intervals for 63 days. Samples were assayed for PRRSV by qRT-PCR and specific antiPRRSV antibodies by ELISA.

Experiment 2 (PCV2): The onset, level, and duration of qPCRdetectable PCV2 and anti-PCV2 antibody in serum and oral fluid were evaluated over time. Four treatment groups ( 6 pigs each) were evaluated: Group 1 - negative control; Group 2 - inoculated IM and IN with PCV2a (ISU- 40895); Group 3 - inoculated IM and IN with PCV2a (ISU-40895) at 0, 4, 8 and 12 weeks; Group 4 inoculated IM and IN with PCV2a (ISU- 40895), then with PCV2b (PVG4072), and with PCV2a (ISU-4838) at weeks 0, 5, and 10, respectively. Serum from each animal and one oral fluid sample per group were collected every other day through day 14 and weekly through week 14 . Serum and oral fluid samples were assayed for PCV2 by qPCR and anti-PCV2 antibody by ELISA. In addition, anti-PCV2 antibody isotypes ( $\lg G, \lg A$, and $\lg M)$ were quantified in oral fluid samples.

\section{Results}

Experiment 1 (PRRSV): PRRSV qRT-PCR results in oral fluid samples were correlated with serum levels. $75 \%$ of the oral fluid samples collected from PRRSV inoculated pens were positive through day 28. Likewise, PRRSV-specific antibody of the lgG, $\lg \mathrm{M}$, and $\lg \mathrm{A}$ isotypes were detected in oral fluid.

Experiment 2 (PCV2): PCV2 was detected in oral fluids in all inoculated groups by day 2 (first sampling) with peak levels between days 10 and 21. Levels declined thereafter, but persisted through the end of the 98 day study. Analysis of PCV2 qPCR results found no statistically significant differences among inoculated groups either in oral fluid or serum specimens. Likewise, there were no significant differences among inoculated groups in the level of anti-PCV2 antibody in serum. Anti-PCV2 antibody was detected in oral fluids and persisted throughout the 98 day study. Anti-PCV2 IgG, IgA, and lgM were present in oral fluid by DPI 14 and persisted through the end of the study.

\section{Discussion}

The results of these two experiments showed that PRRSV and PCV2 infections were detectable in oral fluid specimens using either PCR- or antibody-based assays. These results suggest that oral fluid samples are a viable alternative to serum samples for the surveillance of PRRSV and PCV2. In the field, oral fluid sampling could form the basis of an efficient, cost effective, and practical approach to infectious disease surveillance.

\section{Acknowledgements}

This project was funded in part through Pork Checkoff funds distributed through the National Pork Board (Des Moines, lowa) and the PRRS CAP, USDA-NIFA Award 2008-55620-19132.

\section{References}

Prickett JR, Zimmerman JJ. 2010. The development of oral fluid-based diagnostics and applications in veterinary medicine. Animal Health Research Reviews (in press) 


\title{
P.619
}

\section{Prevalence of lesions found at slaughter among Swedish fattening pigs}

\author{
Nils Lundeheim ${ }^{1}$ Nils Holmgren $^{2}$ \\ 1. Swedish Univ. of Agric. Sci., Uppsala, Sweden; 2. Swedish Animal Health Service, Skara, Sweden
}

\section{Introduction}

Recording of gross lesions at slaughter has for many years been performed in Sweden by the official meat inspectors. This information is stored in a databank run by the Swedish Animal Health Service, and is used to identify herds with health problems, to improve animal welfare, and also for epidemiological studies. In this report, variation in prevalence of, as well as correlations between, gross lesions are analysed.

\section{Material and Methods}

The analyses are based on data on all fattening pigs slaughtered during 2009 at the six largest slaughter plants in Sweden $(n=2.4$ million pigs). Mean prevalence for each herd and slaughter month was calculated for seven pathological lesions (abscesses, arthritis, tail damage, enzootic pneumonia [SEP], pleuropneumonia [PP], pleuritis [PI] and Ascaris [white spots in livers; WS]). In total, final data comprised information on 729 herds and 7241 herd-month averages ( $>50$ pigs in each average).

Analysis of variance was applied, according to a statistical model including the fixed effects of slaughter plant, month and herd size (4 classes: 500-999; 1000-1999; 2000-3999; 4000- fatteners). The statistical model also included the random effect of herd. Correlations between prevalence of different lesions were estimated as residual correlations, when the effect of slaughter plant was adjusted for.

\section{Results}

Slaughter plant had a significant $(p<0.001)$ effect on all prevalences analysed. Herd size had a significant effect on the prevalence of Abscesses $(p<0.05)$ and on PP, PI and WS $(p<0.001)$, see Tab. 1.

Table 1. Mean prevalence (\%) by herd size (LSmeans)

\begin{tabular}{lccccccc}
\hline Size & Absc & Arth & Tail & SEP & PP & Pl & WS \\
\hline $500-$ & 1.5 & 1.4 & 1.3 & 3.1 & 0.2 & 6.6 & 6.5 \\
$1000-$ & 1.5 & 1.3 & 1.3 & 3.4 & 0.5 & 8.4 & 5.8 \\
$2000-$ & 1.6 & 1.3 & 1.5 & 3.6 & 0.8 & 10.5 & 3.9 \\
$4000-$ & 1.6 & 1.2 & 1.5 & 3.7 & 0.9 & 12.5 & 2.5 \\
\hline
\end{tabular}

Month had a significant $(p<0.001)$ effect on all traits analysed, except for PP $(p<0.5)$, see Tab. 2.

\begin{tabular}{llllllll}
\multicolumn{6}{l}{ Table 2. Mean prevalence (\%) by month (LSmeans) } \\
\hline Month & Absc. & Arthr. & Tail & SEP & PP & P1. & WS \\
\hline Jan. & 1.5 & 1.4 & 1.1 & 3.1 & 0.5 & 8.1 & 3.6 \\
Feb. & 1.6 & 1.5 & 1.2 & 3.0 & 0.5 & 8.2 & 3.2 \\
Mars & 1.7 & 1.5 & 1.2 & 3.3 & 0.6 & 9.0 & 3.5 \\
Apr. & 1.7 & 1.5 & 1.2 & 3.4 & 0.5 & 9.3 & 4.0 \\
May & 1.5 & 1.3 & 1.2 & 3.4 & 0.5 & 10.1 & 4.4 \\
June & 1.6 & 1.2 & 1.4 & 4.2 & 0.5 & 10.5 & 5.5 \\
July & 1.5 & 1.1 & 1.5 & 4.7 & 0.4 & 10.2 & 6.7 \\
Aug. & 1.3 & 1.1 & 1.1 & 3.4 & 0.8 & 10.1 & 6.6 \\
Sept. & 1.5 & 1.2 & 1.1 & 3.0 & 0.8 & 9.9 & 5.7 \\
Oct. & 1.5 & 1.3 & 1.5 & 3.2 & 0.6 & 9.6 & 4.6 \\
Nov. & 1.5 & 1.2 & 1.6 & 3.2 & 1.0 & 9.3 & 4.1 \\
Dec. & 1.5 & 1.3 & 2.6 & 3.7 & 0.6 & 9.6 & 4.2 \\
\hline
\end{tabular}

The following correlations ( $r>0.1$ ), between the prevalences of the lesions analysed, were highly significant $(p<0.001)$ :

Tail damage - abscesses: +0.33

Abscesses - arthritis: +0.14

Pleuropneumonia - Pleuritis: +0.30

SEP - Pleuritis: +0.19

SEP-WS: +0.14

\section{Discussion}

This investigation identified 4 primary pathological findings at slaughter which should be focused on to improve herd health and animal welfare. According to Swedish legislation, no tail docking of piglets is allowed. Prophylactic measures against tail biting, such as adequate feed and feed allowance and supply of straw, will prevent secondary abscesses (4).

PP, SEP and PI may be controlled by strict age segregated production (1). Vaccination against Mycoplasma hyopneumoniae reduces SEP and to some extent $\mathrm{PI}$. These measures are especially important in larger herds as they are associated with high prevalences of respiratory diseases $(1,3)$. Condemnations of livers due to white spots can be prevented by strategic pre-farrowing treatments of sows with anthelmintics in age segregated productions systems (2).

\section{References}

1. Beskow P. et al. 2008. Swedish Vet. J. 12;11-18

2. Holmgren N. et al. 1998 15th IPVS Congr 2:111

3. Holmgren N. et al. 201021 st IPVS, Vancouver

4. Schröder-Petersen DL. 2001. The Vet. J. 162;196-210 


\title{
Prevalence of organ lesions in fattening pigs at slaughter recorded in different slaughterhouses in Switzerland
}

\author{
Viviane T. de Vries ${ }^{1}$ Juliane Funke ${ }^{1}$ Roger Stephan ${ }^{2}$ Titus Sydler $^{3}$ Xaver Sidler $^{1}$ \\ 1. Institute of Farm Animals, Division of Swine Medicine, Vetsuisse Faculty, Zürich, Switzerland; 2. Institute for Food Safety and \\ Hygiene, Vetsuisse Faculty, Zürich, Switzerland; 3. Institute of Veterinary Pathology, Vetsuisse Faculty, Zürich, Switzerland
}

\section{Aim of the Study}

Organ lesions of slaughtered pigs can be used effectively to assess the herd health status on preharvest level. The aim of this study was to record the prevalence of organ lesions in fattening pigs at slaughter in different slaughterhouses in Switzerland, to assess their economic impact and to evaluate the introduction of a system based on codes, which will be used in all Swiss slaughterhouses.

\section{Materials and Methods}

Data were collected over the period of June 2008 until May 2009 during meat inspection from 34'706 fattening pigs in four different slaughterhouses in Switzerland. Each lesion was recorded within a code system.

\section{Results}

In total, in 3'101 out of 24'426 organs, which were assessed during meat inspection, lesions were found. The economic loss for the organs was calculated to 15'950 Swiss Francs. The following tables (Table 1-4) summarize the type of lesions in the different organs.

Based on the recorded data during meat inspection, lesions on animal level were statically compared between label and non label pigs and pigs which attend the pig health service (PHS) and those pigs which did not attend. Both label and pig health service slaughtered pigs showed significant less bronchopneumonia (Table 5).

\section{Conclusion}

Based on this project, a system recording systematically meat inspection data should be implemented in the future in Switzerland. The application of the evaluated system would help to monitor the herd health status on the preharvest level in the pig population and allow assessing the impact of the work of the pig health service.
Table 1: Ing

\begin{tabular}{|l|r|r|}
\hline $\begin{array}{l}\text { Lesions recorded during meat } \\
\text { inspection }\end{array}$ & $\begin{array}{r}\text { aumber } \\
\text { of lanss }\end{array}$ & $\begin{array}{r}\text { \% } \\
(\mathrm{n}=24 \mathbf{4 2 6})\end{array}$ \\
\hline no lesions found & 22276 & 91.2 \\
\hline brcachopneumoais & $\mathbf{5 6 7}$ & $\mathbf{3 . 0}$ \\
\hline thrombo-mbolic pneumonia & 56 & 0.2 \\
\hline focal lecions & 133 & 0.5 \\
\hline Post inflarmatory scaring & 1098 & 4.5 \\
\hline cthers & 6 & 0.0 \\
\hline
\end{tabular}

Table 2; heart

\begin{tabular}{|l|r|r|}
\hline $\begin{array}{l}\text { Lesions recorded during meat } \\
\text { inspection }\end{array}$ & $\begin{array}{r}\text { aumber } \\
\text { of hearts }\end{array}$ & $\begin{array}{r}\text { \%6 } \\
\text { (n=24726) }\end{array}$ \\
\hline no lesions found & 23056 & 94.4 \\
\hline pleuritis-pericanditis & 1261 & 5.2 \\
\hline epicarditis & 105 & 0.4 \\
\hline odhers & 4 & 0.0 \\
\hline
\end{tabular}

Table 3: tiver

\begin{tabular}{|c|c|c|}
\hline $\begin{array}{l}\text { Lesioas recorded during meat } \\
\text { inspection }\end{array}$ & $\begin{array}{l}\text { number } \\
\text { of livers }\end{array}$ & $(\mathrm{u}=24 \cdot 426)$ \\
\hline no lesions found & $23 / 316$ & 955 \\
\hline hepatisir pansizaria multiplex & 651 & 2.7 \\
\hline echinococcus & 38 & 0.3 \\
\hline others & 371 & 1.5 \\
\hline
\end{tabular}

Table 4: kidney

\begin{tabular}{|c|c|c|}
\hline $\begin{array}{l}\text { Lesibas mearded during meat } \\
\text { inspection }\end{array}$ & $\begin{array}{r}\text { number } \\
\text { of kidneys }\end{array}$ & $(n=14550)$ \\
\hline no lesions found & $10 \% 24$ & 729 \\
\hline cysts & 1653 & 113 \\
\hline interstitial nephritis & 2'193 & 148 \\
\hline hydrcnephrosis & 63 & 0.4 \\
\hline others & 61 & 0.4 \\
\hline
\end{tabular}

Table 5: PHS pigs versus non PHS pigs

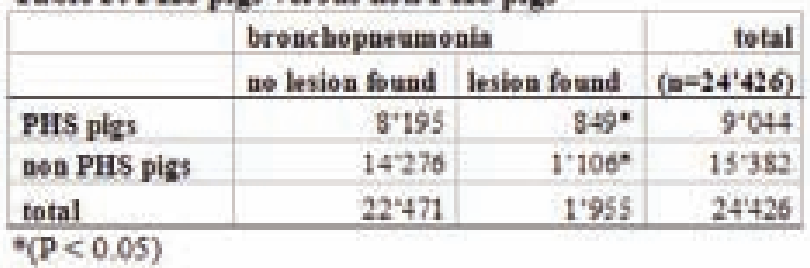




\title{
P.621
}

\section{Gross lesions at slaughter among organic pigs in Sweden}

\author{
Eva Heldmer $^{2}$ Nils Lundeheim ${ }^{1}$ \\ 1. Swedish Univ. of Agric. Sci., Uppsala, Sweden; 2. Swedish Animal Health Service, Uddevalla, Sweden
}

\section{Introduction}

The demand for more organic pigs has increased in Sweden. Production structure has changed from small farrowing-to-finish outdoors organic (OO) herds to larger herds, and also some specialized organic fattening herds. In these herds the pigs are raised indoors $(\mathrm{IO})$ with access to a small area and pasture outside the barn. Thus today a higher proportion of organic pigs in Sweden are kept indoors most of their lives. During 2009 some organic farms started to raise IO pigs in accordance with the EU rules (3). The main difference between these systems is the access to pasture or not. The certified "Swedish" organic pigs must have access to pasture four months of the year, whereas the EU-pigs (EO) must only have access to a small paddock area outside the barn.

The aim with this study was to analyse the prevalence of gross lesions at slaughter over time in organic and in conventional (C) Swedish fattening pigs.

\section{Material and Methods}

The analyses are based on data from a databank, comprising information on all fattening pigs slaughtered in Sweden. This databank is run by the Swedish Animal Health Service. Information on all gross lesions found at slaughter is stored in this databank. The Swedish organic pig herds are limited in number, and for 2009, data was available from 23 IO herds (15900 pigs), and 6 EO herds ( 8000 pigs). Thus, the certified organic pig production in Sweden is $<1 \%$ of the total pig production.

\section{Results}

Prevalence of Swine Enzootic Pneumonia (SEP) decreased continuously in C pigs between 1997 and 2001 (1) and remained at about $4 \%$ until 2009. During the same period, prevalence of SEP increased steadily among OO+IO pigs (2) (Fig.1). In 2009, when EO-pigs were introduced, the prevalence of SEP among these pigs where $7.8 \%$. In C-pigs, joint lesions have remained at a constantly low level (1-2\%) during the last 10 years. However, these lesions are 3-4 times more prevalent among $\mathrm{OO}+\mathrm{IO}$ pigs compared to C-pigs (Fig.2). In 2009, the first year of Swedish EOpigs, the prevalence of joint lesions among them was $4.5 \%$.

\section{Discussion}

$\mathrm{OO}+\mathrm{IO}$-pig production is seldom age segregated and the barns are seldom emptied or cleaned, and older pigs can infect younger ones. The change in raising system from $\mathrm{OO}$ to $\mathrm{IO}$ has increased the prevalence of SEP. The prevalence of joint lesions among $\mathrm{OO}$ and $\mathrm{IO}$ pigs increased 3-6 times during the period studied. The reason why joint lesions are more common in the organic production than in conventional herds is not completely understood. Osteochondrosis and Erysipelas infections are two etiological candidates. In conclusion, prophylactic measures against SEP may be done by applying strict age segregated production and /or vaccination against M.hyo. The etiology of the joint lesions needs to be further investigated.

\section{References}

1. Holmgren N, et.al Swedish Vet. J. 2002, 10 469-474.

2. Heldmer E, et.al. Swedish Vet. J. 2006, 58,13-19

3. COUNSEL REGULATION (EU) nr 834/2007
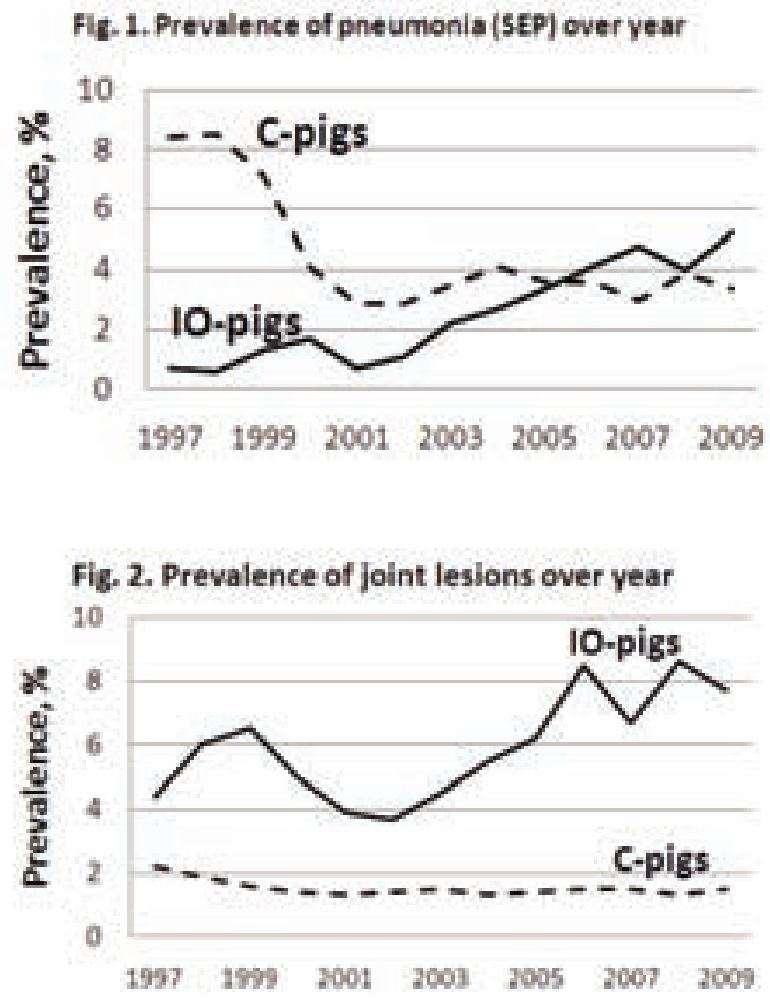


\title{
P.622
}

\section{Correlation of farm and management data with findings in organs and confiscated remains of slaughtered pigs in Swiss slaughterhouses}

\author{
Juliane Funke ${ }^{1}$ Viviane T. de Vries ${ }^{1}$ Gertraud Schüpbach ${ }^{2}$ Xaver Sidler $^{1}$ \\ 1. Institute of Farm Animals, Division of Swine Medicine, Vetsuisse Faculty, Zürich, Switzerland; 2. \\ Department of Clinical Research \& VPH, Vetsuisse Faculty, Bern, Switzerland
}

\section{Introduction}

Pathologic-anatomic findings of lung, pleura, pericard, liver, kidney and carcass in slaughtered pigs can be proper herd health status indicators. The aim of this study was, to determine whether there is a significant relationship between farm management factors, husbandry type and pathologic-anatomic findings in slaughtered pigs.

\section{Materials and Methods}

In the first part of this study, the prevalence of pathologic-anatomic lesions of lung, pleura, pericard, liver, kidney, muscle, joint and skin in 34706 pigs was documented on herd level in four Swiss slaughterhouses.

In the second part 82 pig farms were selected to analyze whether certain farm factors can be linked to the findings in slaughtered pigs. On the basis of their pathologic-anatomic findings, the farms were splitted into "case farms" (more than $30 \%$ of the pigs from one farm had a pathologic-anatomic finding) and "Control farms" (less than 10\% of the pigs from one farm had a pathologic-anatomic finding). The "case farms" were separated into farms with lesions at the pluck (S) (lung, liver, pleura, pericard) and at the carcass (T) (kidney). The category "pluck" had three subcategories: pneumonia (pn), pleuritis/pericarditis (pl/ pc) and milkspots (ms). In order to be allotted into one of these subcategories, the finding had to be present in at least $10 \%$ of the pigs from one farm. The subcategories were formed to find potential risk factors for pathologic-anatomic findings in the different organs. It was not necessary to form subcategories for the farms with findings at the carcass because all "case farms" had only lesions in term of "white spotted" kidneys.

\section{Results}

See tables.

\section{Discussion}

Pathologic-anatomic findings in slaughterhouses can be used as an objective indicator for diseases throughout the fattening period (Blaha, 1993). In Swiss slaughterhouses is a lot of potential herd health data available which could be of interest for the veterinarian and the pig health service. The data could be an assisting tool to monitor diseases, improve the herd health and to increase the food safety. So far, these data are only used for generation of bills. As it was presented before by different authors, this study was able to show that several farmand management factor can be linked to pathologic-anatomic findings.

Table 2 : risk factons (multicariable logistic tegression) (p00.05) fint lesices at the carcass

\begin{tabular}{|c|c|c|c|}
\hline vatiastit & castany & $\mathrm{OR}\left(\mathrm{S}_{3} \mathrm{C}_{\mathrm{Cl}} \mathrm{Cl}\right)$ & P-valut \\
\hline Dodens cotithd & trzalst & $122017-1452)$ & 2049 \\
\hline nateing & yes & $153(1.9-1546)$ & 0.013 \\
\hline
\end{tabular}

\section{References}

Blaha, T. (1993). Fleischwirtsch. 73, 877-8

Table 3 ; risk tacton (multivariable logistic tegression) (p.00.05) for pneumosia

\begin{tabular}{|c|c|c|c|}
\hline Furiable & catepory & $\mathrm{OR}(95 \% \mathrm{CI})$ & pralue \\
\hline coogh & yes & $128(29571)$ & 00009 \\
\hline antibiotic thenpy & no & $118(13.1079)$ & 0029 \\
\hline jeint infoction & $a$ & $45(31.2001)$ & 0032 \\
\hline
\end{tabular}

Table 4: risk factor (multivariable logistic regrestion) (pe0,05) for plemritis pericarditis

\begin{tabular}{|c|c|c|c|}
\hline vasiatic & calezary & $0 R(50 \mathrm{ct})$ & Evalue \\
\hline ecuth & yes & $9+20-455)$ & oods \\
\hline andibierie therpy & as & $106(1.4-504)$ & 8023 \\
\hline fattening dxys & $c=100$ & $11.6(25.543)$ & 0002 \\
\hline $\begin{array}{l}\text { pipeline conten } \\
\text { sefer fooding }\end{array}$ & food waty & $35(1+239)$ & 0.015 \\
\hline $\begin{array}{l}\text { soaking Aloor bever } \\
\text { wahing }\end{array}$ & as & 11.202 .5921 & 8004 \\
\hline
\end{tabular}

Table 5 : risk factoes (multivariable logistic regression) (po0.05) for milkspots

\begin{tabular}{|c|c|c|c|}
\hline vasiatie & satetony & OR $\left(E^{2} \cdot C \mathrm{CH}\right)$ & p-value \\
\hline souce of piglets & changing & $33(1.225)$ & 0042 \\
\hline fresinet maks: & na & $93.7(+1-21993)$ & 0004 \\
\hline inblei & $>2$ & $24.2(1.9 .3001)$ & 0013 \\
\hline chnülent access & yes & $13.4(13.1402)$ & 0.010 \\
\hline
\end{tabular}




\title{
P.623
}

\section{Patterns of condemnation rates in swine from a federally inspected abattoir in relation to disease outbreak information in Ontario (2005 - 2007)}

\author{
Maria R. Amezcua ${ }^{1}$ David L. Pearl ${ }^{1}$ Alejandro Martinez² Robert M. Friendship ${ }^{1}$ \\ 1. University of Guelph, Guelph, ON, Canada; 2. Maple Leaf Pork, CFIA, Burlington, ON, Canada
}

\section{Introduction}

Data sources for animal syndromic surveillance remain relatively unexplored, and may include veterinary records, and/or animal health laboratory submission files. High correlations between clinical signs on farms and the presence of lesions at slaughter have been reported. The objective of this study was to determine if changes in condemnations rates provide an additional data source for the surveillance of disease outbreaks in finisher pigs. We assessed the epidemiological relevance of these data by determining whether the timing of changes for particular pathological lesions were consistent with a documented outbreak of porcine circovirus associated disease (PCVAD) in Ontario that started in 2005 and was largely controlled by the introduction of effective PCV-2 vaccines in 2007.

\section{Material and Methods}

The data were obtained from the database of one large federally inspected packing plant in Ontario from January 2005 to December 2007. Using negative binomial models, we investigated the association between year and quarter with the rate of condemnation for the following lesions that are associated with PCVAD: pneumonia, enteritis and nephritis. Predictive rates for each condemnation category were calculated and graphed by season and year from the final model. Contrasts were performed to determine the difference of rates between season and year.

Retrospective temporal scan statistics were performed to identify the most likely temporal clusters for each condemnation category using a Bernoulli model in SaTScan. The patterns of lesions and temporal clusters were compared to PCV-2 diagnostic reports of the Animal Health Laboratory ( $\mathrm{AHL})$, at the University of Guelph (2005-2007).

\section{Results}

Based on the negative binomial models, a significant decrease in the rates of pneumonia was observed during the study period (Figure 1). In agreement with AHL reports, the pneumonia patterns reflect field infection of PCV-2 in 2005 followed by the increased and widespread use of PCV-2 vaccines in Ontario swine in 2007. In addition, we think that the initial decrease of pneumonia rates in the spring of 2006 may reflect the initiation of PCV-2 vaccines in April of 2006 in Ontario (Figure 1).

Similarly, the most likely temporal clusters for pneumonia occurred from January 2005 to March 2006. However, condemnation rates due to nephritis and enteritis increased significantly in 2007 compared to previous years (Figure 1). The most likely temporal clusters for enteritis and nephritis occurred near the end of the study period.

\section{Discussion}

These results suggest that abattoir data have potential for the surveillance of emerging pathogens as reflected by the pneumonia patterns. However, abattoir data may also suggest areas for enhanced surveillance for unexplained disease patterns not identified by laboratory submissions. Further prospective studies are required to validate the utility of these data. Abattoir data would be of more value if it could be incorporated into a surveillance program, and validated with multiple sources of swine health data.

Figure 1. Predictive rates of condemnations due to pneumonia, enteritis and nephritis from the negative binomial models.

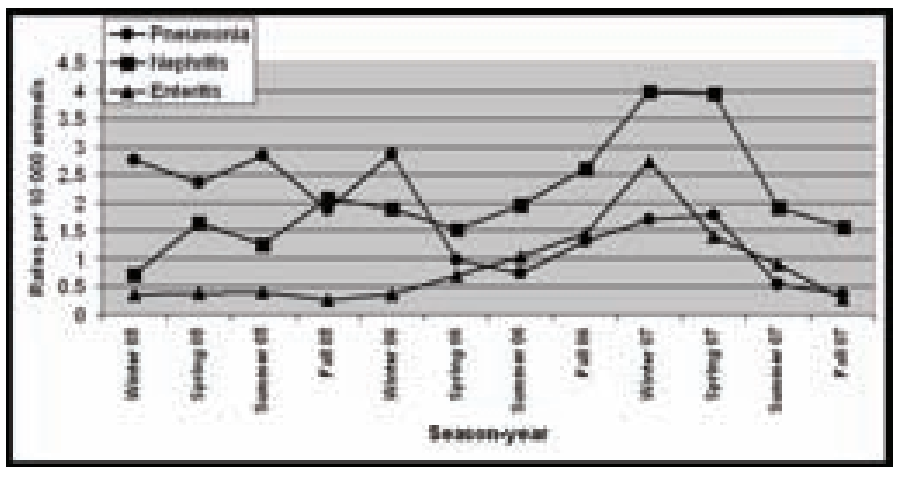




\title{
P.624
}

\section{Comparison of disease trends in swine of Ontario using active practitioner-based surveillance and passive laboratory-based surveillance (2007-2009) as sources of syndromic surveillance data}

\author{
Maria R. Amezcua; David L. Pearl; Robert M. Friendship \\ University of Guelph, Guelph, ON, Canada
}

\section{Introduction}

The growing concern of emerging and re-emerging diseases in swine populations in Ontario in 2005, led to the decision to conduct a pilot study to determine the feasibility of an active veterinary-based surveillance system referred to as the Ontario swine veterinary-based surveillance system (OSVS) (1). The OSVS recorded the occurrence/incidence of different syndromes and investigated potential outbreaks during the study period. However, the integration of OSVS data to additional data sources to validate the disease patterns observed was needed. The main objective of this study was to compare the disease patterns observed in the OSVS system with data obtained from case histories that accompanied laboratory submissions to an Ontario regional laboratory, the Animal Health Laboratory ( $\mathrm{AHL})$. In addition, the passive data obtained from submissions by OSVS members to the AHL were compared to all passive data submitted to the AHL.

\section{Materials and Methods}

Multivariable negative binomial models were used to examine the impact of data stream, season-year and body system affected (i.e., respiratory, digestive and reproductive) on submission rates.

\section{Results}

Our results showed that generally higher rates of submissions were reported to the OSVS for the body systems compared to AHL over the study period. However, over the study both OSVS and $\mathrm{AHL}$ data captured similar long-term trends of disease (e.g., respiratory) (Figure 1a). Yet, the OSVS data were also able to capture potential outbreaks that were not reflected in the laboratory-based submission data, such as a large increase in digestive cases in Summer 2008 (Figure 1b).

\section{Discussion}

Laboratory-based data may be influenced by disease awareness, and more under-reporting compared to active surveillance. In addition, the rates of submissions may be influenced greatly by disease and non-disease factors that influence the total number of submissions to each data stream. The validation of active and passive syndromic surveillance data is necessary, and efforts should be placed on integrating these types of data sources.

\section{References}

1. Amezcua R, Pearl, D.L., Friendship R.M., McNab W.B. Evaluation of a veterinary-based syndromic system implemented for swine. Can J Vet Res. In press

Figure 1 a $\& \mathbf{1 b}$. Predicted rates of swine respiratory and digestive submissions from three data sources (OSVS*, all AHL* and the subset of AHL from OSVS participating members) from Summer 2007 to Spring 2009 in Ontario.

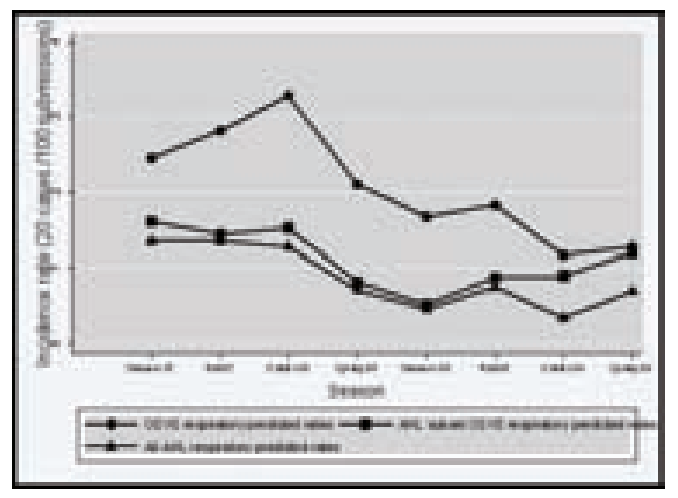

1a. Respiratory

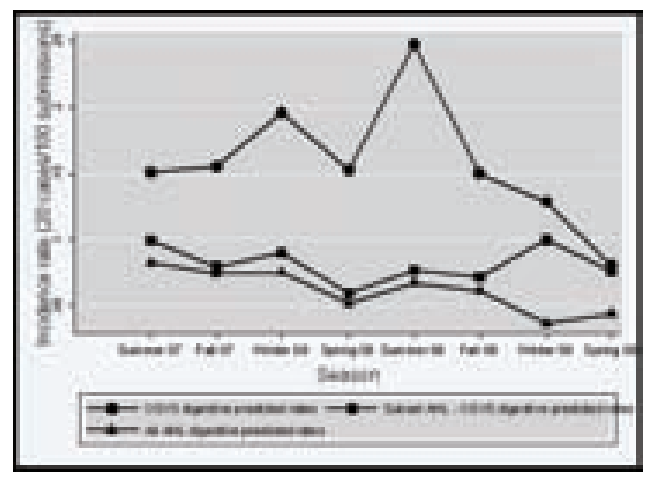

1b. Digestive 


\title{
P.625
}

\section{A New Swine Veterinary Practice Management and Surveillance System for Western Canada}

\author{
Gail L. Cunningham ${ }^{3}$ Brad Chappell ${ }^{1}$ J E. Brockhoff ${ }^{2}$ Pete Pawluk ${ }^{4}$ Chris Misutka ${ }^{2}$ Blaine Tully ${ }^{1}$ Peter Provis ${ }^{1}$ Mike Sheridan ${ }^{1}$ \\ 1. Swine Health Professionals, Steinbach, MB, Canada; 2. Prairie Swine Health Services, Red Deer, AB, Canada; 3. Gail \\ Cunningham Veterinary Services, Kelsey, AB, Canada; 4. Swine Health Centre, Lethbridge, AB, Canada
}

\section{Introduction}

Swine practitioners collect and maintain a large volume of health information as part of daily practice. However, the ability to compile, organize and use this information is often difficult due both to volume and varied methods of collection and reporting. Swine Database Services (SDS) is a company that arose from a shared vision of private industry veterinarians who recognized the need to organize this information and use it to generate knowledge. Together this group has developed a real time online practice management database that collects and organizes data so it can be readily searched and accessed. This system has also allowed for the collection of real time on farm animal health data that can also be used for emerging disease surveillance.

\section{Materials and Methods}

A group of like minded veterinarians from Manitoba and Alberta came together because they shared the need to compile and organize the large volume of health information in their practices. Each group had independently started to develop a data collection database and quickly recognized the synergy to be gained in working together. In merging the two independent databases, the beginning of an innovative information management tool was created. The services of an Information Technology company were contracted to convert it to an online system that could be accessed, used and maintained in real time. The continued development of the database centers on using the data to create knowledge to accomplish the following goals:

- make better decisions for clients

- improve practice management and individual quality of life for swine practitioners

- improve epidemiological data to aid in disease investigation

- create consistent protocols, prescriptions, forms and reporting

- use real time data in the real time surveillance of emerging/reemerging disease

\section{Results}

The SDS database was created primarily to facilitate practice management. Key practice areas were identified and modules created in the database for each area. The current modules in use include:

1. Customer Contact Information

2. Standardized Herd Visit Documentation- veterinary medical records
3. Diagnostic Information - summary of diagnostic information in a searchable format

4. Prescription Generation - injectable and feed which are standardized and error free

5. Producer Medication and Vaccine Usage Plans - provide standardized medication information for clients

6. Quality Assurance Monitoring -notification when CQA need to be done and correction action monitoring.

\section{Export Health Certificates to the United States}

Numerous reports are also available including electronic signature tracking, invoicing and practice efficiency. The practice efficiency can be used to benchmark customer service and overall practice performance. Health information can also be monitored including syndromic data (e.g. coughing), mortality and on farm diagnostics. Monitoring these parameters over time may provide important signals to changes in the health status of an individual farm and/or geographically or otherwise related farms. This allows the veterinarian to react proactively to these changes. The secondary objective of the database was to provide a real time on farm swine surveillance tool. Each farm is given a unique code known only to the veterinarian so if data is shared, the veterinarian becomes the "gatekeeper" of the information and maintains farm confidentiality. A pilot project with the Alberta Veterinary Surveillance Network (AVSN) was conducted with SDS. Blinded data was analyzed starting in February 2009 including key farm attributes \& syndromic information collected during farm visits. The goal of this surveillance is to more rapidly detect and respond to health events in order to protect our industry.

\section{Discussion}

The SDS database has been developed to generate knowledge, establish protocols and devise strategies that can help clients and veterinarians thrive in an increasingly challenging industry. The consumer demand to show a commitment to food safety and public health by monitoring the production of our food supply will continue to drive the need for disease surveillance. The SDS database can produce real time health information and reports that help provide assurance to markets about the health of our pigs. Maintaining this information in one comprehensive practice management database provides high quality data while improving practice life for the veterinarian. 


\title{
P.626
}

\section{Swine veterinary practice surveillance in the Alberta Veterinary Surveillance Network}

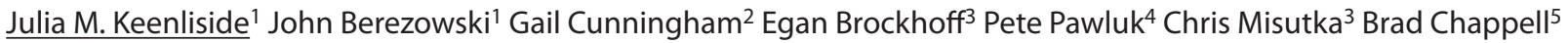 \\ 1. Alberta Agriculture and Rural Development, Edmonton, $A B$, Canada; 2. Gail Cunningham Veterinary Services, Kelsey, $A B, C a n a d a$; \\ 3. Prairie Swine Health Services, Red Deer, AB, Canada; 4. Swine Health Centre, Lethbridge, AB, Canada;
}

5. Swine Health Professionals, Steinbach, MB, Canada

\section{Introduction}

Livestock disease surveillance systems are necessary for countries to meet their OIE reporting requirements. Trading nations must be able to substantiate their claims about disease status and veterinary infrastructure. Surveillance systems must be able to detect OIE listed diseases as well as newly emerging diseases and changing endemic diseases. The Alberta Veterinary Surveillance Network (AVSN) was developed to meet these needs.AVSN has three parts: disease detection, disease response and communication. Veterinary Practice Surveillance (VPS) provides the system for veterinarians to report disease and non-disease data. Analysis of the data provides valuable demographic information and enhances early detection of important disease events.A VPS for cattle has been operational since 2006 with approximately 30 practices participating (1). This paper describes a Veterinary Practice Surveillance (VPS) for Swine Pilot Project begun in 2009.

\section{Materials and Methods}

A contract for data purchase was developed with Swine Database Systems (SDS), a group of swine veterinarians in Western Canada. SDS had recently created an online database system for managing practice and animal health data (2). Three veterinarians from two swine only specialized practices submitted data weekly for one year. Data was provided weekly on all client interactions, including telephone and electronic consultations. Farm name, address, location and phone contacts are present in the SDS database but were not shared. A unique farm and visit identification (ID) code was applied to each farm and only ID codes were included in the data submissions supplied to the AVSN. Definitions for each data field were written in consultation with the SDS veterinarians.

Data were analyzed in Excel and reported to participating veterinarians.

\section{Results}

Data were submitted weekly for one year from visits to farms representing approximately $45 \%$ of the Alberta sow herd. Herd size and pig type on farm were included, but no other farm identifiers. When unusual disease presentations were noted, the reporting veterinarian was contacted and the case discussed. Definitions were developed for the 13 types of submissions (visit reasons), 12 herd/operation types (eg. farrow to finish, wean to finish), 11 disease syndromes and four pig types (sows, piglets, nursery and growing pigs). The 11 disease syndromes reported were: coughing, sneezing, scours, neurological, skin, lameness, unthrifty, vices, sudden death, reproductive, and urogenital. Severity was reported by eight percentage ranges of animals affected in each of four pig types in the syndrome categories: $0 \%$, $<2 \%, 2-5 \%, 5-10 \%, 10-20 \%, 20-40 \%, 40-60 \%$, > 60\%. Total morbidity and mortality for each class of pigs was also reported. This database allowed the description of veterinary infrastructure for swine in Alberta (ie. number of visits per farm, size of farms visited, and reasons for visiting farms). Changes in the number of on-farm visits and types of consults were monitored in response to a declining pork price. Consultations for respiratory disease were monitored during the pandemic H1N1 influenza outbreak.

\section{Discussion}

Because the database was designed primarily for improving practice efficiency, veterinary practitioner compliance and data quality were good. Reporting information back to practitioners can be valuable in assisting their decision making.The VPS for swine has the potential to generate useful information for several purposes. It may be used to identify animal welfare issues and set baselines for welfare measurement. For international trade and food safety issues, this database may provide information on the presence or absence of certain disease syndromes, detection of emerging health problems, setting of baselines for "normal" disease levels, recording the magnitude of health events and the strength of veterinary infrastructure.

\section{References}

1. Berezowski, J. (2010) Advances in Pork Production 21, 87-93.

2. Chappell B, Cunningham G. (2010) Advances in Pork Production 21, 95-98 
P.627

\title{
A descriptive study of biosecurity measures in French farrow-to-finish pig herds
}

\author{
Christelle Fablet; Virginie Dorenlor; Fanny Portier; Fabrice Bidan; Florent Eono; Eric Eveno; Jean-Pierre Jolly; François Madec
} AFSSA-Site de Ploufragan, Ploufragan, France

\section{Introduction}

The term biosecurity in livestock production encompasses a broad spectrum of external and internal farm measures aiming at protecting a herd from infectious agents (viral, bacterial, fungal, parasitic...). Although the importance of effective biosecurity is underlined in numerous studies to reduce disease spread, few data on the implementation of such measures on pig farms are available [1]. The knowledge of currently biosecurity precautions applied in farms might be helpful to determine to what extent appropriate measures are in place. This can in turn provide a baseline of information for veterinarians and producers to build herd health programmes in order to improve the degree of protection. The purpose of the study was to describe the situation vs some biosecurity measures in a sample of farrow-to-finish pig herds.

\section{Materials and Methods}

Data from 121 farrow-to-finish pig herds included in a survey designed to identify risk factors for respiratory diseases were used. Information related to the farm characteristics and the biosecurity measures were collected during a visit to each farm. In a first step of the statistical analysis, an univariate analysis was performed. Secondly, for the variables with sufficient variability (category frequency $>20 \%$ ), a multiple-correspondence analysis method followed by a cluster analysis was used to describe the relationships between the different variables and to reveal groups of pig herds with similar biosecurity practices (SPAD 5.6, DECISIA Inc.).

\section{Results}

Three clusters of farms were constituted. The results of the cluster analysis are presented Tables 1, 2 and 3.

Table 1: Pattern of biosecurity practices of the herds belonging to cluster 1

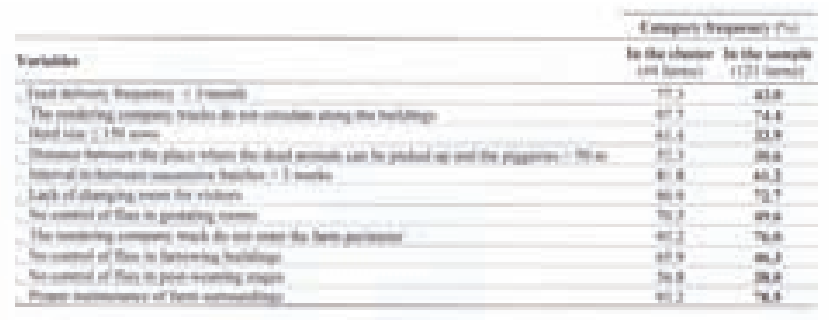

Tables 2 \& 3.

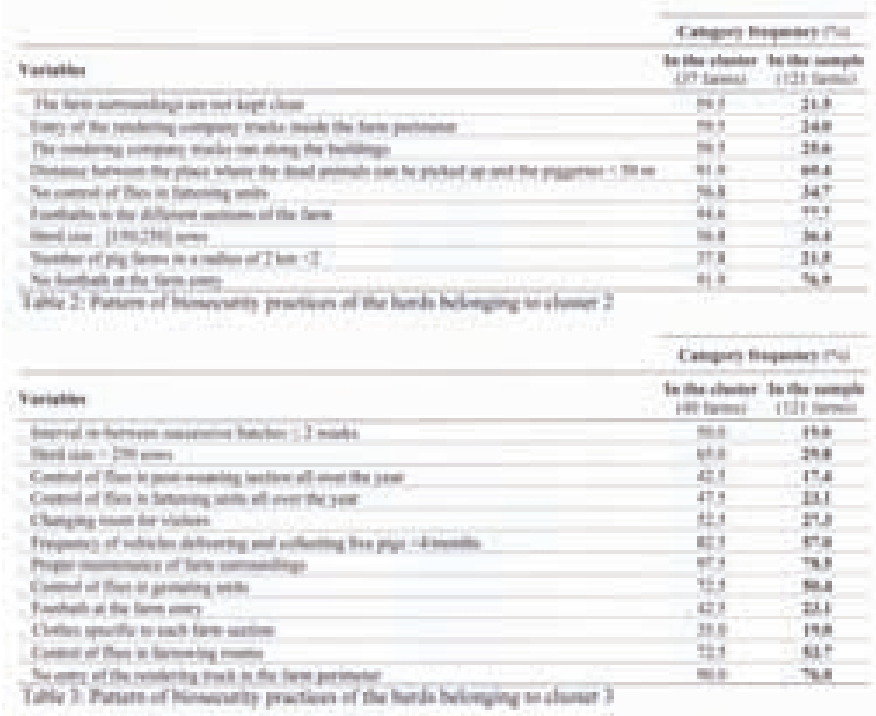

\section{Discussion}

As incoming pigs constitute the main source of pathogens introduction into a herd, when dealing with biosecurity, the policies of replacement stock and quarantine management need to be considered. These factors were collected but were not taken into account in this first approach. They will be added in further analysis. However, the results of the study indicate that the importance given to the other aspects of biosecurity varies among farms, the larger herds paying the highest attention. Improvements are specially needed in medium size herds to reduce the risk of disease introduction and spreading. Ribbens et al., [2] found that procedures for persons entering the herds are better applied in larger herds compared to smaller ones. In our study, the lack of control of flies is a main factor characterizing the groups. Since insects may be vectors of swine infectious agents like PRRS virus special attention must be placed to insect control in the different rearing sections [3].

\section{References}

1. Amass, S.F. and Clark, L.K. (1999). J Swine Health Prod, 7, 217-228.

2. Ribbens, S. et al. (2008). Prev Vet Med, 83, 228-241.

3. Otake, S. et al. (2004). Vet Rec, 154, 80-85. 


\title{
P.628
}

\section{Evaluation of the biosecurity in swine farms in the north of Mexico}

\author{
Pedro J. Pradal-Roa; Diana L. Alonso-Mares; Roberto Martinez-Gamba \\ Universidad Nacional Autónoma de México (UNAM), Facultad de Medicina Veterinaria y Zootecnia, \\ Departamento de Producción Animal: Cerdos, Ciudad, CHIH, Mexico
}

\section{Introduction}

Biosecurity plays an important role in the pig production units because his close relationship with animal health, by ensuring the pig products quality and improvement of production efficiency. It is important to know the level of biosecurity and technification in pig producing areas to know the risk factors present that will lead to the presence of pathogens that cause disease and implement programs of biosecurity to control them (1); being the northwest of Mexico a high pig production area, we carried out an study to help understand the situation prevalent on biosecurity and use it as a guide to improve the health situation and pork production.

\section{Materials and Methods}

Forty-six farms were visited in the municipalities of Cajeme, Navojoa and Hermosillo (15, 14 and 17 respectively). Visual inspection and application of a questionnaire was carried out considering the basic points of biosecurity and technification. Each question was designed with multiple-choice answers with value of $0,5,10,15,20 \& 25$ points each; questions closely related between biosecurity and technifications were considered for both variables (3). The values of each response on biosecurity and technification were summarized independently, getting a final score for each variable in each farm. Statistical analysis in general and by municipality was carried out and Spearman correlation analysis was applied between Biosecurity (B) and Technification ( $\mathrm{T})$.

\section{Results}

Farms with low-level of $B$ and $T$, were not found so four types of farms are identified: MT-MB, MT-HB, HT-MB, HT-HB ( $\mathrm{M}=$ medium, $\mathrm{H}=$ high). The largest number of farms were found to be HT-HB (21). Navojoa has the highest percentage of farms with HB (64\%) and HT (93\%), Cajeme has the lowest HB (53\%) and Hermosillo in HT (53\%). The average Biosecurity in the study was 2481.71 points (HB) and T values were $708.15(\mathrm{HT})$, although by municipality Cajeme has the lowest (2426.67) B points that placed it in a BM level, and Hermosillo has the highest B points (2554.41). A low correlation between $B$ and $T(r=0.0567)$ was found indicating a relative correlation, but not significant for being away from the confidence level $(P>0.05)$.

\section{Discussion}

High homogeneity was found in the pig farms from the north of Mexico, prevailing units with HT (2). Only four types of farms were found because that some state law sets that backyard pig units and all kinds of farms which do not meet the minimum standards of health (4) is prohibited. Correlation between B and $T$ is very low so we can find farms with equal level of technification and biosecurity as farms with higher or lower level of biosecurity, in contrast with their level of technification as found by Zamora (2006), although simple measures of biosecurity, can raise the level of this.

\section{References}

1 Morilla, G. A. Actualidades de Bioseguridad en la Industria Porcina, 9-12, 2008.

2. Hernández, M. M. Estrategias competitivas frente a la globalización, CIAD, 2001.

3. Mujica, R. J. (B.Sc. Thesis). FMVZ-UNAM, 2005.

4. Mazcorro, V. Trueta, R. Procc. VII Congreso Latinoamericano de Sociología Rural, 2006.

5. Zamora, R. S. (B.Sc. Thesis). FMVZ-UNAM, 2006. 


\title{
P.629
}

\section{Seasonal distribution and abundance of invertebrates captured on pig farms in England and Ireland}

\author{
Steven McOrist; Ruth Blunt \\ University of Nottingham, Sutton Bonington, UK
}

\section{Introduction}

Insects on pig farms can cause loss in 4 ways.

1. Biting flies such as Stomoxys stable flies and biting midges are known to be capable of causing direct losses due to lesions of direct damage and immuno-allergic reactions. These lesions cause specific losses due to trim loss and extra abattoir processing times, particularly in the summer months.

2. Many common pests of stored products are found in on-site feed storage and feed mill areas on pig farms, such as in storage and hopper locations. Many insects are attracted to feed components and mixed feedstuffs stored on pig farms. These insects may cause direct losses by feed consumption but also reduced quality of feed.'

3. High numbers of summer insects such as flies may exacerbate minor wounds, disturb feed and rest periods and therefore compromise pig welfare. Very high levels of these pests may also disturb farm workers and nearby residential dwellings or businesses.

4. Insects may spread disease within and between farms. ${ }^{2}$ This may include re-infection of eradication project farms by insects infected with the agent in question.

\section{Materials and Methods}

15 representative pig farm study sites within England and Ireland were selected and studied in 2007 and 2008. Invertebrates were collected every 2 weeks at feed storage, breeder and weaner-finisher pig areas with aerial sticky traps, motorised vacuum devices, sweep nets and walk-in floor traps. Traps were placed in constant numbers and locations throughout farms to assist between-farm comparisons. Invertebrates were counted and sorted into genus and species groups by trained entomologists. The total DNA of insects and/or their intestines was extracted and tested for the presence of eubacterial DNA.

\section{Results}

Comparison of the populations of invertebrates on each study site, via a dendrogram incorporating component similarity coefficients indicated few major differences in the species evident at each site location across England. Despite the high levels of rainfall and lower than average temperatures in 2007 and 2008, invertebrate group numbers peaked during the summer months. Of the Dipteroid flies, Musca domestica (house fly, $\mathrm{n}=14$ farms) was the most common, but also included Muscina stabulans (false stable fly, $n=9$ ), Ophyra spp (garbage fly, $n=10$ ), Drosophila (fruit flies, $n=8$ ), Stomoxys calcitrans (stable fly, $n=2$ ) and Syrphidae containing Eristalis species (hover flies, $n=2$ ). The ground-dwelling beetles found were Carabidae (ground beetles), Curculionoidea (weevils), Staphylinoidea (rove beetles), Tenebrionoidea (darkling beetles) and Bruchidae (bean weevils). A wide variety of Lepidoptera (moths), Acari (mites), Araneae (spiders) and Isopoda (woodlice) species were also present. Other notable findings included Blatta orientalis (oriental cock- roach) on 2 farms. Other insect species found on only one or 2 farms included Siphonaptera (fleas) and parasitic wasps from a variety of families including Ichneumonidae and Chalcidae. PCR products typical of eubacterial DNA were confirmed within the gastrointestinal tract of all fresh cockroaches tested and in over $95 \%$ of all fresh smaller whole insects tested.

\section{Discussion}

A wide range of Dipteriod fly, beetles, and cockroach activity was evident on many British and Irish pig farms, peaking in summer months as expected. Musca domestica was the most common dominant fly type on the pig farms sampled. These often formed close associations with the farm pigs. We only found the garbage fly Ophyra spp to be the dominant dipteroid fly on one farm - these are considered competitive for Musca spp, in terms of fly feeding ecology. We found Drosophila fruit flies to be the dominant fly species on one farm, but these are not competitive for other flies and are not closely associated with the pigs. A range of expected beetles and weevils associated with feed storage products were found. Cockroaches were noted on two farm - they form large indestructible communities on pig farms when they are introduced - usually via food waste or other waste products. Our trapping methods were not adequate for biting midge evaluations in this initial study. Our results indicated that Stomxys was a common but low-level presence on many farms. Some of these insects associated closely with pigs had the likely potential to carry and transmit bacterial and viral enteric pathogens, particularly flies and floor-dwelling insects such as cockroaches or Eristalis sp larvae.

\section{References}

1. Sinclair E. 1982. Population estimates of insect pests of stored products on farms on the Darling Downs, Queensland. Aust J Exp Agric Anim Husbandry 22, 127-132.

2. Medveczky I., Kovacs L., Kovacs K. \& Papp L. 1988. The role of the housefly, Musca domestica, in the spread of Aujesky's disease. Med Vet Entomol 2, 81-86. 


\title{
P.630
}

\section{UV254 inactivation of selected viral pathogens}

\author{
Tim D. Cutler ${ }^{1}$ Steven J. Hoff ${ }^{1}$ Chong Wang ${ }^{1}$ Kristi J. Warren ${ }^{3}$ Fanghong Zhou ${ }^{2}$ Qingsong Qin 4 \\ Cathy Miller ${ }^{4}$ Julia Ridpath ${ }^{5}$ Kyoung-Jin Yoon² Jeffery J. Zimmerman²
}

1. lowa State University, Department of Statistics, Ames, IA, USA; 2. lowa State University, Dept Vet Diagnostic and Production Animal Medicine, Ames, IA, USA; 3. Iowa State University, Ames, IA, USA; 4. Iowa State University, Dept of VMPM, Ames, IA, USA; 5. USDA-ARS-NADC, Ames, IA, USA

\section{Introduction}

UV254 has been a proven method of disinfection since the 1930s (Wells and Brown, 1936). Since then, UV254 inactivation of airborne pathogens has been engineered into areas where people congregate, e.g., by placing UV254 light tube grids into existing ventilation ductwork (Walker and Ko, 2007). Data is available on the inactivation of several human viral pathogens, but little data is available on the UV254 inactivation of viral pathogens of animals.

The goal of this research was to determine the UV254 dose required for inactivation of three common viral pathogens of animals: swine influenza virus (SIV), porcine reproductive and respiratory syndrome virus (PRRSV) and bovine viral diarrhea virus (BVDV). Year One focused on UV254 inactivation of viruses under "static" conditions (virus in liquid medium) using off-theshelf UV hardware from commercial manufacturers (American Ultraviolet Co., Lebanon IN). Year Two (in progress) will focus on inactivation of airborne pathogens.

\section{Materials and Methods}

SIV, PRRSV, BVDV, and Reovirus suspended in media were exposed to specific doses of UV254 where dose is defined as (intensity $x$ exposure time) and intensity is expressed as joules per $\mathrm{cm} 2$. Reovirus, highly resistant to UV254 inactivation, was included for the purpose of process control and comparison.

The effect of UV254 exposure was quantified by comparing virus titers in exposed vs. unexposed controls. Three trials were conducted at 9 exposure doses: $0.3000,0.2500,0.2000,0.1500$, $0.1250,0.1000,0.075,0.050$, and $0.0250 \mathrm{~J} / \mathrm{cm} 2$. Viruses $(1.8 \mathrm{ml} ; 2$ $\mathrm{mm}$ depth) were placed in separate wells of modified Nunclon ${ }^{\circledR}$ MultiDish $^{\text {TM }}$ 8-well plates (Nalge Nunc International, Rochester, NY). Plates were modified by removal of two middle wells to accommodate the placement of a UV254 sensor (Technika, Phoenix, AZ). One well on each plate served as an unexposed control. Following UV254 exposure, the viral suspensions were placed in $2 \mathrm{ml}$ cryovials and frozen at $-80^{\circ} \mathrm{C}$ until assayed for viral infectivity.

Microtitration infectivity assays (TCID50) were conducted on samples in random order. The rate of inactivation was determined by plotting the survival fraction of each viral against the exposure dose.

\section{Results}

\begin{tabular}{|c|c|c|}
\hline Virus & K Value & 95\% CI \\
\hline Reovirus & 0.0118 & $(0.0104,0.0131)$ \\
\hline BVDV & 0.0401 & $(0.0322,0.0480)$ \\
\hline PRRSV & 0.0371 & $(0.0360,0.0483)$ \\
\hline SIV & 0.0298 & $(0.0204,0.0371)$ \\
\hline
\end{tabular}

\section{Discussion}

Larger inactivation constants ( $\mathrm{K}$ value) indicate greater susceptibility to inactivation by UV254. Thus, in contrast to UV-resistant Reovirus (K value $=0.0118$ ), PRRSV, BVDV and SIV were highly susceptible to inactivation by UV254. Year Two (in progress) will focus on inactivation of airborne pathogens using UV254.

\section{References}

Walker CM, Ko G. 2007. Effect of ultraviolet germicidal irradiation on viral aerosols. Environ Sci Technol 41:5460-5465.

Wells WF, Brown HW. 1936. Recovery of influenza virus suspended in air and its destruction by ultraviolet radiation. Am J Hyg 24:407-413.

\section{Acknowledgements}

This project was funded in part through Pork Checkoff funds distributed through the National Pork Board (Des Moines, lowa).

This study was supported in part by the PRRS CAP, USDA-NIFA Award 2008-55620-19132. 


\title{
P.631
}

\section{Classification of farrowing sites according to on-farm biosecurity practices and their geographical distribution in two swine production areas of Quebec, Canada}

\author{
Marie-Ève Lambert ${ }^{1}$ Zvonimir Poljak $^{2}$ Sylvie D'Allaire ${ }^{2}$ \\ 1. University of Montreal, Faculty of Veterinary Medecine, St-Hyacinthe, QC, Canada; \\ 2. University of Guelph, Ontario Veterinary College, Guelph, ON, Canada
}

\section{Introduction}

Compartmentalization based on biosecurity could be an important tool to manage disease within a region. The objectives of this study were to describe biosecurity groups and define their distribution over two regions with different pig-herd densities.

\section{Materials and Methods}

A high (HD: Estrie) and a low density (LD: Monteregie) areas were purposively targeted and all premises containing sows were included within these areas. A questionnaire was developed to assess the principal potential risks factors reported in literature for introduction of PRRS virus on a site or within a herd. The questionnaire was completed by the owner (independent farm) or by the employee (for farm under contract) and filled out by a single interviewer. Answers were obtained by phone and during a farm visit and included information on more than 40 variables about herd characteristics, geographical coordinates of the site and biosecurity requirements (layout of the site, entrance protocol, transportation of animals, dead pig disposal, pest management, feed and semen deliveries, manure management). Biosecurity was described as measures implemented by producer to limit the introduction of diseases on the site and inside units. Differences between regions were assessed through Mantel-Haenszel chi-square, Fisher's exact or Wilcoxon statistic as appropriate $(\mathrm{p} \leq 0.05)$. A two-step clustering procedure (SPSS) was performed to classify the farrowing sites in different groups of biosecurity practices. Geographical distribution of sites belonging to the good biosecurity cluster was assessed in both regions using logistic generalized additive model and predicted values were plotted in commercial software (ArcGIS 9.3).

\section{Results}

Information was gathered on 71 and 54 sites in HD and LD, respectively. Classification of the 125 sites was performed using 22 variables pertaining to biosecurity and revealed two clusters: A ( 86 sites) and B (39 sites). Some biosecurity practices related to the entrance protocol are reported in Table 1 according to cluster and region. Significant differences between HD and LD area were observed for the median sow inventory (180 vs. 235) and the median distance from the nearest site (400 vs. $2750 \mathrm{~m}$ ). Sites belonging to cluster B had a higher proportion of good biosecurity practices and were more frequently located in the LD than in the HD area (62\% vs. $38 \%$ ). Heterogeneity of spatial distribution of sites in cluster B was observed, even in the LD area (Fig. 1).
Table 1: Biosecurity according to region and cluster (\%)

\begin{tabular}{|l|c|c|c|c|}
\hline Entrance protocol variables & LD & HD & A & B \\
\hline No-entry sign on door & $65^{*}$ & 39 & 35 & 84 \\
\hline Locked doors at any time & 31 & 25 & 11 & 67 \\
\hline Doorbell at the entrance & 37 & 31 & 16 & 72 \\
\hline Protocol (4 categories) & & & & \\
\hline 0. Shower at the entrance & $19^{*}$ & 3 & 0 & 31 \\
\hline 1. DE** washing hands & 2 & 4 & 0 & 10 \\
\hline 2. DE** & 9 & 10 & 5 & 20 \\
\hline 3. Washing hands & 7 & 6 & 3 & 13 \\
\hline 4. No protocol & 63 & 77 & 92 & 26 \\
\hline 24 hr downtime for visitors & $59^{*}$ & 18 & 19 & 74 \\
\hline $\begin{array}{l}\text { *Significant difference between regions ( } \mathrm{p} \leq 0.05) \\
\text { **Danish entrance: separated contaminated and clean area }\end{array}$ \\
\hline
\end{tabular}

Figure 1: Predicted probability of observing a site of cluster B in LD area.

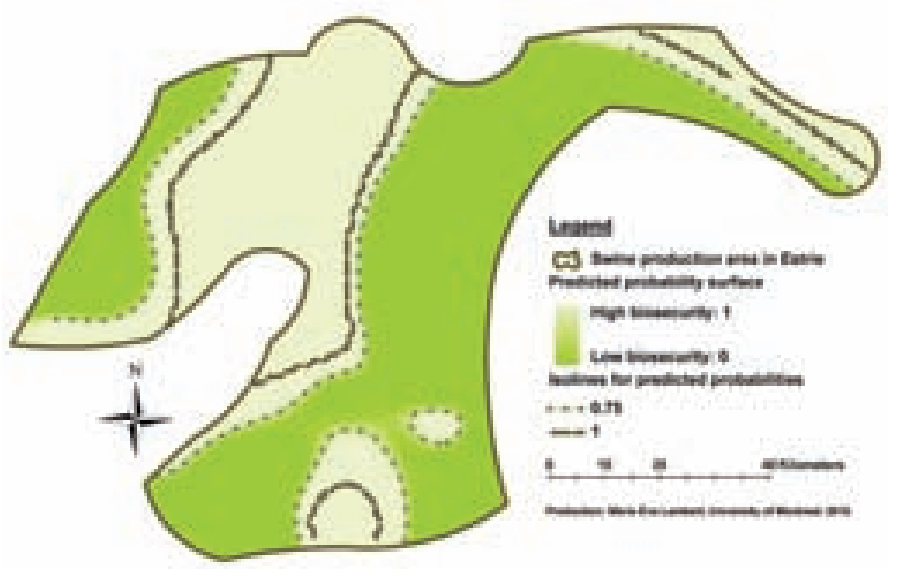

\section{Discussion and Conclusion}

The higher frequency of good entrance protocol practices, the lower pig-site density and the longer distance from neighbours observed in LD may lower the risk of disease transmission compared to HD. Based on entrance protocol and most other biosecurity variables (not shown), sites in cluster B had overall higher standards than sites in cluster A. A knowledge of the distribution of sites with their respective level of biosecurity is important to implement regional control of diseases. 


\title{
P.632
}

\section{The use of an online scoring system for the quantification of the biosecurity status in pig herds}

\author{
Maria Laanen; Stefaan Ribbens; Dominiek Maes; Jeroen Dewulf
}

Faculty of Veterinary Medicine, Ghent University, Belgium, Merelbeke, Belgium

\section{Introduction}

In order to quantify the biosecurity situation on pig farms a biosecurity scoring system was developed by the Veterinary Epidemiology Unit of the faculty of Veterinary Medicine, Ghent University and incorporated in a free online application (www. biocheck.ugent.be).

\section{Materials and Methods}

The scoring system takes both external and internal biosecurity measures into account. Both parts are divided into 6 subcategories each consisting of 2 to 13 questions. Each subcategory received a weight based on literature on pathogen transmission and general knowledge of infection risks. A score between 0 and 100 is obtained for both external and internal biosecurity. The scoring system is adapted to be appropriate for every type of pig unit (fattening herd, breeding herd, mixed herd, etc).

\section{Results and Discussion}

The scoring system has been available for pig farmers in Belgium for one year and 138 herds (i.e. 13 breeding herds, 6 fattening herds and 119 mixed herds) have filled in the questionnaire until January 2010. The average score for external biosecurity is 64 (min 29; $\max 95$ ). The score for internal biosecurity is lower in most farms (91\%) with an average of 51 ( $\min 18$; $\max 89)$. There is a strong positive correlation $(r=0.63)$ between the scores for external and internal biosecurity (see figure below).

Figure 1 The correlation between the scores for external and internal biosecurity on Belgian pig farms.

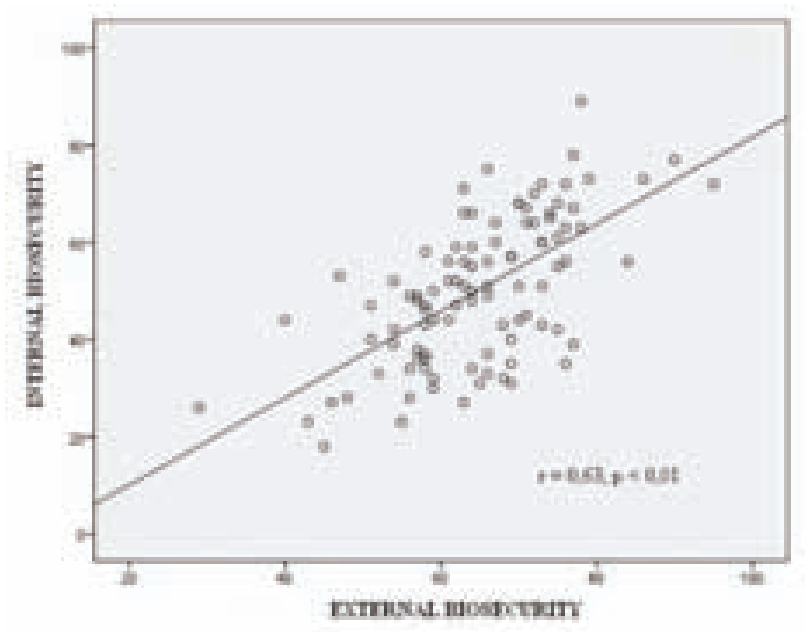

Some selected results relating to external biosecurity show that $81 \%$ of the herds purchasing new breeding animals use quarantine facilities for an average period of 36 days. $96 \%$ provides farm-specific clothing and footwear to visitors, but only $29 \%$ ask visitors to wash and disinfect their hands. Nevertheless, only in $58 \%$ of the herds, the farmer and personnel also carry out these hygienic measures themselves before entering the stables.

Concerning internal biosecurity, only $38 \%$ of the farmers house diseased animals in separate hospital pens and $48 \%$ manipulates the diseased animals after the healthy ones. Suckling piglets are transferred between sows on $98 \%$ of the herds, of which $43 \%$ performs this operation after 4 days post partum. All-in all-out management is practiced in $82 \%$ of the herds in the nursery unit, $70 \%$ of the herds in the fattening unit. Nevertheless, $21 \%$ puts smaller but older pigs together with the younger ones in the nursery and/or fattening unit. From all the herds, $62 \%$ cleans and disinfects every stable after a production round, but only very few (5) verifies the efficiency of these measures. 54\% always applies a sanitary stand empty period after each production round.

The large variation in scores of different farms shows that there is room for improvement in many participating herds. On average, the scores for external biosecurity, which are mainly measures related to infrastructure or measures imposable on other parties, are higher than the scores on internal biosecurity which are more related to the work and management strategies of the farmers themselves. As the results show, there are many biosecurity measures that have become common practice for farmers in Belgium; on the other hand, some effective biosecurity measures should be more frequently practiced. It needs to be emphasized that these 138 farms voluntarily participated in this study and may therefore not be representative for all pig herds. Therefore it is likely that the general results are lower than the results presented here.

The biosecurity scoring system is used in another study (Laanen et al., 2010) to relate biosecurity in a quantitative way with health and production parameters.

\section{References}

- Laanen et al. (2010). Preliminary results on the link between biosecurity status and herd characteristics, daily weight gain and mortality (abstract submitted to IPVS 2010) 


\title{
P.633
}

\section{Preliminary results on the link between biosecurity status and herd characteristics, daily weight gain and mortality}

\author{
Maria Laanen; Stefaan Ribbens; Dominiek Maes; Jeroen Dewulf \\ Faculty of Veterinary Medicine, Ghent University, Belgium, Merelbeke, Belgium
}

\section{Introduction}

It is believed biosecurity influences production results, nevertheless, few studies succeed in demonstrating this link. In order to quantify the biosecurity situation on pig farms a biosecurity scoring system was developed (Laanen et al., 2010). This scoring system was used to study the relation between the biosecurity on a farm and herd characteristics, daily weight gain and mortality.

\section{Materials and Methods}

Thirty one randomly selected Belgian pig herds were visited. The herds had at least 80 sows and 500 fattening pigs. During a herd visit, the biosecurity status of the herd was quantified by means of a biosecurity scoring system (Laanen et al., 2010). At the same time additional data concerning the herd and production characteristics (number of sows, number of piglets, number of fattening pigs, age of the buildings, years of experience of the farmer, daily weight gain and mortality of fattening pigs) were collected.

\section{Results}

Herd characteristics:

A positive correlation was found between the number of sows on a herd and both the external $(r=0.43, p=0.02)$ and internal $(r=0.34, p=0.06)$ biosecurity. Also the number of piglets $(<$ $25 \mathrm{~kg}$ ) and the external biosecurity were positively correlated ( $r$ $=0.39, p=0.03$ ). The correlation with the internal biosecurity was only weakly positive $(r=0.19, p=0.30)$. No correlation was found between the number of fattening pigs on a herd and the external or internal biosecurity.

Both the age of the buildings and the years of experience of the farmer were negatively correlated with the internal $(r=-0.41, p$ $=0.02 ; r=-0.21, p=0.27$ respectively) and external $(r=-0.14, p=$ $0.46 ; r=-0.09, p=0.64$ respectively) biosecurity.

\section{Performance of fattening pigs:}

The daily weight gain of the fattening pigs was positively correlated with the external biosecurity $(r=0.28, p=0.15)$, but no effect of the internal biosecurity $(r=0.03, p=0.86)$ on the daily weight gain was found. No clear effects of the biosecurity status of the herd on the mortality of the fattening pigs were found.

\section{Discussion}

These results clearly indicate the existence of a positive correlation between the number of sows or piglets on a herd and the level of biosecurity, indicating that farmers with larger herds pay more attention to biosecurity than those with smaller herds. The larger a herd becomes, it is more likely to be more professional and well managed. No comparable effect was seen for the number of fattening pigs on the herd. It should also be noticed that the found correlations are moderate to low.

It is also noticeable that the biosecurity increases with a decreasing age of the buildings. This illustrates that in more modern infrastructure more attention is paid to biosecurity. The same holds for the years of experience of the farmer suggesting that younger farmers are more interested in, and willing to apply biosecurity measures.

When evaluating the link with the daily weight gain of the fattening pigs it appears that this increases with an increasing external biosecurity, whereas for mortality no clear link could be identified. This was also seen in a study by Maes et al. (2004).

Several of the found correlations are not statistically significant and therefore need to be interpreted with care. Yet in this study only the results of the first 31 herds out of an ongoing study on 100 herds are presented. Therefore it is believed that with increasing sample size several of the observed trends will become statistically significant. Moreover, the data will also be further explored to identify the parts of biosecurity that are the most influential on health and production parameters.

\section{References}

- Laanen et al. (2010). The use of an online scoring system for the quantification of the biosecurity status in pig herds (abstract submitted to IPVS 2010)

- Maes D., Duchateau L., Larriestra A., Deen J., Morrison R., de Kruif A. (2004). Risk factors for mortality in grow-finishing pigs in Belgium. J. Vet. Med. B., 51, 321-326 


\title{
P.634
}

\section{Evaluation of mortality rate in fattening pig herds and respective correlation on biosecurity measures}

\author{
Giuseppe Martano \\ ASI TO3, Vigone, Italy
}

\section{Introduction}

The aim of this study is to evaluate the mortality rate and correlation with biosecurity level in 26 finishing pig herds and to obtain more knowledge about major risk factors.

Biosecurity is very important in the health management of intensive pig farms, to avoid introduction of pathogenic germs and to reduce microbial circulation in the herd.

Many swine herds could to improve their health standard through improved external and internal biosecurity measures.

\section{Material and Methods}

A survey was performed on 26 fattening pig herds located in Pinerolo District, Northwest Italy to describe their biosecurity status and mortality data, expressed as the number of dead pigs divided by the number of pigs placed in the growing-finishing unit .

The herds size ranged from 420 to 4050 , for a total of 31,000 pigs. All herds introduced pigs at age 70-90 days and the same were slaughtered not before 270 days old.

In order to collect data it has been used a questionnaire divided in different sections regarding internal and external biosecurity, feeding and sanitary treatments .

It was collected data about mortality rate in the last four years ( from 2006 to 2009 ).

\section{Results}

Mortality rate has been evaluated for each farms.

The overall average mortality rate from 2006 to 2009 resulted 4,41 per cent .

\begin{tabular}{|l|c|c|c|c|c|}
\hline & $\mathbf{2 0 0 6}$ & $\mathbf{2 0 0 7}$ & $\mathbf{2 0 0 8}$ & $\mathbf{2 0 0 9}$ & $\mathbf{0 6 - 0 9}$ \\
\hline mortality rate & $4,07 \%$ & $4,77 \%$ & $4,26 \%$ & $4,55 \%$ & $4,41 \%$ \\
\hline standard deviation & $\pm 1,50 \%$ & $\pm 2,68$ & $\pm 2,71 \%$ & $\pm 2,61$ & $\pm 1,74$ \\
\hline
\end{tabular}

No statistical significant difference from year to year for the totality of farms: nevertheless in some herds this index is very variable.

Mortality percentage in the single herds ranged from $1,28 \%$ to $9,12 \%$.
The standard deviation is high compared with the relative mortality rate.

In this study, the season of the year had not a significant impact on the mortality rate in fattening herds.

Many risk factors were investigated but the correlation was proved $(p<0,05)$ only between farms that introduced piglets from more than two origins herds and higher mortality rate.

All in- all out is performed on 56 percent of the farm, in combination with strict separation of age groups.

All farmers perform a cleaning and disinfection after every finishing cycle.

A specific compartment for housing diseased animal and quarantine is prescribed on $100 \%$ of examined farms.

All farms have a refrigerated tank for removal dead pigs and the loading ramp is washed and disinfected every time is used and all of them have a rodent control program.

\section{Discussion}

Some potential risk factors were evaluated about high mortality percentage.

In this work we found farms that introduced piglets from more than two origin herds were associated with higher mortality. Also biosecurity level could influence the mortality.

Biosecurity on farm should be considered a dynamic process, which require recurrent improvements.

In this study, we found that some important strategic practices were not implemented in most of the pig herds.

In all farms the biosecurity level is susceptible of improvements. Reduce mortality in the herds is a target to reach.

\section{References}

Pinto C., Urcelay V Santiago (2003) "Biosecurity practices on intensive pig production system in Chile"Preventive Veterinary medicine, 59,139-145.

Bocklund A.., Alban L., Mortensen S., Houe H., (2004) “ Biosecurity in 116 Danish fattening swine herds : a descriptive results and factor analysis". Prev.Vet.Med.66,49-62.

Casal j., De Manuel A., Mateu., Martin., (2007) “ Biosecurity measures on swine farms in Spain: "Perceptions by farmers and their relationship to current on-farm measures". Prev.Med.Vet 82,138-150 


\title{
P.635
}

\section{A study of cleaning methods on number of bacterial count on farrowing crates in pig farms}

\author{
Pariwat Poolperm; Suwicha Kasemsuwan; Prapha Songjinda; Kanokkan Nunta; Peerakorn Jittrarak; \\ Nisarat lamnoi; Sakarin Praprut; Soraya Na-ranong \\ Kasetsart University, Nakornpathom, Thailand
}

\section{Introduction}

The important risk factor of infection for farrowing sows and piglets is direct exposure to infectious microorganisms. The farrowing crate floor is the main source of bacteria contamination and it is the first surface piglets are directly exposed to after birth (1). Hence, the cleanliness of farrowing crate is vital to reduce the risk of MMA/PHS in farrowing sows and diarrhea in piglets. It is therefore of interest to assess the efficacy of cleaning methods that are using generally in pig farms in Thailand.

The aim of this study was to evaluate the efficacy of different cleaning methods on farrowing crates in pig farm considering the mean of total bacterial count (TC), coliform count (CF) and percentage of E. coli (EC) positive on the floor after cleaning.

\section{Materials and Methods}

A comparison of four different cleaning methods; 1 ) using high pressure and washing with detergent $(C T, n=4), 2)$ same as $C T$ additional with pouring disinfectant one-time ( $T 1, n=10), 3$ ) same as CT additional with pouring disinfectant two-times (T2, $\mathrm{n}=10$ ), and 4) same as CT additional with pouring disinfectant one-time and flaming floor thoroughly $(T 3, n=10)$ were evaluated. The disinfectant (Beta- $\mathrm{Q}^{\circledR}$ ) used in the study was glutaraldehyde base.

Five positions that towards each corner and one in the middle on the farrowing crate for each cleaning method were swabbed using wet and dry technique before cleaning (d-1), at 1-2 hours (d0), 1 (d1), 2 (d2) and 3 days (d3) after cleaning. Pooled samples for each crate were cultured onto PCA agar (Criterian ${ }^{\oplus}$, Santa Maria, CA, USA) and coliform agar (Chromocult ${ }^{\oplus}$, Merck KGaA, Darmstadt, Germany). Colonies of total bacteria and coliform were counted. E.coli was counted and reported as percentage of positive crate. Average of log10 bacterial counts of each cleaning methods were compared using repeated measure ANOVA.

\section{Results}

The results showed that total bacterial count (fig.1) in CT group significantly differed from $\mathrm{T} 1, \mathrm{~T} 2$ and $\mathrm{T} 3(\mathrm{p}=0.02)$. There were no significant differences in the TC and CF among the T1, T2 and T3 methods. Coliform was lowest on day 2 and increased significantly on day 3 after cleaning, which was the same as \% of E.coli positive crate that increased on day 3. The longer period after cleaning, the higher number of coliform and percentage of $E$. coli positive crate were found in this study. The percentage of $\mathrm{E}$. coli positive samples in T1, T2, T3 and CT cleaning method were $40 \%, 30 \%, 15 \%$ and $50 \%$, respectively. T3 method showed the lowest percentage of E.coli.
Figure.1 Average of Log total bacterial count (CFU/cm3) in each round of sampling compared among 4 groups.

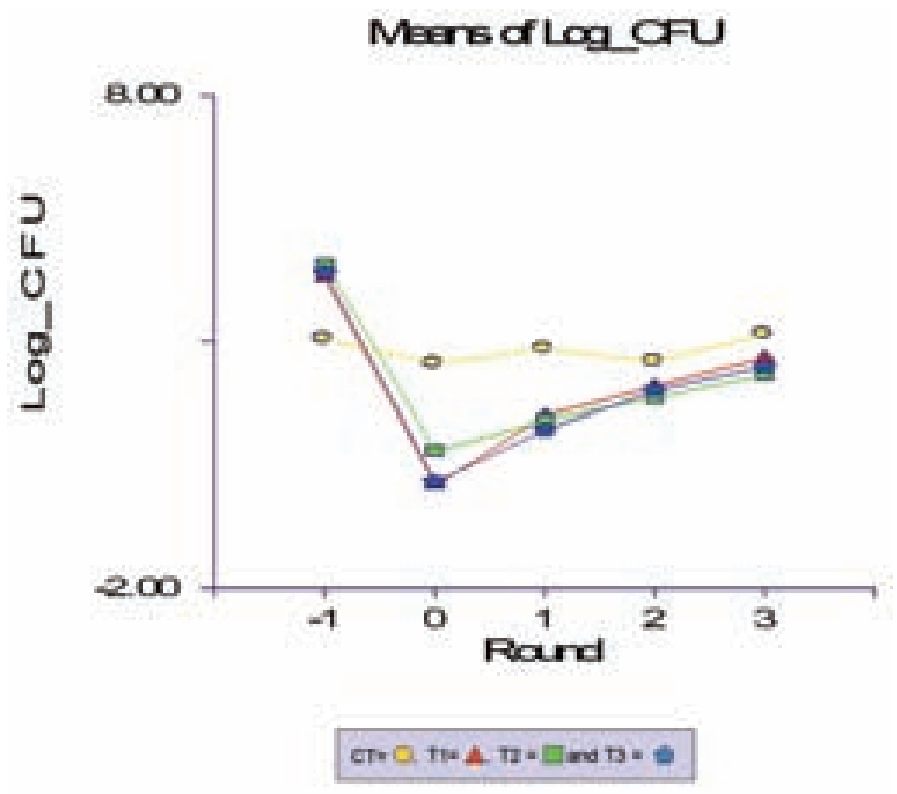

\section{Discussions}

This study demonstrated the benefits of cleaning practices with applying additional disinfectant and/ or flaming step. Without disinfectant the floor was not efficiently clean using only high pressure and detergent washing. The proper cleaning method on farrowing crate could reduce bacterial contamination on the floor and reduce risk of sow being MMA/PHS during farrowing period. The longer period after cleaning was at risk of re-contamination from moving sows in or out of the barn.

\section{Acknowledgement}

This study was funded by Better Pharma Co.Ltd., Betagro group, Thailand.

\section{References}

1. Kihlstorm S.L., et al. (2001). J. Swine Health Prod. 9(2): 65-69 


\title{
P.636
}

\section{Use of the AASV Production Animal Disease Risk Assessment Program to benchmark mortality disposal practices in United States swine production}

\author{
Derald J. Holtkamp; Jamie Melody \\ lowa State University, Ames, IA, USA
}

\section{Introduction}

Frequent transmission of PRRS virus from one premises to another has hampered control and elimination efforts and suggests that current biosecurity practices are often ineffective. Published information on current biosecurity practices in the U.S. is limited. One of the few organizations which does publish such information is the USDA's National Animal Health Monitoring System (NAHMS). 1 The most recent study published by NAHMS, the Swine 2006 study, provided some information on biosecurity practices. Additional information would help producers and veterinarians benchmark biosecurity practices against others in the U.S. and help define industry needs for biosecurity education.

The purpose of this paper is to describe biosecurity practices related to mortality disposal in U.S. swine breeding herds using the database of assessments from the American Association of Swine Veterinarians (AASV) Production Animal Disease Risk Assessment Program (PADRAP). PADRAP is an epidemiologicallybased initiative to help producers and veterinarians manage disease risks. PADRAP is used by veterinarians as a tool to evaluate current biosecurity protocols and/or develop new biosecurity protocols to avoid risk. There are currently two risk assessments available within PADRAP; the PRRS Risk Assessment for the Breeding Herd and PRRS Risk Assessment for Growing Pig Herd.

\section{Methodology}

The PRRS Risk Assessment for the Breeding Herd contains questions regarding introduction of a non-resident PRRS virus (external biosecurity) and management of a resident PRRS virus within a herd (internal biosecurity). Mortality disposal is one of 14 categories of external biosecurity factors in the assessment. As of October 2009, the PRRS Risk Assessment for the Breeding Herd (v2) database had 1749 risk assessments from 1017 premises. Of the 1017 premises, $68.0 \%$ are in the U.S., $18.3 \%$ in Canada, and $13.7 \%$ in Mexico. Only assessments on breeding herd premises in the U.S. completed between May 2005 and October 2009 were included in this analysis.

\section{Results}

The 689 premises included in the analysis were completed for premises in 25 different states and represent 1,567,889 sows, which is approximately $26.8 \%$ of the national inventory $(5,850,000) 2$. A majority $(77.5 \%)$ of the premises are commercial breeding herds, including those with internal multiplication, and $22.5 \%$ are genetic breeding herds producing replacement animals for breeding purposes. Nineteen percent of the premises have fewer than 800 sows, $29 \%$ have 800 to 1999 sows, $31 \%$ have 2000 to 2999 sows, and $21 \%$ have greater than 3000 sows. Sixty percent of the premises are reported to be PRRS positive while $40 \%$ of the premises are PRRS negative.
Of the 689 premises in this analysis, $67.5 \%$ have on-site mortality disposal (i.e. buried, composted, or incinerated). On-site disposal is being used on $73.6 \%$ of the smallest breeding herds, with less than 800 sows, and less frequently (63.5\%) on the largest herds, with more than 3000 sows. Premises that are PRRS negative utilize on-site mortality disposal (79.7\%) of the time, more frequently than PRRS positive premises (59.3\%). Of the premises with off-site mortality disposal the pick-up is usually located at the premises (73.2\%) and $26.8 \%$ have a dedicated location for pick-up greater than $1 / 2$ mile away. Sixty-three percent move dead pigs to another location for pick-up using equipment dedicated to the premises. Animals awaiting pick-up for off-site mortality disposal are usually stored in an enclosed box (77.2\%) and of those, $20.2 \%$ are refrigerated boxes.

\section{Discussion}

The authors are not aware of any studies that have shown that off-site disposal of mortality is associated with greater biosecurity risks but rendering trucks picking up mortality for off-site disposal creates many opportunities for introduction of virus into a herd. If properly managed, on-site disposal by burial, incineration, or composting very likely creates fewer biosecurity risks. For the $32.5 \%$ of the premises in this analysis still using offsite disposal there is an opportunity to improve their biosecurity by switching to on-site disposal. The opportunity is greatest for the largest herds where $36.5 \%$ of premises are using on-site disposal. Adoption of on-site disposal in large herds is complicated by the larger volume of moralities making it increasingly more difficult to manage. For those premises that cannot justify on-site disposal for management or economic reasons, there are still opportunities to reduce biosecurity risks by using enclosed, or in some areas refrigerated, boxes to store mortalities awaiting pickup. This study suggests that there are still significant needs for educational efforts that emphasize the importance of managing biosecurity risks related to mortality disposal practices in U.S. swine production.

\section{References}

1. USDA. 2007. Swine 2006, Part i: Reference of Swine Health and Management Practices in the United States. USDA, APHIS, VS, CEAH, Fort Collins, CO.

2. NASS, 2009. Quarterly Hogs and Pigs, Dec. 30, 2009. Agricultural Statistics Board, USDA, Washington DC. 


\title{
P.637
}

\section{Serology-based classification of pig herds into "zoonoses risk categories" and "herd health categories" by using meat juice as specimen}

\author{
Diana Meemken; Juliane Nobmann; Thomas Blaha \\ Field Station for Epidemiology, University of Veterinary Medicine Hannover, Bakum, Germany
}

\section{Introduction}

The high number of foodborne diseases in humans such as salmonellosis and yersinioses, which leave no apparent or palpable alterations, proves that the traditional meat inspection as end product inspection is not able to control the risks of today (1). Therefore the European Commission has issued a new food safety legislation which regulates the transition of the traditional meat inspection, which demands inspecting each individual carcass in the same way, to a risk-based meat inspection, where the method and the intensity of the inspection are to depend on a risk assessment for human as well as for animal health. This risk assessment is based on the so called food chain information which contains data such as the housing conditions, the use of antibiotics, the results of previous slaughter checks and the results of serological monitoring systems to detect zoonotic pathogens (2). The objective of this paper is to evaluate a set of serological tests, which provides a classification of pig herds into "zoonoses risk categories" as demanded in the regulations and, into "herd health categories" by using meat juice. Collecting meat juice from carcasses at the slaughter line instead of taking blood serum from pigs in the barn by veterinarians implies an increased cost benefit, especially by applying the once taken sample for various ELISA tests.

\section{Material and Methods}

To evaluate the general usability of meat juice as specimen for such ELISA tests, which are solely licensed for blood serum, several test runs with lower dilutions than prescribed for blood serum were conducted. Subsequently, a set of serological tests was selected, which provides results with relevance for human health (measuring antibodies of zoonotic pathogens) as well as for pig herd health (measuring antibodies of infectious pathogens for pigs). Altogether, blood sera and meat juices from 328 pigs were collected at the slaughter line and tested with seven different ELISA-tests, whereas meat juice was ten times fewer diluted than the prescribed dilution for blood serum, respectively.

\section{Results}

The proportion of positive samples and the degree of correlation between the results with serum and meat juice as specimen are shown in Table 1.
Table 1: Correlation between ELISA test results with blood serum and meat juice of 328 slaughter pigs

\begin{tabular}{|l|l|c|c|c|}
\hline $\begin{array}{l}\text { Relevance } \\
\text { for: }\end{array}$ & ELISA-test for & $\begin{array}{c}\text { Blood } \\
\text { serum: } \\
\text { Positive }\end{array}$ & $\begin{array}{c}\text { Meat juice: } \\
\text { Positive }\end{array}$ & $\begin{array}{c}\text { Degree of } \\
\text { Correlation }\end{array}$ \\
\hline Human Health & Salmonella spp. & $17 \%(55)$ & $14 \%(45)$ & $94 \%$ \\
\hline Human Health & Yersinia enterocolitica & $71 \%(233)$ & $69 \%(227)$ & $94 \%$ \\
\hline Human Health & Toxoplasma gondii & $3 \%(9)$ & $2 \%(5)$ & $96 \%$ \\
\hline Human Health & Trichinella spiralis & $0 \%(0)$ & $0 \%(0)$ & $100 \%$ \\
\hline Herd Health & Mycoplasma hyopneumoniae & $57 \%(188)$ & $50 \%(164)$ & $86 \%$ \\
\hline Herd Health & Influenza A (H1N1) & $31 \%(102)$ & $17 \%(55)$ & $84 \%$ \\
\hline Herd Health & Influenza A (H3N2) & $14 \%(45)$ & $8 \%(25)$ & $85 \%$ \\
\hline
\end{tabular}

\section{Discussion}

The demonstrated high degree of correlation between the test results with sera and meat juice shows that it is possible to use meat juice for serologically evaluating the health status of a herd in the framework of the risk-based meat inspection. As for Yersinia enterocolitica a serology-based classification of pig herds may, despite the high seroprevalence of $70 \%$ become the start of a targeted search for risk factors. All in all, this ELISA-based approach with meat juice as specimen is feasible to detect herds with pig health and zoonotic problems. Targeted intervention measures can be established on the farms. The availability of standardised and validated herd health assessment tools is of an increasing importance, since there is a worldwide move from curing disease to prevention.

\section{References}

1) McKenzie and Hathaway (1992), 3rd world Congr. of Foodborne Infections and Intoxications Vol. II; Proc.

2) Blaha et al. (2006), 19th IPVS Congr., Proc. 


\title{
P.638
}

\section{Presence of zoonotic interest bacteria in slaughter pigs}

\author{
Laura Hernandez-Andrade ${ }^{1}$ Paulino Elizalde-Castañeda ${ }^{1}$ Rosa Urrutia ${ }^{1}$ Luis Moles y Cervantes ${ }^{2}$ Efren Díaz-Aparicio ${ }^{1}$ \\ 1. INIFAP, México, DF, Mexico; 2. Universidad Autónoma Metropolitana-Xochimilco, México, DF, Mexico
}

\section{Introduction}

Animal slaughter is one of the professional activities with a higher degree of risk, with a very significant role in the transmission of zoonotic diseases. Slaughterhouse operators, that are in direct contact with carcasses or viscera are the ones that are more at risk (1, 2, 3). Among the main bacterial zoonosis in swine are salmonellosis, brucellosis, yersiniosis and leptospirosis.

\section{Material and Methods}

Since the frequency of these organisms in swine in Mexico is not know, a pilot study with 20 samples was done to estimate infection frequency and based on this estimate the sample size (n) was established with the equation $n=v 2(p)(1-p) / T 2$.

The minimum sample size was estimated in 62 pigs, nevertheless it was increased to 100.

The origin of the animals was the States of Jalisco, Guanajuato, Sonora and Michoacán. From each one of the slaughtered and apparently healthy animals samples were taken from blood, tonsils, ileum, kidney and spleen.

Samples were individually deposited in sterile glass flasks and plastic bags, properly identified and preserved at refrigerator temperature $(40 \mathrm{OC})$. For brucellosis diagnosis, card test and bacteriology from spleen were performed. For leptospirosis a microscopy agglutination technique was used employing 13 serovars of Leptospira interrogans and bacteriology from kidney. Bacteriology study was performed for Salmonella diagnosis using samples from ileum and tonsil samples were used for diagnosis of Yersinia enterocolitica.

\section{Results and Discussion}

Salmonella study results were negative for all animals. Five reactors were found for Brucella by serology (5\%) but no isolation was achieved. Twenty-five (25\%) positive reactors to two serovars of Leptospira were found: L. bratislava 19 animals (19\%) and L. panama 6 (6\%) with no isolation of the bacteria. Twenty-two $(22 \%)$ isolations of Yersinia enterocolitica biotype 1 serotypes 0:3 and 0:9 were obtained; no crossed reaction was detected in the serology for brucella in the sera of the pigs where Yersinia enterocolitica was isolated. Positive animals came from the States of Jalisco and Guanajuato. It is concluded that of the four diseases that were studied, the one that had more relevance was Y. enterocolitica with $22 \%$ of isolations in slaughter swine that are apparently healthy.

\section{References}

1. Boqvist S, Montgomery JM, Hurst M, Thu HT, Engvall EO, Gunnarsson A, Magnusson U. Leptospira in slaughtered fattening pigs in southern Vietnam: presence of the bacteria in the kidneys and association with morphological findings. Vet Microbio. 2003 93: 361-368

2. Campagnolo E R, Warwick M C, Marx H L, Cowart R P, Donnell H D, Bajani M D, Bragg S L, Esteban J E, Alt D P, Tappero J W, Bolin C A, Ashford D A. Analysis of the 1998 outbreak of leptospirosis in Missouri in humans exposed to infected swine. J Am Vet Med Assoc. 2000; 216:676-682.

3. Godfroid J, Cloeckaert A, Liautard JP, Kholer S, Fretin D, Walravens K, Garin BB, Letesson JJ. From the discovery of the Malta fevers agent to the discovery of a marine mammal reservoir, brucellosis has continuously been a re-emerging zoonosis. Vet. Res 36 (2005) 313-326 


\title{
P.639
}

\section{Animal health and public health concerns of stakeholders in pig production}

\author{
Catherine Belloc ${ }^{1}$ Morgane Mennessier ${ }^{1}$ Dominique Pecaud $^{2}$ Etienne Zundel $^{3}$ Henri Seegers $^{1}$ \\ 1. INRA-ONIRIS Vet School, Nantes, France; 2. University of Nantes, Nantes, France; 3. Animal Health Department, INRA, Nouzilly, France
}

\section{Introduction}

Animal health and public health are key issues in pig production for both economical and social reasons. Health management needs the implication of all stakeholders. The latter are more likely to feel involved if they are aware of the importance of the issues they have to control.

The aim of this study was to determine through a survey which issues were regarded as matter of concern by agents involved in pig raising and health control in pig production.

\section{Materials and Methods}

The study took place between June and October 2009 in France. Seventy-three semi-structured interviews were carried out by a single investigator who met 45 veterinarians or scientists, 14 farmers and 14 technicians. Veterinarians/scientists were selected on a geographical basis (all over the north west of France, i.e. the main pig production area in the country). Some of them were asked to select one farmer and one technician they work with. During the interview, the agents were asked to identify what they consider as the main issues for animal health and public health in the pig production chain. They were additionally demanded to explain why a given issue was regarded as a matter of concern.

\section{Results}

Seven items were the most frequently cited by agents. Diseases of pigs were the first issue that they cited, among which the main items were PRRS, Porcine Circovirus Associated Diseases, colibacillosis (mainly oedema disease whose occurrence seems to be increasing), actinobacillosis and enzootic pneumonia. The matter of concern is the improvement of control measures. Salmonella is regarded as a public health concern in the control of which the all production chain should be involved. Many agents insist on the need to reduce the use of antimicrobials for both economical and social reasons. Influenza was categorised as a "mediatic" issue although agents notice that diagnostic tools and vaccines could be improved for this infection. Herd management and biosecurity are cited as key tools for improvement of animal and public health.
Agents think that the pig production chain fails to communicate positively towards consumers.. Welfare regulations (especially sow group housing and piglet castration) are regarded as complex to implement and likely to lower the economic sustainability of the production chain.

Table 1: Items most frequently cited and number of citations per category of agent

\begin{tabular}{|l|c|c|c|}
\hline & Vets (45) & Technicians (14) & Farmers (14) \\
\hline Diseases of pigs & 27 & 11 & 7 \\
\hline Salmonella & 20 & 5 & 8 \\
\hline $\begin{array}{l}\text { Use of anti- } \\
\text { microbials }\end{array}$ & 28 & 9 & 6 \\
\hline Influenza & 21 & 4 & 6 \\
\hline $\begin{array}{l}\text { Management and } \\
\text { biosecurity }\end{array}$ & 12 & 6 & 5 \\
\hline Communication & 19 & 9 & 10 \\
\hline Welfare & 12 & 10 & 9 \\
\hline
\end{tabular}

\section{Discussion}

We were interested in questioning agents involved in animal and public health management at several levels: advisors, supervisors or implementers i.e. vets, technicians and producers respectively.

Apart from pig diseases and their impact on animal health and welfare as well as on farm productivity, many stakeholders highlighted the lack of communication on animal and public health in the pig production chain. Consequently although stakeholders feel more and more involved in the improvement of animal and public health, consumers seem not to be aware that these issues are taken into account. This point was raised as a specific item and was cited for Salmonella, use of antimicrobials, Influenza, welfare regulations.

\section{Bibliography}

Blanchet A \& Gotman A. (2007). L'enquête et ses méthodes: I'entretien. Ed Armand Colin, 126p 


\title{
P.640
}

\section{Use of antibiotics varies in Dutch pig nucleus herds}

\author{
Ellen Meijer ${ }^{1}$ Leo van Leengoed ${ }^{1}$ Inge van Geijlswijk ${ }^{2}$ Geertjan van Groenland ${ }^{3}$ Hanneke Feitsma ${ }^{4}$ Dik Mevius $^{5}$ \\ 1. Department of Farm Animal Health, Faculty of Veterinary Medicine, Utrecht University, Utrecht, Netherlands; \\ 2. Department of Veterinary Pharmacy, Faculty of Veterinary Medicine, Utrecht University, Utrecht, Netherlands; \\ 3. TOPIGS, Vught, Netherlands; 4. Institute for Pig Genetics, Beuningen, Netherlands; 5. Department of Infectious \\ Diseases and Immunology, Faculty of Veterinary Medicine, Utrecht University, Utrecht, Netherlands
}

\section{Introduction}

Despite public opinion to minimize the use of antibiotics in food-producing animals, use of antibiotics in pigs, veal calves and poultry has been increasing during the last decennia (1). The aim of this study was to quantify the use of antibiotics in Dutch nucleus herds in 2007 and 2008.

\section{Materials and Methods}

Thirty-seven nucleus farms of the Dutch breeding association TOPIGS were visited to collect data of the total quantity of antibiotics purchased in 2007 and 2008, and of the number of animals for each category (sows, weaned piglets, nursing piglets, gilts, finishing pigs). Additional data about management and medication regime were included in this survey. The quantity of antibiotics used was expressed in DD/ay (daily doses per animal year), which indicates with how many daily doses an 'average' animal of the farm is treated each year. The farms were divided into categories depending on health status: conventional $(n=33)$ or SPF $(n=4)$. DD/ay was analysed using a linear mixed model (2) allowing for repeated measurements and random effects of farm (nested within health status). Effects of year, health status and farm size were included in the model. Data were not normally distributed and therefore log transformed.

\section{Results}

Average DD/ay did not differ between 2007 and 2008. SPF farms have a lower mean DD/ay $(2,21 \pm 2,29)$ than conventional farms $(14,04 \pm 15,90)(r=0,68, p<0.001)$. DD/ay was positively correlated with the herd size (Figure 1).

Figure $1 \mathrm{Herd}$ size in relation to DD/ay. The dashed line represents the regression line for data from $2007(y=0,0601 x-6,118(R 2=0,4422))$, the solid line for the data from $2008(y=0,0512 x-5,8528(R 2=$ 0,5256)). White triangle $=2007$, black dot $=2008$

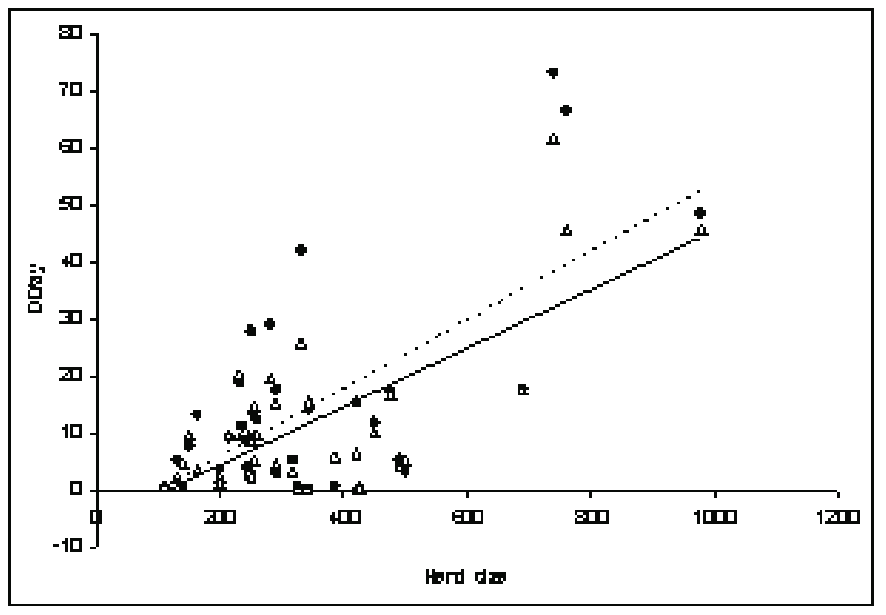

\section{Discussion}

There are huge differences in DD/ay between farms. These can partly be explained by health status and herd size.

As expected, SPF farms have lower DD/ay compared to conventional farms. This might be due to the absence of specific pathogens, and a strict hygiene. Strict hygiene itself may be a reason for lower prevalence of endemic infectious diseases, even in SPF herds. On conventional farms the difference in DD/ay between farms is striking. There is a more than 300-fold difference between the lowest and highest DD/ay.

The number of sows present on the farm is associated with the DD/ay. Data collected from individual farms showed that famers of large herds more often medicate pig groups routinely. Further analysis will be conducted to study the decision making with respect to group medication and its effect upon total DD/ay.

The large differences between herds and the assumed large influence of group medication on the total DD/ay indicates that a strategy is feasible to minimize the use of antibiotics in the future.

\section{References}

1) Bondt N., Toename antibioticagebruik varken en pluimvee in 2005 , LEl, Agrimonitor, 2006

2) SAS 9.1, SAS inst. Inc., Cary, NC 


\title{
P.641
}

\section{Restrictive use of antibiotics: who decides?}

\author{
$\underline{L e o ~ v a n ~ L e e n g o e d ~}^{1}$ Ellen Meijer ${ }^{1}$ Inge van Geijlswijk ${ }^{2}$ Geertjan van Groenland ${ }^{3}$ Merel Postma ${ }^{1}$ Hanneke Feitsma $^{4}$ Dik Mevius $^{5}$ \\ 1. Department of Farm Animal Health, Faculty of Veterinary Medicine, Utrecht University, Utrecht, Netherlands; \\ 2. Department of Veterinary Pharmacy, Faculty of Veterinary Medicine, Utrecht University, Utrecht, Netherlands; \\ 3. TOPIGS, Vught, Netherlands; 4. Institute for Pig Genetics, Beuningen, Netherlands; 5. Department of Infectious \\ Diseases and Immunology, Faculty of Veterinary Medicine, Utrecht University, Utrecht, Netherlands
}

Antibiotics in food-producing animals are often used to allow logistic efficacy in husbandry. This dictates a labor- saving strategy of group-medication to prevent disease. Risk upon infectious diseases, that are endemic, is managed by routine medication, and this preventive use of antibiotics has become a public health threat. The increase in prevalence of multi-resistant bacteria in swine herds that may affect both animals and humans $(1,2)$, urges to reduce the use of antibiotics. In contrast to the awareness of these risks the use of antibiotics in pigs, veal calves and poultry has been increasing during the last decennia (3). Therefore, a decision matrix was designed to get a transparent use of antibiotics in pig herds.

By definition, bacteria are selected for resistance when exposed to an antibiotic. Increase has been found in prevalence of animal-related Methicilline-Resistant Staphylococcus aureus (vMRSA) and Clostridium difficile ribotype 078, that both have a resistant profile that mimics antibiotics used in humans.

Therefore, a convenant between the Royal Dutch Veterinary Association and the Ministry of Agriculture is signed in 2009, and aims to reduce the use of antibiotics in farm animals (5). Veterinarians have economic interest in selling antibiotics to farmers. Farmers have economic interest in preventive use of antibiotics as it can cover up shortcomings of their husbandry system.

Ten TOPIGS nucleus herds were analyzed with respect to antibiotic use. Antibiotic use was expressed as DD/ay, which indicates with how many daily doses an 'average' animal of the farm is treated each year. The daily doses were calculated on label use of the antibiotics purchased at each farm. A huge variation was observed between herds: 0.7-57 DD/ay. High antibiotic use was based upon preventive use in oral medications in feed or drinking water. Routine medication of weaned piglets strongly contributed to the excessive use. However, 4 out of 10 of these nucleus herds had a DD/ay $<7$. Use of cephalosporin or fluoroquinolone, antibiotics that should be restricted to human use, was limited or absent in most of these herds. The observed variation in antibiotic use indicates that reduction in use is feasible. National and EU regulations with respect to antibiotic registration, hygiene conduct and animal welfare intend to limit antibiotic use and to optimize husbandry conditions.

A decision matrix for antibiotic prescription by veterinarians was designed, based upon national Dutch as well as EU regulations.

Routine medications in feed or drinking water account for the major quantity of antibiotics used and routine medication is always preventive. National regulations, however, state that farm animals should be kept in an environment that does not harm their health. This legal obligation is used in the decision matrix as an incentive to improve animal husbandry conditions. Each preventive medication of pig groups is coupled with an assessment of risk factors for infectious diseases present at the herd. Benchmarking of antibiotic use makes comparison of herds possible and transparent. Assessments with respect to working standards and education of farm workers are a consequence of use of the matrix, and essential to achieve reduction of antibiotic use.

\section{References}

1. Bondt N., Toename antibioticagebruik varken en pluimvee in 2005 , LEl, Agrimonitor, 2006

2. Schutte F., Antibioticagebruik in Nederland en Europa, Symposium "Op recept",KNMvD, 19 juni 2007.

3. Wulf, MW, Sørum, M., van Nes, A., et al. Prevalence of methicillin-resistant Staphylococcus aureus among veterinarians: an international study. Clin Microbiol Infect.2008;14:29-34.

4. Debast, S., van Leengoed, L., Goorhuis, A., Harmanus, C., Kuijper, E., Bergwerff, A., Clostridium difficile PCR ribotype 078 toxinotype $V$ found in diarrhoeal pigs identical to isolates from affected humans. Environmental Microbiol. 2009, 11(2):505-511.

5. Antibioticaconvenant www.knmvd.nl 


\title{
P.642
}

\section{The use of registration, analyses and a decision matrix to re-conduct the use of antibiotics on breeding pig farms in the Netherlands}

\author{
Geertjan van Groenland ${ }^{1}$ Eveline Willems ${ }^{1}$ Hanneke Feitsma $^{2}$ Leo van Leengoed ${ }^{3}$ Ellen Meijer $^{3}$ Inge van Geijlswijk $^{4}$ Dik Mevius $^{5}$ \\ 1. TOPIGS S\&D, Helvoirt, Netherlands; 2. Institute for Pig Genetics, Beuningen, Netherlands; 3. Dept. of Farm Animal Health, Faculty of Veterinary \\ Medicine, Utrecht University, Utrecht, Netherlands; 4. Dept. of Veterinary Pharmacy, Faculty of Veterinary Medicine, Utrecht University, Utrecht, \\ Netherlands; 5. Dept. of Infectious Diseases and Immunology, Faculty of Veterinary medicine, Utrecht University, Utrecht, Netherlands
}

\section{Introduction}

The use of antibiotics in food-producing animals has been increasing during the last decades in regions with intensive live stock production. The increase in prevalence of multi-resistant bacteria in swine herds that may affect both animals and humans, urges to reduce the use of antibiotics. In the Netherlands this has lead to an agreement between the most important players in the field of live stock production. This will lead to a national registration of the use of antibiotics per farm expressed in the so called DD/ay (Daily Doses per animal year). These registrations will be followed by analyses and advises to come to a substantial reduction and a better awareness of proper use of antibiotics as a tool to treat diseased animals.

The extensive use of antibiotics can partly be explained by a number of factors among others:

- Use out of habit - "we have always done so"- often not realizing that the original cause of using the treatment has vanished already for a long time.

- Masking management failures. In using antibiotics often necessary changes in the farm and the management are delayed.

- Treatments have been shifted from curative to preventive where often such treatments are given without a real effect or even sometimes with negative side effects.

\section{Registration of the use of antibiotics on farms}

On 45 of the TOPIGS breeding farms in The Netherlands (almost $50 \%$ of the breeding stock) registration of the DD/ay has been done for the years 2007 and 2008. The same will be done for the year 2009. It is done in such a way that more extensive analyses per farm can be done:

- Registration per category of animals present (i.e. sows, suckling and weaned piglets, rearing pigs and if present finishers).

- Registration of the administration route of the medication (i.e. in or over feed via drinking water in case of group medication, routine needle medication, individual treatments based on farm specific formularia).

- Registration of the used groups of type of medication.

\section{Interventions and further actions}

In close co-operation with farmers and their farm veterinarians, a decision matrix will be implemented in order to omit all preventive treatments and mass medications and to exclusively use antibiotics for curative purposes that complies with Good Veterinary Practice. This will be achieved by:

- accurate instruction and training of herdsmen and farm workers with respect to risks, precautions, side effects and administration techniques.

- the before mentioned intensive analysis, bench marking between the various breeding farms,

- a thorough analysis of the causes of disease problems that exist on the farm (with support of laboratory analyses),

- an intensive analysis of managerial improvement items. In this field one can think of:

- internal bio-security improvements like age separation, prevention of mixing animals, working order, prevention of unnecessary contact structures, etc.

- changes in farm lay-out and housing aspects

- a critical evaluation and possibly adaptation of the vaccination program running on the farm

We realize that more factors will be taken into account in adapting the farms to a strategy that must lead to a change of mind in using antibiotics and preventive interventions by antibiotics will be avoided.

As a breeding company, being on top of the pyramid of producing meat for human consumption, we take our responsibility. We have to be ahead on the growing awareness in the Netherlands that social acceptance of meat production goes along with restricted use of antibiotics.

\section{Future developments}

It will be clear that at this very moment of submitting the abstract, implementing of the above described procedures is going on. In June 2010 new data of this project will be available and incorporated in our presentation at IPVS.

\section{References}

Available at the author. 


\title{
P.643
}

\section{Isolation of Methicillin-Resistant Staphylococcus aureus from nasal swabs and Broncho Alveolar Lavage Fluid from flatdeck and fattening pigs in Northwest Germany}

\author{
Christoph Keller $^{1}$ Christian Brüggemann $^{2}$ Rolf Nathaus ${ }^{2}$ Heinrich Wilkes ${ }^{2}$ Ohlinger F. Volker ${ }^{1}$ \\ 1. BioScreen EVDMC GmbH, Münster, Germany; 2. VETeam Reken, Reken, Germany
}

\begin{abstract}
Introduction
Staphylococcus aureus is a Gram-positive Bacterium, that occurs in man and practically all species of animals. Apart from clinically latent colonization of skin and mucous membranes, it is a potentially pathogenic bacterium, that causes a broad range of diseases. Methicillin resistant S. aureus (MRSA) isolates causes severe nosocomial infections in humans. ${ }^{1}$ Recent studies demonstrate, that MRSA has a very high prevalence in pigs. ${ }^{2,3}$ As so far no clinical disease can be linked to MRSA or methicillin sensible S. aureus (MSSA) in pigs, the pathological importance of the high prevalence of $S$. aureus in pigs is not clear. The aim of this study was to investigate the occurrence of MRSA and MSSA in lower respiratory tract of pigs.
\end{abstract}

\section{Materials and Methods}

230 BALF samples and nasal swabs were taken by the same veterinarian on 20 piglet producing farms and 6 connected finisher units. All samples were inoculated on Columbia blood agar plates, Columbia blood agar plates with CNA and a chromogenic selective agar for the isolation of MRSA (MRSA-Ident agar) (heipha Dr. Müller GmbH, Eppelheim, Germany) within $24 \mathrm{~h}$ after sampling. Plates were incubated at $37^{\circ} \mathrm{C}$. Bacterial growth was evaluated after 24 and $48 \mathrm{~h}$ of incubation. All suspicious Staphylococci were isolated. Biochemical identified was done by the use of API ID 32 Staph (bioMérieux Deutschland GmbH, Nürtingen, Germany). S. aureus isolates were additionally identified by a S. aureus specific PCR and tested for the presence of the mecA gene. ${ }^{4}$

\section{Results}

A total of 180 S. aureus were isolated in this study. The majority of these isolates were MRSA (158 out of $180 ; 87,78 \%$ ). MRSA could be isolated from 126 out of 230 nasal swabs (54.8\%), whereas only 32 BALF samples from the same animals were positive for MRSA. A comparable number of both sample types were positive for MSSA: 12 (5.2\%) of the nasal swabs and 10 (4.4 $\%)$ of the BALF samples. Seven nasal swabs were positive for MRSA and MSSA (3.0\%), but only in two BALF samples $(0.9 \%)$ MRSA and MSSA were detected in parallel. One pig was positive for MRSA and MSSA in the nasal swab and the BALF sample. No S. aureus were detected in 99 nasal swabs (43.0\%) and in 190 $(82.6 \%)$ of the BALF samples. In nasal swabs from 23 farms MRSA were detected $(88.5 \%)$, whereas only 12 farms (46.1\%) were positive for MRSA when examining the BALF samples. MSSA were detected in the nasal swabs from seven farms $(26.5 \%)$ and in the BALF from five farms (19.2\%).

\section{Discussion}

The majority of the $S$. aureus isolates found in this study were resistant to methicillin. Interestingly MRSA and MSSA were not present at the same ratio in nasal swabs (12.6:1) and BALF (3.2:1) samples from the same animals. This indicates that MRSA colonizes preferably in the nasal cavity and has no tendency to colonize the lung alveola. Most of the nasal swabs, that were positive for MSSA in the nasal swab were also positive for MRSA (7 out of $12 ; 58.33 \%$ ). This indicates, that antibiotic pressure is not the major cause for the high prevalence of MRSA in pigs. Although the prevalence of MRSA on pig farms and in pigs is very high, in this study $88.5 \%$ and $54.8 \%$ respectively, so far no clinical disease could be linked to MRSA in pigs. Further investigations will be necessary to understand the significance of MRSA in pigs.

\section{References}

1. A. Pantosti and M. Venditti. What is MRSA?. Eur Respir J. 2009: 34, 1190-1196.

2. R. Köck, J. Harlizius, N. Bressan et al. Prevalence and molecular characteristics of methicillin-resitant Staphylococcus aureus (MRSA) among pigs on German farms and import of livestock-related MRSA into hospitals. Eur J Clin Microbiol Infect Dis. 2009: 28, 1375-1382.

3. B.-A. Tenhagen, A. Fetsch, B. Stührenberg et al. Prevalence of MRSA types in slaughter pigs in different German abattoirs. Veterinary Record. 2009: 165, 589-593.

4. F. Martineau, F.J. Picard, N. Lansac et al. Correlation between the Resistance Genotype Determined by Multiplex PCR Assays and the Antibiotic Susceptibility Patterns of Staphylococcus aureus and Staphylococcus epidermidis. Antimicrob Agents Chemother. 2000: $44,231-238$. 


\title{
P.644
}

\section{Occurrence of MRSA isolated from pathologically altered porcine tissues from 2004 to 2007}

\author{
Diana Meemken ${ }^{2}$ Regina Tegeler ${ }^{2}$ Berd-Alois Tenhagen ${ }^{1}$ Beatriz Guerra ${ }^{1}$ Alexandra Fetsch ${ }^{1}$ Thomas Blaha $^{2}$ \\ 1. Federal Institute for Risk Assessment, Department of Biological Safety, Berlin, Germany; 2. Field Station \\ for Epidemiology, University of Veterinary Medicine Hannover, Bakum, Germany
}

\section{Introduction}

Since 2005 data on Methicillin-resistant Staphylococcus aureus (MRSA) isolated from farm animals have been published in various countries $(1,2,3)$. Most of these MRSA are of the spa-types which are associated with the Multilocus Sequence Type (MLST) ST398. This type was also found as nasal colonizer in farmers, veterinarians and slaughterhouse staff $(1,2)$. As in humans, the nasal colonization of MRSA in pigs is in most cases without clinical symptoms. However, there are reports on MRSA as causative agent with clinical symptoms such as exudative dermatitis (4). Triggered by the fact that most researchers assume an emerging occurrence of MRSA ST398 since 2005 in farm animals, our study focuses on two questions:

a) Has MRSA ST398 only started to occur in pigs in 2005?

b) Can MRSA be isolated from pathologically altered tissues in pigs?

This paper reports the results of reculturing S. aureus strains isolated from inflammatory lesions of pigs submitted for diagnostic purposes to the Field Station for Epidemiology of the University for Veterinary Medicine Hannover, Germany.

\section{Material and Methods}

In this study, 138 stored S. aureus isolates, collected between 2004 and 2007 from various pathological lesions of pigs at necropsy, were recultured and potential MRSA were identified by growth on selective chromogenic media. At the Federal Institute for Risk Assessment, Berlin, Germany, all presumptive MRSA isolates were analysed for confirmation by a triplex PCR (5). Furthermore, spa-typing (6) of all confirmed MRSA isolates was performed to determine whether the isolates belong to spa-types associated with the MLST-type ST398. Additionally, all 138 cases were analysed whether $S$. aureus was found in pure or in mixed cultures.

\section{Results}

Out of the 138 S. aureus strains, isolated from pathologically altered porcine tissues between 2004 and 2007, 60 isolates (43\%) turned out to be MRSA (Tab. 1). The majority (57/60) of the MRSA-isolates found were spa-types associated with MRSA ST398. Three MRSA were ST97, a type that has not been described as an MRSA in pigs before. The proportion of MRSA in the tissues are shown in Table 2.
Table 1: Annual distribution of MRSA among 138 S. aureus isolates and No. of MRSA pure in cultures

\begin{tabular}{|l|c|c|c|}
\hline Year & No. of S. aureus & No. of MRSA & No. of MRSA in pure cultures \\
\hline 2004 & 34 & $11(32 \%)$ & $4(36 \%)$ \\
\hline 2004 & 30 & $15(50 \%)$ & $7(47 \%)$ \\
\hline 2006 & 41 & $16(39 \%)$ & $6(38 \%)$ \\
\hline 2007 & 33 & $18(55 \%)$ & $4(22 \%)$ \\
\hline Total & 138 & $60(43 \%)$ & $21(35 \%)$ \\
\hline
\end{tabular}

Table 2: The proportion of MRSA in different tissues and the No. of MRSA in pure cultures

\begin{tabular}{|l|c|c|c|}
\hline Tissue & No. of S. aureus & No. of MRSA & No. of MRSA in pure cultures \\
\hline Skin & 49 & $23(47 \%)$ & $5(22 \%)$ \\
\hline Joint & 25 & $12(48 \%)$ & $10(83 \%)$ \\
\hline CNS & 24 & $8(33 \%)$ & $2(25 \%)$ \\
\hline Lung & 15 & $8(53 \%)$ & $0(0 \%)$ \\
\hline Abscess & 7 & $1(14 \%)$ & $1(100 \%)$ \\
\hline Pericardium & 4 & $2(50 \%)$ & $1(50 \%)$ \\
\hline Others & 14 & $6(43 \%)$ & $2(33 \%)$ \\
\hline Total & 138 & $60(43 \%)$ & $21(35 \%)$ \\
\hline
\end{tabular}

\section{Discussion}

Since MRSA ST398 isolates were found in similar frequency in all four years, we assume that MRSA in pigs may have occurred earlier than 2004 and might be not really emerging, but rather have been overlooked until recently. Furthermore, MRSA ST398 is not only a nasal colonizer, but needs to be discussed as contributor (or even cause) of various lesions in pigs.

\section{References}

(1) Voss et al. (2005), Emerg. Infect. Dis 11:1965-1966

(2) Meemken et al. (2008), Dtsch Tierarztl Wochenschr.115:132-139

(3) Khanna et al. (2008), Vet Microbial 128: 298-303

(4) van Duijkeren et al. (2007), Emerg. Infect Dis 13: 1408-1410

(5) Poulsen et al. (2003), J Antimicrob. Agents Chemother. 51: 419-421

(6) Shopsin et al. (1999), J Clin Microbiol 37: 3556-3563 


\title{
P.645
}

\section{Screening pig farms for MRSA in the Euregio-Region of Lower Saxony part of the MRSA-Safeguard-Project}

\author{
Josef Schulte-Wuelwer ${ }^{1}$ Robin Koeck ${ }^{3}$ Alexander W. Friedrich ${ }^{3}$ Susanne Gundlach ${ }^{2}$ Juergen Apel $^{2}$ Hendrik Nienhoff $^{2}$ \\ 1. Swine Health Service, Chamber of Agriculture Lower Saxony, Oldenburg, Germany; 2. LUFA NRW, Muenster, \\ Germany; 3. Institute of Hygiene, University Hospital Muenster, Muenster, Germany
}

\section{Introduction}

Resistant pathogens, especially coagulase-positive staphylococci are a major challenge in both human- and veterinary medicine. The importance of MRSA in swine production for human medicine has grown in the last years $(1,2)$. The aim of this study was to estimate the prevalence of MRSA in German pig farms in the Dutch-German border region (Euregio) of Lower Saxony, Germany.

\section{Materials and Methods}

From May to Oct. 200954 pig farms were tested for the presence of MRSA. Five barn dust samples or ten nasal swabs were taken per farm. After 24-h enrichment culture in selective broth, all cultures were streaked onto Columbia-blood agar and a chromogenic medium for the detection of MRSA (bioMérieux, Marcy l'Etoile, France). Methicillin-resistance was tested by cefoxitin disc agar diffusion and was confirmed by mecA-specific PCR (5) and all MRSA isolates were typed by $\mathrm{S}$. aureus protein A (spa) sequence-based typing (6).

\section{Results}

31 farm were positive (57,4\%). Among these, 26 farms were detected positive by barn dust, 5 by nasal swabs. $22,2 \%$ of the barn dust samples were positive and $43,6 \%$ of the nasal swabs were positive. The geographic distribution of MRSA positive and negative farms is shown in Fig. 1.

Fig. 1: Location of the sampled and positive pig farms

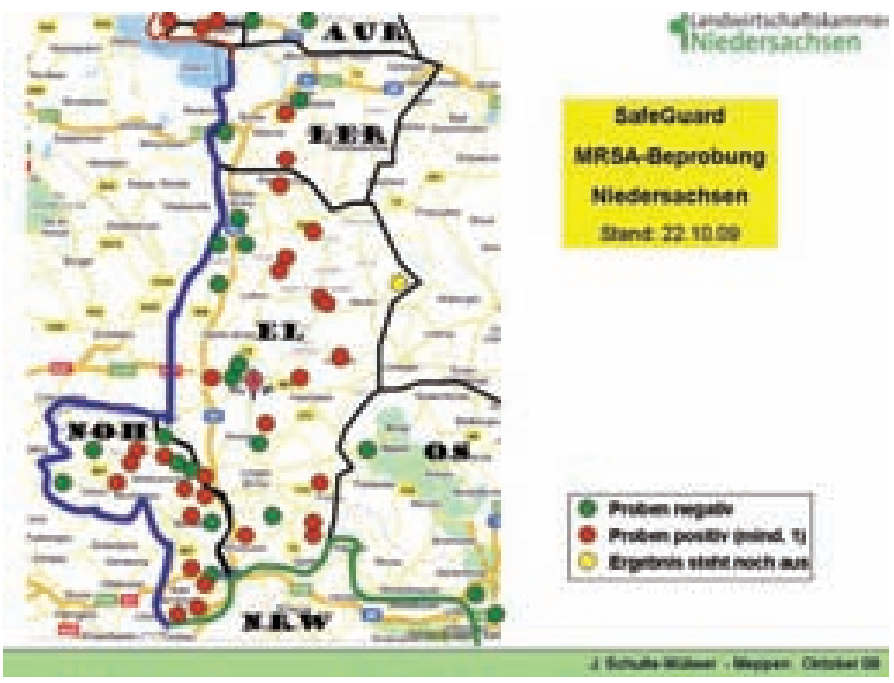

Up to now spa-typing result of 25 farms are available (Table 1)

Table 1: Results of the spa-typing

\begin{tabular}{|l|c|c|}
\hline Spa-type & No. of farms & In combination with \\
\hline t011 & 15 & $1 \times$ t108 \\
\hline t034 & 7 & \\
\hline t108 & 1 & $1 \times$ t011 \\
\hline t1451 & 2 & $1 \times$ t3992 \\
\hline t2576 & 1 & \\
\hline t3992 & 1 & $1 \times \mathrm{t} 1451$ \\
\hline
\end{tabular}

\section{Discussion}

Up to now no correlation between different farms or types of farms could be found. Breeding herds as well as fattening farms had positive results. Also new built farms and high health herds were positive. No regional accumulation in relation to pig density could be found. Our findings, including the spa-typing results, correspond well, to data of other authors $(2,3,4)$.

\section{References}

1. Voss A, Loeffen F, Bakker J, Klaassen C, Wulf M (2005) Emerg Infect Dis 11:1965-1966

2. Huijsdens XW, van Dijke BJ, Spalburg E, van Santen-Verheuvel MG, Heck MEOC, Pluister GN, Voss A, Wannet WJB, de Neeling AJ (2006). Ann Clin Microbiol Antimicrob 5:26

3. Meemken D, Cuny C, Witte W, Eichler U, Staudt R, Blaha T (2008). Dtsch Tierarztl Wochenschr 115:132-139

4. R. Köck, J. Harlizius, N. Bressan, R. Laerberg, L.H. Wieler, W. Witte, R.H. Deurenberg, A. Voss, K. Becker, A.W. Friedrich. Eur. J. Clin. Microbiol. Infect. Dis. 28 (11) 2009 1375-1382

5. Becker K, Pagnier I, Schuhen B, Wenzelburger F, Friedrich AW, Kipp F, Peters G, von Eiff C (2006) J Clin Microbiol 44:229-231

6. Mellmann A, Friedrich AW, Rosenkotter N, Rothganger J, Karch H, Reintjes R, Harmsen D (2006). PLoS Med 3:e33 


\title{
P.646
}

\section{A longitudinal study on MRSA colonization on gilts in supplier farms}

\author{
Jürgen Harlizius ${ }^{1}$ Uwe Heinbach ${ }^{1}$ Susanne Gundlach² Alexander Friedrich ${ }^{3}$ Robin Köck ${ }^{3}$ \\ 1. Animal Health Services, Landwirtschaftskammer NRW, Bonn, Germany; 2. LUFA, Landwirtschaftskammer NRW, Münster, Germany; \\ 3. Universitätsklinikum, Münster, Germany
}

\section{Introduction}

Recently, Methicillin-resistant Staphylococcus aureus (MRSA) have emerged in pigs in most European countries (1,3). Pigs colonized with MRSA are found on $70 \%$ of pig farms in North Rhine-Westphalia, Germany (2). However, the dynamics of colonization among the animals is unclear and strategies for avoiding the spread of MRSA between pig holdings are lacking.

We raised the question when gilts from a nucleus herd documented to be specifically "free" of MRSA get colonized, if they are introduced into MRSA positive farms.

\section{Methods}

Within the framework of the Euregio project SafeGuard VetMednet we identified 2 MRSA free nucleus farms. We chose 3 farms on which MRSA had been detected before in animals or the environment and one farm which was free of MRSA when tested consecutively by pooled samples $(n=5)$. One nucleus herd was build up PaR, PRRS and M.hyo. free after repop/depop in 2007. Since this time the farm is also free of MRSA. We analysed the colonization status of MRSA free gilts introduced into breeding herds known to harbour MRSA colonized pigs and herds which were tested negative for MRSA.

A total of 65 gilts tested negative for MRSA colonization, were introduced into three MRSA positive and one MRSA negative pig holdings. Immediately after the introduction of the gilts into the new herds, nasal swabs were derived from all 65 gilts. During a ten-week-period, swabs were taken 7 times with a sampling interval of 1-3 weeks. MRSA diagnostic was performed using a double enrichment in selective broth (Phenol Red Mannitol Broth with $5 \mathrm{mg} / \mathrm{L}$ ceftizoxime, $75 \mathrm{mg} / \mathrm{L}$ aztreonam) and plating on a chromogenic medium (Brilliance MRSA, Oxoid, Wesel, Germany). MRSA was confirmed using coagulase tube test and mecA-PCR All MRSA isolates were typed using the Staphylococcus aureus protein A (spa) approach (4).

\section{Results}

All gilts tested negative for MRSA colonization in the nucleus farms and introduced into the breeding herd in which MRSA had not been detected before, remained negative.

MRSA negative gilts introduced into MRSA positive herds, the gilts remained MRSA free again into the isolation pens. But immediately after introducing the gilts into the sows of the breeding stock, MRSA was detected. In the first week an overall colonization rate of $61 \%$ was observed, followed by $49 \%$ after 2 weeks, and $73 \%$ after 3 weeks, respectively.

Although colonization rate was fluctuant, overall $88 \%$ of all MRSA negative gilts introduced into positive MRSA holdings were found to acquire MRSA at least intermittently. Clinical symptoms were not seen in any sow. Characterization of spa types identified MRSA t011, t034 and t5838.

\section{Discussion}

We found that there are still MRSA negative pig production chains. Introduction of MRSA free gilts in positive herds led to frequent, but fluctuating colonization of the individual gilts. Therefore, it is unclear to what extent the colonization is transient or persistent. Since there was no clear difference in the use of antimicrobials between the MRSA negative and positive farms, preventive efforts will have to be set up for cleaning and disinfection schemes.

\section{References}

1. The European Food Safety Authority, EFSA Journal 2009; 7(11):1376. [82 pp.]. doi:10.2903/j.efsa.2009.1376. Available online: www.efsa. europa.eu

2. Köck R, Harlizius J, Bressan N, Laerberg R, Wieler L H, Witte W, Deurenberg RH, Voss A, Becker K, Friedrich AW (2009) Eur J Clin Microbiol Infect Dis 28: 1375-1382.

3. Neeling de, A J, van den Broek M J, Spalburg E, C, van Santen-Verheuvel MG, Dam-Deisz W D, Boshuizen HC, van de Giessen AW, van Duijkeren E, Huijsdens XW. (2007) Vet Microbiol, 122:366-372.

4. Mellmann A, Friedrich AW, Rosenkötter N, Rothgänger J, Karch $\mathrm{H}$, Reintjes R, Harmsen D. (2006) PLoS Med, Ma;3(3):e. 


\title{
P.647
}

\section{Longitudinal study on the colonisation dynamics of MRSA ST398 in two German pig herds}

\author{
Diana Meemken ${ }^{1}$ Thomas Blaha ${ }^{1}$ Rolf Nathaus ${ }^{2}$ \\ 1. Field Station for Epidemiology, University of Veterinary Medicine Hannover, Bakum, \\ Germany; 2. Veterinary Clinic Reken, Vet-Team-Reken, Reken, Germany
}

\section{Introduction}

The occurrence of one special clonal line of Methicillin-resistant S. aureus (MRSA) in farm animals, the so called livestock-associated MRSA ST398, is reported in several countries such as The Netherlands (1), Canada (2) and Germany (3). However, there are only few data illustrating the in-herd patterns of colonisation during the pigs' lifetime. Our study presents information about the colonisation dynamics of MRSA in pigs from birth to the end of the nursery in two German pig herds.

\section{Material and Methods}

Data collection took place between November 2008 and May 2009 on two independent pig farms in the Northwest of Germany. Farm $A$ is a two-site production system with 80 sows and farm B is a farrow-to-finish herd with 170 sows. On farm A, 58 piglets (two piglets each from 29 sows) and on farm $B 40$ piglets (two piglets each from 20 sows) were earmarked and tested repeatedly at a three week interval for MRSA by pharyngeal or nasal swab from birth until the end of nursery. Additionally, nasal swabs were taken from the 29 sows in herd $A$ and from the 20 sows in herd $B$ to learn more about the transmission of MRSA between the sow and its offspring.

\section{Results}

The proportion of MRSA-positive animals differed between the two farms remarkably (Tab. 1, Tab. 2).

Tab. 1: Microbiological results of nasal swabs of 29 sows and 58 piglets (two piglets each per sow) in farm $A$

\begin{tabular}{|l|c|c|c|c|}
\hline & $\begin{array}{c}\text { MRSA- } \\
\text { positive }\end{array}$ & $\begin{array}{c}\text { MSSA- } \\
\text { positive }\end{array}$ & $\begin{array}{c}\text { MRSA- and } \\
\text { MSSA-positive }\end{array}$ & $\begin{array}{c}\text { S. aureus- } \\
\text { negative }\end{array}$ \\
\hline sows (peripartal) & $52 \%$ & $7 \%$ & $10 \%$ & $31 \%$ \\
\hline piglets (day 1-3) & $16 \%$ & $17 \%$ & $0 \%$ & $67 \%$ \\
\hline piglets (day 21) & $48 \%$ & $26 \%$ & $0 \%$ & $26 \%$ \\
\hline piglets (day 42) & $19 \%$ & $33 \%$ & $7 \%$ & $41 \%$ \\
\hline piglets (day 63) & $20 \%$ & $33 \%$ & $31 \%$ & $16 \%$ \\
\hline
\end{tabular}

Tab. 2: Microbiological results of nasal swabs of 20 sows and 40 piglets (two piglets each per sow) in farm $B$

\begin{tabular}{|l|c|c|c|c|}
\hline & $\begin{array}{c}\text { MRSA- } \\
\text { positive }\end{array}$ & $\begin{array}{c}\text { MSSA- } \\
\text { positive }\end{array}$ & $\begin{array}{c}\text { MRSA- and } \\
\text { MSSA-positive }\end{array}$ & $\begin{array}{c}\text { S. aureus- } \\
\text { negative }\end{array}$ \\
\hline sows (peripartal) & $0 \%$ & $0 \%$ & $0 \%$ & $100 \%$ \\
\hline piglets (day 1-3) & $0 \%$ & $10 \%$ & $0 \%$ & $90 \%$ \\
\hline piglets (day 21) & $7 \%$ & $13 \%$ & $0 \%$ & $80 \%$ \\
\hline piglets (day 42) & $25 \%$ & $3 \%$ & $0 \%$ & $72 \%$ \\
\hline piglets (day 63) & $8 \%$ & $60 \%$ & $0 \%$ & $32 \%$ \\
\hline
\end{tabular}

While on farm A MRSA was detected in sows and piglets at the first sampling date, positive results on farm $B$ in piglets did not occur before three weeks of life. However, findings of Methicillinsensitive S. aureus (MSSA) in combination with Methicillinresistant $S$. aureus at the same time are possible and can occur in quite high proportions as ascertained on farm A. Although $S$. aureus belongs to the normal porcine skin flora, an absence of S. aureus in the nasal cavity of sows and piglets is detectable in both farms, especially in farm B.

\section{Discussion}

The colonisation dynamics of MRSA and MSSA varies remarkably between farms. The MRSA colonisation status of pigs can change during the animals' lifetime. The MRSA-status of the sow has a strong impact on the MRSA-status of the newborn piglet, and a weaker impact on the colonisation at later points in time during the animals' lifetime. Futhermore, our results support the assumption that an early colonisation with MSSA might have a protective effect due to a phenomenon similar to a competitive exclusion between MSSA and MRSA. To be free from S. aureus early in life seems to lead to more MRSA findings at older ages.

\section{References}

(1) de Neeling et al. (2007), Vet. Microbiol., 122

(2) Khanna et al. (2008), Vet. Microb., 128

(3) Meemken et al. (2008), German Vet. J., 115 


\title{
P.648
}

\section{Survey of methicillin-resistant Staphylococcus aureus (MRSA) from Italian pig finishing holdings}

\author{
Antonio Battisti ${ }^{1}$ Alessia Franco ${ }^{1}$ Henrik Hasman ${ }^{2}$ Manuela lurescia ${ }^{1}$ Raniero Lorenzetti ${ }^{1}$ \\ Fabiola Feltrin ${ }^{1}$ Maurizio Zini ${ }^{1}$ Frank M. Aarestrup ${ }^{2}$ Giuseppe Merialdi ${ }^{3}$ \\ 1. Istituto Zooprofilattico Sperimentale delle Regioni Lazio e Toscana, Rome, Italy; 2. National Food Institute - DTU, Copenhagen, \\ Denmark; 3. Istituto Zooprofilattico Sperimentale della Lombardia e dell'Emilia - Romagna, Bologna, Italy
}

\begin{abstract}
Introduction
Methicillin-resistant Staphylococcus aureus (MRSA) is a leading cause of hospital-associated infections, and has emerged as a significant pathogen in the community. In the last decade, community-acquired MRSA (CA-MRSA) clones have been increasingly detected in companion animals and livestock. Recently, isolates belonging to MRSA Clonal Complex (CC) 398 have emerged in pig farming and it is now prevalent in farm animals in some countries of Central and Northern Europe (de Neeling et al., 2007). MRSA CC398 has also emerged as a cause of colonization and infection in humans and it is responsible for $>20 \%$ of all human MRSA in the Netherlands. The aim of the study was to estimate the herd prevalence of MRSA in finishing pig holdings in Italy, to provide phenotypic and molecular characterization of isolates for epidemiological purposes, and to gather information about their relationships and their possible origin.
\end{abstract}

\section{Materials and Methods}

Herds were selected by a random sampling procedure (20\% exp. prev.; $95 \% \mathrm{CL} ; 7.5 \%$ accuracy) from the national registry of holdings rearing pigs for slaughter, at the base of the pig production pyramid. For among-herd prevalence estimates, 118 holdings from 29 provinces and 10 different regions (Northern, Central and Southern, accounting for $>90 \%$ of the Italian pig population) were enrolled for the survey. Sixty nasal swabs per holding were sampled at slaughter, they were pooled ( 10 per pool) and cultured through a multiple step procedure (pre-enrichment, selective enrichment and selective plating).

\section{Results}

A total of 45 out of 118 (38.1\%, 95\% Cl 29.4\%-46.9\%) holdings were positive for MRSA. Nearly 50\% (22/45) of positive holdings had only one positive pool. Eleven different spa-types were detected, three of which never described before (t4794, t4795, t4838), and belonging to five different MLST profiles, ST398, ST1, ST9, ST97, ST1476. 7.6\% of holdings had their animals positive for 2, 3 or 4 unrelated spa-types belonging to ST1, ST97, ST1476 or ST398 in different combinations. Twenty-eight percent of holdings were positive for pig-associated ST398 lineage and $5.9 \%$ were positive for human-associated MRSA (t127, ST1 SCCmec Type V). Fourteen holdings were positive for other lineages, one for ST9 (t4794), three for t4795, ST97 and ten for t1730. The most prevalent spa-type was t899, ST398, detected in $22 / 45$ (49\%) positive holdings, with an overall holding prevalence of $18.6 \%$. Cattle- and human-associated CCs (CC97 and CC1) were found in $40 \%$ of positive holdings. The most frequent $\mathrm{SCCmec}$ ccr and mec combination was Type V, detected in $77 \%$ of isolates. All isolates were resistant to tetracyclines, and high resistance rates to trimethoprim, erythromycin and ciprofloxacin were detected. A pattern of tetracycline-erythromycin-ciprofloxacin resistance was a common feature among ST1 and ST(CC)97 lineages.

\section{Discussion}

This is the first description of: MRSA among pig population in Italy; three novel spa-types (t4794, ST9; t4795, ST97; t4838, ST398); MRSA of ST1 ST1476 and ST97 lineages among pigs, and the first report of MRSA ST9 in Europe. Spa type t899, ST398, was the most prevalent spa-type, although it had been considered a rare type in pigs until recent description in the Netherlands (van Duijkeren et al. 2008). The presence of unrelated spa-types within the same holdings (up to four spa-types), either belonging to ST398, CC97 or to ST1, suggests subsequent introduction of new strains over time as the most common pattern of colonization and diffusion among animals and holdings. This population study shows that pigs may be a possible reservoir for MRSA of the cattle-associated CC97 lineages, and for the human-associated ST1 (found in 6\% of Italian holdings), and a source of exposure for the community from a zoonotic pathogen that may need little or no adaptation to humans. The antimicrobial resistance profiles suggest that beside the use of beta-lactams, the amount and pattern of usage of other drugs should be investigated as a further important predictor in the spread of MRSA within and among Italian pig holdings.

\section{References}

de Neeling, A.J., van den Broek, M.J., Spalburg, E.C., van SantenVerheuvel, M.G., Dam-Deisz, W.D., Boshuizen, H.C., van de Giessen, A.W., van Duijkeren, E., Huijsdens, X.W., 2007. High prevalence of methicillin resistant Staphylococcus aureus in pigs. Vet. Microbiol. 122, 366-372.

van Duijkeren, E., Ikawaty, R., Broekhuizen-Stins, M.J., Jansen, M.D., Spalburg, E.C., de Neeling, A.J., Allaart, J.G., van Nes, A., Wagenaar, J.A., Fluit, A.C., 2008. Transmission of methicillin-resistant Staphylococcus aureus strains between different kinds of pig farms. Vet. Microbiol. $126,383-389$. 


\title{
P.649
}

\section{Occurrence of MRSA in air and exhaust air of pig barns}

\author{
Anika Friese ${ }^{1}$ Jochen Schulz ${ }^{2}$ Laura Höhle $^{1}$ Alexandra Fetsch ${ }^{3}$ Joerg Hartung ${ }^{2}$ Uwe Roesler $^{1}$ \\ 1. Free University Berlin, Institute of Animal Hygiene and Environmental Health, Berlin, Germany; 2. \\ University of Veterinary Medicine Hannover, Institute for Animal Hygiene, Animal Welfare and Farm Animal \\ Behaviour, Hannover, Germany; 3. Federal Institute for Risk Assessment, Berlin, Germany
}

\section{Introduction}

In recent years a distinction was made between community acquired [1] and animal associated MRSA (methicillin-resistant Staphylococcus aureus), that are also spread in pig production [2]. However, the knowledge on occurrence and transmission are still scarce.

From 2009 to 2011 a nationwide joint research project on MRSA in livestock production in Germany is currently funded by the Federal Ministry of Food, Agriculture and Consumer Protection. This paper gives preliminary results on the incidence of MRSA in animal house air and in exhaust air from pig barns.

\section{Materials and Methods}

\section{Sampled flocks}

A representative number of pig barns of different sizes and management schemes were investigated. MRSA positive and -negative barns were selected by initial screening of dust samples. Most barns were investigated once,some were chosen for longterm studies and will be continuously observed for one year.

\section{Sampling methods}

Environmental samples such as air samples, sock swabs, pooled samples from dust, faces and feed were collected. In addition swab samples of nose and skin of 60 pigs were taken in each barn. Air samples were collected in $1.50 \mathrm{~m}$ height above ground floor at three different locations in the animal house. For sampling All-Glas-Impingers (AGI-30) and IOM samplers (polycarbonate filter) were used simultaneously.

In barns chosen for the long-term-studies air samples of the exhaust air as well as sock swab samples from the ground outside the barn were taken. Air and sock swab samples were taken in different distances from the barn (100 m Luv, as well as $50 \mathrm{~m}$, $150 \mathrm{~m}$ and $300 \mathrm{~m}$ Lee, respectively).

\section{Laboratory methods}

All samples were analysed qualitatively for MRSA. Additionally, quantitative analyses of the nose swabs and all air samples were performed. Swab samples of nose and skin were investigated individually and pooled.

Selective agar (CHROMagar) in combination with a triplex-PCR is used for the identification of MRSA.

\section{Results}

Until now, MRSA was found in dust samples in $51.2 \%$ of 43 initial investigated flocks.

$92.5 \%$ of the individual investigated nasal swabs of all observed flocks ( $n=132$ ) were MRSA positive, with a mean bacteria count of 860 Cfu per swab. However $99.3 \%$ of the pooled samples of nasal swabs ( $n=132$ ), yield positive results. Concerning the skin swabs $88 \%$ of individual analyzed samples $(n=132)$ and $99.3 \%$ of pooled samples $(n=132)$ were MRSA positive.

MRSA could be found in at least one of three air samples of every stable. In general, MRSA could be found in $78 \%$ of the impinger and $47 \%$ of the filter samples from stable air. The mean counts of MRSA were about 435 Cfu / m3 for impinger and 452 Cfu / m3 for filter samples.

MRSA was detected in each dust sample with a mean count of $7.6 \times 104 /$ g. $64 \%$ of fecal samples and $55 \%$ of the feed samples were tested positive for MRSA.

All samples taken of the exhaust air were MRSA negative. However, in sock swab samples outside the barn the detection of MRSA was regularly possible.

\section{Discussion}

The present data show that a high rate of contamination of MRSA in pig barns exists.

Detection of MRSA in the air was possible in each investigated barn. Impingement turned out to be more efficient to detect MRSA than filtration. In the environment around the barn no MRSA could be detected in air samples, however most sock swab samples were positive. It seems that MRSA are emitted from the investigated barns by way of the air and deposited in the environment, however the currently used techniques were unable to sample MRSA directly from the air.

The project continues and future investigations will also include techniques such as typing of MRSA strains which will lead to more information.

\section{References}

[1] Klevens et al. 2006. Community-associated methicillin-resistant Staphylococcus aureus and healthcare risk factors. Emerg Infect Dis 12 (12), 1991-1993

[2] Meemken et al. 2008 [Occurrence of MRSA in pigs and in humans involved in pig production-preliminary results of a study in the northwest of Germany]. DTW 115, 132-139 


\title{
P.650
}

\section{Prevalence of Methicillin-Resistant Staphylococcus aureus (MRSA) in different swine production systems and swine groups}

\author{
Johannes Frick ${ }^{1}$ Stefan Hörmansdorfer ${ }^{2}$ Matthias Eddicks ${ }^{1}$ Susanne Zöls ${ }^{1}$ Andreas Palzer ${ }^{1}$ Mathias Ritzmann ${ }^{3}$ Karl Heinritzi $^{1}$ \\ 1. Clinic for Swine LMU Munich, Oberschleißheim, Germany; 2. Bavarian Health and Food Authority, \\ Oberschleißheim, Germany; 3. Clinic for Swine Vetmeduni Vienna, Vienna, Austria
}

\section{Introduction}

For several years, MRSA are well known for nosocomial infections in hospitals and nursing homes. MRSA can cause wound infections, post operative complications or severe general illness including death. So far, only a few cases of livestock associated MRSA transmission to humans has been reported $(1,2)$. The present study is an epidemiological risk assessment of current MRSA prevalence in Bavarian swine farms, swine producers and their extended family.

\section{Material and Methods}

Animals of Bavarian swine farms, the farm owners and their families were examined for MRSA prevalence. For this purpose, 634 nasal swabs from pigs of 60 farms with different swine production systems as well as nasal swabs from 116 individuals from 57 of these farms were bacteriologically examined. All Staphylococcus aureus isolates were subjected to real time PCR for detecting the mecA-gene and orfX-gene, identifying methicillin-resistance and confirming the species Staphylococcus aureus (3). Strains which were identified by PCR were later characterized using spa-typing (4). A total of 63 strains were subjected to MLST (multilocus sequence typing). Furthermore, the presence of the Panton-Valentine leucocidin-gene (PVL) as a virulence factor for community-aquired MRSA was also examined.

\section{Results}

In the present study the MRSA herd prevalence of bavarian farms was $45.0 \%$ (see tab.1)

Table 1: Prevalence of MRSA positive farms and animals in current production systems in Bavaria

\begin{tabular}{|l|c|c|c|c|}
\hline production system & \multicolumn{2}{|c|}{ total number of } & \multicolumn{2}{c|}{ MRSA prevalence of } \\
\hline & farms & animals & farms & animals \\
\hline nursery & 25 & 285 & $28.0 \%$ & $28.8 \%$ \\
\hline fattening & 19 & 188 & $63.2 \%$ & $41.0 \%$ \\
\hline multi-site & 16 & 161 & $50.0 \%$ & $32.3 \%$ \\
\hline total & 60 & 634 & $45.0 \%$ & $33.3 \%$ \\
\hline
\end{tabular}

Similar results were also reported by Khanna et al. (2008) after investigating farms in Canada (5). According to production systems $28.0 \%$ of the nursery farms, $50.0 \%$ of the multi-site farms and $63.2 \%$ of the fattening farms were MRSA positive and prevalence of nursery and fattening farms differed significantly $(\mathrm{P}=0.006)$. The MRSA prevalence among all animals was $33.3 \%$ but differed considerably between production systems (nursery $28.8 \%$, fattening $41.0 \%$, multi-site $32.3 \%$ ).
The MRSA prevalence of the examined individuals was overall $29.3 \%$ (see tab.2). The prevalence of the owners from multi-site farms was $50.0 \%$ and for the nursery and fattening farm owners $26.9 \%$ and $21.4 \%$ respectively.

Table 2: Prevalence of MRSA positive persons in current production systems in Bavaria

\begin{tabular}{|l|c|c|}
\hline production system & total number & MRSA prevalence \\
\hline nursery & 52 & $26.9 \%$ \\
\hline fattening & 42 & $21.4 \%$ \\
\hline multi-site & 22 & $50.0 \%$ \\
\hline total & 116 & $29.3 \%$ \\
\hline
\end{tabular}

According to spa-typing of the isolated MRSA in pigs, spa-type t011 and spa-type t034 were identified in $86.7 \%$ and $10.0 \%$ of the samples respectively. Similarly, spa-type t011 (88.2\%) and spa-type t034 (9.0\%) were predominantly isolated from farmers. 62 of 63 strains belonged to MLST-type ST398. The Panton-Valentine leucocidin-gene was not identified in any of the isolates. None of the examined animals and persons except for one person with folliculitis showed clinical symptoms indicative of MRSA related diseases.

\section{Conclusion}

The prevalence of $45.0 \%$ in bavarian pig farms and $33.3 \%$ in pigs shows the widespread and the frequent occurrence of MRSA. Due to the examination of the owners, this study confirms that a MRSA transfer between pigs and humans within the production chain is not a rare occurence. The spa-types t011 and t034 dominate in swine population as well as among farm owners. However, in comparison to hospital-acquired and communityacquired MRSA, low virulence is assumed due to the absence of the Panton-Valentine leucocidin-gene. But indiviuals should still consider undergoing a pre-operative MRSA screening before planning an operation to prevent MRSA introduction into hospitals and to minimize the risk of falling ill to a MRSA infection themselves while in hospital.

\section{References}

1) Huijdsens et al. (2006) Ann. Clin. Microbiol. Antimicrob. 5: 26

2) Welinder-Olsson et al. (2008) Emerg. Infect. Dis. 14(8): 1271-2

3) Huletsky et al. (2004) J. Clin. Microbiol. 42(5): 1875-84

4) Frénay et al. (1996) Eur. J. Clin. Microbiol. Infect. Dis. 15: 60-4

5) Khanna et al. (2008) Vet. Microbiol. 128: 298-303 


\title{
Bio-security measures to reduce spread of MRSA in pig production and to humans
}

\author{
Jörg Hartung ${ }^{1}$ Annette Springorum² Jochen Schulz ${ }^{1}$ \\ 1. Institute for Animal Hygiene, Animal Welfare and Farm Animal Behaviour, University of Veterinary \\ Medicine Hannover, Foundation, Hannover, Germany; 2. Institute for Animal Hygiene, Animal Welfare and \\ Farm Animal Behaviour, University of Veterinary Medicine Hannover, Hannover, Germany
}

\section{Introduction}

Staphylococcus aureus including Meticillin-resistant S. aureus (MRSA) is frequently present on the skin, in the nose or in the mouth of human hosts without causing illness (Peacock et al., 2001). However, in some instances, S. aureus can cause disease when it enters wounds or damaged skin leading eventually to abscesses, pneumonia, meningitis, endocarditis and septicaemia preferably in immune suppressed patients. Recently a specific clone (CC398) was identified in nearly all production animals, particularly in pigs, across Europe and world wide that colonises also farmers and veterinarians, and concerns rise that it may enter hospitals and the food chain (EFSA, 2008). Clear abatement strategies are not yet in place in animal production. This paper will describe control options on farms suitable to avoid or reduce transmission of these micro-organisms.

\section{Short characterization of S. aureus}

Staphylococcus is a genus of Gram-positive bacteria that are coccoid (spherical) and approximately 1 micrometer in diameter, have thick peptidoglycan cell walls, have a low $\mathrm{G}+\mathrm{C}$ content, and grow in clusters similar to grapes (staphyl = grape). They are commensals colonising as normal bacterial flora nose, throat, auxilla, rectum, perineum and gastrointestinal tract of mammals, but can also survive in a variety of environments and survive vastly desiccation. There are over 30 species of staphylococci, the most pathogenic species for humans is S. aureus. Infections, carriage or colonisation and contamination have to be carefully distinguished (EFSA, 2008).

\section{Transmission routes}

S. aureus is transmitted from human to human, animals to human and vice versa and through other viable or inanimate vectors (Roberson, 1999) such as flies or ticks (Förster et al., 2007). Mechanical transmission occurs by means of body parts without a multiplication of the infectious agent (Last, 2000). Inanimate vectors could be medical devices, contaminated material or objects, food, milk and dust (Youngster et al., 2008).

The transmission routes of methicillin-resistant S. aureus (MRSA) are probably similar to those of methicillin-sensitive $S$. aureus (MSSA) (Kawada et al., 2003) but there may be differences in the efficiency of host colonization due to genetic diversity. Examples of vector related transmission of MRSA strains will be given.

\section{Prevention measures}

In spite of the presently still limited understanding of the transmission routes it is even more important to apply and constantly improve bio-security measures in order to avoid spread of MRSA. Such general measures on farm level can help to reduce the entrance and the spread of infectious agents in general both within and between herds (Hartung, 2005) and can be described as follows:

- Restriction of traffic of cars and trucks and people transporting in and bringing out animals, feed stuff, equipment, slurry or manure.

- Avoid direct contact with animals. Provide protective clothing, gloves and breathing masks.

- New animals have to undergo veterinary inspection, testing and a quarantine time before entering the herd.

- Store fallen animals in separate and safe containers until removed as soon as possible by specialised companies.

- Reduce contact with rodents, insects and wild birds on the premises in order to avoid transfer of infectious agents and MRSA from farm to farm and between herds in a farm. Prevent pets of the farm to have access to the pigs.

- Check regularly the health status of personnel with regard to MRSA and other zoonotic diseases.

- Apply continuously strict cleaning and disinfecting regimes to all pig houses on the farm.

- In order to avoid airborne transmission of MRSA new farm buildings should be placed in sufficient distance to the next farm or residential dwelling ("safe distances").

- Air cleaners could be applied such as biofilters or bioscrubbers (VDI) guidelines) to reduce or to eliminate bacterial emissions from farms (Seedorf and Hartung, 1999). However, experiences with these techniques are still underway for MRSA control.

There is an urgent need for more research to better understand the transmission pathways and hence be able to develop prevention and control strategies.

\section{References}

EFSA (2008): Assessment of the Public Health significance of meticillin resistant Staphylococcus aureus (MRSA) in animals and foods. Scientific Opinion of the Panel on Biological Hazards. EFSA Journal 993: 1-73

Further references with the authors.. 


\title{
P.652
}

\section{Use of serological monitoring to detect infection with Salmonella spp. in flatdeck piglets in Germany}

\author{
Christoph Keller; Ohlinger F. Volker \\ bioScreen EVDMC GmbH, Münster, Germany
}

\section{Introduction}

Salmonella infections are still one of the most important foodborn diseases in humans. Pig meat is one of the sources for salmonella infections in humans. In many European countries programs are established to monitor and reduce infections with salmonella in pig herds. So far these programs focus on slaughter pigs. ${ }^{1}$ In this study pigs about 10 weeks of age were examined, to get more information about the status of Salmonella infections in the production system prior to the fattening units.

\section{Materials and Methods}

The blood samples were tested for the presence of antibodies against Salmonella spp. using a commercial ELISA kit (HerdChek Swine Salmonella, IDEXX). The ELISA was performed according to the manufactures manual. In brief a 1:20 dilution of the serum sample with sample diluent was incubated at room temperature $\left(18-25^{\circ} \mathrm{C}\right)$ for $30 \mathrm{~min}$ in the antigen coated wells. After washing away of unbound material by three washing steps, $100 \mu \mathrm{l}$ of antiporcine horseradish peroxidase conjugate were added to each well. After a second incubation for $30 \mathrm{~min}$ at room temperature and additional three washing steps, $100 \mu \mathrm{l}$ TMB were added to each well. Colour development was terminated by the addition of $100 \mu \mathrm{l}$ stop solution to each well after $15 \mathrm{~min}$ incubation. The absorbance at $620 \mathrm{~nm}$ was measured for each well. Results were calculated as OD\% in relation to the positive control samples on each plate. Interpretation of results war according to the manual and the German registration of the kit by the FLI (Zul. Nr.- BGVVB305). Samples with OD\% value less than $10 \%$ were considered negative. Samples with OD\% values equal to or greater than $10 \%$ were considered positive.

\section{Results}

A total of 4269 samples were taken from piglets end of flatdeck (about 10 weeks of age). The samples originate from 427 German pig farms. Antibodies against Salmonella spp. were detected in 317 blood samples (7.43\%). The positive blood samples originate from 118 farms ( $27.63 \%$ of the tested farms). Within the positive farms $26.86 \%$ of the samples (317 out of 1180) were positive. In 44 farms (37,3\% of positive farms) only low positive results (OD\% 10\%-20\%) were detected. High positive samples (OD\% > 40\%) were found in 42 farms ( $35.59 \%$ of positive farms; 9.84 of all tested farms).

\section{Discussion}

The ELISA kit used in this survey allows to detect antibodies to a broad range of Salmonella serogroups indicating the exposure of a swine herd to the bacterium. ${ }^{2}$ The reliability and comparability of this ELISA has been verified in an international ring test. ${ }^{3}$

The data presented in this study demonstrate the presence of antibodies specific to Salmonella spp. in German piglets at 10 weeks of age. About $30 \%$ of the herds tested were positive for antibodies against Salmonella spp. at 10 weeks of age. This indicates that infection with Salmonella spp. does not only occur in slaughter pigs, but also earlier in production. So piglets should be considered as potential source of Salmonella intake in finisher units. However this result cannot be compared to the results from monitoring of slaughter pigs, which aim to categorize the health status of the herds. In this study the diagnostic cut off value of the ELISA was used to demonstrate exposure of the pigs to Salmonella spp. early in the production chain.

It can be concluded from this study, that control of Salmonella infection in pigs needs highly sensitive diagnostic tools and has to cover the complete production chain from piglets to slaughter pigs. Serological examination provides a powerful tool to monitor infections with Salmonella, when the diagnostic cut-off value of the ELISA ( $\geq 10 \mathrm{OD} \%$ ) is used. Serological monitoring of herds using diagnostic cut-off values will not only raise awareness of infections with Salmonella spp. in pigs, but also provide a basis for effective disease control.

\section{References}

1. Visscher, CF. Untersuchungen (Feldstudie) zur SalmonellenPrävalenz bei Mastschweinen unter dem Einfluss einer gröberen Futtervermahlung sowie von Futteradditiven (organische Säuren bzw. Kaliumdiformiat). Doctoral thesis, University of Veterinary Medicine Hannover, Germany 2006.

2. van der Wolf PJ. Salmonella in the pork production chain: Feasibility of Salmonella-free pig production. Doctoral thesis, University of Utrecht, Netherlands. 2000. ISBN 90-393-2483-2

3. van der Hejden HMJF. First International Ring Trial of ELISAs for Salmonella-antibody Detection in Swine. Berl. Münch. Tierärztl. Wschr. 114, 389 -392 (2001) 


\title{
P.653
}

\section{Antimicrobial resistance related to class 1 integrons in porcine Salmonella enterica serotype Typhimurium isolates}

\author{
Aeran Kim; Ji Youn Lee; Hee Soo Lee; Suk Kyung Lim; Chun Tae Lim; Suk Chan Jung \\ National Veterinary Research \& Quarantine Service, Anyang, Korea
}

\section{Introduction}

Salmonella enterica serotype Typhimurium is one of the leading causes of food-borne disease in developed countries. Multidrug resistance is generally linked to the presence of integrons and is often related to antibiotic use in food animals. In this work, we investigated the frequency, features and molecular epidemiology of class 1 integron-carrying porcine S. Typhimurium isolates.

\section{Materials and Methods}

A total of $128 \mathrm{~S}$. Typhimurium isolates was collected from pigs and ducks during 2006-2007 in South Korea. Seventeen antimicrobial resistances were determined by disk diffusion method. The isolates were screened for the presence of class 1 integrons by polymerase chain reaction (PCR) amplification using specific primers aimed to detect the int 1 gene. The number of integrons and the size of their variable region (VR) were analysed by PCR employing the $5^{\prime} \mathrm{CS}$ and $3^{\prime} \mathrm{CS}$ set of primers. Genotyping and phage typing of 19 typical class 1 integron-carrying isolates were performed by Pulsed field gel electrophoresis(PFGE) and phage typing.

\section{Results}

The highest rate of resistance was tetracycline (77.3\%), followed by streptomycin (77.3\%), nalidixic acid (76.6\%), ampicillin (50.8\%) and sulfamethoxazole/trimethoprim (46.9\%). A low frequency of resistance was found towards cefazolin (4.7\%) and cefoxitin whereas all isolates were susceptible to amikacin, ceftaxidime, ciprofloxacin and colistin. Only two different VR and two different integron profiles were identified among the 19 strains(14.5\%) exhibiting class 1 integrons. Gene cassettes encoding resistance to streptomycin ( 3 isolates, $\operatorname{aad} \mathrm{A} 1$ ), and trimethoprim (16 isolates, dhfr12) were observed. The PFGE patterns of 19 isolates revealed three different genotypes and irrespective of their integron profile and phage type.

Figure 1. Antimicrobial resistance rate of S. Thyphinmurium isolates

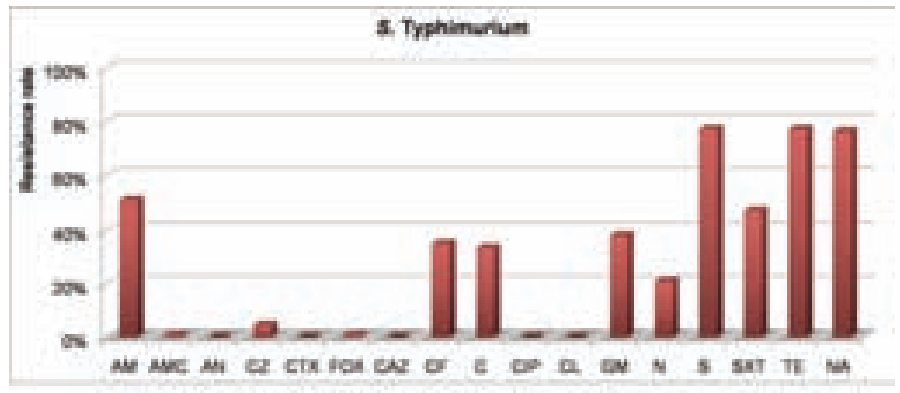

Figure 2. PFGE patterns of class 1 integron contained S. Typhimurium isolates

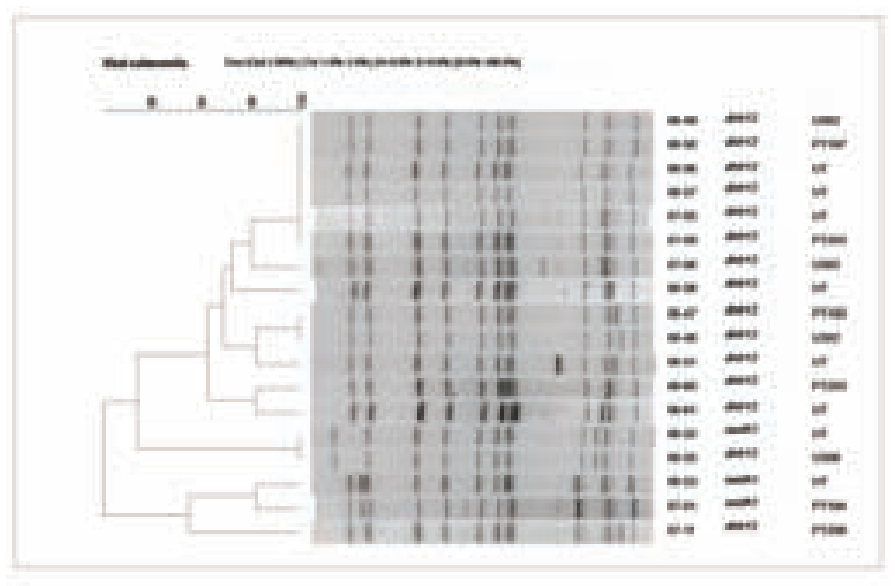

\section{Conclusions}

The results show a high percentage of resistance for some antimcrobials and the presence of integrons in strains of S. Typhimurium isolated from pigs. The prevalence of multidrug resistance in porcine S. Typhimurium isolates is associated with the presence of class 1 integrons, particularly with the integron containing aadA 1 and dhfr $12 \mathrm{VR}$, and is attributable to the spread of a clone.

\section{References}

1. Pérez-Moreno MO, Centelles-Serrano MJ, Cortell-Ortolá M, Ruiz J, Llovet-Lombarte MI, Jardí-Baiges AM, Fort-Gallifa I. Int J Antimicrob Agents. 2009 Oct;34(4):381-3.

2. Martínez N, Mendoza MC, Rodríguez I, Soto S, Bances M, Rodicio MR. J Antimicrob Chemother. 2007 Dec;60(6): 1227-34. 


\title{
P.654
}

\section{Contamination of Salmonella in retail raw meat in Hanoi, Vietnam}

\author{
Thuy N. Do ${ }^{1}$ Huong T. Van ${ }^{1}$ Hang T. Le ${ }^{1}$ Takeshi Koichi' ${ }^{2}$ Yamasaki Eiki ${ }^{2}$ Makino Sou-ichi ${ }^{3}$ \\ 1. National Institute of Veterinary Research, Hanoi, Vietnam; 2. Department of Applied Veterinary Science and Public \\ Health, Obihiro University of Agriculture and Veterinary Medicine, Obihiro, Japan; 3. Laboratory of Food Microbiology \\ and Immunology, Research Center for Animal Hygiene and Food Safety, Obihiro University, Obihiro, Japan
}

\section{Introduction}

Salmonella spp. is one of the most important foodborne pathogens and the causative agent of salmonellosis in humans and animals. Most serotypes can cause gastroenteritis when ingested by eating contaminated foods. Currently, much attention has been focused to improved food safety in Vietnam where most retail meats is sourced directly from abattoirs with no refrigeration, ie the kill during the night and the meat is sold the following morning. Here, we investigated standards of food hygiene in raw meat products as a first step, and based on results, we will investigate hygienic levels of backyard marketplaces and identify epidemiological factors of foodborne salmonellosis due to ingestion of contaminated meat products. The objectives of the present study were therefore to determine the rate of contamination and some characteristics of Salmonella spp. isolated from retail raw meat samples in Hanoi.

\section{Materials and Methods}

Samples $(n=100)$ of retail raw meat (beef, pork and chicken) were collected directly from open markets and supermarkets in Hanoi. The procedures for sample preparations, isolation and identification of Salmonella spp. were based on the FDA method with some modifications according to Department of Applied Veterinary Science and Public Health, Obihiro University of Agriculture and Veterinary Medicine, Japan. PCR tests for rapid screening were performed to amplify DNAs coding for some virulence factors including enterotoxin gene (stn), invasion gene (invA) and $16 \mathrm{~S}$ and $23 \mathrm{~S}$ spacer region of ribosomal RNA (rRNA) of S. Typhimurium DT104 (104SR) according to Kishima et al. (2008)

\section{Results}

Table 1: Prevalence of Salmonella spp. in meat samples

\begin{tabular}{|l|c|c|c|c|c|c|}
\hline & \multicolumn{5}{|c|}{ Origin of samples } \\
\hline Type of samples & \multicolumn{2}{|c|}{ Open markets } & \multicolumn{2}{c|}{ Supermarkets } & \multicolumn{2}{c|}{ Total } \\
\hline & $\begin{array}{c}\text { No. of pos } \\
\text { samples }\end{array}$ & $\%$ & $\begin{array}{c}\text { No. of pos. } \\
\text { sample }\end{array}$ & $\%$ & $\begin{array}{c}\text { No. of pos. } \\
\text { samples }\end{array}$ & $\%$ \\
\hline Beef $(\mathrm{n}=50)$ & $4 / 41$ & 9.8 & $2 / 9$ & 2.2 & $6 / 50$ & 12.0 \\
\hline Pork $(\mathrm{n}=25)$ & $11 / 20$ & 55.0 & $3 / 5$ & 60.0 & $14 / 25$ & 56.0 \\
\hline Chicken $(\mathrm{n}=25)$ & $6 / 17$ & 35.3 & $1 / 8$ & 12.5 & $7 / 25$ & 28.0 \\
\hline Total & $21 / 78$ & 26.9 & $6 / 22$ & 27.3 & $27 / 100$ & 27.0 \\
\hline
\end{tabular}

\section{Acknowledgments}

This work is supported by the Program of Founding Research Centre for Emerging and Reemerging Infectious Disease proposed by the Ministry of Education, Culture, Sports, Science and Technology of Japan.

\section{References}

Do Ngoc Thuy, et al. 2006. Journal of Veterinary Scineces and Technology. Vol XIII, No 3. p 48-54.

Kishima, M., et al. 2008. Zoonoses Public Health. 55, 139-144.

Takeshi, K., et al. 2009. J. Vet. Med Sci. 71 (4), 485-487. 


\title{
P.655
}

\section{Adding of Novobiocin in non-selective pre-enrichment step provide a more suitable starting material for real-time-ttr-PCR assay for the detection of Salmonella enterica spp}

\author{
Talal S. Salih
}

University of Mosul, Mosul, Iraq

Since the production of meat and meat product based foods are multistage processes, the need for on farm food safety risk assessment and quality assurance has greatly increased. Thus, for food safety, the availability of reliable, rapid sensitive and accurate accepted test protocol to detect of Salmonella in examines samples became increasingly important for the agricultural and food industry, as well as the legislative quality control and public.

In this work, 4 Salmonella specific isolation protocols: real-time ttr-PCR, ISO 6579:2002, MSRV and FD CEN/TR 15215-3 were evaluated for their abilities to detect the presence or absence of Salmonella in 275 naturally contaminated animal feed, feces and environmental samples that were collected monthly from two pig farms around the city of Stuttgart, Germany. The main aim was to investigate the effect of addition of $40 \mathrm{mg} / \mathrm{l}$ Novobiocin (NOV) to non-selective buffered peptone water (BPW) for preenrichment of Salmonella in combination with selective enrichment in Rappaport-Vassiliadis soy broth (RVS).

The result showed that all of the 7 samples that were Salmonella-positive by conventional methods were also Salmonellapositive by the ttr-PCR-RVS-NOV method, which also detected 19 additional Salmonella-positive samples. Comparison with the conventional culture methods, the new ttr-PCR-RVS-NOV protocol has a sensitivity of $100 \%$ and no Salmonella false-negative was observed moreover, the McNemar test has showed a significant different. Thus, this method might be used as a meaningful tool in monitoring Salmonella-positive samples at farm level and thus, keeping them out of the food chain. The McNemar test did not show any significant different among the conventional culture methods, also it was no significant different and different agreement values (kappa test) within the samples tested.
Overall, the MSRV method proved to be the most reliable and sensitive method for detecting Salmonella in fecal samples. It detected 2 (50\%) of the positive samples, whereas one (25\%) detected by the both ISO 6579:2002 theFD CEN/TR. The addition of Novobiocin in FD CEN/TR method to BPW gave small but significant increase in the total number of Salmonella isolates from environmental samples. In conclusion, the high sensitivity and the more Salmonella detected in this study maybe caused by the inhibitory influence of Novobiocin on a number of competitive microorganisims in the samples leading to a higher ratio of Salmonella/non-Salmonella, which is likely to provide a more suitable starting material for real-time-ttr-PCR assay.

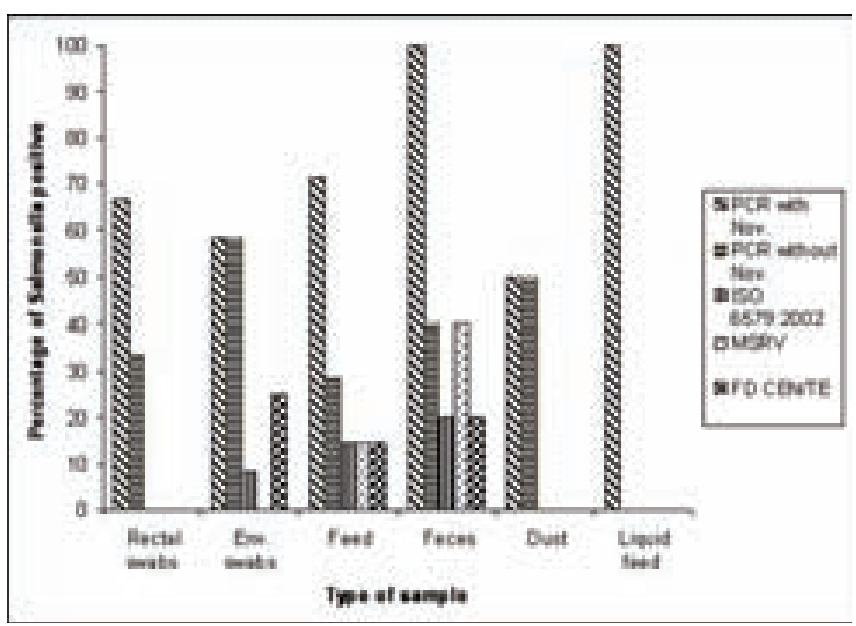




\title{
P.656
}

\section{Effect of K-diformate in fattening diets on Salmonella carriage in high prevalence pig herds}

\author{
Isabelle Corrégé ${ }^{1}$ Anne Hémonic ${ }^{1}$ Eric Royer ${ }^{1}$ Marc Le Roux ${ }^{2}$ \\ 1. IFIP - French Pig Technical Institute, LeRheu, France; 2. BNA Nutrition Animale, Château-Gontier, France
}

\section{Introduction}

The control of Salmonella carriage by pigs is one strategy for reducing the contamination of pig carcasses at slaughter. Several risk factors of high prevalence herds, mainly related to biosecurity and management have been identified. The addition of acids or acid salts in water or feed also appears as a protective factor (5). A study was conducted in farms with high prevalence, to assess the influence of the inclusion of an organic acid salt in fattening feeds on the carriage of Salmonella at the slaughterhouses.

\section{Materials and Methods}

Five farms with high Salmonella prevalence in fatteners were selected to evaluate the influence of $0.6 \% \mathrm{~K}$ - diformate (Formi $\left.{ }^{\oplus}\right)$ addition in growing-finishing pelleted feeds. The Salmonella antibodies were analyzed with the IDEXX serological test on 50 meat juice samples collected from pigs at slaughterhouse during 9 months. An optical density reading of $40 \%$ or above was classified as positive.

Then, the prevalence of three successive batches of pigs fed with the acidified diet during fattening was calculated on 20 samples per batch. After that, the incorporation of K- diformate was stopped and the contamination of 2 new batches was evaluated, respectively 5 and 7 months after stopping. A monitoring was carried out on farms in order to take into account any change of health status, management or housing practices during the treatments.

\section{Results and Discussion}

Three patterns of the evolution of the Salmonella prevalence were obtained (Figure 1).

In the 1 rst pattern (herd A): prevalence significantly decreased during treatment, but increased after cessation and reached a level which was identical to the original one. The survey revealed no change in management that could explain these variations.

In the 2 nd profile (herds B and C): prevalence significantly decreased during treatment but did not increase after cessation of treatment. Two assumptions are made:

1) The acidification limits the shedding and transmission of salmonellae during treatment but also after stopping the treatment.

2) The status improvement may be independent of treatment. Indeed, the Salmonella infection in some farms may vary significantly from one period to another, without any evidence of housing or management changes explaining these variations (1).
In the 3rd pattern (herds D and E), the prevalence was not modified during the treatment. It remained unchanged after the treatment for herd D, whereas it significantly decreased after cessation of the treatment for farm $\mathrm{E}$.

Figure 1: Evolution of Salmonella contamination

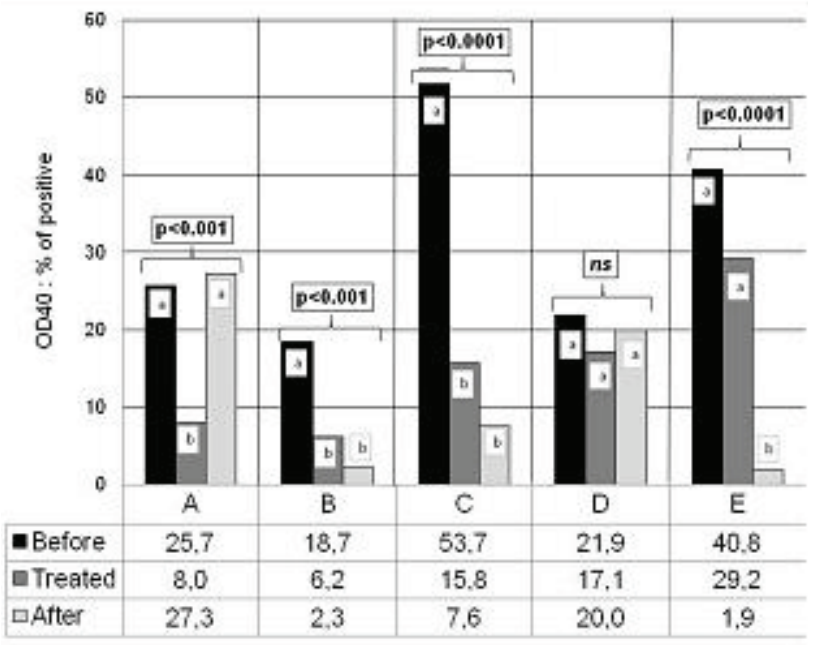

The addition of $0.6 \% \mathrm{~K}$ - diformate to the feed during the fattening period results, in some farms, in a reduction in Salmonella carriage during treatment, and sometimes in a reduced infection after the treatment. In other farms, we do not measure any effect on incidence. These facts confirm previous studies: Papenbrok et al. (3) reported that the inclusion of $1.2 \% \mathrm{~K}$ - diformate in feed can reduce the faecal excretion ratio and shedding duration of infected piglets. However, other trials show that acidifying drinking water or feed has inconsistent effects (4) or sometimes no beneficial effect (2). This study nevertheless illustrates the need for an integrated strategy in which acidification should be included.

\section{References}

1. Corrégé I. et al., 2006. Proc. 19th IPVS, 38, 379-386.

2. Letellier A. et al., 2000. Can. J. Vet. Res., 64, 27-31.

3. Papenbrock S. et al., 2004. Proc. 18th IPVS, 654.

4. Van der Wolf P.J. et al., 2001. Vet. Quaterly, 23, 121-125.

5. Wingstrand A. et al., 1997. Proc. 3rd Int. Symp. Epidem. Control Salmonella in pork, Copenhagen, 170-172. 


\title{
P.657
}

\section{Detection and characterization of Salmonella in lairage, intestines and on pig carcasses in five slaughterhouses using serotyping and macrorestriction genotyping}

\author{
Emily V. De Busser; Dominiek Maes; Jeroen Dewulf; Kurt Houf; Lieven De Zutter \\ Faculty of Veterinary Medicine, Ghent, Merelbeke, Belgium
}

\begin{abstract}
Introduction
In 2008, 3944 Salmonella infections in humans had been registered in Belgium, of which Salmonella Typhimurium (57\%) was the most common serotype (1). As the consumption of pork meat is a major source for human infection, more insight is needed into the epidemiology and spread of this bacterium throughout the production chain.
\end{abstract}

The aims of this study were to investigate the prevalence of Salmonella contamination along the slaughter line and to identify possible critical control points by performing serotyping and macrorestriction genotyping of the isolates.

\section{Materials and Methods}

Five Belgian slaughterhouses (slaughter rate of $170-580$ pigs/ hour) were visited twice. Faecal samples from pigs of different batches were taken at the lairage area using overshoes. At exsanguination, 30 pigs were individually identified (total: 30 pigs * 5 slaughterhouses $* 2=300$ pigs) and swab samples of the oral cavity were taken. These 30 pigs were further investigated at the slaughter line. Carcass swabs were taken after polishing, after splitting and after first chill as well as content of duodenum, ileum and rectum and mesenteric lymph nodes of the corresponding pigs. All the samples were submitted to Salmonella isolation using standard procedures (ISO 6579:2002 FDAM1). Prior to serotyping according to the Kaufmann-White scheme, isolates were clustered by the enterobacterial repetitive intergenic consensus PCR. Characterization at strain level was performed by pulsed-field gel electrophoresis with the restriction endonucleases $\mathrm{Xbal}$ (and Blnl for the serotype Rissen).

\section{Results}

In total, 276 samples (14.4\%) were Salmonella positive, with $48.2 \%$ of the pigs positive in at least one sample. The lairage area was highly contaminated, with a large variation (0-100\% positive) among the slaughterhouses. In total, 13 different serotypes were identified with differences in occurrence between slaughterhouses and slaughterhouse visits. Salmonella Typhimurium (58.7\%) and Salmonella Rissen (17.4\%) were the most common serotypes. The largest variation in serotypes was noticed in lymph nodes, rectum and ileum content. Genotyping of the 273 isolates revealed 46 different strains of which 32 for Salmonella
Typhimurium, 4 for Salmonella Derby and 2 for Salmonella Rissen (by using BInl). The same genotypes could be detected in the lairage as well as in the oral cavity $(94.7 \%)$, in the lairage and in the gut content (65.5\%), in the lairage and on the carcass $(57.9 \%)$, but never in the lairage and the lymph nodes of animals held in these pens. In $82.7 \%$ the same genotype was found in the lymph nodes and gut contents of the same animal, whereas the same genotype was only found in $30 \%$ of the different carcass samples (the other $70 \%$ harbored different serotypes). In $55.5 \%$ the same genotype was found in the gut content and on the carcass. In $47.6 \%$ the same genotype was detected in the oral cavity as on the carcass. In $44.4 \%$ the same genotype was identified in the lymph nodes as on the carcass.

\section{Discussion and conclusions}

Salmonella was commonly present in the lairage area, in the pigs' intestines and on the carcasses along the slaughter line. No positive overshoe samples could be detected in one slaughterhouse where the lairage area consisted of a slatted floor allowing a minimum of contact with the feces. The results showed that the same pig could harbor different serotypes and amongst the same serotype different genotypes in the lymph nodes and gut content. Furthermore the study shows that pigs do take up Salmonella in the lairage area, where Salmonella. was already present in the environment or shed by pen-mates. There was however no association between the genotypes found in the lymph nodes and in the lairage. On the other hand more than the half of the genotypes found on the carcass were also detected in the gut content and in the lairage area. A good fasting procedure, prevention of accidental cutting of intestines and improved cleaning and disinfection of the lairage area are important factors to prevent contamination. As in 30\% of the different carcass samples, the same genotype was detected, cross contamination and contamination out of the slaughterhouse environment does occur and are of major importance to reduce the Salmonella level of the end product.

\section{References}

(1) National Reference Center for Salmonella and Shigella, 2008. http:// bacterio.iph.fgov.be/ 


\title{
P.658
}

\section{Serologic monitoring of salmonella in pigs of different productive systems from Argentina}

\author{
Julián Parada ${ }^{1}$ Alicia I. Carranza Bibiana R. Pelliza' Pablo J. Tamiozzo ${ }^{1}$ Ismael Dolso $^{2}$ Arnaldo Ambrogi ${ }^{1}$ \\ 1. Universidad Nacional de Río Cuarto, Río Cuarto, Argentina; 2. UNRC, Río Cuarto, Argentina
}

\section{Introduction}

The annual increase of human salmonelosis cases related to the consumption of pork has led countries worldwide to implement control programs of Salmonella spp in pork production (Hugas, 2007). The Danish surveillance program for Salmonella in pigs uses a serologic monitoring system with classification of herds in 3 levels: level 1, of low seroprevalence (index $<40 \%$ ), level2, moderate $(40 \% \leq$ index $\leq 70 \%)$, and 3 , high (index $>70 \%)$, using an ELISA evaluated at a cut-off 20\% Optical Density (OD) (Alban, 2002). In Argentina official data about levels of Salmonella infection in herds do not exist.

Due to its importance, this work tries to evaluate the sanitary situation of herds in the zone of greater pig production in Argentina, through serologic monitoring to different ages, in different production systems.

\section{Materials and Methods}

This work was carried out in 5 provinces of Argentina. Nineteen farrow-to-finish pig herds with indoor production systems (SC) $\left(X^{-}=810\right.$ sows) and 11 with outdoors systems (SAL) $\left(X^{-}=266\right.$ sows) were selected. All herds were bred on an intensive basis, with weaning between 21 and 23 days of life, and averages of 22.44 finished pigs/sow/year in SC and 18.05 pigs/sow/year in SAL. From each farm, samples were taken randomly to pigs of 8, 15 and 21 weeks of life. A number of samples was taken necessary to detect the presence of antibodies against Salmonella spp, with $40 \%$ of waited prevalence, $95 \%$ of Confidence and $5 \%$ of error. $40 \%$ represents the minimum prevalence to detect levels 2 and 3 of the Danish system (Alban, 2002).

The serums were analysed using an ELISA Herdchek Swine Salmonella (IDEXX), for detection of antibodies against LPS antigen (serogrupos B, C1 and D) of Salmonella spp, measuring values at $650 \mathrm{~nm}$ of absorbption. The results were evaluated at 3 cut-off values, namely 10,20 and $40 \%$ OD.

\section{Results}

A total of 504 serums were analyzed. The results of positive farms are shown in table 1.

Table 1: Percentage of farms with positive pigs to ELISA for each system, according to age and cut-off value.

\begin{tabular}{|l|c|c|c|c|c|c|c|c|c|}
\hline System & \multicolumn{3}{|c|}{8 weeks } & \multicolumn{3}{c|}{15 weeks } & \multicolumn{3}{c|}{21 weeks } \\
\hline & $10 \%$ OD & $20 \%$ OD & $40 \%$ OD & $10 \%$ OD & $20 \%$ 0D & $40 \%$ 0D & $10 \%$ 0D & $20 \%$ 0D & $40 \%$ 0D \\
\hline SAL & $45 \%$ & $18 \%$ & $9 \%$ & $100 \%$ & $45 \%$ & $27 \%$ & $100 \%$ & $91 \%$ & $73 \%$ \\
\hline SC & $26.3 \%$ & $5 \%$ & $5 \%$ & $47.3 \%$ & $31.5 \%$ & $15.7 \%$ & $89.4 \%$ & $68.4 \%$ & $31.5 \%$ \\
\hline
\end{tabular}

The number of positive pigs by production systems is shown in graphic 1.
Graphic 1: Average positive pigs to ELISA using a cut-off value of $20 \% O D$, with $E E$, according to age and production system.

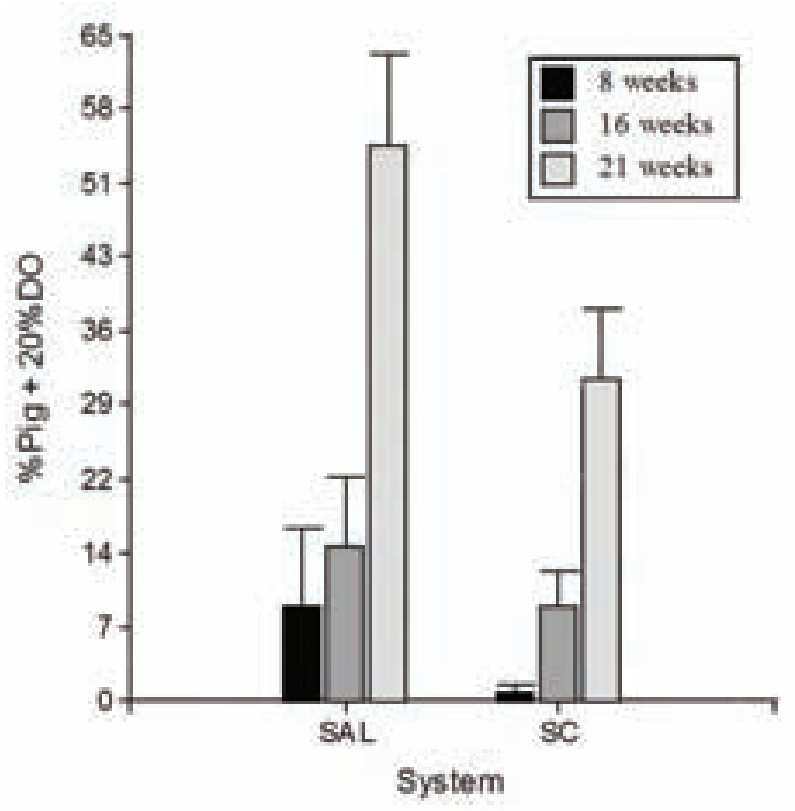

According to Danish classification system, $27 \%$ of SAL were level $1 ; 18 \%$ level 2 and $55 \%$ level 3 . As to SC, $53 \%$ were level $1 ; 16 \%$ level 2 and $32 \%$ level 3.

\section{Discussion}

Considering a cut-off value of $20 \%$ DO, $68.4 \%$ of herds SC (13) and $91 \%$ of herds SAL (10) had positive pigs to Salmonella spp at 21 weeks of life. This seems to indicate an wide distribution of Salmonella in herds of the country. In addition, $40 \%$ (12), of the total herds were classified as level 3 (high risk).

Analyzing the number of positive pigs by herd (non published data), there were no significant differences between systems at 8 and 16 weeks of life. Nevertheless, at 21 weeks of life there was a greater number of positive pigs in SAL than in $S C(p<0.05)$. As Baptista indicates (2009), this could be due to the presence of serotypes of Salmonella enterica of greater virulence in these systems or to a major number of re infections in SAL. The high intra-premises infection levels indicate the necessity to implement control strategies against Salmonella spp in herds from Argentina. Nevertheless, more accurate future works will be necessary to determine the serotypes circulating in the country and the risk factors involved in the dynamics of infection within each system.

\section{References}

Alban et al. 2002. Prev. Vet. Med. 53, 133-146.

Hugas et al. 2007. Int. JF. Microb. 120, 131-135.

Baptista et al. 2009. Prev. Vet. Med. 92, 301-308. 


\title{
P.659
}

\section{Detection of salmonella in finishing pigs on farms in the central zone of Yucatan, Mexico}

\author{
Jorge C. Rodriguez-Buenfil ${ }^{1}$ Eusebio Gonzalez-Reding ${ }^{2}$ Javier Venosa-Peña $^{2}$ Pilar A. Gomez-Ruiz ${ }^{3}$ \\ 1. Facultad de Medicina Veterinaria y Zootecnia Universidad Autonoma de Yucatan, Merida, YUC, Mexico; \\ 2. Novartis, Guadalajara, JAL, Mexico; 3. Private practice, Merida, YUC, Mexico
}

\section{Introduction}

The pig industry is affected by diseases caused by bacteria, virus, parasites and rickettsias. Among the organisms that cause those diseases are the bacteria of the genus Salmonella. Salmonella spp also affect the Public Health. In order to design programs for the control of this pathology, it is necessary to know the presence, distribution, and serotypes. The objectives of this study were: 1) to detect the presence of Salmonella spp in fattening pigs; 2 ) to identify the most frequent serotypes; 3 ) to determine the pattern of resistance to antibiotics.

\section{Materials and Methods}

A cross-sectional epidemiological study was carried out on five pig farms. The farms were located at the central region of Yucatan, Mexico. The sample size to detect the presence of Salmonella spp was calculated considering $5 \%$ prevalence and $95 \%$ confidence level. Feces (1 g/animal) were collected directly for the animal rectum. The samples were analized at FMVZ-UADY using the technique described by Rappapor-Vasiliadis cited by Fedorka et al. (1). The serotyping was carried out at the National Institute for Diagnosis and Epidemiological of Mexico.The antibiogram was conducted at the FMVZ-UADY.

\section{Results}

Sixty percent of the farms in this study result positive (fig. 1). The week of higher risk to detect Salmonella spp was eighteen (fig 2).

Fig1. Distribution of positive animals to Salmonella spp. on five farms, in Yucatan, Mexico

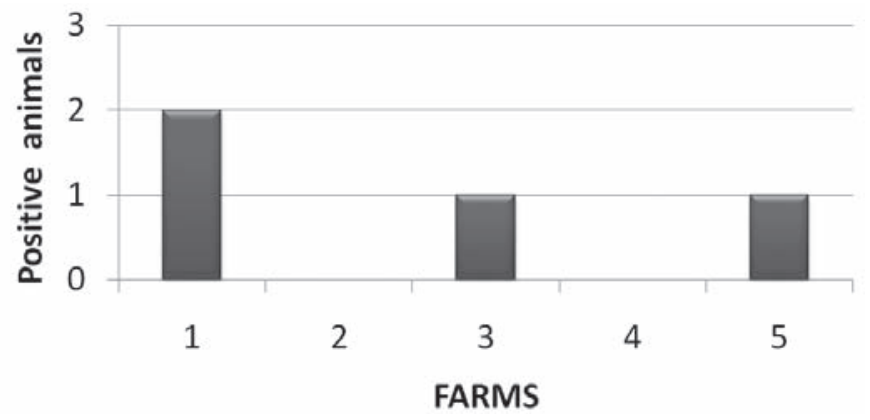

Fig 2. Distribution of positive animals to Salmonella spp according to the age in Yucatan, Mexico

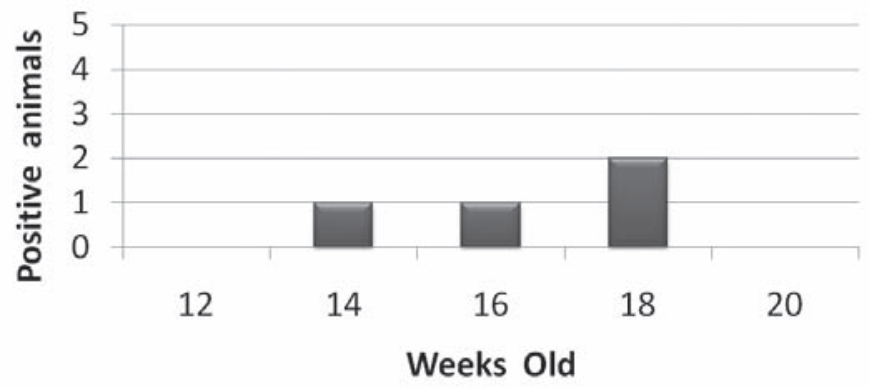

The serotypes most frequent were S. Havana, S.Give y S. Typhymurium. These serotypes showed a different resistant pattern. Only the Amikacin, showed efficacy against the isolates serotypes (Table1).

Table 1. Antibiogram for different serotypes of Salmonella spp

\begin{tabular}{|l|l|c|c|c|c|}
\hline \multicolumn{2}{|l|}{} & $\begin{array}{c}\text { S. Havana } \\
\mathrm{n}=1\end{array}$ & $\begin{array}{c}\text { S. Give } \\
\mathrm{n}=1\end{array}$ & $\begin{array}{c}\text { S. Typhymurium } \\
\mathrm{n}=1\end{array}$ & $\begin{array}{c}\text { S. Typhymurium } \\
\mathrm{n}=2\end{array}$ \\
\hline ANTIBIOTIC & Concentration & 16 weeks age & 18 weeks age & 16 weeks age & 18 weeks age \\
\hline Estreptomicin & $10 \mathrm{mcg}$ & $\mathrm{I}$ & $\mathrm{R}$ & $\mathrm{R}$ & $\mathrm{S}$ \\
\hline Amikacin & $30 \mathrm{mcg}$ & $\mathrm{S}$ & $\mathrm{S}$ & $\mathrm{S}$ & $\mathrm{S}$ \\
\hline Sulfisozasol & $0.25 \mathrm{mcg}$ & $\mathrm{R}$ & $\mathrm{R}$ & $\mathrm{R}$ & $\mathrm{I}$ \\
\hline Ceftriaxone & $30 \mathrm{mcg}$ & $\mathrm{S}$ & $\mathrm{I}$ & $\mathrm{R}$ & $\mathrm{S}$ \\
\hline Gentamicine & $10 \mathrm{mcg}$ & $\mathrm{S}$ & $\mathrm{R}$ & $\mathrm{S}$ & $\mathrm{S}$ \\
\hline Ciprofloxacine & $5 \mathrm{mcg}$ & $\mathrm{R}$ & $\mathrm{S}$ & $\mathrm{S}$ & $\mathrm{S}$ \\
\hline I= Intermediate; $\mathrm{S}=$ Sensible; $\mathrm{R}=$ Resistant & \\
\hline
\end{tabular}

\section{Discussion}

The farms that result positive in this study are indicating that the prevalence of Salmonella spp is $20 \%$ or higher. Those that result negative are indicating that, if Salmonella spp, is present, the prevalence will be below of $20 \%$. Also the serotype S. Thyphymurium has been reported previously en this region (2).On the other hand it is the first time that S. give and S. Havana are reported in this area. These have been reported in other areas and speciecs (3)(4). Finally, it can observe the high percent of resistant of serotypes to varies antibiotics. It can be conclude that salmonellosis is present in the swine industry in this area

\section{References}

1. Fedorka-Cray, P.J. and L. Tollefson. 1997. Salmonella typhimurium definitive phage type (DT) 104. Am. Assoc Swine Practitioner. 219220.

2. Rodriguez-Buenfil J.C. et al 2004 Incidence of Salmonellosis and identification of serogrups and seotypes ina pig commercial farm in Yucatan. Rev. Latin de Microbiol. 48; 10-13

3. Girardin, F et al. 2006. Salmonella serovar Give: an unusual pathogen causinsplenic abscessEur J Clin Microbil Infect dis 25; 272-274

4. Higgins R. et al (1997). Oubreak of Salmonella Give in the 'province of Quebec. Can. Vet. J. ;38 780-781 


\title{
P.660
}

\section{Probiotic effects in swine in different post-weaning periods with Salmonella Typhimurium challenge}

\author{
Larissa J. Parazzi $^{1}$ Esther R. Afonso ${ }^{1}$ Simone M. Martins ${ }^{3,1}$ Juliane D. Magalhães $^{1}$ Daniella C. Donato ${ }^{1}$ \\ João P. Bastos ${ }^{1}$ Andréia G. Arruda Andrea M. Moreno² Aníbal S. Moretti ${ }^{1}$ \\ 1. Laboratory of Swine Research, University of Sao Paulo, Depto. Nutrição e Produção Animal (VNP), Pirassununga, SP, Brazil; \\ 2. Laboratory of Swine Sanity, University of Sao Paulo, Depto. Medicina Veterinária Preventiva e Saúde Animal(VPS), São Paulo, Brazil; \\ 3. Laboratory of Semen Biotechnology and Andrology, University of Sao Paulo, Depto. Reprodução Animal (VRA), Pirassununga, SP, Brazil
}

\section{Introduction}

Probiotics are viable microorganisms that once ingested by humans and animals, produce beneficial physiological effects by assisting in the establishment of an intestinal population 1. The aim of this study was to asses the effect of two probiotics in different inclusion periods during nursery, growing and finishing on the performance of swine.

\section{Materials and Methods}

One hundred sixty pigs from 23 to 138 days of age were used to evaluate two probiotics, one of them was composed by Bacillus subtilis, Bifidobacterium bifidum, Enterococcus faecium, Lactobacillus acidophillus, Lactobacillus casei and Lactobacillus lactis (PA), and the other by Bacillus subtilis and Bacillus toyoi (PB). Both were tested with an inclusion in the diet in 3 different periods characterizing 8 treatments: PA44 (PA until 44 days of age and control diet after), PA65 (PA until 65 days of age and control diet after), PA138 (PA until 138 days of age), PB44 (PB until 44 days of age and control diet after), PB65 (PB until 65 days of age and control diet after), PB138 (PB until 138 days of age), CTR (diet control without additives) and ATB (diet control with an antibiotic as growth promoter). At 51 days of age the programmed challenge with Salmonella Typhimurium (LSS SR41) was given orally. Body weight (BW) at 23, 30, 37, 44, 51, 58, $65,79,106,112,126$ and 138 days of age and feed conversion (FC) corresponding these periods were evaluated. At weaning ( 23 days of age), pigs were blocked by body weight and sex, and placed in a nursery pen (4 pigs/pen). Datas were analyzed by the SAS2, and treatments were compared by orthogonal contrast method. The level of significance considered was $5 \%$.

\section{Results}

The contrast 1 (C1), PA44, PA65 and PA138 vs ATB showed from 37 (8,33Kg vs 9,54Kg, respectively) to 112 days of age $(64,01 \mathrm{Kg}$ vs $70,75 \mathrm{Kg}$, respectively), the highest values for ATB regarding BW. Similar results were obtained for the contrast 2 (C2), PB44, PB65 e PB138 vs ATB from 37 (8,71 Kg vs 9,54Kg, respectively) to 106 days of age ( $60 \mathrm{Kg}$ vs $65,48 \mathrm{Kg}$, respectively), demonstrating the superiority of ATB against probiotics. There were no significant differences from the age of 112 days for $C 1$ and 106 days for C2 until 138 days for neither of the groups. The FC showed significant difference in C1 (1,78 vs 1,55, respectively) and C2 $(1,79$ vs 1,55 , respectively) at nursery period ( 23 to 65 days of age), with lowest values for ATB. During growing-finishing periods ( 66 to 138 days of age) the lowest values were found for probiotics on $C 1$ ( 2,49 vs 2,63 , respectively) and $C 2$ ( 2,53 vs 2,63 , respectively) with $\mathrm{P}=0.06$ and $\mathrm{P}=0.16$, respectively. These values emphasize a numerical advantage (140 and 100 grams for C1 and $\mathrm{C}^{2}$, respectively) in favor of probiotics.

\section{Discussion}

Although there was a significant difference from nursery period until days 106-112 (C2 and C1 respectively) regarding BW, this variable did not show significance until 138 days of age when probiotics and ATB were compared. Similar3 and contradictory 4 results were reported in literature, considering the nursery period, while during growing-finishing few evidences were described5. The treatments PA65 and PB44 (98,87Kg and 97,33Kg respectively) showed equivalent body weights in comparison with the ATB $(99,63 \mathrm{Kg})$, indicating distinct actions depending on the probiotic's inclusion time. In the programmed challenge at 51 days of age, significant effects were observed regarding performance for C1 $(21,74 \mathrm{Kg}$ VS $25,99 \mathrm{Kg}$, respectively) and C2 $(21,91 \mathrm{Kg}$ vs $25,99 \mathrm{Kg}$, respectively), showing the probiotic treatments were affected by the challenge. The modification observed in BW and FC in the growing-finishing suggested a recovery by unknown factors that could be connected to the microflora complex modification. Considering the equivalence of the results on the performance and the economic aspects involving the inclusion of the probiotics, its use until 44 or 65 days was better than until 138 days of age.

\section{References}

1. GUERRA et al., 2007. An Feed Sci Tech, 134: 89-107.

2. SAS User's Guide: statistics. Version 9.1. Cary: 2002-2003.

3. UTIYAMA et al., 2006. R Bras Zoot, v35,6:2359-2367.

4. SILVA et al., 2007. Semina: Ciências Agr, Londrina, v28, n4: 739-746.

5. BRUNO, 2008. Master thesis. FMVZ, University of São Paulo, Brasil.

\section{Acknowledgments}

FAPESP, CNPq, Ouro Fino Saúde Animal Ltda 


\title{
P.661
}

\section{Evaluation of risk factors and proposals for control of infection of Salmonella sp. in different production systems of Brazilian swine}

\author{
Jalusa D. Kich² Patrícia Schwarz ${ }^{1}$ Arlei Coldebella ${ }^{2}$ Luis Gustavo Corbellini ${ }^{1}$ Marisa Cardoso ${ }^{1}$ \\ 1. Universidade Federal do Rio Grande do Sul, Porto Alegre, RS, Brazil; 2. Embrapa Suínos e Aves, Concórdia, SC, Brazil
}

\section{Introduction}

Studies of risk for contamination of meat along the production chain as well as the quantitative representation of their impacts have been conducted around the world (Dahl et al. 2000; Giovaninni et al. 2003). This study was carried out in order to identify factors associated with infection of herds and to suggest alternatives to control the main pig producing regions of Brazil and in different production systems.

\section{Materials and Methods}

In this study, 5 different Brazilian pork production systems were chosen. They were evaluated for Salmonella prevalence. An epidemiological investigation and evaluation of risk factors were infered in 189 farms. The results of seroprevalence and risk factors of each farm were analyzed by logistic regression. The risk factors that were identified were also inserted into a simulation model of intervention in swine.

\section{Results}

The main risk factors associated with high prevalence of Salmonella were pre-pit dirty, other animals on the farm in addition to pigs, the presence of intensive production of broilers in the same farm, post-weaning multisystemic wasting syndrome (PMWS) with high scores of mortality and culling and number of origins of animals housed in the same farm. The simulation models with values clonsidered protective for the risk variables that were found reached 11.20 to $44.89 \%$ prevalence of the mathematival model used.

\section{Discussion}

The predisposing factors for infection of pig herds were determinants variable of biosafety in the evaluated companies. Through simulation models, the combination of risk factors for the intervention of control of Salmonella sp. in farms producing pigs is proposed. In this study, the early phase of Salmonella infection was the finishing one, as previous studies conducted in Brazil by Müller (2007) and Schwarz et al. (2009), therefore this stage was the focus of the epidemiolocical assessment. The risk factor with the highest probability of infection was the presence of intensive production of broilers, indicating the possible contamination of one species to another in the same environment. The Pre-dirty pit was another source of infection for pigs, because the feces in the environment can be directed into the stalls by fomites.

In these cases, the presence of other animals on the farm represents a threat to the swine herds, because the Salmonella sp. is widespread among most animal species (Popoff et al., 2003). PMWS has been identified as an important risk variable in 2 of 5 companies analyzed. This interaction between this risk variable and high seroprevalence may be associated with biosecurity issues common to Salmonella infection and PMWS.

Another risk factor identified in this study was the number of sources of animals housed in the same farm. In Brazil, Kich et al. (2005) found that the number of sources of pigs and other animals on the farm as risk factors for infection with Salmonella $\mathrm{sp}$. The number of sources of piglets can increase the chances of introducing new pathogens in the swine herds (Morés, 2003).

According to Davies et al. (1997), the limited number of days of fallowing (less than 7) was identified as a risk factor in 2 companies, as well as mixing animals from different sources, also described by Quessy et al. (1999), and size of herds, cited by Carstensen \& Christensen (1998).

A comparative analysis of the seroprevalence of Salmonella among the companies in this study showed the differences between production systems $(P<0.05)$, as well as differences in the distribution of identified risk factors.

\section{References}

1. Dahl et al. IPVS, 2000, p.203.

2. Davies et al. Epidem. and Inf., 119:237-244,1997.

3. Carsteensen \& Christensen. Prev. Vet. Med, 34: 191-203, 1998.

4. Giovaninni et al. Food Cont., 15:137-144. 2003.

5. Kich et al. Ciência Rural, 35, 2:398-405, 2005.

6. Morés et al. Arq. Bras. Med. Vet. Zootec, 55,2: 133-140, 2003.

7. Popoff et al. Res. Microbiol., 154: 173-174. 2003.

8. Quessy et al. IPVS 1999, p.165.

9. Schwarz et al. Arq. Bras. Med. Vet. Zootec., 61,5:1028-1034, 2009. 


\title{
P.662
}

\section{Frequency of salmonella seropositive pigs in farms affected by different severity levels of the post-weaning multisystemic wasting syndrome}

\author{
Patrícia Schwarz; Jalusa D. Kich; Arlei Coldebella; Luis Gustavo Corbellini; Marisa Cardoso \\ Embrapa Suínos e Aves, Concórdia, SC, Brazil
}

\section{Introduction}

The post-weaning multisystemic wasting syndrome (PMWS) has caused considerable losses due to mortality and wasting of pigs, and has been often associated with co-infections with other viral or bacterial agents $(2,3)$. The aim of this study was to evaluate the Salmonella seroprevalence in swine herds that are affected by PMWS to different severities.

\section{Materials and Methods}

Productive performance data and most prevalent symptoms were investigated in herds associated to 11 Brazilian swine companies that reported the occurrence of PMWS (1). Herds $(n=188)$ were classified according to the mortality and wasting frequency, and these data were considered as PMWS severity degrees (table1). Blood samples were taken from slaughter pigs of each herd and submitted to an indirect ELISA test against Salmonella sp. Statistical analysis was performed using chi-square test for linear trend to test the hypothesis of increased Salmonella seroprevalence with levels of PMWS severity. The farms were divided in two groups according with the seroprevalence, $\leq 70 \%$ and $>70 \%$. These groups were compared between the levels of PMWS severity. The analysis was performed using the software Epilnfo 6.0 (4).

Table 1: Classification of farms in the intensity levels of involvement by PMWS

\begin{tabular}{|l|c|c|}
\hline Level PMWS & Mortality & Culling \\
\hline Level 1 & below 2\% & below 2\% \\
\hline Level 2 & 2 to 3\% & 2 to 4\% \\
\hline Level 3 & above 3\% & above 4\% \\
\hline
\end{tabular}

\section{Results}

In all herds the appearance of PMWS was associated with a decrease in animal performance and a tendency of a higher frequency of enteric symptoms. Seroprevalence $>70 \%$ against Salmonella was associated to higher PMWS severity $(\mathrm{P}=0.0001)$ (table 2).

\section{Discussion}

This result may be related to the immune impairment caused by PMWS that may predispose to Salmonella infection, as well as to the occurrence of common risk factors to both infections in affected herds (5). These factors include the size of the batch, the mixture of animals of different origins and hygiene facilities (1). In conclusion, a high frequency of Salmonella carrier pigs may be an additional problem in farms severely affected by PMWS.

Table 2: Salmonella seroprevalence in swine herd with different severities of PMWS

\begin{tabular}{|l|c|c|c|}
\hline $\begin{array}{l}\text { Level of PMWS } \\
\text { Sevetity }\end{array}$ & N Herds & $\begin{array}{c}\text { Seroprevalence of } \\
\text { Salmonella (\%) }\end{array}$ & $\begin{array}{c}\text { Seroprevalence of } \\
\text { Salmonella (\%) }\end{array}$ \\
\hline & & $\geq 70 \%$ & $\leq 70 \%$ \\
\hline Level 1 & 32 & $15 / 46.9 \%$ & $17 / 53.15 \%$ \\
\hline Level 2 & 54 & $46 / 85.2 \%$ & $8 / 14.8 \%$ \\
\hline Level 3 & 102 & $81 / 79.4 \%$ & $21 / 20.6 \%$ \\
\hline Total & 188 & $142 / 75.5 \%$ & $46 / 24.5 \%$ \\
\hline
\end{tabular}

\section{References}

1. Beloeil P.A., et al., 2007. Veterinary Research. 38: 835-848.

2. Corrêa, A.M.R, et al., 2006. Pesq. Vet. Bras.26:132-137

3. Desrosiers, R. et al., 2007. Proceedings. 5th Emerging Dis. P.121.

4. Epi-Info 6.0. Center of Disease Control. www.cdc.gov/Epilnfo.

5. Rose, N., et al., 2003. Preventive Veterinary Medicine, 61: 209-225. 


\section{P.663}

\section{Evaluation of a commercial acid mixture administered in drinking water as a control measure in swine salmonelosis}

Héctor Argüello; Jesús Osorio; Álvaro Hidalgo; Sonia Álvarez; Mario Arcos; German Naharro; Ana Carvajal; Pedro Rubio

University of León, León, Spain

\section{Introduccion}

Salmonella control programs have been developed in swine farms from several European countries[1]. Those farms with a high level of contamination will have to establish control measures to reduce the number of Salmonella infected animals [2]. We have carried out an interventional study in a pig fattening unit to assess the effectiveness of an acid treatment administered in drinking water for the control of salmonellosis.

\section{Materials and Methods}

The study was performed in a pig fattening unit infected by Salmonella Typhimurium housing 3,000 pigs distributed in two barns (1,500 pigs each). One of them was chosen as control group and received no treatment while the other was chosen as experimental group. Animals from experimental group were administered a commercial acid, Acidvall ${ }^{\oplus}$, composed of lactic acid $(56 \%)$, formic acid (23\%), propionic acid (13\%) and acetic acid $(5 \%)$, that was added to drinking water during the last 40 days of the fattening period at a concentration of $0.035 \%$. Within each group, 40 animals were randomly selected and sampled through the experiment. Fecal samples and serum were collected the first days of the fattening period, at the beginning of the treatment, 20 days after(only blood samples) as well as at the last day of treatment (previous day to slaughter). Moreover, cecal content and ileocecal lymph nodes were collected from pigs of both experimental groups at the slaughter. Samples were submitted to the infectious animal diseases laboratory and processed. Bacteriological analyses were made using EN-ISO 6579:2002/Amd 1:2007. Serological samples were tested with Idexx herdchek Swine Salmonella kit $^{\circledR}$ according to manufacturer's instructions.

\section{Results}

Results showed significant differences in bacteriology $\left(\mathrm{chi}^{2}=21,4\right.$ $\mathrm{p}<0,001)$ and serology $\left(\mathrm{chi}^{2}=71,45, \mathrm{p}<0,001\right)$ between goups at the end of the fattening period ( $17.5 \%$ shedders and $27.5 \%$ seropositive pigs in treatment group compared to $50 \%$ shedders and $87.5 \%$ seropositive pigs in control group). Similar results, near to significance, were found out at the abattoir where Salmonella was detected in cecal content in $47.5 \%$ of the treated pigs versus $65 \%$ of the control animals. Differences were also found in the prevalence of positive animals in ileocecal lymph nodes $55 \%$ of positive in the treatment group to $70 \%$ in control group.
Figure 1. Fecal shedding of Salmonella during fattening period.

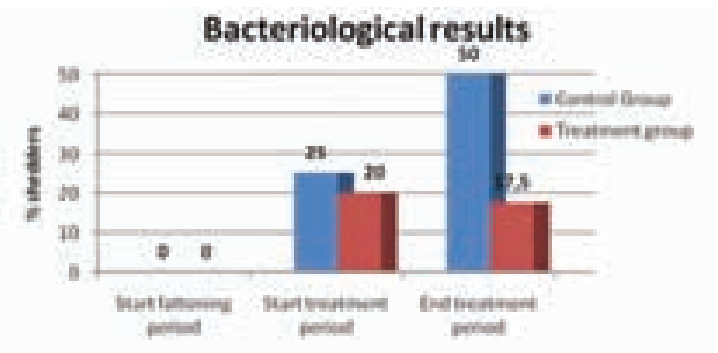

Figure 2. Serological results during fattening period

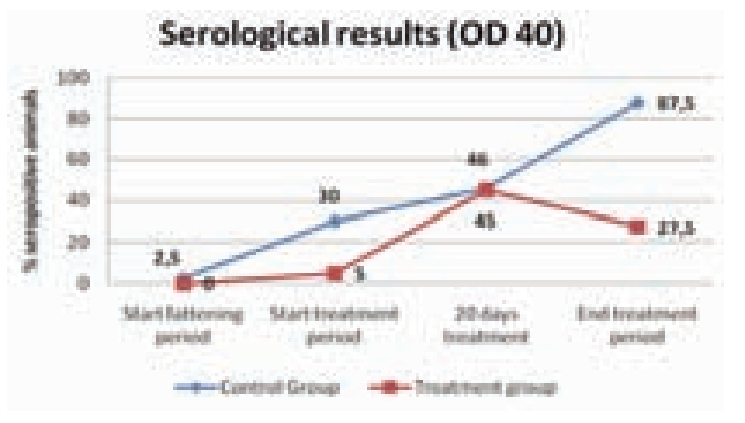

\section{Discussion}

Several studies have demonstrated that organic acids can be useful to reduce Salmonella contamination in swine farms $[2,3]$. In the present study the treatment, which was designed taking into account treatment economic feasibility $(0.035 \%$, during forty days) has been able to demonstrate a reduction of the number of Salmonella shedders. Moreover, the number of Salmonella seropositive animals was also reduced. Despite that this results are promising further studies are required to confirm them.

\section{References}

[1] Mousing et al. (1997) Prev Vet Med.29(4):247-61.

[2] Letellier et al. (1999). Can J Vet Res.64(1):27-31.

[3] Van der Wolf et al. (2001)Vet Q. 2001 Jul;23(3):121-5.

[4] Creus (2007). Zoonoses Public Health. ;54(8):314-9. 


\title{
P.664
}

\section{Salmonella Typhimurium and Derby infection in weaned pigs}

\author{
Jan Matiasovic; Hana Stepanova; Lenka Leva; Hana Havlickova; Frantisek Sisak; Ivan Rychlik; Martin Faldyna \\ Veterinary Research Institute, Brno, Czech Republic
}

\section{Introduction}

Salmonella Typhimurium (STM) and Derby (SD) are most common serovars in pig herds in Europe (EFSA 2008). Studies of STM shedding and serological response to Salmonella infection at herd and individual level were performed (Scherer et al. 2008), but little is known about the course of SD infection. Moreover, no information about competition between STM and SD is available. Here we show the kinetics of Salmonella fecal shedding and development of specific antibody response to SD, STM as well as mixed infection in weaned pigs.

\section{Materials and Methods}

Strains used were isolated from infected sows. STM was of DT104 phagotype. Weaned salmonella-free pigs were divided into groups of five animals for STM, SD and non-infected control. Another group consisting of 10 animals was infected with a mixture of STM and SD. Animals were infected per os with 108 bacteria per animal. Experiment was carried-out for 28 days. Salmonella shedding was investigated in fecal samples by a standard culture method (EN ISO 6579:2002). Presence of antibodies against LPS $O$ antigens (STM and SD share the same O antigens) in blood serum was evaluated by ELISA Salmotype ${ }^{\circledR}$ LDL (Germany). Sampling of feces and blood was done twice a week.

\section{Results}

Shedding of STM was observed in group infected with STM up to $14 \mathrm{DPI}$, in group with mixed infection up to $17 \mathrm{DPI}$. Shedding of SD was observed during all experiment in both groups infected with SD (SD group and group with mixed infection). From $14 \mathrm{DPI}$, the titers of SD in both groups were very similar or the same. Percentage of STM in isolates from mixed infection varied from $0 \%(14 \mathrm{DPI})$ to $50 \%$ (17 DPI).

Antibodies against $\mathrm{O}$ antigens were detected at $14 \mathrm{DPI}$ in three of five animals in group infected with STM. At 28 DPI, four animals reached high antibody titer, but one animal from this group did not develop antibody response. In SD group, a low titer of antibodies was detected only at $28 \mathrm{DPI}$ in three of five animals. In group with mixed infection, antibody titer was measured only at $28 \mathrm{DPl}$; six of ten animals developed antibody response.

\section{Discussion}

Short fecal shedding of STM (up to $14 \mathrm{DPI}$ ) and early developing of antibody response (14 DPI) in group infected with STM was similar as it was previously shown (Scherer et al. 2008). Contrary to STM, the period of SD shedding was prolonged. SD was detected in feces during the entire experiment, but only in a low titer from $21 \mathrm{DPI}$. Contrary to that, antibody response to SD infection was slow and only three of five animals developed low antibody titers at $28 \mathrm{DPI}$. In group with mixed infection, fecal shedding of STM was similar to group infected with STM only. Shedding of SD was nearly the same as in group infected only with SD and was also prolonged during all experiment. From this data we can conclude there is no competition between STM and SD.

\section{References}

EFSA Scientific Report (2008): Report of the Task Force on Zoonoses Data Collection on the Analysis of the baseline survey on the prevalence of Salmonella in slaughter pigs, in the EU, 2006-2007. The EFSA Journal (2008) 135, 1-111.

Scherer K, Szabó I, Rösler U, Appel B, Hensel A, Nöckler K.: Time course of infection with Salmonella typhimurium and its influence on fecal shedding, distribution in inner organs, and antibody response in fattening pigs. J Food Prot. 2008 Apr;71(4):699-705.

The study was supported by the Ministry of Agriculture of the Czech Republic (QH81062 and MZe 0002716202) and Ministry of Education, Youth and Sports of the Czech Republic (CZ.1.05/2.1.00/01.0006 AdmireVet). 


\title{
P.665
}

\section{Efficacy of vaccination with an inactivated vaccine to reduce salmonella prevalence in a pig fattening unit}

\author{
Héctor Argüello; Sonia Álvarez; Álvaro Hidalgo; Jesús Osorio; Mario Arcos; German Naharro; Ana Carvajal; Pedro Rubio \\ University of León, León, Spain
}

\section{Introduction}

Immune response stimulation by vaccines has been a useful mechanism stand up to pathogens [1]. Vaccination can be a useful tool for the control of swine salmonellosis and live vaccines are the first choice, but they have several disadvantages like biosecurity, oral administration and higher prices. In the present study we have evaluated an inactivated vaccine of Salmonella Typhimurium, easy to produce and cheaper than lives vaccines.

\section{Materials and Methods}

The vaccine was prepared from a culture of Salmonella Typhimurium DT104 (109 UFC/ml) inactivated by formol and using hydroxide aluminum $\mathrm{Al}(\mathrm{OH}) 3$ as adjuvant. A pig fattening unit infected by S. Typhimurium and S. 4,5,12:i:- was chosen for the experiment. Farm housed 2960 pigs distributed in two barns (1480 pigs each). One of them was chosen as control group and was not vaccinated while the other was chosen as experimental group(vaccinated animals). Within the farm, animals were housed in two different barns. Pigs from one of the barns, vaccinated group, were injected intramuscularly $2 \mathrm{ml}$ of the inactivated vaccine at the beginning of the fattening period (70 days of life approximately) and 25 days later. Pigs from the second barn served as control group and receive no treatment. Both groups were monitored during the fattening period by collecting serum and fecal samples that were used for serological and bacteriological detection of Salmonella infection. Moreover, cecal content, mesenteric lymph nodes and blood samples were collected after slaughter from both groups. In each sampling forty animals per group were included. Bacteriological analyses were made using EN-ISO 6579:2002/Amd 1:2007: Microbiology of food and animal feeding stuffs- Horizontal method for the detection of Salmonella spp. In animal feces and in environmental samples from the primary production stage. Serological samples were tested with Idexx herdchek Swine Salmonella kit $^{\oplus}$ according to manufacturers' instructions.

\section{Results}

The percentage of Salmonella shedders was significantly lower $\left(\mathrm{Chi}^{2}=67.5 \mathrm{p}<0.001\right)$ among vaccinated animals as compared with control pigs ( $18 \%-65 \%$ in control group versus $0 \%-7.5$ $\%$ in vaccinated group). Abattoir study also revealed significant differences in the prevalence of Salmonella positive samples among cecal content $\left(\mathrm{Chi}^{2}=33.43, \mathrm{p}<0.001\right)(55 \%$ versus 15 $\%)$ and mesenteric lymph nodes $\left(\mathrm{Chi}^{2}=202, \mathrm{p}<0,001\right)(55 \%$ to $22.5 \%$ ). Results of serological analysis were no so consistent although significant differences $\left(\mathrm{Chi}^{2}=49.6 \mathrm{p}<0.001\right)$ in the percentage of seropositive animals were also detected at the end of the study ( $88 \%$ of seropositive animals in control group versus $44 \%$ in vaccinated group).
Figure 1. Fecal shedding of Salmonella during fattening period in vaccinated and unvaccinated animals (day 1 coincides with vaccination date).

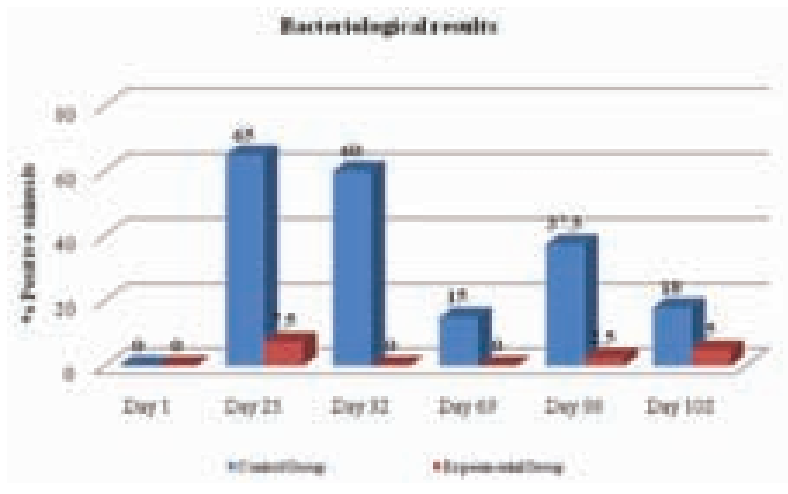

\section{Discusion}

Despite of live vaccines and its cell mediated response provide a better protection $[2,3]$, our study demonstrates that inactivated vaccines can be useful in the control of swine salmonellosis. Further investigations are needed to corroborate these results and particularly in farms infected by other serotypes different to S. Typhimurium.

\section{References}

[1] Denagamage et al. (2007). 4(4):539-49

[2] Lindberg AA, Robertsson JA (1983). Infect Immun. 1983 Aug;41(2):751-7

[3] Lumsden JS, Wilkie BN( 1992). Can J Vet Res. (4):296-302 


\title{
P.666
}

\section{Resistance patterns of Salmonella isolated from pigs and porc in Germany}

\author{
Andreas Schroeter; Annemarie Kaesbohrer; Reiner Helmuth; Christina Dorn; Kirsten Heckenbach; Bernd-Alois Tenhagen \\ Federal Institute for Risk Assessment (BfR), Berlin, Germany
}

\begin{abstract}
Introduction
Antimicrobial resistance in Salmonella spp. is a major public health concern in Europe and world wide. Although in the majority of cases Salmonella is not associated with clinical disease in pigs, studies have shown that they are prevalent at slaughter and enter the food chain. S. Typhimurium from pigs is estimated to be a major source of human salmonella infections. It was the purpose of this study to compare the resistance patterns of Salmonella spp. isolated from porc with those isolated from pigs.
\end{abstract}

\section{Materials and Methods}

Salmonella isolates from pigs and porc submitted to the National Reference Laboratory for Salmonella between 2000 and 2008 were analysed for their susceptibility to antimicrobials using the broth microdilution method according to NCCLS/CLSI standards. Minimum inhibitory concentrations (MIC) were evaluated according to epidemiological cut off values (www.eucast.org). Isolates with MIC above the cut offs were considered resistant, otherwise susceptible.

\section{Results}

Overall 1595 isolates from porc and 3820 isolates from pigs were tested. The majority of isolates from pigs were from three serovars, namely S. Typhimurium (67 \%), S. 1,4[5],12:i:- (9\%) and S. Derby (8\%). The proportion of S. Typhimurium decreased from above $80 \%$ in $2000-2002$ to $44 \%$ in 2008 . In the same period, the proportion of S. 1,4[5],12:i:-increased from 0 to 21 $\%$. S. Derby on the other hand was constant in its share of the submitted isolates.

In porc, the same serovars dominated. S. Typhimurium had a proportion of above $60 \%$ in 2000-2002 that decreased to 43 $\%$ in 2008 . Overall, $53 \%$ of all isolates from porc were S. Typhimurium. S. 1,4[5],12:i:- (9\% of the isolates) was practically not observed in 2000 but had a share of above $20 \%$ in 2007 and 2008. S. Derby ( $10 \%$ of the isolates was fairly constant between 5 and $12 \%$ of the isolates over the years.

The proportion of isolates that were susceptible to all antimicrobials tested increased over the years in animals (10\% in 2000-2002 to $22 \%$ in 2006-2008) and the proportion of isolates resistant against more than one group of antimicrobials decreased slightly (78 vs. $71 \%$ ) in the same time period.
In isolates from porc the changes were less pronounced. The number of isolates that were susceptible against all antimicrobials tested increased only slightly (from 25 to $31 \%$ ). In contrast to the situation in animals, the proportion of isolates resistant against more than one group of substances increased slightly over the years (from 56.6 to $60.2 \%$ ).

In S. Typhimurium from pigs, the resistance situation did not change very much over the years. From 2000-2002 to 2006-2008 the proportion of susceptible strains increased slightly from 6.0 to $10.5 \%$ in pigs, but the high proportion of multiresistant strains remained unchanged ( 86.6 vs $85.2 \%$ ). In food, a different situation was observed. The proportion of susceptible strains decreased from 21.5 to $14.8 \%$ and the proportion of multiresistant strains increased slightly (69.5 to $73.3 \%$ ).

Trends in resistance for S. 1,4[5],12:i:- cannot be described as there were practically no isolates in the first years. However the vast majority of strains from pigs ( $86 \%$ ) and porc (83\%) were multiresistant and only few isolates ( 3 and $4 \%$ ) were susceptible to all antimicrobials tested.

S. Derby was less resistant to antimicrobials. Overall, $47 \%$ of the isolates from pigs and $57 \%$ of the isolates from porc were susceptibel against all tested antimicrobials. The proportion of multiresistant strains was substantially lower than in S. Typhimurium and S. 1,4[5],12:i:- (28\% in pigs, $22 \%$ in porc).

The highest resistance rates were observed for ampicillin, streptomycin, sulfamethoxazole and tetracycline. The resistance rates to these antimicrobials were higher in isolates from pigs (60 to $90 \%$ ) than in isolates from porc (40 to $60 \%$ ). The lowest resistance rates were observed against gentamicin, colistin, and 3rd generation of cephalosporins (0 to $5 \%$ ).

\section{Discussion}

The results underline that colonized or infected pigs are a likely source of contamination for porc indicating the need to control Salmonella pre- and at harvest to reduce this contamination. S. Typhimurium and S. 1,4[5], 12:i:- show multiresistance in the majority of the isolates which hampers treatment of infections in humans. Resistance to antimicrobials that are administered via animal feed, such as tetracycline and amoxicillin is widespread. It has been pointed out, that the use of these antimicrobials is associated with a higher risk of slaughter pigs to harbour S. Typhimurium in their ileocaecal lymph nodes (Tenhagen et al. 2009). 


\title{
P.667
}

\section{Identification of Salmonella Typhimurium genes induced in porcine tonsils and lymph nodes during long-term persistence using in vivo expression technology (IVET)}

\author{
Alexander Van Parys; Filip Boyen; Elin Verbrugghe; Bregje Leyman; Freddy Haesebrouck; Frank Pasmans \\ Department of Pathology, Bacteriology and Avian Diseases, Faculty of Veterinary Medicine, Ghent University, Merelbeke, Belgium
}

\section{Introduction}

Persistent Salmonella Typhimurium infections in pigs often result in asymptomatic carrier pigs and are a major concern for food safety and human health. Tonsils and lymph nodes play a key role in the persistence of Salmonella Typhimurium in pigs, but very little is known about the underlying mechanisms. For the identification of Salmonella Typhimurium genes specifically induced in these organs, a genome-wide screening method was performed using in vivo expression technology (IVET) (Mahan et al, 1993).

\section{Materials and Methods}

We used a spontaneous nalidixic acid resistant derivative of a wild type Salmonella Typhimurium strain isolated from a pig stool sample. A $\triangle$ purA deletion mutant was constructed in this strain using the Datsenko and Wanner method (2000). For proper use in the IVET protocol, we verified that this mutant was significantly impaired in comparison to the wild type strain in a preliminary in vivo mixed infection experiment. We constructed an IVET library as described earlier for Salmonella Enteritidis (Gantois et al., 2008), composed of approximately 12,000 different transformants, representing the major part of the Salmonella Typhimurium genome.

For IVET selection in pigs, 9 pigs were orally inoculated with approximately $10^{8}$ CFU of our IVET library. Three weeks after inoculation, pigs were euthanized and tonsil and lymph node samples were collected, processed and plated on selective MacConkey agar. White colonies, representing in vivo but not in vitro induced genes, were collected and the IVET fusions were sequenced as described before (Gantois et al., 2008). Identification of the sequence of the cloned promoter was done by BLAST analysis.

\section{Results}

From the IVET selection experiment, 19 and 24 different genes were identified in the tonsils and lymph nodes, respectively (see Table 1 for a summary). The majority of these genes plays a role in Salmonella metabolism. One known virulence gene (sifB) was identified, playing a role in formation and maintenance of the Salmonella-containing vacuole. AsmA, a gene important for the invasion of nonphagocytic cells, was upregulated in the lymph nodes. Furthermore, 2 genes were identified that act in stress responses ( $h t p G$ and $d n a K$ ). The flagellin-producing operon fliC/fljB was induced in the tonsils, while $r f a E$, involved in LPSbiosynthesis, was identified in the lymph nodes. Finally, several genes with a yet unknown function were identified in tonsils and lymph nodes.

\section{Discussion}

Using IVET, Huang et al. (2007) already identified several Salmonella Typhimurium genes expressed in porcine tonsils at 2 days post inoculation. These genes differ from the genes that we were able to identify at 3 weeks post inoculation (except for $r p o N)$. This suggests that different sets of Salmonella genes play a role in short- and long-term persistence in pigs.

Although we identified several Salmonella genes that might be important for long-term persistence in pigs, further research is needed to confirm their exact function.

\section{Acknowledgements}

The technical assistance of Nathalie Vanrysselberghe and Roselien Schelfaut is greatly appreciated. This work was supported by the Institute for the Promotion of Innovation by Science and Technology in Flanders (IWT Vlaanderen), Brussels, Belgium (grant IWT Landbouw 040791); the Research Foundation-Flanders (FWO) and the Special Research Fund (BOF), University of Ghent, Belgium.

\section{References}

Datsenko KA and Wanner BL (2000). One-step inactivation of chromosomal genes in Escherichia coli K-12 using PCR products. PNAS 97: 6640-6645.

Gantois I, Ducatelle R, Pasmans F, Haesebrouck F and Van Immerseel F. (2008) Salmonella enterica serovar Enteritidis genes induced during oviduct colonization and egg contamination in laying hens. Applied and environmental microbiology 74: 6616-6622.

Huang Y, Leming CL, Suyemoto M and Altier C (2007) Genome-wide screen of Salmonella genes expressed during infection in pigs, using in vivo expression technology. Applied and environmental microbiology 73: 7522-7530.

Mahan MJ, Slauch JM and Mekalanos JJ (1993) Selection of bacterial virulence genes that are specifically induced in host tissues. Science 259: 686-688.

\section{Results Table.}

\begin{tabular}{|l|c|c|c|c|}
\hline Gene group & \multicolumn{4}{|c|}{ Organ } \\
\hline & \multicolumn{2}{|c|}{ Tonsils } & \multicolumn{2}{c|}{ Lymph nodes } \\
\hline Metabolic & efp & artP & efp & pflD \\
\hline & dnaC/dnaT & kdgK & dnaC/dnaT & fnr \\
\hline & gyrB & lysS & gyrB & rpoZ \\
\hline & rnt & & rnt & nrdB \\
\hline & pflC & & pflC & rpoN \\
\hline & rpsU & & aroK & ssb/asmA \\
\hline Virulence & sifB & & sifB & \\
\hline Stress & htpG & & htpG & \\
\hline & & & dnaK & \\
\hline Motility & fliC/fljB & & & \\
\hline LPS & & & rfaE & \\
\hline Unknown function & ygfA & & ygfA & \\
\hline & ybjP & & citX2/citG2 & \\
\hline & STM4067 & & yaeT & \\
\hline & ydeW & & yadF & \\
\hline & yggE & & yhbG & \\
\hline
\end{tabular}




\title{
P.668
}

\section{Class 1 integrons of "Salmonella enterica" isolated from white or Iberian swine in the Southwest of Europe}

\author{
Gonzalo Palomo ${ }^{1}$ María J. Campos ${ }^{2,1}$ Alberto Quesada ${ }^{3}$ Santiago Vadillo ${ }^{1}$ Anselmo Perea ${ }^{1}$ Carmen Borge ${ }^{4}$ Segundo Píriz ${ }^{1}$ \\ 1. Animal Health Department, Extremadura University, Cáceres, SA, Spain; \\ 2. Grupo de Investigação em Recursos Marinhos (GIRM), Instituto Politécnico de Leiria (IPL), Peniche, Portugal; \\ 3. Biochemistry Department, Extremadura University, Cáceres, Spain; 4. Animal Health Department, Cordoba University, Córdoba, Spain
}

\section{Introduction}

Salmonellosis is the most important food borne disease in the North Hemisphere. There is a mortality of $0.1 \%$ in the European Union, mainly in children, elderly and inmuno-depressed patients, where antimicrobial therapy was no efficient enough. The main sources of infection are pork, eggs and poultry aliments (EFSA, 2009).

\section{Material and Methods}

Our strain collection consists in 144 "Salmonella enterica" strains isolated from slaughter house samples and clinical cases in Spain and Portugal. Minimum inhibitory concentrations (MICs) for ampicillin (Amp), amoxicillin (Amx), streptomycin (Str), spectinomycin (Spt), florphenicol (Fhl), chloramphenicol (Chl), tetracycline (Tet) and sulfametoxazole (Sul) were determined by microdilution broth. The screening for class 1 integrase gene (int1) was performed by PCR (Leverstein-Van Hall et al, 2002). A second PCR was used to amplify the conserved-cassettes (CS) inside the integrons which contains the antibiotic resistance genes. Finally, CS regions were characterized by RFLP analysis and sequencing (Levesque et al, 1995).

\section{Results}

Half of the collection (65 strains) was int 1 positive. Majority of them (92\%) were multidrug resistance (MDR) strains and only three were totally sensible. Among these strains, the most common MDR profiles were: AmpAmxStrSptChISulTet $(n=20)$ found in white swine (WS) and ( $n=10)$ in Iberian ones (IS); AmpAmxStrSptSulTet $(n=6)$ and AmpAmxSptChISulTet $(n=5)$ in WS and AmpAmxStrChISulTet $(n=5)$ in IS. A total of 36 strains has both the penta-resistant phenotype (AmpStrChISulTet) and the class 1 integron. Nine CS profiles were described (from A to I). The conservative-cassettes contain the genes: blaOXA-30, blaPSE1 (amoxicillin and ampicillin resistance); $\operatorname{aad} A 1$, aadA2 y aadA12 (streptomycin and spectinomycin); dfrA1, dfrA12, dfr17 y dhfrVII (trimetoprim, a sulfonamides synergic antimicrobial).

\section{Discussion}

Most class 1 integrons profiles are exclusively distributed among the two swine subpopulations studied, since only profiles D (5 WS and 6 IS) and A (13 WS and 1 IS) are shared between them. The genes associated to the class 1 integrons indicates that these genetic elements might support almost all phenotypical resistance. The finding of new genotype/serovar combinations, as far as (to our knowledge) the identification of dhfrVII in Montevideo; dfr17 in Anatum and Bredeney; and dfrA1 in Hindmarsh, had never been reported.

\section{References}

EFSA, 2009, Trends and sources of zoonoses and zoonotic agents in the European Union in 2007, Parma.

Leverstein-Van Hall, M., A. Paauw, A. Box, H. Blok, J. Verhoef, and A. Fluit, 2002 , Presence of integron-associated resistance in the community is widespread and contributes to multidrug resistance in the hospital: J. Clin. Microbiol., v. 40, p. 3038-40.

Levesque, C., L. Piche, C. Larose, and P. Roy, 1995, PCR mapping of integrons reveals several novel combinations of resistance genes: Antimicrob. Agents. Chemother., v. 39, p. 185-91. 


\title{
P.669
}

\section{Serological Salmonella prevalence in breeding farms}

\author{
Luminita Costinar; Corina Pascu; Viorel Herman \\ Faculty of Veterinary Medicine, Timisoara, Romania
}

\section{Introduction}

Serology is use in the last years to determine the prevalence of Salmonella on pig farms and has been adopted by several countries into national control programs. These programs designed to reduce occurrence of Salmonella on the farm and in pork (4, 6). A national serologic surveillance program has been in place in Denmark since 1993 for breeding stock herds and since 1995 for herds producing slaughter pigs. The prevalence of salmonellae in Danish pork is reported to have declined from 3.5\% in 1993 to $0.7 \%$ in 2000 (2).

The aim of the present study was to monitoring serological prevalence of Salmonella in four sows farms.

\section{Material and Methods}

A longitudinal study was carried out in 4 sows farms. There were tested a number of 304 samples and were made 3 samplings at different times.

Serological monitoring was made October 2008 - May 2009. Samples were analyzed for the presence of antibodies against Salmonella by commercially available ELISA: HerdChek ${ }^{\circledR}$ Swine Salmonella, IDEXX (USA). Samples with OD\% values equal to or greater than $10 \%(S / P=0.25)$ are considered positive. Interpretation results was made with $\mathrm{xChek}$ IDEXX software.

\section{Results}

The HerdChek ${ }^{\circledast}$ Swine Salmonella allows rapid screening for the presence of antibodies to a broad range of Salmonella serogrups indicating swine herd's exposure to the bacteria (1).

Monitoring of the immune status of a herd can be an important tool in the control of the disease. Serological monitoring of Salmonella infection of swine by standardized commercial ELISA is a fast, simple and economical.

Several studies have reported the variability of serologic responses in different groups of animals sampled at the same time and at different times. In the table is presents the samplings results.

The prevalence in each herd at the different time sampling is shown in
Table 1.

\begin{tabular}{|c|c|c|c|c|c|c|}
\hline \multirow{2}{*}{ Farms } & \multicolumn{2}{|c|}{ 1st sampling } & \multicolumn{2}{c|}{ 2nd sampling } & \multicolumn{2}{c|}{ 3rd sampling } \\
\cline { 2 - 7 } & $\begin{array}{c}\text { No. sample } \\
\text { positive }\end{array}$ & $\begin{array}{c}\text { No. sample } \\
\text { tested }\end{array}$ & $\begin{array}{c}\text { No. sample } \\
\text { positive }\end{array}$ & $\begin{array}{c}\text { No. sample } \\
\text { tested }\end{array}$ & $\begin{array}{c}\text { No. sample } \\
\text { positive }\end{array}$ & $\begin{array}{c}\text { No. sample } \\
\text { tested }\end{array}$ \\
\hline farm A & 29 & 30 & 16 & 30 & 29 & 30 \\
\hline farm B & 29 & 30 & 24 & 30 & 20 & 21 \\
\hline farm C & 15 & 15 & 22 & 23 & 27 & 30 \\
\hline farm D & 14 & 19 & 25 & 26 & 20 & 20 \\
\hline TOTAL & 304 & & & & & \\
\hline
\end{tabular}

It could be observed that in all four sows farms are presents the Salmonella antibodies. Between collection of the second and third samples in the farms $A$ the proportions of positive serologic increased.

Epidemiological screening about Salmonella antibodies in sows farms using HerdChek ${ }^{\circledast}$ Swine Salmonella ELISA kit, revealed that all 4 monitorised sows farms were high positive.

Serological tests for Salmonella antibodies in swine are interpreted by associating test results witth a reduction in prevalence of subclinical infection in pigs $(2,6)$ and reduced risk of carcass contamination at slaughter rather than with the presence or absence of disease.

The concept of serologic testing is most useful as an ongoing monitoring surveillance exercise (2). The sensitivity of the test increases with multiple sampling. The serologic response to subclinical Salmonella infection may vary between farms of sows or even within the same group sampled at different times.

\section{References}

1. Ballagi A., Camitz A., Holmquist G. (2003), The 11th International Symp. of the WAVLD and OIE Seminar on Biotechology, Nov. 9-13, 20

2. Harris Turney, I. (2003), J. Swine Health Prod., 11(6), 300-303

3. Mousing J., Thode Jensen P., Halgaard Bager F. et. al. (1997), Prev. Vet. Med. 29:247-261

4. Sorensen L.L., Dahl J., Nielsen B., (2001) Proc. 4th Int. Sym. Eidemiol. Cont. Salmonella Food Path. Pork, 316-318

5. Torremorell M., Turney-Harris I., Gramer M., Donovan T., Harris D.L. (2000), Proc. IPVS Melbourne Australia, 208 


\title{
P.670
}

\section{Serological study on hepatitis E infection in Croatian pig herds}

\author{
Zoran Lipej; Besi Roic'; Dinko Novosel; Z`eljko Mihaljevic' \\ Croatian Veterinary Institute, Zagreb, Croatia
}

\section{Introduction}

Hepatitis E virus (HEV) is one of the most important causes of acute clinical hepatitis in human population. Swine HEV has a cross-reaction with human anti-HEV antibodies. Therefore pigs could be an animal reservoir, rendering hepatitis $E$ as a zoonosis (3). HEV is an important public health problem specially in developing countries (1). The first HEV strain genetically closed to human was described in swine (2). Serological studies reported that swine HEV infection is widespread in many European countries $(4,5)$ The aim of the present study was to determine the prevalence of HEV in domestic pig population at different ages in Croatia.

\section{Material and Methods}

During 2009 serum semples of 200 pigs were collected from four farrow-to-finishing farms located in continental part of Croatia. Blood samples were taken from 50 animals for each farm. Groups of animals were selected in 5 group by age and sex. Group $1(n=10)$ gilts, group $2(n=10)$ sows, group $3(n=10)$ boars, group $4(n=10)$ pigs $<12$ weeks of age and group $5(n=$ 10) pigs $>12$ weeks of age. All blood samples were deliverd to Virology laboratory of the Croatian Veterinary Institute and were stored at -20 o $C$ before testing. Serum specimens were tested for the presence of specific anti-HEV IgG antibodies by enzyme linked immunosorbent assay (ELISA) according to the method decribed by (7) using a commercially available reagents (MP Diagnostics HEV ELISA, MP Biomedicals Suisse S.A., Switzerland) according to manufacturers instructions. The protocol used is designed for detecting HEV antibodies in human sera but adapted to animals using anti-pig IgG horsreadish peroxidase instead of ant-human conjugates.

All swine sera were tested in duplicate at a dilution of 1:21. Serum samples were added to each well and incubated for 30 min at $370 \mathrm{C}$. Plates were washed 6 times with washing buffer and conjugated with goat anti-swine lgG $(\mathrm{H}+\mathrm{L})$ (Jackson ImmunoResearch) was added of $100 \mu \mathrm{l} /$ well. The optimal working dilution of conjugate was 1:1000. After incubation at $370 \mathrm{C}$ for $30 \mathrm{~min}$ and six washes $100 \mu \mathrm{l}$ of substrate solution was added. The reaction was stopped after $15 \mathrm{~min}$ by adding $50 \mu \mathrm{l}$ stop solution. The optical densiti of each serum sample was read at $492 \mathrm{~nm}$ with a photometer and comparasion of the absorbance between test and control wells (Microplate Autoreader TECAN Sunrise -Magellan, Austria). Samples with OD values greater than or equal to the Cut-off Value are considered reactive by the criteria of this HEV antibody ELISA kit.

\section{Results and Discussion}

Among 200 serum samples collected from four pig farms overall seroprevalence was $89,5 \%$ (200/179). Positive rate of HEV-specific lgG in all farms ranging from $86 \%$ to $92 \%$. All four farrow-tofinishing units located in different geographical regions were HEV positive. The highest proportion of $100 \%$ IgG positive animals comprised gilts and finishers. Seroprevalence was $85 \%$ in sows, $90 \%$ in boars and $72,5 \%$ in pigs $<12$ weeks of age.

Table 1. Seroprevalence of HEV in pigs at different ages in four pig production units in Croatia

\begin{tabular}{|l|c|c|c|c|c|c|}
\hline Herd & gilts & sows & boars & $\begin{array}{c}\text { pigs }<12 \\
\text { weeks }\end{array}$ & finishers & No./positive(\%) \\
\hline & $\begin{array}{c}\text { No./ } \\
\text { positive }\end{array}$ & $\begin{array}{c}\text { No./ } \\
\text { positive }\end{array}$ & $\begin{array}{c}\text { No./ } \\
\text { positive }\end{array}$ & $\begin{array}{c}\text { No./ } \\
\text { positive }\end{array}$ & $\begin{array}{c}\text { No./ } \\
\text { positive }\end{array}$ & \\
\hline Farm A & $10 / 10$ & $10 / 9$ & $10 / 10$ & $10 / 7$ & $10 / 10$ & $50 / 46(92 \%)$ \\
\hline Farm B & $10 / 10$ & $10 / 8$ & $10 / 7$ & $10 / 8$ & $10 / 10$ & $50 / 43(86 \%)$ \\
\hline Farm C & $10 / 10$ & $10 / 7$ & $10 / 9$ & $10 / 8$ & $10 / 10$ & $50 / 44(88 \%)$ \\
\hline Farm D & $10 / 10$ & $10 / 10$ & $10 / 10$ & $10 / 6$ & $10 / 10$ & $50 / 46(92 \%)$ \\
\hline TOTAL & $\mathbf{4 0 / 4 0}$ & $\mathbf{4 0 / 3 4}$ & $\mathbf{4 0 / 3 6}$ & $\mathbf{4 0 / 2 9}$ & $\mathbf{4 0 / 4 0}$ & $\mathbf{2 0 0 / 1 7 9}(\mathbf{8 9 , 5 \% )}$ \\
\hline
\end{tabular}

Our results show that HEV is present and widespread among pig population in Croatia at high HEV antibody prevalence (89.5\%). These findings correlate with the results of other studies $(4,5,7)$. This is the first evidence of HEV infection in Croatian domestic swine population.

\section{Acknowledgements}

We are grateful to dr Alain Houde and his group for providing detailed ELISA procedure used in this study.

\section{References}

1. Emerson and Purcell (2003) Rev Med Virol, 13, p.145-154.

2. Meng et al (1997) Proc. Natl. Acad. Sci. U.S.A. 94, p. 9860-9865.

3. Goens and Perdue (2004) Anim Health Res 5, p.145-156.

4. Breum et al (2008) IPVS Congress, Durban, Proccedings, p. 14

5. Casas et al (2008) IPVS Congress, Durban, Proccedings, p. 15

6. Leblanc et al (2007) Int J of Food Microb, 117, p. 160-166.

7. Seminati et al (2008) Vet J, 175, p. 130-132. 


\title{
P.671
}

\section{Comparison of five enzyme immunoassays (EIAs) for detection of anti- hepatitis $E$ virus (HEV) immunoglobulin $\mathbf{G}$ (IgG) in swine}

\author{
Huanrong Zhang ${ }^{1}$ Ulrich Mohn ${ }^{2}$ John Prickett ${ }^{1}$ Shayleen Harrison ${ }^{1}$ Alicia Feagins ${ }^{3}$ Xiang-Jin Meng $^{3}$ Tanja Opriessnig $^{1}$ \\ 1. Iowa State University, Ames, IA, USA; 2. MIKROGEN GmbH, Neuried, Germany; 3. Virginia \\ Polytechnic Institute and State University, Blacksburg, VA, USA
}

\section{Introduction}

Hepatitis E virus (HEV) is a major cause of acute hepatitis in humans but the virus is also widespread in the pig population and HEV is considered a zoonotic agent $(1,4)$. There are currently at least four known genotypes of HEV with one recognized serotype (5). Serological studies in swine are often conducted with in-house assays based on peptides and recombinant proteins of different HEV genotypes and are mainly limited to research laboratories $(2,3)$. The objective of the current study was to determine the diagnostic accuracy of one commercially available and four in-house EIAs on samples from pigs experimentally-infected with genotype 3 human or swine HEV and genotype 4 human HEV.

\section{Materials and Methods}

A total of 72 serum samples (18 obtained from gnotobiotic pigs and 54 obtained from conventional pigs) were tested by five different assays. Twenty-six of the 72 samples were control samples from non-inoculated pigs, 16/72 samples were obtained from pigs experimentally inoculated with genotype 3 human HEV (US-2 strain), 14/72 samples were obtained from pigs experimentally inoculated with genotype 3 swine HEV (Meng strain), and 16/72 samples were obtained from pigs experimentally inoculated with genotype 4 human HEV (Taiwan strain TW6196E). All serum samples were stored at $-80^{\circ} \mathrm{C}$ until processing. For the comparison, all samples above were tested with four enzymelinked immunosorbent assays (ELISAs) and one strip line immunoassay. The antigen used in the different assays varied but all were derived from human HEV genotype 1 or genotype 3 recombinant proteins. Plate-specific cutoffs were used for ELISAs 1-3 and samples in ELISA 4 were considered positive if their optical density (OD) value was equal to or greater than 0.4 . The strip line immunoassay results were read both visually by eyes and digital-interpretation. For the statistical analysis, the optimal cutoff was determined using Receiver operator characteristic (ROC) analysis. The ROC analysis was based on known infection status of each pig and included data from days post inoculation (dpi) 35-56. This analysis provided ROC optimized cut-off values that maximized diagnostic sensitivity and specificity of each assay.

\section{Results}

For the strip line assay samples that were considered positive by visual reading were also found positive when digital analysis was run. All 26 control samples were negative with all five assays. In all experimentally-inoculated pigs, seroconversion was detected at the same dpi for each individual pig across all ElAs. All five EIAs used in this study were found to be able to identify anti-HEV antibodies in pigs experimentally-inoculated with genotype 3 and $4 \mathrm{HEV}$ strains with varying sensitivity and specificity according to ROC analysis. The ROC analysis showed that the assay with the greatest sensitivity and specificity was ELISA 4 when the optimized cutoff was used; however, a small subset of samples was used and results may vary with a greater sample set. The ROC analysis also revealed that from dpi 35 to dpi 56 all four ELISAs had a sensitivity and specificity of $100 \%$.

\section{Discussion}

The similar seroconversion profiles obtained with the five EIAs when used to test sera from the experimentally-infected pigs indicates that (1) each assay could reliably discriminate between the absence and presence of anti-HEV and (2) each antigen reacted with similar levels of sensitivity with different genotypes (genotypes 1, 3 and 4). Since the capture recombinant antigens were from human HEV genotypes 1 and 3, the data suggests that these five EIAs are effective for detecting antibodies against $\mathrm{HEV}$ viruses representing the 4 main genotypes. This is in agreement with previous data indicating that only one HEV serotype exists $(2,3)$.

\section{References}

1. Emerson, S.U. and Purcell, R.H. (2003) Rev. Med. Virol. 13:145-154.

2. Engle, R. E. et al. (2002) J. Clin. Microbiol. 40:4576-4580.

3. Hu, W. P. et al. (2008) Clin. Vaccine Immunol. 15:1151-1157.

4. Meng, X.J. et al. (1997). Proc. Natl. Acad. Sci. USA 94:9860-9865.

5. Schlauder, G.G. et al. (2001) J. Med. Virol. 65:282-292. 


\title{
P.672
}

\section{Presence of hepatitis E virus in slaughter pigs}

\author{
Maribel Casas ${ }^{1}$ Raquel Cortés ${ }^{2}$ Sonia Pina ${ }^{3,4}$ Bibiana Peralta $^{3}$ Martí Cortey ${ }^{3}$ Enric Mateu ${ }^{2,3}$ Jordi Casal $^{2,3}$ Marga Martín $^{2,3}$ \\ 1. CREAL (Centre for Research in Animal Health), Barcelona, Spain; 2. Departament de Sanitat i Anatomia Animals, Barcelona, Spain; 3. Centre \\ de Recerca en Sanitat Animal (CReSA), Barcelona, Spain; 4. Institut de Recerca i Tecnologia Agroalimentàries (IRTA), Barcelona, Spain
}

\section{Introduction}

In Europe, imported human cases of hepatitis E virus (HEV) infection are declining while autochthonous cases although sporadic in nature have become more frequent (1). These autochthonous cases are most often caused by genotypes 3 and 4 of HEV whereas in endemic countries genotypes 1 and 2 are the commonest and cause epidemics transmitted through drinking water. In industrialized countries, the ubiquitous nature of the virus in domestic pigs and the genetic similarity between porcine and human HEV isolates support the notion of a potential zoonotic transmission of the infection. Thus, some human cases have been associated with the consumption of inadequately cooked meat products (2). In Spain, $>90 \%$ of pig farms have HEV-seropositive pigs and the infection is suspected to be present from at least the decade of 1980 (3). The objective of the present study was to determine the percentage of pigs that could reach the abattoir being infected.

\section{Materials and Methods}

A longitudinal survey was conducted in six farrow-to finish swine herds. Farms had been previously diagnosed as HEV positive. Twenty piglets per farm were serologically monitored from nursery to slaughter age $(3,7,13,18$ and 25 weeks (wk) of age). Sera were analyzed for anti-HEV IgM and IgG antibodies using an in-house ELISA. At slaughter, liver $(n=96)$ and bile $(n=80)$ were taken and tested by RT-PCR. In order to compare HEV sequences, serum and faeces collected at $13 \mathrm{wk}$ of age from positive animals at slaughter, were also analyzed by RT-PCR.

\section{Results}

Anti-HEV IgM were firstly detected in pigs of $7 \mathrm{wk}$ of age in five farms whereas in the remaining farm, animals did not show anti HEV lgM until 13wk of age (farm 6). At slaughter age, for the first five farms between $50 \%-100 \%$ of pigs had seroconverted for anti-HEV IgG whereas in farm 6 only $5 \%$ of pigs were lgG seropositive. Six out of 96 livers (6\%) and five out of 80 biles (6\%) were HEV positive by RT-PCR. None of the pigs was simultaneously positive in liver and bile and therefore, the total percentage of infected animals was the sum of both cases (12\%).
Interestingly, positive animals from five farms had already seroconverted at slaughter, whereas positive animals from farm 6 -the one seroconverting late- had not seroconverted yet. All RTPCR product sequences corresponded to HEV genotype 3 and shared 83 5-98.7\% nucleotide similarity among them or with other human or porcine HEV isolates. Three infected pigs (farms $3 \& 4$ ) were also positive in faeces or in serum at $13 \mathrm{wk}$ of age. The sequences from one of them had $85.8 \%$ nucleotide identity between $13 \mathrm{wk}$ and slaughter sequences, whereas another pig had $93.3 \%$ nucleotide identity between them.

\section{Discussion}

The present study demonstrates that HEV could be present in slaughter age pigs (12\%). Other papers reported values ranging from $1.3 \%$ to $11 \%(4-6)$. In the present case, three of the infected animals were already RT-PCR positive at $13 \mathrm{wk}$. This finding suggests either that HEV persisted in that pig longer than expected or that pigs might be infected more than once with the same or a different HEV strain during its productive life. Also, as seen in farm 6, if infections take place late in the pig's life, infected but seronegative pigs could reach the slaughterhouse.

In Spain, consumption of viscera is very low (0-2.9 grams/person/day) and thus, the risk of acquiring HEV by consumption of infected pig livers should be extremely low. However, manipulation or cross-contamination of HEV infected livers could be considered a potential route of transmission.

\footnotetext{
References

1 Nicand et al., 2009. Pathol Biol 57: 203-211.

2 Tei et al., 2003. Lancet 362: 371-373.

3 Casas et al., 2008. Vet Microbiol 135:248-252.

4 Bouwknegt et al., 2007. J Food Prot 70: 2889-2895.

5 Banks et al., 2010. Vet Rec 166: 29.

6 Feagins et al., 2007. J Gen Virol 88: 912-917.
} 


\title{
P.673
}

\section{Surveillance of Shiga toxin-Producing Escherichia coli 0157 and non-0157 from a breeding pig farm in China}

\author{
$\underline{\text { Yaxian Yan }}^{2}$ Dongmei Cao' Luming Xia' Huiying Zhang ${ }^{2}$ Liangke Su$^{2}$ Jianhe Sun $^{2}$ \\ 1. Shanghai Jiaotong University, Shanghai, China; 2. School of Agriculture and Biology, Shanghai, China
}

The prevalence and characterization of Shiga toxin-producing Escherichia coli and Stx-converting phage isolated from a pig farm near Shanghai, China was reported. From randomly collected swine fecal samples (60 per month for 1 year), we isolated 0157 and non-O157 STEC strains and Stx phage, and identified bacterial virulence genes and Stx phage morphologies.

In total, we found 8 STEC 0157 (1.1\%), 33 STEC non-O157 (4.6\%), and 2 stx-negative 0157 (0 3\%) strains. Three virulence gene patterns were observed in the STEC 0157 isolates-stx1-stx2eae (1), stx2-eae-hlyA (4), and stx1-stx2-eae-hlyA (3)—while 6 virulence gene patterns were found in non-0157 STEC, most frequently stx1-stx2. Stx2-eae was in all STEC 0157, whereas stx1 was in most of the non-0157 isolates.

We isolated 15 Stx 1 and 57 Stx2 phages from fecal samples, and induced 1 Stx 1 and 8 Stx 2 phages from STEC 0157 strains. Interestingly, all Stx 1 phages had hexagonal heads with long tails, while Stx2 phages had 3 different structures. Most STEC 0157 strains released more phage and Stx toxin after ciprofloxacin induction. Upon infection with the recombinant phage ØMin27( $\Delta$ stx::cat) for gene mapping, laboratory strains produced lysogenic and lytic phage, while 2 of the 8 O157STEC strains only produced lysogens.
The lysogens from laboratory strains produced infectious particles similar to ØMin27 after norfloxacin induction. The lysogens from STEC 0157 isolates released Stx phage, although free $\varnothing \operatorname{Min} 27(\Delta$ stx::cat) particles were not detected.

Our results showed that breeding pig farms are important reservoirs for 0157 and non-0157 STEC, as well as Stx phage. Residual antibacterial agents may enhance the release of Stx phage and the expression of Stx, while higher virulence of new pathogen may be produced in clinical and experimental strains by phages lysogenic conversion.

Therefore, in STEC monitoring of animal farms, attention must be given to antibiotic residues and Stx phage to improve the safety of animal related products. 


\title{
P.674
}

\section{Confirmation of the presence of Mycobacterium avium infections in two pig herds which had a high risk profile for M. avium as assessed by serologically monitoring}

\author{
Anne Hiller ${ }^{1}$ Henk J. Wisselink ${ }^{2}$ Conny B. van Solt-Smits ${ }^{2}$ Derk Oorburg ${ }^{3}$ Gereon Schulze Althoff ${ }^{4}$ Günter Klein ${ }^{1}$ \\ 1. University of Veterinary Medicine Hannover, Hannover, Germany; 2. Central Veterinary Institute of Wageningen UR, Lelystad, \\ Netherlands; 3. VION Fresh Meat West, Postbus, Boxtel, Netherlands; 4. VION GmbH, Franz-Rennefeld-Weg, Düsseldorf, Germany
}

\section{Introduction}

Diagnosis of Mycobacterium avium (MA) infection in pigs is based on detection of granulomatous lesions in lymph nodes by incision and visual inspection by traditional meat inspection at slaughter. In certain cases MA bacteria can be detected whereas granulomatous lesions are absent (1). On the other hand also Rhodococcus equi has been frequently isolated from lymph nodes with lesions (2). In 2009 a serological monitoring has been implemented within a "risk based meat inspection" pilot in a german slaughterhouse as an alternative for detection of MA infections by traditional meat inspection. From each delivery of pigs from a pig producer to the slaughterhouse, a specific number of blood samples are examined for MA antibody titers (4). Test results from current and several previous deliveries were used to determine the MA risk level for each pig producer. On the basis of this serological monitoring 17 pig herds with a high risk for an MA infection were identified. Here we describe experiments on two of these pig herds to confirm their high risk status for MA infections.

\section{Materials and Methods}

Two farms ( $A$ and $B$ ) with a high risk profile for MA infections were selected. Pigs from both farms $(A: n=58, B: n=19)$ underwent an intradermal tuberculin test with $0,1 \mathrm{ml}$ Avian Tuberculin PPD (25.000 I.U., ASG, Lelystad, The Netherlands). The presence of induration and erythema at the injection site was read $72 \mathrm{~h}$ after injection. After slaughtering, blood serum samples were taken and analysed by MA-ELISA (4). Submaxillary and mesenteric lymph nodes were collected as well at slaughter, and examined pathologically for granulomatous lesions and bacteriologically for M. avium. To identify colonies, Ziehl-Neelson staining (for acid-fast bacilli) and PCR for the presence of IS1245, which is characteristic for MA (3).

\section{Results}

In the tuberculin skin tests $72 \mathrm{~h}$ after injection approximately 44 pigs from farm A 29 and 15 pigs from farm B reacted positive. The skin showed swelling, erythema and partial central necrosis and exsudation. Bacteriological examination of the lymph nodes showed that $66 \%(38 / 58)$ and $32 \%(6 / 19)$ of the pigs were positive for MA (Table 1). In farm A $26 \%$ (15/58) of the pigs tested positive in MA-ELISA and $28 \%(16 / 58)$ by pathological examination. In farm B pigs tested with 16\% (3/19) positivity by serological and pathological examination. The sensitivity of the MA-ELISA in farm A was $21,6 \%$ and the specificity $61,1 \%$, whereas the sensitivity of the pathological examination was $40,5 \%$ and the specificity $94,4 \%$. In farm B the sensitivity of the MA-ELISA and the pathological examination was $66,6 \%$ and the specificity of both examinations was $92,3 \%$.

Table 1: Results of pathological, serological and bacteriological examination of pigs for detection of M. avium infections.

\begin{tabular}{|l|c|c|c|c|}
\hline \multirow{2}{*}{ Examination } & \multicolumn{3}{|c|}{ Number of pigs (\%) tested } & \\
\cline { 2 - 5 } & Negative & Positive & ND & Total (n) \\
\hline Farm A & & & & \\
\hline Bacteriological1 & $17(29)$ & $38(66)$ & $3(5)$ & 58 \\
\hline Pathological ${ }^{2}$ & $42(72)$ & $16(28)$ & & 58 \\
\hline Serological & $43(74)$ & $15(26)$ & & 58 \\
\hline Farm B & & & & 19 \\
\hline Bacteriological1 & $13(68)$ & $6(32)$ & & 19 \\
\hline Pathological ${ }^{1}$ & $16(84)$ & $3(16)$ & & 19 \\
\hline Serological & $16(84)$ & $3(16)$ & & \\
\hline $\begin{array}{l}\text { 1 Positive when M. avium bacteria were detected by bacteriological examination on } \\
\text { submaxillary lymph nodes and mesenteric lymph nodes. }{ }^{2} \text { Positive when granulomatous } \\
\text { lesions were seen in the submaxillary lymph nodes. ND not determined. }\end{array}$ \\
\hline
\end{tabular}

\section{Discussion}

Two pig herds with a high risk profile for MA infections based on the serological monitoring had indeed an MA infection. Results of avian tuberculin testing on both farms showed already the presence of an MA infection, bacteriological examination of lymph nodes confirmed it definitely.

Specificity and sensitivity of the pathological examination for MA infections at slaughter is questioned $(2,4)$. Here we show that serological monitoring for MA infections showed good perspectives to identify succesfully MA positive pig herds.

\section{References}

1. Brown et al., 1979. Appl. Environ. Microbiol. 37, 740-743

2. Komijn et al., 2007. Vet Microbiol. 120: 352-357

3. Van Soolingen et al. 1998. J. Clin. Microb. 36: 3051-3054

4. Wisselink et al. 2009. Vet Microbiol. 2009.11.003 


\title{
P.675
}

\section{Examination of Rhodococcus equi strains isolated from lymph nodes of Dutch slaughter pigs}

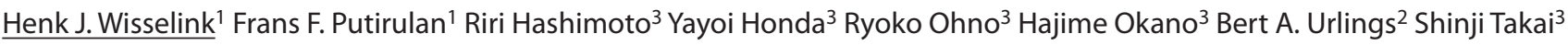 \\ 1. Central Veterinary Institute of Wageningen UR, Lelystad, Netherlands; 2. VION N.V., Best, Netherlands; 3. \\ School of Veterinary Medicine and Animal Sciences, Kitasato University, Towada, Japan
}

\section{Introduction}

Rhodococcus equi can cause disease in horses, especially in young foals. In humans, it mainly causes disease in those infected with HIV (Prescott et al., 1991). R. equi is also frequently isolated from granulomatous lesions in submaxillary lymph nodes of pigs (Komijn et al., 2007). R. equi strains differ in virulence. Virulent $R$. equi strains are isolated from horses and are characterized by the presence of virulence-associated 15- to 17$\mathrm{kDa}$ antigens (VapA). R. equi strains of intermediate virulence are identified by a virulence-associated 20-kDa antigen (VapB) and are found in lymph nodes of pigs (Takai et al., 1996). Avirulent $R$. equi strains show no evidence of virulence-associated antigens or plasmid DNA and are widespread in soil. Humans can be infected with $R$. equi strains of the three virulence levels. The reservoir for infection in humans is not clear. Earlier, the isolation of R. equi from lymph nodes of Dutch slaughter pigs was reported (Komijn et al., 2007). Here we describe the typing of these strains for virulence plasmids.

\section{Materials and Methods}

R. equi strains $(n=46)$ isolated from lymph nodes of slaughter pigs originating from nine farms were used in this study (Komijn et al., 2007). Strain ATCC 33701 (virulent strain) and 27 representative strains of intermediate virulence were used as reference strains (Makrai et al., 2008). Plasmid DNA was extracted by an alkaline lysis method with some modifications and digested with the restriction endonucleases BamHI, EcoRI, EcoT22I and HindIII for detailed comparison and estimation of plasmid sizes (Takai et al., 1993, 1999). Samples of the plasmid preparations were separated in $0.7 \%$ or $1.0 \%$ agarose gels at approx. $5 \mathrm{~V} / \mathrm{cm}$ for $2 \mathrm{~h}$.

\section{Results}

None of the $46 R$. equi isolates contained the vapA gene, 43 isolates (93\%) were positive for the vapB gene showing a 827-bp PCR product of the expected size. The 43 isolates of intermediate virulence contained seven different virulence plasmids including three new plasmid variants (Table 1). On the basis of restriction digestion patterns of plasmid DNAs, we tentatively designated these new variants as type 29, 30 and 31 (Table 1). R. equi strains with virulence plasmid 5 was most widespread, in strains isolated from pigs of eight of the nine farms this plasmid was found. In contrast, strains with plasmid type 1, 9, 29, 30 and 31 were exclusively found in lymph nodes of pigs from single farms.
Table 1 Distribution of VapB-positive and negative plasmid types in R. equi strains isolated from lymph nodes of pigs originating from nine farms

\begin{tabular}{|l|c|c|c|c|c|c|c|c|c|}
\hline Farm & \multicolumn{7}{|c|}{ No. of strains containing plasmid type } & Total \\
\hline No. & 1 & 5 & 9 & 10 & 29 & 30 & 31 & - & \\
\hline 1 & & & & 2 & 3 & & & 1 & 3 \\
\hline 2 & & 2 & & & & & & & 5 \\
\hline 3. & & 2 & & & & & & & 2 \\
\hline 4 & & 2 & & 1 & & & & & 3 \\
\hline 5 & & 3 & & & & & & & 3 \\
\hline 6 & & 4 & & & & 1 & & 2 & 7 \\
\hline 7 & & 1 & 1 & & & & 10 & & 12 \\
\hline 8 & 2 & 4 & & 2 & & & & & 8 \\
\hline 9 & & 3 & & & & & & & 3 \\
\hline Total & 2 & 21 & 1 & 5 & 3 & 1 & 10 & 3 & 46 \\
\hline
\end{tabular}

\section{Discussion}

In agreement with earlier findings the $R$. equi strains isolated from lymph nodes of pigs appeared to be from intermediately virulence (Takai et al., 1996). In addition, compared to other studies, plasmid type 5 is the predominating one (Makrai et al., 2008).

Reports on $R$. equi infections in humans in the Netherlands are scarce. A further surveillance study among Dutch human $R$. equi strains is needed to answer the question to the risk of $R$. equi transmission from pigs to humans.

\section{References}

Prescott et al., 1991. Clin Microbiol Rev. 4, 20-34 Komijn et al., 2007. Vet Microbiol. 120: 352-357 Makrai et al., 2008. Vet Microbiol.131: 318-323 Takai et al., 1993. J Clin Microbiol. 31:1726-1729 Takai et al., 1996. J Clin Microbiol. 34:1034-1037 Takai et al., 1999. J Clin Microbiol. 37:3417-3420 


\title{
P.676
}

\section{Seroprevalence and risk factors associated to Toxoplasma gondii in domestic pigs from Spain}

\author{
Ignacio García-Bocanegra ${ }^{2}$ Meritxell Simon-Grifé ${ }^{3}$ Jitender P. Dubey ${ }^{1}$ Jordi Casal $^{4,3}$ \\ Gerard E. Martín-Valls ${ }^{3}$ Oscar Cabezón ${ }^{5,3}$ Antonio Arenas² Sonia Almería ${ }^{3,5}$ \\ 1. Animal Parasitic Diseases Laboratory, Animal and Natural Resources Institute, Agricultural Research Service, United States Department of \\ Agricultural, Beltsville, MD, USA; 2. Departamento de Sanidad Animal. Facultad de Veterinaria, UCO, Córdoba, Spain; 3. Centre de Recerca \\ en Sanitat Animal (CReSA), UAB-IRTA, Campus de la Universitat Autònoma de Barcelona, Bellaterra, Barcelona, Spain; 4. Departament \\ de Sanitat i Anatomia Animals, Universitat Autònoma de Barcelona, Bellaterra, Barcelona, Spain; 5. Servei d'Ecopatologia de Fauna \\ Salvatge (SEFaS). Departament de Medicina i Cirurgia Animals, Universitat Autònoma de Barcelona, Bellaterra, Barcelona, Spain
}

\section{Introduction}

Toxoplasmosis is a worldwide zoonotic disease caused by Toxoplasma gondii, which infects most warm-blooded animals. In fact, human prevalence has been found worldwide in nearly one-third of the population (Tenter et al., 2000).

The consumption of raw or undercooked pork meat containing tissue cysts is considered among the principal source for human infection (Dubey, 2009). Pigs can get infected with T. gondii through ingestion of sporulated oocysts in soil, vegetation or water, by ingestion of cysts in tissues of infected animals such as rodents, birds and other pigs, or congenitally (Dubey, 2009).

The aim of the present study was to provide recent information on epidemiology and prevalence of T. gondii and the risk factors associated to this protozoan parasite in domestic pigs from the main swine producing regions of Spain.

\section{Material and Methods}

A cross-sectional study was conducted in 100 swine farms (83 farrow-to-finish and 17 piglet production farms) located all over Spain between 2007 and 2009.

Blood samples were collected from a total of 2,970 pigs. Fourteen sows and 20 fattening pigs were randomly selected in each farrow-to-finish farm and samples from 14 sows were obtained from the piglet production farms.

Presence of antibodies to T. gondii was tested by modification agglutination test (MAT). The serum from each pig was tested at dilutions from 1:25, 1:50 and 1:500. Epidemiological data were collected through a structured filled-in questionnaire in order to provide information on exposure levels to potential risk factors. The effect of the exploratory variables on the response variable (seropositivity to T. gondii) was investigated by the method of generalized estimating equations (GEE). The statistical analyses were performed using SPSS v15.0.

\section{Results}

Antibodies (MAT 1:25 or higher) against T. gondii were detected in $492\left(16.6 \% ; \mathrm{Cl}_{95 \%}: 152-18.0\right)$ of 2,970 pigs tested, with titers $1: 25$ in 287 (58.3\%), 1:50 in 171 (34.8\%) and $\geq 1: 500$ in 34 (6.9\%). Farm prevalence was $85.0 \%\left(\mathrm{Cl}_{95 \%}: 78-92\right)$ and within-farm prevalence ranged from $2.9 \%$ to $92.8 \%$ (median= $17.6 \%$ ). Significantly higher seroprevalences were detected in Valencia Community (27.3\%), Extremadura (23.3\%) and Catalonia (21.2\%) with respect to the other sampled regions. Sows with parity number higher than three had significantly higher seropositivity to T. gondii compared to younger sows. The GEE showed that age sows compared to fattening pigs $\left(\mathrm{OR}=2.9 ; \mathrm{Cl}_{95 \%}=1.8-4.5\right)$, no rodent control $\left(\mathrm{OR}=1.9 ; \mathrm{Cl}_{95 \%}=1.04-3.60\right)$ and presence of cats $(\mathrm{OR}=$ $1.6 ; \mathrm{Cl}_{95 \%}=1.12-2.34$ ) were risk factors potentially associated to T. gondii seroprevalence.

\section{Discussion}

The results showed widespread exposure to T. gondii among domestic pigs in Spain, with highly variable prevalence levels among farms and regions. The high seroprevalence rates indicate that raw or undercooked pork could be a source for human infections in Spain and the need of consumption of properly cook pork, especially in pregnant women.

Higher exposure was related to age of pigs, with the highest levels observed in sows, and to poor management practices related to lack of rodent control and to the presence of cats in the farms. Therefore, in order to reduce the risk of infection by T. gondii in pig farms in Spain, adequate rodent control programs and restriction of cats in the farms should be advised, and especial emphasis of avoiding infection should be taken care in the sows.

\section{References}

Dubey, J.P., 2009. Toxoplasmosis in pigs. The last 20 years. Vet. Parasitol. 164, 89-103.

Tenter, A.M., Heckeroth, A.R., and Weiss, L.M., 2000. Toxoplasma gondii: from animals to humans. Int. J. Parasitol. 30:1217-58. 


\title{
P.677
}

\section{Occurrence and antimicrobial susceptibility of Yersinia enterocolitica on liver surfaces of slaughtered pigs}

\author{
Alexandra von Altrock ${ }^{4}$ Uwe Roesler $^{1,2}$ Roswitha Merle ${ }^{3}$ Karl-Heinz Waldmann ${ }^{4}$ \\ 1. Institute of Animal Hygiene and Veterinary Public Health, University of Leipzig, Leipzig, Germany; 2. Institute of Animal \\ Hygiene and Environmental Health, Freie Universitaet, Berlin, Germany; 3. WHO-Collaborating Centre for Research and \\ Training in Veterinary Public Health, University of Veterinary Medicine, Hannover, Germany; 4. Clinic for Swine, Small \\ Ruminants, Forensic Medicine and Ambulatory Service, University of Veterinary Medicine, Hannover, Germany
}

\section{Introduction}

Yersiniosis is the third most frequently reported human foodborne gastroenteritis in Europe caused by Yersinia (Y.) enterocolitica. Bioserotype 4/O:3 is the predominant type, which can be found especially in pigs' tonsils and to a lesser degree in the intestine, the faeces and lymph nodes of slaughtering pigs (4). After evisceration, rinse water enables the spread of $Y$. enterocolitica to the organs of the pluck set, hanging on a hook beneath the tonsils (2).

The purpose of the study was to evaluate the prevalence and the resistant pattern of $Y$. enterocolitica isolated from liver surfaces of slaughtered pigs in order to estimate the risk of the consumer being infected with this zoonotic agent by offal of porcine origin.

\section{Materials and Methods}

In a slaughterhouse in Lower Saxony, 1,500 liver surfaces of pigs from 50 herds (30 livers from each herd) were swabbed during slaughtering process. Y. enterocolitica were grown in irgasanticarcillin-potassium chlorate (ITC) broth, isolated on cefsoludinirgasan-novobiocin (CIN) agar, identified with the API 20E system (bioMérieux, Nuertingen, Germany) and serotyped by slide agglutination (Sifin, Berlin, Germany). Antimicrobial susceptibility was investigated by the broth dilution method following the instructions of the supplier (VetMICTMGN-mo-Plates, SVA, Uppsala, Sweden). Breakpoints to establish resistance were selected, based on CLSI recommendations for Enterobacteriaceae.

\section{Results}

Out of 1,500 swabs from liver surfaces, 70 (4.7\%) were positive for Y. enterocolitica O:3. No other serotype was identified. At herd level, up to seven liver surfaces per herd were positive. From 22 herds (44.0\%), Y. enterocolitica could not be isolated.

All isolates were resistant to ampicillin and sulfamethoxazole. Multi-resistance to four antimicrobials was observed in four strains $(6.8 \%)$.

\section{Discussion}

Isolation of $Y$. enterocolitica O:3 was successful from liver surfaces of pigs from $56 \%$ of the tested herds. However, sampling of liver surfaces does not consequently reflect the prevalence on the farm, since cross-contamination in abattoirs may occur. Altogether, not even five per cent of the livers were contaminated, which does not seem to represent a high risk for consumers. But the psychotropic agent is able to grow at temperatures close to $0^{\circ} \mathrm{C}$. Therefore, low contaminated pork might reach a contamination rate of clinically significant levels during the course of storage period (3).

Monitoring of antimicrobial resistance became compulsory for the EU member states by the directive 2003/99/EC, because resistance of zoonotic pathogens may potentially reduce the effectiveness of antimicrobial treatment of foodborne disease if contracted by humans (1). Altogether, $27.3 \%$ of the strains were resistant to three or more antimicrobials. Nevertheless, antimicrobials used for the therapy of extraintestinal Yersinia infections in humans, where third-generation cephalosporine or fluoroquinolones are recommended, were not affected.

\section{Acknowledgements}

The study was financially supported by the Federal Agency for Agriculture and Food on behalf of the German Federal Ministry for Food, Agriculture and Consumer Protection.

\section{References}

(1) De Jong et al. (2009): J. Antimicrob. Chemother. 63 733-744,

(2) Fredriksson-Ahomaa et al. (2001): Syst. Appl. Microbiol. 24, 457-463,

(3) Hanna et al. (1977). J. Food Sci. 42, 1180-1184,

(4) Nesbakken et al. (2003), Int. J. Food Micriobiol. 80, 231-240

Table 1: Antimicrobial resistance of 65 Y. enterocolitica O:3 isolates

\begin{tabular}{|l|c|c|}
\hline Antimicrobial Agent & Breakpoint (mg/L) & Part of resistant strains \\
\hline Ampicillin & $>32$ & $100 \%$ \\
\hline Ciprofloxacin & $>2$ & 0 \\
\hline Nalidixic acid & $>32$ & 0 \\
\hline Gentamicin & $>16$ & 0 \\
\hline Ceftiofur & $>8$ & 0 \\
\hline Streptomycin & $>16^{*}$ & $18.5 \%$ \\
\hline Tetracyclin & $>16$ & 0 \\
\hline Florfenicol & $>8$ & $13.8 \%$ \\
\hline Kanamycin & $>64$ & $0 \%$ \\
\hline Sulphamethoxazole & $>512^{* *}$ & $100 \%$ \\
\hline Trimethoprim & $>16$ & $7.7 \%$ \\
\hline Chloramphenicol & $>32$ & $0 \%$ \\
\hline Cefotaxime & $>32$ & $0 \%$ \\
\hline *Source: CA-SFM, ${ }^{* * \text { Breakpoint of sulfisoxazole }}$ \\
\hline
\end{tabular}




\title{
P.678
}

\section{Antibiotic sensitivities of fecal E. coli isolates from Austrian pigs}

\author{
Sabine Elicker ${ }^{2}$ Louis Fischer $^{3}$ Daniela Philadelphy ${ }^{1}$ Wolfgang Sipos $^{1}$ \\ 1. University of Veterinary Medicine Vienna, Vienna, Austria; \\ 2. Veterinary Practice Entenfellner, Stössing, Austria; 3. LaboVet GmbH, Vienna, Austria
}

\section{Introduction}

E. coli are the most frequent pathogens causing diarrhea in pigs. Besides diarrhea, they can also cause septicemias and cystitides. Suckling and weaned piglets most often suffer from diarrhea, whereas colitoxin shock is most often found in piglets short after weaning.

E. coli are gram-negative bacteria belonging to the family of Enterobacteriaceae. Classification is based on $\mathrm{O}_{-}, \mathrm{K}-, \mathrm{H}-$-, and $\mathrm{F}-$ antigens. Additionally, E. coli are classified as enterotoxic (ETEC), enteropathogenic (EPEC), necrotoxic (NTEC), and shigatoxin-like producing (STEC) strains due to their pathogenic factors. STECs are further divided into enterohemorrhagic (EHEC) and edema disease causing (EDEC) strains (1).

Due to the clinical importance of these bacteria, large scale usuage of antibiotics is the consequence. As antibiotic usuage is an effective measure in the fight against bacterial infections if administered correctly on the one hand, but may lead to unfavourable side-effects such as increasing antibiotic resistance if given careless on the other hand (2), the following data aim at helping the practitioner in his decision which antibiotic to use.

\section{Materials and Methods}

E. coli isolates out of 444 pigs ( 24 suckling piglets, 314 weaners, 88 fattening pigs, and 13 sows) suffering from diarrhea were tested for their antibiotic sensitivities by routine agar diffusion test. Following antibiotic substances were included: amoxicillin, cefquinome, colistin, enrofloxacin, gentamicin, lincospectin, and sulfamethoxazole-trimethoprim.

\section{Results}

solates were most sensitive to cefquinome and enrofloxacin. Thus, bacterial growth could be inhibited at $98.8 \%$ by cefquinome and at $89.9 \%$ by enrofloxacin. Sensitivities to the other antibiotics were less pronounced (lincospectin: $60.7 \%$, sulfamethoxazole-trimethoprim: $54.9 \%$, gentamicin: $40.9 \%$, colistin: $36.2 \%$, amoxicillin: $21.0 \%$ ). Single isolates, which were additionally tested for their sensitivites against neomycin and tylosine, were completely resistant against these substances.

Additionally, differences in the susceptibilities were found concerning the source, i.e. the different age groups. $100 \%$ of isolates derived from fattening and breeding animals were susceptible to cefquinome and $95.7 \%$ of isolates from fattening pigs alone to enrofloxacine. Nevertheless, susceptibilities to gentamicin and amoxicillin were reduced $(25.5 \%, 14.9 \%)$ in these animals. Isolates of sows were comparably highly susceptible to lincospectin (76.9\%) and those of suckling piglets to lincospectin, sulfamethoxazole-trimethoprim, and gentamicin (91.7\%, $87.5 \%, 65.0 \%)$. However, these age-dependent data are considered preliminary due to the small number of animals in the age groups of suckling piglets and sows.

\section{Discussion}

This study exhibited marked differences in the antibiotic susceptibility of fecal E. coli isolates. Importantly, isolates showed reduced sensitivities against the frequently applied substance colistin and also against the broad-spectrum antibiotic amoxicillin. Cefquinome and enrofloxacin were shown to be most effective against fecal E. coli isolates.

\section{References}

1. Fairbrother and Gyles (2006): Diseases of Swine, 9th ed., pp. 639674.

2. Tenover (2001): Clin Infect Dis 33(S3), S108-S115. 


\title{
P.679
}

\section{Metaphylaxis with butafosfan and vitamin B12 (Catosal ${ }^{\circledR}$ ) in pregnant sows enhanced the immunity of new born piglets that resulted in improved weaning weight}

\author{
Abdulkerim Deniz ${ }^{1}$ Supoj Watanapongchati ${ }^{2}$ Athipoo Nuntaprasert ${ }^{3}$ \\ 1. Bayer Animal Health, Monheim, Germany; 2. Bayer Thai Co.,Ltd. , Bangkok, Thailand; \\ 3. Faculty of Veterinary Science, Chulalongkorn University, Bangkok, Thailand
}

\section{Introduction}

Immunmodulatory effect of the combination butafosfan and vitamin B12 (Catosal ${ }^{\circledR}$ ) in sheep (1), calves (2) and mice (3) was already reported. Beneficial effect of Catosal ${ }^{\circledR}$ on metabolic function in the farrowing sows was also reported (4). The purpose of the present study was to investigate the effect of the metaphylactic treatment with Catosal ${ }^{\circledR}$ in pregnant sows on the immunoglobulines in colostrum and blood of sows and on newborn piglets. Furthermore, production paramaters were also investigated.

\section{Material and method}

Thirty randomly selected pregnant crossbred sows were allocated in one of two study groups equally. All sows were managed and treated with same standard routine applications during the pregnancy. Catosal ${ }^{\circledR}$ group was treated with $20 \mathrm{ml}$ of Catosal ${ }^{\circledR}$ (100 mg butafosfan and 0,05 vitamin B12/ml) at 3 weeks, 2 weeks and 1 week before farrowing start intramuscularly. And control group did not receive Catosal ${ }^{\circledR}$ but $20 \mathrm{ml}$ saline. Colostrum and blood samples from all sows were collected at the start of farrowing to test the immunoglobulines $(\lg G, \lg M, \lg A)$. Blood was collected from new born piglets at 3 days of age to test above mentioned immunoglobulines ( $n=15$ for each group). Farrowing and weaning performance of sows and piglets was evaluated. Commercial Indirect ELISA test kit (Bethyl Laboratories Inc., Montgomery, TX) for analysis of the total immunoglobulins ( $\lg G, \lg M, \lg A)$ in the colostrums and blood was used. Plasma and colostral samples were diluted at 1:10,000 for the analysis. All piglets in the study groups had standard routine treatments. Data were analyzed statistically based on Wilcoxon-Mann-Whitney-U Test, two sided, $95 \% \mathrm{Cl}$.

\section{Results}

Mean number of alive born piglets in Catosal and control group litters was 10.8 and 10.9 respectively. Metaphylaxis with Catosal ${ }^{\circledR}$ had significant beneficial effect for the immunity (Table 1) and weaning weight of liveborn piglets (Figure 1). There was an increase by $6 \%(534 \mathrm{~g})$ in the weaning weight of liveborn piglets in the Catosal group compared to control group $(p<0,05)$. An increase $(2,6 \%)$ in the survivability of alive born piglets up to weaning was observed in the Catosal group $(91,4 \%)$, but this increase could not be proven statistically. In the first week of life, piglets in Catosal ${ }^{\circledR}$ group had better fecal consistency, low diarrhoea incidence.

\section{Conclusion}

Metaphylaxis with Catosal ${ }^{\circledR}$ at prepartum period stimulated the immunity of sows that resulted in increased immunoglobulines in colostrum and consequently in piglets. An increased weaning weight was a significant economic benefit of the treatment.

\begin{tabular}{|l|c|c|c|c|}
\hline & & Catosal & Control & p \\
\hline $\lg \mathrm{G}(\mathrm{mg} / \mathrm{ml})$ & Sow colostrum & $60.46 \pm 3.738$ & $54.54 \pm 4.218$ & $<0.05$ \\
\hline & Sow serum & $23.35 \pm 2.133$ & $21.58 \pm 2.291$ & $<0.05$ \\
\hline & Piglet serum & $23.83 \pm 1.213$ & $23.97 \pm 1.330$ & $>0,05$ \\
\hline $\operatorname{lgA}(\mathrm{mg} / \mathrm{ml})$ & Sow colostrum & $7.78 \pm 1.437$ & $6.53 \pm 1.079$ & $<0.05$ \\
\hline & Sow serum & $2.25 \pm 0.295$ & $1.98 \pm 0.306$ & $>0,05$ \\
\hline & Piglet serum & $2.78 \pm 0.187$ & $2.19 \pm 0.087$ & $=0.0000$ \\
\hline $\operatorname{lgM}(\mathrm{mg} / \mathrm{ml})$ & Sow colostrum & $3.56 \pm 0.061$ & $3.37 \pm 0.265$ & $<0.05$ \\
\hline & Sow serum & $2.98 \pm 0.168$ & $2.92 \pm 0.304$ & $>0,05$ \\
\hline & Piglet serum & $1.33 \pm 0.081$ & $1.28 \pm 0.096$ & $>0,05$ \\
\hline
\end{tabular}

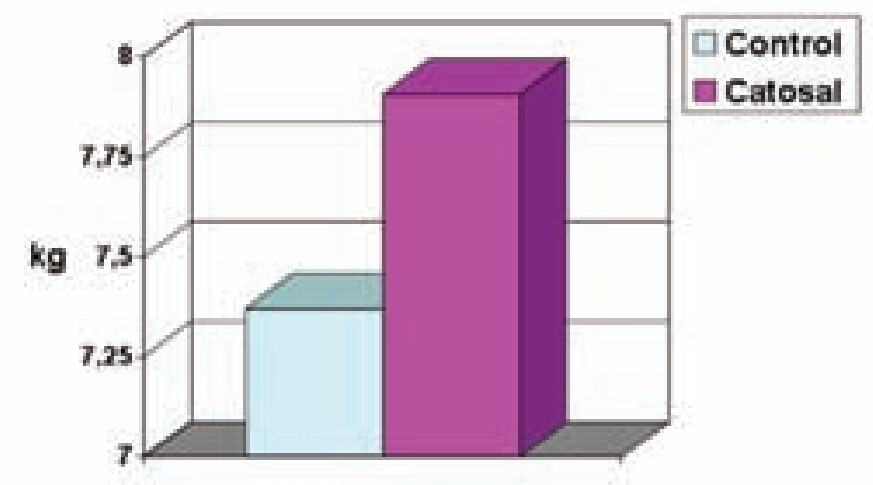

Weaning weight of piglets (in 27 days)

\section{References}

1- Sommer, H (1990): Institut für Pharmakologie und Toxikologie Veterinärmedizinische Universität, Budapest, Hungary. Rep. No. 012765.

2- Semjen G. (1995): University of veterinary science, Budapest. RepNo. 015132.

3- Surong et al. (2005): Chinese Journal of Veterinary Science and Technology, 35 (7): 574-578.

4- Lanfranchi, E. et al. (2008): 20th IPVS2008, Durban, South Africa. 


\title{
P.680
}

\section{Pharmacokinetic / Pharmacodynamic Relationships of Valnemulin $\left(\right.$ Econor $\left.^{\circledR}\right)$ and Lawsonia intracellularis the Cause of 'Ileitis'}

\author{
David G. Burch ${ }^{1}$ Ulrich Klein ${ }^{2}$ \\ 1. Octagon Services Ltd, Old Windsor, Berkshire, UK; 2. Novartis Animal Health Inc., Basel, Switzerland
}

\section{Introduction}

Valnemulin (Econor ${ }^{\oplus}$ - Novartis Animal Health Inc.), a pleuromutilin with exceptional activity against the gut pathogens Brachyspira hyodysenteriae and B. pilosicoli was reported to have good activity against Lawsonia intracellularis (Li), (1) the causal agent of Porcine Proliferative Enteropathy or 'ileitis'. Valnemulin (VAL) has been shown to be highly effective in the treatment of ileitis (1) when given in feed. It was the purpose of this paper to look at the pharmacokinetics (PK) of VAL in the gut contents and relate these to the pharmacodynamics (PD) of VAL and its clinical effect against Li.

\section{Materials and Methods}

\section{A) Pharmacokinetics (PK)}

VAL concentrations were described in colon contents (2) following in feed medication at approximately 75 and 200 ppm for 28 days at 1.6 and $5.2 \mu \mathrm{g} / \mathrm{g}$. The relationship between colon and ileum contents was modelled ( 3 ) and it was estimated that an effective steady state concentration of approximately $29 \%$ of the colon contents was found in the ileum. These figures were used to determine the ileum contents concentration of VAL at 0.49 and $1.5 \mu \mathrm{g} / \mathrm{ml}$, respectively.

\section{B) Pharmacodynamics (PD)}

A recent report (4) showed that VAL had a very low intracellular MIC 90 against 10 isolates of Li from the United States $(n=6)$ and Europe $(n=4)$, at $\leq 0.125 \mu \mathrm{g} / \mathrm{ml}$. This level was much lower than previously reported (1) at $<2 \mu \mathrm{g} / \mathrm{ml}$. The in vitro method was slightly different and used McCoy cells rather than rat enterocytes (IEC-18 cells) to grow the Li. They also used a wider range of different concentrations $(0.125-128 \mu \mathrm{g} / \mathrm{ml})$ than the original study, making a titration of low and high MICs possible.

\section{C) Clinical effect}

In an artificial challenge trial (1), VAL was given in feed at 25, 37.5 \& 50 ppm, from 2 days before challenge with Li strain LR189/5/83, with an intracellular MIC of $<0.125 \mu \mathrm{g} / \mathrm{ml}$, until termination 21 days after infection. In the treatment challenge study (1), VAL was given 7 days after infection for 14 days until termination (see Table 1).

Lesions in the ileum were examined grossly and histologically.

\section{Results and Discussion}

Effective concentrations of VAL are achieved in the ileum contents, which inhibited the development of gross lesions of ileitis at 50 ppm. At 75 ppm VAL and above, no gross or microscopic lesions were observed (see Figure $1 \& 2$ ) following treatment.
Table 1: Necropsy results (ileum) of the prevention (25, 37.5 \& 50 ppm) and treatment (75 \& $125 \mathrm{ppm}$ ) trial

\begin{tabular}{|l|c|c|}
\hline Treatment & Gross lesions & Micro lesions \\
\hline Infected control & $5 / 7$ & $6 / 7$ \\
\hline VAL 25ppm (P) & $2 / 7$ & $6 / 7$ \\
\hline VAL 37.5ppm (P) & $1 / 5$ & $2 / 5$ \\
\hline VAL 50ppm (P) & $0 / 7$ & $1 / 7$ \\
\hline VAL 75ppm (T) & $0 / 7$ & $0 / 7$ \\
\hline VAL 125ppm (T) & $0 / 7$ & $0 / 7$ \\
\hline
\end{tabular}

\section{Figure 1. PK/PD relationship of VAL in ileum contents}

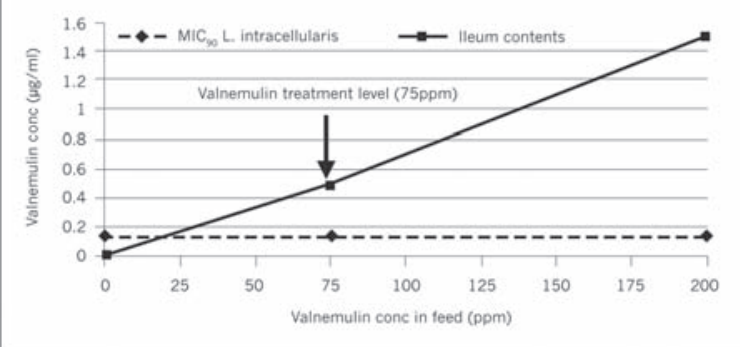

Figure 2. Dose/efficacy relationship with VAL in feed

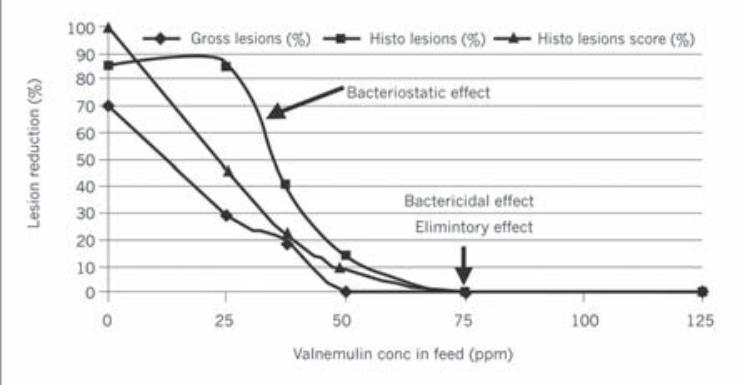

\section{References}

1. McOrist, S. et al (1998) Proc 15th IPVS Congress, Birmingham, UK, vol 3, p 114

2. Novartis Report (1998) No. ECO/INT/0798

3. Burch, D. (2005) Pig Journal, 56, 25-44

4. Wattanaphansak, S et al (2009) Veterinary Microbiology, 134, 305210 


\title{
P.681
}

\section{Effect of Catosal ${ }^{\circledR}$ on appetite and body weight of sows during lactation}

\author{
Eliana O. Dantas ${ }^{1}$ Rogério B. Petri ${ }^{1}$ Stefan A. Rohr ${ }^{2}$ \\ 1. Bayer S.A., São Paulo, Brazil; 2. Integrall Consulting, Belo Horizonte, MG, Brazil
}

\section{Introduction}

Weight loss by the sow should not be more than $12 \%$ during lactating period, which will support good piglet assistance, a fast return to heat after weaning and a good reproductive performance in the subsequent parturition (1). The aim of this trial was to evaluate the impact of Catosal ${ }^{\circledR}$ (Butaphosphan $100 \mathrm{mg}$ and Cyanocobalamin 0,05mg/mL; Bayer Animal Health; in some countries available as Catosal ${ }^{\circledR}$ B12, Coforta ${ }^{\circledR}$ or Phosphorum ${ }^{\circledR}$ B12) in lactating sows on different parameters, including feed intake and weight loss during lactation period.

\section{Materials and Methods}

The experiment was conducted in a Brazilian commercial farm including 360 sows which were equally distributed in blocks according to the parturition order: first parturition, second parturition, and between third and sixth parturition. These blocks were distributed into four treatments, with 90 repetitions, and each animal was considered an experimental unit. Around 110 days of gestation the sows were transferred to the farrowing facility and received the lactation diet in the restricted amount of $2.0 \mathrm{~kg} /$ day. After farrowing, feed was distributed twice in a day, ad libitum. The wet feed consisted of 58\% feed and $42 \%$ water. Sows were weighted in the entrance and in the exit of the farrowing unit, and the feed intake was measured daily. The weaning was proceeded when piglets were 21 days old. The treatment groups were classified as: T1 (negative control), T2 (10mL of Catosal $\left.{ }^{\circledR}\right)$, T3 $\left(15 \mathrm{~mL}\right.$ of Catosal $\left.^{\circledR}\right)$ and T4 (20mL of Catosal $\left.{ }^{\circledR}\right)$. The product was administered intramuscularly after detection of the first signals of parturition.

\section{Results}

Average weekly feed intake was high for all treatments in the first lactation week. However, the T4 group had statistically higher average feed intake $(p<0.05)$ than the control group T1 on lactation week 1, 2 and 4 (Table 1) as well as total feed consumption during lactation of T4 was significantly higher than T1. For absolute values of weight loss, it was observed that the T4 group had a lower weight loss with statistical significance $(p<0.05)$ than the groups T1 and T2. The T3 group also had a lower weight loss with statistical significance than the group T1.

For the percentages of weight loss, only the T4 group had a lower weight loss with statistical significance $(p<0.05)$ than the groups $\mathrm{T} 1$ and $\mathrm{T} 2$.
Table 1: Weekly feed intake ( $\mathrm{kg} /$ day) of lactating sows and average consumption during lactation.

\begin{tabular}{|l|c|c|c|c|c|}
\hline & W1 & W2 & W3 & W4 & AC \\
\hline $\mathrm{T} 1$ & $7.71 \mathrm{~B}$ & $9.54 \mathrm{~B}$ & $10.45 \mathrm{~A}$ & $8.28 \mathrm{BC}$ & $9.28 \mathrm{~B}$ \\
\hline $\mathrm{T} 2$ & $8.00 \mathrm{~A}$ & $9.84 \mathrm{AB}$ & $10.45 \mathrm{~A}$ & $7.86 \mathrm{C}$ & $9.40 \mathrm{AB}$ \\
\hline $\mathrm{T} 3$ & $8.07 \mathrm{~A}$ & $9.76 \mathrm{~B}$ & $10.50 \mathrm{~A}$ & $8.70 \mathrm{AB}$ & $9.43 \mathrm{AB}$ \\
\hline $\mathrm{T} 4$ & $8.21 \mathrm{~A}$ & $10.17 \mathrm{~A}$ & $10.58 \mathrm{~A}$ & $9.00 \mathrm{~A}$ & $9.64 \mathrm{~A}$ \\
\hline
\end{tabular}

$\mathrm{W}=$ average consumption during a specific week of the lactating period; Number after $\mathrm{W}=$ week of the lactation period; $\mathrm{AC}=$ average consumption for the entire period of lactation. Different capital letters in the same column indicate statistically significant difference $(p<0.05)$

Table 2: Sow weight loss during lactation in $\mathrm{kg}$.

\begin{tabular}{|l|c|}
\hline & Weight loss $\mathbf{( k g )}$ \\
\hline $\mathrm{T} 1$ & $27.57 \mathrm{~A}$ \\
\hline $\mathrm{T} 2$ & $26.39 \mathrm{AB}$ \\
\hline $\mathrm{T} 3$ & $24.36 \mathrm{BC}$ \\
\hline $\mathrm{T} 4$ & $22.43 \mathrm{C}$ \\
\hline
\end{tabular}

Different capital letters in the same column indicate statistically significant difference $(p<0.05)$.

\section{Discussion}

Sows that received 15 and $20 \mathrm{~mL}$ of Catosal ${ }^{\circledR}$ at farrowing had lower weight loss during the suckling period with a difference from $T 1$ such as $3.21 \mathrm{~kg}$ and $5.14 \mathrm{~kg}$ respectively. This fact is clearly associated with an increased feed intake (appetite) in these treatment groups. Catosal ${ }^{\circledR}$ application at farrowing stimulates the appetite of sows during lactation and prevents from weight loss at the critical time point where sows should feed newborn piglets.

\section{References}

1. Clowes, E.J. et al. J Anim Sci. 81:753-764, 2003. 


\title{
P.682
}

\section{Effect of butaphosphan+vitamin B12 (Catosal ${ }^{\circledast} /$ Coforta $^{\circledR}$ ) on pig performance}

\author{
Eijalin Z. Bautista' ${ }^{1}$ Zoilo M. Lapus ${ }^{2,4,3}$ Jomer B. Fule$^{1}$ Serafin Jr L. Garciano \\ 1. Bayer Philippines Inc., Laguna, Philippines; 2. Philippine College of Swine Practitioners, Quezon City, Philippines; \\ 3. Asian Pig Veterinary Society, Quezon City, Philippines; 4. Swine Consultant, Quezon City, Philippines
}

\section{Introduction}

In intensive pig production systems, pigs are exposed to multiple stressors such as handling, mixing with unfamiliar conspecifics, and movement to other buildings, all of which may adversely affect performance of the animals. Stress causes the release of catabolic hormones like cortisol which can negatively affect metabolism, leading to reduced weight gain in these growing animals (Ekkel et al., 1995). Coforta ${ }^{\circledR} /$ Catosal $^{\circledR}$ is a metabolic stimulant and tonic supplement containing $100 \mathrm{mg}$ butaphosphan and $0.05 \mathrm{mg}$ of cyanocobalamin per $\mathrm{ml}$ as active ingredients. It has been reported to reduce stress by reducing cortisol levels (van der Staay et al., 2006; de Groot et al., 2003). This field study aimed to investigate the effects of Catosal ${ }^{\circledR}$ on the weight gain of pigs when given strategically during stressful periods such as handling, movement and transfer to other buildings.

\section{Materials and Methods}

This field study was conducted in a 300 sow level farm. Twenty clinically healthy, purebred sows and their piglets were divided into two groups, the treatment group ( $\mathrm{N}=108$ piglets), and control group ( $\mathrm{N}=111$ piglets). In the treatment group, the sows received two injections of Catosal ${ }^{\circledR}$ at $20 \mathrm{ml}$ per dose once at the start of farrowing and the second dose 28 days post-farrowing (weaning day). The corresponding piglets were given Catosal ${ }^{\circledR}$ at the following dosages and schedules: $1 \mathrm{ml}$ at day 3 of age, coinciding with the administration of iron dextran and toltrazuril $0.4 \mathrm{ml} / \mathrm{kg} ; 1 \mathrm{ml}$ at day 28 (weaning day); $2 \mathrm{ml}$ when the piglets were transferred from the farrowing building to the flat deck, and $5 \mathrm{ml}$ upon transfer from the flat deck to the grower buildings.

The sows and piglets from the control group remained untreated with any preparation similar to Catosa ${ }^{\circledR}$, nor with a placebo, apart from a single dose of Catosal ${ }^{\circledR}$ given to the piglets at 10 days of age, which was the usual farm program.

All piglets were weighed at birth, at weaning, during transfers and upon market.

\section{Results and Discussion}

The pigs in the Catosal ${ }^{\circledR}$ group had significantly higher average daily gain (ADG) in the different stages of production compared to the control group. Even by adjusting weight gain at 40 days and 80 days of rearing period in the flat deck and grower-finisher buildings, respectively, and at 150 days birth-to-market rearing period in order to have an equal basis of comparison, weight gain was significantly higher in the group from post-weaning until market. By the end of the trial, the ADG of the Catosal ${ }^{\circledR}$ group was higher by 58 grams per day, which translated to an average of 8.5 kilogram-difference in the weight gain of the Catosal ${ }^{\circledR}$ group.
Table 1.

\begin{tabular}{|l|c|c|c|}
\hline & Control & Catosal & p-value \\
\hline Ave. birth weight (kg) & $1.50(\mathrm{~N}=111)$ & $1.55(\mathrm{~N}=108)$ & $>0.05$ \\
\hline $\begin{array}{l}\text { Ave. birth weight upon transfer to flat } \\
\text { deck (kg) }\end{array}$ & $13.66(\mathrm{~N}=96)$ & $15.31(\mathrm{~N}=96)$ & \\
\hline $\begin{array}{l}\text { Ave. daily weight gain (ADG) flat } \\
\text { deck (kg) }\end{array}$ & 0.547 & 0.627 & $<0.05$ \\
\hline $\begin{array}{l}\text { Adjusted weight gain for 40-day } \\
\text { rearing period in the flat deck (kg) }\end{array}$ & 21.90 & 25.08 & $<0.05$ \\
\hline $\begin{array}{l}\text { Ave. weight upon transfer to the } \\
\text { grower building (kg) }\end{array}$ & $35.7(\mathrm{~N}=96)$ & $40.52(\mathrm{~N}=96)$ & \\
\hline ADG grower-finisher (kg) & 0.761 & 0.831 & $<0.05$ \\
\hline $\begin{array}{l}\text { Adjusted weight gain for 80-day } \\
\text { rearing period in the grower-finisher } \\
\text { building (kg) }\end{array}$ & 60.90 & 66.50 & $<0.05$ \\
\hline Ave. market weight (kg) & $92.49(\mathrm{~N}=96)$ & $102.01(\mathrm{~N}=94)$ & $<0.05$ \\
\hline Ave. market age & 174.32 & 173.23 & $>0.05$ \\
\hline ADG day 0 to market (kg) & 0.523 & 0.581 & $<0.05$ \\
\hline Adjusted weight gain at 150 days (kg) & 78.44 & 87.09 & $<0.05$ \\
\hline
\end{tabular}

\section{Conclusions}

In this field trial, Catosal ${ }^{\circledR}$ given to pigs during stressful periods in production resulted to a significantly increased daily weight gain and market weight of pigs. This resulted, based on the return on investment calculation, to high profitability for the farmers (17.02 USD per head under Philippine conditions). There were no treatment-related side effects neither in piglets nor in pigs.

\section{References}

1. Van der Staay et al., (2006) Proceedings of the 19th IPVS Congress, Copenhagen, Denmark Volume 2.

2. De Groot. J., et al., (2003) J. Vet. Pharmacol. Therap 26 (Suppl. 1), 222223

3. Ekkel, E. D., et al., (1995) Journal of Animal Science, Vol 73, Issue 6 1544-1551. 


\title{
P.683
}

\section{Clinical efficacy of acetyl-salicilic acid as an adjunct to the antibacterial treatment of porcine respiratory disease}

\author{
$\underline{\text { Lorenzo Fraile }}^{1}$ Carles Vilaltaํ Rosa López-Jimenez ${ }^{1}$ María Teresal L. Varela² \\ Sergio Lopez-Soria ${ }^{1}$ Miquel Nofrarias ${ }^{1}$ Tomás Alcala ${ }^{3}$ Sonia Espín ${ }^{4}$ \\ 1. CReSA, Bellaterra, Spain; 2. Laboratorios SYVA, León, Spain; 3. Departamento de Métodos Estadísticos, Zaragoza, Spain; \\ 4. Explotacions Agrícoles i Ramaderes S.L, Bellcaire d'urgell, Spain
}

\section{Introduction}

Infection and environmental conditions make inflammation a manifestation of respiratory disease in pigs that decrease the ability of the lungs to exchange gases. In fact, the inflammatory response may become so overwhelming as to be life threatening in itself $(1,2)$.

The benefits of therapeutic intervention with non-steroideal anti-inflammatory drugs (NSAIDs) are because of their properties as inhibitors of the actions, synthesis or release of inflammatory mediators. In addition, one of the effects of NSAIDs, such as acetylsalicylic acid (AAS), is to act as antipyretics, improve the general clinical status of animals and increase food and water intake. The aim of this study was to investigate the clinical efficacy of AAS as an adjunct to the antibacterial treatment of porcine respiratory disease.

\section{Material and Methods}

142 piglets of 4 months of age were divided in two groups (experimental and control group). These animals were suffering a respiratory disease. The inclusion criteria was the presence of pyrexia $\left(>39.7^{\circ} \mathrm{C}\right)$ in the animals. Experimental group $(A)$ received Doxycline hiclate and AAS by drinking water at a dose of $10 \mathrm{mg} / \mathrm{Kg}$ (doxiporc ${ }^{\circledR}$, Laboratorios Policherm, Spain) and 100 $\mathrm{mg} / \mathrm{kg}$ of body weight (fiebrina porcino ${ }^{\circledR}$, Laboratorios SYVA, Spain) for five consecutive days, respectively whereas control group $(B)$ received only the antibiotic treatment. The animals were clinically examined before treatment (day 1) and at 2, 3, 4,5 and 6 days after treatment for rectal temperature $\left({ }^{\circ} \mathrm{C}\right)$ and abdominal breathing, cough and depression. Each parameter was scored using a scale of $0=$ normal, $1=$ slight or moderate and 2 = severe according to Moore et al. (1986). A statistical analysis was performed by paired t-test using the statistical software SPSS System v15.The alpha level used for determination of significance for all analyses was $P<0.05$.

\section{Results}

The experimental group showed always a mean temperature lower than the negative control group (group B) throughout the trial (see figure) and this fever decrease for animals receiving AAS (group A) is statistically significant at days 2, 5 and 6 of the trial $(p<0.05)$ in comparison with animals not receiving AAS (group B). However, it was not observed significant differences for respiratory symptoms throughout the trial between both groups.

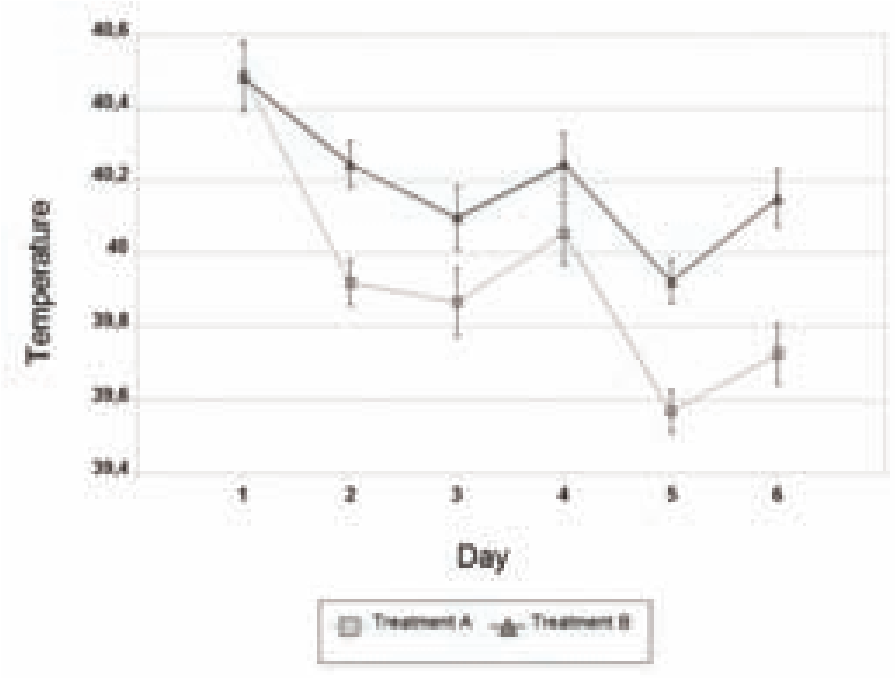

\section{Discussion}

NSAIDs are used to block the production and/or the effects of inflammatory mediators and modulators which may have a deleterious effect on alveolar exchange of gases (3). Results support the idea that AAS is an effective adjunct in the treatment of porcine respiratory disease complex to decrease fever. However, we did not observe a synergic effect to decrease respiratory symptoms in animals receiving antibiotic and AAS. Nevertheless, it was not possible to check the lung inflammation to observe the anti-inflammatory effect at lung level as observed in bovine for other NSAIDs (4).

\section{References}

1. Elitok \& Elitok, 2004. J. Vet. Pharmacol. Therap. 27: 317-320, 2004.

2. Friton et al, 2005 Veter. record 156: 809-811

3. Lees et al, 2004. J. Vet. Pharmacol. Therap. 27, 491-502.

4. Lockwood et al 2003. Veter. Record, 152, 392-394.

5. Moore et a, 1986. Am. J. Vet.Res. 57(2): 224-228. 


\title{
P.684
}

\section{PK and PK/PD of Pharmasin $250 \mathrm{mg} / \mathrm{g}$ Premix (tylosin phosphate) following multiple oral administration in pigs}

\author{
Mariana Karanikolova ${ }^{1}$ Stela Vesselova ${ }^{1}$ Valeri Nazarov${ }^{1}$ Stanislava Ivanova ${ }^{1}$ Dimitar Pashov ${ }^{2}$ Spas Petkov ${ }^{3}$ Alain Kanora $^{3}$ \\ 1. Department of R\&D, Biovet JSC, Peshtera, Bulgaria; 2. Department of Pharmacology, Faculty of \\ Veterinary Medicine, Stara Zagora, Bulgaria; 3. Huvepharma NV, Antwerp, Belgium
}

\section{Introduction}

In this study we determined some pharmacokinetic (PK) parameters of tylosin as Pharmasin $250 \mathrm{mg} / \mathrm{g}$ Premix (Huvepharma $\mathrm{NV}$ ) and related these to its pharmacodynamic (PD) with a view to a substantiated clinical use.

\section{Materials and Methods}

Eleven mixed breed pigs, weighing 20-25kg without a history of macrolide administration were enrolled in the study. Ten animals, equally male and female, received tylosin (as phosphate) at $10 \mathrm{mg} / \mathrm{kg} \mathrm{BW} / 24 \mathrm{~h}$ (Pharmasin $250 \mathrm{mg} / \mathrm{g}$ Premix) via feed offered ad libitum prepared daily at 6.00 am for 5 consecutive days. During the treatment 40 heparinized blood samples were obtained from each pig at predetermined intervals. One pig received no drugs and served as negative control. On the $5^{\text {th }}$ day the treated pigs were euthanized: 2 animals at each of the 5 different time points. Blood, organ and tissue samples from respiratory and Gl tract were collected. Plasma and organ (tissue) tylosin concentrations were measured by validated HPLC method.

\section{Results}

The plasma concentration-time profile of tylosin showed nearly identical daily courses within the treatment period (Fig. 1).

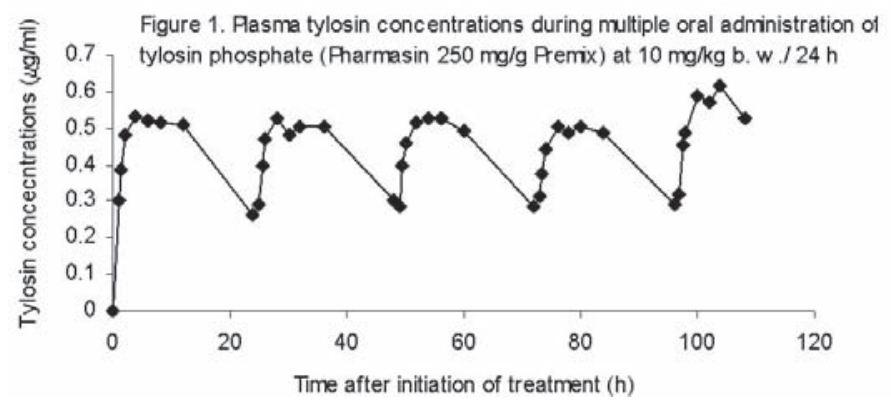

There were no significant differences between the 4 daily peak concentrations $\left(C_{\max }\right)$ and between $C_{\min }$ or AUC values (Repeated measures ANOVA Scheffe post hoc test). However, the individual plasma concentration data were quite variable and showed that in $70 \%$ of the animals, steady state was reached at $54.4 \pm 7.7 \mathrm{~h}$ after administration initiation. After 4 days of treatment $C_{\text {max-ss }}$ was $0.55 \pm 0.04 \mu \mathrm{g} / \mathrm{mL}, C_{\text {min-ss }} 0.30 \pm 0.03 \mu \mathrm{g} / \mathrm{mL}$, $C_{\text {av-ss }} 0.42 \pm 0.03 \mu \mathrm{g} / \mathrm{mL}$ and $A U C_{0-24 \mathrm{~h}} 10.14 \pm 0.84 \mu \mathrm{g} . \mathrm{h} / \mathrm{mL}$. Steady state tylosin concentrations in lung tissue and bronchial mucosa and secretions peaked $4 \mathrm{~h}$ after the $5^{\text {th }}$ administration, being $0.95 \pm 0.05 \mu \mathrm{g} / \mathrm{g}$ and $1.09 \pm 0.06 \mu \mathrm{g} / \mathrm{g}$, respectively, and persisted to the last sampling point ( $12^{\text {th }} \mathrm{h}$ ) being $0.85 \pm 0.06 \mu \mathrm{g} / \mathrm{g}$ and $0.97 \pm 0.04 \mu \mathrm{g} / \mathrm{g}$, respectively. The corresponding tissue/plasma ratios of AUC were 1.81 and 2.04 .
Table 1. Steady state organ (tissue) pharmacokinetic parameters of tylosin (mean $\pm S E M$ ) in pigs, following 4-day p.o. administration at $10 \mathrm{mg} / \mathrm{kg} / 24 \mathrm{~h}$ of tylosin phosphate as Pharmasin ${ }^{\oplus} 250 \mathrm{mg} / \mathrm{g}$ premix (0-12h)

\begin{tabular}{|l|c|c|c|c|c|c|c|}
\hline $\begin{array}{l}\text { Parameters, } \\
\text { units }\end{array}$ & $\begin{array}{c}\text { Blood } \\
\text { plasma }\end{array}$ & $\begin{array}{c}\text { Lung } \\
\text { tissue }\end{array}$ & $\begin{array}{c}\text { Bron- } \\
\text { chial } \\
\text { mu- } \\
\text { cosa* }\end{array}$ & $\begin{array}{c}\text { Ileum } \\
\text { wall }\end{array}$ & $\begin{array}{c}\text { Ileal } \\
\text { content }\end{array}$ & $\begin{array}{c}\text { Colon } \\
\text { wall }\end{array}$ & $\begin{array}{c}\text { Colonic } \\
\text { content }\end{array}$ \\
\hline $\begin{array}{l}\mathrm{AUC}_{0-12{ }^{\prime}} \mu \mathrm{gg} . \mathrm{h} / \\
\mathrm{mL}(\mathrm{g})\end{array}$ & 5.34 & 9.69 & 10.87 & 3.33 & 340.80 & 46.28 & 915.90 \\
\hline $\mathrm{T} / \mathrm{P}^{1}$ ratio of AUC & $\mathrm{NC}$ & 1.81 & 2.04 & 0.62 & 63.82 & 8.67 & 171.52 \\
\hline $\mathrm{C}_{\text {max }} \mu \mathrm{g} / \mathrm{mL}(\mathrm{g})$ & 0.53 & 0.95 & 1.09 & 0.33 & 31.4 & 4.76 & 89.4 \\
\hline $\mathrm{T}_{\max } \mathrm{h}$ & 8.0 & 4.0 & 4.0 & 4.0 & 12.0 & 8.0 & 4.0 \\
\hline $\mathrm{C}_{12 \text { th }} \mathrm{h}, \mu \mathrm{gg} / \mathrm{mL}(\mathrm{g})$ & 0.52 & 0.85 & 0.97 & 0.24 & 31.4 & 3.08 & 67.3 \\
\hline $\mathrm{MRT}, \mathrm{h}$ & 32.3 & 49.0 & 40.1 & 22.4 & 12.9 & 11.9 & 27.6 \\
\hline $\mathrm{T} / \mathrm{P}^{1}$ ratio of $\mathrm{C}_{\max }$ & $\mathrm{NC}$ & 1.79 & 2.05 & 0.62 & 59.2 & 8.98 & 168.67 \\
\hline $\mathrm{T} / \mathrm{P}^{1}$ ratio of $\mathrm{C}_{12 \text { th }} \mathrm{h}$ & $\mathrm{NC}$ & 1.63 & 1.86 & 0.63 & 60.38 & 9.15 & 171.42 \\
\hline
\end{tabular}

*Bronchial mucosa and secretions; 'Tissue/Plasma; NC, Not Calculated (each datum represents mean of 2 animals).

\section{Discussion and Conclusion}

The mean plasma steady state values of $C_{\text {min-ss }}$ and of $C_{a v-s s}$ were higher than the $\mathrm{MIC}_{90}$ described for clinically important porcine bacterial pathogens of respiratory tract: $M$. hyopneumoniae $0.25 \mu \mathrm{g} / \mathrm{mL}^{1}$, E. rhusiopathiae - $<0.13 \mu \mathrm{g} / \mathrm{mL}^{3}$, Leptospira spp. $-0.06 \mu \mathrm{g} / \mathrm{mL}^{3}$. This was valid also for organ (tissue) steady state concentrations in respiratory tract which were higher than those in plasma. The concentration in ileal and colonic content provided active levels against $L$. intracellularis $-\mathrm{MIC}_{90}=4 \mu \mathrm{g} / \mathrm{mL}^{2}$ and some sensitive strains of $B$. hyodysenteriae $-\mathrm{MIC}_{90}>16>256 \mu \mathrm{g} /$ $\mathrm{mL}^{2}$. The efficacy PK-PD breakpoint of macrolide antibiotics is a time above the MIC (T>MIC) of $50-80 \%$ of dosage interval ${ }^{4}$. In this study active tylosin antimicrobial levels persisted throughout the period of administration. Therefore, tylosin phosphate as Pharmasin ${ }^{\circ} 250 \mathrm{mg} / \mathrm{g}$ Premix at the dosage schedule tested complies with the requirements of PK-PD approach. This supports its clinical use in the control of respiratory and gastrointestinal infections.

\section{References}

1. Hannan, P.C. T. et al. (1997). Antimicrob. Agents Chemother. 41: 2037-2040.

2. Page, S.W. (2003). The Role of Enteric Antibiotics in Livestock Production (2003). Avcare Limited, Canberra, ACT 2601, Australia, p. $11-4$.

3. Prescott, J.F. et al. (2000). Antimicrobial Therapy in Veterinary Medicine, 3rd edn. lowa State University Press, Ames, lowa, pp. 237-257.

4. Toutain, P.L. et al. (2002). Res. Vet. Sci. 73:105-114. 


\title{
P.685
}

\section{Pharmacokinetics of Pharmasin $250 \mathrm{mg} / \mathrm{g}$ Premix (tylosin phosphate) following single oral administration in pigs}

\author{
Mariana Karanikolova ${ }^{1}$ Stela Vesselova ${ }^{1}$ Valeri Nazarov${ }^{1}$ Stanislava Ivanova ${ }^{1}$ Dimitar Pashov ${ }^{2}$ Spas Petkov ${ }^{1}$ Alain Kanora $^{3}$ \\ 1. Department of R\&D, Biovet JSC, Peshtera, Bulgaria; 2. Department of Pharmacology, Faculty of \\ Veterinary Medicine, Stara Zagora, Bulgaria; 3. Huvepharma NV, Antwerp, Belgium
}

\section{Introduction}

Tylosin is a macrolide antibiotic that acts mostly against Grampositive bacteria and mycoplasmata. Although it has been used in veterinary medicine for a long time the data on its pharmacokinetic (PK) properties in pigs are incomplete. This study aimed at providing PK data of tylosin as Pharmasin ${ }^{\circledR} 250 \mathrm{mg} / \mathrm{g}$ Premix (Huvepharma NV) that could aid PK-pharmacodynamic (PD) integration as a rational base for its clinical use.

\section{Materials and Methods}

Six healthy pigs, equally male and female, weighing $20-25 \mathrm{~kg}$ without a history of macrolide administration were enrolled in the study. The animals received by intubation a single p.o. dose of tylosin (as phosphate) $10 \mathrm{mg} / \mathrm{kg} \mathrm{BW}$ (Pharmasin $250 \mathrm{mg} / \mathrm{g}$ Premix), as water suspension. Heparinized blood samples $(n=13$ per animal) were taken at predetermined intervals. Plasma tylosin concentrations were measured by a validated HPLC method. The results obtained for tylosin disposition were tested by compartmental method (using Akaike's information criterion) and by non-compartmental approach as well.

\section{Results}

Plasma concentration vs. time data best fitted applying the noncompartmental model. The highest plasma concentration $\left(C_{\max }\right.$ $=1.08 \pm 0.08 \mu \mathrm{g} / \mathrm{mL}$ ) was achieved $2 \mathrm{~h}$ post administration. Thereafter, plasma levels declined remaining higher than $0.38 \pm 0.017$ $\mu \mathrm{g} / \mathrm{mL}$ at $12 \mathrm{~h}$ post administration (Fig. 1). The half-life $\left(\mathrm{t}_{1 / 2 \beta^{\prime}}\right)$ was 6.66h. $\mathrm{AUC}_{0 \text {-tlast }}$ and $\mathrm{AUC}_{0-\infty}$ were $8.2 \pm 0.49 \mu \mathrm{g} . \mathrm{h} / \mathrm{mL}$ and $11.84 \pm 0.58 \mu \mathrm{g} . \mathrm{h} / \mathrm{mL}$, respectively (Table 1 ).

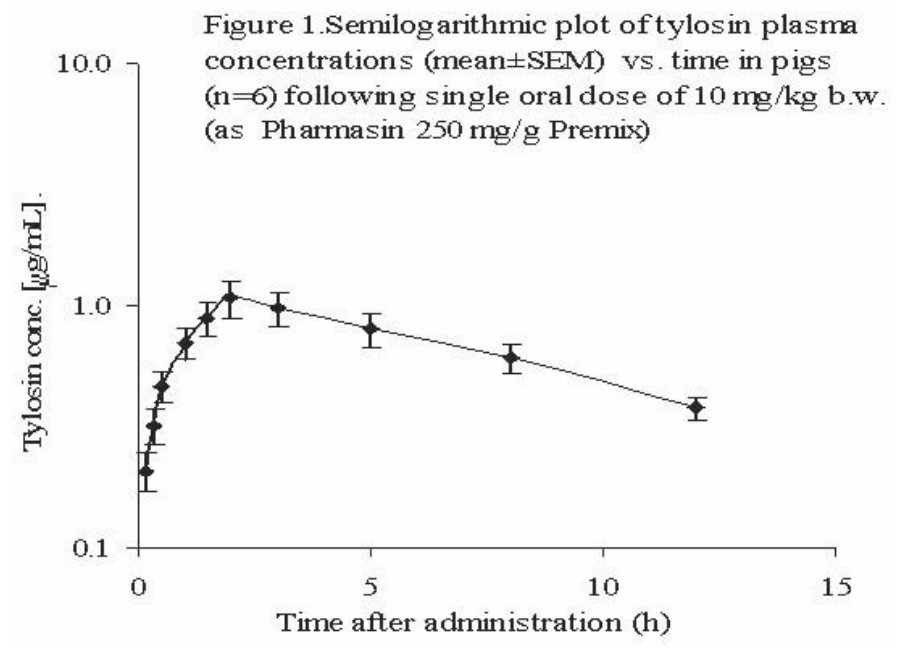

Table 1. Pharmacokinetic parameters of tylosin in pigs following single oral dose of $10 \mathrm{mg} / \mathrm{kg} \mathrm{BW}$

\begin{tabular}{|l|c|}
\hline Parameters, Units & Tylosin, 10 mg/kg BW \\
\hline$\beta^{\prime}\left(\mathrm{h}^{-1}\right)$ & $0.105 \pm 0.002$ \\
\hline $\mathrm{t}_{1 / 2 \beta}(\mathrm{h})$ & $6.66 \pm 0.17 ; 6.64^{*}$ \\
\hline $\mathrm{C}_{\max }(\mu \mathrm{g} . \mathrm{mL})$ & $1.08 \pm 0.08$ \\
\hline $\mathrm{T}_{\max }(\mathrm{h})$ & $2.0 \pm 0$ \\
\hline $\mathrm{AUC}_{\text {tlast }}(\mu \mathrm{g} . \mathrm{h} / \mathrm{mL})$ & $8.2 \pm 0.49$ \\
\hline $\mathrm{AUC}_{0-\infty}(\mu \mathrm{g} . \mathrm{h} / \mathrm{mL})$ & $11.84 \pm 0.58$ \\
\hline$M R T(\mathrm{~h})$ & $10.32 \pm 0.23$ \\
\hline $\mathrm{t}_{\text {last }}(\mathrm{h})$ & 12.0 \\
\hline${ }^{*}$ Harmonic mean, each datum represents the mean \pm SEM \\
\hline
\end{tabular}

\section{Discussion and Conclusion}

The elimination half-life of tylosin, calculated after single oral dose (6.66h), was higher than that reported after i.v. injection $(4.5 \mathrm{~h})^{2}$ and the peak plasma concentration was comparable to the one found after i.m. injection $(1.0 \mu \mathrm{g} / \mathrm{mL})^{2}$. Throughout the period of determination plasma tylosin levels were higher than the $\mathrm{MIC}_{90}$ described for clinically important porcine bacterial pathogens: Mycoplasma hyopneumoniae - $0.25 \mu \mathrm{g} / \mathrm{mL}^{1}$, Erysipelothrix rhusiopathiae $-<0.13 \mu \mathrm{g} / \mathrm{mL}^{3}$, Leptospira spp. $-0.06 \mu \mathrm{g}$ / $\mathrm{mL}^{3}$.

A primary precondition in PK-PD approach for the effectiveness of macrolide antibiotics is a time above the MIC ( $T>M I C)$ of $50-80 \%$ of dosage interval ${ }^{4}$. The results of this study show that tylosin PK at the dosage schedule tested complies with this requirement and can ensure therapeutically effective concentrations throughout the $12 \mathrm{~h}$ period of determination. This supports clinical use of tylosin phosphate as Pharmasin $250 \mathrm{mg} / \mathrm{g}$ Premix in the control of respiratory and gastro-intestinal infections.

\footnotetext{
References

1. Hannan, P.C.T. et al. (1997). Antimicrob. Agents Chemother. 41: 2037-2040.

2. Prats, C. et al. (2002). Res. Vet. Sci. 73: 141-144.

3. Prescott, J. F. et al. (2000). Antimicrobial Therapy in Veterinary Medicine, $3^{\text {rd }}$ edn. lowa State University Press, Ames, lowa, pp. 237-257.

4. Toutain, P. L. et al. (2002). Res. Vet. Sci. 73: 105-114.
} 


\title{
P.686
}

\section{PK and PK/PD of Pharmasin ${ }^{\circ} 100 \%$ w/w WSG (tylosin tartrate) following multiple oral administration in pigs}

\author{
Stela Vesselova ${ }^{1}$ Mariana Karanikolova ${ }^{1}$ Valeri Nazarov ${ }^{1}$ Stanislava Ivanova ${ }^{1}$ Dimitar Pashov² Spas Petkov ${ }^{3}$ Alain Kanora $^{3}$ \\ 1. Department of R\&D, Biovet JSC, Peshtera, Bulgaria; 2. Department of Pharmacology, Faculty of \\ Veterinary Medicine, Stara Zagora, Bulgaria; 3. Huvepharma NV, Antwerp, Belgium
}

\section{Introduction}

In this study we investigated the pharmacokinetic (PK) behavior of tylosin as Pharmasin ${ }^{\circ} 100 \%$ w/w WSG (Huvepharma NV) in pigs in order to explore PK-pharmacodynamic (PD) integration as rational basis for its clinical use.

\section{Materials and Methods}

Eleven mixed breed pigs, weighing 20-25 kg without a history of macrolide administration were enrolled in the study. Ten animals, equally male and female, received tylosin (as tartrate) at 10 mg/kg BW / 24h (Pharmasin 100\% w/w WSG) as medicated drinking water offered ad libitum daily freshly prepared at 6.00 am for 5 consecutive days. During the treatment 40 heparinized blood samples were obtained from each pig at predetermined intervals. One pig did not participate and served as negative control. On the $5^{\text {th }}$ day the treated pigs were euthanized: 2 animals at each of the 5 different time points. Blood, organ and tissue samples from respiratory and GI tract were collected. Plasma and organ (tissue) tylosin concentrations were measured by validated HPLC method.

\section{Results}

The plasma concentration-time profile of tylosin showed nearly identical daily courses within the treatment period (Fig. 1).

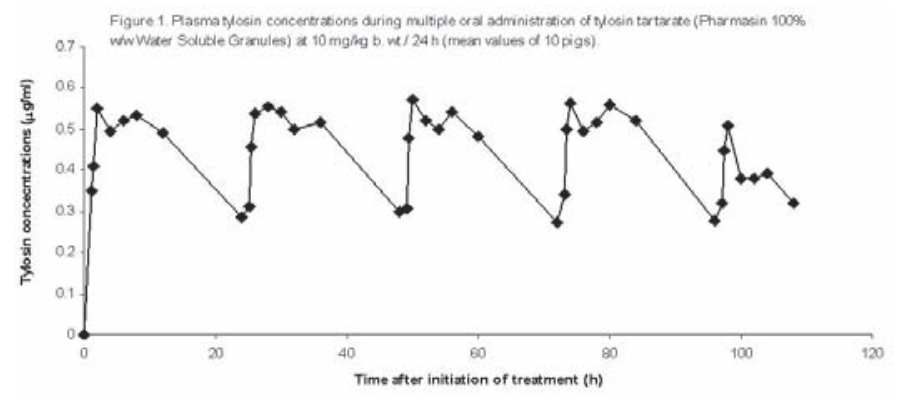

There were no significant differences between the 4 daily peak concentrations $\left(C_{\max }\right)$ and between $C_{\min }$ or AUC values (Repeated measures ANOVA Scheffe post hoc test). However, the individual plasma concentration data were quite variable and showed that in $70 \%$ of the animals steady state was reached at $46.9 \pm 6.33 \mathrm{~h}$ after treatment initiation. After 4 days treatment $\mathrm{C}_{\text {max-ss }}$ was $0.58 \pm 0.04 \mu \mathrm{g} / \mathrm{mL}, C_{\text {min-ss }}-0.28 \pm 0.03 \mu \mathrm{g} / \mathrm{mL}, C_{\text {av-ss }}$ $0.45 \pm 0.04 \mu \mathrm{g} / \mathrm{mL}$, and $\mathrm{AUC}_{0-24 \mathrm{~h}}-10.73 \pm 0.92 \mu \mathrm{g} . \mathrm{h} / \mathrm{mL}$. Steady state tylosin concentrations in lung tissue and bronchial mucosa and secretions peaked $2 \mathrm{~h}$ after the $5^{\text {th }}$ administration, being $0.93 \pm 0.06 \mu \mathrm{g} / \mathrm{g}$ and $1.06 \pm 0.06 \mu \mathrm{g} / \mathrm{g}$, respectively, and persisted to the last sampling point $\left(12^{\text {th }} \mathrm{h}\right)$, being $0.67 \pm 0.12 \mu \mathrm{g} / \mathrm{g}$ and $0.78 \pm 0.13 \mu \mathrm{g} / \mathrm{g}$, respectively. The corresponding tissue/plasma ratios of AUC were 1.88 and 2.17.
Table 1. Steady state organ (tissue) pharmacokinetic parameters of tylosin (mean \pm SEM) in pigs, following 4-day p.o. administration at 10 $\mathrm{mg} / \mathrm{kg} / 24 \mathrm{~h}$ of tylosin tartrate as Pharmasin ${ }^{\circledast} 100 \% \mathrm{w} / \mathrm{w}$ Water Soluble Granules (0-12h)

\begin{tabular}{|c|c|c|c|c|c|c|c|}
\hline $\begin{array}{l}\text { Parameters, } \\
\text { units }\end{array}$ & $\begin{array}{l}\text { Blood } \\
\text { plasma }\end{array}$ & $\begin{array}{l}\text { Lung } \\
\text { tissue }\end{array}$ & $\begin{array}{l}\text { Bron- } \\
\text { chial } \\
\text { mu- } \\
\text { cosa* }\end{array}$ & $\begin{array}{l}\text { Ileum } \\
\text { wall }\end{array}$ & $\begin{array}{c}\text { Ileal } \\
\text { content }\end{array}$ & $\begin{array}{l}\text { Colon } \\
\text { wall }\end{array}$ & $\begin{array}{l}\text { Colonic } \\
\text { content }\end{array}$ \\
\hline $\begin{array}{l}\mathrm{AUC}_{0-12{ }^{\prime}} \mu \mathrm{g} \cdot \mathrm{h} / \\
\mathrm{mL}(\mathrm{g})\end{array}$ & 5.19 & 9.75 & 11.28 & 2.87 & 288.55 & 38.36 & 816.85 \\
\hline$T / P^{1}$ ratio of AUC & NC & 1.88 & 2.17 & 0.55 & 55.6 & 7.39 & 157.39 \\
\hline$C_{\max } \mu g / m L(g)$ & 0.65 & 0.93 & 1.06 & 0.29 & 27.2 & 4.05 & 76.7 \\
\hline$T_{\text {max }} h$ & 2.0 & 2.0 & 2.0 & 8.0 & 4.0 & 4.0 & 4.0 \\
\hline$C_{12 \mathrm{th}} \mathrm{h}, \mu \mathrm{g} / \mathrm{mL}(\mathrm{g})$ & 0.32 & 0.67 & 0.78 & 0.24 & 20.8 & 2.48 & 71.3 \\
\hline MRT, h & 14.1 & 17.0 & 16.2 & 13.1 & 18.0 & 13.6 & 11.1 \\
\hline $\mathrm{T} / \mathrm{P}^{1}$ ratio of $\mathrm{C}_{\max }$ & NC & 1.43 & 1.63 & 0.45 & 41.8 & 6.23 & 118.0 \\
\hline$T / P^{1}$ ratio of $C_{12 \text { th }} h$ & NC & 2.09 & 2.44 & 0.75 & 65.0 & 7.75 & 222.8 \\
\hline
\end{tabular}

*Bronchial mucosa and secretions; 'Tissue/Plasma; NC, Not Calculated (each datum represents mean of 2 animals).

\section{Discussion and Conclusion}

The mean plasma steady state values of $C_{\text {min-ss }}$ and of $C_{a v-s s}$ were higher than the $\mathrm{MIC}_{90}$ described for clinically important porcine bacterial pathogens of respiratory tract: M.hyopneumoniae $0.25 \mu \mathrm{g} / \mathrm{mL}^{1}$, E. rhusiopathiae $-<0.13 \mu \mathrm{g} / \mathrm{mL}^{3}$, Leptospira spp $-0.06 \mu \mathrm{g} / \mathrm{mL}^{3}$. This was valid also for organ (tissue) steady state concentrations in respiratory tract which were higher than those in plasma. The concentration in ileal and colonic content provided active levels against $L$. intracellularis $-\mathrm{MIC}_{90}=4 \mu \mathrm{g} / \mathrm{mL}^{2}$ and some sensitive strains of $B$. hyodysenteria $-\mathrm{MIC}_{90}>16>256$ $\mu \mathrm{g} / \mathrm{mL}^{2}$.

Generally, PK-PD breakpoint for clinical and bacteriological efficacy of macrolide antibiotics is a time above the MIC (T>MIC) of $50-80 \%$ of dosage interval ${ }^{4}$. In this study active tylosin antimicrobial levels persisted throughout the period of administration. Therefore, tylosin tartrate as Pharmasin ${ }^{\circ} 100 \% \mathrm{w} / \mathrm{w}$ WSG at the dosage schedule tested complies with the requirements of PK-PD approach. This supports its clinical use in the control of respiratory and gastro-intestinal infections.

\section{References}

1. Hannan, P.C.T. et al. (1997). Antimicrob. Agents Chemother. 41: 20372040.

2. Page, S.W. (2003). The Role of Enteric Antibiotics in Livestock Production (2003). Avcare Limited, Canberra, ACT 2601, Australia, p. $11-4$.

3. Prescott, J. F. et al. (2000). Antimicrobial Therapy in Veterinary Medicine, 3rd edn. lowa State University Press, Ames, lowa, pp. 237-257.

4. Toutain, P. L. et al. (2002). Res. Vet. Sci. 73:105-114. 


\title{
P.687
}

\section{Pharmacokinetics of Pharmasin $100 \%$ w/w WSG (tylosin tartrate) following single oral administration in pigs}

\author{
Stela Vesselova ${ }^{1}$ Mariana Karanikolova ${ }^{1}$ Valeri Nazarov${ }^{1}$ Stanislava Ivanova ${ }^{1}$ Dimitar Pashov ${ }^{2}$ Spas Petkov ${ }^{3}$ Alain Kanora $^{3}$ \\ 1. Department of R\&D, Biovet JSC, Peshtera, Bulgaria; 2. Department of Pharmacology, Faculty of \\ Veterinary Medicine, Stara Zagora, Bulgaria; 3. Huvepharma NV, Antwerp, Belgium
}

\section{Introduction}

Tylosin is a macrolide antibiotic that acts mostly against Grampositive bacteria and mycoplasma. Although it has been used in veterinary medicine for a long time the data on its pharmacokinetic (PK) properties in pigs are incomplete. The aim of this study was to obtain PK data intended to facilitate PK-pharmacodynamic (PD) integration in clinical use of tylosin as Pharmasin ${ }^{\circ}$ 100\% w/w Water Soluble Granules (Huvepharma NV).

\section{Materials and Methods}

Six healthy pigs, equally male and female, weighing $20-25 \mathrm{~kg}$ without a history of macrolide administration were enrolled in the study. The animals received by intubation a single p.o. dose of tylosin (as tartrate) $10 \mathrm{mg} / \mathrm{kg}$ BW (Pharmasin ${ }^{\circ} 100 \% \mathrm{w} / \mathrm{w}$ Water Soluble Granules), dissolved in water. Heparinized blood samples ( $n=13$ per animal) were taken at predetermined intervals. Plasma tylosin concentrations were measured by a validated HPLC method. The results obtained for tylosin disposition were tested by compartmental method (using Akaike's information criterion) and by non-compartmental approach as well.

\section{Results}

Plasma concentration vs. time data fit best by applying the non-compartmental model. The highest plasma concentration $\left(C_{\max }=1.10 \pm 0.08 \mu \mathrm{g} / \mathrm{mL}\right)$ was achieved $2 \mathrm{~h}$ post administration. Thereafter, plasma levels declined remaining higher than $0.31 \pm 0.2 \mu \mathrm{g} / \mathrm{mL}$ at $12 \mathrm{~h}$ post administration (Fig. 1). The half-life $\left(t_{1 / 2 \beta^{\prime}}\right)$ was 5.6h $A \cup C_{0 \text {-tlast }}$ and $A \cup C_{0-\infty}$ were $7.79 \pm 0.58 \mu \mathrm{g} . \mathrm{h} / \mathrm{mL}$ and $10.31 \pm 0.75 \mu \mathrm{g} . \mathrm{h} / \mathrm{mL}$, respectively (Table 1 ).

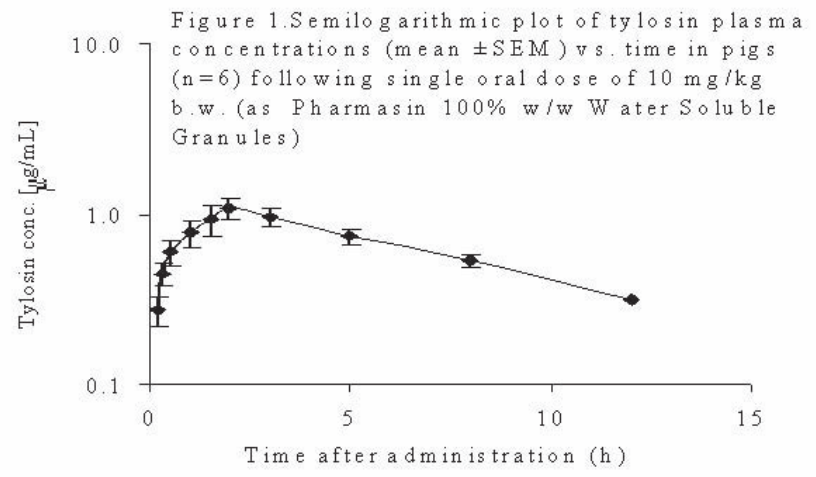

Table 1. Pharmacokinetic parameters of tylosin in pigs following single oral dose of $10 \mathrm{mg} / \mathrm{kg} \mathrm{BW}$

\begin{tabular}{|l|c|}
\hline Parameters, units & Tylosin, $\mathbf{1 0} \mathbf{~ m g} / \mathbf{k g}$ BW \\
\hline$\beta^{\prime}\left(\mathrm{h}^{-1}\right)$ & $0.124 \pm 0.002$ \\
\hline $\mathrm{t}_{1 / 2 \beta}(\mathrm{h})$ & $5.6 \pm 0.08 ; 5.59^{*}$ \\
\hline$C_{\max }(\mu \mathrm{g} / \mathrm{mL})$ & $1.1 \pm 0.08$ \\
\hline $\mathrm{T}_{\max }(\mathrm{h})$ & $2.0 \pm 0$ \\
\hline $\mathrm{AUC}_{\text {tlast }}(\mu \mathrm{g} \cdot \mathrm{h} / \mathrm{mL})$ & $7.79 \pm 0.58$ \\
\hline $\mathrm{AUC}_{0-\infty}(\mu \mathrm{g} \cdot \mathrm{h} / \mathrm{mL})$ & $10.31 \pm 0.75$ \\
\hline MRT $(\mathrm{h})$ & $8.69 \pm 0.1$ \\
\hline $\mathrm{t}_{\text {last }}(\mathrm{h})$ & 12.0 \\
\hline${ }^{*}$ Harmonic mean, each datum represents the mean \pm SEM \\
\hline
\end{tabular}

\section{Discussion and Conclusion}

The elimination half-life of tylosin, calculated after single oral dose (5.6h), was higher than that reported after i.v. injection $(4.5 \mathrm{~h})^{2}$ and the peak plasma concentration was comparable to this found after i.m. injection $(1.0 \mu \mathrm{g} / \mathrm{mL})^{2}$. Throughout the period of determination plasma tylosin levels were higher than the $\mathrm{MIC}_{90}$ described for clinically important porcine bacterial pathogens: Mycoplasma hyopneumoniae - $0.25 \mu \mathrm{g} / \mathrm{mL}^{1}$, Erysipelothrix rhusiopathiae - $<0.13 \mu \mathrm{g} / \mathrm{mL}^{3}$, Leptospira spp. $-0.06 \mu \mathrm{g} / \mathrm{mL}^{3}$.

According to PK-PD approach the breakpoint for clinical effectiveness of macrolide antibiotics is a time above the MIC ( $>$ $>M I C)$ of $50-80 \%$ of dosage interval ${ }^{4}$. The results of this study show that tylosin PK at the dosage schedule tested complies with this requirement and can ensure therapeutically effective concentrations throughout the $12 \mathrm{~h}$ period of determination. This supports clinical use of tylosin tartrate as Pharmasin $100 \%$ w/w Water Soluble Granules in the control of respiratory and gastro-intestinal infections.

\section{References}

1. Hannan, P.C.T. et al. (1997). Antimicrob. Agents Chemother. 41: 2037-2040.

2. Prats, C. et al. (2002). Res. Vet. Sci. 73: 141-144.

3. Prescott, J. F. et al. (2000). Antimicrobial Therapy in Veterinary Medicine, 3rd edn. lowa State University Press, Ames, lowa, pp. 237-257.

4. Toutain, P.L. et al. (2002). Res. Vet. Sci. 73: 105-114. 


\title{
P.688
}

\section{Efficacy of flavomycin to susceptibility of haemolytic Escherichia coli in weaner to finishing pigs}

\author{
Nuvee Prapasarakul ${ }^{1}$ Waree Niyomtuma' ${ }^{1}$ Prapipat Khemsapb ${ }^{2}$ Suthatip Prapatsornpinyo ${ }^{3}$ \\ 1. Department of Veterinary Microbiology, Faculty of Veterinary Science, Chulalongkorn University, Bangkok, Thailand; \\ 2. Huvepharma (Thailand) Ltd. , Bangkok, Thailand; 3. Panuspokphand Pig Farm Co., Ltd., Chonburi, Thailand
}

\section{Introduction}

Escherichia coli is regarded as a commensal and opportunistic pathogen of gastrointestinal tract. a-haemolytic E. coli (HEC) is clearly more potential to induce pathogenic lesion due to possessing of exotoxins (4) This causes colibacillosis and oedema disease and directly impacts to severe economic loss (5)

In Thai pig industrial, intensive use of antimicrobials is a common alternative for control of infectious diseases. There has been a dramatically increasing of resistant bacteria against antimicrobials such as beta-lactams, macrolides, fluoroquinolones, tetracyclines and lincosamides because of use of different several antimicrobials during production periods other than 2 weeks prior to slaughter. However, not only pathogenic bacteria are affected from the administration, but also enteric commensal may rise up the risk of resistance in farm-level (6). Flavomycin is phosphoglycolipid antibiotic in flavophospholipal group that has been approved as a feed additive. It is active against Gram's positive bacteria and also inhibits in vitro conjugation of Gram's negative bacteria such as $\mathrm{E}$. coli.

The current study was to evaluate minimal inhibitory concentration (MIC) to 6 antimicrobials of E. coli derived from weaner to finishing pigs that received flavomycin as a feed additive. We also determined an efficacy of flavomycin to 30 enterotoxogenic E. coli (ETEC) isolated from piglets.

\section{Materials and Methods}

Sample collection. $10 \mathrm{ppm}$ of flavomycin was the addition of routine feed additive during 10 to 21 weeks of pig ages. Pig feces were collected and contained in transport media.

Bacteria. 30 ETEC isolated from piglets with colibacillosis since 2008, were derived from stock isolates of Dept Vet Micro. $63 \mathrm{E}$. coli comprising with 30,16 and 17 isolates that were collected at 5, 15 and 25 week age, respectively. Haemolytic traits on 5\% sheep blood agar were used for colony selective criteria. When HEC was not observed on the primary plate, non-HEC would be selected for MIC determination.

MIC determination. 6 antimicrobials comprising with flavomycin, colistin, chlortetracycline, enrofloxacin, amoxicillin and oxacillin were used. Broth micro-dilution was carried out according to the recommendation of $\operatorname{CLSI}(2,3)$.

\section{Results and Discussion}

Range of MIC distribution is shown in Fig 1. The MIC values of 30 ETEC against amoxicillin, oxacillin, and chlortetracycline, were over $256 \mathrm{ug} / \mathrm{ml}$. They were inhibited by flavomycin at 4-32 ug/ $\mathrm{ml}$. Enrofloxacin and colistin were the effective agents to the tested E. coli.
HEC was mostly isolated from the period before adding of flavomycin and were disappeared in pig aged 15 week. The MIC50 of each batch of isolation is compared in Table 1.

Fig. 1 Distribution of MIC values of 30 ETEC against 6 antimicrobials

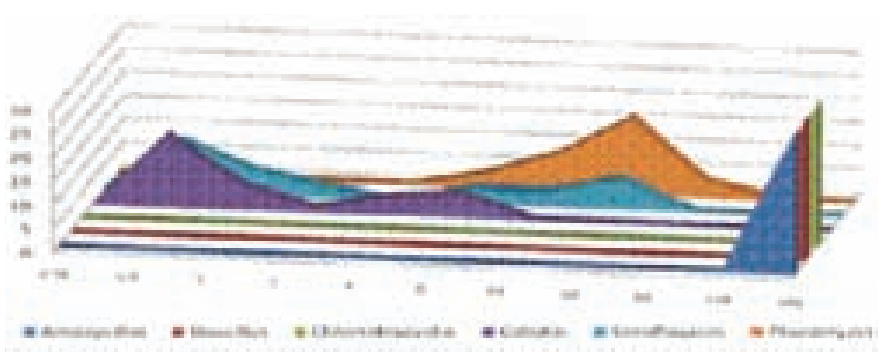

Table 1 Presence of isolation rate and MIC50 of each batch

\begin{tabular}{|l|c|c|c|}
\hline Isolation & & Pig ages (wk) & \\
\hline & $\mathbf{5}$ & $\mathbf{1 5}$ & $\mathbf{2 5}$ \\
\hline HEC & 28 & - & 4 \\
\hline Non-HEC & 2 & 16 & 13 \\
\hline Antimicrobial & & & \\
\hline colistin & 0.5 & 0.5 & 0.5 \\
\hline enrofloxacin & 0.5 & $\geq 0.25$ & $\geq 0.25$ \\
\hline flavomycin & 4 & 16 & 32 \\
\hline chlortetracycline & 256 & 256 & 256 \\
\hline amoxicillin & 512 & 512 & 512 \\
\hline oxacillin & 512 & 512 & 512 \\
\hline
\end{tabular}

The MIC of 63 isolates was consistent to that of ETEC in 2008. beta lactam resistant bacteria may be a native clone in the area of study. Flavomycin clearly reduced HEC in fattening pigs and its susceptibility was lower in the final week. These confirmed a direct efficacy to pathogenic agents (1) and may be able to maintain susceptible level from weaner to finishing.

\section{Acknowledgement}

We thank Meiji pharmaceutical Co. Ltd. (Thailand) for providing colistin sulfate.

\section{References}
1 Aarestrup et al., 1998. APMIS 106, 606.
2 CLSI, 2006. CLSI M7-A7., Wayne, PA.
3 CLSI, 2008. CLSI M100-S18., Wayne, PA.
4 Coldewey et al., 2007. BMC Microbiol 7, 21.
5 Frydendahl, 2002. Vet Microbiol 85, 169.
6 Harada et al., 2008 Microb Drug Resist 14, 239. 


\title{
P.689
}

\section{Pharmacokinetic/pharmacodynamic relationships of HydroDoxx $500 \mathrm{mg} / \mathrm{g}$ Powder (doxycycline) in porcine respiratory disease complex (PRDC)}

\author{
Mariana Karanikolova ${ }^{1}$ Stela Vesselova ${ }^{1}$ Valeri Nazarov${ }^{1}$ Spas Petkov² Koen De Gussem² Alain Kanora ${ }^{2}$ \\ 1. Department of R\&D, Biovet JSC, Peshtera, Bulgaria; 2. Huvepharma NV, Antwerp, Belgium
}

\section{Introduction}

The porcine respiratory disease complex (PRDC) is a leading cause of animal and economic losses in swine production ${ }^{6}$. Because bacteria are involved in this condition, antibiotics are a short-term measure in a multifaceted strategy of disease control. In the swine industry, tetracyclines have been applied particularly for respiratory tract infections ${ }^{7}$. Doxycycline (DOX) is a semi-synthetic tetracycline and a broad-spectrum bacteriostatic antibiotic against Gram-negative and Gram-positive aerobic and anaerobic bacteria, Rickettsiae, Chlamydiae, mycoplasmas and some protozoa ${ }^{10}$. DOX is characterized by a better lipid solubility, greater tissue penetration, improved antimicrobial activity and a different pharmacokinetic profile ${ }^{1,2}$. The objective of this paper was to review existing literature data on pharmacokinetic (PK) and pharmacodynamic (PD) properties of DOX with a view to evaluate their relationships as a base for its clinical effect in PRDC as HydroDoxx 500 mg/g Powder for use in drinking water.

\section{Pharmacokinetics}

The multiple oral administration of DOX at 6.6, 11.5 and 12.9 $\mathrm{mg} / \mathrm{kg}$ BW daily produces steady-state concentrations of respectively $037-0.89,0.56-0.87$ and $0.71-1.14 \mu \mathrm{g} / \mathrm{mL}^{9}$. In another experiment steady-state plasma levels ranging between 0.9 and $1.5 \mu \mathrm{g} / \mathrm{mL}$ have been observed during 8-day oral medication at a dosage regimen of $11.8-13.3 \mathrm{mg} / \mathrm{kg} / \mathrm{day}^{5}$. Administration via drinking water at $10 \mathrm{mg} / \mathrm{kg}$ daily for 5 days results in mean plasma concentration at steady-state of $1.37 \pm 1.21 \mu \mathrm{g} / \mathrm{mL}^{10}$.

\section{Pharmacodynamics}

It was reported that MIC values of DOX for important porcine bacterial respiratory tract pathogens are 0.25 to $05 \mu \mathrm{g} / \mathrm{mL}^{8}$. In other studies, further values were determined ${ }^{3,10}$ (Table 1).

Table 1. MIC values bacterial respiratory tract pathogens

\begin{tabular}{|l|c|c|}
\hline Porcine pathogens & MIC $_{50}$ values, $\boldsymbol{\mu g} / \mathbf{m L}$ & MIC $_{\mathbf{9 0}}$ values, $\boldsymbol{\mu g} / \mathbf{m L}$ \\
\hline M. hyopneumoniae $(\mathrm{Mh})$ & $0.1(10)$ & $0.2(10) ; 1.0(3)$ \\
\hline P. multocida $(\mathrm{Pm})$ & $0.106(10)$ & $0.517(10) ; 1.0(3)$ \\
\hline B. bronchiseptica (Bb) & $0.039(10)$ & $0.053(10)$ \\
\hline A. pleuropneumoniae (Ap) & $1.422(10)$ & $2.387(10) ; 2.0(3)$ \\
\hline
\end{tabular}

\section{PK-PD integration}

Steady-state DOX plasma levels determined after oral medication with DOX at a dose of $11.5 \mathrm{mg} / \mathrm{kg}$ BW daily exceed MIC values 0.25 to $0.5 \mu \mathrm{g} / \mathrm{mL}^{9}$. In conformity with the type of its antimicrobial action, DOX belongs to concentration-time dependent (co-dependent) drugs with integrated PK-PD variables AUC/MIC and $\mathrm{T}>\mathrm{MIC}$ with proposed values of $125 \mathrm{~h}$ and $50-80 \%$. Based on this principle and analyzing the results obtained after 5-day administration of DOX via drinking water at a dose of $10 \mathrm{mg} /$ $\mathrm{kg}$ daily other authors assume that the calculated mean values of $\left(\mathrm{AUC}_{S \mathrm{~S}} / \mathrm{MIC}_{90}\right) 24 \mathrm{~h}$ and \% $>\mathrm{MIC}$ for $\mathrm{Bb}$ and $\mathrm{Mh}$ achieve the breakpoints for these indices ${ }^{10}$. For $\mathrm{Pm}$ the $\% \mathrm{~T}>\mathrm{MIC}$ exceeded $70 \%$. Considering these results the authors conclude that DOX at $10 \mathrm{mg} / \mathrm{kg}$ daily should ensure the plasma concentrations required for successful therapy against $\mathrm{Bb}$ and $\mathrm{Mh}$ and possibly against Pm but probably not against $\mathrm{App}^{10}$.

\section{Clinical effectiveness and conclusion}

The results from clinical investigations of DOX under field conditions in pigs showed that DOX after oral administration at a dose of $10 \mathrm{mg} / \mathrm{kg} \mathrm{BW}$ was effective in controlling PRDC. The effect was demonstrated in the reduction of respiratory disease incidents, decrease in lethality and increase in economically significant zootechnical parameters with significant differences between the treated and untreated groups ${ }^{4,11}$. It can be concluded that HydroDoxx $500 \mathrm{mg} / \mathrm{g}$ Powder for use in drinking water administered to pigs at the recommended rate $(10 \mathrm{mg} / \mathrm{kg}$ BW) is effective to prevent and treat PRDC.

\section{References}

1. Aronson, A.L. (1980), J. Am. Vet. Med. Assoc., 176: 1061.

2. Barza, M. et al (1975), Antimicrob. Agents Chemother., 8: 713.

3. Bousquet, E. et al (1998), Vet. Rec., 141 (2): 37-40.

4. Bousquet, E. et al (1998), Vet. Rec., 143 (10): 269-272.

5. Bousquet, E. et al (1998), Vet. Rec., 29 (5): 475-485.

6. Christensen, G. et al (1999), Diseases of the respiratory system. Diseases of Swine. 8th ed., lowa State Univ. Press, Ames, pp. 913-940.

7. Kunesch, J. P. (1986), Therapeutics, Diseases of Swine (6th ed.), lowa State Univ. Press, Ames, pp. 803-812.

8. Pijpers, A. et al (1989), J. Vet. Pharmacol. Ther., 12: 267.

9. Pijpers, A. et al (1991), J. Anim. Sci., 69: 4512-4522.

10. Prats, C. et al (2005), J. Vet. Pharmacol. Ther., 28: 525-530.

11. Zmudzki, J.et al (2004). Medycyna Weterynaryjna, 60 (7): 743-747. 


\title{
P.690
}

\section{Comparative study on the clinical efficacy of Pharmasin $250 \mathrm{mg} / \mathrm{g}$ Premix and Pharmasin $100 \%$ w/w Water Soluble Granules in pigs with experimentally induced respiratory diseases}

\author{
Stela Vesselova ${ }^{1}$ Mariana Karanikolova ${ }^{1}$ Valeri Nazarov ${ }^{1}$ Valentina Urumova ${ }^{2}$ Mihni \\ Lyutskanov² Marin Aleksandrov ${ }^{3}$ Spas Petkov $^{4}$ Alain Kanora 4 \\ 1. Department of R\&D, Biovet JSC, Peshtera, Bulgaria; 2. Department of Veterinary Microbiology, Infectious and \\ Parasitic Diseases, Faculty of Veterinary Medicine, Stara Zagora, Bulgaria; 3. Institute of Experimental Pathology \\ and Parasitology, Bulgarian Scientific Academy, Sofia, Bulgaria; 4. Huvepharma NV, Antwerp, Belgium
}

\section{Introduction}

The study objective was to determine the clinical efficacy of orally administered tylosin tartrate in growing pigs that have been artificially infected with Mycoplasma hyopneumoniae (Mh), compared to tylosin phosphate.

\section{Materials and Methods}

Four groups of 6 SPF pigs (Danube white), equal number of sexes (10.0-12.0 kg), 10 weeks of age, were used. All groups (I, II and III) were infected with $M h$. Twenty-four hours after the challenge, the infected group (I) was treated via feed with tylosin (as phosphate) (Pharmasin $250 \mathrm{mg} / \mathrm{g}$ Premix) and group II was treated through drinking water with tylosin (as tartrate) (Pharmasin $100 \%$ w/w WSG). Both groups received a dose of 10 mg tylosin per kg BW for 8 consecutive days, followed by $5 \mathrm{mg}$ tylosin per kg BW until the end of the period of risk (14 days). The efficacy of the medication was assessed by observing the rectal temperature, clinical symptoms and gross lung lesions, histology, serology for detection of antibodies against Mh using an ELISA test (Dako Cytomation, Denmark A/S), nasal swabs and lung biopsy for bacteriological examinations (after euthanasia and necropsy of all pigs), bodyweight gain and feed conversion efficiency. The results of the examination were determined according to t-test of Student-Fisher.

\section{Results}

Specific clinical symptoms of respiratory disease and gross lung and histological lesions were observed in the infected unmedicated group (III). Tylosin (as phosphate) administration via feed in pigs of group I and tylosin (as tartrate) administration via water in pigs of group II both lead to a statistically significant decrease in clinical signs and gross lung lesions (Table 1). Bacteriological examinations of nasal swabs and lung showed a statistically significant reduction in re-isolation rate of $M h$ (Table 1).

In both tylosin treatment groups, zootechnical parameters (bodyweight gain and feed conversion ratio) were statistically significantly higher than those recorded in the infected unmedicated group of pigs (Table 2).

Table1. Clinical signs

\begin{tabular}{|l|c|c|c|c|c|}
\hline Groups & $\begin{array}{c}\text { No. of animals } \\
\text { with clinical } \\
\text { signs }\end{array}$ & $\begin{array}{c}\text { Total clinical } \\
\text { scores }\end{array}$ & $\begin{array}{c}\text { No. of animals } \\
\text { with lung lesion / } \\
\text { total number }\end{array}$ & $\begin{array}{c}\text { Lung } \\
\text { lesion } \\
\text { scores }\end{array}$ & $\begin{array}{c}\text { Re-isolation } \\
\text { from nasal } \\
\text { swabs of } \text { Mh }\end{array}$ \\
\hline I & $1 / 6$ & $10^{\mathrm{a}}$ & $1 / 6$ & $2^{\mathrm{a}}$ & $1 / 12^{\mathrm{a}}$ \\
\hline II & $1 / 6$ & $9^{\mathrm{a}}$ & $1 / 6$ & $3^{\mathrm{a}}$ & $2 / 12^{\mathrm{a}}$ \\
\hline III & $5 / 6$ & $52^{\mathrm{b}}$ & $5 / 6$ & $21^{\mathrm{b}}$ & $7 / 12^{\mathrm{b}}$ \\
\hline IV & $0 / 6$ & $0^{\mathrm{a}}$ & $0 / 6$ & $0^{\mathrm{a}}$ & $0 / 12^{\mathrm{a}}$ \\
\hline $\mathrm{a}, \mathrm{b} p \leq 0,05$ & \multicolumn{5}{l}{} \\
\hline
\end{tabular}

Table 2. Zootechnical parameters

\begin{tabular}{|l|c|c|c|c|}
\hline Groups & ADWG, kg & Between groups comparison & p-value & FC, $\mathbf{k g}$ \\
\hline I & $0.280 \pm 0.015$ & $\mathrm{I} / \mathrm{II} ; \mathrm{I} / \mathrm{III} ; \mathrm{I} / \mathrm{IV}$ & NS $^{*} ; 0.05 ; \mathrm{NS}^{*}$ & 2.200 \\
\hline II & $0.282 \pm 0.014$ & $\mathrm{II} / \mathrm{II} ; \mathrm{II} / \mathrm{IV}$ & $0.05 ; \mathrm{NS}^{*}$ & 2.193 \\
\hline III & $0.221 \pm 0.021$ & $\mathrm{III} / \mathrm{IV}$ & 0.05 & 2.463 \\
\hline IV & $0.285 \pm 0.015$ & - & - & 2.170 \\
\hline * NS: not significant; ADWG: Avg. daily weight gain; FC: Feed conversion \\
\hline \multicolumn{5}{|l}{}
\end{tabular}

\section{Discussion and Conclusion}

Tylosin is one of the oldest antibiotics for veterinary medicine that is still frequently being used to treat enzootic pneumonia?. The results show that tylosin (as phosphate) administration via feed and tylosin (as tartrate) administration via water both have a significant metaphylactic and therapeutic effect in experimentally challenged $M h$ infection in pigs. This is further confirmed by the differences in clinical, bacteriological and zootechnical parameters witch were also significantly different compared to group III. The data obtained in this experiment are consistent with the results recorded in previous studies ${ }^{2,5}$. The significant clinical efficacy of tylosin after oral treatment is due to the wide distribution of the drug in pigs and its reaching and accumulation in target tissues ${ }^{3,4}$. These results indicate that the use of tylosin administered as tartrate or phosphate can be successfully used in strategic programs to control enzootic pneumonia caused by $M h$ in pigs.

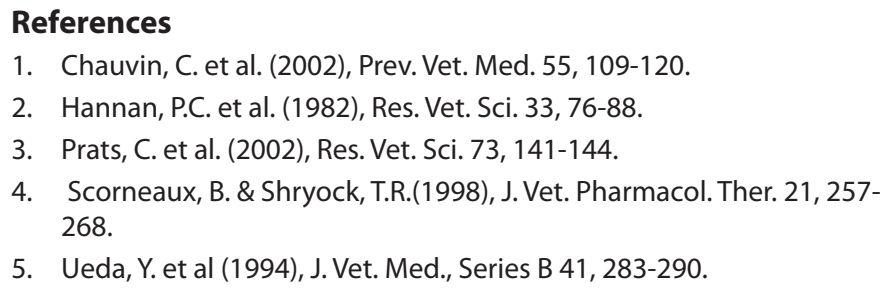

1. Chauvin, C. et al. (2002), Prev. Vet. Med. 55, 109-120.

2. Hannan, P.C. et al. (1982), Res. Vet. Sci. 33, 76-88.

3. Prats, C. et al. (2002), Res. Vet. Sci. 73, 141-144.

4. Scorneaux, B. \& Shryock, T.R.(1998), J. Vet. Pharmacol. Ther. 21, 257268.

5. Ueda, Y. et al (1994), J. Vet. Med., Series B 41, 283-290. 


\title{
P.691
}

\section{Efficacy of Denagard(tiamulin) and amoxycillin combination on porcine mycoplasmosis control in a Thai pig farm}

\author{
Mongkol Lumyai ${ }^{1}$ Sittikorn Traiyarach ${ }^{1}$ Metta Makhanon² Sasiwimon Talummuk ${ }^{2}$ Komkrich Teankum $^{1}$ Roongroje Thanawongnuwech $^{1}$ \\ 1. Chulalongkorn University, Bangkok, Thailand; 2. Novartis (Thailand) Ltd, Bangkok, Thailand
}

\section{Introduction}

Mycoplasmosis is one of major bacterial pneumonia in pigs, usually co-infected with other organisms leading to a complex of respiratory diseases or porcine respiratory disease complex (PRDC)1. Vaccination is a common tool for controlling the disease; however, the elimination of mycoplasma is still incomplete due to the nature of the organism. By administration of tiamulin (Denagard $^{\circledR}$ ), an effective pleuromutilin against mycoplasma2, the infection could be significantly resolved. Additionally, a combination of tiamulin with other antibiotics such as amoxycillin could facilitate the elimination of Mycoplasma hyopneumoniae and other concurrent pathogens. The objective of this study was to evaluate the efficacy of a combination of Denagard ${ }^{\circledR}$ and amoxycillin to control concurrent porcine mycoplasmosis in comparison with lincomycin combined with amoxycillin in a Thai pig farm.

\section{Materials and Methods}

A mycoplasmosis-affected pig farm in Thailand was selected based on clinical signs, bacterial culture results, slaughter check and serological profile. Serologically, pigs were $100 \%$ sero-positive on Mycoplasma indirect ELISA test as early as 16 week-old. Evidently, PRRSV and PCV2 were also detected in this herd. A total of 180 pigs (10 week old) were allocated into 2 groups: Group A $(n=90)$ fed with Denagard ${ }^{\circledR}(100$ ppm) and amoxicillin (300ppm) and Group B ( $\mathrm{n}=90)$ fed with lincomycin (110ppm) and amoxicillin (300ppm). Those pigs were randomly put into each pen in the same housing without the environmental effect. Treatment was conducted in the growing pig feed from 10 to 14 week-old continuously (one week before the presence of the clinical signs). The clinical history and the production parameters were recorded during the study as well as the number of culled pigs until two weeks after the treatment. To measure average daily weigh grain (ADG) pigs were individually weighted each week from 10 and 14 weeks of age.

\section{Results and Discussion}

A higher number of clinically sick and culled pigs were observed in Group B (sick =19, culled pigs=3) than in Group A ( ick =11, culled pigs $=1$ ) $(n=90)$ during the studied period (10-14 weekold). Similarly, ADG of Group A pigs receiving a combination of Denagard $^{\circledR}$ and amoxicillin was significantly better than in Group B using Mann Whitney test $(P<0.01)$ (Fig. 1). Concurrent infection among PRRSV, PCV2, Mycoplasma and possibly other bacteria did exist in the studied farm. Interestingly, vaccination of those mentioned 3 major respiratory pathogens was not practised and the combination of Denagard ${ }^{\circledR}$ and amoxicillin did demonstrate helping those treated pigs growing better than the other medicated group. Minimizing the negative effects of secondary bacterial infection using the drugs of choice in this farm is considered as an important management tool to effectively control PRDC. Unfortunately, the lung scores and other lab results were not available at the time of submission.
Fig. 1 Clinical signs $(A)$ and average daily weigh grain $(A D G)(B)$ in the treatment groups (10-14 $w \mathrm{k})$.

\section{Figure 1A}

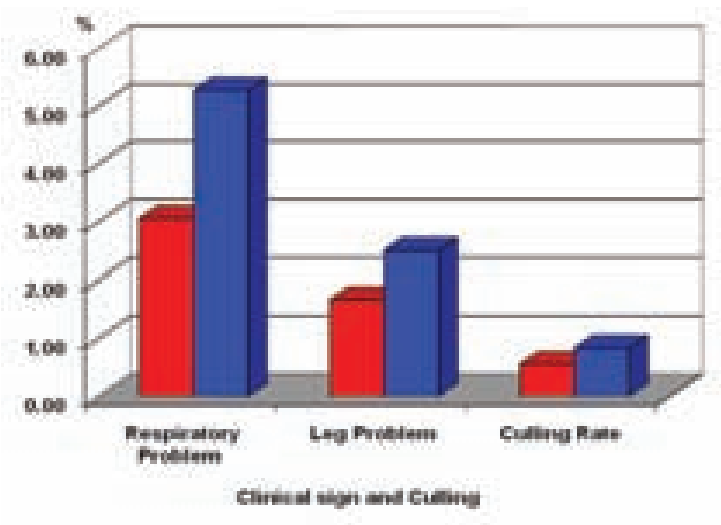

Figure 1B

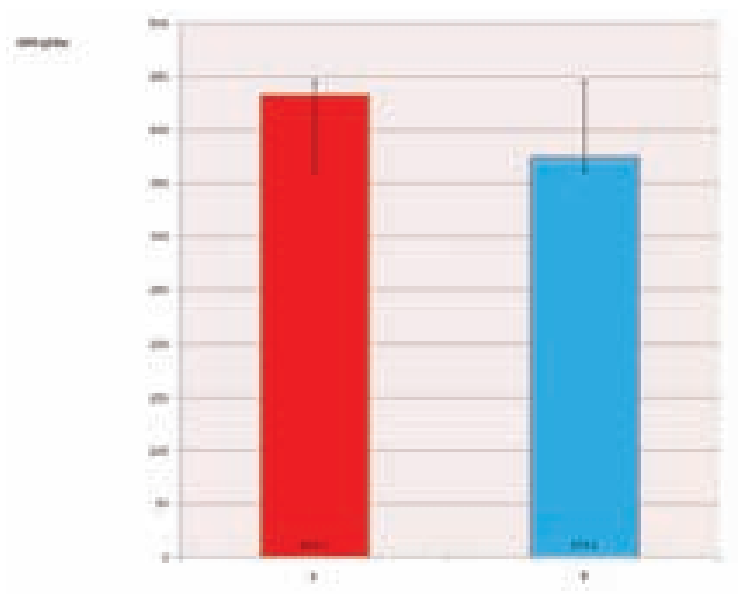

\section{Acknowledgements}

This study was funded by the Novartis Animal Health Inc., Basel, Switzerland.

\section{References}

1. Thacker, E. and Thanawongnuwech, R. 2002. Thai J. Vet. Med. 32:126133.

2. Maes, D. et al., 2008. Vet. Microbiol. 126:297-309. 


\title{
P.692
}

\section{Homogeneity of an oxytetracycline solution administered with a dosing pump}

\author{
Anne Hemonic; Isabelle Corrégé; Nicolas Berthelot \\ IFIP-Institut du porc, LeRheu, France
}

\section{Introduction}

On pig farms, collective treatments can be achieved by oral administration of medicines through drinking water with dosing pumps. Homogeneity of the medicated solution at the drinkers is a key factor for therapeutic success, respect of the maximum residue limits (MRL) in meat and prevention of antimicrobial resistance. The aim of this study was to assess homogeneity of an oxytetracycline (OTC) solution, according to type of stock solution tanks and to solubility of OTC.

\section{Materials and Methods}

\section{Homogeneity in the stock solution tank}

Three types of tanks commonly used on farms (1) were tested:

- One flat-bottomed tank without stirrer,

- One flat-bottomed tank with a motorized propeller,

- One conic-bottomed tank with a pump mixing the solution.

Each tank was filled up with a well-dissolved OTC solution. Sixty samples were taken 3, 5, 7 and 24 hours after the solution had been prepared at 3 heights of the tank (top, middle and bottom).

\section{Homogeneity at the drinker}

Tests were conducted on an experimental pipe, simulating onfarm conditions. An OTC solution prepared in a mixing tank with (solution A) or without solvent (solution B) was administered by a hydraulic pump to the drinkers. Fifty or 60 samples of $50 \mathrm{ml}$ were taken at the drinker continuously or after a break, with 4 combinations of water flows and pressures.

Concentrations of OTC were assessed by colorimetric analysis.

\section{Results and Discussion}

\section{Homogeneity in the stock solution tank}

For the 3 tanks and at a given time, all the coefficients of variation (CV) in the 3 heights and in the entire tank were below $5 \%$. We concluded there was no concentration gradient in tanks at any given time, with or without a stirrer. These results completed those obtained during a test comparing the homogeneity of low or highly soluble preparations of amoxicillin (2).

\section{Homogeneity at the drinker}

With solution B, samples taken after each break showed increases and drops of concentrations (figure 1), certainly matching settling and releasing of OTC in the pipe. This didn't occur with continuous sampling but in both cases, CV were up to $5 \%$ for the 4 combinations of flows and pressures (Figure1). So, without any solvent, the solution wasn't homogeneous at the drinker.

ÁWith continuous sampling of solution A, CV were below $5 \%$ and average concentrations were between 104 and 109\% of the expected concentration $(1 \mathrm{~g} / \mathrm{l})$ (Table 1$)$. This met the quality requirements of an industrial medicated feed.
Figure 1: Discontinuous sampling of solution B at a drinker: variation of OTC concentrations

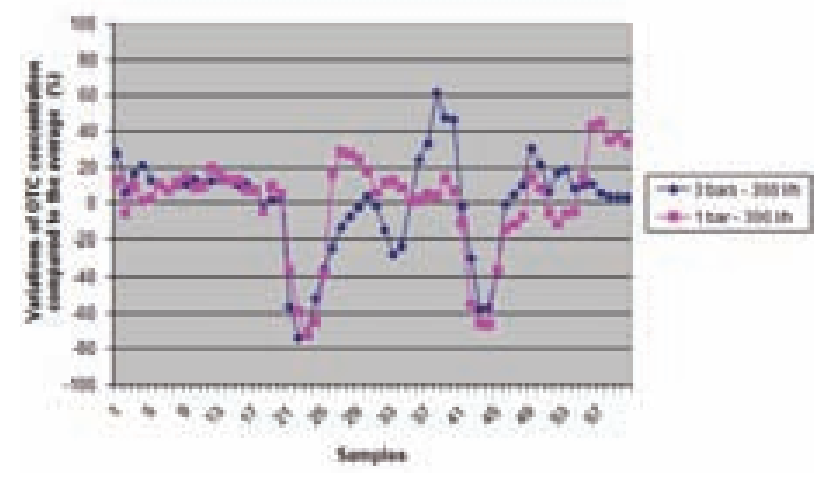

Table 1: Homogeneity of an OTC solution at drinker

\begin{tabular}{|l|c|c|}
\hline $\begin{array}{l}\text { Without any solvent } \\
\text { (solution } \text { B) }\end{array}$ & $\begin{array}{c}\text { Without any solvent } \\
\text { (solution B) }\end{array}$ & $\begin{array}{c}\text { Without any solvent } \\
\text { (solution B) }\end{array}$ \\
\hline Water flow - pressure & CV (\%) of 0TC concentrations & CV (\%) of 0TC concentrations \\
\hline Water flow - pressure & Continuous sampling & Discontinuous sampling \\
\hline $30 \mathrm{I} / \mathrm{h}-0.5$ bars & 14.0 & 85.6 \\
\hline $30 \mathrm{I} / \mathrm{h}-3$ bars & 10.4 & 53.6 \\
\hline $300 \mathrm{I} / \mathrm{h}-1$ bars & 11.7 & 27.0 \\
\hline $300 \mathrm{I} / \mathrm{h}-3$ bars & 7.0 & 27.5 \\
\hline $\begin{array}{l}\text { With a solvent } \\
\text { (solution } \text { ) }\end{array}$ & $\begin{array}{c}\text { With a solvent } \\
\text { (solution A) }\end{array}$ & $\begin{array}{c}\text { With a solvent } \\
\text { (solution A) }\end{array}$ \\
\hline Water flow - pressure & CV (\%) & $\begin{array}{c}\text { Ratio avg. /expected 0TC } \\
\text { concentrations (\%) }\end{array}$ \\
\hline $30 \mathrm{I} / \mathrm{h}-0.5$ bars & 1.4 & 109 \\
\hline $30 \mathrm{I} / \mathrm{h}-3$ bars & 3 & 108 \\
\hline $300 \mathrm{I} / \mathrm{h}-1.5$ bars & 1.6 & 104 \\
\hline $600 \mathrm{I} / \mathrm{h}-3$ bars & 1.4 & 106 \\
\hline
\end{tabular}

After a 12 hour break of solution A, the first samples showed concentration levels between 1.2 and 2.5 times higher than the average levels of the next samples. The involved volume $(400 \mathrm{ml})$, corresponding to the pipe coming down towards the drinker, might globally match the first morning-time drinking of one pig weighting $70 \mathrm{~kg}$ (less than $6 \%$ of its daily water intake). So, this doesn't question the respect of the MRL.

\section{Conclusion}

Our results demonstrate that a well-dissolved medicated solution guarantees a correct homogeneity in the stock solution tank even without a stirrer. At the drinker, the homogeneity of such a solution meets the quality requirements of an industrial medicated feed.

\section{References}

1. Correge I., et al. (2008). Techniporc, 31, 17-21.

2. Hawkins P., et al. (2009). AASV, 223-229. 


\title{
P.693
}

\section{Stability of different amoxicillin medicated premixes in flour feed}

\author{
Eric Bousquet ${ }^{1}$ Mehdi Laraichi ${ }^{1}$ Guillermo Cano ${ }^{2}$ Aurélien Guicherd ${ }^{3}$ Jacques Goutalier $^{3}$ \\ 1. Virbac, Carros, France; 2. Tests and Trials, Lleida, Spain; 3. Phatophy, Lyon, France
}

\section{Introduction}

Interest of a specific coating process of amoxicillin (Suramox ${ }^{\circ}$ ) has been previously demonstrated in order to protect active ingredient degradation in medicated feeds and to insure that adequate amount of active ingredient is available for pigs (1-2). Different comparative studies at laboratory, pilot or industrial scale in Europe showed a better protection of amoxicillin with this coating process against degradation during pelletization and storage of feeds (3).

As medicated feeds are also used in some countries as flour feeds (without pelletization), objective of the present study was to compare stability of amoxicillin in flour feed at industrial scale for different medicated premixes registered in Europe.

\section{Materials and Methods}

Five products containing respectively $5 \%$ (Suramox ${ }^{\circ}, A, B, C$ ) and $10 \%$ (D) of amoxicillin (expressed as base) were tested in the same Spanish feed plant. One batch of a pig grower feed was supplemented by each premix at the concentration of $400 \mathrm{ppm}$ ( $\mathrm{mg}$ of amoxicillin per $\mathrm{kg}$ feed). One blank feed batch was manufactured between 2 medicated batches to avoid cross contamination. Size of medicated feed batches was equal to 3 tons each.

Mixing of raw materials lasted for 2.5 minutes in the mixer where each premix was added manually. Amount of each premix was accurately weighed in order to calculate exact concentration of amoxicillin in feed. Three samples of each medicated feed batch were taken at regular intervals covering the total emptying time of the mixer and stored during 14 days in standardized conditions (respectively $30^{\circ} \mathrm{C} / 65 \%$ of relative humidity $(\mathrm{RH})$ and $40^{\circ} \mathrm{C} / 75 \% \mathrm{RH}$ before assay). Amoxicillin concentrations in feed were determined by High Performance Liquid Chromatography with UV detection. Each sample was analyzed twice and mean concentration was calculated.

\section{Results}

Lower degradation rates were measured with Suramox ${ }^{\circ}(6.1 \%$ and $13.9 \%$ after storage at $30^{\circ} \mathrm{C}$ and $40^{\circ} \mathrm{C}$ respectively) than with the other products (degradation ranging from $30.5 \%$ to $44.4 \%$ and from $45.2 \%$ to $60.3 \%$ after storage at $30^{\circ} \mathrm{C}$ and $40^{\circ} \mathrm{C}$ respectively).
Table: Amoxicillin mean concentrations in feed after storage during 14 days (expressed as percentage of theoretical concentration)

\begin{tabular}{|l|c|c|}
\hline Product & Storage at $\mathbf{3 0}^{\circ} \mathbf{C}$ & Storage at $\mathbf{4 0}{ }^{\circ} \mathbf{C}$ \\
\hline Suramox & $93.9 \%$ & $86.1 \%$ \\
\hline A & $69.5 \%$ & $54.8 \%$ \\
\hline B & $60.4 \%$ & $50.9 \%$ \\
\hline C & $55.6 \%$ & $42.5 \%$ \\
\hline D & $61.4 \%$ & $39.7 \%$ \\
\hline
\end{tabular}

\section{Discussion}

Degradation during storage has been previously shown at laboratory scale when flour feed was stored during 15 days at $40^{\circ} \mathrm{C} / 95 \% \mathrm{RH}$ : degradation was limited to $10 \%$ for Suramox and around $50 \%$ for other products, consistent with present data (3).

This study confirms that amoxicillin may be damaged in feed even without pelletization during transport and storage according to temperature and humidity conditions found in Europe (particularly when feed is stored in silos during summer). A specific coating process of the active ingredient may reduce degradation in such conditions.

\section{References}

1 Derrieu, G. et al (2000). Rev. Med. Vet., 151, 109-117.

2 Bousquet, E. et al (2006). J. Vet. Pharmacol. Therap., 29 (Suppl. 1), 8182.

3 Bousquet, E. et al (2006). Proc. 27th Anaporc Congress, Badajoz Spain, 185-188. 


\title{
P.694
}

\section{Stability of tylosin in liquid feed used in commercial pig farms}

\author{
Alain Kanora; Spas Petkov; Koen De Gussem \\ Huvepharma NV, Antwerp, Belgium
}

\section{Introduction}

In some countries, pig farms are using recyclable foodstuff originating from human consumption and are commonly being added as nutritional basis for liquid feeds. Farmers can also mix commercial feed with water to obtain liquid feed. These two feed systems have the benefit that feed becomes tastier and more acid due to fermentation; both properties will increase consumption. The stability and activity of veterinary medicinal products (VMP) used in liquid feed are often not studied; particularly data on antibiotics are unavailable.

VMD (UK) currently allows the use of in-feed antimicrobials in liquid feed in accordance with the cascade regulations, but veterinarians need to base their decision on the stability of the veterinary specialty first before prescribing the use in liquid feed. In this way issues like overdosing, underdosing, resistance buildup, etc. can be avoided. Therefore, the use of tylosin phosphate (Pharmasin ${ }^{\oplus}$ medicated feed premix) in liquid feed was studied by evaluating the impact on the active substance with regards to stability.

\section{Materials and Methods}

Locally collected dry feed from a GMP certified production site was sourced and diluted at ration 2:1 with tap water to obtain a liquid feed matrix.

Pharmasin $250 \mathrm{mg} / \mathrm{g}$ and Pharmasin $100 \mathrm{mg} / \mathrm{g}$ were added to the liquid feed in dosages providing 100 ppm in the feed based on dry matter.

The mixtures were stored under normal environmental conditions $\left(20-25^{\circ} \mathrm{C}\right.$, ambient humidity) and sampled at 0, 1, 6, 24 and 72 hours.

The samples were analyzed by a microbiologic assay for tylosin content. The method is a four-dose diffusion assay using Micrococcus luteus ATCC 9341 as a test micro-organism.

\section{Results}

The ppm levels of all tested Pharmasin formulations remained perfectly stable for more than 5 hours. The VMD recommendation of $1 \mathrm{~h}$ stability was perfectly matched and also the maximum meal contact time for pigs was prior to onset of a slight activity loss.
Table 1: Tylosin concentration (ppm) in liquid feed samples on dry basis

\begin{tabular}{|l|c|c|c|c|c|}
\hline & \multicolumn{5}{|c|}{ Hours } \\
\hline Product & $\mathbf{0}$ & $\mathbf{1}$ & $\mathbf{6}$ & $\mathbf{2 4}$ & $\mathbf{7 2}$ \\
\hline Pharmasin $^{\circ} 250$ & 115 & 114 & 111 & 102 & 94 \\
\hline Pharmasin $^{\circ} 100$ & 114 & 111 & 112 & 101 & 96 \\
\hline
\end{tabular}

Figure 1. Stability of Pharmasin ${ }^{\circledast}$ in liquid feed

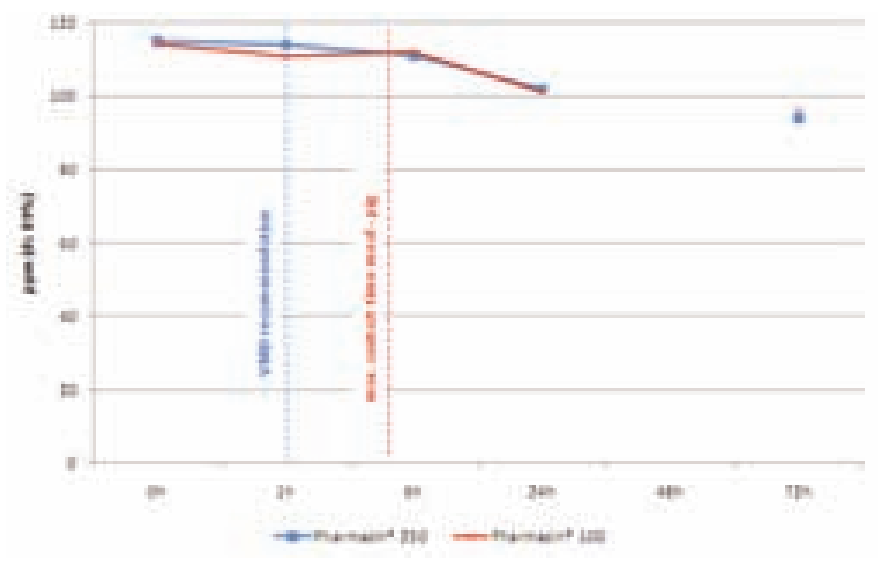

\section{Discussion and Conclusion}

It can be concluded from this study that Pharmasin Medicated Premix used in a responsible way in liquid feed remains perfectly stable and that there is no loss of activity of the used tylosin phosphate. Even after 24 and 72 hours, tylosin activity was in excess of $80 \%$ of the initial value.

\section{References}

1. Hannan, P.C.T. et al. (1997), Antimicrob. Agents Chemother., 41: 2037-2040.

2. Prats, C. et al. (2002), Res. Vet. Sci., 73: 141-144.

3. Prescott, J.F. et al. (2000), Antimicrobial Therapy in Veterinary Medicine, $3^{\text {rd }}$ edn. lowa State University Press, Ames, lowa, pp. 237-257. 


\title{
P.695
}

\section{Pharmacokinetics and tolerance of Catosal ${ }^{\circledR}$ in pigs}

\author{
Bernard H. Schmidt ${ }^{1}$ Ralph Krebber ${ }^{2}$ Martina Rehagen ${ }^{1}$ \\ 1. Bayer Animal Health GmbH, Leverkusen, Germany; 2. Bayer CropScience AG, Monheim, Germany
}

\section{Introduction}

Catosal ${ }^{\circledR}$ is a metabolic stimulant for use in numerous target animal species including the pig in many countries worldwide. It contains $100 \mathrm{mg}$ of butafosfan and $0.05 \mathrm{mg}$ of cyanocobalamin (vitamin B12) per ml. Experimental studies indicated that Catosal ${ }^{\circledR}$ attenuates stress responses of piglets (1), and reduces the prevalence of certain production diseases in sows and piglets $(2,3)$. The present report documents the tolerance and the pharmacokinetics of butafosfan after a single intramuscular (i.m.) injection of Catosal ${ }^{\circledR}$ to piglets.

\section{Materials and Methods}

Two independent studies were performed in an accredited experimental animal centre in Germany. The first one assessed the local and systemic tolerance of Catosal ${ }^{\circledR}$ in healthy German Landrace hybrid piglets (22.5 - 28,5 kg body weight (b.w.)). Catosal ${ }^{\oplus}$ was injected i.m. at the dosages of $0.1,0.3$, and $0.5 \mathrm{ml} /$ $\mathrm{kg}$ ( $\mathrm{N}=6$ per dose group). A control group of 6 piglets received saline at the dose volume of $0.5 \mathrm{ml} / \mathrm{kg}$ b.w. Between injection and the third day thereafter, the animals were closely monitored for clinical signs of intolerance and local reactions at the injection site.

The second study assessed the pharmacokinetics of butafosfan in four Hamshire/Pietrain piglets weighing $36.5-47.0 \mathrm{~kg}$. The animals received Catosal ${ }^{\circledR}$ at a dose volume of $0.1 \mathrm{ml} / \mathrm{kg}$ i.m., corresponding to $10 \mathrm{mg}$ butafosfan/kg b.w. Frequent blood sampling was performed over a period of 24 hours after treatment. Serum was analyzed for butafosfan concentrations using HPLC with tandem mass spectrometric detection. Relevant pharmacokinetic parameters were calculated using non-compartmental analysis and the linear/log trapezoidal rule. All results are given as arithmetic means \pm standard deviation.

\section{Results}

Tolerance: No local reactions at the injection site, immediate systemic reactions or other adverse events were observed in either study after i.m. injection.

Pharmacokinetics: Based on the individual serum concentrations of butafosfan, the concentration-time profiles depicted in Figure 1 were obtained.
Figure 1 Individual butafosfan concentration-time profiles of pigs injected intramuscularly with Catosal ${ }^{\oplus}(0.1 \mathrm{ml} / \mathrm{kg})$

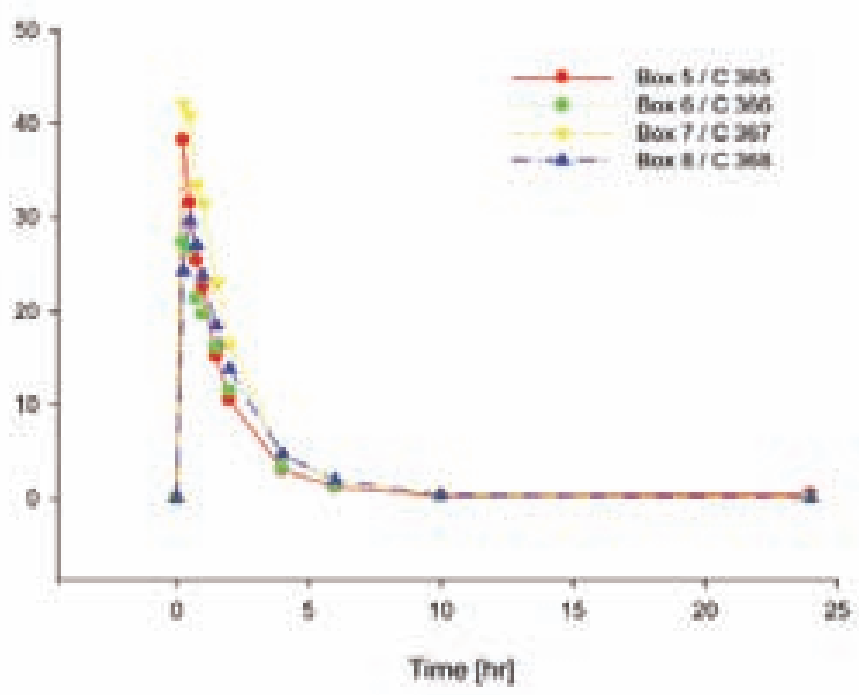

Group mean maximal concentrations of $35 \pm 5.8 \mu \mathrm{g} / \mathrm{L}$ were measured $0.3 \pm 0.1 \mathrm{hrs}$ after administration. The elimination half life time was calculated to $3.5 \pm 0.6 \mathrm{hrs}$. Within 24 hours postadministration, serum butafosfan levels approached the limit of quantitation of $0.02 \mathrm{mg} / \mathrm{L}$. The area under the curve extrapolated ad infinitum was $72.5 \pm 12.6 \mathrm{hr}^{*} \mu \mathrm{g} / \mathrm{L}$. The distribution volume and clearance of the absorbed fraction were $775 \pm 150 \mathrm{~L} / \mathrm{kg}$ and $148 \pm 20.6 \mathrm{~L} / \mathrm{hr} / \mathrm{kg}$, respectively. The mean residence time extrapolated ad infinitum was $2.7 \pm 1.0 \mathrm{hrs}$.

\section{Conclusion}

Intramuscular injection with Catosal $^{\circledR}$ at a dose volume of $0.1 \mathrm{ml} /$ $\mathrm{kg}$ b.w. and 5 times thereof is well tolerated and safe in pigs. The pharmacokinetics of butafosfan show a fast and efficient absorption into blood, a high distribution volume and fairly rapid elimination from the porcine body.

\section{References}

1. Van der Staay, F.J. et al., J. vet. Pharmacol. Therap. 30:410-416 (2007);

2. Nuntaprasert, A. and Watanapongchart, S., Asian Pork Magazine December 2007/January 2008;

3. Nuntaprasert, A. et al., Proc. 20th IPVS Congress, p. 450 (2008) 


\title{
P.696
}

\section{Stability of different amoxicillin medicated premixes in pelleted feed}

\author{
Eric Bousquet ${ }^{1}$ Mehdi Laraichi ${ }^{1}$ Guillermo Cano ${ }^{2}$ Aurélien Guicherd ${ }^{3}$ Jacques Goutalier $^{3}$ \\ 1. Virbac, Carros, France; 2. Tests and Trials, Lleida, Spain; 3. Phatophy, Lyon, France
}

\section{Introduction}

Interest of a specific coating process of amoxicillin (Suramox ${ }^{\circledR}$ ) has been previously demonstrated in order to protect active ingredient degradation in medicated feeds and to insure that adequate amount of active ingredient is available for pigs (1-2). Different comparative studies at laboratory, pilot or industrial scale in Europe showed a better protection of amoxicillin with this coating process against degradation during pelletization and storage of feeds (3-4).

Objective of the present study was to complete stability data of amoxicillin in pelleted feed at industrial scale for different medicated premixes registered in Europe.

\section{Materials and Methods}

Five products containing respectively $5 \%$ (Suramox $, A, B, C$ ) and $10 \%$ (D) of amoxicillin (expressed as base) were tested in the same Spanish feed plant. One batch of a pig grower feed was supplemented by each premix at the concentration of 400 ppm (mg of amoxicillin per kg feed). One blank feed batch was manufactured between 2 medicated batches to avoid cross contamination. Size of medicated feed batches was equal to 3 tons each. Amount of each premix was accurately weighed in order to calculate exact concentration of amoxicillin in feed. Steam pressure was equal to $4 \mathrm{~atm}$ and temperature ranged from 53.7 to $65.5^{\circ} \mathrm{C}$ before pelletization. Temperature ranged from 71.3 to $75.2^{\circ} \mathrm{C}$ after pelletization. Six samples of each medicated feed batch were taken after cooling of the pellets at regular intervals covering the total batch delivery. Samples were analyzed at the time T0 of reception by the laboratory (Phatophy). In the meantime, aliquots of 3 out of 6 samples per batch were stored during 14 days in standardized conditions $\left(25^{\circ} \mathrm{C} / 65 \%\right.$ of relative humidity $(\mathrm{RH})$ before assay at T14). Amoxicillin concentrations in feed were determined by High Performance Liquid Chromatography with UV detection. Each sample was analyzed twice and mean concentration was calculated.

\section{Results}

Mean degradation ranged from $10.2 \%$ to $39.3 \%$ at T0. A further degradation was measured during storage for all products except Suramox ${ }^{\circ}$, leading to a total degradation between $18.3 \%$ and $53.1 \%$ at T14 for products A, B, C and D.

Table Amoxicillin mean concentrations in feed at T0 and after storage during 14 days (expressed as percentage of theoretical concentration)

\begin{tabular}{|l|c|c|}
\hline Product & T0 (n=6) & T14 (n=3) \\
\hline Suramox & $89.8 \%$ & $91.2 \%$ \\
\hline A & $87.6 \%$ & $81.7 \%$ \\
\hline B & $75.6 \%$ & $64 \%$ \\
\hline C & $60.7 \%$ & $46.9 \%$ \\
\hline D & $68.7 \%$ & $56 \%$ \\
\hline
\end{tabular}

\section{Discussion}

Degradation was limited around $10 \%$ for Suramox ${ }^{\circledR}$, without significant evolution during storage (variation within analytical variability). Degradation recorded for the other products was consistent with previous studies following pelletization and eventual storage of pig medicated feeds (3-4).

This study confirms that a specific coating process of the active ingredient may reduce degradation after pelletization and storage of feeds.

\section{References}

1 Derrieu, G. et al (2000). Rev. Med. Vet., 151, 109-117.

2 Bousquet, E. et al (2006). J. Vet. Pharmacol. Therap., 29 (Suppl. 1), 8182.

3 Bousquet, E. et al (2006). Proc. 27th Anaporc Congress, Badajoz Spain, 185-188.

4 Bousquet, E. et al (2008). Proc. 29th Anaporc Congress, Cordoba Spain. 


\title{
P.697
}

\section{Demixing by elutriation of different amoxicillin medicated premixes in feed}

\author{
Eric Bousquet ${ }^{1}$ Mehdi Laraichi $^{1}$ Carine Rousseau ${ }^{1}$ Christine Segot ${ }^{1}$ Aurélien Guicherd ${ }^{2}$ Jacques Goutalier $^{2}$ \\ 1. Virbac, Carros, France; 2. Phatophy, Lyon, France
}

\section{Introduction}

Demixing of medicated feeds is a concern for feed millers and veterinary practitioners, leading to heterogeneous feeds and increase of antimicrobials dosage variability. Elutriation is a demixing phenomenon due to different speeds of powder particles while falling through the air. A standardized test has been developed to assess elutriation of powders from previously described experiments (1). This test has been correlated with demixing of supplemented feeds in plants (2) and previously performed for a range of antimicrobials premixes (3-4). Objective of this study was to complete elutriation tests on 4 amoxicillin premixes registered in Europe.

\section{Materials and Methods}

Four products containing respectively $5 \%$ (Suramox $\left.{ }^{\circ}, \mathrm{A}\right)$ and $10 \%(B, C)$ of amoxicillin (expressed as base) were tested in a prestarter feed (median particle size : $390 \mu \mathrm{m}$ ). Median particle size of the 4 products was determined by laser granulometry. Each product was then tested twice in order to allow statistical analysis. Around $1 \mathrm{~kg}$ of flour feed was supplemented by each product according to incorporation rate recommended (400 ppm of amoxicillin in feed). A tracer was added in each feed sample at the concentration of $500 \mathrm{ppm}$ in order to validate the tests. For each test a $300 \mathrm{ml}$ feed sample was dropped in an $8 \mathrm{~m}$ high tube. After drop, top and buttom $50 \mathrm{ml}$ feed samples were taken for tracer assay by colorimetry and amoxicillin assay by high performance liquid chromatography. In each test, an elutriation index (El) was calculated from previously defined index (5) for the tracer and for amoxicillin (where $\mathrm{Ct}$ and $\mathrm{Cb}$ are respectively concentrations in top and bottom samples):

$$
E I=\frac{C t-C b}{\left[\frac{C t+C b}{2}\right]} \times 100
$$

This index ranges from -200 to +200 , extreme values corresponding to maximal demixing whereas zero value corresponds to lack of demixing. A non parametrical analysis of variance on ranks was performed to compare El between products.

\section{Results}

Amoxicillin El was lower for Suramox ${ }^{\oplus}$ than for the other products.

Table: Median particle size of active ingredient and mean elutriation index of the 4 products tested

\begin{tabular}{|l|c|c|}
\hline Product & Particle size $(\mu \mathrm{m})$ & El \\
\hline Suramox & 380 & 1.4 \\
\hline A & 878 & $-89.4^{*}$ \\
\hline B & 902 & $-137.2^{*}$ \\
\hline C & 188 & $95.1^{*}$ \\
\hline${ }^{* * *}:$ significantly different from Suramox $x^{\circledast}(\operatorname{resp} p<0.05$ and $\mathrm{p}<0.005)$ \\
\hline
\end{tabular}

\section{Discussion}

For products with high negative El, amoxicillin is more concentrated in bottom of the sample after test (amoxicillin particles too large or too dense falling more quickly within large feed particles). For products with high positive El, amoxicillin is more concentrated in top of the sample after test (thin amoxicillin particles falling more slowly within thin feed particles). This is reflected by median particle size higher than feed one for products with high negative El (products A and B) and median particle size lower than feed one for product with high positive El (product C). Lower demixing with Suramox may be due to amoxicillin particle size with specific coating process (median particle size : $380 \mu \mathrm{m}$ ), consistent with feed median particle size (390 $\mu \mathrm{m})$.

\section{Acknowledgements}

The authors thank Fabrice Putier and Marianne Jousselin from Tecaliman (France) for performance of elutriation tests.

\section{References}

1 Amornthewaphat, N. et al (1998). Kansas State University Day Report : 261-263

2 Putier, F. and Bousquet, E. (2004). 4ème Colloque Science et Technologie des poudres, Compiègne, France

3 Bousquet, E. et al (2004). Proc. IPVS, 549

4 Bousquet, E. et al (2006). Proc. IPVS, 429

5 Williams, J.C. and Shields, G. (1969). Powder Technol 1, 134-142 


\title{
P.698
}

\section{Performance improvement of finishing pigs fed Denagard (tiamulin) plus chlortetracycline in feed antibiotic vs. oxytetracycline in feed antibiotic or non-medicated feed}

\author{
J. M. Hammer; Dean Dau \\ Novartis Animal Health US, Greensboro, NC, USA
}

\section{Introduction}

A synergistic effect has been demonstrated when Denagard ${ }^{\circledR}$ (tiamulin) and chlortetracycline (DEN-CTC) are used concurrently in-feed to address the effects of bacterial pneumonia.1 The economic benefit of administering DEN-CTC in-feed has been documented by others.2, 3 The purpose of this study was to evaluate the performance differences in disease control between pigs fed no in-feed antibiotic, OTC (oxytetracycline 440 ppm) or DEN-CTC (38.5 ppm tiamulin; 440 ppm chlortetracycline).

\section{Materials and Methods}

A total of 1344 weaned pigs (avg. age 21 days) were placed in one of two all-in all-out commercial wean to finish barns. Pigs were fed common diets throughout the nursery phase (D1 (weaning)-D39). On day 39, pigs were mixed by gender and allocated to one of three treatment groups ( $3 \times 2$ factorial design): no medication, OTC or DEN-CTC. The in-feed antibiotic was fed for 17 days followed by a subsequent 17 days of no medication (total trial D39-D73). Data analysis for average daily gain (ADG) and feed efficiency (F:G) were done using all weight in, all weight out and total feed consumed. Pen averages were used as the experimental unit with 16 pens/treatment.

\section{Results}

The DEN-CTC group had an ADG advantage over the non-medicated $(0.07 \mathrm{~kg} /$ day $)$ and the OTC $(0.05 \mathrm{~kg} /$ day $)$ groups for the entire 34 day trial ( $p<0.001$; Table 1$)$. This resulted in the DENCTC pigs being significantly heavier than the non-medicated $(2.59 \mathrm{~kg})$ or OTC pigs $(1.77 \mathrm{~kg})$ at the end of the 34 day trial ( $p$ $<0.001)$ period. The DEN-CTC group showed an improved F:G compared to the non-medicated or the OTC group $(p<0.09)$. This resulted in the DEN-CTC pigs requiring $2.54 \mathrm{~kg}$ and $2.81 \mathrm{~kg}$ less feed to reach the same end weight as the non-medicated or OTC pigs, respectively. The in-feed antibiotic increases costs by $\$ 0.12$ USD per head for the OTC pigs and, \$0.61 USD per head for the DEN-CTC pigs. If one assumes a $\$ 140$ USD per ton feed cost and a market price of $\$ 40$ USD per hundred pounds, the DEN-CTC treatment returned \$2.67 USD more per head than non-medicated and $\$ 1.99$ USD more per head than the OTC treatment. Each Dollar spent on Denagard plus chlortetracycline returned \$ 4 USD in improved performance over no medication or oxytetracycline in-feed medication.
Table 1. Performance Effect by in feed antibiotic (D39-D73) application Treatment

\begin{tabular}{|l|c|c|c|}
\hline Parameter & No Med & OTC & DEN-CTC \\
\hline Start weight (D39) & 25.92 & 25.97 & 25.92 \\
\hline ADG & $0.75^{\mathrm{ab}}$ & $0.77^{\mathrm{b}}$ & $0.82^{\mathrm{b}}$ \\
\hline F:G & $2.05^{\mathrm{a}}$ & $2.06^{\mathrm{a}}$ & $1.96^{\mathrm{b}}$ \\
\hline End Weight (D37) & $51.62^{\mathrm{a}}$ & $52.44^{\mathrm{a}}$ & $54.21^{\mathrm{b}}$ \\
\hline Mortality (\%) & 0.70 & 0.70 & 0.90 \\
\hline \multicolumn{4}{|l|}{ different superscripts by row are statistically significant at $\mathrm{p}<0.05$} \\
\hline
\end{tabular}

\section{Discussion}

In the investigated production system the impact of Denagard plus chlortetracycline early in the grow finish phase had a significant impact in improving performance during and after the in feed antibiotic application. The improvements in performance translate into improved return on investment for the production system of \$4 USD per head over either the non medicated or OTC treatments.

\section{References}

1 Burch D, Jones G, Heard T, Truck R. The synergistic activity of Tiamulin and chlortetracycline: In feed treatment of bacterially complicated enzootic pneumonia in fattening pigs. Vet.Rec 1986:119:108-112.

2 Walter D, Holck JT, Sornsen S et al. The effect of metaphylactic pulse dosing in-feed antimicrobial strategy on finishing pig health and performance, Swine Health Prod. 2000; 8(2)65-71.

3 Waddell J, Graham J, Roycroft L. Early finishing performance of pigs fed Denagard plus chlortetracycline vs. oxytetracycline in the grower diet. Proc.AASV 2010 


\title{
P.699
}

\section{Monitoring of antimicrobial usage for pigs in Denmark}

\author{
Helle Stege $^{1}$ Claes Enoe $^{2}$ Erik Jacobsen $^{2}$ Lis Alban $^{3}$ Jens P. Nielsen ${ }^{1}$ \\ 1. KU-Life, Copenhagen, Denmark; 2. Vetinst, Copenhagen, Denmark; 3. VSP, Kjellerup, Denmark
}

\section{Introduction}

The Danish system for monitoring of veterinary drug use, Vetstat, was implemented during 1999 - 2001 (1). Close to $100 \%$ of the drugs used in pig herds are provided on prescription, hence, reported to Vetstat from pharmacies. The aim of this presentation was to present the antimicrobial (AM) usage for pigs during 2004-2009.

\section{Materials and Methods}

The overall use of AM in Denmark (measured as kilo active compound) was downloaded from the Vetstat homepage. In general pigs account for $80 \%$ of the total usage of AM. The number of slaughter pigs produced per year (including export of live growers of $30 \mathrm{~kg}$ ) was obtained from Danish Statistics. When Vetstat data from 2009 were extracted they only included entries until September so the total use of this year was estimated by extrapolation (table 1). When comparing the AM use between farms and/or veterinarians, the total amount of active compound must be converted to "number of Animal Defined Daily Doses, ADD" (2), according to the products' potency and concentration and the animal's weight. The measure used to compare AM usage between farms/ veterinarians is "percentage animals treated per day" which is calculated as: Average number of ADDs used per day divided by the average number of animals present. ADD data on AM usage for pigs were extracted from Vetstat (User page).

\section{Results}

Overall AM usage (kg) in Denmark, 2004-2009 is presented in fig. 1 and table 1. Apart from the increase from 2008 to 2009 (t-test; $p=0.02$ ) there has been no significant changes in the average AM usage ( $\mathrm{g} / \mathrm{pig} /$ year) during the years. The most-used-AMs for pigs were tetracyclines, penicillins, sulpha-tmps, macrolides and tiamulines. The main part of the AM was prescribed for per oral administration to growers for gastro-intestinal disorders. Based on ADD calculations, the present national average is $10-12 \%$ growers treated per day as opposed to $1.5-2 \%$ for breeding stock and $2 \%$ for finishers (data from Vetstat, User page, not presented).

\section{Discussion}

The overall AM usage is reported from the pharmacies (>95\%) by automated transfer to Vetstat, based on their stock managing system. The conversion from actual prescription to amount of active compound is formalized and simple. Hence, these data are very reliable. The AM usage has been stable during 2004-2009 with an average use of $35-4.0 \mathrm{~g}$ AM per produced slaughter pig per year (table 1). Similarly, the usage measured as ADDs or percentage animals treated per day have been reasonably stable during the years. However, ADD data are more prone to errors because of the assumptions and calculations involved.
Fig. 1 Total AM usage $(\mathrm{kg})$ - prescribed by veterinarians

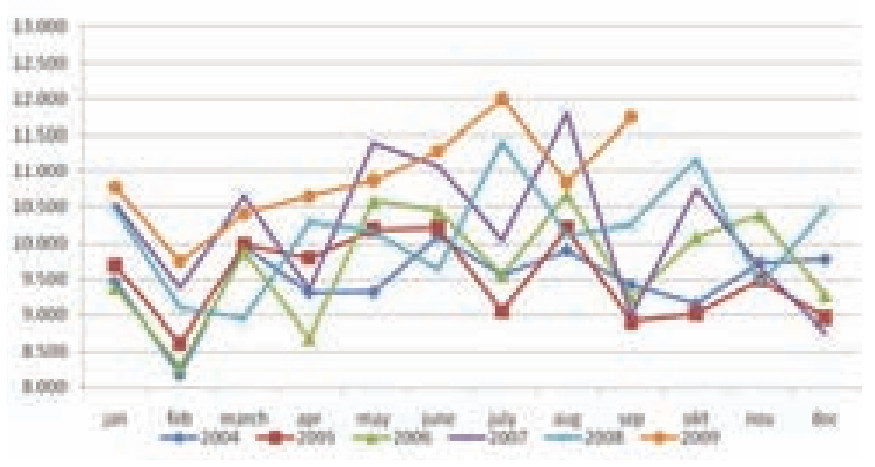

Table 1. Overall use of AM (kg) per year - mean and stddev. Pigs account for $80 \%$ of total use. AM use per pig ( $\mathrm{g} / \mathrm{pig} /$ year) is presented. The apparent increase in AM/pig/year was tested (t-test) comparing 2007 with 2009 and 2008 with 2009, respectively.

\begin{tabular}{|l|c|c|c|c|c|c|}
\hline & $\mathbf{2 0 0 4}$ & $\mathbf{2 0 0 5}$ & $\mathbf{2 0 0 6}$ & $\mathbf{2 0 0 7}$ & $\mathbf{2 0 0 8}$ & $\mathbf{2 0 0 9}$ \\
\hline Total (kgx1000) & 113,897 & 114,173 & 116,475 & 122,438 & 121,435 & 131,132 \\
\hline Mean & 9.491 & 9.514 & 9.706 & 10.203 & 10.120 & 10.928 \\
\hline Stddev & 506,4 & 582,8 & 769,3 & 976,2 & 747,6 & 682,2 \\
\hline Pigs/year & 24,9 & 25,8 & 25,7 & 26,3 & 26 & 26 \\
\hline G/pig/year & 4,57 & 4,43 & 4,53 & 4,66 & 4,67 & 5,04 \\
\hline (x 80\%) & 3,66 & 3,54 & 3,63 & 3,72 & 3,74 & 4,03 \\
\hline & & & & & & \\
\hline ftest & & & & 0,318 & 0,814 & \\
\hline ttest & & & & 0,073 & 0,020 & \\
\hline
\end{tabular}

\section{References}

1. Stege, H., Bager, F., Jacobsen, E. and Thougaard, A., 2003. Prev Vet Med. 57(3):105-15.

2. Jensen, V.F., Jacobsen. E. and Bager, F., 2004. Prev Vet Med. 64:20115. 


\title{
P.700
}

\section{Assessment of exposure of Draxxin ${ }^{\circledR}$ compared to Draxxin plus iron administered to 3 day old piglets}

\author{
Steven P. Lesman ${ }^{1}$ Ian A. Nanjiani ${ }^{2}$ James R. Allison ${ }^{3}$ Ann E. Fielder ${ }^{1}$ Clark D. Smothers ${ }^{1}$ Scott A. Brown ${ }^{1}$ Joseph A. Robinson ${ }^{1}$ \\ 1. Pfizer Animal Health, Kalamazoo, MI, USA; 2. Pfizer Animal Health, Sandwich, UK; 3. Pfizer Animal Health, New York, NY, USA
}

\section{Introduction}

Although undesirable from a pharmaceutical perspective, and usually off-label in terms of product directions, it is common farm practice in some countries for injectable products to be mixed immediately prior to administration. The advantage of this procedure is that it minimizes the number of injections that have to be given to animals. The downside is that it creates the possibility of interactions that could affect drug safety and/ or efficacy, both in the form of pharmacological interactions between the active ingredients and chemical reactions between the formulations. The latter are most likely to affect absorption kinetics from the site of injection and influence drug bioavailability.

Iron injections are commonly given to piglets in the first week of life to counteract the low iron content of the sows' milk. Draxxin $^{\circledast}$ (Pfizer Animal Health) is an injectable formulation of the antibiotic tulathromycin that is sometimes given to piglets of the same age. There have been reports of producers mixing the products prior to injection. Although Draxxin is considered a long-acting product, the duration of activity derives from the intrinsically long half-life of tulathromycin, which accumulates in lung tissue and establishes therapeutic concentrations that persist for many days. The formulation is an aqueous solution that allows rapid absorption of drug from the site of injection. The greatest pharmaceutical risk, therefore, is of an incompatibility that delays or prevents drug uptake and reduces bioavailability. This study was performed to investigate this possibility. Based on previous pharmacokinetic work, lung concentrations of tulathromycin at 24 hours were used to assess the overall outcome of the drug absorption phase.

\section{Materials and Methods}

This two-treatment randomized parallel group study was designed to compare Draxxin (tulathromycin) mixed with iron to Draxxin alone when dosed intramuscularly to 3-day old Landrace/Duroc cross piglets. The doses were Draxxin $2.5 \mathrm{mg} /$ $\mathrm{kg}$ bodyweight and $200 \mathrm{mg}$ iron (Ursoferran ${ }^{\circledR}$ )/animal. Sixteen piglets were randomized to each treatment group according to a generalized block design. Blocking was based on litter and pen location. At $24 \mathrm{hr}$, blood was collected for PK and necropsies performed to harvest lung tissue. The plasma and lung homogenate samples were analyzed for tulathromycin concentrations by an LC-MS/MS procedure.

\section{Results}

The concentration data are summarized in the following table;

\begin{tabular}{|l|c|c|c|c|}
\hline & & & \multicolumn{2}{|c|}{ 95\% Confidence Interval } \\
\hline Matrix & Treatment $^{\dagger}$ & $\begin{array}{c}\text { Least Squares } \\
\text { Mean }\end{array}$ & Lower & Upper \\
\hline Plasma (ng/mL) & T01 & 37.8 & 22.3 & 63.9 \\
\hline Plasma (ng/mL) & T02 & 35.4 & 21.0 & 60.0 \\
\hline Lung (ng/g) & T01 & 3270 & 2990 & 3570 \\
\hline Lung (ng/g) & T02 & 3290 & 3010 & 3600 \\
\hline TT01- Draxxin alone T02- Draxxin + Iron \\
\hline
\end{tabular}

Non-inferiority was demonstrated comparing Draxxin with iron (T02) to Draxxin alone (T01). The 95\% confidence intervals for the ratios of Draxxin with iron to Draxxin alone were 83 - 106\% and $92-110 \%$ for tulathromycin concentrations in plasma and lung homogenate, respectively.

\section{Discussion}

Both plasma and lung tissue concentration data show that T02 (Draxxin administered with iron) was not inferior to T01 (Draxxin alone). This study demonstrated that the mixing of Draxxin with a commonly used commercial injectable iron solution did not adversely affect the lung and plasma tulathromycin concentrations at 24 hours post injection in 3 day old, commercially reared pigs. The study did not specifically address safety, but no adverse events were noted. Unapproved mixing of products is to be discouraged, but this study did not reveal any specific adverse impact.

\section{Reference}

Benchaoui, H. A., et al. Pharmacokinetics and lung tissue concentrations of tulathromycin in swine. J Vet Pharm Therapeutics 2004; 27:203210. 


\title{
P.701
}

\section{In vitro activity of Florfenicol with other antimicrobials}

\author{
Timothy S. Kniffen ${ }^{1}$ Elliot Stevens ${ }^{2}$ Darin Madson ${ }^{3}$ \\ 1. Intervet/Schering-Plough Animal Health, DeSoto, KS, USA; 2. Rural Technologies, Inc., Brookings, SD, USA; 3. Iowa State University, Ames, IA, USA
}

\section{Introduction}

Florfenicol is a broad spectrum antimicrobial approved for use in swine in most pig producing countries. It is approved for use variably as a premix, drinking water concentrate, and/or injectable formulation. Florfenicol is primarily used in swine for the treatment and prevention of respiratory disease. There is no guidance currently available to swine veterinarians concerning the use of florfenicol sequentially with other antimicrobials. A checkerboard dilution technique was utilized to assess the activity of florfenicol with antimicrobial combinations against 4 bacterial pathogens of swine. The results of this checkerboard evaluation provide the first available guidance to swine veterinarians utilizing florfenicol sequentially with other antimicrobials.

\section{Materials and Methods}

\section{Bacterial Isolates:}

Four bacterial isolates were used in this pilot project: Streptococcus suis (Ss), Salmonella choleraesuis (Sc), and Bordetella bronchiseptica (Bb). Escherichia coli (Ec) was also included although it is not one of the respiratory pathogens for which florfenicol has been approved. All bacterial isolates were obtained from swine field cases submitted to a diagnostic laboratory.

\section{Antimicrobials Evaluated:}

Antimicrobial concentrations were tested with florfenicol as serial dilutions in the ranges indicated in Table 1.

\begin{tabular}{|l|c|c|}
\hline Abbreviation & Antimicrobial & Dilution Range \\
\hline $\mathrm{Ffc}$ & florfenicol & $0.25-8 \mu \mathrm{g} / \mathrm{ml}$ \\
\hline $\mathrm{Ce}$ & ceftiofur & $0.12-8 \mu \mathrm{g} / \mathrm{ml}$ \\
\hline $\mathrm{Ctc}$ & chlortetracycline & $0.5-8 \mu \mathrm{g} / \mathrm{ml}$ \\
\hline En & enrofloxacin & $0.12-2 \mu \mathrm{g} / \mathrm{ml}$ \\
\hline Otc & oxytetracycline & $0.5-8 \mu \mathrm{g} / \mathrm{ml}$ \\
\hline $\mathrm{Tc}$ & tetracycline & $4.0-32 \mu \mathrm{g} / \mathrm{ml}$ \\
\hline $\mathrm{Tm}$ & tiamulin & $1.0-32 \mu \mathrm{g} / \mathrm{ml}$ \\
\hline $\mathrm{Ty}$ & tylosin tartrate & $0.5-32 \mu \mathrm{g} / \mathrm{ml}$ \\
\hline $\mathrm{Tu}$ & tulathromycin & $1.0-64 \mu \mathrm{g} / \mathrm{ml}$ \\
\hline
\end{tabular}

\section{Bacterial Susceptibility:}

The minimum inhibitory concentration (MIC) of each antimicrobial tested was determined for each of the four bacterial isolates selected. The checkerboard dilution technique was then utilized to allow calculation of the fractional inhibitory concentration index $(\mathrm{FICl})$ for florfenicol in combination with other antimicrobial agents. The combinations were then categorized based on $\mathrm{FICl}$ results as synergistic (S), antagonistic (A), or indifferent (I).

\section{Fractional Inhibitory Concentration Index}

\begin{tabular}{|c|c|c|c|c|c|c|c|c|}
\hline Ffc & Ce & Ctc & En & Otc & Tc & Tm & Ty & Tu \\
\hline Ss & $\mathrm{I}$ & $\mathrm{I}$ & $\mathrm{I}$ & $\mathrm{I}$ & $\mathrm{I}$ & $\mathrm{I}$ & $\mathrm{I}$ & $\mathrm{I}$ \\
\hline $\mathrm{Sc}$ & $\mathrm{I}$ & $\mathrm{I}$ & $\mathrm{I}$ & $\mathrm{S}$ & $\mathrm{I}$ & $\mathrm{I}$ & $\mathrm{I}$ & $\mathrm{S}$ \\
\hline $\mathrm{Ec}$ & $\mathrm{I}$ & $\mathrm{I}$ & $\mathrm{I}$ & $\mathrm{I}$ & $\mathrm{I}$ & $\mathrm{I}$ & $\mathrm{I}$ & $\mathrm{I}$ \\
\hline $\mathrm{Bb}$ & $\mathrm{I}$ & $\mathrm{I}$ & $\mathrm{I}$ & $\mathrm{I}$ & $\mathrm{I}$ & $\mathrm{I}$ & $\mathrm{I}$ & $\mathrm{S}$ \\
\hline
\end{tabular}

\section{Discussion}

1. Most antimicrobials tested in vitro in combination with florfenicol were indifferent in effect.

2. No antagonistic interactions were found between florfenicol and any tested antimicrobial in vitro.

3. Synergistic relationships between florfenicol and OTC and between florfenicol and tulathromycin were detected in vitro.

4. These in vitro results suggest that antimicrobials commonly used in swine medicine would have no anticipated antagonistic effects when used sequentially with florfenicol.

\section{References}

1. Lorian, (1986) Antibiotics in Laboratory Medicine, 2nd edition, Baltimore: Williams \& Williams

2. Odds, FC. Synergy, Antagonism, \& what the chequerboard puts between them. Journal of Antimicrobial Chemotherapy 2003:52:1

3. Schering-Plough Animal Health Nuflor Technical Monograph 2006 


\title{
P.702
}

\section{Monitoring of enrofloxacine (Baytril ${ }^{\oplus}$ ) sensitivity of clinical field bacterial strains from pigs in the Benelux using $E$ testing}

\author{
Dominique Gevaert \\ Bayer SA-NV, Diegem (Machelen), Belgium
}

\section{Introduction}

In veterinary routine diagnostic laboratories antimicrobial sensitivity testing is performed by agar disc diffusion. The $E$ test ( $A B$ Biodisk, Solna, Sweden) is a newer technique offering technical and veterinary advantages, because the result is expressed as the minimal inhibitory concentration $(\mathrm{MIC}, \mu \mathrm{g} / \mathrm{ml}$ ) rather than sensitive, intermediate or resistant. For concentration dependent antibiotics such as fluoroquinolones, AUC/MIC and Cmax/ MIC ratios have been described as predictive for the clinical outcome and thus valuable indicators in routine veterinary practice (1). Bayer has established with the leading veterinary routine diagnostic laboratories in the Benelux a protocol to use the $E$ test for enrofloxacin (Baytril ${ }^{\circledR}$, Bayer) on all bacteria isolated from routine samples against which Baytril is registered in swine.

\section{Material and Methods}

Between June 2005 and January 2010, the E test for enrofloxacin was carried out routinely on all swine pathogens, isolated by 4 laboratories in the Benelux and falling under the scope of the various marketing authorisations for Baytril in swine. Samples were submitted by practitioners. A bacterial suspension was spread onto Mueller-Hinton plates and the E test for enrofloxacin was put on its surface. Enrofloxacin concentration ranged from 0.002 up to $32 \mu \mathrm{g} / \mathrm{ml}$. After overnight incubation at $37^{\circ} \mathrm{C}$, the MIC was read where the zone of growth inhibition intersected the MIC scale on the strip. The concentration inhibiting growth of at least $90 \%$ (MIC90) of the isolates was calculated.

\section{Results}

In total 2339 swine pathogens examined. The MIC90 value and respective number of strains is as follows: Actinobacillus pleuropneumoniae: $0.094(n=413)$, Bordetella bronchiseptica: 1 ( $n=103)$, Escherichia coli: 1 ( $n=1116)$, Pasteurella/Mannheimia spp.: $0.032(n=475)$, Salmonella spp.: $0.25(n=232)$. A total of 87 isolates with MIC exceeding $2 \mu \mathrm{g} / \mathrm{ml}$ were found (3.7\%). No relevant differences in sensitivity of strains between the participating laboratories were found.

\section{Discussion}

The $E$ test is not often used for routine bacteriology because of the costs of the test. However, routine monitoring of has proven to be feasible under field conditions. It supports swine veterinarians in their treatment schedule: the lower registered dose of 2.5 mg enrofloxacin per $\mathrm{kg}$ bodyweight can still be recommended for infections caused by Actinobacillus pleuropneumoniae and Pasteurella/Mannheimia spp.. The registered higher dose of 5 mg enrofloxacin per $\mathrm{kg}$ bodyweight is recommended in infections caused by Bordetella bronchiseptica, Escherichia coli and Salmonella. Even after more than 20 years of use of Baytril in the Benelux, the registered bacteria remain highly sensitive to enrofloxacin. However, this may change upon the introduction of several cheap generics, because generics have shown to lead to increase in use and, subsequently, the rise of resistance (2).

\section{References}

Meinen J. 1995. Am J Vet Res 56, 1219-1224.

Hellman K. 2005. EMEA/CVMP/SAGAM/184651. 


\title{
P.703
}

\section{Comparison of the efficacy of a florfenicol (Nuflor ${ }^{\oplus}$ ) feed premix with an analogous product in the treatment of Swine Respiratory Disease (SRD)}

\author{
Alberto E. Cevidalli ${ }^{1}$ Ernesto Bongiovanni² Marco Bosetti ${ }^{3}$ \\ 1. Intervet Schering-Plough Animal Health, Peschiera Borromeo, Italy; 2. Practitioner, Casalbellotto, Italy; 3. Practitioner, Soncino, Italy
}

\section{Introduction}

Florfenicol is a broad spectrum antibiotic of the phenicol group. Previous studies have shown it to be highly efficacious in the treatment of SRD, when administered intramuscularly $(1,2,3$,$) .$ The object of this comparative field trial was to evaluate the efficacy of florfenicol administered in feed at a dose rate of $10 \mathrm{mg} /$ $\mathrm{kg}$ body weight for five consecutive days in the treatment of SRD under Italian field conditions.

\section{Materials and Methods}

The trial was carried out on the grower units of two farrow-tofinish herds. If at least $10 \%$ of the pigs in a pen exhibited acute respiratory signs, the pen was included in the trial and randomly allocated to one of the following treatments: florfenicol medicated feed 200 ppm (Nuflor premix Intervet Schering-Plough) or a feed containing a combination of two antibiotics (1400 ppm of sulfadimethoxine and 560 ppm of chlortetracycline). Pens were included independently of each other once the inclusion criteria had been met, and the treatment period was 5 days (days 0-4). The experimental unit was the pen and both populations (clinically ill and exposed) were commingled in each pen.

The parameters on which efficacy was assessed included: rectal temperature, dyspnea, depression and Clinical Illness Index Score (CIIS). The animals were observed for 12 days (Day 0 to Day 11). Success rates, i.e. therapeutic success for the 'ill' population, and the prevention of illness for the 'exposed' population, were recorded on Day 6 and on Day 11.

\section{Results}

Seven hundred and thirty-three pigs were included in the trial on Day 0: 117 were showing acute respiratory signs and 616 belonged to the exposed population. Altogether, 422 pigs were treated with florfenicol-medicated feed and 311 with the control product.

With the exception of prevention in the exposed animals on Day 6 (when the success rates were equal for both groups), the florfenicol-medicated animals always performed better. With respect to the individual parameters (rectal temperature, dyspnea, depression, CIIS), in general, florfenicol always showed better results, in some particulars with statistical significance.

Table 1. Cumulative success rates of the two trial sites

\begin{tabular}{|c|c|c|c|c|}
\hline \multirow[t]{2}{*}{ Group } & \multicolumn{2}{|c|}{ 'Ill' population } & \multicolumn{2}{|c|}{ 'Exposed' population } \\
\hline & $\begin{array}{c}\text { Therapeutic } \\
\text { success } \\
\text { Day } 6\end{array}$ & $\begin{array}{c}\text { Therapentic } \\
\text { success } \\
\text { Day } 11\end{array}$ & $\begin{array}{c}\text { Prevention } \\
\text { success } \\
\text { Day } 6\end{array}$ & $\begin{array}{c}\text { Prevention } \\
\text { success } \\
\text { Day } 11\end{array}$ \\
\hline $\mathrm{FFC}$ & 93 & 87 & 100 & 100 \\
\hline $\begin{array}{c}\text { Sulfa }+ \\
\text { CTC }\end{array}$ & 87 & 73 & 100 & 97 \\
\hline
\end{tabular}

Graph 1. Rectal temp. in clinically ill pigs (on Days 0-5)

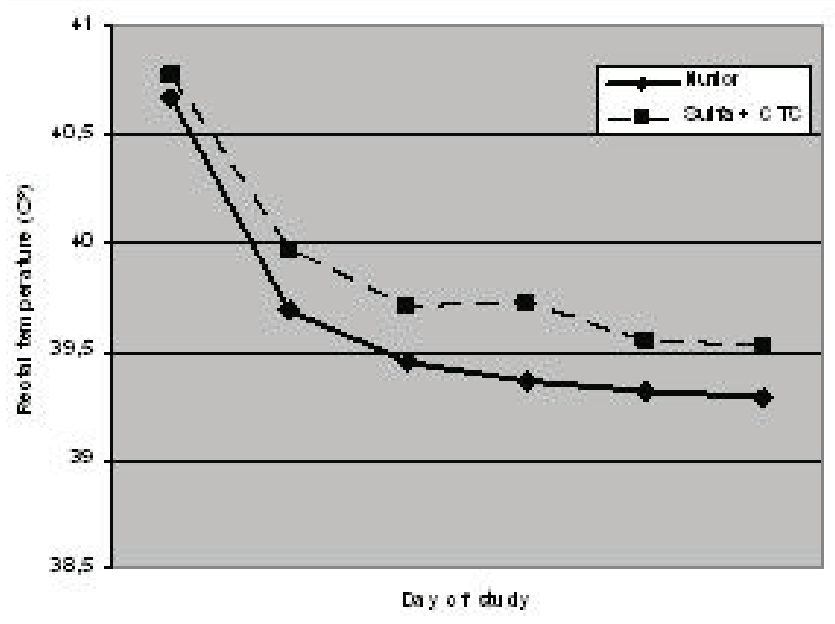

\section{Discussion}

Statistical analysis of the primary end-points (success rates) demonstrated that florfenicol-medicated feed was not inferior to a medicated feed containing high levels of the control product. The analysis of the secondary end-points (clinical parameters) confirms altogether greater efficacy for the florfenicol medication, some of the differences for individual parameters being statistically significant.

In respect of the treatment of ill pigs, the average rectal temperature of the florfenicol-treated group was statistically significantly lower than that of the control group $(p<0.05)$ over the period measured (days 1-5).

\section{References}

1. Glattleider D. et al. (2000) 16th IPVS Proc.: 107.

2. Jackson J.A. et al. (1998) 15th IPVS Proc. vol. 3:187.

3. Terreni M. et al. (2002) 17th IPVS Proc.: 516. 


\title{
Effects on overall production of the treatment of piglets with NUFLOR ${ }^{\oplus}$ PREMIX on a farm with respiratory disease
}

\author{
Manolo Toledo ${ }^{1}$ Rut Menjon ${ }^{2}$ Jesus M. Bollo ${ }^{2}$ Marta Jimenez ${ }^{2}$ \\ 1. Piensos Jimenez S.L.,Lorca, Murcia, Spain; 2. Intervet Schering Plough, Madrid, Spain
}

\section{Introduction}

The aim of the study was to assess the efficacy of the in-feed treatment of piglets with florfenicol to prevent losses caused by swine respiratory disease, during both treatment and subsequent production phases.

Florfenicol (Nuflor ${ }^{\circledast} 40 \mathrm{mg} / \mathrm{g}$ Premix for Swine), a new broadspectrum antimicrobial, is a $3^{\text {rd }}$ generation phenicol, a fluorinated analogue of thiamphenicol obtained by substituting one hydroxyl group with fluorine. A methyl sulfonyl group $\left(\mathrm{CH}_{3}-\mathrm{SO}_{2}\right)$ replaces the p-nitro group $\left(\mathrm{NO}_{2}\right)$ found in chloramphenicol, thus ruling out the risk of aplastic anemia. This also provides a broader spectrum of activity and prevents the chloramphenicol acetyltransferase (CAT) resistance problems of thiamphenicol and chloramphenicol (2).

\section{Materials and Methods}

The trial farm, in a high stock density area of southeast Spain, houses 2,000 sows in three cycles. The farm is positive and unstable for PRRS. Sows are blanket vaccinated four times per year against PRRSv (modified-live vaccine without adjuvant). Replacements are sourced from other units, and all animals entering the farm are vaccinated against PRRSv.

The earliest clinical signs became apparent around the time of weaning. These included an unacceptable percentage of stillborn piglets, a high percentage of runts, non-specific diarrhea, loss of condition, nervous signs, arthritis and an increased mortality rate, all of which failed to respond to treatment with antibiotic (amoxicillin in the drinking water and by injection).

PRRS was confirmed by PCR in 1, 2, 3, 4 and 5 week-old animals. Samples from affected piglets revealed florfenicol-sensitive isolates of Streptococcus suis and Haemophilus parasuis.

The existing in-feed antibiotic treatment (100 ppm tiamulin + 80 ppm colistin in feed for 6 weeks after weaning), a previously tested schedule (1), was changed to $80 \mathrm{ppm}$ florfenicol ( $2 \mathrm{~kg} / \mathrm{Tm}$ Nuflor ${ }^{\oplus} 40 \mathrm{mg} / \mathrm{g}$ Premix for Swine) fed over the same period. The results of the two systems were compared, the previous treatment acting as a control for the florfenicol regime.

\section{Results}

Nursery phase: Mortality rates fell from 3.7\% to 1.8\%. The percentage of runts decreased from $7 \%$ to $2 \%$, and the uniformity of the batches sent to slaughter improved. No differences were found for Average Daily Gain ADG (294 g/day). The economically important results are shown in Table 1. The number and cost of treatments needed to be given via the drinking water or by injection was reduced $(-1.17 €)$.
Table 1: Economic results in the nursery phase

\begin{tabular}{|l|c|c|c|}
\hline Euro /piglet & NUFLOR $^{\odot}$ Premix & Control & Difference \\
\hline Feed & 7.8 & 7.58 & +0.22 \\
\hline $\begin{array}{l}\text { Treatments (water \& } \\
\text { injectable) }\end{array}$ & 1.04 & 2.20 & -1.17 \\
\hline Fixed costs & 3.49 & 3.71 & -0.22 \\
\hline Cost per piglet & 26.34 & 28 & +1.66 \\
\hline TOTAL & 38.67 & 41.48 & $\mathbf{- 2 . 8 2}$ \\
\hline
\end{tabular}

Fattening phase: The mortality rate from historical farm records was 7\%. In the florfenicol-treated group (3600 animals) the mortality rate was reduced by $4.5 \%$. The feed conversion efficiency improved from 2.79 to 2.73 , and the ADG increased from 638 to $700 \mathrm{~g} /$ day. The economically important results are shown in Table 2.

Table 2: Economic results in the fattening phase

\begin{tabular}{|l|c|c|c|}
\hline Euro/piglet & NUFLOR ${ }^{\circledR}$ Premix & Control & Difference \\
\hline Feed & 61.93 & 65.02 & -3.09 \\
\hline $\begin{array}{l}\text { Treatments (water \& } \\
\text { injectable) }\end{array}$ & 1.7 & 2.18 & -0.48 \\
\hline Fixed costs & 14.09 & 15.91 & -1.82 \\
\hline Cost per piglet & 40.99 & 42.15 & -1.16 \\
\hline TOTAL & 118.71 & 125.26 & -6.55 \\
\hline
\end{tabular}

The total saving for the group treated with Nuflor ${ }^{\circledR} 40 \mathrm{mg} / \mathrm{g}$ Premix for Swine, over the course of the whole production cycle, was $9.37 €$ per pig.

\section{Discussion}

Piglets are susceptible to respiratory and digestive conditions in those production pyramids in which PRRSv has led to immune destabilization. This and severe secondary bacterial complications badly affect production.

This trial demonstrated that the use of a broad-spectrum antibiotic such as Nuflor ${ }^{\circledR} 40 \mathrm{mg} / \mathrm{g}$ Premix for Swine, to which the most important respiratory bacteria are highly sensitive, is an effective solution. Both health and productivity improved thoughout the production cycle, resulting in considerable economic benefits.

\section{References \\ 1. Terreni, M. (2002) Proc.17 th IPVS Congress \\ 2. Berro,R.(2004) Proc 18 th IPVS Congress}




\title{
P.705
}

\section{Usage of homeopathy as treatment for several disorders in pigs in an organic production system}

\author{
Roberto G. Martinez; Marisol Esquivel; Enedina Silva; Jorge R. Lopez; Mario E. Haro; Roberto Martinez-Rodriguez \\ Facultad de Medicina Veterinaria y Zootecnia. Universidad nacional Autonoma de Mexico, Mexico, DF, Mexico
}

\section{Introduction}

The necessity of healthy food creates different systems of production; such as the case of organic production. Homeopathy medicine is ideal for this type of production owing to it is a medicine treatment that respects the natural reactions of the organism, and it is considered as a safe therapy par excellence. This is based on "The principle of Similarity", and in order to this principle works it is necessary to have the infinitesimal doses which are: The substances which in considerable doses are able to provoke in healthy individuals clinical manifestations, they are able to eliminate such manifestations in an unhealthy individual if they are prescribed in small doses.

\section{Material and Methods}

This research was carried out in a farm localized $100 \mathrm{~km}$ northwest Mexico City, in an area which was adapted for the hosting sows in an organic production system. In this area were 15 breeding sows with different farrowing number and once farrow the piglets. All the animal were examined twice a day in order to identified any health problem and be able to implement a specific treatment. The following diagnostics and treatments were carried out:

- Sows underwent a change of management and grouping, with treatment based on Aconitum napellus and Carbo vegetailis 30 centasimal (c), administered twice a day, by 18 days.

- Abscesess in teats, in shoulder and in ham of an adult sow. Treatement Silicea terra 30c (three times per day) and Silicea terra 200c (every three days) from 5 to 20 days.

- Banged sows. Arnica Montana 30c (twice a day) per 18 days.

- Sow with severe burn in one foot. Belladona atropa 30c (twice a day) Cantharis 6x (topic) once a day.

- Obstetrical problems, retention of piglets, yellow vaginal secretion with bad smell and almost non appetite. Caulophyllum 30c, Belladona atropa 30c and Arnica Montana 6c (every 2 hours and decreasing the frequency until twice a day).

- Respiratory disorders in piglets froms 3 to 15 day of age and in one adult sow. Aconitum napellus and Belladona atropa $30 \mathrm{c}$ (three times a day per 4 days).

- Suckled piglets from 3 to 20 days, having yellow diarrhea. Arsenicum album 30c (three times a day, form 2 to 3 days).

- Castration injuries, Dye madre de caléndula once a day per 2 days.

- Prevention of anemia ferropriva. Ferrum metalicum 6c (twice a day).

\section{Results}

In connection with the banged and under stress sows after the weaning there were a reaction in all of them (15 animals). Talking about the abscess it was a reaction in 2 out of 3 sows (66.6\%). The burnt sow treated with Belladona and Cantharis responded favorably. All of the sows with postpartum disorders responded favorably (100\%). The combination of Acotinum and Belladona had a response of respiratory disorders in 34 out of 40 piglets (84\%). Arsenicum alba had favorable response in suckled piglets with diarrhea, 14 out of 15 piglets (93\%). None of the piglets had anemia.

\section{Discussion}

The results of this research carried out with the objective, they proved that it is possible to use homeopathy as a treatment of individual disorders in animals in an organic raising system. At the same time it is an alternative to the application of prophylactic treatments in biggest populations of pigs. The implementation of the treatment did not show any problem with management or containment of the animal.

\section{References}

1. Enedina S. Primera edición Propulsora de Homeopatía S.A de C.V. México D.F. 2008: 31.

2. Flavio B. La Homeopatía, Madrid: Dilema, 2006:29,31.

3. León V. 19 edición Porrúa S.A de C.V. México D.F.: 2008. pp 522.

Research financed by PAPIIT IN212008. 


\title{
P.706
}

\section{Efficacy of Denagard $20^{\circledR} \%$ Injection for Treatment of Polyarthritis in Nursery}

\author{
Sasiwimon Talummuk; Penchan Chaiyanate; Metta Makhanon \\ Novartis (Thailand) Limited, Bangkok, Thailand
}

\section{Introduction}

Polyarthritis in suckling pig to nursery pig are very common clinical signs caused by pathogens including Mycoplasma hyorhinis (MHR), Haemophilus parasuis, and Streptococcus suis. These respiratory pathogens are transmitted from sows to piglets by direct contact. Infected piglets can be carriers after weaning (4). Early treatment by injection is the state-of-the art method for effective control of polyarthritis caused by the listed pathogens. Tiamulin provides pronounced effect for treatment of Mycoplasma and Gram positive bacteria like Streptococcus suis due to MIC studies $(2,3)$. However, there are many sources of Tiamulin injection in Thailand. The objective of this study is to compare the efficacy of Denagard ${ }^{\circledR} 20 \%$ injection, original Novartis-Sandoz, and the local generic tiamulin injection for treatment of polyarthritis in nursery (1).

\section{Materials and Methods}

Farm history: A farrow to fattening farm was affected by serious polyarthritis problems, $20-30 \%$ mobility rate and 5-10\% motality rate from suckling piglets to nursery (Fig.1). Preventive program was water treatment with amoxicillin after weaning for 5-7 days. Pigs show minor response.

20 pigs with severe swollen joints and respiratory signs were selected and divided into two groups. Group 1 was treated by Denagard ${ }^{\circledR 2} 2 \%$ injection and Group 2 was treated by generic tiamulin injection for three days. Nasal swabs were collected from all pigs before onset of treatment to confirm mycoplasma infection. Joint diameter of four legs was measured by verneir clipper before the first tiamulin injection (day 1), the third injection (day 3) and two days after withdrawal of treatment (day 5). $\mathrm{pH}$-value of the two different tiamulin injection products was measured. Nursery signs of pain after injection was observed. Statistical analysis of the joint diameter was done by ANOVA at $p<0.05$.

\section{Fig. 1}

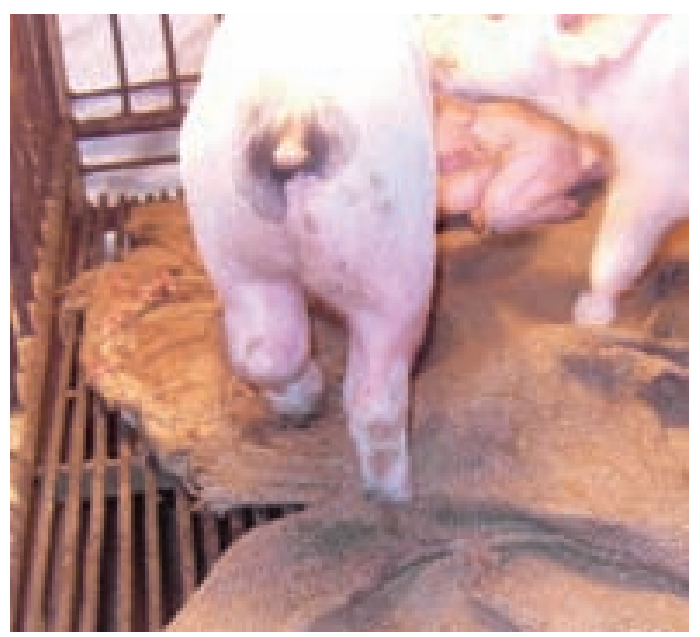

\section{Results}

MHR was isolated from all nasal swab samples. $\mathrm{pH}$ of Denagard ${ }^{\circledR}$ $20 \%$ injection is 8.5 while the generic tiamulin is 22 . Some of the pigs receiving tiamulin generic injection showed sign of pain.. Comparison of the mean joint diameter is summarized in Table 1.

Table 1 Mean Diameter of joint (inch) before, during and after the treatment.

\begin{tabular}{|l|c|c|c|}
\hline Legs & Observed day & \multicolumn{2}{|c|}{ Mean Joint Diameter (inch) } \\
\hline & & Denagard 20\% & Tiamulin generic \\
\hline Right fore leg & Day 1 & $2.73+/-0.33$ & $2.83+/-0.23$ \\
\hline & Day 3 & $2.53+/-0.21^{\text {a }}$ & $2.82+/-0.14^{\mathrm{b}}$ \\
\hline & Day 5 & $2.56+/-0.25$ & $2.78+/-0.20$ \\
\hline Left fore leg & Day 1 & $2.76+/-0.39$ & $2.91+/-0.20$ \\
\hline & Day 3 & $2.70+/-0.34$ & $2.86+/-0.14$ \\
\hline & Day 5 & $2.50+/-0.25^{\mathrm{a}}$ & $2.86+/-0.17^{\mathrm{b}}$ \\
\hline Right hide leg & Day1 & $3.21+/-0.56$ & $3.40+/-0.33$ \\
\hline & Day3 & $2.96+/-0.41$ & $3.24+/-0.18$ \\
\hline & Day5 & $2.90+/-0.38^{\mathrm{a}}$ & $3.30+/-0.17^{\mathrm{b}}$ \\
\hline & Day1 & $3.41+/-0.70$ & $3.33+/-0.25$ \\
\hline & Day3 & $3.12+/-0.59$ & $3.20+/-0.13$ \\
\hline & Day5 & $3.10+/-0.69$ & $3.26+/-0.20$ \\
\hline ab p $<0.05$ & \multicolumn{3}{|l}{} \\
\hline
\end{tabular}

\section{Discussion}

The mean joint diameter of Denagard ${ }^{\circledR} 20 \%$ injection treatment group show a gradual decrease from the first to the last day of evaluation. Significant differences of joint diameter were found from three legs on day 3 and day 5. Interestingly, MHR carriers status can be detected in all pigs from nasal swabs. The acidic $\mathrm{pH}$ in generic formulation and kind of active ingredient used in the generic products can influence the performance and efficacy and cause pain in sick nursery pigs at the site of injection. From this study it can be concluded that based on the higher product performance Denagard ${ }^{\circledR} 20 \%$ Injectable application causes significant reduction of swollen joints in nursery pigs suffered from polyarthritis. Its significant efficacy is showed during three to five days after treatment.

\section{References}

1. Hunt, E. 2000. Pleuromutilins antibiotics. Drugs of the Future. 25(11):1163-1168.

2. Metta, et al. 2009. Proceeding of the 4 th Congress of Asian Pig Veterinary Society. P-008. 410.

3. Nuvee et al. 2009. Proceeding of the 4th Congress of Asian Pig Veterinary Society. P-013. 405.

4. Sibila et al., 2009. Current Perspective on the Diagnosis and Epidemiology of Mycoplasma hyopneumoniae infection. Vet. J. 181:221-231. 


\title{
P.707
}

\section{Effect of Polyfil ${ }^{\mathrm{TM}}$ (8\% Phosphorylcholamine and Vitamin B ${ }_{1,}$ A and E) Injection on Blood Composition and Chemistry of Gilts}

\author{
Chanathip Thammakarn ${ }^{1}$ Jamlong Mitchaothai ${ }^{2}$ \\ 1. School of Animal Production Technology and Fisheries, Faculty of Agricultural Technology, King Mongkut's \\ Institute of Technology Ladkrabang, Ladkrabang, Bangkok, Thailand; 2. Department of Clinic for Swine, Faculty \\ of Veterinary Medicine, Mahanakorn University of Technology, Nong Chok, Bangkok, Thailand
}

\section{Introduction}

Supportive treatments to weak or sick pigs with efficient and feasible methods would lead to minimize the low pig production. In principle, phosphorylcholamine (PC) is a phosphomonoester playing important role in membrane biosynthesis (1). Vitamin A, E and $B_{12}$ are crucial vitamins for the metabolisms of pigs. Hence, this study was performed in order to evaluate the effect of administration of $P C$, vitamin $A, E$ and $B_{12}$ (Polyfil $^{\mathrm{TM}}$ ) on blood composition and chemistry of gilts.

\section{Materials and Methods}

Eighteen gilts were randomly selected for the present study. All experimental pigs were equally allotted into two groups; the control and the treatment. The pigs in the treatment group have got injection of Polyfil ${ }^{\mathrm{TM}}$ (Laboratorios Ovejero, S.A., Spain) $5 \mathrm{ml}$ once a week for 3 times weekly, while the pigs in the control group did not get any administration. Blood samples were collected from all pigs in both experimental groups at before the start of this study and at 3 days after each Polyfil ${ }^{\mathrm{TM}}$ injection. All blood samples were analyzed for blood composition (erythrocyte concentration \& indices and leukocyte concentration \& proportion), thrombocyte concentration and blood chemistry (SGOT, SGPT, BUN, creatinine, calcium, phosphorus and total protein). The statistical analysis is Repeated Measurements.

\section{Results}

The results of the present study have been showed in the Table 1. There was no effect of the administration of Polyfi ${ }^{\mathrm{TM}}$ on erythrocyte concentration and indices $(\mathrm{HB}, \mathrm{MCV}, \mathrm{MCH}$ and $\mathrm{MCHC}$ ) and leukocyte concentration and proportion of leukocytes. The injection of Polyfil ${ }^{\mathrm{TM}}$ significantly increase $(p<0.05)$ the concentration of blood phosphorus and significantly lower $(p<0.05)$ the level of BUN. However, there was no effect of the Polyfil ${ }^{\mathrm{TM}}$ administration on other studied blood chemistry (SGOT, SGPT, Creatinine, Calcium, Total protein).

\section{Discussion}

Most of measured valued fell in the normal rages (2). The composition of blood and erythrocyte indices were not affected by administration of Polyfil ${ }^{\mathrm{TM}}$, this might be explained that the ingredients in Polyfil ${ }^{\mathrm{TM}}$ improve only composition of cell membrane. The increase of phosphorus in blood would be the results of PC containing in Polyfil ${ }^{\mathrm{TM}}$, which directly increase in blood stream or more incorporation into blood cells. The lower BUN of gilts in the treatment group indicated lower urea production, which might be the results of more utilization of amino acids. Therefore, the administration of Polyfil ${ }^{\mathrm{TM}}$ in gilts increased blood phosphorus and lowers BUN.
Table 1 Blood composition and chemistry of all pigs

\begin{tabular}{|c|c|c|c|c|c|c|}
\hline \multirow{2}{*}{ Item } & \multirow{2}{*}{ Group } & \multicolumn{4}{|c|}{ Week } & \multirow{2}{*}{$\begin{array}{c}\text { Signifi- } \\
\text { cance- }\end{array}$} \\
\hline & & 0 & 1 & 2 & 3 & \\
\hline \multirow[t]{2}{*}{$\mathrm{RBC}, \times 10^{3} / \mu \mathrm{l}$} & Cont & 7.10 & 7.39 & 7.50 & 6.91 & NS \\
\hline & Treat & 6.89 & 8.00 & 7.35 & 7.59 & \\
\hline \multirow[t]{2}{*}{$\mathrm{HB}, \mathrm{g} / \mathrm{dl}$} & Cont & 13.33 & 12.93 & 15.36 & 13.76 & NS \\
\hline & Treat & 12.61 & 13.32 & 15.19 & 15.70 & \\
\hline \multirow[t]{2}{*}{$\mathrm{MCV}, \mathrm{fl}$} & Cont & 59.28 & 54.37 & 59.41 & 63.08 & NS \\
\hline & Treat & 61.57 & 51.22 & 59.23 & 65.13 & \\
\hline \multirow[t]{2}{*}{ MCH, pg } & Cont & 18.86 & 17.54 & 20.54 & 20.06 & NS \\
\hline & Treat & 18.42 & 16.58 & 20.64 & 20.71 & \\
\hline \multirow[t]{2}{*}{ MCHC, \% } & Cont & 30.07 & 32.29 & 34.58 & 31.19 & NS \\
\hline & Treat & 29.97 & 32.41 & 34.84 & 31.63 & \\
\hline \multirow[t]{2}{*}{$\mathrm{WBC}, \times 10^{3} / \mu \mathrm{l}$} & Cont & 15.48 & 12.18 & 11.24 & 11.59 & NS \\
\hline & Treat & 12.16 & 12.11 & 14.35 & 14.56 & \\
\hline \multirow[t]{2}{*}{ Neutrophil, \% } & Cont & 27.00 & 32.56 & 39.56 & 38.38 & NS \\
\hline & Treat & 23.44 & 29.22 & 40.13 & 48.50 & \\
\hline \multirow[t]{2}{*}{ Lymphocyte, $\%$} & Cont & 62.44 & 59.22 & 54.22 & 56.75 & NS \\
\hline & Treat & 68.71 & 62.89 & 55.00 & 48.88 & \\
\hline \multirow[t]{2}{*}{ Platelet, $\times 10^{4} / \mu \mathrm{l}$} & Cont & 10.58 & 11.00 & 16.99 & 20.48 & NS \\
\hline & Treat & 6.62 & 11.40 & 19.08 & 21.21 & \\
\hline \multirow[t]{2}{*}{ SGOT,IU/I } & Cont & 38.31 & 34.28 & 28.02 & 33.80 & NS \\
\hline & Treat & 50.13 & 35.35 & 28.45 & 47.22 & \\
\hline \multirow[t]{2}{*}{ SGPT,IU/I } & Cont & 48.50 & 47.10 & 42.68 & 45.26 & NS \\
\hline & Treat & 47.63 & 45.68 & 43.52 & 46.27 & \\
\hline \multirow[t]{2}{*}{ BUN, $\mathrm{mg} / \mathrm{dl}$} & Cont & 20.92 & 19.48 & 16.50 & 12.97 & $p=0.015$ \\
\hline & Treat & 18.18 & 16.57 & 13.75 & 10.25 & \\
\hline \multirow[t]{2}{*}{ Creatinine, mg/dl } & Cont & 2.63 & 2.76 & 2.77 & 2.84 & NS \\
\hline & Treat & 2.75 & 2.72 & 2.85 & 2.73 & \\
\hline \multirow[t]{2}{*}{ Calcium, mg/dl } & Cont & 7.98 & 9.14 & 10.17 & 7.42 & NS \\
\hline & Treat & 8.55 & 9.47 & 10.48 & 6.70 & \\
\hline \multirow[t]{2}{*}{ Phosphorus, mg/dl } & Cont & 7.64 & 7.71 & 7.20 & 7.30 & $p=0.034$ \\
\hline & Treat & 9.02 & 7.90 & 7.80 & 7.52 & \\
\hline \multirow[t]{2}{*}{ Total protein, g/dl } & Cont & 8.24 & 8.11 & 7.59 & 7.56 & NS \\
\hline & Treat & 8.65 & 8.55 & 7.95 & 7.83 & \\
\hline \multicolumn{7}{|c|}{ Cont $=$ Control group, Treat $=$ Treatment group, NS $=$ Not significance } \\
\hline
\end{tabular}

\section{Acknowledgements}

The authors wish to thank Union Agriphar Co., Ltd.

\section{References}

1. Klunk, W.E. et al. 1995. Life Sci. 56: 2377-2383.

2. Aiello, S.E. 1998. The Merck Veterinary Manual 


\title{
P.708
}

\section{Susceptibility of Streptococcus suis strains isolated from diseased pigs in Thai PRRSV positive swine farms to Ceftiofur}

\author{
Angkana Tantituvanont $^{2}$ Walaisiri Muangsiri ${ }^{2}$ Waree Niyomthum ${ }^{1}$ Thitima Tripipat $^{1}$ Indhira Kramongthong $^{1}$ Dachrit Nilubol $^{1}$ \\ 1. Faculty of Veterinary Science, Chulalongkorn University, Bangkok, Thailand; 2. Faculty of \\ Pharmaceutical Sciences, Chulalongkorn University, Bangkok, Thailand
}

\section{Introduction}

Ceftiofur, a broad-spectrum cephalosporin is approved for the treatment of swine respiratory disease including Streptococcus suis (S. suis). Although Ceftiofur effectively reduced mortality associated with PRRSV and S. suis 1, several field investigations in Thailand have reported varying degree of success when ceftiofur is used to control S. suis infection in PRRSV infected pigs. Unsuccessful control in PRRSV infected herds could be due to increased antimicrobial resistance, decreased pharmacokinetic in PRRSV infected pigs 2 and etc. Although the in vitro antimicrobial susceptibility of S. suis has been widely investigated, ceftiofur susceptibility of S. suis isolated from PRRSV positive swine herds has not been reported. The objective of this study was to investigate the Minimum Inhibitory Concentrations (MICs) of ceftiofur againt $\mathrm{S}$. suis isolated from Thai PRRSV positive swine farms.

\section{Materials and Methods}

10 PRRSV positive swine herds located in the Eastern, Western, Northern and Southern regions of Thailand were recruited into the study. The PRRSV status of All 10 herds was considered PRRSV stable/ inactive, which PRRSV viremic phase and clinical diseases displayed during nursery period. Seroconversion against PRRSV as measured by ELISA (Idexx, USA) was 6 - 8 weeks of age. Necropsy examination was performed monthly for 3 consecutive months on pigs displaying clinical diseases associated with PRRSV and S. suis including respiratory distress, swollen joints and convulsion. Lung, tonsil and joint fluid samples were subjected for $\mathrm{S}$. suis isolation.

Bacterial (S. suis) isolation was performed on sheep blood agar. Suspected colonies were separated by colonical morphology and confirmed by biochemical characteristics including API20 and Polymerase Chain Reaction (PCR). Ceftiofur susceptibility testing was performed using an agar dilution technique according to the standardized method described by Clinical and Laboratory Standard Institute (CLSI)3.

\section{Results}

A total of 119 Streptococcus spp. isolates were recovered from 86 diseased pigs. The biochemical characteristics and PCR assay demonstrated that out of 119 Streptococcus spp. isolates, only 17 isolates were S. suis and 2 of 17 were S. suis type 2. MICs values ranged from $0.125-256 \mu \mathrm{g} / \mathrm{mL}$ (Table 1). Considered MICs values of Ceftiofur be $\leq 0.03 \mu \mathrm{g} / \mathrm{mL}$, most of the $S$. suis isolates demonstrated a high degree of ceftiofur resistance. A low degree of resistance was found in only 2 herds.

\begin{tabular}{|l|c|c|}
\hline $\begin{array}{l}\text { No. of } \\
\text { S. suis isolates }\end{array}$ & Regions & MIC $(\mu \mathrm{g} / \mathbf{m L})$ \\
\hline 1 & West & $>256$ \\
\hline 2 & West & $>256$ \\
\hline 3 & West & $>256$ \\
\hline 4 & West & $>256$ \\
\hline 5 & West & $>256$ \\
\hline 6 & West & 32 \\
\hline 7 & West & 16 \\
\hline 8 & West & 256 \\
\hline 9 & West & 2 \\
\hline 10 & West & $>256$ \\
\hline 11 & West & 4 \\
\hline 12 & West & 16 \\
\hline 13 & West & $>256$ \\
\hline 14 & West & 0.125 \\
\hline 15 & West & 256 \\
\hline 16 & West & 4 \\
\hline 17 & South & 0.125 \\
\hline 18 & suis NCTC10234 & 4 \\
\hline
\end{tabular}

\section{*S. suis NCTC 10234: Standard Streptococcus suis capsular type 2}

\section{Discussion}

The results of this study indicated an increased level of ceftiofur resistance of S. suis isolated from Thai PRRSV positive herds especially herds located in high proximity area and high level of antimicrobial usage. Factors associated with increased ceftiofur resistance in PRRSV positive herds should be investigated. In addition, the ceftiofur pharmacokinetics-pharmacodynamics data and its clinical outcomes should be used to justify the optimum therapeutic regimen.

\section{References}

1. Schmitt, C.A. 2001 Vet Microl: $29-37$

2. Tuntituvanont, A. 2009 JAC: $369-373$

3. NCCLS, 1999 


\title{
P.709
}

\section{Pharmacokinetics study of medicated feed containing chlortetracycline (Aurofac ${ }^{\circledR}$ ) with or without $\mathrm{BMD}^{\circledR}$ in concurrent PRRSV and Mycoplasma hyopneumoniae - infected pigs}

\author{
Dachrit Nilubol ${ }^{1}$ Indhira Kramongthong ${ }^{1}$ Thitima Tripipat $^{1}$ Mark Eastaugh ${ }^{4}$ Ratchai Leethochawalit $^{3}$ Angkana Tantituvanont $^{2}$ \\ 1. Faculty of Veterinary Science, Chulalongkorn University, Bangkok, Thailand; 2. Faculty of Pharmaceutical \\ Sciences, Chulalongkorn University, Bangkok, Thailand; 3. Alpharma Pharmaceuticals (Thailand) Ltd., \\ Bangkok, Thailand; 4. Alpharma Animal Health (Beijing) Trading Co, Ltd, Singapore, Singapore
}

\section{Introduction}

Several investigators have reported a synergistic effect for BMD and $C T C$ resulting in decreased resistance of several pathogens $(1,2)$. Our previous studies suggest that the use of CTC in combination with BMD at the day of infection results in the significant reduction of lung lesion associated with Mycoplasma hyopneumoniae induced pneumonia3. The objective of the study was to investigate the pharmacokinetics of Chlortetracycline when fed in combination with BMD in pigs co-infected with PRRSV and Mycoplasma hyopneumoniae.

\section{Materials and Methods}

Ten 3 to 4 week-old PRRSV and Mycoplasma hyopneumoniae negative pigs were randomly assigned with stratification by weight to 2 groups ( $A$ and $B$ ) of 5 pigs each. Pigs in both groups were challenged with both PRRSV and $M$. hyopneumoniae at 0 days post infection (DPI). While PRRSV was administered to pigs intranasally, M. hyopneumoniae was given to pigs intratracheally. While group A received medicated feed containing chlortetracycline (Aurofac ${ }^{\circledast}$, Alpharma Animal Health, Thailand) at dosage $20 \mathrm{mg} / \mathrm{kg} \mathrm{BW}$ and (BMD ${ }^{\circledR}$, Alpharma Animal Health, Thailand) at dosage 33 ppm, group $B$ received medicated feed containing only chlortetracycline. All medicated groups received medicated feed from 0 through 14 DPI (14-day total).

The pigs were gruel-fed twice a day at 600 and $1200 \mathrm{hrs}$. The dosages of CTC and BMD given to the pigs were $20 \mathrm{mg} / \mathrm{kg} \mathrm{BW}$ per day and 33 ppm per kilogram of feed intake per day, respectively. The total amount of the drugs administered per day was calculated and separated into half to provide the pigs twice a day with gruel feed.

On 3 and 7 DPI, blood was serially collected from the same pigs at $0,1,2,4,6,8,12,16$, and 24 hours post medication (starting at $600 \mathrm{hrs}$ and finishing at $600 \mathrm{hrs}$ next day) into heparinized tubes. Plasma was separated and stored in $80^{\circ} \mathrm{C}$ until analysis. All plasma samples were determine the concentration of CTC. The plasma concentrations of CTC were analyzed using a validated high-performance liquid chromatography assay. Plasma concentration data of CTC were subjected to non-compartmental analysis based on statistical moment theory. All pharmacokinetic calculations were performed using linear modeling software (WinNonlin Version 3.2, Pharsight, California, USA).

\section{Results}

Pigs administered with the combination of CTC and BMD showed similar plasma drug concentration to pigs administered with CTC alone on 3 and 7 DPI. Although the average plasma CTC concentration of pigs administered with the combination of CTC and BMD was higher than that of pigs administered with CTC alone, the difference was not statistically significant. The average plasma chlortetracycline concentration of infected pigs was similar to that of non-infected pigs. There was no significant difference in $\mathrm{Cmax}, \mathrm{AUC}, \mathrm{t} 1 / 2 \mathrm{z}$ and $1 \mathrm{z}$ between the two treatment groups ( $p>0.05$ ). No significant differences in pharmacokinetic parameters were observed in pigs administered with either chlortetracycline alone or in combination with BMD ( $p$ $>0.05$ ) on 3 and 7 DPI. However, longer half-life was observed in the non-infected pigs administered with a mixture of CTC and BMD (26.1 h) compared to CTC alone (17.6 h).

\section{Conclusion}

The results showed that administering BMD in combination with chlortetracycline had slightly influence on chlortetracycline absorption. BMD showed to affect the elimination of chlortetracycline in the non-infected pigs, although the half-life values were not significantly different between pigs given CTC alone or in combination with BMD.

\author{
References \\ 1. Winkelman, N. 2001 AASV; 77- 82. \\ 2. Winkelman N. 2003 AASV; 175-179. \\ 3. Nilubol, D. 2008 IPVS
}




\title{
P.710
}

\section{Evaluation of the Pulmotil ${ }^{\otimes}$ premix effect on production parameters of sows and their litters in a commercial farm on the sisal zone in Yucatan, Mexico}

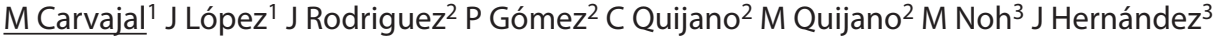 \\ 1. Elanco ${ }^{\circledR}$ Animal Health, León, GTO, Mexico; 1. Facultad de Medicina Veterinaria y Zootecnia Universidad \\ Autonoma de Yucatan, Merida, YUC, Mexico; 3. Private practice, Merida, YUC, Mexico
}

\section{Introduction}

Previous studies have shown that the use of antibiotics in sows during the lactation diets reduces problems associated with infections peripartum and improves reproductive behavior. It reduces the transmission of pathogens of the sows to the piglets improving the health of these (1). This practice increases the survival of piglets and the produced kg per week at weaning and the finishing (2).

The objective of this study was to assess the effect of the use of Pulmotil $^{\circledast}$ (Tilmicosin) in females during the stage of lactation on their litters productive parameters.

\section{Materials and Methods}

A study was conducted in a pig farm with 900 sows in the state of Yucatan, México. Animals were positive to diseases related to respiratory and gastro enteric signs. The total of born piglets from six groups of sows was studied. Each group was formed with 40 sows. The groups were divided according to treatment: 3 groups received $300 \mathrm{~g}$ of Tilmicosin $\left(\right.$ Pulmotil $\left.^{\circledR}\right)+200 \mathrm{~g}$ sulfamethoxazole and $50 \mathrm{~g}$ of Trimethoprin/ton of food. Other 3 groups received a premix based on $400 \mathrm{~g}$ of Oxytetracycline + $200 \mathrm{~g}$ of sulfamethoxazole and $50 \mathrm{~g}$ of Trimethoprin/ton (Other). Both treatments were managed in the diet a week before birth and during lactation. The piglets were individually identified and compared the weight to the birth, weaning, daily gain, and mortality. Data were analyzed in computing programs: SPSS 15.0 and WinEpiscope 2.0.

\section{Results and Discussion}

The average birth weights of piglets from sows of both treatments were compared. There was no significant difference $(p=0.144)$. The mortality rate of piglets of Pulmotil ${ }^{\circledR}$ group and the other group was $2.9 \%$ y $3.4 \%$ respectively. Although there is epidemiologic association between the treatment and mortality rate $(\mathrm{OR}=0.78)$, there was not significant differences because the confidence interval include the number one $(I C=0.49<0 R<1.26)$.

Table 1. Comparison of birth weight and weaning of pigs of both groups

\begin{tabular}{|c|c|c|c|c|c|c|}
\hline & Treatment & N & $\begin{array}{c}\text { Mean } \\
(\mathrm{kg})\end{array}$ & $\begin{array}{c}\text { Estandar } \\
\text { desviation }\end{array}$ & $\begin{array}{c}\text { Coeficient } \\
\text { variation }\end{array}$ & $P$ value \\
\hline \multirow[t]{2}{*}{ Birth } & Pulmotil $^{\infty}$ & 1229 & 1.420 & 0.322 & $22.68 \%$ & \\
\hline & Other & 1092 & 1.440 & 0.352 & $24.88 \%$ & 0.144 \\
\hline \multirow[t]{2}{*}{ Weaning } & Pulmotil $^{\oplus}$ & 1147 & 6.866 & 1.383 & $20.14 \%$ & \\
\hline & Other & 1022 & 6.242 & 1.508 & $24.17 \%$ & 0.000 \\
\hline
\end{tabular}

The average weaning weights of piglets were compared and there was a highly significant difference $(p=0.000)$. The average days of lactation of sows were 20.70 day for Pulmotil ${ }^{\circledR}$ group and 19.98 days for the other group (table 2).

Table 2. Comparison of gain weight of pigs during lactation

\begin{tabular}{|l|c|c|c|c|c|}
\hline Treatment & $\mathbf{N}$ & $\begin{array}{c}\text { Weight gain in } \\
\text { the period }\end{array}$ & $\begin{array}{c}\text { Average daily } \\
\text { gain }\end{array}$ & Lactation days & P value \\
\hline Pulmotil $^{\odot}$ & 1147 & 5.436 & 0.262 & 0.262 & \\
\hline Other & 1022 & 4.790 & 0.239 & 0.239 & 0.000 \\
\hline
\end{tabular}

The weight at 6 weeks of age was compared observing a better performance in the Pulmotil ${ }^{\circledR}$ group $(9.61 \mathrm{~kg})$.in contrast to the other group $(8.37 \mathrm{~kg})$.These weight were fitted to 39.9 days of age and $1.3 \mathrm{~kg}$ of weight birth; $p=0.000$. The mortality rate to 6 week age was lower in the Pulmotil ${ }^{\circledR}$ group although there was not a significant difference.

Table 3. Comparison of weight gain of pigs during growing period.

\begin{tabular}{|l|c|c|c|c|}
\hline Treatment & $\begin{array}{c}\text { Weight average at } \\
\text { sold (6 weeks) }\end{array}$ & $\begin{array}{c}\text { Weights average } \\
\text { at 10 weeks }\end{array}$ & $\begin{array}{c}\text { Daily weight gain } \\
\text { (born to 40 days) }\end{array}$ & P value \\
\hline Pulmotil $^{\oplus}$ & $9.615^{*}$ & $30.85^{* *}$ & $208 \mathrm{gr}$ & \\
\hline Other & $8.370^{*}$ & $30.40^{* *}$ & $175 \mathrm{gr}$ & $<0.05$ \\
\hline
\end{tabular}

* Weight at sold ( 6 weeks) fitted to 39.9 days and considering $1.3 \mathrm{~kg}$ at birth

** Average weight fitted to 74 days considering $1.4 \mathrm{~kg}$ of birth weight.

\section{Conclusion}

It can be concluded that addition of Pulmotil ${ }^{\circledR}$ to the diet of the sows prior and during lactation period had a significant effect in the performance of their litters during lactation and weaning periods. As consequence a better performance is expected in the growing and finishing periods.

\section{References}

1. Alexander,T.I, et al. (1980). Vet. Rec. 106, 114-119.

2. Almond, G.W. et al. (2006). 19th IPVS Congress No: P 34-25.

3. Lehe, K. et al. (2008). Proc. AASV. Pp 229-232 


\title{
P.711
}

\section{Effects of dietary supplementation with a blend of essential oils on growth performance of weaned pigs}

\author{
Tobias Steiner ${ }^{1}$ Edouard Charlemagne ${ }^{2}$ Gaëlle Pichard ${ }^{3}$ Christian Tenier $^{3}$ Diego Padoan $^{1}$ \\ 1. Biomin GmbH, Herzogenburg, Austria; 2. Primex S.A.S., Languidic, France; 3. Biomin France, Ploufragan, France
}

\section{Introduction}

Essential oils (EO) have been gaining growing attention in livestock feeding in the last few years. They are incorporated in animal feed to improve productivity of livestock through amelioration of feed properties and promotion of the animal's production performance. The in vitro antimicrobial, antiviral, antifungal and antioxidant activities of EO are well described and backed up by numerous scientific reports $(1,2)$. In the meanwhile, an increasing number of studies addressing the gastro-intestinal effects of EO under in vivo conditions are available. The intestinal microflora, gut morphology, gastric emptying, activity of endogenous digestive secretions and performance parameters are considered to be influenced by dietary EO (3). In studies with weaned piglets inclusion of the blend of EO investigated herein improved weight gain and feed conversion in comparison with pigs fed negative control diets or diets supplemented with antibiotic growth promoters such as avilamycin, neomycin sulphate and oxytetracycline $(4,5)$. The present study was carried out to determine the effects of a blend of oregano, anis and citrus EO and fructo-oligosaccharides (Biomin ${ }^{\circledR}$ P.E.P. 125) on performance of weaned piglets.

\section{Materials and Methods}

The trial was carried out in two consecutive periods with 168 piglets (Adenia * Pietrain) in total at the experimental station of Primex S.A.S., Languidic, France. Piglets were 42 days old at the beginning of the experiment. In each period piglets were equally assigned to 14 floor pens. There were six piglets in each pen, resulting in 42 piglets per treatment. The treatments were: 1: Control and 2: Supplementation with EO. Piglets in the Control Treatment 1 were fed a starter diet based on wheat, barley and soybean meal. Piglets in Treatment 2 were fed the basal diet supplemented with the phytogenic feed additive Biomin ${ }^{\circ}$ P.E.P. 125 at a dosage of $125 \mathrm{~g} / \mathrm{t}$. Feed and drinking water were provided ad libitum. Individual body weights and pen feed intake were recorded at 42 and 62 days of age. The dataset was subjected to analysis of variance using the software Statgraphics Centurion and an LSD test ( $a=5 \%$ ). Data was analysed using either pen or pig as experimental unit. The model included effects of period and EO supplementation.

\section{Results}

Performance was not affected by period $(P>0.05)$. Initial BW was $12.4 \mathrm{~kg}$ and did not differ between treatments $(P>0.05)$. Using pen as experimental unit, average daily gain was 551 and 530 $\mathrm{g}$ in Treatment 1 and 2, respectively, and did not differ $(P>0.05)$ between treatments. However, using pig as experimental unit revealed a significant increase in average daily gain ( 555 vs. 527 $g, P<0.05)$ in pigs fed EO. Feed intake did not differ between treatments (783 vs. 793 g, P>0.05). Furthermore, supplementation with EO improved feed conversion ratio (feed/gain) by $5.2 \%$ (1.42 vs. $1.50, \mathrm{P}<0.05)$.

\section{Discussion}

Addition of the blend of EO in starter diets for piglets at the age of 42 to 62 days significantly improved growth performance. This data confirms the findings of a study with piglets weaned at 22 days of age, in which the inclusion of the blend of EO investigated herein improved weight gain and feed conversion by 6.2 and $3.5 \%$, respectively, in comparison with a negative control (5). Further studies indicated reduced numbers of intestinal microorganisms, enhanced nutrient digestibility and lower levels of ammonia and biogenic amines in pigs fed the same blend of EO $(4,6)$. Thus, the mode of action of EO is supposed to be related to a relief from the activity of intestinal microorganisms and related intestinal immune stress (3). In conclusion, EO are regarded as potential performance enhancers in feeding programs for weaned pigs.

\section{References}

1. Dorman HJD, Deans SG (2000) J. Appl. Microbiol. 88, 308-316.

2. Helander IM, Alakomi HL, Latva-Kala K, Mattila-Sandholm T, Pol I, Smid EJ, Gorris LGM, von Wright A (1998) J. Agric. Food Chem. 46, 3590-3595.

3. Windisch W, Schedle K, Plitzner C, Kroismayr A (2008) J. Anim. Sci. 86, E140-E148.

4. Kroismayr A, Schedle K, Sehm J, Pfaffl MW, Plitzner C, Foissy H, Ettle T, Mayer H, Schreiner M, Windisch W (2008) Bodenkultur 59, 111-120.

5. Sulabo RC, Jacela JY, DeRouchey JM, Tokach MD, Neher F (2007) J. Anim. Sci. 85(Suppl. 1), 644.

6. Zitterl-Eglseer K, Wetscherek W, Stoni A, Kroismayr A, Windisch W (2008) Bodenkultur 59, 121-129. 


\title{
P.712
}

\section{Estimating the metabolizable energy (ME) of $\mathrm{Albac}^{\oplus}$ in growing and finishing swine diets.}

\author{
Daniel A. Nelson \\ Alpharma Animal Health, East Bethel, MN, USA
}

\section{Introduction}

Albac $^{\bullet}$ (bacitracin-zinc, Alpharma, Inc., Bridgewater, NJ) is approved for increased rate of weight gain and improved feed efficiency when fed at 11 to $55 \mathrm{ppm}$ (10 to $50 \mathrm{~g} / \mathrm{ton}$ ). Theoretically, these improvements can be equated to a metabolizable energy $(\mathrm{ME})$ value contributed by the bacitracin.

\section{Materials and Methods}

A total of 840 pigs ( 5 treatments, 8 repetitions/treatment, 21 pigs/pen) were used during the period from approximately 28.0 to $123.0 \mathrm{~kg}$ body weight. A 6-phase dietary grow-finish program was utilized. All diets were corn-SBM based and maintained a constant available lysine/calorie ratio. Dietary treatments contained 1.0, 2.0, 3.0, and $4.0 \%$ added fat (Animal/Vegetable Blend). An additional treatment contained $1.0 \%$ added fat and 27.5 ppm ( $25 \mathrm{~g} / \mathrm{ton}$ ) Albac. A standard curve was generated from these increasing levels of dietary fat and a regression line was created using average daily gain (ADG) and gain to feed as the response variables.

\section{Results}

Overall ADG, gain to feed and final body weight (BW) increased linearly, and average daily feed intake (ADFI) declined linearly as the level of dietary fat increased in the diets, validating the model used in this experiment to estimate the ME contribution of Albac (Table 1). The cost/kg gain, mortality and removals, carcass backfat and lean yield were not different $(P>0.10)$ as the level of fat increased in the diet. Over the entire experiment, pigs fed the $1.0 \%$ added fat $+27.5 \mathrm{ppm}$ Albac diet had a higher ADG, a heavier final BW, and better feed efficiency than pigs fed the $1.0 \%$ added fat diet (Table 1). Using overall ADG as the response variable, $27.5 \mathrm{ppm}$ Albac was equivalent to $2.47 \%$ added fat or $123.2 \mathrm{kcal} \mathrm{ME} / \mathrm{kg}$ of complete feed. Using overall gain to feed as the response variable, $275 \mathrm{ppm}$ Albac was equivalent to $1.29 \%$ added fat or $64.4 \mathrm{kcal} \mathrm{ME} / \mathrm{kg}$ of complete feed (Figure 1). Nutritionists can assign an ME value for Albac of 257,500 kcal/kg within feed formulation software.
Table 1. Overall pig performance with increasing dietary fat concentrations and $27.5 \mathrm{ppm}$ Albac

\begin{tabular}{|c|c|c|c|c|c|}
\hline Overall trial & $\begin{array}{l}1.0 \% \text { Added } \\
\text { Fat }\end{array}$ & $\begin{array}{l}2.0 \% \text { Added } \\
\text { Fat }\end{array}$ & $\begin{array}{l}3.0 \% \text { Added } \\
\text { Fat }\end{array}$ & $\begin{array}{l}4.0 \% \text { Added } \\
\text { Fat }\end{array}$ & $\begin{array}{c}1.0 \% \text { Added } \\
\text { Fat }+27.5 \\
\text { ppm Albac }\end{array}$ \\
\hline Initial wt, kg & 28.0 & 27.6 & 28.1 & 27.8 & 28.1 \\
\hline$A D G, \mathrm{~kg} / \mathrm{d}^{\mathrm{a}}$ & 0.89 & 0.89 & 0.91 & 0.92 & $.91^{\mathrm{A}}$ \\
\hline $\mathrm{ADFI}, \mathrm{kg} / \mathrm{d}^{\mathrm{b}}$ & 2.43 & 2.39 & 2.41 & 2.33 & 2.43 \\
\hline $\begin{array}{l}\text { Gain to feed, } \\
\mathrm{kg} / \mathrm{kg}^{\mathrm{a}}\end{array}$ & 0.367 & 0.374 & 0.380 & 0.395 & $0.377^{B}$ \\
\hline Final BW, $\mathrm{kg}^{\mathrm{b}}$ & 121.5 & 123.2 & 122.8 & 124.1 & $124.3^{A}$ \\
\hline \multicolumn{6}{|c|}{ a Linear effect of fat, $\mathrm{P}<0.01$; ${ }^{\mathrm{b}}$ Linear effect of fat, $\mathrm{P}<0.05$} \\
\hline \multicolumn{6}{|c|}{$\begin{array}{l}\text { A1.0\% Added Fat+27.5 ppm Albac vs 1.0\% Added Fat, } \mathrm{P}<0.05 ; \mathrm{B} 1.0 \% \text { Added Fat+27.5 ppm } \\
\text { Albac vs } 1.0 \% \text { Added Fat, } \mathrm{P}<0.10\end{array}$} \\
\hline
\end{tabular}

Figure 1. Estimating the ME Value of Albac using Gain/Feed as the Response Variable

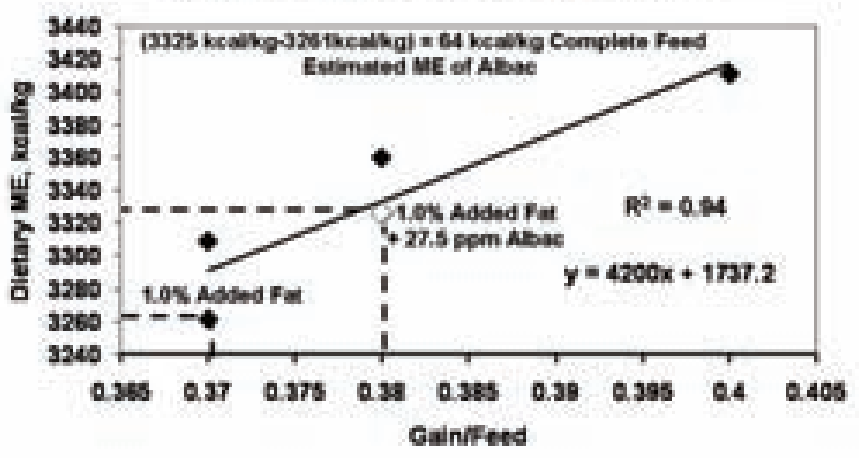




\title{
P.713
}

\section{Effect of an Escherichia coli phytase supplementation on the digestibility and retention of phoshporus and calcium in growing finishing-pigs}

\author{
Alain Kanora ${ }^{1}$ Monita Vereecken ${ }^{1}$ Koen De Gussem ${ }^{1}$ Cezary Purwin² Krzysztof Lipiński² \\ 1. Huvepharma NV, Antwerp, Belgium; 2. University of Warmia and Mazury, Olsztyn, Poland
}

\section{Introduction}

Most commercial diets for fattening pigs are formulated using cereals and oilseed meals, in which the bioavailability of the $P$ is poor, thus requiring additional $P$ supplementation. Phytase enzyme inclusion in pig diets is used to reduce the need for added inorganic phosphorus $(\mathrm{P})$ from feed phosphates and animal byproducts.

In this trial the effect of various doses of an Escherichia coli derived phytase (Optiphos ${ }^{\circ}$ ) on the digestibility and retention of phosphorus and calcium was studied in growing-finishing pigs.

\section{Materials and Methods}

This balance study was carried out with 24 growing pigs with an initial weight of $53 \mathrm{~kg}$, individually housed in metabolic cages to allow quantitative collection of faeces and urine. The four dietary treatments included a positive control (total P $5.1 \mathrm{~g} / \mathrm{kg}$; available P $2.5 \mathrm{~g} / \mathrm{kg}$ and Ca $6.2 \mathrm{~g} / \mathrm{kg}$ ), a negative control (total P 3.6 $\mathrm{g} / \mathrm{kg}$, available $P 1.0$ and $\mathrm{Ca} 4.2 \mathrm{~g} / \mathrm{kg}$ ) and the negative control diet supplemented with 125 or 250 FTU/kg Optiphos ${ }^{\circledR}$ (confirmed by analysis). The experimental diets were fed for 16 days. The study consisted of a 10 days adaptation period followed by a 6 days period of total collection of faeces and urine. Concentrations of $\mathrm{P}$ and $\mathrm{Ca}$ were examined in samples of feed, faeces and urine. Experimental results were processed statistically with the use of one-way analysis of variance and Duncan's test.

\section{Results}

Supplementation of Optiphos to the NC diet resulted in a significant positive effect on phosphorus digestibility for both supplemented enzyme doses. The $P$ retention reflected the $P$ digestibility in a consistent way. The effects of Optiphos ${ }^{\circ}$ on $\mathrm{Ca}$ digestibility and retention showed similar trends as demonstrated for phosphorus showing significant improvements at $125 \mathrm{FTU} / \mathrm{kg}$ or higher.
Table 1: Faecal digestibility and Retention of $\mathrm{P}$ and $\mathrm{Ca}$

\begin{tabular}{|l|c|c|c|c|}
\hline & PC & NC & $\begin{array}{c}\text { NC }+125 \text { FTU } \\
\text { Optiphos }\end{array}$ & $\begin{array}{c}\text { NC+ 250 FTU } \\
\text { Optiphos }\end{array}$ \\
\hline Faecal Digestibility P (\%) & $22.9^{c}$ & $19.7^{c}$ & $28.6^{\mathrm{b}}$ & $36.2^{\mathrm{a}}$ \\
\hline P retention (\%) & $20.8^{\mathrm{c}}$ & $19.0^{\mathrm{c}}$ & $27.6^{\mathrm{b}}$ & $33.5^{\mathrm{a}}$ \\
\hline Faecal Digestibility Ca (\%) & $25.3^{\mathrm{c}}$ & $30.6^{\mathrm{b}}$ & $34.1^{\mathrm{a}}$ & $36.8^{\mathrm{a}}$ \\
\hline Ca retention (\%) & $24.6^{\mathrm{b}}$ & $23.8^{\mathrm{b}}$ & $29.7^{\mathrm{a}}$ & $35.1^{\mathrm{a}}$ \\
\hline a,b,c,d: values in a row not sharing a common superscript differ significantly (P $\leq 0.05)$ \\
\hline
\end{tabular}

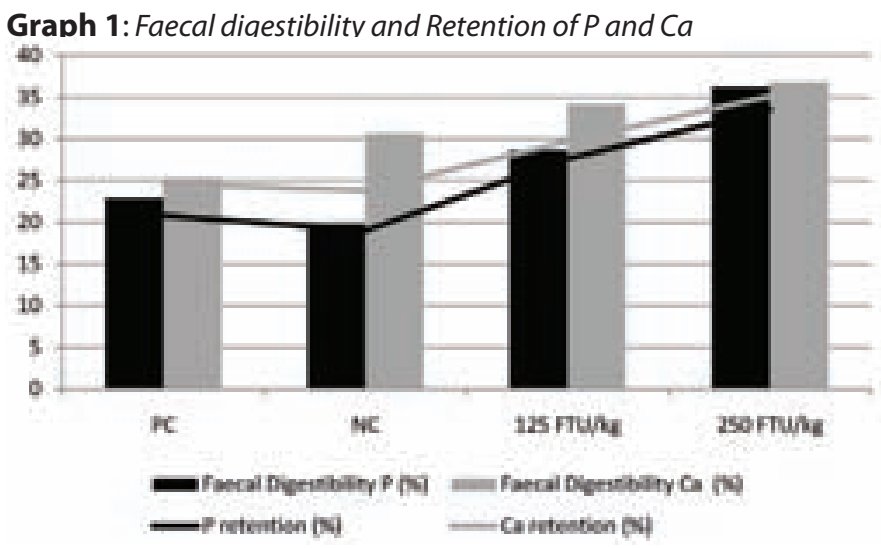

\section{Discussion}

Dietary supplementation with Optiphos ${ }^{\circledR}$ at concentrations of 125 and $250 \mathrm{FTU} / \mathrm{kg}$ improved phosphorus and calcium utilization in growing pigs fed corn-soybean meal diets. Phytase addition increased the total-tract digestibility and the retention of $\mathrm{P}$ and $\mathrm{Ca}$.

\section{References}

Cromwell, G. L. 1992. The biological availability of phosphorus in feedstuffs for pigs. Pig News Inf. 13:75N-78N. 


\title{
P.714
}

\section{Efficacy of a thermo-tolerant probiotic, containing Bacillus subtilis spores, in weaned pigs}

\author{
Spyridon K. Kritas ${ }^{2}$ Evanthia Petridou ${ }^{2}$ Georgios Valergakis ${ }^{1}$ Paschalis Fortomaris ${ }^{1}$ Elinor McCartney ${ }^{3}$ Toshihiro Marubashi $^{4}$ \\ 1. Aristotle University of Thessaloniki, Veterinary Faculty, Dept Animal Husbandry, Thessaloniki, Greece; \\ 2. Aristotle University of Thessaloniki, Veterinary Faculty, Dept Microbiology and Infectious Diseases, Thessaloniki, Greece; \\ 3. Pen \& Tec Consulting SCP, Barcelona, Spain; 4. Calpis Co., Ltd. , Kanagawa, Japan
}

\section{Introduction and Objectives}

Subtherapeutic antimicrobials are customarily used in pigs to improve daily gain and feed utilization (Barton 2000). Despite their beneficial effects, the recent concern on drug residues in food and the potential transfer of antibiotic resistance to human pathogens has directed scientists towards alternative solutions such as probiotics. Probiotics are live cultures of harmless bacteria that equilibrate intestinal microflora to the benefit of the animal (Fuller 1989). Calsporin ${ }^{\circledast}$ is a thermo-tolerant probiotic product based on viable spores of the specifically-selected probiotic strain Bacillus subtilis C-3102. To support EU approval in piglets, the efficacy of Calsporin ${ }^{\circledR}$ was evaluated under typical European conditions in Greece.

\section{Materials and Methods}

This trial was conducted in a commercial farrow-to-finish pig farm with a capacity of 400 sows and its own feed-mill. After weaning at the age of $32 \pm 2$ days, the piglets were transferred in flat decks in pens of 7-11 animals, where they remained until the age of 74 days. To test the efficacy of Calsporin ${ }^{\circledR}, 2$ trial groups of weaned pigs ( 32 to 74 days of age) were formed: a control group (T1) fed basal mash feed with no antimicrobials or probiotics, and a T2 Calsporin ${ }^{\circledR}$ group of piglets that received the same feed but supplemented with 3 x 108 cfu Bacillus subtilis C-3102 per $\mathrm{kg}$ of feed during flat deck period.

Two sets of experiments were performed, having the pen as the experimental unit: a first summer set (May 2 to August 15, 2008), and a second winter set (December 4, 2008 to March 19, 2009). In order to achieve homogeneity of treatment groups and to avoid cross- contamination, a cross-over, blocked design was used. In the summer set of trials, pigs of weaning batch 1 were equally split in 2 rooms (in 6 out of 12 pens per room), while the same was repeated during next week (weaning batch 2 ). At the end of weeks 1 and 2, two treatment rooms each of 12 pens were formed with almost the same population composition with regard to initial bodyweight, age, gender, dam parity and litter of origin. The pigs of room 1 were served as T1 (control) group and those of room 2 as T2 (Calsporin) group. This format was repeated with weaning batches 3 and 4 at a later time block, with the exception that room 1 was then designated as T2 Calsporin $^{\circledR}$ and room 2 as T1 Control. The winter set of experiments employing another pair of experimental groups, had exactly the same design as the first set. Thus, at the end of the trial, a total of 908 weaned pigs were used derived from 2 sets of experiments (summer \& winter) $\times 2$ experimental groups (rooms) $\times 24$ replicates (pens) of weaned piglets.

\begin{tabular}{|l|c|c|}
\hline Parameter & T1 control & T2 Calsporin \\
\hline Dam parity & 5.3 & 5.2 \\
\hline Initial bodyweight, $\mathrm{kg}$ & 7.74 & 7.73 \\
\hline Final bodyweight, $\mathrm{kg}$ & 25.42 & $26.92^{*}$ \\
\hline Average daily gain, $\mathrm{kg}$ & 0.421 & $0.457^{*}$ \\
\hline Average daily feed intake, $\mathrm{kg}$ & 0.802 & 0.788 \\
\hline Feed conversion ratio & 1.907 & $1.725^{*}$ \\
\hline
\end{tabular}

\section{Results and Discussion}

Feed and faecal sampling confirmed the presence of Bacillus subtilis C-3102 in T2 feed and faeces at expected concentrations, and the absence of cross-contamination to T1 Controls. There was no significant difference in mortality or feed intake between groups. However, as presented in the Table 1, significant improvement on production parameters (final bodyweight, average daily gain and feed conversion ratio) were recorded after Calsporin ${ }^{\circledast}$ consumption. Thus, compared to T1 Controls, the T2 Calsporin ${ }^{\circledR}$ piglets were $5.9 \%$ heavier $(P<0.0001)$, exhibited $9.5 \%$ better growth $(P<0.0001)$ and $9.9 \%$ improved feed efficiency $(\mathrm{P}<0.0001)$ after 42 days on trial.

Although these studies were carried out under commercial farm conditions, cross-contamination of Bacillus subtilis C-3102 between Control and Calsporin ${ }^{\circledR}$ piglets was avoided by careful separation of treatments. A cross-over, blocked study design ensured the validity of the data and the best homogeneity of the groups. Calsporin ${ }^{\circledR}$ produced consistent improvements in zootechnical performance over 8 weanings involving around 1,000 piglets, under both summer and winter production conditions. The results of these studies illustrate the contribution of a stable, in-feed probiotic to efficient pig production without antibiotic growth promoters.

\section{References}

1. Barton MD, 2000. Nutrition Research Reviews, 13, 279-299

2. Fuller R 1989. J. Appl. Bacteriol. 66:365-368 


\title{
P.715
}

\section{A survey of free and conjugated deoxynivalenol in feed and feedstuffs from Asian and Pacific countries.}

\author{
Si-Trung Tran; Trevor K. Smith \\ University of Guelph, Guelph, ON, Canada
}

\section{Introduction}

Deoxynivalenol (DON, vomitoxin), one of the most important mycotoxins produced by many Fusarium species, is found as a common contaminant of crops worldwide. DON can reduce production efficiency and cause serious economic losses to livestock and poultry producers. Recent studies have described the presence of conjugated forms of DON (glycosides and fatty acid). The optimal conditions for conjugated DON hydrolysis with trifluoromethanesulfonic acid (TFMSA) have also been determined. The aim of the current study was, therefore, to investigate the natural occurrence of free and conjugated DON in feed and feedstuffs from Asian and Pacific countries.

\section{Materials and Methods}

One hundred forty-nine feed and feedstuffs samples were collected from China, Indonesia, Malaysia, New Zealand, the Philippines, Taiwan, Thailand and Vietnam in 2009.

The conditions for conjugated DON hydrolysis with TFMSA used for corn were $0.5 \mathrm{~N}$ TFMSA, $20 \mathrm{~min}$ and $22^{\circ} \mathrm{C}$ while the conditions used for wheat were $0.5 \mathrm{~N} \mathrm{TFMSA}, 40 \mathrm{~min}$ and $40^{\circ} \mathrm{C}$. Free and conjugated DON in the current study were determined using ELISA kits (AgraQuant ${ }^{\circledR}$ DON test kits, Romer Labs Inc., Union, MO, USA).

\section{Results}

Free DON was found in all samples (116 corn, 21 wheat and 12 feed samples). Conjugated DON, however, was not detected in 39 of 149 samples. Levels of DON ranged from 0.05 to $6.70 \mu \mathrm{g} / \mathrm{g}$ in corn, from 0.02 to $0.71 \mu \mathrm{g} / \mathrm{g}$ in wheat and from 0.06 to 0.47 $\mu \mathrm{g} / \mathrm{g}$ in feed.

The highest levels of free DON were found in corn samples from China and Indonesia while samples from Malaysia, Thailand and Vietnam were less contaminated. Conjugated DON was found mainly in corn from Indonesia with 12 of 20 samples showing high levels of conjugated DON (27-84\%). Lower levels of conjugated DON (12 - $33 \%$ ) were detected in 14 of 20 corn samples from Malaysia. Conjugated DON was not detected in corn from the Philippines and in the majority of corn samples from New Zealand and Vietnam. Similarly, no conjugated DON was seen in 15 of 21 wheat samples while high levels of conjugated DON (up to $67 \%$ ) were found in 9 of 12 feed samples.

\section{Discussion}

In the current study, increases in total DON compared to free DON in corn are of the same magnitude as those reported by Liu et al. (2005) for Fusarium head blight-infected wheat or Zhou et al. (2007) for barley. Samples highly contaminated with DON, however, did not necessarily show a high concentration of conjugated DON, in contrast to the findings of Zhou et al. (2008) in barley and malt. Although significant differences between free DON and total DON concentrations were observed, there was considerable variation between regions. The reasons for the differences between regions could be due to climatic conditions or plant genetics.

The current survey of free and conjugated DON in feed and feedstuffs from Asian and Pacific countries emphasizes the potential challenges in understanding the hazard posed by feed and feedstuffs contaminated with DON. Although much research has been carried out since the original discovery of free and conjugated DON, the significance of these mycotoxins for animal health remains to be determined.

\section{References}

1. Liu et al., 2005. J. Agric. Food Chem. 53, 6864-6869.

2. Zhou et al., 2007. J. Agric. Food Chem. 55, 10141-10149.

3. Zhou et al., 2008. J. Food Prot. 71, 1266-1269. 


\title{
P.716
}

\section{Effect of phytase supplementation on the digestibility of phosphorus and calcium in lactating sows}

\author{
Alain Kanora ${ }^{1}$ János Tossenberger² László Babinszky² Koen De Gussem¹ Monita Vereecken¹ \\ 1. Huvepharma NV, Antwerp, Belgium; 2. Kaposvár University, Department of Animal Nutrition, Kaposvár, Hungary
}

\section{Introduction}

The application of phytase in pig diets has become common practice to improve the bio-availability of phytate $P$ and to reduce phosphorus excretion. The response of phosphorus digestibility to phytase depends on a number of factors, such as dose-rate, animal category and type of diet. In this trial the effect of various doses of an Escherichia coli derived phytase (OptiphOs ${ }^{\circledR}$ ) on the digestibility of phosphorus and calcium was studied in lactating sows.

\section{Materials and Methods}

The trial was conducted with 48 crossbred lactating sows (six treatments, 8 sows/treatment). The six dietary treatments included a positive control (total P $6,8 \mathrm{~g} / \mathrm{kg}$ ), a negative control (total P $4.2 \mathrm{~g} / \mathrm{kg}$ ) and the NC diet supplemented with 125, 250, 500 and 1000 FTU/kg Optiphos ${ }^{\circledR}$ (confirmed by analysis). The experimental diets, formulated on a corn-soybean basis, were fed from about 5 days before farrowing until 27 days post-farrowing. Animals were housed individually in pens to allow grab sampling of faeces between days 18 and 22 of lactation. Faecal collection by marker method was performed and chromic oxide was used as a marker to estimate total tract digestibility of $P$ and Ca. The trial data were analyzed with ANOVA (SAS, 2004). In case of any significant treatment effects the differences among the treatments were checked by LSD-test (SAS, 2004).

Table 1: Calculated P and Ca content of the basal diets

\begin{tabular}{|l|l|l|c|}
\hline & PC & NC & NC+Optiphos dosages \\
\hline Total P in diet $\left(\mathrm{g} \mathrm{kg}^{-1}\right)$ & 6.8 & 4.2 & 4.2 \\
\hline Dig.P in diet $\left(\mathrm{g} \mathrm{kg}^{-1}\right)$ & 3.5 & 1.6 & 1.6 \\
\hline Ca in diet $\left(\mathrm{g} \mathrm{kg}^{-1}\right)$ & 8.1 & 4.8 & 4.8 \\
\hline
\end{tabular}

\section{Results and Discussion}

Supplementation of Optiphos ${ }^{\circledast}$ to the diet resulted in significant positive effect on phosphorus digestibility for all supplemented Optiphos ${ }^{\circledast}$ doses. The digestibility of phosphorus improved up to a phytase dose of $250 \mathrm{FTU} / \mathrm{kg}$. A curvilinear dose response was observed, reaching a plateau at $250 \mathrm{FTU} / \mathrm{kg}$. A similar trend was demonstrated for the effect of Optiphos ${ }^{\circledR}$ on Ca digestibility. (Graph 1and Table 2)
Table 2: Faecal digestibility of $P$ and $C a$ at 18-22 days of lactation after supplementation of different dosages of Optiphos ${ }^{\circledR}$

\begin{tabular}{|c|c|c|c|c|c|c|}
\hline & $P C$ & NC & $\begin{array}{c}\mathrm{NC}+0 \text { ptiphos } \\
\text { at concentration } \\
125 \mathrm{FTU}\end{array}$ & $\begin{array}{c}\mathrm{NC}+0 \text { ptiphos } \\
\text { at concentration } \\
250 \mathrm{FTU}\end{array}$ & $\begin{array}{c}\mathrm{NC}+\text { Optiphos } \\
\text { at concentration } \\
500 \mathrm{FTU}\end{array}$ & $\begin{array}{l}\text { NC + Optiphos } \\
\text { at concentration } \\
1000 \mathrm{FTU}\end{array}$ \\
\hline $\mathrm{P}(\%)$ & $31.7^{c}$ & $25.0^{d}$ & $37.7^{b}$ & $47.4^{\mathrm{a}}$ & $48.0^{\mathrm{a}}$ & $49.9^{a}$ \\
\hline $\mathrm{Ca}(\%)$ & $29.6^{b}$ & $23.7^{c}$ & $29.8^{b}$ & $37.8^{\mathrm{a}}$ & $39.8^{\mathrm{a}}$ & $39.9^{\mathrm{a}}$ \\
\hline
\end{tabular}

Graph 1: Faecal digestibility of $P$ and Ca at 18-22 days of lactation after supplementation of different dosages of Optiphos ${ }^{\circledR}$

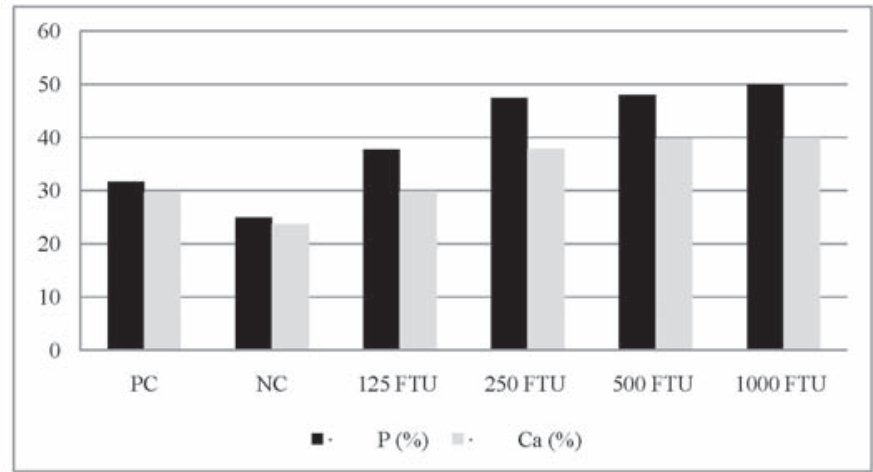

The digestibility of $\mathrm{P}$ and Ca improved up to a dosage of 250 FTU/kg. Phytase doses in excess of that level were not associated with further improvement of $\mathrm{P}$ or Ca digestibility. The poor doseresponse relation is very surprising, because in earlier experiments with other phytase enzymes (Jongbloed et al., 2004) in sows clear dose-response effects were seen.

\section{Conclusion}

Obviously, the enzyme Optiphos ${ }^{\circledast}$ is very efficient in generating digestible $\mathrm{P}$ at a low supplementation level in lactating sows.

\section{Reference}

Jongbloed, A.W., Diepen, J.Th.M. van, Kemme, P.A., Broz, J., 2004. Efficacy of microbial phytase on mineral digestibility in diets for gestating and lactating sows. Livest. Prod. Sci. 91, 143-155 


\title{
P.717
}

\section{Influence on fertility of Lianol ${ }^{\circledR}$ Solapro incorporation in lactation diet}

\author{
José Wavreille ${ }^{1}$ Viviane Planchon ${ }^{1}$ Robert Renaville ${ }^{2}$ Rudi Forier ${ }^{3}$ Richard Agneessens ${ }^{1}$ Alain Kanora ${ }^{4}$ Nicole Bartiaux-Thill ${ }^{1}$ \\ 1. Walloon Agricultural Research Centre (CRA-W), Rue de Liroux 8, Gembloux, Belgium; 2. Gembloux Agro-Biotech Faculty, \\ University of Liège, Liège, Belgium; 3. Ardol BV, Susteren, Netherlands; 4. Huvepharma NV, Antwerp, Belgium
}

\section{Introduction}

At the end of lactation, sows are in a state of negative energy balance (NEB). The metabolic state is the key factor limiting the fertility and embryonic survival. The NEB reduces fertility and reproduction parameters. The inclusion of Lianol ${ }^{\circledR}$ Solapro in the lactation diet improves these parameters.

\section{Material and Methods}

Forty Belgian Landrace sows at CRA-W from three groups were distributed between two feeding treatments upon entering the farrowing unit seven days before expected farrowing: control and supplement. The supplement was Lianol ${ }^{\circledR}$ Solapro, a complementary feeding stuff based on fermented potato protein. It was distributed manually as top feeding on the first diet (14.1 MJ of ME/kg, EVAPIG ${ }^{\circ}$ ) at a rate of $10 \mathrm{~g} /$ day until three days after farrowing and thereafter it was added to the second diet (14.7 MJ of ME/kg, EVAPIG ${ }^{\circ}$ ) at a rate of $0.1 \%$. The piglets are weaned at 4 weeks of age. Total energy requirement (TER, MJ of $\mathrm{ME} / \mathrm{d}$ ) of sows during lactation was calculated ${ }^{1}$. Energy balance (EB) was calculated by subtracting the calculated TER from the intakes. The plasma concentrations of IGF-I were determined in the plasma of sows at weaning. Elisa Kit (Mediagnost ${ }^{\circ}$ ) was used after cryo-precipitation of proteins: acid-ethanol method ${ }^{2}$.

After weaning, sows were managed in the same modalities of breeding until their next farrowing. The sows were scanned pregnant at 4 weeks. At next farrowing, the number of piglets born alive, stillborn and living at day 4 was recorded. Each piglet was weighed at 4 days of age. Data were analyzed by analysis of variance using the GLM procedure (Minitab 15.1.30.0). Model included the effects of feeding treatment and group and the interaction between these 2 factors. A F-test allows comparing intra-litter variance (Excel $\left.{ }^{\oplus}, 2003\right)$; intra-litter variance of control and Lianol $^{\circ}$ corresponds to the residual variance estimated from an analysis of variance with litter and group as factors.

\section{Results}

While the EB was more negative $(P<0.10)$, Lianol ${ }^{\bullet}$ sows had higher plasma IGF-I at weaning. It resulted in higher rates of sows keeping pregnancy until birth. Similarly, Lianol $^{\circ}$ gave better quality embryos that develop in stronger piglets. Indeed, the rate of survival born alive $(P<0.10)$ or living at the fourth day $(\mathrm{P}<0.05)$ were higher for Lianol ${ }^{\bullet}$ sows. The piglets present at day 4 had grown equally well in both groups, but while there were more survivors, the intra-litter variance of weight was lower $(\mathrm{P}<0.05)$; the litters were more homogenous.
Table 1. Reproduction performance

\begin{tabular}{|c|c|c|c|c|}
\hline & Control & $\begin{array}{l}\text { Lianol }^{\oplus} \\
\text { Solapro }\end{array}$ & s.e. & Effect \\
\hline No. of sows at weaning & 22 & 18 & & \\
\hline NEB lactation, MJ EM/d & -22 & -28 & 1.8 & $\mathrm{~T}^{\dagger}$ \\
\hline IGF-I at weaning, ng/ml & 45 & 66 & 3.9 & $T^{* * *}$ \\
\hline No. (\%) of sows inseminated & $19(86 \%)$ & $17(94 \%)$ & / & \\
\hline Weaning to estrus interval, $\mathrm{d}$ & 4.7 & 4.6 & 0.13 & \\
\hline $\begin{array}{l}\text { No. (\%) of sows pregnant at } \\
\text { scanning }\end{array}$ & $17(77 \%)$ & 17 & / & \\
\hline No. of sows that farrow & $16(73 \%)$ & $17(94 \%)$ & I & \\
\hline \multicolumn{5}{|l|}{ Avg. litter } \\
\hline Total piglets born & 12.5 & 12.4 & 0.6 & \\
\hline Piglets born alive & 11.2 & 11.6 & 0.5 & \\
\hline$\%$ of survival/total born & $89 \%$ & $94 \%$ & $1.5 \%$ & $\mathrm{~T}^{\dagger}$ \\
\hline Piglets at $4 \mathrm{~d}$ & 10.4 & 11.1 & 0.5 & \\
\hline$\%$ of survival/total born & $83 \%$ & $91 \%$ & $2.0 \%$ & $T^{*}$ \\
\hline \multicolumn{5}{|l|}{ Piglet BW at $4 \mathrm{~d}, \mathrm{~g}$} \\
\hline Mean & 2,124 & 2,126 & 68 & \\
\hline s.e. intra-litter & 108 & 85 & 6 & $\mathrm{~T}^{*}$ \\
\hline \multicolumn{5}{|c|}{$\begin{array}{l}\text { No standardization of litter size, } \mathrm{T}=\text { Treatment effect, } \mathrm{G}=\text { Group effect, } \mathrm{T} \times \mathrm{G}=\text { Treatment } x \text { Group } \\
\text { interaction }-\dagger=\mathrm{P}<0.10{ }^{*}=\mathrm{P}<0.05 ;{ }^{* * *}=\mathrm{P}<0.001\end{array}$} \\
\hline
\end{tabular}

\section{Conclusion}

Lianol $^{\circledR}$ Solapro is a fermented potato protein which is added to the feed of sows. This study confirms its beneficial effect on several sow fertility parameters and on the quality and survival of the piglets that are born in the subsequent litter.

\section{References}

1. Noblet, J. et al. (1990), Energy utilization in pregnant and lactating sows: modeling of energy requirements. J. Anim. Sci., 68, 562-572.

2. Renaville, R. et al. (1993), Changes in the hypophyso-gonadal axis during the onset of puberty in young bull calves, J. Reprod. Fertil., 99, 443-449. 


\title{
P.718
}

\section{Influence of Lianol ${ }^{\circledR}$ Solapro on sow milk production and piglet weight gain}

\author{
José Wavreille ${ }^{1}$ Viviane Planchon ${ }^{1}$ Robert Renaville ${ }^{2}$ Rudi Forier ${ }^{3}$ Richard Agneessens $^{1}$ Alain Kanora ${ }^{4}$ Nicole Bartiaux-Thill ${ }^{1}$ \\ 1. Walloon Agricultural Research Centre (CRA-W), Rue de Liroux 8, Gembloux, Belgium; \\ 2. Gembloux Agro-Biotech Faculty, University of Liège, Liège, Belgium; 3. Ardol BV, Susteren, Netherlands; 4. Huvepharma NV, Antwerp, Belgium
}

\section{Introduction}

Milk is a pivotal factor in piglet survival, growth, development and pre-weaning piglet body composition. The demonstration that heavier weaning piglets attain market weight faster than lighter weaning piglets ${ }^{1}$ has sparked increasing interest in exploiting the lactation period to enhance overall piglet growth and pork production. The incorporation of Lianol ${ }^{\circ}$ Solapro in the sow's diet increases milk production and feed efficiency.

\section{Material and Methods}

Forty Belgian Landrace sows at CRA-W from 3 groups were divided into a feeding control group and a Lianol ${ }^{\circ}$ Solapro group upon entering the farrowing unit (7 days prior to expected farrowing). Distribution was done manually as top feeding on the first diet (14.1 MJ of ME/kg, EVAPIG ${ }^{\circ}$, rate of $10 \mathrm{~g} /$ day) until 3 days post-farrowing. Thereafter, it was added to the $2^{\text {nd }}$ diet at $0.1 \%$ (14.7 MJ of ME/kg, EVAPIG ${ }^{\circ}$ ). Piglets are weaned at 4 weeks of age. Sows were weighed and backfat thickness was ultrasonographically measured on P2-site ${ }^{1}$ at the beginning and end of trial. Sow weight loss was determined by subtracting uterine weight content ${ }^{2}$. Piglets were weighed at birth and after 4 and 24 days age. Total energy requirements (TER, MJ of ME/d) of sows during lactation were calculated ${ }^{3}$. The Energy Balance (EB) was found by subtracting calculated TER from the intakes. Milk production over the $1^{\text {st }} 3$ weeks was estimated from litter weight gain and litter size ${ }^{4}$. IGF-I concentrations were determined in the sow plasma when entering the farrowing unit and at 4 and 24 days of lactation. Elisa Kit (Mediagnost ${ }^{\circ}$ ) was used after protein cryo-precipitation: acid-ethanol method ${ }^{5}$. Data are processed by variance analysis (GLM procedure, Minitab 15.1.30.0). Included: effects of feeding treatment, of group and corresponding interaction.

\section{Results}

Litter size (total born, born alive, at $24 \mathrm{hrs}$ ) and the average weight of piglets per litter were similar. While the EB was more

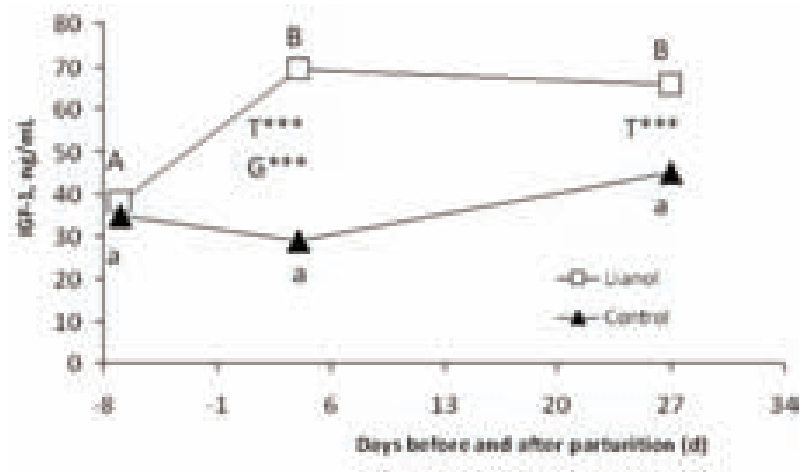

Figure 1. Sow plasma IGF-I negative for Lianol $^{\circledR}$ sows $(\mathrm{P}=0.07)$, they did not lose more weight and backfat thickness, weight of litter at weaning, litter ADG and milk production were not lower. Lianol ${ }^{\circ}$ sows showed optimal feed efficiency. Sow plasma IGF-I was significantly higher $(P<0.001)$ at day 4 of lactation and at weaning.

\section{Conclusion}

Lianol $^{\oplus}$ Solapro added to the feed of sows supports milk production and increases litters of lactating sows.

Table 1. Growth performance

\begin{tabular}{|c|c|c|c|c|}
\hline & Control & $\begin{array}{l}\text { Lianol }^{\circledR} \\
\text { Solapro }\end{array}$ & s.e. & Effect \\
\hline Number of litters & 22 & 18 & & \\
\hline \multicolumn{5}{|l|}{ Avg. litter size } \\
\hline At birth (born alive) & 11.3 & 11.5 & 0.4 & \\
\hline At weaning & 10.0 & 10.4 & 0.3 & \\
\hline \multicolumn{5}{|l|}{ Avg. litter weight, kg } \\
\hline At birth (born alive) & 16.5 & 17.0 & 0.6 & \\
\hline At weaning & 67.5 & 72.0 & 1.8 & \\
\hline Litter ADG, kg/d & 1,956 & 2,129 & 0.063 & \\
\hline TER, MJ EM/d & 74 & 79 & 1.8 & \\
\hline Intake, MJ EM/d & 52 & 51 & 1.8 & \\
\hline EB, MJ EM/d & -22 & -28 & 1.8 & $\mathrm{~T}^{\dagger}$ \\
\hline \multicolumn{5}{|l|}{ BW of sows, $\mathrm{kg}$} \\
\hline Beginning (calculated) & 200 & 204 & 4.4 & \\
\hline At weaning & 184 & 177 & 4.8 & \\
\hline Loss & -26 & -27 & 2.0 & $\mathrm{G}^{* * *}$ \\
\hline \multicolumn{5}{|l|}{ Backfat thickness of sows, mm } \\
\hline Beginning & 18.7 & 18.1 & 0.5 & \\
\hline At weaning & 13.2 & 12.9 & 0.4 & \\
\hline Loss & -5.5 & -5.2 & 0.4 & $\mathrm{G}^{* *}$ \\
\hline Milk Production first 3 wk, kg & 133 & 143 & 3.6 & \\
\hline \multicolumn{5}{|c|}{$\begin{array}{l}\text { No standardization of litter size, } \mathrm{T}=\text { Treatment effect, } \mathrm{G}=\text { Group effect, } \mathrm{TxG}=\text { Treatment } x \text { Group } \\
\text { interaction }-\mathrm{\dagger}=\mathrm{P}<0.01,{ }^{*}=\mathrm{P}<0.05 ;^{* *}=\mathrm{P}<0.01,{ }^{* *}=\mathrm{P}<0.001\end{array}$} \\
\hline
\end{tabular}

\section{References}

1. Dourmad, J.Y. et al. (2001), Measuring backfat depth in sows to optimize feeding strategy, INRA Prod. Anim., 14, 41-50.

2. Dourmad, J.Y. et al. (1997), Prédiction de la composition chimique des truies reproductrices à partir du poids vif et de l'épaisseur de lard dorsal. Application à la définition des besoins énergétiques, Journ. Rech. Porcine, 29, 255-262.

3. Noblet, J. et al. (1990), Energy utilization in pregnant and lactating sows: modeling of energy requirements, J. Anim. Sci., 68, 562-572.

4. Noblet, J., Etienne, M. (1989), Estimation of sow milk nutrient output, J. Anim. Sci., 67: 3352-3359.

5. Renaville R. et al. (1993), Changes in the hypophyso-gonadal axis during the onset of puberty in young bull calves, J. Reprod. Fertil., 99, 443-449 


\title{
P.719
}

\section{Influence of Lianol ${ }^{\circledR}$ Solapro on sow colostrum production}

\author{
José Wavreille ${ }^{1}$ Viviane Planchon ${ }^{1}$ Robert Renaville ${ }^{2}$ Rudi Forier ${ }^{3}$ Alain Kanora ${ }^{4}$ Nicole Bartiaux-Thill ${ }^{1}$ \\ 1. Walloon Agricultural Research Centre (CRA-W), Rue de Liroux 8, Gembloux, Belgium; \\ 2. Gembloux Agro-Biotech Faculty, University of Liège, Liège, Belgium; \\ 3. Ardol BV, Susteren, Netherlands; 4. Huvepharma NV, Antwerp, Belgium
}

\section{Introduction}

Sufficient sow colostrum production and colostrum intake by piglets are vital for the piglet immune status. Due to the increased prolificacy, the average weight of the piglets and their capacity to suckle and stimulate the sow colostrum production has decreased. This results in a diminished colostrum intake and poorer piglet immune status. Lianol ${ }^{\circledR}$ Solapro, a fermented potato protein added to the feed of sows, increases vigor and colostrum intake of piglets and sow colostrum production.

\section{Material and Methods}

Forty Belgian Landrace sows at CRA-W from three groups were divided into a feeding control group and a Lianol ${ }^{\circ}$ Solapro group upon entering the farrowing unit (7 days prior to expected farrowing). Distribution was done manually as top feeding on the first diet (rate of $10 \mathrm{~g} /$ day) until 3 days post-farrowing and thereafter it was added to the $2^{\text {nd }}$ diet at a rate of $0.1 \%$. Farrowings, number of piglets born alive and stillborn and feed intakes were monitored. To determine colostrum intake the interval between birth and first suckling was measured, piglets were weighed at birth and $24 \mathrm{hrs}$ later. The total sow colostrum production is found by adding piglet colostrum intakes ${ }^{1}$. The concentrations of IGF-I were determined in the sow plasma when entering the farrowing unit and at lactation day 4, in plasma of four 4-day old piglets of each litter but also in the colostrum whey (collected at parturition). Centrifugation to $13,000 \mathrm{~g}$ gave the whey. Elisa Kit (Mediagnost ${ }^{\oplus}$ ) was used after cryo-precipitation of proteins: acid-ethanol method ${ }^{2}$. Data were processed by analysis of variance (GLM procedure, Minitab $\left.{ }^{\bullet} 15.1 .30 .0\right)$. Included: effects of feeding treatment, of group and corresponding interaction.

\section{Results}

Litter size (total born, born alive, at $24 \mathrm{hrs}$ ) and the average weight of piglets per litter were similar. During the $1^{\text {st }} 24 \mathrm{hrs}$, Lianol $^{\oplus}$ piglets showed a higher weight gain $(P<0.05)$ and had a higher colostrum intake $(23 \%, \mathrm{P}<0.05)$ than control piglets. Colostrum production was significantly higher in Lianol ${ }^{\circledR}$ sows $(+20 \%, P<0.01)$. They showed a plasma IGF-I concentration which was significantly higher on day 4 of lactation $(P<0.001)$. From entering farrowing to day 4 of lactation, Lianol ${ }^{\circledR}$ sows showed a significant improvement in plasma IGF-I concentration $(\mathrm{P}<0.001)$. IGF-I is known to stimulate lactogenesis ${ }^{3}$. IGF-I in colostrum was not influenced by treatment. However, Lianol ${ }^{\circ}$ piglets had significantly higher plasma IGF-I until 4 days of age $(P<0.001)$. Their daily weight gain was $22 \%$ higher during the first 4 days of life $(P<0.05)$.
Table 1. Growth performance and colostrum intake of neo-natal piglets and IGF-I concentrations

\begin{tabular}{|c|c|c|c|c|}
\hline & Control & $\begin{array}{l}\text { Lianol }^{\odot} \\
\text { Solapro }\end{array}$ & s.e. & Effect \\
\hline Number of litters & 22 & 18 & & \\
\hline \multicolumn{5}{|l|}{ Avg. litter size } \\
\hline Total piglets born & 12.0 & 12.1 & 0.5 & \\
\hline Piglets born alive & 11.3 & 11.5 & 0.4 & \\
\hline Piglets at $24 \mathrm{~h}$ & 11.0 & 10.9 & 0.4 & \\
\hline \multicolumn{5}{|l|}{ Piglet BW, g } \\
\hline At birth & 1,490 & 1,504 & 39 & \\
\hline At $24 \mathrm{~h}$ & 1,556 & 1,629 & 42 & \\
\hline Time first suckling min & 58 & 44 & 5 & \\
\hline Piglet ADG $24 \mathrm{~h}, \mathrm{~g} / \mathrm{d}$ & 63 & 114 & 10 & $T^{*}$ \\
\hline Colostrum intake, $\mathrm{g} / 24 \mathrm{~h}$ & 293 & 353 & 14 & $T^{*}$ \\
\hline Colostrum production, $\mathrm{g} / 24 \mathrm{~h}$ & 3,064 & 3,770 & 141 & $\mathrm{~T}^{* *}, \mathrm{G}^{*}$ \\
\hline \multicolumn{5}{|l|}{ Sow plasma IGF-I, ng/ml } \\
\hline Entering farrowing unit & 35 & 38 & 2.6 & \\
\hline At $4^{\text {th }}$ lactation day & 29 & 69 & 5.5 & $\mathrm{~T}^{* * *}, \mathrm{G}^{* * *}$ \\
\hline \multicolumn{5}{|l|}{ IGF-I concentration, ng/ml } \\
\hline Colostrum whey & 19 & 18 & 0.9 & $\mathrm{G}^{* * *}$ \\
\hline \multicolumn{5}{|l|}{ Piglet plasma IGF-I, ng/ml } \\
\hline Atd 4 & 8 & 15 & 1.2 & $\mathrm{~T}^{* * *}, \mathrm{G}^{* * *}$ \\
\hline Piglet ADG $4 \mathrm{~d}, \mathrm{~g} / \mathrm{d}$ & 143 & 174 & 7 & $T^{*}$ \\
\hline
\end{tabular}

\section{Conclusions}

Lianol ${ }^{\oplus}$ Solapro added to the feed of sows increased piglet vigor as well as colostrum intake and colostrum production.

\section{References}

1. Devillers, N. et al. (2004), Estimation of colostrum intake in the neonatal pig, Anim. Sci., 78, 305-313.

2. Renaville, R. et al. (1993), Changes in the hypophyso-gonadal axis during the onset of puberty in young bull calves, J. Reprod. Fertil., 99, 443-449.

3. Lee, C.Y. et al. (1993), Expression of components of the insulin-like growth factor system in pig mammary glands and serum during pregnancy and pseudopregnancy: Effects of oestrogen, J. Endocrinol., 137, 473-483. 


\title{
P.720
}

\section{Application of Lianol ${ }^{\bullet}$ Ferti in sows to increase the number of piglets per litter}

\author{
Jos Schillebeekx ${ }^{1}$ Rudi Forier ${ }^{2}$ Alain Kanora ${ }^{3}$ Koen De Gussem ${ }^{3}$ \\ 1. DAP Schillebeekx, Koersel, Belgium; 2. Ardol BV, Susteren, Netherlands; 3. Huvepharma NV, Antwerp, Belgium
}

\section{Introduction}

Lianol $^{\bullet}$ Ferti is a highly digestible fermented potato protein of which the use results in prometabolic peptides. The fertility effect of the Negative Energy Balance (NEB) can be countered by feeding Lianol ${ }^{\circledR}$ Ferti to sows, increasing peptide levels and enhancing milk production. These effects are mediated by an elevated IGF-1 level in serum, known to increase these parameters. Lianol ${ }^{\oplus}$ Ferti has the proven ability to enhance the heat to shorten the weaning to insemination period and to reduce the number of empty sows. This trial aimed at confirming the influence of Lianol ${ }^{\ominus}$ Ferti administration to sows around weaning on the number of piglets born in the next litter.

\section{Materials and methods}

A group of $30 \mathrm{~F} 1$ sows (Belgian landrace and French Landrace) on a Belgian breeding farm were equally split in an untreated control group (parity: 3.5) and a Lianol ${ }^{\oplus}$ Ferti group (parity: 3.7 ) receiving $10 \mathrm{~g} \mathrm{Lianol}^{\circledR}$ Ferti per sow per day, during 5 consecutive days, starting on Monday before weaning until Friday after weaning (weaning on Thursday). IGF-1 serum levels were measured at the start on Monday and again the next Monday, at insemination. Secondly, the number of piglets born and weaned in the next litter, were registered.

\section{Results}

The IGF-1 levels of the untreated control group showed an increase of $4.4 \mathrm{ng} / \mathrm{ml}$, being $8.25 \%$. However, the IGF-1 levels of the group having received $10 \mathrm{~g}$ of Lianol $^{\circ}$ Ferti for 5 days showed a noticeable increase of $14.0 \mathrm{ng} / \mathrm{ml}$, which is an increase of $30.2 \%$.

Figure 1. Impact of Lianol ${ }^{\oplus}$ Ferti on IGF-1 levels $(\mathrm{ng} / \mathrm{ml})$

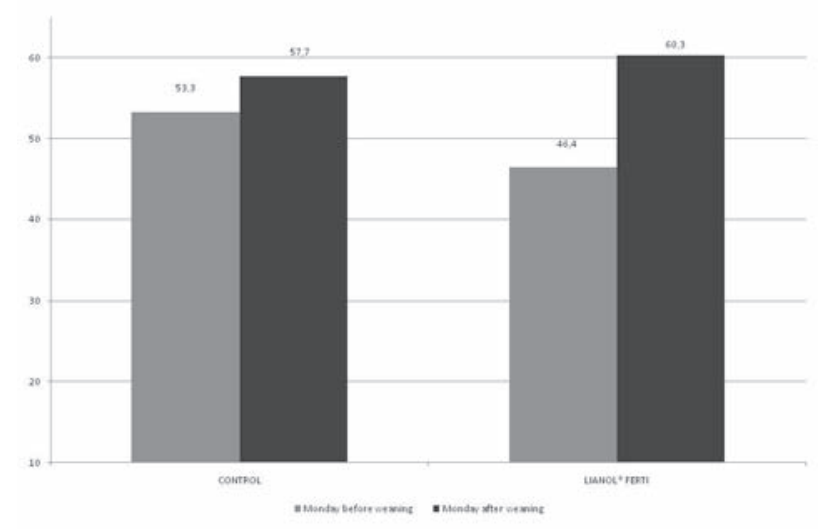

Secondly, the litter size numbers of the consequent parturition were compared in terms of live born piglets, number of piglets weaned and mortality rate. The untreated group showed 11.36 live born piglets whereas the treated group had 12.02 live born piglets, i.e. an increase of $5.8 \%$ or 0.66 piglet more at birth.

When evaluating the number of weaned piglets, the control group had a total of 9.95 piglets, whereas the Lianol ${ }^{\circ}$ Ferti group produced 10.80 weaned piglets, i.e. an improvement of $8.54 \%$ or 0.85 piglet more at weaning.

Moreover, the mortality rate in the Lianol ${ }^{\oplus}$ Ferti group (10.1\%) was considerably lower than that of the untreated control group (12.55\%).

Figure 2. Influence of Lianol ${ }^{\oplus}$ Ferti on litter size

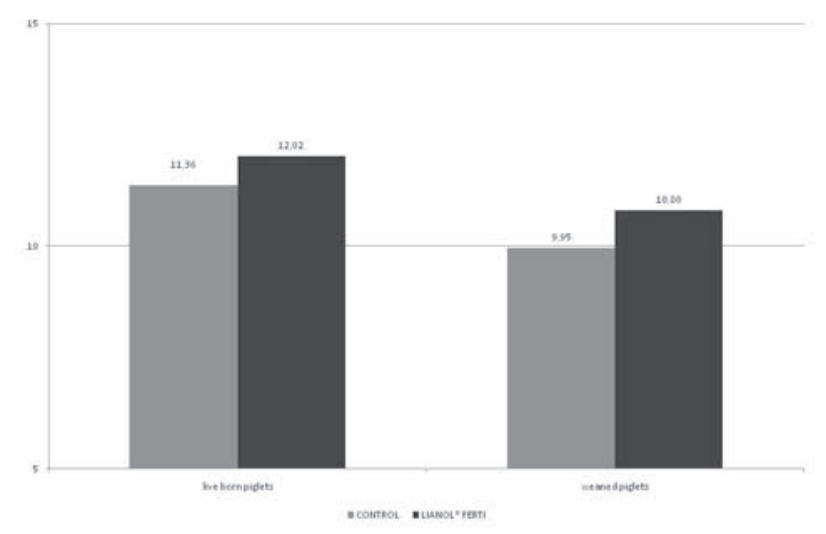

\section{Discussion and conclusion}

IGF-1 influences the ovarian activity by increasing the sensitivity and response of the follicles to FSH and $\mathrm{LH}$. It is also crucial for the survival and nidation of the embryo, but also for its quality. Therefore, the IGF-1 increase at insemination is clearly reflected in the litter size of the parturition following Lianol ${ }^{\circledR}$ Ferti treatment.

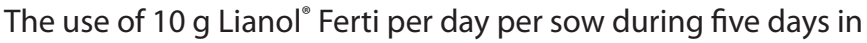
the period around weaning was confirmed to increase the IGF-1 levels and the litter size.

\section{References}

1. Devillers, N. et al. (2004), Estimation of colostrum intake in the neonatal pig, Anim. Sci., 78, 305-313.

2. Renaville, R. et al. (1993), Changes in the hypophyso-gonadal axis during the onset of puberty in young bull calves, J. Reprod. Fertil., 99, 443-449.

3. Lee, C.Y. et al. (1993), Expression of components of the insulin-like growth factor system in pig mammary glands and serum during pregnancy and pseudopregnancy: Effects of oestrogen, J. Endocrinol., 137, 473-483. 


\title{
P.721
}

\section{Effect of phytase supplementation on the digestibility and retention of phosphorus and calcium in weaned piglets}

\author{
Alain Kanora ${ }^{1}$ János Tossenberger² László Babinszky² Koen De Gussem¹ Monita Vereecken \\ 1. Huvepharma NV, Antwerp, Belgium; 2. Kaposvár University, Department of Animal Nutrition, Kaposvár, Hungary
}

\section{Introduction}

Phytase enzyme inclusion in pig diets is used to reduce the need for added inorganic phosphorus (P) from feed phosphates and animal byproducts. In this trial the effect of various doses of an Escherichia coli derived phytase (Optiphos ${ }^{\circ}$ ) on the digestibility and retention of phosphorus and calcium was studied in weaned piglets.

\section{Materials and Methods}

A balance study was carried out with 48 piglets (barrows) with an initial weight of $13.5 \mathrm{~kg}$. Animals were housed individually in metabolic crates to allow collection of faeces and urine. The six dietary treatments included a positive control (total P $6.5 \mathrm{~g} \mathrm{kg-1;}$ Digestible P $3.2 \mathrm{~g} \mathrm{~kg}-1$, Ca $7.1 \mathrm{~g} \mathrm{~kg}-1$ ), a negative control (total P $4.2 \mathrm{~g} \mathrm{~kg}-1$, Digestible P $1.6 \mathrm{~g} \mathrm{~kg}-1$, Ca $4.9 \mathrm{~g} \mathrm{~kg}-1$ ) and the NC diet supplemented with 125, 250, 500 or 1000 FTU kg-1 Optiphos .

Experimental diets were formulated on a corn- soybean basis. The study consisted of a 9 days adaptation period followed by a 5 days period of total collection of faeces and urine. At the end of the collection period the faeces and urine samples were melted, homogenized and prepared for laboratory analysis. The calcium and $P$ content of the diets, the dry matter, $\mathrm{P}$ and calcium content of the faeces, and the $P$ and calcium content of the urine samples were determined in accordance with the provisions of AOAC (1989). The trial data were analyzed with ANOVA (SAS, 2004).

\section{Results}

Supplementation of Optiphos to the NC diet resulted in a significant positive effect on phosphorus digestibility for all the supplemented enzyme doses. The $P$ retention reflected the $P$ digestibility in a consistent way. The effects of Optiphos ${ }^{\bullet}$ on Ca digestibility and retention showed similar trends as demonstrated for phosphorus although significant improvements were noticed at 250 FTU kg-1 and higher. (Table 1 and Graph 1)

Table 1: Faecal digestibility and Retention of $\mathrm{P}$ and $\mathrm{Ca}$

\begin{tabular}{|l|c|c|c|c|c|c|}
\hline & PC & NC & $\begin{array}{c}\text { NC diet + } \\
\text { Optiphos at } \\
\text { concentration } \\
\text { of 125 FTU }\end{array}$ & $\begin{array}{c}\text { NC diet + } \\
\text { Optiphos at } \\
\text { concentration } \\
\text { of 250 FTU }\end{array}$ & $\begin{array}{c}\text { NC diet + } \\
\text { Optiphos at } \\
\text { concentration } \\
\text { of 500 FTU }\end{array}$ & $\begin{array}{c}\text { NC diet + } \\
\text { Optiphos at } \\
\text { concentration } \\
\text { of 1000 FTU }\end{array}$ \\
\hline $\begin{array}{l}\text { Faecal } \\
\text { digestibility } \\
\text { P(\%) }\end{array}$ & $68.5^{\mathrm{bc}}$ & $53.9^{\mathrm{d}}$ & $63.6^{\mathrm{c}}$ & $67.8^{\mathrm{bc}}$ & $74.1^{\mathrm{ab}}$ & $75.8^{\mathrm{a}}$ \\
\hline $\begin{array}{l}\text { Retention } \\
\text { P(\%) }\end{array}$ & $67.2^{\mathrm{bc}}$ & $53.1^{\mathrm{d}}$ & $62.6^{\mathrm{c}}$ & $66.8^{\mathrm{bc}}$ & $73.0^{\mathrm{ab}}$ & $74.7^{\mathrm{a}}$ \\
\hline $\begin{array}{l}\text { Faecal } \\
\text { digestibility } \\
\text { Ca (\%) }\end{array}$ & $72.2^{\text {cd }}$ & $68.8^{\mathrm{d}}$ & $74.2^{\mathrm{cd}}$ & $75.9^{\mathrm{bc}}$ & $80.9^{\mathrm{ab}}$ & $82.1^{\mathrm{a}}$ \\
\hline $\begin{array}{l}\text { Retention } \\
\text { Ca (\%) }\end{array}$ & $68.0^{\mathrm{abc}}$ & $59.4^{\mathrm{d}}$ & $64.9^{\mathrm{cd}}$ & $67.2^{\mathrm{bc}}$ & $73.3^{\mathrm{ab}}$ & $75.1^{\mathrm{a}}$ \\
\hline
\end{tabular}

\section{Graph 1: Faecal digestibility and Retention of $\mathrm{P}$ and $\mathrm{Ca}$}

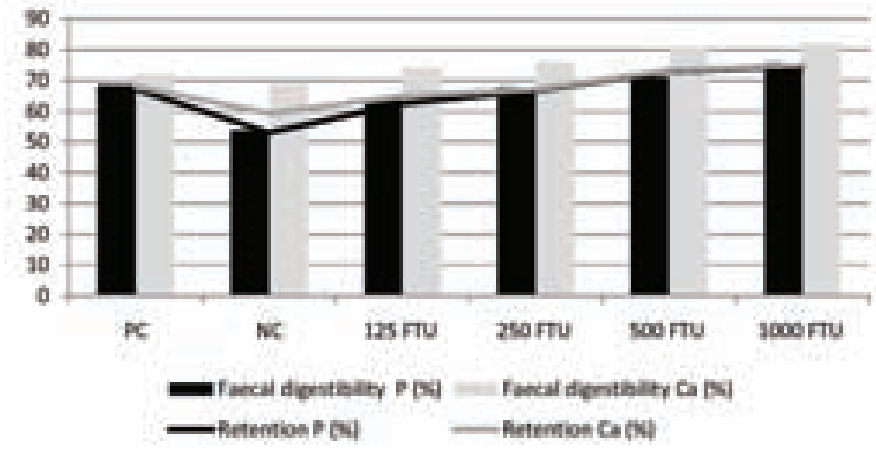

\section{Discussion}

Due to the restricted dietary phosphorus supply, the piglets had a very limited urinary phosphorus excretion (data not shown). As a consequence, the $P$ retention of the piglets showed similar trends to those of phosphorus digestibility. The urinary calcium excretion was higher than the characteristic (physiological) value of weaned pigs (data not shown).

This high urinary Ca excretion (hypercalciuria) indicates, that the digestible phosphorus content of the diets did not cover the requirements of the animals, and consecutively the absorbed $\mathrm{Ca}$ could not be retained in the bones due to a lack of phosphorus in the blood.

As a conclusion supplementation of Optiphos resulted in the improvement of the phosphor and calcium digestibility.

\section{References}

1. AOAC (1989). Association of Official Analytical Chemists. 1989. Official methods of analysis. 3rd ed. AOAC, Washington, DC.

2. SAS, 2004. SAS User's Guide:Statistics Inst., Inc. Cary NC. 


\title{
P.722
}

\section{Age dependent changes in relative and absolute size of leukocyte subsets in the porcine peripheral blood}

\author{
Iwona Markowska-Daniel; Malgorzata Pomorska-Mol \\ National Veterinary Research Institute, Pulawy, Poland
}

\section{Introduction}

The development of the porcine immune system is not complete at birth. Because of its maturation, the amount of leukocyte (LEU) populations vary during life. According to our best knowledge, up today, there are no data about changes in pigs blood LEU during whole fattening period. Therefore, the aim of the study was to analyze data on the changes of LEU subsets from birth up to about 5 months of age.

\section{Materials and Methods}

Study was conducted on healthy pigs $(n=118)$, aged from birth to slaughter. Blood were collected 12 times. Samples were analyzed using hemocytometer and immunophenotyped in order to determine CD3+ (T LYM), CD21+ (B LYM), T LYM subset: CD4+, CD8+ and double positive (DP) - CD4+CD8+. Statistical analyses were performed with Statistica 8.0 (Statsoft, Poland).

\section{Results}

The number of LEU and LYM increased with age from $12.38 \times 109 / \mathrm{L}-1$ on the first day to $25.70 \times 109 / \mathrm{L}-1$ at the 5 month of life and from $5.11 \times 109 / \mathrm{L}-1$ to $9.64 \times 109 / \mathrm{L}-1$ respectively $(\mathrm{p} \leq 0.05)$. The percentage (\%) of LYM was the highest around 14 days of life. The lowest \% of LYM was observed at 84 days of life.

The absolute size of granulocytes (GRA) decreased 1.5-fold just after birth, remained stable until 21 days, and subsequently increased from weaning to 5 months of age. Strong positive correlation (COR) was found between number and \% of GRA and the pigs' age. Starting from 70 day of life the $\%$ of GRA outnumbered about 1.5 -fold the $\%$ of LYM. Just after birth the $\%$ of CD3+ cells increased over $20 \%$ and remained stable until 5 months of age. Positive COR between numbers of CD3+cells and the age was determined. Apart from a significant increase between 14 and 21 day old piglets $(p \leq 0.05)$ no age-related changes were observed in the \% of CD21+ LYM, but positive COR was found between their numbers and age. The numbers of CD3+ and CD21+ cells increased 2-fold from birth to 140 day of life. Just after birth, significant increase of the \% of CD8+was noted $(p \leq 0.05)$. The $\%$ of CD4+ cells were the highest between 14 and 21 days of ages, but these values did not differ significantly from the neighboring age groups. The numbers of DP cells increase with the age $(p \leq 0.05)$ and positive COR was observed between their numbers and age. The absolute number of CD3+ cells increased 2 -fold from birth to 2 weeks after weaning. After this time only CD4+ subset remained stable up to 5 month of life. The amount of remaining populations gradually increased 1.5- and 2.5-fold from 6 week to 5 month of life, respectively for CD8+ and DP cells. Negative COR was evidenced between age and CD4/CD8 ratio.

\section{Discussion}

In pigs, as in different species of animals, the LYM/GRA ratio revealed changes during life. On day first GRA numbers were almost the same as LYM numbers. Over the first 3 weeks of life a predominance of LYM were observed. Starting from 70 day of life GRA outnumbered LYM. Results demonstrated that \% of LYM populations does not reflect their actual size and is therefore of limited value. The increase of CD3+ cells numbers were caused by rising amounts of CD8+ and DP cells. The results of our investigation showed that just after birth the numbers of DP cells, containing activated and memory T cells, increased almost twofold. Directly after weaning the numbers of these cells increase again at about $40 \%$. These data are in agreement with previous results, dealing about age-related changes in LEU counts in pigs up to 41 day of life (3). Till weaning the numbers of LYM were lower than in weaners $(p \leq 0.05)$. From our results it follows that CD4+/CD8+ ratio decreases with age, which is in accordance with other studies $(1,3)$. The amounts of DP LYM in blood of pigs increase with age $(1,3)$. The $\%$ of DP LYM ranges from 4 to $60 \%$ (1). In our study the $\%$ of DP cells increased from $6.63 \%$ at birth to $17.48 \%$ in 20 weeks old pigs, while the absolute number increased almost 5-fold. Age-dependent increase of DP LYM seem to be a result of antigen-dependent maturation of naïve CD4+ Th cells to antigen-specific memory Th cells (1). On the basis of this study we suggested that immunophenotyping of LYM, especially for diagnostic purposes, should base on the absolute size rather than on the \% of LYM subsets, according to appropriate age-matched reference values. Additionally, because there are age-related differences in LYM subsets distribution, there is a need to use appropriate age matched control group (as closely as possible) in the experiment with pigs, especially those which include immunophenotyping.

\section{References}

Stepanova et al. Cell Immunol 2007, 249, 73-792. Joling et al. Vet Immunol Immunopathol 1994, 40,105-108. Borghetti et al. Vet Immunol Immunopathol. 2006, 110, 17-25. 


\title{
P.723
}

\section{Effect of three different antibiotics on commensal intestinal microflora and on some productive traits of weaning piglets}

\author{
Paolo Bosi ${ }^{2}$ Giuseppe Merialdi ${ }^{1}$ Lia Bardasi $^{1}$ Salvatore Scandurra ${ }^{3}$ Mario Vecchi ${ }^{3}$ \\ Stefano Messori ${ }^{2}$ Paolo Ferro ${ }^{3}$ Irma Nisi $^{2}$ Luisa Casini ${ }^{2}$ Paolo Trevisi ${ }^{2}$ \\ 1. Istituto Zooprofilattico Sperimentale della Lombardia e dell'Emilia Romagna, Bologna, Italy; 2. Diproval, University \\ of Bologna, Bologna, Italy; 3. Elanco Animal Health, Div. Eli Lilly Italia, Sesto Fiorentino (FI), Italy
}

\section{Introduction}

Weaning constitutes a critical situation in the life of pigs and it is associated with a number of stress factors, such as separation from the sow and abrupt change from milk to solid feed. These factors may affect the gut physiology, the gut microflora and the immune system of weaning piglets, leading to enteric disorders (1). Weaning piglets are frequently treated with antibiotics for diseases control. Antibiotics can have an additional detrimental effect on gut microflora, leading to decrease in lactic flora and increase in potential pathogenic Enterobacteriaceae concentrations. An in vivo experiment was performed to study the effect of three different antibiotics on gut microflora and on some productive parameters and in weaning piglets.

\section{Materials and Methods}

Seventy-two newly weaned conventional piglets were received by an experimental unit at 24 days of life. They were randomly assigned to 4 different diets: conventional weaning diet without antibiotics $(C)$, diet T with tilmicosin (Pultotil TM $\left.{ }^{\circledast}\right)$ supplementation $(400 \mathrm{mg} / \mathrm{Kg})$, diet A with amoxicillin (Gammamix ${ }^{\circledR}$ ) supplementation $(400 \mathrm{mg} / \mathrm{Kg})$ and diet $\mathrm{D}$ with doxiciclin (Pulmodox $\left.{ }^{\oplus}\right)$ supplementation $(300 \mathrm{mg} / \mathrm{kg}$ ). The experiment had an overall duration of 4 weeks. Antibiotics were added to diets for 3 weeks. During the last week all groups were fed with diet $C$. The body weight and the feed intake of every single pig was recorded weekly. At day 0, 7, 14, 21 a fecal sample was collected from each pig for enumeration of Enterobacteriaceae and lactobacilli. At day 2 a blood sample was collected from each animal for lgM and IgA titration. A fecal score was recorded daily. 12 subjects/ diet and 6 subjects/diet were sacrificed after 3 and 4 weeks respectively and samples were taken to measure full and empty weight of the small intestine; jejunum content for the bacteria counts villus height and crypt depth on villi.

\section{Results}

In the groups with antibiotic diets, average daily gain increased by $34.7 \%$ and by $9.0 \%$, in the 1 st week and in the 3 weeks of treatment, respectively. Feed intake was increased by the anti- biotics in the 1 st week and in the 3 weeks of treatment $(+19 \%$; + 8.3\%). (Table 1). Faecal Enterobacteriaceae counts were changed by the antibiotic treatments, and this effect was linked with diet $\mathrm{T}$ (Table 2). Lactobacilli were lower in A.

Table 1. Effect on ADG and FI

\begin{tabular}{|c|c|c|c|c|c|}
\hline & \multicolumn{4}{|c|}{ Diet } & \multirow{2}{*}{$\frac{p}{C \text { vs }(A+D+T}$} \\
\hline & $\mathrm{C}$ & $A$ & D & $T$ & \\
\hline ADG (g) d 0 to 7 & 120 & 156 & 157 & 169 & $<0,01$ \\
\hline ADG $(\mathrm{g})$ d 0 to 21 & 310 & 332 & 346 & 334 & $<0,05$ \\
\hline $\mathrm{Fl}, \mathrm{g} / \mathrm{d}$ d 0 to 7 & 217 & 251 & 261 & 261 & $<0,01$ \\
\hline $\mathrm{Fl}, \mathrm{g} / \mathrm{d}$ d 0 to 21 & 433 & 457 & 477 & 473 & $<0,05$ \\
\hline
\end{tabular}

Table 2. Feacal bacteria counts

\begin{tabular}{|l|c|c|c|c|c|c|}
\hline & \multicolumn{4}{|c|}{ Diet } & \multicolumn{2}{c|}{ p } \\
\hline & C & A & D & T & T vs A+D & A vs D \\
\hline $\mathrm{d} 21$ & 4,96 & 5,74 & 4,30 & 1,78 & $<0,01$ & $<0,01$ \\
\hline & & & & & & \\
\hline $\mathrm{d} 21$ & 8,48 & 7,78 & 8,45 & 8,50 & 0,051 & $<0,01$ \\
\hline
\end{tabular}

Total IgM content in blood of all pigs after 21 days was averagely reduced in groups with antibiotic diet $(P=0.012)$ while $\lg A$ total contents were not affected (data not shown). No differereces were recorded for other parameters (data not shown)

\section{Discussion}

The presented results suggest that antibiotics can have a different impact on gut micloflora in agreeement with previous observations (2). This should be taken in consideration in the choice of the antibiotic in such a critical moment as weaning.

\section{References}

1. Naaburs M.J.A., 1998, Vet Q., 20, 42-45

2. Collier, C.T. et al. 2003.. J. Anim. Sci. 81:3035-3045 


\title{
P.724
}

\section{Study of the productive benefits of breeding sows due to the dose increase of a commercial organoaluminosilicate}

\author{
Juan C. Medina' Ernesto Ponce Manzano ${ }^{2}$ David Herrera M. ${ }^{1}$ \\ 1. NUTEK S.A de C.V., Tehuacan, PUE, Mexico; 2. Asesor Independiente, Tehuacan, PUE, Mexico
}

\section{Introduction}

Mycotoxins are substances produced by the metabolism of growing fungi. They can be present in grains under favorable conditions in the field, storages and transportacion. Animals exposed to mycotoxins, even in low levels develop a wide range of clinical signs such as weight gain reductions, smaller offspring numbers, teratogenic effects and decrease of productivity in all animal species. The aforementioned represents economic looses for the producers. Council for Agricultural Science and Technology (CAST,2003) refers that potential economic cost of crop losses from mycotoxins in the US only, are large with a mean of \$932 million per year. Mean mitigation costs were estimated to be about $\$ 466$ million and the mean simulated livestock cost were $\$ 6$ millions per year. This study focused on improving productive parameters by using a combination of mycotoxins binders (aluminosilicates (aflatoxins adsorbent) and organoaluminosilicates (zearalenone and mycotoxin adsorbent)). The farm subject of this study did not perform mycotoxins analyses in the past, and the addition of binders was used as a preventative measure. The farm currently adds $2.5 \mathrm{~kg} /$ ton of feed of Zeolex Extra more $1 \mathrm{~kg} / \mathrm{ton}$ of ZEOTEK and use US \# 2 quality grains (sorghum or maize).

\section{Objective}

To demostrate that the addition of $05 \mathrm{~kg} /$ ton of ZEOTEK in the feed of breding sows improves their productive parameters and afford a positive cost-benefit ratio for the production facilities.

\section{Material and Methods}

The company subject of this study has around 4,000 breding sows. The experiment was made using two gropus of 1,800 swine each for fourd months. The first group was given a diet including $1 \mathrm{~kg}$ of ZEOTEK per ton of feed (control group) while the other was given a diet including $1.5 \mathrm{~kg}$ of ZEOTEK per ton of feed (experimental group).

\section{Results}

Statistically significant results were observed in the average feed consumption between the groups involved in the experiment. The rest of the parameters evaluated only had numerical differences (fertility percentaje, piglets per birth, mortality of piglets, average age at weaning, piglest average age at weaning, weaned piglets per sow/yeaar, breding mortality, etc).

\section{Discussion}

The continuous use of binders on preventive doses decreased the effects of mycotoxins presents in the grains used to make feed. Important facts were the sows consumed less feed during nursing ( $3.4 \mathrm{~kg} /$ weaned piglet). The experimental group consumed an average of 9.3 USD less of feed per sow. Thus the addition of higher doses of mycotoxin binders decreased the amount of feed needed to maintain the productive parameters of the farm. These results have been report by many other studies in several species.

\section{References}

CAST: Council for Agricultural Science aand Technology. Task Force Report No. 139. 2003. Ames lowa. USA. 


\title{
P.725
}

\section{Kafirins effect on ileal digestibility of sorghum protein in growing pigs}

\author{
Luis Hernandez $^{2}$ Gerardo Mariscal ${ }^{1}$ Tercia C. Reis de Souza ${ }^{3}$ Juan Becerra ${ }^{1}$ Hector Jimenez ${ }^{1}$ \\ 1. INIFAP - CENID Fisiologia, Ajuchitlan, QRO, Mexico; 2. FES Cuautitlan - UNAM, Cuautitlan, MEX, Mexico; \\ 3. Universidad Autonoma de Queretaro, Queretaro, QRO, Mexico
}

\section{Introduction}

Sorghum is characterized for its low protein digestibility, which is attributed to tannins. However, tannins effect is observed only at high levels (1). Sorghum proteins are classified in: albumins, globulins, prolamins and glutelins. Among them, kafirins (prolamins) are the most abundant. In low tannin sorghums $(<1.0 \%)$ the low digestibility of their protein has been associated to the kafirin content (1). In vitro studies report that digestibility of sorghum protein decreases when kafirin proportion increase (2). The objective of this work was to evaluate in vivo the kafirins effect on ileal apparent (IAD) and ileal standardized (ISD) digestibility of sorghum protein in growing pigs.

\section{Materials and Methods}

A low tannin $(<0.5 \%)$ sorghum hybrid was produced; half of the plot was over fertilized with nitrogen to increase the kafirins level, in order to get the same hybrid with high and low kafirin content. Four Duroc x Landrace pigs weighing $60 \mathrm{~kg}$ were fitted with a "T" cannula at the ileal level. Pigs were placed in metabolic cages and fed twice a day (09:00h and 16:00h), at 2.5 times their Digestible Energy requirement. Experimental diets were fortified sorghum. The experimental period lasted seven days (five days for adaptation and two days for collection). lleal digesta were collected in plastic bags during $24 \mathrm{~h}$ and Frozen at $-20 \mathrm{C}$. At the end of the experimental period ileal digesta were freeze-dried and ground. The next analyses were performed in diets and ileal digesta: Dry matter (DM), crude protein (CP), chromium and tanins. Western Blot analyzes, using an anti kafirinas antibody previously produced, were performed on ileal digesta. IAD of CP and DM and ISD were also analyzed (1) using a "cross over" design with the "Mixed" procedure of SAS (3).

\section{Results}

The CP content was similar, 10.4 and $10.0 \%$, for the high and low kaffirin sorghum respectively. Tannin content was very low in both sorghums $(<0.02 \%)$. However, the relationship between the fractions 2 (alcohol soluble) and 3 (alcohol plus reducing agent) of Landry-Moureaux was different. The low kafirin sorghum 0.73:1 vs high kafirin sorghum 0.5:1. The DM and CP ileal apparent digestibility, as well as CP standardized ileal digestibility were lower in high- as compared with low kafirin sorghum, 0.811 vs $0.839(P<0.10), 0.693$ vs $0.745(P<0.05)$ and, 0.813 vs 0.868 $(P<0.05)$, respectively. These results were supported by western blot analysis.

\section{Discussion}

The lower digestibility in high kaffirin sorghum is due to the higher content of disulfide links, which negatively affect protein digestibility (4).

\section{References}

1. Mariscal-Landín G, et al. 2004. Anim. Feed Sci. Technol. 117, 245-264.

2. Oria, P.M., et al. 1995. J. Cereal Sci. 22, 85-93.

3. SAS. 2002. SAS Institute Inc., Cary, NC, USA.

4. Lallès, J.P., Toullec, R., 1996. INRA Prod. Anim. 9, 255-264. 


\title{
P.726
}

\section{Yeast autolysate: microbiological and immunological properties in vitro and effect on piglet performance in vivo}

\author{
Anja Ganner $^{2}$ Sabine Masching ${ }^{1}$ Gerd Schatzmayr ${ }^{2}$ \\ 1. Biomin Holding GmbH, Herzogenburg, Austria; 2. Biomin Research Center, Tulln, Austria
}

\section{Introduction}

Dietary yeast derivatives such as yeast cell walls, beta-glucan fractions, yeast autolysate and yeast nucleotides have been proposed to improve piglet health by preventing infectious diseases, by modulating the immune system and by controlling pathogenic bacteria.

Although various yeast derivatives have been used as feed additives for a long time, their in vitro and in vivo mode of action to improve swine performance is still not well understood.

Target of the present study was to investigate a yeast autolysate in vitro for its chemical composition, for its microbiological binding capacity and immunological properties and in vivo for its effect on piglet health and performance.

\section{Materials and Methods}

\section{In vitro}

Cell culture studies: As an in vitro model a chicken bone marrowderived macrophage cell line (HD_11) was used. The nitric oxygen (NO) production was determined as an indicator for immune stimulation. It was determined after $48 \mathrm{~h}$ of incubation with yeast products in the supernatant of the cultures using a Griess reagent which provides a colorimetric reaction. Results were compared with the positive control (LPS E. coli 0127; 20ng/ $\mathrm{mL}+/-10 \mu \mathrm{Mol} \mathrm{NO}$ ) and calculated as a \% of the positive control. Additionally the yeast autolysate was examined for its capacity to bind E. coli F4 with a quantitative microbiological microplatebased assay by measuring the optical density as growth parameter of adhering bacteria. The growth rate of adhering bacteria correlates with the amount of adhering bacteria bound to the yeast product. Total mannan and glucan were determined by HPLC using an isocratic method and a Refractive Index (RI) Detector. Polysaccharides were hydrolyzed with $72 \%$ sulfuric acid; with a subsequent Carrez-precipitation interfering materials were removed. Total protein was determined by Total Kjeldahl Nitrogen (TKN).

In vivo

A feeding trial was conducted to evaluate the efficacy of yeast autolysate on performance and health status of weaning piglets in a 56 days study. 40 mixed sexed piglets weaned after 28 days were divided into 2 experimental groups with 2 replicates: control group A, group B (1 kg yeast autolysate product/t feed).

Statistical analyses were performed with t-test, SPSS 18.

\section{Results}

In vitro

Nitric oxygen production was enhanced up to $80 \%$ compared to the positive control. Microbiological assay: E. coli F4 adhered with an amount of $1000000 \mathrm{CFU} / \mathrm{mg}$ to the yeast autolysate. Mannan content was $17 \%$, glucan content $25 \%$ and total protein in dry matter $43 \%$.

In vivo

In the course of the trial a clear positive influence could be observed by the addition of yeast autolysate. Live weight on day $56(43.13 \mathrm{~kg})$ and daily weight gain (604) were improved in comparison to the control (40.92 kg live weight day $56, \mathrm{P}=$ $0.048 ; 505 \mathrm{~g} \mathrm{dwg}, P=0.025)$. Feed consumption was increased ( $P$ $\geq 0.05), F C R(\mathrm{~kg} / \mathrm{kg}$ ) of the trial group was 1.74 , from the control $1.71(P \geq 0.05)$.

\section{Discussion}

The present study revealed that yeast autolysate is an inducer of nitric oxygen production by macrophages. This stimulation might improve animal health by alerting the immune system and preparing it to respond quickly to infections and to counteract the effects of weakened immunity. Additionally yeast autolysate containing mannan-oligosaccharides have been proposed to lessen the effect of enteropathogenic bacteria such as E. coli F4 by displaying alternative adhesion sites and thus binding such pathogens and moving them throughout the gut without colonization. Therefore enteric diseases can be prevented and piglet health maintained. Pathogen binding and modulation of the immune system, as shown in vitro, might have been reasons for the increase in performance of piglets in the feeding trial.

In vitro and in vivo results indicate that this particular yeast autolysate product is a health and performance improving agent. 


\title{
P.727
}

\section{Effects of a synergistic feed additive formulation against gram negative bacteria associated diseases}

\author{
Simone Schaumberger ${ }^{1}$ Sabine Masching ${ }^{2}$ Anja Ganner ${ }^{1}$ Gerd M. Schatzmayr ${ }^{1}$ \\ 1. Biomin Research Center, Tulln, Austria; 2. Biomin Holding GmbH, Herzogenburg, Austria
}

\section{Introduction}

Gram- negative bacteria associated diseases are a present issue in pig health causing economical losses in swine production. Finding a way to protect pigs against overwhelming bacterial growth in the gut and trying to minimize the symptoms is of great importance.

Colistin (Polypeptidantibiotic) is said to be an effective antibiotic against gut infections caused by gram-negative bacteria (E.coli, Salmonella sp.) and in this relation neutralizing endotoxins. Endotoxins are of special interest, as cell wall parts of gram negative bacteria are known to be associated with different disease patterns as MMA and sudden piglet death. Endotoxin associated diseases are very complex in their etiopathology due to different modes of action in inflammation cascades. Therefore it is very important to ensure an overall protection for the animal. On the one hand endotoxin counteracting feed additives should be able to bind toxins and bacteria and on the other hand keep inflammation processes under control and modulate the immune- system.

Aim of this study was to prove the positive effect of a feed formulation containing a yeast-derivate, a clay mineral and a plant extract with anti-inflammatory properties on health status and performance of weaning piglets.

\section{Materials and Methods}

90 piglets chosen from 15 litters were used for this experiment. A $3 \times 3$-trial design was employed, meaning 3 groups with 3 replications each.

The piglets were approx. 4 weeks of age with a mean initial weight of $8.39 \mathrm{~kg}$.

- Group A (control): standard piglet diet without any addition.

- Group B (positive control): standard piglet diet without any addition, but receiving $100 \mathrm{mg}$ Colistin/litre drinking water for 21 days.

- Group C (treated) supplied with $0.2 \%$ of the feed additive formulation over the whole trial period.

A 2-phase feeding system was used, where starter diet was fed between days 1 - 14 and grower diet from day 15 until the end of the trial. Feed and water were supplied ad libitum intake.

Clinical observation was done twice a day and all incidences recorded. Weight of each single animal was recorded at the beginning, at days 14, 42 and 56. The amount of feed distributed, feeding frequency and mixing ratio were recorded automatically per pen and day. All animals were supervised by the responsible veterinarian on a regular basis. All data generated out of the trial was subjected to statistical analysis by means of PASW 18.0.

\section{Results}

On day 56 weight of piglets of group $C$ was improved with a statistical difference of $p=0.019$ compared to the control group. Daily weight gain (dwg) (days 1 - 56) showed significant differences ( $p=0.007$ ) between groups $A$ and $B$ and $A$ and $C$. For feed conversion rate (FCR) and feed intake no statistical differences could be observed, but for group C FCR (1.77) was lower as for group A (1.83) and B (1.88).

In group A one animal died without showing any symptoms. On day 7 single incidences of diarrhea occurred in some pens and lasted for 13 days in changing intensities: group A showed diarrhea for 8 days, group B for 7 days and group C only for 4 days.

\section{Discussion}

The present study shows that a natural feed additive composed of 3 synergistically acting ingredients can replace a feed antibiotic and results in better total end weight, dwg and FCR as well as reduced diarrhea incidences. Although most of the animals showed signs of diarrhea, the growth performance was within the standards. Results indicate that the additive supported the animals in a critical phase of weaning.

Clays binding toxins, yeast derivates binding bacteria as well as acting anti-inflammatory and plant extracts acting anti and proinflammatory might have a synergistic effect in vivo and might have been the mode of action for a better performance in our study.

\section{References:}

L. Malagutti, M. Zannotti, F.Sciaraffia Use of clinoptiolite in piglet diets as a substitute for Colistine 2002; Ital.J.Anim.Sci. Vol 1, 275- 280

H.M.G. van Beers-Schreurs, L.Vellenga, Th. Wensing and H.J. Breukink.The Pathogenesis of the post-weaning syndrome in weanded piglets; A review. 1992; Vet. Quart. Vol 14, 29-34 


\title{
P.728
}

\section{Effect of dietary crude protein level on villous morphology and diarrhea incidence in weaning pigs}

\author{
Tercia C. Reis de Souza ${ }^{1}$ María de Jesús Guerrero Carrillo ${ }^{1}$ Araceli Aguilera Barreyro ${ }^{1}$ María Guadalupe Bernal Santos ${ }^{1}$ \\ Konisgmar Escobar García1 Gerardo Mariscal Landín² \\ 1. Universidad Autónoma de Querétaro, Querétaro, QRO, Mexico; 2. INIFAP, Ajuchitlán - Querétaro, Mexico
}

\section{Introduction}

Post-weaning diarrhea is a problem in nursery pig nutrition and management, mainly after the elimination of in-feed antibiotics (1). The most important nutritional factor for reducing scouring in pigs is to reduce the dietary crude protein (CP) level; because it can reduce the severity and extent of post-weaning diarrhea $(2,3)$. However, if the dietary CP level is too low, it may affect the growth of villi $(4,5)$. The objective of this study was to measure the effect of dietary CP level on villous morphology, and severity of diarrhea during the first 14 days after weaning.

\section{Materials and Methods}

Fifty pigs weaned at $20.2 \pm 2.0 \mathrm{~d}$ of age (initial BW: $6.34 \pm 1.09 \mathrm{~kg}$ ) were allotted to 2 treatment groups based on litter of origin, and BW. There were 5 pigs per pen and 5 pens per treatment. Two antibiotic-free diets were formulated. The HCP diet contained $19.4 \%$ CP, and the LCP diet contained $15.8 \%$ CP. The severity of diarrhea was based on a daily visual faecal consistency score (FS) on a scale from 0 to 3 (6). At the end of the experiment 5 piglets by treatment were slaughtered by exsanguinations from the jugular vein, and the entire intestinal tract was excised for sample collection. One segment of intestine $(5 \mathrm{~cm})$ was removed from the duodenum, middle jejunum, and distal ileum. The intestinal samples were fixed in neutral buffered formaldehyde and embedded in paraffin, sectioned at 5 micron thickness and stained with hematoxylin and eosin (HE). Villous height and width, and crypt depth were measured on the stained sections under microscope. A minimum of 10 straight, intact villi in each intestinal position was measured for each piglet within each treatment. Villous height was measured from the crypt base to villous tip, and villous width at mid-section. All measurements were made in 10 micron increments.

\section{Results and Discussion}

There were significant declines of villous height in the jejunum $(P<0.001)$ and ileum $(P<0.05)$ with the reduction of dietary $C P$ (Fig. 1), however villous width and crypt deep were not affected. The intestinal villi increase the exchange area, thus enhancing both digestion and absorption, and they are very important for piglets to absorb nutrients (5). Decreasing dietary CP from 19.4 to $15.8 \%$, villous height of the jejunum and ileum decrease almost $50 \%$ in the current study, implying that severe protein deficiency may have reduced villous growth $(4,5)$ and functional development of intestinal epithelium (4). Protein content of the diet had a greater effect on villous morphology in the jejunum than in the duodenum in agreement with other observations (4).

Severity of diarrhea was not affected by the dietary CP level. There was a quadratic effect of the post-weaning day $(P<0.0001$, $\mathrm{R} 2=0.94$ ) on FS of piglets fed both diets described by the following equation: $F S=-0.012+0.399 d-0.022 d 2$, where $d=$ postweaning day (Fig. 2). FS increased during the first nine days and decreased until day 15 post-weaning.
Previous studies have reported that reduced CP diets benefit to the health of piglets by reducing the diarrhea incidence $(1,5,6)$. Nevertheless, our results can't confirm the above findings. Probably, the reduction of intestinal functional area in piglets fed the LCP, increased the quantity of nutrient not digested and absorbed, which could be substrate for the fermentation by the microbiota, and consequently diarrhea. In conclusion, LCP diet decreased the villous height in jejunum and ileum and doesn't have a positive effect in diarrhea control.
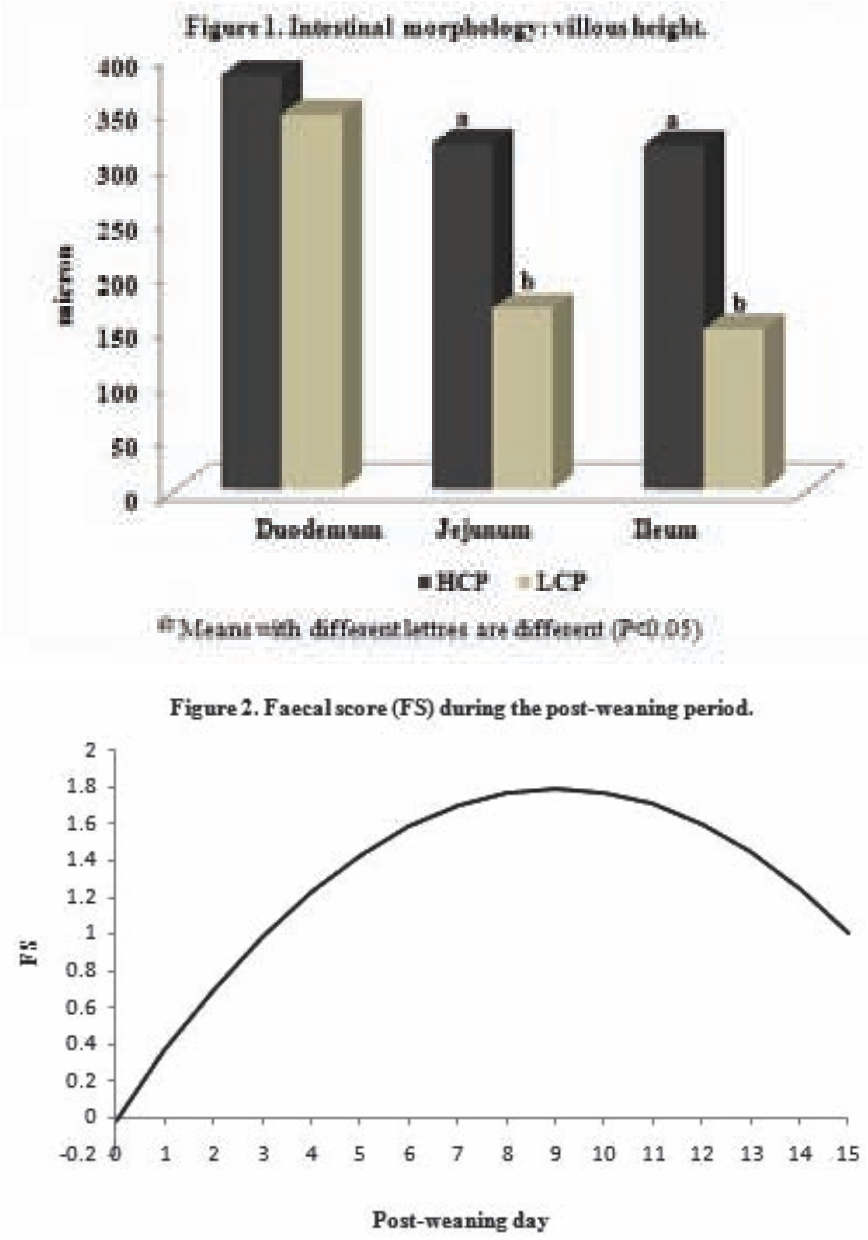

\section{References}

1. Nyachoti CM et al., 2006. J. Anim. Sci. 84, 125-134.

2. Stein $\mathrm{HH}$ et al., 2006. Anim. Biotechnol. 17, 227-231

3. Le Bellego L et al. 2002. Livest. Prod. Sci. 76, 45-58.

4. Gu et al. 2004. Anim. Feed Sci. Technol.114, 113-126.

5. Yue LY et al. 2008. Livest. Sci. 115, 144-152.

6. Ball et al. 1987. Can. J. Anim. Sci. 67, 1105-1115. 


\title{
P.729
}

\section{Effect on the number of stillborn when adding synthetic vitamin E or natural vitamin $E$ to the sow diet}

\author{
Jesper S. Olesen ${ }^{1}$ Claes Enoee $^{2}$ \\ 1. Porcus Swinespecialists, Ringe, Denmark; 2. Veterinærinstitutet, Copenhagen, Denmark
}

\section{Introduction}

An ever rising number of total born piglets in modern swine production make both the number of live born rise but unfortunately also the number of stillborn. When a sow herd experiences a period with many stillborn it is often considered to be an infectious agent that causes the problem. This trial suggests that the cause of many stillborn also could be a physiological one. The thesis was that the muscle of the uterus with big litters was fatigued before the farrowing was finished and that the antioxidant effects of vitamin E could positively influence that process. It was shown earlier that different amounts of selenium and vitamin $\mathrm{E}$ given during gestation can have effects on the number of stillborn (1). It is also shown that sows given a diet with natural vitamin $\mathrm{E}$ (d-alfa-tokoferol-acetate) have a higher concentration of the vitamin $\mathrm{E}$ in plasma and milk compared with sows given a diet with synthetic vitamin E (dl-alfa-tokoferolacetat) (2). Those two studies were background for this trial.

\section{Materials and Methods}

The study was a double blinded clinical trial with 2 treatment groups and 1 control group. The treatment groups received 1000 mg vitamin $\mathrm{E}$ in minimum 7 days just before farrowing. One treatment group had natural vitamin $\mathrm{E}$ and the other treatment group had synthetic vitamin E. The control group received a placebo. In the 3 groups there were a different number of farrowings: in the placebo group 148, in the synthetic group 147, in the natural group 140.

The statistics were done by Claes Enoee in SAS version 9.1.3 in PROC UNIVARIATE and PROC GLIMMIX.

\section{Results}

After analyzing the complete dataset the percent of stillborn was $10 \%$ in the group that received natural vitamin $E$ and $11.7 \%$ in the groups that received synthetic vitamin E and placebo. After the initial descriptive analysis it was clear that the big difference in stillborn within the treatment groups was to be found among the older sows (third parity sows and older).

In the younger sows (first and second parity sows) the difference between placebo and natural vitamin E was not significant $(p=0.37)$. In the older sows the difference between placebo and natural vitamin $E$ was significant $(p=0.03)$. Analyzing the dataset with the older sows alone, the percent of stillborn was $12.4 \%$ in the group that received natural vitamin $\mathrm{E}$ and $14.8 \%$ in the group that received synthetic vitamin E. Among the older parity sows that received placebo the percent of stillborn was $16.7 \%$. See diagram 1.

\section{Diagram 1.}

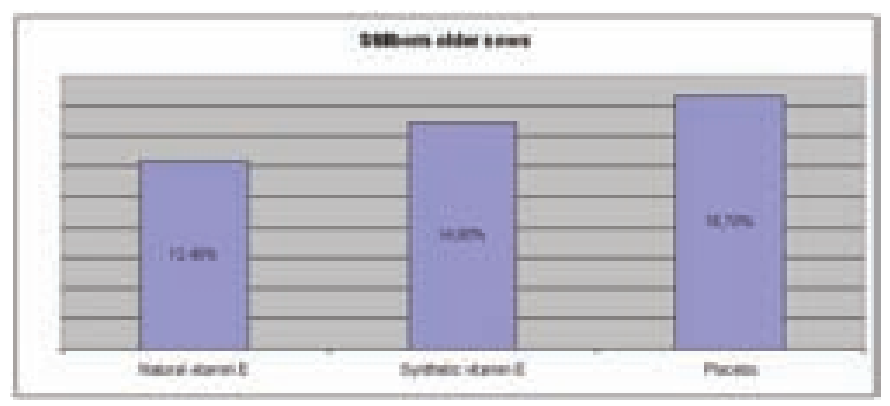

\section{Discussion}

This study showed that in one Danish sow herd adding of 1000 mg natural vitamin E for a minimum of 7 days significantly reduced the number of stillborn in older sows (3 litter sows and older sows).This proves the thesis of the trial that there might also be physiological reasons for high numbers of stillborn not only infectious ones. The number of stillborns was reduced from $16.7 \%$ to $12.4 \%$ in the older sows.

\section{References}

(1). Chavez E. R.; K. L. Patton. (1986): Effect of injectable selenium and vitamin $E$ on reproductive performance of sows receiving a commercial diet. Research reports, Department of Animal Science, McGill University 1986. pp. 52-56.

(2). Lauridsen C.; H. Engel; S. K. Jensen; A. M. Craig. (2002): Lactating sows and suckling piglets preferentially incorporate RRR- over All-rac-alpha-tocofherol into milk, plasma and tissues. Journal of nutrition. 132. pp. 1258-1264.

\section{Acknowledgements}

Hans Aae, Head of nutrition, Vitfoss, Gråsten, Denmark 


\title{
P.730
}

\section{A UK farm study to evaluate productivity parameters following routine iron injection of outdoor piglets}

\author{
Richard B. Pearson \\ George Veterinary Group, Malmesbury, UK
}

\begin{abstract}
Introduction
The vast majority of indoor units administer supplementary iron to piglets in the first few days of life. This is done to prevent iron deficiency anaemia from occurring, which is characterised by hypochromic/microcytic anaemia. Supplementary iron is usually provided in the form of $200 \mathrm{mg}$ of iron as iron dextran delivered by intramuscular injection. In contrast, it is quite rare for outdoor born piglets to receive routine iron, it being widely believed that access to the outdoor environment allows piglets sufficient soil intake to prevent iron deficiency. The aim of this study was to evaluate possible differences in productivity parameters arising from an intervention using routine iron injection of neonatal piglets on a large commercial outdoor unit in Southern England. If differences in productivity parameters were seen then the study was to be extended to include a comparison of haematological parameters between treated and untreated piglets.
\end{abstract}

\section{Materials and Methods}

The unit comprised an 1800 sow outdoor herd which was split into two 900 sow units (Units 1 and 2). Both units had the same management, feed, genetics, average weaning age (26 days) and soil type. Farrowed sows were group managed in paddocks containing 12 uninsulated arcs, with fenders attached. During the summer wallows were established in all farrowing paddocks. Prior to the start of this study iron injection of piglets was not practised. The treatment consisted of $200 \mathrm{mg}$ of iron as iron dextran ( $1 \mathrm{ml}$ of Uniferon- Pharmocosmos). In the first instance Unit 1 was allocated to treatment and Unit 2 to control. For a period of 4 weeks (Batch 1) all piglets were treated on Site 1 at $\sim 48$ hours old. Thereafter, treatment was switched to Site 2 for a period of 4 weeks with Site 1 acting as control (Batch 2). This format was continued for six batches. A total of 9897 piglets were born alive in litters to be iron treated and, 9365 piglets were untreated. Recorded parameters included number born alive, piglet mortality, number weaned, number 'rolled over' (deemed too small and back-fostered) and average weaning weight.

\section{Results}

Mean weaning weight was $7.37 \mathrm{~kg}$ for the iron group and $7.11 \mathrm{~kg}$ for the control group over the 6 month study. This difference was highly statistically significant. There were no significant differences between the two groups for

number weaned per sow, number 'rolled over' or piglet mortality. A strong seasonal effect was noted with batches born in the summer showing significantly poorer results for weaning weight and number weaned per sow. The effect of iron treatment on weaning weight was more pronounced in the first three batches of the trial (piglets born February, March and April). In February the difference between the two groups was just under $0.6 \mathrm{~kg}$. Blood parameters for the two groups showed significant differences for haemoglobin, MCH, MCV, PCV and RBC count.

\section{Discussion}

The overall results suggest a significant benefit from routine iron injection for weaning weight but no benefit for the other parameters measured. Changes in the behaviour of both sows and piglets are suspected to be responsible for the apparent seasonal effects noted. During colder weather sows and piglets spend longer inside their arcs rather than outside. In warmer weather sows, given the option, spend long periods of time wallowing and hence their skin surface is often covered in adherent soil. When these sows return to suckle their litters, the piglets obviously consume relatively large amounts of soil and their iron intake is therefore higher. The improvement in weaning weight would appear to be mediated through improved blood parameters in iron treated piglets. Improved weaning weight is strongly correlated with post weaning performance and on this unit iron treatment was estimated to provide an ROI in excess of 10:1.

\section{References}

Brown, J.M.E., Edwards, S.A., Smith, W.J., Thompson, E. Duncan, J. (1996) Welfare and production implications of teeth clipping and iron injection of piglets in outdoor systems in Scotland. Preventative Veterinary Medicine, 27, 95-105

Egeli, A.K., Framstad, T. and Morberg, H. (1998) Clinical Biochemistry, Haematology and Body Weight in Piglets. Acta Veterinaria Scandinavica, 39, 381-393

Jennings, D.S., (1983) Trials of Iron in Sows and Piglets. The Pig Journal, $10,103-105$

Sansom, B.F., (1984) The Iron Requirements of Young Pigs. The Pig Journal, 11, 67-74

Taylor, D.J. (1995) Piglet Anaemia. In: Pig Diseases 6th Edition, pp 274276 


\title{
P.731
}

\section{The effect of a dietary supplementation of paylean on nitrogen retention and nitrogen excretion in growing pigs}

\author{
Ryozo Takada \\ Niigata University, Faculty of Agriculture, Niigata City, Japan
}

\section{Introduction}

A diet supplemented with Paylean (Ractopamine $\mathrm{HCl}$ ) for growing-finishing pigs is known to increase growth and the protein contents and to decrease the fat contents of dressed carcasses. We conducted a nitrogen balance test in growing pigs to assess the effect of Paylean supplemented in the diet on nitrogen retention and urinary excretion.

\section{Material and Methods}

Ten LWD barrows with a mean weight of about $53 \mathrm{~kg}$ were used in the experiment. The pigs were housed individually in cages. During the first week of the experiment, all pigs were given the control diet ad libitum. In the second week, the pigs were allocated to the control group (5 animals), which was continuously fed with the control diet ad libitum, or the Paylean group ( 5 animals), which was given a diet supplemented 5 ppm of Paylean (Ractopamine $\mathrm{HCl}$ ) into the control diet. During this period, feed intake was measured daily, while body weight was measured weekly. For the last 3 days of the second week, feces and urine were collected separately. Fecal samples were collected from the rectum every morning, while the entire volume of urine was collected every day. Chromium oxide ( $0.1 \%)$ was added to the test diets to determine nitrogen digestibility with the indicator method.

The control diet, a feed for growing pigs, was composed mainly of corn and soybean meal, with a crude protein (CP) level of $15.5 \%$ and a lysine level of $0.92 \%$.

- Urinary nitrogen was calculated by multiplying the urinary nitrogen content determined with an NC coder by the weight of the total urine collected over the 3-day period. Fecal nitrogen was calculated by multiplying the nitrogen intake for the 3-day period by the nitrogen digestibility. Nitrogen retention was calculated using the following equation:

- Nitrogen retention $(g)$ = Nitrogen intake - (Urinary nitrogen + Fecal nitrogen)

\section{Results and Discussion}

In the first week, there were no differences between the control group and the Paylean group. In the second week, however, daily weight gain was significantly higher $(p<0.01)$ in the Paylean group $(1103 \mathrm{~g} / \mathrm{d})$ compared to the control group $(880 \mathrm{~g} / \mathrm{d})$. There were no differences in daily feed intake between the 2 groups. Feed efficiency was significantly higher $(p<0.05)$ in the Paylean group compared to the control group.

- No significant differences were noted in nitrogen intake between the 2 groups, but somewhat higher mean value was observed in the Paylean group. Nitrogen retention ( $g / 3 \mathrm{~d}$ ) was significantly higher $(p<0.05)$ in the Paylean group compared to the control group, and nitrogen retention efficiency (nitrogen retained/nitrogen intake) was more markedly higher in the Paylean group $(p<0.01)$. Although no significant differences were observed in urinary nitrogen excretion $(g / 3$ d), urinary nitrogen excretion (\%) (Urinary nitrogen/nitrogen intake) was definitely lower in the Paylean group compared to the control group $(p=0.01)$. There were no significant differences in nitrogen digestibility between the 2 groups.

- The above results revealed that dietary supplementation of Paylean increased the nitrogen retention and decreased urinary excretion in growing pigs.

Table 1. Nitrogen balance test of growing pigs fed a diet supplemented with Paylean

\begin{tabular}{|l|c|c|}
\hline & Control & Paylean \\
\hline Intake of N (g/3d) & $193 \pm 22$ & $220 \pm 25$ \\
\hline Absorbed N (g/3d) & $155 \pm 22$ & $177 \pm 25$ \\
\hline Retained N (g/3d) & $80 \pm 8$ & $109 \pm 10^{*}$ \\
\hline Retained N/intake N & $0.42 \pm 0.02$ & $0.50 \pm 0.02^{* *}$ \\
\hline Retained N/absorbed N & $0.53 \pm 0.03$ & $0.63 \pm 0.04^{*}$ \\
\hline Urinary N (g/3d) & $75 \pm 13$ & $68 \pm 16$ \\
\hline Urinary N/intake N & $0.38 \pm 0.03$ & $0.29 \pm 0.04^{*}$ \\
\hline Urinary N/absorbed N & $0.47 \pm 0.03$ & $0.37 \pm 0.04^{* *}$ \\
\hline N digestibility & $0.79 \pm 0.03$ & $0.80 \pm 0.02$ \\
\hline Mean \pm SE (n=5) & $* * P<0.01$ & $* P<0.05$ \\
\hline
\end{tabular}




\title{
P.732
}

\section{Defatted corn germ meal as a source of phytic acid associated with phytase enzyme to finishing swine rations}

\author{
Graziela D. Pacheco; Arturo P. Lozano; Caio A. Silva; Ana M. Bridi; Roberta A. Silva; Marina A. Tarsitano \\ Universidade Estadual de Londrina, Londrina, PR, Brazil
}

\section{Introduction}

The phytic acid is a component present in defatted corn germ meal (DCGM) (1), a strong chelation agent with activity on polyvalent cations like calcium, iron, zinc, copper and manganese. So the use of phytic acid can be interesting to improve the pig meat, which has high level of iron, a potent cation involved with meat oxidation, where through the chelation of iron, the phytic acid reduces the damages of meat oxidation (2). Therefore this response also affects the availability of some minerals reducing their absorption, increasing the environmental pollution and affecting the animal performance. The low digestibility of phosphorus phytic increases the cost of ration because it is necessary to use inorganic sources of this mineral or to add phytase enzyme. The objective of this study was to evaluate the influence of phytic acid present mainly in DCGM, associated or no with phytase on the performance, mineral excretion, carcass, meat quality and serum parameters of finishing pigs.

\section{Materials and Methods}

Thirty six commercial pigs, 16 barrows and 16 gilts, with 60,31 $\pm 5,32 \mathrm{Kg} \mathrm{BW}$, located on individual pens were distributed to randomized block design, factorial $2 \times 2 \times 2$ (with and without DCGM, with and without phytase, barrows and gilts) establishing four treatments: T1 - diet without DCGM and without the addition of phytase; T2 - diet without DCGM and with phytase (1000 PU); T3 - diet with 40\% of DCGM and without phytase; T4 - diet with $40 \%$ of DCGM and with phytase (1000 PU). The commercial phytase used is NATUPHOS ${ }^{\circledR} 500$, at 1000 phytase unit (PU). The rations and water were offered ad libitum, been the rations isoenergetic and isonutrients. Were analyzed the performance parameters (daily weight gain, daily feed intake and food conversion), carcass and meat characteristics and the meat oxidation (Longissimus dorsi muscle) through T-BARS method. Also were evaluated the serum values of calcium, phosphorus and iron. To determine the levels of phosphorus and calcium in the feces, was adopted the technique of partial collection of feces, using as marker the chromic oxide $0.3 \%$. At $87.19 \pm 7.08 \mathrm{~kg}$ $\mathrm{BW}$, the pigs were slaughtered and submitted to evaluation of carcass traits and meat quality.

\section{Results and Discussion}

There was no difference in performance parameters and none negative influence between the factors on carcass characteristics $(P>0.05)$. The results of the fecal levels of phosphorus and calcium are showed on the Table 1.

\begin{tabular}{|l|c|c|}
\hline *Factors & Feces & ( $\mathbf{m g} / \mathbf{1 0 0 g})$ \\
\hline DCGM & Fecal phosphorus & Fecal calcium \\
\hline without & & \\
\hline with & $1.55 \mathrm{~b}(0.5)$ & $0.88 \mathrm{~b}(0.2)$ \\
\hline Phytase & $2.10 \mathrm{a}(0.6)$ & $1.03 \mathrm{a}(0.3)$ \\
\hline without & $2.26 \mathrm{a}(0.4)$ & $1.03 \mathrm{a}(0.2)$ \\
\hline with & $1.38 \mathrm{~b}(0.4)$ & $0.88 \mathrm{~b}(0.2)$ \\
\hline Sex & & $1.10 \mathrm{a}(0.2)$ \\
\hline barrows & $1.94(0.5)$ & $0.82 \mathrm{~b}(0.2)$ \\
\hline females & $1.71(0.7)$ & 14.7 \\
\hline VC (\%) & 13.7 & \\
\hline Different letters within the columns means difference by Duncan Test $(\mathrm{P}<0.5)$ \\
\hline
\end{tabular}

Animals that received the DCGM and that were fed with rations with phytase had a greater fecal excretion of phosphorus and calcium ( $P<0.05)$. The last result is according to Simons et al. (4). The serum levels of phosphorus, calcium and iron were similar between the factors, but Gebert et al (5) observed that the pigs that received diets with phytase (1000PU) presented an increased of serum concentration of iron and phosphorus. There were no effects of the treatments on the meat quality, but to the meat oxidation the ration with DCGM (with greater level of phytic acid) shows a lower lipid oxidation than the diet without DCGM ( 0.16 versus $0.21 \mathrm{mg} / \mathrm{kg}$ T-BARS). There results were similar to Harbach et al. (2) findings. The phytase didn't affect the oxidation of the $L$ dorsi muscle.

\section{References}

1. Bohn et al. 2008 J Z U Sci. 9:165-191

2. Harbach et al. 2006. F Chem. 100:1630-1633.

3. Selle et al. 2000. Nut Res Rev. 13:245-248

4. Simons et al. 2005. Brit J Nutr. 93:136-152

5. Gebert et al.1999. j An Phys An Nutr. 81:9-19. 


\section{P.733}

\section{Using enzymatic complex in feed containing defatted corn germ meal for pigs during growth and finishing}

Sylvia L. Vinokurovas; Caio A. Silva; Ana M. Bridi; Arturo P. Lozano; Marina A. Tarsitano; Roberta A. Silva; Danyel B. Dalto

Universidade Estadual de Londrina, Londrina, PR, Brazil

\section{Introduction}

In the Brazilian swine culture, the largest part of production costs is from food, basically constituted by corn and soybean meal. In this scenario, the search for alternative ingredients, associated to enzymatic complexes, is a very common procedure nowadays. Defatted corn germ meal (DCGM) is a co-product from the corn oil extraction, presenting several nutritional attractions and significant availability. Nutritionally, it has been indicated in the feed of pigs as an alternative product (1). However, there are restrictions on the high inclusions of DCGM due to the high levels of fiber and low energy in its composition. The correction of these aspects might increase the cost of the feed. A solution is to reduce the negative effects caused by the excess fiber with the addition of enzymatic complex. Then, the objective of this paper is to study the effects of including $20 \%$ DCGM and an enzymatic complex for pigs' performance, carcass characteristics and meat quality.

\section{Materials and Methods}

A total of 50 pigs (PIC) were used, 25 castrated male and 25 female, with initial average weight of $41.14 \mathrm{~kg}$, kept in two animals per pen. The test was conducted during 83 days, been evaluated the performance, carcass characteristics, meat quality and the cost of the treatments The experimental design was in random blocks, with 5 treatments and 5 repetitions/treatment, being each pen considered an experimental unit. Feed named control was formulated based on corn and soybean meal and the remaining ones were made of corn, soybean meal and $20 \%$ DCGM. Two diets containing DCGM received a commercial enzymatic complex (Allzyme ${ }^{\circledR}$ SSF) on the dose of $0.02 \%$, being that in one of the diets the matrix of the enzymatic complex was considered in the formulation. In the other, the complex was added on top, that is, out of the formulation. Then it was defined 5 treatments: T1- control feed (corn + soybean meal); T2- feed with 20\% DCGM; T3- feed with 20\% DCGM + EC; T4- feed with $20 \%$ DCGM without EC reformulated (discounting the nutritional matrix of the enzymatic complex); T5- feed with 20\% DCGM + EC (in its additive form - on top )

\section{Results and Discussion}

Results for zoo-technical performance are presented in Table 1.

Table 1 - Means of daily weight gain (DWG), daily feed consumption (DFC) and feed conversion (FC) for pigs submitted to feed with and without the inclusion of enzymatic complex

\begin{tabular}{|c|c|c|c|}
\hline \multirow{2}{*}{ Treatments } & \multicolumn{3}{|c|}{ Parameters } \\
\cline { 2 - 4 } & DFC (kg) & DWG (kg) & FC \\
\hline T1 & 2.38 & $0.68 \mathrm{ab}$ & $3.53 \mathrm{~b}$ \\
\hline T2 & 2.43 & $0.71 \mathrm{ab}$ & $3.43 \mathrm{~b}$ \\
\hline T3 & 2.26 & $0.66 \mathrm{ab}$ & $3.40 \mathrm{~b}$ \\
\hline T4 & 2.79 & $0.64 \mathrm{~b}$ & $4.33 \mathrm{a}$ \\
\hline T5 & 2.43 & $0.72 \mathrm{a}$ & $3.36 \mathrm{~b}$ \\
\hline VC $(\%)$ & 15.23 & 6.59 & 14.95 \\
\hline Duncan test $(P<0.05$ & &
\end{tabular}

Significant difference was verified for DWG with advantages for T5 and worst results for T4. For FC, the worst result was verified in T4 $(P<0.05)$ For the parameters related to carcass characteristics, no significant differences $(P>0.05)$ were observed among the treatments. The highest final $\mathrm{pH}$ values for meat were observed for $\mathrm{T} 1$, but influences from this parameter could not be verified (in any of the treatments) on the quality of the meat. According to the conditions of this experiment and the prices for the ingredients, that the best economic and average cost indexes were those obtained for the treatment with DCGM with the inclusion of the enzymatic complex in diets based on soybean and corn in pigs in finishing phase. (2) have observed that the addition of the complex has possibly affected positively the DFC and FC, indicating greater efficiency through the usage of complex in feed, resulting in lower feed cost. The addition of enzymatic complex in diets with $20 \%$ DCGM for pigs in growth and termination phases has improved the mean weight gain, without affecting the characteristics of meat quality, as well as presenting better economic efficiency.

\section{References}

1. Soares et al.. 2004.Bras J Anim Sci 33:1768-1776

2. Araque et al. (2009). Alltech's Annual Symposium on Sc. and Technol in the feed Industry. 25 . 


\title{
P.734
}

\section{Interactive effect of Ractopamine and dietary energy density on performance and characteristics traits of pigs selected for fast weight gain}

\author{
Vinícius S. Cantarelli2 Nikolas O. Amaral ${ }^{2}$ Fernando M. Toledano ${ }^{1}$ Thiago H. Kuribayashi $^{2}$ Tarciso C. Villela $^{2}$ Elias T. Fialho $^{2}$ \\ 1. Ouro Fino Saúde Animal, Cravinhos, SP, Brazil; 2. Universidade Federal de Lavras, Lavras, Brazil
}

\section{Introduction}

Progeny of Large White sire line evaluated in this work differentiate themselves in the market through high intake capacity, result of long of time of selection for feed intake capacity and speed of growth. In the initial period of growing, the high capacity of consumption provides speed up weight profit, however, in the final phase of finishing this can result in excess of fat in the carcass. Thus, supplementation of ractopamine (RAC) in the diet has been an alternative to reduce the feed in this phase and increase the yield of carcass meat (2). However, more studies should be conducted to determine the best nutritional program supplementation with RAC for these animals, especially with regard to energy consumption. Due to changes in genetics and management practices within the swine industry, this study was conducted to evaluate the performance and carcass traits of finishing barrows and gilts, from crossbred sows $x$ Large White terminal sires, receiving diets with different metabolizable energy levels and supplemented with RAC.

\section{Material and Methods}

Animal care and experimental protocols were approved by the University of Lavras Interdepartmental Animal Care and Use Committee before initiation of this experiment. The experiment was conducted at the pig farm of the Department of Animal Science, Federal University of Lavras (UFLA), Lavras, MG, Brazil. A total of 88 crossbred pigs (from crossbred sows $x$ Large White boars), 48 barrows and 40 gilts (initially $75 \pm 6.5 \mathrm{Kg}$ ) were used. The experimental design was randomized blocks with time the treatments (8) were arranged as a $2 \times 4$ factorial (two sexies - barrows and gilts - and four levels of metabolizable energy (ME) - 3000, 3150, 3300 and $3450 \mathrm{Kcal} / \mathrm{kg}$ ) with 6 replicates for barrows and 5 for gilts. There were 2 pigs per pen. All diets (corn-soybean meal) met or exceeded NRC (1998) requirements, were supplemented with 10 ppm of RAC during the $28-d$ feeding trial. The data were subjected to ANOVA was generated using the GLM procedure (SAS Inst. Inc., Cary, NC). Least squares means were generated and separated using the LSD procedures when there was a significant ( $P$ 0.05) F-test for a main effect or interaction.

\section{Results}

The feed conversion (FC) decreased linearly with increased energy density of diet for barrows and gilts. There was no significant interaction between energy level and sex. The other performance variables such as ADG and ADFI as well as carcass traits were not affected $(P>0.05)$ by levels of energy in the diets used in this study. In relation to sex, gilts shown lower final weight (1.83\%), lower average daily gain (6.55\%), lower average daily feed intake (13.73\%), better feed conversion (6.29\%), lower backfat thickness (26.62\%), larger longissimus muscle $(9.40 \%)$ and higher meat yield (4.25\%) all those signficative by $(\mathrm{P}<0.05)$.

\section{Discussion}

The increase in energy density in the finishing phase makes the voluntary intake of animals reduced, thus resulting in improved of feed conversion. However, as observed in other studies (1, 3 ), increasing the energy level of diets supplemented with RAC improved feed conversion of barrows and gilts without affecting feed intake. In relation to sex, the result shown that the efficiency of gilts to deposit lean tissue in the carcass should be due to the influence of hormonal activity, that increase basal metabolism and lowered the feed intake capacity. Results of this study indicate that including ractopamine (10 ppm) with higher ME levels in the diets could lower FC in both barrows and gilts in the finishing phase. However the inclusion of RAC with higher energy concentration in the late-finishing diet did not affect the others variables of growth performance as well as the carcass.

\section{References}

1. Aplle, J.K.; Maxwell, C.V.; Brown, D.C. et al. Effects of dietary lysine and energy density on performance and carcass characteristics of finishing pigs fed ractopamine. J. Anim Sci, v.82, p. 3277-3287, 2004.

2. Cantarelli, V.S.; Fialho, E.T.; Almeida, E.C. et al. Ractopamine for finish barrows fed restricted or ad libitum diets: performance and nitrogen balance. Brazilian Journal of Animal Science, v. 39, p. 1870-1880, 2009.

3. Dunshea, F. R.; King, R. H.; Eason, P. J. et al. Interrelationships between dietary ractopamine, energy intake, and sex in pigs. Aust. J. Agric. Res. 49:565-574, 1998. 


\title{
P.735
}

\section{Performance and carcass traits of pigs selected for feed efficiency fed diets containing Ractopamine with different energy density}

\author{
Nikolas O. Amaral ${ }^{2}$ Vinícius S. Cantarelli² Fernando M. Toledano ${ }^{1}$ Tarciso C. Villela $^{2}$ Thiago H. Kuribayashi $^{2}$ Elias T. Fialho $^{2}$ \\ 1. Ouro Fino Saúde Animal, Cravinhos, SP, Brazil; 2. Universidade Federal de Lavras, Lavras, Brazil
}

\section{Introduction}

Soft pork fat has become an economical concern of the Brazilian pork industry. Accordingly, progeny of Pietrain sire line evaluated in this work is characterized by the excellent resulting in conformation and lean meat content in the carcass, result of the intense selection auto-restriction of feed. Moreover, including Ractopamine (RAC) in swine finishing diets produces more muscular carcasses as well improve feed conversion including those with restrictive feeding regimes (1). It is well known, however, that the differences in feed intake and efficiency of protein deposition between barrows and gilts resultant of crosses between different strains, especially with regard to energy consumption can result in different responses of these finishing pigs to RAC supplementation in the diet. The objective of this work was to evaluate the performance and carcass traits of finishing barrows and gilts, from crossbred sows $x$ Pietrain sires, receiving diets with different metabolizable energy levels and supplemented with RAC.

\section{Materials and Methods}

Animal care and experimental protocols were approved by the University of Lavras Interdepartmental Animal Care and Use Committee before initiation of this experiment. The experiment was conducted at the pig farm of the Department of Animal Science, Federal University of Lavras (UFLA), Lavras, MG, Brazil. A total of 88 crossbred pigs (crossbred sows $x$ Pietrain terminal sires), 48 barrows and 40 gilts (initially $75 \pm 5.5 \mathrm{~kg}$ ) were used. The experimental design was randomized blocks with time the treatments (8) were arranged as a $2 \times 4$ factorial (two sex - barrows and gilts) and four levels of metabolizable energy $-\mathrm{ME}$ (3000, 3150, 3300 and $3450 \mathrm{Kcal}$ ME / kg) with 6 replicates for barrows and 5 for gilts. There were 2 pigs per pen. All diets (cornsoybean meal) met or exceeded NRC (1998) requirements, were supplemented with 10 ppm of RAC during the $28-d$ feeding trial. The data were analyzed by ANOVA by using the GLM procedure (SAS Inst. Inc., Cary, NC), with the main affects of level ME and sex as well the Energy $x$ sex interaction, included in the statistical model. Least squares means were generated and separated using the LSD procedures when there was a significant (P 0.05) F-test for a main effect or interaction.

\section{Results}

The data shown a significant interaction $(P<0.05)$ between sex and ME on feed conversion (FC). For barrows, the FC decreased linearly with increased ME of diet. However, we found nonsignificant difference $(P>0.05)$ of energy levels on the others performance and carcass traits variables. In relation to sex, gilts shown lower final weight (1.63\%), lower average daily gain (5.26\%), lower average daily feed intake (15.82\%), better feed conversion (10.2\%), less backfat thickness (17.79\%) and higher meat yield (3.27\%) all those signficative by $(P<0.05)$.

\section{Discussion}

According to (3), regardless of supplementation with RAC, Increasing the energy density of swine diets has been shown to have no affect on feed intake, resulting in improvements in feed efficiency F:G. However, in the present experiment this effect was only observed for barrows. A possible explanation for this result would be a physical limitation of intake capacity of gilts as a observed by the smaller numerical variation in feed consumption as a function of energy density. The greater efficiency in lean deposition and lower voluntary feed consumption of gilts should be explaining the other results (2). It is therefore suggested that supplementation of diets with RAC (10 ppm) with higher ME levels could lower FC in the finishing barrows. However the inclusion of RAC (10 ppm or $10 \mathrm{mg} / \mathrm{Kg}$ ) with higher energy concentration in the late-finishing diet did not affect the others variables of growth performance as well as carcass traits of barrows therefore gilts shown better carcass traits in finishing phase.

\section{References}

1. Almeida, E. C. Níveis de lisina e ractopamina em rações para suínos em terminação. Tese. Lavras : UFLA, 2008. 90 p.

2. Amaral, N.O.; Fialho, E.T.; Cantarelli, V.S. et al. Ractopamine hydrochloride in formulated rations for barrows or gilts from 94 to $130 \mathrm{~kg}$. Revista Brasileira de Zootecnia, v. 38, p. 1494-1501, 2009.

3. Aplle, J.K.; Maxwell, C.V.; Brown, D.C. et al. Effects of dietary lysine and energy density on performance and carcass characteristics of finishing pigs fed ractopamine. J. Anim Sci, v.82, p. 3277-3287, 2004. 


\title{
P.736
}

\section{Assesment of body condition and body composition during the transition period between confinement and organic production system in pregnant sows in Mexico}

\author{
Ernesto Hurtado-Guerrero; Marco A. Herradora-Lozano; Roberto A. Martínez-Gamba \\ Departamento de Producción Animal: Cerdos de la Facultad de Medicina Veterinaria y Zootecnia \\ de la Universidad Nacional Autónoma de México, México, DF, Mexico
}

\section{Introduction}

To date only few farms are using organic pig production in Mexico. One of the main challenges facing producers during the process of conversion to organic is the transition period, in which a decreased productivity is perceived in relation to the production parameters obtained before the process1. The major obstacle comes from the difficulty to fulfill all the regulations needed to obtain the organic certification, like feeding and management2. The sows body condition and composition, can explain why the productive performance of the sows are affected during the transition period between a confinement system to a free range with alternative feeding system 1,3 . The objective of this study was to evaluate the body condition and composition of gestating breeding sows during the transition period of tie used to implement a organic production system in a pig farm from the centre of Mexico. The production conditions including the feeding and management systems were also monitored, to find out how this change in procedures affects the sows.

\section{Material and Methods}

Fifteen York-Landrace sows with different parity were allocated into 3 groups with different treatments; organic managementalternative feeding $(\mathrm{O}-\mathrm{A})$, organic management- conventional feeding (O-C), and conventional management - conventional feeding (C-C). Two types of isoenergetic and isoproteic diets were used, one was "conventional" with sorghum and soy cake, and the other "alternative" based on lentil sprouts and bananapotato silage. The organic management was carried out in accordance with the established by KRAV2. All sows were weighed, the back fat (BF) measured, and the body condition (BC) registered in four different times, at the beginning of the study $(\mathrm{m} 1)$, at the second third of pregnancy (m2), at the third (m3), and on 112 (m4). Two equations were employed to calculate the body fat (BoF) and the protean mass (PM). The data analysis was carried by means of orthogonal contrasts, and for the body condition a non-parametric test Kruskal-Wallis test was used.

\section{Results}

As for weigh and BF no differences were found throughout pregnancy $(p>0.05)$; however, the total weight gain showed some differences $(p=0.001)$ in contrast "management effect" (O-C, O-A vs. C-C), with O-C (26.15 $\pm 19.3 \mathrm{~kg}), \mathrm{O}-\mathrm{A}(10.62 \pm 14.7 \mathrm{~kg})$ and $C-C(48.5 \pm 11.9 \mathrm{~kg})$. The BF measurements did not showed differences between groups ( $p>0.05)$, until $\mathrm{m} 4$ in which the contrast "feed effect" (O-A vs. O-C) $(p=0.035)$ obtained for $C-C$ $(21.16 \pm 1.69 \mathrm{~mm})$ was higher in comparison to O-C $(20.7 \pm 2.2 \mathrm{~mm})$ and O-A (18 $\pm 2.12 \mathrm{~mm})$. The body composition (BoF, PM, the relationship between them and maternal gain $M G)$, did not show differences ( $p>0.05)$; however, maternal fat gain showed differences for both contrasts $(p<0.05)$ with $O-C(9.8 \pm 5.7 \mathrm{~kg})$, O-A (1.55 $\pm 6.24 \mathrm{~kg})$ and C-C (17.27 $\pm 1.7 \mathrm{~kg})$.

\section{Discussion}

Sows maintained with organic management showed less weight gain, from which a considerable portion was BoF. Despite the diets were formulated according to the established requirements of NRC 1998, they were not sufficient to satisfy the energetic demands of the animals, principally at the end of pregnancy, period of greatest nutrient demand of the products owing to their fast growth. Therefore, it can be concluded that it is possible to maintain an adequate body condition and composition with an alternative diet during the transition period to organic, as long as the feed's energetic contribution is increased but not the protein, in such a way that these can be adjusted to the activity and thermoneutrality in this system.

Research supported by PAPPIT Project IN. 202128

\section{References}

1. Hermansen J.E. 2003. Liv. Prod. Sci.; 80: 3-15.

2. KRAV. KRAV 2008. Standards. Uppsala, (Sweden): January 2008 http://www.krav.se/Documents/Regler/englishEditions/Standards_ July_2008.pdf

3. Noblet J.Y., et al., 1997. J. of Anim. Sci.; 75: 2708-2714. 


\title{
P.737
}

\section{Effect of two different feed phosphates on the content of phosphorus and calcium in blood and bones of finishing pigs}

\author{
Zbigniew Dobrzański ${ }^{1}$ Roman Kołacz ${ }^{1}$ Daniel Korniewicz ${ }^{2}$ \\ 1. Wrocław University of Environmental and Life Sciences, Wrocław, Poland; 2. LNB POLAND Ltd, Kiszkowo, Poland
}

\section{Introduction}

Phosphorus (P), except calcium (Ca), is the most important component of an organism. Pigs body contains about $0.45 \%$ of phosphorus, and $80 \%$ of that amount is present in bones, considerable concentration is in blood (6).

Mono- (MCP) and dicalcium phosphates (DCP) are the most often used in pigs feeding, but phosphorus digestibility is very differentiated, e.g. 51-80\%. (5). Very important, except $P$, is calcium content in feed $(1,3)$.

The aim of the present study was to determine an influence of two different feed phosphates (MCP and DCP) on P and Ca content in blood serum and thigh bones of finishing pigs.

\section{Materials and Methods}

The material were 40 piglets (sow - Large Polish White $x$ Polish Landrace) $\mathrm{x}$ boar (hampshire $\mathrm{x}$ pietrain) The fattening lasted 85 days, i.e. to the body mass of about $110 \mathrm{~kg}$. Group I was given MCP (Ca (H2PO4)2,, Finnish product) while group II - DCP (CaH$\mathrm{PO} 4 \times 2 \mathrm{H} 2 \mathrm{O}$, Polish product). The contribution of particular feed phosphates resulted from the optimization of $P$ content in feed mixtures. It was assumed that mineral phosphorus makes up $30 \%$ of the demand for an overall phosphorus. The nutritional value of starter, grower and finisher mixtures, with mineral components was similar in I and II group (3).

Blood for analysis was collected at 85th day of pigs life from the zygomatic vein. When determining the level of an inorganic phosphorus, the UV-VIS method was used, and while determining calcium level - photometric method was applied.

Thigh bones were collected after the slaughter. Ring shaped samples of a thickness of $3 \mathrm{~mm}$ were collected from each bone. Double weighted amount, of a mass of $0.5-0.6 \mathrm{~g}$ each, were prepared. Samples subjected to analysis were mineralized using a microwave furnace. Determination of $\mathrm{P}$ and $\mathrm{Ca}$ content was conducted using atomic emission spectrometry (ICP - OES). The results were worked out statistically (Duncan's test).

\section{Results and Discussion}

The feed phosphates used differed considerably as regards their physico-chemical features (2), but the level of $\mathrm{P}$ (and Ca as well) was similar in pigs diet (3). Phosphorus level in blood serum of pigs was similar in both groups (about $2.5 \mathrm{mmol} / \mathrm{l}$ ), what was also observed in the case of calcium (about $3.1 \mathrm{mmol} / \mathrm{l}$ ) (Table 1). The values were close to an upper limit of reference values (8). In the case of a bit lighter pigs $(85 \mathrm{~kg})$ the considerably higher values of $\mathrm{P}$ were obtained (over $4 \mathrm{mmol} / \mathrm{l}$ ), but similar ones of $\mathrm{Ca}(7)$. Statistically higher $(\mathrm{p}<0.05)$ content of $\mathrm{P}(142,2)$ and $\mathrm{Ca}$ $(261,2 \mathrm{~g} / \mathrm{kg})$ was in thigh bones in II group (DCP) as compared to group I (MCP). Another data in this range may be found in the literature $(1,4)$, that proves numerous factors influencing $P$ accumulation in pigs bones.

Table 1. The content of $P$ and $C a$ in pigs blood serum $(n=12)$ and thigh bones $(n=10)(x \pm S D)$

\begin{tabular}{|c|c|c|c|c|}
\hline \multirow{2}{*}{ Element } & \multicolumn{2}{|c|}{ Blood (mmol/I) } & \multicolumn{2}{c|}{ Bone (g/kg) } \\
\cline { 2 - 5 } & I-MCP & II-DCP & I-MCP & II-DCP \\
\hline$C a$ & $2.58 \pm 0.13$ & $2.48 \pm 0.15$ & $250.6 \mathrm{~b} \pm 7.5$ & $262.1 \mathrm{a} \pm 9.1$ \\
\hline $\mathrm{P}$ & $3.03 \pm 0.14$ & $3.09 \pm 0.38$ & $118.6 \mathrm{~b} \pm 3.5$ & $142.2 \mathrm{a} \pm 2.4$ \\
\hline $\mathrm{a}-\mathrm{b} \mathrm{p}<0.05$ & \multicolumn{3}{|c}{} \\
\hline
\end{tabular}

Generally it should be stated that the two different phosphates used in the experiment did not significantly influence the differentiation of $\mathrm{P}$ and $\mathrm{Ca}$ of blood of pigs, while the highest concentrations $\mathrm{P}$ and $\mathrm{Ca}$ in thigh bones of pigs that were given DCP may prove the better bioavailability of those building elements in pigs, that is of a special significance in the fast-growing animals.

\section{References}

1. Fernández J.A. (1995) Livest. Prod. Sci., 41, 243-254.

2. Hoffmann J. et. al (2008) Chem. Agric., 9, 25-30.

3. Korniewicz D., Hoffmann J., Korniewicz A., Dobrzański Z. (2010) J. Anim. Feed Sci., (in press).

4. Hinson R.B. et al. (2009) J. Anim. Sci., 87, 1502-1517

5. Poulsen H.D. (2007) Livest. Sci., 109, 247-250.

6. Rymarz A., Fandrejewski H., Kielanowski J. (1992) Livest. Prod. Sci., 9, 399-407.

7. Tao X., Xu Z.R., Wang Y.Z. (2006) Turk. J. Vet. Anim. Sci., 30, 65-70.

8. Winnicka A. (2008) Reference values of basic laboratory analysis in veterinary medicine. Ed. SGGW, Warszawa. 


\title{
P.738
}

\section{Decreasing pork's carbon footprint: greenhouse gas emissions savings from feeding ractopamine (Paylean ${ }^{\circledR}$ ) to the US swine herd}

\author{
Garth Boyd ${ }^{1}$ David B. Anderson² Allen L. Sutton ${ }^{3}$ Casey V. Hogan' Marks-Callahan Amy ${ }^{4}$ \\ 1. Camco, Broomfield, CO, USA; 2. Elanco Animal Health, retired, Loveland, USA; 3. Purdue University Department of Animal Sciences, \\ West Lafayette, IN, USA; 4. Elanco Animal Health, Greenfield, USA
}

\section{Introduction}

In response to global concern about climate change, there is pressure on the pork industry from the retail sector to measure, and over time, reduce the carbon intensity of pork. The National Pork Board is currently assessing the carbon footprint of US produced pork. Ractopamine (RAC; Paylean ${ }^{\circledR}$ ) is a feed ingredient that when fed the last 28 days to finishing pigs results in enhanced feed efficiency, increased growth rate, and increased leanness(1). These benefits impact pork production by decreasing feed utilization, days to market and by reducing the population of pigs needed to supply the same quantity of protein produced from non-RAC-fed pigs. The purpose of this study was to quantify the cradle-to-gate GHG emissions savings resulting from the theoretical feeding of RAC to the 109.2 million head of pigs harvested in the US in 2008.

\section{Materials and Methods}

Using 2008 U.S. pork production statistics, the environmental benefits associated with the production efficiency of RAC were quantified and assumed implementation of RAC in all finishing pig diets in the U.S. at 5 or $10 \mathrm{mg} / \mathrm{kg} \operatorname{diet}(2)$. The derived benefit of the increased leanness per pig resulted in fewer animals needed to produce the same amount of pork protein. In addition, pigs experienced greater feed efficiency as a result of RAC, and reach their market weight sooner for harvest(2). The model used to assess GHG emissions savings followed the same methodology as a 'cradle-to-gate' Life Cycle Assessment and included all upstream and on-farm GHG emission savings.

\section{Results and Discussion}

The leanness response of 109.2 million head of finishing pigs being fed 5 or $10 \mathrm{mg} / \mathrm{kg}$ RAC resulted in the same amount of pork protein being produced in 2008 with 5.8 and 6.9 million fewer finishing pigs and 310,000 and 369,000 fewer sows, respectively. The RAC-fed pigs reached market weight in 3.00 or 2.76 fewer days for the 5 or $10 \mathrm{mg} / \mathrm{kg}$ dose respectively.

The reduced population of sows and finishing pigs, improved feed efficiency, and reduced number of days to market, resulted in GHG savings measured on a $\mathrm{CO}_{2}$-equivalent basis for the three major categories of production activity changes shown in Table 1.
Table 1. Summary, Annual GHG Emissions Savings

\begin{tabular}{|c|c|c|}
\hline Source of Emissions & $\begin{array}{l}\text { GHG emissions savings for } \\
5 \mathrm{mg} / \mathrm{kg} \text { Dose }\left(\mathrm{tCO}_{2} \mathrm{e} / \mathrm{yr}\right)\end{array}$ & $\begin{array}{l}\text { GHG emissions savings for } \\
10 \mathrm{mg} / \mathrm{kg} \text { Dose }\left(\mathrm{tCO}_{2} \mathrm{e} / \mathrm{yr}\right)\end{array}$ \\
\hline Feed Production & 586,246 & 738,828 \\
\hline Manure & $1,204,221$ & $1,367,944$ \\
\hline Pig Housing & 78,949 & 94,350 \\
\hline Paylean ${ }^{\circledR}$ GHG LCA & -6.3 & -12 \\
\hline Total & $1,869,410$ & $2,201,110$ \\
\hline
\end{tabular}

The avoidance of methane emissions from manure was over $60 \%$ of the overall GHG emissions savings for both the 5 and 10 $\mathrm{mg} / \mathrm{kg}$ dose of RAC (due to its 25 times higher global warming potential compared to $\mathrm{CO}_{2}$ ). Over $30 \%$ of the $\mathrm{GHG}$ savings associated with both doses resulted from decreased upstream emissions resulting from reduced feed needed. The avoided emissions from pig housing energy demands was the third most substantial contributor to the over GHG savings for both doses with approximately $4 \%$ of the total savings. Overall, this is a very conservative estimate of the GHG emissions savings because none of the downstream GHG emissions savings were included such as post farm transport and processing of pigs. The carbon footprint of the manufacturing and distribution of Paylean to the entire pig population resulted in a fraction of GHG emissions attributable to pork production.

\section{References}

1. Apple, J.K., P.J. Rincker, F.K. McKeith, S.N. Carr, T.A. Armstrong and P.D. Matzat. 2007. Meta-analysis of the ractopamine response in finishing swine. Prof. Anim. Sci. 23:179-196.

2. Anderson, D.B., A.L. Woods, T.A. Armstrong T.E. Elam, A. L. Sutton. 2010. Environmental benefits of Paylean ${ }^{\circledast}$ use in United States finisher swine. AAVS Proceedings (in press). 


\title{
P.739
}

\section{A comparative study on route and number of times of iron administration in piglets on hematological values}

\author{
Pariwat Poolperm; Prapha Songjinda; Piyapol Tiangladdawong; Peerapat Krittanan; Patthanan
}

Paprakorb; Wimwiga Sakchainanon; Yaowaluk Panavajkitkul; Apichart Subsiribhaiboon

Kasetsart University, Nakornpathom, Thailand

\section{Introduction}

Piglets are most likely to develop anemia after birth since the iron requirement for red blood cell production is about 7-10 $\mathrm{mg} /$ day while there is insufficient amount of iron at $1 \mathrm{mg} /$ day available in sow milk (1). Therefore, the supplementation of iron to piglets at a few days of age is of importance for anemia prevention. The iron supplementation can be given by oral or parenteral route, and its effectiveness was varied. Two doses of intramuscular injection showed higher hematocrit and hemoglobin than two doses of oral administration and one dose of injection (2). The larger dosage of iron intramuscular injection promoting haemoglobin formation was also confirmed (3). In the other hand, Miller, et al (4) showed that oral administration gave significantly higher hematocrit than intramuscular injection.

The aim of this study was to investigate the effect of iron administration methods (oral versus intramuscular route) and number of times of iron administration ( 1 time versus 2 times dosage) on hematological values of piglets. Hematocrit (Hct), haemoglobin concentration $(\mathrm{Hb})$ and erythrocyte count $(\mathrm{RBC})$ were determined.

\section{Materials and Methods}

In this study, new born piglets $(n=45)$ were randomly assigned into 5 groups. Each group was given iron supplementation as follows: control group received no iron supplementation (C, $\mathrm{n}=5$ ); piglets intramuscularly injected with iron dextran (Abidex $\left.100^{\circledR}\right) 200 \mathrm{mg}$ on day $4(\mathrm{IM} 1, \mathrm{n}=10)$, on day 4 and 18 after birth (IM2, n=10); piglets given $200 \mathrm{mg}$ ferrous sulfate (Ferrous Booster $^{\circledast}$ ) orally on day $4(\mathrm{OR} 1, \mathrm{n}=10)$, on day 4 and 18 after birth (OR2, n=10).

Blood from the piglets were collected weekly in EDTA, as anticoaggulant, for the period of 4 weeks. The blood samples were then examined for hematological indices including hematocrit, haemoglobin concentration and RBC count using Automated hematology analyzer (Abacus Junior Vet, Austria). The results were statistically analyzed by repeated measures analysis of variance.

\section{Results}

The hematological values of all the treatment groups were significantly different from that of the $C$ group $(P<0.05)$, except the RBC count of OR2 group. Meanwhile, Hct and $\mathrm{Hb}$ were similar for all treatment groups. Compared within route of iron administration, one-time and two-times administration had the same hematological values.

Table 1. Values of Hematocrit (\%), Hemoglobin concentration $(\mathrm{g} / \mathrm{dl})$ and $R B C$ count $\left(\times 10^{6} / \mu l\right)$ in trial groups. (mean \pm SEM)

\begin{tabular}{|l|c|c|c|}
\hline Group & Hematocrit & Hb conc. & RBC count \\
\hline Control & $25.3 \pm 1.0^{\mathrm{a}}$ & $7.4 \pm 0.3^{\mathrm{a}}$ & $5.1 \pm 0.2^{\mathrm{a}}$ \\
\hline IM1 & $30.6 \pm 0.7^{\mathrm{b}}$ & $9.6 \pm 0.2^{\mathrm{b}}$ & $5.7 \pm 0.1^{\mathrm{b}}$ \\
\hline IM2 & $32.1 \pm 0.7^{\mathrm{b}}$ & $9.7 \pm 0.2^{\mathrm{b}}$ & $5.7 \pm 0.1^{\mathrm{b}}$ \\
\hline OR1 & $29.7 \pm 0.7^{\mathrm{b}}$ & $9.2 \pm 0.3^{\mathrm{b}}$ & $5.6 \pm 0.1^{\mathrm{b}, \mathrm{c}}$ \\
\hline OR2 & $29.9 \pm 0.7^{\mathrm{b}}$ & $9.2 \pm 0.2^{\mathrm{b}}$ & $5.3 \pm 0.1^{\mathrm{a}, \mathrm{c}}$ \\
\hline $\mathrm{a}, \mathrm{b}, \mathrm{c}=$ significant difference within column $(\mathrm{p}<0.05)$ \\
\hline
\end{tabular}

\section{Discussion}

The results from this study indicated that iron supplementation to piglets was benefit to piglet health on haematological values regardless of routes or number of times that piglets received iron supplementation. Oral administration of iron might be compromised with animal welfare for those piglets.

\section{References}

1. Svoboda, M. and Drabek J. (2002). Czech J. Anim. Sci. 47: 213-218.

2. Manner, J.H., et al. (1959). J. Anim. Sci. 18: 1373-1377.

3. Haugegaard, J., Kristensen, P.J., Wachmann, H. (2006). IPVS Proceeding vol. l, p.302.

4. Miller, E.R., et al. (2008). J. Nutrition 24: 187-193. 


\title{
P.740
}

\section{Serum micro-element reference values for gestating and lactating sows}

\author{
Frédéric A. Vangroenweghe ${ }^{1}$ Tamara Vandersmissen $^{2}$ Ellen Van Driessche $^{1}$ Dominiek Maes $^{3}$ Ellen De Jongh $^{3}$ \\ 1. DGZ-Vlaanderen, Torhout, Belgium; 2. DGZ-Vlaanderen, Lier, Belgium; 3. Faculty of Veterinary Medicine-Ghent University, Merelbeke, Belgium
}

\section{Introduction and Objective}

The measurement of serum macro-element parameters of farm animals can provide important information on health and metabolism of several organ systems $(1,2)$ Recently, an episode of reproductive problems was observed in several pig herds in Flanders, although no specific infectious causes could be identified. Besides infectious causes, feeding strategies and feed composition could have an impact on reproductive performance of the sows. Several components such as vitamin E, selenium (Se) and minerals ( $\mathrm{Ca}, \mathrm{Mg}, \mathrm{Mn}, \mathrm{Cu}$ and $\mathrm{Zn}$ ) could have an influence on reproduction (1). However, to interpret the obtained results in serum macro- and micro-element analysis, reliable reference values should be available. Therefore, blood was collected from primiparous and multiparous sows in several farms with no reproductive problems in order to establish reference values for several minerals and vitamin $\mathrm{E}$. The effect of stage of reproductive cycle was also evaluated.

\section{Materials and Methods}

Seven pig farms with at least 100 sows in a batch management system (BMS) were selected based on the absence of reproductive problems. Samples were collected from the same sows at $60 \pm 5 \mathrm{~d}$ of gestation, $3 \pm 1 \mathrm{~d}$ after farrowing and at $3 \pm 1 \mathrm{~d}$ after weaning. Analysis was performed as follows: Se $(\mu \mathrm{g} / \mathrm{l})$ and $\mathrm{Mn}$ $(\mu \mathrm{g} / \mathrm{l})$ using an atomic absorption technique and vitamin $\mathrm{E}(\mathrm{mg} /$ dl) using HPLC-analysis.

\section{Results}

Results expressed as mean and range (2.5-percentile and 97.5-percentile) are given for primiparous and multiparous sows in Tables 1-3.

Table 1. Reference values for serum macro-elements of the parity-one sows $(n=14)$ and parity $\geq 2$ sows $(n=31)$ from 7 commercial pig herds at $3 \pm 1$ d of lactation

\begin{tabular}{|l|c|c|c|c|}
\hline & \multicolumn{2}{|c|}{ Parity-one sows } & \multicolumn{2}{c|}{ Parity $\geq 2$ sows } \\
\hline & Mean & Range & Mean & Range \\
\hline $\mathrm{Mn}(\mu \mathrm{g} / \mathrm{l})$ & 1.07 & $0.45-2.14$ & 1.24 & $0.34-2.70$ \\
\hline $\mathrm{Se}(\mu \mathrm{g} / \mathrm{l})$ & 174.64 & $125.75-239.50$ & 166.42 & $125.75-237.75$ \\
\hline Vit $\mathrm{E}(\mathrm{mg} / \mathrm{dl})$ & 2.01 & $1.34-2.65$ & 1.93 & $0.80-2.93$ \\
\hline
\end{tabular}

Table 2. Reference values for serum macro-elements of the parity-one sows $(n=14)$ and parity $\geq 2$ sows $(n=31)$ from 7 commercial pig herds at $3 \pm 1$ d after weaning

\begin{tabular}{|l|c|c|c|c|}
\hline & \multicolumn{2}{|c|}{ Parity-one sows } & \multicolumn{2}{c|}{ Parity $\geq 2$ sows } \\
\hline & Mean & Range & Mean & Range \\
\hline $\mathrm{Mn}(\mu \mathrm{g} / \mathrm{l})$ & 2.02 & $0.64-4.70$ & 2.30 & $0.61-6.13$ \\
\hline $\mathrm{Se}(\mu \mathrm{g} / \mathrm{l})$ & 188.73 & $141.75-237.50$ & 177.84 & $135.75-234.25$ \\
\hline $\operatorname{Vit} \mathrm{E}(\mathrm{mg} / \mathrm{dl})$ & 3.61 & $1.88-5.12$ & 3.44 & $1.72-5.32$ \\
\hline
\end{tabular}

Table 3. Reference values for serum macro-elements of the parity-one sows $(n=14)$ and parity $\geq 2$ sows $(n=31)$ from 7 commercial pig herds at $60 \pm 5$ d of gestation

\begin{tabular}{|l|c|c|c|c|}
\hline & \multicolumn{2}{|c|}{ Parity-one sows } & \multicolumn{2}{c|}{ Parity $\geq 2$ sows } \\
\hline & Mean & Range & Mean & Range \\
\hline $\mathrm{Mn}(\mu \mathrm{g} / \mathrm{l})$ & 1.93 & $0.68-3.49$ & 5.66 & $0.39-23.94$ \\
\hline $\mathrm{Se}(\mu \mathrm{g} / \mathrm{l})$ & 168.27 & $143.50-198.75$ & 173.58 & $135.00-210.50$ \\
\hline Vit E $(\mathrm{mg} / \mathrm{dl})$ & 2.56 & $1.15-2.95$ & 2.50 & $1.08-3.68$ \\
\hline
\end{tabular}

\section{Discussion and Conclusions}

It is difficult to compare the obtained results with previous results due to differences in analytical techniques (3). The reference values obtained for vitamin $\mathrm{E}$ were lowest at parturition and increased at weaning. Selenium reference values had a quite stable kinetics with slightly higher values at $3 \mathrm{~d}$ after weaning. For $\mathrm{Mn}$, the lowest reference values were observed at $3 \mathrm{~d}$ of lactation. Due to one farm with very high Mn-levels at $60 \mathrm{~d}$ of gestation, the reference value and range is much higher in multiparous sows. Overall, primiparous sows had lower reference values as compared to multiparous sows in all three production stages.

\section{References}

1. Friendship R.M., Henry S.C. 1992. In: Leman A.D., Straw B.E., Mengeling W.L., d'Allaire S., Taylor D.J. (Eds.), Diseases of Swine, 7th ed., lowa State University Press, Ames, IA, pp. 3-11

2. Verheyen A. et al. 2007. The Veterinary Journal, 174: 92-98

3. Newland H.W., David G.K. 1961. J. Anim. Sci., 20: 15-17 


\title{
Serum macro-element reference values for gestating and lactating sows
}

\author{
Frédéric A. Vangroenweghe ${ }^{1}$ Tamara Vandersmissen $^{2}$ Ellen De Jongh $^{3}$ Ellen Van Driessche $^{1}$ Dominiek Maes $^{3}$ \\ 1. DGZ-Vlaanderen, Torhout, Belgium; 2. DGZ-Vlaanderen, Lier, Belgium; 3. Faculty of Veterinary Medicine-Ghent University, Merelbeke, Belgium
}

\section{Introduction and Objective}

The measurement of serum macro-element parameters of farm animals can provide important information on health and metabolism of several organ systems (1) and is a practical diagnostic tool for assessing pathological conditions in the live animal or for monitoring the health status of groups of animals (2). Recently, an episode of reproductive problems was observed in several pig herds in Flanders, although no specific infectious causes could be identified. Also no major shortcomings were observed regarding management and housing conditions. Besides infectious causes of the reproductive problems, feeding strategies and feed composition could have an impact on reproductive performance of the sows. Several components such as vitamin $\mathrm{E}$, selenium ( $\mathrm{Se}$ ) and minerals ( $\mathrm{Ca}, \mathrm{Mg}, \mathrm{Mn}, \mathrm{Cu}$ and $\mathrm{Zn}$ ) could have an influence on reproduction (1). However, to interpret the obtained results in serum macro- and micro-element analysis, reliable reference values should be available. Therefore, blood was collected from primiparous and multiparous sows in several farms with no reproductive problems in order to establish reference values for several minerals and vitamin E. The effect of stage of reproductive cycle was also evaluated.

\section{Materials and Methods}

Seven pig farms with at least 100 sows in a batch management system (BMS) (3-, 4- or 5- week BMS) were selected based on the absence of reproductive problems. The herds had between 200-450 sows. Samples were collected from the same sows at $60 \pm 5 \mathrm{~d}$ of gestation, $3 \pm 1 \mathrm{~d}$ after farrowing and at $3 \pm 1 \mathrm{~d}$ after weaning. All samples were stored at $-20^{\circ} \mathrm{C}$ until the end of the sampling period and analysed subsequently in one single batch. Analysis was performed as follows: $\mathrm{Na}(\mathrm{mmol} / \mathrm{l}), \mathrm{Cl}(\mathrm{mmol} / \mathrm{l}), \mathrm{K}$ (mmol/l) using Vetlyte (Idexx), P (mmol/l) using Vettest (Idexx), Fe ( $\mu \mathrm{mol} / \mathrm{l})$ using Diasys $\mathrm{nl}$ Iron FS Ferene (Konelab TX20), Cu ( $\mu \mathrm{mol} / \mathrm{l})$ using Randox (Konelab TX20) and $\mathrm{Ca}(\mathrm{mmol} / \mathrm{l})$ and $\mathrm{Mg}$ (mmol/l) using Siemens Medical Solutions (Konelab TX20) and $\mathrm{Zn}(\mu \mathrm{g} / \mathrm{dl})$ using an atomic absorption technique.

\section{Results}

Results expressed as mean and range (2.5-percentile and 97.5-percentile) are given for primiparous and multiparous sows in Table 1-2. The results of $3 \mathrm{~d}$ after weaning were comparable to those at $60 \mathrm{~d}$ of gestation and were therefore not presented here.

\section{Discussion and Conclusions}

It is difficult to compare the obtained results with previous results due to differences in analytical techniques (3). The reference values obtained for most of the determined elements were slightly higher at $60 \mathrm{~d}$ of gestation as compared to $3 \mathrm{~d}$ in lactation. Moreover, the ranges are higher and narrower at $60 \mathrm{~d}$ in gestation, indicating less variation within the animal population. Overall, primiparous sows had higher reference values as compared to multiparous sows in lactation and during gestation.

\section{References}

1. Friendship R.M., Henry S.C. 1992. Cardiovascular system, hematology and clinical chemistry. In: Leman A.D., Straw B.E., Mengeling W.L., d'Allaire S., Taylor D.J. (Eds.), Diseases of Swine, 7th ed., lowa State University Press, Ames, IA, pp. 3-11

2. Verheyen A., Maes D., Mateusen B., Deprez P., Janssens G., de Lange L., Counotte G. 2007. Serum biochemical reference values for gestating and lactating sows. The Veterinary Journal, 174: 92-98

3. Newland H.W., David G.K. (1961). Placental transfer of Mg in swine. J. Anim. Sci., 20: 15-17.

Table 1. Reference values for serum macro-elements of the parity-one sows $(n=14)$ and parity $\geq 2$ sows $(n=31)$ from 7 commercial pig herds at $3 \pm 1$ d of lactation

\begin{tabular}{|l|c|c|c|c|}
\hline & \multicolumn{2}{|c|}{ Parity-one sows } & \multicolumn{2}{c|}{ Parity $\geq 2$ sows } \\
\hline & Mean & Range & Mean & Range \\
\hline $\mathrm{Ca}(\mathrm{mmol} / \mathrm{l})$ & 2.58 & $1.97-2.88$ & 2.63 & $1.65-3.07$ \\
\hline $\mathrm{P}(\mathrm{mmol} / \mathrm{l})$ & 2.13 & $1.44-2.62$ & 2.03 & $1.23-2.62$ \\
\hline $\mathrm{Mg}(\mathrm{mmol} / \mathrm{l})$ & 1.13 & $0.75-1.52$ & 0.99 & $0.39-1.26$ \\
\hline $\mathrm{Na}(\mathrm{mmol} / \mathrm{l})$ & 138.3 & $101.5-151.0$ & 139.2 & $84.2-154.3$ \\
\hline $\mathrm{Cl}(\mathrm{mmol} / \mathrm{l})$ & 99.45 & $76.75-105.50$ & 99.13 & $64.50-110.25$ \\
\hline $\mathrm{K}(\mathrm{mmol} / \mathrm{l})$ & 7.85 & $5.12-12.23$ & 6.75 & $3.60-47.13$ \\
\hline $\mathrm{Cu}(\mu \mathrm{mol} / \mathrm{l})$ & 37.36 & $25.92-48.02$ & 36.49 & $21.67-47.13$ \\
\hline $\mathrm{Zn}(\mu \mathrm{g} / \mathrm{dl})$ & 78.64 & $49.25-111.00$ & 71.16 & $44.75-116.25$ \\
\hline $\mathrm{Fe}(\mu \mathrm{mol} / \mathrm{l})$ & 41.39 & $14.02-88.50$ & 32.67 & $14.82-60.75$ \\
\hline
\end{tabular}

Table 2. Reference values for serum macro-elements of the parity-one sows $(n=14)$ and parity $\geq 2$ sows $(n=31)$ from 7 commercial pig herds at $60 \pm 5 d$ of gestation

\begin{tabular}{|l|c|c|c|c|}
\hline & \multicolumn{2}{|c|}{ Parity-one sows } & \multicolumn{2}{c|}{ Parity $\geq 2$ sows } \\
\hline & Mean & Range & Mean & Range \\
\hline $\mathrm{Ca}(\mathrm{mmol} / \mathrm{l})$ & 2.67 & $2.32-3.01$ & 2.74 & $2.44-2.98$ \\
\hline $\mathrm{P}(\mathrm{mmol} / \mathrm{l})$ & 2.68 & $2.14-3.44$ & 2.38 & $1.77-3.70$ \\
\hline $\mathrm{Mg}(\mathrm{mmol} / \mathrm{l})$ & 1.19 & $1.06-1.32$ & 1.14 & $0.98-1.39$ \\
\hline $\mathrm{Na}(\mathrm{mmol} / \mathrm{l})$ & 145.3 & $137.5-152.3$ & 144.5 & $133.2-150.3$ \\
\hline $\mathrm{Cl}(\mathrm{mmol} / \mathrm{l})$ & 104.0 & $101.0-107.0$ & 104.2 & $97.25-108.25$ \\
\hline $\mathrm{K}(\mathrm{mmol} / \mathrm{l})$ & 9.00 & $6.70-12.77$ & 8.17 & $5.07-13.25$ \\
\hline $\mathrm{Cu}(\mu \mathrm{mol} / \mathrm{l})$ & 37.28 & $28.00-43.70$ & 34.39 & $27.52-41.65$ \\
\hline $\mathrm{Zn}(\mu \mathrm{g} / \mathrm{dl})$ & 84.64 & $66.45-118.25$ & 77.26 & $52.50-100.75$ \\
\hline $\mathrm{Fe}(\mu \mathrm{mol} / \mathrm{l})$ & 42.55 & $32.47-70.88$ & 40.10 & $21.15-75.08$ \\
\hline
\end{tabular}




\title{
P.742
}

\section{Growth performance response to different sources and batches of Ractopamine}

\author{
Diego Brana Varela ${ }^{1}$ Cesar A. Mejía Guadarrama1 Jose J. Lopez Espinoza ${ }^{2}$ \\ 1. INIFAP, National research institute for Forestry, Agriculture and Livestock, Queretaro, QRO, Mexico; 2. Elanco, Guadalajara, JAL, Mexico
}

\section{Introduction}

Ractopamine (RAC) is a phenethanolamine $\beta$-adrenergic receptor agonist that is commercially available (Paylean ${ }^{\circledR}, \mathrm{PAY}$ ) for enhanced muscle protein growth in pigs. After the success of RAC hydrochloride $\left(\mathrm{PAY}^{\circledR}\right)$, several companies have commercialized generic versions of the product. This trial was done to study growth performance and consistency of response between brands of Ractopamine (generic "GEN" vs. PAY), and also to determine the uniformity of response when comparing different production batches of the same brand.

\section{Materials and Methods}

The experiment was conducted as a Randomized Complete Block Experiment. RAC source and batches (treatments) were present in all the blocks. A total of 240 pigs (half gilts and half barrows), were individually contained providing $2.09 \mathrm{~m} 2$ space per pig and were fed one of the RAC products (at $5 \mathrm{ppm}$ ) or a Control diet (C; with the same formula) during the last $28 \mathrm{~d}$ of the finishing period. There were 48 pigs for the control diet and 24 for each of the 4 product batches within RAC source. Thus the Ractopamine source effect included 96 experimental units, and the source * batch (lot) interaction, 24. All Treatments were present in 4 blocks (block=week of birth). Animals were individually feed twice a day, weighed every week, and average fat free lean tissue growth (FFLTG) was estimated by real time ultrasound (Aloka 550 SD) measurements. Data was statisticallyanalyzed under the restriction of a complete random block model, with 9 treatments and 4 blocks. General Linear Models (GLM, SAS) were used. Least square means from the variance analysis were presented.

\section{Results}

Initial body weight $(76.6 \pm 8.32 \mathrm{~kg})$ was similar $(\mathrm{P}<0.9)$ between Treatments. After $28 \mathrm{~d}$, average daily gain (ADG) differed between brands (1030; 1153, and $1115 \pm 202 \mathrm{~g} / \mathrm{d} ; \mathrm{P}<0.0001)$ respectively for $C$, PAY and GEN. Interestingly, ADG response was not the same within the GEN batches: (C: 1033; PAY: 1161; 1149; 1171; 1138; GEN: $1128 ; 1169 ; 1076 ; 1089 \pm 32 \mathrm{~g} / \mathrm{d}$ ), respectively for the mean of each one of the production batches within Trt. Similar response was observed for feed efficiency (gain over feed; FE), where overall the use of RAC improved FE, but the response was dependent not only on the brand, but also was dependent on the production batch for the GEN (C: 361 ; PAY: 394; 404; 406; 407; GEN: 382; 390; 374; 373 $\pm 8.4 ; P<0.0001)$. The FFLTG was improved by RAC (C: 355; PAY: 415; RAC: $394 \pm 6.8 \mathrm{~g} / \mathrm{d}$; P<0.0001).

\section{Discussion}

Overall treatment responses were consistent $(P<0.0001)$ for ADG, FE and FFLTG, although the response was better and more consistent when the PAY product was used. But the evaluation should also be done in probabilistic terms. Interestingly, two batches of the GEN product out of four, when compared to the Control, where not different $(P>0.6)$; meaning that the response to the GEN product could not be observed in $50 \%$ of the times. Differences in feed intake and ADG can be relatively small and hard to detect at the farm because of the variation inherent to the process. Feed efficiency is a more sensitive and reliable variable. Additionally, in times of expensive feed, feed efficiency is one of the most relevant variables in profitability. Finally, as demonstrated in this trial, product consistency is a feature that producers should consider when evaluating different products. 


\title{
P.743
}

\section{Effects of feed withdrawal/fasting prior to slaughter on dressing percentage and meat $\mathrm{pH}$}

\author{
Lorenzo Encinares \\ CEFN Genetics Australia Inc., Ortigas, Pasig City, Philippines
}

This study explored the effect of pre-slaughter fasting on the pigs' carcass value and weight.

The optimum time of feed withdrawal before slaughter was determined. Findings will be useful to the pork industry in minimizing the decrease in carcass value particularly weight and therefore increase the amount of money a producer may receive, without compromising carcass value.

Ninety (90) market weight pigs were divided into 3 treatments: 0,12 and 24 hour feed withdrawal prior to slaughter without restriction to water access. Each treatment was allocated with 30 pigs irrespective of sex. The experiment was conducted in 6 slaughter days, wherein 15 heads were slaughtered on each slaughter day. Carcass weight was recorded, along with the weight of body organs and digestive tract full and emptied. Meat $\mathrm{pH}$ was also measured 45 minutes post-slaughter to determine the effect of feed withdrawal on meat.

There was an observed $2.6 \mathrm{~kg}$ body weight loss in the first 12 hour of fasting which increased to $4.7 \mathrm{~kg}$ after 24 hour of continued feed withdrawal. Total shrinkage of $55.3 \%$ occurred during the first 12 hours, which was reduced to only $44.7 \%$ in the next 12 hours of fasting. Dressing percentage was observed to be higher in fasted animals compared to pigs not fasted before slaughter $(P<0.01)$. It was estimated that the weight loss for every hour of feed withdrawal was $0.2791 \mathrm{~kg}$ loss. This study found that a considerable live weight loss occurred when pigs are fasted over a 24 hour period. This loss translates to monetary losses to the producer. Within 12-24 hour period of feed withdrawal, carcass losses also entail monetary losses on the part of the buyer.
Regarding the current trend of buying and selling of finishing hogs in the market today, it seems that a lot of money is wasted due to improper fasting time of finishing pigs. In order to avoid unnecessary monetary losses, pigs should be fasted not more than 12 hours before the actual time of slaughter. It is also important to note that this study is limited to the effect of feed withdrawal only, so it could be assumed that additional weight losses can be incurred if transportation parameter is included. Ordinarily buyers purchase their pigs at long distances so drastic losses are expected. Highly significant differences $(\mathrm{P}<0.0001)$ were observed in fasted and non fasted groups for the digestive tract, (full and emptied), residual gutfill, and liver weight.

After 24 hour of feed withdrawal, when the digestive tract is fully empty, there was a progressive loss in liver weight with increased fasting time. Except for the liver being significantly bigger in barrows than in gilts, there were no other carcass traits observed to be significant between genders. No significant changes were observed among body organs in the three treatments. There are also no significant differences in the initial $\mathrm{pH}$ of the meat at 45 minutes post-mortem among fasted and non fasted animal. 


\title{
P.744
}

\section{Determination of the quantity of water used to produce a pig of $100 \mathbf{~ k g}$}

\author{
Roberto M. Rodríguez ${ }^{1}$ Ismael C. Cabreraํㅡㄹ Marco A. Vega-López ${ }^{2}$ \\ 1. Universidad Nacional Autónoma de México, México City, DF, Mexico; 2. Cinvesta-IPN, México City, DF, Mexico
}

\section{Introduction}

The steady increase in demand by the world population explosion, the unbalanced regional distribution and various forms of pollution have made water a scarce and precious resource. Nowadays, the use of water is an issue of great concern in all human activities due to its growing scarcity. Therefore all efforts are relevant to optimize its use. Unfortunately, there is very little information on water use in swine production.

\section{Aims}

To determine the amount of water needed to produce a finished $100 \mathrm{~kg}$ live weight pig, taking into account the water used by the pigs and cleaning activities on the farm.

\section{Material and Methods}

The study was conducted at the experimental farm of the Universidad Nacional Autónoma de México, wich is a 120 sows farrow to finish operation located in central México, with an average temperature of $18^{\circ} \mathrm{C}$ and average rainfall of $608 \mathrm{~mm}$. The feed were based on sorghum and soybean meal. The pigs in the weaning and finishing stages are fed ad libitum. In all areas of pigs were used nipple type drinkers and had free access to water. Water meters were used for metrological class $C$, which measure $100 \mathrm{ml}$ fractions. The washing of the pens was made with a pressure washing machine. Only in the area of early gestation was performed daily washing with a hose. During the study, said there were no leaks in the hydraulic installation of the farm. In the areas of breeding pigs, gilts, gestation and lactation was measured amount of water used and calculated how much each area contributes to produce a 21 days weaned pig. In the stages of weaning and fattening, was calculated the individual average water used by a pig during each stage. The amounts were added to the average water used for washing pig facilities in each stage, to finally get the amount of water used for production of a pig of $100 \mathrm{~kg}$. The results were analyzed with descriptive statistics.

\section{Results}

Table 1 shows the average water used per individual in each stage of production and the individual average of water used for washing facilities. The average water used to produce a 21 days weaned pig was 89423 liters. The average water used by the weaning and finishing pig was 1705.44 liters. The sum of these amounts represents the average water used for production of a pig of $100 \mathrm{~kg}$, which was 2599.67 liters.

\section{Discussion}

The results obtained should not be taken as a universal measure, but as a reference for estimating water needs for a pig farm, and to seek to reduce water consumption by specific strategies. Results of the study discovered areas where water use is too high, wich allows to identify critical points in wich to work to establish corrective actions. These measurements can be implemented relatively easily in pig farms. Future studies will be necessary to include the water used in bathrooms and offices of the pig farms to obtain more complete information. Is suggested to perform jobs that are water ways to optimize every step of the production process of the pigs, which is an important objective in relation to water use in the swine industry worldwide.

Table 1. Individual average daily liters of water used in each stage of production (AWP) and average water used for washing facilities (AWF).

\begin{tabular}{|c|c|c|}
\hline STAGE & AWP & AWF \\
\hline Boars & 19.54 & 95 \\
\hline Gilts & 20.79 & 95 \\
\hline Early pregnancy & 63.46 & 297 \\
\hline Late pregnancy & 36.14 & 95 \\
\hline Farrow & 36.64 & 277.5 \\
\hline Nursery & 4.06 & 43.4 \\
\hline Fattening & 12.78 & 36.8 \\
\hline
\end{tabular}

\section{References}

1. Straw B, et al, 2006. Diseases of Swine. Blackwell Publishing. USA.

2. León F., 2005. Agua. Ed. La Jornada. México.

3. Shaw M, 2006. J. Anim. Sci. 84:3123-3132.

4. Close $W$ and Cole D, 2000. Nutrition of sows and boars. Nottingham University Press. UK. 


\title{
P.745
}

\section{Effect of feeding newborn piglets with a fermented bioprotein (Lianol ${ }^{\circledR}$ ) in mortality before weaning}

\author{
Daniel Villalba ${ }^{1}$ Guillermo Cano ${ }^{2}$ Ruddy Forier ${ }^{3}$ Ramon Gatnau $^{4}$ Alberto Morillo $^{2}$ \\ 1. University of Lleida, Lleida, Spain; 2. Tests and Trials, Lleida, Spain; 3. Ardol, Susteren, Netherlands; 4. Molimen, Barcelona, Spain
}

\section{Introduction and objectives}

Piglets with low weights at birth are related with a decreased livability falling into hypothermia, starvation and death ${ }^{1}$. Lianol ${ }^{\circledR}$ (a fermented bioprotein by Ardol, The Netherlands) is supposed to have a positive effect in new born piglets from an increase of circulating IGF-I. The aim of the study was to evaluate the effect of supply orally Lianol ${ }^{\circledR}$ at first day of live in mortality from born to weaning in piglets and to evaluate if this improvement could be done by and increase of serum IGF-I.

\section{Material and Methods}

391 newborn piglets were tagged, weighed and assigned to one of two groups during the first 12 hours after birth depending on BW: piglets with BW higher or equal to $1.2 \mathrm{~kg}$ (BIG) or with BW lower than $12 \mathrm{~kg}$ (SMALL). Treatment were: i) $2 \mathrm{ml}$ of Lianol ${ }^{\circledR}$ given orally in the mouth during first 12 hours after birth (Lianol2), ii) $1 \mathrm{ml}$ of Lianol ${ }^{\circledR}$ during first 12 hours after birth plus 1 $\mathrm{ml} 24$ hours later (Lianol1+1) and iii) a negative control group (Control). Piglets were randomized to be assigned onto each treatment within BW group and sex. All animals were weighed again on days 3, 7 and 20 of live.

181 randomized piglets were bled at the day 1, 7 and 20 of live. Number of piglets in every group and treatment is shown in table 1.

Blood samples were tested by enzyme-linked immunosorbent assay (ELISA) using established methods.

Piglet was the experimental unit. IGF-I, BW and ADG were analyzed with ANOVA. Mortality was analyzed using a logistic regression. Piglet uniformity was assessed from the coefficients of variation of the individual BW and differences between treatment groups were analyzed using $\mathrm{F}$ test. The level of significance was $5 \%$.

\section{Results and Discussion}

Table 1 shows number of piglets included and mortality levels. Mortality was not statistically different between treatments. However, we have observed a relevant reduction of $50 \%$ between Control and Lianol2 in BIG group and $46 \%$ between Control and Lianol 2 in SMALL group. These values were not statistically different but are a relevant data under commercial situations.

We found no difference between treatments in IGF-I levels any day of the study. Values of IGF-I have a high variability even when we compare same piglets in same circumstances. IGF-I values were agreed with scientific literature values. At day 0 , IGF-I values were $59.56 \pm 5.05 ; 62.63 \pm 5.18$ and $60.60 \pm 5.12 \mathrm{ng} /$ $\mathrm{ml}$ in BIG group for Lianol2, Lianol1+1 and Control respectively and $57.24 \pm 7.16 ; 44.63 \pm 8.48$ and $61.33 \mathrm{ng} / \mathrm{ml}$ in the SMALL group for Lianol2, Lianol1+1 and Control. At day 20 IGF-I values were $76.28 \pm 5.01 ; 79.02 \pm 5.06$ and $78.80 \mathrm{ng} / \mathrm{ml}$ in BIG group for Lianol2, Lianol $1+1$ and Control respectively and 79.77 \pm 7.07 ; $80.98 \pm 9.01$ and $82.37 \mathrm{ng} / \mathrm{ml}$ in the SMALL group for Lianol2, Lianol $1+1$ and Control. Circulating IGF-I levels in piglets has a high variability depending on factors such as nutrition, season, management techniques and sex².
We found neither statistical difference in BW nor in ADG. Piglet uniformity was not different between treatments at day 20 .

Table 1. Piglet inclasion and mortality absolute values and percentage.

\begin{tabular}{|c|c|c|c|}
\hline Group & LhanoL2 & Lianel1+1 & Control \\
\hline $\begin{array}{c}\text { BIG } \\
\text { Included }\end{array}$ & 78 & 76 & 79 \\
\hline $\begin{array}{c}\text { BIG } \\
\text { Mortality }\end{array}$ & 3 & 2 & 6 \\
\hline $\begin{array}{c}\text { BIG } \\
\text { Mortality, \% }\end{array}$ & $3.85 \%$ & $2.63 \%$ & $7.59 \%$ \\
\hline $\begin{array}{c}\text { SMAL } \\
\text { Included }\end{array}$ & 56 & 51 & 51 \\
\hline $\begin{array}{c}\text { SMALL } \\
\text { Mortality }\end{array}$ & 10 & 15 & 14 \\
\hline $\begin{array}{c}\text { SMALL } \\
\text { Mortality. \% }\end{array}$ & $17.58 \% 6$ & $29.41 \%$ & $27.45 \%$ \\
\hline
\end{tabular}

\section{Implications and Conclusions}

The supply of Lianol ${ }^{\circledR}$ after birth did not improve mortality statistically but the numerical improvement was relevant under commercial situations mainly in BIG piglets. IGF-I values, BW and ADG were not improved. More investigations should be done in order to know the relationship between IGF-I levels and piglet performances.

\section{References}

1. Louveau I, Dauncey MJ, LeDividich J. (2000). Regulation of development by nutrition and by the somatotrophic and thyroid axes in the neonatal pig. Livest.Prod.Sci.66,121-131.

2. Harrell RJ, Thomas MJ, Boyd RD, Czerwinski SM, Steele NC, Bauman DE (1999). Ontogenic maturation of the somatotropin/insulin-like growth factor axis. J Anim Sci. 1999;77(11):2934-41. 


\title{
P.746
}

\section{Effect of dietary cysteamine on growth performance and growth hormone of starter-finishing pigs}

\author{
Jamlong Mitchaothai ${ }^{1}$ Chanathip Thammakarn² Suaysuda Ninlaworn ${ }^{3}$ \\ 1. Faculty of Veterinary Medicine, Mahanakorn University of Technology, Nong Chok, Bangkok, Thailand; \\ 2. School of Animal Production Technology and Fisheries, Faculty of Agricultural Technology, King Mongkut's Institute of Technology \\ Ladkrabang, Bangkok, Thailand; 3. Technical and Product Department (Veterinarian), Grandsiam.Co., Ltd., Bangna, Bangkok, Thailand
}

\section{Introduction}

Cysteamine (CS) can be used as a feed additive for improving growth performance of growing pigs. Pigs obtaining CS would lead to lower level of somatostatin, resulting in elevation of growth hormone (GH) level (1). It has been known that $\mathrm{GH}$ increases muscle growth (2) and decreases deposition of fat in pigs (3). In finishing pigs, dietary CS supplementation improved protein deposition (4). However, the effects of dietary supplementation on growing pigs raised under field condition have not been reported. Thus, the current study aims to determine effect of dietary supplementation on growth performance and serum $\mathrm{GH}$ of starter-finishing pigs.

\section{Materials and Methods}

Three hundred and seventy five starter pigs were randomly selected from a commercial pig farm for the current study. All experimental pigs were equally allotted into three groups; the control (CT), recommended level of CS (RC) and high level of CS (HC). Each experimental group contained 125 pigs, equally housed in 5 pens. The pigs in the CT were offered control diet, which is normally used in the farm, while the pigs in the RC and $\mathrm{HC}$ were offered the control diet with supplementation of CS at 300 and 500 ppm, respectively. The pigs were fed ad libitum. Live body weight of the pigs of each pen was measured at the start and the end of the study. Feed intake of the pigs was recorded throughout the experiment. Blood samples of 10 pigs from each group were randomly collected at 13.00 hours in order to quantify level of GH with RIA method. At the conclusion of the study, ADG, FCR and feed cost per body weight gain (FCG) were calculated. The statistical analysis is one way ANOVA with Duncan's multiple comparison.

\section{Results}

The results of the growth performance have been demonstrated in the Table 1. There was no effect of dietary CS supplementation on ADG and FCG. However, the pigs fed on the RC diet had significant lowest FCR $(p<0.05)$, whereas the pigs fed on the CT and $\mathrm{HC}$ had no significant difference. The levels of $\mathrm{GH}$ have been shown in the Table 2. The change of GH was greatest (42\%) in value for the pigs fed on the CT diet, while the GH change in the pigs fed on the RC (22\%) and HC (18\%) diets were around half of the change of the CT group.
Table 1 Growth performance of the pig

\begin{tabular}{|c|c|c|c|}
\hline Item & CT & RC & HC \\
\hline ADG (g/day) & 625.17 & 670.75 & 654.11 \\
\hline FCR & $3.19^{\mathrm{b}}$ & $2.81^{\mathrm{a}}$ & $3.09^{\mathrm{b}}$ \\
\hline FCG (Bath/kg) & 29.26 & 28.92 & 31.35 \\
\hline
\end{tabular}

Table 2 Level and change of GH

\begin{tabular}{|c|c|c|c|}
\hline Item & CT & RC & HC \\
\hline Initial GH $(\mathrm{ng} / \mathrm{ml})$ & 0.19 & 0.41 & 0.17 \\
\hline Final GH (ng/ml) & 0.11 & 0.32 & 0.14 \\
\hline Changed GH $(\mathrm{ng} / \mathrm{ml})$ & 42.11 & 21.95 & 17.65 \\
\hline
\end{tabular}

\section{Discussion}

In the current study, the FCR of the pigs fed on the dietary CS supplementation at recommended level, while there was no effect of the supplementation at recommended and high level on ADG and FCG. The difference of the results of ADG from earlier report (Yang et al, 2005) might be the results of wider range of pig age and uncontrollable environment conditions in the current study. However, dietary CS supplementation at recommended level had the best improvement of growth performance values, which it is the similar direction with reports previously $(1,4)$. Serum GH levels in this study was dramatically lower than previous experiments (5). This would be the results of more light exposure during blood sample collection (6). However, dietary CS supplementation at both levels had smaller values in decreasing $\mathrm{GH}$, which might be used to explain improved values of growth performance.

\section{Acknowledgements}

The authors wish to thank Grandsiam Co., Ltd.

\section{References}

1. Yang, C.B., et al., 2005. J. Sci. Food Agric. 85: 1947-1952.

2. Machlin, L.J., 1972. J. Anim. Sci. 35: 749-800.

3. Pursel, V.G., et al., 1990. J. Reprod. Fertil. 41: 77-87.

4. Lui, Gua, et al., 2009. Livest. Sci. 122: 86-89.

5. McElwain, K.V., et al. 1999. Life Sci. 64 (24): 2233-2238.

6. Kasuya, E., et al., 2008. J. Anim. Sci. 1799-1807. 


\title{
P.747
}

\section{The effects of a naturally contaminated diet with and without a mycotoxin deactivator on blood parameters of weaning piglets}

\author{
Ursula Hofstetter; Sabine Masching \\ Biomin Holding GmbH, Herzogenburg, Austria
}

\section{Introduction}

When ingested, mycotoxins cause insidious losses, ill thrift and increase disease incidence due to their immune-suppressive effects. In spite of all efforts to prevent formation of mycotoxins in feeds, significant contaminations still occur. While the use of products based on aluminosilicates gave good results in counteracting aflatoxins (1), the adsorptive deactivation of other toxins failed under field conditions (2). A further efficient way of detoxification of non-adsorbable mycotoxins is microbial or enzymatic biotransformation. It has been reported that an anaerobic rumen bacterium (Eubacterium BBSH797) is able to deactivate trichothecenes by biotransformation of their epoxide ring (3). A trial with weaning piglets was carried out to investigate the negative effects of deoxynivalenol (DON) on blood parameters and immune globulins and to prove the efficacy of a feed additive based on different strategies to counteract these effects.

\section{Materials and Methods}

The trial was conducted at the Center for Applied Animal Nutrition (CAN) in Austria with 14 pens for 10 piglets each, equipped with slatted floor, cup drinkers and a computer - operated feeding system (Spotmix). Directly after housing all piglets were fed a prestarter for approx. 3 days and afterwards changed over to the experimental diets. A 2-phase feeding system was used, where starter diet was fed between d4-14 and grower diet from d15. Water and feed were provided ad libitum.

\begin{tabular}{|l|l|}
\hline CG(-) & Negative control group; no detectable mycotoxins, no additive. \\
\hline ToG & Toxin group; 2 ppm deoxynivalenol (DON). \\
\hline TG & Trial group; 2 ppm DON and $2 \mathrm{~kg} / \mathrm{mt}$ feed additive. \\
\hline $\mathbf{C G}(+)$ & Positive control group; no toxin but $2 \mathrm{~kg} / \mathrm{mt}$ feed additive. \\
\hline
\end{tabular}

140 weaning piglets (mixed sex; age approx. 4 weeks, average weight approx. $8 \mathrm{~kg}$ ) were delivered from one farm. All animals were ear-tagged, the weight of the animals recorded individually and the ten smallest and heaviest animals were separated from the trial to reduce the standard deviation. The remaining 120 animals were allocated to 12 pens of 10 piglets each. All piglets were of the Austrian genotype Ö-HYB-F1[(Landrace $x$ Large White) $x$ Pietrain]. From day 6 on animals started with feed refusal and diarrhea and were treated with Enteroxid (colin sulfate + zinc oxide) for 4 days. Due to the fact that the general condition of the animals did not improve, all animals had to be treated with Paracillin (Amoxicillin-antibiotic; effective against Streptococci sp.). At day 38 animals started coughing and were treated with Pulmodox (Tetracycline-AB) for 8 days. For the rest of the trial period all animals remained stable, but were in a bad general condition.

\section{Results}

Table1 shows all blood parameters with statistically significant differences between the groups. The hematocrit value is an indicator for the concentration of erythrocytes and general water balance. In general all groups revealed hematocrit values close to the lower level of the reference values. This may be due to interference in water balance caused by diarrhea. The hemoglobin value is an indicator for the concentration of the iron-containing blood pigment. The high level of DON in the naturally contaminated diet caused an extreme activation of the immune system. In the toxin group the IgA value was significantly increased. IgA is with approx. $15 \%$ the main immune globulin in serum. In the trial group the immune suppression caused by DON was counteracted.

\begin{tabular}{|l|c|c|c|}
\hline & Hematocrit [l/l] & Hemoglobin [g/l] & IgA [mg/dl] \\
\hline CG(-) & $0.35^{\mathrm{a}}$ & $106.67^{\mathrm{a}}$ & $86.64^{\mathrm{a}}$ \\
\hline ToG & $0.34^{\mathrm{a}}$ & $104.67^{\mathrm{a}}$ & $638.22^{\mathrm{b}}$ \\
\hline TG & $0.35^{\mathrm{a}}$ & $111.00^{\mathrm{a}}$ & $126.44^{\mathrm{a}}$ \\
\hline $\mathbf{C G}(+)$ & $0.38^{\mathrm{b}}$ & $120.67^{\mathrm{b}}$ & $215.20^{\mathrm{ab}}$ \\
\hline Reference value & $0.33-8.10$ & $108-148$ & $50-500$ \\
\hline \multicolumn{4}{|l|}{$\mathrm{a}, \mathrm{b} \ldots$ significant difference (P<0.05) } \\
\hline
\end{tabular}

\section{Discussion}

Hematocrit values in the non-contaminated group with the feed additive were significantly improved compared to the control and the naturally contaminated group. Hemoglobin values in the feed additive group were significantly better compared to the control and all other groups. In the toxin group, DON activated the immune system and significantly increased the $\lg A$ values. The feed additive counteracted the immune suppression caused by deoxynivalenol.

\section{References}

1. Scott, P.M. (1998). Revue Méd. Vét. 149, 543-548.

2. Huff, W.E. et al. (1992). Poult. Sc. 71, 64-69.

3. Binder E.M. et al (2001). 10th Int. IUPAC Symposium on Mycotoxins and Phycotoxins, 271 - 277. 


\title{
P.748
}

\section{Early feeding of flaxseed leads to a permanent retention of $\mathbf{n - 3}$ fatty acids in pork tissues}

\author{
Hector R. Martinez-Ramirez ${ }^{1}$ John K. Kramer² Cornelius F. de Lange ${ }^{1}$ \\ 1. University of Guelph, Guelph, ON, Canada; 2. Agriculture and Agri-Food Canada, Guelph, ON, Canada
}

\section{Introduction}

Value added pork products may be produced by enriching pork with health benefits providing $n-3$ fatty acids (FA), such as eicosapentaenoic acid (EPA; 20:5n-3), docosapentaenoic acid (DPA, 22 5n-3) and docosahexaenoic acid (DHA, 22:6n-3) when these n-3 FA are incorporated into inter- and intra-muscular fats depots.

Several experiments have been conducted to assess the extent of incorporation of n-3 FA in pork products. However, little attention has been paid to determine the effect of feeding n-3 FA at different stages of growth to pigs.

\section{Objective}

To determine the incorporation of $n-3$ FA such as 18:3n-3 (ALA) and highly unsaturated n-3 FA (HUFA; 18:4n-3, 20:3n-3, 20:4n-3, EPA, DPA and DHA) in trimmed loin (L) and whole belly (B) of pigs fed flaxseed that contains high levels of ALA.

\section{Materials and Methods}

A total of 24 purebred individually housed Yorkshire female pigs, weighing approximately $25 \mathrm{~kg}$ BW were allotted in one of three feeding programs $(n=8): T 1$, diet with $10 \%$ FS diet fed between 25 and $50 \mathrm{~kg} \mathrm{BW}$, and control diets (C) low in n-3 FA fed thereafter until $110 \mathrm{~kg} \mathrm{BW}$; T2, C fed between 25 and $85 \mathrm{~kg} \mathrm{BW}$, and diet containing $6 \%$ FS fed thereafter until $110 \mathrm{~kg} \mathrm{BW}$; and T3, fed $C$ between 25 and $110 \mathrm{~kg} \mathrm{BW}$. Feed intake was fixed at $95 \%$ of the voluntary feed intake according to NRC (1998). Fat samples were extracted from $5 \mathrm{~g}$ of fresh $\mathrm{L}$ and $\mathrm{B}$ samples using chloroform/methanol (1:1 v/v) and then methylated using anhydrous methanol-HCl 5\%. FA methyl esters were analyzed using a gas chromatography (Aligent Technolgies Model 6890N) equipped with a $100 \mathrm{~m}$ CP-Sil 88 fused capillary column. The FA content in the tissues of pigs slaughtered at $110 \mathrm{~kg} \mathrm{BW}$ was expressed as $\mathrm{mg} / 100 \mathrm{~g}$ of fresh tissue. Results were subjected to analysis of variance using the Proc Mixed procedure of SAS v9.1 (SAS, Inst., Cary, NC). Pigs and block (pigs upon arrival, $n=2$ ) were used as a random effect.

\section{Results and Discussion}

No treatment effect was observed on growth performance, carcass characteristics or loin meat quality $(P>0.05)$. Pigs on $\mathrm{T} 1$ and $\mathrm{T} 2$ consumed equal lifetime amounts of FS (5.1 vs. 5.2 $\mathrm{kg}, \mathrm{P}>0.10)$. In $\mathrm{L}, \mathrm{ALA}$ was higher $(\mathrm{P}<0.05)$ for $\mathrm{T} 2$ than $\mathrm{T} 1$ and T3 (143, 76.4 and 37.0, respectively). In B, ALA was similar for T1 and T2 (667 vs. 752; P > 0.10) and both were higher than T3 (207; $P<0.01$ ). The main HUFA's were $20: 3 n-3$ (in B similar for T1 and T2, P > 0.10, and higher than T3: 104, 102, 31, respectively; and in $\mathrm{L}$ similar for $\mathrm{T} 1$ and $\mathrm{T} 2, \mathrm{P}>0.10$, and higher than T3: 12.5, 21, 6.0), DHA (in B similar for T1 and T2, P > 0.10, and higher than T3: $10,8.3,6.9$, respectively; in $L$ no treatment effect, $2.1,2.0,1.8 ; P$ $>0.05$ ) and DPA (in B similar for T1 and T2, P > 0.10, and higher than T3: 40, 34, 20; in L similar for T1 and T2, P > 0.10, and higher than T3: 9.7, 11, 6.2). Total HUFA content was larger $(P<0.05)$ for $T 1$ and $T 2$ compared to $T 3$ in $L(30,42,18)$ and $B(179,168,71)$. In $\mathrm{T} 1$ and $\mathrm{T} 2$ the $\mathrm{n}-6 / \mathrm{n}-3$ ratio was lower than $\mathrm{T} 3$ in $\mathrm{L}(5.9,4.0$, 12.7) and $B(4.9,3.8,133 ; P<0.001)$. These results indicate that feeding modest amounts of FS leads to a significant enrichment of ALA and HUFA, in particular 20:3n-3 and DPA, in pork. The enrichment of $n-3$ FA appears to be independent of time of feeding n-3 FA and provides flexibility as to when n-3 FA can be fed for generating $\mathrm{n}-3$ enriched pork.

Key words: Pork growth, n-3 fatty acid, pigs

\section{References}

NRC, 1998. Nutrient Requirements of Swine. 10th Rev. Ed. Natl. Acad. Press, Washington, DC, USA.

Author's mails: hmartine@uoguelph.ca, john.kramer@agr.gc.ca Corresponding author: cdelange@uoguelph.ca 


\title{
P.749
}

\section{Effects of carbohydralase supplementation on gut microbiota and immunology of growing pigs}

\author{
Joseane Willamil ${ }^{1}$ Judith González ${ }^{1}$ David Torrallardona² Pierre-André Geraert ${ }^{3}$ Estelle Devillard $^{3}$ Ignacio Badiola $^{1}$ \\ 1. Centre de Recerca en Sanitat Animal (CRESA), Barcelona, Spain; 2. Institut de Recerca i Tecnologia Agroalimentàries - IRTA, Tarragona, Spain; \\ 3. ADISSEO France SAS, Commentry, France
}

Exogenous carbohydrolase enzymes used in feed to improve animal performance, hydrolyse non-starch polysaccharides (PNA) from raw materials. A study conducted in our laboratory has shown that this type of enzymes modify the intestinal microbiota of growing pigs (dos Santos et al. 2009). These changes in microbiota could play a positive effect on intestinal health as suggested by Kim et al. (2003). The aim of our study was to determine the effects of enzyme supplementation on gut microbiota and immunological parameters of growing pigs.

Thirty-six pigs ( $25 \mathrm{~kg} \mathrm{BW.)} \mathrm{were} \mathrm{fed} \mathrm{during} 28$ days with one of 4 different treatments based on the type of diet (WBR or C) and the supplementation or not ( + or - ) with enzymes. The pigs were slaughtered to obtain samples of serum, bile and ileal mucosa for immunological studies. In addition, samples of ileal and caecal digesta and ileal mucosa were collected for microbiota studies. The microbiota composition was studied by RFLP-PCR. The serum $\lg A$, IgG and $\lg M$ and bile $\lg A$ were examined by ELISA. The terminal ileum was used to study some immunological cellular markers by immunofluorescence (Table 1) and some morphological parameters by HE and PAS stains.

Table 1. Primary monoclonal and secondary conjugated antibodies used in the immunefluorescence technique.

\begin{tabular}{|c|c|c|c|c|}
\hline & \multicolumn{2}{|c|}{ Primary $\mathrm{mAbs}$} & \multicolumn{2}{|c|}{ Secondary mAbs } \\
\hline & Mouse Isotype & MoAb (Clon) & Anti-mouse conjugate & Dilution \\
\hline $\mathrm{CD}^{*}$ & $\lg G 2 B$ & BB23-8E6 & $\lg G 2 b-C y 3$ & $1: 500$ \\
\hline CD4* & $\lg G 2 B$ & $74-12-4$ & $\lg G 2 b-C y 3$ & $1: 500$ \\
\hline CD163* & $\lg G 1$ & $2 \mathrm{~A} 10 / 11$ & $\lg G 1-C y 3$ & $1: 500$ \\
\hline CD172a(SWC3)* & $\lg G 1$ & BA1C11 & $\lg G 1-C \mathrm{3}$ & $1: 500$ \\
\hline SCW8* & $\lg M$ & MIL-3 & IgGM-PE & $1: 200$ \\
\hline IgA secretory & $\lg G 1$ & K60/1F1 & $\lg G 1-C y 3$ & $1: 100$ \\
\hline
\end{tabular}

WBR+ diet presented an increase in the mucosal biodiversity and in the villous height:crypt depth ratio compared to WBR- diet (Table 2). These results are in accordance with the gut health concept: a higher microbiota biodiversity promotes a lower aggression on the mucosa. The corn based diet supplemented with enzymes promoted an increase of neutral Goblet cells, a reduction of secretory IgA, intra epithelial lymphocytes (IEL) and CD3+ cells of villi compared to corn based diet without enzymes (Figure 1). An increase of secretory $\lg A$, an increase of IEL and an increase in $\mathrm{CD} 3+$ cells can be indicative of a higher aggression of gut mucosa in the corn based diet without enzyme supplementation.
Table 2. Effects of the treatments on ileal immunological parameters.

\begin{tabular}{|l|c|c|c|c|c|c|c|}
\hline & \multicolumn{7}{|c|}{ Diets } \\
\cline { 2 - 9 } & $c-$ & $c+$ & WBR- & WBR+ & p-feed & $\begin{array}{c}\text { p- } \\
\text { enzyme }\end{array}$ & $\begin{array}{c}\text { p-feed* } \\
\text { enzyme }\end{array}$ \\
\hline Villous height $(\mu \mathrm{m})$ & 412 & 420 & 367 & 428 & 0.4074 & 0.0523 & 0.1253 \\
\hline Crypt Depth $(\mu \mathrm{m})$ & 318 & 319 & 324 & 300 & 0.7398 & 0.2592 & 0.2973 \\
\hline VH:DC ratio & $1.31^{\mathrm{ab}}$ & $1.32^{\mathrm{ab}}$ & $1.18^{\mathrm{b}}$ & $1.46^{\mathrm{a}}$ & 0.8789 & 0.0113 & 0.0271 \\
\hline Goblet cells* & 9.5 & 9.3 & 8.0 & 9.4 & 0.2261 & 0.2425 & 0.1543 \\
\hline IEL** & $4.8^{\mathrm{a}}$ & $3.1^{\mathrm{b}}$ & $2.8^{\mathrm{b}}$ & $3.2^{\mathrm{b}}$ & 0.0002 & 0.0144 & 0.0002 \\
\hline Neutral Goblet cells* & 1.7 & 2.7 & 2.3 & 2.3 & 0.3141 & 0.0802 & 0.6641 \\
\hline Neutral Goblet cells** & 7.7 & 8.8 & 8.7 & 9.7 & 0.2483 & 0.2725 & 0.9889 \\
\hline${ }^{*}$ number of cells per $100 \mu \mathrm{m}$ of the crypt; ${ }^{* *}$ number of cells per $100 \mu \mathrm{m}$ of the villous \\
\hline
\end{tabular}

Figure 1. Immunofluorescence of the gut cells $C D 3+(A)$ and $s-\lg A+S C W 8(B)$.
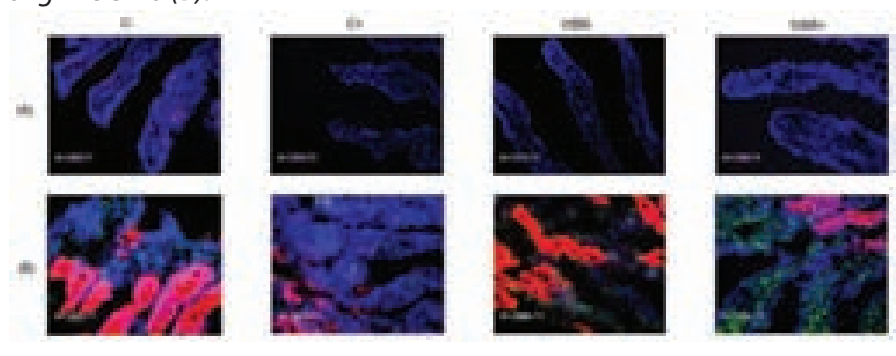

The biodiversity degree of ileal mucosa presented significant correlation with the bile $\lg A$ concentration $(R=-0.42, p=0.001)$, the IEL of villi $(R=-0.43, p=0.008)$ and neutral Goblet cells $(R=0.53, p=0.03)$.

Our results suggest that a more complex intestinal microbiota could play a positive effect on intestinal health. Changes on microbiota components can be induced by changes of feed components and by the addition of exogenous enzymes. The beneficial effects of enzymes could be related with an improvement of the adaptive capacity of commensal microbiota as a natural barrier defence against the overgrowth of pathogens.

\section{References}

Dos Santos J., Badiola I., Torrallardona D., Geraert P.A., Devillard E., 2009. Effet d'un complexe carbohydrolase sur la microflore intestinale de porcs. Journées Rech. Porcine, 41, 159-162

Kim S.W., Knabe D.A., Hong K.J., Easter R.A., 2003. Use of carbohydrases in corn-soybean meal-based nursery diets. J. Anim. Sci., 81, 2496-2504. 


\title{
P.750
}

\section{Effect of the inclusion of enramycin and bacitracin methylene disalicylate over the productive performance of growing $(30-55 \mathrm{~kg})$ pigs}

\author{
Erandi Toledo ${ }^{1}$ Juan M. Palacios² Francisco Rosales² Arturo G. Borbolla1 \\ 1. Departamento de Producción Animal: Cerdos. Facultad de Medicina Veterinaria y Zootecnia. Universidad Nacional \\ Autónoma de México., Coyoacan, DF, Mexico; 2. Shering Plough Intervet S.A. de C.V., México, DF, Mexico
}

\section{Introduction}

The use of feed antibiotics as growth promotants (AGP), in farm animals has been a practice used for several decades. From a production standpoint, these molecules have been consistently shown to improve weight gain and feed efficiency (Gaskins et al., 2002). Despite of these benefits, there has been a tendency to ban AGP in animal feed, which would have important economic implications in the cost of production. In the EU the ban of AGP was related to the alleged selection of antibioticresistant bacteria (RB), which might infect human beings and produce non-treatable diseases, particularly with Gram negative bacteria (Butaye et al., 2003). However, the development of RB has not being conclusively related to the use of AGP. The correct use of AGP could reduce the risk of producing RB. Therefore, it is important to evaluate continuously different molecules and administration programs that allow a correct use of APC in farms. The objective of this study was to compare the effect of enramycin and bacitracin methylene disalicylate over some important production parameters in growing pigs $(30-55 \mathrm{Kg})$.

\section{Materials and Methods}

Four hundred and fifty mixed pigs ( $\mathrm{N}=45$ animals/pen, 255 pigs/ treatment, 5 repetitions/treatment), were assigned randomly to two treatments. Control group (C) was the basal diet plus $500 \mathrm{~g}$ of bacitracin methylene disalicylate/ton and the experimental group (E) contained the basal diet plus $120 \mathrm{~g}$ of enramycin per ton. The trial was conducted during the first 35 days of the growing phase $(30-55 \mathrm{~kg})$. At the beginning of the study, 5 pigs/ pen were tagged and weight at the beginning and end of the trial. These data were used to evaluate average daily gain (ADG) average daily feed intake (ADFI) and feed conversion (F/G). These data were used to analyze the main effect of each treatment by mean comparison using the statistical program SPSS Statistics 17 , with a level of significance of $(P<0.05)$.

\section{Results}

Production parameters are shown in table 1 and figure 1. The initial and final body weights during the growing phase were simi$\operatorname{lar}(\mathrm{P}>0.05)$ for both treatments ( 31.4 and $54.4 \mathrm{~kg}$ vs. 30.0 and $55.7 \mathrm{~kg}$ for $\mathrm{C}$ and $\mathrm{E}$ pigs, respectively). It implies a mathematical improvement of $4 \%$ in weight gain for the E pigs. The ADFI was smaller $(P<0.05)$ for the $C$ than the $E$ animals $(1.5$ vs. $1.6 \mathrm{~kg} / \mathrm{d}$, respectively). However, the ADG was greater $(P=0.08)$, for the E group $(0.74 \mathrm{Kg} / \mathrm{d})$, than the $C$ group $(0.66 \mathrm{Kg} / \mathrm{d})$. Nevertheless, $\mathrm{F}: \mathrm{G}$ ratio was similar $(\mathrm{P}>0.05)$ for both treatments.
Table 1. Production parameters per treatment for pigs between 30 and $55 \mathrm{Kg}$.

\begin{tabular}{|l|c|c|c|}
\hline \multirow{2}{*}{ BW } & \multicolumn{3}{|c|}{ Treatment } \\
\cline { 2 - 4 } & $\mathbf{C ( B a c i t r a c i n )}$ & $\mathbf{E}$ (Enramycin) & $\mathbf{P}$ \\
\hline Initial,Kg & $31.4 \pm 0.9$ & $30.0 \pm 0.9$ & 0.12 \\
\hline Final,Kg & $54.4 \pm 1.8$ & $55.7 \pm 1.8$ & 0.44 \\
\hline ADFI,Kg & $\mathbf{1 , 5} \pm \mathbf{0 . 1}$ & $\mathbf{1 . 6} \pm \mathbf{0 . 1}$ & $\mathbf{0 . 0 1}$ \\
\hline ADG,Kb & $\mathbf{0 . 6 6} \pm \mathbf{0 . 1}$ & $0.74 \pm 0.1$ & $\mathbf{0 . 0 8}$ \\
\hline F.G & $2.3 \pm 0.1$ & $2.2 \pm 0.2$ & 0.41 \\
\hline a,b Means with different superscript letter differ $(\mathrm{P}<0.05)$ & \\
\hline
\end{tabular}

Figure 1. $A D F I, A D G$ and $F: G$ ratio for pigs during the growing phase.

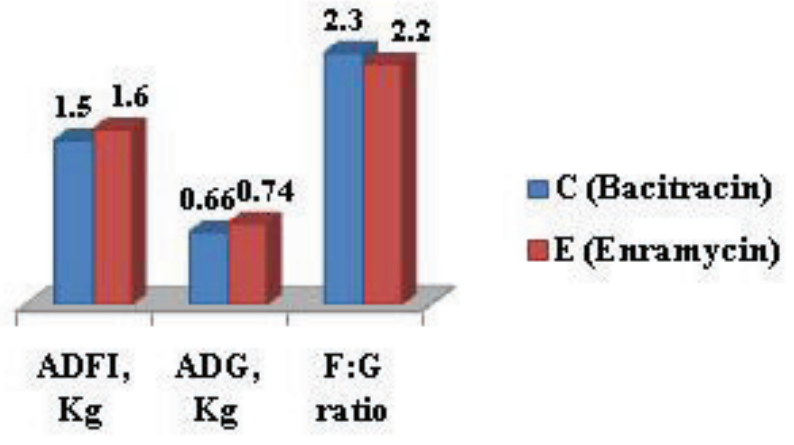

\section{Discussion}

The addition of bacitracin and enramycin in the diet of growing pigs in this trial showed similar results to those observed by Silva et al. (2003), who reported that the addition of enramycin reduced $A D F I$ and increased $A D G$; therefore improving $F: G$ ratio. In this study, however, the improvement in $\mathrm{F}: \mathrm{G}$ was not observed. Therefore, it is conclude that enramycin used as a growth promotant improves the performance parameters of pigs during the growing phase.

\section{References}

Butaye et al. 2003. Clin.Microbiol.Rev. 16:175-188. Gaskins et al. 2002. ANIMAL BIOTECHNOLOGY. Vol. 13, No. 1, pp. 29-42. Silva et al. 2003. XI ABRAVSES: 285-286 


\title{
P.751
}

\section{Effect of peroral iron supplementation in piglets on health and production}

\author{
Caroline Vanderhaeghe ${ }^{1}$ Jeroen Dewulf ${ }^{1}$ Frédéric Vangroenweghe ${ }^{2}$ Maarten Steyaert ${ }^{1}$ Ellen de \\ Jong ${ }^{1}$ Irris Villareal ${ }^{1}$ Alfonso Lopez ${ }^{1}$ Ruben Del Pozo Sacristan ${ }^{1}$ Dominiek Maes $^{1}$ \\ 1. Facutly of Veterinary Medicine, Department of Reproduction, Obstetrics and Herd Health, \\ Merelbeke, Belgium; 2. Animal Health Care Flanders, Torhout, Belgium
}

\section{Objectives}

Piglets are born with only minor iron reserves and also sow milk contains very low amounts of iron. Therefore, if piglets in intensive pig herds are not provided with extra iron during the first week after birth, they will become anaemic (1). Iron is commonly provided by means of intramuscular injection. However, if iron could be supplemented orally, it could save labour and also, the stress of manipulating pigs and injecting them could be avoided. The objective of this field study was to investigate the efficacy of peroral iron (Fe) supplementation by means of health, performance and haematological parameters. Additionally, the correlation between the concentration of haemoglobin $(=\mathrm{Hb})$ measured in the laboratory and measured on-farm using the HemoCue $^{\circledR}$ test (HemoCue ${ }^{\circledR}$ Diagnostics B.V., Waalre, The Netherlands), was assessed.

\section{Material and Methods}

Three commercial pig herds (>300 sows) participated in the study. Within each herd, 40 sows with their litters were included. Blood samples of the sows for $\mathrm{Hb}$ concentration were obtained one week before farrowing. A droplet of blood was absorbed in a microcuvette by capillary action for quick $\mathrm{Hb}$-determination by the HemoCue ${ }^{\circledR}$. Within each herd, the litters of the 40 sows were randomly divided in two groups. Group 1 (G1) received the peroral iron supplement (40g for a litter of 12 piglets) (HemOral ${ }^{\circledR}$, Biofiber-Damino, Gesten, Denmark) on day 2-4, 5-7 and 8-12. Group 2 (G2) received an intramuscular injection with $200 \mathrm{mg}$ Fe3+ on day 2-3 (Bastion 20\% ${ }^{\circledR}$, Intervet, Brussels, Belgium). Within each litter, 3 piglets were randomly selected and blood sampled just prior to iron injection and at weaning. Additionally, a droplet of blood was absorbed in a microcuvette by capillary action for quick $\mathrm{Hb}$-determination $\left(\mathrm{HemoCue}^{\circledR}\right.$ ). All piglets were weighted at these two time-points, and the total feed intake was recorded for each group. Farrowing data of the sows and performance of the piglets during lactation were measured. Statistical analysis was performed by means of analysis of variance (SPSS 16.0), in which the effect of the herd was taken into account.

\section{Results}

The sow farrowing data and the performance of the piglets are shown in Table 1. None of the parameters differed significantly between the two groups $(P>0.05)$. The time needed to treat a litter was significantly lower in the $\mathrm{HemOral}^{\circledR}(\mathrm{G} 1)$ group than in the Bastion $20 \%{ }^{\circledR}(\mathrm{G} 2)$ group $(\mathrm{P}<0.0001)$, but the difference was very small.

Table 1. Sow farrowing data and performance of the piglets (mean \pm SD) receiving $\mathrm{HemOral}^{\circledR}(\mathrm{G} 1)$ or Bastion $20 \%{ }^{\circledR}(G 2)$

\begin{tabular}{|l|c|c|}
\hline Parameters & G1 $(\mathbf{n = 4 4 )}$ & G2 $(\mathbf{n = 4 4 )}$ \\
\hline No of live born piglets & $13.2(3.21)$ & $12.6(2.69)$ \\
\hline No of stillborn piglets & $1.7(1.15)$ & $1.5(0.66)$ \\
\hline No of mummified piglets & $1.4(0.79)$ & $1.5(0.69)$ \\
\hline Pre-weaning mortality (\%) & $17.9(14.65)$ & $19.9(22.63)$ \\
\hline No of weaned piglets & $10.5(1.52)$ & $10.4(1.78)$ \\
\hline Daily weight gain (g) & $213.1(54.7)$ & $207.9(50.8)$ \\
\hline Feed intake (g) & $165.1(120.6)$ & $196.0(166.6)$ \\
\hline Hb concentration & $92.4(24.6)$ & $89.5(21.0)$ \\
\hline
\end{tabular}

The correlation between the $\mathrm{Hb}$ concentration measured in the laboratory and measured on-farm using the HemoCue ${ }^{\circledR}$ test $(n=$ 1036 piglets) was 0.79 .

\section{Discussion and conclusions}

The present study did not show significant differences between oral or intramuscular supplementation of iron on performance of piglets during lactation. As oral supplementation is less time consuming and does not require individual injections, it may be the preferential method. It also appeared that the quick on-farm testing of $\mathrm{Hb}$ can be used reliably to assess the $\mathrm{Hb}$ concentration of the piglets.

\section{References}

1. Szabo-P, Bilkei G., 2002. J. Vet. Med. A Physiol. Pathol. Clin. Med. 49, 390-391. 


\title{
P.752
}

\section{Evaluation of three feeding systems during gestation and its effect on reproductive performance of sows}

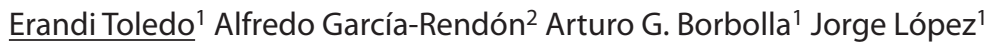 \\ 1. Departamento de Producción Animal: Cerdos. Facultad de Medicina Veterinaria y Zootecnia. Universidad Nacional \\ Autónoma de México., Coyoacan, DF, Mexico; 2. Granjas Covadonga, Estado de México, Mexico
}

\section{Introduction}

In sows, body reserves (protein and fat tissues), at farrowing and feed consumption during lactation determine milk production capacity and future reproductive performance (McNamara and Pettigrew, 2002). Therefore, an inadequate feeding program during the gestation and lactation periods, affects sow productivity and finally, farm output (Gill, 2006). However, in the majority of commercial farms, feed allowance during gestation is generally not focused on sow's body reserves, but in easiness of delivery. Therefore, most sows are offered a homogeneous amount of feed; practice that in short time, alter the reserves of nutrients in the body. The objective of this study was to evaluate three feeding systems during gestation and their effect on body reserves of sows at farrowing and weaning

\section{Material and Methods}

Four hundred sows from parity 0 to 5 were assigned randomly to three treatments: Tr. 1. feed allowance based on visual body condition. Tr. 2. Feed allowance based on body condition evaluated by weight and backfat using ultrasound (Aloha 500), techniques. Tr. 3. Feeding was based on the expected weight gain (net maternal gain), during gestation established by the modeling software from the NRC (1998). Body condition was determined using Noblet (1990) formulas. The amount of feed distributed to each sow did not consider parity number. These data were analyzed by ANOVA using the statistical program SPSS 15.0, and with a significance level of $P<0.05$.

\section{Results and Discussion}

In general, sows from Tr. 2 and 1 consumed the same amount of feed over gestation, which was lower than sows in Tr. 3. Weight and protein loss after the lactation period were reduced in sows of Tr. 2 and 3 (Table 1). Fat loss however, was higher in sows of Tr. 2. Reproductive performance seemed to be not affected at farrowing or weaning by any of the dietary treatments (Table 2). However, sows in Tr. 3 shown better $(P>0.05)$ numbers in total pigs born.

Body weight gain in gestating sows depends highly on the feeding level. Therefore at a higher feed intake there is a higher weight gain. In the present study, however, sows of Tr. 3 had a greater feed intake $(P<0.05)$ than sows of Tr. 1 and 2; but maternal weight gain did not show any differences for the three dietary treatments (Table 1). It was observed however, that sows in Tr. 3 had greater energy retention (fat), which agrees with results reported by Noblet et al. (1990), and Kusina et al (1999). As reported by Revell et al (1998) and Sinclair et al (2001), there were no effects of feeding level and feed intake in gestation over piglets birth weight, because an increase in gestation feed intake enhances maternal weight gain but has little influence over piglets weight.
Table 1. Effect of feeding system on feed intake, body weight gain and body composition of sows during gestation.

\begin{tabular}{|c|c|c|c|c|}
\hline & Gestation day & Treatment 1 & Treatment 2 & Treatment 3 \\
\hline Feed intake, $\mathrm{Kg}$ & & $263.7 \pm 1.3^{\mathrm{a}}$ & $269.1 \pm 3.4^{b}$ & $287.8 \pm 1.2^{c}$ \\
\hline ME Intake, Mcal & & $817.4 \pm 4.11^{\mathrm{a}}$ & $834.2 \pm 10.2^{b}$ & $892.1 \pm 3.8^{c}$ \\
\hline $\begin{array}{l}\text { Excess of ME vs } \\
\text { Requirements, } \%\end{array}$ & & $2.4 \pm 1.5^{\mathrm{a}}$ & $2.9 \pm 1.5^{\mathrm{a}}$ & $11.3 \pm 1.5^{b}$ \\
\hline \multirow[t]{7}{*}{ Body Weight, Kg } & Gestation day, 0 & $193.7 \pm 3.0$ & $197.6 \pm 3.6$ & $198.0 \pm 3.5$ \\
\hline & Gestation day, 112 & $253.8 \pm 4.9$ & $257.5 \pm 4.7$ & $260.3 \pm 4.9$ \\
\hline & Total gain & $60.2 \pm 2.1$ & $59.5 \pm 2$ & $62.3 \pm 4.9$ \\
\hline & Maternal weight & $228.5 \pm 4.7$ & $231.9 \pm 4.7$ & $234.9 \pm 4.9$ \\
\hline & Maternal gain & $34.8 \pm 2.0$ & $34.3 \pm 2.0$ & $36.9 \pm 2.8$ \\
\hline & Weaning weight & $210.9 \pm 2.8$ & $218.5 \pm 3.5$ & $219.8 \pm 2.9$ \\
\hline & Weight loss (F-W) & $17.6 \pm 1.6$ & $13.4 \pm 1.4$ & $15.1 \pm 2.0$ \\
\hline \multirow[t]{2}{*}{ Body protein, $\mathrm{Kg}$} & Gestation gain & $6.3 \pm 0.7$ & $6.2 \pm 0.3$ & $5.8 \pm 0.5$ \\
\hline & F-W loss & $2.7 \pm 0.2$ & $2.2 \pm 0.2$ & $2.6 \pm 0.3$ \\
\hline \multirow[t]{2}{*}{ Body Fat, $\mathrm{Kg}$} & Gestation gain & $6.4 \pm 0.7^{\mathrm{a}}$ & $9.1 \pm 0.8^{b}$ & $8.5 \pm 0.8^{\mathrm{a}, \mathrm{b}}$ \\
\hline & F-W loss & $6.8 \pm 0.6$ & $7.2 \pm 0.5$ & $5.9 \pm 0.7$ \\
\hline
\end{tabular}

Table 2. Effect of feeding system over the reproductive performance.

\begin{tabular}{|l|c|c|c|}
\hline & Treatment 1 & Treatment 2 & Treatment 3 \\
\hline Total born & $10.8 \pm 0.2$ & $10.6 \pm 0.2$ & $11.0 \pm 0.2$ \\
\hline Born alive & $9.7 \pm 0.2$ & $9.6 \pm 0.2$ & $10.2 \pm 0.2$ \\
\hline Pigs weaned & $9.4 \pm 0.1$ & $9.6 \pm 0.1$ & $9.7 \pm 0.1$ \\
\hline Litter birth weight, $\mathbf{K g}$ & $14.6 \pm 0.3$ & $14.6 \pm 0.3$ & $15.3 \pm 0.3$ \\
\hline Individual birth weight, $\mathbf{K g}$ & $1.53 \pm 0.02$ & $1.54 \pm 0.02$ & $1.52 \pm 0.01$ \\
\hline Weaning weight, $\mathbf{K g}$ & $56.9 \pm 1.9$ & $58.7 \pm 2.7$ & $62.6 \pm 1.3$ \\
\hline Weaning- Breeding Interval, d & $4.2 \pm 0.1$ & $4.1 \pm 0.1$ & $4.5 \pm 0.1$ \\
\hline
\end{tabular}

\section{References}

Noblet et al. 1990. J.Anim:Sci.68:562-572.

McNamara and Pettigrew. 2002. J. Anim. Sci. 80:2442-2451.

Revell et al. 1998. J.Anim.Sci.76:1738-1744.

Sinclair et al. 2001. J.Anim.Sci.79:2397-2405.

Gill. 2006. J. Anim. Sci. 84:1926-1934. 


\title{
P.753
}

\section{Galactogeno use (product based organic fennel, and juniper alcarabea) as a stimulant in dairy production sows in maternity}

\author{
Oscar Huerta Alva; FJC Crespo; N. Vázquez \\ Laboratorios Sanfer SA de CV, Mexico, DF, Mexico
}

\section{Introduction}

The improvement of productive and reproductive performance in pig farms is the work of every day, sometimes we have to use the tools available to improve results. Milk production allows us to have a good weaning weight of our pigs by ensuring better growth during the fattening.

The galactógena, natural product made from fennel, caraway and juniper promotes glandular environment free of pathogens (bacteria, viruses and fungi) and ensures the rhythmic discharge from the alveoli, increasing milk production and thus improve weight weaning.

\section{Materials and Methods}

On a farm with a capacity of 1,200 bellies in western Mexico, it randomly selects a maternity ward with a capacity of 28 deliveries, where the control and treated group received the same diet for 21 days, the treated group was given daily directly in the food 30 grams of galactógeno, divided into two served. The following table describes the groups:

\begin{tabular}{|l|c|c|c|c|c|}
\hline Group & \# of gilts & $\begin{array}{c}\text { Average } \\
\text { parity }\end{array}$ & \# piglets & $\begin{array}{c}\text { Initial } \\
\text { Weight }\end{array}$ & $\begin{array}{c}\text { Average } \\
\text { weight }\end{array}$ \\
\hline Control & 13 & 6.14 & 119 & 224.30 & 1.69 \\
\hline Treated & 13 & 4.28 & 128 & 231.95 & 1.62 \\
\hline
\end{tabular}

\section{Results}

The following table integrating the final results.

Group total initial weight final weight total \# piglets

\begin{tabular}{|l|c|c|c|c|c|}
\hline Group & $\begin{array}{c}\text { Initial } \\
\text { weight }\end{array}$ & $\begin{array}{c}\text { Final } \\
\text { Weight }\end{array}$ & $\begin{array}{c}\text { \# of } \\
\text { piglets }\end{array}$ & $\begin{array}{c}\text { Average } \\
\text { initial weight }\end{array}$ & $\begin{array}{c}\text { Average } \\
\text { final weight }\end{array}$ \\
\hline Control & 239.40 & 748 & 119 & 1.69 & 6.28 \\
\hline Treated & 231.95 & 824.5 & 128 & 1.61 & 6.44 \\
\hline Dif & -7.45 & 76.5 & 9 & -0.08 & 0.161 \\
\hline \multicolumn{7}{|l|}{ Total final difference $(0.080+0.161)$} & & 0.241 \\
\hline
\end{tabular}

As can be seen, the final margin rate was $0241 \mathrm{~g}$ per piglet, considering the total number of piglets weaned, gained $30.9 \mathrm{~kg}$ piglet weight at weaning.

The difference in these herds $0.241 \mathrm{gr}$ weaning piglets, has a direct impact on market day, feed conversion and daily weight gain, as demonstrated by Mahan 2005.

\section{Conclusions}

Based on the results, where female piglets children were treated with a final weight Galctogeno accumulated $0241 \mathrm{~kg}$, compared to children of female piglets of control, it is concluded that use of this product helps in improving the accumulated weight weaning.

\section{References}

Technical sheet, Laboratorios Ovejero, León, Spain, 2009. 


\title{
P.754
}

\section{Use of a product based organic fennel, and juniper alcarabea (galactogeno) as a stimulant in dairy production sows in maternity and a farm in the west of Mexico}

\author{
Oscar Huerta Alva ${ }^{1}$ FJC CrespoํN N. Vázquez ${ }^{1}$ M Jimenez ${ }^{2}$ \\ 1. Laboratorios Sanfer SA de CV, Mexico, DF, Mexico; 2. Private practice, Mexico, DF, Mexico
}

\section{Introduction}

The improvement of productive and reproductive performance in pig farms is the work of every day, sometimes we have to use the tools available to improve results. Milk production allows us to have a good weaning weight of our pigs by ensuring better growth during the fattening.

The galactógena, natural product made from fennel, caraway and juniper promotes glandular environment free of pathogens (bacteria, viruses and fungi) and ensures the rhythmic discharge from the alveoli, increasing milk production and thus improve weight weaning.

\section{Materials and Methods}

On a farm with a capacity of 850 stomachs in western Mexico, it randomly selects a maternity ward with a capacity of 22 births, designates a row for each group and treated control group received the same diet for 28 days on average, the treated group was given daily live in food 30 grams of galactógena, divided into two served.

\section{Results}

The following table integrating the final results.

\section{Treated Group}

\begin{tabular}{|c|c|c|c|c|c|c|}
\hline \# Gilts & \# farrow & @ piglets & $\begin{array}{c}\text { Initial } \\
\text { weight }\end{array}$ & @ weaned & $\begin{array}{c}\text { Final } \\
\text { weight }\end{array}$ & $\begin{array}{c}\text { Lactation } \\
\text { days }\end{array}$ \\
\hline 574 & 3 & 10 & 17 & 10 & 86 & 27 \\
\hline 50 & 2 & 11 & 16.3 & 10 & 89 & 31 \\
\hline 862 & 4 & 11 & 16.1 & 10 & 87 & 28 \\
\hline 65 & 2 & 12 & 17.4 & 10 & 95 & 27 \\
\hline 550 & 6 & 11 & 17.5 & 10 & 83 & 29 \\
\hline 804 & 5 & 12 & 18.9 & 8 & 87 & 28 \\
\hline 1066 & 5 & 11 & 16.5 & 11 & 97 & 30 \\
\hline 871 & 7 & 9 & 16.2 & 9 & 90 & 30 \\
\hline 378 & 6 & 10 & 17.2 & 10 & 88 & 29 \\
\hline 532 & 6 & 11 & 15.3 & 6 & 71 & 30 \\
\hline 772 & 5 & 11 & 15.3 & 8 & 76 & 28 \\
\hline Total & $\mathbf{5 1}$ & $\mathbf{1 1 9}$ & $\mathbf{1 8 3 . 7}$ & $\mathbf{1 0 2}$ & $\mathbf{9 4 9}$ & $\mathbf{3 1 7}$ \\
\hline Average & $\mathbf{4 . 6 4}$ & $\mathbf{1 0 . 8 1}$ & $\mathbf{1 . 5 4}$ & $\mathbf{9 . 1 7}$ & $\mathbf{9 . 3}$ & $\mathbf{2 8 . 8 2}$ \\
\hline
\end{tabular}

\section{Control Group}

\begin{tabular}{|c|c|c|c|c|c|c|}
\hline \# Gilts & \# farrow & @piglets & $\begin{array}{c}\text { Initial } \\
\text { weight }\end{array}$ & @ weaned & $\begin{array}{c}\text { Final } \\
\text { weight }\end{array}$ & $\begin{array}{c}\text { Lactation } \\
\text { days }\end{array}$ \\
\hline 2014 & 5 & 10 & 16.9 & 10 & 75 & 31 \\
\hline 2004 & 5 & 10 & 19.3 & 9 & 73.8 & 28 \\
\hline 144 & 5 & 10 & 19.6 & 9 & 92 & 27 \\
\hline 612 & 5 & 10 & 21.1 & 8 & 1 & 28 \\
\hline 844 & 4 & 10 & 17.8 & 7 & 47 & 27 \\
\hline 112 & 5 & 10 & 16.5 & 10 & 99 & 28 \\
\hline 417 & 5 & 11 & 17.3 & 10 & 77 & 28 \\
\hline 385 & 4 & 12 & 20.4 & 9 & 85 & 28 \\
\hline 1053 & 3 & 12 & 18.1 & 11 & 75 & 28 \\
\hline 555 & 5 & 10 & 17.3 & 9 & 96 & 28 \\
\hline 853 & 4 & 11 & 16.9 & 11 & 95 & 28 \\
\hline Total & $\mathbf{5 0}$ & $\mathbf{1 1 6}$ & $\mathbf{2 0 1 . 2}$ & $\mathbf{1 0 3}$ & $\mathbf{8 9 5 . 8}$ & $\mathbf{3 0 9}$ \\
\hline Average & $\mathbf{4 . 5 5}$ & $\mathbf{1 0 . 5 4}$ & $\mathbf{1 . 7 3}$ & $\mathbf{9 . 3 6}$ & $\mathbf{8 . 7 0}$ & $\mathbf{2 8 . 0 9}$ \\
\hline
\end{tabular}

\begin{tabular}{|l|c|c|c|c|c|}
\hline Group & $\begin{array}{c}\text { Initial } \\
\text { weight }\end{array}$ & $\begin{array}{c}\text { Final } \\
\text { weight }\end{array}$ & $\begin{array}{c}\text { \# Piglets } \\
\text { weaned }\end{array}$ & $\begin{array}{c}\text { Average } \\
\text { initial weight }\end{array}$ & $\begin{array}{c}\text { Average } \\
\text { final weight }\end{array}$ \\
\hline Treated & 183.7 & 949 & 102 & 1.540 & 9.300 \\
\hline Control & 201.2 & 895.8 & 103 & 1.730 & 8.700 \\
\hline Dif. & -17.5 & 53.2 & -1 & -0.29 & 0.610 \\
\hline Average total final difference (0.290+0.610) & & $\mathbf{0 . 8 0 1}$ \\
\hline \multicolumn{4}{|l}{ Total final difference (17-5+53.2) } & & $\mathbf{7 0 . 7} \mathbf{~ k g}$ \\
\hline
\end{tabular}

As can be seen, the final margin rate was $0801 \mathrm{~g}$ per piglet, considering the initial and final average, because the piglets in the treated group started with lower weight. Considering the difference of the total initial weight and final weight total $(17.5+53.2)$ and divide by the total piglets have a difference of $0.693 \mathrm{~kg}$ of difference for children piglets treated females.

\section{Conclusions}

Based on the results, where children piglets galactógena treated females had the most weight at weaning compared to piglets from control female children, it is concluded that use of this product helps in improving the accumulated weight at weaning.

\section{References:}

Technical sheet, Laboratorios Ovejero, León, Spain, 2009. 


\title{
P.755
}

\section{TOPIGS: Feeding pigs according to the (genetic) requirements}

\author{
Michiel Westerhof \\ KNMvD, Helvoirt, Netherlands
}

\section{Introduction}

For a major pig breeding company like TOPIGS, it is crucial that genetic progress achieved by breeding is expressed under practical conditions, the so called phenotypic expression. This phenotypic expression is a product of genotype and environment of the pig. The phenotype consists of the observable characteristics or traits, the genotype is the inherited instructions within the genetic code. By selection, TOPIGS is gradually changing the genotype, for instance into the direction of higher growth and a lower feed conversion. The problem is that environment $(E)$ is not controlled by a genetics company, while $E$ is very important to benefit from the genetic progress.

Most important factors in environment of the pig are: farm management, health, feeding and climate. In relation to feeding, TOPIGS decided to redefine the protein deposition capacity of the animals. Protein deposition is defined as $P D=f(F I, W, N H \mid$ sire line, sex, ...) where Fl stands for Feed Intake, W for weight and $\mathrm{NH}$ for nutritional history. When PD is estimated, one can calculate the lysine and energy requirements and thus the composition of the feed needed to achieve best results. PD is described by the Linear Plateau theory (Whittemore and Fawcett, de Greef), see figure 1.

Figure 1. Protein and fat deposition as function of energy intake.

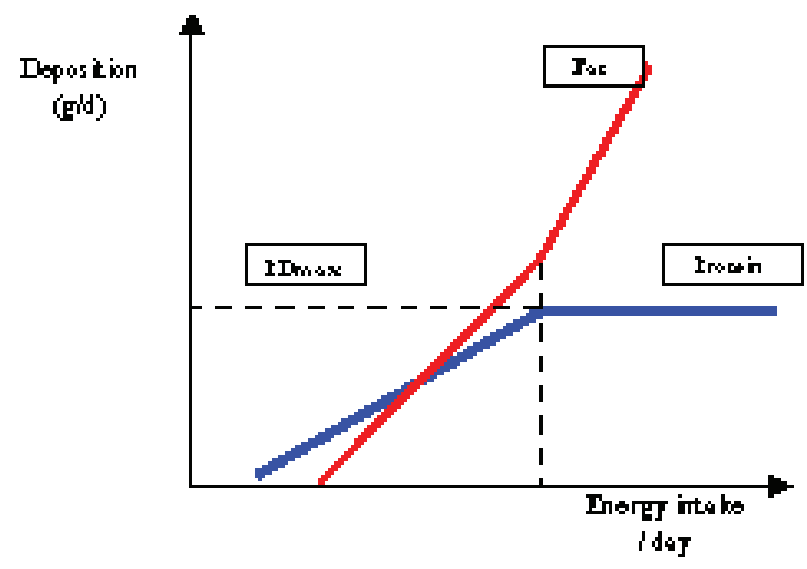

\section{Materials \& Methods}

The TOPIGS specific protein deposition model is established by using the records of 15,000 animals in the breeding data base. Since TOPIGS is a worldwide operating breeding company, we collect data for breeding purposes in over 25 countries. At individual animal level, feed intake, back fat and weight is measured and recorded and slaughter house data are available.

\section{Results}

Protein deposition for the different sire lines and the sexes were estimated and the lysine and energy requirements were calculated. Figure 2 shows one example, differences between gilts and barrows In the TOPIGS feeding manuals for finishers these requirements are expressed for the different genetic lines, the different sexes and different age groups.

Figure 2. Protein deposition for barrows and gilts at different age.

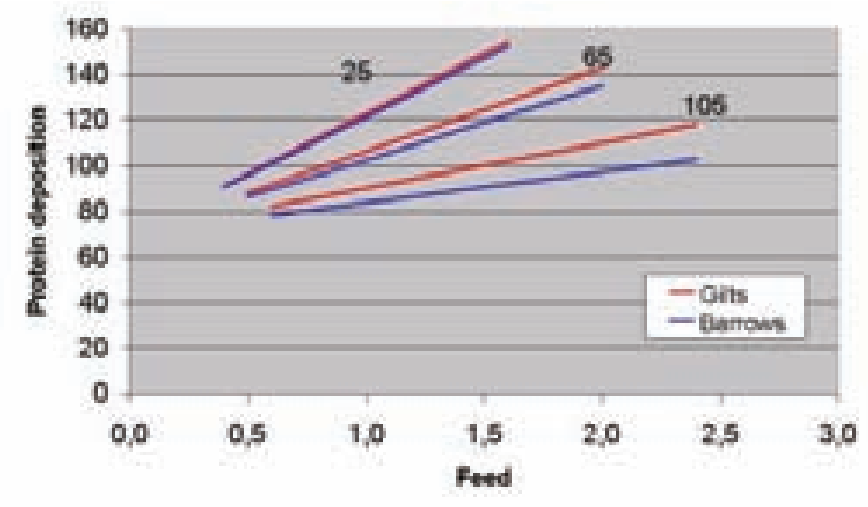

\section{Discussion}

Feeding is an important aspect of getting optimal expression of genetic potential. If an animal is not fed according to its requirements it is not only unfavorable for economic results but it might also have negative impact on health and welfare. By having good estimations of the protein deposition capacity in different stages of the life of the pig, the feed industry can manufacture feeds to fully exploit the genetic potential.

TOPIGS is in the process of using the data also to predict the effects of health status, of the use of growth promoters, of immuno-castration and of climate on the protein deposition capacity of our animals .

\section{References}

Available on request 


\title{
P.756
}

\section{Impact of fineness of grinding of corn and high-moisture corn on growth performance, carcass characteristics and stomach lesions in liquid-fed growing-finishing pigs}

\author{
Cuilan Zhu; Doug Wey; Robert Friendship; Cornelis F. de Lange \\ University of Guelph, Guelph, ON, Canada
}

\section{Introduction}

To minimize the energy cost in cereal grain milling and maximize feed utilization, much attention has been drawn to defining the optimum particle size for pig diets. In various studies it has been shown that reducing mean particle size of typical corn and soybean meal based diets to $<600 \mu \mathrm{m}$ result in improvements in nutrient digestibility and feed efficiency in growing-finishing pigs (Wondra, 1995). Research conducted in the UK indicates that the optimum particle size for barley-based diets used in liquid feeding systems is larger than that in conventional dry feeding (MLC, 2005), and thus reduces energy costs of liquid feed preparation. No research has been conducted to establish the optimum fineness of grinding of dry and high-moisture corn (HMCorn) that is used in swine liquid feeding. Two experiments were conducted to determine the impact of fineness of grinding of corn and HMcorn on growth performance, carcass characteristics and stomach lesions.

\section{Materials and Methods}

In experiment 1, a total of 96 Yorkshire pigs with initial BW 32.3 $\mathrm{kg}$ (SEM 1.4) were randomly assigned to 6 pens per treatment and 8 pigs per pen. Two complete corn and soybean meal-based diets were formulated with dry corn hammer milled to two different particle sizes. Titanium was added as marker for determining fecal digestibility. Pigs were liquid fed using a computer controlled feeding system according to a two phase feeding program until pigs reached market weight $(110 \mathrm{~kg})$. Body weight was monitored at biweekly intervals and fecal samples were collected during the growing period. Carcass quality was evaluated according to the standard Canadian carcass grading scheme.

In experiment 2, 160 Yorkshire pigs with initial BW $33.8 \mathrm{~kg}$ (SEM 0.63) were randomly assigned to 10 pens per treatment and 8 pigs per pen. HMCorn was ground to two different particle sizes, transferred to large fermentation tanks, mixed with water to a target of $26 \%$ dry matter (DM), and stored for a maximum of seven days until feeding. The feeding program and pig management were as in experiment 1. The pigs' stomachs were collected at the commercial slaughtering house and scored for lesions: 1=normal, 2=erosions, $3=$ ulcers, and 4=severe ulcers.

Statistic analysis was done by using the GLM procedure of SAS v9.2 (SAS, Inst., Cary, NC). Initial BW used as covariate.

\section{Results and Discussion}

In experiment 1, dry corn particle sizes, coarse vs. fine, were 652 $\mu \mathrm{m}( \pm 47.5)$ vs. $525 \mu \mathrm{m}( \pm 22.6)$. Average daily gain (ADG), average daily feed intake (ADFI), and gain:feed were not affected by fineness of grinding ( $P>0.5$ ) with means of ADG $1.11 \mathrm{~kg} / \mathrm{d}, \mathrm{ADFI} 2.46$ $\mathrm{kg} / \mathrm{d}$, and gain:feed 2.22. There were no differences $(P>0.05)$ in apparent fecal digestibilities of DM, crude protein, and ash, with means of $85.8 \%, 76.0 \%$ and $58.8 \%$, respectively. Carcass weight and estimated carcass lean yield were not affected by treatment $(P>0.05)$.

In experiment 2, increasing mean particle size of ground HMCorn from 355 to $918 \mu \mathrm{m}$, did not impact growth performance ( $P>0.1)$, with mean ADG, ADFI and gain:feed of $1.03 \mathrm{~kg} / \mathrm{d}$, $2.22 \mathrm{~kg} / \mathrm{d}$ and 2.15, respectively. Carcass characteristics were not affected $(P>0.1)$ either. However, more severe stomach lesions were observed when pigs were fed finely ground HMcorn. Of the 135 stomachs that were evaluated, for pigs fed the fine diet (65 stomachs), the number of observations for scores 1 to 4 were $9,11,14$ and 31, respectively; for pigs fed the coarse diet (70 stomachs), the number of observations were $23,20,11$ and 16 , respectively.

In contrast to the conventional dry feed practices (Wondra, 1995), manipulating particle size appears to have little influence on performance of liquid-fed pigs. Coarsely grinding HMCorn has the benefit of reducing stomach lesions.

\section{Implication}

The optimum particle size of ground corn is larger in liquid feeding than conventional dry feeding. Increasing HMCorn particle size to $950 \mu \mathrm{m}$ did not influence growth performance and carcass characteristics, while the incidence of stomach lesions was reduced.

\section{References}

MLC (2005) Finishing pigs - systems research. Final report to Defra, August 2005.

Wondra, K.J J. Anim. Sci. 1995. 73:2564-2573. 


\title{
P.757
}

\section{Route of iron supplementation to piglets: effects on hemoglobin, piglet growth and infectious diseases}

\author{
Dan Rantzer; Mats Andersson; Jos Botermans; Anne-Charlotte Olsson; Jørgen Svendsen \\ Swedish University of Agricultural Sciences, Faculty of Landscape Planning, Horticulture and Agricultural Sciences, Alnarp, Sweden
}

\section{Introduction}

In modern production systems newborn pigs have no natural access to iron (Fe) in the environment and to avoid iron deficiency they need an Fe supplementation which may be given as an injection or be based on voluntary consumption. It is generally accepted ${ }^{1}$ that hemoglobin $(\mathrm{Hb})$ levels in piglets should be above $80-90 \mathrm{~g} / 1000 \mathrm{ml}$ blood and it is considered that higher levels should be aimed for. While early conventional Fe injection is a safe way of securing sufficient iron to all the suckling piglets, there is some evidence ${ }^{23}$ that early Fe injection as opposed to voluntary oral Fe intake may increase the risk of joint infections and generalized infectious diseases in the piglets. The aim of the present study was to further elucidate the effect the route of Fe supplementation may have on infectious diseases in piglets, and the relationship between $\mathrm{Hb}$ levels during suckling and growth from birth to slaughter.

\section{Materials and Methods}

The treatments were a) early (d 1-4) and b) late (d 8-12) Fe inj (Pigeron ${ }^{\circledR}$ vet, Leo), 20 litters, c) oral Fe supplementation (Porcofer, Svenska foder, 20 litters, and TorvoJärn Extra, Lantmännen, 20 litters) starting on d 3 and d) oral suppl. (as under c) and an early Fe injection.

Observations: The pigs were individually marked, weighed and blood sampled on d 2, 9, 20 and at weaning (d 33), respectively. $\mathrm{Hb}$ level was determined (Hemo Cue ${ }^{\circledR} \mathrm{B}$-Hemoglobin Fotometer [Hemo Cue AB, Ângelholm, Sweden]). The presence of joint infections was checked daily and on d 9 more thoroughly. The pigs were individually identified and recorded throughout and at slaughter. Mortality, treatments and morbidity were noted daily and all dead pigs in the trial were post-mortem examined.

\section{Results and Discussion}

There were significantly more treatments for joint infections in pigs receiving an early Fe inj. than in the littermates receiving a late inj. (Table 1). Also, pigs only receiving Fe by oral supplementation (Table 2) had significantly fewer joint infections than littermates also given an early Fe inj. Hb was significantly higher on $\mathrm{d} 9$ and $\mathrm{d} 20$ in pigs receiving an early iron inj. than in littermates receiving a late Fe inj. (Table 1) or only oral suppl., respectively (table 2 ). For the orally suppl. pigs, daily growth to slaughter, and mortality was the same for both treatment groups (Table 2); however, for the inj. groups (Table 1), growth from day 2 to slaughter tended to be lower for the late injection treatment group. The results support the proposal that early Fe inj. increases the risk of piglet joint infections usually occurring in the second week of life. There are no indications in this study that pigs with high $\mathrm{Hb}$ levels perform better than pigs with levels of 80-110.
Table 1. Early vs late Fe inj. Split litter design. Individ. obs. corr. for start weight and litter. Means \pm st. errors

\begin{tabular}{|c|c|c|c|}
\hline & Early & Late & Sign. \\
\hline No. pigs at day 2 & 114 & 110 & \\
\hline \multicolumn{4}{|l|}{ During suckling } \\
\hline $\mathrm{Hb}^{\mathrm{a})}$ day 2 & $88 \pm 2$ & $89 \pm 2$ & n.s. \\
\hline $\mathrm{Hb}^{\mathrm{a})}$ day 9 & $98 \pm 1$ & $57 \pm 1$ & $* * *$ \\
\hline $\mathrm{Hb}^{\mathrm{a})}$ day 20 & $120 \pm 1$ & $112 \pm 1$ & $* * *$ \\
\hline With joint inf. (\%) & 17.5 & 8.2 & * \\
\hline Deaths due to inf. (\%) Day 2 to slaughter & 0.9 & 0.9 & \\
\hline Growth (g/d) & $625 \pm 7$ & $605 \pm 7$ & + \\
\hline Mortality (\%) & 8.8 & 9.1 & n.s. \\
\hline
\end{tabular}

Table 2. Oral supp. vs oral + early Fe inj. (oral+). Split litter design. Individ. obs. corr. for start weight and litter. Means \pm st. errors

\begin{tabular}{|c|c|c|c|}
\hline & Oral & Oral+ & Sign. \\
\hline No. pigs at day 2 & 223 & 228 & \\
\hline \multicolumn{4}{|l|}{ During suckling } \\
\hline $\mathrm{Hb}^{\mathrm{a})}$ day 2 & $85 \pm 2$ & $84 \pm 2$ & n.s. \\
\hline $\mathrm{Hb}^{\mathrm{a})}$ day 9 & $95 \pm 1$ & $100 \pm 1$ & $* * *$ \\
\hline $\mathrm{Hb}^{\mathrm{a})}$ day 20 & $113 \pm 1$ & $126 \pm 1$ & $* * *$ \\
\hline With joint inf. (\%) & 17.5 & 8.2 & * \\
\hline Deaths due to inf. (\%) & 9.2 & 16.4 & $*$ \\
\hline Day 2 to slaughter & 1.3 & 2.6 & n.s. \\
\hline Growth $(\mathrm{g} / \mathrm{d})$ & $632 \pm 6$ & $634 \pm 6$ & n.s. \\
\hline Mortality (\%) & 12.1 & 11.8 & n.s. \\
\hline \multicolumn{4}{|c|}{$\begin{array}{l}+p \leq 0,1 ;{ }^{*} p \leq 0,05 ;{ }^{* *} p \leq 0,01 ;{ }^{* *} p \leq 0,001 ; \text { n.s. } p>0,1 \text {, } \\
\text { a) Hemoglobin contents (g/1000 ml) }\end{array}$} \\
\hline
\end{tabular}

\section{References}

1. National Research Council (NRC) (1979) Iron. Committee on medical and biological effects of environmental pollutants. Sub-committee on iron. Univ. Park Publ., Baltimore, U.S.A

2. Svendsen, J. \& Andreasson, B. 1981. (Investigations into the supplying of liquids to piglets and to weaned pigs: Liquid consumption and production results). Dept. Farm Buildings, SLU, Report 14. Lund

3. Holmgren, N. 1996. (Iron as a cause of joint inflammation in nursing pigs). Pig, No. 4, Skara. 


\title{
P.758
}

\section{Effect of dietary supplementation of humic substances on the concentration of some elements and antioxidant status in the small intestine of weaned pigs}

\author{
V. Petrovič ${ }^{1}$ J. Novotný ${ }^{2} L^{\vee}$. Lenhardt ${ }^{3}$ J. Bernardy $^{4}$ G. Kováč ${ }^{1}$ \\ 1. University of Veterinary Medicine and Pharmacology, Košice, Slovak Republic; 2. Clinic of Ruminants, Košice, Slovak Republic; \\ 3. Department of Pathological Anatomy, Košice, Slovak Republic; 4. University of Veterinary and Pharmaceutical Sciences, Brno, Czech Republic
}

\section{Introduction}

Humic substances (HS) have relatively high molecular weight and include different amounts of humic acids, fulvic acids and humins. HS contain up to $80 \%$ of organic carbon. The variety of molecular structures are presented in HS, such as alkylaromatic, quinoide and aliphatic structures in the core, amino acid and carbohydrates structures and carbonyl-, carboxyl-, phenyl-, hydroxyl groups in the periphery. These functional groups modulate their enzymes activities (Zeichmann 1996; Nardi et al., 2002). In addition, they are capable to alter the bioavailability of nutrients. Herzig et al. (2001) and Laurberg et al. (2003) found the thyroid system affected by HS via significantly reduced iodine bioavailability. The aim of this study was to determine the effect of dietary supplementation of humic substances on the concentration of $\mathrm{Se}, \mathrm{Zn}, \mathrm{Fe}, \mathrm{Cu}, \mathrm{Mg}$, $\mathrm{Ca}$ and malondialdehyde, the number of Goblet cell and the activity of alkaline phosphatase in the duodenum and jejunum of weaned pigs.

\section{Material and Methods}

The twelve piglets Large White, were weaned at the age of 32 nd day and were randomly divided into 2 groups. Control group was fed with basal diet for weaned pigs and experimental group of pigs received the same basal diet supplemented with humic substances in the dose $5 \mathrm{~g}$ per $\mathrm{kg}$ DM for 60 days. At the age of 92 days, the pigs were weighed (the average body mass was $30 \mathrm{~kg}$ per animal). After the slaughter of pigs, the samples of duodenum and jejunum were taken, processed and stored under $-60^{\circ} \mathrm{C}$ till analyzed the concentration of studied elements and malondialdehyde. To obtain reliable microscopic pictures, the small excisions of the proximal duodenum and middle part of the jejunum were immediately harvested from carcass, the lumen rinsed in physiological solution, fixed in $4 \%$ formaldehyde for 2 hours, processed for paraffin embedding and sectioned to slides at a thickness of 5-7 microns. The Goblet cells were counted in 10 longitudinal sections of intact duodenal and jejunal villi. Determination of alkaline phosphatase activity was performed by using a cytophotometrical method at a wavelength of $480 \mathrm{~nm}$.

\section{Results}

The concentration of $\mathrm{Se}, \mathrm{Zn}, \mathrm{Fe}, \mathrm{Cu}, \mathrm{Mg}$ and $\mathrm{Ca}$ in duodenum did not reveal the statistical difference between both groups of pigs. However, a significantly lower concentration of Fe $(p<0.05)$ was determined in the jejunum of pigs fed basal diet supplemented with humic substances as compared with that of the control group of pigs receiving only basal diet. Similarly, no statistical differences in the number of Goblet cell and in the concentration of malondialdehyde were found in the duodenum of pigs in both groups. Whereas, the higher number of Goblet cell $(p<0.01)$, higher concentration of malondialdehyde $(p<0.001)$ and significantly lower activity of alkaline phosphatase $(p<0.001)$ were found in the jejunum of pigs fed with the diet supplemented with humic substances.

\section{Conclusions}

Taken together, the presented data suggest that the supplementation of humic substances into the diet for pigs decreases the concentration of iron in the jejunum. On the other hand, humic substances could possess the property to increase the intermediate metabolism in the jejunum of weaned pigs. A further research on pigs is needed before clearer conclusive statements can be made about the way how humic substances may cause the above mentioned changes.

\section{References}

Ziechmann, W. 1996: Huminstoffe und ihre Wirkungen. Spektrum Akademischer Verlag, Heidelberg

Nardi, S., Pizzeghello, D., Muscolo, A., Vianello, A. 2002: Physiological effects of humic substances on higher plants. Soil Biol. Biochem. 34, 1527-1536

Laurberg, P., Andersen, S., Pedersen, I.B., Ovesen, L., Knudsen, N. 2003: Humic substances in drinking water and the epidemiology of thyroid disease. Biofactors. 19, 145-153

Herzig I, Pisarikova B, Kursa J, Bendova J. 2001: Effects of humine compounds on iodine utilisation and retention and on the function of the thyroid gland. Vet. Med. 46, 61-64

*This study was supported by Grant Agency for Science, VEGA of the Slovak Republic, Grant no. 1/0614/09 


\title{
P.759
}

\section{Effect of temperature and packing method on storage of boar semen}

\author{
Ellen de Jong ${ }^{1}$ Jeroen Dewulf ${ }^{1}$ Ann Van Soom ${ }^{1}$ Candida Adriaens ${ }^{1}$ Wouter Deley ${ }^{2}$ Aart de Kruif ${ }^{1}$ Dominiek Maes $^{1}$ \\ 1. Ghent University, Faculty of Veterinary Medicine, Department of Reproduction, Obstetrics and Herd Health, Merelbeke, Belgium; \\ 2. Al Centre Hypor, Olsene, Belgium
}

\section{Introduction and objectives}

Storage of semen has an irreversible effect on its quality (1). Boar spermatozoa are very sensitive to "cold shock" (2). If semen temperature suddenly drops below $15^{\circ} \mathrm{C}$, most spermatozoa do not survive $(3,4)$. After dilution, semen is cooled until $17^{\circ} \mathrm{C}$. At this temperature, it can be stored without causing damage $(5,6)$. The purpose of this study was to investigate whether the storage of boar semen at different temperatures and in different packages influences sperm quality.

\section{Materials and Methods}

The experiment was performed in April 2009. Ejaculates (dilution with Minitub BTS ${ }^{\otimes}$ until $2.5 \times 10^{9}$ at $\left.26^{\circ} \mathrm{C}\right)$ of 6 Piétrain boars from a commercial Al centre (1 ejaculate/boar), were packed in 2 dissimilar assemblages: blisters (most frequently used: $85 \%$ market share) and Gédis ${ }^{\circledR}$ (Genes Diffusion: 12\% market share). The semen samples were transported to the laboratory of the Faculty of Veterinary Medicine in Merelbeke, kept at room temperature for two more hours and then stored at 3 distinct temperatures: $14^{\circ} \mathrm{C}, 17^{\circ} \mathrm{C}$ (standard) and $20^{\circ} \mathrm{C}$. To determine the quality of the spermatozoa, the following aspects were investigated: motility, progressive motility, percentage normal and percentage live spermatozoa. The first two parameters were analysed using the CASA (Computer Assisted Semen Analyzer) daily from D0 (semen collection) to D4. The percentage of normal and live spermatozoa was also examined daily in a blinded way using eosin-nigrosin staining. Statistical analysis was performed by means of a linear mixed effects model, in which boar was the random effect $(\mathrm{S}+)$.

\section{Results}

Table I shows the outcomes (mean over the five days) of the four investigated parameters of the semen, stored at different temperatures in blisters. Table II shows the same, but with semen packaged in Gédis ${ }^{\circledR}$.

Over the five days, a significant lower percentage live and normal spermatozoa was seen in semen packed in Gédis ${ }^{\circledR}$ in comparison to that in blisters at $17^{\circ} \mathrm{C}(\mathrm{p}<0.05)$.

In case of extended preservation of the semen ( $>2$ days), a significant decrease in the percentage live (90.3\% vs. $86.1 \%)$ and normal spermatozoa ( $84.7 \%$ vs. $80.4 \%)$, motility $(67.1 \%$ vs. $57.4 \%$ ) and progressive motility (45.9\% vs. $32.5 \%$ ) was observed in both packing methods and at all temperatures $(p<0.01)$.
Table I: Mean outcomes of semen stored in blisters

\begin{tabular}{|l|c|c|c|}
\hline & $\mathbf{1 4}^{\circ} \mathbf{C}($ ref. $)$ & $\mathbf{1 7}^{\circ} \mathbf{C}$ & $\mathbf{2 0}^{\circ} \mathbf{C}$ \\
\hline \% live spermatozoa & 89.4 & $91.5^{*}$ & 91 \\
\hline \% motile spermatozoa & 63.5 & 60.3 & $57.9^{*}$ \\
\hline \% progressive motile spermatozoa & 36.1 & 37.3 & 37.3 \\
\hline \% normal spermatozoa & 83.3 & 85.7 & 86.1 \\
\hline *: significantly different from reference category within row $(\mathrm{p}<0.05)$ \\
\hline
\end{tabular}

Table II: Mean outcomes of semen stored in Gédis

\begin{tabular}{|l|c|c|c|}
\hline & $\mathbf{1 4}^{\circ} \mathbf{C}$ (ref.) & $\mathbf{1 7}^{\circ} \mathbf{C}$ & $\mathbf{2 0}^{\circ} \mathbf{C}$ \\
\hline \% live spermatozoa & 83.7 & $87.1^{*}$ & 84.1 \\
\hline$\%$ motile spermatozoa & 64.6 & 61.9 & $59.4^{*}$ \\
\hline \% progressive motile spermatozoa & 40.4 & 37.4 & 38.6 \\
\hline \% normal spermatozoa & 78 & 81.5 & 78.6 \\
\hline *: significantly different from reference category within row $(\mathrm{p}<0.05)$ \\
\hline
\end{tabular}

\section{Discussion and conclusion}

These results show that the quality of boar semen decreases when stored for a longer period than two days as well as when it is preserved at temperatures lower or higher than $17^{\circ} \mathrm{C}$. The present study demonstrates also a better longevity when spermatozoa are packed in blisters than in Gédis. To investigate the fertility results of semen stored in these conditions, more in vivo studies are needed.

\section{Acknowledgements}

The authors are grateful to Al centre Hypor to provide us with boar semen.

\section{References}

(1) Waberski et al., 1994, Theriogenology 41, 1367-77.

(2) Pursel et al., 1973, Journal of Animal Science 34, 278-83.

(3) Eriksson, 2000, Doctoral Thesis, Swedish University of Agriculture Sciences, Uppsala.

(4) Johnson et al., 2000, Animal Reproduction Science 62, 143-72.

(5) Althouse et al., 1998, Theriogenology 50, 535-43.

(6) Paulenz et al., Proc. 15th IPVS, Birmingham, 63. 


\title{
P.760
}

\section{Adrenocortical activity of boars exposed to heat stress, supplemented with organic and inorganic trace mineral and its effect on semen quality}

\author{
Daiane Donin; Aline D. Viott \\ Universidade Federal do Paraná, Palotina, PR, Brazil
}

Seasonal high temperatures or inadequate nutrition can decrease reproductive efficiency in boars, especially through both a reduction in the number of ejaculate spermatozoal and abnormal morphology. In these situations, animals are under stress, which can be defined as a body reaction to an adverse condition of the environment in an attempt to achieve thermoregulation and homeostasis. Several hormones, including adrenocorticotropic hormone (ACTH), glucocorticoids, catecholamines and prolactin are involved in stress response and can be measured to quantify this response. Environmental factors seem to be primarily responsible for the reproductive performance of boar and, among these factors stands out nutrition. Organic minerals contain a chain of minerals and amino acids and this association enhances the absorption of minerals in the small intestine by protecting the metal in a complex in an inert chemical form due to covalent and ionic bonds between the ligands and amino acids.

The aim of this study was to evaluate the effect of organic and inorganic trace mineral supplementation on adrenocortical activity in boars exposed to high environmental temperatures in Southern Brazil.

Thirteen boars ( \pm 2 years of age) were divided into three groups to receive inorganic ( $G \ln , n=4)$, organic (GOr, $n=4)$ mineral supplementation or a lactation control diet ( $G C O, n=5)$. Inorganic and organic diets were made of a premix of inorganic and organic trace minerals, respectively, with the same quantity of each trace mineral, based on NRC (nutritional requirements for boars). The lactation diet was based on NRC requirements for lactating sows and contained a higher level of inorganic trace minerals, protein and metabolic energy. For the cortisol analysis 124 fecal samples were collected from organic and inorganic groups and 155 samples from the control group. To determine the concentrations of fecal corticoids, samples collected from the latest feces of the day from males from groups GIn, GOr and GCo, weekly, throughout the experiment from 11:00 to 12:00 h.

Even though the fecal analysis represent a pool of concentrations of metabolites, the time of collection was observed to avoid possible changes in cortisol concentration related with circadian variations,. The sample processing (extraction and analysis by radioimmunoassay) was performed at the Laboratory of Hormone levels (LDH), University of São Paulo (USP), following the pattern described by Graham et al. (2001). Radioimmunoassay (RIA I125) was biologically validated for swine feces by administration of adrenocorticotropic hormone (ACTH) in two boars and fecal samples were collected prior to and one day after administration. Feces collection was done at the same as test animals stool collection. Fecal corticosterone metabolites were quantified by radioimmunoassay double antibody, using commercial diagnostic kit, developed to measure corticosterone in human serum. Values of hormone metabolites were corrected for weight and dilution and expressed in nanograms per gram of wet feces (ng /g). Maximum mean environmental temperatures were higher than the normal thermal comfort temperature for boars $(26 \circ C)$ during the experimental period, and were associated with a reduction in semen quality.

Results are expressed as mean \pm SEM. Mean fecal corticoid concentrations, assessed by RIA in wet samples, were similar among groups (GIn 10,932.77 $\pm 12,332.00 \mathrm{ng} / \mathrm{g}$; GOr 9,088.60 \pm 8,748.61 $\mathrm{ng} / \mathrm{g}$ and GCo 9,988.20 \pm 10,072.27 ng/g). However, negative correlations were found between fecal corticoids and normal spermatozoa percentage $(\mathrm{P}<0.05)$. Percentage of normal spermatozoa, evaluated by humid preparation technique (KRAUSE, 1966), was higher in the Organic group when compared with both Inorganic and Lactation groups (93.31 $\pm 5.20 \%$ versus 78.48 $\pm 12.15 \%$ and $82.59 \pm 17.27 \%$, respectively; $P=0.00021$ ). Levels of cortisol's metabolites were found in the stools with a significant negative correlation with the percentage of normal sperm $(-0,353, p<0,05)$ so with the increased adrenocortical activity, there is a reduction in the production of normal sperm, with a consequent increase in the number of sperm morphological defects, when boars were subjected to a stressful situation. Organic mineral supplementation did not influence adrenocortical response to the stressor agent (heat stress) in this experiment, but was beneficial to seminal quality, reducing some of the heat stress effects in exposed boars. 


\title{
P.761
}

\section{Effects of organic selenium supplementation on mitochondrial potential and integrity of plasma and acrossomal membranes in boar sperm}

\author{
Simone M. Martins ${ }^{1,2}$ André F. Andrade ${ }^{1}$ Larissa J. Parazzi ${ }^{2}$ Fabiane G. Zaffalon ${ }^{1}$ \\ Carolina I. Silva² Andres M. Gallego' ${ }^{1}$ Aníbal S. Moretti ${ }^{2}$ Rubens P. Arruda ${ }^{1}$ \\ 1. Laboratory of Semen Biotechnology and Andrology, University of Sao Paulo, Depto. Reprodução Animal (VRA), Pirassununga, SP, Brazil; \\ 2. Laboratory of Swine Research, University of Sao Paulo, Depto. Nutrição e Produção Animal (VNP), Pirassununga, SP, Brazil
}

\section{Introduction}

Selenium (Se) is an essential element playing an important role in animal reproduction. The selenoproteins participate in regulation of various physiological functions including antioxidant protection, redox regulation of gene expression and sperm structure integrity maintenance1. Although there are some studies regarding the influence of the addition of selenium on the quality of boar semen, researchers did not examine the effects of selenium on membrane integrity of sperm cells and mitochondrial potential. Therefore, the aim of this study was to investigate the effects of organic selenium in spermatozoa quality of boars at $0,24,48$ and 72 hours after diluting with a semen extender.

\section{Materials and Methods}

Fifteen boars were kept on the same conditions and were fed the same standard diet during training. The animals were fed with three experimental diets: $0.3 p p m$ sodium selenite (Control); $0.5 \mathrm{ppm}$ sodium selenite (Inorganic) and $0.5 \mathrm{ppm}$ selenium yeast (Organic). Ejaculates were collected from each boar once per week using the gloved-hand technique. Semen was diluted with standard extender to $3 \times 109$ cells/dose and stored for 3 days at $18^{\circ} \mathrm{C}$. For flow cytometry assessment of the integrity of plasma and acrosomal membranes and mitochondrial function, semen samples were diluted in a $37^{\circ} \mathrm{C}$ prewarmed TALP sperm medium at a concentration of $5 \times 106$ sperms $/ \mathrm{mL}$. Thereafter, two aliquots $(150 \mu \mathrm{L})$ were transferred to microcentrifuge tubes. One of these tubes was supplemented with $3 \mu \mathrm{L}$ of propidium iodide-PI $(0.5$ $\mathrm{mg} / \mathrm{mL}$ ) and $10 \mu \mathrm{L}$ of fluorescein isothiocyanate-conjugated Psium sativum agglutinin-FITC-PSA $(100 \mu \mathrm{g} / \mathrm{mL})$ to evaluate the integrity of plasma and acrosomal membranes, whereas the other received $2 \mu \mathrm{L}$ of $\mathrm{JC}-1(153 \mu \mathrm{M})$ to evaluate mitochondrial membrane potential. Samples were incubated in the dark for 8 min at $37^{\circ} \mathrm{C}$, and then TALP was added to achieve an appropriate sperm concentration ( $2.5 \times 106$ sperms $/ \mathrm{mL}) 2,3$. The experimental design was a randomised block. Data were analyzed using the PROC repeated from SAS4 and outcomes were compared among the three treatments by the orthogonal contrast method with $\mathrm{p}<0.05$ level of significance.

\section{Results}

Cells with both plasma membrane and acrosome intact (AIMI) did not differ between treatments, but significance was detected according to time. The higher percentage of the AIMI cells was observed in 0 hours. Mitochondrial potential interaction between treatment and time was not observed, but there was a significant effect for time. There was also a lower percentage of cells in the 24 and 48 hours after dilution.

\section{Discussion}

Some studies have reported that the diet supplemented with sodium selenite has improved the quality of boar semen5. For the reason of environmental and toxicity concerns, however, the FDA6 allows a maximum of only $0.3 \mathrm{ppm}$ Se in swine diets, but studies have shown that $0.5 \mathrm{ppm}$ Se has improved the percentage of normal and motile sperm cells of boars5. This result is in agreement with our results, but when we assess more specifically the sperm membranes we found that selenium supplementation did not affect these variables. In conclusion, the selenium yeast showed no better results in comparison to others treatments in the seminal analyses.

\footnotetext{
References

1 Dimitrov et al. 2007 Anim. Reprod. Sci. 100:311-317

2 Andrade et al 2007 Reprod. Dom. Anim. 42:190-4

3 Celeghini et al. 2007 Reprod. Dom Anim. 42 479-488

4 SAS: software, 9.0. SAS Institute (2002-2003).

5 Marin-Guzman et al 1997 J. Anim. Sci. 75:2994-3003

6 Food and Drug Administration, 1983
}

\section{Acknowledgements}

FAPESP Proc nº 07/55613-6, Alltech ${ }^{\circledR}$ Inc., Agroceres ${ }^{\circledR}$ Nutrição Animal and Vet life ${ }^{\circledR}$ Veterinary Products 


\title{
P.762
}

\section{Sperm production in Duroc, Landrace, Yorkshire, Berkshire and Pietrain boars kept in evaporative cooling system in Thailand}

\author{
Chanyuth Tretipskul ${ }^{1}$ Padet Tummaruk' Seri Koonjaenak² Mongkol Techakumphu ${ }^{1}$ \\ 1. Department of Obstetrics, Gynaecology and Reproduction, Faculty of Veterinary Science, Chulalongkorn University, \\ Bangkok, Thailand 10330, Bangkok, Thailand; 2. Kasetsart University, Bangkhen Bangkok, Thailand
}

\section{Introduction}

The sperm production of the boars is affected by several factors such as, breed, season, nutrition and housing (1). Since the environmental temperature in Thailand is often over $30^{\circ} \mathrm{C}$ for several months of the years, a reduction of pig production and the reproductive performance are observed during hot periods. Nowadays, the evaporative cooling system (EVAP) is normally used for housing boars in Thailand. It has been suggested that this system help to reduce the seasonal variation of the sperm production in Duroc boars (2). However, the variation of sperm production among different boar breeds used in Thailand has not been evaluated. The objective of the present study was to determine the sperm production of Duroc (D), Landrace (L), Yorkshire $(\mathrm{Y})$, Berkshire $(\mathrm{B})$ and Pietrain $(\mathrm{P})$ boars kept in EVAP in Thailand.

\section{Materials and Methods}

Semen production data from 377 boars used during a period from January 2003 to December 2009 in a commercial boar stud with different breeds (i.e., 184 D, 45 L, 77 Y, 23 B and 48 P) were analyzed. Each boar was kept in each pen ( $9 \mathrm{~m}^{2} /$ boar) and was fed with 2.2-3.0 kg/day of a commercial feed containing $14-18 \%$ crude protein, and 3,000-3,200 kcal DE/kg. All boars were accessed to water ad libitum. The data set consisted of 20,643 ejaculates of semen. Semen parameters including volume $(\mathrm{ml})$, sperm concentration $\left(x 10^{6} \mathrm{spz} / \mathrm{ml}\right)$ measured by Spermacue (Minitube, Tiefenbach, Germany), subjective motility and total number of sperm per ejaculates $\left(x 10^{9} \mathrm{spz}\right)$ were evaluated. The sperm production data were analysed using general linear mixed model procedure (MIXED) of SAS version 9.0. The models included boar breeds, year and month in which the semen were collected as fixed effect and included boar identity nested within breed as random effect. Least-squared means were obtained and compared among breeds using Tukey-Kramer test. $\mathrm{P}<0.05$ was regarded as a significant difference.

\section{Result and Discussion}

The sperm production in each breed is presented in Table 1. Across the breeds, volume, concentration, total sperm per ejaculates and subjective motility were $239.6 \pm 94.6 \mathrm{ml}, 319.8 \pm 97.9 \mathrm{x}$ $10^{6} \mathrm{sperm} / \mathrm{ml}, 71.7 \pm 25.4 \times 10^{9} \mathrm{sperm} /$ ejaculate and $73.4 \pm 8.8 \%$ of motility, respectively.
Table 1 Means, standard deviation (SD) and range of sperm production in Duroc (D), Landrace (L), Yorkshire (Y), Berkshire (B) and Pietrain $(P)$ boars

\begin{tabular}{|c|c|c|c|c|}
\hline Parameter & Breed & $\mathrm{N}$ & Mean \pm SD & Range \\
\hline \multirow{5}{*}{$\begin{array}{c}\text { Volume } \\
\text { (ml) }\end{array}$} & D & 6,889 & $169.7 \pm 66.6^{c}$ & $50-497$ \\
\hline & $\mathrm{L}$ & 2,553 & $294.2 \pm 76.7^{\mathrm{a}}$ & $52-500$ \\
\hline & $Y$ & 8,858 & $278.4 \pm 88.2^{\mathrm{a}}$ & $50-500$ \\
\hline & B & 292 & $168.7 \pm 68.5^{c}$ & $56-481$ \\
\hline & $P$ & 1,725 & $250.7 \pm 76.1^{b}$ & $58-500$ \\
\hline \multirow{5}{*}{$\begin{array}{l}\text { Concentration } \\
(\times 106 / \mathrm{ml})\end{array}$} & $D$ & 6,317 & $375.990 .2^{\mathrm{a}}$ & $75-600$ \\
\hline & $\mathrm{L}$ & 2,405 & $297.0 \pm 77.7^{c}$ & $96.8-600$ \\
\hline & $Y$ & 8,729 & $288.8 \pm 93.0^{c}$ & $51-599$ \\
\hline & $B$ & 217 & $295.5 \pm 108^{\mathrm{ab}}$ & $135-600$ \\
\hline & $\mathrm{P}$ & 1,610 & $305.3 \pm 82.7^{b}$ & $97-600$ \\
\hline \multirow{5}{*}{$\begin{array}{l}\text { Total Sperm } \\
\text { (x109) }\end{array}$} & $D$ & 6,280 & $59.7 \pm 21.7^{\mathrm{a}}$ & $12-255$ \\
\hline & $\mathrm{L}$ & 2,383 & $85.3 \pm 24.0^{\mathrm{a}}$ & $17-25$ \\
\hline & $Y$ & 8,564 & $76.8 \pm 25.1^{b}$ & $4-235$ \\
\hline & $B$ & 216 & $49.7 \pm 28.1^{\mathrm{ab}}$ & 16-184 \\
\hline & $\mathrm{P}$ & 1,601 & $73.8 \pm 21.1^{\mathrm{ab}}$ & $20-230$ \\
\hline
\end{tabular}

The sperm production of boars differed among the breeds. D and $B$ boars had a lower semen volume and total sperm per ejaculate than $L$ and $Y$ boars. It has been demonstrated that both volume and concentration of the boar semen had a heritability of 0.58 and 0.49 , respectively (3). Selection of boars, used in tropical climates, based on sperm production is therefore important. In conclusions, the semen production of $L$ and $Y$ boars kept in evaporative cooling system in Thailand were superior to D, B and P boars.

\section{References}

1. Kunavongkrit, A. et al. (2005). Theriogenology 63, 657-667.

2. Suriyasomboon, A. et al. (2004). Livest. Prod. Sci. 89: 19-31.

3. Smital, J. (2005). Anim. Reprod. Sci. 86, 119-130. 


\title{
P.763
}

\section{Seasonal influence on sperm production in boars kept in evaporative cooling system in Thailand}

\author{
Chanyuth Tretipskul ${ }^{1}$ Padet Tummaruk' ${ }^{1}$ Seri Koonjaenak² Mongkol Techakumphu \\ 1. Department of Obstetrics, Gynaecology and Reproduction, Faculty of Veterinary Science, Chulalongkorn University, Bangkok, Thailand; \\ 2. Kasetsart University, Bangkhen Bangkok, Thailand
}

\section{Introduction}

Fluctuation of temperature and humidity cause a decrease of semen output in boar (1). It was found that high sperm production was observed in autumn and winter but low sperm production was observed in summer (2). In Thailand, evaporative cooling system (EVAP) is commonly used for boar housing in order to minimize the seasonal effect of the sperm production. An earlier study in Thailand has demonstrated that the total number of sperm per ejaculate was $72.2 \times 10^{9}$ and $74.9 \times 10^{9}$ sperm in boars kept in evaporative and in conventional open housing system, respectively and minor difference in the seasonal pattern was found between the two housing system in Thailand (3). However, high humidity negatively affect both semen volume and total sperm output (3). The objective of the present study was to investigate the seasonal influence on sperm production of boars kept in evaporative cooling system in Thailand.

\section{Materials and Methods}

Semen production data from 20,502 ejaculates of 554 boars (164 Duroc, 14 Yorkshire, 23 Berkshire, 31 Pietrain, 54 crossbred Pietrain x Duroc and 268 crossbred Landrace x Yorkshire) collected during January 2006 - December 2009 in a commercial boar station were included. Each boar was kept individual pen (9 $\mathrm{m}^{2} /$ boar) and was fed with 2.2-3.0 kg/day of a commercial feed containing $14-18 \%$ crude protein, and 3,000-3,200 kcal DE/kg. The boars were accessed to water ad libitum. Semen parameters including volume $(\mathrm{ml})$, sperm concentration $\left(x 10^{6} \mathrm{spz} / \mathrm{ml}\right)$ measured by Spermacue ${ }^{\circ}$, subjective motility and total number of sperm per ejaculates ( $\left.\times 10^{9} \mathrm{spz}\right)$ were evaluated. On average, the temperature $\left({ }^{\circ} \mathrm{C}\right)$ and Humidity (\%) in EVAP from Jan to Dec were 22.5/85.7, 23.8/82.2, 25/81.6, 25/82, 24.9/86, 25.1/85, 25/86.3, 25/85.2, 24.8/85.4, 24.5/86.1, 22.6/ 85.6 and 21.8/85.5, respectively. The sperm production data were analysed using general linear mixed model procedure (MIXED) of SAS version 9.0. The models included boar breeds, year and month in which the semen were collected as fixed effect and included boar identity nested within breed as random effect. Least-squared means were obtained and compared among breeds using Tukey-Kramer test. $p<0.05$ was regarded as a significant difference.

\section{Result and Discussion}

On average, the semen volume, concentration, total sperm per ejaculates and individual motility were $249.4 \pm 97.9 \mathrm{ml}$, $334.6 \pm 96.3 \times 10^{6} \mathrm{sperm} / \mathrm{ml}, 78.7 \pm 28.6 \times 10^{9} \mathrm{sperm} /$ ejaculate, and $73.9 \pm 11.1 \%$ subjective motility, respectively. The sperm production by months is presented in Figure 1.

Figure 1 Least-squared means and standard error of total number of sperm/ejaculation by months.

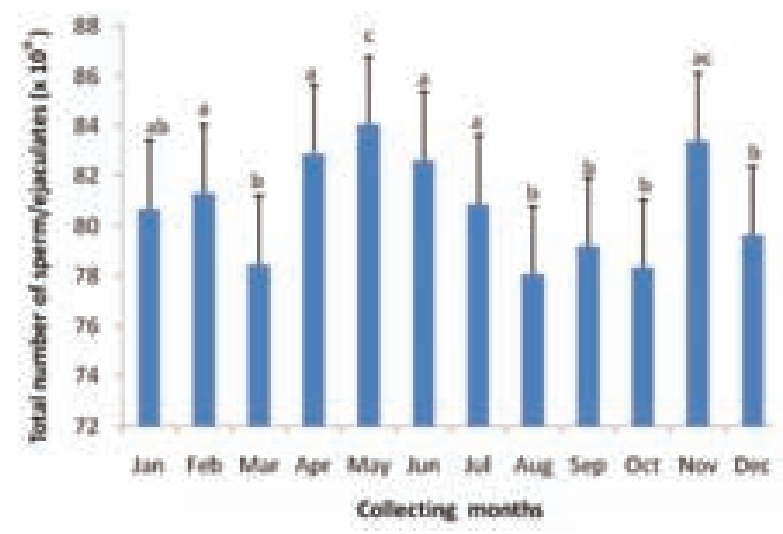

a,b different superscripts are significantly difference $(p<0.05)$.

The total number of sperm production in March, August, September and October was significantly lower than April, May and November (Figure 1). Since spermatogenesis spent 35 - 40 days for completing process, short day length during August-October might cause the decrease of sperm production. Furthermore, high humidity during rainy season (Jul-Oct) might also contribute to the seasonal influence on the sperm output of boars kept in EVAP in Thailand. It could be concluded that the seasonal influence on the sperm production was still observed in boars kept in EVAP in Thailand.

\section{References}

1. Kunavongkrit, A. et al. (2005). Theriogenology 63, 657-667.

2. Ciereszko et al. (2000). Anim. Reprod. Sci. 64: 89-96.

3. Suriyasomboon, A., Lundeheim, N., Kunavongkrit, A., Einarsson, S., 2004. Livest. Prod. Sci. 89: 19-31. 


\title{
Reproductive failure associated with poor Al dilution
}

\author{
John Carr \\ Portec Australia, Belmont, WA, Australia
}

\section{Introduction}

The preparation of diluent used in artificial insemination is a key component in processing semen.

Failure to achieve the correct osmotic pressure by incorrectly diluting the extender is more difficult to detect during the semen examination. This paper reports a failure to achieve the correct dilution and subsequent effect on reproduction.

\section{Clinical outline}

A 32 sow 2 week batch farm practiced on-farm Al. Following a change in stockpeople, the farm suffered a massive drop in farrowing rate.

Problems started in batch 22 and ended in batch 33 with a change in Al practice. Statistical process control clearly demonstrates the production issue.

Detailed analysis of the semen processing revealed that the stockpeople has misread their instructions and were not diluting the extender properly. In general they were over-diluting - adding 1.5 litres of distilled water to $50 \mathrm{~g}$ of diluents instead of only 1 litre.

Al semen samples were examined without any obvious abnormality being detected. There were no change in live to dead ratios, semen abnormalities or semen movement. Visual analysis of the semen immediately post-dilution, the semen appeared to be stunned and did not move properly. However, within an hour, their movement became visually normal.

The diluents was examined for conductivity $\mathrm{mS} / \mathrm{cm} 17^{\circ} \mathrm{C}$ where the failure of adequate dilution was confirmed. The conductivity at various dilutions:

\begin{tabular}{|l|c|c|c|c|c|}
\hline Water I & $\mathbf{0 . 5}$ & $\mathbf{1 . 0}$ & $\mathbf{1 . 5}$ & $\mathbf{2 . 0}$ & Water \\
\hline Cond. $\mathrm{mS} / \mathrm{cm}$ & 12.83 & 8.01 & 5.88 & 4.60 & 0.01 \\
\hline
\end{tabular}

The initial concern was noticed the stockpeople failing to detect 3 and 4 week returns. The stockpeople subsequently mislead management about pregnancy results rather than believe the extreme poor performance.

\section{Consequence and cost of the Al failure}

The farm lost 780 weaners associated with this reproductive failure. At $\$ A U 3.40$ per kg deadweight costs this resulted in a $\$ 75,000$ increase in fixed costs - or $\$ 9$ increase per pig sold over the next year.

Please visit our website www.portec.com.au

\section{Detailed production results}

The batch farrowing rate $\%$

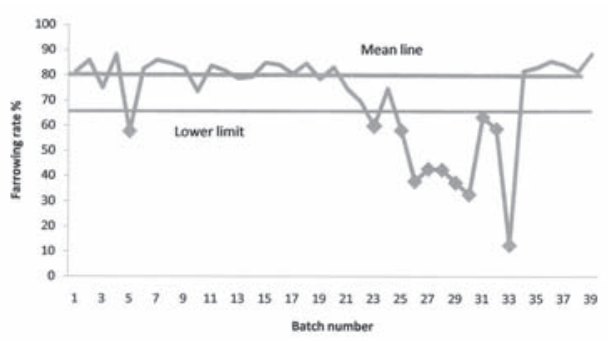

The upper line represents the mean, the lower line 2sd below the mean

$\begin{array}{lll}\text { Pre-batch 22 } & \text { Mean } 80 & \text { Standard deviation 6.6 } \\ \text { Batch } 22 \text { to } 33 & \text { Mean } 49 & \text { Standard deviation } 17\end{array}$

Statistical process control indicates that from batch 23 the system was out of control 10/11 batches. Stastistical analysis indicate a $\mathrm{p}<0.001$ difference between batches 22 and 33 and normal.

Piglets weaned per farrowing sow

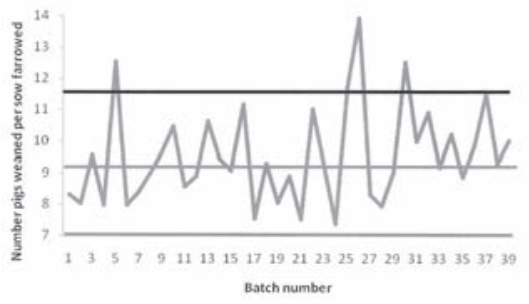

The middle line is the mean. The two other lines represents $2 \mathrm{sd}$ above and below the mean

Pre-batch 22 Mean 9.1 Standard deviation 1.3

Batch 22 to 33 Mean 10.1 Standard deviation 2.0

Statistical process control indicates that from batch 22 to 33 the system was never out of control. Stastistical analysis indicate $p=0.14$. There is no difference in numbers weaned between batches 22 and 33 and normal.

\section{Discussion}

Failure to adequately dilute semen resulted in a catestrophic failure of reproduction. The farrowing rate collapsed. The litter size - numbers weaned - did not statistically change. Those sows who became pregnant had normal litters.

The failure of dilution was confirmed by monitoring the conductivity of the diluent. As osmolarity is difficult to measure in the field, conductivity should be included in the standard Al investigation.

The stockpeople did not initially accept their failing and attempted to cover up the problems. 


\title{
P.765
}

\section{Retention rate and boar productivity in two genetic transfer centers managed under an all-in all-out system in central western México}

\author{
Arturo Juarez-Martinez ${ }^{1}$ Joaquin Becerril ${ }^{2}$ Raul Ortega ${ }^{3}$ Pedro Aceves ${ }^{4}$ Pedro Vargas ${ }^{4}$ Jesus Gonzalez $^{1}$ \\ 1. PIC Mexico, Queretaro, QRO, Mexico; 2. LAPISA, La Piedad, MICH, Mexico; \\ 3. Independent Consultan, Morelia, MICH, Mexico; 4. CTG Sinergia, S.P.R. de R.L, La Piedad, MICH, Mexico
}

\section{Introduction}

It has been demostrated $(1,2)$, the effect of environmental and genetic factors on the productivity of boars at two Genetic Transfer Centers (GTC), managed under an all-in all-out systems (AIAO), as well as some estimates of longevity, productive life and seminal output repeatability (3). The aim was to obtain comparative estimates for retention rate, live sperm production, and semen doses produced, in two GTC managed under an AIAO system, that were filled with boars sourced from a single genetic supplier.

\section{Materials and Methods}

Data was obtained from two GTC established in Michoacan, Mexico, and located at $102015^{\prime} \mathrm{N}$ and 190 50'W. GTC One (GTC1), operated from Sept 1, 2004 until Jan 31, 2007, with an initial inventory of 66 boars. GTC Two (GTC2), operated from Jun 1st, 2006 until Dec 31 st 2008, with an initial inventory of 77 boars. Retention rate (RR) was analyzed by trimesters, in order to obtain Lineal Regression Models for each GTC. Sperm production analysis was carried out considering total sperm live concentration per ejaculate (TSLCE) and based on the interval between collections (CI). Annual semen doses production was calculated for each one of the GTC and the boar dose production was carried out by percentile $(100,75,50,25)$. The whole analysis was performed using SAS System SAS (Ver.8.0, 1999).

\section{Results}

For GTC1 and GTC2, 10, 086 and 11,997 ejaculates were processed respectively. All boars were introduced on the same day and were first ejaculated at an average age of $230.6 \pm 28.7$ days. For GTC1, the obtained models for RR were $108.49^{* *}-7.21^{* *}$ (trimester); R2=0.97, and for GTC2, RR were $109.27^{* *}-4.97^{* *}$ (trimester); $R 2=0.98$. The obtained models for TSLCE did not explain the variation observed: $\mathrm{GTC} 1$ : $\mathrm{TSLCE}=47114^{*}+95.19^{*}(\mathrm{Cl}) ; \mathrm{R} 2=0.003$; and for GTC2: TSLCE $=46840^{* *}+194.3^{* *}(\mathrm{Cl}) ; \mathrm{R} 2=0.008$, although, all coefficients were significatively different, indicating that for a longer collection interval, there will be an increase on TSLCE, therefore the importance of follow an adequate $\mathrm{Cl}$ protocol.

\section{Discussion}

For $\mathrm{RR}$ and considering regression trend, there was the same direction for both GTC, but not its magnitude, neither on its time-dependent distribution. Also, it was possible to see improvement in CTG2, maybe due to a better understanding and more experience gained. Regarding TSLCE and although, all coefficients were significatively different we conclude that for a longer collection interval, there will be an increase on doses produced, therefore the importance of follow up an adequate $\mathrm{Cl}$ protocol. At each GTC, there were also productivity differences between periods and the same happened when doses produced per boar during his lifetime were evaluated (Table 2), where a declining trend was observed between RR, ejaculates processed and total doses produced at each GTC. In a previous study, it was established that a peak of doses produced was reached at 953 days of age, with an average of 20.3 doses per ejaculated (1).

\section{References}

1. Juarez, M.A., et al. 2007. Memorias del XLII Congreso AMVEC. Pag.209.

2 Juarez, M.A., et al. 2009. Memorias del XLIV Congreso AMVEC. Pag. 168.

3. Ortega, G.R. et al. 2009. Memorias del XLIV Congreso AMVEC. Pag. 169.

\section{Table1.}

\begin{tabular}{|c|c|c|c|c|c|}
\hline GTC 1 & Ejaculates & Doses & GTC2 & Ejaculates & Doses \\
\hline 2004 & 1,003 & 14,746 & - & - & - \\
\hline 2005 & 4,333 & 75,609 & 2006 & 2,128 & 34,732 \\
\hline 2006 & 4,608 & 75,604 & 2007 & 5,564 & 79,008 \\
\hline 2007 & 142 & 2,630 & 2008 & 4,305 & 57,809 \\
\hline Total & 10,086 & 168,319 & & 11,997 & 171,550 \\
\hline
\end{tabular}

\section{Table2.}

\begin{tabular}{|l|c|c|c|c|}
\hline & GTC1 & GTC1 & GTC2 & GTC2 \\
\hline Percentile & Ejaculates & Doses & Ejaculates & Doses \\
\hline Top 25 & 256 & 7,874 & 233 & 4,782 \\
\hline Averange & 218 & 5,400 & 220 & 3,033 \\
\hline Low 25 & 174 & 4,100 & 165 & 2,263 \\
\hline
\end{tabular}




\title{
P.766
}

\section{High levels of dietary Zn-Methionate increase sperm DNA fragmentation in the boar: A preliminary investigation}

\author{
A García-Contreras ${ }^{1}$ Y De Loera ${ }^{2,1}$ C García- A ${ }^{2} \underline{\text { A Palomo }}^{2} \mathrm{~J}_{\text {Guevara-G }}{ }^{3} \mathrm{~J}$ Herrera- $\mathrm{H}^{4}$ C López -F 5 J Gosálvez ${ }^{5}$ \\ 1. POLIVET-AZ, DCBS-DPAA UAM-Xochimilco, Coyoacán, DF, Mexico; \\ 2. Facultad de Veterinaria. Universidad Complutense de Madrid, Madrid, Spain; \\ 3. MVZ FES-C UNAM, Cuautitlán, MEX, Mexico; 4. Ganadería Colegio de Posgraduados, Montecillos, MEX, Mexico; \\ 5. Dpto. Biol. U. Genética Universidad Autónoma de Madrid, Madrid, Spain
}

\section{Introduction}

Zinc (Zn) is as a member of metalloenzymes (GP, SOD) whereby it operates as an essential trace element important to a range of the reproductive processes in mammalian species (1). This mineral is necessary for the stability of sperm chromatin and embryonic development (2). Studies have demonstrated an increase in a range of abnormal seminal parameters linked to deficiencies in $\mathrm{Zn}$ concentration. The boar is susceptible to the adverse effects of $Z n$ deficiency, so that there is increasing interest to understand the relationship between dietary levels of $\mathrm{Zn}$ sources and its effect on fertility. Rojas et al. (3) shown that there is a difference in the bioavailability of $Z n$ source, well as the rate of absorption, transfer mechanisms, metabolism and storage (4). The aim of this investigation was to assess the influence of elevated concentrations of dietary Zn-Methionate (Zn-Met) on sperm DNA fragmentation (SDF).

\section{Material and Methods}

This experiment was conducted using ten individually housed York-Landrace breed boars with an age average of 8 months. Five boars received a standard food ration (SFR) containing 25 ppm Zn and were used as a control. The SFR was balanced according to NRC requirements (1998) using a premix of grain supplemented with soybean, vitamins and minerals. The experimental group consisted of 5 boars that were fed on a SFR containing a supplement of 200 ppm Zn (Zn-Met200). Boars were assessed for sperm DNA fragmentation (SDF \%) over 6 consecutive weeks. The levels of SDF were measured using the Sperm-Sus-Halomax assay (DNA Halotech SL, Madrid, Spain). SDF mean values were compared with Tukey's test.

Fig. 1. Graphic presentation of the varying levels of SDF in Control (grey line) and Zn-Met200 treated individuals (black line). Mean \pm SE obtained for group are showed.

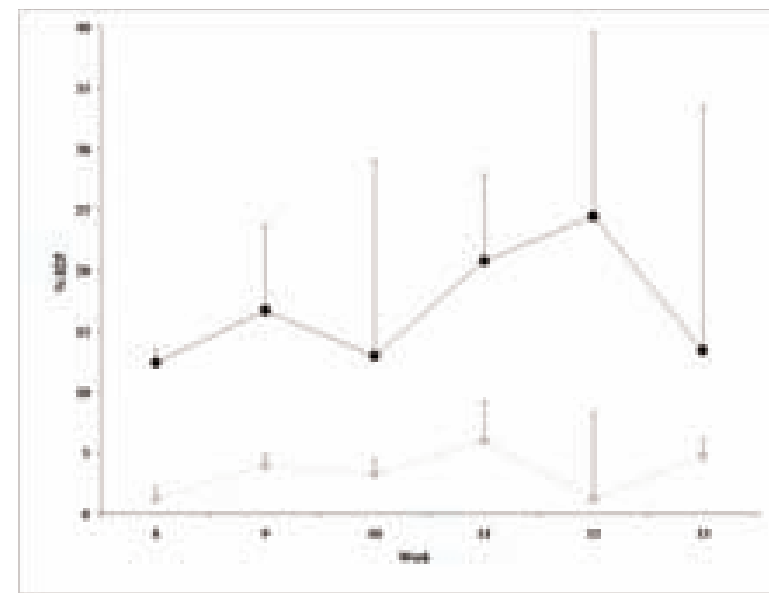

\section{Results and Discussion}

Fig. 1 shows the effect of increased dietary Zn on the SDF of boar semen collected over 6 consecutive weeks. On average the level of SDF for the control group was $2.91 \pm 3.04 \%$; this value is likely to be with the normal range for this species, i.e. $<5 \%(5,6)$. However, boars receiving the $\mathrm{Zn}$-Met200 diet showed average values for the SDF of greater than $15.01 \pm 3.10 \%$, which was in the order of four times more intense that those values found in the Control $(P=0.003)$ and values previously reported for boars (6). An interesting unexpected observation was the weekly rhythmic (10 to 20\%) expression of SDF throughout the collection period (Fig.1). Although dietary Zn-Met200 in this experiment resulted in significant damage to the sperm DNA during the period of feeding, further studies are required to determine whether this effect is reversible if the animal is returned to a normal diet or whether the initial exposure to high levels of dietary $Z n$ may interfere with future normal sperm development. Since a diet containing 200 ppm of organic $Z n$ (Zn-Met) resulted in a significant increase in boar sperm DNA damage, new ongoing experiments have been conducted to investigate the maximum level of $\mathrm{Zn}$ which does not interfere with the baseline level of SDF.

\section{References}

1. Hedemann MS., Jensen BB., Pulsen HD. 2006. Influence of dietary Zn and $\mathrm{Cu}$ on digestive enzyme activity and intestinal morphology in weaned pigs. J. Anim. Sci. 84:3310-3320

2. Evenson DP., Wixon R. 2006. Clinical aspects of sperm DNA fragmentation detection and male infertility. Theriogenology. 65:979-991.

3. Rojas LX., McDowell LR., Cousins RJ., Martint FG., Wilkinson NS:, Johnsons AB., Velasquez JB. 1995. Relative bioavailability of two organic and two inorganic $Z n$ sources fed to sheep. J. Anim. Sci. 73:1202-1207.

4. Krebs NF. 2000. Overview of Zn absorption and excretion in the human gastrointestinal tract. J. Nut. 130:1374S-1377S.

5. Enciso M., López-Fernández C., Fernández JL., García P., Gosálbez A., Gosálvez J. 2006. A new method to analyze boar sperm DNA fragmentation under brigh-field or fluorescence microscopy. Theriogenology 65:308-316.

6. López-Fernández C., Pérez-Llano B., García C. P., Sala R., Gosálbez A:, Arroyo F., Fernández JL., Gosálvez J. 2008. Sperm DNA fragmentation in a random simple of the spanish boar livestock. Anim. Rep. Sci. 103:87-98. 


\title{
P.767
}

\section{Fertility of sows inseminated with extended semen stored at $+5^{\circ} \mathrm{C}$ or $+17^{\circ} \mathrm{C}$}

\author{
Caterine Braga ${ }^{1}$ Maristela Palhares ${ }^{1}$ Amanda Siqueira $^{1}$ Mário Moraes $^{2}$ José Monteiro Silva Filho $^{1}$ \\ 1. Federal University of Minas Gerais, Belo Horizonte, MG, Brazil; 2. DB-Dan Bred, Patos de Minas, MG, Brazil
}

\section{Introduction}

Cooling boar semen to lower temperatures is necessary for transport over long distances and exchange of material genetic as sperm cryopreservation remains suboptimal in this species. Moreover, temperature reduction has been used to prolong the sperm viability, as a result of decreased cellular metabolic processes and bacterial growth reduction. However, there are few studies related to the preservation of boar sperm at $+5^{\circ} \mathrm{C}$ and its effectiveness "in vivo", involving the measurement of fertility parameters, such as farrowing rate, return to oestrous and litter size. Thus, the objective of the research was to evaluate the reproductive performance of sows inseminated with semen diluted in glycine-egg yolk extender (1) and cooled to $+5^{\circ} \mathrm{C}$.

\section{Material and Methods}

A total of 70 primiparous and multiparous sows were distributed uniformly, after grouping by breed and parity, into two treatments: $\mathrm{TI}(\mathrm{n}=35)$ - sows inseminated with semen diluted in BTS extender, submitted to stabilization period at room temperature $\left(23 \pm 2^{\circ} \mathrm{C}\right)$ for 2 hours, with subsequent storage at $+17^{\circ} \mathrm{C}$ (control treatment); and TII $(n=35)$ - sows inseminated semen diluted in glycine-egg yolk extender (1), submitted to a stabilization period at room temperature $\left(23 \pm 2^{\circ} \mathrm{C}\right)$ for 4 hours, before cooled at $+5^{\circ} \mathrm{C}$ in previously described container (2). The females of both treatments were inseminated with doses containing $3 \times 10^{9}$ viable sperm. The first insemination was performed 12 hours after the onset of oestrous, and the subsequent every 12 hours, for up to three insemination. If still in oestrous within 24 hours of the third insemination, the sows also received a fourth insemination. The data were analyzed using the statistical program "Statistical Analysis System", Version 5 - Microsoft ${ }^{\circ}$, with a significance level of $95 \%$ ( $p<0.05)$. The data were submitted to the procedure GLM (General Linear Models) and the quantitative variables analyzed by SNK or Student's t test, and proportional variables, by chi-square test $\left(x^{2}\right)$.

\section{Results}

The semen extended in glycine-egg yolk (TII) was superior $(p<0.05)$ with regard to the maintenance of the physical characteristics (motility and vigor), after 19.79 \pm 7.06 hours of storage $(\mathrm{Tl}=68.17 \pm 5.83 \%$ and $3.19 \pm 0.43 ; \mathrm{TI}=75.47 \pm 3.78 \%$ and $3.60 \pm 0.42$ ). Regarding the sows reproductive performance, there was an influence $(p<0.05)$ of treatment on the farrowing rate, from $77.14 \%$ with semen diluted in glycine-egg yolk and stored at $+5^{\circ} \mathrm{C}$ in special container to $97.14 \%$ when using the semen diluted in $\mathrm{BTS}^{\circ}$ and stored at $+17^{\circ} \mathrm{C}$. However, there were no significant differences $(p>0.05)$ between treatments regarding the average of total number of born piglets ( $14.87 \pm 2.85$ vs. $10.72 \pm 3.94)$ and born alive (13.26 \pm 2.89 and $10.12 \pm 3.53)$, for $\mathrm{TI}$ and TII, respectively.

\section{Discussion}

Studies related to cooling boar spermatozoa at $+5^{\circ} \mathrm{C}$ showed a farrowing rate of $85 \%$ (4) or $63 \%$ (1). Thus, the results found here are in agreement with those showed in the literature. Boar spermatozoa are sensitive to cooling below $+15^{\circ} \mathrm{C}$ (3), and membrane damages increasing with the storage time reducing the sperm longevity (5). Thus, one alternative to use of cooled boar semen will be the change in the site of sperm deposition in the female genital tract as have been made with cryopreserved semen in this specie.

\section{Conclusion}

The use of swine semen cooled to $+5^{\circ} \mathrm{C}$ proved to be viable using the glycine-egg yolk extender and container proposed (2). However, caution is recommended when used on a large scale in commercial herds, especially considering the excellent reproductive performance of sows submitted to intracervical insemination with sperm diluted in $\mathrm{BTS}^{\circ}$ and kept at $+17^{\circ} \mathrm{C}$.

\section{Reference}

1. Foote, R.H. Reprod. Dom. Anim., v.37, p.61-63, 2002.

2. Roner, M.N.B. Arq. Bras. Med. Vet. Zootec., v.58, n.1, p.78-86, 2006.

3. Watson, P.F. and Plummer, J.M. In: International Conference on Deep Freezing of Boar Semen 1, 1985, Uppsala, Proc... Uppsala: 1985, p.113-125.

4. Park, C.S. et al., Reprod. Dom. Anim., v.31, p.269, 1996.

5. Waberski, D. Proceedings Annual Meeting of the EU AI VETs, Ghent, Belgium, 2009.

This work received financial support from FAPEMIG- Minas Gerais Research Support Foundation and DB-Danbred of Brazil. 


\title{
P.768
}

\section{Main bacterial species isolated from commercial seminal doses and antibiotic activity}

\author{
René Mozo-Martín; Yahya Dahmani; Carmen Larraz' Juan Luís Úbeda \\ $R \& D$ department, Magapor SL, Ejea de los Caballeros. Zaragoza, Spain;
}

\section{Introduction}

Presence of bacterial contamination in pig seminal doses occurs with some frequency in artificial insemination centres, and may have a negative effect on its durability, even using ejaculates with good spermatic quality. Normally the source of this contamination is the own boar. However, distilled water used to elaborate the seminal doses can also be an important source of bacterial contamination. To overcome this problem it is essential to have a good hygiene and disinfection protocol. Moreover it is necessary to use extenders with an effective antibiotic combination.The aim of this study is to observe the main bacterial species isolated from seminal doses and to assess the activity of antibiotics commonly used against these microorganisms, in the seminal doses production and swine reproductive infections.

\section{Material and Methods}

The study was carried out with more than 170 diluted sperm samples from artificial insemination centres located throughout the Spanish geography. The sample cultures were routinely performed within 24 hours post-dilution (without any previous manipulation). Bacterial isolation and identification were performed in TSA agar (incubation 24 hours at $37^{\circ} \mathrm{C} \pm 1^{\circ} \mathrm{C}$ ). A kit API-20E was used to identify isolated bacteria, while the antibiogram was performed by diffusion in Mueller-Hinton agar (incubation 24 hours at $37^{\circ} \mathrm{C} \pm 1{ }^{\circ} \mathrm{C}$ ). The antibiogram test was carried out using antibiotics routinely included in the elaboration of seminal doses or pig reproduction pathology chemotherapy. Statistical analysis was developed with relative frequency description for bacterial isolates (StatView 5.0 statistical package) and Chi-Square for antibiotics activity (WinEpi: Working in Epidemiology).

\section{Results}

The most commonly bacterial species isolated in extended semen samples were those shown in Table 1.

Figure 1 show the frequency which bacterial isolates present sensitivity (S), resistance (R) or intermediate-acting (I) against antibiotics commonly used in pig reproduction (differences among antibiotic activity: $\mathrm{p}<0.0001$ )

Figure 1. Antibiotic activity against isolated bacteria.

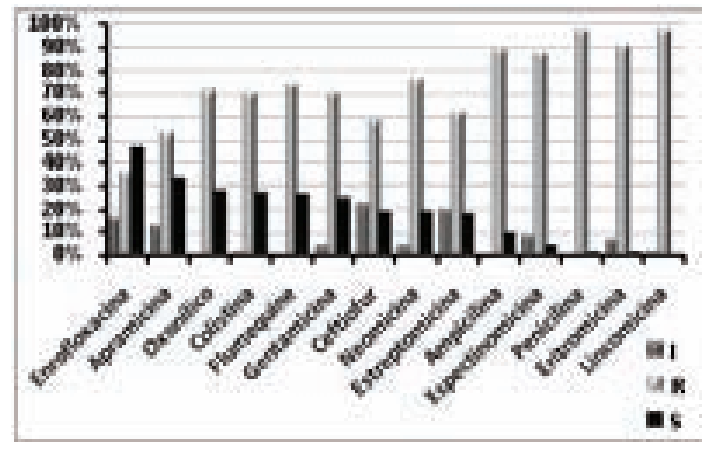

Table 1. Main bacterial isolates in extended semen frequency

\begin{tabular}{|l|c|}
\hline Bacteria & $\%$ \\
\hline Serratia marcescens & 24.0 \\
\hline Klebsiella oxytoca & 6.0 \\
\hline Proteus vulgaris & 5.3 \\
\hline Pseudomona fluorescens & 5.3 \\
\hline Escherichia coli & 4.0 \\
\hline Pseudomona aeruginosa & 4.0 \\
\hline Stenotrophomonas maltophilia & 4.0 \\
\hline Enterobacter asburiae & 2.7 \\
\hline Proteus mirabilis & 2.7 \\
\hline Serratia liquefaciens & 2.7 \\
\hline Serratia odorifera & 2.7 \\
\hline Brevibacillus brevis & 2.0 \\
\hline Burkholderia cepacia & 2.0 \\
\hline Cedecea davisae & 2.0 \\
\hline Citrobacter freundii & 2.0 \\
\hline Enterobacter cloacae & 2.0 \\
\hline Pseudomona oryzihabitans & 2.0 \\
\hline Staphylococcus equorum & 2.0 \\
\hline Others... & 22.7 \\
\hline
\end{tabular}

Discussion:Isolation of bacteria in seminal doses treated with antibiotics, indicate that it is produced a selection of bacterial species according to their susceptibility (resistance) to this antibiotics (i.e.: S. marcescens). Therefore, we consider essential to make an accurate bacterial type diagnostic and quantification, in addition to, a proper antibiotic selection to use. Taking into account frequent apparition of bacterial species with resistance or intermediate susceptibility to some antibiotics, no randomly selected antibiotic combination with synergic activity may support a proper tool to guarantee the minimum detrimental effect caused by bacterial contamination in the seminal dose conservative period. In short, With the objective of find more efficient antibiotic combinations, we consider essential assessing the activity and the MIC (Minimum Inhibitory Concentration) to each antibiotic about no treated seminal doses.

\section{References.}

Althouse GC, Lu KG. Bacteriospermia in extended porcine semen. Theriogenology. 2005 Jan 15;63(2):573-84.

Althouse GC, Pierdon MS, Lu KG. Thermotemporal dynamics of contaminant bacteria and antimicrobials in extended porcine semen. Theriogenology. 2008 Nov;70(8):1317-23. 


\title{
P.769
}

\section{Correlation between sperm quality traits in seminal doses distributed by boar stud to farms in Spain}

\author{
Yahya Dahmani; René Mozo-Martín; Carmen Larraz; Juan Luís Úbeda \\ R\&D Department, Magapor SL, Ejea de los Caballeros. Zaragoza, Spain
}

\begin{abstract}
Introduction
Fertilizing potential assessment of an ejaculate or semen doses destinated for swine artificial insemination (AI) includes evaluation of sperm quality traits like morphology, concentration, motility and functionality of sperm membrane. Nowadays, in Spain there are more than 60 boar stud supplying boar semen doses for farms, and in some cases, the distance between centres and farms is large. Nevertheless, semen doses should exhibit adequate fertilizing predictive parameters comply with standard limits. The aim of the present study was to assess sperm quality traits of seminal doses supplied by boar studs in Spain during last 5 years, and to investigate the correlations between these sperm parameters.
\end{abstract}

\section{Materials and Methods}

8728 semen doses were obtained from 56 commercial Al boar studs between January 2004 and December 2009. Semen quality parameters: Motility, Concentration, Whole acrosoms, functionality of sperm membrane (Host), sperm abnormal forms (head, tail, mid-piece, proximal and distal droplets) and temperature of semen doses reception in laboratory were checked. All analyses were carried out by the same person. Data were processed by StatView 5.0 software (1). Model of Multiple Regressions was used to test the correlation between all parameters and ANOVA test was applied to confirm differences of variances. Significant differences were considered when $p<0.05$.

\section{Results}

All sperm parameters analyzed were acceptable and according standard limits, excluding, sperm abnormal forms which expressed mean value of $32 \pm 7$, superior to standard limit value (30\%). Regression coefficients between parameters are illustrated in table 1 . Motility showed a correlation $(R=0.4)$ with whole acrosoms and negative slight correlation with total sperm abnormal forms and with abnormal tails. A great logical correlation was observed between sperm abnormal forms and abnormal tail, proximal droplets and distal droplets.

In what concern temperature of semen doses reception in the laboratory, ANOVA analyses showed increased values from June until September, and decreased values from December to Mars. In parallel, functionality of sperm membrane (Host) changed similarly and inversely $(P<0.0001$, figure 1$)$. Correlation between both parameters was negatively significant $R=-0.4$ (Table 1 ).

\section{Discussion}

In this study, we observed some correlations between different sperm quality parameters. Motility demonstrated a correlation with intact acrosoms and negative correlation with total abnormal forms and specifically with spermatozoa with abnormal tails which appear coherent with requirements for propulsion and movement of sperm. Furthermore, sperm abnormal forms were clearly correlated with abnormal tails and presence of proximal and distal droplets. This finding corroborate that any change or influence of total abnormal forms of sperm can be explained by these three classes of sperm morphoanomalies: abnormal tails, distal and proximal droplets. It's of utmost importance the good checking of abnormal forms, because negative association between morphological anomalies and fertility was observed in several studies $(2,3)$. Other parameter which showed a significant variation is the temperature reception of seminal doses. Some differences found in Host values could demonstrate the importance of minimize the temperature variation during sperm processing and transportation, even the temperature inside de boar stud, because a significant fall in this paramether during hot months can take place. Any change in this area can affect functionality of sperm membrane and consequently the potential fertility of semen doses.

Table 1. Regression coefficients matrix between all parameters studied.

\begin{tabular}{|c|c|c|c|c|c|c|c|c|c|c|c|}
\hline & $\mathbf{T}$ & $M$ & $c$ & A & $\mathrm{Ht}$ & $\mathbf{A F}$ & H & $T$ & MP & PD & \\
\hline $\mathrm{T}^{\circ}$ & 1 & & & & -0.4 & & & & & & \\
\hline M & & 1 & & 0.4 & & -0.2 & & -0.3 & & & \\
\hline$C$ & & & 1 & & & 0.2 & & 0.2 & & & \\
\hline$A$ & & 0.4 & & 1 & & & & & & & \\
\hline HT & -0.4 & & & & & 0.2 & & 0.2 & & & 0.2 \\
\hline AF & & -0.2 & 0.2 & & & 1 & 0.2 & 0.6 & 0.2 & 0.6 & 0.6 \\
\hline $\mathrm{H}$ & & & & & & 0.2 & 1 & & 0.2 & & \\
\hline$T$ & & -0.3 & 0.2 & & 0.2 & 0.6 & & 1 & & & \\
\hline MP & & & & & & 0.2 & 0.2 & & 1 & & \\
\hline$P D$ & & & & & & 0.6 & 0.2 & & & 1 & \\
\hline DD & & & & & 0.2 & 0.6 & & & & & 1 \\
\hline \multicolumn{12}{|c|}{$\begin{array}{l}\mathrm{T}^{\circ} \text { : temperature of semen doses reception, M: sperm motility, C: concentration, A: whole } \\
\text { acrosoms, Ht: Host: }\end{array}$} \\
\hline & erm & bnorm & & & : tail, & $\mathrm{P}: \mathrm{m}$ & biece & & & & distal \\
\hline
\end{tabular}

Figure 1. Mean values of Host and temperature $\left(T^{\circ}\right)$ of semen doses reception among months.

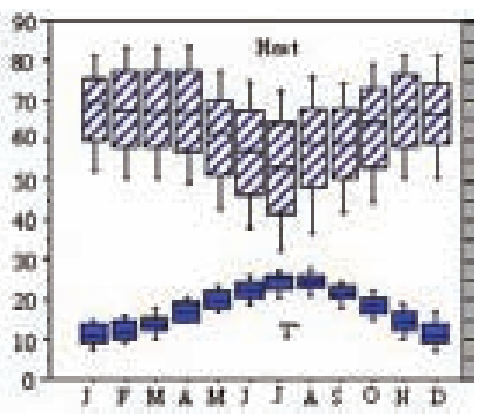

\section{References}

1. StatView, SAS institute Inc. Version 5.0

2. Waberski D., et al., 1994. Anim. Reprod. Sc. 36, 145-151

3. Xu X., et al., 1998. J. Anim. Sc. 76, 3079-3089. 


\title{
P.770
}

\section{Fertility and prolificacy of boar semen extended with Duragen ${ }^{\circledast}$ and BTS}

\author{
René Mozo-Martín; Yahya Dahmani; Carmen Larraz; Juan Luís Úbeda
}

$R \& D$ department, Magapor SL, Ejea de los Caballeros. Zaragoza, Spain

IntroductionThe productivity of the swine industry is highly dependent on artificial insemination and consequently on the production and transport of boar semen. Technologies of swine reproduction has developed semen extenders based manly on salts and sugars denominated short term extenders, long-term and extra long-term extenders. Many of the commercial extenders have been investigated in vitro for their fertilizing potential using some sperm parameters like motility.

Duragen ${ }^{\circledR}$ is an example of these extenders, and it's the unique extender which can preserve sperm parameters for 2 weeks. It has been demonstrated that Duragen ${ }^{\circledR}$ maintains a high degree of sperm fertility for more than 12 days (1) and increase the farrowing rate also at 1 day of sperm extension. Some studies in vitro about quality of sperm motility in extended semen with Duragen $^{\circledast}$ (data not shown) suggest a down-regulation of sperm motility at 24 hours, since it was observed that sperm exhibit value of average head orientation change (AOC) lower than 5 , being characterized as immotile. The aim of this study is to investigate the fertilizing potential of extra-long term extender Duragen ${ }^{\circledR}$ after 24 hours of semen extension.

\section{Materials and Methods}

The sperm rich fraction of ejaculates from fertile boars were collected, evaluated and pooled. Seminal heterospermic doses of 3 billion spermatozoa in $90 \mathrm{ml}$ were prepared with Duragen ${ }^{\circledR}$ and BTS extenders, and stored at $17^{\circ} \mathrm{C}$ before being used for artificial insemination at 24 hours. 4464 multiparous crossbred sows (Landrance $x$ Large White) were randomly divided in tow groups. Group1: BTS N=2303 and Group2: Duragen ${ }^{\circledR}, \mathrm{N}=2161$. Cervical Insemination was performed at 0 and 24 hours after external estrus signs observation. 2303 inseminations were carried out with semen extended with BTS and 2161 with Duragen ${ }^{\circledR}$. Farrowing rate (FR) and total born piglets were checked. Data were processed by StatView 5.0 software (2). ANOVA test and post hoc analyse were performed to confirm differences in variances of FR. Significant differences are considered when $p<0.05$.

\section{Results}

1996 sows inseminated with semen extended with BTS get to farrows, showing a fertility rate of $86,67 \%$. In parallel to this data, 1917 sows inseminated with semen extended with Duragen ${ }^{\circledR}$ a higher significant fertility rate $88,7 \%(P=0,03)$ Tabe 1 . In what concern total number of piglets and mean of born piglets per sow, no differences was found between both groups Duragen ${ }^{\circledR}$ and BTS $(P=0.13)$.

Table 1. Farrowing rate and mean of born piglets per sow obtained in Duragen ${ }^{\circledR}$ and BTS groups

\begin{tabular}{|l|c|c|c|c|c|c|}
\hline Extender & Total AI & $\begin{array}{c}\text { Farrowing } \\
\text { rate N }\end{array}$ & $\begin{array}{c}\text { Farrowing } \\
\text { rate } \%\end{array}$ & Post hoc & $\begin{array}{c}\text { Piglets } \\
\text { /sow }\end{array}$ & $\mathbf{1 1 . 3 \pm 2 . 5}$ \\
\hline Duragen $^{\circledast}$ & 2161 & 1917 & 88.67 & $\mathrm{P}=0.03$ & $11.2 \pm 2.4$ & $\mathrm{P}=0.13$ \\
\hline BTS & 2303 & 1996 & 88.7 & & $11.3 \pm 2.5$ & \\
\hline
\end{tabular}

\section{Discussion}

Fertility rate of sows inseminated with semen extended for 24 hours with BTS and Duragen ${ }^{\circledR}$ was higher in Duragen ${ }^{\circledR}$ group. This funding demonstrate that Duragen ${ }^{\circledast}$ can be used at 24 hours as short-term extender, even it is developed for extra-long term conservation of boar sperm. In addition, this exhaustive study with 4464 sows, demonstrate strongly the functionality of Duragen ${ }^{\circledast}$ at 24 hours excluding any doubt about fertilizing potential of Duragen ${ }^{\circledast}$ at $24 \mathrm{~h}$ even the observation on quality of sperm motility at $24 \mathrm{~h}$.

\section{References}

1. Gómez-Rincón et al., 2009. Proc. VIII Int. Conf. Pig reproduction. P.128

2. StatView, SAS institute Inc. Version 5.0 


\title{
Boar semen cryopreservation: effects of straws types, number of spermatozoa, insemination site
}

\author{
Pierre Thilmant \\ Centre Provincial liègeoid e de productions animales, Argenteau, Belgium
}

\section{Introduction}

The objectives of this study are to check 2 types of French straws for boar semen cryopreservation: medium or $0.5 \mathrm{ml}$ versus fine or $0.25 \mathrm{ml}$; the number of straws per dose $(10,5,4$ or 3); 2 insemination sites: intracervical versus intrauterine (corpus); 2 insemination volumes (100 versus $50 \mathrm{ml}$ ) with intracervical insemination. The goal is to decrease the number of spermatozoa per insemination dose and to have good results on the field.

\section{Materials and Methods}

210 sows have been inseminated with frozen semen in 2 farms. 24 ejaculates of 7 Pietrain boar have been frozen with own method $(1,2)$. Each 0.5 and $0.25 \mathrm{ml}$ straws contained respectively $0.72 \times 109$ and $0.32 \times 109$ spermatozoa. The $0.5 \mathrm{ml}$ straws were thawed by immersing in a hot water bath at $55^{\circ} \mathrm{C}$ during 12 seconds and the $0.25 \mathrm{ml}$ straws in a water bath at $38^{\circ} \mathrm{C}$ during \pm 60 seconds (no risks of overheat). After that, the contents were directly poured into BTS at $38^{\circ} \mathrm{C}$. Semen was thawed just before the insemination. Double AI has been made 5 days postweaning during the standing reflex. We have used a foam tip insemination catheter for intracervical insemination (IC) and the same catheter equipped with a flexible tube for the post cervical insemination (IU). 2 parameters have been measured: pregnancy rate with an ultrasound scan between 23 - 42 days post Al and the litter size or total piglets born per litter. Three trials were successively performed. The first tested the effect of straws types and number of spermatozoa per dose (from 3.6 to $1.0 \times 109$ ); the second and third trials tested these same alternatives and insemination sites (trial 2) or insemination volumes (trial 3) on sows' pregnancy rates and litters size.

\section{Results}

With IC insemination and $100 \mathrm{ml}$ insemination volume, the pregnancy rate $(92.9 \% ; n=141)$ was not influenced by the straws types and the number of spermatozoa. However it has significantly decreased when IU inseminations were performed (60.0; $n=30$ versus $91.7 \% ; n=180 ; p<0.05)$ and particularly with $1 x$ 109 spermatozoa or 3 fine straws $(50.0 \% ; n=10)$. There was no significant difference between the 2 volumes when IC inseminations were performed $92.9 ; n=141$ versus $87.2 \% ; n=39$ ) whatever the straws type and the number of spermatozoa per dose. The litter size was significantly higher when sows were intracervically inseminated using $3.2 \times 109$ (10 fine straws) versus $1.0 \times 109$ spermatozoa ( $12.3 \pm 0.6 ; n=20$ versus $10.6 \pm 0.5 ; n=31 ; p<0.05)$. In the same way, the litter size tended to decrease when IU rather than IC insemination was performed $(11.5 \pm 0.2 ; n=156$ versus $10.2 \pm 0.7 ; n=16 ; p=0.08)$. There was no significant difference between results obtained when using $100 \mathrm{ml}$ insemination volume (11.5 $\pm 0.2 ; n=139)$ versus $50(11.0 \pm 05 ; n=33)$ when IC inseminations were performed, whatever the straws type and the number of spermatozoa.

\section{Discussion}

With IC insemination, the pregnancy rate is always above $86.8 \%$ despite the spermatozoa number and straw type, lower litter size is with $1 \times 109$ spermatozoa / dose. The IC insemination is not influenced by the volume (100 or $50 \mathrm{ml}$ ). With IU insemination, pregnancy rate and litter size are lower than IC insemination.

So, with frozen semen, we recommend intracervical insemination with a minimum $1.3 \times 109$ spermatozoa / dose or 4 fine straws / dose

\section{References}

1. Thilmant P., 1997. Ann. Méd. Vét., 141, 457-462.

2. Thilmant P., 2001. Journées Rech. Porcine en France, 33, 151-156. 


\title{
P.772
}

\section{Using autologous and heterologous boar sperm supernatant from freezing processes as post-thawing solution: its effect on sperm motility}

\author{
Kampon Kaeoket ${ }^{1}$ Panida Chanapiwat ${ }^{1,2}$ Padet Tummaruk ${ }^{2}$ Mongkol Techakumphu² Annop Kunavongkrit ${ }^{2}$ \\ 1. Faculty of Veterinary Science, Mahidol University, Phutthamonthon, Nakorn-pathom, Thailand; \\ 2. Faculty of Veterinary Science, Chulalongkorn University, Bangkok, Thailand
}

\section{Introduction}

Cryopreservation process damage the sperm plasma membrane by a number of reason, e.g. cold-shock effect, osmotic stress and oxidative stress from reactive oxygen species (ROS) which result in a decrease of sperm motility, viability and fertilizing ability (Kaeoket et al., 2008). It is evidence that seminal plasma able to arrest or reverse cryoinjury and perhaps extend the longevity of the sperm by inhibiting or reversing capacitation and acrosome reaction, and also by its antioxidant activity (Vadnais and Roberts, 2007). The aim of this study was to evaluate the effect of post-thawing dilution with autologous and heterologous sperm supernatant on motility of frozen-thawed boar spermatozoa.

\section{Materials and Methods}

During the cryopreservation, sperm supernatant (a combination of seminal plasma and semen extender, $50 \% \mathrm{v} / \mathrm{v}$ ) or seminal plasma from 9 boars (Duroc, Large White and Landrace; 3 in each) was collected by centrifugation and stored frozen until using as post-thawing solution. Sperm pellet were further processed and cryopreserved using control rate freezer and was thawed at $50^{\circ} \mathrm{C}$ for $12 \mathrm{sec}$. After thawing, frozen thawed semen was diluted with seminal plasma (group A), supernatant from Landrace (group B), supernatant from Large White (group C), supernatant from Duroc (group D) and Modena ${ }^{\circledR}$ semen extender (group E). Post-thawing motility was evaluated using a phase contrast microscope after thawing at 1, 10, 20 and $30 \mathrm{~min}$. The present results show that at $1 \mathrm{~min}$, a significantly higher percentage $(P \leq 0.001)$ of progressive motility was found in groups $B(53.3 \%)$ and $C(53.9 \%)$ than the other groups. At $10 \mathrm{~min}$, the highest $(P \leq 0.001)$ progressive motility was found in groups $B$ $(65 \%)$ and $C(61 \%)$. At 20 and 30 min, a significantly higher percentage $(P \leq 0.001)$ of progressive motility was found in groups $B(58.9 \%), C(53.5 \%)$ and $D(45.6 \%)$ than groups A $(3.9 \%)$ and $E$ (20.6\%).

\section{Discussion}

It is clearly showed, in the present study, that using autologous or heterologous sperm supernatant from cryopreservation process as post-thawing solution yielded a superior frozen-thawed boar sperm motility (as high as 70\%). This is in accordance with the studies in dog (Rota et al., 2007) and boar (Okazaki et al., 2009) in which seminal plasma and prostatic fluid, respectively, were added in post-thawing solution.

\section{Conclusion}

It can be concluded that supernatant from freezing processes (consisting of seminal plasma and Modena ${ }^{\circledR}, 50 \% \mathrm{v} / \mathrm{v}$ ) had beneficial effect on post-thawing progressive motility of frozen boar semen.

\section{References}

Kaeoket K et al. (2008) Reprod. Dom. Anim. In press Okazaki T et al., (2009) Theriogenelogy 71, 491-497. Rota A et al. (2007) Theriogenology 67, 520-525. 


\title{
P.773
}

\section{Post cervical artificial inseminastion use of small volumes and sperms number with cervical stimulation}

\author{
Javier Gil ${ }^{1}$ Marcos Nieto ${ }^{2}$ \\ 1. JGPAsesor S.L.P., Segovia, Spain; 2. COPESE S.A., Coca, Spain
}

\section{Introduction}

Since 2000 when Gil et al. (1) published the first reproductive results obtained by using Post Cervical Artificial Insemination (PCAI), was proved that the number of sperms that guarantees similar reproductive results to those used in the standard $\mathrm{Al}$ is 1.0 billion (2). Also it is possible to obtain excellent results using doses of $15 \mathrm{ml}$ and half billion sperms, but is it necessary to increase the inseminated volume by infusing $15 \mathrm{ml}$ of extender after the semen introduction(3).

In 2006 we improved the technique by adding the cervical stimulation (4). The results obtained confirm that this management improves litter size.

After semen introduction, cervical stimulation could help the preovulatory pulsatile $\mathrm{LH}$ release that lead to the ovulation process (5) and advance the ovulation or perhaps promote passive sperm transport by enhancement of uterine contractions.

Is it possible to inseminate less than $30 \mathrm{ml}$ and obtain good results when stimulate the uterus by cervical stimulation? This paper shows the results.

\section{Material and Methods}

The trial was carried out in a 1700 sows farm. In this farm PCAI was used in weaned sows in a habitual way. After standing oestrus control, sows were randomly allocated to three treatment groups. In group Mono-phase 30 (MPh30), sows were inseminated with doses of $30 \mathrm{ml}$ and 1.0 billion sperms. In group Bi-phase 30 (BPh30) sows were inseminated with doses of $18 \mathrm{ml}$ and 0.6 billion sperms and $12 \mathrm{ml}$ of extender added after the infusion of the dose. In group Mono-phase 18 (MPh18) sows were inseminated only with doses of $18 \mathrm{ml}$ and 0.6 billion sperms.

To apply the semen it was used a combined catheter-cannula kit (Soft \& Quick, Import-vet, Spain). In all groups, the sows received a cervical massage. After the infusion of the dose in groups MPh30 and MPh18 or the dose and the extender in group BPh30, only the cannula was removed and then with the catheter, still placed in the cervix, shaking it in a rotational way, the neck of the womb was massaged during five to ten seconds. Once the massage was finished the catheter was also removed.

\section{Results}

Table 1 shows that there isn't relationship between the treatments with the fertility and prolificity levels. Farrowing rate is very similar in all groups, $89.74 \%, 92.11 \%$ and $89.74 \%$ in $\mathrm{MPh} 30$, BPh30 and MPh18. The best litter size, 12.20 total born and 12.11 born alive, takes place in the MPh18 group, being very similar,+ 0.17 total born and +0.25 born alive, in MPh30 group and being considerably better, +0.51 total born and +0.65 born alive, than the BPh30 group, 11.69 total born and 11.46 born alive.

Table 2 shows the percentage of litters according to the number of total born. It attracts our attention the difference in percentage of short litters, 11.5 vs. 5.8 in BPh30 and MPh18 respectively. The smaller number of short litters in MPh18 group increases the percentage of large litters $28.6 \%$ vs. $20.10 \%$ in BPh30 group.

Table 1 Reproductive results.

\begin{tabular}{|l|c|c|c|c|c|}
\hline Group & Sows $^{\mathbf{0}}$ & Pregnant \% & F. Rate \% & Born alive & Total born \\
\hline MPh30 & 39 & 94.87 & 89.74 & 11.86 & 12.03 \\
\hline BPh30 & 38 & 92.11 & 92.11 & 11.46 & 11.69 \\
\hline MPh18 & 39 & 92.30 & 89.74 & 12.11 & 12.20 \\
\hline
\end{tabular}

Table 2 Total born litter scatter \%

\begin{tabular}{|l|c|c|c|c|c|c|c|}
\hline Group & $\mathbf{5 8}$ & $\mathbf{9}$ & $\mathbf{1 0}$ & $\mathbf{1 1}$ & $\mathbf{1 2}$ & $\mathbf{1 3}$ & $\mathbf{2 1 4}$ \\
\hline MPh30 & 8.6 & 8.6 & 17.1 & 8.6 & 17.1 & 11.4 & 28.7 \\
\hline BPh30 & 11.5 & 5.7 & 11.4 & 17.1 & 22.9 & 11.4 & 20.1 \\
\hline MPh18 & 5.8 & 8.6 & 11.4 & 8.6 & 14.3 & 22.9 & 28.6 \\
\hline
\end{tabular}

\section{Discussion}

The reduction of the percentage of short litters leads up to improve the litter size in MPh18 group.

These results suggest us that isn't necessary a large volume so the uterus can absorb the semen when it's properly stimulated. Cervical stimulation seems to promote passive sperm transport and allow excellent reproductive results with small doses of semen.

\section{References}

1. Gil, J. et al. (2000). 16th IPVS: 399.

2. Gil, J. et al. (2002). 17th IPVS: 493.

3. Gil, J. et al. (2004). 18th IPVS: 456.

4. Gil, J. et al. (2006). 19th IPVS: 116.

5. Ziecik, A. et al. (1981) J. Animal Science 53:434-438 


\title{
P.774
}

\section{Post cervical artificial inseminastion use of different volumes and sperms number with cervical stimulation}

\author{
Javier Gil ${ }^{1}$ Ana M. del Valle ${ }^{2}$ Antonio Roset $^{3}$ \\ 1. JGPAsesor S.L.P., Segovia, Spain; 2. Pinsos YAK, Lleida, Spain; 3. ARVET Veterinaria S.L., Lleida, Spain
}

\section{Introduction}

Since 2000 when Gil et al. (1) published the first reproductive results obtained by using Post Cervical Artificial Insemination (PCAI), was proved that the number of sperms that guarantees similar reproductive results to those used in the standard $\mathrm{Al}$ is 1.0 billion (2). Also it is possible to obtain excellent results using doses of $15 \mathrm{ml}$ and half billion sperms, but is it necessary to increase the inseminated volume by infusing $15 \mathrm{ml}$ of extender after the semen introduction (3).

In 2006 we improved the technique by adding the cervical stimulation (4). The results obtained confirm that this management improves litter size.

After semen introduction, cervical stimulation could help the preovulatory pulsatile $\mathrm{LH}$ release that lead to the ovulation process (5) or perhaps promote passive sperm transport by enhancement of uterine contractions.

Is it possible to inseminate less than $30 \mathrm{ml}$ and obtain good results when stimulate the uterus by cervical stimulation?

\section{Material and Methods}

The trial was carried out in a 760 sows farm. PCAI was used in gilts and weaned sows in a habitual way. The trial was conducted in three consecutive periods. In the first, from 09/02/09 to $15 / 03 / 09$, sows were inseminated with doses of $45 \mathrm{ml}$ and 1.4 billion sperms. In the second, from 16/03/09 to 19/04/09, sows were inseminated with doses of $20 \mathrm{ml}$ and 0.6 billion sperms. In the third, from 20/04/09 to 21/05/09, sows were inseminated with doses of $30 \mathrm{ml}$ and 0.9 billion sperms. In all groups the first insemination was done half a day after heat detection. In the first group sows were inseminated three times every half a day. In the other groups sows were inseminated four times every half a day.

To apply the semen it was used a combined catheter-cannula kit (Soft \& Quick, Import-vet, Spain). In all groups, the sows received a cervical massage. After semen infusion only the cannula was removed and then with the catheter, still placed in the cervix, shaking it in a rotational way, the neck of the womb was massaged during 5-10 seconds. Once the massage was finished the catheter was also removed.

\section{Results}

Table 1 shows that the fertility level and the farrowing rate are very similar in all groups, $90.55 \%, 89.29$ and $91.79 \%$ respectively. However, there is a difference in litter size according to the treatment. The best litter size, 12.55 total born and 11.58 born alive, takes place in the $20 \mathrm{ml}$ group, followed by $45 \mathrm{ml}$ and $30 \mathrm{ml}$ groups with 12.02 and 11.78 total born and 11.20 and 10,98 born alive respectively.

Table 2 shows the litter size by parity. In $45 \mathrm{ml}$ and $20 \mathrm{ml}$ groups litter size was very similar in each parity. In group $30 \mathrm{ml}$ litter size was also very similar to other groups from 3 to 6 parities but was considerably lowest in parities 1 and 2.

Table 1 Reproductive results.

\begin{tabular}{|l|c|c|c|c|c|}
\hline Group & Sows $^{\mathbf{0}}$ & Pregnant $\%$ & F. Rate $\%$ & Born alive & Total born \\
\hline $45 \mathrm{ml}$ & 201 & 92.57 & 90.55 & 11.20 & 12.02 \\
\hline $20 \mathrm{ml}$ & 196 & 91.83 & 89.29 & 11.58 & 12.55 \\
\hline $30 \mathrm{ml}$ & 195 & 93.33 & 91.79 & 10.98 & 11.78 \\
\hline
\end{tabular}

Table 2 Total born by parity

\begin{tabular}{|l|c|c|c|c|c|c|c|}
\hline Parity & $\mathbf{1}$ & $\mathbf{2}$ & $\mathbf{3}$ & $\mathbf{4}$ & $\mathbf{5}$ & $\mathbf{6}$ & $\mathbf{7}$ \\
\hline $45 \mathrm{ml}$ & 11.8 & 11.4 & 12.9 & 13.4 & 12.8 & 11.9 & 16.0 \\
\hline $20 \mathrm{ml}$ & 10.2 & 10.8 & 12.7 & 13.1 & 12.5 & 11.1 & - \\
\hline $30 \mathrm{ml}$ & 11.6 & 11.4 & 12.7 & 12.7 & 11.4 & 12.0 & - \\
\hline & & & & & & & \\
\hline
\end{tabular}

\section{Discussion}

Inadequate management of nulliparous and primiparous sows during the period of $30 \mathrm{ml}$ group may have been responsible for this drop in production in parities 1 and 2.

The $20 \mathrm{ml}$ group results, better than those obtained in group $45 \mathrm{ml}$, suggest us that isn't necessary a large volume so the uterus can absorb the semen when it's properly stimulated. Inseminate with low number of sperm in a normal dilution ratio, without the need of increasing the total volume, greatly facilitates the success of PCAI with low number of sperms.
References
1. Gil, J. et al. (2000). 16th IPVS: 399.
2. Gil, J. et al. (2002). 17th IPVS: 493.
3. Gil, J. et al. (2004). 18th IPVS: 456.
4. Gil, J. et al. (2006). 19th IPVS: 116.
5. Ziecik, A. et al. (1981) J. Animal Science 53:434-438 


\title{
P.775
}

\section{Use of P.G. $600^{\circledR}$ in replacement gilts with unknown reproductive status: Ovarian, estrual and fertility responses}

\author{
Robert V. Knox \\ University of Illinois, Urbana, IL, USA
}

\section{Introduction}

Effective methods to induce and synchronize estrus in replacement gilts are essential for controlling costs related to size of the gilt pool, days from entry to service, and fertility. We performed two experiments to evaluate ovarian and estrual responses to use of P.G. $600^{\circledR}$ in gilts with known or unknown reproductive status.

\section{Materials and Methods}

Experiment 1

PIC gilts $(n=15)>6$ mo. of age and $\sim 140 \mathrm{~kg}$ but of unknown reproductive status, were treated with MATRIXTM for 14 days. Following last MATRIX ${ }^{\mathrm{TM}}$ feeding (LMF) gilts were checked for estrus and ovarian status determined using ultrasound. All gilts diagnosed with or without corpora lutea $(C L)$ were treated with P.G. $600^{\circledR}(\mathrm{d} 0)$. Ultrasound was performed every other day for the next 6 days and estrous detection was performed for the next 22 days. Reproductive tracts were assessed at day 25. Experiment 2

This study was performed in replicates using 123 prepubertal PIC terminal-cross gilts. All gilts were given P.G. $600^{\circledR}$ for initial induction of estrus at $168 \mathrm{~d}$ of age and $114 \mathrm{~kg}$. Estrous detection was performed daily. Ten days following P.G. $600^{\circledR}$, gilts were synchronized using MATRIXTM. In replicates 1-3, gilts received no P.G. $600^{\circledR}$ following LMF, and in replicates 4-6, all gilts received an injection of P.G. $600^{\circledR} 24 \mathrm{~h}$ following LFM. Estrous detection began after LMF and transrectal ultrasound was initiated at estrus to observe follicles and time of ovulation. Numbers of follicles $>5.0 \mathrm{~mm}$ were counted and digitally recorded at estrus for confirmation of follicle number. All estrus gilts were inseminated and reproductive tracts collected at $\mathrm{d} 35$.

\section{Results}

Experiment 1

For gilts displaying estrus following $L M F$, all (12/15) had $C L$ on their ovaries at time of P.G. $600^{\circledR}(\mathrm{d} 0)$. None of these gilts had large follicles at time of treatment ( $\mathrm{d} 0$ ) but $75 \%$ developed large follicles over the next 6 days. All gilts maintained their $C L$ for the next $6 \mathrm{~d}$. Two gilts had cysts on $\mathrm{d} 0$, and 3 had cysts on days $2-4$, but none had cysts by day 6 . None of the gilts showed estrus within 6 days. The interval from P.G. $600^{\circledR}$ to estrus averaged 12 days and cyclic gilts treated with P.G. $600^{\circledR}$ showed an inter-estrus interval that averaged 22 days. The two gilts that did not display estrus following LMF and did not have CL on their ovaries, developed large follicles, ovulated, and expressed estrus within 3 days of P.G. $600^{\circledR}$. The one gilt that failed to show estrus following LMF, but that did have CL at time of P.G. $600^{\circledR}$ treatment, developed large follicles, but did not display estrus until $13 \mathrm{~d}$ after P.G. $600^{\circledR}$. At the end of this study, $92 \%$ of all gilts had expressed estrus, and 100\% were cyclic.Experiment 2

Initial induction with P.G. $600^{\circledR}$ was $66 \%$ with an interval to estrus of $100 \mathrm{~h}$, and an estrous duration of $42 \mathrm{~h}$. All gilts were treated with MATRIXTM regardless of whether they expressed estrus or not. In replicates 1-3, gilts received no treatment following LMF but in replicates $4-6$, P.G. $600^{\circledR}$ was given at $24 \mathrm{~h}$ following LMF to improve estrous synchrony. Expression of estrus following LMF was $>85 \%$ for all replicates. Use of P.G. $600^{\circledR}$ following LMF shortened the interval from LMF to estrus $(P<0.0001)$ in replicates 4-6 (130 h) compared to replicates 1-3 (148 h). P.G. $600^{\circledR}$ following LMF also shortened the duration of estrus $(P<0.0005)$ in replicates 4-6 (42 h) compared to replicates 1-3 (52 h). However, the interval from onset of estrus to ovulation was not influenced by P.G. $600^{\circledR}(P>0.20)$, and averaged $33 \mathrm{~h}$ in replicates $1-3$, and $34 \mathrm{~h}$ in replicates $4-6$. The number of large follicles ( $>5 \mathrm{~mm}$ ) counted using ultrasound at estrus was highly correlated to the digital recording ( $r=0.90,19$ follicles), but was unrelated to the final number of CL at slaughter (17). There was no effect of P.G. $600^{\circledR}$ following LMF on pregnancy at slaughter (74\%).

\section{Discussion}

The results suggest that P.G. $600^{\circledR}$ can be used in gilts of unknown reproductive status which are cyclic or acyclic gilts to improve farm estrus expression and synchrony without detrimental effect on fertility. Also, use of P.G. $600^{\oplus}$ in a puberty induction program and followed by MATRIXTM regardless of estrus induction results in estrus expression and synchrony in $>85 \%$ of replacement females. 


\title{
P.776
}

\section{Single fixed-time insemination protocol in weaned sows: synchronizing ovulation with porcine luteinizing hormone}

\author{
Louisa J. Zak ${ }^{1}$ Jennifer Patterson² George R. Foxcroft ${ }^{2}$ John Hancock ${ }^{3}$ Dragan Rogan ${ }^{1}$ \\ 1. Bioniche Animal Health, Belleville, ON, Canada; 2. University of Alberta, Edmonton, AB, Canada; 3. Picton Animal Hospital, Picton, ON, Canada
}

\section{Introduction}

Optimal sow fertility is achieved when insemination occurs up to 24 hours before ovulation. In practice, multiple inseminations are necessary to ensure good sow fertility as estrus to ovulation interval is unpredictable and depends on the wean to estrus interval (WEI) and estrus duration (ED).

The inability to predict the moment of ovulation causes inefficiencies in current artificial insemination (Al) technology. Multiple inseminations necessitate extensive labour demands and result in inefficient use of semen - which directly limits the wider application of genetically superior boars. Variation in the timing of the pre-ovulatory luteinizing hormone (LH) surge relative to onset of estrus causes the variation in time of ovulation. Ovulation is synchronized in sows ${ }^{1}$ and gilts ${ }^{2}$ by administration of porcine LH (pLH; Lutropin, Bioniche Animal Health, Belleville, Ontario, Canada), and occurs 38 \pm 2.8 h later $^{1}$. Recently Zak et al., ${ }^{3}$ showed that administration of Lutropin at onset of standing heat $(\mathrm{SH})$ in the weaned sow, followed by a double fixed time Al (FTAI) within the period of optimal sow fertility, resulted in more pigs born compared to a conventional Al protocol. The present study in the sow, compared effectiveness of combining Lutropin administration at first detection of $\mathrm{SH}$ followed by a single FTAI to results from sows conventionally managed and inseminated until they were no longer in $\mathrm{SH}$.

\section{Materials and Methods}

Sows were randomly assigned to treatment at weaning. Twicedaily boar exposure facilitated estrus detection (8AM and 2PM). As was usual farm practice, untreated control sows (CONT) were inseminated at least twice, based on WEI and ED. Treated sows (LUT) were administered $5 \mathrm{mg}$ Lutropin IM concomitant with detection of $\mathrm{SH}$. Sows in estrus in the morning or afternoon were inseminated once, 24 hours after Lutropin injection. Semen was $<3$ days old when used, and contained a minimum of $3^{*} 10^{9}$ live spermatozoa per $80-\mathrm{mL}$ dose. Effect of treatment on WEI, number of inseminations, total pigs born and litter weight were analyzed using a linear mixed effect model. Proportions of sows that were bred and farrowed were analyzed using a chi-squared test.

\section{Results}

Results are shown for sows having a WEI between 3 and 7 days. Lactation length ranged from 14 to 24 days. Quality for all inseminations was routinely excellent.

Table 1. Reproductive traits for sows bred according to a conventional insemination protocol (CONT) compared to sows administered Lutropin at onset of SH and inseminated once 24 hours later (LUT).

\begin{tabular}{|l|c|c|c|}
\hline Variable & \multicolumn{2}{|c|}{ Treatment } & P-Value \\
\hline & CONT & LUT & Treatment \\
\hline $\mathrm{n}$ & 129 & 109 & NA \\
\hline WEl (d) & $4.8 \pm 0.07$ & $4.7 \pm 0.07$ & NS \\
\hline Number of inseminations & $2.07 \pm 0.02$ & $1.00 \pm 0.02$ & $<0.001$ \\
\hline Total pigs born & $12.21 \pm 0.28$ & $12.23 \pm 0.30$ & NS \\
\hline Litter Weight $(\mathrm{kg})$ & $17.9 \pm 0.40$ & $18.2 \pm 0.42$ & NS \\
\hline Farrowing rate (\%) & 85.3 & 90.6 & NS \\
\hline
\end{tabular}

Figure 1. Total number of pigs born per Al dose for sows bred multiple times (CONT) or once (LUT). ab differ $P<0.001$

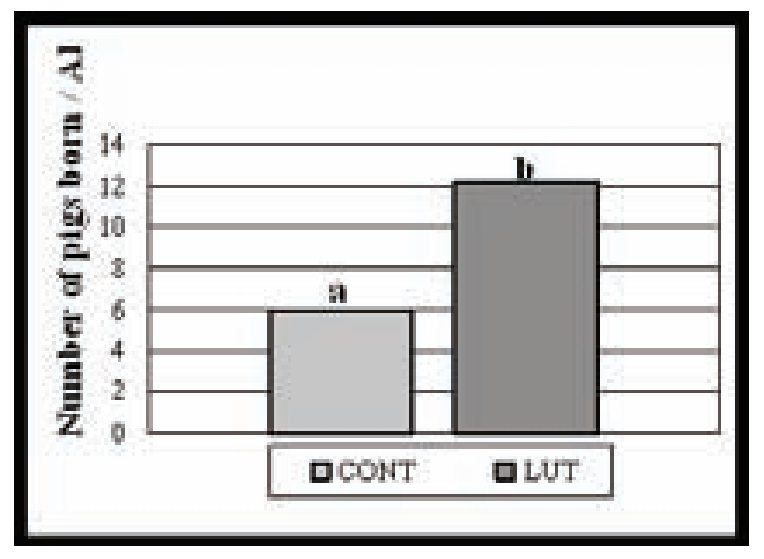

\section{Discussion}

The control of ovulation using Lutropin followed by a SingleFTAI is a breeding technology that can be easily applied at the farm level. Single-FTAI saves on labour and semen usage. It is a tool that increases the number of females bred per boar, allowing wider application of the best boar genetics within a breeding pyramid.

\footnotetext{
References

1 Cassar et al., J Swine Health Prod. 2005;13: 254-258.

2 Degenstein et al., Theriogenology. 2008;70:1075-1085.

3 Zak et al., J Swine Health Prod (accepted for publication).
} 


\title{
P.777
}

\section{Improving gilt utilization on commercial swine farms}

\author{
Jeremy S. Pittman ${ }^{1}$ Chris M. Holt ${ }^{1}$ Keith D. Allen ${ }^{1}$ Charles J. Francisco ${ }^{2}$ \\ 1. Murphy-Brown, LLC, Waverly, VA, USA; 2. Intervet/Schering-Plough Animal Health, Desoto, KS, USA
}

\section{Introduction}

Gilt management should be a major focus for commercial sow farms. Too often, gilts are neglected and their reproductive performance is poor, resulting in inefficiencies. P.G. $600^{\circ}$ is often incorrectly used to compensate for "poor gilt performance", compounding the poor performance and a vicious cycle ensues. This scenario results in lost confidence in gilts and P.G. $600^{\circ}$. Proper gilt management is even more essential with start-ups, depoprepops, breeding projects and parity segregated production. This paper describes implementation of gilt development and estrus induction with P.G. $600^{\circ}$ to improve gilt utilization and performance in a parity segregated commercial gilt herd.

\section{System overview}

The farm was a 1,000-gilt one-site farrow-to-finish commercial unit. The farm internally multiplied all replacement animals. It was essential that gilt breeding remain predictable, consistent and efficient. This was not the case the first year of breeding internal gilts, leading to poor gilt utilization, inefficient use of employee time and poor reproductive performance.

\section{Gilt development and selection}

All matings were with maternal semen, thus all progeny were potential replacements. Every 3 weeks, 180, 22 to 24 week old gilts were selected from finishing, sized into three equal groups, assigned to a "synch" week and moved to an acclimation barn. The heaviest group was the first to be induced, allowing additional grow time for smaller groups. Gilts remained on a development diet until breeding. A key component to the program was the controlled lack of boar exposure to insure that gilts are pre-pubertal prior to entering the next phase.

\section{Estrus induction and synchronization}

Each Wednesday, a new "synch" group was moved from acclimation into a "synchronization" barn. Pens of gilts (10-15) were taken to an Intense Boar Exposure area (IBE), based on the BEAR system. ${ }^{1}$ In each IBE, 2 boar crates were placed in the middle of the pens, allowing 360 degree gilt-boar exposure. Boar exposure was given from entry to Friday (3 days) for 15-20 minutes. Each gilt was observed for signs of heat during this period and recorded. On Friday, all gilts received a $5 \mathrm{ml}$ dose of P.G. $600^{\circ}$. It has been shown that 2-3 days of boar exposure may improve the percentage of gilts responding to P.G. $600^{\circ} .^{2}$ The group of gilts received casual boar exposure over the weekend. The following week, each pen of gilts would return daily to the IBE for 15-20 min. Daily heat scores were recorded for each gilt $(0=$ no response; 1 = slight interest; 2 = interest, swollen vulva, no locking stance; 3 = full locking heat). Gilts achieving a score of 2 or 3 were identified as Heat No Service (HNS) and remained in groups for 2 weeks before being moved to the breeding barn. Gilts scoring $<2$ were mixed and provided physical boar contact until in heat was identified or culled.

\section{Results}

Key performance parameters were compared between 52-weeks of the old program and the new "synch" program (Table 1).

Table 1. Comparison of of gilt performance between programs.

\begin{tabular}{|l|c|c|c|}
\hline Parameter & Old Program & "Synch" Program & Dif \\
\hline 1 st services/wk $^{\text {Services/wk }}$ & 49.1 & 55.8 & +6.7 \\
\hline Pct multi matings & 56.6 & 60.6 & +4.0 \\
\hline Pos preg check & 70.3 & 94.4 & $+24.3 \%$ \\
\hline Gilts farrowed/wk & 79.9 & 87.9 & $+8.0 \%$ \\
\hline Farrowing Rate & 41.6 & 48.8 & +7.2 \\
\hline Total pigs/litter & 73.1 & 80.9 & $+7.8 \%$ \\
\hline Born alive/litter & 11.9 & 12.5 & +0.6 \\
\hline Pigs weaned/wk & 10.7 & 11.0 & +0.3 \\
\hline Pigs wnd/female/yr ${ }^{\text {a }}$ & 346.0 & 398.8 & +52.8 \\
\hline Pigs wnd/gilt entered & 10.9 & 12.5 & +1.6 \\
\hline Gilts entered/wk & 4.8 & 6.1 & +1.3 \\
\hline Gilts transferred/wk & 71.5 & 65.9 & -5.6 \\
\hline Gilt retention ${ }^{\text {b }}$ & 38.8 & 45.9 & +7.1 \\
\hline anote females are removed from farm after first farrowing. & & $+9.0 \%$ \\
\hline b Gilt retention = (Gilts farrowed/Gilts entered)*100 & & \\
\hline
\end{tabular}

\section{Discussion}

Under the "Synchronization" system, gilt flow, pig flow and downstream P1 supply was more predictable, consistent and efficient than the previous system. The reproductive improvements seen were most likely due to several factors associated with the P.G. $600^{\circ}$ induction program. Estrus was induced and synchronized in large numbers of gilts, allowing for more predictable time and number of matings per week. Heat detection and timing of insemination was more accurate with induced HNS. Under this program P.G. $600^{\circ}$ was being utilized consistent with the label, in gilts with a known reproductive state and not being misused or wasted.

\section{Acknowledgements}

The authors would like to thank the farm employees for their dedication to the program.

\section{References}

1. Beltanena E., et al. 2005. Alberta Pork Industry News.

2. Breen S.M., et al. J Anim Sci 2005. 83:460-465. 


\title{
P.778
}

\section{Use of a new GnRH to control reproduction in gilts}

\author{
Silke Engl ${ }^{1}$ Ralf Bischoff ${ }^{2}$ Wolfgang Zaremba ${ }^{1}$ \\ 1. Veyx-Pharma GmbH, Schwarzenborn, Germany; 2. Veterinary Practice for Farm Animals, Lammersbrink, Germany
}

\section{Introduction}

In pig production units controlling reproduction is widely used to optimize farm work flow and fertility performance of animals. This leads to better hygiene, supervision, health and output capacity. A new GnRH has been discovered in the sea lamprey (I-GnRH-III, INN: Peforelin) with proven selectivity for FSH release from male pigs' hypophysis (1). Peforelin was registered last year in Europe and in some other countries for the indications oestrus induction in gilts after cycle blockade and in sows after weaning. This paper reports about some experiences with Peforelin in mature gilts following a cycle blockade.

\section{Materials and Methods}

This surveillance was conducted from August 2008 to May 2009 in a 220 sows unit in Germany. In total 110 sexually mature, healthy gilts (Danish landrace $x$ Yorkshire) were included. Each gilt received $20 \mathrm{mg}$ of Altrenogest per day orally for a period of $18 \mathrm{~d}$ and was then randomly allocated to either Group I $(\mathrm{n}=52)$ : $48 \mathrm{~h}$ after last treatment $150 \mu \mathrm{g}$ Peforelin (=2 ml Maprelin $\left.{ }^{\circ}\right)$ i.m. or Group II $(n=58)$ : no further hormonal treatment. Oestrus detection was performed starting $\mathrm{d} 3$ after cycle blockade twice daily in the presence of a mature boar. Gilts were artificially inseminated up to 3 times in intervals of $12 \mathrm{~h}$. Sonographic pregnancy detection was performed $4 \mathrm{wk}$ after insemination. Oestrus rate $\left(\mathrm{OR}_{8 \mathrm{~d}}=\right.$ on heat by $\left.\mathrm{d} 8\right)$, last Altrenogest-to-oestrusinterval $\left(\mathrm{AO}_{8 \mathrm{~d}}=\right.$ on heat by $\mathrm{d} 8$ and $\mathrm{AO}_{\mathrm{all}}=$ all gilts $)$, oestrus duration (OD), pregnancy rate ( $P R=$ percentage of mated sows that have been sonographically detected pregnant), farrowing rate $(F R=$ percentage of inseminated gilts that farrowed), total born piglets per litter (TBP) and piglets born alive per litter (PBA) were recorded.

\section{Results}

OR was higher and AO was lower in group I than in group II ( $p$ $\leq 0.05$; see table) and the distribution of first inseminations was more concentrated in group I (see figure). Taking into account only those gilts displaying oestrus within $8 \mathrm{~d}$ after cycle blockade the $A_{8 \mathrm{~d}}$ was shortened by $0.45 \mathrm{~d}$. $\mathrm{AO}_{\text {all }}$ was shortened by $1.88 d(p \leq 0.05)$. There were no significant differences in PR between the groups; however, there was a tendency for higher PR in group I. From all sows inseminated $\left(\mathrm{AO}_{\text {all }}\right), 9$ gilts of each group returned to oestrus, 3 had abortions in group I and 5 in group II (PCV and PRRS were diagnosed). From the gilts on heat by $\mathrm{d} 8,38$ litters were born in group I and 32 in group II. The FR did not differ between the two groups and was at a low level. The litter size (TBP and PBA) was similar in both groups ( $p>$ 0.05).

\section{Distribution of first inseminations}

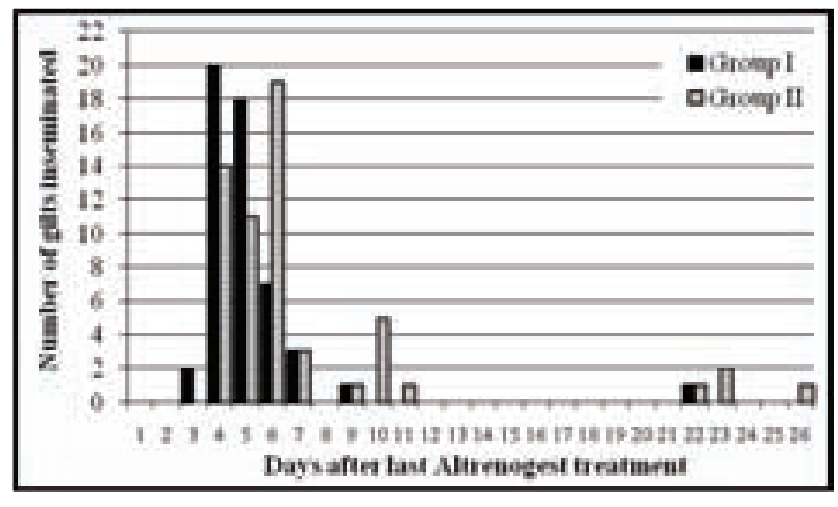

\begin{tabular}{|c|c|c|}
\hline & Group I & Group II \\
\hline $0 \mathrm{R}_{8 \mathrm{~d}}{ }^{*}$ & $96.2 \%^{\mathrm{a}}$ & $81.0 \%^{b}$ \\
\hline $\mathrm{AO}_{8 \mathrm{~d}}(\text { mean } \pm \mathrm{SD})^{*}$ & $4.78 \pm 0.95^{b}$ & $5.23 \pm 0.96^{a}$ \\
\hline $\mathrm{AO}_{\mathrm{all}}($ mean $\pm \mathrm{SD})$ & $5.19 \pm 2.62^{b}$ & $7.07 \pm 4.87^{\mathrm{a}}$ \\
\hline$O D(\text { mean } \pm S D)^{*}$ & $2.30 \pm 0.61$ & $2.30 \pm 0.53$ \\
\hline $\mathrm{PR}^{*}$ & $88.0 \%$ & $83.0 \%$ \\
\hline $\mathrm{FR}^{*}$ & $76.0 \%$ & $68.1 \%$ \\
\hline $\operatorname{TBP}(\text { mean } \pm S D)^{*}$ & $14.11 \pm 3.95$ & $13.81 \pm 2.35$ \\
\hline $\mathrm{PBA}(\text { mean } \pm \mathrm{SD})^{*}$ & $13.03 \pm 3.61$ & $12.22 \pm 3.55$ \\
\hline \multicolumn{3}{|l|}{${ }^{*}$ gilts on heat by $d 8$} \\
\hline
\end{tabular}

\section{Discussion}

In comparison to untreated animals after the inhibition of cycle the Maprelin -treatment yielded a significantly higher oestrus rate, a significant reduction of last Altrenogest-treatment-tooestrus-interval and thus an induction of oestrus with a clear concentration of insemination times. These results confirm previous results $(2,3)$. In the following period a suitable vaccination programme was established that yielded in a considerably decreased number of returns and abortions.

\section{References}

1. Kauffold J et al. 2005. Reprod Domest Anim 40(5):475-9.

2. Hühn U, Rothe K 1992. Mh Veterinaermed 47:615-21.

3. Engl S et al. 2004. Proc IPVS Congress 2:466. 


\title{
P.779
}

\section{Practical experience with Peforelin in a large sow herd: data from primiparous sows}

\author{
Silke Engl ${ }^{1}$ Heiko Zepperitz ${ }^{2}$ Reinhold Rath ${ }^{3}$ Wolfgang Zaremba ${ }^{1}$ \\ 1. Veyx-Pharma GmbH, Schwarzenborn, Germany; 2. Veterinary Practice Zepperitz, Neubukow, Germany; \\ 3. Tierzucht Gut Losten GmbH \& Co. KG, Bad Kleinen, Germany
}

\section{Introduction}

Primiparous sows (first litter sows) represent an age group of animals with special needs. Particularly in the summer time they may show a depression in fertility performance. Thus, especially in large sow herds, this group is often treated with pharmaceuticals to control reproduction. For oestrus induction a new $\mathrm{GnRH}$ is available. The substance originates from the sea lamprey (I-GnRH-III, INN: Peforelin) and has proven selectivity for FSH release from male pigs' hypophysis (1). Peforelin is registered in Europe and in some other countries for inducing oestrus in gilts after a cycle blockade and in sows after weaning off the piglets. This paper reports about practical experiences from primiparous sows and shows an example of a large farm.

\section{Materials and Methods}

The herd consists of sows of Danish genetics and is managed in a weekly-batch-farrowing system with 3 wk of lactation (weaning: Thursday, $10.30 \mathrm{~h}$ ). The farm managers used to apply equine chorion gonadotropin (eCG) for inducing oestrus in the weaned sows and started using the new Peforelin for this indication in January 2009. Thus, here a comparison of two production periods is presented. The data of a total of 3498 primiparous sows are included.

Group I: From January to July 2008 the sows received $24 \mathrm{~h}$ after weaning 1000 IU eCG (Pregmagon ${ }^{\circ}$ ) i.m. $(n=2062)$.

Group II: From January to July 2009 the sows received $24 \mathrm{~h}$ after weaning $37.5 \mu \mathrm{g}$ Peforelin (=0.5 ml Maprelin ${ }^{\circ}$ i.m. $(n=1436)$.

The following parameters were recorded: Oestrus rate $(\mathrm{OR}=$ proportion of sows with standing reflex on Tuesday or Wednesday after weaning), farrowing rate ( $F R=$ percentage of inseminated sows that farrowed), total born piglets per litter (TBP), piglets born alive per litter (PBA) and piglet index $\left(\mathrm{PI}_{\mathrm{TBP}}=\mathrm{TBP} / 100\right.$ inseminated sows; $\mathrm{PI}_{\mathrm{PBA}}=\mathrm{PBA} / 100$ inseminated sows). The results were compared between the groups and in addition between 2 seasons ( $A$ = insemination January to mid May, B = insemination mid May to July).

\section{Results}

In group II OR was significantly higher than in group I. The farrowing parameters including litter sizes were similar in both groups.

\begin{tabular}{|l|c|c|}
\hline & Group I & Group II \\
\hline Treatment & eCG & Peforelin \\
\hline Sows mated & 2062 & 1436 \\
\hline OR & $80.5 \%^{\mathrm{b}}$ & $87.0 \%^{\mathrm{a}}$ \\
\hline FR & $88.3 \%$ & $89.6 \%$ \\
\hline TBP (mean) & 13.79 & 14.62 \\
\hline PBA (mean) & 12.89 & 13.43 \\
\hline PI & 1218 & 1310 \\
\hline TBP & 1138 & 1203 \\
\hline a, b: Means between groups differ significantly $(p<0.05)$ & \\
\hline
\end{tabular}

In the comparison between seasons both groups had better results for OR in the cold compared to the warmer, while the farrowing parameters including litter sizes did not differ between seasons. There was a tendency for better results for group I in the cold season and for group II in the warmer season.

\begin{tabular}{|l|c|c|c|c|}
\hline & \multicolumn{2}{|c|}{ Group I } & \multicolumn{2}{c|}{ Group II } \\
\hline Treatment & eCG & & Peforelin & \\
\hline Season & $\mathrm{A}$ & $\mathrm{B}$ & $\mathrm{A}$ & $\mathrm{B}$ \\
\hline Sows mated & 1291 & 771 & 901 & 535 \\
\hline OR & $85.8 \%^{\mathrm{b}}$ & $71.6 \%^{\mathrm{c}}$ & $89.9 \%^{\mathrm{a}}$ & $82.2 \% \mathrm{~b}$ \\
\hline FR & $88.5 \%$ & $88.1 \%$ & $88.8 \%$ & $91.0 \%$ \\
\hline TBP (mean) & 13.85 & 13.68 & 14.47 & 14.86 \\
\hline PBA (mean) & 12.95 & 12.78 & 13.27 & 13.70 \\
\hline $\mathrm{PI}_{\text {TBP }}$ & 1225 & 1205 & 1284 & 1353 \\
\hline $\mathrm{PI}_{\mathrm{PBA}}$ & 1146 & 1125 & 1178 & 1247 \\
\hline $\mathrm{a}, \mathrm{b}, \mathrm{c}:$ Means between groups differ significantly $(\mathrm{p}<0.05)$ \\
\hline
\end{tabular}

\section{Discussion}

The results show at least equal efficacy of Peforelin in comparison to eCG and are in line with previous results yielded in Peforelin treated animals (2). In addition, Peforelin seems to be highly potent to overcome a possible depression of fertility performance in the warmer season.

Of course, the comparison of two years is limited for a scientific evaluation, because, e. g., climate and feeding conditions may vary between years. But it is a comparison of practice and should be allowed, because a producing company will always make comparisons between two years of production in order to assess its progress.

\section{References}

1. Kauffold J et al. 2005. Reprod Domest Anim 40(5):475-9.

2. Engl S et al. 2004. Proc IPVS Congress 2:466. 


\title{
P.780
}

\section{Practical experience with Peforelin in a large sow herd: data from pluriparous sows}

\author{
Silke Engl ${ }^{1}$ Heiko Zepperitz ${ }^{2}$ Reinhold Rath ${ }^{3}$ Wolfgang Zaremba ${ }^{1}$ \\ 1. Veyx-Pharma GmbH, Schwarzenborn, Germany; 2. Veterinary Practice Zepperitz, Neubukow, Germany; \\ 3. Tierzucht Gut Losten GmbH \& Co. KG, Bad Kleinen, Germany
}

\section{Introduction}

Controlling reproduction is crucial for economic success especially in large sow herds. Application of management tools like optimized feeding, batch farrowing, light regimes etc. alone are not in every case sufficient to meet the high performance expectations of farmers. Thus, pharmaceuticals to control reproduction are widely used to increase fertility performance of animals and to optimize economics. Today, a new $\mathrm{GnRH}$ is available for inducing oestrus in gilts after a cycle blockade and in sows after weaning off the piglets. The substance was discovered in the sea lamprey (I-GnRH-III, INN: Peforelin) and has proven selectivity for FSH release from male pigs' hypophysis (1). Peforelin is registered in Europe and in some other countries. This paper reports about practical experiences from pluriparous sows on a large farm.

\section{Materials and Methods}

The herd consists of sows of Danish genetics and is managed in a weekly-batch-farrowing system with 3 wk of lactation (weaning: Thursday, $10.30 \mathrm{~h}$ ). The farm managers used to apply equine chorion gonadotropin (eCG) for inducing oestrus in the weaned sows and started using the new Peforelin for this indication in January 2009. Thus, here a comparison of two production periods is presented. The data of a total of 11852 pluriparous sows (more than 1 litter born) are included.

Group I: From January to July 2008 the sows received $24 \mathrm{~h}$ after weaning $800 \mathrm{IU}$ eCG (Pregmagon $\left.{ }^{\circ}\right)$ i.m. $(n=6430)$.

Group II: From January to July 2009 the sows received $24 \mathrm{~h}$ after weaning $150 \mu \mathrm{g}$ Peforelin (= $2 \mathrm{ml}$ Maprelin $\left.{ }^{\circ}\right)$ i.m. $(\mathrm{n}=5422)$.

Sonographic pregnancy detection was performed $4 \mathrm{wk}$ after insemination. The following parameters were recorded: Number of sows showing standing reflex on Tuesday or Wednesday after weaning (SR), Oestrus rate (OR = percentage of sows showing standing reflex on Tuesday or Wednesday after weaning), Pregnancy rate ( $P R=$ percentage of inseminated sows that have been sonographically detected pregnant), number of litters born, farrowing rate ( $F R=$ percentage of inseminated sows that farrowed), total born piglets per litter (TBP), piglets born alive per litter $(\mathrm{PBA})$ and piglet index $\left(\mathrm{PI}_{\mathrm{TBP}}=\mathrm{TBP} / 100\right.$ inseminated sows; $\mathrm{PI}_{\mathrm{PBA}}=\mathrm{PBA} / 100$ inseminated sows).

\section{Results}

OR and PR were significantly higher in group II than in group I. The farrowing parameters including litter sizes were similar in both groups.

\begin{tabular}{|l|c|c|}
\hline & Group I & Group II \\
\hline Treatment & eCG & Peforelin \\
\hline Sows mated & 6430 & 5422 \\
\hline SR & 5837 & 4983 \\
\hline OR & $90.8 \%^{\mathrm{b}}$ & $91.9 \%^{\mathrm{a}}$ \\
\hline Sows pregnant & 5999 & 5147 \\
\hline PR & $93.3 \%^{\mathrm{b}}$ & $94.9 \%^{\mathrm{a}}$ \\
\hline Litters born & 5768 & 4883 \\
\hline FR & $89.7 \%$ & $90.1 \%$ \\
\hline TBP (mean) & 14.44 & 14.89 \\
\hline PBA (mean) & 12.94 & 13.17 \\
\hline PI & 1295 & 1341 \\
\hline PBP & 1161 & 1186 \\
\hline a, b. Means between groups differ significantly $(\mathrm{p}<0.05)$ \\
\hline
\end{tabular}

\section{Discussion}

The results show at least equal efficacy of Peforelin in comparison to eCG and are in line with previous results yielded in Peforelin treated animals (2).

Of course, the comparison of two years is limited for a scientific evaluation, because, e. g., climate and feeding conditions may vary between years. But it is a comparison of practice and should be allowed, because a producing company will always make comparisons between two years of production in order to assess its progress.

\section{References}

1. Kauffold J et al. 2005. Reprod Domest Anim 40(5):475-9.

2. Engl S et al. 2004. Proc IPVS Congress 2:466. 


\title{
Al monitoring to improve the fertility of sows
}

\author{
Steffen Hoy ${ }^{1}$ Stefan Viebahn² \\ 1. SVIFT Consultancy, Marienheide, Germany; 2. Justus-Liebig-Universität Gießen, Gießen, Germany
}

\section{Introduction}

At the moment the discussion strikes large waves around the fertility rate of different breeds. But, the goal must be to use the achievement potential of each existing genetic optimally. So, a method (Al monitoring) was developed to examine and improve the success of the artificial insemination.

Material and methodsThe Al monitoring represents a control of the insemination times by the evaluation of the rate of farrowing sows (based on the number of inseminated sows), litter size total and alive born piglets and piglet index in sows inseminated at certain times (HOY 2007). The precondition for a meaningful application of the Al monitoring is an accurate execution and documentation of the result of heat detection (standing: yes or no). The heat controls should begin at the third day after weaning. When weaning is accomplished on Thursday morning the heat detection has to begin on Sunday afternoon or evening! The determination of the duration of heat is the crucial condition to estimate (afterwards) whether the insemination took place in the biologically optimal period ( $16 \mathrm{~h}$ before to $4 \mathrm{~h}$ after the ovulation). Al monitoring was tested in 8 farms with approximately 3000 inseminations. It is to be recognized that actually in each farm specific conditions are prevailed. All data provided by the farms were registered into an Excel table and statistically analysed. The Al data were divided in categories: too early, optimally, too late. Beyond that we computed three goal sizes:

- percentage of sows with farrowing (based on all sows inseminated),

- litter size total and alive born piglets and

- piglet index for the sows with different time of AI (too early, optimally, too late - at least related to the first Al).

\section{Results}

Exemplarily the results of a farm are presented, which provides a very good documentation. A total of 829 inseminations of sows and 195 of gilts were evaluated. It could be shown that sows inseminated within the optimal time window (for $\mathrm{Al} 1$ ) yielded the best fertility - both related to the farrowing rate $(89.4 \%)$ and to the litter size (113) thus leading to the highest piglet index (1006). A comparatively small difference of approximately 3 percent in the farrowing rate led together with a lower litter size (around 0.83 piglets) to a difference of more than 100 alive born piglets per 100 inseminated sows (comparison: too early and at the optimal time inseminated sows). At this farm despite altogether very good achievements in the analysis period almost a fifth of the sows was nevertheless inseminated too early - with appropriate effects on the fertility rate. The frequency of Al which was too late was low, led however also to clearly worse achievements (farrowing rate $=66.7 \%$, piglet index $=760)(p<$ 0.05).

A further evaluation was concentrated on the number of inseminations because about $12 \%$ of the sows $(n=97)$ was inseminated three times. Sows, which were inseminated twice, obtained the best achievement. With three inseminations the farrowing rate, litter size and therefore also the piglet index were lower than with two Al. However, this must not be the case in each farm.

The first evaluations at $195 \mathrm{Al}$ in gilts showed, that gilts inseminated three times had clearly worse performance data (farrowing rate $=70.9 \%$, piglet index $=749$ ) than gilts inseminated twice (farrowing rate $=76.5 \%$, piglet index $=832$ ). Here, further investigations must be done to analyse the cause.

\section{Discussion}

Clear differences between the farms occurred in the insemination management (times, quality and documentation of heat detection; times and number of Al). The heat control (standing reflex) (including also the registration of a possible standing after the last insemination as the precondition for the calculation of the duration of heat) is (more or less) in need of improvement at the farms but at the same time a compelling requirement for the Al monitoring. The determination of farrowing rate, litter size and piglet index related to the onset and duration of heat and to the insemination date (in particular Al 1) allows clear statements for the optimization of the Al.

\section{Reference}

HOY, ST. (2007): Monitoring insemination success. Pig International 37

(2007) 6, S. 10-11 


\title{
P.782
}

\section{Fertility performance of estrus-blocked and not-estrus-blocked sows after 3 weeks length of lactation}

\author{
Stefan Viebahn ${ }^{1}$ Steffen Hoy ${ }^{2}$ \\ 1. SVIFT Consultancy, Marienheide, Germany; 2. Justus-Liebig-Universität Gießen, Gießen, Germany
}

\begin{abstract}
Introduction
The effect of a progestagen (Regumate ${ }^{\circ}$ ) induced prolongation of the lactation anoestrus to reproductive parameters in the subsequent parity was evaluated. The objective of the monitoring was to find out whether Regumate is indicated for the synchronisation of sow groups in a protocol to change the production rhythm and to analyse the effect of the blockade on the fertility performance in the subsequent parity considering the lengths of lactation.
\end{abstract}

\section{Material and methods}

In a 800 sow farm the change from a 1-week-group-management-system to a 2-week-group-management-system was monitored. The total of 20 groups was reduced to a total of 10 groups. This was realized by blocking the first oestrus after weaning in every second group with a progestagen (Regumate - Altrenogest) given up to one day before weaning the consecutive group. The application started $24 \mathrm{~h}$ before weaning. The dosage was $20 \mathrm{mg}$ Altrenogest per day (= $5 \mathrm{ml}$ Regumate $/$ day) applicated 8 times. All sows of the new established group were artificially inseminated in their first standing heat. Data records of 9 groups including 517 sows in the final 2-week-system were analysed. As a benchmark fertility records out of an insemination monitoring programme conducted over 2 previous years in that farm including 659 sows with lengths of lactation between 19 and 23 days were used. Statistical analyses were obtained by using the SPSS 15.0 statistical programme. The differences between the groups concerning litter size were tested by the multiple t-test. Differences in farrowing rates were tested by the Pearson's chi-square test. The analysis of variance (ANOVA) was used to test the parameter litter size total born piglets under consideration of Regumate treatment (yes/no), parity and length of lactation as independent factors to evaluate also interfering effects.

\section{Results}

Regumate ${ }^{\bullet}$ treated animals reached a significantly higher farrowing rate $(+8.5 \%, p<0.05)$ and an increased litter size $(+0.65$ total born piglets, $\mathrm{p}<0.052$ ) compared with non-treated sows. The difference in piglet index was +157 in favour of the treated sows. The litter size of progestagen treated primiparous sows was significantly higher in comparison to non-treated primiparous sows ( +2.02 total born piglets, $p<0.07$ ). The farrowing rate of the treated primiparous sows was increased by $12.8 \%$ in contrast to the untreated primiparous sows $(p>0.05)$. Referring to the piglet index with Regumate treated primiparous sows generated 300 total born piglets more per 100 first inseminations in contrast to the not synchronized animals. In the group with very short length of lactation ( $<21$ days) treated sows realized a $12.8 \%$ higher farrowing rate compared to the not treated sows. Treated sows with more than 21 days length of lactation performed $3.7 \%$ more in the farrowing rate in relation to the not treated animals $(p>0.05)$. Pharmacologically synchronized sows generated 193 total born piglets more per 100 inseminations after a lactation length of 13-20 days than not-estrus-blocked sows. No differences in estrus behaviour or weaning-service interval between the treated and untreated group were found.

\section{Discussion}

he conclusion is that Regumate is indicated for the cycle synchronisation of sows in a protocol in order to change the production rhythm. The prolongation of the lactational anoestrus by applicating Regumate ${ }^{\circledR}$ can also be recommended to ensure fertility performance after a previous very short length of lactation especially in primiparous sows. This study confirms that a prolongation of the lactation anoestrus can be induced by starting the Altrenogest application one day before weaning. This result is in accordance with the results of other authors. It has to be respected that Regumate is not registered for that indication. Local or national guidelines must be considered by veterinarians and by farmers.

\section{References}

WALLENHORST CHR. K. (1998): Feldversuche zur hormonellen Zyklussteuerung und methodische Arbeiten zum Embryotransfer beim Schwein. University of. Göttingen/Germany ,Thesis.

PATTERSON J. et al (2008): Responses to delayed estrus after weaning in sows using oral progestagen treatment. J. Anim Sci., http:/jas.fass. org. doi: 10.2527/jas.2007-0440. 


\title{
P.783
}

\section{A descriptive questionnaire on weaning management in commercial pig herds in Belgium}

\author{
Ellen de Jong ${ }^{1}$ Jeroen Dewulf ${ }^{1}$ Maria Laanen ${ }^{1}$ Jan Jourquin$^{2}$ Dominiek Maes ${ }^{1}$ \\ 1. Ghent University, Faculty of Veterinary Medicine, Department of Reproduction, Obstetrics and \\ Herd Health, Merelbeke, Belgium; 2. Janssen Animal Health, Beerse, Belgium
}

\section{Introduction and objectives}

Suboptimal reproduction results in modern pig herds are not only disease related. Many different non-infectious factors such as length of lactation, body condition, season, and weaning management practices have an influence on the fertility of sows (1). The purpose of this epidemiological study was to investigate how weaning management influences different reproduction parameters in commercial pig herds in Belgium.

\section{Materials and Methods}

In August 2009, a questionnaire was sent by conventional mail to 150 randomly selected pig herds in Flanders, Belgium, with exclusion of farms smaller than 80 sows. After one and a half months, all farms were contacted by phone and asked whether they were willing to cooperate. All cooperating farms were then visited individually by the same interviewer, starting in October 2009. This paper shows the data gathered until January 2010. The questionnaire contained a variety of questions, related to gilt management, batch management system, weaning practices, heat stimulation and detection, insemination, farrowing, housing, climate, feeding, hygiene, vaccinations and fertility parameters. Each visit lasted approximately one hour.

\section{Results}

Table 1 shows the descriptive results of important parameters of 30 herds located in three provinces in Flanders. These provinces represent $94 \%$ of the Belgian pig production. Of the 30 herds, $37 \%$ were farrow-to-finish farms. An equal number of farms purchased gilts or selected their own gilts. Farm size distribution was as follows: $10 \%$ less than 150 sows, $63 \%$ between 150 and 300 sows and $27 \%$ more than 300 sows. The majority (57\%) of the farms used a batch management system, mainly 3 and 4 week systems.

Table 2 shows the mean, standard deviation and range (minimum, maximum) of the major fertility parameters.

\section{Discussion and conclusion}

As these are preliminary results on a limited number of farms, correlations are not yet clearly visible. By mid 2010, the study will be finalized and the final results and a risk factor analysis will be presented.
Table 1: Descriptive results of 30 herds

\begin{tabular}{|c|c|c|}
\hline \multicolumn{2}{|c|}{ Parameters } & \multirow{2}{*}{$\begin{array}{c}\% \\
7\end{array}$} \\
\hline Weaning & $<21$ days & \\
\hline & 21-24 days & 43 \\
\hline & $25-28$ days & 50 \\
\hline \multirow[t]{4}{*}{ Number of teaser boars per 20 sows } & $<1$ & 23 \\
\hline & 1 & 40 \\
\hline & 2 & 23 \\
\hline & $>2$ & 13 \\
\hline \multirow[t]{3}{*}{ Heat stimulation starting from } & the day of weaning & 23 \\
\hline & day 1 after weaning & 50 \\
\hline & $>$ day 1 after weaning & 27 \\
\hline \multirow[t]{3}{*}{ Heat stimulation } & $<2 x /$ day & 43 \\
\hline & 2x/day & 53 \\
\hline & $>2 x /$ day & 3 \\
\hline \multirow[t]{3}{*}{ First heat detection } & $<$ day 4 after weaning & 13 \\
\hline & day 4 after weaning & 70 \\
\hline & $>$ day 4 after weaning & 17 \\
\hline \multirow[t]{2}{*}{ Heat detection } & $<2 x /$ day & 17 \\
\hline & 2x/day & 83 \\
\hline $\begin{array}{l}\text { Light at sow-height in insemination unit } \\
\qquad>150 \text { lux }\end{array}$ & & 50 \\
\hline \multirow[t]{3}{*}{ Feed weaned sows } & pregnancy feed & 57 \\
\hline & lactation feed & 33 \\
\hline & other & 10 \\
\hline
\end{tabular}

Table 2: Major reproduction parameters of 30 herds

\begin{tabular}{|l|c|c|c|c|}
\hline & Mean & $\begin{array}{c}\text { Standard } \\
\text { deviation }\end{array}$ & Minimum & Maximum \\
\hline Weaned piglets/sow/year & 25.82 & 2.78 & 21.32 & 31.44 \\
\hline Weaning-to-oestrus interval & 6.18 & 1.19 & 4.64 & 9.27 \\
\hline Litters/100 inseminations & 82.6 & 4.39 & 75.1 & 90.8 \\
\hline Pregnancy rate after 1st insemination & 91.78 & 5.95 & 71.9 & 97.6 \\
\hline
\end{tabular}

\section{Acknowledgements}

Janssen Animal Health is acknowledged for financial support of this study.

\section{References}

(1) Koketsu and Dial, 1997, Theriogenology 47, 1445-1461 


\title{
P.784
}

\section{Comparison of the effect of $\mathrm{PG} 600^{\circledR}$ and other gonadotropins administered at weaning}

\author{
Carlos Cantin ${ }^{2}$ Rut Menjon ${ }^{1}$ Jesus M. Bollo ${ }^{1}$ Jesus V. Lopez ${ }^{1}$ Marta Jimenez ${ }^{1}$ \\ 1. Intervet Schering Plough Animal Health, Alcobendas (Madrid), Spain; 2. Veterinay Practitioner, Zaragoza, Spain
}

\section{Introduction}

Weaning to estrus interval is a major measure of reproductive efficiency in a breeding herd. Failure to return, or delay in returning to estrus, after weaning increases the number of non-productive days, resulting in economic losses. Many studies have shown that the use of PG600 ${ }^{\circledR}$ (400 IU of PMSG and $200 \mathrm{IU}$ of hCG) at weaning increases the number of sows returning to fertile estrus and shortens the estrus to weaning interval (1).

In Spain, there are other commercial products with the same composition and indication as $\mathrm{PG} 600^{\circledR}$. Previous data have shown that products with the same composition do not always perform as well as each other (2). The aim of this study was to compare the efficacy of $\mathrm{PG} 600^{\circledR}$ in terms of the rate of return to estrus with two other commercial products with the same composition and indication.

\section{Materials and Methods}

The trial was undertaken in July, August, September and October in two breeding herds of 750 and 3500 sows, in the northeast of Spain. Due to the high summer temperatures in this area, these farms had a history of extended weaning to estrus intervals during these months. In an attempt to address this, all sows were routinely treated at weaning with $\mathrm{PG} 600^{\circledR}$ during the summer months.

A total of 1910 multiparous sows were included in the trial over the period. Every week, sows were divided into three study groups, equalized for parity, in total numbers as follows:

- PG600 Group: 856 sows treated with PG600.

- Group A: 728 sows treated with product A (400 IU of PMSG and $200 \mathrm{IU}$ of hCG)

- Group B: 326 sows treated with product B (400 IU of PMSG and $200 \mathrm{IU}$ of hCG)

All products were administered on the day of weaning.

The number of sows in estrus in the 10 days following treatment was recorded, as well as the number of induced estrus which proved fertile and the number of piglets born at the farrowing following the induced estrus.

The data obtained were subjected to statistical analysis using the Fisher's exact test.

\section{Results}

Sows treated with $\mathrm{PG} 600^{\circledR}$ at weaning came into estrus in statistically significantly greater numbers than the sows treated with either of the other two commercial gonadotropins $(p<0.001)$ (Table 1).

Table 1. Reproductive data after treatments

\begin{tabular}{|l|c|c|c|}
\hline & PG600 $^{\circ}$ & Product A & Product B \\
\hline No sows & 856 & 728 & 326 \\
\hline № sows in estrus & 788 & 589 & 273 \\
\hline \% estrus & $92.05^{\mathrm{a}}$ & $80.90^{\mathrm{b}}$ & $83.74^{\mathrm{b}}$ \\
\hline № sows pregnant & 662 & 466 & 221 \\
\hline$\%$ fertility & $84.01^{\mathrm{a}}$ & $79.11^{\mathrm{b}}$ & $80.95^{\mathrm{a}}$ \\
\hline Total Born & 11.02 & 10.76 & 10.84 \\
\hline $\begin{array}{l}\text { a, b: values with different superscripts in the same row represent statistically significant } \\
\text { differences ( }<<0.001)\end{array}$ & \multicolumn{4}{l}{} \\
\hline
\end{tabular}

Sows treated with $\mathrm{PG} 600^{\oplus}$ also exhibited significantly better fertility rates than those treated with product $A(p<0.001)$, and better than those treated with product $B$, though the latter difference was not statistically significant $(p=0.26)$.

\section{Discussion}

The results obtained in this trial are evidence that $\mathrm{PG} 600^{\circledR}$ administered at weaning is the most effective treatment to induce estrus in multiparous sows in herds with increased weaning to estrus intervals due to high temperatures. Estrus induced by $\mathrm{PG} 600^{\circledR}$ are also more fertile than those induced by the two other tested products with the same composition, with consequent improvements in the economic performance of the herd. $\mathrm{PG} 600^{\circledR}$ is thus the best choice when induction of estrus is required in these circumstances.

\section{References}

1. Kirkwood, RN. et al. 1998. Swine Health and Production. V.6.

2. Ruiz, S et al 1990. Proceedings 5as Jornadas Internacionales de Reproducción Animal e Inseminación Artificial. 


\title{
P.785
}

\section{Improving sow fertility to insemination with aged semen}

\author{
Am-in Nutthee ${ }^{2}$ Wichai Tantasuparuk ${ }^{1}$ David Sprecher ${ }^{1}$ Roy Kirkwood $^{1}$ \\ 1. Michigan State University, East Lansing, MI, USA; \\ 2. Department of Obstetrics, Gynaecology and Reproduction, Faculty of Veterinary Science, Chulalongkorn University, Bangkok, Thailand
}

\section{Introduction}

Semen quality deteriorates during storage which can result in poorer sow fertility to artificial insemination (Al). To counter this, the usual recommendation is that semen should not be used beyond 48-72 hours from collection. This has a negative economic effect since it may result in the discarding of semen doses. When using aged semen, fertility affects can be reduced by inseminating am/pm, which increases costs from semen and labor but also implies that the semen doses do have viable sperm but presumably fewer of them. We hypothesized that the application of technologies that allow for Al of fewer sperm, such as intrauterine insemination (1), will permit the maintenance of fertility with once daily $\mathrm{Al}$ of aged sperm. The aim of this present study was to test this hypothesis.

\section{Material and methods}

In 3 replicates, sows were induced into estrus by PG600 to remove confounding effects of WEl and sows estrous on days 4 or 5 after weaning were assigned by parity to cervical Al on consecutive days with 1 and 2 or 2 and 3-d old semen (Control, $n=29)$, or cervical Al with 4 and 5 or 5 and 6 - $d$ old semen (group1; $\mathrm{n}=30$ ) or intrauterine (Deep Golden Pig; IMV) Al with 4 and 5 or 5 and 6 -d old semen (group2; $n=29$ ). Semen was extended in BTS and semen quality was assessed for days 2 to 6 after collection. Sow performance was compared on the basis of pregnancy rate and subsequent litter size (at writing only 2 replicates farrowed) using Fisher's exact test and ANOVA in SAS 9.1.

\section{Results}

On days $2,3,4,5$, and 6 , percent sperm motility was $83,73,63,63$ and $60 \%$; percent live sperm was $94,83,77,74$, and $72 \%$ and percent normal sperm was $93,93,84,84$, and $83 \%$, respectively. Compared to the Control group, pregnancy rate of sows receiving a cervical $\mathrm{Al}$ of aged semen was lower $(\mathrm{P}=0.05)$. However, sows receiving an intrauterine $\mathrm{Al}$ with aged semen maintained their pregnancy rate (Fig. 1). Based on limited farrowings, litter size was lower $(P=0.06)$ for sows receiving aged semen $(11.4 \pm 0.6$, $8.7 \pm 1.0$ and $8.6 \pm 0.8$ for control and groups 1 and 2 , respectively).
Fig.1 Pregnancy rate of the sows in each group

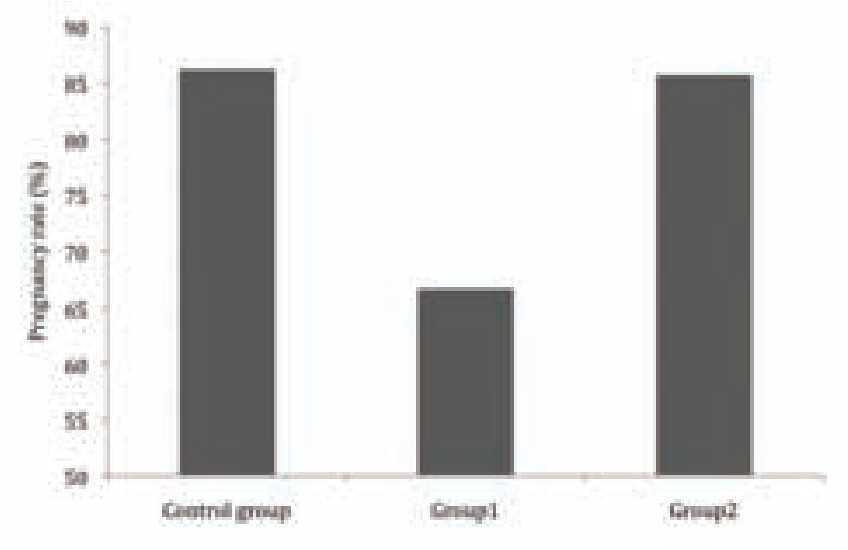

\section{Discussions}

The results of the present study confirm that measures of sperm quality are lower by 2 or 3 days after collection and further declined to reach lowest quality by 4 days when extended in a short-term extender such as BTS. As noted by others (2) this age-associated effect on sperm quality was further evidenced by depressions in sow fertility when the sperm were deposited conventionally (ie. intra-cervical) and sperm was 4 days from collection. One explanation for this effect is that as the sperm age there is a growing proportion of sperm that are less able to support a normal fertilization, possibly because of ageassociated DNA damage such as may result from inadequate anti-oxidant capacity (Am-in et al. unpublished data). That viable sperm were still available in 4 and 5-day old semen is supported by the normal pregnancy rates when aged sperm were inseminated intrauterine. We suggest that this is similar to the effect documented previously (1) whereby conventional insemination of fewer sperm resulted in poorer performance, which effect was prevented by intrauterine insemination. Interestingly, based on incomplete data collected to date, litter size was reduced in both aged semen groups. The etiology of the differential effects on pregnancy and litter size are not known.

\section{References}

1. Watson and Behan 2002. Theriogenology 57: 1683-1693.

2. Waberski et al. 1994. Theriogenology. 41:1367-1377. 


\title{
P.786
}

\section{Energy intake and exogenous gonadotrophins (eCG and LH) on embryo viability}

\author{
Octávio H. Eckhardt ${ }^{1}$ Felipe C. Horta ${ }^{1}$ Larissa J. Parazzi ${ }^{1}$ Esther R. Afonso ${ }^{1}$ Simone M. Martins ${ }^{1}$ \\ Flávia R. Barros ${ }^{2}$ Carolina I. Silva ${ }^{1}$ José A. Visintin $^{1}{ }^{\text {Aníbal S. Moretti }}{ }^{1}$ \\ 1. Laboratory of Swine Research, University of Sao Paulo, Depto. Nutrição e Produção Animal (VNP), Pirassununga, SP, Brazil; 2. Laboratory \\ of In Vitro, Cloning and Animal Transgenesis, University of Sao Paulo, Depto. Reprodução Animal (VRA), São Paulo, Brazil
}

\section{Introduction}

Greater energy intake during late gestation have been linked to lower feed intake during lactation 1, which in turn can lead to lower gonadotropin secretion 2 and an unsatisfactory reproductive outcome. The aim of the study was to investigate the effects of gestational energy intake and exogenous gonadotropins at weaning on primiparous sow's reproductive performance.

\section{Materials and Methods}

Twenty three out of thirty hybrid gilts, with 75 days of gestation, were fed according to 2 treatments until farrowing: $(\mathrm{P}, \mathrm{n}=12)$ $2,9 \mathrm{~kg}$ of a pre-lactation diet (3203 kcalME/ $\mathrm{kg}, 17,25 \% \mathrm{CP}$ ), and $(\mathrm{G}, \mathrm{n}=11) 2,5 \mathrm{~kg}$ of a gestation diet (2930 kcalEM/kg, 16,43\%CP). At weaning, animals were split into 2 other groups: $(H ; n=12)$ administration of 600IU of eCG and, after 72 hours, $2,5 \mathrm{mg}$ of $\mathrm{LH}$, and $(C ; n=10)$ control group, without hormonal protocol. Animals were weighed at the beginning of the trial, before and after parturition and at weaning $(23,7 \pm 2,2$ days after farrowing $)$ and were fed to appetite during lactation. Pos-weaning estrus was determined by standing reflexes in the presence of adult boar, following artificial insemination. After 4,56 $\pm 0,92$ days, sows were slaughtered and had their reproductive tracts collected. Ovulation rate was established and embryos were recovered and stained with Hoechst 33342 and propidium iodide for evaluation of viable embryo cells through epifluorescence microscopy. Experimental design was entirely casualized in a $2 \times 2$ factorial arrangement, analyzed with the PROC GLM of SAS5

\section{Results}

Gilts fed the gestation diet $(G)$ weighed $186,4 \pm 9,6$ and $220,1 \pm 13,7 \mathrm{~kg}$ at the beginning of the trial and at farrowing whereas treatment $P$ gilts weighed $183,8 \pm 7,1$ and $229,1 \pm 10,2$ in the same periods $(p>0,05)$. Daily weight gains during late gestation were $0,915 \pm 0,159$ and $1,23 \pm 0,158 \mathrm{~kg} /$ day for $\mathrm{G}$ and $P$, respectively $(p<0,001)$. Post farrowing and weaning weights were, respectively, 194,1 $\pm 10,4$ and $197 \pm 15,2$ for treatment $G$ and $199,7 \pm 8,8$ and $195,3 \pm 12,2$ for $P(p>0,05)$. Mean lactational weight change was $-4,7 \pm-12,2 \mathrm{~kg}$ for $\mathrm{G}$ and $-5,3 \pm-9,3$ for P. Post weaning performance and embryo evaluation is presented in Table 1. The results indicated an interaction between factors for the percentage of fecundated structures, with values of $98,55 \%$, 78, 97\%, 96, 88 and 99, 09 for GC, GH, PC and $\mathrm{PH}$, respectively

\section{Discussion}

The level of energy intake did not interfere on lactation weight losses, although researches have shown different results 1,2. Unlike other reports3,4, hormonal treatment did not influence WEI or enhance the ovulation rate. The literature4 mentions that an increase in the serum gonadotropins levels due to eCG and LH administration, could have led to the ovulation of immature follicles, resulting in oocytes and, hence, embryos of lower quality. At the same time, the higher weight at farrowing could have provided sufficient body reserves for the sows to cope with the metabolic demands of lactation, resulting in a lower impact of the hormonal protocol on the reproductive outcome signaled by the percentage of fertilized structures. Similar findings were reported previously4 when using the same hormonal protocol combined with flushing in gilts. In conclusion, the condition associating body reserves at weaning and hormonal pattern in primarous sows can affect embryo viability. Further information is needed regarding the mechanism involved.

Table 1. Post-weaning performance and embryo quality for treatments GC, GH, PC and PH

\begin{tabular}{|l|c|c|c|c|c|}
\hline & GC & GH & PC & PH & CV (\%) \\
\hline $\mathrm{n} 1$ & 3 & 6 & 4 & 5 & \\
\hline WEI (days) 1 & 4.3 & 4.3 & 4.3 & 4.2 & 10.8 \\
\hline Ovulation Rate 1 & 24.0 & 18.2 & 20.8 & 19.8 & 29.6 \\
\hline Number of Structures 1 & 22.7 & 15.7 & 15.8 & 14.8 & 41.6 \\
\hline Fertilized Structures (\%) 2 & 98.6 & 79.0 & 96.9 & 99.1 & 19.3 \\
\hline Total Embryo Cells 1 & 29.1 & 14.7 & 17.8 & 21.7 & 143.7 \\
\hline Total Live Cells (\%) 1 & 81.0 & 97.8 & 94.5 & 96.5 & 146.0 \\
\hline 1- $p>0,5 ; 2-p<0,05$ for interaction between factors \\
\hline
\end{tabular}

\section{Acknowledgement}

\section{FAPESP}

\author{
References \\ 1 Weldon et al., 1994. J Anim Sci, 72:387-394. \\ 2 Einarsson and Rojkittikhun, 1993. J Repr Fert Supl, 48:229-239. \\ 3 Candini, 2001. Master thesis, University of São Paulo. \\ 4 Lago et al., 2005. Reprod Dom Anim, 40:141-144. \\ 5 SAS: software, Version 9.0. Cary: SAS Institute, 2002
}




\title{
P.787
}

\section{Improving oestrus behavior with peforelin, a specific FSH releasing GnRH}

\author{
Jan Arnauts ${ }^{2}$ Jan Jourquin ${ }^{1}$ Lieve Goossens ${ }^{1}$ \\ 1. Janssen Animal Heatlh, Beerse, Belgium; 2. Katholieke Universiteit Leuven, Leuven, Belgium
}

\section{Introduction}

During oestrus, the expression of the oestrus symptoms is very important for the heat detection and timing of insemination. Oestrus symptoms are induced by the estrogens from the growing follicles. They prepare the sows for mating. During follicle growth, the oestrus production in the follicles increases and will eventually cause a luteinizing hormone (LH) peak that leads to ovulation. Follicular growth is regulated by the hypothalamus. Gonadorelin releasing hormones $(\mathrm{GnRH})$ induce the release of follicle stimulating hormone (FSH) and LH from the pituitary. These hormones are responsible for the recruitment, selection and growth of the follicles. Peforelin (Maprelin ${ }^{\circ}$ ) is a synthetic $\mathrm{GnRH}$ that specifically induces the release of FSH is pigs (1). The product is used for the induction of oestrus (2). The aim of this study was to measure the effect on the timing and intensity of the heat symptoms in a high productive farm.

\section{Materials and Methods}

In a 2400 sows farm, 224 sows and 224 gilts were included in the study. All gilts were synchronized with a daily dose of $20 \mathrm{mg}$ altrenogest (Regumate) during 18 days directly in the mouth. The last dose of altrenogest was given the day before weaning of the sows. 48 hours after the last altrenogest dose, 112 randomly chosen gilts were injected intramuscular with $150 \mu \mathrm{g}$ of peforelin ( $2 \mathrm{ml}$ Maprelin ${ }^{\circ}$ ) and 112 randomly chosen sows were injected with peforelin 24 hours after weaning. Primiparous sows received $37.5 \mu \mathrm{g}$ peforelin $\left(0.5 \mathrm{ml}\right.$ Maprelin $\left.{ }^{\circ}\right)$ and multiparous sows received $150 \mu \mathrm{g}$ of peforelin ( $2 \mathrm{ml}$ Maprelin $\left.{ }^{\circ}\right)$. From day 3 after weaning, twice daily oestrus control was carried out. A score from 1 to 5 was given: 1 no heat, 2 first heat symptoms, 3 pre-oestrus, 4 oestrus but not yet perfect and 5 for perfect oestrus. The weaning to oestrus interval (WOI) and the weaning to insemination interval (WII) was recorded. At the moment of submission of this abstracts, only partial pregnancy information testing and no farrowing data were available yet. The Wilcoxon Mann-Whitney $\mathrm{U}$ test between groups was performed in SAS ${ }^{\circ}$ (version 9.1).

\section{Results}

The numbers of gilts coming in heat within 7 days after last altrenogest dose was $95.5 \%$ for the peforelin treated group versus $92.0 \%$ for the non-treated group. In multiparous sows, $96.4 \%$ of both groups came on heat within 6 days after weaning. The pregnancy rate was slightly better for the peforelin treated group, both for the gilts ( $85.7 \%$ vs. $84.0 \%)$ and sows $(92.4 \%$ vs. $91.0 \%)$. The WOI and WII of sows and gilts coming in heat within the set range are shown in table 1.
Table 1: Impact peforelin treatment on WOI and WII

\begin{tabular}{|l|c|c|c|}
\hline & Number of sows & WOI (days) & WII (days) \\
\hline Non-treated sows & 108 & $4.41^{\mathrm{a}}$ & $4.86^{\mathrm{c}}$ \\
\hline Peforelin treated sows & 108 & $4.15^{\mathrm{b}}$ & $4.63^{\mathrm{d}}$ \\
\hline Non-treated gilts & 103 & 4.92 & 5.03 \\
\hline Peforelin treated gilts & 107 & 5.03 & 5.16 \\
\hline $\begin{array}{l}\text { ab:statistical difference } p<0.0148 \\
\text { (based on ranked values) }\end{array}$ & & & \\
\hline $\begin{array}{l}\text { cd: } \text { statistical difference } p<0.0135 \\
\text { (based on ranked values) }\end{array}$ & & & \\
\hline
\end{tabular}

Peforelin treated sows come into heat earlier and are inseminated earlier. The difference in gilts is not significant $(p>0.10)$. Figure 1 shows the oestrus score curve for both sows groups. The oestrus of peforelin treated sows peaks earlier and finishes earlier.

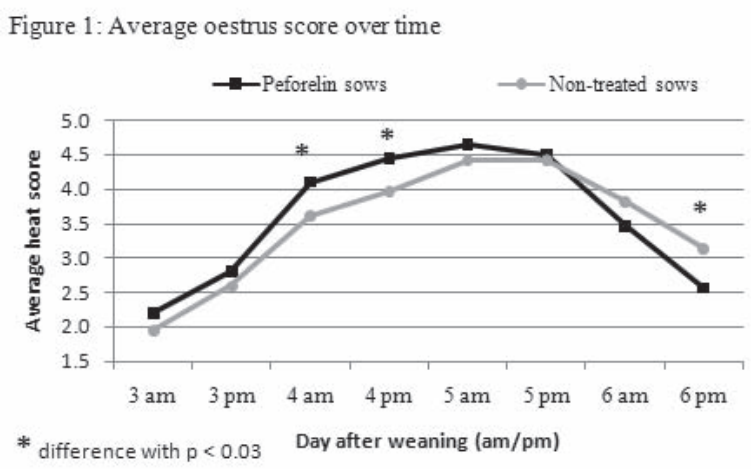

\section{Discussion}

The results of this study show that the peforelin treatment has a positive effect on timing and intensity of oestrus in high performing farms. Improving results in high productive farms is a challenge. Early onset of a good quality oestrus facilitates the breeding management and often results in improved reproductive performance. With only few animals remaining one week after weaning or synchronization, it is easier to cull and to reduce empty days. While in gilts, the use of peforelin seems to reduce the number of gilts not bred, this is hardly possible in the sows on this farm. In sows, WOI is shortened and more concentrated after the use of peforelin.

\section{References}

1. J. Kauffold; Reprod. Dom. Anim. 2005, 40, 475-479

2. S. Engl; IPVS 2006, vol. 2, p 466 


\title{
P.788
}

\section{Infertility investigation in Scottish pig herds by examination of reproductive tracts}

\author{
Allan Ward ${ }^{2}$ Mandy Nevel ${ }^{3}$ Jill R. Thomson $^{1}$ \\ 1. Scottish Agricultural College, Edinburgh, UK; 2. Quality Meat Scotland, Edinburgh, UK; 3. Royal Veterinary College, London, UK
}

\section{Introduction}

Examination of the reproductive tracts of infertile sows and gilts is recognised as a valuable diagnostic aid $(1,2)$. Between 2006 and 2008, Scottish pig farmers were offered examinations of reproductive tracts of sows and gilts culled for infertility reasons, including multiple returns to oestrus, pregnancy failure/not in pig and suspected anoestrus. The aims were three-fold; firstly to assist farmers and their veterinary advisors with understanding the causes of infertility on their units, secondly to improve the reproductive performance of herds and thirdly, to carry out surveillance for any previously unrecognised or any new and emerging reproductive conditions.

\section{Materials and Methods}

After slaughter, the tracts were bagged, sealed, identified by herd and animal number and delivered to one of two diagnostic laboratories within 24 hours. Similar protocols were used by both laboratories including inspection of all parts of the reproductive tract and bladder (if present), counting the number of follicles, and other features on the ovaries (corpora lutea, corpora albicans, corpora haemorrhagica, cystic lesions), testing oviducts for patency, examination of uterine horns for pregnancy, inflammation or other lesions, the cervical structure, the vagina and bladder for inflammation or abnormalities. Swabbing of the uterine body anterior to the cervix for bacterial culture was done routinely by one laboratory, and only in the event of gross inflammation or abnormal uterine contents, by the other. Selected cases were tested for Chlamydia species by PCR. Representative samples were collected for histopathology.

\section{Results}

In total, 142 tracts were examined from 31 units. No abnormalities were evident in 58 of the tracts ( 23 units). These tracts showed normal ovarian activity with presence of corpora lutea. Nineteen tracts were pregnant (14 units); 11 were in early pregnancy (up to 6 weeks gestation), 4 were in mid-pregnancy ( 7 - 13 weeks gestation) and 4 were in late pregnancy ( 14 weeks or more, gestation).Two gilts were found to have congenital defects of the cervix or uterine horns. Twelve sows had cystic ovaries (10 units). Uterine bacterial infection was detected in 32 tracts (13 units). Some cases showed gross inflammatory changes associated with infection. Infections involved were as follows: E.coli (8 tracts, 5 units), Strep. dysgalactiae (6 tracts, 3 units), other Streptococcus species (3 tracts, 3 units), Actinomyces pyogenes ( 6 tracts, 1 unit), other infections including mixed environmental-type organisms, Fusobacterium species or Clostridium perfringens ( 8 tracts, 6 units). Abscessation of the uterine wall was present in one sow. Evidence of anoestrus was found in 17 tracts (7 units). One anoestrus sow also had totally blocked oviducts. Two sows had severe cystitis. Both yielded the same bacterial isolates from their uterus and bladder suggesting a link between cystitis and the endometrial infection. No Chlamydia $\mathrm{sp}$. were detected in any of the uteri tested.
Table 1. Findings in reproductive tracts of 142 sows/gilts culled for infertility from 31 units.

\begin{tabular}{|l|c|c|}
\hline Feature & Number of pigs & $\%$ \\
\hline Normal tract & 58 & 41 \\
\hline Pregnant & 19 & 13 \\
\hline Congenital defect & 2 & $<1$ \\
\hline Cystic ovaries & 12 & 8 \\
\hline Uterine bacterial infection & 32 & 22 \\
\hline Anoestrus & 17 & 12 \\
\hline Cystitis & 2 & $<1$ \\
\hline & & \\
\hline Discussion & &
\end{tabular}

This study allowed reports to be provided for individual sows and gilts including assessments in relation to last oestrus and service dates. Where the cause of infertility could not be determined from the slaughter examination, other means of investigation including serology were suggested. The culling of pregnant animals highlighted the need for better pregnancy testing and/or training on some farms. In herds with endometritis, there was a tendency for particular organisms to be prevalent, for example streptococci or $A$. pyogenes. Coliforms and other environmental bacteria associated with endometritis suggested hygiene issues on some units. Happily, the study did not demonstrate any previously unrecognised or any new and emerging conditions on the farms.

\section{References}

1. Almond, G.W. and Richards, R.G. 1992. Compend. Cont. Ed. Pract. Vet. 14, $542-546$.

2. Dalin, A-M et al. 1997. Acta Vet. Scand. 38, 253-262. 


\title{
P.789
}

\section{Effect of different sexual stimuli on the estrous cycle and reproductive performance of artificially inseminated sows}

\author{
Flavea Reis ${ }^{2}$ Ronaldo Reis ${ }^{1}$ Maristela Palhares ${ }^{1}$ Amanda Siqueira $^{1}$ G. B. Mourão ${ }^{1}$ C. Malm¹ José Monteiro Silva Filho ${ }^{1}$ \\ 1. Federal University of Minas Gerais, Belo Horizonte, MG, Brazil; 2. Elanco Animal Health, São Paulo, Brazil
}

\section{Introduction}

Deficiencies in management, particularly concerning estrus detection, have been reported as the main cause of low reproductive performance when using artificial insemination (A.I.). It is known that the boar influences many physiological and reproductive processes in the female, thus having a positive effect on reproduction. Aiming to use the copulative and seminal plasma stimuli, while loosing none of the genetic benefits of A.I., many studies have been conducted to assess the effectiveness of teaser or infertile males on increasing the fertility rates of inseminated females. The objective of this study was to evaluate the influence of using vasectomized boars or boars with deviated preputial on estrus detection and reproductive performance of artificially inseminated sows.

\section{Material and Methods}

A total of 110 primiparous and multiparous females (parity 1 to 11) and 12 boar teaser were used (five were vasectomized and seven had preputial ostium deviation). Four fertile boars were used as semen donors. The vasectomized males presented olfactory, visual, auditive and tactile (external and internal - by penis penetration) stimuli, in addition to the action of the seminal plasma. The males with preputial ostium deviation, on the other hand, provided no internally contact or seminal plasma. The females were randomly distributed to the three pre-insemination treatments: $\mathrm{TI}(\mathrm{n}=35)$ - females inseminated without direct male stimulation (control); TII $(n=38)$ - female stimulated and mated by males with preputial ostium deviation, immediately before each insemination; TIII $(n=37)$ - female stimulated and copulated with a vasectomized male, immediately before each insemination. Estrus detection was done three times a day - in TI was done with the females housed in individual pens, by the back pressure test in the presence of a boar (nose-to-nose contact); in TII and TIII the boar was kept in physical contact with the sows in the pen. Were performed up to three inseminations using insemination doses of $3 \times 10^{9}$ viable sperm, diluted in the MR-A extender and stored at $17^{\circ} \mathrm{C}$ for up to 12 hours. The data were analyzed by Analysis of Variance, and the averages compared by SNK test.

\section{Results}

The average duration of estrus was $67.53 \pm 16.42$ hours, showing a numerical superiority when identified in the male's pens (treatment II and III). The average weaning-estrus-interval was $3.98 \pm 0.85$ days, with a significantly higher percentage $(p<0.05)$ of females showing estrus between 1-3 days after weaning in the treated groups (24.66\%), compared with the controls (8.57\%). The percentage of females that showed estrus in the morning was $69.09 \%$. Regarding sows reproductive performance, no statistically significant differences ( $p>0.05)$ were found in any of the parameters measured. The farrowing rate was $88.18 \%$ and the average total number of piglets born was $11.22 \pm 2.77$.

\section{Discussion}

The combination of the tactile, visual, auditive and olfactory stimuli increased the proportion of females in heat in accordance with others studies (1). The group exposed to vasectomized males presented the worst dates. Probably the amount of seminal plasma altered the sperm concentration in the doses and reduced the fertility. Also, these females had full contact with the boars and so, were exposed to stressful conditions (2).

\section{Conclusion}

The contact with the teaser boars immediately before the insemination not resulted in improvements in the reproductive performance in the females. Probably, the effect of vasectomized boar use is not evident in farms that use artificial insemination and show rates of fertility and prolificacy close to ideal.

\section{References}

1. SIGNORET, J.P. Reproductive behavior of pigs in relation to fertility. Vet. Rec., v.88, p.34-38, 1971.

2. VARLEY, M.; STEDMAN, R. Stress and reproduction. In: COLE, D.J.A.;WISEMAN, J.; VARLEY, M.A. Principles of Pig Science, Loughborough: Nottingham University Press, p.277-296,1994 


\title{
P.790
}

\section{The rate of gilts become pregnant at the age of 220-240 days}

\author{
Marijan Sviben ${ }^{1}$ Pero Gnjidic'2 \\ 1. Freelance consultant, Zagreb, Croatia; 2. Biotim KG, Županja, Croatia
}

\section{Introduction}

Gilts may be covered naturally or inseminated artificially when they reached the live weight of $130 \mathrm{kgs}$ being old 220 days at least (Hühn, 1999). Recently it was recommended the gilts would be covered old 220-240 days in order to farrow at the age of 335-355 days (Zootechnical Dpt., Biotechnical Faculty, University of Ljubljana, 2009). We decided to establish how many gilts became pregnant at the age of 220-240 days.

\section{Materials and Methods}

Data collected at the Family Farm Glavas'ic' in Bizovac, Republic of Croatia, were the material used during this research. The farm bought 65 hybrid PIC gilts from farm "Belje" where they had been born during the period of August 21st till September 14th 2008. Gilts were covered naturally or inseminated artificially with the boar's semen obtained from the A.I. Center in Osijek so that they became pregnant during the period from February 18th till September 14th 2009. Litters from those females dropped in the period from June 11 th 2009 till January 7th 2010. It was accounted how many days particular gilts was old at the start of her successful pregnancy and how many days particular female was old at the 1st farrowing. Numbers were elaborated using the methods described by Serdar (1959).

\section{Results}

Ten gilts from 65 or $1538 \%$ of all observed females became pregnant at the age of 225-240 days giving the average of 234.9 days.

\section{Figure 1}

OBSERVED AND ADAPTED TO NORMAL FREQUENCY DISTRIBUTION CURVE OF THE AGE OF GILTS AT THE START OF SUCCESSFUL PREGNANCY $\Sigma \mathrm{f}=65$

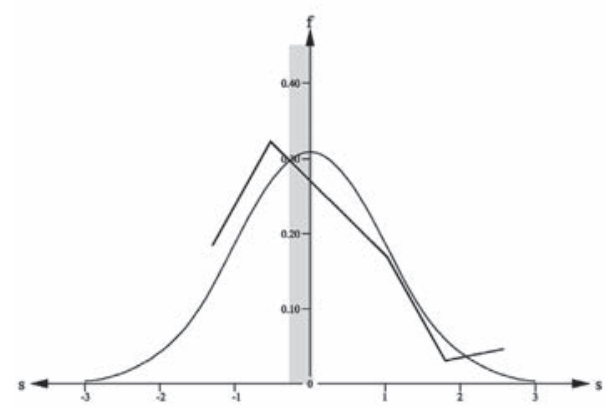

These gilts farrowed at the age of 342-354 days, old 350.1 days on an average. The frequency distribution curves on the age of 65 gilts at the start of successful pregnancy observed and adapted to normal one can be seen at Figure 1. Gilts became pregnant old 172-386, 238.4 days on an average. They farrowed at the age of 287-501, giving the mean of 352.4 days.

\section{Discussion}

Gilts became sows as yearlings covered at the age of 225-240 days or at the age of 172-386 days but in former case less than $16 \%$ of all females in our research. Successful pregnancy of 65 gilts in 2009 started at the age what was observed with the data on the age at the first successful services of gilts born in 1960-1962 (169-401 days - Sviben, 1967), when gilts reached $89 \mathrm{~kg}$ at the age of 212 days on an average. Contemporarily bred pigs in appropriate circumstances reach the live weight of almost $140 \mathrm{~kg}$ on an average at the age of 154-175 days (Sviben, 2007) when gilts are sexually mature. Pigmen have an interest to service gilts as early as possible what means at the age of six months (Legault and Dagorn, 1973).

\section{References}

1. Hühn, U., 1999: Jungsauen fit machen für die erste Zuchtbenutzung. Veredlung Produktion 4(2)36-37.

2. Legault, C., J. Dagorn, 1973: Incidence de l'age a la première mise bas sur la productivité de la truie. Ann. Zootech. 22 (3) 387-388.

3. Serdar, V., 1959: Udz ‘̌benik statistike. S`kolska knjiga. Zagreb

4. viben, M. 1967: Realnost imaginacije ritma odabiranja prema toku života i iskoris`tavanja svinja. Zbornik III kongresa veterinara i veterinarskih tehnic ara Jugoslavije. Str 323-327. Sarajevo.

5. Sviben, M., 2007: Količina energija krme procijenjena potrebnom i utrošena u tovu svinja 22-140 kg žive vage. Krmiva 2007. Str. 32. Opatija.

6. Zootechnical Dpt., Biotechnical Faculty, University of Ljubljana, 2009: Plodnost prašičev. Domžale 


\title{
P.791
}

\section{A systematic approach to assess reproductive failure at slaughter}

\author{
Eduardo J. Kwiecien ${ }^{1}$ Carolina Gutierrez ${ }^{2}$ Patricia Mora² Evelyn Cadenas ${ }^{2}$ \\ 1. EKM Servicios Veterinarios, Maracay, Venezuela; 2. Carval Venezuela S.A., Valencia, Venezuela
}

\section{Introduction}

Reproductive failure continues to be one of the most common reasons for culling sows from pig farms1. Reproductive slaughter checks have been conducted to determine the causes of urogenital infections, discharging sows and reproductive failures2,3,4. Nevertheless, a few studies been done on the presence of gross reproductive lesions at slaughterhouse and their application to solve reproductive problems in the Pigfarms. The aim of this paper was to schedule a systematic protocol to assess reproductive failure by using a spreadsheet as a procedure to analyze and to reduce reproductive problem in farms.

\section{Material and Methods}

Three Pigfarms with historical reproductive failure were selected (Farm $A=4.000$ sows, Farm $B=7.000$ sows, Farm $C=2500$ sows). A longitudinal study was performed to determine the main reproductive problems in each farm. 20 to 30 culled sows or replacement were examine at slaughterhouse during April - November 2009 period. A systematic monthly or every twothree weeks inspection was performed to each pigfarms to assess the evolution of each case. Post-mortem examination of the genital organs was carried out immediately after slaughtering. A detailed protocol was used to examine every part of the organs by using a technical inspection guide. Sample of several tissues were taken for histopathological and bacteriological studies. The inspection outcome were transferred to a specific spreadsheet designed to analyze the pathological findings.

\section{Results}

Nine reproductive inspections were performed in 244 sows or replacement belongs to the three Pigfarms. The pathological findings are shown in the Table 1. The main problem we found was a high percentage of pregnancy $(13,5 \%)$, follow by inactive ovaries (13\%) and pyometra and cystitis (10,60\% each one). $8.6 \%$ of ovary inspected had multiple large cysts. Lithiasis was a nonexpected finding for us since it was higher in Farm A than the other ones (Fig 1). Farm $C$ had the mayor reproductive problems with a high percentage of pyometra and pregnancy (data not shown). E. coli, Streptococcus spp and Proteus spp were the predominant bacteria isolated, respectively.

\section{Discussion and Conclusion}

The results were similar to those previous report3,4, however, the prevalence of pregnancy, cystitis and pyometra were higher. The systematic evaluation conducted in the reproductive tract on sow from the three pig farms in this study showed to be a worth tool to detect the main reproductive problems in those farms and to introduce management strategies to reduce the reproductive problems in this herds.

\section{References}

1. Karveliene, B., Zilinskas H, et al. 2007. Reproduction in Domestic Animals. 42(3): 275 - 281.

2. Almond, GW and Richards, RG. 1992. Evaluation of Reproductive Failure by the use of salughtercheck. Compend Con. Edu. Vol. 14, No. 4, p 542-546.

3. Villamizar-Galvis, L.A. et al. 2000. Pathological findings present in uterus and ovaries of slaughtered sows. 16th International Pig Veterinary Society Congress, 17-20 Sept, Melbourne, Australia.

4. Madec, F. 1982. A Survey of Urogenital Lesions in Culled Sows (Observations made at Slaughterhouse). Abstract of J. Rech. Porc. En France. p342.

Table 1

\begin{tabular}{|l|c|}
\hline Type of Pathology & $\%$ \\
\hline Inactive Ovaries & 13,00 \\
\hline Unilateral Cysts & 6,90 \\
\hline Bilateral Cysts & 6,30 \\
\hline Multiple Ovarian Cysts & 8,60 \\
\hline Hydrosalpinx & 3,50 \\
\hline Pyosalpinx & 0,00 \\
\hline Endometritis & 7,50 \\
\hline Endometrial Cysts & 6,80 \\
\hline Metritis & 5,50 \\
\hline Pyometra & 10,60 \\
\hline Vaginitis & 0,00 \\
\hline Cystitis & 10,60 \\
\hline Lithiasis & 7,20 \\
\hline Pregnancy & 13,50 \\
\hline TOTAL & 100,00 \\
\hline
\end{tabular}

\section{Figure 1}

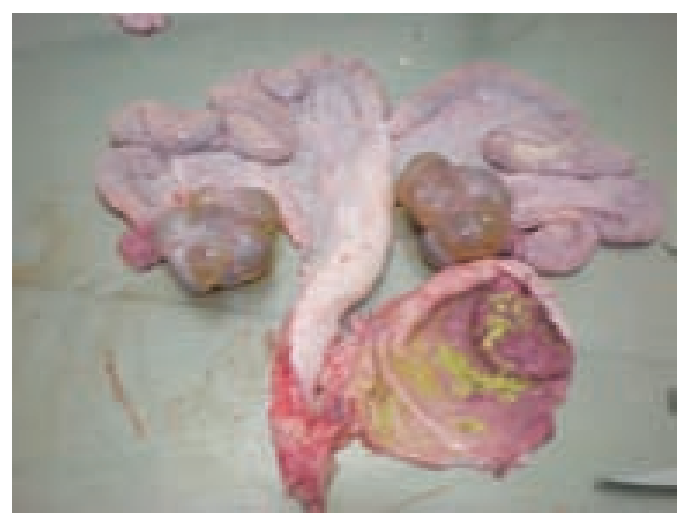




\title{
P.792
}

\section{Responses to delayed breeding protocols in weaned parity 1 sows}

\author{
Jennifer Patterson ${ }^{1}$ Ana Paula Mellagi ${ }^{2}$ Merel Verhoeven ${ }^{1}$ Meghan McCarty ${ }^{1}$ Tanja Minder ${ }^{1}$ Barbra Behiels ${ }^{1}$ George Foxcroft $^{1}$ \\ 1. University of Alberta, Edmonton, AB, Canada; 2. Setor de Suinos, Porto Alegre, RS, Brazil
}

\section{Introduction}

In today's well managed sow farms, approaching $90 \%$ of sows can be detected in estrus within 3 to 5 days after weaning and pregnancy rates in these sows can be better than $90 \%{ }^{1,2}$. However, the results of our recent studies suggest that although modern, primiparous sows can be consistently bred soon after weaning, the emerging pre-ovulatory follicles are of poorer quality, which may affect both the number and quality of pigs born. This study examined the effect of delayed breeding after weaning in primiparous sows, using either "skip-a-heat" breeding or treatment with oral progestagen, on subsequent reproductive performance.

\section{Materials and Methods}

In this study, $1^{\text {st }}$ parity sows restricted to $60 \%$ of their ad libitum intake, and induced to lose $17.0 \pm 4.1 \mathrm{~kg}$ of body weight during the last week of a 21-d lactation, were allocated as trios to one of 3 breeding strategies at weaning (weaning weight $=160.2 \mathrm{~kg}$ ): 1) CON $(n=25)$ bred at the first post-weaning estrus; 2$)$ SKIP $(n=23)$ bred at second post-weaning estrus; 3$)$ ALT $(n=23)$ oral progestagen (altrenogest: ALT) treatment for $14 \mathrm{~d}$ starting the $\mathrm{d}$ of weaning and bred at the first, but delayed, post-weaning estrus after ALT withdrawal. After weaning, sows were heat checked twice daily and bred by Al (using single sire Al doses, evenly assigned across trio). At day 30 of gestation, reproductive tracts were recovered after euthanasia and dissected on-site.

\section{Results}

At breeding, SKP and ALT accumulated more non-productive days as measured by the wean-to-service interval (WSI) but the proportion of sows bred was similar (PBRD). ALT sows were bred as a more synchronous group (VAR) than either SKP or CON. Sow body weight at weaning, and total energy balance during lactation did not differ among treatments. The longer delay to breeding resulted in greater weight at breeding (ALT: $169.4 \pm$ 21.4; SKP: $177.1 \pm 22.3$; CON: $162.1 \pm 21.0 \mathrm{~kg})$ and a more positive energy balance at breeding (ALT: $423.9 \pm 12$ 2; SKP: 633.3 \pm 14.0; CON: $196.2 \pm 11.5$ ) in SKP and ALT sows. Pregnancy rate (PR) was improved in SKP and CON sows. An improvement in follicular quality and corpus luteum function (total luteal weight (CLWT) and progesterone concentration (PCON)) was observed in ALT sows. Delaying breeding from $1^{\text {st }}$ to $2^{\text {nd }}$ estrus in SKIP sows increased the size of the pre-ovulatory follicle (FOLL) At $\mathrm{d} 30$ of gestation, no differences in ovulation rate (OR), live embryos (LE), embryo survival, embryo size (LEWT) or placental fluid volume (PVOL) were detected (Table 3). A Fertility index (FI) was calculated as the percent of sows bred and pregnant per 100 sows weaned $x$ live embryos at $\mathrm{d} 30$.
Results show that as a result of the exceptional performance of CON sows, the FI of ALT (1179) and SKP (1215) did not meet the performance achieved by CON (1406) sows.

Table 1: Effects of treatment in primiparous sows at weaning (ALT, SKP, $(O N)$, on various reproductive parameters at breeding and day 30 of gestation for those sows bred.

\begin{tabular}{|c|c|c|c|}
\hline & ALT & SKP & $\mathrm{CON}$ \\
\hline \multicolumn{4}{|l|}{ Breeding } \\
\hline WSI, d** & $19.4 \pm .2^{\mathrm{a}}$ & $26.5 \pm .3^{b}$ & $5.5 \pm .2^{c}$ \\
\hline PBRD, \% & 90.1 & 80.8 & 91.3 \\
\hline VAR & $0.42^{\mathrm{a}}$ & $1.42^{b}$ & $1.20^{\mathrm{b}}$ \\
\hline FOLL, mm ${ }^{* *}$ & $7.5 \pm .3^{\mathrm{a}}$ & $7.6 \pm .3^{\mathrm{a}}$ & $6.8 \pm .4^{b}$ \\
\hline $\mathrm{PCON}, \mathrm{ng} / \mathrm{ml}^{*}$ & $11.4 \pm 0.8^{\mathrm{a}}$ & $9.3 \pm 0.8^{a b}$ & $8.7 \pm 0.8^{b}$ \\
\hline$P R, \% *$ & $79.2^{\mathrm{a}}$ & $95.2^{2 \mathrm{ab}}$ & $100.0^{\mathrm{b}}$ \\
\hline \multicolumn{4}{|c|}{ Day 30 of gestation } \\
\hline $\mathrm{OR}, \#$ & $20.0 \pm 0.6$ & $18.4 \pm 0.6$ & $19.1 \pm 0.5$ \\
\hline $\mathrm{LE}, \#$ & $15.4 \pm 0.7$ & $15.8 \pm 0.8$ & $15.1 \pm 0.7$ \\
\hline LEWT, g & $1.8 \pm 0.08$ & $1.8 \pm 0.07$ & $2.0 \pm 0.06$ \\
\hline PVOL, ml & $220.3 \pm 9.8$ & $211.5 \pm 9.8$ & $243.8 \pm 9.6$ \\
\hline CLWT, $g^{*}$ & $7.7 \pm 0.3^{\mathrm{a}}$ & $7.0 \pm 0.3 \mathrm{ab}$ & $6.9 \pm 0.2^{b}$ \\
\hline
\end{tabular}

\section{Discussion}

Delayed breeding allows for additional time for the sow to recover weight lost due to lactation catabolism. Altrenogest treatment effectively synchronizes estrus after weaning and reduces the number of days in the breeding week. Delayed breeding, improves follicular quality, corpus luteum function and the synchrony of sows bred. In the present study, the performance of Control sows, as measured by the Fertility Index was exceptional and no gain in estimated productivity was achieved by delaying breeding after weaning in sows.

\section{References}

1 Poleze E. et al., Swine Health Prod. 2006;103:124-130.

2 Vinsky M., et al., Reprod, Fert and Development 2006;18:347-355. 


\title{
P.793
}

\section{Performance of sows in rural villages of Western Kenya}

\author{
Florence K. Mutua ${ }^{1}$ Cate Dewey ${ }^{2}$ Samuel Arimi ${ }^{3}$ Michael Levy² William Ogara ${ }^{3}$ Esther Schelling ${ }^{4}$ \\ 1. International Livestock Research Institute, Nairobi, Kenya; 2. University of Guelph, Guelph, ON, Canada; \\ 3. University of Nairobi, Nairobi, Kenya; 4. Swiss Tropical Institute, Basel, Switzerland
}

\section{Introduction}

Local pig farming is an important source of livelihood in many rural communities of Western Kenya $(1,2)$. Sows are important in sustaining this important sector. Data on their performance is currently lacking. Such information is crucial for the pig farmer to identify target areas for farm improvement. Studies on the performance of pigs have only been done on commercial farms keeping exotic breeds (3). The purpose of this study was to provide baseline information on the reproductive performance of sows raised by small-holder pig farmers in rural villages of Busia and Kakamega districts, Western Kenya.

\section{Materials and Methods}

We studied a random sample of 288 local pig farmers in the two districts of Western Kenya. Farms were randomly identified and visited three times in the course of the study period (June 2006 to October 2008). Information on sow productivity was gathered by means of questionnaires administered to sow owners in face to face interviews. All data were managed and analysed in Stata ${ }^{\circledR}$ statistical software. Student's t-tests were used to compare data between districts.

\section{Results}

The mean number of sows owned per farm per visit was 1.3 $(\mathrm{SD}=0.62)$ on farms where at least one sow was present when the farm visit was made. Farmers in Kakamega bred their sows more times (2.4) compared to farmers in Busia (1.8) $(p<0.05)$. The average prices for sows purchased as piglets ( $\leq 4$ weeks), growing pigs ( 4 - 8 weeks) or adults were Ksh 619 (SD=178), Ksh 813 (SD=457) and Ksh 3060 (SD=684) respectively. Piglets were cheaper in Busia (Ksh 509 SD=57) than in Kakamega (Ksh $777 \mathrm{SD}=174)(p<0.05)$. Pigs were weaned at an average age of $5.4(\mathrm{SD}=3.3)$ weeks. The mean number of piglets born alive was
7.87(SD 2.67) and the mean number of piglets weaned was 6.60 (SD 3.25). A median and mean pre-wean mortality of $0 \%$ and $13 \%(S D=26)$ was reported. The median and mean age for sows at first breeding was 6 and $8.25(\mathrm{SD}=4.8)$ months, respectively. Sows were bred 1.951 .64 months after piglets were weaned. Sows were sold at Ksh 2286 (SD=934), Ksh 2603 (SD=996) and Ksh 2830 (SD=1114) for the lowest, highest and expected price respectively. Most sows (91\%; 74/81) were sold to pork butcher men who slaughtered them for immediate local consumption. Many sows were sold immediately after their first litter (56\%; $33 / 59$ ). Sources of breeding boars included; boars that were borrowed from a neighbour (77\%), farmer's-own boar (14\%), group-owned boars $(0.4 \%)$ and boars that were free-roaming in the villages (2\%).

\section{Discussion}

This study has provided important production data that was previously lacking. Increasing the productivity and profitability of small-holder pigs directly contributes to the social and economic wellbeing of households in low-income countries. Some of the opportunities that may increase sow productivity include; improved sow feeding, shorter wean to breed interval, breeding more than one in an estrus, keeping sows for more than one litter by lowering the female culling rate, introduction of improved genetics with better litter size. There is need for more detailed research particularly on potential management factors influencing sow productivity (number of born alive piglets, farrowing rate, sows age at first farrowing and piglet mortality).

\footnotetext{
References

Mutua et al (2007). J Swine HIth Prod, 15(4); 206-212

Githigia et al (2005). The Kenya Veterinarian, 29: 37-39.

Wabacha et al (2004). Pre Vet Med, 63; 197-210
} 


\title{
P.794
}

\section{Reproductive parameters in pregnant sows adapted from a conventional system to an organic system}

\author{
Roberto G. Martinez; Yadira Miranda; Jorge R. Lopez; Roberto Martinez-Rodriguez; Mario E. Haro \\ Facultad de Medicina Veterinaria y Zootecnia. Universidad nacional Autonoma de Mexico, Mexico, DF, Mexico
}

An alternative to the industrial pork production is the development of market niches. An example of this is the production of organic pork meat, in order that a product goes through one system to another, it is necessary a conversion process. However, there is not study that indicates any change in breeding parameter with sows which have been adapted from one system to the other. It is known that sows in conventional system could have breeding problems when there are some food changes or environmental changes; that why the reason of knowing the breeding behavior in sows which have been adapted from a conventional system to an organic one.

\section{Material and Methods}

It was carried out in a pig farm localized $100 \mathrm{~km}$ northeast from México City in a separated area from the rest of the farm (term established by IFOAM). Six yard with concrete floor $(28.26 \mathrm{~m} 2$ each one) and an outside area with land floor (197.62m2) were used for this research. This space fulfills the specifications that are established by certifying organizations $(2.5 \mathrm{~m} 2 / \mathrm{sow}$ in inside area, and $1.9 \mathrm{~m} 2 /$ sow in outside areas). It was used 17 adult sows, 11 of them were placed in yards with outside area and the other 6 in confinement.

At the moment of weaning they were separated in 3 groups. A. 6 sows in an organic management and also organic food; B. 6 sows in equal conditions but conventional food. C. 6 sows in an intensive management.

It was registered number of births, number of services, length of estrus, length of gestation, physical conditions at the moment of birth, dorsal grease at the moment of birth, length of the birth, internal between birth, number of mail piglets meconium, total number of newborn piglets, number of dead piglets and weight at the moment of birth. Each variable was analyzed using ANDEVA in a new design completely randomized.

\section{Results}

The result are presented in the following table.

Table 1.

\begin{tabular}{|l|c|c|c|}
\hline Group & A & B & C \\
\hline Services & 1.0 & 1.4 & 1.0 \\
\hline Estrus (hrs) & 46.0 & 45.6 & 38.0 \\
\hline Gestation length (days) & $116.3 \mathrm{a}$ & $115.4 \mathrm{a}$ & $113.8 \mathrm{~b}$ \\
\hline Backfat mm & $17.8 \mathrm{a}$ & $20.7 \mathrm{~b}$ & $20.7 \mathrm{~b}$ \\
\hline Colostrum (hrs) & 26.1 & 37.4 & 12.1 \\
\hline Farrow lenght (hrs) & $4.03 \mathrm{ab}$ & $6.6 \mathrm{a}$ & $2.9 \mathrm{~b}$ \\
\hline Birth Interval (hrs) & $37.7 \mathrm{~A}$ & $31.9 \mathrm{a}$ & $17.1 \mathrm{~b}$ \\
\hline Total born average & $9.66 \mathrm{~A}$ & $12.6 \mathrm{ab}$ & $13.5 \mathrm{~b}$ \\
\hline Dead born average & 1.33 & 0.8 & 2.0 \\
\hline L.Meconium & $0.33 \mathrm{a}$ & $0.8 \mathrm{a}$ & $3.33 \mathrm{~b}$ \\
\hline Weigh (kg) & 1.58 & 1.55 & 1.60 \\
\hline Different literals in the same line indicate $(\mathrm{P}<0.05)$ & & \\
\hline
\end{tabular}

\section{Discussion}

The sows in the organic system with both types of diet had similar parameters to the control sows, except with parameters related with the length of birth, they were higher than the controls; the previous is related with less stress conditions at birth.

\section{References}

1. Estienne, J.M et al. J. Journal of Swine Health and Production. 14 (5): 241-246. 2006.

2. Mc Glone; et al. Professional Animal Scientist. 4: 2004.

3. CERTIMEX, 2005. http://www.certimexsc.com/docs/NormasCERTIME Xactualizadas2005\%5B4\%5D.pdf

4. Kuehl RO. (2001). Thomsom Learning México. México D.F. .2001

\section{Acknowledge}

PAPIIT IN202108 


\title{
P.795
}

\section{The removal of gilts and primiparous sows from swine breeding herds in Thailand}

\author{
Atthaporn Roongsitthichai ${ }^{1}$ Em-on Olanratmanee ${ }^{1}$ Seri Koonjaenak ${ }^{2}$ Mongkol Techakumphu ${ }^{1}$ Padet Tummaruk $^{1}$ \\ 1. Chulalongkorn University, Bangkok, Thailand; 2. Kasetsart University, Bangkok, Thailand
}

\section{Introduction}

In general, the removal of sows from breeding herds consisted of planned (i.e., old and low productivity) and unplanned (e.g., reproductive failure, lameness, sudden dead) reasons (1). It has been demonstrated that at least three litters are required from sows before a positive cash flow could be obtained (2). Unfortunately, $15-20 \%$ of the removal sows produced only one litter (1, 2) and most of these were culled due to unplanned reasons (1). The objective of the present study was to evaluate the pattern and reasons for culling gilts and primiparous sows in swine herds of Thailand.

\section{Materials and methods}

Data from 38,323 gilts and sows culled from eight swine herds in Thailand between 2006 and 2009 were included. The herds are located in the eastern, western, middle, and northeastern parts of Thailand between latitude $14-17^{\circ} \mathrm{N}$ and longitude $102-103^{\circ} \mathrm{E}$. The sow on production in the herds was between 1,600 and 4,000 sows. The sow breeds were chiefly Landrace $x$ Yorkshire (LY) crossbred and some were $L$ and $Y$. Conventional artificial insemination (AI) was used in all herds. Most gilts and sows were housed in a conventional open-housing system with water sprinklers and fans. They were kept in individual stalls during gestation and in individual farrowing pens during lactation. All herds produced replacement gilts within the herd by their own grandparent (GP) stocks ( $L$ and $Y$ ). In general, the gilts were mated at $\geq 32$ weeks of age with $\geq 135 \mathrm{~kg}$ of body weight at the second or later observed estrus. Vaccination for gilts and sows included foot-and-mouth disease, classical swine fever, Aujeszky's disease, porcine Parvovirus, and arthrophic rhinitis. The gilts and sows received ad libitum water via water nipples. The feed was provided once or twice a day (about $1.5-3.5 \mathrm{~kg} /$ day $/$ head during gestation and $5.0-7.0 \mathrm{~kg} /$ day/head during lactation). The feed was rice-maize-soybean-fish base containing 150-180 g crude protein, 12.1-13.4 MJ metabolizable energy and 8-10 g lysine/kg. Generally, the planned removal was performed after the sows reached the sixth parity.

\section{Results and Discussion}

Of all slaughtered females, $14.5 \%(n=5,543)$ and $13.8 \%(n=5,302)$ were removed as gilts and primiparous sows respectively (Figure 1). Of all the removed females, $93.2 \%$ was sent to slaughterhouse, $6.8 \%$ was found dead, and $0.02 \%$ was euthanized. The reasons for culling of gilts and primiparous sows are demonstrated in Table 1.
Figure 1 Percentage of gilts and sows removed from herds by parity number at removal ( $n=38,383$ females)

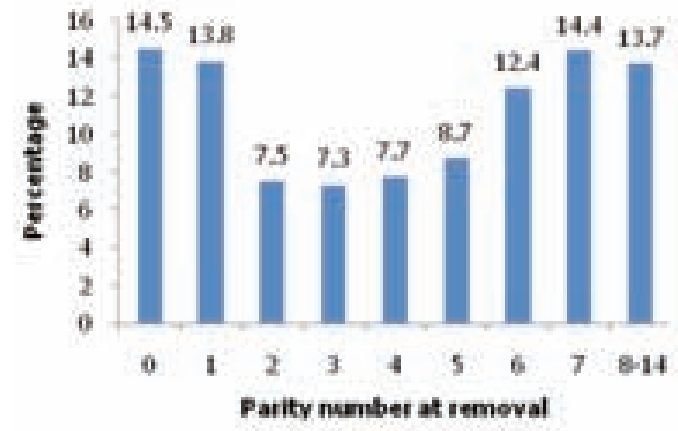

Table 1 Reasons for culling in gilts $(n=5,543)$ and primiparous sows $(n=5,302)$ in 8 swine herds in Thailand

\begin{tabular}{|l|c|c|}
\hline Reasons for culling & Gilts (\%) & $\begin{array}{c}\text { Primiparous sows } \\
\text { (\%) }\end{array}$ \\
\hline Anestrus & 26.5 & 14.3 \\
\hline Not pregnancy & 22.2 & 10.4 \\
\hline Vaginal discharge & 14.0 & 15.1 \\
\hline Abortion & 11.3 & 6.1 \\
\hline Lameness & 9.1 & 17.3 \\
\hline Illness & 4.6 & 11.9 \\
\hline Others & 12.2 & 25.0 \\
\hline & & \\
\hline
\end{tabular}

The present study indicated that reproductive disorders are the most common reason for culling gilts and primiparous sows, in agreement with earlier studies $(1,2)$. Most of the reproductive problems of the female included anestrus, not pregnancy, vaginal discharge, and abortion. In addition, it also indicates that at least $28.3 \%$ of the female produced only one litter or less when they were culled.

\section{References}

1. Engblom et al., 2007. Livest. Sci. 106: 76-86.

2. Lucia et al., 2000. Livest. Prod. Sci. 63: 213-222. 


\title{
P.796
}

\section{Productive life and reasons for removal of sows in four pig farms in Yucatan}

\author{
Jesús E. Ek-Mex; José Segura-Correa; Alejandro Alzina-López; Joaquín Rodríguez \\ Faculty of Veterinary Medicine and Zootechny, University of Yucatan, Merida, YUC, Mexico
}

\section{Introduction}

An alternative to increase the profitability of hog farms is to increase the length of productive life (PL) of sows, which is interrupted by different causes that affect their productivity. The establishment of the causes of removal of sows is critical in the culling and replacement programs to implement preventive measures. The aim of this study was to determine the effect of farm, number of piglets born alive at first farrowing (NBA) and age at first farrowing (AFF) on the length of the PL and number of parity (NP), and the main causes of removal sows.

\section{Materials and Methods}

We used data from four pig farms representative of the central area of Yucatan Mexico. The farms are of the intensive system and technically. Production records to estimate PL and causes of removal of sows were the year 2003-2009 and were obtained from Pigchamp program. The variables used were: identification of farms and sows, NP, NBA, AFF, date and cause of removal. The $\mathrm{PL}$ was defined as the number of days between first farrowing and removal, or termination of data collection (1). To determine the length of the PL and NP will use a model with fixed effects of farm, NBA and AFF. The data of the causes of removal were analyzed in general and by NP using descriptive statistics.

\section{Resulted and Discussion}

Table 1 shows the results for PL and NP of sows per farm, NBA and AFF. Other studies also reported an effect of these factors on the PL and NP $(1,2)$. The main causes of removal were reproductive problems $(26.75 \%)$, which agree with other studies $(3,4)$. The highest percentage of removal occurred in the parities 6 to 14 with $28.7 \%$, of which $83 \%$ were removal because of advanced age. In the party 0 the $51.3 \%$ of sows were removal for reproductive reasons (Table 2). Similar results have been reported by other authors (3).
Table 1. Least Square Means and standard errors ( $\pm E E)$ for NP and PL of sow removal.

\begin{tabular}{|c|c|c|c|}
\hline $\begin{array}{l}\text { Sources of } \\
\text { variation }\end{array}$ & n & $\mathrm{NP}$ ( $\pm \mathrm{EE})$ & $\mathrm{PL}( \pm \mathrm{EE})$ \\
\hline Farm 1 & 3327 & $4.31(0.04) \mathrm{a}$ & $0.6534 .86(6.32) \mathrm{a} 0 \%$ \\
\hline Farm 2 & 128 & $5.25(0.17) c$ & $806.04(25.80) \mathrm{c}$ \\
\hline Farm 3 & 81 & 4.38(0.32)ab & $751.47(49.32) \mathrm{bc}$ \\
\hline Farm 4 & 210 & $4.73(0.11) \mathrm{b}$ & $714.30(16.59) c$ \\
\hline $\mathrm{NBA} \leq 8$ & 2069 & $4.49(0.10) \mathrm{a}$ & 675.11(15.15)a \\
\hline NBA9-12 & 746 & $4.75(0.10) \mathrm{b}$ & $715.24(16.22) \mathrm{b}$ \\
\hline$N B A \geq 13$ & 931 & $4.75(0.10) \mathrm{b}$ & $714.65(16.10) \mathrm{b}$ \\
\hline $\mathrm{AFF} \leq 330$ & 1007 & $4.81(0.11) \mathrm{b}$ & $726.36(16.70) c$ \\
\hline AFF331-347 & 1844 & $4.73(0.10) \mathrm{b}$ & 709.05(16.15)b \\
\hline AFF $\geq 348$ & 895 & $4.45(0.09) \mathrm{a}$ & 669.58(14.82)a \\
\hline \multicolumn{4}{|c|}{ Different literal in column indicates significant difference $(\mathrm{P}<0.05)$. } \\
\hline
\end{tabular}

Table 2. Frequency (\%) of removal reason of sows by parity number (NP) at removal

\begin{tabular}{|c|c|c|c|c|c|}
\hline \multirow[t]{2}{*}{ Reasons of removal } & \multicolumn{4}{|c|}{ NP at removal } & \multirow{2}{*}{\begin{tabular}{|c|} 
Total \\
$\%$ \\
\end{tabular}} \\
\hline & 0 & 1 & $2-5$ & 6-14 & \\
\hline Reproduction & 8.05 & 5.64 & 11.07 & 1.98 & 26.75 \\
\hline Locomotion & 3.12 & 3.61 & 7.69 & 0.78 & 15.20 \\
\hline Productivity & 0.04 & 2.59 & 9.27 & 0.72 & 12.62 \\
\hline Diseases & 3.04 & 2.58 & 6.30 & 0.63 & 12.92 \\
\hline Old age & 0.01 & 0.01 & 1.11 & 23.81 & 24.96 \\
\hline Miscellaneous & 1.04 & 1.93 & 3.84 & 0.74 & 7.55 \\
\hline No. of Sows & 1156 & 1206 & 2898 & 2116 & 7376 \\
\hline Total (\%) & 15.67 & 16.35 & 39.65 & 28.68 & 100 \\
\hline
\end{tabular}

In conclusion difference was found between farms, NBA and AFF on the length of the PL and NP of the sows. The main causes of removal of sows were reproductive problems.

\section{References}

1. Engblom et al., (2008) J. Anim. Sci. 86:432-411

2. Serenius and Stalder. (2007) Animal. 1:745-750

3. Lucia et al., (2000) Livest.Prod.Sci.63: 213-222

4. Engblom et al., (2007) Livest.Sci.106: 76-86 


\title{
Relationship among age, body weight and number of ovulation in Landrace $x$ Yorkshire crossbred replacement gilts
}

\author{
Padet Tummaruk; Sawang Kesdangsakonwut; Atthaporn Roongsitthichai; Annop Kunavongkrit
}

Chulalongkorn University, Bangkok, Thailand

\section{Introduction}

On average, Landrace $x$ Yorkshire (LY) crossbred gilts in Thailand attain puberty at 196 days and $106 \mathrm{~kg}$ body weight (1). In practice, replacement gilts are usually mated at the second or later observed estrus, about 7-9 months of age. The objective of the present study was to investigate the relationship among number of ovulation, age and body weight in LY crossbred replacement gilts in Thailand.

\section{Materials and Methods}

Genital organs from 316 culled LY replacement gilts from 6 swine herds in Thailand were collected at slaughterhouses between May 2005 and September 2008. The organs, including ovaries, oviducts, uteri, cervixes and vaginas-vestibules-vulvas were collected, placed on ice and transported to the laboratory within $24 \mathrm{~h}$ after culling. Historical data included herd, gilt identity, breed, date of birth, culling date and body weight at culling were collected. Age at culling was calculated. The ovaries were weighed by using an electronic balance (Precisa Instruments Ltd., Switzerland). Corpora lutea (CL) and corpora albicantia (CA) were counted. Number of ovulations was defined as total number of $\mathrm{CL}$ from both ovaries. Number of $\mathrm{CA}$ was regarded to the number of ovulations in the previous reproductive cycle. The gilts were classified according to body weight (Table 1) and age at culling (Table 2). Number of ovulations was compared among groups by using one way analysis of variance.

\section{Results and discussion}

On average, the gilts were culled at $302.4 \pm 51.5$ days of age and the body weight at culling was $144.0 \pm 20.8 \mathrm{~kg}$. Of the 316 gilts, 23 gilts (7.3\%) had multiple cysts, 17 gilts (5.4\%) had single cyst, 2 gilts (0.6\%) had unilateral ovo-testis and 7 gilts (2.2\%) had miscellaneous abnormalities. Gilts with abnormal ovaries were excluded and 261 gilts (82.5\%) were remained for further investigation. Of these gilts, 107 gilts did not ovulate (86 prepubertal and 21 follicular phase) and 154 gilts (59\%) having at least one $C L$ were investigated. On average, the number of ovulations was $15.9 \pm 3.5$ and the number of CA was 15.3 \pm 6.1 . The number of ovulations was $8.1 \pm 2.9$ on the left ovary and $7.8 \pm 3.2$ on the right ovary $(P=0.380)$. The diameter of the $C L$ and CA was $9.3 \pm 2.2 \mathrm{~mm}$ and $4.5 \pm 1.4 \mathrm{~mm}$, respectively. The weight of the ovaries was $6.6 \pm 2.4$ gram. The number of ovulations in relation to body weight of gilts is presented in Table 1. Gilts with body weight of $92-130 \mathrm{~kg}$ and $131-140 \mathrm{~kg}$ had 3.2 and 2.0 ova, respectively, which were less than those of $141-150 \mathrm{~kg}$ (Table 1). Number of ovulation of gilts with a body weight of 141 to 205 kg did not differ significantly (Table 1). The relationship between age at culling and number of ovulation are presented in Table 2. Gilts aged 30-35 weeks had a lower number of ovulations than gilts aged 40-50 weeks (Table 2). The present study indicated that maximal number of ovulation of LY gilts in Thailand was obtained at $>141 \mathrm{~kg}$ body weight and at $>36$ weeks of age. Therefore, to achieve high prolificacy, the gilts are recommended to be bred at a body weight of at least $141 \mathrm{~kg}$.

Table 1 Number of ovulations in LY crossbred gilts in relation to body weight at culling

\begin{tabular}{|c|c|c|c|}
\hline Body weight (kg) & N & CL & CA \\
\hline $92-130$ & 65 & $13.5 \mathrm{a}$ & $11.2 \mathrm{a}$ \\
\hline $131-140$ & 38 & $14.7 \mathrm{ab}$ & $15.2 \mathrm{~b}$ \\
\hline $141-150$ & 50 & $16.7 \mathrm{~b}$ & $16.7 \mathrm{bc}$ \\
\hline $151-160$ & 49 & $16.6 \mathrm{~b}$ & $18.5 \mathrm{c}$ \\
\hline $161-205$ & 52 & $16.9 \mathrm{~b}$ & $14.3 \mathrm{ab}$ \\
\hline
\end{tabular}

Table 2 Number of ovulations in LY crossbred gilts in relation to age at culling

\begin{tabular}{|c|c|c|c|}
\hline Age (wk) & N & CL & CA \\
\hline $30-35$ & 14 & $13.8 \mathrm{a}$ & $9.3 \mathrm{a}$ \\
\hline $36-40$ & 31 & $15.5 \mathrm{ab}$ & $14.4 \mathrm{~b}$ \\
\hline $41-45$ & 60 & $16.6 \mathrm{~b}$ & $16.4 \mathrm{~b}$ \\
\hline $46-50$ & 23 & $16.3 \mathrm{~b}$ & $15.6 \mathrm{~b}$ \\
\hline $51-72$ & 22 & $15.9 \mathrm{ab}$ & $17.3 \mathrm{~b}$ \\
\hline a,b,c different superscript within column differed significantly $(\mathrm{P}<0.05)$ \\
\hline
\end{tabular}

\section{References}

1. Tummaruk, P., Tantasuparuk, W., Techakumphu, M., Kunavongkrit, A. 2007. Anim. Reprod. Sci. 99:167-181. 


\title{
P.798
}

\section{Association between lived weight and size of the uterus in Landrace $\mathbf{x}$ Yorkshire crossbred gilts}

\author{
Padet Tummaruk; Sawang Kesdangsakonwut; Atthaporn Roongsitthichai; Annop Kunavongkrit \\ Chulalongkorn University, Bangkok, Thailand
}

\section{Introduction}

In Thailand, replacement gilts are usually mated at the second or later observed estrus, about 7-9 months of age. The objective of the present study was to investigate an association between lived weight and size of the uterus (i.e., weight and length) in Landrace $x$ Yorkshire (LY) crossbred replacement gilts in Thailand.

\section{Materials and Methods}

The study was conducted between May 2005 and September 2008. Genital organs of 316 LY crossbred replacement gilts from six swine commercial herds in Thailand were examined. The herds, where the samples were collected, were located between latitude $13^{\circ}$ and $17^{\circ} \mathrm{N}$ and between longitude $100^{\circ}$ and $104^{\circ} \mathrm{E}$. The gilts entered the gilt pool at body weight of $80-100 \mathrm{~kg}$ and were housed in an open-housing system. The genital organs were collected, placed on ice and transported to the laboratory within $24 \mathrm{~h}$ after culling. Data including herd, gilt identity, breed, date of birth, culling date and body weight at culling were collected. Age at culling was calculated. The genital tract including ovaries, uteri and cervixes were dissected from the mesometrium and examined macroscopically. The uterus from uterotubal junction to the uterine body was dissected and weighed by using an electronic balance. The length of the uterine horns was measured by using a metrical scale plastic tape. The measurement was done by placing one end of the plastic tape at the junction of the uterine horn and uterine body and threading the tape along the horn between the thumb and fingers. The measurement was performed on both sides of the uterine horn and the uterine body. The sum of the length of each part was regarded as total length of the uterus. Stages of the reproductive cycle were defined according to an appearance of the ovaries, i.e., corpora lutea (CL), corpora albicantia (CA) and follicles (1). The gilts were classified according to their lived weight at culling (Table 1). Weight and length of the uterus were compared among groups by using multiple ANOVA.

\section{Results and discussion}

Of the 316 gilts, 36 gilts (11.4\%) had endometritis, 15 gilts (4.8\%) had congenital abnormalities and 9 gilts (2.9\%) had miscellaneous abnormalities. Gilts with abnormal uterus were excluded; therefore $\mathbf{2 5 5}$ gilts were remained for further analyses. The average age at culling was $297.0 \pm 46.5$ days and the body weight at culling was $144.6 \pm 20.5 \mathrm{~kg}$. Of these gilts, 147 gilts (57.6\%) were in luteal phase, 31 gilts (12.2\%) were in follicular phase and 77 gilts (30.2\%) were in pre-pubertal phase. The weight of the uterus was $689.0 \pm 219.0,727.6 \pm 315.0$ and $127.7 \pm 70.8 \mathrm{~g}$ in luteal, follicular and pre-pubertal phases, respectively. The length of the uterus was $263.2 \pm 61.2,200.4 \pm 35.2$ and $123.3 \pm 25.4 \mathrm{~cm}$ in luteal, follicular and pre-pubertal phases, respectively. Since both weight and length of the uterus in pre-pubertal gilts was significantly lower than in cyclic gilts, they were not included in the analyses. The weight and length of the uterus in relation to body weight at culling in cyclic gilts is presented in Table 1. Gilts with body weight of 92-130 kg had smaller uterus than those of $141-150 \mathrm{~kg}$ (Table 1). Gilts with body weight of $161-205 \mathrm{~kg}$ had significantly heavier and longer uterus than those of $92-160 \mathrm{~kg}$ (Table 1). The present study indicated that the maximum size of the uterus of LY gilts in Thailand was obtained at body weight of $>161 \mathrm{~kg}$. Therefore, breeding gilts at body weight of $>161 \mathrm{~kg}$ may increase the number of piglets born per litter since a maximum uterine capacity was achieved.

Table 1 Weight $(\mathrm{kg})$ and length $(\mathrm{cm})$ of the uterus in LY crossbred gilts by body weight at culling $(\mathrm{kg})$

\begin{tabular}{|l|c|c|c|}
\hline Body weight & N & Uterine weight & Uterine length \\
\hline $92-130$ & 27 & $573.4 \mathrm{a}$ & $204.8 \mathrm{a}$ \\
\hline $131-140$ & 28 & $624.4 \mathrm{ab}$ & $226.9 \mathrm{a}$ \\
\hline $141-150$ & 38 & $681.5 \mathrm{bc}$ & $231.5 \mathrm{a}$ \\
\hline $151-160$ & 35 & $736.3 \mathrm{c}$ & $230.9 \mathrm{a}$ \\
\hline $161-205$ & 41 & $872.3 \mathrm{~d}$ & $264.6 \mathrm{~b}$ \\
\hline $\mathrm{a}, \mathrm{b}, \mathrm{c}, \mathrm{d} d$ different superscript within column differed significantly $(\mathrm{P}<0.05)$ \\
\hline
\end{tabular}

\section{References}

1. Tummaruk, P., Kesdangsakonwut, S., Kunavongkrit, A. 2009. Theriogenology. 71:369-375. 


\title{
P.799
}

\section{Effect of genotype of sows kept under the same housing conditions on piglet litter size}

\author{
Mario Ostovic ${ }^{1}$ Anamaria Ekert-Kabalin ${ }^{1}$ Sven Mencik ${ }^{1}$ Tomislav Balenovic ${ }^{1}$ Dinka Milic Zeljko Pavicic $^{1}$ \\ 1. Faculty of Veterinary Medicine University of Zagreb, Zagreb, Croatia; 2. Dubravica Pig-Breeding Farm, Ltd, Dubravica, Croatia
}

\section{Introduction}

Litter size in pigs is an important component of production efficiency (1) and sow genotype is one of the factors demonstrated to have significant effect on piglet litter size (2). However, other non-genetic factors including sow housing can cover up this benefit. The aim of this study was to determine association between piglet litter size and genetic type of sows kept under the same housing conditions in order to eliminate the possible influence of housing conditions.

\section{Material and Methods}

Data were collected at a commercial pig farm and included two groups of 40 sows (Swedish Landrace, SL and Segers hybrid, SG) representing a part of the farm population (groups 1 and 2). Group 3 of 28 sows (Swedish Landrace and Large White crossbreeds, $S L^{*} \mathrm{LW}$ ) represented the whole population of this genotype on the farm. All sows were kept under the same housing conditions during pregnancy and lactation. The average length of lactation was 25 days. Semen from the same boars was used for insemination of sows in all groups. Data on litter size included six parity records and were expressed as number of piglets born in total (NBT), number of live-born piglets (NBA) and number of still-born piglets per litter (NSB). Statistical analysis was performed using the Statistica 8.0 (Statsoft Inc., 2008) statistical software and methods of variance analysis (one-way ANOVA and ANOVA Repeated Measures).

\section{Results}

Results are presented in Table 1.

\section{Discussion}

NBT was highest in the SL*LW sow population in sixth parity and significantly differed from sixth parity of sow groups 1 and $2(P<0.05)$. Group 3 NBT differed significantly in sixth parity due to the first parity NBT in the same group $(P<0.05)$. In all groups, NBA and NSB differed within the six parity cycles observed due to the sow genotype. Study results indicated the genotype of sows kept under the same housing conditions to influence the piglet litter size.

\section{References}

1. Johnson, R. K., M. K. Nielsen, D. S. Casey (1999): Responses in ovulation rate, embryonal survival, and litter traits in swine to 14 generations of selection to increase litter size. J. Anim. Sci. 77, 541-557.

2. Uremovic, M., Z. Lukovic, D. Skorput, Z. Mahnet (2009): Litter size of pigs at large scale farms in Croatia. Proc. 44th Croatian and 4th International Symposium on Agriculture, 240-241.

Supported by the Ministry of Science, Education and Sports of the Republic of Croatia (Grant No. 053-0532265-2242 and 053-0532265-2238).

Table 1. Litter size characteristics of different genetic type of sows kept under the same housing conditions

\begin{tabular}{|c|c|c|c|c|c|c|c|c|c|}
\hline & $S L(n=40)$ & & & $S G(n=40)$ & & & SL LW (n=28) & & \\
\hline Parity & NBT & NBA & NSB & NBT & NBA & NSB & NBT & NBA & NSB \\
\hline 1 & $10.93 \pm 2.32$ & $10.53 \pm 2.36$ & $0.40 \pm 0.63$ & $10.08 \pm 2.65$ & $9.55 \pm 2.58$ & $0.53 \pm 1.13$ & $10.68^{*} \pm 2.86$ & $10.25^{*} \pm 2.78$ & $0.43 \pm 0.74$ \\
\hline 2 & $12.30^{\mathrm{a}} \pm 2.74$ & $11.88^{b} \pm 2.66$ & $0.43 \pm 0.71$ & $10.48^{\mathrm{a}} \pm 3.15$ & $10.13^{b} \pm 3.00$ & $0.35 \pm 0.62$ & $11.32 \pm 2.18$ & $11.11 \pm 2.01$ & $0.21 \pm 0.50$ \\
\hline 3 & $12.13 \pm 2.95$ & $11.83 \pm 2.93$ & $0.30 \pm 0.56$ & $11.05 \pm 2.17$ & $10.73 \pm 2.00$ & $0.33 \pm 0.86$ & $12.18 \pm 2.44$ & $11.82 \pm 2.23$ & $0.36 \pm 0.49$ \\
\hline 4 & $11.75 \pm 3.30$ & $11.50 \pm 3.17$ & $0.25 \pm 0.54$ & $11.68 \pm 2.32$ & $11.20 \pm 2.20$ & $0.48 \pm 0.82$ & $11.71 \pm 2.59$ & $11.29 \pm 2.40$ & $0.43 \pm 0.74$ \\
\hline 5 & $11.93 \pm 3.10$ & $11.25 \pm 3.11$ & $0.68 \pm 1.12$ & $11.90 \pm 2.66$ & $11.25 \pm 2.52$ & $0.65 \pm 1.03$ & $12.86 \pm 3.23$ & $12.54^{*} \pm 3.21$ & $0.32 \pm 0.61$ \\
\hline
\end{tabular}




\title{
P.800
}

\section{Assessment of sow births synchronised with oxytotocin and long-acting oxytocin (carbetocin)}

\author{
Carlo Cerati ${ }^{2,3}$ Alessandro Palese $^{1}$ Fausto Gamba ${ }^{2,3}$ Paolo Benaglia ${ }^{2,3}$ Paolo Bonilauri ${ }^{4}$ Lucio Nisoli $^{1}$ \\ 1. Bayer HealthCare, Milano, Italy; 2. Bompieri Farm, Piubega, Mantova, Italy; \\ 3. Gruppo Suinicolo Mantovano, Mantova, Italy; 4. Istituto Zooprofilattico Sperimentale Reggio Emilia, Reggio Emilia, Italy
}

\section{Introduction}

The purpose of farrowing synchronisation in sows is to concentrate births into a short period of time, benefiting both production and farm management. Grouping births during working hours is useful to enable proper assistance, reducing perinatal piglet mortality [1] and improving fostering. The duration of farrowing influences the rate of stillbirths: a prolonged farrowing $(>3 \mathrm{~h}$ ) is more likely to result in stillborn piglets than a shorter farrowing [2]. This study compared two synchronisation protocols, using a long-acting oxytocin (carbetocin) and a synthetic oxytocin in association with prostaglandin.

\section{Materials and Methods}

The test was carried out in a 2,700 sow farm with weekly births in the Po valley in Italy. Births from 240 sows were analysed: the sows were divided into 3 groups (80 sows per group) at random:

- Group 1: 2 mg of alfaprostol (Gabbrostim ${ }^{\circledR}$ ) i.m. at 11 am on the day before the expected birth date (approximately day 113 of gestation) and $0.35 \mu \mathrm{g}$ of carbetocin (Hypohysin ${ }^{\circledR}$ LA) i.m. at 7 a.m. the next day;

- Group 2: 2 mg of alfaprostol (Gabbrostim ${ }^{\circledR}$ ) i.m at 11 am on the day before the expected birth date and 10 iu of oxytocin i.m. at 7 a.m. the next day;

- Group 3: 2 mg of alfaprostol (Gabbrostim ${ }^{\circledR}$ ) i.m. at 11 am on the day before the expected birth date.

The following parameters were analysed: duration of farrowing (from expulsion of first piglet until expulsion of placenta), interval between administration of the drugs and time farrowing started, number of liveborn piglets, weight of litter/number of liveborn piglets, stillborns, mummified foetuses, inspections during farrowing (vaginal palpation if birth per piglet was more than $30 \mathrm{~min}$ ) and mortality during lactation.

\section{Results}

The duration of farrowing was significantly longer in group 2 than in the other groups. The mean duration in group 1 was $3 \mathrm{~h}$ $41 \mathrm{~min}$, the mean duration in group 2 was $4 \mathrm{~h} 40 \mathrm{~min}$, and the mean duration in group 3 was $4 \mathrm{~h} 4 \mathrm{~min}$. The interval between administration of the carbetocin or oxytocin and the start of parturition was $1 \mathrm{~h} 59 \mathrm{~min}$ and $2 \mathrm{~h} 47 \mathrm{~min}$ respectively. The mean numbers of liveborn in groups 1, 2 and 3 were: 11.83, 11.66,
11.63 , respectively. The mean weight of the litter/number of liveborn piglets in each of groups 1, 2 and 3, expressed in $\mathrm{kg}$, were $1.70,1.69$ and 1.52 respectively. The mean number of stillborns and mummified foetuses per group was 0.68 . 0.88 and 0.74 , for groups 1, 2 and 3, respectively. The mean number of stillbirths per litter for groups 1, 2 and 3 were $0.39,0,55$ and 0.61 , respectively. Inspections during farrowing were significantly fewer in group $1(p<0.01)$ with 14 inspections during 80 farrowing compared to 55 and 33 inspections in 80 farrowing for groups 2 and 3 respectively. The mean number of piglets that died during lactation in groups 1, 2 and 3 were: $0.40,0.56$ and 0.65 .

\begin{tabular}{|l|c|c|}
\hline & Duration of farrowing $\mathbf{h}$ $\min$ & No inspections/labours \\
\hline Group 1 & $3.41 \mathrm{a}$ & $14 / 80 \mathrm{a}$ \\
\hline Group 2 & $4.40 \mathrm{~b}$ & $55 / 80 \mathrm{~b}$ \\
\hline Group 3 & $4.04 \mathrm{a}$ & $33 / 80 \mathrm{~b}$ \\
\hline ab: indicates that the groups differ significantly $(\mathrm{p}<0.05)$ & \\
\hline
\end{tabular}

\begin{tabular}{|l|c|c|c|c|c|}
\hline & Liveborn & $\begin{array}{c}\text { Mummies + } \\
\text { stillborn }\end{array}$ & Stillborn & Total born & $\begin{array}{c}\text { \% stillborn / } \\
\text { total born }\end{array}$ \\
\hline Group 1 & 11.83 & 0.68 & 0.39 & 12.51 & $3.11 \%$ \\
\hline Group 2 & 11.66 & 0.88 & 0.55 & 12.54 & $4.38 \%$ \\
\hline Group 3 & 11.63 & 0.74 & 0.61 & 12.37 & $4.93 \%$ \\
\hline & & & & & \\
\hline
\end{tabular}

\section{Discussion}

The use of carbetocin in a birth synchronisation protocol showed a significant reduction in duration of farrowing and number of inspections, compared to oxytocin. Besides this, there was a tendency for less stillborn piglets per litter after using carbetocin (3.11\%, 4.38\%, 4,93\% in Groups 1, 2 and 3, respectively). This test confirmed [3] the efficacy of this molecule in inducing farrowing and positively influencing the productive performance of the sows.

\section{References}

1. Holyoake et al. (1995) J Anim Sci 73:3543-3551.

2. Borges V.F. et al. (2005) Prev Vet Med 165-176.

3. Hühn U. et al. (1992) Berl Munch Tierarztl Wochenschr 345-9. 


\title{
Randomised double blind comparison of oxytocin and long acting oxytocin (carbetocin) in the synchronisation of farrowing in batch-breeding sows: evaluation of production parameters
}

\author{
Claudio Mazzoni ${ }^{2}$ Elena Borri ${ }^{2}$ Valeria Raffi ${ }^{2}$ Stefano Bertacchini ${ }^{3}$ Paolo Bonilauri ${ }^{4}$ Alessandro Palese $^{1}$ Lucio Nisoli $^{1}$ \\ 1. Bayer HealthCare, Milano, Italy; 2. Suivet, Reggio Emilia, Italy; 3. II Girasole Farm, Reggio Emilia, \\ Italy; 4. Istituto Zooprofilattico Sperimentale Reggio Emilia, Reggio Emilia, Italy
}

\section{Introduction}

In maternity units, synchronising farrowings is undoubtedly advantageous. Concentrating farrowings into working hours and attendance at the farrowings reduces stillborns [1] and means that a higher number of sows are available for matching operations. The objective of this study was to compare two farrowing synchronisation programmes (prostaglandin associated with oxytocin or long-acting oxytocin), examining the most important production parameters, and specifically, weight at weaning, a fundamental piece of information for sites 1 (maternity unit).

\section{Materials and Methods}

The test was carried out in a 360 sow farm in the Po valley in Italy operating three weekly batch farrowing. A randomised double blind study was planned, in which the sows were divided into two groups:

- Group 1 ( $n=52)$ : intramuscular cloprostenol (Estrumate ${ }^{\circledR}$ ) was administered the day before the scheduled farrowing day (approximately day 113 of gestation), and $35 \mu \mathrm{g}$ of intramuscular carbetocin (Hypophysin ${ }^{\circledast}$ LA) the day after ( $24 \mathrm{~h}$ after cloprostenol).

- Group 2 ( $n=59$ ): intramuscular cloprostenol (Estrumate ${ }^{\circledR}$ ) was administered the day before the scheduled farrowing day (approximately day 113 of gestation), and 20 IU of intramuscular oxytocin (Izossitocina ${ }^{\circledR}$ ) the day after ( $24 \mathrm{~h}$ after cloprostenol).

The following parameters were evaluated: duration of farrowing (the time from expulsion of first piglet until expulsion of last piglet of the litter), number of liveborn piglets and weight on weaning; the weight determinations were performed every three batches, and the litters were selected randomly. A total of 33 litters of piglets were weighed. The differences observed in duration of farrowing, number of liveborns and mean weight at weaning between the two groups were compared within ANOVA and the Kruskal-Wallis test with a level of significance of $\mathrm{p}<0.05$.

\section{Results}

The mean duration of farrowing was 122.12 minutes and 127.21 minutes in group 1 and group 2 respectively; the mean number of liveborns was 13.71 and 12.73 in group 1 and group 2 respectively (table 1 ). The mean piglet weight on weaning was statistically $(p<0.05)$ higher in group 1 than in group 2 , with 7.43 and $6.80 \mathrm{~kg}$ respectively.

Table 1

\begin{tabular}{|l|c|c|}
\hline & Duration of farrowing $(\mathbf{m i n})$ & No. liveborn piglets per litter \\
\hline Group 1: & 122.12 & 13.71 \\
\hline Group 2: & 127.12 & 12.73 \\
\hline
\end{tabular}

\section{Table 2}

\begin{tabular}{|l|c|}
\hline & Mean weight at weaning per piglet $(\mathbf{K g})$ \\
\hline Group 1: & $7.43^{\mathrm{a}} \pm 1.85$ \\
\hline Group 2: & $6.80^{\mathrm{b}} \pm 1.39$ \\
\hline ab: indicates that the groups differ significantly $(\mathrm{p}<0.05)$ \\
\hline
\end{tabular}

\section{Discussion}

In this test, synchronising farrowings with prostaglandin associated with long acting oxytocin resulted in a weight at weaning that was significantly higher than the association with oxytocin. An appropriate farrowing synchronisation protocol combined with the assistance required as necessary can increase piglet survival $[1,2]$; in addition, the short duration of birth ensuring the litter receives higher quantities of immunoglobulin, protein and fat, may explain the increased weight of the litter. This hypothesis could be tested in the future.

\section{References}

1. Holyoake et al. (1995) J Anim Sci 73:3543-3551.

2. Hühn U. et al. (1992) Berl Munch Tierarztl Wochenschr 345-9. 


\title{
P.802
}

\section{Comparison of the effects of cloprostenol (Planate ${ }^{\circledR}$ ) and dinoprost on the smooth muscle of sow's uterus}

\author{
Maria V. Falceto ${ }^{1}$ Juan Luis Ubeda ${ }^{1}$ Divina Murillo² Ruth Menjon ${ }^{3}$ Marta Jimenez ${ }^{3}$ Jesus M. Bollo $^{3}$ Jesus V. Lopez $^{3}$ \\ 1. Dept of Reproduction. Faculty of Veterinary Medicine, Zaragoza, Spain; \\ 2. Faculty of Veterinary Medicine, Zaragoza, Spain; 3. Intervet/Schering-Plough AH, Cantabria, Madrid, Spain
}

\section{Introduction}

Besides its luteolytic effects, prostaglandin F2 a (PGF2 a) exerts contractile effect on uterine smooth muscle due to reduction of intracellular cyclic-AMP levels and increase of intracellular calcium concentration (1). In sows, these contractions can be useful in facilitating elimination of uterine secretions and prevention of vaginal discharge (2). Aim of present study was to compare effects of cloprostenol (Planate ${ }^{\circledR}$ ), a synthetic analogue of PGF2 a, on uterine smooth muscle of the sow with dinoprost, a product used for this indication.

\section{Materials and methods}

Seven 8-month old LDxLW prepubertal gilts were included in the study. After induction of first estrus with 400 IU PMSG and 200 IU HCG (PG600 $\left.{ }^{\circledR}\right)$, gilts were humanely killed on first day of estrus (confirmed by standing reflex). Effects of cloprostenol and dinoprost on uterine smooth muscle was evaluated using in vitro organ bath technique.

Eight $10 \times 5 \mathrm{~mm}$ strips of myometrium were removed from each gilt, using the same protocol, and placed in Petri dishes in sterile saline. After recording exact weight and length of each strip, four were mounted in direction of the longitudinal fibers and four in direction of circular fibers, and placed in the organ bath vessels attached to an isometric transducer (Pioden UF1, Graham Bell House, Canterbury, UK). Krebs Ringer solution ( $5 \mathrm{ml}$ ) was then added to each vessel. The following protocol was followed:

1. Study of spontaneous motility of longitudinal and circular uterine smooth muscle in Krebs Ringer solution. After the stabilization period, the spontaneous motility of the preparations was recorded over about 10 minutes

2. Study of the longitudinal and circular smooth muscle motility in response to dinoprost $(571 \mathrm{mcg} / \mathrm{ml}$. 10 minutes) and cloprostenol (Planate ${ }^{\circledR}$ ) (10 mcg/ml 10 minutes) (3). The concentration was selected in the light of experience from previous studies, with similar proportions to those used systemically under field conditions for both substances.

The mechanical activity of the preparations was amplified (The MacLab Bridge Amp, AD Instruments Inc, Milford, Mass, EE.UU) and filtered (4). This mechanical signal was sent to an analog-todigital converter, and the data recorded numerically on computer by a data acquisition and processing program (MacLab System), which was used to measure amplitude, frequency and area under the curve of induced uterine motility per unit of time (Interval/time/surface, Maximum-minimum, Interval/time) for all recorded preparations.

Statistical study included the Kolmogorov Smirnov test to assess the goodness of fit of the variables to a normal distribution. An ANOVA test was then performed, and subsequently non-parametric tests (Friedman test and chi-square test).

\section{Results}

Contractions of the circular fibers of uterine smooth muscle were quantified (intensity, rhythm and duration) in the first place, and no significant differences were found for any of the parameters studied.

Table 1: Average ( $\pm S D$ ) motility of circular fibers.

\begin{tabular}{|l|c|c|c|}
\hline & Interval/time/surface & Max-Min & Interval/Time \\
\hline Cloprostenol & $0.023 \pm 0.006$ & $0.99 \pm 0.19$ & $0.99 \pm 0.17$ \\
\hline Dinoprost & $0.023 \pm 0.004$ & $1.18 \pm 0.25$ & $1.09 \pm 0.145$ \\
\hline & $\mathrm{p}=0.804$ & $0=0.548$ & $\mathrm{p}=0.666$ \\
\hline
\end{tabular}

Nor were any significant differences found in the quantified contractions of the longitudinal fibers.

Table 2: Average $( \pm S D)$ motility of longitudinal fibers.

\begin{tabular}{|l|c|c|c|}
\hline & Interval/Time/Surface & Max-Min & Interval/Time \\
\hline Cloprostenol & $0.059 \pm 0.011$ & $3.89 \pm 1.03$ & $2.7 \pm 0.46$ \\
\hline Dinoprost & $0.044 \pm 0.006$ & $3.29 \pm 0.76$ & $2.14 \pm 0.36$ \\
\hline & $\mathrm{p}=0.548$ & $\mathrm{p}=0.603$ & $\mathrm{p}=0.58$ \\
\hline
\end{tabular}

Finally, the values obtained for each of the gilts included in the study were compared, and again, no significant differences were found between them.

\section{Discussion}

According to these results, cloprostenol produced the same effect as dinoprost on the contractions of uterine smooth muscle of sows, for both circular and longitudinal fibers. This means that cloprostenol (Planate ${ }^{\circledR}$ ) is a candidate for post partum use for prevention of vaginal discharge, as has been shown in previous field studies (5). The fact that no significant differences were found between the animals included in the study indicates the consistent repeatability of the results obtained with both treatments.

\section{References}

1. Vanderhaeglhe,C. (2008). Reproduction in domestic animals $43,484-$ 489.

2. Kurowicka, B. (2005). Biology of Reproduction

3. Hirsbrunner G. (1998) Theriogenology 50: 445-455

4. Grasa, L (2006) Prostaglandins \& other mediators 79

5. Lopez, J.V. (2009) Veterinary Record 164: 807-809 


\title{
P.803
}

\section{Effect of the postpartum administration of Cloprostenol (PLANATE ${ }^{\circledR}$ ) on production and reproduction performance indices}

\author{
Juan C. Ezpeleta ${ }^{1}$ Rut Menjon ${ }^{2}$ Marta Jimenez ${ }^{2}$ Jesus M. Bollo ${ }^{2}$ Jesus V. Lopez $^{2}$ Maria T. Tejedor ${ }^{3}$ \\ 1. Grupo AN, Pamplona, Spain; 2. Intervet Schering Plough, Madrid, Spain; 3. Dept. of Anatomy, Embryology \\ and Genetics Unit of Genetics - Faculty of Veterinary Medicine, Zaragoza, Spain
}

\section{Introduction}

Among its other properties, such as its luteolytic effect, prostaglandin $\mathrm{F}_{2 a}\left(\mathrm{PGF}_{2 a}\right)$ induces contractions of the smooth muscle of the uterus. It has been reported that, in the sow, postpartum $\mathrm{PGF}_{2 a}$ injection may cause a more rapid return to estrus after weaning, may increase prolificacy in subsequent farrowings and prevent uterine discharge, thus assisting in the reduction of premature culling or mortality. Furthermore, it may increase milk production and consequently reduce neonatal mortality(1). The aim of the present study was to assess the effects of postpartum cloprostenol (Planate ${ }^{\circledast}$ ), a synthetic analogue of $\mathrm{PGF}_{12 \text { a }}$ compared with dinoprost (Dinolytic ${ }^{\oplus}$ ), another product with the same indication.

\section{Materials and Methods}

The study was undertaken on a 1400-sow farm on two sites in Navarra (northern Spain), which transfers two lots of 80 sows per week into the farrowing barn, and practises early weaning at average of 19-21 days of age. The herd was negative for PRRSv, but had severe reproductive problems. In February 2009, the farm records showed that about $17.5 \%$ of sows returned to estrus after weaning, and 8-10\% exhibited an abnormal postfarrowing uterine discharge.

452 sows were allocated to one of two groups: A Planate ${ }^{\circledR}$ group: 220 sows, injected IM with $2 \mathrm{ml}$ cloprostenol $(875 \mu \mathrm{g} / \mathrm{ml})$; and a Dinolytic ${ }^{\circledR}$ group: 232 sows, injected IM with $2 \mathrm{ml}$ dinoprost $(5 \mathrm{mg} / \mathrm{ml})$. Untreated animals acted as controls using the farm's historical data. In any week, all the sows which farrowed were treated 24-36h later with one or other product, the products being alternated week by week.

The following parameters were recorded: number of sows with abnormal vulval discharge during lactation and weaning, duration of lactation, number of weaned piglets, weaning to service interval, weaning to fertile service interval, and fertility. Sows were individually identified and cross-fostering was only allowed among sows from the same treatment group. No oxytocics or NSAIDs were used during or after farrowing.

Statistical analysis was carried out using the General Linear Model (GLM-SPSS 15.0 for Windows). The Pearson's chi-square test and the Breslow test were used for the comparison of means.

\section{Results}

A comparison, between groups, of the parities of the sows within them showed that they were fully comparable: $86.45 \%$ multiparous and $13.6 \%$ primiparous sows in the Planate ${ }^{\circledR}$ group, and $88.8 \%$ and $11.2 \%$, respectively, in the Dinolytic ${ }^{\circledR}$ group, $(p=0.1522)$.
Table 1: Comparative results for primiparous animals

\begin{tabular}{|l|c|c|c|}
\hline & Dinoprost & Cloprostenol & \\
\hline No.weaned & $11.31 \pm 0.34$ & $10.97 \pm 0.2$ & $\mathrm{p}=0.63$ \\
\hline Lactation days & 19.08 & 18.98 & \\
\hline$\%$ Fertility & 96 & 96.6 & $\mathrm{p}=0.93$ \\
\hline$\%$ Vulvar discharge & 4 & 0 & $\mathrm{p}>0.05$ \\
\hline WSI (fertile) & $\mathbf{6 . 4}$ & $\mathbf{6}$ & $\mathrm{p}=0.31$ \\
\hline
\end{tabular}

Table 2: Comparative results for multiparous animals

\begin{tabular}{|l|c|c|c|}
\hline & Dinoprost & Cloprostenol & \\
\hline No. weaned & $11.14 \pm 0.2$ & $11.24 \pm 0.1$ & $\mathrm{p}=0.102$ \\
\hline Lactation days & 19.08 & 18.98 & \\
\hline$\%$ Fertility & 87.9 & 88.1 & $\mathrm{p}=0.19$ \\
\hline$\%$ Vulvar discharge & 2.5 & 1.7 & $\mathrm{p}=0.73$ \\
\hline WSI (fertile) & $\mathbf{1 2 . 7}$ & $\mathbf{1 1 . 9}$ & $\mathrm{p}=0.18$ \\
\hline
\end{tabular}

The mean number of weaned piglets in untreated animals was 10.8. In the month before treatment, vulval discharge was present in $7 \%$ of untreated sows, and $60 \%$ of these returned to estrus.

\section{Discussion}

Postpartum reproductive parameters improved with both treatments, especially fertility, weaning to fertile service interval, and number of weaned piglets, the most notable feature being the nearly complete absence of vulval discharges. As a result, an improvement in prolificacy could be expected in the next cycle (2).

Therefore, the use of $\mathrm{PGF}_{2 a}$ analogues after farrowing on a farm with a high prevalence of uterine discharge can be a very valuable treatment option, although other factors such as hygiene and general management still require proper attention.

There were no significant differences between the treatment groups, thus it can be concluded that post farrowing treatment with cloprostenol (Planate ${ }^{\circledast}$ ) is an option for the prevention of this type of reproductive disorder.

\section{References}

1. Vanderhaeghe,C. (2008) Reprod.Dom.Anim.43(484-489)

2. Lopez, J.V. (2009) Veterinary Record 


\title{
P.804
}

\section{Interest of Pracetam ${ }^{\circledast}$ used in peripartum in sows - field trial in Northern Italy}

\author{
$\underline{\text { P Martelli }}{ }^{1}$ Nathalie Capdevielle ${ }^{2}$ J Beranger ${ }^{2}$ \\ 1. University of Parma, Depatment of Animal Health, Parma, Italy; 2. Laboratoire SOGEVAL, Laval, France
}

\section{Introduction}

Paracetamol has antipyretic and antalgic properties but has a specific pharmacological profile $(1,2)$ which explains paracetamol's good level of tolerance in pigs. The demonstration of the paracetamol's safety for gestating and lactating sows(3) has allowed the inclusion of these two categories in the summary of product characteristics (SPC) for Pracetam ${ }^{\circledR} 10 \%$ premix. A field trial has been carried out by the University of Parma within a conventional breeding unit to measure the interest of paracetamol during peripartum period.

\section{Materials and Methods}

A hundred sows have been included in the trial, divided into two batches, control and treated. The treated batch $(n=50)$ received a medicated feedstuff containing paracetamol (Pracetam ${ }^{\circledR} 10 \%$ premix / 30mg of paracetamol per kg of body weight per day) during the peripartum period, over 6 days, 4 days before farrowing and 2 days after. The control batch $(n=50)$ has received the same feedstuff with no paracetamol. The sows have been intensively observed for the 6 days prior to farrowing, on the day of farrowing and during the post-partum period. In order to describe each criterion, they were marked 0 , when normal and 1 when they were considered as abnormal. For daily monitoring, the scores were totalled over the entire period covered. Unless stated to the contrary, the statistical analysis used the MannWhitney test (Wilcoxon).RESULTS

On the day of inclusion in the study, none of the observed parameters was significantly different between the two batches. In addition, no difference was noted during the monitoring of the farrowing. The lactation anomalies were marked individually and two classes were created: normal lactation and moderately abnormal lactation. For these two classes, there was no difference between the two batches (Chi 2 test, $p=0.137$ ). Three classes were created to mark the sows' appetite: the appetite was significantly less disturbed in the batch treated with Pracetam $^{\circledast}$ than in the control batch (Chi 2 test, $p=0.033$ ). In addition, the phenomenon of constipation was significantly less frequent, particularly during the first three days post-partum, in the treated batch (Chi 2 test, $p<0.001$ ). Moreover, the number of sows having presented abnormal faeces was significantly lower than in the treated batch (Chi 2 test, $p=0.010$ ). The number of sows whose condition required medical intervention is also lower than in the treated batch (particularly during the first three days post-partum, Chi 2 test $p=0.044$ ) (see figure 1).

With regard to the piglets, the litters from the two batches presented no significant difference in terms of mortality. Nor was there any significant difference between the two batches with regard to the health of the piglets at three days of age, but there is a trend (Chi 2 test, $p=0.07$ ) of better health in piglets born of sows treated with Pracetam ${ }^{\circledR}$. The same is also valid for the incidence of diarrhoea (Chi 2 test, $p=0.059$ ). Moreover, the number of piglets having to receive an individual treatment was significantly weaker in the treated batch, particularly during the first three days of life (55 compared with 36 piglets, Chi 2 test, $p=0.037)$.

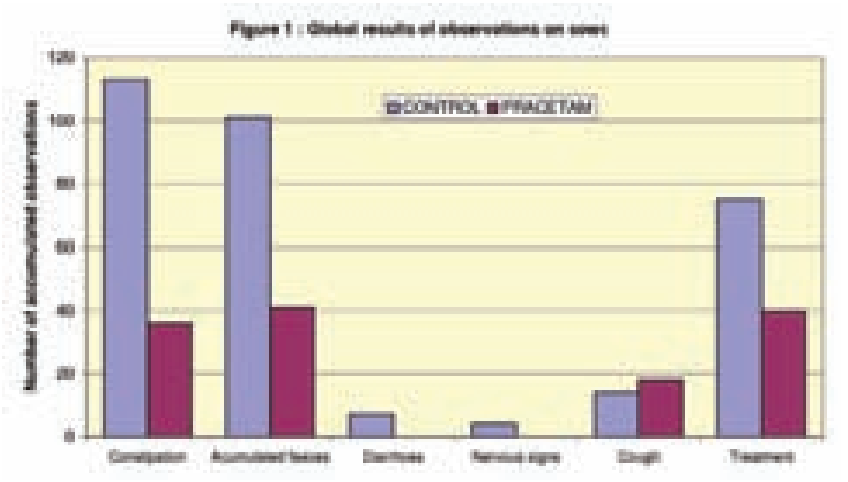

\section{Discussion and Conclusion}

The significant differences observed in the sows from the treated batch (state of health, number of medical interventions) may be attributed to the effects of the paracetamol. As for the significant differences recorded in piglets (state of health, number of treatments), they may be interpreted as a indirect consequent of the positive effects observed in the sows; the sows being in better health in post-partum period (less fever, less constipation, appetite regained earlier), they more voluntarily provided piglets access to the teat, which had a direct consequence on their health and their development.

\section{References}

1) Flower RJ, Vane JR. Inhibition of prostaglandin synthetase in the brain explains the anti-pyretic activity of paracetamol Nature. 1972 $\operatorname{dec} 15 ; 240$ (53813) : 410-1

2) Chandrasekharan NV and coll., COX-3, a cyclo-oxygenase-1 variant inhibited by acetaminophen. Proc Natl Acad Sci USA 2002 Oct 15:99 (21): $13926-31$

3) POBEL T 2004, Study of the tolerance of Pracetam premix in sows during pregnancy and lactation. Registration file. 


\title{
P.805
}

\section{Porcine circovirus type 2 (PCV2) prevalence in abortions in France}

\author{
Guillaume Perreul $^{1}$ Bernard Fily ${ }^{1}$ Sophie Longo ${ }^{2}$ Thaïs Vila $^{3}$ Jean-Bernard Herin ${ }^{1}$ Julie Venet $^{3}$ François Joisel $^{3}$ \\ 1. Merial S.A.S., Ancenis, France; 2. BPC, Labruguière, France; 3. Merial S.A.S., Lyon, France
}

\section{Introduction}

The role of porcine circovirus type 2 (PCV2) in reproductive disorders has been studied (1). The present paper reviews the results of the lab diagnosis collected through a MERIAL protocol « pack avortons » from June 2008 to June 2009.

\section{Material and Methods}

Aborted foetuses or weak piglets (AF-WP) include stillborns and weak piglets which breathed and/or suckled some colostrum before death.

Macroscopic aspect was detailed for 271 AF-WP. Due to poor conservation, 25 AF-WP were not submitted for any analyses.

PCV2, PPV and PRRSV diagnostics were performed on 296 AF-WP from 93 sows in 30 farrow-to-wean and farrow-to-finish non-PCV2-vaccinated herds.

Lab analyses for PRRSV and/or PPV were performed in 15 herds out of 30 on lung pools. In each herd, 1 pool (=3 AF-WP) for each test: all pools were PCR-negative.

Diagnostics for PCV2 were completed in 266 AF-WP as follows:

- by PCR on liver and/or heart for all pooled AF-WP (101 pools : 1 pool=max 3 AF-WP from the same dam)

- in frozen AF-WP which did not drink or breathe totally: antiPCV2 antibody test performed on 135 AF-WP using thoracic and/or abdominal liquid (SERELISA ${ }^{\circledR}$ PCV2 Ab, Synbiotics, Lyon, France)

- by immunohistochemistry (IHC): $36 \mathrm{IHC}$ were performed from individual AF-WP samples: 11 on livers and 25 on hearts.

A farm was considered as facing PCV2 reproduction disorders when at least 1 AF-WP was PCV2 positive using at least 1 method.

\section{Results}

Table 1: $P C V 2$ prevalence in herds

\begin{tabular}{|l|c|c|}
\hline No/Total & Percentage (\%) & \\
\hline PCV2-positive farms & $12 / 30$ & 40 \\
\hline PCV2-positive AF-WP & $66 / 266$ & 24.8 \\
\hline
\end{tabular}

Table 2: Distribution of PCV2-positive AF-WP according to organ and analysis techniques

\begin{tabular}{|l|c|c|c|}
\hline $\begin{array}{l}\text { Clinical aspect of positive } \\
\text { AF-WP(No) }\end{array}$ & $\begin{array}{c}\text { \%age + liver } \\
\text { (PCR \&/or IHC) }\end{array}$ & $\begin{array}{c}\text { \%age +heart } \\
\text { (PCR \&/or IHC) }\end{array}$ & $\begin{array}{c}\text { Abdom. \&/or thor. } \\
\text { liquid (ELISA) }\end{array}$ \\
\hline No lesion (31) & 34.6 & 51.6 & 69.6 \\
\hline Lysed (11) & 0 & 85.7 & 50 \\
\hline Mummified (8) & 100 & 75 & 0 \\
\hline Stillborn (8) & 0 & 0 & 100 \\
\hline Lysed/discolored liver (3) & 0 & 100 & 0 \\
\hline Breathed/No drink (5) & 66.7 & 20 & 75 \\
\hline Total positive AF-WP (66) & 29.1 & 51.6 & 61.5 \\
\hline
\end{tabular}

In the 66 PCV2-positive/266 AF-WP (24.8\%) PCV2 was found most frequently in hearts, except in stillborn AF-WP in which anti-PCV2 antibodies were found. Anti-PCV2 antibodies were found in all AF-WP types except in mummified AF-WP and in AFWP with lysed/discoloured liver.

\section{Discussion}

The «pack avortons » was put in place to systematically confirm or infirm the involvement of the major reproductive pathogens, i.e. PPV, PRRSV and PCV2 in case they could be suspected. All analysis methods taken together, these results report a high PCV2 prevalence. Indeed PCV2 was evidenced in AF-WP in $40 \%$ (often several aborted litters per case) of herds where reproduction disorders were investigated.

In addition, these results enabled to define a diagnosis strategy: mummified foetuses are more likely to be found PCR positive (mumified piglets are often dry so no liquid can be harvested from them to perform ELISA) especially when they are not lysed whereas stillborn foetuses are more often ELISA-positive (i.e. anti-PCV2 antibodies detection). It is well-known that foetuses become immunocompetent from the last third of the gestation, i.e. about 70 days onwards. So age ( $>70$ days of age, i.e. about $17 \mathrm{~cm}$ long) and denaturation status of the conspectus could be used as choice criteria for the diagnosis method.

\section{Conclusion}

PCV2 appears to be involved in a large number of reproductive disorder cases and has to be included in the differential diagnosis.

\section{Acknowledgements}

The authors wish to thank the LDA22 laboratory.

\section{References}

1. Park JS et al., (2005) Journal of Comparative Pathology, Volume 132, 139-144

๑ CIRCOVAC is a registered trademark of Merial in European Union and elsewhere.

๑ SERELISA is a registered trademark of Synbiotics Corporation. 


\title{
P.806
}

\section{Investigation of reproductive failures caused by porcine circovirus type 2 in large pig production units in Croatia}

\author{
Zoran Lipej; Dinko Novosel; Jelena Balatinec; Besi Roic' \\ Croatian Veterinary Institute, Zagreb, Croatia
}

Introduction Porcine circovirus type 2 (PCV2) considered the causal agent of PMWS, has also been demonstrated to be involved in reproductive failure [1]. Congestive heart failure, excess fluid in the thoracic and abdominal cavity, dilated and hypertrophied heart, firm and enlarged liver was frequent and the most prominent gross pathological finding in aborted and stillborn piglets [2]. Histologically, there were severe non-suppurative myocarditis and myocardial necrosis with fibrosis [1, 2].

The objective of this study was to investigate role of PCV2 in aborted and premature piglets connected with reproductive disorders on large pig production units in Croatia.

Material and Methods Eight farrow-to-finish farms with previous history of PMWS and reproductive failures were selected for the study. A total of 83 fetuses aborted in the last third of gestation and premature piglets submitted to the Croatian Veterinary Institute, were included in this study.

Briefly, a post-mortem examination was performed and samples of heart were collected in 10\% buffered formalin for histopathological examination and immunohistochemistry (IHC) and in situ hybridization (ISH). For PCR detection samples of lungs, spleen and liver were pooled and stored on $-70^{\circ} \mathrm{C}$. Tissue in fixative was routinely processed and stained with $\mathrm{H} \& \mathrm{E}$. Myocardium sections were proceeded by IHC detection with anti-PCV2 ORF2 monoclonal antibodies (Ingenasa ${ }^{\circledast}$ ) according to previously described protocol[3]. In situ hybridization was performed with digoxigenin labelled 41 base oligonucleotide probe on myocardium tissue according previously described protocol [4]. For detection of PCV2 ORF 1 is used Q-RT-PCR as previously described protocol [5].

Results Salient gross findings demonstrated in aborted or stillborn piglets were diffuse subcutaneous oedema, oedema and segmental haemorrhages of umbilical cord, hydrothorax, ascites, subepicardial haemorrhages and haemorrhages on kidney cortex. Histopathologically, in 21 (25.30\%) fetuses mild myocardial degeneration and mild myocardial necrosis without lymphocytic inflammatory reaction were observed. All of tested samples of heart were negative for presence of PCV2 antigen by IHC and all of tested myocardium tissue was negative for presence of PCV2 genome by ISH. In all 83 tested samples PCV2 genomic DNA is detected.
Discussion In this study gross lesions on fetuses characterized by severe, nonsuppurative myocarditis and myocardial necrosis with fibrosis reported as specific for PCV2 associated reproductive failure, were not found.

Positive PCR findings in all 83 samples of myocardial tissue are unexpected and surprising and in contrast with negative IHC and ISH results in same heart tissues. Therefore, all PCR positive results are considered as false positive or result of secondary contamination. Because of that sequencing of PCR products still need to be performed to confirm it. According to absence of specific gross lesions on examined fetuses, no characteristic histopathological lesions and negative results of $\mathrm{IHC}$ and ISH in myocardial tissue we consider there is no evidence that PCV2 caused reproductive failure in Croatian large pig production units. Based on our results, the lack of evidence of association between PCV2 and reproductive failure suggests that PCV2 is probably not an important abortifacient pathogen in Croatia. These results are in accordance with Spain researchers [6]

\section{References}

1. O'Connor, B., et al. (2001): Can Vet J,. 42: p. 551-3.

2. West, K.H. et al. (1999): J Vet Diagn Invest,. 11: p. 530-2.

3. Sorden, S.D., et al. (1999): J Vet Diagn Invest,. 11: p. 528-30.

4. Rosell, C., et al. (1999): J Comp Pathol,. 120: p. 59-78.

5. Jemersic, L., et al. (2004): Res Vet Sci,. 77: p. 171-5.

6. Maldonado, J., et al.. (2005): Vet J,. 169: p. 454-6. 


\title{
P.807
}

\section{Detection and prevalence of viral pathogens from aborted fetuses and stillborn piglets in Korea}

\author{
Won Gwnag Lee; Tae Hun Woo; Jeong Hee Han \\ School of Veterinary Medicine and Institute of Veterinary Science, Kangwon National University, Chuncheon-si, Korea
}

\section{Introduction}

Reproductive disease during the gestation in sows is usually manifested by abortion or stillbirth. Two major classes of reproductive failure are recognised in swine. A first category is represented by infectious agents (considered responsible for $30-40 \%$ of abortions, mummified fetuses, and stillbirths) that cause primary infection of the reproductive tract and most of them are viruses. A second group includes toxins, environmental and nutritional stresses, and systemic disease in the sow (considered responsible for the rest of the reproductive failure). A large number of viruses have been reported to affect porcine reproductive performance, and it is likely that any virus capable of causing clinical illness in adult swine, or crossing the placental barrier to infect the porcine conceptus, is a potential reproductive pathogen. This experiment was conducted to investigate the presence of recognised abortifacient viruses from aborted fetuses and stillborn in cases of reproductive failure in sows.

\section{Materials and Methods}

A total of 219 samples of aborted fetuses or stillborn, submitted to the Department of Veterinary Pathology of Kangwon National University between 2006 and 2009 May, were collected from 97 commercial swine farms located in the five provinces(Gyunggi, Gangwon, Chungcheong, Jeolla, Gyeongsang)

in Korea. Each samples were tested for abortifacient viruses such as PRRSV, PPV, ADV, JEV, EMCV, SIV and PCV2. The presence of virus in fetal tissues was determined by PCR or RT-PCR.

\section{Results}

Abortifacient virus infections were detected in 82 out of 219 aborted fetuses or stillborn(37.4\%) as well as on 39 out of 97 pig farms $(40.2 \%)$. The major viral infection were procine circovirus type 2 (PCV2), porcine reproductive and respiratory syndrome virus (PRRSV) and Aujesky's disease virus (ADV) for which 46 (21\%), 19 (8.6\%) and 16 (7.3\%) were positive, respectively, with 9 fetuses had complicated infection that 4 for PCV2+PRRSV, 3 for PCV2 + ADV, 2 for PCV2 + PRRSV + ADV. As minor viral infection SIV, PPV, JEV for which 8(3.6\%), 3(1.3\%), 1(0.4\%) were detected. And EMCV was not identified.

\section{Conclusions}

The result suggest that PCV2, PRRSV, ADV is apparently the most important viral infectious agents associated with fetal infection leading to abortion or stillbirth in Korea. Moreover, SIV, PPV and JEV might have a minor impact on reproductive disease.

\section{References}

1. Straw BE et al. 1999. Diseases of Swine, 8th ed. lowa State University Press, Ames, lowa, 41-89.

2. Vannier P. 1999. Reprod DomAnim 34, 367-376.

3. O' Connor B et al. 2001. Can. Vet. J., 42, 551-553. 


\title{
P.808
}

\section{Gross morphology of the oviducts in slaughtered replacement gilts}

\author{
Padet Tummaruk; Sawang Kesdangsakonwut; Paisan Tienthai \\ Chulalongkorn University, Bangkok, Thailand
}

\section{Introduction}

Retrospective studies based on slaughterhouse materials are a useful tool for the clinical diagnosis of reproductive disturbance in pigs $(1,2)$. In the pig oviduct, a lot of important reproductive events such as sperm capacitation, fertilization and early embryonic development take place (3). In Thailand, $47 \%$ of all culling replacement gilts are culled due to reproductive problems, i.e., anestrus, repeat breeding, not being pregnant, vaginal discharge and abortion (4). The objective of the present study, therefore, was to investigate gross morphology of the oviducts in gilts culled due to reproductive failure in Thailand.

\section{Materials and Methods}

Genital organs from 337 replacement gilts from 6 swine herds in Thailand were collected at slaughterhouses between May 2005 and September 2008. The organs, including ovaries, oviducts, uteri, cervices and vaginas-vestibules-vulvae were collected, placed on ice and transported to the laboratory within $24 \mathrm{~h}$ after culling. Historical data included herd, gilt identity, breed, date of birth, culling date, body weight at culling and reason for culling were collected. Age at culling was calculated. The reproductive cycle of the gilts were classified according to the appearance of the ovary as inactive, follicular and luteal phases (2). The abnormalities of the oviduct were investigated and analyzed by using frequency analysis. Pearson's correlation was used to analyze the relationship between the length of the oviduct and body weight, uterine weight, length of the uterine horns, length of other genital organs and number of ovulations. Length of the oviduct was compared between left and right sides by using paired t-test. The influence of reproductive cycle and body weight on the length of the oviduct was analyzed by using multiple ANOVA.

\section{Results and discussion}

On average, the gilts were culled at $300.9 \pm 49.3$ days of age and the body weight at culling was $145.5 \pm 20.2 \mathrm{~kg}$. The reason for culling included anestrus (154 gilts), abnormal vaginal discharge $(n=62)$, repeat breeding $(n=41)$, abortion $(n=27)$, not pregnant $(n=20)$, non-reproductive problems, e.g., lameness, illness, and poor conformation $(n=21)$ and miscellaneous $(n=8)$. Of these gilts, 294 gilts (88.0\%) had normal oviducts, 29 gilts (8.7\%) had moderate to severe congestion of the oviducts, 4 gilts (1.2\%) had salpingitis, 3 gilts had pyosalpinx (0.9\%), 3 gilts $(0.9 \%)$ had congenital abnormalities, i.e., unilateral hypoplasia, oviductal diverticulum and a remaining of epididymis in a true hermapodite gilt, and 1 gilt had blood clot obstruction in the oviduct. Of the anestrus gilts, 134 had normal oviduct, 12 had oviduct congestion, 3 had congenital abnormalities, 2 had salpingitis and 1 had pyosalpinx. Of the gilts culled due to vaginal discharge, 54 had normal oviduct, 6 had oviduct congestion and 2 had pyosalpinx. Of the repeat breeding gilts, 33 had normal oviduct, 6 had oviduct congestion, 1 had salpingitis, and 1 had blood obstruction. Of the aborted gilts, 25 had normal oviduct and 2 had oviduct congestion. Of the non-pregnant gilts, 17 had normal oviduct, 2 had oviduct congestion and 1 had salpingitis. All of the gilts culled due to non-reproductive and miscellaneous causes had normal oviduct. Of the gilts with normal and congested oviducts $(n=323)$, the length of the oviducts was $27.9 \pm 6.7 \mathrm{~cm}$. on the left and $26.2 \pm 6.2 \mathrm{~cm}$. on the right sides. The left oviduct was $1.7 \mathrm{~cm}$ longer than the right oviduct $(P<0.001)$. The length of the oviducts during inactive phase was shorter than luteal and follicular phases (21.1 versus 28.7 and $31.3 \mathrm{~cm}$, respectively $(\mathrm{P}<0.001)$. Gilts with a body weight of $92-130 \mathrm{~kg}$ had a significantly shorter oviduct than gilts with a body weight of above $150 \mathrm{~kg}(\mathrm{P}<0.008)$. The length of the oviduct was significantly correlated with body weight $(r=0.34, P<0.001)$, uterine weight $(r=0.62, P<0.001)$, length of the uterine horns $(r=0.54, P<0.001)$, length of the uterine body $(r=0.44, P<0.001)$, length of the cervix $(r=0.26$, $P<0.001)$, length of the vagina $(r=0.31, P<0.001)$ and number of ovulation ( $r=0.21, P=0.003$ ). The present study demonstrated some important gross morphology of the oviduct in slaughtered replacement gilts in Thailand.

\section{References}

1. Dalin A-M, Gidlund K, Eliasson-Selling L. 1997. Acta vet. scand. 38:253-262.

2. Tummaruk, P., Kesdangsakonwut, S., Kunavongkrit, A. 2009. Theriogenology. 71:369-375.

3. Jiwakanon, J., Persson, E., Kaeoket, K., Dalin, A.-M., 2005. Reprod. Domest. Anim. 40: 28-39.

4. Tummaruk, P., Sukamphaichit, N., Kitiarpornchai, W., Musikjearanan, S., Tantasuparuk, W. 2006. Proc 19th IPVS Congress, Copenhagen, Denmark, 2006, p. 498.. 


\title{
P.809
}

\section{Urinary tract infections in sow herds with and without frequent puerperal diseases}

\author{
Michael Wendt ${ }^{1}$ Jutta Verspohl$^{2}$ \\ 1. Clinic for Swine and Small Ruminants, Hannover, Germany; 2. Institute for Microbiology, Hannover, Germany
}

\section{Introduction}

Uncomplicated infection of the lower urinary tract frequently occurs in sow herds. Clinical symptoms in sows are very mild and include urinary anomalies and vulvar discharges. However, urinary tract infections (UTI) are often associated with the development of puerperal endometritis and reduced fertility accompanied by increased rates of regular returns to estrus and repeat-breeder sows (1).

The aim of this study was to investigate the prevalence of UTI in sow herds with or without frequent puerperal diseases.

\section{Materials and Methods}

During farm visits as many mid-stream urine samples as possible were collected from a total of 34 sow herds by spontaneous micturition of sows in dry sow, gestation and farrowing areas (herd size: group 1-50: $n=3$, group 51-100: $n=8$, group 101-250: $n=16$, group 250-500: $\mathrm{n}=7$ ).

Urine samples were first investigated by macroscopic examination (turbidity, irregular contents). Visually altered samples were selected and further controlled by test strips ( $\mathrm{pH}$, nitrite, protein, blood) as well as by microscopic investigation (crystalluria). Aerobic bacterial counts were estimated by dip slides, $10^{5} \mathrm{cfu} /$ $\mathrm{ml}$ urine were taken as a threshold for a significant bacteriuria (=UTI). Specification of bacterial isolates was carried out by aerobic culture techniques in 129 samples (4 per farm on average).

\section{Results}

In total 1194 urine samples were collected; the mean percentage of sampled sows per farm was $20.3 \%$, ranging from 8.2 to $64.8 \%$. Macroscopic changes were detected in 500 samples (41.9\%), on 8 farms $<30 \%$ of samples were altered, on 21 farms $30-60 \%$ and on 5 farms $>60 \%$. 435 out of 500 visually altered urine samples were investigated using dip slides, revealing 213 samples with significant bacteriuria (49.0\%). Severe crystalluria was a common finding in visually altered samples (37.7\%; unspecific phosphates, triple phosphates, calcium oxalate), but only one third was accompanied by a bacteriuria.

The mean percentage of UTI for all herds was 20.9\%; on farms without frequent puerperal problems UTI were detected, in 13.0 $(+8.1) \%$ of the herd, on farms with frequent MMA problems UTI were present in $25.3(+12.6) \%$ of the herd. However, sample size for a prevalence survey (with $90 \%$ confidence interval, $10 \%$ precision, estimated prevalence $20 \%$ >> sows per herd/sows to be sampled: 50/24, 100/31, 250/37, 500/40, >500/43) was fulfilled only in 15 herds. The mean percentage of UTI for these 15 farms was $18.0(+9.6) \%, 5$ herds without MMA problems and 10 herds with frequent MMA problems revealing $10.6(+6.0) \%$ and 21.6 $(+9.1) \%$ UTI, respectively $(p<0.05)$.

Results of test strips with regard to nitrite-contents were compared to results of bacterial counts (dip slides). Only $40.3 \%$ of urine samples showing significant bacteriuria were nitritepositive.

Bacteriological investigation of 129 samples demonstrated in 110 cases only one species with a bacterial count $>10^{5} \mathrm{cfu} / \mathrm{ml}$; in 19 cases two species with a high bacterial count were found. $E$. coli was isolated most frequently $(n=96)$ followed by Enterococcus spp. $(n=14)$ and alpha-hemolytic streptococci $(n=7)$.

\section{Discussion}

Intensive sampling is necessary to obtain a sufficient number of urine samples for a conclusive UTI prevalence survey. The mean UTI percentage seemed to be overestimated when sample size was too small. UTI has to be supposed as an important risk factor for frequent MMA problems, if the prevalence in a herd exceeds $20 \%$. Ascending infection of the uterus follows uncomplicated UTI after farrowing (2). The bacterial flora found in the infected bladder in this study is often detected in puerperal endometritis of sows (3).

As significant bacteriuria rarely occurs in urine samples without visual changes, only visually altered samples were selected for dip slide investigation; for this reason the percentage of UTI may be slightly underestimated. However, severe crystalluria was also a common reason for turbidity.

Nitrite content alone was not a sufficient diagnostic parameter for UTI, because $60 \%$ of UTI cases were not detected by this test.

\section{References}

1. Dial, G.D., MacLachlan, N.J. (1988), Comp. Cont. Ed. Pract. Vet. 10, 63-71.

2. Berner, H. (1984), Tieraerztl. Umsch. 39, 450-458.

3. De Winter, P.J.J. et al. (1994), Anim. Reprod. Sci. 37, 325-335. 


\title{
P.810
}

\section{Relationship between seminal quality and leucocytes presence in ejaculate of experimentally infected boars with porcine rubulavirus}

\author{
SG Estrada-B1 ML Galicia-G ${ }^{1}$ JF Rivera-Benitez ${ }^{1}{ }^{1}$ J Herrera-H ${ }^{3}$ H Ramirez-Mendoza ${ }^{4}$ A García-Contreras ${ }^{1}$ \\ 1. POLIVET-AZ, DCBS-DPAA UAM-Xochimilco, Coyoacán, DF, Mexico; 2. Ganadería Colegio de Posgraduados, Montecillos, MEX, \\ Mexico; 3. Ganadería Colegio de Posgraduados, Montecillos, MEX, Mexico; 4. Lab. Virología, FMVZ-UNAM, Coyoán, Mexico
}

\section{Introduction}

The leukocytes presence analyze, in boar semen, as a routine technique for quality control of seminal doses is not commune, however, in human is performed with strict care, because of its association with low fertility patients. Ramírez et al. (1) and Solís et al. (2) have indicated that the porcine rubulavirus pig has affinity with epididymis and prostate declining the semen quality on boars, so a study was conducted to determine the relationship between leukocytes presence and ejaculate quality on boars.

\section{Material and methods}

Nine York-Landrace boars, aged 12 months, serologically free of reproductive diseases underwent two experimental phases: Phase I, before-infection, where 10 ejaculates per animal were obtained, and, Phase II, after-infection where boars were inoculated intranasally with $5 \mathrm{ml} 10^{5} \mathrm{TCID}_{50} / \mathrm{ml}$ of strain PAC-3 Jalisco/1992, scoring 21 ejaculates per animal. The boars ejaculates were evaluated without fractions: Volume $(\mathrm{ml})$, sperm concentration (spz ml-1) motility progressive (\% MTP), abnormalities, and live sperm (\% SL) were recorder. The leucocytes count ( $\mathrm{L} x$ $10^{3} \mathrm{~mL}$ ) was conducted in two phase, using a Neubauer counting camera (3), and stained slides with Wright solution. Statistical analysis was a repeated measure design with SAS Mixed Model.

\section{Results}

Table 1 shows the quality and production values of boar's sperm obtained during the evaluated phases. There were significant difference $(\mathrm{P}<0.05)$ on abnormalities, spz ml-1 and leukocytes count. The volume, motility and SL were not different $(P>0.05)$.

\begin{tabular}{|l|c|c|}
\hline Semen Quality & Phase I Before-Infection & Phase II After-Infection \\
\hline Volumen $\mathrm{ml}$ & $158 \pm 151$ & $152.33 \pm 35$ \\
\hline Spz. $\mathrm{ml}^{-1}$ & $829 \pm 241$ & $246-483 \pm 78.25$ \\
\hline$\%$ MTP & $85 \pm 3.8$ & $84.75 \pm 6.0$ \\
\hline Abnormalities \% & $18.4 \pm 2.5$ & $41.8 \pm 6.6$ \\
\hline SL \% & $80 \pm 2.11$ & $80.8 \pm 6.5$ \\
\hline L x $10^{3}$ ml & - & $30.2 \pm 21.66$ \\
\hline Least square means within a row with different subscript letter differ $(\mathrm{P}<0.05)$ \\
\hline
\end{tabular}

\section{Discussion}

The production and semen quality of boars during Phase I was similar to healthy boars and weekly work rhythm, reported for Kolenbrander et al.(4). However, during Phase II, sperm production (spz ml-1) decreased 66\%, abnormalities rose twice, and the leukocytes presence beginning at eight days after-infection $(P<0.05)$.There was a significant increase on Leukocytes weekly, where its highest value occur at the eighth week, to gradually decline thereafter, reaching a level of 20 leukocyte $\times 10^{3} \mathrm{ml}$, holding constant these values the last five weeks of the experimental work.

\section{Conclusion}

The inoculation of porcine rubulavirus had a negative effect on sperm concentration and abnormalities increase, but volume, live spermatozoa's and motility remained without changes. In addition, leucospermia showed, which at least not disappear during the period under investigation and the semen quality never recovers.

\section{References}

1. Ramírez, M. H., Hernández, J. P., Reyes, L. J., Zenteno, E., Moreno, L. J., Kennedy, S., 1997. Lesions in the productive tract of boar experimentally infected with porcine rubulavirus J. Comp. Pathology., 117:237-252

2. Solís M., Espinosa H. S., Mercado G. C., Hernández L. J., Ramírez H. 2007. Semen alterations porcine rubulavirus infected boars are related to viral excretion and have implications for artificial insemination. Res. Vet Sci.

3. Henry, J. B. 2001. Diagnóstico y tratamiento clínico por el laboratorio. $10^{a}$ edición. Editorial Masson Salvat., pp. 582-603

4. Kolenbrander B., Feitsma H., Grooten HJ. 1993. Optimizing semen production for artificial insemination in swine. Journal Reproduction Fertility Suppl., 48: 207-215 


\title{
P.811
}

\section{Influence of maternal antibodies on PCV2 vaccine efficacy}

\author{
Brad J. Thacker; Warren D. Wilson \\ Intervet/Schering-Plough Animal Health, Desoto, KS, USA
}

\section{Introduction}

Vaccine interference due to maternally-derived antibodies (MDA) has been shown to occur in a number of species and with a number of diseases. ${ }^{1}$ The data reported in this paper was derived from a previously reported study where Circumvent ${ }^{\circ} \mathrm{PCV}$ (Intervet Schering Plough Animal Health) was shown to be effective in controlling PCVD in a herd with a history of moderate PCVD. ${ }^{2}$ In this paper, we report the influence of MDA at weaning (time of first vaccinatin) on the growth performance and mortality of vaccinated and non-vaccinated pigs.

\section{Materials and Methods}

The study was conducted in a 1700 sow herd that had experienced a mild increase in mortality rates and a severe increase in cull rates due to PCVAD. This herd had not previously vaccinated for PCV2 so the dams of the pigs used for this study had never been vaccinated. Pigs were weighed, tagged and their gender was determined one day prior to weaning at approximately 23 days of age. The pigs were allocated to vaccinated or control groups with stratification by weight, gender and litter. The pigs were vaccinated per label directions at 3 and 6 weeks of age (VAC) or left as non-vaccinated controls (CON). The pigs were weighed at weaning with a final weight at 21 weeks of age.

One pig per litter (the largest pig) was bled at weaning and periodically thereafter. Antibody titers to PCV2 were measured using an indirect immunofluoresence assay (IFA). Titers are reported as the highest dilution that inhibited fluorescence. The IFA titer at weaning of the tested pig was used as a surrogate MDA titer for litter mates. Not all litters contained a pig that was bled and those litters were not included in the analysis. The growth rate and mortality from weaning to 21 weeks of age were evaluated by linear regression and Fisher's exact test for each titer level, respectively. Previous studies indicated that an MDA titer of $>=640$ may interfere with vaccination based on post-vaccination serological responses so the data was grouped by MDA titers $<=320$ or $>=640 .{ }^{3}$ These titer groupings were similarly evaluated by ANOVA and Chi Square.

\section{Results}

The number and percentage of pigs with each MDA titer is presented in Table 1. Table 2 presents growth and mortality data for vaccinates and controls overall and grouped by MDA titers $<=320$ or $>=640$. There was no influence of MDA titer on either growth or mortality rates. Overall, vaccination improved growth rate and decreased mortality.
Table 1. PCV2 MDA titers of pigs at weaning

\begin{tabular}{|l|c|c|c|}
\hline MDA titer & No. (\%) & MDA titer & No. $(\%)$ \\
\hline$<1: 20$ & $43(9.9)$ & $1: 160$ & $98(22.5)$ \\
\hline $1: 20$ & $38(8.7)$ & $1: 320$ & $74(17.0)$ \\
\hline $1: 40$ & $51(11.7)$ & $1: 640$ & $59(13.6)$ \\
\hline $1: 80$ & $52(12.0)$ & $>=1: 1280$ & $20(4.6)$ \\
\hline
\end{tabular}

Table 2. Growth and mortality by MDA titer and vaccination status

\begin{tabular}{|c|c|c|c|c|}
\hline Group & Parameter & $M D A<=320$ & $M D A>=640$ & Overal \\
\hline \multirow[t]{3}{*}{ VAC } & No. of pigs & 178 & 39 & 217 \\
\hline & Daily gain & 1.358 & 1.335 & $1.354^{\mathrm{a}}$ \\
\hline & Mortality & $6.2 \%$ & $5.1 \%$ & $6.0 \%^{c}$ \\
\hline \multirow[t]{3}{*}{$\mathrm{CON}$} & No. of pigs & 178 & 40 & 218 \\
\hline & Daily gain & 1.313 & 1.329 & $1.316^{\mathrm{b}}$ \\
\hline & Mortality & $11.2 \%$ & $7.5 \%$ & $10.6 \%^{d}$ \\
\hline \multicolumn{5}{|c|}{$\mathrm{a}, \mathrm{b} P$ value $=0.019 ; c, \mathrm{~d} P$ value $=0.060$} \\
\hline
\end{tabular}

\section{Discussion}

MDA titer at weaning (time of first vaccination) with Circumvent ${ }^{\circ}$ PCV appeared to have no impact on the subsequent performance of the pigs. Growth rate and mortality in the pigs that were sampled was not impacted by MDA titer as well (data not shown). The dams of the pigs used in this study had never been vaccinated. Both dam titers and pig MDA titers have been shown to be increased by vaccination of the dams prior to farrowing. ${ }^{3}$ Vaccination of dams was not evaluated in this study. However, serological evaluations of young pigs from sows that were vaccinated as young pigs have yielded similar IFA titer patterns as reported in Table 1 of this study.

\section{References}

1. Thacker, B.J., et al. Proc. AASV Ann. Mtg.,Nashville, Tennessee, pp. 513516, 2001.

2. Thacker BJ, et al. Proc IPVS, Durban, South Africa, p. 90, 2008.

3. Thacker, BJ, et al. Proc. AASV Ann. Mtg., San Diego, California, pp. 153$156,2008$. 


\title{
P.812
}

\section{Validation of a novel real-time PCR for detection of Leptospira DNA in clinical samples}

\author{
Adolf Steinrigl ${ }^{1}$ Maria Müller ${ }^{1}$ Louis Fischer $^{2}$ Friedrich Schmoll ${ }^{3}$ Sandra Revilla-Fernández Petra Winter $^{1}$ \\ 1. Austrian Agency for Health and Food Safety (AGES), Institute for Veterinary Disease Control Mödling, Mödling, Austria; \\ 2. Labovet GmbH - Laboratory for Veterinary Diagnostics and Hygiene, Vienna, Austria; \\ 3. Institute for Veterinary Public Health, University of Veterinary Medicine Vienna, Vienna, Austria
}

\section{Introduction}

Leptospirosis is considered an important zoonosis as well as an emerging infectious disease. Pigs are among those domestic animals mostly affected by clinical disease, resulting from infection with pathogenic leptospires. Clinical signs of leptospirosis in pigs mainly include unspecific symptoms related to reproductive disorders, such as abortion, stillbirth, mummification and birth of weak piglets [1]. Similar symptoms may also be caused by a variety of common infectious agents, making laboratory testing for leptospirosis essential for both clinical diagnosis as well as eradication of the agent from affected livestock. Importantly, Leptospira-infected animals may proceed to an asymptomatic carrier state, shedding infectious leptospires into the environment and thus providing a source of infection for humans [2]. In order to develop a rapid, simple and sensitive method for direct demonstration of leptospires in clinical materials, polymerase chain reaction (PCR) based methods have been propagated in recent years. Among these, real-time PCR methods are especially attractive for clinical applications, because they allow not only qualitative, but also (semi)-quantitative detection of leptospiral DNA. However, only a few published real-time PCR protocols have been validated using clinical material from humans or livestock [3-5]. Here, we describe the design and validation of a novel real-time PCR specific for the detection of DNA from pathogenic Leptospira spp. and our experience with testing a variety of porcine clinical samples.

\section{Materials and Methods}

The LipL32 coding region that is only present in pathogenic Leptospira spp. was chosen as target region for the design of primers and hydrolysis probe for real-time PCR. Special care was taken to minimize the number of mismatches between primers/ probe and the available LipL32 target sequences. Real-time PCR kinetics and analytical sensitivity were evaluated by amplifying serially diluted DNA obtained from a culture of L. interrogans. Analytical specificity was determined by analyzing nucleic acid extracted from non-pathogenic leptospires and Leptospirarelated bacteria as well as from a variety of viral, bacterial and protozoan infectious agents involved in clinical disorders of swine. Clinical material from pigs, such as EDTA-blood, serum and tissue samples was obtained in the course of routine testing, from private veterinary practitioners and from a private veterinary laboratory.

\section{Results}

Alignment of all Leptospira LipL32 sequences ( $n=106)$, which were available from GenBank at the time of assay design revealed a maximum of 1 mismatch to the forward primer (13 sequences) and 4 mismatches to the reverse primer ( 1 sequence). Eight sequences showed a single mismatch to the hydrolysis probe and 2 mismatches to the probe were evident in 9 sequences, all of which belonged to L. santarosai. Real time PCR analysis of a serial dilution of leptospiral DNA showed high sensitivity, linear amplification within 5 orders of magnitude and acceptable real-time PCR kinetics $\left(R^{2}>0.99\right.$; PCR efficiency $=92-$ 94\%), independent whether leptospiral DNA was diluted in yeast tRNA or in pig genomic DNA. The assay was capable of detecting all serovars of pathogenic leptospires tested $(n=13)$, while no positive signal was observed with any non-pathogenic member of the Leptospiraceae, or with any of the other infectious agents tested. Leptospiral DNA was detected in several clinical samples obtained from pigs.

\section{Discussion}

The real-time PCR assay described here was shown to be specific for the detection of DNA from pathogenic leptospires. The presence of pig genomic DNA in serial dilutions of leptospiral DNA did not influence real-time PCR performance, suggesting that the test can also be used for demonstration of leptospires in tissues from infected pigs. This is further supported by detection of leptospiral DNA in porcine clinical samples. Straightforward detection and quantification of leptospiral DNA is necessary for clinical diagnosis and may also increase our understanding of the epidemiology of leptospirosis.

\author{
References \\ 1. Adler B, de la Pena Moctezuma A. 2010; Vet Microbiol 140:287-96. \\ 2. Levett PN. 2001; Clin Microbiol Rev 14:296-326. \\ 3. Smythe LD, et al. 2002; BMC Infect Dis 2:13. \\ 4. Merien F, et al. 2005; FEMS Microbiol Lett 249:139-147. \\ 5. Fearnley C, et al. 2008; Res Vet Sci 85:8-16.
}




\title{
P.813
}

\section{Diagnostic investigations on mycobacterial abortion in a sow herd}

\author{
Benjamin Schade ${ }^{1}$ Britta Janowetz ${ }^{1}$ Heike Köhler ${ }^{2}$ Irmgard Moser ${ }^{2}$ Ulrike Mittermeier ${ }^{1}$ Reinhard Nothum ${ }^{3}$ Jens Böttcher $^{1}$ \\ 1. Tiergesundheitsdienst Bayern e.V., Poing, Germany; 2. Friedrich-Loeffler Institute, Institute of Molecular Pathogenesis, Jena, Germany; \\ 3. Veterinary Practice Dr. Reinhard Nothum, Haselbach, Germany
}

\section{Introduction}

Swine abortion associated with a mycobacterial infection is an uncommon finding (Ellsworth et al.). Since intradermal testing is time-consuming and therefore cost-intensive, alternative testsystems are needed to get an economically justifiable overview of the situation in an affected sow herd.

\section{Materials and Methods}

Case history: In March 2009, three neonatal porcine fetuses were submitted for diagnostic investigations. The sow was necropsied one month later.

Histopathology: Samples were fixed in 10\% buffered formalin. 4$\mu \mathrm{m}$-thick sections were cut and stained with haematoxylin and eosin, as well as Ziehl-Neelseńs stain.

PCR: DNA was extracted from fetal and maternal tissue with the DNeasy Blood and Tissue Kit (Qiagen, Hilden, Germany) following the manufacturer's instructions. Presence of mycobacteria of the Mycobacterium-tuberculosis-complex and Mycobacteriumavium-complex were examined using the primers according to Rodriguez et al., Guerrero et al. and Kunze et al..

Immunological tests: Blood samples were collected from 30 sows and 10 suckling, growing and fattening pigs, respectively. They were stimulated with phosphate-buffered saline (PBS), Avian PPD (Prionics) and poke-weed mitogen (Sigma), respectively. Thereafter plasma was analyzed for IFN- $\gamma$ with Quantikine ${ }^{\circledR}$ Porcine IFN- $\gamma$ Immunoassay (R\&D Systems, Wiesbaden; Cut-off $40 \mathrm{pg} / \mathrm{ml}$ ). Serum was tested for antibodies with two prototype ELISA ID-Screen ${ }^{\circledast}$ Paratuberculosis Indirect Multispecies (IDVetMAP) and Mycobacterium avium hominissuis (IDVet-MAHS) ELISA (kindly provided by Philippe Pourquier, Montpellier).

\section{Results}

Necropsy and histopathology: The fetuses showed numerous, small (Ø 2-6 mm), white foci in the liver. Sow: Several white, small, firm nodules projected from the surface of the endometrium. Several lymph nodes were enlarged. Multifocal, small ( $\varnothing$ 2-4 mm), white, firm nodules were seen on the surface of the lungs and in the kidneys. Fetal livers and the affected organs of the sow showed a mild to severe, multifocal, granulomatous inflammation, multinucleated giant cells with single to numerous, intracytoplasmatic, acid-fast and rod-shaped bacteria.

PCR: M. avium ssp. hominissuis was detected in fetal liver and organs of the sow including endometrium, lung, kidney, tonsil and several lymph nodes. Mycobacteria of the Mycobacteriumtuberculosis-complex were not detectable.
Immunological tests: Eleven of 27 sows ( $3 x>1000 ; 4 x$ 100-1000 and $4 x 40-100 \mathrm{pg} / \mathrm{ml}$ ) and only one suckling piglet $(104 \mathrm{pg} / \mathrm{ml})$ responded to Avian PPD. Three sows had detectable antibodies (one positive in both ELISAs had to be excluded from IFN- $\gamma$-test; one positive in IDVet-MAHS and IFN- $\gamma$-test; one positive in IDVet-MAP and negative in IFN- $\gamma$-test). None of the other animals showed a serological reaction.

\section{Discussion}

Tuberculous lymphadenitis caused by M. avium ssp. hominissuis is common in swine but genital and/or systemic infections seem to be rare. Detection of subclinically infected animals is essential, as they could be a source of infection for others. Frequent infection of sows was demonstrated by IFN- $\gamma$-test (41\%). In contrast, antibodies were detectable only in single cases. Preliminary data are promising, but further investigations are necessary to validate this new test system.

\section{Acknowledgements}

This study was financially supported by the Free State of Bavaria and the Bavarian Joint Founding Scheme for the Control and Eradication of contagious Livestock Diseases.

\section{References}

Ellsworth SR et al., Mycobacterium avium abortion in a sow, Vet. Pathol. 16: 310-317 (1979)

Guerrero $C$ et al., A novel insertion element from Mycobacterium avium, IS1245, is a specific target for analysis of strain relatedness, J. clin. Microbiol. 33: 304-307 (1995)

Kunze ZM et al., Biologically distinct subtypes of Mycobacterium avium differ in possession of insertion sequence IS901, J. Clin. Microbiol. 30: 2366-2372 (1992)

Rodriguez JG et al., Species-specific identification of Mycobacterium bovis by PCR, Microbiol. 141: 2131-2138 (1995) 


\title{
P.814
}

\section{A case report of porcine circovirus type 2 (PCV2) infection with many mummified in a newly started sow herd}

\author{
Jesper S. Olesen \\ Porcus Swinespecialists, Ringe, Denmark
}

\section{Introduction}

This case report fully proves that PCV2 infection can result in many mummified fetuses at farrowing and be a costly problem for the producer. The report describes in details a PCV2 outbreak in a newly started 550 head sow herd in Denmark autumn 2008. PCV2 is also described in the literature as the cause of return to estrus as well as mummified and stillborn (1).

\section{Materials and Methods}

Data material consists of the production results from the first 399 inseminated gilts in a newly started sow herd. The results for each animal includes: identification number, date of insemination, date of farrowing, liveborn, stillborn, and mummified.Thirty mummified fetuses from four different sows were sent to the Veterinary Institute, Copenhagen for proper pathological testing. The mummified fetuses were tested in the following ways: 1) bacteriological, 2) porcine parvovirus virus (PPV) by antigenELISA, 3) PPV antibodies by immunofluorescence, 4) amount of lgG by ELISA, and 5) PCV2 by immunohistochemistry. Three out of the four sows with mummified fetuses sent to the laboratory were also blood tested. The blood was analyzed for: 1) PCV2 antibodies by ELISA, 2) PPV antibodies by ELISA, and 3) PCV2 by PCR.

\section{Results}

Out of the 399 inseminated gilts 356 farrowed (farrowing rate of $89.2 \%)$. The number of total born was 14.5 piglets, live born was $12.8(88.5 \%)$, stillborn was 1.0 (6.8\%), and mummified was 0.7 (4.7\%).

The average duration of gestation was 117 ( \pm 1.7 days).There were mummified fetuses in $86(24.2 \%)$ of the litters. The number of mummified fetuses ranged from 1 to 15 . More than $75 \%$ of the sows had from 1 to 3 mummified fetuses. The number of mummified fetuses per litter was observed to be highest in a period of $4-6$ weeks. There were stillborn in $182(51.1 \%)$ of the litters ranging from 1 to 10 piglets. More than $75 \%$ of the sows had 1 to 2 stillborn.Out of the 30 mummified fetuses was: 3 examined bacteriological all negative for pathogene bacteria, 6 examined for PPV virus all negative, 8 examined for PPV antibodies all negative, 3 examined for the amount of IgG all normal, 9 examined for PCV2 in the heart musculature all 9 was positive. The 3 blood tested sows had low antibody titers for PCV2 and PPV infections. In the PCV2 PCR test two sows were negative and one sow had a low reaction.

\section{Discussion}

The case report shows PCV2 can case many mummified fetuses in a newly started sow herd. The diagnostics is best in the heart musculature on the mummified fetuses using immunohistochemistry. A differential diagnosis that should be taken into account in Denmark typically includes PPV. Blood testing of the sow with many mummified fetuses is not a good choice if you suspect PCV2.With the production losses described in this abstract caused by a PCV2 infection, vaccinating gilts for PCV2 before mating must be seriously considered when initially populating a newly started sow herd.

\section{References}

(1). Nauwynck H.; D. Lefebvre; G. Misinzo ; P. Meerts ; B. Mateusen ; R. Sanchez ; P. Delputte. (2007): Pathogenesis of porcine circovirus 2 infections. American Association of Swine Veterinarians annual meeting 2007. pp. $489-496$.

\section{Acknowledgements}

L. Lau, Technical Services swine, Merial, Denmark

J. Venet \& F. Joisel, GSM, Merial SAS, France

S. Longo, BPC, Labruguire, France 


\title{
P.815
}

\section{Detection of viral antigen in mummified fetuses and its association with sow reproductive performance}

\author{
Alongkot Boonsoongnern ${ }^{1}$ Pariwat Poolperm ${ }^{1}$ Preeyaphan Udomprasert ${ }^{1}$ Narut Tananthong ${ }^{1}$ \\ Preeda Lertwatcharasarakul² Kitcha Urairong ${ }^{1}$ \\ 1. Department of Farm Resources and Production Medicine, Faculty of Veterinary Medicine, Kasetsart University, \\ Kampaengsaen campus, Nakhonpathom, Thailand; 2. Department of Pathology, Faculty of Veterinary Medicine, \\ Kasetsart University, Kampaengsaen campus, Nakhonpathom, Thailand
}

\section{Introduction}

Mummified fetus is one problem that affects number of piglets born alive. Uterine space has been reported to be a factor increasing in percentage of mummified fetuses $(1,2)$. Diseases could associate with mummy's appearance in lower litter size sows (2). Several diseases cause fetal death including Porcine reproductive and respiratory syndrome virus (PRRSv), Porcine parvovirus (PPV), Porcine circovirus type 2 (PCV-2) and Japanese encephalitis virus (JEV). These viruses could be detected in mummified fetuses by molecular biology methods such as polymerase chain reaction (PCR) and reverse transcription PCR (RT-PCR) (3). The objectives of this study were to investigate the presence of viruses in mummified fetuses collected from sows using appropriated molecular biology methods and to study an association of the viruses found in sample with reproductive performance of those sows.

\section{Materials and Methods}

A total of 66 mummified fetuses were collected from 5 commercial pig farms, with percentage of mummies greater than 3. The mummified fetuses then were necropsied and collected tissue sample from lung, liver, spleen, kidney and brain. Lysate of collected tissue were extracted for RNA for PRRSv and DNA for PPV, PCV-2 and JEV. Specific primers were used in each reaction as follow; for PRRSv: W7PRRS-1 (ATGGCCAGCCAGTCAATCAGC), W7PRRS-2 (TCGCCCTAATTGAATAGGTGAC), W7USA (TGGAGTTTAGTTTGCCKACGCA) and W7EU (AAAACTGACCTTCCCGCTGGA), for PCV-2: PCV-2F (TAGGTTAGGGCTGTGGCCTT) and PCV-2R (CCGCACCTTCGGATATACTG), for PPV: NS1-142F (AGCCAAAAATGCAAACCCCAATA) and NS1-142R (CTCCACGGCTCCAAGGCTAAAG), for JEV: PWN - JE1 (GTGAAYATGACCAGCCAGGT) and PWN - JE2 (CATGAACCARATGGCYCTGC). All samples were required to run for beta-actin primer specific to nuclear receptor co-repressor 1 (NCOR 1) and mito-primer specific to cytochrome $B(C B)$ to proof the presence of RNA and DNA, respectively. Sample products that had negative results for each primer were excluded from corresponded test.

The reproductive performance parameters, including total born/ litter, born alive/litter, \% stillbirth, average daily litter weight gain, wean to first service interval and number of pig wean/litter, of sows that mummified fetuses were collected from were recorded and analyzed for association with viral antigens found in those mummified fetuses.

\section{Results}

The results showed that $80 \%$ (4/5) of farm was positive to PRRSv and $60 \%(3 / 5)$ was positive to PPV. There were only 2 farms found both viruses. The results from PCR and RT-PCR showed that $17.95 \%$ (7/39) of the samples were positive to PRRSV and $7.69 \%$ (3/65) were positive to PPV. All fetuses had negative results for PCV-2 and JEV.

Statistical analysis revealed only an association between litter sizes at 13 or lower and the positive samples $(p=0.033)$. Moreover, sows with litter size of 13 or lower had an odd ratio $=2.4$ to have positive results comparing to sows with bigger litter size.

\section{Discussion}

The results showed that PRRSv and PPV antigen were able to be detected in mummified fetal organ. There was no evidence of PCV-2 and JEV in the participated farms. Meanwhile, there was no association of viral antigen found with sow's breeding performances. However, mummified fetuses in lower litter size $(\leq 13)$ sows associated with number of positive samples (odd ratio $=2.4$ ). Virus infection might play a role in being an additional cause of fetal death during gestation in low litter size sows.

\section{References}

1. Wu, M.C., Hentzel, M.D., Dziuk, P.J., 1988. J. Anim. Sci. 66 (12), 3202-3207

2. Vladimir, F. B., Mari, L. B., Fernando, P. B. and Ivo Wentz, 2005. Preventive Veterinary Medicine. 70: 165-176.

3. Horter, D.C., Pogranichniy, R.C., Evans, R.B., Yoon, K.J., Zimmermann, J.J. 2002. Vet. Microbiol. 86:213-218. 


\title{
Differential serological screening of reproductive infectious agents important for surveillance
}

\author{
Elena Souza de Lima ${ }^{2,3}$ Camila Sa Rocha ${ }^{2,3}$ Giseli A. Ritterbusch ${ }^{2}$ Nelson Mores ${ }^{3}$ Armando L. Amaral ${ }^{3}$ \\ Lauren Ventura ${ }^{4}$ Regia M. Dambros ${ }^{4}$ Suzana S. Kuchiishi ${ }^{4}$ Maria Isabel B. Vieira ${ }^{5}$ Janice R. Ciacci-Zanella $^{1}$ \\ 1. Virus and Prion Diseases Research Unit, NADC, ARS, USDA, Ames, IA, USA; 2. Agroveterinarian Science Center - CAV - University of Santa Catarina \\ State - UDESC, Lages, SC, Brazil; 3. Embrapa Swine and Poultry Research Center, Animal Health Laboratory, Concordia, SC, Brazil; 4. CEDISA - Center for \\ Diagnostic in Animal Health, Concordia, SC, Brazil; 5. College of Agronomy and Veterinary Medicine - University of Passo Fundo, Passo Fundo, RS, Brazil
}

Clinical manifestation of reproductive failures is highly variable and may be presented by either a subclinical infection or by the termination of pregnancy. The etiology of abortions is usually difficult and a conclusive diagnostic occurs in only $30-40 \%$ of cases (1). Several infectious agents are associated with abortion and reproductive failures in swine production. In Brazil the porcine circovirus 2 (PCV2) associated diseases (PCVAD) are the most economically important viral disease for swine production and it is associated with reproductive losses. The objective of this study was to perform differential serology for pathogens that cause reproductive failure in sows from farms with a history of severe PCVAD in the last 12 months before the sampling.

\section{Material and Methods}

Blood samples were collected from 120 sows which presented reproductive problems (mummified, stillborn or unviable piglets). Samples were collected from 27 swine farms and up to 15 days following the farrowing or abortion. Analyzes were performed by routinely standardized laboratory tests and / or recommended by veterinarian surveillance authorities and OIE. Tests were performed at CEDISA, Embrapa Swine and Poultry Research Center or Veterinary Hospital from University of Passo Fundo, RS, Brazil. Serological tests used in this work included in house tests like immunocytochemistry or ICC for PCV2, Haemagglutination inhibition (HI) test for porcine parvovirus or PPV; Rose Bengal test (RBT) and the buffered plate agglutination test (BPAT) to identify antibodies against Brucella suis; Microscopic agglutination test (MAT) for diagnostic of leptospirosis (antigens selected for use in the MAT included representative strains of the serogroups known to exist in the region) and commercially available tests such as the Elisa screening for Pseudorabies virus (IDEXX HerdChek* PRV gB Antibody Test Kit), followed by serum neutralization test; IDEXX HerdChek* Porcine Reproductive and Respiratory Syndrome Antibody Test Kit and for classical swine fever virus the IDEXX HerdChek* CSFV Antibody Test. For Toxoplasma gondii antibody detection the indirect hemagglutination commercial kit Imuno-Hai from the Wama-Diagnostica Laboratories (SP, Brazil) was used (2).

\section{Results and Discussion}

Antibodies against viruses (PCV2, PRRSV, PPV, PRV, CSFV); specific bacterial agents (Leptospira sp., Brucella suis) and protozoa (Toxoplasma gondii) from selected sows with reproductive problems were investigated. All sows tested for PCV2 presented a positive reaction at ICC, but with different titers (Table 1). Titers varied between 1:20 - 1:160 (+), 1:320 - 1:2560(++) and above or greater than 1:5120 (+++). PPV antibodies were also very frequent whereas most of the sows (113 or $94.2 \%$ ) were positive. High positive titers for PPV varied from 1:128 to 1:1024 (++) and 1:2048 to $>1: 8192(+++)$ (Table 1$)$. In contrast, $117(97.5 \%)$ of sows presented no titer to leptospirosis, and 3 were reagent to serovar L. Bratislava with different titers, one sample 1:100 and two samples at 1:400. All sows were negative for brucellosis, PRRSV, PRV and CSFV, confirming the control and surveillance programs enforced in the country. No T. gondii antibodies were detected in the tested samples.

Table 1 - Distribution of antibody titers for PCV2 and PPV

\begin{tabular}{|c|c|c|c|c|}
\hline & Pos (+++) & Pos (++) & Pos (+) & $\operatorname{Neg}(-)$ \\
\hline PCV2 & $4(3.3 \%)^{\mathrm{a}}$ & $84(70 \%)^{b}$ & $32(26.7 \%)^{c}$ & $0^{\mathrm{d}}$ \\
\hline PPV & $44(36.7 \%)^{\mathrm{e}}$ & $69(57.5)^{f}$ & $5(4.15 \%)^{g}$ & $2(1.65 \%)^{h}$ \\
\hline \multicolumn{5}{|c|}{ 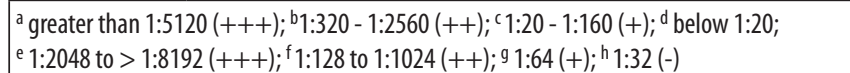 } \\
\hline
\end{tabular}

This study analyzed antibody response of individual sows with reproductive problems in order to investigate the possible infectious cause. Results indicate indicated a low frequency of antibodies for bacterial agents and a high frequency of antibodies for endemic viral agents such as PCV2 and PPV.

\section{References}

1. Sobestiansky, J.and Barcellos, D. 2007. Doenças de Suínos. Goiânia: Cânone Editorial, 770p.

2. Vieira, M.I.B, et al. 2008. Hora Vet. 163:34-36 


\title{
P.817
}

\section{Reproductive disorders in Iberian sows in extensive farms caused by Chlamydophila abortus}

\author{
Carmen Borge ${ }^{1}$ Nieves Ortega ${ }^{2}$ Maria José Rangel ${ }^{1}$ Alfonso Carbonero ${ }^{1}$ Jesús Salinas ${ }^{2}$ Anselmo Perea ${ }^{1}$ Maria Rosa Caro ${ }^{2}$ \\ 1. Department Animal Health, Veterinary Faculty, Córdoba, Spain; 2. Animal Heath Department, Veterinary Faculty, Murcia, Spain
}

\section{Introduction}

Members of Chlamydiaceae family are obligate intracellular pathogens with a broad host spectrum. They have been associated with a wide range of diseases, including enteritis, pneumonia, conjunctivitis, pericarditis, perinatal mortality, and reproductive disorders, particularly abortion and endometritis. Chlamydial species detected in natural infections in swine include Chlamydophila (Cp.) abortus, $\mathrm{Cp}$. pecorum and a recently proposed species, Chlamydia (C.) suis, strains phylogenetically related to human C. trachomatis (Everett K.D. et al., 1999). The present study describes an outbreak of reproductive disorders associated to Chlamydophila abortus in a farm of Iberian pigs in Southwest Spain.

\section{Materials and Methods}

Farm characteristics.- The outbreak was conducted on a multisite farrow-to-finish farm with 2060 Iberian sows (nulliparous) introduced from a free disease's farm in 2008. Animals were distributed weekly in 22 flocks. Natural mating is used and pregnancy is confirmed by ultrasound between 21 and 24 days postinsemination. The pigs were reared in semi extensive systems.

Outbreak description.- Disorders were initially detected from flock 10 onwards in October 2009. They consisted of an decreased in farrowing rates (from $92 \%$ to $54 \%$ ); decrease in piglets born alive/litter (from 7.03 to 5.69), increase in piglets born dead/litter and with low viability (from $14.05 \%$ to $25.05 \%$ ) and decrease in the weaned piglets per sow (from 6.62 to 5.62). The last 3 flocks showed a higher rate in the return to oestrus, cyclic and acyclic (from $19.1 \%$ to $60.6 \%$ ).

Bacteriology and virilogical examination.- Six fetuses were examinated for bacteriological and virological pathogens by standards methods. When the other aetiological agents were discarded, a screening for chlamydiae by PCR was performance, follow by the typing of chlamydial DNA. To get the DNA, a DNAeasy tissue kit (QIAGEN. Berlin. Germany) was used. DNA was amplified with different protocols and primers. Firstly, a family-specific PCR were carry out using CHYF and CHYR primers which target 16S rRNA gene of the Chlamydiaceae family, following the protocol described in Condon and Oakey (2007). After that, a specific PCR Chlamydophila abortus using primers pmp-F and pmp-R821 which target pmp 90/91 gene were used following the protocol described by Berri et al., (2009).

Serological examination:A total of 53 sera from aborted sows with reproductive disorders were tested by an ELISA- rPOMP specific to Cp. abortus using the ELISA kit commercialized pby IDDEX Laboratories. An anti-Pig lgG- Peroxidase conjugated was used (Sigma). The results were expressed as optical densities (DO). In addition, 13 sera were tested a second time 17 days after abortion looking for sero-conversions.

\section{Results}

All six abortion cases tested were positive in stamp stain.

The PCR showed $3 / 6$ positive samples (samples $a, b, c$ ) and $3 / 6$ doubtful samples (samples d, e, f) when DNA was amplified with a family-specific PCR for the gene of the Chlamydiaceae family. However, when these samples were tested with a specific PCR $C p$. abortus we only detected $2 / 6$ positive results (samples e, $f$ ).

Fifty nine per cent of the sera were positive for specific antibodies against $\mathrm{Cp}$. abortus. Seroconversions 17 days post-abortion were detected in $83 \%$ of the cases.

\section{Discussion}

The results suggest that $\mathrm{Cp}$. abortus may be a possible cause of returns to oestrus, abortion and increase of incidence of litters with stillborn piglets and piglets with low viability in Iberian sows (Camenisch et al., 2004). However, the positive samples amplified with a family-specific PCR for the gene of the Chlamydiaceae family (samples $a, b, c)$ and negative when were tested with a specific PCR Cp. abortus (samples a, b, c, d) suggest the involvement of other species included in the family Chlamydiaceae (C. suis, Cp. pecorum) as pathogen in porcine reproductive disorders.

The role of domestic or wild ruminants like sources of infection and their importance for transmission must be also studied.

\section{References}

- Berri M. et al. 2009. BMC Microbiology 2009. 9:130.

- Camenisch, U et al., Vet. Rec. 2004. 155:593-6.

- Condon K. and Oakey J. 2007. Letters in Applied Microbiology (45): 121-127.

- $\quad$ Everett K.D. et al. Int J Syst Bacteriol. 1999 Apr;49 Pt 2:415-40. 


\title{
P.818
}

\section{PCV2 subtype and co-agents involved in reproductive failure occurring in PCV2-positive swine farms in São Paulo state, Brazil}

\author{
Alessandra M. Castro ${ }^{1}$ Tatiana M. Kanashiro ${ }^{2}$ Vanessa R. Salgado ${ }^{1}$ Sheila O. Souza ${ }^{1}$ Taís F. Cruz ${ }^{2}$ \\ Karen L. Ferrari ${ }^{1}$ João J. Pessoa ${ }^{2}$ Paulo E. Brandão ${ }^{1}$ Leonardo J. Richtzenhain ${ }^{1}$ \\ 1. Faculdade de Medicina Veterinária e Zootecnia da Universidade de São Paulo, São Paulo, Brazil; 2. UNESP-Campus de Botucatu, Botucatu, Brazil
}

\section{Introduction}

Porcine circovirus type 2 (PCV2) is widespread and has been associated with several disease complexes (3). Naturally occurring reproductive diseases related to PCV2 infection have been reported and intra-fetal inoculation of PCV2 at various stages of gestation showed that virus replicates highly in fetal tissue eventually leading to fetal death $(1,2)$. The aim of this study was to identify the subtype of PCV2 and the co-agents involved in the reproductive failure in São Paulo state, Brazil.

\section{Materials and Methods}

One hundred sixty-eight samples from reproductive failure (44 aborted and 124 stillborns) collected in positive PCV2 swine farms were examined between 2007 and 2009. The homogenized pools of tissue were analyzed for PCV2 by PCR and the positive samples tested for PPV, Leptospira spp and Brucella spp by PCR to verify the presence of co-infection. To determine the extent of genetic diversity among PCV2 detected, a target segment encompassing the open reading frame 2 (ORF2) of the PCV2 genome was sequenced from nine samples.

\section{Results}

PCV was detected in 18 (10.7\%) out of the 168 samples examined. Among the 18 PCV2-positive samples, 14 were aborted fetuses and 4 stillborns piglets. The others agents detected were PPV (2/18) and Brucella spp (10/18). The Brucella spp (3/10) and PPV (2/2) positive samples were sequenced and compared with sequences contained at the GenBank database demonstrating a nucleotide identity of $\geq 95 \%$. The phylogenetic relationship of nine PCV2 positive samples showed nucleotide and amino acid identity values of 92.2 to $99.8 \%$ and 91.3 to $99.5 \%$, respectively. A Neighbour-Joining tree was assembled and showed that six samples had been classified in subgroup PCV2-1 (A and B) and one in the PCV2-2E. Considering the phylogenetic analysis of representative sequences from the three Swedish genogroups (SG1-3), all samples were grouped in genogroup 3 (SG-3) (Fig. 1).

\section{Discussion}

The surprised results are that Brucella spp. is the main co-agent present in co-infection, since the São Paulo state participates of the national controlling program for Brucelosis. Therefore the positive farms are being oriented about this zoonosis with the objective to negative them. However PPV, the main virus involved in PCV2-associated disease since PRRVS is absent in Brazil, was detected in only two of 12 co-infected animals which demonstrates that the farms follow active vaccination program for the control of these viral infections. The almost PCV2-positive samples involved in reproductive disorders of sows in São Paulo state, Brazil are grouped in PCV2-1 subtype and SG-3.
Figure 1: Neighbour-Joining tree of the PCV2 ORF2 for genogroup (A) and subgroup (B) classifications with PCV1 as outgroup. Numbers indicates values calculated on 1000 repeats of the alignment with the heuristic method. Strains are indicated by their GenBank access number and subgroup or genogroup identification between parentheses.

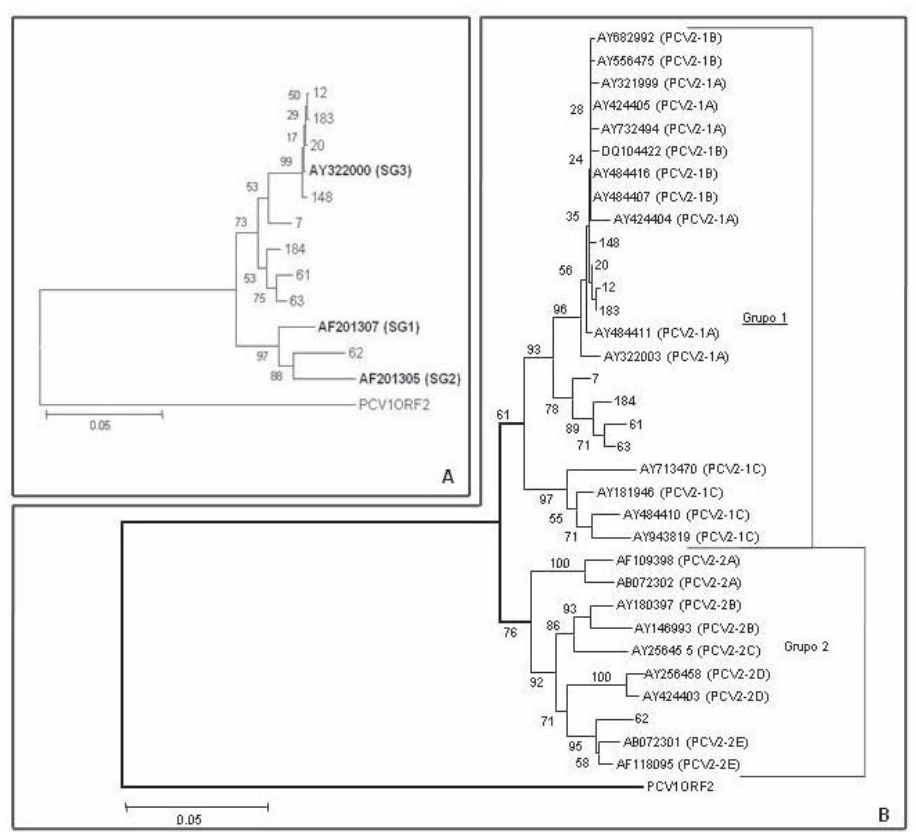

\section{Acknowledgements}

This research is supported by FAPESP (process number: 07/50003-5).

\section{References}

1. Farnham, M. D. W. et al. (2003). Can. J. Vet. Res., 67, 108-113.

2. Maldonado, J. et al. (2005). Vet. J., 169, 454-456.

3. Opriessnig, T. et al. (2007). J. Vet. Diagn. Invest., 19, 591-615. 


\title{
P.819
}

\section{Effect of vaccination against Swine Eryripelas in \% of piglets born dead on a farm in Guanajuato State in Mexico}

\author{
Oscar Huerta Alva; A. Ayllón \\ Laboratorios Sanfer SA de CV, Mexico, DF, Mexico
}

\section{Introduction}

The improved production is a constant that requires us competitive in any market, the pig is no exception and towards more pigs sent per female flea per year, directly depends on the number of piglets born alive per litter and these is reduced by the $\%$ of stillborn piglets and mummies, the latter two are caused by a myriad of causes and in the specific case of stillborn piglets may be involved the Swine Erysipelas (personal communication from Drs C. Sanchez [2005] and R. Treviso [2007])

The Swine Erysipelas is an actual disease, as reported Schwarts (2002), Sierra (2006), Perea (2006) and Hopkins (2006-2008), is a systemic disease and has a direct effect on blood vessels, causing more fragile cord cord, same as the time of delivery breaks more easily.

\section{Materials and Methods}

By delivering a live vaccine against swine erysipelas 2 or 3 weeks before delivery reduced the number of piglets born dead (personal communication of Dr. C. Sanchez [2005], R. Treviso [2007], in Monterrey 2007 staff report) on this basis, the aim of this study is to confirm the personal experiences of Sanchez and Huerta Treviso applying erysipelas live vaccine strain in two groups, one 2 weeks before delivery and another 3 weeks before delivery, on a farm of 650 stomachs located in the State of Guanajuato, as main problem is the number of piglets stillborn birth. The groups 16 and 17 were interviewed with the swine erysipelas live vaccine and compared against two previous groups (14 and 15) and two subsequent groups (18 and 19). The farm was an outbreak of Swine Erysipelas 5 months ago.

\section{Results}

In the table below we can see the results of group 14 to 19:

\begin{tabular}{|c|c|c|c|c|c|c|}
\hline Week & $\mathbf{1 4}$ & $\mathbf{1 5}$ & $\mathbf{1 6}$ & $\mathbf{1 7}$ & $\mathbf{1 9}$ & $\mathbf{1 9}$ \\
\hline Farrows & 30 & 31 & 29 & 30 & 29 & 30 \\
\hline TPborn & 340 & 358 & 340 & 346 & 344 & 340 \\
\hline TPBorn L & 295 & 318 & 312 & 324 & 309 & 309 \\
\hline Deads & 29 & 31 & 15 & 14 & 23 & 22 \\
\hline Mummy & 13 & 9 & 13 & 8 & 12 & 9 \\
\hline Ave TPB & 11.3 & 11.5 & 11.7 & 11.5 & 11.8 & 11.3 \\
\hline Ave TPBL & 9.93 & 10.26 & 10.76 & 10.8 & 10.66 & 10.3 \\
\hline \% deads & 8.53 & 8.66 & 4.41 & 4.05 & 6.69 & 6.47 \\
\hline \% mummy & 3.82 & 2.51 & 3.82 & 2.31 & 3.49 & 2.65 \\
\hline
\end{tabular}

\section{Discussion}

In the weeks 16 and 17 where sows were vaccinated with a live vaccine strain vs. rhusiopathiae Erisipelothrix Koganei 65-0.15, females at birth had about $50 \%$ less stillborn piglets, compared with the two previous groups (14 and 15) and $36 \%$ less than groups 18 and 19. Consequently increased the number of piglets born alive.

\section{Conclusion}

According to these results I conclude that vaccinating pregnant sows 2 to 3 weeks before delivery with a live vaccine against Erisipelothrix rhusiopathiae strain Koganei 65-0.15, declined by $50 \%$ the number of piglets born dead.

We must remember that the swine erysipelas is a systemic disease with high affinity to blood vessels (become friable) and at delivery, the umbilical cord to get power out piglets and is likely to be broken by the stress of labor.

\section{References}

1. Perea, $\mathrm{CML}$, et al. Determinación de anticuerpos contra Erisipelothrix rhusiopathiae.en sueros de cerdos utilizando una prueba sencilla de aglitunación., Congreso AMVEC, 2006.

2. Huerta, AOF; Montaño MB, Reporte de casos de Erisipela porcina en el Estado de Sonora, Congreso AMVEC, 2006

3. Huerta, AOF; Montaño MB, Reporte de casos de Erisipela porcina en el Estado de Sonora, IPVS, 2008.

4. Schwartz, K.J.,2002, Erysipelas: an old disease returns to the swine industry or something NEW?, American Association of Swine Veterinarians.

5. Sierra, N., Multietiologías complejas de interacción entre PRRS y otros patógenos. Congreso AMVEC, 2006.

6. Wood, R.L., 1999, Erysipelas. In Diseases of Swine 8th Ed, ISU press, Ames lowa 


\title{
P.820
}

\section{Induction of ovulation in weaned sows: how much Buserelin is needed to get a normal LH surge and normal embryonic development in primiparous or multiparous females?}

\author{
H.M.J.M. Swarts ${ }^{3}$ N.M. Soede ${ }^{1}$ J. van der Lelij ${ }^{1}$ M.A. Driancourt ${ }^{2}$ \\ 1. Dept. Animal Science Wageningen University, Wageningen, Netherlands; \\ 2. ISPAH R\&D Pharmaceuticals, Beaucouze, France; 3. ISPAH, Global Marketing Swine, Boxmeer, Netherlands
}

\section{Introduction:}

While the ability of $10 \mu \mathrm{g}$ Buserelin to induce ovulation in gilts has already been demonstrated, the dose of Buserelin needed to induce ovulation in weaned sows has not been established. The aim of this experiment was to assess the ability of different amounts of Buserelin to induce a normal LH surge, ovulation and early embryo development and monitor whether parity or genetic line were modulating the response to Buserelin.

\section{Materials \& methods}

On 77 hours after weaning, 64 sows received an intra-muscular injection of either $0 \mu \mathrm{g}$, or $6 \mu \mathrm{g}$, or $10 \mu \mathrm{g}$, or $16 \mu \mathrm{g}$ of Buserelin. Sows were equally allocated to treatments based on parity (primiparous: parity 1 and multiparous: parity 3 to 6) and breed (TOPIGS20 and TOPIGS40, both from Topigs, Netherlands). Subgroups [dose of Buserelin * breed * parity] were therefore set to include four animals.

The end points used were (1) the detection of ovulation by ultrasonography at a specific time window (32-44h) after treatment, (2) the detection of an LH surge (and its magnitude) in blood samples collected 1, 2.5, 4, 12 and $24 \mathrm{~h}$ following treatment, (3) the number of corpora lutea and viable embryos at slaughter 5 days after the expected ovulation and (4) the progesterone secretion by the corpora lutea at slaughter.

\section{Results}

As 2 sows had already ovulated within $85 \mathrm{~h}$ after weaning and as 5 others ovulated within $32 \mathrm{~h}$ of injection, data from 15 control sows as well as 15, 11 and 12 sows treated with 6,10 or $16 \mu \mathrm{g}$ Buserelin were used for statistical analyses.

Table 1: timing of ovulation

\begin{tabular}{|l|c|c|c|c|}
\hline Timing* & Controls & & Treated & \\
\hline & primiparous & multiparous & primiparous & multiparous \\
\hline $109-121$ & 1 & 1 & 11 & 18 \\
\hline $121-133$ & 4 & 3 & & 0 \\
\hline $133-145$ & 1 & 3 & 5 & 0 \\
\hline $145-157$ & 1 & 0 & & \\
\hline$>157$ & 1 & 0 & 4 & \\
\hline$*=$ Hrs after weaning &
\end{tabular}

Of the Buserelin treated sows $76 \%$ were synchronized to ovulate between $32-44 \mathrm{~h}$ after treatment. The proportion of sows successfully induced to ovulate was unrelated to the dose of Buserelin applied $(73,73$ and $83 \%$ in sows receiving 6, 10 or $16 \mu \mathrm{g}$ Buserelin). Parity however had a clear effect on treatment's efficacy as $100 \%$ multiparous sows ovulated during this time window, while only 50,50 and $67 \%$ of the primiparous gilts ovulated following injection of 6, 10 or $16 \mu \mathrm{g}$ Buserelin

In all sows that ovulated during the $32-44 \mathrm{~h}$ time window, a clear increase in LH concentrations was observed. Its magnitude $(2.4 \mathrm{ng} / \mathrm{ml})$ was similar to that observed in the control group ( 2.8 $\mathrm{ng} / \mathrm{ml}$ ) and unaffected by the amounts of Buserelin injected. In control and treated sows that ovulated at the expected time, neither parity nor breed affected the features of ovulatory LH surge. In contrast, sows that ovulated late had a blunted increase in LH concentrationsThe number of corpora lutea (23.5 on average) was unaffected by treatment (control vs treated) and by the amounts of Buserelin injected. Cyst formation was more common in primiparous gilts (4/8 and 5/7) injected with 6 and $16 \mu \mathrm{g}$ compared to those receiving $10 \mu \mathrm{g}$ Buserelin (1/6). Cyst number reached $7.3 \pm 8.5$ in the $6 \mu \mathrm{g}$ Buserelin group.

In contrast, the average number of good embryos (18.7) was unaffected by treatment and by the dose of Buserelin applied.

No interaction between breed or parity and treatment was detected for progesterone concentrations on day 5 post ovulation.

\section{Discussion and conclusion}

It is concluded that:

(1) In all multiparous sows of either breed, administration of Buserelin at $77 \mathrm{~h}$ after weaning successfully triggered a full ovulatory process generating a normal number of good embryos.

(2) In primiparous sows, injection of Buserelin may have to be delayed past 77h after weaning.

(3) $10 \mu \mathrm{g}$ Buserelin appeared the optimal dosage based on the occurrence and number of cystic follicles. 


\title{
Treatment of urinary tract infections in sows associated with E. coli
}

\author{
$\underline{\text { Isabel Radeloff }}{ }^{1}$ Klaus Hellmann ${ }^{1}$ Heinrich Greife ${ }^{2}$ Bernd Stephan $^{2}$ \\ 1. KLIFOVET AG, Muenchen, Germany; 2. Bayer Animal Health GmbH, Leverkusen, Germany
}

\begin{abstract}
Urinary tract infections (UTI) are a major health concern in breeding herds and one of the major causes of death or culling of female breeding pigs. Infections may be caused by ascending bacterial pathogens from the external genitalia to the bladder and kidneys as a consequence of coitus and parturition, and incomplete closure of the vulva and/or damaged or relaxed urethra. Intensive housing conditions where vulvas get in direct contact with faeces may lead to the infection with faecal flora. Several farms in Germany, the Netherlands and Slovenia, known to have a history of urinary tract infections in sows were included in the study. Housing and management conditions were maintained stable during the clinical trial.
\end{abstract}

Sows (sows and gilts) showing urinary tract infections associated with Escherichia (E.) coli were treated with Baytril ${ }^{\circledR}$ (enrofloxacin) in a dosage of $5.0 \mathrm{mg} / \mathrm{kg}$ body weight. One group (T1) was treated for three consecutive days, $24 \mathrm{hrs}$ apart, and one group (T2) was treated twice, $48 \mathrm{hrs}$ apart. Both groups were compared to a control group (T3), dosed with $15.0 \mathrm{mg} / \mathrm{kg}$ bodyweight amoxicillin (Duphamox ${ }^{\circledR}$ LA) twice, $48 \mathrm{hrs}$ apart.

Sows were included in the clinical trial if at least 105 colony forming units (cfu) were detected per $\mathrm{mL}$ mid-stream urine, and at least one criterion related to macroscopic appearance of the urine was abnormal ( $\mathrm{pH} \geq 8.0$ or smell or cloudiness). The minimum bacterial count had to be fulfilled in one of two systems: either in urine analysis of a native urine sample or in the Uricult ${ }^{\circledR}$ dip stick agar system. In addition, the general health was observed and bacterial isolation and identification was performed to identify the associated pathogen. E. coli (including haemolytic and mucoid growth) was identified in $76.8 \%$ of the positive animals. Other bacterial species identified were Enterobacter spp., Enterococcus spp., Klebsiella spp., Staphylococcus spp. and Streptococcus spp.

Pre-treatment clinical signs (general health) and urine assessment were compared to post-treatment assessment of the same criteria three and 14 days after the start of treatment. The success criterion was the bacteriological cure rate on day 14 of animals bacteriologically positive for $\mathrm{E}$. coli on Day 0 , but negative on day 14 (animals with a bacteriological result of less than $105 \mathrm{cfu} / \mathrm{mL}$ in both, Uricult ${ }^{\circledR}$ dip stick agar system and urinary analysis).
E. coli was eradicated in $46.4 \%$ of the animals in treatment group $\mathrm{T} 1$, in $53.1 \%$ of the animals in treatment group $\mathrm{T} 2$ and in $44.8 \%$ of the animals of the control group (T3). For the subgroup where mucoid growth of E. coli was identified, the rate of eradication was $80.0 \%$ in T1 and $100.0 \%$ in T2 compared to $42.9 \%$ in the control group. Similar results were observed using the Uricult ${ }^{\circledR}$ dip stick agar system: E. coli was eradicated in $45.5 \%$ of the animals in the T1 group, in $36.8 \%$ of the animals in the T2 group and in $43.2 \%$ of the animals in the control group. For the subgroup where mucoid growth of E. coli was identified, the rate of eradication was $83.3 \%$ in $\mathrm{T} 1$ and $100.0 \%$ in $\mathrm{T} 2$ compared to $33.3 \%$ in the control group.

Assessing the urine pre (Day 0) and post treatment (Day 3 to day 14) using the Macroscopic Urine Score (MUS), calculated as sum of scores of the cloudiness (score 0 to 3 ) and foul smelling (score 0 to 2) of the urine, a significant reduction was observed $(p<0.05)$. On day 3 and day 14 the MUS was reduced significantly within each treatment group as compared to day 0 . General health was good at pre-treatment and was kept at this stage during the study.

Based on the results observed Baytril ${ }^{\circledR} 10 \%$ used as an antimicrobial therapy for sows showing clinical signs of E. coli urinary tract infection, was shown to reduce the infection pressure on animal and farm level and can help to interrupt infection cycles. Therefore, it should be part of an eradication program including improvement of housing conditions, herd management and gestation programs in farrowing and nursing pig farms. 


\title{
P.822
}

\section{Consumer research on attitudes to vaccination against boar taint in Denmark}

\author{
Jens Christian E. Jensen \\ Orion Pharma Animal Health, Nivaa, Denmark
}

\section{Introduction}

The vaccine against boar taint - Improvac ${ }^{\circledR}$ - was approved through central registration in the European Union in May 2009. The European licensing procedure includes a full assessment of food safety and the product is considered safe for consumers of pork. In many countries, however, including Denmark, informed consumers develop their own opinions about the use of new technical approaches in food production, taking into account both the possible effect on food and the impact on areas of ethical concern, such as animal welfare. To investigate consumer attitudes market research has earlier been carried out in other countries 1,2. The aim of this investigation was to learn the reaction of Danish consumers to different ways of controlling boar taint: castration without anesthesia, castration with pain relief (NSAID), castration with local anesthesia, and vaccination against boar taint.

\section{Material and Methods}

Capacent, the leading Nordic business consultancy company was in charge of collecting, analyzing and reporting the results of computer assisted telephone interviews of 1012 persons with an age of 18 years or older. A random sample of consumers was secured and only one person per household could be interviewed. A short factual description of each method was developed in collaboration with industry stakeholders and presented to the interviewed persons. Interviews took place between August 3rd and 16th 2009

\section{Results}

Asked how acceptable do you find:

1. Castration of piglets without anesthesia or pain relief (named castration in the chart), the answers were $65 \%$ unacceptable, $12 \%$ neutral, $23 \%$ acceptable and don't know $1 \%$.

2. Castration of piglets with pain relief (named NSAID in the chart), the answers were $44 \%$ unacceptable, $16 \%$ neutral, $39 \%$ acceptable and don't know $2 \%$.

3. Castration of piglets with local anesthetics (named Lidocaine in the chart), the answers were $43 \%$ unacceptable, $18 \%$ neutral, 37\% acceptable and don't know $2 \%$.

4. Vaccination instead of castration, the answers were $16 \%$ unacceptable, $9 \%$ neutral, $73 \%$ acceptable and don't know $3 \%$.

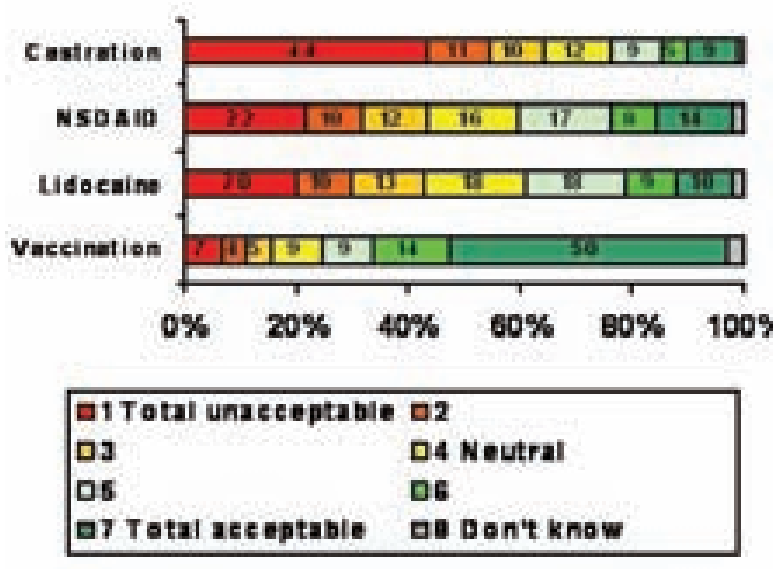

Asked which of the methods they would prefer for control of boar taint, vaccination was by far the preferred approach.

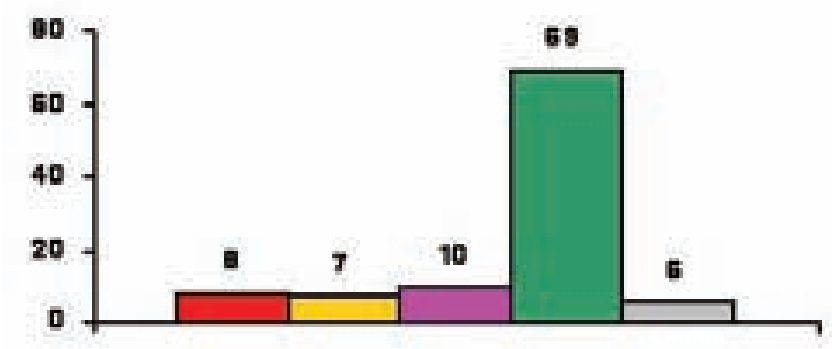

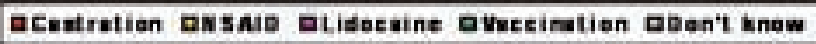

\section{Discussion}

Modern, educated consumers may question the use of new products in food production. However, there is also growing consumer concern about the pain and stress suffered by piglets during and after physical castration. As in other consumer acceptance investigations 1, 2 the results here confirm that when fully informed consumers prefer vaccination as the way of controlling boar taint, with their concerns about pork quality and animal welfare outweighing their caution about the use of a new technology.

\section{References}

1) J. Allison et al. Consumer Acceptance of the use of Vaccination to Control Boar Taint. Proceedings EAAP, 2008 Vilnius

2) C. J. Lagerquist et al. AgBioForum, 2006. 9(1): 51-58 


\title{
Growth performance and health status of pigs vaccinated with gonadotropin- releasing factor vaccine (Improvac ${ }^{\mathrm{TM}}$ ) in comparison with surgically castrated pigs and boars under conventionally managed conditions
}

\author{
Thilo Fuchs ${ }^{1} \underline{\text { Heiko Nathues }}^{1}$ Annika Koehrmann² Stuart Andrews ${ }^{3}$ Fiona Brock ${ }^{3}$ Guenter Klein ${ }^{4}$ Elisabeth Grosse Beilage $^{1}$ \\ 1. Field Station for Epidemiology in Bakum, University of Veterinary Medicine Hannover, Bakum, Germany; \\ 2. Pfizer Animal Health, Berlin, Germany; 3. Pfizer Animal Health, Sandwich, UK; 4. Institute for Food \\ Quality and Food Safety, University of Veterinary Medicine Hannover, Hannover, Germany
}

\section{Introduction}

Surgical castration of male piglets is still a common procedure worldwide and is primarily done to prevent entire boars from developing an unpleasant taste and flavour of meat. For animal welfare reasons, several countries are enacting new laws to ban surgical castration without anaesthesia (2). Immunisation with two doses of a gonadotrophin releasing factor (GnRF) vaccine against boar taint, the second dose given 4 to 5 weeks prior to slaughter, suppresses testicular function through the induction of antibodies against GnRF (1). The timing of vaccination makes it possible to maintain the economic advantages of boars for most of the fattening period, while also avoiding the presence of boar taint at slaughter. The objective of this study was to determine the effect of using a GnRF vaccine on growth performance of male pigs. Vaccinated pigs were compared with surgically castrated pigs and entire males under conventionally managed farm conditions.

\section{Materials and Methods}

Male pigs were randomly assigned to three treatment groups: pigs surgically castrated during the first week of life (group " $C^{\prime \prime}$, $n=274$ ), pigs immunised twice during the fattening period with a GnRF vaccine (Improvac, Pfizer Animal Health), the first time when 13-14 weeks of age and the second time when 20-21 weeks of age (group „V", $\mathrm{n}=280$ ) and entire males (group " $\mathrm{E}^{\prime \prime}$, $\mathrm{n}=56$ ). Average daily weight gain (ADG), mortality and medical treatment were measured during the study. Additionally, feed conversion ratio (FCR) was assessed during the fattening period.

\section{Results}

No difference in the ADG during the fattening period could be determined between " $\mathrm{C}^{\prime \prime}$ and " $\mathrm{V}^{\prime \prime}$ (Tab. 1). From the start of the study until slaughter at study day $160 / 173$ the ADG in group „ $\mathrm{E}^{\prime \prime}$ was lower (625 g/day; $\mathrm{P})$ than in group " $\mathrm{C}^{\prime \prime}(681 \mathrm{~g} /$ day; $\mathrm{P}<0.001)$ and group " $V^{\prime \prime}(675 \mathrm{~g} /$ day; $P<0.01)$. The FCR during the fattening period until study day 160 , three days before the first batch of pigs were slaughtered at 24-25 weeks of age, was higher in group "C" (2.72 kg feed intake/ kg weight gain) compared with group " $V^{\prime \prime}(2.44 \mathrm{~kg}$ feed intake/ kg weight gain; $\mathrm{P}<0.001)$ and group "E" (2.44 kg feed intake/kg weight gain; $P<0.01)$. Pigs

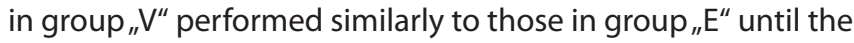
second vaccination (day 135) after which time they showed a substantial increase in feed intake and ADG, while retaining an FCR advantage over group C (Tab. 2). The mortality from the start to the end was $10.6 \%, 11.8 \%$ and $8.9 \%$ in groups ${ }^{\prime \prime} \mathrm{C}^{\prime \prime}{ }_{1}, \mathrm{~V}^{\prime \prime}$ and ${ } \mathrm{E}$ ", respectively. During the same time period, pigs in groups " $\mathrm{C}$, "V" and „E" were medically treated $0.51,0.63$ and 0.29 times per animal.
Tab. 1: ADG (Means in g/day)

\begin{tabular}{|c|c|c|c|}
\hline Period & "C" & "V" & "E" \\
\hline Study start to fattening unit entry & $430.2^{\mathrm{a}}$ & $405.3^{b}$ & $412.1^{b}$ \\
\hline Study start to second vaccination & $631.8^{\mathrm{a}}$ & $582.4^{\mathrm{b}}$ & $588.4^{b}$ \\
\hline Study start to cut-off & $675.1^{\mathrm{a}}$ & $657.4^{\mathrm{a}}$ & $625.3^{b}$ \\
\hline Study start to first/second slaughter & $681.4^{\mathrm{a}}$ & $674.9^{\mathrm{a}}$ & $625.3^{b}$ \\
\hline $\begin{array}{l}\text { Fattening unit entry to second } \\
\text { vaccination }\end{array}$ & $1012.8^{a}$ & $918.7^{\mathrm{b}}$ & $925.5^{b}$ \\
\hline Fattening unit entry to cut-off & $977.5^{\mathrm{a}}$ & $970.6^{\mathrm{a}}$ & $890.9^{b}$ \\
\hline $\begin{array}{l}\text { Fattening unit entry to first/second } \\
\text { slaughter }\end{array}$ & $971.2^{\mathrm{a}}$ & $982.6^{\mathrm{a}}$ & $890.9^{b}$ \\
\hline Second vaccination to cut-off & $897.2^{\mathrm{a}}$ & $1056.5^{b}$ & $826.0^{c}$ \\
\hline $\begin{array}{l}\text { Second vaccination to first/second } \\
\text { slaughter }\end{array}$ & $887.9^{\mathrm{a}}$ & $1064.0^{b}$ & $825.9^{c}$ \\
\hline
\end{tabular}

Tab. 2: FCR (Means in kg feed intake per kg weight gain)

\begin{tabular}{|l|c|c|c|}
\hline Period & "C" & "V" & "E" \\
\hline $\begin{array}{l}\text { Entry to fattening unit until second } \\
\text { vaccination }\end{array}$ & $2.39^{\mathrm{a}}$ & $2.20^{\mathrm{b}}$ & $2.19^{\mathrm{b}}$ \\
\hline Second vaccination until cut-off & $3.31^{\mathrm{a}}$ & $2.71^{\mathrm{b}}$ & $2.96^{\mathrm{b}}$ \\
\hline Entry to fattening unit until cut-off & $2.72^{\mathrm{a}}$ & $2.44^{\mathrm{b}}$ & $2.44^{\mathrm{b}}$ \\
\hline
\end{tabular}

\section{Discussion}

The present study supports in part earlier findings that, by replacing surgical castration early in life with vaccination of pigs against GnRF during the fattening period, the feed conversion advantages of boars could be retained for pig production (1). Higher ADG of vaccinated animals compared with castrated animals could only be detected after the second vaccination. No effects of vaccination could be observed on the mortality or need of medical treatment over the whole lifetime. To take greater benefit from the effect of vaccination on ADG, the second vaccination could be brought forward to the earliest possible point of time, although this study did not examine the possible negative effects of doing this, such as increased carcass fatness.

\section{References}

(1) Dunshea FR,et al. (2001) J. Anim. Sci. 79: 2524-2535

(2) Zöls, S. (2006) Diss. med. vet., München 


\title{
Influence of Improvac on FSH and LH concentrations and on the development of testicles and the expression of boar taint in male pigs
}

\author{
Thilo Fuchs ${ }^{1}$ Rico Thun ${ }^{5}$ Nahid Parvizi ${ }^{6}$ Heiko Nathues $^{1}$ Annika Koehrmann ${ }^{2}$ Stuart Andrews ${ }^{3}$ \\ Fiona Brock ${ }^{3}$ Guenter Klein ${ }^{4}$ Nadine Sudhaus ${ }^{4}$ Elisabeth Grosse Beilage ${ }^{1}$ \\ 1. Field Station for Epidemiology in Bakum, University of Veterinary Medicine Hannover, Bakum, Germany; 2. Pfizer Animal Health, \\ Berlin, Germany; 3. Pfizer Animal Health, Sandwich, UK; 4. Institute for Food Quality and Food Safety, University of Veterinary \\ Medicine Hannover, Hannover, Germany; 5. Clinic of Reproductive Medicine, University of Zuerich, Zuerich, Switzerland; 6. Federal \\ Agriculture Research Centre, Institute for Animal Breeding, Functional Genetics and Bioregulation, Neustadt, Germany
}

\section{Introduction}

Surgical castration of male pigs is frequently performed due to the fact that entire male pigs have the potential to develop an unpleasant taste and flavour of the meat. Vaccination with Improvac (Pfizer Animal Health) suppresses testicular function through the induction of antibodies against gonadotropinreleasing factor (GnRF). GnRF is released from the hypothalamus and stimulates the pituitary gland to release luteinising hormone (LH) and follicle stimulating hormone (FSH) which controls the production and release of testicular steroids, including androstenone. Antibodies against GnRF disrupt this hypothalamic-pituitary-gonad axis and thereby reduce the production of testicular steroids (2). The objective of this study was to evaluate the effect of Improvac vaccination on the concentrations of $\mathrm{LH}$ and FSH in blood, the development of the testicles, and the suppression of boar taint (3).

\section{Material and Methods}

A total of 610 males were randomly assigned to three treatment groups (T): 274 were surgically castrated in the first week of life (T1), 258 (number then remaining) were vaccinated twice with Improvac during the fattening period, at 13-14 and at 20-21 weeks of age, respectively (T2) and 56 were left entire (T3). From a subgroup of both T1 and T2 and from all pigs of T3, blood samples were collected immediately before second vaccination (T2) and again before slaughter at either 24-25 or 26-27 weeks of life to determine the serum concentrations of $\mathrm{LH}$ and $\mathrm{FSH}$. Meat and fat samples were examined with the cold cooking and the melting tests. Length, width, weight and volume of testicles were determined after slaughter.

\section{Results}

Immediately before the second vaccination (T2 only) LH and FSH concentrations were not significantly different between $\mathrm{T} 2$ and T3 (Tab. 1). However, LH and FSH concentrations were significantly higher in $\mathrm{T} 1$ compared with $\mathrm{T} 2$ and $\mathrm{T} 3$ at this time as well as before both slaughter dates. Before the first slaughter date $\mathrm{LH}$ and FSH concentrations were significantly lower in group T2 than in T3.

Tab. 1: Concentration of LH \& FSH in plasma (LS means)

\begin{tabular}{|l|c|c|c|}
\hline Time & T1 & T2 & T3 \\
\hline LH: Before second vaccination & $1.84^{\mathrm{a}}$ & $1.08^{\mathrm{b}}$ & $1.02^{\mathrm{b}}$ \\
\hline LH: Before first slaughter batch & $1.78^{\mathrm{a}}$ & $0.75^{\mathrm{b}}$ & $1.05^{\mathrm{c}}$ \\
\hline LH: Before second slaughter batch & $1.66^{\mathrm{a}}$ & $0.67^{\mathrm{b}}$ & \\
\hline FSH: Before second vaccination & $3.75^{\mathrm{a}}$ & $0.60^{\mathrm{b}}$ & $0.55^{\mathrm{b}}$ \\
\hline FSH: Before first slaughter batch & $3.76^{\mathrm{a}}$ & $0.45^{\mathrm{b}}$ & $0.55^{\mathrm{c}}$ \\
\hline FSH: Before second slaughter batch & $4.17^{\mathrm{a}}$ & $0.44^{\mathrm{b}}$ & \\
\hline
\end{tabular}

Testicle size, weight and volume were significantly lower in T2 compared to T3 (Tab. 2). In the cold cooking test as well as in the fat melting test $100 \%$ ( $25 \%$ of total T1) of the samples of T1 were rated negative for boar taint. In T2, 98\% were rated negative for boar taint, whereas in T3 94\% were rated positive. In the fat melting test $97 \%$ of $\mathrm{T} 2$ were rated negative and $3 \%$ were rated positive, including the pigs testing positive in the cold cooking test. In T3 94\% were rated positive. All pigs in T2 which were positive for boar taint in the cooking or melting test and that were tested had androstenone and skatole concentrations in backfat below threshold levels of $1 \mu \mathrm{g} / \mathrm{g}$ and $0.2 \mu \mathrm{g} / \mathrm{g}$, respectively.

Tab. 2: Testicles weight, volume, length and width (LS Means)

\begin{tabular}{|l|c|c|c|}
\hline Variable & Side & T2 & T3 \\
\hline Length $(\mathrm{mm})$ & Left & 79 & 107 \\
\hline Length $(\mathrm{mm})$ & Right & 77 & 103 \\
\hline Width $(\mathrm{mm})$ & Left & 46 & 64 \\
\hline Width $(\mathrm{mm})$ & Right & 46 & 63 \\
\hline Weight $(\mathrm{g})$ & Left & 86 & 239 \\
\hline Weight $(\mathrm{g})$ & Right & 83 & 226 \\
\hline Volume $(\mathrm{mL})$ & Left & 114 & 270 \\
\hline Volume $(\mathrm{mL})$ & Right & 110 & 257 \\
\hline
\end{tabular}

\section{Discussion}

After vaccination with Improvac levels of LH and FSH in blood as well as testes size were significantly reduced, consistent with mode of action. Of the vaccinated pigs, 98\% (235/239) were rated negative for boar taint in the cold cooking test and $97 \%$ $(232 / 239)$ in the fat melting test. The androstenone and skatole levels of the pigs testing positive for boar taint $(7 / 239,3 \%)$ were below the commonly accepted threshold levels for these substances (1).

\section{References}

(1) Banon S,et al. (2003) Food Qual Pref 15: 293-300

(2) Hoffman B, (2003) Andrologie LOB.de: 68-84

(3) Zamaratskaia, G, et al. (2008) Reprod. Dom. Anim. 43(3):351-359 


\title{
P.825
}

\section{Impact of raising Improvac ${ }^{\oplus}$-vaccinated boars on growth, carcass quality and meat quality compared to physical castrates under field conditions}

\author{
Aloyzas Januskauskas $^{1}$ H. Zilinskas² N. Sutkeviciene ${ }^{2}$ R. Bilskis ${ }^{2}$ I. Szabo ${ }^{1}$ K. Garlaite ${ }^{1}$ \\ 1. Pfizer Luxembourg SARL, Vilnius, Lithuania; 2. Lithuanian Veterinary Academy, Vilnius, Lithuania
}

\section{Introduction}

Boar taint is related to sexual maturity and male hormones and is usually controlled by surgical castration. With growing focus on animal welfare, surgical castration without anesthesia has become controversial. Alternative approaches include immunization against gonadotropin releasing factor (GnRF)1, which disrupts the hypothalamic-pituitary-gonad axis, thereby temporarily suppressing testicular function and steroid synthesis. Our aim was to compare productivity, carcass quality and meat quality of pigs vaccinated against GnRF using Improvac against those of surgically castrated pigs under field conditions.

\section{Materials and Methods}

The trial was conducted in a commercial farm in Lithuania. 402 cross-bred pigs were split into two groups. Pigs in group 1 (204) were castrated at 3 days of age. Pigs in group 2 (198) were vaccinated with Improvac at day 95 and day 140 of life. All pigs were fed the same farm-made diet depending on the growth phase. Animals were weighed in groups of 20-30 at weaning (d.28) entry to the fattening stage (d.60); first vaccination (d.95), second vaccination (d.140) and on the day of slaughter (d.180) and daily weight gain (DWG) was calculated. Mortality was recorded and necropsies performed. Fat was measured using a Fat-o-Meater according to the routine slaughterhouse procedures. Samples of longissimus dorsi were tested to assess meat quality.

Means, standard deviations and coefficients of variation were calculated for the variables assessed. The unpaired t-test (MS Excell 2003) was used to test differences between the mean values of groups. $\mathrm{P}<0.05$ was used as the significance level.

\section{Results}

Castrates were significantly heavier $(74.1 \mathrm{~kg}$ v $66.5 \mathrm{~kg}, \mathrm{P}<0.05)$ at day 140 but other differences were not significant. Overall mortality rates from farrowing (Gp 1, 14.71\%, Gp 2, 14.41\%) were not significantly different. Lifetime DWG in vaccinated pigs was $629.1 \mathrm{~g}$ compared with $631 \mathrm{~g}$ in controls, with the differences being $+69.73 g,-40.56 g,-143.85$ and $+125.07 g$ for the four periods (Fig 1).

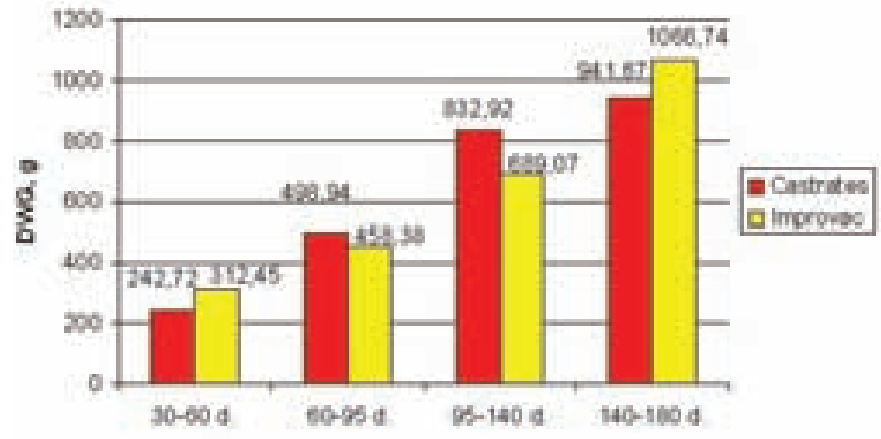

Vaccinated animals had reduced carcass fat thickness $(P<0.001)$ as measured on 2 points of the carcass $(22.79 \pm 3.93$ and $17.17 \pm 2.78$ v. $26.2 \pm 5.2$ and $19.2 \pm 3.7 \mathrm{~mm})$. Lean meat in vaccinated animals was greater by 1.9 percentage points $(56.7 \pm 2.4 \%$ v 54.8 $\pm 3.1 \%, P<0.001$ ). Meat quality parameters did not differ between the two groups (Table 1).

\begin{tabular}{|l|c|c|c|}
\hline Animals & Improvac & Control & $\mathbf{P}$ \\
\hline Dry matter $\%$ & $26.48 \pm 1.2$ & $26.52 \pm 1.2$ & N.S. \\
\hline $\mathrm{pH}$ & $5.48 \pm 0.04$ & $5.56 \pm 0.11$ & N.S. \\
\hline Color & $56.4 \pm 1.5$ & $52.2 \pm 2.5$ & N.S. \\
\hline Water binding \% & $55.6 \pm 5.5$ & $55.8 \pm 5.1$ & N.S. \\
\hline Cooking loss & $26.1 \pm 2.13$ & $25.0 \pm 2.0$ & N.S. \\
\hline IM.fat \% & $2.34 \pm 0.73$ & $1.9 \pm 0.45$ & N.S. \\
\hline
\end{tabular}

\section{Discussion}

Compared to boars, castrates are known to be fatter and less efficient. Feed conversion was not assessed in this study but DWG showed distinct differences between the groups. It was initially lower in vaccinated pigs compared to castrates, but after second vaccination weight gain was superior and at slaughter pigs were heavier (not significant). Other studies have reported a higher final live weight in vaccinated pigs2. The higher growth rate following second vaccination was probably due to higher feed intake reflecting reduced sexual and aggressive activity2. Use of vaccination reduced fat thickness and had a positive effect on muscling. Vaccination did not affect objective meat quality parameters.

Under the conditions of this study, Improvac appears to offer an alternative to physical castration that reduces back-fat and increases lean meat percent, while having no impact on meat quality. Further work may allow the differences in growth patterns to be exploited to improve overall performance.

\section{References}

1. Einarsson S. Acta Vet Scand 2006; 48 (Suppl. 1):S10.

2. Cronin, G.M., et al. (2003). Applied Animal Behaviour Science. 81:111-126. 


\title{
P.826
}

\section{A comparison of the carcase characteristics of pigs immunized with a gonadotrophin- releasing factor (GnRF) vaccine against boar taint with physically castrated pigs}

\author{
Thilo Fuchs ${ }^{1} \underline{\text { Heiko Nathues }}^{1}$ Annika Koehrmann ${ }^{2}$ Stuart Andrews ${ }^{3}$ Fiona Brock ${ }^{3}$ \\ Nadine Sudhaus ${ }^{4}$ Guenter Klein ${ }^{4}$ Elisabeth Grosse Beilage ${ }^{1}$ \\ 1. Field Station for Epidemiology in Bakum, University of Veterinary Medicine Hannover, Bakum, Germany; \\ 2. Pfizer Animal Health, Berlin, Germany; 3. Pfizer Animal Health, Sandwich, UK; 4. Institute for Food \\ Quality and Food Safety, University of Veterinary Medicine Hannover, Hannover, Germany
}

\section{Introduction}

Entire male pigs are known to be superior to physical castrates with regards to lean meat production and growth performance, which could lead to more profitable pig production (1, 2). Nevertheless, physical castration of male pigs is still a common procedure in many countries in order to prevent an unpleasant taste and flavour of the meat of sexually mature boars. The main objective of this comparative study was to investigate the carcase characteristics of physically castrated pigs and pigs immunized with a gonadotrophin-releasing factor (GnRF) vaccine, which acts via the hypothalamic-pituitary-gonad axis and suppresses testicular function through the induction of antibodies against GnRF. Pigs were raised under field conditions in a conventionally managed farm in Germany.

\section{Materials and Methods}

Male pigs were randomly assigned to three treatment groups: pigs surgically castrated during the first week of life (group T1, $\mathrm{n}=274$ ), pigs immunised twice during the fattening period with a GnRF vaccine (Improvac, Pfizer Animal Health), the first time when 13-14 weeks of age and the second time when 20-21 weeks of age (group T2, n=280) and entire males (group T3, $\mathrm{n}=56$ ). All pigs were weighed individually three days before slaughter in either the 24th or 26th week of life. Pigs of groups T1 and T2 that weighed $110 \mathrm{~kg}$ or more on day 160 were slaughtered in the first batch. Overall, 242 pigs met these criteria. The remaining 241 pigs were slaughtered in the 26th week. At slaughter, testes were removed and hot carcase weight determined. Lean meat percentage, thickness of backfat and backmuscle was measured. The boned ham, boned shoulder, loin and belly weights alongside the lean meat percentage of belly were measured with the AutoFOM ultrasound system. Carcases were categorised, using the EUROP grading system.

\section{Results}

There was neither significant difference between the groups in terms of average liveweight nor in the hot carcase weight (Tab. 1). The mean dressing percentage was $1.5 \%$ higher for $T 1$ than for T2 $(P<0.0001)$. The lean meat percentage was significantly higher in T2 $(P<0.0001)$. Backfat and backmuscle thickness were significantly higher in $\mathrm{T} 1$ ( $\mathrm{P}<0.0001$ and $\mathrm{P}=0.0099$, respectively). Within the EUROP grading (Tab. 1) vaccinated pigs were in favour $(P=0.0034)$. There were no significant differences using the AutoFOM system: weights of the boned ham, boned shoulder and loin ( $\mathrm{P}=0.5102, \mathrm{P}=0.8881$ and $\mathrm{P}=0.1919$, respectively). The weight of the belly was significantly higher $(P=0.0042)$ in $T 1$ while the lean meat percentage of belly was significantly higher $(P<0.0001)$ in $\mathrm{T} 2$.

Tab. 1: Effect of immunization with a GnRF vaccine on average liveweight and carcase weight.

\begin{tabular}{|l|c|c|c|c|c|}
\hline Parameter & T1 / LS mean & T1/SE & T2 / LS mean & T2 / SE & P-value \\
\hline $\begin{array}{l}\text { Liveweight, 24th } \\
\text { week (kg) }\end{array}$ & 110.2 & 0.97 & 107.6 & 1.01 & 0.0648 \\
\hline $\begin{array}{l}\text { Liveweight, 26th } \\
\text { week (kg) }\end{array}$ & 120.7 & 1.00 & 121.3 & 1.07 & 0.6999 \\
\hline Carcase weight (kg) & 91.5 & 0.62 & 89.8 & 0.62 & 0.0682 \\
\hline
\end{tabular}

Tab. 2: Effect of immunization with a GnRF vaccine on EUROP grading of carcase; number of pigs (\%).

\begin{tabular}{|l|c|c|}
\hline Grading & Physical castrates (T1) & GnRF vaccinates (T2) \\
\hline E & $87(36 \%)$ & $118(49 \%)$ \\
\hline U & $133(55 \%)$ & $113(47 \%)$ \\
\hline R & $22(9 \%)$ & $9(4 \%)$ \\
\hline 0 & - & - \\
\hline P & - & - \\
\hline
\end{tabular}

\section{Discussion}

In this study differences in carcase characteristics between physically castrated pigs and vaccinated pigs were evaluated. There were no statistically significant differences between the physically castrated pigs and those immunized with a GnRF vaccine in terms of the average live bodyweight of pigs before slaughter. The immunization with a GnRF vaccine improves lean meat content, EUROP grading score and reduces backfat thickness. Despite a lower dressing percentage, no adverse effects on the weights of valuable parts of the carcase such as ham, loin and shoulder could be detected with the exception of a lower belly weight and a lower back muscle thickness. In conclusion, the use of a GnRF vaccine to control boar taint could contribute to producing pigs with leaner and more muscular carcasses.

\section{References}

(1) Bussen T. et al. (1997) Liv. Prod. Sci. 51

(2) Dunshea FR. et al. (2001) J Anim. Sci. 79 


\title{
P.827
}

\section{Welfare consequences of slaughter strategy in entire male pig production}

\author{
Bente Fredriksen; Cathrine Hexeberg; Ola Nafstad \\ Animalia, Oslo, Norway
}

\section{Introduction}

Aggressive and sexual behaviour is more frequent in entire male pigs than in castrates, and might represent an animal welfare problem (EFSA 2004). While the incidence of unwanted behaviour might be kept on an acceptable level by measures in the herd, like sibling groups, the conditions during transport and lairage might be harder to control. In Norway, and probably also in other countries, a common practice is that the animals are sent to slaughter as they reach a certain weight. Consequently, not all animals from a pen are delivered to slaughter at the same time (split marketing). This practice causes a considerable mixing of unfamiliar animals during transport and lairage. The aim of the study was to investigate the practical and animal welfare consequences of different slaughter strategies in entire male pig production.

\section{Materials and Methods}

The study was performed as a field study with two replicates in one herd in the period June 2008 - January 2009. A total of 134 entire male pigs and 139 castrates, all Noroc hybrids (LYXLD) were included. The animals were regrouped by sex into pens of 9 animals when transferred to the growing/fattening unit. In half of the pens within each sex, the animals were slaughtered by traditional split marketing while the other half were slaughtered pen-wise, without mixing with unfamiliar pigs during transport and lairage. Skin lesions were recorded regularly for all animals in the herd and also at the slaughter house. A four point scale was used for front, middle and back on both sides of the animals. Both total score and max-score were used for the statistical analyses. Behavour was also recorded both in the herd and at the slaughter house. A sexual index (S-Index) and an aggression index (A-Index) per pen were calculated in the following way:

Sexual index $=((($ number of flank pushes $+2 x$ number of attempts to mount $+3 x$ number of mountings)/number of males in the pen)/number of minutes recorded) $\times 60$.

Aggression index $=(($ number of head knocks $+2 x$ number of bites $+3 x$ number of fightings)/number of pigs in the pen)/num-

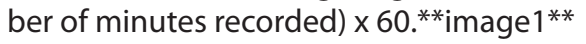

Figure 1. Mean total score for skin lesions in the herd at the different registrations for castrates and entire males.

$B=$ last registration before first slaughter in the pen, $A=$ first registration after first slaughter in the pen.

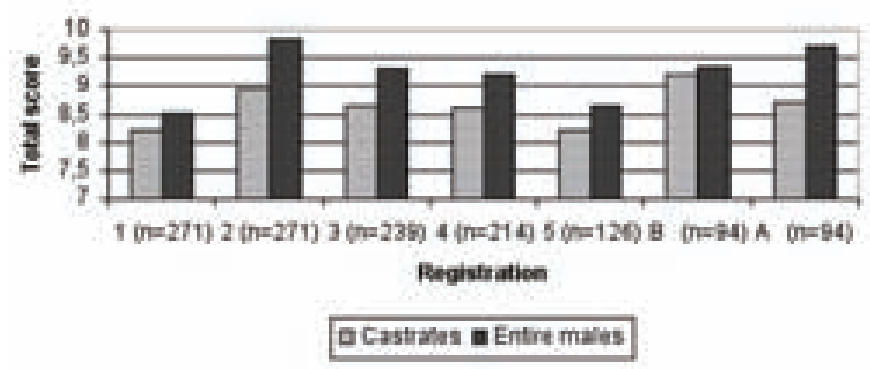

Figure 2. Aggressive behaviour recorded at the slaughter house, given as index perpen.

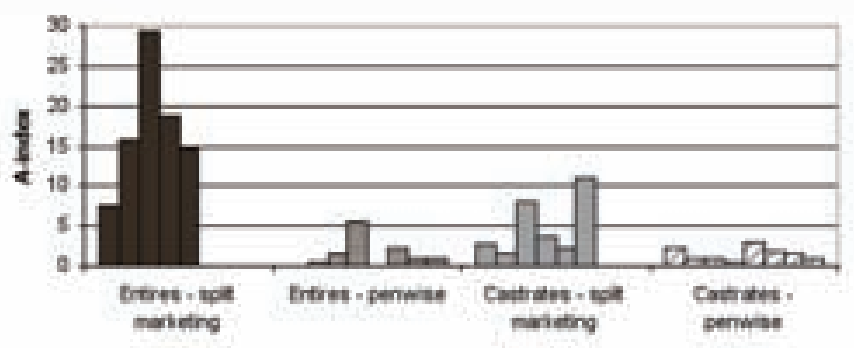

\section{Discussion}

Even though higher skin lesion scores as well as higher A- and $S$-indexes were recorded for entire males than for castrates, the effect of sex on parameters recorded in the herd was moderate. At the slaughter house, the effects of both sex and slaughter strategy were pronounced (Figure 2). Split marketing seems to have a negative effect on animal welfare, especially for entire male pigs. In addition, the fighting might affect meat quality and result in downgrading of carcasses because of skin lesions. When the animals were kept penwise during transport and lairage in the same units as in the herd, aggressive and sexual behaviour in entire males were reduced significantly.

\section{References}

EFSA 2004. Welfare aspects of the castration of piglets. Scientific Report of the Scientific Panel for Animal Health and Welfare on a request from the Commission related to welfare aspects of the castration of Piglets. The EFSA Journal (2004) 91, 1-18. 


\title{
P.828
}

\section{Impact of vaccination against boar taint on production variables in Poland, Czech Republic and Hungary}

\author{
Zygmunt Pejsak ${ }^{1}$ Jacek Zmudzki ${ }^{1}$ Marian Porowski² Marek Lesiak ${ }^{3}$ Marek Zizlavsky ${ }^{4}$ Robert Travnicek $^{5}$ Tamas Abonyi $^{6}$ \\ 1. National Veterinary Research Institute, Pulawy, Poland; 2. Vet Clinic Animal, Pobiedziska, Poland; \\ 3. Pfizer Animal Health, Warsaw, Poland; 4. Sevaron, Brno, Czech Republic; \\ 5. Pfizer Animal Health, Praha, Czech Republic; 6. Pfizer Animal Health, Budapest, Hungary
}

\section{Introduction}

The meat industry is highly competitive and is subject to two major drivers of change: new legislation and technological innovations. For meat processors, immunological castration provides better quality carcasses, with less fat, less wastage and thus higher value (1). The objective of this study was to evaluate the efficacy and production benefits of vaccination with Improvac for the control of boar taint under field conditions in Poland, Czech Republic and Hungary.

\section{Materials and Methods}

The study was performed at 4 commercial pig farms located in Poland (PL) (2 farms: PL-L and PL-S), Czech Republic (CZ) and Hungary (H). Male piglets (PL-L, 108; PL-S, 60; CZ, 119; and H, 83) were surgically castrated (T01). On each farm, the same number of piglets were vaccinated with Improvac subcutaneously twice: initially at ages 9-10 weeks (PL), 11-13 (CZ),

20-23 $(\mathrm{H})$ then again at ages: $20(\mathrm{PL}), 22-24(\mathrm{CZ})$ and 24-27 $(\mathrm{H})$ weeks (T02). Pigs were slaughtered at ages 24-26 (PL), 27-29 $(\mathrm{CZ})$ and 28-31 (H) weeks. Average body weight (ABW) at castration, 1 st and 2 nd vaccination, and slaughter was determined and average daily body weight gain (ADG) was determined for the periods: castration to 1 st vaccination, castration to slaughter, 1 st vaccination to slaughter and 2 nd vaccination to slaughter. Lean meat percentage was estimated at slaughter.

\section{Results}

Compared with barrows (T01), ABW of pigs vaccinated with Improvac (T02) was comparable at castration, and 1st and 2nd vaccination, but 7\% higher at slaughter on farm PL-L On farms PL-S, $\mathrm{CZ}$ and $\mathrm{H}, \mathrm{ABW}$ was similar at castration, 1 st and 2 nd vaccination and slaughter (Table 1a).From castration to 1st vaccination, ADG of barrows (T01) and vaccinated pigs (T02) was comparable on all farms. From castration to slaughter, 1st vaccination to slaughter, and 2nd vaccination to slaughter, ADG was 7\%, 9\% and $32 \%$ higher respectively in vaccinated pigs than barrows on farm $\mathrm{PL}-\mathrm{S}$, and $5 \%, 9 \%$ and $7 \%$ higher respectively in vaccinated pigs than barrows on farm $\mathrm{H}$. On farm L, ADG was marginally lower in vaccinated pigs compared with barrows from castration to slaughter and 1 st vaccination to slaughter, but $5 \%$ higher in vaccinated pigs than barrows from 2 nd vaccination to slaughter (Table 1b).Compared with barrows (T01), lean meat percentage of vaccinated pigs (T02) was $3 \%$ higher on farm CZ and $5 \%$ higher on farm $\mathrm{H}$ (Table 1c).
Table 1a. Average body weight.

\begin{tabular}{|l|c|c|c|c|c|c|c|c|}
\hline \multirow{2}{*}{ Time } & \multicolumn{8}{|c|}{ Average Body Weight (kg) } \\
\cline { 2 - 10 } & \multicolumn{2}{|c|}{ Poland Farm L } & \multicolumn{2}{|c|}{ Poland Farm S } & \multicolumn{2}{c|}{ Czech } & \multicolumn{2}{c|}{ Hungary } \\
\hline & T01 & T02 & T01 & T02 & T01 & T02 & T01 & T02 \\
\hline C & 2.3 & 2.3 & 3.1 & 3.2 & 2.2 & 2.2 & 2.7 & 2.7 \\
\hline V1 & 23.4 & 23.1 & 30.6 & 30.6 & 32.2 & 33.1 & 76.4 & 73.9 \\
\hline V2 & 88.7 & 84.4 & 101 & 100 & 98.8 & 100 & 103 & 100 \\
\hline S & 114 & 111 & 131 & 140 & 122 & 124 & 127 & 127 \\
\hline
\end{tabular}

Table $\mathbf{1 b}$. Average daily body weight gain.

\begin{tabular}{|l|c|c|c|c|c|c|c|c|}
\hline \multirow{2}{*}{ Period } & \multicolumn{7}{|c|}{ Average Daily Body Weight Gain (g) } \\
\cline { 2 - 10 } & \multicolumn{1}{|c|}{ Poland Farm L } & \multicolumn{2}{|c|}{ Poland Farm S } & \multicolumn{2}{c|}{ Czech } & \multicolumn{2}{c|}{ Hungary } \\
\hline & T01 & T02 & T01 & T02 & T01 & T02 & T01 & T02 \\
\hline C to V1 & 339 & 334 & 443 & 442 & 369 & 379 & n.a. & n.a. \\
\hline C to S & 661 & 643 & 757 & 810 & 610 & 616 & 605 & 604 \\
\hline V1 to S & 848 & 823 & 939 & 1024 & 777 & 792 & 894 & 941 \\
\hline V2 to S & 819 & 859 & 971 & 1288 & 691 & 738 & 864 & 943 \\
\hline
\end{tabular}

Table 1c. Lean meat percentage.

\begin{tabular}{|l|c|c|c|c|c|c|c|c|}
\hline Carcass Variable & \multicolumn{7}{|c|}{ Mean } \\
\hline & \multicolumn{2}{|c|}{ Poland Farm L } & \multicolumn{2}{|c|}{ Poland Farm S } & \multicolumn{2}{c|}{ Czech } & \multicolumn{2}{c|}{ Hungary } \\
\hline & T01 & T02 & T01 & T02 & T01 & T02 & T01 & T02 \\
\hline $\begin{array}{l}\text { Lean Meat Percent- } \\
\text { age }\end{array}$ & 57.2 & 57.7 & 55.3 & 56.2 & 55.6 & 57.3 & 53.4 & 56.0 \\
\hline
\end{tabular}

T01, barrows; T02, pigs vaccinated with Improvac; C, castration; V1, 1st vaccination; V2, second vaccination; $S$, slaughter; n.a., not measured.

\section{Discussion}

ADBWG was higher in pigs vaccinated with Improvac compared with barrows, especially following the 2 nd vaccination. Vaccination resulted in a significant increase in lean meat percentage.

\section{References}

1. Mackinnon JD, Pearce MC. Pig J. 2007;59:68-90. 


\title{
P.829
}

\section{Hematology of entire boars and boars vaccinated with Improvac ${ }^{\circledR}$}

\author{
Tatjana Sattler ${ }^{1}$ Ruth Wipf ${ }^{1}$ Franziska Sauer ${ }^{1}$ Kerstin Lösel ${ }^{2}$ Friedrich Schmoll ${ }^{1,3}$ \\ 1. Large Animal Clinic for Internal Medicine, Leipzig, Germany; 2. Pfizer Animal Health, Sandwich, UK; \\ 3. Institute for Veterinary Public Health, VetmedUni, Vienna, Austria
}

\section{Introduction}

Hematological reference values for slaughter age boars are rare and none are published for boars vaccinated with Improvac ${ }^{\circledR}$ (GnRH-analogue vaccine against boar taint (1). The objective of the study was to determine and compare mean hematological values at slaughter in boars vaccinated with Improvac and controls among two crossbreeds.

\section{Materials and Methods}

A total of 549 blood samples were collected by exsanguination from 410 Piétrain cross ( 96 entire boars and 314 boars vaccinated with Improvac (1)) and 139 Duroc cross (42 entire boars and 97 Improvac) boars. Mean body weights at slaughter were between 118.6 kg (Piétrain) and 119.9 kg (Duroc). Blood cells were counted using an ADVIA120 hematological counter (Bayer). Differential white blood cell counts were estimated by microscopy.

\section{Results}

Piétrain crosses had lower hemoglobin, MCV, MCH and MCHC than Duroc crosses. In Piétrain crosses, leucocyte count was higher in entire boars compared with boars vaccinated with Improvac driven by a higher lymphocyte, monocyte and eosinophil count in this group. The platelet count in Piétrain crosses was higher in entire boars than vaccinated pigs (table 1 ).

\section{Discussion}

Mean values for all groups were within existing physio-logical ranges of swine (2) except raised leucocytes in entire boars of both breeds. Further examination is needed to determine if this effect is boar specific.

The vaccination of boars with Improvac had no impact on hematology results.
Table 1: Hematology of entire boars and pigs vaccinated with Improvac at slaughter, by breed (mean; \pm standard deviation).

\begin{tabular}{|l|c|c|c|c|}
\hline & \multicolumn{2}{|c|}{ Piétrain Cross } & \multicolumn{2}{c|}{ Duroc Cross } \\
\hline & Boars & Improvac & Boars & Improvac \\
\hline \multicolumn{1}{|c|}{$\mathbf{n}$} & $\mathbf{9 6}$ & $\mathbf{3 1 4}$ & $\mathbf{4 2}$ & $\mathbf{9 7}$ \\
\hline erythrocytes (T/I) & $8.05 \pm 0.61$ & $8.02 \pm 0.62$ & $7.9 \pm 0.52$ & $8.07 \pm 0.53$ \\
\hline hematocrit (I/I) & $0.42 \pm 0.09$ & $0.43 \pm 0.03$ & $0.43 \pm 0.03$ & $0.43 \pm 0.02$ \\
\hline hemoglobin (mmol/I) & $8.7 \pm 0.6$ & $8.9 \pm 0.5$ & $9.0 \pm 0.6$ & $9.1 \pm 0.5$ \\
\hline MCV (fl) & $52.5 \pm 2.9$ & $53.4 \pm 2.9$ & $54.3 \pm 2.6$ & $53.9 \pm 2.5$ \\
\hline MCH (fmol) & $1.08 \pm 0.06$ & $1.11 \pm 0.06$ & $1.14 \pm 0.05$ & $1.14 \pm 0.06$ \\
\hline MCHC (mmol/I) & $20.5 \pm 1.2$ & $20.8 \pm 0.6$ & $21.0 \pm 0.6$ & $21.1 \pm 0.6$ \\
\hline platelets (G/I) & $426 \pm 131$ & $386 \pm 117$ & $267 \pm 120$ & $318 \pm 121$ \\
\hline leukocytes (G/I) & $23.6 \pm 5.8$ & $20.9 \pm 5.2$ & $22.3 \pm 6.2$ & $21.1 \pm 5.4$ \\
\hline neutrophiles (G/I) & $12.2 \pm 6.3$ & $11.2 \pm 5.3$ & $10.7 \pm 6.9$ & $11.2 \pm 5.4$ \\
\hline lymphocytes (G/I) & $9.2 \pm 2.5$ & $7.8 \pm 2.4$ & $9.4 \pm 2.6$ & $8.2 \pm 1.9$ \\
\hline monocytes (G/I) & $1.5 \pm 0.5$ & $1.2 \pm 0.8$ & $1.5 \pm 0.7$ & $1.1 \pm 0.5$ \\
\hline eosinophils (G/I) & $0.4 \pm 0.3$ & $0.3 \pm 0.2$ & $0.3 \pm 0.2$ & $0.3 \pm 0.2$ \\
\hline basophils (G/I) & $0.2 \pm 0.1$ & $0.2 \pm 0.1$ & $0.2 \pm 0.1$ & $0.2 \pm 0.1$ \\
\hline neutrophils (\%) & $49 \pm 14$ & $52 \pm 15$ & $46 \pm 18$ & $51 \pm 13$ \\
\hline Iymphocytes (\%) & $41 \pm 13$ & $40 \pm 13$ & $45 \pm 17$ & $41 \pm 11$ \\
\hline monocytes (\%) & $6.5 \pm 2.2$ & $6.1 \pm 4.2$ & $7.0 \pm 3.4$ & $5.3 \pm 3.1$ \\
\hline
\end{tabular}

\section{References}

1. Schmoll F. et al. J Swine Health Prod. 2009;17:250-255.

2. Heinritzi and Plonait 2001: In: Lehrbuch der Schweinekrankheiten 4th Ed. 170-196. 


\title{
P.830
}

\section{Clinical chemistry in entire boars and boars vaccinated with Improvac ${ }^{\circ}$}

\author{
Tatjana Sattler ${ }^{1}$ Ruth Wipf ${ }^{1}$ Franziska Sauer ${ }^{1}$ Kerstin Lösel ${ }^{2}$ Friedrich Schmoll ${ }^{1,3}$ \\ 1. Large Animal Clinic for Internal Medicine, Leipzig, Germany; 2. Pfizer Animal Health, Sandwich, UK; \\ 3. Institute for Veterinary Public Health, VetmedUni, Vienna, Austria
}

\section{Introduction}

Blood markers of protein and mineral metabolism in swine are likely to be different between breeds and gender (1). Reference values for slaughter age boars are rarely described and none are published for boars vaccinated with Improvac $(\mathrm{GnRH}$-analogue vaccine against boar taint). The objective of the study was to determine and compare mean values of blood markers for protein and mineral metabolism at slaughter in boars vaccinated with Improvac and controls among two crossbreeds.

\section{Materials and Methods}

A total of 569 blood samples were collected by exsanguination of 425 Piétrain cross ( 96 entire boars and 329 Improvac) and 144 Duroc cross (44 entire boars and 100 Improvac) male pigs. Boars had a mean body weight of $118.9 \mathrm{~kg}$ and a mean age of 180 days at slaughter. The analysis of alkaline phosphatase (AP), measured at $37^{\circ} \mathrm{C}$, urea, creatinine, inorganic phosphate $(P)$ and calcium (Ca) were determined with an analysis automate Hitachi 704 (Boehringer Mannheim).

\section{Results}

Boars from both breeds had higher serum AP concentrations as well as lower serum creatinine and serum urea concentrations than boars vaccinated with Improvac.

Improvac vaccinated Duroc boars showed higher AP activities and lower $\mathrm{Ca}$ and $\mathrm{P}$ concentrations than the vaccinated Piétrain boars. The creatinine concentration was over all higher in Duroc boars than in the Piétrain boars (table 1).

\section{Discussion}

Mean values for all groups were within previously reported physiological ranges of swine $(2,3)$ and were consistent with published reference intervals for boars from different German boar studs and breeds (1). Pigs in this study were fed a standard diet (optimised for barrows and females and not for boars) but there was no evidence of an undersupply of nutrients. There was no evidence that vaccination with Improvac affected the variables measured.

Table 1. Hematological values of entire boars and pigs vaccinated with Improvac, by breed (mean; \pm standard deviation).

\begin{tabular}{|l|c|c|c|c|}
\hline & \multicolumn{2}{|c|}{ Piétrain Cross } & \multicolumn{2}{c|}{ Duroc Cross } \\
\hline & Boars & Improvac & Boars & Improvac \\
\hline \multicolumn{1}{|c|}{$\mathbf{n}$} & $\mathbf{9 6}$ & $\mathbf{3 2 9}$ & $\mathbf{4 4}$ & $\mathbf{1 0 0}$ \\
\hline AP U/I & $162 \pm 47$ & $138 \pm 43$ & $171 \pm 49$ & $150 \pm 48$ \\
\hline Ca mmol/I & $2.65 \pm 0.1$ & $2.67 \pm 0.1$ & $2.65 \pm 0.1$ & $2.63 \pm 1.2$ \\
\hline P mmol/I & $3.04 \pm 0.3$ & $3.04 \pm 0.3$ & $3.02 \pm 0.4$ & $2.88 \pm 0.3$ \\
\hline creatinine $\mu \mathrm{mol} / \mathbf{I}$ & $148 \pm 16$ & $153 \pm 19$ & $136 \pm 13$ & $145 \pm 19$ \\
\hline urea mmol/I & $5.6 \pm 1.2$ & $6.6 \pm 1.2$ & $6.0 \pm 1.1$ & $6.8 \pm 1.3$ \\
\hline
\end{tabular}

\section{References}

1. Nerbas 2008: Thesis, Hannover, Germany 1-240.

2. Heinritzi and Plonait 2001: In: Lehrbuch der Schweinekrankheiten 4th Ed. 170-196.

3. Baumgartner 2005: In: Klinische Propädeutik, 6th Ed. 207-224. 


\title{
P.831
}

\section{A comparison of feed consumption and zootechnical performance of entire boars, boars vaccinated with Improvac ${ }^{\mathrm{Tm}}$, barrows and gilts fattened on low and high energy diets}

\author{
$\underline{\text { Herman G. Prust }}^{1}$ Evelien Hangoor ${ }^{2}$ Fiona Brock ${ }^{3}$ Michael Pearce ${ }^{3}$ David Lamot $^{2}$ \\ 1. Pfizer Animal Health, Capelle a/d IJssel, Netherlands; 2. Provimi BV, Rotterdam, Netherlands; 3. Pfizer Animal HealthVMRD, Sandwich, UK
}

\section{Introduction}

Typically, male pigs are physically castrated in Europe to control boar taint ${ }^{1}$. Use of Improvac allows male pigs to grow as efficiently as boars until the time of second vaccination. This study examined the impact of feeding low and high density diets to entire boars, barrows, vaccinates and gilts after the second administration of Improvac to vaccinates.

\section{Materials and Methods}

A total of $179 \mathrm{~F} 2$ crossbred pigs aged 66 days from Yorkshire $\mathrm{x}$ Dutch Landrace sows sired by Piétrain boars were enrolled: 48 gilts, 47 barrows, 42 entire boars and 42 boars vaccinated with Improvac at enrolment (day 0, 66 days of age) and day 84, which was approximately 6 weeks before slaughter (day 123). Pigs were grouped by weight within sex, and groups randomly allocated to pens so that there were 6 pens per sex fed a low energy $(9.04$ $\mathrm{MJ})$ and 6 pens fed the high energy $(10.01 \mathrm{MJ})$ finishing diet. These diets were fed from day 84 , after being fed the same boar ration. Pigs were weighed on days $0,12,34,62,84$ and 123 . Feed intake was monitored using a computerised feeding system and residual feed was weighed when pigs were weighed.

\section{Results}

Before the second injection of Improvac vaccinated pigs performed similarly to boars: they were significantly lighter than castrates, but also had significantly lower feed intake and feed conversion. After administration of the second dose of Improvac and partitioning of sex groups into low and high density diets, feed intake and average daily gain (ADG) of vaccinates increased markedly and their performance diverged from entire boars. Thus, final bodyweights and overall study ADG values for barrows $(874 \mathrm{~g} / \mathrm{d})$ and vaccinates $(875 \mathrm{~g} / \mathrm{d})$ were similar. Overall, feed conversion ratio $(F C R)$ was significantly different $(p<0.05)$ between vaccinates (2.34) and barrows (2.66). Boars had the lowest overall FCR (2.26). From day 84 to 123, there was a significant effect of sex $(P<0.0001)$ but not diet on ADG which was highest in vaccinated pigs. From day 84 to 123 , the difference in ADG and feed intake between vaccinates on the LE and HE diets was not significant but there was a significant effect of sex $(p<0.001)$ and diet $(p<0.0001)$ on FCR.. From day 84 to 123 , boars had the best feed conversion and barrows the worst, with FCR in vaccinated boars being $17 \%$ lower than barrows on the LE or HE diets. In vaccinates and barrows, the HE diet resulted in a significantly lower FCR. In barrows this was associated with a significantly increased $A D G$, but in vaccinates it reflected a small increase in $\mathrm{ADG}$ and a reduction in $\mathrm{FI}$.
Table 1

\begin{tabular}{|c|c|c|c|c|c|}
\hline \multicolumn{2}{|l|}{ Variable } & Barrow & Boar & Improvac & Gilt \\
\hline \multicolumn{6}{|c|}{ Bodyweight(kg) } \\
\hline \multicolumn{2}{|l|}{ Day 0} & $25.0^{\mathrm{a}}$ & $23.6^{\mathrm{b}}$ & $23.7^{a b}$ & $24.6^{\mathrm{ab}}$ \\
\hline \multicolumn{2}{|l|}{ Day 84} & $97.7^{\mathrm{a}}$ & $88.2^{b}$ & $87.3^{b}$ & $88.8^{b}$ \\
\hline \multicolumn{2}{|l|}{ Day 123} & $132.3^{\mathrm{a}}$ & $127.4^{\mathrm{ab}}$ & $131.3^{\mathrm{ab}}$ & $122.9^{c}$ \\
\hline \multicolumn{6}{|l|}{ Days 0 to 84} \\
\hline \multicolumn{2}{|l|}{ ADG (g/day) } & $869^{a}$ & $770^{b}$ & $757^{b}$ & $765^{b}$ \\
\hline \multicolumn{2}{|l|}{$\mathrm{FI}$ (g/day) } & $2068^{a}$ & $1637^{b c}$ & $1592^{c}$ & $1720^{b}$ \\
\hline \multicolumn{2}{|l|}{ FCR } & $2.38^{\mathrm{a}}$ & $2.13^{c}$ & $2.10^{c}$ & $2.25^{b}$ \\
\hline \multicolumn{6}{|c|}{ Days 84 to 123} \\
\hline \multirow[t]{3}{*}{ ADG 9g/day) } & LE & $832^{1}$ & 1003 & 1115 & 864 \\
\hline & HE & $933^{2}$ & 1005 & 1140 & 888 \\
\hline & all & $883^{a}$ & $1004^{\mathrm{b}}$ & $1128^{\mathrm{C}}$ & $876^{a}$ \\
\hline \multirow[t]{3}{*}{$\mathrm{FI}$ (g/day) } & LE & 2865 & 2573 & 3180 & 2458 \\
\hline & HE & 2878 & 2402 & 2929 & 2503 \\
\hline & all & $2871^{b}$ & $2487^{c}$ & $3054^{a}$ & $2481^{C}$ \\
\hline \multirow[t]{3}{*}{ FCR } & LE & $3.44^{1}$ & 2.57 & $2.84^{1}$ & 2.85 \\
\hline & HE & $3.10^{2}$ & 2.41 & $2.57^{2}$ & 2.80 \\
\hline & all & $3.27^{\mathrm{a}}$ & $2.49^{\circ}$ & $270^{b}$ & $2.82^{b}$ \\
\hline \multicolumn{6}{|c|}{$\begin{array}{l}\text { All values are LS means.. LE=low energy diet, } \mathrm{HE}=\text { high energy diet, } \mathrm{All}=\text { both } \\
\mathrm{a}, \mathrm{b}, \mathrm{c} \text { different superscripts within row differ significantly }(\mathrm{p}<0.05) \\
1,2 \text { - different superscripts within NE/HE pair differ significantly }\end{array}$} \\
\hline
\end{tabular}

\section{Discussion and Conclusion}

After the 2nd Improvac vaccination feed intake increased tremendously, even exceeding that of castrates. This was associated with a high ADG and favourable FCR, particularly when fed the high energy diet

\section{References}

1. Report on welfare aspects of castration of pigs (2004) EFSA Journal, $91,1-18$

2. Cronin GM et al. (2003). App. Anim. Behav. Sci;81:111-126 


\title{
P.832
}

\section{A comparison of carcass characteristics of entire boars, boars vaccinated with Improvac ${ }^{\mathrm{TM}}$, barrows and gilts fattened on low and high energy diets}

\author{
Herman G. Prust $^{1}$ Evelien Hangoor ${ }^{2}$ Fiona Brock ${ }^{3}$ Michael Pearce ${ }^{3}$ David Lamot $^{2}$ \\ 1. Pfizer Animal Health, Capelle a/d IJssel, Netherlands; 2. Provimi BV, Rotterdam, Netherlands; 3. Pfizer Animal Health VMRD, Sandwich, UK
}

\section{Introduction}

Typically, male pigs are physically castrated in Europe to control boar taint ${ }^{1}$. However, entire male pigs have better feed conversion and produce a leaner carcase than physically castrated pigs and gilts ${ }^{2}$. Use of Improvac allows male pigs to grow as efficiently as entire males until the time of second vaccination. This study examined the impact of feeding low and high energy diets to entire boars, barrows, vaccinates and gilts after the time of the second administration of Improvac to vaccinates.

\section{Materials and Methods}

A total of $179 \mathrm{~F} 2$ crossbred pigs aged 66 days from Yorkshire $\mathrm{x}$ Dutch Landrace sows sired by Piétrain boars were enrolled: 48 gilts, 47 barrows, 42 entire boars and 42 boars vaccinated with Improvac at enrolment and again 6 weeks before slaughter. Pigs were grouped by weight within sex, and groups randomly allocated to pens so that there were 6 pens per sex fed the low energy (9.04 MJ) and 6 pens fed the high energy (10.01 MJ) finishing diet. Pigs were fed the same diet until administration of the second dose of Improvac. Pre-slaughter live weight was taken before transport after 8 hours of fasting. Slaughter was at a commercial abattoir. Carcass lean meat and back fat were measured using a Hennessy Grading Probe. Lean meat \% was calculated using a formula in EU Directive 2005/627/EG.

\section{Results}

Overall, liveweight and hot carcase weights were highest in barrows and vaccinated pigs ( $P \leq 0.0041)$; killing out percentages were highest in barrows and gilts $(\mathrm{P}<0.0001)$; $\mathrm{P} 2$ backfat thickness was highest in barrows, and lowest in entire boars and gilts $(P<0.0001)$; and meat percentage was highest in boars and gilts, and lowest in barrows $(P=0.0024)$. In both the latter cases, Improvac vaccinated pigs were significantly different from other groups.

In gilts, lean meat percentage was 1.5 percentage points higher in those fed the low energy diet $(P=0.018)$ and backfat was $14 \%$ thicker in those fed the high energy diet $(P=0.0348)$ There was a significant difference $(P=0.033)$ in the killing out percentage between vaccinated pigs fed the low energy diet (79.5\%) and those fed the high energy diet $(80.5 \%)$ and in the carcass lean meat percentage (55.4\% compared with $56.7 \%$ respectively).
Table 1

\begin{tabular}{|c|c|c|c|c|c|}
\hline \multicolumn{2}{|c|}{ Carcass Variable } & \multirow{2}{*}{$\begin{array}{c}\text { Barrow } \\
128.2 \\
\end{array}$} & \multirow{2}{*}{$\begin{array}{l}\text { Boar } \\
128.0 \\
\end{array}$} & \multirow{2}{*}{$\begin{array}{c}\text { Improvac } \\
127.3 \\
\end{array}$} & \multirow{2}{*}{$\begin{array}{c}\text { Gilt } \\
119.9 \\
\end{array}$} \\
\hline Empty Live & LE & & & & \\
\hline \multirow[t]{2}{*}{ Weight(kg) } & HE & 131.6 & 123.5 & 129.8 & 123.2 \\
\hline & All & $129.9^{\mathrm{a}}$ & $125.8^{\mathrm{ab}}$ & $128.5^{\mathrm{a}}$ & $121.6^{b}$ \\
\hline Hot & LE & 105.2 & 103.1 & 101.5 & 98.1 \\
\hline carcassweight & $\mathrm{HE}$ & 108.2 & 99.0 & 104.5 & 100.6 \\
\hline$(\mathrm{kg})^{1}$ & All & $106.7^{\mathrm{a}}$ & $101.0^{b}$ & $103.0^{\mathrm{ab}}$ & $99.4^{b}$ \\
\hline \multirow[t]{3}{*}{ Killing out $\%$} & LE & 81.9 & 80.5 & $79.5^{1}$ & 81.8 \\
\hline & HE & 82.2 & 80.2 & $80.5^{2}$ & 81.6 \\
\hline & All & $82.1^{\mathrm{a}}$ & $80.3^{b}$ & $80.0^{b}$ & $81.7^{\mathrm{a}}$ \\
\hline \multirow[t]{3}{*}{ Backfat (mm) } & LE & 20.1 & 15.0 & 18.0 & $14.0^{1}$ \\
\hline & HE & 21.2 & 15.0 & 17.0 & $16.0^{2}$ \\
\hline & All & $20.6^{\mathrm{a}}$ & $15.0^{c}$ & $17.5^{b}$ & $15.0^{c}$ \\
\hline Meat & LE & 54.3 & 58.1 & $55.4^{1}$ & $59.0^{1}$ \\
\hline \multirow[t]{2}{*}{ Percentage } & HE & 53.5 & 57.9 & $56.7^{2}$ & $57.5^{2}$ \\
\hline & All & $53.9^{c}$ & $58.0^{\mathrm{a}}$ & $56.1^{b}$ & $58.2^{\mathrm{a}}$ \\
\hline Loin thickness & LE & 63.3 & 62.8 & 60.2 & 63.3 \\
\hline \multirow[t]{2}{*}{$(\mathrm{mm})$} & $\mathrm{HE}$ & 62.5 & 61.2 & 63.2 & 64.1 \\
\hline & All & $62.9^{\mathrm{a}}$ & $62.0^{\mathrm{a}}$ & $61.7^{\mathrm{a}}$ & $63.7^{\mathrm{a}}$ \\
\hline \multicolumn{6}{|c|}{$\begin{array}{l}\mathrm{LE}=\text { low energy diet, } \mathrm{HE}=\text { high energy diet, } \mathrm{All}=\text { both } \\
\mathrm{a}, \mathrm{b}, \mathrm{c} \text { different superscripts within row differ significantly }(\mathrm{p}<0.05) \\
1,2 \text { different superscripts within NE/HE pair differ significantly }\end{array}$} \\
\hline
\end{tabular}

\section{Discussion and Conclusion}

Differences between sex groups are consistent with published literature. The high energy diet was predicted to result in increased fatness in the Improvac pigs but this did not occur. In fact fat was numerically less and lean meat \% significantly increased. Dressing percentage was also improved, perhaps associated with the increased loin thickness.

\section{References}

1. Report on welfare aspects of castration of pigs (2004) EFSA Journal, $91,1-18$

2. Cronin GM et al. (2003). App. Anim. Behav. Sci;81:111-126 


\title{
P.833
}

\section{A comparison of mortality (animal withdrawal) rates in male fattening pigs reared using either physical castration or vaccination with $\operatorname{Improvac}^{\circledR}$ as the method to reduce boar taint}

\author{
James R. Allison ${ }^{1}$ Michael Pearce ${ }^{1}$ Fiona Brock ${ }^{1}$ John Crane ${ }^{2}$ \\ 1. Pfizer Animal Health, New York, NY, USA; 2. Pfizer Animal Health, Sandwich, UK
}

\begin{abstract}
Introduction
In most of Europe, male piglets are physically castrated early in life. The procedure is traumatic and leaves an open wound, creating opportunities for bacterial infection and other complications. Improvac is a vaccine alternative where male piglets are left entire but are administered two doses of vaccine later in life, the second 4 to 6 weeks before slaughter. The second dose results in a temporary suppression of testicular function and a reduction in boar taint. At this time pig behaviour also changes to become similar to that of physical castrates. The objective of this analysis was to compare the impact of these two approaches on pig mortality at various stages of the production cycle.
\end{abstract}

\section{Materials and Methods}

A meta-analysis was conducted on data from 15 European field studies comparing physically castrated and Improvac vaccinated pigs. The studies were primarily conducted to compare efficacy in the reduction of boar taint or to look at the impact of the two approaches on growth performance. In all cases piglets were selected and randomly allocated to treatment groups at the time of physical castration. As part of normal procedure, records were kept of all animals that died or were withdrawn from the studies until the trials closed at slaughter, resulting in a database of 4393 animal records, well balanced between physically castrated and vaccinated pigs. In general, study withdrawals were of animals considered too sick to continue or in such poor condition that a producer would not normally retain them, so would equate to mortality in commercial practice. To avoid introducing subjective judgment all withdrawals were included in the analysis except those due to over-recruitment or logistical reasons.

Data on mortalities-cum-withdrawals were analysed for four time periods: castration to weaning (C-W), weaning to 1 st vaccination (W-V1) (typically entry to fattening), 1st vaccination to 2nd vaccination (V1-V2), and second vaccination to slaughter (V2-S). he number and percentage of withdrawals were summarised by study and overall. Only those pigs present at the start of a period were eligible for withdrawal within that period.

Until first vaccination the experimental unit was the pig and withdrawals were analysed in two pooled analyses within PROC GLIMMIX (SAS v9.1.3), with treatment as a fixed effect, and study and study by treatment interaction as random effects. A binomial distribution and a logit link were used to force logistic regression for the model due to the dichotomous response for completion of a period. The ilink option was used to transform the LS mean model estimates into the estimated percentage removed for each treatment group during each time period.
From first vaccination onwards, the experimental unit was the fattening pen and the percentage removals within each fattening pen was analysed with linear mixed models (PROC MIXED, SAS v9.1.3) for each study with treatment as a fixed effect and random effects for fattening pen and where appropriate study site and fattening pen within study site, where the study was conducted in multiple locations. The LS means from each of these models were then used in meta-analyses to estimate the percentage removals from each treatment group. Three studies were not appropriate for these analyses due to the study design and data limitations.

\section{Results}

Records were available for 2196 physical castrates and 2197 vaccinated pigs. Comparative mortality-cum-withdrawal rates for the four periods are shown below. as percentages of the number of pigs entering that phase. The mortality-cum-withdrawal rate pre-weaning was significantly higher in the castrate group. Differences in other periods were not significantly different.

Table 1: Pig Mortality-cum-Withdrawal by Production Period

\begin{tabular}{|l|c|c|c|}
\hline & Physical. Cast. & Improvac & p-value \\
\hline C-W & $5.7 \pm 1.08$ & $4.1 \pm 0.81$ & 0.0245 \\
\hline W-V1 & $3.3 \pm 0.89$ & $3.5 \pm 0.93$ & 0.8384 \\
\hline V1-V2 & $1.6 \pm 0.26$ & $1.6 \pm 0.29$ & 0.7674 \\
\hline V2-S & $1.9 \pm 0.37$ & $1.3 \pm 0.35$ & 0.2566 \\
\hline \multicolumn{4}{|l}{ (back-transformed least squares means \pm SE) (\%) } \\
\hline
\end{tabular}

\section{Discussion and Conclusion}

The design of Improvac field studies and the large number of studies undertaken provide a unique database of pigs monitored from a few days of age until slaughter. This analysis confirms anecdotal reports that physical castration increases pre-weaning mortality in male pigs 


\title{
P.834
}

\section{Impact of surgical castration of piglets on growth performance, feed efficiency and health parameters}

\author{
Djane Dallanora ${ }^{1}$ Karine G. Pasinato ${ }^{2}$ Roniê Pinheiro ${ }^{1}$ Glauber Machado ${ }^{1}$ Cleber Henn ${ }^{3}$ \\ 1. Integrall Soluções em Produção Animal Ltda, Patos de Minas, MG, Brazil; \\ 2. Universidade do Oeste de Santa Catarina, Xanxerê, Brazil; 3. Pfizer Animal Health, São Paulo, Brazil
}

\section{Introduction}

During the pre-weaning period, piglets are exposed to several challenges which can limit weight gain and also increase wasting and mortality indexes. Surgical castration is a traumatic procedure that has been associated with inflammatory conditions and impact on immune system development $(1,2)$. Behavioral and physiological responses of castrates indicate that suffering and pain are not limited to the few hours after castration and are still present up to 4 days after the surgical procedure (3). The goal of this study was to evaluate the impact of surgical castration on the growth performance of young pigs from pre-weaning till the end of the nursery period.

\section{Materials and Methods}

The experiment was carried out in a commercial farm with 3400 sows, in Santa Catarina state, Brazil. A total of 1024 four-day old piglets were individually weighed and randomly allocated into 2 treatment groups: T1 (entire males) and T2 (surgically castrated males, castrated at 4 days of age). Weaning took place at 21 days of age, and the end of nursery was on day 63 of age. After weaning, piglets were distributed into 16 pens (experimental units) within each treatment, being five pens with 20 piglets and 11 pens with 34 piglets each. During pre-weaning and nursery phases, the main parameters evaluated were piglet body weight at $4^{\text {th }}, 21^{\text {st }}, 35^{\text {th }}, 49^{\text {th }}$ and $63^{\text {rd }}$ days of age, daily weight gain (DWG), mortality rate, incidence of wasting (runts), incidence of arthritis and diarrhea, mortality rate and number of piglets receiving additional injections treatment. For all these parameters the experimental unit was the individual pig. Also during the nursery phase feed consumption and feed conversion (experimental unit was the pen). Analysis of Variance was performed for comparison between treatment means. For non-parametric data, Chi-square test and Kruskall-Wallis were used.

\section{Results and Discussion}

Piglets which were surgically castrated required higher use of additional injectable treatments with antibiotics when compared to $T 1$ during nursery phase $(p=0,016)$ and also throughout the whole trial period $(p=0,004)$, as seen in Table 1 . Mortality rate was lower for entire males before weaning $(p=0,087)$ and also during the complete trial period $(p=0,059)$. Weaning weight was higher for entire males when compared to surgically castrated males $(p=0,071)$, with averages of $5,57 \mathrm{~kg}$ and $5,42 \mathrm{~kg}$ respectively.
Table 1 - Mortality, additional injectable medication and weight at weaning for both treatments.

\begin{tabular}{|l|c|c|c|}
\hline Group & $\begin{array}{c}\text { Mortality } \\
(\mathbf{D 4 - D 6 3 )}\end{array}$ & $\begin{array}{c}\text { Addition. } \\
\text { Inject. }\end{array}$ & $\begin{array}{c}\text { Weight at D-21 } \\
\text { (kG) }\end{array}$ \\
\hline T1 (boars) & $6.4 \%^{\mathrm{a}}$ & $53,3 \%^{\mathrm{a}}$ & $5,57^{\mathrm{a}}$ \\
\hline T2 (castrates) & $9.7 \%^{\mathrm{b}}$ & $62,3 \%^{\mathrm{b}}$ & $5,42^{\mathrm{b}}$ \\
\hline \multicolumn{4}{|c|}{ Different superscripts in the same column mean significantly different values $(\mathrm{p}<0.10)}$. \\
\hline
\end{tabular}

There was no effect of treatments on the incidence of diarrhea and arthritis before weaning. During the nursery phase, there was also no effect of treatments on the occurrence of arthritis, but the incidence of diarrhea was significantly higher for surgically castrated pigs ( $p=0,002)$. No statistical differences between treatments were detected for weight at 35th, 49th and 63 rd days of age, daily gain, feed intake and feed efficiency. A higher frequency of light-weight pigs was found among castrated pigs, both at weaning $(p=0,017)$ and at the end of nursery period $(p=0,052)$, as seen on table 2 .

Table 2. Incidence of light-weight pigs at weaning (D-21) and at the end of nursery period (D-63)

\begin{tabular}{|c|c|c|}
\hline Treatment & D-21 & D-63 \\
\hline T1 & $4,8 \%^{\mathrm{a}}$ & $17 \%^{\mathrm{a}}$ \\
\hline $\mathrm{T} 2$ & $8,7 \%^{\mathrm{b}}$ & $22,2 \%^{\mathrm{b}}$ \\
\hline \multicolumn{2}{|c|}{$\begin{array}{l}\text { Different superscripts in the same column mean significantly different values } \\
(\mathrm{p}<0.10) .\end{array}$} \\
\hline
\end{tabular}

Under the conditions of this study, surgical castration had a negative impact on weaning weight, mortality rate and incidence of light weight pigs. Surgical castration at day 4 also increased the need for additional injectable medication before and after weaning, as well as the occurrence of diarrhea during the nursery phase.

\section{References}

1 de Kruijf, J.M., and Welling, A.A. (1988) .Tijd. voor Diergeneeskunde, 113, 415-417.

2 Lessard, M. et al (2002). Can J Animal Sci., 82, 519-526

3 Hay et al. (2003). Appl. Anim. Behav. Sci., 83:201-218 


\title{
P.835
}

\section{Continuous throughput compared with 'all in-all out' in the management of a farrowing unit in Thailand}

\author{
Alexander A. Eggen ${ }^{1}$ Suraphan Boonyawatan ${ }^{2}$ \\ 1. Intervet/Schering-Plough, Boxmeer, Netherlands; 2. Intervet/Schering-Plough, Bangkok, Thailand
}

\section{Introduction}

An all in-all out production system minimizes the transmission of infection between different age groups and batches of pigs. For a farm operating a continuous flow production system it is normally difficult to change to and manage an all in-all out system, but the economic benefits can be significant. Regumate ${ }^{\circledR}$ can be used to synchronize insemination and farrowing as a major step towards establishing an all in-all out system.

The aim of this study was to compare the production performance of a farrowing unit managed by continuous flow and by an all in-all out system.

\section{Materials and Methods}

This study was performed on a 400-sow farm arranged as follows. There were two farrowing houses each with 65 farrowing pens, and a dry sow house with 520 pens. Sows were served by Al (19-26 sows per week), and between 12 and 24 sows farrowed per week. The farm manager realized that this system did not allow for the proper cleaning and disinfection of the farrowing accommodation.

A batch farrowing system was instituted in January 2008 with estrus synchronized on a 3-week cycle using Regumate ${ }^{\circledR}$. Thereafter 68-72 sows were inseminated every 3 weeks, and 55-65 sows farrowed in the same period. So the first batch of sows and gilts that was inseminated farrowed in March 2008 and their piglets were weaned in April 2008.

It is possible therefore to compare the performance of the farrowing unit between April 2007 and April 2008 (continuous production) and April 2008-April 2009 (All in-all out management) (Fig 1).

\section{Results}

Twenty-one weeks after the change to batch farrowing, the farm could be divided into seven batches of 55-65 sows per batch. All the sows had been through the system, so the farrowing accommodation could be emptied, cleaned and disinfected. Many performance indicators improved in 2008 even though Thailand experienced a PED outbreak.

The performance in the farrowing unit is shown in Table 1.

\begin{tabular}{|l|c|c|c|}
\hline & Continuous & All In - All Out & Difference \\
\hline No of sows Al & 1,189 & 1,176 & $-13(-1 \%)$ \\
\hline No of weaned pigs & 8,353 & 9,870 & $1,517(+18 \%)$ \\
\hline Farrowing rate (\%) & 84,4 & 86,3 & 1.9 \\
\hline Pigs born alive & 10.4 & 11 & 0.6 \\
\hline Weaned pigs & 9.3 & 9.9 & 0.6 \\
\hline Weaned weight $(\mathrm{kg})$ & 7.5 & 7.5 & 0 \\
\hline \% loss before weaning & 18.7 & 11.2 & $-7.5 \%$ \\
\hline
\end{tabular}

\section{Discussion}

After implementing all in-all out management a lot of improvements were seen on this farm.

In spite of $1 \%$ fewer sow inseminations, $18 \%$ more pigs were weaned. The farm was easier to manage. Inseminations were better planned and more controlled, and the labor force involved were more focused and less stressed.

The farrowing house could be properly cleaned and disinfected, and one of most important improvements was that the farm manager could organize and plan all aspects of production according to a reliable timetable.

\section{References}

1. Gonyou H, Lemay S and Zhang Y 1999 Diseases of Swine, Editors Straw B Mengeling W D'Allaire S an Taylor D, p1024

2. Gonyou H, Lemay S and Zhang Y 1999 Diseases of Swine, Editors Straw B Mengeling W D'Allaire S an Taylor D, p1087-1088

3. J. Carr., et al 2004 Proceedings 18th IPVS Congress, Hamburg, Germany p822

4. Busch, ME. et al. 2006 Proceeding of 19th IPVS congress, Copenhagen, Denmark 0.37-02

5. Pijoan C. et al. 2004 Proceedings 18th IPVS Congress, Hamburg, Germany

Fig 1. Stock management before and after the change (each pig icon above represents five pigs)
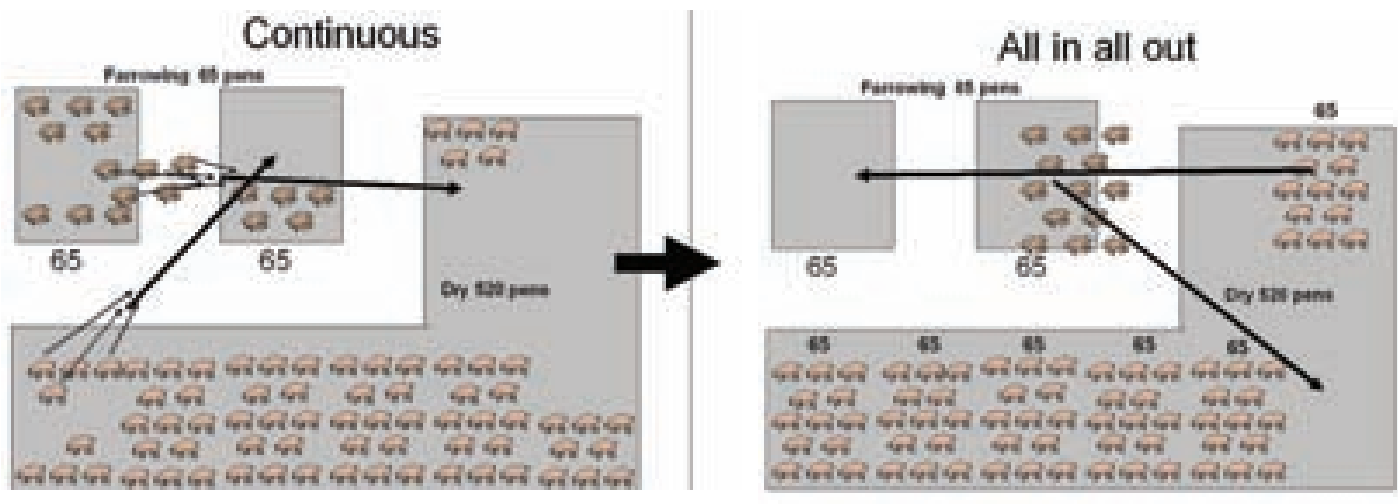


\title{
P.836
}

\section{Pig Flow - Managing the health of the business}

\author{
John Carr
}

Portec Australia, Belmont, WA, Australia

Setting formal pig flow targets through batching allows a unique opportunity for veterinarians to help manage the health of their client's pig and keep their own businesses healthy.

Batch farming sets targets, minimises variation and reduces under and overstocking - a major cause of disease, reduced performance and increase in costs.

A key control of the expression of the clinical signs of disease is through vaccination and prophylactic medication.

But - on many farms while a highly efficient preventative medicine programme is designed by the veterinarian, it is on trust that this programme is used and implemented by the farm staff.

Setting pig flow targets allow at least purchases of health items to be monitored within pre-agreed parameters by the whole health team.

To set a pig flow model, the easiest initial point of reference is in the organisation of the farrowing house - the number of farrowing places per batch.

Example of a pig flow medicine monitoring system using an Excel spreadsheet:

\begin{tabular}{|l|c|c|}
\hline Average herd size & $\mathbf{1 7 7 3}$ & sows/gilts \\
\hline Farrowing places & $\mathbf{7 5}$ & per batch \\
\hline Batch time & 1 & weeks \\
\hline Wean/farrow place & 10 & weaners \\
\hline Gilt replacement rate & 40 & $\%$ \\
\hline Pigs per batch & Year & \\
\hline Sows & 61 & 3172 \\
\hline Gilts & 14 & 728 \\
\hline Weaners & 750 & 39000 \\
\hline Compliance required & 95 & $\%$ \\
\hline
\end{tabular}

\begin{tabular}{|l|c|c|c|c|c|}
\hline & \multicolumn{5}{|c|}{ Programme } \\
\hline Vaccine/medication & Dose mls & Bottle (mls) & Gilts & Sows & Weaner \\
\hline Parvovirus & 2 & 100 & 1 & 0 & \\
\hline Erysipelas/Lepto. & 2.5 & 250 & 1 & 0 & 0 \\
\hline E. coli & 4 & 500 & 2 & 1 & \\
\hline HPS & 2 & 100 & 2 & 1 & 0 \\
\hline PCV2 & 1 & 50 & 0 & 0 & 0 \\
\hline Mycoplasma & 2 & 500 & 0 & & 1 \\
\hline Ileitis & 2 & 100 & 0 & & 0 \\
\hline Coccidiosis & 1 & 1000 & & & 1 \\
\hline PRRSv & 2 & 100 & 0 & 0 & 0 \\
\hline SIV & 2 & 100 & 0 & 0 & 0 \\
\hline Improvac* & 2 & 500 & & & 1 \\
\hline
\end{tabular}

\begin{tabular}{|l|c|c|c|c|}
\hline & \multicolumn{4}{|c|}{ Actual use and comment } \\
\hline Vaccine/medication & \multicolumn{1}{|c|}{ Q1 } & Q2 & Q3 & Q4 \\
\hline Parvovirus & 4 OK & $80 \mathrm{~K}$ & $40 \mathrm{~K}$ & $40 \mathrm{~K}$ \\
\hline Erysipelas/Lepto. & 1 Low & $30 \mathrm{~K}$ & 6 High & $20 \mathrm{~K}$ \\
\hline E. coli & $140 \mathrm{~K}$ & 6 Low & 7 Low & $120 \mathrm{~K}$ \\
\hline HPS & 21 Low & 10 Low & $240 \mathrm{~K}$ & $240 \mathrm{~K}$ \\
\hline PCV2 & & & & \\
\hline Mycoplasma & $580 \mathrm{~K}$ & 29 Low & $400 \mathrm{~K}$ & $400 \mathrm{~K}$ \\
\hline Ileitis & & & & \\
\hline Coccidiosis & 6 Low & $150 \mathrm{~K}$ & $120 \mathrm{~K}$ & $80 \mathrm{~K}$ \\
\hline PRRSV & & & & \\
\hline SIV & & & & \\
\hline Improvac & 35 Low & 36 Low & $390 \mathrm{~K}$ & $400 \mathrm{~K}$ \\
\hline
\end{tabular}

\section{Discussion}

The records clearly show that:

1. Medicine compliance. The farm is PRRSv and PMWS free and vaccines are not used.

2. There was a period of non-compliance in quarter 1 and 2 which was associated with a particular stockperson and this was resolved with a training opportunity.

3. The non compliance was associated with a reduction in health and performance.

4. The Improvac errors are serious as they impact slaughter value (boar taint) and sales.

5. There was a overuse of Lepto/ery vaccine in quarter 3 - farm playing catch up.

6. Veterinary sales were lost due to poor compliance and this was rectified. $17 \mathrm{HSP}$ vaccine and 4 Improvac. This was a loss of $\$ 4000$ Aus sales.

7. The client's satisfaction was ensured because of the proactive health management and attention to details provided.

8. The system is easy to run and modify to other health product sales such as injection, water, feed medications or disinfectants.

Please visit our website www.portec.com.au 


\title{
P.837
}

\section{Hygiene and the relevance of Endotoxins on swine producing farms}

\author{
Simone Schaumberger ${ }^{1}$ Christine Ratzinger ${ }^{1}$ Lukas Krüger $^{1}$ Sabine Masching ${ }^{2}$ Gerd M. Schatzmayr ${ }^{1}$ \\ 1. Biomin Research Center, Tulln, Austria; 2. Biomin Holding GmbH, Herzogenburg, Austria
}

\section{Introduction}

Endotoxin associated diseases are a result of permanent existing endotoxin values in the gut and through outside influences like dust, feces, food and other organic materials. Small amounts stimulate the immune- system in a positive way but excess amounts lead to influenza like symptoms, shock and death.

As endotoxins are known to play a significant role in different pig associated diseases, especially in MMA in sows and the postweaning syndrome in piglets, a screening on piglet producing farms was performed to find out if there is any correlation between detected endotoxin levels and the incidence of illnesses in the animals.

\section{Material and methods}

16 farms with documented MMA problems were chosen. 32 mother sows ( 2 sows/farm) were sampled between the first and third lactating week. One sow was without medical treatment and the other sow of the same farm was on treatment (antibiotic) against MMA. Samples taken of the sow were milk, feces and urine. Environmental samples were taken from water, feed and air. Additionally temperature and humidity (EL USB-2 Data logger, RS Components) were measured. For air collection a WISA suction pump with pyrogen free glass fiber filters (Whatman) was used. All samples of the sows and environmental samples were tested with the kinetic chromogen Limulus- Amoebycat Lysat test (Charles River Laboratories). Feces, air and feed were extracted with Tween 20 for 1 hour and dilution rows of all the samples were prepared within 12 hours after sampling. Dilution of the samples tested was chosen depending on the sample matrix, for example water samples were tested in a 1:10 dilution and feces samples in 1:10.000. All used material was proved pyrogen free. Performance Standards were valid with r2 $>0.97$ and the recovery of all samples ranged between $50-200 \%$. Statistical analysis was done with SPSS 17.0 (Box plots, p-value, Regression).

\section{Results}

Levels of endotoxin in the air revealed significant differences $(p=0.003)$ between farms with dry and wet feeding systems. Airborne endotoxin concentrations of farms washing their sows before moving them to the weaning boxes were lower compared to those not washing their sows. Disinfection of the stables before moving the sows seemed to reduce air endotoxin values as well, but there was no significant difference compared to farms without disinfection. No positive correlations of endotoxin air levels and levels in feces $(p=-0.052)$ or urine $(p=0.124)$ could be observed. No correlation between stable temperature and air endotoxin values ( $p=0.641)$ nor humidity and air endotoxin values $(p=0.617)$ could be found. Although water samples of one farm widely varied in their endotoxin values ( $S D=14.39$ ), the design of the drinking troughs did not reveal any correlation $(p=0.342)$ with detected endotoxins .

\section{Discussion}

Management and hygiene have an impact on endotoxin air values and further consequences on humans and animals. Especially endotoxin levels in the air are an important issue not only for the animals, but also for the farmers because they spend a lot of time in the stable.

In this study some interesting approaches for measuring possible risk factors could be observed. Dust seems to be a major source of endotoxin in the air according to the results of farms with different feeding systems. Dry feeding produces more dust than wet feeding. Besides feeding, hygiene of the sows plays a major role as well. Disinfection of the stable does not seem to be as important as washing the sows before moving them into the weaning boxes.

This study could not prove the coherence of endotoxin values and the incidence of illness but showed clear trends concerning hygiene and endotoxin values. Results of this study will be verified in follow up studies on different farms.

\section{References}

KrügerM., W. Schrödel, T. Seidler u. D. Fritsche (2001a): Endotoxinassoziierte Erkrankungen landwirtschaftlicher Nutztiere unter besonderer Berücksichtigung des Schweines.

in: Handbuch der tierischen Veredlung 26. Auflage, Verlag Kamlage $\mathrm{GmbH} \&$ Co, S.251 - 264 


\title{
P.838
}

\section{Application of mineral sorbents for reduction of odorous volatile compounds emitted from pig manure}

\author{
Roman Kolacz; Mariusz Korczynski; Sebastian Opalinski; Zbigniew Dobrzanski \\ Wroclaw University of Environmental and Life Sciences, Department of Environmental Hygiene and Animal Welfare, \\ Chelmonskiego 38C, 51-630 Wroclaw, Wroclaw, Poland
}

\section{Introduction}

Odors are specific air pollutants, since human nose is able to detect and differentiate odorous compounds even when their concentrations are below the threshold detectable by technical facilities, e.g. gas chromatography. It has been estimated that odor detection threshold by the human nose is as low as $10^{8}-10^{9}$ odorant molecules, while $1 \mathrm{~g}$ of ethyl mercaptan in the air contains $10^{16}$ molecules of this gas, which is $10^{7}-10^{8}$ higher than the detection threshold [4,5]. Major odorants emitted from animal breeding include ammonia and its derivatives, sulfur compounds (hydrogen sulfide, mercaptans), volatile fatty acids and phenolic compounds, adversely affecting human and animal health $[1,2]$.

Many efforts have been made to reduce the emission of odors from animal manure. One of the potential means of limiting odorants in the air of livestock buildings is the application of alluminosilicate sorbents.

The aim of the investigation was to study under laboratory conditions the influence of bentonite and halloisyte, added to pig manure, on the reduction of odorous volatile compounds emission.

\section{Materials and Methods}

The samples of fresh pig manure $(50 \mathrm{~g})$ were placed in 9 coneflasks $(500 \mathrm{ml})$. The flasks were divided into 3 groups and the sorbents were added as follows: group I - control - no sorbents, group II - raw bentonite (20\% of the manure mass), group III - raw halloisyte ( $20 \%$ of the manure mass). The odorous compounds were extracted (sampling time $=30 \mathrm{~min}$.) from the headspace of each flask at $30^{\circ} \mathrm{C}$ after $\mathrm{d} 1,4$ and 8 . The extraction of the substances was carried out using a manual SPME holder with StableFlex 50/30 $\mathrm{mm}$ divinylobenzene/ carboxen/polydimethylosiloxan. DVB/CAR/PDMS fibers were used (Supelco, USA). The analysis of air samples was performed using a GC/MS method (Thermo Electron, Finnigan Focus Polaris Q type). Identification of volatile odor substances was based on comparative analysis of mass spectra and NIST spectra database.

\section{Results and Discussion}

40 volatile odor compounds (VOCs) were identified in the samples collected from the air immediately above the manure. They included 4 sulfur organic compounds and alcohols, ketones and other derivatives, e.g. benzene and xylene. The identified substances contained 7 compounds very poisonous to humans [3], i.e. trimethyloamine, cyclohexanone, toluene, methylpentanol, 2-heptanone, chlorobenzene and 1,4-dichlorobenzene.

The sorbents used in the study, especially halloisyte, markedly reduced the emission of odorants from pig manure (Table 1). The greatest efficiency of the sorbents was observed on $\mathrm{d} 8$. Reductions of volatile odor compounds accounted for $65.62 \%$ (bentonite) and $83.14 \%$ (halloisyte). On d 1 and 4 of the experiment, halloisyte was the only sorbent that reduced the emission of odorants from the manure. In contrast, bentonite, applied at the same time, increased the quantity of VOCs. Significant reductions were also observed by Turan et al. [4] who added $10 \%$ of aluminosilicate and vermiculite to poultry slurry. The reduction reported by them was comparable to the results observed in our study with the use of bentonite (61.53\%). Summing up, it can be concluded that the greatest efficiency in reducing VOC emissions from pig manure was achieved with the use of halloisyte (from $5 \%$ after $d 1$ to $83 \%$ after $d 8$ ).

Table 1. The effects of bentonite and halloisyte on reductions (-) and increases (+) (\%) in VOC's concentrations.

\begin{tabular}{|c|c|c|c|c|c|c|}
\hline Control group (I) & \multicolumn{3}{|c|}{ Bentonite (Group II) } & \multicolumn{3}{c|}{ Halloisyte (Group III) } \\
\hline after d 1 & +43 & & & -5 & & \\
\hline after d 4 & & +34 & & & -40 & \\
\hline after d 8 & & & -66 & & & -83 \\
\hline
\end{tabular}

\section{References}

1. O'Neil, D and Phillips, V A (1992):, Journal of Agricultural Engineering Research, 53, 23-50.

2. Rappert, S. and Muller, R. (2005):, Waste Management, 24, 887-907.

3. Schiffman S. S., (1998): Journal of Animal Science, 76, (5), 1343-1355.

4. Turan, N G; Akdemir, A and Ergun, O N (2009):, Bioresources Technology, 100, 798-803.

5. Zahn, J; Hatfield, J; Do, Y; DiSpirito, A; Laird, D and Pfeiffer, R (1997):, Journal of Environmental Quality, 26, 1687-96. 


\title{
P.839
}

\section{Impacts of technological level on production efficiency and economic variables in pork farms in the States of Guanajuato, Jalisco, Sonora and Yucatan, México}

\author{
Juan Nava; Rafael Trueta \\ Universidad Nacional Autonoma De Mexico, Mexico, DF, Mexico
}

\section{Introduction}

The objective of the present study was technological level grading (high, intermediate and low) and its impact on production efficiency, defined as the number of animals sent to market per sow per year and on the main economic indicators, of a random sample of complete cycle pork farms in the four states of Mexico showing greater production. $31.1 \%$ of farms use high technology, $39.9 \%$ use low technology and the remaining $29.5 \%$ can be described as semi-technified. The Kruskall-Wallis non parametric statistical analysis provides conclusive evidence that high level yields a lower cost, MXP 9.45 vs 11.20 and 11.84 for the intermediate and low technology levels, respectively $(P<0.01)$.

\section{Materials and Methods}

Data on technical, animal health, production and economic variables was obtained through surveys performed in a random sample of complete cycle swine farms. Farms were graded in accordance with their technological level taking into account the following parameters: a) biosafety, b) reproduction parameters, c) production parameters.

\section{Results}

Production efficiency

When farms were grouped by technological level, the number of animals sent to market yearly per sow was $18.60,15.97$ and $11.67(P<0.01)$.for high, intermediate and low technological levels, respectively.

\section{Economic production variables}

\section{Production cost}

Average cost of production per kg live weight when farms were grouped by technological level, those in the high technology bracket showed a lower CKg, MXP 9.45, while those in the intermediate and low levels, showed MXP 11.20 and 11.84, respectively.

Revenue

Profit per average $\mathrm{kg}$ live weight sold for the 61 farms was MXP 4.93. At the high technology level Pkg was MXP 5.52, MXP 4.77 for intermediate and MXP 4.59 for low technology.

Profitability

Average profitability per kg sold (RKg), the high technology level showed the higher Rkg, $64.9 \%$, well above the $49.1 \%$ and $49.9 \%$ found for the intermediate and low technology levels, respectively.

\section{Discussion}

Obtaining profits (income - costs) is the most important goal of commercial farmers. One of the determining factors for improving economic efficiency is technology that manifests through multiple expressions. Results obtained in the present study confirm this notion, because production costs of swine show an inverse relationship to the technological level of the farm.

In the interest to share this experience with other producers, we list the deficiencies found in low technology farms studied: 100 $\%$ did not have perimeter wall, $83.3 \%$ did not have a sanitary moat; $66.6 \%$ does not compel workers to bathe; $83.3 \%$ allows animals to enter the premises; $66.7 \%$ did not use $\mathrm{Al}$ and $66.7 \%$ do not use balanced feed. Besides, none of them have systematic parasite and vaccination programs. Low technology farms acquire more competitive advantages when introducing technology than those of a higher level.

\section{References}

- SAGARPA. Dinámica de la producción de carne de porcino en México. México. 2005.

- OECD. The pig sector. 1era ed. Paris, Francia: OECD Publications, 2003.

- Hadley D. Patterns in technical efficiency and technical change at the farm-level in England and Wales, 1982-2002. J Agric Economics 2006;57(1):81-100.

- Conover WJ. Practical nonparametric statistcs. 2nd ed. Texas, USA: Texas Tech; 1980.

- Sierra ML, Ortiz-Rosa J, et. al. Estructura del mercado y comportamiento del precio de la carne de cerdo en Yucatán 1990-2003. Téc Pecu Mex 2005;43(3):347-360.

- Mascorro E, Trueta S. La organización de porcicultores de ciclo completo en Sonora, México" [resumen]. VII Congreso Latinoamericano de Sociología Rural, Quito, Ecuador. 2006:265. 


\title{
P.840
}

\section{The effect of standard deviation reduction on optimal average slaughter weight}

\author{
$\underline{\text { Dennis DiPietre }}^{3}$ Eric Lewandowski $^{2}$ Remy Jagu ${ }^{2}$ Matthias J. Adam $^{1}$ \\ 1. Boehringer Ingelheim Animal Health, Ingelheim am Rhein, Germany; 2. Boehringer Ingelheim Vetmedica, Pace, France; \\ 3. KnowledgeVentures, LLC, Columbia, MO, USA
}

\section{Introduction}

In a previous paper(1) we show the economic impact of reducing the standard deviation of slaughter weights on overall farm profitability as measured by return over feed costs. In that paper we present a side-by-side trial, where pigs were randomly assigned at weaning to two treatment groups, $\operatorname{Circovac}^{\circledR}(\mathrm{CV})$ or Ingelvac CircoFLEX ${ }^{\circledR}$ (FLEX). Pigs of both groups were kept in separate pens, but in the same rooms(2). The producer was blind to the treatments and employed his standard selection and marketing procedures to both groups. Though market weights for the two groups were almost identical, the standard deviation of market weights was $16.7 \%$ less for the FLEX pigs. This was estimated to produce $a € 1.85 /$ head advantage for the FLEX group. The amount of the increase in profitability depends on the market price, cost of feed, and the particular buying matrix used. In this paper, we extend the analysis by examining how changes in standard deviation of marketing weights also change the optimal average weight of the distribution of pigs marketed.

\section{Materials and Methods}

A stochastic simulation method was used to estimate the average carcass weight which maximizes expected return over feed costs for each group based on its distribution of slaughter weight at the time of marketing. Distributions were fitted to the actual observations for carcass weight and lean percent in each group. These distributions were correlated to match the correlation between weight and lean in the actual data. 10,000 pair of observations on carcass weight and correlated lean percent were then randomly drawn from each group's estimated distribution and used in the analysis. The optimal average weight for each group of 10,000 observations was determined through the use of a detailed simulation model employing growth and average daily feed intake functions matched to the farm and utilizing the actual pricing matrix in which the pigs were originally sold. The average feed cost per Mton wean-to-finish was $\$ 200$ and the base carcass price was $\$ 1.30$ per $\mathrm{kg}$. The estimated optimal average weight was compared to the marketing weight chosen by the producer. In the original case, the producer was blind to the treatments and chose to market the pigs in both groups at essentially the same average weight.
The model employed can be represented as follows:

Find mean average carcass weight $\mathrm{Q}_{j}{ }^{*}$ of the distribution of weights marketed which maximizes return over feed cost:

$\operatorname{Max} P j=\Sigma_{i} P Q_{Q i, L i} N_{j}\left(Q_{i, L i}\right)-T F C_{Q i, L i}$

Where:

$\mathrm{J}=$ the treatment group

$\mathrm{P}_{\mathrm{Q} i, \mathrm{Li}}=$ Grid determined price/kg (a function of current base carcass price and premium or discount associated with carcass weight and lean percent)

$\left(Q_{r, L, i}\right)=$ Randomly selected carcass weight $(\mathrm{kg})$ with its randomly selected, correlated lean percent from treatment group $\mathrm{j}$

$\mathrm{N}()=$. Correlated distributions of carcass weights $(\mathrm{kg})$ and associated lean percents estimated from the sales data for each of the treatment groups

$\mathrm{TFC}_{\mathrm{Q}, \mathrm{Li}}=$ Estimated total feed cost to produce carcass $\mathrm{Q}_{\mathrm{i}}, \mathrm{L}_{\mathrm{i}}$

Results

Table 1: Economic returns over feed/hd for both groups

\begin{tabular}{|l|c|c|c|}
\hline & FLEX Group & CV Group & Difference per head \\
\hline As Marketed Avg carcass (kg) & 91.04 & 90.50 & $0.60 \%$ \\
\hline As Marketed StDev & 6.06 & 7.28 & $-16.7 \%$ \\
\hline As Marketed Return over Feed/hd & 0.40 & 68.55 & 1.85 \\
\hline Optimized Carcass (kg) & 96.40 & 95.00 & $1.4 \mathrm{~kg}$ \\
\hline Optimized Return over feed/hd & $€ 71.98$ & $€ 70.23$ & 1.75 \\
\hline
\end{tabular}

\section{Discussion}

Profit optimizing average weight results in an improvement in return over feed costs for each group. In this case, Q*FLEX = $96.4 \mathrm{~kg}$ with average return over feed costs per head of $€ 71.98 /$ hd and $Q^{*} C V=95.0 \mathrm{~kg}$ with return over feed costs equal to $€ 70.23 / \mathrm{hd}$. This is an improvement from "as marketed" of $€ 158 /$ hd and $€ 1.68 /$ hd respectively. In conclusion optimized carcass weights would generate substantial additional returns irrespective of treatment groups.

\section{References}

1. DiPietre D., and al. (2010).IPVS (in review)

2. Guillaume, J.M., and al. (2009) ESPHM, p52 


\title{
The analysis of farm management factors affecting the MSY in Korea's hog industry
}

\author{
Hwa soon Kang \\ Cargill Agri Purina, Inc., Seongnam-si, Korea
}

The purpose of the hog farm operation is to maximize a profit. But, compared to the advanced countries for pork production, Korean hog industry has faced the challenge to be sustainable and to be developed due to lower productivity. In addition, as the trade barrier between countries has been gradually lowered, Korean hog industry has been challenged seriously. Under the existing circumstances, to accomplish the objective of the hog farm operation and to grow sustainably, it is necessary to analyze the performance, to operate efficiently and to maximize the productivity level in each production stage. Therefore, it is necessary to analyze the factors affection the farm productivity to improve farm productivity, to grow sustainably, and to increase the competitiveness of the industry.

Previous studies on the farm productivity and the competitiveness of the industry were conducted to measure the productivity level and recommend the ways for improvement by analyzing farm facility, farm management, disease control, management capability, each component adoption, profit, and so on. However, in this study, it is assumed that all the factors affecting farm productivity level are compounded, as the hog industry has been integrated and been complex, and they are interconnected with one another. Additionally, biological indicators, such as PSY and quantitative indicators, such as income and spending are limited to measure farm performance level and to represent financial performance, respectively. And, there is a tendency that qualitative factors, i.e., technology adaptability and management capability, are individually analyzed to be indicated in a quantitative manner.

In this study, a diverse array of factors, such as facility, technology, workforce, and management, are analyzed by considering effect of each factor on the farm productivity and qualitative feature. An indicator for farm productivity is determined to be an MSY, because there is no one solid standard to determine farm productivity, because it has not been feasible to collect realistic data for profitability and to use factors such as income and spending for evaluating productivity under the circumstance that every farm's management type and financial status are different. Moreover, considering the accessibility and objectivity to the information, MSY, the number of pigs alive from birth to market per sow per year, is determined the most suitable indicator for productivity effectiveness. The data used for analysis were gathered through a set of in-depth interviews with hog industry expert and farm owner while visiting on-site. The data collected were specifically on farm productivity level, farm workforce status, farm owner's characteristics, facility and disease status.

Through the Decision Tree used for the analysis, broad impacts of the factors mentioned above on productivity and interactions between factors were identified. And, using the result from the Decision Tree analysis, variables for CARTEG using optimal scaling were selected and using the optimal scaling while considering that the data from the interviews are three different types, i.e. category, sequence, numeric value are blended, the conclusion for CARTEG was drawn.
Based on the this result, after setting average MSY as a primary basis to categorize the data set into two groups, the higher and the lower, respectively, the factors to influence on hog farm productivity were identified. In addition, while the higher and the lower groups were divided by MSY value, the variables affecting hog farm productivity for each group were figured out and the strategies to increase MSY value for each group were provided.

For those farms on the bottom, they need to establish business plans while focusing more on avoiding exclusive management dependent on experience, systematic and regular participation in training, farm consulting, workforce management by capability, implementation of incentive program to promote longer years of service and high performance, broadening workforce benefits program, facility management, such as specialized access control system, all-in all-out system as well as site management, regular service call by specialized veterinarians, and prevention of antibiotics' abuse. And then, to be more competitively in the future, farm owners or farmers'group have to set business plans and implement them while especially bearing in mind following areas: self-designed training program, customized training, business direction setting with farm consultants, farm analysis and record maintenance, long-term strategy for sustainable growth including operating principles and succession plans, as well as modernized and environmentally-friendly farm.

These kinds of strategies for higher MSY are useful not only to increase competitiveness of individual farms or farmers'groups, but also to establish the foundation of Korean hog industry to compete against the imports in the era of FTAs. Accordingly, primary purpose of this study is to enable individual farms to choose necessary strategies to manage farms effectively, leading to more income. And, the ultimate purpose of this study is to propose strategies for securitizing competitiveness of overall hog industry and for further development of the industry. keyword: Hogindustry, Factors, MSY, CATREG, Decisiontree, Strategy 


\title{
P.842
}

\section{Measuring full value pigs}

\author{
Paul D. Matzat ${ }^{1}$ Dennis DiPietre ${ }^{2}$ \\ 1. Elanco Animal Health, Greenfield, IN, USA; 2. Knowledge Ventures, LLC, Columbia, MO, USA
}

\section{Introduction}

The concept of capturing maximum profit or full value from every pig that comes into a producer's care has been discussed and developed over the last few years by Elanco Animal Health. All production outcomes less than full value are defined as lost opportunity, deviation from optimal or as attrition for a contemporary group. Optimizing profitability for a group of pigs means identifying the point at which the group's marginal input cost equals marginal revenue. This requires knowledge of the production function to estimate the cohort's marginal cost of gain and jointly determined market value. Although the underlying determination of profit maximization is the same, the process for estimating it for a group is much more complex than for a single pig.

\section{Materials and Methods}

The slaughterhouse is the only place in the current swine production chain where every animal is individually weighed and assessed for quality. Information is often reported to producers in summary form, such as total animals sold, average weight, average price, average discount or premium and other assessments. Generally there is little or no reporting of group outcomes and the impact of variation on lost opportunity. The premium and discount schedules that buying programs provide vary across geographies and between pork processing companies and thus must be clearly understood prior to developing a marketing strategy or standard marketing procedure. Figure 1 is an example of actual market weight and selling price outcomes.

Figure 1. Actual Marketing Outcomes

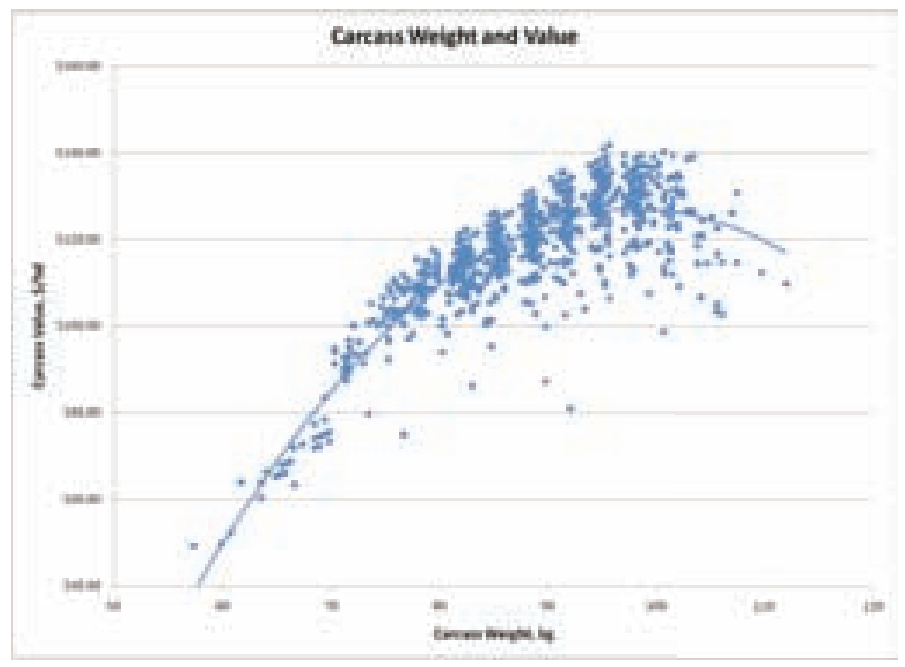

\section{Results}

The marketing decision for a single animal can be easily calculated and demonstrated by determining if adding another kilo of weight prior to marketing will add profit margin. However, making the marketing decision for a truck load, building or entire site can be difficult due to discounts imposed on pigs in the tails of the target weight distribution. Producers can accurately identify weights of pigs to initiate the marketing process. This may be done by scaling pigs, visual appraisal or use of calendars and growth curves to trigger marketing of animals within a group or barn. Accuracy of selection, both from an optimal weight perspective and minimizing variation within the group of pigs can be metrics used to score how effectively marketing decisions were implemented for each load and for the entire population of pigs evaluated.

\section{Discussion}

Developing targets or report cards for the team responsible for executing the marketing plan is critical for continuous process improvement and capturing opportunity costs. First, those who physically identify or mark pigs must have rapid and accurate feedback reporting achievement. Second, the load out crew must receive reports demonstrating how effective they are at placing the correct pigs on the transport vehicle. Third, the transportation team must have an understanding of transport losses that could impact financial outcomes. And finally, farm managers responsible for marketing activities and financial results must have reports indicating profits and identifying value opportunities.

Creating a grade, metric or score that allows various levels of the farm organization to quickly and easily see how well the marketing activity is being executed is important to drive continuous improvement. We propose the metric, Full Value Pigs, to mean all animals marketed within a contemporary group which achieve at least $90 \%$ of the single pig optimal margin over feed cost. Establishing a score or rating allows a better understanding of system achievement and helps the entire team responsible for the marketing activities target and capture opportunities for continuous improvement.

\section{References}

Dipietre, D. 2009. Optimizing Marginal Rates or Return. Proceedings of the Annual Meeting for AASV, Dallas TX. Pp. 547-548.

Matzat, P.D., R. Arentson and M. Ritter. 2010. Full Value PigTM (FVP): Uncovering profit opportunities in production systems. Proceedings for the Annual Meeting for AASV, Omaha, NE. 


\title{
P.843
}

\section{Description of the economic impact of the political crisis on small- scale pig farmers and butchers in Western Kenya}

\author{
Cate E. Dewey ${ }^{1}$ Jared Wohlgemut ${ }^{1}$ Florence Mutua ${ }^{2}$ Mike Levy ${ }^{1}$ \\ 1. University of Guelph, Guelph, ON, Canada; 2. International Livestock Research Institute, Nairobi, Kenya
}

\section{Introduction}

Politically based violence followed the 2007 federal election in Kenya. This crisis caused the Nairobi Stock Exchange to drop by $\$ 600$ million USD. In the first week, the crisis caused the Nairobi Stock exchange to drop $\$ 600$ million USD and costs due to crisisrelated damage was $\$ 1$ billion US. Tourism was severely affected. Hotel occupancy rates dropped from above $80 \%$ to under $20 \%$ and 120,000 tourism-related jobs were lost. The purpose of this research was to describe the economic impact of this crisis on the smallholder pig farmers and pig butchers in Western Kenya.

\section{Materials and Methods}

In June 2008, face-to-face interviews were used to collect information from 100 farmers, 38 pig butchers, and 17 government staff in Busia and Kakamega Districts of Western Kenya. Farmers were randomly selected but butchers and government staff represented a census. Farmers were asked what livestock they owned in June 2007 and June 2008 and the price they paid for raw meat before and after the crisis. Everyone was asked to describe the effect of the crisis on their businesses. The 2007 and 2008 livestock numbers were compared with a paired t-test.

The cost of meat before and after the crisis and from Busia to Kakamega was compared using Student's t-tests.

\section{Results}

Food became more expensive, additional family members moved in with the farmers to escape violence in the city, farm inputs became expensive and scarce, it was impossible to travel outside the village and off-farm jobs were lost. Farmers sold livestock to feed their families and because they had no food for the pigs. In Busia, ownership of sows, boars, growing pigs and piglets decreased by $41 \%, 32 \%, 42 \%$, and $40 \%$ respectively. In Kakamega, sows and boars decreased by $27 \%$ and $46 \%$; growing pigs and piglets decreased by $55 \%$ and $70 \%$ respectively.

Many butchers (52\%) had less business due to the crisis. More butchers in Kakemega (42\%) closed for longer periods of time $(40+/-19 \mathrm{~d})$ than in Busia where $18 \%$ of butchers closed for 18 $+/-19 \mathrm{~d}(\mathrm{P}<0.001)$. Pork price increased from 105 to $119 \mathrm{ksh} / \mathrm{kg}$ in Busia and from 116 to $146 \mathrm{ksh} / \mathrm{kg}$ in Kakamega after the crisis $(\mathrm{P}<0.0001)$. The live pig price did not increase.

Extension workers were unable to travel to farms. Pig mortality increased to due malnutrition and untreated diseases.

\section{Discussion}

The pig industry in this area of Western Kenya relies on trade within and between villages. The transport of pigs is done either on foot or using bicycles. Therefore, this drastic decrease in the numbers of pigs in the region will have a large impact on the industry. Sows are 12-14 months old when they wean their first litter and pigs are 8-12 months old when they reach market weight. It will take more than a year to replace the pig herd to return to the previous population.

The closure of butcher businesses has left the butchers with little to no financial reserve. There will be few pigs for the butchers to buy, which will put pressure on them to purchase smaller weight market pigs. With less pork to sell, the fixed costs of slaughter will result in low to no profit.

The crisis had a severe impact on the pig industry's sustainability, shocking market equilibriums. The farmers needed to sell pigs to support their families when food prices were high. The current low breeding population has reduced the supply of pigs, forcing upward pressure on prices and jeopardizing long term sustainability. Improved breeding efficiency is now essential to ensure a renewed availability of pigs. The political crisis has negatively affected all of the industry's stakeholders.

\section{References}

Kiai, M. (2008) 'The Political Crisis in Kenya: A Call for Justice \& Peaceful Resolution', Review of African Political Economy, 35: 140-4.

Klopp, J., Kamungi, P. (2008) 'Violence and Elections: Will Kenya Collapse?', World Policy Journal, 24: 11-8. 


\title{
P.844
}

\section{Economic income of small pig production systems in Mexico.}

\author{
Enriquez Celestino; Giovanna Penuelas; Francisco E. Martinez Castañeda
}

Universidad Autonoma del Estado de Mexico, Toluca, MEX, Mexico

\section{Introduction}

$53 \%$ of Mexican population lives with some level of poverty and haungry. Backyard breeding animals is a central elemnt of estrategic and improving diversificating economies all around the world. The aim of this study was to evaluate the economic performance of small pig production systems in Texcoco México.

\section{Materials and Methods}

With participative techniques we analyzed 53 small pig producers in Texcoco, Central Valley of Mexico. Three Strata were defined according the number of sow and with a proportional allocation model: Strata 1: from 1 to 4 sow; Strata 2: form 5 to 10 sow; Strata 3: from 11 to 16.

\section{Results}

Strata 1 had $80 \%$ of small production units with an economic income of $16 \%$ of total family income. Strata 2 got $12 \%$ of all producers and the economic income was 17\%. Finally, Strata 3 represent $8 \%$ only and $28 \%$ of economic income (Fig 1).

The animal inventory was 173 sow and 23 boars. 90\% of producers live in a nuclear family and only $10 \%$ were singles. Age average was 51 years old and we detected producers with more than 70 years old.

$33 \%$ of all population analyzed produce complete cycle, $33 \%$ sell piglets, $14 \%$ finished piglets to market and less than $3 \%$ transform pigmeat.

\section{Discussion}

The best economic performance and income was for the bigger Strata 3. It is known as big is the enterprise more possibilities and access to resources can get. It is difficult to establish scale economies in this kind of production systems, but it is also true that more animals in market, represents less variables costs.

One amazing data is the average of producers, because it seems that in general this economies strategies represents an important diversification of incomes due to this producers are at the end of this working age. That way, small pig production systems can be not only an economic way of life.

Several authors mentioned the importance of this systems. We want to add the fact that the historical way of production is very important in the occupational health of families.
Figure 1. Income of small pig production systems.

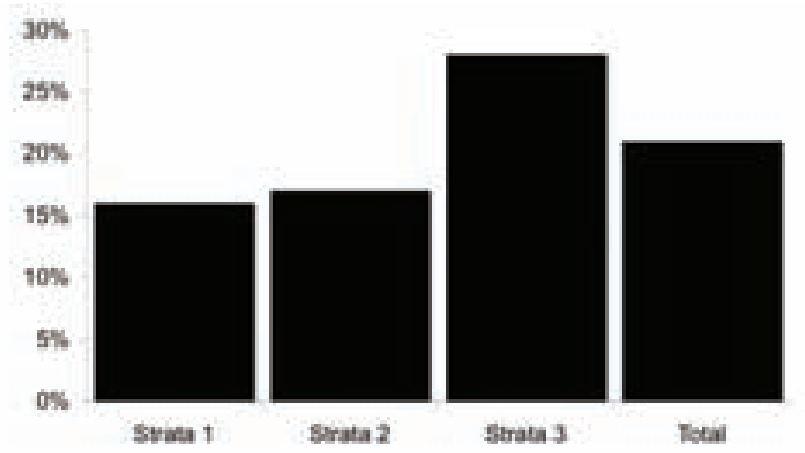




\title{
P.845
}

\section{A combination of azaperone/butorphanole/ketamine to anesthetize piglets for castration}

\author{
Iwan Nussbaumer; Niklaus Indermühle; Werner Zimmermann; Friederike Zeeh; Yvonne Leist
}

VITAL AG, Oberentfelden, Switzerland

\section{Introduction}

Since January 2010 surgical castration of male piglets without anesthesia is forbidden by federal law for the protection of animals in Switzerland.

Due to ignorance to altenative methods avoiding surgical castration by the swiss meat industry, pig breeders are forced to perform surgical castration under general anesthesia. Pig producers can choose between using an inhalation device with isoflurane or anesthesia by intramuscular injection performed by the veterinarian.

Azaperon and Ketamin is the only registered drug combination to anesthetize pigs in Switzerland. The surgical tolerance of this combination is considered to be not sufficient for painful procedures. Our own experiences confirm this estimation, especially if these drugs are given intramuscularly. We observed also adverse reaction like excitement, hyperaesthesia and convulsions.

Consideration concerning improvement of this combination to a more balanced anesthetic protocol with better analgetic action led to a triple combination where a potent analgetic drug (e.g. opioid) by off label us was added.

Because of good experiences with this drug, butor-phanole was chosen.In a field trial the security and efficacy of a combination of azaperone, butorphanole and ketamine for general anesthesia by intramuscular injection to allow castration of piglets was tested.

\section{Materials and Methods}

Out of another study the optimal doses for the combination were found: $5 \mathrm{mg}$ per $\mathrm{kg}$ bodyweight of azaperone, $0.2 \mathrm{mg}$ per $\mathrm{kg}$ bodyweight of butorphanole and $15 \mathrm{mg}$ per $\mathrm{kg}$ bodyweight of ketamine.

From three different pig breeding units 140 male pigs (suckling piglets and weaners) received an intramuscular injection of the combination mentioned above, mixed in the same syringe and performed by the veterinarian. After reaching an adequate depth of anesthesia they were castrated by the farmer in the habitual manner.
For piglets older than 3 weeks of age an emasculator was used. Before administration of the drugs the pigs were shortly clinically examinated and alterations in health status were recorded. Introduction, surgical tolerance and recovery from anesthesia were judged using a score system $(A=$ very good, $B=$ good, $C=$ fair) to evaluate the characteristics and the acceptability of this protocol for this purpose.

\section{Results}

Out of 140, 138 pigs reached within 5 to 10 minutes an adequate depth of anesthesia. Two pigs received an additional dose of $2 / 3$ of the original dose.

Eighteen, especially piglets weighing between 2.5 and $3 \mathrm{~kg}$ showed moderate struggling during tension on the funiculus. After a maximum of two hours all pigs recovered uneventful. There were no losses with coherence to the anesthesia.

The older the pig was, it was more likely to be judged by score A. None of the pigs were judged by score $C$.

\section{Discussion}

This anesthesia protocol showed to be valuable for use in practice to provide general anesthesia allowing surgical castration of piglets. The experiences made showed relevant advantages in comparison of experiences made with azaperone/ketamine alone.

The ideal point in time to perform this procedure showed to be after a piglet's age of three weeks.

\section{References}

1. Brodbelt, D.C. \& Taylor P.M,. (1999) Vet Rec 145: 283-287

2. Lahrmann, K.H. et al. (2006) Prakt. Tierarzt 10: 802-809

3. Nussbaumer, I. et al. (2008) Vet Rec 163: 720-721

4. Sakaguchi, M. et al. (1996) Am J Vet Res: 529-534

5. Saers, S. et al. (2005) Tierärztl. Praxis 33 (G): 432-437

6. Walker, B. et al. (2004) J Vet Med A 51: 150-154 


\title{
P.846
}

\section{Clinical effects of NSAID treatment in piglets with arthritis}

\author{
$\underline{\text { Helena Frohm }}^{1}$ Magdalena Jacobson ${ }^{1}$ Mate Zoric ${ }^{2}$ \\ 1. Dept. of Clinical Sciences, SLU, Uppsala, Sweden; 2. National Veterinary Institute, Uppsala, Sweden
}

\section{Introduction}

In Sweden, approximately $10 \%$ of all piglets are treated against arthritis. The disease is mainly caused by hemolytic streptococci and Staphylococcus spp $(1,4)$ and the piglets are usually treated with intramuscular injections of penicillin once daily for five days. The disease causes suffering to the affected piglets and it has become increasingly common to also include NSAIDs in the treatment. This regimen has however not been evaluated. The purpose of this study was to investigate the clinical effects of combining treatment of lame piglets with NSAID-drugs and penicillin and compare it to treatment with penicillin only.

\section{Materials and Methods}

In this study 40 piglets (Group 1) with arthritis from four different herds in Sweden were treated with benzylpenicillinprokain (20 mg/kg bw) i.m. once daily for five days. 43 piglets (Group 2) were treated similarly but with the addition of a subcutaneous injection of meloxicam $(0.4 \mathrm{mg} / \mathrm{kg} \mathrm{bw})$ the first day of treatment. The piglets included were below three weeks of age with evident lameness. The rectal temperature, weight, degree of lameness and degree of joint swelling were recorded day 1, 2 and 6 (the day after the final treatment). The degree of lameness and joint swelling were scored none (0), mild (1), moderate (2) or severe (3) by a person blinded for treatments. In each pen, a matched, apparently healthy piglet was included as weight gain control. The results were analyzed by MannWhitney Rank Sum test (rectal temperature and weight), and Fischer's exact test (lameness and joint swelling). Comparisons were made between the two treatment groups, between piglets included day 1-2 (i.e. pigs that might have been lame before the start of the study; $\mathrm{n}$ $=48 ; 58 \%$ ) and those included day 3-7 (i.e. pigs that developed arthritis during the study), as well as between piglets with joint swelling in the claws and those with swelling of other joints.

\section{Results}

In both groups, a decrease in rectal temperature was noted between day 1 and 2 (Group 1; P<0.01, Group 2; $\mathrm{P}<0.001$ ) and day 1 and 6 (Group 1; $P<0.05$, Group 2; $P<0.001$ ). The differences between the groups were not significant. Further, there was no difference between the groups in the decrease of swelling of the joints (Table 1). In both groups, the swelling persisted to a larger degree on day 6 among piglets included day 1-2 $(P<0.01)$ than among those included later. Within groups, the swelling was more pronounced day 6 among the piglets in group 1, included day 1-2 ( $P=0.05)$. Swelling of the claw joints were more severe day 6 as compared to other joints $(P<0.001)$. There were no differences with respect to weight gain or lameness between the groups (Table 1).
Table 1.

\begin{tabular}{|c|c|c|c|c|c|c|c|c|c|}
\hline & \multicolumn{3}{|c|}{ Weight (kg) } & \multicolumn{2}{c|}{ Temp $\left({ }^{\circ} \mathbf{C}\right)$} & \multicolumn{2}{c|}{ Swollen } & \multicolumn{2}{c|}{ Lame } \\
\hline Group & $\mathbf{1}$ & $\mathbf{2}$ & $\mathbf{3}$ & $\mathbf{1}$ & $\mathbf{2}$ & $\mathbf{1}$ & $\mathbf{2}$ & $\mathbf{1}$ & $\mathbf{2}$ \\
\hline Day 1 & 2.2 & 2.2 & 2.3 & 39.7 & 39.7 & 1 & 1 & 1 & 2 \\
\hline Day 6 & 3.2 & 2.9 & 3.3 & 39.4 & 39.5 & 1 & 1 & 0 & 0 \\
\hline
\end{tabular}

Group $1=$ piglets treated with penicillin only for 5 days, Group $2=$ piglets treated with penicillin for 5 days + metacam the first day of treatment. Group 3 = healthy weight gain control. Day $1=$ median values measured before the first treatment, Day $6=$ median values measured the day after the last treatment

\section{Discussion}

Initiating treatment early in the course of disease is important to avoid persistent infection, haematogenous spread and further damage to the joints (3). This was also emphasized in the present study by the persistent swelling of the joints among piglets included early in the study, as these piglets may have suffered from a more progressed infection. The results also indicate that the use of meloxicam may reduce joint swelling in these progressed infections. The significance of persistent joint swelling needs further investigation but the degree of synovitis and amount of exudates may influence the ability to eliminate the infection with antibiotics (2). Further studies are also needed to investigate the poor response to therapy of piglets with claw joint swelling. No clinical signs of adverse effects were noted and in conclusion usage of meloxicam in piglets with arthritis can neither be supported nor rejected based on this study.

\section{References}

1. Hill, B.D. et al 1996. Austr Vet J 73:4, pp. 179-180

2. Radostits, O.M. et al 2007. Veterinary Medicine 10. ed. W.B. Saunders Company

3. Straw, B.E. et al 1999. Diseases of Swine 8 ed. USA

4. Zoric, M. et al 2008. Acta Vet Scand 50:37 


\title{
P.847
}

\section{Efficacy of NSAIDs in treatments of lame piglets}

\author{
Mate Zoric $^{1}$ Eva Norling ${ }^{2}$ Ulla Schmidt ${ }^{2}$ Per Wallgren ${ }^{1}$ \\ 1. National Veterinary Institute, Uppsala, Sweden; 2. Swedish University of Agricultural Sciences, Uppsala, Sweden
}

\section{Introduction}

Joint swelling and lameness are the most obvious and persistent clinical signs of infectious arthritis in piglets (1). Prompt treatment with antibiotics is required to achieve a positive treatment effect in lame piglets that limp due to pain (2). Hitherto pain-reducing drugs have rarely been used within veterinary medicine, but the potential of recently introduced non steroid anti-inflammatory drugs (NSAID) is apparent from welfare aspects. However, pain prevents activity and pain-reducing may lead to over-activities with chronic injuries of affected joints as a consequence. Therefore, the aim of this study was to validate the clinical effects of concurrent treatment of lame piglets with NSAID-drugs and antibiotics to that of using antibiotics solely.

\section{Materials \& Methods}

The study was carried out at the research station at FunboLövsta, Swedish University of Agricultural Sciences. The farrowto-finish herd comprised 110 sows and piglets were given individual identities at birth. The piglets were weighed at birth and at five (weaning) and nine weeks of age. Records of disease and treatments were kept for each pig.

All piglets born alive during six months were included in the study. Piglets attended with lameness were randomly allotted into one of two groups. Piglets in group I were injected intramuscularly with 20 mg benzyl penicillin (Penovet vet., Boehringer IngelheimVetmedica) per kg bodyweight once a day for five days. Piglets in group II were additionally injected with 3 mg ketoprofen (Romefen vet., Merial) per kg bodyweight once a day for three days.

The occurrence of lameness was registered until the age of 5 weeks and the clinical efficacies of treatment were assessed daily. Lameness and/or visibly swollen joints, as well as general condition were scored as 0 (good), 1 (almost good), 2 (not good), 3 (not good at all). Groups were compared by Chi2-tests using two-by-two frequency tables.

\section{Results}

In total 93 out of 1572 liveborn piglets were diagnosed with lameness (5.9\%). Around $85 \%$ of these diagnoses took place during the first 3 weeks of life. As shown in Figure 1, the clinical response differed significantly $(p<0.05)$ between the two treatment groups. The clinical status of penicillin and ketoprofen treated piglets ( $n=49$ ) was improved daily during the first four treatment days $(p<0.05$ to 0.01$)$, whereas a significant $(p<0.01)$ improvement only was recorded between days 2 and 3 of piglets solely treated with penicillin $(n=44)$.
Figure 1. The clinical efficacy of treatment for lameness.

\begin{tabular}{|c|c|c|c|c|c|c|c|}
\hline \multicolumn{8}{|c|}{ 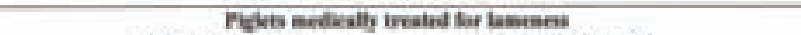 } \\
\hline & \multicolumn{3}{|c|}{ Granlin $=49$} & \multicolumn{3}{|c|}{ 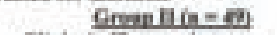 } & \multirow{3}{*}{ 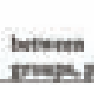 } \\
\hline & \multicolumn{2}{|c|}{ cinical emerasy } & \multirow{2}{*}{$\begin{array}{l}\text { Detaen } \\
\text { tays p }\end{array}$} & \multicolumn{2}{|c|}{ Clinical atilleary } & \multirow{2}{*}{$\begin{array}{l}\text { betwers } \\
\text { Aojap }\end{array}$} & \\
\hline & 0.1 & $1+3$ & & $3-1$ & $2-3$ & & \\
\hline Way 1 & 9 & 1232 & $=$ & 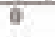 & 175 & + & $m$ \\
\hline Day 2 & 0 & $20-24$ & $H_{l=m i}$ & 5 & 44 & mise & - \\
\hline Day 3 & 7 & n & $=\lim *$ & it & $\mathbf{n}$ & $=\lim _{\min }$ & $\bullet$ \\
\hline Das 4 & B & 31 & 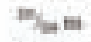 & 28 & 21 & $\omega_{h i=*}$ & $\bullet$ \\
\hline Day 5 & $a$ & 24 & 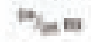 & $x$ & it & Fan & + \\
\hline
\end{tabular}

The weights recorded at birth, five and nine weeks of age are shown in Figure 2. Piglets that remained healthy were about 1.1 $\mathrm{kg}$ heavier than piglets attended with lameness at 5 and 9 weeks of age. There was no difference between weights of the piglets treated for lameness regardless of treatment strategy.

Figure 2. Means bodyweight of piglets born alive.

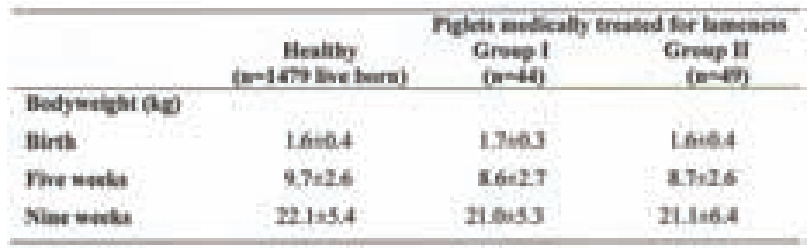

\section{Discussion \& Conclusions}

Like other diseases (3), lameness reduced the growth rate of the piglets. Despite the low number of pigs in this study, our results clearly indicated an earlier recovery among lame piglets treated with both antimicrobials and NSAIDs. Use of NSAIDs in combination with antibiotics as treatment for lameness in piglets ought therefore be considered for animal welfare issues.

No difference in weight gain between the treated groups were recorded, but the earlier recovery of piglets given NSAIDs may contribute to higher activity including suckling which could improve the general condition of affected piglets. This will be scrutinised in enlarged studies. However, it should be emphasized that pharmacological treatment of pain in sick piglets not can replace the management routines, floor quality and good care.
References
1. Zoric et al., 2003. Vet Rec. 153:323-328.
2. Dewey., 2006. Diseases of Swine. 9:87-111.
3. Wallgren., 2000. Sw Vet J. 13:685-694. 


\title{
P.848
}

\section{Effect of ibuprofen on pigs during routine vaccination and willingness to approach behavior}

\author{
Peggy Anne Hawkins; Michael Strobel; Leah M. Erickson \\ Veterinary Provisions, Inc., Northfield, MN, USA
}

\section{Introduction}

Vaccinations are a necessary part of producing healthy pigs. Today, nearly all pigs receive some vaccinations during the nursery and/or grower phases of production. The objective of this study was to determine if ibuprofen administered in the water could reduce fever associated with vaccination of pigs as measured by rectal temperature. Additionally, a pilot study on the behavior known as willingness to approach was evaluated in pigs with and without ibuprofen at the time of vaccination. The ibuprofen group had significantly lower rectal temperatures as compared to the non-medicated group.

\section{Materials and Methods}

One of two rooms was randomly assigned to receive the ibuprofen medication. And, fifty (50) pigs within one randomly chosen pen in each of the two rooms were selected. Pigs were tagged for identification 5 days prior to vaccination day. At 3:00p.m. the day prior to vaccination, a stock solution of ibuprofen (Ve$\left.t R x^{\circledast}\right)$ was prepared according to label directions: $160 z$ (473ml) q.s. with water to 1 gallon and metered to the pigs at 1:128. Ibuprofen remained available to the pigs for 24 hours post-vaccination. The next morning at 9:00a.m., pigs were vaccinated, booster dose, per veterinary recommendation $\left(\mathrm{M}+\mathrm{Pac}{ }^{\circ}\right.$ and $\mathrm{Cir}-$ cumvent ${ }^{\circ}$ PCV2; Intervet/Schering). Pigs were crowed in the pen and injected in the neck muscle. Immediately following vaccination individual rectal temperatures (RT) were measured. About 5 hours after vaccination, the pigs were crowded in the pen again and RT were taken again. Pigs in the remaining pens of the room were used to evaluate willingness to approach as described by Baumert et al. (1). The baseline or pre-vaccination evaluation for the approach study was done the afternoon before vaccination. The post-vaccination evaluation was done the following afternoon about 5 hours post-vaccination.

\section{Results}

The non-medicated control pigs had an average RT $105.7^{\circ} \mathrm{F}$ and the ibuprofen group was $105.6^{\circ} \mathrm{F}$ immediately following vaccination. At 5 hours post-vaccination, non-medicated control pigs had an average RT $105.5^{\circ} \mathrm{F}$ and the ibuprofen group was significantly lower at $104.8^{\circ} \mathrm{F}$. Additionally, there was no difference between the immediate RT of either group and the RT of the control group after 5 hours (Fig. 1). Plots with different letters are different $(\mathrm{P}<0.05)$.

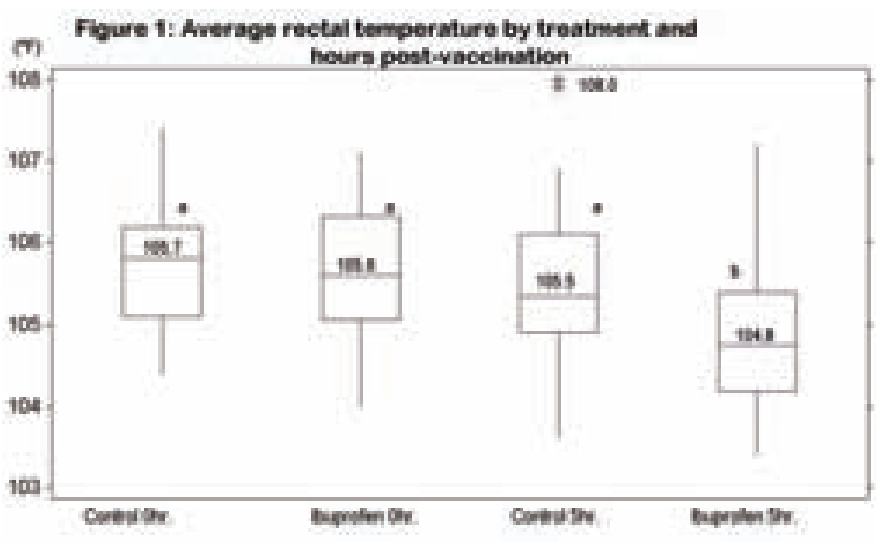

There was a significant decrease in willingness to approach post-vaccination within both treatment groups. However, there was no difference in the decrease in willingness to approach between groups. The control pigs' willingness to approach reduced by $16.6 \%$ and the ibuprofen pigs' was reduced by $12.2 \%$. There were too few pens in this pilot study to show a difference. All data were analyzed using Wilcoxon rank sum test. A $P$ value of $<0.05$ was considered significant.

\section{Discussion}

Vaccines stimulate and activate the immune system of pigs (2). The inflammatory response is an important part of this development (3). A febrile response is expected when a vaccine has done a good job of stimulating immunity. However, a very high fever response $\left(>105.0^{\circ} \mathrm{F}\right)$ may do more harm than good for the animal. Ibuprofen is used effectively in human medicine to control vaccine induced fever. In this study, the increase in RT due to the stress of the vaccination process (crowding and injection) was not affected by having ibuprofen in the drinking water. But, at 5 hours after vaccination, the non-medicated animals continued to have an increasing RT with some as high at $108.0^{\circ} \mathrm{F}$. Pigs with a high fever were less likely to exhibit willingness to approach behavior than pigs with RTs controlled by ibuprofen. Further work will be presented at the meeting.

\section{References}

1- Baumert D, Fangman T, Edler R, DuBois P. Willingness to approach behavior and feed disappearance of weaned pigs following vaccination with Mycoplasma vaccines, IPVS Proceedings, Durban, South Africa, 2008, P02.030

2- Chase C. Immunology primer for practitioners, Swine Disease Conference Proceedings, Ames, lowa, 2009, P:13-15

3- Mueller K. Special Section: Innate Immunity, Science 2010 Vol 3277 


\title{
P.849
}

\section{Investigations about the use of topical cryogen spray and local anesthetics for the castration of male suckling piglets}

\author{
Daniela Schiele ${ }^{1}$ Tim Kilchling $^{1}$ Susanne Zöls ${ }^{1}$ Mathias Ritzmann² Andreas Palzer ${ }^{1}$ Karl Heinritzi ${ }^{1}$ \\ 1. Clinic for Swine, LMU Munich, Oberschleißheim, Germany; 2. Clinic for Swine Diseases, VU Vienna, Vienna, Austria
}

\section{Introduction}

In many European countries, the search for a reasonable alternative to piglet castration without analgesic treatment is ongoing. In Austria a new method was developed in 2008. It combines the application of topical cryogen spray on the scrotal area before skin incision and spraying local anesthetics (LA) on the fixed spermatic cord after removal of the testes. Therefore the spermatic cord is fixed with a clamp (1). The intent of the present study was to evaluate the impact of cryogen spray application, intraoperative fixation of the spermatic cord and intraoperative application of local anesthetics on castration induced stress and pain. Similar to other studies, the dimension of stress was evaluated by cortisol measurements $(2,3)$.

\section{Materials and Methods}

A total of 221 clinically healthy male suckling piglets were randomly assigned to eight different treatments and groups (Table 1).

Table 1: Treatment groups 1-8 and number of animals

\begin{tabular}{|c|l|c|}
\hline group & \multicolumn{1}{|c|}{ Treatment } & number \\
\hline $\mathbf{1}$ & Fixation & 29 \\
\hline $\mathbf{2}$ & Fixation, cryogen spray & 29 \\
\hline $\mathbf{3}$ & Fixation, castration & 28 \\
\hline $\mathbf{4}$ & Fixation, cryogen spray, castration & 28 \\
\hline $\mathbf{5}$ & Fixation, castration, LA dropped into wound & 28 \\
\hline $\mathbf{6}$ & Fixation, cryogen spray, castration, LA dropped into wound & 29 \\
\hline $\mathbf{7}$ & $\begin{array}{l}\text { Fixation, cryogen spray, castration, LA dropped into wound LA intrates- } \\
\text { ticular, fixation, castration }\end{array}$ & 29 \\
\hline $\mathbf{8}$ & $\begin{array}{l}\text { Fixation, cryogen spray, castration, fixation of spermatic cord with clamp, } \\
\text { LA dropped on fixed cord }\end{array}$ & 21 \\
\hline
\end{tabular}

The cryogen spray (ethyl chloride) was applied on the scrotal skin from a distance of $10 \mathrm{~cm}$ until a skin temperature up to $-12^{\circ} \mathrm{C}$ was achieved. Temperature of the scrotal skin was measured by a pyrometer. The local anesthetics ( $40 \mathrm{mg}$ procainehydrochloride with $0.05 \mathrm{mg}$ epinephrine) were either dropped into the wound after removal of the testes (Group 5 and 6), injected into the testes 10 minutes prior to castration (Group 7) or dropped on the fixed spermatic cord after removal of the testes (Group 8). Blood samples taken from the cranial vena cava were collected from each piglet half an hour before and half an hour, one hour, four hours and one day after treatment. Based on cortisol measurements, the impact of the various treatments on pain and stress was examined.

\section{Results}

The average values of cortisol concentration of all groups are shown in Figure 1.

Figure 1: Average values of cortisol concentration before and $1 / 2 h, 1$ $h, 4 h, 24 h$ after treatment

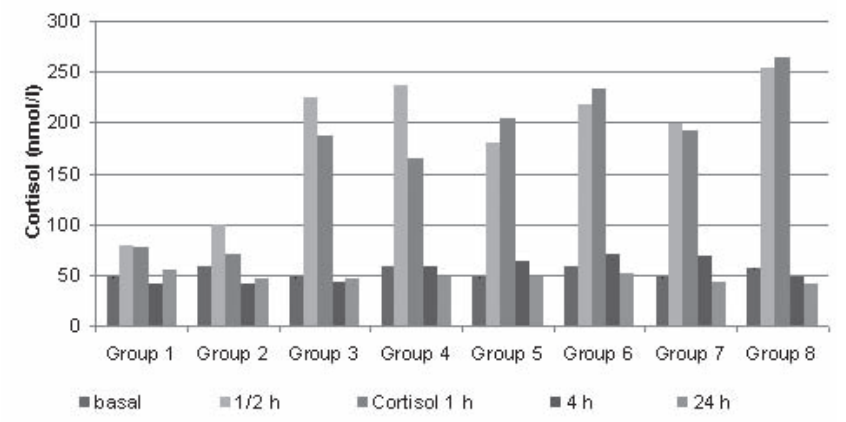

Fixation and application of cryogen spray (group 2) caused half an hour after treatment significantly higher average cortisol concentrations than fixation alone (group 1$)(p \leq 0.05)$. A minimizing effect on castration induced stress using a cryogen spray prior to castration was not evident (group 3 vs. group 4, p > 0.05).

Four hours after castration, the average cortisol concentration of Group 5, 6 and 7 were significantly higher than that of Group 3 ( $p \leq 0.05$ ). The application of cryogen spray combined with the use of a clamp to fix the spermatic cord (Group 8) lead to a significant increase in serum cortisol concentration one hour after castration compared with serum cortisol concentration of Group 3 ( $p \leq 0.05)$.

The results of the cortisol measurements indicated that none of the examined castration methods (Groups 4-8) induced a significant minor stress reaction than the conventional method of castration (Group 3).

\section{Discussion}

Results indicate that neither cryogen spray nor local anesthetics as applied in this study cause an appropriate analgesia for piglet castration. Therefore, those techniques are not feasible as alternative to conventional piglet castration.

\section{References}

(1) Gasteiner et al. (2008) Bericht LFZ Raumberg Gumpenstein: 9-17

(2) Prunier et al. (2005) Journal of Animal Science 83: 216-222

(3) Zöls et al. (2006) Berliner Münchner Tierärztliche Wochenschrift 119: 193-196 


\title{
P.850
}

\section{Evaluation of different topical anaesthetics for pain reduction during surgical castration of male piglets}

\author{
Karl-Heinz Waldmann ${ }^{1}$ Dina Rittershaus ${ }^{1}$ Manfred Kietzmann² Birger Puppe ${ }^{3}$ \\ 1. Clinic for Swine and Small Ruminants, Hannover, Germany; 2. Department of Pharmacology, Toxicology and Pharmacy, \\ University for Veterinary Medicine, Hannover, Germany; 3. Research Institute for the Biology of Farm Animals, Dummerstorf, Germany
}

\section{Introduction}

$80 \%$ of the male piglets in the EU and almost all male piglets in Germany are castrated to avoid unpleasant odour and taste of the meat of entire mal pigs [1]. According to European law, castration can be performed without anaesthesia during the first 7 days of the piglets' lives. Various studies however documented painfulness of this procedure. Therefore different research projects were started to find an alternative to conventional castration of piglets. However, general anaesthesia showed some disadvantages [2] and for antiphlogistics, positive effects could be found merely on postoperative pain [3]. This study intended to test the ability of different topical anaesthetic techniques for pain reduction during castration of male suckling piglets.

\section{Material and Methods}

193 male piglets at 6 or 7 days of age were included in the study and divided into 8 different groups. There were 5 castration groups, in which the piglets were castrated conventionally or after use of chlorethyl spray, a combination of chlorethyl and lidocaine spray or EMLA-cream, which is an euthectic mixture of lidocaine and prilocaine. One group of piglets, which were castrated after intratesticular application of local anaesthesia in combination with an NSAID served as positive control. Additionally, piglets of three control groups were either not medicated or treated with chlorethyl spray or EMLA-cream and fixated but not castrated. For measurement of the impact of castration on the piglets, blood samples were taken pre OP and 1 and 24 hours after castration or fixation with cortisol levels being measured afterwards. Vocalizations of the piglets were recorded and analyzed to detect pain during castration/fixation. The piglets were followed up (wound control/weighing) for three weeks.

\section{Results}

In piglets castrated without anaesthesia, a significant rise of cortisol levels from the basal value to the value $1 \mathrm{~h}$ after castration could be registered $(p<0.05)$. Piglets which underwent topical anaesthesia had almost equally high cortisol levels ( $p>0.05)$. In contrast, piglets which received local anaesthesia before castration and uncastrated piglets had almost no increase of plasma cortisol levels. Animals of the castration groups showed distinct changes of vocalisation during the possibly painful surgery stages, which could not be found before or after castration. Although sound analysis indicated pain reduction during surgery by use of chlorethyl spray, it also showed that application of chlorethyl spray itself is painful. Regarding EMLA-cream, sound analysis was suggestive of some anaesthetic effects on the skin, but not on the spermatic cord. On the contrary, in piglets, which were castrated under local anaesthesia, almost no changes of vocalization were detected. Wound healing showed no difference between the control group and the treatment groups ( $p>0.05$ ) except for piglets, which had received an injection of a local anaesthetic prior to castration, which had significantly $(p<0.05)$ increased wound healing disorders.

\section{Discussion}

On the basis of increase of plasma cortisol levels, no stress reduction by the use of chlorethyl spray and just a small but not significant improvement by using a combination of chlorethyl and lidocaine spray or EMLA-cream were discovered. Although analysis of the piglets' vocalisations indicated some pain reduction during the skin incision and the emasculation by use of chlorethyl spray, it also showed that application of chlorethyl spray itself is painful. Regarding the usage of EMLA-cream, sound analysis was suggestive of some anaesthetic effects on the skin but not on the spermatic cord.

Altogether the results of this study show that none of the topical anaesthetic techniques is as effective for pain reduction as an intratesticularly applied local anaesthetic. Since local anaesthesia prior to castration produced increased wound healing disorders, further research is required to find a practical and efficient alternative to conventional castration of piglets.

\section{References}

[1] EFSA (2004): Opinion of the Scientific Panel on Animal Health and Welfare on a request from the Commission related to welfare aspects of the castration of piglets. The EFSA Journal, 91, 1-18

[2] Hodgson, D.S. (2007): Comparison of isoflurane and sevoflurane for short-term anesthesia in piglets. Vet Anaesth Analg, 34, 117-24

[3] Zöls, S., Ritzmann, M. and Heinritzi, K. (2006): Effect of analgesics on castration of male piglets. Berl Munch Tierarztl Wochenschr, 119, 193-96 


\section{Possibilities of pain reduction in tail docking in piglets}

Tim Kilchling; Daniela Schiele; Susanne Zöls; Andreas Palzer; Matthias Eddicks; Karl Heinritzi

Clinic for Swine, LMU Munich, Oberschleißheim, Germany

\section{Introduction}

Tail docking is performed as a precautionary move to prevent tail biting in pigs (1). According to the German Animal Rights Law, tail docking is only allowed without anaesthesia and pain medication until the fourth day of life. However, this procedure,appears to be painful for piglets.

The aim of this present study was to identify the pain caused by tail docking and the possibility of reducing this neuroendocrine stress reaction by administering a nonsteroidal anti-inflammatory drug (NSAID) before tail docking. A further study evaluated the pain caused by tail docking based on cortisol values (2).

The evaluation of cortisol permits the measurement of the neuroendocrine stress reaction in animals (3).

\section{Materials and Methods}

A total of 219 one-day old clinically healthy piglets were randomly assigned to seven different groups. A general overview of the seven different groups, number of piglets per group, the tail docking method and the drug administered is depicted in Table 1. Piglets from Group 1 served as controls and were manually restrained in the same position used for tail docking without having their tails removed.

Table 1: Treatment groups and tail docking method

\begin{tabular}{|c|c|c|c|c|}
\hline & Group & Tail docking method & Treatment & $\mathbf{n}$ \\
\hline $\mathbf{1}$ & Control & none & none & 32 \\
\hline $\mathbf{2}$ & Cauterisation & Cauterisation & none & 32 \\
\hline $\mathbf{3}$ & Meloxicam / cauterisation & Cauterisation & $0.4 \mathrm{mg} / \mathrm{kg}$ Meloxicam & 31 \\
\hline $\mathbf{4}$ & Flunixin / cauterisation & Cauterisation & $2.2 \mathrm{mg} / \mathrm{kg}$ Flunixin & 31 \\
\hline $\mathbf{5}$ & Side cutting pliers & Side cutting pliers & none & 31 \\
\hline $\mathbf{6}$ & $\begin{array}{c}\text { Meloxicam / side cutting } \\
\text { pliers }\end{array}$ & Side cutting pliers & $0.4 \mathrm{mg} / \mathrm{kg}$ Meloxicam & 31 \\
\hline $\mathbf{7}$ & $\begin{array}{c}\text { Flunixin } / \text { side cutting } \\
\text { pliers }\end{array}$ & Side cutting pliers & $2.2 \mathrm{mg} / \mathrm{kg}$ Flunixin & 31 \\
\hline & & & & \\
\hline
\end{tabular}

The tail docking was always performed on the third day of life. On that day, approximately 2-3 $\mathrm{ml}$ of blood was collected from each piglet via the vena cava cranialis thirty minutes before tail docking. The cortisol concentration, determined from the serum samples taken 30 minutes before tail docking served as the base value. Following blood collection, piglets from Groups 3, 4, 6 and 7 were administered their respective NSAIDs. After drug administration, the tails from piglets of all groups, with the exception of Group 1, were removed using the corresponding instrument. Thereafter, blood was collected from all piglets 30 minutes, 60 minutes, four and 24 hours for cortisol measurement.

\section{Results and Discussion}

The average values of the serum cortisol concentration of all groups is shown in Figure 1.

Figure 1: Average values of Cortisol concentration (with 95\% confidence intervals) before, 30 and 60 minutes, four and 24 hours after tail docking

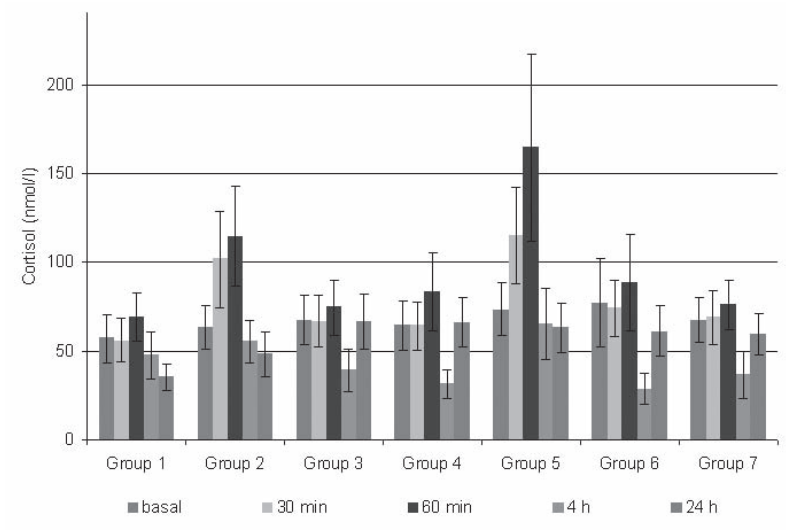

No difference could be found in the basal value ( $p>0.05$ ) of each group. The cortisol concentration of Group 2 and Group 5 was significantly $(p<0.05)$ increased 30 and 60 minutes after tail docking when compared to the control Group 1. The cortisol concentration, measured 30 minutes after tail docking, was significantly $(p<0.05)$ lower in all groups that received NSAID prior to tail docking when compared to Groups 2 and 5.60 minutes after tail docking, the cortisol concentration of Group 3, which received Meloxicam, was significantly lower than that of Group 2. Additionally, the cortisol concentration of Group 6 was also significantly lower when compared to Group 5. After Flunixin administration, the cortisol concentration of Group 7 was significantly reduced in comparison to Group 5.

Significant differences between the both tail docking methods were not observed at any of the examined times. The serum cortisol concentration of Group 5 tends to be higher than that of Group 2.

\section{Conclusion}

The results of the study infer that the cortisol concentration caused by the neuroendocrine stress reaction is not influenced by handling or blood-sampling, but significantly raised due to pain and distress like tail docking without any treatment. It can be reduced by the prior administration of an analgesic such as Meloxicam or Flunixin.

\section{References}

1) Hunter et al. (2001) Veterinary Journal 161:72-79

2) Sutherland et al. (2008) Animal 2: 292-297

3) Zöls et al. (2006) Berliner Münchner Tierärztliche Wochenschrift 119: 193-196 


\title{
P.852
}

\section{A non-penetrating captive bolt (modified Zephyr) is effective for euthanasia of neonatal piglets}

\author{
Teresa M. Casey-Trott ${ }^{1}$ Suzanne T. Millman² Penny Lawlis ${ }^{3}$ Tina M. Widowski ${ }^{1}$ \\ 1. University of Guelph, Guelph, ON, Canada; 2. lowa State University, Ames, IA, USA; \\ 3. Ontario Ministry of Agriculture, Food and Rural Affairs, Guelph, ON, Canada
}

\section{Introduction}

On-farm euthanasia of low viability piglets is an inevitable task for producers, and it is essential that a humane and cost effective method be established. Any euthanasia method must ensure minimal pain, fear and distress as well as include considerations for the overall aesthetic acceptance of the procedure (AVMA, 2007). Currently acceptable methods for piglets up to $12 \mathrm{lbs}$ include carbon dioxide, anesthetic overdose, non-penetrating captive bolt, and the most common method, blunt force trauma (NPB-04259-01/09; AVMA, 2007). To be considered effective without a secondary step, the non-penetrating captive bolt must cause sufficient depression of the central nervous system and destruction of brain tissue to cause both immediate insensibility and rapid death (AVMA, 2007).Recent work comparing a nonpenetrating captive bolt (Zephyr, round head, $120 \mathrm{PSI}$ ) to blunt force trauma found that both methods rendered piglets immediately insensible, but some piglets showed signs of returning to consciousness when the Zephyr was used (Widowski et al, 2008). Variation in depth of depression resulting from different guns used in that study may have contributed to this finding. For the current study, the design of the nylon bolt head attachment for the Zephyr gun was modified to a convex shape to increase the depth of depression when used at 120 PSI without causing penetration.Methods

All procedures were approved by the Animal Care Committee at the University of Guelph. Ten experienced stock people were trained to use the Zephyr, and each stock person euthanized 10 low-viability or compromised piglets $<72 \mathrm{~h}$ of age. Each stock person manually restrained the piglet on its sternum on a hard, flat surface. Two shots were administered rapid fire on the frontal bone (NPB-04259-01/09), followed immediately by one shot delivered to the back of the skull behind one ear. Piglets were assessed for signs of consciousness using brainstem and spinal reflexes: presence or absence of corneal reflex, pupillary light response, jaw tone, and response to nose prick. Reflexes were repeatedly checked in that order every 15 seconds for at least 4 minutes. Clonic and tonic neuromuscular spasms (convulsions) were monitored along with the presence of breathing and heartbeat. Termination of tonic convulsions was used to estimate time of death (ETOD). Macroscopic scoring was used to assess skull fracture and hemorrhage at necropsy. Skull fractures (SK) were recorded on a 5 point scale; 1 (no fracture) - 5 (fully fragmented). Subcutaneous (SC), Subdural Dorsal (SD-D) and Subdural Ventral (SD-V) scores used a 6 point scale; 1 (no hemorrhage) - 6 (complete coverage). A brief questionnaire was provided to each stock person to assess individual experience and rate the method on a 10 point scale; 1 (completely ineffective) - 10 (highly effective).

\section{Results}

Data from 98 piglets showed that $100 \%$ were insensible immediately, and no piglets showed signs of regaining consciousness. ETOD averaged $225.9 \pm 88.6 \mathrm{~s}( \pm$ S.D). Five of the 98 piglets required a secondary step (exsanguination) for cessation of movement and were not included in the ETOD average. Macroscopic scores averaged $3.3 \pm 0.5$ (SK); $4.5 \pm 1.1$ (SC); $4.2 \pm 0.9$ (SD-D); and $4.4 \pm 0.8(\mathrm{SD}-\mathrm{V})$. Stock personnel rated the effectiveness of the Zephyr at $8.7 \pm 1.6$.

\section{Discussion}

The modified Zephyr used in this trial was a highly effective method of euthanasia for neonatal piglets less than 72 hours of age. The Zephyr caused immediate insensibility without return to consciousness in all 98 piglets and led to death without a secondary step in $95 \%$ of piglets. Macroscopic scoring indicated that moderate to severe damage was inflicted to the skull and brain tissue of all piglets. The potential of the Zephyr for on-farm euthanasia was supported by a reasonable average ETOD (3m45s) and high stock personnel ratings. With additional modifications for safety and portability, the Zephyr has potential to be an effective and practical method of on-farm euthanasia within the swine industry.

\section{References}

American Veterinary Medical Association, 2007. Guidelines on Euthanasia http://www.avma.org/issues/ animalwelfare/euthanasia.pdf, Accessed Feb 08, 2010.

National Pork Board, 2009. On farm euthanasia- options for the producer. Pub. 04259-01/09, Des Moines, IA.

Widowski, T.M., et al (2008) Assessing the effectiveness of a non-penetrating captive bolt for euthanasia of newborn piglets. Proc. Allen D. Leman Swine Conf, pp 107-111. 


\title{
P.853
}

\section{Reduction of the discomfort at castration of piglets with azaperone}

\author{
Bert Driessens ${ }^{2}$ Jan Jourquin ${ }^{1}$ Sanne Van Beirendonck ${ }^{2}$ Rony Geers ${ }^{3}$ Lieve Goossens $^{1}$ \\ 1. Janssen Animal Heatlh, Beerse, Belgium; 2. Cluster Animal Welfare, K.H.Kempen, Geel, Belgium; \\ 3. Laboratory of Quality Care in Animal Production, KULeuven, Leuven, Belgium
}

\section{Introduction}

Castration of piglets is widely practiced throughout the world to avoid boar taint. Castration is performed at very young age and is recognized to be a painful experience for the piglets. To improve welfare, castration has been banned (UK) or is only allowed when the piglets receive an analgesic (EU) or under local (Norway) or complete (The Netherlands) anesthesia. Today, there is also the option of immunocastration.

A distinction is made between the direct pain during the act of castration and the pain after the procedure. Farmers are not allowed to use anesthetics which raises the need for an alternative to reduce the discomfort at castration.

The aim of this study was to investigate if the use of azaperone $\left(\right.$ Stresnil ${ }^{\circ}$ ), a sedative registered for pigs and allowed to be used by farmers, could be a valid alternative to anesthesia of the piglets.

\section{Materials and Methods}

In the experimental farm of Lovenjoel, 392 piglets were included in the trial. As a reference group, 258 piglets were sham castrated (REF). The negative control group consisted of 71 piglets castrated without any treatment (NC) and 63 piglets were injected with $2 \mathrm{mg} / \mathrm{kg}$ azaperone IM 15 minutes before castration (AZA). Castration was performed during the first week of life. Weight gain was measured at 23 and 63 days post castration. In $21 \mathrm{NC}$ and 16 AZA piglets, vocalization at handling, injection and castration was measured with a decibel meter. The behavior of the piglets was recorded every minute during 10 minutes after castration. Walking, sitting, standing, huddled up position, isolated position, trembling and udder massage were categorized as negative behavior, while suckling, sleeping, lateral and ventral lying was categorized as positive behavior.

The generalized linear model approach was perform in SAS 9.1 for the statistical analysis.

\section{Results}

There was no difference in weight gain 23 and 63 days post castration between the three groups. The vocalization results are shown in Table 1. Injection caused a significant increase of screaming intensity compared to only handling, but the screaming intensity was lower compared to screaming at castration. The screaming intensity at castration was significantly reduced in the AZA compared to NC.
Table 1: Vocalization (average decibel)

\begin{tabular}{|c|c|c|c|c|}
\hline Treatment & No. piglets & Handling & Injection & Castration \\
\hline NC & 21 & 75.9 & - & $99.2^{\mathrm{c}}$ \\
\hline AZA & 16 & $73.3^{\mathrm{a}}$ & $89.0^{\mathrm{b}}$ & $92.1^{\mathrm{d}}$ \\
\hline $\begin{array}{l}\mathrm{ab}: \text { significant difference } \mathrm{p}<0.05 \\
\mathrm{~cd}: \text { significant difference } \mathrm{p}<0.05\end{array}$ \\
\hline
\end{tabular}

Figure 1 shows the evolution of negative behavior scores in the three groups during the 10 minutes after castration. Both REF and AZA show a significantly lower number of negative behavior scores $(p<0.05)$ compared to $\mathrm{NC}$, while there is overall no difference between REF and AZA. There were more REF piglets standing and less huddled up however.

Figure 1: Negative behavior score evolution

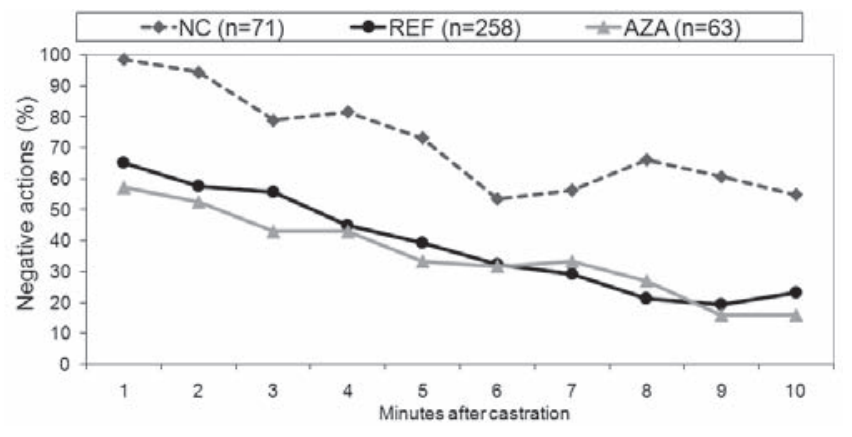

All groups are significantly different for positive behavior. Both REF and AZA behave significantly better than NC. REF sleeps and suckles more than AZA while more AZA are lying in ventral position.

\section{Discussion}

An injection of azaperone 15 minutes before castration diminishes the screaming intensity at castration. The behavior of the piglet immediately after castration resembles that of a sham castrated piglet and is significantly better than conventionally castrated piglets.

Azaperone (Stresnil ${ }^{\circ}$ ) is a registered product for pigs and can be applied by the farmer. With a cost of less than 3 Eurocent per piglet, there is no economical constraint.

In conclusion, the use of azaperone prior to castration could be taken into account as an alternative to anesthesia. 


\title{
Biopsies for grading of shoulder ulcerations in sows
}

\author{
Henrik E. Jensen; Kirstin Dahl-Pedersen \\ University of Copenhagen, Frederiksberg, Denmark
}

\section{Introduction}

The presence of shoulder ulcerations in sows commonly develop and signal poor animal welfare (1). Presence of shoulder ulcerations is due to pressure and is significantly related to the laying bout duration $(2,3)$. Clinically, it may be difficult to state the presence of an ulceration. Moreover, it is not always straight forward to deduct the progression of shoulder ulcerations (4). With reference to national strategies initiated towards limiting the presence of shoulder ulcerations, an intervention must be initiated when the ulceration has penetrated into the subcutaneous tissue. Therefore, different para-clinical techniques should be evaluated for the characterization of ulcerations. Such techniques should enable one to state the presence or absence of an ulceration and preferably aide for grading the ulceration.

Based on pathology, shoulder ulcerations are graded 1-4 according to the progression into the different anatomical structures of the shoulder region with the following characteristics $(1,3)$ : Stage 1) the ulceration is limited to the epidermis (necrotic or sloughed off), and may be covered with a scab. Stage 2) the dermis is ulcerated and may be covered with a scab. Usually a small amount of granulation tissue or fibrosis is bordering the ulcer. Stage 3) the subcutaneous tissue is ulcerating and may be covered with a scab. It is accomplished by heavy formation of granulation tissue or fibrosis towards the surroundings. Stage 4) an ulceration with exposed bone (tuber spina scapula). Stage 4 ulcerations are always accompanied by a heavy proliferation of new osseous tissue. Shoulders free of ulcerations are scored as stage 0 (3).

In the present study, the diagnosis of shoulder ulcerations based on the examination of a single biopsy taken from the skin overlaying tuber spina scapula, i.e. centrally from the ulcer when present, was compared with the classical method implying examination of cross-sectioned shoulder areas during necropsy.

\section{Materials and Methods}

Shoulder regions from 33 sows admitted to the department for necropsy were evaluated. Before cross-sectioning and grading the shoulder ulcerations, a punch biopsy ( $8 \mathrm{~mm}$ in diameter, KRUUSE, Denmark) was taken from the skin overlaying tuber spina scapula from animals without ulcerations, and centrally from the ulcer when present. Tissue specimens were fixed in $10 \%$ neutral buffered formalin for 7 days, processed through graded concentrations of ethanol and xylen and embedded in paraffin wax. Tissue sections were cut at 4-5 $\mu \mathrm{m}$ and stained with haematoxylin and eosin ( $\mathrm{HE})$. The grading of ulceration stages from the biopsies was compared with the grading based on the cross-section method (3).

\section{Results}

The cross-section method revealed the following pattern of shoulder ulcerations: stage $0=16$; stage $1=0$; stage $2=4$; stage $3=13$; and stage $4=0$. In five cases, the stage of ulceration was based on gross examination because the sections taken for histology were all bordered by granulation tissue, i.e. the involved tissues of the skin could not be judged histologically. Presence $(n=17)$ or absence $(n=16)$ of an ulceration, as assessed by cross-sectioning the shoulders, was in all cases verified in the biopsies. In the biopsies, four cases of both stage 2 and stage 3 ulcerations were confirmed, too. However, in four and five cases of stage 3 ulcerations the biopsies deducted the ulcerations being stage 2 or at least stage 2 ulcerations, respectively.

\section{Discussion}

Examination of a single biopsy taken from the skin overlaying tuber spina scapula in all cases enabled one to settle whether an ulceration was present or not. Moreover, in eight sows with ulcerations a correct stage was documented in the biopsies.

In four out of nine stage 3 ulcerations, however, the biopsies showed a stage 2 ulceration, and in five out of nine stage 3 ulcerations the biopsies revealed stage 2 ulceration at least. Due to the limited tissue sampled by a punch biopsy, however, some ulcerations are difficult to grade because the material is mainly made up by granulation tissue.

In conclusion, a single biopsy is sufficient for stating the presence of an ulceration and when a stage 3 ulceration is documented in a single biopsy it is in accordance with the crosssection method. Moreover, a major advantage of punch biopsies is that they can be sampled from living animals.

\section{References}

1. Lund et al. (2003). Danish Vet. J. 86, 8-11.

2. Jensen (2009). Vet. Rec. 165, 171-174.

3. Rolandsdotter et al. (2009). Acta Vet. Scand. 51, 44-59.

4. Vestergaard et al. (2007) Danish Pig production Medd. 798, 1-12 


\title{
P.855
}

\section{Clinical assessment of shoulder lesions in Danish sows}

\author{
Helle Stege $^{1}$ Jens Strathe ${ }^{2}$ Anders R. Kristensen ${ }^{1}$ Jens P. Nielsen ${ }^{1}$ \\ 1. KU-Life, Copenhagen, Denmark; 2. Hyovet, Bigum, Denmark
}

\section{Introduction}

Decubital shoulder lesions in lactating sows may compromise animal welfare. Lesions with adherent crust/scar to the tuber spina scapula (TSS) and lesions penetrating to exposed bone are notifiable in Denmark and are here defined as pathological lesions. The severity of shoulder lesions may be difficult to determine by clinical inspection and palpation ante mortem because the wound can be undermined and be more extensive than the mere penetration of the skin shows. The aim of this study was to identify combinations of simple clinical signs as markers of pathological shoulder lesions for veterinarians in an advisory situation.

\section{Materials and Methods}

The study was observational and included 363 Danish culled sows sent to an abattoir during a two week period. Each sow was registered by sow- and herd-number and date of registration. All sows were examined ante mortem, a visual body condition score (1-5) was assigned and the following clinical signs were recorded: Skin-covered shoulder, i.e. "Normal” (no sign of epithelial deficit); Skin alterations (Erythema; redness in the skin overlaying the TSS or peripheral to a crust/swelling and Crust; dried exudate overlying an epithelial deficit); Swelling (soft or firm elevation of the skin); Adherence to the TSS (scar/ crust or swelling adherent to the underlying bony foundations by palpation); Scar (intact skin with scar tissue apparent at the site). Diameters of swellings and diameters of crust/scar (the largest width) were measured with callipers, using a hierarchy of swellings rather than crust/scar when both signs were apparent. After slaughter, the following pathological signs were recorded by post mortem examination: No lesions of the skin; lesions limited to the epidermis with or without a moderate crust; lesions involving the dermis with or without extensive crusting and with little fibrosis and/or granulation tissue; lesions penetrating to the subcutis with intense formation of granulation tissue; ulcer penetration to the bone or ulcer development with periosteal bony proliferation. A Bayesian network (1) modelling the causal relations between pathological shoulder lesions and ante mortem clinical signs was constructed. Furthermore, the body condition score (normal-to-fat vs. thin) was included as a risk factor.

\section{Results}

According to the post mortem examinations, 83 sows (22.9\%) had shoulder lesions. Hereof 76 sows (20.9\%) had mild lesions and 7 sows (1.9\%) had pathological lesions. Among the 76 sows with milder lesions, 39 sows $(51.3 \%)$ had no ante mortem findings, 26 (34.2\%) had skin alterations, 7 (9.2\%) had skin alterations and firm swelling, $2(2.6 \%)$ had skin alterations and adherence and 1 (1.3\%) had firm swelling and adherence. Among the 7 sows with pathological lesions, 1 (14.3\%) had skin alterations and firm swelling and $6(85.7 \%)$ had skin alterations, firm swelling and adherence. The probability of having a pathological lesion (as determined by post mortem examination) was calculated in the Bayesian model for all clinical signs, recorded ante mortem: Skin alteration $=11.7 \%$; firm swelling $=21.8 \%$; skin alteration + firm swelling $=71.1 \%$. The probability of a shoulder lesion being pathological increased from $35.5 \%$ to $81.7 \%$ when increasing the width of the swelling from $30-35 \mathrm{~mm}$ to $60-65$ $\mathrm{mm}$. Including Adherence resulted in a very high probability of the shoulder lesion being pathological (96.7\%). Finally, when known state of the body condition was added to the evidence of a severe shoulder lesion the probability of having a pathological lesion by post mortem examination increased from $96.5 \%$ (normal-to-fat) to $98.4 \%$ (thin).

\section{Discussion}

Based on calculations in the Bayesian network, the best predictor of pathological shoulder lesions were; Firm swellings with a fair extent ( $>50 \mathrm{~mm}$ in width) and adherence of lesions to the underlying bony foundation. In summary, the condition of the skin (crust/scar) in itself was of little value if not compared with the palpable findings in the tissue underneath, where especially firm larger swellings and adherence to the underlying bony foundation (TSS) were the most useful markers of pathological lesions. All the milder shoulder lesions were considerably smaller in extent than the pathological lesions, suggesting that pathological shoulder lesions may be differentiated from milder shoulder lesions by the size of the swelling involved.

\section{References}

1. Jensen, F.V., 1996. UCL Press, London. pp. 3 -74 


\title{
P.856
}

\section{Rubber mats in farrowing pens as prevention of sow shoulder lesions}

\author{
Lisbeth B. Petersen; Mai Britt F. Nielsen \\ Pig Research Centre, Danish Agricultural and Food Council, Copenhagen, Denmark
}

\section{Introduction}

Shoulder lesions in sows are a common disorder in most countries with intensive pig production. It has been shown that rubber mats have a positive therapeutic effect compared with fully slatted floors in farrowing pens (1) (2). It has therefore been assumed that mats also are a way of preventing shoulder lesions from developing. The objective of this trial was to determine if rubber mats under the sow in farrowing pens were better than solid concrete floor in terms of prevention of shoulder lesions.

\section{Material and methods}

The trial was carried out in two herds with crossbred sows (LY): herd A with 1700 sows and herd B with 800 sows. The farrowing pens had crates, liquid feed and partly slatted floor. The floor in the main part of the pen was made of solid concrete. The back of the pen had slatted floor (cast iron). The sows were placed in the pens app. one week prior to farrowing and remained there until weaning of the piglets 4-5 weeks after farrowing.Three groups were compared: Group 1: solid concrete floor (control). Group 2: rubber mat, solid, thickness: $17 \mathrm{~mm}$. Group 3: rubber mat with a core of foam, thickness: $45 \mathrm{~mm}$ (centre), $17 \mathrm{~mm}$ (edges).

Mats in groups 2 and 3 were placed on the solid floor under the sow during her entire stay in the farrowing pen.The effects of the flooring systems were compared with respect to shoulder lesions at weaning, body condition score (BCS) and scar tissue on the sow's shoulder when entering the farrowing compartment.

\section{Results}

A total of 888 sows were included in the trial. The over-all prevalence of shoulder lesions was 16.3 percent in herd A and 15.2 percent in herd B. Sows with poor BCS had a higher prevalence of shoulder lesions compared with sows in good body condition $(p=0.07$ in herd A and $p=0.02$ in herd B). Sows with scar tissue had a higher prevalence of shoulder lesions than sows without scar tissue $(p<0.0001$ in herd $A$ and $p=0.002$ in herd B). Overall, 681 sows were regarded as "sows not at risk", i.e. good BCS and no scar tissue. On average, only $10 \%$ of these sows developed shoulder lesions. The combina-tion of low BCS and scar tissue (207 sows) resulted in a prevalence of $35 \%$. The effect of soft floor on the preva-lence of shoulder lesions was significant in herd B, but not in A. However, the effect in herd B was primarily related to sows at risk (table 2 ).

\section{Discussion}

Rubber mats in farrowing pens was effective in one of two trial herds in respect to prevention of shoulder le-sions. Only a minority of sows that are not at risk of developing a shoulder lesion, do in fact develop a lesion. In contrast, app. one third of sows at risk develop a lesion. Therefore, rubber mats are primarily relevant for sows at risk.

\section{References}

(1) Zurbrigg, K., 2006. Sow shoulder lesions: Risk factors and treatment effects on an Ontario farm. J. Anim. Sci., 84: 2509-2514.

(2) Kaiser, M. et al., 2007. [The effect of rubber mats on sow shoulder lesions]. Gummimåtters effekt på skuldersår. Danish Pig Production, Report no. 783.

Table 1: Percentage of sows that develop a shoulder le-sion. Herd A. () = cases out of total no. of sows.

\begin{tabular}{|l|c|c|c|c|}
\hline & Group 1 & Group 2 & Group 3 & p-value \\
\hline Sows at risk & $53 \mathrm{a}(18 / 34)$ & $44 \mathrm{a}(15 / 34)$ & $29 \mathrm{a}(8 / 28)$ & NS \\
\hline Sows not at risk & $13 \mathrm{~b}(19 / 146)$ & $11 \mathrm{~b}(16 / 149)$ & $8 \mathrm{~b}(11 / 142)$ & NS \\
\hline & & & & \\
\hline
\end{tabular}

Table 2: Percentage of sows that develop a shoulder lesion. Herd B. () = cases out of total no. of sows.

\begin{tabular}{|l|c|c|c|c|}
\hline & Group 1 & Group 2 & Group 3 & p-value \\
\hline Sows at risk & $47 a(16 / 34)$ & 32a (13/40) & $8 \mathrm{~b}(3 / 37)$ & $\mathrm{P}<0,05$ \\
\hline Sows not at risk & $15 \mathrm{c}(13 / 84)$ & $10 \mathrm{c}(8 / 78)$ & $\operatorname{0d}(0 / 82)$ & $\mathrm{P}<0,05$ \\
\hline
\end{tabular}




\title{
P.857
}

\section{Pathological and haematological findings in tail biters, bitten finishing pigs and their controls}

\author{
Camilla Munsterhjelm², 3 Pälvi Palander ${ }^{3}$ Emma Brunberg ${ }^{2}$ Hanna-Kaisa Sihvo ${ }^{3}$ Anna Valros ${ }^{3}$ Linda Keeling $^{2}$ Mari Heinonen $^{1}$ \\ 1. University of Helsinki, Faculty of Veterinary Medicine, Saarentaus, Finland; 2. Swedish University of Agricultural Sciences, Uppsala, Sweden; \\ 3. University of Helsinki, Faculty of Veterinary Medicine, Helsinki, Finland
}

\section{Introduction}

Tail biting continues to be a well-known and important problem in pig production. It is associated with considerable economic losses to the whole industry and decreased animal welfare. It has been found to be associated with carcass condemnations due to abscesses. Tail biting leads to the spread of infection and activation of the acute phase response (1). This study was carried out in order to describe pathological and haematological findings in tail biters, victims and control pigs.

\section{Materials and Methods}

Tail biting and control pens were identified with direct observation in a finishing herd with an ongoing problem with tail biting. Quartets of age and gender matched individuals were chosen: tail biter (TB, $n=16)$, victim $(V, n=16)$, a control pig in the pen with tail biting ( $\mathrm{Ctb}, \mathrm{N}=9$ ) or control pig in pen without tail biting $(C, n=14)$. Pigs were injected intramuscularly with midazolam, ketamine and butorphanol, after which a blood sample was collected from the vena cava cranialis while the animal was in dorsal recumbency. Immediately afterwards pigs were euthanized with an intracardial injection of pentobarbital and taken to autopsy, which was performed the next day. The pathological findings were analysed pair-wise using McNemar's test for binomial and Wilcoxon rank sum test for continuous variables. Haematological values were analysed using a mixed model.

\section{Results}

The general trend seemed to be that pigs in all groups, except the controls in pens with no tail biting, had several pathological findings. The haematological values did not differ between groups (Table 1).

\begin{tabular}{|c|c|c|c|}
\hline & TB & $\mathbf{V}$ & $\mathbf{C t b}+\mathbf{C}$ \\
\hline $\mathbf{W B C}, \mathbf{x ~ 1 0 ^ { 9 }} / \mathbf{I}$ & $19.5+5.8$ & $23.3+6.9$ & $21.4+5.6$ \\
\hline $\mathbf{R B C}, \mathbf{x} \mathbf{1 0}^{12} / \mathbf{I}$ & $6.4+0.8$ & $6.4+0.7$ & $6.3+0.5$ \\
\hline HCT & $33.9+3.9$ & $33.8+4.0$ & $33.5+2.1$ \\
\hline HB, $\mathbf{g} / \mathbf{I}$ & $104.7+11.4$ & $104.0+13.1$ & $103.1+7.2$ \\
\hline
\end{tabular}

TB tended to be heavier than Ctb $(43.7 \pm 3.6$ vs $42.4 \pm 4.9 \mathrm{~kg}$, $\mathrm{p}=0.09)$. More $\mathrm{V}$ tended to have changes in the stomach wall (ulcers or mild lesions) than TB ( 38 vs $6 \%$ of the animals, $p=0.06$ ). A score classifying the severity of joint lesions (cartilage and joint capsule) tended to be greater in both TB $(0.5+0.3)$ and $\mathrm{V}(0.3+0.2)$ pigs compared to $\mathrm{C}(0.0+0.0 ; \mathrm{p}=0.1)$ pigs. The $\mathrm{Ctb}$ $(0.4+0.3)$ pigs had a high level of joint lesions even if this difference was not significant. Infections in respiratory organs were more frequent in $\mathrm{V}$ than $\mathrm{Ctb}$ ( 63 vs $13 \%$ of individuals, $\mathrm{p}=0.03$ ). Although scores of acute tail lesions ( $0=$ healthy to $3=$ severe) were significantly worse in $\mathrm{V}$ (median (range) 3(1-3)) than TB $(1(1-3), p=0.01)$ these scores were not significantly lower in Ctb than $\mathrm{V}$ despite the fact that $\mathrm{Ctb}$ received significantly fewer bites to the tail during the behavioural observations than $\mathrm{V}$ (bites received $\mathrm{Ctb}=0(0-1)$ vs $\mathrm{V}=11(1-31), \mathrm{p}<0.01)$.

\section{Discussion}

The pathological findings were more frequent than anticipated, even considering that this farm had ongoing problem with tail biting. The pigs in tail biting pens seemed to suffer not only from tail biting but also from other health problems (stomach ulcers, joint lesions). However, cause and effect were not separated in this study.

Haematology did not reveal problems in the organs. The variation in haematological values was large and the lack of a difference here may reflect the relatively small sample size in the study. In contrast to our findings, differences in blood values have been found (2), mostly in clinical chemistry. However, the same parameters were not investigated in our study.

\section{References}

1. Heinonen M., Orro T., Kokkonen T., Munsterhjelm C., Peltoniemi O., Valros A.Vet. J. doi: 10.1016/j.tvjl.2009.02.021.

2. Holmgren $N_{\Perp}$, Keeling L., Lundeheim N. 18th IPVS Congress Proceedings, Hamburg 2004, 608. 


\title{
P.858
}

\section{Effect of zinc organic supplementation on feet lameness of sows}

\author{
Annop Suriyasomboon ${ }^{1}$ Nualprae Seanbungkhor ${ }^{2}$ Sutep Luengyotluechakul ${ }^{3}$ Supunnee Jiarakhun ${ }^{3}$ \\ 1. Chulalongkorn University, Bangkok, Thailand; 2. Laemthong Hybrid Co., Ltd., Bangkok, Thailand; 3. Zincpro Co., LTD., Bangkok, Thailand
}

\section{Introduction}

Feet and leg problems of sows were the major economic losses in pig industry. Many lameness sows usually were not seen in herds and underestimated. These lameness sows were affecting on the herd productivity $[1,2,3]$. There were several factors contribute to lameness such as bacterial infections, genetics, management, and nutrition [1, 2, 3, 4]. Nutritional factors may contribute to lameness. The aim of this study was to investigate the effect of zinc supplementation on feet lesions of sows.

\section{Materials and Methods}

Sixty crossbred (LR x LW) sows were divided into two groups, 20 sows in control group (C) and 40 sows in treatment group (T). Sows in $\mathrm{C}$ and $\mathrm{T}$ group were accordingly classified to parity number and location. Group C was gilt (P0) and sow in parity 1 , and located in unit B. Group T was sow in parity 2 or more, and located in unit E. All sows were directly examined for the feet performances during the lactating or gestating periods. The feet lesions were classified into three groups: heel over-growth and erosion (HOE), heel-sole crack (HSC), and white line (WL). The level of lesion scores were classified into three levels: no lesion (0), mild (1), moderate (2), and severe (3). Sows had feet lesions were identified and randomly selected for testing the feed additive supplement, Avila-Zn 120. The selected sows were taken pictures of the feet lesions and examined the lesion scores before giving feed additive. Sows in group $C$ were received conventional diets, and sows in group T were received daily of $40 \mathrm{ppm}$ of Avila-Zn 120 by mixing in the conventional diets for 90 days. Feet performances and feet lesion scores were monitored at 1, 2, and 3 months after Avila-Zn 120 supplementation. The repeated sows and/or culled sows will be examined and taken pictures for checking the feet lesion improvement. Chi-square analysis (PROC FREQ) was used to analyze the frequency distribution of feet lesion scores.

\section{Results and Discussion}

This study was investigated the effect of zinc supplementation on feet lesions of sows. At least one feet lesion was found in sows in both groups. Some sows had more than one feet lesions. In general, gilts and sows in parity 1 had lower feet lesions than sows in parity 2 or more. There was a trend $(P=0.09)$ for higher proportion number of sows with more feet lesions in the $C$ group (i.e. HOE). Moreover, there were lower proportion of sows with HSC in the T group $(\mathrm{P}<0.001)$. This improvement might be explained by the higher bioavailability of zinc in the feed. This is in agreement with a study performed in Danish sows herd [5]. In conclusion, the supplement of zinc organic might be an alternative choice to improve the feet quality of sows.
Table 1. The proportion of sows with different feet lesions.

\begin{tabular}{|c|c|c|c|c|c|c|c|c|c|c|c|c|c|}
\hline \multirow[b]{2}{*}{ Month } & \multirow[b]{2}{*}{ Score* } & \multicolumn{4}{|c|}{$\mathrm{HOE}$} & \multicolumn{4}{|c|}{ HSC } & \multicolumn{4}{|c|}{ WL } \\
\hline & & 0 & 1 & 2 & 3 & 0 & 1 & 2 & 3 & 0 & 1 & 2 & 3 \\
\hline 1 & & 15 & 5 & 0 & 0 & 12 & 5 & 3 & 0 & 13 & 5 & 2 & 0 \\
\hline 2 & & 8 & 9 & 2 & 0 & 8 & 7 & 4 & 0 & 10 & 7 & 2 & 0 \\
\hline 3 & Control & 9 & 6 & 4 & 0 & 5 & 8 & 5 & 1 & 6 & 10 & 3 & 0 \\
\hline 1 & & 10 & 10 & 16 & 4 & 11 & 4 & 17 & 8 & 16 & 7 & 9 & 8 \\
\hline 2 & & 10 & 14 & 11 & 4 & 10 & 15 & 12 & 2 & 23 & 9 & 7 & 0 \\
\hline 3 & Treatment & 14 & 17 & 7 & 1 & 18 & 17 & 4 & 0 & 30 & 8 & 1 & 0 \\
\hline
\end{tabular}

* $0=$ no lesion, $1=$ mild, $2=$ moderate, and $3=$ severe

Figure 1. Feet lesions of control and treatment groups.
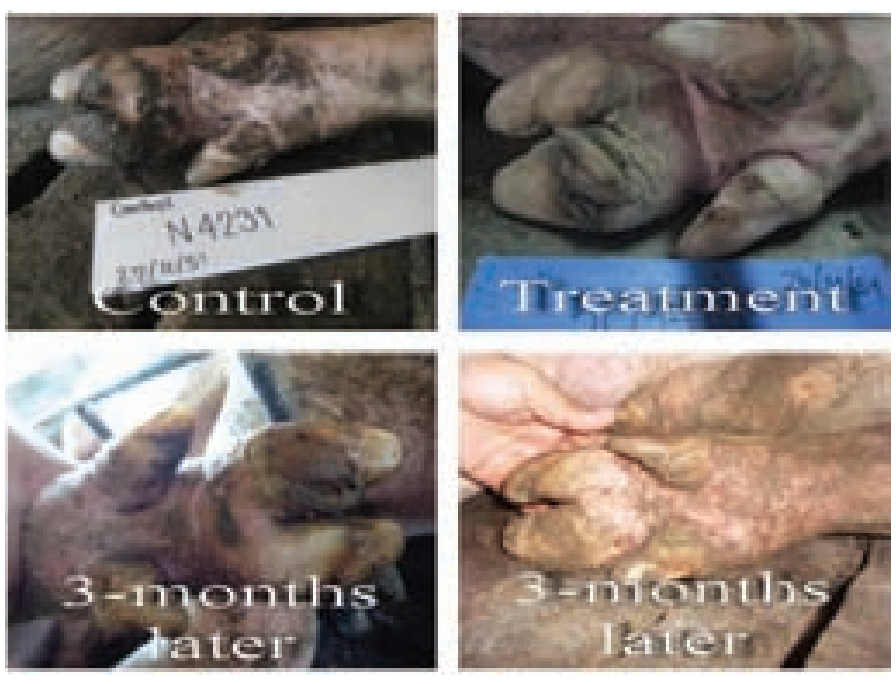

\section{References}

1. The Veterinary Record. 159:383-387.

2. Journal of Animal Sciences. 86:432-441.

3. Journal of the American Veterinary Medical Association. 235:734-738.

4. Journal of Swine Health and Production. 14:202-206.

5. FeetFirst Conference. 


\title{
P.859
}

\section{Use of a subjective scoring system to describe leg and claw conformation and gait in gilts and sows}

\author{
Elisabeth O. Nielsen; Flemming Thorup; Hansen U. Lisbeth \\ Pig Research Centre, Danish Agricultural \& Food Council, Copenhagen, Denmark
}

\section{Introduction}

Lameness and claw lesions are frequent causes of discomfort or pain in gilts and sows (1-3). Lameness in sows reduces productivity levels and the longevity. Describing leg conformation and gait is necessary when selecting gilts to achieve strong breeding animals. Furthermore, descriptions of gait and leg lesions are important to identify individuals in pain or discomfort, indicating a need for treatment and movement to a hospital pen. Despite the inclusion of leg soundness in the Danish breeding index, the producers need an individual evaluation of each gilt to know which gilt to breed and which one to cull. The farmer must also evaluate the leg soundness of the sows before the next breeding cycle.

The purpose of this study was to design a subjective scoring system to describe leg and claw conformation and gait in gilts and sows

\section{Materials and Methods}

The subjective scoring system was based on previous descriptions (4-6) of leg conformation and gait. The scoring system is used in ongoing studies. A training day for standardization is planned every six months throughout the studies. This paper presents scoring results for gilts and sows. A total of 255 gilts aged 9 months and 219 loose-housed gestating sows of different parities were scored. One technician scored gilts in one herd, and another technician scored gestating sows in another herd. Both herds had problems with lameness. All scorings was performed inside the pen. Scoring of one animal was completed in two to four minutes. The animal was evaluated both standing and moving slowly. Animals were not scored moving at high speed, because mild cases of lameness would not be easily seen. The pen floor was non-slip slats or solid floor. The animals' feet were not cleaned before scoring the claws.

\section{Results}

The subjective scoring system includes 12 categories (Table 1). The categories describe different features in claw and leg conformation. Length of claws and dewclaws are described as normal, long and very long. The movement categories (swaggering gait, stride length and lameness) describe increasing abnormality. Lameness ranged from normal $=0$ to "not able to rise" $=3$. The scoring results of 255 gilts and 219 sows are presented in Table 1. A total of $18 \%$ of the gilts were lame to some degree, and $15 \%$ of the sows were lame to some degree. If the animal was lame, it was noted which limb(s) was affected, and acute lesions of the limbs were also noted (data not shown).
Table 1: Leg, claw and gait scoring in gilts and sows

\begin{tabular}{|l|c|c|c|}
\hline & Scores $\mathbf{0}=$ normal & 255 gilts scores & 219 sows scores \\
\hline Fore limbs, front & $0 ; 1 ; 2 ; 3$ & $246 ; 0 ; 8 ; 1$ & $185 ; 0 ; 33 ; 1$ \\
\hline Fore limbs, side & $0 ; 1 ; 2 ; 3$ & $211 ; 0 ; 44 ; 0$ & $137 ; 6 ; 67 ; 9$ \\
\hline Hind limbs, back & $0 ; 1 ; 2$ & $246 ; 1 ; 8$ & $216 ; 1 ; 2$ \\
\hline Hind limbs, side & $0 ; 1 ; 2 ; 3$ & $196 ; 1 ; 56 ; 2$ & $101 ; 1 ; 117 ; 0$ \\
\hline Back & $0 ; 1 ; 2$ & $253 ; 1 ; 1$ & $214 ; 2 ; 3$ \\
\hline Claws even size & $0 ; 1$ & $221 ; 34$ & $191 ; 28$ \\
\hline Claw length & $0 ; 1 ; 2$ & $254 ; 1 ; 0$ & $183 ; 30 ; 6$ \\
\hline Dewclaw length & $0 ; 1 ; 2$ & $255 ; 0 ; 0$ & $140 ; 67 ; 12$ \\
\hline Missing dewclaws & $0 ; 1 ; 2$ & $254 ; 1 ; 0$ & $206 ; 10 ; 3$ \\
\hline Swaggering gait & $0 ; 1 ; 2$ & $238 ; 17 ; 0$ & $200 ; 19 ; 0$ \\
\hline Stride length & $0 ; 1 ; 2$ & $208 ; 43 ; 4$ & $193 ; 23 ; 3$ \\
\hline Lame & $0 ; 1 ; 2 ; 3$ & $216 ; 36 ; 3$ & $190 ; 25 ; 4 ; 0$ \\
\hline
\end{tabular}

\section{Discussion}

A subjective scoring system was chosen, because it is easy to use and no instruments are needed. Although subjective scoring has inherent problems with repeatability $(5,6)$, these problems can be overcome through repeated training of the observers. The scoring system must be simple, with clear definitions and good illustrations. Video sequences of the animals' gait are useful. We plan to correlate the leg, claw and gait scores with the longevity of the gilts and sows. The scoring will then be refined and simplified for practical use in herds.

\section{References}

1. Anil S. et al., JAVMA (2009) $235,734-82$

2. Heinonen M. et al., Vet. Rec. (2006) 159, 383-7

3. Kirk R. et al, J. Vet. Med. (2005) 52, 423-8

4. Jørgensen B., Acta vet. scand. (2000) 41,105-121

5. Main D.et al., Vet. Rec. (2000) $147,574-6$

6. Anil L. et al., IPVS (2008) p615 


\title{
P.860
}

\section{Three-year study of lameness in piglets and piglets mortality at a herd following reparation of floors}

\author{
Mate Zoric ${ }^{1}$ Sigbrit Mattsson ${ }^{1}$ Cecilia Kellerman ${ }^{2}$ Petter Kjellerby ${ }^{2}$ Per Wallgren ${ }^{1}$ \\ 1. National Veterinary Institute, Uppsala, Sweden; 2. Vissgärde Farming, Uppsala, Sweden
}

\section{Introduction}

Many piglets die or are medically treated before they are weaned. The main causes of mortality are emaciation or crushing by the sow when lying down (1). Lameness in suckling piglets is another welfare issue in farrowing enterprises contributing to ill-thrift, piglet mortality, decreased growth and increased antimicrobial use (2). Environment can improve piglet survival (1), and the present study compared the prevalence of lameness and mortality in piglets during three years in farrowing units with different floor surfaces.

\section{Materials \& Methods}

The study was carried out at a herd with four identical farrowing units with solid concrete floor, embedded with $1 \mathrm{~kg}$ chopped straw per sow and $1 \mathrm{hg}$ per piglet and day. Two units were dedicated as controls ( $\mathbf{\square} ; \mathbf{\Delta})$. In the third and fourth farrowing units, the floor surfaces were repaired with two-component materials; $\bullet=$ Thorocrete SL ${ }^{\bullet}$ (Växa Halland, Sweden), a powder with cement, graded sand and additives and an acryl polymer emulsion, and; $\bullet=$ Piglet Floor (Flowcrete Sweden AB, Sweden), an epoxy free two-component solvent solution with natural quartz.

All of the 17,474 piglets born alive during three years were studied ( $\mathbf{\square =} 338$ litters \& 4425 piglets; $\boldsymbol{\Delta}=341$ \& 4333; $\bullet=343$ $\& 4525 ; \bullet=323 \& 4191)$. Twenty-three farrowing batches were studied in each unit. Records of mortality and medical treatments for lameness were kept for each piglet until the age of 4 weeks.

\section{Results}

The mortality was defined as crushing and other causes of death. Up to $85 \%$ of the piglet losses occurred during the first week of life and the majority of the deaths were attributed to crushing. There were large differences in the prevalence of piglets found dead on the different types of floor (Fig. 1). Piglet born on the repaired floors had the lowest prevalence of mortality during the three-year study $(\bullet p<0.05 ; \bullet p<0.01)$.

The mortality during the first three days of life was high, but not correlated to lameness. In total, 971 piglets were treated for lameness, corresponding to $5.5 \%$ of the live born piglets. Approximately $85 \%$ of these diagnoses were made during the first three weeks of life. As seen in Fig. 2, the prevalence of lameness was lower in the units with repaired floor $(\bullet p<0.05 ; \bullet p<0.01)$ than in the control units ( $\bullet$ and $\boldsymbol{\Delta}$ ).
Figure 1. Mortality pre-weaning (\%) in four identical farrowing units with different types of floor surface during three years.

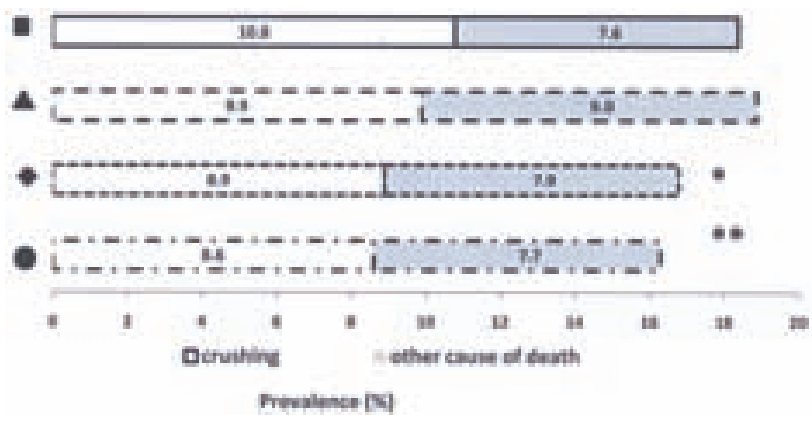

Figure 2. The prevalence of lameness in piglets during the first four weeks of life in identical farrowing units with different types of floor surface during three years.

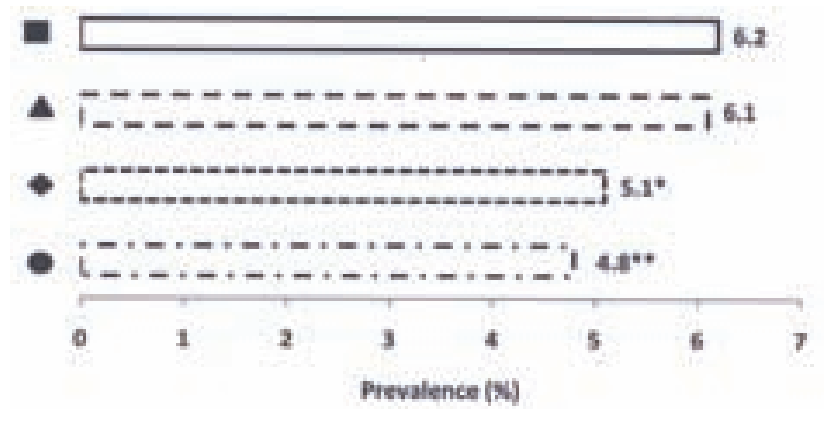

\section{Discussion \& Conclusions}

The mortality was highest during the first week, and to be crushed under the sow when lying down was the most common source of death. Also the frequency of skin lesions was highest during the first week. Lameness is associated to many factors out of which the floor is one (3). Maintaining the surface of concrete floors with these two commercially available solutions decreased the mortality and the incidence of lameness in piglets.Thus, a proper maintenance of the floor will improve roughness and abrasiveness of the floor which in turn can contribute significantly to prevention of lameness and mortality in suckling piglets. The floor can also affect lying down behaviour of the sow (4), and this study revealed that the piglets mortality was lower in the pens with repaired floors. The results suggest that reparations of the floor and sustainability in three years will pay off for both piglets and pig farmers.

\section{References}

1. Christison et al., 1987. Vet Rec. 121:37-41.

2. Zoric et al., 2003. Vet Rec. 153:323-328.

3. Zoric et al., 2009. Acta Vet Scand. 51:23.

4. Damm et al., 2005. App Anim Beh Sci. 90:3-20. 


\title{
Analysis of the healing effect of complexed trace minerals on claw lesions of gestating sows housed in group pens with electronic sow feeders (ESF)
}

\author{
Sukumarannair S. Anil ${ }^{2}$ Leena Anil ${ }^{1}$ John Deen ${ }^{2}$ Sam K. Baidoo ${ }^{1}$ Mark E. Wilson ${ }^{3}$ Christof Rapp ${ }^{4}$ Terry L. Ward ${ }^{3}$ \\ 1. University of Minnesota, Southern Research and Outreach Center, Waseca, MN, USA; 2. University of Minnesota, Veterinary Population \\ Medicine, St Paul, MN, USA; 3. Zinpro Corporation, Eden Prairie, MN, USA; 4. Zinpro Performance Minerals, Boxmeer, Netherlands
}

\section{Introduction}

Claw lesions are reported to be an important underlying cause for lameness in pigs in breeding herds. However, not all claw lesions cause lameness in pigs. The area affected and the severity of lesion may determine the progression towards lameness. Previous studies have indicated the positive relationship of side wall and white line lesions with lameness in pigs 1,2. Housing and nutritional factors are associated with the development of claw lesions in pigs. Trace minerals such as $\mathrm{Cu}, \mathrm{Zn}$ and $\mathrm{Mn}$ are important in the keratinization process3. The bioavailability of trace minerals depends on the quantity and the form (organic or inorganic) of these minerals. Despite the high prevalence of claw lesions in pigs, attempts to minimize the incidence of claw lesions or to reduce the severity of lesions are not implemented widely in swine herds. The objective of the present study was to understand the effect of feeding complexed trace minerals (CTM; $\mathrm{Cu}, \mathrm{Zn}$ and $\mathrm{Mn}$ ) in decreasing the severity of claw lesions in breeding female pigs housed in group pens with ESF in comparison to those fed with trace minerals in inorganic form (ITM).

\section{Material and Methods}

This study included 104 sows of mixed parities (ITM diet, $n=59$ and CTM diet, $n=45$ ). Trace minerals supplemented in the ITM diet were Zn, 125 ppm, Mn, 40 ppm and Cu, 15 ppm, while CTM was a partial substitution of the inorganic minerals with complexed minerals ( $Z n, 50$ ppm, Mn, 20 ppm and Cu, 10 ppm) with isolvels of total trace mineral supplementation. The sows were fed the respective diets for a period of 3 parities. The first claw observation was performed at mid-gestation in the first parity. The claws of these sows were re-examined by the same person at mid-gestation in the subsequent third parity during the study period. Claw examination was facilitated by a mechanical restraint designed for the purpose. Different claw areas (side wall, heel, white line, sole, heel-sole junction and overgrown toes and dew claws) were examined for lesions on a severity scale of 0 (no lesions noted) to 3 (severe) following the Zinpro FeetFirst claw lesion scoring system. The total score for each area was obtained by adding the scores for that area in different claws of front and hind limbs. The proportions of sows showing either no change in lesion scores or a reduction in lesion scores in different claw areas in the CTM and ITM groups were compared using 2-sample proportion test (SAS v 9.1).

\section{Results}

The results indicated a higher proportion $(P<0.05)$ of sows fed CTM (91\%) had either a reduction or similar lesion score for vertical side wall cracks in the second claw examination compared to sows fed ITM (73\%). The proportions of sows with similar or lower scores for total medial claw lesions, heel lesions and total side wall lesions were also higher in the sows fed CTM, though not statistically significant.

\section{Discussion}

The present results are similar to a previous trial which indicated a negative relationship between complexed trace mineral supplementation and number of lesions in different claw areas4. However, this study had not considered the severity of lesions. The previous study showed that sows receiving inorganic trace minerals had more lesions on the hind limbs than the sows receiving complexed trace minerals4. The same study also showed that sows consuming inorganic trace minerals had more lesions on the sole than sows fed with complexed trace minerals. The results of this study indicated a beneficial effect of feeding complexed trace minerals in reducing the severity or preventing the worsening of vertical side wall cracks in breeding female pigs.

\section{References}

1. Anil, S.S., et.al., (2007). Factors associated with claw lesions in gestating sows. Journal of Swine Health and Production 15: 78-83.

2. Anil, L. et al., (2007). Analysis of the association of claw lesions with lameness in breeding sows. J. Anim. Sci. Vol. 85, Suppl. 1/J. Dairy Sci. Vol. 90, Suppl. 1/Poult. Sci. Vol. 86, Suppl. 1

3. Tomlinson, D. J. et al. (2004). Formation of keratins in the bovine claw: Roles of hormones, minerals, and vitamins in functional claw integrity. J. Dairy Sci. 87:7907-809.

4. Anil, S.S. et al. (2009). Evaluation of the supplementation of complexed trace minerals on the number of claw lesions in breeding sows. XII, Australasian Pig Science Association. Cairns, Australia, p. 108. 


\title{
P.862
}

\section{Comparison of the production performance of stall-housed sows receiving complexed trace minerals}

\author{
Sukumarannair S. Anil ${ }^{2}$ Leena Anil' ${ }^{1}$ Sam K. Baidoo' John Deen ${ }^{2}$ Mark E. Wilson ${ }^{3}$ Christof Rapp ${ }^{4}$ Terry L. Ward $^{3}$ \\ 1. University of Minnesota, Southern Research and Outreach Center, Waseca, MN, USA; 2. University of Minnesota, Veterinary Population \\ Medicine, St Paul, MN, USA; 3. Zinpro Corporation, Eden Prairie, MN, USA; 4. Zinpro Performance Minerals, Boxmeer, Netherlands
}

\section{Introduction}

The beneficial role of trace minerals in improving the performance of breeding females has previously been described1,2,3. The bioavailability of trace minerals depends on both the quantity and form (organic or inorganic). Though the mechanism is not well understood, it has been reported that organic minerals are more soluble and may cross the intestinal wall more easily4. The objective of the present study was to compare the production performance of stall-housed sows receiving a supplementation of complexed trace minerals (CTM) with those receiving trace minerals in inorganic form (ITM).

\section{Material and Methods}

This study conducted at the Southern Research and Outreach Center of the University of Minnesota at Waseca involved 229 sows (ITM $n=113$, CTM $n=116$ ) housed in conventional gestation stalls. The sows were allocated randomly to two groups and fed either ITM (inorganic sulphate minerals, Zn, 125 ppm, Mn, 40 ppm and $\mathrm{Cu}, 15 \mathrm{ppm}$ ) or CTM (containing complexed trace minerals as a partial substitution of inorganic minerals: $Z n, 50$ ppm, Mn, 20 ppm and Cu, 10 ppm fed at isolevels of total trace mineral supplementation) diet. The analysis involved 399 parity records (ITM $n=199$, CTM $n=200$ ) of these sows pertaining to 1 or 2 farrowings during the study period. Information on farrowing and weaning performances and lactation feed intake were collected from the PigCHAMP database of the research unit and sow cards and compared using 2 sample t-test (SAS V. 9.1).

\section{Results}

Sows fed CTM had more piglets born alive $(P<0.05)$ compared to sows fed ITM (11.07 and 10.44 piglets per litter, respectively). However, the number of stillborn piglets per litter was higher in the sows fed CTM $(1.0+0.12$ vs. $0.70+0.09)$. Litter birth weight tended $(P<0.07)$ to be higher in the sows fed CTM $(37.45+0.68$ vs. 35.64+ $0.72 \mathrm{lbs})$. Other parameters such as average mummies per litter, wean litter weight, weight of the sow at weaning and average lactation feed intake were similar, regardless of trace mineral form.

\section{Discussion}

A previous study involving supplementation with complexed trace minerals indicated higher litter birth weights in supplemented sows 3 though the number of live born piglets was not different $(P=0.2)$ in control (12.89) and treatment (13.37) groups. A higher number of stillborn piglets per litter in the sows fed CTM observed in the present study could be related to the higher number live born piglets per litter. Similarly, the higher litter birth weight in the sows fed CTM could also be related to higher number of live born piglets.

\section{Conclusion}

This study indicated a beneficial effect of feeding complexed trace minerals in terms of higher number of piglets born alive per litter.

\section{References}

1. Mahan, D. (2005). Feeding the sow and piglet to achieve maximum antioxidant and immunity protection. [Book chapter] Re-defining mineral nutrition. Nottingham University Press, Nottingham, UK: 63-73.

2. Peters, J.C. and Mahan, D.C. (2008). Effects of dietary organic and inorganic trace mineral levels on sow reproductive performances and daily mineral intakes over six parities. J Anim Sci 86:2247-2260.

3. Bradley et al., (2009). The influence of complexed minerals fed to developing maternal gilts through three parities on reproductive and lactation performance. Midwest ASAS meeting, March 16-18. Abstract 219

4 Rompala, R. E. and Halley, J.T. (1995). Explaining the absorption of chelated trace minerals: the Trojan horse of nutrition. Feed Management 46:52. 


\title{
P.863
}

\section{Effect of the supplementation of complexed trace minerals on the healing of claw lesions in two sow herds}

\author{
A. Da Silva; S.S. Anil; J. Deen; R.B. Morrison \\ University of Minnesota, Saint Paul, MN, USA
}

\section{Introduction}

Claw lesions are very common in sows. Claw lesions are reported to be associated with lameness (1), an important reason for premature removal of sows in swine breeding herds. Nutrition may play an important role in the development of claw lesions, since the quality of the claw depends on its physical structure and this is controlled by cellular processes affected by nutritional status (2). The importance of trace minerals in the keratinization process has been reported previously (3). Both the quantity and the form (organic or inorganic) of these minerals are crucial (4). However, the effect of these trace minerals in ameliorating claw lesions in pigs has not been well demonstrated. The objective of this study was to understand the effect of feeding complexed trace minerals ( $\mathrm{Cu}, \mathrm{Zn}$ and $\mathrm{Mn}$ ) in reducing the severity of claw lesions in breeding female pigs.

\section{Materials and Methods}

Data for this study were collected from a convenience sample of 43 stall-housed sows (parities 1 to 6 ) belonging to two sow herds (Herd $A, n=28$ and Herd $B, n=15$ ) which had high prevalence of claw lesions. These sows were scored for lesions in different claw areas (side wall, heel, including overgrown heels, white line, heel-sole junction and overgrown toes and dew claws) twice. The first observation was done in May 2009 while the sows were in the farrowing crates, prior to the start of feeding complexed trace minerals ( $\mathrm{Zn}, 50 \mathrm{ppm}, \mathrm{Mn}, 20 \mathrm{ppm}$ and $\mathrm{Cu}$ at $10 \mathrm{ppm}$ ). The second one was done when these sows were in farrowing crates in their subsequent parity (approximately after 140 days). The claws were examined while the sows were in a recumbent position as there was no mechanical restraint to facilitate lesion scoring. The proportions of sows showing either no change in lesion scores or a reduction in lesion scores in different claw areas were analyzed using one sample proportion test (SAS v 9.1).

Table 1: Comparison of the number of sows showing improvement / no change in lesion scores in different claw areas

\begin{tabular}{|l|c|c|}
\hline \multirow{2}{*}{ Claw area } & Herd $\mathbf{A}(\mathbf{n}=\mathbf{2 8})$ & Herd B ( $=\mathbf{1 5})$ \\
\cline { 2 - 3 } & Improvement / no change & Improvement / no change \\
\hline Toe & $27^{*}$ & $12^{*}$ \\
\hline Dew claw & $22^{*}$ & 10 \\
\hline Heel overgrown erosion & $21^{*}$ & 10 \\
\hline White line & $25^{*}$ & $12^{*}$ \\
\hline Side wall horizintal crack & $25^{*}$ & 9 \\
\hline Side wall vertical crack & $28^{*}$ & $15^{*}$ \\
\hline Total claw lesion & $21^{*}$ & $14^{*}$ \\
\hline * P-value $<0.05$ & $24^{*}$ & 8 \\
\hline
\end{tabular}

\section{Results}

In herd A, the proportions of sows with either similar or reduced severity of lesion scores in all claw areas were higher (Table 1) at the second claw examination $(\mathrm{P}<0.05$ for all). However, in herd $B$, similar results were shown only in the case of overgrown toes, heel-sole junction lesions and vertical and horizontal side wall cracks $(P<0.05)$.

\section{Discussion}

The present results are similar to a previous trial which indicated that sows receiving inorganic trace minerals had more lesions on the hind limbs than the sows receiving complexed trace minerals (5). However, that study did not assess the effect on severity of lesions. The same study also showed that sows consuming inorganic trace minerals had more lesions on the sole than sows fed with complexed trace minerals. The non-significant effects in herd B could be related to the small sample size. All the sows with baseline data could not be evaluated a second time in both farms as there was no mechanical device to facilitate lesion scoring in live animals. The results of this study are indicative of a preventive effect of feeding complexed trace minerals on claw lesions in breeding female pigs.

\section{References}

1. Anil, S.S. et al. 2008. Association between claw lesions and sow lameness. Proc 20th IPVS. 1:p.282.

2. Ossent, P. et al. 1998. Bovine laminitis: the lesions and their pathogenesis. In Practice. 20:415-427.

3. Tomlinson, D. J. et al. 2004. Formation of keratins in the bovine claw: Roles of hormones, minerals, and vitamins in functional claw integrity. J. Dairy Sci. 87:7907-809.

4. Miles, R.D. et al. Relative trace minerals bioavailability. http://www. revistas.ufg.br/index.php/vet/article/viewFile/252/223

5. Anil, S.S. et al. 2009. Evaluation of the supplementation of complexed trace minerals on the number of claw lesions in breeding sows. XII, Australasian Pig Science Association. p108.

\section{Acknowledgements}

The authors thank the assistance of Barry Nienhaus for claw lesion scoring. 


\title{
P.864
}

\section{Melatonin release patterns in pigs kept under different light regimens}

\author{
Arie van Nes ${ }^{1}$ Maaike Pannekoek ${ }^{2,1}$ Inge van Geijlswijk ${ }^{2}$ \\ 1. Department of Farm Animal Health, Utrecht, Netherlands; 2. Veterinary Pharmacy Division, Utrecht, Netherlands
}

\section{Introduction}

Melatonin is a hormone controlling the biorhythm of mammals, birds and fish and might be an indicator for harmony between individuals and their environment. The major 'zeitgeber' for the release of melatonin is environmental light. It synchronizes the melatonin secretion with the 24- hour day/night cycle. Normally, melatonin levels are high in the night and low during the day.

Melatonin is not only synthesized in the pineal gland, it's also synthesized in the gastrointestinal tract, retina, skin, lymphocytes, platelets and bone marrow. Despite this, the level of circulating melatonin is almost totally derived from the pineal gland. Melatonin is not stored in the pineal gland and the liver metabolizes the circulating melatonin rapidly. So levels of melatonin and its metabolite 6-sulfatoxymelatonin in blood plasma and saliva are representative for pineal biosynthetic activity (1).

In Dutch pig husbandry, it is obligatory to have minimally eight hours light per day. Some pig owners have these lighthours divided in several periods per day (multiphase lightregime).

The goal of this study is: Do pigs in multiple phase light regimes have other melatonin release patterns compared to single phase lightregimes? Therefore, we measured the melatonin release pattern in pigs kept under a a single phase light regime to the melatonin release pattern in pigs kept under a multiple phase light regime.

\section{Material and methods}

The pigs kept under a single phase light regime had twelve hours of natural light per day. The other group is kept under a multiple phased light regimen, three periods of three hours of light spread over 24 hours.

Each group of pigs were sampled during a 24 hour period, by a saliva sample taken every hour. Saliva samples are taken because levels of melatonin or its metabolite 6-sulfatoxymelatonin in saliva (or blood plasma) are reflective of pineal biosynthetic activity, since there is no storage of melatonin in the pineal gland and circulating melatonin is rapidly metabolised in the liver.

Before the 24 hour sampling period, the pigs were trained for 3 days by test sampling and animal handling, to prevent stress in the pigs during the experiment.
In the experiment we used animals of about five months old. The animals were kept from the age of about two months under the multiple phase light regime. The pigs kept under the single phase light regimen, were at the time of sampling housed from birth under this light regimen.

Piglets at an age of ten weeks already show a normal regular melatonin release pattern (1).

The samples were collected by having the pigs chew on salivettes $^{\circledR}$ for 30 seconds. A salivette ${ }^{\circledR}$ is a roll-shaped saliva collector made by Sarstedt bv Etten Leur. The samples were stored at a temperature of $4^{\circ} \mathrm{C}$ and centrifuged within 24 hours and then stored at a temperature of $-18^{\circ} \mathrm{C}$. The samples were analyzed by Radio Immuno Assay (RIA) at hospital "Gelderse vallei" in Ede.

\section{Results}

There was no difference in the mean melatonin concentrations of the two different light regimens. The mean melatonin concentration in the single phase group was $12.34 \pm 3.51 \mathrm{pg} / \mathrm{ml}$, the mean melatonin concentration in the multiple phase group was $12.95 \pm 2.48 \mathrm{pg} / \mathrm{ml}$. Mean concentration of melatonin during the light hours in the single phase group was $13.75 \pm 11.08 \mathrm{ng} / \mathrm{ml}$, versus $15.01 \pm 9.95 \mathrm{ng} / \mathrm{ml}$ in the multiple phase group. During the dark phases the mean melatonin level was $11.15 \pm 6.08 \mathrm{ng} /$ $\mathrm{ml}$ versus $11.07 \pm 5.36 \mathrm{ng} / \mathrm{ml}$. All concentrations were not significantly different.

There was no difference in melatonin concentrations during the light hours compared to the dark hours in both groups.

\section{Discussion}

Unfortunately, there was no nocturnal rise of melatonin like we expected to find in the control group, based on found literature $(1,2)$. The curves were not flat but all pigs had a different moment of rise in melatonin concentration. Most peaks were found during daytime, when lights were on, which makes the melatonin concentration significant higher during daytime than during the night. These results are in contrast to melatonin curves found in other studies in pigs $(1,2)$ and found in other animals $(3,4)$ and in humans $(5)$. But other studies found melatonin curves in pigs like we found in our experiment.

\section{References}

1. Anderson. Acta vet. scand. 2001, 42:287-294.

2. Griffith and Minton. J Anim Sci 1992. 70:492-498.

3. Tast et al.,. Animal Reproduction Science 200165 283-290

4. Diekman et al. Domestic Animal Endocrinology 1992 9, 2:161-167 


\title{
P.865
}

\section{The influence of close confinement of pregnant sows on cortisol and acute phase protein concentrations}

\author{
Roman Kolacz; Michal Kulok; Zbigniew Dobrzanski \\ Wroclaw University of Environmental and Life Sciences, Department of Environmental Hygiene and Animal Welfare, Wroclaw, Poland
}

\section{Introduction}

Confinement of pregnant sows to individual gestation stalls can be a major factor inducing stress responses, consequently affecting animal health and causing disorders in reproduction (1). The results of extensive studies carried out on acute phase protein (APP) show a lot of discrepancies. Changes in APP concentrations have been reported as a response to close confinement or hierarching within a group $(3,4)$, commonly known to be stressful for animals $(5,6)$, or a reaction to transport stress $(2,5)$.

The purpose of the present investigation was to study the impact of immobilization of sows during gestation on their condition, measured by cortisol concentrations and APP profile of the animals' blood.

\section{Materials and Methods}

The experiment was carried out on two farms with varied housing systems with respect to animal mobility.

Experimental group (EG). The sows in this group were kept in unbedded individual stalls, with a pen floor area of $1.3 \mathrm{~m}^{2}$, from $\mathrm{d}$ 1 to $d 100$ of gestation. On d 100, the sows were moved to a farrowing stall and kept there for the next 28 days. Getting up and lying down were the only movements possible for the animals.

Control group (CG). The sows in this group were also kept in unbedded pens, from $\mathrm{d} 1$ to $\mathrm{d} 100$ of gestation, but in groups of 10 , with a pen floor area of $2.25 \mathrm{~m}^{2}$ per animal. On d 100 the sows were also moved to a farrowing stall, where they had comparatively reasonable space for exercising. Blood samples were collected 4 times in each group: 1) on d 30 of gestation, 2) on $\mathrm{d} 98$ ( $36 \mathrm{~h}$ before moving the animals to the farrowing stalls and 3 ) on $\mathrm{d} 100$ after mating ( $12 \mathrm{~h}$ after moving the animals to the farrowing stalls).

The laboratory measurements included:

a) cortisol in the serum determined using ELISA kits (Diagnostic Systems Laboratories, Inc. USA),

b) acute phase protein profile; haptoglobin, amyloid $A$ and C-reactive protein, determined using ELISA kits (Tridelta Ltd. Ireland).

Both the cortisol concentrations and APP profile were determined using a Bio Tek, Power Wave analyzer.

\section{Results and Discussion}

The results obtained in the study showed that keeping the sows in individual pens resulted in stress responses manifested by increased cortisol concentrations only in the first month of gestation. In the next months, the cortisol concentrations were higher in the sows kept in groups, which was likely due to more frequent hierarchy conflicts (Table1).

Table 1. Cortisol concentrations of the sows' serum [ng/ml]

\begin{tabular}{|l|c|c|c|c|c|}
\hline \multirow{2}{*}{ Group } & \multirow{2}{*}{$\mathbf{n}$} & & \multicolumn{3}{|c|}{ Days of sample collection } \\
\cline { 4 - 6 } & & & $\mathbf{3 0}$ & $\mathbf{9 8}$ & $\mathbf{1 0 0}$ \\
\hline EG & 10 & $\mathrm{x}$ & 29,85 (A 1.4) & 20,07 (a 1.2) & 29,65 ( A 2.3) \\
\hline & & SD & 5.001 & 2.200 & 4.398 \\
\hline CG & 10 & $x$ & $20,05\left(\mathrm{~A}^{*}\right)$ & $23,18(\mathrm{a} 2)$ & $38,62(\mathrm{~A} 1,2,3)$ \\
\hline & & SD & 2.893 & 2.423 & 4.497 \\
\hline & & & & & \\
\hline
\end{tabular}

The values in the same column designated with the same capital letters show significant differences at $P \leq 0.01$. The values designated with small letters show significant differences at $P \leq 0.05$. The values in the same rows, designated with the same numbers, show significant differences at $P \leq 0.01$, or * $P$ $\leq 0.05$. Besides, the results showed that moving the sows from pig barns to the farrowing stalls (d 100 of gestation) induced a rapid elevation of cortisol concentrations (Table1). For the entire gestation period, haptoglobin concentrations in the two groups ranged from 1.1 to $1.6 \mathrm{mg} / \mathrm{ml}$; c-reactive protein ranged from 35 to $37 \mathrm{mg} / \mathrm{ml}$; while serum amyloid A (SAA) was found within the ranges from 21 to $25 \mathrm{mg} / \mathrm{ml}$. No significant differences in APP concentrations between the two groups and in consecutive months of gestation were observed.

\section{References}

1. Broom DM.; Pig News Inf 1996, 17, 109-114.

2. Kołacz et al.; Proc. ISAH, Mexico, 2003

3. Petersen et al.; Appl. Anim. Behav. Sci. 1989, 23, 223-236.

4. Pineiro et al. ;Vet J. $2007,173(3): 669-74$.

5. Swanson 1995, Swanson J.C.; J. Anim. Sci. 1995, 73, 2744-2751.

6. Salak-Johnson et al.; J. Anim Sci. 2007, 85, 81-88 


\title{
P.866
}

\section{Oral interferon-alpha treatment and environmental adaptation of swine}

\author{
Elisabetta Razzuoli; Silvia Dotti; Riccardo Villa; Enrico Sossi; Paolo Candotti; Massimo Amadori \\ Istituto Zooprofilattico Sperimentale Lombardia Emilia Romagna, Brescia, Italy
}

\begin{abstract}
Introduction
Weaning is a critical phase for piglets, in which infectious and non-infectious stressors exert their negative effects. Farmers are prone, for different causes, to weaning 3-weeks old piglets, even though the above age does not seem appropriate with regard to inadequate immune system development and intestinal mucosal functions $(1,2)$. The aim of this study was to investigate the effects of an oral IFN- a treatment on the environmental adaptation response in pigs weaned at 22 days of age.
\end{abstract}

\section{Materials and Methods}

The study was carried out on 40, Landrace $x$ Large White, randomly selected piglets of four litters. Groups 1 and 2 were formed by 20 piglets each; all animals were weaned at 22 days of age: group 2 animals were orally treated with human interferon alpha at low-dose (1 IU Kg b.w./day) in drinking water for the first 10 days after weaning. Also, all piglets were given the same starter feed containing amoxicillin (400 ppm), colistin (120 ppm) and zinc oxide (2000 ppm) over the first 10 days after weaning. Pigs were clinically inspected, weighed, and blood samples in vacuum tubes without anti-coagulant were collected at days 0 , $+6,+12$ and +26 with respect to weaning, in order to investigate the most common viral infections and IFN- $a$, IL-6, TNF- $a$. Viral infections were evaluated by PCR and antibody assays whereas cytokines were investigated by bio-assays. In particular, IFN- $a$ was measured in serum samples by a CPE inhibition assay on MDBK cells (3); TNF- $a$ and IL-6 were determined by a cytotoxicity assay on WEHI 164 cells (4) and a proliferation test on 7TD1 cells (5), respectively. TNF- $a$ and IL-6 concentrations ( $\mathrm{pg} / \mathrm{ml})$ are shown hereunder as $75 \%$ group percentile values.

\section{Results}

All pigs remained healthy during the whole period of observation. In particular, there was no detectable clinical difference under satisfactory hygiene and welfare conditions for all the pigs under study. No animals were infected by the usual viral agents of swine (PRRSV, SIV, PCV2, PRV) in the first 2 weeks after weaning. The period from 22 to 48 days of age was divided into 3 phases (22-28, 28-34, 34-48 days). In the first one, weight gain was not significantly different between the two groups; in the second one, the daily mean weight gain of group 1 was greater than that of group 2, and vice versa in the 3rd period (Table 1). On the whole, IFN- a treated piglets showed on average $1.5 \mathrm{Kg}$ more at 48 days of age $(P<0.001)$. All piglets showed an IL- 6 response between day 0 and +6 with respect to weaning, then the levels decreased until day +26 . The trend of TNF- a was opposite to that of IL- 6 , the highest concentrations being revealed at day +26 . At this time point, all pigs of group 1 and $65 \%$ of group 2 showed high serum concentrations of this cytokine; moreover concentrations were much higher in group 1 (12050 pg/ml) compared with group $2(2613 \mathrm{pg} / \mathrm{ml})$. The sera of all piglets in group 1 and of 18 animals in group 2 were IFN - a positive at day 6 after weaning. This result was confirmed by a neutralization assay of IFN- $\mathrm{a}$ on MDBK cells by a monoclonal antibody to porcine IFN- $a 1$.

Table 1: Daily mean weight gains (grams) are shown as mean $\pm S D$

\begin{tabular}{|l|c|c|c|}
\hline Animals & $\mathbf{2 2 - 2 8}$ days & $\mathbf{2 1 - 3 4}$ days & 34-48 days \\
\hline Group 1 & $239 \pm 123 \mathrm{~g}$ & $171 \pm 103 \mathrm{~g}$ & $361 \pm 99 \mathrm{~g}$ \\
\hline Group 2 & $246 \pm 72 \mathrm{~g}$ & $123 \pm 94 \mathrm{~g}$ & $404 \pm 105 \mathrm{~g}$ \\
\hline & & & \\
\hline
\end{tabular}

\section{Discussion}

Our results indicate that an oral, low-dose IFN-alpha treatment can affect the adaptation response of piglets to the weaning stress. In fact, the treatment determined a better growth till day 26 after weaning, probably related to a lesser TNF- a response. As in SPF pigs, oral administration of IFN-alpha is likely to reduce the IFN-gamma response at weaning and to control the related detrimental effects in terms of intestinal permeability in the post-weaning phase (2). These findings highlight the importance of type I IFNs as homeostatic agents in the response to environmental stressors.

\section{Acknoweledgements}

This study was supported by the Italian Ministry of Health, grant PRC2005002.

\section{References}

1. Pié $S$ et al. 2004. J Nutr, 134:641-647.

2. Amadori $M$ et al. 2009. J. Interferon Cytokine Res, 29: 241-247.

3. Meager A 1987. In: Clemens MJ, Morris AG, Gearing, AJH, eds. Lymphokines and interferons, a practical approach, 129-147.

4. Asai T et al. 1993. Vet Immunol Immunopathol, 38: 253-260.

5. Grenett HE et al. 1991. Gene, 101: 267-271. 


\title{
P.867
}

\section{Evaluation of the pig welfare and its association with overcrowding}

\author{
Martina Tarantola ${ }^{1}$ Laura Tomassone $^{1}$ Igor Ferenaz ${ }^{1}$ Giuseppe Martano $^{2}$ \\ 1. University of Turin, Grugliasco (TO), Italy; 2. ASI TO3, Vigone, Italy
}

\section{Introduction}

Intensive swine breeding shows several critical points that can decrease animal welfare, such as microclimate, food management, mutilation, staff training, structure of buildings.

In the last years, different methods for the evaluation of animal welfare have been studied and developed, mainly based on the observation of the animals and their reactions to stimulus and environmental variations (Smulders et al 2006).

Different behaviour anomalies and injuries have been recognized to be stress indicators (Faucitano et al 2008, Velarde et al 2007). Questions concerning the minimum space requirements for intensively bred pigs in relation to their welfare have been an issue of interest for many decades (Ekkel et al 2003).

The aim of this study was to evaluate pig welfare in farms in the North of Italy, using the method proposed by Candotti et al (2007), and to verify its association with overcrowding.

\section{Materials and Methods}

We tested 33 breeding units within two Italian Provinces: 9 farrow to breed herds and 24 fattening herds. The sample was further divided into: A) weaning piglets (28-55 days old) and B) pigs (55-120 days of life). A form containing specific items related to typical abnormal behaviour and presence of injuries was compiled. For each item, the frequency, prevalence and association with overcrowding was recorded (Directives 91/630/ EEC and 2001/93/EC lay down space requirements for pigs)

Finally, a "Global Score" was awarded to the studied herds, using the check list described by Candotti et al (2007), that assigns a score for each animal welfare item.

Higher scores always implied "better" welfare.

\section{Results}

Sample A ( 9 breeding units) included 3 overcrowded breeding units $(33.3 \%$, 95\% Cl: 7.5-70.1).

Injuries against other pigs was significantly more frequent in the overcrowded farms ( $p<0.05$; Fisher Exact Test), while no association between other welfare items and overcrowding was detected. The mean welfare score in group A was $8.11 \pm 2.96$, ranging from 4.4 to 13.4. This score was significantly higher in farms with adequate animal density (t test, $p<0.01$ ), having a mean value of 9.81 versus 4.70 in overcrowded breedings.

Sample B (29 breeding units) included 9 overcrowded farms (31.0\%, 95\% Cl: 15.3-50.8). No association between overcrowding and welfare items was found. The mean welfare score was $3.80 \pm 1.6$, ranging from 1.3 to 7.7. Again, the welfare score was higher in farms with adequate densities (t test, $p<0.001$; mean value: 4.49 versus 2.70 ).

In both groups abnormal behaviours were observed: ear biting (sample A: $78 \%$; sample B: $93 \%$ ); tail biting (sample A: 67\%, sample B: $59 \%$ ); ears lesions (sample A: 78\%, sample B: $76 \%$ ); tails lesions (sample A: 67\%). Moreover, $55 \%$ of the group B units showed aggressive behaviour and 52\% bar biting.

\section{Discussion}

In our survey, a high percentage of anomalous behaviours was detected, indicating poor welfare. This result has a multi-factorial origin, but lack in space is one of the main causes.

The association between poor welfare, measured as a "Global Score", and overcrowding, underlines the importance of adequate room for this animal species.

\section{References}

Smulders D., Verbeke G., Mormede P., Geers R., (2006). "Validation of a behavioural observation tool to assess pig welfare". Physiology \& Behaviour 89. 438-447

Faucitano L., Schaefer A. L. (2008). Welfare of Pigs From Birth to Slaughter. Wageningen Academic Publishers

Velarde A., Geers R., (2007). On farm monitoring of pig welfare. Wageningen Academic Publishers pp 207

Ekkel D., Spoolder H., Hulsegge I., Hopste H, (2003). Lying characteristics as determinants for space requirements in pigs. Applied Animal Behaviour Science 80, 19-30

Candotti P., Rota Nodari S., Tranquillo M., Mattiello S., Verga M., (2007). "A new approach to welfare measurement in weaned pigslets: the behaviour rating scale. Preliminary results." SIPAS, Modena, 317-325. 


\title{
P.868
}

\section{The over-muscled sow syndrome: a new emerging syndrome in a hyperprolific sow herds: Clinical consequences: preliminary observations}

\author{
Thierry Solignac $^{1}$ G-P Martineau ${ }^{2}$ \\ 1. Coopagri Bretagne, Landerneau, France; 2. Ecole Vétérinaire, Toulouse, France
}

\section{Introduction}

We report emergence of a new body building syndrome that we called "Over-muscled sow syndrome" (Solignac, 2009). "Overmuscled sow syndrome" is the indirect consequence of genetic improvement for lean deposition and for hyperprolificacy (Solignac, 2008).

As a syndrome, there are consequences at different levels such as farrowing (Solignac et al., 2010) or prolificay (Solignac and Martineau, 2010).

\section{Materials and Methods}

A follow-up has been performed in 199 litters ( $\geq 15 \mathrm{~TB} /$ litter) from multiparous sows and categorized according to presence or absence of piglets less than $1 \mathrm{~kg} \mathrm{BW}$ (Table 1). These sows belong to 14 herds with different genetic lines.

For each sow, we measured back fat (BF) and back lean (BL) (Noveko AC037L, 3.5 MHtz) at 4 different period starting 3 weeks after farrowing, then 4 weeks after Al, 3 weeks before farrowing, and at the next farrowing (all measures at $\mathrm{P} 2$ site).

Table 1: Litter performances in multiparous sows according to presence of absence of piglets $\leq 1 \mathrm{~kg} \mathrm{BW}$

\begin{tabular}{|l|c|c|}
\hline At birth,piglets $<=1 \mathbf{k g}$ BW/litter & No & Yes \\
\hline No sows & 124 & 75 \\
\hline Total born & 17 & 17.3 \\
\hline Born alive & 15.7 & 16 \\
\hline Stillborn & 1.20 & 1.29 \\
\hline Weaned & 11.4 & 11.5 \\
\hline No piglets $<=1 \mathrm{~kg}$ BW/litter & 0 & 2.47 \\
\hline
\end{tabular}

\section{Results and Discussion}

Results are presented in Figure 1.

We report observations on some litter characteristics to propose, in a second time, some characteristics associated with morphotypes according to BF and BL.

Beside hyperprolificacy, lean deposition is of major importance. However, as $B L$ is not measured on a regular basis, we have only information on Back fat (BF). Therefore, there is no mention of this syndrome even if it is probably present in many countries. In France, during the last 2 years, we have been confronted to different herd problems around farrowing considered from the sow point of view (Solignac et al., 2010) and the litter point of view.

According to the classification used for describe the effect of BF and $B L$ on duration of farrowing (Solignac et al., 2010a), we propose a lecture of some common problems encountered in herds characterized by high level of performances, high technicity, and high management skills (Tables 2 ).
Figure 1: Evolution of $B F(A)$ and $B L(B)$ in 199 sows classified according to presence of piglets less than $1 \mathrm{~kg} B M$

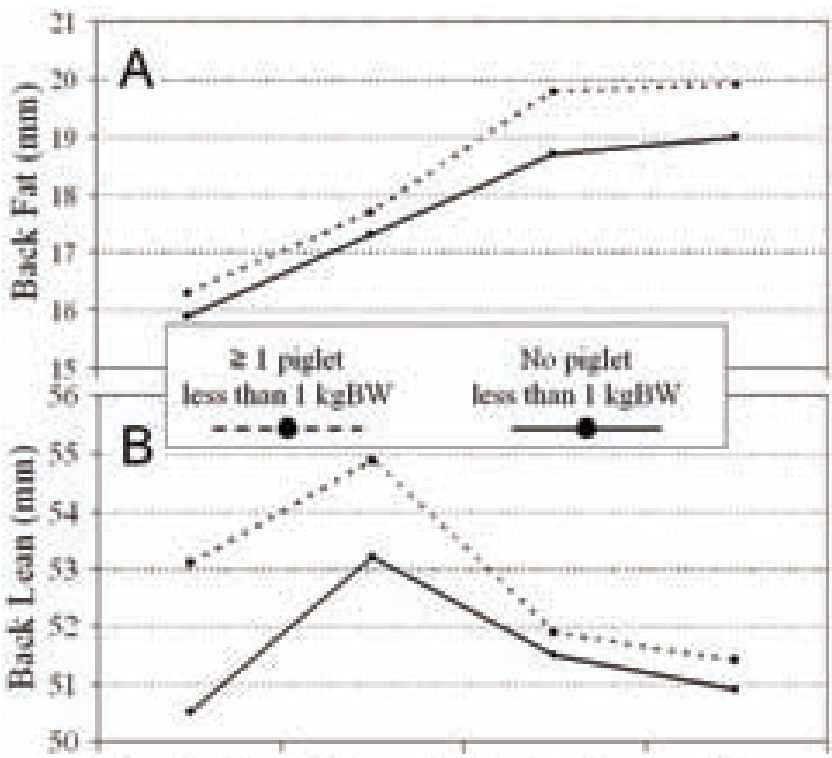

Far +3 weeks Al +4 weeks Far-3wedss Far

Table 2: Morphotypes and some characteristics around farrowing and in lactation

\begin{tabular}{|l|c|c|c|c|c|}
\hline $\begin{array}{l}\text { Morphotypes } \\
\text { at farrowing }\end{array}$ & $\begin{array}{c}\text { Prepartum } \\
\text { catabolism }\end{array}$ & $\begin{array}{c}\text { Duration of } \\
\text { farrowing }\end{array}$ & $\begin{array}{c}\text { Neonatal piglets' } \\
\text { quality }\end{array}$ & $\begin{array}{c}\text { Feed in } \\
\text { lactation }\end{array}$ & $\begin{array}{c}\text { Milk pro- } \\
\text { duction }\end{array}$ \\
\hline BF+/BF+ & No & Increased & Variable & Low & Low \\
\hline BF+/BF- & Moderate & Normal & Good & Normal & Normal \\
\hline BF-/BF+ & Increased & Increased & Bad & Low & Low \\
\hline BF-/BL- & Increased & Increased & Mean & High & High \\
\hline
\end{tabular}

\section{Conclusion}

Simultaneous BF and BL evaluation allows the combination of zootechnical and clinical evaluation of a sow herd. However, it questions the classical approach based on the sow nutritional needs. Therefore, we have to include an objective of BF/BL ratio to reconcile productivity, rusticity, and longevity.

\section{References}

Solignac T, Porc Magazine, 2008, septembre, 140-143

Solignac T et al., Proceedings IPVS 2010

Solignac T and Martineau G-P, Proceedings IPVS 2010 


\title{
P.869
}

\section{The over-muscled sow syndrome: a new emerging syndrome in a hyperprolific sow herds: Effect of back fat and back lean on prolificacy}

\author{
Thierry Solignac $^{1}$ G-P Martineau ${ }^{2}$ \\ 1. Coopagri Bretagne, Landerneau, France; 2. Ecole Vétérinaire, Toulouse, France
}

\section{Introduction}

In another paper, we reported the effect of the "over-muscled sow syndrome" on farrowing duration and on some clinical parameters around farrowing and in lactation (Solignac et al., 2010; Solignac and Martineau, 2010).

The objective is to report observational data on the effect of morphotype on prolificacy in herds with hyperprolific sows.

\section{Materials and Methods}

Litter characteristics were obtained from 491 sows of 14 commercial herds in Brittany (France) with different hyperprolific genetic (see Solignac et al., 2010a). These sows are representative of the sow herds in Coopagri Bretagne (150 herds) (Table 1).

Data were categorized according to littersize : $<13$ TB, between 13 and 15 TB and >15 TB per litter.

For each sow, we measured back fat (BF) and back lean (BL) (Noveko AC037L, 3.5 MHtz) at P2 site at 4 different period starting 3 weeks after farrowing, then 4 weeks after Al, 3 weeks before farrowing, and at the next farrowing.

Table 1: Representativity of the choosen sample of 483 sows from Coopagri Bretagne (Brittany, France)

\begin{tabular}{|l|c|c|}
\hline & Sample of 483 sows & Coopagri $\mathbf{1 1 5}$ herds \\
\hline Mean Total born & 14.35 & 14.3 \\
\hline Mean born alive & 13.46 & 13.1 \\
\hline Mean stillborn & 0.9 & 1 \\
\hline Mean weaned & 11.5 & 11.3 \\
\hline
\end{tabular}

\section{Results and Discussion}

Globally, sows with litter $<13$ piglets were from morphotype $\mathrm{BF}+/ \mathrm{BL}+$ (Figure 1) and sows with litter $>15$ piglets were from morphotype BF-/BL-.

In sows with $>15 \mathrm{~TB}$, a muscular catabolism is starting as soon as mi-gestation until end of gestation

The negative consequences on prolificacy in fat sows are probably an indirect consequence via the decrease of feed intake in lactation.

Therefore, management of lean deposition and fat deposition is of major importance during mi-gestation.

Moreover, the management of lean and fat deposition is of crucial importance as soon as in the gilt development unit.

\section{Conclusion}

Consequences of the "over-muscled sow syndrome" are not limited at farrowing and in lactation. We agree with Foxcroft et al. (2005): "Accepting the risk of being considered some what heretical, most of our recent experiments with the lactating and weaned sow lead to the conclusion "that from a fertility and prolificacy perspective, fatness is simply not the key risk factor".

There is a dynamic of lean deposition, starting at the gilt level. We have to globally consider lean and fat deposition and to reconsider our paradigms regarding sow nutrition taking into account according to is the indirect consequence of genetic improvement for lean deposition and for hyperprolificacy.

\section{References}

Solignac T et al., Proceedings IPVS, 2010

Solignac T et Martineau G-P, IPVS, 2010

Figure 1: Evolution of Back fat (top, A) and back lean (bottom, B) at four different stages starting 3 weeks after farrowing, then 4 weeks after Al, 3 weeks before farrowing, and at the next farrowing

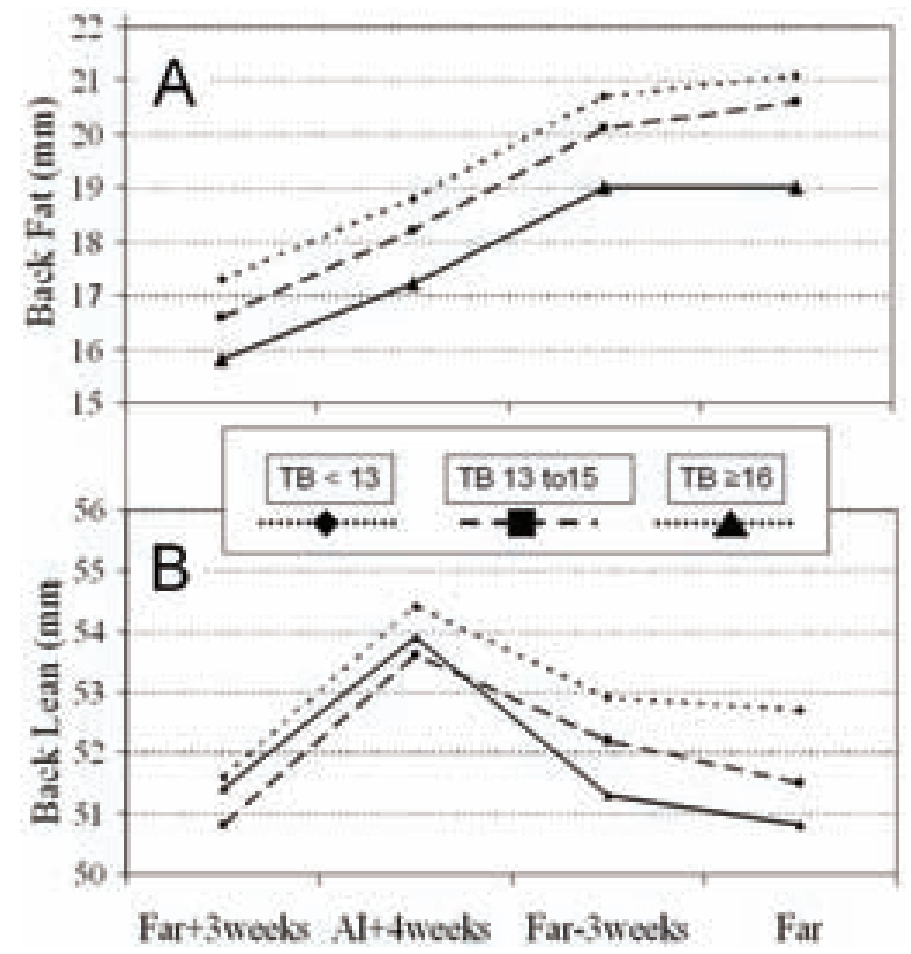




\title{
P.870
}

\section{Bacteriological analysis from milk of sows affected and non-affected by PDS (Postpartum Dysgalactia Syndrome) with special emphasis on Escherichia coli}

\author{
Imke Gerjets ${ }^{1}$ Kerstin Reiners ${ }^{2}$ Nicole Kemper $^{1}$ \\ 1. Institute for Animal Breeding and Husbandry, Christian-Albrechts-University Kiel, Kiel, Germany; \\ 2. PIC Germany GmbH, Schleswig, Germany
}

\section{Introduction}

The Postpartum Dysgalactia Syndrome (PDS) is an important disease in sows after farrowing. Serious economic losses come along with the disease due to reduced performance of the sows and increased mortality of the piglets. Prevalence in herds can be as high as $60 \%$ [1]. The etiology of PDS is multifactorial. Amongst others, management, feeding, hygiene and bacterial pathogens are regarded as influencing factors. This study examines the spectrum of pathogens involved in PDS und analyses risk factors concerning this complex puerperal disease.

\section{Materials and Methods}

Milk samples of 800 sows with PDS and 800 non-infected sows of different age were taken on four piglet rearing and fattening units. Sows were identified as PDS-affected when the measured rectal temperature was above $39.5^{\circ} \mathrm{C}$. Additional criteria like the clinical appearance of the mammary gland and the behavior of the piglets were considered. Bacteria involved in the pathogenesis of the MMA syndrome were identified by advanced bacteriological analysis including molecular methods like PCR. The isolated Escherichia coli were analyzed for various virulence genes with two multiplex PCRs [2].

\section{Results}

A wide spectrum of pathogens was isolated, belonging mainly to Enterobacteriaceae, Staphylococcaceae, Streptococcaceae and Enterococcaceae. Escherichia coli played the most important role with isolation rates over $70 \%$ in both PDS-affected and non-affected sows. With regard to the virulence genes, significant differences between strains from affected and non-affected sows were assessed for the virulence genes iron, kpsMTII, fimC and traT.

\section{Discussion}

Concerning the analysed bacteria spectrum, all species have been isolated in porcine mastitis before [3]. Coliform bacteria were found more often than the others pathogens. In particular, Escherichia coli represented the major part of all isolated bacteria. These results are in accordance with several other investigations, showing that these pathogens are one causing agent in the etiology of PDS $[3,4]$. Concerning the significant virulence factors detected in Escherichia coli, iroN and fimC are associated with urinary tract infections in humans [5]. Escherichia coli isolated from bovine mastitis possessed the gene traT [6], indicating a possible effect of this virulence factor.

Bacterial, environmental and animal factors may change the susceptibility for PDS. These factors are interdependent and the relative influence of each factor is related to the type of pathogens. Therefore, more comprehensive studies on virulence patterns are needed. However, the equal bacterial spectrum in diseased and healthy sows stresses the importance of the individual resistance of the single sow.

\section{References}

1. Bäckström L. et al., 1984. JAVMA, 185(1): 70-73.

2. Ewers C. et al., 2007. IJMM, 297: 163-176.

3. Awad Masalmeh M. et al., 1990. Tierärztl. Umschau, 45:526-535.

4. Hirsch A.C. et al., 2003. J. Vet. Pharmacol. Ther., 26(5): 355-360.

5. Wiles T. et al., 2008: Exp. Molecular Path., 85: 11-19.

6. Kaipainen T. et al., 2002. Vet. Microbiol., 85: 37-46.

This study is part of the FUGATO-plus-project 'geMMA', funded by the Federal Ministry of Education and Research (BMBF), Germany. (www.gemma-kiel.de). 


\title{
P.871
}

\section{The effect of air temperatures in farrowing house for sows on occurrence of stillborn piglets}

\author{
Svetlana Odehnalová1 Jiří Odehnal ${ }^{1}$ Marek Žižlavský Linda Czanderlová1 Pavel Novák² Josef Drábek² Antonín Vinkler² \\ 1. SEVARON s.r.o., Brno, Czech Republic; 2. University of Veterinary and Pharmaceutical Sciences, Brno, Czech Republic
}

\section{Introduction}

Of the piglets classified as stillborn, $70-75 \%$ on average die during the delivery, the rest of them die shortly after the parturition. In about $30 \%$ cases, pathogenic agents are identified as a cause of stillbirth (Vanroose et al., 2000), $70 \%$ stillbirths are caused by various factors such as litter size, age and condition of the sow, birth weight of the piglet as well as internal temperature in the farrowing house. The purpose of this study was to analyse the influence of different air temperature of housing environment in the farrowing house for sows on the occurrence of stillborn piglets in purebred Landrace sows and gilts.

\section{Materials and Methods}

Raw data were analysed for 236 litters during 14 month period. Pregnant sows were moved to farrowing pens cca 14 days before parturition. Sows were fed two times per day and quality of feed was not changed during the trial. Sows were not fed on the farrowing day. The air temperature was measured at hourly intervals by Datalogger (COMET), kept in the farrowing house in the sow living zone. Sow living zone is characterized by height of sow head ( $35 \mathrm{~cm}$ above floor). Temperature was measured every hour. From the values measured, average daily air temperature was calculated. The internal air temperature values were divided into the following intervals: optimal $16-23^{\circ} \mathrm{C}$, elevated 23,1 $-27,9^{\circ} \mathrm{C}$, and high above $28^{\circ} \mathrm{C}$, for the lactating sow. Reproduction parameters were evaluated with regard to average internal air temperature on the day of delivery - total number of piglets born, number of live born piglets, and number of stillbirths. The statistical evaluation of data observed was made by STATISTICA. CZ software; method of ANOVA was used.

\section{Results}

The highest numbers of stillborn piglets occurred in the deliveries that took place under high room temperatures $(p<0,01)$; the lowest one was found in those taking place under the optimal room temperature. If the numerical values were converted into percentage values there were $18.3 \%$ stillbirths at high stable temperatures, $11.6 \%$ stillbirths at elevated temperatures, and $6.9 \%$ stillbirths at the optimal temperature, out of total number of piglets born.

Evaluation of the influence of average daily temperature in the parturition day on the piglet looses is mentioned in table.1.

\begin{tabular}{|l|c|c|c|}
\hline Internal air temperature & High above $\mathbf{2 8}^{\circ} \mathbf{C}$ & Elevated 23,1-27,9 & Optimal $\mathbf{1 6 - 2 2 ^ { \circ } \mathbf { C }}$ \\
\hline Total born [pcs] & 9,46 & 9,52 & 10,00 \\
\hline Live born [pcs] & 7,73 & 8,42 & 9,31 \\
\hline Stillborn [pcs] & 1,73 & 1,10 & 0,69 \\
\hline Stillborn [\%] & 18,3 & 11,6 & 6,9 \\
\hline
\end{tabular}

\section{Discussion}

Real impact of high air temperatures of housing environment on the occurrence of stillborn piglets was improved by observing room temperatures in time of farrowing. By comparing these data with English and Edwards (1996) suggested an acceptable occurrence of stillbirth of 4-8\% out of total number of piglets born; this level was not exceeded only in the case of deliveries running at the optimal room temperature for lactating sows. Already by elevated temperature for lactating sows $\left(23,1^{\circ} \mathrm{C}-\right.$ $27,9^{\circ} \mathrm{C}$ ) was ratio for stillborn piglets double $(11,66 \%)$ and by high temperature nearly triple (18,3\%).

Elevated room temperatures exert stress on the parturient sow. Adrenalin, excreted in the alarm phase, causes vasoconstriction of vessels in the smooth muscle via the postsynaptic a1-receptors, consequently also in the uterine smooth muscle (Ruckebusch et al., 1991). Vasoconstriction prevents oxytocin from performing its uterotonic effect. Consequently, the parturition is prolonged, and the secondary uterine hypocontractility develops.

\section{Acknowledgement}

The study was supported from the project VZ MSM 6215712403 from Ministry of Education, Youth and Sports of the Czech Republic.

\section{References}

Vanroose, G. et al.(2000): Embryonic mortality and embryo-pathogen interactions. Anim. Reprod. Sci. 60-61, 131-143.

English, P.R., Edwards, S.A. (1996): Management of the nursing sow and her liter. In: Dunkin, A.C., Taverner, M. (Eds. Pig Production. World Animal Science, Vol. C10. Elsevier, Amsterdam. 113-140.

Ruckebusch, Y. et al. (1991): Physiology of small and Large Animals. B.C. Decker Inc. Philadelphia. 401. 


\title{
P.872
}

\section{The effect of air temperatures in farrowing house for sows on occurrence of puerperal complications}

\author{
Svetlana Odehnalová ${ }^{1,2}$ Jiří Odehnal ${ }^{1}$ Marek Žižlavský ${ }^{1}$ Linda Czanderlová1 Antonín Vinkler² Pavel Novák² Josef Drábek ${ }^{2}$ \\ 1. SEVARON s.r.o., Brno, Czech Republic; 2. University of Veterinary and Pharmaceutical Sciences, Brno, Czech Republic
}

\section{Introduction}

In recent years was mentioned big improvement in production parameters of sows - number of produced pigs per sow per year (Whittemore, 2006). Nowadays epidemiologic data about occurrence of postpartal dysgalactic syndrome and existing possibilities of risk factors related to management and feeding practice are narrow (Papadopoulos et al., 2009). The aim of this study was to analyse the influence of different air temperature of housing environment in the farrowing house for sows and consequent changes in purebred Landrace dams.

\section{Materials and Methods}

The observations were made in a commercial pig farm with during 14 month period. The external air temperature was measured by Datalogger (COMET), located in the farm premises. Temperature was measured at hourly intervals. Average daily temperature was calculated from 24 values measured. The period of mild winter (MW) was characterized by temperatures $-10^{\circ} \mathrm{C}$ to $0^{\circ} \mathrm{C}$, spring - autumn $(\mathrm{SA}) 0^{\circ} \mathrm{C}$ to $10^{\circ} \mathrm{C}$, mild summer (MS) $10^{\circ} \mathrm{C}$ to $20^{\circ} \mathrm{C}$, and hot summer (HS) over $20^{\circ} \mathrm{C}$. The internal air temperature was also measured by Datalogger (COMET), kept in the farrowing house in the sow living zone $(35 \mathrm{~cm}$ above floor). Temperature was measured every hour. There were chosen groups of 12 sows farrowing in each external temperature period for periodic daily checking health status and measuring the rectal temperature in first 5 days after farrowing.

\section{Results}

Measured data about external and internal temperatures in each period are noticed in table 1 .

\begin{tabular}{|l|c|c|c|c|}
\hline Period & HS & MS & SA & MW \\
\hline External temperatures & & & & \\
\hline Max. $\left[{ }^{\circ} \mathrm{C}\right]$ & 39,2 & 28 & 16,7 & 12,0 \\
\hline Min. $\left[^{\circ} \mathrm{C}\right]$ & 8,5 & 0,6 & $-2,5$ & $-16,3$ \\
\hline Avg. $\left[{ }^{\circ} \mathrm{C}\right]$ & 24,6 & 13,1 & 6,5 & $-2,5$ \\
\hline Internal temperature & & & & \\
\hline Max. $\left[{ }^{\circ} \mathrm{C}\right]$ & 33,0 & 28,9 & 25,2 & 23,6 \\
\hline Min. $\left[{ }^{\circ} \mathrm{C}\right]$ & 18,7 & 15,2 & 13,9 & 13,2 \\
\hline Avg. $\left[{ }^{\circ} \mathrm{C}\right]$ & 26,4 & 23,1 & 18,7 & 20,0 \\
\hline
\end{tabular}

\section{Acknowledgement}

The study was supported from the project VZ MSM 6215712403 from Ministry of Education, Youth and Sports of the Czech Republic.

\section{References}

Becker, B.A., Misfeldt, M.L. (1995): Effects of constant and cycling hot environments on mitogen-stimulated proliferation of peripheral lymphocytes from sows and litters. Journal of Thermal Biology 20 (6), 485-488.

Papadopoulos, G.A. et al. (2009): Risk factors associated with postpartum dysgalactia syndrome in sows. The Veterinary Journal, Article in Press, doi:10.1016/j.tvjl.2009.01.010

Whittemore, C. (2006): Introduction. In: Kyriazakis, I., Whittemore, C.T. (Eds.), Whittemore's Science and Practice of Pig Production, third ed. Blackwell, Publishing, pp. 127-134. 


\title{
P.873
}

\section{Blood cord cortisol is not a good indicator of piglet vitality in hyperprolific sows}

\author{
Audrey Giraudo ${ }^{1}$ Yannig Le Treut ${ }^{1}$ Eric Chevaux ${ }^{1}$ Guy-Pierre Martineau ${ }^{2}$ \\ 1. LALLEMAND SAS, Blagnac, France; 2. Veterinary School of Toulouse, Toulouse, France
}

\section{Introduction}

For hyperprolific sows, piglets vitality at birth is particularly important and is influenced by the quality of the farrowing process: the longer the worst it is for the piglets which face an increased risk of hypoxia. The reduction of oxygen supply to the fetus blood generates the set-up of an anaerobic metabolism with some subsequent modifications in the blood metabolites balance. Cortisol is usually associated to stress (van de Kar et al., 1999) and used as an indicator of vitality (Canario et al., 2006).

\section{Materials and Methods}

Herd: a typical French herd (400 sows) with hyperprolific sows $(\mathrm{LWxL}) \mathrm{x}(\mathrm{PxL}), 195$ piglets (188 born alive +7 stillborn) from 70 sows were sampled for umbilical cord mixed blood within the first minute after birth. Serum cortisol was analyzed according to two classical techniques: radioimmunoassay (RIA) and luminoimmunoassay (LIA). The relationships with piglets status at birth (live weight, presence or absence of meconium, cord status, position at birth, birth rank) were established by analysis of variance and correlations between parameters with the Pearson test (SPSS 17.0). Blood data exceeding the mean \pm 3 standard deviations were considered as outliers $(n=4)$ and eliminated for the statistical analyses $(P<0.05)$.

\section{Results}

The straightforward outcome concerns the dispersion of the analyzed values for both techniques (Figure 1), the latter's remaining well.

Figure 1: Correlation between analytical techniques (RIA and LIA) $(r=0.747, P<0.01)$.

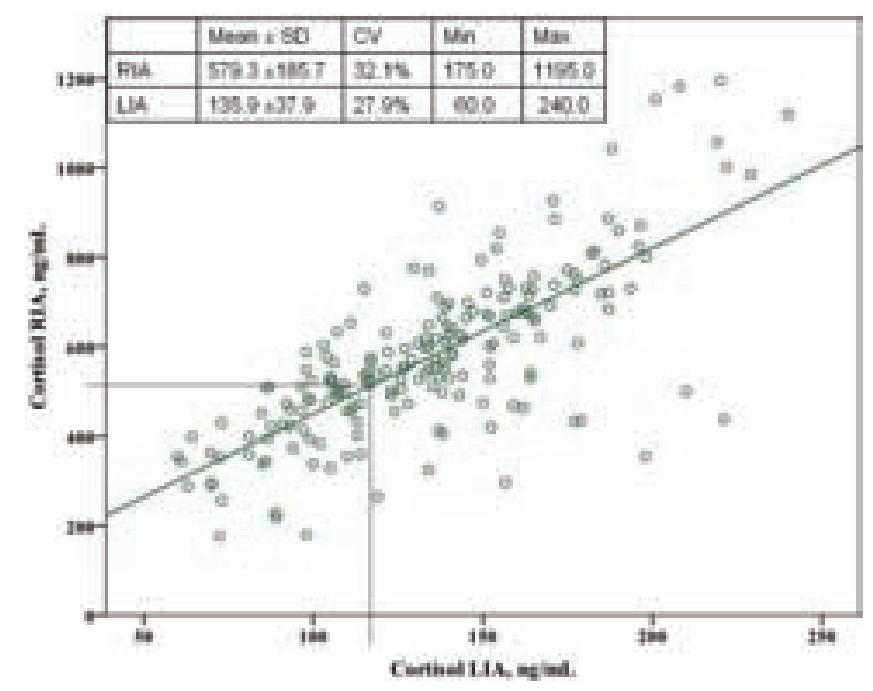

Some weak $(r<0.3)$ correlations $(P<0.05)$ are measured with a higher cortisol level for the lightest piglets and for those born from the largest litters (Table 1).

Live piglets displayed higher $(\mathrm{P}<0.05)$ cortisol content than the stillborn (585.1 \pm 13.7 vs $427.5 \pm 32.9 ; 137.1 \pm 2.7$ vs $104.2 \pm 13.7$ $\mathrm{mg} / \mathrm{mL}$ for RIA and LIA, respectively). Cortisol RIA was increased $(P<0.05)$ for the 115 vs 114 days gestation $(648.8 \pm 28.6$ vs $560.0 \pm 15.3 \mathrm{mg} / \mathrm{mL})$.

Table 1: Umbilical cord cortisol $(\mathrm{mg} / \mathrm{mL})$ according to piglet weight (top) and total born litter size (bottom) (mean \pm SEM; a,b differ for $p<0.05)$

\begin{tabular}{|l|c|c|}
\hline & Cortisol RIA & Cortisol LIA \\
\hline$<1 \mathrm{~kg}(\mathrm{n}=20)$ & $715.7 \pm 40.4^{\mathrm{a}}$ & $162.0 \pm 8.3^{\mathrm{a}}$ \\
\hline $1-1.2 \mathrm{~kg}(\mathrm{n}=27)$ & $559.0 \pm 35.4^{\mathrm{b}}$ & $137.2 \pm 7.1^{\mathrm{b}}$ \\
\hline$>1.2 \mathrm{~kg}(\mathrm{n}=147)$ & $564.3 \pm 14.9^{\mathrm{b}}$ & $132.1 \pm 3.1^{\mathrm{b}}$ \\
\hline & & \\
\hline$<14$ TB $(n=52)$ & $494.9 \pm 30.0^{\mathrm{a}}$ & $120.8 \pm 6.1^{\mathrm{a}}$ \\
\hline $14-16$ TB $(n=88)$ & $583.2 \pm 17.6^{\mathrm{b}}$ & $134.1 \pm 3.6^{\mathrm{a}}$ \\
\hline$>16$ TB $(n=54)$ & $639.7 \pm 25.2^{\mathrm{b}}$ & $151.0 \pm 5.0^{\mathrm{b}}$ \\
\hline
\end{tabular}

The presence of blood together with the meconium on the piglets at birth indicated higher $(P<0.05)$ cortisol LIA $(140.0 \pm 38.1$ vs $132.3 \pm 37.3)$, but no effect of the rank at birth was measured, nor for the piglet's position at birth (head vs back).

\section{Discussion}

Weak vitality is often reported as a problem in small piglets, more numerous in hyperprolific sows. Thus, a strong correlation could be expected with the cortisol and the indicators of low vitality. The absence of link between the cortisol and the piglet vitality, its rank at birth, the quality of the cord (regular vs twisted) or the presence of meconium does not support our initial hypothesis. Qualifying cord blood right after birth was quite innovative for this study, but it may also explain the high dispersion of the values as the level gets more stable after birth (Mc Cauley et al., 1984), where the few available studies were focused on. Therefore, cortisol cord level at birth does not appear relevant to characterize piglet vitality.

\section{References}

Canario et al. (2006), J. Anim. Sci., 84:3185-3196.

McCauley et al. (1984), Res. Vet. Sci., 37(2):234-241.

van de Kar et al. (1999), Front. Neuroendocrinol. 20:1-48 


\title{
P.874
}

\section{Blood cord metabolites as indicators of piglet's vitality in hyperprolific sows}

\author{
Audrey Giraudo ${ }^{1}$ Yannig Le Treut ${ }^{1}$ Eric Chevaux ${ }^{1}$ Guy-Pierre Martineau ${ }^{2}$ \\ 1. LALLEMAND SAS, Blagnac, France; 2. Veterinary School of Toulouse, Toulouse, France
}

\section{Introduction}

For hyperprolific sows, piglets' vitality at birth is particularly influenced by the quality of the farrowing process: the longer the worst it is for the piglets which face an increased risk of hypoxia. The reduction of oxygen supply to the fetus blood generates the set-up of an anaerobic metabolism with some subsequent modifications in the blood metabolites balance.

\section{Materials and Methods}

Herd: a typical French herd (400 sows) with hyperprolific sows (LWxL)x(PxL), 195 piglets (188 born alive +7 stillborn) from 70 sows were sampled for umbilical cord mixed blood within the first minute after birth (Giraudeau et al., 2009). Blood was then centrifuged (2900g, $12 \mathrm{~min}$ ), plasma freezed until analyses.

Vitality has been evaluated according to scale developed by Baxter et al., 2008 (grade 0 to 3).

Analysis: $\mathrm{Na}, \mathrm{Cl}, \mathrm{K}, \mathrm{Ca}$, P, glucose (Glu), bicarbonate (HCO3), proteins, urea, uric acid and creatinin. The relationships with piglets status at birth (live weight, presence or absence of meconium, cord status, position at birth, birth rank) were established by analysis of variance (SPSS 17.0). Blood data exceeding the mean \pm 3 standard deviations were considered as outliers $(n=4)$ and eliminated for the statistical analyses $(P<0.05)$.

\section{Results}

Results (Table 1) are in accordance with other data (Tuchscherer et al., 2000) except phosphorus which was below (1.8 vs 4 $\mathrm{mmol} / \mathrm{L})$.

Table 1: Averages of the analyzed cord blood parameters

\begin{tabular}{|l|c|c|c|c|c|c|}
\hline & $\mathbf{N a}$ & $\mathbf{K}$ & $\mathbf{C l}$ & Urea & Creat. & Uric ac. \\
\hline Mean & 138.4 & 5.1 & 99.7 & 4.6 & 259.6 & 29.8 \\
\hline SD & 8.6 & 1.3 & 5.8 & 3.1 & 246.0 & 39.1 \\
\hline & & & & & & \\
\hline & Glu & $\mathrm{HCO}_{3}$ & $\mathrm{Ca}$ & P & Prot. & \\
\hline Mean & 2.7 & 23.8 & 3.2 & 1.8 & 21.8 & \\
\hline SD & 1.0 & 4.4 & 0.6 & 0.3 & 3.3 & \\
\hline
\end{tabular}

Piglets with very low vitality had higher glucose and $\mathrm{P}$, and lower $\mathrm{HCO} 3$ than the more vigorous ones $\mathrm{Ca}$ and urea were higher $(p<0.05)$ for the vitalities 0 and 1 as opposed to 2 and 3 .

The effect of birth order is reported in Figure 1. Glucose and $P$ content increase for the last third of the litter as does $\mathrm{K}$, where more weak piglets are usually reported.
Figure 1: $K, P$ and Glu blood cord level $(\mathrm{mmol} / \mathrm{L})$ per third of the litter (mean $\pm S E M) ; a, b, c$ differ for $p<0.05$.

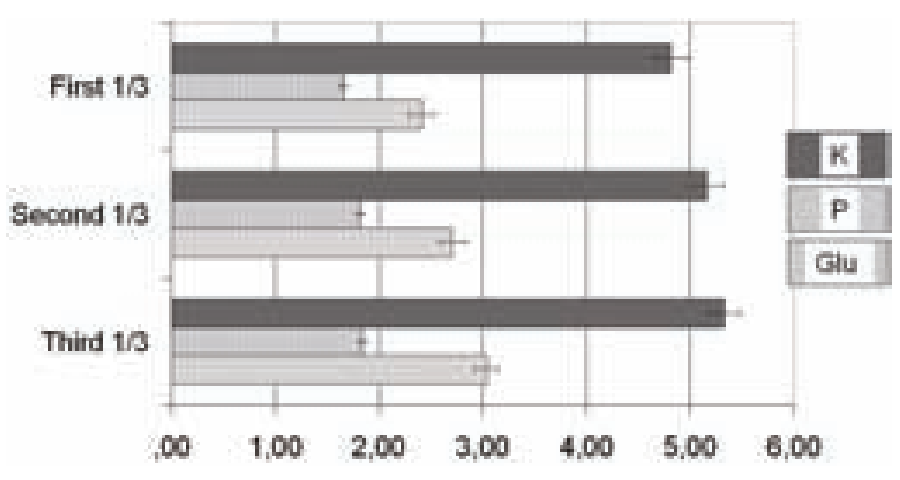

In stillborn, there are higher $(\mathrm{p}<0.05) \mathrm{K}, \mathrm{P}$ and Glu and a lower $(\mathrm{p}<0.05) \mathrm{HCO}$.

In hyperprolificacy litter $(\geq 16), \mathrm{K}$ is lower $(\mathrm{p}<0.05)$ as well as in light piglets $(\leq 1 \mathrm{~kg})$, whereas $\mathrm{Na}$ increases with the litter size.

In piglets with intact cords, K, Glu and urea are lower $(\mathrm{p}<0.01)$ as opposed to the broken ones, the figure being intermediate for the twisted cords.

In piglets with presence of blood on the skin matches with higher ( $\mathrm{p}<0.05$ ) urea, creatinin, $\mathrm{Ca}$, Glu and lower $\mathrm{Na}, \mathrm{Cl}, \mathrm{HCO}$;

In piglets with meconium, there is less urea and more protein $(p<0.05)$ in the blood than the others.

\section{Discussion}

Vitality scoring is confirmed through the cord blood profile. Weak vitality is often reported as a problem in small piglets, also more likely to suffer from hypoxia (Herpin et al., 1996). Low $\mathrm{HCO} 3$, high Glu and $\mathrm{K}$ are good predictors of the level of intrapartum anoxia (Trujillo-Ortega et al., 2007). When combined with the presence of meconium, the cord integrity, the rank at birth and the live weight, piglet vitality is well correlated with the cord blood profile.

\section{References}

Baxter et al. (2008), Theriogenology 69(6), 773-783.

Herpin et al. (2006), J. Anim. Sci. 74(9), 2067-2075.

Trujillo-Ortega et al. (2007), Acta Biomed. 78(1), 29-35.

Tuchscherer et al. (2000), Theriogenology 54(3), 371-88. 


\title{
P.875
}

\section{Inverted teats (Mammillae invertitae) in sows - effect on piglet survival and growth rate}

\author{
Helena Chalkias ${ }^{1}$ Nils Lundeheim ${ }^{1}$ Magdalena Jacobson $^{2}$ \\ 1. Dept of Animal Breeding and Genetics, Uppsala, Sweden; \\ 2. Dept. of Clinical Sciences, Faculty of Veterinary Medicine and Animal Science, Swedish University of Agricultural Sciences, Uppsala, Sweden
}

\section{Introduction}

Piglet mortality during lactation is a large problem in pig production. The nursing and suckling behavior of piglets follow a complex scheme and the milk ejection lasts for only 10-20 seconds, once per hour. There is no transfer of antibodies across the placenta and the piglets are born with low immunity. Each piglet must thus have access to a functional teat for nourishment and growth (3). One example of a non-functional teat is the inverted teat. The degree of inversion may differ from slightly to totally inverted. The aim of this study was to evaluate the function of inverted teats identified at $100 \mathrm{~kg}$ live weight, and to analyze differences between the mammary glands of inverted teats vs. normal teats. Further, the piglets' ability to suckle an inverted teat and differences in daily weight gain that might be related to the presence of inverted teats was investigated.

\section{Material and Methods}

Two Yorkshire gilts $(a, b)$ and one Yorkshire*Landrace gilt (c) with 4, 4 and 8 inverted teats at $100 \mathrm{~kg}$ live weight were inseminated. One week before expected farrowing the teats were palpated. After farrowing, to ensure that each piglet only had access to one teat, all excessive, functional teats (above the number of liveborn piglets) were blocked with adhesive bandage, beginning with the posterior teats (1). The health status of the piglets and gilts were recorded daily. The piglets were individually marked and the teat order of the piglets was registered by videotaping. The piglets were weighed individually each day during the first week and thereafter once a week until weaning at 4 weeks of age. The gilts were euthanized immediately after weaning and the mammary gland for each teat was weighed individually.

\section{Results}

One week before farrowing, two of the gilts $(b, c)$ only had one single inverted teat left and gilt (a) had no inverted teats remaining. All three gilts had 13 liveborn piglets each. The two piglets that suckled the inverted teats were euthanized 5 and 8 days after farrowing, respectively, due to loss of body weight. Both these piglets were still fighting for other teats the day they were euthanized, but necropsy revealed empty intestines and sparse amount of feed in the stomach. Both piglets still had some fat remaining in the coronary groove, but one piglet had serous atrophy of the bone marrow. Strong significant positive correlations $\left(0.66^{* * *}, 0.64^{* * *}, 0.62^{* *}\right)$ between weight of mammary gland and daily gain of the piglets during the second to fourth week of lactation could be found. However, no significant differences in piglet weight gain between normal and previously inverted teats could be seen. The average mammary gland weight for normal teats was 606 gram and for previously inverted 570 gram.

Table 1. Piglet weight by type of teat used

\begin{tabular}{|c|c|c|}
\hline & \multicolumn{2}{|c|}{ Average piglet weight (kg) } \\
\hline Week & Normal teats & Previously inverted teats \\
\hline 1 & 2.45 & 2.21 \\
\hline 2 & 4.45 & 4.18 \\
\hline 3 & 6.45 & 6.20 \\
\hline 4 & 8.66 & 8.51 \\
\hline & & \\
\hline
\end{tabular}

\section{Discussion}

This study clearly showed that inverted teats at farrowing are of low functional value and this does emphasize the importance of functional teats. Inverted teats can sometimes protrude close to farrowing (2), but it was unexpected that most of the inverted teats were normalized weeks before farrowing. To be able to demonstrate relationship between previously inverted teats and piglet daily weight gain, more data is needed.

\section{Acknowledgement}

This study was financed by grants from the Swedish Farmers' Foundation for Agricultural Research.

\section{References}

1. Fraser, D., et al. (1992) Anim. Prod. 55:419-424

2. Nordby, J.E. (1934). J. Hered. 25: 499-502

3. Verstegen, M.W.A., et al. (1998). The lactating sow. Wageningen, The Netherlands: $300-308$ 


\title{
P.876
}

\section{Use of a new technology based on thermography to monitor health and risk factors in newborn piglets}

\author{
Joquin Morales ${ }^{1}$ Alberto Manso ${ }^{1}$ Maria Aparicio ${ }^{1,2}$ Carlos Piñeiro $^{1}$ \\ 1. PigCHAMP Pro Europa, Segovia, Spain; 2. Centro de Experimentación y de Formación en Porcino, Segovia, Spain
}

\section{Introduction}

Perinatal mortality still plagues the swine industry despite the improving knowledge in neonatal physiology. Hypothermia is one of the most important factors, affecting colostrum intake and hence, its later health status and performance.

In this study, thermography was used as a new tool to determine hypothermia and its evolution in newborn piglets. Thermography might provide high advantages in swine clinical practice, allowing the inspection of a high number of animals in few time, revealing lesions and risk factors that would remain hidden with other diagnostic systems (Friendship et al., 2009).

\section{Materials and Methods}

Twenty-two piglets from two different litters entered the study at birth time. In each litter, half of the piglets were immediately dry using an absorbent material (paper). The other half was not manipulated at birth. A heat lamp and extra-paper $(2 \mathrm{~kg}$ ) were supplemented in both litters.

Skin temperature was recorded from each piglet at birth and every 10 minute's time during 1.5 hours using a thermographic camera (Fluke Ti45). Piglets were weighed at birth and at 2, 4 and 9 days of life.

In each picture, the average temperature in the back was measured. In figure 1 some examples of thermographic pictures are shown.

Figure 1 - Thermographic pictures of newborn piglet

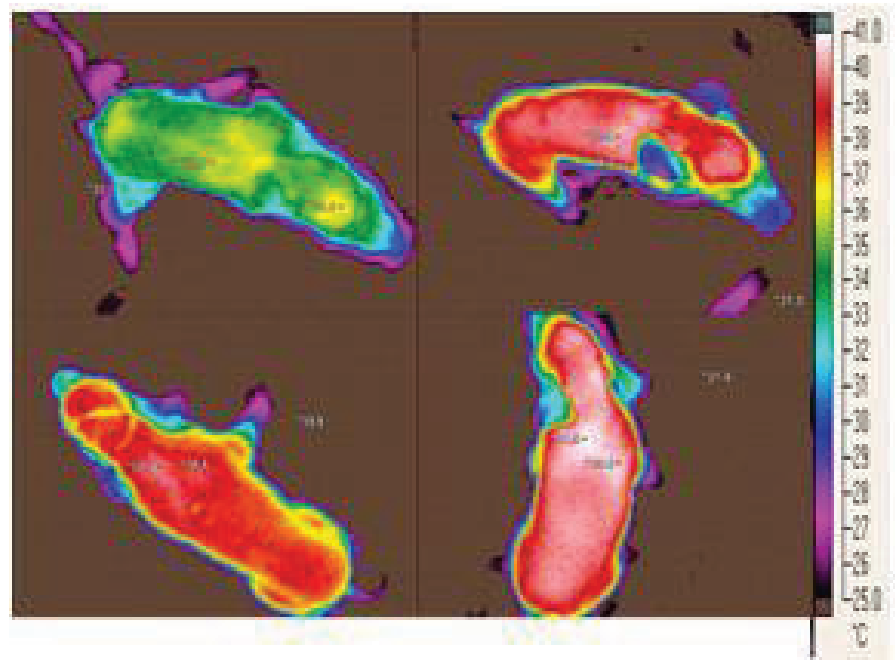

Temperature and body weight (BW) evolution were analysed using the MIXED procedure of SAS ( $v 9.00)$. In the temperature analysis, the statistical model included the fixed effects of treatment (control vs drying with paper), time (10 minutes' intervals) and their interaction and the block effect of litter within treatment. In the BW analysis, the statistical model included the fixed effect of treatment and the block effect of litter within treatment. Initial BW was included as covariate in both models.

\section{Results}

Drying piglets immediately after birth increased the skin temperature in the first $90 \mathrm{~min}$ of life $\left(39.3^{\circ} \mathrm{C}\right.$ vs $37.8^{\circ} \mathrm{C}$ in dried and control piglets, respectively; $P=0.048$ ). Evolution of the skin temperature was also different between treatment ( $P$ treatment $x$ time $=0.0001)$. Initial temperature was $39.3 \pm 0.79{ }^{\circ} \mathrm{C}$, and was kept almost constant in the dried-piglets in the 90 min-interval. In the control group (non-attended piglets) skin temperature immediately decreased after birth and then increased progressively and 90 min after farrowing temperature did not differ between groups $(P=0.27)$. Evolution of skin temperature is shown in the figure 2.

Figure 2 - Skin temperature evolution in the back

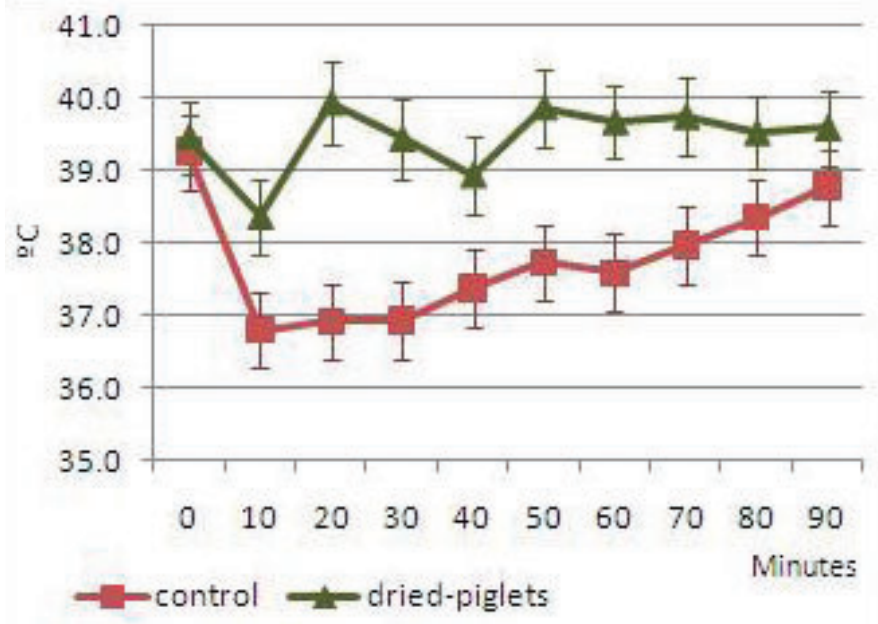

Body weight evolution did not differ between treatments (2.71 $\mathrm{kg} \mathrm{BW}$ at 9 days of life).

\section{Discussion}

Drying piglets immediately after birth was effective to keep the body temperature. Preventing the perinatal hypothermia provides the piglet more possibilities to survive and reaching higher BW at weaning. Infrared thermography demonstrated enough accuracy to be considered as a new tool to complement other diagnostic tools.

\section{References}

Friendship R, Poljak Z, McIntosh K. 2009. Use of infrared thermography for early detection of disease causing sudden death in a swine finishing barn. 28th Centralia Swine Research Update, Ontario. January 2009. p |27-I28. 


\title{
P.877
}

\section{The effect on pre-weaning piglet mortality of tilmicosin oral administration when used in pre-farrowing sows}

\author{
Alain Kanora ${ }^{1}$ Patrick Rabaeys ${ }^{2}$ Henk Lecluyse$^{1}$ Spas Petkov ${ }^{3}$ Koen De Gussem ${ }^{1}$ \\ 1. Huvepharma NV, Antwerp, Belgium; 2. Danis, Knijffelingstraat 15, Koolskamp, Belgium; 3. Department of R\&D, Biovet JSC, Peshtera, Bulgaria
}

\section{Introduction}

Tilmicosin is a semi-synthetic macrolide antibiotic which is affecting bacterial protein synthesis. Macrolides have a proven benefit as treatment for a wide range of infectious diseases, with an increased interest for respiratory infections.

The aim of this study was to evaluate the effect of tilmicosin (Tilmovet 250 mg/ml Concentrate for Oral Solution - Huvepharma) treatments and its capacity to prevent neo-natal piglet mortality.

\section{Materials and methods}

To set up this trial in a sow farm $(n=350), 60$ pregnant and healthy sows (without health issues during the last gestation and regardless of parity) of around 110 days of gestation were provided a dose of $9 \mathrm{ml}$ of Tilmovet $250 \mathrm{mg} / \mathrm{ml}$ Concentrate for Oral Solution per sow per day for minimum 5 to maximum 7 days through an individual medication system / sow. The total dose was split up and distributed twice a day ( $4.5 \mathrm{ml}$ per dose).

The final day of treatment was the farrowing day. After farrowing and starting on the day of farrowing (day 0), milk samples were collected on day 2 and 4 with a minimum of $10 \mathrm{ml}$ per sample. Samples were directly frozen after collection. From these samples, tilmicosin was extracted by means of liquid-liquid parturition, purified by C18 SPE and identified and quantified through HPLC method. The HPLC method has been developed and validated for analysis of tilmicosin in milk.

Piglet mortality and weight gain figures were recorded daily for a period of 14 days.

\section{Results}

The treated group of sows had $9.4 \%$ more piglets born (15.2 vs 13.9) than the untreated sows (Table 1 and Figure 1). They also had $10 \%$ more live born piglets (14.3 vs 13.0 ) and the preweaning mortality was clearly lower than in the control group (13.28\% vs $16.92 \%)$.

The number of weaned piglets was similar in both groups, yet the Tilmovet ${ }^{\circledR}$ piglets were nearly $9.5 \%$ heavier at weaning.
Table 1. Piglet birth and growth

\begin{tabular}{|c|c|c|}
\hline & \multicolumn{2}{|c|}{ Average piglet weight (kg) } \\
\hline Week & Normal teats & Previously inverted teats \\
\hline 1 & 2.45 & 2.21 \\
\hline 2 & 4.45 & 4.18 \\
\hline 3 & 6.45 & 6.20 \\
\hline 4 & 8.66 & 8.51 \\
\hline & & \\
\hline
\end{tabular}

Figure 1. Pre-weaning piglet mortality and weight at weaning

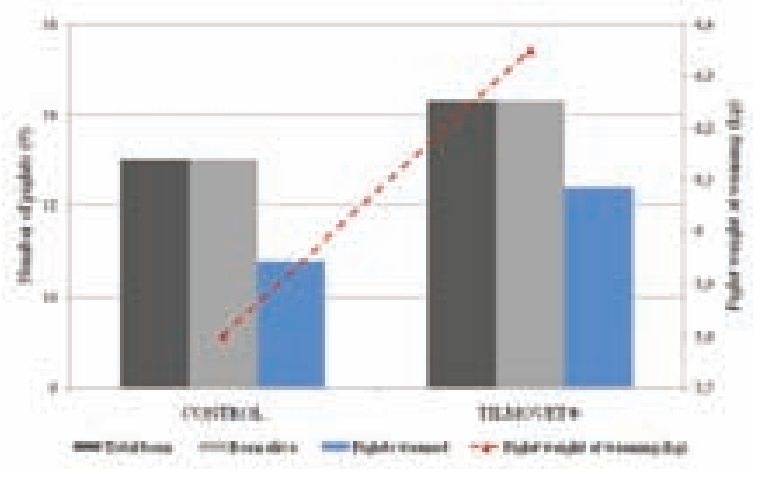

\section{Discussion and conclusion}

Earlier research ${ }^{1,2}$ has demonstrated the beneficial effect of tilmicosin treatment on sow productivity and the prevention of postpartum infections through reduction of piglet pathogen transmission. The current study confirms these findings and indicates that gestating sows being treated with Tilmovet ${ }^{\circ}$ for 5 to 7 days prior to farrowing suffered less from neo-natal infections. This is a result from the tilmicosin concentration in the sow milk and consequent colostrum transmission. The impact of the effect in the treated group can be noticed in the higher individual piglet weights at weaning.

\section{References}

1. Biancotto, G. et al. (2002), Tilmicosin excretion in sow milk after oral administration, Int. Conf. on Antimicrobial Agents in Veterinary Medicine, Bethesda, Maryland, 27-29/06/2002.

2. Almond, G.W. et al. (2006), Assessment of the therapeutic effect of tilmicosin in lactation feed, IPVS Congress 2006, Proceedings Vol. 2, p. 523. 


\title{
P.878
}

\section{Piglet survival depends on relative birth weight}

\author{
Flemming Thorup ${ }^{1}$ Susanne L. Musse ${ }^{2}$ \\ 1. Center for Pig Production, Agriculture and Food Council, Copenhagen, Denmark; 2. PORCUS Svinefagdyrlæger \& Agronomer, Ringe, Denmark
}

\section{Introduction}

Large size at birth is the primary indicator of high piglet survival (1). For high litter survival, uniform birth weights are more important. The Meishan show high piglet survival with low but uniform birth weights (2). We investigated piglet survival, when birth weights were decreased due to eighter low parity or high litter size.

\section{Materials and Methods}

Trial one included 1401 DanBred DxLY piglets born in two herds. Trial two included 1182 DanBred YxL female piglets born in one herd. Piglets were weighed and ear tagged at birth. Survival was registered at weaning.

\section{Results}

Piglets born to 1 st litter sows were 150 to 200 gram lighter than piglets born to older sows (Table 1). Piglet survival by birth weight and parity in trial 2 is shown in figure 1. Birth weights and piglet survival for piglets born in small litters (10-14 total born) and large litters (18-22 total born) is given in table 2 . Piglets born in litters of 1-9, 15-17 or $>22$ are excluded. Piglets born in large litters were app. 150 gram lighter than piglets born in small litters. Survival by birth weight and litter size in trial 2 is given in fig. 2. More figures will be presented.

\section{Discussion}

Despite having the same genotype, piglets born in 1st litter and piglets born in large litters were app. 150 gram lighter than piglets born in sow litters and piglets born in small litters. Restricted uterine capacity may cause this "piglet phenotype", 150 gram leighter, than the genetic merit would allow for under better uterine conditions. There were only small differences in piglet survival between the groups. Both 1 st litter piglets and large litter piglets reached 100 per cent survival at a lower birth weight than piglets born by sows or born is small litters (see fig. 1 and 2). A level of survival is correlated to birth weight (genotype) and is still the same, if a piglets achieves a lower birth weight (phenotype), when born in a gilt litter or in a large litter. A reduced birth weight in first parity or due to high litter size did not effect piglet survival.

\section{References}

(1). Foxcroft G. R. et al., Soc. Reprod. Fertil. Suppl. (2009), 66, 213-31.

(2). Ford. S. P., Reprod. Fertil. Suppl. (1997), 52, 165-76.
Table 1. Effect of parity on birth weight and piglet survival

\begin{tabular}{|l|c|c|c|c|c|c|}
\hline Parity & $\mathbf{1}$ & $\mathbf{2 - 9}$ & $\mathbf{1}$ & $\mathbf{2 - 9}$ & $\mathbf{1}$ & $\mathbf{2 - 9}$ \\
\hline Factor & \multicolumn{2}{|c|}{ No. piglets } & \multicolumn{2}{|c|}{ Birth weight, kg } & \multicolumn{2}{|c|}{ Survival \% } \\
\hline Trial one & 360 & 1244 & 1.24 & 1.41 & 93 & 87 \\
\hline Troal two & 343 & 889 & 1.28 & 1.48 & 89 & 89 \\
\hline
\end{tabular}

Table 2. effect of litter size on weight and piglet survival

\begin{tabular}{|l|c|c|c|c|c|c|}
\hline Litter size & $\mathbf{1 1 - 1 4}$ & $\mathbf{1 8 - 2 2}$ & $\mathbf{1 1 - 1 4}$ & $\mathbf{1 8 - 2 2}$ & $\mathbf{1 1 - 1 4}$ & $\mathbf{1 8 - 2 2}$ \\
\hline Factor & \multicolumn{2}{|c|}{ No. piglets } & \multicolumn{2}{|c|}{ Birth weight, $\mathbf{k g}$} & \multicolumn{2}{|c|}{ Survival \% } \\
\hline Trial one & 360 & 566 & 1.47 & 1.32 & 89 & 84 \\
\hline Trial two & 343 & 332 & 1.45 & 1.34 & 89 & 88 \\
\hline
\end{tabular}

Figure 1.

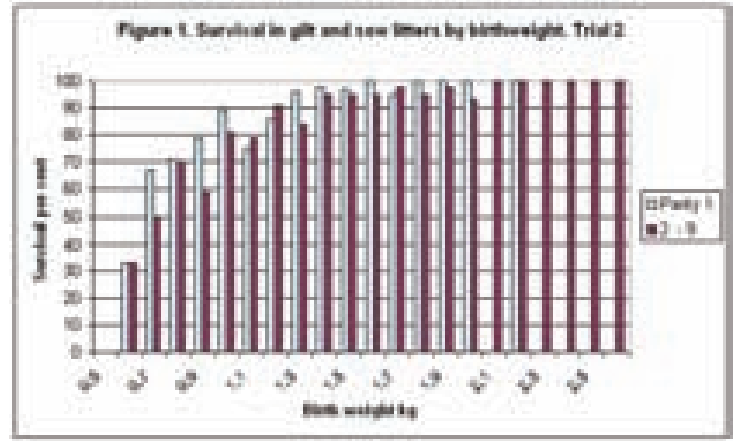

Figure 2.

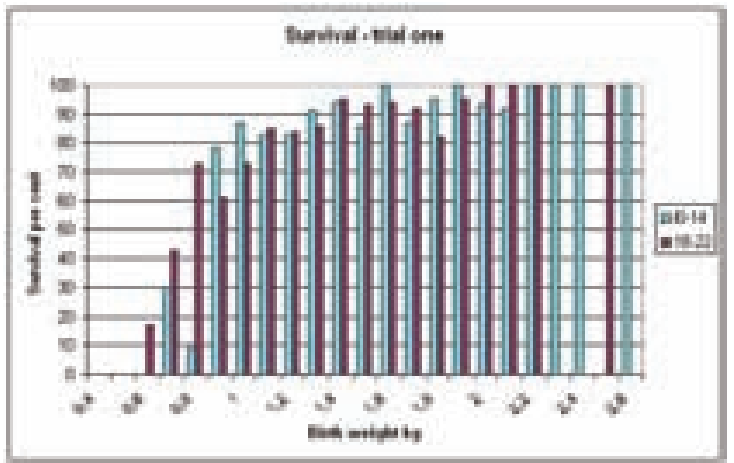




\title{
P.879
}

\section{Extreme uterine crowding in Prolific French York (PFY) sows}

\author{
John Harding ${ }^{1}$ Crissie Auckland ${ }^{1}$ Jennifer L. Patterson ${ }^{2}$ George R. Foxcroft ${ }^{2}$ \\ 1. University of Saskatchewan, Saskatoon, SK, Canada; 2. University of Alberta, Edmonton, AB, Canada
}

\section{Introduction}

The selection for litter size without concurrent selection for uterine capacity will intensify the adverse affects of uterine crowding, particularly in prolific and mid- to high-parity sows. Recently, a prolific French York (PFY) dam line was imported from France into North America as a way of increasing the prolificacy of commercial sows. The objective of this study was to evaluate various reproductive attributes of the PFY dam-line at the end of the first trimester.

\section{Materials and Methods}

Gravid reproductive tracts, harvested from 34 healthy, vaccinated (parvo, lepto, erysipelas) PFY sows of various parities, were slaughtered 26 to 40 days following insemination. All sows were designated for culling for non-reproductive reasons and had been bred specifically for this study. After humane slaughter the gravid uteri were removed and stored frozen at $-20^{\circ} \mathrm{C}$. After thawing, the corpora lutea $(\mathrm{CL})$ were counted, the uterus incised, each conceptus removed, excess fluid drained from the chorioallantois, and the conceptus (embryo, amnion + drained chorioallantois) weighed. The embryos were removed from placental membranes, weighed and the crow-rump length (CRL) measured. The conceptus was classified as degenerate if the embryo, placental membranes, or both, showed gross signs of degeneration as previously described ${ }^{1}$. In the event of twinning, each embryo was counted and separate embryo mass and crown-rump lengths were obtained. Analysis: The total number $\mathrm{CL}$ on both ovaries was considered the ovulation rate. The estimated live embryonic survival rate was calculated (number assumed live embryos/number $\mathrm{CL}$ ). The average live embryo CRL and weight, and the wet placental weight (total conceptus weight less embryonic weight) were calculated for each litter. Litters were categorized by gestation age (26-30, 31-35, 36-40), and group differences in the median scores for each reproductive attribute compared using a Kruskal-Wallis analysis of variance. $P<.05$ was considered significant.

\section{Results}

The median parity across all sows was 3 (range 1 to 7). Ovulation rate and total embryo number ranged from 17-33 and 9-29 respectively (Figure 1). Embryo and placental weight, and CRL increased with gestation age (Table 1), but the number of total and live embryos, and embryo survival rate were not affected.
Figure 1. Distribution of ovulation rate (black) and total embryo number (grey) in 34 prolific French York sows

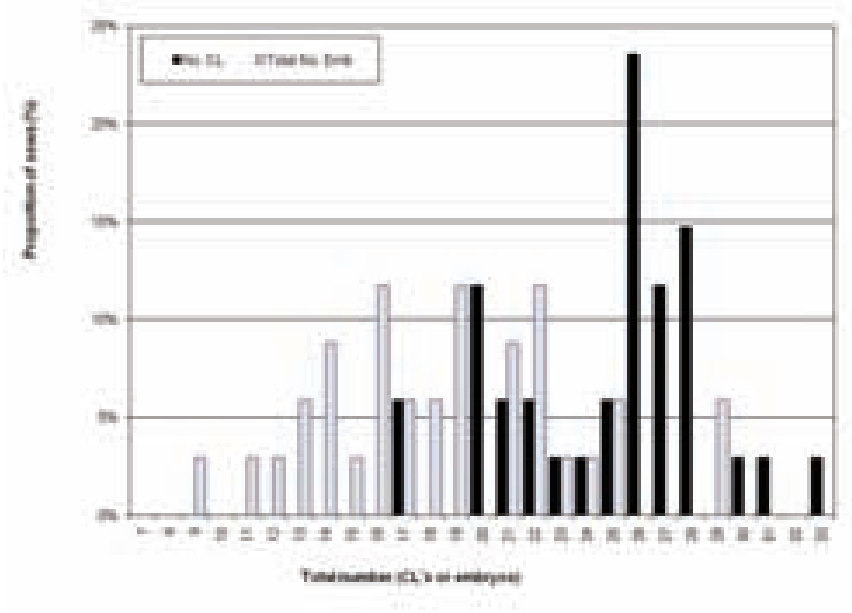

Table 1. Reproductive attributes by gestation age in 34 prolific French York sows

\begin{tabular}{|l|c|c|c|c|}
\hline & \multicolumn{5}{|c|}{ Gestation age (days) } \\
\hline Variable & $\mathbf{2 6 - 3 0}$ & $\mathbf{3 1 - 3 5}$ & $\mathbf{3 6 - 4 0}$ & Total \\
\hline No. sows & $\mathbf{1 6}$ & $\mathbf{1 1}$ & $\mathbf{7}$ & $\mathbf{3 4}$ \\
\hline No. CL & 26 & 25 & 26 & 26 \\
\hline No. total embryos & 21 & 16 & 19 & 18.5 \\
\hline No. live embryos & 17.5 & 15 & 16 & 16.5 \\
\hline Embryo survival rate \% & 68.5 & 67.0 & 73.0 & 69.5 \\
\hline Avg placental wt (g) & $16.5^{\mathrm{a}}$ & $32.6^{\mathrm{b}}$ & $50.2^{\mathrm{b}}$ & 23.2 \\
\hline Avg. embryo wt (g) & $2.0^{\mathrm{a}}$ & $4.0^{\mathrm{b}}$ & $6.8^{\mathrm{c}}$ & 3.3 \\
\hline Avg. embryo CRL (mm) & $25.6^{\mathrm{a}}$ & $33.1^{\mathrm{b}}$ & $45.5^{\mathrm{c}}$ & 30.2 \\
\hline \multicolumn{5}{|l|}{ Superscripted letters within rows differ statistically P $<.05$} \\
\hline
\end{tabular}

\section{Discussion}

Previous research by our team demonstrated that when the number of viable embryos at gestation day 30 exceeded 9 to10, fetal development was adversely affected ${ }^{2}$. Our present results confirm the combination of high ovulation and embryo survival rates in prolific French York sows results in extreme intrauterine crowding during the first trimester. Its impact on postnatal performance warrants continued investigation.

\section{References}

1. Geisert, RD et al (2007) Reprod Domest Anim. 42(3),333

2. Town, SC et al (2004) Reprod (Cambridge).128(4), 443-454. 


\title{
P.880
}

\section{Bacterial pathogens present in the milk of healthy sows and sows with PDS (Postpartum Dysgalactia Syndrome): detailed analysis of isolated Aerococcus spp}

\author{
Andrea Menrath ${ }^{1}$ Imke Gerjets ${ }^{1}$ Kerstin Reiners ${ }^{2}$ Nicole Kemper $^{1}$ \\ 1. Institute for Animal Breeding and Husbandry, Christian-Albrechts-University Kiel, Kiel, Germany; 2. PIC Germany GmbH, Schleswig, Germany
}

\section{Introduction}

The porcine Postpartum Dysgalactia Syndrome (PDS) is a well known disease in sows 24 to $48 \mathrm{~h}$ post partum. The main symptom, the mastitis, is accompanied by fever and reduced milk production. Hence, decreased rates of growth and a higher mortality in piglets are the results.

The disease is multifactorial due to influences of management and feeding, but additionally, there is a relevance of individual factors of each animal and of the occurrence of different pathogens in the infected mammary glands.

In the geMMA-project, the spectrum of pathogens in the milk of PDS-diseased and healthy sows was compared. In this special part of the study, the isolated Aerococcus (Ac.) species (spp.) were further investigated.

\section{Materials and Methods}

The sows were defined as PDS-diseased by several criteria.

The identification and subtyping of all isolates were performed by classical bacteriology, the API-system ${ }^{\circledR}$ of Biomerieux (Nürtingen, Germany) and PCR.

\section{Results}

In the milk of 344 (22.9\%) of 1,500 examined sows, both PDSdiseased and healthy, Aerococcus spp. were isolated.

This is the first description of the isolation of Aerococcus spp. in the milk of sows. In this study, the isolated Aerococcus spp. belonged to Ac. viridians ( 285 isolates) and Ac. urinae (59 isolates) by biochemical differentiation. Further results of the advanced PCR-analyses will be represented.

\section{Discussion and Conclusion}

Aerococcus spp. are ubiquitous in the environment and also in the air. The Genus Aerococcus includes seven species: Ac. viridans, Ac. urinae, Ac. sanguinicola, Ac. christensensii, Ac. urinaeequi, Ac. urinaehominis and Ac. suis. These Gram-positive, catalase-negative Cocci have been associated with disease in human (Ac. viridans und Ac. urinae), swine, and cattle (Ac. viridans und Ac. suis) $(1,2)$. In swine, Ac. viridans has been described in association with arthritis, meningitis and pneumonia (2). Most recently, Ac. suis has been shown to cause arthritis, enteritis, meningitis and pneumonia in swine (3).

Due to the relation of Aerococcus spp. with mastitis in cows (1), the role of this pathogen in the pathogenesis of PDS is clearly worth further examinations, with this study as a first step in this direction.

This study is part of the BMBF-supported FUGATO-plus-project 'geMMA - structural and functional analysis of the genetic variation of the MMA-syndrome'.

\section{References}

1. Devriese, L. A., J. Hommez, H. Laevens, B. Pot, P. Vandamme, and F. Haesebrouck. 1999. Identification of aesculin-hydrolyzing streptococci, lactococci, aerococci and enterococci from subclinical intramammary infections in dairy cows. Vet Microbiol 70:87-94.

2. Martin, V., A. I. Vela, M. Gilbert, J. Cebolla, J. Goyache, L. Dominguez, and J. F. Fernandez-Garayzabal. 2007. Characterization of Aerococcus viridans isolates from swine clinical specimens. J Clin Microbiol 45:3053-7.

3. Vela, A. I., N. Garcia, M. V. Latre, A. Casamayor, C. Sanchez-Porro, V. Briones, A. Ventosa, L. Dominguez, and J. F. Fernandez-Garayzabal. 2007. Aerococcus suis sp. nov., isolated from clinical specimens from swine. Int J Syst Evol Microbiol 57:1291-4. 


\title{
P.881
}

\section{Facilitating nursing behavior of sows at farrowing has a positive effect on colostrum distribution}

\author{
Johan Biermann ${ }^{2}$ Jan Jourquin ${ }^{1}$ Rainier van Gelderen ${ }^{1}$ Lieve Goossens ${ }^{1}$ \\ 1. Janssen Animal Heatlh, Beerse, Belgium; 2. DAP Helmond, Helmond, Netherlands
}

\section{Introduction}

Farrowing is a stressful event for sows, especially first parity sows. In the most severe cases this leads to aggression and savaging of newborn piglets. Next to clear aggression, there is a range of less explicit unwanted behavior, negatively impacting the possibility for the piglets to take up colostrum. Bottle fed piglets ingest more colostrum in the first day than naturally nursed piglets (1), indicating that the sows is a limiting factor for colostrum intake.

Our investigations show that colostrum distribution is correlated with piglet survival. We were also able to demonstrate that with an injection of azaperone of the sow just after farrowing, resulted in an increase of calm behavior and in an increase of number of piglets suckling during the first hours after farrowing.

The aim of this pilot study was to investigate if an injection of azaperone (Stresnil ${ }^{\circ}$ ) at the end of the partus had a positive effect on colostrum distribution.

\section{Materials and Methods}

In a commercial sow farm, 193 first and second parity litters were included in the study. The day time litters $(n=98)$ were injected $320 \mathrm{mg}$ of azaperone IM ( $8 \mathrm{ml}$ Stresnil $\left.{ }^{\circ}\right)$ at the moment of expulsion of the placenta. The night time litters $(n=95)$ were not treated. Gestation length and the number of piglets born total, born alive, fostered $( \pm)$, dead and weaned were recorded. All normal farm procedures were continued. At tail docking (day 3 post partum) a blood drop of the remaining tail bud from 6 randomly chosen litter mates was sampled. The samples were analyzed with a DAS-ELISA test (2) (Colostrum Quality CounterTM). Piglet serum IgG concentration after gut closure can be used as a parameter for colostrum intake (3). In order to compare the distribution of colostrum between sows, the coefficient of variation of the piglet serum IgG (CV lgG) was calculated. This is the standard deviation divided with the average of the litter.

The statistical analysis was performed with the SAS ${ }^{\oplus}$ program. A t-test on the log-transformed data was performed.

\section{Results}

The mean ( \pm standard deviation) of colostrum distribution (CV $\mathrm{lgG}$ ) of the piglets of treated sows was 46.9 ( \pm 20.4 ) compared to 55.2 ( \pm 32.2) for non-treated sows. The difference is nearly statistically significant $(p<0.07)$. There was no difference in colostrum distribution between first and second parity sows. Figure 1 shows the relation of variation in colostrum intake and the number of piglets born alive. The variation increases with litter size in non-treated sows, while the variation does not differ between litter sizes in the azaperone treated sows.

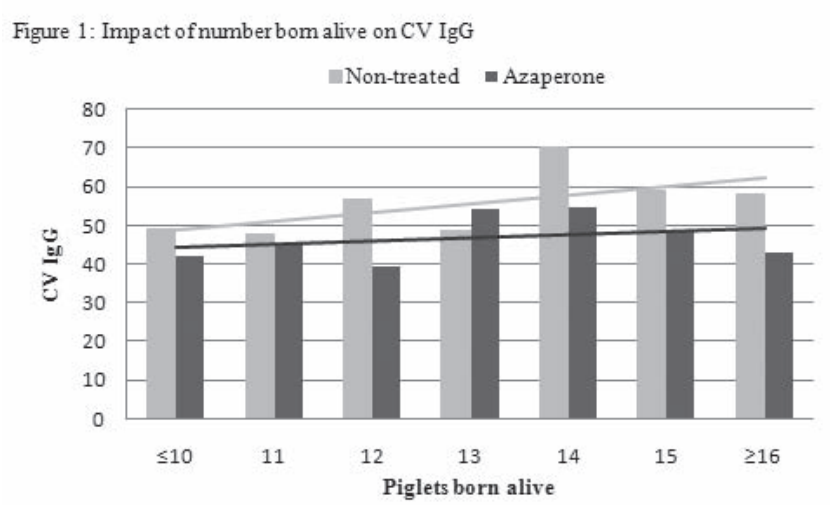

\section{Discussion}

The results demonstrate that the behavioral change of the sow after injection of azaperone results in a better distribution of the available colostrum amongst the piglets. Increasing number of piglets born alive per litter had a negative impact on colostrum distribution in the non-treated group. In litters where the sow was treated with azaperone, the distribution of colostrum was not affected by the number of piglets born alive. This confirms earlier findings that the sow is a limiting factor for colostrum distribution.

In this study, it was shown that one injection of azaperone to the sow at the moment of expulsion of the placenta, improves colostrum distribution amongst her piglets. The challenges caused by poor colostrum distribution are more relevant in high productive farms.

\section{References}

1. N. Devillers et al., Animal Science 2004, 78, 305-313

2. A. Bianchi et al., Veterinary Immunology and Immunopathology 1995, 44, p 309-317

3. J.P. Nielson et al., Sammendrag 2008 


\title{
P.882
}

\section{Facilitation of nursing behavior in sows during colostrum phase improves piglet condition}

\author{
Steven Tseng ${ }^{2}$ Jan Jourquin ${ }^{1}$ Lieve Goossens ${ }^{1}$ \\ 1. Janssen Animal Heatlh, Beerse, Belgium; 2. China Chemical \& Pharmaceutical Company, Tainan, Taiwan
}

\section{Introduction}

The development and the wellbeing of the piglet is determined by its ability to fulfill its need for homeostasis, growth and its ability to fight off the sanitary challenges. Early colostrum intake is the key for success. For the first 2 days of their lives, colostrum provides the nutrients to the piglets and during their first weeks, the immunoglobulin $(\mathrm{lg})$ fraction passively protects the piglets from disease (1). Critical factors for early life survival are the ability of the piglet to suckle, the available volume of colostrum, the udder quality and the behavior of the sow during the colostrum phase. At parturition, the behavior of about $25 \%$ of sows prevents their piglets from suckling. 6 hours later this increases even to $30 \%$. With an injection of azaperone (Stresnil ${ }^{\circ}$ ) at the end of the partus, sows become calmer and allow the piglets easy access to the udder.

In this study, the effect of an injection of azaperone at the end of the partus of all parity sows on piglet development was investigated.

\section{Materials and Methods}

In a 3000 sow farm in Taiwan, 50 sows of all parities were included in the study. They were randomly allocated to a treated group ( 25 sows) and a non treated reference group ( 25 sows). The treated sows were injected IM $2 \mathrm{mg} / \mathrm{kg}$ of azaperone $(5 \mathrm{ml}$ Stresnil $^{\bullet}$ per $100 \mathrm{~kg}$ body weight) at the moment of expulsion of the placenta. To assess the condition and health status in field conditions, early mortality rate and weight gain were used as parameters. The piglets were weighed and identified individually at birth and at weaning. Fostering was allowed and recorded. Farrowing and weaning date, numbers born alive, pre-weaning mortality and numbers weaned was recorded.

A linear mixed model was fit using the procedure MIXED in SAS ${ }^{\circ}$ version 9.1.

\section{Results}

The number piglets born alive was 10.8 for the non treated group and 9.7 for the treated group. Pre-weaning mortality rate was similar for both groups (11.74\% and $11.64 \%)$. Weight data are presented in Table 1. All litters were weaned at 28 days. While individual birth weights were not different between both groups, weaning weight was significantly higher in the azaperone treated group.
Table 1: Impact of azaperone treatment on weight gain

\begin{tabular}{|l|c|c|c|}
\hline & Birth weight $\mathbf{( k g )}$ & Weaning weight $\mathbf{( k g )}$ & Weight gain $\mathbf{( k g )}$ \\
\hline Azaperone & 1.64 & 7.07 & 5.43 \\
\hline Non-treated & 1.61 & 6.55 & 4.94 \\
\hline p-value & 0.7478 & 0.0313 & 0.0132 \\
\hline
\end{tabular}

In Figure 1, the impact of birth weight on weight gain is shown. Weight gain increases with birth weight.

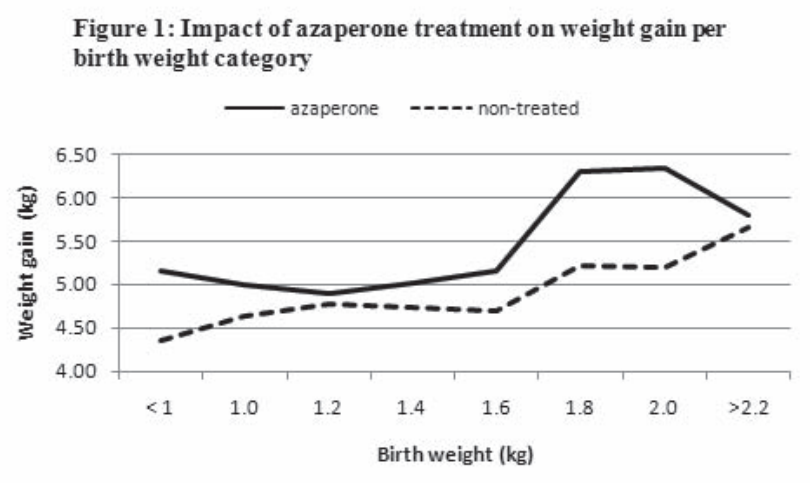

The observed growth improvement in the treated group was seen in all birth weight classes.

\section{Discussion}

The present study showed the benefit of an azaperone injection of sows at the end of the partus. Piglets thrive better, resulting in a $9.9 \%$ weight gain increase.

The sedative effect of azaperone is limited to a few hours. The origin of any difference therefore lies in the early intake of colostrum. If enough colostrum is available, a good distribution increases the chances of all piglets. Stronger piglets increase the suckling impulse, which leads to a higher milk production. The antibodies acquired from the colostrum protect the piglets against disease. In this study, it results in a significant increase of weaning weight of the piglets.

\section{References}

1. J. Le Dividich et al., Journal of Agricultural Science 2005, 143, 469485 


\title{
P.883
}

\section{Analysis of oligosaccharides in pigs' colostral milk}

\author{
Regine Preissler ${ }^{1}$ Nicole Kemper $^{1}$ Karl Klisch $^{2}$ \\ 1. Institute for Animal Breeding and Husbandry, Christian-Albrechts-University Kiel, Kiel, Germany; \\ 2. School of Veterinary Medicine and Science. University of Nottingham, Nottingham, UK
}

\section{Introduction}

Milk oligosaccharides (MOs) have been investigated in humans as well as in several other mammals, but studies of sows' milk are lacking (Urashima et al., 2001).

Their prebiotic and antiadhesive effect for the newborns is well described for human milk oligosaccharides (Bode, 2009) and can be assumed for other species, too. Because of increasing litter sizes and the rising demand for milk substitutes in farrowing units, the composition of sows' milk, including oligosaccharides as functional ingredient, is of peculiar interest. Furthermore, a possible anti-adhesive effect in sows' mammary glands may play a role in the pathogenesis of mastitis as part of the Postpartum Dysgalactia Syndrome (PDS).

This study therefore analysed MOs in sows' colostral milk, considering also the clinical health status of these sows in the periparturient period and bacteriological results of their milk samples.

\section{Material and Methods}

At the Institute's research farm, 17 sows were examined clinically at the expected farrowing date and milk samples were taken. Five sows showed clinical signs of mastitis during the sampling time or developed mastitis post partum.

All sows were sampled during or directly after parturition, and all milk samples were obtained by hand milking without oxytocin injection. Bacteriological analysis and species differentiation were performed by routine bacteriological diagnostics. At least one $\mathrm{ml}$ milk was stored at $-20^{\circ} \mathrm{C}$ and MOs were isolated, reduced and permethylated. Analysis was performed by MALDI-TOF mass spectrometry.

\section{Results}

All detected MOs were sialylated with terminal sialic acid (Neu5Ac). No fucosylated MOs were detected. The reducing end of the oligosaccharides is generally formed by lactose (Gal $\beta 1-4 \mathrm{Glc}$ ) and in much lower abundance by lactosamine (Gal $\beta 1$ 4GIcNAc). Sialyllactose (Neu5Ac2-[3/6]Gal $\beta 1-4 G l c)$ was the most abundant structure in all samples. The carbohydrate portion of GM2-ganglioside (GalNAc $\beta 1-4[\mathrm{Neu} 5 \mathrm{Ac} 2-3] \mathrm{Gal} \beta 1-4 \mathrm{Glc}$ ) revealed the second highest peak in most samples, but was completely absent in one sample and just detectable in one other sample. Biantennary oligosaccharides formed the highmolecular fraction with monosialylated lacto-N-hexaose (Neu5Ac-Gal-GlcNAc-[GalGlcNAc-]Gal $\beta 1-4 \mathrm{Glc}$ ) being the third most abundant structure in most samples.

\section{Discussion}

This is the first study analysing MOs in pigs. The porcine MOs show some similarities with those of cattle (Tao et al., 2008). In both species sialylated MOs dominate and fucosylated MOs are absent. One main difference is the presence of high quantities of the GM2-ganglioside carbohydrate in pig MOs. Only traces of this structure are found in bovine MOs. Pig's milk contains more complex high molecular weight biantennary MOs than bovine milk.

Like in other species the MOs in pigs are likely to act as prebiotics by supporting the development of a beneficial gut flora in piglets. The knowledge of the natural composition of porcine MOs could be the basis for further functional studies and also for a rational composition of food supplements for piglets and weaners.

Possible changes in oligosaccharide patterns between healthy and diseased animals should be investigated in further studies.

\section{References}

Bode, L., 2009. Human milk oligosaccharides: prebiotics and beyond. Nutrition Reviews 67, S183-S191.

Tao, N., Depeters, E.J., Freeman, S., German, J.B.,

Grimm, R., Lebrilla, C.B., 2008. Bovine milk glycome. Journal of Dairy Science 91, 3768-3778.

Urashima, T., Saito, T., Nakamura, T., Messer, M., 2001. Oligosaccharides of milk and colostrum in non-human mammals. Glycoconjugate Journal 18, 357-371.•

\section{Acknowledgement}

The authors want to thank Anne Dell and Poh-Choo Pang (Division of Molecular Biosciences, Faculty of Natural Sciences, Imperial College London) for the mass spectrometric analysis. 


\title{
P.884
}

\section{Impact of uterine crowding on fetal development and lactational performance}

\author{
John Harding; Shrijana Dhakal; Yanyun Huang; Crissie Auckland \\ University of Saskatchewan, Saskatoon, SK, Canada
}

\section{Introduction}

Prenatal programming is a process by which stimuli during gestation establish permanent responses in the fetus that impact physiologic function later in life. In pigs, low birth weight or body mass index are associated with altered metabolism, fat deposition and stress response ${ }^{1,2}$. Uterine overcrowding reduces fetal weight, impairs myogenesis and organ development ${ }^{3}$. We are investigating the effects of uterine crowding on postnatal health and performance. Reported herein are descriptive statistics highlighting the effects of uterine crowding on fetal development and lactational performance.

\section{Materials and Methods:}

Right oviductal cauterization was performed 2 weeks post weaning on 13 multiparous PIC sows (CAUT). CAUT and 12 control sows (CTR) delivered 10 to 17 total born on their previous parity and were in good body condition at weaning. After surgery, sows were heat synchronized and inseminated. Corpus lutea and embryo number were counted via laparotomy at 36 \pm 2 days of gestation. At farrowing, litter size, gender, individual birth weight, crown rump length (CRL), heart girth was recorded for live and stillborn pigs. Judicious cross-fostering between treatments ensured CAUT and CTR sows suckled piglets of the opposite group. Non-trial pigs were fostered onto trial sows as appropriate to ensure CAUT and CTR litter sizes were similar. Piglets were re-weighed at day 21 . Sows were weaned and rebred, and farrowed a subsequent litter if they conceived. Laparotomies were not performed, but performance was monitored. Parametric and non parametric outcomes were compared between group using Student's t-test or Mann-Whitney $U$ test as appropriate.

\section{Results:}

The significantly lower embryo numbers in CAUT (Table 1) provided an enriched uterine environment resulting in increased embryo survival, heavier and longer piglets with greater girth and BMI at birth (Table 2). The CAUT offspring had superior 21 day survivability even though litter sizes after cross- fostering were numerically similar in CTR and CAUT sows. Lactational ADG and weaning weight were significantly higher in CAUT offspring.
Table 1: Ovulation rate, embryo number and survivability in unilateral cauterized and control sows

\begin{tabular}{|l|c|c|}
\hline Variable & CAUT & CTR \\
\hline No. sows & 13 & 12 \\
\hline Ovulation rate & $23.9 \pm 4.9$ & $27.0 \pm 4.1$ \\
\hline Ovulation rate (available ovary) & $12.9 \pm 3.5^{\mathrm{d}}$ & $27.0 \pm 4.1$ \\
\hline Total no. embryos & $9.1 \pm 3^{\mathrm{d}}$ & $15.3 \pm 4.3$ \\
\hline Embryo survival \% (available ovary) & $66 \pm .20 .4^{\mathrm{a}}$ & $53 \pm 16$ \\
\hline a $\mathrm{P}<0.05 ;{ }^{\mathrm{b}} \mathrm{P}<0.01,{ }^{\mathrm{C}} \mathrm{P}<0.005, \mathrm{~d} \mathrm{P}<0.001$ & \\
\hline
\end{tabular}

Table 2: Farrowing performance of unilateral cauterized and control sows and their offspring

\begin{tabular}{|c|c|c|}
\hline Variable & CAUT & CTR \\
\hline No. sows & 19 & 21 \\
\hline Total born/L & $7.9 \pm 2.9 c$ & $11.2 \pm 3.2$ \\
\hline Live born/L & $7.6 \pm 2.9^{b}$ & $10.4 \pm 3$ \\
\hline Birth wt (kg) & $1.7 \pm 0.3^{\mathrm{d}}$ & $1.4 \pm 0.3$ \\
\hline $\mathrm{DO} C \mathrm{CR}(\mathrm{cm})$ & $30.3 \pm 1.9^{c}$ & $28.4 \pm 2.3$ \\
\hline DO Girth $(\mathrm{cm})$ & $25.6 \pm 1.6^{c}$ & $24.1 \pm 1.5$ \\
\hline DO BMI & $0.18 \pm .01^{\mathrm{a}}$ & $0.17 \pm .02$ \\
\hline D0 post foster litter size & $10.1 \pm 1.7$ & $11 \pm 1.8$ \\
\hline Pigs/litter weaned & $9.2 \pm 1.7$ & $8.9 \pm 1.4$ \\
\hline D21 survival (no. pigs/L) & $8.2 \pm 2.3$ & $7.0 \pm 2.6$ \\
\hline D21survival (\% of live born) & $92.9 \pm 11.6^{c}$ & $82.1 \pm 2.5$ \\
\hline D21 weight $(\mathrm{kg})$ & $7.9 \pm .8^{c}$ & $7.0 \pm 0.9$ \\
\hline ADG 0-21 (kg/d) & $0.3 \pm 0.04^{\mathrm{a}}$ & $0.3 \pm 0.04$ \\
\hline
\end{tabular}

\section{Discussion:}

These preliminary results indicate fetal development was attenuated in the relatively crowded CTR sows resulting in disproportionate fetal growth and the birth of thin and slightly shorter pigs that had lower day 21 survival, weaning weight and lactational ADG. Additional analysis is planned to determine if these effects are dependent or independent of birth weight, and if uterine crowding affects post weaning health, stress response, growth and fat deposition.

\section{References:}

1. Poore, KR et al (2003) J Physiol (Lond). 547(1), 107-116.

2. Poore, KR et al (2004) J Physiol (Lond). 558(1), 295-304.

3. Town, SC et al (2004) Reprod (Cambridge). 128(4), 443-454 


\title{
P.885
}

\section{Evaluation of the in feed use of Larch sawdust in sows for treatment and prophylaxis of Postpartum Dysgalactia Syndrome}

\author{
Eleni D. Tzika ${ }^{1}$ Panagiotis D. Tassis ${ }^{1}$ Vassilios G. Papatsiros ${ }^{2}$ Dimitrios Koylialis ${ }^{1}$ \\ Asimoula Siochu ${ }^{3}$ Panagiota Toyplikioti ${ }^{3}$ Costas Alexopoulos ${ }^{4}$ Spiros C. Kyriakis ${ }^{5}$ \\ 1. Clinic of Farm Animal Medicine, Faculty of Veterinary Medicine, Aristotle University of Thessaloniki, Thessaloniki, Greece; \\ 2. Clinic of Medicine, School of Veterinary Medicine, University of Thessaly, Karditsa, Greece; 3. Microbiology Laboratory, Thessaloniki, Greece; \\ 4. Deceased author, Thessaloniki, Greece; 5. Foundation of Biomedical Research, Academy of Athens, Athens, Greece
}

\section{Introduction}

The Postpartum Dysgalactia Syndrome (PPDS) causes important economical losses in the global swine industry. The clinical signs are characterized mainly by disorders of lactation and health status of sows, as well as from decreased litter performance (1).

The aim of this field study was the assessment of possible anti-inflammatory activity of Larch sawdust, Echinacea, Pumpkin, Salix, Sinupret and Grape Seed based on the reduction of PPDS prevalence in sows. This trial was part of the SAFEWASTES specific targeted research project financed by the European Union (6th Framework Project).

\section{Materials and Methods}

The trial farm had 900 sows under production and previous history of PPDS occurrence. Genetically similar sows were selected in terms of achieving homogenity of parity, bodyweight, previous production parameters and health status. Thus, 40 sows with no clinical signs of disease were randomly allocated in 8 groups of 5 sows each. The trial period was 35 days (from 7 days prior to farrow up to weaning day). Six compounds at the inclusion level of $1 \%$ were added to sow feed in order to be tested for anti-inflammatory activity (gestation and lactation feed) as follows: 1) Control and Positive group: 99\% Basic diet and 1\% Corn starch, 2) Larch group: 99\% Basic diet and 1\% Larch, 3) Echinacea group: 99\% Basic diet and 1\% Echinacea, 4) Pumpkin group: 99\% Basic diet and 1\% Pumpkin, 5) Salix group: 99\% Basic diet and 1\% Salix, 6) Sinupret group: 99\% Basic diet and 1\% Sinupret, 7) Grape seed group: 99\% Basic diet and 1\% Grape seed.

The positive controls were injected with a single $2 \mathrm{ml}$ dosage of an anti-inflammatory drug (meloxicam) at parturition day. The animals of all the other groups were injected with the same dose of normal saline at the same day. The milk production index was calculated. Production and health parameters of sows and their litters were also recorded. Blood samples were collected from all sows at allocation day and at 24 and 72 hours post partum (PP) for inflammation markers evaluation (serum tumor necrosisfactor-2/TNF-a, plasma interleukin-6/IL-6). The cytokines results were expressed as $n$-fold increase of cytokine expression in relation to untreated sows at allocation day.

\section{Results and Discussion}

Results showed that larch sawdust had particular anti-inflammatory effect.
Figure 1: Mean rectal temperatures (OC) of sows 12 and 36 hours post farrowing

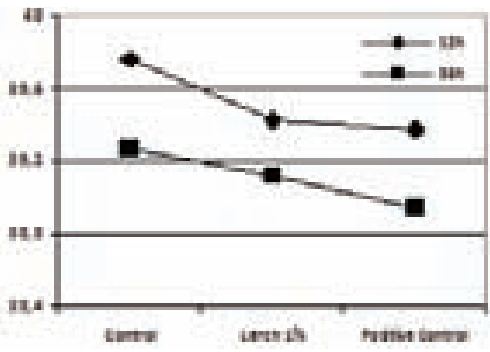

Figures 2a, 2b: Average litter body weight at 21 days of age $(\mathrm{kg})$ and average litter body weight gain $(\mathrm{kg})$
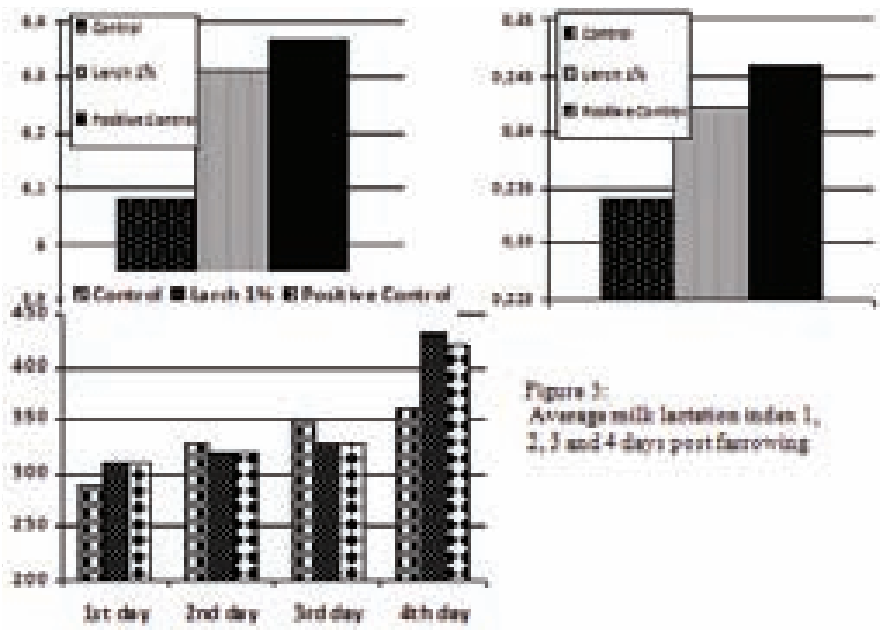

\section{Pipare 3}

Awerapoith lavtwien istan 1 .

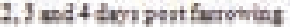

Table 1. Serum IL6 and plasma TNF- $a$ at sows $24 h$ and $72 h$ PP

\begin{tabular}{|c|c|c|c|}
\hline Sampling & Group & IL6 & TNF- $a$ \\
\hline \multirow[t]{3}{*}{24 hours PP } & Controls & $4.05 \pm 1.07^{\mathrm{a}}$ & $3.69 \pm 1.62^{\mathrm{a}}$ \\
\hline & Larch 1\% & $2.96 \pm 1.02^{\mathrm{ab}}$ & $2.11 \pm 0.71^{b}$ \\
\hline & Positive controls & $1.86 \pm 0.92^{b}$ & $0.86 \pm 0.29^{b}$ \\
\hline \multirow[t]{3}{*}{72 hours PP } & Controls & $2.48 \pm 1.71^{\mathrm{a}}$ & $2.68 \pm 1.32^{\mathrm{a}}$ \\
\hline & Larch 1\% & $1.59 \pm 0.86^{\mathrm{a}}$ & $1.07 \pm 0.55^{b}$ \\
\hline & Positive controls & $1.12 \pm 0.72^{\mathrm{a}}$ & $0.98 \pm 0.77^{b}$ \\
\hline
\end{tabular}

Sows fed with larch sawdust had an improvement tendency in performance and health parameters (eg. average litter body weight, PP temperatures) (Fig. 2a,b) compared to the control group. Additionally, there was a significant decrease of serum IL6 and plasma TNF a concentrations at 24 and 72 hours PP (Tab. 1), probably due to larch sawdust anti-inflammatory activity.

\section{References}

1. Papatsiros V.G. et al.J. Hellenic Vet. Med. Soc., 58:61-75, 2007. 


\title{
P.886
}

\section{The effects of an enhanced uterine environment on immune competence in pigs}

\author{
Jamie M. Wilkinson ${ }^{1}$ Susan Novak${ }^{1}$ Michael K. Dyck ${ }^{1}$ Walter T. Dixon ${ }^{1}$ George R. Foxcroft ${ }^{1}$ John C. Harding ${ }^{2}$ \\ 1. University of Alberta, Edmonton, AB, Canada; \\ 2. University of Saskatchewan, Western College of Veterinary Medicine, Saskatoon, SK, Canada
}

\section{Introduction}

The 'fetal programming' hypothesis states that the gestational environment of the embryo affects its development, and that this can have long-term consequences for the animal postnatally. In swine, low birth weight animals exhibit an altered metabolism and stress response over a range of ages, and this could be programmed in utero ${ }^{1-2}$. To date, the potential of the uterine environment to influence the immune system of pigs has not been investigated. In this study, immune competence in the offspring of control sows from a commercial dam line was compared to immune competence in the offspring of sows from the same line that had undergone unilateral oviductal cauterization surgery to artificially reduce the number of embryos in utero and provide an 'enhanced' uterine environment.

\section{Materials and Methods}

The experiment used PIC Line 42 parity 3-5 sows in good health and body condition that farrowed between 10 and 17 total born on their most recent parity. Right oviductal cauterization was performed on a group of 10 sows (CAUT). The oviducts of 11 age-matched controls were not cauterized (CTRL). Following surgery, sows in both groups were estrus synchronized by treatment with oral progestagen and bred. Farrowing was induced by cloprostenol to obtain a gestation length of 114 to 116 days. Individual gender and birth weight data were recorded for all piglets born. Blood samples were collected from 2 male and 2 female piglets from each litter at 4, 9, and 15 weeks of age. For each sex, one piglet representative of above and one representative of below average litter birth weight was chosen. Animals at the extremes of birth weight were excluded. Lymphocytes were isolated by density gradient centrifugation using 'Lymphoprep' (Axis-Shield), and $1 \times 10^{5}$ cells in triplicate were used to determine immune competence by measuring lymphocyte proliferation with or without addition of the B and T cell mitogen concanavalin A. Cells were grown in RPMI medium for 72 hours. Tritiated thymidine was then added and the cells were cultured for a further 18 hours, harvested, and incorporation of labeled thymidine was measured with a scintillation counter (TopPlate, Packard). Proliferation data were normalized by $\log _{2}$ transformation. Statistical analyses were performed using the MIXED procedure in Statistical Analysis Software (SAS 9.2, SAS Inc.).

\section{Results}

- Litter average birth weight was greater in CAUT $(1.74 \mathrm{Kg})$ than CTRL $(1.40 \mathrm{Kg})$ litters $(p=0.004)$.

- There was no overall effect of cauterization treatment on lymphocyte proliferation ( $p=0.402)$.

- Across all ages there was a positive correlation between birth weight and lymphocyte proliferation for the CAUT group $(r=$ $0.421 ; p=0.008)$ but not for the CTRL group $(r=-0.061 ; p=$ $0.636)$.

Figure 1. Relationship between birth weight and lymphocyte proliferation in response to concanavalin A stimulation over a 90-hour culture period.

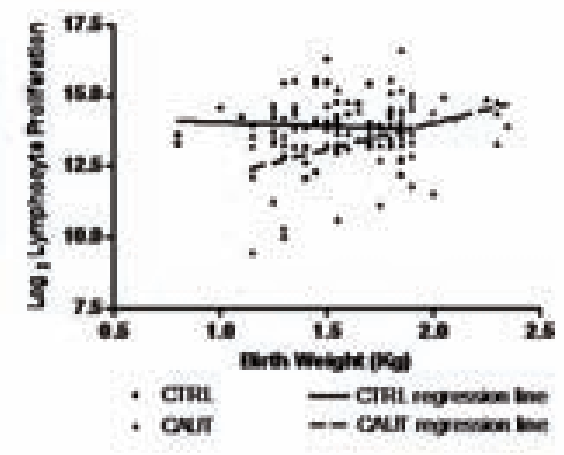

\section{Discussion}

The larger average birth weight of the CAUT compared to the CTRL pigs indicates that oviductal ligation successfully provided a uterine environment enhancing fetal growth of CAUT offspring. Although no overall difference in lymphocyte proliferation in the offspring of CAUT and CTRL sows was identified in this study, the positive correlation between birth weight and lymphocyte proliferation in the CAUT offspring and its absence in the CTRL offspring suggests that a combination of genetic and epigenetic factors may influence the post-natal immune capacity of pigs.

\section{References}

1. Poore et al. Diabetologia. 2002; 45: 1247-1254.

2. Klemke et al. J. Anim. Sci. 1993; 71: 1010-1018. 


\title{
P.887
}

\section{Improving sow behavior at farrowing}

\author{
Jan Jourquin ${ }^{1}$ Ilse Vanthillo ${ }^{3}$ Everson Zotti ${ }^{2}$ Lieve Goossens ${ }^{1}$ \\ 1. Janssen Animal Heatlh, Beerse, Belgium; 2. Sanphar SA, Campinas, SP, Brazil; 3. KHK Geel, Geel, Belgium
}

\section{Introduction}

Farrowing is a stressful event for sows, especially first parity sows. In the worst cases this leads to aggression and savaging of newborn piglets. Next to clear aggression, there is a range of less explicit unwanted behavior negatively impacting the possibility for the piglets to take up colostrum. Bottle fed piglets ingest more colostrum in the first day then naturally nursed piglets (1), indicating that the sows is a limiting factor for colostrum intake. The aim of two studies was to investigate if an injection of azaperone (Stresnil $\left.{ }^{\circ}\right)$ at the end of the partus has a positive effect on sow behavior and on sow-piglet interaction.

\section{Materials and Methods}

In a first study, 69 sows of all parities were injected with 320 $\mathrm{mg}$ of azaperone ( $8 \mathrm{ml}$ of Stresnil $\left.{ }^{\oplus}\right)$ at the moment of expulsion of the placenta. Simultaneously in the same environment 81 sows of all parities were enclosed in the study as non treated controls. At the expulsion of the placenta ( 0 hours) and 6 hours later, when the sedative effect of azaperone has worn out, the behavior of the sows was scored as follows. Calm: the sow is relaxed, lies on her side and the piglets have free access to the udder. Alert: the sow is alert, lies or sits. Stressed: the sow shows signs of unrest, rises and vocalizes at the slightest noise. Aggressive sows during parturition were injected with azaperone on the spot and excluded from the trial. On each time point, statistical comparison between groups on the ordinal scores was performed by means of the Wilcoxon Mann-Whitney U-test.

In the second study, 22 sows of all parities were treated in the same way as the first study and compared to 11 non treated sows. At 2, 4, 6 and 8 hours after expulsion of placenta, the position of the sow (standing, lying on flank or belly), the accessibility of the udder (fully, half or not accessible) and the number of piglets suckling was recorded. The statistical analysis was performed with $S A S^{\ominus}$ version 9.1. A generalized linear model was performed to do statistical comparison between groups.

\section{Results}

The outcome of the first study is shown in Table 1.

\begin{tabular}{|c|c|c|c|c|}
\hline \multirow{2}{*}{$\begin{array}{l}\text { Table 1: Behavior score } \\
\text { Behavior }\end{array}$} & \multicolumn{2}{|c|}{0 hours } & \multicolumn{2}{c|}{6 hours } \\
\cline { 2 - 6 } & Azaperone & Non-treated & Azaperone & Non-treated \\
\hline Calm & $68 \%$ & $78 \%$ & $87 \%$ & $69 \%$ \\
\hline Alert & $20 \%$ & $14 \%$ & $13 \%$ & $27 \%$ \\
\hline Stressed & $12 \%$ & $9 \%$ & $0 \%$ & $4 \%$ \\
\hline p-valve & \multicolumn{2}{|c|}{0.2121} & \multicolumn{2}{c|}{0.0078} \\
\hline
\end{tabular}

Non-treated sows show a decrease of calm behavior after farrowing, while the opposite is seen in the treated group.

In the second study, no difference was recorded in positioning of sow except for a decreased number of azaperone treated sows lying on their belly compared to non-treated sows ( $14 \%$ vs. $27 \%)$ at 4 hours. This also reflected in less hidden udders in the azaperone treated group.

Figure 1 shows the evolution of percentage of piglets suckling at observation.

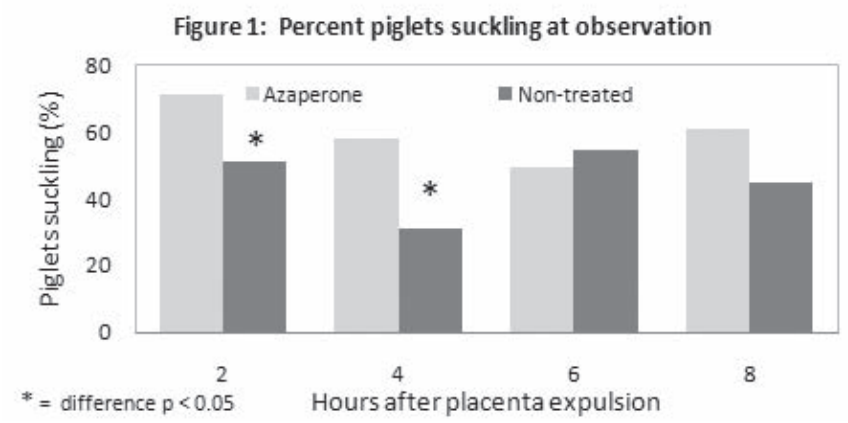

At 2 and 4 hours, there is a statistically significant increase in piglets that are suckling in the azaperone treated group.

\section{Discussion}

The results indicate that sow behavior during the first critical hours after farrowing influences the accessibility of the udder for the piglets. The azaperone treated sows allowed more piglets to suckle during the first critical hours. This may impact the survival rate and piglet growth. The effect was independent of parity. The present studies showed that an injection of azaperone at expulsion of placenta has positive effect on sows behavior and on sow-piglet interaction, facilitating the piglets to suckle during the early colostrum phase.

\section{References}

1. N. Devillers et al., Animal Science 2004, 78, 305-313 


\section{Author Index}

Aakerblom, Simon....................................... 80

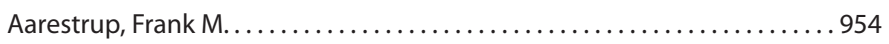

Aastrup, Peter ........................................... 105

Abad, Francesc X...................................... 76

Abad, Miguel.................................667, 668, 669, 683

Abbott, Elizabeth M................................ 720, 721

Abizanda, Teresa........................................... 772

Abonyi, Tamas.......................................... 1134

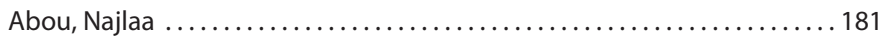

Abrahante, Juan ......................................... 313

Abs-EL Osta, Youssef......................... 138, 624, 625, 626, 628

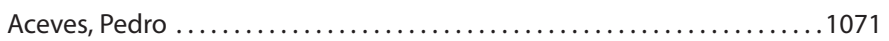

Achacha, Maamar ..........................................

Acutis, Pierluigi ........................................... 337

Adachi, Yoshikazu ...............................726, 734, 915

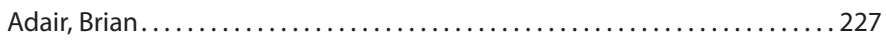

Adam, Matthias J.......................... 281, 400, 440, 716, 1146

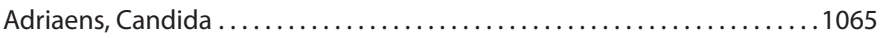

Afonso, Esther R.................................60, 966, 1092

Agneessens, Richard............................... 1023, 1024

Agnoletti, Fabrizio ...................................... 83

Agten, Sonja ....................................... 421

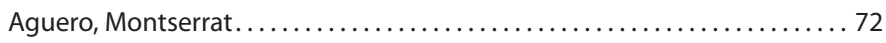

Aguilar-Calvo, Patricia .............................. 151, 547

Aguilar, Francisco...................................... 444

Aguilera Barreyro, Araceli ................................. 1034

Ahmad, Sardar ....................................... 843

Alapi, István ............................................... 614

Alarcon, Pablo.......................................... 57

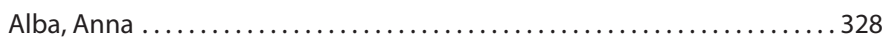

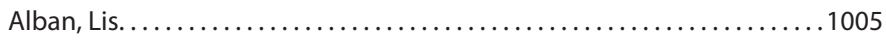

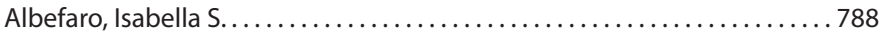

Alborali, Loris G. .................................463, 483, 585

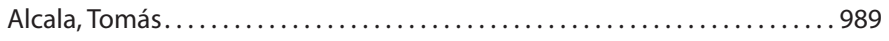

Alegre, Ana............................................ 651

Aleksandrov, Marin ....................................... 996

Alemany, Reina................................... 523, 866

Alex, Michaela.................................... 146, 304

Alexa, Pavel ........................................... 291

Alexopoulos, Costas ......................634, 711, 780, 782, 1191

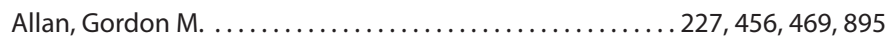

Allemandou, Aude...................................... 52

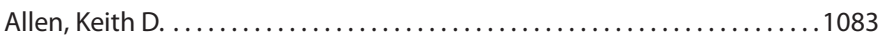

Allison, James R..................................1006, 1139

Almeida, Lissandro . ....................................... 359

Almería, Sonia....................................... 982

Almond, Glen W............................ 81, 87, 108, 635, 921

Alonso-Mares, Diana L.................................... 934

Alonso, Carmen ..................................... 98, 746

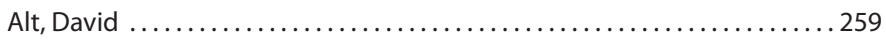

Alt, Katja .............................................. 119

Alt, Michael ........................................... 558

Altamirano, Mariana ..................................... 59

Althouse, Gary C......................................... 103

Alujas, Alberto M........................................ 95

Alvarado-Cesar, Gabriela................................... 198

Álvarez, Sonia .................................742, 969, 971

Alves, Guilherme G.................................... 788

Alzina-López, Alejandro................................. 1102

Amadori, Massimo..........................490, 919, 920, 1172
Amaral, Armando L. ..................................... 862, 1122

Amaral, Nikolas O. .................................... 1040, 1041

Ambrogi, Arnaldo .................................621, 731, 964

Ambrose, Divakar J....................................... 132

Åmdal, Solfrid ........................................... 256

Amezcua, Maria R..............................251, 306, 929, 930

Amonsin, Alongkorn..................................... 589

Amy, Marks-Callahan .................................... 1044

An, Dong-Jun . . . . . . . . . . . . . . . . . . . . . . . . . . . . 864

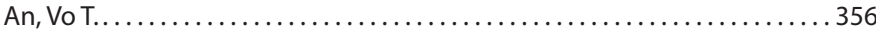

Andaluz, Daniel ......................................... 485

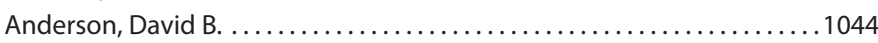

Anderson, Janet ........................................... 704

Andersson, Mats............................................ 1063

Andrade, André F. . . . . . . . . . . . . . . . . 1067

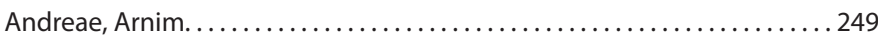

Andreasen, Margit........................................ 121

Andreu, David ........................................ 225

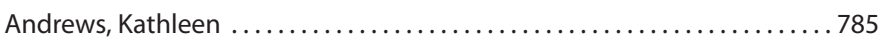

Andrews, Stuart ............................1129, 1130, 1132

Angen, Øystein .................... 91, 284, 464, 596, 597, 702, 756

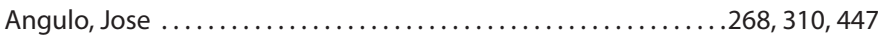

Anil, Leena .................................... 292, 1167, 1168

Anil, Sukumarannair S............149, 292, 293, 295, 510, 1167, 1168, 1169

Antillano, Carlos....................................... 830

Antonelli, Andrea....................................... 909

Ao, Zhuogui .......................................... 517

Aparicio, Maria ....................................... 212, 1182

Apel, Jürgen..................................... 122, 951

Appel, Bernd ........................................... 119

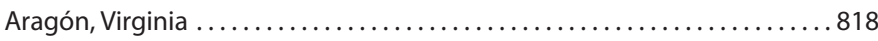

Aramouni, Mario ............................... 231, 232, 880, 883

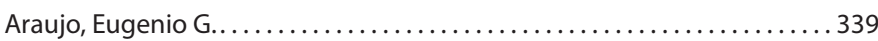

Arcelia, Alvarado I........................................ 855

Archambault, Marie ................................. 612, 641

Archetti, Ivonne ........................................ 296

Arcos, Mario....................................... 969, 971

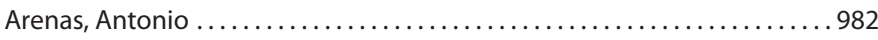

Arevalo, Pablo ...........................................677

Argilaguet, Jordi M.................................. 261, 846

Argüello, Héctor........................ 724, 739, 742, 969, 971

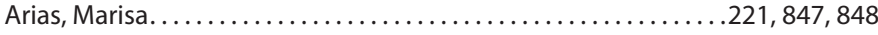

Arimi, Samuel ...................................... 278, 1099

Ariza-Nieto, Claudia ..................................... 61

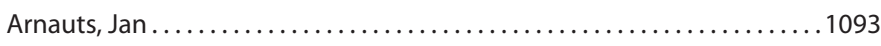

Arrospide, Silvio ........................................... 692

Arruda, Andréia G........................................ 966

Arruda, Rubens P................................. 70, 1067

Arsenault, Julie ....................................... 494

Arturo, Garcia F. . . . . . . . . . . . . . . . . . . . . . . . . . . . . 855

Ascanio, Elias......................................... 448

Astrup, Peter .......................... 354, 355, 360, 751, 889

Atanasova, K........................................ 54

Auckland, Crissie ........................127, 128, 922, 1185, 1190

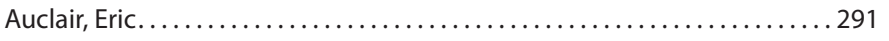

Auerbach, Horst ..................................... 174

Austin-Busse, Rose-Leah ............................... 819

Auvigne, Vincent ......................203, 213, 413, 500, 697, 698

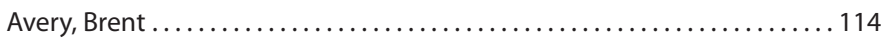

Ayllón, A............................................. 1125 
Baadsgaard, Niels Peter Babinszky, László .44

Babiuk, Lorne

1022,1027

Baborenko, Elena P.

Bacci, B. ........................................ 283, 458

Bacci, M.L...................................... 283, 458

Badiola, Ignacio ........................................... 1055

Bae, Chae Wun ................................ 445, 584, 814

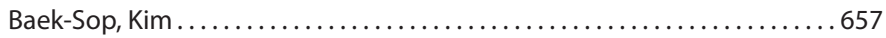

Bækbo, Poul.................................... 384, 843

Baekbo, Poul .................................237, 241, 707

Baekbo, Poul ....................................... 327

Baidoo, Samuel K. .................. 61, 292, 295, 787, 1167, 1168

Baier, Sylvia ........................................... 553

Bak, Hanne .................................. 340, 347, 706

Baker, Rodney ......................................... 894

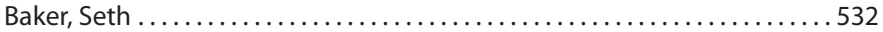

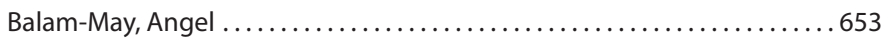

Balantakbo, Jose R. .................................. 371, 373

Balasch, Mònica ......................................... 314

Balasch, Sam ....................................... 917

Balatinec, Jelena. .................................... 1112

Baldin, Cíntia M......................... 340, 474, 885, 887, 888

Balenovic, Tomislav ..................................... 1105

Balis, Bart M........................................ 421

Balka, Gyula ..................................................................... 528

Ballarà, Isaac R. ................................ 486, 522

Ballester, Maria ................................... 846, 880

Bano, Luca ......................................... 83

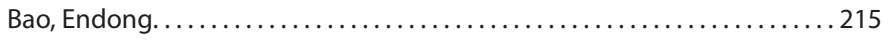

Barbé, F............................................. 54

Barbosa, Clara N. ......................................... 336

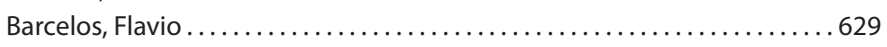

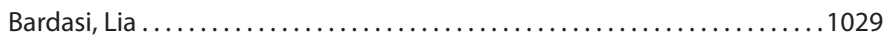

Barmettler, Thomas...................................... 553

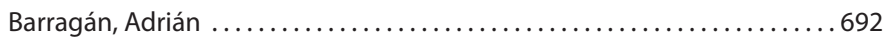

Barranco, Inmaculada..............................147, 508, 560

Barros, Flávia R....................................... 1092

Barthasson, Denise L................................ 339, 473

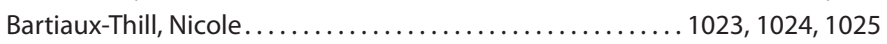

Bartol, Frank F . . . . . . . . . . . . . . . . . . . . . . . . . . . . . 211

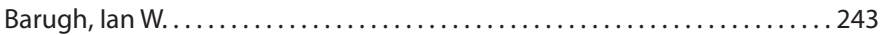

Barz, Andrea.................................... 197, 649

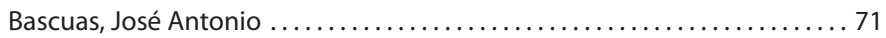

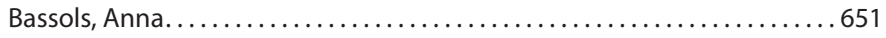

Bastos, João P. . . . . . . . . . . . . . . . . . . . . . . . . . . . . . . . . . 966

Batista, Laura .................................. 273, 274

Battistacci, Lorenzo ................................. 252, 919

Battisti, Antonio . ......................................... 954

Bauer, Sharon A.................................. 720, 721

Bautista, Criselda T. ............................... 540, 541

Bautista, Eijalin Z................................... 988

Bautista, Marc ........................................ 348

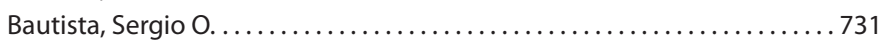

Baybikov, Taufik Z.................................... 527, 610

Bayegan, Keywan.................................... 904

Bayles, Darrell O................................ 259, 822

Baynes, Ronald ...................................... 81

Bayon-Auboyer, Marie Helene.............................. 820

Baysinger, Angela ................................... 308, 307, 659

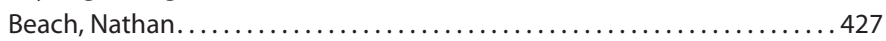

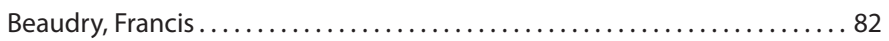

Becerra, Juan........................................ 1031

Becerril, Joaquin.................................... 1071

Becker, Petra M. ....................................... 617
Beckler, Dana C......................................... 709

Beers-Schreurs, Hetty van.................................. 305

Begg, Douglas........................................ 129

Behan, Stephen K................................... 75,258

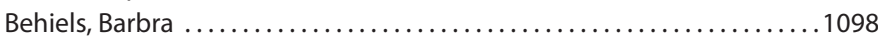

Behringer, Wilhelm ............................... 904, 906

Belák, Katinka ....................................... 316

Belik, E..............................................610

Bellini, Silvia ..................................... 483

Bello, Natalia ......................................... 276

Belloc, Catherine ............................266, 413, 761, 945

Beltran-Figueroa, Rolando ................................ 570

Beltranena, Eduardo.................................... 254

Benaglia, Paolo.........................................1106

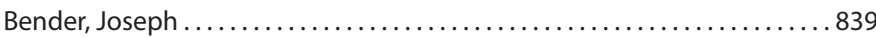

Bensaid, Albert ........................................ 76

Bensari, Abdelhak ....

Benyeda, János........................614, 616, 868, 870, 873, 874

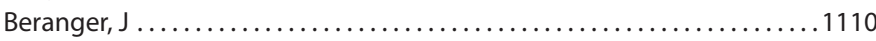

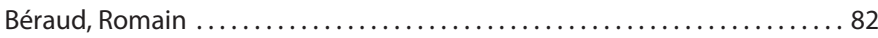

Berezowski, John ..................................... 932

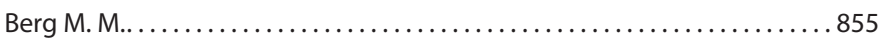

Berges, Ana Cristina .................................... 648

Bergsjø, Bjarne ..................................... 773

Bernabé, Antonio.................................. 147, 508

Bernal Santos, María Guadalupe.............................. 1034

Bernardy, Jan.......................... 163, 291, 619, 779, 1064

Bernier, Dave ....................................... 82

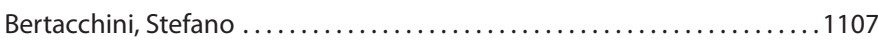

Bertani, Giovani R..................................... 336

Berthelot, Nicolas................................ 218, 998

Bertone, Judhit ................................693, 794, 795

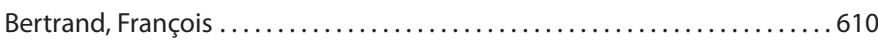

Bertrand, Marianne .................................... 761

Bertsch, Natalie. ...................................... 913

Bertucat, Bénédicte.................................... 213

Bessone, Fernando A.................................., 594

Beth, Young ...................................... 496, 497

Bey, Russ ........................................... 193

Beyerbach, Martin ..................................... 481

Bidan, Fabrice .................................... 933

Biermann, Johan .................................... 294, 1187

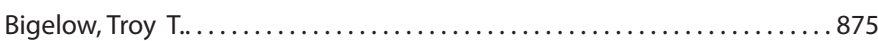

Biksi, Imre ..................................... 443, 614

Billard, Caroline....................................... 922

Bille-Hansen, Vivi. ................................ 316, 327

Bilskis, R. ............................................ 1131

Biryuchenkov, Dmitri ................................ 610

Bischoff, Ralf ........................................... 1084

Bishop, R.P. .................................. 847, 848

Blackwell, Tim ................................189, 303, 923

Blaha, Thomas........................118, 123, 175, 943, 950, 953

Blanco, Renny ........................................ 830

Blecha, Frank .......................................... 600

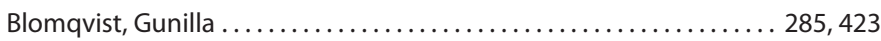

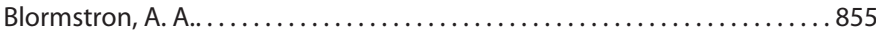

Blunt, Ruth ................................ 93, 95, 689, 935

Bobadilla, Ernesto ................................... 586

Bochicchio, Davide ................................... 277

Böcker, Anita ........................................ 122

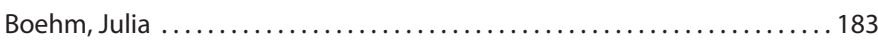

Boehmer, Jan................................ 481, 821

Boettcher, Jens ................................. 146, 304

Bogner, Péter......................................... 654

Boilletot, Eric .....................................684 
Bollo, Jesus M............. 392, 395, 398, 399, 401, 402, 409, 410, 415, 422 535, 632, 646, 677, 772, 829, 1010, 1090, 1108, 1109

Bolsius, Nadine.

234,690

Bonde, Marianne ........................................... 277

Bonet, Jordi ................................... 668, 669

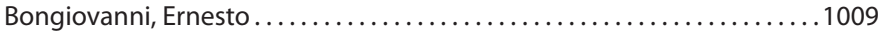

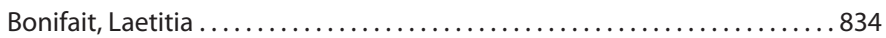

Bonilauri, Paolo............ 283, 407, 432, 458, 735, 791, 826, 1106, 1107

Boniotti, Beatrice ................................... 483

Boonsoongnern, Alongkot.............................. 1121

Boonyawatan, Suraphan ................................. 1141

Boonyawatana, Suraphan................................ 380

Borbolla, Arturo G................................ 1056, 1058

Bordin, Edson .............................. 376, 629, 630, 631

Borge, Carmen .................................... 974, 1123

Borges, Elizabeth L................................... 235

Borghetti, Paolo ................................. 397, 407, 408

Bories, Pauline...................................... 125

Borisov, Vladimir.......................................610

Borobia Belsué, Jesus ................................48, 583

Borri, Elena ......................................... 1107

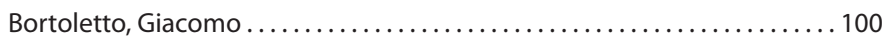

Bortoletto, Giacomo ................................... 208

Bosetti, Marco ........................................... 1009

Bosi, Paolo ................................................. 1029

Botermans, Jos .......................................... 1063

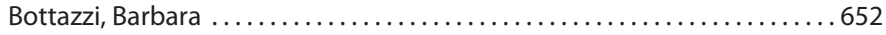

Böttcher, Jens ........................................... 1119

Boulanger, Andres................................. 4481, 830

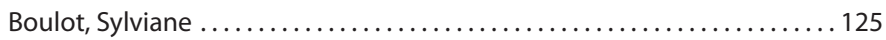

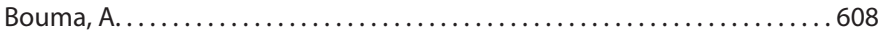

Bourdieu, Laetitia................................... 921

Bourgoin, Aude...................................... 277

Bourguignon, Patrick ...................................6 633

Bousquet, Eric ............................ 999, 1002, 1003

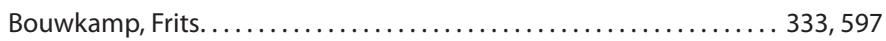

Bower, Leslie ...................................... 302

Boyd, Garth ......................................... 1044

Boyen, Filip........................................ 973

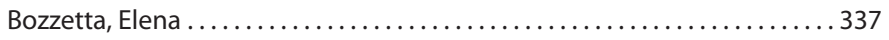

Bradaric, Zrinjka ...................................... 429

Braga, Caterine ......................................... 1073

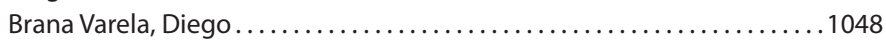

Brandão, Paulo E....................... 340, 474, 885, 887, 888, 1124

Brase, Katja........................................ 877

Brauer, Carsten .................................... 662, 663

Bräunig, Juliane ...................................... 119

Bresaola, Marcello ......................................... 884

Breuer, Wolfram ......................................... 905

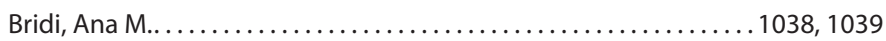

Bringas, Joaquim ..................................... 441

Brini, Elisa .......................................... 909

Brinkmann, Ulrich ..................................... 99

Briscolini, Sara ............................................ 331

Brito, Wilia M.................................339, 473, 620

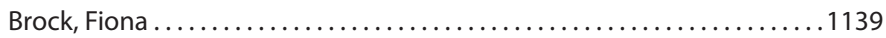

Brock, Fiona ........................ 1129, 1130, 1132, 1137, 1138

Brockers, Birgit ..................................... 118

Brockhoff, Egan................................. 931, 932

Brockmeier, Susan L........................... 192, 262, 264, 427

Broens, Els M............................................ 120

Bronnert, Bastian ................................ 107, 308

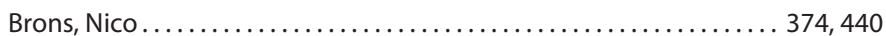

Brooks, Tiffanie A..................................... 250

Brown, I I . ........................................ 73
Brown, Karen K........................................ 571

Brown, Scott A......................................... 1006

Brüggemann, Christian ................................... 949

Brugnera, Enrico........................ 405, 418, 419, 455, 1117

Bruguera, Sergi D. .............. 276, 486, 519, 520, 521, 522, 523, 525

$526,530,533,539,540,541,543,866$

Brun, Edgar. ......................................... 256

Brunberg, Emma ....................................... 1163

Brunier, Eric ..................................... 226, 615

Brunori, Jorge ...

Bruns, Georg ................................... 43, 899, 900

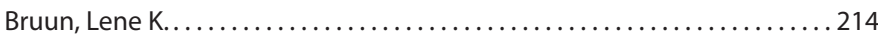

Bublot, M. .......................................... 565

Buergi, Esther ........................405, 418, 419, 455, 1117

Buitrago, Dolores..................................... 72

Bulnes Goicochea, Carlos ................................ 472

Bunpapong, Napawan.................................... 551

Burch, David G....................................675, 732, 986

Burrell, Angela M..................................... 568

Burrin, Douglas........................................ 11

Burzio, Giorgio ......................................... 871

Busch, Marie Erika ...................................44, 45

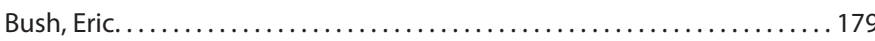

Busquets, Nuria. ........................... 76, 77, 261, 590

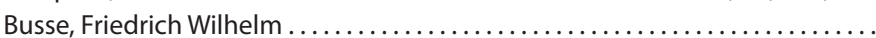

Byun, Jae W....................................... 763

Bzdawka, Marcin ............................................ 593

Caballero, José M. . ...................................... 86

Cabanes, J. Q....................................... 493, 561

Cabezón, Oscar..................................... 982

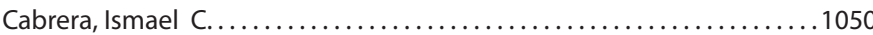

Cabrera, Rafael ............................................. 781

Cadenas, Evelyn ....................................... 898, 1097

Cadogan, David .......................................... 781

Caldwell, Brian ............................................ 106

Caldwell, Brian ................................................. 394

Caleffi, Antonio.................................. 397, 407

Callen, Antonio............................493, 529, 561, 863

Calus, Dries.............................................. 136

Cameron, Audrey C. . . . . . . . . . . .

Campbell, Joy .................................. 338, 560

Campos, Fábia S. ..................................... 460

Campos, Johanna M..................................... 180

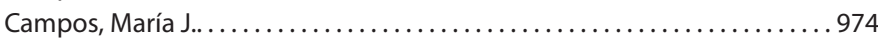

Cancer, Jose L............................................. 692

Candotti, Paolo................................. 296, 457, 1172

Canning, Paisley................................... 189, 303

Cano, G............................................ 863

Cano, Gonzalo..................................426, 683, 710

Cano, Guillermo .............................62, 999, 1002, 1051

Cano, Jean P. ............................... 149, 273, 448, 510

Cantarelli, Vinícius S.................................... 1040, 1041

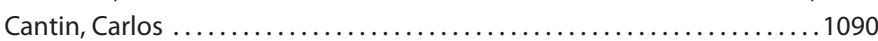

Cao, Dongmei ............................................... 979

Capalvo, Santiago .................................... 587

Capdevielle, Nathalie ......................................1110

Cappuccio, Javier A. ..............................563, 587, 692

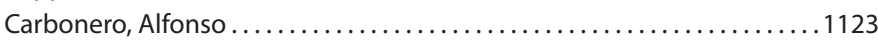

Cardoso, Marisa ................................... 967, 968

Careddu, Maria E.......................................... 337

Cariolet, Roland ......................................... 52

Carmen, Susy................................... 892, 923

Carmichael, Brent................................... 204, 206

Caro, Maria Rosa.......................................... 1123

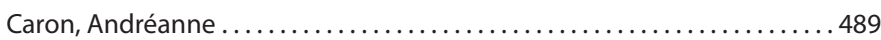


Carr, John

Carranza, Alicia I.

$142,461,1070,1142$

Carrasco, Librado . .

$621,731,964$

Carreon-Napoles, Rosalba.

229,239

Cartaud, Gérald.

.. 277

Carvajal, Ana

$724,739,742,969,971$

Carvajal, M

...1016

Carvalho, Luiz Fernando O..

.. 485

Casal, Jordi . ...

$.77,260,590,978,982$

Casas, Maribel .

442,978

Casey-Trott, Teresa M..................................... 1158

Casini, Luisa ........................................... 1029

Cassar, Glen ........................................ 251

Castro-Peralta, Felipa ................................... 228

Castro, Alessandra M................................ 340, 474, 1124

Castro, Joao Paulo S..................................... 645

Castro, José María ......................... 151, 498, 505, 546, 547

Castryck, Frans ................................. 155, 279, 777

Catalano, Antonella..................................... 46

Catana, Nicolae. ....................................... 491

Catelli, Anna............................................ 585

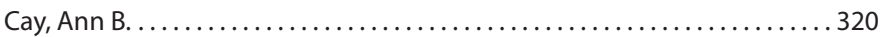

Celer, Vladimir ......................................... 886

Celestino, Enriquez ..................................... 1150

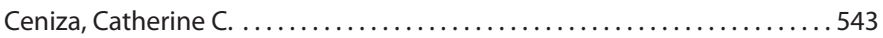

Cerati, Carlo ............................................ 1106

Cerne, Malin..................................... 747, 748

Cerón, José J................................... 749, 750

Cevidalli, Alberto E................................ 397, 407, 1009

Cha, Sangho......................................... 504

Cha, Seung-Bin ................................... 604, 605

Cha, Seungbin......................................... 775

Chabros, Katarzyna................................... 145, 503

Chae, Chan Hee .................................... 309, 316

Chai, Weidong .................................... 454, 813

Chaiyakul, Mark .......................................... 53

Chaiyanate, Penchan .............................. 551, 1012

Chalkias, Helena ............................................ 1181

Chanapiwat, Panida ....................................... 1078

Chang, Chia Y. . . . . . . . . . . . . . . . . . . . . . . . . . . . . 224

Chang, Nai-Yun .................................. 601, 603

Chang, Tsung C. .......................................685

Chang, Wei-Ming ........................................... 878

Chang, Yu-Mei....................................... 57

Channarong, Jitjaroen ...............................113, 349, 414

Chantong, Rawat .......................................226

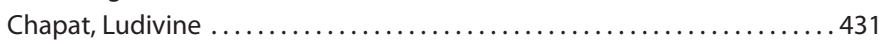

Chappell, Brad.................................. 931, 932

Charlebois, Audrey .......................................641

Charlemagne, Edouard .................................. 1017

Charoenvisal, Nataya ...................................... 564

Charreyre, Catherine...................... 205, 431, 454, 749, 750

Chauvin, Alban ............................................ 94

Chayomchai, Ampol ................................... 681, 691

Chen, Pin..............................................607 607

Chen, Shumin ......................................... 865

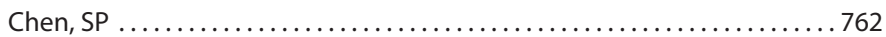

Chen, Ter-Hsin ..........................................601

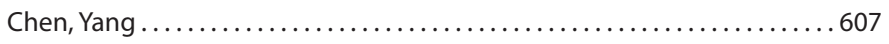

Chen, Zeng-Weng ...................................601, 603, 858

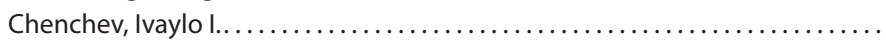

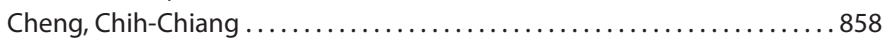

Cheung, Andrew K................................... 332

Chevalier, Mathieu....................... 205, 433, 437, 439, 442

Chevaux, Eric.............................. 209, 1179, 1180
Chevez, Jean Claude.................................. 310, 447

Chiapponi, C......................................... 565

Chien, Maw-Sheng ..............................601, 603, 858

Chien, MS ........................................... 446

Chiers, Koen........................................... 117

Chiou, Ming T. ............................................ 685

Chiou, Ming-Tang. ...................................... 511

Chirino-Trejo, Manuel....................................... 740

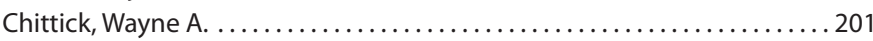

Chittick, Wayne....................................... 484

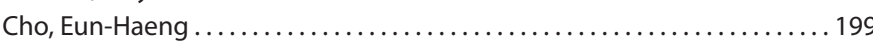

Cho, Ji-Eun .............................................. 605

Cho, Yongil .......................................... 504

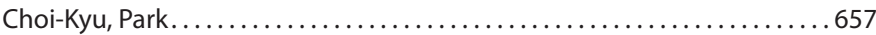

Choi, Dae-Gun. .........................................605

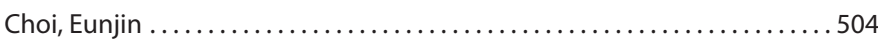

Choi, In-Soo ...................................... 199, 816

Choi, Young-Ki.......................................... 592

Chong, Daine......................................... 472

Choo, Pow Y...................................... 563, 526, 627

Chorfi, Younès............................................ 489

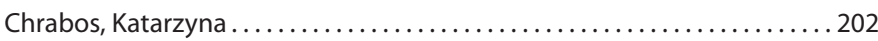

Christian, Nielsen K. ...................................... 80

Christiansen, Michael G. ................................. 214

Christopher-Hennings, Jane . ........................... 13

Ciacci-Zanella, Janice R..... . 74, 253, 262, 299, 336, 466, 468, 471, 862, 1122

Cinar, Mehmet U..................................... 914

Ciprian-Carrasco, Abel ...................138, 507, 624, 625, 626, 902

Ciuti, Francesca......................................... 331

Claerhout, Lieven....................................... 737

Clark, Amber ............................................ 661

Clavijo, Maria J............................................ 817

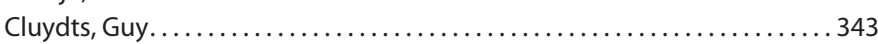

Coddens, Annelies.................................. 287, 764

Coldebella, Arlei ................................... 967, 968

Colin, Rafael......................................... 318, 319

Coll, Anna ........................................... 477

Collell, Miquel .........................................6 632

Collins, Alison M. ......................129, 233, 234, 688, 690, 723

Colomer, Susana. ........................................611

Comprido, Henrique v................................... 385

Concepción, Gómez ..................................... 221

Connor, Joseph F........................................., 133

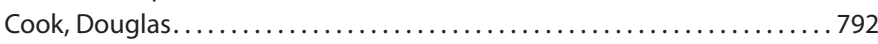

Cools, An ............................................ 64

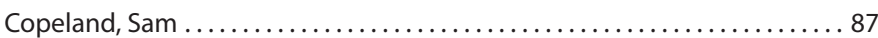

Corbellini, Luis Gustavo ................................... 967, 968

Cordioli, Paolo....................................... 585

Cordoba, Lorena. ..................................... 76

Corona-Barrera, Enrique ................................. 738

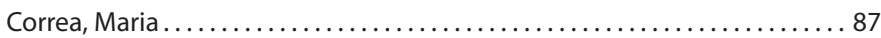

Corrégé, Isabelle. ..................................218, 962, 998

Cortes-Fernandez, Refugio............................ 228, 315

Cortés, Raquel............................................ 978

Cortey, Martí ............232, 286, 314, 316, 326, 328, 881, 882, 883, 978

Cortez, Adriana ................................... 334, 335

Cortiñas Abrahantes, Jose. . ................................... 764

Corzo, Cesar A. ............................................ 549

COSI Consortium, The .................................. 257

Costa, Adrienny T. ....................................334, 335, 460

Costa, Mirella C. . . . . . . . . . . . . . . . . . . . . . . . . . . . . . . . . . 701

Costarelli, Silva .................................... 46

Costinar, Luminita . ....................491, 513, 606, 618, 658, 975

Cottura, German. . ...

Couacy-Hymann, E....................................... 847 
Couteau, Mathieu .633

Coutinho, Tânia A. .. 888

Cox, Eric.

287,764

Cozette, Valérie.

205, 430

Craig, María I. .

563, 587

Crane, John P.

184,1139

Crenshaw, Joe.

.. 560

Crespo, FJC. . .

1059,1060

Crisci, Elisa

$.76,652$

Cristani, José . . .

$84,783,784,903$

Crooke, Helen

859,860

Cruijsen, Toine.

$272,450,554,555$

Cruz Muñoz, Eduardo................................... 764

Cruz, Celene.......................................... 239

Cruz, Eduardo C. Júnior...............................722, 757, 788

Cruz, Taís F......................................... 1124

Csank, Tomas......................................... 330

Cucco, Lucilla....................................... 728, 735

Cucurull, Glòria........................................ 917

Cui, Shangjin ..................................... 479, 876

Cui, Wenxiao ....................................... 322

Cunningham, Gail L................................220, 931, 932

Curado, Paulo H........................................6 620

Curiel, Fernando........................................ 389

Cutler, Tim D..................................... 506, 936

Czach, Jenifer L. ..................................... 75,258

Czanderlova, Linda .........................644, 815, 1177, 1178

Czub, Markus........................................ 53

D'Allaire, Sylvie .......................... 150, 489, 494, 501, 937

D'Angelo, Gloria ...................................... 735

D'Avino, Nicoletta ...................................... 728

Da Silva, A. . . . . . . . . . . . 29. 295, 1169

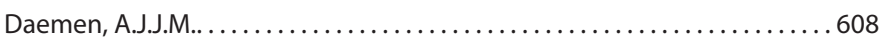

Dahl-Pedersen, Kirstin ................................. 1160

Dahmani, Yahya ......................... 69, 71, 1074, 1075, 1076

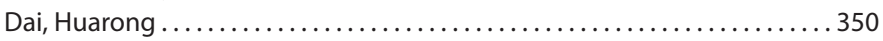

Daignault, Danielle ...................................... 114

Daigneault, Josee. ....................................... 185

Dallanora, Djane................................... 396, 1140

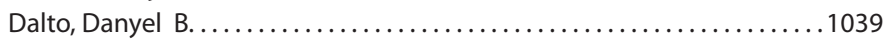

Dambros, Regia M.................................... 1122

Dan, Tran T. ......................................... 356

Danguy-des-Déserts, Rosine ............................... 820

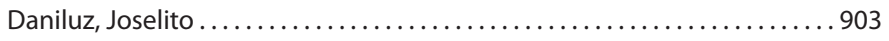

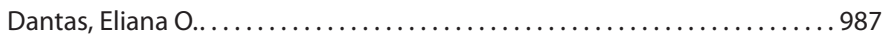

Dardari, Rkia........................................... 53

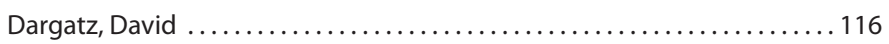

Darji, Ayub ........................................ 261

Darwich, Laila .................................. 529, 530

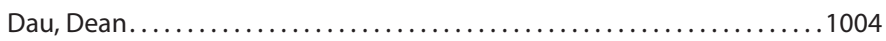

Davies, Peter .............................15, 106, 324, 394, 549

Davis, Brittany L....................................... 250

de Almeida, Kialane C.................................... 903

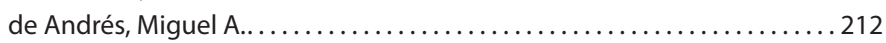

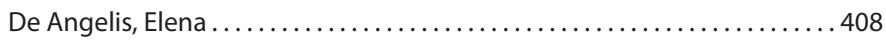

De Backer, Peggy ....................................... 343

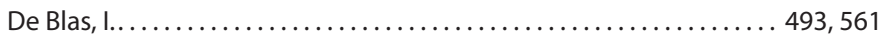

De Busser, Emily V.................................... 963

de Castro, Alessandra M. M. G........................ 885, 887, 888

de Castro, Fernando G..................................... 474

De Graef, Evelyne.............................. 155, 736, 737, 777

de Grau, Francisco ........................... 282, 341, 364, 435

de Groot, Mark W. .................................... 450

De Gussem, Koen....673, 676, 729, 995, 1000, 1019, 1022, 1026, 1027, 1183

de Jong, Ellen ..................... 1046, 1047, 1057, 1065, 1089 de Kruif, Aart ........................................... 1065

de Lange, Cornelius F................................. 1054, 1062

De Loera, Y .............................................. 1072

de Mi, G. ............................................. 847

De Paz Solanes, Xavier ..................................... 698

de Paz, Xavier ................................667, 668, 669, 683

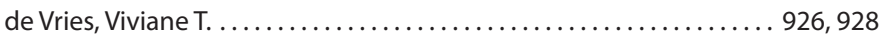

De Zutter, Lieven . . . . . . . . . . . . . . . . . . . . . . . . . . . . . . . . . . 963

Deardon, Rob ................................... 270, 492

Deckert, Anne E................................... 114, 115

Dedeurwaerdere, Audrey ........................... 679, 680

Dee, Scott............................. 149, 153, 510, 532, 549

Deen, John ..................153, 292, 293, 295, 298, 1167, 1168, 1169

deFoucauld, Jean..............................868, 870, 873

Degenstein, Karen ......................................... 836

Deitmer, Ricarda.................................. 712, 716

del Castillo, Jérôme R..................................... 82

Del Castillo, Susan V................................. 448, 830

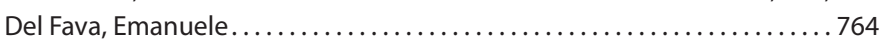

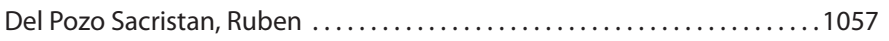

del Pozo, M. . ........................................... 848

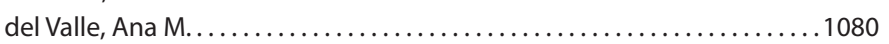

Delahaye, Bernard ........................................ 390

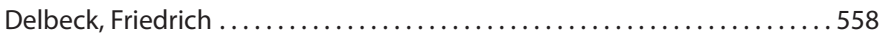

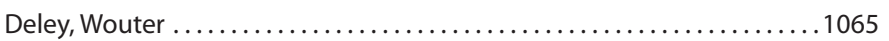

Delgadillo-Alvarez, Joaquin ....................... 138, 624, 625, 626

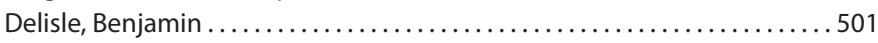

Delleur, Valery........................................ 802

Delvecchio, Andrea ........................... 205, 436, 630, 631

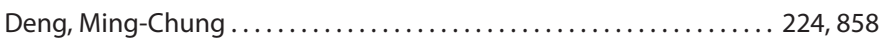

Denicourt, Martine ..................................... 489

Deniz, Abdulkerim......................... 797, 798, 801, 985

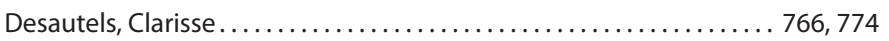

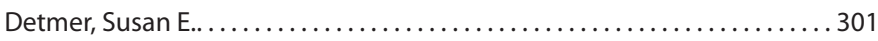

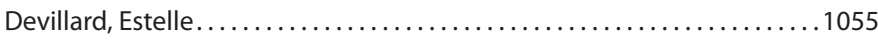

Deville, Sébastien.........................................610 610

Dewey, Cate.......................42, 85, 178, 270, 275, 278, 282, 364

$492,496,497,892,923,1099,1149$

Dewulf, Jeroen ..........47, 67, 117, 135, 938, 939, 963, 1057, 1065, 1089

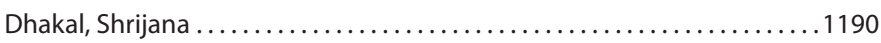

Di Cola, Gabriel..................................693, 794, 795

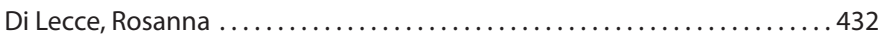

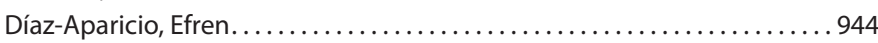

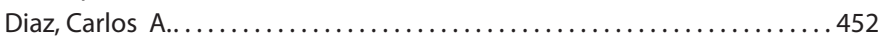

Diaz, Carmen ..................................... 448, 830

Diaz, Edgar ............................. 168, 169, 307, 310, 447

Diaz, Ivan....................................276, 529, 530

Díaz, Ivan...................................477, 560, 590

Dick, Joan ........................................... 741

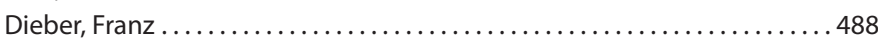

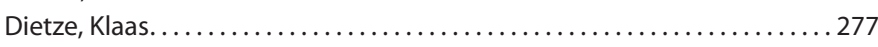

Díez-Fuertes, Francisco ................... 151, 498, 505, 546, 547

Dijkman, Remco.................................. 823, 824

Diniz-Mendes, Leonardo............................... 466, 471

Dion, Kate........................................... 267

DiPietre, Dennis ............................281, 400, 1146, 1148

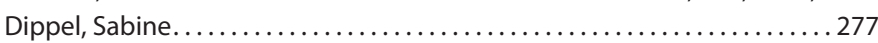

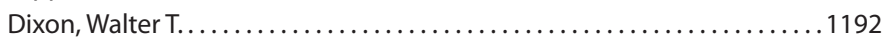

Do, Thuy N..................................766, 774, 960

Dobrzanski, Zbigniew ...............................1144, 1171

Doherr, Marcus G. . . . . . . . . . . . 148, 808

Dolores, Eva .......................................... 918

Dolso, Ismael M.............................693, 794, 795, 964

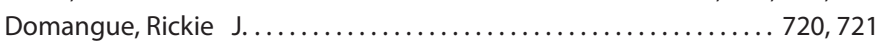

Domingo, Mariano ................................... 76,225 
Dominguez Perez, Patricia

Dominguez Punaro, Marycruz

Donato, Daniella C. .

Doncecchi, Paolo

Done, Stanley H.

Donin, Daiane

Doolittle, Kent.

Dorazio, Carly

Dorenlor, Virginie.

Dorn, Christina

Dotti, Silvia. . .

Dottori, M.

Dottori, Michele...

Drábek, Josef.

Drew, Trevor.

Driancourt, M.A.

Driessens, Bert

Drigo, Ilenia

Drigo, Michele.

Drungowski, Mario

Du, Yijun

Duangwhae, Nathaya

Dubey, Jitender P..

Ducatelle, Richard

Duchateau, Luc.

Ducommun, María de la Luz .

Duffy, Catherine

Duim, B....

Duinhof, Tom. ...

Dung, Bui T. ...................................... 533

Dupuis, Jeanne ....................................... 94

Dupuis, Laurent .......................................6 610

Duran, Leticia ......................................... 59

Duran, Oliver ................................... 647, 717

Dürrwald, Ralf ..........78, 297, 572, 573, 574, 576, 577, 578, 579, 580, 581

Dušek, Pavel. ............................................. 381

Dutil, Lucie ........................................... 114

Duvigneau, Catharina.................................... 904

Duyet, Hoang Nghia ..................................... 765

Dvorak, Ron ........................................ 781

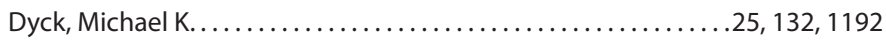

Eastaugh, Mark........................................ 1015

Echave, Alicia. ....................................... 402

Echeveste-Garcia de Alba, Ruben ............138, 228, 229, 315, 624, 625

$626,849,850,851,852,853,854$

Echeveste, Ruben. .................................... 507

Eckhardt, Octávio H............................... 60, 1092

Eddicks, Matthias................................... 956, 1157

Edler, Roy .......................................... 169, 307

Eger, Sabine ....................................... 553

Eggen, Alex ..110, 166, 167, 312, 341, 344, 356, 391, 393, 403, 404, 686, 1141

Ehlorsson, Johan ...............................423, 424, 598

Eicher, Susan D......................................... 176

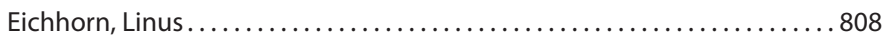

Eiki, Yamasaki ....................................... 960

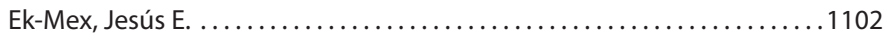

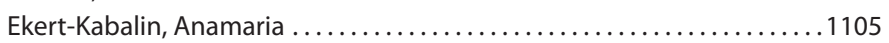

El-Attrache, J........................................ 482

Elicker, Sabine ............................ 509, 760, 905, 984

Eliopoulos, Chris D.............................. 720, 721

Elizalde-Castañeda, Paulino................................. 944

Ellert, J............................................ 344

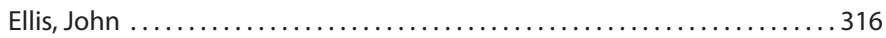

Ellsworth, Michael A........................................ 75

Elvstrøm, Anders ....................................... 207
Emanuelson, Ulf ......................................... 700

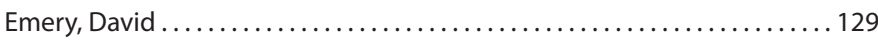

Encinares, Lorenzo. ......................................... 1049

Engels, Monika ....................................... 1117

Engl, Silke ............................... 1084, 1085, 1086

Engle, Mark J.......................................... 201, 512

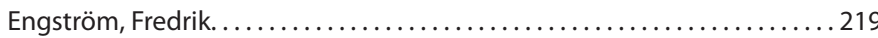

Enoe, Claes.................................... 327, 1005, 1035

Enriquez, Karina ................................ 467, 470

Ensley, Steve ......................................... 100

Eono, Florent ...............................660, 684, 933

Er, Chiek............................................ 256

Erawati, Arisa M.........................................

Erickson, Leah M. ......................................... 1154

Erlandson, Keith ........................................... 169

Escalada, David...................................... 422

Escobar García, Konisgmar.................................. 1034

Escobar-Lopez, Ana Cecilia.................................... 857

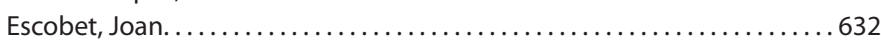

Espín, Sonia ............................................ 989

Espinoza, Angelica.................................... 586

Espinoza, Salvador..................................... 444

Espunya, Enric......................................... 72

Esquivel, Marisol. ........................................ 1011

Essen, S C......................................... 73

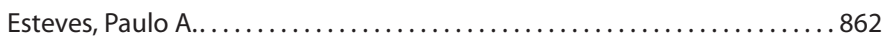

Estrada-B, SG ....................................... 1116

Evans, Charlotte M. ................................... 152

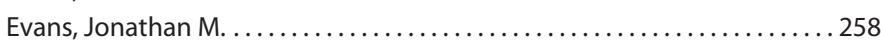

Evelsizer, Robert W................................ 743, 781

Eveno, Eric .................................660, 684, 933

Everett, Helen E. . . . . . . . . . . . . . . . . . . . . . . . . . . . . . . . . . 8 859, 860

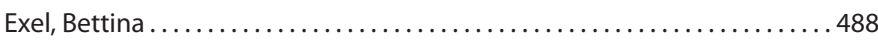

Ezpelata, Juan C......................................... 402

Ezpeleta, Juan C.........................................1109

Fablet, Christelle..............................660, 684, 933

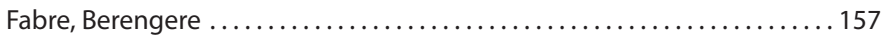

Faccenda, Marco ........................................... 337

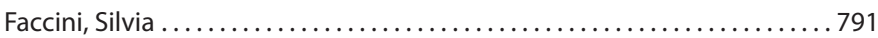

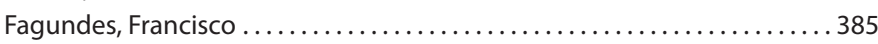

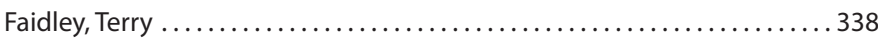

Fairbrother, John M. .........................766, 769, 774

Falceto, María Victoria ................................71, 648, 1108

Faldyna, Martin............................... 89, 841, 970

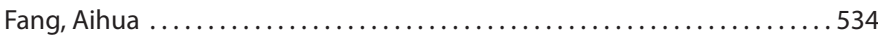

Fang, Brook ......................................... 350, 357

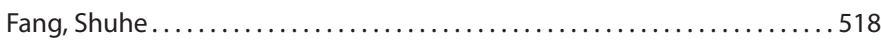

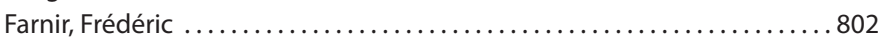

Farreres, Josep. ................................. 409, 410

Farzan, Vahab ......................................... 240

Faur, Bogdan ............................... 491, 513, 618, 658

Favero, Cíntia M........................... 340, 474, 885, 887, 888

Feagins, Alicia ........................................ 977

Feitsma, Hanneke ...........................68, 946, 947, 948

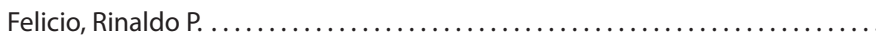

Fell, Shayne .................................234, 690, 723

Feltrin, Fabiola ........................................ 954

Ferchaud, S. ........................................ 134

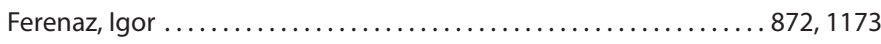

Fernández-Pinero, Jovita. ............................... 221

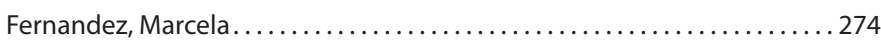

Fernandez, Marta.......................................611

Fernando, Champika ................................... 740

Ferrara, D. ...................................... 283, 458

Ferrari, Karen L.................................. 340, 1124 


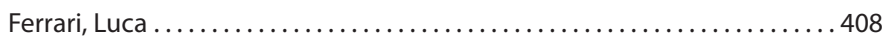

Ferrari, Maura ........................................ 884

Ferro, Paolo .......................................666, 1029

Fetsch, Alexandra...............................119, 950, 955

Fialho, Elias T......................................... 1040, 1041

Fielder, Ann E. . . . . . . . . . . . . . . . . . . . . . . . . . . . . . . . . 1006

Fierro, Jose A... . . . . . . . . . . . . . . . . . . . . . . . . . . . . . 59

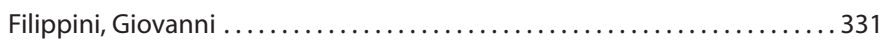

Fily, Bernard ................................... 436, 1111

Finestra, Albert ........................................ 829

Fischer, Laurent. ................................. 433, 437, 439

Fischer, Louis ....................... 476, 509, 760, 893, 984, 1118

Fischer, Susanne..................................... 118

Flahou, Bram ............................................ 117

Fleury, Roselyne ....................................... 346

Fomin, Alexander E................................... 527

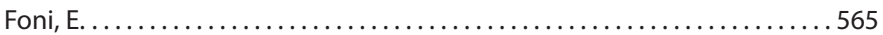

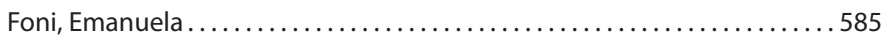

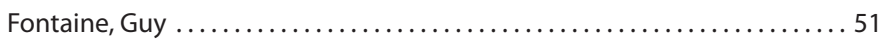

Fontana, Maria C......................................... 909

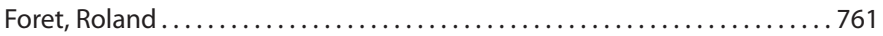

Forgie, Sarah ....................................... 254

Forier, Rudi .......................... 1023, 1024, 1025, 1026, 1051

Forster, Zenon ........................................ 740

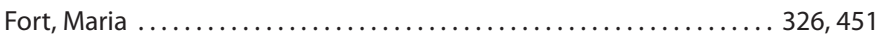

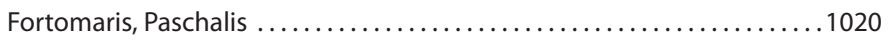

Fortunati, Manfredo ..................................... 331

Fossum, Caroline ................................ 285, 599

Fourchon, Pascal ..................................... 413

Foxcroft, George R. ............. 25, 58, 132, 133, 1082, 1098, 1185, 1192

Fraile, Lorenzo....................76, 86, 225, 327, 442, 651, 652, 989

Framstad, Tore ....................................... 190

Franchi, Luigi ............................................. 826

Francisco, Charles J........................... 806, 807, 1083

Franco, Alessia....................................... 954

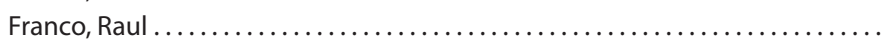

Franssen, Paul J.M........................................... 559

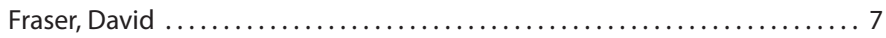

Fredriksen, Bente................................ 773, 1133

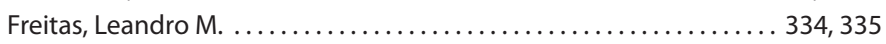

Fresen, Christina................................ 107, 308

Frey, Barbara ......................................... 725

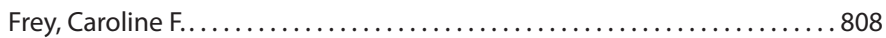

Frick, Johannes ...................................... 956

Friedrich, Alexander W. ...........................122, 951, 952

Friendship, Robert M................. 42, 85, 240, 251, 270, 306, 496

$497,892,923,929,930,1062$

Friese, Anika.................................... 123, 955

Frohm, Helena........................................ 1152

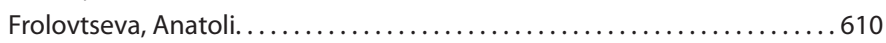

Fuchs, Thilo................................1129, 1130, 1132

Fugato Consortium RePoRI ................... 196, 602, 609, 656, 913

Fule, Jomer B. . . . . . . . . . . . . . . . . . . . . . . . . . . . . . . . 988

Funke, Juliane .................................. 926, 928

Furtado, Manohar .................................... 568

Fusaro, Laura ......................................283, 458, 909

G. De la Torre, Beatriz ..................................... 225

Gabardo, Michelle de Paula .................................. 903

Gagnon, Carl A................................... 51, 501, 641

Galeati, G. .......................................... 458

Galicia-G, ML ......................................... 1116

Galina-Pantoja, Lucina.................................... 194

Galinari, Grazielle C. . ..................................... 460

Galindo, Ivan .................... 225, 276, 317, 451, 530, 560, 846

Gallardo, C....................................... 847
Gallardo, Carmina .................................. 221, 846

Gallego, Andres M. ............................... 70, 1067

Galletti, Elena ........................................ 909

Gamba, Fausto ...................................... 1106

Gambade, Patrick....................................... 514

Gambova, Marcela................................. 644, 815

Gamlem, Hans. .......................................... 588

Ganges, LLilianne...................................... 225

Gangl, Armin ................................... 146, 304

Ganner, Anja ................................. 908, 1032, 1033

Garbay, Jhanina ............................................ 918

García-Artiga, Carlo..................................... 498

García-Bocanegra, Ignacio ........................... 260, 982

Garcia-Camacho, Lucia A............................444, 467, 470

García-Contreras, Adelfa Del Carmen................856, 857, 1072, 1116

García-Rendón, Alfredo ................................... 1058

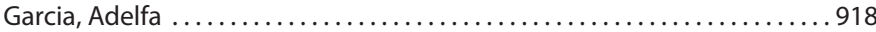

Garcia, Gustavo. ............................................ 525

Garcia, Jose M............................................ 535

Garciano, Serafin L. Jr ................................... 988

Garlaite, K. ............................................ 1131

Garrido, Angel.................................. 416, 417

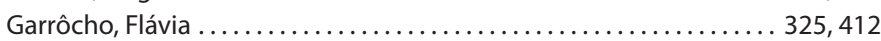

Gasse, Hagen........................................ 600

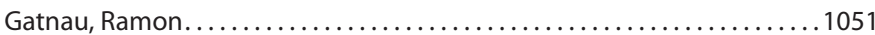

Gauger, Phillip C...........................74, 79, 255, 299, 332

Gaustad, Ann Helen ...................................... 190

Gautier, Nathalie. ....................................... 550

Gauvreau, Henry .....................................49, 50

Gava, Danielle......................................... 253

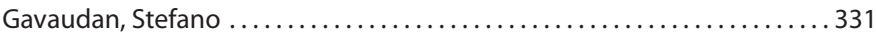

Ge, Xinna......................................... 322, 534

Gebhart, Connie J................... 236, 689, 701, 704, 709, 718, 719

Geers, Rony .......................................... 1159

Geiger, Jerome O......................................... 640

Geijlswijk, Inge van ............................946, 947, 948, 1170

Geldhof, M....................................... 54

Geldhof, Marc F. .................................... 544

Gelmetti, Daniela ........................................ 791

Geraert, Pierre-André .................................... 1055

Gerards, Joep. ..................................... 101

Gerber, Priscilla................................... 325, 412

Gerjets, Imke ..................................... 1176, 1186

Gerlach, Gerald F............................ 196, 600, 656, 662

German, Colmenares V. . . . . . . . . . . . . . . . . . . . . . . . . . . . 855

Germundsson, Anna...................................... 256

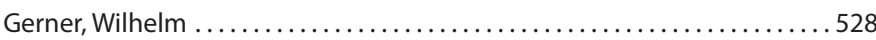

Gerth, Christel......................................... 122

Geudeke, Theo ........................................ 210

Geurts, Victor....................................... 272, 450

Gevaert, Dominique ................................ 802, 1008

Gherpelli, Yuri ....................................... 735

Giacomini, Enrico.................................... 483

Gibelli, Lucia................................................ 791

Gibert, Xavier....................................... 776, 778

Gil, Javier......................................... 1079, 1080

Gillespie, Tom ............................................. 377

Giménez, Merce ............................................651

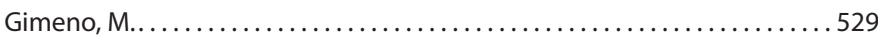

Gimeno, Mariona .................................... 276, 530

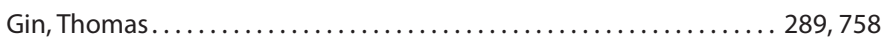

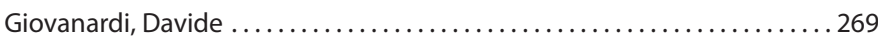

Giovannini, Stefano................................. 463, 483

Giraudo, Audrey..................................1179, 1180

Gispert, Marina ........................................... 917 
Gjerset, Britt

Gjestvang, Mona

256,588

Gnjidi , Pero ...

643

1096

Gnozzio, Mark J.

720,721

Goddeeris, Bruno M.

... 764

Goebel, Amke .

277,759

Goldstein, David

Golinar Oven, Irena

Gómez-Laguna, Jaime

147,508

Gomez-Ruiz, Pilar A.

... 965

Gómez, P.

1016

Gómez, Serafín ...

749,750

Gondol, Daniel .

.. 801

Goñi, Carlos

621,731

Gonsalves, Jocelyn ....................................... 723

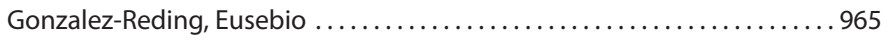

Gonzalez, Jesus...................................... 1071

González, José M........................................ 416, 417

González, Judith. .......................................1055

Goossens, Lieve ...........162, 294, 810, 811, 1093, 1159, 1187, 1188, 1193

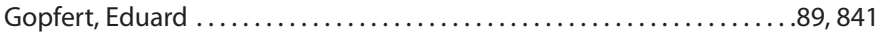

Göransson, Leif....................................... 598

Gorp, Stefaan Van ................................... 421

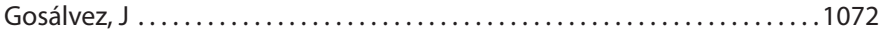

Gotter, Verena .......................................... 175

Gottschalk, Marcelo .................86, 595, 596, 641, 671, 832, 833, 834

Gottstein, Bruno...................................... 808

Goubier, Anne....................................... 431

Gouré, Julien ............................................612

Gourgues, Hervé ....................................... 203

Goutalier, Jacques ............................. 999, 1002, 1003

Gouvars, Benoît ......................................... 500

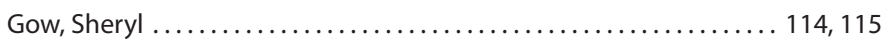

Goyvaerts, Els ....................................... 279

Gozio, Stefano.................................. 497, 407, 408

Graat, Elisabeth A. ................................... 120

Grabarević, Željko ..........................................696

Gradassi, Matteo ................................463, 483, 585

Graf, Alexandra ................................... 904, 906

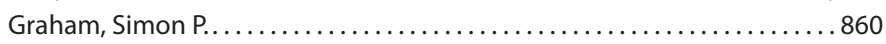

Gramer, Marie R........................ 258, 259, 263, 298, 301

Grandia, Juan........................................6 648

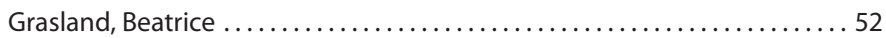

Grassi, Paula......................................... 917

Grau-Roma, Llorenç ........................ 317, 327, 328, 442, 818

Green, Laura E. . . . . . . . . . . . . . . . . . . . . . . . . . . . . . . . . . . 152

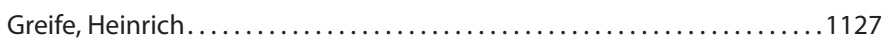

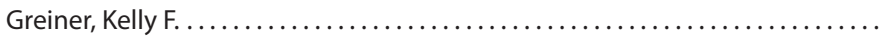

Greiner, Laura ....................................... 133

Greiser-Wilke, Irene ..................................... 148

Grenier, Daniel ...................................... 834

Grimbeek, Pieter J..................................... 372

Grodzycki, Mario ....................................... 245

Groenland, Geertjan van.................... 272, 559, 946, 947, 948

Grönlund-Andersson, Ulrika ................................ 897

Groot, Mark de ........................................ 101

Grosse Beilage, Elisabeth............ 131, 148, 622, 727, 1129, 1130, 1132

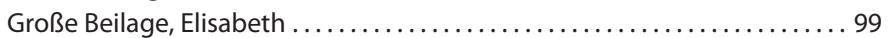

Grosse Liesner, Bernd .................................. 712

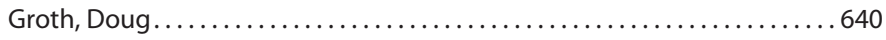

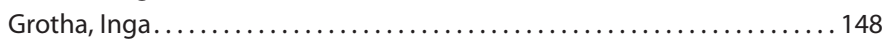

Guazzetti, Stefano ............................................ 497, 408

Guedes, Roberto M............... 235, 242, 316, 396, 701, 722, 757, 788

Guerra, Beatriz ................................ 119, 950

Guerrero Carrillo, María de Jesús ............................ 1034

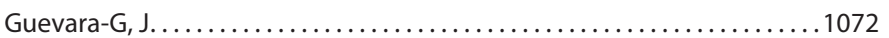

Guicherd, Aurélien. ............................. 999, 1002, 1003

Guillermo, Jose L................................. 318, 319

Guillouet, P. ........................................ 134

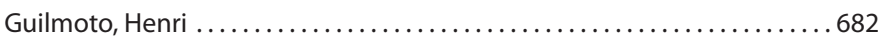

Guimaraes, Walter V.............................459, 645, 910

Gundlach, Susanne ..............................122, 951, 952

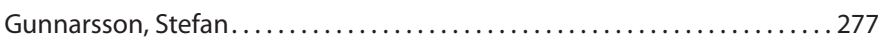

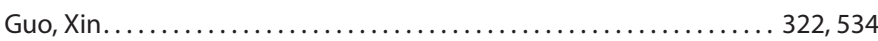

Gustafsson, Gösta ...................................... 598

Gutierrez, Carolina..................................... 898, 1097

Gutzwiller, A......................................... 896

Haas, Katharina....................................... 906

Habernoll, Henrike................................73, 191, 650

Haesebrouck, Freddy ...................... 117, 136, 137, 636, 973

Hagemann, Guntram .................................... 579

Haines, Felicity ....................................... 860

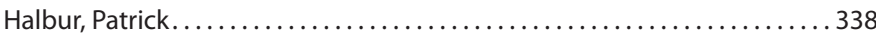

Haley, Charles A..................................116, 179, 449

Hall, William F........................................ 243

Hamaekers, Veerle...................................... 797

Hamilton, David . .......................................... 688

Hammer, Mark J...................... 635, 639, 709, 718, 719, 1004

Hammerl, Jens A. ........................................ 119

Hampson, David J.............................. 724, 725

Han, J H............................................ 403

Han, Jae-Yeon .......................... 478, 480, 569, 812, 891

Han, Jang-Hyuck . ...................................... 199

Han, Jeong Hee................. 365, 366, 367, 368, 369, 370, 901, 1113

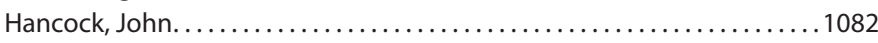

Hancox, Laura ........................................ 93

Hangoor, Evelien ....................................1137, 1138

Hansen, Claus .............................................. 65

Hansen, Lisbeth U.................................. 187, 214

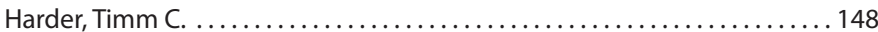

Harding, John C.............. 49, 50, 127, 128, 740, 922, 1185, 1190, 1192

Harel, Josée .....................................51, 595, 612

Harland, Michelle L.................................... 259

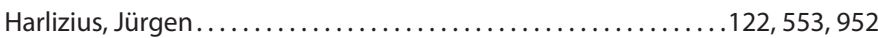

Harmon, Karen ............................................ 302

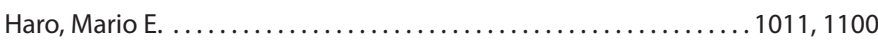

Harris, DL Hank.............................. 1, 263, 300, 545

Harrison, Shayleen.................................338, 894, 977

Hartl, Romana ....................................... 904

Hartsook, Greg .......................... 204, 206, 267, 475, 659

Hartung, Jörg ............................... 123, 215, 955, 957

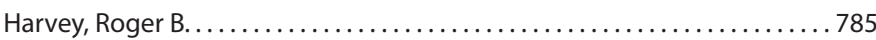

Hashimoto, Riri ..................................... 981

Hasman, Henrik ...................................... 954

Hatherell, Avril. ............................................ 53

Haugegaard, Svend.................................. 354, 360

Haugum, Magne ....................................... 773

Havlickova, Hana ...................................... 970

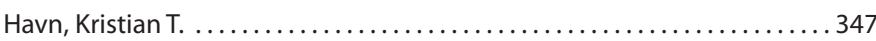

Hawkins, Peggy Anne................................ 575, 1154

Hayden, John. ....................................... 165

Hayes, M. Anthony..................................... 916

He, Qigai ...................................454, 607, 813

Heckenbach, Kirsten ................................... 972

Hedges, Jim .......................................... 781

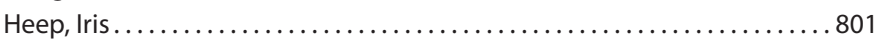

Hegelund, Lene ................................... 277

Heinbach, Uwe ....................................... 952

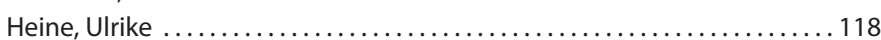

Heinemann, Marcos B............................334, 335, 460 
Heinonen, Mari.

.1163

Heinritzi, Karl. ... $166,247,819,828,956,1155,1157$

Heissenberger, Bernhard.

111,329

Heldmer, Eva .

.927

Hélie, Pierre ....

641

Hellmann, Klaus

714,1127

Helmuth, Reiner

Hémonic, Anne

.. 962

Hemonic, Anne.

218,998

Henderson, Lorraine E.

Henn, Cleber

1140

Hennig-Pauka, Isabel . . .

600,663

Henriques, Mayka R. . .

459,645

Henry, Steve.

... 462

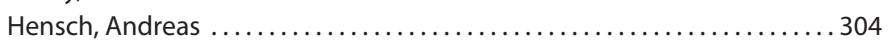

Herczeg, József.

$614,616,868,870,873,874$

Heres, Lourens

Herin, Jean-Bernard

$321,390,436,565,1111$

Herman, Viorel

$491,513,606,618,975$

Hernandez Lopez, Jesus ............................... 856, 857

Hernandez-Andrade, Laura .............................. 944

Hernández-Baumgarten, Eliseo ......................... 507, 902

Hernández, Ivonne I....................................... 827

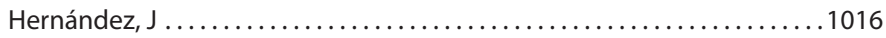

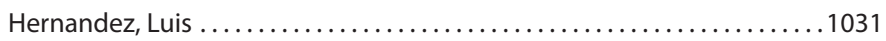

Herradora-Lozano, Marco A. ............................. 570, 1042

Herrera M., David ..................................... 1030

Herrera-H, J ................................... 1072, 1116

Herrera, Jose ......................................... 918

Herrero-Medrano, Juan M. .............................. 417

Hertwig, Stefan............................................ 119

Hexeberg, Cathrine ....................................... 1133

Heydenreich, Annette V. . ............................... 844

Hidalgo, Álvaro.......................... 724, 739, 742, 969, 971

Hill, Delores ............................................ 179

Hill, Janet E. ........................................ 740

Hillen, Sonja...........................................694

Hiller, Anne........................................ 980

Hirschberger, Johannes................................. 905

Hjertner, Bernt ................................. 227, 456

Hjulsager, Charlotte K...........80, 105, 207, 256, 284, 327, 360, 464, 702

Hoang, Quoc ........................................ 568

Hoeltig, Doris..........................196, 600, 602, 656, 662, 663

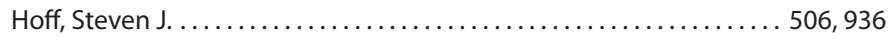

Hofshagen, Merete .................................... 256

Hofstetter, Ursula .............................43, 899, 900, 1053

Hogan, Casey V. . . . . . . . . . . . . . . . . . . . . . . . . . . . . . . . 1044

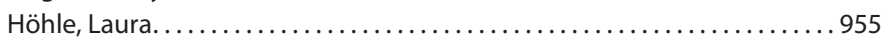

Hohloch, Corinna.............................. 107, 308

Holmgren, Nils ...............................158, 598, 925

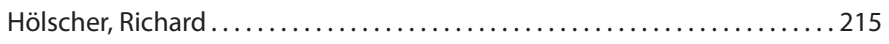

Holt, Chris M........................................ 1083

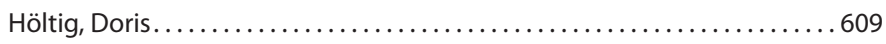

Holtkamp, Derald J..............................265, 270, 942

Holtslag, Hans ........................................ 404

Holyoake, Patricia K....................... 129, 233, 284, 464, 688

Holzer, Michael ........................................ 906

Homuth, Matthias ............................... 481, 821

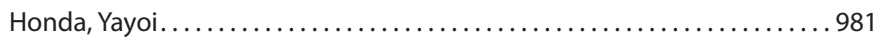

Hoo-Don, Joo ............................................ 657

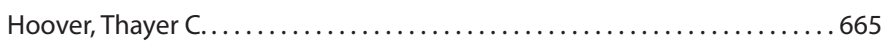

Hoppe, Maren .......................................... 249

Hörmansdorfer, Stefan. ................................ 956

Hornillos-Gumiel, Lidia ...........................151, 505, 546

Horta, Felipe C...............................60, 1092
Hortua, Myriam A. .................................. 180

Horváth, Ágnes. . . . . . . . . . . . . . . . . . . . . . . . . . . . . . 786

Houben, Manon ................................... 101, 555

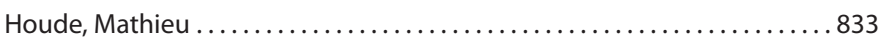

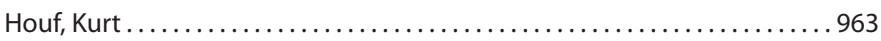

Howells, Mark J. ...................................... 732

Hoy, Steffen ........................................ 1087, 1088

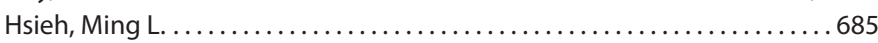

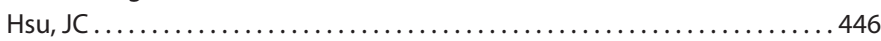

Hsu, Karolynn ...................................... 53

Hsuan, Shih-Ling ...............................446, 601, 603

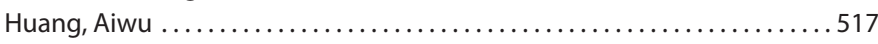

Huang, Chienjin ................................. 446, 858

Huang, Chin-Cheng ................................. 224, 878

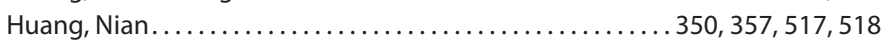

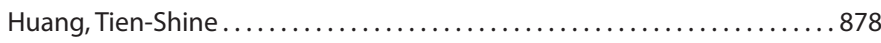

Huang, Yanyun ............................... 49, 50, 1190

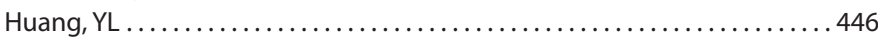

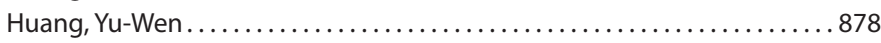

Hübner, Katrin........................................ 244

Huerta Alva, Oscar........................ 840, 1059, 1060, 1125

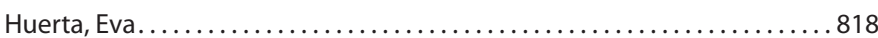

Hughes, Allison M...................................... 440

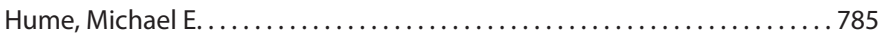

Huneault, Louis. ....................................... 82

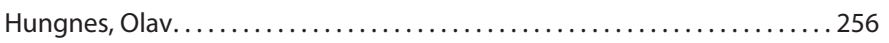

Hunt, Brian ........................................ 836

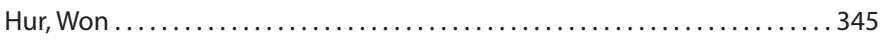

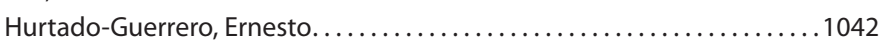

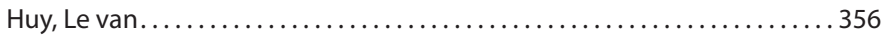

Hwang, Min-A.................................. 199, 816

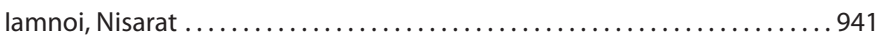

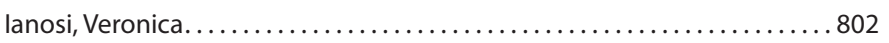

Ibrahim, Ibrahim N..................................... 485

leremie, Sorina ................................................... 513

Illanes, Natalia V....................................... 731

Indermühle, Niklaus ....................................... 1151

Inman, Melissa ......................................... 75

Inoue, Alberto Yocyitaca . . . . . . . . . . . . . . . . . . . . . . . . . . 359

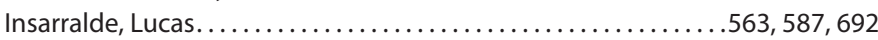

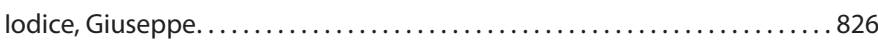

lonkoff, Ghislaine .....................................6 610

IRAS Fugato Consortium............................... 196, 656

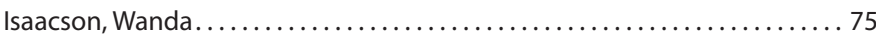

Iseki, Hiroshi. ......................................... 538

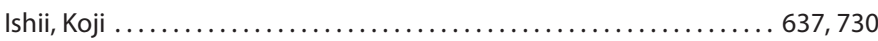

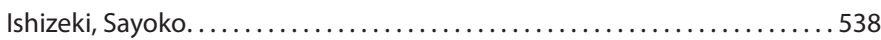

Ishizuka, Masaio Mizuno ................................... 359

Iswhikawa, Hiromichi . . . . . . . . . . . . . . . . . . . . . . . . . . 538

lurescia, Manuela..................................... 954

Ivanova, Emilia R. .

Ivanova, Stanislava .......................... 990, 991, 992, 993

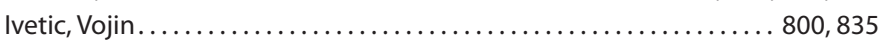

Jackova, Anna ................................................................................. 502

Jacobs, Jos ...................................... 810, 811

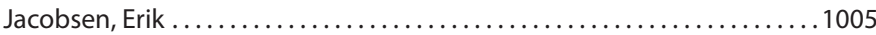

Jacobson, Magdalena............................. 700, 1152, 1181

Jacobson, Mark.................................. 220, 740

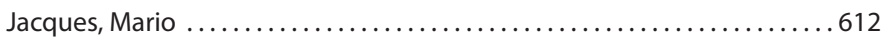

Jafarikia, Mohsen ........................................ 916

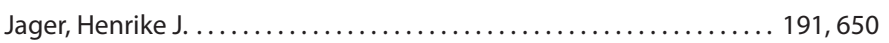

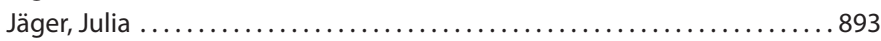

Jagu, Remy...................................... 400, 1146

Jaime, Jairo.................................... 313, 452

Jakobsen, Jeanne T..............................842, 843, 844 
Janata, Andreas

904,906

Jang, Hyun ....

499,838

Janke, Bruce $\mathrm{H}$.

$.74,299$

Janowetz, Britta

$146,304,1119$

Janssen, Rick 210

Janssens, Geert........................................ 64

Januskauskas, Aloyzas .................................... 1131

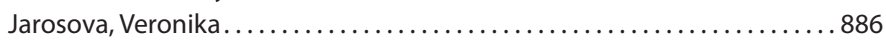

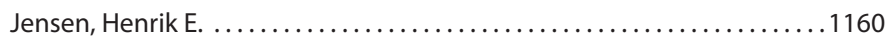

Jensen, Inger M. ..................................... 45

Jensen, Jens Christian E. ......................137, 670, 678, 1128

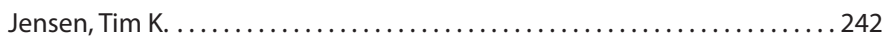

Jeong, PilSoo ........................................ 770

Jeong, Wooseog........................................ 864

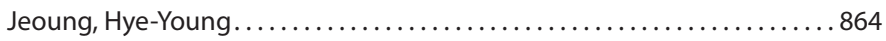

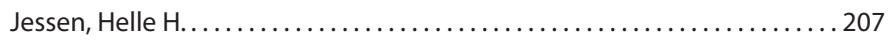

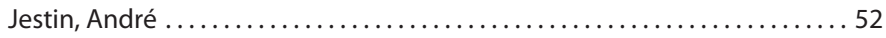

Jiarakhun, Supunnee .................................. 1164

Jimenez, Hector ........................................... 1031

Jimenez, M........................................ 1060

Jimenez, Marta ............ 392, 395, 398, 399, 401, 402, 409, 410, 415, 422

.................535, 632, 646, 677, 772, 829, 1010, 1090, 1108, 1109

Jimenez, Miguel A. ................................... 422

Jin, Wen ......................................... 901

Jittimanee, Suphattra................................ 311, 567

Jittrarak, Peerakorn .................................. 941

Joachim, Anja ....................................... 796

Johansen, Markku ............................237, 284, 464, 707

Johansson, Sivert..................................... 598

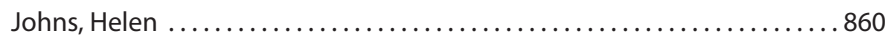

Johnson, Jeremy F................................ 201, 512

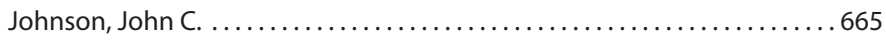

Johnson, John K. ........................ 217, 351, 382, 661, 924

Joisel, François .................. 165, 205, 283, 321, 362, 374, 376, 390

$.429,430,431,433,434,436,437,439,458$

$493,529,561,565,630,631,749,750,1111$

Jolly, Jean-Pierre

684,933

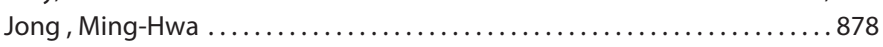

Joo, Han Soo ........................... 106, 298, 323, 324, 394

Jorge De Moura, D. ................................ 630, 631

Jorsal, Sven E. ........................... 241, 242, 327, 751, 756

Joung, Kang Soeng ...................................................... 345

Jourquin, Jan.....................67, 135, 156, 162, 294, 810, 811 $1089,1093,1159,1187,1188,1193$

Jørgensen, Anne .................................. 643, 773

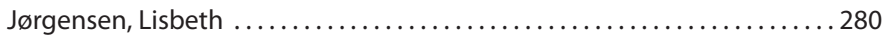

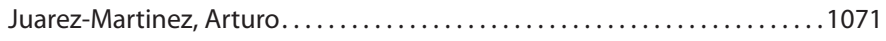

Jung, Bock-Gie ........................................ 556

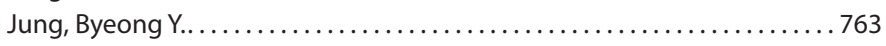

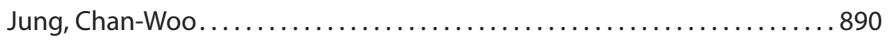

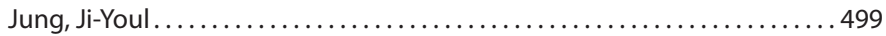

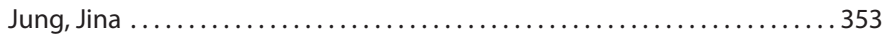

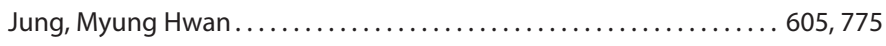

Jung, Suk Chan ..................................... 655, 959

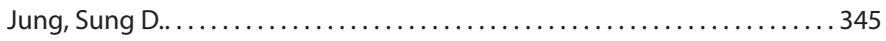

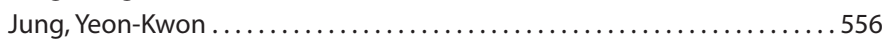

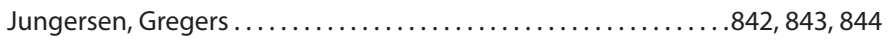

Jungi , Andreja ........................................ 202

Junior, José E. ...................................... 60

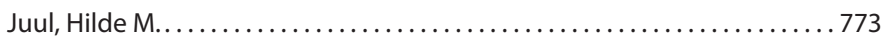

Juvanteny, Agnès..................................... 917

Kadra, Benaouda ..............................614, 615, 616, 786

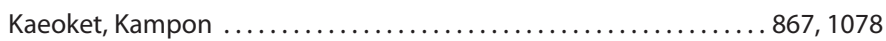

Kaesbohrer, Annemarie............................ 119, 972

Kaeser, Tobias ........................................ 528
Kaestner, Sabine. ...................................... 249

Kaevska, Marija ..................................... 841

Kagawa, M........................................... 171

Kamieniecka, Katarzyna ................................ 861

Kamp, Johan ....................................... 733

Kanashiro, Tatiana M. .................................. 1124

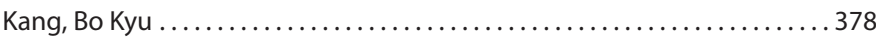

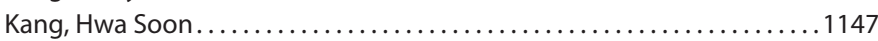

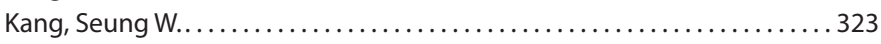

Kang, Shienyoung ....................................... 814

Kanora, Alain .......62, 673, 674, 676, 729, 737, 990, 991, 992, 993, 995, 996 $.1000,1019,1022,1023,1024,1025,1026,1027,1183$

Karamon, Jacek. ....................................... 799

Karanikolova, Mariana ....................990, 991, 992, 993, 995, 996

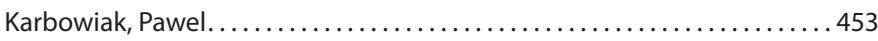

Karlsson, Katarina .............................. 747, 748

Karniychuk, U.U.................................... 54

Kars-Hendriksen, Susan .............................. 733

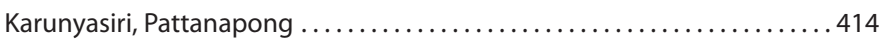

Kasemsuwan, Suwicha.................................. 941

Kauffold, Johannes ...............................103, 183, 211

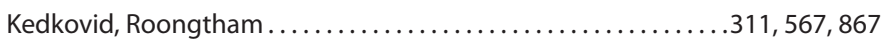

Keeling, Linda ......................................1163

Keeling, Matt ......................................... 266

Keenliside, Julia M. ......................................... 254, 932

Kehrli, Marcus E. Jr............................74, 79, 299, 332, 875

Keirstead, Natalie D. ................................... 916

Keita, Alassane ............................. 124, 248, 362, 761

Kekarainen, Tuija ............... 231, 232, 649, 879, 880, 881, 882, 883

Keller, Christoph.................................104, 949, 958

Kellerman, Cecilia..................................... 1166

Kellnerova, Dita .................................... 644, 815

Kemper, Nicole ................................1176, 1186, 1189

Kéranflec'h, André ......................................... 52

Kern, Douglas R........................................ 582

Kesdangsakonwut, Sawang........................1103, 1104, 1114

Kesl, Lyle D............................................. 144

Keum, Hyun-Ok ....................... 478, 480, 569, 812, 891

Khan, Amir S........................................ 582

Khemsapb , Prapipat......................................... 994

Kheyar, Ali.....

Kich, Jalusa D. . . . . . . . . . . . . . . . 968

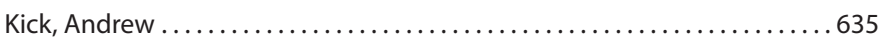

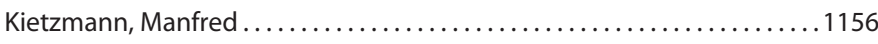

Kilchling, Tim .................................... 1155, 1157

Kim, Aeran ....................................... 655, 959

Kim, Byeong-Hak ............................... 864, 890

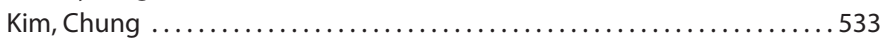

Kim, Dae-Hyuk ......................................... 605

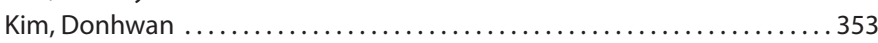

Kim, Ha Y............................................ 763

Kim, Hye-Kwon ......................... 478, 480, 569, 812, 891

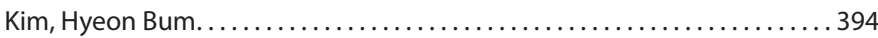

Kim, Hyoung S....................................... 763

Kim, Jae-Hoon...................................... 890

Kim, Jong Man . ............................................ 378

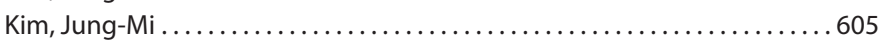

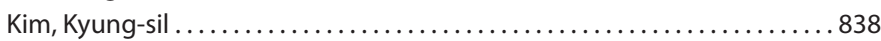

Kim, Myung Hyee......................... 365, 367, 368, 369, 403

Kim, Seong-Hee .......................... 445, 537, 584, 814

Kim, Wonil....................................100, 302, 504

Kim, Yeong Hun ................ 365, 366, 367, 368, 369, 370, 403, 901

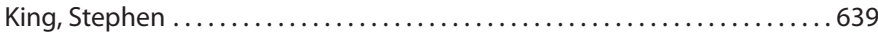

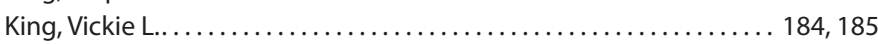

Kircher, P............................................ 896 
Kirkby, Nikolai

Kirkland, Peter

Kirkwood, Roy

Kis, Tamás E. ...

Kitikoon, Pravina

Kitkha, Supatee.

Kittawornrat, Apisit

Kixmoeller, Marion. .

Kjeldsen, Niels Jorgen

Kjellerby, Petter. ...

Klein, Günter

Klein, Ulrich ...

Klien, Kerstin

Klinkenberg, D.

Klisch, Karl

Klopfenstein, Christian.

Kmiec, Magdalena. ..

Knap, Inge

Knauf, Dina

Kneblewski, Piotr.

Kniffen, Timothy S..

Knotigova, Pavlina.

Knox, Robert V.

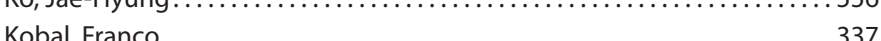

Kobisch, Marylène.................................. 641, 660

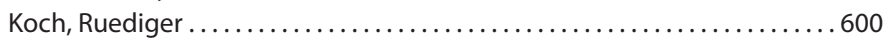

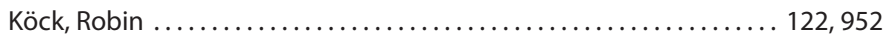

Koeck, Robin ........................................... 951

Koehrmann, Annika ........................... 1129, 1130, 1132

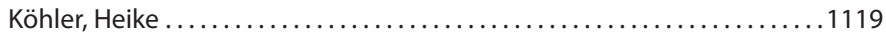

Koichi, Takeshi........................................ 960

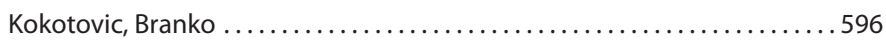

Kolacz, Roman................................... 1144, 1171

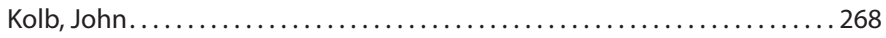

Kolb, Katharina ......................................... 828

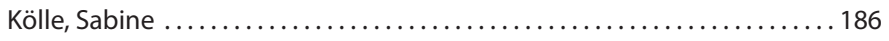

Kolpa, Bas ........................................... 210

Kołacz, Roman. ......................................... 1043

Kongtes, Sopon ................................113, 349, 414

Koonjaenak, Seri............................ 1068, 1069, 1101

Korczynski, Mariusz........................................1144

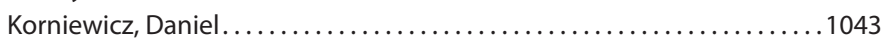

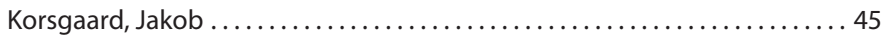

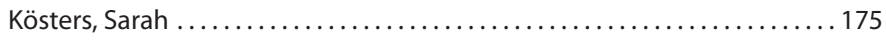

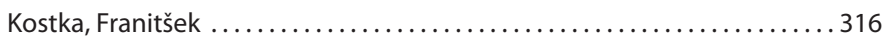

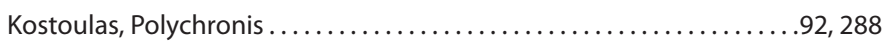

Kováč, G......................................... 1064

Kovács, Márk ........................................ 443

Kovács, Melinda .......................................... 654

Kowalczyk, Andrzej.................................... 566

Koylialis, Dimitrios............................ 711, 780, 1191

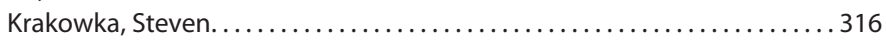

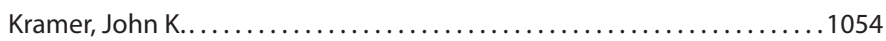

Kramongthong, Indhira..............................1014, 1015

Krassóvári, Diana .................................. 870, 874

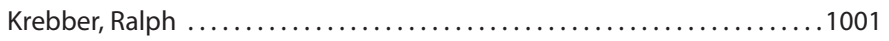

Kreienbrock, Lothar................................... 481

Kreienbrock, Lothar.............................573, 578, 579

Krejči, Roman ........................... 616, 868, 870, 873, 874

Kristensen, Anders R. .................................... 1161

Kristensen, Charlotte S.......................80, 121, 237, 327, 384

Kristensen, Heine ..................................... 214

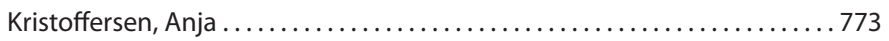

Kritas, Spyridon K............................. 711, 1020
Krittanan, Peerapat ....................................... 1045

Krizanac, Danica.......................................... 904

Krol, Ed............................................. 220

Krüger, Lukas......................................... 1143

Krumbholz, Andi ................................297, 572, 577

Kubiak, Rene ....................................... 622

Kuchiishi, Suzana S. ....................................... 1122

Kudlackova, Hana ..................................... 89, 841

Kuhn, Mike ......................................... 139

Kühnel, K. .......................................... 344

Kukushkin, Sergey A. ............................... 527, 610

Kuller, Wikke......................................... 210

Kulok, Michal....................................... 1171

Kumar, Smitha........................................ 117

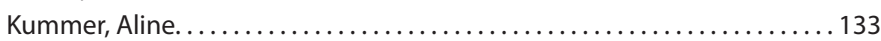

Kumprechtova, Dana ................................. 291, 779

Kunalintip, Rapeepat .................................... 745

Kunavongkrit, Annop......................... 542, 1078, 1103, 1104

Kupcsulik, Bálint..................................... 614, 616

Kureljusic, Branislav.................................... 800

Kuribayashi, Thiago H. ............................. 1040, 1041

Kurmann, Jeremias .................................. 405, 418

Kuroda, Tomoko .................................... 771

Kurtz, Sherry ............................................ 326

Kuzemtseva, Liudmila ..................................... 560

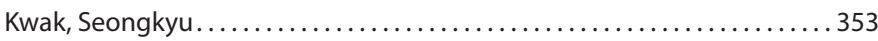

Kwang, Jimmy ........................................ 428

Kwiecien, Eduardo J................................ 898, 1097

Kyriakis, Spiros C. ........................ 634, 711, 780, 782, 1191

Kyung-Kee, Lee......................................... 657

Laanen, Maria .............................. 938, 939, 1089

Labarque, Geoffrey .......................................633

Labuscagne, Annemarie ..................................... 744

Lacouture, Sonia. ............................. 595, 596, 832, 834

Ladinig, Andrea ................. 111, 197, 329, 476, 528, 649, 717, 819

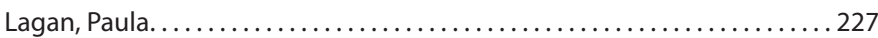

Lage, Andrey P. . . . . . . . . . . . . . . . . . . . . . . . . . . . . . . . . 722

Lager, Kelly M................ 55, 74, 79, 253, 255, 259, 299, 332, 875

Laitat, Martine......................................... 802

Lambert, Marie-Ève...............................150, 494, 937

Lambrecht, Claudia .................................. 122

Lamichhane, Chinta M. ..................................... 383

Lamot, David ........................................ 1137, 1138

Lang, Christiane ...............43, 111, 166, 329, 476, 649, 828, 899, 900

Langhoff, Rebecca................................. 197, 649

Lantz, Robert........................................... 792

Laplante , Benoit ...................................... 341

Lapuente, Susana.......................667, 668, 669, 683, 697, 698

Lapus, Zoilo M. ................................540, 541, 988

Lara-Puente, Horacio . ................ 138, 228, 229, 315, 507, 624, 625

$626,849,850,851,852,853,854,902$

Laraichi, Mehdi .............................. 999, 1002, 1003

Larraz, Carmen ................................69, 1074, 1075, 1076

Larsen, Claus B........................................... 360

Larsen, Inge ......................................... 159

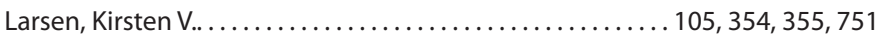

Larsen, Lars E. ............ 80, 105, 207, 284, 327, 360, 464, 588, 702, 751

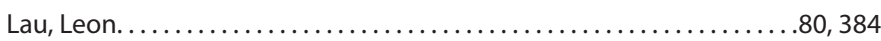

Lauritsen, Klara T. ...............................842, 843, 844

Lautrou, Yannick........................................ 752

Laval, Arlette .............................. 213, 514, 679, 680

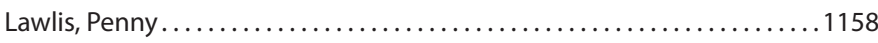

Lay, Donald C. Jr....................................... 176

Lazaroto, Juliana. ................................. 783, 784

Lazo, Gloria.............................................653 
Le Galludec, Herve. ..................................... 821

Le Guennec, Jean. . . . . . . . . . . . . . . . . . . . . . . . . . . 758

Le Roux, Marc ........................................ 962

Le Treut, Yannig................................ 209, 1179, 1180

Le, Hang T. . . . . . . . . . . . . . . . . . . . . . . . . . . . . . . . . . 960

Le, Hien T. ......................................... 492

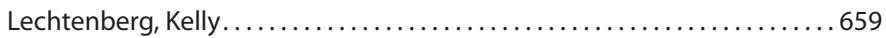

Leclair, Daniel .......................................... 114

Lecluyse, Henk .............................673, 674, 737, 1183

Lecours, Marie-Pier ................................. 832, 833

Lee, Bong-Joo ........................................ 556

Lee, Hee Soo ......................................... 959

Lee, Ji Youn..................................... 655, 959

Lee, Joong-Bok ................................... 199, 816

Lee, Jung-Ah .................................... 199, 816

Lee, K-K .................................... 537, 584

Lee, Kichan .......................................... 655

Lee, Kyoung Ki. . . . . . . . . . . . . . . . . . . . . . . . . . . . . 445

Lee, Kyoungki .......................................... 814

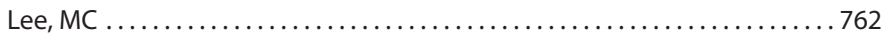

Lee, O Soo............................. 445, 537, 584, 763, 814

Lee, Pill Soo ............................................. 345

Lee, Sang-Won ........................................... 199

Lee, SeungYoon ........................................ 770

Lee, WC .......................................... 446

Lee, Wei-Cheng......................................... 603

Lee, Won Gwnag ....................................... 1113

Lee, Won-Jung.................................... 604, 605

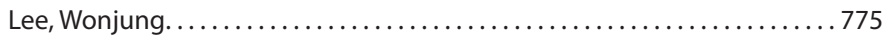

Leeb, Christine ..................................... 277

Leengoed, Leo van ....................... 101, 555, 946, 947, 948

Leenhouwers, Jascha .................................. 68

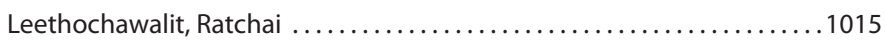

Lefebvre, DJ ....................................... 54, 320

Lefebvre, Herve..................................... 157

Leger, David..................................... 114, 115

Lehrbach, Philip R.......................... 112, 230, 371, 373

Leist, Yvonne .......................................... 1151

Lelij, J. van der........................................ 1126

Lelli, D. ............................................ 458

Lemus, Clemente ................................... 918

Leneveu, Philippe ..................................... 761

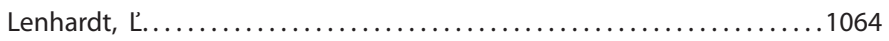

Lenz, M. Corinne...................................... 75

Leontides, Leonidas ..................................92, 288

Leotti, Giorgio ................................... 283, 458

Lertwatcharasarakul, Preeda .............................. 1121

Lesiak, Marek.......................................... 1134

Leskova, Valeria........................................ 502

Lesman, Steven P...................................... 1006

Letellier, Ann . . . . . . . . . . . . . . . . . . . . . . . . . . . . . . . 82

Leva, Lenka...................................... 89, 970

Levy, Michael...............................178, 278, 1099, 1149

Levy, Nyssa......................................... 139, 845

Lewandowski, Eric................................. 400, 1146

Lewerin, Susanna S........................................ 173

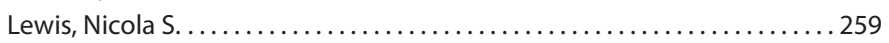

Leyman, Bregje..................................... 973

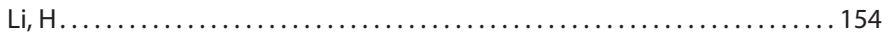

LI, Wentao........................................... 454

Liaño, Nicolas G...................................... 776, 778

Liao, Chih-Ming ................................... 601, 603

Liao, Jiunn-Wang ........................................6 603

Liao, JW ............................................ 446

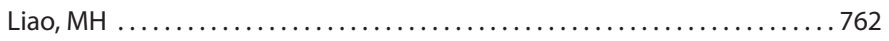

Liebstein, Martin ............................... 108, 635, 921

Liesner, Bernd G.. . . . . . . . . . . . . . . . . . . . . . . . . . . . . 420

Lillie, Brandon N. ....................................... 916

Lim, Ban K. ...................................363, 526, 627

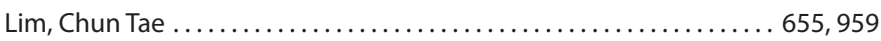

Lim, H.C. ..................................... 363, 627

Lim, Hang C......................................... 526

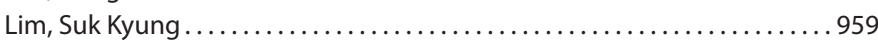

Lima, Civilo Q.................................... 371, 373

Lin, Boh C.......................................... 571

Lin, Cheng-Chung ............................................... 601

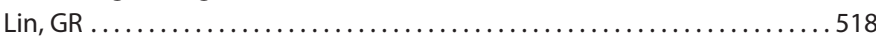

Lin, Guang-Jan ..................................... 858

Lin, Susan Yun Fan . . . . . . . . . . . . . . . . . . . . . . . . . . . . . . 878

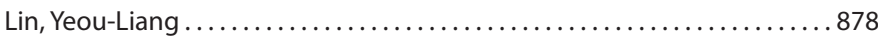

Lin, Yu J........................................... 224

Lindberg, Maria ........................................ 897

Lindgren, Kristina......................................... 277

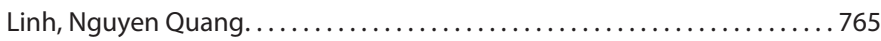

Linhares, Daniel ......................... 143, 339, 473, 515, 620

Lipej, Zoran ......................... 202, 503, 696, 837, 976, 1112

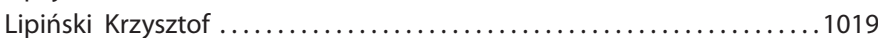

Lisbeth, Hansen U........................................... 1165

Liu, Hui.............................................. 607

Liu, Sidang ...................................... 154, 352

Liu, Ting-Yu........................................... 858

Lium, Bjørn................................. 256, 588, 643, 773

Llanes, Nuria ...................................98, 746

Llopart, David V....... 276, 486, 519, 520, 521, 522, 530, 539, 540, 541, 543

Llorens, Anna M...................................... 231

Lo, DY........................................ 446

Lobato, Francisco F................................ 757, 788

Lobato, Zélia Inês P. ......................325, 334, 335, 396, 412, 460

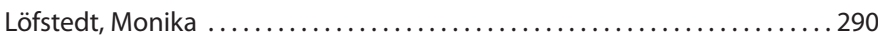

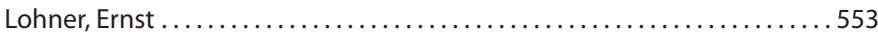

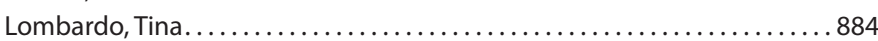

Longo, Sophie................................374, 429, 434, 1111

Longo, Sophie................................. 749, 750

Looft, Christian .......................................... 914

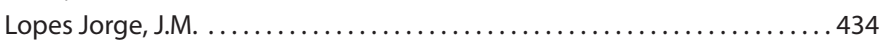

Lopes, João C. . ............................................ 385

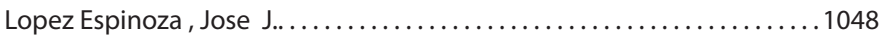

Lopez Rodriguez, Alfonso . . . . . . . . . . . . . . . . . . . . . . . . . . . . . 47

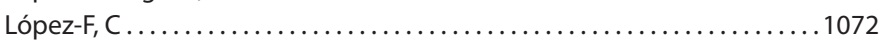

Lopez-Jimenez, Rosa.................................... 442

López-Jimenez, Rosa............................... 86, 651, 989

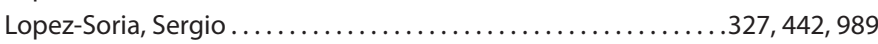

López-Soria, Sergio .................................. 261

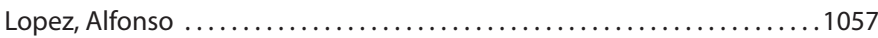

López, J .................................................. 1016

Lopez, Jesus V................... 392, 395, 398, 399, 409, 410, 535, 632

............................646, 677, 772, 829, 1090, 1108, 1109

Lopez, Jorge R............................ 767, 1011, 1100

López, Jorge.............................................. 1058

Lopez, Rosa ........................................ 225

Lorca, Cristina ....................................... 560

Lorenzetti, Raniero ............................................ 954

Lorenzo, Jose L................................................................. 425, 667

Lorusso, Alessio ..................................74, 259, 299

Lösel, Kerstin ........................................ 1135, 1136

Losson, Bertrand .......................................... 802

Loving, Crystal L. ...............................192, 255, 264

Lowe, James F.

Loynachan, Alan. ......................................... 100 
Lozano-Dubernard, Bernardo

$138,228,229,315,624,625,626$ $.849,850,851,852,853,854$

Lozano, Arturo P.

1038,1039

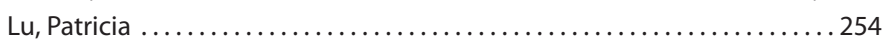

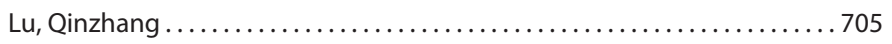

Lucas, Merlyn..........................................664 664

Lucena, Marcos L........................................ 620

Lückstädt, Christian.................................... 174

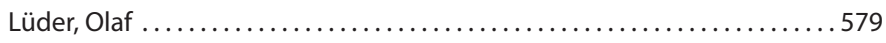

Luengyotluechakul, Sutep .................................. 1164

Lumyai, Mongkol ........................................ 997

Lundeheim, Nils ........................ 158, 598, 925, 927, 1181

Luppi, Andrea ..................................432, 791, 826

Lurette, Amandine..................................... 266

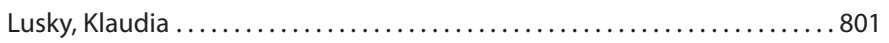

Lyoo, Kwang Soo ............................. 106, 323, 324, 394

Lyutskanov, Mihni ...................................... 996

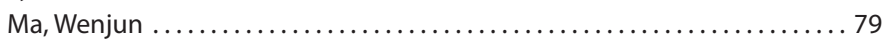

Macaraig, Ruel C.................................. 520,543

MacDougald, Doug...................................... 275

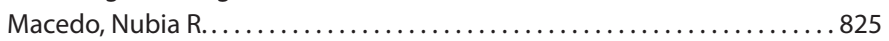

Macera, Lisa.................................... 881, 882

Machado, Glauber S. ........................184, 376, 396, 1140

Machuca, Mariana ...............................563, 587, 692

Madec, François ............................... 52, 660, 684, 933

Madonna, Riccardo ....................................... 337

Madson, Darin..................................56, 427, 1007

Maes, Dominiek ...............30, 47, 64, 67, 135, 136, 137, 634, 636, 938

$.939,963,1046,1047,1057,1065,1089$

Magalhães, Juliane D................................... 966

Magalhães, Wagner Carlos S. ......................... 334, 335

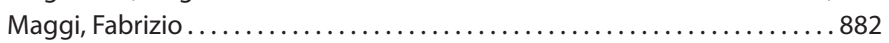

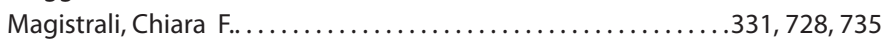

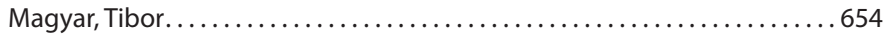

Mahlberg, Jon ........................................ 193

Main, Rodger........................................ 661

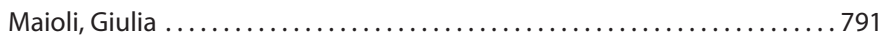

Majó, Natàlia .......................................76, 261

Makhanon, Metta ...................564, 638, 745, 803, 804, 997, 1012

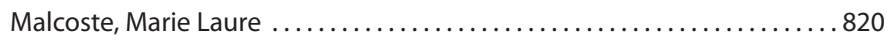

Maldonado, Jaime ............................ 232, 231, 276, 530, 611

Malherbe, Cyrille ............................... 679, 680

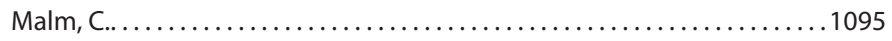

Manabat, Angel Antonio C. ............................... 536

Manavalan, Anil ...................................... 723

Mancilla, Florencio. ............................................ 387

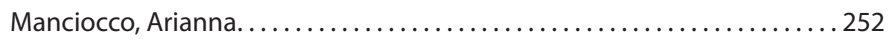

Mandelli, Claudio........................................666

Manrique Ramirez, Paula C. ................................ 827

Manso, Alberto ........................................ 1182

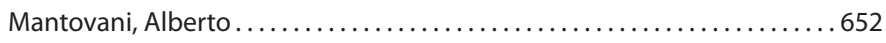

Manuel, Redentor A...............................519, 540, 541

Manzano, Rodrigo M. .

Manzo, Janett ....................................................................... 830

March, Ricard..................................... 276, 530

Marchant-Forde, Jeremy N. ............................... 36

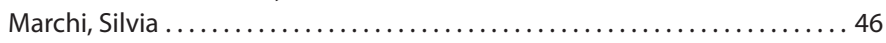

Marco, Enric ................................... 415, 632

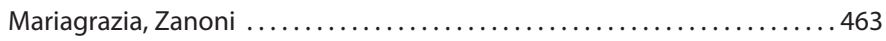

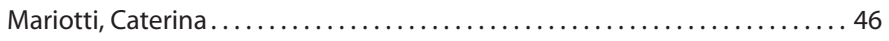

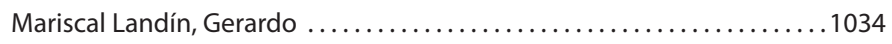

Mariscal, Enrique ........................................ 387

Mariscal, Gerardo...................................... 1031

Markowska-Daniel, Iwona........................ 566, 687, 1028

Marois, Corinne.................................. 641, 660
Maroto Martin, Luis O. . . . . . . . . . . . . . . . . . . . . . . . . . . . 764

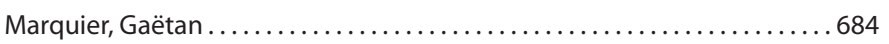

Marr, Greg V....................................... 713, 715

Marshall, Frank ....................................... 53

Martano, Giuseppe .................................... 1173

Martano, Giuseppe .............................. 871, 872, 940

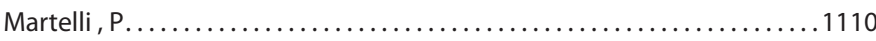

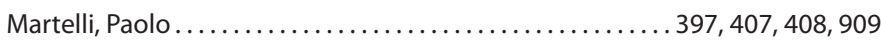

Martens, Marc ..................................... 312, 686

Martin del Campo, Carlos ................................. 444

Martín-Valls, Gerard E. .................... 77, 260, 261, 590, 652, 982

Martin-Valls, Gerard E. ............................ 76, 232, 880

Martín, E...................................... 847, 848

Martín, Margarita............................ 77, 260, 590, 978

Martín, R............................................ 847

Martineau, Guy-Pierre ............... 124, 125, 157, 209, 289, 752, 753

$.758,921,1174,1175,1179,1180$

Martinez Castañeda, Francisco E......................... 586, 1150

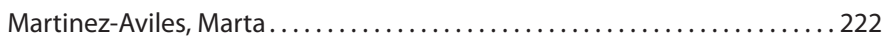

Martinez-Bautista, Rebeca ........................... 856, 857

Martínez-Gamba, Roberto A................................... 1042

Martinez-Gamba, Roberto .................................. 934

Martínez-Guinó, Laura............................... 231, 879

Martínez-Lobo, Francisco Javier ................ 151, 498, 505, 546, 547

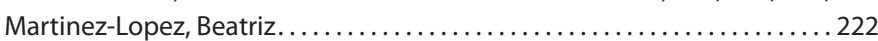

Martinez-Ramirez, Hector R.. . . .............................. 1054

Martinez-Rodriguez, Roberto ......................5570, 1011, 1100

Martinez, Alejandro. ...................................... 929

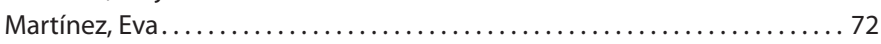

Martinez, Eva......................................... 530

Martinez, Roberto G...............................1011, 1100

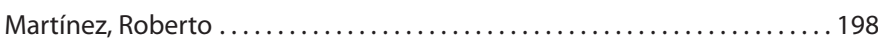

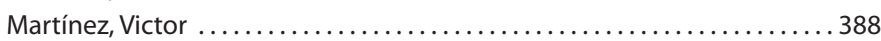

Martini, Marco........................................ 208

Martins, Simone M. ...................... 60, 70, 966, 1067, 1092

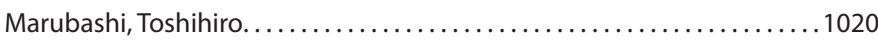

Masaříková, Martina ................................... 790, 793

Masching, Sabine......................... 1032, 1033, 1053, 1143

Mason, Sharon ........................................... 81

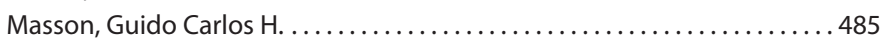

Mastin, Alexander ..................................... 257

Mateu, Enric................ 77, 260, 276, 451, 477, 529, 530, 590, 978

Mathur, Sheerin ..................................184, 185, 301

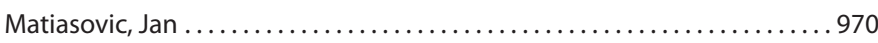

Mattsson, Sigbrit ............................... 290, 768, 1166

Matzat, Paul D. . . . . . . . . . . . . . . . . . . . . . . . . . . . . 1148

Mayor, Ivan......................................... 422

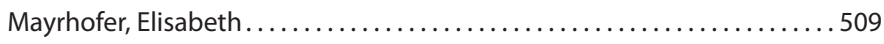

Mazzoni, Claudio .............................. 432, 791, 1107

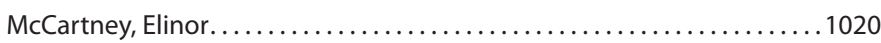

McCarty, Meghan.......................................... 1098

McClintock, Julie ........................................ 895

McCrone, IS ......................................... 73

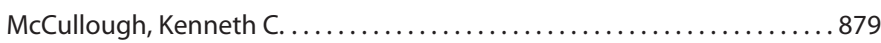

McEwen, Bev.................................... 892, 923

McEwen, Scott ........................................... 115

McGlone, John J. ........................................... 250

McGonigle, Jack D. ..................................... 571

McKay, Karen .......................................... 227

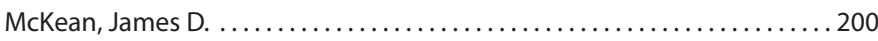

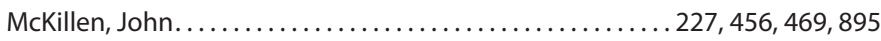

McKinley, TJ ..................................... 191, 650

McMenamy, Michael........................... 227, 456, 469, 895

McNab, W. Bruce. ....................................... 306

McNair, Irene ..............................227, 456, 469, 895 
McNeilly, Francis

McOrist, Steven.

Medina, Juan C.

Medley, Graham F.

Meedacha, Lerdtida

Meemken, Diana

Meijer, Ellen

Mejía Guadarrama, Cesar A.

Melendez-Perez, Rosalia

Melin, Lennart. ...

Mellagi, Ana Paula

Mellencamp, Martha A

Mellits, Ken.

Melody, Jamie

Mencik, Sven ....

Mendez, Jorge.

Mendoza-Elvira, Susana. . .

Meng, Xiang-Jin

Menjon, Rut.....

$\ldots$

$138,507,624,625,626,902$

427,977

392, 395, 398, 399, 401, 402, 409, 410, 415, 422, 535 $632,646,677,772,829,1010,1090,1108,1109$

Mennessier, Morgane.

1186

Menrath, Andrea

826

Merenda, Marianna

$735,826,909,954,1029$

Merialdi, Giuseppe

Merle, Roswitha

Méroc, Estelle ........................................ 177

Merza, Malik......................................... 700

Messori, Stefano........................................... 1029

Mevius, Dik.................................. 946, 947, 948

Meyer, Lars.......................................... 118

Mieli, Luc........................................... 514

Mihaljevic, Zeljko................................... 837, 976

Milic, Dinka............................................... 1105

Miljkovic, Vladan . ........................................ 717

Millar, Andrena ........................................... 227

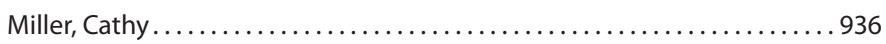

Miller, Helen M.......................................... 109

Miller, Laura C. ...................................... 79, 875

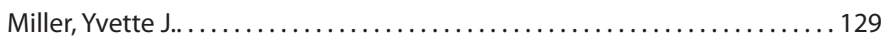

Millman, Suzanne T. ..................................... 1158

Minani, Alphonse . .................................... 476

Minder, Tanja.......................................... 1098

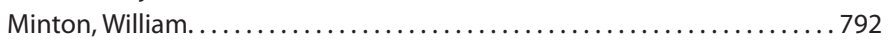

Miorcec, Aude ........................................ 413

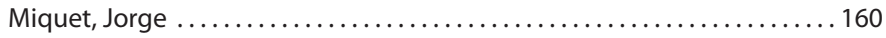

Miramontes, Jesus...................................... 274

Miranda, Raquel.................................... 318, 319

Miranda, Yadira.......................................... 1100

Miry, Cora ............................ 320, 736, 777, 823, 824

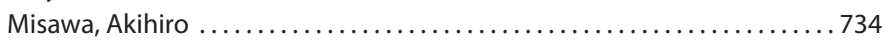

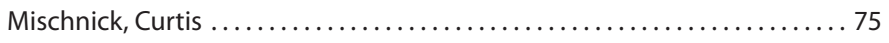

Misener, Martin.................................... 275, 342

Misutka, Chris ................................. 931, 932

Mitchaothai, Jamlong.................................1013, 1052

Mittermeier, Ulrike...................................... 1119

Miyasaka, Daniela ................................. 783, 784

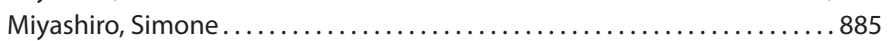

Miyashita, Mali .................................................................... 172

Mogler, Mark A...................................263, 300, 545

Mogollon, Jose D.............................. 313, 361, 452, 827

Mohn, Ulrich ......................................... 977

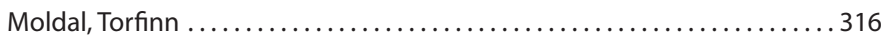

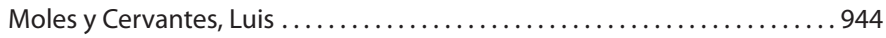

Mondaca, Enrique .....................273, 274, 475, 524, 531, 532

Mondok, József. ...................................... 654
Monsó, Marta .......................................... 225

Montoya, Maria.................................. 76, 652, 833

Moon, Hyoung-Joon. ....................378, 478, 480, 569, 812, 891

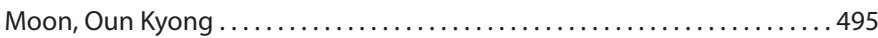

Moon, Seong-cheol......................................... 499

Moon, Seong-Hwan ..................................... 890

Mora, Mercedes .................................. 77, 225, 260

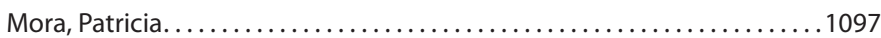

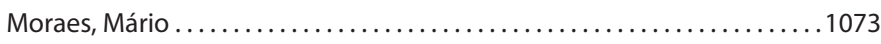

Morales, Joaquin ...........................212, 670, 678, 1182

Morandi, Federico ................................... 283, 458

Moreau, Isabelle ........................................ 275

Moreno Martin, Anna ................................... 585

Moreno-Morales, Yolanda........................... 138, 624, 625, 626

Moreno-Ramos, Carolina................................ 507, 902

Moreno, Andrea M. ................................ 888, 966

Moreno, Veronica......................................... 59

MorenoMartinez , Ernesto . .......................... 507, 902

Mores, Nelson ........................... 466, 468, 471, 862, 1122

Moretti, Aníbal S....................... 60, 70, 966, 1067, 1092

Morgan, Colin .......................................... 741

Morilla-González, Antonio ...................................... 562

Morillo, Alberto..................................... 62, 1051

Morillo, Alberto. ........................................ 863

Morrison, R.B............................................ 293, 1169

Morrison, Robert B....................................... 549

Morrison, Robert ................................... 149, 510

Morvan, Herve .............................316, 758, 761, 820

Moscati, Livia............................................ 252, 919

Moser, Irmgard ........................................ 1119

Mottram, Malcolm...................................... 781

Mouat, Denise............................................ 781

Mourão, G. B............................................... 1095

Mozo-Martín, René ........................69, 71, 1074, 1075, 1076

Muangsiri, Walaisiri ...................................... 1014

Muangyai, Manop .................................. 803, 804

Mühlbauer, Isabel ........................................... 247

Mul, Monique F. . . . . . . . . . . . . . . . . . . . . . . . . . . . . 617

Mulholland, Catherine .................................. 456

Mullan, Bruce....................................... 233

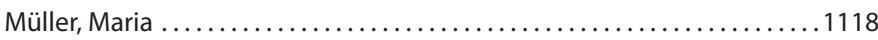

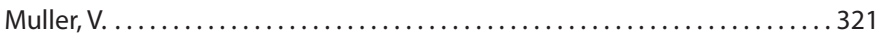

Mullins, Michael A................................. 192, 822

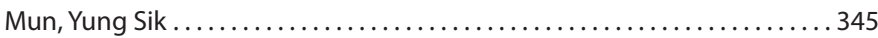

Mundt, Hans-Christian. ............................. 796, 801

Munguía, Jesús......................................... 738

Muñoz, Antonio ......................... 416, 417, 431, 749, 750

Muñoz, Iván .......................................... 225

Munoz, J. A. ..................................... 493, 561

Muñoz, Marta .......................................... 225

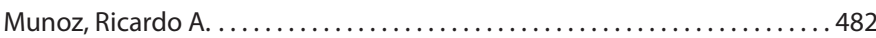

Munsterhjelm, Camilla.................................... 1163

Mur, Lina .............................................. 222

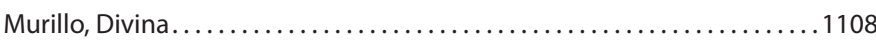

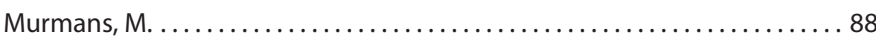

Murray, Brian P. ......................................... 741

Murtaugh, Michael ......................................... 313

Musella, Chiara ........................................... 337

Music, Nedzad........................................ 51

Musiksilp, Nantasak. ....................................... 567

Mussá, Tufária .....................................76, 652, 833

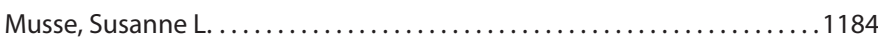

Mutua, Florence K.........................178, 278, 1099, 1149

Myers, Gil H..................................... 805, 806, 807

Na-ranong, Soraya .................................... 941 
Nadeau, Eric.

$.766,769,774$

Nafstad, Ola ..1133

Nagahama, Chihiro

Nagatomo, Hiroshi

771

Naharro, German ....

Nakajima, Hiromi . .

$724,742,969,971$

Nakanishi, Nobuo

637,730

Nam, Nguyen T... .

Nanjiani, Ian A. . .

Napp, Sebastian

Nardelli, Stefano.

Nascimento, Ernane F.

Nascimento, Juliana Amália F.

Nassuato, Claudia

Nathaus, Rolf...

Nathues, Heiko . . .

Nauwynck, Hans J...

Nava, Juan

Navas, Jesús.

Nazarov, Valeri.

Nechvatalova, Katerina

Nell, Tom .

Nelson, Daniel A.

Nemet, Zoltan

Nerem, Joel

Nes, Arie van

Neto, Ricardo.

Neumann, Eric J.

Nevel, Amanda .

$\mathrm{Ng}$, Fook K. . .

$\mathrm{Nga}$, Ho T.

Ngoc, Le H.

Nguyen, Van-Giap

Nicholson, Tracy L.

Nie, L. . . .

Niel, Christian

Nielsen, Elisabeth O.

Nielsen, Jens P.

....................

Nielsen, Mai-Britt F.

Nielsen, Uffe.

Niemeyer, Hermann

Nienhoff, Hendrik.

Nieto, David.

Nieto, Marcos.

Nieto, R.

Nignol, Anne-Cécile

Nilubol, Dachrit. ...

Ninh, Nguyen T.

Ninlaworn, Suaysuda

Nishikata, Taís

Nisi, Irma . ...

Nisoli, Lucio

Nissen, Hanne .

Nitzel, Gregory P. . . .

Niyomtuma, Waree

Nkambua, Tshibwabwa P. . .

Nobmann, Juliane

Noé, Thomas

Nofrarias, Miquel

Noh, M.

Noirrit, Michel ....

$99,131,148,622,727,1129,1130,1132$

........... 356

$.137,670,678,1006$

328

208

460

$.334,335,460$

457,483

949,953

$54,320,544$

1145

..611

$990,991,992,993,995,996$

.89

634

$787,789,1018$

.......... 443

170

.1170

165,374

243

$.57,102,1094$

.. 112

356

356

$478,480,569,812,891$

192

.. 154

466,471

$121,214,280,843,1165$

$80,91,207,237,241,242,284,464$

$.702,754,755,756,842,1005,1161$

$384,707,1162$

$146,304,553$

553,951

Norby, Bo ........................................ 785

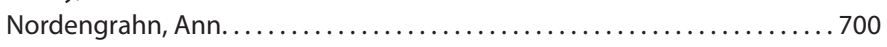

Norling, Eva .

1153
Norman, Keri N. ....................................... 785

Normand, Valérie ..................................... 213

Nörregård, Erik ......................................... 140

Nothum, Reinhard .................................... 1119

Novák, Pavel..................................... 1177, 1178

Novak, Susan........................................ 1192

Novosel, Dinko ..................... 202, 503, 696, 837, 976, 1112

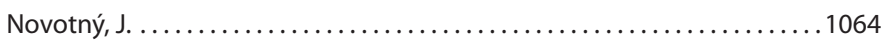

Nunes, Rogério F...................................... 84

Nunez, Alejandro .................................. 859, 860

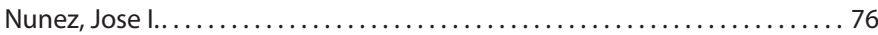

Nunta, Kanokkan .................................... 941

Nuntaprasert, Athipoo................................... 985

Nuntawan Na Ayudhya, Suparlark...................... 564, 867

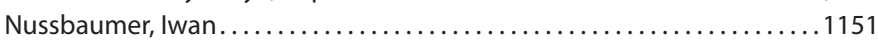

Nutsch, Robert ........................................ 664

Nutthee, Am-in . .......................................... 1091

O'Connor, Brendan .......................................... 50

O'Donoghue, Rose A..................................... 58

O'Sullivan, Terri L.................................. 892, 923

Ocak, Marion ......................................... 798

Ochiai, Susumu. ..................................... 726

Odehnal, Jiří..............................644, 815, 1177, 1178

Odehnalová, Svetlana ......................644, 815, 1177, 1178

Ogara, William........................................278, 1099

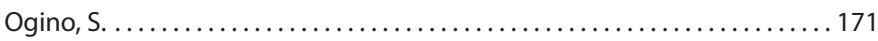

Ogris, Mario .......................................43, 899

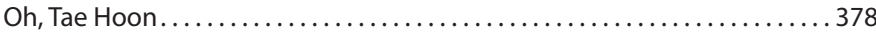

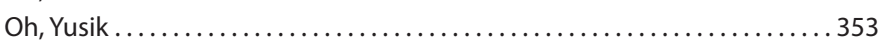

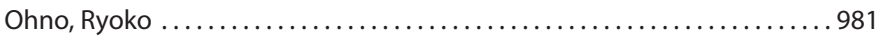

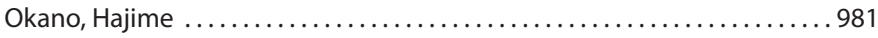

Okones, Jeff ............................................... 169

Okoth, E. ......................................... 847, 848

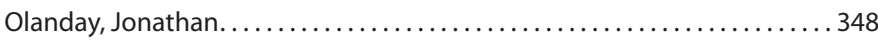

Olanratmanee, Em-on .............................. 542, 1101

Olea-Popelka, Francisco. ...................................... 116

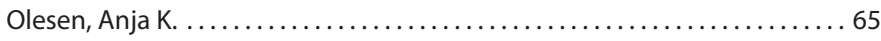

Olesen, Jesper S.................................... 1035, 1120

Oliveira, F. ......................................630, 631

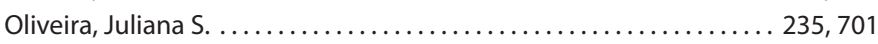

Oliveira, Simone ....................... 143, 153, 193, 817, 825

Oliver, Maria Angels ..................................... 917

Olsen, Chris W.......................................... 201, 512

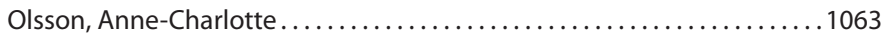

Olvera, Alex ......................................... 823, 824

Ontanillas, Jaspearl T. ................................... 543

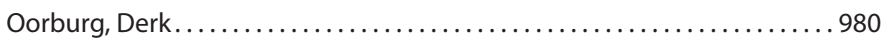

Opalinski, Sebastian ....................................1144

Opriessnig, Tanja ........... 55, 56, 100, 316, 338, 427, 839, 894, 924, 977

Oraveerakul, Kanisak ................................... 589

Orchard, Beverley.................................. 284, 464

Országh, Georges...................614, 615, 616, 868, 870, 873, 874

Ortega, Nieves ...................................... 1123

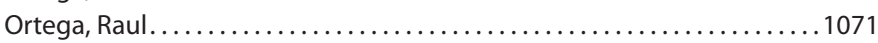

Orveillon, Francois-Xavier .................................... 346

Osorio, Jesús ......................... 724, 739, 742, 969, 971

Ostanello, F. ...................................... 283, 458

Österberg, Julia....................................... 173

Ostmeier, Miriam ....................................... 118

Ostovic, Mario ........................................... 1105

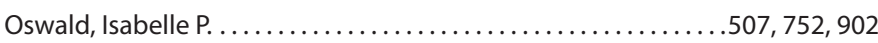

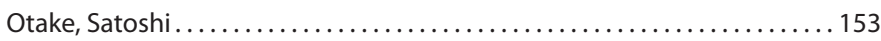

Otaki, Hiroshi.................................... 637, 730

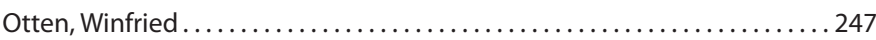

Ovelhey, Amely........................................ 175 
Pacciarini, Maria ...................................... 483

Pacheco, Graziela D................................... 1038

Padoan, Diego............................................. 1017

Pagot, Eric................................. 124, 248, 289, 362

Pakingan, Karen Anne A................................. 543

Pal, Narinder ............................................ 894

Palacios, Juan M...................239, 386, 387, 388, 389, 438, 1056

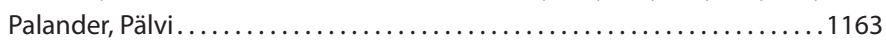

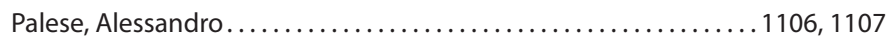

Palhares, Maristela................................... 1073, 1095

Pallarés, Francisco J. ................. 147, 416, 417, 431, 508, 749, 750

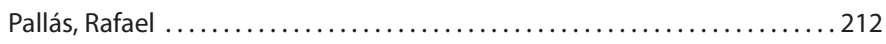

Palomo, A ............................................ 1072

Palomo, Antonio................................... 535, 677

Palomo, Gonzalo ...................................... 974

Palzer, Andreas .................166, 197, 247, 819, 828, 956, 1155, 1157

Panarese, S. .................................... 283, 458

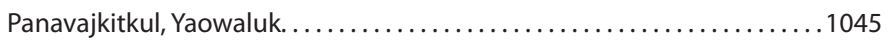

Panek, Robert ........................................ 522

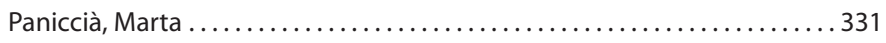

Pannekoek, Maaike ...................................... 1170

Panzieri, Carmen ....................................... 46

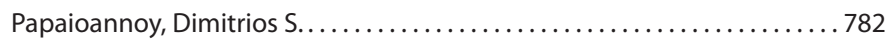

Papatsiros, Vassilios G. .....................634, 711, 780, 782, 1191

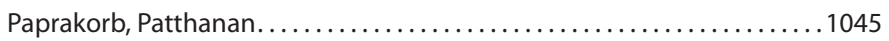

Parada, Julián ....................................... 731, 964

Paradis, Marie Anne..................................... 552

Parazzi, Larissa J........................ 60, 70, 966, 1067, 1092

Park, Bong-Kyun............................ 478, 480, 569, 812, 891

Park, Choi-Kyu...............................445, 537, 584

Park, Choikyu.......................................... 814

Park, Jeonghwa .......................................... 42

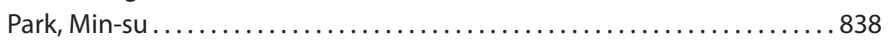

Park, Sang-Shin ....................................... 199

Park, Seong-Jun ......................... 478, 480, 569, 812, 891

Park, Seung-Yong................................... 199, 816

Park, Sung-Su ........................................ 499

Parvizi, Nahid....................................... 1130

Pascu, Corina .........................491, 513, 606, 618, 658, 975

Pashov, Dimitar............................... 990, 991, 992, 993

Pasinato, Karine G................................... 1140

Pasmans, Frank......................... 117, 136, 137, 636, 973

Pasqualon, Hellen P. ..................................... 910

Patterson, Jennifer L............... 25, 58, 132, 133, 254, 1082, 1098, 1185

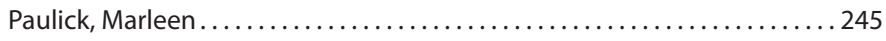

Pavicic, Zeljko .......................................... 1105

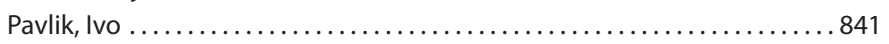

Pavlovic, Ivan.................................... 800, 835

Pawluk, Pete................................. 931, 932

Pearce, Gareth P................................... 191, 650

Pearce, Michael................................. 1137, 1138, 1139

Pearl, David L. ........................... 306, 892, 923, 929, 930

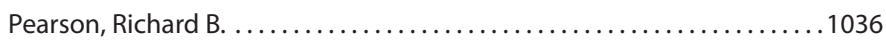

Pecaud, Dominique. ................................... 945

Pedersen, Ken S............ 91, 237, 241, 242, 284, 464, 702, 754, 755, 756

Pedersen, Klaus.................................702, 754, 755

Pedro, Mejia S......................................... 855

Peerboom, Refke ................................ 823, 824

Peinhart, Eva-Maria ..................................... 488

Peitzmeier, Eva Ursula . . .................................. 877

Pejsak, Zygmunt................. 145, 465, 566, 687, 703, 799, 861, 1134

Pelayo, V...................................... 847, 848

Pelenc, Frederic ........................................ 753

Pelliza, Bibiana R. ................................ 621, 964

Pelz-Studlar, Manuela................................... 908
Pena, Lindomar. .......................................... 563

Penrose, Lindsay........................................ 690

Penuelas, Giovanna..................................... 918

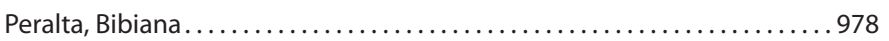

Percedo, Maria Iriam ..................................... 472

Pere, K................................................ 134

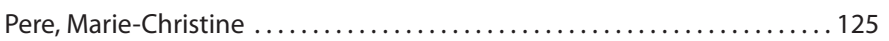

Perea, Anselmo..................................... 974, 1123

Pereda, Ariel..................................... 563, 587

Pérez de Rozas, Anna .................................... 451

Perez-Brena, Maria L. ..................................... 646

Perez-Franco, Ruben ................................... 59

Perez-Maillo, Monica....................................76, 317

Perez-Razo, Miguel A..................................... 467

Pérez-Simó, Marta .......................................... 76

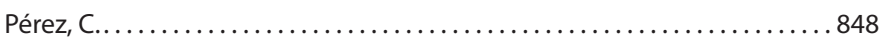

Perez, Daniel .......................................... 563

Perez, Lester Josue. . . . . . . . . . . . . . . . . . . . . . . . . . . . . . . 472

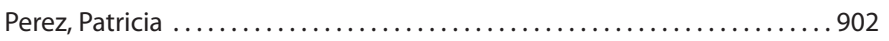

Perfumo, Carlos J...................................563, 587, 692

Peroni, Luiz G. .......................................... 184

Perreul, Guillaume.......................... 321, 390, 436, 565, 1111

Perrin, Hugues. . . . . . . . . . . . . . . . . . . . . . . . . . . . . . . 213

Persson, Maria................................................................ 428, 831

Pertoci, Alojz ......................................... 487

Pesente, Patrizia .............................................. 269

Pessoa, João J.............................................. 1124

Petersen, Lisbeth B.................................. 1162

Petkov, Spas.................. 990, 991, 992, 993, 995, 996, 1000, 1183

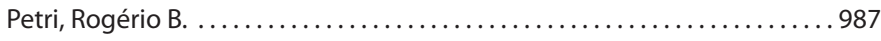

Petridou, Evanthia ....................................... 1020

Petrini, Stefano . . . . . . . . . . . . . . . . . . . . . . . . . . . . . . . 331

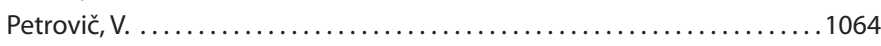

Pezzotti, Giovanni .................................. 331, 728

Pfeiffer, Dirk U............................................ 57

Phatsara, Chirawath ........................................ 914

Philips, Reid ............................ 268, 273, 516, 531, 659

Phipps, Jenny ....................................... 922

Phipps, Michel.......................................... 922

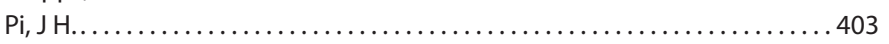

Picard, J. A. ......................................... 744

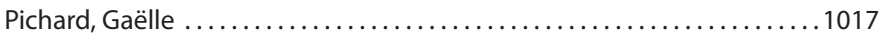

Pietschmann, Peter ............................................ 907

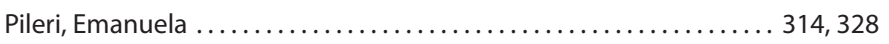

Pillet, Florence........................................... 633

Pina, Sonia ...................................... 76,978

Piñeiro, Carlos ............................ 212, 395, 670, 678, 1182

Piñeros Duque, Ricardo J. ............................... 827

Ping-Cheng, David Y.................................... 230

Pinheiro, Roniê W. . ............................376, 396, 629, 1140

Pinsard, Jean-Louis . . ................................. 203

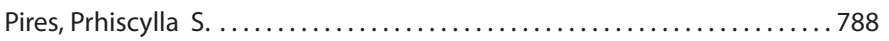

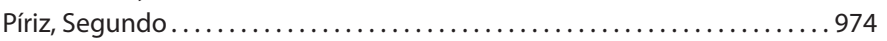

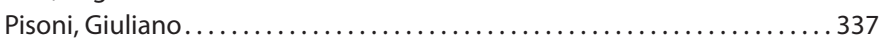

Pistl, Juraj . ........................................... 330

Pittman, Jeremy S............... 216, 217, 351, 382, 805, 806, 807, 1083

Plana, Joan ............................................. 314

Planchon, Viviane...................................1023, 1024, 1025

Planz, Christina L....................................99, 727

Pliego, Ramon......................................... 274

Pluym, Liesbet M.......................................... 47

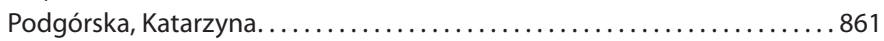

Poezevara, Typhaine ......................................660

Pogranichniy, Roman ........................................661

Policarpio, Renato T. ...................................... 539 
Poljak, Zvonimir ...... 42, 85, 150, 270, 275, 282, 364, 492, 494, 496, 497, 937 Pollakova, Jana ....................................... 330 Pollock, Geoffrey P. ................................. 713, 715 Polo, Javier .............................................. 560 Polson, Dale D. ........... 204, 206, 265, 267, 273, 475, 524, 531, 532, 659 Pomorska-Mol, Malgorzata ............................... 1028 Ponce Manzano, Ernesto.................................... 1030 Poolperm, Pariwat........................ 188, 557, 941, 1045, 1121 Popa, Virgilia ........................................ 658

Porowski, Marian ..................................... 1134

Portier, Fanny.......................................... 933

Pósa, Roland .......................................... 654

Postma, Merel......................................... 947

Poulsen, Hanne D. . . . . . . . . . . . . . . . . . . . . . . . . . . . . . . . . 63

Pradal-Roa, Pedro J.......................... 562, 570, 738, 934

Prapasarakul, Nuvee .................................. 638, 994

Prapatsornpinyo, Suthatip................................ 994

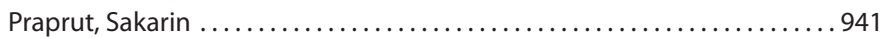

Preissler, Regine ..........................................1189

Prickett, John R. .....................201, 484, 512, 661, 924, 977

Prieto, Cinta ........................... 151, 498, 505, 546, 547

Probst, Inga ..........................................602

Provis, Peter ........................................... 931

Prunier, Armelle ..................................... 277

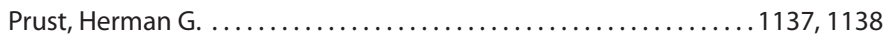

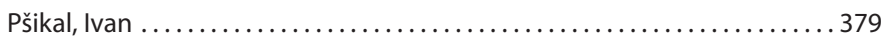

Puig, Daniel ...................................... 409, 410

Pujols, Joan .................................... 314, 560

Puppe, Birger...........................................1156

Purwin, Cezary .......................................... 1019

Püschel, Christiane ................................. 111, 329

Putirulan, Frans F....................................... 981

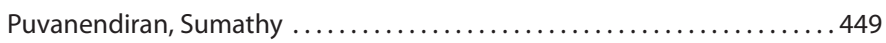

Pyrek, Rosmarie ...................................... 900

Qin, Qingsong .......................................... 936

Quereda, Juan J............................ 416, 431, 749, 750

Quesada, Alberto..................................... 974

Quezada-Monroy, Francisco............. 138, 228, 229, 315, 624, 625, 626

$849,850,851,852,853,854$

Quezada, Francisco ............................... 507, 902

Quezada, Manuel........................................... 316

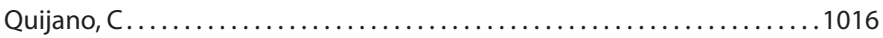

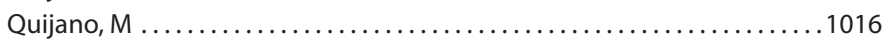

Quilitis, Michael E........................519, 520, 539, 540, 541, 543

Quiniou, Nathalie...................................... 752

Quintal, Monica ........................................ 318, 319

Quintero, Victor ................................444, 467, 470

Quiroga, María Alejandra ........................ 316, 563, 587, 692

Rabaeys, Patrick ..................................... 1183

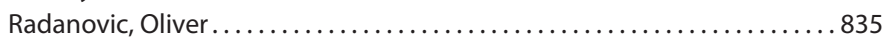

Radeloff, Isabel ................................... 714, 1127

Raes, Maurice ........................................ 110

Raffi, Valeria ...................................... 791, 1107

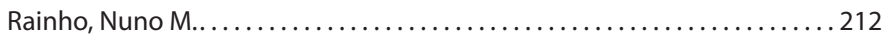

Ramamoorthy, Sheela ............................... 351, 382

Ramírez-Estudillo, Carmen ............................... 198, 653

Ramirez-Mendoza, Humberto. ...................472, 856, 857, 1116

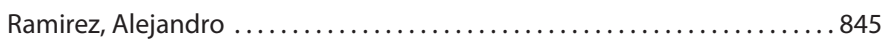

Ramirez, Gloria C................................. 313, 452

Ramis, Guillermo ......................... 416, 417, 431, 749, 750

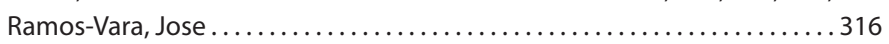

Rangel-Rodriguez, Ignacio C.............................. 467

Rangel, Maria José....................................... 1123

Rantzer, Dan.............................................. 1063

Raphanaphraiwan, Chanunda........................ 681, 691
Rapp-Gabrielson, Vicki J........................... 75, 258, 301

Rapp, Christof .................................. 1167, 1168

Rasmussen, Anette ......................................... 354

Rasmussen, Josephine S.................................. 843

Rath, Reinhold................................... 1085, 1086

Rathkjen, Poul Henning S................................ 706

Ratzinger, Christine..................................... 1143

Rau, Franziska ......................................... 245

Rauner, Martina ...................................... 907

Raymakers, Rudolf.................................... 101

Razzuoli, Elisabetta ............................. 490, 920, 1172

Rebollo, Belén ......................................... 477

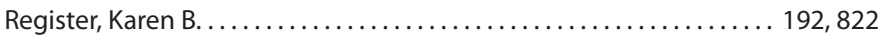

Rehagen, Martina ....................................... 1001

Reicks, Darwin L........................................ 271

Reid-Smith, Richard................................ 114, 115

Reidl, Melissa.......................................... 282

Reinacher, Manfred ...................................... 107, 308

Reindl, Melissa ............................................. 364

Reiner, Gerald ................. 107, 244, 308, 694, 695, 911, 912, 913

Reiners, Kerstin ....................................1176, 1186

Reis de Souza, Tercia C. ............................. 1031, 1034

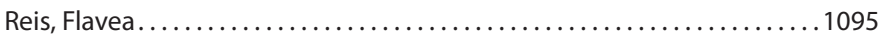

Reis, Kledna C. P.................................. 459, 645

Reis, Ronaldo............................................... 1095

Renaville, Robert ............................... 1023, 1024, 1025

Rendón, Alfredo G. . ................................... 386

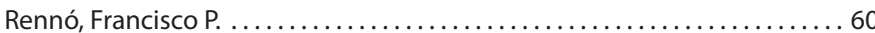

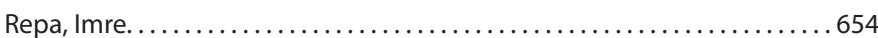

Revilla-Fernández, Sandra .............................. 1118

Reyes-Leyva, Julio Roberto.......................... 856, 857

Reyes, Libertad S................................... 371, 373

Reynolds, Kristen . . . . . . . . . . . . . . . . . . . . . . . . . . . . . 85

Rho, Se-Mi ........................ 478, 480, 569, 812, 891

Ribbens, Stefaan. ..................................... 938, 939

Riber, Ulla ...................................... 842, 843, 844

Richardson, John S................................ 411

Richardson, Karen . . . . . . . . . . . . . . . . . . . . . . 364

Richt, Juergen A........................................ 79

Richter, Susanne. . . . . . . . . . . . . . . . . . . . . . . . . . . . . . . . . 893

Richtzenhain, Leonardo José........ 334, 335, 340, 474, 885, 887, 888, 1124

Ridpath, Julia........................................ 936

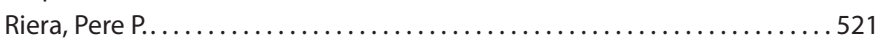

Rigaut, Martial........................................... 514

Rimondi, Agustina.................................. 563, 587

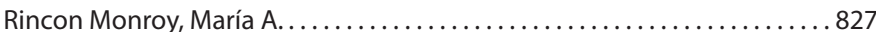

Rincón, María A. .............................................. 313

Rioja-Lang, Fiona C.................................. 246

Riquelme, Ramón G........................................ 521

Risso, Adriana ............................................. 587

Rist, Beate .......................................... 166

Ristoski, Trpe ............................................. 316

Ritchie, Catriona M......................................... 97

Ritterbusch, Giseli A....................... 466, 468, 471, 862, 1122

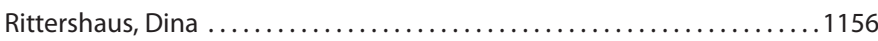

Ritzmann, Mathias................43, 111, 166, 197, 247, 329, 476, 528

$649,717,819,828,899,900,956,1155$

Rivas, Raquel ...................................... 261

Rivera-Benitez, JF. . .......................472, 856, 857, 1116

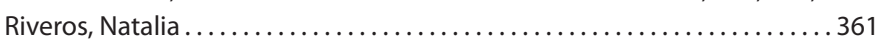

Rivest, Joël ............................................ 489

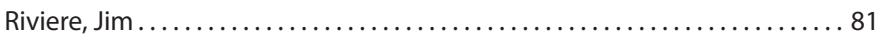

Robbins, Rebecca..................................... 87

Robert, Desrosiers ......................................672

Roberta, Pavesi ................................... 463, 483 


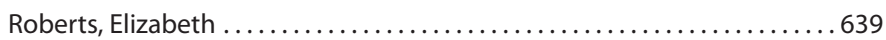

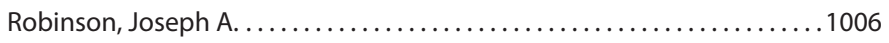

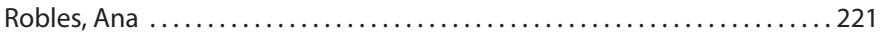

Robles, Francisco ................................. 310, 447

Roca, Merce ................................... 276, 530

Roca, Mercè .......................................... 776

Roche, Paulo M. ......................................... 473

Rodriguez-Buenfil, Jorge C. ................................ 965

Rodríguez-Estévez, Vicente ............................. 212

Rodríguez-Gómez, Irene M........................147, 508, 560

Rodriguez, Elizabeth..................................... 59

Rodríguez, Fernando ...................................... 261

Rodriguez, Fernando .................................. 846

Rodriguez, J........................................ 1016

Rodriguez, Javier M. . .................................. 398, 846

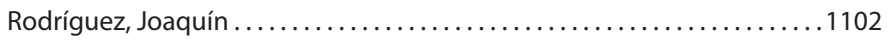

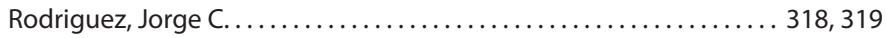

Rodriguez, Manuel ................................... 361, 830

Rodriguez, María N. .................................... 452

Rodriguez, Pedro Yoelvys de la Fe ............................. 764

Rodríguez, Roberto M................................... 1050

Roe, Cassandra ........................................ 139, 845

Roerink, Frank.................................. 167, 391

Roesler, Uwe .................................. 955, 983

Roesner, Patricia...................................... 404

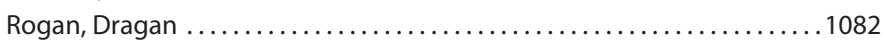

Roh, Yumi .......................................604, 605, 775

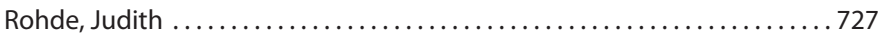

Rohr, Stefan A............................................. 987

Rohrmann, Hartmut ................................... 183

Roić, Besi.................................. 202, 976, 1112

Rojas, Reyna........................................ 918

Rojo, Carlos ......................................... 525

Romagosa, Anna ...................................... 298

Romanini, Silvia ...............................693, 794, 795

Romero, Yolanda .................................. 467, 470

Roongsitthichai, Atthaporn .......................1101, 1103, 1104

Roozen, Mark......................................... 647

Rosales, Francisco ........................................ 1056

Rosas, Marisa . . . . . . . . . . . . . . . . . . . . . . . . . . . . . 425

Rose, Nicolas ........................................ 52, 660

Rosell, Rosa .......................................... 225

Rosendal, Thomas ................................ 496, 497

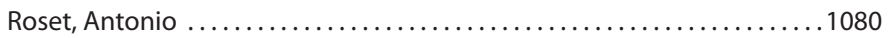

Rosignoli, Carlo........................................ 791

Rösler, Uwe......................................... 123

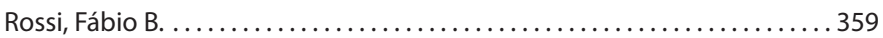

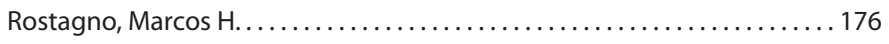

Rota Nodari, Sara ................................. 296, 457

Rothkamp, Anja ...............................597, 823, 824

Rotto, Hans. . . . . . . . . . . . . . . . . . . . . . . . . . . . . 613

Rough, Niels H. ......................................6 676

Rousseau, Carine .......................................... 1003

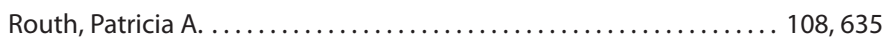

Rovira, Albert.............................. 149, 510, 515, 524

Rowland, R.R..................................... 482

Roy, Olivier ...........................................633

Roycroft, Linda ...........................639, 709, 718, 719

Royer, Eric ........................................ 962

Rubio, Pedro .......................... 724, 739, 742, 969, 971

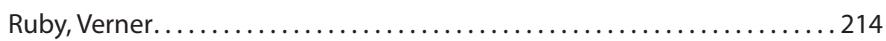

Ruiz, Alvaro ....................................... 438

Rukkwamsuk, Teera............................................ 188

Runcharoen, Achinee ................................... 226

Rusaleev, Vladimir .....................................6 610
Russell, Brandon........................................ 545

Russell, Louis .................................... 338, 560

Rustvold, Justin. . . . .................................... 169

Rusvai, Miklós ........................................ 528

Rychlik, Ivan......................................... 970

Rycroft, Andrew ...................................... 102

Sa Rocha, Camila ........................... 466, 468, 471, 862, 1122

Saalmueller, Armin ..................................... 528

Saco, Yolanda.......................................... 651

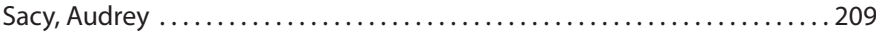

Saegerman, Claude...................................... 802

Saha, Dipongkor..................................... 54

Saito, Mere E...................................... 84

Saito, Y.............................................. 171

Saives, Annick ............................ 94, 550, 697, 698, 699

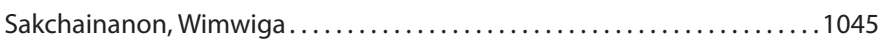

Sala, Vittorio............................................. 337

Sales, Tatyane P. ........................................ 339, 620

Salgado, Vanessa R...................................... 1124

Salguero, Francisco J........................ 147, 508, 859, 860

Salih, Talal S.......................................... 961

Salinas, Jesús.............................................. 1123

Salogni, Cristian ................................. 463, 585

Salvarani, Felipe M. ............................... 757, 788

Salwén, Thersa ......................................... 90

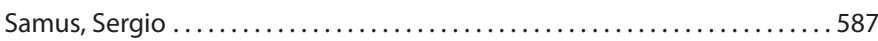

Sánchez-Betancourt, Ivan.......................... 562, 570

Sánchez-Vizcaíno, JM..................................... 847

Sanchez-Vizcaino, Jose Manuel. . . . . . . . . . . . . . . . . . . . . . . . . . . 222

Sánchez, MA......................................... 847

Sánchez, Pedro U. ...................................... 521

Sanchez, Romeo........................................ 615

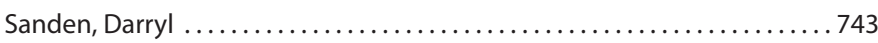

Sandra, Cuevas R...................................... 855

Sandri, Giampietro ................................ 269, 490

Sang-Gassanee, Kridtasak. .................................. 380

Sanguinetti, Ramón .......................................... 587

Sanjosé, Helena ................................... 416, 417

Sanmartin, Joan ...............................426, 683, 710

Santos, Daniel L......................................4459, 645, 910

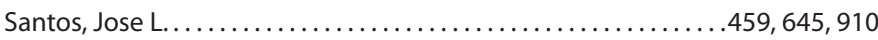

Santos, Lucas F.................................459, 645, 910

Santos, Raquel L. . . . . . . . . . . . . . . . . . . . . . . . . . . . . . . . . . . . 396

Santos, Y. L.......................................... 840

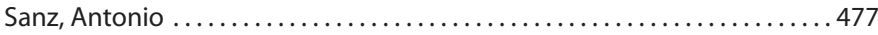

Sarasola, Patxi .......................................... 442

Sarfati-Mizrahi, David......................138, 228, 229, 315, 624, 625

$626,849,850,851,852,853,854$

Sargent, Carole A............................................ 194

Sarli, Giuseppe ...............................283, 458, 463, 909

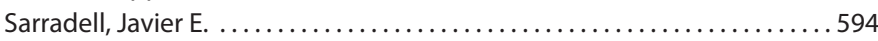

Sarubbi, J..................................... 630, 631

Sattler, Tatjana................................ 893, 1135, 1136

Sauer, Franziska ...................................1135, 1136

Savic, Bozidar................................... 800, 835

Sayvaw, Paitoon ...................................... 414

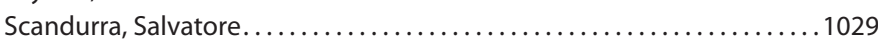

Scanlan, Mack ....................................... 785

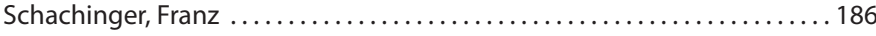

Schade, Ariane ........................................ 429

Schade, Benjamin ........................................... 1119

Schatzmayr, Gerd M........................ 908, 1032, 1033, 1143

Schaumberger, Simone........................... 908, 1033, 1143

Schawalder, P. ....................................... 896

Scheidt, Alan ....................................... 81 
Schellander, Karl Schelling, Esther .. 914

Scherba, Gail ... 278,1099

Scherer, Nina 509

Schieber, Teresa 75

Schiele, Daniela

1155,1157

Schilcher, Franz.

509,760

Schillebeekx, Jos ............................................ 1026

Schiochet, Marisete F. ............................... 336

Schlegel, Michael..........297, 572, 573, 574, 576, 577, 578, 579, 580, 581

Schmidt, Bernard H. ...................................... 1001

Schmidt, Tatjana........................................ 245

Schmidt, Ulla ........................................ 1153

Schmidt, Ulrike .............................110, 167, 391, 393

Schmoll, Friedrich ................. 103, 186, 476, 893, 1118, 1135, 1136

Schneider, Marc ....................................... 86

Schoder, Gottfried ....................................... 553

Schoos, Jean ............................................. 553

Schott, Rydell ........................................ 133

Schouten, Chris..................................... 450

Schreiber, Frank ...................................... 420

Schroeter, Andreas ............................................ 972

Schulte-Wuelwer, Josef .............................. 122, 951

Schultz, Roy A........................................ 789

Schulz, Jochen ............................. 123, 215, 955, 957

Schulze Althoff, Gereon.................................. 980

Schulze-Horsel, Theo .................................. 122

Schüpbach, Gertraud ................................. 928

Schuttert-Wilps, Rita ...................................... 733

Schwartz, Kent J. ................................... 201, 512

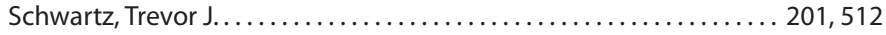

Schwarz, Patrícia .................................. 967, 968

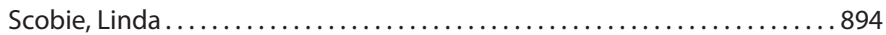

Scott, Morgan ......................................... 785

Seanbungkhor, Nualprae ................................. 1164

Seegers, Henri............................................. 945

Segales, Joaquim ..................76, 98, 231, 232, 261, 276, 286, 314

$.316,317,326,327,328,442,451,530,649$

$651,746,818,846,879,880,881,882,883$

Segot, Christine . . ..................................... 1003

Segura-Correa, José ....................................1102

Segura, Mariela........................................ 833

Seifert, Hermann . ......................................663 663

Selbitz, Hans-Joachim . ...... 297, 572, 573, 574, 576, 577, 578, 579, 580, 581

Selke, Martin ........................................... 656

Sellal, Eric ........................................... 500

Senn, Michael ...........................................664 664

Senne, Dennis A......................................... 79

Sensi, Marco................................. 46, 252, 331, 919

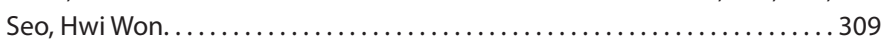

Sequera, Abel ....................................... 361, 830

Shah, R............................................... 482

Shah, Rohan........................................ 568

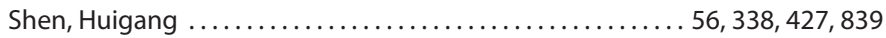

Shepherd, Gene ............................... 805, 806, 807

Sheppard, Cara ........................................ 115

Sheridan, Mike ......................................... 931

Shimomura, Yasuko.................................... 172

Shin, Dong H........................................ 763

Shin, Min-kyoung...............................604, 605, 775

Shin, Yeun-Kyung................................ 591, 592

Shkedy, Ziv........................................... 764

Shryock, Tom . . . . . . . . . . . . . . . . . . . . . . . . . . . . . 552

Sialelli, Jean-Noel. ................................. 125, 752

Sibila, Marina .................. 232, 314, 326, 327, 328, 442, 451, 818
Sick, Fred............................................. 531

Siddiqi, Saba ...................................... 922

Sidler, Xaver ................. 405, 418, 419, 420, 455, 926, 928, 1117

Siedlik, Leann M. . . ......................................... 571

Siemers, Soelen ................................... 796, 801

Sierra, Pascale ................................... 670, 678

Sievert, Kai ....................................551, 803, 804

Siewert, Carsten ........................................663

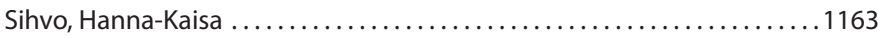

Siklódi, Botond ...............................614, 616, 786

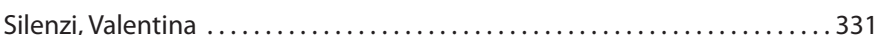

Silva Filho, José Monteiro ............................. 1073, 1095

Silva, Caio A................................... 1038, 1039

Silva, Carolina I..............................60, 70, 1067, 1092

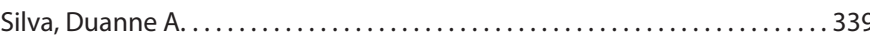

Silva, Enedina ........................................... 1011

Silva, Priscyla C....................................... 84

Silva, Roberta A. ................................ 1038, 1039

Silva, Rodrigo Otávio S. ............................ 757, 788

Simarro, Isabel......................... 151, 498, 505, 546, 547

Simon-Grifé, Meritxell. ..................... 76, 77, 260, 590, 652, 982

Simón, A............................................. 847, 848

Simon, Neide $L \ldots \ldots \ldots \ldots \ldots \ldots \ldots \ldots \ldots \ldots \ldots \ldots$ 466, 468, 471, 862

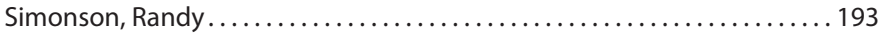

Singer, Randall ........................................... 704

Sinha, Avanti .......................................... 55

Sinovas, Nuria ........................................ 442

Siochu, Asimoula .......................................... 1191

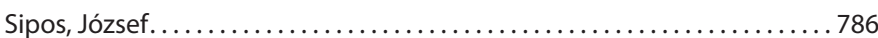

Sipos, Wolfgang .............476, 488, 509, 760, 904, 905, 906, 907, 984

Siqueira, Amanda...................................1073, 1095

Sisak, Frantisek ......................................... 970

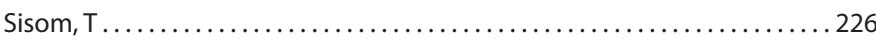

Sistac, Jorge . ....................................... 772

Sitjá, Marta ..................................... 776, 778

Sittinuntn, Susichol....................................... 226

Sjölund, Marie.................................... 599, 897

Skampardonis, Vasilis ................................92, 288

Skov, Robert......................................... 121

Skrzypiec, Ewelina ........................................ 145

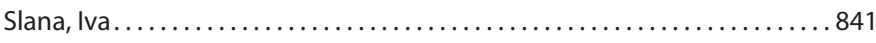

Slavic, Durda ........................................... 923

Smeets, Jos.....................................166, 344, 404

Smit, Miranda N....................................... 58

Smith, Taylor ............................................. 133

Smith, Trevor K............................................. 1021

Smits, Rob J.............................................. 129

Smola, Ji í ........................................... 790, 793

Smothers, Clark D.......................................... 1006

Snider, Timothy P............................................ 642

Sno, Melanie ............................................. 393

So, Nam H. ........................................... 324

Soares, Patricia .................................339, 473, 620

Sobestiansky, Jurij ...................................339, 473, 620

Sobrino, Francisco ...................................... 225

Soede, N.M. ...........................................1126

Sogbe, Elias J. .................................. 461, 448, 830

Sogbe, Miguel E....................................... 448

Soler, Alejandro ................................... 221,847

Soler, Joaquim. ........................................ 917

Solignac, Thierry............................... 124, 1174, 1175

Söllner, Heidi ............................................ 649

Sombor, Erzsébet................................ 868, 873

Song, Chang-Seon. ......................................... 199

Song, Chang-Sun .................................... 816 
Song, Dae Sub

Song, Fei . . .

Song, Jae-Young

Song, Kyung-Ok ...

Song, Y-K.

Song, Yong ....

Song, Yun Kyung

Songjinda, Prapha

Sonil, Pak. ...

Sosan, Olubukola.

Sossi, Enrico.

Sotiraki, Smaragda

Soto-Priante, Ernesto

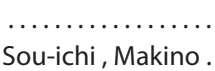

Soukup, Ivan

Souza de Lima, Elena

Souza, Keili M.

Souza, Sheila O..

Sozzi, Enrica.

Sørensen, Gunner

Sørensen, Jens

Sørensen, Kristina U.

Spaggiari, Brunella

Spencer, B. T.

Spergser, Joachim

Spessatto, Daiane D.

Spiru, Daniel

Sprecher, David.

Spricigo, Gilberto.

Springorum, Annette

Spronk, Gordon

Squires, E. James

Sreta, Donruethai.

Sriyong, Yongyot ...

St-Hilaire, Manon

Stadejek, Tomasz

Stahl, Marie

Ståhl, Marie

Stampe, Michael.

Stancioli, Edel F. ...

Stankevicius, Arunas.

Stärk, Katharina D.C.

Stege, Helle

Stegeman, J.A...

Steigmann, Maria.

Steiner, Tobias

Steinmetz, Henriette.

Steinrigl, Adolf ....

Stensland, Wendy

Stepanova, Hana ...

Stephan, Bernd...

Stephan, Katharina .

Stephan, Roger...

Stepniewska, Katarzyna.

Sterz, Fritz.

Stevens, Elliot

Steyaert, Maarten

Stich, H.

Stigger, Evelyn.

Stine, Doug

Stockhofe-Zurwieden, Norbert.

Stockmarr, Anders

Stoiber, Christian .

Stoiber, Christian ...

Proceedings of the 21st IPVS Congress, Vancouver, Canada - July 18-21, 2010
454,813

.. 592

.890

537,584

... 725

445,814

941,1045

.657

860

$490,884,920,1172$

..92, 288

$138,228,229,315,624,625$

$626,849,850,851,852,853,854$

379,381

...1122

339,473

340,1124

... 585

65,214

.714

.. 63

791,909

744

197,819

.722

798

1091

359

957

516

916

$564,567,589$

691

672

$145,202,465,500,503,703,861$

284,464

702

290

.757

503

57

$80,207,237,241,242,284,464$

$702,754,755,756,1005,1161$

608

249

1017

280

1118

484

970

1127

211

926

687

904,906

1007

1057

.896

254

193

$01,316,617$

237,327
Stone, Suzanne....................................... 924

Strait, Erin L.................................139, 144, 484, 845

Strathe, Jens........................................ 1161

Straw, Barbara .......................................... 164

Stricker, Teija M. .................................... 828

Strobel, Michael .................................. 575, 1154

Strubbe, Marieke ........................................ 177

Strugnell, Benjamin............................... 191, 650

Strutzberg-Minder, Katrin.........................481, 821, 828

Studnitz, Merete. ...................................... 214

Stukelj, Marina ......................................... 548

Su, Liangke............................................. 979

Suarez, David L...................................... 79

Subsiribhaiboon, Apichart ............................... 1045

Sudhaus, Nadine ...................................1130, 1132

Sueyoshi, Masuo ....................................... 771

Sukhumavasi, Woraporn .............................. 803, 804

Sullivan, Brian ........................................ 916

Suls, Leo ......................................... 156, 279

Summerfield, Artur ....................................... 879

Sun, Dong.......................................... 504

Sun, Jianhe ......................................... 979

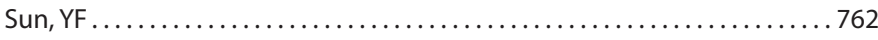

Sundrum, Albert..............................277, 759, 809

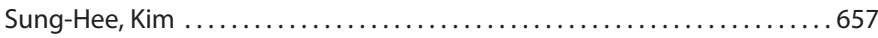

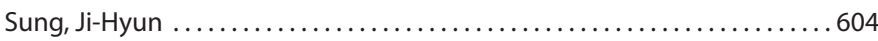

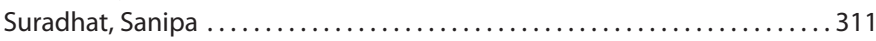

Suriyasomboon, Annop.............................. 130, 1164

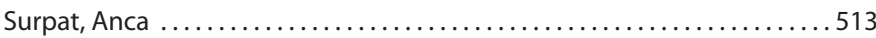

Surprenant, Charles........................... 341, 671, 832, 833

Sutherland, Mhairi A. .................................. 250

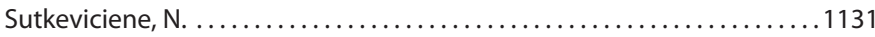

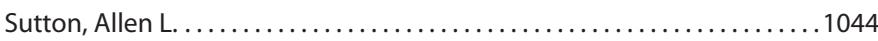

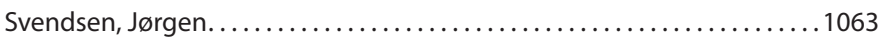

Sviben, Marijan ......................................... 1096

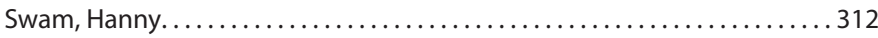

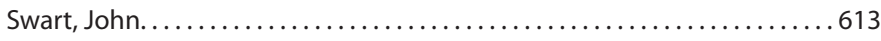

Swarts, H.M.J.M. . ................................88, 134, 1126

Swayne, David E. .................................... 79

Swenson, Sabrina L. . . . . . . . . . . . . . . . . . . . . . . . . . . . 259, 875

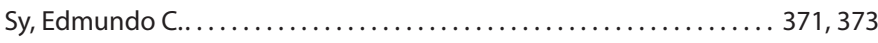

Sydler, Titus .....................316, 405, 418, 419, 455, 926, 1117

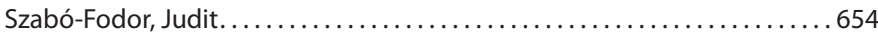

Szabo, I..........................................1131

Szalai, Tamás ................................ 868, 870, 873, 874

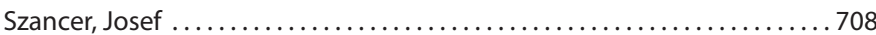

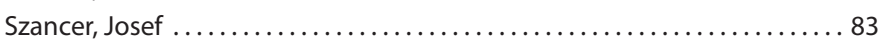

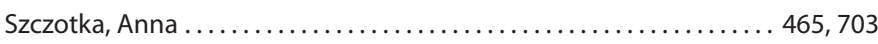

Szucs, Judit. ........................................... 874

Takada, Ryozo ....................................... 1037

Takagi, Michihiro ........................................ 538

Takahashi, Y......................................... 172

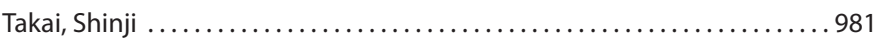

Talummuk, Sasiwimon.................551, 638, 745, 803, 804, 997, 1012

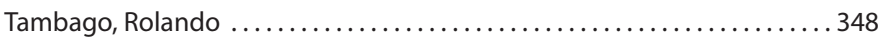

Tamiozzo, Pablo J. ...............................621, 731, 964

Tananthong, Narut ..................................... 1121

Tanghe, Lieven ....................................... 674

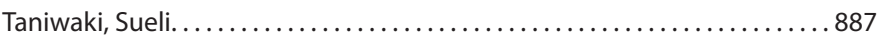

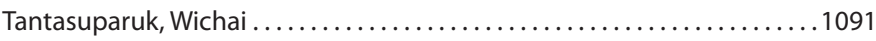

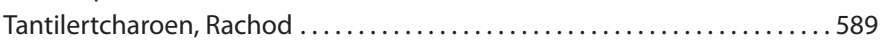

Tantituvanont, Angkana ................................ 1014, 1015

Tarantola, Martina ..................................... 1173

Tarasiuk, Kazimierz ........................................ 593

Tarradas, Joan ...................................... 225 
Tarsitano, Marina A.

1038,1039

Tasker, John B.

647,714

Tassis, Panagiotis D.

$634,711,780,782,1191$

Taylor, Lucas P.

75,258

Taylor, Micah

.. 659

Teankum, Komkrich

311,997

Techakumphu, Mongkol

$130,1068,1069,1078,1101$

Tegeler, Regina $118,131,622,950$

Tejedor, Maria T. ... $392,646,1109$

Teneberg, Susann

$119,950,972$

Tenhagen, Bernd-Alois . .

...... 1017

Tenier, Christian

$.614,615,616$

Tenk, Miklos...

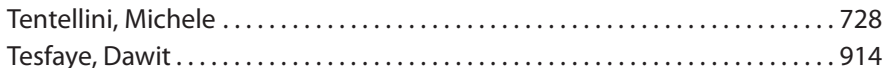

Testa, Marcela ........................................ 587

Thacker, Brad J............ 144, 217, 341, 351, 382, 383, 406, 805, 806, 807

Thacker, Jennifer

783,784

Thaler Neto, André

Thammakarn, Chanathip .......................... 1013, 1052

Thanawongnuwech, Roongroje...... 311, 542, 551, 564, 567, 589, 867, 997

Theis, Kara M........................................ 462

Theobald, Peter ....................................... 174

Thevenon, Jérôme...................614, 615, 616, 868, 870, 873, 874

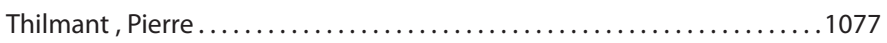

Thingnes, Signe Lovise. ................................... 190

Tholen, Ernst ......................................... 914

Thomas, Jessica ....................................... 346

Thomas, Paul............................................. 100

Thomaz, Maria Cristina ............................485, 783, 784

Thomson, Cindy ......................................... 383

Thomson, Jill R.............................738, 741, 836, 1094

Thomson, Peter...................................... 688

Thongkamkoon, Pacharee ................................ 638

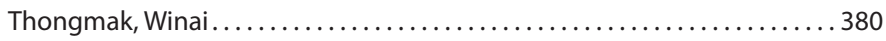

Thontiravong, Aunyaratana .............................. 589

Thorup, Flemming.......................65, 66, 159, 214, 1165, 1184

Thun, Rico........................................ 1130

Thuy, Nguyen Thi ...................................... 765

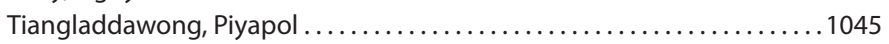

Tibau, Joan .......................................... 917

Tienthai, Paisan....................................... 1114

Tinaro, Miriam . . . . . . . . . . . . . . . . . . . . . . . . . . . . . . . 46

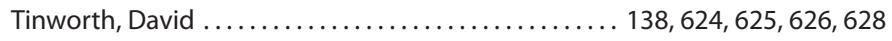

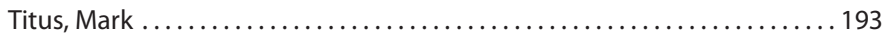

Toan, Nguyen T. ....................................... 356

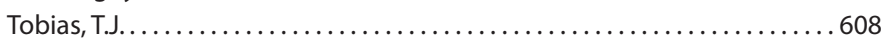

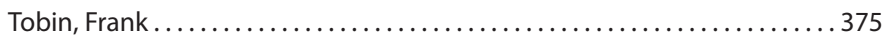

Tokach, Lisa ........................................ 462

Tokuyama, Keiri ....................................... 171

Toledano, Fernando M. ............................ 84, 1040, 1041

Toledo, Erandi ..................................... 1056, 1058

Toledo, Manuel................................. 416, 417, 1010

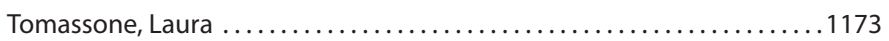

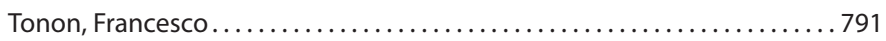

Too, Henry ........................................... 627

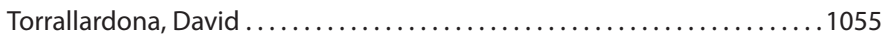

Torrelles, Hernan ................................... 668, 669

Torremorell, Montserrat...................... 143, 204, 298, 515, 549

Torrents, Dani G..............486, 519, 520, 521, 523, 525, 543, 778, 866

Torres, Ma. Immaculada T.........................559, 540, 541, 543

Torres, Marco A. ..................................... 318, 319

Torres, Maria I..................................... 519, 520

Torrison, Jerry ........................................... 394

Tortora-Perez, Jorge ............................. 507, 902
Tossenberger, János ..................................... 1022, 1027

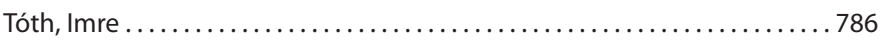

Toyplikioti, Panagiota..................................... 1191

Traiyarach, Sittikorn ....................................... 997

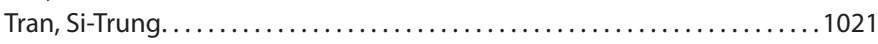

Traverso, Sandra D.............................783, 784, 903

Travnicek, Robert.................................... 1134

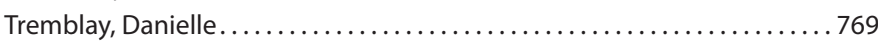

Tremblay, Donald.......................................51, 595

Tretipskul, Chanyuth...............................1068, 1069

Trevisi, Paolo .................................................. 1029

Tripipat, Thitima ............................. 681, 1014, 1015

Trotel, Anne ......................................... 362

Trott, Darren J...................................... 766, 774

Trueta, Rafael............................................. 1145

Trujillo-Ceballos, David .................................. 507

Trujillo-Ortega, Maria E.................................... 570

Trujillo, Maria........................................... 586

Tschentscher, Astrid . .................................. 481

Tseng, Steven ......................................... 1188

Tuanudom, Ranida ................................ 567, 589

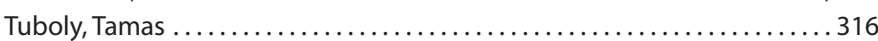

Tucker, Alexander W...........................73, 191, 194, 650, 894

Tully, Blaine ........................................ 931

Tummaruk, Padet. . 542, 803, 804, 1068, 1069, 1078, 1101, 1103, 1104, 1114

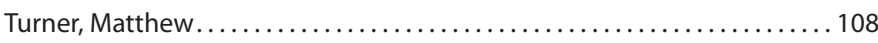

Tuyttens, Frank ...................................... 47

Tzika, Eleni D.........................634, 711, 780, 782, 1191

Ubeda, Juan Luis ........... 69, 71, 398, 399, 401, 1074, 1075, 1076, 1108

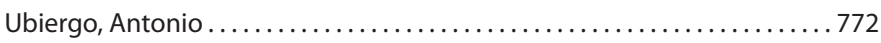

Uddin, Muhammad J.................................. 914

Udomprasert, Preeyaphan ....................... 188, 557, 1121

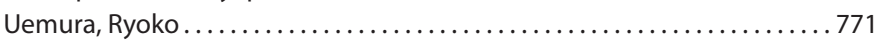

Urairong, Kitcha ................................... 557, 1121

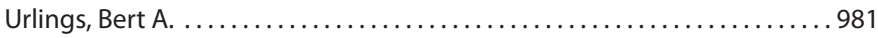

Urrutia, Rosa ......................................... 944

Urumova, Valentina...................................... 996

Utama, Prasetya P. . . . . . . . . . . . . . . . . . . . . . . . . . . . . . .

Utrera, Vitelio....................................361, 448, 830

Vadillo, Santiago. ..................................... 974

Vaduva, loana ................................ 491, 513, 618, 658

Valdes-Martinez, Sara ................................. 902

Valencak, Zdravko .................................195, 487, 548

Valencia, L. ......................................... 840

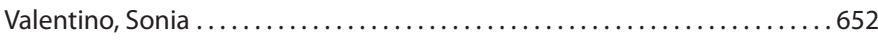

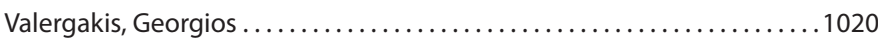

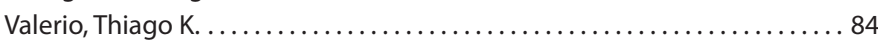

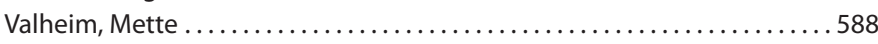

Valls, Laura ....................................... 72,611

Valros, Anna .......................................... 1163

Van Beirendonck, Sanne .................................... 1159

van de Giessen, Arjen A. .................................. 120

van der Heijden, Harold M.J.F........................ 823, 824

van der Peet-Schwering, Carola ............................... 617

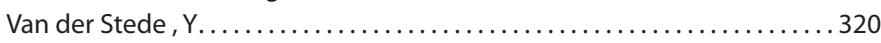

Van Der Stede, Yves..................................... 177

van der Wolf, Peter........................................ 120

Van Doorsselaere, Jan. ..................................54, 544

Van Driessche, Ellen ......................155, 177, 279, 1046, 1047

van Engen, Marrit. . . . . . . . . . . . . . . . . . . . . . . . . . . . . 162

van Esch, Eric. ....................................597, 823, 824

van Gelderen, Rainier................................ 294, 1187

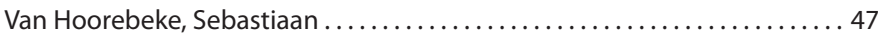

Van Leemput, Leo .................................. 810, 811

Van Parys, Alexander ................................... 973 
van Solt-Smits, Conny B.

Van Soom, Ann...

Van Steelant, Jan ............................................. 674

van Straaten, Jolien................................... 234, 690

Van, Huong T.......................................... 960

Vander Veen, Ryan L................................263, 300, 545

Vanderhaeghe, Caroline ...........................67, 135, 1057

Vandersmissen, Tamara ..........................155, 279, 1046, 1047

VanderVoort, Gordon E..................................... 916

Vangroenweghe, Frédéric A...............47, 155, 177, 279, 553, 736

$.737,777,1046,1047,1057$

Vanhee, Merijn ....................................... 544

Vanhonacker, Filiep..................................... 182

Vannucci, Fabio A.................................235, 236, 701

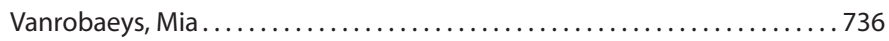

Vanthillo, Ilse ...........................................1193

Varela, María Teresal L. ....................................... 989

Varello, Katia ............................................ 337

Vargas, Alejandro........................................ 198

Vargas, Pedro......................................... 1071

Vaughn, Eric............................................. 475

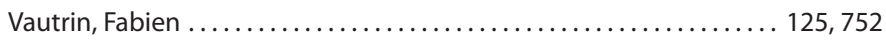

Vazquez, Mercedes .................................... 794, 795

Vázquez, N.............................................1059, 1060

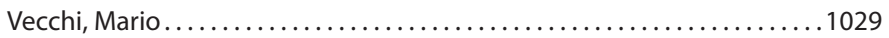

Vega-López, Marco A............................. 198, 653, 1050

Velasco-J, Mario . . ........................................ 562

Velasova, Martina....................................... 57

Veljkovic, Veljko ........................................ 261

Venegas-Vargas, Cristina.................................... 164

Venet, Julie........................ 376, 390, 429, 436, 529, 1111

Venosa-Peña, Javier....................................... 965

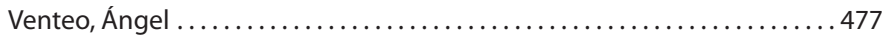

Ventura, Lauren .......................................... 1122

Vera, Víctor........................................ 313, 452

Verbeke, Wim....................................... 182

Verbrugghe, Elin..................................... 973

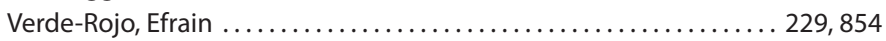

Vereecken, Monita............................ 1019, 1022, 1027

Vergara-Alert, Julia .................................... 261

Vergara, Helga......................................... 553

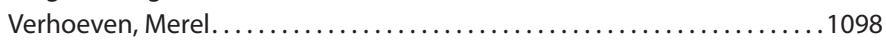

Verspohl, Jutta ...................................... 1115

Vesna, Milicevic. ....................................... 503

Vesselova, Stela.........................990, 991, 992, 993, 995, 996

Vestergaard, Kaj ................................161, 214, 384

Viana, Gustavo . ....................................... 160

Viancelli, Aline........................................ 862

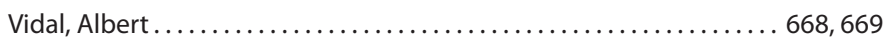

Viebahn, Stefan...................................1087, 1088

Vieira, Maria Isabel B. ................................... 1122

Viekilde, Kristian. ....................................... 161

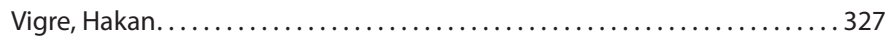

Vila, Thaïs .................205, 283, 321, 374, 390, 429, 430, 434, 436

$458,565,573,574,576,578,579,580,581,1111$

Vilaca, Kevin...................................... 282, 364

Vilalta, Carles.......................................8, 989

Vilar, Maria J. ...................................... 77,260

Vilček, Stefan ........................................ 330, 502

Villa, Riccardo .............................490, 884, 920, 1172

Villalba, Daniel ................................... 62, 1051

Villarreal, Iris..............................136, 137, 636, 1057

Villela, Tarciso C. ...................................1040, 1041

Vincent, Amy L................. 74, 79, 253, 255, 259, 262, 264, 299, 332
Vink, Daan ............................................. 243

Vinkler, Antonín ..................................1177, 1178

Vinokurovas, Sylvia L....................................... 1039

Vinter, Jens ......................................... 187

Viott, Aline M. ................................ 701, 722, 1066

Visintin, José A......................................... 1092

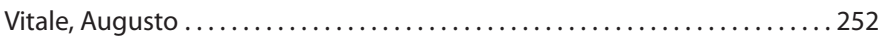

Vivanco, E. V. . . . . . . . . . . . . . . . . . . . . . . . . . . . . . . . . 840

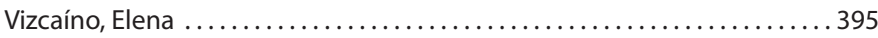

Vlasakova, Michaela ................................ 330, 502

Voglmayr, Thomas................................43, 899, 900

Voisin, Florian ......................................... 362

Volker, Ohlinger F. ............................. 94, 949, 958

von Altrock, Alexandra. .................................. 983

von Berg, Stephan ................................... 107, 308

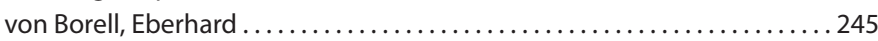

von Rüden, Stefan .................................... 166, 404

Vranckx, Katleen....................................136, 137, 636

Vrijenhoek, Mieke P. ..................................... 450

Vyt, Philip ...................................... 238, 729

Waddell, John ........................................ 168

Waddilove, Jake . . . . . . . . . . . . . . . . . . . . . . . . . . . . . . . . 358

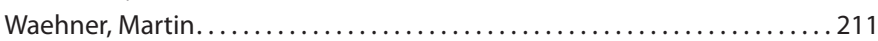

Wagenaar, J.A.......................................... 608

Wagner, Bruce A. ................................. 116, 449

Wajjwalku, Worawidh..................................... 557

Waldmann, Karl-Heinz ...............196, 249, 600, 656, 662, 983, 1156

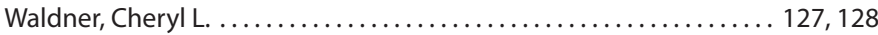

Walker, Roger D. ................................... 61

Wallgren, Per .. 90, 96, 173, 285, 290, 423, 424, 599, 700, 768, 831, 1153, 1166

Wang, Aiguo . . . . . . . . . . . . . . . . . . . . . . . . . . . . . . . . 865

Wang, Chong.......................... 55, 201, 484, 506, 512, 936

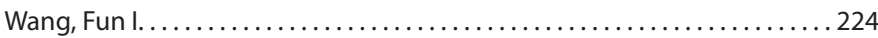

Wang, Pei-Ling .......................................... 511

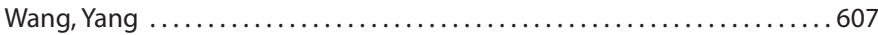

Ward, Allan................................................. 1094

Ward, Terry L........................................ 292, 1167, 1168

Warren, Kristi J..................................... 936

Watanapongchati, Supoj................................... 985

Wathes, Christopher M. ..................................... 102

Wattanaphansak, Suphot......................... 236, 704

Wavreille, José.................................. 1023, 1024, 1025

Waxenecker, Franz................................43, 899, 900

Webby, Richard...................................... 254

Webel, Steve K..................................... 58

Webster, Grace .......................................... 836

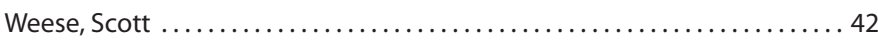

Wehrend, Axel. ...................................... 183

Weibel, Helen .................................... 419, 420

Weihs, Wolfgang. ...................................... 904, 906

Weissenböck, Herbert ....................43, 509, 528, 649, 760, 899

Wellenberg, Gerard J..................... 333, 554, 597, 823, 824

Welsh, Michael ........................................... 895

Welti, Sandra ......................................... 455

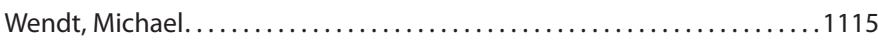

Weng, Chung-Nan .....................................623

Werling, Dirk .......................................... 102

Werner, Christina ................................... 759, 809

Werner, Ralf ......................................... 404

Westerhof, Michiel. ............................................. 1061

Wettlaufer-Zimmer, Uta....................................... 553

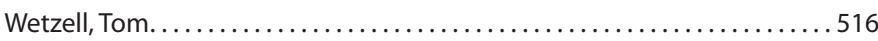

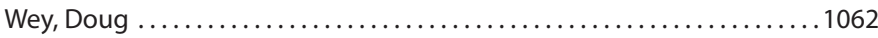

White, Mark E. ....................................... 411

Whitney, Daniel J........................................ 201, 512 
Wiberg, Sofia .

Wicklund, Eric .. 277

Widowski, Tina M.

.75, 258

Wieland, Barbara

1158

Wielen, John van de

$57,102,257$

Wilczynski, Krzysztof. ...

.555

Wilkes, Heinrich

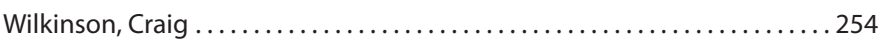

Wilkinson, Jamie M..

194

Willamil, Joseane ....

.. 1055

Willems, Eveline

Willems, Hermann

68,948

Willemsen, Peter...

$107,244,308,694,695,912,913$

Williams, M. C. . .

Williamson, Susanna M.

Wilson, Jeff B...

Wilson, Mark E.

Wilson, Warren D...

Winkelman, Nathan

Winkelmann, Melina.

Winter, Petra

Wipf, Ruth

Wisselink, Henk J..

Wittkowski, Gerd ....

Witvliet, Maarten

Wohlgemut, Jared

Wolf, Peter van der

Woo-Chang, Kim ...

Woo, Tae Hun.

Wood, James L...

Woodger, Nicholas

Woods, Jennifer

Woolfall, David

Woolfenden, Nigel J.

Wootton, Sarah K.

Worliczek, Hanna L.

Wu, Chi-Ming.

Xia, Luming ...

Xie, D . . .

Xu, David.

$X u$, Lili.

Xu, Weiwei

Yaciuk, Raúl

Yamaguchi, Takeshi

Yan, Yaxian

Yang, Cheng-Yao

Yang, Hanchun

Yang, Hyoung-Seok

Yang, II-Yung ...

Yang, Ping-Cheng

Yang, Seung Hyuk ...

Yao-Horng, Wang

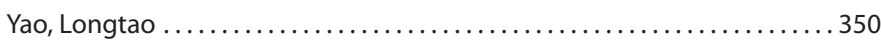

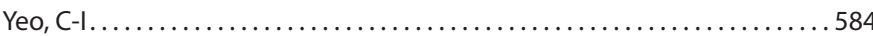

Yeo, Changil.................................... 445, 814

Yeom, Min Joo................................................... 378

Yeske, Paul E. . . . . . . . . . . . . . . . . . . . . . . . . . . . . 141

Yong, Ming ........................................ 112, 230

Yonkers, Therese K. .................................. 75,258

Yoo, Dongwan. ........................................ 552

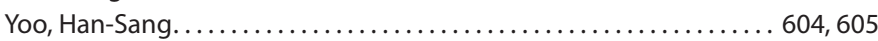

Yoo, Hansang. .......................................... 775

Yoon, Kyoung-Jin ............................... 302, 484, 504, 936

Yoon, Soon-Seek ........................................ 591

Yoon, Sorah . ........................................ 591

Youil, Rima .............................. 138, 624, 625, 626, 628

Yu, Joey ....................................... 601, 603

Yuill, K............................................ 73

Zaffalon, Fabiane G. ............................. 70, 1067

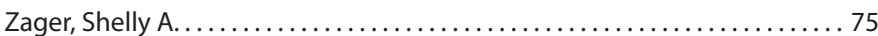

Zak, Louisa J. ........................................... 1082

Zanella, Eraldo L. ..................... 74, 253, 262, 299, 466, 875

Zanni, I. ............................................. 565

Zanoni, Mariagrazia ............................................ 585

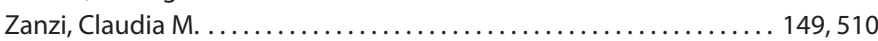

Zappulla, Daniele...................................... 296

Zaremba, Wolfgang.............................. 1084, 1085, 1086

Zeeh, Friederike ............................ 808, 896, 1151

Zell, Roland .................................297, 572, 577

Zenker, Isabella ........................................ 905

Zepperitz, Heiko.................................. 1085, 1086

Zhang, Huanrong. ..................................... 977

Zhang, Huiying .......................................... 979

Zhang, Kun................................................. 813

Zhou, Fanghong....................................... 936

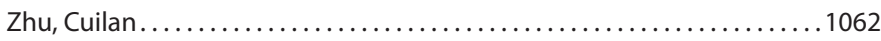

Zhu, Liande ................................... 352, 865, 869

Zielinski, Gustavo C. ..................................., 594

Zilinskas, H. ........................................ 1131

Zimmerman, Jeffery J. ............. 201, 482, 484, 506, 512, 661, 924, 936

Zimmermann, Barbara H. ................................. 180

Zimmermann, W. .................................. 808, 896

Zimmermann, Werner ................................. 1151

Zini, Maurizio......................................... 954

Žižlavský, Marek........................644, 815, 1134, 1177, 1178

Zmudzki, Jacek............................... 465, 703, 1134

Zoels, Susanne ..................................... 828

Zöls, Susanne...............................247, 956, 1155, 1157

Zoric, Mate ............................ 831, 1152, 1153, 1166

Zotti, Everson ..................................... 1193

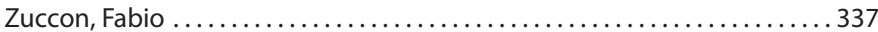

Zundel, Etienne ....................................... 945

Zutic, Milenko ................................. 800, 835 


\section{Industry Partners}

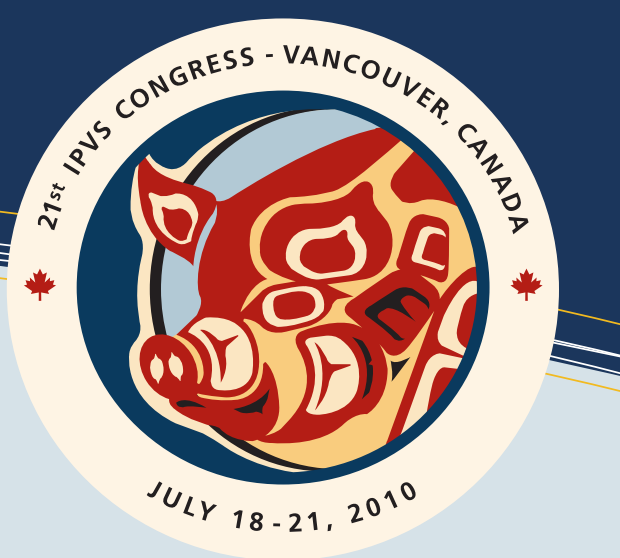

(ANA) Bayer (illi) Boehringer

\section{Intervet}

Schering-Plough Animal Health

(1) NOVARTIS Pfizer Animal Health

\section{Industry Supporters}

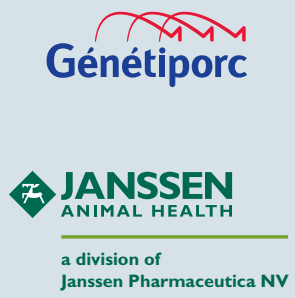

hu harrisvaccines

Hypoir

NEWSHAM

Choice Genetics

Wir uniferon"

IPVS 2010

\section{CONGRESS SECRETARIAT}

c/o Advance Group Conference Management Inc.

Suite 101 - 1444 Alberni Street

Vancouver, BC V6G 2 Z4 Canada

Phone: +1.604.688.9655 ext. 2

Fax: +1.604.685.3521

Email: ipvs2010@advance-group.com 